Argonne

\title{
Summary of Operations and Performance of the Murdock Site Restoration Project in 2008
}

\author{
Environmental Science Division
}


About Argonne National Laboratory

Argonne is a U.S. Department of Energy laboratory managed by UChicago Argonne, LLC under contract DE-AC02-06CH11357. The Laboratory's main facility is outside Chicago, at 9700 South Cass Avenue, Argonne, Illinois 60439. For information about Argonne and its pioneering science and technology programs, see www.anl.gov.

\section{Availability of This Report}

This report is available, at no cost, at http://www.osti.gov/bridge. It is also available on paper to the U.S. Department of Energy and its contractors, for a processing fee, from:

U.S. Department of Energy

Office of Scientific and Technical Information

P.O. Box 62

Oak Ridge, TN 37831-0062

phone (865) 576-8401

fax (865) 576-5728

reports@adonis.osti.gov

\section{Disclaimer}

This report was prepared as an account of work sponsored by an agency of the United States Government. Reference herein to any specific commercial product, process, or service by trade name, trademark, manufacturer, or otherwise, does not necessarily constitute or imply its endorsement, recommendation, or favoring by the United States Government or any agency thereof. The views and opinions of document authors expressed herein do not necessarily state or reflect those of the United States Government or any agency thereof, Argonne National Laboratory, or UChicago Argonne, LLC. 


\section{Summary of Operations and Performance of the Murdock Site Restoration Project in 2008}

by

Applied Geosciences and Environmental Management Section

Environmental Science Division, Argonne National Laboratory

June 2009 


\section{Contents}

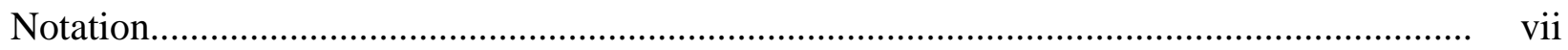

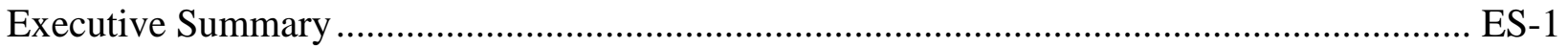

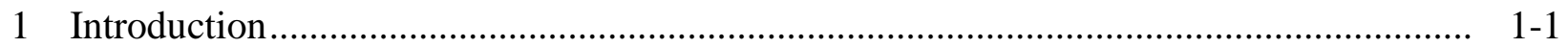

2 Overview of the Treatment Facilities at Murdock .................................................... 2-1

2.1 Well GWEX-1 and the Spray Irrigation Treatment Unit .................................... 2-1

2.2 Phytoremediation and Buffer Plantation Area ....................................................... 2-1

2.3 Constructed Wetlands Area ................................................................................ 2-2

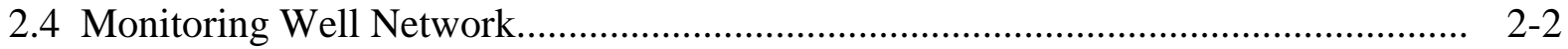

3 Overview of System Operations and Site Activities............................................. $3-1$

3.1 Well GWEX-1 and the Spray Irrigation Treatment Unit....................................... 3-1

3.2 Phytoremediation and Buffer Areas...................................................................... 3-1

3.3 Constructed Wetlands Area .............................................................................. 3-6

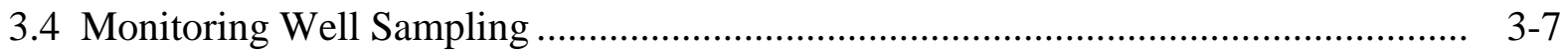

3.5 Monitoring of Surface Waters ........................................................................... 3-7

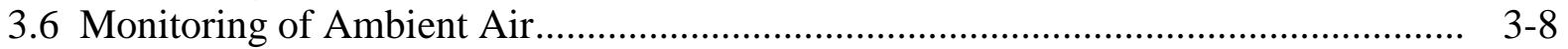

4 Results of Groundwater, Surface Water, Vegetation, and Atmospheric Monitoring ........ 4-1

4.1 Climatic and Hydrologic Factors Affecting the Murdock Site in 2008.................. 4-1

4.2 Results for GWEX-1 and Compliance Monitoring of the Spray Irrigation

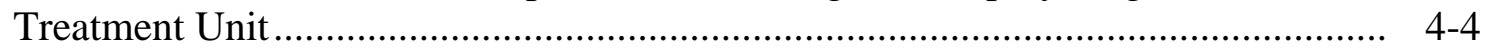

4.3 Results for the Phytoremediation Area ............................................................. 4-6

4.3.1 Evidence of Possible Hydraulic "Pumping" Effects..................................... 4-6

4.3.2 Evidence of Carbon Tetrachloride Uptake and Possible Degradation by the Phytoremediation Plantings

4.3.3 Evidence of Possible Enhanced Microbial Degradation................................. 4-16

4.3.4 Summary of Results for the Phytoremediation Area ................................... 4-18

4.4 Results for Vegetation Sampling in the Wetlands Area ........................................ 4-20

4.5 Results for Compliance Monitoring at Surface Water Locations SWM1, SWM2, and SWM3............................................................................. 4-21

4.6 Results for Atmospheric Compliance Monitoring ............................................... 4-21

4.7 Quality Control for Monitoring Activities ..................................................... 4-23 


\section{Contents (Cont.)}

5 Operation, Maintenance, and System Modifications ................................................ $5-1$

5.1 Well GWEX-1 and the Spray Irrigation Treatment Unit.................................... 5-1

5.2 Phytoremediation Planting and Buffer Areas .................................................. 5-1

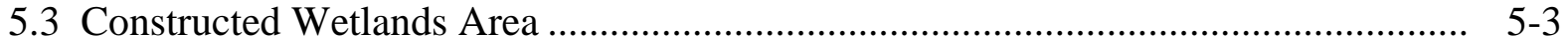

5.4 Monitoring Well Network........................................................................................... 5-3

5.5 Operating and Maintenance Costs for the Current Review Period............................ 5-3

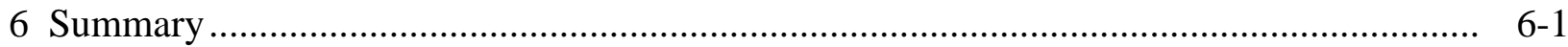

6.1 Performance of the Removal Action Systems .................................................. 6-1

6.2 Regulatory Compliance of the Removal Action Systems....................................... 6-2

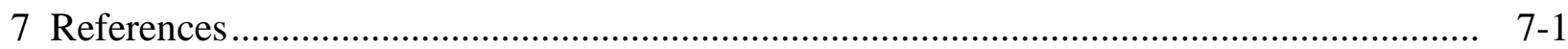

Appendix A: Photo Gallery …................................................................................. A-1

Appendix B: Vegetation Analysis Results............................................................. B-1

Supplement 1: Water Level Recorder Data .............................................................. (on CD)

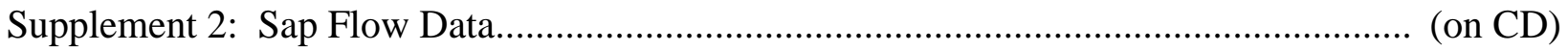

\section{Figures}

1.1 Locations of Murdock, the carbon tetrachloride plume in groundwater, and the treatment facilities

2.1 Locations of the GWEX-1 well, the spray irrigation treatment area, the shallow and deep phytoremediation planting areas, the constructed wetlands, the former $\mathrm{CCC} / \mathrm{USDA}$ grain storage facility, and the carbon tetrachloride plume in groundwater

2.2 Specially designed mobile spray treatment unit on the athletic field at Murdock........ 2-6

2.3 Distribution of the 19 monitoring wells used for twice yearly groundwater sampling and the 16 wells used for water level monitoring

3.1 Tree counts in the waterlogged and dry areas of the phytoremediation plantings....... 3-9 


\section{Figures (Cont.)}

3.2 Thermal dissipation probe (sap flow meter) array in the southeast corner of the tree planting area.

3.3 A thermal dissipation probe (sap flow meter) installed in a cottonwood tree

3.4 "What Is Phytoremediation" sign, newly installed in 2006 and faded in August 2008

3.5 Surface water sampling locations

3.6 Ambient air sampling locations

4.1 Weather station in old location and new location

4.2 Monthly rainfall for the Lincoln-Murdock area, 2006-2008

4.3 Hydrographs for upgradient wells, January 2007 through December 2008

4.4 Hydrographs for phytoremediation area wells near the headwaters of the tributary creek, January 2007 through December 2008

4.5 Hydrographs for wells in the downstream portion of the phytoremediation area, January 2007 through December 2008

4.6 Hydrographs for phytoremediation area wells near the headwaters of the tributary creek, May to October 2008

4.7 Hydrographs for wells in the downstream portion of the phytoremediation area, May to October 2008.

4.8 Calculated surface water discharge rates determined from the V-notch weir at the wetlands outfall, May to October 2008.

4.9 Distribution of blue dye in a freshly cut log from a harvested cottonwood tree, confirming that the entire trunk inside the bark actively took up water

4.10 Carbon tetrachloride concentrations in branch samples, March 2008 


\section{Figures (Cont.)}

4.11 Carbon tetrachloride concentrations in branch samples, July 2008

4.12 Carbon tetrachloride concentrations in branch samples, October 2008

4.13 Geostatistical comparison of carbon tetrachloride concentrations in branch samples, July 2007 and July 2008

4.14 Tree diameter measurements by species as a function of planting depth and surface soil moisture in 2008

4.15 Carbon tetrachloride concentrations in grasses and other vegetation in July 2008

4.16 Vertical distribution of carbon tetrachloride in tissues of harvested eastern cottonwood tree W417EC1

4.17 Maximum carbon tetrachloride concentrations in groundwater samples collected from permanent monitoring points in May 2008

4.18 Maximum carbon tetrachloride concentrations in groundwater samples collected from permanent monitoring points in July 2008

4.19 Carbon tetrachloride concentrations in groundwater samples collected from the shallow monitoring wells in the headwaters area, July 2005-July 2008

B.1 Tree sampling locations in 2008

\section{Tables}

2.1 Plantings in the Murdock phytoremediation and wetlands areas

4.1 Analytical results for volatile organic compounds in samples of untreated water collected from extraction well GWEX-1 at Murdock, 2005-2008

4.2 Analytical results for volatile organic compounds in samples of treated spray collected at Murdock, 2005-2008 


\section{Tables (Cont.)}

4.3 Analytical results for $\mathrm{pH}$ in samples of treated spray collected at Murdock, 2005-2008

4.4 Calculated mean daily sap flow values, summer 2008

4.5 Field-measured biomass parameters (June and July 2008), per tree

4.6 Analytical results for volatile organic compounds in groundwater samples collected from monitoring wells at Murdock, 2005-2008

4.7 Geochemical parameters measured in water samples collected from monitoring wells at Murdock, 2005-2008

4.8 Analytical results for volatile organic compounds in surface water samples collected at Murdock, 2005-2008

4.9 Analytical results for volatile organic compounds in ambient air at Murdock in 2006-2008

5.1 Operations and maintenance costs for the Murdock site restoration project, January-December 2008

B.1 Results of analyses for volatile organic compounds in vegetation samples collected at Murdock in 2008 


\section{Notation}

AGEM Applied Geosciences and Environmental Management

BGL below ground level

CCC Commodity Credit Corporation

CD compact disc

$\mathrm{cm} \quad$ centimeter(s)

d $\quad \operatorname{day}(\mathrm{s})$

DBH diameter at breast height

DO dissolved oxygen

EPA U.S. Environmental Protection Agency

$\mathrm{Fe}^{2+} \quad$ reduced iron

$\mathrm{ft} \quad$ foot (feet)

g $\operatorname{gram}(\mathrm{s})$

gal gallon(s)

gpm gallon(s) per minute

GWEX groundwater extraction well

hr hour(s)

in. inch(es)

$\mathrm{kg} \quad$ kilogram(s)

L $\quad$ liter(s)

$\mu \mathrm{g} / \mathrm{kg} \quad$ microgram(s) per kilogram

$\mu \mathrm{g} / \mathrm{L} \quad \operatorname{microgram}(\mathrm{s})$ per liter

$\mu \mathrm{g} / \mathrm{m}^{3} \quad$ microgram(s) per cubic meter

$\mathrm{mm} \quad$ millimeter(s)

MRL minimal risk level

ND not detected

NDEQ Nebraska Department of Environmental Quality

O\&M operating and maintenance

ORP oxidation-reduction potential

USDA U.S. Department of Agriculture

VOC volatile organic compound

$\mathrm{yr}$

year(s) 


\section{Summary of Operations and Performance of the Murdock Site Restoration Project in 2008}

\section{Executive Summary}

This document reports on the performance in calendar year 2008 of the remediation systems installed by the Commodity Credit Corporation of the U.S. Department of Agriculture (CCC/USDA) at Murdock, Nebraska. The data presented here indicate that the remediation systems are functioning successfully as designed. Specific indicators of this success are as follows:

- Seasonal operation of the upgradient groundwater extraction well captures elevated concentrations of carbon tetrachloride near the location of the former CCC/USDA grain storage facility. The water is treated by spray irrigation equipment and is beneficially reused to improve the quality of the local school's athletic fields. The treated spray has consistently met the established compliance criteria. This part of the remediation system was not operated in 2008, however, because unusually heavy rainfall prevented the school from accepting the discharged spray throughout the review period.

- Uptake of carbon tetrachloride and chloroform by the planted stand of some 2,000 trees has been confirmed through the seasonal detection of these contaminants in branch tissues. The extent of demonstrated contaminant uptake in 2008 remained similar to that observed in 2007 and is consistent with the estimated extent of the groundwater plume. The concentrations detected have declined slightly since the 2007 season.

- Carbon tetrachloride levels in surface waters decrease rapidly along the flow path through the phytoremediation and wetlands treatment areas. The surface water discharged from the treatment system has consistently met the established compliance criteria.

- No carbon tetrachloride has been detected at unacceptable levels in ambient air encountered by workers and visitors in the phytoremediation and wetlands treatment areas. The levels of carbon tetrachloride detected in the ambient air at sampling points AA1-AA3 were significantly below the regulatory target 
level $\left(192 \mu \mathrm{g} / \mathrm{m}^{3}\right)$ adopted for this contaminant by the U.S. Environmental Protection Agency (by approximately two orders of magnitude).

- Noteworthy results regarding the performance of the remediation systems at Murdock during the current review period include the following:

- The GWEX1 extraction well and spray irrigation treatment unit were not operated during the current review period as a consequence of frequent heavy rainfalls and resulting persistent wet surface conditions at Murdock throughout much of the spring, summer, and fall. These conditions precluded the use of these components of the Murdock remediation systems throughout the seasonal operating window during which the spray irrigation treatment process can be effectively employed.

- Distinct seasonal fluctuations in groundwater levels were observed in 2008 at all monitoring locations in the phytoremediation area. The fluctuations appeared to be linked to diurnal effects of groundwater "pumping" by the phytoremediation plantings and nearby crops. Limited estimates based on measurements of discharge from the wetlands suggest that this effect reduced net surface water flow by $30-40 \mathrm{gpm}$ during maximum daily water usage at the height of the 2008 growing season, calculated as the greatest difference between observed maximum and minimum flows for a single day. This is an increase in maximum daily water usage from the values of 15-20 gpm reported for 2007.

Average daily water uptake by the remedial system was measured by using thermal dissipation probes to quantify sap flow and thus water use by the trees. The tree stand appears to have taken up, at the height of the growth season, 8,000-20,000 gal/d of water (average 14,000 gal/d), 84\% of which was used by willows. The estimated rate represents approximately $50-67 \%$ of the average daily reduction (per 24-h day) in discharge observed at the wetlands outfall during the height of the growing season. 
- The increased ratio of chloroform to carbon tetrachloride observed in plant tissues versus groundwater suggests that carbon tetrachloride degradation is occurring in the phytoremediation system. The degradation could be associated with the plant tissue activity, microbial activity in the root zones of the plantings, or both.

- Geochemical data obtained from the monitoring well network during the 2008 growing season indicate that conditions were slightly more oxidizing than in 2006 and 2007. In none of these years did the results indicate widespread development of reducing conditions in the aquifer that might facilitate localized degradation of carbon tetrachloride in the subsurface.

- Increased carbon tetrachloride concentrations detected in groundwater in summer 2007 and throughout 2008 in three wells with shallow completions in the upgradient (headwaters) portion of the phytoremediation area might reflect the movement of more highly contaminated water from the deeper portion of the aquifer into the shallower portion at these locations, in association with the observed rise in groundwater levels due to heavy rainfall.

- Carbon tetrachloride was consistently measured, often at elevated concentrations, in branch tissues of cottonwoods planted where the depth to groundwater is greatest. These results indicate that the roots of these trees have reached the contaminant plume. Sampling in 2008 confirmed the 2007 findings.

- Heavy precipitation during the 2007 and 2008 review periods raised the groundwater levels at Murdock significantly, relative to the levels observed during construction of the phytoremediation and wetlands areas in 2005. The heavy precipitation fostered abundant growth of the phytoremediation plantings and might have placed groundwater within reach of the developing root systems. The continuing (potentially detrimental) effects of these high levels will bear close observation. 


\section{Introduction}

This document summarizes the performance of the groundwater and surface water restoration systems installed by the Commodity Credit Corporation of the U.S. Department of Agriculture (CCC/USDA) at the former CCC/USDA grain storage facility in Murdock, Nebraska, during the third full year of system operation, from January 1 through December 31, 2008. Performance in June 2005 through December 2007 was reported previously (Argonne 2007, 2008).

In the Murdock project, several innovative technologies are being used to remove carbon tetrachloride contamination from a shallow aquifer underlying the town, as well as from water naturally discharged to the surface at the headwaters of a small creek (a tributary to Pawnee Creek) north of the town (Figure 1.1). The restoration activities at Murdock are being conducted by the CCC/USDA as a non-time-critical removal action under the regulatory authority and supervision of the U.S. Environmental Protection Agency (EPA), Region VII. Argonne National Laboratory assisted the CCC/USDA by providing technical oversight for the restoration effort and facilities during this review period.

Included in this report are the results of all sampling and monitoring activities performed in accord with the EPA-approved Monitoring Plan for this site (Argonne 2006), as well as additional investigative activities conducted during the review period.

The annual performance reports for the Murdock project assemble information that will become part of the five-year review and evaluation of the remediation effort. This review will occur in 2010.

This document presents overviews of the treatment facilities (Section 2) and site operations and activities (Section 3), then describes the groundwater, surface water, vegetation, and atmospheric monitoring results (Section 4) and modifications and costs during the review period (Section 5). Section 6 summarizes the current period of operation. A gallery of photographs of the Murdock project is in Appendix A. 


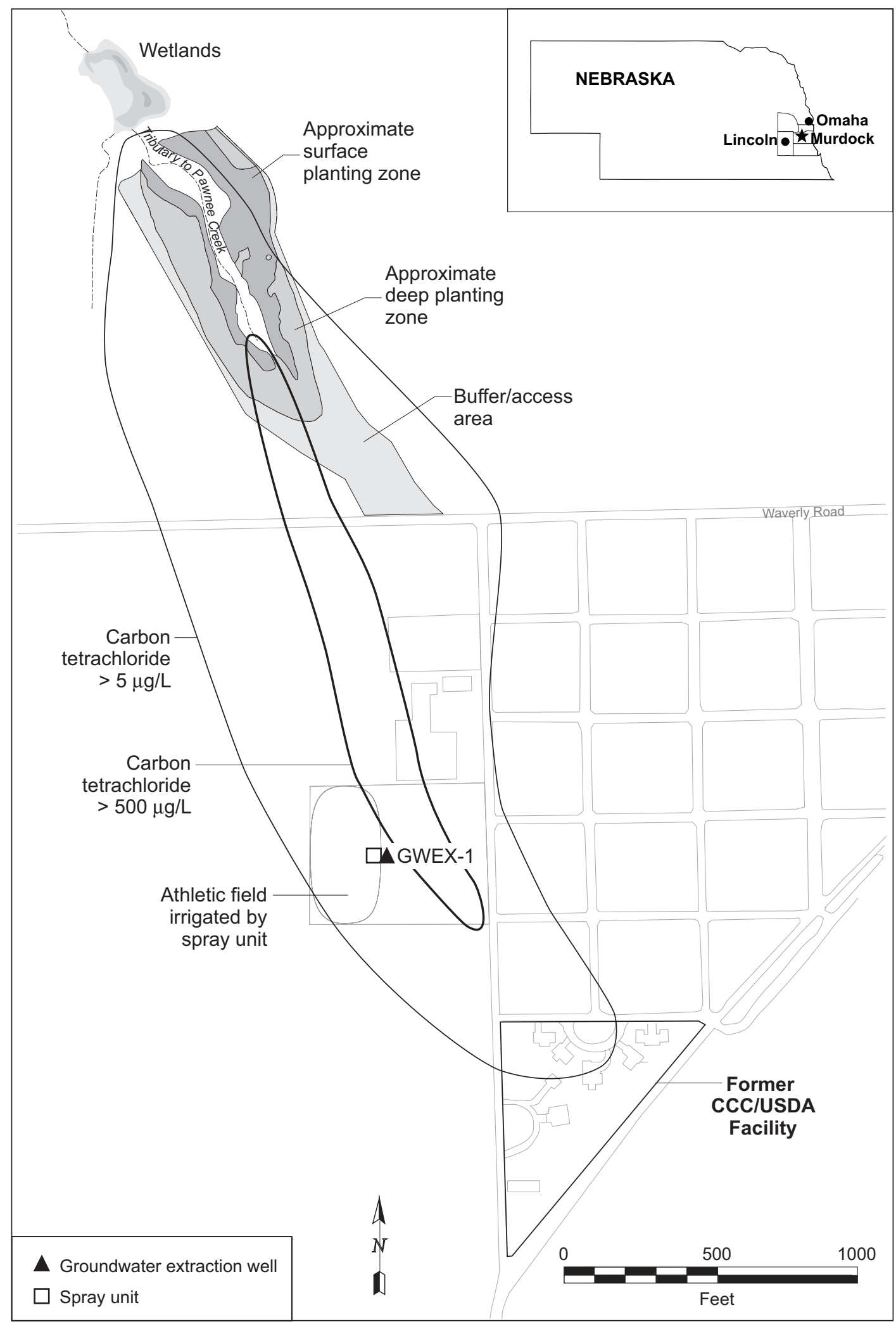

FIGURE 1.1 Locations of Murdock, the carbon tetrachloride plume in groundwater, and the treatment facilities. 


\section{Overview of the Treatment Facilities at Murdock}

The removal action implemented at Murdock employs several innovative technologies in sequence along the contaminant migration pathway to decrease carbon tetrachloride levels in groundwater and in water naturally discharged to the surface at the headwaters of the tributary creek. The principal elements of this restoration approach are described below. The facilities include (1) a groundwater extraction well and spray irrigation treatment system, (2) a phytoremediation and buffer vegetation planting area, and (3) constructed wetlands (Figure 2.1).

\subsection{Well GWEX-1 and the Spray Irrigation Treatment Unit}

Groundwater extraction well GWEX-1 (Figure 2.1) removes contaminated groundwater from the upgradient, more concentrated portion of the carbon tetrachloride plume, before the high contaminant concentrations reach the headwaters of the tributary creek. A specially designed mobile spray treatment unit (Figure 2.2) is used simultaneously to volatilize carbon tetrachloride from the produced groundwater and to irrigate property (athletic fields) owned by the Elmwood-Murdock Public School system.

Well GWEX-1 is operated seasonally, subject to local weather conditions and the irrigation needs of the school system. Routine operation of the well and spray unit is performed by representatives of the school system, under Argonne supervision.

\subsection{Phytoremediation and Buffer Plantation Area}

Selected vegetation types planted in the headwaters area of the tributary creek decrease carbon tetrachloride concentrations in the relatively shallow groundwater and surface waters via phytoremediation processes. Some 2,000 trees representing six species (Niobe willow, black willow, eastern cottonwood, hybrid poplar, green ash, and northern catalpa) cover an area of approximately 4.5 acres (Figure 2.1). Removal of the carbon tetrachloride occurs as a result of (1) uptake, transpiration, and volatilization of the contaminated groundwater by the trees; (2) degradation of the carbon tetrachloride within the plant tissues; and (3) enhanced microbial activity in the root zone created by the plantings. At locations where the static groundwater level is typically more than 4-5 ft below ground level (BGL) - in the "deep" planting zone (Figure 2.1) - a special technique was used to plant the trees in 24-in.-diameter boreholes lined 
with plastic sleeves. This TreeWell ${ }^{\circledR}$ technique (http://www.treemediation.com/) limits the availability of shallow soil water and direct precipitation to the trees, and hence it promotes vertical root growth and uptake of the deeper contaminated groundwater. In the remaining "surface" planting zone (Figure 2.1), the trees were installed without borehole liners to give the roots access to contaminated groundwater typically encountered at depths of less than $4 \mathrm{ft}$ BGL.

Additional vegetation, including a mixture of native prairie grasses, wildflowers, and other species (Table 2.1), was planted between the trees and in the adjacent areas (Figure 2.1). These cover plantings (1) enhance erosion control, (2) intercept local precipitation and runoff and hence promote the uptake of deeper contaminated groundwater by the trees, (3) help protect the trees from physical damage, (4) provide a transitional buffer zone between the tree planting areas and the surrounding croplands, and (5) create a barrier to herbicide drift.

\subsection{Constructed Wetlands Area}

Shallow wetlands constructed downstream from the main planting area provide an additional phytoremediation "polishing" stage for the water discharged to the tributary creek (Figure 2.1). In the wetlands, surface water flow is directed through a sequence of shallow ponds of various depths before it returns to the natural tributary creek channel via an adjustable water control structure. By increasing the residence time and surface exposure area of the flow entering the downstream portion of the tributary, the wetlands promote further carbon tetrachloride evaporation and degradation by water-loving plants and associated microbes.

\subsection{Monitoring Well Network}

A suite of 19 permanent monitoring wells established at the Murdock restoration site facilitates sampling of the groundwater for volatile organic compounds (VOCs) analyses and for the measurement of selected geochemical parameters indicative of possible contaminant degradation within the aquifer (Figure 2.3; see also Section 4.3.3.2). At each location, wells have been installed either individually or as clusters screened to permit sampling from multiple vertical intervals through the saturated zone. Construction information for these wells is in the Monitoring Plan (Appendix A in Argonne 2006). 
Groundwater levels are measured automatically and continuously, at 4-hr intervals, for wells in both the phytoremediation and upgradient areas. The measurements are made by downhole pressure transducers and data loggers installed at 16 locations along the contaminant migration pathway. These locations are highlighted in Figure 2.3. 
TABLE 2.1 Plantings in the Murdock phytoremediation and wetlands areas.
Common Name
Scientific Name

Buffer, Upland, and Tree Area Seeding

Big Bluestem

Indiangrass

Switchgrass

Little Bluestem

Sideoats Grama

Western Wheatgrass

Virginia Wildrye

Blue Grama

Buffalograss

Canada Wildrye

Hard Fescue

Sheep Fescue

Annual Ryegrass

Baby's Breath

Blackeyed Susan

Blanket Flower

Blue Flax

California Poppy

Clarkia

Clasping Coneflower

Cornflower

Corn Poppy

Dwarf Red Coreopsis

Illinois Bundleflower

Indian Blanket

Lance Leaved

Coreopsis

Lead Plant

Lemon Mint

Mexican Red Hat

Coneflower

Missouri Primrose

Partridgepea

Purple Prairieclover

Plains Coreopsis

Perennial Lupine

Purple Coneflower

Rosy Red Yarrow

Shasta Daisy

Upright Coneflower

Catchfly

Drummond Phlox

Siberian Wallflower

Scarlet Flax

Alfalfa

Red Clover
Andropogon gerardii

Sorghastrum nutans

Panicum virgatum

Schizachyrium scoparium

Bouteloua curtipendula

Agropyron smithii

Elymus virginicus

Bouteloua gracilis

Buchloe dactyloides

Elymus Canadensis

Festuca longifolia

Festuca ovina

Lolium multiflorum

Gypsophila elegans

Rudbeckia hirta

Gaillardia aristata

Linum lewisii

Eschscholzia californica

Clarkia amoena

Rudbeckia amplexicaulis

Centaurea cyanus

Papaver rhoeas

Coreopsis tintoria, red

Desmanthus illinoensis

Gaillardia pulchella

Coreopsis lanceolata

Amorpha canescens

Monarda citriodora

Ratibida columnifera, red

Oenothera missouriensis

Cassia chamaecrista

Petalostemum or Dalea

purpurea

Coreopsis tinctoria

Lupinus perennis

Echinacea purpurea

Achillea millefolium, rubra

Chrysanthemum

maximum

Ratibida columnifera

Silene ameria

Phlox drummondii

Cheiranthus allioni

Linum grandiflorum

rubrum

Medicago sativa

Trifolium pratense
Common Name

Scientific Name

Wetlands Seeding

Alumroot

Aster, New England

Aster, Silky

Aster, Swamp

Blackeyed Susan, Sweet Rudbeckia subtromentosa

Blue Flag Iris

Blue Vervain

Boneset

Bottle Gentian

Cardinal Flower

Coreopsis, Tall

Duck Potato

False Dragonhead

Fowl Mannagrass

Great Blue Lobelia

Great Angelica

Joe Pye Weed

Marsh Betony

Marsh Blazing Star

Marsh Milkweed

Nodding Bur Marigold

Primrose, Showy

Evening

Sneezeweed

Heuchera richardsonii

Aster novae-angliae

Aster sericeus

Aster puniceus

Iris veriscolor

Verbena hastate

Eupatorium perfoliatum

Gentiana andrewsii

Lobelia cardinalis

Coreopsis tripteris

Sagittaria latifolia

Physostegia virginiana

Glyceria striata

Lobelia siphiltica

Angelica atropurpurea

Eupatorium maculatum

Stachys palustris

Liatris spicata

Ascelpias incarnata

Bidens cernua

Oenothera speciosa

Helenium autumnale

Blue Joint Grass

Bulrush, Hardstem

Bulrush, Dark Green

Bulrush, Softstem

Bur Reed

Rush, Inland

Rush, Creeping Spike

Rush, Spike

Rush, Soft

Sedge, Bebb's

Sedge, Bristly

Sedge, Long Beaked

Sedge, Porcupine

Sedge, Three Way

Sedge, Tussock

Woolgrass

Calamagrostis canadensis

Scirpus acutus

Scirpus atrovirens

Scirpus validus

Sparganium eurycarpum

Juncus interior

Eleocharis palustris

Eleocharis calva

Juncus effuses

Carex bebbii

Carex comosa

Carex sprengelii

Carex hystericina

Dulichium arundinaceum

Carex stricta

Scirpus cyperinus

Wetlands Transplanting

Broadleaf Arrowhead

Bulrush, Green

Bulrush, Hardstem

Bulrush, River

Bulrush, Softstem

Flatstem Spikerush
Sagittaria latifolia

Scirpus atrovirens

Scirpus acutus

Scirpus fluviatilis

Scirpus validus

Eleocharis compressa 


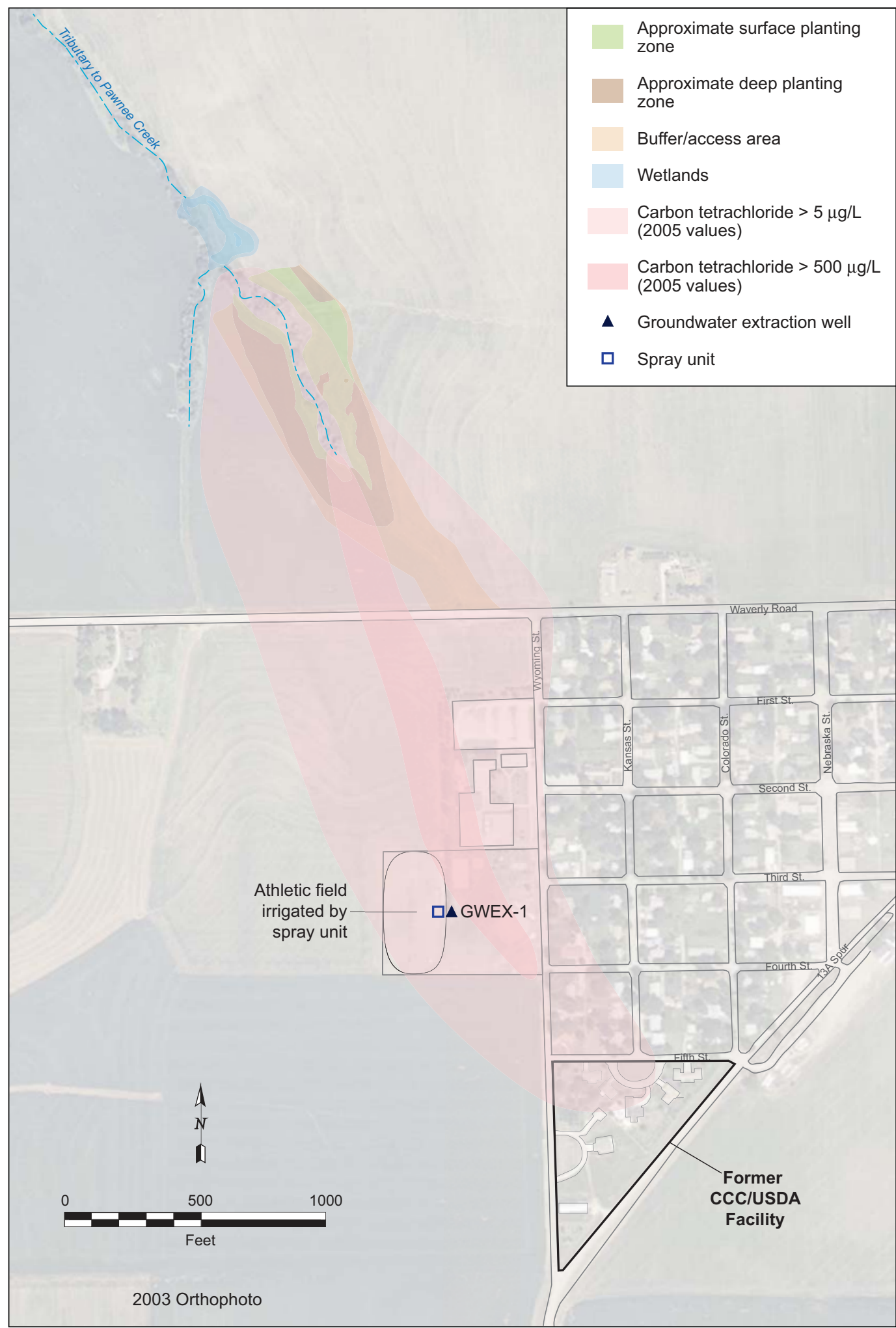

FIGURE 2.1 Locations of the GWEX-1 well, the spray irrigation treatment area, the shallow and deep phytoremediation planting areas, the constructed wetlands, the former CCC/USDA grain storage facility, and the carbon tetrachloride plume in groundwater. Source of photograph: NAIP (2003). 


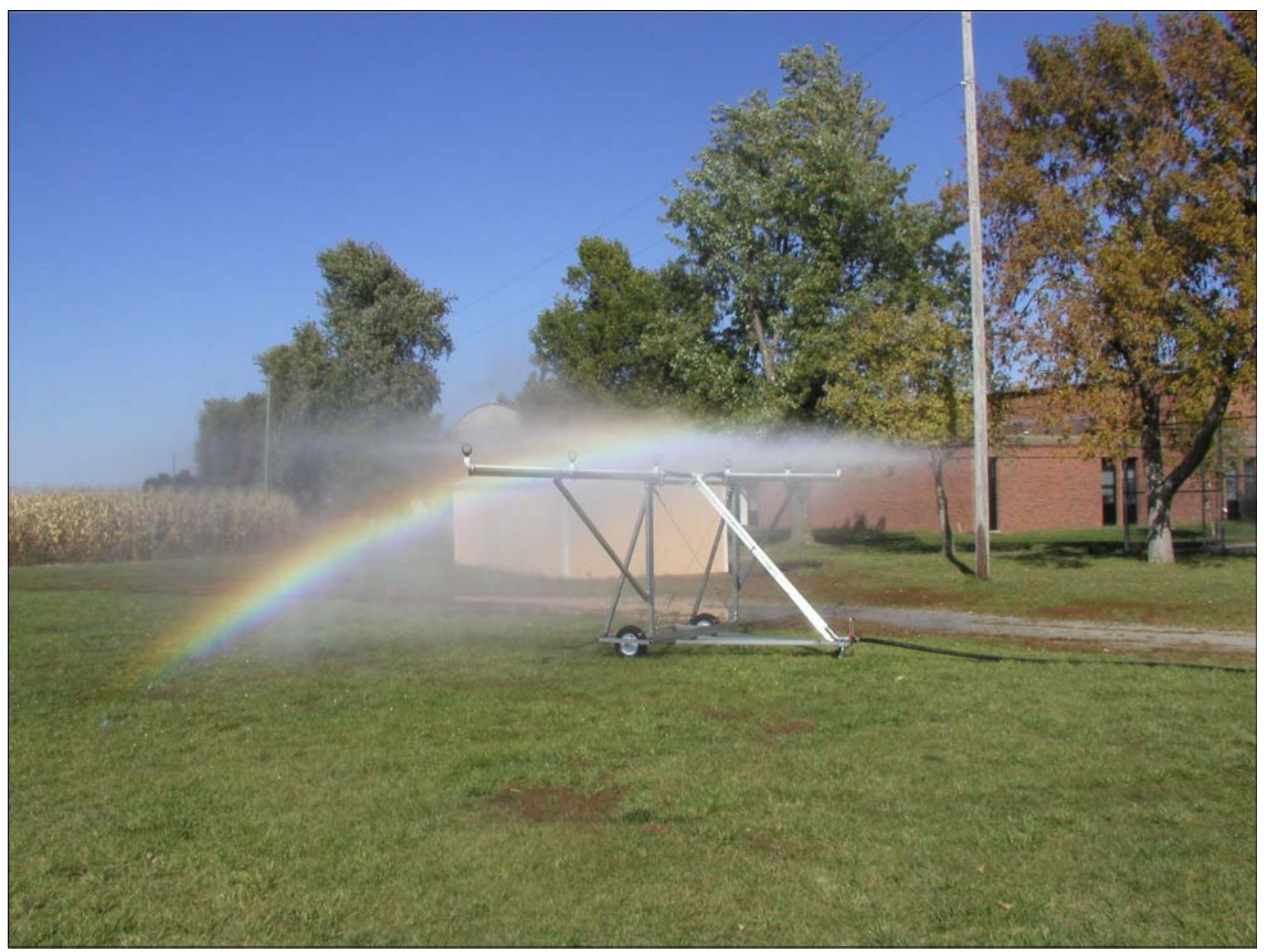

FIGURE 2.2 Specially designed mobile spray treatment unit on the athletic field at Murdock. 


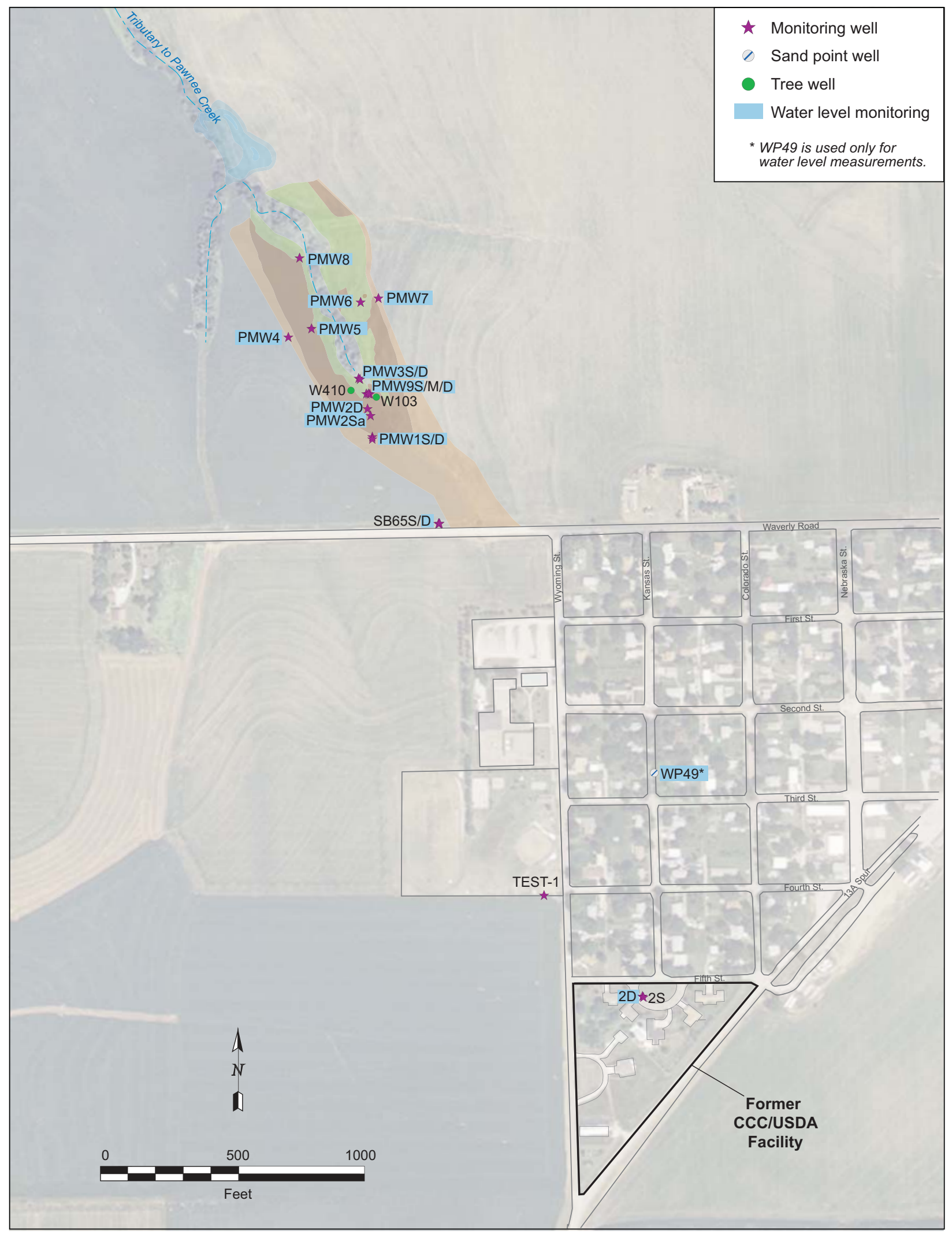

FIGURE 2.3 Distribution of the 19 monitoring wells used for twice yearly groundwater sampling and the 16 wells used for water level monitoring. Source of photograph: NAIP (2003). 


\section{Overview of System Operations and Site Activities}

This section briefly summarizes the operations and related activities at the Murdock restoration site during the review period.

\subsection{Well GWEX-1 and the Spray Irrigation Treatment Unit}

Well GWEX-1 was installed on August 2, 2004. Pilot testing was carried out in October 2004 to determine the well's sustainable production capacity and to demonstrate the viability of the spray irrigation treatment technology at this site (Argonne 2006). With the approval of the EPA, the use of GWEX-1 and the spray irrigation treatment unit as one component of the removal action at Murdock began in 2005.

The extraction well and treatment unit were not operated during the current review period, at the specific request of the Elmwood-Murdock Public School administration, as a consequence of frequent heavy rainfalls and resulting persistent wet surface conditions at Murdock throughout much of the spring and summer. These conditions, coupled with the need for daily use of the school property for athletic and other school activities during the later summer and fall, precluded the use of GWEX-1 and the spray treatment unit throughout the potential 2008 irrigation season.

During the normal seasonal operation of GWEX-1, samples of the untreated groundwater and the treated effluent from the spray irrigation unit are collected quarterly for VOCs analyses (Argonne 2006). Because GWEX-1 and the spray treatment unit did not operate in 2008, no monitoring events occurred, or were required, during the present review period. The results of previous (2005-2007) monitoring activities conducted at GWEX-1 and the spray treatment unit are briefly summarized in Section 4.2.

\subsection{Phytoremediation and Buffer Areas}

Trees were planted in the main phytoremediation area in April and May 2005. Results of preliminary sampling during baseline studies in the phytoremediation area in July 2005 indicated that the plantings had already begun taking up carbon tetrachloride from the contaminated groundwater and surface waters at that time. Monitoring of vegetation in the phytoremediation 
planting area in accord with the EPA-approved Monitoring Plan (Argonne 2006) began in August 2005. All required planting in the phytoremediation and buffer zone areas was completed in spring 2006.

Unusually high rainfalls throughout the late spring and much of the summer of 2007 raised groundwater levels at Murdock and increased surface runoff to the tributary creek. Shallow flooding of the land surface was consequently observed in the phytoremediation area throughout much of 2007. Locally pooled surface water as much as several inches deep, flowing groundwater seepage, and surface runoff were persistently noted, particularly in the western and central portions of the shallow planting area near the headwaters of the tributary creek and along both sides of the tributary creek in the more downstream portion of the shallow planting area. These conditions persisted throughout the current review period, because local groundwater levels remained high during the winter of 2007-2008, then rose farther in response to heavy rains in spring and early summer 2008.

As a result of the unusually wet conditions, the following specific activities were conducted to monitor and maintain the health of the plantings and the integrity of the visitor amenities in the phytoremediation area:

- To help control and enhance the patterns of surface drainage through the phytoremediation planting, the main channel leading into the phytoremediation area at the headwaters of the tributary creek was regraded and stabilized (with gravel), additional drainage conduits were installed at selected locations in the phytoremediation area, and the pedestrian walking path was redressed in several low portions of the headwaters area.

- The phytoremediation planting area was inspected frequently throughout the growing season for possible indicators of plant stress or other detrimental conditions associated with the wet surface environment. In March 2008, the conditions at the site were reviewed by Argonne personnel in consultation with a representative of the Nebraska state forester's office.

- Mowing, trimming, and weeding were conducted from late June through early October 2008 to control overabundant growth of the groundcover species in 
the phytoremediation area and to facilitate access to the trees for vegetation sampling and monitoring.

- In 2007, temporary support was provided for a number of trees that had begun to lean unacceptably during the late spring and summer because of soft soil conditions that developed in several areas of persistent standing surface water. Inspection of the phytoremediation plantings throughout 2008 showed that additional cottonwood trees were leaning. Investigations beginning in June revealed that most of the affected trees were in waterlogged areas and that the root systems of these trees had decayed significantly because of their continued submersion. In addition, the soil surrounding these trees had lost its structural properties and was thus unable to support the tree weight. As a consequence of these factors, the trees were both sinking into the ground and leaning sideways. Selected trees (almost exclusively cottonwoods) in the areas of continued standing water were therefore removed. Several of the trees removed were dissected for study of internal structure and selective tissue sampling at various heights to determine the distribution of carbon tetrachloride in the tree. Entire trees, including leaves, were weighed to arrive at a biomass estimate that could be applied to the entire plantation with some confidence. In addition, a basal portion of the trunk was placed in dyed water to visually assess the extent of sapwood actively taking up water. Photographs of these activities are in Appendix A.

- The trees in areas with standing water ("wet areas") and in dry areas were counted in October to delineate the extent of the areas affected by the wet conditions and to determine additional effects on tree growth. The count showed 264 tree pairs in wet areas and 740 tree pairs in dry areas (Figure 3.1). The results are discussed further in Section 4.3.2.3.

- As in 2007, some trees were affected by fungi in 2008, partly as a result of the prolonged wet conditions at the site and the possible restriction of local air flow by the dense canopy, particularly in the shallow-groundwater areas planted with willows. Some thinning occurred after consultation with the Nebraska state forester, Richard Lodes, during his annual visit to the site. 
Twice yearly vegetation sampling (for VOCs analyses) from a representative suite of trees in the phytoremediation area, on an approximate grid pattern, was recommended in the approved Monitoring Plan (Argonne 2006) to assess the seasonal uptake of groundwater and carbon tetrachloride by the plantings. Vegetation sampling was proposed (1) in early spring, before the onset of significant plant growth, and (2) in late summer or early fall, at the peak or near the end of the growth cycle.

Vegetation sampling was conducted during the current review period as follows:

- March 28, 2008 - partial sampling for a subset of the grid locations identified in the Monitoring Plan (Argonne 2006) and selected preexisting native and background trees, to determine contaminant levels while the trees were dormant.

- June 23, 2008 - harvesting of one tree for root system inspection, total tree carbon tetrachloride analysis, and a biomass metrics study (yield, size).

- July 21-23, 2008 — primary annual sampling for approximately the full grid pattern, as well as for background trees, to indicate contaminant uptake by the trees at the peak of the growing season. Whole-tree analysis for carbon tetrachloride in two additional cottonwood trees was conducted at this time.

- October 7-9, 2008 - supplemental sampling for VOCs analyses at selected locations in the far northeastern and the northwestern portions of the phytoremediation area (at tile drain outfalls), as well as at several other locations where carbon tetrachloride concentrations had been observed to be lower than in 2007.

Measurements of tree trunk diameter were made for all trees on October 7-9, 2008, at the onset of the dormant season. The purpose was to provide a basis for (1) stand-wide calculation of water consumption from sap flow data and (2) correlation of tree growth with planting method, response to groundwater and surface water levels, and the observed patterns of carbon tetrachloride uptake. 
Total biomass yield data were collected for several cottonwoods to correlate total standing biomass with diameter measurements (allometric measurements). The purpose of calculating the standing biomass was to provide a basis for (1) future determination of carbon tetrachloride removal by the tree stand and (2) estimation of total nitrogen use by the phytoremediation stand.

Growth and nitrogen analysis data are discussed in Section 4.3.2.3.

The herbaceous groundcover stand in the phytoremediation area was sampled during the July event to determine whether the deep-rooted grasses growing in this area are taking up contaminants and thus are rooting sufficiently deeply to draw water from the contaminant plume. The results of these analyses are discussed in Section 4.3.2.1.

Thermal dissipation probes were installed from July 23 to August 27, 2008, for direct measurement of water use by 18 trees clustered in the southwestern corner of the tree planting area (Figure 3.2). Instrumented trees included cottonwoods and willows of comparable size, as well as one green ash. Thermal dissipation probes are two-pronged, small-diameter probes that are inserted into the tree trunk at a standardized height, to record the sap flux (in milliliters per second) as the velocity of heat dissipation from a heated probe compared to the baseline temperature sensor. Probes are connected to a continuous data logger, and the system is powered by a solar panel charging a marine battery. By indexing the sap flux to the area of active sapwood of the instrumented tree, a measure of sap flow (in liters per day) is obtained. Figure 3.3 shows a thermal dissipation probe installed in a cottonwood tree. The results of the sap flow analyses are discussed in Section 4.3.1.2.

On October 9, a field trip organized by the Great Plains Society of American Foresters brought 24 visitors to observe the phytoremediation plantings. The forestry society chapter, which includes members from Nebraska and Kansas, was holding its annual meeting in Lincoln. A representative of the chapter wrote, "I wanted to thank you for the excellent presentation. I received a lot of comments that it was extremely interesting."

A final activity in the phytoremediation area in 2008 concerned the trail signs along the pedestrian path. Some of the signs were observed to have faded noticeably since their installation (Figure 3.4). The vendor agreed to replace the signs at no cost. The replacements are being stored for the winter before installation in spring 2009. 


\subsection{Constructed Wetlands Area}

Construction of the wetlands treatment area started in the summer of 2005 and was substantially completed in the spring of 2006. In late spring and early summer of 2006, additional work was completed to control unexpected surface erosion observed initially along the southern margin of the wetlands. The following corrective measures were implemented:

- Removal of excess sediment from the southern wetlands basin

- Regrading of the southern wetlands margin

- Seeding of additional groundcover

- Planting of wetlands species (rushes and sedges) to promote development and stabilization of the wetlands environment

Localized surface channeling was observed near the southwestern margin of the wetlands during early spring of 2007, as a result of runoff associated with heavy spring rains. Little further impact was observed in this area in 2007, however, after seasonal groundcover and crop growth began, respectively, along the wetlands margins and in the agricultural fields west of the wetlands. In 2008, minor regrading was performed adjacent to the walking pathway in this area in conjunction with the replacement of several conduits with larger-diameter units beneath the pathway, for better control of the surface drainage into the wetlands from the west.

Under all but extreme flood conditions, discharge from the phytoremediation and wetlands areas is returned to the Pawnee Creek tributary channel via a water control structure (stop logs) installed at the downstream edge of the wetlands basin. With the approval of the CCC/USDA, a weir system was implemented at the control structure in April 2007 to facilitate quantitative monitoring of the rates and volumes of discharge from the wetlands (Argonne 2008). Flow rates through the weir system are calculated on the basis of certain fixed parameters determined by the construction of the weir, in conjunction with measurements of the flowing water levels observed upstream of the weir system. The water level measurements required for the flow calculations are obtained by using automatic water level recorders installed at two points upstream from the weir system. The results of the flow rate monitoring are discussed in Sections 4.1 and 4.3.1. 
Proper functioning of the weir system requires a free fall of water from the weir outlet. In spring 2008, the "standing" water level in the stream channel immediately downstream of the water control structure rose to the approximate height of the weir outfall, as a result of vegetation growth and natural obstructions (tree limbs, etc.) that had accumulated in the downstream portion of the channel after frequent spring rains. To correct this problem, the channel downstream of the weir was cleared and regraded in May of 2008.

To investigate for evidence of possible contaminant uptake by the developing plant community in the Murdock wetlands, vegetation samples for VOCs analyses were collected during the summer growth period at 20 locations and from 9 plant types (bulrush, cattail, arrowhead, lemna, switchgrass, knotweed, reed canary grass, and adventitious willow and cottonwood saplings) in the water and at the water's edge along the perimeter of the wetlands. The wetlands sampling was performed in conjunction with the vegetation sampling in the phytoremediation area on July 21-23, 2008. The results of the wetlands vegetation sampling are discussed in Section 4.4.

\subsection{Monitoring Well Sampling}

Groundwater samples are collected twice annually from the full suite of 19 permanent monitoring wells shown in Figure 2.3, for VOCs analyses and the determination of selected inorganic geochemical parameters including total nitrogen (Argonne 2006). During the current review period, the monitoring wells were sampled on May 8-13 and July 21-23, 2008.

Samples of shallow subsurface water are also collected for VOCs analyses and geochemical parameter determinations from the vent tubes installed in the borehole liners at two deep tree planting locations (W103 and W410) in the phytoremediation area. Sampling at these locations during the current review period occurred on May 13 and July 26, 2008.

The analytical results for the subsurface water samples are presented in Section 4.3.3.

\subsection{Monitoring of Surface Waters}

Surface water samples for VOCs analyses are collected quarterly (Argonne 2006) along the tributary creek at three locations (Figure 3.5). One location (SWM1) is near the upgradient 
margin of the phytoremediation area, the second (SWM2) is at the upgradient margin of the wetlands area, and the third (SWM3) is directly downgradient from the outfall from the wetlands area. Surface water was sampled at the designated locations on February 13, May 5, July 21, and November 17, 2008. The results of the analyses are in Section 4.5.

\subsection{Monitoring of Ambient Air}

Ambient air samples for VOCs analyses are collected twice annually (Argonne 2006) at three locations (Figure 3.6). Two locations (AA1, AA2) are in the phytoremediation area, and one (AA3) is in the wetlands area. Sampling at these locations occurred on May 13-15 and July 22-24, 2008. In each of these events, a comparison sample of "background" air was collected at location BA1, to the southwest and upgradient/upstream from the tributary creek headwaters area. The analytical results for these events are discussed in Section 4.6. 


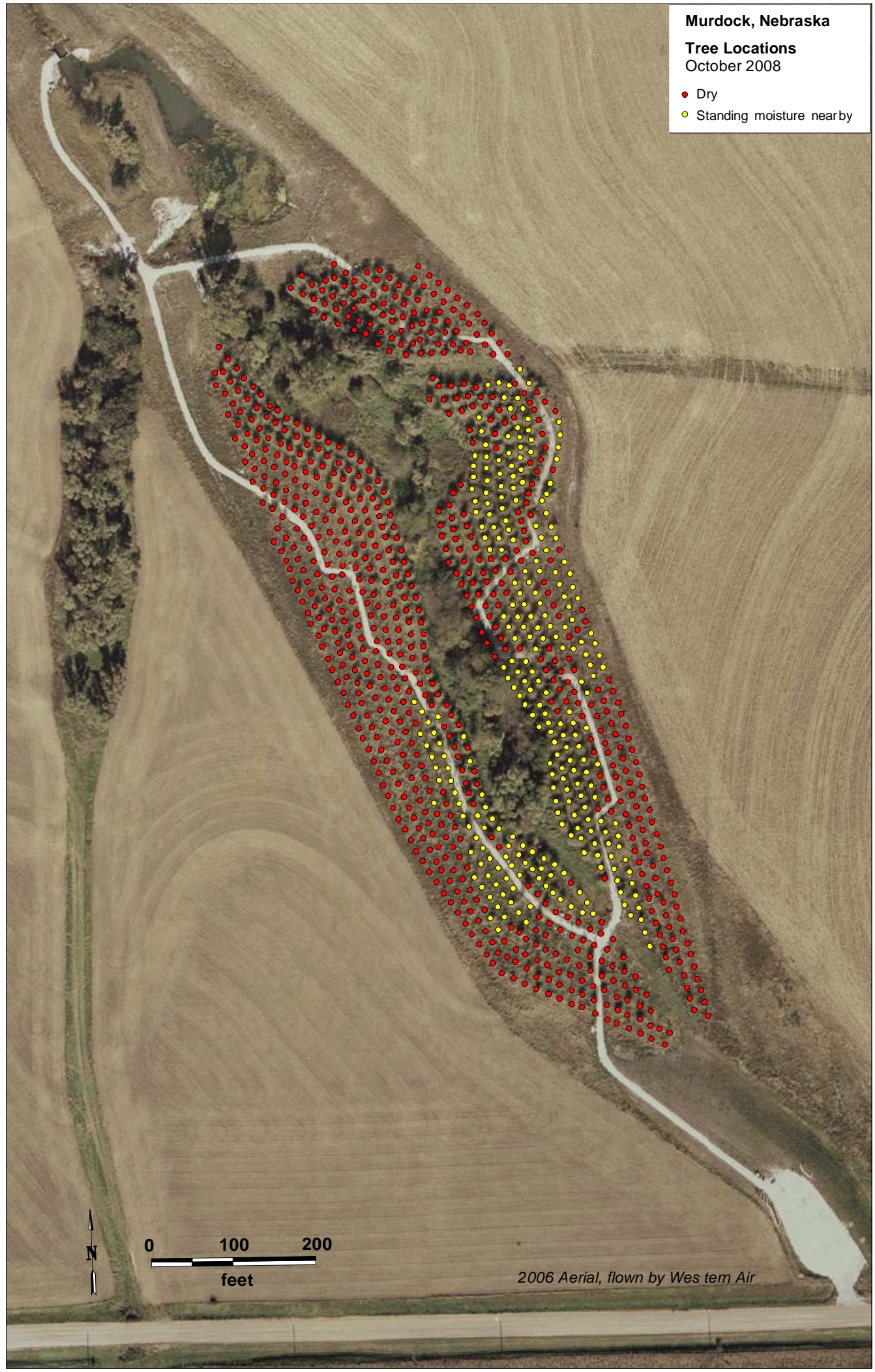

FIGURE 3.1 Tree counts in the waterlogged and dry areas of the phytoremediation plantings. 

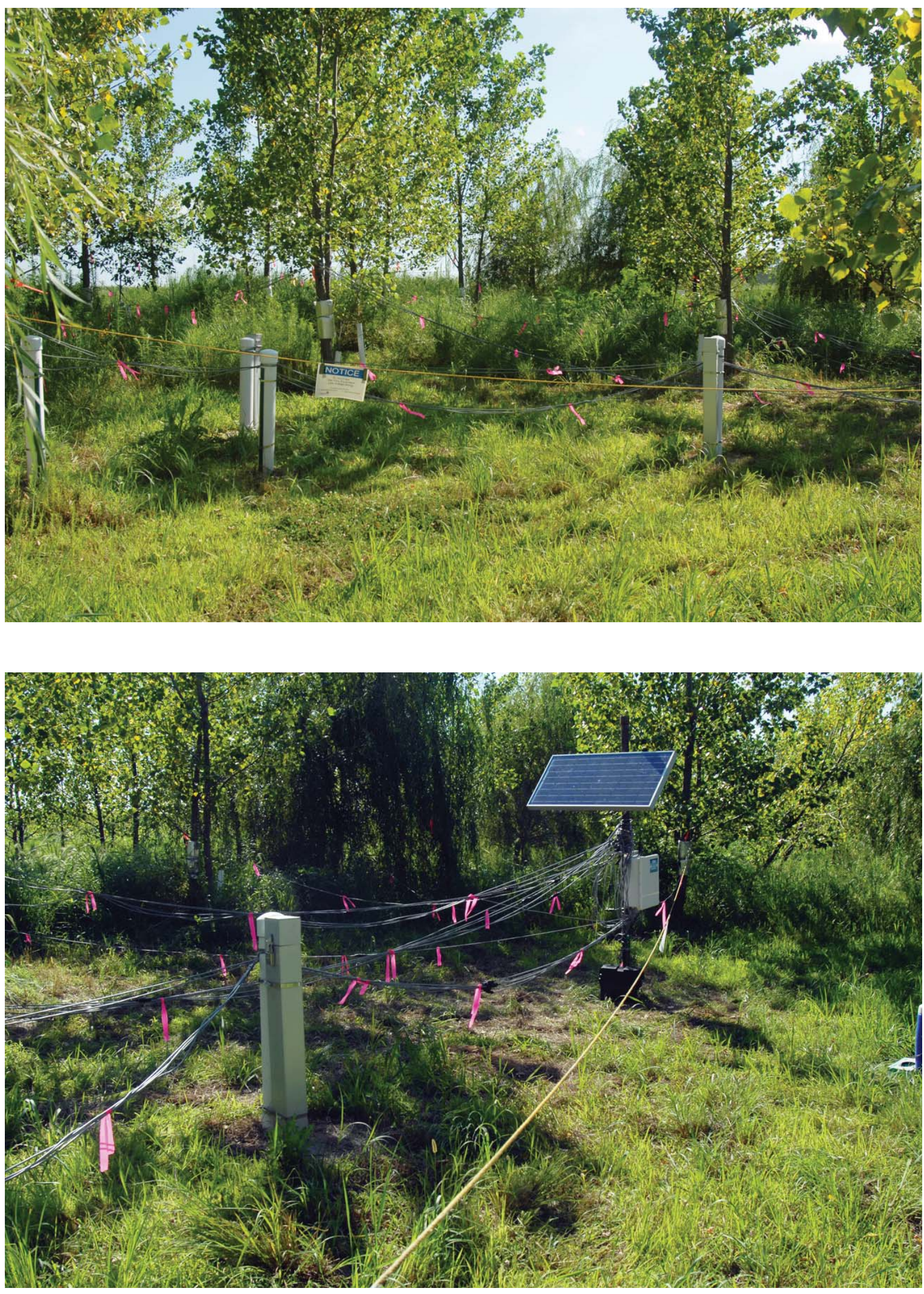

FIGURE 3.2 Thermal dissipation probe (sap flow meter) array in the southeast corner of the tree planting area. 


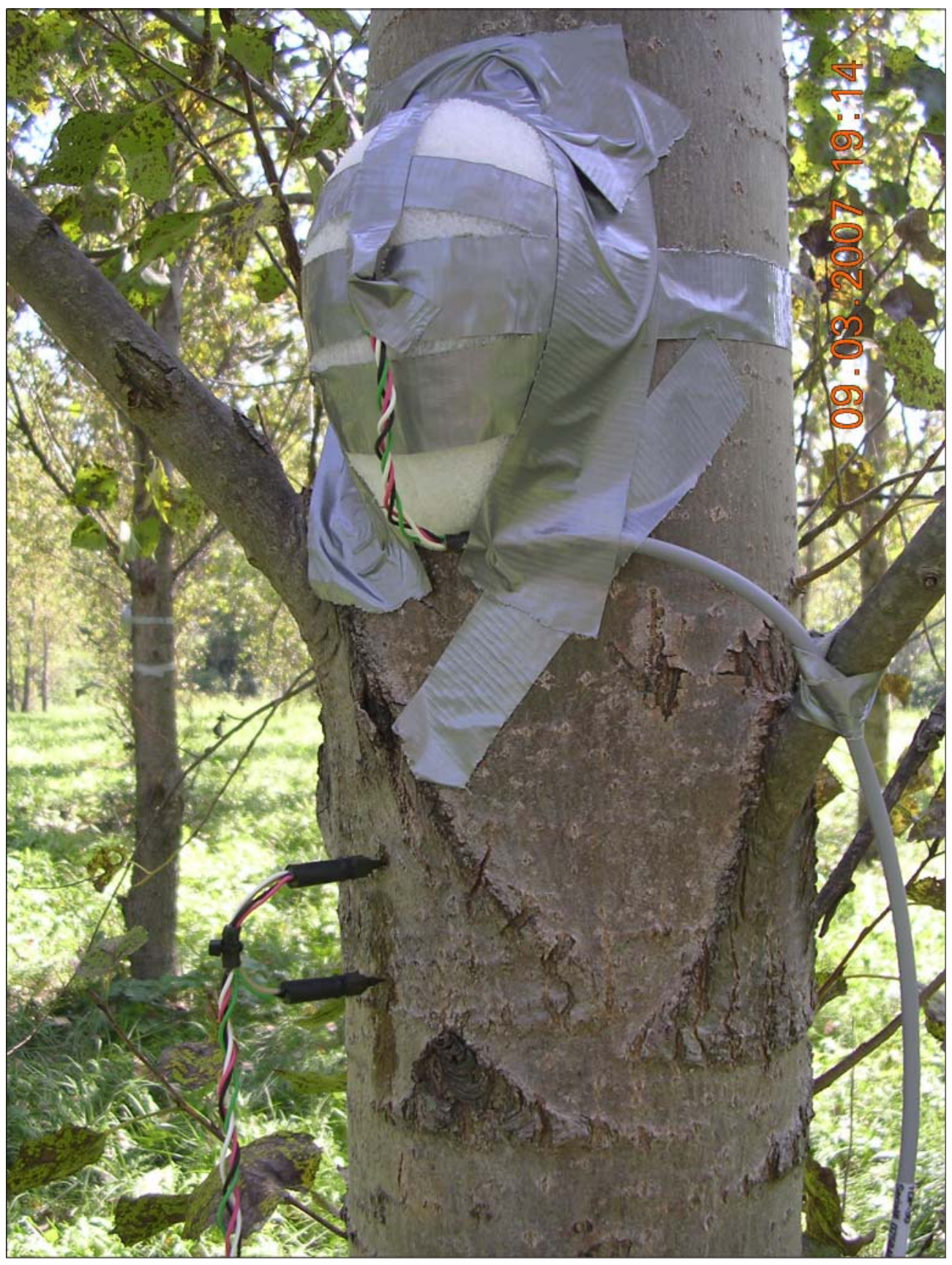

FIGURE 3.3 A thermal dissipation probe (sap flow meter) installed in a cottonwood tree. 

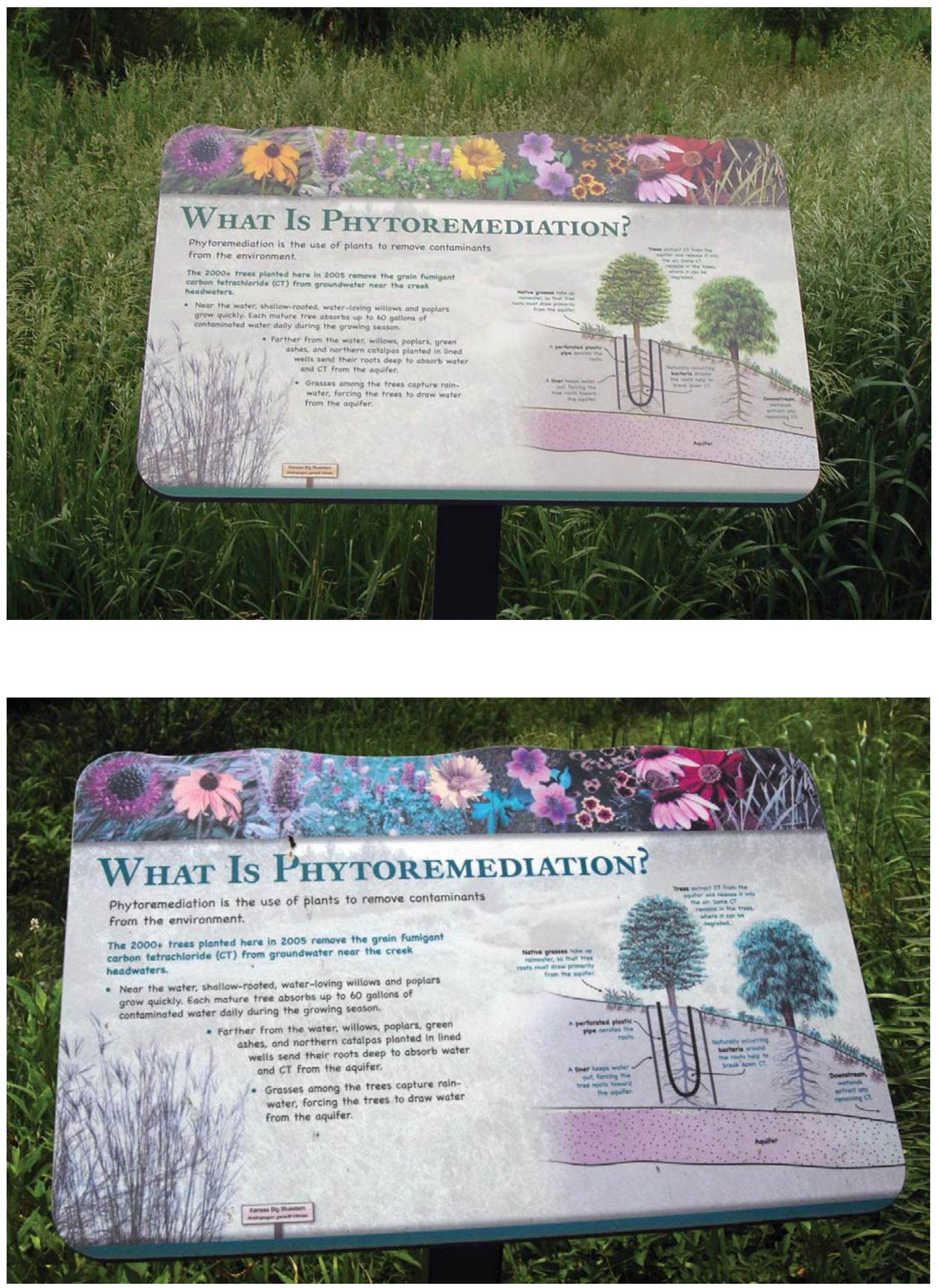

FIGURE 3.4 "What Is Phytoremediation" sign, newly installed in 2006 (top) and faded in August 2008 (bottom). 


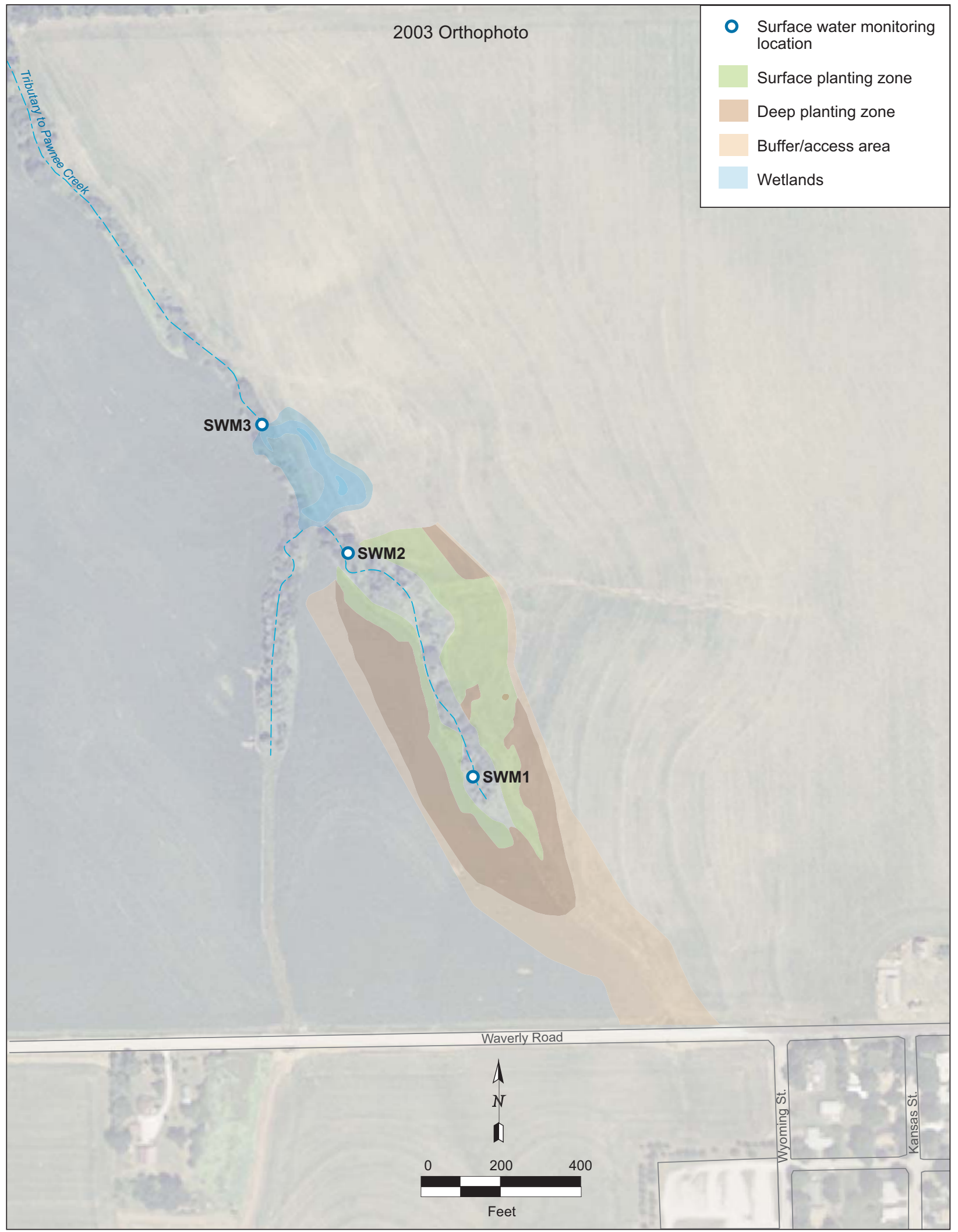

FIGURE 3.5 Surface water sampling locations. Source of photograph: NAIP (2003). 


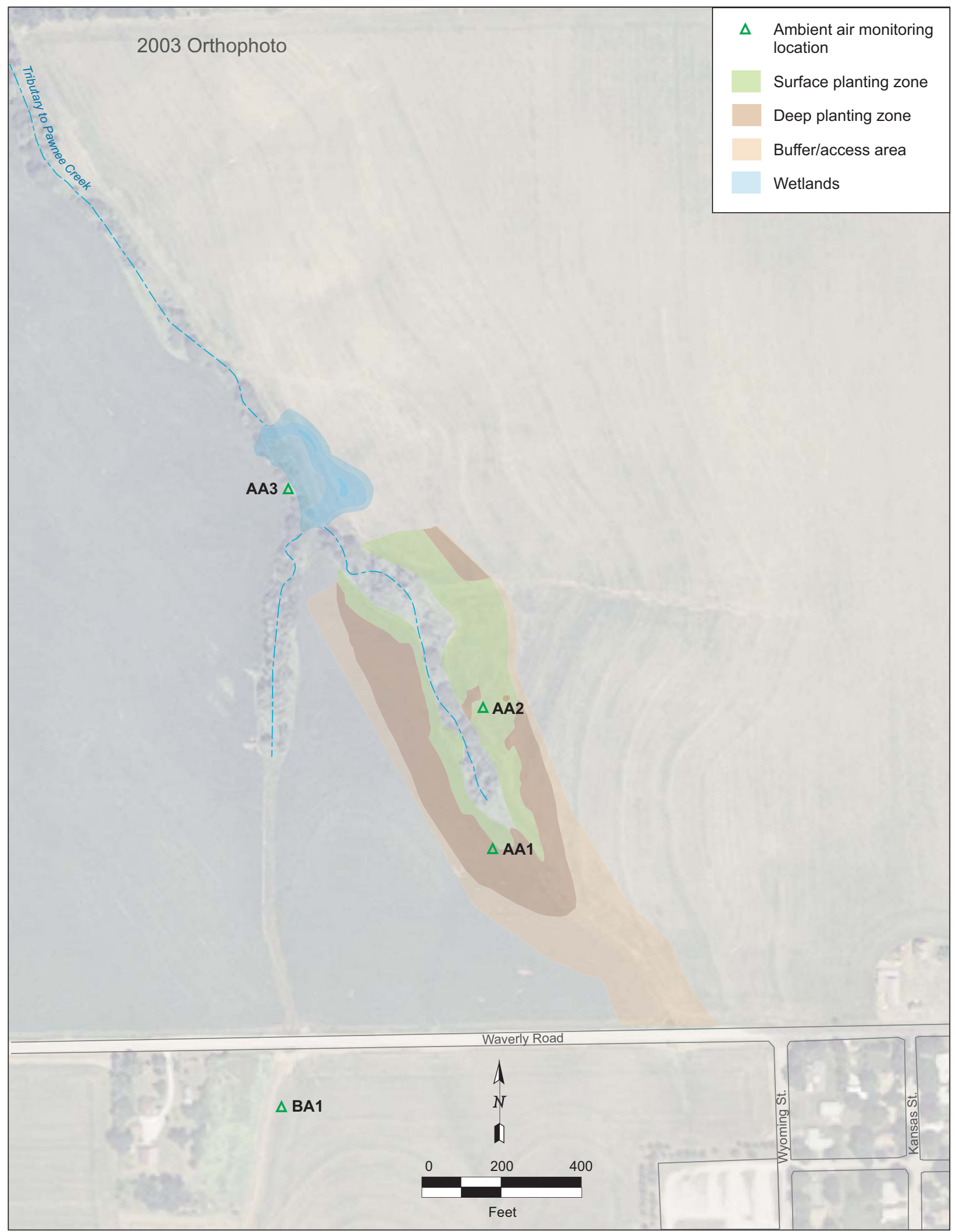

FIGURE 3.6 Ambient air sampling locations. Source of photograph: NAIP (2003). 


\section{Results of Groundwater, Surface Water, Vegetation, and Atmospheric Monitoring}

\subsection{Climatic and Hydrologic Factors Affecting the Murdock Site in 2008}

During the 2007 and current (2008) review periods, the Murdock site experienced significantly wetter conditions than in several previous years. The impacts of these wet conditions on the performance of the individual treatment system elements at Murdock are addressed below.

Climate data indicate that from 1999 until 2006, southeastern Nebraska, including the Murdock area, was under designated drought conditions. Except in 2001, annual rainfalls in the Lincoln, Nebraska, area in 1999-2006 were consistently below the long-term average of $28.37 \mathrm{in./yr}$ for 1976-2005. At the end of 2006, a total deficit of approximately $19.3 \mathrm{in}$. of precipitation was reported for the Lincoln area, with approximately $3.7 \mathrm{in}$. of the shortfall occurring in 2006 (UNL 2009).

Automated monitoring of on-site weather conditions at Murdock began in mid 2005, in conjunction with the aquifer and surface water restoration program. Data recorded at the headwaters area of the tributary creek indicated a total rainfall of 26.4 in. for Murdock in 2006, roughly 2 in. below the Lincoln annual average. In response to this sparse precipitation, groundwater levels in both the upgradient portion of the Murdock groundwater plume and the more downgradient phytoremediation treatment area declined slowly from fall 2005 to spring 2006, then remained fairly stable or declined very slowly through the end of 2006.

In 2007, the Murdock on-site weather station recorded 35.8 in. of rainfall, in close agreement with the total reported for the greater Lincoln area (35.4 in.; UNL 2009). The observed total was approximately $25 \%$ greater than the $30-\mathrm{yr}$ annual average for this area (28.51 in. for 1977-2006).

In 2008, the on-site weather station recorded an apparent total rainfall of only $21.8 \mathrm{in}$.; however, these data are incomplete. Although the available data suggest that most rainfall events at the site were detected, interference with the rainfall sensor caused by encroachment of the surrounding trees at the station's original location near the headwaters of the tributary creek caused rainfall values recorded during the spring and early summer to be lower than the actual 
amounts. The weather station was subsequently moved to a more open location immediately south of the wetlands (Figure 4.1); however, a number of rainfall events were not recorded during the relocation period. The rainfall total of 34.9 in. (6.4 in. above the long-term average) reported for the Lincoln area in 2008 (UNL 2009) is comparable to the rainfall observed at Murdock in 2007 and is believed to be more representative of the total rainfall at the Murdock site than the amounts measured at the site in 2008. Approximately $25 \%$ of the total rainfall for the current review period fell in June 2008, while in 2007 unusually heavy rains occurred in both May and August (Figure 4.2).

Groundwater levels are recorded automatically at 16 locations at Murdock (Figure 2.3) to monitor the patterns of water level variations along the contaminant migration pathway, as well as within the phytoremediation treatment zone, that might influence the fate and transport of carbon tetrachloride. Complete data recovered from the water level recorders are in Supplement 1 (on the compact disc [CD] inside the back cover of this report); these data for 2007-2008 are summarized in Figure 4.3 and Figure 4.4 for upgradient wells and wells near the headwaters of the tributary creek, respectively. The method used to calculate the discharge rates in Table S1.5 (Supplement 1) was described previously (Argonne 2008).

Figure 4.3 shows that the groundwater levels at monitoring locations upgradient of the phytoremediation area (wells D2, WP49, and SB65D; Figure 2.3) rose by approximately 10-12 ft in 2007 and then declined slowly and by only $4-5 \mathrm{ft}$ during the fall and winter, because of frequent rains during the later part of 2007. In 2008, the groundwater levels at these locations again rose by 10-12 ft, peaking in July, but decreased more rapidly in the late summer and fall because of generally drier conditions. Throughout 2008, however, the groundwater levels at any given time were higher than the corresponding levels in 2007. A net increase in groundwater elevations of approximately $2 \mathrm{ft}$ was observed at these locations from January 1 to December 31 , 2008.

Groundwater levels in the headwaters area of the tributary creek (monitoring well locations PMW1-PMW3 and PMW9; Figure 4.4) showed a pattern of seasonal rise and fall similar to that observed in the upgradient wells, with increases of $2-5 \mathrm{ft}$ in the spring and early summer followed by declines in the late summer and fall. At the end of 2008, groundwater levels in the headwaters area were higher by approximately $0.5-1 \mathrm{ft}$ than at the beginning of the year. The groundwater level recorders originally installed at monitoring locations PMW3S and 
PMW3D (Figure 2.3) were removed in 2007 to prevent damage due to artesian flow that began in these wells in spring 2007. Artesian flow continued at these locations throughout 2008.

The downgradient monitoring wells in the phytoremediation area (PMW4-PMW8; Figure 2.3) showed the smallest absolute responses, in both 2007 and 2008, because of the probable controlling influence at these locations of groundwater seepage to the tributary creek (Figure 4.5). The greatest increases (approximately $3 \mathrm{ft}$ ) during the current review period were observed at the outermost flanking wells (PMW4, PMW7), as well as at PMW5 on the west side of the creek. The groundwater at location PMW6 rose to the approximate land surface elevation in early 2007; the ground surface in this area has remained locally saturated since that time.

Results of quantitative monitoring indicate that the total surface water flow from the phytoremediation and wetlands treatment areas during summer and fall 2008 (June-November; approximately 28.8 million gallons, excluding very brief storm flows) was almost twice the discharge for the corresponding period in 2007 (approximately 15.0 million gallons). To some degree this increase in discharge might reflect differences in the timing of the precipitation in 2007 and 2008; however, the observations suggest that a greater fraction of the available moisture in 2007 might have replenished subsurface storage that had been depleted in the previous drought years. In 2007 and 2008 the initial spring increases in groundwater levels were comparable, but the higher levels observed throughout 2008 appear to have contributed to combined groundwater seepage and runoff rates that were also significantly higher.

Because of the timing of the 2008 rains (Figure 4.2), much of the soybean crop in the cultivated fields west of the phytoremediation and wetlands areas failed or grew very poorly in 2008. This crop failure might have decreased the local demand for surface and shallow groundwater in these fields relative to 2007 and hence also contributed to increased groundwater and surface water flow into the phytoremediation and wetlands treatment areas in 2008. This hypothesis is supported by an approximately twofold increase in the combined metered discharges from tile drains TD3 and TD4 (which drain the western agricultural fields) in JuneNovember 2008, relative to the corresponding period in 2007 (6.4 million gallons versus 3.0 million gallons, respectively). A similar increase was not observed in the flows for the tile drains (TD1 and TD5) that drain the fields east of the phytoremediation area, which were planted in corn in 2008. 


\subsection{Results for GWEX-1 and Compliance Monitoring of the Spray Irrigation Treatment Unit}

The treated effluent from extraction well GWEX-1 and the spray treatment unit is used for irrigation of the Elmwood-Murdock Public School system's athletic fields when local weather conditions permit effective operation of the spray-irrigation treatment technology (generally, mid spring to late fall). Per agreement with the school system, the well and treatment system are operated in consultation with the local school personnel, subject to the identified watering needs for these fields. Frequent heavy rains and persistent wet surface conditions at Murdock prevented the operation of GWEX-1 and the treatment unit throughout the spring and much of the summer of 2008. At the request of the school administration, these systems were subsequently not operated for the remainder of 2008 to facilitate use of the athletic fields for scheduled school and community sports activities throughout the late summer and fall. Consequently, no groundwater was extracted by GWEX-1 or treated and discharged during the present review period.

Unusually wet conditions also affected the operation of GWEX-1 and the spray irrigation treatment unit during 2007 (Argonne 2008). In 2007, sporadic operation of GWEX-1 and the spray treatment system was possible only from early July until September 12; a total of 141,867 gal of groundwater was extracted, treated, and discharged to the surface during that year. In comparison, approximately 627,400 gal of groundwater was treated and discharged during the 2005 operating period, along with 400,800 gal during the 2006 operating period.

The spray irrigation treatment unit and well GWEX-1 are operated in accord with a statement of discharge requirements (National Pollutant Discharge Elimination System Tracking No. NE0137464) issued by the Nebraska Department of Environmental Quality (NDEQ) (Appendix B in Argonne 2006). In keeping with the NDEQ requirements, samples of the treated effluent are collected quarterly, when the systems are in operation, and analyzed for carbon tetrachloride, chloroform, and $\mathrm{pH}$ levels. The NDEQ statement of discharge requirements places no restrictions on the quality of the groundwater supplied to the spray irrigation unit before treatment occurs, and it does not require analysis of the untreated flow. Nevertheless, well GWEX-1 is sampled for VOCs analyses to document the concentrations of carbon tetrachloride in the groundwater captured by the well.

Because GWEX-1 and the spray treatment unit were not operated in 2008, no monitoring events were required or performed during the present review period. Analytical results for the 
untreated groundwater from GWEX-1 for all previous (2005-2007) sampling events are in Table 4.1.

The concentrations of carbon tetrachloride in two samples of the untreated groundwater from GWEX-1 collected (in a single sampling event) in 2007 were $285 \mu \mathrm{g} / \mathrm{L}$ and $336 \mu \mathrm{g} / \mathrm{L}$. These results indicate an apparent increase in 2007 relative to concentrations in 2005 and 2006. The observed concentrations in the untreated groundwater indicate that GWEX-1 was effectively capturing relatively high contaminant concentrations in the plume before they reached the phytoremediation and wetlands treatment areas. Low levels of chloroform $(2.3-4.5 \mu \mathrm{g} / \mathrm{L})$ were also detected in the untreated groundwater during the previous sampling events.

The outfall from the spray irrigation treatment unit is identified by the NDEQ as a land application; no specific targets for the removal of carbon tetrachloride (or chloroform) have therefore been established. The NDEQ requirements qualitatively note, however, that the discharge may not be toxic to aquatic life in surface waters of the state outside the mixing zones allowed under NDEQ Title 177 - Nebraska Surface Water Quality Standard. This standard defines a maximum acceptable level of $44.2 \mu \mathrm{g} / \mathrm{L}$ for carbon tetrachloride in surface water bodies. The NDEQ requirements further identify an acceptable $\mathrm{pH}$ range of 6.0-9.0 for the treated groundwater. These targets were accepted by the EPA (2005) in conjunction with the Murdock Monitoring Plan (Argonne 2006).

Samples of treated groundwater discharged by the irrigation treatment unit are collected from multiple locations throughout the spray cloud during each sampling event. Spray accumulation rates at each collection point may differ for each event, depending on the local wind and site conditions at the time of sample collection. A minimum of four individual spray samples are analyzed, however, for each event, to account for the potential variability.

Because GWEX-1 and the spray treatment unit were not operated in 2008, no spray samples were required or collected during the present review period. Carbon tetrachloride concentrations detected in individual samples of the spray discharge for all previous (2005-2007) sampling events are in Table 4.2. The results indicate that the levels of carbon tetrachloride in the spray outfall to date have been far below the acceptable limit established by the EPA and the $N D E Q$. Chloroform and methylene chloride were not detected in any of the spray samples analyzed. 
The maximum and minimum $\mathrm{pH}$ levels detected in samples of the treated spray discharge for the 2005-2007 sampling events are in Table 4.3. The observed levels ranged from 6.54 to 7.88, and thus they complied with the $\mathrm{pH}$ requirements (6.0-9.0) specified by the NDEQ.

\subsection{Results for the Phytoremediation Area}

Carbon tetrachloride removal in the phytoremediation treatment area is expected to occur through the combined effects of

- Hydraulic "pumping" of the contaminated groundwater as a result of plant uptake,

- Transpiration and degradation of carbon tetrachloride within plant tissues, and

- Enhanced microbial degradation (reductive dechlorination) in the root zone created by the plants.

The results of the monitoring activities targeted to investigate these remedial processes (Argonne 2006, Section 3.1.2) are summarized below.

\subsubsection{Evidence of Possible Hydraulic “Pumping” Effects}

\subsubsection{Evidence from Monitoring of Groundwater Levels and Creek Discharge}

The overall patterns of variations in groundwater levels at Murdock during the current review period are discussed in Section 4.1. Figures 4.6 and 4.7 illustrate the small-scale oscillations apparent in the hydrographs - for all of the monitoring wells, completed at both shallow and deeper depths in the phytoremediation area - from early May to mid October 2008. The observed patterns strongly suggest that the fluctuations are associated with the diurnal cycle of groundwater uptake by the phytoremediation plantings (and other nearby vegetation) during the annual growing season. The diurnal oscillations reached an apparent maximum amplitude (up to approximately $0.6 \mathrm{ft}$ at PMW8) in July and August, suggesting that the use of groundwater by the phytoremediation and native plantings was greatest at this time. The magnitude of the 
oscillations recorded at most of the monitored locations showed little or no increase in 2008 relative to 2007 observations and decreased at one location (PMW9S).

Estimated flow rates for water discharged from the wetlands outfall in May to mid October 2008, obtained from monitoring data for the V-notch weir, are shown in Figure 4.8. (The full data set is in Supplement 1, Table S1.5.) The results suggest that water flow rates from the wetlands generally declined during the above period, with maximum flow rates of 110-150 gpm (except for storm responses) occurring daily at 9:00-12:00 and minimum rates of 70-120 gpm at 18:00-21:00. The indicated rate variations correspond closely to the inferred diurnal fluctuations observed in groundwater levels in the phytoremediation area. This observed correlation strongly suggests that the evapotranspiration "pumping" action of the phytoremediation plantings, native plants, and nearby crops (in conjunction with possible direct evaporation processes) reduced the water flow reaching (and hence exiting) the wetlands by as much as 30-40 gpm (or 15-20 gpm averaged over a 24-hr period) at the peak of each daily water use cycle.

In 2008, the estimated maximum effect of the inferred diurnal evapotranspiration "pumping" processes in the phytoremediation, wetlands, and surrounding crop areas (a transient maximum reduction in flow rates of 30-40 gpm) reflected an approximate $27 \%$ decrease in the surface discharge to the tributary creek. In 2007 the estimated diurnal effect of the evapotranspiration "pumping" was smaller in magnitude (approximately 15-20 gpm at maximum); however, the relative impact on the observed surface flow rates in 2007 was greater, representing a 33-43\% decrease. In conjunction with the observations outlined in Section 4.1, the available data suggest that although the combined water demands of the phytoremediation plantings, farm crops, and natural vegetation at Murdock increased during the current review period, these demands did not keep pace with the greater quantities of groundwater, surface seepage, and runoff available to the plant communities during the current review period.

\subsubsection{Evidence from Monitoring of Tree Sap Flow Rates}

Measurement of sap flow in 18 trees for approximately one month at the peak of the transpiration season (July-August) yielded direct measurements of water use by the trees in the phytoremediation planting. The results are summarized in Table 4.4. The complete data set is in Supplement 2 (on CD). Sap flux data (in milliliters of sap per centimeter-hour) were obtained from thermal dissipation probes installed in the trees. These values were multiplied by the 
surface area of the active sapwood per tree, and hence for the tree stand, to obtain estimates of total daily tree water use. The active sapwood area was determined by allowing a freshly cut tree $\log$ to imbibe an aqueous solution of nontoxic dye and observing the presence of dye at the opposite end of the log. This test confirmed that at this growth stage the entire trunk diameter of a cottonwood (minus the bark) was actively taking up water. The results are illustrated by the blue stain in the axial section of the tree trunk in Figure 4.9. Whereas the 2008 evaluation focused primarily on cottonwoods, the 2009 evaluation will focus on willows.

Sap flow data show that, because of their faster sap flux rate, larger diameter, and higher total number at the site, willows were the predominant water users among the different tree species. Niobe willows accounted for approximately $84 \%$ of the total estimated water uptake by the tree stand. Cottonwoods represented an additional $15 \%$ of the total uptake, while the remaining $1 \%$ was contributed by all of the other species combined. On a daily basis, total water usage by the tree stand during the study period was estimated to average $14,200 \mathrm{gal} / \mathrm{d}$, with a two-standard-deviation range (5th to 95th percentile) of 8,200-20,200 gal/d. On a yearly basis, for an assumed growing season of $180 \mathrm{~d}$ with peak water use in June-September and $50 \%$ of peak flow in May and October, the average tree stand water use was calculated at 2.21 million gallons per year. The calculated average daily tree water "pumping rate" (approximately $9.9 \mathrm{gpm}$ ) therefore empirically accounts for approximately 50-67\% of the average daily (24-h) reduction in flow rate $(15-20 \mathrm{gpm})$ observed at the wetlands outfall during the period of peak water use in July-August (Section 4.3.1.1).

Although indirect evidence provided by the presence of carbon tetrachloride in the tree tissue supports the hypothesis that at least a portion of the total water used by the tree stand is drawn from groundwater, the data obtained so far do not permit quantitative estimation of the relative percentage of water drawn from groundwater versus surface water sources. Also, the water removal totals for the phytoremediation trees do not reflect additional water use associated with both direct surface water evaporation and evapotranspiration from the herbaceous groundcover plants, native trees, and agricultural crops growing within the boundaries of the creek tributary hydrologic system, which might also affect the observed discharge rates at the wetlands outfall. 


\subsubsection{Evidence of Carbon Tetrachloride Uptake and Possible Degradation by the Phytoremediation Plantings}

The presence and concentrations of volatile solvents in plant tissues have been shown to be related to the presence and concentrations of the contaminants in the water source(s) used to support plant growth. At Murdock, carbon tetrachloride has been identified in the subsurface groundwater, as well as in groundwater seeping from the contaminated aquifer to the surface waters of the tributary creek. At this site, vegetation sampling (primarily of branch and leaf tissues) for VOCs analyses is therefore employed in accord with the EPA-approved Monitoring Plan (Argonne 2006) as an indicator of groundwater use and contaminant uptake by the phytoremediation plantings.

A review of the VOCs data for vegetation samples collected at Murdock in 2005-2006 (Argonne 2007) showed that evidence for uptake of carbon tetrachloride by trees occurs far more reliably in branch tissues than in leaf tissues. Consequently, in both 2007 and 2008, leaf samples were collected only during the July sampling event, at a limited subset of locations (some near the air sampling locations). Leaf samples were not collected during the March and October sampling events, which occurred too early and too late in the growing season, respectively, for healthy leaves to be reliably present.

Complete results for the 2008 vegetation sampling and VOCs analyses are in Table B.1, Appendix B. Tree sampling locations in 2008 are shown in Figure B.1, Appendix B. The analytical results for carbon tetrachloride are discussed in Section 4.3.2.1. Results for chloroform are discussed in Section 4.3.2.2.

The results for carbon tetrachloride in branch samples collected during the major sampling events in 2005-2007 were illustrated in the summary report for performance in 2007 (Argonne 2008), as follows:

- July 2005, baseline sampling

- September 2005, end of first growing season

- January 2006, first winter sampling 
- March-April 2006, first spring sampling

- August 2006, peak of second growing season

- April 2007, second spring sampling

- July 2007, peak of third growing season

- October 2007, end of third growing season

In the current document, the results for carbon tetrachloride in branch samples collected during the major sampling events in 2008 are illustrated as follows:

- Figure 4.10: March 2008, spring sampling

- Figure 4.11: July 2008, peak of fourth growing season

- Figure 4.12: October 2008, end of fourth growing season

Figure 4.13 compares the concentrations of carbon tetrachloride in tree branch samples collected in the July sampling events in 2007 and 2008.

Tree diameter measurements in 2008 are illustrated in Figure 4.14. Figure 4.15 shows sampling locations and concentrations of carbon tetrachloride in the herbaceous cover and wetlands plants.

\subsubsection{Results of Vegetation Analyses for Carbon Tetrachloride}

Previous results (Argonne 2007, 2008) showed that quantifiable levels of carbon tetrachloride in branch samples have been detected in increasing numbers of trees as the phytoremediation plantings have grown since spring 2005. Carbon tetrachloride concentrations in branch samples were lowest in the dormant seasons (winter, early spring), then increased and occurred over a wider area of the phytoremediation zone during successive summer growth 
periods. Furthermore, measurable amounts of carbon tetrachloride were initially found in shallower plantings, then identified subsequently in deeper plantings as time progressed.

In 2008, the results of the sampling and analyses followed trends similar to those observed previously (Argonne 2007, 2008). The identified concentrations of carbon tetrachloride in branch tissues were lowest in March (Figure 4.10), when most samples had no detectable contamination. The 2008 monitoring, thus, further confirmed that carbon tetrachloride uptake by vegetation in the phytoremediation area is seasonal; the contaminant does not accumulate in plant tissues from year to year, but instead is eliminated fairly rapidly from the tissues when the annual growing season ends and the trees become dormant.

By the peak of the 2008 growing season (July 2008; Figure 4.11), the areal extent of the trees with measurable carbon tetrachloride encompassed almost the entire planting area, and measured concentrations ranged up to $2,396 \mu \mathrm{g} / \mathrm{kg}$. In July 2008, carbon tetrachloride was detected in both shallow- and deep-planted trees, with an areal distribution similar to that observed in 2007. In July 2008, however, the carbon tetrachloride levels identified were slightly lower than those in July 2007. Figure 4.13 shows the results of geostatistical comparison of carbon tetrachloride concentrations in samples collected in 2007 and 2008. The lower observed concentrations in tree branch tissues in July 2008 (lighter color in the figure) might reflect the influence of one or more possible processes, including dilution of the near-surface groundwater, natural temporal fluctuations, enhanced (presumably anaerobic) microbial activity in the locally flooded portions of the phytoremediation area, reduced sap flow transport in flooded areas (where roots might have decayed), and preferential use by the trees of their more surficial roots in flooded areas. All of these processes might be qualitatively linked to the exceptional levels of precipitation that began at the Murdock site in 2007 and continued in 2008 (Section 4.1).

Twice during the summer of 2008 (June 23 and July 22), an entire cottonwood tree was harvested, and tissue samples were collected to estimate the spatial distribution of carbon tetrachloride and chloroform throughout the entire tree. The trees sampled (W417EC1 and W421EC2) were selected from those slated for removal because of leaning; they were also used to estimate total biomass (Section 4.3.2.3). The spatial distribution of the carbon tetrachloride detected in trunk core and branch samples from tree W417EC1 (harvested in July) is shown in Figure 4.16. The results for tree W421EC2 (harvested in June) showed similar trends. As previously reported in literature sources (Ma and Burken 2004), the identified concentrations of 
carbon tetrachloride tended to decrease with height above the ground, as well as with distance from the tree trunk, probably because of diffusion from the sap tissue through the bark.

The lower concentrations of carbon tetrachloride detected for the lowest sampling locations above the ground from the base of the tree (Figure 4.16; cores and branches) deviated from the expected general trend. Sampling procedures could possibly have contributed to this result by permitting progressive losses to occur upward from the cut base of the (still transpiring) tree as it awaited sampling. For comparison, a carbon tetrachloride concentration approximately three times greater was obtained for a sample from this tree at a similar height above ground (91 cm compared to $84 \mathrm{~cm}$ and $87 \mathrm{~cm}$ ) before the tree was harvested. The results suggest that a modification of the harvesting and sampling strategy used in 2008 might be required to minimize analyte losses from harvested trees.

Eleven leaf samples were collected during the July 2008 sampling event, some near the locations of the twice yearly air monitoring (Section 4.6). Contaminant levels in all leaf samples were low (below $1 \mu \mathrm{g} / \mathrm{kg}$ ) or undetectable. These results confirm that leaf analyses are of little value in understanding VOCs uptake by trees. Leaf analyses may, however, be useful in future interpretation, in conjunction with air monitoring results, of possible VOCs concentration changes in ambient air.

Samples of the herbaceous groundcover were also analyzed during the July 2008 event, collected in the western portion of the phytoremediation tree stand (Figure 4.15). Most of the samples showed no detectable concentrations of carbon tetrachloride. The exceptions were as follows (Table B.1):

- One blanket flower sample (Grass-31) collected near tree W707 showed a carbon tetrachloride concentration of $43 \mu \mathrm{g} / \mathrm{kg}$.

- Three grass samples (Grass-1, Grass-28, and Grass-33) collected near locations W118, SWM2, and W407, respectively, contained carbon tetrachloride at the relatively low level of $5 \mu \mathrm{g} / \mathrm{kg}$. 


\subsubsection{Results of Vegetation Analyses for Chloroform}

Reductive dechlorination of carbon tetrachloride will result in the generation of progressively more reduced degradation products, including chloroform and dichloromethane. The identification of elevated levels of these degradation products in association with carbon tetrachloride in groundwater or plant materials at Murdock might therefore provide empirical evidence for possible reductive dechlorination, on either the macroscale or the microscale, in the phytoremediation environment.

Concentrations of chloroform ranging up to $7 \mu \mathrm{g} / \mathrm{kg}$ were detected in branch tissue samples collected at locations throughout the phytoremediation area during the spring sampling event (March 2008; Table B.1). In July, chloroform detections occurred throughout the phytoremediation planting area, with levels ranging up to $68 \mu \mathrm{g} / \mathrm{kg}$ (at location W606). Background locations outside the planted area had non-detectable chloroform levels.

Samples of groundcover collected in the planting area and along the margins of the wetlands predominantly showed no detectable chloroform; a few samples contained low concentrations of chloroform (Table B.1), as follows:

- A wild rye sample collected at W118 (Grass-01) contained chloroform at $2 \mu \mathrm{g} / \mathrm{kg}$.

- A switch grass sample collected between trees W505 and W606 (Grass-13) contained chloroform at $1 \mu \mathrm{g} / \mathrm{kg}$.

- A sample of switch grass near W407 (Grass-33) contained chloroform at $7 \mu \mathrm{g} / \mathrm{kg}$.

- A sample of blanket flower near W707 (Grass-31) contained chloroform at $6 \mu \mathrm{g} / \mathrm{kg}$. 


\subsubsection{Tree Growth, Depth to Water, and Nitrogen Use}

The patterns of carbon tetrachloride uptake outlined above are qualitatively consistent with measurements of tree growth (trunk diameter at breast height or DBH) recorded in the phytoremediation area in April 2007, November 2007, and October 2008. As in the previous reporting period, the current data (Figure 4.14) indicate that for cottonwoods, willows, and ashes the most pronounced growth has occurred in the shallow planting zone, where a relatively unlimited supply of water is readily available. This difference was found to be statistically significant $(\mathrm{P}=0.05)$. Growth of northern catalpa was similar for deep- and shallow-planted locations; however, very few catalpas were planted at shallow locations. Anecdotally, catalpas appeared to benefit significantly from their association with cottonwoods for wind shielding. Niobe willows and cottonwoods growing in the deeper planting zone had diameters in fall 2008 that were comparable to or larger than those for the same species in the shallow planting zone in fall 2007. These observations indicate that a developmental lag of approximately one growing season for the trees in the deep planting zone persisted in the fourth (2008) growth season.

Overall, tree survival since the 2005 planting was approximately 93\%. The highest relative losses were recorded for black willows and green ashes (two of the four species planted in smaller numbers). The black willows, particularly, did not always compete successfully with their nearest neighbors. Survival rates for the most represented trees were, respectively, $94 \%$ for eastern cottonwoods and $93 \%$ for Niobe willows. These survival rates are within typical ranges for large-scale plantings.

The slower tree growth observed in the deep planting areas upslope from the creek also suggests that the specialized planting technique (TreeWell ${ }^{\circledR}$ ) and the growth of groundcover species in the deep planting zone are achieving the intended effect of limiting the availability of direct precipitation and surface runoff to the deeply planted trees, while encouraging growth of their root systems toward the groundwater. The similarity of patterns of carbon tetrachloride and chloroform in trees in the deep planting zone to patterns in previous reporting periods confirms that the roots of the deeply planted trees are accessing the contaminated groundwater resource.

The presence of standing water in parts of the phytoremediation area since the spring of 2007 has caused ongoing concern over the health of the tree stand. When tree diameters were recorded in fall 2008, the presence of standing water in the vicinity of each location was documented. Figure 3.1 illustrates the survey findings. Wet ground was found near 264 
locations, while dry soil was found near 740 locations. The condition of the wet-ground soils ranged from saturated to submerged below several centimeters of standing water. Wet areas in the planting occurred predominantly on the east side of the tributary creek, and to a lesser degree in the southernmost area of the west side.

The effects of presence or absence of wet ground (Figure 3.1) on tree growth were evaluated. Unlike the effects of deep versus shallow planting, the presence of wet versus dry soil conditions did not result in statistically significant growth differences. This interpretation might have been influenced, however, by the relatively short duration (approximately two growing seasons thus far) of the unusually wet conditions at the site, as well as by the dissimilar numbers of observation points available for the wet and dry conditions.

Three cottonwood trees were harvested in June and July 2008 to obtain information indicative of standing biomass yield and metrics. The tree harvest and measurement activities are documented in the Photo Gallery (Appendix A). The 2007 total Kjeldahl nitrogen analysis results for branches and leaves were used to estimate nitrogen use by the trees at the peak of the growing season. The values (Table 4.5) were compared with literature data. Trees harvested were similar in $\mathrm{DBH}$ to the stand-wide average for cottonwoods and were thus considered representative of a typical cottonwood at the site. The average cottonwood woody biomass (moist) measured from harvested trees was $26.6 \mathrm{~kg}$ per tree, and the leaf biomass was $4.3 \mathrm{~kg}$ per tree. Average tree height was $797 \mathrm{~cm}$. Allometric equations for determining standing biomass from DBH measurements were investigated and checked against the field data. In particular, equations and coefficients reported by Ter-Mikaelian and Korzukhin (1997) were tested, as they were the only authors who reported values for the tree species represented at Murdock. Because data derived from literature coefficients were significantly different from the field-measured data, calculations of standing biomass were made by using Murdock field data only. Reasons for this discrepancy are unknown at this time, but finding large differences is not unusual in attempts to fit broadly derived coefficients in a specific context. Nitrogen use data in Table 4.5 were conservative in comparison to available literature data, as the concentrations recorded in the 2007 samples were generally at the lower end of the range reported by Hansen (1994).

Extrapolation of the average cottonwood biomass to the 745 standing cottonwoods and poplars gave a total standing biomass for these two species of 19.8 metric tons. Although the collection of biomass data for willows was deferred until the 2009 growing season, applying the same per-tree biomass values would provide a very conservative estimate of 24.5 tons for the 
924 Niobe willows standing. Biomass from other tree species (green ash and catalpa) is still too small to be significant, and - because of their small numbers - measurement by harvesting is not recommended at this time.

\subsubsection{Evidence of Possible Enhanced Microbial Degradation}

Direct measurement of the microbial activity in the root zone created by the phytoremediation plantings is neither easy nor economical to employ at this time as a periodic monitoring tool. In accordance with the approved Monitoring Plan (Argonne 2006), twice yearly sampling and analysis of the groundwater at 19 permanent monitoring wells within and upgradient of the phytoremediation area (Figure 2.3) is performed as a logistically viable alternative for monitoring for (1) changes in the concentrations of carbon tetrachloride in groundwater and (2) the possible development of reducing/anaerobic conditions within the aquifer required for the microbial degradation of carbon tetrachloride by reductive dechlorination.

\subsubsection{VOCs Analysis Results for Groundwater}

The analytical results for VOCs in groundwater samples collected during the review period are summarized in Table 4.6 and in Figures 4.17 and 4.18. Table 4.6 includes results from previous sampling events for comparison.

Table 4.6 indicates that the observed concentrations of carbon tetrachloride in the Murdock aquifer have remained relatively stable in the years 2005-2008 at most of the monitoring well locations, showing no consistent trend of increase or decrease. The highest concentrations of carbon tetrachloride in groundwater (since monitoring began in 2005) have consistently been identified at monitoring locations PMW1, PMW2, and PMW3 near the tributary creek headwaters, as well as at location SB65, upgradient (south) of the phytoremediation planting area. The highest maximum concentrations consistently detected during all sampling events have occurred at monitoring wells PMW1D (641-3,077 $\mu \mathrm{g} / \mathrm{L})$ and PMW2D (568-3,827 $\mu \mathrm{g} / \mathrm{L})$, although concentrations exceeding 1,000 $\mu \mathrm{g} / \mathrm{L}$ were also observed at location PMW3 during the July 2007 and July 2008 sampling events (Table 4.6). Figures 4.17 and 4.18, respectively, show the analytical results for groundwater samples in the May and July 2008 sampling events. 
Figure 4.19 highlights carbon tetrachloride results for three wells with shallow completions (PMW1S, PMW2SA, PMW3S) in the upgradient, headwaters portion of the phytoremediation area. As Figure 4.19 illustrates, the carbon tetrachloride concentrations in these three shallow wells were generally similar in April 2006, August 2006, and April 2007. In the July 2007 sampling event, however, dramatic increases in the levels of carbon tetrachloride were observed in all three wells, and relatively elevated levels persisted at these locations in both the May 2008 and July 2008 sampling events. The observed increases are believed to reflect the movement of more highly contaminated groundwater from the deeper portion of the aquifer into the shallower portion at these locations, in association with the 2007 and 2008 rises in groundwater levels described in Section 4.1. Persistent seepage to the land surface (presumably groundwater seepage) was observed during much of 2007 and throughout 2008 in the vicinity of these monitoring wells, further suggesting upward movement of contaminated groundwater in this area subsequent to the April 2007 sampling event.

Groundwater monitoring for VOCs has consistently demonstrated that the measured carbon tetrachloride concentrations in groundwater decrease rapidly northward and downgradient from the creek headwaters area; the 2008 sampling results continue this trend. The maximum concentrations identified at the northernmost groundwater sampling location, PMW8, ranged from $16 \mu \mathrm{g} / \mathrm{L}$ to $26 \mu \mathrm{g} / \mathrm{L}$ between the original Murdock baseline sampling event in July 2005 and the event in May 2008; a slight increase, to $44 \mu \mathrm{g} / \mathrm{L}$, was observed in the July 2008 sampling event (Table 4.6).

Chloroform concentrations identified in the groundwater samples from each of the monitoring events in 2008 (Table 4.6) were generally low $(<10 \mu \mathrm{g} / \mathrm{L})$, with the consistent exception of the TEST-1 well on the Murdock public school property $(17 \mu \mathrm{g} / \mathrm{L}$ and $16 \mu \mathrm{g} / \mathrm{L}$ in the May 2008 and July 2008 sampling events, respectively).

Comparison of the carbon tetrachloride and chloroform concentrations in the groundwater samples collected in the area with elevated carbon tetrachloride concentrations (> $50 \mu \mathrm{g} / \mathrm{L}$ ) indicated that, except for the TEST-1 well, the ratios of chloroform to carbon tetrachloride were consistently $<3 \%$, and mostly $<1 \%$. 


\subsubsection{Geochemical Analysis Results for Groundwater}

The inorganic geochemical parameters dissolved oxygen (DO), reduced iron $\left(\mathrm{Fe}^{2+}\right)$, and oxidation-reduction potential (ORP) are measured in each of the monitoring wells as possible indicators of the development of oxygen-depleted, chemically reducing conditions that would promote anaerobic degradation of the carbon tetrachloride within the Murdock aquifer. The results of these measurements for the present review period are in Table 4.7, together with data from previous sampling events. With few exceptions, the results show no clear patterns of significantly decreased DO or ORP levels or increased $\mathrm{Fe}^{2+}$ in conjunction with the annual growing season that would suggest the widespread onset of anaerobic conditions in the groundwater as a result of plant growth or microbial activity. The measured concentrations of dissolved $\mathrm{Fe}^{2+}$ increased very slightly at all monitoring well locations (except for PMW4 and SB65D) in July 2008 relative to May 2008; however, the concentrations of $\mathrm{Fe}^{2+}$ remained at trace levels (generally $\leq 0.2 \mathrm{mg} / \mathrm{L}$ ) at all locations during both sampling events.

At most of the sampled locations, the observed DO and ORP levels variably showed minor increases or decreases between the May 2008 and July 2008 sampling events. More significant decreases in DO in July were noted at locations PMW5, PMW6, and PMW7 in the downstream portion of the phytoremediation area; however, increased DO levels were observed at nearby downgradient locations PMW4 and PMW8. Curiously, with only one exception (at PMW9D), a consistent inverse relationship was observed for these geochemical parameters at each groundwater monitoring point for the 2008 sampling events, in that increased DO concentrations (from May to July) were associated with decreased ORP levels and vice-versa. These results are in contrast to data for the 2006 and 2007 monitoring events (Table 4.7), which showed an apparent seasonal influence on ORP values measured at all well locations in the phytoremediation area, with relatively lower ORP values occurring during the summer periods of active plant growth. The 2008 geochemical measurements therefore provide little evidence for the possible development of locally more reducing conditions in groundwater during the 2008 summer growth period. These observations may in part reflect the influence of a greater flux of recently infiltrated shallow groundwater and surface runoff through the Murdock flow system during 2008, as noted in Section 4.1.

\subsubsection{Summary of Results for the Phytoremediation Area}

The results of the phytoremediation monitoring activities during the present review period support the following observations: 
- Distinct seasonal fluctuations in groundwater levels observed at all monitoring well locations in the phytoremediation area appear linked to the diurnal effects of groundwater "pumping" by the phytoremediation plantings, native vegetation, and nearby crops during the annual growing season. Limited estimates suggest that this effect reduced net water flow in the tributary creek by $30-40 \mathrm{gpm}$ during peak daily water usage in the driest period of the summer growing season.

- In contrast to results obtained in 2006 and 2007, geochemical data for 2008 suggest little development of increasingly reducing conditions in the Murdock aquifer during the annual summer growing period that might stimulate the localized degradation of carbon tetrachloride in the subsurface.

- Uptake of carbon tetrachloride by the planted tree stand has been confirmed by the seasonal presence of this contaminant, along with chloroform, in branch tissue at increasing concentrations and over an expanding areal extent, both in the shallow planting area and in the deep planting area. Concentrations of carbon tetrachloride and chloroform appeared to be slightly lower than in 2007 for reasons that are not yet fully understood.

- The increased ratio of chloroform to carbon tetrachloride observed in plant tissues versus groundwater suggests that carbon tetrachloride degradation is occurring in the phytoremediation system. The degradation could be associated with the plant tissue activity, microbial activity in the root zones of the plantings, or both.

- Native grasses sampled in 2008 did not show significant carbon tetrachloride and chloroform concentrations.

- Evidence of groundwater and contaminant uptake was found in the trees planted deepest, indicating that these trees might have reached or be close to reaching the target rooting depth. Higher-than-normal groundwater elevations recorded in 2008 may, however, have contributed to enhanced contact between the tree roots and the groundwater. If this is true, a decline in uptake in future years might reflect the effect of a declining water level rather than a 
failure of the system to accomplish groundwater restoration. As the water level recedes to more typical elevations in the future, the tree roots will extend downward to follow the declining water table. A relatively gradual decline in the water level is not expected to stress the trees.

- Significant tree growth during the 2008 growing season indicates the potential for substantial water use by the trees. This potential was measured as tree water use through sap flow monitoring and was also reflected in the decrease in measured discharge from the phytoremediation-wetlands system. Sap flow monitoring indicated an estimated tree water use of 8,000-20,000 gal/d. Decreases in the measured discharge exiting the wetlands area showed that, averaged over $24 \mathrm{hr}$, the estimated combined water usage (15-20 gpm) associated with uptake and treatment by the phytoremediation plantings, groundcover species, natural vegetation, and adjacent farm crops, as well as possible direct evaporation from these areas, in 2008 would amount to $21,600-28,800 \mathrm{gal} / \mathrm{d}$ during the active growing season, or approximately 3.24-5.18 million gallons in a growing season of 150-180 days.

\subsection{Results for Vegetation Sampling in the Wetlands Area}

Selected herbaceous and woody vegetation samples were collected during the July 2008 sampling event, in the water and at the water's edge along the wetlands perimeter. Species collected included young adventitious willows and cottonwoods, as well as arrowhead, blanket flower, bluestem, bulrush, cattail, knotweed, lemna, reeds, switch grass, and wild rye. No carbon tetrachloride or chloroform was found in these samples (Table B.1), except for the trace levels $(<1 \mu \mathrm{g} / \mathrm{kg}$ ) of carbon tetrachloride detected in two samples (Grass-24 and Grass-37) collected just south of the wetlands outfall and south of the west side weir wall. As the plantings become better established with time, convincing evidence of carbon tetrachloride uptake by the wetlands vegetation may be found if the wetlands water concentrations are elevated enough to make carbon tetrachloride detectable in plant tissue. 


\subsection{Results for Compliance Monitoring at Surface Water Locations SWM1, SWM2, and SWM3}

Surface water sampling point SWM3 lies directly downstream of the outfall from the phytoremediation and wetlands treatment zones (Figure 3.5), and hence it reflects the final contaminant levels achieved in the effluent from the combined treatment processes. Location SWM3 was therefore identified in the EPA-accepted Monitoring Plan (Argonne 2006) as the primary point for quantitative assessment of the net performance of the Murdock treatment efforts. A maximum target carbon tetrachloride concentration of $44.2 \mu \mathrm{g} / \mathrm{L}$ was adopted for this discharge, as established by the NDEQ for surface waters of the state under NDEQ Title 117 Nebraska Surface Water Quality Standard.

The results of the 2008 surface water sampling and analyses are in Table 4.8, along with previous data for comparison. Carbon tetrachloride contamination was detected in all surface water samples collected at SWM3 in 2008, at levels exceeding the instrument detection limit of $0.1 \mu \mathrm{g} / \mathrm{L}$. The levels identified $(1.0-2.9 \mu \mathrm{g} / \mathrm{L})$, however, were below the regulatory target concentration $(44.2 \mu \mathrm{g} / \mathrm{L})$ promulgated for this outfall by an order of magnitude. Carbon tetrachloride levels detected in 2008 at upstream sampling location SWM2 ranged from 3.6 $\mu \mathrm{g} / \mathrm{L}$ to $9.0 \mu \mathrm{g} / \mathrm{L}$, also falling well below the identified target level for surface waters. Carbon tetrachloride levels identified in the surface waters sampled at upstream location SWM1 ranged from $100 \mu \mathrm{g} / \mathrm{L}$ in February 2008 to $20 \mu \mathrm{g} / \mathrm{L}$ in July 2008; these values were consistently lower than the concentrations observed for the corresponding quarterly monitoring events in 2007.

The results demonstrate almost complete removal of carbon tetrachloride contamination from the surface water, as well as the groundwater seepage, that passed through the phytoremediation and wetlands treatment zones during the review period. The system met the EPA's compliance goals.

\subsection{Results for Atmospheric Compliance Monitoring}

The EPA has adopted the Agency for Toxic Substances and Disease Registry's intermediate inhalation minimal risk level (MRL) of $192 \mu \mathrm{g} / \mathrm{m}^{3}$ as a target maximum concentration for carbon tetrachloride in air in the phytoremediation and wetlands treatment areas. This concentration will be protective of health for both workers and members of the general public who might periodically visit these areas. The intermediate MRL is defined as an 
estimate of the daily human exposure that is likely to be without appreciable risk of adverse noncancer health effects for an exposure duration of 15-364 d (EPA 2005).

Ambient air is sampled at three locations (Figure 3.6) - two in the phytoremediation area (AA1, AA2) and one in the wetlands (AA3) - as specified in the EPA-approved Monitoring Plan (Argonne 2006). Samples are collected by using special evacuated canisters fitted with metered inlet valves. The valves allow air to enter the canisters at a preset, slow rate. At Murdock, an air sample is collected at each location over an 8-hr period, on each of three consecutive days, for each sampling event. This procedure yields "average" samples of the air to which a visitor at the site might be exposed. The purpose is to compensate for unusual wind or other weather conditions that might affect the air sampled on any one given day. For comparison, samples of "background" air are collected during each sampling event from a location southwest of the phytoremediation and wetlands areas (BA1; Figure 3.6), by using the same procedures. The air samplers were placed at an inlet height of approximately $5.5 \mathrm{ft}$ above ground level, roughly corresponding to the breathing zone.

The results of the 2008 air sampling and analyses are compared to data for previous sampling events in Table 4.9. Carbon tetrachloride was detected at low levels in the ambient air sampled at location AA1, in the upstream portion of the phytoremediation area (Figure 3.6), during both the May 2008 and July 2008 sampling events, at concentrations of 2.0-4.5 $\mu \mathrm{g} / \mathrm{m}^{3}$ and ND (not detected) to $2.5 \mu \mathrm{g} / \mathrm{m}^{3}$, respectively. Carbon tetrachloride was also detected at levels slightly above the indicated reporting limit of $1.3 \mu \mathrm{g} / \mathrm{m}^{3}$ in one sample of ambient air collected at location AA2 $\left(1.4 \mu \mathrm{g} / \mathrm{m}^{3}\right.$, on May 15) and in one sample of air at location AA3 $\left(1.5 \mu \mathrm{g} / \mathrm{m}^{3}\right.$, on May 1) during the May 2008 sampling event; however, these results were not corroborated by the samples collected at these locations on the remaining days of that event. No carbon tetrachloride was detected at the background air collection point (BA1), and no chloroform was identified (at an indicated reporting limit of $0.98 \mu \mathrm{g} / \mathrm{m}^{3}$ ) in the ambient air at locations BA1 or AA1-AA3, during the current review period. The levels of carbon tetrachloride detected in the ambient air at Murdock sampling points AA1-AA3 were below the regulatory target level $\left(192 \mu \mathrm{g} / \mathrm{m}^{3}\right)$ adopted for this contaminant by the EPA, by approximately two orders of magnitude.

Several other compounds, including chloromethane, acetone, and chlorofluoromethanes, were identified at low or trace concentrations in many of the air samples collected in the phytoremediation and wetlands areas, at levels comparable to those found at background 
sampling location BA1 (Table 4.9). The presence of these compounds appears unrelated to the former CCC/USDA grain storage activities at Murdock or to the groundwater and surface water restoration processes that are presently in progress.

\subsection{Quality Control for Monitoring Activities}

A comprehensive quality assurance/quality control program was implemented to confirm the reliability and representativeness of all analytical results as they were accumulated. Sample collection and handling activities were monitored by documenting samples as they were collected and using chain-of-custody forms and custody seals to ensure sample integrity during handling and shipment. Field blanks, equipment rinsates, and trip blanks were collected as appropriate to ensure that cross-contamination did not occur during sample collection and handling. To monitor the consistency of the sampling methodology and provide a measure of analytical precision, blind replicate samples were collected, and other samples were selected for duplicate analyses.

Groundwater, surface water, and spray irrigation effluent samples were analyzed for VOCs by the Applied Geosciences and Environmental Management (AGEM) Laboratory at Argonne, Illinois. The AGEM Laboratory used a modification of EPA Method 524.2 to achieve a quantitation limit of $1.0 \mu \mathrm{g} / \mathrm{L}$. In this purge-and-trap method with analysis on a gas chromatograph-mass spectrometer system, VOCs are extracted (purged) from the sample matrix by bubbling an inert gas through each sample. The purged components are then trapped in a specified sorbent tube. After the purging, the sorbent tube is heated and backflushed with an inert gas to desorb the components into the analytical system. The compounds eluting from the gas chromatography column are identified by retention time and through comparison with reference library spectra. The concentration of each component is calculated by comparison of the mass spectrometer response for the quantitation ion to corresponding calibration curves, the responses for internal standards, or both.

Vegetation samples were analyzed for carbon tetrachloride and chloroform by the AGEM Laboratory with a modification of the protocol in EPA Method 5021 (headspace analysis on a gas chromatograph with electron capture detection) to achieve the low detection limits required. An 11-point calibration of the gas chromatograph system was established on the basis of the mass of known quantities of carbon tetrachloride and chloroform. Consistency in the results for 
secondary quality control analyses provides support for the sampling and analytical methodologies.

To aid in characterization of the water-bearing zone, groundwater samples were analyzed for geochemical parameters including total nitrogen, $\mathrm{DO}, \mathrm{Fe}^{2+}, \mathrm{ORP}, \mathrm{pH}$, and conductivity. Samples were analyzed for nitrate/nitrite-nitrogen by TestAmerica Laboratories (University Park, Illinois) with EPA Method 353.2, an automated colorimetric method based on cadmium reduction. Commercial field test kits and instruments were used for measurement of the other geochemical parameters of interest.

Air-monitoring samples were analyzed at TestAmerica Laboratories (South Burlington, Vermont) by EPA Method TO-15 on a gas chromatograph-mass spectrometer system. The atmospheric air samples were drawn into specially prepared, preevacuated stainless steel canisters. For the analysis, the sample (a known volume of air) was directed from the canister through a multisorbent concentrator. After the concentration and drying steps were complete, the VOCs were thermally desorbed, entrained in a carrier gas stream, and directed through a multisorbent trap. The sample was then released by thermal desorption and carried onto the gas chromatography column for separation. 
TABLE 4.1 Analytical results for volatile organic compounds in samples of untreated water collected from extraction well GWEX-1 at Murdock, 2005-2008. ${ }^{a}$

\begin{tabular}{|c|c|c|c|c|c|}
\hline \multirow[b]{2}{*}{ Sample } & \multirow[b]{2}{*}{$\begin{array}{l}\text { Sample } \\
\text { Date }\end{array}$} & \multirow[b]{2}{*}{$\begin{array}{l}\text { Depth } \\
\text { (ft BGL) }\end{array}$} & \multicolumn{3}{|c|}{ Concentration $(\mu \mathrm{g} / \mathrm{L})$} \\
\hline & & & $\begin{array}{c}\text { Carbon } \\
\text { Tetrachloride }\end{array}$ & Chloroform & $\begin{array}{c}\text { Methylene } \\
\text { Chloride }\end{array}$ \\
\hline MUGWEX-W-18312 & $7 / 18 / 05$ & $47.0-77.0$ & 205 & 2.3 & $N^{b}$ \\
\hline MUTAP-W-15717 & $9 / 22 / 05$ & $47.0-77.0$ & 232 & 2.7 & ND \\
\hline MUTAP-W-15718c & 9/22/05 & $47.0-77.0$ & 223 & 2.4 & ND \\
\hline MUTAP-W-18128 & 6/8/06 & $47.0-77.0$ & 262 & 3.2 & ND \\
\hline MUTAP-W-18129c & 6/8/06 & $47.0-77.0$ & 270 & 3.3 & ND \\
\hline MUGWX1-W-18151 & $8 / 15 / 06$ & $47.0-77.0$ & 262 & 4.5 & ND \\
\hline MUGWX1-W-18152c & $8 / 15 / 06$ & $47.0-77.0$ & 179 & 2.7 & ND \\
\hline MUGWX1-W-23082 & $9 / 12 / 07$ & $47.0-77.0$ & 336 & 4.2 & ND \\
\hline MUGWX1-W-23083 ${ }^{c}$ & $9 / 12 / 07$ & $47.0-77.0$ & 285 & 3.7 & ND \\
\hline
\end{tabular}

a Well GWEX-1 was not operated in 2008 because of wet conditions at the site.

b ND, not detected at instrument detection limit of $0.1 \mu \mathrm{g} / \mathrm{L}$.

c Quality control replicate. 
TABLE 4.2 Analytical results for volatile organic compounds in samples of treated spray collected at Murdock, 2005-2008. a

\begin{tabular}{|c|c|c|c|c|}
\hline Sample & $\begin{array}{l}\text { Sample } \\
\text { Date }\end{array}$ & $\begin{array}{c}\text { Carbon } \\
\text { Tetrachloride }\end{array}$ & Chloroform & $\begin{array}{c}\text { Methylene } \\
\text { Chloride }\end{array}$ \\
\hline MUTEST1-W-B & $6 / 2 / 05$ & $0.8 \mathrm{~J}^{\mathrm{b}}$ & $N^{c}$ & ND \\
\hline MUTEST1-W-C & 6/2/05 & 1.8 & ND & ND \\
\hline MUTEST1-W-D & $6 / 2 / 05$ & 1.1 & ND & ND \\
\hline MUTEST1-W-E & $6 / 2 / 05$ & $0.7 \mathrm{~J}$ & ND & ND \\
\hline MUTEST2-W-B & $6 / 2 / 05$ & 1.3 & ND & ND \\
\hline MUTEST2-W-C & $6 / 2 / 05$ & 2.1 & ND & ND \\
\hline MUTEST2-W-D & 6/2/05 & 2.2 & ND & ND \\
\hline MUTEST2-W-E & $6 / 2 / 05$ & $5.9 \mathrm{~S}^{\mathrm{d}}$ & ND & ND \\
\hline MU-C-W-15722 & $9 / 22 / 05$ & 1.9 & ND & ND \\
\hline MU-D-W-15723 & $9 / 22 / 05$ & 2.9 & ND & ND \\
\hline MU-E-W-15719 & $9 / 22 / 05$ & 4.7 & ND & ND \\
\hline MU-F-W-15720 & $9 / 22 / 05$ & 3.0 & ND & ND \\
\hline MU-G-W-15721 & $9 / 22 / 05$ & 4.0 & ND & ND \\
\hline MUB-W-18134 & 6/8/06 & 5.2 & ND & ND \\
\hline MUC-W-18133 & $6 / 8 / 06$ & 1.7 & ND & ND \\
\hline MUD-W-18130 & 6/8/06 & 3.0 & ND & ND \\
\hline MUD-W-18131e & 6/8/06 & 3.1 & ND & ND \\
\hline MUE-W-18132 & 6/8/06 & 2.7 & ND & ND \\
\hline MUB-W-18153 & $8 / 15 / 06$ & 5.2 & ND & ND \\
\hline MUC-W-18154 & $8 / 15 / 06$ & $0.9 \mathrm{~J}$ & ND & ND \\
\hline MUD-W-18155 & $8 / 15 / 06$ & 2.6 & ND & ND \\
\hline MUD-W-18156e & $8 / 15 / 06$ & 1.7 & ND & ND \\
\hline MUE-W-18157 & $8 / 15 / 06$ & 2.5 & ND & ND \\
\hline MUF-W-18158 & $8 / 15 / 06$ & 2.3 & ND & ND \\
\hline MUB-W-23090 & $9 / 12 / 07$ & 2.1 & ND & ND \\
\hline MUC-W-23088 & $9 / 12 / 07$ & 2.4 & ND & ND \\
\hline MUC-W-23089 & $9 / 12 / 07$ & 2.3 & ND & ND \\
\hline MUD-W-23086 & $9 / 12 / 07$ & 4.0 & ND & ND \\
\hline MUD-W-23087 & $9 / 12 / 07$ & 4.3 & ND & ND \\
\hline MUE-W-23085 & $9 / 12 / 07$ & 2.7 & ND & ND \\
\hline MUF-W-23084 & $9 / 12 / 07$ & 6.5 & ND & ND \\
\hline
\end{tabular}

a The spray treatment unit was not operated in 2008 because of wet conditions at the site.

b Qualifier $\mathrm{J}$ indicates an estimated concentration below the method quantitation limit of $1.0 \mu \mathrm{g} / \mathrm{L}$.

c ND, not detected at instrument detection limit of $0.1 \mu \mathrm{g} / \mathrm{L}$.

d Qualifier S indicates that the surrogate recovery was outside the quality control range.

e Quality control replicate. 
TABLE 4.3 Analytical results for $\mathrm{pH}$ in samples of treated spray collected at Murdock, 2005-2008. ${ }^{a}$

\begin{tabular}{ccc}
\hline & \multicolumn{2}{c}{$\mathrm{pH}$} \\
\cline { 2 - 3 } $\begin{array}{c}\text { Sample } \\
\text { Date }\end{array}$ & Minimum & Maximum \\
\hline 6/2/05 & 7.76 & 7.88 \\
$9 / 22 / 05$ & 6.57 & 7.33 \\
$6 / 8 / 06$ & 6.54 & 6.55 \\
$8 / 15 / 06$ & 7.60 & 7.66 \\
$9 / 12 / 07$ & 7.24 & 7.33 \\
\hline
\end{tabular}

a The spray treatment unit was not operated in 2008 because of wet conditions at the site. 
TABLE 4.4 Calculated mean daily sap flow values, summer 2008 (July 22-August 26).

\begin{tabular}{cccc}
\hline Probe & $\begin{array}{c}\text { Tree } \\
(\mathrm{n}=18)\end{array}$ & $\begin{array}{c}\text { Diameter at } \\
\text { Breast Height, } \\
\text { July 2008 } \\
(\mathrm{cm})\end{array}$ & $\begin{array}{c}\text { Calculated Total } \\
\text { Wood Sap Flow } \\
(\mathrm{L} / \mathrm{d})\end{array}$ \\
\hline & & & \\
1 & W506EC & 10.9 & 26.3 \\
2 & W610WN1 & 11.9 & 51.7 \\
3 & W609EC2 & 6.4 & 16.5 \\
4 & W608EC2 & 10.2 & 38.8 \\
5 & W608EC2 & 10.2 & 29.2 \\
6 & W709EC & 11.4 & 21.0 \\
7 & W607EC1 & 10.7 & 19.5 \\
8 & W505EC1 & 9.1 & 33.3 \\
9 & W505EC1 & 9.1 & 28.7 \\
10 & W504WN1 & 10.7 & 45.8 \\
11 & W504WN2 & 10.9 & 39.4 \\
12 & W605WN1 & 13.7 & 82.2 \\
13 & W605WN1 & 13.7 & 102.7 \\
14 & W606EC1 & 9.7 & 17.9 \\
15 & W706EC2 & 9.4 & 17.9 \\
16 & W707EC2 & 11.2 & 24.8 \\
17 & W810EC1 & 10.0 & 37.6 \\
18 & W809EC1 & 10.9 & 24.6 \\
19 & W708EC1 & 7.1 & 8.7 \\
20 & W707GA & 2.5 & 0.7 \\
21 & W708EC2 & 11.2 & 62.9 \\
& & & \\
\hline
\end{tabular}

a Tree species abbreviations: EC, eastern cottonwood; GA, green ash; WN, Niobe willow. 
TABLE 4.5 Field-measured biomass parameters (June and July 2008), per tree.

\begin{tabular}{lcccc}
\hline & \multicolumn{3}{c}{ Values for each Tree } & \\
\cline { 2 - 4 } & W421EC1 & W421EC2 & W417EC1 & Average Values \\
\cline { 2 - 4 } & & & & \\
Height (cm) & 866 & 838 & 688 & 797.3 \\
Diameter at breast height (mm) & 100 & 92 & 85 & 92.3 \\
Total wood weight (kg) & 32.4 & 24.5 & 23 & 26.6 \\
Leaf weight (kg) & 5.9 & 2.9 & 4 & 4.3 \\
Moisture in wood (\%) & 43 & 43 & 43 & 43 \\
Moisture in leaf (\%) & 54 & 54 & 54 & 54 \\
Nitrogen (\%) in wood, moist basis ${ }^{\text {a }}$ & 0.15 & 0.15 & 0.15 & 0.15 \\
Nitrogen (\%) in leaf, moist basis & 0.6 & 0.6 & 0.6 & 0.6 \\
Total nitrogen mass in wood (g) & 48.6 & 36.75 & 34.5 & 39.95 \\
Total nitrogen mass in leaf (g) & 35.4 & 17.4 & 24 & 25.6 \\
& & & & \\
Dry yield wood mass (kg/tree) & 18.47 & 13.97 & 13.11 & 15.18 \\
Dry yield leaf mass (kg/tree) & 2.71 & 1.33 & 1.84 & 1.96 \\
& & & & \\
\hline
\end{tabular}

a Data from 2007 Murdock biomass sampling. 
TABLE 4.6 Analytical results for volatile organic compounds in groundwater samples collected from monitoring wells at Murdock, 2005-2008.

\begin{tabular}{|c|c|c|c|c|c|c|}
\hline \multirow[b]{2}{*}{ Location } & \multirow[b]{2}{*}{ Sample } & \multirow[b]{2}{*}{$\begin{array}{c}\text { Sample } \\
\text { Date }\end{array}$} & \multirow[b]{2}{*}{$\begin{array}{l}\text { Depth } \\
\text { (ft BGL) }\end{array}$} & \multicolumn{3}{|c|}{ Concentration $(\mu \mathrm{g} / \mathrm{L})$} \\
\hline & & & & $\begin{array}{c}\text { Carbon } \\
\text { Tetrachloride }\end{array}$ & Chloroform & $\begin{array}{c}\text { Methylene } \\
\text { Chloride }\end{array}$ \\
\hline \multicolumn{7}{|c|}{ Wells in phytoremediation treatment area } \\
\hline PMW1S & MUPMW1S-W-18318 & $7 / 19 / 05$ & $4.6-14.6$ & 9.4 & $0.4 \mathrm{Ja}$ & ND \\
\hline PMW1S & MUPMW1S-W-15720 & 4/6/06 & 4.6-14.6 & 4.1 & $0.3 \mathrm{~J}$ & ND \\
\hline PMW1S & MUPMW1S-W-18168 & $8 / 17 / 06$ & $4.6-14.6$ & $0.2 \mathrm{~J}$ & $N D^{b}$ & ND \\
\hline PMW1S & MUPMW1S-W-18205 & $4 / 12 / 07$ & $4.6-14.6$ & $0.9 \mathrm{~J}$ & ND & ND \\
\hline PMW1S & MUPMW1S-W-22846 & $7 / 24 / 07$ & 4.6-14.6 & 820 & 2.7 & ND \\
\hline PMW1S & MUPMW1S-W-22923 & 5/8/08 & 4.6-14.6 & 106 & 1.0 & ND \\
\hline PMW1S & MUPMW1S-W-19183 & $7 / 22 / 08$ & $4.6-14.6$ & 640 & 3.4 & ND \\
\hline PMW1D & MUPMW1D-W-18313 & $7 / 19 / 05$ & $24.6-34.6$ & 1233 & 5.2 & ND \\
\hline PMW1D & MUPMW1D-W-15721 & $4 / 6 / 06$ & $24.6-34.6$ & 3077 & 1.6 & ND \\
\hline PMW1D & MUPMW1D-W-18166 & $8 / 17 / 06$ & $24.6-34.6$ & 2241 & 9.3 & ND \\
\hline PMW1D & MUPMW1D-W-18206 & $4 / 12 / 07$ & $24.6-34.6$ & 1876 & 4.8 & ND \\
\hline PMW1D & MUPMW1D-W-22845 & $7 / 24 / 07$ & $24.6-34.6$ & 1706 & $N^{c}$ & ND \\
\hline PMW1D & MUPMW1D-W-22924 & $5 / 8 / 08$ & $24.6-34.6$ & 901 & 7.1 & ND \\
\hline PMW1D & MUPMW1D-W-19184 & $7 / 22 / 08$ & $24.6-34.6$ & 641 & 4.8 & ND \\
\hline PMW2SA & MUPMW2SA-W-15717 & 4/6/06 & $4.6-14.6$ & 359 & 2.8 & ND \\
\hline PMW2SA & MUPMW2SA-W-18163 & $8 / 17 / 06$ & $4.6-14.6$ & 385 & 3.5 & ND \\
\hline PMW2SA & MUPMW2SA-W-18196 & $4 / 12 / 07$ & 4.6-14.6 & 603 & 6.1 & ND \\
\hline PMW2SA & MUPMW2SA-W-22839 & $7 / 23 / 07$ & $4.6-14.6$ & 2742 & 7.9 & ND \\
\hline PMW2SA & MUPMW2SA-W-22925 & $5 / 12 / 08$ & 4.6-14.6 & 758 & 6.7 & ND \\
\hline PMW2SA & MUPMW2SA-W-19185 & $7 / 23 / 08$ & $4.6-14.6$ & 361 & 3.9 & ND \\
\hline PMW2SB & MUPMW2SB-W-18334 & $7 / 21 / 05$ & $4.6-14.6$ & 377 & 6.4 & ND \\
\hline PMW2SB & MUPMW2SB-W-18194 & $4 / 12 / 07$ & $4.6-14.6$ & 586 & 4.2 & ND \\
\hline PMW2D & MUPMW2D-W-18314 & $7 / 19 / 05$ & $19.6-29.6$ & 1564 & 4.8 & ND \\
\hline PMW2D & MUPMW2D-W-15718 & 4/6/06 & $19.6-29.6$ & 3353 & 9.6 & ND \\
\hline PMW2D & MUPMW2D-W-18164 & $8 / 17 / 06$ & $19.6-29.6$ & 1336 & 7.4 & ND \\
\hline PMW2D & MUPMW2D-W-18193 & $4 / 12 / 07$ & $19.6-29.6$ & 1273 & 5.9 & ND \\
\hline PMW2D & MUPMW2D-W-22841 & $7 / 23 / 07$ & $19.6-29.6$ & 1009 & 8.2 & ND \\
\hline PMW2D & MUPMW2D-W-22926 & $5 / 12 / 08$ & $19.6-29.6$ & 763 & 5.4 & ND \\
\hline PMW2D & MUPMW2D-W-19186 & $7 / 23 / 08$ & 19.6-29.6 & 568 & 3.8 & ND \\
\hline PMW3S & MUPMW3S-W-18331 & $7 / 21 / 05$ & $4.5-14.5$ & 394 & 2.4 & ND \\
\hline PMW3S & MUPMW3S-W-15673 & $4 / 5 / 06$ & $4.5-14.5$ & 65 & $0.7 \mathrm{~J}$ & ND \\
\hline PMW3S & MUPMW3S-W-18159 & $8 / 15 / 06$ & $4.5-14.5$ & 85 & 1.0 & ND \\
\hline PMW3S & MUPMW3S-W-18201 & $4 / 12 / 07$ & $4.5-14.5$ & 90 & ND & ND \\
\hline PMW3S & MUPMW3S-W-22834 & $7 / 23 / 07$ & $4.5-14.5$ & 1133 & 12 & ND \\
\hline PMW3S & MUPMW3S-W-22927 & $5 / 13 / 08$ & $4.5-14.5$ & 417 & 2.9 & ND \\
\hline PMW3S & MUPMW3S-W-19187 & $7 / 23 / 08$ & $4.5-14.5$ & 207 & 1.3 & ND \\
\hline PMW3D & MUPMW3D-W-18316 & $7 / 19 / 05$ & $19.5-24.5$ & 801 & 4.9 & ND \\
\hline PMW3D & MUPMW3D-W-15671 & 4/5/06 & $19.5-24.5$ & 346 & ND & ND \\
\hline PMW3D & MUPMW3D-W-18161 & $8 / 15 / 06$ & $19.5-24.5$ & 444 & 4.4 & ND \\
\hline PMW3D & MUPMW3D-W-18202 & $4 / 12 / 07$ & $19.5-24.5$ & 382 & 3.0 & ND \\
\hline PMW3D & MUPMW3D-W-22833 & $7 / 23 / 07$ & 19.5 & 352 & 2.9 & ND \\
\hline PMW3D & MUPMW3D-W-22928 & $5 / 13 / 08$ & $19.5-24.5$ & 867 & 6.2 & ND \\
\hline PMW3D & MUPMW3D-W-19188 & $7 / 23 / 08$ & $19.5-24.5$ & 1152 & 8.3 & ND \\
\hline
\end{tabular}


TABLE 4.6 (Cont.)

\begin{tabular}{|c|c|c|c|c|c|c|}
\hline \multirow[b]{2}{*}{ Location } & \multirow[b]{2}{*}{ Sample } & \multirow[b]{2}{*}{$\begin{array}{l}\text { Sample } \\
\text { Date }\end{array}$} & \multirow[b]{2}{*}{$\begin{array}{l}\text { Depth } \\
\text { (ft BGL) }\end{array}$} & \multicolumn{3}{|c|}{ Concentration $(\mu \mathrm{g} / \mathrm{L})$} \\
\hline & & & & $\begin{array}{c}\text { Carbon } \\
\text { Tetrachloride }\end{array}$ & Chloroform & $\begin{array}{r}\text { Methyle } \\
\text { Chlorid }\end{array}$ \\
\hline \multicolumn{7}{|c|}{ Wells in phytoremediation treatment area (cont.) } \\
\hline PMW4 & MUPMW4-W-18321 & $7 / 20 / 05$ & $19.5-24.5$ & 52 & 1.8 & ND \\
\hline PMW4 & MUPMW4-W-15728 & $4 / 10 / 06$ & $19.5-24.5$ & 31 & $0.6 \mathrm{~J}$ & ND \\
\hline PMW4 & MUPMW4-W-18346 & $8 / 21 / 06$ & $19.5-24.5$ & 35 & $0.6 \mathrm{~J}$ & ND \\
\hline PMW4 & MUPMW4-W-18188 & 4/9/07 & $19.5-24.5$ & 65 & 1.0 & ND \\
\hline PMW4 & MUPMW4-W-22849 & $7 / 24 / 07$ & $19.5-24.5$ & 75 & 1.1 & ND \\
\hline PMW4 & MUPMW4-W-22929 & $5 / 12 / 08$ & $19.5-24.5$ & 17 & $0.9 \mathrm{~J}$ & ND \\
\hline PMW4 & MUPMW4-W-19189 & $7 / 21 / 08$ & $19.5-24.5$ & 118 & 2.0 & ND \\
\hline PMW5 & MUPMW5-W-18322 & $7 / 20 / 05$ & $4.5-14.5$ & 118 & 1.0 & ND \\
\hline PMW5 & MUPMW5-W-15727 & $4 / 10 / 06$ & $4.5-14.5$ & 178 & 1.5 & ND \\
\hline PMW5 & MUPMW5-W-18347 & $8 / 21 / 06$ & $4.5-14.5$ & 34 & $0.2 \mathrm{~J}$ & ND \\
\hline PMW5 & MUPMW5-W-18204 & $4 / 12 / 07$ & $4.5-14.5$ & 355 & 2.9 & ND \\
\hline PMW5 & MUPMW5-W-22850 & $7 / 24 / 07$ & $4.5-14.5$ & 372 & 3.6 & ND \\
\hline PMW5 & MUPMW5-W-22930 & $5 / 12 / 08$ & $4.5-14.5$ & 168 & 3.7 & ND \\
\hline PMW5 & MUPMW5-W-19190 & $7 / 21 / 08$ & $4.5-14.5$ & 260 & 3.9 & ND \\
\hline PMW6 & MUPMW6-W-18333 & $7 / 21 / 05$ & $4.5-14.5$ & 9.6 & $0.4 \mathrm{~J}$ & ND \\
\hline PMW6 & MUPMW6-W-15724 & $4 / 10 / 06$ & $4.5-14.5$ & 8.7 & $0.4 \mathrm{~J}$ & ND \\
\hline PMW6 & MUPMW6-W-18174 & $8 / 23 / 06$ & $4.5-14.5$ & 9.4 & $0.6 \mathrm{~J}$ & ND \\
\hline PMW6 & MUPMW6-W-18190 & $4 / 10 / 07$ & $4.5-14.5$ & 5.5 & $0.4 \mathrm{~J}$ & ND \\
\hline PMW6 & MUPMW6-W-22853 & $7 / 24 / 07$ & $4.5-14.5$ & 6.9 & $0.2 \mathrm{~J}$ & ND \\
\hline PMW6 & MUPMW6-W-22931 & $5 / 12 / 08$ & $4.5-14.5$ & 3.1 & $0.4 \mathrm{~J}$ & ND \\
\hline PMW6 & MUPMW6-W-19191 & $7 / 22 / 08$ & $4.5-14.5$ & 1.8 & $0.2 \mathrm{~J}$ & ND \\
\hline PMW7 & MUPMW7-W-18332 & $7 / 21 / 05$ & $14.5-19.5$ & 3.5 & $0.6 \mathrm{~J}$ & ND \\
\hline PMW7 & MUPMW7-W-15722 & $4 / 10 / 06$ & $14.5-19.5$ & 3.7 & $0.9 \mathrm{~J}$ & ND \\
\hline PMW7 & MUPMW7-W-18172 & $8 / 23 / 06$ & $14.5-19.5$ & 5.4 & 1.1 & ND \\
\hline PMW7 & MUPMW7-W-18192 & $4 / 10 / 07$ & $14.5-19.5$ & 3.4 & $0.7 \mathrm{~J}$ & ND \\
\hline PMW7 & MUPMW7-W-22854 & $7 / 24 / 07$ & $14.5-19.5$ & 2.5 & $0.7 \mathrm{~J}$ & ND \\
\hline PMW7 & MUPMW7-W-22932 & $5 / 12 / 08$ & $14.5-19.5$ & 2.3 & $0.4 \mathrm{~J}$ & ND \\
\hline PMW7 & MUPMW7-W-19192 & $7 / 22 / 08$ & $14.5-19.5$ & $0.8 \mathrm{~J}$ & ND & ND \\
\hline PMW8 & MUPMW8-W-18323 & $7 / 20 / 05$ & $4.5-14.5$ & 23 & $0.8 \mathrm{~J}$ & ND \\
\hline PMW8 & MUPMW8-W-15726 & $4 / 10 / 06$ & $4.5-14.5$ & 16 & $0.6 \mathrm{~J}$ & ND \\
\hline PMW8 & MUPMW8-W-18169 & $8 / 21 / 06$ & $4.5-14.5$ & 19 & $0.7 \mathrm{~J}$ & ND \\
\hline PMW8 & MUPMW8-W-18189 & $4 / 9 / 07$ & $4.5-14.5$ & 17 & $0.5 \mathrm{~J}$ & ND \\
\hline PMW8 & MUPMW8-W-22851 & $7 / 24 / 07$ & $4.5-14.5$ & 26 & $0.5 \mathrm{~J}$ & ND \\
\hline PMW8 & MUPMW8-W-22933 & $5 / 12 / 08$ & $4.5-14.5$ & 19 & $0.4 \mathrm{~J}$ & ND \\
\hline PMW8 & MUPMW8-W-19193 & $7 / 21 / 08$ & $4.5-14.5$ & 44 & $0.8 \mathrm{~J}$ & ND \\
\hline PMW9S & MUPMW9S-W-18328 & $7 / 20 / 05$ & $5.0-9.0$ & 10 & 14 & ND \\
\hline PMW9S & MUPMW9S-W-15712 & 4/5/06 & $5.0-9.0$ & 8.1 & 14 & ND \\
\hline PMW9S & MUPMW9S-W-17206 & $8 / 16 / 06$ & $5.0-9.0$ & 11 & 8.7 & ND \\
\hline PMW9S & MUPMW9S-W-18197 & $4 / 12 / 07$ & $5.0-9.0$ & 11 & 24 & ND \\
\hline PMW9S & MUPMW9S-W-22837 & $7 / 23 / 07$ & $5.0-9.0$ & 17 & 5.2 & ND \\
\hline PMW9S & MUPMW9S-W-22934 & $5 / 12 / 08$ & $5.0-9.0$ & 17 & 2.7 & ND \\
\hline PMW9S & MUPMW9S-W-19194 & $7 / 23 / 08$ & $5.0-9.0$ & 12 & $0.4 \mathrm{~J}$ & ND \\
\hline PMW9M & MUPMW9M-W-18329 & $7 / 20 / 05$ & $11.0-15.0$ & 60 & 2.0 & ND \\
\hline PMW9M & MUPMW9M-W-15674 & $4 / 5 / 06$ & $11.0-15.0$ & 60 & 1.8 & ND \\
\hline PMW9M & MUPMW9M-W-17207 & $8 / 16 / 06$ & $11.0-15.0$ & 44 & 1.6 & ND \\
\hline
\end{tabular}


TABLE 4.6 (Cont.)

\begin{tabular}{|c|c|c|c|c|c|c|}
\hline \multirow[b]{2}{*}{ Location } & \multirow[b]{2}{*}{ Sample } & \multirow[b]{2}{*}{$\begin{array}{l}\text { Sample } \\
\text { Date }\end{array}$} & \multirow[b]{2}{*}{$\begin{array}{l}\text { Depth } \\
\text { (ft BGL) }\end{array}$} & \multicolumn{3}{|c|}{ Concentration $(\mu \mathrm{g} / \mathrm{L})$} \\
\hline & & & & $\begin{array}{c}\text { Carbon } \\
\text { Tetrachloride }\end{array}$ & Chloroform & $\begin{array}{c}\text { Methylene } \\
\text { Chloride }\end{array}$ \\
\hline \multicolumn{7}{|c|}{ Wells in phytoremediation treatment area (cont.) } \\
\hline PMW9M & MUPMW9M-W-18200 & $4 / 12 / 07$ & $11.0-15.0$ & 28 & 1.2 & ND \\
\hline PMW9M & MUPMW9M-W-22835 & $7 / 23 / 07$ & $11.0-15.0$ & 24 & $0.5 \mathrm{~J}$ & ND \\
\hline PMW9M & MUPMW9M-W-22935 & $5 / 12 / 08$ & $11.0-15.0$ & 6.5 & ND & ND \\
\hline PMW9M & MUPMW9M-W-19195 & $7 / 23 / 08$ & $11.0-15.0$ & 8.1 & ND & ND \\
\hline PMW9D & MUPMW9D-W-18315 & $7 / 20 / 05$ & $19.5-29.5$ & 4.6 & $0.9 \mathrm{~J}$ & ND \\
\hline PMW9D & MUPMW9D-W-15713 & 4/6/06 & $19.5-29.5$ & ND & $0.4 \mathrm{~J}$ & ND \\
\hline PMW9D & MUPMW9D-W-18345 & $8 / 16 / 06$ & $19.5-29.5$ & $0.5 \mathrm{~J}$ & $0.3 \mathrm{~J}$ & ND \\
\hline PMW9D & MUPMW9D-W-18198 & $4 / 12 / 07$ & $19.5-29.5$ & ND & $0.3 \mathrm{~J}$ & ND \\
\hline PMW9D & MUPMW9D-W-22842 & $7 / 23 / 07$ & $19.5-29.5$ & $0.7 \mathrm{~J}$ & $0.5 \mathrm{~J}$ & ND \\
\hline PMW9D & MUPMW9D-W-22936 & $5 / 13 / 08$ & $19.5-29.5$ & ND & $0.3 \mathrm{~J}$ & ND \\
\hline PMW9D & MUPMW9D-W-19196 & $7 / 23 / 08$ & $19.5-29.5$ & $0.9 \mathrm{~J}$ & $0.2 \mathrm{~J}$ & ND \\
\hline W103 & MU578-W-18260 & $7 / 19 / 05$ & $2.9-5.0$ & ND & ND & ND \\
\hline W103 & MUW103-W-17200 & $4 / 13 / 06$ & $2.9-5.0$ & ND & ND & ND \\
\hline W103 & MUW103-W-18182 & $8 / 24 / 06$ & $2.9-5.0$ & ND & ND & ND \\
\hline W103 & MUW103-W-18208 & $4 / 12 / 07$ & $2.9-5.0$ & ND & ND & ND \\
\hline W103 & MUW103-W-22867 & $8 / 2 / 07$ & $2.9-5.0$ & ND & ND & ND \\
\hline W103 & MUW103-W-22937 & $5 / 13 / 08$ & $2.9-5.0$ & ND & ND & ND \\
\hline W103 & MUW103-W-19197 & $7 / 26 / 08$ & $2.9-5.0$ & 1.6 & ND & ND \\
\hline W410 & MUTW444-W-18261 & $7 / 23 / 05$ & $2.5-4.9$ & 38 & 31 & ND \\
\hline W410 & MUW410-W-18127 & $4 / 13 / 06$ & $2.5-4.9$ & 1.6 & 3.6 & ND \\
\hline W410 & MUW410-W-22872 & $8 / 2 / 07$ & $2.5-4.9$ & 15 & 23 & ND \\
\hline W410 & MUW410-W-22938 & $5 / 13 / 08$ & $2.5-4.9$ & 242 & 77 & 1.4 \\
\hline W410 & MUW410-W-19198 & $7 / 26 / 08$ & $2.5-4.9$ & 59 & 54 & 2.3 \\
\hline \multicolumn{7}{|c|}{ Wells in upgradient plume area } \\
\hline $2 S$ & MU2S-W-18308 & $7 / 18 / 05$ & $70.5-80.5$ & 85 & 2.5 & ND \\
\hline $2 S$ & MUS2-W-17202 & $4 / 18 / 06$ & $70.5-80.5$ & 88 & 1.6 & ND \\
\hline $2 S$ & MUS2-W-18179 & $8 / 24 / 06$ & $70.5-80.5$ & 130 & 2.8 & ND \\
\hline $2 S$ & MU2S-W-18213 & $4 / 13 / 07$ & $70.5-80.5$ & 100 & 2.4 & ND \\
\hline $2 S$ & MU2S-W-22861 & $7 / 25 / 07$ & $70.5-80.5$ & 102 & 2.3 & ND \\
\hline $2 S$ & MU2S-W-22917 & $5 / 8 / 08$ & $70.5-80.5$ & 59 & 1.8 & ND \\
\hline $2 S$ & MUS2-W-19178 & $7 / 24 / 08$ & $70.5-80.5$ & 54 & 1.6 & ND \\
\hline $2 \mathrm{D}$ & MUD2-W-18344 & $7 / 22 / 05$ & $85.0-95.0$ & 10 & 3.4 & ND \\
\hline $2 \mathrm{D}$ & MUD2-W-17201 & $4 / 17 / 06$ & $85.0-95.0$ & 2.2 & 4.6 & ND \\
\hline $2 \mathrm{D}$ & MUD2-W-18181 & $8 / 24 / 06$ & $85.0-95.0$ & 36 & 4.1 & ND \\
\hline $2 \mathrm{D}$ & MU2D-W-18212 & $4 / 13 / 07$ & $85.0-95.0$ & 20 & 4.9 & ND \\
\hline $2 \mathrm{D}$ & MU2D-W-22859 & $7 / 25 / 07$ & $85.0-95.0$ & 17 & 5.3 & ND \\
\hline $2 \mathrm{D}$ & MU2D-W-22918 & $5 / 8 / 08$ & $85.0-95.0$ & 1.3 & 6.0 & ND \\
\hline $2 \mathrm{D}$ & MUD2-W-19179 & $7 / 24 / 08$ & $85.0-95.0$ & 2.8 & 4.4 & ND \\
\hline SB65S & MUSB65S-W-18335 & $7 / 21 / 05$ & $23.7-38.7$ & ND & ND & ND \\
\hline SB65S & MUSB65S-W-15732 & $4 / 13 / 06$ & $23.7-38.7$ & $0.5 \mathrm{~J}$ & ND & ND \\
\hline SB65S & MUSB65S-W-18176 & $8 / 23 / 06$ & $23.7-38.7$ & $0.7 \mathrm{~J}$ & ND & ND \\
\hline SB65S & MUSB65S-W-18209 & $4 / 13 / 07$ & $23.7-38.7$ & ND & ND & ND \\
\hline SB65S & MUSB65S-W-22855 & $7 / 25 / 07$ & $23.7-38.7$ & ND & ND & ND \\
\hline
\end{tabular}


TABLE 4.6 (Cont.)

\begin{tabular}{|c|c|c|c|c|c|c|}
\hline \multirow[b]{2}{*}{ Location } & \multirow[b]{2}{*}{ Sample } & \multirow[b]{2}{*}{$\begin{array}{l}\text { Sample } \\
\text { Date }\end{array}$} & \multirow[b]{2}{*}{$\begin{array}{l}\text { Depth } \\
\text { (ft BGL) }\end{array}$} & \multicolumn{3}{|c|}{ Concentration $(\mu \mathrm{g} / \mathrm{L})$} \\
\hline & & & & $\begin{array}{c}\text { Carbon } \\
\text { Tetrachloride }\end{array}$ & Chloroform & $\begin{array}{c}\text { Methylene } \\
\text { Chloride }\end{array}$ \\
\hline \multicolumn{7}{|c|}{ Wells in upgradient plume area (cont.) } \\
\hline SB65S & MUSB65S-W-22919 & 5/8/08 & 23.7-38.7 & ND & ND & ND \\
\hline SB65S & MUSB65S-W-19181 & $7 / 24 / 08$ & $23.7-38.7$ & $0.9 \mathrm{~J}$ & ND & ND \\
\hline SB65D & MU65D-W-18309 & $7 / 18 / 05$ & $38.0-53.0$ & 201 & 1.0 & ND \\
\hline SB65D & MUSB65D-W-15733 & 4/13/06 & $38.0-53.0$ & 582 & 3.6 & ND \\
\hline SB65D & MUSB65D-W-18175 & $8 / 23 / 06$ & $38.0-53.0$ & 595 & 4.9 & ND \\
\hline SB65D & MUSB65D-W-18210 & $4 / 13 / 07$ & $38.0-53.0$ & 457 & 2.7 & ND \\
\hline SB65D & MUSB65D-W-22857 & $7 / 25 / 07$ & $38.0-53.0$ & 122 & $\mathrm{ND}^{\mathrm{C}}$ & ND \\
\hline SB65D & MUSB65D-W-22920 & $5 / 8 / 08$ & $38.0-53.0$ & 3.1 & ND & ND \\
\hline SB65D & MUSB65D-W-19182 & $7 / 24 / 08$ & $38.0-53.0$ & 3.1 & ND & ND \\
\hline GWEXTEST1 & MUTEST1-W-18311 & 7/18/05 & $60.0-65.0$ & 81 & 49 & ND \\
\hline GWEXTEST1 & MUTEST1-W-18125 & $4 / 13 / 06$ & $60.0-65.0$ & 79 & 24 & ND \\
\hline GWEXTEST1 & MUTST1-W-18177 & $8 / 23 / 06$ & $60.0-65.0$ & 84 & 24 & $0.4 \mathrm{~J}$ \\
\hline GWEXTEST1 & MUGWEXTEST1-W-18214 & $4 / 13 / 07$ & $60.0-65.0$ & 138 & 18 & ND \\
\hline GWEXTEST1 & MUGWTXT-W-22862 & $7 / 25 / 07$ & $60.0-65.0$ & 187 & 14 & ND \\
\hline GWEXTEST1 & MUGWEXTEST1-W-22921 & 5/8/08 & $60.0-65.0$ & 361 & 17 & ND \\
\hline GWEXTEST1 & MUTEST1-W-19180 & $7 / 26 / 08$ & $60.0-65.0$ & 457 & 16 & ND \\
\hline
\end{tabular}

a Qualifier $\mathrm{J}$ indicates an estimated concentration below the method quantitation limit of $1.0 \mu \mathrm{g} / \mathrm{L}$.

b ND, not detected at instrument detection limit of $0.1 \mu \mathrm{g} / \mathrm{L}$.

c No analysis at dilution to detect chloroform. 
TABLE 4.7 Geochemical parameters measured in water samples collected from monitoring wells at Murdock, 2005-2008.

\begin{tabular}{|c|c|c|c|c|c|c|c|c|c|c|}
\hline Location & Sample & $\begin{array}{l}\text { Sample } \\
\text { Date }\end{array}$ & $\begin{array}{l}\text { Depth } \\
\text { (ft BGL) }\end{array}$ & $\begin{array}{l}\text { Temperature } \\
\left({ }^{\circ} \mathrm{C}\right)\end{array}$ & $\mathrm{pH}$ & $\begin{array}{l}\text { Conductivity } \\
(\mu \mathrm{S} / \mathrm{cm})\end{array}$ & $\begin{array}{c}\text { Dissolved } \\
\text { Oxygen } \\
(\mathrm{mg} / \mathrm{L})\end{array}$ & $\begin{array}{l}\text { Iron II } \\
\text { (mg/L) }\end{array}$ & $\begin{array}{l}\text { Oxidation- } \\
\text { Reduction } \\
\text { Potential } \\
(\mathrm{mV})\end{array}$ & $\begin{array}{l}\text { Nitrate- } \\
\text { Nitrite } \\
\text { Nitrogen } \\
\text { (mg/L) }\end{array}$ \\
\hline \multicolumn{11}{|c|}{ Monitoring wells in phytoremediation treatment area } \\
\hline PMW1S & MUPMW1S-W-18318 & $7 / 19 / 05$ & $4.6-14.6$ & 19.8 & 6.45 & 463 & $N R^{b}$ & NR & NR & $N A^{c}$ \\
\hline PMW1S & MUPMW1S-W-15720 & $4 / 6 / 06$ & $4.6-14.6$ & 10.7 & 6.81 & 470 & 8.29 & 0.09 & 177 & 15.4 \\
\hline PMW1S & MUPMW1S-W-18168 & $8 / 17 / 06$ & $4.6-14.6$ & 18.7 & 6.68 & 377 & 0.13 & 0.07 & 27 & 1.98 \\
\hline PMW1S & MUPMW1S-W-18205 & $4 / 12 / 07$ & $4.6-14.6$ & 8.9 & 7.05 & 328 & 2.71 & 0.10 & 311 & 4.40 \\
\hline PMW1S & MUPMW1S-W-22846 & $7 / 24 / 07$ & $4.6-14.6$ & 16.6 & 6.33 & 442 & 6.01 & 0.01 & 73 & 9.50 \\
\hline PMW1S & MUPMW1S-W-22923 & $5 / 8 / 08$ & $4.6-14.6$ & 13.1 & 6.74 & 486 & 6.65 & 0.02 & 121 & 11.0 \\
\hline PMW1S & MUPMW1S-W-19183 & $7 / 22 / 08$ & $4.6-14.6$ & 15.6 & 6.21 & 473 & 6.27 & 0.11 & 149 & 8.20 \\
\hline PMW1D & MUPMW1D-W-18313 & 7/19/05 & $24.6-34.6$ & 14.6 & 6.58 & 416 & 8.31 & 0.00 & 215 & 3.84 \\
\hline PMW1D & MUPMW1D-W-15721 & $4 / 6 / 06$ & $24.6-34.6$ & 12.2 & 6.74 & 430 & 8.04 & 0.12 & 188 & 4.25 \\
\hline PMW1D & MUPMW1D-W-18166 & $8 / 17 / 06$ & $24.6-34.6$ & 14.9 & 6.42 & 403 & 7.17 & 0.15 & 84 & 4.23 \\
\hline PMW1D & MUPMW1D-W-18206 & $4 / 12 / 07$ & $24.6-34.6$ & 10.4 & 6.76 & 446 & 3.86 & 0.00 & 210 & 4.90 \\
\hline PMW1D & MUPMW1D-W-22845 & $7 / 24 / 07$ & $24.6-34.6$ & 15.8 & 6.27 & 494 & 7.91 & 0.06 & 65 & 5.90 \\
\hline PMW1D & MUPMW1D-W-22924 & $5 / 8 / 08$ & $24.6-34.6$ & 14.0 & 6.67 & 457 & 6.44 & 0.01 & 186 & 5.40 \\
\hline PMW1D & MUPMW1D-W-19184 & $7 / 22 / 08$ & $24.6-34.6$ & 15.9 & 6.19 & 509 & 6.81 & 0.12 & 144 & 4.50 \\
\hline PMW2SA & MUPMW2SA-W-15717 & 4/6/06 & $4.6-14.6$ & 10.0 & 6.87 & 439 & 8.91 & 0.09 & 189 & 8.28 \\
\hline PMW2SA & MUPMW2SA-W-18163 & $8 / 17 / 06$ & $4.6-14.6$ & 17.8 & 6.42 & 443 & 5.62 & 0.06 & 96 & 7.45 \\
\hline PMW2SA & MUPMW2SA-W-18196 & $4 / 12 / 07$ & $4.6-14.6$ & 9.6 & 6.90 & 511 & 4.44 & 0.33 & 276 & 13.0 \\
\hline PMW2SA & MUPMW2SA-W-22839 & $7 / 23 / 07$ & $4.6-14.6$ & 16.5 & 6.44 & 416 & 9.48 & 0.20 & 188 & 5.80 \\
\hline PMW2SA & MUPMW2SA-W-22925 & $5 / 12 / 08$ & $4.6-14.6$ & 12.8 & 6.29 & 547 & 6.07 & 0.03 & 234 & 6.10 \\
\hline PMW2SA & MUPMW2SA-W-19185 & $7 / 23 / 08$ & $4.6-14.6$ & 14.4 & 6.15 & 619 & 6.35 & 0.21 & 224 & 6.40 \\
\hline PMW2SB & MUPMW2SB-W-18334 & $7 / 21 / 05$ & $4.6-14.6$ & 16.0 & 6.54 & 540 & 7.67 & 0.55 & NR & 13.0 \\
\hline PMW2SB & MUPMW2SB-W-18194 & $4 / 12 / 07$ & $4.6-14.6$ & 9.5 & 6.84 & 521 & 5.60 & 1.14 & 289 & 14.0 \\
\hline PMW2D & MUPMW2D-W-18314 & $7 / 19 / 05$ & $19.6-29.6$ & 12.0 & 6.56 & 500 & 8.36 & 0.00 & 220 & 4.48 \\
\hline PMW2D & MUPMW2D-W-15718 & $4 / 6 / 06$ & $19.6-29.6$ & 11.8 & 6.68 & 435 & 7.26 & 0.13 & 202 & 5.75 \\
\hline PMW2D & MUPMW2D-W-18164 & $8 / 17 / 06$ & $19.6-29.6$ & 14.9 & 6.40 & 461 & 7.00 & 0.23 & 73 & 5.45 \\
\hline PMW2D & MUPMW2D-W-18193 & $4 / 12 / 07$ & $19.6-29.6$ & 10.2 & 6.78 & 444 & 3.75 & 0.13 & 296 & 7.50 \\
\hline PMW2D & MUPMW2D-W-22841 & $7 / 23 / 07$ & $19.6-29.6$ & 17.8 & 6.43 & 454 & 8.94 & 0.10 & 114 & 5.50 \\
\hline PMW2D & MUPMW2D-W-22926 & $5 / 12 / 08$ & $19.6-29.6$ & 13.8 & 6.68 & 579 & 6.36 & 0.07 & 219 & 7.50 \\
\hline PMW2D & MUPMW2D-W-19186 & $7 / 23 / 08$ & $19.6-29.6$ & 15.0 & 6.10 & 544 & 6.27 & 0.19 & 226 & 7.10 \\
\hline
\end{tabular}


Monitoring wells in phytoremediation treatment area (cont.)

\begin{tabular}{|c|c|c|c|c|c|c|c|c|c|c|}
\hline PMW3S & MUPMW3S-W-18331 & $7 / 21 / 05$ & 4.5-14.5 & 13.0 & 6.16 & 407 & 8.17 & 0.20 & NR & 6.12 \\
\hline PMW3S & MUPMW3S-W-15673 & 4/5/06 & 4.5-14.5 & 10.6 & 6.45 & 419 & 8.49 & 1.51 & 207 & 8.80 \\
\hline PMW3S & MUPMW3S-W-18159 & 8/15/06 & 4.5-14.5 & 15.8 & 6.96 & 403 & 7.48 & 0.13 & 72 & 6.20 \\
\hline PMW3S & MUPMW3S-W-18201 & $4 / 12 / 07$ & $4.5-14.5$ & 10.6 & 6.75 & 432 & 5.18 & 0.23 & 265 & 9.00 \\
\hline PMW3S & MUPMW3S-W-22834 & $7 / 23 / 07$ & $4.5-14.5$ & 14.0 & 6.25 & 528 & 7.57 & 0.00 & 115 & 5.50 \\
\hline PMW3S & MUPMW3S-W-22927 & $5 / 13 / 08$ & 4.5-14.5 & 11.7 & 6.62 & 522 & 6.80 & 0.00 & 222 & 7.30 \\
\hline PMW3S & MUPMW3S-W-19187 & $7 / 23 / 08$ & $4.5-14.5$ & 12.1 & 6.27 & 511 & 6.56 & 0.11 & 233 & 6.90 \\
\hline PMW3D & MUPMW3D-W-18316 & 7/19/05 & $19.5-24.5$ & 12.1 & 6.44 & 595 & 7.17 & 0.00 & 215 & 5.06 \\
\hline PMW3D & MUPMW3D-W-15671 & $4 / 5 / 06$ & $19.5-24.5$ & 11.6 & 6.86 & 638 & 5.13 & 0.00 & 196 & 5.76 \\
\hline PMW3D & MUPMW3D-W-18161 & $8 / 15 / 06$ & $19.5-24.5$ & 14.0 & 7.32 & 620 & 5.01 & 0.15 & 77 & 5.45 \\
\hline PMW3D & MUPMW3D-W-18202 & $4 / 12 / 07$ & $19.5-24.5$ & 10.8 & 7.03 & 646 & 2.40 & 0.16 & 279 & 6.30 \\
\hline PMW3D & MUPMW3D-W-22833 & $7 / 23 / 07$ & $19.5-24.5$ & 18.7 & 6.07 & 586 & 6.14 & 0.17 & 62 & 5.80 \\
\hline PMW3D & MUPMW3D-W-22928 & $5 / 13 / 08$ & $19.5-24.5$ & 11.9 & 6.81 & 607 & 6.45 & 0.02 & 230 & 6.80 \\
\hline PMW3D & MUPMW3D-W-19188 & $7 / 23 / 08$ & $19.5-24.5$ & 12.7 & 6.23 & 592 & 6.65 & 0.16 & 218 & 5.10 \\
\hline PMW4 & MUPMW4-W-18321 & $7 / 20 / 05$ & $19.5-24.5$ & 13.0 & 6.36 & 395 & 7.97 & NR & 210 & 9.08 \\
\hline PMW4 & MUPMW4-W-15728 & $4 / 10 / 06$ & $19.5-24.5$ & 11.3 & 6.80 & 411 & 8.05 & 0.31 & 207 & 9.98 \\
\hline PMW4 & MUPMW4-W-18346 & $8 / 21 / 06$ & $19.5-24.5$ & 14.9 & 6.37 & 410 & 2.97 & 0.05 & 102 & 11.8 \\
\hline PMW4 & MUPMW4-W-18188 & $4 / 9 / 07$ & $19.5-24.5$ & 11.2 & 6.86 & 429 & 2.01 & 0.04 & 235 & 11.0 \\
\hline PMW4 & MUPMW4-W-22849 & $7 / 24 / 07$ & $19.5-24.5$ & 20.2 & 6.27 & 379 & 3.02 & 0.33 & 72 & 10.0 \\
\hline PMW4 & MUPMW4-W-22929 & $5 / 12 / 08$ & $19.5-24.5$ & 16.8 & 6.53 & 423 & 2.86 & 0.00 & 187 & 19.0 \\
\hline PMW4 & MUPMW4-W-19189 & $7 / 21 / 08$ & $19.5-24.5$ & 16.8 & 6.17 & 377 & 3.44 & 0.00 & 139 & 6.80 \\
\hline PMW5 & MUPMW5-W-18322 & $7 / 20 / 05$ & $4.5-14.5$ & 14.6 & 6.56 & 520 & 6.74 & 0.00 & NR & 4.58 \\
\hline PMW5 & MUPMW5-W-15727 & $4 / 10 / 06$ & 4.5-14.5 & 10.2 & 6.86 & 591 & 7.16 & 0.26 & 191 & 5.17 \\
\hline PMW5 & MUPMW5-W-18347 & 8/21/06 & $4.5-14.5$ & 16.6 & 6.58 & 572 & 6.42 & 0.38 & 86 & 5.02 \\
\hline PMW5 & MUPMW5-W-18204 & $4 / 12 / 07$ & $4.5-14.5$ & 9.7 & 6.96 & 587 & 5.14 & 0.44 & 301 & 5.20 \\
\hline PMW5 & MUPMW5-W-22850 & $7 / 24 / 07$ & $4.5-14.5$ & 18.2 & 6.52 & 491 & 7.36 & 0.01 & 58 & 4.80 \\
\hline PMW5 & MUPMW5-W-22930 & $5 / 12 / 08$ & 4.5-14.5 & 15.5 & 6.64 & 482 & 9.28 & 0.05 & 157 & 5.60 \\
\hline PMW5 & MUPMW5-W-19190 & $7 / 21 / 08$ & $4.5-14.5$ & 17.2 & 5.44 & 479 & 5.44 & 0.19 & 199 & 4.50 \\
\hline
\end{tabular}


TABLE 4.7 (Cont.)

\begin{tabular}{|c|c|c|c|c|c|c|c|c|c|c|}
\hline Location & Sample & $\begin{array}{l}\text { Sample } \\
\text { Date }\end{array}$ & $\begin{array}{c}\text { Depth } \\
\text { (ft BGL) }\end{array}$ & $\begin{array}{c}\text { Temperature } \\
\left({ }^{\circ} \mathrm{C}\right)\end{array}$ & $\mathrm{pH}$ & $\begin{array}{l}\text { Conductivity } \\
\qquad(\mu \mathrm{S} / \mathrm{cm})\end{array}$ & $\begin{array}{c}\text { Dissolved } \\
\text { Oxygen } \\
(\mathrm{mg} / \mathrm{L})\end{array}$ & $\begin{array}{l}\text { Iron II } \\
\text { (mg/L) }\end{array}$ & $\begin{array}{l}\text { Oxidation- } \\
\text { Reduction } \\
\text { Potential } \\
(\mathrm{mV})\end{array}$ & $\begin{array}{r}\text { Nitrate } \\
\text { Nitrite } \\
\text { Nitroge } \\
\text { (mg/L }\end{array}$ \\
\hline \multicolumn{11}{|c|}{ Monitoring wells in phytoremediation treatment area (cont.) } \\
\hline PMW6 & MUPMW6-W-18333 & $7 / 21 / 05$ & $4.5-14.5$ & 18.4 & 6.52 & 884 & NR & NR & NR & NA \\
\hline PMW6 & MUPMW6-W-15724 & $4 / 10 / 06$ & $4.5-14.5$ & 10.7 & 6.72 & 863 & 5.15 & 0.17 & 222 & 10.7 \\
\hline PMW6 & MUPMW6-W-18174 & $8 / 23 / 06$ & $4.5-14.5$ & 17.3 & 6.58 & 835 & 3.16 & 0.82 & 68 & 9.33 \\
\hline PMW6 & MUPMW6-W-18190 & $4 / 10 / 07$ & 4.5-14.5 & 8.4 & 7.03 & 792 & 4.04 & 0.00 & 83 & 16.0 \\
\hline PMW6 & MUPMW6-W-22853 & $7 / 24 / 07$ & $4.5-14.5$ & 15.7 & 6.34 & 808 & 7.15 & 0.20 & 49 & 12.0 \\
\hline PMW6 & MUPMW6-W-22931 & $5 / 12 / 08$ & $4.5-14.5$ & 14.6 & 6.65 & 962 & 8.85 & 0.04 & 194 & 18.0 \\
\hline PMW6 & MUPMW6-W-19191 & $7 / 22 / 08$ & $4.5-14.5$ & 16.1 & 5.43 & 1067 & 4.18 & 0.13 & 254 & 13.0 \\
\hline PMW7 & MUPMW7-W-18332 & $7 / 21 / 05$ & $14.5-19.5$ & 14.6 & 6.31 & 691 & NR & NR & NR & NA \\
\hline PMW7 & MUPMW7-W-15722 & $4 / 10 / 06$ & $14.5-19.5$ & 11.3 & 6.36 & 604 & 8.65 & 0.00 & 174 & 10.8 \\
\hline PMW7 & MUPMW7-W-18172 & $8 / 23 / 06$ & $14.5-19.5$ & 13.9 & 6.41 & 642 & 9.26 & 0.07 & 96 & 14.9 \\
\hline PMW7 & MUPMW7-W-18192 & $4 / 10 / 07$ & $14.5-19.5$ & 9.0 & 7.12 & 695 & 6.28 & 0.06 & 160 & 18.0 \\
\hline PMW7 & MUPMW7-W-22854 & $7 / 24 / 07$ & $14.5-19.5$ & 15.7 & 6.43 & 640 & 8.50 & 0.00 & 114 & 15.0 \\
\hline PMW7 & MUPMW7-W-22932 & $5 / 12 / 08$ & $14.5-19.5$ & 14.4 & 6.63 & 837 & 7.15 & 0.02 & 162 & 17.0 \\
\hline PMW7 & MUPMW7-W-19192 & $7 / 22 / 08$ & $14.5-19.5$ & 16.5 & 5.86 & 855 & 3.26 & 0.11 & 188 & 14.0 \\
\hline PMW8 & MUPMW8-W-18323 & $7 / 20 / 05$ & $4.5-14.5$ & 15.8 & 6.46 & 418 & 2.79 & 0.60 & NR & 4.63 \\
\hline PMW8 & MUPMW8-W-15726 & $4 / 10 / 06$ & $4.5-14.5$ & 10.1 & 6.82 & 435 & 5.42 & 0.07 & 181 & 5.37 \\
\hline PMW8 & MUPMW8-W-18169 & $8 / 21 / 06$ & $4.5-14.5$ & 16.2 & 6.52 & 425 & 5.93 & 0.00 & 100 & 6.43 \\
\hline PMW8 & MUPMW8-W-18189 & $4 / 9 / 07$ & $4.5-14.5$ & 9.1 & 6.91 & 453 & 3.73 & 0.03 & 147 & 5.90 \\
\hline PMW8 & MUPMW8-W-22851 & $7 / 24 / 07$ & $4.5-14.5$ & 17.3 & 6.41 & 417 & 7.08 & 0.08 & 138 & 9.30 \\
\hline PMW8 & MUPMW8-W-22933 & $5 / 12 / 08$ & $4.5-14.5$ & 11.2 & 6.59 & 417 & 3.75 & 0.09 & 99 & 3.80 \\
\hline PMW8 & MUPMW8-W-19193 & $7 / 21 / 08$ & $4.5-14.5$ & 15.6 & 5.31 & 479 & 6.31 & 0.12 & 279 & 8.30 \\
\hline PMW9S & MUPMW9S-W-18328 & $7 / 20 / 05$ & $5.0-9.0$ & 21.3 & 6.41 & 508 & NR & NR & NR & NA \\
\hline PMW9S & MUPMW9S-W-15712 & $4 / 5 / 06$ & $5.0-9.0$ & 10.1 & 6.87 & 474 & 5.36 & 0.13 & 203 & 12.3 \\
\hline PMW9S & MUPMW9S-W-17206 & $8 / 16 / 06$ & $5.0-9.0$ & 19.5 & 6.30 & 454 & 4.54 & 0.05 & 111 & 11.2 \\
\hline PMW9S & MUPMW9S-W-18197 & $4 / 12 / 07$ & $5.0-9.0$ & 8.5 & 6.79 & 542 & 2.41 & 0.02 & 251 & 15.0 \\
\hline PMW9S & MUPMW9S-W-22837 & $7 / 23 / 07$ & $5.0-9.0$ & 19.6 & 6.32 & 466 & 1.34 & 0.00 & 120 & 12.0 \\
\hline PMW9S & MUPMW9S-W-22934 & $5 / 12 / 08$ & $5.0-9.0$ & 10.4 & 6.48 & 474 & 3.16 & 0.00 & 195 & 14.0 \\
\hline PMW9S & MUPMW9S-W-19194 & $7 / 23 / 08$ & $5.0-9.0$ & 17.0 & 5.16 & 493 & 3.70 & 0.16 & 266 & 12.0 \\
\hline PMW9M & MUPMW9M-W-18329 & $7 / 20 / 05$ & $11.0-15.0$ & 14.8 & 6.24 & 466 & NR & NR & NR & NA \\
\hline PMW9M & MUPMW9M-W-15674 & $4 / 5 / 06$ & $11.0-15.0$ & 10.3 & 6.62 & 502 & 4.55 & 0.06 & 206 & 12.3 \\
\hline
\end{tabular}


TABLE 4.7 (Cont.)

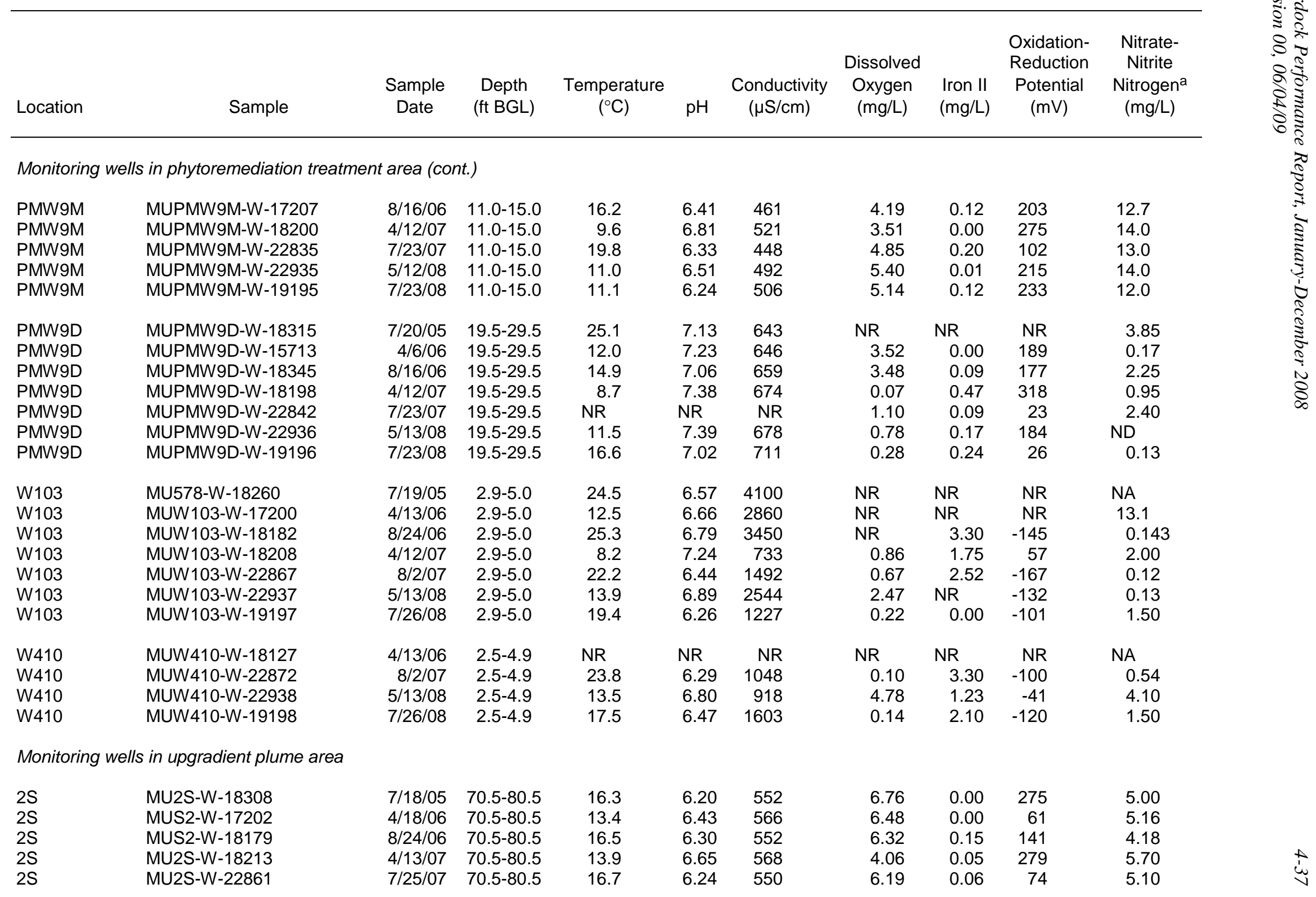


TABLE 4.7 (Cont.)

\begin{tabular}{|c|c|c|c|c|c|c|c|c|c|c|}
\hline Location & Sample & $\begin{array}{l}\text { Sample } \\
\text { Date }\end{array}$ & $\begin{array}{c}\text { Depth } \\
\text { (ft BGL) }\end{array}$ & $\begin{array}{c}\text { Temperature } \\
\left({ }^{\circ} \mathrm{C}\right)\end{array}$ & $\mathrm{pH}$ & $\begin{array}{l}\text { Conductivity } \\
\qquad(\mu \mathrm{S} / \mathrm{cm})\end{array}$ & $\begin{array}{c}\text { Dissolved } \\
\text { Oxygen } \\
(\mathrm{mg} / \mathrm{L})\end{array}$ & $\begin{array}{l}\text { Iron II } \\
\text { (mg/L) }\end{array}$ & $\begin{array}{c}\text { Oxidation- } \\
\text { Reduction } \\
\text { Potential } \\
(\mathrm{mV})\end{array}$ & $\begin{array}{c}\text { Nitrate- } \\
\text { Nitrite } \\
\text { Nitrogen } \\
\text { (mg/L) }\end{array}$ \\
\hline \multicolumn{11}{|c|}{ Monitoring wells in upgradient plume area (cont.) } \\
\hline $\begin{array}{l}2 S \\
2 S\end{array}$ & $\begin{array}{l}\text { MU2S-W-22917 } \\
\text { MUS2-W-19178 }\end{array}$ & $\begin{array}{r}5 / 8 / 08 \\
7 / 24 / 08\end{array}$ & $\begin{array}{l}70.5-80.5 \\
70.5-80.5\end{array}$ & $\begin{array}{l}15.2 \\
18.5\end{array}$ & $\begin{array}{l}6.49 \\
6.35\end{array}$ & $\begin{array}{l}568 \\
615\end{array}$ & $\begin{array}{l}7.30 \\
4.12\end{array}$ & $\begin{array}{l}0.00 \\
0.09\end{array}$ & $\begin{array}{l}156 \\
163\end{array}$ & $\begin{array}{l}5.40 \\
6.10\end{array}$ \\
\hline $\begin{array}{l}2 D \\
2 D \\
2 D \\
2 D \\
2 D \\
2 D \\
2 D\end{array}$ & $\begin{array}{l}\text { MUD2-W-18344 } \\
\text { MUD2-W-17201 } \\
\text { MUD2-W-18181 } \\
\text { MU2D-W-18212 } \\
\text { MU2D-W-22859 } \\
\text { MU2D-W-22918 } \\
\text { MUD2-W-19179 }\end{array}$ & $\begin{array}{r}7 / 22 / 05 \\
4 / 17 / 06 \\
8 / 24 / 06 \\
4 / 13 / 07 \\
7 / 25 / 07 \\
5 / 8 / 08 \\
7 / 24 / 08\end{array}$ & $\begin{array}{l}85.0-95.0 \\
85.0-95.0 \\
85.0-95.0 \\
85.0-95.0 \\
85.0-95.0 \\
85.0-95.0 \\
85.0-95.0\end{array}$ & $\begin{array}{l}\text { Dry } \\
13.1 \\
21.2 \\
14.9 \\
22.8 \\
16.8 \\
19.5\end{array}$ & $\begin{array}{l}\text { Dry } \\
8.21 \\
6.92 \\
9.17 \\
6.88 \\
8.46 \\
8.27\end{array}$ & $\begin{array}{l}\text { Dry } \\
481 \\
598 \\
260 \\
609 \\
577 \\
546\end{array}$ & $\begin{array}{l}\text { Dry } \\
0.95 \\
6.69 \\
0.10 \\
0.86 \\
1.76 \\
0.40\end{array}$ & $\begin{array}{l}\text { Dry } \\
0.12 \\
0.33 \\
0.14 \\
0.00 \\
0.03 \\
0.08\end{array}$ & $\begin{array}{r}\text { Dry } \\
140 \\
113 \\
140 \\
116 \\
136 \\
-6\end{array}$ & $\begin{array}{l}\text { Dry } \\
1.64 \\
2.61 \\
4.40 \\
4.90 \\
1.20 \\
0.75\end{array}$ \\
\hline $\begin{array}{l}\text { SB65S } \\
\text { SB65S } \\
\text { SB65S } \\
\text { SB65S } \\
\text { SB65S } \\
\text { SB65S } \\
\text { SB65S }\end{array}$ & $\begin{array}{l}\text { MUSB65S-W-18335 } \\
\text { MUSB65S-W-15732 } \\
\text { MUSB65S-W-18176 } \\
\text { MUSB65S-W-18209 } \\
\text { MUSB65S-W-22855 } \\
\text { MUSB65S-W-22919 } \\
\text { MUSB65S-W-19181 }\end{array}$ & $\begin{array}{r}7 / 21 / 05 \\
4 / 13 / 06 \\
8 / 23 / 06 \\
4 / 13 / 07 \\
7 / 25 / 07 \\
5 / 8 / 08 \\
7 / 24 / 08\end{array}$ & $\begin{array}{l}23.7-38.7 \\
23.7-38.7 \\
23.7-38.7 \\
23.7-38.7 \\
23.7-38.7 \\
23.7-38.7 \\
23.7-38.7\end{array}$ & $\begin{array}{l}15.3 \\
12.5 \\
17.7 \\
12.9 \\
18.3 \\
14.2 \\
15.5\end{array}$ & $\begin{array}{l}6.28 \\
6.59 \\
6.18 \\
6.49 \\
5.93 \\
6.26 \\
5.79\end{array}$ & $\begin{array}{l}403 \\
371 \\
403 \\
401 \\
331 \\
371 \\
382\end{array}$ & $\begin{array}{l}\text { NR } \\
7.24 \\
7.17 \\
3.85 \\
6.29 \\
6.70 \\
6.74\end{array}$ & $\begin{array}{l}\text { NR } \\
0.00 \\
0.17 \\
0.00 \\
0.10 \\
0.00 \\
0.13\end{array}$ & $\begin{array}{l}\text { NR } \\
228 \\
120 \\
318 \\
111 \\
208 \\
231\end{array}$ & $\begin{array}{l}\text { NA } \\
9.17 \\
9.98 \\
8.50 \\
13.0 \\
16.0 \\
16.0\end{array}$ \\
\hline $\begin{array}{l}\text { SB65D } \\
\text { SB65D } \\
\text { SB65D } \\
\text { SB65D } \\
\text { SB65D } \\
\text { SB65D } \\
\text { SB65D }\end{array}$ & $\begin{array}{l}\text { MU65D-W-18309 } \\
\text { MUSB65D-W-15733 } \\
\text { MUSB65D-W-18175 } \\
\text { MUSB65D-W-18210 } \\
\text { MUSB65D-W-22857 } \\
\text { MUSB65D-W-22920 } \\
\text { MUSB65D-W-19182 }\end{array}$ & $\begin{array}{r}7 / 18 / 05 \\
4 / 13 / 06 \\
8 / 23 / 06 \\
4 / 13 / 07 \\
7 / 25 / 07 \\
5 / 8 / 08 \\
7 / 24 / 08\end{array}$ & $\begin{array}{l}38.0-53.0 \\
38.0-53.0 \\
38.0-53.0 \\
38.0-53.0 \\
38.0-53.0 \\
38.0-53.0 \\
38.0-53.0\end{array}$ & $\begin{array}{l}15.1 \\
16.2 \\
17.2 \\
13.4 \\
16.9 \\
14.1 \\
14.6\end{array}$ & $\begin{array}{l}6.28 \\
6.59 \\
6.51 \\
6.70 \\
6.33 \\
6.60 \\
6.14\end{array}$ & $\begin{array}{l}510 \\
494 \\
489 \\
514 \\
493 \\
444 \\
452\end{array}$ & $\begin{array}{l}8.76 \\
\text { NR } \\
8.06 \\
2.16 \\
5.62 \\
6.03 \\
5.89\end{array}$ & $\begin{array}{l}0.00 \\
\text { NR } \\
0.00 \\
0.00 \\
0.05 \\
0.13 \\
0.13\end{array}$ & $\begin{array}{l}235 \\
203 \\
102 \\
248 \\
169 \\
125 \\
188\end{array}$ & $\begin{array}{l}4.74 \\
5.96 \\
5.67 \\
5.50 \\
5.70 \\
5.30 \\
4.10\end{array}$ \\
\hline $\begin{array}{l}\text { GWEXTEST1 } \\
\text { GWEXTEST1 } \\
\text { GWEXTEST1 } \\
\text { GWEXTEST1 } \\
\text { GWEXTEST1 } \\
\text { GWEXTEST1 } \\
\text { GWEXTEST1 }\end{array}$ & $\begin{array}{l}\text { MUTEST1-W-18311 } \\
\text { MUTEST1-W-18125 } \\
\text { MUTST1-W-18177 } \\
\text { MUGWEXTEST1-W-18214 } \\
\text { MUGWTXT-W-22862 } \\
\text { MUGWEXTEST1-W-22921 } \\
\text { MUTEST1-W-19180 }\end{array}$ & $\begin{array}{r}7 / 18 / 05 \\
4 / 13 / 06 \\
8 / 23 / 06 \\
4 / 13 / 07 \\
7 / 25 / 07 \\
5 / 8 / 08 \\
7 / 26 / 08\end{array}$ & $\begin{array}{l}60.0-65.0 \\
60.0-65.0 \\
60.0-65.0 \\
60.0-65.0 \\
60.0-65.0 \\
60.0-65.0 \\
60.0-65.0\end{array}$ & $\begin{array}{l}12.7 \\
16.0 \\
16.5 \\
14.1 \\
16.4 \\
11.8 \\
16.5\end{array}$ & $\begin{array}{l}6.56 \\
6.71 \\
6.61 \\
4.48 \\
6.45 \\
7.45 \\
7.00\end{array}$ & $\begin{array}{l}663 \\
631 \\
616 \\
647 \\
615 \\
579 \\
592\end{array}$ & $\begin{array}{l}5.14 \\
\text { NR } \\
6.82 \\
2.70 \\
6.43 \\
8.04 \\
4.43\end{array}$ & $\begin{array}{l}\text { NR } \\
\text { NR } \\
0.02 \\
0.10 \\
0.11 \\
0.00 \\
0.03\end{array}$ & $\begin{array}{r}245 \\
\text { NR } \\
126 \\
286 \\
244 \\
178 \\
85\end{array}$ & $\begin{array}{l}6.13 \\
7.12 \\
7.15 \\
8.30 \\
7.30 \\
6.40 \\
6.40\end{array}$ \\
\hline
\end{tabular}


TABLE 4.7 (Cont.)

a July 2005 samples were analyzed for nitrate as nitrogen.

b NR, not recorded.

c NA, not analyzed 
TABLE 4.8 Analytical results for volatile organic compounds in surface water samples collected at Murdock, 2005-2008.

\begin{tabular}{|c|c|c|c|c|c|}
\hline \multirow[b]{2}{*}{ Location } & \multirow[b]{2}{*}{ Sample } & \multirow[b]{2}{*}{$\begin{array}{c}\text { Sample } \\
\text { Date }\end{array}$} & \multicolumn{3}{|c|}{ Concentration $(\mu \mathrm{g} / \mathrm{L})$} \\
\hline & & & $\begin{array}{c}\text { Carbon } \\
\text { Tetrachloride }\end{array}$ & Chloroform & $\begin{array}{c}\text { Methylene } \\
\text { Chloride }\end{array}$ \\
\hline SWM1 & MUSWM1-W-15730 & 4/10/06 & 151 & 8.3 & $0.4 \mathrm{~J}$ \\
\hline SWM1 & MUSWM1-W-18147 & $8 / 14 / 06$ & 380 & 6.3 & ND \\
\hline SWM1 & MUSWM1-W-15724 & $11 / 8 / 06$ & 259 & 12 & 1.6 \\
\hline SWM1 & MUSWM1-W-18184 & $2 / 23 / 07$ & 237 & 12 & ND \\
\hline SWM1 & MUSWM1-W-18250 & $4 / 18 / 07$ & 149 & 8.5 & ND \\
\hline SWM1 & MUSWM1-W-22863 & $8 / 1 / 07$ & 77 & 1.9 & ND \\
\hline SWM1 & MUSWM1-W-18954 & $11 / 8 / 07$ & 52 & 1.4 & ND \\
\hline SWM1 & MUSWM1-W-19101 & $2 / 13 / 08$ & 100 & 5.9 & ND \\
\hline SWM1 & MUSWM1-W-22912 & $5 / 5 / 08$ & 45 & 1.5 & ND \\
\hline SWM1 & MUSWM1-W-19199 & $7 / 21 / 08$ & 20 & $0.5 \mathrm{~J}$ & ND \\
\hline SWM1 & MUSWM1-W-27026 & $12 / 2 / 08$ & 25 & $0.8 \mathrm{~J}$ & ND \\
\hline SWM2 & MUSWM2-W-15729 & $4 / 10 / 06$ & 3.6 & $0.4 \mathrm{~J}$ & ND \\
\hline SWM2 & MUSWM2-W-18148 & $8 / 14 / 06$ & 1.4 & $0.2 \mathrm{~J}$ & ND \\
\hline SWM2 & MUSWM2-W-15725 & $11 / 8 / 06$ & 6.1 & $0.6 \mathrm{~J}$ & ND \\
\hline SWM2 & MUSWM2-W-18185 & $2 / 23 / 07$ & 7.5 & $0.6 \mathrm{~J}$ & ND \\
\hline SWM2 & MUSWM2-W-18251 & $4 / 18 / 07$ & 3.5 & $0.4 \mathrm{~J}$ & ND \\
\hline SWM2 & MUSWM2-W-22865 & $8 / 1 / 07$ & 4.4 & $0.3 \mathrm{~J}$ & ND \\
\hline SWM2 & MUSWM2-W-18956 & $11 / 8 / 07$ & 19 & $0.7 \mathrm{~J}$ & ND \\
\hline SWM2 & MUSWM2-W-19102 & 2/13/08 & 9.0 & $0.8 \mathrm{~J}$ & ND \\
\hline SWM2 & MUSWM2-W-22914 & $5 / 5 / 08$ & 3.6 & $0.3 \mathrm{~J}$ & ND \\
\hline SWM2 & MUSWM2-W-19200 & $7 / 21 / 08$ & 4.6 & $0.2 \mathrm{~J}$ & ND \\
\hline SWM2 & MUSWM2-W-27027 & $12 / 2 / 08$ & 7.7 & $0.3 \mathrm{~J}$ & ND \\
\hline SWM3 & MU-W-15670 & $10 / 19 / 05$ & ND & ND & ND \\
\hline SWM3 & MUSWM3-W-15731 & $4 / 10 / 06$ & ND & ND & ND \\
\hline SWM3 & MUSWM3-W-18149 & $8 / 14 / 06$ & ND & ND & ND \\
\hline SWM3 & MUSWM3-W-15726 & $11 / 8 / 06$ & $0.7 \mathrm{~J}$ & ND & ND \\
\hline SWM3 & MUSWM3-W-18186 & $2 / 23 / 07$ & 2.4 & $0.2 \mathrm{~J}$ & ND \\
\hline SWM3 & MUSWM3-W-18252 & 4/18/07 & $0.3 \mathrm{~J}$ & ND & ND \\
\hline SWM3 & MUSWM3-W-22866 & $8 / 1 / 07$ & 1.0 & ND & ND \\
\hline SWM3 & MUSWM3-W-18957 & $11 / 8 / 07$ & 4.4 & ND & ND \\
\hline SWM3 & MUSWM3-W-19103 & $2 / 13 / 08$ & 2.9 & ND & ND \\
\hline SWM3 & MUSWM3-W-22915 & $5 / 5 / 08$ & 1.0 & ND & ND \\
\hline SWM3 & MUSWM3-W-19201 & $7 / 21 / 08$ & 1.2 & ND & ND \\
\hline SWM3 & MUSWM3-W-27028 & $12 / 2 / 08$ & 2.1 & ND & ND \\
\hline
\end{tabular}

a Qualifier $\mathrm{J}$ indicates an estimated concentration below the method quantitation limit of $1.0 \mu \mathrm{g} / \mathrm{L}$.

b ND, not detected at instrument detection limit of $0.1 \mu \mathrm{g} / \mathrm{L}$. 


\begin{tabular}{|c|c|c|c|c|c|c|c|c|c|c|c|c|c|c|c|c|c|c|c|c|c|c|c|}
\hline \multirow[b]{2}{*}{ 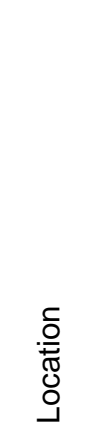 } & \multirow[b]{2}{*}{ 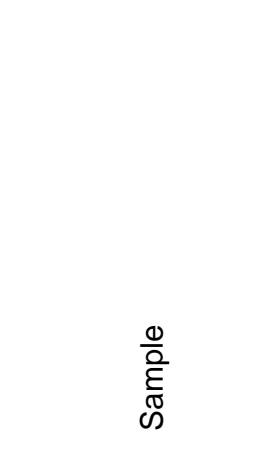 } & \multirow[b]{2}{*}{ 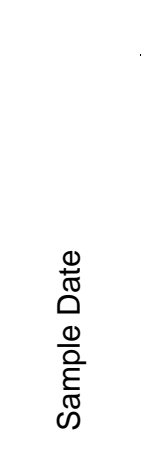 } & \multicolumn{21}{|c|}{ Concentration $\left(\mu \mathrm{g} / \mathrm{m}^{3}\right)$} \\
\hline & & & 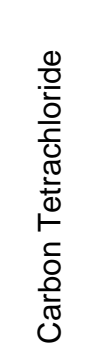 & $\begin{array}{l}\frac{E}{\bar{D}} \\
\text { 잉 } \\
\text { 을 }\end{array}$ & 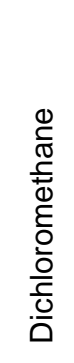 & 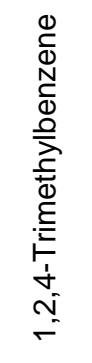 & 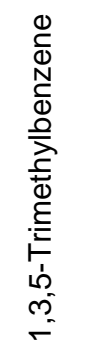 & 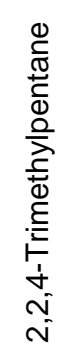 & 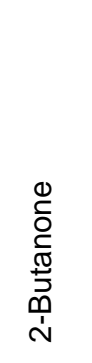 & 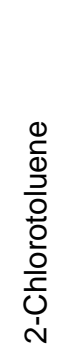 & 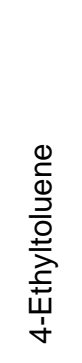 & $\begin{array}{l}\frac{0}{5} \\
\stackrel{5}{0} \\
\frac{0}{4}\end{array}$ & 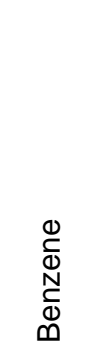 & 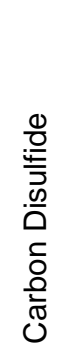 & 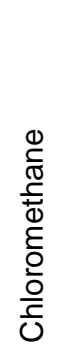 & 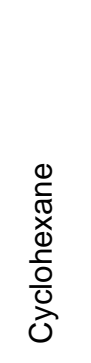 & 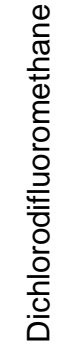 & 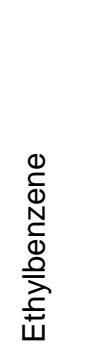 & 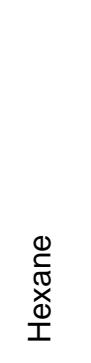 & $\begin{array}{l}\frac{\mathscr{\Phi}}{\bar{\Phi}} \\
\stackrel{\overline{0}}{\circ}\end{array}$ & 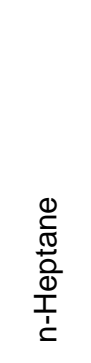 & 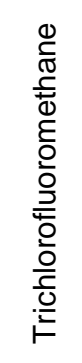 & 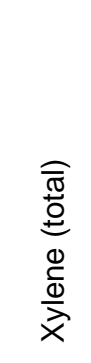 \\
\hline \multicolumn{2}{|c|}{ Detection Limit } & & 1.3 & 0.98 & 1.7 & 0.98 & 0.98 & 0.93 & 1.5 & 1.0 & 0.98 & 12 & 0.64 & 1.6 & 1.0 & 0.69 & 2.5 & 0.87 & 1.8 & 0.75 & 0.82 & 1.1 & 0.87 \\
\hline \multicolumn{24}{|c|}{ Location AA1 } \\
\hline AA1 & MUAA1-G-15785 & $5 / 17 / 06$ & - & - & - & - & - & - & - & - & - & - & - & - & 1.4 & - & 3.3 & - & - & 0.94 & - & 1.6 & - \\
\hline AA1 & MUAA1-G-15786 & $5 / 18 / 06$ & - & - & - & - & - & - & - & - & - & - & - & - & 1.5 & - & 3.4 & - & - & 0.9 & - & 1.7 & - \\
\hline AA1 & MUAA1-G-15787 & $5 / 19 / 06$ & - & - & - & - & - & - & - & - & - & $=$ & - & - & 1.5 & - & 3.2 & - & - & 1.8 & - & 1.5 & - \\
\hline AA1 & MUAA1-G-18138 & 8/14/06 & - & - & - & - & - & 1.1 & 2.1 & - & - & 15 & 1.2 & - & 1.4 & - & 3.2 & - & - & 4.5 & - & 1.5 & 2.3 \\
\hline AA1 & MUAA1-G-18139 & 8/15/06 & - & - & - & - & - & - & - & - & - & - & - & - & 1.5 & - & 3.4 & - & - & 0.75 & - & 1.6 & - \\
\hline AA1 & MUAA1-G-18140 & 8/16/06 & - & - & - & - & - & - & 2.8 & - & - & 15 & - & 1.6 & 1.9 & - & 3.8 & - & - & 1.6 & - & 1.7 & - \\
\hline AA1 & MUAA1-G-18170 & 8/23/06 & - & - & - & - & - & - & 3.8 & - & - & 36 & - & - & 1.8 & - & 3.8 & - & - & 0.87 & - & 1.6 & - \\
\hline AA1 & MUAA1-G-18241 & $4 / 17 / 07$ & - & - & - & - & - & - & 1.8 & - & - & 15 & - & - & 1.2 & - & 2.5 & - & - & - & - & 1.2 & - \\
\hline AA1 & MUAA1-G-18242 & 4/18/07 & - & - & - & - & - & - & 1.6 & - & - & 14 & - & - & 1.3 & - & 2.6 & - & - & - & - & 1.2 & - \\
\hline AA1 & MUAA1-G-18243 & 4/19/07 & - & - & - & - & - & - & 3.8 & - & - & 31 & - & - & 1.4 & - & 2.7 & - & - & - & - & 1.2 & - \\
\hline AA1 & MUAA1-G-22229 & $7 / 23 / 07$ & - & - & - & - & - & - & - & - & - & - & - & - & - & - & - & - & - & - & - & 1.1 & - \\
\hline AA1 & MUAA1-G-22230 & 7/24/07 & - & - & - & - & - & - & 2.4 & - & - & 16 & - & - & 1.1 & - & - & - & - & - & - & 1.2 & - \\
\hline AA1 & MUAA1-G-22231 & 7/25/07 & - & - & - & - & - & - & 2.9 & 3.2 & - & 14 & - & 5.9 & 2.9 & - & 3.0 & - & - & - & - & 1.2 & - \\
\hline AA1 & MUAA1-G-22944 & $5 / 13 / 08$ & 2.0 & - & - & - & - & - & - & - & - & - & - & - & 1.3 & - & 2.9 & - & - & - & - & 1.3 & - \\
\hline AA1 & MUAA1-G-22948 & $5 / 14 / 08$ & 2.3 & - & - & - & - & - & 3.2 & - & - & 15 & - & - & 1.3 & - & 2.9 & - & - & - & - & 1.2 & - \\
\hline AA1 & MUAA1-G-229 & $5 / 15 / 0$ & 4.3 & - & - & - & - & - & -0 & - & $\overline{2}$ & - & $\bar{z}_{0}$ & 3.1 & 1.1 & - & 3.0 & -2 & $\overline{7}$ & 1.2 & -7 & 1.4 & - \\
\hline AA1 & MUAA1-G-19230 & $7 / 22 / \mathrm{C}$ & - & - & - & 2.0 & - & 4.1 & 3.8 & - & 2.0 & 26 & 7.3 & - & 1.2 & 2.3 & - & 3.2 & 7.0 & 24 & 2.7 & 2.2 & 13 \\
\hline AA1 & MUAA1-G-19231 & 7/23/08 & 2.3 & - & - & - & - & - & - & - & - & - & 0.7 & - & 1.2 & - & - & - & - & 2.0 & - & 1.2 & - \\
\hline AA1 & MUAA1-G-19232 & $7 / 24 / 08$ & 2.5 & - & - & - & - & - & 1.6 & - & - & - & - & - & 1.2 & - & - & - & - & 1.0 & - & - & - \\
\hline \multicolumn{24}{|c|}{ Location AA2 } \\
\hline AA2 & MUAA2-G-15791 & $5 / 17 / 06$ & - & - & - & - & - & - & - & - & - & - & - & - & 1.8 & - & 3.6 & - & - & 2.5 & - & 1.7 & - \\
\hline AA2 & MUAA2-G-157 & 18/06 & 1.4 & - & - & - & - & - & - & - & - & - & - & - & 1.6 & - & 3.6 & - & - & & - & & - \\
\hline AA2 & MUAA2-G-157 & $5 / 19$ & - & - & - & - & - & - & - & - & - & - & - & - & 1.8 & - & 3.5 & - & - & 1.8 & - & 1.7 & - \\
\hline AA2 & MUAA2-G-18 & & - & - & - & 1.7 & - & 1.1 & - & - & 1.4 & 22 & 1.2 & - & 1.6 & - & & 1.7 & - & 5.7 & - & 6 & 6.9 \\
\hline AA2 & MUAA2-G-18 & & - & - & - & - & - & - & - & - & - & - & - & - & 1.6 & - & 3.7 & - & - & - & - & 6 & - \\
\hline AA2 & MUAA2-G-181 & & - & - & - & - & - & - & 2.3 & - & - & 19 & - & - & $1 . \varepsilon$ & - & 3.7 & - & - & 0.9 & - & 7 & - \\
\hline AA2 & MUAA2-G-181 & & - & - & - & - & - & - & 4.1 & - & - & 38 & - & - & 1. & - & 3.9 & - & - & 0.79 & - & & - \\
\hline AA2 & MUAA2-G-182 & & - & - & - & - & - & - & 2.3 & - & - & 20 & - & - & 1. & - & 2.6 & - & - & - & - & 2 & - \\
\hline AA2 & MUAA2-G-182 & & - & - & - & - & - & - & - & - & - & - & - & - & 1.3 & - & - & - & - & - & - & & - \\
\hline AA2 & MUAA2-G-182 & $4 / 1$ & - & - & - & - & - & - & - & - & - & - & - & - & 1.1 & - & - & - & - & 1.1 & - & 2 & - \\
\hline AA2 & MUAA2-G-222 & $7 / 2$ & - & - & - & - & - & - & 2.1 & - & - & 13 & - & - & 1. & - & - & - & - & - & - & 1 & - \\
\hline AA2 & MUAA2-G-2223 & $7 / 24 / 0$ & - & - & - & - & - & - & - & - & - & - & - & - & 1.1 & - & - & - & - & - & - & 1 & - \\
\hline AA2 & MUAA2-G-2223 & $7 / 25 / 0$ & - & - & - & - & - & - & 1.6 & - & - & - & - & - & 1.4 & - & 3.0 & - & - & - & - & 2 & - \\
\hline AA2 & MUAA2-G-229 & $5 / 13 / 08$ & - & - & - & - & - & - & - & - & - & - & - & - & 1.2 & - & 2.8 & - & - & - & - & 2 & - \\
\hline AA2 & MUAA2-G-22949 & $5 / 14 / 08$ & - & - & - & - & - & - & - & - & - & - & - & - & 1.3 & - & 2.9 & - & - & 0.75 & - & 2 & - \\
\hline AA2 & MUAA2-G-22953 & $5 / 15 / 08$ & 1.4 & - & - & 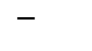 & - & - & - & - & - & - & - & 8.1 & 1.5 & - & 2.8 & - & - & 1.5 & - & 1.2 & - \\
\hline $\begin{array}{l}A A 2 \\
A A 2\end{array}$ & $\begin{array}{l}\text { MUAA2-G-19233 } \\
\text { MUAA2-G-19234 }\end{array}$ & $\begin{array}{l}7 / 22 / 08 \\
7 / 23 / 8\end{array}$ & - & - & - & - & - & - & - & - & - & $\begin{array}{ll}- \\
-\end{array}$ & - & - & $\begin{array}{l}1.2 \\
1.2\end{array}$ & - & 2.5 & - & - & $\begin{array}{l}1.4 \\
0.83\end{array}$ & - & $\begin{array}{l}1.2 \\
1.1\end{array}$ & - \\
\hline $\begin{array}{l}\text { AA2 } \\
\text { AA2 }\end{array}$ & MUAA2-G-19235 & $7 / 24 / 08$ & - & - & - & & & & 4.7 & & & 20 & & - & 1.1 & & - & & & 29 & & $\begin{array}{l}.1 \\
2.3\end{array}$ & $2 \overline{0}$ \\
\hline
\end{tabular}


Concentration $\left(\mu \mathrm{g} / \mathrm{m}^{3}\right)$

\begin{tabular}{|c|c|c|c|c|c|c|c|c|c|c|c|c|c|c|c|c|c|c|c|c|c|c|c|}
\hline 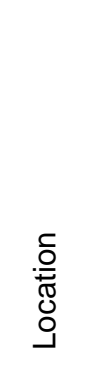 & 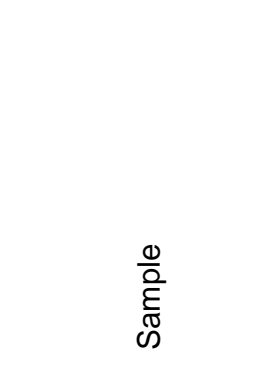 & 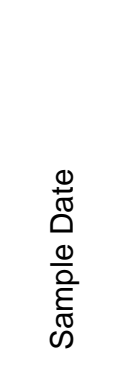 & 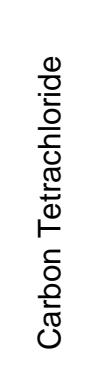 & $\begin{array}{l}\xi \\
\frac{\varepsilon}{\bar{D}} \\
\frac{\mathrm{D}}{\mathrm{o}} \\
\frac{\mathrm{c}}{\mathrm{J}}\end{array}$ & 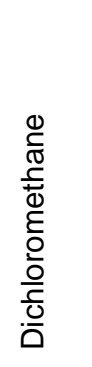 & 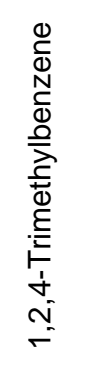 & 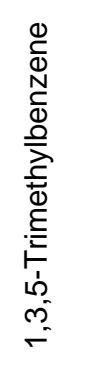 & 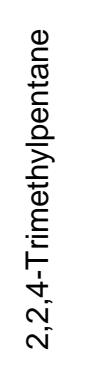 & 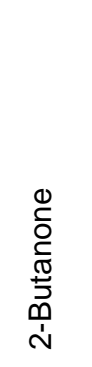 & 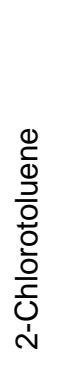 & 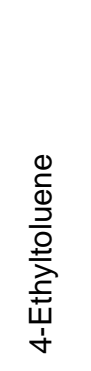 & 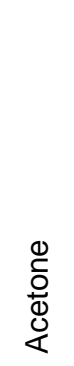 & 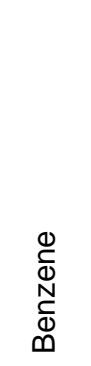 & 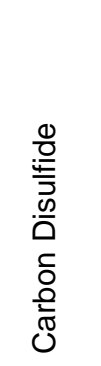 & 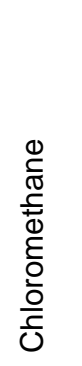 & 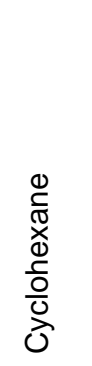 & 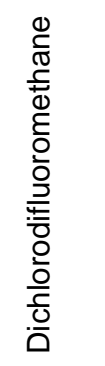 & 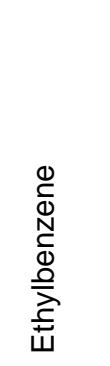 & 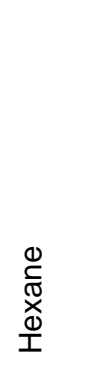 & $\begin{array}{l}\frac{\mathscr{\Phi}}{\mathrm{\Phi}} \\
\stackrel{\overline{0}}{\circ}\end{array}$ & 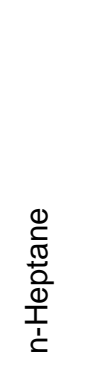 & 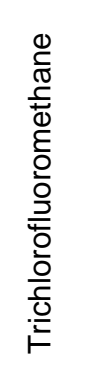 & 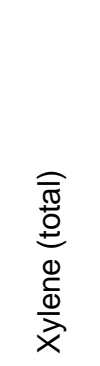 \\
\hline \multicolumn{2}{|c|}{ Detection Limit } & & 1.3 & 0.98 & 1.7 & 0.98 & 0.98 & 0.93 & 1.5 & 1.0 & 0.98 & 12 & 0.64 & 1.6 & 1.0 & 0.69 & 2.5 & 0.87 & 1.8 & 0.75 & 0.82 & 1.1 & 0.87 \\
\hline \multicolumn{24}{|c|}{ Location AA3 } \\
\hline AA3 & MUAA3-G-15788 & $5 / 17 / 06$ & - & - & - & - & - & - & - & - & - & - & - & - & 1.7 & - & 3.3 & - & - & 1.2 & - & 1.6 & - \\
\hline AA3 & MUAA3-G-15789 & $5 / 18 / 06$ & - & - & - & - & - & - & - & - & - & - & - & - & 1.4 & - & 3.2 & - & - & 0.87 & - & 1.5 & - \\
\hline AA3 & MUAA3-G-15790 & $5 / 19 / 06$ & - & - & - & - & - & - & 1.5 & - & - & - & 0.77 & - & 2.0 & - & 3.9 & - & - & 3.4 & - & 2 & - \\
\hline AA3 & MUAA3-G-18141 & $8 / 14 / 06$ & - & - & - & - & - & - & - & - & - & - & - & - & 1.6 & - & 3.5 & - & - & 1.3 & - & 1.6 & - \\
\hline AA3 & MUAA3-G-18142 & $8 / 15 / 06$ & - & - & - & - & - & - & - & - & - & - & - & - & 1.7 & - & 3.7 & - & - & 0.9 & - & 1.8 & - \\
\hline AA3 & MUAA3-G-18143 & $8 / 16 / 06$ & $2.5 \mathrm{U}^{\mathrm{a}}$ & $2 U$ & $3.5 \mathrm{U}$ & $2 U$ & - & $1.9 \mathrm{U}$ & 27 & - & $2 U$ & 170 & $1.3 \mathrm{U}$ & $3.1 \mathrm{U}$ & 4.3 & - & $4.9 \mathrm{U}$ & $1.7 \mathrm{U}$ & - & $1.5 \mathrm{U}$ & 2.5 & $2.2 \mathrm{U}$ & $1.7 \mathrm{U}$ \\
\hline AA3 & MUAA3-G-18244 & $4 / 17 / 07$ & - & - & - & - & - & - & 1.9 & - & - & 13 & 0.73 & - & 1.2 & - & 2.9 & - & - & 1.9 & - & 1.3 & - \\
\hline $\mathrm{AA3}$ & MUAA3-G-18245 & $4 / 18 / 07$ & - & - & - & - & - & - & - & - & - & 15 & - & - & 1.3 & - & 2.7 & - & - & - & - & 1.2 & - \\
\hline AA3 & MUAA3-G-18246 & $4 / 19 / 07$ & - & - & - & - & - & - & 6.2 & - & - & 33 & - & & 1.1 & - & - & - & - & - & - & 1.1 & - \\
\hline AA3 & MUAA3-G-22232 & 7/23/07 & - & - & - & - & - & - & - & - & - & - & - & - & - & - & - & - & - & - & - & 1.2 & - \\
\hline AA3 & MUAA3-G-22233 & $7 / 24 / 07$ & - & - & - & - & - & - & 1.5 & - & - & 17 & - & - & 1.1 & - & - & - & - & - & - & 1.1 & - \\
\hline AA3 & MUAA3-G-2223 & 7/25/07 & - & - & - & - & - & - & 1.9 & - & - & 13 & - & - & 1.3 & - & 3.0 & - & - & - & - & 1.1 & - \\
\hline AA3 & MUAA3-G-2294 & $5 / 13 / 08$ & - & - & - & - & - & - & - & - & - & - & - & - & 1.2 & - & 2.8 & - & - & - & - & 1.2 & - \\
\hline AA3 & MUAA3-G-22950 & $4 / 08$ & 1.5 & - & - & - & - & - & - & - & - & - & - & - & 1.2 & - & 3.1 & - & - & - & - & 1.3 & - \\
\hline AA3 & MUAA3-G-2295 & $5 / 15 / 08$ & - & - & - & - & - & - & - & - & - & - & - & 34 & 1.2 & - & 2.8 & - & - & 5.7 & - & 1.2 & - \\
\hline AA3 & MUAA3-G-19236 & 7/22/08 & - & - & - & - & - & - & - & - & - & - & - & - & 1.2 & - & 2.5 & - & - & 2.0 & - & 1.2 & - \\
\hline AA3 & MUAA3-G-19237 & 7/23/08 & - & - & - & - & - & 1.4 & 2.0 & - & - & - & 2.5 & - & 1.1 & - & - & 1.1 & 2.1 & 7.2 & - & 1.4 & 4.8 \\
\hline AA3 & MUAA3-G-19238 & 7/24/08 & - & - & - & 0.98 & - & 1.5 & 2.1 & - & 0.98 & - & 2.5 & - & 1.1 & - & - & 1.4 & 2.3 & 7.9 & 0.82 & 1.4 & 6.1 \\
\hline
\end{tabular}

\section{Location BA1}

BA1 MUBA1-G-17203

$\begin{array}{ll}\text { BA1 } & \text { MUBA1-G-17204 } \\ \text { BA1 } & \text { MUBA1-G-17205 }\end{array}$

BA1 MUBA1-G-18135

BA1 MUBA1-G-18137

BA1 MUBA1-G-18238

BA1 MUBA1-G-18239

BA1 MUBA1-G-2222

BA1 MUBA1-G-22228

BA1 MUBA1-G-22943

BA1 MUBA1-G-22947

BA1 MUBA1-G-19227

BA1 MUBA1-G-19228

$5 / 17 / 06$
$5 / 18 / 06$
$5 / 19 / 06$
$8 / 4 / 106$
$8 / 15 / 06$
$8 / 16 / 06$
$4 / 17 / 07$
$4 / 18 / 07$
$4 / 19 / 07$
$7 / 23 / 07$
$7 / 24 / 07$
$7 / 25 / 07$
$5 / 1308$
$5 / 14 / 08$
$5 / 15 / 108$
$7 / 22 / 108$
$7 / 23 / 08$
$7 / 24 / 08$

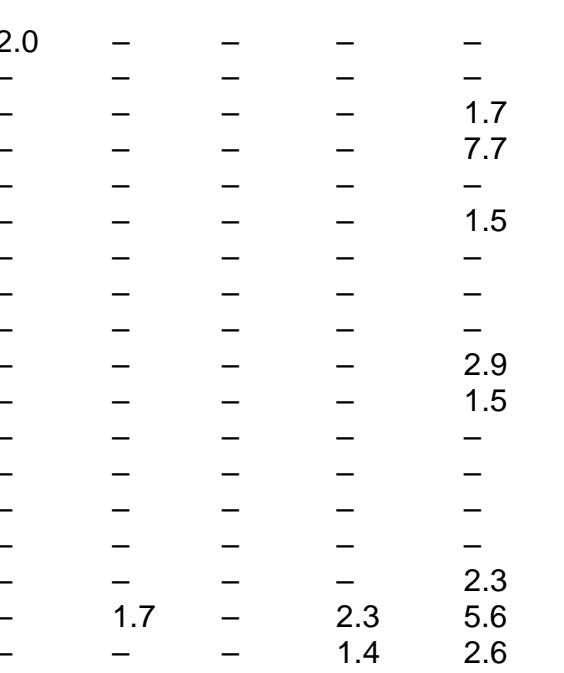

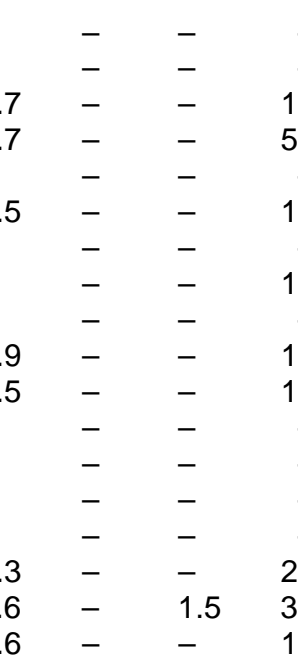
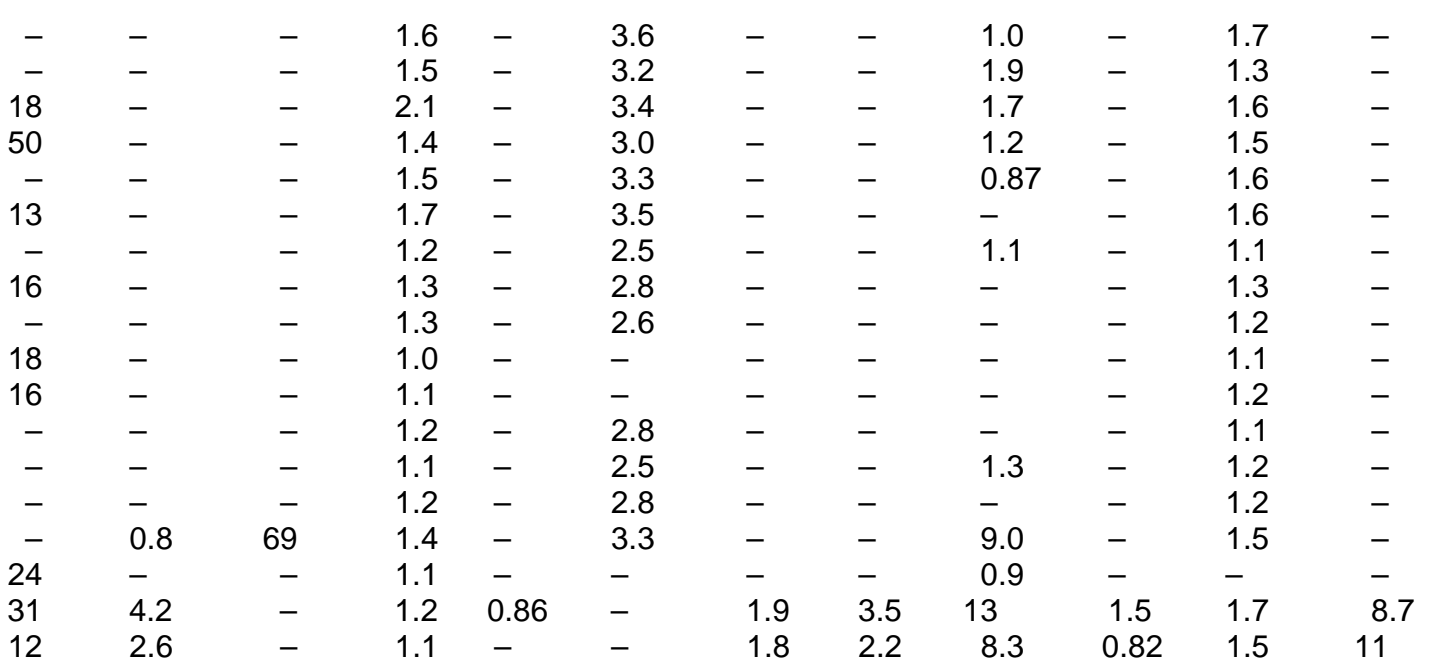

$\mathrm{U}$, not detected at indicated reporting limit. Apparent elevation in concentration is due to dilution of the sample to quantify other constituents. 

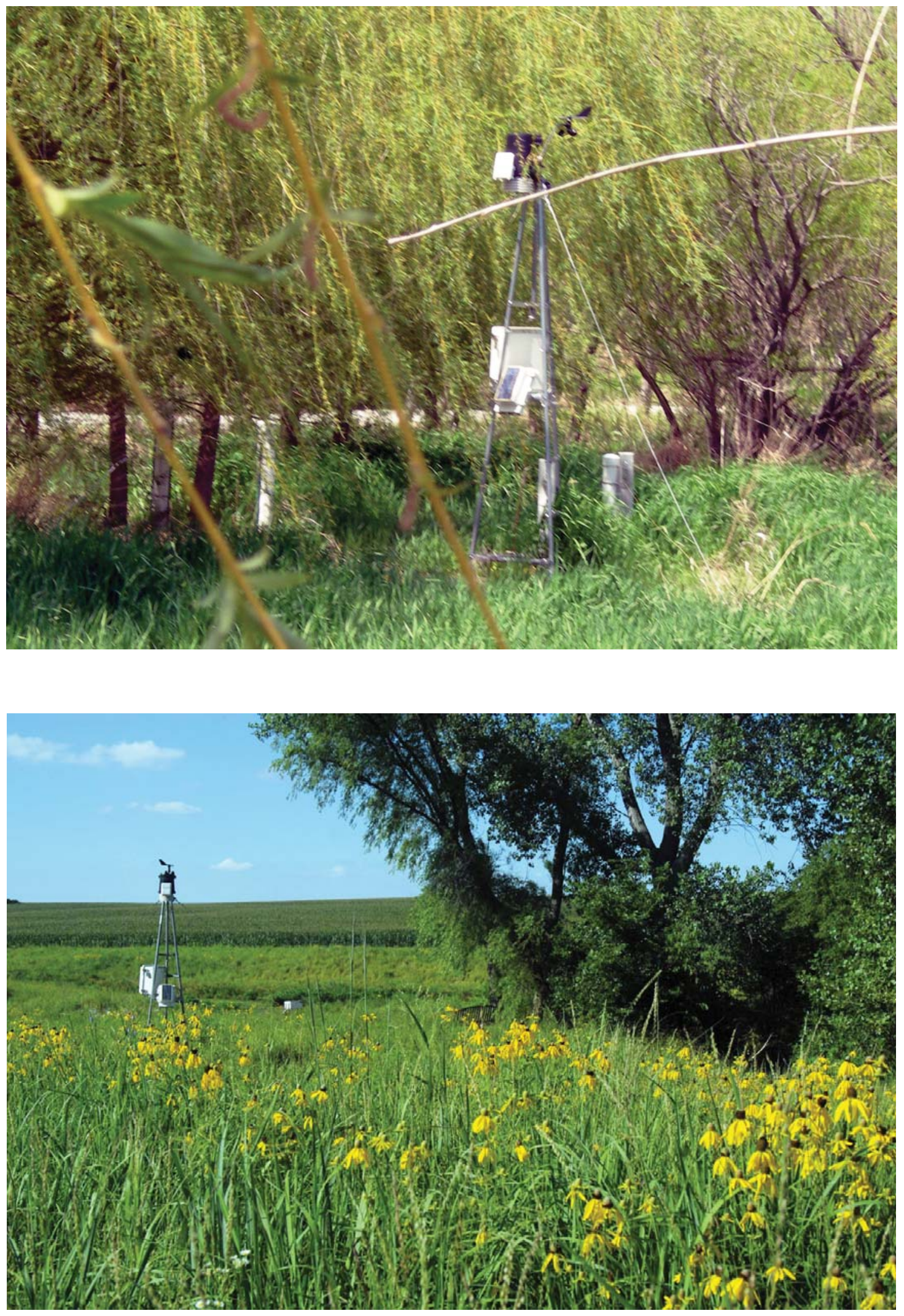

FIGURE 4.1 Weather station in old location (May 2008; top) and new location (July 2008; bottom). 
Rainfall Comparison for the Lincoln-Murdock Area

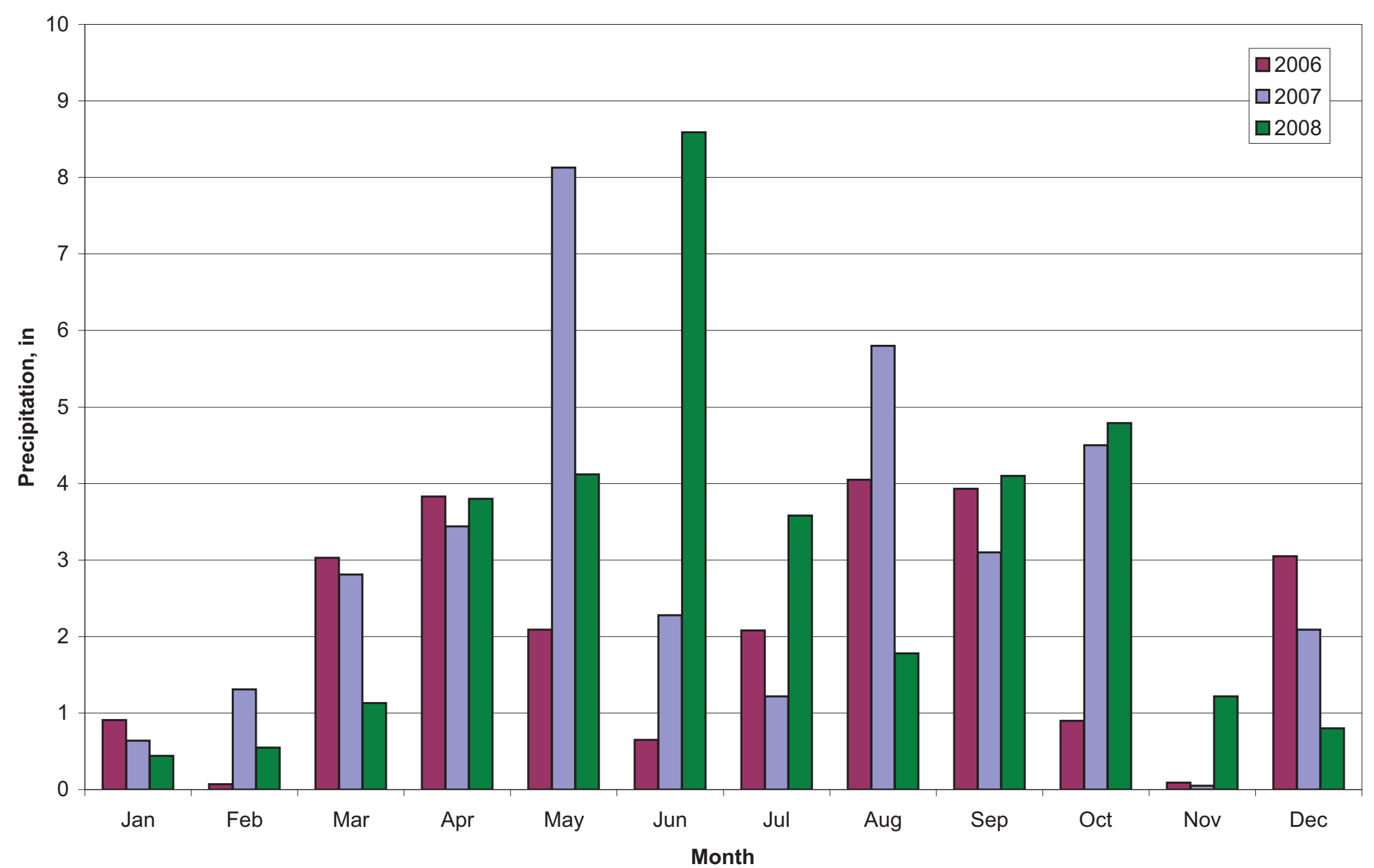

FIGURE 4.2 Monthly rainfall for the Lincoln-Murdock area, 2006-2008. 
Hydrographs for Upgradient Town Wells

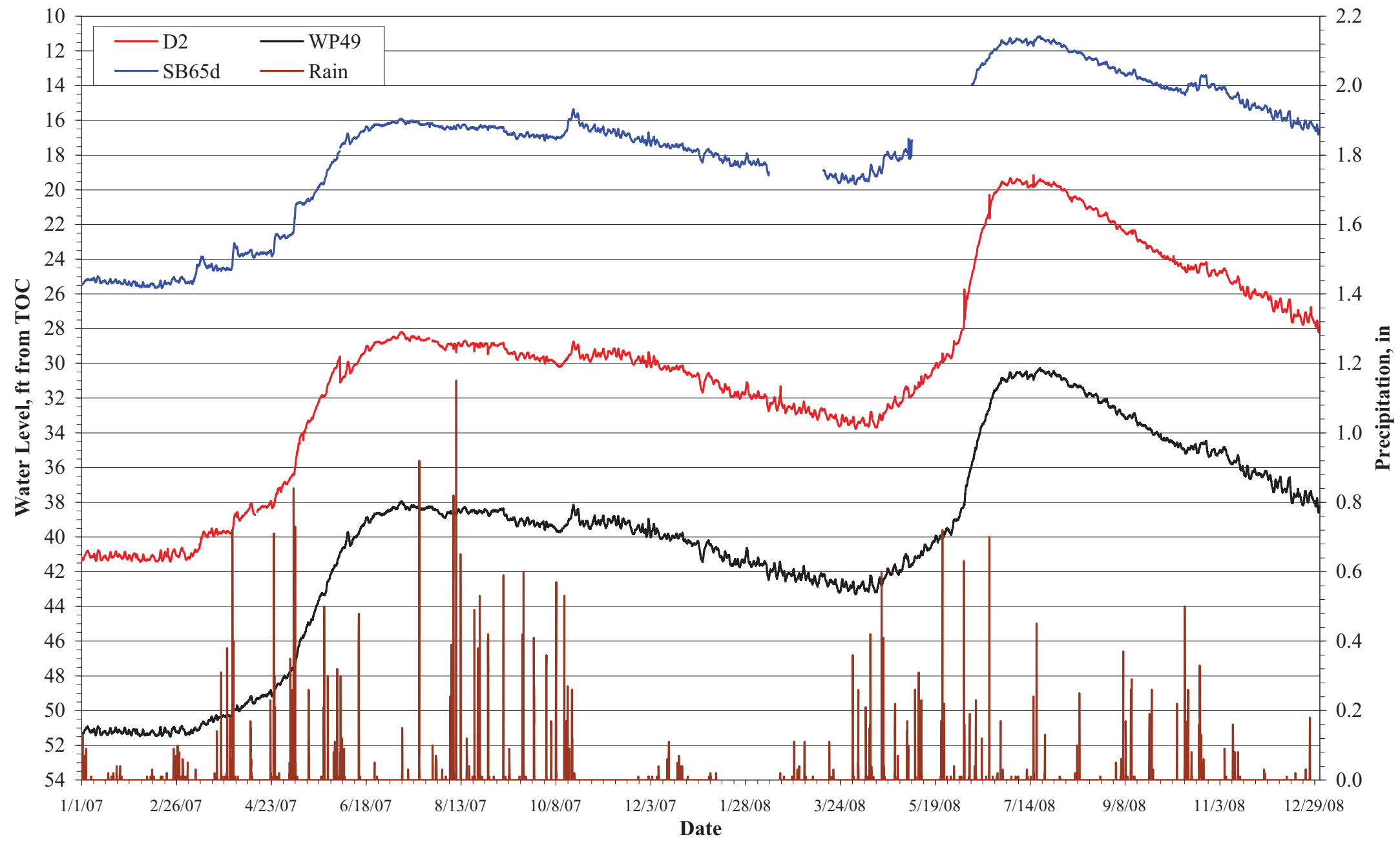

FIGURE 4.3 Hydrographs for upgradient wells, January 2007 through December 2008. 


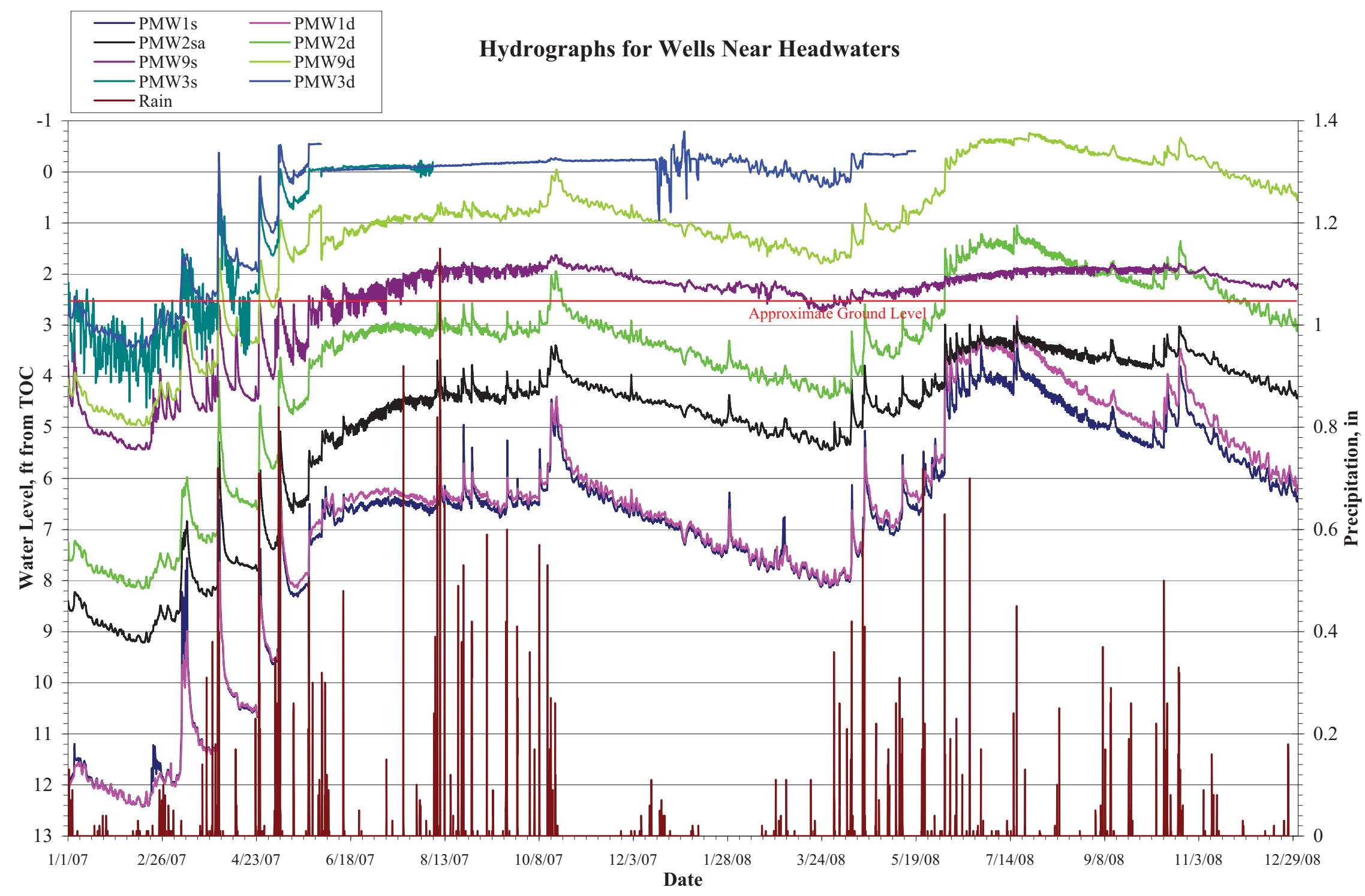

FIGURE 4.4 Hydrographs for phytoremediation area wells near the headwaters of the tributary creek, January 2007 through December 2008. 


\section{Hydrographs for Wells Along E-W Transect}

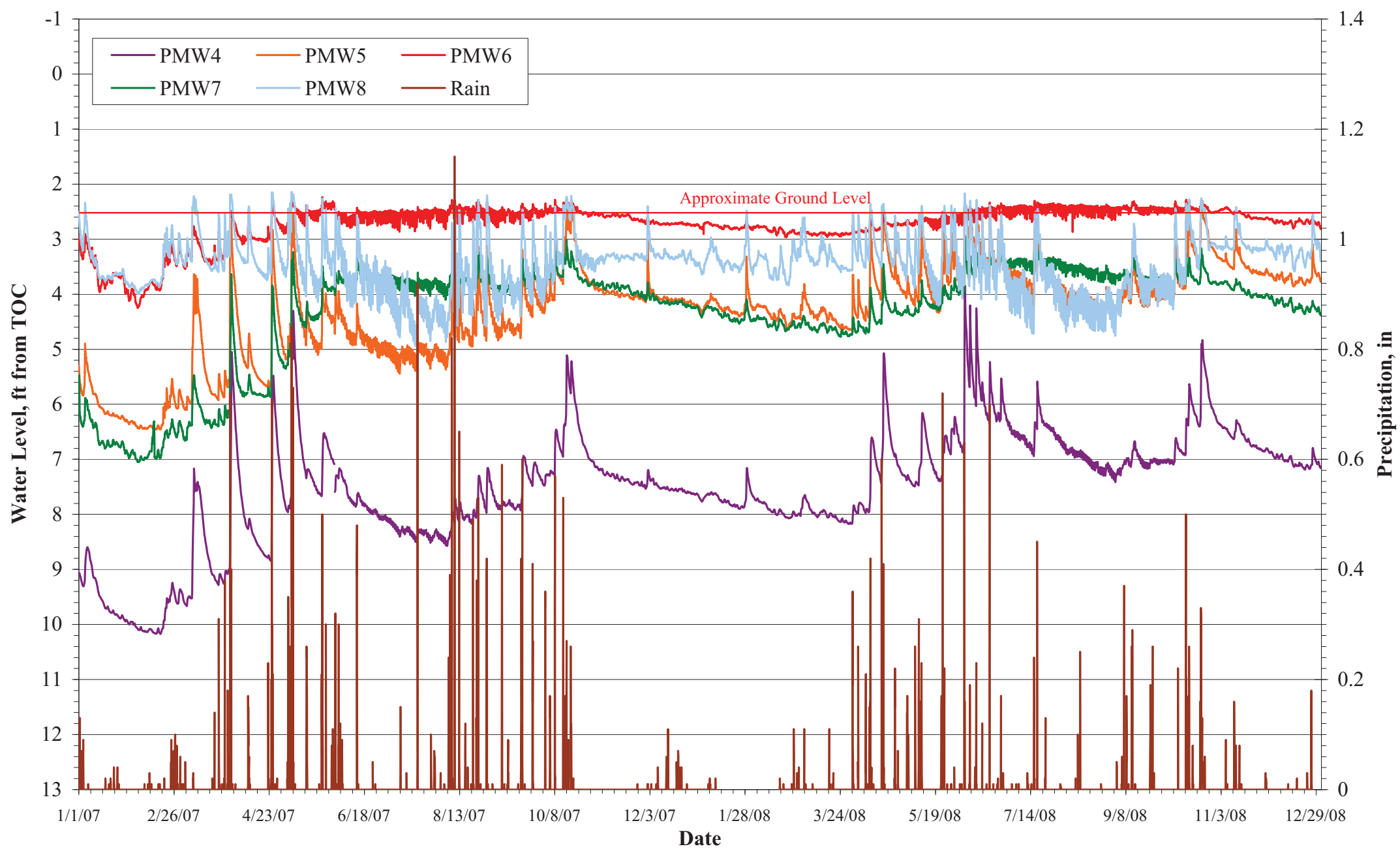

FIGURE 4.5 Hydrographs for wells in the downstream portion of the phytoremediation area, January 2007 through December 2008. 


\section{Hydrographs for Wells Near Headwaters}

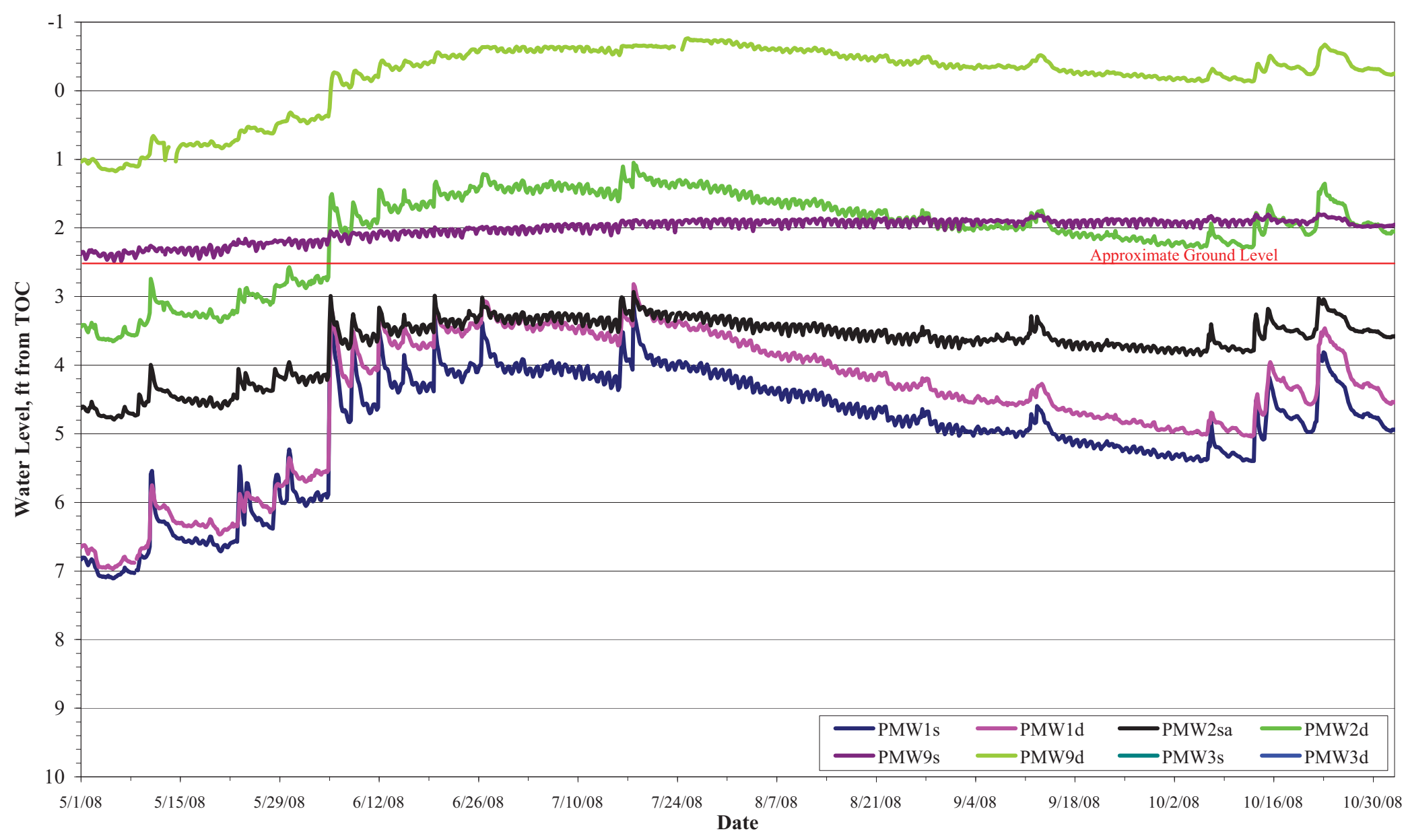

FIGURE 4.6 Hydrographs for phytoremediation area wells near the headwaters of the tributary creek, May to October 2008. 


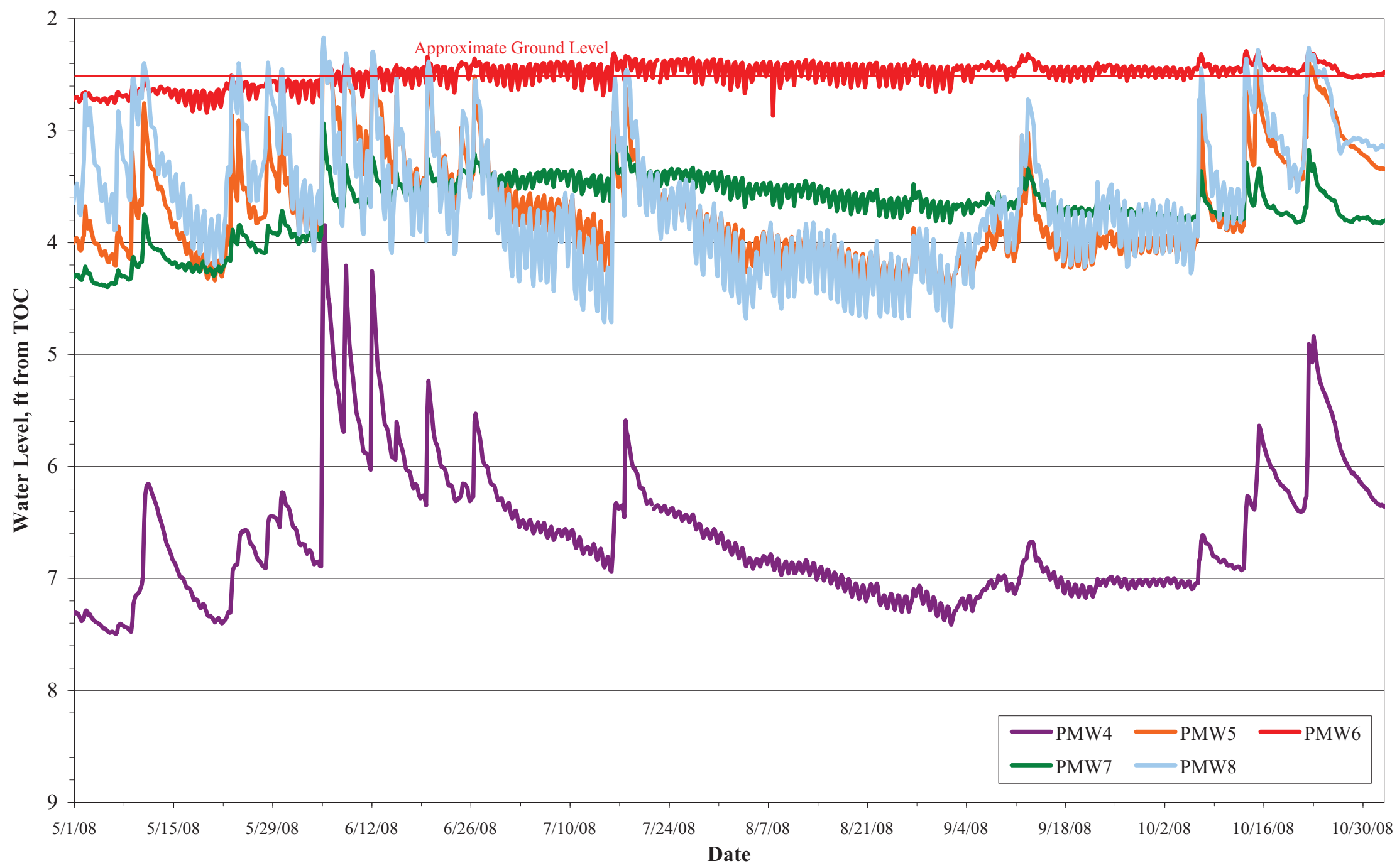

FIGURE 4.7 Hydrographs for wells in the downstream portion of the phytoremediation area, May to October 2008. 
Calculated Discharge Rates from theWetlands

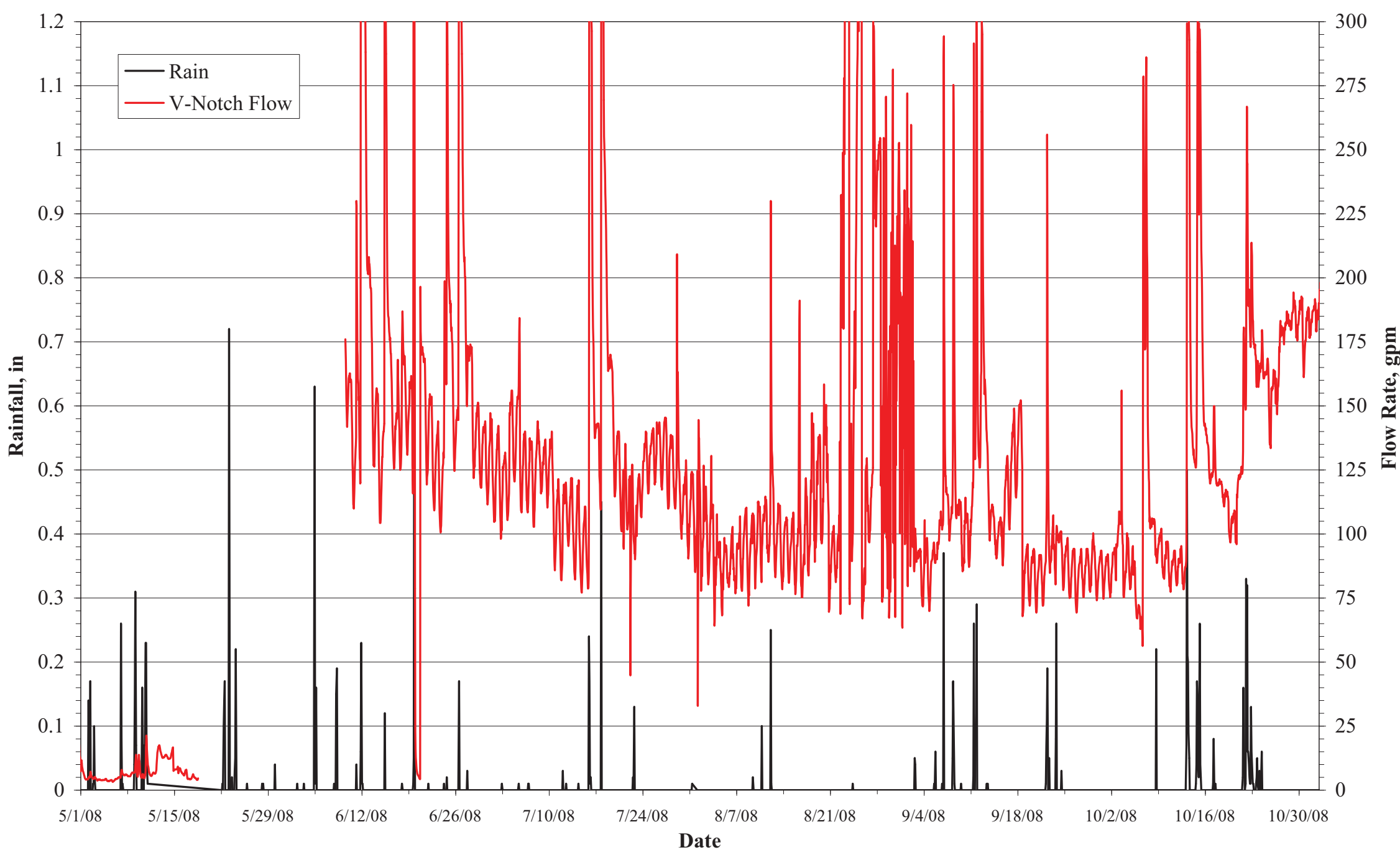

FIGURE 4.8 Calculated surface water discharge rates determined from the V-notch weir at the wetlands outfall, May to October 2008. 


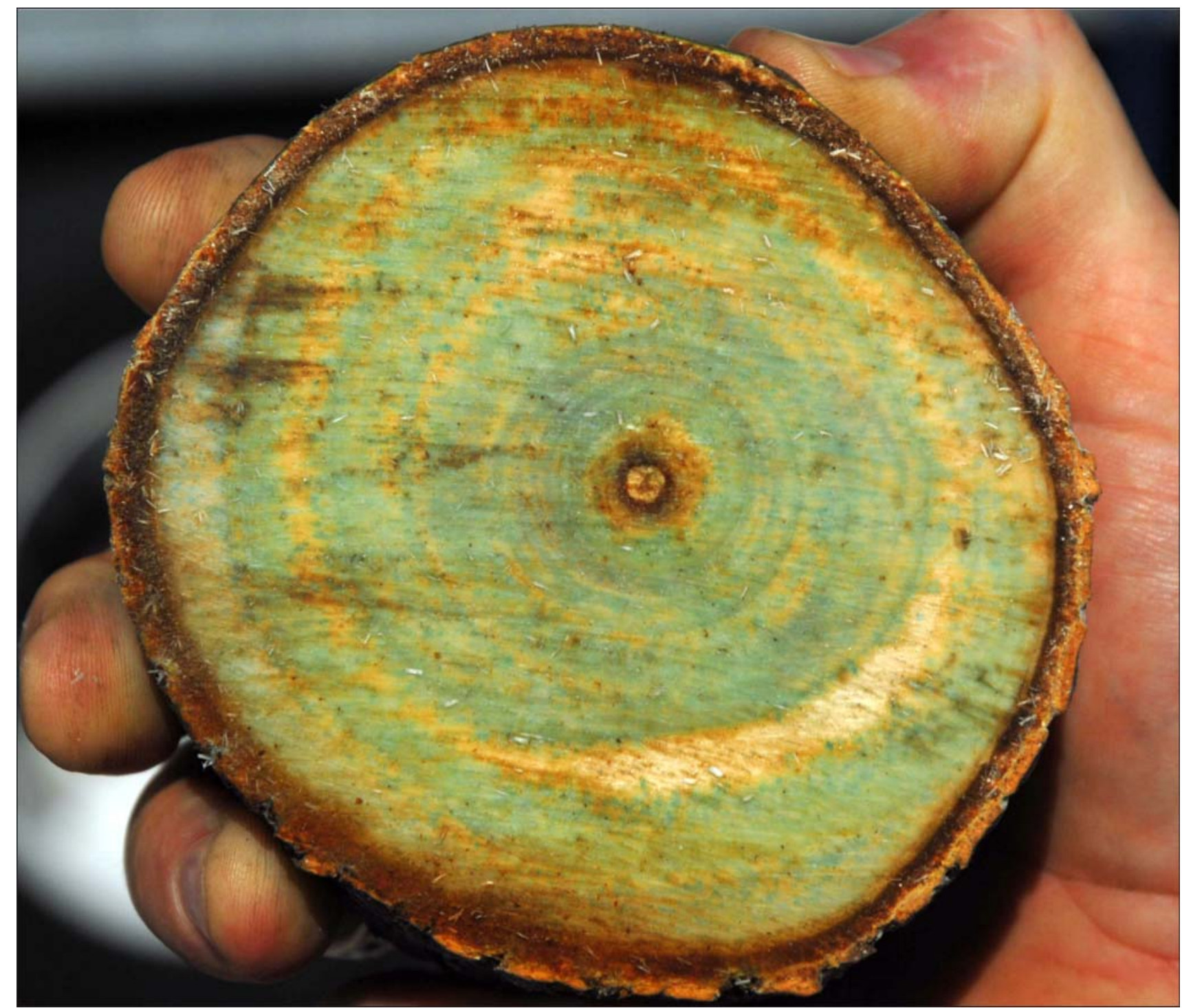

FIGURE 4.9 Distribution of blue dye in a freshly cut log from a harvested cottonwood tree, confirming that the entire trunk inside the bark actively took up water. 


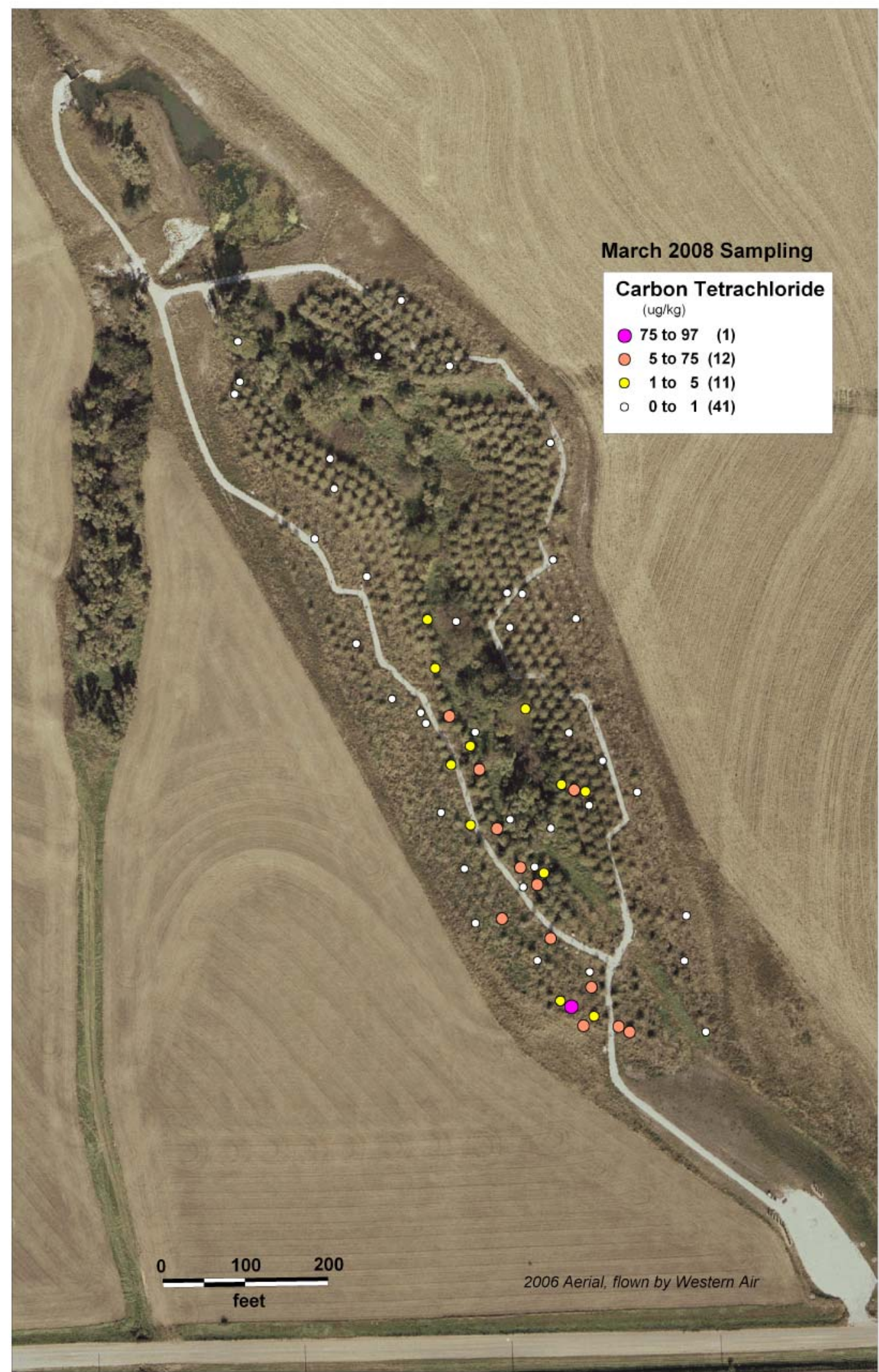

FIGURE 4.10 Carbon tetrachloride concentrations in branch samples, March 2008. 


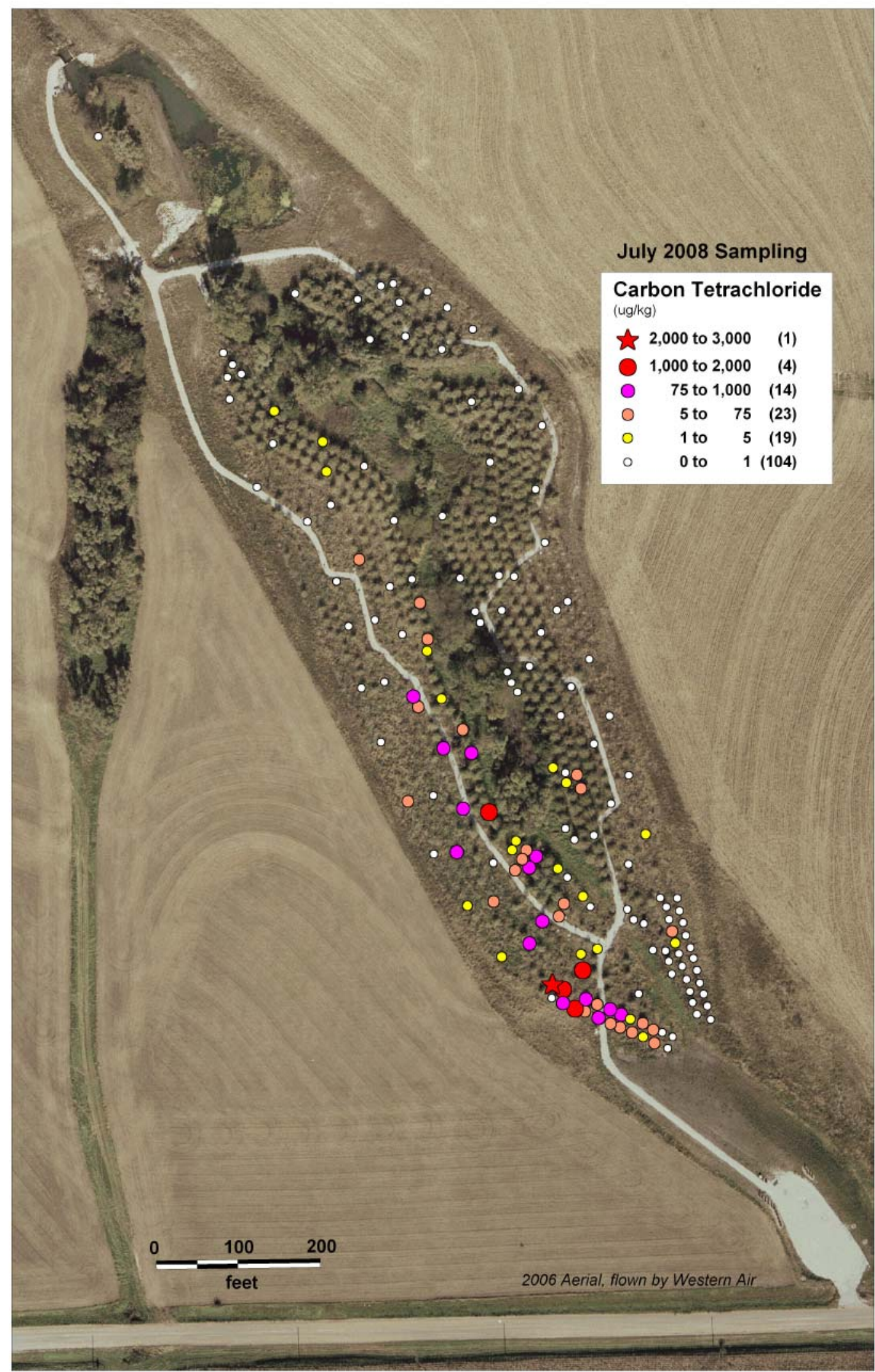

FIGURE 4.11 Carbon tetrachloride concentrations in branch samples, July 2008. 


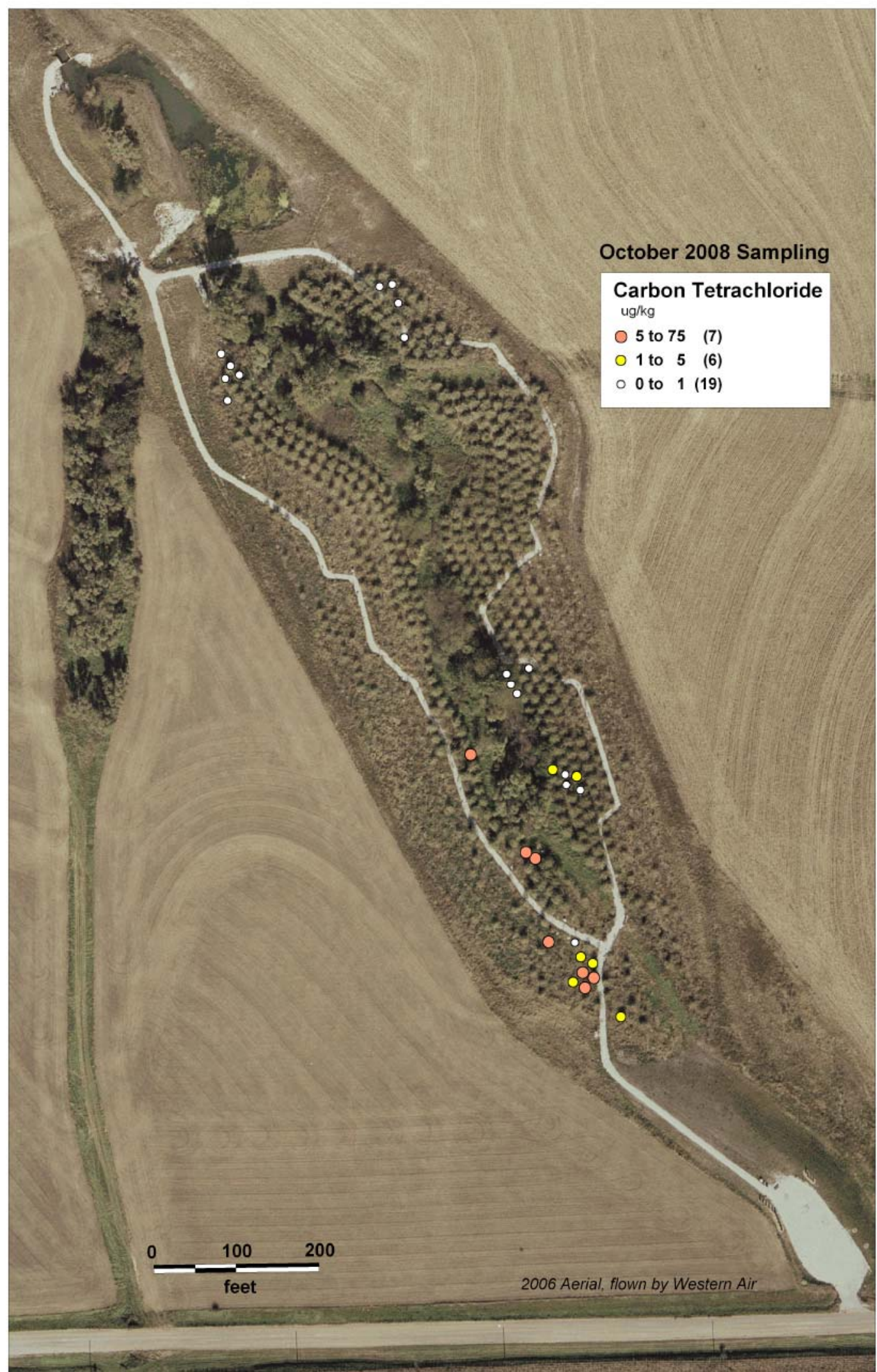

FIGURE 4.12 Carbon tetrachloride concentrations in branch samples, October 2008. 


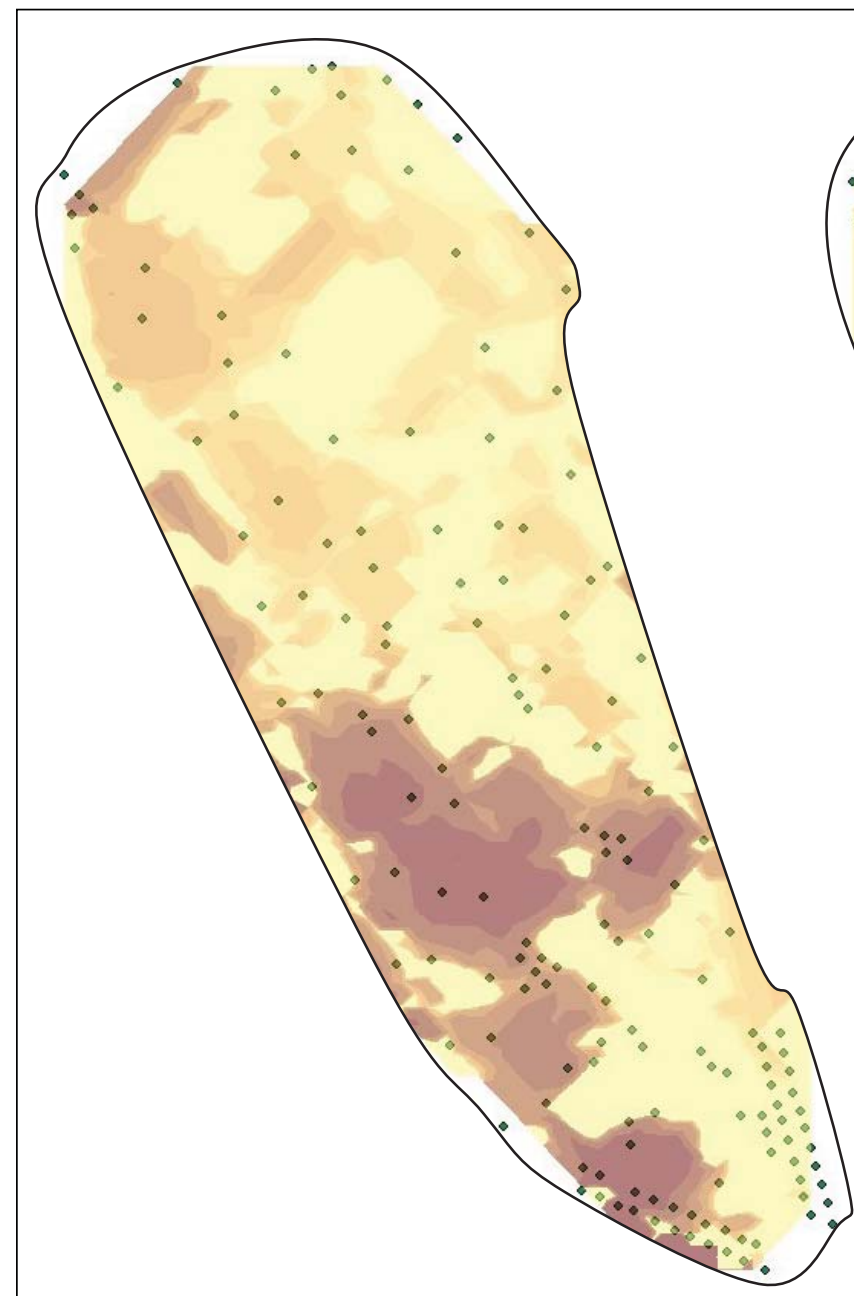

2007 Data

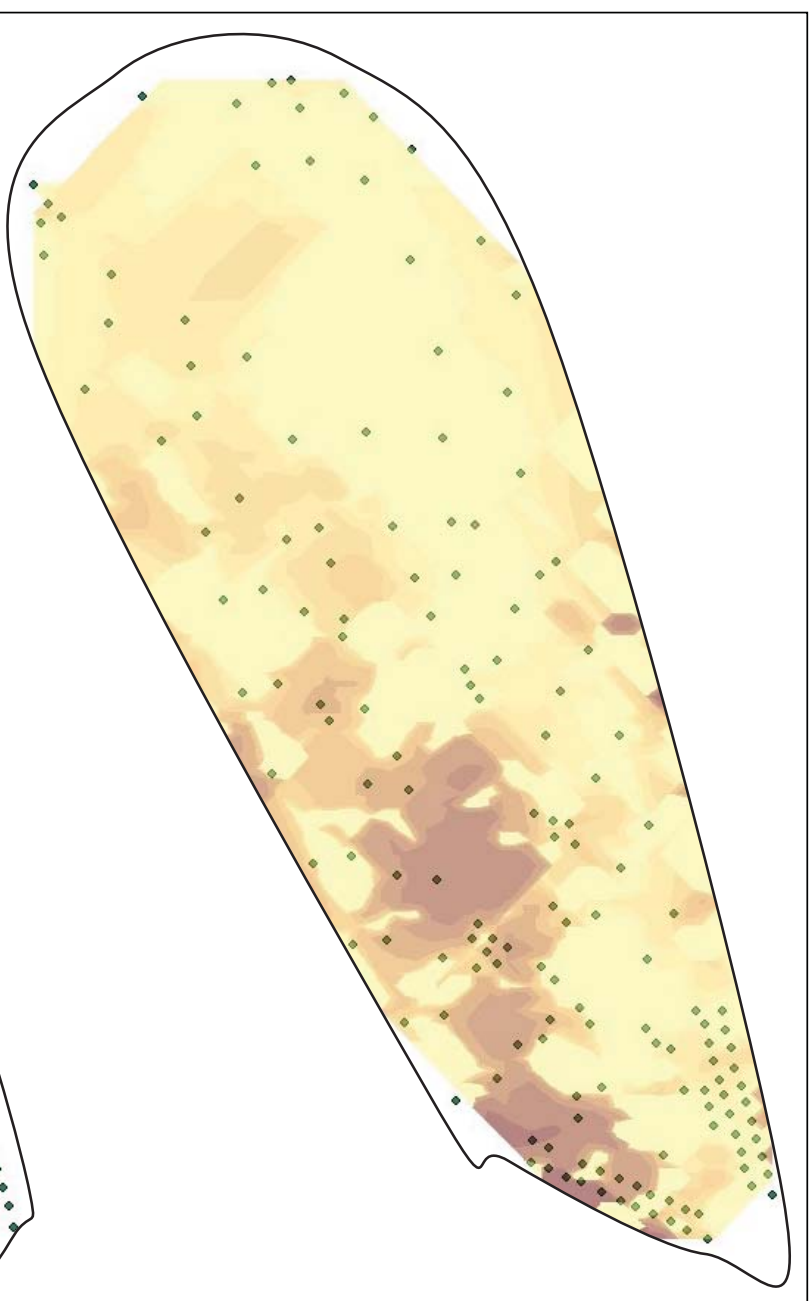

2008 Data

Carbon tetrachloride concentrations $(\mu \mathrm{g} / \mathrm{kg})$

\begin{tabular}{|l|l|}
\hline & $0.000000-0.266279$ \\
$0.266279-1.034359$ \\
$1.034359-3.249879$ \\
$3.249879-9.640533$ \\
$9.640533-28.074326$ \\
$28.074326-81.246468$ \\
$81.246468-234.621124$ \\
$234.621124-677.029175$ \\
\hline $677.029175-1953.151978$ \\
\hline $1953.151978-2395.560059$ \\
\hline
\end{tabular}

FIGURE 4.13 Geostatistical comparison of carbon tetrachloride concentrations in branch samples, July 2007 and July 2008. 


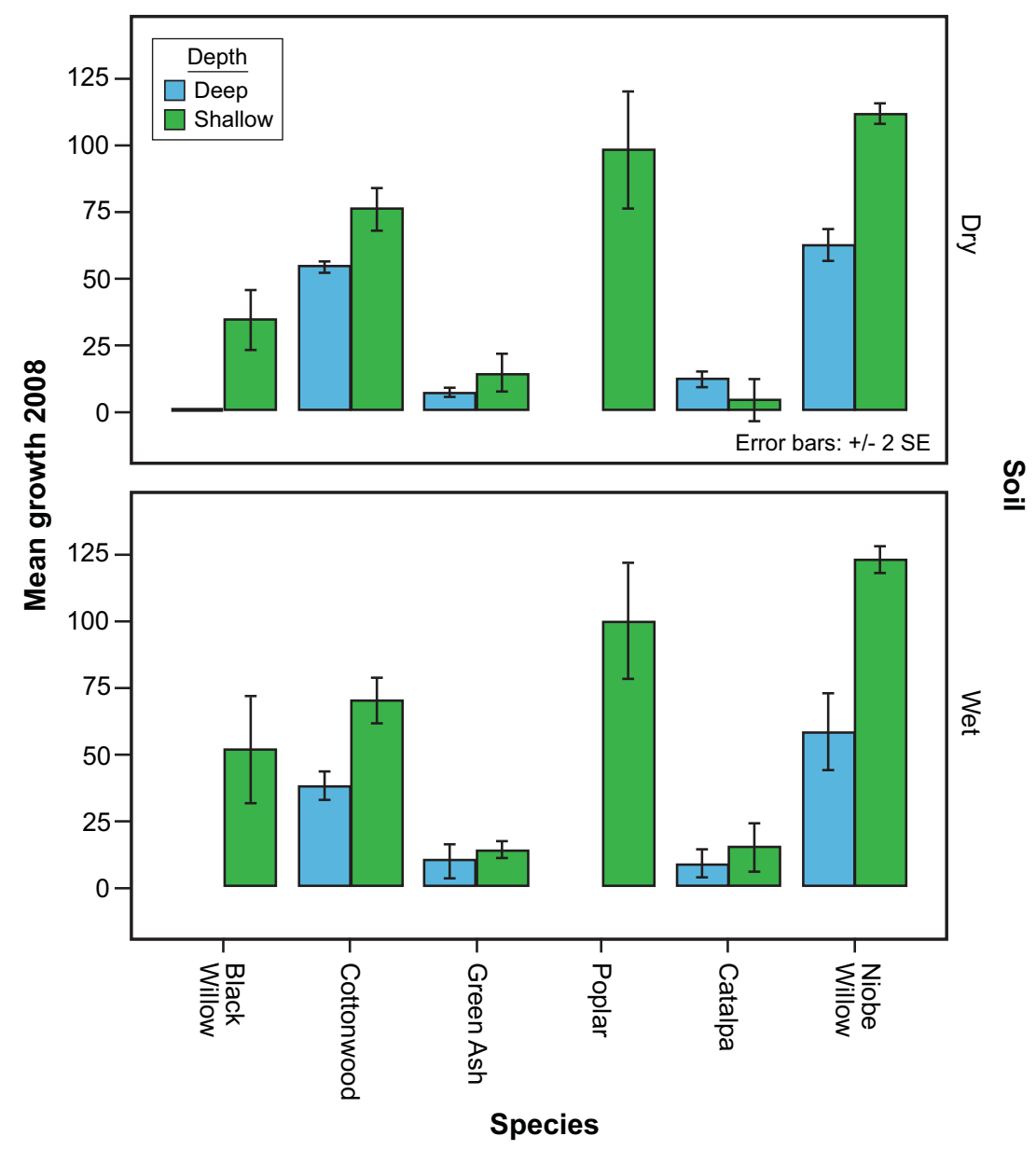

FIGURE 4.14 Tree diameter measurements by species as a function of planting depth and surface soil moisture in 2008. 


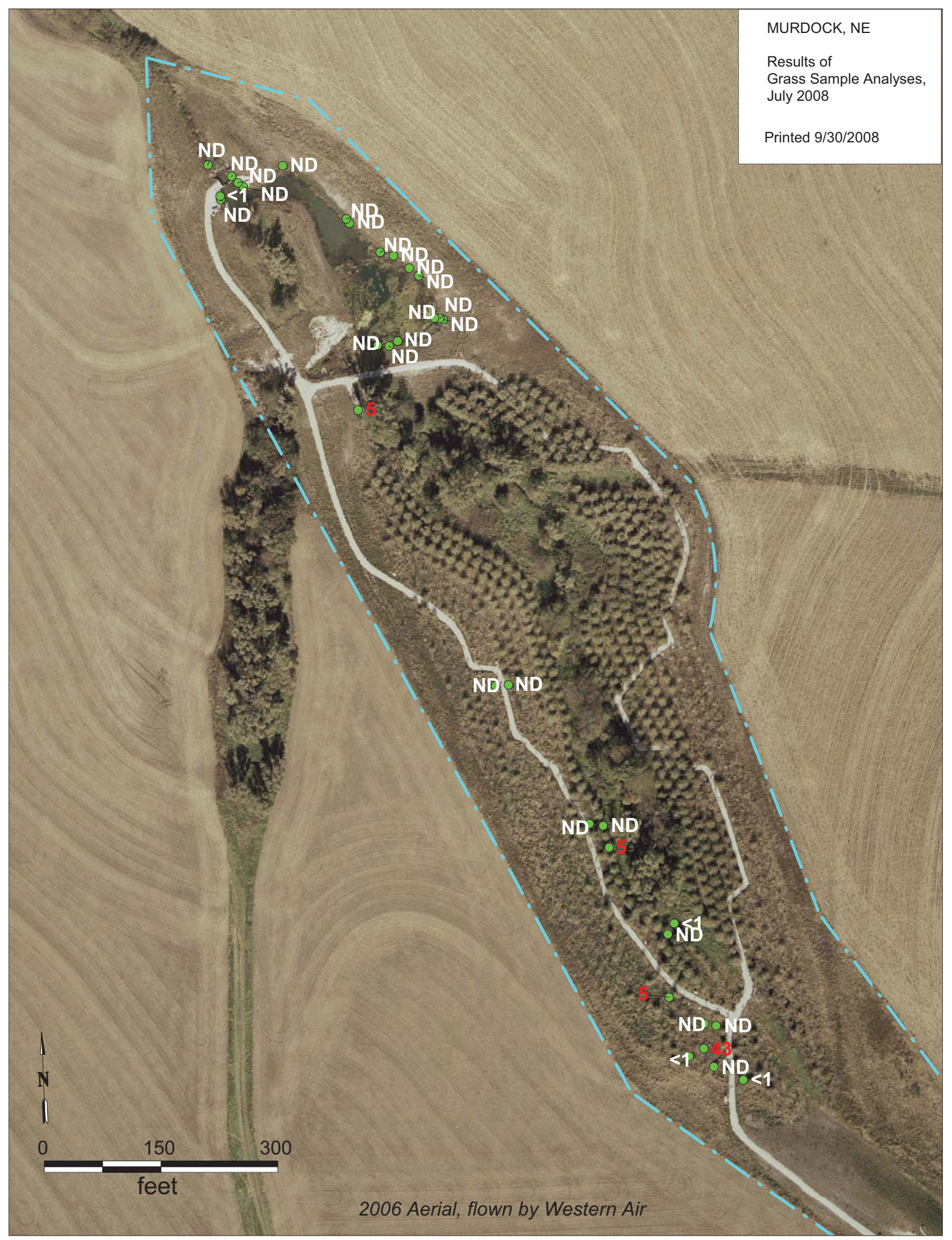

FIGURE 4.15 Carbon tetrachloride concentrations in grasses and other vegetation in July 2008. 


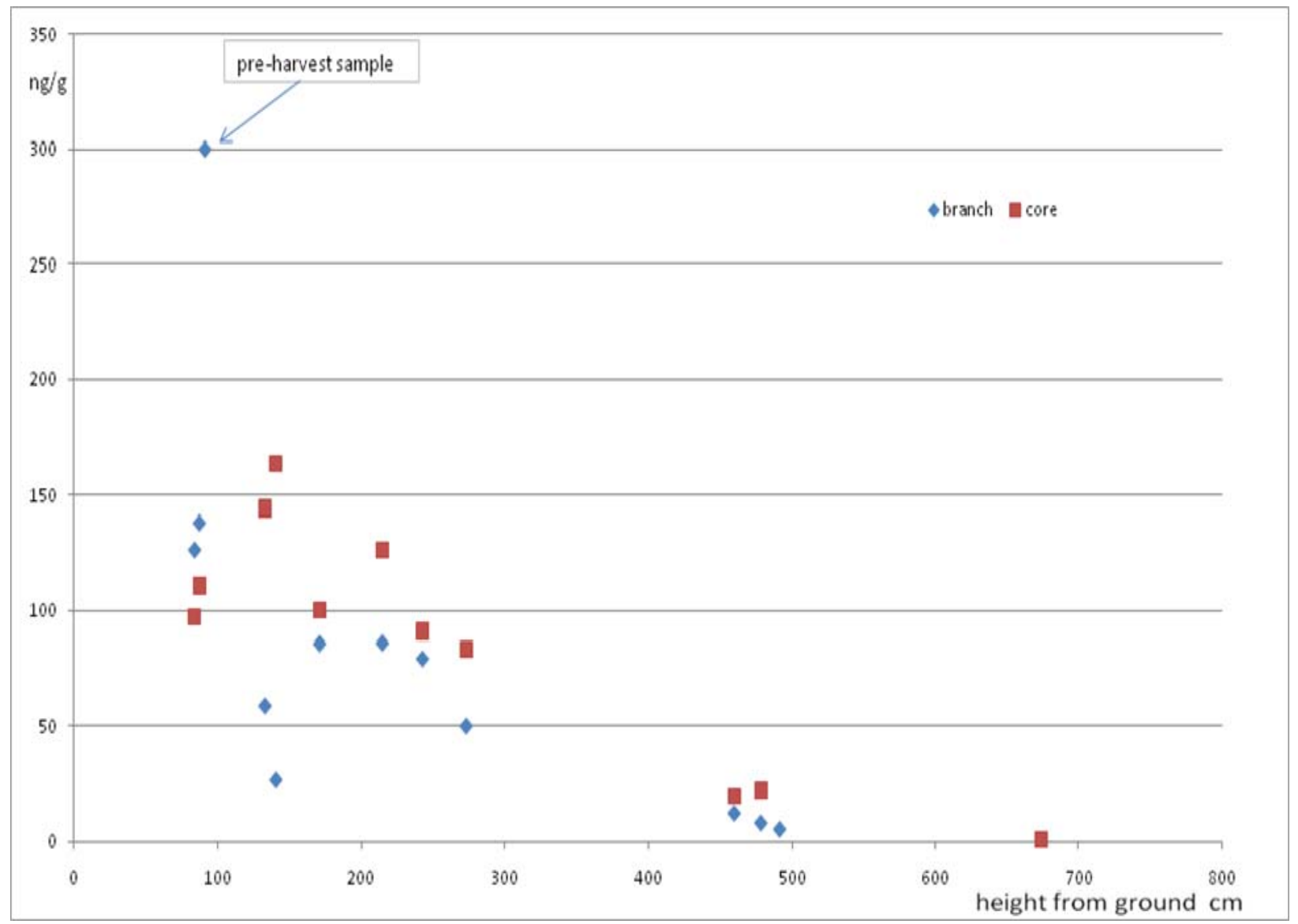

FIGURE 4.16 Vertical distribution of carbon tetrachloride in tissues of harvested eastern cottonwood tree W417EC1. 


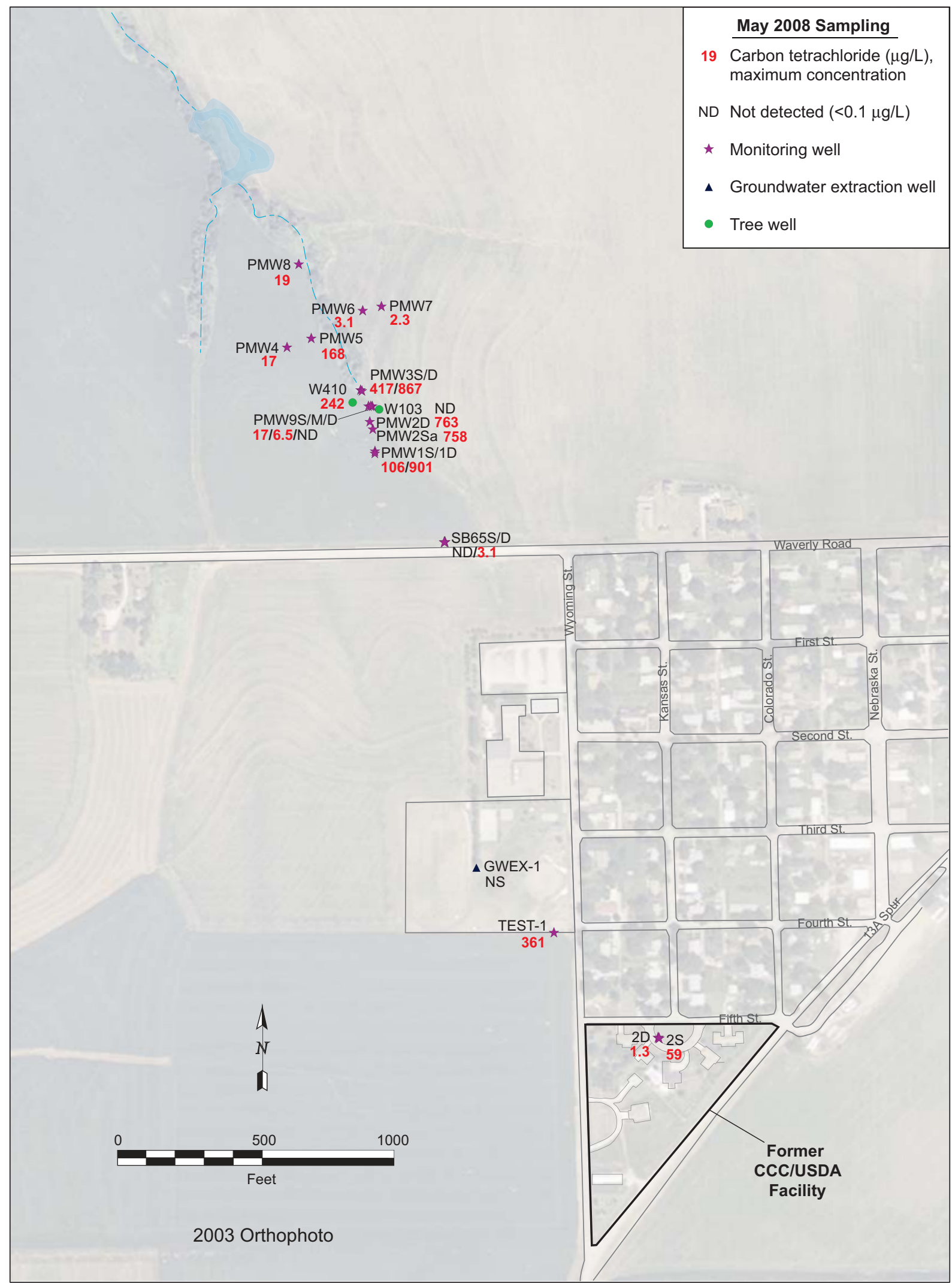

FIGURE 4.17 Maximum carbon tetrachloride concentrations in groundwater samples collected from permanent monitoring points in May 2008. Source of photograph: NAIP (2003). 


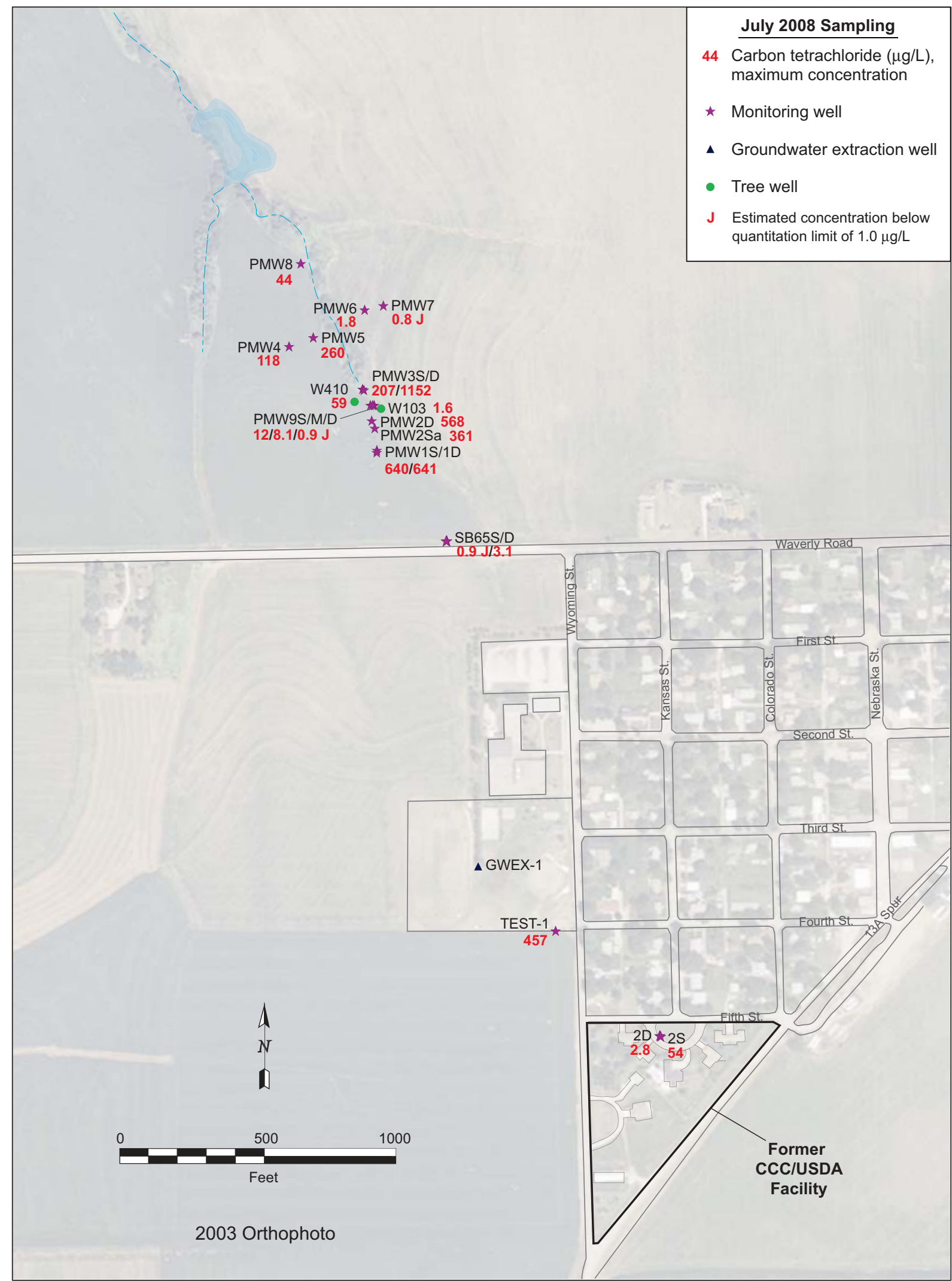

FIGURE 4.18 Maximum carbon tetrachloride concentrations in groundwater samples collected from permanent monitoring points in July 2008. Source of photograph: NAIP (2003). 


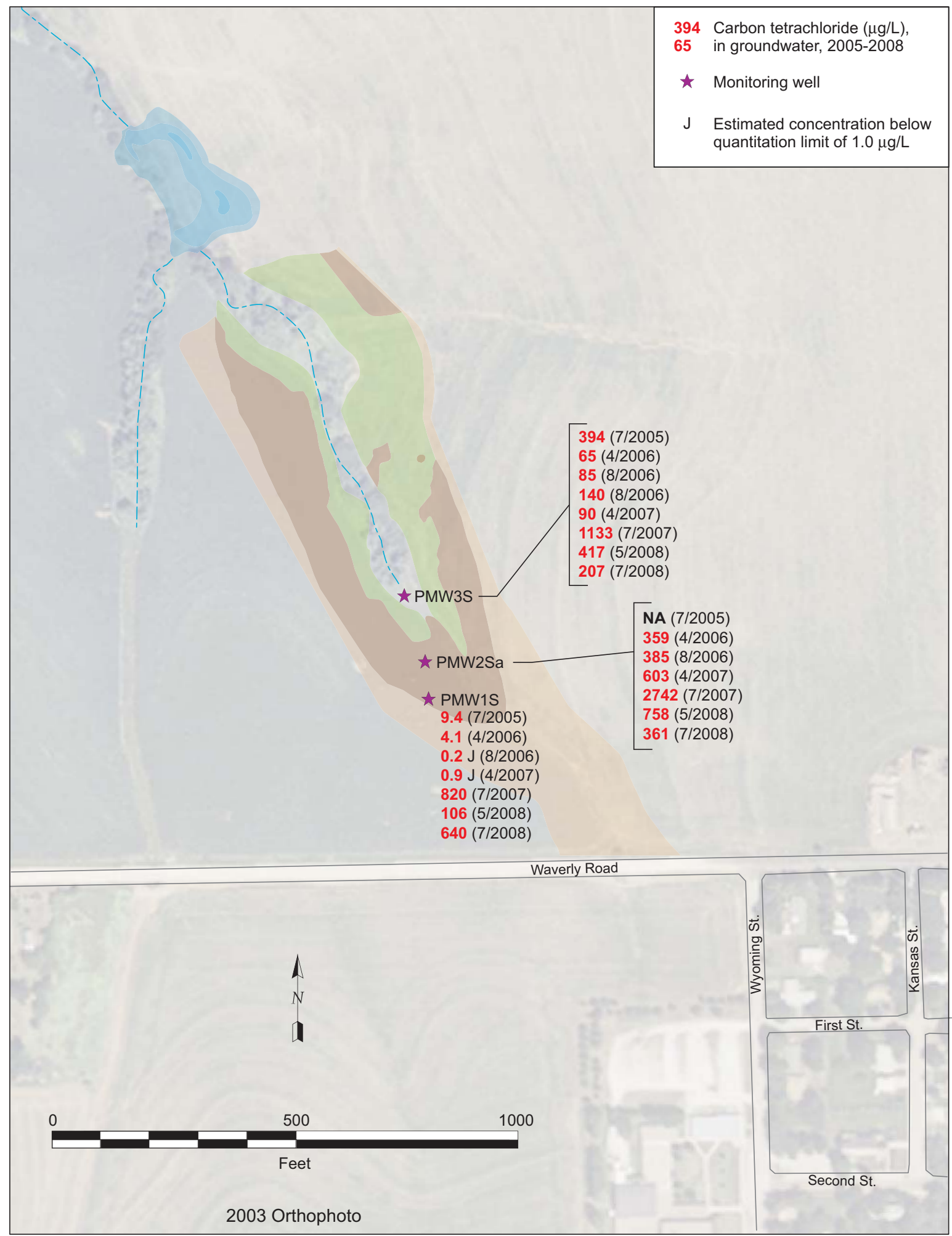

FIGURE 4.19 Carbon tetrachloride concentrations in groundwater samples collected from the shallow monitoring wells in the headwaters area, July 2005-July 2008. Source of photograph: NAIP (2003). 


\section{Operation, Maintenance, and System Modifications}

\subsection{Well GWEX-1 and the Spray Irrigation Treatment Unit}

No service or maintenance was required for the GWEX-1 extraction well or the spray irrigation treatment unit during the review period.

\subsection{Phytoremediation Planting and Buffer Areas}

Unusually high rainfalls at Murdock in 2007 resulted in shallow flooding of the land surface in portions of the phytoremediation area throughout much of that year. Local pooling of surface water, groundwater seepage, and increased surface runoff were particularly noted in the western and central portions of the shallow planting area near the headwaters of the tributary creek, as well as along both sides of the tributary creek in the more downstream portion of the shallow planting area (Figure 2.1). These surface conditions persisted during 2008, as a result of continued greater-than-normal rainfall (particularly in the spring).

The following specific activities were implemented in 2008 to maintain the health of the plantings and the integrity of the visitor amenities in the phytoremediation area:

- The phytoremediation planting area was inspected frequently throughout the growing season for possible indicators of plant stress or other detrimental conditions associated with the wet surface environment.

- Argonne representatives met with the Nebraska state forester (Richard Lodes) early in 2008, for continued consideration of the condition of the phytoremediation plantings and development of both (1) a long-term tree management plan and (2) immediate actions needed to foster the healthy development of the tree stand during the 2008 growing season.

- As in 2007, some trees were affected by fungi, partly because of the prolonged wet conditions and the dense canopy growth that restricted local air flow and promoted the stagnation of moist air. Some thinning occurred in 2008, after consultation with Richard Lodes during his annual visit to the site. 
- Mowing, trimming, and weeding were conducted throughout the summer and early fall of 2008 to control vigorous growth of the groundcover species in the phytoremediation area and to facilitate access to the trees for vegetation sampling and monitoring.

- Temporary support was provided in 2007 for a number of trees that began to lean unacceptably during the late spring and summer because of soft soil conditions that had developed in several areas of persistent standing surface water. In 2008, selected trees were removed in these areas and dissected to provide additional data regarding contaminant uptake in the tree tissues.

- Activities to help control and enhance the patterns of surface drainage through the phytoremediation plantings included (1) regrading and stabilization (with gravel) of the main channel leading into the phytoremediation area at the headwaters of the tributary creek, (2) the installation of additional drainage conduits at selected locations in the phytoremediation area, and (3) redressing of the pedestrian walking path in several low places in the headwaters area.

- Localized erosion was observed near the headwaters of the tributary creek, at the southernmost end of the phytoremediation area, as a result of the heavy spring rains. Representatives from Olsson Associates, the engineering firm that assisted in the original design and construction of erosion control measures for the phytoremediation and wetlands areas, visited the site to observe and consult regarding potentially continuing detrimental erosional effects. Little additional downcutting was observed during the remainder of the review period, however, and no further actions were taken.

- The on-site weather station was relocated from its original site near the headwaters of the tributary creek (in the phytoremediation area) to a new location at the southern margin of the wetlands area. The station was moved to prevent encroachment on the weather sensors by the neighboring trees as they continue to increase in size. 


\subsection{Constructed Wetlands Area}

In May 2008, the weir structure (water control structure) at the outlet of the wetlands was repositioned at a slightly higher level beneath the water control stop logs. In conjunction with this modification, the stream channel immediately downstream of the weir was cleared of heavy plant growth and other natural flow obstructions, and the channel was regraded slightly to improve the surface water flow leaving the wetlands. These actions were taken to ensure continuation of a free fall of water from the weir outlet, which is required for the weir to function properly in the estimation of discharge flow rates from the wetlands.

In summer 2008, minor regrading was performed adjacent to the walking pathway near the southwestern margin of the wetlands, to mitigate the effects of localized runoff and surface erosion observed previously (primarily in spring 2007) at this location. Several conduits beneath the pathway in this area were also replaced with larger-diameter units, to improve control of sporadic surface drainage into the wetlands from the agricultural fields immediately to the west.

\subsection{Monitoring Well Network}

No repairs to or modifications of the monitoring well network were required during 2008. Routine data recovery and battery replacement for the water level recorders installed at the site were performed approximately quarterly.

\subsection{Operating and Maintenance Costs for the Current Review Period}

The operating and maintenance (O\&M) costs for the 12-month period from January to December 2008 are summarized in Table 5.1. The costs for this period of operation include onetime expenses associated with the following:

- The erosion control measures described in Section 3.3.

- Purchase and deployment of some of the thermal dissipation probes and sap flow meters described in Section 3.2. (Additional thermal dissipation probes were on loan from another Argonne program.) 
- Performance of a separate investigation authorized by the CCC/USDA concerning the potential for mitigation of nitrate contamination by the constructed wetlands.

TABLE 5.1 Operations and maintenance costs for the Murdock site restoration project, January-December 2008.

Item

$\operatorname{Cost}^{\mathrm{a}}(\$)$

Routine O\&M

General management and technical oversight

20,297

Logistics and field support

67,846

Remediation monitoring and reporting

316,165

Total routine O\&M

404,309

One-Time Expenses

Erosion control

16,726

Purchase and deployment of thermal dissipation probes and sap flow meters

16,500

CCC/USDA wetlands study at Murdock

Total one-time expenses

a Numbers rounded; totals may not be equal to the sum of individual items. 


\section{Summary}

\subsection{Performance of the Removal Action Systems}

The results of the baseline sampling performed at Murdock in July 2005 indicated that the intended outcome of the integrated remedial approach designed for this site is achievable. Subsequent monitoring conducted during the third full year of operation (January to December 2008) of the remediation systems demonstrated the following:

- Seasonal operation of well GWEX-1 captures elevated concentrations of carbon tetrachloride in the upgradient portion of the groundwater plume and reduces the contaminant load approaching the tributary creek headwaters. The associated spray irrigation treatment unit decreases contaminant concentrations in the spray discharge to acceptable levels and improves the quality of the Elmwood-Murdock Public School athletic fields. Well GWEX-1 and the spray irrigation treatment unit could not be operated during 2008, however, because of unusually wet conditions at the site throughout much of the year.

- Highly contaminated water in the more downgradient portion of the groundwater plume continues to approach the phytoremediation and wetlands treatment areas.

- Carbon tetrachloride levels in surface waters show a rapid drop along the flow pathway established through the phytoremediation and wetlands treatment areas; no unacceptable levels of this contaminant have been detected in the final surface discharge from the wetlands.

- Carbon tetrachloride was not detected at unacceptable levels in ambient air in the phytoremediation and wetlands treatment zones at the "breathing" level among the planted and preexisting native trees. 
- The total costs for operation and maintenance of the removal action systems at Murdock during the review period were $\$ 520,325$. This amount includes several one-time costs.

\subsection{Regulatory Compliance of the Removal Action Systems}

Under the EPA-approved Monitoring Plan (Argonne 2006), site-specific monitoring requirements and compliance criteria governing the removal action at Murdock have been established by the EPA and the NDEQ. These requirements are as follows:

- For GWEX-1 and the spray irrigation treatment system:

- Sample the discharge quarterly during periods of seasonal operation.

- Demonstrate an acceptable carbon tetrachloride level $(44.2 \mu \mathrm{g} / \mathrm{L})$ in the discharge.

- $\quad$ Demonstrate an acceptable $\mathrm{pH}$ range (6.0-9.0) in the discharge.

- Report the actual volume of groundwater treated and discharged.

- Report results quarterly.

- For surface water discharged from the combined phytoremediation and wetlands treatment zones:

- Sample surface flow quarterly at locations SWM1-SWM3.

- Demonstrate an acceptable carbon tetrachloride level $(44.2 \mu \mathrm{g} / \mathrm{L})$ at location SWM3.

- Report results quarterly.

- For ambient air in the phytoremediation and wetlands treatment zones: 
- Sample twice yearly at locations AA1-AA3.

- Demonstrate an acceptable carbon tetrachloride level $\left(192 \mu \mathrm{g} / \mathrm{m}^{3}\right)$ in the ambient air.

- Report results annually.

The results of all compliance monitoring performed during the current review period are summarized as follows:

- No sampling of treated groundwater from the spray irrigation treatment system was required or performed during the current review period, as the GWEX-1 extraction well and spray system did not operate in 2008.

- Trace to low levels of carbon tetrachloride contamination $(1.0-2.9 \mu \mathrm{g} / \mathrm{L})$ were detected in the surface water samples collected at the surface water compliance point, location SWM3. The acceptable limit is $44.2 \mu \mathrm{g} / \mathrm{L}$.

- Carbon tetrachloride was detected at low levels $\left(2.0-4.5 \mu \mathrm{g} / \mathrm{m}^{3}\right.$ and ND-2.5 $\mu \mathrm{g} / \mathrm{m}^{3}$, respectively) in the ambient air sampled at location AA1 in the upstream portion of the phytoremediation area during both the May 2008 and July 2008 sampling events. Carbon tetrachloride was also detected at trace levels in one sample of ambient air collected at location AA2 $\left(1.4 \mu \mathrm{g} / \mathrm{m}^{3}\right.$, on May 15) and in one sample of air at location AA3 $\left(1.5 \mu \mathrm{g} / \mathrm{m}^{3}\right.$, on May 1$)$ during the May 2008 sampling event. The target maximum concentration is $192 \mu \mathrm{g} / \mathrm{m}^{3}$.

All monitoring and reporting requirements and compliance criteria were met during the review period. 


\section{References}

Argonne, 2006, Final Monitoring Plan for Site Restoration at Murdock, Nebraska, ANL/EVS/AGEM/TR-05-04, prepared for the Commodity Credit Corporation, U.S. Department of Agriculture, Washington, D.C., by Argonne National Laboratory, Argonne, Illinois, February.

Argonne, 2007, Summary of Operations and Performance of the Murdock Site Restoration Project in June 2005-December 2006, ANL/EVS/AGEM/TR-07-05, prepared for the Commodity Credit Corporation, U.S. Department of Agriculture, Washington, D.C., by Argonne National Laboratory, Argonne, Illinois, May.

Argonne, 2008, Summary of Operations and Performance of the Murdock Site Restoration Project in 2007, ANL/EVS/AGEM/TR-08-07, prepared for the Commodity Credit Corporation, U.S. Department of Agriculture, Washington, D.C., by Argonne National Laboratory, Argonne, Illinois, March.

EPA, 2005, memorandum from M. Beringer (Data Interpretation and Support Operations Branch, Environmental Services Division, U.S. Environmental Protection Agency Region VII, Kansas City, Kansas) to J. Field (Drinking Water Management Branch; Water, Wetlands, and Pesticides Division, U.S. Environmental Protection Agency Region VII, Kansas City, Kansas), December 21.

Hansen, E., 1994, A Guide for Determining When To Fertilize Hybrid Poplar Plantations, Forest Service Research Paper NC-319, U.S. Department of Agriculture, St. Paul, Minnesota, July 15.

Ma, X., and J. Burken, 2004, "Modeling of TCE Diffusion to the Atmosphere and Distribution in Plant Stems," Environmental Science and Technology 38(17):4580-4586.

NAIP, 2003, aerial photograph of Murdock, Nebraska, National Agricultural Imagery Program, U.S. Department of Agriculture, http://www.apfo.usda.gov/NAIP.html.

Ter-Mikaelian, M.T., and M.D. Korzukhin, 1997, "Biomass Equations for Sixty-Five North American Tree Species," Forest Ecology and Management 97:1-24. 
UNL, 2009, Lincoln, NE, Weather and Climate, Applied Climate Sciences Department, School of Natural Resources, University of Nebraska-Lincoln, www.hprcc.unl.edu/nebraska/ lincoln.html, accessed March 3. 


\section{Appendix A:}

\section{Photo Gallery}



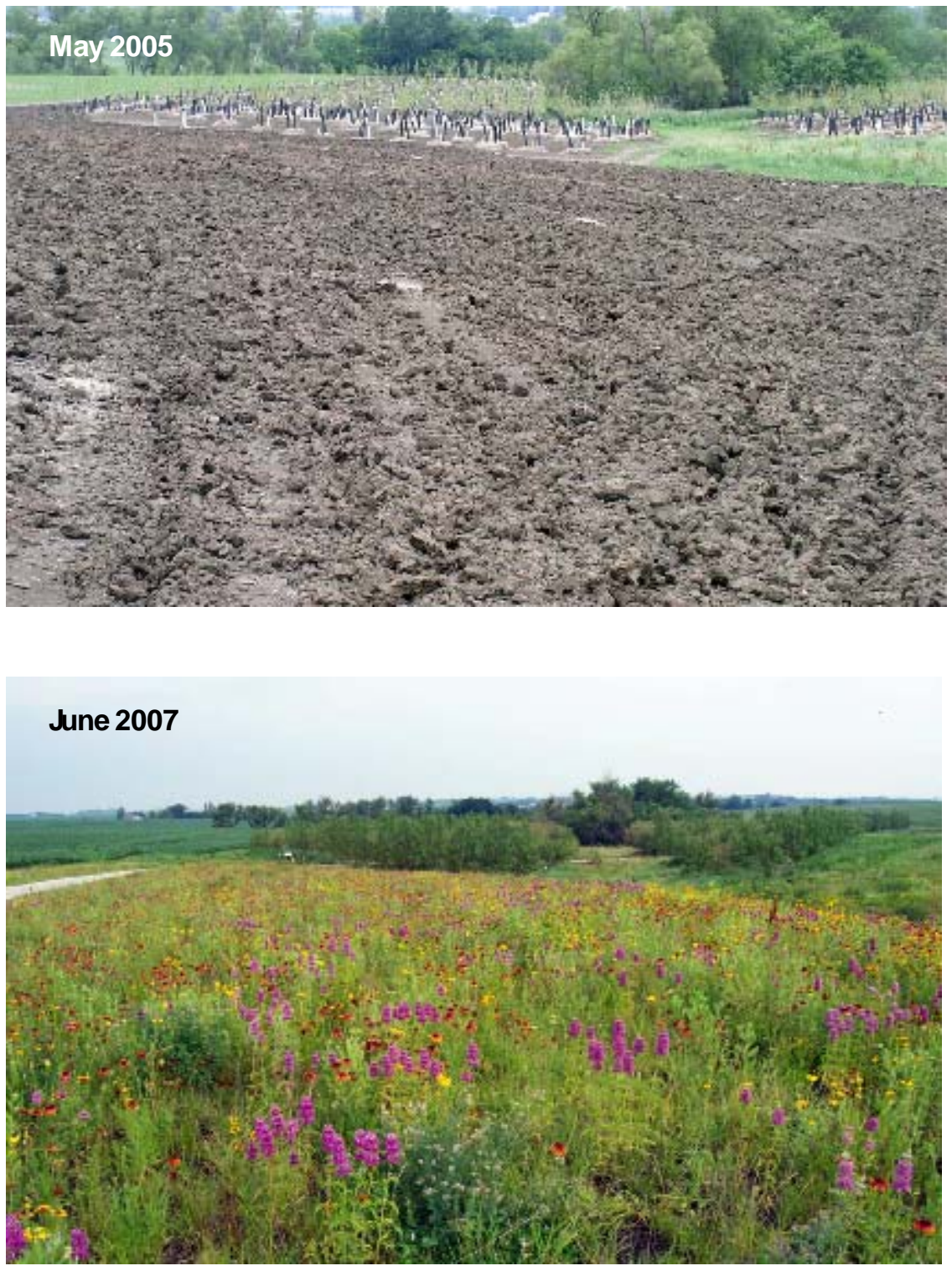

Phytoremediation plantation, 2005 to 2008.

\section{September 2005}
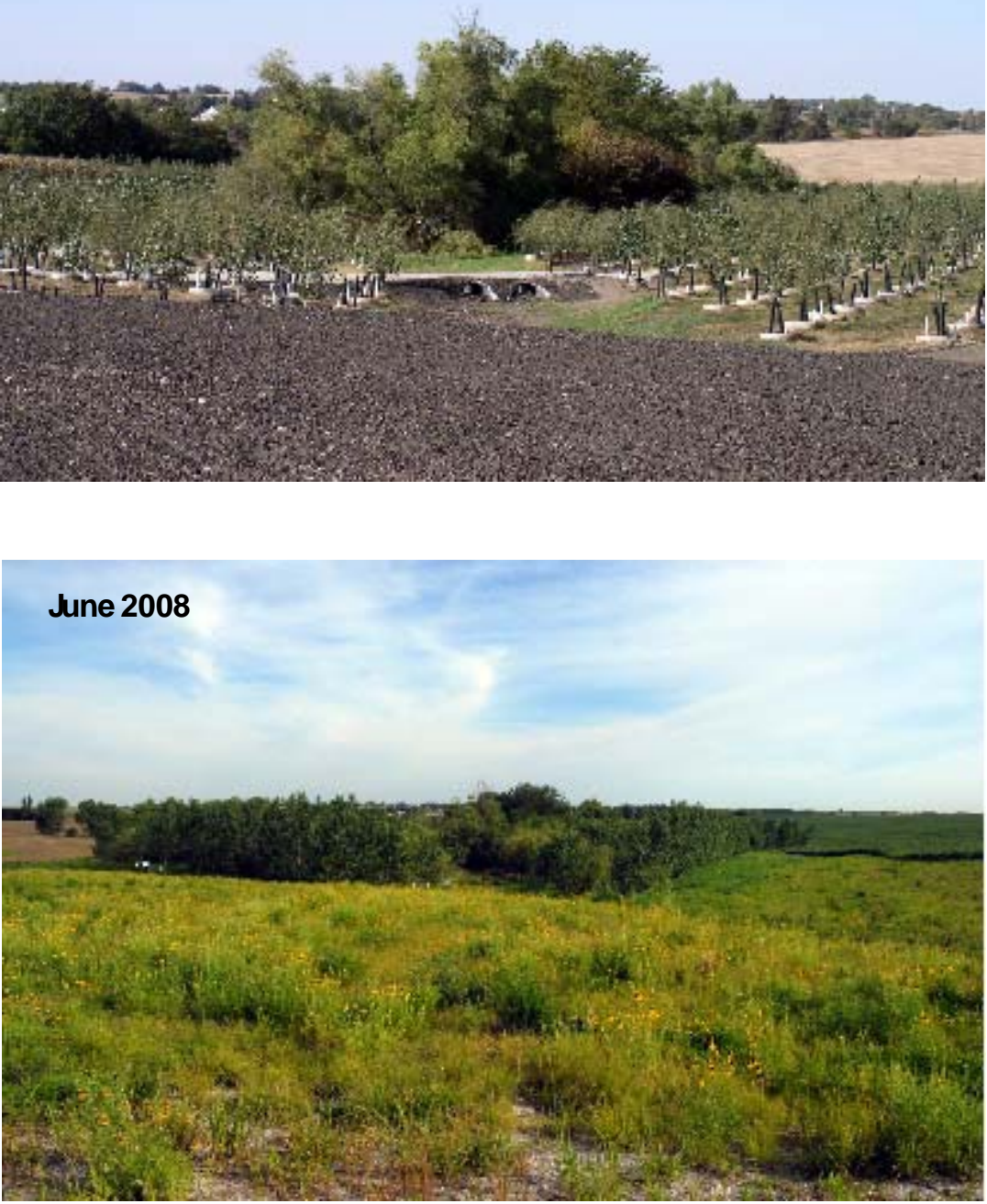

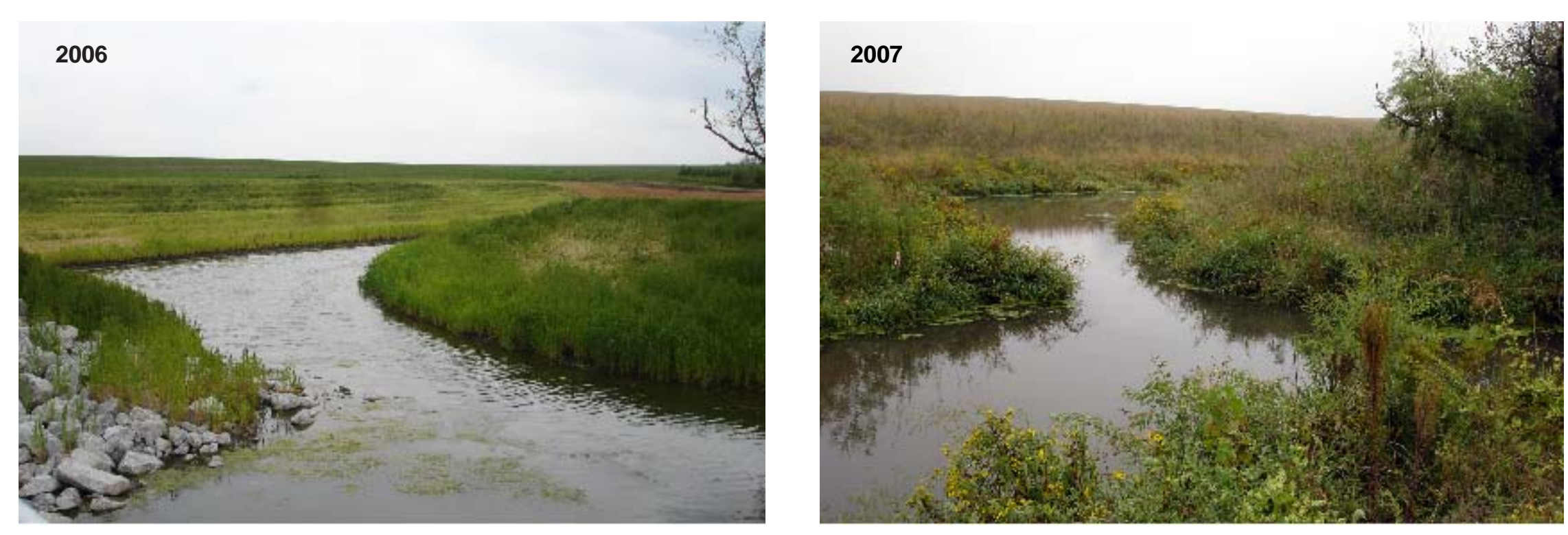

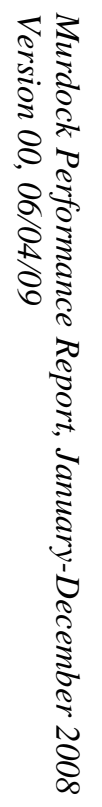

2008

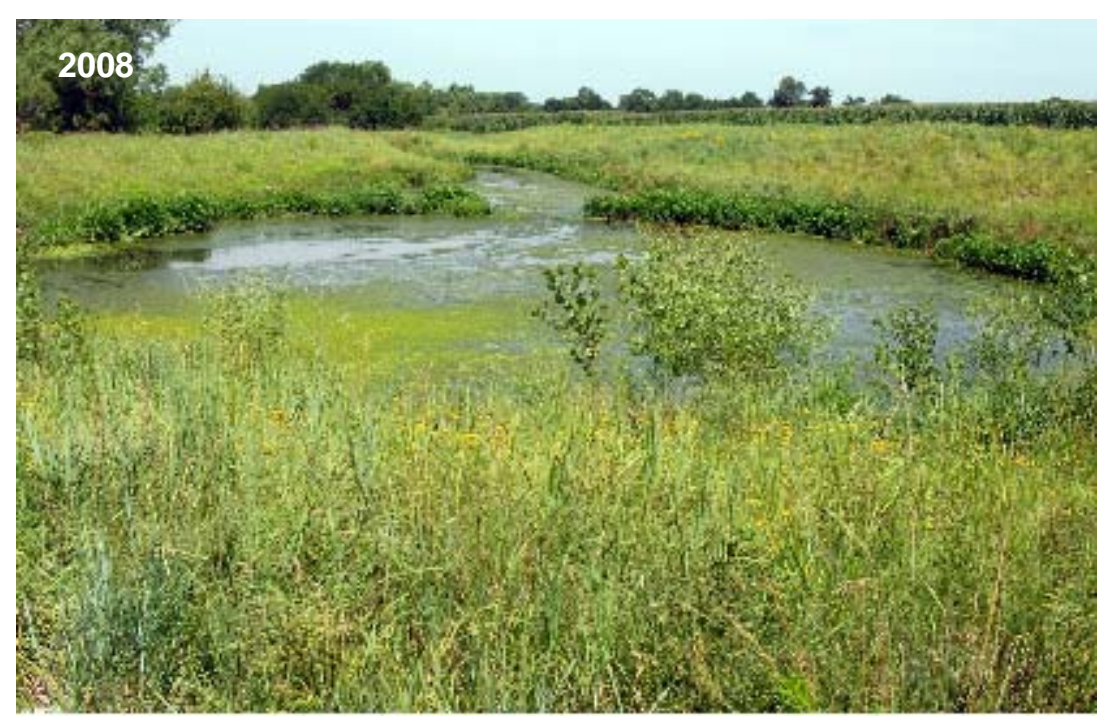

Wetlands view from north end, 2006-2008. 


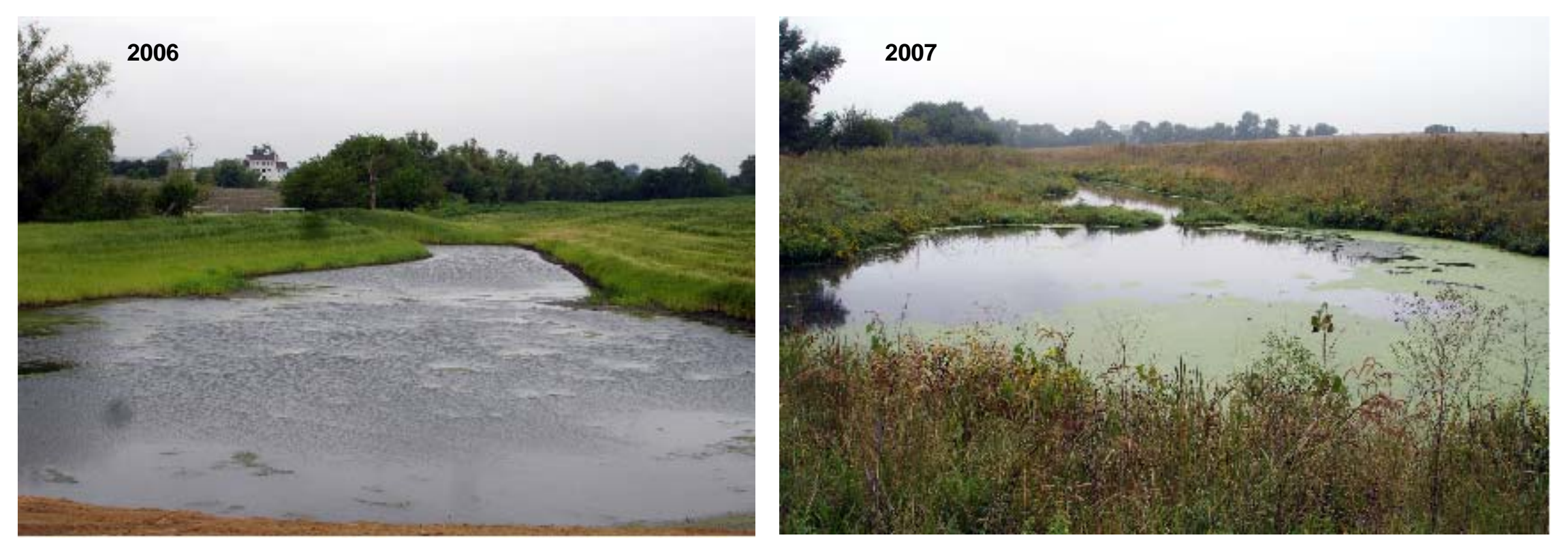

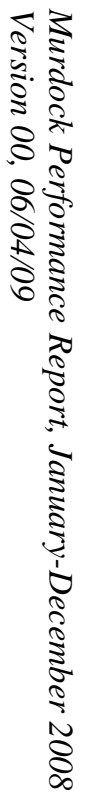

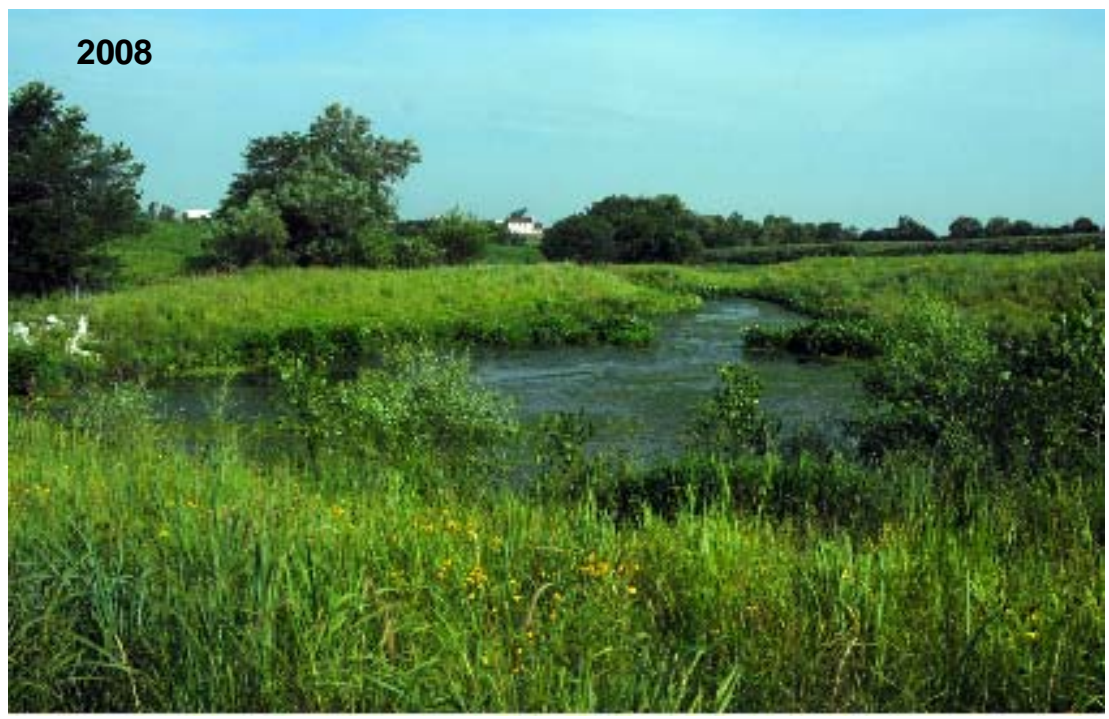

Wetlands view from south end, 2006-2008. 

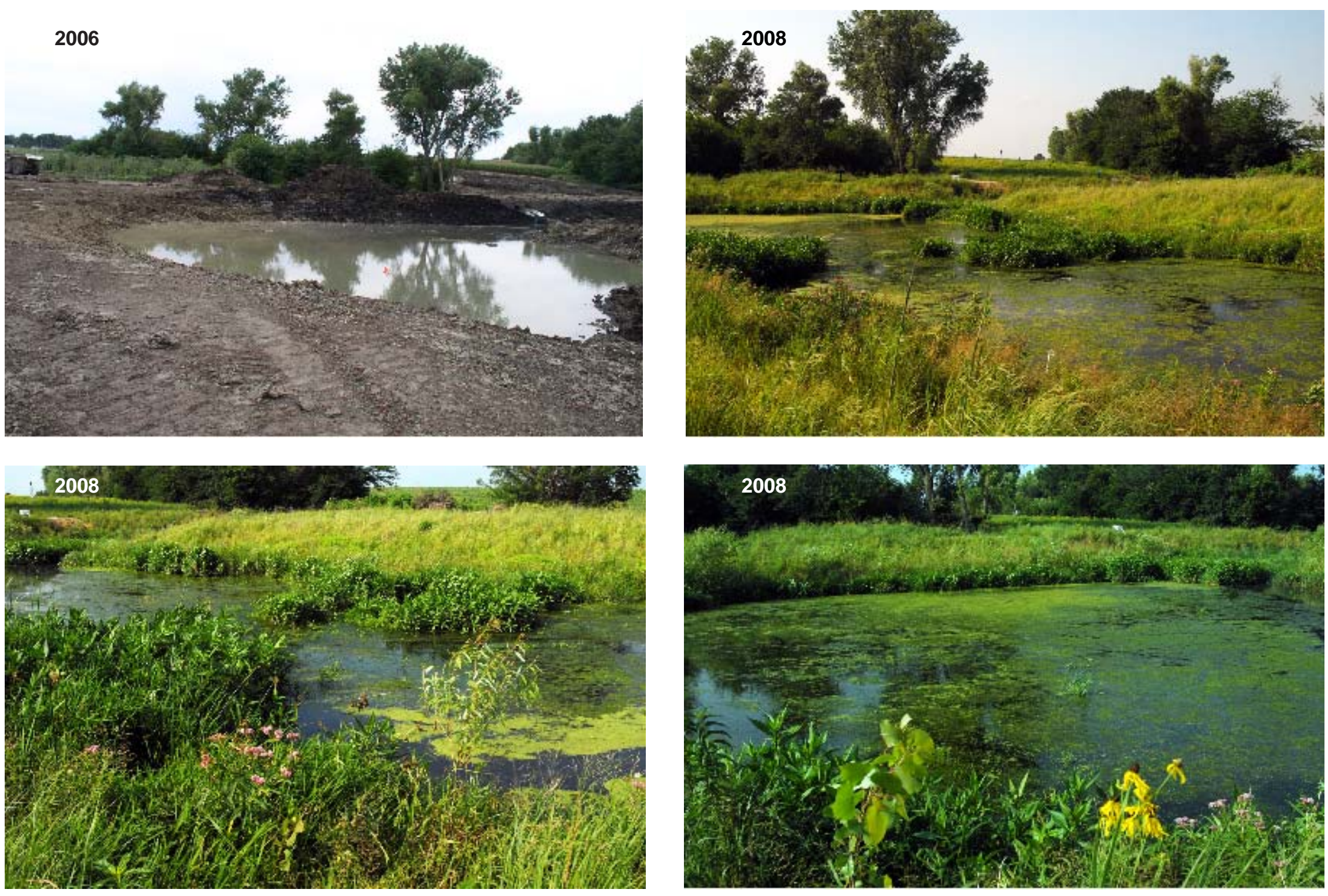

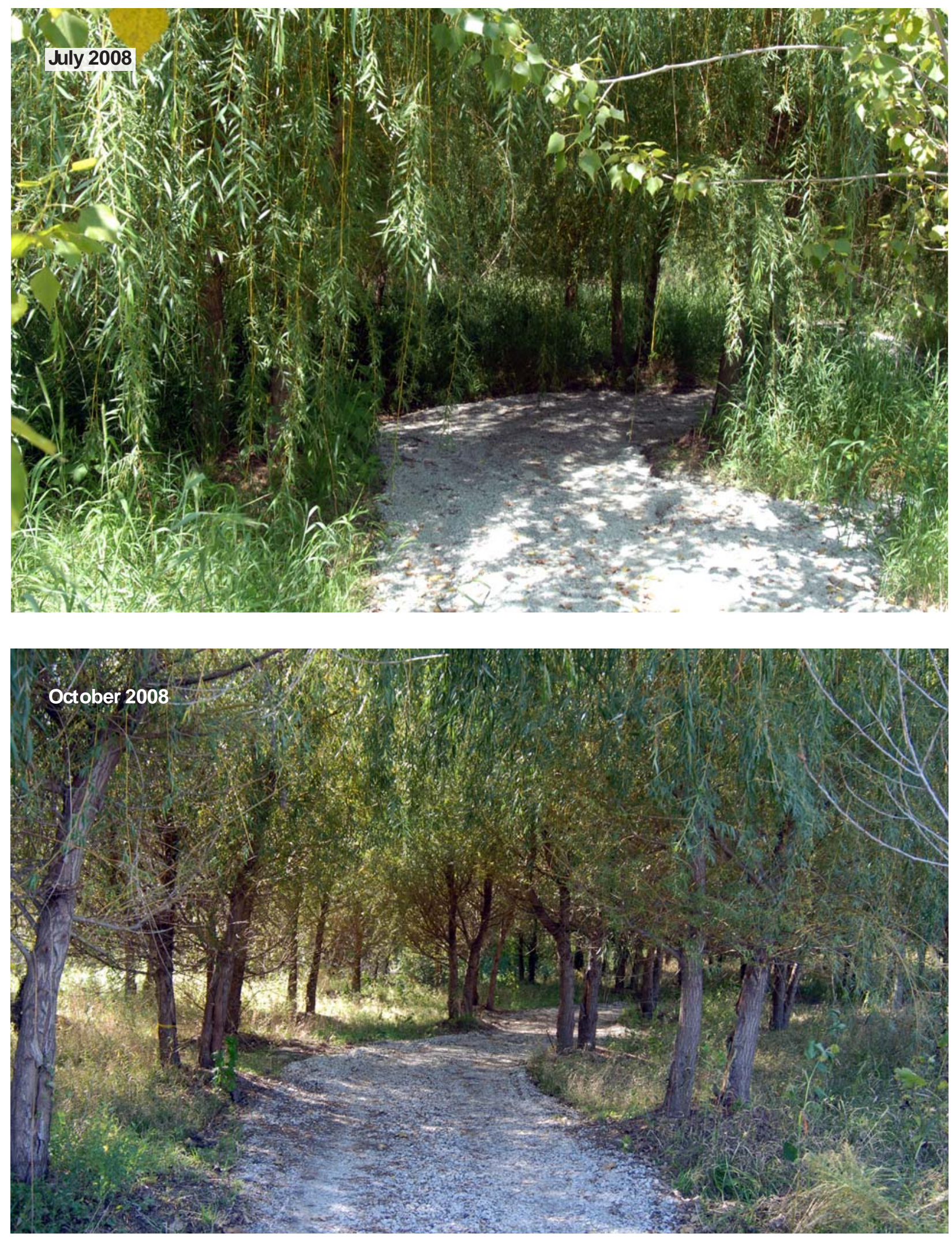

Effect of tree trimming and path reconstruction, 2008. 

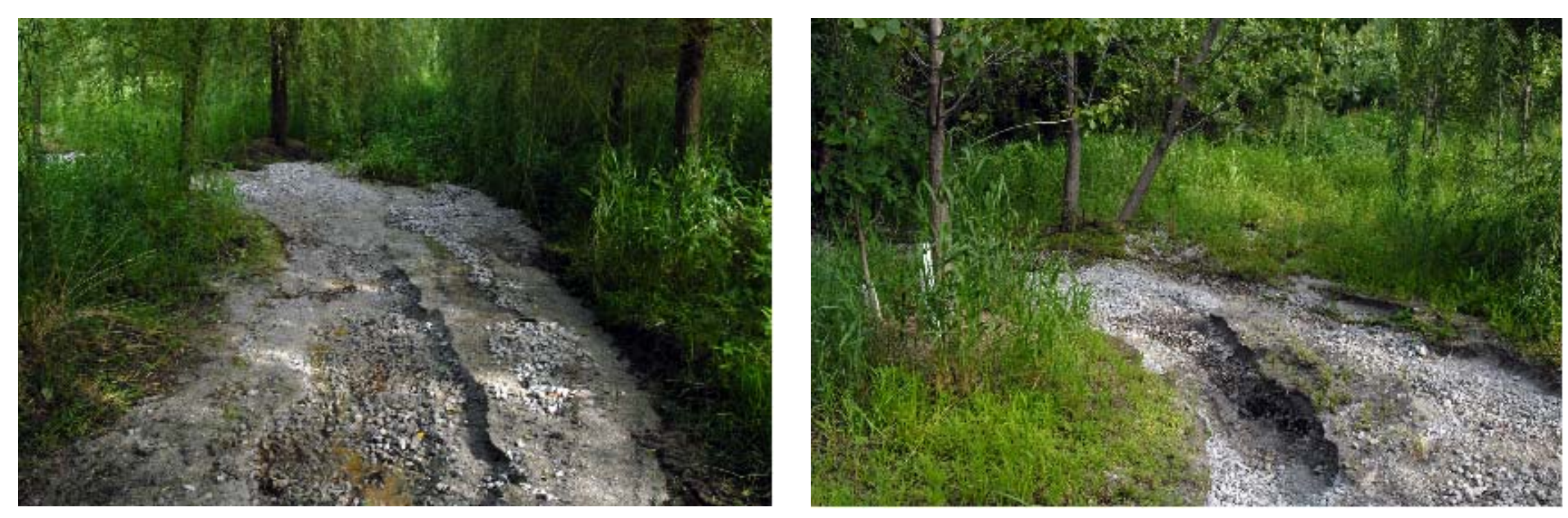

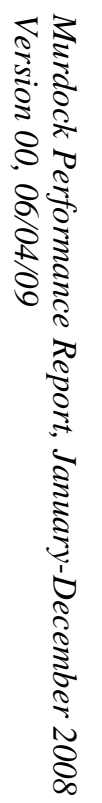
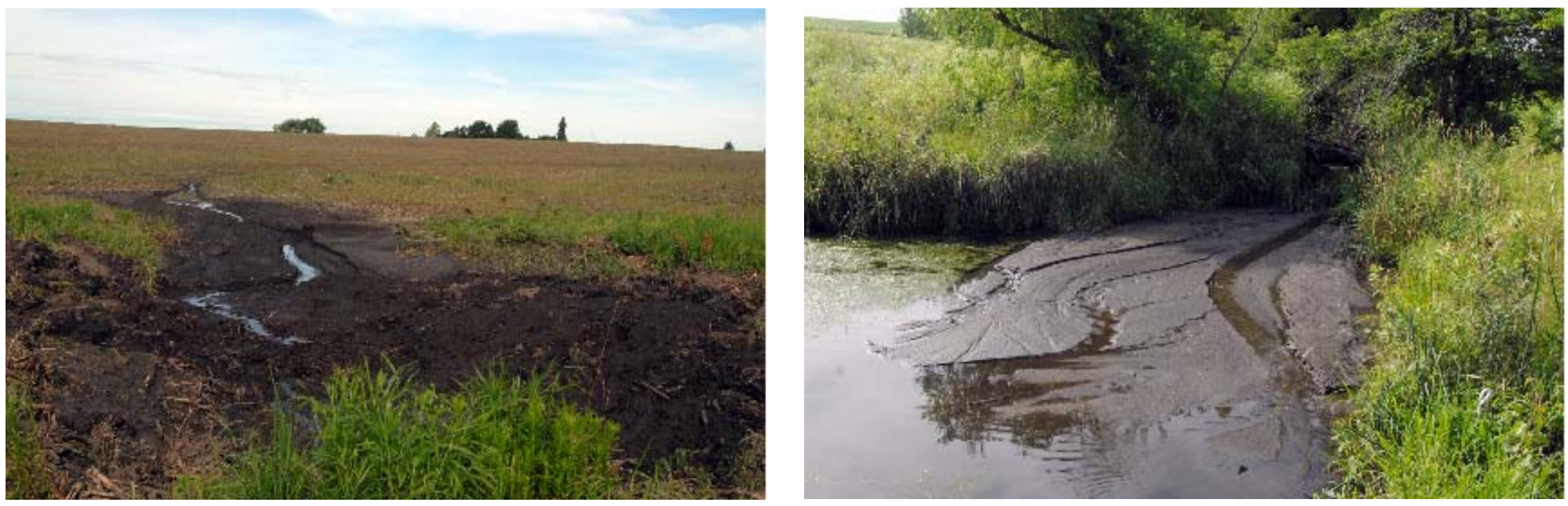

Erosion, June 2008. 

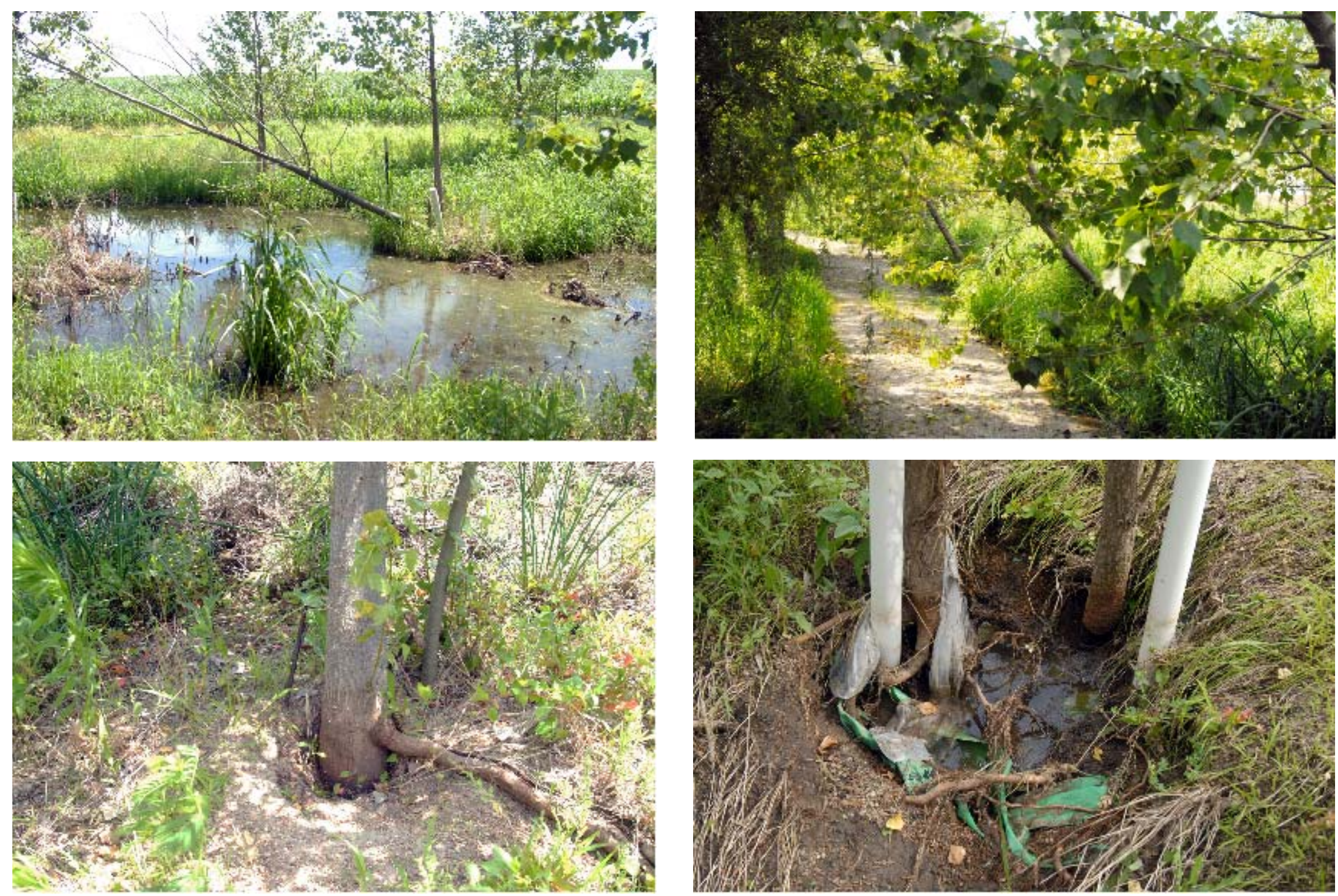

Wet ground and leaning trees, June 2008. 

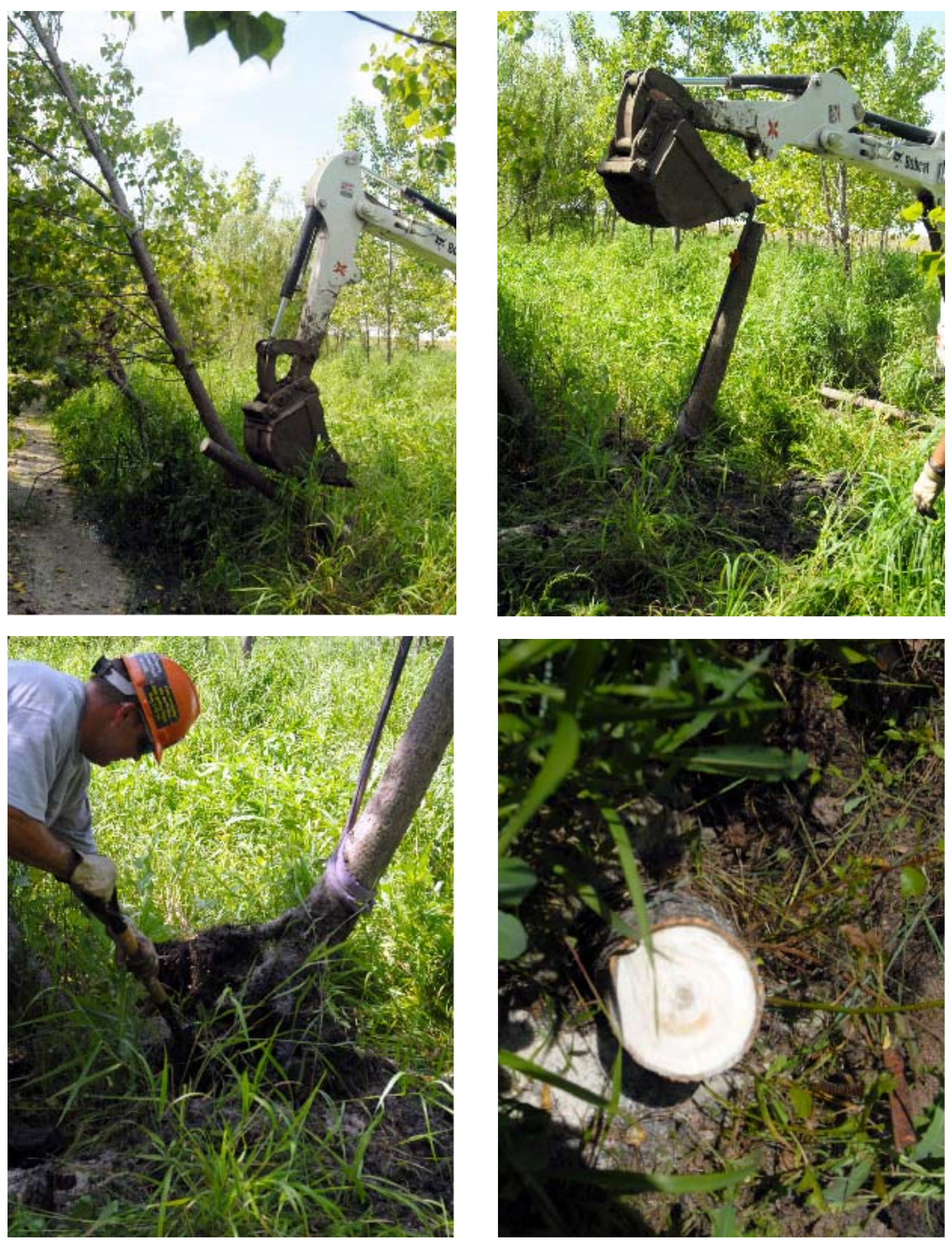

Tree harvest, June 2008. 

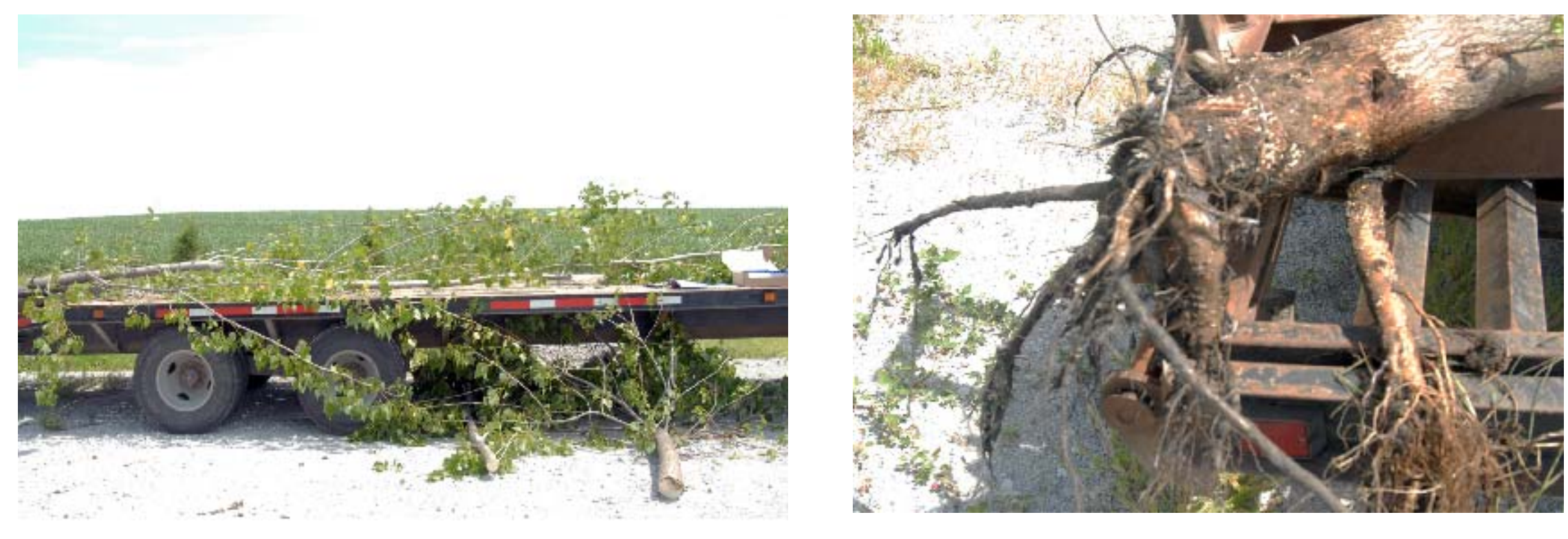

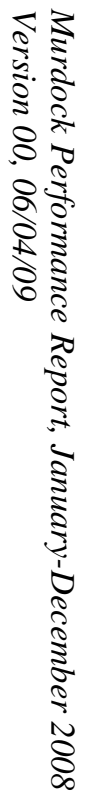
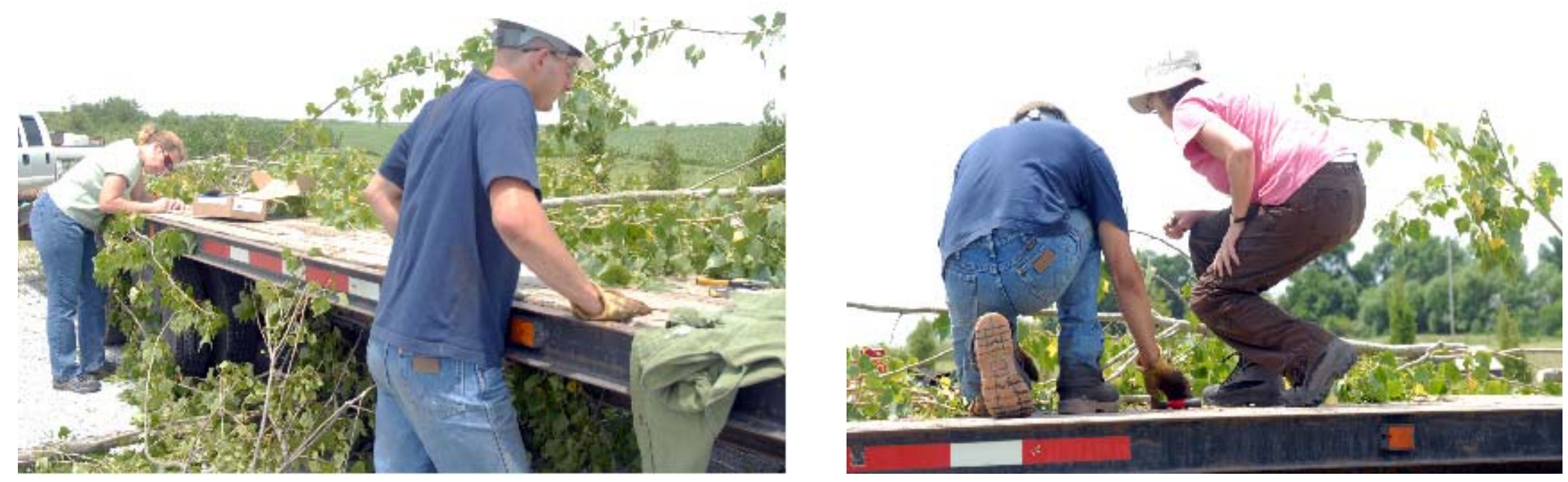

Preparing the harvested tree for weighing, June 2008. 

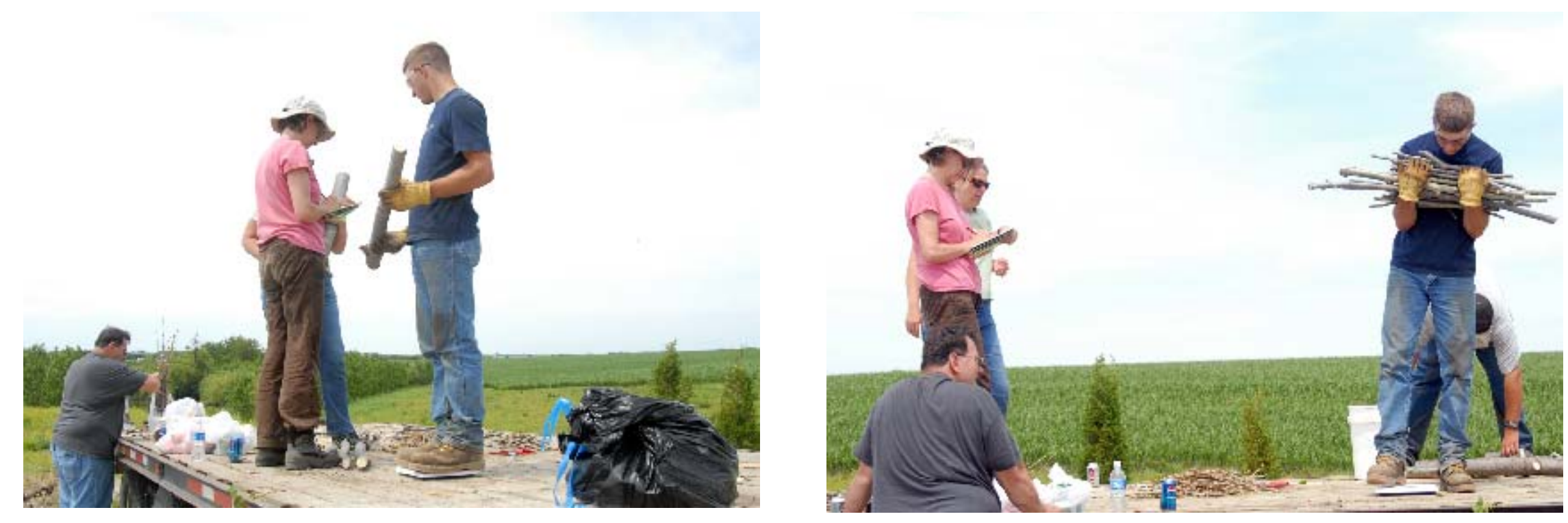

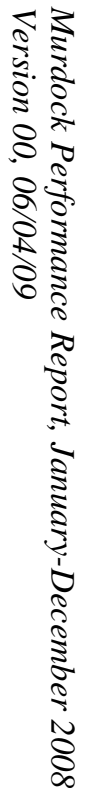
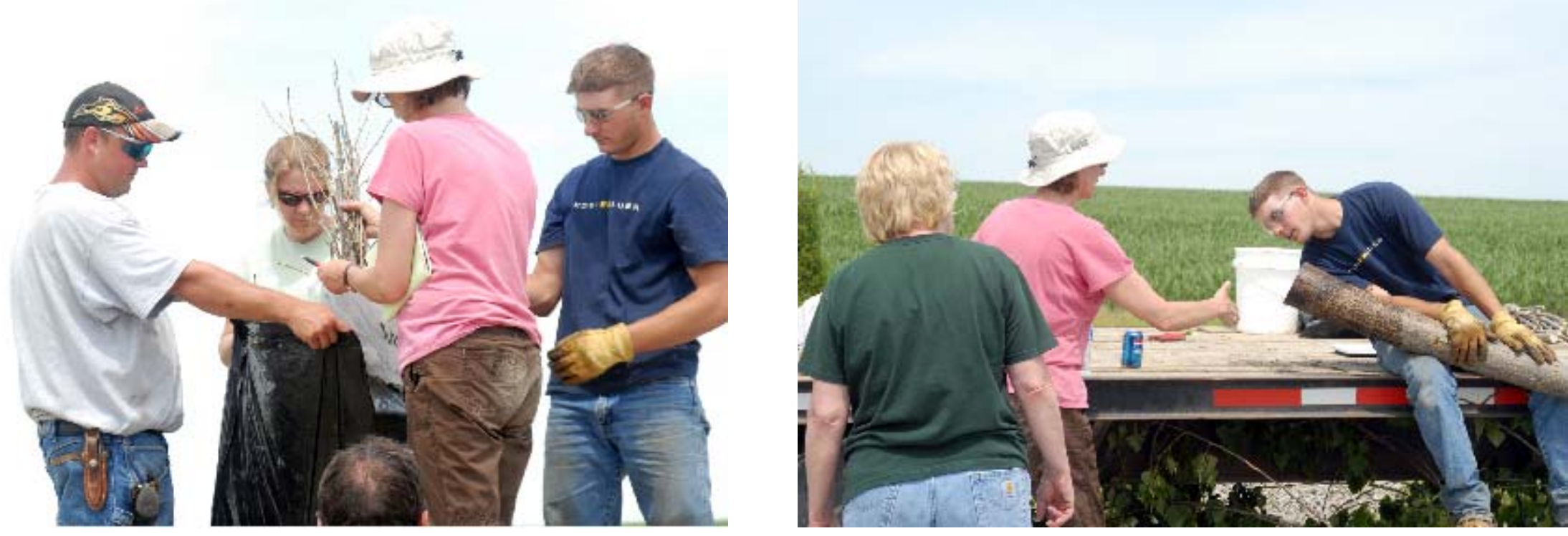

Weighing the tree and dyeing the log, June 2008. 

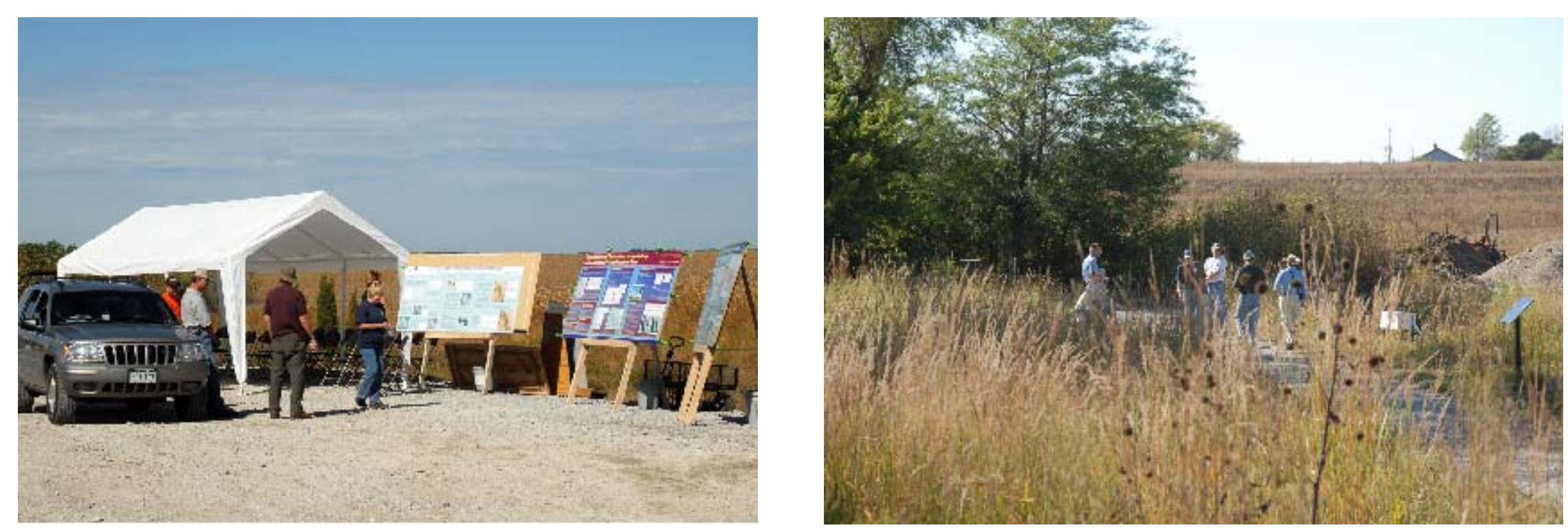

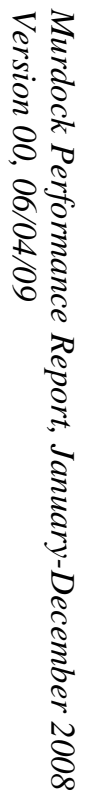
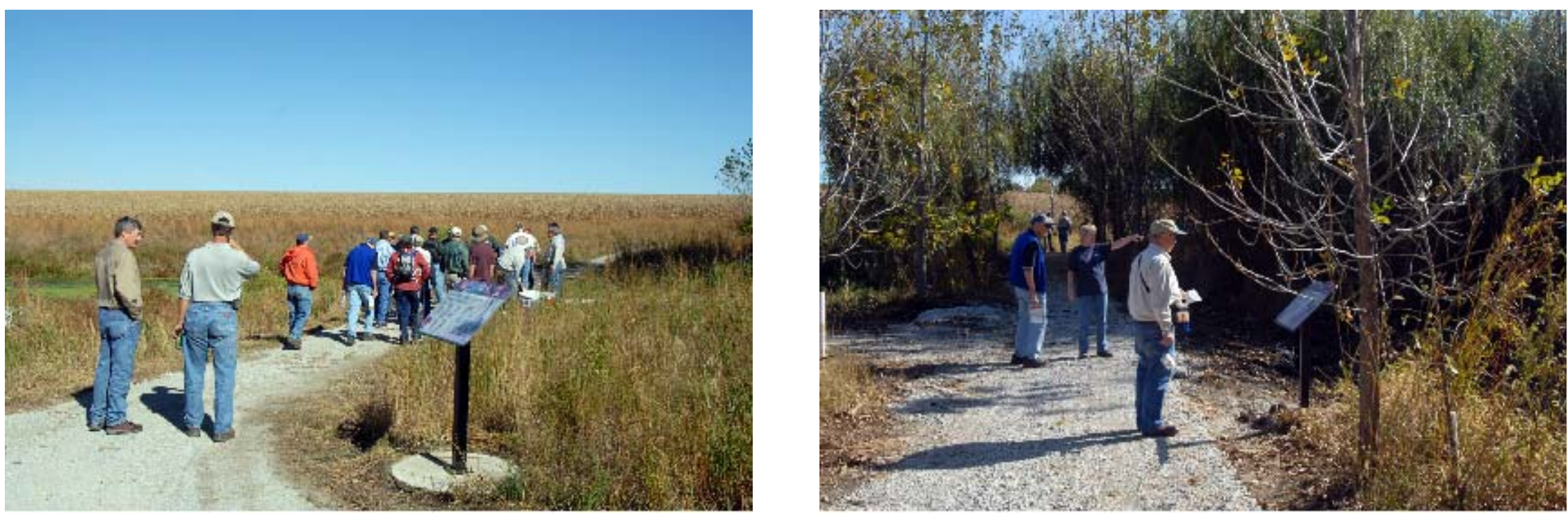

Field trip, Great Plains Society of American Foresters, October 2008. 


\section{Appendix B:}

\section{Vegetation Analysis Results}




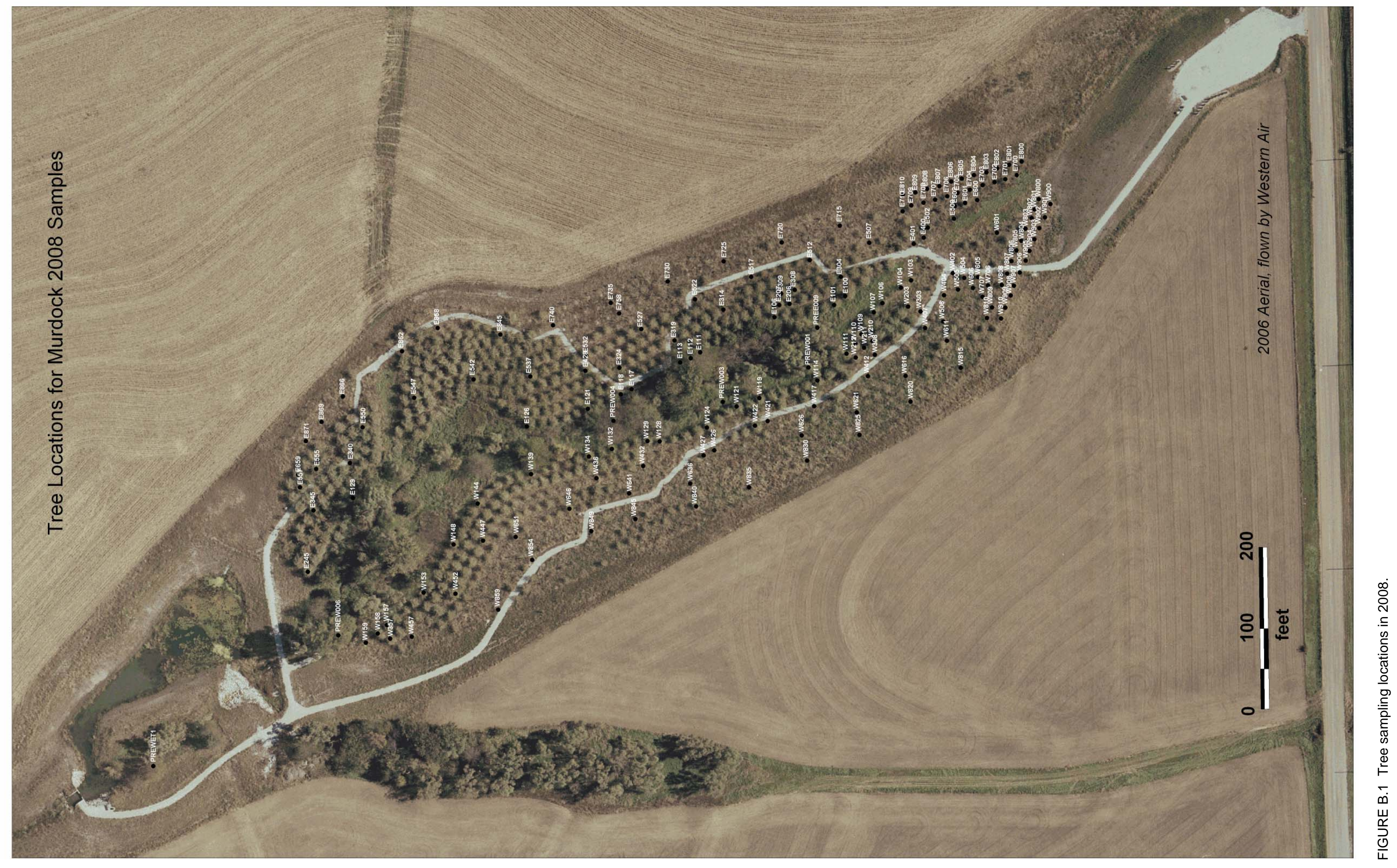


TABLE B.1 Results of analyses for volatile organic compounds in vegetation samples collected at Murdock in 2008.

\begin{tabular}{|c|c|c|c|c|c|c|c|c|c|}
\hline \multirow[b]{2}{*}{ Location } & \multirow[b]{2}{*}{ Species } & \multirow[b]{2}{*}{ Sample } & \multirow[b]{2}{*}{ Date } & \multirow[b]{2}{*}{ Time } & \multirow[b]{2}{*}{ Type } & \multicolumn{2}{|c|}{ Concentration $^{\mathrm{b}}(\mu \mathrm{g} / \mathrm{kg})$} & \multirow[b]{2}{*}{$\begin{array}{l}\text { Height } \\
(\mathrm{cm})\end{array}$} & \multirow[b]{2}{*}{ Sample Description } \\
\hline & & & & & & $\begin{array}{c}\text { Carbon } \\
\text { Tetrachloride }\end{array}$ & Chloroform & & \\
\hline \multicolumn{10}{|c|}{ Sampling in March 2008} \\
\hline E106 & WN1 & MUE106WN1-B-25030 & $3 / 27 / 08$ & $11: 10$ & Branch & 1 & $N D^{d}$ & 10 & $\begin{array}{l}\text { Branch } 4 \text { in. above ground, west } \\
\text { orientation. }\end{array}$ \\
\hline E111 & WN1 & MUE111WN1-B-25031 & $3 / 27 / 08$ & $11: 15$ & Branch & 1 & ND & 91 & $\begin{array}{l}\text { Branch } 36 \text { in. above ground, south } \\
\text { orientation. }\end{array}$ \\
\hline E129 & WN1 & MUE129WN1-B-25032 & $3 / 27 / 08$ & $11: 56$ & Branch & ND & ND & 10 & $\begin{array}{l}\text { Branch } 4 \text { in. above ground, north } \\
\text { orientation. }\end{array}$ \\
\hline E207 & WN1 & MUE207WN1-B-25033 & $3 / 27 / 08$ & $11: 36$ & Branch & 5 & ND & 122 & $\begin{array}{l}\text { Branch } 4 \mathrm{ft} \text { above ground, north } \\
\text { orientation. }\end{array}$ \\
\hline E207 & WN2 & MUE207WN2-B-25034 & $3 / 27 / 08$ & $11: 35$ & Branch & 12 & 2 & 122 & $\begin{array}{l}\text { Branch } 4 \mathrm{ft} \text { above ground, north } \\
\text { orientation. }\end{array}$ \\
\hline E308 & WN1 & MUE308WN1-B-25035 & $3 / 27 / 08$ & $11: 30$ & Branch & $<1^{\mathrm{e}}$ & ND & 15 & $\begin{array}{l}\text { Branch } 6 \text { in. above ground, west } \\
\text { orientation. }\end{array}$ \\
\hline E308 & WN2 & MUE308WN2-B-25036 & $3 / 27 / 08$ & $11: 31$ & Branch & $<1$ & ND & 15 & $\begin{array}{l}\text { Branch } 6 \text { in. above ground, west } \\
\text { orientation. }\end{array}$ \\
\hline E309 & WN1 & MUE309WN1-B-25037 & $3 / 27 / 08$ & $11: 25$ & Branch & $<1$ & ND & 15 & $\begin{array}{l}\text { Branch } 6 \text { in. above ground, south } \\
\text { orientation. }\end{array}$ \\
\hline E309 & WN2 & MUE309WN2-B-25038 & $3 / 27 / 08$ & $11: 26$ & Branch & 1 & ND & 10 & $\begin{array}{l}\text { Branch } 4 \text { in. above ground, south } \\
\text { orientation. }\end{array}$ \\
\hline E314 & HP1 & MUE314HP1-B-25039 & $3 / 27 / 08$ & $11: 20$ & Branch & $<1$ & ND & 152 & $\begin{array}{l}\text { Branch } 5 \mathrm{ft} \text { above ground, } \\
\text { northeast orientation. Sample } \\
\text { collected from secondary } \\
\text { branch } 12 \text { in. from trunk insert. }\end{array}$ \\
\hline E324 & WN1 & MUE324WN1-B-25040 & $3 / 27 / 08$ & $11: 45$ & Branch & ND & ND & 3 & $\begin{array}{l}\text { Branch } 1 \text { in. above ground, north } \\
\text { orientation. Sickly tree. }\end{array}$ \\
\hline E428 & WN1 & MUE428WN1-B-25041 & $3 / 27 / 08$ & $11: 46$ & Branch & $<1$ & ND & 122 & $\begin{array}{l}\text { Branch } 4 \mathrm{ft} \text { above ground, north } \\
\text { orientation. }\end{array}$ \\
\hline E517 & WN1 & MUE517WN1-B-25042 & $3 / 27 / 08$ & $11: 40$ & Branch & ND & ND & 122 & $\begin{array}{l}\text { Branch } 4 \mathrm{ft} \text { above ground, east } \\
\text { orientation. }\end{array}$ \\
\hline E532 & WN1 & MUE532WN1-B-25054 & $3 / 27 / 08$ & $11: 50$ & Branch & $<1$ & ND & 152 & $\begin{array}{l}\text { Branch } 5 \mathrm{ft} \text { above ground, east } \\
\text { orientation. Sample from } \\
\text { secondary branch } 6 \text { in. off trunk. }\end{array}$ \\
\hline E550 & WN1 & MUE550WN1-B-25043 & $3 / 27 / 08$ & $11: 51$ & Branch & ND & ND & 122 & $\begin{array}{l}\text { Branch } 4 \mathrm{ft} \text { above ground, east } \\
\text { orientation. }\end{array}$ \\
\hline
\end{tabular}




\begin{tabular}{|c|c|c|c|c|c|c|c|c|c|}
\hline & & & & & & \multicolumn{2}{|c|}{ Concentration $^{\mathrm{b}}(\mu \mathrm{g} / \mathrm{kg})$} & \multirow[b]{2}{*}{$\begin{array}{l}\text { Heightc } \\
(\mathrm{cm})\end{array}$} & \multirow[b]{2}{*}{ Sample Description } \\
\hline Location & Species $^{a}$ & Sample & Date & Time & Type & $\begin{array}{c}\text { Carbon } \\
\text { Tetrachloride }\end{array}$ & Chloroform & & \\
\hline
\end{tabular}

Sampling in March 2008 (cont.)

\begin{tabular}{|c|c|c|c|c|c|c|c|c|c|}
\hline E659 & WN1 & MUE659WN1-B-25044 & $3 / 27 / 08$ & $11: 55$ & Branch & ND & ND & 20 & $\begin{array}{l}\text { Branch } 8 \text { in. above ground, south } \\
\text { orientation. }\end{array}$ \\
\hline E700 & EC1 & MUE700EC1-B-25050 & $3 / 27 / 08$ & $11: 00$ & Branch & ND & ND & 30 & $\begin{array}{l}\text { Secondary branch } 12 \text { in. above } \\
\text { ground, north orientation. }\end{array}$ \\
\hline E706 & WN2 & MUE706WN2-B-25051 & $3 / 27 / 08$ & $11: 01$ & Branch & $<1$ & ND & 0 & $\begin{array}{l}\text { Branch sample at base of tree, } \\
\text { south orientation. Tree labeled } \\
\text { EC is actually WN2. }\end{array}$ \\
\hline E720 & EC & MUE720EC-B-25045 & $3 / 27 / 08$ & $12: 12$ & Branch & ND & ND & 122 & $\begin{array}{l}\text { Branch } 4 \mathrm{ft} \text { above ground, east } \\
\text { orientation. }\end{array}$ \\
\hline E720 & GA & MUE720GA-B-25046 & $3 / 27 / 08$ & $12: 13$ & Branch & ND & ND & 122 & $\begin{array}{l}\text { Branch } 4 \mathrm{ft} \text { above ground, east } \\
\text { orientation. }\end{array}$ \\
\hline E735 & EC1 & MUE735EC1-B-25047 & $3 / 27 / 08$ & $12: 10$ & Branch & ND & ND & 122 & $\begin{array}{l}\text { Branch } 4 \mathrm{ft} \text { above ground, west } \\
\text { orientation. Wet area. }\end{array}$ \\
\hline E735 & EC2 & MUE735EC2-B-25048 & $3 / 27 / 08$ & $12: 11$ & Branch & ND & $<1$ & 152 & $\begin{array}{l}\text { Branch } 5 \mathrm{ft} \text { above ground, east } \\
\text { orientation. Secondary branch } \\
6 \text { in. from main stem. }\end{array}$ \\
\hline E740 & EC & MUE740EC-B-25049 & $3 / 27 / 08$ & $12: 01$ & Branch & ND & $<1$ & 91 & $\begin{array}{l}\text { Branch } 3 \mathrm{ft} \text { above ground, west } \\
\text { orientation. }\end{array}$ \\
\hline E810 & EC1 & MUE810EC1-B-25052 & $3 / 27 / 08$ & $11: 05$ & Branch & ND & ND & 25 & $\begin{array}{l}\text { Branch } 10 \text { in. above ground, east } \\
\text { orientation. }\end{array}$ \\
\hline E858 & HP & MUE858HP-B-25053 & $3 / 27 / 08$ & $12: 00$ & Branch & ND & ND & 152 & $\begin{array}{l}\text { Branch } 5 \mathrm{ft} \text { above ground, north } \\
\text { orientation. Secondary branch } \\
8 \text { in. from main stem. }\end{array}$ \\
\hline W109 & WN1 & MUW109WN1-B-25060 & $3 / 27 / 08$ & $13: 25$ & Branch & 2 & 2 & 15 & $\begin{array}{l}\text { Branch } 6 \text { in. above ground, south } \\
\text { orientation. }\end{array}$ \\
\hline W109 & WN2 & MUW109WN2-B-25061 & $3 / 27 / 08$ & $13: 26$ & Branch & $<1$ & ND & 30 & $\begin{array}{l}\text { Branches } 6 \text { in and } 12 \text { in. above } \\
\text { ground, south orientation. Took } \\
2 \text { small branches near each } \\
\text { other. }\end{array}$ \\
\hline W110 & WN1 & MUW110WN1-B-25062 & $3 / 27 / 08$ & $13: 30$ & Branch & $<1$ & ND & 3 & $\begin{array}{l}\text { Basal shoot } 1 \text { in. above ground, } \\
\text { west orientation. }\end{array}$ \\
\hline W110 & WN2 & MUW110WN2-B-25063 & $3 / 27 / 08$ & $13: 31$ & Branch & $<1$ & $<1$ & 3 & $\begin{array}{l}\text { Basal shoot } 1 \text { in. above ground, } \\
\text { west orientation. }\end{array}$ \\
\hline
\end{tabular}




\begin{tabular}{|c|c|c|c|c|c|c|c|c|c|}
\hline & & & & & & \multicolumn{2}{|c|}{ Concentration $^{\mathrm{b}}(\mu \mathrm{g} / \mathrm{kg})$} & \multirow[b]{2}{*}{$\begin{array}{l}\text { Heightc }^{c} \\
(\mathrm{~cm})\end{array}$} & \multirow[b]{2}{*}{ Sample Description } \\
\hline Location & Species $^{a}$ & Sample & Date & Time & Type & $\begin{array}{c}\text { Carbon } \\
\text { Tetrachloride }\end{array}$ & Chloroform & & \\
\hline
\end{tabular}

Sampling in March 2008 (cont.)

\begin{tabular}{|c|c|c|c|c|c|c|c|c|c|}
\hline W114 & WN2 & MUW114WN2-B-25064 & $3 / 27 / 08$ & $13: 32$ & Branch & 6 & $<1$ & 61 & $\begin{array}{l}\text { Branch } 24 \text { in. above ground, } \\
\text { south orientation. }\end{array}$ \\
\hline W119 & WN1 & MUW119WN1-B-25065 & 3/27/08 & $13: 35$ & Branch & 50 & 2 & 91 & $\begin{array}{l}\text { Branch } 3 \mathrm{ft} \text { above ground, east } \\
\text { orientation. Secondary branch } \\
8 \text { in. from main stem. }\end{array}$ \\
\hline W119 & WN2 & MUW119WN2-B-25066 & $3 / 27 / 08$ & $13: 36$ & Branch & 3 & 1 & 10 & $\begin{array}{l}\text { Branch } 4 \text { in. above ground, west } \\
\text { orientation. }\end{array}$ \\
\hline W121 & WN2 & MUW121WN2-B-25067 & $3 / 27 / 08$ & $13: 40$ & Branch & 3 & ND & 61 & $\begin{array}{l}\text { Branch } 24 \text { in. above ground, west } \\
\text { orientation. Secondary branch } \\
4 \text { in. from main trunk. }\end{array}$ \\
\hline W124 & WN2 & MUW124WN2-B-25068 & $3 / 27 / 08$ & $13: 41$ & Branch & 15 & 2 & 122 & $\begin{array}{l}\text { Branch } 4 \mathrm{ft} \text { above ground, south } \\
\text { orientation. }\end{array}$ \\
\hline W128 & WN1 & MUW128WN1-B-25069 & $3 / 27 / 08$ & $13: 42$ & Branch & 3 & ND & 20 & $\begin{array}{l}\text { Branch } 8 \text { in. above ground, east } \\
\text { orientation. }\end{array}$ \\
\hline W128 & WN2 & MUW128WN2-B-25070 & $3 / 27 / 08$ & $13: 43$ & Branch & 1 & ND & 61 & $\begin{array}{l}\text { Branch } 24 \text { in. above ground, } \\
\text { south orientation. }\end{array}$ \\
\hline W132 & WN1 & MUW132WN1-B-25071 & $3 / 27 / 08$ & $13: 45$ & Branch & $<1$ & ND & 91 & $\begin{array}{l}\text { Branch } 36 \text { in. above ground, } \\
\text { south orientation. Secondary } \\
\text { branch } 8 \text { in. from main stem. }\end{array}$ \\
\hline W132 & WN2 & MUW132WN2-B-25072 & 3/27/08 & $13: 46$ & Branch & 1 & ND & 122 & $\begin{array}{l}\text { Branch } 48 \text { in. above ground, } \\
\text { south orientation. }\end{array}$ \\
\hline W148 & WN2 & MUW148WN2-B-25073 & $3 / 27 / 08$ & $13: 47$ & Branch & $<1$ & ND & 152 & $\begin{array}{l}\text { Branch } 5 \mathrm{ft} \text { above ground, south } \\
\text { orientation. }\end{array}$ \\
\hline W158 & WN1 & MUW158WN1-B-25074 & $3 / 27 / 08$ & $13: 50$ & Branch & ND & ND & 15 & $\begin{array}{l}\text { Branch } 6 \text { in. above ground, west } \\
\text { orientation. }\end{array}$ \\
\hline W158 & WN2 & MUW158WN2-B-25075 & $3 / 27 / 08$ & $13: 51$ & Branch & ND & ND & 30 & $\begin{array}{l}\text { Branch } 12 \text { in. above ground, west } \\
\text { orientation. }\end{array}$ \\
\hline W210 & WN1 & MUW210WN1-B-25076 & $3 / 27 / 08$ & $14: 31$ & Branch & 23 & 3 & 122 & $\begin{array}{l}\text { Branch } 48 \text { in. above ground, } \\
\text { south orientation. Secondary } \\
\text { branch } 4 \text { in. from main stem. }\end{array}$ \\
\hline W210 & WN2 & MUW210WN2-B-25077 & $3 / 27 / 08$ & $14: 32$ & Branch & $<1$ & 1 & 20 & $\begin{array}{l}\text { Branch } 8 \text { in. above ground, west } \\
\text { orientation. }\end{array}$ \\
\hline
\end{tabular}




\begin{tabular}{|c|c|c|c|c|c|c|c|c|c|}
\hline & & & & & & \multicolumn{2}{|c|}{ Concentration $^{\mathrm{b}}(\mu \mathrm{g} / \mathrm{kg})$} & \multirow[b]{2}{*}{$\begin{array}{l}\text { Heightc } \\
(\mathrm{cm})\end{array}$} & \multirow[b]{2}{*}{ Sample Description } \\
\hline Location & Species $^{a}$ & Sample & Date & Time & Type & $\begin{array}{c}\text { Carbon } \\
\text { Tetrachloride }\end{array}$ & Chloroform & & \\
\hline
\end{tabular}

Sampling in March 2008 (cont.)

\begin{tabular}{|c|c|c|c|c|c|c|c|c|c|}
\hline W212 & WN2 & MUW212WN2-B-25078 & $3 / 27 / 08$ & $14: 40$ & Branch & 10 & 2 & 91 & $\begin{array}{l}\text { Branch } 36 \text { in. above ground, east } \\
\text { orientation. Secondary branch } \\
4 \text { in. from main stem. }\end{array}$ \\
\hline W308 & EC1 & MUW308EC1-B-25080 & $3 / 27 / 08$ & 14:46 & Branch & $<1$ & ND & 91 & $\begin{array}{l}\text { Branch } 36 \text { in. above ground, east } \\
\text { orientation. Cottonwood. }\end{array}$ \\
\hline W308 & $\mathrm{NC}$ & MUW308EC-B-25079 & $3 / 27 / 08$ & $14: 45$ & Branch & ND & ND & 122 & $\begin{array}{l}\text { Branch } 48 \text { in. above ground, east } \\
\text { orientation. This is a catalpa } \\
\text { tree. }\end{array}$ \\
\hline W357 & WN1 & MUW357WN1-B-25081 & $3 / 27 / 08$ & 13:55 & Branch & ND & ND & 0 & $\begin{array}{l}\text { Base sucker collected at ground } \\
\text { level, north orientation. }\end{array}$ \\
\hline W407 & WN1 & MUW407WN1-B-25082 & $3 / 27 / 08$ & 14:47 & Branch & 31 & 3 & 152 & $\begin{array}{l}\text { Branch } 5 \mathrm{ft} \text { above ground, south } \\
\text { orientation. }\end{array}$ \\
\hline W407 & WN2 & MUW407WN2-B-25083 & $3 / 27 / 08$ & $14: 48$ & Branch & 62 & 2 & 122 & $\begin{array}{l}\text { Branch } 48 \text { in. above ground, } \\
\text { south orientation. Secondary } \\
\text { branch } 6 \text { in. from main stem. }\end{array}$ \\
\hline W417 & EC1 & MUW417EC1-B-25084 & $3 / 27 / 08$ & $14: 50$ & Branch & 3 & $<1$ & 152 & $\begin{array}{l}\text { Branch } 5 \mathrm{ft} \text { above ground, east } \\
\text { orientation. }\end{array}$ \\
\hline W422 & EC2 & MUW422EC2-B-25086 & $3 / 27 / 08$ & 14:51 & Branch & $<1$ & ND & 152 & $\begin{array}{l}\text { Branch } 5 \mathrm{ft} \text { above ground, west } \\
\text { orientation. Secondary branch } \\
8 \text { in. from main stem. }\end{array}$ \\
\hline W426 & EC2 & MUW426EC2-B-25085 & $3 / 27 / 08$ & 14:52 & Branch & $<1$ & $<1$ & 152 & $\begin{array}{l}\text { Branch } 5 \mathrm{ft} \text { above ground, west } \\
\text { orientation. }\end{array}$ \\
\hline W427 & EC & MUW427EC-B-25100 & $3 / 27 / 08$ & 14:55 & Branch & $<1$ & ND & 183 & $\begin{array}{l}\text { Branch } 6 \mathrm{ft} \text { above ground, west } \\
\text { orientation. Secondary branch } \\
12 \text { in. from main trunk. Labeled } \\
\text { in field as EC2. }\end{array}$ \\
\hline W427 & GA & MUW427GA-B-25101 & $3 / 27 / 08$ & 14:56 & Branch & ND & ND & 122 & $\begin{array}{l}\text { Branch } 48 \text { in. above ground, } \\
\text { south orientation. Distal branch } \\
\text { tip. }\end{array}$ \\
\hline W447 & WN & MUW447WN-B-25102 & $3 / 27 / 08$ & $15: 00$ & Branch & ND & $<1$ & 61 & $\begin{array}{l}\text { Branch } 24 \text { in. above ground, } \\
\text { south orientation. }\end{array}$ \\
\hline W505 & EC1 & MUW505EC1-B-25103 & $3 / 27 / 08$ & $15: 32$ & Branch & $<1$ & 2 & 152 & $\begin{array}{l}\text { Branch } 5 \mathrm{ft} \text { above ground, west } \\
\text { orientation. Secondary branch } \\
16 \text { in. from main stem. }\end{array}$ \\
\hline
\end{tabular}




\begin{tabular}{|c|c|c|c|c|c|c|c|c|c|}
\hline & & & & & & \multicolumn{2}{|c|}{ Concentration $^{\mathrm{b}}(\mu \mathrm{g} / \mathrm{kg})$} & \multirow[b]{2}{*}{$\begin{array}{l}\text { Height }^{\mathrm{C}} \\
(\mathrm{cm})\end{array}$} & \multirow[b]{2}{*}{ Sample Description } \\
\hline Location & Species $^{a}$ & Sample & Date & Time & Type & $\begin{array}{c}\text { Carbon } \\
\text { Tetrachloride }\end{array}$ & Chloroform & & \\
\hline
\end{tabular}

Sampling in March 2008 (cont.)

\begin{tabular}{|c|c|c|c|c|c|c|c|c|c|}
\hline W505 & EC2 & MUW505EC2-B-25104 & $3 / 27 / 08$ & $15: 33$ & Branch & $<1$ & 1 & 122 & $\begin{array}{l}\text { Branch } 48 \text { in. above ground, west } \\
\text { orientation. }\end{array}$ \\
\hline W606 & EC1 & MUW606EC1-B-25105 & $3 / 27 / 08$ & $15: 30$ & Branch & 41 & 7 & 122 & $\begin{array}{l}\text { Branch } 48 \text { in. above ground, east } \\
\text { orientation. }\end{array}$ \\
\hline W606 & EC2 & MUW606EC2-B-25106 & $3 / 27 / 08$ & $15: 31$ & Branch & 35 & 4 & 152 & $\begin{array}{l}\text { Branch } 5 \mathrm{ft} \text { above ground, east } \\
\text { orientation. }\end{array}$ \\
\hline W611 & EC1 & MUW611EC1-B-25107 & $3 / 27 / 08$ & $15: 37$ & Branch & $<1$ & 2 & 91 & $\begin{array}{l}\text { Branch } 36 \text { in. above ground, east } \\
\text { orientation. Secondary branch } \\
2 \text { in. from main stem. }\end{array}$ \\
\hline W616 & EC1 & MUW616EC1-B-25108 & $3 / 27 / 08$ & $15: 26$ & Branch & 6 & 3 & 10 & $\begin{array}{l}\text { Branch } 4 \text { in. above ground, west } \\
\text { orientation. }\end{array}$ \\
\hline W621 & EC & MUW621EC-B-25109 & $3 / 27 / 08$ & $15: 25$ & Branch & $<1$ & 4 & 122 & $\begin{array}{l}\text { Branch } 48 \text { in. above ground, west } \\
\text { orientation. }\end{array}$ \\
\hline W626 & EC & MUW626EC-B-25110 & $3 / 27 / 08$ & $15: 21$ & Branch & ND & 2 & 122 & $\begin{array}{l}\text { Branch } 48 \text { in. above ground, } \\
\text { south orientation. Secondary } \\
\text { branch } 6 \text { in. from main stem. }\end{array}$ \\
\hline W636 & EC & MUW636EC-B-25111 & $3 / 27 / 08$ & $15: 20$ & Branch & $<1$ & ND & 122 & $\begin{array}{l}\text { Branch } 48 \text { in. above ground, } \\
\text { south orientation. Secondary } \\
\text { branch } 12 \text { in. off main stem. }\end{array}$ \\
\hline W646 & WN1 & MUW646WN1-B-25112 & $3 / 27 / 08$ & $15: 15$ & Branch & ND & 1 & 15 & $\begin{array}{l}\text { Branch } 6 \text { in. above ground, south } \\
\text { orientation. }\end{array}$ \\
\hline W805 & WN1 & MUW805WN1-B-25090 & $3 / 27 / 08$ & $14: 20$ & Branch & 5 & 1 & 10 & $\begin{array}{l}\text { Branch } 4 \text { in. above ground, south } \\
\text { orientation. }\end{array}$ \\
\hline W806 & EC2 & MUW806EC2-B-25113 & $3 / 27 / 08$ & $14: 16$ & Branch & 18 & 2 & 122 & $\begin{array}{l}\text { Branch } 48 \text { in. above ground, } \\
\text { south orientation. }\end{array}$ \\
\hline W808 & WN2 & MUW808WN2-B-25089 & $3 / 27 / 08$ & $14: 21$ & Branch & 3 & ND & 122 & $\begin{array}{l}\text { Branch } 48 \text { in. above ground, } \\
\text { south orientation. Tree is } \\
\text { mislabeled as W807. }\end{array}$ \\
\hline W809 & EC2 & MUW809EC2-B-25091 & $3 / 27 / 08$ & $14: 30$ & Branch & 96 & 2 & 152 & $\begin{array}{l}\text { Branch } 5 \mathrm{ft} \text { above ground, west } \\
\text { orientation. }\end{array}$ \\
\hline W810 & EC2 & MUW810EC2-B-25114 & $3 / 27 / 08$ & $14: 15$ & Branch & 4 & ND & 122 & $\begin{array}{l}\text { Branch } 48 \text { in. above ground, east } \\
\text { orientation. }\end{array}$ \\
\hline
\end{tabular}




\begin{tabular}{|c|c|c|c|c|c|c|c|c|c|}
\hline & & & & & & \multicolumn{2}{|c|}{ Concentration $^{\mathrm{b}}(\mu \mathrm{g} / \mathrm{kg})$} & \multirow[b]{2}{*}{$\begin{array}{l}\text { Heightc }^{c} \\
(\mathrm{~cm})\end{array}$} & \multirow[b]{2}{*}{ Sample Description } \\
\hline Location & Species $^{a}$ & Sample & Date & Time & Type & $\begin{array}{c}\text { Carbon } \\
\text { Tetrachloride }\end{array}$ & Chloroform & & \\
\hline
\end{tabular}

Sampling in March 2008 (cont.)

\begin{tabular}{|c|c|c|c|c|c|c|c|c|c|}
\hline W820 & $\mathrm{EC} 1$ & MUW820EC1-B-25115 & $3 / 27 / 08$ & $14: 10$ & Branch & ND & ND & 122 & $\begin{array}{l}\text { Branch } 48 \text { in. above ground, west } \\
\text { orientation. Secondary branch } \\
3 \text { in. from main stem. }\end{array}$ \\
\hline W820 & EC2 & MUW820EC2-B-25116 & $3 / 27 / 08$ & $14: 11$ & Branch & ND & ND & 122 & $\begin{array}{l}\text { Branch } 48 \text { in. above ground, west } \\
\text { orientation. }\end{array}$ \\
\hline W845 & EC1 & MUW845EC1-B-25117 & $3 / 27 / 08$ & $14: 05$ & Branch & ND & ND & 91 & $\begin{array}{l}\text { Branch } 36 \text { in. above ground, east } \\
\text { orientation. }\end{array}$ \\
\hline W854 & EC & MUW854EC-B-25118 & $3 / 27 / 08$ & $14: 00$ & Branch & ND & ND & 122 & $\begin{array}{l}\text { Branch } 4 \mathrm{ft} \text { above ground, north } \\
\text { orientation. }\end{array}$ \\
\hline W854 & GA & MUW854GA-B-25119 & $3 / 27 / 08$ & $14: 01$ & Branch & ND & ND & 0 & $\begin{array}{l}\text { Basal sucker collected at ground } \\
\text { level, west orientation. }\end{array}$ \\
\hline W908 & EC1 & MUW908EC1-B-25088 & $3 / 27 / 08$ & $15: 35$ & Branch & 12 & 3 & 122 & $\begin{array}{l}\text { Branch } 48 \text { in. above ground, west } \\
\text { orientation. Secondary branch } \\
2 \text { in. from main stem. }\end{array}$ \\
\hline PREW001 & Willow & MUPREW001-B-25120 & $3 / 27 / 08$ & $15: 45$ & Branch & $<1$ & 3 & 122 & $\begin{array}{l}\text { Sucker at base collected } 48 \text { in. } \\
\text { above ground, west orientation. }\end{array}$ \\
\hline PREW003 & Willow-Ash & MUPREW003-B-25121 & $3 / 27 / 08$ & $15: 50$ & Branch & ND & 2 & 122 & $\begin{array}{l}\text { Willow closest to creek bed is in } \\
\text { bad shape, looks dead, no } \\
\text { easy branches. Sampled } \\
\text { nearby ash which had ribbon } \\
\text { on. Samples at } 48 \text { in. above } \\
\text { ground. Fourth-order branch } \\
7 \mathrm{ft} \text { from main stem. }\end{array}$ \\
\hline PREW004 & Ash & MUPREW004-B-25122 & $3 / 27 / 08$ & $15: 51$ & Branch & $<1$ & 3 & 122 & $\begin{array}{l}\text { Branch } 48 \text { in. above ground, west } \\
\text { orientation. Ash, small, } \\
\text { between two cottonwoods. }\end{array}$ \\
\hline PREW006 & Cottonwood & MUPREW006-B-25123 & $3 / 27 / 08$ & $15: 55$ & Branch & ND & 2 & 183 & $\begin{array}{l}\text { Branch } 6 \mathrm{ft} \text { above ground, } \\
\text { northwest orientation. } \\
\text { Cottonwood, fourth-order } \\
\text { branch } 6 \mathrm{ft} \text { off main trunk. }\end{array}$ \\
\hline
\end{tabular}




\begin{tabular}{|c|c|c|c|c|c|c|c|c|c|}
\hline & & & & & & \multicolumn{2}{|c|}{ Concentration $^{\mathrm{b}}(\mu \mathrm{g} / \mathrm{kg})$} & & \\
\hline Location & Species $^{a}$ & Sample & Date & Time & Type & $\begin{array}{c}\text { Carbon } \\
\text { Tetrachloride }\end{array}$ & Chloroform & $\begin{array}{l}\text { Height }^{\mathrm{C}} \\
(\mathrm{cm})\end{array}$ & Sample Description \\
\hline
\end{tabular}

Sampling in March 2008 (cont.)

Willow

MUPREE009-B-25124

$3 / 27 / 08$

16:10 Branch

$<1$

3

ND

Bruttig-

MUBRUTTIG1-B-25126

$3 / 27 / 08$

16:21 Branch

ND

ND

ND

School-1

$$
\text { Ash }
$$

MUSCHOOL1-B-25125

$3 / 27 / 08$

16:15 Branch

Sampling in June 2008 (tree sacrifice)

$\begin{array}{lll}\text { W421 } & \text { EC2 } & \text { MUW421-EC2-R1-19128 } \\ \text { W421 } & \text { EC2 } & \text { MUW421-EC2-R2-19129 } \\ \text { W421 } & \text { EC2 } & \text { MUW421-EC2-R3-19130 } \\ \text { W421 } & \text { EC2 } & \text { MUW421-EC2-S1-19131 } \\ \text { W421 } & \text { EC2 } & \text { MUW421-EC2-S2-19132 } \\ \text { W421 } & \text { EC2 } & \text { MUW421-EC2-B1-19133 }\end{array}$

$\begin{array}{lll}6 / 23 / 08 & 12: 15 & \text { Root } \\ 6 / 23 / 08 & 12: 23 & \text { Root } \\ 6 / 23 / 08 & 12: 25 & \text { Root } \\ 6 / 23 / 08 & 12: 30 & \text { Soil } \\ 6 / 23 / 08 & 12: 32 & \text { Soil } \\ 6 / 23 / 08 & 12: 35 & \text { Branch }\end{array}$

$<1$

$<1$

$<1$

$<1$

$<1$

$6 / 23 / 08$
$<1$

2

1

ND

ND

$\begin{array}{llllrrr}\text { W421 } & \text { EC2 } & \text { MUW421-EC2-B2-19134 } & 6 / 23 / 08 & 12: 36 \text { Branch } & 41 & 16 \\ \text { W421 } & \text { EC2 } & \text { MUW421-EC2-B3-19135 } & 6 / 23 / 08 & 12: 40 \text { Branch } & 3 & 6 \\ \text { W421 } & \text { EC2 } & \text { MUW421-EC2-L1-19136 } & 6 / 23 / 08 & 12: 42 \text { Leaf } & \text { ND } & 2\end{array}$

122 Willow on east side of creek.

(Moved ribbon from tree on

west side to one on east side.)

Sucker 48 in. above ground, east orientation.

Branch $5 \mathrm{ft}$ above ground, west orientation. Tertiary branch 12 in. from main stem. Pin Oak.

Branch $6 \mathrm{ft}$ above ground, east orientation. Fourth-order branch $3 \mathrm{ft}$ from main trunk.

Basal shoot 1 in. above ground, north orientation. Ash.

Surface roots. Lateral roots; deep roots at site rotten. Live root.

Surface roots. Live lateral root.

Surface root. Live root.

Soil from roots.

Soil from roots rhizosphere.

Primary branch, 5 in. off ground line. (SOP deviation:

Orientation not recorded for branch samples, as tree had been cut off.)

Primary branch, 2 in. up second log.

Secondary branch, 2 in. up second log.

Leaf sample from branch sampled as B3-19135. 


\begin{tabular}{|c|c|c|c|c|c|c|c|c|c|}
\hline & & & & & & \multicolumn{2}{|c|}{ Concentration ${ }^{\mathrm{b}}(\mu \mathrm{g} / \mathrm{kg})$} & & \\
\hline Location & Species $^{a}$ & Sample & Date & Time & Type & $\begin{array}{c}\text { Carbon } \\
\text { Tetrachloride }\end{array}$ & Chloroform & $\begin{array}{l}\text { Height }^{\mathrm{C}} \\
(\mathrm{cm})\end{array}$ & Sample Description \\
\hline
\end{tabular}

Sampling in June 2008 (tree sacrifice) (cont.)

$\begin{array}{lllllllr}\text { W421 } & \text { EC2 } & \text { MUW421-EC2-B4-19137 } & 6 / 23 / 08 & 12: 44 \text { Branch } & 46 & 21 \\ \text { W421 } & \text { EC2 } & \text { MUW421-EC2-L2-19138 } & 6 / 23 / 08 & 12: 45 \text { Leaf } & \text { ND } & 1 \\ \text { W421 } & \text { EC2 } & \text { MUW421-EC2-B5-19139 } & 6 / 23 / 08 & 12: 48 \text { Branch } & 16 & 12 \\ \text { W421 } & \text { EC2 } & \text { MUW421-EC2-L3-19140 } & 6 / 23 / 08 & 12: 50 \text { Leaf } & \text { ND } & 2 \\ \text { W421 } & \text { EC2 } & \text { MUW421-EC2-B7-19142 } & 6 / 23 / 08 & 12: 55 \text { Branch } & <1 & 2 \\ \text { W421 } & \text { EC2 } & \text { MUW421-EC2-B6-19141 } & 6 / 23 / 08 & 12: 55 \text { Branch } & \text { ND } & <1 \\ & & & & & & \\ \text { W421 } & \text { EC2 } & \text { MUW421-EC2-L4-19143 } & 6 / 23 / 08 & 12: 57 \text { Leaf } & \text { ND } & 2 \\ \text { W421 } & \text { EC2 } & \text { MUW421-EC2-B8-19144 } & 6 / 23 / 08 & 12: 59 \text { Branch } & 17 & 15 \\ \text { W421 } & \text { EC2 } & \text { MUW421-EC2-B9-19145 } & 6 / 23 / 08 & 13: 02 \text { Branch } & 4 & 6 \\ \text { W421 } & \text { EC2 } & \text { MUW421-EC2-B10-19146 } & 6 / 23 / 08 & 13: 05 \text { Branch } & 3 & 4 \\ \text { W421 } & \text { EC2 } & \text { MUW421-EC2-L5-19147 } & 6 / 23 / 08 & 13: 06 \text { Leaf } & \text { ND } & 1 \\ \text { W421 } & \text { EC2 } & \text { MUW421-EC2-B11-19148 } & 6 / 23 / 08 & 13: 08 \text { Branch } & 2 & 2 \\ \text { W421 } & \text { EC2 } & \text { MUW421-EC2-B12-19149 } & 6 / 23 / 08 & 13: 10 \text { Branch } & <1 & 3 \\ \text { W421 } & \text { EC2 } & \text { MUW421-EC2-L6-19150 } & 6 / 23 / 08 & 13: 12 \text { Leaf } & \text { ND } & 1 \\ \text { W421 } & \text { EC2 } & \text { MUW421-EC2-B13-19151 } & 6 / 23 / 08 & 13: 14 \text { Branch } & <1 & 5 \\ \text { W421 } & \text { EC2 } & \text { MUW421-EC2-L7-19152 } & 6 / 23 / 08 & 13: 15 \text { Leaf } & \text { ND } & 2\end{array}$

rimary branch from second log, 18 in. up, same side as sample B1-19133.

Leaf sample from branch sampled as B4-19137.

Primary branch, 52 in. from bottom of second log, 45 deg off first secondary branch.

Leaf from branch sampled as B519139.

Sample 42 in. higher than B619141.

Secondary branch 65 in. from second cut; 6 in. off primary, first cut.

Leaf sample from branch sampled as B7-19142.

Primary branch 6 in. from third piece at intersection.

Sample 36 in. up from B8-19144, same branch.

Primary branch 3 in. below last cut.

Leaf sample from B10-19146.

Primary branch 6 in. from up fourth cut.

Branch sample 28 in. from log 4.

Leaf sample from branch sampled as B12-19149.

Primary branch $47 \mathrm{in.} \mathrm{off} \mathrm{fourth}$ log.

Leaf sample from branch sampled as B13-19151. 


\begin{tabular}{|c|c|c|c|c|c|c|c|c|c|}
\hline & & & & & & \multicolumn{2}{|c|}{ Concentration ${ }^{\mathrm{b}}(\mu \mathrm{g} / \mathrm{kg})$} & & \\
\hline Location & Species $^{a}$ & Sample & Date & Time & Type & $\begin{array}{c}\text { Carbon } \\
\text { Tetrachloride }\end{array}$ & Chloroform & $\begin{array}{l}\text { Height }^{\mathrm{C}} \\
(\mathrm{cm})\end{array}$ & Sample Description \\
\hline
\end{tabular}

Sampling in June 2008 (tree sacrifice) (cont.)

\begin{tabular}{|c|c|c|c|c|c|c|c|}
\hline W421 & EC2 & MUW421-EC2-B14-19153 & $6 / 23 / 08$ & $13: 17$ & Branch & $<1$ & 2 \\
\hline W421 & EC2 & MUW421-EC2-B15-19154 & $6 / 23 / 08$ & $13: 20$ & Branch & $<1$ & ND \\
\hline W421 & EC2 & MUW421-EC2-B16-19155 & 6/23/08 & 13:22 & Branch & ND & ND \\
\hline W421 & EC2 & MUW421-EC2-L8-19156 & 6/23/08 & $13: 25$ & Leaf & ND & 1 \\
\hline W421 & EC2 & MUW421-EC2-L9-19157 & 6/23/08 & $13: 30$ & Leaf & $N A^{f}$ & NA \\
\hline W421 & EC2 & MUW421-EC2-L10-19158 & 6/23/08 & 13:31 & Leaf & NA & NA \\
\hline W421 & EC2 & MUW421-EC2-B17-19159 & 6/23/08 & 13:32 & Branch & NA & NA \\
\hline W421 & EC2 & MUW421-EC2-C1-19160 & $6 / 23 / 08$ & $13: 40$ & Core & 53 & 8 \\
\hline W421 & EC2 & $\begin{array}{l}\text { MUW421-EC2-C2-19161- } \\
\text { edge }\end{array}$ & 6/23/08 & 13:45 & Core & 109 & 25 \\
\hline W421 & EC2 & $\begin{array}{l}\text { MUW421-EC2-C3-19162- } \\
\text { edge }\end{array}$ & 6/23/08 & $13: 46$ & Core & 13 & 6 \\
\hline W421 & EC2 & $\begin{array}{l}\text { MUW421-EC2-C4-19163- } \\
\text { edge }\end{array}$ & 6/23/08 & 13:50 & Core & 4 & 4 \\
\hline W421 & EC2 & $\begin{array}{l}\text { MUW421-EC2-C5-19164- } \\
\text { edge }\end{array}$ & 6/23/08 & 14:00 & Core & 2 & 3 \\
\hline
\end{tabular}

Branch 14 is 57 in. off begin fourth.

Apical branch 36 in. higher than B14-19153.

Top of tree, 3 in. below top distal leaves.

Apical leaves, leaves from B1619155.

Sample of leaves in plastic bag for moisture analysis.

Large leaf sample for moisture analysis and leaf area analysis.

Large branch sample for moisture analysis.

Core 1; beginning of second log; 1 -in.-thick slice cut off with saw for VOCs analyses.

Core 2; 31 in. from beginning of $\log 2 ; 1$-in.-thick slice for VOCs analyses. Sample aliquot removed from edge of core.

Core 3; 66 in. from beginning of $\log 2$ for VOCs analyses. Sample aliquot removed from edge of core.

Core 4; 36 in. up on third log for VOCs analyses. Sample aliquot removed from edge of core.

Core 5; 72 in. up on third log for VOCs analyses. Sample aliquot removed from edge of core. 


\begin{tabular}{|c|c|c|c|c|c|c|c|c|c|}
\hline & & & & & & \multicolumn{2}{|c|}{ Concentration $^{\mathrm{b}}(\mu \mathrm{g} / \mathrm{kg})$} & & \\
\hline Location & Species $^{a}$ & Sample & Date & Time & Type & $\begin{array}{c}\text { Carbon } \\
\text { Tetrachloride }\end{array}$ & Chloroform & $\begin{array}{l}\text { Height }^{\mathrm{c}} \\
(\mathrm{cm})\end{array}$ & Sample Description \\
\hline
\end{tabular}

Sampling in June 2008 (tree sacrifice) (cont.)

\begin{tabular}{|c|c|c|c|c|c|c|c|}
\hline W421 & EC2 & $\begin{array}{l}\text { MUW421-EC2-C5-19164- } \\
\text { center }\end{array}$ & $6 / 23 / 08$ & $14: 00$ & Core & 9 & 7 \\
\hline W421 & EC2 & $\begin{array}{l}\text { MUW421-EC2-C4-19163- } \\
\text { center }\end{array}$ & $6 / 23 / 08$ & $14: 00$ & Core & 34 & 13 \\
\hline W421 & EC2 & $\begin{array}{l}\text { MUW421-EC2-C6-19165- } \\
\text { edge }\end{array}$ & $6 / 23 / 08$ & $14: 01$ & Core & 1 & 3 \\
\hline W421 & EC2 & $\begin{array}{l}\text { MUW421-EC2-C6-19165- } \\
\text { center }\end{array}$ & $6 / 23 / 08$ & $14: 01$ & Core & 1 & 4 \\
\hline W421 & EC2 & MUW421-EC2-C7-19166 & $6 / 23 / 08$ & $14: 02$ & Core & $<1$ & 2 \\
\hline W421 & EC2 & MUW421-EC2-C8-19167 & $6 / 23 / 08$ & $14: 45$ & Core & NA & NA \\
\hline W421 & EC2 & MUW421-EC2-C9-19168 & $6 / 23 / 08$ & $15: 00$ & Core & NA & NA \\
\hline W421 & EC2 & MUW421-EC2-C10-19169 & $6 / 23 / 08$ & $15: 01$ & Core & NA & NA \\
\hline W421 & EC1 & MUW421-EC1-C1-19170 & $6 / 23 / 08$ & $16: 00$ & Core & NA & NA \\
\hline
\end{tabular}

Sampling in July 2008

E100 WN1 MUE100-WN1-B-19178 7/21/08 10:45 Branch
ND

A

Core 5; 72 in. up on third log for VOCs analyses. Sample aliquot removed from center of core.

Core $4 ; 36$ in. up on third log for VOCs analyses. Sample aliquot removed from center of core.

Core $6 ; 36$ in. up on fourth log for VOCs analyses. Sample aliquot removed from edge of core.

Core 6; 36 in. up on fourth log for VOCs analyses. Sample aliquot removed from center of core.

Core $7 ; 72$ in. up on fourth log for VOCs analyses.

Core 8; core with stain collected at tree base, ground level, for sapwood stain.

Log 2 base for density analysis. Large wood.

Core 10 for density analysis. Smaller wood.

Second tree at location for sap stain. Core sample 1 from base

ND of tree. stem. Sample collected at ground level, south orientation. 


\begin{tabular}{|c|c|c|c|c|c|c|c|c|c|}
\hline & & & & & & \multicolumn{2}{|c|}{ Concentration ${ }^{\mathrm{b}}(\mu \mathrm{g} / \mathrm{kg})$} & & \\
\hline Location & Species $^{a}$ & Sample & Date & Time & Type & $\begin{array}{c}\text { Carbon } \\
\text { Tetrachloride }\end{array}$ & Chloroform & $\begin{array}{l}\text { Height } \\
\text { (cm) }\end{array}$ & Sample Description \\
\hline
\end{tabular}

Sampling in July 2008 (cont.)

\begin{tabular}{|c|c|c|c|c|c|c|c|c|c|}
\hline E101 & WN1 & MUE101-WN1-B-19179 & $7 / 21 / 08$ & 10:47 & Branch & $<1$ & ND & 0 & $\begin{array}{l}\text { Secondary branch } 1.5 \mathrm{ft} \text { off main } \\
\text { stem. Sample collected at } \\
\text { ground level, south orientation. }\end{array}$ \\
\hline E106 & WN1 & MUE106-WN1-B-19180 & $7 / 21 / 08$ & $11: 30$ & Branch & 3 & 2 & 46 & $\begin{array}{l}\text { Primary branch. Sample collected } \\
1.5 \mathrm{ft} \text { above ground, east } \\
\text { orientation. }\end{array}$ \\
\hline E111 & WN1 & MUE111-WN1-B-19181 & $7 / 21 / 08$ & $11: 31$ & Branch & $<1$ & ND & 91 & $\begin{array}{l}\text { Secondary branch } 6 \text { in. off main } \\
\text { stem. Sample collected } 3 \mathrm{ft} \\
\text { above ground, north } \\
\text { orientation. Wet ground. }\end{array}$ \\
\hline E112 & WN2 & MUE112-WN2-B-19183 & $7 / 21 / 08$ & 11:35 & Branch & $<1$ & ND & 46 & $\begin{array}{l}\text { Primary branch. Sample collected } \\
1.5 \mathrm{ft} \text { above ground, northwest } \\
\text { orientation. }\end{array}$ \\
\hline E112 & WN2 & MUE112-WN2-L-19182 & $7 / 21 / 08$ & $11: 36$ & Leaf & ND & 1 & 46 & $\begin{array}{l}\text { Leaf sample from same branch as } \\
\text { sample ID } 19183 \text {. }\end{array}$ \\
\hline E113 & WN1 & MUE113-WN1-B-19185 & $7 / 21 / 08$ & $11: 40$ & Branch & ND & ND & 30 & $\begin{array}{l}\text { Primary branch. Sample collected } \\
1 \mathrm{ft} \text { above ground, west } \\
\text { orientation. }\end{array}$ \\
\hline E113 & WN1 & MUE113-WN1-L-19184 & $7 / 21 / 08$ & $11: 41$ & Leaf & ND & $<1$ & 30 & $\begin{array}{l}\text { Leaf sample from same branch as } \\
\text { sample ID } 19185 .\end{array}$ \\
\hline E117 & WN2 & MUE117-WN2-B-19186 & $7 / 21 / 08$ & $11: 42$ & Branch & ND & ND & 46 & $\begin{array}{l}\text { Primary branch. Sample collected } \\
1.5 \mathrm{ft} \text { above ground, south } \\
\text { orientation. Tree E116W is } \\
\text { dead. Unclear from log if this is } \\
\text { the previously sampled WN2 } \\
\text { tree. }\end{array}$ \\
\hline E118 & WN1 & MUE118-WN1-B-19187 & $7 / 21 / 08$ & $11: 45$ & Branch & $<1$ & 1 & 122 & $\begin{array}{l}\text { Secondary branch. Sample } \\
\text { collected } 4 \mathrm{ft} \text { above ground, } \\
\text { north orientation. }\end{array}$ \\
\hline E121 & WN1 & MUE121-WN1-B-19188 & $7 / 21 / 08$ & $11: 46$ & Branch & ND & 1 & 30 & $\begin{array}{l}\text { Primary branch. Sample collected } \\
1 \mathrm{ft} \text { above ground, west } \\
\text { orientation. }\end{array}$ \\
\hline
\end{tabular}




\begin{tabular}{|c|c|c|c|c|c|c|c|c|c|}
\hline & & & & & & \multicolumn{2}{|c|}{ Concentration $^{\mathrm{b}}(\mu \mathrm{g} / \mathrm{kg})$} & \multirow[b]{2}{*}{$\begin{array}{l}\text { Height }^{\mathrm{C}} \\
(\mathrm{cm})\end{array}$} & \multirow[b]{2}{*}{ Sample Description } \\
\hline Location & Species $^{a}$ & Sample & Date & Time & Type & $\begin{array}{c}\text { Carbon } \\
\text { Tetrachloride }\end{array}$ & Chloroform & & \\
\hline
\end{tabular}

Sampling in July 2008 (cont.)

\begin{tabular}{|c|c|c|c|c|c|c|c|c|c|}
\hline E126 & WN1 & MUE126-WN1-B-19189 & $7 / 21 / 08$ & $11: 50$ & Branch & ND & 1 & 30 & $\begin{array}{l}\text { Secondary branch. Sample } \\
\text { collected } 1 \mathrm{ft} \text { above ground, } \\
\text { west orientation. }\end{array}$ \\
\hline E129 & WN1 & MUE129-WN1-B-19190 & $7 / 21 / 08$ & $12: 00$ & Branch & ND & $<1$ & 61 & $\begin{array}{l}\text { Primary branch. Sample collected } \\
2 \mathrm{ft} \text { above ground, east } \\
\text { orientation. Tree is broken } \\
\text { approximately } 2 \mathrm{ft} \text { above } \\
\text { ground. }\end{array}$ \\
\hline E206 & WN2 & MUE206-WN2-B-19191 & $7 / 21 / 08$ & $12: 05$ & Branch & $<1$ & ND & 30 & $\begin{array}{l}\text { Primary branch. Sample collected } \\
1 \mathrm{ft} \text { above ground, west } \\
\text { orientation. }\end{array}$ \\
\hline E207 & WN1 & MUE207-WN1-B-19192 & $7 / 21 / 08$ & $12: 08$ & Branch & ND & $<1$ & 30 & $\begin{array}{l}\text { Primary branch. Sample collected } \\
1 \mathrm{ft} \text { above ground, west } \\
\text { orientation. Small tree. }\end{array}$ \\
\hline E207 & WN2 & MUE207-WN2-B-19193 & $7 / 21 / 08$ & $12: 06$ & Branch & $<1$ & 1 & 91 & $\begin{array}{l}\text { Primary branch. Sample collected } \\
3 \mathrm{ft} \text { above ground, west } \\
\text { orientation. }\end{array}$ \\
\hline E245 & WN1 & MUE245-WN1-B-19194 & $7 / 21 / 08$ & $12: 02$ & Branch & ND & ND & 5 & $\begin{array}{l}\text { Primary branch. Sample collected } \\
2 \text { in. above ground, south } \\
\text { orientation. }\end{array}$ \\
\hline E300 & WN1 & MUE300WN1-C-26294 & $7 / 23 / 08$ & $8: 55$ & Core & NA & NA & & $\begin{array}{l}\text { Core for sapwood area analysis. } \\
\text { Density, agronomy analyses; } \\
\text { no VOCs analyses. }\end{array}$ \\
\hline E302 & WN1 & MUE302WN1-C-26295 & $7 / 23 / 08$ & $8: 56$ & Core & NA & NA & & $\begin{array}{l}\text { Core for sapwood area analysis. } \\
\text { Density, agronomy analysis; no } \\
\text { VOCs analyses. }\end{array}$ \\
\hline E304 & WN2 & MUE304-WN2-B-19195 & $7 / 21 / 08$ & $10: 50$ & Branch & ND & ND & 91 & $\begin{array}{l}\text { Primary branch. Sample collected } \\
3 \mathrm{ft} \text { above ground, south } \\
\text { orientation. Wet ground. }\end{array}$ \\
\hline E308 & WN1 & MUE308-WN1-B-19196 & $7 / 21 / 08$ & $10: 55$ & Branch & $<1$ & ND & 0 & $\begin{array}{l}\text { Secondary branch } 6 \text { in. off main } \\
\text { stem. Sample collected at } \\
\text { ground level, west orientation. }\end{array}$ \\
\hline
\end{tabular}




\begin{tabular}{|c|c|c|c|c|c|c|c|c|c|}
\hline & & & & & & \multicolumn{2}{|c|}{ Concentration $^{\mathrm{b}}(\mu \mathrm{g} / \mathrm{kg})$} & \multirow[b]{2}{*}{$\begin{array}{l}\text { Heightc }^{c} \\
(\mathrm{~cm})\end{array}$} & \multirow[b]{2}{*}{ Sample Description } \\
\hline Location & Species $^{a}$ & Sample & Date & Time & Type & $\begin{array}{c}\text { Carbon } \\
\text { Tetrachloride }\end{array}$ & Chloroform & & \\
\hline
\end{tabular}

Sampling in July 2008 (cont.)

\begin{tabular}{|c|c|c|c|c|c|c|c|c|c|}
\hline E308 & WN2 & MUE308-WN2-B-19197 & $7 / 21 / 08$ & $10: 56$ & Branch & 12 & ND & 30 & $\begin{array}{l}\text { Secondary branch } 3 \text { in. off main } \\
\text { stem. Sample collected } 1 \mathrm{ft} \\
\text { above ground, west orientation. }\end{array}$ \\
\hline E309 & WN1 & MUE309-WN1-B-19198 & $7 / 21 / 08$ & $10: 58$ & Branch & 11 & $<1$ & 15 & $\begin{array}{l}\text { Primary branch. Sample collected } \\
6 \text { in. above ground, east } \\
\text { orientation. }\end{array}$ \\
\hline E309 & WN2 & MUE309-WN2-B-19199 & $7 / 21 / 08$ & $11: 00$ & Branch & $<1$ & ND & 46 & $\begin{array}{l}\text { Primary branch. Sample collected } \\
1.5 \mathrm{ft} \text { above ground, southeast } \\
\text { orientation. Wet ground. }\end{array}$ \\
\hline E314 & HP1 & MUE314-HP1-B-19200 & $7 / 21 / 08$ & $11: 05$ & Branch & $<1$ & ND & 91 & $\begin{array}{l}\text { Secondary branch. Sample } \\
\text { collected } 3 \mathrm{ft} \text { above ground, } \\
\text { east orientation. Wet ground. }\end{array}$ \\
\hline E319 & WN2 & MUE319-WN2-B-19201 & $7 / 21 / 08$ & $12: 15$ & Branch & ND & ND & 13 & $\begin{array}{l}\text { Secondary branch } 5 \text { in. off main } \\
\text { stem. Sample collected } 5 \text { in. } \\
\text { above ground, south } \\
\text { orientation. }\end{array}$ \\
\hline E324 & WN1 & MUE324-WN1-B-19202 & $7 / 21 / 08$ & $12: 18$ & Branch & ND & ND & 30 & $\begin{array}{l}\text { Primary branch. Sample collected } \\
1 \mathrm{ft} \text { above ground, south } \\
\text { orientation. }\end{array}$ \\
\hline E340 & WN2 & MUE340-WN2-B-19203 & $7 / 21 / 08$ & $12: 20$ & Branch & ND & ND & 11 & $\begin{array}{l}\text { Secondary branch } 2 \mathrm{ft} \text { off main } \\
\text { stem. Sample collected } 4.5 \text { in. } \\
\text { above ground, north } \\
\text { orientation. }\end{array}$ \\
\hline E345 & WN1 & MUE345-WN1-B-19204 & $7 / 21 / 08$ & $12: 22$ & Branch & ND & ND & 46 & $\begin{array}{l}\text { Primary branch. Sample collected } \\
1.5 \mathrm{ft} \text { above ground, north } \\
\text { orientation. }\end{array}$ \\
\hline E400 & EC1 & MUE400-EC1-B-19206 & $7 / 21 / 08$ & $14: 12$ & Branch & ND & ND & 46 & $\begin{array}{l}\text { Primary branch. Sample collected } \\
1.5 \mathrm{ft} \text { above ground, west } \\
\text { orientation. NOTE: true E400 } \\
\text { tree. }\end{array}$ \\
\hline
\end{tabular}




\begin{tabular}{|c|c|c|c|c|c|c|c|c|c|}
\hline & & & & & & \multicolumn{2}{|c|}{ Concentration $^{\mathrm{b}}(\mu \mathrm{g} / \mathrm{kg})$} & \multirow[b]{2}{*}{$\begin{array}{l}\text { Heightc } \\
(\mathrm{cm})\end{array}$} & \multirow[b]{2}{*}{ Sample Description } \\
\hline Location & Species $^{a}$ & Sample & Date & Time & Type & $\begin{array}{c}\text { Carbon } \\
\text { Tetrachloride }\end{array}$ & Chloroform & & \\
\hline
\end{tabular}

Sampling in July 2008 (cont.)

E401

E40

E428

E428

E500

EC

MUE500-EC1-B-19209

$7 / 21 / 08$

14:20 Branch

ND

$<1$

ND

ND

ND

ND

NA

NA

E502

E507

E512

E517
WN1

MUE512-WN1-B-19212

$7 / 21 / 08$

14:26 Branch

$7 / 21 / 08$

14:27 Branch
WN1 MUE517-WN1-B-19213
ND

ND

ND

ND

ND

ND

$7 / 21 / 08$

46

Primary branch. Sample collected

$1.5 \mathrm{ft}$ above ground, west orientation. NOTE: tree is mislabeled as E400 in field, but is really E401.

Core for sapwood area analysis.

Density, agronomy analysis; no VOCs analyses.

Secondary branch. Sample collected $5 \mathrm{ft}$ above ground, west orientation.

Primary branch. Sample collected $1 \mathrm{ft}$ above ground, south orientation.

Secondary branch 8 in. off primary branch. Sample collected $1 \mathrm{ft}$ above ground, west orientation. Tree EC2 is not there.

Primary branch. Sample collected at ground level, west orientation.

Secondary branch 6 in. off main stem. Sample collected $3 \mathrm{ft}$ above ground, south orientation.

Primary branch. Sample collected 2 in. above ground, south orientation.

Primary branch. Sample collected 6 in. above ground, east orientation. 


\begin{tabular}{|c|c|c|c|c|c|c|c|c|c|}
\hline & & & & & & \multicolumn{2}{|c|}{ Concentration $^{\mathrm{b}}(\mu \mathrm{g} / \mathrm{kg})$} & \multirow[b]{2}{*}{$\begin{array}{l}\text { Heightc } \\
(\mathrm{cm})\end{array}$} & \multirow[b]{2}{*}{ Sample Description } \\
\hline Location & Species $^{a}$ & Sample & Date & Time & Type & $\begin{array}{c}\text { Carbon } \\
\text { Tetrachloride }\end{array}$ & Chloroform & & \\
\hline
\end{tabular}

Sampling in July 2008 (cont.)

\begin{tabular}{|c|c|c|c|c|c|c|c|c|c|}
\hline E522 & EC & MUE522-EC-B-19214 & $7 / 21 / 08$ & $14: 31$ & Branch & $<1$ & ND & 91 & $\begin{array}{l}\text { Primary branch. Sample collected } \\
3 \mathrm{ft} \text { above ground, north } \\
\text { orientation. }\end{array}$ \\
\hline E527 & WN2 & MUE527-WN2-B-19215 & $7 / 21 / 08$ & $14: 35$ & Branch & ND & ND & 122 & $\begin{array}{l}\text { Secondary branch. Sample } \\
\text { collected } 4 \mathrm{ft} \text { above ground, } \\
\text { west orientation. }\end{array}$ \\
\hline E532 & WN1 & MUE532-WN1-B-19216 & $7 / 21 / 08$ & $14: 45$ & Branch & ND & ND & 168 & $\begin{array}{l}\text { Secondary branch } 1 \mathrm{ft} \text { from main } \\
\text { branch. Sample collected } 5.5 \mathrm{ft} \\
\text { above ground, east orientation. }\end{array}$ \\
\hline E537 & WN1 & MUE537-WN1-B-19217 & $7 / 21 / 08$ & $14: 55$ & Branch & ND & ND & 5 & $\begin{array}{l}\text { Secondary branch } 6 \text { in. off main } \\
\text { stem. Sample collected } 2 \text { in. } \\
\text { above ground, south } \\
\text { orientation. }\end{array}$ \\
\hline E542 & WN & MUE542-WN-B-19218 & $7 / 21 / 08$ & $15: 10$ & Branch & ND & ND & 152 & $\begin{array}{l}\text { Secondary branch } 6 \text { in. off main } \\
\text { stem. Sample collected } 5 \mathrm{ft} \\
\text { above ground, north } \\
\text { orientation. }\end{array}$ \\
\hline E547 & WN2 & MUE547-WN2-B-19219 & $7 / 21 / 08$ & $15: 11$ & Branch & ND & ND & 122 & $\begin{array}{l}\text { Primary branch. Sample collected } \\
4 \mathrm{ft} \text { above ground, north } \\
\text { orientation. }\end{array}$ \\
\hline E550 & WN1 & MUE550-WN1-B-19220 & $7 / 21 / 08$ & $15: 15$ & Branch & ND & ND & 152 & $\begin{array}{l}\text { Secondary branch. Sample } \\
\text { collected } 5 \mathrm{ft} \text { above ground, } \\
\text { east orientation. Tree location } \\
\text { is mislabeled in field as E556; } \\
\text { true location is E550. }\end{array}$ \\
\hline E555 & WN1 & MUE555-WN1-B-19221 & $7 / 21 / 08$ & $15: 20$ & Branch & ND & ND & 152 & $\begin{array}{l}\text { Secondary branch } 1.5 \mathrm{ft} \text { off main } \\
\text { stem. Sample collected } 5 \mathrm{ft} \\
\text { above ground, east orientation. }\end{array}$ \\
\hline E557 & WN1 & MUE557-WN1-B-19222 & $7 / 21 / 08$ & $15: 21$ & Branch & ND & ND & 168 & $\begin{array}{l}\text { Secondary branch } 2 \text { in. off main } \\
\text { stem. Sample collected } 5.5 \mathrm{ft} \\
\text { above ground, south } \\
\text { orientation. }\end{array}$ \\
\hline
\end{tabular}




\begin{tabular}{|c|c|c|c|c|c|c|c|c|c|}
\hline & & & & & & \multicolumn{2}{|c|}{ Concentration ${ }^{\mathrm{b}}(\mu \mathrm{g} / \mathrm{kg})$} & & \\
\hline Location & Species $^{a}$ & Sample & Date & Time & Type & $\begin{array}{c}\text { Carbon } \\
\text { Tetrachloride }\end{array}$ & Chloroform & $\begin{array}{l}\text { Height } \\
\text { (cm) }\end{array}$ & Sample Description \\
\hline
\end{tabular}

Sampling in July 2008 (cont.)

\begin{tabular}{|c|c|c|c|c|c|c|c|c|c|}
\hline E600 & EC2 & MUE600-EC2-B-19223 & $7 / 21 / 08$ & $16: 15$ & Branch & ND & ND & 91 & $\begin{array}{l}\text { Secondary branch } 1 \mathrm{ft} \text { off main } \\
\text { stem. Sample collected } 3 \mathrm{ft} \\
\text { above ground, north } \\
\text { orientation. }\end{array}$ \\
\hline E601 & WN1 & MUE601-WN1-B-19224 & $7 / 21 / 08$ & $16: 16$ & Branch & ND & ND & 5 & $\begin{array}{l}\text { Primary branch. Sample collected } \\
2 \text { in. above ground, northwest } \\
\text { orientation. }\end{array}$ \\
\hline E602 & EC2 & MUE602-EC2-B-19225 & $7 / 21 / 08$ & $16: 20$ & Branch & ND & ND & 5 & $\begin{array}{l}\text { Primary branch. Sample collected } \\
2 \text { in. above ground, west- } \\
\text { northwest orientation. }\end{array}$ \\
\hline E659 & WN1 & MUE659-WN1-B-19226 & $7 / 21 / 08$ & $15: 30$ & Branch & $<1$ & ND & 15 & $\begin{array}{l}\text { Primary branch. Sample collected } \\
6 \text { in. above ground, west } \\
\text { orientation. }\end{array}$ \\
\hline E700 & EC1 & MUE700-EC1-B-19227 & $7 / 21 / 08$ & $16: 21$ & Branch & ND & ND & 122 & $\begin{array}{l}\text { Secondary branch } 6 \text { in. off main } \\
\text { stem. Sample collected } 4 \mathrm{ft} \\
\text { above ground, north } \\
\text { orientation. }\end{array}$ \\
\hline E701 & WN1 & MUE701-WN1-B-19228 & $7 / 21 / 08$ & $16: 25$ & Branch & ND & ND & 5 & $\begin{array}{l}\text { Primary branch. Sample collected } \\
2 \text { in. above ground, west } \\
\text { orientation. }\end{array}$ \\
\hline E702 & EC2 & MUE702-EC2-B-19229 & $7 / 21 / 08$ & $16: 26$ & Branch & ND & ND & 15 & $\begin{array}{l}\text { Primary branch. Sample collected } \\
6 \text { in. above ground, south } \\
\text { orientation. }\end{array}$ \\
\hline E703 & EC2 & MUE703-EC2-B-19230 & 7/21/08 & $16: 30$ & Branch & ND & ND & 15 & $\begin{array}{l}\text { Primary branch. Sample collected } \\
6 \text { in. above ground, south } \\
\text { orientation. }\end{array}$ \\
\hline E704 & EC & MUE704-EC-B-19231 & $7 / 21 / 08$ & $16: 31$ & Branch & ND & ND & 15 & $\begin{array}{l}\text { Primary branch. Sample collected } \\
6 \text { in. above ground, southwest } \\
\text { orientation. }\end{array}$ \\
\hline E705 & EC2 & MUE705-EC2-B-19232 & $7 / 21 / 08$ & $16: 35$ & Branch & $<1$ & ND & 152 & $\begin{array}{l}\text { Primary branch. Sample collected } \\
5 \mathrm{ft} \text { above ground, east } \\
\text { orientation. }\end{array}$ \\
\hline
\end{tabular}


TABLE B.1 (Cont.)

\begin{tabular}{|c|c|c|c|c|c|c|c|c|c|}
\hline \multirow[b]{2}{*}{ Location } & \multirow[b]{2}{*}{ Species $^{a}$} & \multirow[b]{2}{*}{ Sample } & \multirow[b]{2}{*}{ Date } & \multirow[b]{2}{*}{ Time } & \multirow[b]{2}{*}{ Type } & \multicolumn{2}{|c|}{ Concentration $^{\mathrm{b}}(\mu \mathrm{g} / \mathrm{kg})$} & \multirow[b]{2}{*}{$\begin{array}{l}\text { Height }^{\mathrm{C}} \\
(\mathrm{cm})\end{array}$} & \multirow[b]{2}{*}{ Sample Description } \\
\hline & & & & & & $\begin{array}{c}\text { Carbon } \\
\text { Tetrachloride }\end{array}$ & Chloroform & & \\
\hline
\end{tabular}

Sampling in July 2008 (cont.)

\begin{tabular}{|c|c|c|c|c|c|c|c|c|c|}
\hline E706 & WN2 & MUE706-WN2-B-19233 & $7 / 21 / 08$ & $16: 36$ & Branch & 1 & ND & 0 & $\begin{array}{l}\text { Primary branch. Sample collected } \\
\text { at ground level, west } \\
\text { orientation. }\end{array}$ \\
\hline E707 & EC1 & MUE707-EC1-B-19234 & $7 / 21 / 08$ & $16: 40$ & Branch & 6 & 1 & 122 & $\begin{array}{l}\text { Secondary branch } 6 \text { in. off main } \\
\text { stem. Sample collected } 4 \mathrm{ft} \\
\text { above ground, west orientation }\end{array}$ \\
\hline E708 & EC2 & MUE708-EC2-B-19235 & $7 / 21 / 08$ & $16: 41$ & Branch & ND & ND & 15 & $\begin{array}{l}\text { Primary branch. Sample collected } \\
6 \text { in. above ground, east } \\
\text { orientation. }\end{array}$ \\
\hline E709 & WN1 & MUE709-WN1-B-19236 & $7 / 21 / 08$ & $16: 45$ & Branch & ND & ND & 0 & $\begin{array}{l}\text { Primary branch. Sample collected } \\
\text { at ground level, west } \\
\text { orientation. }\end{array}$ \\
\hline E710 & EC & MUE710-EC-B-19237 & 7/21/08 & $16: 46$ & Branch & ND & ND & 122 & $\begin{array}{l}\text { Secondary branch } 1 \mathrm{ft} \text { off main } \\
\text { stem. Sample collected } 4 \mathrm{ft} \\
\text { above ground, north } \\
\text { orientation. }\end{array}$ \\
\hline E715 & EC1 & MUE715-EC1-B-19238 & $7 / 21 / 08$ & $17: 21$ & Branch & 4 & $<1$ & 122 & $\begin{array}{l}\text { Secondary branch } 10 \text { in. off main } \\
\text { stem. Sample collected } 4 \mathrm{ft} \\
\text { above ground, south } \\
\text { orientation. }\end{array}$ \\
\hline E715 & EC2 & MUE715-EC2-B-19239 & $7 / 21 / 08$ & $17: 22$ & Branch & $<1$ & ND & 91 & $\begin{array}{l}\text { Secondary branch } 1 \mathrm{ft} \text { off main } \\
\text { stem. Sample collected } 3 \mathrm{ft} \\
\text { above ground, east orientation. }\end{array}$ \\
\hline E720 & EC & MUE720-EC-B-19240 & 7/21/08 & $17: 25$ & Branch & ND & ND & 137 & $\begin{array}{l}\text { Primary branch. Sample collected } \\
4.5 \mathrm{ft} \text { above ground, north } \\
\text { orientation. }\end{array}$ \\
\hline E720 & GA & MUE720-GA-B-19241 & $7 / 21 / 08$ & $17: 26$ & Branch & ND & ND & 15 & $\begin{array}{l}\text { Primary branch. Sample collected } \\
6 \text { in. above ground, south } \\
\text { orientation. }\end{array}$ \\
\hline E725 & EC & MUE725-EC-B-19242 & $7 / 21 / 08$ & $17: 30$ & Branch & ND & ND & 122 & $\begin{array}{l}\text { Primary branch. Sample collected } \\
4 \mathrm{ft} \text { above ground, west } \\
\text { orientation. }\end{array}$ \\
\hline
\end{tabular}




\begin{tabular}{|c|c|c|c|c|c|c|c|c|c|}
\hline & & & & & & \multicolumn{2}{|c|}{ Concentration ${ }^{\mathrm{b}}(\mu \mathrm{g} / \mathrm{kg})$} & & \\
\hline Location & Species $^{a}$ & Sample & Date & Time & Type & $\begin{array}{c}\text { Carbon } \\
\text { Tetrachloride }\end{array}$ & Chloroform & $\begin{array}{l}\text { Height }^{\mathrm{C}} \\
(\mathrm{cm})\end{array}$ & Sample Description \\
\hline
\end{tabular}

Sampling in July 2008 (cont.)

\begin{tabular}{|c|c|c|c|c|c|c|c|c|c|}
\hline E730 & EC & MUE730-EC-B-19243 & $7 / 21 / 08$ & $17: 31$ & Branch & ND & ND & 152 & $\begin{array}{l}\text { Secondary branch } 3 \text { in. off main } \\
\text { stem. Sample collected } 5 \mathrm{ft} \\
\text { above ground, northwest } \\
\text { orientation. }\end{array}$ \\
\hline E735 & EC1 & MUE735-EC1-B-19244 & $7 / 21 / 08$ & $17: 35$ & Branch & ND & ND & 122 & $\begin{array}{l}\text { Primary branch. Sample collected } \\
4 \mathrm{ft} \text { above ground, east } \\
\text { orientation. Wet area. }\end{array}$ \\
\hline E735 & EC2 & MUE735-EC2-B-19245 & $7 / 21 / 08$ & $17: 36$ & Branch & $<1$ & ND & 152 & $\begin{array}{l}\text { Secondary branch } 3 \text { in. off main } \\
\text { stem. Sample collected } 5 \mathrm{ft} \\
\text { above ground, east orientation. } \\
\text { EC2 is bending down. }\end{array}$ \\
\hline E740 & EC & MUE740-EC-B-19246 & $7 / 21 / 08$ & $17: 40$ & Branch & ND & ND & 122 & $\begin{array}{l}\text { Primary branch. Sample collected } \\
1 \mathrm{ft} \text { from insertion, } 4 \mathrm{ft} \text { above } \\
\text { ground, west orientation. }\end{array}$ \\
\hline E758 & EC1 & MUE758-EC1-B-19247 & $7 / 21 / 08$ & 15:32 & Branch & ND & ND & 137 & $\begin{array}{l}\text { Secondary branch } 4 \text { in. off main } \\
\text { stem. Sample collected } 4.5 \mathrm{ft} \\
\text { above ground, west orientation. } \\
\text { No tags on tree. Sample } \\
\text { collected from larger of two } \\
\text { trees. }\end{array}$ \\
\hline E800 & EC2 & MUE800-EC2-B-19248 & $7 / 21 / 08$ & $17: 20$ & Branch & ND & ND & 91 & $\begin{array}{l}\text { Secondary branch } 6 \text { in. off main } \\
\text { stem. Sample collected } 3 \mathrm{ft} \\
\text { above ground, west orientation. }\end{array}$ \\
\hline E801 & WN1 & MUE801-WN1-B-19249 & $7 / 21 / 08$ & $17: 16$ & Branch & ND & ND & 0 & $\begin{array}{l}\text { Primary branch. Sample collected } \\
\text { at ground level, east } \\
\text { orientation. }\end{array}$ \\
\hline E802 & EC & MUE802-EC-B-19250 & $7 / 21 / 08$ & $17: 15$ & Branch & $<1$ & ND & 10 & $\begin{array}{l}\text { Primary branch. Sample collected } \\
2 \mathrm{ft} \text { from insertion, } 4 \text { in. above } \\
\text { ground, north orientation. }\end{array}$ \\
\hline E803 & EC2 & MUE803-EC2-B-19251 & $7 / 21 / 08$ & $17: 12$ & Branch & $<1$ & ND & 91 & $\begin{array}{l}\text { Secondary branch } 1.5 \mathrm{ft} \text { off main } \\
\text { stem. Sample collected } 3 \mathrm{ft} \\
\text { above ground, west orientation. }\end{array}$ \\
\hline
\end{tabular}




\begin{tabular}{|c|c|c|c|c|c|c|c|c|c|}
\hline & & & & & & \multicolumn{2}{|c|}{ Concentration $^{\mathrm{b}}(\mu \mathrm{g} / \mathrm{kg})$} & \multirow[b]{2}{*}{$\begin{array}{l}\text { Heightc } \\
(\mathrm{cm})\end{array}$} & \multirow[b]{2}{*}{ Sample Description } \\
\hline Location & Species $^{a}$ & Sample & Date & Time & Type & $\begin{array}{c}\text { Carbon } \\
\text { Tetrachloride }\end{array}$ & Chloroform & & \\
\hline
\end{tabular}

Sampling in July 2008 (cont.)

E80

E805

E806

E807

E808

E809

EC

MUE809-EC-B-19257

EC1

MUE810-EC1-B-25130

$\mathrm{WN}$

MUE845-WN1-B-25131

$7 / 21 / 08$

15:51 Branch

E845

WN2

MUE845-WN2-B-25132

$7 / 21 / 08$

$7 / 21 / 08$

15:50 Branch
HP MUE858-HP-B-25133
ND

ND

ND

ND

ND

ND

ND

ND

ND
ND

ND

ND

$<1$

107

ND

107

ND

ND

ND

ND
107

10

107

91

91

91

137

152

152
Primary branch. Sample collected $2 \mathrm{ft}$ from insertion, 4 in. above ground, west orientation. Tree location is mislabeled in the field as E802, E804 is correct.

Primary branch. Sample collected

$2 \mathrm{ft}$ from insertion, $3.5 \mathrm{ft}$ above ground, west orientation.

Secondary branch $1.5 \mathrm{ft}$ off main stem. Sample collected $3 \mathrm{ft}$

above ground, west orientation.

Primary branch. Sample collected $3.5 \mathrm{ft}$ above ground, north orientation.

Secondary branch 3 in. off main stem. Sample collected $3.5 \mathrm{ft}$ above ground, south orientation.

Secondary branch 1 in. off main stem. Sample collected $3.5 \mathrm{ft}$ above ground, west orientation. Primary branch. Sample collected $3 \mathrm{ft}$ above ground, east orientation.

Primary branch. Sample collected $4.5 \mathrm{ft}$ above ground, north orientation.

Primary branch. Sample collected $5 \mathrm{ft}$ above ground, west orientation.

Secondary branch $1 \mathrm{ft}$ off main stem. Sample collected $5 \mathrm{ft}$ above ground, west orientation. 


\begin{tabular}{|c|c|c|c|c|c|c|c|c|c|}
\hline & & & & & & \multicolumn{2}{|c|}{ Concentration ${ }^{\mathrm{b}}(\mu \mathrm{g} / \mathrm{kg})$} & & \\
\hline Location & Species $^{a}$ & Sample & Date & Time & Type & $\begin{array}{c}\text { Carbon } \\
\text { Tetrachloride }\end{array}$ & Chloroform & $\begin{array}{l}\text { Height } \\
\text { (cm) }\end{array}$ & Sample Description \\
\hline
\end{tabular}

Sampling in July 2008 (cont.)

\begin{tabular}{|c|c|c|c|c|c|c|c|c|c|}
\hline E862 & EC & MUE862-EC-B-25134 & $7 / 21 / 08$ & $15: 45$ & Branch & $<1$ & ND & 91 & $\begin{array}{l}\text { Secondary branch } 8 \text { in. off main } \\
\text { stem. Sample collected } 3 \mathrm{ft} \\
\text { above ground, east orientation. }\end{array}$ \\
\hline E866 & EC & MUE866-EC-B-25135 & $7 / 21 / 08$ & $15: 35$ & Branch & ND & ND & 183 & $\begin{array}{l}\text { Primary branch. Sample collected } \\
6 \mathrm{ft} \text { above ground, west } \\
\text { orientation. }\end{array}$ \\
\hline E866 & GA & MUE866-GA-B-25136 & $7 / 21 / 08$ & $15: 43$ & Branch & ND & ND & 122 & $\begin{array}{l}\text { Secondary branch } 2 \text { in. off main } \\
\text { stem. Sample collected } 4 \mathrm{ft} \\
\text { above ground, east orientation. }\end{array}$ \\
\hline E869 & EC & MUE869-EC-B-25137 & $7 / 21 / 08$ & $15: 40$ & Branch & ND & ND & 183 & $\begin{array}{l}\text { Secondary branch } 6 \text { in. off main } \\
\text { stem. Sample collected } 6 \mathrm{ft} \\
\text { above ground, west orientation. }\end{array}$ \\
\hline E871 & EC & MUE871-EC-B-25138 & $7 / 21 / 08$ & $15: 33$ & Branch & ND & ND & 152 & $\begin{array}{l}\text { Primary branch. Sample collected } \\
5 \mathrm{ft} \text { above ground, west } \\
\text { orientation. }\end{array}$ \\
\hline W103 & EC1 & MUW103-EC1-B-25140 & $7 / 22 / 08$ & $8: 45$ & Branch & ND & ND & 107 & $\begin{array}{l}\text { Primary branch. Sample collected } \\
6 \text { in. from insertion, } 3.5 \mathrm{ft} \\
\text { above ground, east orientation. }\end{array}$ \\
\hline W104 & WN1 & MUW104-WN1-B-25141 & $7 / 22 / 08$ & $8: 46$ & Branch & $<1$ & ND & 5 & $\begin{array}{l}\text { Primary branch. Sample collected } \\
2 \text { in. above ground, east } \\
\text { orientation. }\end{array}$ \\
\hline W106 & WN & MUW106-WN-B-25142 & $7 / 22 / 08$ & $8: 47$ & Branch & $<1$ & ND & 0 & $\begin{array}{l}\text { Primary branch. Sample collected } \\
\text { at ground level, east } \\
\text { orientation. Wet soil. }\end{array}$ \\
\hline W107 & WN2 & MUW107-WN2-B-25143 & $7 / 22 / 08$ & $8: 50$ & Branch & 4 & 7 & 15 & $\begin{array}{l}\text { Primary branch. Sample collected } \\
6 \text { in. above ground, east } \\
\text { orientation. Wet soil. }\end{array}$ \\
\hline W109 & WN1 & MUW109-WN1-B-25144 & $7 / 22 / 08$ & $8: 51$ & Branch & 428 & 64 & 0 & $\begin{array}{l}\text { Primary branch. Sample collected } \\
\text { at ground level, east } \\
\text { orientation. }\end{array}$ \\
\hline W109 & WN1 & MUW109-WN1-L-25145 & $7 / 22 / 08$ & $8: 52$ & Leaf & ND & ND & 0 & $\begin{array}{l}\text { Leaf sample from branch sampled } \\
\text { as ID } 25144 \text {. }\end{array}$ \\
\hline
\end{tabular}




\begin{tabular}{|c|c|c|c|c|c|c|c|c|c|}
\hline & & & & & & \multicolumn{2}{|c|}{ Concentration ${ }^{\mathrm{b}}(\mu \mathrm{g} / \mathrm{kg})$} & & \\
\hline Location & Species $^{a}$ & Sample & Date & Time & Type & $\begin{array}{c}\text { Carbon } \\
\text { Tetrachloride }\end{array}$ & Chloroform & $\begin{array}{l}\text { Height } \\
\text { (cm) }\end{array}$ & Sample Description \\
\hline
\end{tabular}

Sampling in July 2008 (cont.)

\begin{tabular}{|c|c|c|c|c|c|c|c|c|c|}
\hline W109 & WN2 & MUW109-WN2-B-25146 & $7 / 22 / 08$ & $8: 53$ & Branch & 29 & 4 & 5 & $\begin{array}{l}\text { Primary branch. Sample collected } \\
2 \text { in. above ground, east } \\
\text { orientation. }\end{array}$ \\
\hline W109 & WN2 & MUW109-WN2-L-25147 & $7 / 22 / 08$ & $8: 54$ & Leaf & ND & 2 & 5 & $\begin{array}{l}\text { Leaf sample from branch sampled } \\
\text { as ID } 25146 \text {. }\end{array}$ \\
\hline W110 & WN1 & MUW110-WN1-B-25148 & $7 / 22 / 08$ & $8: 55$ & Branch & 8 & 7 & 15 & $\begin{array}{l}\text { Primary branch. Sample collected } \\
6 \text { in. above ground, west } \\
\text { orientation. }\end{array}$ \\
\hline W110 & WN1 & MUW110-WN1-L-25149 & $7 / 22 / 08$ & $8: 56$ & Leaf & $<1$ & 1 & 15 & $\begin{array}{l}\text { Leaf sample from branch sampled } \\
\text { as ID } 25148 \text {. }\end{array}$ \\
\hline W110 & WN2 & MUW110-WN2-B-26206 & $7 / 22 / 08$ & $8: 57$ & Branch & 1 & 3 & 15 & $\begin{array}{l}\text { Primary branch. Sample collected } \\
6 \text { in. above ground, east } \\
\text { orientation. }\end{array}$ \\
\hline W111 & WN1 & MUW111-WN1-B-25151 & $7 / 22 / 08$ & 9:01 & Branch & 3 & 2 & 15 & $\begin{array}{l}\text { Primary branch. Sample collected } \\
6 \text { in. above ground, west } \\
\text { orientation. }\end{array}$ \\
\hline W111 & WN1 & MUW111-WN1-L-25150 & $7 / 22 / 08$ & 9:00 & Leaf & $<1$ & 1 & 15 & $\begin{array}{l}\text { Leaf sample from branch sampled } \\
\text { as ID } 25151 .\end{array}$ \\
\hline W111 & WN2 & MUW111-WN2-B-25153 & $7 / 22 / 08$ & 9:03 & Branch & $<1$ & ND & 15 & $\begin{array}{l}\text { Primary branch. Sample collected } \\
6 \text { in. above ground, west } \\
\text { orientation. }\end{array}$ \\
\hline W111 & WN2 & MUW111-WN2-L-25152 & 7/22/08 & 9:02 & Leaf & ND & $<1$ & 15 & $\begin{array}{l}\text { Leaf sample from branch sampled } \\
\text { as ID } 25153 \text {. }\end{array}$ \\
\hline W114 & WN2 & MUW114-WN2-B-25154 & $7 / 22 / 08$ & 9:05 & Branch & 1708 & 25 & 15 & $\begin{array}{l}\text { Primary branch. Sample collected } \\
6 \text { in. above ground, west } \\
\text { orientation. Wet ground. }\end{array}$ \\
\hline W119 & WN1 & MUW119-WN1-B-25155 & $7 / 22 / 08$ & 9:06 & Branch & 114 & 27 & 122 & $\begin{array}{l}\text { Secondary branch } 2 \mathrm{ft} \text { off main } \\
\text { stem. Sample collected } 4 \mathrm{ft} \\
\text { above ground, west orientation. } \\
\text { Wet ground. }\end{array}$ \\
\hline W119 & WN2 & MUW119-WN2-B-25156 & 7/22/08 & 9:07 & Branch & 225 & 30 & 152 & $\begin{array}{l}\text { Primary branch. Sample collected } \\
1.5 \mathrm{ft} \text { from intersect with main, } \\
5 \mathrm{ft} \text { above ground, west } \\
\text { orientation. Wet ground. }\end{array}$ \\
\hline
\end{tabular}




\begin{tabular}{|c|c|c|c|c|c|c|c|c|c|}
\hline & & & & & & \multicolumn{2}{|c|}{ Concentration $^{\mathrm{b}}(\mu \mathrm{g} / \mathrm{kg})$} & \multirow[b]{2}{*}{$\begin{array}{l}\text { Height }^{\mathrm{C}} \\
(\mathrm{cm})\end{array}$} & \multirow[b]{2}{*}{ Sample Description } \\
\hline Location & Species $^{a}$ & Sample & Date & Time & Type & $\begin{array}{c}\text { Carbon } \\
\text { Tetrachloride }\end{array}$ & Chloroform & & \\
\hline
\end{tabular}

Sampling in July 2008 (cont.)

\begin{tabular}{|c|c|c|c|c|c|c|c|c|c|}
\hline W121 & WN2 & MUW121-WN2-B-25157 & 7/22/08 & $9: 15$ & Branch & 6 & 1 & 15 & $\begin{array}{l}\text { Primary branch. Sample collected } \\
6 \text { in. above ground, west } \\
\text { orientation. Wet ground. }\end{array}$ \\
\hline W124 & WN2 & MUW124-WN2-B-25158 & $7 / 22 / 08$ & $9: 16$ & Branch & 1 & ND & 15 & $\begin{array}{l}\text { Primary branch. Sample collected } \\
6 \text { in. above ground, west } \\
\text { orientation. Wet ground. }\end{array}$ \\
\hline W128 & WN1 & MUW128-WN1-B-25159 & 7/22/08 & 9:20 & Branch & 3 & 2 & 152 & $\begin{array}{l}\text { Secondary branch } 2 \mathrm{ft} \mathrm{from} \\
\text { insertion point. Sample } \\
\text { collected } 5 \mathrm{ft} \text { above ground, } \\
\text { east orientation. Wet around. }\end{array}$ \\
\hline W128 & WN2 & MUW128-WN2-B-26120 & 7/22/08 & 9:21 & Branch & 2 & 3 & 15 & $\begin{array}{l}\text { Primary branch. Sample collected } \\
6 \text { in. above ground, west } \\
\text { orientation. }\end{array}$ \\
\hline W129 & WN2 & MUW129-WN2-B-26121 & $7 / 22 / 08$ & 9:22 & Branch & 7 & 6 & 152 & $\begin{array}{l}\text { Tertiary branch, } 12 \text { in. from } \\
\text { insertion, } 1 \text { in. off main stem. } \\
\text { Sample collected } 5 \mathrm{ft} \text { above } \\
\text { ground, east orientation. }\end{array}$ \\
\hline W132 & WN1 & MUW132-WN1-B-26122 & 7/22/08 & 9:25 & Branch & 2 & 2 & 122 & $\begin{array}{l}\text { Secondary branch } 2 \mathrm{ft} \text { from } \\
\text { insertion point. Sample } \\
\text { collected } 4 \mathrm{ft} \text { above ground, } \\
\text { west orientation. Wet ground } \\
\text { but accessible. }\end{array}$ \\
\hline W132 & WN2 & MUW132-WN2-B-26123 & 7/22/08 & 9:26 & Branch & 10 & 4 & 122 & $\begin{array}{l}\text { Primary branch. Sample collected } \\
4 \mathrm{ft} \text { above ground, north } \\
\text { orientation. }\end{array}$ \\
\hline W134 & WN1 & MUW134-WN1-B-26124 & $7 / 22 / 08$ & 9:30 & Branch & $<1$ & ND & 91 & $\begin{array}{l}\text { Secondary branch at insertion } \\
\text { point. Sample collected } 3 \mathrm{ft} \\
\text { above ground, northwest } \\
\text { orientation. }\end{array}$ \\
\hline W139 & WN1 & MUW139-WN1-B-26125 & 7/22/08 & 9:35 & Branch & ND & ND & 152 & $\begin{array}{l}\text { Secondary branch } 6 \text { in. off main } \\
\text { stem. Sample collected } 5 \mathrm{ft} \\
\text { above ground, west orientation } \\
\text { Tree WN1 was sampled; tree } \\
\text { WN2 is dead. }\end{array}$ \\
\hline
\end{tabular}




\begin{tabular}{|c|c|c|c|c|c|c|c|c|c|}
\hline & & & & & & \multicolumn{2}{|c|}{ Concentration $^{\mathrm{b}}(\mu \mathrm{g} / \mathrm{kg})$} & & \\
\hline Location & Species $^{a}$ & Sample & Date & Time & Type & $\begin{array}{c}\text { Carbon } \\
\text { Tetrachloride }\end{array}$ & Chloroform & $\begin{array}{l}\text { Heightc } \\
(\mathrm{cm})\end{array}$ & Sample Description \\
\hline
\end{tabular}

Sampling in July 2008 (cont.)

\begin{tabular}{|c|c|c|c|c|c|c|c|c|c|}
\hline W144 & WN2 & MUW144-WN2-B-26126 & $7 / 22 / 08$ & 9:36 & Branch & ND & ND & 152 & $\begin{array}{l}\text { Secondary branch } 2 \mathrm{ft} \text { from main } \\
\text { insertion. Sample collected } 5 \mathrm{ft} \\
\text { above ground, south } \\
\text { orientation. No water on } \\
\text { ground; dry area. }\end{array}$ \\
\hline W148 & WN2 & MUW148-WN2-B-26127 & $7 / 22 / 08$ & $9: 40$ & Branch & 3 & 3 & 152 & $\begin{array}{l}\text { Secondary branch } 6 \text { in. off main } \\
\text { stem. Sample collected } 5 \mathrm{ft} \\
\text { above ground, southwest } \\
\text { orientation. No tag on tree; } \\
\text { there is a ribbon. Wet ground. }\end{array}$ \\
\hline W153 & WN2 & MUW153-WN2-B-26128 & $7 / 22 / 08$ & $9: 41$ & Branch & $<1$ & 1 & 122 & $\begin{array}{l}\text { Primary branch. Sample collected } \\
4 \mathrm{ft} \text { above ground, northeast } \\
\text { orientation. Only tree at this } \\
\text { location. Dry soil. }\end{array}$ \\
\hline W157 & WN1 & MUW157-WN1-B-26129 & $7 / 22 / 08$ & $9: 45$ & Branch & $<1$ & ND & 152 & $\begin{array}{l}\text { Secondary branch } 1 \text { in. off main } \\
\text { stem. Sample collected } 5 \mathrm{ft} \\
\text { above ground, east orientation. } \\
\text { Dry ground. }\end{array}$ \\
\hline W158 & WN1 & MUW158-WN1-B-26130 & $7 / 22 / 08$ & $9: 46$ & Branch & $<1$ & 2 & 122 & $\begin{array}{l}\text { Secondary branch } 2.5 \mathrm{ft} \text { from } \\
\text { insertion. Sample collected } 4 \mathrm{ft} \\
\text { above ground, east orientation. } \\
\text { Dry ground. }\end{array}$ \\
\hline W158 & WN2 & MUW158-WN2-B-26131 & $7 / 22 / 08$ & $9: 47$ & Branch & $<1$ & 2 & 152 & $\begin{array}{l}\text { Secondary branch } 2.5 \mathrm{ft} \text { from } \\
\text { main insertion. Sample } \\
\text { collected } 5 \mathrm{ft} \text { above ground, } \\
\text { west orientation. }\end{array}$ \\
\hline W159 & EC2 & MUW159-EC2-B-26132 & $7 / 22 / 08$ & $9: 50$ & Branch & ND & ND & 30 & $\begin{array}{l}\text { Secondary branch } 1.5 \mathrm{ft} \text { from } \\
\text { insertion point. Sample } \\
\text { collected } 1 \mathrm{ft} \text { above ground, } \\
\text { south orientation. }\end{array}$ \\
\hline W203 & WN1 & MUW203-WN1-B-26133 & $7 / 22 / 08$ & $10: 00$ & Branch & 58 & 8 & 5 & $\begin{array}{l}\text { Primary branch. Sample collected } \\
2 \text { in. above ground, west } \\
\text { orientation. }\end{array}$ \\
\hline
\end{tabular}


TABLE B.1 (Cont.)

\begin{tabular}{|c|c|c|c|c|c|c|c|c|c|}
\hline & & & & & & \multicolumn{2}{|c|}{ Concentration $^{\mathrm{b}}(\mu \mathrm{g} / \mathrm{kg})$} & & \\
\hline Location & Species $^{a}$ & Sample & Date & Time & Type & $\begin{array}{c}\text { Carbon } \\
\text { Tetrachloride }\end{array}$ & Chloroform & $\begin{array}{l}\text { Height }^{\mathrm{c}} \\
(\mathrm{cm})\end{array}$ & Sample Description \\
\hline
\end{tabular}

Sampling in July 2008 (cont.)

\begin{tabular}{|c|c|c|c|c|c|c|c|c|c|}
\hline W210 & WN1 & MUW210-WN1-B-26134 & $7 / 22 / 08$ & $10: 05$ & Branch & 40 & 3 & 5 & $\begin{array}{l}\text { Primary branch. Sample collected } \\
2 \text { in. above ground, west } \\
\text { orientation. }\end{array}$ \\
\hline W210 & WN2 & MUW210-WN2-B-26135 & $7 / 22 / 08$ & 10:06 & Branch & 91 & 15 & 5 & $\begin{array}{l}\text { Primary branch. Sample collected } \\
2 \text { in. above ground, west } \\
\text { orientation. Wet ground. }\end{array}$ \\
\hline W211 & EC & MUW211-EC-B-26136 & $7 / 22 / 08$ & $10: 10$ & Branch & 19 & 20 & 107 & $\begin{array}{l}\text { Secondary branch at insertion } \\
\text { point. Sample collected } 3.5 \mathrm{ft} \\
\text { above ground, east orientation. } \\
\text { Wet ground. }\end{array}$ \\
\hline W211 & EC & MUW211-EC-L-26207 & $7 / 22 / 08$ & $10: 16$ & Leaf & ND & $<1$ & 0 & $\begin{array}{l}\text { Leaf sample from same tree as } \\
\text { the branch sampled as ID } \\
26136, \text { but not from the same } \\
\text { branch. Sample of leaves } \\
\text { closest to the air sampler. }\end{array}$ \\
\hline W212 & WN2 & MUW212-WN2-B-26137 & $7 / 22 / 08$ & $10: 15$ & Branch & 4 & 3 & 152 & $\begin{array}{l}\text { Secondary branch } 6 \text { in. from } \\
\text { insertion point. Sample } \\
\text { collected } 5 \mathrm{ft} \text { above ground, } \\
\text { east orientation. Aphids on } \\
\text { tree; wet ground. }\end{array}$ \\
\hline W303 & EC1 & MUW303-EC1-B-26138 & $7 / 22 / 08$ & $10: 19$ & Branch & 54 & 10 & 107 & $\begin{array}{l}\text { Primary branch. Sample collected } \\
3.5 \mathrm{ft} \text { above ground, west } \\
\text { orientation. }\end{array}$ \\
\hline W308 & EC & MUW308-EC-B-26140 & $7 / 22 / 08$ & $10: 18$ & Branch & 31 & 17 & 122 & $\begin{array}{l}\text { Secondary branch } 2 \mathrm{ft} \text { from } \\
\text { insertion point. Sample } \\
\text { collected } 4 \mathrm{ft} \text { above ground, } \\
\text { west orientation. }\end{array}$ \\
\hline W308 & EC & MUW308-EC-L-26139 & $7 / 22 / 08$ & $10: 17$ & Leaf & $<1$ & ND & 122 & $\begin{array}{l}\text { Secondary branch } 2 \mathrm{ft} \text { from } \\
\text { insertion point. Sample } \\
\text { collected } 4 \mathrm{ft} \text { above ground, } \\
\text { west orientation. }\end{array}$ \\
\hline
\end{tabular}


TABLE B.1 (Cont.)

\begin{tabular}{|c|c|c|c|c|c|c|c|c|c|}
\hline & & & & & & \multicolumn{2}{|c|}{ Concentration $^{\mathrm{b}}(\mu \mathrm{g} / \mathrm{kg})$} & \multirow[b]{2}{*}{$\begin{array}{l}\text { Heightc } \\
(\mathrm{cm})\end{array}$} & \multirow[b]{2}{*}{ Sample Description } \\
\hline Location & Species $^{a}$ & Sample & Date & Time & Type & $\begin{array}{c}\text { Carbon } \\
\text { Tetrachloride }\end{array}$ & Chloroform & & \\
\hline
\end{tabular}

Sampling in July 2008 (cont.)

\begin{tabular}{|c|c|c|c|c|c|c|c|}
\hline W357 & WN1 & MUW357-WN1-B-26141 & $7 / 22 / 08$ & $10: 51$ & Branch & ND & ND \\
\hline W402 & EC2 & MUW402-EC2-B-26142 & $7 / 22 / 08$ & $10: 20$ & Branch & 2 & 3 \\
\hline W407 & WN1 & MUW407-WN1-B-26143 & $7 / 22 / 08$ & $10: 21$ & Branch & 52 & 9 \\
\hline W407 & WN2 & MUW407-WN2-B-26144 & $7 / 22 / 08$ & $10: 22$ & Branch & 460 & 30 \\
\hline W412 & EC2 & MUW412-EC2-B-26145 & $7 / 22 / 08$ & $10: 25$ & Branch & ND & ND \\
\hline W417 & EC1 & MUW417EC1-B9-26232 & $7 / 22 / 08$ & $16: 42$ & Branch & 5 & 14 \\
\hline W417 & EC1 & MUW417EC1-B11-26234 & $7 / 22 / 08$ & $16: 45$ & Branch & 8 & 15 \\
\hline W417 & EC1 & MUW417EC1-B10-26233 & $7 / 22 / 08$ & $16: 43$ & Branch & 12 & 17 \\
\hline W417 & EC1 & MUW417EC1-C10-26244 & $7 / 22 / 08$ & $17: 22$ & Core & 20 & 22 \\
\hline W417 & EC1 & MUW417EC1-C11-26245 & $7 / 22 / 08$ & $17: 23$ & Core & 22 & 27 \\
\hline W417 & EC1 & MUW417EC1-B4-26227 & $7 / 22 / 08$ & $16: 33$ & Branch & 27 & 16 \\
\hline W417 & EC1 & MUW417EC1-B8-26231 & $7 / 22 / 08$ & $16: 41$ & Branch & 50 & 27 \\
\hline W417 & EC1 & MUW417EC1-B3-26226 & $7 / 22 / 08$ & $16: 32$ & Branch & 59 & 30 \\
\hline W417 & EC1 & MUW417EC1-B7-26230 & $7 / 22 / 08$ & $16: 40$ & Branch & 79 & 33 \\
\hline W417 & EC1 & MUW417EC1-C8-26243 & $7 / 22 / 08$ & $17: 21$ & Core & 84 & 37 \\
\hline W417 & EC1 & MUW417EC1-B5-26228 & $7 / 22 / 08$ & $16: 35$ & Branch & 86 & 31 \\
\hline W417 & EC1 & MUW417EC1-B6-26229 & $7 / 22 / 08$ & $16: 36$ & Branch & 86 & 35 \\
\hline W417 & EC1 & MUW417EC1-C7-26242 & $7 / 22 / 08$ & $17: 20$ & Core & 91 & 35 \\
\hline W417 & EC1 & MUW417EC1-C1-26236 & $7 / 22 / 08$ & $17: 00$ & Core & 97 & 21 \\
\hline W417 & EC1 & MUW417EC1-C5-26240 & $7 / 22 / 08$ & $17: 11$ & Core & 100 & 31 \\
\hline
\end{tabular}

152 Secondary branch $1.5 \mathrm{ft}$ from insertion point. Sample collected $5 \mathrm{ft}$ above ground, north orientation. Dry ground.

Secondary branch 6 in. from insertion point. Sample collected $4 \mathrm{ft}$ above ground, north orientation. Dry ground.

$0 \quad$ Primary branch. Sample collected at ground level, north orientation. Dry ground.

$0 \quad$ Primary branch. Sample collected at ground level, north orientation.

61 Secondary branch $2 \mathrm{ft}$ from insertion point. Sample collected $2 \mathrm{ft}$ above ground, southeast orientation. Wet ground.

At 86 in. from B8.

At 7.3 in. from B10.

At 10 in. from base of log 3.

Core cross section at B10.

Core cross section at B11.

At 3 in. above B3.

At 12 in. above B7.

At 18 in. above B2.

At 11 in above $B 6$.

Core cross section at B8.

At 12 in. above B4.

At 3.5 in. from base of log 2 .

Core cross section at B7.

Core cross section at B1.

Core cross section at B5. 


\begin{tabular}{|c|c|c|c|c|c|c|c|c|c|}
\hline & & & & & & \multicolumn{2}{|c|}{ Concentration $^{\mathrm{b}}(\mu \mathrm{g} / \mathrm{kg})$} & \multirow[b]{2}{*}{$\begin{array}{l}\text { Heightc } \\
(\mathrm{cm})\end{array}$} & \multirow[b]{2}{*}{ Sample Description } \\
\hline Location & Species $^{a}$ & Sample & Date & Time & Type & $\begin{array}{c}\text { Carbon } \\
\text { Tetrachloride }\end{array}$ & Chloroform & & \\
\hline
\end{tabular}

Sampling in July 2008 (cont.)

\begin{tabular}{|c|c|c|c|c|c|c|c|c|c|}
\hline W417 & EC1 & MUW417EC1-C2-26237 & $7 / 22 / 08$ & $17: 01$ & Core & 111 & 30 & & Core cross section at B2. \\
\hline W417 & EC1 & MUW417EC1-C6-26241 & $7 / 22 / 08$ & $17: 15$ & Core & 126 & 38 & & Core cross section at $B 6$. \\
\hline W417 & EC1 & MUW417EC1-B1-26224 & $7 / 22 / 08$ & $16: 30$ & Branch & 126 & 27 & & $\begin{array}{l}\text { Begin sampling harvested tree } \\
\text { W417-EC1. Sample collected } \\
36 \text { in. from base of log } 1 .\end{array}$ \\
\hline W417 & EC1 & MUW417EC1-B2-26225 & $7 / 22 / 08$ & $16: 31$ & Branch & 138 & 35 & & At 1.3 in. above B1, west side. \\
\hline W417 & EC1 & MUW417EC1-C3-26238 & $7 / 22 / 08$ & $17: 05$ & Core & 144 & 34 & & Core cross section at B3. \\
\hline W417 & EC1 & MUW417EC1-C4-26239 & $7 / 22 / 08$ & $17: 10$ & Core & 163 & 45 & & Core cross section at B4. \\
\hline W417 & EC1 & MUW417-EC1-B-26146 & $7 / 22 / 08$ & $10: 26$ & Branch & 300 & 36 & 91 & $\begin{array}{l}\text { Primary branch } 6 \text { in. from main. } \\
\text { Sample collected } 3 \mathrm{ft} \text { above } \\
\text { ground, east orientation. Wet } \\
\text { ground. Tree collapsed. }\end{array}$ \\
\hline W417 & EC1 & MUW417EC1-B12-26235 & $7 / 22 / 08$ & $16: 46$ & Branch & $<1$ & 9 & & At 77 in. from B11. \\
\hline W417 & EC1 & MUW417EC1-BB-26247 & $7 / 22 / 08$ & $17: 25$ & Branch & NA & NA & & $\begin{array}{l}\text { Representative small branch } \\
\text { samples for density, agronomy } \\
\text { analyses; no VOCs analyses. }\end{array}$ \\
\hline W417 & EC1 & MUW417EC1-L1-26246 & $7 / 22 / 08$ & $17: 24$ & Leaf & NA & NA & & $\begin{array}{l}\text { Leaf sample for LAI; no VOCs } \\
\text { analyses. }\end{array}$ \\
\hline W417 & EC1 & MUW417EC1-T-26248 & $7 / 22 / 08$ & $17: 30$ & Core & NA & NA & & $\begin{array}{l}\text { Representative trunk sample for } \\
\text { density, agronomy analyses; } \\
\text { no VOCs analyses. }\end{array}$ \\
\hline W422 & EC2 & MUW422-EC2-B-26147 & $7 / 22 / 08$ & $10: 30$ & Branch & 104 & 20 & 152 & $\begin{array}{l}\text { Secondary branch } 2 \mathrm{ft} \text { from main } \\
\text { insertion point. Sample } \\
\text { collected } 5 \mathrm{ft} \text { above ground, } \\
\text { north orientation. }\end{array}$ \\
\hline W426 & EC2 & MUW426-EC2-B-26148 & $7 / 22 / 08$ & $10: 31$ & Branch & 7 & 8 & 152 & $\begin{array}{l}\text { Primary branch. Sample collected } \\
5 \mathrm{ft} \text { above ground, } 1 \mathrm{ft} \text { from } \\
\text { main stem, south orientation. }\end{array}$ \\
\hline W427 & EC & MUW427-EC-B-26149 & $7 / 22 / 08$ & $10: 32$ & Branch & 149 & 34 & 152 & $\begin{array}{l}\text { Secondary branch } 1.5 \mathrm{ft} \text { from } \\
\text { insertion point. Sample } \\
\text { collected } 5 \mathrm{ft} \text { above ground, } \\
\text { south orientation. }\end{array}$ \\
\hline
\end{tabular}




\begin{tabular}{|c|c|c|c|c|c|c|c|c|c|}
\hline & & & & & & \multicolumn{2}{|c|}{ Concentration $^{\mathrm{b}}(\mu \mathrm{g} / \mathrm{kg})$} & & \\
\hline Location & Species $^{a}$ & Sample & Date & Time & Type & $\begin{array}{c}\text { Carbon } \\
\text { Tetrachloride }\end{array}$ & Chloroform & $\begin{array}{l}\text { Height } \\
\text { (cm) }\end{array}$ & Sample Description \\
\hline
\end{tabular}

Sampling in July 2008 (cont.)

\begin{tabular}{|c|c|c|c|c|c|c|c|c|c|}
\hline W427 & GA & MUW427-GA-B-26150 & $7 / 22 / 08$ & 10:33 & Branch & 2 & 4 & 122 & $\begin{array}{l}\text { Primary branch. Sample collected } \\
4 \mathrm{ft} \text { above ground, } 2.5 \mathrm{ft} \text { from } \\
\text { main insert, east orientation. } \\
\text { Wet area. }\end{array}$ \\
\hline W432 & EC & MUW432-EC-B-26151 & $7 / 22 / 08$ & 10:40 & Branch & $<1$ & 2 & 152 & $\begin{array}{l}\text { Primary branch. Sample collected } \\
5 \mathrm{ft} \text { above ground, east } \\
\text { orientation. }\end{array}$ \\
\hline W436 & WN2 & MUW436-WN2-B-26152 & $7 / 22 / 08$ & 10:41 & Branch & $<1$ & 1 & 0 & $\begin{array}{l}\text { Primary branch. Sample collected } \\
\text { at ground level, south } \\
\text { orientation. }\end{array}$ \\
\hline W447 & WN & MUW447-WN-B-26153 & $7 / 22 / 08$ & 10:45 & Branch & 2 & 2 & 15 & $\begin{array}{l}\text { Primary branch. Sample collected } \\
6 \text { in. above ground, east } \\
\text { orientation. Dry ground. }\end{array}$ \\
\hline W452 & WN2 & MUW452-WN2-B-26154 & $7 / 22 / 08$ & 10:46 & Branch & $<1$ & ND & 15 & $\begin{array}{l}\text { Primary branch. Sample collected } \\
6 \text { in. above ground, west } \\
\text { orientation. }\end{array}$ \\
\hline W457 & EC & MUW457-EC-B-26155 & $7 / 22 / 08$ & 10:50 & Branch & ND & ND & 107 & $\begin{array}{l}\text { Primary branch. Sample collected } \\
3.5 \mathrm{ft} \text { above ground, north } \\
\text { orientation. }\end{array}$ \\
\hline W505 & EC1 & MUW505-EC1-B-26156 & $7 / 22 / 08$ & $8: 25$ & Branch & 2 & 2 & 168 & $\begin{array}{l}\text { Secondary branch } 1 \mathrm{ft} \text { off main } \\
\text { stem. Sample collected } 5.5 \mathrm{ft} \\
\text { above ground, west orientation. }\end{array}$ \\
\hline W505 & EC1 & MUW505-EC1-L-26157 & $7 / 22 / 08$ & $8: 27$ & Leaf & ND & 2 & 168 & $\begin{array}{l}\text { Leaf sample from branch sampled } \\
\text { as ID } 26156 \text {. }\end{array}$ \\
\hline W505 & EC2 & MUW505-EC2-B-26158 & $7 / 22 / 08$ & $8: 26$ & Branch & 2 & 2 & 122 & $\begin{array}{l}\text { Primary branch. Sample collected } \\
4 \mathrm{ft} \text { above ground, north } \\
\text { orientation. }\end{array}$ \\
\hline W505 & EC2 & MUW505-EC2-L-26159 & 7/22/08 & $8: 28$ & Leaf & ND & $<1$ & 122 & $\begin{array}{l}\text { Leaf sample from branch sampled } \\
\text { as ID } 26158 \text {. }\end{array}$ \\
\hline W601 & EC & MUW601-EC-B-26160 & 7/22/08 & $8: 40$ & Branch & $<1$ & ND & 91 & $\begin{array}{l}\text { Secondary branch } 1 \mathrm{ft} \text { off main } \\
\text { stem. Sample collected } 3 \mathrm{ft} \\
\text { above ground, west orientation. }\end{array}$ \\
\hline
\end{tabular}




\begin{tabular}{|c|c|c|c|c|c|c|c|c|c|}
\hline & & & & & & \multicolumn{2}{|c|}{ Concentration $^{\mathrm{b}}(\mu \mathrm{g} / \mathrm{kg})$} & \multirow[b]{2}{*}{$\begin{array}{l}\text { Heightc } \\
(\mathrm{cm})\end{array}$} & \multirow[b]{2}{*}{ Sample Description } \\
\hline Location & Species $^{a}$ & Sample & Date & Time & Type & $\begin{array}{c}\text { Carbon } \\
\text { Tetrachloride }\end{array}$ & Chloroform & & \\
\hline
\end{tabular}

Sampling in July 2008 (cont.)

\begin{tabular}{|c|c|c|c|c|c|c|c|c|c|}
\hline W606 & EC1 & MUW606-EC1-B-26161 & $7 / 22 / 08$ & $8: 30$ & Branch & 1274 & 58 & 152 & $\begin{array}{l}\text { Primary branch. Sample collected } \\
5 \mathrm{ft} \text { above ground, south } \\
\text { orientation. }\end{array}$ \\
\hline W606 & EC2 & MUW606-EC2-B-26162 & $7 / 22 / 08$ & $8: 31$ & Branch & 465 & 68 & 168 & $\begin{array}{l}\text { Primary branch. Sample collected } \\
5.5 \mathrm{ft} \text { above ground, south } \\
\text { orientation. }\end{array}$ \\
\hline W611 & EC1 & MUW611-EC1-B-26163 & $7 / 22 / 08$ & $8: 35$ & Branch & 122 & 5 & 76 & $\begin{array}{l}\text { Primary branch. Sample collected } \\
2.5 \mathrm{ft} \text { above ground, south } \\
\text { orientation. }\end{array}$ \\
\hline W616 & EC1 & MUW616-EC1-B-26164 & $7 / 22 / 08$ & $12: 15$ & Branch & 34 & 27 & 122 & $\begin{array}{l}\text { Primary branch at } 6 \text { in. from } \\
\text { insertion. Sample collected } 4 \mathrm{ft} \\
\text { above ground, east orientation. } \\
\text { Wet ground. Tree fell over but } \\
\text { is still alive. EC2 tree is dead. }\end{array}$ \\
\hline W621 & EC & MUW621-EC-B-26165 & $7 / 22 / 08$ & $12: 20$ & Branch & 123 & 16 & 5 & $\begin{array}{l}\text { Secondary branch } 2 \text { in. from main } \\
\text { stem. Sample collected } 2 \text { in. } \\
\text { above ground, south } \\
\text { orientation. }\end{array}$ \\
\hline W626 & EC & MUW626-EC-B-26166 & $7 / 22 / 08$ & $12: 21$ & Branch & $<1$ & ND & 122 & $\begin{array}{l}\text { Secondary branch } 3 \text { in. from } \\
\text { insertion. Sample collected } 4 \mathrm{ft} \\
\text { above ground, south } \\
\text { orientation. }\end{array}$ \\
\hline W636 & EC & MUW636-EC-B-26167 & $7 / 22 / 08$ & $12: 25$ & Branch & $<1$ & $<1$ & 122 & $\begin{array}{l}\text { Secondary branch } 1.5 \mathrm{ft} \text { from } \\
\text { insertion. Sample collected } 4 \mathrm{ft} \\
\text { above ground, east orientation. }\end{array}$ \\
\hline W641 & EC1 & MUW641-EC1-B-26168 & $7 / 22 / 08$ & $12: 30$ & Branch & ND & ND & 152 & $\begin{array}{l}\text { Primary branch. Sample collected } \\
5 \mathrm{ft} \text { above ground, east } \\
\text { orientation. }\end{array}$ \\
\hline W646 & WN1 & MUW646-WN1-B-26169 & $7 / 22 / 08$ & $12: 31$ & Branch & 9 & 4 & 5 & $\begin{array}{l}\text { Primary branch. Sample collected } \\
2 \text { in. above ground, south } \\
\text { orientation. Dry ground. }\end{array}$ \\
\hline W651 & WN2 & MUW651-WN2-B-26170 & 7/22/08 & $12: 35$ & Branch & ND & 1 & 15 & $\begin{array}{l}\text { Primary branch. Sample collected } \\
6 \text { in. above ground, south } \\
\text { orientation. Dry ground. }\end{array}$ \\
\hline
\end{tabular}




\begin{tabular}{|c|c|c|c|c|c|c|c|c|c|}
\hline & & & & & & \multicolumn{2}{|c|}{ Concentration ${ }^{\mathrm{b}}(\mu \mathrm{g} / \mathrm{kg})$} & & \\
\hline Location & Species $^{a}$ & Sample & Date & Time & Type & $\begin{array}{c}\text { Carbon } \\
\text { Tetrachloride }\end{array}$ & Chloroform & $\begin{array}{l}\text { Height }^{\mathrm{C}} \\
(\mathrm{cm})\end{array}$ & Sample Description \\
\hline
\end{tabular}

Sampling in July 2008 (cont.)

\begin{tabular}{|c|c|c|c|c|c|c|c|c|c|}
\hline W800 & EC1 & MUW800-EC1-B-26171 & $7 / 22 / 08$ & $11: 20$ & Branch & ND & ND & 15 & $\begin{array}{l}\text { Secondary branch at insertion } \\
\text { point. Sample collected } 6 \text { in. } \\
\text { above ground, south } \\
\text { orientation. Dry ground. }\end{array}$ \\
\hline W801 & EC2 & MUW801-EC2-B-26172 & $7 / 22 / 08$ & $11: 21$ & Branch & $<1$ & ND & 137 & $\begin{array}{l}\text { Primary branch. Sample collected } \\
4.5 \mathrm{ft} \text { above ground, approx } 3 \mathrm{ft} \\
\text { from insert, east orientation. } \\
\text { Dry ground. }\end{array}$ \\
\hline W802 & EC2 & MUW802-EC2-B-26173 & $7 / 22 / 08$ & $11: 25$ & Branch & 15 & 3 & 137 & $\begin{array}{l}\text { Primary branch. Sample collected } \\
4.5 \mathrm{ft} \text { above ground, } 4 \text { in. from } \\
\text { main insert, east orientation. } \\
\text { Dry ground. }\end{array}$ \\
\hline W803 & WN1 & MUW803-WN1-B-26174 & $7 / 22 / 08$ & $11: 26$ & Branch & 5 & 2 & 5 & $\begin{array}{l}\text { Primary branch. Sample collected } \\
2 \text { in. above ground, east } \\
\text { orientation. Dry ground. }\end{array}$ \\
\hline W804 & EC & MUW804-EC-B-26175 & $7 / 22 / 08$ & $11: 30$ & Branch & 1 & 1 & 5 & $\begin{array}{l}\text { Secondary branch } 6 \text { in. off main } \\
\text { trunk. Sample collected } 2 \text { in. } \\
\text { above ground, west orientation. }\end{array}$ \\
\hline W805 & WN1 & MUW805-WN1-B-26176 & $7 / 22 / 08$ & $11: 31$ & Branch & 671 & 12 & 5 & $\begin{array}{l}\text { Primary branch. Sample collected } \\
2 \text { in. above ground, east } \\
\text { orientation. Dry ground. }\end{array}$ \\
\hline W806 & EC2 & MUW806-EC2-B-26177 & $7 / 22 / 08$ & $11: 35$ & Branch & 383 & 16 & 122 & $\begin{array}{l}\text { Secondary branch } 6 \text { in. off main } \\
\text { stem. Sample collected } 4 \mathrm{ft} \\
\text { above ground, east orientation. }\end{array}$ \\
\hline W807 & WN & MUW807-WN-B-26178 & $7 / 22 / 08$ & $11: 40$ & Branch & 60 & 8 & 0 & $\begin{array}{l}\text { Primary branch. Sample collected } \\
\text { at ground level, west } \\
\text { orientation. }\end{array}$ \\
\hline W808 & WN2 & MUW808-WN2-B-26179 & $7 / 22 / 08$ & $11: 41$ & Branch & 85 & 5 & 30 & $\begin{array}{l}\text { Primary branch. Sample collected } \\
1 \mathrm{ft} \text { above ground, north } \\
\text { orientation. Dry ground. }\end{array}$ \\
\hline W809 & EC2 & MUW809-EC2-B-26180 & $7 / 22 / 08$ & $11: 42$ & Branch & 1303 & 55 & 122 & $\begin{array}{l}\text { Primary branch. Sample collected } \\
4 \mathrm{ft} \text { above ground, south } \\
\text { orientation. }\end{array}$ \\
\hline
\end{tabular}




\begin{tabular}{|c|c|c|c|c|c|c|c|c|c|}
\hline \multirow[b]{2}{*}{ Location } & \multirow[b]{2}{*}{ Species $^{a}$} & \multirow[b]{2}{*}{ Sample } & \multirow[b]{2}{*}{ Date } & \multirow[b]{2}{*}{ Time } & \multirow[b]{2}{*}{ Type } & \multicolumn{2}{|c|}{ Concentration $^{\mathrm{b}}(\mu \mathrm{g} / \mathrm{kg})$} & \multirow[b]{2}{*}{$\begin{array}{l}\text { Heightc } \\
(\mathrm{cm})\end{array}$} & \multirow[b]{2}{*}{ Sample Description } \\
\hline & & & & & & $\begin{array}{c}\text { Carbon } \\
\text { Tetrachloride }\end{array}$ & Chloroform & & \\
\hline \multicolumn{10}{|c|}{ Sampling in July 2008 (cont.) } \\
\hline W810 & EC2 & MUW810-EC2-B-26182 & $7 / 22 / 08$ & $11: 46$ & Branch & 1528 & 31 & 122 & $\begin{array}{l}\text { Secondary branch } 1 \mathrm{ft} \text { from } \\
\text { insertion. Sample collected } 4 \mathrm{ft} \\
\text { above ground, west orientation. }\end{array}$ \\
\hline W810 & EC2 & MUW810-EC2-B-26181 & $7 / 22 / 08$ & $11: 45$ & Branch & 2396 & 68 & 5 & $\begin{array}{l}\text { Primary branch. Sample collected } \\
2 \text { in. above ground, north } \\
\text { orientation. Dry ground. }\end{array}$ \\
\hline W815 & EC2 & MUW815-EC2-B-26183 & $7 / 22 / 08$ & 13:01 & Branch & $<1$ & ND & 152 & $\begin{array}{l}\text { Secondary branch at } 1.5 \mathrm{ft} \text { from } \\
\text { main insertion. Sample } \\
\text { collected } 5 \mathrm{ft} \text { above ground, } \\
\text { east orientation. Wet. }\end{array}$ \\
\hline W820 & EC1 & MUW820-EC1-B-26184 & $7 / 22 / 08$ & $13: 00$ & Branch & 1 & ND & 168 & $\begin{array}{l}\text { Primary branch. Sample collected } \\
5.5 \mathrm{ft} \text { above ground, east } \\
\text { orientation. }\end{array}$ \\
\hline W820 & EC2 & MUW820-EC2-B-26185 & $7 / 22 / 08$ & $12: 55$ & Branch & $<1$ & ND & 152 & $\begin{array}{l}\text { Primary branch. Sample collected } \\
5 \mathrm{ft} \text { above ground, north } \\
\text { orientation. }\end{array}$ \\
\hline W825 & EC1 & MUW825-EC1-B-26186 & $7 / 22 / 08$ & $12: 53$ & Branch & $<1$ & ND & 122 & $\begin{array}{l}\text { Primary branch. Sample collected } \\
4 \mathrm{ft} \text { above ground, east } \\
\text { orientation. }\end{array}$ \\
\hline W830 & $\mathrm{EC}$ & MUW830-EC-B-26187 & $7 / 22 / 08$ & $12: 52$ & Branch & 8 & 2 & 137 & $\begin{array}{l}\text { Secondary branch } 6 \text { in. from } \\
\text { main. Sample collected } 4.5 \mathrm{ft} \\
\text { above ground, north } \\
\text { orientation. }\end{array}$ \\
\hline W835 & EC & MUW835-EC-B-26188 & $7 / 22 / 08$ & $12: 51$ & Branch & $<1$ & ND & 91 & $\begin{array}{l}\text { Secondary branch at } 1 \mathrm{ft} \text { from } \\
\text { main insertion. Sample } \\
\text { collected } 3 \mathrm{ft} \text { above ground, } \\
\text { south orientation. }\end{array}$ \\
\hline W840 & WN1 & MUW840-WN1-B-26189 & $7 / 22 / 08$ & $12: 50$ & Branch & ND & ND & 15 & $\begin{array}{l}\text { Primary branch. Sample collected } \\
6 \text { in. above ground, south } \\
\text { orientation. }\end{array}$ \\
\hline W845 & EC1 & MUW845-EC1-B-26190 & $7 / 22 / 08$ & $12: 46$ & Branch & ND & ND & 152 & $\begin{array}{l}\text { Primary branch. Sample collected } \\
5 \mathrm{ft} \text { above ground, east } \\
\text { orientation. }\end{array}$ \\
\hline
\end{tabular}




\begin{tabular}{|c|c|c|c|c|c|c|c|c|c|}
\hline \multirow[b]{2}{*}{ Location } & \multirow[b]{2}{*}{ Species $^{a}$} & \multirow[b]{2}{*}{ Sample } & \multirow[b]{2}{*}{ Date } & \multirow[b]{2}{*}{ Time } & \multirow[b]{2}{*}{ Type } & \multicolumn{2}{|c|}{ Concentration $^{\mathrm{b}}(\mu \mathrm{g} / \mathrm{kg})$} & \multirow[b]{2}{*}{$\begin{array}{l}\text { Height }^{\mathrm{c}} \\
(\mathrm{cm})\end{array}$} & \multirow[b]{2}{*}{ Sample Description } \\
\hline & & & & & & $\begin{array}{c}\text { Carbon } \\
\text { Tetrachloride }\end{array}$ & Chloroform & & \\
\hline \multicolumn{10}{|c|}{ Sampling in July 2008 (cont.) } \\
\hline W849 & EC1 & MUW849-EC1-B-26191 & $7 / 22 / 08$ & $12: 45$ & Branch & ND & ND & 137 & $\begin{array}{l}\text { Primary branch } 6 \text { in. from main } \\
\text { trunk. Sample collected } 4.5 \mathrm{ft} \\
\text { above ground, north } \\
\text { orientation. }\end{array}$ \\
\hline W854 & EC & MUW854-EC-B-26192 & $7 / 22 / 08$ & $12: 41$ & Branch & $<1$ & ND & 46 & $\begin{array}{l}\text { Primary branch. Sample collected } \\
1.5 \mathrm{ft} \text { above ground, south } \\
\text { orientation. }\end{array}$ \\
\hline W854 & GA & MUW854-GA-B-26193 & $7 / 22 / 08$ & $12: 40$ & Branch & ND & ND & 0 & $\begin{array}{l}\text { Secondary branch at } 6 \text { in. from } \\
\text { main insertion. Sample } \\
\text { collected at ground level, south } \\
\text { orientation. }\end{array}$ \\
\hline W859 & EC & MUW859-EC-B-26194 & $7 / 22 / 08$ & $12: 36$ & Branch & $<1$ & ND & 91 & $\begin{array}{l}\text { Primary branch at } 1 \mathrm{ft} \text { from } \\
\text { insertion. Sample collected } 3 \mathrm{ft} \\
\text { above ground, north } \\
\text { orientation. }\end{array}$ \\
\hline W900 & EC1 & MUW900-EC1-B-26195 & $7 / 22 / 08$ & $12: 06$ & Branch & $<1$ & ND & 122 & $\begin{array}{l}\text { Primary branch at } 2.5 \mathrm{ft} \text { from } \\
\text { intersect. Sample collected } 4 \mathrm{ft} \\
\text { above ground, east orientation. } \\
\text { Dry ground. }\end{array}$ \\
\hline W901 & $\mathrm{EC} 1$ & MUW901-EC1-B-26196 & $7 / 22 / 08$ & 12:05 & Branch & 12 & 3 & 137 & $\begin{array}{l}\text { Secondary branch } 1.5 \mathrm{ft} \text { from } \\
\text { main stem. Sample collected } \\
4.5 \mathrm{ft} \text { above ground, east } \\
\text { orientation. }\end{array}$ \\
\hline W902 & EC2 & MUW902-EC2-B-26197 & $7 / 22 / 08$ & $12: 02$ & Branch & 1 & $<1$ & 137 & $\begin{array}{l}\text { Secondary branch } 1.5 \mathrm{ft} \text { from } \\
\text { main stem. Sample collected } \\
4.5 \mathrm{ft} \text { above ground, west } \\
\text { orientation. }\end{array}$ \\
\hline W903 & EC & MUW903-EC-B-26198 & $7 / 22 / 08$ & 12:01 & Branch & 49 & 4 & 30 & $\begin{array}{l}\text { Secondary branch } 6 \text { in. from } \\
\text { insertion. Sample collected } 1 \mathrm{ft} \\
\text { above ground, west orientation }\end{array}$ \\
\hline W904 & EC2 & MUW904-EC2-B-26199 & $7 / 22 / 08$ & $12: 00$ & Branch & 14 & 4 & 122 & $\begin{array}{l}\text { Secondary branch } 1 \mathrm{ft} \text { from } \\
\text { insertion. Sample collected } 4 \mathrm{ft} \\
\text { above ground, south } \\
\text { orientation. Dry. }\end{array}$ \\
\hline
\end{tabular}




\begin{tabular}{|c|c|c|c|c|c|c|c|c|c|}
\hline & & & & & & \multicolumn{2}{|c|}{ Concentration $^{\mathrm{b}}(\mu \mathrm{g} / \mathrm{kg})$} & & \\
\hline Location & Species $^{a}$ & Sample & Date & Time & Type & $\begin{array}{c}\text { Carbon } \\
\text { Tetrachloride }\end{array}$ & Chloroform & $\begin{array}{l}\text { Height }^{\mathrm{C}} \\
(\mathrm{cm})\end{array}$ & Sample Description \\
\hline
\end{tabular}

Sampling in July 2008 (cont.)

\begin{tabular}{|c|c|c|c|c|c|c|c|c|c|}
\hline W905 & EC1 & MUW905-EC1-B-26200 & $7 / 22 / 08$ & $11: 56$ & Branch & 56 & 4 & 91 & $\begin{array}{l}\text { Secondary branch } 1 \mathrm{ft} \text { from } \\
\text { insertion. Sample collected } 3 \mathrm{ft} \\
\text { above ground, east orientation. }\end{array}$ \\
\hline W906 & EC2 & MUW906-EC2-B-26201 & $7 / 22 / 08$ & $11: 55$ & Branch & 229 & 17 & 137 & $\begin{array}{l}\text { Primary branch. Sample collected } \\
4.5 \mathrm{ft} \text { above ground, north } \\
\text { orientation. }\end{array}$ \\
\hline W907 & EC2 & MUW907-EC2-B-26202 & $7 / 22 / 08$ & $11: 51$ & Branch & 69 & 9 & 137 & $\begin{array}{l}\text { Secondary branch } 1 \mathrm{ft} \text { from } \\
\text { insertion. Sample collected } 4.5 \\
\mathrm{ft} \text { above ground, east } \\
\text { orientation. }\end{array}$ \\
\hline W908 & EC1 & MUW908-EC1-B-26203 & $7 / 22 / 08$ & $11: 50$ & Branch & 1660 & 58 & 152 & $\begin{array}{l}\text { Secondary branch } 6 \text { in. from } \\
\text { insertion. Sample collected } 5 \mathrm{ft} \\
\text { above ground, south } \\
\text { orientation. Dry ground. No tag } \\
\text { on tree. }\end{array}$ \\
\hline W909 & EC1 & MUW909-EC1-B-26204 & $7 / 22 / 08$ & $11: 48$ & Branch & 207 & 14 & 91 & $\begin{array}{l}\text { Primary branch. Sample collected } \\
3 \mathrm{ft} \text { above ground, south } \\
\text { orientation. Dry ground. }\end{array}$ \\
\hline W910 & EC2 & MUW910-EC2-B-26205 & $7 / 22 / 08$ & $11: 47$ & Branch & $<1$ & ND & 30 & $\begin{array}{l}\text { Primary branch. Sample collected } \\
1 \mathrm{ft} \text { above ground, east } \\
\text { orientation. Dry ground. }\end{array}$ \\
\hline REWET1 & & MUPREWET1-B-26251 & $7 / 23 / 08$ & $9: 01$ & Branch & ND & ND & 183 & $\begin{array}{l}\text { Secondary branch } 2 \mathrm{ft} \text { from } \\
\text { insertion. Sample collected } 6 \mathrm{ft} \\
\text { above ground, west orientation. } \\
\text { Same location as } 2007 \text { sample. }\end{array}$ \\
\hline REWET1 & & MUPREWET1-L-26250 & $7 / 23 / 08$ & 9:00 & Leaf & ND & 1 & 183 & Leaf sample. \\
\hline Bruttig-3 & Ash & MUBRUTTIG3-B-26208 & $7 / 22 / 08$ & $14: 20$ & Branch & ND & ND & 244 & $\begin{array}{l}\text { Fifth order branch, } 1 \mathrm{ft} \text { from main } \\
\text { stem. Sample collected } 8 \mathrm{ft} \\
\text { above ground, south } \\
\text { orientation. }\end{array}$ \\
\hline School-1 & Ash & MUSCHOOL1-B-26209 & $7 / 22 / 08$ & $14: 21$ & Branch & ND & ND & 305 & $\begin{array}{l}\text { Tertiary branch. Sample collected } \\
10 \mathrm{ft} \text { above ground, north } \\
\text { orientation. }\end{array}$ \\
\hline Grass-01 & & MUGRASS01-G-26210 & $7 / 22 / 08$ & $15: 35$ & $\mathrm{~N}$ & 5 & 2 & & Wild rye underneath W118. \\
\hline
\end{tabular}


TABLE B.1 (Cont.)

\begin{tabular}{|c|c|c|c|c|c|c|c|c|c|}
\hline & & & & & & \multicolumn{2}{|c|}{ Concentration $^{\mathrm{b}}(\mu \mathrm{g} / \mathrm{kg})$} & & \\
\hline Location & Species $^{a}$ & Sample & Date & Time & Type & $\begin{array}{c}\text { Carbon } \\
\text { Tetrachloride }\end{array}$ & Chloroform & $\begin{array}{l}\text { Heightc } \\
(\mathrm{cm})\end{array}$ & Sample Description \\
\hline
\end{tabular}

Sampling in July 2008 (cont.)

\begin{tabular}{|c|c|c|c|c|c|c|}
\hline Grass-02 & MUGRASS02-G-26211 & 7/22/08 & $15: 41$ & $\mathrm{~N}$ & ND & ND \\
\hline Grass-03 & MUGRASS03-G-26212 & 7/22/08 & $15: 40$ & $\mathrm{~N}$ & ND & ND \\
\hline Grass-04 & MUGRASS04-G-26213 & 7/22/08 & $15: 46$ & $\mathrm{~N}$ & ND & ND \\
\hline Grass-05 & MUGRASS05-G-26214 & $7 / 22 / 08$ & $15: 45$ & $\mathrm{~N}$ & ND & ND \\
\hline Grass-06 & MUGRASS06-G-26252 & $7 / 23 / 08$ & $9: 10$ & $\mathrm{~N}$ & ND & ND \\
\hline Grass-09 & MUGRASS09-G-26255 & $7 / 23 / 08$ & $10: 30$ & $\mathrm{~N}$ & ND & ND \\
\hline Grass-10 & MUGRASS10-G-26215 & $7 / 22 / 08$ & $15: 30$ & $\mathrm{~N}$ & $<1$ & ND \\
\hline Grass-11 & MUGRASS11-G-26216 & $7 / 22 / 08$ & $15: 31$ & $\mathrm{~N}$ & ND & ND \\
\hline Grass-12 & MUGRASS12-G-26217 & $7 / 22 / 08$ & $15: 00$ & $\mathrm{~N}$ & ND & ND \\
\hline Grass-13 & MUGRASS13-G-26218 & $7 / 22 / 08$ & 15:01 & $\mathrm{N}$ & ND & 1 \\
\hline Grass-14 & MUGRASS14-G-26256 & $7 / 23 / 08$ & $9: 15$ & $\mathrm{~N}$ & ND & ND \\
\hline Grass-15 & MUGRASS15-G-26257 & $7 / 23 / 08$ & $9: 25$ & $\mathrm{~N}$ & ND & ND \\
\hline Grass-16 & MUGRASS16-G-26258 & $7 / 23 / 08$ & $9: 45$ & $\mathrm{~N}$ & ND & ND \\
\hline Grass-17 & MUGRASS17-G-26259 & $7 / 23 / 08$ & 9:30 & $\mathrm{N}$ & ND & ND \\
\hline Grass-18 & MUGRASS18-G-26260 & $7 / 23 / 08$ & $9: 35$ & $\mathrm{~N}$ & ND & ND \\
\hline Grass-19 & MUGRASS19-G-26261 & $7 / 23 / 08$ & $9: 41$ & $\mathrm{~N}$ & ND & ND \\
\hline Grass-20 & MUGRASS20-G-26262 & $7 / 23 / 08$ & $9: 50$ & $\mathrm{~N}$ & ND & ND \\
\hline Grass-21 & MUGRASS21-G-26263 & $7 / 23 / 08$ & $9: 55$ & $\mathrm{~N}$ & ND & ND \\
\hline Grass-22 & MUGRASS22-G-26264 & $7 / 23 / 08$ & $10: 05$ & $\mathrm{~N}$ & ND & ND \\
\hline
\end{tabular}

Grass not identified.

Wild rye.

Switch grass near W643.

Wild rye near W744.

Switch grass at outfall of TD5 approximately $10 \mathrm{ft}$ up from water edge.

Bulrush at 2007 Grass-9 GPS point (stake).

Wild rye $2 \mathrm{ft}$ west of W109 piezometer.

Bulrush $5 \mathrm{ft}$ south of W109.

Bluestem $2 \mathrm{ft}$ east of piezometer W505.

Switch grass $5 \mathrm{ft}$ east of W505 EC2 tree.

Knotweed.

Young willow at water's edge at same location as 2007 sample.

Leaves collected from water surface at location 16.

Cattail just south of north-south wetland partitioning.

Bulrush at sample location Grass18 in 2007. Found 2007 stake.

Bulrush at sample location Grass19 in 2007.

Cattail at 2007 Grass-20 location. Stake found.

Willow at water's edge same location as Grass-20.

Reed on east side of north wetland just south of concrete wall. 
TABLE B.1 (Cont.)

\begin{tabular}{|c|c|c|c|c|c|c|c|c|c|}
\hline & & & & & & \multicolumn{2}{|c|}{ Concentration $^{\mathrm{b}}(\mu \mathrm{g} / \mathrm{kg})$} & \multirow[b]{2}{*}{$\begin{array}{l}\text { Height }^{\mathrm{C}} \\
(\mathrm{cm})\end{array}$} & \multirow[b]{2}{*}{ Sample Description } \\
\hline Location & Species $^{a}$ & Sample & Date & Time & Type & $\begin{array}{c}\text { Carbon } \\
\text { Tetrachloride }\end{array}$ & Chloroform & & \\
\hline
\end{tabular}

Sampling in July 2008 (cont.)

\begin{tabular}{|c|c|c|c|c|}
\hline Grass-23 & MUGRASS23-G-26265 & 7/23/08 & $10: 25$ & ND \\
\hline Grass-24 & MUGRASS24-G-26266 & 7/23/08 & $10: 26$ & $<1$ \\
\hline Grass-25 & MUGRASS25-B-26267 & 7/23/08 & 10:15 & ND \\
\hline $\begin{array}{l}\text { Grass-26 } \\
\text { Grass-27 }\end{array}$ & $\begin{array}{l}\text { MUGRASS26-G-26268 } \\
\text { MUGRASS27-G-26269 }\end{array}$ & $\begin{array}{l}7 / 23 / 08 \\
7 / 23 / 08\end{array}$ & $\begin{array}{l}10: 20 \\
10: 10\end{array}$ & $\begin{array}{l}\text { ND } \\
\text { ND }\end{array}$ \\
\hline Grass-28 & MUGRASS28-G-26270 & 7/23/08 & 10:21 & 5 \\
\hline Grass-29 & MUGRASS29-G-26219 & 7/22/08 & 15:05 & $<1$ \\
\hline Grass-30 & MUGRASS30-G-26220 & 7/22/08 & $15: 15$ & ND \\
\hline Grass-31 & MUGRASS31-G-26221 & 7/22/08 & $15: 16$ & 43 \\
\hline Grass-32 & MUGRASS32-G-26222 & 7/22/08 & $15: 20$ & $<1$ \\
\hline Grass-33 & MUGRASS33-G-26223 & 7/22/08 & $15: 25$ & 5 \\
\hline Grass-34 & MUGRASS34-B-26271 & $7 / 23 / 08$ & $9: 16$ & ND \\
\hline Grass-35 & MUGRASS35-B-26272 & 7/23/08 & 9:31 & ND \\
\hline Grass-36 & MUGRASS36-B-26273 & 7/23/08 & $9: 40$ & ND \\
\hline Grass-37 & MUGRASS37-G-26274 & 7/23/08 & 10:31 & $<1$ \\
\hline
\end{tabular}

Willow at water's edge, same Grass-23 2007 location, GPS

Bulrush, same location as Grass23.

Cottonwood at GPS location Grass-25 in 2007. (No arrowhead found.)

Arrowhead ??

Cattail at GPS location Grass-27 in 2007.

Reed at Grass-28 2007 location. Found stake, GPS.

Switch grass $8 \mathrm{ft}$ southeast of W806, equidistant with W804.

Switch grass $2 \mathrm{ft}$ northwest of W808 EC2.

Blanket flower $1 \mathrm{ft}$ from W707 EC2.

Wild rye between W809 and W810.

Switch grass $8 \mathrm{ft}$ southwest of W407.

Cottonwood sapling growing at water's edge, same location as Grass-14. Stems collected at 6 in. above ground.

Willow growing at water's edge halfway between Grass-15 and Grass-17.

Willow at water's edge same location as Grass-18.

Leaves from water surface just south of concrete wall on west side of weir. 


\begin{tabular}{|c|c|c|c|c|c|c|c|c|c|}
\hline & & & & & & \multicolumn{2}{|c|}{ Concentration $^{\mathrm{b}}(\mu \mathrm{g} / \mathrm{kg})$} & & \\
\hline Location & Species $^{a}$ & Sample & Date & Time & Type & $\begin{array}{c}\text { Carbon } \\
\text { Tetrachloride }\end{array}$ & Chloroform & $\begin{array}{l}\text { Heightc } \\
(\mathrm{cm})\end{array}$ & Sample Description \\
\hline
\end{tabular}

Sampling in October 2008

\begin{tabular}{|c|c|c|c|c|c|c|c|c|c|}
\hline E106 & WN1 & MUE106WN1-B-27429 & $10 / 7 / 08$ & $12: 00$ & Branch & $<1$ & ND & 152 & $\begin{array}{l}\text { Secondary branch } 5 \mathrm{ft} \text { above } \\
\text { ground; } 6 \text { in. from main. East } \\
\text { orientation. }\end{array}$ \\
\hline E111 & WN1 & MUE111WN1-B-27425 & $10 / 7 / 08$ & $11: 50$ & Branch & ND & ND & 122 & $\begin{array}{l}\text { Branch tip } 4 \mathrm{ft} \text { above ground. } \\
\text { North orientation. Bad looking } \\
\text { tree. }\end{array}$ \\
\hline E112 & WN2 & MUE112WN2-B-27426 & $10 / 7 / 08$ & $11: 51$ & Branch & ND & ND & 15 & $\begin{array}{l}\text { Basal sucker } 6 \text { in. above ground. } \\
\text { Northeast orientation. }\end{array}$ \\
\hline E113 & WN1 & MUE113WN1-B-27427 & $10 / 7 / 08$ & $11: 52$ & Branch & ND & ND & 30 & $\begin{array}{l}\text { Sucker off main stem } 1 \mathrm{ft} \text { above } \\
\text { ground. South orientation. }\end{array}$ \\
\hline E206 & WN1 & MUE206WN1-B-27430 & $10 / 7 / 08$ & $12: 05$ & Branch & ND & ND & 61 & $\begin{array}{l}\text { Primary sucker off main trunk } 2 \mathrm{ft} \\
\text { above ground. North } \\
\text { orientation. Water is flowing in } \\
\text { the area. }\end{array}$ \\
\hline E207 & WN1 & MUE207WN1-B-27431 & $10 / 7 / 08$ & $12: 06$ & Branch & ND & ND & 122 & $\begin{array}{l}\text { Branch tip } 4 \mathrm{ft} \text { above ground. } \\
\text { North orientation. Bad tree. }\end{array}$ \\
\hline E207 & WN2 & MUE207WN2-B-27432 & $10 / 7 / 08$ & $12: 07$ & Branch & $<1$ & ND & 122 & $\begin{array}{l}\text { Branch tip } 4 \mathrm{ft} \text { above ground. } \\
\text { North orientation. Bad tree. }\end{array}$ \\
\hline E308 & WN2 & MUE308WN2-B-27433 & $10 / 7 / 08$ & $12: 08$ & Branch & ND & ND & 213 & $\begin{array}{l}\text { Secondary branch } 7 \mathrm{ft} \text { above } \\
\text { ground; } 2 \mathrm{ft} \text { from main stem. } \\
\text { West orientation. }\end{array}$ \\
\hline E309 & WN1 & MUE309WN1-B-27434 & $10 / 7 / 08$ & $12: 10$ & Branch & $<1$ & ND & 15 & $\begin{array}{l}\text { Basal sucker } 6 \text { in. above ground. } \\
\text { South orientation. }\end{array}$ \\
\hline E319 & WN2 & MUE319WN2-B-27428 & $10 / 7 / 08$ & $11: 55$ & Branch & ND & ND & 183 & $\begin{array}{l}\text { Secondary branch } 6 \mathrm{ft} \text { above } \\
\text { ground; } 6 \text { in. from main stem. } \\
\text { Northeast orientation. }\end{array}$ \\
\hline E340 & WN2 & MUE340WN2-B-27423 & $10 / 7 / 08$ & $11: 36$ & Branch & $<1$ & ND & 122 & $\begin{array}{l}\text { Secondary branch } 4 \mathrm{ft} \text { above } \\
\text { ground; } 6 \text { in. from main stem. } \\
\text { West orientation. }\end{array}$ \\
\hline E555 & WN1 & MUE555WN1-B-27421 & $10 / 7 / 08$ & $11: 31$ & Branch & $<1$ & ND & 198 & $\begin{array}{l}\text { Secondary branch } 6.5 \mathrm{ft} \text { above } \\
\text { ground; } 2 \mathrm{ft} \text { from main stem. } \\
\text { East orientation. }\end{array}$ \\
\hline
\end{tabular}




\begin{tabular}{|c|c|c|c|c|c|c|c|c|c|}
\hline & & & & & & \multicolumn{2}{|c|}{ Concentration ${ }^{\mathrm{b}}(\mu \mathrm{g} / \mathrm{kg})$} & & \\
\hline Location & Species $^{a}$ & Sample & Date & Time & Type & $\begin{array}{c}\text { Carbon } \\
\text { Tetrachloride }\end{array}$ & Chloroform & $\begin{array}{l}\text { Height }^{\mathrm{C}} \\
(\mathrm{cm})\end{array}$ & Sample Description \\
\hline
\end{tabular}

Sampling in October 2008 (cont.)

\begin{tabular}{|c|c|c|c|c|c|c|c|c|c|}
\hline E557 & WN1 & MUE557WN1-B-27420 & $10 / 7 / 08$ & $11: 30$ & Branch & $<1$ & ND & 183 & $\begin{array}{l}\text { Secondary branch; } 6 \mathrm{ft} \text { above } \\
\text { ground; } 12 \text { in. from main. East } \\
\text { orientation. }\end{array}$ \\
\hline E659 & WN1 & MUE659WN1-B-27422 & 10/7/08 & $11: 35$ & Branch & ND & ND & 20 & $\begin{array}{l}\text { Sucker off main stem; } 8 \text { in. above } \\
\text { ground. North orientation. }\end{array}$ \\
\hline E659 & WN2 & MUE659WN2-B-27424 & $10 / 7 / 08$ & $11: 37$ & Branch & $<1$ & ND & 15 & $\begin{array}{l}\text { Sucker off main stem; } 6 \text { in. above } \\
\text { ground. South orientation. } \\
\text { Large diameter tree. }\end{array}$ \\
\hline W109 & WN1 & MUW109WN1-B-27437 & 10/7/08 & $13: 20$ & Branch & 15 & ND & 20 & $\begin{array}{l}\text { Primary sucker } 8 \text { in. above } \\
\text { ground. East orientation. }\end{array}$ \\
\hline W109 & WN2 & MUW109WN2-B-27438 & $10 / 7 / 08$ & $13: 21$ & Branch & 22 & 2 & 30 & $\begin{array}{l}\text { Primary sucker } 12 \text { in. above } \\
\text { ground. North orientation. }\end{array}$ \\
\hline W110 & WN1 & MUW110WN1-B-27439 & $10 / 7 / 08$ & $13: 25$ & Branch & 7 & 2 & 122 & $\begin{array}{l}\text { Secondary branch } 4 \mathrm{ft} \text { above } \\
\text { ground; } 12 \text { in. from main stem. } \\
\text { Northeast orientation. }\end{array}$ \\
\hline W110 & WN2 & MUW110WN2-B-27440 & $10 / 7 / 08$ & $13: 26$ & Branch & ND & ND & 122 & $\begin{array}{l}\text { Secondary branch } 4 \mathrm{ft} \text { above } \\
\text { ground; } 12 \text { in. from main stem. } \\
\text { Northeast orientation. }\end{array}$ \\
\hline W119 & WN1 & MUW119WN1-B-26312 & $10 / 7 / 08$ & $11: 10$ & Branch & 17 & 1 & 183 & $\begin{array}{l}\text { Primary branch } 6 \mathrm{ft} \text { above ground. } \\
\text { East orientation. }\end{array}$ \\
\hline W119 & WN1 & MUW119WN1-B-27445 & $10 / 8 / 08$ & $9: 25$ & Branch & 6 & 2 & 122 & $\begin{array}{l}\text { Daily time series. Secondary } \\
\text { branch } 4 \mathrm{ft} \text { above ground; } 1 \mathrm{ft} \\
\text { from main. North orientation. }\end{array}$ \\
\hline W119 & WN1 & MUW119WN1-B-27461 & $10 / 9 / 08$ & $9: 10$ & Branch & 5 & 1 & 183 & $\begin{array}{l}\text { Daily time series. Secondary } \\
\text { branch } 6 \mathrm{ft} \text { above ground; } 8 \text { in. } \\
\text { from main stem. East } \\
\text { orientation. }\end{array}$ \\
\hline W119 & WN2 & MUW119WN2-B-26313 & $10 / 7 / 08$ & $11: 11$ & Branch & 1 & $<1$ & 183 & $\begin{array}{l}\text { Primary branch } 6 \mathrm{ft} \text { above ground. } \\
\text { East orientation. }\end{array}$ \\
\hline W119 & WN2 & MUW119WN2-B-27446 & 10/8/08 & $9: 26$ & Branch & 4 & 1 & 213 & $\begin{array}{l}\text { Daily time series. Primary branch } \\
\text { tip } 7 \mathrm{ft} \text { above ground. } \\
\text { Northeast orientation. }\end{array}$ \\
\hline
\end{tabular}




\begin{tabular}{|c|c|c|c|c|c|c|c|c|c|}
\hline & & & & & & \multicolumn{2}{|c|}{ Concentration $^{\mathrm{b}}(\mu \mathrm{g} / \mathrm{kg})$} & \multirow[b]{2}{*}{$\begin{array}{l}\text { Heightc }^{c} \\
(\mathrm{~cm})\end{array}$} & \multirow[b]{2}{*}{ Sample Description } \\
\hline Location & Species $^{a}$ & Sample & Date & Time & Type & $\begin{array}{c}\text { Carbon } \\
\text { Tetrachloride }\end{array}$ & Chloroform & & \\
\hline
\end{tabular}

Sampling in October 2008 (cont.)

\begin{tabular}{|c|c|c|c|c|c|c|c|c|c|}
\hline W119 & WN2 & MUW119WN2-B-27462 & $10 / 9 / 08$ & $9: 11$ & Branch & 9 & 1 & 213 & $\begin{array}{l}\text { Daily time series. Primary branch } \\
7 \mathrm{ft} \text { above ground. North } \\
\text { orientation. }\end{array}$ \\
\hline W157 & WN1 & MUW157WN1-B-26314 & 10/7/08 & $11: 15$ & Branch & ND & ND & 152 & $\begin{array}{l}\text { Primary branch } 5 \mathrm{ft} \text { above ground. } \\
\text { East orientation. }\end{array}$ \\
\hline W158 & WN1 & MUW158WN1-B-26315 & $10 / 7 / 08$ & $11: 16$ & Branch & ND & ND & 20 & $\begin{array}{l}\text { Suckers off trunk; } 8 \text { in. above } \\
\text { ground. East orientation. }\end{array}$ \\
\hline W158 & WN2 & MUW158WN2-B-26316 & $10 / 7 / 08$ & $11: 17$ & Branch & ND & ND & 20 & $\begin{array}{l}\text { Sucker off main; } 8 \text { in. above } \\
\text { ground. East orientation. }\end{array}$ \\
\hline W159 & EC2 & MUW159EC2-B-26317 & $10 / 7 / 08$ & $11: 18$ & Branch & ND & ND & 183 & $\begin{array}{l}\text { Primary branch } 6 \mathrm{ft} \text { above ground. } \\
\text { Northeast orientation. Very few } \\
\text { leaves left on tree. }\end{array}$ \\
\hline W357 & WN1 & MUW357WN1-B-26318 & $10 / 7 / 08$ & $11: 20$ & Branch & ND & ND & 15 & $\begin{array}{l}\text { Sucker off main stem; } 6 \text { in. above } \\
\text { ground. East orientation. }\end{array}$ \\
\hline W404 & EC1 & MUW404EC1-B-26296 & 10/7/08 & $10: 40$ & Branch & $<1$ & ND & 122 & $\begin{array}{l}\text { Secondary branch } 4 \mathrm{ft} \text { up; } 6 \text { in. } \\
\text { from main stem. South } \\
\text { orientation. }\end{array}$ \\
\hline W404 & EC2 & MUW404EC2-B-26297 & $10 / 7 / 08$ & $10: 41$ & Branch & $<1$ & $<1$ & 122 & $\begin{array}{l}\text { Primary branch } 4 \mathrm{ft} \text { above ground. } \\
\text { East orientation. }\end{array}$ \\
\hline W457 & EC & MUW457EC-B-26319 & 10/7/08 & $11: 21$ & Branch & $<1$ & ND & 183 & $\begin{array}{l}\text { Secondary branch } 6 \mathrm{ft} \text { up; } 6 \text { in. } \\
\text { from main stem. North } \\
\text { orientation. Tree in bad shape } \\
\text { with canker; leaves eaten up } \\
\text { by caterpillars. Much smaller } \\
\text { tree than those at nearby } \\
\text { locations. }\end{array}$ \\
\hline W504 & WN1 & MUW504WN1-B-26302 & 10/7/08 & $10: 47$ & Branch & 4 & $<1$ & 15 & $\begin{array}{l}\text { Basal sucker; } 6 \text { in. above ground. } \\
\text { Northeast orientation. }\end{array}$ \\
\hline W504 & WN2 & MUW504WN2-B-26303 & 10/7/08 & $10: 48$ & Branch & 1 & ND & 15 & $\begin{array}{l}\text { Basal sucker; } 6 \text { in. above ground. } \\
\text { Northeast orientation. }\end{array}$ \\
\hline W505 & EC1 & MUW505EC1-B-26298 & 10/7/08 & $10: 44$ & Branch & $<1$ & ND & 183 & $\begin{array}{l}\text { Primary branch; } 6 \mathrm{ft} \text { above } \\
\text { ground. West orientation. }\end{array}$ \\
\hline
\end{tabular}


TABLE B.1 (Cont.)

\begin{tabular}{|c|c|c|c|c|c|c|c|c|c|}
\hline & & & & & & \multicolumn{2}{|c|}{ Concentration $^{\mathrm{b}}(\mu \mathrm{g} / \mathrm{kg})$} & \multirow[b]{2}{*}{$\begin{array}{l}\text { Height }^{\mathrm{C}} \\
(\mathrm{cm})\end{array}$} & \multirow[b]{2}{*}{ Sample Description } \\
\hline Location & Species $^{a}$ & Sample & Date & Time & Type & $\begin{array}{c}\text { Carbon } \\
\text { Tetrachloride }\end{array}$ & Chloroform & & \\
\hline
\end{tabular}

Sampling in October 2008 (cont.)

\begin{tabular}{|c|c|c|c|c|c|c|c|c|c|}
\hline W505 & EC1 & MUW505EC1-B-27441 & $10 / 8 / 08$ & $9: 15$ & Branch & $<1$ & $<1$ & 152 & $\begin{array}{l}\text { Daily time series on second day } \\
\text { of sampling. Secondary branch } \\
5 \mathrm{ft} \text { above ground; } 10 \mathrm{in} \text {. off } \\
\text { main stem. West orientation. }\end{array}$ \\
\hline W505 & EC1 & MUW505EC1-B-27447 & $10 / 9 / 08$ & 9:00 & Branch & $<1$ & 1 & 183 & $\begin{array}{l}\text { Daily time series on third day of } \\
\text { sampling. Primary branch } 6 \mathrm{ft} \\
\text { above ground. West } \\
\text { orientation. }\end{array}$ \\
\hline W505 & EC2 & MUW505EC2-B-26299 & $10 / 7 / 08$ & $10: 45$ & Branch & ND & ND & 183 & $\begin{array}{l}\text { Secondary branch; } 6 \mathrm{ft} \text { above } \\
\text { ground; } 6 \text { in. from main. South } \\
\text { orientation. }\end{array}$ \\
\hline W505 & EC2 & MUW505EC2-B-27448 & $10 / 9 / 08$ & 9:01 & Branch & ND & ND & 183 & $\begin{array}{l}\text { Daily time series. Primary branch } \\
6 \mathrm{ft} \text { above ground. East } \\
\text { orientation. }\end{array}$ \\
\hline W506 & EC & MUW506EC-B-26300 & $10 / 7 / 08$ & $10: 46$ & Branch & 67 & 4 & 137 & $\begin{array}{l}\text { Primary branch; } 4.5 \mathrm{ft} \text { above } \\
\text { ground. East orientation. }\end{array}$ \\
\hline W605 & WN1 & MUW605WN1-B-26304 & $10 / 7 / 08$ & $10: 49$ & Branch & $<1$ & ND & 30 & $\begin{array}{l}\text { Suckers off main stem; } 12 \text { in. } \\
\text { above ground. North } \\
\text { orientation. }\end{array}$ \\
\hline W605 & WN2 & MUW605WN2-B-26305 & $10 / 7 / 08$ & $10: 50$ & Branch & 11 & 2 & 30 & $\begin{array}{l}\text { Sucker off main stem; } 12 \text { in. } \\
\text { above ground. North } \\
\text { orientation. }\end{array}$ \\
\hline W606 & EC1 & MUW606EC1-B-26306 & $10 / 7 / 08$ & $10: 51$ & Branch & ND & ND & 91 & $\begin{array}{l}\text { Secondary branch; } 3 \mathrm{ft} \text { above } \\
\text { ground; } 1 \mathrm{ft} \text { from main stem. } \\
\text { North orientation. }\end{array}$ \\
\hline W606 & EC1 & MUW606EC1-B-27444 & 10/8/08 & $9: 21$ & Branch & 21 & 1 & 152 & $\begin{array}{l}\text { Daily time series. Secondary } \\
\text { branch } 5 \mathrm{ft} \text { above ground; } 8 \text { in. } \\
\text { from main stem. West } \\
\text { orientation. }\end{array}$ \\
\hline W606 & EC1 & MUW606EC1-B-27449 & 10/9/08 & 9:02 & Branch & 18 & 2 & 152 & $\begin{array}{l}\text { Daily time series. Primary branch } \\
5 \mathrm{ft} \text { above ground. East } \\
\text { orientation. }\end{array}$ \\
\hline
\end{tabular}


TABLE B.1 (Cont.)

\begin{tabular}{|c|c|c|c|c|c|c|c|c|c|}
\hline \multirow[b]{2}{*}{ Location } & \multirow[b]{2}{*}{ Species $^{a}$} & \multirow[b]{2}{*}{ Sample } & \multirow[b]{2}{*}{ Date } & \multirow[b]{2}{*}{ Time } & \multirow[b]{2}{*}{ Type } & \multicolumn{2}{|c|}{ Concentration $^{\mathrm{b}}(\mu \mathrm{g} / \mathrm{kg})$} & \multirow[b]{2}{*}{$\begin{array}{l}\text { Heightc }^{c} \\
(\mathrm{~cm})\end{array}$} & \multirow[b]{2}{*}{ Sample Description } \\
\hline & & & & & & $\begin{array}{c}\text { Carbon } \\
\text { Tetrachloride }\end{array}$ & Chloroform & & \\
\hline \multicolumn{10}{|c|}{ Sampling in October 2008 (cont.) } \\
\hline W606 & EC2 & MUW606EC2-B-26307 & $10 / 7 / 08$ & $10: 52$ & Branch & 7 & 2 & 183 & $\begin{array}{l}\text { Secondary branch; } 6 \mathrm{ft} \text { above } \\
\text { ground; } 1 \mathrm{ft} \text { from main stem. } \\
\text { East orientation. }\end{array}$ \\
\hline W606 & EC2 & MUW606EC2-B-27443 & 10/8/08 & 9:20 & Branch & 25 & 2 & 183 & $\begin{array}{l}\text { Daily time series. Secondary } \\
\text { branch } 6 \mathrm{ft} \text { above ground; } 8 \text { in. } \\
\text { from main stem. West } \\
\text { orientation. }\end{array}$ \\
\hline W606 & EC2 & MUW606EC2-B-27460 & 10/9/08 & 9:05 & Branch & 11 & 1 & 168 & $\begin{array}{l}\text { Daily time series. Primary branch } \\
5.5 \mathrm{ft} \text { above ground. West } \\
\text { orientation. }\end{array}$ \\
\hline W706 & EC1 & MUW706EC1-B-26308 & $10 / 7 / 08$ & $10: 55$ & Branch & 2 & $<1$ & 122 & $\begin{array}{l}\text { Secondary branch; } 4 \mathrm{ft} \text { above } \\
\text { ground; } 6 \text { in. from main. South } \\
\text { orientation. }\end{array}$ \\
\hline W706 & EC2 & MUW706EC2-B-26309 & $10 / 7 / 08$ & 10:56 & Branch & 23 & 1 & 122 & $\begin{array}{l}\text { Primary branch } 4 \mathrm{ft} \text { above ground. } \\
\text { East orientation. }\end{array}$ \\
\hline W707 & EC & MUW707EC-B-26310 & 10/7/08 & $11: 00$ & Branch & 1 & ND & 122 & $\begin{array}{l}\text { Primary branch } 4 \mathrm{ft} \text { above ground. } \\
\text { North orientation. }\end{array}$ \\
\hline W805 & EC2 & MUW805EC2-B-27442 & $10 / 8 / 08$ & 9:16 & Branch & $<1$ & ND & 152 & $\begin{array}{l}\text { Daily time series. Primary branch } \\
5 \mathrm{ft} \text { above ground. North } \\
\text { orientation. }\end{array}$ \\
\hline Bruttig-3 & Ash & MUBRUTTIG3-B-27435 & $10 / 7 / 08$ & 13:05 & Branch & ND & ND & 183 & $\begin{array}{l}\text { Tertiary branch } 6 \mathrm{ft} \text { above ground; } \\
6 \mathrm{ft} \text { from main stem. West } \\
\text { orientation. Ash tree. }\end{array}$ \\
\hline School-1 & Ash & MUSCHOOL1-B-27436 & $10 / 7 / 08$ & 13:10 & Branch & ND & ND & 122 & $\begin{array}{l}\text { Sucker off main stem } 4 \mathrm{ft} \text { above } \\
\text { ground. South orientation. Ash } \\
\text { tree. }\end{array}$ \\
\hline
\end{tabular}


a Tree species: EC, eastern cottonwood; GA, green ash; HP, hybrid poplar; NC, northern catalpa; WN, Niobe willow. Numerals 1 and 2 distinguish two trees of the same species at a single location.

b Concentrations are rounded to whole numbers.

c Sample height is measured from the branch insertion point to ground level.

d ND, contaminant not detected at reporting limits of $0.1 \mu \mathrm{g} / \mathrm{kg}$ for carbon tetrachloride and $0.75 \mu \mathrm{g} / \mathrm{kg}$ for chloroform.

e $<1$, concentration below the method quantitation limit of $1.0 \mu \mathrm{g} / \mathrm{kg}$.

f NA, not analyzed for this constituent. 


\section{Supplementary Material for Summary of Operations and Performance of the Murdock Site Restoration Project in 2008 ANL/EVSIAGEM/TR-09-06}

\section{Contents}

\section{Supplement 1: Water Level Recorder Data}

Supplement 1 contains water level data recorded automatically during 2008 in the 16 monitoring wells identified in Figure 2.3. The data are presented as follows:

- Table S1.1: Rainfall data from the on-site weather station

- Table S1.2: Water level data for upgradient wells

- Table S1.3: Water level data for headwaters area wells

- Table S1.4: Water level data for downgradient wells

- Table S1.5: Water level measurements at the wetlands outfall and calculated discharge flow rates

The discharge flow rates presented in Table S1.5 were calculated as discussed in Supplement 1 of the previous performance report (Argonne 2008).

\section{Supplement 2: Sap Flow Data}

Sap flux data (in milliliters of sap per centimeter-hour) were obtained from thermal dissipation probes installed in 18 trees in the phytoremediation planting area at Murdock for approximately one month at the peak of the 2008 transpiration season (July-August). The complete data set is in Table S2.1. The measured data were multiplied by the surface area of the active sapwood per tree, and hence for the tree stand, to obtain estimates of total daily tree water use. The results are discussed in Section 4.3.1.2 and are summarized in Table 4.4. 
TABLE S1.1 Rainfall data collected by the on-site weather station in 2008 (values used to plot rainfall in figures).

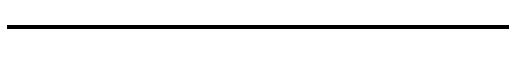

\begin{tabular}{|c|c|c|}
\hline Date & Time & Rain (in.) \\
\hline 1/6/08 & $6: 00$ & 0 \\
\hline 1/6/08 & 8:00 & 0.01 \\
\hline 1/6/08 & $10: 00$ & 0 \\
\hline 1/7/08 & 2:00 & 0 \\
\hline 1/7/08 & $4: 00$ & 0.02 \\
\hline 1/7/08 & $6: 00$ & 0 \\
\hline 1/7/08 & 8:00 & 0 \\
\hline 1/7/08 & $10: 00$ & 0.01 \\
\hline 1/7/08 & $12: 00$ & 0 \\
\hline 1/10/08 & $12: 00$ & 0 \\
\hline 1/10/08 & 14:00 & 0.02 \\
\hline 1/10/08 & $16: 00$ & 0 \\
\hline 2/17/08 & $6: 00$ & 0 \\
\hline 2/17/08 & 8:00 & 0.01 \\
\hline 2/17/08 & $10: 00$ & 0.02 \\
\hline 2/17/08 & $12: 00$ & 0 \\
\hline 2/19/08 & $12: 00$ & 0 \\
\hline 2/19/08 & $14: 00$ & 0.01 \\
\hline 2/19/08 & $16: 00$ & 0 \\
\hline 2/24/08 & 8:00 & 0 \\
\hline 2/24/08 & 10:00 & 0.01 \\
\hline 2/24/08 & $12: 00$ & 0 \\
\hline 2/25/08 & $12: 00$ & 0 \\
\hline 2/25/08 & $14: 00$ & 0.11 \\
\hline $2 / 25 / 08$ & $16: 00$ & 0.06 \\
\hline 2/25/08 & 18:00 & 0.01 \\
\hline 2/25/08 & 20:00 & 0 \\
\hline 2/27/08 & $10: 00$ & 0 \\
\hline 2/27/08 & $12: 00$ & 0.03 \\
\hline 2/27/08 & $14: 00$ & 0.02 \\
\hline 2/27/08 & $16: 00$ & 0 \\
\hline 2/28/08 & $10: 00$ & 0 \\
\hline 2/28/08 & $12: 00$ & 0.01 \\
\hline 2/28/08 & $14: 00$ & 0.04 \\
\hline 2/28/08 & $16: 00$ & 0 \\
\hline 3/2/08 & $16: 00$ & 0 \\
\hline $3 / 2 / 08$ & $18: 00$ & 0.11 \\
\hline $3 / 2 / 08$ & 20:00 & 0 \\
\hline $3 / 2 / 08$ & 22:00 & 0.01 \\
\hline $3 / 3 / 08$ & 0:00 & 0 \\
\hline 3/3/08 & $12: 00$ & 0 \\
\hline
\end{tabular}

\begin{tabular}{|c|c|c|}
\hline Date & Time & Rain (in.) \\
\hline 1/6/08 & $6: 00$ & 0 \\
\hline 1/6/08 & 8:00 & 0.01 \\
\hline 1/6/08 & $10: 00$ & 0 \\
\hline 1/7/08 & $2: 00$ & 0 \\
\hline 1/7/08 & $4: 00$ & 0.02 \\
\hline 1/7/08 & $6: 00$ & 0 \\
\hline 1/7/08 & $8: 00$ & 0 \\
\hline 1/7/08 & $10: 00$ & 0.01 \\
\hline 1/7/08 & $12: 00$ & 0 \\
\hline 1/10/08 & $12: 00$ & 0 \\
\hline 1/10/08 & 14:00 & 0.02 \\
\hline 1/10/08 & $16: 00$ & 0 \\
\hline 2/17/08 & 6:00 & 0 \\
\hline 2/17/08 & 8:00 & 0.01 \\
\hline 2/17/08 & 10:00 & 0.02 \\
\hline 2/17/08 & $12: 00$ & 0 \\
\hline 2/19/08 & $12: 00$ & 0 \\
\hline 2/19/08 & 14:00 & 0.01 \\
\hline 2/19/08 & $16: 00$ & 0 \\
\hline 2/24/08 & 8:00 & 0 \\
\hline 2/24/08 & 10:00 & 0.01 \\
\hline $2 / 24 / 08$ & $12: 00$ & 0 \\
\hline 2/25/08 & $12: 00$ & 0 \\
\hline 2/25/08 & $14: 00$ & 0.11 \\
\hline $2 / 25 / 08$ & $16: 00$ & 0.06 \\
\hline 2/25/08 & 18:00 & 0.01 \\
\hline 2/25/08 & 20:00 & 0 \\
\hline 2/27/08 & $10: 00$ & 0 \\
\hline 2/27/08 & $12: 00$ & 0.03 \\
\hline 2/27/08 & $14: 00$ & 0.02 \\
\hline 2/27/08 & $16: 00$ & 0 \\
\hline 2/28/08 & 10:00 & 0 \\
\hline 2/28/08 & $12: 00$ & 0.01 \\
\hline 2/28/08 & $14: 00$ & 0.04 \\
\hline 2/28/08 & $16: 00$ & 0 \\
\hline 3/2/08 & $16: 00$ & 0 \\
\hline $3 / 2 / 08$ & $18: 00$ & 0.11 \\
\hline $3 / 2 / 08$ & 20:00 & 0 \\
\hline $3 / 2 / 08$ & $22: 00$ & 0.01 \\
\hline $3 / 3 / 08$ & 0:00 & 0 \\
\hline 3/3/08 & $12: 00$ & 0 \\
\hline
\end{tabular}

Table S1.1 (Cont.)

\begin{tabular}{|c|c|c|c|c|c|}
\hline Date & Time & Rain (in.) & Date & Time & Rain (in.) \\
\hline $3 / 3 / 08$ & $14: 00$ & 0.01 & $4 / 9 / 08$ & $6: 00$ & 0 \\
\hline $3 / 3 / 08$ & $16: 00$ & 0 & 4/9/08 & $22: 00$ & 0 \\
\hline $3 / 7 / 08$ & $14: 00$ & 0 & 4/10/08 & $0: 00$ & 0.05 \\
\hline $3 / 7 / 08$ & $16: 00$ & 0.01 & $4 / 10 / 08$ & $2: 00$ & 0.15 \\
\hline $3 / 7 / 08$ & $18: 00$ & 0 & 4/10/08 & $4: 00$ & 0.04 \\
\hline 3/17/08 & 8:00 & 0 & 4/10/08 & $6: 00$ & 0.05 \\
\hline $3 / 17 / 08$ & $10: 00$ & 0.11 & $4 / 10 / 08$ & $8: 00$ & 0.03 \\
\hline 3/17/08 & $12: 00$ & 0 & 4/10/08 & $10: 00$ & 0.05 \\
\hline 3/17/08 & $18: 00$ & 0 & 4/10/08 & $12: 00$ & 0.16 \\
\hline 3/17/08 & 20:00 & 0.01 & 4/10/08 & $14: 00$ & 0.1 \\
\hline 3/17/08 & $22: 00$ & 0.01 & 4/10/08 & $16: 00$ & 0.42 \\
\hline 3/18/08 & $0: 00$ & 0 & $4 / 10 / 08$ & $18: 00$ & 0 \\
\hline 3/19/08 & $6: 00$ & 0 & 4/10/08 & $20: 00$ & 0 \\
\hline 3/19/08 & $8: 00$ & 0.01 & 4/10/08 & $22: 00$ & 0.02 \\
\hline 3/19/08 & $10: 00$ & 0 & 4/11/08 & $0: 00$ & 0.01 \\
\hline 3/19/08 & $18: 00$ & 0 & 4/11/08 & $2: 00$ & 0.01 \\
\hline $3 / 19 / 08$ & $20: 00$ & 0.03 & $4 / 11 / 08$ & $4: 00$ & 0.01 \\
\hline 3/19/08 & $22: 00$ & 0 & 4/11/08 & $6: 00$ & 0.04 \\
\hline 3/31/08 & $2: 00$ & 0 & 4/11/08 & 8:00 & 0.02 \\
\hline $3 / 31 / 08$ & $4: 00$ & 0.19 & $4 / 11 / 08$ & $10: 00$ & 0 \\
\hline 3/31/08 & $6: 00$ & 0.36 & 4/11/08 & $16: 00$ & 0 \\
\hline 3/31/08 & 8:00 & 0.08 & 4/11/08 & $18: 00$ & 0.01 \\
\hline 3/31/08 & $10: 00$ & 0.06 & 4/11/08 & 20:00 & 0 \\
\hline 3/31/08 & $12: 00$ & 0 & $4 / 12 / 08$ & 8:00 & 0 \\
\hline 3/31/08 & $16: 00$ & 0 & $4 / 12 / 08$ & $10: 00$ & 0.01 \\
\hline 3/31/08 & $18: 00$ & 0.01 & $4 / 12 / 08$ & $12: 00$ & 0 \\
\hline 3/31/08 & $20: 00$ & 0 & 4/17/08 & $4: 00$ & 0 \\
\hline $4 / 3 / 08$ & $6: 00$ & 0 & $4 / 17 / 08$ & $6: 00$ & 0.6 \\
\hline $4 / 3 / 08$ & $8: 00$ & 0.05 & $4 / 17 / 08$ & $8: 00$ & 0.24 \\
\hline $4 / 3 / 08$ & $10: 00$ & 0.02 & 4/17/08 & $10: 00$ & 0.01 \\
\hline $4 / 3 / 08$ & $12: 00$ & 0.26 & $4 / 17 / 08$ & $12: 00$ & 0 \\
\hline $4 / 3 / 08$ & $14: 00$ & 0.06 & $4 / 17 / 08$ & $14: 00$ & 0.02 \\
\hline $4 / 3 / 08$ & $16: 00$ & 0 & 4/17/08 & $16: 00$ & 0.06 \\
\hline $4 / 7 / 08$ & $18: 00$ & 0 & $4 / 17 / 08$ & $18: 00$ & 0.02 \\
\hline $4 / 7 / 08$ & $20: 00$ & 0.01 & 4/17/08 & $20: 00$ & 0.02 \\
\hline $4 / 7 / 08$ & $22: 00$ & 0.21 & 4/17/08 & $22: 00$ & 0.13 \\
\hline $4 / 8 / 08$ & $0: 00$ & 0.03 & $4 / 18 / 08$ & $0: 00$ & 0.02 \\
\hline $4 / 8 / 08$ & $2: 00$ & 0 & 4/18/08 & $2: 00$ & 0 \\
\hline $4 / 8 / 08$ & $4: 00$ & 0.01 & 4/18/08 & $4: 00$ & 0.06 \\
\hline $4 / 8 / 08$ & $6: 00$ & 0 & 4/18/08 & $6: 00$ & 0.25 \\
\hline $4 / 8 / 08$ & $10: 00$ & 0 & $4 / 18 / 08$ & $8: 00$ & 0.41 \\
\hline $4 / 8 / 08$ & $12: 00$ & 0.01 & $4 / 18 / 08$ & $10: 00$ & 0.01 \\
\hline $4 / 8 / 08$ & $14: 00$ & 0.01 & 4/18/08 & $12: 00$ & 0.01 \\
\hline $4 / 8 / 08$ & $16: 00$ & 0 & $4 / 18 / 08$ & $14: 00$ & 0.04 \\
\hline $4 / 9 / 08$ & $2: 00$ & 0 & $4 / 18 / 08$ & $16: 00$ & 0 \\
\hline 4/9/08 & 4:00 & 0.01 & 4/18/08 & $22: 00$ & 0 \\
\hline
\end{tabular}

Table S1.1 (Cont.)

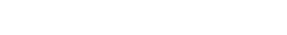


Table S1.1 (Cont.)

\begin{tabular}{|c|c|c|}
\hline Date & Time & Rain (in.) \\
\hline 4/19/08 & 0:00 & 0.01 \\
\hline 4/19/08 & $2: 00$ & 0 \\
\hline 4/24/08 & $18: 00$ & 0 \\
\hline $4 / 24 / 08$ & $20: 00$ & 0.02 \\
\hline 4/24/08 & $22: 00$ & 0 \\
\hline $4 / 25 / 08$ & $0: 00$ & 0 \\
\hline $4 / 25 / 08$ & $2: 00$ & 0.15 \\
\hline 4/25/08 & $4: 00$ & 0.22 \\
\hline $4 / 25 / 08$ & $6: 00$ & 0.01 \\
\hline $4 / 25 / 08$ & 8:00 & 0 \\
\hline $4 / 25 / 08$ & $10: 00$ & 0.01 \\
\hline $4 / 25 / 08$ & $12: 00$ & 0 \\
\hline $4 / 26 / 08$ & $18: 00$ & 0 \\
\hline $4 / 26 / 08$ & $20: 00$ & 0.07 \\
\hline $4 / 26 / 08$ & $22: 00$ & 0.07 \\
\hline $4 / 27 / 08$ & 0:00 & 0.01 \\
\hline $4 / 27 / 08$ & $2: 00$ & 0 \\
\hline $5 / 2 / 08$ & $2: 00$ & 0 \\
\hline $5 / 2 / 08$ & $4: 00$ & 0.14 \\
\hline $5 / 2 / 08$ & $6: 00$ & 0 \\
\hline $5 / 2 / 08$ & $8: 00$ & 0.05 \\
\hline $5 / 2 / 08$ & $10: 00$ & 0.17 \\
\hline $5 / 2 / 08$ & $12: 00$ & 0 \\
\hline $5 / 2 / 08$ & $18: 00$ & 0 \\
\hline $5 / 2 / 08$ & $20: 00$ & 0.01 \\
\hline $5 / 2 / 08$ & $22: 00$ & 0.01 \\
\hline $5 / 3 / 08$ & 0:00 & 0.1 \\
\hline $5 / 3 / 08$ & $2: 00$ & 0.02 \\
\hline $5 / 3 / 08$ & $4: 00$ & 0 \\
\hline $5 / 6 / 08$ & $22: 00$ & 0 \\
\hline $5 / 7 / 08$ & $0: 00$ & 0.26 \\
\hline $5 / 7 / 08$ & $2: 00$ & 0.08 \\
\hline $5 / 7 / 08$ & $4: 00$ & 0 \\
\hline $5 / 7 / 08$ & $6: 00$ & 0.01 \\
\hline $5 / 7 / 08$ & 8:00 & 0 \\
\hline $5 / 8 / 08$ & $22: 00$ & 0 \\
\hline 5/9/08 & $0: 00$ & 0.04 \\
\hline $5 / 9 / 08$ & $2: 00$ & 0.15 \\
\hline $5 / 9 / 08$ & $4: 00$ & 0.31 \\
\hline 5/9/08 & $6: 00$ & 0.07 \\
\hline $5 / 9 / 08$ & 8:00 & 0 \\
\hline 5/10/08 & $2: 00$ & 0 \\
\hline 5/10/08 & $4: 00$ & 0.16 \\
\hline 5/10/08 & $6: 00$ & 0 \\
\hline $5 / 10 / 08$ & $10: 00$ & 0 \\
\hline $5 / 10 / 08$ & $12: 00$ & 0.07 \\
\hline
\end{tabular}

Table S1.1 (Cont.)

Date Time Rain (in.)

Table S1.1 (Cont.)

Date Time Rain (in)

\begin{tabular}{|c|c|c|c|c|c|}
\hline $5 / 10 / 08$ & $14: 00$ & 0.07 & $6 / 5 / 08$ & $0: 00$ & 0.01 \\
\hline 5/10/08 & $16: 00$ & 0.23 & $6 / 5 / 08$ & $2: 00$ & 0.03 \\
\hline 5/10/08 & $18: 00$ & 0.23 & 6/5/08 & $4: 00$ & 0.16 \\
\hline 5/10/08 & $20: 00$ & 0.04 & $6 / 5 / 08$ & $6: 00$ & 0 \\
\hline $5 / 10 / 08$ & $22: 00$ & 0.03 & $6 / 7 / 08$ & $18: 00$ & 0 \\
\hline $5 / 11 / 08$ & 0:00 & 0.01 & $6 / 7 / 08$ & $20: 00$ & 0.01 \\
\hline $5 / 11 / 08$ & $2: 00$ & 0 & $6 / 7 / 08$ & $22: 00$ & 0 \\
\hline $5 / 22 / 08$ & $2: 00$ & 0 & $6 / 8 / 08$ & $2: 00$ & 0 \\
\hline $5 / 22 / 08$ & $4: 00$ & 0.01 & $6 / 8 / 08$ & $4: 00$ & 0.15 \\
\hline $5 / 22 / 08$ & $6: 00$ & 0 & $6 / 8 / 08$ & $6: 00$ & 0.19 \\
\hline $5 / 22 / 08$ & 8:00 & 0.1 & 6/8/08 & $8: 00$ & 0 \\
\hline $5 / 22 / 08$ & $10: 00$ & 0.14 & 6/11/08 & $2: 00$ & 0 \\
\hline $5 / 22 / 08$ & $12: 00$ & 0.17 & 6/11/08 & $4: 00$ & 0.04 \\
\hline $5 / 22 / 08$ & $14: 00$ & 0 & 6/11/08 & $6: 00$ & 0 \\
\hline $5 / 23 / 08$ & $2: 00$ & 0 & 6/11/08 & $18: 00$ & 0 \\
\hline $5 / 23 / 08$ & $4: 00$ & 0.72 & 6/11/08 & $20: 00$ & 0.06 \\
\hline $5 / 23 / 08$ & $6: 00$ & 0.02 & 6/11/08 & $22: 00$ & 0.23 \\
\hline $5 / 23 / 08$ & 8:00 & 0 & 6/12/08 & 0:00 & 0.01 \\
\hline $5 / 23 / 08$ & $10: 00$ & 0.01 & 6/12/08 & $2: 00$ & 0.01 \\
\hline $5 / 23 / 08$ & $12: 00$ & 0.01 & 6/12/08 & $4: 00$ & 0 \\
\hline $5 / 23 / 08$ & $14: 00$ & 0.02 & 6/15/08 & 8:00 & 0 \\
\hline $5 / 23 / 08$ & $16: 00$ & 0 & 6/15/08 & $10: 00$ & 0.12 \\
\hline $5 / 23 / 08$ & $22: 00$ & 0 & 6/15/08 & $12: 00$ & 0 \\
\hline $5 / 24 / 08$ & 0:00 & 0.03 & 6/17/08 & $22: 00$ & 0 \\
\hline $5 / 24 / 08$ & $2: 00$ & 0.05 & 6/18/08 & $0: 00$ & 0.01 \\
\hline $5 / 24 / 08$ & $4: 00$ & 0.22 & 6/18/08 & $2: 00$ & 0 \\
\hline $5 / 24 / 08$ & $6: 00$ & 0 & 6/19/08 & $14: 00$ & 0 \\
\hline $5 / 25 / 08$ & $18: 00$ & 0 & 6/19/08 & $16: 00$ & 0.01 \\
\hline $5 / 25 / 08$ & $20: 00$ & 0.01 & 6/19/08 & $18: 00$ & 0.05 \\
\hline $5 / 25 / 08$ & $22: 00$ & 0 & 6/19/08 & $20: 00$ & 0.7 \\
\hline 5/28/08 & 0:00 & 0 & 6/19/08 & $22: 00$ & 0 \\
\hline 5/28/08 & $2: 00$ & 0.01 & $6 / 21 / 08$ & $20: 00$ & 0 \\
\hline $5 / 28 / 08$ & $4: 00$ & 0 & 6/21/08 & $22: 00$ & 0.01 \\
\hline 5/28/08 & $6: 00$ & 0.01 & $6 / 22 / 08$ & $0: 00$ & 0 \\
\hline $5 / 28 / 08$ & 8:00 & 0 & 6/24/08 & $4: 00$ & 0 \\
\hline $5 / 29 / 08$ & $22: 00$ & 0 & 6/24/08 & $6: 00$ & 0.01 \\
\hline $5 / 30 / 08$ & 0:00 & 0.04 & $6 / 24 / 08$ & $8: 00$ & 0 \\
\hline $5 / 30 / 08$ & $2: 00$ & 0 & $6 / 24 / 08$ & $14: 00$ & 0 \\
\hline 6/2/08 & $6: 00$ & 0 & 6/24/08 & $16: 00$ & 0.02 \\
\hline $6 / 2 / 08$ & $8: 00$ & 0.01 & 6/24/08 & $18: 00$ & 0 \\
\hline $6 / 2 / 08$ & $10: 00$ & 0 & 6/26/08 & $10: 00$ & 0 \\
\hline $6 / 3 / 08$ & $6: 00$ & 0 & 6/26/08 & $12: 00$ & 0.17 \\
\hline $6 / 3 / 08$ & 8:00 & 0.01 & 6/26/08 & $14: 00$ & 0 \\
\hline $6 / 3 / 08$ & $10: 00$ & 0 & $6 / 27 / 08$ & $16: 00$ & 0 \\
\hline $6 / 4 / 08$ & $20: 00$ & 0 & 6/27/08 & $18: 00$ & 0.03 \\
\hline $6 / 4 / 08$ & $22: 00$ & 0.63 & 6/27/08 & $20: 00$ & 0 \\
\hline
\end{tabular}


Table S1.1 (Cont.)

\begin{tabular}{|c|c|c|}
\hline Date & Time & Rain (in.) \\
\hline 7/2/08 & $20: 00$ & 0 \\
\hline $7 / 2 / 08$ & $22: 00$ & 0.01 \\
\hline $7 / 3 / 08$ & 0:00 & 0 \\
\hline 7/5/08 & 8:00 & 0 \\
\hline 7/5/08 & $10: 00$ & 0.01 \\
\hline 7/5/08 & $12: 00$ & 0 \\
\hline 7/6/08 & $18: 00$ & 0 \\
\hline $7 / 6 / 08$ & 20:00 & 0.01 \\
\hline 7/6/08 & $22: 00$ & 0.01 \\
\hline 7/7/08 & $0: 00$ & 0 \\
\hline $7 / 11 / 08$ & $22: 00$ & 0 \\
\hline $7 / 12 / 08$ & 0:00 & 0.03 \\
\hline $7 / 12 / 08$ & $2: 00$ & 0 \\
\hline $7 / 12 / 08$ & $10: 00$ & 0 \\
\hline $7 / 12 / 08$ & $12: 00$ & 0.01 \\
\hline $7 / 12 / 08$ & $14: 00$ & 0 \\
\hline $7 / 14 / 08$ & $6: 00$ & 0 \\
\hline $7 / 14 / 08$ & 8:00 & 0.01 \\
\hline $7 / 14 / 08$ & $10: 00$ & 0 \\
\hline $7 / 15 / 08$ & $20: 00$ & 0 \\
\hline $7 / 15 / 08$ & $22: 00$ & 0.24 \\
\hline $7 / 16 / 08$ & $0: 00$ & 0.17 \\
\hline 7/16/08 & $2: 00$ & 0.01 \\
\hline 7/16/08 & $4: 00$ & 0.02 \\
\hline 7/16/08 & $6: 00$ & 0 \\
\hline $7 / 17 / 08$ & $16: 00$ & 0 \\
\hline $7 / 17 / 08$ & $18: 00$ & 0.45 \\
\hline $7 / 17 / 08$ & 20:00 & 0 \\
\hline $7 / 22 / 08$ & $10: 00$ & 0 \\
\hline $7 / 22 / 08$ & $12: 00$ & 0.02 \\
\hline $7 / 22 / 08$ & $14: 00$ & 0 \\
\hline $7 / 22 / 08$ & $16: 00$ & 0.13 \\
\hline $7 / 22 / 08$ & $18: 00$ & 0 \\
\hline 7/31/08 & $6: 00$ & 0 \\
\hline 7/31/08 & 8:00 & 0.01 \\
\hline 8/1/08 & $2: 00$ & 0 \\
\hline 8/9/08 & 8:00 & 0 \\
\hline 8/9/08 & $10: 00$ & 0.02 \\
\hline 8/9/08 & $12: 00$ & 0 \\
\hline 8/10/08 & $16: 00$ & 0 \\
\hline 8/10/08 & $18: 00$ & 0.1 \\
\hline 8/10/08 & $20: 00$ & 0 \\
\hline 8/12/08 & 0:00 & 0 \\
\hline 8/12/08 & $2: 00$ & 0.25 \\
\hline 8/12/08 & $4: 00$ & 0.01 \\
\hline 8/12/08 & $6: 00$ & 0 \\
\hline
\end{tabular}

Table S1.1 (Cont.)

Date Time Rain (in.)

\begin{tabular}{rrc}
\hline 8/24/08 & $6: 00$ & 0 \\
8/24/08 & $8: 00$ & 0.01 \\
8/24/08 & $10: 00$ & 0 \\
9/2/08 & $12: 00$ & 0 \\
$9 / 2 / 08$ & $14: 00$ & 0.05 \\
$9 / 2 / 08$ & $16: 00$ & 0.04 \\
$9 / 2 / 08$ & $18: 00$ & 0 \\
$9 / 5 / 08$ & $10: 00$ & 0 \\
$9 / 5 / 08$ & $12: 00$ & 0.01 \\
$9 / 5 / 08$ & $14: 00$ & 0 \\
$9 / 5 / 08$ & $16: 00$ & 0.06
\end{tabular}

$9 / 5 / 08 \quad 18: 00 \quad 0$

9/6/08 14:00 0

9/6/08 $16: 00 \quad 0.01$

9/6/08 18:00 0

9/6/08 20:00 0

$9 / 6 / 08 \quad 22: 00 \quad 0.37$

$9 / 7 / 08 \quad 0: 00 \quad 0$

$9 / 8 / 08 \quad 4: 00 \quad 0$

9/8/08 $\quad 6: 00 \quad 0.05$

$9 / 8 / 08 \quad 8: 00 \quad 0.17$

$9 / 8 / 08 \quad 10: 00 \quad 0.1$

$9 / 8 / 08 \quad 12: 00 \quad 0.01$

$9 / 8 / 08$

9/9/08

9/9/08

9/9/08

$9 / 11 / 08$

9/11/08

9/11/08

$9 / 11 / 08$

9/11/08

9/11/08

$9 / 11 / 08$

9/11/08

9/11/08

$9 / 13 / 08$

$9 / 13 / 08$

9/13/08

$9 / 13 / 08$

$9 / 13 / 08$

$9 / 22 / 08$

$9 / 22 / 08$

$9 / 22 / 08$

$9 / 22 / 08$

9/22/08
14:00 0

10:00 0

$12: 00 \quad 0.01$

$14: 00$

6:00

8:00

10:00

$12: 00$

14:00

16:00

18:00

20:00

22:00

6:00

8:00

10:00

12:00

14:00

2:00

4:00

6:00

8:00

10:00
0

0

0.02

0.26

0

0.08

0.12

0.29

0

0
0.01

0

0.01

0

0.01

0.07

0.11

0.19
Table S1.1 (Cont.)

\begin{tabular}{|c|c|c|}
\hline Date & Time & Rain (in.) \\
\hline 9/22/08 & $12: 00$ & 0 \\
\hline 9/22/08 & $14: 00$ & 0 \\
\hline 9/22/08 & $16: 00$ & 0.05 \\
\hline 9/22/08 & $18: 00$ & 0 \\
\hline 9/23/08 & $14: 00$ & 0 \\
\hline 9/23/08 & $16: 00$ & 0.08 \\
\hline 9/23/08 & $18: 00$ & 0.26 \\
\hline 9/23/08 & $20: 00$ & 0 \\
\hline 9/24/08 & $10: 00$ & 0 \\
\hline 9/24/08 & $12: 00$ & 0.03 \\
\hline 9/24/08 & $14: 00$ & 0 \\
\hline 10/8/08 & $14: 00$ & 0 \\
\hline 10/8/08 & $16: 00$ & 0.22 \\
\hline 10/8/08 & $18: 00$ & 0 \\
\hline $10 / 13 / 08$ & $2: 00$ & 0 \\
\hline $10 / 13 / 08$ & $4: 00$ & 0.07 \\
\hline 10/13/08 & $6: 00$ & 0.5 \\
\hline $10 / 13 / 08$ & 8:00 & 0.21 \\
\hline 10/13/08 & $10: 00$ & 0.2 \\
\hline $10 / 13 / 08$ & $12: 00$ & 0.09 \\
\hline 10/13/08 & $14: 00$ & 0.05 \\
\hline $10 / 13 / 08$ & $16: 00$ & 0 \\
\hline 10/14/08 & $12: 00$ & 0 \\
\hline $10 / 14 / 08$ & $14: 00$ & 0.01 \\
\hline $10 / 14 / 08$ & $16: 00$ & 0.04 \\
\hline $10 / 14 / 08$ & $18: 00$ & 0.17 \\
\hline $10 / 14 / 08$ & 20:00 & 0.14 \\
\hline $10 / 14 / 08$ & $22: 00$ & 0.06 \\
\hline 10/15/08 & $0: 00$ & 0.02 \\
\hline 10/15/08 & $2: 00$ & 0.12 \\
\hline 10/15/08 & $4: 00$ & 0.26 \\
\hline 10/15/08 & $6: 00$ & 0.04 \\
\hline 10/15/08 & 8:00 & 0.02 \\
\hline $10 / 15 / 08$ & $10: 00$ & 0 \\
\hline 10/17/08 & $2: 00$ & 0 \\
\hline 10/17/08 & $4: 00$ & 0.01 \\
\hline 10/17/08 & $6: 00$ & 0.08 \\
\hline 10/17/08 & 8:00 & 0 \\
\hline 10/17/08 & $10: 00$ & 0 \\
\hline 10/17/08 & $12: 00$ & 0.01 \\
\hline 10/17/08 & $14: 00$ & 0 \\
\hline $10 / 21 / 08$ & $12: 00$ & 0 \\
\hline $10 / 21 / 08$ & $14: 00$ & 0.02 \\
\hline $10 / 21 / 08$ & $16: 00$ & 0.16 \\
\hline $10 / 21 / 08$ & 18:00 & 0.08 \\
\hline $10 / 21 / 08$ & $20: 00$ & 0.03 \\
\hline
\end{tabular}


Table S1.1 (Cont.)

\begin{tabular}{|c|c|c|c|c|c|}
\hline Date & Time & Rain (in.) & Date & Time & Rain (in.) \\
\hline $10 / 21 / 08$ & $22: 00$ & 0 & $11 / 11 / 08$ & $8: 00$ & 0 \\
\hline $10 / 22 / 08$ & 0:00 & 0.01 & 11/11/08 & $10: 00$ & 0.03 \\
\hline $10 / 22 / 08$ & $2: 00$ & 0.33 & 11/11/08 & $12: 00$ & 0.05 \\
\hline $10 / 22 / 08$ & 4:00 & 0.18 & 11/11/08 & $14: 00$ & 0.08 \\
\hline $10 / 22 / 08$ & $6: 00$ & 0.32 & $11 / 11 / 08$ & $16: 00$ & 0.06 \\
\hline $10 / 22 / 08$ & 8:00 & 0.06 & 11/11/08 & $18: 00$ & 0.08 \\
\hline $10 / 22 / 08$ & $10: 00$ & 0.06 & 11/11/08 & $20: 00$ & 0 \\
\hline $10 / 22 / 08$ & $12: 00$ & 0.04 & $11 / 13 / 08$ & $12: 00$ & 0 \\
\hline $10 / 22 / 08$ & $14: 00$ & 0.02 & 11/13/08 & $14: 00$ & 0.01 \\
\hline $10 / 22 / 08$ & $16: 00$ & 0.01 & $11 / 13 / 08$ & $16: 00$ & 0.01 \\
\hline $10 / 22 / 08$ & $18: 00$ & 0.01 & $11 / 13 / 08$ & $18: 00$ & 0.08 \\
\hline $10 / 22 / 08$ & 20:00 & 0.13 & 11/13/08 & 20:00 & 0 \\
\hline $10 / 22 / 08$ & $22: 00$ & 0.06 & $11 / 14 / 08$ & $18: 00$ & 0 \\
\hline 10/23/08 & 0:00 & 0.03 & $11 / 14 / 08$ & $20: 00$ & 0.01 \\
\hline $10 / 23 / 08$ & $2: 00$ & 0.01 & $11 / 14 / 08$ & $22: 00$ & 0.01 \\
\hline $10 / 23 / 08$ & 4:00 & 0.01 & 11/15/08 & $0: 00$ & 0 \\
\hline 10/23/08 & $6: 00$ & 0 & $11 / 29 / 08$ & $0: 00$ & 0 \\
\hline $10 / 23 / 08$ & $12: 00$ & 0 & $11 / 29 / 08$ & $2: 00$ & 0.03 \\
\hline 10/23/08 & $14: 00$ & 0.01 & $11 / 29 / 08$ & $4: 00$ & 0.01 \\
\hline $10 / 23 / 08$ & $16: 00$ & 0.02 & $11 / 29 / 08$ & $6: 00$ & 0 \\
\hline $10 / 23 / 08$ & $18: 00$ & 0.05 & $11 / 29 / 08$ & 8:00 & 0.01 \\
\hline $10 / 23 / 08$ & $20: 00$ & 0.01 & $11 / 29 / 08$ & $10: 00$ & 0.01 \\
\hline $10 / 23 / 08$ & $22: 00$ & 0 & $11 / 29 / 08$ & $12: 00$ & 0.02 \\
\hline $10 / 24 / 08$ & $0: 00$ & 0 & $11 / 29 / 08$ & $14: 00$ & 0.01 \\
\hline $10 / 24 / 08$ & $2: 00$ & 0.03 & $11 / 29 / 08$ & $16: 00$ & 0 \\
\hline $10 / 24 / 08$ & $4: 00$ & 0 & $12 / 12 / 08$ & 8:00 & 0 \\
\hline $10 / 24 / 08$ & $6: 00$ & 0 & $12 / 12 / 08$ & $10: 00$ & 0.01 \\
\hline $10 / 24 / 08$ & $8: 00$ & 0.01 & $12 / 12 / 08$ & $12: 00$ & 0 \\
\hline $10 / 24 / 08$ & $10: 00$ & 0.06 & $12 / 17 / 08$ & $12: 00$ & 0 \\
\hline $10 / 24 / 08$ & $12: 00$ & 0 & $12 / 17 / 08$ & $14: 00$ & 0.02 \\
\hline 11/5/08 & $16: 00$ & 0 & $12 / 17 / 08$ & $16: 00$ & 0 \\
\hline $11 / 5 / 08$ & $18: 00$ & 0.09 & $12 / 23 / 08$ & $12: 00$ & 0 \\
\hline $11 / 5 / 08$ & $20: 00$ & 0 & $12 / 23 / 08$ & $14: 00$ & 0.03 \\
\hline 11/6/08 & $14: 00$ & 0 & $12 / 23 / 08$ & $16: 00$ & 0.03 \\
\hline $11 / 6 / 08$ & $16: 00$ & 0.01 & $12 / 23 / 08$ & $18: 00$ & 0 \\
\hline 11/6/08 & $18: 00$ & 0 & $12 / 25 / 08$ & $20: 00$ & 0 \\
\hline $11 / 10 / 08$ & $12: 00$ & 0 & $12 / 25 / 08$ & $22: 00$ & 0.01 \\
\hline $11 / 10 / 08$ & $14: 00$ & 0.02 & $12 / 26 / 08$ & $0: 00$ & 0.06 \\
\hline $11 / 10 / 08$ & $16: 00$ & 0.16 & $12 / 26 / 08$ & $2: 00$ & 0.18 \\
\hline 11/10/08 & $18: 00$ & 0.08 & $12 / 26 / 08$ & $4: 00$ & 0.12 \\
\hline $11 / 10 / 08$ & 20:00 & 0.02 & $12 / 26 / 08$ & $6: 00$ & 0.01 \\
\hline $11 / 10 / 08$ & $22: 00$ & 0.04 & $12 / 26 / 08$ & 8:00 & 0 \\
\hline $11 / 11 / 08$ & $0: 00$ & 0 & & & \\
\hline 11/11/08 & $2: 00$ & 0.01 & & & \\
\hline $11 / 11 / 08$ & $4: 00$ & 0 & & & \\
\hline 11/11/08 & $6: 00$ & 0.05 & & & \\
\hline
\end{tabular}

Table S1.1 (Cont.) 
TABLE S1.2 Automatically measured water levels in upgradient wells in 2008.

\begin{tabular}{|c|c|c|c|c|c|c|c|c|c|}
\hline \multirow[b]{3}{*}{ Date } & \multirow[b]{3}{*}{ Time } & \multirow{2}{*}{\multicolumn{3}{|c|}{$\begin{array}{l}\text { Depth to Water (ft below top of } \\
\text { casing) at Indicated Well }\end{array}$}} & \multirow{3}{*}{ Date } & \multirow{3}{*}{ Time } & \multicolumn{3}{|c|}{$\begin{array}{l}\text { Depth to Water (ft below top of } \\
\text { casing) at Indicated Well }\end{array}$} \\
\hline & & & & & & & & & \\
\hline & & D2 & WP49 & SB65d & & & & & \\
\hline & & & & & $1 / 8 / 08$ & 0:00 & 30.874 & 40.55 & 17.886 \\
\hline $1 / 1 / 08$ & $0: 00$ & 31.088 & 40.855 & 18.094 & $1 / 8 / 08$ & $4: 00$ & 30.876 & 40.541 & 17.869 \\
\hline $1 / 1 / 08$ & $4: 00$ & 31.193 & 40.972 & 18.169 & 1/8/08 & $8: 00$ & 30.949 & 40.653 & 17.965 \\
\hline $1 / 1 / 08$ & 8:00 & 31.282 & 41.054 & 18.209 & 1/8/08 & $12: 00$ & 31.023 & 40.731 & 18.014 \\
\hline $1 / 1 / 08$ & $12: 00$ & 31.387 & 41.169 & 18.284 & $1 / 8 / 08$ & $16: 00$ & 31.072 & 40.787 & 18.052 \\
\hline $1 / 1 / 08$ & $16: 00$ & 31.431 & 41.193 & 18.284 & $1 / 8 / 08$ & $20: 00$ & 31.151 & 40.867 & 18.101 \\
\hline $1 / 1 / 08$ & $20: 00$ & 31.492 & 41.263 & 18.321 & $1 / 9 / 08$ & $0: 00$ & 31.193 & 40.899 & 18.113 \\
\hline $1 / 2 / 08$ & $0: 00$ & 31.539 & 41.312 & 18.351 & $1 / 9 / 08$ & $4: 00$ & 31.179 & 40.85 & 18.066 \\
\hline $1 / 2 / 08$ & $4: 00$ & 31.569 & 41.331 & 18.358 & $1 / 9 / 08$ & $8: 00$ & 31.14 & 40.801 & 18.021 \\
\hline $1 / 2 / 08$ & $8: 00$ & 31.615 & 41.385 & 18.396 & $1 / 9 / 08$ & $12: 00$ & 31.142 & 40.808 & 18.028 \\
\hline $1 / 2 / 08$ & $12: 00$ & 31.678 & 41.439 & 18.426 & $1 / 9 / 08$ & $16: 00$ & 31.03 & 40.651 & 17.902 \\
\hline $1 / 2 / 08$ & $16: 00$ & 31.634 & 41.345 & 18.34 & $1 / 9 / 08$ & $20: 00$ & 30.997 & 40.651 & 17.919 \\
\hline $1 / 2 / 08$ & $20: 00$ & 31.569 & 41.277 & 18.284 & 1/10/08 & 0:00 & 30.993 & 40.644 & 17.916 \\
\hline $1 / 3 / 08$ & $0: 00$ & 31.501 & 41.2 & 18.232 & $1 / 10 / 08$ & $4: 00$ & 30.953 & 40.602 & 17.888 \\
\hline $1 / 3 / 08$ & $4: 00$ & 31.394 & 41.061 & 18.129 & $1 / 10 / 08$ & 8:00 & 30.932 & 40.59 & 17.881 \\
\hline $1 / 3 / 08$ & $8: 00$ & 31.27 & 40.911 & 18.026 & 1/10/08 & $12: 00$ & 30.932 & 40.604 & 17.904 \\
\hline $1 / 3 / 08$ & $12: 00$ & 31.179 & 40.827 & 17.989 & $1 / 10 / 08$ & $16: 00$ & 30.928 & 40.592 & 17.9 \\
\hline $1 / 3 / 08$ & $16: 00$ & 31.058 & 40.695 & 17.904 & 1/10/08 & $20: 00$ & 30.986 & 40.667 & 17.956 \\
\hline $1 / 3 / 08$ & $20: 00$ & 31.004 & 40.66 & 17.895 & 1/11/08 & $0: 00$ & 31.028 & 40.712 & 17.989 \\
\hline $1 / 4 / 08$ & $0: 00$ & 30.96 & 40.623 & 17.874 & $1 / 11 / 08$ & $4: 00$ & 31.039 & 40.71 & 17.982 \\
\hline $1 / 4 / 08$ & $4: 00$ & 30.916 & 40.571 & 17.846 & $1 / 11 / 08$ & $8: 00$ & 31.035 & 40.7 & 17.97 \\
\hline $1 / 4 / 08$ & $8: 00$ & 30.872 & 40.534 & 17.83 & 1/11/08 & $12: 00$ & 31.044 & 40.7 & 17.965 \\
\hline $1 / 4 / 08$ & $12: 00$ & 30.86 & 40.52 & 17.823 & $1 / 11 / 08$ & $16: 00$ & 31.007 & 40.66 & 17.932 \\
\hline $1 / 4 / 08$ & $16: 00$ & 30.783 & 40.428 & 17.759 & $1 / 11 / 08$ & $20: 00$ & 31.032 & 40.714 & 17.986 \\
\hline $1 / 4 / 08$ & $20: 00$ & 30.755 & 40.412 & 17.759 & $1 / 12 / 08$ & $0: 00$ & 31.077 & 40.761 & 18.024 \\
\hline $1 / 5 / 08$ & $0: 00$ & 30.711 & 40.353 & 17.72 & $1 / 12 / 08$ & $4: 00$ & 31.135 & 40.836 & 18.082 \\
\hline $1 / 5 / 08$ & $4: 00$ & 30.671 & 40.318 & 17.699 & $1 / 12 / 08$ & $8: 00$ & 31.217 & 40.939 & 18.162 \\
\hline $1 / 5 / 08$ & $8: 00$ & 30.643 & 40.299 & 17.699 & $1 / 12 / 08$ & $12: 00$ & 31.317 & 41.04 & 18.23 \\
\hline $1 / 5 / 08$ & $12: 00$ & 30.648 & 40.297 & 17.699 & $1 / 12 / 08$ & $16: 00$ & 31.354 & 41.052 & 18.218 \\
\hline $1 / 5 / 08$ & $16: 00$ & 30.592 & 40.234 & 17.652 & $1 / 12 / 08$ & $20: 00$ & 31.413 & 41.132 & 18.279 \\
\hline $1 / 5 / 08$ & $20: 00$ & 30.573 & 40.222 & 17.649 & $1 / 13 / 08$ & $0: 00$ & 31.448 & 41.153 & 18.281 \\
\hline $1 / 6 / 08$ & $0: 00$ & 30.55 & 40.187 & 17.624 & $1 / 13 / 08$ & $4: 00$ & 31.466 & 41.162 & 18.283 \\
\hline $1 / 6 / 08$ & $4: 00$ & 30.538 & 40.198 & 17.647 & $1 / 13 / 08$ & $8: 00$ & 31.473 & 41.169 & 18.288 \\
\hline $1 / 6 / 08$ & 8:00 & 30.589 & 40.28 & 17.72 & $1 / 13 / 08$ & $12: 00$ & 31.499 & 41.19 & 18.295 \\
\hline $1 / 6 / 08$ & $12: 00$ & 30.666 & 40.367 & 17.78 & $1 / 13 / 08$ & $16: 00$ & 31.441 & 41.096 & 18.213 \\
\hline $1 / 6 / 08$ & $16: 00$ & 30.713 & 40.412 & 17.809 & $1 / 13 / 08$ & $20: 00$ & 31.452 & 41.15 & 18.269 \\
\hline $1 / 6 / 08$ & $20: 00$ & 30.771 & 40.468 & 17.844 & $1 / 14 / 08$ & $0: 00$ & 31.522 & 41.244 & 18.351 \\
\hline $1 / 7 / 08$ & $0: 00$ & 30.79 & 40.454 & 17.818 & $1 / 14 / 08$ & $4: 00$ & 31.578 & 41.303 & 18.386 \\
\hline $1 / 7 / 08$ & $4: 00$ & 30.776 & 40.463 & 17.837 & $1 / 14 / 08$ & $8: 00$ & 31.632 & 41.347 & 18.414 \\
\hline $1 / 7 / 08$ & $8: 00$ & 30.729 & 40.4 & 17.776 & $1 / 14 / 08$ & $12: 00$ & 31.688 & 41.415 & 18.459 \\
\hline $1 / 7 / 08$ & $12: 00$ & 30.771 & 40.447 & 17.813 & $1 / 14 / 08$ & $16: 00$ & 31.674 & 41.364 & 18.403 \\
\hline $1 / 7 / 08$ & $16: 00$ & 30.792 & 40.47 & 17.834 & $1 / 14 / 08$ & $20: 00$ & 31.667 & 41.357 & 18.393 \\
\hline $1 / 7 / 08$ & $20: 00$ & 30.853 & 40.557 & 17.902 & $1 / 15 / 08$ & $0: 00$ & 31.641 & 41.317 & 18.361 \\
\hline
\end{tabular}

. 
Table S1.2 (Cont.)

\begin{tabular}{|c|c|c|c|c|c|c|c|c|c|}
\hline \multirow[b]{2}{*}{ Date } & \multirow[b]{2}{*}{ Time } & \multicolumn{3}{|c|}{$\begin{array}{l}\text { Depth to Water (ft below top of } \\
\text { casing) at Indicated Well }\end{array}$} & \multirow[b]{2}{*}{ Date } & \multirow[b]{2}{*}{ Time } & \multicolumn{3}{|c|}{$\begin{array}{l}\text { Depth to Water (ft below top of } \\
\text { casing) at Indicated Well }\end{array}$} \\
\hline & & D2 & WP49 & SB65d & & & D2 & WP49 & SB65d \\
\hline 1/15/08 & $4: 00$ & 31.578 & 41.239 & 18.297 & 1/22/08 & $8: 00$ & 31.877 & 41.579 & 18.581 \\
\hline $1 / 15 / 08$ & 8:00 & 31.485 & 41.132 & 18.223 & $1 / 22 / 08$ & $12: 00$ & 31.879 & 41.563 & 18.56 \\
\hline $1 / 15 / 08$ & $12: 00$ & 31.408 & 41.028 & 18.148 & $1 / 22 / 08$ & $16: 00$ & 31.797 & 41.439 & 18.454 \\
\hline 1/15/08 & $16: 00$ & 31.268 & 40.862 & 18.026 & 1/22/08 & $20: 00$ & 31.741 & 41.397 & 18.426 \\
\hline 1/15/08 & $20: 00$ & 31.203 & 40.834 & 18.033 & 1/23/08 & 0:00 & 31.692 & 41.338 & 18.389 \\
\hline 1/16/08 & $0: 00$ & 31.182 & 40.817 & 18.028 & 1/23/08 & 4:00 & 31.643 & 41.289 & 18.356 \\
\hline $1 / 16 / 08$ & $4: 00$ & 31.226 & 40.904 & 18.113 & $1 / 23 / 08$ & 8:00 & 31.606 & 41.265 & 18.356 \\
\hline 1/16/08 & 8:00 & 31.31 & 41.012 & 18.199 & 1/23/08 & $12: 00$ & 31.662 & 41.357 & 18.436 \\
\hline 1/16/08 & $12: 00$ & 31.413 & 41.127 & 18.283 & 1/23/08 & $16: 00$ & 31.713 & 41.425 & 18.492 \\
\hline 1/16/08 & $16: 00$ & 31.457 & 41.143 & 18.281 & 1/23/08 & $20: 00$ & 31.867 & 41.603 & 18.627 \\
\hline 1/16/08 & 20:00 & 31.499 & 41.195 & 18.323 & 1/24/08 & 0:00 & 31.947 & 41.661 & 18.66 \\
\hline 1/17/08 & 0:00 & 31.522 & 41.2 & 18.316 & 1/24/08 & 4:00 & 31.968 & 41.678 & 18.665 \\
\hline 1/17/08 & $4: 00$ & 31.494 & 41.15 & 18.279 & 1/24/08 & 8:00 & 32.003 & 41.711 & 18.681 \\
\hline 1/17/08 & $8: 00$ & 31.445 & 41.092 & 18.23 & 1/24/08 & $12: 00$ & 32.047 & 41.753 & 18.712 \\
\hline 1/17/08 & $12: 00$ & 31.45 & 41.117 & 18.258 & $1 / 24 / 08$ & $16: 00$ & 32.01 & 41.678 & 18.637 \\
\hline 1/17/08 & $16: 00$ & 31.401 & 41.038 & 18.183 & 1/24/08 & $20: 00$ & 31.977 & 41.657 & 18.623 \\
\hline $1 / 17 / 08$ & $20: 00$ & 31.38 & 41.033 & 18.19 & $1 / 25 / 08$ & 0:00 & 31.97 & 41.638 & 18.609 \\
\hline 1/18/08 & $0: 00$ & 31.373 & 41.033 & 18.19 & $1 / 25 / 08$ & 4:00 & 31.914 & 41.554 & 18.534 \\
\hline $1 / 18 / 08$ & $4: 00$ & 31.378 & 41.04 & 18.195 & $1 / 25 / 08$ & 8:00 & 31.83 & 41.46 & 18.473 \\
\hline 1/18/08 & $8: 00$ & 31.427 & 41.132 & 18.286 & 1/25/08 & $12: 00$ & 31.779 & 41.406 & 18.435 \\
\hline 1/18/08 & $12: 00$ & 31.597 & 41.364 & 18.475 & 1/25/08 & $16: 00$ & 31.69 & 41.303 & 18.363 \\
\hline 1/18/08 & $16: 00$ & 31.681 & 41.404 & 18.475 & $1 / 25 / 08$ & $20: 00$ & 31.676 & 41.329 & 18.396 \\
\hline 1/18/08 & $20: 00$ & 31.76 & 41.493 & 18.536 & 1/26/08 & 0:00 & 31.662 & 41.307 & 18.384 \\
\hline $1 / 19 / 08$ & 0:00 & 31.865 & 41.612 & 18.618 & 1/26/08 & 4:00 & 31.641 & 41.293 & 18.382 \\
\hline 1/19/08 & $4: 00$ & 31.891 & 41.605 & 18.583 & 1/26/08 & 8:00 & 31.653 & 41.314 & 18.403 \\
\hline 1/19/08 & $8: 00$ & 31.895 & 41.608 & 18.583 & 1/26/08 & $12: 00$ & 31.725 & 41.406 & 18.482 \\
\hline 1/19/08 & $12: 00$ & 31.94 & 41.661 & 18.625 & 1/26/08 & $16: 00$ & 31.732 & 41.399 & 18.471 \\
\hline 1/19/08 & $16: 00$ & 31.9 & 41.575 & 18.536 & 1/26/08 & $20: 00$ & 31.786 & 41.467 & 18.527 \\
\hline 1/19/08 & $20: 00$ & 31.877 & 41.565 & 18.534 & $1 / 27 / 08$ & 0:00 & 31.797 & 41.462 & 18.513 \\
\hline $1 / 20 / 08$ & 0:00 & 31.879 & 41.572 & 18.541 & $1 / 27 / 08$ & 4:00 & 31.797 & 41.455 & 18.506 \\
\hline 1/20/08 & $4: 00$ & 31.879 & 41.579 & 18.56 & 1/27/08 & 8:00 & 31.804 & 41.462 & 18.513 \\
\hline $1 / 20 / 08$ & 8:00 & 31.902 & 41.615 & 18.59 & $1 / 27 / 08$ & $12: 00$ & 31.793 & 41.436 & 18.496 \\
\hline 1/20/08 & $12: 00$ & 31.949 & 41.664 & 18.625 & 1/27/08 & $16: 00$ & 31.692 & 41.272 & 18.349 \\
\hline 1/20/08 & $16: 00$ & 31.886 & 41.549 & 18.52 & 1/27/08 & $20: 00$ & 31.604 & 41.193 & 18.3 \\
\hline 1/20/08 & $20: 00$ & 31.884 & 41.596 & 18.571 & $1 / 28 / 08$ & $0: 00$ & 31.525 & 41.103 & 18.239 \\
\hline $1 / 21 / 08$ & 0:00 & 31.881 & 41.556 & 18.531 & $1 / 28 / 08$ & 4:00 & 31.417 & 40.979 & 18.15 \\
\hline 1/21/08 & $4: 00$ & 31.811 & 41.464 & 18.461 & $1 / 28 / 08$ & $8: 00$ & 31.301 & 40.857 & 18.07 \\
\hline 1/21/08 & $8: 00$ & 31.746 & 41.399 & 18.412 & $1 / 28 / 08$ & $12: 00$ & 31.198 & 40.733 & 17.982 \\
\hline 1/21/08 & $12: 00$ & 31.704 & 41.354 & 18.389 & $1 / 28 / 08$ & $16: 00$ & 31.081 & 40.616 & 17.886 \\
\hline 1/21/08 & $16: 00$ & 31.634 & 41.27 & 18.333 & $1 / 28 / 08$ & 20:00 & 31.128 & 40.721 & 17.958 \\
\hline 1/21/08 & $20: 00$ & 31.657 & 41.359 & 18.421 & $1 / 29 / 08$ & 0:00 & 31.142 & 40.759 & 17.965 \\
\hline $1 / 22 / 08$ & $0: 00$ & 31.758 & 41.479 & 18.52 & $1 / 29 / 08$ & 4:00 & 31.207 & 40.848 & 18.017 \\
\hline $1 / 22 / 08$ & 4:00 & 31.846 & 41.568 & 18.583 & 1/29/08 & 8:00 & 31.359 & 41.071 & 18.188 \\
\hline
\end{tabular}


Table S1.2 (Cont.)

\begin{tabular}{|c|c|c|c|c|c|c|c|c|c|}
\hline \multirow[b]{2}{*}{ Date } & \multirow[b]{2}{*}{ Time } & \multicolumn{3}{|c|}{$\begin{array}{l}\text { Depth to Water (ft below top of } \\
\text { casing) at Indicated Well }\end{array}$} & \multirow[b]{2}{*}{ Date } & \multirow[b]{2}{*}{ Time } & \multicolumn{3}{|c|}{$\begin{array}{l}\text { Depth to Water (ft below top of } \\
\text { casing) at Indicated Well }\end{array}$} \\
\hline & & D2 & WP49 & SB65d & & & D2 & WP49 & SB65d \\
\hline 1/29/08 & $12: 00$ & 31.515 & 41.204 & 18.251 & $2 / 5 / 08$ & $16: 00$ & 31.942 & 41.563 & 18.524 \\
\hline 1/29/08 & $16: 00$ & 31.606 & 41.286 & 18.295 & $2 / 5 / 08$ & $20: 00$ & 31.97 & 41.617 & 18.578 \\
\hline 1/29/08 & $20: 00$ & 31.727 & 41.422 & 18.386 & $2 / 6 / 08$ & 0:00 & 31.972 & 41.596 & 18.55 \\
\hline 1/30/08 & 0:00 & 31.8 & 41.481 & 18.414 & $2 / 6 / 08$ & $4: 00$ & 31.937 & 41.556 & 18.522 \\
\hline $1 / 30 / 08$ & 4:00 & 31.835 & 41.5 & 18.419 & $2 / 6 / 08$ & $8: 00$ & 31.923 & 41.549 & 18.52 \\
\hline 1/30/08 & 8:00 & 31.853 & 41.511 & 18.424 & $2 / 6 / 08$ & $12: 00$ & 31.956 & 41.603 & 18.566 \\
\hline 1/30/08 & $12: 00$ & 31.902 & 41.568 & 18.457 & $2 / 6 / 08$ & $16: 00$ & 31.935 & 41.558 & 18.524 \\
\hline 1/30/08 & $16: 00$ & 31.825 & 41.46 & 18.361 & $2 / 6 / 08$ & $20: 00$ & 31.963 & 41.612 & 18.574 \\
\hline 1/30/08 & $20: 00$ & 31.865 & 41.537 & 18.436 & $2 / 7 / 08$ & 0:00 & 32 & 41.647 & 18.597 \\
\hline 1/31/08 & 0:00 & 31.937 & 41.619 & 18.506 & $2 / 7 / 08$ & $4: 00$ & 31.991 & 41.608 & 18.557 \\
\hline 1/31/08 & $4: 00$ & 31.972 & 41.643 & 18.52 & $2 / 7 / 08$ & 8:00 & 31.968 & 41.596 & 18.555 \\
\hline 1/31/08 & $8: 00$ & 32.021 & 41.701 & 18.566 & $2 / 7 / 08$ & $12: 00$ & 31.977 & 41.61 & 18.569 \\
\hline 1/31/08 & $12: 00$ & 32.059 & 41.732 & 18.581 & $2 / 7 / 08$ & $16: 00$ & 31.926 & 41.528 & 18.494 \\
\hline 1/31/08 & $16: 00$ & 32.019 & 41.65 & 18.501 & $2 / 7 / 08$ & $20: 00$ & 31.902 & 41.516 & 18.492 \\
\hline 1/31/08 & $20: 00$ & 32.005 & 41.654 & 18.515 & 2/8/08 & 0:00 & 31.858 & 41.446 & 18.435 \\
\hline 2/1/08 & 0:00 & 31.975 & 41.61 & 18.48 & $2 / 8 / 08$ & $4: 00$ & 31.788 & 41.382 & 18.396 \\
\hline $2 / 1 / 08$ & $4: 00$ & 31.928 & 41.568 & 18.457 & $2 / 8 / 08$ & $8: 00$ & 31.769 & 41.389 & 18.414 \\
\hline $2 / 1 / 08$ & 8:00 & 31.886 & 41.516 & 18.421 & 2/8/08 & $12: 00$ & 31.835 & 41.479 & 18.492 \\
\hline $2 / 1 / 08$ & $12: 00$ & 31.863 & 41.502 & 18.419 & $2 / 8 / 08$ & $16: 00$ & 31.851 & 41.483 & 18.489 \\
\hline $2 / 1 / 08$ & $16: 00$ & 31.788 & 41.387 & 18.323 & $2 / 8 / 08$ & $20: 00$ & 31.895 & 41.549 & 18.545 \\
\hline $2 / 1 / 08$ & $20: 00$ & 31.767 & 41.401 & 18.358 & $2 / 9 / 08$ & $0: 00$ & 31.93 & 41.561 & 18.543 \\
\hline $2 / 2 / 08$ & $0: 00$ & 31.769 & 41.399 & 18.356 & $2 / 9 / 08$ & $4: 00$ & 31.935 & 41.558 & 18.545 \\
\hline $2 / 2 / 08$ & $4: 00$ & 31.776 & 41.415 & 18.377 & $2 / 9 / 08$ & 8:00 & 31.963 & 41.619 & 18.599 \\
\hline $2 / 2 / 08$ & 8:00 & 31.807 & 41.481 & 18.445 & $2 / 9 / 08$ & $12: 00$ & 32.098 & 41.797 & 18.742 \\
\hline $2 / 2 / 08$ & $12: 00$ & 31.893 & 41.579 & 18.527 & $2 / 9 / 08$ & $16: 00$ & 32.252 & 41.957 & 18.85 \\
\hline $2 / 2 / 08$ & $16: 00$ & 31.94 & 41.612 & 18.543 & 2/9/08 & $20: 00$ & 32.38 & 42.088 & 18.934 \\
\hline $2 / 2 / 08$ & $20: 00$ & 31.968 & 41.643 & 18.566 & 2/10/08 & $0: 00$ & 32.492 & 42.198 & 19.004 \\
\hline $2 / 3 / 08$ & $0: 00$ & 32.007 & 41.659 & 18.574 & 2/10/08 & $4: 00$ & 32.576 & 42.287 & 19.048 \\
\hline $2 / 3 / 08$ & $4: 00$ & 31.989 & 41.631 & 18.55 & $2 / 10 / 08$ & $8: 00$ & 32.667 & 42.384 & 19.109 \\
\hline $2 / 3 / 08$ & $8: 00$ & 31.963 & 41.591 & 18.513 & $2 / 10 / 08$ & $12: 00$ & 32.756 & 42.475 & 19.163 \\
\hline $2 / 3 / 08$ & $12: 00$ & 31.937 & 41.554 & 18.482 & 2/10/08 & $16: 00$ & 32.716 & 42.374 & 19.051 \\
\hline $2 / 3 / 08$ & $16: 00$ & 31.832 & 41.427 & 18.377 & $2 / 10 / 08$ & $20: 00$ & 32.663 & 42.301 & 18.995 \\
\hline $2 / 3 / 08$ & $20: 00$ & 31.788 & 41.411 & 18.393 & $2 / 11 / 08$ & $0: 00$ & 32.597 & 42.229 & \\
\hline $2 / 4 / 08$ & $0: 00$ & 31.769 & 41.373 & 18.358 & 2/11/08 & $4: 00$ & 32.511 & 42.126 & \\
\hline $2 / 4 / 08$ & $4: 00$ & 31.648 & 41.2 & 18.227 & $2 / 11 / 08$ & $8: 00$ & 32.408 & 42.025 & \\
\hline $2 / 4 / 08$ & 8:00 & 31.592 & 41.181 & 18.23 & 2/11/08 & $12: 00$ & 32.369 & 41.971 & \\
\hline $2 / 4 / 08$ & $12: 00$ & 31.622 & 41.246 & 18.297 & 2/11/08 & $16: 00$ & 32.266 & 41.861 & \\
\hline $2 / 4 / 08$ & $16: 00$ & 31.653 & 41.291 & 18.342 & $2 / 11 / 08$ & $20: 00$ & 32.278 & 41.919 & \\
\hline $2 / 4 / 08$ & $20: 00$ & 31.765 & 41.446 & 18.471 & $2 / 12 / 08$ & $0: 00$ & 32.301 & 41.95 & \\
\hline $2 / 5 / 08$ & $0: 00$ & 31.881 & 41.551 & 18.541 & 2/12/08 & $4: 00$ & 32.308 & 41.95 & \\
\hline $2 / 5 / 08$ & $4: 00$ & 31.94 & 41.636 & 18.616 & $2 / 12 / 08$ & $8: 00$ & 32.31 & 41.962 & \\
\hline $2 / 5 / 08$ & 8:00 & 31.977 & 41.643 & 18.597 & $2 / 12 / 08$ & $12: 00$ & 32.345 & 41.999 & \\
\hline $2 / 5 / 08$ & $12: 00$ & 31.998 & 41.636 & 18.578 & 2/12/08 & $16: 00$ & 32.294 & 41.912 & \\
\hline
\end{tabular}


Table S1.2 (Cont.)

Depth to Water (ft below top of casing) at Indicated Well

$\begin{array}{llll}\text { Date Time } & \text { D2 } & \text { WP49 SB65d }\end{array}$

Table S1.2 (Cont.)

Depth to Water (ft below top of casing) at Indicated Well

\begin{tabular}{|c|c|c|c|c|c|c|c|c|c|}
\hline Date & Time & D2 & WP49 & SB65d & Date & Time & D2 & WP49 & SB65d \\
\hline 2/12/08 & 20:00 & 32.296 & 41.936 & & 2/20/08 & $0: 00$ & 32.551 & 42.231 & \\
\hline 2/13/08 & 0:00 & 32.273 & 41.891 & & 2/20/08 & $4: 00$ & 32.674 & 42.353 & \\
\hline 2/13/08 & 4:00 & 32.238 & 41.858 & & 2/20/08 & 8:00 & 32.772 & 42.466 & \\
\hline 2/13/08 & 8:00 & 32.194 & 41.807 & & 2/20/08 & $12: 00$ & 32.877 & 42.559 & \\
\hline 2/13/08 & $12: 00$ & 32.175 & 41.79 & & 2/20/08 & $16: 00$ & 32.83 & 42.461 & \\
\hline 2/13/08 & $16: 00$ & 32.056 & 41.619 & & $2 / 20 / 08$ & 20:00 & 32.807 & 42.442 & \\
\hline 2/13/08 & 20:00 & 31.977 & 41.53 & & 2/21/08 & 0:00 & 32.781 & 42.393 & \\
\hline 2/14/08 & 0:00 & 31.93 & 41.504 & & 2/21/08 & $4: 00$ & 32.73 & 42.325 & \\
\hline 2/14/08 & 4:00 & 31.961 & 41.582 & & $2 / 21 / 08$ & 8:00 & 32.637 & 42.229 & \\
\hline 2/14/08 & 8:00 & 31.996 & 41.631 & & 2/21/08 & $12: 00$ & 32.623 & 42.231 & \\
\hline 2/14/08 & $12: 00$ & 32.117 & 41.793 & & 2/21/08 & $16: 00$ & 32.539 & 42.112 & \\
\hline 2/14/08 & $16: 00$ & 32.278 & 41.969 & & 2/21/08 & $20: 00$ & 32.502 & 42.107 & \\
\hline 2/14/08 & 20:00 & 32.432 & 42.135 & & $2 / 22 / 08$ & 0:00 & 32.502 & 42.107 & \\
\hline 2/15/08 & 0:00 & 32.558 & 42.257 & & $2 / 22 / 08$ & $4: 00$ & 32.481 & 42.083 & \\
\hline 2/15/08 & $4: 00$ & 32.616 & 42.301 & & $2 / 22 / 08$ & $8: 00$ & 32.488 & 42.105 & \\
\hline 2/15/08 & 8:00 & 32.672 & 42.346 & & $2 / 22 / 08$ & $12: 00$ & 32.511 & 42.126 & \\
\hline 2/15/08 & $12: 00$ & 32.73 & 42.402 & & $2 / 22 / 08$ & $16: 00$ & 32.483 & 42.074 & \\
\hline 2/15/08 & $16: 00$ & 32.672 & 42.294 & & $2 / 22 / 08$ & $20: 00$ & 32.467 & 42.076 & \\
\hline $2 / 15 / 08$ & $20: 00$ & 32.621 & 42.245 & & $2 / 23 / 08$ & $0: 00$ & 32.495 & 42.105 & \\
\hline 2/16/08 & 0:00 & 32.593 & 42.198 & & 2/23/08 & $4: 00$ & 32.511 & 42.121 & \\
\hline 2/16/08 & 4:00 & 32.53 & 42.121 & & 2/23/08 & 8:00 & 32.511 & 42.109 & \\
\hline 2/16/08 & 8:00 & 32.448 & 42.039 & & 2/23/08 & $12: 00$ & 32.537 & 42.151 & \\
\hline 2/16/08 & $12: 00$ & 32.362 & 41.915 & & 2/23/08 & $16: 00$ & 32.537 & 42.137 & \\
\hline 2/16/08 & $16: 00$ & 32.215 & 41.748 & & 2/23/08 & $20: 00$ & 32.567 & 42.198 & \\
\hline $2 / 16 / 08$ & $20: 00$ & 32.117 & 41.659 & & $2 / 24 / 08$ & $0: 00$ & 32.616 & 42.238 & \\
\hline 2/17/08 & 0:00 & 32.038 & 41.575 & & $2 / 24 / 08$ & $4: 00$ & 32.632 & 42.241 & \\
\hline $2 / 17 / 08$ & 4:00 & 31.926 & 41.446 & & $2 / 24 / 08$ & $8: 00$ & 32.609 & 42.205 & \\
\hline 2/17/08 & 8:00 & 31.863 & 41.42 & & $2 / 24 / 08$ & $12: 00$ & 32.607 & 42.203 & \\
\hline 2/17/08 & $12: 00$ & 31.951 & 41.577 & & $2 / 24 / 08$ & $16: 00$ & 32.553 & 42.13 & \\
\hline 2/17/08 & $16: 00$ & 31.319 & 41.687 & & $2 / 24 / 08$ & 20:00 & 32.504 & 42.083 & \\
\hline $2 / 17 / 08$ & $20: 00$ & 32.149 & 41.814 & & $2 / 25 / 08$ & $0: 00$ & 32.464 & 42.03 & \\
\hline 2/18/08 & 0:00 & 32.205 & 41.833 & & $2 / 25 / 08$ & $4: 00$ & 32.383 & 41.912 & \\
\hline 2/18/08 & 4:00 & 32.285 & 41.926 & & $2 / 25 / 08$ & 8:00 & 32.31 & 41.856 & \\
\hline 2/18/08 & 8:00 & 32.308 & 41.936 & & $2 / 25 / 08$ & $12: 00$ & 32.299 & 41.865 & \\
\hline 2/18/08 & $12: 00$ & 32.387 & 42.037 & & $2 / 25 / 08$ & $16: 00$ & 32.355 & 41.976 & \\
\hline 2/18/08 & $16: 00$ & 32.448 & 42.102 & & $2 / 25 / 08$ & $20: 00$ & 32.53 & 42.198 & \\
\hline $2 / 18 / 08$ & $20: 00$ & 32.506 & 42.161 & & $2 / 26 / 08$ & $0: 00$ & 32.677 & 42.348 & \\
\hline 2/19/08 & 0:00 & 32.558 & 42.203 & & 2/26/08 & $4: 00$ & 32.772 & 42.419 & \\
\hline 2/19/08 & 4:00 & 32.574 & 42.208 & & 2/26/08 & $8: 00$ & 32.851 & 42.508 & \\
\hline 2/19/08 & 8:00 & 32.539 & 42.14 & & 2/26/08 & $12: 00$ & 32.933 & 42.585 & \\
\hline 2/19/08 & $12: 00$ & 32.478 & 42.072 & & 2/26/08 & $16: 00$ & 32.938 & 42.55 & \\
\hline 2/19/08 & $16: 00$ & 32.385 & 41.962 & & 2/26/08 & 20:00 & 32.886 & 42.475 & \\
\hline 2/19/08 & $20: 00$ & 32.411 & 42.048 & & $2 / 27 / 08$ & 0:00 & 32.856 & 42.442 & \\
\hline
\end{tabular}


Table S1.2 (Cont.)

Depth to Water (ft below top of casing) at Indicated Well

Date Time D2 WP49 SB65d
Table S1.2 (Cont.)
Depth to Water (ft below top of casing) at Indicated Well

\begin{tabular}{|c|c|c|c|c|c|c|c|}
\hline $2 / 27 / 08$ & 4:00 & 32.823 & 42.416 & 3/5/08 & $8: 00$ & 32.546 & 42.158 \\
\hline 2/27/08 & 8:00 & 32.821 & 42.419 & 3/5/08 & $12: 00$ & 32.702 & 42.339 \\
\hline $2 / 27 / 08$ & $12: 00$ & 32.83 & 42.428 & 3/5/08 & $16: 00$ & 32.772 & 42.391 \\
\hline 2/27/08 & $16: 00$ & 32.786 & 42.363 & 3/5/08 & $20: 00$ & 32.835 & 42.461 \\
\hline $2 / 27 / 08$ & 20:00 & 32.758 & 42.341 & 3/6/08 & $0: 00$ & 32.907 & 42.522 \\
\hline 2/28/08 & $0: 00$ & 32.737 & 42.313 & 3/6/08 & 4:00 & 32.903 & 42.489 \\
\hline 2/28/08 & 4:00 & 32.693 & 42.259 & 3/6/08 & 8:00 & 32.903 & 42.503 \\
\hline 2/28/08 & $8: 00$ & 32.623 & 42.18 & 3/6/08 & $12: 00$ & 32.945 & 42.55 \\
\hline 2/28/08 & $12: 00$ & 32.593 & 42.17 & 3/6/08 & $16: 00$ & 32.893 & 42.456 \\
\hline 2/28/08 & $16: 00$ & 32.537 & 42.09 & 3/6/08 & $20: 00$ & 32.858 & 42.442 \\
\hline 2/28/08 & 20:00 & 32.532 & 42.114 & 3/7/08 & $0: 00$ & 32.896 & 42.501 \\
\hline 2/29/08 & $0: 00$ & 32.567 & 42.158 & 3/7/08 & 4:00 & 32.98 & 42.604 \\
\hline 2/29/08 & $4: 00$ & 32.628 & 42.252 & 3/7/08 & 8:00 & 33.047 & 42.684 \\
\hline 2/29/08 & 8:00 & 32.751 & 42.402 & 3/7/08 & $12: 00$ & 33.113 & 42.733 \\
\hline 2/29/08 & $12: 00$ & 32.851 & 42.489 & 3/7/08 & $16: 00$ & 33.082 & 42.663 \\
\hline 2/29/08 & $16: 00$ & 32.868 & 42.473 & 3/7/08 & $20: 00$ & 33.026 & 42.599 \\
\hline 2/29/08 & 20:00 & 32.87 & 42.468 & 3/8/08 & $0: 00$ & 32.991 & 42.562 \\
\hline $3 / 1 / 08$ & 0:00 & 32.854 & 42.428 & 3/8/08 & 4:00 & 32.912 & 42.454 \\
\hline $3 / 1 / 08$ & $4: 00$ & 32.781 & 42.325 & 3/8/08 & $8: 00$ & 32.828 & 42.37 \\
\hline 3/1/08 & 8:00 & 32.667 & 42.18 & 3/8/08 & $12: 00$ & 32.753 & 42.276 \\
\hline 3/1/08 & $12: 00$ & 32.583 & 42.102 & 3/8/08 & $16: 00$ & 32.688 & 42.234 \\
\hline 3/1/08 & $16: 00$ & 32.495 & 42.006 & 3/8/08 & $20: 00$ & 32.698 & 42.287 \\
\hline 3/1/08 & 20:00 & 32.422 & 41.94 & 3/9/08 & $0: 00$ & 32.758 & 42.36 \\
\hline $3 / 2 / 08$ & 0:00 & 32.369 & 41.877 & 3/9/08 & 4:00 & 32.812 & 42.409 \\
\hline $3 / 2 / 08$ & $4: 00$ & 32.278 & 41.755 & 3/9/08 & $8: 00$ & 32.877 & 42.503 \\
\hline $3 / 2 / 08$ & 8:00 & 32.18 & 41.659 & 3/9/08 & $12: 00$ & 32.963 & 42.595 \\
\hline $3 / 2 / 08$ & $12: 00$ & 32.212 & 41.772 & 3/9/08 & $16: 00$ & 33.001 & 42.611 \\
\hline $3 / 2 / 08$ & $16: 00$ & 32.334 & 41.919 & 3/9/08 & $20: 00$ & 33.045 & 42.663 \\
\hline $3 / 2 / 08$ & 20:00 & 32.448 & 42.06 & 3/10/08 & $0: 00$ & 33.117 & 42.747 \\
\hline $3 / 3 / 08$ & $0: 00$ & 32.569 & 42.198 & 3/10/08 & 4:00 & 33.164 & 42.775 \\
\hline $3 / 3 / 08$ & $4: 00$ & 32.66 & 42.262 & 3/10/08 & $8: 00$ & 33.201 & 42.827 \\
\hline $3 / 3 / 08$ & 8:00 & 32.753 & 42.367 & 3/10/08 & $12: 00$ & 33.255 & 42.871 \\
\hline $3 / 3 / 08$ & $12: 00$ & 32.854 & 42.482 & 3/10/08 & $16: 00$ & 33.19 & 42.752 \\
\hline $3 / 3 / 08$ & $16: 00$ & 32.849 & 42.435 & 3/10/08 & $20: 00$ & 33.124 & 42.684 \\
\hline $3 / 3 / 08$ & $20: 00$ & 32.814 & 42.4 & $3 / 11 / 08$ & $0: 00$ & 33.092 & 42.656 \\
\hline $3 / 4 / 08$ & 0:00 & 32.795 & 42.358 & 3/11/08 & 4:00 & 33.022 & 42.557 \\
\hline $3 / 4 / 08$ & $4: 00$ & 32.744 & 42.297 & 3/11/08 & $8: 00$ & 32.942 & 42.477 \\
\hline $3 / 4 / 08$ & $8: 00$ & 32.705 & 42.259 & 3/11/08 & $12: 00$ & 32.875 & 42.402 \\
\hline $3 / 4 / 08$ & $12: 00$ & 32.665 & 42.212 & 3/11/08 & $16: 00$ & 32.786 & 42.294 \\
\hline $3 / 4 / 08$ & $16: 00$ & 32.581 & 42.107 & 3/11/08 & $20: 00$ & 32.725 & 42.252 \\
\hline 3/4/08 & 20:00 & 32.532 & 42.081 & 3/12/08 & $0: 00$ & 32.709 & 42.241 \\
\hline 3/5/08 & 0:00 & 32.525 & 42.083 & $3 / 12 / 08$ & 4:00 & 32.672 & 42.182 \\
\hline 3/5/08 & $4: 00$ & 32.492 & 42.034 & 3/12/08 & $8: 00$ & 32.616 & 42.13 \\
\hline
\end{tabular}


Table S1.2 (Cont.)

\begin{tabular}{|c|c|c|c|c|c|c|c|c|c|}
\hline \multirow[b]{2}{*}{ Date } & \multirow[b]{2}{*}{ Time } & \multicolumn{3}{|c|}{$\begin{array}{l}\text { Depth to Water (ft below top of } \\
\text { casing) at Indicated Well }\end{array}$} & \multirow[b]{2}{*}{ Date } & \multirow[b]{2}{*}{ Time } & \multicolumn{3}{|c|}{$\begin{array}{l}\text { Depth to Water (ft below top of } \\
\text { casing) at Indicated Well }\end{array}$} \\
\hline & & D2 & WP49 & SB65d & & & D2 & WP49 & SB65d \\
\hline $3 / 12 / 08$ & $12: 00$ & 32.579 & 42.09 & & 3/19/08 & $16: 00$ & 33.099 & 42.62 & 19.202 \\
\hline $3 / 12 / 08$ & $16: 00$ & 32.574 & 42.116 & & 3/19/08 & $20: 00$ & 33.08 & 42.627 & 19.254 \\
\hline $3 / 12 / 08$ & $20: 00$ & 32.628 & 42.194 & & 3/20/08 & 0:00 & 33.127 & 42.677 & 19.265 \\
\hline 3/13/08 & $0: 00$ & 32.665 & 42.224 & & $3 / 20 / 08$ & $4: 00$ & 33.143 & 42.679 & 19.261 \\
\hline 3/13/08 & $4: 00$ & 32.7 & 42.252 & & $3 / 20 / 08$ & 8:00 & 33.152 & 42.709 & 19.296 \\
\hline 3/13/08 & $8: 00$ & 32.702 & 42.25 & & 3/20/08 & $12: 00$ & 33.178 & 42.707 & 19.27 \\
\hline 3/13/08 & $12: 00$ & 32.725 & 42.276 & & $3 / 20 / 08$ & $16: 00$ & 33.071 & 42.555 & 19.148 \\
\hline $3 / 13 / 08$ & $16: 00$ & 32.67 & 42.177 & & 3/20/08 & $20: 00$ & 33.001 & 42.489 & 19.104 \\
\hline 3/13/08 & $20: 00$ & 32.642 & 42.17 & 18.905 & 3/21/08 & 0:00 & 32.949 & 42.447 & 19.071 \\
\hline 3/14/08 & 0:00 & 32.618 & 42.137 & 18.865 & $3 / 21 / 08$ & $4: 00$ & 32.935 & 42.442 & 19.076 \\
\hline $3 / 14 / 08$ & $4: 00$ & 32.609 & 42.135 & 18.868 & $3 / 21 / 08$ & $8: 00$ & 33.017 & 42.588 & 19.223 \\
\hline $3 / 14 / 08$ & $8: 00$ & 32.63 & 42.168 & 18.889 & 3/21/08 & $12: 00$ & 33.148 & 42.738 & 19.345 \\
\hline $3 / 14 / 08$ & $12: 00$ & 32.663 & 42.217 & 18.926 & $3 / 21 / 08$ & $16: 00$ & 33.208 & 42.773 & 19.343 \\
\hline $3 / 14 / 08$ & $16: 00$ & 32.658 & 42.189 & 18.905 & 3/21/08 & $20: 00$ & 33.269 & 42.852 & 19.42 \\
\hline $3 / 14 / 08$ & $20: 00$ & 32.709 & 42.269 & 18.982 & $3 / 22 / 08$ & 0:00 & 33.341 & 42.916 & 19.453 \\
\hline 3/15/08 & $0: 00$ & 32.807 & 42.391 & 19.074 & $3 / 22 / 08$ & $4: 00$ & 33.383 & 42.944 & 19.46 \\
\hline 3/15/08 & $4: 00$ & 32.858 & 42.43 & 19.097 & $3 / 22 / 08$ & 8:00 & 33.43 & 43.012 & 19.516 \\
\hline 3/15/08 & 8:00 & 32.907 & 42.494 & 19.144 & $3 / 22 / 08$ & $12: 00$ & 33.497 & 43.085 & 19.56 \\
\hline 3/15/08 & $12: 00$ & 32.984 & 42.573 & 19.205 & $3 / 22 / 08$ & $16: 00$ & 33.509 & 43.073 & 19.532 \\
\hline $3 / 15 / 08$ & $16: 00$ & 32.991 & 42.55 & 19.167 & $3 / 22 / 08$ & $20: 00$ & 33.551 & 43.131 & 19.588 \\
\hline $3 / 15 / 08$ & $20: 00$ & 33.036 & 42.62 & 19.235 & $3 / 23 / 08$ & $0: 00$ & 33.607 & 43.192 & 19.623 \\
\hline 3/16/08 & $0: 00$ & 33.113 & 42.7 & 19.284 & $3 / 23 / 08$ & 4:00 & 33.612 & 43.171 & 19.6 \\
\hline $3 / 16 / 08$ & $4: 00$ & 33.141 & 42.724 & 19.293 & 3/23/08 & $8: 00$ & 33.609 & 43.174 & 19.605 \\
\hline 3/16/08 & 8:00 & 33.204 & 42.803 & 19.359 & 3/23/08 & $12: 00$ & 33.635 & 43.202 & 19.621 \\
\hline 3/16/08 & $12: 00$ & 33.257 & 42.855 & 19.387 & 3/23/08 & $16: 00$ & 33.6 & 43.141 & 19.556 \\
\hline 3/16/08 & $16: 00$ & 33.243 & 42.806 & 19.329 & $3 / 23 / 08$ & $20: 00$ & 33.574 & 43.115 & 19.544 \\
\hline 3/16/08 & $20: 00$ & 33.206 & 42.749 & 19.282 & $3 / 24 / 08$ & 0:00 & 33.558 & 43.092 & 19.523 \\
\hline 3/17/08 & $0: 00$ & 33.204 & 42.763 & 19.308 & $3 / 24 / 08$ & $4: 00$ & 33.528 & 43.047 & 19.49 \\
\hline $3 / 17 / 08$ & $4: 00$ & 33.162 & 42.686 & 19.228 & $3 / 24 / 08$ & 8:00 & 33.458 & 42.967 & 19.427 \\
\hline $3 / 17 / 08$ & $8: 00$ & 33.122 & 42.67 & 19.221 & $3 / 24 / 08$ & $12: 00$ & 33.357 & 42.829 & 19.312 \\
\hline 3/17/08 & $12: 00$ & 33.08 & 42.618 & 19.179 & $3 / 24 / 08$ & $16: 00$ & 33.18 & 42.609 & 19.148 \\
\hline $3 / 17 / 08$ & $16: 00$ & 32.982 & 42.489 & 19.095 & $3 / 24 / 08$ & $20: 00$ & 33.059 & 42.517 & 19.104 \\
\hline $3 / 17 / 08$ & $20: 00$ & 32.954 & 42.482 & 19.092 & $3 / 25 / 08$ & $0: 00$ & 32.977 & 42.437 & 19.05 \\
\hline 3/18/08 & $0: 00$ & 32.952 & 42.494 & 19.109 & $3 / 25 / 08$ & $4: 00$ & 32.947 & 42.445 & 19.09 \\
\hline $3 / 18 / 08$ & $4: 00$ & 32.931 & 42.468 & 19.083 & $3 / 25 / 08$ & $8: 00$ & 33.078 & 42.658 & 19.268 \\
\hline 3/18/08 & 8:00 & 32.94 & 42.489 & 19.123 & $3 / 25 / 08$ & $12: 00$ & 33.171 & 42.731 & 19.317 \\
\hline 3/18/08 & $12: 00$ & 32.984 & 42.527 & 19.141 & $3 / 25 / 08$ & $16: 00$ & 33.201 & 42.738 & 19.319 \\
\hline 3/18/08 & $16: 00$ & 32.968 & 42.496 & 19.106 & $3 / 25 / 08$ & $20: 00$ & 33.248 & 42.803 & 19.375 \\
\hline 3/18/08 & $20: 00$ & 33.001 & 42.557 & 19.174 & $3 / 26 / 08$ & $0: 00$ & 33.308 & 42.857 & 19.408 \\
\hline 3/19/08 & $0: 00$ & 33.071 & 42.627 & 19.216 & $3 / 26 / 08$ & $4: 00$ & 33.339 & 42.874 & 19.417 \\
\hline 3/19/08 & $4: 00$ & 33.068 & 42.606 & 19.209 & $3 / 26 / 08$ & 8:00 & 33.336 & 42.864 & 19.401 \\
\hline $3 / 19 / 08$ & $8: 00$ & 33.075 & 42.641 & 19.247 & $3 / 26 / 08$ & $12: 00$ & 33.339 & 42.871 & 19.399 \\
\hline 3/19/08 & $12: 00$ & 33.131 & 42.691 & 19.272 & $3 / 26 / 08$ & $16: 00$ & 33.269 & 42.745 & 19.286 \\
\hline
\end{tabular}


Table S1.2 (Cont.)

\begin{tabular}{|c|c|c|c|c|c|c|c|c|c|}
\hline \multirow[b]{2}{*}{ Date } & \multirow[b]{2}{*}{ Time } & \multicolumn{3}{|c|}{$\begin{array}{l}\text { Depth to Water (ft below top of } \\
\text { casing) at Indicated Well }\end{array}$} & \multirow[b]{2}{*}{ Date } & \multirow[b]{2}{*}{ Time } & \multicolumn{3}{|c|}{$\begin{array}{l}\text { Depth to Water (ft below top of } \\
\text { casing) at Indicated Well }\end{array}$} \\
\hline & & D2 & WP49 & SB65d & & & D2 & WP49 & SB65d \\
\hline $3 / 26 / 08$ & 20:00 & 33.197 & 42.679 & 19.249 & 4/3/08 & $0: 00$ & 33.5 & 42.977 & 19.406 \\
\hline $3 / 27 / 08$ & 0:00 & 33.15 & 42.63 & 19.209 & $4 / 3 / 08$ & $4: 00$ & 33.437 & 42.895 & 19.34 \\
\hline $3 / 27 / 08$ & $4: 00$ & 33.103 & 42.564 & 19.165 & $4 / 3 / 08$ & 8:00 & 33.39 & 42.871 & 19.34 \\
\hline 3/27/08 & 8:00 & 33.103 & 42.611 & 19.233 & $4 / 3 / 08$ & $12: 00$ & 33.376 & 42.871 & 19.357 \\
\hline $3 / 27 / 08$ & $12: 00$ & 33.131 & 42.653 & 19.261 & $4 / 3 / 08$ & $16: 00$ & 33.341 & 42.82 & 19.303 \\
\hline $3 / 27 / 08$ & $16: 00$ & 33.183 & 42.705 & 19.289 & $4 / 3 / 08$ & $20: 00$ & 33.367 & 42.888 & 19.364 \\
\hline 3/27/08 & $20: 00$ & 33.243 & 42.794 & 19.371 & $4 / 4 / 08$ & 0:00 & 33.423 & 42.932 & 19.38 \\
\hline $3 / 28 / 08$ & 0:00 & 33.334 & 42.892 & 19.455 & $4 / 4 / 08$ & $4: 00$ & 33.413 & 42.906 & 19.34 \\
\hline $3 / 28 / 08$ & $4: 00$ & 33.406 & 42.958 & 19.502 & $4 / 4 / 08$ & 8:00 & 33.434 & 42.944 & 19.373 \\
\hline 3/28/08 & 8:00 & 33.481 & 43.068 & 19.584 & $4 / 4 / 08$ & $12: 00$ & 33.451 & 42.939 & 19.354 \\
\hline $3 / 28 / 08$ & $12: 00$ & 33.549 & 43.115 & 19.598 & $4 / 4 / 08$ & $16: 00$ & 33.385 & 42.843 & 19.268 \\
\hline $3 / 28 / 08$ & $16: 00$ & 33.523 & 43.038 & 19.518 & $4 / 4 / 08$ & $20: 00$ & 33.336 & 42.799 & 19.249 \\
\hline 3/28/08 & $20: 00$ & 33.502 & 43.028 & 19.525 & $4 / 5 / 08$ & $0: 00$ & 33.332 & 42.799 & 19.251 \\
\hline 3/29/08 & 0:00 & 33.528 & 43.068 & 19.56 & $4 / 5 / 08$ & $4: 00$ & 33.308 & 42.768 & 19.233 \\
\hline 3/29/08 & $4: 00$ & 33.516 & 43.028 & 19.518 & $4 / 5 / 08$ & 8:00 & 33.311 & 42.78 & 19.249 \\
\hline 3/29/08 & 8:00 & 33.495 & 43.019 & 19.513 & $4 / 5 / 08$ & $12: 00$ & 33.299 & 42.749 & 19.207 \\
\hline 3/29/08 & $12: 00$ & 33.458 & 42.946 & 19.436 & $4 / 5 / 08$ & $16: 00$ & 33.215 & 42.634 & 19.118 \\
\hline 3/29/08 & $16: 00$ & 33.343 & 42.813 & 19.338 & $4 / 5 / 08$ & $20: 00$ & 33.171 & 42.613 & 19.127 \\
\hline 3/29/08 & $20: 00$ & 33.288 & 42.787 & 19.343 & $4 / 6 / 08$ & 0:00 & 33.166 & 42.611 & 19.116 \\
\hline 3/30/08 & $0: 00$ & 33.313 & 42.829 & 19.375 & $4 / 6 / 08$ & $4: 00$ & 33.124 & 42.552 & 19.085 \\
\hline 3/30/08 & $4: 00$ & 33.329 & 42.841 & 19.387 & $4 / 6 / 08$ & 8:00 & 33.129 & 42.588 & 19.123 \\
\hline 3/30/08 & 8:00 & 33.383 & 42.92 & 19.453 & $4 / 6 / 08$ & $12: 00$ & 33.178 & 42.672 & 19.207 \\
\hline 3/30/08 & $12: 00$ & 33.416 & 42.944 & 19.469 & $4 / 6 / 08$ & $16: 00$ & 33.225 & 42.698 & 19.205 \\
\hline 3/30/08 & $16: 00$ & 33.388 & 42.892 & 19.42 & $4 / 6 / 08$ & $20: 00$ & 33.306 & 42.829 & 19.338 \\
\hline 3/30/08 & $20: 00$ & 33.383 & 42.888 & 19.422 & $4 / 7 / 08$ & 0:00 & 33.437 & 42.96 & 19.425 \\
\hline 3/31/08 & 0:00 & 33.369 & 42.86 & 19.392 & $4 / 7 / 08$ & $4: 00$ & 33.474 & 42.965 & 19.406 \\
\hline 3/31/08 & $4: 00$ & 33.229 & 42.611 & 19.179 & $4 / 7 / 08$ & 8:00 & 33.509 & 43.007 & 19.446 \\
\hline 3/31/08 & 8:00 & 33.117 & 42.599 & 19.184 & $4 / 7 / 08$ & $12: 00$ & 33.584 & 43.092 & 19.492 \\
\hline 3/31/08 & $12: 00$ & 33.068 & 42.564 & 19.176 & $4 / 7 / 08$ & $16: 00$ & 33.539 & 42.991 & 19.396 \\
\hline $3 / 31 / 08$ & $16: 00$ & 33.089 & 42.611 & 19.212 & $4 / 7 / 08$ & $20: 00$ & 33.553 & 43.073 & 19.495 \\
\hline 3/31/08 & $20: 00$ & 33.204 & 42.754 & 19.324 & $4 / 8 / 08$ & $0: 00$ & 33.56 & 43.045 & 19.455 \\
\hline 4/1/08 & $0: 00$ & 33.341 & 42.906 & 19.429 & $4 / 8 / 08$ & $4: 00$ & 33.546 & 43.019 & 19.406 \\
\hline $4 / 1 / 08$ & $4: 00$ & 33.444 & 43.01 & 19.497 & $4 / 8 / 08$ & $8: 00$ & 33.511 & 42.96 & 19.373 \\
\hline $4 / 1 / 08$ & 8:00 & 33.567 & 43.164 & 19.619 & 4/8/08 & $12: 00$ & 33.53 & 43.028 & 19.432 \\
\hline $4 / 1 / 08$ & $12: 00$ & 33.682 & 43.263 & 19.67 & $4 / 8 / 08$ & $16: 00$ & 33.525 & 42.993 & 19.389 \\
\hline $4 / 1 / 08$ & $16: 00$ & 33.721 & 43.27 & 19.651 & $4 / 8 / 08$ & $20: 00$ & 33.521 & 43.005 & 19.42 \\
\hline $4 / 1 / 08$ & $20: 00$ & 33.735 & 43.272 & 19.651 & 4/9/08 & 0:00 & 33.584 & 43.092 & 19.49 \\
\hline $4 / 2 / 08$ & $0: 00$ & 33.766 & 43.307 & 19.677 & $4 / 9 / 08$ & $4: 00$ & 33.607 & 43.092 & 19.483 \\
\hline $4 / 2 / 08$ & $4: 00$ & 33.745 & 43.256 & 19.614 & $4 / 9 / 08$ & 8:00 & 33.626 & 43.122 & 19.509 \\
\hline $4 / 2 / 08$ & 8:00 & 33.721 & 43.23 & 19.605 & $4 / 9 / 08$ & $12: 00$ & 33.651 & 43.143 & 19.518 \\
\hline $4 / 2 / 08$ & $12: 00$ & 33.698 & 43.195 & 19.567 & $4 / 9 / 08$ & $16: 00$ & 33.614 & 43.08 & 19.448 \\
\hline $4 / 2 / 08$ & $16: 00$ & 33.607 & 43.061 & 19.446 & $4 / 9 / 08$ & $20: 00$ & 33.514 & 42.953 & 19.364 \\
\hline $4 / 2 / 08$ & $20: 00$ & 33.516 & 42.986 & 19.41 & 4/10/08 & $0: 00$ & 33.481 & 42.927 & 19.336 \\
\hline
\end{tabular}


Table S1.2 (Cont.)

\begin{tabular}{|c|c|c|c|c|c|c|c|c|c|}
\hline \multirow[b]{2}{*}{ Date } & \multirow[b]{2}{*}{ Time } & \multicolumn{3}{|c|}{$\begin{array}{l}\text { Depth to Water (ft below top of } \\
\text { casing) at Indicated Well }\end{array}$} & \multirow[b]{2}{*}{ Date } & \multirow[b]{2}{*}{ Time } & \multicolumn{3}{|c|}{$\begin{array}{l}\text { Depth to Water (ft below top of } \\
\text { casing) at Indicated Well }\end{array}$} \\
\hline & & D2 & WP49 & SB65d & & & D2 & WP49 & SB65d \\
\hline 4/10/08 & $4: 00$ & 33.364 & 42.777 & 19.212 & 4/17/08 & 8:00 & 33.022 & 42.787 & 19.043 \\
\hline 4/10/08 & 8:00 & 33.218 & 42.611 & 19.088 & 4/17/08 & $12: 00$ & 33.276 & 42.787 & 18.931 \\
\hline 4/10/08 & $12: 00$ & 33.031 & 42.393 & 18.917 & 4/17/08 & $16: 00$ & 33.239 & 42.731 & 18.821 \\
\hline 4/10/08 & $16: 00$ & 32.847 & 42.215 & 18.758 & 4/17/08 & $20: 00$ & 33.185 & 42.67 & 18.765 \\
\hline 4/10/08 & $20: 00$ & 32.8 & 42.234 & 18.61 & 4/18/08 & 0:00 & 33.131 & 42.62 & 18.683 \\
\hline 4/11/08 & $0: 00$ & 32.781 & 42.229 & 18.545 & 4/18/08 & $4: 00$ & 33.045 & 42.515 & 18.561 \\
\hline $4 / 11 / 08$ & $4: 00$ & 32.798 & 42.269 & 18.556 & 4/18/08 & $8: 00$ & 32.863 & 42.468 & 18.472 \\
\hline 4/11/08 & 8:00 & 32.872 & 42.384 & 18.641 & 4/18/08 & $12: 00$ & 32.926 & 42.428 & 18.236 \\
\hline 4/11/08 & $12: 00$ & 32.991 & 42.513 & 18.718 & 4/18/08 & $16: 00$ & 32.893 & 42.402 & 18.112 \\
\hline 4/11/08 & $16: 00$ & 33.096 & 42.62 & 18.795 & 4/18/08 & $20: 00$ & 32.865 & 42.386 & 18.06 \\
\hline 4/11/08 & $20: 00$ & 33.208 & 42.731 & 18.868 & 4/19/08 & 0:00 & 32.851 & 42.365 & 18.014 \\
\hline $4 / 12 / 08$ & 0:00 & 33.281 & 42.782 & 18.891 & 4/19/08 & $4: 00$ & 32.819 & 42.327 & 17.993 \\
\hline $4 / 12 / 08$ & $4: 00$ & 33.311 & 42.792 & 18.896 & 4/19/08 & 8:00 & 32.828 & 42.355 & 18.039 \\
\hline $4 / 12 / 08$ & 8:00 & 33.35 & 42.845 & 18.947 & 4/19/08 & $12: 00$ & 32.842 & 42.351 & 18.039 \\
\hline $4 / 12 / 08$ & $12: 00$ & 33.411 & 42.911 & 18.999 & 4/19/08 & $16: 00$ & 32.786 & 42.266 & 17.983 \\
\hline $4 / 12 / 08$ & $16: 00$ & 33.451 & 42.939 & 19.017 & 4/19/08 & $20: 00$ & 32.723 & 42.205 & 17.974 \\
\hline $4 / 12 / 08$ & $20: 00$ & 33.495 & 42.988 & 19.06 & $4 / 20 / 08$ & 0:00 & 32.709 & 42.196 & 17.988 \\
\hline 4/13/08 & $0: 00$ & 33.539 & 43.04 & 19.106 & $4 / 20 / 08$ & $4: 00$ & 32.672 & 42.151 & 17.974 \\
\hline $4 / 13 / 08$ & $4: 00$ & 33.565 & 43.049 & 19.111 & $4 / 20 / 08$ & 8:00 & 32.625 & 42.102 & 17.946 \\
\hline 4/13/08 & 8:00 & 33.616 & 43.124 & 19.179 & 4/20/08 & $12: 00$ & 32.553 & 42.001 & 17.878 \\
\hline $4 / 13 / 08$ & $12: 00$ & 33.679 & 43.188 & 19.226 & 4/20/08 & $16: 00$ & 32.434 & 41.877 & 17.808 \\
\hline $4 / 13 / 08$ & $16: 00$ & 33.672 & 43.153 & 19.186 & 4/20/08 & $20: 00$ & 32.343 & 41.809 & 17.803 \\
\hline 4/13/08 & $20: 00$ & 33.649 & 43.122 & 19.172 & 4/21/08 & 0:00 & 32.364 & 41.865 & 17.869 \\
\hline $4 / 14 / 08$ & 0:00 & 33.661 & 43.148 & 19.207 & $4 / 21 / 08$ & $4: 00$ & 32.343 & 41.837 & 17.864 \\
\hline $4 / 14 / 08$ & $4: 00$ & 33.658 & 43.136 & 19.2 & $4 / 21 / 08$ & 8:00 & 32.366 & 41.889 & 17.929 \\
\hline 4/14/08 & 8:00 & 33.675 & 43.174 & 19.24 & 4/21/08 & $12: 00$ & 32.443 & 41.999 & 18.032 \\
\hline $4 / 14 / 08$ & $12: 00$ & 33.679 & 43.148 & 19.209 & $4 / 21 / 08$ & $16: 00$ & 32.474 & 42.011 & 18.037 \\
\hline $4 / 14 / 08$ & $16: 00$ & 33.605 & 43.045 & 19.116 & 4/21/08 & $20: 00$ & 32.464 & 41.999 & 18.037 \\
\hline $4 / 14 / 08$ & $20: 00$ & 33.514 & 42.944 & 19.048 & $4 / 22 / 08$ & 0:00 & 32.509 & 42.069 & 18.098 \\
\hline 4/15/08 & $0: 00$ & 33.483 & 42.935 & 19.057 & $4 / 22 / 08$ & $4: 00$ & 32.513 & 42.076 & 18.112 \\
\hline $4 / 15 / 08$ & $4: 00$ & 33.416 & 42.848 & 18.992 & $4 / 22 / 08$ & 8:00 & 32.56 & 42.147 & 18.173 \\
\hline $4 / 15 / 08$ & 8:00 & 33.348 & 42.78 & 18.952 & $4 / 22 / 08$ & $12: 00$ & 32.602 & 42.187 & 18.203 \\
\hline 4/15/08 & $12: 00$ & 33.255 & 42.66 & 18.861 & 4/22/08 & $16: 00$ & 32.583 & 42.147 & 18.168 \\
\hline 4/15/08 & $16: 00$ & 33.11 & 42.487 & 18.739 & $4 / 22 / 08$ & $20: 00$ & 32.551 & 42.121 & 18.166 \\
\hline $4 / 15 / 08$ & $20: 00$ & 33.019 & 42.43 & 18.718 & 4/23/08 & 0:00 & 32.579 & 42.166 & 18.215 \\
\hline 4/16/08 & 0:00 & 32.989 & 42.395 & 18.702 & $4 / 23 / 08$ & $4: 00$ & 32.574 & 42.151 & 18.208 \\
\hline 4/16/08 & $4: 00$ & 32.912 & 42.316 & 18.657 & 4/23/08 & 8:00 & 32.576 & 42.166 & 18.224 \\
\hline 4/16/08 & 8:00 & 32.931 & 42.402 & 18.769 & 4/23/08 & $12: 00$ & 32.555 & 42.114 & 18.173 \\
\hline 4/16/08 & $12: 00$ & 33.017 & 42.508 & 18.854 & 4/23/08 & $16: 00$ & 32.436 & 41.962 & 18.065 \\
\hline $4 / 16 / 08$ & $16: 00$ & 33.085 & 42.566 & 18.893 & 4/23/08 & $20: 00$ & 32.366 & 41.908 & 18.035 \\
\hline 4/16/08 & $20: 00$ & 33.15 & 42.651 & 18.975 & 4/24/08 & 0:00 & 32.341 & 41.91 & 18.053 \\
\hline $4 / 17 / 08$ & 0:00 & 33.262 & 42.761 & 19.038 & $4 / 24 / 08$ & $4: 00$ & 32.273 & 41.819 & 17.995 \\
\hline $4 / 17 / 08$ & $4: 00$ & 33.257 & 42.707 & 19.008 & 4/24/08 & 8:00 & 32.224 & 41.783 & 17.983 \\
\hline
\end{tabular}


Table S1.2 (Cont.)

\begin{tabular}{|c|c|c|c|c|c|c|c|c|c|}
\hline \multirow[b]{2}{*}{ Date } & \multirow[b]{2}{*}{ Time } & \multicolumn{3}{|c|}{$\begin{array}{l}\text { Depth to Water (ft below top of } \\
\text { casing) at Indicated Well }\end{array}$} & \multirow[b]{2}{*}{ Date } & \multirow[b]{2}{*}{ Time } & \multicolumn{3}{|c|}{$\begin{array}{l}\text { Depth to Water (ft below top of } \\
\text { casing) at Indicated Well }\end{array}$} \\
\hline & & D2 & WP49 & SB65d & & & D2 & WP49 & SB65d \\
\hline 4/24/08 & $12: 00$ & 32.173 & 41.729 & 17.95 & $5 / 1 / 08$ & $16: 00$ & 31.319 & 41.003 & 17.695 \\
\hline 4/24/08 & $16: 00$ & 32.108 & 41.661 & 17.911 & $5 / 1 / 08$ & 20:00 & 31.408 & 41.122 & 17.77 \\
\hline $4 / 24 / 08$ & $20: 00$ & 32.075 & 41.643 & 17.908 & $5 / 2 / 08$ & 0:00 & 31.499 & 41.232 & 17.836 \\
\hline 4/25/08 & 0:00 & 32.045 & 41.603 & 17.894 & $5 / 2 / 08$ & 4:00 & 31.438 & 41.143 & 17.796 \\
\hline $4 / 25 / 08$ & 4:00 & 31.907 & 41.575 & 17.801 & $5 / 2 / 08$ & 8:00 & 31.375 & 41.078 & 17.754 \\
\hline $4 / 25 / 08$ & 8:00 & 32.042 & 41.697 & 18.007 & $5 / 2 / 08$ & $12: 00$ & 31.359 & 41.087 & 17.766 \\
\hline $4 / 25 / 08$ & $12: 00$ & 32.131 & 41.783 & 18.06 & $5 / 2 / 08$ & $16: 00$ & 31.394 & 41.127 & 17.808 \\
\hline 4/25/08 & $16: 00$ & 32.182 & 41.828 & 18.091 & $5 / 2 / 08$ & 20:00 & 31.45 & 41.214 & 17.883 \\
\hline $4 / 25 / 08$ & $20: 00$ & 32.24 & 41.896 & 18.145 & $5 / 3 / 08$ & 0:00 & 31.578 & 41.364 & 17.995 \\
\hline 4/26/08 & 0:00 & 32.285 & 41.94 & 18.173 & $5 / 3 / 08$ & $4: 00$ & 31.676 & 41.476 & 17.052 \\
\hline $4 / 26 / 08$ & $4: 00$ & 32.292 & 41.929 & 18.152 & $5 / 3 / 08$ & $8: 00$ & 31.818 & 41.645 & 18.194 \\
\hline 4/26/08 & 8:00 & 32.334 & 42.006 & 18.224 & $5 / 3 / 08$ & $12: 00$ & 31.928 & 41.739 & 17.221 \\
\hline $4 / 26 / 08$ & $12: 00$ & 32.397 & 42.067 & 18.273 & $5 / 3 / 08$ & $16: 00$ & 31.944 & 41.715 & 17.184 \\
\hline $4 / 26 / 08$ & $16: 00$ & 32.406 & 42.072 & 18.271 & $5 / 3 / 08$ & 20:00 & 31.933 & 41.692 & 17.17 \\
\hline $4 / 26 / 08$ & $20: 00$ & 32.434 & 42.119 & 18.309 & $5 / 4 / 08$ & 0:00 & 31.956 & 41.72 & 18.206 \\
\hline 4/27/08 & 0:00 & 32.462 & 42.14 & 18.32 & $5 / 4 / 08$ & 4:00 & 31.935 & 41.69 & 18.18 \\
\hline $4 / 27 / 08$ & 4:00 & 32.481 & 42.163 & 18.341 & $5 / 4 / 08$ & 8:00 & 31.933 & 41.704 & 17.184 \\
\hline $4 / 27 / 08$ & 8:00 & 32.495 & 42.18 & 18.365 & $5 / 4 / 08$ & $12: 00$ & 31.937 & 41.69 & 17.163 \\
\hline $4 / 27 / 08$ & $12: 00$ & 32.478 & 42.14 & 18.313 & $5 / 4 / 08$ & $16: 00$ & 31.872 & 41.603 & 18.114 \\
\hline $4 / 27 / 08$ & $16: 00$ & 32.392 & 42.02 & 18.215 & $5 / 4 / 08$ & 20:00 & 31.823 & 41.565 & 18.098 \\
\hline $4 / 27 / 08$ & $20: 00$ & 32.299 & 41.926 & 18.154 & $5 / 5 / 08$ & 0:00 & 31.853 & 41.631 & 17.151 \\
\hline $4 / 28 / 08$ & 0:00 & 32.252 & 41.901 & 18.152 & $5 / 5 / 08$ & 4:00 & 31.853 & 41.619 & 17.144 \\
\hline 4/28/08 & $4: 00$ & 32.257 & 41.929 & 18.196 & $5 / 5 / 08$ & $8: 00$ & 31.86 & 41.633 & 17.16 \\
\hline $4 / 28 / 08$ & 8:00 & 32.294 & 41.99 & 18.255 & $5 / 5 / 08$ & $12: 00$ & 31.863 & 41.626 & \\
\hline $4 / 28 / 08$ & $12: 00$ & 32.313 & 42.006 & 18.259 & $5 / 5 / 08$ & $16: 00$ & 31.814 & 41.551 & \\
\hline $4 / 28 / 08$ & $16: 00$ & 32.296 & 41.969 & 18.234 & $5 / 5 / 08$ & 20:00 & 31.751 & 41.488 & \\
\hline $4 / 28 / 08$ & $20: 00$ & 32.266 & 41.94 & 18.222 & $5 / 6 / 08$ & $0: 00$ & 31.746 & 41.49 & \\
\hline 4/29/08 & 0:00 & 32.24 & 41.908 & 18.196 & $5 / 6 / 08$ & 4:00 & 31.704 & 41.443 & \\
\hline $4 / 29 / 08$ & 4:00 & 32.219 & 41.901 & 18.201 & $5 / 6 / 08$ & 8:00 & 31.664 & 41.406 & \\
\hline 4/29/08 & 8:00 & 32.217 & 41.905 & 18.217 & $5 / 6 / 08$ & $12: 00$ & 31.629 & 41.361 & \\
\hline 4/29/08 & $12: 00$ & 32.18 & 41.851 & 18.163 & $5 / 6 / 08$ & $16: 00$ & 31.527 & 41.232 & \\
\hline $4 / 29 / 08$ & $16: 00$ & 32.068 & 41.701 & 18.049 & $5 / 6 / 08$ & $20: 00$ & 31.438 & 41.155 & \\
\hline 4/29/08 & $20: 00$ & 31.982 & 41.631 & 18.014 & $5 / 7 / 08$ & 0:00 & 31.289 & 41.188 & \\
\hline 4/30/08 & 0:00 & 31.93 & 41.591 & 17.997 & $5 / 7 / 08$ & $4: 00$ & 31.375 & 41.117 & \\
\hline 4/30/08 & $4: 00$ & 31.837 & 41.467 & 17.906 & $5 / 7 / 08$ & 8:00 & 31.413 & 41.204 & \\
\hline 4/30/08 & 8:00 & 31.751 & 41.401 & 17.88 & $5 / 7 / 08$ & $12: 00$ & 31.422 & 41.209 & \\
\hline 4/30/08 & $12: 00$ & 31.671 & 41.31 & 17.815 & $5 / 7 / 08$ & $16: 00$ & 31.448 & 41.225 & \\
\hline 4/30/08 & $16: 00$ & 31.573 & 41.202 & 17.749 & $5 / 7 / 08$ & 20:00 & 31.431 & 41.197 & \\
\hline $4 / 30 / 08$ & $20: 00$ & 31.501 & 41.143 & 17.721 & $5 / 8 / 08$ & $0: 00$ & 31.445 & 41.218 & \\
\hline 5/1/08 & 0:00 & 31.48 & 41.143 & 17.742 & $5 / 8 / 08$ & 4:00 & 31.443 & 41.223 & \\
\hline $5 / 1 / 08$ & 4:00 & 31.41 & 41.054 & 17.679 & $5 / 8 / 08$ & $8: 00$ & 31.441 & 41.211 & \\
\hline $5 / 1 / 08$ & 8:00 & 31.38 & 41.042 & 17.686 & $5 / 8 / 08$ & $12: 00$ & & 41.19 & \\
\hline $5 / 1 / 08$ & $12: 00$ & 31.333 & 40.986 & 17.642 & $5 / 8 / 08$ & $16: 00$ & 31.378 & 41.106 & \\
\hline
\end{tabular}


Table S1.2 (Cont.)

\begin{tabular}{|c|c|c|c|c|c|c|c|c|c|}
\hline \multirow[b]{2}{*}{ Date } & \multirow[b]{2}{*}{ Time } & \multicolumn{3}{|c|}{$\begin{array}{l}\text { Depth to Water (ft below top of } \\
\text { casing) at Indicated Well }\end{array}$} & \multirow[b]{2}{*}{ Date } & \multirow[b]{2}{*}{ Time } & \multicolumn{3}{|c|}{$\begin{array}{l}\text { Depth to Water (ft below top of } \\
\text { casing) at Indicated Well }\end{array}$} \\
\hline & & D2 & WP49 & SB65d & & & D2 & WP49 & SB65d \\
\hline $5 / 8 / 08$ & $20: 00$ & 31.312 & 41.094 & & $5 / 16 / 08$ & 0:00 & 30.713 & 40.649 & \\
\hline $5 / 9 / 08$ & 0:00 & 31.31 & 41.127 & & 5/16/08 & $4: 00$ & 30.685 & 40.613 & \\
\hline $5 / 9 / 08$ & 4:00 & 31.06 & 41.092 & & 5/16/08 & 8:00 & 30.68 & 40.625 & \\
\hline $5 / 9 / 08$ & 8:00 & 31.298 & 41.164 & & 5/16/08 & $12: 00$ & 30.664 & 40.592 & \\
\hline $5 / 9 / 08$ & $12: 00$ & 31.352 & 41.211 & & $5 / 16 / 08$ & $16: 00$ & 30.592 & 40.501 & \\
\hline $5 / 9 / 08$ & $16: 00$ & 31.336 & 41.169 & & $5 / 16 / 08$ & $20: 00$ & 30.505 & 40.412 & \\
\hline $5 / 9 / 08$ & $20: 00$ & 31.319 & 41.148 & & $5 / 17 / 08$ & $0: 00$ & 30.454 & 40.379 & \\
\hline 5/10/08 & $0: 00$ & 31.308 & 41.125 & & $5 / 17 / 08$ & $4: 00$ & 30.396 & 40.33 & \\
\hline $5 / 10 / 08$ & $4: 00$ & 31.261 & 41.059 & & 5/17/08 & 8:00 & 30.403 & 40.363 & \\
\hline 5/10/08 & 8:00 & 31.233 & 41.042 & & $5 / 17 / 08$ & $12: 00$ & 30.386 & 40.337 & \\
\hline $5 / 10 / 08$ & $12: 00$ & 31.119 & 40.878 & & $5 / 17 / 08$ & $16: 00$ & 30.319 & 40.252 & \\
\hline $5 / 10 / 08$ & $16: 00$ & 30.729 & 40.827 & & $5 / 17 / 08$ & 20:00 & 30.272 & 40.229 & \\
\hline $5 / 10 / 08$ & $20: 00$ & 30.979 & 40.834 & & $5 / 18 / 08$ & $0: 00$ & 30.284 & 40.255 & \\
\hline $5 / 11 / 08$ & $0: 00$ & 31.067 & 40.93 & & 5/18/08 & 4:00 & 30.263 & 40.238 & \\
\hline $5 / 11 / 08$ & $4: 00$ & 31.109 & 40.967 & & $5 / 18 / 08$ & 8:00 & 30.286 & 40.285 & \\
\hline $5 / 11 / 08$ & 8:00 & 31.172 & 41.035 & & $5 / 18 / 08$ & $12: 00$ & 30.291 & 40.269 & \\
\hline $5 / 11 / 08$ & $12: 00$ & 31.198 & 41.038 & & 5/18/08 & $16: 00$ & 30.23 & 40.184 & \\
\hline $5 / 11 / 08$ & $16: 00$ & 31.163 & 40.97 & & $5 / 18 / 08$ & $20: 00$ & 30.139 & 40.088 & \\
\hline $5 / 11 / 08$ & $20: 00$ & 31.091 & 40.878 & & $5 / 19 / 08$ & $0: 00$ & 30.095 & 40.06 & \\
\hline $5 / 12 / 08$ & $0: 00$ & 31.058 & 40.853 & & 5/19/08 & $4: 00$ & 30.004 & 39.922 & \\
\hline $5 / 12 / 08$ & $4: 00$ & 30.979 & 40.761 & & 5/19/08 & 8:00 & 29.976 & 39.957 & \\
\hline $5 / 12 / 08$ & 8:00 & 30.918 & 40.695 & & 5/19/08 & $12: 00$ & 29.988 & 39.985 & \\
\hline $5 / 12 / 08$ & $12: 00$ & 30.827 & 40.588 & & 5/19/08 & $16: 00$ & 29.983 & 39.969 & \\
\hline $5 / 12 / 08$ & $16: 00$ & 30.697 & 40.438 & & $5 / 19 / 08$ & $20: 00$ & 29.964 & 39.966 & \\
\hline $5 / 12 / 08$ & $20: 00$ & 30.638 & 40.426 & & $5 / 20 / 08$ & $0: 00$ & 30.048 & 40.093 & \\
\hline $5 / 13 / 08$ & $0: 00$ & 30.666 & 40.484 & & 5/20/08 & $4: 00$ & 30.095 & 40.135 & \\
\hline $5 / 13 / 08$ & $4: 00$ & 30.636 & 40.438 & & $5 / 20 / 08$ & $8: 00$ & 30.205 & 40.285 & \\
\hline $5 / 13 / 08$ & 8:00 & 30.643 & 40.494 & & $5 / 20 / 08$ & $12: 00$ & 30.261 & 40.306 & \\
\hline $5 / 13 / 08$ & $12: 00$ & 30.669 & 40.522 & & $5 / 20 / 08$ & $16: 00$ & 30.223 & 40.231 & \\
\hline $5 / 13 / 08$ & $16: 00$ & 30.706 & 40.578 & & $5 / 20 / 08$ & $20: 00$ & 30.153 & 40.161 & \\
\hline $5 / 13 / 08$ & $20: 00$ & 30.762 & 40.649 & & $5 / 21 / 08$ & $0: 00$ & 30.121 & 40.135 & \\
\hline $5 / 14 / 08$ & $0: 00$ & 30.841 & 40.747 & & $5 / 21 / 08$ & $4: 00$ & 30.1 & 40.123 & \\
\hline $5 / 14 / 08$ & $4: 00$ & 30.844 & 40.731 & & $5 / 21 / 08$ & 8:00 & 30.086 & 40.119 & \\
\hline $5 / 14 / 08$ & $8: 00$ & 30.862 & 40.778 & & $5 / 21 / 08$ & $12: 00$ & 30.048 & 40.065 & \\
\hline $5 / 14 / 08$ & $12: 00$ & 30.879 & 40.771 & & $5 / 21 / 08$ & $16: 00$ & 29.992 & 39.994 & \\
\hline $5 / 14 / 08$ & $16: 00$ & 30.823 & 40.693 & & $5 / 21 / 08$ & $20: 00$ & 29.918 & 39.931 & \\
\hline $5 / 14 / 08$ & $20: 00$ & 30.783 & 40.677 & & $5 / 22 / 08$ & $0: 00$ & 29.901 & 39.926 & \\
\hline $5 / 15 / 08$ & $0: 00$ & 30.783 & 40.691 & & $5 / 22 / 08$ & 4:00 & 29.892 & 39.929 & \\
\hline $5 / 15 / 08$ & $4: 00$ & 30.757 & 40.663 & & $5 / 22 / 08$ & $8: 00$ & 29.878 & 39.929 & \\
\hline $5 / 15 / 08$ & $8: 00$ & 30.767 & 40.695 & & $5 / 22 / 08$ & $12: 00$ & 29.904 & 39.948 & \\
\hline $5 / 15 / 08$ & $12: 00$ & 30.781 & 40.714 & & $5 / 22 / 08$ & $16: 00$ & 29.878 & 39.917 & \\
\hline $5 / 15 / 08$ & $16: 00$ & 30.741 & 40.646 & & $5 / 22 / 08$ & $20: 00$ & 29.817 & 39.856 & \\
\hline $5 / 15 / 08$ & $20: 00$ & 30.713 & 40.639 & & $5 / 23 / 08$ & $0: 00$ & 29.848 & 39.912 & \\
\hline
\end{tabular}


Table S1.2 (Cont.)

\begin{tabular}{|c|c|c|c|c|c|c|c|c|c|}
\hline \multirow[b]{2}{*}{ Date } & \multirow[b]{2}{*}{ Time } & \multicolumn{3}{|c|}{$\begin{array}{l}\text { Depth to Water (ft below top of } \\
\text { casing) at Indicated Well }\end{array}$} & \multirow[b]{2}{*}{ Date } & \multirow[b]{2}{*}{ Time } & \multicolumn{3}{|c|}{$\begin{array}{l}\text { Depth to Water (ft below top of } \\
\text { casing) at Indicated Well }\end{array}$} \\
\hline & & D2 & WP49 & SB65d & & & D2 & WP49 & SB65d \\
\hline 5/23/08 & 4:00 & 29.428 & 40.001 & & $5 / 30 / 08$ & $8: 00$ & 28.887 & 38.991 & \\
\hline 5/23/08 & 8:00 & 29.862 & 39.98 & & 5/30/08 & $12: 00$ & 28.878 & 39.007 & \\
\hline 5/23/08 & $12: 00$ & 29.936 & 40.046 & & 5/30/08 & $16: 00$ & 28.859 & 38.986 & \\
\hline $5 / 23 / 08$ & $16: 00$ & 29.918 & 39.999 & & 5/30/08 & 20:00 & 28.85 & 38.993 & \\
\hline $5 / 23 / 08$ & $20: 00$ & 29.904 & 39.987 & & $5 / 31 / 08$ & $0: 00$ & 28.871 & 39.033 & \\
\hline $5 / 24 / 08$ & $0: 00$ & 29.89 & 39.962 & & $5 / 31 / 08$ & 4:00 & 28.88 & 39.036 & \\
\hline $5 / 24 / 08$ & $4: 00$ & 29.727 & 39.713 & & $5 / 31 / 08$ & 8:00 & 28.894 & 39.064 & \\
\hline $5 / 24 / 08$ & 8:00 & 29.792 & 39.849 & & $5 / 31 / 08$ & $12: 00$ & 28.91 & 39.078 & \\
\hline $5 / 24 / 08$ & $12: 00$ & 29.794 & 39.854 & & 5/31/08 & $16: 00$ & 28.873 & 39.021 & \\
\hline $5 / 24 / 08$ & $16: 00$ & 29.745 & 39.779 & & 5/31/08 & 20:00 & 28.822 & 38.979 & \\
\hline $5 / 24 / 08$ & $20: 00$ & 29.699 & 39.739 & & 6/1/08 & $0: 00$ & 28.826 & 39.007 & \\
\hline $5 / 25 / 08$ & $0: 00$ & 29.678 & 39.706 & & $6 / 1 / 08$ & $4: 00$ & 28.794 & 38.977 & \\
\hline $5 / 25 / 08$ & $4: 00$ & 29.626 & 39.643 & & 6/1/08 & 8:00 & 28.815 & 39.017 & \\
\hline $5 / 25 / 08$ & 8:00 & 29.608 & 39.638 & & $6 / 1 / 08$ & $12: 00$ & 28.798 & 38.993 & \\
\hline $5 / 25 / 08$ & $12: 00$ & 29.542 & 39.542 & & $6 / 1 / 08$ & $16: 00$ & 28.749 & 38.935 & \\
\hline $5 / 25 / 08$ & $16: 00$ & 29.489 & 39.495 & & 6/1/08 & 20:00 & 28.689 & 38.883 & \\
\hline $5 / 25 / 08$ & $20: 00$ & 29.451 & 39.53 & & $6 / 2 / 08$ & $0: 00$ & 28.686 & 38.904 & \\
\hline $5 / 26 / 08$ & $0: 00$ & 29.451 & 39.488 & & $6 / 2 / 08$ & 4:00 & 28.626 & 38.825 & \\
\hline $5 / 26 / 08$ & $4: 00$ & 29.447 & 39.521 & & $6 / 2 / 08$ & 8:00 & 28.598 & 38.799 & \\
\hline $5 / 26 / 08$ & $8: 00$ & 29.47 & 39.549 & & $6 / 2 / 08$ & $12: 00$ & 28.542 & 38.733 & \\
\hline $5 / 26 / 08$ & $12: 00$ & 29.54 & 39.629 & & $6 / 2 / 08$ & $16: 00$ & 28.437 & 38.606 & \\
\hline $5 / 26 / 08$ & $16: 00$ & 29.538 & 39.601 & & $6 / 2 / 08$ & $20: 00$ & 28.367 & 38.578 & \\
\hline $5 / 26 / 08$ & $20: 00$ & 29.505 & 39.563 & & $6 / 3 / 08$ & $0: 00$ & 28.297 & 38.489 & \\
\hline $5 / 27 / 08$ & $0: 00$ & 29.538 & 39.626 & & $6 / 3 / 08$ & 4:00 & 28.192 & 38.403 & \\
\hline $5 / 27 / 08$ & 4:00 & 29.526 & 39.61 & & $6 / 3 / 08$ & 8:00 & 28.159 & 38.379 & \\
\hline $5 / 27 / 08$ & 8:00 & 29.568 & 39.673 & & $6 / 3 / 08$ & $12: 00$ & 28.134 & 38.36 & \\
\hline $5 / 27 / 08$ & $12: 00$ & 29.636 & 39.755 & & $6 / 3 / 08$ & $16: 00$ & 28.103 & 38.335 & \\
\hline $5 / 27 / 08$ & $16: 00$ & 29.659 & 39.776 & & $6 / 3 / 08$ & 20:00 & 28.068 & 38.316 & \\
\hline $5 / 27 / 08$ & $20: 00$ & 29.675 & 39.8 & & $6 / 4 / 08$ & $0: 00$ & 28.061 & 38.313 & \\
\hline $5 / 28 / 08$ & $0: 00$ & 29.696 & 39.821 & & $6 / 4 / 08$ & $4: 00$ & 28.05 & 38.304 & \\
\hline $5 / 28 / 08$ & 4:00 & 29.706 & 39.835 & & $6 / 4 / 08$ & 8:00 & 28.029 & 38.311 & \\
\hline $5 / 28 / 08$ & 8:00 & 29.703 & 39.87 & & $6 / 4 / 08$ & $12: 00$ & 28.006 & 38.271 & \\
\hline $5 / 28 / 08$ & $12: 00$ & 29.708 & 39.847 & & $6 / 4 / 08$ & $16: 00$ & 27.957 & 38.203 & \\
\hline 5/28/08 & $16: 00$ & 29.64 & 39.753 & & $6 / 4 / 08$ & $20: 00$ & 27.87 & 38.116 & \\
\hline $5 / 28 / 08$ & $20: 00$ & 29.556 & 39.645 & & $6 / 5 / 08$ & $0: 00$ & 27.52 & 38.091 & \\
\hline $5 / 29 / 08$ & $0: 00$ & 29.491 & 39.589 & & $6 / 5 / 08$ & 4:00 & 25.741 & 38.154 & \\
\hline $5 / 29 / 08$ & 4:00 & 29.402 & 39.486 & & $6 / 5 / 08$ & $8: 00$ & 27.46 & 37.957 & \\
\hline $5 / 29 / 08$ & 8:00 & 29.337 & 39.418 & & $6 / 5 / 08$ & $12: 00$ & 27.285 & 37.741 & \\
\hline $5 / 29 / 08$ & $12: 00$ & 29.244 & 39.3 & & $6 / 5 / 08$ & $16: 00$ & 27.084 & 37.498 & \\
\hline $5 / 29 / 08$ & $16: 00$ & 29.109 & 39.139 & & $6 / 5 / 08$ & 20:00 & 26.816 & 37.279 & \\
\hline $5 / 29 / 08$ & $20: 00$ & 28.987 & 39.031 & & $6 / 6 / 08$ & 0:00 & 26.655 & 37.075 & \\
\hline $5 / 30 / 08$ & $0: 00$ & 28.707 & 39.089 & & $6 / 6 / 08$ & 4:00 & 26.406 & 37.017 & \\
\hline $5 / 30 / 08$ & 4:00 & 28.92 & 39.05 & & $6 / 6 / 08$ & 8:00 & 26.189 & 37.064 & \\
\hline
\end{tabular}


Table S1.2 (Cont.)

\begin{tabular}{|c|c|c|c|c|c|c|c|c|c|}
\hline \multirow[b]{2}{*}{ Date } & \multirow[b]{2}{*}{ Time } & \multicolumn{3}{|c|}{$\begin{array}{l}\text { Depth to Water (ft below top of } \\
\text { casing) at Indicated Well }\end{array}$} & \multirow[b]{2}{*}{ Date } & \multirow[b]{2}{*}{ Time } & \multicolumn{3}{|c|}{$\begin{array}{l}\text { Depth to Water (ft below top of } \\
\text { casing) at Indicated Well }\end{array}$} \\
\hline & & D2 & WP49 & SB65d & & & D2 & WP49 & SB65d \\
\hline $6 / 6 / 08$ & $12: 00$ & 26.147 & 37.083 & & 6/13/08 & $16: 00$ & 23.097 & 34.22 & 13.028 \\
\hline 6/6/08 & $16: 00$ & 26.121 & 37.01 & & 6/13/08 & $20: 00$ & 23.003 & 34.131 & 12.998 \\
\hline 6/6/08 & $20: 00$ & 26.277 & 36.932 & & 6/14/08 & 0:00 & 22.943 & 34.079 & 12.981 \\
\hline $6 / 7 / 08$ & $0: 00$ & 26.224 & 36.881 & & 6/14/08 & $4: 00$ & 22.859 & 33.992 & 12.942 \\
\hline $6 / 7 / 08$ & $4: 00$ & 26.093 & 36.714 & & $6 / 14 / 08$ & 8:00 & 22.824 & 33.981 & 12.967 \\
\hline $6 / 7 / 08$ & $8: 00$ & 25.979 & 36.616 & & 6/14/08 & $12: 00$ & 22.772 & 33.927 & 12.946 \\
\hline $6 / 7 / 08$ & $12: 00$ & 25.911 & 36.557 & & 6/14/08 & $16: 00$ & 22.693 & 33.826 & 12.878 \\
\hline $6 / 7 / 08$ & $16: 00$ & 25.823 & 36.464 & & 6/14/08 & 20:00 & 22.586 & 33.718 & 12.818 \\
\hline $6 / 7 / 08$ & $20: 00$ & 25.741 & 36.414 & & 6/15/08 & 0:00 & 22.516 & 33.671 & 12.811 \\
\hline 6/8/08 & 0:00 & 25.697 & 36.389 & & 6/15/08 & 4:00 & 22.451 & 33.606 & 12.785 \\
\hline 6/8/08 & $4: 00$ & 25.531 & 36.325 & & 6/15/08 & $8: 00$ & 22.425 & 33.587 & 12.801 \\
\hline 6/8/08 & $8: 00$ & 25.487 & 36.26 & & 6/15/08 & $12: 00$ & 22.343 & 33.606 & 12.785 \\
\hline $6 / 8 / 08$ & $12: 00$ & 25.415 & 36.208 & & 6/15/08 & $16: 00$ & 22.318 & 33.54 & 12.703 \\
\hline $6 / 8 / 08$ & $16: 00$ & 25.31 & 36.126 & & 6/15/08 & $20: 00$ & 22.241 & 33.477 & 12.691 \\
\hline $6 / 8 / 08$ & $20: 00$ & 25.219 & 36.046 & & 6/16/08 & 0:00 & 22.229 & 33.5 & 12.747 \\
\hline $6 / 9 / 08$ & $0: 00$ & 25.158 & 36.02 & & 6/16/08 & $4: 00$ & 22.208 & 33.463 & 12.738 \\
\hline $6 / 9 / 08$ & $4: 00$ & 25.086 & 35.936 & & $6 / 16 / 08$ & 8:00 & 22.208 & 33.491 & 12.801 \\
\hline $6 / 9 / 08$ & 8:00 & 25.025 & 35.891 & & 6/16/08 & $12: 00$ & 22.206 & 33.477 & 12.794 \\
\hline $6 / 9 / 08$ & $12: 00$ & 24.967 & 35.838 & & 6/16/08 & $16: 00$ & 22.148 & 33.402 & 12.75 \\
\hline $6 / 9 / 08$ & $16: 00$ & 24.883 & 35.746 & 13.929 & $6 / 16 / 08$ & $20: 00$ & 22.092 & 33.355 & 12.731 \\
\hline $6 / 9 / 08$ & $20: 00$ & 24.78 & 35.65 & 13.873 & $6 / 17 / 08$ & $0: 00$ & 22.073 & 33.357 & 12.754 \\
\hline 6/10/08 & $0: 00$ & 24.743 & 35.643 & 13.913 & $6 / 17 / 08$ & $4: 00$ & 22.036 & 33.315 & 12.729 \\
\hline 6/10/08 & $4: 00$ & 24.682 & 35.58 & 13.885 & 6/17/08 & 8:00 & 21.994 & 33.284 & 12.738 \\
\hline 6/10/08 & 8:00 & 24.65 & 35.556 & 13.896 & 6/17/08 & $12: 00$ & 21.982 & 33.256 & 12.717 \\
\hline 6/10/08 & $12: 00$ & 24.601 & 35.5 & 13.873 & $6 / 17 / 08$ & $16: 00$ & 21.891 & 33.137 & 12.621 \\
\hline 6/10/08 & $16: 00$ & 24.491 & 35.362 & 13.779 & $6 / 17 / 08$ & $20: 00$ & 21.802 & 33.066 & 12.595 \\
\hline $6 / 10 / 08$ & $20: 00$ & 24.382 & 35.27 & 13.739 & 6/18/08 & $0: 00$ & 21.739 & 32.968 & 12.549 \\
\hline 6/11/08 & $0: 00$ & 24.312 & 35.212 & 13.718 & 6/18/08 & $4: 00$ & 21.667 & 32.937 & 12.523 \\
\hline 6/11/08 & $4: 00$ & 24.207 & 35.113 & 13.653 & $6 / 18 / 08$ & 8:00 & 21.655 & 32.954 & 12.565 \\
\hline $6 / 11 / 08$ & $8: 00$ & 24.148 & 35.076 & 13.66 & 6/18/08 & $12: 00$ & 21.646 & 32.944 & 12.572 \\
\hline 6/11/08 & $12: 00$ & 24.067 & 34.977 & 13.599 & 6/18/08 & $16: 00$ & 21.578 & 32.855 & 12.499 \\
\hline 6/11/08 & $16: 00$ & 23.99 & 34.904 & 13.566 & $6 / 18 / 08$ & $20: 00$ & 21.504 & 32.79 & 12.462 \\
\hline $6 / 11 / 08$ & $20: 00$ & 23.883 & 34.848 & 13.585 & $6 / 19 / 08$ & $0: 00$ & 21.471 & 32.766 & 12.469 \\
\hline $6 / 12 / 08$ & $0: 00$ & 23.88 & 34.982 & 13.41 & 6/19/08 & $4: 00$ & 21.443 & 32.736 & 12.46 \\
\hline $6 / 12 / 08$ & $4: 00$ & 23.81 & 34.865 & 13.3 & $6 / 19 / 08$ & 8:00 & 21.42 & 32.717 & 12.453 \\
\hline $6 / 12 / 08$ & 8:00 & 23.729 & 34.832 & 13.225 & 6/19/08 & $12: 00$ & 21.404 & 32.696 & 12.443 \\
\hline $6 / 12 / 08$ & $12: 00$ & 23.605 & 34.698 & 13.155 & 6/19/08 & $16: 00$ & 21.348 & 32.621 & 12.397 \\
\hline $6 / 12 / 08$ & $16: 00$ & 23.465 & 34.553 & 13.066 & $6 / 19 / 08$ & $20: 00$ & 20.289 & 32.647 & 12.389 \\
\hline $6 / 12 / 08$ & $20: 00$ & 23.355 & 34.466 & 13.052 & $6 / 20 / 08$ & $0: 00$ & 21.653 & 32.567 & 12.226 \\
\hline $6 / 13 / 08$ & $0: 00$ & 23.281 & 34.412 & 13.049 & $6 / 20 / 08$ & $4: 00$ & 21.231 & 32.471 & 12.191 \\
\hline $6 / 13 / 08$ & $4: 00$ & 23.232 & 34.356 & 13.035 & $6 / 20 / 08$ & 8:00 & 21.04 & 32.379 & 12.186 \\
\hline $6 / 13 / 08$ & 8:00 & 23.197 & 34.351 & 13.08 & $6 / 20 / 08$ & $12: 00$ & 20.923 & 32.295 & 12.167 \\
\hline 6/13/08 & $12: 00$ & 23.181 & 34.328 & 13.096 & $6 / 20 / 08$ & $16: 00$ & 20.751 & 32.178 & 12.118 \\
\hline
\end{tabular}


Table S1.2 (Cont.)

\begin{tabular}{|c|c|c|c|c|c|c|c|c|c|}
\hline \multirow[b]{2}{*}{ Date } & \multirow[b]{2}{*}{ Time } & \multicolumn{3}{|c|}{$\begin{array}{l}\text { Depth to Water (ft below top of } \\
\text { casing) at Indicated Well }\end{array}$} & \multirow[b]{2}{*}{ Date } & \multirow[b]{2}{*}{ Time } & \multicolumn{3}{|c|}{$\begin{array}{l}\text { Depth to Water (ft below top of } \\
\text { casing) at Indicated Well }\end{array}$} \\
\hline & & D2 & WP49 & SB65d & & & D2 & WP49 & SB65d \\
\hline 6/20/08 & $20: 00$ & 20.657 & 32.1 & 12.102 & 6/28/08 & $0: 00$ & 19.601 & 30.944 & 11.538 \\
\hline $6 / 21 / 08$ & 0:00 & 20.639 & 32.1 & 12.139 & 6/28/08 & $4: 00$ & 19.599 & 30.912 & 11.496 \\
\hline 6/21/08 & $4: 00$ & 20.592 & 32.046 & 12.13 & 6/28/08 & $8: 00$ & 19.615 & 30.933 & 11.526 \\
\hline $6 / 21 / 08$ & 8:00 & 20.585 & 32.039 & 12.146 & 6/28/08 & $12: 00$ & 19.657 & 30.973 & 11.561 \\
\hline $6 / 21 / 08$ & $12: 00$ & 20.566 & 32.002 & 12.125 & 6/28/08 & $16: 00$ & 19.641 & 30.935 & 11.524 \\
\hline $6 / 21 / 08$ & $16: 00$ & 20.501 & 31.906 & 12.06 & 6/28/08 & $20: 00$ & 19.613 & 30.902 & 11.507 \\
\hline $6 / 21 / 08$ & $20: 00$ & 20.422 & 31.828 & 12.034 & $6 / 29 / 08$ & 0:00 & 19.627 & 30.928 & 11.538 \\
\hline $6 / 22 / 08$ & $0: 00$ & 20.378 & 31.789 & 12.003 & 6/29/08 & 4:00 & 19.65 & 30.954 & 11.564 \\
\hline $6 / 22 / 08$ & $4: 00$ & 20.333 & 31.753 & 11.999 & $6 / 29 / 08$ & $8: 00$ & 19.683 & 30.998 & 11.608 \\
\hline $6 / 22 / 08$ & 8:00 & 20.308 & 31.723 & 11.987 & 6/29/08 & $12: 00$ & 19.722 & 31.034 & 11.634 \\
\hline $6 / 22 / 08$ & $12: 00$ & 20.282 & 31.683 & 11.961 & 6/29/08 & $16: 00$ & 19.718 & 31.001 & 11.594 \\
\hline $6 / 22 / 08$ & $16: 00$ & 20.228 & 31.608 & 11.91 & 6/29/08 & $20: 00$ & 19.692 & 30.97 & 11.573 \\
\hline $6 / 22 / 08$ & $20: 00$ & 20.165 & 31.556 & 11.893 & 6/30/08 & 0:00 & 19.713 & 31.008 & 11.613 \\
\hline $6 / 23 / 08$ & 0:00 & 20.182 & 31.601 & 11.943 & 6/30/08 & $4: 00$ & 19.72 & 31.008 & 11.608 \\
\hline $6 / 23 / 08$ & $4: 00$ & 20.154 & 31.561 & 11.922 & $6 / 30 / 08$ & $8: 00$ & 19.741 & 31.031 & 11.629 \\
\hline $6 / 23 / 08$ & 8:00 & 20.158 & 31.575 & 11.943 & 6/30/08 & $12: 00$ & 19.748 & 31.02 & 11.615 \\
\hline $6 / 23 / 08$ & $12: 00$ & 20.177 & 31.573 & 11.938 & 6/30/08 & $16: 00$ & 19.704 & 30.942 & 11.55 \\
\hline $6 / 23 / 08$ & $16: 00$ & 20.163 & 31.545 & 11.924 & $6 / 30 / 08$ & $20: 00$ & 19.643 & 30.879 & 11.505 \\
\hline $6 / 23 / 08$ & $20: 00$ & 20.116 & 31.486 & 11.882 & 7/1/08 & 0:00 & 19.624 & 30.869 & 11.505 \\
\hline $6 / 24 / 08$ & 0:00 & 20.135 & 31.531 & 11.931 & $7 / 1 / 08$ & $4: 00$ & 19.594 & 30.832 & 11.47 \\
\hline $6 / 24 / 08$ & $4: 00$ & 20.091 & 31.453 & 11.87 & $7 / 1 / 08$ & 8:00 & 19.568 & 30.799 & 11.458 \\
\hline $6 / 24 / 08$ & 8:00 & 20.065 & 31.418 & 11.851 & $7 / 1 / 08$ & $12: 00$ & 19.531 & 30.731 & 11.393 \\
\hline $6 / 24 / 08$ & $12: 00$ & 20.067 & 31.446 & 11.861 & $7 / 1 / 08$ & $16: 00$ & 19.463 & 30.654 & 11.346 \\
\hline $6 / 24 / 08$ & $16: 00$ & 20.032 & 31.388 & 11.821 & $7 / 1 / 08$ & 20:00 & 19.386 & 30.574 & 11.299 \\
\hline $6 / 24 / 08$ & $20: 00$ & 19.99 & 31.338 & 11.788 & $7 / 2 / 08$ & 0:00 & 19.356 & 30.548 & 11.287 \\
\hline $6 / 25 / 08$ & $0: 00$ & 19.96 & 31.313 & 11.776 & $7 / 2 / 08$ & $4: 00$ & 19.326 & 30.513 & 11.252 \\
\hline $6 / 25 / 08$ & $4: 00$ & 19.927 & 31.277 & 11.76 & $7 / 2 / 08$ & 8:00 & 19.323 & 30.551 & 11.316 \\
\hline $6 / 25 / 08$ & 8:00 & 19.932 & 31.273 & 11.755 & $7 / 2 / 08$ & $12: 00$ & 19.347 & 30.558 & 11.327 \\
\hline $6 / 25 / 08$ & $12: 00$ & 19.913 & 31.245 & 11.73 & $7 / 2 / 08$ & $16: 00$ & 19.37 & 30.586 & 11.351 \\
\hline $6 / 25 / 08$ & $16: 00$ & 19.878 & 31.191 & 11.697 & $7 / 2 / 08$ & $20: 00$ & 19.44 & 30.717 & 11.496 \\
\hline $6 / 25 / 08$ & $20: 00$ & 19.846 & 31.16 & 11.676 & $7 / 3 / 08$ & $0: 00$ & 19.512 & 30.783 & 11.536 \\
\hline $6 / 26 / 08$ & $0: 00$ & 19.836 & 31.167 & 11.685 & $7 / 3 / 08$ & $4: 00$ & 19.561 & 30.804 & 11.535 \\
\hline $6 / 26 / 08$ & $4: 00$ & 19.818 & 31.118 & 11.643 & $7 / 3 / 08$ & 8:00 & 19.613 & 30.855 & 11.575 \\
\hline $6 / 26 / 08$ & 8:00 & 19.79 & 31.092 & 11.636 & $7 / 3 / 08$ & $12: 00$ & 19.636 & 30.853 & 11.564 \\
\hline $6 / 26 / 08$ & $12: 00$ & 19.701 & 31.16 & 11.648 & $7 / 3 / 08$ & $16: 00$ & 19.634 & 30.832 & 11.54 \\
\hline $6 / 26 / 08$ & $16: 00$ & 19.762 & 31.092 & 11.561 & $7 / 3 / 08$ & $20: 00$ & 19.606 & 30.787 & 11.498 \\
\hline $6 / 26 / 08$ & $20: 00$ & 19.662 & 30.947 & 11.435 & $7 / 4 / 08$ & 0:00 & 19.61 & 30.811 & 11.526 \\
\hline $6 / 27 / 08$ & 0:00 & 19.58 & 30.867 & 11.374 & $7 / 4 / 08$ & $4: 00$ & 19.617 & 30.804 & 11.512 \\
\hline $6 / 27 / 08$ & $4: 00$ & 19.547 & 30.832 & 11.36 & $7 / 4 / 08$ & $8: 00$ & 19.613 & 30.794 & 11.512 \\
\hline $6 / 27 / 08$ & 8:00 & 19.524 & 30.839 & 11.409 & $7 / 4 / 08$ & $12: 00$ & 19.62 & 30.792 & 11.505 \\
\hline $6 / 27 / 08$ & $12: 00$ & 19.543 & 30.869 & 11.444 & $7 / 4 / 08$ & $16: 00$ & 19.585 & 30.729 & 11.449 \\
\hline $6 / 27 / 08$ & $16: 00$ & 19.54 & 30.834 & 11.426 & $7 / 4 / 08$ & $20: 00$ & 19.54 & 30.687 & 11.421 \\
\hline $6 / 27 / 08$ & $20: 00$ & 19.547 & 30.872 & 11.468 & $7 / 5 / 08$ & 0:00 & 19.547 & 30.712 & 11.447 \\
\hline
\end{tabular}


Table S1.2 (Cont.)

\begin{tabular}{|c|c|c|c|c|c|c|c|c|c|}
\hline \multirow[b]{2}{*}{ Date } & \multirow[b]{2}{*}{ Time } & \multicolumn{3}{|c|}{$\begin{array}{l}\text { Depth to Water (ft below top of } \\
\text { casing) at Indicated Well }\end{array}$} & \multirow[b]{2}{*}{ Date } & \multirow[b]{2}{*}{ Time } & \multicolumn{3}{|c|}{$\begin{array}{l}\text { Depth to Water (ft below top of } \\
\text { casing) at Indicated Well }\end{array}$} \\
\hline & & D2 & WP49 & SB65d & & & D2 & WP49 & SB65d \\
\hline $7 / 5 / 08$ & $4: 00$ & 19.526 & 30.673 & 11.407 & $7 / 12 / 08$ & 8:00 & 19.809 & 30.855 & 11.65 \\
\hline $7 / 5 / 08$ & 8:00 & 19.517 & 30.684 & 11.428 & $7 / 12 / 08$ & $12: 00$ & 19.862 & 30.905 & 11.685 \\
\hline $7 / 5 / 08$ & $12: 00$ & 19.491 & 30.63 & 11.388 & $7 / 12 / 08$ & $16: 00$ & 19.86 & 30.844 & 11.603 \\
\hline $7 / 5 / 08$ & $16: 00$ & 19.445 & 30.551 & 11.32 & $7 / 12 / 08$ & $20: 00$ & 19.822 & 30.783 & 11.55 \\
\hline $7 / 5 / 08$ & $20: 00$ & 19.403 & 30.52 & 11.297 & 7/13/08 & 0:00 & 19.82 & 30.794 & 11.566 \\
\hline $7 / 6 / 08$ & $0: 00$ & 19.4 & 30.52 & 11.309 & $7 / 13 / 08$ & $4: 00$ & 19.816 & 30.78 & 11.55 \\
\hline $7 / 6 / 08$ & $4: 00$ & 19.382 & 30.499 & 11.288 & 7/13/08 & $8: 00$ & 19.822 & 30.794 & 11.566 \\
\hline $7 / 6 / 08$ & 8:00 & 19.365 & 30.49 & 11.294 & 7/13/08 & $12: 00$ & 19.839 & 30.801 & 11.566 \\
\hline $7 / 6 / 08$ & $12: 00$ & 19.403 & 30.525 & 11.325 & $7 / 13 / 08$ & $16: 00$ & 19.802 & 30.733 & 11.507 \\
\hline $7 / 6 / 08$ & $16: 00$ & 19.421 & 30.532 & 11.332 & 7/13/08 & $20: 00$ & 19.75 & 30.675 & 11.472 \\
\hline $7 / 6 / 08$ & $20: 00$ & 19.428 & 30.539 & 11.351 & 7/14/08 & 0:00 & 19.743 & 30.684 & 11.486 \\
\hline $7 / 7 / 08$ & 0:00 & 19.449 & 30.56 & 11.369 & $7 / 14 / 08$ & $4: 00$ & 19.739 & 30.682 & 11.489 \\
\hline $7 / 7 / 08$ & $4: 00$ & 19.447 & 30.544 & 11.358 & $7 / 14 / 08$ & 8:00 & 19.757 & 30.705 & 11.512 \\
\hline $7 / 7 / 08$ & 8:00 & 19.442 & 30.537 & 11.351 & 7/14/08 & $12: 00$ & 19.767 & 30.696 & 11.498 \\
\hline $7 / 7 / 08$ & $12: 00$ & 19.449 & 30.532 & 11.341 & $7 / 14 / 08$ & $16: 00$ & 19.739 & 30.651 & 11.47 \\
\hline $7 / 7 / 08$ & $16: 00$ & 19.435 & 30.506 & 11.327 & $7 / 14 / 08$ & $20: 00$ & 19.713 & 30.623 & 11.454 \\
\hline $7 / 7 / 08$ & $20: 00$ & 19.419 & 30.501 & 11.337 & $7 / 15 / 08$ & 0:00 & 19.755 & 30.705 & 11.557 \\
\hline $7 / 8 / 08$ & 0:00 & 19.454 & 30.548 & 11.379 & $7 / 15 / 08$ & $4: 00$ & 19.755 & 30.691 & 11.526 \\
\hline $7 / 8 / 08$ & $4: 00$ & 19.48 & 30.558 & 11.372 & $7 / 15 / 08$ & 8:00 & 19.769 & 30.696 & 11.54 \\
\hline $7 / 8 / 08$ & 8:00 & 19.503 & 30.602 & 11.414 & $7 / 15 / 08$ & $12: 00$ & 19.832 & 30.769 & 11.585 \\
\hline $7 / 8 / 08$ & $12: 00$ & 19.547 & 30.635 & 11.437 & $7 / 15 / 08$ & $16: 00$ & 19.846 & 30.762 & 11.571 \\
\hline $7 / 8 / 08$ & $16: 00$ & 19.552 & 30.616 & 11.407 & $7 / 15 / 08$ & $20: 00$ & 19.836 & 30.743 & 11.55 \\
\hline $7 / 8 / 08$ & $20: 00$ & 19.573 & 30.656 & 11.454 & 7/16/08 & 0:00 & 19.146 & 30.933 & 11.713 \\
\hline $7 / 9 / 08$ & 0:00 & 19.627 & 30.719 & 11.505 & $7 / 16 / 08$ & $4: 00$ & 19.578 & 30.874 & 11.592 \\
\hline $7 / 9 / 08$ & $4: 00$ & 19.641 & 30.701 & 11.479 & $7 / 16 / 08$ & 8:00 & 19.783 & 30.729 & 11.407 \\
\hline $7 / 9 / 08$ & 8:00 & 19.678 & 30.75 & 11.526 & 7/16/08 & $12: 00$ & 19.813 & 30.776 & 11.463 \\
\hline $7 / 9 / 08$ & $12: 00$ & 19.699 & 30.759 & 11.522 & $7 / 16 / 08$ & $16: 00$ & 19.781 & 30.691 & 11.4 \\
\hline $7 / 9 / 08$ & $16: 00$ & 19.685 & 30.719 & 11.477 & $7 / 16 / 08$ & $20: 00$ & 19.718 & 30.602 & 11.346 \\
\hline $7 / 9 / 08$ & $20: 00$ & 19.645 & 30.673 & 11.454 & $7 / 17 / 08$ & 0:00 & 19.727 & 30.621 & 11.381 \\
\hline $7 / 10 / 08$ & $0: 00$ & 19.662 & 30.708 & 11.482 & $7 / 17 / 08$ & $4: 00$ & 19.711 & 30.583 & 11.362 \\
\hline $7 / 10 / 08$ & $4: 00$ & 19.638 & 30.656 & 11.433 & $7 / 17 / 08$ & 8:00 & 19.692 & 30.555 & 11.36 \\
\hline $7 / 10 / 08$ & 8:00 & 19.657 & 30.691 & 11.463 & 7/17/08 & $12: 00$ & 19.692 & 30.546 & 11.358 \\
\hline $7 / 10 / 08$ & $12: 00$ & 19.636 & 30.649 & 11.426 & 7/17/08 & $16: 00$ & 19.659 & 30.492 & 11.313 \\
\hline $7 / 10 / 08$ & $16: 00$ & 19.603 & 30.593 & 11.386 & $7 / 17 / 08$ & $20: 00$ & 19.573 & 30.48 & 11.255 \\
\hline $7 / 10 / 08$ & $20: 00$ & 19.566 & 30.555 & 11.362 & 7/18/08 & 0:00 & 19.589 & 30.487 & 11.245 \\
\hline $7 / 11 / 08$ & 0:00 & 19.568 & 30.576 & 11.395 & $7 / 18 / 08$ & $4: 00$ & 19.571 & 30.459 & 11.21 \\
\hline $7 / 11 / 08$ & $4: 00$ & 19.568 & 30.569 & 11.381 & 7/18/08 & $8: 00$ & 19.547 & 30.443 & 11.213 \\
\hline $7 / 11 / 08$ & 8:00 & 19.552 & 30.541 & 11.36 & 7/18/08 & $12: 00$ & 19.54 & 30.433 & 11.213 \\
\hline $7 / 11 / 08$ & $12: 00$ & 19.536 & 30.515 & 11.339 & 7/18/08 & $16: 00$ & 19.515 & 30.394 & 11.182 \\
\hline $7 / 11 / 08$ & $16: 00$ & 19.501 & 30.464 & 11.299 & 7/18/08 & 20:00 & 19.463 & 30.344 & 11.164 \\
\hline $7 / 11 / 08$ & $20: 00$ & 19.489 & 30.464 & 11.325 & 7/19/08 & 0:00 & 19.482 & 30.391 & 11.213 \\
\hline $7 / 12 / 08$ & $0: 00$ & 19.601 & 30.687 & 11.522 & 7/19/08 & $4: 00$ & 19.461 & 30.351 & 11.182 \\
\hline 7/12/08 & $4: 00$ & 19.673 & 30.719 & 11.547 & 7/19/08 & 8:00 & 19.456 & 30.368 & 11.215 \\
\hline
\end{tabular}


Table S1.2 (Cont.)

\begin{tabular}{|c|c|c|c|c|c|c|c|c|c|}
\hline \multirow[b]{2}{*}{ Date } & \multirow[b]{2}{*}{ Time } & \multicolumn{3}{|c|}{$\begin{array}{l}\text { Depth to Water (ft below top of } \\
\text { casing) at Indicated Well }\end{array}$} & \multirow[b]{2}{*}{ Date } & \multirow[b]{2}{*}{ Time } & \multicolumn{3}{|c|}{$\begin{array}{l}\text { Depth to Water (ft below top of } \\
\text { casing) at Indicated Well }\end{array}$} \\
\hline & & D2 & WP49 & SB65d & & & D2 & WP49 & SB65d \\
\hline $7 / 19 / 08$ & $12: 00$ & 19.461 & 30.354 & 11.199 & $7 / 26 / 08$ & $16: 00$ & 19.767 & 30.661 & 11.482 \\
\hline 7/19/08 & $16: 00$ & 19.407 & 30.276 & 11.14 & $7 / 26 / 08$ & $20: 00$ & 19.715 & 30.607 & 11.442 \\
\hline $7 / 19 / 08$ & $20: 00$ & 19.379 & 30.269 & 11.147 & $7 / 27 / 08$ & $0: 00$ & 19.699 & 30.605 & 11.449 \\
\hline $7 / 20 / 08$ & 0:00 & 19.403 & 30.326 & 11.213 & $7 / 27 / 08$ & $4: 00$ & 19.669 & 30.572 & 11.419 \\
\hline $7 / 20 / 08$ & $4: 00$ & 19.435 & 30.363 & 11.241 & $7 / 27 / 08$ & 8:00 & 19.662 & 30.567 & 11.428 \\
\hline $7 / 20 / 08$ & 8:00 & 19.466 & 30.398 & 11.274 & $7 / 27 / 08$ & $12: 00$ & 19.655 & 30.555 & 11.414 \\
\hline $7 / 20 / 08$ & $12: 00$ & 19.51 & 30.447 & 11.327 & $7 / 27 / 08$ & $16: 00$ & 19.608 & 30.487 & 11.365 \\
\hline $7 / 20 / 08$ & $16: 00$ & 19.512 & 30.424 & 11.29 & $7 / 27 / 08$ & $20: 00$ & 19.552 & 30.433 & 11.327 \\
\hline $7 / 20 / 08$ & $20: 00$ & 19.477 & 30.379 & 11.253 & $7 / 28 / 08$ & 0:00 & 19.568 & 30.487 & 11.372 \\
\hline $7 / 21 / 08$ & 0:00 & 19.468 & 30.394 & 11.267 & $7 / 28 / 08$ & $4: 00$ & 19.561 & 30.485 & 11.381 \\
\hline $7 / 21 / 08$ & $4: 00$ & 19.515 & 30.454 & 11.318 & $7 / 28 / 08$ & $8: 00$ & 19.641 & 30.593 & 11.482 \\
\hline $7 / 21 / 08$ & 8:00 & 19.489 & 30.422 & 11.313 & $7 / 28 / 08$ & $12: 00$ & 19.671 & 30.583 & 11.465 \\
\hline $7 / 21 / 08$ & $12: 00$ & 19.51 & 30.433 & 11.311 & $7 / 28 / 08$ & $16: 00$ & 19.671 & 30.583 & 11.47 \\
\hline $7 / 21 / 08$ & $16: 00$ & 19.512 & 30.426 & 11.304 & 7/28/08 & $20: 00$ & 19.664 & 30.567 & 11.454 \\
\hline $7 / 21 / 08$ & $20: 00$ & 19.494 & 30.417 & 11.304 & $7 / 29 / 08$ & $0: 00$ & 19.725 & 30.673 & 11.543 \\
\hline $7 / 22 / 08$ & 0:00 & 19.517 & 30.447 & 11.337 & $7 / 29 / 08$ & $4: 00$ & 19.739 & 30.675 & 11.543 \\
\hline $7 / 22 / 08$ & $4: 00$ & 19.545 & 30.485 & 11.369 & $7 / 29 / 08$ & 8:00 & 19.732 & 30.661 & 11.536 \\
\hline $7 / 22 / 08$ & $8: 00$ & 19.568 & 30.508 & 11.383 & $7 / 29 / 08$ & $12: 00$ & 19.79 & 30.731 & 11.604 \\
\hline $7 / 22 / 08$ & $12: 00$ & 19.599 & 30.534 & 11.402 & $7 / 29 / 08$ & $16: 00$ & 19.802 & 30.712 & 11.573 \\
\hline $7 / 22 / 08$ & $16: 00$ & 19.61 & 30.53 & 11.4 & $7 / 29 / 08$ & $20: 00$ & 19.785 & 30.698 & 11.559 \\
\hline $7 / 22 / 08$ & $20: 00$ & 19.596 & 30.52 & 11.393 & $7 / 30 / 08$ & 0:00 & 19.809 & 30.729 & 11.578 \\
\hline $7 / 23 / 08$ & 0:00 & 19.638 & 30.583 & 11.454 & $7 / 30 / 08$ & $4: 00$ & 19.806 & 30.724 & 11.578 \\
\hline $7 / 23 / 08$ & $4: 00$ & 19.655 & 30.59 & 11.449 & $7 / 30 / 08$ & $8: 00$ & 19.818 & 30.738 & 11.589 \\
\hline $7 / 23 / 08$ & 8:00 & 19.669 & 30.602 & 11.461 & $7 / 30 / 08$ & $12: 00$ & 19.825 & 30.724 & 11.582 \\
\hline $7 / 23 / 08$ & $12: 00$ & 19.666 & 30.583 & 11.442 & $7 / 30 / 08$ & $16: 00$ & 19.816 & 30.698 & 11.557 \\
\hline $7 / 23 / 08$ & $16: 00$ & 19.641 & 30.544 & 11.407 & 7/30/08 & $20: 00$ & 19.774 & 30.654 & 11.529 \\
\hline $7 / 23 / 08$ & $20: 00$ & 19.62 & 30.525 & 11.4 & $7 / 31 / 08$ & $0: 00$ & 19.809 & 30.717 & 11.592 \\
\hline $7 / 24 / 08$ & 0:00 & 19.606 & 30.506 & 11.383 & 7/31/08 & $4: 00$ & 19.802 & 30.696 & 11.561 \\
\hline $7 / 24 / 08$ & $4: 00$ & 19.587 & 30.499 & 11.379 & $7 / 31 / 08$ & 8:00 & 19.816 & 30.717 & 11.585 \\
\hline $7 / 24 / 08$ & 8:00 & 19.592 & 30.504 & 11.383 & $7 / 31 / 08$ & $12: 00$ & 19.846 & 30.745 & 11.587 \\
\hline $7 / 24 / 08$ & $12: 00$ & 19.589 & 30.492 & 11.372 & $7 / 31 / 08$ & $16: 00$ & 19.811 & 30.689 & 11.561 \\
\hline $7 / 24 / 08$ & $16: 00$ & 19.736 & 30.473 & 11.355 & 7/31/08 & $20: 00$ & 19.816 & 30.712 & 11.587 \\
\hline $7 / 24 / 08$ & $20: 00$ & 19.594 & 30.48 & 11.374 & 8/1/08 & 0:00 & 19.874 & 30.799 & 11.66 \\
\hline $7 / 25 / 08$ & 0:00 & 19.599 & 30.522 & 11.412 & 8/1/08 & $4: 00$ & 19.906 & 30.825 & 11.681 \\
\hline $7 / 25 / 08$ & $4: 00$ & 19.599 & 30.511 & 11.395 & 8/1/08 & 8:00 & 19.962 & 30.9 & 11.751 \\
\hline $7 / 25 / 08$ & 8:00 & 19.634 & 30.574 & 11.465 & 8/1/08 & $12: 00$ & 20.025 & 30.944 & 11.765 \\
\hline $7 / 25 / 08$ & $12: 00$ & 19.699 & 30.647 & 11.512 & 8/1/08 & $16: 00$ & 20.028 & 30.902 & 11.739 \\
\hline $7 / 25 / 08$ & $16: 00$ & 19.727 & 30.656 & 11.503 & $8 / 1 / 08$ & $20: 00$ & 20.009 & 30.891 & 11.725 \\
\hline $7 / 25 / 08$ & $20: 00$ & 19.722 & 30.644 & 11.498 & $8 / 2 / 08$ & 0:00 & 20.037 & 30.942 & 11.772 \\
\hline $7 / 26 / 08$ & 0:00 & 19.76 & 30.712 & 11.55 & $8 / 2 / 08$ & $4: 00$ & 20.049 & 30.944 & 11.763 \\
\hline $7 / 26 / 08$ & $4: 00$ & 19.776 & 30.71 & 11.543 & $8 / 2 / 08$ & $8: 00$ & 20.044 & 30.926 & 11.739 \\
\hline $7 / 26 / 08$ & 8:00 & 19.788 & 30.726 & 11.554 & 8/2/08 & $12: 00$ & 20.03 & 30.893 & 11.711 \\
\hline $7 / 26 / 08$ & $12: 00$ & 19.816 & 30.764 & 11.582 & $8 / 2 / 08$ & $16: 00$ & 19.983 & 30.839 & 11.653 \\
\hline
\end{tabular}


Table S1.2 (Cont.)

\begin{tabular}{|c|c|c|c|c|c|c|c|c|c|}
\hline \multirow[b]{2}{*}{ Date } & \multirow[b]{2}{*}{ Time } & \multicolumn{3}{|c|}{$\begin{array}{l}\text { Depth to Water (ft below top of } \\
\text { casing) at Indicated Well }\end{array}$} & \multirow[b]{2}{*}{ Date } & \multirow[b]{2}{*}{ Time } & \multicolumn{3}{|c|}{$\begin{array}{l}\text { Depth to Water (ft below top of } \\
\text { casing) at Indicated Well }\end{array}$} \\
\hline & & D2 & WP49 & SB65d & & & D2 & WP49 & SB65d \\
\hline $8 / 2 / 08$ & $20: 00$ & 19.944 & 30.825 & 11.643 & 8/10/08 & $0: 00$ & 20.436 & 31.263 & 12.057 \\
\hline 8/3/08 & 0:00 & 19.965 & 30.881 & 11.697 & 8/10/08 & $4: 00$ & 20.452 & 31.266 & 12.048 \\
\hline $8 / 3 / 08$ & $4: 00$ & 19.986 & 30.898 & 11.711 & 8/10/08 & $8: 00$ & 20.482 & 31.32 & 12.102 \\
\hline 8/3/08 & 8:00 & 20.007 & 30.912 & 11.732 & 8/10/08 & $12: 00$ & 20.515 & 31.331 & 12.102 \\
\hline 8/3/08 & $12: 00$ & 20.035 & 30.923 & 11.737 & 8/10/08 & $16: 00$ & 20.503 & 31.301 & 12.071 \\
\hline 8/3/08 & $16: 00$ & 20.03 & 30.902 & 11.728 & 8/10/08 & $20: 00$ & 20.489 & 31.291 & 12.067 \\
\hline $8 / 3 / 08$ & $20: 00$ & 20.014 & 30.888 & 11.723 & $8 / 11 / 08$ & $0: 00$ & 20.543 & 31.369 & 12.135 \\
\hline 8/4/08 & 0:00 & 20.039 & 30.933 & 11.772 & 8/11/08 & $4: 00$ & 20.541 & 31.348 & 12.102 \\
\hline 8/4/08 & $4: 00$ & 20.065 & 30.949 & 11.781 & 8/11/08 & 8:00 & 20.555 & 31.367 & 12.13 \\
\hline 8/4/08 & $8: 00$ & 20.098 & 30.991 & 11.83 & 8/11/08 & $12: 00$ & 20.566 & 31.367 & 12.121 \\
\hline 8/4/08 & $12: 00$ & 20.13 & 31.022 & 11.852 & 8/11/08 & $16: 00$ & 20.536 & 31.313 & 12.069 \\
\hline $8 / 4 / 08$ & $16: 00$ & 20.151 & 31.034 & 11.854 & 8/11/08 & $20: 00$ & 20.492 & 31.268 & 12.041 \\
\hline 8/4/08 & $20: 00$ & 20.156 & 31.031 & 11.863 & 8/12/08 & $0: 00$ & 20.499 & 31.294 & 12.083 \\
\hline 8/5/08 & 0:00 & 20.21 & 31.102 & 11.926 & 8/12/08 & $4: 00$ & 20.471 & 31.266 & 12.06 \\
\hline 8/5/08 & $4: 00$ & 20.268 & 31.172 & 11.968 & $8 / 12 / 08$ & $8: 00$ & 20.503 & 31.301 & 12.085 \\
\hline 8/5/08 & 8:00 & 20.319 & 31.219 & 11.99 & 8/12/08 & $12: 00$ & 20.543 & 31.345 & 12.118 \\
\hline 8/5/08 & $12: 00$ & 20.373 & 31.282 & 12.053 & 8/12/08 & $16: 00$ & 20.538 & 31.315 & 12.083 \\
\hline 8/5/08 & $16: 00$ & 20.403 & 31.277 & 12.036 & 8/12/08 & $20: 00$ & 20.522 & 31.303 & 12.074 \\
\hline 8/5/08 & $20: 00$ & 20.403 & 31.266 & 12.015 & 8/13/08 & $0: 00$ & 20.548 & 31.345 & 12.125 \\
\hline 8/6/08 & 0:00 & 20.412 & 31.299 & 12.05 & 8/13/08 & $4: 00$ & 20.576 & 31.369 & 12.125 \\
\hline 8/6/08 & $4: 00$ & 20.431 & 31.301 & 12.041 & 8/13/08 & 8:00 & 20.594 & 31.39 & 12.151 \\
\hline 8/6/08 & 8:00 & 20.499 & 31.327 & 12.067 & 8/13/08 & $12: 00$ & 20.62 & 31.404 & 12.149 \\
\hline 8/6/08 & $12: 00$ & 20.517 & 31.352 & 12.067 & 8/13/08 & $16: 00$ & 20.615 & 31.385 & 12.132 \\
\hline 8/6/08 & $16: 00$ & 20.559 & 31.287 & 12.018 & 8/13/08 & $20: 00$ & 20.604 & 31.383 & 12.142 \\
\hline 8/6/08 & $20: 00$ & 20.475 & 31.277 & 12.02 & 8/14/08 & $0: 00$ & 20.653 & 31.463 & 12.238 \\
\hline 8/7/08 & $0: 00$ & 20.436 & 31.322 & 12.055 & 8/14/08 & $4: 00$ & 20.636 & 31.427 & 12.184 \\
\hline $8 / 7 / 08$ & $4: 00$ & 20.583 & 31.303 & 12.032 & $8 / 14 / 08$ & 8:00 & 20.681 & 31.481 & 12.217 \\
\hline 8/7/08 & 8:00 & 20.592 & 31.331 & 12.064 & 8/14/08 & $12: 00$ & 20.723 & 31.519 & 12.24 \\
\hline 8/7/08 & $12: 00$ & 20.69 & 31.355 & 12.071 & 8/14/08 & $16: 00$ & 20.737 & 31.521 & 12.242 \\
\hline 8/7/08 & $16: 00$ & 20.562 & 31.317 & 12.029 & 8/14/08 & $20: 00$ & 20.758 & 31.549 & 12.27 \\
\hline 8/7/08 & $20: 00$ & 20.452 & 31.28 & 12.008 & 8/15/08 & $0: 00$ & 20.823 & 31.641 & 12.341 \\
\hline 8/8/08 & $0: 00$ & 20.461 & 31.313 & 12.041 & $8 / 15 / 08$ & $4: 00$ & 20.865 & 31.667 & 12.352 \\
\hline 8/8/08 & $4: 00$ & 20.471 & 31.32 & 12.048 & 8/15/08 & 8:00 & 20.902 & 31.711 & 12.39 \\
\hline 8/8/08 & 8:00 & 20.485 & 31.329 & 12.064 & 8/15/08 & $12: 00$ & 20.975 & 31.786 & 12.444 \\
\hline 8/8/08 & $12: 00$ & 20.492 & 31.322 & 12.06 & 8/15/08 & $16: 00$ & 20.986 & 31.765 & 12.408 \\
\hline 8/8/08 & $16: 00$ & 20.447 & 31.245 & 11.99 & 8/15/08 & $20: 00$ & 20.986 & 31.779 & 12.434 \\
\hline 8/8/08 & $20: 00$ & 20.394 & 31.195 & 11.964 & 8/16/08 & $0: 00$ & 21.054 & 31.864 & 12.495 \\
\hline 8/9/08 & 0:00 & 20.387 & 31.2 & 11.973 & 8/16/08 & $4: 00$ & 21.072 & 31.864 & 12.483 \\
\hline 8/9/08 & $4: 00$ & 20.375 & 31.188 & 11.971 & 8/16/08 & $8: 00$ & 21.091 & 31.882 & 12.5 \\
\hline 8/9/08 & 8:00 & 20.38 & 31.2 & 11.987 & 8/16/08 & $12: 00$ & 21.117 & 31.899 & 12.5 \\
\hline 8/9/08 & $12: 00$ & 20.389 & 31.205 & 11.99 & 8/16/08 & $16: 00$ & 21.077 & 31.824 & 12.427 \\
\hline 8/9/08 & $16: 00$ & 20.399 & 31.205 & 11.997 & 8/16/08 & $20: 00$ & 21.03 & 31.784 & 12.401 \\
\hline 8/9/08 & $20: 00$ & 20.389 & 31.193 & 11.997 & 8/17/08 & $0: 00$ & 21.033 & 31.798 & 12.418 \\
\hline
\end{tabular}


Table S1.2 (Cont.)

\begin{tabular}{|c|c|c|c|c|c|c|c|c|c|}
\hline \multirow[b]{2}{*}{ Date } & \multirow[b]{2}{*}{ Time } & \multicolumn{3}{|c|}{$\begin{array}{l}\text { Depth to Water (ft below top of } \\
\text { casing) at Indicated Well }\end{array}$} & \multirow[b]{2}{*}{ Date } & \multirow[b]{2}{*}{ Time } & \multicolumn{3}{|c|}{$\begin{array}{l}\text { Depth to Water (ft below top of } \\
\text { casing) at Indicated Well }\end{array}$} \\
\hline & & D2 & WP49 & SB65d & & & D2 & WP49 & SB65d \\
\hline $8 / 17 / 08$ & $4: 00$ & 21.021 & 31.782 & 12.408 & 8/24/08 & 8:00 & 21.537 & 32.286 & 12.809 \\
\hline 8/17/08 & 8:00 & 21.03 & 31.798 & 12.425 & 8/24/08 & $12: 00$ & 21.551 & 32.281 & 12.797 \\
\hline 8/17/08 & $12: 00$ & 21.037 & 31.784 & 12.411 & 8/24/08 & $16: 00$ & 21.525 & 32.234 & 12.75 \\
\hline $8 / 17 / 08$ & $16: 00$ & 20.995 & 31.723 & 12.355 & 8/24/08 & $20: 00$ & 21.495 & 32.206 & 12.729 \\
\hline 8/17/08 & $20: 00$ & 20.949 & 31.69 & 12.341 & 8/25/08 & 0:00 & 21.499 & 32.232 & 12.759 \\
\hline 8/18/08 & $0: 00$ & 20.961 & 31.721 & 12.373 & 8/25/08 & $4: 00$ & 21.504 & 32.229 & 12.759 \\
\hline 8/18/08 & $4: 00$ & 20.968 & 31.723 & 12.378 & $8 / 25 / 08$ & 8:00 & 21.516 & 32.236 & 12.769 \\
\hline 8/18/08 & $8: 00$ & 20.979 & 31.744 & 12.397 & 8/25/08 & $12: 00$ & 21.537 & 32.255 & 12.78 \\
\hline 8/18/08 & $12: 00$ & 21.005 & 31.763 & 12.418 & 8/25/08 & $16: 00$ & 21.485 & 32.166 & 12.71 \\
\hline 8/18/08 & $16: 00$ & 21.007 & 31.744 & 12.401 & 8/25/08 & $20: 00$ & 21.455 & 32.143 & 12.696 \\
\hline 8/18/08 & $20: 00$ & 20.995 & 31.744 & 12.404 & 8/26/08 & 0:00 & 21.46 & 32.164 & 12.729 \\
\hline 8/19/08 & 0:00 & 21.021 & 31.786 & 12.444 & 8/26/08 & $4: 00$ & 21.457 & 32.157 & 12.715 \\
\hline 8/19/08 & $4: 00$ & 21.042 & 31.803 & 12.46 & 8/26/08 & 8:00 & 21.467 & 32.171 & 12.736 \\
\hline 8/19/08 & 8:00 & 21.079 & 31.852 & 12.495 & 8/26/08 & $12: 00$ & 21.481 & 32.175 & 12.738 \\
\hline 8/19/08 & $12: 00$ & 21.114 & 31.871 & 12.509 & 8/26/08 & $16: 00$ & 21.432 & 32.086 & 12.656 \\
\hline 8/19/08 & $16: 00$ & 21.11 & 31.842 & 12.474 & 8/26/08 & $20: 00$ & 21.401 & 32.072 & 12.656 \\
\hline 8/19/08 & $20: 00$ & 21.089 & 31.828 & 12.472 & 8/27/08 & 0:00 & 21.432 & 32.119 & 12.699 \\
\hline 8/20/08 & 0:00 & 21.112 & 31.868 & 12.504 & 8/27/08 & $4: 00$ & 21.432 & 32.117 & 12.696 \\
\hline 8/20/08 & $4: 00$ & 21.119 & 31.873 & 12.509 & 8/27/08 & 8:00 & 21.443 & 32.18 & 12.771 \\
\hline 8/20/08 & 8:00 & 21.145 & 31.901 & 12.532 & 8/27/08 & $12: 00$ & 21.299 & 32.189 & 12.743 \\
\hline 8/20/08 & $12: 00$ & 21.159 & 31.894 & 12.518 & 8/27/08 & $16: 00$ & 21.425 & 32.098 & 12.666 \\
\hline 8/20/08 & $16: 00$ & 21.121 & 31.828 & 12.465 & 8/27/08 & $20: 00$ & 21.369 & 32.03 & 12.614 \\
\hline 8/20/08 & $20: 00$ & 21.1 & 31.821 & 12.469 & 8/28/08 & 0:00 & 21.352 & 32.018 & 12.612 \\
\hline 8/21/08 & 0:00 & 21.107 & 31.838 & 12.49 & 8/28/08 & $4: 00$ & 21.341 & 32.023 & 12.64 \\
\hline 8/21/08 & $4: 00$ & 21.103 & 31.826 & 12.472 & 8/28/08 & 8:00 & 21.383 & 32.075 & 12.678 \\
\hline 8/21/08 & 8:00 & 21.096 & 31.819 & 12.469 & 8/28/08 & $12: 00$ & 21.485 & 32.196 & 12.78 \\
\hline 8/21/08 & $12: 00$ & 21.096 & 31.819 & 12.469 & 8/28/08 & $16: 00$ & 21.544 & 32.246 & 12.811 \\
\hline 8/21/08 & $16: 00$ & 21.072 & 31.772 & 12.427 & 8/28/08 & $20: 00$ & 21.576 & 32.267 & 12.823 \\
\hline 8/21/08 & $20: 00$ & 21.035 & 31.744 & 12.404 & 8/29/08 & 0:00 & 21.674 & 32.398 & 12.935 \\
\hline $8 / 22 / 08$ & 0:00 & 21.056 & 31.789 & 12.441 & 8/29/08 & $4: 00$ & 21.725 & 32.44 & 12.949 \\
\hline $8 / 22 / 08$ & $4: 00$ & 21.072 & 31.803 & 12.46 & 8/29/08 & 8:00 & 21.76 & 32.468 & 12.968 \\
\hline $8 / 22 / 08$ & 8:00 & 21.089 & 31.826 & 12.488 & 8/29/08 & $12: 00$ & 21.814 & 32.518 & 12.991 \\
\hline $8 / 22 / 08$ & $12: 00$ & 21.126 & 31.861 & 12.507 & 8/29/08 & $16: 00$ & 21.812 & 32.49 & 12.954 \\
\hline $8 / 22 / 08$ & $16: 00$ & 21.147 & 31.868 & 12.525 & 8/29/08 & $20: 00$ & 21.786 & 32.464 & 12.94 \\
\hline $8 / 22 / 08$ & $20: 00$ & 21.205 & 31.962 & 12.607 & 8/30/08 & 0:00 & 21.833 & 32.532 & 12.996 \\
\hline 8/23/08 & 0:00 & 21.306 & 32.096 & 12.706 & 8/30/08 & $4: 00$ & 21.84 & 32.527 & 12.984 \\
\hline 8/23/08 & $4: 00$ & 21.378 & 32.171 & 12.748 & 8/30/08 & 8:00 & 21.868 & 32.569 & 13.024 \\
\hline 8/23/08 & 8:00 & 21.467 & 32.267 & 12.827 & 8/30/08 & $12: 00$ & 21.893 & 32.569 & 13.005 \\
\hline $8 / 23 / 08$ & $12: 00$ & 21.516 & 32.269 & 12.802 & 8/30/08 & $16: 00$ & 21.861 & 32.511 & 12.951 \\
\hline 8/23/08 & $16: 00$ & 21.49 & 32.213 & 12.748 & 8/30/08 & 20:00 & 21.83 & 32.49 & 12.947 \\
\hline 8/23/08 & $20: 00$ & 21.469 & 32.201 & 12.743 & 8/31/08 & 0:00 & 21.847 & 32.525 & 12.975 \\
\hline $8 / 24 / 08$ & $0: 00$ & 21.499 & 32.243 & 12.776 & 8/31/08 & $4: 00$ & 21.849 & 32.52 & 12.97 \\
\hline $8 / 24 / 08$ & $4: 00$ & 21.492 & 32.232 & 12.757 & 8/31/08 & 8:00 & 21.851 & 32.525 & 12.975 \\
\hline
\end{tabular}


Table S1.2 (Cont.)

\begin{tabular}{|c|c|c|c|c|c|c|c|c|c|}
\hline \multirow[b]{2}{*}{ Date } & \multirow[b]{2}{*}{ Time } & \multicolumn{3}{|c|}{$\begin{array}{l}\text { Depth to Water (ft below top of } \\
\text { casing) at Indicated Well }\end{array}$} & \multirow[b]{2}{*}{ Date } & \multirow[b]{2}{*}{ Time } & \multicolumn{3}{|c|}{$\begin{array}{l}\text { Depth to Water (ft below top of } \\
\text { casing) at Indicated Well }\end{array}$} \\
\hline & & D2 & WP49 & SB65d & & & D2 & WP49 & SB65d \\
\hline 8/31/08 & $12: 00$ & 21.858 & 32.518 & 12.963 & 9/7/08 & $16: 00$ & 22.425 & 33.05 & 13.314 \\
\hline 8/31/08 & $16: 00$ & 21.812 & 32.45 & 12.904 & 9/7/08 & 20:00 & 22.43 & 33.066 & 13.34 \\
\hline 8/31/08 & $20: 00$ & 21.781 & 32.429 & 12.904 & $9 / 8 / 08$ & 0:00 & 22.476 & 33.118 & 13.379 \\
\hline 9/1/08 & 0:00 & 21.819 & 32.494 & 12.97 & 9/8/08 & $4: 00$ & 22.481 & 33.106 & 13.363 \\
\hline 9/1/08 & $4: 00$ & 21.835 & 32.499 & 12.97 & 9/8/08 & $8: 00$ & 22.521 & 33.177 & 13.447 \\
\hline 9/1/08 & 8:00 & 21.854 & 32.529 & 12.998 & 9/8/08 & $12: 00$ & 22.544 & 33.212 & 13.436 \\
\hline 9/1/08 & $12: 00$ & 21.872 & 32.522 & 12.984 & 9/8/08 & $16: 00$ & 22.549 & 33.186 & 13.398 \\
\hline 9/1/08 & $16: 00$ & 21.851 & 32.49 & 12.956 & 9/8/08 & $20: 00$ & 22.539 & 33.165 & 13.379 \\
\hline 9/1/08 & $20: 00$ & 21.83 & 32.471 & 12.944 & 9/9/08 & 0:00 & 22.565 & 33.195 & 13.41 \\
\hline $9 / 2 / 08$ & 0:00 & 21.861 & 32.529 & 13.01 & 9/9/08 & $4: 00$ & 22.563 & 33.183 & 13.393 \\
\hline $9 / 2 / 08$ & $4: 00$ & 21.879 & 32.534 & 13 & 9/9/08 & $8: 00$ & 22.57 & 33.195 & 13.407 \\
\hline $9 / 2 / 08$ & 8:00 & 21.942 & 32.654 & 13.127 & 9/9/08 & $12: 00$ & 22.572 & 33.184 & 13.384 \\
\hline $9 / 2 / 08$ & $12: 00$ & 22.066 & 32.785 & 13.22 & 9/9/08 & $16: 00$ & 22.518 & 33.099 & 13.309 \\
\hline $9 / 2 / 08$ & $16: 00$ & 22.166 & 32.832 & 13.216 & 9/9/08 & 20:00 & 22.474 & 33.057 & 13.291 \\
\hline $9 / 2 / 08$ & $20: 00$ & 22.164 & 32.844 & 13.225 & 9/10/08 & 0:00 & 22.483 & 33.071 & 13.3 \\
\hline $9 / 3 / 08$ & $0: 00$ & 22.22 & 32.912 & 13.274 & 9/10/08 & $4: 00$ & 22.46 & 33.045 & 13.291 \\
\hline $9 / 3 / 08$ & $4: 00$ & 22.248 & 32.928 & 13.279 & 9/10/08 & $8: 00$ & 22.465 & 33.062 & 13.316 \\
\hline $9 / 3 / 08$ & 8:00 & 22.269 & 32.949 & 13.283 & $9 / 10 / 08$ & $12: 00$ & 22.465 & 33.041 & 13.279 \\
\hline 9/3/08 & $12: 00$ & 22.266 & 32.921 & 13.251 & 9/10/08 & $16: 00$ & 22.418 & 32.982 & 13.239 \\
\hline $9 / 3 / 08$ & $16: 00$ & 22.206 & 32.827 & 13.152 & 9/10/08 & 20:00 & 22.404 & 32.984 & 13.265 \\
\hline 9/3/08 & $20: 00$ & 22.145 & 32.771 & 13.117 & 9/11/08 & 0:00 & 22.418 & 33.001 & 13.274 \\
\hline 9/4/08 & 0:00 & 22.129 & 32.762 & 13.12 & 9/11/08 & $4: 00$ & 22.406 & 32.973 & 13.26 \\
\hline 9/4/08 & $4: 00$ & 22.117 & 32.743 & 13.11 & 9/11/08 & 8:00 & 22.437 & 33.033 & 13.316 \\
\hline 9/4/08 & 8:00 & 22.092 & 32.719 & 13.096 & 9/11/08 & $12: 00$ & 22.465 & 33.083 & 13.344 \\
\hline $9 / 4 / 08$ & $12: 00$ & 22.087 & 32.712 & 13.082 & 9/11/08 & $16: 00$ & 22.474 & 33.066 & 13.319 \\
\hline $9 / 4 / 08$ & $16: 00$ & 22.05 & 32.658 & 13.04 & $9 / 11 / 08$ & $20: 00$ & 22.329 & 33.097 & 13.342 \\
\hline $9 / 4 / 08$ & $20: 00$ & 22.047 & 32.672 & 13.075 & 9/12/08 & 0:00 & 22.521 & 33.153 & 13.361 \\
\hline $9 / 5 / 08$ & $0: 00$ & 22.087 & 32.729 & 13.124 & 9/12/08 & $4: 00$ & 22.535 & 33.139 & 13.312 \\
\hline $9 / 5 / 08$ & $4: 00$ & 22.117 & 32.752 & 13.15 & $9 / 12 / 08$ & 8:00 & 22.53 & 33.123 & 13.298 \\
\hline 9/5/08 & 8:00 & 22.155 & 32.813 & 13.204 & 9/12/08 & $12: 00$ & 22.53 & 33.101 & 13.276 \\
\hline 9/5/08 & $12: 00$ & 22.215 & 32.876 & 13.241 & 9/12/08 & $16: 00$ & 22.439 & 33.005 & 13.181 \\
\hline 9/5/08 & $16: 00$ & 22.25 & 32.902 & 13.251 & 9/12/08 & $20: 00$ & 22.397 & 32.947 & 13.124 \\
\hline $9 / 5 / 08$ & $20: 00$ & 22.273 & 32.921 & 13.267 & 9/13/08 & 0:00 & 22.371 & 32.921 & 13.096 \\
\hline $9 / 6 / 08$ & $0: 00$ & 22.294 & 32.935 & 13.274 & 9/13/08 & $4: 00$ & 22.332 & 32.872 & 13.064 \\
\hline 9/6/08 & $4: 00$ & 22.297 & 32.923 & 13.248 & 9/13/08 & $8: 00$ & 22.332 & 32.883 & 13.085 \\
\hline $9 / 6 / 08$ & 8:00 & 22.292 & 32.94 & 13.272 & 9/13/08 & $12: 00$ & 22.355 & 32.905 & 13.103 \\
\hline $9 / 6 / 08$ & $12: 00$ & 22.35 & 32.998 & 13.305 & 9/13/08 & $16: 00$ & 22.346 & 32.876 & 13.089 \\
\hline $9 / 6 / 08$ & $16: 00$ & 22.315 & 32.94 & 13.241 & 9/13/08 & $20: 00$ & 22.381 & 32.937 & 13.157 \\
\hline 9/6/08 & $20: 00$ & 22.313 & 32.942 & 13.255 & 9/14/08 & 0:00 & 22.448 & 33.019 & 13.225 \\
\hline 9/7/08 & 0:00 & 22.341 & 33.008 & 13.309 & 9/14/08 & $4: 00$ & 22.488 & 33.045 & 13.241 \\
\hline 9/7/08 & $4: 00$ & 22.357 & 32.996 & 13.288 & $9 / 14 / 08$ & $8: 00$ & 22.558 & 33.127 & 13.323 \\
\hline 9/7/08 & $8: 00$ & 22.397 & 33.055 & 13.342 & 9/14/08 & $12: 00$ & 22.626 & 33.219 & 13.396 \\
\hline 9/7/08 & $12: 00$ & 22.434 & 33.083 & 13.351 & 9/14/08 & $16: 00$ & 22.71 & 33.291 & 13.44 \\
\hline
\end{tabular}


Table S1.2 (Cont.)

\begin{tabular}{|c|c|c|c|c|c|c|c|c|c|}
\hline \multirow[b]{2}{*}{ Date } & \multirow[b]{2}{*}{ Time } & \multicolumn{3}{|c|}{$\begin{array}{l}\text { Depth to Water (ft below top of } \\
\text { casing) at Indicated Well }\end{array}$} & \multirow[b]{2}{*}{ Date } & \multirow[b]{2}{*}{ Time } & \multicolumn{3}{|c|}{$\begin{array}{l}\text { Depth to Water (ft below top of } \\
\text { casing) at Indicated Well }\end{array}$} \\
\hline & & D2 & WP49 & SB65d & & & D2 & WP49 & SB65d \\
\hline $9 / 14 / 08$ & $20: 00$ & 22.756 & 33.345 & 13.489 & 9/22/08 & $0: 00$ & 23.262 & 33.805 & 13.754 \\
\hline 9/15/08 & 0:00 & 22.842 & 33.437 & 13.557 & 9/22/08 & $4: 00$ & 23.269 & 33.814 & 13.772 \\
\hline 9/15/08 & $4: 00$ & 22.889 & 33.477 & 13.574 & $9 / 22 / 08$ & $8: 00$ & 23.255 & 33.798 & 13.763 \\
\hline 9/15/08 & 8:00 & 22.933 & 33.528 & 13.609 & 9/22/08 & $12: 00$ & 23.274 & 33.812 & 13.765 \\
\hline 9/15/08 & $12: 00$ & 22.98 & 33.559 & 13.62 & 9/22/08 & $16: 00$ & 23.232 & 33.746 & 13.705 \\
\hline 9/15/08 & $16: 00$ & 22.936 & 33.479 & 13.536 & 9/22/08 & 20:00 & 23.227 & 33.758 & 13.73 \\
\hline 9/15/08 & $20: 00$ & 22.901 & 33.448 & 13.517 & 9/23/08 & 0:00 & 23.26 & 33.793 & 13.758 \\
\hline 9/16/08 & 0:00 & 22.922 & 33.486 & 13.557 & 9/23/08 & $4: 00$ & 23.283 & 33.814 & 13.78 \\
\hline 9/16/08 & $4: 00$ & 22.922 & 33.477 & 13.543 & 9/23/08 & $8: 00$ & 23.314 & 33.852 & 13.808 \\
\hline 9/16/08 & 8:00 & 22.922 & 33.486 & 13.557 & 9/23/08 & $12: 00$ & 23.335 & 33.87 & 13.817 \\
\hline 9/16/08 & $12: 00$ & 22.94 & 33.493 & 13.557 & 9/23/08 & $16: 00$ & 23.318 & 33.835 & 13.772 \\
\hline 9/16/08 & $16: 00$ & 22.898 & 33.425 & 13.492 & 9/23/08 & $20: 00$ & 23.339 & 33.882 & 13.836 \\
\hline 9/16/08 & $20: 00$ & 22.875 & 33.42 & 13.501 & 9/24/08 & 0:00 & 23.407 & 33.964 & 13.885 \\
\hline 9/17/08 & 0:00 & 22.905 & 33.467 & 13.548 & 9/24/08 & $4: 00$ & 23.453 & 34.006 & 13.906 \\
\hline 9/17/08 & $4: 00$ & 22.929 & 33.488 & 13.567 & 9/24/08 & $8: 00$ & 23.509 & 34.07 & 13.964 \\
\hline 9/17/08 & 8:00 & 22.952 & 33.519 & 13.597 & 9/24/08 & $12: 00$ & 23.561 & 34.114 & 13.983 \\
\hline 9/17/08 & $12: 00$ & 22.989 & 33.554 & 13.618 & 9/24/08 & $16: 00$ & 23.544 & 34.065 & 13.927 \\
\hline 9/17/08 & $16: 00$ & 22.975 & 33.512 & 13.569 & 9/24/08 & $20: 00$ & 23.526 & 34.051 & 13.915 \\
\hline 9/17/08 & $20: 00$ & 22.964 & 33.509 & 13.578 & 9/25/08 & 0:00 & 23.556 & 34.1 & 13.962 \\
\hline 9/18/08 & 0:00 & 22.996 & 33.561 & 13.625 & 9/25/08 & $4: 00$ & 23.558 & 34.089 & 13.939 \\
\hline 9/18/08 & $4: 00$ & 23.017 & 33.577 & 13.634 & 9/25/08 & 8:00 & 23.572 & 34.11 & 13.955 \\
\hline 9/18/08 & 8:00 & 23.031 & 33.596 & 13.651 & 9/25/08 & $12: 00$ & 23.577 & 34.095 & 13.939 \\
\hline 9/18/08 & $12: 00$ & 23.059 & 33.613 & 13.653 & 9/25/08 & $16: 00$ & 23.526 & 34.023 & 13.871 \\
\hline 9/18/08 & $16: 00$ & 23.034 & 33.561 & 13.599 & $9 / 25 / 08$ & $20: 00$ & 23.498 & 34.004 & 13.871 \\
\hline 9/18/08 & $20: 00$ & 23.008 & 33.545 & 13.597 & 9/26/08 & 0:00 & 23.509 & 34.025 & 13.889 \\
\hline 9/19/08 & $0: 00$ & 23.022 & 33.568 & 13.62 & 9/26/08 & $4: 00$ & 23.498 & 34.002 & 13.864 \\
\hline 9/19/08 & $4: 00$ & 23.036 & 33.575 & 13.627 & 9/26/08 & 8:00 & 23.5 & 34.014 & 13.889 \\
\hline 9/19/08 & 8:00 & 23.048 & 33.601 & 13.651 & 9/26/08 & $12: 00$ & 23.512 & 34.011 & 13.873 \\
\hline 9/19/08 & $12: 00$ & 23.064 & 33.603 & 13.644 & 9/26/08 & $16: 00$ & 23.463 & 33.948 & 13.819 \\
\hline 9/19/08 & $16: 00$ & 23.036 & 33.552 & 13.595 & 9/26/08 & $20: 00$ & 23.446 & 33.943 & 13.838 \\
\hline 9/19/08 & $20: 00$ & 23.02 & 33.552 & 13.602 & 9/27/08 & 0:00 & 23.477 & 33.99 & 13.88 \\
\hline 9/20/08 & 0:00 & 23.055 & 33.606 & 13.651 & $9 / 27 / 08$ & $4: 00$ & 23.495 & 34.004 & 13.894 \\
\hline 9/20/08 & $4: 00$ & 23.078 & 33.624 & 13.66 & 9/27/08 & 8:00 & 23.523 & 34.039 & 13.929 \\
\hline 9/20/08 & 8:00 & 23.108 & 33.667 & 13.702 & 9/27/08 & $12: 00$ & 23.582 & 34.103 & 13.971 \\
\hline 9/20/08 & $12: 00$ & 23.148 & 33.697 & 13.716 & 9/27/08 & $16: 00$ & 23.589 & 34.098 & 13.962 \\
\hline 9/20/08 & $16: 00$ & 23.127 & 33.648 & 13.67 & $9 / 27 / 08$ & $20: 00$ & 23.614 & 34.135 & 13.995 \\
\hline 9/20/08 & $20: 00$ & 23.39 & 33.725 & 13.691 & 9/28/08 & 0:00 & 23.661 & 34.187 & 14.032 \\
\hline 9/21/08 & 0:00 & 23.218 & 33.756 & 13.74 & 9/28/08 & $4: 00$ & 23.68 & 34.192 & 14.025 \\
\hline 9/21/08 & $4: 00$ & 23.218 & 33.76 & 13.747 & 9/28/08 & $8: 00$ & 23.698 & 34.217 & 14.049 \\
\hline 9/21/08 & 8:00 & 23.234 & 33.781 & 13.768 & 9/28/08 & $12: 00$ & 23.726 & 34.234 & 14.042 \\
\hline 9/21/08 & $12: 00$ & 23.248 & 33.788 & 13.768 & 9/28/08 & $16: 00$ & 23.682 & 34.168 & 13.985 \\
\hline 9/21/08 & $16: 00$ & 23.355 & 33.791 & 13.707 & 9/28/08 & $20: 00$ & 23.628 & 34.098 & 13.918 \\
\hline 9/21/08 & $20: 00$ & 23.244 & 33.758 & 13.707 & 9/29/08 & $0: 00$ & 23.605 & 34.079 & 13.955 \\
\hline
\end{tabular}


Table S1.2 (Cont.)

\begin{tabular}{|c|c|c|c|c|c|c|c|c|c|}
\hline \multirow[b]{2}{*}{ Date } & \multirow[b]{2}{*}{ Time } & \multicolumn{3}{|c|}{$\begin{array}{l}\text { Depth to Water (ft below top of } \\
\text { casing) at Indicated Well }\end{array}$} & \multirow[b]{2}{*}{ Date } & \multirow[b]{2}{*}{ Time } & \multicolumn{3}{|c|}{$\begin{array}{l}\text { Depth to Water (ft below top of } \\
\text { casing) at Indicated Well }\end{array}$} \\
\hline & & D2 & WP49 & SB65d & & & D2 & WP49 & SB65d \\
\hline 9/29/08 & $4: 00$ & 23.673 & 34.18 & 14.004 & 10/6/08 & 8:00 & 24.127 & 34.593 & 14.28 \\
\hline 9/29/08 & 8:00 & 23.75 & 34.292 & 14.114 & 10/6/08 & $12: 00$ & 24.146 & 34.6 & 14.273 \\
\hline 9/29/08 & $12: 00$ & 23.838 & 34.375 & 14.163 & 10/6/08 & $16: 00$ & 23.803 & 34.539 & 14.224 \\
\hline 9/29/08 & $16: 00$ & 23.838 & 34.344 & 14.123 & 10/6/08 & $20: 00$ & 24.057 & 34.571 & 14.217 \\
\hline $9 / 29 / 08$ & $20: 00$ & 23.843 & 34.351 & 14.123 & $10 / 7 / 08$ & $0: 00$ & 24.116 & 34.607 & 14.217 \\
\hline 9/30/08 & $0: 00$ & 23.862 & 34.374 & 14.142 & 10/7/08 & $4: 00$ & 24.048 & 34.67 & 14.247 \\
\hline 9/30/08 & $4: 00$ & 23.88 & 34.386 & 14.142 & 10/7/08 & $8: 00$ & 24.225 & 34.745 & 14.252 \\
\hline 9/30/08 & 8:00 & 23.901 & 34.414 & 14.163 & 10/7/08 & $12: 00$ & 24.274 & 34.764 & 14.24 \\
\hline 9/30/08 & $12: 00$ & 23.918 & 34.414 & 14.147 & 10/7/08 & $16: 00$ & 24.256 & 34.703 & 14.182 \\
\hline 9/30/08 & $16: 00$ & 23.862 & 34.328 & 14.067 & 10/7/08 & $20: 00$ & 24.244 & 34.691 & 14.184 \\
\hline 9/30/08 & $20: 00$ & 23.827 & 34.3 & 14.056 & 10/8/08 & 0:00 & 24.237 & 34.658 & 14.161 \\
\hline 10/1/08 & 0:00 & 23.822 & 34.297 & 14.06 & 10/8/08 & $4: 00$ & 24.214 & 34.628 & 14.137 \\
\hline 10/1/08 & $4: 00$ & 23.801 & 34.278 & 14.049 & 10/8/08 & $8: 00$ & 24.207 & 34.625 & 14.149 \\
\hline 10/1/08 & 8:00 & 23.848 & 34.361 & 14.135 & 10/8/08 & $12: 00$ & 24.186 & 34.579 & 14.114 \\
\hline 10/1/08 & $12: 00$ & 23.929 & 34.443 & 14.189 & 10/8/08 & $16: 00$ & 24.155 & 34.555 & 14.109 \\
\hline 10/1/08 & $16: 00$ & 23.931 & 34.414 & 14.154 & 10/8/08 & $20: 00$ & 24.197 & 34.632 & 14.201 \\
\hline 10/1/08 & $20: 00$ & 23.927 & 34.414 & 14.161 & 10/9/08 & $0: 00$ & 24.267 & 34.708 & 14.264 \\
\hline $10 / 2 / 08$ & 0:00 & 23.943 & 34.426 & 14.161 & 10/9/08 & $4: 00$ & 24.316 & 34.747 & 14.278 \\
\hline $10 / 2 / 08$ & $4: 00$ & 23.941 & 34.417 & 14.149 & 10/9/08 & 8:00 & 24.375 & 34.818 & 14.346 \\
\hline $10 / 2 / 08$ & $8: 00$ & 23.936 & 34.419 & 14.152 & $10 / 9 / 08$ & $12: 00$ & 24.403 & 34.827 & 14.336 \\
\hline $10 / 2 / 08$ & $12: 00$ & 23.936 & 34.405 & 14.13 & 10/9/08 & $16: 00$ & 24.34 & 34.729 & 14.24 \\
\hline $10 / 2 / 08$ & $16: 00$ & 23.869 & 34.297 & 14.037 & 10/9/08 & $20: 00$ & 24.293 & 34.686 & 14.222 \\
\hline $10 / 2 / 08$ & $20: 00$ & 23.829 & 34.278 & 14.037 & 10/10/08 & 0:00 & 24.277 & 34.677 & 14.219 \\
\hline 10/3/08 & 0:00 & 23.843 & 34.309 & 14.072 & 10/10/08 & $4: 00$ & 24.272 & 34.665 & 14.21 \\
\hline 10/3/08 & $4: 00$ & 23.859 & 34.325 & 14.095 & 10/10/08 & $8: 00$ & 24.272 & 34.672 & 14.231 \\
\hline 10/3/08 & 8:00 & 23.894 & 34.379 & 14.142 & $10 / 10 / 08$ & $12: 00$ & 24.272 & 34.656 & 14.215 \\
\hline 10/3/08 & $12: 00$ & 23.957 & 34.44 & 14.177 & 10/10/08 & $16: 00$ & 24.228 & 34.6 & 14.177 \\
\hline 10/3/08 & $16: 00$ & 23.955 & 34.414 & 14.154 & 10/10/08 & $20: 00$ & 24.244 & 34.656 & 14.24 \\
\hline $10 / 3 / 08$ & $20: 00$ & 23.962 & 34.433 & 14.18 & $10 / 11 / 08$ & $0: 00$ & 24.33 & 34.757 & 14.322 \\
\hline $10 / 4 / 08$ & $0: 00$ & 24.015 & 34.506 & 14.24 & $10 / 11 / 08$ & $4: 00$ & 24.372 & 34.79 & 14.346 \\
\hline $10 / 4 / 08$ & $4: 00$ & 24.053 & 34.529 & 14.245 & 10/11/08 & $8: 00$ & 24.419 & 34.85 & 14.4 \\
\hline $10 / 4 / 08$ & 8:00 & 24.055 & 34.532 & 14.245 & $10 / 11 / 08$ & $12: 00$ & 24.472 & 34.904 & 14.418 \\
\hline $10 / 4 / 08$ & $12: 00$ & 24.076 & 34.536 & 14.226 & $10 / 11 / 08$ & $16: 00$ & 24.47 & 34.897 & 14.397 \\
\hline $10 / 4 / 08$ & $16: 00$ & 24.006 & 34.435 & 14.14 & 10/11/08 & $20: 00$ & 24.484 & 34.921 & 14.414 \\
\hline $10 / 4 / 08$ & $20: 00$ & 23.976 & 34.417 & 14.14 & $10 / 12 / 08$ & $0: 00$ & 24.508 & 34.93 & 14.414 \\
\hline 10/5/08 & 0:00 & 24.004 & 34.464 & 14.182 & 10/12/08 & $4: 00$ & 24.507 & 34.937 & 14.43 \\
\hline 10/5/08 & $4: 00$ & 24.004 & 34.45 & 14.156 & 10/12/08 & $8: 00$ & 24.526 & 34.956 & 14.439 \\
\hline $10 / 5 / 08$ & $8: 00$ & 24.013 & 34.473 & 14.187 & $10 / 12 / 08$ & $12: 00$ & 24.549 & 34.963 & 14.437 \\
\hline 10/5/08 & $12: 00$ & 24.036 & 34.494 & 14.198 & $10 / 12 / 08$ & $16: 00$ & 24.517 & 34.895 & 14.371 \\
\hline 10/5/08 & $16: 00$ & 24.015 & 34.457 & 14.175 & 10/12/08 & $20: 00$ & 24.528 & 34.951 & 14.439 \\
\hline $10 / 5 / 08$ & $20: 00$ & 24.039 & 34.496 & 14.215 & $10 / 13 / 08$ & $0: 00$ & 24.57 & 34.977 & 14.446 \\
\hline $10 / 6 / 08$ & $0: 00$ & 24.078 & 34.548 & 14.259 & $10 / 13 / 08$ & $4: 00$ & 24.577 & 35.008 & 14.484 \\
\hline 10/6/08 & $4: 00$ & 24.102 & 34.557 & 14.252 & 10/13/08 & 8:00 & 24.442 & 35.08 & 14.54 \\
\hline
\end{tabular}


Table S1.2 (Cont.)

\begin{tabular}{|c|c|c|c|c|c|c|c|c|c|}
\hline \multirow[b]{2}{*}{ Date } & \multirow[b]{2}{*}{ Time } & \multicolumn{3}{|c|}{$\begin{array}{l}\text { Depth to Water (ft below top of } \\
\text { casing) at Indicated Well }\end{array}$} & \multirow[b]{2}{*}{ Date } & \multirow[b]{2}{*}{ Time } & \multicolumn{3}{|c|}{$\begin{array}{l}\text { Depth to Water (ft below top of } \\
\text { casing) at Indicated Well }\end{array}$} \\
\hline & & D2 & WP49 & SB65d & & & D2 & WP49 & SB65d \\
\hline 10/13/08 & $12: 00$ & 24.661 & 35.19 & 14.491 & $10 / 20 / 08$ & $16: 00$ & 24.743 & 35.111 & 14.217 \\
\hline 10/13/08 & $16: 00$ & 24.694 & 35.181 & 14.378 & 10/20/08 & $20: 00$ & 24.759 & 35.139 & 14.25 \\
\hline $10 / 13 / 08$ & $20: 00$ & 24.703 & 35.174 & 14.374 & 10/21/08 & $0: 00$ & 24.78 & 35.158 & 14.245 \\
\hline 10/14/08 & 0:00 & 24.745 & 35.198 & 14.383 & $10 / 21 / 08$ & $4: 00$ & 24.787 & 35.155 & 14.24 \\
\hline $10 / 14 / 08$ & $4: 00$ & 24.738 & 35.158 & 14.341 & $10 / 21 / 08$ & 8:00 & 24.771 & 35.125 & 14.212 \\
\hline $10 / 14 / 08$ & 8:00 & 24.736 & 35.165 & 14.353 & $10 / 21 / 08$ & $12: 00$ & 24.738 & 35.066 & 14.177 \\
\hline 10/14/08 & $12: 00$ & 24.729 & 35.122 & 14.315 & 10/21/08 & $16: 00$ & 24.636 & 34.942 & 14.051 \\
\hline 10/14/08 & $16: 00$ & 24.654 & 35.005 & 14.233 & 10/21/08 & 20:00 & 24.573 & 34.904 & 14.042 \\
\hline 10/14/08 & $20: 00$ & 24.5 & 34.963 & 14.224 & 10/22/08 & 0:00 & 24.549 & 34.881 & 14.018 \\
\hline 10/15/08 & 0:00 & 24.556 & 34.921 & 14.128 & 10/22/08 & $4: 00$ & 24.44 & 34.808 & 13.925 \\
\hline 10/15/08 & $4: 00$ & 24.323 & 34.867 & 14.039 & 10/22/08 & $8: 00$ & 24.307 & 34.675 & 13.576 \\
\hline 10/15/08 & 8:00 & 24.466 & 34.874 & 13.922 & $10 / 22 / 08$ & $12: 00$ & 24.277 & 34.644 & 13.501 \\
\hline 10/15/08 & $12: 00$ & 24.517 & 34.935 & 13.939 & $10 / 22 / 08$ & $16: 00$ & 24.251 & 34.623 & 13.471 \\
\hline 10/15/08 & $16: 00$ & 24.514 & 34.895 & 13.904 & 10/22/08 & $20: 00$ & 24.242 & 34.609 & 13.471 \\
\hline $10 / 15 / 08$ & $20: 00$ & 24.524 & 34.904 & 13.932 & $10 / 23 / 08$ & $0: 00$ & 24.225 & 34.59 & 13.424 \\
\hline 10/16/08 & 0:00 & 24.568 & 34.947 & 13.974 & $10 / 23 / 08$ & $4: 00$ & 24.232 & 34.581 & 13.396 \\
\hline $10 / 16 / 08$ & $4: 00$ & 24.58 & 34.937 & 13.964 & $10 / 23 / 08$ & 8:00 & 24.253 & 34.607 & 13.426 \\
\hline 10/16/08 & 8:00 & 24.591 & 34.947 & 13.992 & 10/23/08 & $12: 00$ & 24.288 & 34.628 & 13.454 \\
\hline 10/16/08 & $12: 00$ & 24.601 & 34.944 & 13.974 & 10/23/08 & $16: 00$ & 24.286 & 34.616 & 13.445 \\
\hline 10/16/08 & $16: 00$ & 24.524 & 34.839 & 13.889 & 10/23/08 & $20: 00$ & 24.302 & 34.632 & 13.466 \\
\hline 10/16/08 & $20: 00$ & 24.47 & 34.799 & 13.885 & $10 / 24 / 08$ & 0:00 & 24.316 & 34.635 & 13.471 \\
\hline 10/17/08 & 0:00 & 24.487 & 34.822 & 13.911 & $10 / 24 / 08$ & $4: 00$ & 24.272 & 34.569 & 13.426 \\
\hline 10/17/08 & $4: 00$ & 24.445 & 34.747 & 13.836 & $10 / 24 / 08$ & $8: 00$ & 24.256 & 34.562 & 13.429 \\
\hline 10/17/08 & 8:00 & 24.412 & 34.743 & 13.857 & $10 / 24 / 08$ & $12: 00$ & 24.27 & 34.59 & 13.461 \\
\hline 10/17/08 & $12: 00$ & 24.414 & 34.757 & 13.885 & $10 / 24 / 08$ & $16: 00$ & 24.277 & 34.6 & 13.473 \\
\hline 10/17/08 & $16: 00$ & 24.584 & 34.82 & 13.925 & $10 / 24 / 08$ & $20: 00$ & 24.3 & 34.63 & 13.506 \\
\hline $10 / 17 / 08$ & $20: 00$ & 24.517 & 34.888 & 13.999 & $10 / 25 / 08$ & $0: 00$ & 24.314 & 34.635 & 13.501 \\
\hline 10/18/08 & 0:00 & 24.552 & 34.916 & 14.028 & $10 / 25 / 08$ & $4: 00$ & 24.279 & 34.581 & 13.457 \\
\hline $10 / 18 / 08$ & $4: 00$ & 24.566 & 34.921 & 14.025 & $10 / 25 / 08$ & 8:00 & 24.26 & 34.569 & 13.454 \\
\hline 10/18/08 & $8: 00$ & 24.577 & 34.94 & 14.044 & $10 / 25 / 08$ & $12: 00$ & 24.242 & 34.539 & 13.429 \\
\hline 10/18/08 & $12: 00$ & 24.594 & 34.944 & 14.042 & $10 / 25 / 08$ & $16: 00$ & 24.169 & 34.454 & 13.375 \\
\hline 10/18/08 & $16: 00$ & 24.514 & 34.829 & 13.927 & 10/25/08 & $20: 00$ & 24.176 & 34.492 & 13.422 \\
\hline 10/18/08 & $20: 00$ & 24.456 & 34.78 & 13.906 & 10/26/08 & 0:00 & 24.181 & 34.489 & 13.496 \\
\hline 10/19/08 & 0:00 & 24.431 & 34.759 & 13.892 & 10/26/08 & $4: 00$ & 24.298 & 34.661 & 13.618 \\
\hline 10/19/08 & $4: 00$ & 24.396 & 34.714 & 13.864 & 10/26/08 & $8: 00$ & 24.454 & 34.855 & 13.777 \\
\hline 10/19/08 & 8:00 & 24.361 & 34.691 & 13.859 & 10/26/08 & $12: 00$ & 24.601 & 35.012 & 13.892 \\
\hline 10/19/08 & $12: 00$ & 24.358 & 34.686 & 13.859 & 10/26/08 & $16: 00$ & 24.661 & 35.064 & 13.939 \\
\hline 10/19/08 & $16: 00$ & 24.314 & 34.637 & 13.838 & 10/26/08 & $20: 00$ & 24.783 & 35.204 & 14.032 \\
\hline 10/19/08 & $20: 00$ & 24.368 & 34.729 & 13.936 & 10/27/08 & 0:00 & 24.857 & 35.258 & 14.053 \\
\hline 10/20/08 & 0:00 & 24.44 & 34.815 & 14.006 & 10/27/08 & $4: 00$ & 24.871 & 35.256 & 14.039 \\
\hline 10/20/08 & $4: 00$ & 24.524 & 34.909 & 14.1 & 10/27/08 & $8: 00$ & 24.916 & 35.322 & 14.098 \\
\hline $10 / 20 / 08$ & $8: 00$ & 24.636 & 35.038 & 14.205 & $10 / 27 / 08$ & $12: 00$ & 24.981 & 35.38 & 14.126 \\
\hline $10 / 20 / 08$ & $12: 00$ & 24.738 & 35.146 & 14.273 & $10 / 27 / 08$ & $16: 00$ & 24.946 & 35.315 & 14.058 \\
\hline
\end{tabular}


Table S1.2 (Cont.)

\begin{tabular}{|c|c|c|c|c|c|c|c|c|c|}
\hline \multirow[b]{2}{*}{ Date } & \multirow[b]{2}{*}{ Time } & \multicolumn{3}{|c|}{$\begin{array}{l}\text { Depth to Water (ft below top of } \\
\text { casing) at Indicated Well }\end{array}$} & \multirow[b]{2}{*}{ Date } & \multirow[b]{2}{*}{ Time } & \multicolumn{3}{|c|}{$\begin{array}{l}\text { Depth to Water (ft below top of } \\
\text { casing) at Indicated Well }\end{array}$} \\
\hline & & D2 & WP49 & SB65d & & & D2 & WP49 & SB65d \\
\hline $10 / 27 / 08$ & $20: 00$ & 24.932 & 35.312 & 14.065 & $11 / 4 / 08$ & $0: 00$ & 24.783 & 35.137 & 14.247 \\
\hline 10/28/08 & 0:00 & 24.948 & 35.331 & 14.084 & $11 / 4 / 08$ & $4: 00$ & 24.79 & 35.129 & 14.238 \\
\hline 10/28/08 & $4: 00$ & 24.941 & 35.319 & 14.077 & $11 / 4 / 08$ & $8: 00$ & 24.78 & 35.104 & 14.212 \\
\hline $10 / 28 / 08$ & $8: 00$ & 24.937 & 35.317 & 14.079 & $11 / 4 / 08$ & $12: 00$ & 24.755 & 35.073 & 14.184 \\
\hline 10/28/08 & $12: 00$ & 24.937 & 35.308 & 14.07 & $11 / 4 / 08$ & $16: 00$ & 24.671 & 34.951 & 14.095 \\
\hline 10/28/08 & $16: 00$ & 24.836 & 35.167 & 13.95 & $11 / 4 / 08$ & $20: 00$ & 24.631 & 34.935 & 14.095 \\
\hline $10 / 28 / 08$ & $20: 00$ & 24.773 & 35.122 & 13.939 & $11 / 5 / 08$ & $0: 00$ & 24.624 & 34.921 & 14.088 \\
\hline 10/29/08 & 0:00 & 24.745 & 35.09 & 13.918 & 11/5/08 & $4: 00$ & 24.566 & 34.848 & 14.034 \\
\hline 10/29/08 & $4: 00$ & 24.692 & 35.029 & 13.885 & $11 / 5 / 08$ & $8: 00$ & 24.531 & 34.827 & 14.03 \\
\hline 10/29/08 & 8:00 & 24.654 & 34.996 & 13.887 & 11/5/08 & $12: 00$ & 24.526 & 34.82 & 14.041 \\
\hline 10/29/08 & $12: 00$ & 24.689 & 35.057 & 13.948 & 11/5/08 & $16: 00$ & 24.51 & 34.818 & 14.051 \\
\hline 10/29/08 & $16: 00$ & 24.65 & 34.989 & 13.896 & 11/5/08 & $20: 00$ & 24.54 & 34.883 & 14.128 \\
\hline 10/29/08 & $20: 00$ & 24.64 & 34.993 & 13.924 & 11/6/08 & 0:00 & 24.626 & 34.979 & 14.182 \\
\hline 10/30/08 & 0:00 & 24.64 & 34.989 & 13.932 & 11/6/08 & $4: 00$ & 24.647 & 34.97 & 14.182 \\
\hline 10/30/08 & $4: 00$ & 24.636 & 34.987 & 13.939 & 11/6/08 & $8: 00$ & 24.629 & 34.937 & 14.152 \\
\hline $10 / 30 / 08$ & $8: 00$ & 24.622 & 34.97 & 13.936 & $11 / 6 / 08$ & $12: 00$ & 24.647 & 34.986 & 14.201 \\
\hline $10 / 30 / 08$ & $12: 00$ & 24.631 & 34.989 & 13.96 & $11 / 6 / 08$ & $16: 00$ & 24.678 & 35.022 & 14.245 \\
\hline 10/30/08 & $16: 00$ & 24.587 & 34.919 & 13.899 & 11/6/08 & $20: 00$ & 24.801 & 35.202 & 14.397 \\
\hline $10 / 30 / 08$ & $20: 00$ & 24.612 & 34.982 & 13.978 & 11/7/08 & $0: 00$ & 24.904 & 35.291 & 14.442 \\
\hline $10 / 31 / 08$ & $0: 00$ & 24.664 & 35.043 & 14.041 & $11 / 7 / 08$ & $4: 00$ & 24.92 & 35.277 & 14.409 \\
\hline 10/31/08 & $4: 00$ & 24.724 & 35.111 & 14.1 & 11/7/08 & $8: 00$ & 24.918 & 35.275 & 14.404 \\
\hline $10 / 31 / 08$ & $8: 00$ & 24.79 & 35.186 & 14.168 & $11 / 7 / 08$ & $12: 00$ & 24.944 & 35.312 & 14.435 \\
\hline 10/31/08 & $12: 00$ & 24.862 & 35.261 & 14.219 & 11/7/08 & $16: 00$ & 24.981 & 35.359 & 14.47 \\
\hline 10/31/08 & $16: 00$ & 24.869 & 35.237 & 14.194 & 11/7/08 & $20: 00$ & 25.051 & 35.441 & 14.526 \\
\hline 10/31/08 & $20: 00$ & 24.897 & 35.284 & 14.245 & 11/8/08 & $0: 00$ & 25.086 & 35.467 & 14.528 \\
\hline 11/1/08 & 0:00 & 24.923 & 35.298 & 14.252 & $11 / 8 / 08$ & $4: 00$ & 25.102 & 35.483 & 14.542 \\
\hline 11/1/08 & $4: 00$ & 24.932 & 35.303 & 14.25 & 11/8/08 & $8: 00$ & 25.137 & 35.526 & 14.573 \\
\hline $11 / 1 / 08$ & $8: 00$ & 24.932 & 35.305 & 14.257 & 11/8/08 & $12: 00$ & 25.2 & 35.603 & 14.633 \\
\hline $11 / 1 / 08$ & $12: 00$ & 24.948 & 35.317 & 14.259 & $11 / 8 / 08$ & $16: 00$ & 25.228 & 35.617 & 14.636 \\
\hline $11 / 1 / 08$ & $16: 00$ & 24.888 & 35.219 & 14.177 & $11 / 8 / 08$ & $20: 00$ & 25.275 & 35.673 & 14.68 \\
\hline $11 / 1 / 08$ & $20: 00$ & 24.857 & 35.2 & 14.177 & 11/9/08 & $0: 00$ & 25.314 & 35.706 & 14.694 \\
\hline $11 / 2 / 08$ & $0: 00$ & 24.839 & 35.174 & 14.163 & $11 / 9 / 08$ & $4: 00$ & 25.326 & 35.713 & 14.697 \\
\hline $11 / 2 / 08$ & $4: 00$ & 24.806 & 35.148 & 14.147 & $11 / 9 / 08$ & 8:00 & 25.345 & 35.73 & 14.708 \\
\hline $11 / 2 / 08$ & $8: 00$ & 24.783 & 35.125 & 14.142 & $11 / 9 / 08$ & $12: 00$ & 25.38 & 35.772 & 14.725 \\
\hline $11 / 2 / 08$ & $12: 00$ & 24.787 & 35.134 & 14.152 & $11 / 9 / 08$ & $16: 00$ & 25.356 & 35.727 & 14.683 \\
\hline $11 / 2 / 08$ & $16: 00$ & 24.722 & 35.036 & 14.084 & $11 / 9 / 08$ & $20: 00$ & 25.352 & 35.727 & 14.692 \\
\hline $11 / 2 / 08$ & $20: 00$ & 24.694 & 35.026 & 14.093 & 11/10/08 & 0:00 & 25.394 & 35.791 & 14.75 \\
\hline $11 / 3 / 08$ & $0: 00$ & 24.699 & 35.029 & 14.107 & $11 / 10 / 08$ & $4: 00$ & 25.419 & 35.809 & 14.76 \\
\hline $11 / 3 / 08$ & $4: 00$ & 24.68 & 35.003 & 14.093 & 11/10/08 & 8:00 & 25.419 & 35.805 & 14.755 \\
\hline $11 / 3 / 08$ & 8:00 & 24.675 & 35.005 & 14.114 & $11 / 10 / 08$ & $12: 00$ & 25.419 & 35.793 & 14.753 \\
\hline $11 / 3 / 08$ & $12: 00$ & 24.703 & 35.045 & 14.154 & $11 / 10 / 08$ & $16: 00$ & 25.391 & 35.762 & 14.706 \\
\hline $11 / 3 / 08$ & $16: 00$ & 24.692 & 35.019 & 14.14 & $11 / 10 / 08$ & $20: 00$ & 25.373 & 35.744 & 14.704 \\
\hline $11 / 3 / 08$ & $20: 00$ & 24.729 & 35.087 & 14.212 & 11/11/08 & 0:00 & 25.347 & 35.702 & 14.664 \\
\hline
\end{tabular}


Table S1.2 (Cont.)

\begin{tabular}{|c|c|c|c|c|c|c|c|c|c|}
\hline \multirow[b]{2}{*}{ Date } & \multirow[b]{2}{*}{ Time } & \multicolumn{3}{|c|}{$\begin{array}{l}\text { Depth to Water (ft below top of } \\
\text { casing) at Indicated Well }\end{array}$} & \multirow[b]{2}{*}{ Date } & \multirow[b]{2}{*}{ Time } & \multicolumn{3}{|c|}{$\begin{array}{l}\text { Depth to Water (ft below top of } \\
\text { casing) at Indicated Well }\end{array}$} \\
\hline & & D2 & WP49 & SB65d & & & D2 & WP49 & SB65d \\
\hline $11 / 11 / 08$ & $4: 00$ & 25.305 & 35.655 & 14.619 & 11/18/08 & 8:00 & 26.098 & 36.456 & 15.202 \\
\hline 11/11/08 & 8:00 & 25.272 & 35.636 & 14.629 & 11/18/08 & $12: 00$ & 26.072 & 36.421 & 15.162 \\
\hline 11/11/08 & $12: 00$ & 25.282 & 35.652 & 14.64 & 11/18/08 & $16: 00$ & 25.911 & 36.196 & 14.966 \\
\hline 11/11/08 & $16: 00$ & 25.244 & 35.601 & 14.612 & $11 / 18 / 08$ & $20: 00$ & 25.781 & 36.079 & 14.9 \\
\hline 11/11/08 & $20: 00$ & 25.258 & 35.636 & 14.645 & $11 / 19 / 08$ & 0:00 & 25.681 & 35.973 & 14.823 \\
\hline $11 / 12 / 08$ & $0: 00$ & 25.298 & 35.68 & 14.654 & $11 / 19 / 08$ & $4: 00$ & 25.587 & 35.887 & 14.795 \\
\hline $11 / 12 / 08$ & $4: 00$ & 25.305 & 35.676 & 14.65 & $11 / 19 / 08$ & $8: 00$ & 25.583 & 35.92 & 14.846 \\
\hline $11 / 12 / 08$ & 8:00 & 25.312 & 35.678 & 14.645 & 11/19/08 & $12: 00$ & 25.657 & 36.03 & 14.949 \\
\hline $11 / 12 / 08$ & $12: 00$ & 25.345 & 35.716 & 14.666 & $11 / 19 / 08$ & $16: 00$ & 25.713 & 36.091 & 15.019 \\
\hline $11 / 12 / 08$ & $16: 00$ & 25.305 & 35.645 & 14.598 & 11/19/08 & $20: 00$ & 25.865 & 36.285 & 15.176 \\
\hline $11 / 12 / 08$ & $20: 00$ & 25.275 & 35.612 & 14.575 & $11 / 20 / 08$ & 0:00 & 25.977 & 36.388 & 15.239 \\
\hline 11/13/08 & 0:00 & 25.235 & 35.542 & 14.521 & $11 / 20 / 08$ & $4: 00$ & 26.051 & 36.461 & 15.286 \\
\hline $11 / 13 / 08$ & $4: 00$ & 25.144 & 35.434 & 14.453 & $11 / 20 / 08$ & $8: 00$ & 26.119 & 36.529 & 15.338 \\
\hline 11/13/08 & 8:00 & 25.065 & 35.355 & 14.404 & $11 / 20 / 08$ & $12: 00$ & 26.205 & 36.628 & 15.394 \\
\hline 11/13/08 & $12: 00$ & 25.025 & 35.317 & 14.39 & $11 / 20 / 08$ & $16: 00$ & 26.226 & 36.614 & 15.373 \\
\hline $11 / 13 / 08$ & $16: 00$ & 24.986 & 35.284 & 14.399 & $11 / 20 / 08$ & $20: 00$ & 26.266 & 36.663 & 15.406 \\
\hline $11 / 13 / 08$ & $20: 00$ & 25.032 & 35.378 & 14.47 & $11 / 21 / 08$ & $0: 00$ & 26.275 & 36.658 & 15.377 \\
\hline $11 / 14 / 08$ & 0:00 & 25.081 & 35.418 & 14.519 & $11 / 21 / 08$ & $4: 00$ & 26.264 & 36.642 & 15.368 \\
\hline $11 / 14 / 08$ & $4: 00$ & 25.121 & 35.46 & 14.552 & $11 / 21 / 08$ & 8:00 & 26.231 & 36.592 & 15.324 \\
\hline $11 / 14 / 08$ & 8:00 & 25.214 & 35.587 & 14.673 & $11 / 21 / 08$ & $12: 00$ & 26.201 & 36.548 & 15.275 \\
\hline $11 / 14 / 08$ & $12: 00$ & 25.352 & 35.741 & 14.788 & $11 / 21 / 08$ & $16: 00$ & 26.1 & 36.424 & 15.181 \\
\hline $11 / 14 / 08$ & $16: 00$ & 25.452 & 35.835 & 14.844 & $11 / 21 / 08$ & $20: 00$ & 26.033 & 36.37 & 15.155 \\
\hline $11 / 14 / 08$ & $20: 00$ & 25.55 & 35.955 & 14.931 & $11 / 22 / 08$ & 0:00 & 25.998 & 36.33 & 15.139 \\
\hline 11/15/08 & 0:00 & 25.653 & 36.058 & 14.994 & $11 / 22 / 08$ & $4: 00$ & 25.939 & 36.267 & 15.09 \\
\hline $11 / 15 / 08$ & $4: 00$ & 25.713 & 36.109 & 15.017 & $11 / 22 / 08$ & $8: 00$ & 25.881 & 36.208 & 15.062 \\
\hline 11/15/08 & 8:00 & 25.746 & 36.131 & 15.015 & $11 / 22 / 08$ & $12: 00$ & 25.869 & 36.215 & 15.08 \\
\hline 11/15/08 & $12: 00$ & 25.783 & 36.166 & 15.029 & $11 / 22 / 08$ & $16: 00$ & 25.865 & 36.215 & 15.094 \\
\hline $11 / 15 / 08$ & $16: 00$ & 25.755 & 36.112 & 14.973 & $11 / 22 / 08$ & $20: 00$ & 25.907 & 36.271 & 15.143 \\
\hline $11 / 15 / 08$ & $20: 00$ & 25.753 & 36.114 & 14.966 & $11 / 23 / 08$ & $0: 00$ & 25.897 & 36.236 & 15.118 \\
\hline $11 / 16 / 08$ & $0: 00$ & 25.708 & 36.044 & 14.898 & $11 / 23 / 08$ & $4: 00$ & 25.86 & 36.196 & 15.087 \\
\hline $11 / 16 / 08$ & $4: 00$ & 25.625 & 35.943 & 14.811 & $11 / 23 / 08$ & $8: 00$ & 25.823 & 36.14 & 15.057 \\
\hline $11 / 16 / 08$ & 8:00 & 25.569 & 35.903 & 14.809 & $11 / 23 / 08$ & $12: 00$ & 25.792 & 36.109 & 15.036 \\
\hline 11/16/08 & $12: 00$ & 25.629 & 36.013 & 14.914 & $11 / 23 / 08$ & $16: 00$ & 25.715 & 36.032 & 15.001 \\
\hline $11 / 16 / 08$ & $16: 00$ & 25.669 & 36.039 & 14.928 & $11 / 23 / 08$ & $20: 00$ & 25.79 & 36.163 & 15.118 \\
\hline $11 / 16 / 08$ & $20: 00$ & 25.732 & 36.126 & 15.012 & $11 / 24 / 08$ & $0: 00$ & 25.888 & 36.269 & 15.202 \\
\hline 11/17/08 & 0:00 & 25.809 & 36.206 & 15.069 & $11 / 24 / 08$ & $4: 00$ & 25.951 & 36.332 & 15.246 \\
\hline 11/17/08 & $4: 00$ & 25.872 & 36.274 & 15.113 & $11 / 24 / 08$ & $8: 00$ & 26.007 & 36.389 & 15.289 \\
\hline $11 / 17 / 08$ & 8:00 & 25.944 & 36.351 & 15.169 & $11 / 24 / 08$ & $12: 00$ & 26.047 & 36.405 & 15.291 \\
\hline 11/17/08 & $12: 00$ & 26.037 & 36.454 & 15.244 & $11 / 24 / 08$ & $16: 00$ & 26.007 & 36.346 & 15.23 \\
\hline $11 / 17 / 08$ & $16: 00$ & 26.044 & 36.428 & 15.202 & $11 / 24 / 08$ & $20: 00$ & 26.007 & 36.36 & 15.244 \\
\hline $11 / 17 / 08$ & $20: 00$ & 26.068 & 36.461 & 15.228 & $11 / 25 / 08$ & $0: 00$ & 26.033 & 36.396 & 15.277 \\
\hline $11 / 18 / 08$ & $0: 00$ & 26.103 & 36.496 & 15.244 & $11 / 25 / 08$ & $4: 00$ & 26.044 & 36.407 & 15.282 \\
\hline 11/18/08 & $4: 00$ & 26.121 & 36.506 & 15.246 & $11 / 25 / 08$ & 8:00 & 26.065 & 36.426 & 15.296 \\
\hline
\end{tabular}


Table S1.2 (Cont.)

\begin{tabular}{|c|c|c|c|c|c|c|c|c|c|}
\hline \multirow[b]{2}{*}{ Date } & \multirow[b]{2}{*}{ Time } & \multicolumn{3}{|c|}{$\begin{array}{l}\text { Depth to Water (ft below top of } \\
\text { casing) at Indicated Well }\end{array}$} & \multirow[b]{2}{*}{ Date } & \multirow[b]{2}{*}{ Time } & \multicolumn{3}{|c|}{$\begin{array}{l}\text { Depth to Water (ft below top of } \\
\text { casing) at Indicated Well }\end{array}$} \\
\hline & & D2 & WP49 & SB65d & & & D2 & WP49 & SB65d \\
\hline $11 / 25 / 08$ & $12: 00$ & 26.075 & 36.424 & 15.286 & $12 / 2 / 08$ & $16: 00$ & 26.119 & 36.431 & 15.31 \\
\hline $11 / 25 / 08$ & $16: 00$ & 26.007 & 36.316 & 15.197 & 12/2/08 & $20: 00$ & 26.119 & 36.447 & 15.333 \\
\hline $11 / 25 / 08$ & $20: 00$ & 25.981 & 36.32 & 15.218 & $12 / 3 / 08$ & 0:00 & 26.145 & 36.485 & 15.373 \\
\hline $11 / 26 / 08$ & $0: 00$ & 25.981 & 36.316 & 15.204 & $12 / 3 / 08$ & $4: 00$ & 26.264 & 36.653 & 15.529 \\
\hline 11/26/08 & $4: 00$ & 25.97 & 36.311 & 15.214 & 12/3/08 & 8:00 & 26.401 & 36.801 & 15.637 \\
\hline 11/26/08 & $8: 00$ & 25.984 & 36.342 & 15.244 & $12 / 3 / 08$ & $12: 00$ & 26.567 & 36.993 & 15.759 \\
\hline $11 / 26 / 08$ & $12: 00$ & 26.03 & 36.393 & 15.289 & $12 / 3 / 08$ & $16: 00$ & 26.63 & 37.021 & 15.754 \\
\hline $11 / 26 / 08$ & $16: 00$ & 26.019 & 36.358 & 15.253 & 12/3/08 & $20: 00$ & 26.704 & 37.108 & 15.799 \\
\hline 11/26/08 & $20: 00$ & 26.016 & 36.36 & 15.267 & $12 / 4 / 08$ & 0:00 & 26.758 & 37.151 & 15.82 \\
\hline 11/27/08 & 0:00 & 26.04 & 36.384 & 15.284 & $12 / 4 / 08$ & $4: 00$ & 26.791 & 37.181 & 15.836 \\
\hline 11/27/08 & $4: 00$ & 26.044 & 36.391 & 15.305 & $12 / 4 / 08$ & $8: 00$ & 26.865 & 37.275 & 15.894 \\
\hline 11/27/08 & $8: 00$ & 26.063 & 36.414 & 15.338 & 12/4/08 & $12: 00$ & 26.975 & 37.408 & 15.969 \\
\hline $11 / 27 / 08$ & $12: 00$ & 26.163 & 36.548 & 15.42 & $12 / 4 / 08$ & $16: 00$ & 26.991 & 37.378 & 15.923 \\
\hline 11/27/08 & $16: 00$ & 26.17 & 36.529 & 15.403 & 12/4/08 & $20: 00$ & 27.017 & 37.415 & 15.953 \\
\hline 11/27/08 & $20: 00$ & 26.196 & 36.553 & 15.41 & 12/5/08 & 0:00 & 27.012 & 37.394 & 15.911 \\
\hline $11 / 28 / 08$ & $0: 00$ & 26.194 & 36.546 & 15.401 & $12 / 5 / 08$ & $4: 00$ & 26.956 & 37.308 & 15.843 \\
\hline $11 / 28 / 08$ & $4: 00$ & 26.196 & 36.543 & 15.391 & $12 / 5 / 08$ & $8: 00$ & 26.87 & 37.2 & 15.759 \\
\hline 11/28/08 & 8:00 & 26.18 & 36.524 & 15.377 & 12/5/08 & $12: 00$ & 26.772 & 37.073 & 15.651 \\
\hline $11 / 28 / 08$ & $12: 00$ & 26.184 & 36.524 & 15.37 & $12 / 5 / 08$ & $16: 00$ & 26.567 & 36.815 & 15.473 \\
\hline $11 / 28 / 08$ & $16: 00$ & 26.114 & 36.419 & 15.282 & $12 / 5 / 08$ & $20: 00$ & 26.431 & 36.703 & 15.431 \\
\hline 11/28/08 & $20: 00$ & 26.089 & 36.414 & 15.286 & 12/6/08 & 0:00 & 26.385 & 36.698 & 15.45 \\
\hline $11 / 29 / 08$ & $0: 00$ & 26.089 & 36.419 & 15.298 & $12 / 6 / 08$ & $4: 00$ & 26.392 & 36.726 & 15.487 \\
\hline 11/29/08 & $4: 00$ & 26.056 & 36.365 & 15.251 & 12/6/08 & 8:00 & 26.455 & 36.827 & 15.593 \\
\hline 11/29/08 & 8:00 & 26.005 & 36.309 & 15.218 & 12/6/08 & $12: 00$ & 26.597 & 37.01 & 15.735 \\
\hline $11 / 29 / 08$ & $12: 00$ & 25.974 & 36.281 & 15.204 & $12 / 6 / 08$ & $16: 00$ & 26.672 & 37.059 & 15.764 \\
\hline 11/29/08 & $16: 00$ & 25.9 & 36.187 & 15.134 & $12 / 6 / 08$ & $20: 00$ & 26.758 & 37.151 & 15.834 \\
\hline 11/29/08 & $20: 00$ & 25.9 & 36.222 & 15.181 & 12/7/08 & 0:00 & 26.802 & 37.172 & 15.831 \\
\hline $11 / 30 / 08$ & $0: 00$ & 25.911 & 36.227 & 15.188 & $12 / 7 / 08$ & $4: 00$ & 26.786 & 37.108 & 15.763 \\
\hline $11 / 30 / 08$ & $4: 00$ & 25.886 & 36.194 & 15.158 & $12 / 7 / 08$ & 8:00 & 26.728 & 37.054 & 15.717 \\
\hline $11 / 30 / 08$ & $8: 00$ & 25.883 & 36.203 & 15.181 & $12 / 7 / 08$ & $12: 00$ & 26.66 & 36.963 & 15.637 \\
\hline $11 / 30 / 08$ & $12: 00$ & 25.925 & 36.262 & 15.225 & 12/7/08 & $16: 00$ & 26.527 & 36.813 & 15.532 \\
\hline $11 / 30 / 08$ & $16: 00$ & 25.935 & 36.26 & 15.23 & $12 / 7 / 08$ & $20: 00$ & 26.49 & 36.803 & 15.551 \\
\hline $11 / 30 / 08$ & $20: 00$ & 25.995 & 36.349 & 15.3 & $12 / 8 / 08$ & $0: 00$ & 26.462 & 36.766 & 15.532 \\
\hline 12/1/08 & $0: 00$ & 26.049 & 36.405 & 15.349 & 12/8/08 & $4: 00$ & 26.413 & 36.707 & 15.499 \\
\hline $12 / 1 / 08$ & $4: 00$ & 26.121 & 36.485 & 15.413 & $12 / 8 / 08$ & 8:00 & 26.359 & 36.651 & 15.476 \\
\hline 12/1/08 & 8:00 & 26.205 & 36.583 & 15.473 & $12 / 8 / 08$ & $12: 00$ & 26.317 & 36.604 & 15.438 \\
\hline 12/1/08 & $12: 00$ & 26.301 & 36.693 & 15.546 & 12/8/08 & $16: 00$ & 26.243 & 36.522 & 15.405 \\
\hline $12 / 1 / 08$ & $16: 00$ & 26.322 & 36.679 & 15.518 & $12 / 8 / 08$ & $20: 00$ & 26.264 & 36.578 & 15.473 \\
\hline $12 / 1 / 08$ & $20: 00$ & 26.331 & 36.679 & 15.501 & $12 / 9 / 08$ & $0: 00$ & 26.34 & 36.689 & 15.562 \\
\hline $12 / 2 / 08$ & $0: 00$ & 26.317 & 36.656 & 15.469 & $12 / 9 / 08$ & $4: 00$ & 26.452 & 36.82 & 15.658 \\
\hline $12 / 2 / 08$ & $4: 00$ & 26.277 & 36.588 & 15.406 & $12 / 9 / 08$ & $8: 00$ & 26.604 & 37.01 & 15.789 \\
\hline $12 / 2 / 08$ & 8:00 & 26.182 & 36.478 & 15.328 & $12 / 9 / 08$ & $12: 00$ & 26.786 & 37.211 & 15.941 \\
\hline $12 / 2 / 08$ & $12: 00$ & 26.156 & 36.473 & 15.338 & $12 / 9 / 08$ & $16: 00$ & 26.872 & 37.272 & 15.958 \\
\hline
\end{tabular}


Table S1.2 (Cont.)

Depth to Water (ft below top of casing) at Indicated Well

\begin{tabular}{|c|c|c|c|c|c|c|c|c|c|}
\hline \multirow[b]{2}{*}{ Date } & \multirow[b]{2}{*}{ Time } & \multicolumn{3}{|c|}{$\begin{array}{l}\text { Depth to Water (ft below top of } \\
\text { casing) at Indicated Well }\end{array}$} & \multirow[b]{2}{*}{ Date } & \multirow[b]{2}{*}{ Time } & \multicolumn{3}{|c|}{$\begin{array}{l}\text { Depth to Water (ft below top of } \\
\text { casing) at Indicated Well }\end{array}$} \\
\hline & & D2 & WP49 & SB65d & & & D2 & WP49 & SB65d \\
\hline 12/9/08 & 20:00 & 26.979 & 37.397 & 16.037 & 12/17/08 & $0: 00$ & 27.168 & 37.519 & 16.056 \\
\hline 12/10/08 & 0:00 & 27.047 & 37.436 & 16.037 & 12/17/08 & $4: 00$ & 27.194 & 37.558 & 16.093 \\
\hline $12 / 10 / 08$ & $4: 00$ & 27.049 & 37.425 & 16.011 & $12 / 17 / 08$ & $8: 00$ & 27.241 & 37.617 & 16.149 \\
\hline 12/10/08 & 8:00 & 27.045 & 37.415 & 15.99 & $12 / 17 / 08$ & $12: 00$ & 27.287 & 37.676 & 16.185 \\
\hline $12 / 10 / 08$ & $12: 00$ & 27.035 & 37.394 & 15.965 & $12 / 17 / 08$ & $16: 00$ & 27.278 & 37.624 & 16.14 \\
\hline 12/10/08 & $16: 00$ & 26.958 & 37.282 & 15.878 & $12 / 17 / 08$ & $20: 00$ & 27.266 & 37.629 & 16.161 \\
\hline $12 / 10 / 08$ & $20: 00$ & 26.94 & 37.284 & 15.892 & $12 / 18 / 08$ & $0: 00$ & 27.297 & 37.657 & 16.175 \\
\hline $12 / 11 / 08$ & 0:00 & 26.919 & 37.256 & 15.869 & 12/18/08 & $4: 00$ & 27.278 & 37.629 & 16.159 \\
\hline 12/11/08 & $4: 00$ & 26.886 & 37.223 & 15.848 & 12/18/08 & $8: 00$ & 27.29 & 37.657 & 16.182 \\
\hline 12/11/08 & 8:00 & 26.874 & 37.225 & 15.864 & $12 / 18 / 08$ & $12: 00$ & 27.287 & 37.652 & 16.18 \\
\hline $12 / 11 / 08$ & $12: 00$ & 26.912 & 37.268 & 15.906 & 12/18/08 & $16: 00$ & 27.199 & 37.502 & 16.051 \\
\hline 12/11/08 & $16: 00$ & 26.895 & 37.242 & 15.88 & 12/18/08 & 20:00 & 27.117 & 37.413 & 16.016 \\
\hline $12 / 11 / 08$ & $20: 00$ & 26.942 & 37.305 & 15.948 & 12/19/08 & $0: 00$ & 26.963 & 37.225 & 15.869 \\
\hline $12 / 12 / 08$ & 0:00 & 26.998 & 37.373 & 16.007 & 12/19/08 & $4: 00$ & 26.914 & 37.249 & 15.955 \\
\hline $12 / 12 / 08$ & $4: 00$ & 27.052 & 37.422 & 16.025 & $12 / 19 / 08$ & $8: 00$ & 27.08 & 37.481 & 16.133 \\
\hline $12 / 12 / 08$ & 8:00 & 27.045 & 37.394 & 16.002 & $12 / 19 / 08$ & $12: 00$ & 27.245 & 37.666 & 16.248 \\
\hline $12 / 12 / 08$ & $12: 00$ & 27.047 & 37.39 & 15.983 & $12 / 19 / 08$ & $16: 00$ & 27.257 & 37.598 & 16.164 \\
\hline $12 / 12 / 08$ & $16: 00$ & 26.935 & 37.225 & 15.852 & 12/19/08 & 20:00 & 27.278 & 37.64 & 16.206 \\
\hline $12 / 12 / 08$ & $20: 00$ & 26.856 & 37.146 & 15.789 & $12 / 20 / 08$ & 0:00 & 27.266 & 37.605 & 16.173 \\
\hline $12 / 13 / 08$ & 0:00 & 26.763 & 37.036 & 15.719 & $12 / 20 / 08$ & $4: 00$ & 27.187 & 37.495 & 16.086 \\
\hline $12 / 13 / 08$ & $4: 00$ & 26.618 & 36.855 & 15.6 & $12 / 20 / 08$ & 8:00 & 27.217 & 37.568 & 16.149 \\
\hline $12 / 13 / 08$ & 8:00 & 26.499 & 36.757 & 15.553 & $12 / 20 / 08$ & $12: 00$ & 27.294 & 37.683 & 16.264 \\
\hline $12 / 13 / 08$ & $12: 00$ & 26.445 & 36.707 & 15.529 & $12 / 20 / 08$ & $16: 00$ & 27.346 & 37.725 & 16.283 \\
\hline 12/13/08 & $16: 00$ & 26.35 & 36.602 & 15.473 & $12 / 20 / 08$ & $20: 00$ & 27.448 & 37.842 & 16.358 \\
\hline $12 / 13 / 08$ & $20: 00$ & 26.361 & 36.649 & 15.527 & $12 / 21 / 08$ & $0: 00$ & 27.523 & 37.922 & 16.405 \\
\hline $12 / 14 / 08$ & 0:00 & 26.338 & 36.597 & 15.487 & $12 / 21 / 08$ & $4: 00$ & 27.569 & 37.966 & 16.428 \\
\hline $12 / 14 / 08$ & $4: 00$ & 26.298 & 36.562 & 15.471 & $12 / 21 / 08$ & $8: 00$ & 27.595 & 37.98 & 16.423 \\
\hline $12 / 14 / 08$ & $8: 00$ & 26.415 & 36.789 & 15.705 & $12 / 21 / 08$ & $12: 00$ & 27.649 & 38.051 & 16.475 \\
\hline $12 / 14 / 08$ & $12: 00$ & 26.672 & 37.087 & 15.894 & $12 / 21 / 08$ & $16: 00$ & 27.7 & 38.102 & 16.5 \\
\hline $12 / 14 / 08$ & $16: 00$ & 26.814 & 37.223 & 15.979 & $12 / 21 / 08$ & $20: 00$ & 27.749 & 38.149 & 16.521 \\
\hline $12 / 14 / 08$ & $20: 00$ & 26.991 & 37.415 & 16.107 & $12 / 22 / 08$ & $0: 00$ & 27.779 & 38.182 & 16.531 \\
\hline $12 / 15 / 08$ & $0: 00$ & 27.173 & 37.624 & 16.241 & $12 / 22 / 08$ & $4: 00$ & 27.763 & 38.149 & 16.5 \\
\hline $12 / 15 / 08$ & $4: 00$ & 27.348 & 37.798 & 16.344 & $12 / 22 / 08$ & $8: 00$ & 27.733 & 38.1 & 16.454 \\
\hline $12 / 15 / 08$ & 8:00 & 27.485 & 37.936 & 16.386 & $12 / 22 / 08$ & $12: 00$ & 27.695 & 38.044 & 16.416 \\
\hline $12 / 15 / 08$ & $12: 00$ & 27.604 & 38.051 & 16.442 & $12 / 22 / 08$ & $16: 00$ & 27.546 & 37.835 & 16.257 \\
\hline $12 / 15 / 08$ & $16: 00$ & 27.637 & 38.051 & 16.414 & $12 / 22 / 08$ & $20: 00$ & 27.42 & 37.72 & 16.189 \\
\hline $12 / 15 / 08$ & 20:00 & 27.642 & 38.046 & 16.395 & $12 / 23 / 08$ & $0: 00$ & 27.287 & 37.556 & 16.11 \\
\hline $12 / 16 / 08$ & $0: 00$ & 27.632 & 38.023 & 16.36 & $12 / 23 / 08$ & $4: 00$ & 27.171 & 37.432 & 16.014 \\
\hline 12/16/08 & $4: 00$ & 27.574 & 37.936 & 16.283 & $12 / 23 / 08$ & 8:00 & 27.073 & 37.354 & 15.993 \\
\hline $12 / 16 / 08$ & 8:00 & 27.457 & 37.793 & 16.178 & $12 / 23 / 08$ & $12: 00$ & 27.068 & 37.371 & 16.021 \\
\hline $12 / 16 / 08$ & $12: 00$ & 27.339 & 37.664 & 16.093 & $12 / 23 / 08$ & $16: 00$ & 27.047 & 37.357 & 16.016 \\
\hline $12 / 16 / 08$ & $16: 00$ & 27.203 & 37.509 & 16.007 & $12 / 23 / 08$ & $20: 00$ & 27.117 & 37.474 & 16.135 \\
\hline $12 / 16 / 08$ & $20: 00$ & 27.161 & 37.507 & 16.042 & $12 / 24 / 08$ & $0: 00$ & 27.222 & 37.596 & 16.196 \\
\hline
\end{tabular}

Table S1.2 (Cont.)

Depth to Water (ft below top of casing) at Indicated Well 
Table S1.2 (Cont.)

\begin{tabular}{|c|c|c|c|c|c|c|c|c|c|}
\hline \multirow[b]{2}{*}{ Date } & \multirow[b]{2}{*}{ Time } & \multicolumn{3}{|c|}{$\begin{array}{l}\text { Depth to Water (ft below top of } \\
\text { casing) at Indicated Well }\end{array}$} & \multirow[b]{2}{*}{ Date } & \multirow[b]{2}{*}{ Time } & \multicolumn{3}{|c|}{$\begin{array}{l}\text { Depth to Water (ft below top of } \\
\text { casing) at Indicated Well }\end{array}$} \\
\hline & & D2 & WP49 & SB65d & & & D2 & WP49 & SB65d \\
\hline $12 / 24 / 08$ & $4: 00$ & 27.264 & 37.622 & 16.227 & 12/31/08 & 8:00 & 28.199 & 38.59 & 16.821 \\
\hline $12 / 24 / 08$ & 8:00 & 27.346 & 37.72 & 16.304 & $12 / 31 / 08$ & $12: 00$ & 28.213 & 38.578 & 16.788 \\
\hline $12 / 24 / 08$ & $12: 00$ & 27.462 & 37.863 & 16.402 & $12 / 31 / 08$ & $16: 00$ & 28.078 & 38.367 & 16.615 \\
\hline $12 / 24 / 08$ & $16: 00$ & 27.527 & 37.912 & 16.426 & 12/31/08 & $20: 00$ & 27.929 & 38.201 & 16.5 \\
\hline $12 / 24 / 08$ & $20: 00$ & 27.602 & 37.971 & 16.444 & & & & & \\
\hline $12 / 25 / 08$ & $0: 00$ & 27.632 & 38.002 & 16.477 & & & & & \\
\hline $12 / 25 / 08$ & $4: 00$ & 27.637 & 37.987 & 16.444 & & & & & \\
\hline $12 / 25 / 08$ & $8: 00$ & 27.6 & 37.926 & 16.383 & & & & & \\
\hline $12 / 25 / 08$ & $12: 00$ & 27.551 & 37.868 & 16.341 & & & & & \\
\hline $12 / 25 / 08$ & $16: 00$ & 27.388 & 37.643 & 16.168 & & & & & \\
\hline $12 / 25 / 08$ & $20: 00$ & 27.299 & 37.577 & 16.154 & & & & & \\
\hline $12 / 26 / 08$ & 0:00 & 27.222 & 37.49 & 16.091 & & & & & \\
\hline $12 / 26 / 08$ & $4: 00$ & 27.164 & 37.451 & 16.086 & & & & & \\
\hline $12 / 26 / 08$ & $8: 00$ & 27.147 & 37.441 & 16.096 & & & & & \\
\hline $12 / 26 / 08$ & $12: 00$ & 27.133 & 37.415 & 16.075 & & & & & \\
\hline $12 / 26 / 08$ & $16: 00$ & 26.742 & 37.35 & 16.03 & & & & & \\
\hline $12 / 26 / 08$ & $20: 00$ & 27.091 & 37.408 & 16.042 & & & & & \\
\hline $12 / 27 / 08$ & $0: 00$ & 27.203 & 37.582 & 16.196 & & & & & \\
\hline $12 / 27 / 08$ & $4: 00$ & 27.313 & 37.683 & 16.248 & & & & & \\
\hline $12 / 27 / 08$ & 8:00 & 27.378 & 37.723 & 16.25 & & & & & \\
\hline $12 / 27 / 08$ & $12: 00$ & 27.385 & 37.715 & 16.255 & & & & & \\
\hline $12 / 27 / 08$ & $16: 00$ & 27.371 & 37.69 & 16.213 & & & & & \\
\hline $12 / 27 / 08$ & $20: 00$ & 27.423 & 37.767 & 16.294 & & & & & \\
\hline $12 / 28 / 08$ & $0: 00$ & 27.485 & 37.83 & 16.332 & & & & & \\
\hline $12 / 28 / 08$ & $4: 00$ & 27.534 & 37.882 & 16.367 & & & & & \\
\hline 12/28/08 & $8: 00$ & 27.583 & 37.929 & 16.402 & & & & & \\
\hline $12 / 28 / 08$ & $12: 00$ & 27.656 & 38.016 & 16.456 & & & & & \\
\hline 12/28/08 & $16: 00$ & 27.646 & 37.98 & 16.411 & & & & & \\
\hline $12 / 28 / 08$ & $20: 00$ & 27.663 & 37.999 & 16.43 & & & & & \\
\hline $12 / 29 / 08$ & $0: 00$ & 27.66 & 37.976 & 16.409 & & & & & \\
\hline $12 / 29 / 08$ & $4: 00$ & 27.635 & 37.95 & 16.402 & & & & & \\
\hline $12 / 29 / 08$ & $8: 00$ & 27.707 & 38.079 & 16.519 & & & & & \\
\hline $12 / 29 / 08$ & $12: 00$ & 27.849 & 38.238 & 16.615 & & & & & \\
\hline $12 / 29 / 08$ & $16: 00$ & 27.875 & 38.227 & 16.585 & & & & & \\
\hline $12 / 29 / 08$ & $20: 00$ & 27.884 & 38.22 & 16.575 & & & & & \\
\hline $12 / 30 / 08$ & $0: 00$ & 27.803 & 38.088 & 16.458 & & & & & \\
\hline 12/30/08 & $4: 00$ & 27.667 & 37.91 & 16.334 & & & & & \\
\hline $12 / 30 / 08$ & 8:00 & 27.523 & 37.767 & 16.236 & & & & & \\
\hline $12 / 30 / 08$ & $12: 00$ & 27.539 & 37.868 & 16.36 & & & & & \\
\hline $12 / 30 / 08$ & $16: 00$ & 27.644 & 38.016 & 16.493 & & & & & \\
\hline 12/30/08 & $20: 00$ & 27.847 & 38.288 & 16.702 & & & & & \\
\hline $12 / 31 / 08$ & $0: 00$ & 28.05 & 38.475 & 16.788 & & & & & \\
\hline $12 / 31 / 08$ & $4: 00$ & 28.157 & 38.569 & 16.821 & & & & & \\
\hline
\end{tabular}

Table S1.2 (Cont.)

Depth to Water (ft below top of
Depth to Wa

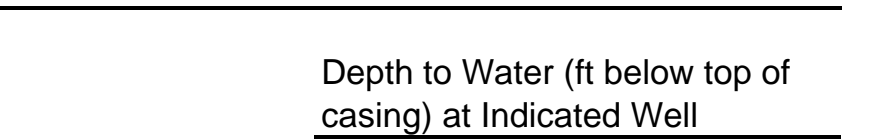

$\sqrt{2}$

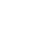


TABLE S1.3 Automatically measured water levels in headwaters area wells in 2008.

Depth to Water (feet below top of casing) in Indicated Wells

Date Time PMW1s PMW1d PMW2sa PMW2d PMW9s PMW9d PMW3d

\begin{tabular}{|c|c|c|c|c|c|c|c|c|}
\hline $1 / 1 / 08$ & 0:00 & 7.119 & 7.063 & 4.69 & 3.624 & 2.236 & 1.121 & -0.356 \\
\hline 1/1/08 & 4:00 & 7.152 & 7.102 & 4.709 & 3.655 & 2.246 & 1.154 & -0.563 \\
\hline 1/1/08 & 8:00 & 7.176 & 7.128 & 4.725 & 3.671 & 2.253 & 1.175 & -0.617 \\
\hline 1/1/08 & $12: 00$ & 7.206 & 7.162 & 4.746 & 3.702 & 2.277 & 1.203 & -0.603 \\
\hline 1/1/08 & $16: 00$ & 7.22 & 7.174 & 4.751 & 3.706 & 2.26 & 1.205 & -0.477 \\
\hline 1/1/08 & 20:00 & 7.241 & 7.195 & 4.763 & 3.723 & 2.276 & 1.224 & -0.669 \\
\hline $1 / 2 / 08$ & 0:00 & 7.26 & 7.213 & 4.774 & 3.737 & 2.293 & 1.22 & -0.769 \\
\hline $1 / 2 / 08$ & 4:00 & 7.27 & 7.22 & 4.779 & 3.741 & 2.316 & 1.236 & -0.774 \\
\hline $1 / 2 / 08$ & 8:00 & 7.286 & 7.239 & 4.791 & 3.758 & 2.337 & 1.241 & -0.795 \\
\hline $1 / 2 / 08$ & $12: 00$ & 7.302 & 7.258 & 4.802 & 3.77 & 2.346 & 1.264 & -0.578 \\
\hline $1 / 2 / 08$ & $16: 00$ & 7.279 & 7.227 & 4.774 & 3.73 & 2.279 & 1.241 & -0.194 \\
\hline $1 / 2 / 08$ & 20:00 & 7.258 & 7.197 & 4.756 & 3.704 & 2.255 & 1.21 & -0.244 \\
\hline 1/3/08 & 0:00 & 7.234 & 7.172 & 4.744 & 3.688 & 2.265 & 1.201 & -0.211 \\
\hline $1 / 3 / 08$ & 4:00 & 7.197 & 7.123 & 4.716 & 3.645 & 2.256 & 1.156 & -0.024 \\
\hline $1 / 3 / 08$ & $8: 00$ & 7.15 & 7.072 & 4.683 & 3.601 & 2.244 & 1.131 & 0.156 \\
\hline $1 / 3 / 08$ & $12: 00$ & 7.126 & 7.046 & 4.671 & 3.584 & 2.244 & 1.135 & 0.528 \\
\hline $1 / 3 / 08$ & $16: 00$ & 7.094 & 7.007 & 4.641 & 3.549 & 2.188 & 1.121 & -0.286 \\
\hline $1 / 3 / 08$ & 20:00 & 7.072 & 6.988 & 4.634 & 3.54 & 2.167 & 1.098 & -0.395 \\
\hline $1 / 4 / 08$ & $0: 00$ & 7.058 & 6.972 & 4.624 & 3.531 & 2.159 & 1.082 & -0.4 \\
\hline $1 / 4 / 08$ & 4:00 & 7.047 & 6.958 & 4.615 & 3.517 & 2.15 & 1.056 & -0.412 \\
\hline $1 / 4 / 08$ & 8:00 & 7.033 & 6.947 & 4.615 & 3.512 & 2.162 & 1.047 & -0.414 \\
\hline $1 / 4 / 08$ & $12: 00$ & 7.028 & 6.942 & 4.613 & 3.509 & 2.16 & 1.051 & -0.416 \\
\hline $1 / 4 / 08$ & $16: 00$ & 7.011 & 6.916 & 4.594 & 3.486 & 2.141 & 1.056 & -0.344 \\
\hline $1 / 4 / 08$ & 20:00 & 7.002 & 6.912 & 4.592 & 3.484 & 2.139 & 1.051 & -0.435 \\
\hline $1 / 5 / 08$ & $0: 00$ & 6.988 & 6.896 & 4.58 & 3.465 & 2.122 & 1.03 & -0.358 \\
\hline 1/5/08 & 4:00 & 6.976 & 6.884 & 4.573 & 3.46 & 2.118 & 1.009 & -0.325 \\
\hline $1 / 5 / 08$ & 8:00 & 6.969 & 6.877 & 4.577 & 3.458 & 2.127 & 0.997 & -0.281 \\
\hline $1 / 5 / 08$ & $12: 00$ & 6.972 & 6.879 & 4.58 & 3.458 & 2.141 & 1.018 & -0.134 \\
\hline $1 / 5 / 08$ & $16: 00$ & 6.955 & 6.858 & 4.568 & 3.444 & 2.155 & 1.007 & -0.103 \\
\hline $1 / 5 / 08$ & $20: 00$ & 6.951 & 6.854 & 4.563 & 3.442 & 2.169 & 0.995 & -0.003 \\
\hline $1 / 6 / 08$ & $0: 00$ & 6.939 & 6.842 & 4.556 & 3.43 & 2.162 & 0.993 & -0.251 \\
\hline 1/6/08 & 4:00 & 6.941 & 6.847 & 4.563 & 3.437 & 2.171 & 0.99 & -0.253 \\
\hline $1 / 6 / 08$ & 8:00 & 6.964 & 6.879 & 4.585 & 3.47 & 2.178 & 0.997 & -0.256 \\
\hline $1 / 6 / 08$ & $12: 00$ & 6.997 & 6.914 & 4.606 & 3.495 & 2.181 & 1.014 & -0.256 \\
\hline $1 / 6 / 08$ & $16: 00$ & 7.016 & 6.935 & 4.613 & 3.509 & 2.195 & 1.028 & -0.258 \\
\hline $1 / 6 / 08$ & 20:00 & 7.03 & 6.954 & 4.617 & 3.519 & 2.211 & 1.04 & -0.251 \\
\hline $1 / 7 / 08$ & $0: 00$ & 7.03 & 6.949 & 4.61 & 3.509 & 2.209 & 1.047 & -0.258 \\
\hline $1 / 7 / 08$ & 4:00 & 7.03 & 6.951 & 4.62 & 3.517 & 2.222 & 1.051 & -0.256 \\
\hline $1 / 7 / 08$ & 8:00 & 7.002 & 6.919 & 4.603 & 3.521 & 2.218 & 1.042 & -0.256 \\
\hline $1 / 7 / 08$ & $12: 00$ & 7.023 & 6.942 & 4.615 & 3.509 & 2.227 & 1.047 & -0.249 \\
\hline $1 / 7 / 08$ & $16: 00$ & 7.03 & 6.949 & 4.606 & 3.507 & 2.225 & 1.051 & -0.249 \\
\hline $1 / 7 / 08$ & $20: 00$ & 7.049 & 6.977 & 4.61 & 3.519 & 2.246 & 1.058 & -0.258 \\
\hline 1/8/08 & $0: 00$ & 7.049 & 6.974 & 4.613 & 3.514 & 2.248 & 1.063 & -0.263 \\
\hline 1/8/08 & 4:00 & 7.044 & 6.965 & 4.613 & 3.514 & 2.25 & 1.063 & -0.253 \\
\hline
\end{tabular}


TABLE S1.3 (Cont.)

Depth to Water (feet below top of casing) in Indicated Wells

Date Time PMW1s PMW1d PMW2sa PMW2d PMW9s PMW9d PMW3d

\begin{tabular}{|c|c|c|c|c|c|c|c|c|}
\hline 1/8/08 & 8:00 & 7.082 & 7.009 & 4.645 & 3.561 & 2.265 & 1.082 & -0.253 \\
\hline $1 / 8 / 08$ & $12: 00$ & 7.107 & 7.039 & 4.664 & 3.582 & 2.272 & 1.098 & -0.253 \\
\hline $1 / 8 / 08$ & $16: 00$ & 7.129 & 7.06 & 4.674 & 3.596 & 2.272 & 1.112 & -0.251 \\
\hline 1/8/08 & 20:00 & 7.15 & 7.088 & 4.681 & 3.615 & 2.286 & 1.126 & -0.249 \\
\hline $1 / 9 / 08$ & $0: 00$ & 7.164 & 7.097 & 4.69 & 3.627 & 2.288 & 1.147 & -0.253 \\
\hline $1 / 9 / 08$ & 4:00 & 7.15 & 7.081 & 4.681 & 3.606 & 2.272 & 1.14 & -0.242 \\
\hline $1 / 9 / 08$ & $8: 00$ & 7.131 & 7.058 & 4.671 & 3.589 & 2.258 & 1.128 & -0.239 \\
\hline $1 / 9 / 08$ & $12: 00$ & 7.136 & 7.06 & 4.676 & 3.601 & 2.253 & 1.133 & -0.146 \\
\hline $1 / 9 / 08$ & $16: 00$ & 7.084 & 7.002 & 4.638 & 3.54 & 2.248 & 1.114 & 0.071 \\
\hline $1 / 9 / 08$ & 20:00 & 7.077 & 6.995 & 4.643 & 3.547 & 2.262 & 1.103 & 0.076 \\
\hline 1/10/08 & 0:00 & 7.075 & 6.995 & 4.641 & 3.547 & 2.267 & 1.1 & 0.078 \\
\hline 1/10/08 & 4:00 & 7.061 & 6.981 & 4.634 & 3.535 & 2.262 & 1.093 & 0.132 \\
\hline 1/10/08 & 8:00 & 7.056 & 6.972 & 4.634 & 3.533 & 2.263 & 1.089 & 0.163 \\
\hline 1/10/08 & $12: 00$ & 7.063 & 6.981 & 4.641 & 3.545 & 2.265 & 1.089 & 0.123 \\
\hline 1/10/08 & 16:00 & 7.061 & 6.984 & 4.641 & 3.545 & 2.267 & 1.086 & -0.256 \\
\hline 1/10/08 & 20:00 & 7.089 & 7.012 & 4.655 & 3.568 & 2.272 & 1.096 & -0.258 \\
\hline $1 / 11 / 08$ & 0:00 & 7.108 & 7.032 & 4.664 & 3.582 & 2.274 & 1.119 & -0.242 \\
\hline 1/11/08 & 4:00 & 7.11 & 7.035 & 4.669 & 3.584 & 2.267 & 1.121 & -0.279 \\
\hline $1 / 11 / 08$ & 8:00 & 7.107 & 7.032 & 4.671 & 3.584 & 2.26 & 1.114 & -0.342 \\
\hline 1/11/08 & $12: 00$ & 7.112 & 7.032 & 4.678 & 3.589 & 2.26 & 1.11 & -0.365 \\
\hline 1/11/08 & $16: 00$ & 7.096 & 7.016 & 4.664 & 3.57 & 2.251 & 1.107 & -0.244 \\
\hline 1/11/08 & 20:00 & 7.114 & 7.039 & 4.678 & 3.594 & 2.263 & 1.112 & -0.256 \\
\hline $1 / 12 / 08$ & $0: 00$ & 7.133 & 7.06 & 4.688 & 3.61 & 2.267 & 1.121 & -0.258 \\
\hline $1 / 12 / 08$ & 4:00 & 7.159 & 7.09 & 4.706 & 3.634 & 2.275 & 1.138 & -0.249 \\
\hline $1 / 12 / 08$ & 8:00 & 7.19 & 7.128 & 4.734 & 3.669 & 2.288 & 1.154 & -0.253 \\
\hline $1 / 12 / 08$ & $12: 00$ & 7.22 & 7.162 & 4.756 & 3.697 & 2.293 & 1.173 & -0.242 \\
\hline $1 / 12 / 08$ & $16: 00$ & 7.236 & 7.172 & 4.753 & 3.697 & 2.291 & 1.182 & -0.249 \\
\hline $1 / 12 / 08$ & 20:00 & 7.255 & 7.197 & 4.77 & 3.718 & 2.303 & 1.201 & -0.246 \\
\hline $1 / 13 / 08$ & 0:00 & 7.267 & 7.206 & 4.772 & 3.725 & 2.298 & 1.212 & -0.342 \\
\hline $1 / 13 / 08$ & 4:00 & 7.272 & 7.213 & 4.779 & 3.727 & 2.307 & 1.222 & -0.26 \\
\hline $1 / 13 / 08$ & 8:00 & 7.279 & 7.218 & 4.786 & 3.73 & 2.3 & 1.224 & -0.267 \\
\hline 1/13/08 & $12: 00$ & 7.283 & 7.223 & 4.788 & 3.734 & 2.3 & 1.231 & -0.26 \\
\hline $1 / 13 / 08$ & $16: 00$ & 7.25 & 7.186 & 4.758 & 3.695 & 2.267 & 1.21 & -0.281 \\
\hline $1 / 13 / 08$ & 20:00 & 7.269 & 7.206 & 4.774 & 3.72 & 2.282 & 1.208 & -0.262 \\
\hline $1 / 14 / 08$ & $0: 00$ & 7.297 & 7.241 & 4.795 & 3.751 & 2.291 & 1.222 & -0.248 \\
\hline $1 / 14 / 08$ & 4:00 & 7.314 & 7.26 & 4.807 & 3.77 & 2.3 & 1.236 & -0.237 \\
\hline $1 / 14 / 08$ & 8:00 & 7.33 & 7.278 & 4.821 & 3.781 & 2.319 & 1.259 & -0.227 \\
\hline $1 / 14 / 08$ & $12: 00$ & 7.349 & 7.297 & 4.838 & 3.798 & 2.335 & 1.269 & -0.213 \\
\hline $1 / 14 / 08$ & $16: 00$ & 7.342 & 7.285 & 4.821 & 3.781 & 2.298 & 1.255 & -0.23 \\
\hline $1 / 14 / 08$ & 20:00 & 7.342 & 7.283 & 4.821 & 3.777 & 2.298 & 1.259 & -0.232 \\
\hline $1 / 15 / 08$ & $0: 00$ & 7.33 & 7.269 & 4.821 & 3.767 & 2.321 & 1.25 & -0.241 \\
\hline 1/15/08 & 4:00 & 7.304 & 7.237 & 4.805 & 3.744 & 2.333 & 1.229 & -0.262 \\
\hline $1 / 15 / 08$ & 8:00 & 7.272 & 7.197 & 4.784 & 3.713 & 2.333 & 1.215 & -0.283 \\
\hline $1 / 15 / 08$ & $12: 00$ & 7.241 & 7.162 & 4.76 & 3.678 & 2.324 & 1.21 & -0.307 \\
\hline
\end{tabular}


TABLE S1.3 (Cont.)

Depth to Water (feet below top of casing) in Indicated Wells

Date Time PMW1s PMW1d PMW2sa PMW2d PMW9s PMW9d PMW3d

\begin{tabular}{|c|c|c|c|c|c|c|c|c|}
\hline $1 / 15 / 08$ & $16: 00$ & 7.192 & 7.107 & 4.72 & 3.627 & 2.249 & 1.227 & -0.344 \\
\hline $1 / 15 / 08$ & 20:00 & 7.173 & 7.088 & 4.716 & 3.624 & 2.233 & 1.182 & -0.346 \\
\hline 1/16/08 & 0:00 & 7.168 & 7.083 & 4.711 & 3.62 & 2.214 & 1.161 & -0.351 \\
\hline $1 / 16 / 08$ & $4: 00$ & 7.196 & 7.118 & 4.737 & 3.657 & 2.223 & 1.156 & -0.325 \\
\hline 1/16/08 & 8:00 & 7.236 & 7.167 & 4.767 & 3.697 & 2.221 & 1.18 & -0.297 \\
\hline 1/16/08 & $12: 00$ & 7.279 & 7.213 & 4.795 & 3.734 & 2.221 & 1.175 & -0.271 \\
\hline 1/16/08 & $16: 00$ & 7.295 & 7.227 & 4.793 & 3.739 & 2.209 & 1.201 & -0.271 \\
\hline 1/16/08 & $20: 00$ & 7.312 & 7.248 & 4.807 & 3.751 & 2.223 & 1.21 & -0.26 \\
\hline $1 / 17 / 08$ & 0:00 & 7.318 & 7.253 & 4.805 & 3.751 & 2.218 & 1.208 & -0.26 \\
\hline $1 / 17 / 08$ & 4:00 & 7.3 & 7.232 & 4.791 & 3.73 & 2.212 & 1.198 & -0.271 \\
\hline $1 / 17 / 08$ & $8: 00$ & 7.276 & 7.202 & 4.774 & 3.709 & 2.207 & 1.184 & -0.288 \\
\hline $1 / 17 / 08$ & $12: 00$ & 7.286 & 7.216 & 4.784 & 3.723 & 2.217 & 1.194 & -0.281 \\
\hline $1 / 17 / 08$ & $16: 00$ & 7.255 & 7.181 & 4.76 & 3.69 & 2.205 & 1.175 & -0.304 \\
\hline $1 / 17 / 08$ & 20:00 & 7.253 & 7.179 & 4.765 & 3.692 & 2.209 & 1.175 & -0.302 \\
\hline $1 / 18 / 08$ & 0:00 & 7.253 & 7.179 & 4.763 & 3.692 & 2.211 & 1.177 & -0.302 \\
\hline 1/18/08 & $4: 00$ & 7.255 & 7.181 & 4.765 & 3.695 & 2.207 & 1.18 & -0.297 \\
\hline 1/18/08 & 8:00 & 7.29 & 7.223 & 4.798 & 3.737 & 2.23 & 1.208 & -0.269 \\
\hline 1/18/08 & $12: 00$ & 7.361 & 7.306 & 4.852 & 3.812 & 2.253 & 1.262 & -0.215 \\
\hline 1/18/08 & $16: 00$ & 7.379 & 7.327 & 4.849 & 3.814 & 2.24 & 1.262 & -0.215 \\
\hline $1 / 18 / 08$ & $20: 00$ & 7.403 & 7.35 & 4.866 & 3.835 & 2.251 & 1.285 & -0.197 \\
\hline $1 / 19 / 08$ & 0:00 & 7.433 & 7.388 & 4.884 & 3.866 & 2.256 & 1.294 & -0.171 \\
\hline $1 / 19 / 08$ & 4:00 & 7.438 & 7.39 & 4.882 & 3.856 & 2.251 & 1.29 & -0.178 \\
\hline $1 / 19 / 08$ & 8:00 & 7.443 & 7.392 & 4.887 & 3.859 & 2.265 & 1.297 & -0.173 \\
\hline $1 / 19 / 08$ & $12: 00$ & 7.457 & 7.408 & 4.903 & 3.875 & 2.284 & 1.315 & -0.159 \\
\hline $1 / 19 / 08$ & $16: 00$ & 7.433 & 7.378 & 4.875 & 3.842 & 2.265 & 1.297 & -0.192 \\
\hline $1 / 19 / 08$ & 20:00 & 7.431 & 7.374 & 4.877 & 3.842 & 2.265 & 1.297 & -0.19 \\
\hline $1 / 20 / 08$ & 0:00 & 7.438 & 7.378 & 4.884 & 3.847 & 2.274 & 1.297 & -0.185 \\
\hline $1 / 20 / 08$ & 4:00 & 7.445 & 7.388 & 4.896 & 3.859 & 2.291 & 1.304 & -0.178 \\
\hline $1 / 20 / 08$ & 8:00 & 7.462 & 7.406 & 4.908 & 3.873 & 2.289 & 1.32 & -0.162 \\
\hline $1 / 20 / 08$ & $12: 00$ & 7.473 & 7.42 & 4.915 & 3.889 & 2.282 & 1.329 & -0.152 \\
\hline $1 / 20 / 08$ & $16: 00$ & 7.438 & 7.374 & 4.88 & 3.842 & 2.249 & 1.297 & -0.192 \\
\hline $1 / 20 / 08$ & $20: 00$ & 7.45 & 7.392 & 4.898 & 3.866 & 2.254 & 1.306 & -0.171 \\
\hline $1 / 21 / 08$ & 0:00 & 7.443 & 7.381 & 4.887 & 3.849 & 2.249 & 1.299 & -0.185 \\
\hline $1 / 21 / 08$ & 4:00 & 7.412 & 7.346 & 4.866 & 3.816 & 2.242 & 1.271 & -0.213 \\
\hline $1 / 21 / 08$ & 8:00 & 7.389 & 7.32 & 4.852 & 3.795 & 2.235 & 1.262 & -0.232 \\
\hline $1 / 21 / 08$ & $12: 00$ & 7.375 & 7.304 & 4.845 & 3.786 & 2.242 & 1.257 & -0.243 \\
\hline $1 / 21 / 08$ & $16: 00$ & 7.351 & 7.276 & 4.828 & 3.76 & 2.226 & 1.234 & -0.264 \\
\hline $1 / 21 / 08$ & 20:00 & 7.375 & 7.306 & 4.854 & 3.8 & 2.247 & 1.252 & -0.232 \\
\hline $1 / 22 / 08$ & 0:00 & 7.422 & 7.364 & 4.887 & 3.845 & 2.265 & 1.29 & -0.19 \\
\hline $1 / 22 / 08$ & $4: 00$ & 7.461 & 7.406 & 4.91 & 3.873 & 2.279 & 1.308 & -0.164 \\
\hline $1 / 22 / 08$ & 8:00 & 7.471 & 7.415 & 4.912 & 3.877 & 2.287 & 1.311 & -0.164 \\
\hline $1 / 22 / 08$ & $12: 00$ & 7.469 & 7.406 & 4.908 & 3.87 & 2.298 & 1.313 & -0.169 \\
\hline $1 / 22 / 08$ & $16: 00$ & 7.424 & 7.355 & 4.875 & 3.819 & 2.289 & 1.285 & -0.213 \\
\hline $1 / 22 / 08$ & 20:00 & 7.405 & 7.334 & 4.863 & 3.807 & 2.289 & 1.276 & -0.225 \\
\hline
\end{tabular}


TABLE S1.3 (Cont.)

Depth to Water (feet below top of casing) in Indicated Wells

Date Time PMW1s PMW1d PMW2sa PMW2d PMW9s PMW9d PMW3d

\begin{tabular}{|c|c|c|c|c|c|c|c|c|}
\hline 1/23/08 & $0: 00$ & 7.384 & 7.313 & 4.852 & 3.788 & 2.294 & 1.262 & -0.239 \\
\hline $1 / 23 / 08$ & $4: 00$ & 7.37 & 7.297 & 4.842 & 3.779 & 2.303 & 1.255 & -0.25 \\
\hline $1 / 23 / 08$ & 8:00 & 7.361 & 7.29 & 4.845 & 3.777 & 2.314 & 1.257 & -0.25 \\
\hline $1 / 23 / 08$ & $12: 00$ & 7.393 & 7.325 & 4.873 & 3.816 & 2.324 & 1.283 & -0.218 \\
\hline $1 / 23 / 08$ & $16: 00$ & 7.422 & 7.357 & 4.891 & 3.84 & 2.321 & 1.297 & -0.197 \\
\hline $1 / 23 / 08$ & $20: 00$ & 7.487 & 7.429 & 4.934 & 3.896 & 2.324 & 1.336 & -0.145 \\
\hline $1 / 24 / 08$ & 0:00 & 7.506 & 7.452 & 4.941 & 3.91 & 2.333 & 1.35 & -0.131 \\
\hline $1 / 24 / 08$ & $4: 00$ & 7.513 & 7.459 & 4.948 & 3.915 & 2.349 & 1.353 & -0.127 \\
\hline $1 / 24 / 08$ & $8: 00$ & 7.525 & 7.469 & 4.957 & 3.924 & 2.368 & 1.357 & -0.117 \\
\hline $1 / 24 / 08$ & $12: 00$ & 7.539 & 7.485 & 4.971 & 3.938 & 2.392 & 1.381 & -0.108 \\
\hline $1 / 24 / 08$ & $16: 00$ & 7.52 & 7.457 & 4.948 & 3.908 & 2.364 & 1.357 & -0.136 \\
\hline $1 / 24 / 08$ & $20: 00$ & 7.508 & 7.448 & 4.945 & 3.903 & 2.357 & 1.353 & -0.138 \\
\hline $1 / 25 / 08$ & 0:00 & 7.506 & 7.443 & 4.941 & 3.898 & 2.354 & 1.35 & -0.143 \\
\hline $1 / 25 / 08$ & 4:00 & 7.48 & 7.408 & 4.92 & 3.868 & 2.343 & 1.329 & -0.171 \\
\hline $1 / 25 / 08$ & 8:00 & 7.447 & 7.374 & 4.898 & 3.84 & 2.34 & 1.308 & -0.194 \\
\hline $1 / 25 / 08$ & $12: 00$ & 7.433 & 7.355 & 4.887 & 3.823 & 2.328 & 1.297 & -0.211 \\
\hline $1 / 25 / 08$ & $16: 00$ & 7.4 & 7.318 & 4.863 & 3.791 & 2.305 & 1.271 & -0.239 \\
\hline $1 / 25 / 08$ & $20: 00$ & 7.4 & 7.32 & 4.87 & 3.802 & 2.303 & 1.264 & -0.227 \\
\hline $1 / 26 / 08$ & 0:00 & 7.393 & 7.316 & 4.863 & 3.793 & 2.298 & 1.269 & -0.234 \\
\hline $1 / 26 / 08$ & $4: 00$ & 7.389 & 7.311 & 4.863 & 3.793 & 2.3 & 1.264 & -0.239 \\
\hline $1 / 26 / 08$ & 8:00 & 7.398 & 7.318 & 4.868 & 3.8 & 2.308 & 1.271 & -0.229 \\
\hline $1 / 26 / 08$ & $12: 00$ & 7.431 & 7.357 & 4.896 & 3.835 & 2.322 & 1.292 & -0.199 \\
\hline $1 / 26 / 08$ & $16: 00$ & 7.433 & 7.362 & 4.894 & 3.83 & 2.305 & 1.266 & -0.206 \\
\hline $1 / 26 / 08$ & $20: 00$ & 7.459 & 7.388 & 4.908 & 3.854 & 2.31 & 1.28 & -0.185 \\
\hline $1 / 27 / 08$ & 0:00 & 7.459 & 7.388 & 4.903 & 3.849 & 2.307 & 1.285 & -0.19 \\
\hline $1 / 27 / 08$ & $4: 00$ & 7.457 & 7.383 & 4.901 & 3.845 & 2.31 & 1.292 & -0.194 \\
\hline $1 / 27 / 08$ & 8:00 & 7.461 & 7.388 & 4.901 & 3.847 & 2.31 & 1.299 & -0.192 \\
\hline $1 / 27 / 08$ & $12: 00$ & 7.452 & 7.378 & 4.898 & 3.838 & 2.307 & 1.292 & -0.199 \\
\hline $1 / 27 / 08$ & $16: 00$ & 7.4 & 7.316 & 4.842 & 3.77 & 2.256 & 1.276 & -0.243 \\
\hline $1 / 27 / 08$ & $20: 00$ & 7.363 & 7.276 & 4.807 & 3.716 & 2.249 & 1.241 & -0.248 \\
\hline $1 / 28 / 08$ & 0:00 & 7.332 & 7.244 & 4.788 & 3.69 & 2.254 & 1.215 & -0.253 \\
\hline $1 / 28 / 08$ & $4: 00$ & 7.295 & 7.202 & 4.763 & 3.655 & 2.24 & 1.187 & -0.264 \\
\hline $1 / 28 / 08$ & 8:00 & 7.255 & 7.158 & 4.73 & 3.617 & 2.226 & 1.161 & -0.267 \\
\hline $1 / 28 / 08$ & $12: 00$ & 7.206 & 7.109 & 4.692 & 3.568 & 2.191 & 1.138 & -0.262 \\
\hline $1 / 28 / 08$ & $16: 00$ & 6.924 & 6.958 & 4.477 & 3.392 & 2.177 & 1.089 & -0.274 \\
\hline $1 / 28 / 08$ & $20: 00$ & 6.45 & 6.728 & 4.371 & 3.301 & 2.221 & 1.035 & -0.281 \\
\hline $1 / 29 / 08$ & 0:00 & 6.278 & 6.61 & 4.397 & 3.32 & 2.258 & 0.995 & -0.27 \\
\hline $1 / 29 / 08$ & $4: 00$ & 6.331 & 6.629 & 4.449 & 3.374 & 2.282 & 0.993 & -0.267 \\
\hline $1 / 29 / 08$ & 8:00 & 6.521 & 6.759 & 4.54 & 3.481 & 2.308 & 1.04 & -0.256 \\
\hline $1 / 29 / 08$ & $12: 00$ & 6.72 & 6.879 & 4.592 & 3.542 & 2.28 & 1.084 & -0.351 \\
\hline $1 / 29 / 08$ & $16: 00$ & 6.909 & 6.96 & 4.636 & 3.587 & 2.27 & 1.117 & -0.353 \\
\hline $1 / 29 / 08$ & $20: 00$ & 7.062 & 7.044 & 4.685 & 3.643 & 2.277 & 1.156 & -0.316 \\
\hline $1 / 30 / 08$ & 0:00 & 7.135 & 7.095 & 4.718 & 3.674 & 2.275 & 1.18 & -0.297 \\
\hline $1 / 30 / 08$ & $4: 00$ & 7.175 & 7.125 & 4.739 & 3.69 & 2.296 & 1.203 & -0.285 \\
\hline
\end{tabular}


TABLE S1.3 (Cont.)

Depth to Water (feet below top of casing) in Indicated Wells

Date Time PMW1s PMW1d PMW2sa PMW2d PMW9s PMW9d PMW3d

\begin{tabular}{|c|c|c|c|c|c|c|c|c|}
\hline $1 / 30 / 08$ & 8:00 & 7.203 & 7.148 & 4.76 & 3.704 & 2.324 & 1.227 & -0.276 \\
\hline $1 / 30 / 08$ & $12: 00$ & 7.233 & 7.174 & 4.779 & 3.73 & 2.333 & 1.248 & -0.262 \\
\hline $1 / 30 / 08$ & $16: 00$ & 7.217 & 7.148 & 4.765 & 3.702 & 2.273 & 1.21 & -0.285 \\
\hline $1 / 30 / 08$ & $20: 00$ & 7.25 & 7.188 & 4.795 & 3.742 & 2.273 & 1.229 & -0.255 \\
\hline 1/31/08 & $0: 00$ & 7.283 & 7.225 & 4.821 & 3.772 & 2.314 & 1.255 & -0.234 \\
\hline 1/31/08 & $4: 00$ & 7.306 & 7.246 & 4.833 & 3.788 & 2.329 & 1.269 & -0.222 \\
\hline $1 / 31 / 08$ & $8: 00$ & 7.332 & 7.276 & 4.854 & 3.809 & 2.34 & 1.287 & -0.206 \\
\hline 1/31/08 & $12: 00$ & 7.353 & 7.292 & 4.866 & 3.821 & 2.357 & 1.315 & -0.199 \\
\hline $1 / 31 / 08$ & $16: 00$ & 7.337 & 7.271 & 4.845 & 3.791 & 2.268 & 1.264 & -0.225 \\
\hline 1/31/08 & $20: 00$ & 7.346 & 7.278 & 4.852 & 3.807 & 2.235 & 1.264 & -0.213 \\
\hline 2/1/08 & 0:00 & 7.339 & 7.269 & 4.847 & 3.791 & 2.252 & 1.264 & -0.222 \\
\hline $2 / 1 / 08$ & $4: 00$ & 7.332 & 7.26 & 4.854 & 3.786 & 2.296 & 1.271 & -0.225 \\
\hline $2 / 1 / 08$ & 8:00 & 7.32 & 7.246 & 4.847 & 3.777 & 2.322 & 1.266 & -0.232 \\
\hline $2 / 1 / 08$ & $12: 00$ & 7.32 & 7.248 & 4.849 & 3.777 & 2.362 & 1.283 & -0.232 \\
\hline $2 / 1 / 08$ & $16: 00$ & 7.287 & 7.204 & 4.816 & 3.737 & 2.226 & 1.224 & -0.269 \\
\hline $2 / 1 / 08$ & $20: 00$ & 7.297 & 7.216 & 4.826 & 3.749 & 2.212 & 1.217 & -0.257 \\
\hline $2 / 2 / 08$ & $0: 00$ & 7.299 & 7.218 & 4.823 & 3.749 & 2.179 & 1.217 & -0.26 \\
\hline $2 / 2 / 08$ & $4: 00$ & 7.313 & 7.232 & 4.831 & 3.756 & 2.172 & 1.226 & -0.253 \\
\hline $2 / 2 / 08$ & $8: 00$ & 7.337 & 7.26 & 4.852 & 3.786 & 2.186 & 1.231 & -0.227 \\
\hline $2 / 2 / 08$ & $12: 00$ & 7.37 & 7.302 & 4.877 & 3.819 & 2.209 & 1.259 & -0.201 \\
\hline $2 / 2 / 08$ & $16: 00$ & 7.386 & 7.318 & 4.882 & 3.828 & 2.221 & 1.198 & -0.189 \\
\hline $2 / 2 / 08$ & $20: 00$ & 7.398 & 7.332 & 4.891 & 3.84 & 2.235 & 1.25 & -0.183 \\
\hline $2 / 3 / 08$ & $0: 00$ & 7.409 & 7.343 & 4.889 & 3.84 & 2.214 & 1.285 & -0.18 \\
\hline $2 / 3 / 08$ & $4: 00$ & 7.405 & 7.336 & 4.887 & 3.831 & 2.186 & 1.278 & -0.19 \\
\hline $2 / 3 / 08$ & $8: 00$ & 7.393 & 7.323 & 4.88 & 3.819 & 2.184 & 1.264 & -0.201 \\
\hline $2 / 3 / 08$ & $12: 00$ & 7.386 & 7.309 & 4.873 & 3.809 & 2.191 & 1.25 & -0.21 \\
\hline $2 / 3 / 08$ & $16: 00$ & 7.341 & 7.255 & 4.835 & 3.763 & 2.165 & 1.21 & -0.253 \\
\hline $2 / 3 / 08$ & $20: 00$ & 7.337 & 7.253 & 4.842 & 3.765 & 2.189 & 1.215 & -0.255 \\
\hline $2 / 4 / 08$ & $0: 00$ & 7.327 & 7.239 & 4.828 & 3.753 & 2.177 & 1.21 & -0.269 \\
\hline $2 / 4 / 08$ & $4: 00$ & 7.278 & 7.183 & 4.786 & 3.697 & 2.147 & 1.18 & -0.32 \\
\hline $2 / 4 / 08$ & 8:00 & 7.264 & 7.174 & 4.786 & 3.697 & 2.161 & 1.173 & -0.323 \\
\hline $2 / 4 / 08$ & $12: 00$ & 7.287 & 7.216 & 4.807 & 3.725 & 2.179 & 1.173 & -0.297 \\
\hline $2 / 4 / 08$ & $16: 00$ & 7.301 & 7.225 & 4.816 & 3.739 & 2.193 & 1.182 & -0.281 \\
\hline $2 / 4 / 08$ & $20: 00$ & 7.355 & 7.285 & 4.849 & 3.786 & 2.242 & 1.205 & -0.262 \\
\hline $2 / 5 / 08$ & $0: 00$ & 7.4 & 7.332 & 4.875 & 3.824 & 2.259 & 1.238 & -0.225 \\
\hline $2 / 5 / 08$ & $4: 00$ & 7.426 & 7.364 & 4.898 & 3.854 & 2.273 & 1.264 & -0.196 \\
\hline $2 / 5 / 08$ & $8: 00$ & 7.43 & 7.367 & 4.894 & 3.849 & 2.254 & 1.283 & -0.196 \\
\hline $2 / 5 / 08$ & $12: 00$ & 7.43 & 7.367 & 4.889 & 3.84 & 2.254 & 1.283 & -0.203 \\
\hline $2 / 5 / 08$ & $16: 00$ & 7.409 & 7.343 & 4.875 & 3.817 & 2.243 & 1.257 & -0.227 \\
\hline $2 / 5 / 08$ & $20: 00$ & 7.43 & 7.367 & 4.896 & 3.842 & 2.256 & 1.273 & -0.206 \\
\hline $2 / 6 / 08$ & $0: 00$ & 7.426 & 7.357 & 4.884 & 3.833 & 2.24 & 1.266 & -0.215 \\
\hline $2 / 6 / 08$ & $4: 00$ & 7.409 & 7.339 & 4.877 & 3.819 & 2.237 & 1.262 & -0.227 \\
\hline $2 / 6 / 08$ & $8: 00$ & 7.407 & 7.336 & 4.875 & 3.817 & 2.238 & 1.264 & -0.227 \\
\hline $2 / 6 / 08$ & $12: 00$ & 7.43 & 7.364 & 4.891 & 3.84 & 2.245 & 1.28 & -0.206 \\
\hline
\end{tabular}


TABLE S1.3 (Cont.)

Depth to Water (feet below top of casing) in Indicated Wells

Date Time PMW1s PMW1d PMW2sa PMW2d PMW9s PMW9d PMW3d

\begin{tabular}{|c|c|c|c|c|c|c|c|c|}
\hline $2 / 6 / 08$ & $16: 00$ & 7.416 & 7.346 & 4.88 & 3.824 & 2.238 & 1.271 & -0.225 \\
\hline 2/6/08 & 20:00 & 7.438 & 7.371 & 4.896 & 3.847 & 2.25 & 1.287 & -0.201 \\
\hline 2/7/08 & $0: 00$ & 7.447 & 7.381 & 4.903 & 3.856 & 2.245 & 1.294 & -0.192 \\
\hline 2/7/08 & 4:00 & 7.437 & 7.369 & 4.889 & 3.838 & 2.233 & 1.287 & -0.208 \\
\hline $2 / 7 / 08$ & 8:00 & 7.433 & 7.367 & 4.889 & 3.84 & 2.235 & 1.283 & -0.208 \\
\hline $2 / 7 / 08$ & $12: 00$ & 7.442 & 7.376 & 4.898 & 3.849 & 2.238 & 1.292 & -0.201 \\
\hline $2 / 7 / 08$ & $16: 00$ & 7.412 & 7.341 & 4.873 & 3.814 & 2.228 & 1.264 & -0.232 \\
\hline 2/7/08 & 20:00 & 7.405 & 7.334 & 4.87 & 3.814 & 2.228 & 1.264 & -0.231 \\
\hline 2/8/08 & $0: 00$ & 7.386 & 7.311 & 4.854 & 3.791 & 2.217 & 1.257 & -0.252 \\
\hline 2/8/08 & 4:00 & 7.36 & 7.283 & 4.842 & 3.772 & 2.217 & 1.241 & -0.266 \\
\hline 2/8/08 & 8:00 & 7.362 & 7.288 & 4.849 & 3.781 & 2.224 & 1.243 & -0.259 \\
\hline 2/8/08 & $12: 00$ & 7.395 & 7.327 & 4.877 & 3.817 & 2.228 & 1.259 & -0.229 \\
\hline 2/8/08 & $16: 00$ & 7.405 & 7.334 & 4.88 & 3.819 & 2.219 & 1.273 & -0.229 \\
\hline 2/8/08 & 20:00 & 7.428 & 7.362 & 4.891 & 3.84 & 2.229 & 1.273 & -0.215 \\
\hline 2/9/08 & $0: 00$ & 7.435 & 7.369 & 4.891 & 3.84 & 2.226 & 1.278 & -0.21 \\
\hline $2 / 9 / 08$ & 4:00 & 7.435 & 7.369 & 4.894 & 3.842 & 2.223 & 1.285 & -0.21 \\
\hline $2 / 9 / 08$ & 8:00 & 7.449 & 7.39 & 4.91 & 3.863 & 2.233 & 1.297 & -0.189 \\
\hline 2/9/08 & $12: 00$ & 7.491 & 7.443 & 4.948 & 3.917 & 2.245 & 1.304 & -0.143 \\
\hline $2 / 9 / 08$ & $16: 00$ & 7.536 & 7.497 & 4.973 & 3.955 & 2.25 & 1.334 & -0.108 \\
\hline $2 / 9 / 08$ & 20:00 & 7.573 & 7.543 & 4.999 & 3.985 & 2.259 & 1.376 & -0.08 \\
\hline 2/10/08 & $0: 00$ & 7.604 & 7.578 & 5.018 & 4.013 & 2.259 & 1.404 & -0.052 \\
\hline 2/10/08 & 4:00 & 7.634 & 7.61 & 5.037 & 4.037 & 2.263 & 1.423 & -0.031 \\
\hline 2/10/08 & $8: 00$ & 7.665 & 7.643 & 5.06 & 4.06 & 2.264 & 1.442 & -0.007 \\
\hline 2/10/08 & $12: 00$ & 7.698 & 7.673 & 5.083 & 4.086 & 2.294 & 1.467 & 0.014 \\
\hline 2/10/08 & $16: 00$ & 7.684 & 7.647 & 5.069 & 4.053 & 2.27 & 1.435 & -0.016 \\
\hline 2/10/08 & 20:00 & 7.67 & 7.624 & 5.06 & 4.039 & 2.268 & 1.432 & -0.028 \\
\hline 2/11/08 & 0:00 & 7.648 & 7.596 & 5.053 & 4.023 & 2.289 & 1.423 & -0.044 \\
\hline 2/11/08 & 4:00 & 7.623 & 7.569 & 5.039 & 3.999 & 2.32 & 1.411 & -0.066 \\
\hline 2/11/08 & $8: 00$ & 7.595 & 7.536 & 5.025 & 3.976 & 2.35 & 1.411 & -0.087 \\
\hline 2/11/08 & $12: 00$ & 7.583 & 7.517 & 5.011 & 3.959 & 2.364 & 1.402 & -0.103 \\
\hline 2/11/08 & $16: 00$ & 7.552 & 7.48 & 4.983 & 3.927 & 2.303 & 1.364 & -0.133 \\
\hline 2/11/08 & 20:00 & 7.564 & 7.499 & 4.999 & 3.955 & 2.273 & 1.369 & -0.108 \\
\hline 2/12/08 & $0: 00$ & 7.585 & 7.52 & 5.016 & 3.974 & 2.284 & 1.39 & -0.091 \\
\hline $2 / 12 / 08$ & 4:00 & 7.592 & 7.524 & 5.02 & 3.978 & 2.296 & 1.395 & -0.089 \\
\hline 2/12/08 & 8:00 & 7.602 & 7.538 & 5.03 & 3.988 & 2.31 & 1.397 & -0.082 \\
\hline 2/12/08 & $12: 00$ & 7.62 & 7.557 & 5.046 & 4.006 & 2.359 & 1.435 & -0.07 \\
\hline $2 / 12 / 08$ & $16: 00$ & 7.594 & 7.527 & 5.018 & 3.969 & 2.294 & 1.381 & -0.098 \\
\hline $2 / 12 / 08$ & 20:00 & 7.604 & 7.534 & 5.03 & 3.983 & 2.276 & 1.383 & -0.084 \\
\hline 2/13/08 & 0:00 & 7.595 & 7.52 & 5.023 & 3.971 & 2.292 & 1.388 & -0.098 \\
\hline 2/13/08 & 4:00 & 7.585 & 7.508 & 5.016 & 3.962 & 2.317 & 1.397 & -0.105 \\
\hline $2 / 13 / 08$ & 8:00 & 7.569 & 7.492 & 5.004 & 3.948 & 2.306 & 1.376 & -0.117 \\
\hline 2/13/08 & $12: 00$ & 7.566 & 7.485 & 4.999 & 3.941 & 2.348 & 1.388 & -0.126 \\
\hline 2/13/08 & $16: 00$ & 7.524 & 7.434 & 4.955 & 3.892 & 2.256 & 1.325 & -0.173 \\
\hline 2/13/08 & $20: 00$ & 7.496 & 7.397 & 4.927 & 3.849 & 2.21 & 1.346 & -0.21 \\
\hline
\end{tabular}


TABLE S1.3 (Cont.)

Depth to Water (feet below top of casing) in Indicated Wells

Date Time PMW1s PMW1d PMW2sa PMW2d PMW9s PMW9d PMW3d

\begin{tabular}{|c|c|c|c|c|c|c|c|c|}
\hline $2 / 14 / 08$ & $0: 00$ & 7.48 & 7.385 & 4.917 & 3.847 & 2.198 & 1.315 & -0.213 \\
\hline $2 / 14 / 08$ & $4: 00$ & 7.491 & 7.406 & 4.936 & 3.873 & 2.214 & 1.306 & -0.187 \\
\hline $2 / 14 / 08$ & 8:00 & 7.505 & 7.422 & 4.945 & 3.892 & 2.205 & 1.308 & -0.178 \\
\hline 2/14/08 & $12: 00$ & 7.562 & 7.487 & 4.99 & 3.95 & 2.233 & 1.329 & -0.124 \\
\hline 2/14/08 & $16: 00$ & 7.627 & 7.564 & 5.037 & 4.004 & 2.249 & 1.376 & -0.075 \\
\hline $2 / 14 / 08$ & $20: 00$ & 7.681 & 7.622 & 5.079 & 4.051 & 2.259 & 1.421 & -0.03 \\
\hline 2/15/08 & $0: 00$ & 7.723 & 7.666 & 5.107 & 4.086 & 2.264 & 1.439 & 0 \\
\hline 2/15/08 & $4: 00$ & 7.74 & 7.685 & 5.121 & 4.1 & 2.305 & 1.456 & 0.014 \\
\hline 2/15/08 & $8: 00$ & 7.759 & 7.703 & 5.142 & 4.116 & 2.378 & 1.486 & 0.03 \\
\hline 2/15/08 & $12: 00$ & 7.782 & 7.722 & 5.158 & 4.128 & 2.469 & 1.547 & 0.042 \\
\hline 2/15/08 & $16: 00$ & 7.756 & 7.689 & 5.135 & 4.093 & 2.378 & 1.484 & 0.007 \\
\hline 2/15/08 & $20: 00$ & 7.738 & 7.668 & 5.123 & 4.077 & 2.317 & 1.453 & -0.005 \\
\hline 2/16/08 & 0:00 & 7.726 & 7.652 & 5.109 & 4.06 & 2.296 & 1.451 & -0.019 \\
\hline 2/16/08 & $4: 00$ & 7.702 & 7.622 & 5.09 & 4.037 & 2.31 & 1.442 & -0.038 \\
\hline 2/16/08 & 8:00 & 7.674 & 7.594 & 5.081 & 4.018 & 2.343 & 1.428 & -0.054 \\
\hline 2/16/08 & $12: 00$ & 7.644 & 7.555 & 5.051 & 3.974 & 2.374 & 1.437 & -0.096 \\
\hline 2/16/08 & $16: 00$ & 7.594 & 7.499 & 5.006 & 3.922 & 2.28 & 1.357 & -0.143 \\
\hline 2/16/08 & $20: 00$ & 7.557 & 7.462 & 4.971 & 3.887 & 2.233 & 1.348 & -0.18 \\
\hline $2 / 17 / 08$ & $0: 00$ & 7.526 & 7.429 & 4.943 & 3.859 & 2.191 & 1.327 & -0.199 \\
\hline $2 / 17 / 08$ & $4: 00$ & 7.489 & 7.385 & 4.915 & 3.824 & 2.163 & 1.301 & -0.231 \\
\hline $2 / 17 / 08$ & $8: 00$ & 7.468 & 7.374 & 4.912 & 3.821 & 2.175 & 1.283 & -0.236 \\
\hline $2 / 17 / 08$ & $12: 00$ & 7.505 & 7.425 & 4.95 & 3.877 & 2.215 & 1.287 & -0.187 \\
\hline $2 / 17 / 08$ & $16: 00$ & 7.54 & 7.464 & 4.945 & 3.894 & 2.203 & 1.308 & -0.182 \\
\hline $2 / 17 / 08$ & $20: 00$ & 7.576 & 7.504 & 4.969 & 3.924 & 2.219 & 1.32 & -0.147 \\
\hline 2/18/08 & 0:00 & 7.59 & 7.517 & 4.983 & 3.934 & 2.217 & 1.334 & -0.138 \\
\hline 2/18/08 & $4: 00$ & 7.618 & 7.548 & 5.011 & 3.967 & 2.224 & 1.357 & -0.11 \\
\hline 2/18/08 & 8:00 & 7.627 & 7.557 & 5.016 & 3.969 & 2.214 & 1.362 & -0.11 \\
\hline 2/18/08 & $12: 00$ & 7.655 & 7.587 & 5.041 & 3.999 & 2.24 & 1.404 & -0.082 \\
\hline 2/18/08 & $16: 00$ & 7.679 & 7.615 & 5.06 & 4.02 & 2.231 & 1.381 & -0.059 \\
\hline 2/18/08 & $20: 00$ & 7.698 & 7.638 & 5.083 & 4.044 & 2.235 & 1.411 & -0.04 \\
\hline 2/19/08 & 0:00 & 7.714 & 7.654 & 5.095 & 4.058 & 2.231 & 1.414 & -0.028 \\
\hline 2/19/08 & $4: 00$ & 7.724 & 7.659 & 5.107 & 4.063 & 2.259 & 1.416 & -0.023 \\
\hline 2/19/08 & $8: 00$ & 7.707 & 7.636 & 5.1 & 4.044 & 2.35 & 1.449 & -0.04 \\
\hline 2/19/08 & $12: 00$ & 7.684 & 7.61 & 5.088 & 4.02 & 2.415 & 1.465 & -0.063 \\
\hline 2/19/08 & $16: 00$ & 7.646 & 7.571 & 5.06 & 3.985 & 2.338 & 1.399 & -0.091 \\
\hline 2/19/08 & $20: 00$ & 7.667 & 7.599 & 5.079 & 4.016 & 2.322 & 1.414 & -0.065 \\
\hline 2/20/08 & 0:00 & 7.735 & 7.68 & 5.13 & 4.088 & 2.305 & 1.458 & -0.002 \\
\hline $2 / 20 / 08$ & $4: 00$ & 7.77 & 7.719 & 5.156 & 4.121 & 2.278 & 1.453 & 0.026 \\
\hline $2 / 20 / 08$ & $8: 00$ & 7.801 & 7.75 & 5.184 & 4.154 & 2.378 & 1.479 & 0.061 \\
\hline 2/20/08 & $12: 00$ & 7.829 & 7.782 & 5.21 & 4.175 & 2.584 & 1.657 & 0.084 \\
\hline 2/20/08 & $16: 00$ & 7.817 & 7.761 & 5.203 & 4.149 & 2.463 & 1.537 & 0.063 \\
\hline 2/20/08 & $20: 00$ & 7.817 & 7.759 & 5.208 & 4.156 & 2.406 & 1.505 & 0.07 \\
\hline $2 / 21 / 08$ & $0: 00$ & 7.803 & 7.74 & 5.201 & 4.142 & 2.432 & 1.54 & 0.058 \\
\hline $2 / 21 / 08$ & $4: 00$ & 7.784 & 7.722 & 5.186 & 4.121 & 2.481 & 1.572 & 0.04 \\
\hline
\end{tabular}


TABLE S1.3 (Cont.)

Depth to Water (feet below top of casing) in Indicated Wells

Date Time PMW1s PMW1d PMW2sa PMW2d PMW9s PMW9d PMW3d

\begin{tabular}{|c|c|c|c|c|c|c|c|c|}
\hline $2 / 21 / 08$ & $8: 00$ & 7.752 & 7.685 & 5.168 & 4.095 & 2.49 & 1.551 & 0.019 \\
\hline $2 / 21 / 08$ & $12: 00$ & 7.754 & 7.685 & 5.172 & 4.1 & 2.523 & 1.554 & 0.019 \\
\hline $2 / 21 / 08$ & $16: 00$ & 7.719 & 7.647 & 5.142 & 4.065 & 2.453 & 1.516 & -0.012 \\
\hline 2/21/08 & $20: 00$ & 7.712 & 7.64 & 5.14 & 4.067 & 2.381 & 1.46 & -0.009 \\
\hline 2/22/08 & 0:00 & 7.714 & 7.645 & 5.14 & 4.067 & 2.367 & 1.467 & -0.005 \\
\hline $2 / 22 / 08$ & $4: 00$ & 7.707 & 7.636 & 5.135 & 4.063 & 2.361 & 1.46 & -0.009 \\
\hline $2 / 22 / 08$ & $8: 00$ & 7.716 & 7.65 & 5.147 & 4.077 & 2.371 & 1.456 & 0.005 \\
\hline $2 / 22 / 08$ & $12: 00$ & 7.726 & 7.659 & 5.154 & 4.079 & 2.469 & 1.558 & 0.002 \\
\hline $2 / 22 / 08$ & $16: 00$ & 7.716 & 7.643 & 5.144 & 4.063 & 2.369 & 1.458 & -0.009 \\
\hline $2 / 22 / 08$ & 20:00 & 7.714 & 7.643 & 5.137 & 4.06 & 2.32 & 1.451 & -0.009 \\
\hline 2/23/08 & 0:00 & 7.726 & 7.654 & 5.14 & 4.07 & 2.276 & 1.458 & 0.002 \\
\hline 2/23/08 & $4: 00$ & 7.73 & 7.661 & 5.137 & 4.072 & 2.247 & 1.439 & 0.002 \\
\hline 2/23/08 & 8:00 & 7.726 & 7.657 & 5.13 & 4.063 & 2.25 & 1.435 & -0.007 \\
\hline $2 / 23 / 08$ & $12: 00$ & 7.74 & 7.675 & 5.149 & 4.079 & 2.325 & 1.488 & 0.007 \\
\hline 2/23/08 & $16: 00$ & 7.74 & 7.671 & 5.14 & 4.067 & 2.289 & 1.444 & -0.016 \\
\hline 2/23/08 & $20: 00$ & 7.754 & 7.689 & 5.144 & 4.084 & 2.282 & 1.451 & 0.005 \\
\hline 2/24/08 & 0:00 & 7.77 & 7.708 & 5.158 & 4.1 & 2.259 & 1.458 & 0.023 \\
\hline $2 / 24 / 08$ & 4:00 & 7.773 & 7.712 & 5.158 & 4.1 & 2.238 & 1.449 & 0.028 \\
\hline 2/24/08 & $8: 00$ & 7.758 & 7.694 & 5.147 & 4.081 & 2.224 & 1.463 & 0.014 \\
\hline $2 / 24 / 08$ & $12: 00$ & 7.754 & 7.689 & 5.142 & 4.079 & 2.208 & 1.444 & 0.012 \\
\hline $2 / 24 / 08$ & $16: 00$ & 7.636 & 7.636 & 5.102 & 4.039 & 2.187 & 1.432 & -0.04 \\
\hline $2 / 24 / 08$ & 20:00 & 7.536 & 7.592 & 5.06 & 4.004 & 2.182 & 1.421 & -0.061 \\
\hline $2 / 25 / 08$ & 0:00 & 7.491 & 7.559 & 5.046 & 3.99 & 2.168 & 1.404 & -0.065 \\
\hline 2/25/08 & $4: 00$ & 7.442 & 7.513 & 5.018 & 3.95 & 2.142 & 1.383 & -0.096 \\
\hline $2 / 25 / 08$ & $8: 00$ & 7.411 & 7.494 & 5.004 & 3.938 & 2.147 & 1.364 & -0.103 \\
\hline $2 / 25 / 08$ & $12: 00$ & 7.397 & 7.478 & 5.001 & 3.939 & 2.159 & 1.353 & -0.103 \\
\hline $2 / 25 / 08$ & $16: 00$ & 7.416 & 7.49 & 5.008 & 3.964 & 2.187 & 1.353 & -0.096 \\
\hline $2 / 25 / 08$ & $20: 00$ & 7.467 & 7.492 & 5.06 & 4.035 & 2.226 & 1.371 & -0.026 \\
\hline 2/26/08 & 0:00 & 7.531 & 7.439 & 5.102 & 4.089 & 2.252 & 1.421 & 0.023 \\
\hline $2 / 26 / 08$ & 4:00 & 7.578 & 7.364 & 5.133 & 4.117 & 2.256 & 1.456 & 0.051 \\
\hline $2 / 26 / 08$ & $8: 00$ & 7.641 & 7.334 & 5.161 & 4.145 & 2.27 & 1.488 & 0.079 \\
\hline 2/26/08 & $12: 00$ & 7.723 & 7.766 & 5.189 & 4.17 & 2.277 & 1.514 & 0.103 \\
\hline 2/26/08 & $16: 00$ & 7.732 & 7.761 & 5.186 & 4.156 & 2.261 & 1.509 & 0.089 \\
\hline 2/26/08 & 20:00 & 7.721 & 7.736 & 5.17 & 4.131 & 2.254 & 1.5 & 0.065 \\
\hline $2 / 27 / 08$ & 0:00 & 7.744 & 7.731 & 5.163 & 4.119 & 2.256 & 1.5 & 0.056 \\
\hline $2 / 27 / 08$ & $4: 00$ & 7.756 & 7.729 & 5.161 & 4.117 & 2.252 & 1.484 & 0.051 \\
\hline $2 / 27 / 08$ & $8: 00$ & 7.77 & 7.733 & 5.168 & 4.124 & 2.254 & 1.486 & 0.056 \\
\hline $2 / 27 / 08$ & $12: 00$ & 7.782 & 7.747 & 5.179 & 4.133 & 2.263 & 1.484 & 0.061 \\
\hline $2 / 27 / 08$ & $16: 00$ & 7.695 & 7.705 & 5.149 & 4.095 & 2.247 & 1.453 & 0.016 \\
\hline $2 / 27 / 08$ & $20: 00$ & 7.608 & 7.678 & 5.13 & 4.088 & 2.254 & 1.458 & 0.016 \\
\hline $2 / 28 / 08$ & 0:00 & 7.573 & 7.659 & 5.128 & 4.081 & 2.256 & 1.463 & 0.019 \\
\hline $2 / 28 / 08$ & 4:00 & 7.54 & 7.636 & 5.111 & 4.065 & 2.25 & 1.46 & 0.005 \\
\hline $2 / 28 / 08$ & $8: 00$ & 7.516 & 7.601 & 5.09 & 4.042 & 2.24 & 1.449 & -0.016 \\
\hline $2 / 28 / 08$ & $12: 00$ & 7.514 & 7.594 & 5.088 & 4.042 & 2.245 & 1.439 & -0.014 \\
\hline
\end{tabular}


TABLE S1.3 (Cont.)

Depth to Water (feet below top of casing) in Indicated Wells

Date Time PMW1s PMW1d PMW2sa PMW2d PMW9s PMW9d PMW3d

\begin{tabular}{|c|c|c|c|c|c|c|c|c|}
\hline 2/28/08 & $16: 00$ & 7.479 & 7.566 & 5.062 & 4.011 & 2.226 & 1.425 & -0.044 \\
\hline 2/28/08 & $20: 00$ & 7.416 & 7.562 & 5.058 & 4.011 & 2.242 & 1.421 & -0.047 \\
\hline 2/29/08 & 0:00 & 7.399 & 7.571 & 5.065 & 4.025 & 2.252 & 1.423 & -0.028 \\
\hline 2/29/08 & $4: 00$ & 7.411 & 7.601 & 5.093 & 4.063 & 2.275 & 1.437 & 0.007 \\
\hline 2/29/08 & $8: 00$ & 7.469 & 7.666 & 5.14 & 4.119 & 2.305 & 1.46 & 0.058 \\
\hline 2/29/08 & $12: 00$ & 7.486 & 7.703 & 5.165 & 4.147 & 2.306 & 1.488 & 0.08 \\
\hline 2/29/08 & $16: 00$ & 7.356 & 7.668 & 5.102 & 4.105 & 2.287 & 1.498 & 0.03 \\
\hline 2/29/08 & $20: 00$ & 7.031 & 7.585 & 5.048 & 4.058 & 2.273 & 1.488 & -0.002 \\
\hline $3 / 1 / 08$ & 0:00 & 6.818 & 7.52 & 5.039 & 4.046 & 2.282 & 1.472 & 0.005 \\
\hline $3 / 1 / 08$ & $4: 00$ & 6.779 & 7.473 & 5.018 & 4.018 & 2.275 & 1.456 & -0.019 \\
\hline $3 / 1 / 08$ & 8:00 & 6.851 & 7.45 & 4.985 & 3.978 & 2.259 & 1.437 & -0.054 \\
\hline $3 / 1 / 08$ & $12: 00$ & 7.349 & 7.485 & 4.983 & 3.96 & 2.261 & 1.418 & -0.077 \\
\hline $3 / 1 / 08$ & $16: 00$ & 6.972 & 7.411 & 4.896 & 3.892 & 2.254 & 1.399 & -0.145 \\
\hline $3 / 1 / 08$ & $20: 00$ & 6.76 & 7.313 & 4.828 & 3.831 & 2.259 & 1.367 & -0.189 \\
\hline $3 / 2 / 08$ & $0: 00$ & 7.26 & 7.336 & 4.83 & 3.821 & 2.268 & 1.339 & -0.191 \\
\hline $3 / 2 / 08$ & $4: 00$ & 7.31 & 7.325 & 4.826 & 3.8 & 2.261 & 1.318 & -0.213 \\
\hline $3 / 2 / 08$ & $8: 00$ & 7.32 & 7.309 & 4.828 & 3.789 & 2.278 & 1.299 & -0.227 \\
\hline $3 / 2 / 08$ & $12: 00$ & 7.365 & 7.355 & 4.866 & 3.835 & 2.317 & 1.304 & -0.191 \\
\hline $3 / 2 / 08$ & $16: 00$ & 7.419 & 7.413 & 4.898 & 3.878 & 2.343 & 1.32 & -0.154 \\
\hline $3 / 2 / 08$ & $20: 00$ & 7.372 & 7.432 & 4.842 & 3.866 & 2.329 & 1.336 & -0.198 \\
\hline $3 / 3 / 08$ & 0:00 & 7.329 & 7.457 & 4.882 & 3.901 & 2.35 & 1.346 & -0.131 \\
\hline $3 / 3 / 08$ & $4: 00$ & 7.442 & 7.487 & 4.919 & 3.936 & 2.364 & 1.364 & -0.096 \\
\hline $3 / 3 / 08$ & 8:00 & 7.536 & 7.543 & 4.983 & 3.988 & 2.383 & 1.4 & -0.049 \\
\hline $3 / 3 / 08$ & $12: 00$ & 7.604 & 7.601 & 5.032 & 4.042 & 2.387 & 1.432 & 0 \\
\hline $3 / 3 / 08$ & $16: 00$ & 7.613 & 7.596 & 5.025 & 4.028 & 2.371 & 1.442 & -0.019 \\
\hline $3 / 3 / 08$ & $20: 00$ & 7.614 & 7.594 & 5.029 & 4.025 & 2.392 & 1.442 & -0.016 \\
\hline $3 / 4 / 08$ & $0: 00$ & 7.618 & 7.587 & 5.034 & 4.021 & 2.385 & 1.453 & -0.021 \\
\hline $3 / 4 / 08$ & $4: 00$ & 7.614 & 7.576 & 5.039 & 4.014 & 2.376 & 1.442 & -0.03 \\
\hline $3 / 4 / 08$ & 8:00 & 7.614 & 7.566 & 5.041 & 4.011 & 2.362 & 1.432 & -0.033 \\
\hline $3 / 4 / 08$ & $12: 00$ & 7.607 & 7.559 & 5.037 & 4.002 & 2.343 & 1.428 & -0.044 \\
\hline $3 / 4 / 08$ & $16: 00$ & 7.576 & 7.522 & 5.006 & 3.967 & 2.329 & 1.432 & -0.077 \\
\hline $3 / 4 / 08$ & $20: 00$ & 7.572 & 7.515 & 5.008 & 3.971 & 2.359 & 1.437 & -0.072 \\
\hline $3 / 5 / 08$ & 0:00 & 7.579 & 7.522 & 5.018 & 3.978 & 2.369 & 1.425 & -0.065 \\
\hline $3 / 5 / 08$ & $4: 00$ & 7.572 & 7.513 & 5.011 & 3.964 & 2.364 & 1.414 & -0.079 \\
\hline $3 / 5 / 08$ & 8:00 & 7.614 & 7.569 & 5.053 & 4.023 & 2.397 & 1.444 & -0.028 \\
\hline $3 / 5 / 08$ & $12: 00$ & 7.677 & 7.636 & 5.097 & 4.081 & 2.411 & 1.481 & 0.026 \\
\hline $3 / 5 / 08$ & $16: 00$ & 7.703 & 7.664 & 5.107 & 4.098 & 2.406 & 1.495 & 0.035 \\
\hline $3 / 5 / 08$ & $20: 00$ & 7.729 & 7.692 & 5.128 & 4.119 & 2.427 & 1.519 & 0.056 \\
\hline $3 / 6 / 08$ & 0:00 & 7.757 & 7.717 & 5.147 & 4.138 & 2.416 & 1.54 & 0.075 \\
\hline $3 / 6 / 08$ & $4: 00$ & 7.762 & 7.717 & 5.147 & 4.131 & 2.397 & 1.528 & 0.068 \\
\hline $3 / 6 / 08$ & 8:00 & 7.773 & 7.729 & 5.165 & 4.145 & 2.395 & 1.528 & 0.077 \\
\hline $3 / 6 / 08$ & $12: 00$ & 7.792 & 7.747 & 5.175 & 4.161 & 2.388 & 1.53 & 0.089 \\
\hline $3 / 6 / 08$ & $16: 00$ & 7.769 & 7.715 & 5.144 & 4.121 & 2.367 & 1.5 & 0.051 \\
\hline $3 / 6 / 08$ & $20: 00$ & 7.759 & 7.712 & 5.144 & 4.121 & 2.388 & 1.512 & 0.054 \\
\hline
\end{tabular}


TABLE S1.3 (Cont.)

Depth to Water (feet below top of casing) in Indicated Wells

Date Time PMW1s PMW1d PMW2sa PMW2d PMW9s PMW9d PMW3d

\begin{tabular}{|c|c|c|c|c|c|c|c|c|}
\hline $3 / 7 / 08$ & 0:00 & 7.79 & 7.745 & 5.175 & 4.152 & 2.411 & 1.53 & 0.082 \\
\hline $3 / 7 / 08$ & 4:00 & 7.818 & 7.777 & 5.193 & 4.182 & 2.418 & 1.554 & 0.11 \\
\hline $3 / 7 / 08$ & 8:00 & 7.842 & 7.808 & 5.214 & 4.208 & 2.43 & 1.568 & 0.133 \\
\hline $3 / 7 / 08$ & $12: 00$ & 7.862 & 7.828 & 5.224 & 4.22 & 2.416 & 1.587 & 0.14 \\
\hline $3 / 7 / 08$ & $16: 00$ & 7.851 & 7.81 & 5.2 & 4.189 & 2.378 & 1.568 & 0.115 \\
\hline $3 / 7 / 08$ & $20: 00$ & 7.832 & 7.784 & 5.191 & 4.173 & 2.386 & 1.554 & 0.098 \\
\hline $3 / 8 / 08$ & 0:00 & 7.823 & 7.773 & 5.193 & 4.168 & 2.39 & 1.554 & 0.094 \\
\hline $3 / 8 / 08$ & 4:00 & 7.794 & 7.736 & 5.172 & 4.135 & 2.376 & 1.528 & 0.066 \\
\hline $3 / 8 / 08$ & 8:00 & 7.771 & 7.705 & 5.158 & 4.119 & 2.376 & 1.514 & 0.049 \\
\hline $3 / 8 / 08$ & $12: 00$ & 7.748 & 7.678 & 5.14 & 4.089 & 2.36 & 1.479 & 0.021 \\
\hline $3 / 8 / 08$ & $16: 00$ & 7.729 & 7.659 & 5.125 & 4.077 & 2.353 & 1.481 & 0.012 \\
\hline $3 / 8 / 08$ & $20: 00$ & 7.738 & 7.678 & 5.137 & 4.098 & 2.362 & 1.493 & 0.028 \\
\hline $3 / 9 / 08$ & 0:00 & 7.769 & 7.712 & 5.163 & 4.131 & 2.369 & 1.519 & 0.056 \\
\hline $3 / 9 / 08$ & 4:00 & 7.795 & 7.738 & 5.179 & 4.147 & 2.364 & 1.54 & 0.073 \\
\hline $3 / 9 / 08$ & 8:00 & 7.827 & 7.78 & 5.21 & 4.182 & 2.378 & 1.563 & 0.105 \\
\hline $3 / 9 / 08$ & $12: 00$ & 7.865 & 7.815 & 5.228 & 4.213 & 2.388 & 1.563 & 0.129 \\
\hline $3 / 9 / 08$ & $16: 00$ & 7.872 & 7.831 & 5.229 & 4.215 & 2.378 & 1.565 & 0.129 \\
\hline $3 / 9 / 08$ & $20: 00$ & 7.886 & 7.842 & 5.24 & 4.227 & 2.4 & 1.582 & 0.145 \\
\hline 3/10/08 & 0:00 & 7.907 & 7.87 & 5.259 & 4.253 & 2.42 & 1.603 & 0.166 \\
\hline 3/10/08 & 4:00 & 7.921 & 7.882 & 5.268 & 4.26 & 2.413 & 1.626 & 0.171 \\
\hline 3/10/08 & 8:00 & 7.938 & 7.9 & 5.285 & 4.278 & 2.416 & 1.631 & 0.187 \\
\hline 3/10/08 & $12: 00$ & 7.954 & 7.919 & 5.294 & 4.288 & 2.404 & 1.619 & 0.194 \\
\hline 3/10/08 & $16: 00$ & 7.924 & 7.877 & 5.257 & 4.239 & 2.383 & 1.608 & 0.15 \\
\hline 3/10/08 & 20:00 & 7.895 & 7.849 & 5.238 & 4.217 & 2.404 & 1.598 & 0.131 \\
\hline 3/11/08 & 0:00 & 7.891 & 7.84 & 5.243 & 4.215 & 2.418 & 1.591 & 0.131 \\
\hline $3 / 11 / 08$ & 4:00 & 7.865 & 7.808 & 5.222 & 4.187 & 2.407 & 1.582 & 0.103 \\
\hline 3/11/08 & $8: 00$ & 7.837 & 7.777 & 5.205 & 4.166 & 2.397 & 1.57 & 0.087 \\
\hline 3/11/08 & $12: 00$ & 7.813 & 7.752 & 5.182 & 4.142 & 2.376 & 1.554 & 0.063 \\
\hline 3/11/08 & $16: 00$ & 7.781 & 7.717 & 5.142 & 4.103 & 2.393 & 1.535 & 0.026 \\
\hline 3/11/08 & $20: 00$ & 7.757 & 7.696 & 5.118 & 4.086 & 2.404 & 1.516 & 0.012 \\
\hline $3 / 12 / 08$ & 0:00 & 7.748 & 7.687 & 5.118 & 4.086 & 2.421 & 1.507 & 0.014 \\
\hline $3 / 12 / 08$ & $4: 00$ & 7.738 & 7.671 & 5.114 & 4.072 & 2.421 & 1.502 & 0.005 \\
\hline $3 / 12 / 08$ & 8:00 & 7.722 & 7.657 & 5.109 & 4.063 & 2.413 & 1.495 & -0.004 \\
\hline $3 / 12 / 08$ & $12: 00$ & 7.708 & 7.64 & 5.095 & 4.049 & 2.397 & 1.486 & -0.018 \\
\hline $3 / 12 / 08$ & $16: 00$ & 7.708 & 7.645 & 5.079 & 4.06 & 2.409 & 1.481 & -0.016 \\
\hline $3 / 12 / 08$ & 20:00 & 7.724 & 7.668 & 5.076 & 4.068 & 2.427 & 1.481 & -0.007 \\
\hline 3/13/08 & $0: 00$ & 7.736 & 7.68 & 5.093 & 4.075 & 2.465 & 1.488 & 0.005 \\
\hline 3/13/08 & 4:00 & 7.752 & 7.694 & 5.116 & 4.091 & 2.481 & 1.5 & 0.017 \\
\hline 3/13/08 & $8: 00$ & 7.755 & 7.696 & 5.128 & 4.096 & 2.484 & 1.512 & 0.021 \\
\hline 3/13/08 & $12: 00$ & 7.766 & 7.705 & 5.128 & 4.1 & 2.442 & 1.519 & 0.026 \\
\hline 3/13/08 & $16: 00$ & 7.731 & 7.666 & 5.088 & 4.058 & 2.435 & 1.512 & -0.016 \\
\hline $3 / 13 / 08$ & 20:00 & 7.715 & 7.654 & 5.072 & 4.047 & 2.458 & 1.498 & -0.023 \\
\hline $3 / 14 / 08$ & 0:00 & 7.703 & 7.64 & 5.069 & 4.04 & 2.486 & 1.488 & -0.03 \\
\hline $3 / 14 / 08$ & $4: 00$ & 7.703 & 7.638 & 5.079 & 4.044 & 2.514 & 1.488 & -0.028 \\
\hline
\end{tabular}


TABLE S1.3 (Cont.)

Depth to Water (feet below top of casing) in Indicated Wells

Date Time PMW1s PMW1d PMW2sa PMW2d PMW9s PMW9d PMW3d

\begin{tabular}{|c|c|c|c|c|c|c|c|c|}
\hline 3/14/08 & 8:00 & 7.715 & 7.652 & 5.097 & 4.06 & 2.528 & 1.498 & -0.004 \\
\hline 3/14/08 & $12: 00$ & 7.733 & 7.673 & 5.109 & 4.077 & 2.495 & 1.507 & 0 \\
\hline 3/14/08 & $16: 00$ & 7.724 & 7.664 & 5.093 & 4.061 & 2.479 & 1.509 & -0.014 \\
\hline 3/14/08 & 20:00 & 7.748 & 7.694 & 5.111 & 4.086 & 2.509 & 1.512 & 0.01 \\
\hline 3/15/08 & $0: 00$ & 7.79 & 7.74 & 5.149 & 4.133 & 2.547 & 1.528 & 0.047 \\
\hline 3/15/08 & 4:00 & 7.813 & 7.761 & 5.17 & 4.147 & 2.577 & 1.547 & 0.061 \\
\hline 3/15/08 & 8:00 & 7.835 & 7.787 & 5.196 & 4.173 & 2.601 & 1.57 & 0.084 \\
\hline 3/15/08 & $12: 00$ & 7.865 & 7.819 & 5.217 & 4.199 & 2.573 & 1.591 & 0.108 \\
\hline 3/15/08 & $16: 00$ & 7.867 & 7.817 & 5.217 & 4.192 & 2.556 & 1.603 & 0.098 \\
\hline 3/15/08 & 20:00 & 7.886 & 7.842 & 5.233 & 4.217 & 2.589 & 1.612 & 0.124 \\
\hline 3/16/08 & 0:00 & 7.917 & 7.87 & 5.261 & 4.248 & 2.643 & 1.638 & 0.15 \\
\hline 3/16/08 & 4:00 & 7.933 & 7.889 & 5.287 & 4.264 & 2.663 & 1.659 & 0.164 \\
\hline 3/16/08 & $8: 00$ & 7.959 & 7.914 & 5.315 & 4.295 & 2.675 & 1.682 & 0.194 \\
\hline 3/16/08 & $12: 00$ & 7.978 & 7.933 & 5.313 & 4.302 & 2.598 & 1.701 & 0.199 \\
\hline 3/16/08 & $16: 00$ & 7.966 & 7.919 & 5.285 & 4.274 & 2.539 & 1.668 & 0.173 \\
\hline 3/16/08 & 20:00 & 7.945 & 7.896 & 5.273 & 4.255 & 2.547 & 1.661 & 0.157 \\
\hline 3/17/08 & 0:00 & 7.952 & 7.905 & 5.287 & 4.264 & 2.572 & 1.661 & 0.166 \\
\hline 3/17/08 & 4:00 & 7.931 & 7.875 & 5.268 & 4.241 & 2.568 & 1.657 & 0.143 \\
\hline 3/17/08 & 8:00 & 7.921 & 7.863 & 5.268 & 4.239 & 2.577 & 1.65 & 0.145 \\
\hline 3/17/08 & $12: 00$ & 7.895 & 7.88 & 5.205 & 4.196 & 2.47 & 1.638 & 0.133 \\
\hline 3/17/08 & $16: 00$ & 7.849 & 7.791 & 5.175 & 4.152 & 2.512 & 1.605 & 0.063 \\
\hline 3/17/08 & 20:00 & 7.839 & 7.782 & 5.179 & 4.152 & 2.533 & 1.591 & 0.066 \\
\hline 3/18/08 & 0:00 & 7.837 & 7.763 & 5.182 & 4.157 & 2.542 & 1.587 & 0.07 \\
\hline 3/18/08 & 4:00 & 7.83 & 7.761 & 5.179 & 4.152 & 2.554 & 1.582 & 0.066 \\
\hline 3/18/08 & 8:00 & 7.837 & 7.773 & 5.191 & 4.166 & 2.57 & 1.584 & 0.059 \\
\hline 3/18/08 & $12: 00$ & 7.86 & 7.805 & 5.21 & 4.18 & 2.568 & 1.596 & 0.089 \\
\hline 3/18/08 & $16: 00$ & 7.851 & 7.794 & 5.2 & 4.168 & 2.556 & 1.598 & 0.077 \\
\hline 3/18/08 & $20: 00$ & 7.872 & 7.822 & 5.219 & 4.194 & 2.577 & 1.601 & 0.096 \\
\hline 3/19/08 & 0:00 & 7.902 & 7.852 & 5.243 & 4.22 & 2.612 & 1.615 & 0.122 \\
\hline 3/19/08 & 4:00 & 7.903 & 7.849 & 5.252 & 4.22 & 2.635 & 1.624 & 0.12 \\
\hline 3/19/08 & 8:00 & 7.914 & 7.866 & 5.273 & 4.241 & 2.661 & 1.647 & 0.141 \\
\hline 3/19/08 & $12: 00$ & 7.938 & 7.886 & 5.271 & 4.25 & 2.568 & 1.652 & 0.147 \\
\hline 3/19/08 & $16: 00$ & 7.914 & 7.861 & 5.257 & 4.225 & 2.593 & 1.647 & 0.124 \\
\hline 3/19/08 & 20:00 & 7.915 & 7.868 & 5.264 & 4.229 & 2.598 & 1.643 & 0.126 \\
\hline 3/20/08 & 0:00 & 7.931 & 7.884 & 5.261 & 4.241 & 2.586 & 1.643 & 0.138 \\
\hline 3/20/08 & 4:00 & 7.938 & 7.886 & 5.271 & 4.246 & 2.612 & 1.647 & 0.143 \\
\hline 3/20/08 & 8:00 & 7.95 & 7.9 & 5.287 & 4.262 & 2.64 & 1.654 & 0.157 \\
\hline 3/20/08 & $12: 00$ & 7.952 & 7.9 & 5.282 & 4.257 & 2.608 & 1.664 & 0.155 \\
\hline 3/20/08 & $16: 00$ & 7.905 & 7.847 & 5.254 & 4.208 & 2.629 & 1.652 & 0.105 \\
\hline 3/20/08 & 20:00 & 7.877 & 7.815 & 5.238 & 4.187 & 2.624 & 1.638 & 0.089 \\
\hline 3/21/08 & 0:00 & 7.856 & 7.791 & 5.221 & 4.173 & 2.612 & 1.626 & 0.075 \\
\hline 3/21/08 & 4:00 & 7.853 & 7.789 & 5.219 & 4.173 & 2.61 & 1.619 & 0.077 \\
\hline 3/21/08 & 8:00 & 7.898 & 7.849 & 5.261 & 4.229 & 2.635 & 1.626 & 0.129 \\
\hline 3/21/08 & $12: 00$ & 7.954 & 7.91 & 5.303 & 4.281 & 2.649 & 1.652 & 0.176 \\
\hline
\end{tabular}


Depth to Water (feet below top of casing) in Indicated Wells

Date Time PMW1s PMW1d PMW2sa PMW2d PMW9s PMW9d PMW3d

\begin{tabular}{|c|c|c|c|c|c|c|c|c|}
\hline 3/21/08 & $16: 00$ & 7.98 & 7.933 & 5.325 & 4.293 & 2.675 & 1.673 & 0.185 \\
\hline 3/21/08 & 20:00 & 7.997 & 7.956 & 5.339 & 4.318 & 2.68 & 1.694 & 0.208 \\
\hline 3/22/08 & 0:00 & 8.018 & 7.977 & 5.348 & 4.335 & 2.675 & 1.71 & 0.22 \\
\hline $3 / 22 / 08$ & $4: 00$ & 8.029 & 7.989 & 5.355 & 4.339 & 2.673 & 1.722 & 0.227 \\
\hline 3/22/08 & $8: 00$ & 8.048 & 8.009 & 5.374 & 4.36 & 2.684 & 1.734 & 0.246 \\
\hline $3 / 22 / 08$ & $12: 00$ & 8.067 & 8.033 & 5.383 & 4.377 & 2.654 & 1.748 & 0.26 \\
\hline 3/22/08 & $16: 00$ & 8.074 & 8.033 & 5.388 & 4.37 & 2.673 & 1.75 & 0.255 \\
\hline 3/22/08 & 20:00 & 8.088 & 8.054 & 5.404 & 4.391 & 2.694 & 1.76 & 0.274 \\
\hline 3/23/08 & $0: 00$ & 8.107 & 8.072 & 5.416 & 4.41 & 2.694 & 1.774 & 0.29 \\
\hline 3/23/08 & 4:00 & 8.112 & 8.074 & 5.421 & 4.405 & 2.699 & 1.781 & 0.288 \\
\hline 3/23/08 & $8: 00$ & 8.116 & 8.079 & 5.432 & 4.414 & 2.724 & 1.788 & 0.295 \\
\hline 3/23/08 & $12: 00$ & 8.126 & 8.091 & 5.43 & 4.419 & 2.652 & 1.795 & 0.295 \\
\hline $3 / 23 / 08$ & $16: 00$ & 8.111 & 8.07 & 5.425 & 4.4 & 2.708 & 1.785 & 0.278 \\
\hline 3/23/08 & 20:00 & 8.109 & 8.063 & 5.428 & 4.4 & 2.708 & 1.788 & 0.278 \\
\hline 3/24/08 & $0: 00$ & 8.104 & 8.056 & 5.428 & 4.398 & 2.717 & 1.788 & 0.276 \\
\hline 3/24/08 & 4:00 & 8.097 & 8.047 & 5.43 & 4.389 & 2.731 & 1.785 & 0.271 \\
\hline 3/24/08 & 8:00 & 8.074 & 8.016 & 5.416 & 4.368 & 2.733 & 1.778 & 0.255 \\
\hline 3/24/08 & $12: 00$ & 8.034 & 7.968 & 5.36 & 4.316 & 2.589 & 1.764 & 0.206 \\
\hline $3 / 24 / 08$ & $16: 00$ & 7.969 & 7.898 & 5.308 & 4.248 & 2.635 & 1.722 & 0.143 \\
\hline $3 / 24 / 08$ & 20:00 & 7.926 & 7.856 & 5.294 & 4.227 & 2.661 & 1.689 & 0.124 \\
\hline 3/25/08 & $0: 00$ & 7.9 & 7.826 & 5.271 & 4.204 & 2.626 & 1.671 & 0.103 \\
\hline $3 / 25 / 08$ & 4:00 & 7.886 & 7.822 & 5.271 & 4.208 & 2.624 & 1.654 & 0.108 \\
\hline $3 / 25 / 08$ & 8:00 & 7.945 & 7.893 & 5.315 & 4.281 & 2.645 & 1.664 & 0.173 \\
\hline 3/25/08 & $12: 00$ & 7.98 & 7.931 & 5.341 & 4.307 & 2.663 & 1.68 & 0.194 \\
\hline 3/25/08 & $16: 00$ & 7.999 & 7.945 & 5.36 & 4.316 & 2.712 & 1.696 & 0.201 \\
\hline $3 / 25 / 08$ & 20:00 & 8.02 & 7.97 & 5.378 & 4.339 & 2.701 & 1.717 & 0.22 \\
\hline 3/26/08 & 0:00 & 8.041 & 7.991 & 5.385 & 4.353 & 2.668 & 1.731 & 0.234 \\
\hline 3/26/08 & 4:00 & 8.051 & 8.003 & 5.39 & 4.353 & 2.649 & 1.738 & 0.234 \\
\hline $3 / 26 / 08$ & $8: 00$ & 8.051 & 8 & 5.385 & 4.351 & 2.64 & 1.738 & 0.232 \\
\hline 3/26/08 & $12: 00$ & 8.055 & 8.003 & 5.392 & 4.356 & 2.666 & 1.738 & 0.236 \\
\hline 3/26/08 & $16: 00$ & 8.015 & 7.956 & 5.369 & 4.316 & 2.696 & 1.731 & 0.199 \\
\hline $3 / 26 / 08$ & 20:00 & 7.989 & 7.926 & 5.35 & 4.297 & 2.673 & 1.722 & 0.18 \\
\hline 3/27/08 & $0: 00$ & 7.971 & 7.907 & 5.329 & 4.276 & 2.635 & 1.71 & 0.164 \\
\hline $3 / 27 / 08$ & 4:00 & 7.952 & 7.884 & 5.31 & 4.255 & 2.619 & 1.694 & 0.147 \\
\hline $3 / 27 / 08$ & $8: 00$ & 7.957 & 7.898 & 5.325 & 4.274 & 2.626 & 1.685 & 0.164 \\
\hline $3 / 27 / 08$ & $12: 00$ & 7.971 & 7.917 & 5.334 & 4.293 & 2.623 & 1.685 & 0.175 \\
\hline $3 / 27 / 08$ & $16: 00$ & 7.992 & 7.935 & 5.343 & 4.307 & 2.591 & 1.692 & 0.192 \\
\hline $3 / 27 / 08$ & $20: 00$ & 8.022 & 7.972 & 5.371 & 4.342 & 2.607 & 1.699 & 0.22 \\
\hline 3/28/08 & $0: 00$ & 8.06 & 8.014 & 5.395 & 4.372 & 2.614 & 1.717 & 0.246 \\
\hline 3/28/08 & 4:00 & 8.086 & 8.042 & 5.416 & 4.391 & 2.621 & 1.736 & 0.264 \\
\hline 3/28/08 & 8:00 & 8.114 & 8.074 & 5.439 & 4.426 & 2.642 & 1.753 & 0.295 \\
\hline $3 / 28 / 08$ & $12: 00$ & 8.133 & 8.095 & 5.446 & 4.433 & 2.623 & 1.767 & 0.302 \\
\hline 3/28/08 & $16: 00$ & 8.123 & 8.074 & 5.442 & 4.41 & 2.656 & 1.769 & 0.281 \\
\hline $3 / 28 / 08$ & 20:00 & 8.121 & 8.072 & 5.444 & 4.412 & 2.651 & 1.774 & 0.283 \\
\hline
\end{tabular}


Depth to Water (feet below top of casing) in Indicated Wells

Date Time PMW1s PMW1d PMW2sa PMW2d PMW9s PMW9d PMW3d

\begin{tabular}{|c|c|c|c|c|c|c|c|c|}
\hline 3/29/08 & $0: 00$ & 8.135 & 8.091 & 5.451 & 4.426 & 2.635 & 1.781 & 0.295 \\
\hline 3/29/08 & $4: 00$ & 8.126 & 8.077 & 5.442 & 4.412 & 2.623 & 1.781 & 0.281 \\
\hline 3/29/08 & $8: 00$ & 8.121 & 8.072 & 5.442 & 4.412 & 2.623 & 1.776 & 0.281 \\
\hline $3 / 29 / 08$ & $12: 00$ & 8.102 & 8.047 & 5.425 & 4.386 & 2.626 & 1.767 & 0.26 \\
\hline $3 / 29 / 08$ & $16: 00$ & 8.055 & 7.993 & 5.402 & 4.349 & 2.668 & 1.75 & 0.222 \\
\hline 3/29/08 & $20: 00$ & 8.039 & 7.979 & 5.395 & 4.344 & 2.656 & 1.741 & 0.22 \\
\hline 3/30/08 & $0: 00$ & 8.051 & 7.996 & 5.397 & 4.356 & 2.625 & 1.741 & 0.232 \\
\hline 3/30/08 & $4: 00$ & 8.058 & 8.003 & 5.397 & 4.361 & 2.605 & 1.739 & 0.234 \\
\hline 3/30/08 & $8: 00$ & 8.084 & 8.035 & 5.416 & 4.389 & 2.604 & 1.746 & 0.26 \\
\hline 3/30/08 & $12: 00$ & 8.097 & 8.049 & 5.428 & 4.4 & 2.618 & 1.75 & 0.267 \\
\hline 3/30/08 & $16: 00$ & 8.086 & 8.033 & 5.421 & 4.386 & 2.64 & 1.75 & 0.25 \\
\hline 3/30/08 & $20: 00$ & 8.083 & 8.03 & 5.421 & 4.382 & 2.625 & 1.753 & 0.248 \\
\hline 3/31/08 & 0:00 & 8.077 & 8.019 & 5.409 & 4.37 & 2.602 & 1.75 & 0.239 \\
\hline 3/31/08 & $4: 00$ & 8.013 & 7.938 & 5.348 & 4.288 & 2.553 & 1.727 & 0.164 \\
\hline 3/31/08 & $8: 00$ & 7.929 & 7.956 & 4.933 & 4.065 & 2.415 & 1.661 & 0.024 \\
\hline 3/31/08 & $12: 00$ & 7.821 & 7.828 & 4.896 & 4.007 & 2.448 & 1.566 & -0.072 \\
\hline 3/31/08 & $16: 00$ & 7.8 & 7.782 & 4.997 & 4.044 & 2.483 & 1.521 & -0.046 \\
\hline 3/31/08 & $20: 00$ & 7.844 & 7.828 & 5.081 & 4.119 & 2.504 & 1.526 & 0.019 \\
\hline 4/1/08 & 0:00 & 7.905 & 7.891 & 5.151 & 4.19 & 2.52 & 1.554 & 0.08 \\
\hline $4 / 1 / 08$ & $4: 00$ & 7.95 & 7.938 & 5.203 & 4.236 & 2.537 & 1.587 & 0.122 \\
\hline $4 / 1 / 08$ & 8:00 & 7.999 & 7.991 & 5.264 & 4.297 & 2.558 & 1.619 & 0.176 \\
\hline $4 / 1 / 08$ & $12: 00$ & 8.041 & 8.035 & 5.301 & 4.335 & 2.576 & 1.657 & 0.213 \\
\hline $4 / 1 / 08$ & $16: 00$ & 8.065 & 8.051 & 5.324 & 4.347 & 2.59 & 1.682 & 0.222 \\
\hline $4 / 1 / 08$ & $20: 00$ & 8.076 & 8.058 & 5.339 & 4.361 & 2.59 & 1.701 & 0.232 \\
\hline $4 / 2 / 08$ & $0: 00$ & 8.09 & 8.072 & 5.36 & 4.379 & 2.593 & 1.715 & 0.25 \\
\hline $4 / 2 / 08$ & $4: 00$ & 8.088 & 8.061 & 5.362 & 4.365 & 2.586 & 1.722 & 0.239 \\
\hline $4 / 2 / 08$ & 8:00 & 8.083 & 8.056 & 5.367 & 4.368 & 2.586 & 1.724 & 0.241 \\
\hline $4 / 2 / 08$ & $12: 00$ & 8.079 & 8.044 & 5.367 & 4.361 & 2.588 & 1.724 & 0.234 \\
\hline $4 / 2 / 08$ & $16: 00$ & 8.041 & 7.998 & 5.341 & 4.321 & 2.597 & 1.717 & 0.201 \\
\hline $4 / 2 / 08$ & $20: 00$ & 8.011 & 7.968 & 5.331 & 4.307 & 2.6 & 1.703 & 0.187 \\
\hline $4 / 3 / 08$ & $0: 00$ & 8.008 & 7.965 & 5.329 & 4.307 & 2.579 & 1.701 & 0.187 \\
\hline $4 / 3 / 08$ & $4: 00$ & 7.987 & 7.94 & 5.313 & 4.286 & 2.562 & 1.689 & 0.164 \\
\hline $4 / 3 / 08$ & 8:00 & 7.975 & 7.91 & 5.308 & 4.262 & 2.548 & 1.678 & 0.155 \\
\hline $4 / 3 / 08$ & $12: 00$ & 7.966 & 7.947 & 5.278 & 4.302 & 2.485 & 1.664 & 0.15 \\
\hline $4 / 3 / 08$ & $16: 00$ & 7.886 & 7.854 & 5.011 & 4.091 & 2.443 & 1.61 & -0.004 \\
\hline $4 / 3 / 08$ & $20: 00$ & 7.87 & 7.854 & 5.079 & 4.133 & 2.488 & 1.575 & 0.035 \\
\hline $4 / 4 / 08$ & $0: 00$ & 7.884 & 7.866 & 5.123 & 4.159 & 2.497 & 1.573 & 0.059 \\
\hline $4 / 4 / 08$ & $4: 00$ & 7.877 & 7.852 & 5.142 & 4.161 & 2.504 & 1.57 & 0.061 \\
\hline $4 / 4 / 08$ & 8:00 & 7.886 & 7.863 & 5.172 & 4.206 & 2.518 & 1.58 & 0.082 \\
\hline $4 / 4 / 08$ & $12: 00$ & 7.893 & 7.866 & 5.186 & 4.192 & 2.527 & 1.587 & 0.087 \\
\hline $4 / 4 / 08$ & $16: 00$ & 7.87 & 7.835 & 5.179 & 4.171 & 2.546 & 1.584 & 0.066 \\
\hline $4 / 4 / 08$ & $20: 00$ & 7.856 & 7.822 & 5.184 & 4.169 & 2.551 & 1.584 & 0.066 \\
\hline $4 / 5 / 08$ & 0:00 & 7.858 & 7.817 & 5.189 & 4.173 & 2.536 & 1.584 & 0.071 \\
\hline $4 / 5 / 08$ & $4: 00$ & 7.849 & 7.81 & 5.186 & 4.168 & 2.529 & 1.584 & 0.068 \\
\hline
\end{tabular}


TABLE S1.3 (Cont.)

Depth to Water (feet below top of casing) in Indicated Wells

Date Time PMW1s PMW1d PMW2sa PMW2d PMW9s PMW9d PMW3d

\begin{tabular}{|c|c|c|c|c|c|c|c|c|}
\hline $4 / 5 / 08$ & 8:00 & 7.854 & 7.812 & 5.196 & 4.18 & 2.527 & 1.582 & 0.078 \\
\hline 4/5/08 & $12: 00$ & 7.851 & 7.808 & 5.198 & 4.176 & 2.56 & 1.582 & 0.073 \\
\hline $4 / 5 / 08$ & $16: 00$ & 7.832 & 7.775 & 5.193 & 4.15 & 2.609 & 1.58 & 0.049 \\
\hline 4/5/08 & 20:00 & 7.814 & 7.766 & 5.196 & 4.152 & 2.592 & 1.58 & 0.052 \\
\hline 4/6/08 & 0:00 & 7.814 & 7.759 & 5.189 & 4.15 & 2.553 & 1.58 & 0.054 \\
\hline 4/6/08 & 4:00 & 7.8 & 7.747 & 5.175 & 4.133 & 2.527 & 1.573 & 0.038 \\
\hline 4/6/08 & 8:00 & 7.804 & 7.754 & 5.186 & 4.15 & 2.527 & 1.568 & 0.049 \\
\hline 4/6/08 & $12: 00$ & 7.83 & 7.787 & 5.214 & 4.182 & 2.55 & 1.573 & 0.08 \\
\hline $4 / 6 / 08$ & $16: 00$ & 7.851 & 7.798 & 5.231 & 4.197 & 2.592 & 1.582 & 0.091 \\
\hline 4/6/08 & 20:00 & 7.893 & 7.852 & 5.271 & 4.251 & 2.634 & 1.605 & 0.136 \\
\hline 4/7/08 & 0:00 & 7.938 & 7.905 & 5.296 & 4.286 & 2.599 & 1.636 & 0.169 \\
\hline $4 / 7 / 08$ & 4:00 & 7.957 & 7.914 & 5.303 & 4.288 & 2.574 & 1.654 & 0.169 \\
\hline 4/7/08 & 8:00 & 7.973 & 7.935 & 5.32 & 4.304 & 2.574 & 1.664 & 0.185 \\
\hline $4 / 7 / 08$ & $12: 00$ & 8.001 & 7.963 & 5.339 & 4.328 & 2.578 & 1.678 & 0.206 \\
\hline $4 / 7 / 08$ & $16: 00$ & 7.98 & 7.931 & 5.32 & 4.295 & 2.569 & 1.678 & 0.173 \\
\hline $4 / 7 / 08$ & $20: 00$ & 8.004 & 7.926 & 5.348 & 4.295 & 2.574 & 1.682 & 0.187 \\
\hline 4/8/08 & $0: 00$ & 7.973 & 7.942 & 5.205 & 4.279 & 2.447 & 1.668 & 0.122 \\
\hline 4/8/08 & 4:00 & 7.948 & 7.912 & 5.219 & 4.237 & 2.48 & 1.638 & 0.124 \\
\hline 4/8/08 & 8:00 & 7.936 & 7.891 & 5.226 & 4.227 & 2.49 & 1.622 & 0.115 \\
\hline 4/8/08 & $12: 00$ & 7.957 & 7.912 & 5.257 & 4.265 & 2.499 & 1.626 & 0.141 \\
\hline 4/8/08 & $16: 00$ & 7.95 & 7.91 & 5.252 & 4.248 & 2.504 & 1.629 & 0.131 \\
\hline 4/8/08 & $20: 00$ & 7.957 & 7.917 & 5.266 & 4.265 & 2.522 & 1.631 & 0.143 \\
\hline 4/9/08 & $0: 00$ & 7.992 & 7.954 & 5.299 & 4.302 & 2.534 & 1.645 & 0.176 \\
\hline $4 / 9 / 08$ & 4:00 & 8.004 & 7.961 & 5.308 & 4.307 & 2.537 & 1.657 & 0.18 \\
\hline $4 / 9 / 08$ & 8:00 & 8.013 & 7.975 & 5.324 & 4.321 & 2.548 & 1.666 & 0.204 \\
\hline $4 / 9 / 08$ & $12: 00$ & 8.028 & 7.989 & 5.336 & 4.33 & 2.553 & 1.678 & 0.201 \\
\hline 4/9/08 & $16: 00$ & 8.009 & 7.963 & 5.329 & 4.311 & 2.578 & 1.678 & 0.187 \\
\hline $4 / 9 / 08$ & $20: 00$ & 7.966 & 7.917 & 5.299 & 4.272 & 2.562 & 1.668 & 0.15 \\
\hline 4/10/08 & 0:00 & 7.955 & 7.886 & 5.289 & 4.248 & 2.517 & 1.664 & 0.136 \\
\hline 4/10/08 & 4:00 & 7.879 & 7.905 & 5.097 & 4.234 & 2.401 & 1.624 & 0.078 \\
\hline $4 / 10 / 08$ & $8: 00$ & 7.783 & 7.824 & 4.98 & 4.136 & 2.396 & 1.556 & -0.004 \\
\hline 4/10/08 & $12: 00$ & 7.65 & 7.715 & 4.704 & 3.92 & 2.35 & 1.484 & -0.203 \\
\hline 4/10/08 & $16: 00$ & 6.697 & 7.065 & 4.069 & 3.123 & 2.312 & 1.329 & -0.355 \\
\hline $4 / 10 / 08$ & $20: 00$ & 6.129 & 6.64 & 4.271 & 3.152 & 2.357 & 1.098 & -0.351 \\
\hline 4/11/08 & $0: 00$ & 6.457 & 6.622 & 4.369 & 3.285 & 2.359 & 1.019 & -0.351 \\
\hline 4/11/08 & 4:00 & 6.742 & 6.698 & 4.46 & 3.302 & 2.373 & 1.019 & -0.358 \\
\hline $4 / 11 / 08$ & 8:00 & 6.835 & 6.872 & 4.467 & 3.447 & 2.368 & 1.04 & -0.346 \\
\hline 4/11/08 & $12: 00$ & 6.927 & 6.947 & 4.559 & 3.529 & 2.401 & 1.068 & -0.341 \\
\hline 4/11/08 & $16: 00$ & 7.016 & 6.981 & 4.634 & 3.587 & 2.415 & 1.112 & -0.339 \\
\hline 4/11/08 & $20: 00$ & 7.096 & 7.086 & 4.685 & 3.662 & 2.42 & 1.154 & -0.343 \\
\hline $4 / 12 / 08$ & $0: 00$ & 7.148 & 7.125 & 4.72 & 3.693 & 2.424 & 1.192 & -0.329 \\
\hline $4 / 12 / 08$ & 4:00 & 7.18 & 7.153 & 4.748 & 3.712 & 2.433 & 1.215 & -0.289 \\
\hline $4 / 12 / 08$ & 8:00 & 7.223 & 7.19 & 4.781 & 3.749 & 2.44 & 1.238 & -0.261 \\
\hline 4/12/08 & $12: 00$ & 7.267 & 7.232 & 4.816 & 3.789 & 2.455 & 1.262 & -0.233 \\
\hline
\end{tabular}


TABLE S1.3 (Cont.)

Depth to Water (feet below top of casing) in Indicated Wells

Date Time PMW1s PMW1d PMW2sa PMW2d PMW9s PMW9d PMW3d

\begin{tabular}{|c|c|c|c|c|c|c|c|c|}
\hline $4 / 12 / 08$ & $16: 00$ & 7.3 & 7.258 & 4.837 & 3.808 & 2.457 & 1.285 & -0.212 \\
\hline $4 / 12 / 08$ & 20:00 & 7.335 & 7.292 & 4.861 & 3.834 & 2.459 & 1.308 & -0.186 \\
\hline 4/13/08 & 0:00 & 7.37 & 7.323 & 4.884 & 3.864 & 2.462 & 1.325 & -0.163 \\
\hline 4/13/08 & $4: 00$ & 7.394 & 7.341 & 4.896 & 3.873 & 2.462 & 1.343 & -0.151 \\
\hline 4/13/08 & 8:00 & 7.429 & 7.378 & 4.924 & 3.909 & 2.476 & 1.36 & -0.119 \\
\hline 4/13/08 & $12: 00$ & 7.464 & 7.415 & 4.947 & 3.934 & 2.492 & 1.381 & -0.095 \\
\hline 4/13/08 & $16: 00$ & 7.476 & 7.418 & 4.954 & 3.932 & 2.506 & 1.395 & -0.098 \\
\hline 4/13/08 & $20: 00$ & 7.478 & 7.42 & 4.961 & 3.937 & 2.494 & 1.402 & -0.098 \\
\hline 4/14/08 & 0:00 & 7.497 & 7.439 & 4.971 & 3.951 & 2.48 & 1.411 & -0.084 \\
\hline 4/14/08 & 4:00 & 7.509 & 7.446 & 4.978 & 3.955 & 2.48 & 1.418 & -0.079 \\
\hline 4/14/08 & 8:00 & 7.528 & 7.466 & 4.999 & 3.979 & 2.494 & 1.428 & -0.058 \\
\hline 4/14/08 & $12: 00$ & 7.535 & 7.469 & 4.992 & 3.972 & 2.483 & 1.432 & -0.067 \\
\hline 4/14/08 & $16: 00$ & 7.514 & 7.439 & 4.983 & 3.946 & 2.506 & 1.428 & -0.088 \\
\hline 4/14/08 & 20:00 & 7.488 & 7.408 & 4.968 & 3.925 & 2.499 & 1.421 & -0.109 \\
\hline 4/15/08 & 0:00 & 7.493 & 7.415 & 4.971 & 3.932 & 2.478 & 1.416 & -0.102 \\
\hline 4/15/08 & $4: 00$ & 7.471 & 7.39 & 4.954 & 3.909 & 2.459 & 1.407 & -0.126 \\
\hline 4/15/08 & 8:00 & 7.457 & 7.374 & 4.945 & 3.897 & 2.454 & 1.393 & -0.137 \\
\hline 4/15/08 & $12: 00$ & 7.431 & 7.341 & 4.936 & 3.871 & 2.489 & 1.379 & -0.161 \\
\hline 4/15/08 & $16: 00$ & 7.387 & 7.288 & 4.915 & 3.831 & 2.548 & 1.365 & -0.198 \\
\hline 4/15/08 & $20: 00$ & 7.371 & 7.269 & 4.915 & 3.831 & 2.564 & 1.355 & -0.196 \\
\hline 4/16/08 & 0:00 & 7.368 & 7.265 & 4.898 & 3.822 & 2.492 & 1.353 & -0.21 \\
\hline 4/16/08 & 4:00 & 7.354 & 7.244 & 4.882 & 3.801 & 2.464 & 1.336 & -0.224 \\
\hline 4/16/08 & 8:00 & 7.378 & 7.281 & 4.912 & 3.845 & 2.468 & 1.332 & -0.189 \\
\hline 4/16/08 & $12: 00$ & 7.425 & 7.334 & 4.95 & 3.887 & 2.489 & 1.346 & -0.151 \\
\hline 4/16/08 & $16: 00$ & 7.455 & 7.369 & 4.973 & 3.913 & 2.515 & 1.365 & -0.128 \\
\hline 4/16/08 & 20:00 & 7.49 & 7.408 & 4.997 & 3.946 & 2.522 & 1.388 & -0.105 \\
\hline 4/17/08 & 0:00 & 7.528 & 7.448 & 5.011 & 3.974 & 2.491 & 1.409 & -0.079 \\
\hline 4/17/08 & $4: 00$ & 7.518 & 7.439 & 4.999 & 3.951 & 2.463 & 1.418 & -0.1 \\
\hline 4/17/08 & 8:00 & 6.981 & 7.29 & 4.378 & 3.611 & 2.337 & 1.36 & -0.341 \\
\hline 4/17/08 & $12: 00$ & 6.762 & 7.012 & 4.516 & 3.527 & 2.382 & 1.236 & -0.343 \\
\hline 4/17/08 & $16: 00$ & 6.953 & 7.042 & 4.507 & 3.562 & 2.354 & 1.173 & -0.343 \\
\hline 4/17/08 & $20: 00$ & 6.965 & 6.993 & 4.523 & 3.513 & 2.363 & 1.131 & -0.346 \\
\hline 4/18/08 & 0:00 & 6.88 & 6.926 & 4.413 & 3.442 & 2.349 & 1.098 & -0.348 \\
\hline 4/18/08 & 4:00 & 6.81 & 6.817 & 4.449 & 3.36 & 2.356 & 1.054 & -0.355 \\
\hline 4/18/08 & 8:00 & 5.47 & 5.953 & 3.79 & 2.723 & 2.297 & 0.951 & -0.367 \\
\hline 4/18/08 & $12: 00$ & 5.069 & 5.406 & 3.854 & 2.589 & 2.316 & 0.745 & -0.362 \\
\hline 4/18/08 & $16: 00$ & 5.202 & 5.438 & 3.91 & 2.653 & 2.316 & 0.647 & -0.358 \\
\hline 4/18/08 & 20:00 & 5.346 & 5.51 & 4.011 & 2.767 & 2.328 & 0.621 & -0.353 \\
\hline 4/19/08 & 0:00 & 5.517 & 5.619 & 4.088 & 2.854 & 2.335 & 0.638 & -0.353 \\
\hline 4/19/08 & $4: 00$ & 5.728 & 5.724 & 4.151 & 2.92 & 2.342 & 0.666 & -0.351 \\
\hline 4/19/08 & 8:00 & 5.935 & 5.847 & 4.217 & 2.999 & 2.354 & 0.703 & -0.348 \\
\hline 4/19/08 & $12: 00$ & 6.068 & 5.937 & 4.261 & 3.049 & 2.359 & 0.743 & -0.344 \\
\hline 4/19/08 & $16: 00$ & 6.132 & 5.981 & 4.287 & 3.067 & 2.365 & 0.768 & -0.348 \\
\hline 4/19/08 & 20:00 & 6.184 & 6.023 & 4.313 & 3.091 & 2.363 & 0.789 & -0.346 \\
\hline
\end{tabular}


TABLE S1.3 (Cont.)

Depth to Water (feet below top of casing) in Indicated Wells

Date Time PMW1s PMW1d PMW2sa PMW2d PMW9s PMW9d PMW3d

\begin{tabular}{|c|c|c|c|c|c|c|c|c|}
\hline $4 / 20 / 08$ & $0: 00$ & 6.237 & 6.074 & 4.336 & 3.119 & 2.354 & 0.806 & -0.346 \\
\hline 4/20/08 & $4: 00$ & 6.275 & 6.106 & 4.35 & 3.138 & 2.351 & 0.822 & -0.341 \\
\hline 4/20/08 & 8:00 & 6.305 & 6.132 & 4.362 & 3.149 & 2.351 & 0.834 & -0.341 \\
\hline 4/20/08 & $12: 00$ & 6.313 & 6.132 & 4.362 & 3.138 & 2.356 & 0.836 & -0.348 \\
\hline 4/20/08 & $16: 00$ & 6.317 & 6.125 & 4.374 & 3.135 & 2.384 & 0.836 & -0.346 \\
\hline $4 / 20 / 08$ & $20: 00$ & 6.329 & 6.137 & 4.385 & 3.147 & 2.384 & 0.836 & -0.351 \\
\hline $4 / 21 / 08$ & $0: 00$ & 6.378 & 6.19 & 4.406 & 3.189 & 2.368 & 0.853 & -0.362 \\
\hline 4/21/08 & $4: 00$ & 6.399 & 6.216 & 4.413 & 3.194 & 2.358 & 0.864 & -0.341 \\
\hline 4/21/08 & 8:00 & 6.447 & 6.264 & 4.439 & 3.231 & 2.368 & 0.874 & -0.358 \\
\hline 4/21/08 & $12: 00$ & 6.507 & 6.336 & 4.474 & 3.283 & 2.377 & 0.897 & -0.358 \\
\hline 4/21/08 & $16: 00$ & 6.543 & 6.371 & 4.491 & 3.299 & 2.382 & 0.92 & -0.355 \\
\hline 4/21/08 & $20: 00$ & 6.564 & 6.39 & 4.495 & 3.311 & 2.377 & 0.934 & -0.339 \\
\hline $4 / 22 / 08$ & 0:00 & 6.608 & 6.438 & 4.519 & 3.344 & 2.377 & 0.955 & -0.339 \\
\hline $4 / 22 / 08$ & $4: 00$ & 6.632 & 6.466 & 4.528 & 3.353 & 2.375 & 0.962 & -0.355 \\
\hline $4 / 22 / 08$ & 8:00 & 6.672 & 6.508 & 4.549 & 3.388 & 2.377 & 0.979 & -0.343 \\
\hline $4 / 22 / 08$ & $12: 00$ & 6.704 & 6.543 & 4.568 & 3.412 & 2.396 & 0.995 & -0.346 \\
\hline $4 / 22 / 08$ & $16: 00$ & 6.716 & 6.552 & 4.577 & 3.412 & 2.421 & 1.005 & -0.343 \\
\hline $4 / 22 / 08$ & $20: 00$ & 6.728 & 6.561 & 4.585 & 3.421 & 2.41 & 1.016 & -0.348 \\
\hline 4/23/08 & 0:00 & 6.754 & 6.594 & 4.592 & 3.435 & 2.384 & 1.026 & -0.344 \\
\hline 4/23/08 & $4: 00$ & 6.768 & 6.605 & 4.596 & 3.44 & 2.381 & 1.033 & -0.344 \\
\hline 4/23/08 & 8:00 & 6.784 & 6.624 & 4.601 & 3.454 & 2.379 & 1.035 & -0.344 \\
\hline 4/23/08 & $12: 00$ & 6.786 & 6.619 & 4.606 & 3.449 & 2.41 & 1.04 & -0.343 \\
\hline 4/23/08 & $16: 00$ & 6.759 & 6.582 & 4.599 & 3.417 & 2.444 & 1.035 & -0.343 \\
\hline 4/23/08 & $20: 00$ & 6.749 & 6.573 & 4.589 & 3.409 & 2.407 & 1.035 & -0.351 \\
\hline 4/24/08 & $0: 00$ & 6.763 & 6.591 & 4.596 & 3.421 & 2.384 & 1.03 & -0.344 \\
\hline 4/24/08 & $4: 00$ & 6.742 & 6.564 & 4.577 & 3.395 & 2.37 & 1.021 & -0.346 \\
\hline $4 / 24 / 08$ & 8:00 & 6.742 & 6.564 & 4.58 & 3.398 & 2.367 & 1.012 & -0.346 \\
\hline 4/24/08 & $12: 00$ & 6.737 & 6.559 & 4.58 & 3.393 & 2.377 & 1.005 & -0.348 \\
\hline $4 / 24 / 08$ & $16: 00$ & 6.728 & 6.55 & 4.58 & 3.384 & 2.384 & 1 & -0.348 \\
\hline 4/24/08 & $20: 00$ & 6.73 & 6.547 & 4.575 & 3.386 & 2.358 & 0.998 & -0.343 \\
\hline $4 / 25 / 08$ & 0:00 & 6.728 & 6.517 & 4.575 & 3.379 & 2.356 & 0.993 & -0.343 \\
\hline $4 / 25 / 08$ & $4: 00$ & 6.679 & 6.552 & 4.385 & 3.304 & 2.307 & 0.972 & -0.348 \\
\hline $4 / 25 / 08$ & 8:00 & 6.728 & 6.538 & 4.51 & 3.348 & 2.36 & 0.958 & -0.351 \\
\hline $4 / 25 / 08$ & $12: 00$ & 6.775 & 6.622 & 4.559 & 3.402 & 2.365 & 0.977 & -0.346 \\
\hline $4 / 25 / 08$ & $16: 00$ & 6.798 & 6.65 & 4.587 & 3.433 & 2.377 & 0.993 & -0.346 \\
\hline 4/25/08 & $20: 00$ & 6.841 & 6.689 & 4.617 & 3.47 & 2.386 & 1.016 & -0.343 \\
\hline 4/26/08 & $0: 00$ & 6.864 & 6.719 & 4.634 & 3.489 & 2.379 & 1.037 & -0.336 \\
\hline $4 / 26 / 08$ & $4: 00$ & 6.874 & 6.721 & 4.634 & 3.487 & 2.372 & 1.049 & -0.35 \\
\hline 4/26/08 & $8: 00$ & 6.904 & 6.756 & 4.657 & 3.524 & 2.386 & 1.063 & -0.35 \\
\hline 4/26/08 & $12: 00$ & 6.932 & 6.793 & 4.681 & 3.548 & 2.402 & 1.079 & -0.341 \\
\hline 4/26/08 & $16: 00$ & 6.949 & 6.807 & 4.69 & 3.559 & 2.412 & 1.093 & -0.343 \\
\hline $4 / 26 / 08$ & $20: 00$ & 6.967 & 6.761 & 4.678 & 3.468 & 2.374 & 1.107 & -0.339 \\
\hline $4 / 27 / 08$ & 0:00 & 6.972 & 6.777 & 4.648 & 3.543 & 2.365 & 1.105 & -0.341 \\
\hline 4/27/08 & $4: 00$ & 6.989 & 6.782 & 4.674 & 3.508 & 2.379 & 1.105 & -0.339 \\
\hline
\end{tabular}


Depth to Water (feet below top of casing) in Indicated Wells

Date Time PMW1s PMW1d PMW2sa PMW2d PMW9s PMW9d PMW3d

\begin{tabular}{|c|c|c|c|c|c|c|c|c|}
\hline $4 / 27 / 08$ & 8:00 & 7 & 6.847 & 4.69 & 3.58 & 2.383 & 1.112 & -0.339 \\
\hline 4/27/08 & $12: 00$ & 6.998 & 6.858 & 4.688 & 3.569 & 2.388 & 1.117 & -0.334 \\
\hline $4 / 27 / 08$ & $16: 00$ & 6.963 & 6.817 & 4.678 & 3.543 & 2.411 & 1.112 & -0.346 \\
\hline $4 / 27 / 08$ & $20: 00$ & 6.942 & 6.789 & 4.669 & 3.524 & 2.397 & 1.105 & -0.341 \\
\hline $4 / 28 / 08$ & $0: 00$ & 6.937 & 6.786 & 4.666 & 3.524 & 2.376 & 1.098 & -0.339 \\
\hline 4/28/08 & $4: 00$ & 6.958 & 6.81 & 4.681 & 3.543 & 2.379 & 1.098 & -0.346 \\
\hline $4 / 28 / 08$ & $8: 00$ & 6.989 & 6.849 & 4.699 & 3.576 & 2.383 & 1.105 & -0.339 \\
\hline 4/28/08 & $12: 00$ & 7.003 & 6.861 & 4.709 & 3.585 & 2.4 & 1.117 & -0.334 \\
\hline $4 / 28 / 08$ & $16: 00$ & 7.005 & 6.861 & 4.716 & 3.585 & 2.421 & 1.124 & -0.336 \\
\hline 4/28/08 & $20: 00$ & 7.005 & 6.858 & 4.713 & 3.583 & 2.407 & 1.128 & -0.339 \\
\hline 4/29/08 & 0:00 & 6.998 & 6.851 & 4.702 & 3.571 & 2.381 & 1.128 & -0.338 \\
\hline 4/29/08 & $4: 00$ & 7 & 6.854 & 4.704 & 3.576 & 2.383 & 1.124 & -0.341 \\
\hline 4/29/08 & 8:00 & 7.01 & 6.863 & 4.711 & 3.583 & 2.381 & 1.124 & -0.339 \\
\hline 4/29/08 & $12: 00$ & 7 & 6.849 & 4.706 & 3.571 & 2.4 & 1.124 & -0.345 \\
\hline 4/29/08 & $16: 00$ & 6.961 & 6.8 & 4.692 & 3.538 & 2.423 & 1.114 & -0.346 \\
\hline 4/29/08 & $20: 00$ & 6.942 & 6.777 & 4.685 & 3.527 & 2.416 & 1.107 & -0.348 \\
\hline 4/30/08 & $0: 00$ & 6.93 & 6.77 & 4.671 & 3.515 & 2.379 & 1.098 & -0.348 \\
\hline 4/30/08 & $4: 00$ & 6.897 & 6.733 & 4.645 & 3.48 & 2.36 & 1.082 & -0.355 \\
\hline $4 / 30 / 08$ & $8: 00$ & 6.878 & 6.71 & 4.641 & 3.468 & 2.365 & 1.063 & -0.353 \\
\hline 4/30/08 & $12: 00$ & 6.862 & 6.689 & 4.638 & 3.456 & 2.388 & 1.051 & -0.35 \\
\hline 4/30/08 & $16: 00$ & 6.843 & 6.659 & 4.638 & 3.442 & 2.411 & 1.042 & -0.348 \\
\hline 4/30/08 & $20: 00$ & 6.831 & 6.65 & 4.636 & 3.44 & 2.404 & 1.037 & -0.35 \\
\hline $5 / 1 / 08$ & $0: 00$ & 6.832 & 6.654 & 4.627 & 3.44 & 2.367 & 1.035 & -0.355 \\
\hline $5 / 1 / 08$ & $4: 00$ & 6.808 & 6.631 & 4.606 & 3.414 & 2.35 & 1.023 & -0.351 \\
\hline $5 / 1 / 08$ & $8: 00$ & 6.808 & 6.631 & 4.608 & 3.416 & 2.35 & 1.012 & -0.353 \\
\hline $5 / 1 / 08$ & $12: 00$ & 6.806 & 6.624 & 4.617 & 3.414 & 2.385 & 1.005 & -0.351 \\
\hline $5 / 1 / 08$ & $16: 00$ & 6.832 & 6.65 & 4.655 & 3.449 & 2.456 & 1.012 & -0.348 \\
\hline $5 / 1 / 08$ & $20: 00$ & 6.876 & 6.703 & 4.671 & 3.492 & 2.43 & 1.042 & -0.35 \\
\hline $5 / 2 / 08$ & $0: 00$ & 6.918 & 6.752 & 4.678 & 3.52 & 2.378 & 1.063 & -0.348 \\
\hline $5 / 2 / 08$ & $4: 00$ & 6.876 & 6.689 & 4.622 & 3.454 & 2.325 & 1.051 & -0.355 \\
\hline $5 / 2 / 08$ & 8:00 & 6.86 & 6.689 & 4.62 & 3.447 & 2.346 & 1.028 & -0.353 \\
\hline $5 / 2 / 08$ & $12: 00$ & 6.827 & 6.67 & 4.533 & 3.381 & 2.322 & 1 & -0.346 \\
\hline $5 / 2 / 08$ & $16: 00$ & 6.85 & 6.696 & 4.592 & 3.424 & 2.355 & 0.993 & -0.355 \\
\hline $5 / 2 / 08$ & $20: 00$ & 6.888 & 6.705 & 4.629 & 3.461 & 2.362 & 1.007 & -0.355 \\
\hline $5 / 3 / 08$ & $0: 00$ & 6.944 & 6.721 & 4.629 & 3.412 & 2.336 & 1.028 & -0.355 \\
\hline $5 / 3 / 08$ & $4: 00$ & 6.972 & 6.837 & 4.645 & 3.527 & 2.357 & 1.044 & -0.353 \\
\hline $5 / 3 / 08$ & $8: 00$ & 7.029 & 6.898 & 4.697 & 3.588 & 2.378 & 1.07 & -0.345 \\
\hline $5 / 3 / 08$ & $12: 00$ & 7.066 & 6.942 & 4.727 & 3.613 & 2.402 & 1.1 & -0.341 \\
\hline $5 / 3 / 08$ & $16: 00$ & 7.076 & 6.942 & 4.737 & 3.616 & 2.413 & 1.119 & -0.336 \\
\hline $5 / 3 / 08$ & $20: 00$ & 7.076 & 6.942 & 4.744 & 3.618 & 2.406 & 1.133 & -0.336 \\
\hline $5 / 4 / 08$ & $0: 00$ & 7.085 & 6.949 & 4.741 & 3.623 & 2.381 & 1.14 & -0.341 \\
\hline $5 / 4 / 08$ & $4: 00$ & 7.078 & 6.942 & 4.737 & 3.613 & 2.371 & 1.142 & -0.339 \\
\hline $5 / 4 / 08$ & $8: 00$ & 7.085 & 6.951 & 4.746 & 3.625 & 2.376 & 1.142 & -0.336 \\
\hline $5 / 4 / 08$ & $12: 00$ & 7.092 & 6.954 & 4.758 & 3.632 & 2.427 & 1.145 & -0.336 \\
\hline
\end{tabular}


Depth to Water (feet below top of casing) in Indicated Wells

Date Time PMW1s PMW1d PMW2sa PMW2d PMW9s PMW9d PMW3d

\begin{tabular}{|c|c|c|c|c|c|c|c|c|}
\hline $5 / 4 / 08$ & $16: 00$ & 7.078 & 6.928 & 4.763 & 3.62 & 2.465 & 1.152 & -0.338 \\
\hline $5 / 4 / 08$ & 20:00 & 7.068 & 6.919 & 4.758 & 3.616 & 2.439 & 1.156 & -0.348 \\
\hline $5 / 5 / 08$ & 0:00 & 7.09 & 6.951 & 4.763 & 3.634 & 2.395 & 1.159 & -0.343 \\
\hline $5 / 5 / 08$ & 4:00 & 7.087 & 6.949 & 4.753 & 3.627 & 2.378 & 1.157 & -0.345 \\
\hline $5 / 5 / 08$ & $8: 00$ & 7.097 & 6.96 & 4.76 & 3.637 & 2.376 & 1.152 & -0.338 \\
\hline 5/5/08 & $12: 00$ & 7.108 & 6.97 & 4.784 & 3.653 & 2.441 & 1.157 & -0.327 \\
\hline $5 / 5 / 08$ & $16: 00$ & 7.104 & 6.954 & 4.793 & 3.646 & 2.483 & 1.164 & -0.292 \\
\hline $5 / 5 / 08$ & 20:00 & 7.085 & 6.933 & 4.774 & 3.627 & 2.434 & 1.173 & -0.32 \\
\hline 5/6/08 & 0:00 & 7.08 & 6.935 & 4.756 & 3.62 & 2.385 & 1.166 & -0.324 \\
\hline 5/6/08 & 4:00 & 7.064 & 6.916 & 4.739 & 3.602 & 2.367 & 1.152 & -0.327 \\
\hline 5/6/08 & 8:00 & 7.052 & 6.905 & 4.732 & 3.592 & 2.359 & 1.138 & -0.327 \\
\hline $5 / 6 / 08$ & $12: 00$ & 7.054 & 6.898 & 4.751 & 3.597 & 2.423 & 1.128 & -0.324 \\
\hline 5/6/08 & $16: 00$ & 7.038 & 6.87 & 4.76 & 3.585 & 2.483 & 1.128 & -0.327 \\
\hline $5 / 6 / 08$ & 20:00 & 7.012 & 6.842 & 4.737 & 3.564 & 2.444 & 1.133 & -0.329 \\
\hline $5 / 7 / 08$ & $0: 00$ & 7.01 & 6.8 & 4.655 & 3.468 & 2.331 & 1.119 & -0.336 \\
\hline $5 / 7 / 08$ & 4:00 & 6.949 & 6.791 & 4.608 & 3.44 & 2.327 & 1.077 & -0.339 \\
\hline $5 / 7 / 08$ & 8:00 & 6.98 & 6.837 & 4.659 & 3.52 & 2.35 & 1.065 & -0.339 \\
\hline $5 / 7 / 08$ & $12: 00$ & 6.989 & 6.847 & 4.681 & 3.534 & 2.359 & 1.065 & -0.339 \\
\hline $5 / 7 / 08$ & $16: 00$ & 7.008 & 6.861 & 4.695 & 3.548 & 2.373 & 1.075 & -0.336 \\
\hline $5 / 7 / 08$ & 20:00 & 7.012 & 6.861 & 4.704 & 3.55 & 2.373 & 1.082 & -0.336 \\
\hline 5/8/08 & 0:00 & 7.022 & 6.875 & 4.706 & 3.559 & 2.357 & 1.089 & -0.339 \\
\hline 5/8/08 & 4:00 & 7.024 & 6.879 & 4.709 & 3.564 & 2.355 & 1.089 & -0.336 \\
\hline $5 / 8 / 08$ & $8: 00$ & 7.024 & 6.877 & 4.706 & 3.562 & 2.35 & 1.091 & -0.336 \\
\hline 5/8/08 & $12: 00$ & 7.031 & 6.879 & 4.727 & 3.569 & 2.408 & 1.093 & -0.334 \\
\hline 5/8/08 & $16: 00$ & & & 4.734 & 3.562 & 2.441 & 1.1 & -0.336 \\
\hline $5 / 8 / 08$ & 20:00 & 6.987 & 6.824 & 4.727 & 3.559 & 2.397 & 1.105 & -0.336 \\
\hline 5/9/08 & $0: 00$ & 6.991 & 6.775 & 4.72 & 3.503 & 2.357 & 1.105 & -0.336 \\
\hline 5/9/08 & 4:00 & 6.886 & 6.775 & 4.428 & 3.395 & 2.28 & 1.082 & -0.343 \\
\hline $5 / 9 / 08$ & 8:00 & 6.759 & 6.696 & 4.446 & 3.342 & 2.315 & 1.009 & -0.346 \\
\hline 5/9/08 & $12: 00$ & 6.782 & 6.67 & 4.514 & 3.363 & 2.329 & 0.977 & -0.344 \\
\hline $5 / 9 / 08$ & $16: 00$ & 6.792 & 6.668 & 4.559 & 3.391 & 2.355 & 0.972 & -0.346 \\
\hline $5 / 9 / 08$ & 20:00 & 6.806 & 6.67 & 4.585 & 3.412 & 2.352 & 0.979 & -0.344 \\
\hline 5/10/08 & 0:00 & 6.806 & 6.666 & 4.587 & 3.417 & 2.331 & 0.986 & -0.344 \\
\hline $5 / 10 / 08$ & 4:00 & 6.792 & 6.636 & 4.542 & 3.395 & 2.298 & 0.984 & -0.346 \\
\hline 5/10/08 & 8:00 & 6.768 & 6.652 & 4.531 & 3.37 & 2.317 & 0.967 & -0.344 \\
\hline 5/10/08 & $12: 00$ & 6.733 & 6.58 & 4.531 & 3.323 & 2.331 & 0.953 & -0.348 \\
\hline $5 / 10 / 08$ & $16: 00$ & 6.644 & 6.524 & 4.273 & 3.238 & 2.27 & 0.925 & -0.348 \\
\hline $5 / 10 / 08$ & 20:00 & 5.589 & 5.872 & 3.997 & 2.742 & 2.261 & 0.797 & -0.346 \\
\hline $5 / 11 / 08$ & 0:00 & 5.542 & 5.749 & 4.062 & 2.81 & 2.273 & 0.689 & -0.339 \\
\hline 5/11/08 & 4:00 & 5.783 & 5.828 & 4.156 & 2.908 & 2.284 & 0.659 & -0.341 \\
\hline $5 / 11 / 08$ & 8:00 & 6.044 & 5.953 & 4.24 & 3.004 & 2.298 & 0.677 & -0.344 \\
\hline $5 / 11 / 08$ & $12: 00$ & 6.161 & 6.025 & 4.306 & 3.053 & 2.329 & 0.708 & -0.353 \\
\hline $5 / 11 / 08$ & $16: 00$ & 6.222 & 6.06 & 4.341 & 3.079 & 2.34 & 0.736 & -0.353 \\
\hline $5 / 11 / 08$ & 20:00 & 6.25 & 6.069 & 4.35 & 3.084 & 2.319 & 0.754 & -0.351 \\
\hline
\end{tabular}


TABLE S1.3 (Cont.)

Depth to Water (feet below top of casing) in Indicated Wells

Date Time PMW1s PMW1d PMW2sa PMW2d PMW9s PMW9d PMW3d

\begin{tabular}{|c|c|c|c|c|c|c|c|c|}
\hline $5 / 12 / 08$ & $0: 00$ & 6.276 & 6.088 & 4.355 & 3.093 & 2.296 & 0.764 & -0.351 \\
\hline $5 / 12 / 08$ & $4: 00$ & 6.276 & 6.081 & 4.353 & 3.084 & 2.296 & 0.761 & -0.351 \\
\hline $5 / 12 / 08$ & $8: 00$ & 6.281 & 6.076 & 4.355 & 3.084 & 2.294 & 0.761 & -0.353 \\
\hline $5 / 12 / 08$ & $12: 00$ & 6.285 & 6.062 & 4.374 & 3.084 & 2.345 & 0.757 & -0.348 \\
\hline $5 / 12 / 08$ & $16: 00$ & 6.278 & 6.039 & 4.376 & 3.199 & & 0.759 & -0.341 \\
\hline $5 / 12 / 08$ & $20: 00$ & 6.295 & 6.058 & 4.385 & 3.093 & 2.387 & 1.012 & -0.351 \\
\hline $5 / 13 / 08$ & $0: 00$ & 6.316 & 6.093 & 4.378 & 3.102 & 2.317 & 0.92 & -0.351 \\
\hline $5 / 13 / 08$ & $4: 00$ & 6.311 & 6.083 & 4.367 & 3.088 & 2.296 & 0.85 & -0.351 \\
\hline $5 / 13 / 08$ & $8: 00$ & 6.344 & 6.12 & 4.393 & 3.123 & 2.305 & 0.82 & -0.348 \\
\hline $5 / 13 / 08$ & $12: 00$ & 6.372 & 6.148 & 4.411 & 3.142 & 2.317 & & -0.348 \\
\hline $5 / 13 / 08$ & $16: 00$ & 6.41 & 6.188 & 4.444 & 3.18 & 2.347 & & -0.407 \\
\hline $5 / 13 / 08$ & $20: 00$ & 6.445 & 6.227 & 4.453 & 3.203 & 2.324 & & -0.407 \\
\hline $5 / 14 / 08$ & 0:00 & 6.482 & 6.271 & 4.465 & 3.229 & 2.31 & & -0.404 \\
\hline $5 / 14 / 08$ & $4: 00$ & 6.485 & 6.274 & 4.456 & 3.22 & 2.296 & & -0.407 \\
\hline $5 / 14 / 08$ & $8: 00$ & 6.504 & 6.294 & 4.47 & 3.241 & 2.307 & 1.03 & -0.402 \\
\hline $5 / 14 / 08$ & $12: 00$ & 6.522 & 6.306 & 4.489 & 3.25 & 2.338 & 0.916 & -0.404 \\
\hline $5 / 14 / 08$ & $16: 00$ & 6.525 & 6.297 & 4.505 & 3.248 & 2.364 & 0.857 & -0.407 \\
\hline $5 / 14 / 08$ & $20: 00$ & 6.532 & 6.301 & 4.505 & 3.252 & 2.338 & 0.829 & -0.409 \\
\hline $5 / 15 / 08$ & 0:00 & 6.527 & 6.306 & 4.484 & 3.245 & 2.298 & 0.808 & -0.407 \\
\hline $5 / 15 / 08$ & $4: 00$ & 6.515 & 6.294 & 4.47 & 3.231 & 2.289 & 0.787 & -0.404 \\
\hline $5 / 15 / 08$ & 8:00 & 6.534 & 6.315 & 4.482 & 3.245 & 2.293 & 0.776 & -0.407 \\
\hline $5 / 15 / 08$ & $12: 00$ & 6.569 & 6.343 & 4.526 & 3.28 & 2.363 & 0.778 & -0.404 \\
\hline $5 / 15 / 08$ & $16: 00$ & 6.576 & 6.339 & 4.535 & 3.281 & 2.387 & 0.792 & -0.407 \\
\hline $5 / 15 / 08$ & $20: 00$ & 6.574 & 6.345 & 4.526 & 3.281 & 2.342 & 0.801 & -0.407 \\
\hline $5 / 16 / 08$ & 0:00 & 6.567 & 6.345 & 4.505 & 3.269 & 2.305 & 0.797 & -0.404 \\
\hline $5 / 16 / 08$ & $4: 00$ & 6.553 & 6.332 & 4.486 & 3.252 & 2.289 & 0.785 & -0.409 \\
\hline $5 / 16 / 08$ & 8:00 & 6.562 & 6.343 & 4.493 & 3.259 & 2.289 & 0.776 & -0.407 \\
\hline 5/16/08 & $12: 00$ & 6.588 & 6.352 & 4.538 & 3.288 & 2.363 & 0.776 & -0.407 \\
\hline $5 / 16 / 08$ & $16: 00$ & 6.595 & 6.341 & 4.561 & 3.292 & 2.403 & 0.79 & -0.407 \\
\hline 5/16/08 & $20: 00$ & 6.574 & 6.325 & 4.542 & 3.269 & 2.363 & 0.799 & -0.411 \\
\hline $5 / 17 / 08$ & 0:00 & 6.541 & 6.299 & 4.496 & 3.236 & 2.305 & 0.787 & -0.411 \\
\hline $5 / 17 / 08$ & $4: 00$ & 6.518 & 6.281 & 4.479 & 3.222 & 2.289 & 0.769 & -0.411 \\
\hline $5 / 17 / 08$ & 8:00 & 6.534 & 6.301 & 4.486 & 3.236 & 2.284 & 0.754 & -0.409 \\
\hline $5 / 17 / 08$ & $12: 00$ & 6.584 & 6.327 & 4.556 & 3.278 & 2.38 & 0.757 & -0.409 \\
\hline $5 / 17 / 08$ & $16: 00$ & 6.602 & 6.332 & 4.592 & 3.299 & 2.45 & 0.78 & -0.407 \\
\hline $5 / 17 / 08$ & $20: 00$ & 6.602 & 6.339 & 4.58 & 3.299 & 2.415 & 0.806 & -0.411 \\
\hline $5 / 18 / 08$ & $0: 00$ & 6.574 & 6.332 & 4.526 & 3.269 & 2.331 & 0.806 & -0.407 \\
\hline 5/18/08 & $4: 00$ & 6.555 & 6.318 & 4.503 & 3.25 & 2.298 & 0.79 & -0.407 \\
\hline $5 / 18 / 08$ & 8:00 & 6.572 & 6.341 & 4.51 & 3.264 & 2.291 & 0.776 & -0.407 \\
\hline 5/18/08 & $12: 00$ & 6.612 & 6.362 & 4.564 & 3.299 & 2.359 & 0.778 & -0.407 \\
\hline $5 / 18 / 08$ & $16: 00$ & 6.623 & 6.359 & 4.596 & 3.311 & 2.389 & 0.79 & \\
\hline $5 / 18 / 08$ & $20: 00$ & 6.591 & 6.327 & 4.561 & 3.278 & 2.342 & 0.797 & \\
\hline $5 / 19 / 08$ & 0:00 & 6.546 & 6.299 & 4.507 & 3.236 & 2.281 & 0.783 & \\
\hline 5/19/08 & $4: 00$ & 6.497 & 6.243 & 4.467 & 3.184 & 2.246 & 0.757 & \\
\hline
\end{tabular}


TABLE S1.3 (Cont.)

Depth to Water (feet below top of casing) in Indicated Wells

Date Time PMW1s PMW1d PMW2sa PMW2d PMW9s PMW9d PMW3d

\begin{tabular}{|c|c|c|c|c|c|c|c|}
\hline 5/19/08 & 8:00 & 6.502 & 6.257 & 4.477 & 3.203 & 2.263 & 0.736 \\
\hline 5/19/08 & $12: 00$ & 6.574 & 6.311 & 4.566 & 3.273 & 2.361 & 0.743 \\
\hline $5 / 19 / 08$ & $16: 00$ & 6.616 & 6.343 & 4.608 & 3.306 & 2.431 & 0.768 \\
\hline 5/19/08 & $20: 00$ & 6.619 & 6.355 & 4.594 & 3.304 & 2.398 & 0.801 \\
\hline $5 / 20 / 08$ & 0:00 & 6.621 & 6.383 & 4.566 & 3.309 & 2.33 & 0.811 \\
\hline $5 / 20 / 08$ & 4:00 & 6.621 & 6.392 & 4.547 & 3.302 & 2.293 & 0.806 \\
\hline $5 / 20 / 08$ & 8:00 & 6.663 & 6.445 & 4.571 & 3.341 & 2.293 & 0.806 \\
\hline $5 / 20 / 08$ & $12: 00$ & 6.699 & 6.468 & 4.608 & 3.367 & 2.337 & 0.815 \\
\hline $5 / 20 / 08$ & $16: 00$ & 6.715 & 6.464 & 4.634 & 3.369 & 2.396 & 0.829 \\
\hline $5 / 20 / 08$ & $20: 00$ & 6.699 & 6.45 & 4.62 & 3.358 & 2.377 & 0.841 \\
\hline $5 / 21 / 08$ & 0:00 & 6.647 & 6.415 & 4.552 & 3.306 & 2.288 & 0.832 \\
\hline $5 / 21 / 08$ & 4:00 & 6.626 & 6.399 & 4.528 & 3.285 & 2.263 & 0.811 \\
\hline $5 / 21 / 08$ & 8:00 & 6.619 & 6.392 & 4.521 & 3.281 & 2.255 & 0.79 \\
\hline $5 / 21 / 08$ & $12: 00$ & 6.652 & 6.401 & 4.575 & 3.313 & 2.328 & 0.785 \\
\hline $5 / 21 / 08$ & $16: 00$ & 6.656 & 6.394 & 4.589 & 3.311 & 2.351 & 0.792 \\
\hline $5 / 21 / 08$ & 20:00 & 6.631 & 6.376 & 4.564 & 3.292 & 2.316 & 0.799 \\
\hline $5 / 22 / 08$ & 0:00 & 6.6 & 6.362 & 4.524 & 3.262 & 2.265 & 0.79 \\
\hline $5 / 22 / 08$ & 4:00 & 6.586 & 6.352 & 4.505 & 3.252 & 2.244 & 0.768 \\
\hline $5 / 22 / 08$ & 8:00 & 6.586 & 6.308 & 4.505 & 3.206 & 2.239 & 0.752 \\
\hline $5 / 22 / 08$ & $12: 00$ & 6.572 & 6.355 & 4.428 & 3.257 & 2.204 & 0.743 \\
\hline $5 / 22 / 08$ & $16: 00$ & 6.57 & 6.339 & 4.463 & 3.213 & 2.244 & 0.726 \\
\hline $5 / 22 / 08$ & $20: 00$ & 6.558 & 6.322 & 4.474 & 3.213 & 2.248 & 0.717 \\
\hline $5 / 23 / 08$ & 0:00 & 6.574 & 6.35 & 4.486 & 3.231 & 2.244 & 0.722 \\
\hline $5 / 23 / 08$ & 4:00 & 6.03 & 6.232 & 4.055 & 3.051 & 2.169 & 0.705 \\
\hline $5 / 23 / 08$ & 8:00 & 5.474 & 5.877 & 4.158 & 2.899 & 2.213 & 0.607 \\
\hline $5 / 23 / 08$ & $12: 00$ & 5.592 & 5.918 & 4.229 & 2.988 & 2.216 & 0.581 \\
\hline $5 / 23 / 08$ & $16: 00$ & 5.927 & 6 & 4.268 & 3.023 & 2.22 & 0.577 \\
\hline $5 / 23 / 08$ & 20:00 & 6.237 & 6.088 & 4.313 & 3.063 & 2.225 & 0.591 \\
\hline $5 / 24 / 08$ & 0:00 & 6.323 & 6.134 & 4.348 & 3.081 & 2.225 & 0.603 \\
\hline $5 / 24 / 08$ & 4:00 & 5.936 & 5.958 & 4.114 & 2.889 & 2.155 & 0.589 \\
\hline $5 / 24 / 08$ & 8:00 & 5.716 & 5.863 & 4.158 & 2.873 & 2.199 & 0.544 \\
\hline $5 / 24 / 08$ & $12: 00$ & 5.726 & 5.863 & 4.212 & 2.927 & 2.229 & 0.525 \\
\hline $5 / 24 / 08$ & $16: 00$ & 5.82 & 5.895 & 4.271 & 2.962 & 2.265 & 0.528 \\
\hline $5 / 24 / 08$ & 20:00 & 6.056 & 5.932 & 4.28 & 2.976 & 2.234 & 0.544 \\
\hline $5 / 25 / 08$ & 0:00 & 6.122 & 5.944 & 4.273 & 2.967 & 2.206 & 0.546 \\
\hline $5 / 25 / 08$ & 4:00 & 6.159 & 5.939 & 4.266 & 2.957 & 2.201 & 0.542 \\
\hline $5 / 25 / 08$ & 8:00 & 6.185 & 5.958 & 4.28 & 2.969 & 2.204 & 0.539 \\
\hline $5 / 25 / 08$ & $12: 00$ & 6.209 & 5.953 & 4.32 & 2.983 & 2.276 & 0.537 \\
\hline $5 / 25 / 08$ & $16: 00$ & 6.248 & 5.97 & 4.369 & 3.018 & 2.337 & 0.556 \\
\hline $5 / 25 / 08$ & 20:00 & 6.263 & 5.937 & 4.369 & 2.962 & 2.304 & 0.582 \\
\hline $5 / 26 / 08$ & 0:00 & 6.225 & 5.967 & 4.311 & 2.988 & 2.218 & 0.582 \\
\hline $5 / 26 / 08$ & 4:00 & 6.237 & 5.981 & 4.318 & 3.004 & 2.218 & 0.57 \\
\hline $5 / 26 / 08$ & 8:00 & 6.253 & 6.004 & 4.322 & 3.009 & 2.213 & 0.567 \\
\hline $5 / 26 / 08$ & $12: 00$ & 6.295 & 6.041 & 4.355 & 3.051 & 2.241 & 0.574 \\
\hline
\end{tabular}


TABLE S1.3 (Cont.)

\begin{tabular}{|c|c|c|c|c|c|c|c|c|}
\hline \multirow[b]{2}{*}{ Date } & \multirow[b]{2}{*}{ Time } & \multicolumn{7}{|c|}{ Depth to Water (feet below top of casing) in Indicated Wells } \\
\hline & & PMW1s & PMW1d & PMW2sa & PMW2d & PMW9s & PMW9d & PMW3d \\
\hline $5 / 26 / 08$ & $16: 00$ & 6.335 & 6.062 & 4.402 & 3.079 & 2.292 & 0.591 & \\
\hline $5 / 26 / 08$ & $20: 00$ & 6.314 & 6.048 & 4.369 & 3.053 & 2.248 & 0.61 & \\
\hline $5 / 27 / 08$ & 0:00 & 6.324 & 6.067 & 4.362 & 3.063 & 2.225 & 0.612 & \\
\hline $5 / 27 / 08$ & $4: 00$ & 6.312 & 6.058 & 4.353 & 3.051 & 2.215 & 0.607 & \\
\hline $5 / 27 / 08$ & 8:00 & 6.331 & 6.081 & 4.364 & 3.074 & 2.225 & 0.605 & \\
\hline 5/27/08 & $12: 00$ & 6.359 & 6.09 & 4.371 & 3.072 & 2.218 & 0.614 & \\
\hline $5 / 27 / 08$ & $16: 00$ & 6.368 & 6.146 & 4.374 & 3.119 & 2.217 & 0.619 & \\
\hline $5 / 27 / 08$ & $20: 00$ & 6.378 & 6.109 & 4.374 & 3.086 & 2.217 & 0.621 & \\
\hline 5/28/08 & 0:00 & 6.382 & 6.102 & 4.367 & 3.088 & 2.213 & 0.624 & \\
\hline $5 / 28 / 08$ & $4: 00$ & 6.148 & 6.081 & 4.224 & 3.013 & 2.192 & 0.603 & \\
\hline $5 / 28 / 08$ & $8: 00$ & 5.704 & 5.823 & 4.055 & 2.854 & 2.178 & 0.551 & \\
\hline 5/28/08 & $12: 00$ & 5.599 & 5.754 & 4.107 & 2.828 & 2.192 & 0.5 & \\
\hline 5/28/08 & $16: 00$ & 5.595 & 5.733 & 4.147 & 2.849 & 2.208 & 0.479 & \\
\hline $5 / 28 / 08$ & $20: 00$ & 5.642 & 5.735 & 4.156 & 2.845 & 2.192 & 0.472 & \\
\hline 5/29/08 & 0:00 & 5.902 & 5.761 & 4.168 & 2.854 & 2.187 & 0.467 & \\
\hline $5 / 29 / 08$ & $4: 00$ & 5.986 & 5.765 & 4.172 & 2.845 & 2.185 & 0.462 & \\
\hline $5 / 29 / 08$ & 8:00 & 6.007 & 5.761 & 4.182 & 2.842 & 2.185 & 0.455 & \\
\hline 5/29/08 & $12: 00$ & 6.005 & 5.742 & 4.184 & 2.828 & 2.196 & 0.451 & \\
\hline $5 / 29 / 08$ & $16: 00$ & 6.012 & 5.717 & 4.21 & 2.826 & 2.241 & 0.444 & \\
\hline $5 / 29 / 08$ & $20: 00$ & 5.986 & 5.691 & 4.184 & 2.802 & 2.206 & 0.446 & \\
\hline 5/30/08 & 0:00 & 5.965 & 5.714 & 4.09 & 2.793 & 2.201 & 0.437 & \\
\hline $5 / 30 / 08$ & $4: 00$ & 5.346 & 5.457 & 3.999 & 2.603 & 2.175 & 0.38 & \\
\hline 5/30/08 & 8:00 & 5.229 & 5.355 & 3.955 & 2.571 & 2.154 & 0.336 & \\
\hline 5/30/08 & $12: 00$ & 5.328 & 5.387 & 4.02 & 2.629 & 2.192 & 0.317 & \\
\hline 5/30/08 & $16: 00$ & 5.639 & 5.466 & 4.116 & 2.702 & 2.252 & 0.331 & \\
\hline $5 / 30 / 08$ & $20: 00$ & 5.81 & 5.536 & 4.149 & 2.744 & 2.227 & 0.362 & \\
\hline 5/31/08 & 0:00 & 5.838 & 5.559 & 4.123 & 2.739 & 2.187 & 0.373 & \\
\hline 5/31/08 & $4: 00$ & 5.852 & 5.566 & 4.118 & 2.737 & 2.175 & 0.378 & \\
\hline 5/31/08 & 8:00 & 5.88 & 5.594 & 4.137 & 2.758 & 2.18 & 0.383 & \\
\hline 5/31/08 & $12: 00$ & 5.937 & 5.631 & 4.196 & 2.802 & 2.231 & 0.395 & \\
\hline 5/31/08 & $16: 00$ & 5.991 & 5.656 & 4.25 & 2.838 & 2.294 & 0.418 & \\
\hline $5 / 31 / 08$ & $20: 00$ & 5.991 & 5.659 & 4.233 & 2.826 & 2.245 & 0.444 & \\
\hline 6/1/08 & 0:00 & 5.958 & 5.647 & 4.182 & 2.8 & 2.189 & 0.444 & \\
\hline $6 / 1 / 08$ & $4: 00$ & 5.941 & 5.631 & 4.168 & 2.781 & 2.182 & 0.427 & \\
\hline $6 / 1 / 08$ & 8:00 & 5.958 & 5.652 & 4.175 & 2.8 & 2.184 & 0.423 & \\
\hline $6 / 1 / 08$ & $12: 00$ & 6.024 & 5.689 & 4.259 & 2.854 & 2.278 & 0.43 & \\
\hline 6/1/08 & $16: 00$ & 6.052 & 5.7 & 4.292 & 2.877 & 2.322 & 0.455 & \\
\hline $6 / 1 / 08$ & $20: 00$ & 6.031 & 5.689 & 4.252 & 2.849 & 2.257 & 0.474 & \\
\hline $6 / 2 / 08$ & 0:00 & 5.998 & 5.677 & 4.205 & 2.821 & 2.203 & 0.467 & \\
\hline $6 / 2 / 08$ & $4: 00$ & 5.958 & 5.638 & 4.175 & 2.781 & 2.182 & 0.444 & \\
\hline $6 / 2 / 08$ & 8:00 & 5.941 & 5.675 & 4.158 & 2.821 & 2.175 & 0.425 & \\
\hline $6 / 2 / 08$ & $12: 00$ & 5.944 & 5.612 & 4.177 & 2.767 & 2.194 & 0.411 & \\
\hline $6 / 2 / 08$ & $16: 00$ & 5.986 & 5.619 & 4.243 & 2.803 & 2.273 & 0.413 & \\
\hline $6 / 2 / 08$ & $20: 00$ & 5.965 & 5.612 & 4.21 & 2.793 & 2.231 & 0.427 & \\
\hline
\end{tabular}


TABLE S1.3 (Cont.)

Depth to Water (feet below top of casing) in Indicated Wells

Date Time PMW1s PMW1d PMW2sa PMW2d PMW9s PMW9d PMW3d

\begin{tabular}{|c|c|c|c|c|c|c|c|}
\hline 6/3/08 & 0:00 & 5.902 & 5.561 & 4.147 & 2.732 & 2.18 & 0.411 \\
\hline 6/3/08 & $4: 00$ & 5.864 & 5.531 & 4.13 & 2.704 & 2.182 & 0.383 \\
\hline 6/3/08 & 8:00 & 5.852 & 5.568 & 4.118 & 2.699 & 2.17 & 0.366 \\
\hline 6/3/08 & $12: 00$ & 5.892 & 5.54 & 4.175 & 2.737 & 2.229 & 0.362 \\
\hline 6/3/08 & $16: 00$ & 5.96 & 5.584 & 4.25 & 2.798 & 2.282 & 0.38 \\
\hline 6/3/08 & 20:00 & 5.967 & 5.598 & 4.238 & 2.798 & 2.25 & 0.409 \\
\hline $6 / 4 / 08$ & 0:00 & 5.909 & 5.568 & 4.165 & 2.746 & 2.179 & 0.409 \\
\hline $6 / 4 / 08$ & 4:00 & 5.89 & 5.556 & 4.142 & 2.725 & 2.165 & 0.392 \\
\hline $6 / 4 / 08$ & 8:00 & 5.888 & 5.561 & 4.142 & 2.73 & 2.172 & 0.378 \\
\hline $6 / 4 / 08$ & $12: 00$ & 5.876 & 5.54 & 4.135 & 2.716 & 2.17 & 0.366 \\
\hline $6 / 4 / 08$ & $16: 00$ & 5.932 & 5.561 & 4.217 & 2.765 & 2.245 & 0.366 \\
\hline $6 / 4 / 08$ & 20:00 & 5.888 & 5.529 & 4.161 & 2.72 & 2.186 & 0.376 \\
\hline 6/5/08 & 0:00 & 4 & 4.459 & 3.385 & 1.938 & 2.109 & 0.25 \\
\hline 6/5/08 & 4:00 & 3.071 & 3.516 & 2.99 & 1.549 & 2.046 & 0.004 \\
\hline 6/5/08 & 8:00 & 3.317 & 3.194 & 3.196 & 1.507 & 2.069 & -0.183 \\
\hline 6/5/08 & $12: 00$ & 3.568 & 3.324 & 3.313 & 1.631 & 2.091 & -0.255 \\
\hline 6/5/08 & $16: 00$ & 3.753 & 3.449 & 3.404 & 1.72 & 2.123 & -0.267 \\
\hline 6/5/08 & 20:00 & 3.899 & 3.53 & 3.413 & 1.748 & 2.095 & -0.26 \\
\hline 6/6/08 & 0:00 & 3.964 & 3.574 & 3.376 & 1.713 & 2.055 & -0.258 \\
\hline 6/6/08 & 4:00 & 4.117 & 3.7 & 3.451 & 1.793 & 2.088 & -0.248 \\
\hline 6/6/08 & 8:00 & 4.269 & 3.834 & 3.524 & 1.889 & 2.116 & -0.227 \\
\hline 6/6/08 & $12: 00$ & 4.445 & 3.974 & 3.606 & 1.978 & 2.165 & -0.181 \\
\hline 6/6/08 & $16: 00$ & 4.593 & 4.078 & 3.673 & 2.043 & 2.193 & -0.131 \\
\hline 6/6/08 & 20:00 & 4.67 & 4.145 & 3.69 & 2.062 & 2.158 & -0.092 \\
\hline 6/7/08 & 0:00 & 4.675 & 4.176 & 3.645 & 2.036 & 2.109 & -0.082 \\
\hline 6/7/08 & 4:00 & 4.656 & 4.159 & 3.622 & 1.994 & 2.09 & -0.094 \\
\hline 6/7/08 & 8:00 & 4.682 & 4.185 & 3.634 & 2.001 & 2.1 & -0.106 \\
\hline $6 / 7 / 08$ & $12: 00$ & 4.762 & 4.241 & 3.711 & 2.071 & 2.186 & -0.099 \\
\hline $6 / 7 / 08$ & $16: 00$ & 4.823 & 4.28 & 3.76 & 2.104 & 2.235 & -0.071 \\
\hline 6/7/08 & 20:00 & 4.83 & 4.31 & 3.741 & 2.102 & 2.179 & -0.045 \\
\hline 6/8/08 & 0:00 & 4.807 & 4.306 & 3.68 & 2.067 & 2.107 & -0.054 \\
\hline 6/8/08 & $4: 00$ & 4.021 & 3.997 & 3.374 & 1.846 & 2.078 & -0.099 \\
\hline 6/8/08 & 8:00 & 3.636 & 3.512 & 3.257 & 1.628 & 2.067 & -0.225 \\
\hline 6/8/08 & $12: 00$ & 3.859 & 3.6 & 3.36 & 1.671 & 2.074 & -0.286 \\
\hline 6/8/08 & $16: 00$ & 4.011 & 3.681 & 3.43 & 1.746 & 2.085 & -0.293 \\
\hline 6/8/08 & 20:00 & 4.14 & 3.756 & 3.481 & 1.802 & 2.097 & -0.276 \\
\hline 6/9/08 & 0:00 & 4.223 & 3.825 & 3.493 & 1.83 & 2.083 & -0.258 \\
\hline 6/9/08 & 4:00 & 4.276 & 3.853 & 3.495 & 1.83 & 2.074 & -0.248 \\
\hline 6/9/08 & 8:00 & 4.342 & 3.897 & 3.516 & 1.856 & 2.083 & -0.241 \\
\hline 6/9/08 & $12: 00$ & 4.455 & 3.962 & 3.591 & 1.914 & 2.141 & -0.225 \\
\hline 6/9/08 & $16: 00$ & 4.558 & 4.015 & 3.645 & 1.952 & 2.165 & -0.195 \\
\hline 6/9/08 & 20:00 & 4.586 & 4.029 & 3.624 & 1.943 & 2.116 & -0.174 \\
\hline 6/10/08 & 0:00 & 4.57 & 4.041 & 3.584 & 1.919 & 2.085 & -0.171 \\
\hline 6/10/08 & $4: 00$ & 4.558 & 4.036 & 3.568 & 1.903 & 2.081 & -0.185 \\
\hline
\end{tabular}


TABLE S1.3 (Cont.)

Depth to Water (feet below top of casing) in Indicated Wells

Date Time PMW1s PMW1d PMW2sa PMW2d PMW9s PMW9d PMW3d

\begin{tabular}{|c|c|c|c|c|c|c|c|}
\hline 6/10/08 & 8:00 & 4.574 & 4.053 & 3.575 & 1.912 & 2.078 & -0.19 \\
\hline 6/10/08 & $12: 00$ & 4.659 & 4.104 & 3.671 & 1.98 & 2.172 & -0.183 \\
\hline 6/10/08 & $16: 00$ & 4.703 & 4.122 & 3.713 & 1.999 & 2.209 & -0.159 \\
\hline 6/10/08 & 20:00 & 4.687 & 4.118 & 3.673 & 1.973 & 2.146 & -0.138 \\
\hline 6/11/08 & 0:00 & 4.624 & 4.078 & 3.603 & 1.919 & 2.088 & -0.15 \\
\hline 6/11/08 & 4:00 & 4.574 & 4.062 & 3.54 & 1.884 & 2.066 & -0.183 \\
\hline 6/11/08 & 8:00 & 4.57 & 4.039 & 3.559 & 1.875 & 2.083 & -0.204 \\
\hline 6/11/08 & $12: 00$ & 4.586 & 4.036 & 3.587 & 1.879 & 2.101 & -0.22 \\
\hline 6/11/08 & $16: 00$ & 4.652 & 4.076 & 3.657 & 1.931 & 2.158 & -0.211 \\
\hline 6/11/08 & 20:00 & 4.617 & 4.055 & 3.598 & 1.856 & 2.106 & -0.199 \\
\hline 6/12/08 & 0:00 & 3.486 & 3.284 & 3.16 & 1.441 & 2.052 & -0.309 \\
\hline 6/12/08 & 4:00 & 3.606 & 3.287 & 3.205 & 1.45 & 2.041 & -0.396 \\
\hline 6/12/08 & 8:00 & 3.709 & 3.315 & 3.254 & 1.486 & 2.045 & -0.438 \\
\hline 6/12/08 & $12: 00$ & 3.84 & 3.382 & 3.341 & 1.556 & 2.085 & -0.442 \\
\hline 6/12/08 & $16: 00$ & 3.976 & 3.458 & 3.425 & 1.631 & 2.115 & -0.419 \\
\hline 6/12/08 & 20:00 & 4.044 & 3.514 & 3.439 & 1.659 & 2.092 & -0.389 \\
\hline 6/13/08 & 0:00 & 4.044 & 3.526 & 3.409 & 1.64 & 2.059 & -0.382 \\
\hline 6/13/08 & 4:00 & 4.058 & 3.537 & 3.402 & 1.638 & 2.05 & -0.379 \\
\hline 6/13/08 & 8:00 & 4.115 & 3.586 & 3.427 & 1.668 & 2.069 & -0.379 \\
\hline 6/13/08 & $12: 00$ & 4.223 & 3.656 & 3.512 & 1.741 & 2.139 & -0.358 \\
\hline 6/13/08 & $16: 00$ & 4.305 & 3.695 & 3.559 & 1.771 & 2.174 & -0.328 \\
\hline 6/13/08 & 20:00 & 4.331 & 3.711 & 3.533 & 1.757 & 2.115 & -0.302 \\
\hline 6/14/08 & 0:00 & 4.272 & 3.679 & 3.463 & 1.701 & 2.059 & -0.314 \\
\hline 6/14/08 & 4:00 & 4.241 & 3.651 & 3.444 & 1.671 & 2.052 & -0.337 \\
\hline 6/14/08 & $8: 00$ & 4.274 & 3.684 & 3.467 & 1.696 & 2.073 & -0.351 \\
\hline 6/14/08 & $12: 00$ & 4.359 & 3.735 & 3.552 & 1.757 & 2.153 & -0.342 \\
\hline 6/14/08 & $16: 00$ & 4.401 & 3.753 & 3.582 & 1.778 & 2.171 & -0.318 \\
\hline 6/14/08 & 20:00 & 4.375 & 3.735 & 3.542 & 1.746 & 2.108 & -0.302 \\
\hline 6/15/08 & 0:00 & 4.3 & 3.695 & 3.47 & 1.689 & 2.061 & -0.321 \\
\hline $6 / 15 / 08$ & 4:00 & 4.267 & 3.672 & 3.446 & 1.661 & 2.052 & -0.346 \\
\hline 6/15/08 & 8:00 & 4.26 & 3.67 & 3.439 & 1.657 & 2.043 & -0.365 \\
\hline 6/15/08 & $12: 00$ & 3.852 & 3.414 & 3.264 & 1.45 & 2.038 & -0.417 \\
\hline 6/15/08 & $16: 00$ & 3.972 & 3.477 & 3.388 & 1.575 & 2.094 & -0.442 \\
\hline 6/15/08 & 20:00 & 4.045 & 3.523 & 3.413 & 1.612 & 2.073 & -0.428 \\
\hline 6/16/08 & 0:00 & 4.059 & 3.554 & 3.397 & 1.617 & 2.054 & -0.419 \\
\hline 6/16/08 & 4:00 & 4.073 & 3.565 & 3.39 & 1.61 & 2.042 & -0.417 \\
\hline 6/16/08 & 8:00 & 4.131 & 3.621 & 3.42 & 1.647 & 2.061 & -0.412 \\
\hline 6/16/08 & $12: 00$ & 4.206 & 3.67 & 3.474 & 1.692 & 2.096 & -0.396 \\
\hline 6/16/08 & $16: 00$ & 4.274 & 3.707 & 3.516 & 1.722 & 2.12 & -0.375 \\
\hline 6/16/08 & 20:00 & 4.288 & 3.723 & 3.498 & 1.713 & 2.08 & -0.356 \\
\hline 6/17/08 & 0:00 & 4.26 & 3.714 & 3.451 & 1.682 & 2.049 & -0.363 \\
\hline 6/17/08 & 4:00 & 4.256 & 3.7 & 3.434 & 1.661 & 2.045 & -0.377 \\
\hline 6/17/08 & 8:00 & 4.274 & 3.714 & 3.442 & 1.664 & 2.054 & -0.386 \\
\hline 6/17/08 & $12: 00$ & 4.359 & 3.758 & 3.524 & 1.727 & 2.124 & -0.379 \\
\hline
\end{tabular}


TABLE S1.3 (Cont.)

Depth to Water (feet below top of casing) in Indicated Wells

Date Time PMW1s PMW1d PMW2sa PMW2d PMW9s PMW9d PMW3d

\begin{tabular}{|c|c|c|c|c|c|c|c|}
\hline 6/17/08 & $16: 00$ & 4.399 & 3.769 & 3.554 & 1.736 & 2.136 & -0.358 \\
\hline 6/17/08 & 20:00 & 4.385 & 3.762 & 3.514 & 1.713 & 2.082 & -0.349 \\
\hline 6/18/08 & 0:00 & 4.286 & 3.697 & 3.42 & 1.622 & 2.019 & -0.368 \\
\hline 6/18/08 & $4: 00$ & 4.253 & 3.672 & 3.409 & 1.614 & 2.035 & -0.398 \\
\hline 6/18/08 & $8: 00$ & 4.267 & 3.691 & 3.42 & 1.628 & 2.04 & -0.41 \\
\hline 6/18/08 & $12: 00$ & 4.342 & 3.737 & 3.493 & 1.682 & 2.096 & -0.41 \\
\hline 6/18/08 & $16: 00$ & 4.38 & 3.749 & 3.526 & 1.701 & 2.122 & -0.396 \\
\hline 6/18/08 & 20:00 & 4.356 & 3.739 & 3.488 & 1.678 & 2.07 & -0.386 \\
\hline 6/19/08 & 0:00 & 4.291 & 3.702 & 3.432 & 1.629 & 2.042 & -0.4 \\
\hline 6/19/08 & $4: 00$ & 4.267 & 3.684 & 3.413 & 1.61 & 2.033 & -0.417 \\
\hline 6/19/08 & $8: 00$ & 4.263 & 3.681 & 3.409 & 1.607 & 2.028 & -0.431 \\
\hline 6/19/08 & $12: 00$ & 4.324 & 3.718 & 3.472 & 1.652 & 2.087 & -0.428 \\
\hline 6/19/08 & $16: 00$ & 4.382 & 3.742 & 3.521 & 1.694 & 2.119 & -0.414 \\
\hline 6/19/08 & 20:00 & 3.25 & 3.215 & 2.987 & 1.364 & 1.991 & -0.473 \\
\hline 6/20/08 & 0:00 & 3.547 & 3.173 & 3.156 & 1.326 & 2.012 & -0.533 \\
\hline 6/20/08 & 4:00 & 3.705 & 3.247 & 3.221 & 1.392 & 2.023 & -0.559 \\
\hline $6 / 20 / 08$ & 8:00 & 3.791 & 3.301 & 3.256 & 1.425 & 2.028 & -0.559 \\
\hline 6/20/08 & $12: 00$ & 3.89 & 3.359 & 3.329 & 1.483 & 2.077 & -0.545 \\
\hline 6/20/08 & $16: 00$ & 3.974 & 3.41 & 3.381 & 1.525 & 2.103 & -0.519 \\
\hline 6/20/08 & 20:00 & 3.993 & 3.428 & 3.357 & 1.511 & 2.051 & -0.501 \\
\hline 6/21/08 & 0:00 & 3.991 & 3.442 & 3.336 & 1.507 & 2.03 & -0.503 \\
\hline 6/21/08 & $4: 00$ & 3.993 & 3.445 & 3.329 & 1.497 & 2.03 & -0.51 \\
\hline 6/21/08 & 8:00 & 4.017 & 3.463 & 3.334 & 1.507 & 2.026 & -0.51 \\
\hline 6/21/08 & $12: 00$ & 4.087 & 3.505 & 3.406 & 1.558 & 2.098 & -0.503 \\
\hline 6/21/08 & $16: 00$ & 4.141 & 3.526 & 3.442 & 1.582 & 2.124 & -0.477 \\
\hline 6/21/08 & $20: 00$ & 4.108 & 3.507 & 3.381 & 1.53 & 2.047 & -0.473 \\
\hline $6 / 22 / 08$ & 0:00 & 4.066 & 3.451 & 3.336 & 1.493 & 2.018 & -0.489 \\
\hline $6 / 22 / 08$ & 4:00 & 4.066 & 3.465 & 3.329 & 1.483 & 2.021 & -0.512 \\
\hline 6/22/08 & 8:00 & 4.059 & 3.456 & 3.327 & 1.476 & 2.018 & -0.524 \\
\hline $6 / 22 / 08$ & $12: 00$ & 4.12 & 3.491 & 3.392 & 1.525 & 2.091 & -0.524 \\
\hline 6/22/08 & $16: 00$ & 4.174 & 3.519 & 3.439 & 1.561 & 2.119 & -0.503 \\
\hline $6 / 22 / 08$ & 20:00 & 4.162 & 3.516 & 3.406 & 1.542 & 2.065 & -0.487 \\
\hline 6/23/08 & 0:00 & 4.12 & 3.503 & 3.357 & 1.511 & 2.028 & -0.494 \\
\hline 6/23/08 & $4: 00$ & 4.089 & 3.477 & 3.336 & 1.483 & 2.021 & -0.515 \\
\hline 6/23/08 & $8: 00$ & 4.101 & 3.489 & 3.343 & 1.495 & 2.035 & -0.526 \\
\hline 6/23/08 & $12: 00$ & 4.185 & 3.54 & 3.434 & 1.563 & 2.119 & -0.517 \\
\hline 6/23/08 & $16: 00$ & 4.249 & 3.584 & 3.479 & 1.605 & 2.14 & -0.482 \\
\hline 6/23/08 & $20: 00$ & 4.197 & 3.554 & 3.411 & 1.551 & 2.067 & -0.468 \\
\hline $6 / 24 / 08$ & 0:00 & 4.155 & 3.54 & 3.364 & 1.521 & 2.032 & -0.48 \\
\hline 6/24/08 & 4:00 & 4.11 & 3.493 & 3.338 & 1.483 & 2.021 & -0.505 \\
\hline 6/24/08 & 8:00 & 4.085 & 3.507 & 3.308 & 1.502 & 1.997 & -0.524 \\
\hline $6 / 24 / 08$ & $12: 00$ & 4.087 & 3.479 & 3.32 & 1.467 & 2.021 & -0.54 \\
\hline 6/24/08 & $16: 00$ & 4.031 & 3.475 & 3.207 & 1.465 & 1.995 & -0.555 \\
\hline 6/24/08 & 20:00 & 3.967 & 3.389 & 3.252 & 1.385 & 2.007 & -0.58 \\
\hline
\end{tabular}


TABLE S1.3 (Cont.)

Depth to Water (feet below top of casing) in Indicated Wells

Date Time PMW1s PMW1d PMW2sa PMW2d PMW9s PMW9d PMW3d

\begin{tabular}{|c|c|c|c|c|c|c|c|}
\hline 6/25/08 & 0:00 & 3.979 & 3.391 & 3.268 & 1.399 & 2.009 & -0.599 \\
\hline 6/25/08 & 4:00 & 3.986 & 3.389 & 3.271 & 1.404 & 2.013 & -0.604 \\
\hline $6 / 25 / 08$ & $8: 00$ & 3.995 & 3.41 & 3.273 & 1.404 & 2.007 & -0.601 \\
\hline 6/25/08 & $12: 00$ & 4.024 & 3.412 & 3.306 & 1.422 & 2.037 & -0.599 \\
\hline 6/25/08 & $16: 00$ & 4.101 & 3.454 & 3.374 & 1.472 & 2.086 & -0.566 \\
\hline $6 / 25 / 08$ & 20:00 & 4.101 & 3.458 & 3.35 & 1.46 & 2.042 & -0.559 \\
\hline 6/26/08 & 0:00 & 4.045 & 3.428 & 3.296 & 1.422 & 2.009 & -0.569 \\
\hline 6/26/08 & 4:00 & 4.005 & 3.393 & 3.273 & 1.392 & 1.997 & -0.601 \\
\hline 6/26/08 & 8:00 & 4 & 3.387 & 3.275 & 1.387 & 1.997 & -0.613 \\
\hline 6/26/08 & $12: 00$ & 3.381 & 3.129 & 3.013 & 1.256 & 1.985 & -0.634 \\
\hline 6/26/08 & $16: 00$ & 3.505 & 3.055 & 3.13 & 1.214 & 2.025 & -0.634 \\
\hline 6/26/08 & 20:00 & 3.583 & 3.071 & 3.153 & 1.228 & 2.004 & -0.636 \\
\hline $6 / 27 / 08$ & 0:00 & 3.609 & 3.073 & 3.151 & 1.221 & 1.988 & -0.636 \\
\hline 6/27/08 & 4:00 & 3.644 & 3.089 & 3.16 & 1.23 & 1.983 & -0.639 \\
\hline 6/27/08 & 8:00 & 3.7 & 3.131 & 3.189 & 1.265 & 2.004 & -0.639 \\
\hline $6 / 27 / 08$ & $12: 00$ & 3.81 & 3.21 & 3.278 & 1.343 & 2.072 & -0.632 \\
\hline $6 / 27 / 08$ & $16: 00$ & 3.881 & 3.254 & 3.313 & 1.378 & 2.076 & -0.615 \\
\hline $6 / 27 / 08$ & $20: 00$ & 3.843 & 3.252 & 3.24 & 1.338 & 2.004 & -0.62 \\
\hline 6/28/08 & 0:00 & 3.855 & 3.273 & 3.238 & 1.345 & 2.002 & -0.634 \\
\hline 6/28/08 & 4:00 & 3.836 & 3.254 & 3.221 & 1.324 & 1.99 & -0.636 \\
\hline 6/28/08 & $8: 00$ & 3.86 & 3.273 & 3.231 & 1.336 & 1.992 & -0.639 \\
\hline 6/28/08 & $12: 00$ & 3.963 & 3.338 & 3.317 & 1.415 & 2.076 & -0.618 \\
\hline 6/28/08 & $16: 00$ & 4.014 & 3.368 & 3.348 & 1.434 & 2.083 & -0.573 \\
\hline 6/28/08 & 20:00 & 4 & 3.356 & 3.306 & 1.404 & 2.03 & -0.585 \\
\hline 6/29/08 & 0:00 & 3.974 & 3.347 & 3.273 & 1.385 & 2.002 & -0.611 \\
\hline 6/29/08 & 4:00 & 3.974 & 3.354 & 3.273 & 1.383 & 2.002 & -0.618 \\
\hline 6/29/08 & $8: 00$ & 3.993 & 3.375 & 3.292 & 1.401 & 2.011 & -0.618 \\
\hline 6/29/08 & $12: 00$ & 4.073 & 3.428 & 3.362 & 1.465 & 2.072 & -0.578 \\
\hline $6 / 29 / 08$ & $16: 00$ & 4.11 & 3.449 & 3.388 & 1.483 & 2.083 & -0.566 \\
\hline 6/29/08 & $20: 00$ & 4.094 & 3.44 & 3.357 & 1.462 & 2.037 & -0.562 \\
\hline 6/30/08 & 0:00 & 4.045 & 3.419 & 3.308 & 1.425 & 2.001 & -0.569 \\
\hline 6/30/08 & 4:00 & 4.017 & 3.403 & 3.282 & 1.406 & 1.999 & -0.599 \\
\hline 6/30/08 & $8: 00$ & 4.021 & 3.407 & 3.282 & 1.408 & 2.004 & -0.606 \\
\hline 6/30/08 & $12: 00$ & 4.094 & 3.449 & 3.367 & 1.467 & 2.081 & -0.58 \\
\hline 6/30/08 & $16: 00$ & 4.125 & 3.458 & 3.395 & 1.483 & 2.086 & -0.566 \\
\hline 6/30/08 & 20:00 & 4.089 & 3.433 & 3.345 & 1.446 & 2.032 & -0.564 \\
\hline 7/1/08 & 0:00 & 4.017 & 3.389 & 3.285 & 1.392 & 1.999 & -0.58 \\
\hline 7/1/08 & 4:00 & 3.977 & 3.354 & 3.261 & 1.364 & 1.987 & -0.625 \\
\hline 7/1/08 & 8:00 & 3.965 & 3.338 & 3.261 & 1.354 & 1.99 & -0.643 \\
\hline $7 / 1 / 08$ & $12: 00$ & 4.04 & 3.373 & 3.343 & 1.408 & 2.071 & -0.627 \\
\hline $7 / 1 / 08$ & $16: 00$ & 4.094 & 3.4 & 3.392 & 1.448 & 2.097 & -0.58 \\
\hline $7 / 1 / 08$ & 20:00 & 4.061 & 3.38 & 3.343 & 1.411 & 2.041 & -0.583 \\
\hline $7 / 2 / 08$ & 0:00 & 3.96 & 3.315 & 3.256 & 1.338 & 1.983 & -0.625 \\
\hline $7 / 2 / 08$ & 4:00 & 3.911 & 3.275 & 3.228 & 1.308 & 1.973 & -0.639 \\
\hline
\end{tabular}


TABLE S1.3 (Cont.)

Depth to Water (feet below top of casing) in Indicated Wells

Date Time PMW1s PMW1d PMW2sa PMW2d PMW9s PMW9d PMW3d

\begin{tabular}{|c|c|c|c|c|c|c|c|}
\hline $7 / 2 / 08$ & $8: 00$ & 3.923 & 3.294 & 3.238 & 1.322 & 1.985 & -0.639 \\
\hline $7 / 2 / 08$ & $12: 00$ & 4.007 & 3.345 & 3.322 & 1.385 & 2.055 & -0.634 \\
\hline $7 / 2 / 08$ & $16: 00$ & 4.106 & 3.419 & 3.397 & 1.462 & 2.095 & -0.59 \\
\hline $7 / 2 / 08$ & 20:00 & 4.134 & 3.477 & 3.385 & 1.488 & 2.062 & -0.566 \\
\hline 7/3/08 & 0:00 & 4.078 & 3.452 & 3.32 & 1.441 & 2.013 & -0.576 \\
\hline 7/3/08 & 4:00 & 4.047 & 3.433 & 3.294 & 1.415 & 1.999 & -0.601 \\
\hline $7 / 3 / 08$ & 8:00 & 4.052 & 3.445 & 3.299 & 1.422 & 2.006 & -0.606 \\
\hline $7 / 3 / 08$ & $12: 00$ & 4.106 & 3.472 & 3.357 & 1.462 & 2.05 & -0.592 \\
\hline $7 / 3 / 08$ & $16: 00$ & 4.169 & 3.51 & 3.413 & 1.507 & 2.076 & -0.571 \\
\hline $7 / 3 / 08$ & $20: 00$ & 4.12 & 3.477 & 3.353 & 1.458 & 2.05 & -0.564 \\
\hline $7 / 4 / 08$ & 0:00 & 4.064 & 3.449 & 3.299 & 1.42 & 2.006 & -0.578 \\
\hline $7 / 4 / 08$ & $4: 00$ & 4.033 & 3.424 & 3.275 & 1.394 & 1.982 & -0.618 \\
\hline $7 / 4 / 08$ & 8:00 & 4.019 & 3.412 & 3.271 & 1.387 & 1.978 & -0.627 \\
\hline $7 / 4 / 08$ & $12: 00$ & 4.094 & 3.456 & 3.348 & 1.444 & 2.031 & -0.618 \\
\hline $7 / 4 / 08$ & $16: 00$ & 4.16 & 3.489 & 3.402 & 1.483 & 2.069 & -0.569 \\
\hline 7/4/08 & 20:00 & 4.134 & 3.475 & 3.364 & 1.458 & 2.017 & -0.569 \\
\hline $7 / 5 / 08$ & 0:00 & 4.064 & 3.44 & 3.299 & 1.411 & 1.973 & -0.601 \\
\hline 7/5/08 & 4:00 & 4.012 & 3.398 & 3.266 & 1.371 & 1.959 & -0.629 \\
\hline 7/5/08 & 8:00 & 4.014 & 3.398 & 3.273 & 1.38 & 1.973 & -0.643 \\
\hline $7 / 5 / 08$ & $12: 00$ & 3.996 & 3.377 & 3.266 & 1.357 & 1.959 & -0.639 \\
\hline $7 / 5 / 08$ & $16: 00$ & 4.066 & 3.405 & 3.348 & 1.411 & 2.043 & -0.627 \\
\hline $7 / 5 / 08$ & 20:00 & 4.064 & 3.407 & 3.32 & 1.401 & 2.003 & -0.613 \\
\hline $7 / 6 / 08$ & 0:00 & 3.991 & 3.366 & 3.256 & 1.347 & 1.961 & -0.636 \\
\hline $7 / 6 / 08$ & 4:00 & 3.951 & 3.335 & 3.233 & 1.319 & 1.954 & -0.639 \\
\hline $7 / 6 / 08$ & $8: 00$ & 3.939 & 3.324 & 3.231 & 1.317 & 1.954 & -0.641 \\
\hline 7/6/08 & $12: 00$ & 4.047 & 3.398 & 3.324 & 1.397 & 2.01 & -0.636 \\
\hline $7 / 6 / 08$ & $16: 00$ & 4.118 & 3.449 & 3.364 & 1.446 & 2.043 & -0.601 \\
\hline 7/6/08 & 20:00 & 4.099 & 3.451 & 3.334 & 1.425 & 1.996 & -0.601 \\
\hline 7/7/08 & $0: 00$ & 4.021 & 3.407 & 3.266 & 1.373 & 1.954 & -0.629 \\
\hline 7/7/08 & 4:00 & 3.986 & 3.377 & 3.247 & 1.347 & 1.95 & -0.636 \\
\hline 7/7/08 & $8: 00$ & 3.972 & 3.363 & 3.242 & 1.336 & 1.95 & -0.639 \\
\hline 7/7/08 & $12: 00$ & 4.087 & 3.431 & 3.353 & 1.418 & 2.036 & -0.627 \\
\hline 7/7/08 & $16: 00$ & 4.148 & 3.477 & 3.39 & 1.46 & 2.059 & -0.58 \\
\hline 7/7/08 & 20:00 & 4.045 & 3.414 & 3.287 & 1.378 & 1.971 & -0.618 \\
\hline $7 / 8 / 08$ & 0:00 & 4.014 & 3.407 & 3.264 & 1.366 & 1.956 & -0.634 \\
\hline 7/8/08 & 4:00 & 3.989 & 3.38 & 3.242 & 1.352 & 1.938 & -0.639 \\
\hline 7/8/08 & 8:00 & 4 & 3.4 & 3.254 & 1.359 & 1.949 & -0.639 \\
\hline 7/8/08 & $12: 00$ & 4.038 & 3.428 & 3.287 & 1.387 & 1.968 & -0.632 \\
\hline 7/8/08 & $16: 00$ & 4.099 & 3.465 & 3.35 & 1.432 & 2.008 & -0.625 \\
\hline 7/8/08 & $20: 00$ & 4.094 & 3.475 & 3.317 & 1.425 & 1.98 & -0.618 \\
\hline 7/9/08 & 0:00 & 4.061 & 3.465 & 3.28 & 1.406 & 1.954 & -0.625 \\
\hline $7 / 9 / 08$ & 4:00 & 4.024 & 3.435 & 3.256 & 1.376 & 1.942 & -0.636 \\
\hline 7/9/08 & $8: 00$ & 4.038 & 3.454 & 3.266 & 1.39 & 1.942 & -0.634 \\
\hline $7 / 9 / 08$ & $12: 00$ & 4.118 & 3.498 & 3.345 & 1.444 & 2.01 & -0.625 \\
\hline
\end{tabular}


TABLE S1.3 (Cont.)

Depth to Water (feet below top of casing) in Indicated Wells

Date Time PMW1s PMW1d PMW2sa PMW2d PMW9s PMW9d PMW3d

\begin{tabular}{|c|c|c|c|c|c|c|c|}
\hline 7/9/08 & 16:00 & 4.186 & 3.537 & 3.39 & 1.486 & 2.031 & -0.578 \\
\hline $7 / 9 / 08$ & 20:00 & 4.087 & 3.477 & 3.299 & 1.404 & 1.954 & -0.613 \\
\hline 7/10/08 & 0:00 & 4.059 & 3.463 & 3.278 & 1.39 & 1.947 & -0.632 \\
\hline 7/10/08 & 4:00 & 4.014 & 3.424 & 3.254 & 1.359 & 1.942 & -0.641 \\
\hline 7/10/08 & $8: 00$ & 4.024 & 3.433 & 3.259 & 1.369 & 1.945 & -0.641 \\
\hline 7/10/08 & $12: 00$ & 4.118 & 3.482 & 3.36 & 1.439 & 2.024 & -0.632 \\
\hline 7/10/08 & $16: 00$ & 4.209 & 3.54 & 3.432 & 1.5 & 2.078 & -0.573 \\
\hline 7/10/08 & 20:00 & 4.186 & 3.533 & 3.39 & 1.476 & 2.017 & -0.571 \\
\hline $7 / 11 / 08$ & 0:00 & 4.094 & 3.484 & 3.31 & 1.411 & 1.97 & -0.604 \\
\hline $7 / 11 / 08$ & 4:00 & 4.045 & 3.445 & 3.273 & 1.378 & 1.952 & -0.639 \\
\hline 7/11/08 & $8: 00$ & 4.012 & 3.417 & 3.259 & 1.357 & 1.947 & -0.641 \\
\hline 7/11/08 & $12: 00$ & 4.115 & 3.47 & 3.367 & 1.432 & 2.033 & -0.629 \\
\hline 7/11/08 & $16: 00$ & 4.202 & 3.528 & 3.439 & 1.495 & 2.087 & -0.58 \\
\hline 7/11/08 & 20:00 & 4.186 & 3.535 & 3.39 & 1.472 & 2.024 & -0.569 \\
\hline $7 / 12 / 08$ & 0:00 & 4.172 & 3.505 & 3.36 & 1.444 & 2.005 & -0.576 \\
\hline $7 / 12 / 08$ & 4:00 & 4.139 & 3.547 & 3.334 & 1.465 & 1.97 & -0.594 \\
\hline $7 / 12 / 08$ & 8:00 & 4.165 & 3.561 & 3.341 & 1.476 & 1.966 & -0.583 \\
\hline $7 / 12 / 08$ & $12: 00$ & 4.183 & 3.6 & 3.36 & 1.495 & 1.973 & -0.587 \\
\hline 7/12/08 & $16: 00$ & 4.235 & 3.616 & 3.413 & 1.528 & 2.012 & -0.58 \\
\hline $7 / 12 / 08$ & $20: 00$ & 4.24 & 3.614 & 3.404 & 1.521 & 1.986 & -0.569 \\
\hline 7/13/08 & 0:00 & 4.155 & 3.568 & 3.329 & 1.46 & 1.949 & -0.573 \\
\hline 7/13/08 & 4:00 & 4.115 & 3.535 & 3.303 & 1.429 & 1.942 & -0.611 \\
\hline 7/13/08 & $8: 00$ & 4.108 & 3.53 & 3.303 & 1.427 & 1.942 & -0.625 \\
\hline 7/13/08 & $12: 00$ & 4.209 & 3.593 & 3.404 & 1.504 & 2.024 & -0.587 \\
\hline 7/13/08 & $16: 00$ & 4.284 & 3.633 & 3.465 & 1.554 & 2.059 & -0.573 \\
\hline 7/13/08 & $20: 00$ & 4.268 & 3.628 & 3.432 & 1.53 & 2.003 & -0.552 \\
\hline 7/14/08 & 0:00 & 4.16 & 3.563 & 3.334 & 1.451 & 1.944 & -0.569 \\
\hline $7 / 14 / 08$ & $4: 00$ & 4.118 & 3.533 & 3.308 & 1.425 & 1.94 & -0.606 \\
\hline 7/14/08 & 8:00 & 4.108 & 3.526 & 3.306 & 1.425 & 1.942 & -0.627 \\
\hline $7 / 14 / 08$ & $12: 00$ & 4.233 & 3.598 & 3.434 & 1.519 & 2.054 & -0.587 \\
\hline $7 / 14 / 08$ & $16: 00$ & 4.333 & 3.663 & 3.512 & 1.584 & 2.108 & -0.562 \\
\hline 7/14/08 & $20: 00$ & 4.305 & 3.658 & 3.458 & 1.556 & 2.026 & -0.529 \\
\hline $7 / 15 / 08$ & 0:00 & 4.228 & 3.63 & 3.383 & 1.511 & 1.97 & -0.538 \\
\hline $7 / 15 / 08$ & 4:00 & 4.172 & 3.584 & 3.345 & 1.467 & 1.954 & -0.569 \\
\hline 7/15/08 & $8: 00$ & 4.158 & 3.568 & 3.334 & 1.451 & 1.944 & -0.587 \\
\hline $7 / 15 / 08$ & $12: 00$ & 4.27 & 3.651 & 3.444 & 1.547 & 2.028 & -0.58 \\
\hline $7 / 15 / 08$ & $16: 00$ & 4.364 & 3.714 & 3.516 & 1.612 & 2.07 & -0.552 \\
\hline $7 / 15 / 08$ & 20:00 & 4.319 & 3.693 & 3.446 & 1.57 & 1.996 & -0.519 \\
\hline 7/16/08 & 0:00 & 3.886 & 3.537 & 3.064 & 1.331 & 1.923 & -0.557 \\
\hline 7/16/08 & 4:00 & 3.642 & 3.315 & 3.011 & 1.237 & 1.897 & -0.648 \\
\hline 7/16/08 & 8:00 & 3.52 & 3.085 & 3.025 & 1.104 & 1.89 & -0.653 \\
\hline $7 / 16 / 08$ & $12: 00$ & 3.747 & 3.199 & 3.142 & 1.226 & 1.918 & -0.65 \\
\hline 7/16/08 & $16: 00$ & 3.881 & 3.296 & 3.233 & 1.305 & 1.96 & -0.648 \\
\hline 7/16/08 & 20:00 & 3.937 & 3.328 & 3.252 & 1.322 & 1.932 & -0.643 \\
\hline
\end{tabular}


TABLE S1.3 (Cont.)

Depth to Water (feet below top of casing) in Indicated Wells

Date Time PMW1s PMW1d PMW2sa PMW2d PMW9s PMW9d PMW3d

\begin{tabular}{|c|c|c|c|c|c|c|c|}
\hline 7/17/08 & 0:00 & 3.932 & 3.345 & 3.226 & 1.317 & 1.911 & -0.646 \\
\hline 7/17/08 & 4:00 & 3.916 & 3.331 & 3.21 & 1.303 & 1.902 & -0.648 \\
\hline $7 / 17 / 08$ & $8: 00$ & 3.918 & 3.335 & 3.214 & 1.301 & 1.902 & -0.648 \\
\hline $7 / 17 / 08$ & $12: 00$ & 4.003 & 3.391 & 3.294 & 1.366 & 1.965 & -0.643 \\
\hline 7/17/08 & $16: 00$ & 4.066 & 3.426 & 3.345 & 1.406 & 2.005 & -0.634 \\
\hline $7 / 17 / 08$ & 20:00 & 3.107 & 2.82 & 2.933 & 1.048 & 1.89 & -0.657 \\
\hline 7/18/08 & 0:00 & 3.306 & 2.857 & 3.025 & 1.09 & 1.89 & -0.657 \\
\hline 7/18/08 & 4:00 & 3.393 & 2.95 & 3.057 & 1.09 & 1.883 & -0.66 \\
\hline 7/18/08 & 8:00 & 3.473 & 3.015 & 3.083 & 1.153 & 1.893 & -0.657 \\
\hline 7/18/08 & $12: 00$ & 3.562 & 3.069 & 3.137 & 1.197 & 1.921 & -0.653 \\
\hline 7/18/08 & $16: 00$ & 3.66 & 3.124 & 3.193 & 1.242 & 1.951 & -0.653 \\
\hline 7/18/08 & 20:00 & 3.698 & 3.15 & 3.179 & 1.235 & 1.93 & -0.653 \\
\hline 7/19/08 & 0:00 & 3.705 & 3.161 & 3.156 & 1.228 & 1.902 & -0.655 \\
\hline 7/19/08 & 4:00 & 3.712 & 3.157 & 3.149 & 1.212 & 1.897 & -0.657 \\
\hline 7/19/08 & 8:00 & 3.759 & 3.18 & 3.16 & 1.223 & 1.902 & -0.657 \\
\hline 7/19/08 & $12: 00$ & 3.815 & 3.21 & 3.212 & 1.261 & 1.951 & -0.653 \\
\hline $7 / 19 / 08$ & $16: 00$ & 3.888 & 3.238 & 3.266 & 1.296 & 1.986 & -0.648 \\
\hline 7/19/08 & $20: 00$ & 3.904 & 3.261 & 3.249 & 1.301 & 1.956 & -0.648 \\
\hline $7 / 20 / 08$ & 0:00 & 3.862 & 3.252 & 3.203 & 1.27 & 1.913 & -0.653 \\
\hline 7/20/08 & 4:00 & 3.848 & 3.25 & 3.189 & 1.265 & 1.902 & -0.655 \\
\hline $7 / 20 / 08$ & 8:00 & 3.855 & 3.259 & 3.198 & 1.272 & 1.902 & -0.657 \\
\hline $7 / 20 / 08$ & $12: 00$ & 3.946 & 3.326 & 3.278 & 1.343 & 1.965 & -0.646 \\
\hline 7/20/08 & $16: 00$ & 4 & 3.359 & 3.32 & 1.378 & 2 & -0.643 \\
\hline $7 / 20 / 08$ & 20:00 & 3.982 & 3.347 & 3.282 & 1.35 & 1.937 & -0.648 \\
\hline $7 / 21 / 08$ & 0:00 & 3.914 & 3.308 & 3.224 & 1.303 & 1.906 & -0.653 \\
\hline $7 / 21 / 08$ & 4:00 & 3.916 & 3.319 & 3.224 & 1.315 & 1.906 & -0.641 \\
\hline $7 / 21 / 08$ & 8:00 & 3.893 & 3.301 & 3.217 & 1.294 & 1.904 & -0.643 \\
\hline $7 / 21 / 08$ & $12: 00$ & 3.958 & 3.34 & 3.275 & 1.345 & 1.96 & -0.639 \\
\hline $7 / 21 / 08$ & $16: 00$ & 4.019 & 3.384 & 3.324 & 1.392 & 1.983 & -0.636 \\
\hline 7/21/08 & $20: 00$ & 3.977 & 3.363 & 3.271 & 1.354 & 1.927 & -0.639 \\
\hline $7 / 22 / 08$ & 0:00 & 3.935 & 3.342 & 3.231 & 1.324 & 1.902 & -0.641 \\
\hline $7 / 22 / 08$ & 4:00 & 3.93 & 3.345 & 3.228 & 1.322 & 1.897 & -0.643 \\
\hline $7 / 22 / 08$ & $8: 00$ & 3.928 & 3.345 & 3.221 & 1.322 & 1.897 & -0.646 \\
\hline $7 / 22 / 08$ & $12: 00$ & 4.007 & 3.4 & 3.303 & 1.39 & 1.974 & -0.639 \\
\hline $7 / 22 / 08$ & $16: 00$ & 4.069 & 3.433 & 3.364 & & 2.014 & -0.632 \\
\hline $7 / 22 / 08$ & 20:00 & 4.057 & 3.433 & 3.329 & 1.42 & 1.955 & -0.618 \\
\hline 7/23/08 & 0:00 & 4.003 & 3.414 & 3.271 & 1.373 & 1.906 & -0.641 \\
\hline 7/23/08 & 4:00 & 3.977 & 3.393 & 3.254 & 1.347 & 1.892 & -0.643 \\
\hline $7 / 23 / 08$ & 8:00 & 3.968 & 3.387 & 3.247 & 1.34 & 1.892 & -0.646 \\
\hline $7 / 23 / 08$ & $12: 00$ & 4.024 & 3.421 & & 1.385 & 1.958 & -0.639 \\
\hline $7 / 23 / 08$ & $16: 00$ & 4.071 & 3.447 & 3.357 & 1.408 & 2.065 & \\
\hline $7 / 23 / 08$ & 20:00 & 4.014 & 3.412 & 3.289 & 1.354 & 1.941 & \\
\hline $7 / 24 / 08$ & 0:00 & 3.965 & 3.377 & 3.249 & 1.315 & 1.906 & \\
\hline $7 / 24 / 08$ & 4:00 & 3.944 & 3.363 & 3.238 & 1.305 & 1.899 & \\
\hline
\end{tabular}


TABLE S1.3 (Cont.)

Depth to Water (feet below top of casing) in Indicated Wells

Date Time PMW1s PMW1d PMW2sa PMW2d PMW9s PMW9d PMW3d

\begin{tabular}{|c|c|c|c|c|c|c|c|}
\hline $7 / 24 / 08$ & 8:00 & 3.939 & 3.356 & 3.233 & 1.298 & 1.894 & \\
\hline $7 / 24 / 08$ & $12: 00$ & 3.97 & 3.373 & 3.278 & 1.326 & 1.92 & \\
\hline $7 / 24 / 08$ & $16: 00$ & 4.036 & 3.412 & 3.327 & 1.369 & 1.95 & -0.599 \\
\hline $7 / 24 / 08$ & $20: 00$ & 4.036 & 3.424 & 3.303 & 1.366 & 1.922 & -0.653 \\
\hline $7 / 25 / 08$ & 0:00 & 3.989 & 3.405 & 3.259 & 1.336 & 1.892 & -0.718 \\
\hline $7 / 25 / 08$ & 4:00 & 3.956 & 3.377 & 3.238 & 1.308 & 1.883 & -0.753 \\
\hline $7 / 25 / 08$ & 8:00 & 3.977 & 3.407 & 3.254 & 1.326 & 1.883 & -0.76 \\
\hline $7 / 25 / 08$ & $12: 00$ & 4.003 & 3.431 & 3.266 & 1.352 & 1.89 & -0.765 \\
\hline $7 / 25 / 08$ & $16: 00$ & 4.073 & 3.475 & 3.331 & 1.404 & 1.929 & -0.753 \\
\hline $7 / 25 / 08$ & $20: 00$ & 4.09 & 3.491 & 3.327 & 1.408 & 1.913 & -0.735 \\
\hline $7 / 26 / 08$ & 0:00 & 4.05 & 3.477 & 3.282 & 1.383 & 1.89 & -0.744 \\
\hline $7 / 26 / 08$ & $4: 00$ & 4.022 & 3.458 & 3.263 & 1.359 & 1.878 & -0.732 \\
\hline $7 / 26 / 08$ & 8:00 & 4.019 & 3.456 & 3.261 & 1.359 & 1.88 & -0.742 \\
\hline $7 / 26 / 08$ & $12: 00$ & 4.061 & 3.489 & 3.301 & 1.394 & 1.911 & -0.739 \\
\hline $7 / 26 / 08$ & $16: 00$ & 4.092 & 3.489 & 3.341 & 1.404 & 1.934 & -0.739 \\
\hline $7 / 26 / 08$ & 20:00 & 4.085 & 3.484 & 3.324 & 1.399 & 1.918 & -0.732 \\
\hline $7 / 27 / 08$ & 0:00 & 4.012 & 3.44 & 3.259 & 1.345 & 1.873 & -0.735 \\
\hline $7 / 27 / 08$ & 4:00 & 3.977 & 3.407 & 3.24 & 1.317 & 1.871 & -0.735 \\
\hline $7 / 27 / 08$ & 8:00 & 3.965 & 3.398 & 3.233 & 1.31 & 1.866 & -0.737 \\
\hline $7 / 27 / 08$ & $12: 00$ & 4.047 & 3.445 & 3.32 & 1.376 & 1.941 & -0.73 \\
\hline $7 / 27 / 08$ & $16: 00$ & 4.125 & 3.493 & 3.385 & 1.427 & 1.983 & -0.723 \\
\hline $7 / 27 / 08$ & 20:00 & 4.101 & 3.477 & 3.341 & 1.397 & 1.92 & -0.723 \\
\hline $7 / 28 / 08$ & 0:00 & 4.026 & 3.445 & 3.273 & 1.347 & 1.88 & -0.73 \\
\hline $7 / 28 / 08$ & $4: 00$ & 3.989 & 3.417 & 3.252 & 1.326 & 1.88 & -0.732 \\
\hline $7 / 28 / 08$ & $8: 00$ & 4.024 & 3.456 & 3.275 & 1.364 & 1.892 & -0.732 \\
\hline $7 / 28 / 08$ & $12: 00$ & 4.108 & 3.51 & 3.357 & 1.422 & 1.966 & -0.725 \\
\hline $7 / 28 / 08$ & $16: 00$ & 4.193 & 3.563 & 3.423 & 1.486 & 2.001 & -0.695 \\
\hline $7 / 28 / 08$ & 20:00 & 4.167 & 3.556 & 3.371 & 1.453 & 1.924 & -0.688 \\
\hline 7/29/08 & $0: 00$ & 4.113 & 3.542 & 3.317 & 1.425 & 1.887 & -0.723 \\
\hline $7 / 29 / 08$ & 4:00 & 4.073 & 3.512 & 3.289 & 1.392 & 1.875 & -0.735 \\
\hline $7 / 29 / 08$ & $8: 00$ & 4.057 & 3.498 & 3.287 & 1.38 & 1.88 & -0.735 \\
\hline $7 / 29 / 08$ & $12: 00$ & 4.136 & 3.556 & 3.35 & 1.439 & 1.915 & -0.727 \\
\hline $7 / 29 / 08$ & $16: 00$ & 4.188 & 3.588 & 3.388 & 1.472 & 1.938 & -0.711 \\
\hline 7/29/08 & 20:00 & 4.162 & 3.577 & 3.35 & 1.448 & 1.906 & -0.716 \\
\hline 7/30/08 & $0: 00$ & 4.101 & 3.54 & 3.296 & 1.408 & 1.875 & -0.732 \\
\hline 7/30/08 & 4:00 & 4.073 & 3.519 & 3.28 & 1.387 & 1.868 & -0.737 \\
\hline 7/30/08 & $8: 00$ & 4.066 & 3.517 & 3.28 & 1.387 & 1.87 & -0.735 \\
\hline 7/30/08 & $12: 00$ & 4.136 & 3.554 & 3.353 & 1.437 & 1.934 & -0.727 \\
\hline 7/30/08 & $16: 00$ & 4.214 & 3.607 & 3.416 & 1.495 & 1.962 & -0.697 \\
\hline 7/30/08 & 20:00 & 4.2 & 3.6 & 3.381 & 1.474 & 1.917 & -0.69 \\
\hline 7/31/08 & 0:00 & 4.134 & 3.572 & 3.317 & 1.432 & 1.88 & -0.723 \\
\hline $7 / 31 / 08$ & 4:00 & 4.087 & 3.53 & 3.289 & 1.399 & 1.868 & -0.735 \\
\hline 7/31/08 & $8: 00$ & 4.078 & 3.526 & 3.282 & 1.394 & 1.87 & -0.735 \\
\hline 7/31/08 & $12: 00$ & 4.169 & 3.581 & 3.374 & 1.465 & 1.943 & -0.725 \\
\hline
\end{tabular}


TABLE S1.3 (Cont.)

Depth to Water (feet below top of casing) in Indicated Wells

Date Time PMW1s PMW1d PMW2sa PMW2d PMW9s PMW9d PMW3d

\begin{tabular}{|c|c|c|c|c|c|c|c|}
\hline $7 / 31 / 08$ & $16: 00$ & 4.265 & 3.642 & 3.456 & 1.53 & 1.999 & -0.688 \\
\hline 7/31/08 & 20:00 & 4.254 & 3.653 & 3.409 & 1.511 & 1.924 & -0.669 \\
\hline 8/1/08 & 0:00 & 4.188 & 3.628 & 3.348 & 1.474 & 1.887 & -0.678 \\
\hline 8/1/08 & 4:00 & 4.153 & 3.607 & 3.322 & 1.451 & 1.877 & -0.699 \\
\hline 8/1/08 & 8:00 & 4.162 & 3.626 & 3.329 & 1.465 & 1.884 & -0.713 \\
\hline 8/1/08 & $12: 00$ & 4.251 & 3.677 & 3.42 & 1.535 & 1.957 & -0.688 \\
\hline 8/1/08 & $16: 00$ & 4.336 & 3.73 & 3.493 & 1.589 & 2.013 & -0.662 \\
\hline 8/1/08 & 20:00 & 4.315 & 3.725 & 3.439 & 1.568 & 1.943 & -0.634 \\
\hline $8 / 2 / 08$ & 0:00 & 4.237 & 3.686 & 3.369 & 1.514 & 1.894 & -0.643 \\
\hline $8 / 2 / 08$ & 4:00 & 4.197 & 3.656 & 3.338 & 1.481 & 1.88 & -0.667 \\
\hline 8/2/08 & 8:00 & 4.174 & 3.63 & 3.331 & 1.462 & 1.875 & -0.683 \\
\hline $8 / 2 / 08$ & $12: 00$ & 4.247 & 3.667 & 3.411 & 1.516 & 1.95 & -0.685 \\
\hline 8/2/08 & $16: 00$ & 4.324 & 3.711 & 3.477 & 1.565 & 1.994 & -0.667 \\
\hline 8/2/08 & 20:00 & 4.305 & 3.709 & 3.434 & 1.549 & 1.94 & -0.643 \\
\hline 8/3/08 & 0:00 & 4.228 & 3.672 & 3.364 & 1.497 & 1.889 & -0.653 \\
\hline 8/3/08 & 4:00 & 4.193 & 3.644 & 3.338 & 1.479 & 1.886 & -0.674 \\
\hline 8/3/08 & 8:00 & 4.183 & 3.64 & 3.334 & 1.472 & 1.877 & -0.685 \\
\hline 8/3/08 & $12: 00$ & 4.312 & 3.718 & 3.463 & 1.565 & 1.982 & -0.678 \\
\hline 8/3/08 & $16: 00$ & 4.401 & 3.783 & 3.535 & 1.636 & 2.045 & -0.641 \\
\hline 8/3/08 & 20:00 & 4.387 & 3.786 & 3.491 & 1.61 & 1.966 & -0.601 \\
\hline 8/4/08 & 0:00 & 4.303 & 3.742 & 3.411 & 1.556 & 1.903 & -0.613 \\
\hline $8 / 4 / 08$ & 4:00 & 4.273 & 3.718 & 3.388 & 1.533 & 1.893 & -0.634 \\
\hline $8 / 4 / 08$ & 8:00 & 4.268 & 3.728 & 3.388 & 1.533 & 1.889 & -0.648 \\
\hline 8/4/08 & $12: 00$ & 4.345 & 3.774 & 3.472 & 1.598 & 1.954 & -0.65 \\
\hline 8/4/08 & $16: 00$ & 4.401 & 3.816 & 3.507 & 1.633 & 1.954 & -0.625 \\
\hline 8/4/08 & 20:00 & 4.394 & 3.82 & 3.474 & 1.624 & 1.924 & -0.599 \\
\hline 8/5/08 & 0:00 & 4.329 & 3.79 & 3.413 & 1.579 & 1.889 & -0.606 \\
\hline 8/5/08 & 4:00 & 4.317 & 3.79 & 3.402 & 1.577 & 1.889 & -0.615 \\
\hline 8/5/08 & $8: 00$ & 4.31 & 3.788 & 3.395 & 1.572 & 1.884 & -0.625 \\
\hline 8/5/08 & $12: 00$ & 4.383 & 3.841 & 3.465 & 1.626 & 1.926 & -0.622 \\
\hline 8/5/08 & $16: 00$ & 4.401 & 3.853 & 3.47 & 1.633 & 1.919 & -0.601 \\
\hline 8/5/08 & 20:00 & 4.42 & 3.865 & 3.472 & 1.645 & 1.921 & -0.59 \\
\hline 8/6/08 & 0:00 & 4.362 & 3.837 & 3.418 & 1.605 & 1.896 & -0.597 \\
\hline 8/6/08 & 4:00 & 4.333 & 3.816 & 3.399 & 1.582 & 1.889 & -0.608 \\
\hline 8/6/08 & 8:00 & 4.329 & 3.814 & 3.395 & 1.579 & 1.891 & -0.618 \\
\hline 8/6/08 & $12: 00$ & 4.397 & 3.855 & 3.463 & 1.633 & 1.952 & -0.618 \\
\hline 8/6/08 & $16: 00$ & 4.425 & 3.865 & 3.491 & 1.647 & 1.949 & -0.608 \\
\hline 8/6/08 & 20:00 & 4.423 & 3.872 & 3.467 & 1.64 & 1.921 & -0.592 \\
\hline 8/7/08 & 0:00 & 4.373 & 3.846 & 3.418 & 1.608 & 1.891 & -0.594 \\
\hline 8/7/08 & 4:00 & 4.333 & 3.814 & 3.392 & 1.575 & 1.881 & -0.611 \\
\hline 8/7/08 & 8:00 & 4.331 & 3.816 & 3.395 & 1.582 & 1.886 & -0.62 \\
\hline 8/7/08 & $12: 00$ & 4.423 & 3.874 & 3.491 & 1.65 & 1.973 & -0.618 \\
\hline 8/7/08 & $16: 00$ & 4.505 & 3.918 & 3.549 & 1.701 & 2.015 & -0.59 \\
\hline 8/7/08 & 20:00 & 4.481 & 3.909 & 3.5 & 1.671 & 1.945 & -0.562 \\
\hline
\end{tabular}


TABLE S1.3 (Cont.)

Depth to Water (feet below top of casing) in Indicated Wells

Date Time PMW1s PMW1d PMW2sa PMW2d PMW9s PMW9d PMW3d

\begin{tabular}{|c|c|c|c|c|c|c|c|}
\hline 8/8/08 & $0: 00$ & 4.401 & 3.865 & 3.427 & 1.617 & 1.9 & -0.576 \\
\hline 8/8/08 & $4: 00$ & 4.369 & 3.844 & 3.406 & 1.596 & 1.886 & -0.594 \\
\hline 8/8/08 & 8:00 & 4.355 & 3.834 & 3.397 & 1.589 & 1.886 & -0.611 \\
\hline 8/8/08 & $12: 00$ & 4.444 & 3.881 & 3.491 & 1.652 & 1.965 & -0.611 \\
\hline 8/8/08 & $16: 00$ & 4.538 & 3.939 & 3.575 & 1.715 & 2.008 & -0.58 \\
\hline 8/8/08 & $20: 00$ & 4.5 & 3.923 & 3.514 & 1.68 & 1.942 & -0.555 \\
\hline 8/9/08 & 0:00 & 4.406 & 3.865 & 3.432 & 1.612 & 1.891 & -0.569 \\
\hline 8/9/08 & 4:00 & 4.364 & 3.832 & 3.406 & 1.584 & 1.877 & -0.594 \\
\hline 8/9/08 & $8: 00$ & 4.355 & 3.821 & 3.402 & 1.58 & 1.881 & -0.615 \\
\hline 8/9/08 & $12: 00$ & 4.357 & 3.82 & 3.409 & 1.58 & 1.879 & -0.625 \\
\hline 8/9/08 & $16: 00$ & 4.467 & 3.89 & 3.514 & 1.664 & 1.965 & -0.618 \\
\hline 8/9/08 & $20: 00$ & 4.472 & 3.906 & 3.486 & 1.661 & 1.919 & -0.587 \\
\hline 8/10/08 & 0:00 & 4.413 & 3.883 & 3.43 & 1.624 & 1.884 & -0.587 \\
\hline 8/10/08 & $4: 00$ & 4.373 & 3.853 & 3.402 & 1.596 & 1.874 & -0.599 \\
\hline 8/10/08 & $8: 00$ & 4.378 & 3.862 & 3.406 & 1.603 & 1.881 & -0.611 \\
\hline 8/10/08 & $12: 00$ & 4.458 & 3.909 & 3.491 & 1.664 & 1.954 & -0.606 \\
\hline 8/10/08 & $16: 00$ & 4.542 & 3.962 & 3.561 & 1.718 & 1.982 & -0.576 \\
\hline 8/10/08 & $20: 00$ & 4.453 & 3.916 & 3.451 & 1.643 & 1.89 & -0.559 \\
\hline 8/11/08 & $0: 00$ & 4.432 & 3.911 & 3.432 & 1.636 & 1.881 & -0.571 \\
\hline 8/11/08 & 4:00 & 4.399 & 3.881 & 3.409 & 1.605 & 1.869 & -0.59 \\
\hline 8/11/08 & 8:00 & 4.39 & 3.876 & 3.409 & 1.603 & 1.872 & -0.604 \\
\hline 8/11/08 & $12: 00$ & 4.453 & 3.909 & 3.472 & 1.65 & 1.925 & -0.604 \\
\hline 8/11/08 & $16: 00$ & 4.507 & 3.939 & 3.507 & 1.673 & 1.932 & -0.587 \\
\hline 8/11/08 & 20:00 & 4.467 & 3.916 & 3.465 & 1.643 & 1.9 & -0.58 \\
\hline 8/12/08 & 0:00 & 4.416 & 3.89 & 3.423 & 1.61 & 1.876 & -0.59 \\
\hline 8/12/08 & 4:00 & 4.366 & 3.874 & 3.388 & 1.591 & 1.872 & -0.615 \\
\hline 8/12/08 & 8:00 & 4.362 & 3.846 & 3.385 & 1.575 & 1.865 & -0.627 \\
\hline 8/12/08 & $12: 00$ & 4.43 & 3.895 & 3.456 & 1.633 & 1.921 & -0.622 \\
\hline 8/12/08 & $16: 00$ & 4.509 & 3.944 & 3.516 & 1.683 & 1.942 & -0.597 \\
\hline 8/12/08 & 20:00 & 4.495 & 3.944 & 3.481 & 1.668 & 1.902 & -0.576 \\
\hline 8/13/08 & 0:00 & 4.434 & 3.916 & 3.427 & 1.626 & 1.872 & -0.58 \\
\hline 8/13/08 & 4:00 & 4.406 & 3.89 & 3.406 & 1.61 & 1.86 & -0.594 \\
\hline 8/13/08 & 8:00 & 4.397 & 3.888 & 3.404 & 1.603 & 1.865 & -0.608 \\
\hline 8/13/08 & $12: 00$ & 4.465 & 3.927 & 3.472 & 1.652 & 1.918 & -0.604 \\
\hline 8/13/08 & $16: 00$ & 4.58 & 3.995 & 3.566 & 1.732 & 1.977 & -0.578 \\
\hline 8/13/08 & 20:00 & 4.566 & 3.999 & 3.523 & 1.713 & 1.914 & -0.545 \\
\hline 8/14/08 & 0:00 & 4.502 & 3.981 & 3.47 & 1.678 & 1.881 & -0.548 \\
\hline 8/14/08 & 4:00 & 4.448 & 3.932 & 3.432 & 1.636 & 1.869 & -0.569 \\
\hline 8/14/08 & $8: 00$ & 4.444 & 3.932 & 3.427 & 1.638 & 1.865 & -0.583 \\
\hline 8/14/08 & $12: 00$ & 4.486 & 3.96 & 3.467 & 1.671 & 1.897 & -0.585 \\
\hline 8/14/08 & $16: 00$ & 4.591 & 4.029 & 3.559 & 1.743 & 1.956 & -0.564 \\
\hline 8/14/08 & 20:00 & 4.596 & 4.043 & 3.531 & 1.741 & 1.918 & -0.531 \\
\hline 8/15/08 & $0: 00$ & 4.538 & 4.027 & 3.479 & 1.713 & 1.886 & -0.531 \\
\hline 8/15/08 & 4:00 & 4.512 & 4.011 & 3.456 & 1.69 & 1.872 & -0.54 \\
\hline
\end{tabular}


TABLE S1.3 (Cont.)

Depth to Water (feet below top of casing) in Indicated Wells

Date Time PMW1s PMW1d PMW2sa PMW2d PMW9s PMW9d PMW3d

\begin{tabular}{|c|c|c|c|c|c|c|c|}
\hline $8 / 15 / 08$ & 8:00 & 4.514 & 4.015 & 3.453 & 1.694 & 1.876 & -0.55 \\
\hline 8/15/08 & $12: 00$ & 4.584 & 4.067 & 3.531 & 1.753 & 1.942 & -0.543 \\
\hline 8/15/08 & $16: 00$ & 4.662 & 4.111 & 3.596 & 1.804 & 1.977 & -0.515 \\
\hline 8/15/08 & 20:00 & 4.657 & 4.122 & 3.566 & 1.797 & 1.93 & -0.487 \\
\hline 8/16/08 & 0:00 & 4.608 & 4.106 & 3.509 & 1.765 & 1.893 & -0.487 \\
\hline 8/16/08 & 4:00 & 4.575 & 4.08 & 3.484 & 1.736 & 1.881 & -0.501 \\
\hline 8/16/08 & 8:00 & 4.568 & 4.076 & 3.479 & 1.732 & 1.886 & -0.512 \\
\hline 8/16/08 & $12: 00$ & 4.645 & 4.12 & 3.563 & 1.79 & 1.965 & -0.512 \\
\hline 8/16/08 & $16: 00$ & 4.709 & 4.152 & 3.624 & 1.83 & 1.991 & -0.484 \\
\hline 8/16/08 & 20:00 & 4.674 & 4.136 & 3.566 & 1.793 & 1.925 & -0.463 \\
\hline 8/17/08 & 0:00 & 4.603 & 4.094 & 3.505 & 1.746 & 1.89 & -0.48 \\
\hline 8/17/08 & 4:00 & 4.568 & 4.064 & 3.479 & 1.718 & 1.883 & -0.503 \\
\hline 8/17/08 & 8:00 & 4.559 & 4.06 & 3.474 & 1.713 & 1.888 & -0.519 \\
\hline 8/17/08 & $12: 00$ & 4.648 & 4.108 & 3.57 & 1.774 & 1.965 & -0.519 \\
\hline 8/17/08 & $16: 00$ & 4.713 & 4.148 & 3.622 & 1.818 & 1.979 & -0.491 \\
\hline 8/17/08 & 20:00 & 4.688 & 4.141 & 3.575 & 1.797 & 1.923 & -0.47 \\
\hline 8/18/08 & 0:00 & 4.61 & 4.097 & 3.509 & 1.743 & 1.888 & -0.484 \\
\hline 8/18/08 & 4:00 & 4.573 & 4.067 & 3.484 & 1.72 & 1.878 & -0.505 \\
\hline 8/18/08 & 8:00 & 4.559 & 4.06 & 3.474 & 1.711 & 1.876 & -0.522 \\
\hline 8/18/08 & $12: 00$ & 4.66 & 4.122 & 3.58 & 1.788 & 1.963 & -0.522 \\
\hline 8/18/08 & $16: 00$ & 4.756 & 4.187 & 3.659 & 1.861 & 1.995 & -0.484 \\
\hline 8/18/08 & 20:00 & 4.725 & 4.18 & 3.594 & 1.828 & 1.928 & -0.454 \\
\hline 8/19/08 & 0:00 & 4.648 & 4.141 & 3.528 & 1.776 & 1.888 & -0.466 \\
\hline 8/19/08 & 4:00 & 4.613 & 4.118 & 3.505 & 1.753 & 1.878 & -0.482 \\
\hline 8/19/08 & $8: 00$ & 4.61 & 4.118 & 3.5 & 1.753 & 1.881 & -0.496 \\
\hline 8/19/08 & $12: 00$ & 4.695 & 4.166 & 3.591 & 1.821 & 1.96 & -0.498 \\
\hline 8/19/08 & $16: 00$ & 4.767 & 4.213 & 3.645 & 1.868 & 1.981 & -0.47 \\
\hline 8/19/08 & 20:00 & 4.753 & 4.215 & 3.61 & 1.851 & 1.934 & -0.44 \\
\hline 8/20/08 & 0:00 & 4.678 & 4.173 & 3.542 & 1.8 & 1.89 & -0.449 \\
\hline 8/20/08 & 4:00 & 4.641 & 4.145 & 3.514 & 1.774 & 1.878 & -0.47 \\
\hline 8/20/08 & 8:00 & 4.631 & 4.141 & 3.507 & 1.769 & 1.883 & -0.487 \\
\hline 8/20/08 & $12: 00$ & 4.695 & 4.176 & 3.58 & 1.811 & 1.951 & -0.487 \\
\hline 8/20/08 & $16: 00$ & 4.777 & 4.222 & 3.652 & 1.868 & 1.995 & -0.463 \\
\hline 8/20/08 & 20:00 & 4.749 & 4.215 & 3.598 & 1.847 & 1.932 & -0.435 \\
\hline 8/21/08 & 0:00 & 4.676 & 4.173 & 3.538 & 1.793 & 1.888 & -0.449 \\
\hline $8 / 21 / 08$ & 4:00 & 4.631 & 4.136 & 3.505 & 1.76 & 1.871 & -0.473 \\
\hline 8/21/08 & 8:00 & 4.608 & 4.115 & 3.491 & 1.741 & 1.867 & -0.496 \\
\hline 8/21/08 & $12: 00$ & 4.615 & 4.115 & 3.505 & 1.748 & 1.874 & -0.51 \\
\hline 8/21/08 & $16: 00$ & 4.685 & 4.15 & 3.573 & 1.795 & 1.913 & -0.503 \\
\hline 8/21/08 & 20:00 & 4.69 & 4.155 & 3.559 & 1.793 & 1.897 & -0.491 \\
\hline $8 / 22 / 08$ & 0:00 & 4.631 & 4.129 & 3.502 & 1.755 & 1.867 & -0.496 \\
\hline $8 / 22 / 08$ & 4:00 & 4.606 & 4.111 & 3.486 & 1.739 & 1.86 & -0.508 \\
\hline $8 / 22 / 08$ & $8: 00$ & 4.603 & 4.111 & 3.486 & 1.739 & 1.857 & -0.519 \\
\hline $8 / 22 / 08$ & $12: 00$ & 4.711 & 4.178 & 3.596 & 1.823 & 1.962 & -0.512 \\
\hline
\end{tabular}


TABLE S1.3 (Cont.)

Depth to Water (feet below top of casing) in Indicated Wells

Date Time PMW1s PMW1d PMW2sa PMW2d PMW9s PMW9d PMW3d

\begin{tabular}{|c|c|c|c|c|c|c|c|}
\hline 8/22/08 & $16: 00$ & 4.81 & 4.255 & 3.669 & 1.898 & 1.993 & -0.468 \\
\hline 8/22/08 & 20:00 & 4.798 & 4.275 & 3.631 & 1.893 & 1.93 & -0.426 \\
\hline 8/23/08 & $0: 00$ & 4.76 & 4.273 & 3.584 & 1.872 & 1.899 & -0.424 \\
\hline 8/23/08 & 4:00 & 4.756 & 4.273 & 3.573 & 1.868 & 1.89 & -0.431 \\
\hline 8/23/08 & $8: 00$ & 4.765 & 4.294 & 3.575 & 1.877 & 1.895 & -0.433 \\
\hline 8/23/08 & $12: 00$ & 4.826 & 4.322 & 3.643 & 1.919 & 1.96 & -0.424 \\
\hline 8/23/08 & $16: 00$ & 4.878 & 4.35 & 3.687 & 1.954 & 1.972 & -0.398 \\
\hline 8/23/08 & $20: 00$ & 4.847 & 4.338 & 3.641 & 1.922 & 1.92 & -0.382 \\
\hline $8 / 24 / 08$ & 0:00 & 4.786 & 4.306 & 3.58 & 1.879 & 1.883 & -0.393 \\
\hline 8/24/08 & $4: 00$ & 4.751 & 4.275 & 3.556 & 1.854 & 1.878 & -0.417 \\
\hline 8/24/08 & 8:00 & 4.751 & 4.285 & 3.556 & 1.854 & 1.885 & -0.428 \\
\hline 8/24/08 & $12: 00$ & 4.824 & 4.322 & 3.634 & 1.905 & 1.965 & -0.431 \\
\hline 8/24/08 & $16: 00$ & 4.896 & 4.361 & 3.697 & 1.959 & 1.983 & -0.405 \\
\hline 8/24/08 & 20:00 & 4.871 & 4.354 & 3.652 & 1.933 & 1.923 & -0.377 \\
\hline 8/25/08 & $0: 00$ & 4.803 & 4.315 & 3.589 & 1.886 & 1.888 & -0.391 \\
\hline 8/25/08 & 4:00 & 4.763 & 4.287 & 3.561 & 1.858 & 1.878 & -0.414 \\
\hline 8/25/08 & 8:00 & 4.744 & 4.273 & 3.545 & 1.844 & 1.874 & -0.433 \\
\hline 8/25/08 & $12: 00$ & 4.831 & 4.324 & 3.641 & 1.91 & 1.965 & -0.433 \\
\hline $8 / 25 / 08$ & $16: 00$ & 4.899 & 4.361 & 3.701 & 1.957 & 2 & -0.407 \\
\hline 8/25/08 & 20:00 & 4.868 & 4.352 & 3.648 & 1.931 & 1.925 & -0.379 \\
\hline 8/26/08 & 0:00 & 4.793 & 4.308 & 3.582 & 1.879 & 1.885 & -0.393 \\
\hline 8/26/08 & $4: 00$ & 4.751 & 4.273 & 3.547 & 1.847 & 1.871 & -0.417 \\
\hline 8/26/08 & 8:00 & 4.737 & 4.264 & 3.54 & 1.837 & 1.878 & -0.438 \\
\hline 8/26/08 & $12: 00$ & 4.796 & 4.294 & 3.605 & 1.882 & 1.934 & -0.442 \\
\hline $8 / 26 / 08$ & $16: 00$ & 4.854 & 4.322 & 3.659 & 1.915 & 1.967 & -0.424 \\
\hline 8/26/08 & 20:00 & 4.845 & 4.326 & 3.631 & 1.907 & 1.913 & -0.403 \\
\hline 8/27/08 & $0: 00$ & 4.784 & 4.299 & 3.573 & 1.865 & 1.871 & -0.41 \\
\hline $8 / 27 / 08$ & 4:00 & 4.749 & 4.268 & 3.545 & 1.842 & 1.864 & -0.428 \\
\hline 8/27/08 & $8: 00$ & 4.76 & 4.289 & 3.566 & 1.865 & 1.892 & -0.445 \\
\hline 8/27/08 & $12: 00$ & 4.638 & 4.192 & 3.439 & 1.739 & 1.848 & -0.484 \\
\hline $8 / 27 / 08$ & $16: 00$ & 4.744 & 4.234 & 3.563 & 1.823 & 1.927 & -0.491 \\
\hline 8/27/08 & 20:00 & 4.735 & 4.231 & 3.542 & 1.816 & 1.881 & -0.475 \\
\hline 8/28/08 & 0:00 & 4.683 & 4.199 & 3.505 & 1.781 & 1.857 & -0.484 \\
\hline 8/28/08 & $4: 00$ & 4.676 & 4.196 & 3.507 & 1.786 & 1.857 & -0.496 \\
\hline 8/28/08 & $8: 00$ & 4.676 & 4.206 & 3.502 & 1.788 & 1.848 & -0.501 \\
\hline 8/28/08 & $12: 00$ & 4.76 & 4.278 & 3.577 & 1.863 & 1.904 & -0.487 \\
\hline 8/28/08 & $16: 00$ & 4.866 & 4.352 & 3.664 & 1.945 & 1.976 & -0.449 \\
\hline 8/28/08 & 20:00 & 4.859 & 4.361 & 3.627 & 1.924 & 1.904 & -0.412 \\
\hline 8/29/08 & $0: 00$ & 4.831 & 4.368 & 3.591 & 1.917 & 1.88 & -0.405 \\
\hline 8/29/08 & 4:00 & 4.817 & 4.359 & 3.573 & 1.903 & 1.873 & -0.41 \\
\hline 8/29/08 & $8: 00$ & 4.814 & 4.361 & 3.573 & 1.898 & 1.876 & -0.417 \\
\hline 8/29/08 & $12: 00$ & 4.892 & 4.405 & 3.657 & 1.959 & 1.958 & -0.41 \\
\hline $8 / 29 / 08$ & $16: 00$ & 4.969 & 4.454 & 3.725 & 2.015 & 2 & -0.377 \\
\hline 8/29/08 & 20:00 & 4.946 & 4.447 & 3.669 & 1.98 & 1.92 & -0.351 \\
\hline
\end{tabular}


TABLE S1.3 (Cont.)

Depth to Water (feet below top of casing) in Indicated Wells

Date Time PMW1s PMW1d PMW2sa PMW2d PMW9s PMW9d PMW3d

\begin{tabular}{|c|c|c|c|c|c|c|c|}
\hline 8/30/08 & 0:00 & 4.896 & 4.431 & 3.622 & 1.952 & 1.89 & -0.358 \\
\hline 8/30/08 & 4:00 & 4.859 & 4.401 & 3.591 & 1.922 & 1.876 & -0.375 \\
\hline 8/30/08 & 8:00 & 4.859 & 4.405 & 3.594 & 1.926 & 1.885 & -0.389 \\
\hline 8/30/08 & $12: 00$ & 4.936 & 4.447 & 3.678 & 1.98 & 1.967 & -0.386 \\
\hline 8/30/08 & $16: 00$ & 5.007 & 4.482 & 3.741 & 2.032 & 2.032 & -0.356 \\
\hline 8/30/08 & 20:00 & 4.974 & 4.475 & 3.69 & 2.006 & 1.941 & -0.325 \\
\hline 8/31/08 & 0:00 & 4.91 & 4.44 & 3.624 & 1.957 & 1.895 & -0.339 \\
\hline 8/31/08 & 4:00 & 4.873 & 4.412 & 3.598 & 1.926 & 1.88 & -0.361 \\
\hline 8/31/08 & $8: 00$ & 4.857 & 4.398 & 3.589 & 1.917 & 1.871 & -0.384 \\
\hline 8/31/08 & $12: 00$ & 4.925 & 4.431 & 3.664 & 1.966 & 1.951 & -0.389 \\
\hline 8/31/08 & $16: 00$ & 5.002 & 4.477 & 3.737 & 2.022 & 2.011 & -0.363 \\
\hline 8/31/08 & 20:00 & 4.976 & 4.475 & 3.69 & 2.001 & 1.941 & -0.337 \\
\hline 9/1/08 & 0:00 & 4.917 & 4.449 & 3.631 & 1.966 & 1.887 & -0.344 \\
\hline 9/1/08 & 4:00 & 4.88 & 4.424 & 3.601 & 1.936 & 1.871 & -0.365 \\
\hline 9/1/08 & 8:00 & 4.868 & 4.417 & 3.594 & 1.929 & 1.869 & -0.382 \\
\hline 9/1/08 & $12: 00$ & 4.941 & 4.454 & 3.676 & 1.978 & 1.944 & -0.386 \\
\hline 9/1/08 & $16: 00$ & 5.03 & 4.51 & 3.758 & 2.05 & 2.021 & -0.356 \\
\hline 9/1/08 & 20:00 & 5.004 & 4.503 & 3.709 & 2.025 & 1.944 & -0.321 \\
\hline 9/2/08 & 0:00 & 4.943 & 4.48 & 3.648 & 1.985 & 1.89 & -0.33 \\
\hline $9 / 2 / 08$ & 4:00 & 4.903 & 4.447 & 3.612 & 1.95 & 1.869 & -0.351 \\
\hline 9/2/08 & 8:00 & 4.925 & 4.484 & 3.634 & 1.987 & 1.892 & -0.363 \\
\hline 9/2/08 & $12: 00$ & 4.976 & 4.528 & 3.669 & 2.029 & 1.909 & -0.354 \\
\hline 9/2/08 & $16: 00$ & 4.955 & 4.517 & 3.631 & 2.006 & 1.871 & -0.346 \\
\hline 9/2/08 & 20:00 & 4.969 & 4.528 & 3.65 & 2.006 & 1.895 & -0.349 \\
\hline 9/3/08 & 0:00 & 4.974 & 4.542 & 3.643 & 2.013 & 1.887 & -0.344 \\
\hline 9/3/08 & 4:00 & 4.969 & 4.54 & 3.638 & 2.006 & 1.887 & -0.342 \\
\hline 9/3/08 & 8:00 & 4.967 & 4.538 & 3.636 & 2.004 & 1.887 & -0.346 \\
\hline 9/3/08 & $12: 00$ & 4.997 & 4.545 & 3.68 & 2.022 & 1.958 & -0.346 \\
\hline $9 / 3 / 08$ & $16: 00$ & 5.03 & 4.549 & 3.713 & 2.039 & 1.967 & -0.33 \\
\hline 9/3/08 & 20:00 & 4.995 & 4.526 & 3.669 & 2.006 & 1.916 & -0.321 \\
\hline $9 / 4 / 08$ & 0:00 & 4.941 & 4.489 & 3.627 & 1.968 & 1.88 & -0.335 \\
\hline $9 / 4 / 08$ & 4:00 & 4.913 & 4.47 & 3.603 & 1.945 & 1.878 & -0.354 \\
\hline 9/4/08 & 8:00 & 4.892 & 4.449 & 3.591 & 1.931 & 1.878 & -0.372 \\
\hline 9/4/08 & $12: 00$ & 4.925 & 4.463 & 3.638 & 1.959 & 1.923 & -0.379 \\
\hline 9/4/08 & $16: 00$ & 4.993 & 4.501 & 3.701 & 2.008 & 1.955 & -0.365 \\
\hline 9/4/08 & 20:00 & 4.993 & 4.519 & 3.676 & 2.011 & 1.93 & -0.337 \\
\hline 9/5/08 & 0:00 & 4.946 & 4.501 & 3.631 & 1.982 & 1.897 & -0.337 \\
\hline 9/5/08 & 4:00 & 4.932 & 4.491 & 3.62 & 1.968 & 1.887 & -0.349 \\
\hline 9/5/08 & 8:00 & 4.939 & 4.505 & 3.622 & 1.978 & 1.899 & -0.354 \\
\hline 9/5/08 & $12: 00$ & 4.978 & 4.533 & 3.652 & 2.006 & 1.918 & -0.351 \\
\hline 9/5/08 & $16: 00$ & 4.993 & 4.552 & 3.662 & 2.018 & 1.911 & -0.342 \\
\hline 9/5/08 & $20: 00$ & 4.988 & 4.552 & 3.648 & 2.011 & 1.904 & -0.337 \\
\hline 9/6/08 & 0:00 & 4.969 & 4.542 & 3.631 & 1.992 & 1.89 & -0.339 \\
\hline 9/6/08 & 4:00 & 4.95 & 4.521 & 3.615 & 1.975 & 1.883 & -0.346 \\
\hline
\end{tabular}


TABLE S1.3 (Cont.)

Depth to Water (feet below top of casing) in Indicated Wells

Date Time PMW1s PMW1d PMW2sa PMW2d PMW9s PMW9d PMW3d

\begin{tabular}{|c|c|c|c|c|c|c|c|}
\hline 9/6/08 & 8:00 & 4.948 & 4.521 & 3.622 & 1.982 & 1.899 & -0.354 \\
\hline 9/6/08 & $12: 00$ & 4.976 & 4.545 & 3.638 & 1.999 & 1.906 & -0.349 \\
\hline 9/6/08 & $16: 00$ & 4.969 & 4.528 & 3.634 & 1.987 & 1.906 & -0.349 \\
\hline 9/6/08 & $20: 00$ & 4.957 & 4.521 & 3.62 & 1.978 & 1.897 & -0.351 \\
\hline 9/7/08 & 0:00 & 4.925 & 4.466 & 3.552 & 1.919 & 1.885 & -0.363 \\
\hline 9/7/08 & 4:00 & 4.92 & 4.498 & 3.575 & 1.943 & 1.883 & -0.375 \\
\hline 9/7/08 & 8:00 & 4.946 & 4.526 & 3.601 & 1.973 & 1.897 & -0.372 \\
\hline 9/7/08 & $12: 00$ & 4.955 & 4.538 & 3.605 & 1.98 & 1.895 & -0.365 \\
\hline 9/7/08 & $16: 00$ & 4.99 & 4.549 & 3.641 & 1.999 & 1.916 & -0.358 \\
\hline 9/7/08 & 20:00 & 5.004 & 4.568 & 3.648 & 2.011 & 1.909 & -0.346 \\
\hline 9/8/08 & $0: 00$ & 4.993 & 4.57 & 3.631 & 2.008 & 1.902 & -0.337 \\
\hline 9/8/08 & $4: 00$ & 4.983 & 4.565 & 3.627 & 1.992 & 1.899 & -0.339 \\
\hline 9/8/08 & 8:00 & 4.988 & 4.549 & 3.577 & 1.94 & 1.89 & -0.342 \\
\hline 9/8/08 & $12: 00$ & 4.927 & 4.586 & 3.535 & 2.011 & 1.887 & -0.365 \\
\hline 9/8/08 & $16: 00$ & 4.974 & 4.552 & 3.61 & 1.978 & 1.932 & -0.368 \\
\hline 9/8/08 & $20: 00$ & 4.988 & 4.563 & 3.615 & 1.987 & 1.909 & -0.356 \\
\hline 9/9/08 & 0:00 & 4.981 & 4.568 & 3.61 & 1.99 & 1.902 & -0.349 \\
\hline 9/9/08 & $4: 00$ & 4.971 & 4.556 & 3.605 & 1.98 & 1.902 & -0.349 \\
\hline 9/9/08 & 8:00 & 4.976 & 4.561 & 3.61 & 1.99 & 1.904 & -0.349 \\
\hline 9/9/08 & $12: 00$ & 5.009 & 4.572 & 3.652 & 2.011 & 1.958 & -0.344 \\
\hline 9/9/08 & $16: 00$ & 5.047 & 4.589 & 3.694 & 2.036 & 1.976 & -0.325 \\
\hline 9/9/08 & $20: 00$ & 5.028 & 4.577 & 3.662 & 2.015 & 1.937 & -0.314 \\
\hline 9/10/08 & 0:00 & 4.988 & 4.559 & 3.622 & 1.99 & 1.904 & -0.318 \\
\hline 9/10/08 & $4: 00$ & 4.971 & 4.542 & 3.615 & 1.98 & 1.909 & -0.332 \\
\hline 9/10/08 & 8:00 & 4.969 & 4.545 & 3.615 & 1.982 & 1.909 & -0.339 \\
\hline 9/10/08 & $12: 00$ & 4.986 & 4.545 & 3.636 & 1.987 & 1.911 & -0.344 \\
\hline 9/10/08 & $16: 00$ & 5.03 & 4.565 & 3.678 & 2.02 & 1.958 & -0.335 \\
\hline 9/10/08 & $20: 00$ & 4.997 & 4.556 & 3.638 & 1.997 & 1.906 & -0.328 \\
\hline 9/11/08 & 0:00 & 4.969 & 4.542 & 3.615 & 1.975 & 1.888 & -0.337 \\
\hline 9/11/08 & 4:00 & 4.946 & 4.519 & 3.591 & 1.952 & 1.873 & -0.351 \\
\hline 9/11/08 & 8:00 & 4.96 & 4.538 & 3.605 & 1.973 & 1.878 & -0.358 \\
\hline 9/11/08 & $12: 00$ & 4.92 & 4.51 & 3.533 & 1.919 & 1.864 & -0.375 \\
\hline 9/11/08 & $16: 00$ & 4.925 & 4.51 & 3.545 & 1.98 & 1.86 & -0.384 \\
\hline 9/11/08 & 20:00 & 4.714 & 4.389 & 3.287 & 1.783 & 1.829 & -0.41 \\
\hline $9 / 12 / 08$ & 0:00 & 4.784 & 4.361 & 3.451 & 1.795 & 1.853 & -0.44 \\
\hline 9/12/08 & 4:00 & 4.821 & 4.424 & 3.491 & 1.87 & 1.853 & -0.44 \\
\hline 9/12/08 & 8:00 & 4.847 & 4.424 & 3.514 & 1.875 & 1.855 & -0.435 \\
\hline 9/12/08 & $12: 00$ & 4.833 & 4.466 & 3.474 & 1.915 & 1.836 & -0.433 \\
\hline $9 / 12 / 08$ & $16: 00$ & 4.594 & 4.343 & 3.294 & 1.776 & 1.801 & -0.466 \\
\hline 9/12/08 & $20: 00$ & 4.617 & 4.306 & 3.355 & 1.76 & 1.817 & -0.503 \\
\hline 9/13/08 & 0:00 & 4.648 & 4.299 & 3.39 & 1.76 & 1.824 & -0.515 \\
\hline 9/13/08 & 4:00 & 4.66 & 4.287 & 3.404 & 1.753 & 1.822 & -0.519 \\
\hline 9/13/08 & $8: 00$ & 4.688 & 4.271 & 3.425 & 1.743 & 1.827 & -0.512 \\
\hline 9/13/08 & $12: 00$ & 4.718 & 4.301 & 3.456 & 1.802 & 1.834 & -0.501 \\
\hline
\end{tabular}


TABLE S1.3 (Cont.)

Depth to Water (feet below top of casing) in Indicated Wells

Date Time PMW1s PMW1d PMW2sa PMW2d PMW9s PMW9d PMW3d

\begin{tabular}{|c|c|c|c|c|c|c|c|}
\hline 9/13/08 & $16: 00$ & 4.81 & 4.357 & 3.549 & 1.87 & 1.895 & -0.48 \\
\hline 9/13/08 & 20:00 & 4.84 & 4.398 & 3.547 & 1.893 & 1.869 & -0.447 \\
\hline 9/14/08 & $0: 00$ & 4.842 & 4.419 & 3.538 & 1.9 & 1.855 & -0.433 \\
\hline 9/14/08 & 4:00 & 4.845 & 4.424 & 3.531 & 1.9 & 1.843 & -0.426 \\
\hline 9/14/08 & $8: 00$ & 4.885 & 4.463 & 3.566 & 1.94 & 1.869 & -0.414 \\
\hline 9/14/08 & $12: 00$ & 4.925 & 4.503 & 3.594 & 1.971 & 1.89 & -0.396 \\
\hline 9/14/08 & $16: 00$ & 4.969 & 4.545 & 3.627 & 2.006 & 1.92 & -0.372 \\
\hline 9/14/08 & $20: 00$ & 5 & 4.579 & 3.641 & 2.029 & 1.916 & -0.344 \\
\hline 9/15/08 & 0:00 & 5.004 & 4.598 & 3.627 & 2.032 & 1.895 & -0.33 \\
\hline 9/15/08 & $4: 00$ & 5.009 & 4.607 & 3.624 & 2.032 & 1.895 & -0.323 \\
\hline 9/15/08 & 8:00 & 5.021 & 4.621 & 3.634 & 2.041 & 1.907 & -0.316 \\
\hline 9/15/08 & $12: 00$ & 5.054 & 4.644 & 3.673 & 2.067 & 1.953 & -0.311 \\
\hline $9 / 15 / 08$ & $16: 00$ & 5.086 & 4.651 & 3.709 & 2.081 & 1.97 & -0.293 \\
\hline 9/15/08 & 20:00 & 5.075 & 4.644 & 3.676 & 2.06 & 1.93 & -0.281 \\
\hline 9/16/08 & $0: 00$ & 5.058 & 4.642 & 3.652 & 2.05 & 1.913 & -0.286 \\
\hline 9/16/08 & 4:00 & 5.035 & 4.626 & 3.636 & 2.032 & 1.899 & -0.295 \\
\hline 9/16/08 & 8:00 & 5.03 & 4.621 & 3.634 & 2.027 & 1.899 & -0.304 \\
\hline 9/16/08 & $12: 00$ & 5.079 & 4.654 & 3.694 & 2.069 & 1.963 & -0.304 \\
\hline 9/16/08 & $16: 00$ & 5.122 & 4.668 & 3.739 & 2.097 & 1.995 & -0.283 \\
\hline 9/16/08 & 20:00 & 5.103 & 4.665 & 3.692 & 2.074 & 1.923 & -0.265 \\
\hline 9/17/08 & 0:00 & 5.07 & 4.654 & 3.662 & 2.053 & 1.902 & -0.274 \\
\hline 9/17/08 & $4: 00$ & 5.056 & 4.647 & 3.648 & 2.046 & 1.89 & -0.286 \\
\hline 9/17/08 & 8:00 & 5.056 & 4.651 & 3.645 & 2.046 & 1.892 & -0.295 \\
\hline 9/17/08 & $12: 00$ & 5.119 & 4.691 & 3.723 & 2.102 & 1.967 & -0.293 \\
\hline $9 / 17 / 08$ & $16: 00$ & 5.168 & 4.714 & 3.772 & 2.135 & 2.002 & -0.262 \\
\hline 9/17/08 & 20:00 & 5.145 & 4.712 & 3.718 & 2.107 & 1.93 & -0.241 \\
\hline 9/18/08 & $0: 00$ & 5.11 & 4.7 & 3.685 & 2.086 & 1.897 & -0.253 \\
\hline 9/18/08 & 4:00 & 5.093 & 4.688 & 3.669 & 2.076 & 1.892 & -0.267 \\
\hline 9/18/08 & $8: 00$ & 5.089 & 4.686 & 3.664 & 2.072 & 1.892 & -0.276 \\
\hline 9/18/08 & $12: 00$ & 5.143 & 4.716 & 3.727 & 2.114 & 1.967 & -0.276 \\
\hline 9/18/08 & $16: 00$ & 5.187 & 4.737 & 3.772 & 2.142 & 2 & -0.253 \\
\hline 9/18/08 & 20:00 & 5.161 & 4.728 & 3.727 & 2.118 & 1.937 & -0.23 \\
\hline 9/19/08 & 0:00 & 5.119 & 4.707 & 3.687 & 2.09 & 1.897 & -0.239 \\
\hline 9/19/08 & $4: 00$ & 5.098 & 4.693 & 3.669 & 2.071 & 1.888 & -0.258 \\
\hline 9/19/08 & $8: 00$ & 5.091 & 4.691 & 3.666 & 2.072 & 1.888 & -0.269 \\
\hline 9/19/08 & $12: 00$ & 5.133 & 4.714 & 3.72 & 2.104 & 1.951 & -0.274 \\
\hline 9/19/08 & $16: 00$ & 5.183 & 4.735 & 3.762 & 2.135 & 1.979 & -0.258 \\
\hline 9/19/08 & 20:00 & 5.161 & 4.73 & 3.723 & 2.118 & 1.923 & -0.241 \\
\hline 9/20/08 & $0: 00$ & 5.131 & 4.721 & 3.692 & 2.097 & 1.895 & -0.248 \\
\hline 9/20/08 & 4:00 & 5.115 & 4.712 & 3.678 & 2.086 & 1.883 & -0.262 \\
\hline 9/20/08 & $8: 00$ & 5.117 & 4.716 & 3.676 & 2.088 & 1.883 & -0.269 \\
\hline 9/20/08 & $12: 00$ & 5.164 & 4.744 & 3.732 & 2.125 & 1.944 & -0.267 \\
\hline 9/20/08 & $16: 00$ & 5.215 & 4.77 & 3.786 & 2.163 & 1.991 & -0.244 \\
\hline 9/20/08 & 20:00 & 5.192 & 4.767 & 3.739 & 2.142 & 1.923 & -0.223 \\
\hline
\end{tabular}


TABLE S1.3 (Cont.)

Depth to Water (feet below top of casing) in Indicated Wells

Date Time PMW1s PMW1d PMW2sa PMW2d PMW9s PMW9d PMW3d

\begin{tabular}{|c|c|c|c|c|c|c|c|}
\hline 9/21/08 & 0:00 & 5.168 & 4.763 & 3.711 & 2.128 & 1.893 & -0.23 \\
\hline 9/21/08 & 4:00 & 5.15 & 4.751 & 3.692 & 2.111 & 1.886 & -0.241 \\
\hline 9/21/08 & $8: 00$ & 5.145 & 4.751 & 3.69 & 2.109 & 1.883 & -0.248 \\
\hline 9/21/08 & $12: 00$ & 5.18 & 4.77 & 3.732 & 2.135 & 1.935 & -0.253 \\
\hline 9/21/08 & $16: 00$ & 5.225 & 4.784 & 3.781 & 2.165 & 1.977 & -0.237 \\
\hline 9/21/08 & $20: 00$ & 5.197 & 4.774 & 3.737 & 2.144 & 1.918 & -0.223 \\
\hline 9/22/08 & 0:00 & 5.176 & 4.772 & 3.711 & 2.13 & 1.89 & -0.227 \\
\hline 9/22/08 & 4:00 & 5.161 & 4.763 & 3.697 & 2.114 & 1.886 & -0.241 \\
\hline 9/22/08 & $8: 00$ & 5.138 & 4.753 & 3.671 & 2.109 & 1.869 & -0.258 \\
\hline 9/22/08 & $12: 00$ & 5.107 & 4.721 & 3.631 & 2.057 & 1.865 & -0.279 \\
\hline 9/22/08 & $16: 00$ & 5.164 & 4.742 & 3.716 & 2.104 & 1.946 & -0.276 \\
\hline 9/22/08 & 20:00 & 5.173 & 4.756 & 3.713 & 2.114 & 1.911 & -0.258 \\
\hline 9/23/08 & 0:00 & 5.152 & 4.753 & 3.687 & 2.102 & 1.876 & -0.26 \\
\hline 9/23/08 & 4:00 & 5.147 & 4.751 & 3.683 & 2.097 & 1.869 & -0.262 \\
\hline 9/23/08 & 8:00 & 5.15 & 4.758 & 3.683 & 2.104 & 1.874 & -0.267 \\
\hline 9/23/08 & $12: 00$ & 5.197 & 4.786 & 3.739 & 2.142 & 1.939 & -0.267 \\
\hline 9/23/08 & $16: 00$ & 5.19 & 4.726 & 3.711 & 2.041 & 1.902 & -0.246 \\
\hline 9/23/08 & $20: 00$ & 5.168 & 4.777 & 3.69 & 2.116 & 1.876 & -0.248 \\
\hline 9/24/08 & 0:00 & 5.178 & 4.791 & 3.692 & 2.13 & 1.879 & -0.253 \\
\hline 9/24/08 & 4:00 & 5.18 & 4.795 & 3.692 & 2.132 & 1.874 & -0.251 \\
\hline 9/24/08 & $8: 00$ & 5.194 & 4.816 & 3.701 & 2.149 & 1.879 & -0.248 \\
\hline 9/24/08 & $12: 00$ & 5.215 & 4.832 & 3.72 & 2.16 & 1.9 & -0.241 \\
\hline 9/24/08 & $16: 00$ & 5.255 & 4.844 & 3.769 & 2.186 & 1.946 & -0.227 \\
\hline 9/24/08 & 20:00 & 5.241 & 4.842 & 3.741 & 2.172 & 1.902 & -0.211 \\
\hline 9/25/08 & 0:00 & 5.227 & 4.842 & 3.725 & 2.165 & 1.883 & -0.213 \\
\hline 9/25/08 & 4:00 & 5.208 & 4.825 & 3.709 & 2.144 & 1.874 & -0.223 \\
\hline 9/25/08 & $8: 00$ & 5.208 & 4.828 & 3.706 & 2.149 & 1.879 & -0.232 \\
\hline 9/25/08 & $12: 00$ & 5.241 & 4.842 & 3.748 & 2.17 & 1.935 & -0.232 \\
\hline 9/25/08 & $16: 00$ & 5.272 & 4.846 & 3.79 & 2.191 & 1.975 & -0.218 \\
\hline 9/25/08 & $20: 00$ & 5.251 & 4.839 & 3.751 & 2.17 & 1.916 & -0.204 \\
\hline 9/26/08 & 0:00 & 5.218 & 4.823 & 3.718 & 2.144 & 1.881 & -0.216 \\
\hline 9/26/08 & 4:00 & 5.187 & 4.798 & 3.697 & 2.123 & 1.872 & -0.232 \\
\hline 9/26/08 & $8: 00$ & 5.183 & 4.795 & 3.697 & 2.123 & 1.874 & -0.244 \\
\hline 9/26/08 & $12: 00$ & 5.229 & 4.821 & 3.753 & 2.16 & 1.951 & -0.246 \\
\hline 9/26/08 & $16: 00$ & 5.274 & 4.839 & 3.802 & 2.191 & 1.998 & -0.225 \\
\hline 9/26/08 & 20:00 & 5.255 & 4.837 & 3.758 & 2.172 & 1.918 & -0.204 \\
\hline 9/27/08 & 0:00 & 5.225 & 4.828 & 3.727 & 2.151 & 1.883 & -0.213 \\
\hline 9/27/08 & 4:00 & 5.206 & 4.816 & 3.711 & 2.142 & 1.867 & -0.227 \\
\hline 9/27/08 & 8:00 & 5.204 & 4.821 & 3.706 & 2.142 & 1.872 & -0.239 \\
\hline 9/27/08 & $12: 00$ & 5.236 & 4.849 & 3.744 & 2.175 & 1.904 & -0.234 \\
\hline 9/27/08 & $16: 00$ & 5.29 & 4.879 & 3.8 & 2.217 & 1.956 & -0.216 \\
\hline 9/27/08 & $20: 00$ & 5.29 & 4.89 & 3.776 & 2.212 & 1.914 & -0.195 \\
\hline 9/28/08 & 0:00 & 5.267 & 4.886 & 3.744 & 2.196 & 1.881 & -0.195 \\
\hline 9/28/08 & 4:00 & 5.251 & 4.874 & 3.73 & 2.177 & 1.872 & -0.204 \\
\hline
\end{tabular}


TABLE S1.3 (Cont.)

Depth to Water (feet below top of casing) in Indicated Wells

Date Time PMW1s PMW1d PMW2sa PMW2d PMW9s PMW9d PMW3d

\begin{tabular}{|c|c|c|c|c|c|c|c|}
\hline 9/28/08 & 8:00 & 5.251 & 4.877 & 3.732 & 2.179 & 1.876 & -0.213 \\
\hline 9/28/08 & $12: 00$ & 5.272 & 4.886 & 3.755 & 2.196 & 1.918 & -0.216 \\
\hline 9/28/08 & $16: 00$ & 5.309 & 4.895 & 3.8 & 2.219 & 1.961 & -0.202 \\
\hline 9/28/08 & 20:00 & 5.272 & 4.865 & 3.755 & 2.177 & 1.904 & -0.197 \\
\hline 9/29/08 & 0:00 & 5.229 & 4.835 & 3.718 & 2.146 & 1.869 & -0.211 \\
\hline 9/29/08 & 4:00 & 5.241 & 4.83 & 3.723 & 2.121 & 1.869 & -0.218 \\
\hline 9/29/08 & 8:00 & 5.274 & 4.902 & 3.746 & 2.2 & 1.879 & -0.216 \\
\hline 9/29/08 & $12: 00$ & 5.307 & 4.932 & 3.781 & 2.235 & 1.921 & -0.206 \\
\hline 9/29/08 & $16: 00$ & 5.342 & 4.946 & 3.816 & 2.254 & 1.956 & -0.185 \\
\hline 9/29/08 & 20:00 & 5.335 & 4.948 & 3.793 & 2.245 & 1.923 & -0.169 \\
\hline 9/30/08 & 0:00 & 5.312 & 4.937 & 3.767 & 2.224 & 1.893 & -0.171 \\
\hline 9/30/08 & 4:00 & 5.302 & 4.93 & 3.755 & 2.214 & 1.888 & -0.181 \\
\hline 9/30/08 & 8:00 & 5.302 & 4.935 & 3.755 & 2.219 & 1.895 & -0.185 \\
\hline 9/30/08 & $12: 00$ & 5.323 & 4.941 & 3.788 & 2.233 & 1.946 & -0.188 \\
\hline 9/30/08 & $16: 00$ & 5.347 & 4.939 & 3.823 & 2.24 & 1.982 & -0.176 \\
\hline 9/30/08 & 20:00 & 5.323 & 4.928 & 3.786 & 2.219 & 1.926 & -0.164 \\
\hline 10/1/08 & 0:00 & 5.286 & 4.907 & 3.751 & 2.196 & 1.895 & -0.178 \\
\hline 10/1/08 & 4:00 & 5.272 & 4.893 & 3.741 & 2.184 & 1.9 & -0.195 \\
\hline 10/1/08 & 8:00 & 5.29 & 4.918 & 3.755 & 2.21 & 1.905 & -0.199 \\
\hline 10/1/08 & $12: 00$ & 5.342 & 4.962 & 3.807 & 2.254 & 1.963 & -0.188 \\
\hline 10/1/08 & $16: 00$ & 5.37 & 4.974 & 3.83 & 2.268 & 1.977 & -0.167 \\
\hline 10/1/08 & 20:00 & 5.356 & 4.972 & 3.8 & 2.252 & 1.933 & -0.155 \\
\hline $10 / 2 / 08$ & 0:00 & 5.326 & 4.953 & 3.769 & 2.226 & 1.907 & -0.162 \\
\hline $10 / 2 / 08$ & 4:00 & 5.307 & 4.937 & 3.753 & 2.212 & 1.902 & -0.174 \\
\hline $10 / 2 / 08$ & $8: 00$ & 5.297 & 4.93 & 3.751 & 2.207 & 1.905 & -0.183 \\
\hline 10/2/08 & $12: 00$ & 5.319 & 4.937 & 3.79 & 2.226 & 1.961 & -0.188 \\
\hline $10 / 2 / 08$ & $16: 00$ & 5.337 & 4.93 & 3.816 & 2.231 & 1.977 & -0.178 \\
\hline $10 / 2 / 08$ & 20:00 & 5.316 & 4.921 & 3.779 & 2.212 & 1.928 & -0.169 \\
\hline 10/3/08 & 0:00 & 5.297 & 4.914 & 3.76 & 2.203 & 1.912 & -0.178 \\
\hline $10 / 3 / 08$ & 4:00 & 5.29 & 4.911 & 3.753 & 2.198 & 1.902 & -0.188 \\
\hline 10/3/08 & 8:00 & 5.295 & 4.925 & 3.758 & 2.21 & 1.902 & -0.19 \\
\hline 10/3/08 & $12: 00$ & 5.349 & 4.967 & 3.814 & 2.254 & 1.958 & -0.183 \\
\hline $10 / 3 / 08$ & $16: 00$ & 5.389 & 4.988 & 3.851 & 2.282 & 1.991 & -0.157 \\
\hline $10 / 3 / 08$ & 20:00 & 5.377 & 4.99 & 3.819 & 2.266 & 1.94 & -0.141 \\
\hline $10 / 4 / 08$ & 0:00 & 5.37 & 4.997 & 3.802 & 2.266 & 1.919 & -0.143 \\
\hline $10 / 4 / 08$ & 4:00 & 5.356 & 4.99 & 3.786 & 2.254 & 1.909 & -0.148 \\
\hline $10 / 4 / 08$ & 8:00 & 5.347 & 4.983 & 3.779 & 2.242 & 1.905 & -0.157 \\
\hline $10 / 4 / 08$ & $12: 00$ & 5.37 & 4.993 & 3.809 & 2.261 & 1.949 & -0.159 \\
\hline 10/4/08 & $16: 00$ & 5.387 & 4.986 & 3.842 & 2.268 & 1.993 & -0.152 \\
\hline $10 / 4 / 08$ & 20:00 & 5.363 & 4.974 & 3.807 & 2.245 & 1.93 & -0.141 \\
\hline 10/5/08 & 0:00 & 5.351 & 4.976 & 3.79 & 2.242 & 1.907 & -0.15 \\
\hline 10/5/08 & 4:00 & 5.33 & 4.955 & 3.767 & 2.224 & 1.886 & -0.164 \\
\hline 10/5/08 & $8: 00$ & 5.326 & 4.96 & 3.765 & 2.221 & 1.886 & -0.176 \\
\hline 10/5/08 & $12: 00$ & 5.356 & 4.979 & 3.805 & 2.247 & 1.93 & -0.178 \\
\hline
\end{tabular}


TABLE S1.3 (Cont.)

Depth to Water (feet below top of casing) in Indicated Wells

Date Time PMW1s PMW1d PMW2sa PMW2d PMW9s PMW9d PMW3d

\begin{tabular}{|c|c|c|c|c|c|c|c|}
\hline $10 / 5 / 08$ & $16: 00$ & 5.403 & 5.002 & 3.856 & 2.285 & 2.005 & -0.159 \\
\hline $10 / 5 / 08$ & $20: 00$ & 5.396 & 5.011 & 3.828 & 2.28 & 1.937 & -0.138 \\
\hline $10 / 6 / 08$ & $0: 00$ & 5.387 & 5.013 & 3.812 & 2.273 & 1.905 & -0.138 \\
\hline 10/6/08 & 4:00 & 5.37 & 5.002 & 3.79 & 2.254 & 1.886 & -0.145 \\
\hline 10/6/08 & 8:00 & 5.368 & 5.006 & 3.786 & 2.257 & 1.886 & -0.155 \\
\hline $10 / 6 / 08$ & $12: 00$ & 5.382 & 5.011 & 3.805 & 2.266 & 1.907 & -0.159 \\
\hline 10/6/08 & $16: 00$ & 5.368 & 4.974 & 3.748 & 2.196 & 1.888 & -0.162 \\
\hline $10 / 6 / 08$ & 20:00 & 5.129 & 4.809 & 3.549 & 2.067 & 1.839 & -0.227 \\
\hline $10 / 7 / 08$ & 0:00 & 5.185 & 4.849 & 3.641 & 2.111 & 1.855 & -0.253 \\
\hline $10 / 7 / 08$ & 4:00 & 4.831 & 4.691 & 3.402 & 1.936 & 1.825 & -0.288 \\
\hline $10 / 7 / 08$ & 8:00 & 4.993 & 4.702 & 3.535 & 2.01 & 1.846 & -0.318 \\
\hline $10 / 7 / 08$ & $12: 00$ & 5.1 & 4.774 & 3.617 & 2.083 & 1.883 & -0.309 \\
\hline $10 / 7 / 08$ & $16: 00$ & 5.166 & 4.809 & 3.673 & 2.121 & 1.905 & -0.283 \\
\hline $10 / 7 / 08$ & $20: 00$ & 5.197 & 4.837 & 3.683 & 2.142 & 1.881 & -0.26 \\
\hline $10 / 8 / 08$ & $0: 00$ & 5.194 & 4.835 & 3.673 & 2.132 & 1.865 & -0.253 \\
\hline 10/8/08 & 4:00 & 5.194 & 4.832 & 3.676 & 2.128 & 1.868 & -0.251 \\
\hline 10/8/08 & 8:00 & 5.206 & 4.839 & 3.685 & 2.139 & 1.877 & -0.248 \\
\hline $10 / 8 / 08$ & $12: 00$ & 5.22 & 4.842 & 3.711 & 2.153 & 1.945 & -0.246 \\
\hline $10 / 8 / 08$ & $16: 00$ & 5.246 & 4.858 & 3.744 & 2.174 & 1.963 & -0.225 \\
\hline 10/8/08 & $20: 00$ & 5.267 & 4.893 & 3.741 & 2.196 & 1.912 & -0.206 \\
\hline $10 / 9 / 08$ & 0:00 & 5.279 & 4.916 & 3.734 & 2.205 & 1.898 & -0.199 \\
\hline $10 / 9 / 08$ & 4:00 & 5.286 & 4.928 & 3.732 & 2.21 & 1.889 & -0.195 \\
\hline $10 / 9 / 08$ & $8: 00$ & 5.309 & 4.955 & 3.748 & 2.231 & 1.903 & -0.185 \\
\hline $10 / 9 / 08$ & $12: 00$ & 5.333 & 4.969 & 3.779 & 2.252 & 1.963 & -0.178 \\
\hline 10/9/08 & $16: 00$ & 5.335 & 4.951 & 3.79 & 2.24 & 1.982 & -0.164 \\
\hline 10/9/08 & $20: 00$ & 5.312 & 4.937 & 3.76 & 2.219 & 1.921 & -0.16 \\
\hline $10 / 10 / 08$ & $0: 00$ & 5.293 & 4.923 & 3.741 & 2.205 & 1.898 & -0.171 \\
\hline 10/10/08 & 4:00 & 5.276 & 4.911 & 3.725 & 2.191 & 1.884 & -0.188 \\
\hline 10/10/08 & $8: 00$ & 5.276 & 4.914 & 3.727 & 2.191 & 1.881 & -0.195 \\
\hline $10 / 10 / 08$ & $12: 00$ & 5.297 & 4.918 & 3.753 & 2.203 & 1.931 & -0.199 \\
\hline 10/10/08 & $16: 00$ & 5.307 & 4.916 & 3.774 & 2.214 & 1.965 & -0.188 \\
\hline 10/10/08 & $20: 00$ & 5.319 & 4.941 & 3.767 & 2.228 & 1.919 & -0.174 \\
\hline $10 / 11 / 08$ & $0: 00$ & 5.333 & 4.967 & 3.765 & 2.245 & 1.902 & -0.169 \\
\hline 10/11/08 & $4: 00$ & 5.333 & 4.976 & 3.76 & 2.24 & 1.888 & -0.167 \\
\hline $10 / 11 / 08$ & $8: 00$ & 5.349 & 4.995 & 3.772 & 2.256 & 1.893 & -0.164 \\
\hline 10/11/08 & $12: 00$ & 5.37 & 5.013 & 3.788 & 2.273 & 1.916 & -0.16 \\
\hline 10/11/08 & $16: 00$ & 5.391 & 5.02 & 3.814 & 2.287 & 1.958 & -0.148 \\
\hline 10/11/08 & 20:00 & 5.387 & 5.025 & 3.795 & 2.282 & 1.912 & -0.136 \\
\hline $10 / 12 / 08$ & $0: 00$ & 5.372 & 5.018 & 3.779 & 2.271 & 1.893 & -0.141 \\
\hline $10 / 12 / 08$ & $4: 00$ & 5.372 & 5.02 & 3.779 & 2.268 & 1.888 & -0.148 \\
\hline $10 / 12 / 08$ & $8: 00$ & 5.375 & 5.025 & 3.776 & 2.268 & 1.881 & -0.15 \\
\hline $10 / 12 / 08$ & $12: 00$ & 5.391 & 5.032 & 3.79 & 2.282 & 1.902 & -0.15 \\
\hline $10 / 12 / 08$ & $16: 00$ & 5.391 & 5.018 & 3.802 & 2.273 & 1.947 & -0.145 \\
\hline $10 / 12 / 08$ & $20: 00$ & 5.398 & 5.039 & 3.802 & 2.287 & 1.909 & -0.136 \\
\hline
\end{tabular}


TABLE S1.3 (Cont.)

Depth to Water (feet below top of casing) in Indicated Wells

Date Time PMW1s PMW1d PMW2sa PMW2d PMW9s PMW9d PMW3d

\begin{tabular}{|c|c|c|c|c|c|c|c|}
\hline $10 / 13 / 08$ & $0: 00$ & 5.389 & 5.034 & 3.783 & 2.278 & 1.881 & -0.136 \\
\hline 10/13/08 & 4:00 & 5.396 & 5.02 & 3.788 & 2.249 & 1.884 & -0.141 \\
\hline 10/13/08 & $8: 00$ & 4.852 & 4.73 & 3.385 & 1.877 & 1.832 & -0.19 \\
\hline 10/13/08 & $12: 00$ & 4.512 & 4.503 & 3.261 & 1.783 & 1.807 & -0.307 \\
\hline 10/13/08 & $16: 00$ & 4.613 & 4.422 & 3.348 & 1.807 & 1.821 & -0.391 \\
\hline 10/13/08 & $20: 00$ & 4.85 & 4.549 & 3.481 & 1.942 & 1.853 & -0.393 \\
\hline 10/14/08 & 0:00 & 4.967 & 4.64 & 3.549 & 2.02 & 1.863 & -0.358 \\
\hline 10/14/08 & 4:00 & 5.021 & 4.677 & 3.58 & 2.053 & 1.865 & -0.328 \\
\hline $10 / 14 / 08$ & $8: 00$ & 5.068 & 4.714 & 3.615 & 2.095 & 1.884 & -0.302 \\
\hline $10 / 14 / 08$ & $12: 00$ & 5.086 & 4.726 & 3.622 & 2.102 & 1.884 & -0.279 \\
\hline 10/14/08 & $16: 00$ & 5.075 & 4.707 & 3.608 & 2.076 & 1.868 & -0.274 \\
\hline $10 / 14 / 08$ & 20:00 & 4.892 & 4.663 & 3.437 & 1.999 & 1.84 & -0.29 \\
\hline 10/15/08 & 0:00 & 4.662 & 4.401 & 3.381 & 1.931 & 1.833 & -0.354 \\
\hline 10/15/08 & 4:00 & 4.39 & 4.243 & 3.181 & 1.727 & 1.803 & -0.412 \\
\hline $10 / 15 / 08$ & $8: 00$ & 4.176 & 4.002 & 3.205 & 1.671 & 1.817 & -0.494 \\
\hline 10/15/08 & $12: 00$ & 4.233 & 3.957 & 3.268 & 1.694 & 1.845 & -0.512 \\
\hline 10/15/08 & $16: 00$ & 4.305 & 3.995 & 3.331 & 1.748 & 1.891 & -0.494 \\
\hline 10/15/08 & 20:00 & 4.373 & 4.048 & 3.367 & 1.8 & 1.877 & -0.466 \\
\hline 10/16/08 & 0:00 & 4.449 & 4.106 & 3.392 & 1.837 & 1.873 & -0.44 \\
\hline 10/16/08 & 4:00 & 4.517 & 4.143 & 3.406 & 1.853 & 1.876 & -0.419 \\
\hline $10 / 16 / 08$ & $8: 00$ & 4.589 & 4.185 & 3.434 & 1.879 & 1.885 & -0.403 \\
\hline $10 / 16 / 08$ & $12: 00$ & 4.634 & 4.213 & 3.456 & 1.896 & 1.927 & -0.386 \\
\hline 10/16/08 & $16: 00$ & 4.643 & 4.208 & 3.458 & 1.882 & 1.915 & -0.377 \\
\hline $10 / 16 / 08$ & 20:00 & 4.657 & 4.215 & 3.46 & 1.886 & 1.903 & -0.375 \\
\hline 10/17/08 & 0:00 & 4.671 & 4.236 & 3.46 & 1.9 & 1.894 & -0.368 \\
\hline 10/17/08 & $4: 00$ & 4.655 & 4.213 & 3.444 & 1.877 & 1.885 & -0.372 \\
\hline 10/17/08 & 8:00 & 4.66 & 4.185 & 3.434 & 1.853 & 1.88 & -0.377 \\
\hline 10/17/08 & $12: 00$ & 4.678 & 4.241 & 3.458 & 1.896 & 1.901 & -0.379 \\
\hline 10/17/08 & $16: 00$ & 4.709 & 4.268 & 3.484 & 1.921 & 1.913 & -0.37 \\
\hline 10/17/08 & 20:00 & 4.744 & 4.31 & 3.498 & 1.95 & 1.908 & -0.351 \\
\hline $10 / 18 / 08$ & 0:00 & 4.758 & 4.329 & 3.498 & 1.957 & 1.901 & -0.342 \\
\hline 10/18/08 & 4:00 & 4.765 & 4.338 & 3.5 & 1.959 & 1.897 & -0.335 \\
\hline $10 / 18 / 08$ & 8:00 & 4.782 & 4.357 & 3.509 & 1.973 & 1.904 & -0.328 \\
\hline $10 / 18 / 08$ & $12: 00$ & 4.786 & 4.361 & 3.507 & 1.971 & 1.899 & -0.323 \\
\hline 10/18/08 & $16: 00$ & 4.77 & 4.329 & 3.505 & 1.945 & 1.92 & -0.33 \\
\hline 10/18/08 & 20:00 & 4.763 & 4.324 & 3.498 & 1.938 & 1.903 & -0.33 \\
\hline $10 / 19 / 08$ & 0:00 & 4.756 & 4.317 & 3.493 & 1.933 & 1.892 & -0.337 \\
\hline $10 / 19 / 08$ & $4: 00$ & 4.744 & 4.308 & 3.484 & 1.924 & 1.887 & -0.344 \\
\hline 10/19/08 & 8:00 & 4.744 & 4.306 & 3.486 & 1.921 & 1.89 & -0.351 \\
\hline 10/19/08 & $12: 00$ & 4.772 & 4.326 & 3.512 & 1.94 & 1.927 & -0.349 \\
\hline 10/19/08 & $16: 00$ & 4.789 & 4.333 & 3.53 & 1.952 & 1.955 & -0.339 \\
\hline 10/19/08 & 20:00 & 4.817 & 4.378 & 3.535 & 1.982 & 1.912 & -0.323 \\
\hline $10 / 20 / 08$ & 0:00 & 4.835 & 4.408 & 3.54 & 1.999 & 1.896 & -0.316 \\
\hline $10 / 20 / 08$ & 4:00 & 4.868 & 4.447 & 3.556 & 2.027 & 1.905 & -0.304 \\
\hline
\end{tabular}


TABLE S1.3 (Cont.)

Depth to Water (feet below top of casing) in Indicated Wells

Date Time PMW1s PMW1d PMW2sa PMW2d PMW9s PMW9d PMW3d

\begin{tabular}{|c|c|c|c|c|c|c|c|}
\hline 10/20/08 & 8:00 & 4.908 & 4.498 & 3.58 & 2.062 & 1.908 & -0.286 \\
\hline 10/20/08 & $12: 00$ & 4.953 & 4.545 & 3.61 & 2.097 & 1.936 & -0.267 \\
\hline 10/20/08 & 16:00 & 4.967 & 4.549 & 3.615 & 2.092 & 1.95 & -0.251 \\
\hline 10/20/08 & 20:00 & 4.976 & 4.566 & 3.61 & 2.102 & 1.927 & -0.241 \\
\hline 10/21/08 & 0:00 & 4.976 & 4.57 & 3.603 & 2.097 & 1.915 & -0.241 \\
\hline 10/21/08 & 4:00 & 4.976 & 4.572 & 3.601 & 2.092 & 1.908 & -0.239 \\
\hline 10/21/08 & 8:00 & 4.969 & 4.566 & 3.591 & 2.081 & 1.899 & -0.244 \\
\hline 10/21/08 & $12: 00$ & 4.957 & 4.552 & 3.584 & 2.062 & 1.899 & -0.248 \\
\hline 10/21/08 & $16: 00$ & 4.901 & 4.528 & 3.516 & 2.048 & 1.866 & -0.267 \\
\hline 10/21/08 & 20:00 & 4.835 & 4.501 & 3.472 & 2.024 & 1.866 & -0.302 \\
\hline 10/22/08 & 0:00 & 4.821 & 4.431 & 3.477 & 1.964 & 1.864 & -0.323 \\
\hline 10/22/08 & 4:00 & 4.406 & 4.268 & 3.233 & 1.788 & 1.824 & -0.37 \\
\hline 10/22/08 & 8:00 & 3.841 & 3.711 & 3.025 & 1.476 & 1.796 & -0.501 \\
\hline 10/22/08 & $12: 00$ & 3.876 & 3.681 & 3.069 & 1.507 & 1.806 & -0.59 \\
\hline 10/22/08 & 16:00 & 3.895 & 3.523 & 3.092 & 1.434 & 1.813 & -0.625 \\
\hline 10/22/08 & 20:00 & 3.94 & 3.577 & 3.111 & 1.413 & 1.81 & -0.632 \\
\hline 10/23/08 & 0:00 & 3.811 & 3.496 & 3.041 & 1.373 & 1.804 & -0.655 \\
\hline 10/23/08 & 4:00 & 3.825 & 3.463 & 3.069 & 1.354 & 1.808 & -0.671 \\
\hline 10/23/08 & 8:00 & 3.893 & 3.512 & 3.116 & 1.481 & 1.825 & -0.662 \\
\hline 10/23/08 & $12: 00$ & 3.958 & 3.565 & 3.158 & 1.525 & 1.839 & -0.641 \\
\hline 10/23/08 & $16: 00$ & 4.017 & 3.572 & 3.196 & 1.535 & 1.85 & -0.62 \\
\hline 10/23/08 & 20:00 & 4.05 & 3.598 & 3.196 & 1.528 & 1.846 & -0.601 \\
\hline 10/24/08 & 0:00 & 4.097 & 3.66 & 3.207 & 1.582 & 1.846 & -0.587 \\
\hline 10/24/08 & 4:00 & 4.111 & 3.663 & 3.2 & 1.579 & 1.841 & -0.585 \\
\hline 10/24/08 & $8: 00$ & 4.148 & 3.679 & 3.217 & 1.584 & 1.846 & -0.583 \\
\hline 10/24/08 & $12: 00$ & 4.146 & 3.711 & 3.203 & 1.621 & 1.846 & -0.576 \\
\hline 10/24/08 & $16: 00$ & 4.176 & 3.709 & 3.226 & 1.603 & 1.855 & -0.573 \\
\hline 10/24/08 & 20:00 & 4.214 & 3.742 & 3.249 & 1.628 & 1.865 & -0.562 \\
\hline 10/25/08 & $0: 00$ & 4.235 & 3.76 & 3.254 & 1.638 & 1.86 & -0.552 \\
\hline 10/25/08 & 4:00 & 4.24 & 3.758 & 3.252 & 1.631 & 1.853 & -0.55 \\
\hline 10/25/08 & 8:00 & 4.259 & 3.776 & 3.263 & 1.64 & 1.86 & -0.548 \\
\hline 10/25/08 & $12: 00$ & 4.27 & 3.783 & 3.273 & 1.645 & 1.876 & -0.543 \\
\hline 10/25/08 & $16: 00$ & 4.282 & 3.786 & 3.287 & 1.643 & 1.895 & -0.541 \\
\hline 10/25/08 & 20:00 & 4.31 & 3.816 & 3.299 & 1.668 & 1.869 & -0.531 \\
\hline 10/26/08 & $0: 00$ & 4.32 & 3.832 & 3.296 & 1.671 & 1.862 & -0.526 \\
\hline 10/26/08 & 4:00 & 4.397 & 3.92 & 3.345 & 1.739 & 1.883 & -0.503 \\
\hline 10/26/08 & $8: 00$ & 4.47 & 4.004 & 3.385 & 1.809 & 1.904 & -0.473 \\
\hline 10/26/08 & $12: 00$ & 4.535 & 4.076 & 3.427 & 1.86 & 1.937 & -0.438 \\
\hline 10/26/08 & 16:00 & 4.585 & 4.122 & 3.451 & 1.891 & 1.958 & -0.41 \\
\hline 10/26/08 & 20:00 & 4.631 & 4.18 & 3.47 & 1.924 & 1.951 & -0.377 \\
\hline 10/27/08 & $0: 00$ & 4.653 & 4.208 & 3.472 & 1.928 & 1.944 & -0.354 \\
\hline 10/27/08 & 4:00 & 4.664 & 4.22 & 3.472 & 1.928 & 1.945 & -0.344 \\
\hline $10 / 27 / 08$ & 8:00 & 4.692 & 4.255 & 3.491 & 1.954 & 1.957 & -0.332 \\
\hline 10/27/08 & $12: 00$ & 4.718 & 4.285 & 3.502 & 1.971 & 1.971 & -0.318 \\
\hline
\end{tabular}


TABLE S1.3 (Cont.)

Depth to Water (feet below top of casing) in Indicated Wells

Date Time PMW1s PMW1d PMW2sa PMW2d PMW9s PMW9d PMW3d

\begin{tabular}{|c|c|c|c|c|c|c|c|}
\hline 10/27/08 & $16: 00$ & 4.714 & 4.273 & 3.498 & 1.954 & 1.975 & -0.314 \\
\hline 10/27/08 & 20:00 & 4.728 & 4.289 & 3.507 & 1.964 & 1.975 & -0.309 \\
\hline 10/28/08 & $0: 00$ & 4.739 & 4.303 & 3.512 & 1.973 & 1.973 & -0.302 \\
\hline 10/28/08 & 4:00 & 4.744 & 4.31 & 3.509 & 1.973 & 1.975 & -0.3 \\
\hline 10/28/08 & 8:00 & 4.751 & 4.32 & 3.514 & 1.978 & 1.98 & -0.297 \\
\hline 10/28/08 & $12: 00$ & 4.76 & 4.326 & 3.516 & 1.978 & 1.985 & -0.293 \\
\hline 10/28/08 & 16:00 & 4.718 & 4.278 & 3.493 & 1.938 & 1.98 & -0.3 \\
\hline 10/28/08 & 20:00 & 4.718 & 4.275 & 3.495 & 1.935 & 1.98 & -0.314 \\
\hline 10/29/08 & $0: 00$ & 4.714 & 4.273 & 3.488 & 1.933 & 1.975 & -0.316 \\
\hline 10/29/08 & 4:00 & 4.704 & 4.262 & 3.481 & 1.921 & 1.968 & -0.323 \\
\hline 10/29/08 & 8:00 & 4.706 & 4.266 & 3.479 & 1.919 & 1.968 & -0.33 \\
\hline 10/29/08 & $12: 00$ & 4.742 & 4.308 & 3.505 & 1.949 & 1.985 & -0.323 \\
\hline 10/29/08 & 16:00 & 4.732 & 4.294 & 3.495 & 1.933 & 1.982 & -0.318 \\
\hline 10/29/08 & 20:00 & 4.749 & 4.31 & 3.505 & 1.947 & 1.975 & -0.318 \\
\hline 10/30/08 & 0:00 & 4.756 & 4.32 & 3.505 & 1.949 & 1.975 & -0.316 \\
\hline 10/30/08 & 4:00 & 4.76 & 4.326 & 3.505 & 1.954 & 1.971 & -0.316 \\
\hline 10/30/08 & 8:00 & 4.76 & 4.331 & 3.505 & 1.949 & 1.961 & -0.316 \\
\hline 10/30/08 & $12: 00$ & 4.779 & 4.35 & 3.519 & 1.963 & 1.975 & -0.311 \\
\hline 10/30/08 & 16:00 & 4.765 & 4.331 & 3.507 & 1.945 & 1.97 & -0.314 \\
\hline 10/30/08 & 20:00 & 4.798 & 4.371 & 3.528 & 1.978 & 1.968 & -0.311 \\
\hline 10/31/08 & $0: 00$ & 4.821 & 4.398 & 3.54 & 1.999 & 1.965 & -0.302 \\
\hline 10/31/08 & 4:00 & 4.845 & 4.429 & 3.552 & 2.022 & 1.966 & -0.288 \\
\hline 10/31/08 & $8: 00$ & 4.875 & 4.466 & 3.563 & 2.043 & 1.97 & -0.279 \\
\hline 10/31/08 & $12: 00$ & 4.906 & 4.498 & 3.58 & 2.062 & 1.982 & -0.262 \\
\hline 10/31/08 & $16: 00$ & 4.913 & 4.508 & 3.582 & 2.057 & 1.977 & -0.253 \\
\hline 10/31/08 & 20:00 & 4.934 & 4.531 & 3.591 & 2.078 & 1.977 & -0.246 \\
\hline 11/1/08 & $0: 00$ & 4.943 & 4.542 & 3.591 & 2.081 & 1.97 & -0.241 \\
\hline 11/1/08 & 4:00 & 4.948 & 4.549 & 3.591 & 2.078 & 1.971 & -0.237 \\
\hline 11/1/08 & 8:00 & 4.955 & 4.559 & 3.594 & 2.078 & 1.966 & -0.237 \\
\hline $11 / 1 / 08$ & $12: 00$ & 4.964 & 4.568 & 3.596 & 2.083 & 1.971 & -0.232 \\
\hline 11/1/08 & $16: 00$ & 4.941 & 4.535 & 3.58 & 2.05 & 1.966 & -0.239 \\
\hline 11/1/08 & 20:00 & 4.943 & 4.542 & 3.582 & 2.055 & 1.961 & -0.246 \\
\hline 11/2/08 & $0: 00$ & 4.941 & 4.54 & 3.58 & 2.05 & 1.954 & -0.248 \\
\hline 11/2/08 & 4:00 & 4.936 & 4.533 & 3.575 & 2.045 & 1.952 & -0.255 \\
\hline 11/2/08 & 8:00 & 4.936 & 4.535 & 3.575 & 2.043 & 1.949 & -0.26 \\
\hline 11/2/08 & $12: 00$ & 4.95 & 4.549 & 3.584 & 2.053 & 1.954 & -0.258 \\
\hline 11/2/08 & $16: 00$ & 4.929 & 4.519 & 3.57 & 2.024 & 1.949 & -0.262 \\
\hline 11/2/08 & 20:00 & 4.932 & 4.526 & 3.568 & 2.031 & 1.944 & -0.269 \\
\hline 11/3/08 & $0: 00$ & 4.934 & 4.533 & 3.566 & 2.031 & 1.933 & -0.272 \\
\hline 11/3/08 & 4:00 & 4.929 & 4.528 & 3.561 & 2.027 & 1.923 & -0.274 \\
\hline $11 / 3 / 08$ & 8:00 & 4.941 & 4.54 & 3.566 & 2.031 & 1.926 & -0.276 \\
\hline $11 / 3 / 08$ & $12: 00$ & 4.962 & 4.566 & 3.58 & 2.05 & 1.93 & -0.274 \\
\hline 11/3/08 & $16: 00$ & 4.962 & 4.566 & 3.584 & 2.05 & 1.933 & -0.269 \\
\hline $11 / 3 / 08$ & 20:00 & 4.99 & 4.596 & 3.601 & 2.076 & 1.935 & -0.262 \\
\hline
\end{tabular}


TABLE S1.3 (Cont.)

Depth to Water (feet below top of casing) in Indicated Wells

Date Time PMW1s PMW1d PMW2sa PMW2d PMW9s PMW9d PMW3d

\begin{tabular}{|c|c|c|c|c|c|c|c|}
\hline $11 / 4 / 08$ & 0:00 & 5.004 & 4.614 & 3.603 & 2.088 & 1.928 & -0.253 \\
\hline 11/4/08 & 4:00 & 5.007 & 4.619 & 3.598 & 2.083 & 1.916 & -0.251 \\
\hline $11 / 4 / 08$ & $8: 00$ & 5.002 & 4.612 & 3.591 & 2.071 & 1.909 & -0.253 \\
\hline $11 / 4 / 08$ & $12: 00$ & 4.997 & 4.605 & 3.591 & 2.064 & 1.914 & -0.255 \\
\hline 11/4/08 & $16: 00$ & 4.969 & 4.566 & 3.573 & 2.031 & 1.911 & -0.269 \\
\hline 11/4/08 & 20:00 & 4.962 & 4.563 & 3.573 & 2.031 & 1.904 & -0.276 \\
\hline 11/5/08 & 0:00 & 4.96 & 4.566 & 3.57 & 2.029 & 1.895 & -0.283 \\
\hline 11/5/08 & 4:00 & 4.941 & 4.54 & 3.554 & 2.01 & 1.888 & -0.29 \\
\hline 11/5/08 & $8: 00$ & 4.936 & 4.538 & 3.556 & 2.01 & 1.886 & -0.3 \\
\hline $11 / 5 / 08$ & $12: 00$ & 4.946 & 4.545 & 3.559 & 2.01 & 1.876 & -0.304 \\
\hline $11 / 5 / 08$ & $16: 00$ & 4.95 & 4.552 & 3.568 & 2.022 & 1.883 & -0.302 \\
\hline $11 / 5 / 08$ & 20:00 & 4.971 & 4.586 & 3.57 & 2.036 & 1.881 & -0.302 \\
\hline 11/6/08 & 0:00 & 5.002 & 4.617 & 3.587 & 2.067 & 1.886 & -0.29 \\
\hline 11/6/08 & 4:00 & 5 & 4.619 & 3.584 & 2.055 & 1.874 & -0.283 \\
\hline 11/6/08 & $8: 00$ & 4.99 & 4.607 & 3.58 & 2.045 & 1.867 & -0.283 \\
\hline 11/6/08 & $12: 00$ & 5.014 & 4.631 & 3.601 & 2.074 & 1.889 & -0.281 \\
\hline 11/6/08 & $16: 00$ & 5.03 & 4.654 & 3.615 & 2.085 & 1.898 & -0.274 \\
\hline 11/6/08 & 20:00 & 5.079 & 4.709 & 3.645 & 2.144 & 1.917 & -0.248 \\
\hline 11/7/08 & 0:00 & 5.11 & 4.744 & 3.652 & 2.158 & 1.917 & -0.227 \\
\hline 11/7/08 & 4:00 & 5.11 & 4.742 & 3.645 & 2.144 & 1.913 & -0.22 \\
\hline 11/7/08 & $8: 00$ & 5.11 & 4.749 & 3.645 & 2.144 & 1.92 & -0.218 \\
\hline 11/7/08 & $12: 00$ & 5.126 & 4.76 & 3.659 & 2.16 & 1.929 & -0.209 \\
\hline 11/7/08 & $16: 00$ & 5.143 & 4.779 & 3.669 & 2.174 & 1.939 & -0.199 \\
\hline 11/7/08 & 20:00 & 5.171 & 4.807 & 3.683 & 2.198 & 1.953 & -0.185 \\
\hline 11/8/08 & 0:00 & 5.178 & 4.818 & 3.685 & 2.2 & 1.955 & -0.174 \\
\hline 11/8/08 & 4:00 & 5.187 & 4.83 & 3.694 & 2.205 & 1.96 & -0.169 \\
\hline 11/8/08 & $8: 00$ & 5.201 & 4.846 & 3.697 & 2.221 & 1.965 & -0.162 \\
\hline 11/8/08 & $12: 00$ & 5.227 & 4.874 & 3.718 & 2.242 & 1.981 & -0.145 \\
\hline $11 / 8 / 08$ & $16: 00$ & 5.234 & 4.886 & 3.718 & 2.245 & 1.986 & -0.136 \\
\hline 11/8/08 & 20:00 & 5.255 & 4.907 & 3.73 & 2.263 & 1.995 & -0.124 \\
\hline 11/9/08 & 0:00 & 5.269 & 4.921 & 3.734 & 2.268 & 1.995 & -0.117 \\
\hline 11/9/08 & 4:00 & 5.276 & 4.928 & 3.737 & 2.27 & 2 & -0.108 \\
\hline 11/9/08 & $8: 00$ & 5.286 & 4.937 & 3.746 & 2.277 & 2.007 & -0.106 \\
\hline $11 / 9 / 08$ & $12: 00$ & 5.297 & 4.953 & 3.751 & 2.282 & 2.009 & -0.094 \\
\hline $11 / 9 / 08$ & $16: 00$ & 5.288 & 4.939 & 3.741 & 2.27 & 2.007 & -0.096 \\
\hline 11/9/08 & 20:00 & 5.297 & 4.946 & 3.748 & 2.277 & 2.019 & -0.096 \\
\hline 11/10/08 & $0: 00$ & 5.316 & 4.972 & 3.765 & 2.301 & 2.026 & -0.089 \\
\hline 11/10/08 & 4:00 & 5.321 & 4.979 & 3.765 & 2.303 & 2.026 & -0.08 \\
\hline 11/10/08 & 8:00 & 5.326 & 4.983 & 3.765 & 2.301 & 2.03 & -0.078 \\
\hline 11/10/08 & $12: 00$ & 5.328 & 4.986 & 3.765 & 2.296 & 2.03 & -0.073 \\
\hline 11/10/08 & $16: 00$ & 5.316 & 4.955 & 3.746 & 2.275 & 2.023 & -0.08 \\
\hline 11/10/08 & 20:00 & 5.279 & 4.981 & 3.68 & 2.284 & 2.016 & -0.094 \\
\hline 11/11/08 & 0:00 & 5.251 & 4.986 & 3.666 & 2.27 & 2.007 & -0.11 \\
\hline 11/11/08 & 4:00 & 5.213 & 4.997 & 3.648 & 2.291 & 2.002 & -0.129 \\
\hline
\end{tabular}


TABLE S1.3 (Cont.)

Depth to Water (feet below top of casing) in Indicated Wells

Date Time PMW1s PMW1d PMW2sa PMW2d PMW9s PMW9d PMW3d

\begin{tabular}{|c|c|c|c|c|c|c|c|}
\hline $11 / 11 / 08$ & 8:00 & 5.208 & 4.958 & 3.648 & 2.282 & 2.012 & -0.138 \\
\hline 11/11/08 & $12: 00$ & 5.213 & 4.997 & 3.655 & 2.289 & 2.014 & -0.141 \\
\hline 11/11/08 & $16: 00$ & 5.131 & 4.928 & 3.568 & 2.214 & 1.995 & -0.157 \\
\hline $11 / 11 / 08$ & $20: 00$ & 5.014 & 4.814 & 3.523 & 2.151 & 2.002 & -0.183 \\
\hline $11 / 12 / 08$ & 0:00 & 5.049 & 4.754 & 3.575 & 2.099 & 2.014 & -0.195 \\
\hline $11 / 12 / 08$ & $4: 00$ & 5.1 & 4.791 & 3.615 & 2.134 & 2.019 & -0.192 \\
\hline $11 / 12 / 08$ & 8:00 & 5.14 & 4.821 & 3.645 & 2.163 & 2.028 & -0.181 \\
\hline 11/12/08 & $12: 00$ & 5.18 & 4.853 & 3.666 & 2.191 & 2.033 & -0.167 \\
\hline $11 / 12 / 08$ & $16: 00$ & 5.178 & 4.844 & 3.666 & 2.177 & 2.026 & -0.16 \\
\hline $11 / 12 / 08$ & $20: 00$ & 5.18 & 4.844 & 3.669 & 2.177 & 2.024 & -0.157 \\
\hline $11 / 13 / 08$ & 0:00 & 5.173 & 4.828 & 3.657 & 2.163 & 2.012 & -0.157 \\
\hline $11 / 13 / 08$ & $4: 00$ & 5.15 & 4.802 & 3.65 & 2.139 & 2.007 & -0.169 \\
\hline 11/13/08 & $8: 00$ & 5.131 & 4.781 & 3.641 & 2.125 & 2.002 & -0.178 \\
\hline $11 / 13 / 08$ & $12: 00$ & 5.129 & 4.772 & 3.641 & 2.127 & 1.998 & -0.185 \\
\hline $11 / 13 / 08$ & $16: 00$ & 5.124 & 4.772 & 3.641 & 2.127 & 1.995 & -0.192 \\
\hline 11/13/08 & $20: 00$ & 5.15 & 4.786 & 3.641 & 2.104 & 2 & -0.188 \\
\hline $11 / 14 / 08$ & $0: 00$ & 5.164 & 4.823 & 3.652 & 2.163 & 2.002 & -0.181 \\
\hline 11/14/08 & $4: 00$ & 5.187 & 4.844 & 3.669 & 2.179 & 2.002 & -0.171 \\
\hline $11 / 14 / 08$ & 8:00 & 5.227 & 4.9 & 3.699 & 2.223 & 2.019 & -0.153 \\
\hline 11/14/08 & $12: 00$ & 5.281 & 4.955 & 3.73 & 2.273 & 2.03 & -0.127 \\
\hline $11 / 14 / 08$ & $16: 00$ & 5.316 & 4.995 & 3.753 & 2.296 & 2.035 & -0.101 \\
\hline $11 / 14 / 08$ & $20: 00$ & 5.354 & 5.034 & 3.774 & 2.331 & 2.047 & -0.08 \\
\hline $11 / 15 / 08$ & 0:00 & 5.382 & 5.071 & 3.783 & 2.355 & 2.052 & -0.057 \\
\hline $11 / 15 / 08$ & $4: 00$ & 5.405 & 5.092 & 3.8 & 2.366 & 2.059 & -0.04 \\
\hline 11/15/08 & $8: 00$ & 5.417 & 5.106 & 3.802 & 2.369 & 2.056 & -0.033 \\
\hline 11/15/08 & $12: 00$ & 5.433 & 5.12 & 3.814 & 2.376 & 2.064 & -0.024 \\
\hline $11 / 15 / 08$ & $16: 00$ & 5.419 & 5.102 & 3.8 & 2.355 & 2.059 & -0.024 \\
\hline $11 / 15 / 08$ & 20:00 & 5.426 & 5.106 & 3.805 & 2.359 & 2.061 & -0.022 \\
\hline 11/16/08 & 0:00 & 5.403 & 5.078 & 3.788 & 2.336 & 2.054 & -0.029 \\
\hline 11/16/08 & 4:00 & 5.37 & 5.041 & 3.769 & 2.306 & 2.05 & -0.04 \\
\hline 11/16/08 & 8:00 & 5.363 & 5.03 & 3.772 & 2.303 & 2.057 & -0.054 \\
\hline 11/16/08 & $12: 00$ & 5.405 & 5.078 & 3.807 & 2.35 & 2.073 & -0.045 \\
\hline $11 / 16 / 08$ & $16: 00$ & 5.419 & 5.097 & 3.809 & 2.359 & 2.07 & -0.036 \\
\hline 11/16/08 & $20: 00$ & 5.447 & 5.132 & 3.828 & 2.388 & 2.085 & -0.019 \\
\hline 11/17/08 & 0:00 & 5.469 & 5.157 & 3.84 & 2.409 & 2.089 & -0.003 \\
\hline 11/17/08 & $4: 00$ & 5.49 & 5.183 & 3.851 & 2.427 & 2.096 & 0.011 \\
\hline 11/17/08 & $8: 00$ & 5.511 & 5.206 & 3.863 & 2.441 & 2.101 & 0.027 \\
\hline 11/17/08 & $12: 00$ & 5.541 & 5.241 & 3.879 & 2.467 & 2.108 & 0.044 \\
\hline 11/17/08 & $16: 00$ & 5.546 & 5.243 & 3.877 & 2.458 & 2.103 & 0.051 \\
\hline 11/17/08 & 20:00 & 5.558 & 5.257 & 3.884 & 2.474 & 2.108 & 0.058 \\
\hline 11/18/08 & 0:00 & 5.572 & 5.269 & 3.891 & 2.479 & 2.113 & 0.065 \\
\hline 11/18/08 & 4:00 & 5.579 & 5.276 & 3.894 & 2.479 & 2.115 & 0.07 \\
\hline $11 / 18 / 08$ & $8: 00$ & 5.572 & 5.264 & 3.886 & 2.465 & 2.111 & 0.07 \\
\hline 11/18/08 & $12: 00$ & 5.562 & 5.255 & 3.877 & 2.453 & 2.108 & 0.067 \\
\hline
\end{tabular}


TABLE S1.3 (Cont.)

\begin{tabular}{|c|c|c|c|c|c|c|c|c|}
\hline \multirow[b]{2}{*}{ Date } & \multirow[b]{2}{*}{ Time } & \multicolumn{7}{|c|}{ Depth to Water (feet below top of casing) in Indicated Wells } \\
\hline & & PMW1s & PMW1d & PMW2sa & PMW2d & PMW9s & PMW9d & PMW3d \\
\hline 11/18/08 & $16: 00$ & 5.497 & 5.171 & 3.828 & 2.38 & 2.087 & 0.042 & \\
\hline 11/18/08 & 20:00 & 5.452 & 5.127 & 3.816 & 2.352 & 2.087 & 0.016 & \\
\hline 11/19/08 & 0:00 & 5.422 & 5.088 & 3.795 & 2.327 & 2.078 & -0.008 & \\
\hline 11/19/08 & 4:00 & 5.398 & 5.062 & 3.786 & 2.313 & 2.076 & -0.029 & \\
\hline 11/19/08 & $8: 00$ & 5.408 & 5.076 & 3.8 & 2.334 & 2.085 & -0.031 & \\
\hline $11 / 19 / 08$ & $12: 00$ & 5.452 & 5.127 & 3.83 & 2.376 & 2.099 & -0.019 & \\
\hline 11/19/08 & $16: 00$ & 5.478 & 5.167 & 3.851 & 2.404 & 2.108 & -0.005 & \\
\hline 11/19/08 & $20: 00$ & 5.546 & 5.239 & 3.891 & 2.467 & 2.124 & 0.023 & \\
\hline 11/20/08 & 0:00 & 5.581 & 5.28 & 3.903 & 2.491 & 2.125 & 0.049 & \\
\hline $11 / 20 / 08$ & 4:00 & 5.605 & 5.308 & 3.915 & 2.507 & 2.132 & 0.072 & \\
\hline $11 / 20 / 08$ & 8:00 & 5.626 & 5.334 & 3.929 & 2.521 & 2.134 & 0.086 & \\
\hline 11/20/08 & $12: 00$ & 5.649 & 5.364 & 3.94 & 2.545 & 2.146 & 0.102 & \\
\hline 11/20/08 & $16: 00$ & 5.659 & 5.369 & 3.938 & 2.537 & 2.141 & 0.112 & \\
\hline $11 / 20 / 08$ & $20: 00$ & 5.673 & 5.385 & 3.952 & 2.554 & 2.148 & 0.121 & \\
\hline 11/21/08 & 0:00 & 5.675 & 5.382 & 3.947 & 2.547 & 2.144 & 0.126 & \\
\hline $11 / 21 / 08$ & 4:00 & 5.68 & 5.385 & 3.952 & 2.549 & 2.151 & 0.126 & \\
\hline 11/21/08 & $8: 00$ & 5.666 & 5.369 & 3.945 & 2.533 & 2.149 & 0.123 & \\
\hline $11 / 21 / 08$ & $12: 00$ & 5.652 & 5.35 & 3.933 & 2.516 & 2.139 & 0.119 & \\
\hline $11 / 21 / 08$ & $16: 00$ & 5.612 & 5.304 & 3.903 & 2.474 & 2.132 & 0.105 & \\
\hline $11 / 21 / 08$ & $20: 00$ & 5.593 & 5.28 & 3.898 & 2.467 & 2.137 & 0.086 & \\
\hline 11/22/08 & 0:00 & 5.581 & 5.269 & 3.886 & 2.453 & 2.133 & 0.079 & \\
\hline $11 / 22 / 08$ & 4:00 & 5.562 & 5.246 & 3.877 & 2.439 & 2.13 & 0.067 & \\
\hline $11 / 22 / 08$ & 8:00 & 5.548 & 5.232 & 3.875 & 2.427 & 2.13 & 0.058 & \\
\hline 11/22/08 & $12: 00$ & 5.548 & 5.236 & 3.879 & 2.437 & 2.135 & 0.053 & \\
\hline $11 / 22 / 08$ & $16: 00$ & 5.555 & 5.243 & 3.884 & 2.441 & 2.137 & 0.053 & \\
\hline $11 / 22 / 08$ & $20: 00$ & 5.579 & 5.269 & 3.896 & 2.465 & 2.139 & 0.063 & \\
\hline 11/23/08 & 0:00 & 5.576 & 5.264 & 3.889 & 2.458 & 2.137 & 0.065 & \\
\hline $11 / 23 / 08$ & 4:00 & 5.565 & 5.252 & 3.887 & 2.446 & 2.137 & 0.06 & \\
\hline $11 / 23 / 08$ & 8:00 & 5.551 & 5.239 & 3.877 & 2.43 & 2.133 & 0.056 & \\
\hline $11 / 23 / 08$ & $12: 00$ & 5.544 & 5.227 & 3.87 & 2.42 & 2.13 & 0.049 & \\
\hline $11 / 23 / 08$ & $16: 00$ & 5.525 & 5.211 & 3.865 & 2.409 & 2.13 & 0.037 & \\
\hline $11 / 23 / 08$ & $20: 00$ & 5.567 & 5.257 & 3.896 & 2.458 & 2.146 & 0.044 & \\
\hline $11 / 24 / 08$ & 0:00 & 5.609 & 5.304 & 3.919 & 2.498 & 2.149 & 0.063 & \\
\hline $11 / 24 / 08$ & 4:00 & 5.633 & 5.334 & 3.931 & 2.512 & 2.156 & 0.079 & \\
\hline $11 / 24 / 08$ & 8:00 & 5.654 & 5.357 & 3.943 & 2.53 & 2.158 & 0.095 & \\
\hline $11 / 24 / 08$ & $12: 00$ & 5.663 & 5.366 & 3.943 & 2.526 & 2.154 & 0.105 & \\
\hline 11/24/08 & $16: 00$ & 5.642 & 5.341 & 3.929 & 2.505 & 2.151 & 0.1 & \\
\hline $11 / 24 / 08$ & $20: 00$ & 5.647 & 5.345 & 3.933 & 2.512 & 2.153 & 0.1 & \\
\hline $11 / 25 / 08$ & 0:00 & 5.661 & 5.362 & 3.943 & 2.528 & 2.158 & 0.102 & \\
\hline $11 / 25 / 08$ & 4:00 & 5.666 & 5.369 & 3.945 & 2.53 & 2.158 & 0.107 & \\
\hline $11 / 25 / 08$ & 8:00 & 5.675 & 5.378 & 3.954 & 2.54 & 2.161 & 0.112 & \\
\hline $11 / 25 / 08$ & $12: 00$ & 5.675 & 5.375 & 3.947 & 2.535 & 2.158 & 0.116 & \\
\hline $11 / 25 / 08$ & $16: 00$ & 5.637 & 5.334 & 3.919 & 2.491 & 2.146 & 0.107 & \\
\hline $11 / 25 / 08$ & $20: 00$ & 5.633 & 5.331 & 3.924 & 2.498 & 2.154 & 0.098 & \\
\hline
\end{tabular}


TABLE S1.3 (Cont.)

Depth to Water (feet below top of casing) in Indicated Wells

Date Time PMW1s PMW1d PMW2sa PMW2d PMW9s PMW9d PMW3d

\begin{tabular}{|c|c|c|c|c|c|c|c|}
\hline 11/26/08 & 0:00 & 5.633 & 5.329 & 3.924 & 2.5 & 2.151 & 0.095 \\
\hline 11/26/08 & 4:00 & 5.633 & 5.331 & 3.929 & 2.5 & 2.151 & 0.093 \\
\hline 11/26/08 & 8:00 & 5.647 & 5.348 & 3.938 & 2.516 & 2.159 & 0.098 \\
\hline 11/26/08 & $12: 00$ & 5.673 & 5.373 & 3.952 & 2.533 & 2.161 & 0.105 \\
\hline 11/26/08 & 16:00 & 5.663 & 5.357 & 3.94 & 2.519 & 2.156 & 0.1 \\
\hline 11/26/08 & 20:00 & 5.661 & 5.359 & 3.94 & 2.519 & 2.153 & 0.098 \\
\hline 11/27/08 & 0:00 & 5.673 & 5.369 & 3.945 & 2.53 & 2.156 & 0.102 \\
\hline 11/27/08 & 4:00 & 5.677 & 5.378 & 3.952 & 2.535 & 2.159 & 0.107 \\
\hline 11/27/08 & 8:00 & 5.684 & 5.389 & 3.959 & 2.542 & 2.166 & 0.109 \\
\hline 11/27/08 & $12: 00$ & 5.712 & 5.422 & 3.978 & 2.575 & 2.173 & 0.123 \\
\hline 11/27/08 & $16: 00$ & 5.717 & 5.424 & 3.973 & 2.566 & 2.17 & 0.13 \\
\hline 11/27/08 & 20:00 & 5.724 & 5.431 & 3.978 & 2.575 & 2.172 & 0.135 \\
\hline 11/28/08 & 0:00 & 5.724 & 5.429 & 3.976 & 2.568 & 2.17 & 0.137 \\
\hline 11/28/08 & 4:00 & 5.722 & 5.429 & 3.973 & 2.57 & 2.168 & 0.137 \\
\hline 11/28/08 & $8: 00$ & 5.717 & 5.422 & 3.973 & 2.563 & 2.17 & 0.137 \\
\hline 11/28/08 & $12: 00$ & 5.72 & 5.424 & 3.976 & 2.563 & 2.173 & 0.135 \\
\hline 11/28/08 & 16:00 & 5.682 & 5.385 & 3.947 & 2.526 & 2.161 & 0.123 \\
\hline 11/28/08 & 20:00 & 5.68 & 5.378 & 3.95 & 2.528 & 2.163 & 0.116 \\
\hline 11/29/08 & 0:00 & 5.682 & 5.38 & 3.952 & 2.53 & 2.163 & 0.114 \\
\hline 11/29/08 & 4:00 & 5.666 & 5.364 & 3.936 & 2.523 & 2.154 & 0.107 \\
\hline 11/29/08 & $8: 00$ & 5.644 & 5.343 & 3.924 & 2.54 & 2.149 & 0.095 \\
\hline 11/29/08 & $12: 00$ & 5.635 & 5.329 & 3.917 & 2.505 & 2.152 & 0.086 \\
\hline 11/29/08 & $16: 00$ & 5.607 & 5.297 & 3.898 & 2.479 & 2.142 & 0.074 \\
\hline 11/29/08 & 20:00 & 5.614 & 5.308 & 3.91 & 2.474 & 2.149 & 0.067 \\
\hline 11/30/08 & $0: 00$ & 5.621 & 5.315 & 3.915 & 2.481 & 2.149 & 0.07 \\
\hline 11/30/08 & 4:00 & 5.612 & 5.304 & 3.908 & 2.472 & 2.142 & 0.067 \\
\hline 11/30/08 & 8:00 & 5.616 & 5.313 & 3.917 & 2.481 & 2.145 & 0.065 \\
\hline 11/30/08 & $12: 00$ & 5.637 & 5.338 & 3.931 & 2.502 & 2.152 & 0.072 \\
\hline 11/30/08 & $16: 00$ & 5.642 & 5.341 & 3.931 & 2.498 & 2.149 & 0.077 \\
\hline $11 / 30 / 08$ & $20: 00$ & 5.673 & 5.375 & 3.95 & 2.528 & 2.159 & 0.086 \\
\hline 12/1/08 & $0: 00$ & 5.694 & 5.399 & 3.964 & 2.547 & 2.164 & 0.095 \\
\hline 12/1/08 & $4: 00$ & 5.724 & 5.436 & 3.98 & 2.57 & 2.175 & 0.107 \\
\hline 12/1/08 & $8: 00$ & 5.745 & 5.461 & 3.992 & 2.601 & 2.18 & 0.123 \\
\hline 12/1/08 & $12: 00$ & 5.773 & 5.496 & 4.015 & 2.626 & 2.189 & 0.144 \\
\hline 12/1/08 & $16: 00$ & 5.778 & 5.496 & 4.006 & 2.615 & 2.182 & 0.151 \\
\hline 12/1/08 & 20:00 & 5.778 & 5.496 & 4.001 & 2.608 & 2.18 & 0.156 \\
\hline $12 / 2 / 08$ & $0: 00$ & 5.771 & 5.482 & 4.001 & 2.601 & 2.18 & 0.156 \\
\hline $12 / 2 / 08$ & 4:00 & 5.75 & 5.454 & 3.985 & 2.577 & 2.176 & 0.149 \\
\hline $12 / 2 / 08$ & 8:00 & 5.71 & 5.41 & 3.964 & 2.54 & 2.169 & 0.133 \\
\hline $12 / 2 / 08$ & $12: 00$ & 5.708 & 5.408 & 3.966 & 2.547 & 2.171 & 0.126 \\
\hline 12/2/08 & $16: 00$ & 5.694 & 5.392 & 3.954 & 2.533 & 2.168 & 0.114 \\
\hline $12 / 2 / 08$ & 20:00 & 5.698 & 5.401 & 3.961 & 2.54 & 2.173 & 0.112 \\
\hline 12/3/08 & $0: 00$ & 5.712 & 5.42 & 3.973 & 2.558 & 2.178 & 0.114 \\
\hline $12 / 3 / 08$ & 4:00 & 5.773 & 5.489 & 4.015 & 2.622 & 2.199 & 0.135 \\
\hline
\end{tabular}


TABLE S1.3 (Cont.)

Depth to Water (feet below top of casing) in Indicated Wells

Date Time PMW1s PMW1d PMW2sa PMW2d PMW9s PMW9d PMW3d

\begin{tabular}{|c|c|c|c|c|c|c|c|}
\hline $12 / 3 / 08$ & 8:00 & 5.811 & 5.538 & 4.034 & 2.657 & 2.199 & 0.158 \\
\hline $12 / 3 / 08$ & $12: 00$ & 5.858 & 5.594 & 4.062 & 2.699 & 2.213 & 0.189 \\
\hline $12 / 3 / 08$ & $16: 00$ & 5.874 & 5.612 & 4.065 & 2.699 & 2.206 & 0.205 \\
\hline $12 / 3 / 08$ & $20: 00$ & 5.895 & 5.635 & 4.076 & 2.718 & 2.218 & 0.221 \\
\hline $12 / 4 / 08$ & 0:00 & 5.912 & 5.652 & 4.086 & 2.725 & 2.22 & 0.236 \\
\hline $12 / 4 / 08$ & 4:00 & 5.923 & 5.666 & 4.095 & 2.734 & 2.225 & 0.243 \\
\hline $12 / 4 / 08$ & 8:00 & 5.945 & 5.689 & 4.109 & 2.758 & 2.235 & 0.257 \\
\hline $12 / 4 / 08$ & $12: 00$ & 5.975 & 5.728 & 4.13 & 2.788 & 2.244 & 0.275 \\
\hline $12 / 4 / 08$ & $16: 00$ & 5.98 & 5.726 & 4.118 & 2.769 & 2.23 & 0.282 \\
\hline $12 / 4 / 08$ & $20: 00$ & 5.994 & 5.74 & 4.13 & 2.786 & 2.241 & 0.287 \\
\hline $12 / 5 / 08$ & 0:00 & 5.991 & 5.733 & 4.125 & 2.779 & 2.239 & 0.289 \\
\hline $12 / 5 / 08$ & 4:00 & 5.966 & 5.703 & 4.114 & 2.748 & 2.23 & 0.282 \\
\hline $12 / 5 / 08$ & 8:00 & 5.935 & 5.661 & 4.093 & 2.718 & 2.221 & 0.271 \\
\hline $12 / 5 / 08$ & $12: 00$ & 5.898 & 5.617 & 4.065 & 2.68 & 2.204 & 0.247 \\
\hline $12 / 5 / 08$ & $16: 00$ & 5.827 & 5.533 & 4.013 & 2.603 & 2.183 & 0.212 \\
\hline 12/5/08 & 20:00 & 5.792 & 5.494 & 4.004 & 2.582 & 2.188 & 0.186 \\
\hline 12/6/08 & 0:00 & 5.776 & 5.482 & 4.001 & 2.587 & 2.199 & 0.168 \\
\hline 12/6/08 & 4:00 & 5.78 & 5.494 & 4.008 & 2.601 & 2.202 & 0.165 \\
\hline $12 / 6 / 08$ & 8:00 & 5.816 & 5.536 & 4.034 & 2.638 & 2.216 & 0.172 \\
\hline 12/6/08 & $12: 00$ & 5.879 & 5.612 & 4.076 & 2.699 & 2.23 & 0.2 \\
\hline 12/6/08 & $16: 00$ & 5.907 & 5.638 & 4.079 & 2.706 & 2.225 & 0.217 \\
\hline 12/6/08 & 20:00 & 5.935 & 5.675 & 4.097 & 2.734 & 2.237 & 0.235 \\
\hline 12/7/08 & 0:00 & 5.949 & 5.686 & 4.1 & 2.739 & 2.235 & 0.25 \\
\hline 12/7/08 & 4:00 & 5.935 & 5.666 & 4.083 & 2.713 & 2.225 & 0.254 \\
\hline 12/7/08 & $8: 00$ & 5.916 & 5.642 & 4.081 & 2.701 & 2.228 & 0.25 \\
\hline 12/7/08 & $12: 00$ & 5.891 & 5.612 & 4.062 & 2.676 & 2.216 & 0.236 \\
\hline $12 / 7 / 08$ & $16: 00$ & 5.844 & 5.556 & 4.032 & 2.631 & 2.206 & 0.217 \\
\hline 12/7/08 & 20:00 & 5.832 & 5.547 & 4.032 & 2.629 & 2.216 & 0.2 \\
\hline $12 / 8 / 08$ & 0:00 & 5.823 & 5.533 & 4.025 & 2.617 & 2.209 & 0.193 \\
\hline 12/8/08 & 4:00 & 5.806 & 5.519 & 4.011 & 2.605 & 2.209 & 0.184 \\
\hline 12/8/08 & $8: 00$ & 5.792 & 5.508 & 4.006 & 2.594 & 2.209 & 0.175 \\
\hline 12/8/08 & $12: 00$ & 5.778 & 5.489 & 3.997 & 2.582 & 2.202 & 0.165 \\
\hline 12/8/08 & $16: 00$ & 5.759 & 5.468 & 3.985 & 2.561 & 2.202 & 0.154 \\
\hline $12 / 8 / 08$ & 20:00 & 5.769 & 5.485 & 3.999 & 2.582 & 2.211 & 0.154 \\
\hline $12 / 9 / 08$ & $0: 00$ & 5.806 & 5.529 & 4.027 & 2.624 & 2.22 & 0.163 \\
\hline $12 / 9 / 08$ & 4:00 & 5.853 & 5.584 & 4.053 & 2.664 & 2.23 & 0.184 \\
\hline 12/9/08 & $8: 00$ & 5.914 & 5.649 & 4.09 & 2.727 & 2.247 & 0.214 \\
\hline 12/9/08 & $12: 00$ & 5.975 & 5.724 & 4.13 & 2.776 & 2.256 & 0.247 \\
\hline 12/9/08 & $16: 00$ & 5.998 & 5.751 & 4.14 & 2.786 & 2.254 & 0.271 \\
\hline $12 / 9 / 08$ & 20:00 & 6.031 & 5.786 & 4.161 & 2.818 & 2.263 & 0.292 \\
\hline $12 / 10 / 08$ & 0:00 & 6.048 & 5.802 & 4.165 & 2.826 & 2.254 & 0.31 \\
\hline $12 / 10 / 08$ & 4:00 & 6.052 & 5.802 & 4.168 & 2.819 & 2.247 & 0.313 \\
\hline 12/10/08 & $8: 00$ & 6.05 & 5.798 & 4.165 & 2.818 & 2.238 & 0.303 \\
\hline 12/10/08 & $12: 00$ & 6.048 & 5.789 & 4.161 & 2.807 & 2.226 & 0.308 \\
\hline
\end{tabular}


TABLE S1.3 (Cont.)

Depth to Water (feet below top of casing) in Indicated Wells

Date Time PMW1s PMW1d PMW2sa PMW2d PMW9s PMW9d PMW3d

\begin{tabular}{|c|c|c|c|c|c|c|c|}
\hline 12/10/08 & $16: 00$ & 6.005 & 5.744 & 4.125 & 2.76 & 2.219 & 0.303 \\
\hline $12 / 10 / 08$ & 20:00 & 6.003 & 5.742 & 4.128 & 2.762 & 2.233 & 0.292 \\
\hline $12 / 11 / 08$ & $0: 00$ & 5.991 & 5.731 & 4.121 & 2.755 & 2.23 & 0.282 \\
\hline 12/11/08 & 4:00 & 5.982 & 5.719 & 4.118 & 2.748 & 2.235 & 0.28 \\
\hline 12/11/08 & 8:00 & 5.984 & 5.721 & 4.123 & 2.755 & 2.245 & 0.275 \\
\hline 12/11/08 & $12: 00$ & 6.003 & 5.744 & 4.132 & 2.769 & 2.244 & 0.28 \\
\hline 12/11/08 & 16:00 & 5.996 & 5.735 & 4.123 & 2.76 & 2.242 & 0.282 \\
\hline 12/11/08 & 20:00 & 6.019 & 5.765 & 4.14 & 2.772 & 2.256 & 0.289 \\
\hline $12 / 12 / 08$ & $0: 00$ & 6.043 & 5.793 & 4.154 & 2.783 & 2.265 & 0.301 \\
\hline $12 / 12 / 08$ & 4:00 & 6.059 & 5.812 & 4.158 & 2.816 & 2.265 & 0.313 \\
\hline $12 / 12 / 08$ & 8:00 & 6.052 & 5.8 & 4.154 & 2.804 & 2.263 & 0.313 \\
\hline $12 / 12 / 08$ & $12: 00$ & 6.05 & 5.798 & 4.151 & 2.802 & 2.263 & 0.315 \\
\hline $12 / 12 / 08$ & $16: 00$ & 5.996 & 5.733 & 4.114 & 2.744 & 2.249 & 0.303 \\
\hline $12 / 12 / 08$ & 20:00 & 5.966 & 5.698 & 4.095 & 2.715 & 2.249 & 0.282 \\
\hline $12 / 13 / 08$ & 0:00 & 5.933 & 5.659 & 4.079 & 2.69 & 2.247 & 0.264 \\
\hline $12 / 13 / 08$ & 4:00 & 5.886 & 5.603 & 4.043 & 2.643 & 2.233 & 0.24 \\
\hline 12/13/08 & $8: 00$ & 5.853 & 5.566 & 4.036 & 2.624 & 2.235 & 0.217 \\
\hline 12/13/08 & $12: 00$ & 5.834 & 5.55 & 4.029 & 2.615 & 2.23 & 0.203 \\
\hline $12 / 13 / 08$ & 16:00 & 5.808 & 5.524 & 4.013 & 2.591 & 2.228 & 0.184 \\
\hline 12/13/08 & 20:00 & 5.813 & 5.531 & 4.018 & 2.605 & 2.235 & 0.182 \\
\hline $12 / 14 / 08$ & $0: 00$ & 5.801 & 5.515 & 4.008 & 2.589 & 2.225 & 0.175 \\
\hline $12 / 14 / 08$ & 4:00 & 5.794 & 5.505 & 4.006 & 2.587 & 2.225 & 0.17 \\
\hline $12 / 14 / 08$ & 8:00 & 5.855 & 5.589 & 4.062 & 2.671 & 2.256 & 0.182 \\
\hline $12 / 14 / 08$ & $12: 00$ & 5.951 & 5.698 & 4.118 & 2.762 & 2.268 & 0.224 \\
\hline 12/14/08 & $16: 00$ & 6.001 & 5.754 & 4.144 & 2.795 & 2.273 & 0.268 \\
\hline $12 / 14 / 08$ & 20:00 & 6.059 & 5.826 & 4.182 & 2.844 & 2.28 & 0.299 \\
\hline $12 / 15 / 08$ & 0:00 & 6.111 & 5.886 & 4.219 & 2.891 & 2.287 & 0.338 \\
\hline $12 / 15 / 08$ & 4:00 & 6.158 & 5.942 & 4.245 & 2.929 & 2.294 & 0.373 \\
\hline $12 / 15 / 08$ & 8:00 & 6.195 & 5.981 & 4.266 & 2.954 & 2.297 & 0.408 \\
\hline 12/15/08 & $12: 00$ & 6.228 & 6.018 & 4.287 & 2.975 & 2.292 & 0.42 \\
\hline $12 / 15 / 08$ & $16: 00$ & 6.242 & 6.025 & 4.285 & 2.968 & 2.259 & 0.425 \\
\hline $12 / 15 / 08$ & 20:00 & 6.247 & 6.025 & 4.289 & 2.968 & 2.269 & 0.422 \\
\hline $12 / 16 / 08$ & $0: 00$ & 6.242 & 6.016 & 4.289 & 2.961 & 2.269 & 0.411 \\
\hline 12/16/08 & 4:00 & 6.219 & 5.986 & 4.273 & 2.933 & 2.25 & 0.392 \\
\hline $12 / 16 / 08$ & $8: 00$ & 6.174 & 5.932 & 4.25 & 2.891 & 2.243 & 0.362 \\
\hline 12/16/08 & $12: 00$ & 6.137 & 5.886 & 4.226 & 2.854 & 2.196 & 0.315 \\
\hline 12/16/08 & 16:00 & 6.092 & 5.835 & 4.198 & 2.809 & 2.159 & 0.287 \\
\hline $12 / 16 / 08$ & 20:00 & 6.08 & 5.83 & 4.2 & 2.814 & 2.154 & 0.287 \\
\hline $12 / 17 / 08$ & 0:00 & 6.085 & 5.837 & 4.2 & 2.821 & 2.147 & 0.294 \\
\hline $12 / 17 / 08$ & 4:00 & 6.101 & 5.858 & 4.214 & 2.837 & 2.159 & 0.303 \\
\hline $12 / 17 / 08$ & $8: 00$ & 6.127 & 5.884 & 4.233 & 2.858 & 2.161 & 0.315 \\
\hline 12/17/08 & $12: 00$ & 6.151 & 5.912 & 4.245 & 2.884 & 2.164 & 0.32 \\
\hline $12 / 17 / 08$ & $16: 00$ & 6.139 & 5.895 & 4.233 & 2.856 & 2.149 & 0.313 \\
\hline $12 / 17 / 08$ & 20:00 & 6.141 & 5.898 & 4.236 & 2.861 & 2.166 & 0.317 \\
\hline
\end{tabular}


TABLE S1.3 (Cont.)

Depth to Water (feet below top of casing) in Indicated Wells

Date Time PMW1s PMW1d PMW2sa PMW2d PMW9s PMW9d PMW3d

\begin{tabular}{|c|c|c|c|c|c|c|c|}
\hline 12/18/08 & 0:00 & 6.155 & 5.916 & 4.243 & 2.868 & 2.177 & 0.334 \\
\hline 12/18/08 & 4:00 & 6.148 & 5.907 & 4.243 & 2.863 & 2.185 & 0.336 \\
\hline 12/18/08 & 8:00 & 6.158 & 5.916 & 4.254 & 2.879 & 2.196 & 0.341 \\
\hline 12/18/08 & $12: 00$ & 6.162 & 5.919 & 4.257 & 2.882 & 2.194 & 0.336 \\
\hline 12/18/08 & 16:00 & 6.113 & 5.863 & 4.217 & 2.826 & 2.175 & 0.301 \\
\hline 12/18/08 & 20:00 & 6.085 & 5.826 & 4.2 & 2.797 & 2.185 & 0.28 \\
\hline 12/19/08 & 0:00 & 6.033 & 5.765 & 4.158 & 2.741 & 2.168 & 0.235 \\
\hline 12/19/08 & 4:00 & 6.029 & 5.775 & 4.182 & 2.762 & 2.194 & 0.259 \\
\hline 12/19/08 & 8:00 & 6.097 & 5.856 & 4.224 & 2.84 & 2.21 & 0.308 \\
\hline 12/19/08 & $12: 00$ & 6.158 & 5.928 & 4.254 & 2.891 & 2.222 & 0.35 \\
\hline 12/19/08 & $16: 00$ & 6.148 & 5.909 & 4.229 & 2.856 & 2.203 & 0.329 \\
\hline 12/19/08 & 20:00 & 6.165 & 5.928 & 4.245 & 2.875 & 2.22 & 0.341 \\
\hline 12/20/08 & 0:00 & 6.153 & 5.912 & 4.233 & 2.858 & 2.222 & 0.336 \\
\hline 12/20/08 & 4:00 & 6.118 & 5.87 & 4.21 & 2.823 & 2.22 & 0.315 \\
\hline 12/20/08 & $8: 00$ & 6.137 & 5.898 & 4.231 & 2.851 & 2.231 & 0.329 \\
\hline $12 / 20 / 08$ & $12: 00$ & 6.174 & 5.946 & 4.259 & 2.893 & 2.248 & 0.355 \\
\hline 12/20/08 & 16:00 & 6.191 & 5.963 & 4.266 & 2.903 & 2.243 & 0.366 \\
\hline 12/20/08 & 20:00 & 6.223 & 6 & 4.285 & 2.936 & 2.248 & 0.385 \\
\hline 12/21/08 & 0:00 & 6.247 & 6.025 & 4.299 & 2.957 & 2.253 & 0.399 \\
\hline 12/21/08 & 4:00 & 6.263 & 6.044 & 4.308 & 2.968 & 2.252 & 0.406 \\
\hline 12/21/08 & $8: 00$ & 6.27 & 6.048 & 4.315 & 2.973 & 2.243 & 0.406 \\
\hline 12/21/08 & $12: 00$ & 6.284 & 6.074 & 4.334 & 2.99 & 2.25 & 0.434 \\
\hline 12/21/08 & $16: 00$ & 6.303 & 6.088 & 4.341 & 3.001 & 2.243 & 0.432 \\
\hline 12/21/08 & 20:00 & 6.319 & 6.104 & 4.348 & 3.013 & 2.234 & 0.439 \\
\hline $12 / 22 / 08$ & $0: 00$ & 6.329 & 6.113 & 4.357 & 3.02 & 2.225 & 0.446 \\
\hline $12 / 22 / 08$ & 4:00 & 6.326 & 6.104 & 4.353 & 3.013 & 2.218 & 0.439 \\
\hline 12/22/08 & 8:00 & 6.312 & 6.086 & 4.343 & 2.994 & 2.208 & 0.415 \\
\hline $12 / 22 / 08$ & $12: 00$ & 6.296 & 6.065 & 4.336 & 2.98 & 2.185 & 0.418 \\
\hline $12 / 22 / 08$ & 16:00 & 6.237 & 5.995 & 4.292 & 2.915 & 2.152 & 0.362 \\
\hline $12 / 22 / 08$ & $20: 00$ & 6.198 & 5.949 & 4.275 & 2.884 & 2.145 & 0.336 \\
\hline 12/23/08 & $0: 00$ & 6.155 & 5.905 & 4.245 & 2.837 & 2.129 & 0.308 \\
\hline 12/23/08 & 4:00 & 6.118 & 5.856 & 4.226 & 2.809 & 2.112 & 0.275 \\
\hline 12/23/08 & $8: 00$ & 6.092 & 5.83 & 4.217 & 2.793 & 2.117 & 0.268 \\
\hline 12/23/08 & $12: 00$ & 6.09 & 5.833 & 4.219 & 2.8 & 2.115 & 0.273 \\
\hline 12/23/08 & $16: 00$ & 6.085 & 5.828 & 4.219 & 2.797 & 2.119 & 0.271 \\
\hline 12/23/08 & 20:00 & 6.12 & 5.874 & 4.245 & 2.842 & 2.134 & 0.28 \\
\hline $12 / 24 / 08$ & $0: 00$ & 6.162 & 5.921 & 4.268 & 2.879 & 2.134 & 0.31 \\
\hline 12/24/08 & 4:00 & 6.183 & 5.944 & 4.282 & 2.886 & 2.134 & 0.331 \\
\hline $12 / 24 / 08$ & $8: 00$ & 6.214 & 5.981 & 4.303 & 2.917 & 2.143 & 0.35 \\
\hline $12 / 24 / 08$ & $12: 00$ & 6.256 & 6.03 & 4.334 & 2.957 & 2.155 & 0.385 \\
\hline $12 / 24 / 08$ & $16: 00$ & 6.282 & 6.058 & 4.346 & 2.971 & 2.154 & 0.385 \\
\hline $12 / 24 / 08$ & 20:00 & 6.301 & 6.074 & 4.348 & 2.983 & 2.145 & 0.397 \\
\hline $12 / 25 / 08$ & $0: 00$ & 6.317 & 6.09 & 4.36 & 2.997 & 2.15 & 0.408 \\
\hline $12 / 25 / 08$ & 4:00 & 6.315 & 6.086 & 4.355 & 2.987 & 2.14 & 0.404 \\
\hline
\end{tabular}


TABLE S1.3 (Cont.)

\begin{tabular}{|c|c|c|c|c|c|c|c|c|}
\hline \multirow[b]{2}{*}{ Date } & \multirow[b]{2}{*}{ Time } & \multicolumn{7}{|c|}{ Depth to Water (feet below top of casing) in Indicated Wells } \\
\hline & & PMW1s & PMW1d & PMW2sa & PMW2d & PMW9s & PMW9d & PMW3d \\
\hline $12 / 25 / 08$ & $8: 00$ & 6.294 & 6.06 & 4.348 & 2.966 & 2.131 & 0.385 & \\
\hline $12 / 25 / 08$ & $12: 00$ & 6.275 & 6.037 & 4.336 & 2.95 & 2.126 & 0.378 & \\
\hline $12 / 25 / 08$ & $16: 00$ & 6.212 & 5.96 & 4.294 & 2.877 & 2.115 & 0.32 & \\
\hline $12 / 25 / 08$ & $20: 00$ & 6.183 & 5.928 & 4.287 & 2.861 & 2.124 & 0.308 & \\
\hline $12 / 26 / 08$ & $0: 00$ & 6.158 & 5.9 & 4.268 & 2.84 & 2.119 & 0.301 & \\
\hline 12/26/08 & $4: 00$ & 6.143 & 5.886 & 4.264 & 2.833 & 2.126 & 0.301 & \\
\hline $12 / 26 / 08$ & 8:00 & 6.139 & 5.881 & 4.261 & 2.83 & 2.124 & 0.299 & \\
\hline $12 / 26 / 08$ & $12: 00$ & 6.127 & 5.872 & 4.24 & 2.814 & 2.108 & 0.289 & \\
\hline $12 / 26 / 08$ & $16: 00$ & 6.052 & 5.812 & 4.1 & 2.706 & 2.094 & 0.271 & \\
\hline $12 / 26 / 08$ & $20: 00$ & 5.869 & 5.758 & 4.095 & 2.692 & 2.138 & 0.243 & \\
\hline $12 / 27 / 08$ & 0:00 & 5.834 & 5.786 & 4.137 & 2.751 & 2.18 & 0.24 & \\
\hline 12/27/08 & $4: 00$ & 5.883 & 5.826 & 4.179 & 2.795 & 2.196 & 0.259 & \\
\hline $12 / 27 / 08$ & $8: 00$ & 5.988 & 5.87 & 4.222 & 2.828 & 2.199 & 0.294 & \\
\hline $12 / 27 / 08$ & $12: 00$ & 6.075 & 5.902 & 4.243 & 2.844 & 2.206 & 0.313 & \\
\hline $12 / 27 / 08$ & $16: 00$ & 6.108 & 5.902 & 4.252 & 2.847 & 2.22 & 0.32 & \\
\hline $12 / 27 / 08$ & 20:00 & 6.155 & 5.942 & 4.275 & 2.884 & 2.231 & 0.376 & \\
\hline $12 / 28 / 08$ & $0: 00$ & 6.19 & 5.972 & 4.289 & 2.903 & 2.203 & 0.392 & \\
\hline $12 / 28 / 08$ & 4:00 & 6.214 & 5.997 & 4.301 & 2.922 & 2.187 & 0.404 & \\
\hline 12/28/08 & $8: 00$ & 6.237 & 6.021 & 4.315 & 2.938 & 2.171 & 0.415 & \\
\hline $12 / 28 / 08$ & $12: 00$ & 6.263 & 6.051 & 4.334 & 2.966 & 2.168 & 0.425 & \\
\hline 12/28/08 & $16: 00$ & 6.268 & 6.046 & 4.329 & 2.954 & 2.183 & 0.385 & \\
\hline 12/28/08 & $20: 00$ & 6.275 & 6.055 & 4.332 & 2.964 & 2.201 & 0.404 & \\
\hline $12 / 29 / 08$ & $0: 00$ & 6.275 & 6.051 & 4.329 & 2.957 & 2.199 & 0.415 & \\
\hline 12/29/08 & $4: 00$ & 6.268 & 6.044 & 4.327 & 2.947 & 2.201 & 0.418 & \\
\hline $12 / 29 / 08$ & $8: 00$ & 6.296 & 6.086 & 4.357 & 2.99 & 2.215 & 0.432 & \\
\hline $12 / 29 / 08$ & $12: 00$ & 6.338 & 6.132 & 4.376 & 3.029 & 2.222 & 0.453 & \\
\hline $12 / 29 / 08$ & $16: 00$ & 6.345 & 6.139 & 4.371 & 3.02 & 2.232 & 0.465 & \\
\hline $12 / 29 / 08$ & $20: 00$ & 6.347 & 6.139 & 4.367 & 3.015 & 2.246 & 0.469 & \\
\hline $12 / 30 / 08$ & $0: 00$ & 6.308 & 6.086 & 4.336 & 2.966 & 2.236 & 0.462 & \\
\hline $12 / 30 / 08$ & $4: 00$ & 6.256 & 6.025 & 4.303 & 2.915 & 2.222 & 0.444 & \\
\hline 12/30/08 & $8: 00$ & 6.209 & 5.967 & 4.285 & 2.879 & 2.218 & 0.42 & \\
\hline $12 / 30 / 08$ & $12: 00$ & 6.23 & 6.004 & 4.315 & 2.926 & 2.243 & 0.415 & \\
\hline $12 / 30 / 08$ & $16: 00$ & 6.282 & 6.067 & 4.346 & 2.973 & 2.259 & 0.427 & \\
\hline $12 / 30 / 08$ & $20: 00$ & 6.357 & 6.16 & 4.388 & 3.055 & 2.295 & 0.458 & \\
\hline $12 / 31 / 08$ & $0: 00$ & 6.404 & 6.211 & 4.407 & 3.09 & 2.288 & 0.509 & \\
\hline 12/31/08 & $4: 00$ & 6.434 & 6.246 & 4.421 & 3.107 & 2.274 & 0.535 & \\
\hline 12/31/08 & $8: 00$ & 6.448 & 6.257 & 4.43 & 3.114 & 2.253 & 0.537 & \\
\hline $12 / 31 / 08$ & $12: 00$ & 6.455 & 6.257 & 4.432 & 3.109 & 2.243 & 0.558 & \\
\hline 12/31/08 & $16: 00$ & 6.399 & 6.185 & 4.395 & 3.041 & 2.194 & 0.493 & \\
\hline $12 / 31 / 08$ & $20: 00$ & 6.347 & 6.123 & 4.364 & 2.994 & 2.18 & 0.462 & \\
\hline
\end{tabular}


TABLE S1.4 Automatically measured water levels in downgradient wells in 2008.
Table S1.4 (Cont.)

Depth to Water (ft below top of casing) in Indicated Well

in Indicated Wel

Date Time PMW4 PMW5 PMW6 PMW7 PMW8

\begin{tabular}{|c|c|c|c|c|c|c|c|c|c|c|c|c|c|}
\hline & & & & & & & $1 / 9 / 08$ & $4: 00$ & 7.639 & 4.091 & 2.753 & 4.292 & 3.144 \\
\hline $1 / 1 / 08$ & $0: 00$ & 7.693 & 4.231 & 2.763 & 4.311 & 3.423 & $1 / 9 / 08$ & $8: 00$ & 7.632 & 4.098 & 2.749 & 4.285 & 3.195 \\
\hline $1 / 1 / 08$ & $4: 00$ & 7.7 & 4.243 & 2.774 & 4.33 & 3.437 & $1 / 9 / 08$ & $12: 00$ & 7.639 & 4.112 & 2.749 & 4.288 & 3.233 \\
\hline $1 / 1 / 08$ & 8:00 & 7.711 & 4.259 & 2.791 & 4.346 & 3.493 & $1 / 9 / 08$ & $16: 00$ & 7.618 & 4.089 & 2.735 & 4.26 & 3.207 \\
\hline $1 / 1 / 08$ & $12: 00$ & 7.721 & 4.276 & 2.805 & 4.365 & 3.531 & $1 / 9 / 08$ & 20:00 & 7.618 & 4.084 & 2.742 & 4.264 & 3.17 \\
\hline $1 / 1 / 08$ & $16: 00$ & 7.728 & 4.278 & 2.786 & 4.371 & 3.528 & 1/10/08 & 0:00 & 7.618 & 4.087 & 2.744 & 4.264 & 3.167 \\
\hline $1 / 1 / 08$ & $20: 00$ & 7.737 & 4.287 & 2.798 & 4.383 & 3.545 & $1 / 10 / 08$ & $4: 00$ & 7.614 & 4.087 & 2.742 & 4.255 & 3.17 \\
\hline $1 / 2 / 08$ & 0:00 & 7.744 & 4.299 & 2.805 & 4.397 & 3.573 & 1/10/08 & 8:00 & 7.614 & 4.089 & 2.742 & 4.255 & 3.172 \\
\hline $1 / 2 / 08$ & $4: 00$ & 7.751 & 4.311 & 2.807 & 4.404 & 3.596 & 1/10/08 & $12: 00$ & 7.616 & 4.098 & 2.744 & 4.26 & 3.177 \\
\hline $1 / 2 / 08$ & 8:00 & 7.758 & 4.325 & 2.812 & 4.413 & 3.622 & $1 / 10 / 08$ & $16: 00$ & 7.616 & 4.096 & 2.742 & 4.262 & 3.151 \\
\hline $1 / 2 / 08$ & $12: 00$ & 7.767 & 4.336 & 2.814 & 4.423 & 3.643 & $1 / 10 / 08$ & $20: 00$ & 7.63 & 4.098 & 2.746 & 4.271 & 3.135 \\
\hline $1 / 2 / 08$ & $16: 00$ & 7.765 & 4.32 & 2.784 & 4.399 & 3.612 & 1/11/08 & 0:00 & 7.637 & 4.112 & 2.749 & 4.278 & 3.172 \\
\hline $1 / 2 / 08$ & $20: 00$ & 7.758 & 4.306 & 2.779 & 4.388 & 3.577 & 1/11/08 & $4: 00$ & 7.642 & 4.129 & 2.753 & 4.281 & 3.244 \\
\hline $1 / 3 / 08$ & 0:00 & 7.749 & 4.308 & 2.781 & 4.381 & 3.601 & 1/11/08 & 8:00 & 7.644 & 4.15 & 2.758 & 4.283 & 3.321 \\
\hline $1 / 3 / 08$ & $4: 00$ & 7.735 & 4.299 & 2.777 & 4.362 & 3.612 & 1/11/08 & $12: 00$ & 7.656 & 4.168 & 2.756 & 4.288 & 3.377 \\
\hline $1 / 3 / 08$ & 8:00 & 7.716 & 4.283 & 2.767 & 4.339 & 3.615 & $1 / 11 / 08$ & $16: 00$ & 7.651 & 4.159 & 2.744 & 4.278 & 3.34 \\
\hline $1 / 3 / 08$ & $12: 00$ & 7.714 & 4.283 & 2.902 & 4.332 & 3.617 & $1 / 11 / 08$ & $20: 00$ & 7.66 & 4.159 & 2.751 & 4.29 & 3.307 \\
\hline $1 / 3 / 08$ & $16: 00$ & 7.704 & 4.262 & 2.725 & 4.306 & 3.557 & $1 / 12 / 08$ & $0: 00$ & 7.667 & 4.166 & 2.753 & 4.299 & 3.298 \\
\hline $1 / 3 / 08$ & $20: 00$ & 7.7 & 4.25 & 2.716 & 4.297 & 3.508 & $1 / 12 / 08$ & $4: 00$ & 7.681 & 4.18 & 2.763 & 4.313 & 3.326 \\
\hline $1 / 4 / 08$ & 0:00 & 7.693 & 4.234 & 2.714 & 4.285 & 3.473 & $1 / 12 / 08$ & 8:00 & 7.695 & 4.201 & 2.774 & 4.334 & 3.384 \\
\hline $1 / 4 / 08$ & $4: 00$ & 7.686 & 4.217 & 2.709 & 4.278 & 3.449 & $1 / 12 / 08$ & $12: 00$ & 7.714 & 4.22 & 2.781 & 4.351 & 3.403 \\
\hline $1 / 4 / 08$ & 8:00 & 7.679 & 4.215 & 2.714 & 4.274 & 3.445 & $1 / 12 / 08$ & $16: 00$ & 7.718 & 4.217 & 2.774 & 4.353 & 3.379 \\
\hline $1 / 4 / 08$ & $12: 00$ & 7.677 & 4.213 & 2.714 & 4.271 & 3.452 & $1 / 12 / 08$ & $20: 00$ & 7.73 & 4.222 & 2.781 & 4.367 & 3.368 \\
\hline $1 / 4 / 08$ & $16: 00$ & 7.667 & 4.194 & 2.7 & 4.257 & 3.421 & 1/13/08 & 0:00 & 7.737 & 4.229 & 2.784 & 4.369 & 3.382 \\
\hline $1 / 4 / 08$ & $20: 00$ & 7.663 & 4.187 & 2.702 & 4.255 & 3.396 & 1/13/08 & $4: 00$ & 7.739 & 4.245 & 2.793 & 4.376 & 3.445 \\
\hline $1 / 5 / 08$ & $0: 00$ & 7.653 & 4.171 & 2.697 & 4.243 & 3.368 & 1/13/08 & $8: 00$ & 7.746 & 4.257 & 2.795 & 4.381 & 3.464 \\
\hline $1 / 5 / 08$ & $4: 00$ & 7.646 & 4.161 & 2.7 & 4.236 & 3.347 & 1/13/08 & $12: 00$ & 7.753 & 4.262 & 2.793 & 4.383 & 3.48 \\
\hline $1 / 5 / 08$ & $8: 00$ & 7.642 & 4.159 & 2.702 & 4.234 & 3.351 & $1 / 13 / 08$ & $16: 00$ & 7.737 & 4.238 & 2.774 & 4.36 & 3.431 \\
\hline $1 / 5 / 08$ & $12: 00$ & 7.642 & 4.164 & 2.7 & 4.232 & 3.368 & $1 / 13 / 08$ & $20: 00$ & 7.746 & 4.243 & 2.781 & 4.376 & 3.398 \\
\hline $1 / 5 / 08$ & $16: 00$ & 7.632 & 4.147 & 2.69 & 4.22 & 3.34 & $1 / 14 / 08$ & 0:00 & 7.76 & 4.252 & 2.788 & 4.393 & 3.387 \\
\hline $1 / 5 / 08$ & $20: 00$ & 7.63 & 4.138 & 2.693 & 4.218 & 3.307 & $1 / 14 / 08$ & $4: 00$ & 7.765 & 4.266 & 2.798 & 4.399 & 3.431 \\
\hline $1 / 6 / 08$ & $0: 00$ & 7.623 & 4.129 & 2.686 & 4.208 & 3.279 & $1 / 14 / 08$ & 8:00 & 7.772 & 4.287 & 2.812 & 4.414 & 3.503 \\
\hline $1 / 6 / 08$ & $4: 00$ & 7.621 & 4.133 & 2.69 & 4.213 & 3.263 & $1 / 14 / 08$ & $12: 00$ & 7.784 & 4.306 & 2.821 & 4.427 & 3.536 \\
\hline $1 / 6 / 08$ & $8: 00$ & 7.63 & 4.147 & 2.7 & 4.227 & 3.263 & $1 / 14 / 08$ & $16: 00$ & 7.781 & 4.292 & 2.8 & 4.416 & 3.501 \\
\hline $1 / 6 / 08$ & $12: 00$ & 7.644 & 4.164 & 2.704 & 4.241 & 3.267 & $1 / 14 / 08$ & $20: 00$ & 7.784 & 4.292 & 2.805 & 4.416 & 3.492 \\
\hline $1 / 6 / 08$ & $16: 00$ & 7.649 & 4.152 & 2.702 & 4.243 & 3.235 & $1 / 15 / 08$ & 0:00 & 7.784 & 4.306 & 2.816 & 4.418 & 3.552 \\
\hline $1 / 6 / 08$ & $20: 00$ & 7.649 & 4.124 & 2.709 & 4.246 & 3.176 & $1 / 15 / 08$ & $4: 00$ & 7.777 & 4.313 & 2.814 & 4.409 & 3.583 \\
\hline $1 / 7 / 08$ & 0:00 & 7.639 & 4.115 & 2.716 & 4.241 & 3.174 & $1 / 15 / 08$ & 8:00 & 7.765 & 4.311 & 2.809 & 4.395 & 3.599 \\
\hline $1 / 7 / 08$ & $4: 00$ & 7.632 & 4.117 & 2.728 & 4.246 & 3.19 & 1/15/08 & $12: 00$ & 7.76 & 4.304 & 2.793 & 4.379 & 3.599 \\
\hline $1 / 7 / 08$ & $8: 00$ & 7.621 & 4.103 & 2.721 & 4.234 & 3.193 & $1 / 15 / 08$ & $16: 00$ & 7.737 & 4.271 & 2.756 & 4.341 & 3.536 \\
\hline $1 / 7 / 08$ & $12: 00$ & 7.625 & 4.105 & 2.723 & 4.241 & 3.202 & $1 / 15 / 08$ & $20: 00$ & 7.732 & 4.255 & 2.753 & 4.334 & 3.485 \\
\hline $1 / 7 / 08$ & $16: 00$ & 7.621 & 4.042 & 2.711 & 4.234 & 3.072 & 1/16/08 & 0:00 & 7.728 & 4.246 & 2.751 & 4.327 & 3.445 \\
\hline $1 / 7 / 08$ & $20: 00$ & 7.609 & 3.993 & 2.725 & 4.236 & 2.967 & 1/16/08 & $4: 00$ & 7.742 & 4.255 & 2.763 & 4.348 & 3.44 \\
\hline $1 / 8 / 08$ & $0: 00$ & 7.597 & 4.014 & 2.73 & 4.236 & 3.004 & $1 / 16 / 08$ & 8:00 & 7.758 & 4.271 & 2.772 & 4.372 & 3.454 \\
\hline $1 / 8 / 08$ & $4: 00$ & 7.595 & 4.033 & 2.728 & 4.236 & 3.051 & 1/16/08 & $12: 00$ & 7.777 & 4.281 & 2.774 & 4.39 & 3.454 \\
\hline $1 / 8 / 08$ & 8:00 & 7.611 & 4.066 & 2.742 & 4.264 & 3.097 & 1/16/08 & $16: 00$ & 7.779 & 4.273 & 2.767 & 4.386 & 3.431 \\
\hline $1 / 8 / 08$ & $12: 00$ & 7.623 & 4.082 & 2.749 & 4.278 & 3.13 & $1 / 16 / 08$ & $20: 00$ & 7.784 & 4.273 & 2.772 & 4.397 & 3.419 \\
\hline $1 / 8 / 08$ & $16: 00$ & 7.635 & 4.08 & 2.749 & 4.288 & 3.093 & $1 / 17 / 08$ & 0:00 & 7.784 & 4.267 & 2.77 & 4.397 & 3.412 \\
\hline $1 / 8 / 08$ & $20: 00$ & 7.639 & 4.061 & 2.756 & 4.295 & 3.039 & $1 / 17 / 08$ & $4: 00$ & 7.772 & 4.257 & 2.765 & 4.39 & 3.403 \\
\hline $1 / 9 / 08$ & $0: 00$ & 7.644 & 4.08 & 2.758 & 4.299 & 3.095 & 1/17/08 & $8: 00$ & 7.76 & 4.245 & 2.76 & 4.381 & 3.394 \\
\hline
\end{tabular}


Table S1.4 (Cont.)

Depth to Water (ft below top of casing) in Indicated Well

Date Time PMW4 PMW5 PMW6 PMW7 PMW8

\begin{tabular}{|c|c|c|c|c|c|c|}
\hline $1 / 17 / 08$ & $12: 00$ & 7.765 & 4.25 & 2.765 & 4.386 & 3.401 \\
\hline 1/17/08 & $16: 00$ & 7.753 & 4.232 & 2.756 & 4.367 & 3.389 \\
\hline 1/17/08 & $20: 00$ & 7.751 & 4.234 & 2.761 & 4.369 & 3.384 \\
\hline 1/18/08 & 0:00 & 7.753 & 4.234 & 2.763 & 4.367 & 3.396 \\
\hline 1/18/08 & 4:00 & 7.753 & 4.238 & 2.767 & 4.374 & 3.417 \\
\hline 1/18/08 & 8:00 & 7.772 & 4.257 & 2.779 & 4.397 & 3.436 \\
\hline $1 / 18 / 08$ & $12: 00$ & 7.8 & 4.29 & 2.796 & 4.437 & 3.452 \\
\hline 1/18/08 & $16: 00$ & 7.805 & 4.28 & 2.788 & 4.437 & 3.44 \\
\hline 1/18/08 & $20: 00$ & 7.814 & 4.288 & 2.796 & 4.451 & 3.433 \\
\hline 1/19/08 & 0:00 & 7.823 & 4.297 & 2.807 & 4.467 & 3.447 \\
\hline 1/19/08 & 4:00 & 7.826 & 4.292 & 2.805 & 4.465 & 3.468 \\
\hline $1 / 19 / 08$ & 8:00 & 7.826 & 4.304 & 2.814 & 4.472 & 3.503 \\
\hline 1/19/08 & $12: 00$ & 7.835 & 4.323 & 2.819 & 4.483 & 3.543 \\
\hline $1 / 19 / 08$ & $16: 00$ & 7.828 & 4.313 & 2.805 & 4.465 & 3.529 \\
\hline 1/19/08 & $20: 00$ & 7.83 & 4.313 & 2.805 & 4.467 & 3.508 \\
\hline 1/20/08 & 0:00 & 7.835 & 4.325 & 2.812 & 4.472 & 3.531 \\
\hline $1 / 20 / 08$ & 4:00 & 7.84 & 4.337 & 2.821 & 4.479 & 3.562 \\
\hline $1 / 20 / 08$ & 8:00 & 7.847 & 4.344 & 2.823 & 4.486 & 3.566 \\
\hline 1/20/08 & $12: 00$ & 7.854 & 4.348 & 2.821 & 4.495 & 3.562 \\
\hline $1 / 20 / 08$ & $16: 00$ & 7.84 & 4.327 & 2.805 & 4.469 & 3.52 \\
\hline 1/20/08 & $20: 00$ & 7.849 & 4.339 & 2.814 & 4.486 & 3.496 \\
\hline $1 / 21 / 08$ & 0:00 & 7.847 & 4.327 & 2.802 & 4.476 & 3.475 \\
\hline $1 / 21 / 08$ & $4: 00$ & 7.833 & 4.318 & 2.8 & 4.462 & 3.466 \\
\hline $1 / 21 / 08$ & 8:00 & 7.826 & 4.318 & 2.798 & 4.455 & 3.468 \\
\hline $1 / 21 / 08$ & $12: 00$ & 7.823 & 4.323 & 2.796 & 4.451 & 3.478 \\
\hline $1 / 21 / 08$ & $16: 00$ & 7.816 & 4.311 & 2.786 & 4.437 & 3.457 \\
\hline $1 / 21 / 08$ & $20: 00$ & 7.828 & 4.325 & 2.796 & 4.455 & 3.452 \\
\hline $1 / 22 / 08$ & 0:00 & 7.851 & 4.341 & 2.803 & 4.479 & 3.454 \\
\hline $1 / 22 / 08$ & $4: 00$ & 7.863 & 4.351 & 2.807 & 4.493 & 3.466 \\
\hline $1 / 22 / 08$ & 8:00 & 7.863 & 4.351 & 2.809 & 4.495 & 3.487 \\
\hline $1 / 22 / 08$ & $12: 00$ & 7.863 & 4.351 & 2.807 & 4.488 & 3.513 \\
\hline $1 / 22 / 08$ & $16: 00$ & 7.847 & 4.339 & 2.793 & 4.467 & 3.501 \\
\hline $1 / 22 / 08$ & $20: 00$ & 7.84 & 4.332 & 2.791 & 4.46 & 3.485 \\
\hline $1 / 23 / 08$ & 0:00 & 7.833 & 4.33 & 2.789 & 4.453 & 3.482 \\
\hline $1 / 23 / 08$ & 4:00 & 7.83 & 4.33 & 2.793 & 4.448 & 3.496 \\
\hline $1 / 23 / 08$ & 8:00 & 7.83 & 4.337 & 2.796 & 4.451 & 3.515 \\
\hline $1 / 23 / 08$ & $12: 00$ & 7.849 & 4.358 & 2.802 & 4.472 & 3.527 \\
\hline $1 / 23 / 08$ & $16: 00$ & 7.861 & 4.362 & 2.805 & 4.481 & 3.511 \\
\hline $1 / 23 / 08$ & $20: 00$ & 7.879 & 4.372 & 2.81 & 4.504 & 3.499 \\
\hline $1 / 24 / 08$ & 0:00 & 7.886 & 4.374 & 2.814 & 4.511 & 3.494 \\
\hline $1 / 24 / 08$ & $4: 00$ & 7.889 & 4.379 & 2.821 & 4.516 & 3.524 \\
\hline $1 / 24 / 08$ & 8:00 & 7.893 & 4.388 & 2.826 & 4.525 & 3.569 \\
\hline $1 / 24 / 08$ & $12: 00$ & 7.9 & 4.402 & 2.831 & 4.535 & 3.601 \\
\hline $1 / 24 / 08$ & $16: 00$ & 7.896 & 4.393 & 2.817 & 4.518 & 3.585 \\
\hline $1 / 24 / 08$ & 20:00 & 7.893 & 4.393 & 2.814 & 4.514 & 3.559 \\
\hline $1 / 25 / 08$ & 0:00 & 7.896 & 4.39 & 2.812 & 4.511 & 3.552 \\
\hline $1 / 25 / 08$ & 4:00 & 7.884 & 4.383 & 2.805 & 4.495 & 3.557 \\
\hline $1 / 25 / 08$ & 8:00 & 7.872 & 4.374 & 2.807 & 4.488 & 3.55 \\
\hline $1 / 25 / 08$ & $12: 00$ & 7.868 & 4.367 & 2.8 & 4.479 & 3.539 \\
\hline $1 / 25 / 08$ & $16: 00$ & 7.856 & 4.353 & 2.789 & 4.46 & 3.506 \\
\hline
\end{tabular}

Table S1.4 (Cont.)

Depth to Water (ft below top of casing) in Indicated Well

Date Time PMW4 PMW5 PMW6 PMW7 PMW8

\begin{tabular}{|c|c|c|c|c|c|c|}
\hline $1 / 25 / 08$ & $20: 00$ & 7.856 & 4.353 & 2.789 & 4.463 & 3.485 \\
\hline 1/26/08 & 0:00 & 7.856 & 4.348 & 2.786 & 4.456 & 3.459 \\
\hline 1/26/08 & 4:00 & 7.849 & 4.344 & 2.786 & 4.453 & 3.445 \\
\hline 1/26/08 & 8:00 & 7.854 & 4.348 & 2.791 & 4.458 & 3.455 \\
\hline $1 / 26 / 08$ & $12: 00$ & 7.87 & 4.365 & 2.8 & 4.476 & 3.473 \\
\hline $1 / 26 / 08$ & $16: 00$ & 7.868 & 4.358 & 2.793 & 4.472 & 3.45 \\
\hline $1 / 26 / 08$ & 20:00 & 7.877 & 4.355 & 2.793 & 4.472 & 3.41 \\
\hline $1 / 27 / 08$ & 0:00 & 7.875 & 4.348 & 2.793 & 4.467 & 3.399 \\
\hline $1 / 27 / 08$ & 4:00 & 7.87 & 4.344 & 2.793 & 4.463 & 3.392 \\
\hline $1 / 27 / 08$ & 8:00 & 7.87 & 4.344 & 2.793 & 4.465 & 3.387 \\
\hline $1 / 27 / 08$ & $12: 00$ & 7.865 & 4.339 & 2.791 & 4.46 & 3.389 \\
\hline $1 / 27 / 08$ & $16: 00$ & 7.84 & 4.255 & 2.756 & 4.407 & 3.256 \\
\hline $1 / 27 / 08$ & 20:00 & 7.805 & 4.183 & 2.749 & 4.348 & 3.116 \\
\hline $1 / 28 / 08$ & 0:00 & 7.781 & 4.197 & 2.747 & 4.33 & 3.13 \\
\hline 1/28/08 & 4:00 & 7.763 & 4.19 & 2.74 & 4.309 & 3.135 \\
\hline 1/28/08 & 8:00 & 7.742 & 4.157 & 2.73 & 4.279 & 3.093 \\
\hline $1 / 28 / 08$ & $12: 00$ & 7.714 & 4.087 & 2.709 & 4.234 & 3.035 \\
\hline 1/28/08 & $16: 00$ & 7.525 & 3.359 & 2.667 & 4.13 & 2.561 \\
\hline 1/28/08 & 20:00 & 7.227 & 3.319 & 2.677 & 4.097 & 2.482 \\
\hline $1 / 29 / 08$ & 0:00 & 7.159 & 3.517 & 2.698 & 4.116 & 2.543 \\
\hline 1/29/08 & 4:00 & 7.166 & 3.648 & 2.714 & 4.153 & 2.615 \\
\hline 1/29/08 & 8:00 & 7.22 & 3.758 & 2.744 & 4.216 & 2.704 \\
\hline 1/29/08 & $12: 00$ & 7.269 & 3.816 & 2.737 & 4.251 & 2.799 \\
\hline $1 / 29 / 08$ & $16: 00$ & 7.311 & 3.87 & 2.749 & 4.283 & 2.89 \\
\hline 1/29/08 & 20:00 & 7.357 & 3.919 & 2.765 & 4.323 & 2.979 \\
\hline 1/30/08 & 0:00 & 7.392 & 3.961 & 2.775 & 4.348 & 3.074 \\
\hline 1/30/08 & 4:00 & 7.418 & 3.998 & 2.782 & 4.369 & 3.191 \\
\hline 1/30/08 & 8:00 & 7.446 & 4.04 & 2.786 & 4.388 & 3.296 \\
\hline 1/30/08 & $12: 00$ & 7.476 & 4.075 & 2.784 & 4.407 & 3.364 \\
\hline 1/30/08 & $16: 00$ & 7.483 & 4.082 & 2.777 & 4.407 & 3.364 \\
\hline 1/30/08 & 20:00 & 7.513 & 4.108 & 2.791 & 4.432 & 3.387 \\
\hline 1/31/08 & 0:00 & 7.539 & 4.138 & 2.8 & 4.453 & 3.448 \\
\hline 1/31/08 & 4:00 & 7.56 & 4.162 & 2.807 & 4.467 & 3.494 \\
\hline 1/31/08 & 8:00 & 7.583 & 4.192 & 2.821 & 4.486 & 3.534 \\
\hline 1/31/08 & $12: 00$ & 7.607 & 4.218 & 2.824 & 4.493 & 3.567 \\
\hline 1/31/08 & $16: 00$ & 7.616 & 4.213 & 2.8 & 4.477 & 3.539 \\
\hline 1/31/08 & 20:00 & 7.628 & 4.218 & 2.786 & 4.481 & 3.504 \\
\hline $2 / 1 / 08$ & $0: 00$ & 7.637 & 4.227 & 2.796 & 4.474 & 3.522 \\
\hline 2/1/08 & 4:00 & 7.646 & 4.255 & 2.821 & 4.484 & 3.585 \\
\hline 2/1/08 & 8:00 & 7.656 & 4.271 & 2.835 & 4.481 & 3.618 \\
\hline 2/1/08 & $12: 00$ & 7.67 & 4.288 & 2.831 & 4.486 & 3.641 \\
\hline 2/1/08 & $16: 00$ & 7.667 & 4.269 & 2.8 & 4.456 & 3.595 \\
\hline 2/1/08 & 20:00 & 7.677 & 4.236 & 2.791 & 4.456 & 3.546 \\
\hline $2 / 2 / 08$ & 0:00 & 7.684 & 4.246 & 2.775 & 4.446 & 3.504 \\
\hline $2 / 2 / 08$ & 4:00 & 7.691 & 4.257 & 2.768 & 4.444 & 3.471 \\
\hline $2 / 2 / 08$ & 8:00 & 7.702 & 4.271 & 2.777 & 4.456 & 3.462 \\
\hline $2 / 2 / 08$ & $12: 00$ & 7.718 & 4.288 & 2.784 & 4.474 & 3.483 \\
\hline $2 / 2 / 08$ & $16: 00$ & 7.725 & 4.278 & 2.791 & 4.479 & 3.485 \\
\hline 2/2/08 & 20:00 & 7.735 & 4.257 & 2.796 & 4.481 & 3.462 \\
\hline $2 / 3 / 08$ & 0:00 & 7.742 & 4.248 & 2.791 & 4.477 & 3.445 \\
\hline
\end{tabular}


Table S1.4 (Cont.)

Depth to Water (ft below top of casing) in Indicated Well

Date Time PMW4 PMW5 PMW6 PMW7 PMW8

\begin{tabular}{|c|c|c|c|c|c|c|}
\hline $2 / 3 / 08$ & $4: 00$ & 7.744 & 4.239 & 2.786 & 4.47 & 3.438 \\
\hline $2 / 3 / 08$ & 8:00 & 7.746 & 4.241 & 2.782 & 4.463 & 3.462 \\
\hline $2 / 3 / 08$ & $12: 00$ & 7.749 & 4.234 & 2.777 & 4.458 & 3.478 \\
\hline $2 / 3 / 08$ & $16: 00$ & 7.732 & 4.213 & 2.763 & 4.435 & 3.448 \\
\hline 2/3/08 & 20:00 & 7.732 & 4.211 & 2.77 & 4.435 & 3.413 \\
\hline $2 / 4 / 08$ & 0:00 & 7.732 & 4.206 & 2.763 & 4.421 & 3.394 \\
\hline $2 / 4 / 08$ & $4: 00$ & 7.709 & 4.183 & 2.747 & 4.39 & 3.366 \\
\hline $2 / 4 / 08$ & 8:00 & 7.709 & 4.185 & 2.756 & 4.39 & 3.354 \\
\hline $2 / 4 / 08$ & $12: 00$ & 7.723 & 4.199 & 2.765 & 4.402 & 3.35 \\
\hline $2 / 4 / 08$ & $16: 00$ & 7.732 & 4.187 & 2.754 & 4.402 & 3.296 \\
\hline $2 / 4 / 08$ & 20:00 & 7.746 & 4.183 & 2.768 & 4.423 & 3.212 \\
\hline 2/5/08 & 0:00 & 7.758 & 4.204 & 2.772 & 4.432 & 3.238 \\
\hline 2/5/08 & $4: 00$ & 7.765 & 4.22 & 2.789 & 4.456 & 3.27 \\
\hline 2/5/08 & 8:00 & 7.772 & 4.22 & 2.777 & 4.451 & 3.282 \\
\hline $2 / 5 / 08$ & $12: 00$ & 7.772 & 4.215 & 2.77 & 4.446 & 3.285 \\
\hline $2 / 5 / 08$ & $16: 00$ & 7.763 & 4.211 & 2.77 & 4.435 & 3.282 \\
\hline $2 / 5 / 08$ & 20:00 & 7.774 & 4.222 & 2.777 & 4.449 & 3.292 \\
\hline 2/6/08 & 0:00 & 7.774 & 4.218 & 2.77 & 4.444 & 3.294 \\
\hline 2/6/08 & $4: 00$ & 7.772 & 4.213 & 2.772 & 4.437 & 3.294 \\
\hline 2/6/08 & $8: 00$ & 7.774 & 4.218 & 2.775 & 4.437 & 3.296 \\
\hline 2/6/08 & $12: 00$ & 7.784 & 4.225 & 2.782 & 4.444 & 3.306 \\
\hline $2 / 6 / 08$ & $16: 00$ & 7.781 & 4.218 & 2.77 & 4.437 & 3.301 \\
\hline 2/6/08 & 20:00 & 7.788 & 4.227 & 2.779 & 4.453 & 3.31 \\
\hline $2 / 7 / 08$ & 0:00 & 7.793 & 4.229 & 2.779 & 4.456 & 3.315 \\
\hline $2 / 7 / 08$ & $4: 00$ & 7.791 & 4.222 & 2.772 & 4.444 & 3.315 \\
\hline $2 / 7 / 08$ & 8:00 & 7.793 & 4.229 & 2.779 & 4.446 & 3.32 \\
\hline $2 / 7 / 08$ & $12: 00$ & 7.8 & 4.239 & 2.779 & 4.456 & 3.334 \\
\hline $2 / 7 / 08$ & $16: 00$ & 7.788 & 4.225 & 2.768 & 4.442 & 3.334 \\
\hline $2 / 7 / 08$ & 20:00 & 7.791 & 4.229 & 2.77 & 4.442 & 3.34 \\
\hline 2/8/08 & 0:00 & 7.784 & 4.22 & 2.763 & 4.428 & 3.336 \\
\hline 2/8/08 & $4: 00$ & 7.774 & 4.213 & 2.763 & 4.423 & 3.338 \\
\hline $2 / 8 / 08$ & 8:00 & 7.777 & 4.222 & 2.772 & 4.428 & 3.348 \\
\hline 2/8/08 & $12: 00$ & 7.8 & 4.241 & 2.777 & 4.446 & 3.368 \\
\hline 2/8/08 & $16: 00$ & 7.8 & 4.241 & 2.772 & 4.446 & 3.364 \\
\hline $2 / 8 / 08$ & $20: 00$ & 7.807 & 4.222 & 2.775 & 4.449 & 3.268 \\
\hline 2/9/08 & 0:00 & 7.807 & 4.225 & 2.772 & 4.451 & 3.294 \\
\hline 2/9/08 & $4: 00$ & 7.809 & 4.229 & 2.775 & 4.451 & 3.31 \\
\hline 2/9/08 & $8: 00$ & 7.814 & 4.246 & 2.784 & 4.465 & 3.331 \\
\hline 2/9/08 & $12: 00$ & 7.828 & 4.267 & 2.798 & 4.493 & 3.364 \\
\hline 2/9/08 & $16: 00$ & 7.847 & 4.276 & 2.805 & 4.514 & 3.38 \\
\hline 2/9/08 & $20: 00$ & 7.858 & 4.285 & 2.814 & 4.533 & 3.378 \\
\hline 2/10/08 & 0:00 & 7.872 & 4.292 & 2.824 & 4.544 & 3.39 \\
\hline 2/10/08 & $4: 00$ & 7.882 & 4.302 & 2.826 & 4.56 & 3.413 \\
\hline 2/10/08 & 8:00 & 7.893 & 4.316 & 2.838 & 4.579 & 3.476 \\
\hline 2/10/08 & $12: 00$ & 7.91 & 4.339 & 2.847 & 4.6 & 3.551 \\
\hline 2/10/08 & $16: 00$ & 7.91 & 4.339 & 2.838 & 4.593 & 3.562 \\
\hline 2/10/08 & 20:00 & 7.91 & 4.334 & 2.842 & 4.591 & 3.572 \\
\hline 2/11/08 & 0:00 & 7.912 & 4.344 & 2.842 & 4.586 & 3.616 \\
\hline 2/11/08 & $4: 00$ & 7.91 & 4.355 & 2.849 & 4.579 & 3.658 \\
\hline 2/11/08 & $8: 00$ & 7.907 & 4.365 & 2.863 & 4.572 & 3.688 \\
\hline
\end{tabular}

Table S1.4 (Cont.)

Depth to Water (ft below top of casing) in Indicated Well

Date Time PMW4 PMW5 PMW6 PMW7 PMW8

\begin{tabular}{|c|c|c|c|c|c|c|}
\hline 2/11/08 & $12: 00$ & 7.914 & 4.372 & 2.854 & 4.565 & 3.704 \\
\hline 2/11/08 & $16: 00$ & 7.912 & 4.36 & 2.842 & 4.549 & 3.679 \\
\hline 2/11/08 & 20:00 & 7.926 & 4.36 & 2.847 & 4.565 & 3.651 \\
\hline 2/12/08 & 0:00 & 7.933 & 4.402 & 2.859 & 4.572 & 3.658 \\
\hline 2/12/08 & 4:00 & 7.94 & 4.418 & 2.863 & 4.572 & 3.684 \\
\hline 2/12/08 & 8:00 & 7.945 & 4.43 & 2.87 & 4.581 & 3.705 \\
\hline 2/12/08 & $12: 00$ & 7.954 & 4.446 & 2.875 & 4.588 & 3.726 \\
\hline 2/12/08 & $16: 00$ & 7.952 & 4.442 & 2.852 & 4.572 & 3.707 \\
\hline 2/12/08 & 20:00 & 7.956 & 4.446 & 2.856 & 4.579 & 3.684 \\
\hline 2/13/08 & $0: 00$ & 7.959 & 4.446 & 2.859 & 4.568 & 3.7 \\
\hline 2/13/08 & 4:00 & 7.961 & 4.453 & 2.863 & 4.563 & 3.728 \\
\hline 2/13/08 & 8:00 & 7.956 & 4.453 & 2.861 & 4.558 & 3.733 \\
\hline 2/13/08 & $12: 00$ & 7.963 & 4.453 & 2.852 & 4.556 & 3.742 \\
\hline 2/13/08 & $16: 00$ & 7.94 & 4.414 & 2.812 & 4.519 & 3.677 \\
\hline 2/13/08 & 20:00 & 7.917 & 4.348 & 2.791 & 4.493 & 3.56 \\
\hline 2/14/08 & $0: 00$ & 7.91 & 4.355 & 2.789 & 4.479 & 3.544 \\
\hline 2/14/08 & 4:00 & 7.917 & 4.374 & 2.796 & 4.491 & 3.534 \\
\hline 2/14/08 & 8:00 & 7.921 & 4.376 & 2.793 & 4.488 & 3.52 \\
\hline 2/14/08 & $12: 00$ & 7.945 & 4.397 & 2.814 & 4.516 & 3.516 \\
\hline 2/14/08 & $16: 00$ & 7.966 & 4.418 & 2.826 & 4.547 & 3.518 \\
\hline 2/14/08 & 20:00 & 7.977 & 4.432 & 2.84 & 4.575 & 3.523 \\
\hline 2/15/08 & $0: 00$ & 7.989 & 4.437 & 2.856 & 4.595 & 3.537 \\
\hline 2/15/08 & 4:00 & 7.996 & 4.451 & 2.882 & 4.612 & 3.595 \\
\hline 2/15/08 & 8:00 & 8 & 4.463 & 2.912 & 4.635 & 3.679 \\
\hline 2/15/08 & $12: 00$ & 8.014 & 4.481 & 2.929 & 4.654 & 3.737 \\
\hline 2/15/08 & $16: 00$ & 8.012 & 4.467 & 2.905 & 4.642 & 3.737 \\
\hline 2/15/08 & 20:00 & 8.007 & 4.453 & 2.894 & 4.63 & 3.707 \\
\hline 2/16/08 & 0:00 & 8.007 & 4.442 & 2.882 & 4.614 & 3.677 \\
\hline 2/16/08 & 4:00 & 8 & 4.435 & 2.884 & 4.602 & 3.677 \\
\hline 2/16/08 & 8:00 & 7.993 & 4.439 & 2.896 & 4.598 & 3.709 \\
\hline 2/16/08 & $12: 00$ & 7.989 & 4.428 & 2.88 & 4.577 & 3.735 \\
\hline 2/16/08 & $16: 00$ & 7.968 & 4.383 & 2.854 & 4.544 & 3.693 \\
\hline 2/16/08 & 20:00 & 7.945 & 4.299 & 2.826 & 4.514 & 3.588 \\
\hline 2/17/08 & 0:00 & 7.928 & 4.302 & 2.805 & 4.488 & 3.544 \\
\hline $2 / 17 / 08$ & 4:00 & 7.912 & 4.297 & 2.791 & 4.463 & 3.513 \\
\hline 2/17/08 & 8:00 & 7.905 & 4.304 & 2.791 & 4.449 & 3.495 \\
\hline 2/17/08 & $12: 00$ & 7.919 & 4.325 & 2.803 & 4.472 & 3.492 \\
\hline 2/17/08 & $16: 00$ & 7.933 & 4.267 & 2.786 & 4.47 & 3.392 \\
\hline 2/17/08 & 20:00 & 7.933 & 4.276 & 2.8 & 4.474 & 3.117 \\
\hline 2/18/08 & 0:00 & 7.928 & 4.304 & 2.798 & 4.465 & 3.135 \\
\hline 2/18/08 & 4:00 & 7.94 & 4.332 & 2.807 & 4.477 & 3.212 \\
\hline 2/18/08 & 8:00 & 7.942 & 4.339 & 2.807 & 4.477 & 3.271 \\
\hline 2/18/08 & $12: 00$ & 7.959 & 4.346 & 2.812 & 4.493 & 3.317 \\
\hline 2/18/08 & $16: 00$ & 7.968 & 4.365 & 2.819 & 4.512 & 3.352 \\
\hline 2/18/08 & 20:00 & 7.979 & 4.379 & 2.835 & 4.533 & 3.385 \\
\hline 2/19/08 & $0: 00$ & 7.989 & 4.381 & 2.835 & 4.542 & 3.411 \\
\hline 2/19/08 & 4:00 & 7.993 & 4.395 & 2.854 & 4.554 & 3.457 \\
\hline 2/19/08 & 8:00 & 7.993 & 4.416 & 2.875 & 4.561 & 3.541 \\
\hline 2/19/08 & $12: 00$ & 7.998 & 4.437 & 2.891 & 4.565 & 3.616 \\
\hline 2/19/08 & $16: 00$ & 7.986 & 4.409 & 2.877 & 4.554 & 3.635 \\
\hline
\end{tabular}


Table S1.4 (Cont.)

Depth to Water (ft below top of casing) in Indicated Well

Date Time PMW4 PMW5 PMW6 PMW7 PMW8

\begin{tabular}{|c|c|c|c|c|c|c|}
\hline 2/19/08 & $20: 00$ & 7.998 & 4.381 & 2.875 & 4.565 & 3.553 \\
\hline 2/20/08 & 0:00 & 8.017 & 4.395 & 2.887 & 4.596 & 3.553 \\
\hline 2/20/08 & 4:00 & 8.026 & 4.397 & 2.877 & 4.605 & 3.562 \\
\hline 2/20/08 & 8:00 & 8.035 & 4.423 & 2.919 & 4.635 & 3.618 \\
\hline 2/20/08 & $12: 00$ & 8.047 & 4.479 & 2.957 & 4.67 & 3.709 \\
\hline 2/20/08 & $16: 00$ & 8.049 & 4.505 & 2.957 & 4.668 & 3.744 \\
\hline 2/20/08 & $20: 00$ & 8.054 & 4.509 & 2.961 & 4.672 & 3.747 \\
\hline 2/21/08 & 0:00 & 8.054 & 4.533 & 2.964 & 4.665 & 3.763 \\
\hline 2/21/08 & 4:00 & 8.054 & 4.579 & 2.964 & 4.658 & 3.789 \\
\hline 2/21/08 & $8: 00$ & 8.052 & 4.598 & 2.959 & 4.651 & 3.805 \\
\hline $2 / 21 / 08$ & $12: 00$ & 8.061 & 4.617 & 2.95 & 4.656 & 3.824 \\
\hline $2 / 21 / 08$ & $16: 00$ & 8.059 & 4.619 & 2.926 & 4.633 & 3.819 \\
\hline $2 / 21 / 08$ & 20:00 & 8.059 & 4.624 & 2.91 & 4.626 & 3.8 \\
\hline $2 / 22 / 08$ & 0:00 & 8.061 & 4.628 & 2.898 & 4.621 & 3.793 \\
\hline $2 / 22 / 08$ & 4:00 & 8.061 & 4.633 & 2.891 & 4.619 & 3.803 \\
\hline $2 / 22 / 08$ & 8:00 & 8.063 & 4.642 & 2.903 & 4.626 & 3.819 \\
\hline $2 / 22 / 08$ & $12: 00$ & 8.075 & 4.649 & 2.903 & 4.635 & 3.838 \\
\hline $2 / 22 / 08$ & $16: 00$ & 8.07 & 4.619 & 2.894 & 4.624 & 3.821 \\
\hline $2 / 22 / 08$ & 20:00 & 8.068 & 4.565 & 2.891 & 4.612 & 3.77 \\
\hline 2/23/08 & 0:00 & 8.068 & 4.551 & 2.887 & 4.605 & 3.73 \\
\hline 2/23/08 & $4: 00$ & 8.066 & 4.533 & 2.873 & 4.596 & 3.695 \\
\hline 2/23/08 & $8: 00$ & 8.059 & 4.528 & 2.861 & 4.584 & 3.679 \\
\hline 2/23/08 & $12: 00$ & 8.068 & 4.563 & 2.88 & 4.596 & 3.695 \\
\hline 2/23/08 & $16: 00$ & 8.066 & 4.535 & 2.866 & 4.584 & 3.63 \\
\hline 2/23/08 & $20: 00$ & 8.054 & 4.505 & 2.873 & 4.577 & 3.348 \\
\hline $2 / 24 / 08$ & 0:00 & 8.056 & 4.519 & 2.877 & 4.579 & 3.315 \\
\hline $2 / 24 / 08$ & $4: 00$ & 8.054 & 4.512 & 2.873 & 4.577 & 3.35 \\
\hline $2 / 24 / 08$ & $8: 00$ & 8.047 & 4.493 & 2.863 & 4.568 & 3.378 \\
\hline $2 / 24 / 08$ & $12: 00$ & 8.042 & 4.479 & 2.859 & 4.568 & 3.401 \\
\hline $2 / 24 / 08$ & $16: 00$ & 8.031 & 4.444 & 2.838 & 4.54 & 3.257 \\
\hline $2 / 24 / 08$ & $20: 00$ & 7.993 & 4.358 & 2.84 & 4.519 & 3.019 \\
\hline 2/25/08 & $0: 00$ & 7.975 & 4.39 & 2.842 & 4.509 & 3.084 \\
\hline $2 / 25 / 08$ & $4: 00$ & 7.959 & 4.393 & 2.833 & 4.493 & 3.142 \\
\hline $2 / 25 / 08$ & 8:00 & 7.949 & 4.397 & 2.838 & 4.486 & 3.196 \\
\hline $2 / 25 / 08$ & $12: 00$ & 7.951 & 4.404 & 2.842 & 4.488 & 3.233 \\
\hline $2 / 25 / 08$ & $16: 00$ & 7.961 & 4.351 & 2.838 & 4.491 & 3.073 \\
\hline $2 / 25 / 08$ & 20:00 & 7.965 & 4.362 & 2.87 & 4.523 & 3.033 \\
\hline 2/26/08 & 0:00 & 7.977 & 4.4 & 2.884 & 4.558 & 3.119 \\
\hline 2/26/08 & $4: 00$ & 7.993 & 4.421 & 2.889 & 4.575 & 3.187 \\
\hline 2/26/08 & 8:00 & 8.005 & 4.435 & 2.896 & 4.596 & 3.252 \\
\hline 2/26/08 & $12: 00$ & 8.024 & 4.437 & 2.898 & 4.612 & 3.296 \\
\hline 2/26/08 & $16: 00$ & 8.026 & 4.435 & 2.898 & 4.61 & 3.317 \\
\hline 2/26/08 & $20: 00$ & 8.019 & 4.435 & 2.901 & 4.603 & 3.331 \\
\hline $2 / 27 / 08$ & 0:00 & 8.017 & 4.428 & 2.903 & 4.598 & 3.348 \\
\hline $2 / 27 / 08$ & $4: 00$ & 8.014 & 4.421 & 2.905 & 4.596 & 3.362 \\
\hline $2 / 27 / 08$ & 8:00 & 8.021 & 4.428 & 2.905 & 4.6 & 3.378 \\
\hline $2 / 27 / 08$ & $12: 00$ & 8.028 & 4.442 & 2.908 & 4.605 & 3.394 \\
\hline $2 / 27 / 08$ & $16: 00$ & 8.021 & 4.428 & 2.894 & 4.584 & 3.233 \\
\hline $2 / 27 / 08$ & $20: 00$ & 8.012 & 4.418 & 2.896 & 4.57 & 3.168 \\
\hline 2/28/08 & 0:00 & 8.003 & 4.428 & 2.901 & 4.568 & 3.205 \\
\hline
\end{tabular}

Table S1.4 (Cont.)

Depth to Water (ft below top of casing) in Indicated Well

Date Time PMW4 PMW5 PMW6 PMW7 PMW8

\begin{tabular}{|c|c|c|c|c|c|c|}
\hline 2/28/08 & 4:00 & 7.998 & 4.425 & 2.901 & 4.561 & 3.261 \\
\hline 2/28/08 & 8:00 & 7.989 & 4.418 & 2.894 & 4.549 & 3.296 \\
\hline $2 / 28 / 08$ & $12: 00$ & 7.991 & 4.421 & 2.894 & 4.551 & 3.329 \\
\hline 2/28/08 & $16: 00$ & 7.982 & 4.409 & 2.88 & 4.535 & 3.31 \\
\hline 2/28/08 & 20:00 & 7.982 & 4.395 & 2.88 & 4.53 & 3.161 \\
\hline 2/29/08 & 0:00 & 7.982 & 4.402 & 2.889 & 4.537 & 3.163 \\
\hline 2/29/08 & 4:00 & 7.993 & 4.416 & 2.905 & 4.558 & 3.212 \\
\hline 2/29/08 & 8:00 & 8.019 & 4.442 & 2.924 & 4.589 & 3.278 \\
\hline 2/29/08 & $12: 00$ & 8.033 & 4.449 & 2.924 & 4.605 & 3.313 \\
\hline 2/29/08 & $16: 00$ & 8.012 & 4.285 & 2.901 & 4.572 & 2.991 \\
\hline 2/29/08 & $20: 00$ & 7.931 & 4.197 & 2.908 & 4.554 & 2.797 \\
\hline $3 / 1 / 08$ & $0: 00$ & 7.905 & 4.248 & 2.91 & 4.551 & 2.911 \\
\hline $3 / 1 / 08$ & 4:00 & 7.896 & 4.276 & 2.903 & 4.542 & 3.028 \\
\hline 3/1/08 & 8:00 & 7.889 & 4.285 & 2.894 & 4.523 & 3.1 \\
\hline $3 / 1 / 08$ & $12: 00$ & 7.884 & 4.285 & 2.884 & 4.51 & 3.093 \\
\hline $3 / 1 / 08$ & $16: 00$ & 7.816 & 4.038 & 2.868 & 4.47 & 2.702 \\
\hline 3/1/08 & 20:00 & 7.746 & 3.97 & 2.866 & 4.44 & 2.615 \\
\hline $3 / 2 / 08$ & $0: 00$ & 7.716 & 4.029 & 2.866 & 4.428 & 2.718 \\
\hline $3 / 2 / 08$ & 4:00 & 7.698 & 4.05 & 2.852 & 4.407 & 2.799 \\
\hline $3 / 2 / 08$ & 8:00 & 7.688 & 4.066 & 2.845 & 4.393 & 2.867 \\
\hline $3 / 2 / 08$ & $12: 00$ & 7.695 & 4.057 & 2.852 & 4.419 & 2.832 \\
\hline $3 / 2 / 08$ & $16: 00$ & 7.693 & 4.047 & 2.859 & 4.437 & 2.802 \\
\hline $3 / 2 / 08$ & 20:00 & 7.67 & 3.818 & 2.842 & 4.414 & 2.606 \\
\hline 3/3/08 & $0: 00$ & 7.651 & 3.912 & 2.859 & 4.449 & 2.688 \\
\hline 3/3/08 & $4: 00$ & 7.646 & 3.98 & 2.866 & 4.472 & 2.807 \\
\hline 3/3/08 & 8:00 & 7.67 & 4.045 & 2.88 & 4.517 & 2.944 \\
\hline 3/3/08 & $12: 00$ & 7.707 & 4.101 & 2.901 & 4.551 & 3.049 \\
\hline 3/3/08 & $16: 00$ & 7.718 & 4.08 & 2.882 & 4.542 & 2.949 \\
\hline 3/3/08 & 20:00 & 7.723 & 4.087 & 2.891 & 4.549 & 2.951 \\
\hline 3/4/08 & $0: 00$ & 7.737 & 4.113 & 2.889 & 4.549 & 3.056 \\
\hline 3/4/08 & $4: 00$ & 7.746 & 4.141 & 2.887 & 4.549 & 3.135 \\
\hline $3 / 4 / 08$ & 8:00 & 7.76 & 4.166 & 2.882 & 4.551 & 3.198 \\
\hline 3/4/08 & $12: 00$ & 7.774 & 4.19 & 2.877 & 4.547 & 3.238 \\
\hline 3/4/08 & $16: 00$ & 7.774 & 4.171 & 2.866 & 4.519 & 3.208 \\
\hline $3 / 4 / 08$ & 20:00 & 7.781 & 4.18 & 2.875 & 4.519 & 3.205 \\
\hline 3/5/08 & $0: 00$ & 7.791 & 4.192 & 2.88 & 4.526 & 3.233 \\
\hline 3/5/08 & 4:00 & 7.798 & 4.194 & 2.875 & 4.519 & 3.252 \\
\hline $3 / 5 / 08$ & 8:00 & 7.816 & 4.22 & 2.894 & 4.549 & 3.289 \\
\hline 3/5/08 & $12: 00$ & 7.842 & 4.241 & 2.905 & 4.577 & 3.313 \\
\hline 3/5/08 & $16: 00$ & 7.861 & 4.239 & 2.898 & 4.586 & 3.299 \\
\hline 3/5/08 & $20: 00$ & 7.875 & 4.25 & 2.91 & 4.598 & 3.303 \\
\hline 3/6/08 & $0: 00$ & 7.889 & 4.269 & 2.91 & 4.61 & 3.334 \\
\hline 3/6/08 & 4:00 & 7.898 & 4.297 & 2.901 & 4.61 & 3.357 \\
\hline $3 / 6 / 08$ & 8:00 & 7.907 & 4.33 & 2.903 & 4.624 & 3.39 \\
\hline 3/6/08 & $12: 00$ & 7.924 & 4.36 & 2.905 & 4.631 & 3.406 \\
\hline 3/6/08 & $16: 00$ & 7.921 & 4.306 & 2.898 & 4.603 & 3.369 \\
\hline 3/6/08 & 20:00 & 7.924 & 4.295 & 2.908 & 4.605 & 3.324 \\
\hline 3/7/08 & $0: 00$ & 7.933 & 4.311 & 2.919 & 4.624 & 3.341 \\
\hline 3/7/08 & $4: 00$ & 7.945 & 4.325 & 2.926 & 4.638 & 3.362 \\
\hline $3 / 7 / 08$ & 8:00 & 7.959 & 4.337 & 2.936 & 4.652 & 3.385 \\
\hline
\end{tabular}


Table S1.4 (Cont.)

Depth to Water (ft below top of casing) in Indicated Well

Date Time PMW4 PMW5 PMW6 PMW7 PMW8

\begin{tabular}{|c|c|c|c|c|c|c|}
\hline $3 / 7 / 08$ & $12: 00$ & 7.97 & 4.344 & 2.929 & 4.656 & 3.401 \\
\hline $3 / 7 / 08$ & $16: 00$ & 7.973 & 4.323 & 2.922 & 4.642 & 3.366 \\
\hline $3 / 7 / 08$ & 20:00 & 7.97 & 4.316 & 2.922 & 4.635 & 3.348 \\
\hline $3 / 8 / 08$ & 0:00 & 7.97 & 4.341 & 2.919 & 4.635 & 3.383 \\
\hline $3 / 8 / 08$ & $4: 00$ & 7.966 & 4.365 & 2.905 & 4.621 & 3.415 \\
\hline $3 / 8 / 08$ & 8:00 & 7.966 & 4.388 & 2.901 & 4.614 & 3.45 \\
\hline $3 / 8 / 08$ & $12: 00$ & 7.968 & 4.432 & 2.887 & 4.6 & 3.478 \\
\hline $3 / 8 / 08$ & $16: 00$ & 7.968 & 4.395 & 2.896 & 4.593 & 3.485 \\
\hline $3 / 8 / 08$ & 20:00 & 7.977 & 4.383 & 2.901 & 4.603 & 3.467 \\
\hline $3 / 9 / 08$ & 0:00 & 7.987 & 4.386 & 2.908 & 4.617 & 3.462 \\
\hline $3 / 9 / 08$ & $4: 00$ & 7.996 & 4.383 & 2.91 & 4.626 & 3.455 \\
\hline $3 / 9 / 08$ & 8:00 & 8.005 & 4.397 & 2.915 & 4.645 & 3.457 \\
\hline $3 / 9 / 08$ & $12: 00$ & 8.014 & 4.411 & 2.917 & 4.659 & 3.469 \\
\hline $3 / 9 / 08$ & $16: 00$ & 8.019 & 4.39 & 2.91 & 4.649 & 3.453 \\
\hline $3 / 9 / 08$ & 20:00 & 8.026 & 4.381 & 2.917 & 4.652 & 3.418 \\
\hline 3/10/08 & 0:00 & 8.033 & 4.395 & 2.929 & 4.668 & 3.422 \\
\hline 3/10/08 & $4: 00$ & 8.04 & 4.418 & 2.926 & 4.673 & 3.429 \\
\hline 3/10/08 & $8: 00$ & 8.047 & 4.444 & 2.926 & 4.689 & 3.448 \\
\hline 3/10/08 & $12: 00$ & 8.056 & 4.467 & 2.929 & 4.694 & 3.464 \\
\hline 3/10/08 & $16: 00$ & 8.045 & 4.397 & 2.912 & 4.661 & 3.439 \\
\hline 3/10/08 & 20:00 & 8.033 & 4.367 & 2.915 & 4.645 & 3.387 \\
\hline 3/11/08 & 0:00 & 8.031 & 4.374 & 2.922 & 4.649 & 3.385 \\
\hline 3/11/08 & $4: 00$ & 8.024 & 4.383 & 2.912 & 4.64 & 3.39 \\
\hline 3/11/08 & 8:00 & 8.014 & 4.4 & 2.912 & 4.633 & 3.399 \\
\hline $3 / 11 / 08$ & $12: 00$ & 8.01 & 4.376 & 2.905 & 4.614 & 3.394 \\
\hline 3/11/08 & $16: 00$ & 7.998 & 4.355 & 2.889 & 4.584 & 3.287 \\
\hline 3/11/08 & 20:00 & 7.982 & 4.32 & 2.887 & 4.57 & 3.133 \\
\hline $3 / 12 / 08$ & 0:00 & 7.973 & 4.318 & 2.891 & 4.57 & 3.15 \\
\hline $3 / 12 / 08$ & $4: 00$ & 7.966 & 4.323 & 2.891 & 4.568 & 3.201 \\
\hline $3 / 12 / 08$ & 8:00 & 7.961 & 4.334 & 2.894 & 4.563 & 3.245 \\
\hline $3 / 12 / 08$ & $12: 00$ & 7.956 & 4.323 & 2.882 & 4.547 & 3.229 \\
\hline $3 / 12 / 08$ & $16: 00$ & 7.954 & 4.288 & 2.875 & 4.538 & 3.028 \\
\hline 3/12/08 & $20: 00$ & 7.952 & 4.243 & 2.884 & 4.538 & 2.921 \\
\hline 3/13/08 & 0:00 & 7.945 & 4.252 & 2.896 & 4.547 & 3.01 \\
\hline 3/13/08 & $4: 00$ & 7.945 & 4.278 & 2.903 & 4.563 & 3.11 \\
\hline $3 / 13 / 08$ & 8:00 & 7.947 & 4.304 & 2.908 & 4.573 & 3.194 \\
\hline $3 / 13 / 08$ & $12: 00$ & 7.959 & 4.311 & 2.898 & 4.568 & 3.161 \\
\hline $3 / 13 / 08$ & $16: 00$ & 7.942 & 4.264 & 2.882 & 4.538 & 3.028 \\
\hline 3/13/08 & $20: 00$ & 7.931 & 4.234 & 2.884 & 4.526 & 2.975 \\
\hline $3 / 14 / 08$ & 0:00 & 7.919 & 4.243 & 2.889 & 4.521 & 3.042 \\
\hline 3/14/08 & $4: 00$ & 7.917 & 4.262 & 2.896 & 4.528 & 3.117 \\
\hline 3/14/08 & 8:00 & 7.924 & 4.292 & 2.898 & 4.542 & 3.194 \\
\hline $3 / 14 / 08$ & $12: 00$ & 7.935 & 4.297 & 2.898 & 4.547 & 3.164 \\
\hline $3 / 14 / 08$ & $16: 00$ & 7.931 & 4.269 & 2.882 & 4.538 & 3.091 \\
\hline 3/14/08 & $20: 00$ & 7.938 & 4.269 & 2.894 & 4.547 & 3.098 \\
\hline $3 / 15 / 08$ & 0:00 & 7.949 & 4.292 & 2.908 & 4.57 & 3.156 \\
\hline $3 / 15 / 08$ & $4: 00$ & 7.959 & 4.318 & 2.912 & 4.582 & 3.212 \\
\hline 3/15/08 & $8: 00$ & 7.973 & 4.344 & 2.926 & 4.605 & 3.261 \\
\hline 3/15/08 & $12: 00$ & 7.991 & 4.362 & 2.922 & 4.619 & 3.273 \\
\hline $3 / 15 / 08$ & $16: 00$ & 7.994 & 4.36 & 2.915 & 4.612 & 3.25 \\
\hline
\end{tabular}

Table S1.4 (Cont.)

Depth to Water (ft below top of casing) in Indicated Well

Date Time PMW4 PMW5 PMW6 PMW7 PMW8

\begin{tabular}{|c|c|c|c|c|c|c|}
\hline 3/15/08 & $20: 00$ & 8.005 & 4.372 & 2.922 & 4.629 & 3.266 \\
\hline 3/16/08 & $0: 00$ & 8.021 & 4.407 & 2.938 & 4.652 & 3.341 \\
\hline 3/16/08 & $4: 00$ & 8.033 & 4.458 & 2.95 & 4.673 & 3.42 \\
\hline 3/16/08 & 8:00 & 8.047 & 4.509 & 2.961 & 4.696 & 3.49 \\
\hline 3/16/08 & $12: 00$ & 8.059 & 4.514 & 2.931 & 4.689 & 3.432 \\
\hline 3/16/08 & $16: 00$ & 8.054 & 4.444 & 2.919 & 4.659 & 3.301 \\
\hline 3/16/08 & 20:00 & 8.042 & 4.432 & 2.924 & 4.652 & 3.308 \\
\hline 3/17/08 & 0:00 & 8.049 & 4.446 & 2.931 & 4.663 & 3.343 \\
\hline 3/17/08 & 4:00 & 8.042 & 4.442 & 2.922 & 4.649 & 3.355 \\
\hline 3/17/08 & $8: 00$ & 8.04 & 4.451 & 2.924 & 4.652 & 3.369 \\
\hline 3/17/08 & $12: 00$ & 8.031 & 4.379 & 2.884 & 4.603 & 3.103 \\
\hline 3/17/08 & $16: 00$ & 8.001 & 4.336 & 2.896 & 4.584 & 3.096 \\
\hline 3/17/08 & 20:00 & 7.991 & 4.346 & 2.901 & 4.587 & 3.142 \\
\hline 3/18/08 & 0:00 & 7.984 & 4.344 & 2.903 & 4.587 & 3.154 \\
\hline 3/18/08 & 4:00 & 7.98 & 4.351 & 2.903 & 4.582 & 3.194 \\
\hline 3/18/08 & 8:00 & 7.984 & 4.365 & 2.908 & 4.591 & 3.234 \\
\hline 3/18/08 & $12: 00$ & 7.994 & 4.376 & 2.903 & 4.596 & 3.252 \\
\hline 3/18/08 & $16: 00$ & 7.989 & 4.367 & 2.898 & 4.591 & 3.238 \\
\hline 3/18/08 & $20: 00$ & 8.001 & 4.386 & 2.903 & 4.605 & 3.257 \\
\hline 3/19/08 & 0:00 & 8.012 & 4.407 & 2.908 & 4.617 & 3.303 \\
\hline 3/19/08 & 4:00 & 8.017 & 4.425 & 2.915 & 4.629 & 3.35 \\
\hline 3/19/08 & $8: 00$ & 8.026 & 4.46 & 2.931 & 4.652 & 3.406 \\
\hline 3/19/08 & $12: 00$ & 8.042 & 4.458 & 2.905 & 4.642 & 3.35 \\
\hline 3/19/08 & $16: 00$ & 8.035 & 4.437 & 2.901 & 4.624 & 3.348 \\
\hline 3/19/08 & 20:00 & 8.035 & 4.453 & 2.908 & 4.629 & 3.369 \\
\hline 3/20/08 & 0:00 & 8.042 & 4.444 & 2.898 & 4.617 & 3.348 \\
\hline $3 / 20 / 08$ & 4:00 & 8.045 & 4.456 & 2.903 & 4.624 & 3.385 \\
\hline 3/20/08 & 8:00 & 8.052 & 4.474 & 2.915 & 4.643 & 3.418 \\
\hline 3/20/08 & $12: 00$ & 8.056 & 4.472 & 2.896 & 4.633 & 3.397 \\
\hline 3/20/08 & $16: 00$ & 8.038 & 4.458 & 2.905 & 4.619 & 3.383 \\
\hline 3/20/08 & 20:00 & 8.026 & 4.458 & 2.894 & 4.612 & 3.392 \\
\hline 3/21/08 & 0:00 & 8.021 & 4.453 & 2.887 & 4.598 & 3.394 \\
\hline 3/21/08 & 4:00 & 8.024 & 4.46 & 2.884 & 4.596 & 3.413 \\
\hline 3/21/08 & 8:00 & 8.045 & 4.484 & 2.896 & 4.624 & 3.434 \\
\hline 3/21/08 & $12: 00$ & 8.063 & 4.5 & 2.905 & 4.649 & 3.45 \\
\hline 3/21/08 & $16: 00$ & 8.066 & 4.507 & 2.91 & 4.661 & 3.448 \\
\hline 3/21/08 & 20:00 & 8.075 & 4.53 & 2.91 & 4.682 & 3.462 \\
\hline $3 / 22 / 08$ & 0:00 & 8.084 & 4.535 & 2.908 & 4.684 & 3.476 \\
\hline $3 / 22 / 08$ & 4:00 & 8.089 & 4.537 & 2.908 & 4.691 & 3.485 \\
\hline $3 / 22 / 08$ & 8:00 & 8.094 & 4.547 & 2.917 & 4.708 & 3.502 \\
\hline $3 / 22 / 08$ & $12: 00$ & 8.103 & 4.549 & 2.91 & 4.71 & 3.497 \\
\hline 3/22/08 & $16: 00$ & 8.108 & 4.546 & 2.915 & 4.715 & 3.492 \\
\hline $3 / 22 / 08$ & $20: 00$ & 8.112 & 4.561 & 2.924 & 4.729 & 3.506 \\
\hline 3/23/08 & 0:00 & 8.122 & 4.568 & 2.924 & 4.743 & 3.518 \\
\hline $3 / 23 / 08$ & 4:00 & 8.124 & 4.572 & 2.926 & 4.747 & 3.523 \\
\hline $3 / 23 / 08$ & 8:00 & 8.129 & 4.593 & 2.943 & 4.764 & 3.546 \\
\hline 3/23/08 & $12: 00$ & 8.133 & 4.591 & 2.917 & 4.75 & 3.511 \\
\hline $3 / 23 / 08$ & $16: 00$ & 8.133 & 4.589 & 2.929 & 4.747 & 3.513 \\
\hline $3 / 23 / 08$ & $20: 00$ & 8.133 & 4.596 & 2.931 & 4.754 & 3.53 \\
\hline $3 / 24 / 08$ & $0: 00$ & 8.136 & 4.605 & 2.936 & 4.759 & 3.551 \\
\hline
\end{tabular}


Table S1.4 (Cont.)

Depth to Water (ft below top of casing) in Indicated Well

Date Time PMW4 PMW5 PMW6 PMW7 PMW8

\begin{tabular}{|c|c|c|c|c|c|c|}
\hline $3 / 24 / 08$ & $4: 00$ & 8.138 & 4.621 & 2.947 & 4.766 & 3.579 \\
\hline 3/24/08 & 8:00 & 8.138 & 4.635 & 2.952 & 4.766 & 3.602 \\
\hline 3/24/08 & $12: 00$ & 8.124 & 4.605 & 2.901 & 4.717 & 3.5 \\
\hline $3 / 24 / 08$ & $16: 00$ & 8.098 & 4.554 & 2.901 & 4.673 & 3.476 \\
\hline $3 / 24 / 08$ & 20:00 & 8.084 & 4.554 & 2.898 & 4.666 & 3.486 \\
\hline $3 / 25 / 08$ & 0:00 & 8.077 & 4.547 & 2.889 & 4.649 & 3.481 \\
\hline $3 / 25 / 08$ & $4: 00$ & 8.073 & 4.551 & 2.889 & 4.649 & 3.483 \\
\hline 3/25/08 & $8: 00$ & 8.103 & 4.575 & 2.903 & 4.684 & 3.506 \\
\hline 3/25/08 & $12: 00$ & 8.117 & 4.584 & 2.908 & 4.698 & 3.511 \\
\hline $3 / 25 / 08$ & $16: 00$ & 8.124 & 4.605 & 2.926 & 4.712 & 3.527 \\
\hline $3 / 25 / 08$ & 20:00 & 8.136 & 4.628 & 2.922 & 4.724 & 3.539 \\
\hline $3 / 26 / 08$ & 0:00 & 8.14 & 4.628 & 2.912 & 4.724 & 3.541 \\
\hline $3 / 26 / 08$ & $4: 00$ & 8.143 & 4.621 & 2.91 & 4.724 & 3.539 \\
\hline $3 / 26 / 08$ & 8:00 & 8.145 & 4.617 & 2.905 & 4.722 & 3.539 \\
\hline 3/26/08 & $12: 00$ & 8.147 & 4.614 & 2.912 & 4.724 & 3.532 \\
\hline 3/26/08 & $16: 00$ & 8.131 & 4.607 & 2.917 & 4.712 & 3.528 \\
\hline 3/26/08 & $20: 00$ & 8.122 & 4.605 & 2.905 & 4.701 & 3.53 \\
\hline $3 / 27 / 08$ & 0:00 & 8.117 & 4.596 & 2.894 & 4.687 & 3.521 \\
\hline $3 / 27 / 08$ & 4:00 & 8.11 & 4.584 & 2.884 & 4.673 & 3.516 \\
\hline $3 / 27 / 08$ & $8: 00$ & 8.117 & 4.593 & 2.891 & 4.687 & 3.527 \\
\hline $3 / 27 / 08$ & $12: 00$ & 8.122 & 4.598 & 2.891 & 4.689 & 3.535 \\
\hline $3 / 27 / 08$ & $16: 00$ & 8.133 & 4.6 & 2.889 & 4.696 & 3.535 \\
\hline $3 / 27 / 08$ & $20: 00$ & 8.143 & 4.61 & 2.896 & 4.712 & 3.546 \\
\hline 3/28/08 & 0:00 & 8.15 & 4.619 & 2.898 & 4.729 & 3.556 \\
\hline $3 / 28 / 08$ & 4:00 & 8.154 & 4.621 & 2.903 & 4.74 & 3.565 \\
\hline $3 / 28 / 08$ & 8:00 & 8.164 & 4.631 & 2.912 & 4.761 & 3.576 \\
\hline 3/28/08 & $12: 00$ & 8.171 & 4.633 & 2.905 & 4.757 & 3.567 \\
\hline $3 / 28 / 08$ & $16: 00$ & 8.166 & 4.631 & 2.915 & 4.754 & 3.558 \\
\hline $3 / 28 / 08$ & $20: 00$ & 8.168 & 4.642 & 2.912 & 4.759 & 3.572 \\
\hline 3/29/08 & $0: 00$ & 8.173 & 4.647 & 2.905 & 4.764 & 3.576 \\
\hline 3/29/08 & $4: 00$ & 8.171 & 4.642 & 2.898 & 4.752 & 3.572 \\
\hline 3/29/08 & 8:00 & 8.173 & 4.642 & 2.901 & 4.754 & 3.572 \\
\hline 3/29/08 & $12: 00$ & 8.166 & 4.631 & 2.898 & 4.74 & 3.558 \\
\hline 3/29/08 & $16: 00$ & 8.15 & 4.628 & 2.912 & 4.736 & 3.56 \\
\hline $3 / 29 / 08$ & 20:00 & 8.147 & 4.642 & 2.905 & 4.733 & 3.569 \\
\hline 3/30/08 & 0:00 & 8.159 & 4.645 & 2.894 & 4.729 & 3.567 \\
\hline $3 / 30 / 08$ & 4:00 & 8.161 & 4.64 & 2.889 & 4.726 & 3.562 \\
\hline $3 / 30 / 08$ & 8:00 & 8.173 & 4.642 & 2.891 & 4.74 & 3.562 \\
\hline 3/30/08 & $12: 00$ & 8.173 & 4.64 & 2.894 & 4.731 & 3.555 \\
\hline 3/30/08 & $16: 00$ & 8.171 & 4.642 & 2.898 & 4.729 & 3.567 \\
\hline 3/30/08 & $20: 00$ & 8.168 & 4.645 & 2.891 & 4.729 & 3.569 \\
\hline $3 / 31 / 08$ & 0:00 & 8.166 & 4.638 & 2.884 & 4.722 & 3.565 \\
\hline 3/31/08 & $4: 00$ & 8.14 & 4.605 & 2.861 & 4.678 & 3.537 \\
\hline $3 / 31 / 08$ & 8:00 & 8.047 & 3.655 & 2.758 & 4.459 & 2.522 \\
\hline $3 / 31 / 08$ & $12: 00$ & 7.912 & 3.653 & 2.791 & 4.421 & 2.538 \\
\hline 3/31/08 & $16: 00$ & 7.849 & 3.886 & 2.817 & 4.459 & 2.625 \\
\hline 3/31/08 & $20: 00$ & 7.835 & 4.026 & 2.828 & 4.501 & 2.713 \\
\hline 4/1/08 & $0: 00$ & 7.84 & 4.11 & 2.84 & 4.545 & 2.802 \\
\hline $4 / 1 / 08$ & $4: 00$ & 7.854 & 4.173 & 2.849 & 4.575 & 2.888 \\
\hline $4 / 1 / 08$ & $8: 00$ & 7.877 & 4.229 & 2.866 & 4.617 & 2.972 \\
\hline
\end{tabular}

Table S1.4 (Cont.)

Depth to Water (ft below top of casing) in Indicated Well

Date Time PMW4 PMW5 PMW6 PMW7 PMW8

\begin{tabular}{|c|c|c|c|c|c|c|}
\hline & $2: 00$ & & & & & \\
\hline & & & & & & \\
\hline & 0:00 & & & & & \\
\hline 2/08 & 0:00 & 94 & 351 & .88 & & .22 \\
\hline & :00 & & & & & .262 \\
\hline & :00 & & & & & .294 \\
\hline $2 / 08$ & $: 00$ & & 4.39 & .88 & & .315 \\
\hline | & 00 & & 44 & .88 & & .332 \\
\hline $2 / 08$ & 20:00 & 966 & & 88 & .68 & 357 \\
\hline $3 / 08$ & 0:00 & 975 & 23 & .873 & & .383 \\
\hline & $4: 00$ & & & & & .392 \\
\hline $3 / 08$ & :00 & 977 & 3 & 863 & & .397 \\
\hline & :00 & & & 828 & & 182 \\
\hline & 00 & & & 793 & & 564 \\
\hline 3/08 & D:00 & 814 & & .826 & & .655 \\
\hline & 0:00 & & & & & .746 \\
\hline & $4: 00$ & & & & & 2.842 \\
\hline /4/08 & :00 & 786 & 36 & .842 & & 2.935 \\
\hline 14/ & 00 & & & & & 998 \\
\hline & & & & & & .063 \\
\hline & 20:00 & & & & & 3.136 \\
\hline & $0: 00$ & & & & & .189 \\
\hline & $4: 00$ & & & & & .231 \\
\hline & 8:00 & & & & & .266 \\
\hline & & & & & & .287 \\
\hline & & & & 863 & & 325 \\
\hline & :00 & & & & & .364 \\
\hline & $0: 00$ & & & & & .383 \\
\hline & & & & & & 392 \\
\hline & & & & & & .406 \\
\hline & & & & & & 42 \\
\hline & & & & & & .441 \\
\hline & & & & & & .481 \\
\hline & & & & & & \\
\hline & & & & & & .504 \\
\hline & & & & .863 & & 511 \\
\hline & & & & & & 507 \\
\hline & & & & & & .511 \\
\hline & & & & & & .521 \\
\hline & & & & & & 2.814 \\
\hline & & & & & & .933 \\
\hline & & 898 & & & & .028 \\
\hline & & & & & & .091 \\
\hline & & & & & & .112 \\
\hline & 20:00 & & & & & .182 \\
\hline & & & & & 4.664 & .25 \\
\hline & & & & 2.847 & & 3.297 \\
\hline & & & & & & 3.334 \\
\hline & & & & & & .346 \\
\hline & & 1.00 & $4.7<4$ & 2.863 & 4.689 & 3.362 \\
\hline
\end{tabular}


Table S1.4 (Cont.)

Depth to Water (ft below top of casing) in Indicated Well

Date Time PMW4 PMW5 PMW6 PMW7 PMW8

\begin{tabular}{|c|c|c|c|c|c|c|}
\hline $4 / 9 / 08$ & $20: 00$ & 7.949 & 4.425 & 2.856 & 4.675 & 3.388 \\
\hline 4/10/08 & $0: 00$ & 7.952 & 4.425 & 2.833 & 4.664 & 3.392 \\
\hline 4/10/08 & $4: 00$ & 7.9 & 4.138 & 2.756 & 4.54 & 2.744 \\
\hline 4/10/08 & $8: 00$ & 7.826 & 3.991 & 2.747 & 4.475 & 2.618 \\
\hline 4/10/08 & $12: 00$ & 7.723 & 3.233 & 2.688 & 4.321 & 2.452 \\
\hline 4/10/08 & $16: 00$ & 7.397 & 2.652 & 2.628 & 3.884 & 2.366 \\
\hline 4/10/08 & $20: 00$ & 6.896 & 2.941 & 2.681 & 3.935 & 2.473 \\
\hline 4/11/08 & $0: 00$ & 6.709 & 3.074 & 2.688 & 4 & 2.501 \\
\hline 4/11/08 & 4:00 & 6.653 & 3.167 & 2.7 & 4.061 & 2.527 \\
\hline 4/11/08 & $8: 00$ & 6.616 & 3.037 & 2.691 & 4.07 & 2.478 \\
\hline 4/11/08 & $12: 00$ & 6.602 & 3.205 & 2.719 & 4.126 & 2.548 \\
\hline 4/11/08 & $16: 00$ & 6.607 & 3.307 & 2.735 & 4.184 & 2.606 \\
\hline 4/11/08 & $20: 00$ & 6.626 & 3.37 & 2.747 & 4.228 & 2.648 \\
\hline $4 / 12 / 08$ & 0:00 & 6.637 & 3.415 & 2.754 & 4.263 & 2.697 \\
\hline 4/12/08 & $4: 00$ & 6.66 & 3.457 & 2.763 & 4.296 & 2.758 \\
\hline 4/12/08 & $8: 00$ & 6.693 & 3.503 & 2.777 & 4.326 & 2.814 \\
\hline $4 / 12 / 08$ & $12: 00$ & 6.733 & 3.545 & 2.791 & 4.359 & 2.87 \\
\hline $4 / 12 / 08$ & $16: 00$ & 6.77 & 3.578 & 2.793 & 4.382 & 2.926 \\
\hline $4 / 12 / 08$ & 20:00 & 6.807 & 3.613 & 2.8 & 4.405 & 2.972 \\
\hline 4/13/08 & 0:00 & 6.849 & 3.643 & 2.807 & 4.429 & 3.01 \\
\hline 4/13/08 & $4: 00$ & 6.882 & 3.667 & 2.812 & 4.443 & 3.045 \\
\hline 4/13/08 & $8: 00$ & 6.926 & 3.704 & 2.826 & 4.471 & 3.087 \\
\hline 4/13/08 & $12: 00$ & 6.963 & 3.734 & 2.835 & 4.484 & 3.112 \\
\hline 4/13/08 & $16: 00$ & 6.989 & 3.755 & 2.842 & 4.491 & 3.145 \\
\hline 4/13/08 & $20: 00$ & 7.019 & 3.779 & 2.835 & 4.505 & 3.182 \\
\hline $4 / 14 / 08$ & 0:00 & 7.05 & 3.8 & 2.833 & 4.515 & 3.21 \\
\hline 4/14/08 & $4: 00$ & 7.078 & 3.818 & 2.833 & 4.522 & 3.234 \\
\hline 4/14/08 & 8:00 & 7.11 & 3.849 & 2.845 & 4.545 & 3.259 \\
\hline 4/14/08 & $12: 00$ & 7.134 & 3.853 & 2.835 & 4.536 & 3.238 \\
\hline 4/14/08 & $16: 00$ & 7.15 & 3.872 & 2.84 & 4.526 & 3.269 \\
\hline 4/14/08 & $20: 00$ & 7.166 & 3.886 & 2.833 & 4.522 & 3.299 \\
\hline 4/15/08 & 0:00 & 7.19 & 3.902 & 2.826 & 4.524 & 3.315 \\
\hline 4/15/08 & $4: 00$ & 7.204 & 3.907 & 2.819 & 4.512 & 3.32 \\
\hline 4/15/08 & 8:00 & 7.22 & 3.917 & 2.814 & 4.503 & 3.327 \\
\hline 4/15/08 & $12: 00$ & 7.229 & 3.931 & 2.814 & 4.499 & 3.336 \\
\hline 4/15/08 & $16: 00$ & 7.234 & 3.954 & 2.828 & 4.489 & 3.376 \\
\hline 4/15/08 & 20:00 & 7.257 & 3.984 & 2.821 & 4.489 & 3.413 \\
\hline 4/16/08 & 0:00 & 7.278 & 3.984 & 2.798 & 4.473 & 3.418 \\
\hline 4/16/08 & $4: 00$ & 7.285 & 3.982 & 2.791 & 4.466 & 3.409 \\
\hline 4/16/08 & 8:00 & 7.315 & 4.008 & 2.798 & 4.482 & 3.418 \\
\hline 4/16/08 & $12: 00$ & 7.343 & 4.038 & 2.807 & 4.499 & 3.439 \\
\hline 4/16/08 & $16: 00$ & 7.371 & 4.071 & 2.819 & 4.515 & 3.476 \\
\hline 4/16/08 & $20: 00$ & 7.399 & 4.094 & 2.821 & 4.536 & 3.504 \\
\hline $4 / 17 / 08$ & 0:00 & 7.427 & 4.094 & 2.812 & 4.54 & 3.497 \\
\hline 4/17/08 & $4: 00$ & 7.434 & 4.082 & 2.8 & 4.531 & 3.476 \\
\hline 4/17/08 & $8: 00$ & 7.213 & 2.747 & 2.663 & 4.184 & 2.396 \\
\hline 4/17/08 & $12: 00$ & 6.998 & 3.104 & 2.716 & 4.205 & 2.503 \\
\hline 4/17/08 & $16: 00$ & 6.91 & 3.128 & 2.679 & 4.177 & 2.487 \\
\hline 4/17/08 & $20: 00$ & 6.842 & 3.209 & 2.691 & 4.156 & 2.522 \\
\hline 4/18/08 & $0: 00$ & 6.73 & 2.932 & 2.672 & 4.068 & 2.471 \\
\hline
\end{tabular}

Table S1.4 (Cont.)

Depth to Water (ft below top of casing) in Indicated Well

Date Time PMW4 PMW5 PMW6 PMW7 PMW8

\begin{tabular}{|c|c|c|c|c|c|c|}
\hline 4/18/08 & 4:00 & 6.614 & 3.055 & 2.679 & 4.051 & 2.501 \\
\hline 4/18/08 & 8:00 & 5.565 & 2.512 & 2.558 & 3.453 & 2.361 \\
\hline 4/18/08 & $12: 00$ & 5.092 & 2.584 & 2.6 & 3.492 & 2.445 \\
\hline 4/18/08 & $16: 00$ & 5.071 & 2.6 & 2.611 & 3.588 & 2.452 \\
\hline 4/18/08 & 20:00 & 5.141 & 2.656 & 2.637 & 3.702 & 2.492 \\
\hline 4/19/08 & $0: 00$ & 5.239 & 2.703 & 2.653 & 3.795 & 2.524 \\
\hline 4/19/08 & $4: 00$ & 5.339 & 2.743 & 2.665 & 3.865 & 2.555 \\
\hline 4/19/08 & 8:00 & 5.446 & 2.787 & 2.681 & 3.94 & 2.587 \\
\hline 4/19/08 & $12: 00$ & 5.537 & 2.834 & 2.695 & 3.989 & 2.622 \\
\hline 4/19/08 & $16: 00$ & 5.614 & 2.899 & 2.698 & 4.026 & 2.699 \\
\hline 4/19/08 & 20:00 & 5.693 & 2.948 & 2.702 & 4.061 & 2.781 \\
\hline $4 / 20 / 08$ & $0: 00$ & 5.77 & 2.983 & 2.702 & 4.086 & 2.823 \\
\hline 4/20/08 & 4:00 & 5.84 & 3.018 & 2.702 & 4.11 & 2.858 \\
\hline $4 / 20 / 08$ & 8:00 & 5.905 & 3.053 & 2.702 & 4.124 & 2.884 \\
\hline $4 / 20 / 08$ & $12: 00$ & 5.954 & 3.083 & 2.707 & 4.126 & 2.905 \\
\hline $4 / 20 / 08$ & $16: 00$ & 6.006 & 3.156 & 2.721 & 4.145 & 2.984 \\
\hline 4/20/08 & 20:00 & 6.066 & 3.205 & 2.723 & 4.161 & 3.061 \\
\hline $4 / 21 / 08$ & 0:00 & 6.138 & 3.233 & 2.716 & 4.177 & 3.082 \\
\hline 4/21/08 & 4:00 & 6.187 & 3.254 & 2.716 & 4.184 & 3.094 \\
\hline $4 / 21 / 08$ & 8:00 & 6.253 & 3.293 & 2.719 & 4.205 & 3.11 \\
\hline $4 / 21 / 08$ & $12: 00$ & 6.323 & 3.342 & 2.726 & 4.231 & 3.14 \\
\hline $4 / 21 / 08$ & $16: 00$ & 6.369 & 3.38 & 2.73 & 4.247 & 3.18 \\
\hline $4 / 21 / 08$ & 20:00 & 6.416 & 3.408 & 2.73 & 4.254 & 3.215 \\
\hline $4 / 22 / 08$ & 0:00 & 6.469 & 3.436 & 2.733 & 4.266 & 3.224 \\
\hline $4 / 22 / 08$ & $4: 00$ & 6.507 & 3.452 & 2.73 & 4.273 & 3.227 \\
\hline $4 / 22 / 08$ & 8:00 & 6.558 & 3.482 & 2.735 & 4.291 & 3.238 \\
\hline $4 / 22 / 08$ & $12: 00$ & 6.602 & 3.52 & 2.744 & 4.308 & 3.264 \\
\hline $4 / 22 / 08$ & $16: 00$ & 6.635 & 3.564 & 2.763 & 4.317 & 3.343 \\
\hline $4 / 22 / 08$ & 20:00 & 6.67 & 3.585 & 2.758 & 4.321 & 3.402 \\
\hline 4/23/08 & $0: 00$ & 6.709 & 3.594 & 2.744 & 4.324 & 3.371 \\
\hline 4/23/08 & $4: 00$ & 6.735 & 3.604 & 2.742 & 4.324 & 3.35 \\
\hline $4 / 23 / 08$ & 8:00 & 6.768 & 3.62 & 2.74 & 4.331 & 3.343 \\
\hline $4 / 23 / 08$ & $12: 00$ & 6.791 & 3.643 & 2.751 & 4.331 & 3.399 \\
\hline $4 / 23 / 08$ & $16: 00$ & 6.805 & 3.678 & 2.768 & 4.331 & 3.488 \\
\hline $4 / 23 / 08$ & $20: 00$ & 6.833 & 3.683 & 2.749 & 4.321 & 3.497 \\
\hline $4 / 24 / 08$ & $0: 00$ & 6.859 & 3.683 & 2.742 & 4.322 & 3.437 \\
\hline 4/24/08 & 4:00 & 6.87 & 3.676 & 2.73 & 4.308 & 3.395 \\
\hline $4 / 24 / 08$ & 8:00 & 6.889 & 3.688 & 2.728 & 4.303 & 3.369 \\
\hline $4 / 24 / 08$ & $12: 00$ & 6.905 & 3.702 & 2.726 & 4.298 & 3.378 \\
\hline $4 / 24 / 08$ & $16: 00$ & 6.924 & 3.725 & 2.73 & 4.298 & 3.458 \\
\hline $4 / 24 / 08$ & $20: 00$ & 6.94 & 3.713 & 2.716 & 4.289 & 3.385 \\
\hline $4 / 25 / 08$ & $0: 00$ & 6.952 & 3.718 & 2.716 & 4.284 & 3.341 \\
\hline $4 / 25 / 08$ & 4:00 & 6.903 & 3.109 & 2.639 & 4.156 & 2.569 \\
\hline $4 / 25 / 08$ & 8:00 & 6.898 & 3.403 & 2.695 & 4.207 & 2.629 \\
\hline $4 / 25 / 08$ & $12: 00$ & 6.919 & 3.524 & 2.705 & 4.24 & 2.72 \\
\hline $4 / 25 / 08$ & $16: 00$ & 6.942 & 3.604 & 2.712 & 4.27 & 2.844 \\
\hline $4 / 25 / 08$ & 20:00 & 6.977 & 3.664 & 2.721 & 4.298 & 2.961 \\
\hline $4 / 26 / 08$ & $0: 00$ & 7.003 & 3.699 & 2.721 & 4.315 & 3.04 \\
\hline $4 / 26 / 08$ & 4:00 & 7.017 & 3.72 & 2.719 & 4.317 & 3.096 \\
\hline $4 / 26 / 08$ & 8:00 & 7.045 & 3.753 & 2.733 & 4.34 & 3.143 \\
\hline
\end{tabular}


Table S1.4 (Cont.)

Depth to Water (ft below top of casing) in Indicated Well

Date Time PMW4 PMW5 PMW6 PMW7 PMW8

\begin{tabular}{|c|c|c|c|c|c|c|}
\hline $4 / 26 / 08$ & $12: 00$ & 7.073 & 3.783 & 2.742 & 4.352 & 3.18 \\
\hline $4 / 26 / 08$ & $16: 00$ & 7.094 & 3.807 & 2.744 & 4.359 & 3.255 \\
\hline $4 / 26 / 08$ & 20:00 & 7.113 & 3.762 & 2.714 & 4.347 & 3.133 \\
\hline $4 / 27 / 08$ & $0: 00$ & 7.11 & 3.657 & 2.707 & 4.315 & 2.879 \\
\hline $4 / 27 / 08$ & 4:00 & 7.12 & 3.725 & 2.723 & 4.333 & 2.984 \\
\hline $4 / 27 / 08$ & 8:00 & 7.134 & 3.769 & 2.733 & 4.35 & 3.063 \\
\hline $4 / 27 / 08$ & $12: 00$ & 7.143 & 3.786 & 2.73 & 4.35 & 3.105 \\
\hline $4 / 27 / 08$ & $16: 00$ & 7.143 & 3.818 & 2.737 & 4.343 & 3.21 \\
\hline $4 / 27 / 08$ & 20:00 & 7.152 & 3.837 & 2.733 & 4.34 & 3.297 \\
\hline $4 / 28 / 08$ & $0: 00$ & 7.164 & 3.842 & 2.728 & 4.34 & 3.308 \\
\hline $4 / 28 / 08$ & 4:00 & 7.18 & 3.853 & 2.73 & 4.352 & 3.318 \\
\hline $4 / 28 / 08$ & 8:00 & 7.201 & 3.872 & 2.733 & 4.366 & 3.329 \\
\hline $4 / 28 / 08$ & $12: 00$ & 7.215 & 3.891 & 2.744 & 4.375 & 3.343 \\
\hline $4 / 28 / 08$ & $16: 00$ & 7.229 & 3.917 & 2.754 & 4.384 & 3.413 \\
\hline $4 / 28 / 08$ & 20:00 & 7.243 & 3.931 & 2.744 & 4.377 & 3.458 \\
\hline $4 / 29 / 08$ & $0: 00$ & 7.252 & 3.919 & 2.73 & 4.368 & 3.434 \\
\hline $4 / 29 / 08$ & 4:00 & 7.262 & 3.921 & 2.733 & 4.373 & 3.42 \\
\hline $4 / 29 / 08$ & 8:00 & 7.273 & 3.926 & 2.733 & 4.375 & 3.411 \\
\hline $4 / 29 / 08$ & $12: 00$ & 7.28 & 3.942 & 2.74 & 4.375 & 3.441 \\
\hline $4 / 29 / 08$ & $16: 00$ & 7.278 & 3.963 & 2.747 & 4.364 & 3.521 \\
\hline 4/29/08 & 20:00 & 7.287 & 3.975 & 2.742 & 4.357 & 3.567 \\
\hline 4/30/08 & 0:00 & 7.294 & 3.959 & 2.726 & 4.345 & 3.514 \\
\hline $4 / 30 / 08$ & 4:00 & 7.283 & 3.94 & 2.716 & 4.326 & 3.467 \\
\hline $4 / 30 / 08$ & 8:00 & 7.285 & 3.942 & 2.714 & 4.322 & 3.444 \\
\hline $4 / 30 / 08$ & $12: 00$ & 7.287 & 3.961 & 2.719 & 4.315 & 3.502 \\
\hline $4 / 30 / 08$ & $16: 00$ & 7.292 & 3.989 & 2.73 & 4.319 & 3.607 \\
\hline $4 / 30 / 08$ & 20:00 & 7.304 & 4.003 & 2.723 & 4.315 & 3.651 \\
\hline $5 / 1 / 08$ & $0: 00$ & 7.313 & 3.984 & 2.709 & 4.305 & 3.574 \\
\hline 5/1/08 & 4:00 & 7.306 & 3.961 & 2.702 & 4.289 & 3.509 \\
\hline $5 / 1 / 08$ & 8:00 & 7.311 & 3.961 & 2.698 & 4.287 & 3.469 \\
\hline $5 / 1 / 08$ & $12: 00$ & 7.315 & 3.987 & 2.714 & 4.287 & 3.57 \\
\hline 5/1/08 & $16: 00$ & 7.336 & 4.054 & 2.742 & 4.319 & 3.735 \\
\hline $5 / 1 / 08$ & $20: 00$ & 7.364 & 4.073 & 2.723 & 4.329 & 3.756 \\
\hline $5 / 2 / 08$ & $0: 00$ & 7.381 & 4.054 & 2.709 & 4.333 & 3.656 \\
\hline $5 / 2 / 08$ & 4:00 & 7.369 & 3.956 & 2.663 & 4.287 & 3.423 \\
\hline $5 / 2 / 08$ & 8:00 & 7.348 & 3.938 & 2.684 & 4.28 & 3.255 \\
\hline $5 / 2 / 08$ & $12: 00$ & 7.297 & 3.676 & 2.656 & 4.214 & 2.671 \\
\hline $5 / 2 / 08$ & $16: 00$ & 7.285 & 3.807 & 2.679 & 4.242 & 2.825 \\
\hline $5 / 2 / 08$ & 20:00 & 7.301 & 3.872 & 2.688 & 4.273 & 2.954 \\
\hline $5 / 3 / 08$ & $0: 00$ & 7.327 & 3.811 & 2.663 & 4.268 & 2.804 \\
\hline 5/3/08 & 4:00 & 7.32 & 3.804 & 2.684 & 4.277 & 2.795 \\
\hline $5 / 3 / 08$ & $8: 00$ & 7.341 & 3.884 & 2.707 & 4.322 & 2.928 \\
\hline $5 / 3 / 08$ & $12: 00$ & 7.357 & 3.933 & 2.718 & 4.345 & 3.042 \\
\hline $5 / 3 / 08$ & $16: 00$ & 7.369 & 3.975 & 2.725 & 4.357 & 3.175 \\
\hline $5 / 3 / 08$ & 20:00 & 7.381 & 4.005 & 2.725 & 4.364 & 3.294 \\
\hline $5 / 4 / 08$ & $0: 00$ & 7.39 & 4.008 & 2.714 & 4.366 & 3.308 \\
\hline $5 / 4 / 08$ & 4:00 & 7.392 & 4.008 & 2.711 & 4.366 & 3.315 \\
\hline $5 / 4 / 08$ & $8: 00$ & 7.399 & 4.017 & 2.716 & 4.373 & 3.327 \\
\hline $5 / 4 / 08$ & $12: 00$ & 7.409 & 4.05 & 2.732 & 4.377 & 3.42 \\
\hline $5 / 4 / 08$ & $16: 00$ & 7.416 & 4.092 & 2.742 & 4.377 & 3.565 \\
\hline
\end{tabular}

Table S1.4 (Cont.)

Depth to Water (ft below top of casing) in Indicated Well

Date Time PMW4 PMW5 PMW6 PMW7 PMW8

\begin{tabular}{|c|c|c|c|c|c|c|}
\hline $5 / 4 / 08$ & 20:00 & 7.43 & 4.113 & 2.73 & 4.373 & 3.63 \\
\hline $5 / 5 / 08$ & $0: 00$ & 7.444 & 4.096 & 2.718 & 4.382 & 3.556 \\
\hline $5 / 5 / 08$ & $4: 00$ & 7.444 & 4.078 & 2.711 & 4.378 & 3.504 \\
\hline $5 / 5 / 08$ & 8:00 & 7.451 & 4.075 & 2.711 & 4.382 & 3.476 \\
\hline $5 / 5 / 08$ & $12: 00$ & 7.46 & 4.122 & 2.742 & 4.394 & 3.656 \\
\hline $5 / 5 / 08$ & $16: 00$ & 7.472 & 4.169 & 2.758 & 4.396 & 3.805 \\
\hline $5 / 5 / 08$ & 20:00 & 7.479 & 4.176 & 2.73 & 4.38 & 3.819 \\
\hline $5 / 6 / 08$ & 0:00 & 7.485 & 4.141 & 2.709 & 4.371 & 3.693 \\
\hline $5 / 6 / 08$ & 4:00 & 7.479 & 4.117 & 2.704 & 4.364 & 3.614 \\
\hline $5 / 6 / 08$ & 8:00 & 7.474 & 4.103 & 2.702 & 4.359 & 3.563 \\
\hline $5 / 6 / 08$ & $12: 00$ & 7.481 & 4.141 & 2.725 & 4.366 & 3.71 \\
\hline $5 / 6 / 08$ & $16: 00$ & 7.488 & 4.199 & 2.751 & 4.371 & 3.889 \\
\hline $5 / 6 / 08$ & 20:00 & 7.495 & 4.201 & 2.725 & 4.357 & 3.878 \\
\hline $5 / 7 / 08$ & $0: 00$ & 7.483 & 3.947 & 2.662 & 4.296 & 3.187 \\
\hline $5 / 7 / 08$ & 4:00 & 7.423 & 3.856 & 2.655 & 4.247 & 2.825 \\
\hline $5 / 7 / 08$ & 8:00 & 7.411 & 3.935 & 2.676 & 4.277 & 2.935 \\
\hline $5 / 7 / 08$ & $12: 00$ & 7.404 & 3.968 & 2.683 & 4.289 & 3.014 \\
\hline $5 / 7 / 08$ & $16: 00$ & 7.413 & 4.003 & 2.69 & 4.303 & 3.133 \\
\hline $5 / 7 / 08$ & 20:00 & 7.42 & 4.033 & 2.695 & 4.298 & 3.252 \\
\hline $5 / 8 / 08$ & $0: 00$ & 7.43 & 4.043 & 2.686 & 4.301 & 3.271 \\
\hline $5 / 8 / 08$ & 4:00 & 7.434 & 4.05 & 2.683 & 4.308 & 3.287 \\
\hline $5 / 8 / 08$ & 8:00 & 7.434 & 4.054 & 2.681 & 4.31 & 3.299 \\
\hline $5 / 8 / 08$ & $12: 00$ & 7.444 & 4.087 & 2.714 & 4.326 & 3.418 \\
\hline $5 / 8 / 08$ & $16: 00$ & 7.455 & 4.134 & 2.735 & 4.331 & 3.595 \\
\hline $5 / 8 / 08$ & 20:00 & 7.469 & 4.143 & 2.711 & 4.326 & 3.619 \\
\hline $5 / 9 / 08$ & $0: 00$ & 7.476 & 4.115 & 2.688 & 4.319 & 3.521 \\
\hline 5/9/08 & 4:00 & 7.385 & 3.193 & 2.595 & 4.149 & 2.536 \\
\hline $5 / 9 / 08$ & 8:00 & 7.227 & 3.382 & 2.63 & 4.128 & 2.524 \\
\hline $5 / 9 / 08$ & $12: 00$ & 7.176 & 3.573 & 2.648 & 4.168 & 2.606 \\
\hline $5 / 9 / 08$ & $16: 00$ & 7.15 & 3.688 & 2.669 & 4.194 & 2.744 \\
\hline $5 / 9 / 08$ & 20:00 & 7.143 & 3.76 & 2.672 & 4.212 & 2.888 \\
\hline $5 / 10 / 08$ & 0:00 & 7.131 & 3.781 & 2.66 & 4.219 & 2.944 \\
\hline $5 / 10 / 08$ & 4:00 & 7.117 & 3.639 & 2.623 & 4.189 & 2.718 \\
\hline $5 / 10 / 08$ & 8:00 & 7.085 & 3.618 & 2.641 & 4.177 & 2.641 \\
\hline $5 / 10 / 08$ & $12: 00$ & 7.054 & 3.664 & 2.658 & 4.173 & 2.711 \\
\hline 5/10/08 & $16: 00$ & 6.982 & 2.946 & 2.571 & 4.047 & 2.426 \\
\hline 5/10/08 & 20:00 & 6.5 & 2.754 & 2.55 & 3.749 & 2.396 \\
\hline 5/11/08 & $0: 00$ & 6.253 & 2.89 & 2.571 & 3.765 & 2.454 \\
\hline $5 / 11 / 08$ & $4: 00$ & 6.171 & 3.025 & 2.59 & 3.837 & 2.517 \\
\hline $5 / 11 / 08$ & 8:00 & 6.157 & 3.125 & 2.611 & 3.919 & 2.566 \\
\hline $5 / 11 / 08$ & $12: 00$ & 6.159 & 3.212 & 2.637 & 3.97 & 2.648 \\
\hline 5/11/08 & $16: 00$ & 6.183 & 3.298 & 2.653 & 4.007 & 2.818 \\
\hline $5 / 11 / 08$ & 20:00 & 6.213 & 3.333 & 2.641 & 4.024 & 2.961 \\
\hline $5 / 12 / 08$ & $0: 00$ & 6.25 & 3.34 & 2.63 & 4.038 & 2.965 \\
\hline $5 / 12 / 08$ & 4:00 & 6.274 & 3.347 & 2.627 & 4.047 & 2.975 \\
\hline $5 / 12 / 08$ & 8:00 & 6.304 & 3.366 & 2.63 & 4.059 & 2.986 \\
\hline $5 / 12 / 08$ & $12: 00$ & 6.337 & 3.431 & 2.665 & & 3.082 \\
\hline $5 / 12 / 08$ & $16: 00$ & 6.374 & 3.482 & 2.672 & 4.056 & 3.308 \\
\hline $5 / 12 / 08$ & 20:00 & 6.416 & 3.522 & 2.653 & 4.07 & 3.39 \\
\hline $5 / 13 / 08$ & 0:00 & 6.46 & 3.506 & 2.618 & 4.07 & 3.283 \\
\hline
\end{tabular}


Table S1.4 (Cont.)

Depth to Water (ft below top of casing) in Indicated Well

Date Time PMW4 PMW5 PMW6 PMW7 PMW8

\begin{tabular}{|c|c|c|c|c|c|c|}
\hline 5/13/08 & 4:00 & 6.483 & 3.499 & 2.606 & 4.065 & 3.224 \\
\hline 5/13/08 & 8:00 & 6.523 & 3.524 & 2.616 & 4.091 & 3.201 \\
\hline 5/13/08 & $12: 00$ & 6.56 & 3.562 & 2.632 & 4.105 & 3.25 \\
\hline $5 / 13 / 08$ & $16: 00$ & 6.605 & 3.622 & 2.66 & 4.128 & 3.402 \\
\hline $5 / 13 / 08$ & $20: 00$ & 6.649 & 3.662 & 2.646 & 4.14 & 3.392 \\
\hline $5 / 14 / 08$ & 0:00 & 6.691 & 3.685 & 2.63 & 4.152 & 3.334 \\
\hline $5 / 14 / 08$ & $4: 00$ & 6.709 & 3.692 & 2.62 & 4.149 & 3.292 \\
\hline $5 / 14 / 08$ & $8: 00$ & 6.74 & 3.716 & 2.627 & 4.163 & 3.273 \\
\hline $5 / 14 / 08$ & $12: 00$ & 6.763 & 3.76 & 2.672 & 4.177 & 3.392 \\
\hline $5 / 14 / 08$ & $16: 00$ & 6.796 & 3.83 & 2.704 & 4.186 & 3.577 \\
\hline $5 / 14 / 08$ & 20:00 & 6.831 & 3.865 & 2.686 & 4.189 & 3.633 \\
\hline $5 / 15 / 08$ & 0:00 & 6.849 & 3.835 & 2.648 & 4.182 & 3.5 \\
\hline $5 / 15 / 08$ & 4:00 & 6.863 & 3.823 & 2.634 & 4.173 & 3.427 \\
\hline $5 / 15 / 08$ & 8:00 & 6.887 & 3.832 & 2.639 & 4.184 & 3.388 \\
\hline $5 / 15 / 08$ & $12: 00$ & 6.917 & 3.912 & 2.721 & 4.217 & 3.6 \\
\hline $5 / 15 / 08$ & $16: 00$ & 6.947 & 3.949 & 2.746 & 4.226 & 3.784 \\
\hline $5 / 15 / 08$ & 20:00 & 6.973 & 3.968 & 2.693 & 4.217 & 3.763 \\
\hline $5 / 16 / 08$ & 0:00 & 6.989 & 3.933 & 2.648 & 4.201 & 3.611 \\
\hline $5 / 16 / 08$ & 4:00 & 6.991 & 3.912 & 2.639 & 4.191 & 3.527 \\
\hline $5 / 16 / 08$ & $8: 00$ & 7.005 & 3.914 & 2.637 & 4.198 & 3.471 \\
\hline $5 / 16 / 08$ & $12: 00$ & 7.026 & 3.987 & 2.732 & 4.231 & 3.684 \\
\hline 5/16/08 & $16: 00$ & 7.057 & 4.068 & 2.777 & 4.245 & 3.908 \\
\hline $5 / 16 / 08$ & 20:00 & 7.082 & 4.099 & 2.728 & 4.224 & 3.919 \\
\hline $5 / 17 / 08$ & $0: 00$ & 7.087 & 4.04 & 2.658 & 4.191 & 3.744 \\
\hline $5 / 17 / 08$ & $4: 00$ & 7.085 & 4.012 & 2.646 & 4.182 & 3.651 \\
\hline $5 / 17 / 08$ & 8:00 & 7.099 & 4.003 & 2.644 & 4.191 & 3.583 \\
\hline $5 / 17 / 08$ & $12: 00$ & 7.124 & 4.108 & 2.763 & 4.235 & 3.854 \\
\hline 5/17/08 & $16: 00$ & 7.157 & 4.201 & 2.828 & 4.259 & 4.048 \\
\hline $5 / 17 / 08$ & 20:00 & 7.192 & 4.229 & 2.784 & 4.252 & 4.052 \\
\hline 5/18/08 & 0:00 & 7.192 & 4.15 & 2.676 & 4.214 & 3.861 \\
\hline $5 / 18 / 08$ & $4: 00$ & 7.187 & 4.101 & 2.648 & 4.201 & 3.747 \\
\hline $5 / 18 / 08$ & 8:00 & 7.192 & 4.082 & 2.651 & 4.21 & 3.674 \\
\hline $5 / 18 / 08$ & $12: 00$ & 7.215 & 4.159 & 2.751 & 4.245 & 3.875 \\
\hline $5 / 18 / 08$ & $16: 00$ & 7.252 & 4.25 & 2.804 & 4.261 & 4.101 \\
\hline $5 / 18 / 08$ & 20:00 & 7.266 & 4.25 & 2.749 & 4.231 & 4.08 \\
\hline $5 / 19 / 08$ & 0:00 & 7.255 & 4.171 & 2.66 & 4.194 & 3.882 \\
\hline $5 / 19 / 08$ & 4:00 & 7.227 & 4.115 & 2.639 & 4.168 & 3.77 \\
\hline $5 / 19 / 08$ & 8:00 & 7.236 & 4.106 & 2.651 & 4.182 & 3.712 \\
\hline $5 / 19 / 08$ & $12: 00$ & 7.273 & 4.206 & 2.777 & 4.242 & 3.98 \\
\hline $5 / 19 / 08$ & $16: 00$ & 7.306 & 4.302 & 2.839 & 4.268 & 4.185 \\
\hline 5/19/08 & $20: 00$ & 7.332 & 4.32 & 2.786 & 4.254 & 4.176 \\
\hline $5 / 20 / 08$ & 0:00 & 7.339 & 4.25 & 2.695 & 4.242 & 3.982 \\
\hline $5 / 20 / 08$ & $4: 00$ & 7.332 & 4.204 & 2.662 & 4.231 & 3.866 \\
\hline $5 / 20 / 08$ & 8:00 & 7.343 & 4.183 & 2.665 & 4.245 & 3.789 \\
\hline $5 / 20 / 08$ & $12: 00$ & 7.355 & 4.227 & 2.735 & 4.275 & 3.908 \\
\hline $5 / 20 / 08$ & $16: 00$ & 7.374 & 4.309 & 2.8 & 4.294 & 4.139 \\
\hline 5/20/08 & $20: 00$ & 7.392 & 4.337 & 2.781 & 4.28 & 4.204 \\
\hline $5 / 21 / 08$ & 0:00 & 7.376 & 4.241 & 2.662 & 4.231 & 3.971 \\
\hline $5 / 21 / 08$ & $4: 00$ & 7.362 & 4.19 & 2.639 & 4.217 & 3.842 \\
\hline $5 / 21 / 08$ & 8:00 & 7.353 & 4.159 & 2.632 & 4.214 & 3.756 \\
\hline
\end{tabular}

Table S1.4 (Cont.)

Depth to Water (ft below top of casing) in Indicated Well

Date Time PMW4 PMW5 PMW6 PMW7 PMW8

\begin{tabular}{|c|c|c|c|c|c|c|}
\hline $5 / 21 / 08$ & $12: 00$ & 7.371 & 4.234 & 2.753 & 4.254 & 3.989 \\
\hline $5 / 21 / 08$ & $16: 00$ & 7.39 & 4.288 & 2.786 & 4.259 & 4.139 \\
\hline $5 / 21 / 08$ & 20:00 & 7.402 & 4.302 & 2.725 & 4.238 & 4.176 \\
\hline $5 / 22 / 08$ & 0:00 & 7.39 & 4.227 & 2.655 & 4.21 & 3.971 \\
\hline $5 / 22 / 08$ & $4: 00$ & 7.376 & 4.18 & 2.632 & 4.198 & 3.854 \\
\hline $5 / 22 / 08$ & $8: 00$ & 7.367 & 4.148 & 2.627 & 4.168 & 3.765 \\
\hline $5 / 22 / 08$ & $12: 00$ & 7.348 & 3.851 & 2.574 & 4.156 & 3.455 \\
\hline $5 / 22 / 08$ & $16: 00$ & 7.306 & 3.944 & 2.616 & 4.133 & 3.327 \\
\hline $5 / 22 / 08$ & $20: 00$ & 7.301 & 4.01 & 2.627 & 4.145 & 3.397 \\
\hline $5 / 23 / 08$ & 0:00 & 7.306 & 4.026 & 2.618 & 4.161 & 3.373 \\
\hline $5 / 23 / 08$ & $4: 00$ & 7.162 & 2.852 & 2.508 & 4.003 & 2.622 \\
\hline $5 / 23 / 08$ & $8: 00$ & 6.931 & 3.228 & 2.55 & 3.884 & 2.513 \\
\hline $5 / 23 / 08$ & $12: 00$ & 6.903 & 3.431 & 2.567 & 3.944 & 2.613 \\
\hline $5 / 23 / 08$ & $16: 00$ & 6.882 & 3.503 & 2.578 & 3.97 & 2.683 \\
\hline $5 / 23 / 08$ & $20: 00$ & 6.875 & 3.578 & 2.578 & 3.998 & 2.781 \\
\hline $5 / 24 / 08$ & 0:00 & 6.873 & 3.625 & 2.585 & 4.014 & 2.842 \\
\hline $5 / 24 / 08$ & $4: 00$ & 6.742 & 2.908 & 2.522 & 3.849 & 2.394 \\
\hline $5 / 24 / 08$ & $8: 00$ & 6.626 & 3.186 & 2.541 & 3.842 & 2.475 \\
\hline $5 / 24 / 08$ & $12: 00$ & 6.591 & 3.326 & 2.581 & 3.868 & 2.618 \\
\hline $5 / 24 / 08$ & $16: 00$ & 6.579 & 3.461 & 2.648 & 3.916 & 2.877 \\
\hline $5 / 24 / 08$ & 20:00 & 6.584 & 3.524 & 2.602 & 3.933 & 2.989 \\
\hline $5 / 25 / 08$ & $0: 00$ & 6.57 & 3.513 & 2.571 & 3.933 & 2.949 \\
\hline $5 / 25 / 08$ & $4: 00$ & 6.567 & 3.51 & 2.564 & 3.94 & 2.949 \\
\hline $5 / 25 / 08$ & 8:00 & 6.574 & 3.524 & 2.569 & 3.954 & 2.963 \\
\hline $5 / 25 / 08$ & $12: 00$ & 6.591 & 3.608 & 2.658 & 3.989 & 3.215 \\
\hline $5 / 25 / 08$ & $16: 00$ & 6.644 & 3.734 & 2.72 & 4.024 & 3.588 \\
\hline $5 / 25 / 08$ & 20:00 & 6.688 & 3.779 & 2.66 & 4.033 & 3.63 \\
\hline $5 / 26 / 08$ & 0:00 & 6.695 & 3.699 & 2.588 & 3.996 & 3.42 \\
\hline $5 / 26 / 08$ & $4: 00$ & 6.712 & 3.692 & 2.59 & 4.012 & 3.345 \\
\hline $5 / 26 / 08$ & 8:00 & 6.726 & 3.681 & 2.59 & 4.019 & 3.273 \\
\hline $5 / 26 / 08$ & $12: 00$ & 6.763 & 3.72 & 2.625 & 4.045 & 3.324 \\
\hline $5 / 26 / 08$ & $16: 00$ & 6.8 & 3.828 & 2.716 & 4.086 & 3.625 \\
\hline $5 / 26 / 08$ & $20: 00$ & 6.821 & 3.823 & 2.646 & 4.065 & 3.621 \\
\hline $5 / 27 / 08$ & 0:00 & 6.84 & 3.797 & 2.609 & 4.068 & 3.495 \\
\hline $5 / 27 / 08$ & $4: 00$ & 6.842 & 3.781 & 2.602 & 4.068 & 3.425 \\
\hline $5 / 27 / 08$ & 8:00 & 6.863 & 3.788 & 2.604 & 4.079 & 3.392 \\
\hline $5 / 27 / 08$ & $12: 00$ & 6.882 & 3.779 & 2.595 & 4.086 & 3.352 \\
\hline $5 / 27 / 08$ & $16: 00$ & 6.891 & 3.774 & 2.602 & 4.089 & 3.296 \\
\hline $5 / 27 / 08$ & $20: 00$ & 6.903 & 3.769 & 2.595 & 4.091 & 3.257 \\
\hline $5 / 28 / 08$ & $0: 00$ & 6.908 & 3.723 & 2.588 & 4.086 & 3.154 \\
\hline $5 / 28 / 08$ & $4: 00$ & 6.763 & 3.193 & 2.546 & 3.972 & 2.582 \\
\hline $5 / 28 / 08$ & 8:00 & 6.509 & 2.883 & 2.525 & 3.849 & 2.391 \\
\hline $5 / 28 / 08$ & $12: 00$ & 6.455 & 3.095 & 2.548 & 3.877 & 2.489 \\
\hline $5 / 28 / 08$ & $16: 00$ & 6.446 & 3.235 & 2.574 & 3.902 & 2.631 \\
\hline $5 / 28 / 08$ & $20: 00$ & 6.441 & 3.296 & 2.564 & 3.905 & 2.699 \\
\hline $5 / 29 / 08$ & 0:00 & 6.446 & 3.34 & 2.553 & 3.914 & 2.741 \\
\hline $5 / 29 / 08$ & $4: 00$ & 6.448 & 3.363 & 2.555 & 3.912 & 2.776 \\
\hline $5 / 29 / 08$ & 8:00 & 6.458 & 3.391 & 2.557 & 3.916 & 2.813 \\
\hline $5 / 29 / 08$ & $12: 00$ & 6.465 & 3.424 & 2.578 & 3.916 & 2.9 \\
\hline $5 / 29 / 08$ & $16: 00$ & 6.483 & 3.517 & 2.641 & 3.933 & 3.133 \\
\hline
\end{tabular}


Table S1.4 (Cont.)

Depth to Water (ft below top of casing) in Indicated Well

Date Time PMW4 PMW5 PMW6 PMW7 PMW8

\begin{tabular}{|c|c|c|c|c|c|c|}
\hline $5 / 29 / 08$ & 20:00 & 6.511 & 3.545 & 2.583 & 3.919 & 3.203 \\
\hline $5 / 30 / 08$ & $0: 00$ & 6.542 & 3.174 & 2.543 & 3.888 & 3 \\
\hline $5 / 30 / 08$ & 4:00 & 6.29 & 3.011 & 2.518 & 3.758 & 2.463 \\
\hline $5 / 30 / 08$ & 8:00 & 6.229 & 2.981 & 2.504 & 3.714 & 2.449 \\
\hline $5 / 30 / 08$ & $12: 00$ & 6.234 & 3.146 & 2.56 & 3.758 & 2.603 \\
\hline 5/30/08 & $16: 00$ & 6.281 & 3.363 & 2.65 & 3.819 & 2.984 \\
\hline 5/30/08 & $20: 00$ & 6.341 & 3.48 & 2.611 & 3.844 & 3.212 \\
\hline $5 / 31 / 08$ & $0: 00$ & 6.353 & 3.433 & 2.553 & 3.849 & 3.075 \\
\hline $5 / 31 / 08$ & 4:00 & 6.36 & 3.422 & 2.541 & 3.856 & 3.037 \\
\hline $5 / 31 / 08$ & 8:00 & 6.383 & 3.436 & 2.546 & 3.877 & 3.033 \\
\hline $5 / 31 / 08$ & $12: 00$ & 6.425 & 3.543 & 2.604 & 3.914 & 3.257 \\
\hline $5 / 31 / 08$ & $16: 00$ & 6.486 & 3.692 & 2.695 & 3.954 & 3.632 \\
\hline $5 / 31 / 08$ & 20:00 & 6.532 & 3.737 & 2.648 & 3.944 & 3.702 \\
\hline $6 / 1 / 08$ & $0: 00$ & 6.546 & 3.648 & 2.571 & 3.923 & 3.485 \\
\hline 6/1/08 & 4:00 & 6.549 & 3.608 & 2.56 & 3.921 & 3.378 \\
\hline $6 / 1 / 08$ & 8:00 & 6.57 & 3.608 & 2.56 & 3.935 & 3.322 \\
\hline 6/1/08 & $12: 00$ & 6.614 & 3.746 & 2.685 & 3.989 & 3.632 \\
\hline $6 / 1 / 08$ & $16: 00$ & 6.667 & 3.863 & 2.751 & 4.012 & 3.926 \\
\hline $6 / 1 / 08$ & 20:00 & 6.7 & 3.856 & 2.662 & 3.986 & 3.908 \\
\hline $6 / 2 / 08$ & 0:00 & 6.702 & 3.769 & 2.585 & 3.961 & 3.681 \\
\hline $6 / 2 / 08$ & 4:00 & 6.691 & 3.72 & 2.564 & 3.942 & 3.56 \\
\hline $6 / 2 / 08$ & 8:00 & 6.695 & 3.699 & 2.55 & 3.937 & 3.478 \\
\hline $6 / 2 / 08$ & $12: 00$ & 6.695 & 3.709 & 2.615 & 3.947 & 3.443 \\
\hline $6 / 2 / 08$ & $16: 00$ & 6.744 & 3.881 & 2.718 & 3.986 & 3.891 \\
\hline $6 / 2 / 08$ & 20:00 & 6.779 & 3.888 & 2.622 & 3.963 & 3.903 \\
\hline 6/3/08 & 0:00 & 6.758 & 3.795 & 2.569 & 3.926 & 3.688 \\
\hline 6/3/08 & 4:00 & 6.747 & 3.755 & 2.562 & 3.914 & 3.572 \\
\hline 6/3/08 & 8:00 & 6.751 & 3.737 & 2.55 & 3.907 & 3.492 \\
\hline 6/3/08 & $12: 00$ & 6.777 & 3.814 & 2.634 & 3.944 & 3.623 \\
\hline 6/3/08 & $16: 00$ & 6.831 & 3.972 & 2.748 & 3.993 & 3.975 \\
\hline 6/3/08 & 20:00 & 6.873 & 4.012 & 2.685 & 3.984 & 4.073 \\
\hline $6 / 4 / 08$ & 0:00 & 6.866 & 3.902 & 2.574 & 3.935 & 3.819 \\
\hline 6/4/08 & 4:00 & 6.852 & 3.846 & 2.553 & 3.923 & 3.681 \\
\hline $6 / 4 / 08$ & $8: 00$ & 6.854 & 3.821 & 2.555 & 3.928 & 3.595 \\
\hline $6 / 4 / 08$ & $12: 00$ & 6.847 & 3.8 & 2.548 & 3.919 & 3.537 \\
\hline 6/4/08 & $16: 00$ & 6.887 & 3.937 & 2.692 & 3.975 & 3.835 \\
\hline $6 / 4 / 08$ & 20:00 & 6.891 & 3.919 & 2.588 & 3.933 & 3.828 \\
\hline $6 / 5 / 08$ & 0:00 & 4.978 & 2.458 & 2.417 & 3.274 & 2.316 \\
\hline 6/5/08 & 4:00 & 3.917 & 2.278 & 2.315 & 2.936 & 2.169 \\
\hline 6/5/08 & 8:00 & 3.843 & 2.402 & 2.378 & 3.053 & 2.316 \\
\hline $6 / 5 / 08$ & $12: 00$ & 4.127 & 2.444 & 2.427 & 3.188 & 2.414 \\
\hline 6/5/08 & $16: 00$ & 4.344 & 2.5 & 2.483 & 3.29 & 2.575 \\
\hline $6 / 5 / 08$ & 20:00 & 4.488 & 2.507 & 2.445 & 3.344 & 2.557 \\
\hline 6/6/08 & $0: 00$ & 4.551 & 2.467 & 2.401 & 3.323 & 2.382 \\
\hline 6/6/08 & 4:00 & 4.693 & 2.528 & 2.436 & 3.411 & 2.433 \\
\hline 6/6/08 & 8:00 & 4.824 & 2.582 & 2.462 & 3.486 & 2.501 \\
\hline 6/6/08 & $12: 00$ & 4.961 & 2.673 & 2.536 & 3.56 & 2.748 \\
\hline 6/6/08 & $16: 00$ & 5.094 & 2.813 & 2.594 & 3.609 & 3.152 \\
\hline 6/6/08 & 20:00 & 5.211 & 2.894 & 2.559 & 3.63 & 3.376 \\
\hline $6 / 7 / 08$ & $0: 00$ & 5.274 & 2.817 & 2.492 & 3.621 & 3.149 \\
\hline
\end{tabular}

Table S1.4 (Cont.)

Depth to Water (ft below top of casing) in Indicated Well

Date Time PMW4 PMW5 PMW6 PMW7 PMW8

\begin{tabular}{|c|c|c|c|c|c|c|}
\hline $6 / 7 / 08$ & 4:00 & 5.311 & 2.787 & 2.469 & 3.611 & 3.054 \\
\hline $6 / 7 / 08$ & 8:00 & 5.372 & 2.796 & 2.48 & 3.628 & 3.014 \\
\hline $6 / 7 / 08$ & $12: 00$ & 5.474 & 2.974 & 2.576 & 3.679 & 3.462 \\
\hline $6 / 7 / 08$ & $16: 00$ & 5.577 & 3.163 & 2.646 & 3.716 & 3.826 \\
\hline $6 / 7 / 08$ & 20:00 & 5.658 & 3.212 & 2.587 & 3.707 & 3.852 \\
\hline 6/8/08 & 0:00 & 5.691 & 3.044 & 2.497 & 3.67 & 3.504 \\
\hline 6/8/08 & 4:00 & 4.91 & 2.444 & 2.417 & 3.474 & 2.571 \\
\hline 6/8/08 & 8:00 & 4.204 & 2.421 & 2.401 & 3.309 & 2.307 \\
\hline 6/8/08 & $12: 00$ & 4.526 & 2.472 & 2.424 & 3.383 & 2.375 \\
\hline 6/8/08 & $16: 00$ & 4.728 & 2.542 & 2.459 & 3.441 & 2.466 \\
\hline 6/8/08 & $20: 00$ & 4.906 & 2.607 & 2.487 & 3.488 & 2.608 \\
\hline 6/9/08 & $0: 00$ & 5.029 & 2.633 & 2.457 & 3.516 & 2.592 \\
\hline 6/9/08 & 4:00 & 5.115 & 2.656 & 2.452 & 3.528 & 2.615 \\
\hline 6/9/08 & 8:00 & 5.209 & 2.689 & 2.457 & 3.551 & 2.655 \\
\hline 6/9/08 & $12: 00$ & 5.316 & 2.815 & 2.564 & 3.604 & 2.935 \\
\hline 6/9/08 & $16: 00$ & 5.432 & 3.009 & 2.622 & 3.637 & 3.329 \\
\hline $6 / 9 / 08$ & 20:00 & 5.518 & 3.072 & 2.562 & 3.628 & 3.411 \\
\hline 6/10/08 & $0: 00$ & 5.565 & 2.985 & 2.494 & 3.618 & 3.21 \\
\hline 6/10/08 & 4:00 & 5.595 & 2.962 & 2.48 & 3.616 & 3.121 \\
\hline 6/10/08 & 8:00 & 5.644 & 2.967 & 2.478 & 3.625 & 3.082 \\
\hline 6/10/08 & $12: 00$ & 5.721 & 3.163 & 2.604 & 3.679 & 3.46 \\
\hline 6/10/08 & $16: 00$ & 5.805 & 3.356 & 2.692 & 3.709 & 3.894 \\
\hline 6/10/08 & 20:00 & 5.868 & 3.387 & 2.608 & 3.686 & 3.924 \\
\hline 6/11/08 & $0: 00$ & 5.88 & 3.275 & 2.513 & 3.646 & 3.639 \\
\hline 6/11/08 & 4:00 & 5.877 & 3.006 & 2.469 & 3.607 & 3.406 \\
\hline 6/11/08 & 8:00 & 5.889 & 3.079 & 2.48 & 3.609 & 3.18 \\
\hline 6/11/08 & $12: 00$ & 5.919 & 3.165 & 2.527 & 3.621 & 3.212 \\
\hline 6/11/08 & $16: 00$ & 5.989 & 3.363 & 2.611 & 3.665 & 3.632 \\
\hline 6/11/08 & 20:00 & 6.029 & 3.312 & 2.531 & 3.623 & 3.681 \\
\hline 6/12/08 & $0: 00$ & 4.253 & 2.404 & 2.382 & 3.232 & 2.302 \\
\hline $6 / 12 / 08$ & 4:00 & 4.374 & 2.425 & 2.392 & 3.25 & 2.293 \\
\hline $6 / 12 / 08$ & 8:00 & 4.558 & 2.467 & 2.396 & 3.295 & 2.349 \\
\hline $6 / 12 / 08$ & $12: 00$ & 4.745 & 2.556 & 2.464 & 3.36 & 2.494 \\
\hline 6/12/08 & $16: 00$ & 4.95 & 2.705 & 2.543 & 3.427 & 2.876 \\
\hline $6 / 12 / 08$ & 20:00 & 5.108 & 2.778 & 2.515 & 3.453 & 3.07 \\
\hline 6/13/08 & $0: 00$ & 5.181 & 2.738 & 2.448 & 3.453 & 2.921 \\
\hline 6/13/08 & 4:00 & 5.248 & 2.743 & 2.436 & 3.465 & 2.888 \\
\hline 6/13/08 & 8:00 & 5.32 & 2.775 & 2.455 & 3.493 & 2.895 \\
\hline 6/13/08 & $12: 00$ & 5.43 & 2.955 & 2.566 & 3.551 & 3.28 \\
\hline 6/13/08 & $16: 00$ & 5.539 & 3.167 & 2.639 & 3.586 & 3.698 \\
\hline 6/13/08 & $20: 00$ & 5.623 & 3.237 & 2.576 & 3.576 & 3.791 \\
\hline 6/14/08 & $0: 00$ & 5.642 & 3.104 & 2.478 & 3.534 & 3.492 \\
\hline $6 / 14 / 08$ & $4: 00$ & 5.661 & 3.055 & 2.455 & 3.523 & 3.352 \\
\hline 6/14/08 & 8:00 & 5.707 & 3.079 & 2.473 & 3.541 & 3.308 \\
\hline 6/14/08 & $12: 00$ & 5.782 & 3.27 & 2.592 & 3.595 & 3.702 \\
\hline 6/14/08 & $16: 00$ & 5.861 & 3.431 & 2.646 & 3.618 & 4.057 \\
\hline 6/14/08 & 20:00 & 5.917 & 3.459 & 2.564 & 3.588 & 4.092 \\
\hline 6/15/08 & $0: 00$ & 5.919 & 3.331 & 2.478 & 3.546 & 3.768 \\
\hline 6/15/08 & $4: 00$ & 5.924 & 3.268 & 2.455 & 3.53 & 3.595 \\
\hline 6/15/08 & 8:00 & 5.938 & 3.237 & 2.445 & 3.525 & 3.481 \\
\hline
\end{tabular}


Table S1.4 (Cont.)

Depth to Water (ft below top of casing) in Indicated Well

Date Time PMW4 PMW5 PMW6 PMW7 PMW8

\begin{tabular}{|c|c|c|c|c|c|c|}
\hline 6/15/08 & $12: 00$ & 5.602 & 2.684 & 2.417 & 3.371 & 2.522 \\
\hline $6 / 15 / 08$ & $16: 00$ & 5.663 & 2.974 & 2.527 & 3.458 & 2.923 \\
\hline 6/15/08 & $20: 00$ & 5.747 & 3.095 & 2.51 & 3.481 & 3.124 \\
\hline 6/16/08 & $0: 00$ & 5.791 & 3.067 & 2.447 & 3.476 & 3.014 \\
\hline 6/16/08 & $4: 00$ & 5.814 & 3.06 & 2.441 & 3.479 & 2.986 \\
\hline 6/16/08 & $8: 00$ & 5.859 & 3.093 & 2.452 & 3.509 & 3.026 \\
\hline 6/16/08 & $12: 00$ & 5.912 & 3.214 & 2.522 & 3.544 & 3.257 \\
\hline 6/16/08 & $16: 00$ & 5.978 & 3.37 & 2.587 & 3.572 & 3.56 \\
\hline 6/16/08 & $20: 00$ & 6.027 & 3.408 & 2.536 & 3.562 & 3.623 \\
\hline $6 / 17 / 08$ & 0:00 & 6.034 & 3.321 & 2.464 & 3.537 & 3.411 \\
\hline 6/17/08 & $4: 00$ & 6.038 & 3.279 & 2.445 & 3.525 & 3.31 \\
\hline 6/17/08 & $8: 00$ & 6.048 & 3.272 & 2.457 & 3.53 & 3.261 \\
\hline $6 / 17 / 08$ & $12: 00$ & 6.096 & 3.422 & 2.571 & 3.579 & 3.574 \\
\hline $6 / 17 / 08$ & $16: 00$ & 6.15 & 3.569 & 2.618 & 3.597 & 3.922 \\
\hline $6 / 17 / 08$ & 20:00 & 6.192 & 3.583 & 2.548 & 3.574 & 3.933 \\
\hline 6/18/08 & $0: 00$ & 6.159 & 3.412 & 2.452 & 3.509 & 3.658 \\
\hline 6/18/08 & $4: 00$ & 6.155 & 3.349 & 2.438 & 3.497 & 3.481 \\
\hline 6/18/08 & $8: 00$ & 6.169 & 3.34 & 2.441 & 3.504 & 3.38 \\
\hline 6/18/08 & $12: 00$ & 6.211 & 3.464 & 2.527 & 3.548 & 3.576 \\
\hline 6/18/08 & $16: 00$ & 6.255 & 3.597 & 2.59 & 3.581 & 3.87 \\
\hline 6/18/08 & 20:00 & 6.285 & 3.606 & 2.515 & 3.546 & 3.901 \\
\hline 6/19/08 & $0: 00$ & 6.269 & 3.499 & 2.45 & 3.507 & 3.656 \\
\hline 6/19/08 & $4: 00$ & 6.26 & 3.45 & 2.438 & 3.495 & 3.527 \\
\hline 6/19/08 & 8:00 & 6.255 & 3.422 & 2.433 & 3.49 & 3.443 \\
\hline 6/19/08 & $12: 00$ & 6.295 & 3.534 & 2.513 & 3.528 & 3.639 \\
\hline 6/19/08 & $16: 00$ & 6.348 & 3.681 & 2.576 & 3.562 & 3.987 \\
\hline 6/19/08 & $20: 00$ & 5.498 & 2.432 & 2.338 & 3.241 & 2.459 \\
\hline 6/20/08 & 0:00 & 5.232 & 2.549 & 2.382 & 3.288 & 2.382 \\
\hline $6 / 20 / 08$ & $4: 00$ & 5.369 & 2.644 & 2.401 & 3.339 & 2.454 \\
\hline $6 / 20 / 08$ & 8:00 & 5.456 & 2.71 & 2.405 & 3.365 & 2.51 \\
\hline 6/20/08 & $12: 00$ & 5.556 & 2.845 & 2.492 & 3.416 & 2.755 \\
\hline $6 / 20 / 08$ & $16: 00$ & 5.665 & 3.06 & 2.548 & 3.453 & 3.184 \\
\hline $6 / 20 / 08$ & $20: 00$ & 5.742 & 3.095 & 2.473 & 3.439 & 3.226 \\
\hline $6 / 21 / 08$ & 0:00 & 5.782 & 3.051 & 2.426 & 3.43 & 3.084 \\
\hline $6 / 21 / 08$ & 4:00 & 5.807 & 3.046 & 2.422 & 3.43 & 3.033 \\
\hline 6/21/08 & $8: 00$ & 5.842 & 3.055 & 2.422 & 3.434 & 3.014 \\
\hline $6 / 21 / 08$ & $12: 00$ & 5.901 & 3.219 & 2.527 & 3.483 & 3.348 \\
\hline $6 / 21 / 08$ & $16: 00$ & 5.971 & 3.389 & 2.592 & 3.513 & 3.723 \\
\hline $6 / 21 / 08$ & $20: 00$ & 6.003 & 3.352 & 2.487 & 3.469 & 3.611 \\
\hline $6 / 22 / 08$ & 0:00 & 6.003 & 3.265 & 2.433 & 3.437 & 3.413 \\
\hline $6 / 22 / 08$ & 4:00 & 6.015 & 3.237 & 2.431 & 3.434 & 3.308 \\
\hline $6 / 22 / 08$ & $8: 00$ & 6.024 & 3.223 & 2.431 & 3.43 & 3.245 \\
\hline $6 / 22 / 08$ & $12: 00$ & 6.066 & 3.359 & 2.538 & 3.479 & 3.506 \\
\hline $6 / 22 / 08$ & $16: 00$ & 6.124 & 3.517 & 2.625 & 3.523 & 3.889 \\
\hline $6 / 22 / 08$ & $20: 00$ & 6.169 & 3.541 & 2.559 & 3.502 & 3.919 \\
\hline 6/23/08 & $0: 00$ & 6.171 & 3.45 & 2.461 & 3.465 & 3.663 \\
\hline $6 / 23 / 08$ & $4: 00$ & 6.166 & 3.401 & 2.443 & 3.441 & 3.523 \\
\hline $6 / 23 / 08$ & 8:00 & 6.176 & 3.389 & 2.445 & 3.448 & 3.441 \\
\hline 6/23/08 & $12: 00$ & 6.225 & 3.545 & 2.583 & 3.511 & 3.758 \\
\hline $6 / 23 / 08$ & $16: 00$ & 6.285 & 3.695 & 2.66 & 3.558 & 4.101 \\
\hline
\end{tabular}

Table S1.4 (Cont.)

Depth to Water (ft below top of casing) in Indicated Well

Date Time PMW4 PMW5 PMW6 PMW7 PMW8

\begin{tabular}{|c|c|c|c|c|c|c|}
\hline 6/23/08 & 20:00 & 6.311 & 3.667 & 2.557 & 3.513 & 4.08 \\
\hline 6/24/08 & 0:00 & 6.306 & 3.564 & 2.468 & 3.472 & 3.817 \\
\hline $6 / 24 / 08$ & $4: 00$ & 6.283 & 3.503 & 2.443 & 3.444 & 3.665 \\
\hline $6 / 24 / 08$ & $8: 00$ & 6.285 & 3.361 & 2.417 & 3.416 & 3.541 \\
\hline $6 / 24 / 08$ & $12: 00$ & 6.271 & 3.375 & 2.424 & 3.42 & 3.408 \\
\hline $6 / 24 / 08$ & $16: 00$ & 6.239 & 2.971 & 2.389 & 3.353 & 3.154 \\
\hline $6 / 24 / 08$ & $20: 00$ & 6.152 & 3.111 & 2.398 & 3.348 & 2.823 \\
\hline 6/25/08 & 0:00 & 6.155 & 3.179 & 2.403 & 3.36 & 2.857 \\
\hline 6/25/08 & 4:00 & 6.159 & 3.205 & 2.405 & 3.362 & 2.888 \\
\hline 6/25/08 & $8: 00$ & 6.173 & 3.226 & 2.405 & 3.367 & 2.909 \\
\hline 6/25/08 & $12: 00$ & 6.194 & 3.303 & 2.457 & 3.39 & 3.042 \\
\hline 6/25/08 & $16: 00$ & 6.26 & 3.499 & 2.541 & 3.434 & 3.487 \\
\hline 6/25/08 & 20:00 & 6.311 & 3.555 & 2.494 & 3.427 & 3.595 \\
\hline 6/26/08 & 0:00 & 6.297 & 3.464 & 2.426 & 3.39 & 3.378 \\
\hline 6/26/08 & 4:00 & 6.278 & 3.412 & 2.412 & 3.371 & 3.273 \\
\hline 6/26/08 & 8:00 & 6.269 & 3.394 & 2.41 & 3.367 & 3.221 \\
\hline 6/26/08 & $12: 00$ & 5.598 & 2.514 & 2.352 & 3.206 & 2.587 \\
\hline 6/26/08 & $16: 00$ & 5.528 & 2.724 & 2.422 & 3.269 & 2.547 \\
\hline 6/26/08 & 20:00 & 5.616 & 2.859 & 2.419 & 3.288 & 2.746 \\
\hline $6 / 27 / 08$ & 0:00 & 5.661 & 2.882 & 2.391 & 3.283 & 2.722 \\
\hline $6 / 27 / 08$ & 4:00 & 5.71 & 2.92 & 2.384 & 3.285 & 2.75 \\
\hline $6 / 27 / 08$ & $8: 00$ & 5.763 & 2.971 & 2.398 & 3.309 & 2.811 \\
\hline 6/27/08 & $12: 00$ & 5.849 & 3.162 & 2.501 & 3.371 & 3.165 \\
\hline 6/27/08 & $16: 00$ & 5.938 & 3.361 & 2.55 & 3.409 & 3.564 \\
\hline $6 / 27 / 08$ & 20:00 & 5.971 & 3.233 & 2.433 & 3.36 & 3.324 \\
\hline 6/28/08 & 0:00 & 5.992 & 3.202 & 2.41 & 3.357 & 3.189 \\
\hline 6/28/08 & 4:00 & 5.992 & 3.176 & 2.398 & 3.343 & 3.116 \\
\hline 6/28/08 & 8:00 & 6.01 & 3.186 & 2.398 & 3.348 & 3.084 \\
\hline 6/28/08 & $12: 00$ & 6.069 & 3.361 & 2.508 & 3.416 & 3.443 \\
\hline 6/28/08 & $16: 00$ & 6.127 & 3.499 & 2.557 & 3.444 & 3.777 \\
\hline 6/28/08 & 20:00 & 6.155 & 3.487 & 2.492 & 3.416 & 3.751 \\
\hline 6/29/08 & 0:00 & 6.162 & 3.415 & 2.431 & 3.39 & 3.541 \\
\hline 6/29/08 & 4:00 & 6.166 & 3.38 & 2.419 & 3.383 & 3.429 \\
\hline 6/29/08 & 8:00 & 6.18 & 3.375 & 2.431 & 3.395 & 3.368 \\
\hline 6/29/08 & $12: 00$ & 6.222 & 3.513 & 2.529 & 3.453 & 3.578 \\
\hline 6/29/08 & $16: 00$ & 6.271 & 3.627 & 2.59 & 3.488 & 3.886 \\
\hline 6/29/08 & $20: 00$ & 6.302 & 3.639 & 2.538 & 3.469 & 3.914 \\
\hline 6/30/08 & 0:00 & 6.297 & 3.529 & 2.452 & 3.423 & 3.686 \\
\hline 6/30/08 & 4:00 & 6.283 & 3.471 & 2.424 & 3.402 & 3.553 \\
\hline 6/30/08 & 8:00 & 6.283 & 3.445 & 2.424 & 3.399 & 3.466 \\
\hline 6/30/08 & $12: 00$ & 6.323 & 3.578 & 2.538 & 3.458 & 3.716 \\
\hline 6/30/08 & $16: 00$ & 6.364 & 3.704 & 2.601 & 3.495 & 4.04 \\
\hline 6/30/08 & 20:00 & 6.388 & 3.69 & 2.543 & 3.471 & 4.064 \\
\hline $7 / 1 / 08$ & 0:00 & 6.364 & 3.569 & 2.45 & 3.406 & 3.807 \\
\hline 7/1/08 & $4: 00$ & 6.339 & 3.503 & 2.419 & 3.381 & 3.66 \\
\hline $7 / 1 / 08$ & 8:00 & 6.325 & 3.475 & 2.419 & 3.374 & 3.567 \\
\hline $7 / 1 / 08$ & $12: 00$ & 6.376 & 3.653 & 2.55 & 3.437 & 3.919 \\
\hline 7/1/08 & $16: 00$ & 6.437 & 3.809 & 2.639 & 3.499 & 4.232 \\
\hline $7 / 1 / 08$ & 20:00 & 6.465 & 3.818 & 2.569 & 3.474 & 4.299 \\
\hline $7 / 2 / 08$ & $0: 00$ & 6.432 & 3.657 & 2.447 & 3.39 & 4.008 \\
\hline
\end{tabular}


Table S1.4 (Cont.)

Depth to Water (ft below top of casing) in Indicated Well

Date Time PMW4 PMW5 PMW6 PMW7 PMW8

\begin{tabular}{|c|c|c|c|c|c|c|}
\hline & $4: 00$ & & & & & \\
\hline $7 / 2 / 08$ & $8: 00$ & & & & & \\
\hline$/ 2 / 08$ & $2: 00$ & .427 & & & & 010 \\
\hline /2/08 & $6: 00$ & .5 & & & & \\
\hline /2/08 & $0: 00$ & & & & & \\
\hline & 0:00 & & & & & \\
\hline $3 / 08$ & :00 & & & & & .87 \\
\hline 3/08 & 8:00 & & & & & 1.10 \\
\hline 3/08 & $12: 00$ & .495 & & & & .896 \\
\hline /3/08 & $6: 00$ & 549 & & & & 4.227 \\
\hline 7/3/08 & 0:00 & & & & &.$+ \angle 2 \angle$ \\
\hline $7 / 4 / 08$ & :00 & & & & & 3.963 \\
\hline /4/08 & $: 00$ & & & & & .802 \\
\hline 4/08 & 8:00 & & & & & 69 \\
\hline $4 / 08$ & $12: 00$ & & & & & .91 \\
\hline $4 / 08$ & $6: 00$ & & & & & 4.297 \\
\hline & & & & & & \\
\hline $7 / 5 / 08$ & 0:00 & & & & & \\
\hline & 00 & & & & & .924 \\
\hline & 00 & & & & & \\
\hline /5/08 & $12: 00$ & & & & & 3.707 \\
\hline /5/08 & $6: 00$ & & & & & \\
\hline /5/08 & :00 & & & & & \\
\hline $6 / 08$ & & & & & & 68 \\
\hline & & & & & & 365 \\
\hline /6/08 & 00 & & & & & .725 \\
\hline$/ 6 / 08$ & $12: 00$ & & & & & 3.984 \\
\hline & & & & & & \\
\hline & & & & & & \\
\hline 7/7/08 & & & & & & $06 \varepsilon$ \\
\hline 7/7/08 & & & & & & 368 \\
\hline $7 / 7 / 08$ & & & & & & \\
\hline 7/7/08 & $12: 00$ & & & & & \\
\hline סחורו & & & & & & 4.432 \\
\hline 7/7/08 & & & & & & 4.232 \\
\hline & & & & & & \\
\hline & & & & & & \\
\hline & & & & & & \\
\hline 7/8/08 & & & & & & \\
\hline $7 / 8 / 08$ & & & & & & \\
\hline $7 / 8 / 08$ & 20:00 & & & & & 3.994 \\
\hline & & & & & & 3.777 \\
\hline & & & & & & \\
\hline & & & & & & \\
\hline & & & & & & 3.798 \\
\hline & & & & & & 4.211 \\
\hline & 20:00 & & & & & 3.99 \\
\hline & & & & & & 3.809 \\
\hline & & & & & & 3.669 \\
\hline & & & & & 3.355 & 3571 \\
\hline
\end{tabular}

Table S1.4 (Cont.)

Depth to Water (ft below top of casing) in Indicated Well

Date Time PMW4 PMW5 PMW6 PMW7 PMW8

\begin{tabular}{|c|c|c|c|c|c|c|}
\hline 7/10/08 & $12: 00$ & 6.602 & 3.788 & 2.517 & 3.434 & 3.975 \\
\hline 7/10/08 & $16: 00$ & 6.686 & 4.005 & 2.62 & 3.539 & 4.416 \\
\hline 7/10/08 & $20: 00$ & 6.728 & 4.035 & 2.564 & 3.52 & 4.502 \\
\hline 7/11/08 & 0:00 & 6.7 & 3.874 & 2.445 & 3.429 & 4.276 \\
\hline $7 / 11 / 08$ & $4: 00$ & 6.663 & 3.776 & 2.408 & 3.385 & 4.071 \\
\hline 7/11/08 & 8:00 & 6.63 & 3.711 & 2.398 & 3.364 & 3.903 \\
\hline 7/11/08 & $12: 00$ & 6.665 & 3.874 & 2.529 & 3.453 & 4.236 \\
\hline 7/11/08 & $16: 00$ & 6.742 & 4.068 & 2.641 & 3.567 & 4.549 \\
\hline 7/11/08 & $20: 00$ & 6.779 & 4.087 & 2.568 & 3.541 & 4.6 \\
\hline $7 / 12 / 08$ & 0:00 & 6.758 & 3.946 & 2.471 & 3.485 & 4.4 \\
\hline $7 / 12 / 08$ & $4: 00$ & 6.737 & 3.846 & 2.436 & 3.439 & 4.199 \\
\hline $7 / 12 / 08$ & 8:00 & 6.723 & 3.806 & 2.424 & 3.436 & 4.064 \\
\hline $7 / 12 / 08$ & $12: 00$ & 6.716 & 3.785 & 2.429 & 3.439 & 3.959 \\
\hline $7 / 12 / 08$ & $16: 00$ & 6.74 & 3.921 & 2.531 & 3.497 & 4.227 \\
\hline $7 / 12 / 08$ & $20: 00$ & 6.784 & 4.005 & 2.52 & 3.502 & 4.421 \\
\hline $7 / 13 / 08$ & 0:00 & 6.754 & 3.867 & 2.419 & 3.429 & 4.185 \\
\hline $7 / 13 / 08$ & $4: 00$ & 6.716 & 3.783 & 2.403 & 3.402 & 4.005 \\
\hline $7 / 13 / 08$ & 8:00 & 6.691 & 3.739 & 2.403 & 3.397 & 3.875 \\
\hline $7 / 13 / 08$ & $12: 00$ & 6.726 & 3.893 & 2.538 & 3.483 & 4.176 \\
\hline $7 / 13 / 08$ & $16: 00$ & 6.796 & 4.086 & 2.629 & 3.572 & 4.507 \\
\hline $7 / 13 / 08$ & $20: 00$ & 6.835 & 4.14 & 2.564 & 3.551 & 4.614 \\
\hline $7 / 14 / 08$ & 0:00 & 6.796 & 3.958 & 2.44 & 3.446 & 4.376 \\
\hline $7 / 14 / 08$ & $4: 00$ & 6.758 & 3.86 & 2.412 & 3.408 & 4.183 \\
\hline $7 / 14 / 08$ & 8:00 & 6.733 & 3.806 & 2.405 & 3.399 & 4.026 \\
\hline $7 / 14 / 08$ & $12: 00$ & 6.782 & 4.012 & 2.552 & 3.499 & 4.372 \\
\hline $7 / 14 / 08$ & $16: 00$ & 6.866 & 4.222 & 2.685 & 3.627 & 4.668 \\
\hline 7/14/08 & 20:00 & 6.9 & 4.243 & 2.62 & 3.602 & 4.712 \\
\hline $7 / 15 / 08$ & $0: 00$ & 6.875 & 4.075 & 2.482 & 3.502 & 4.509 \\
\hline $7 / 15 / 08$ & $4: 00$ & 6.835 & 3.97 & 2.436 & 3.448 & 4.348 \\
\hline 7/15/08 & $8: 00$ & 6.805 & 3.911 & 2.431 & 3.427 & 4.22 \\
\hline $7 / 15 / 08$ & $12: 00$ & 6.842 & 4.042 & 2.536 & 3.516 & 4.39 \\
\hline $7 / 15 / 08$ & $16: 00$ & 6.919 & 4.238 & 2.638 & 3.63 & 4.668 \\
\hline $7 / 15 / 08$ & 20:00 & 6.942 & 4.226 & 2.559 & 3.585 & 4.712 \\
\hline $7 / 16 / 08$ & $0: 00$ & 6.803 & 3.006 & 2.354 & 3.222 & 3.739 \\
\hline 7/16/08 & $4: 00$ & 6.574 & 2.826 & 2.307 & 3.127 & 2.773 \\
\hline $7 / 16 / 08$ & $8: 00$ & 6.348 & 2.899 & 2.324 & 3.157 & 2.523 \\
\hline $7 / 16 / 08$ & $12: 00$ & 6.327 & 3.134 & 2.368 & 3.264 & 2.638 \\
\hline $7 / 16 / 08$ & $16: 00$ & 6.343 & 3.375 & 2.457 & 3.355 & 2.941 \\
\hline 7/16/08 & 20:00 & 6.376 & 3.503 & 2.447 & 3.376 & 3.179 \\
\hline 7/17/08 & $0: 00$ & 6.374 & 3.461 & 2.394 & 3.355 & 3.111 \\
\hline $7 / 17 / 08$ & 4:00 & 6.357 & 3.431 & 2.377 & 3.341 & 3.076 \\
\hline 7/17/08 & 8:00 & 6.348 & 3.421 & 2.38 & 3.341 & 3.06 \\
\hline $7 / 17 / 08$ & $12: 00$ & 6.395 & 3.566 & 2.471 & 3.408 & 3.34 \\
\hline $7 / 17 / 08$ & $16: 00$ & 6.453 & 3.722 & 2.547 & 3.481 & 3.76 \\
\hline $7 / 17 / 08$ & $20: 00$ & 5.588 & 2.616 & 2.333 & 3.141 & 2.477 \\
\hline 7/18/08 & 0:00 & 5.693 & 2.782 & 2.345 & 3.203 & 2.456 \\
\hline 7/18/08 & 4:00 & 5.738 & 2.85 & 2.342 & 3.222 & 2.484 \\
\hline 7/18/08 & 8:00 & 5.812 & 2.917 & 2.345 & 3.248 & 2.526 \\
\hline $7 / 18 / 08$ & $12: 00$ & 5.868 & 3.018 & 2.398 & 3.299 & 2.628 \\
\hline $7 / 18 / 08$ & $16: 00$ & 5.938 & 3.207 & 2.461 & 3.364 & 2.936 \\
\hline
\end{tabular}


Table S1.4 (Cont.)

Depth to Water (ft below top of casing) in Indicated Well

Date Time PMW4 PMW5 PMW6 PMW7 PMW8

\begin{tabular}{|c|c|c|c|c|c|c|}
\hline 7/18/08 & $20: 00$ & 5.994 & 3.263 & 2.429 & 3.362 & 3.065 \\
\hline 7/19/08 & $0: 00$ & 6.017 & 3.202 & 2.373 & 3.325 & 2.974 \\
\hline $7 / 19 / 08$ & $4: 00$ & 6.02 & 3.169 & 2.359 & 3.306 & 2.922 \\
\hline $7 / 19 / 08$ & $8: 00$ & 6.045 & 3.169 & 2.361 & 3.311 & 2.913 \\
\hline $7 / 19 / 08$ & $12: 00$ & 6.064 & 3.263 & 2.438 & 3.362 & 3.072 \\
\hline 7/19/08 & $16: 00$ & 6.12 & 3.44 & 2.517 & 3.429 & 3.487 \\
\hline 7/19/08 & $20: 00$ & 6.185 & 3.512 & 2.473 & 3.427 & 3.662 \\
\hline $7 / 20 / 08$ & $0: 00$ & 6.194 & 3.41 & 2.398 & 3.369 & 3.431 \\
\hline $7 / 20 / 08$ & $4: 00$ & 6.194 & 3.356 & 2.377 & 3.346 & 3.312 \\
\hline $7 / 20 / 08$ & 8:00 & 6.197 & 3.337 & 2.368 & 3.341 & 3.244 \\
\hline $7 / 20 / 08$ & $12: 00$ & 6.239 & 3.463 & 2.466 & 3.413 & 3.391 \\
\hline $7 / 20 / 08$ & $16: 00$ & 6.29 & 3.603 & 2.547 & 3.485 & 3.706 \\
\hline 7/20/08 & $20: 00$ & 6.33 & 3.606 & 2.484 & 3.46 & 3.755 \\
\hline $7 / 21 / 08$ & 0:00 & 6.316 & 3.496 & 2.408 & 3.39 & 3.545 \\
\hline $7 / 21 / 08$ & $4: 00$ & 6.318 & 3.461 & 2.391 & 3.373 & 3.447 \\
\hline $7 / 21 / 08$ & $8: 00$ & 6.299 & 3.428 & 2.382 & 3.36 & 3.368 \\
\hline $7 / 21 / 08$ & $12: 00$ & 6.337 & 3.526 & 2.466 & 3.418 & 3.473 \\
\hline $7 / 21 / 08$ & $16: 00$ & & 3.659 & 2.529 & 3.49 & 3.727 \\
\hline $7 / 21 / 08$ & $20: 00$ & 6.381 & 3.592 & 2.449 & 3.436 & 3.69 \\
\hline $7 / 22 / 08$ & $0: 00$ & 6.364 & 3.508 & 2.389 & 3.383 & 3.538 \\
\hline $7 / 22 / 08$ & $4: 00$ & 6.353 & 3.468 & 2.377 & 3.369 & 3.443 \\
\hline $7 / 22 / 08$ & 8:00 & 6.346 & 3.445 & 2.37 & 3.362 & 3.382 \\
\hline $7 / 22 / 08$ & $12: 00$ & 6.353 & 3.566 & & 3.413 & 3.552 \\
\hline $7 / 22 / 08$ & $16: 00$ & 6.369 & 3.72 & 2.568 & 3.495 & 3.886 \\
\hline $7 / 22 / 08$ & $20: 00$ & 6.402 & 3.729 & 2.522 & 3.476 & 3.963 \\
\hline 7/23/08 & $0: 00$ & 6.385 & 3.617 & 2.407 & 3.401 & 3.748 \\
\hline $7 / 23 / 08$ & $4: 00$ & 6.362 & 3.557 & 2.382 & 3.371 & 3.608 \\
\hline $7 / 23 / 08$ & $8: 00$ & 6.35 & 3.529 & 2.377 & 3.359 & 3.529 \\
\hline $7 / 23 / 08$ & $12: 00$ & 6.374 & 3.629 & 2.466 & 3.418 & 3.65 \\
\hline 7/23/08 & $16: 00$ & 6.418 & 3.753 & 2.533 & 3.478 & 3.909 \\
\hline $7 / 23 / 08$ & $20: 00$ & 6.423 & 3.685 & 2.44 & 3.411 & 3.828 \\
\hline $7 / 24 / 08$ & 0:00 & 6.402 & 3.615 & 2.389 & 3.366 & 3.683 \\
\hline $7 / 24 / 08$ & $4: 00$ & 6.383 & 3.578 & 2.375 & 3.35 & 3.583 \\
\hline $7 / 24 / 08$ & $8: 00$ & 6.374 & 3.55 & 2.366 & 3.339 & 3.51 \\
\hline $7 / 24 / 08$ & $12: 00$ & 6.374 & 3.594 & 2.426 & 3.371 & 3.543 \\
\hline $7 / 24 / 08$ & $16: 00$ & 6.42 & 3.727 & 2.498 & 3.429 & 3.795 \\
\hline $7 / 24 / 08$ & $20: 00$ & 6.453 & 3.75 & 2.454 & 3.422 & 3.874 \\
\hline $7 / 25 / 08$ & 0:00 & 6.439 & 3.652 & 2.377 & 3.369 & 3.681 \\
\hline 7/25/08 & $4: 00$ & 6.411 & 3.592 & 2.363 & 3.343 & 3.552 \\
\hline $7 / 25 / 08$ & 8:00 & 6.404 & 3.573 & 2.363 & 3.345 & 3.48 \\
\hline $7 / 25 / 08$ & $12: 00$ & 6.409 & 3.573 & 2.377 & 3.357 & 3.457 \\
\hline $7 / 25 / 08$ & $16: 00$ & 6.448 & 3.711 & 2.459 & 3.429 & 3.669 \\
\hline $7 / 25 / 08$ & $20: 00$ & 6.483 & 3.774 & 2.459 & 3.443 & 3.804 \\
\hline $7 / 26 / 08$ & $0: 00$ & 6.472 & 3.676 & 2.384 & 3.392 & 3.629 \\
\hline 7/26/08 & $4: 00$ & 6.444 & 3.617 & 2.361 & 3.364 & 3.515 \\
\hline $7 / 26 / 08$ & 8:00 & 6.43 & 3.587 & 2.359 & 3.355 & 3.44 \\
\hline $7 / 26 / 08$ & $12: 00$ & 6.441 & 3.622 & 2.405 & 3.387 & 3.496 \\
\hline $7 / 26 / 08$ & $16: 00$ & 6.465 & 3.746 & 2.482 & 3.439 & 3.741 \\
\hline 7/26/08 & $20: 00$ & 6.5 & 3.795 & 2.47 & 3.446 & 3.877 \\
\hline $7 / 27 / 08$ & $0: 00$ & 6.476 & 3.685 & 2.38 & 3.373 & 3.676 \\
\hline
\end{tabular}

Table S1.4 (Cont.)

Depth to Water (ft below top of casing) in Indicated Well

Date Time PMW4 PMW5 PMW6 PMW7 PMW8

\begin{tabular}{|c|c|c|c|c|c|c|}
\hline $7 / 27 / 08$ & $4: 00$ & 6.446 & 3.624 & 2.361 & 3.343 & 3.545 \\
\hline $7 / 27 / 08$ & 8:00 & 6.427 & 3.589 & 2.349 & 3.329 & 3.459 \\
\hline $7 / 27 / 08$ & $12: 00$ & 6.458 & 3.718 & 2.473 & 3.411 & 3.678 \\
\hline $7 / 27 / 08$ & $16: 00$ & 6.525 & 3.904 & 2.571 & 3.508 & 4.082 \\
\hline $7 / 27 / 08$ & 20:00 & 6.563 & 3.93 & 2.508 & 3.478 & 4.185 \\
\hline $7 / 28 / 08$ & 0:00 & 6.544 & 3.795 & 2.403 & 3.394 & 3.935 \\
\hline 7/28/08 & 4:00 & 6.511 & 3.718 & 2.375 & 3.359 & 3.765 \\
\hline $7 / 28 / 08$ & $8: 00$ & 6.514 & 3.694 & 2.389 & 3.364 & 3.671 \\
\hline $7 / 28 / 08$ & $12: 00$ & 6.544 & 3.825 & 2.494 & 3.446 & 3.949 \\
\hline $7 / 28 / 08$ & $16: 00$ & 6.605 & 3.995 & 2.585 & 3.548 & 4.248 \\
\hline $7 / 28 / 08$ & 20:00 & 6.637 & 4 & 2.522 & 3.513 & 4.287 \\
\hline $7 / 29 / 08$ & 0:00 & 6.618 & 3.869 & 2.419 & 3.439 & 4.059 \\
\hline $7 / 29 / 08$ & $4: 00$ & 6.586 & 3.79 & 2.384 & 3.397 & 3.884 \\
\hline $7 / 29 / 08$ & 8:00 & 6.558 & 3.743 & 2.373 & 3.378 & 3.758 \\
\hline $7 / 29 / 08$ & $12: 00$ & 6.579 & 3.811 & 2.421 & 3.413 & 3.844 \\
\hline 7/29/08 & $16: 00$ & 6.628 & 3.935 & 2.489 & 3.471 & 4.103 \\
\hline $7 / 29 / 08$ & 20:00 & 6.644 & 3.93 & 2.449 & 3.45 & 4.131 \\
\hline 7/30/08 & $0: 00$ & 6.611 & 3.816 & 2.379 & 3.394 & 3.893 \\
\hline 7/30/08 & $4: 00$ & 6.581 & 3.75 & 2.363 & 3.369 & 3.741 \\
\hline 7/30/08 & 8:00 & 6.558 & 3.715 & 2.363 & 3.364 & 3.648 \\
\hline 7/30/08 & $12: 00$ & 6.574 & 3.818 & 2.459 & 3.429 & 3.856 \\
\hline 7/30/08 & $16: 00$ & 6.649 & 3.993 & 2.559 & 3.532 & 4.21 \\
\hline 7/30/08 & $20: 00$ & 6.684 & 4.016 & 2.503 & 3.504 & 4.301 \\
\hline 7/31/08 & 0:00 & 6.658 & 3.881 & 2.403 & 3.425 & 4.047 \\
\hline 7/31/08 & $4: 00$ & 6.618 & 3.802 & 2.37 & 3.383 & 3.858 \\
\hline 7/31/08 & $8: 00$ & 6.595 & 3.755 & 2.365 & 3.369 & 3.734 \\
\hline 7/31/08 & $12: 00$ & 6.618 & 3.867 & 2.463 & 3.441 & 3.968 \\
\hline 7/31/08 & $16: 00$ & 6.695 & 4.077 & 2.587 & 3.562 & 4.369 \\
\hline 7/31/08 & $20: 00$ & 6.74 & 4.098 & 2.517 & 3.536 & 4.423 \\
\hline 8/1/08 & 0:00 & 6.719 & 3.963 & 2.424 & 3.457 & 4.196 \\
\hline 8/1/08 & $4: 00$ & 6.684 & 3.879 & 2.386 & 3.418 & 4.01 \\
\hline 8/1/08 & 8:00 & 6.665 & 3.83 & 2.382 & 3.411 & 3.867 \\
\hline 8/1/08 & $12: 00$ & 6.688 & 3.93 & 2.498 & 3.494 & 4.1 \\
\hline 8/1/08 & $16: 00$ & 6.758 & 4.119 & 2.638 & 3.616 & 4.432 \\
\hline 8/1/08 & $20: 00$ & 6.789 & 4.114 & 2.571 & 3.583 & 4.479 \\
\hline 8/2/08 & 0:00 & 6.754 & 3.972 & 2.438 & 3.485 & 4.236 \\
\hline $8 / 2 / 08$ & $4: 00$ & 6.716 & 3.893 & 2.398 & 3.439 & 4.047 \\
\hline $8 / 2 / 08$ & 8:00 & 6.684 & 3.839 & 2.389 & 3.42 & 3.909 \\
\hline 8/2/08 & $12: 00$ & 6.702 & 3.951 & 2.496 & 3.487 & 4.14 \\
\hline $8 / 2 / 08$ & $16: 00$ & 6.777 & 4.135 & 2.606 & 3.599 & 4.441 \\
\hline 8/2/08 & 20:00 & 6.819 & 4.161 & 2.559 & 3.583 & 4.537 \\
\hline 8/3/08 & $0: 00$ & 6.789 & 4.021 & 2.435 & 3.483 & 4.317 \\
\hline 8/3/08 & $4: 00$ & 6.756 & 3.937 & 2.4 & 3.441 & 4.14 \\
\hline 8/3/08 & 8:00 & 6.726 & 3.883 & 2.391 & 3.422 & 3.988 \\
\hline 8/3/08 & $12: 00$ & 6.772 & 4.065 & 2.526 & 3.534 & 4.329 \\
\hline 8/3/08 & $16: 00$ & 6.852 & 4.25 & 2.666 & 3.678 & 4.628 \\
\hline 8/3/08 & 20:00 & 6.891 & 4.261 & 2.608 & 3.643 & 4.679 \\
\hline 8/4/08 & $0: 00$ & 6.866 & 4.119 & 2.475 & 3.536 & 4.485 \\
\hline $8 / 4 / 08$ & 4:00 & 6.84 & 4.047 & 2.438 & 3.49 & 4.341 \\
\hline 8/4/08 & 8:00 & 6.817 & 4.002 & 2.417 & 3.471 & 4.231 \\
\hline
\end{tabular}


Table S1.4 (Cont.)

Depth to Water (ft below top of casing) in Indicated Well

Date Time PMW4 PMW5 PMW6 PMW7 PMW8

\begin{tabular}{|c|c|c|c|c|c|c|}
\hline 8/4/08 & $12: 00$ & 6.831 & 4.082 & 2.517 & 3.546 & 4.322 \\
\hline 8/4/08 & $16: 00$ & 6.886 & 4.189 & 2.564 & 3.608 & 4.495 \\
\hline 8/4/08 & 20:00 & 6.914 & 4.208 & 2.538 & 3.606 & 4.576 \\
\hline 8/5/08 & 0:00 & 6.882 & 4.075 & 2.435 & 3.52 & 4.371 \\
\hline 8/5/08 & $4: 00$ & 6.849 & 4.002 & 2.414 & 3.494 & 4.21 \\
\hline 8/5/08 & $8: 00$ & 6.826 & 3.953 & 2.396 & 3.473 & 4.082 \\
\hline 8/5/08 & $12: 00$ & 6.828 & 4 & 2.463 & 3.525 & 4.159 \\
\hline 8/5/08 & $16: 00$ & 6.861 & 4.06 & 2.484 & 3.55 & 4.289 \\
\hline 8/5/08 & 20:00 & 6.893 & 4.11 & 2.505 & 3.571 & 4.401 \\
\hline 8/6/08 & 0:00 & 6.856 & 4.002 & 2.417 & 3.508 & 4.166 \\
\hline 8/6/08 & 4:00 & 6.821 & 3.937 & 2.393 & 3.478 & 4.007 \\
\hline 8/6/08 & $8: 00$ & 6.798 & 3.902 & 2.386 & 3.466 & 3.914 \\
\hline 8/6/08 & $12: 00$ & 6.807 & 3.965 & 2.47 & 3.527 & 4.079 \\
\hline 8/6/08 & $16: 00$ & 6.835 & 4.056 & 2.512 & 3.569 & 4.24 \\
\hline 8/6/08 & 20:00 & 6.87 & 4.074 & 2.494 & 3.56 & 4.282 \\
\hline 8/7/08 & 0:00 & 6.847 & 3.974 & 2.412 & 3.499 & 4.056 \\
\hline 8/7/08 & $4: 00$ & 6.805 & 3.906 & 2.386 & 3.466 & 3.904 \\
\hline 8/7/08 & 8:00 & 6.782 & 3.871 & 2.386 & 3.462 & 3.823 \\
\hline 8/7/08 & $12: 00$ & 6.803 & 3.988 & 2.508 & 3.55 & 4.098 \\
\hline 8/7/08 & $16: 00$ & 6.884 & 4.18 & 2.864 & 3.662 & 4.439 \\
\hline 8/7/08 & 20:00 & 6.924 & 4.194 & 2.538 & 3.625 & 4.478 \\
\hline 8/8/08 & 0:00 & 6.891 & 4.06 & 2.433 & 3.534 & 4.25 \\
\hline 8/8/08 & 4:00 & 6.854 & 3.983 & 2.403 & 3.499 & 4.068 \\
\hline 8/8/08 & $8: 00$ & 6.824 & 3.932 & 2.391 & 3.483 & 3.935 \\
\hline 8/8/08 & $12: 00$ & 6.842 & 4.037 & 2.515 & 3.56 & 4.184 \\
\hline 8/8/08 & $16: 00$ & 6.933 & 4.259 & 2.62 & 3.685 & 4.53 \\
\hline 8/8/08 & 20:00 & 6.97 & 4.264 & 2.566 & 3.648 & 4.59 \\
\hline 8/9/08 & 0:00 & 6.931 & 4.119 & 2.447 & 3.546 & 4.38 \\
\hline 8/9/08 & 4:00 & 6.893 & 4.037 & 2.414 & 3.501 & 4.222 \\
\hline 8/9/08 & $8: 00$ & 6.866 & 3.99 & 2.403 & 3.485 & 4.1 \\
\hline 8/9/08 & $12: 00$ & 6.847 & 3.972 & 2.405 & 3.48 & 4.04 \\
\hline 8/9/08 & $16: 00$ & 6.898 & 4.142 & 2.543 & 3.597 & 4.359 \\
\hline 8/9/08 & 20:00 & 6.956 & 4.198 & 2.522 & 3.599 & 4.485 \\
\hline 8/10/08 & 0:00 & 6.928 & 4.072 & 2.421 & 3.527 & 4.259 \\
\hline 8/10/08 & $4: 00$ & 6.887 & 3.99 & 2.391 & 3.492 & 4.077 \\
\hline 8/10/08 & 8:00 & 6.856 & 3.948 & 2.389 & 3.485 & 3.953 \\
\hline 8/10/08 & $12: 00$ & 6.87 & 4.035 & 2.496 & 3.564 & 4.18 \\
\hline 8/10/08 & $16: 00$ & 6.952 & 4.224 & 2.594 & 3.671 & 4.481 \\
\hline 8/10/08 & $20: 00$ & 6.952 & 4.126 & 2.447 & 3.564 & 4.348 \\
\hline 8/11/08 & 0:00 & 6.924 & 4.037 & 2.412 & 3.525 & 4.154 \\
\hline 8/11/08 & 4:00 & 6.882 & 3.972 & & 3.492 & 3.998 \\
\hline 8/11/08 & 8:00 & 6.856 & 3.932 & 2.389 & 3.483 & 3.897 \\
\hline 8/11/08 & $12: 00$ & 6.868 & 4.011 & 2.466 & 3.543 & 4.098 \\
\hline 8/11/08 & $16: 00$ & 6.924 & 4.137 & 2.512 & 3.592 & 4.334 \\
\hline 8/11/08 & $20: 00$ & 6.949 & 4.13 & 2.47 & 3.557 & 4.348 \\
\hline 8/12/08 & 0:00 & 6.919 & 4.035 & 2.41 & 3.506 & 4.135 \\
\hline 8/12/08 & 4:00 & 6.868 & 3.911 & 2.377 & 3.448 & 3.944 \\
\hline 8/12/08 & $8: 00$ & 6.835 & 3.885 & 2.37 & 3.448 & 3.832 \\
\hline 8/12/08 & $12: 00$ & 6.847 & 3.965 & 2.452 & 3.515 & 3.946 \\
\hline 8/12/08 & $16: 00$ & 6.921 & 4.14 & 2.538 & 3.606 & 4.308 \\
\hline
\end{tabular}

Table S1.4 (Cont.)

Depth to Water (ft below top of casing) in Indicated Well

Date Time PMW4 PMW5 PMW6 PMW7 PMW8

\begin{tabular}{|c|c|c|c|c|c|c|}
\hline 8/12/08 & 20:00 & 6.968 & 4.161 & 2.489 & 3.585 & 4.35 \\
\hline 8/13/08 & $0: 00$ & 6.931 & 4.044 & 2.41 & 3.513 & 4.1 \\
\hline 8/13/08 & 4:00 & 6.893 & 3.972 & 2.384 & 3.483 & 3.93 \\
\hline 8/13/08 & 8:00 & 6.861 & 3.927 & 2.375 & 3.471 & 3.82 \\
\hline 8/13/08 & $12: 00$ & 6.868 & 3.993 & 2.454 & 3.527 & 3.977 \\
\hline 8/13/08 & $16: 00$ & 6.959 & 4.207 & 2.571 & 3.65 & 4.373 \\
\hline 8/13/08 & 20:00 & 7.008 & 4.231 & 2.531 & 3.625 & 4.453 \\
\hline 8/14/08 & $0: 00$ & 6.977 & 4.107 & 2.438 & 3.552 & 4.222 \\
\hline 8/14/08 & 4:00 & 6.928 & 4.025 & 2.405 & 3.508 & 4.035 \\
\hline 8/14/08 & 8:00 & 6.9 & 3.974 & 2.391 & 3.494 & 3.907 \\
\hline 8/14/08 & $12: 00$ & 6.896 & 4 & 2.431 & 3.525 & 3.977 \\
\hline 8/14/08 & $16: 00$ & 6.968 & 4.184 & 2.55 & 3.636 & 4.329 \\
\hline 8/14/08 & 20:00 & 7.019 & 4.207 & 2.526 & 3.632 & 4.436 \\
\hline 8/15/08 & $0: 00$ & 6.989 & 4.093 & 2.433 & 3.562 & 4.191 \\
\hline 8/15/08 & 4:00 & 6.952 & 4.018 & 2.4 & 3.529 & 4.007 \\
\hline 8/15/08 & $8: 00$ & 6.919 & 3.974 & 2.398 & 3.52 & 3.886 \\
\hline 8/15/08 & $12: 00$ & 6.926 & 4.028 & 2.475 & 3.585 & 4.035 \\
\hline 8/15/08 & $16: 00$ & 7.008 & 4.214 & 2.585 & 3.683 & 4.408 \\
\hline 8/15/08 & 20:00 & 7.047 & 4.235 & 2.54 & 3.662 & 4.502 \\
\hline 8/16/08 & $0: 00$ & 7.017 & 4.116 & 2.442 & 3.59 & 4.266 \\
\hline 8/16/08 & 4:00 & 6.977 & 4.042 & 2.407 & 3.552 & 4.07 \\
\hline 8/16/08 & 8:00 & 6.945 & 3.995 & 2.398 & 3.541 & 3.93 \\
\hline 8/16/08 & $12: 00$ & 6.959 & 4.077 & 2.501 & 3.615 & 4.154 \\
\hline 8/16/08 & $16: 00$ & 7.05 & 4.28 & 2.603 & 3.718 & 4.511 \\
\hline 8/16/08 & $20: 00$ & 7.08 & 4.268 & 2.529 & 3.671 & 4.532 \\
\hline 8/17/08 & $0: 00$ & 7.038 & 4.142 & 2.433 & 3.587 & 4.313 \\
\hline $8 / 17 / 08$ & $4: 00$ & 6.998 & 4.07 & 2.403 & 3.55 & 4.137 \\
\hline 8/17/08 & $8: 00$ & 6.968 & 4.021 & 2.398 & 3.536 & 3.995 \\
\hline 8/17/08 & $12: 00$ & 6.994 & 4.144 & 2.496 & 3.613 & 4.259 \\
\hline 8/17/08 & $16: 00$ & 7.087 & 4.329 & 2.582 & 3.708 & 4.569 \\
\hline 8/17/08 & 20:00 & 7.115 & 4.329 & 2.54 & 3.678 & 4.611 \\
\hline 8/18/08 & $0: 00$ & 7.078 & 4.198 & 2.438 & 3.59 & 4.406 \\
\hline 8/18/08 & $4: 00$ & 7.036 & 4.116 & 2.407 & 3.552 & 4.238 \\
\hline 8/18/08 & $8: 00$ & 7.003 & 4.06 & 2.398 & 3.536 & 4.084 \\
\hline 8/18/08 & $12: 00$ & 7.029 & 4.184 & 2.501 & 3.622 & 4.317 \\
\hline 8/18/08 & $16: 00$ & 7.129 & 4.392 & 2.615 & 3.746 & 4.623 \\
\hline 8/18/08 & 20:00 & 7.162 & 4.373 & 2.543 & 3.697 & 4.637 \\
\hline 8/19/08 & $0: 00$ & 7.12 & 4.233 & 2.442 & 3.608 & 4.429 \\
\hline 8/19/08 & $4: 00$ & 7.078 & 4.151 & 2.412 & 3.569 & 4.266 \\
\hline 8/19/08 & $8: 00$ & 7.043 & 4.095 & 2.403 & 3.555 & 4.112 \\
\hline 8/19/08 & $12: 00$ & 7.059 & 4.184 & 2.508 & 3.634 & 4.301 \\
\hline 8/19/08 & $16: 00$ & 7.141 & 4.345 & 2.599 & 3.725 & 4.548 \\
\hline 8/19/08 & $20: 00$ & 7.185 & 4.38 & 2.557 & 3.706 & 4.655 \\
\hline 8/20/08 & $0: 00$ & 7.145 & 4.242 & 2.449 & 3.615 & 4.455 \\
\hline 8/20/08 & 4:00 & 7.099 & 4.158 & 2.417 & 3.58 & 4.284 \\
\hline 8/20/08 & 8:00 & 7.061 & 4.102 & 2.407 & 3.564 & 4.128 \\
\hline 8/20/08 & $12: 00$ & 7.073 & 4.177 & 2.494 & 3.627 & 4.296 \\
\hline 8/20/08 & $16: 00$ & 7.159 & 4.371 & 2.612 & 3.739 & 4.588 \\
\hline 8/20/08 & $20: 00$ & 7.203 & 4.387 & 2.55 & 3.701 & 4.667 \\
\hline 8/21/08 & $0: 00$ & 7.164 & 4.259 & 2.447 & 3.615 & 4.469 \\
\hline
\end{tabular}


Table S1.4 (Cont.)

Depth to Water (ft below top of casing) in Indicated Well

Date Time PMW4 PMW5 PMW6 PMW7 PMW8

\begin{tabular}{|c|c|c|c|c|c|c|}
\hline 8/21/08 & 4:00 & 7.117 & 4.172 & 2.41 & 3.571 & 4.303 \\
\hline 8/21/08 & $8: 00$ & 7.078 & 4.114 & 2.398 & 3.55 & 4.147 \\
\hline 8/21/08 & $12: 00$ & 7.05 & 4.098 & 2.414 & 3.552 & 4.079 \\
\hline 8/21/08 & $16: 00$ & 7.096 & 4.24 & 2.491 & 3.622 & 4.364 \\
\hline 8/21/08 & $20: 00$ & 7.148 & 4.294 & 2.489 & 3.622 & 4.466 \\
\hline $8 / 22 / 08$ & $0: 00$ & 7.115 & 4.179 & 2.414 & 3.562 & 4.252 \\
\hline 8/22/08 & 4:00 & 7.075 & 4.114 & 2.393 & 3.538 & 4.093 \\
\hline $8 / 22 / 08$ & 8:00 & 7.043 & 4.07 & 2.393 & 3.529 & 3.986 \\
\hline $8 / 22 / 08$ & $12: 00$ & 7.082 & 4.221 & 2.517 & 3.632 & 4.326 \\
\hline $8 / 22 / 08$ & $16: 00$ & 7.199 & 4.431 & 2.605 & 3.76 & 4.63 \\
\hline 8/22/08 & $20: 00$ & 7.241 & 4.427 & 2.54 & 3.72 & 4.662 \\
\hline 8/23/08 & $0: 00$ & 7.21 & 4.301 & 2.456 & 3.653 & 4.459 \\
\hline 8/23/08 & $4: 00$ & 7.175 & 4.224 & 2.431 & 3.627 & 4.308 \\
\hline 8/23/08 & 8:00 & 7.148 & 4.177 & 2.433 & 3.622 & 4.177 \\
\hline 8/23/08 & $12: 00$ & 7.159 & 4.249 & 2.519 & 3.687 & 4.352 \\
\hline 8/23/08 & $16: 00$ & 7.245 & 4.429 & 2.601 & 3.771 & 4.611 \\
\hline 8/23/08 & $20: 00$ & 7.269 & 4.406 & 2.535 & 3.725 & 4.623 \\
\hline $8 / 24 / 08$ & 0:00 & 7.22 & 4.277 & 2.442 & 3.646 & 4.413 \\
\hline 8/24/08 & $4: 00$ & 7.171 & 4.198 & 2.419 & 3.611 & 4.242 \\
\hline $8 / 24 / 08$ & 8:00 & 7.136 & 4.147 & 2.41 & 3.601 & 4.098 \\
\hline $8 / 24 / 08$ & $12: 00$ & 7.145 & 4.217 & 2.519 & 3.676 & 4.294 \\
\hline 8/24/08 & $16: 00$ & 7.231 & 4.401 & 2.612 & 3.774 & 4.569 \\
\hline 8/24/08 & $20: 00$ & 7.28 & 4.42 & 2.568 & 3.743 & 4.658 \\
\hline 8/25/08 & 0:00 & 7.234 & 4.291 & 2.463 & 3.66 & 4.452 \\
\hline $8 / 25 / 08$ & $4: 00$ & 7.187 & 4.212 & 2.424 & 3.618 & 4.282 \\
\hline 8/25/08 & 8:00 & 7.15 & 4.156 & 2.407 & 3.599 & 4.126 \\
\hline 8/25/08 & $12: 00$ & 7.161 & 4.245 & 2.522 & 3.678 & 4.331 \\
\hline 8/25/08 & $16: 00$ & 7.257 & 4.434 & 2.626 & 3.781 & 4.618 \\
\hline $8 / 25 / 08$ & $20: 00$ & 7.299 & 4.443 & 2.556 & 3.743 & 4.679 \\
\hline 8/26/08 & 0:00 & 7.25 & 4.305 & 2.456 & 3.655 & 4.469 \\
\hline 8/26/08 & $4: 00$ & 7.201 & 4.224 & 2.417 & 3.611 & 4.305 \\
\hline 8/26/08 & $8: 00$ & 7.161 & 4.165 & 2.405 & 3.594 & 4.151 \\
\hline 8/26/08 & $12: 00$ & 7.161 & 4.221 & 2.477 & 3.643 & 4.287 \\
\hline 8/26/08 & $16: 00$ & 7.236 & 4.38 & 2.573 & 3.725 & 4.543 \\
\hline 8/26/08 & $20: 00$ & 7.297 & 4.434 & 2.542 & 3.713 & 4.66 \\
\hline 8/27/08 & $0: 00$ & 7.259 & 4.315 & 2.447 & 3.636 & 4.464 \\
\hline $8 / 27 / 08$ & $4: 00$ & 7.215 & 4.24 & 2.421 & 3.608 & 4.315 \\
\hline 8/27/08 & $8: 00$ & 7.185 & 4.203 & 2.435 & 3.618 & 4.198 \\
\hline $8 / 27 / 08$ & $12: 00$ & 7.094 & 3.869 & 2.375 & 3.478 & 3.937 \\
\hline $8 / 27 / 08$ & $16: 00$ & 7.117 & 4.112 & 2.51 & 3.601 & 4.235 \\
\hline $8 / 27 / 08$ & 20:00 & 7.155 & 4.175 & 2.479 & 3.599 & 4.263 \\
\hline 8/28/08 & $0: 00$ & 7.115 & 4.102 & 2.414 & 3.555 & 4.042 \\
\hline 8/28/08 & $4: 00$ & 7.087 & 4.072 & 2.412 & 3.55 & 3.937 \\
\hline 8/28/08 & $8: 00$ & 7.064 & 4.037 & 2.393 & 3.543 & 3.871 \\
\hline 8/28/08 & $12: 00$ & 7.085 & 4.112 & 2.461 & 3.618 & 3.983 \\
\hline 8/28/08 & $16: 00$ & 7.173 & 4.277 & 2.591 & 3.725 & 4.34 \\
\hline 8/28/08 & 20:00 & 7.229 & 4.296 & 2.529 & 3.694 & 4.354 \\
\hline 8/29/08 & 0:00 & 7.196 & 4.193 & 2.449 & 3.643 & 4.119 \\
\hline 8/29/08 & $4: 00$ & 7.155 & 4.126 & 2.421 & 3.618 & 3.969 \\
\hline 8/29/08 & $8: 00$ & 7.117 & 4.084 & 2.414 & 3.61 & 3.876 \\
\hline
\end{tabular}

Table S1.4 (Cont.)

Depth to Water (ft below top of casing) in Indicated Well

Date Time PMW4 PMW5 PMW6 PMW7 PMW8

\begin{tabular}{|c|c|c|c|c|c|c|}
\hline 8/29/08 & $12: 00$ & 7.131 & 4.165 & 2.498 & 3.676 & 4.079 \\
\hline 8/29/08 & $16: 00$ & 7.238 & 4.364 & 2.608 & 3.774 & 4.427 \\
\hline 8/29/08 & 20:00 & 7.285 & 4.366 & 2.545 & 3.732 & 4.45 \\
\hline 8/30/08 & $0: 00$ & 7.25 & 4.263 & 2.466 & 3.673 & 4.252 \\
\hline 8/30/08 & $4: 00$ & 7.201 & 4.189 & 2.426 & 3.634 & 4.074 \\
\hline 8/30/08 & 8:00 & 7.166 & 4.144 & 2.426 & 3.627 & 3.969 \\
\hline 8/30/08 & $12: 00$ & 7.192 & 4.242 & 2.519 & 3.699 & 4.212 \\
\hline 8/30/08 & $16: 00$ & 7.299 & 4.431 & 2.65 & 3.806 & 4.532 \\
\hline 8/30/08 & 20:00 & 7.336 & 4.448 & 2.582 & 3.764 & 4.606 \\
\hline 8/31/08 & $0: 00$ & 7.301 & 4.329 & 2.479 & 3.685 & 4.403 \\
\hline 8/31/08 & 4:00 & 7.259 & 4.254 & 2.445 & 3.648 & 4.247 \\
\hline 8/31/08 & 8:00 & 7.22 & 4.2 & 2.428 & 3.631 & 4.114 \\
\hline 8/31/08 & $12: 00$ & 7.241 & 4.287 & 2.514 & 3.69 & 4.315 \\
\hline 8/31/08 & $16: 00$ & 7.339 & 4.476 & 2.626 & 3.801 & 4.62 \\
\hline 8/31/08 & $20: 00$ & 7.374 & 4.487 & 2.577 & 3.764 & 4.695 \\
\hline 9/1/08 & $0: 00$ & 7.343 & 4.373 & 2.479 & 3.69 & 4.506 \\
\hline 9/1/08 & 4:00 & 7.292 & 4.289 & 2.438 & 3.65 & 4.343 \\
\hline 9/1/08 & $8: 00$ & 7.25 & 4.231 & 2.426 & 3.636 & 4.198 \\
\hline 9/1/08 & $12: 00$ & 7.269 & 4.317 & 2.512 & 3.694 & 4.382 \\
\hline 9/1/08 & $16: 00$ & 7.378 & 4.518 & 2.652 & 3.82 & 4.695 \\
\hline 9/1/08 & 20:00 & 7.413 & 4.532 & 2.591 & 3.783 & 4.753 \\
\hline $9 / 2 / 08$ & 0:00 & 7.376 & 4.408 & 2.486 & 3.706 & 4.562 \\
\hline 9/2/08 & 4:00 & 7.322 & 4.317 & 2.44 & 3.659 & 4.399 \\
\hline $9 / 2 / 08$ & 8:00 & 7.292 & 4.273 & 2.442 & 3.671 & 4.275 \\
\hline $9 / 2 / 08$ & $12: 00$ & 7.287 & 4.273 & 2.459 & 3.701 & 4.256 \\
\hline $9 / 2 / 08$ & $16: 00$ & 7.262 & 4.217 & 2.419 & 3.659 & 4.128 \\
\hline $9 / 2 / 08$ & $20: 00$ & 7.245 & 4.207 & 2.452 & 3.676 & 4.118 \\
\hline 9/3/08 & $0: 00$ & 7.227 & 4.177 & 2.431 & 3.669 & 4.027 \\
\hline 9/3/08 & 4:00 & 7.201 & 4.149 & 2.424 & 3.659 & 3.971 \\
\hline 9/3/08 & 8:00 & 7.18 & 4.121 & 2.419 & 3.652 & 3.918 \\
\hline $9 / 3 / 08$ & $12: 00$ & 7.178 & 4.167 & 2.496 & 3.694 & 4.1 \\
\hline 9/3/08 & $16: 00$ & 7.243 & 4.307 & 2.554 & 3.748 & 4.361 \\
\hline 9/3/08 & 20:00 & 7.276 & 4.3 & 2.512 & 3.715 & 4.373 \\
\hline 9/4/08 & $0: 00$ & 7.234 & 4.207 & 2.447 & 3.664 & 4.16 \\
\hline 9/4/08 & $4: 00$ & 7.192 & 4.151 & 2.421 & 3.638 & 4.023 \\
\hline 9/4/08 & 8:00 & 7.157 & 4.107 & 2.419 & 3.622 & 3.922 \\
\hline 9/4/08 & $12: 00$ & 7.159 & 4.153 & 2.475 & 3.662 & 4.069 \\
\hline $9 / 4 / 08$ & $16: 00$ & 7.236 & 4.317 & 2.563 & 3.739 & 4.377 \\
\hline 9/4/08 & 20:00 & 7.292 & 4.343 & 2.519 & 3.72 & 4.401 \\
\hline 9/5/08 & 0:00 & 7.25 & 4.235 & 2.452 & 3.669 & 4.17 \\
\hline 9/5/08 & $4: 00$ & 7.208 & 4.172 & 2.428 & 3.648 & 4.018 \\
\hline 9/5/08 & 8:00 & 7.178 & 4.13 & 2.426 & 3.645 & 3.927 \\
\hline 9/5/08 & $12: 00$ & 7.166 & 4.132 & 2.442 & 3.659 & 3.946 \\
\hline 9/5/08 & $16: 00$ & 7.173 & 4.151 & 2.456 & 3.673 & 4.004 \\
\hline 9/5/08 & 20:00 & 7.168 & 4.132 & 2.44 & 3.666 & 3.948 \\
\hline 9/6/08 & $0: 00$ & 7.143 & 4.088 & 2.419 & 3.648 & 3.848 \\
\hline 9/6/08 & 4:00 & 7.122 & 4.06 & 2.412 & 3.634 & 3.803 \\
\hline 9/6/08 & $8: 00$ & 7.099 & 4.044 & 2.421 & 3.638 & 3.785 \\
\hline 9/6/08 & $12: 00$ & 7.096 & 4.044 & 2.426 & 3.65 & 3.801 \\
\hline 9/6/08 & $16: 00$ & 7.101 & 4.074 & 2.445 & 3.657 & 3.892 \\
\hline
\end{tabular}


Table S1.4 (Cont.)

Depth to Water (ft below top of casing) in Indicated Well

Date Time PMW4 PMW5 PMW6 PMW7 PMW8

\begin{tabular}{|c|c|c|c|c|c|c|}
\hline 9/6/08 & $20: 00$ & 7.089 & 4.039 & 2.431 & 3.641 & 3.806 \\
\hline 9/7/08 & 0:00 & 7.059 & 3.855 & 2.389 & 3.559 & 3.712 \\
\hline 9/7/08 & $4: 00$ & 7.033 & 3.908 & 2.402 & 3.582 & 3.694 \\
\hline 9/7/08 & 8:00 & 7.024 & 3.929 & 2.419 & 3.608 & 3.689 \\
\hline 9/7/08 & $12: 00$ & 7.017 & 3.932 & 2.419 & 3.62 & 3.675 \\
\hline 9/7/08 & $16: 00$ & 7.052 & 4.013 & 2.463 & 3.652 & 3.836 \\
\hline 9/7/08 & $20: 00$ & 7.085 & 4.055 & 2.461 & 3.664 & 3.904 \\
\hline 9/8/08 & 0:00 & 7.068 & 4.011 & 2.435 & 3.648 & 3.771 \\
\hline 9/8/08 & $4: 00$ & 7.045 & 3.985 & 2.426 & 3.643 & 3.701 \\
\hline 9/8/08 & 8:00 & 7.029 & 3.843 & 2.402 & 3.606 & 3.654 \\
\hline 9/8/08 & $12: 00$ & 6.977 & 3.687 & 2.382 & 3.552 & 3.556 \\
\hline 9/8/08 & $16: 00$ & 6.989 & 3.857 & 2.458 & 3.608 & 3.729 \\
\hline 9/8/08 & 20:00 & 7.015 & 3.932 & 2.447 & 3.622 & 3.789 \\
\hline 9/9/08 & 0:00 & 6.998 & 3.915 & 2.423 & 3.62 & 3.67 \\
\hline 9/9/08 & $4: 00$ & 6.982 & 3.901 & 2.419 & 3.617 & 3.612 \\
\hline 9/9/08 & $8: 00$ & 6.973 & 3.894 & 2.426 & 3.622 & 3.589 \\
\hline 9/9/08 & $12: 00$ & 6.987 & 3.948 & 2.477 & 3.657 & 3.696 \\
\hline 9/9/08 & $16: 00$ & 7.068 & 4.095 & 2.542 & 3.704 & 4.06 \\
\hline 9/9/08 & 20:00 & 7.11 & 4.123 & 2.507 & 3.685 & 4.086 \\
\hline 9/10/08 & 0:00 & 7.089 & 4.055 & 2.449 & 3.648 & 3.887 \\
\hline 9/10/08 & 4:00 & 7.066 & 4.03 & 2.444 & 3.638 & 3.806 \\
\hline 9/10/08 & $8: 00$ & 7.047 & 4.004 & 2.437 & 3.636 & 3.738 \\
\hline 9/10/08 & $12: 00$ & 7.057 & 4.034 & 2.458 & 3.65 & 3.771 \\
\hline 9/10/08 & $16: 00$ & 7.129 & 4.165 & 2.512 & 3.694 & 4.069 \\
\hline 9/10/08 & 20:00 & 7.138 & 4.13 & 2.468 & 3.662 & 3.983 \\
\hline 9/11/08 & 0:00 & 7.103 & 4.06 & 2.44 & 3.634 & 3.822 \\
\hline 9/11/08 & 4:00 & 7.066 & 4.011 & 2.426 & 3.617 & 3.715 \\
\hline 9/11/08 & 8:00 & 7.047 & 3.992 & 2.421 & 3.622 & 3.668 \\
\hline 9/11/08 & $12: 00$ & 6.989 & 3.698 & 2.389 & 3.538 & 3.514 \\
\hline 9/11/08 & $16: 00$ & 6.977 & 3.757 & 2.389 & 3.545 & 3.554 \\
\hline 9/11/08 & 20:00 & 6.891 & 3.042 & 2.328 & 3.422 & 3.04 \\
\hline 9/12/08 & 0:00 & 6.847 & 3.437 & 2.365 & 3.426 & 3.178 \\
\hline 9/12/08 & $4: 00$ & 6.835 & 3.582 & 2.377 & 3.499 & 3.266 \\
\hline 9/12/08 & $8: 00$ & 6.833 & 3.647 & 2.382 & 3.522 & 3.313 \\
\hline 9/12/08 & $12: 00$ & 6.817 & 3.491 & 2.3 & & 3.18 \\
\hline 9/12/08 & $16: 00$ & 6.73 & 3.014 & 2.314 & 3.34 & 2.72 \\
\hline 9/12/08 & $20: 00$ & 6.691 & 3.208 & 2.337 & 3.382 & 2.739 \\
\hline 9/13/08 & 0:00 & 6.677 & 3.343 & 2.351 & 3.415 & 2.797 \\
\hline 9/13/08 & $4: 00$ & 6.67 & 3.397 & 2.354 & 3.431 & 2.83 \\
\hline 9/13/08 & $8: 00$ & 6.674 & 3.465 & 2.356 & 3.457 & 2.898 \\
\hline 9/13/08 & $12: 00$ & 6.693 & 3.53 & 2.372 & 3.485 & 2.958 \\
\hline 9/13/08 & $16: 00$ & 6.786 & 3.733 & 2.458 & 3.552 & 3.271 \\
\hline 9/13/08 & $20: 00$ & 6.84 & 3.794 & 2.435 & 3.568 & 3.367 \\
\hline 9/14/08 & 0:00 & 6.835 & 3.771 & 2.398 & 3.564 & 3.325 \\
\hline 9/14/08 & 4:00 & 6.821 & 3.759 & 2.386 & 3.561 & 3.306 \\
\hline 9/14/08 & 8:00 & 6.84 & 3.794 & 2.416 & 3.594 & 3.348 \\
\hline 9/14/08 & $12: 00$ & 6.868 & 3.843 & 2.43 & 3.617 & 3.42 \\
\hline 9/14/08 & $16: 00$ & 6.905 & 3.904 & 2.463 & 3.645 & 3.546 \\
\hline 9/14/08 & 20:00 & 6.94 & 3.948 & 2.472 & 3.666 & 3.621 \\
\hline 9/15/08 & $0: 00$ & 6.931 & 3.904 & 2.437 & 3.659 & 3.53 \\
\hline
\end{tabular}

Table S1.4 (Cont.)

Depth to Water (ft below top of casing) in Indicated Well

Date Time PMW4 PMW5 PMW6 PMW7 PMW8

\begin{tabular}{|c|c|c|c|c|c|c|}
\hline 9/15/08 & 4:00 & 6.91 & 3.873 & 2.428 & 3.657 & 3.472 \\
\hline 9/15/08 & 8:00 & 6.9 & 3.859 & 2.433 & 3.664 & 3.444 \\
\hline 9/15/08 & $12: 00$ & 6.924 & 3.911 & 2.482 & 3.694 & 3.514 \\
\hline 9/15/08 & $16: 00$ & 6.982 & 4.046 & 2.531 & 3.717 & 3.84 \\
\hline 9/15/08 & 20:00 & 7.008 & 4.046 & 2.493 & 3.694 & 3.826 \\
\hline 9/16/08 & $0: 00$ & 6.989 & 3.985 & 2.458 & 3.68 & 3.698 \\
\hline 9/16/08 & $4: 00$ & 6.961 & 3.941 & 2.435 & 3.666 & 3.609 \\
\hline 9/16/08 & 8:00 & 6.94 & 3.911 & 2.43 & 3.664 & 3.544 \\
\hline 9/16/08 & $12: 00$ & 6.98 & 3.999 & 2.486 & 3.701 & 3.705 \\
\hline 9/16/08 & $16: 00$ & 7.052 & 4.151 & 2.549 & 3.738 & 4.072 \\
\hline 9/16/08 & 20:00 & 7.078 & 4.132 & 2.498 & 3.708 & 4.015 \\
\hline 9/17/08 & $0: 00$ & 7.045 & 4.046 & 2.449 & 3.683 & 3.812 \\
\hline 9/17/08 & 4:00 & 7.015 & 3.999 & 2.428 & 3.671 & 3.698 \\
\hline 9/17/08 & 8:00 & 6.991 & 3.967 & 2.426 & 3.669 & 3.621 \\
\hline 9/17/08 & $12: 00$ & 7.031 & 4.058 & 2.491 & 3.715 & 3.812 \\
\hline 9/17/08 & $16: 00$ & 7.113 & 4.214 & 2.554 & 3.755 & 4.167 \\
\hline 9/17/08 & 20:00 & 7.134 & 4.191 & 2.505 & 3.724 & 4.125 \\
\hline 9/18/08 & $0: 00$ & 7.094 & 4.097 & 2.456 & 3.696 & 3.91 \\
\hline 9/18/08 & 4:00 & 7.061 & 4.044 & 2.435 & 3.685 & 3.777 \\
\hline 9/18/08 & 8:00 & 7.033 & 4.006 & 2.426 & 3.68 & 3.686 \\
\hline 9/18/08 & $12: 00$ & 7.054 & 4.074 & 2.496 & 3.722 & 3.871 \\
\hline 9/18/08 & $16: 00$ & 7.141 & 4.226 & 2.561 & 3.759 & 4.202 \\
\hline 9/18/08 & 20:00 & 7.159 & 4.212 & 2.514 & 3.731 & 4.186 \\
\hline 9/19/08 & 0:00 & 7.122 & 4.118 & 2.458 & 3.699 & 3.969 \\
\hline 9/19/08 & $4: 00$ & 7.082 & 4.06 & 2.433 & 3.685 & 3.822 \\
\hline 9/19/08 & $8: 00$ & 7.054 & 4.02 & 2.433 & 3.68 & 3.724 \\
\hline 9/19/08 & $12: 00$ & 7.064 & 4.072 & 2.477 & 3.71 & 3.861 \\
\hline 9/19/08 & $16: 00$ & 7.136 & 4.207 & 2.528 & 3.745 & 4.165 \\
\hline 9/19/08 & 20:00 & 7.164 & 4.207 & 2.493 & 3.722 & 4.162 \\
\hline 9/20/08 & $0: 00$ & 7.124 & 4.118 & 2.451 & 3.699 & 3.943 \\
\hline 9/20/08 & $4: 00$ & 7.089 & 4.062 & 2.433 & 3.687 & 3.808 \\
\hline 9/20/08 & 8:00 & 7.061 & 4.025 & 2.428 & 3.689 & 3.719 \\
\hline 9/20/08 & $12: 00$ & 7.071 & 4.072 & 2.477 & 3.72 & 3.847 \\
\hline 9/20/08 & $16: 00$ & 7.152 & 4.233 & 2.556 & 3.762 & 4.216 \\
\hline 9/20/08 & 20:00 & 7.173 & 4.214 & 2.505 & 3.736 & 4.181 \\
\hline 9/21/08 & $0: 00$ & 7.138 & 4.13 & 2.458 & 3.715 & 3.969 \\
\hline 9/21/08 & 4:00 & 7.103 & 4.072 & 2.437 & 3.699 & 3.833 \\
\hline 9/21/08 & 8:00 & 7.075 & 4.032 & 2.428 & 3.696 & 3.735 \\
\hline 9/21/08 & $12: 00$ & 7.075 & 4.065 & 2.472 & 3.722 & 3.84 \\
\hline 9/21/08 & $16: 00$ & 7.138 & 4.198 & 2.547 & 3.759 & 4.174 \\
\hline 9/21/08 & 20:00 & 7.162 & 4.188 & 2.5 & 3.731 & 4.158 \\
\hline 9/22/08 & $0: 00$ & 7.131 & 4.114 & 2.454 & 3.71 & 3.955 \\
\hline 9/22/08 & 4:00 & 7.101 & 4.062 & 2.437 & 3.701 & 3.826 \\
\hline 9/22/08 & 8:00 & 7.066 & 3.99 & 2.409 & 3.673 & 3.71 \\
\hline $9 / 22 / 08$ & $12: 00$ & 7.005 & 3.733 & 2.393 & 3.617 & 3.455 \\
\hline 9/22/08 & $16: 00$ & 7.029 & 3.95 & 2.491 & 3.671 & 3.857 \\
\hline 9/22/08 & $20: 00$ & 7.061 & 4.03 & 2.477 & 3.685 & 3.917 \\
\hline 9/23/08 & 0:00 & 7.047 & 3.999 & 2.435 & 3.673 & 3.749 \\
\hline 9/23/08 & 4:00 & 7.026 & 3.967 & 2.426 & 3.671 & 3.647 \\
\hline 9/23/08 & 8:00 & 7.01 & 3.946 & 2.421 & 3.675 & 3.584 \\
\hline
\end{tabular}


Table S1.4 (Cont.)

Depth to Water (ft below top of casing) in Indicated Well

Date Time PMW4 PMW5 PMW6 PMW7 PMW8

\begin{tabular}{|c|c|c|c|c|c|c|}
\hline 9/23/08 & $12: 00$ & 7.024 & 4.013 & 2.468 & 3.708 & 3.742 \\
\hline 9/23/08 & $16: 00$ & 7.064 & 4.048 & 2.449 & 3.685 & 3.91 \\
\hline 9/23/08 & $20: 00$ & 7.029 & 3.943 & 2.421 & 3.668 & 3.693 \\
\hline 9/24/08 & $0: 00$ & 7.012 & 3.922 & 2.414 & 3.675 & 3.593 \\
\hline 9/24/08 & $4: 00$ & 6.994 & 3.904 & 2.409 & 3.675 & 3.525 \\
\hline 9/24/08 & $8: 00$ & 6.987 & 3.899 & 2.414 & 3.687 & 3.49 \\
\hline 9/24/08 & $12: 00$ & 6.982 & 3.899 & 2.426 & 3.701 & 3.479 \\
\hline 9/24/08 & $16: 00$ & 7.022 & 4.016 & 2.496 & 3.731 & 3.808 \\
\hline 9/24/08 & $20: 00$ & 7.047 & 4.034 & 2.477 & 3.717 & 3.852 \\
\hline 9/25/08 & $0: 00$ & 7.026 & 3.976 & 2.44 & 3.708 & 3.686 \\
\hline 9/25/08 & $4: 00$ & 7.003 & 3.941 & 2.419 & 3.694 & 3.593 \\
\hline 9/25/08 & $8: 00$ & 6.989 & 3.922 & 2.421 & 3.696 & 3.537 \\
\hline 9/25/08 & $12: 00$ & 6.998 & 3.978 & 2.458 & 3.72 & 3.675 \\
\hline 9/25/08 & $16: 00$ & 7.05 & 4.104 & 2.521 & 3.743 & 4.036 \\
\hline 9/25/08 & 20:00 & 7.073 & 4.102 & 2.491 & 3.722 & 4.034 \\
\hline 9/26/08 & $0: 00$ & 7.05 & 4.032 & 2.442 & 3.703 & 3.833 \\
\hline 9/26/08 & $4: 00$ & 7.022 & 3.983 & 2.428 & 3.689 & 3.705 \\
\hline 9/26/08 & $8: 00$ & 7.001 & 3.953 & 2.426 & 3.689 & 3.626 \\
\hline 9/26/08 & $12: 00$ & 7.012 & 4.023 & 2.475 & 3.717 & 3.838 \\
\hline 9/26/08 & $16: 00$ & 7.071 & 4.158 & 2.554 & 3.752 & 4.216 \\
\hline 9/26/08 & 20:00 & 7.094 & 4.151 & 2.505 & 3.731 & 4.204 \\
\hline 9/27/08 & $0: 00$ & 7.071 & 4.074 & 2.454 & 3.71 & 3.978 \\
\hline 9/27/08 & $4: 00$ & 7.045 & 4.02 & 2.43 & 3.699 & 3.826 \\
\hline 9/27/08 & $8: 00$ & 7.022 & 3.985 & 2.421 & 3.701 & 3.719 \\
\hline 9/27/08 & $12: 00$ & 7.017 & 3.997 & 2.447 & 3.724 & 3.742 \\
\hline 9/27/08 & $16: 00$ & 7.061 & 4.109 & 2.519 & 3.757 & 4.095 \\
\hline 9/27/08 & 20:00 & 7.087 & 4.118 & 2.496 & 3.748 & 4.123 \\
\hline 9/28/08 & 0:00 & 7.064 & 4.044 & 2.444 & 3.729 & 3.887 \\
\hline 9/28/08 & $4: 00$ & 7.033 & 3.992 & 2.435 & 3.72 & 3.747 \\
\hline 9/28/08 & 8:00 & 7.015 & 3.964 & 2.43 & 3.722 & 3.658 \\
\hline 9/28/08 & $12: 00$ & 7.01 & 3.974 & 2.458 & 3.736 & 3.71 \\
\hline 9/28/08 & $16: 00$ & 7.045 & 4.095 & 2.514 & 3.759 & 4.076 \\
\hline 9/28/08 & $20: 00$ & 7.057 & 4.081 & 2.484 & 3.731 & 4.05 \\
\hline 9/29/08 & 0:00 & 7.031 & 4.018 & 2.444 & 3.708 & 3.864 \\
\hline 9/29/08 & $4: 00$ & 7.022 & 3.974 & 2.423 & 3.708 & 3.735 \\
\hline 9/29/08 & $8: 00$ & 7.012 & 3.953 & 2.435 & 3.729 & 3.651 \\
\hline 9/29/08 & $12: 00$ & 7.015 & 3.974 & 2.461 & 3.752 & 3.705 \\
\hline 9/29/08 & $16: 00$ & 7.05 & 4.079 & 2.514 & 3.773 & 4.05 \\
\hline 9/29/08 & $20: 00$ & 7.068 & 4.086 & 2.489 & 3.762 & 4.034 \\
\hline 9/30/08 & 0:00 & 7.043 & 4.02 & 2.451 & 3.748 & 3.822 \\
\hline 9/30/08 & 4:00 & 7.024 & 3.978 & 2.437 & 3.741 & 3.7 \\
\hline 9/30/08 & $8: 00$ & 7.008 & 3.953 & 2.435 & 3.745 & 3.623 \\
\hline 9/30/08 & $12: 00$ & 7.008 & 3.976 & 2.468 & 3.762 & 3.714 \\
\hline 9/30/08 & $16: 00$ & 7.04 & 4.088 & 2.528 & 3.778 & 4.088 \\
\hline 9/30/08 & $20: 00$ & 7.061 & 4.093 & 2.496 & 3.755 & 4.074 \\
\hline 10/1/08 & 0:00 & 7.033 & 4.018 & 2.454 & 3.738 & 3.852 \\
\hline 10/1/08 & $4: 00$ & 7.015 & 3.983 & 2.44 & 3.729 & 3.749 \\
\hline $10 / 1 / 08$ & 8:00 & 7.008 & 3.962 & 2.444 & 3.745 & 3.672 \\
\hline 10/1/08 & $12: 00$ & 7.019 & 3.999 & 2.484 & 3.776 & 3.812 \\
\hline 10/1/08 & $16: 00$ & 7.052 & 4.086 & 2.521 & 3.79 & 4.097 \\
\hline
\end{tabular}

Table S1.4 (Cont.)

Depth to Water (ft below top of casing) in Indicated Well

Date Time PMW4 PMW5 PMW6 PMW7 PMW8

\begin{tabular}{|c|c|c|c|c|c|c|}
\hline 10/1/08 & 20:00 & 7.066 & 4.081 & 2.491 & 3.773 & 4.067 \\
\hline 10/2/08 & $0: 00$ & 7.04 & 4.009 & 2.447 & 3.755 & 3.838 \\
\hline 10/2/08 & 4:00 & 7.015 & 3.967 & 2.435 & 3.745 & 3.707 \\
\hline 10/2/08 & 8:00 & 6.996 & 3.939 & 2.435 & 3.748 & 3.621 \\
\hline $10 / 2 / 08$ & $12: 00$ & 6.998 & 3.967 & 2.472 & 3.762 & 3.719 \\
\hline 10/2/08 & $16: 00$ & 7.031 & 4.076 & 2.517 & 3.771 & 4.083 \\
\hline 10/2/08 & 20:00 & 7.045 & 4.074 & 2.479 & 3.748 & 4.046 \\
\hline 10/3/08 & $0: 00$ & 7.029 & 4.016 & 2.451 & 3.738 & 3.857 \\
\hline 10/3/08 & 4:00 & 7.01 & 3.976 & 2.435 & 3.736 & 3.735 \\
\hline 10/3/08 & $8: 00$ & 6.998 & 3.948 & 2.437 & 3.745 & 3.647 \\
\hline 10/3/08 & $12: 00$ & 7.012 & 3.997 & 2.486 & 3.778 & 3.803 \\
\hline 10/3/08 & $16: 00$ & 7.061 & 4.118 & 2.531 & 3.797 & 4.174 \\
\hline 10/3/08 & 20:00 & 7.08 & 4.107 & 2.498 & 3.78 & 4.13 \\
\hline 10/4/08 & $0: 00$ & 7.066 & 4.048 & 2.47 & 3.778 & 3.938 \\
\hline 10/4/08 & 4:00 & 7.045 & 3.997 & 2.449 & 3.771 & 3.791 \\
\hline 10/4/08 & 8:00 & 7.022 & 3.964 & 2.444 & 3.769 & 3.689 \\
\hline 10/4/08 & $12: 00$ & 7.024 & 3.988 & 2.484 & 3.785 & 3.819 \\
\hline 10/4/08 & $16: 00$ & 7.054 & 4.102 & 2.549 & 3.804 & 4.197 \\
\hline 10/4/08 & 20:00 & 7.073 & 4.1 & 2.503 & 3.778 & 4.176 \\
\hline 10/5/08 & $0: 00$ & 7.064 & 4.058 & 2.47 & 3.771 & 4.015 \\
\hline 10/5/08 & 4:00 & 7.04 & 4.009 & 2.444 & 3.757 & 3.861 \\
\hline 10/5/08 & 8:00 & 7.024 & 3.971 & 2.44 & 3.755 & 3.752 \\
\hline 10/5/08 & $12: 00$ & 7.026 & 4.004 & 2.465 & 3.773 & 3.875 \\
\hline 10/5/08 & $16: 00$ & 7.073 & 4.137 & 2.531 & 3.799 & 4.274 \\
\hline 10/5/08 & $20: 00$ & 7.096 & 4.139 & 2.5 & 3.789 & 4.249 \\
\hline 10/6/08 & $0: 00$ & 7.087 & 4.081 & 2.465 & 3.778 & 4.076 \\
\hline 10/6/08 & $4: 00$ & 7.064 & 4.032 & 2.444 & 3.766 & 3.922 \\
\hline 10/6/08 & $8: 00$ & 7.047 & 3.997 & 2.437 & 3.771 & 3.805 \\
\hline 10/6/08 & $12: 00$ & 7.04 & 3.997 & 2.451 & 3.776 & 3.838 \\
\hline 10/6/08 & $16: 00$ & 7.043 & 3.871 & 2.449 & 3.738 & 3.945 \\
\hline 10/6/08 & 20:00 & 6.847 & 3.178 & 2.356 & 3.487 & 2.722 \\
\hline 10/7/08 & $0: 00$ & 6.807 & 3.458 & 2.377 & 3.573 & 2.83 \\
\hline 10/7/08 & 4:00 & 6.663 & 2.846 & 2.316 & 3.361 & 2.442 \\
\hline 10/7/08 & 8:00 & 6.612 & 3.147 & 2.356 & 3.48 & 2.575 \\
\hline 10/7/08 & $12: 00$ & 6.618 & 3.35 & 2.395 & 3.564 & 2.715 \\
\hline 10/7/08 & $16: 00$ & 6.653 & 3.511 & 2.43 & 3.615 & 2.963 \\
\hline 10/7/08 & 20:00 & 6.686 & 3.593 & 2.426 & 3.638 & 3.082 \\
\hline 10/8/08 & $0: 00$ & 6.688 & 3.607 & 2.405 & 3.647 & 3.093 \\
\hline 10/8/08 & $4: 00$ & 6.693 & 3.624 & 2.402 & 3.661 & 3.114 \\
\hline 10/8/08 & 8:00 & 6.707 & 3.642 & 2.409 & 3.673 & 3.142 \\
\hline 10/8/08 & $12: 00$ & 6.726 & 3.696 & 2.449 & 3.699 & 3.32 \\
\hline 10/8/08 & $16: 00$ & 6.768 & 3.785 & 2.489 & 3.72 & 3.588 \\
\hline 10/8/08 & 20:00 & 6.8 & 3.801 & 2.463 & 3.724 & 3.551 \\
\hline 10/9/08 & $0: 00$ & 6.805 & 3.773 & 2.437 & 3.727 & 3.439 \\
\hline 10/9/08 & 4:00 & 6.803 & 3.754 & 2.426 & 3.729 & 3.383 \\
\hline 10/9/08 & $8: 00$ & 6.812 & 3.754 & 2.433 & 3.748 & 3.362 \\
\hline 10/9/08 & $12: 00$ & 6.821 & 3.785 & 2.477 & 3.771 & 3.504 \\
\hline 10/9/08 & $16: 00$ & 6.845 & 3.859 & 2.517 & 3.773 & 3.796 \\
\hline 10/9/08 & $20: 00$ & 6.854 & 3.862 & 2.486 & 3.757 & 3.756 \\
\hline 10/10/08 & 0:00 & 6.852 & 3.822 & 2.454 & 3.75 & 3.609 \\
\hline
\end{tabular}


Table S1.4 (Cont.)

Depth to Water (ft below top of casing) in Indicated Well

Date Time PMW4 PMW5 PMW6 PMW7 PMW8

\begin{tabular}{|c|c|c|c|c|c|c|}
\hline 10/10/08 & 4:00 & 6.838 & 3.794 & 2.433 & 3.741 & 3.52 \\
\hline $10 / 10 / 08$ & 8:00 & 6.831 & 3.775 & 2.43 & 3.743 & 3.46 \\
\hline 10/10/08 & $12: 00$ & 6.842 & 3.806 & 2.458 & 3.755 & 3.562 \\
\hline 10/10/08 & $16: 00$ & 6.861 & 3.883 & 2.51 & 3.773 & 3.875 \\
\hline $10 / 10 / 08$ & $20: 00$ & 6.882 & 3.89 & 2.486 & 3.769 & 3.828 \\
\hline 10/11/08 & 0:00 & 6.884 & 3.855 & 2.454 & 3.766 & 3.667 \\
\hline 10/11/08 & 4:00 & 6.877 & 3.824 & 2.437 & 3.764 & 3.569 \\
\hline $10 / 11 / 08$ & 8:00 & 6.877 & 3.815 & 2.442 & 3.773 & 3.516 \\
\hline 10/11/08 & $12: 00$ & 6.882 & 3.827 & 2.447 & 3.785 & 3.525 \\
\hline 10/11/08 & 16:00 & 6.903 & 3.904 & 2.489 & 3.799 & 3.831 \\
\hline 10/11/08 & 20:00 & 6.912 & 3.89 & 2.468 & 3.792 & 3.775 \\
\hline $10 / 12 / 08$ & $0: 00$ & 6.903 & 3.852 & 2.44 & 3.78 & 3.646 \\
\hline 10/12/08 & 4:00 & 6.896 & 3.834 & 2.44 & 3.782 & 3.569 \\
\hline $10 / 12 / 08$ & 8:00 & 6.891 & 3.815 & 2.433 & 3.78 & 3.516 \\
\hline $10 / 12 / 08$ & $12: 00$ & 6.894 & 3.831 & 2.447 & 3.792 & 3.548 \\
\hline $10 / 12 / 08$ & $16: 00$ & 6.91 & 3.892 & 2.477 & 3.792 & 3.814 \\
\hline $10 / 12 / 08$ & 20:00 & 6.926 & 3.899 & 2.47 & 3.799 & 3.782 \\
\hline 10/13/08 & 0:00 & 6.914 & 3.864 & 2.44 & 3.785 & 3.665 \\
\hline 10/13/08 & $4: 00$ & 6.91 & 3.841 & 2.433 & 3.787 & 3.597 \\
\hline 10/13/08 & $8: 00$ & 6.642 & 2.699 & 2.316 & 3.373 & 2.447 \\
\hline $10 / 13 / 08$ & $12: 00$ & 6.371 & 2.646 & 2.288 & 3.282 & 2.356 \\
\hline 10/13/08 & $16: 00$ & 6.26 & 2.8 & 2.321 & 3.375 & 2.435 \\
\hline 10/13/08 & 20:00 & 6.269 & 2.986 & 2.372 & 3.501 & 2.554 \\
\hline $10 / 14 / 08$ & $0: 00$ & 6.299 & 3.115 & 2.384 & 3.571 & 2.634 \\
\hline 10/14/08 & 4:00 & 6.323 & 3.196 & 2.393 & 3.61 & 2.701 \\
\hline 10/14/08 & $8: 00$ & 6.355 & 3.266 & 2.409 & 3.65 & 2.769 \\
\hline $10 / 14 / 08$ & $12: 00$ & 6.376 & 3.308 & 2.409 & 3.668 & 2.818 \\
\hline 10/14/08 & $16: 00$ & 6.385 & 3.299 & 2.402 & 3.659 & 2.834 \\
\hline 10/14/08 & 20:00 & 6.257 & 2.72 & 2.344 & 3.531 & 2.4 \\
\hline 10/15/08 & 0:00 & 6.11 & 2.786 & 2.339 & 3.461 & 2.4 \\
\hline 10/15/08 & 4:00 & 5.8 & 2.468 & 2.286 & 3.389 & 2.279 \\
\hline 10/15/08 & 8:00 & 5.635 & 2.622 & 2.321 & 3.34 & 2.361 \\
\hline 10/15/08 & $12: 00$ & 5.67 & 2.732 & 2.36 & 3.417 & 2.438 \\
\hline 10/15/08 & 16:00 & 5.714 & 2.823 & 2.409 & 3.498 & 2.587 \\
\hline 10/15/08 & 20:00 & 5.773 & 2.888 & 2.412 & 3.552 & 2.68 \\
\hline 10/16/08 & $0: 00$ & 5.817 & 2.93 & 2.407 & 3.594 & 2.727 \\
\hline $10 / 16 / 08$ & $4: 00$ & 5.852 & 2.963 & 2.416 & 3.619 & 2.769 \\
\hline 10/16/08 & $8: 00$ & 5.887 & 3 & 2.43 & 3.65 & 2.811 \\
\hline 10/16/08 & $12: 00$ & 5.922 & 3.035 & 2.449 & 3.675 & 2.869 \\
\hline 10/16/08 & $16: 00$ & 5.945 & 3.094 & 2.461 & 3.68 & 3.012 \\
\hline 10/16/08 & $20: 00$ & 5.975 & 3.119 & 2.454 & 3.687 & 3.049 \\
\hline 10/17/08 & $0: 00$ & 6.003 & 3.133 & 2.442 & 3.692 & 3.042 \\
\hline $10 / 17 / 08$ & $4: 00$ & 6.013 & 3.133 & 2.435 & 3.687 & 3.047 \\
\hline 10/17/08 & $8: 00$ & 6.024 & 3.005 & 2.421 & 3.671 & 2.848 \\
\hline 10/17/08 & $12: 00$ & 6.043 & 3.073 & 2.44 & 3.692 & 2.881 \\
\hline $10 / 17 / 08$ & $16: 00$ & 6.075 & 3.152 & 2.461 & 3.71 & 3.044 \\
\hline 10/17/08 & 20:00 & 6.106 & 3.194 & 2.456 & 3.727 & 3.075 \\
\hline 10/18/08 & $0: 00$ & 6.124 & 3.208 & 2.447 & 3.734 & 3.065 \\
\hline 10/18/08 & 4:00 & 6.136 & 3.217 & 2.442 & 3.736 & 3.058 \\
\hline 10/18/08 & 8:00 & 6.155 & 3.229 & 2.447 & 3.748 & 3.056 \\
\hline
\end{tabular}

Table S1.4 (Cont.)

Depth to Water (ft below top of casing) in Indicated Well

Date Time PMW4 PMW5 PMW6 PMW7 PMW8

\begin{tabular}{|c|c|c|c|c|c|c|}
\hline $10 / 18 / 08$ & $12: 00$ & 6.169 & 3.234 & 2.451 & 3.752 & 3.047 \\
\hline 10/18/08 & $16: 00$ & 6.171 & 3.259 & 2.47 & 3.745 & 3.175 \\
\hline 10/18/08 & 20:00 & 6.187 & 3.28 & 2.463 & 3.741 & 3.21 \\
\hline 10/19/08 & 0:00 & 6.199 & 3.278 & 2.447 & 3.736 & 3.18 \\
\hline 10/19/08 & $4: 00$ & 6.206 & 3.276 & 2.442 & 3.731 & 3.159 \\
\hline $10 / 19 / 08$ & $8: 00$ & 6.213 & 3.283 & 2.44 & 3.734 & 3.154 \\
\hline $10 / 19 / 08$ & $12: 00$ & 6.229 & 3.315 & 2.461 & 3.748 & 3.268 \\
\hline 10/19/08 & $16: 00$ & 6.257 & 3.376 & 2.498 & 3.757 & 3.513 \\
\hline 10/19/08 & $20: 00$ & 6.288 & 3.395 & 2.475 & 3.761 & 3.483 \\
\hline 10/20/08 & 0:00 & 6.306 & 3.383 & 2.454 & 3.764 & 3.378 \\
\hline $10 / 20 / 08$ & $4: 00$ & 6.323 & 3.385 & 2.456 & 3.775 & 3.329 \\
\hline $10 / 20 / 08$ & $8: 00$ & 6.343 & 3.397 & 2.461 & 3.794 & 3.301 \\
\hline $10 / 20 / 08$ & $12: 00$ & 6.364 & 3.418 & 2.479 & 3.817 & 3.336 \\
\hline 10/20/08 & $16: 00$ & 6.378 & 3.453 & 2.503 & 3.82 & 3.548 \\
\hline 10/20/08 & $20: 00$ & 6.395 & 3.462 & 2.489 & 3.817 & 3.523 \\
\hline $10 / 21 / 08$ & 0:00 & 6.399 & 3.441 & 2.47 & 3.815 & 3.42 \\
\hline $10 / 21 / 08$ & $4: 00$ & 6.404 & 3.434 & 2.465 & 3.81 & 3.376 \\
\hline $10 / 21 / 08$ & $8: 00$ & 6.404 & 3.43 & 2.458 & 3.806 & 3.341 \\
\hline $10 / 21 / 08$ & $12: 00$ & 6.397 & 3.43 & 2.461 & 3.801 & 3.336 \\
\hline 10/21/08 & $16: 00$ & 6.376 & 3.157 & 2.414 & 3.75 & 3.238 \\
\hline $10 / 21 / 08$ & 20:00 & 6.295 & 2.975 & 2.402 & 3.685 & 2.694 \\
\hline $10 / 22 / 08$ & $0: 00$ & 6.267 & 3.061 & 2.405 & 3.687 & 2.671 \\
\hline $10 / 22 / 08$ & $4: 00$ & 5.901 & 2.555 & 2.332 & 3.463 & 2.326 \\
\hline $10 / 22 / 08$ & 8:00 & 4.906 & 2.391 & 2.281 & 3.17 & 2.26 \\
\hline $10 / 22 / 08$ & $12: 00$ & 4.938 & 2.454 & 2.314 & 3.27 & 2.328 \\
\hline $10 / 22 / 08$ & $16: 00$ & 4.982 & 2.506 & 2.33 & 3.324 & 2.37 \\
\hline $10 / 22 / 08$ & $20: 00$ & 5.069 & 2.487 & 2.328 & 3.363 & 2.372 \\
\hline $10 / 23 / 08$ & 0:00 & 4.836 & 2.433 & 2.312 & 3.296 & 2.323 \\
\hline 10/23/08 & $4: 00$ & 4.901 & 2.499 & 2.332 & 3.349 & 2.384 \\
\hline 10/23/08 & 8:00 & 5.006 & 2.559 & 2.356 & 3.415 & 2.435 \\
\hline $10 / 23 / 08$ & $12: 00$ & 5.092 & 2.601 & 2.374 & 3.47 & 2.475 \\
\hline $10 / 23 / 08$ & $16: 00$ & 5.162 & 2.627 & 2.388 & 3.501 & 2.514 \\
\hline 10/23/08 & $20: 00$ & 5.215 & 2.608 & 2.379 & 3.508 & 2.468 \\
\hline $10 / 24 / 08$ & $0: 00$ & 5.257 & 2.643 & 2.384 & 3.529 & 2.496 \\
\hline $10 / 24 / 08$ & $4: 00$ & 5.281 & 2.643 & 2.381 & 3.531 & 2.489 \\
\hline $10 / 24 / 08$ & 8:00 & 5.318 & 2.669 & 2.391 & 3.55 & 2.517 \\
\hline $10 / 24 / 08$ & $12: 00$ & 5.337 & 2.627 & 2.379 & 3.538 & 2.451 \\
\hline $10 / 24 / 08$ & $16: 00$ & 5.358 & 2.669 & 2.398 & 3.561 & 2.498 \\
\hline $10 / 24 / 08$ & $20: 00$ & 5.393 & 2.699 & 2.407 & 3.582 & 2.547 \\
\hline $10 / 25 / 08$ & $0: 00$ & 5.425 & 2.716 & 2.405 & 3.591 & 2.577 \\
\hline $10 / 25 / 08$ & $4: 00$ & 5.449 & 2.734 & 2.402 & 3.594 & 2.608 \\
\hline $10 / 25 / 08$ & $8: 00$ & 5.479 & 2.753 & 2.409 & 3.601 & 2.64 \\
\hline $10 / 25 / 08$ & $12: 00$ & 5.509 & 2.772 & 2.419 & 3.612 & 2.694 \\
\hline $10 / 25 / 08$ & $16: 00$ & 5.537 & 2.804 & 2.447 & 3.619 & 2.846 \\
\hline $10 / 25 / 08$ & $20: 00$ & 5.581 & 2.823 & 2.428 & 3.624 & 2.897 \\
\hline $10 / 26 / 08$ & 0:00 & 5.609 & 2.835 & 2.419 & 3.624 & 2.918 \\
\hline 10/26/08 & $4: 00$ & 5.663 & 2.87 & 2.435 & 3.659 & 2.944 \\
\hline 10/26/08 & 8:00 & 5.719 & 2.902 & 2.454 & 3.692 & 2.988 \\
\hline 10/26/08 & $12: 00$ & 5.77 & 2.947 & 2.475 & 3.727 & 3.068 \\
\hline $10 / 26 / 08$ & $16: 00$ & 5.803 & 3.003 & 2.498 & 3.75 & 3.175 \\
\hline
\end{tabular}


Table S1.4 (Cont.)

Depth to Water (ft below top of casing) in Indicated Well

Date Time PMW4 PMW5 PMW6 PMW7 PMW8

\begin{tabular}{|c|c|c|c|c|c|c|}
\hline $10 / 26 / 08$ & $20: 00$ & 5.849 & 3.04 & 2.496 & 3.761 & 3.203 \\
\hline $10 / 27 / 08$ & $0: 00$ & 5.875 & 3.047 & 2.489 & 3.768 & 3.177 \\
\hline $10 / 27 / 08$ & $4: 00$ & 5.896 & 3.052 & 2.489 & 3.771 & 3.145 \\
\hline 10/27/08 & $8: 00$ & 5.926 & 3.07 & 2.5 & 3.789 & 3.124 \\
\hline $10 / 27 / 08$ & $12: 00$ & 5.954 & 3.084 & 2.51 & 3.803 & 3.11 \\
\hline 10/27/08 & 16:00 & 5.966 & 3.094 & 2.51 & 3.796 & 3.098 \\
\hline 10/27/08 & 20:00 & 5.989 & 3.11 & 2.514 & 3.803 & 3.114 \\
\hline $10 / 28 / 08$ & $0: 00$ & 6.01 & 3.122 & 2.514 & 3.806 & 3.107 \\
\hline $10 / 28 / 08$ & $4: 00$ & 6.027 & 3.129 & 2.517 & 3.808 & 3.093 \\
\hline $10 / 28 / 08$ & $8: 00$ & 6.045 & 3.143 & 2.521 & 3.813 & 3.088 \\
\hline 10/28/08 & $12: 00$ & 6.064 & 3.14 & 2.526 & 3.813 & 3.079 \\
\hline $10 / 28 / 08$ & $16: 00$ & 6.054 & 3.138 & 2.519 & 3.792 & 3.06 \\
\hline 10/28/08 & 20:00 & 6.073 & 3.154 & 2.519 & 3.789 & 3.077 \\
\hline $10 / 29 / 08$ & $0: 00$ & 6.087 & 3.159 & 2.512 & 3.782 & 3.075 \\
\hline 10/29/08 & $4: 00$ & 6.094 & 3.164 & 2.51 & 3.775 & 3.07 \\
\hline 10/29/08 & $8: 00$ & 6.106 & 3.171 & 2.51 & 3.773 & 3.065 \\
\hline 10/29/08 & $12: 00$ & 6.134 & 3.187 & 2.521 & 3.792 & 3.07 \\
\hline 10/29/08 & $16: 00$ & 6.138 & 3.194 & 2.514 & 3.78 & 3.072 \\
\hline $10 / 29 / 08$ & $20: 00$ & 6.157 & 3.208 & 2.514 & 3.782 & 3.098 \\
\hline $10 / 30 / 08$ & $0: 00$ & 6.169 & 3.213 & 2.507 & 3.778 & 3.098 \\
\hline $10 / 30 / 08$ & 4:00 & 6.18 & 3.22 & 2.505 & 3.78 & 3.093 \\
\hline $10 / 30 / 08$ & 8:00 & 6.187 & 3.224 & 2.503 & 3.778 & 3.086 \\
\hline 10/30/08 & $12: 00$ & 6.204 & 3.236 & 2.51 & 3.787 & 3.086 \\
\hline $10 / 30 / 08$ & $16: 00$ & 6.206 & 3.238 & 2.503 & 3.771 & 3.102 \\
\hline $10 / 30 / 08$ & $20: 00$ & 6.229 & 3.259 & 2.507 & 3.785 & 3.137 \\
\hline $10 / 31 / 08$ & 0:00 & 6.248 & 3.269 & 2.5 & 3.794 & 3.135 \\
\hline 10/31/08 & 4:00 & 6.267 & 3.28 & 2.498 & 3.801 & 3.135 \\
\hline 10/31/08 & $8: 00$ & 6.283 & 3.295 & 2.505 & 3.813 & 3.137 \\
\hline 10/31/08 & $12: 00$ & 6.299 & 3.304 & 2.507 & 3.824 & 3.133 \\
\hline 10/31/08 & $16: 00$ & 6.306 & 3.311 & 2.505 & 3.808 & 3.142 \\
\hline $10 / 31 / 08$ & $20: 00$ & 6.32 & 3.325 & 2.5 & 3.82 & 3.17 \\
\hline 11/1/08 & $0: 00$ & 6.329 & 3.33 & 2.496 & 3.82 & 3.165 \\
\hline 11/1/08 & $4: 00$ & 6.336 & 3.332 & 2.491 & 3.82 & 3.156 \\
\hline 11/1/08 & $8: 00$ & 6.343 & 3.339 & 2.493 & 3.824 & 3.151 \\
\hline $11 / 1 / 08$ & $12: 00$ & 6.353 & 3.339 & 2.491 & 3.829 & 3.14 \\
\hline 11/1/08 & $16: 00$ & 6.343 & 3.332 & 2.489 & 3.81 & 3.126 \\
\hline 11/1/08 & 20:00 & 6.353 & 3.341 & 2.489 & 3.808 & 3.151 \\
\hline $11 / 2 / 08$ & $0: 00$ & 6.357 & 3.341 & 2.477 & 3.801 & 3.147 \\
\hline $11 / 2 / 08$ & $4: 00$ & 6.36 & 3.341 & 2.475 & 3.799 & 3.137 \\
\hline $11 / 2 / 08$ & 8:00 & 6.364 & 3.346 & 2.4 & 3.794 & 3.128 \\
\hline $11 / 2 / 08$ & $12: 00$ & 6.374 & 3.353 & 2.475 & 3.801 & 3.128 \\
\hline $11 / 2 / 08$ & $16: 00$ & 6.367 & 3.353 & 2.475 & 3.787 & 3.151 \\
\hline $11 / 2 / 08$ & $20: 00$ & 6.378 & 3.365 & 2.47 & 3.789 & 3.177 \\
\hline $11 / 3 / 08$ & $0: 00$ & 6.385 & 3.362 & 2.461 & 3.787 & 3.163 \\
\hline $11 / 3 / 08$ & $4: 00$ & 6.385 & 3.358 & 2.456 & 3.782 & 3.144 \\
\hline $11 / 3 / 08$ & $8: 00$ & 6.392 & 3.362 & 2.456 & 3.785 & 3.133 \\
\hline $11 / 3 / 08$ & $12: 00$ & 6.404 & 3.372 & 2.463 & 3.796 & 3.13 \\
\hline $11 / 3 / 08$ & $16: 00$ & 6.411 & 3.386 & 2.463 & 3.799 & 3.163 \\
\hline $11 / 3 / 08$ & 20:00 & 6.425 & 3.4 & 2.468 & 3.813 & 3.196 \\
\hline $11 / 4 / 08$ & $0: 00$ & 6.432 & 3.4 & 2.458 & 3.815 & 3.186 \\
\hline
\end{tabular}

Table S1.4 (Cont.)

Depth to Water (ft below top of casing) in Indicated Well

Date Time PMW4 PMW5 PMW6 PMW7 PMW8

\begin{tabular}{|c|c|c|c|c|c|c|}
\hline $11 / 4 / 08$ & 4:00 & 6.434 & 3.395 & 2.456 & 3.81 & 3.168 \\
\hline $11 / 4 / 08$ & 8:00 & 6.432 & 3.39 & 2.454 & 3.803 & 3.154 \\
\hline $11 / 4 / 08$ & $12: 00$ & 6.432 & 3.39 & 2.454 & 3.801 & 3.142 \\
\hline $11 / 4 / 08$ & $16: 00$ & 6.416 & 3.39 & 2.454 & 3.782 & 3.151 \\
\hline $11 / 4 / 08$ & 20:00 & 6.427 & 3.393 & 2.449 & 3.782 & 3.163 \\
\hline $11 / 5 / 08$ & $0: 00$ & 6.432 & 3.393 & 2.442 & 3.778 & 3.158 \\
\hline $11 / 5 / 08$ & $4: 00$ & 6.425 & 3.386 & 2.433 & 3.766 & 3.147 \\
\hline $11 / 5 / 08$ & 8:00 & 6.425 & 3.39 & 2.433 & 3.764 & 3.142 \\
\hline 11/5/08 & $12: 00$ & 6.434 & 3.39 & 2.433 & 3.761 & 3.135 \\
\hline 11/5/08 & $16: 00$ & 6.437 & 3.404 & 2.437 & 3.771 & 3.144 \\
\hline $11 / 5 / 08$ & $20: 00$ & 6.441 & 3.26 & 2.426 & 3.766 & 2.948 \\
\hline 11/6/08 & $0: 00$ & 6.451 & 3.332 & 2.426 & 3.775 & 2.988 \\
\hline $11 / 6 / 08$ & 4:00 & 6.448 & 3.362 & 2.421 & 3.773 & 3.025 \\
\hline 11/6/08 & 8:00 & 6.444 & 3.376 & 2.421 & 3.768 & 3.044 \\
\hline $11 / 6 / 08$ & $12: 00$ & 6.453 & 3.4 & 2.43 & 3.782 & 3.072 \\
\hline 11/6/08 & $16: 00$ & 6.465 & 3.416 & 2.433 & 3.785 & 3.093 \\
\hline 11/6/08 & $20: 00$ & 6.49 & 3.446 & 2.454 & 3.82 & 3.128 \\
\hline 11/7/08 & $0: 00$ & 6.504 & 3.451 & 2.449 & 3.822 & 3.142 \\
\hline $11 / 7 / 08$ & 4:00 & 6.502 & 3.446 & 2.442 & 3.82 & 3.14 \\
\hline 11/7/08 & $8: 00$ & 6.507 & 3.451 & 2.445 & 3.822 & 3.142 \\
\hline $11 / 7 / 08$ & $12: 00$ & 6.523 & 3.46 & 2.456 & 3.831 & 3.149 \\
\hline 11/7/08 & $16: 00$ & 6.523 & 3.472 & 2.461 & 3.838 & 3.156 \\
\hline 11/7/08 & 20:00 & 6.539 & 3.479 & 2.466 & 3.848 & 3.168 \\
\hline $11 / 8 / 08$ & $0: 00$ & 6.544 & 3.481 & 2.466 & 3.848 & 3.168 \\
\hline 11/8/08 & $4: 00$ & 6.544 & 3.486 & 2.473 & 3.857 & 3.168 \\
\hline $11 / 8 / 08$ & 8:00 & 6.558 & 3.493 & 2.482 & 3.869 & 3.175 \\
\hline 11/8/08 & $12: 00$ & 6.57 & 3.505 & 2.494 & 3.883 & 3.182 \\
\hline 11/8/08 & $16: 00$ & 6.574 & 3.502 & 2.496 & 3.883 & 3.177 \\
\hline $11 / 8 / 08$ & $20: 00$ & 6.586 & 3.514 & 2.505 & 3.894 & 3.186 \\
\hline $11 / 9 / 08$ & $0: 00$ & 6.591 & 3.514 & 2.508 & 3.901 & 3.186 \\
\hline 11/9/08 & $4: 00$ & 6.595 & 3.512 & 2.512 & 3.908 & 3.182 \\
\hline $11 / 9 / 08$ & $8: 00$ & 6.6 & 3.521 & 2.519 & 3.915 & 3.184 \\
\hline 11/9/08 & $12: 00$ & 6.607 & 3.521 & 2.524 & 3.915 & 3.179 \\
\hline $11 / 9 / 08$ & $16: 00$ & 6.604 & 3.516 & 2.522 & 3.906 & 3.161 \\
\hline 11/9/08 & $20: 00$ & 6.611 & 3.53 & 2.529 & 3.913 & 3.17 \\
\hline 11/10/08 & $0: 00$ & 6.623 & 3.542 & 2.538 & 3.924 & 3.182 \\
\hline 11/10/08 & 4:00 & 6.628 & 3.544 & 2.536 & 3.92 & 3.184 \\
\hline 11/10/08 & $8: 00$ & 6.632 & 3.544 & 2.54 & 3.924 & 3.179 \\
\hline $11 / 10 / 08$ & $12: 00$ & 6.635 & 3.542 & 2.543 & 3.927 & 3.17 \\
\hline $11 / 10 / 08$ & $16: 00$ & 6.63 & 3.479 & 2.531 & 3.908 & 3.109 \\
\hline 11/10/08 & 20:00 & 6.6 & 3.096 & 2.51 & 3.864 & 2.634 \\
\hline $11 / 11 / 08$ & $0: 00$ & 6.53 & 3.138 & 2.503 & 3.848 & 2.582 \\
\hline 11/11/08 & 4:00 & 6.504 & 3.209 & 2.501 & 3.838 & 2.596 \\
\hline $11 / 11 / 08$ & 8:00 & 6.493 & 3.178 & 2.505 & 3.831 & 2.587 \\
\hline $11 / 11 / 08$ & $12: 00$ & 6.488 & 3.176 & 2.508 & 3.834 & 2.592 \\
\hline $11 / 11 / 08$ & $16: 00$ & 6.427 & 2.9 & 2.482 & 3.778 & 2.466 \\
\hline $11 / 11 / 08$ & $20: 00$ & 6.313 & 2.826 & 2.48 & 3.726 & 2.417 \\
\hline $11 / 12 / 08$ & $0: 00$ & 6.288 & 2.959 & 2.498 & 3.759 & 2.503 \\
\hline $11 / 12 / 08$ & 4:00 & 6.29 & 3.05 & 2.515 & 3.787 & 2.568 \\
\hline $11 / 12 / 08$ & 8:00 & 6.304 & 3.124 & 2.519 & 3.808 & 2.631 \\
\hline
\end{tabular}


Table S1.4 (Cont.)

Depth to Water (ft below top of casing) in Indicated Well

Date Time PMW4 PMW5 PMW6 PMW7 PMW8

\begin{tabular}{|c|c|c|c|c|c|c|}
\hline $11 / 12 / 08$ & $12: 00$ & 6.327 & 3.178 & 2.526 & 3.827 & 2.692 \\
\hline $11 / 12 / 08$ & $16: 00$ & 6.332 & 3.204 & 2.522 & 3.822 & 2.739 \\
\hline $11 / 12 / 08$ & $20: 00$ & 6.346 & 3.232 & 2.519 & 3.82 & 2.79 \\
\hline $11 / 13 / 08$ & $0: 00$ & 6.35 & 3.246 & 2.51 & 3.815 & 2.832 \\
\hline $11 / 13 / 08$ & $4: 00$ & 6.35 & 3.258 & 2.51 & 3.808 & 2.862 \\
\hline $11 / 13 / 08$ & 8:00 & 6.355 & 3.269 & 2.505 & 3.806 & 2.881 \\
\hline $11 / 13 / 08$ & $12: 00$ & 6.367 & 3.279 & 2.501 & 3.801 & 2.904 \\
\hline 11/13/08 & 16:00 & 6.371 & 3.286 & 2.503 & 3.801 & 2.914 \\
\hline $11 / 13 / 08$ & $20: 00$ & 6.39 & 3.153 & 2.487 & 3.78 & 2.715 \\
\hline $11 / 14 / 08$ & $0: 00$ & 6.39 & 3.213 & 2.491 & 3.801 & 2.762 \\
\hline $11 / 14 / 08$ & 4:00 & 6.402 & 3.26 & 2.496 & 3.792 & 2.832 \\
\hline $11 / 14 / 08$ & 8:00 & 6.43 & 3.307 & 2.508 & 3.836 & 2.895 \\
\hline $11 / 14 / 08$ & $12: 00$ & 6.458 & 3.337 & 2.519 & 3.866 & 2.942 \\
\hline $11 / 14 / 08$ & $16: 00$ & 6.479 & 3.356 & 2.519 & 3.876 & 2.967 \\
\hline $11 / 14 / 08$ & $20: 00$ & 6.5 & 3.379 & 2.533 & 3.896 & 2.998 \\
\hline 11/15/08 & $0: 00$ & 6.518 & 3.384 & 2.531 & 3.913 & 3.002 \\
\hline $11 / 15 / 08$ & $4: 00$ & 6.53 & 3.402 & 2.54 & 3.922 & 3.03 \\
\hline 11/15/08 & $8: 00$ & 6.542 & 3.414 & 2.545 & 3.929 & 3.051 \\
\hline 11/15/08 & $12: 00$ & 6.551 & 3.423 & 2.55 & 3.938 & 3.063 \\
\hline 11/15/08 & 16:00 & 6.546 & 3.423 & 2.547 & 3.929 & 3.061 \\
\hline 11/15/08 & 20:00 & 6.556 & 3.43 & 2.55 & 3.931 & 3.075 \\
\hline 11/16/08 & $0: 00$ & 6.551 & 3.43 & 2.543 & 3.92 & 3.079 \\
\hline $11 / 16 / 08$ & 4:00 & 6.539 & 3.426 & 2.536 & 3.903 & 3.079 \\
\hline 11/16/08 & $8: 00$ & 6.546 & 3.437 & 2.543 & 3.908 & 3.086 \\
\hline $11 / 16 / 08$ & $12: 00$ & 6.572 & 3.454 & 2.559 & 3.934 & 3.093 \\
\hline $11 / 16 / 08$ & $16: 00$ & 6.584 & 3.458 & 2.559 & 3.936 & 3.089 \\
\hline 11/16/08 & 20:00 & 6.6 & 3.472 & 2.568 & 3.95 & 3.105 \\
\hline $11 / 17 / 08$ & $0: 00$ & 6.614 & 3.479 & 2.571 & 3.959 & 3.114 \\
\hline $11 / 17 / 08$ & 4:00 & 6.625 & 3.491 & 2.5 & 3.971 & 3.126 \\
\hline 11/17/08 & $8: 00$ & 6.639 & 3.498 & 2.585 & 3.978 & 3.135 \\
\hline $11 / 17 / 08$ & $12: 00$ & 6.653 & 3.512 & 2.594 & 3.997 & 3.147 \\
\hline 11/17/08 & $16: 00$ & 6.653 & 3.512 & 2.592 & 3.992 & 3.142 \\
\hline 11/17/08 & 20:00 & 6.665 & 3.521 & 2.599 & 4.004 & 3.156 \\
\hline $11 / 18 / 08$ & $0: 00$ & 6.672 & 3.528 & 2.594 & 4.008 & 3.168 \\
\hline 11/18/08 & 4:00 & 6.679 & 3.531 & 2.597 & 4.011 & 3.168 \\
\hline 11/18/08 & 8:00 & 6.677 & 3.526 & 2.594 & 4.006 & 3.166 \\
\hline 11/18/08 & $12: 00$ & 6.681 & 3.526 & 2.592 & 3.999 & 3.163 \\
\hline 11/18/08 & $16: 00$ & 6.644 & 3.498 & 2.573 & 3.962 & 3.126 \\
\hline $11 / 18 / 08$ & $20: 00$ & 6.635 & 3.498 & 2.571 & 3.948 & 3.124 \\
\hline 11/19/08 & 0:00 & 6.625 & 3.491 & 2.559 & 3.929 & 3.121 \\
\hline 11/19/08 & 4:00 & 6.623 & 3.493 & 2.559 & 3.92 & 3.119 \\
\hline 11/19/08 & 8:00 & 6.642 & 3.512 & 2.566 & 3.931 & 3.135 \\
\hline $11 / 19 / 08$ & $12: 00$ & 6.67 & 3.531 & 2.58 & 3.957 & 3.149 \\
\hline $11 / 19 / 08$ & $16: 00$ & 6.686 & 3.542 & 2.587 & 3.969 & 3.145 \\
\hline 11/19/08 & 20:00 & 6.707 & 3.561 & 2.601 & 3.997 & 3.177 \\
\hline 11/20/08 & $0: 00$ & 6.721 & 3.568 & 2.601 & 4.011 & 3.187 \\
\hline $11 / 20 / 08$ & 4:00 & 6.73 & 3.573 & 2.608 & 4.018 & 3.196 \\
\hline $11 / 20 / 08$ & $8: 00$ & 6.74 & 3.58 & 2.615 & 4.029 & 3.198 \\
\hline $11 / 20 / 08$ & $12: 00$ & 6.751 & 3.589 & 2.625 & 4.048 & 3.205 \\
\hline $11 / 20 / 08$ & $16: 00$ & 6.754 & 3.587 & 2.622 & 4.046 & 3.201 \\
\hline
\end{tabular}

Table S1.4 (Cont.)

Depth to Water (ft below top of casing) in Indicated Well

Date Time PMW4 PMW5 PMW6 PMW7 PMW8

\begin{tabular}{|c|c|c|c|c|c|c|}
\hline $11 / 20 / 08$ & $20: 00$ & 6.763 & 3.594 & 2.632 & 4.057 & 3.212 \\
\hline 11/21/08 & $0: 00$ & 6.763 & 3.596 & 2.627 & 4.055 & 3.217 \\
\hline 1/21/08 & 4:00 & 6.768 & 3.608 & 2.632 & 4.064 & 3.231 \\
\hline L/21/08 & 8:00 & 6.765 & 3.622 & 2.634 & 057 & 3.25 \\
\hline 1/21/08 & $12: 00$ & 6.765 & 3.61 & 2.622 & 4.05 & 3.236 \\
\hline 1/21/08 & $16: 00$ & 6.747 & 3.552 & 2.615 & 4.029 & 3.157 \\
\hline 1/21/08 & $20: 00$ & 6.742 & 3.557 & 2.618 & 4.022 & 3.152 \\
\hline $1 / 22 / 08$ & $0: 00$ & 6.744 & & 2.613 & & 3.157 \\
\hline $11 / 22 / 08$ & 4:00 & 6.74 & 68 & 2.613 & & 3.161 \\
\hline $11 / 22 / 08$ & 8:00 & 6.74 & 3.575 & 2.611 & & 3.164 \\
\hline $11 / 22 / 08$ & $12: 00$ & 6.749 & 3.58 & 2.613 & & 3.168 \\
\hline $1 / 22 / 08$ & $16: 00$ & 6.754 & 3.578 & 2.615 & 004 & 3.164 \\
\hline $1 / 22 / 08$ & 20:00 & 6.768 & 3.594 & 2.615 & 08 & 3.18 \\
\hline $1 / 23 / 08$ & $0: 00$ & 6.768 & & & & 3.187 \\
\hline 11/23/08 & $4: 00$ & 6.763 & 3.5 & 2.613 & & 3.189 \\
\hline 11/23/08 & 8:00 & 6.756 & 96 & 2.608 & & 3.189 \\
\hline 11/23/08 & $12: 00$ & 6.758 & 3.58 & 504 & & 3.171 \\
\hline $1 / 23 / 08$ & $16: 00$ & 6.744 & 3.566 & 2.606 & 87 & 3.145 \\
\hline $1 / 23 / 08$ & 20:00 & 6.772 & 3.594 & 2.618 & 08 & 3.166 \\
\hline $1 / 24 / 08$ & $0: 00$ & 6.789 & 3.6 & 2.6 & & 3.187 \\
\hline $11 / 24 / 08$ & 4:00 & 6.796 & 3.6 & 2.622 & & 3.194 \\
\hline $11 / 24 / 08$ & 8:00 & 803 & 3.622 & 2.629 & & 3.206 \\
\hline $11 / 24 / 08$ & $12: 00$ & 6.803 & 3.617 & 2.625 & & 3.196 \\
\hline $11 / 24 / 08$ & $16: 00$ & 6.793 & 3.603 & & & 3.171 \\
\hline $11 / 24 / 08$ & 20:00 & 6.8 & 3.613 & 2.627 & & 3.182 \\
\hline $11 / 25 / 08$ & $0: 00$ & 6.81 & 3.622 & 2.629 & & 3.196 \\
\hline $11 / 25 / 08$ & 4:00 & 6.81 & 3.6 & 2.632 & & 3.208 \\
\hline $11 / 25 / 08$ & $8: 00$ & 817 & 3.6 & 2.634 & & 3.222 \\
\hline $11 / 25 / 08$ & $12: 00$ & 821 & 3.6 & 2.627 & & 3.208 \\
\hline $11 / 25 / 08$ & $16: 00$ & 6.8 & 3.589 & 2.62 & 25 & 3.166 \\
\hline 11/25/08 & 20:00 & 6.803 & 3.6 & & & 3.171 \\
\hline 11/26/08 & $0: 00$ & 6.807 & 3.613 & & & 3.178 \\
\hline 11/26/08 & $4: 00$ & & 3.6 & & & 3.182 \\
\hline 11/26/08 & $8: 00$ & & & & & 96 \\
\hline $11 / 26 / 08$ & $12: 00$ & 828 & 3.631 & 2.634 & & 3.194 \\
\hline $11 / 26 / 08$ & $16: 00$ & 6.821 & 3.608 & 2.627 & & 3.164 \\
\hline 11/26/08 & 20:00 & 6.826 & & & & 3.166 \\
\hline 11/27/08 & $0: 00$ & 6.831 & 3.629 & 2.622 & & 3.178 \\
\hline 11/27/08 & $4: 00$ & 6.835 & 3.6 & & & 3.185 \\
\hline 11/27/08 & $8: 00$ & & & & & 3.194 \\
\hline $11 / 27 / 08$ & $12: 00$ & 6.851 & 3.645 & 2.639 & & 3.201 \\
\hline 11/27/08 & $16: 00$ & 6.849 & 3.629 & 2.639 & & 3.178 \\
\hline 11/27/08 & $20: 00$ & 6.852 & 3.636 & & & 3.182 \\
\hline $11 / 28 / 08$ & $0: 00$ & 6.854 & 3.641 & 2.639 & 64 & 3.182 \\
\hline $11 / 28 / 08$ & $4: 00$ & 6.854 & 3.641 & & 64 & 3.187 \\
\hline & 8:00 & & & & & 3.192 \\
\hline 11/28/08 & $12: 00$ & & 3.65 & & & 3.199 \\
\hline 11/28/08 & $16: 00$ & 6.838 & 3.613 & 2.632 & 4.043 & 3.161 \\
\hline 11/28/08 & 20:00 & 6.838 & 3.622 & 2.634 & & 3.164 \\
\hline $11 / 29 / 08$ & $0: 00$ & 6.842 & 3.627 & 2.634 & 4.043 & 3.166 \\
\hline
\end{tabular}


Table S1.4 (Cont.)

Depth to Water (ft below top of casing) in Indicated Well

Date Time PMW4 PMW5 PMW6 PMW7 PMW8

\begin{tabular}{|c|c|c|c|c|c|c|}
\hline $11 / 29 / 08$ & 4:00 & 6.835 & 3.599 & 2.622 & 4.029 & 3.124 \\
\hline $11 / 29 / 08$ & 8:00 & 6.824 & 3.566 & 2.618 & 4.018 & 3.05 \\
\hline $11 / 29 / 08$ & $12: 00$ & 6.817 & 3.561 & 2.613 & 4.008 & 3.05 \\
\hline $11 / 29 / 08$ & $16: 00$ & 6.798 & 3.538 & 2.606 & 3.997 & 3.003 \\
\hline $11 / 29 / 08$ & $20: 00$ & 6.803 & 3.561 & 2.611 & 4.004 & 3.024 \\
\hline $11 / 30 / 08$ & 0:00 & 6.81 & 3.575 & 2.611 & 4.006 & 3.052 \\
\hline $11 / 30 / 08$ & $4: 00$ & 6.807 & 3.582 & 2.611 & 4.004 & 3.073 \\
\hline $11 / 30 / 08$ & 8:00 & 6.812 & 3.599 & 2.613 & 4.008 & 3.096 \\
\hline $11 / 30 / 08$ & $12: 00$ & 6.826 & 3.613 & 2.618 & 4.02 & 3.117 \\
\hline $11 / 30 / 08$ & $16: 00$ & 6.828 & 3.61 & 2.618 & 4.018 & 3.12 \\
\hline 11/30/08 & 20:00 & 6.845 & 3.624 & 2.627 & 4.034 & 3.138 \\
\hline $12 / 1 / 08$ & $0: 00$ & 6.854 & 3.629 & 2.627 & 4.039 & 3.143 \\
\hline $12 / 1 / 08$ & $4: 00$ & 6.863 & 3.641 & 2.634 & 4.057 & 3.155 \\
\hline $12 / 1 / 08$ & $8: 00$ & 6.872 & 3.652 & 2.639 & 4.069 & 3.169 \\
\hline $12 / 1 / 08$ & $12: 00$ & 6.884 & 3.662 & 2.646 & 4.085 & 3.185 \\
\hline 12/1/08 & $16: 00$ & 6.882 & 3.65 & 2.641 & 4.078 & 3.176 \\
\hline $12 / 1 / 08$ & 20:00 & 6.884 & 3.655 & 2.641 & 4.078 & 3.176 \\
\hline $12 / 2 / 08$ & $0: 00$ & 6.882 & 3.666 & 2.641 & 4.08 & 3.19 \\
\hline $12 / 2 / 08$ & 4:00 & 6.875 & 3.662 & 2.637 & 4.071 & 3.197 \\
\hline $12 / 2 / 08$ & $8: 00$ & 6.861 & 3.643 & 2.634 & 4.053 & 3.178 \\
\hline $12 / 2 / 08$ & $12: 00$ & 6.865 & 3.646 & 2.632 & 4.053 & 3.171 \\
\hline $12 / 2 / 08$ & $16: 00$ & 6.861 & 3.625 & 2.627 & 4.029 & 3.145 \\
\hline $12 / 2 / 08$ & 20:00 & 6.866 & 3.641 & 2.632 & 4.039 & 3.157 \\
\hline $12 / 3 / 08$ & $0: 00$ & 6.875 & 3.653 & 2.632 & 4.048 & 3.173 \\
\hline $12 / 3 / 08$ & $4: 00$ & 6.896 & 3.671 & 2.655 & 4.083 & 3.197 \\
\hline $12 / 3 / 08$ & 8:00 & 6.907 & 3.678 & 2.66 & 4.101 & 3.208 \\
\hline $12 / 3 / 08$ & $12: 00$ & 6.928 & 3.695 & 2.674 & 4.125 & 3.232 \\
\hline $12 / 3 / 08$ & $16: 00$ & 6.931 & 3.681 & 2.669 & 4.127 & 3.215 \\
\hline $12 / 3 / 08$ & 20:00 & 6.94 & 3.697 & 2.679 & 4.139 & 3.232 \\
\hline $12 / 4 / 08$ & $0: 00$ & 6.947 & 3.711 & 2.683 & 4.153 & 3.253 \\
\hline $12 / 4 / 08$ & $4: 00$ & 6.952 & 3.723 & 2.69 & 4.162 & 3.276 \\
\hline $12 / 4 / 08$ & $8: 00$ & 6.963 & 3.734 & 2.697 & 4.176 & 3.297 \\
\hline $12 / 4 / 08$ & $12: 00$ & 6.975 & 3.744 & 2.711 & 4.197 & 3.316 \\
\hline $12 / 4 / 08$ & $16: 00$ & 6.975 & 3.713 & 2.697 & 4.188 & 3.26 \\
\hline $12 / 4 / 08$ & 20:00 & 6.982 & 3.727 & 2.711 & 4.199 & 3.267 \\
\hline $12 / 5 / 08$ & 0:00 & 6.984 & 3.746 & 2.714 & 4.202 & 3.32 \\
\hline $12 / 5 / 08$ & $4: 00$ & 6.977 & 3.753 & 2.707 & 4.192 & 3.348 \\
\hline $12 / 5 / 08$ & $8: 00$ & 6.968 & 3.753 & 2.697 & 4.176 & 3.369 \\
\hline $12 / 5 / 08$ & $12: 00$ & 6.956 & 3.73 & 2.679 & 4.15 & 3.346 \\
\hline $12 / 5 / 08$ & $16: 00$ & 6.924 & 3.667 & 2.653 & 4.104 & 3.236 \\
\hline $12 / 5 / 08$ & 20:00 & 6.905 & 3.65 & 2.658 & 4.092 & 3.173 \\
\hline $12 / 6 / 08$ & $0: 00$ & 6.903 & 3.643 & 2.66 & 4.087 & 3.141 \\
\hline $12 / 6 / 08$ & $4: 00$ & 6.912 & 3.643 & 2.662 & 4.09 & 3.122 \\
\hline $12 / 6 / 08$ & $8: 00$ & 6.931 & 3.657 & 2.676 & 4.108 & 3.124 \\
\hline $12 / 6 / 08$ & $12: 00$ & 6.959 & 3.683 & 2.693 & 4.143 & 3.15 \\
\hline $12 / 6 / 08$ & $16: 00$ & 6.966 & 3.664 & 2.688 & 4.143 & 3.12 \\
\hline $12 / 6 / 08$ & 20:00 & 6.977 & 3.69 & 2.7 & 4.16 & 3.148 \\
\hline $12 / 7 / 08$ & $0: 00$ & 6.982 & 3.72 & 2.7 & 4.164 & 3.211 \\
\hline $12 / 7 / 08$ & $4: 00$ & 6.98 & 3.72 & 2.693 & 4.155 & 3.236 \\
\hline $12 / 7 / 08$ & 8:00 & 6.973 & 3.725 & 2.697 & 4.155 & 3.26 \\
\hline
\end{tabular}

Table S1.4 (Cont.)

Depth to Water (ft below top of casing) in Indicated Well

Date Time PMW4 PMW5 PMW6 PMW7 PMW8

\begin{tabular}{|c|c|c|c|c|c|c|}
\hline $12 / 7 / 08$ & $12: 00$ & 6.963 & 3.723 & 2.688 & 4.141 & 3.274 \\
\hline 12/7/08 & $16: 00$ & 6.945 & 3.671 & 2.674 & 4.113 & 3.19 \\
\hline $12 / 7 / 08$ & 20:00 & 6.938 & 3.657 & 2.676 & 4.111 & 3.145 \\
\hline $12 / 8 / 08$ & $0: 00$ & 6.935 & 3.648 & 2.672 & 4.099 & 3.134 \\
\hline $12 / 8 / 08$ & $4: 00$ & 6.928 & 3.641 & 2.669 & 4.09 & 3.122 \\
\hline $12 / 8 / 08$ & $8: 00$ & 6.921 & 3.643 & 2.669 & 4.085 & 3.122 \\
\hline 12/8/08 & $12: 00$ & 6.917 & 3.634 & 2.667 & 4.076 & 3.118 \\
\hline 12/8/08 & $16: 00$ & 6.905 & 3.601 & 2.658 & 4.064 & 3.087 \\
\hline 12/8/08 & 20:00 & 6.912 & 3.622 & 2.665 & 4.071 & 3.099 \\
\hline $12 / 9 / 08$ & $0: 00$ & 6.931 & 3.65 & 2.674 & 4.09 & 3.127 \\
\hline $12 / 9 / 08$ & $4: 00$ & 6.952 & 3.678 & 2.681 & 4.108 & 3.157 \\
\hline $12 / 9 / 08$ & 8:00 & 6.982 & 3.713 & 2.695 & 4.139 & 3.199 \\
\hline 12/9/08 & $12: 00$ & 7.001 & 3.739 & 2.714 & 4.178 & 3.239 \\
\hline 12/9/08 & $16: 00$ & 7.008 & 3.737 & 2.718 & 4.19 & 3.241 \\
\hline $12 / 9 / 08$ & 20:00 & 7.017 & 3.76 & 2.73 & 4.213 & 3.283 \\
\hline 12/10/08 & 0:00 & 7.026 & 3.776 & 2.73 & 4.223 & 3.33 \\
\hline 12/10/08 & $4: 00$ & 7.029 & 3.786 & 2.732 & 4.227 & 3.374 \\
\hline $12 / 10 / 08$ & $8: 00$ & 7.036 & 3.795 & 2.73 & 4.23 & 3.407 \\
\hline $12 / 10 / 08$ & $12: 00$ & 7.038 & 3.781 & 2.714 & 4.22 & 3.39 \\
\hline 12/10/08 & $16: 00$ & 7.022 & 3.734 & 2.695 & 4.19 & 3.297 \\
\hline $12 / 10 / 08$ & 20:00 & 7.019 & 3.72 & 2.702 & 4.188 & 3.243 \\
\hline 12/11/08 & 0:00 & 7.012 & 3.711 & 2.697 & 4.176 & 3.225 \\
\hline 12/11/08 & $4: 00$ & 7.01 & 3.725 & 2.704 & 4.178 & 3.255 \\
\hline $12 / 11 / 08$ & 8:00 & 7.019 & 3.751 & 2.711 & 4.188 & 3.295 \\
\hline 12/11/08 & $12: 00$ & 7.031 & 3.741 & 2.709 & 4.197 & 3.272 \\
\hline $12 / 11 / 08$ & $16: 00$ & 7.024 & 3.706 & 2.702 & 4.185 & 3.181 \\
\hline $12 / 11 / 08$ & $20: 00$ & 7.029 & 3.695 & 2.711 & 4.192 & 3.136 \\
\hline $12 / 12 / 08$ & 0:00 & 7.038 & 3.702 & 2.721 & 4.204 & 3.141 \\
\hline $12 / 12 / 08$ & $4: 00$ & 7.04 & 3.713 & 2.723 & 4.211 & 3.169 \\
\hline $12 / 12 / 08$ & $8: 00$ & 7.036 & 3.718 & 2.718 & 4.202 & 3.213 \\
\hline $12 / 12 / 08$ & $12: 00$ & 7.038 & 3.732 & 2.716 & 4.199 & 3.241 \\
\hline $12 / 12 / 08$ & $16: 00$ & 7.012 & 3.685 & 2.702 & 4.171 & 3.164 \\
\hline $12 / 12 / 08$ & $20: 00$ & 6.996 & 3.669 & 2.702 & 4.153 & 3.143 \\
\hline 12/13/08 & 0:00 & 6.984 & 3.667 & 2.697 & 4.139 & 3.155 \\
\hline $12 / 13 / 08$ & $4: 00$ & 6.966 & 3.655 & 2.683 & 4.113 & 3.15 \\
\hline $12 / 13 / 08$ & $8: 00$ & 6.954 & 3.662 & 2.683 & 4.101 & 3.162 \\
\hline $12 / 13 / 08$ & $12: 00$ & 6.952 & 3.662 & 2.679 & 4.092 & 3.16 \\
\hline $12 / 13 / 08$ & $16: 00$ & 6.942 & 3.622 & 2.667 & 4.076 & 3.108 \\
\hline $12 / 13 / 08$ & 20:00 & 6.945 & 3.629 & 2.672 & 4.078 & 3.118 \\
\hline $12 / 14 / 08$ & 0:00 & 6.942 & 3.634 & 2.665 & 4.067 & 3.125 \\
\hline $12 / 14 / 08$ & $4: 00$ & 6.935 & 3.634 & 2.662 & 4.064 & 3.125 \\
\hline $12 / 14 / 08$ & 8:00 & 6.966 & 3.674 & 2.693 & 4.108 & 3.16 \\
\hline $12 / 14 / 08$ & $12: 00$ & 7.012 & 3.723 & 2.704 & 4.155 & 3.218 \\
\hline $12 / 14 / 08$ & $16: 00$ & 7.033 & 3.751 & 2.709 & 4.176 & 3.267 \\
\hline $12 / 14 / 08$ & 20:00 & 7.052 & 3.793 & 2.735 & 4.213 & 3.335 \\
\hline 12/15/08 & 0:00 & 7.071 & 3.818 & 2.76 & 4.251 & 3.412 \\
\hline $12 / 15 / 08$ & $4: 00$ & 7.087 & 3.832 & 2.781 & 4.281 & 3.468 \\
\hline 12/15/08 & 8:00 & 7.103 & 3.844 & 2.795 & 4.304 & 3.509 \\
\hline $12 / 15 / 08$ & $12: 00$ & 7.119 & 3.858 & 2.805 & 4.32 & 3.547 \\
\hline $12 / 15 / 08$ & $16: 00$ & 7.127 & 3.842 & 2.774 & 4.318 & 3.521 \\
\hline
\end{tabular}


Table S1.4 (Cont.)

Depth to Water (ft below top of casing) in Indicated Well

Date Time PMW4 PMW5 PMW6 PMW7 PMW8

\begin{tabular}{|c|c|c|c|c|c|c|}
\hline $12 / 15 / 08$ & $20: 00$ & 7.131 & 3.844 & 2.781 & 4.325 & 3.547 \\
\hline $12 / 16 / 08$ & 0:00 & 7.136 & 3.849 & 2.784 & 4.327 & 3.572 \\
\hline 12/16/08 & $4: 00$ & 7.131 & 3.846 & 2.774 & 4.316 & 3.579 \\
\hline $12 / 16 / 08$ & $8: 00$ & 7.117 & 3.832 & 2.767 & 4.297 & 3.577 \\
\hline $12 / 16 / 08$ & $12: 00$ & 7.108 & 3.816 & 2.723 & 4.271 & 3.533 \\
\hline 12/16/08 & $16: 00$ & 7.089 & 3.783 & 2.686 & 4.241 & 3.454 \\
\hline 12/16/08 & 20:00 & 7.092 & 3.774 & 2.677 & 4.237 & 3.402 \\
\hline $12 / 17 / 08$ & $0: 00$ & 7.094 & 3.769 & 2.677 & 4.237 & 3.372 \\
\hline 12/17/08 & $4: 00$ & 7.101 & 3.786 & 2.684 & 4.248 & 3.377 \\
\hline 12/17/08 & 8:00 & 7.115 & 3.797 & 2.691 & 4.26 & 3.386 \\
\hline 12/17/08 & $12: 00$ & 7.126 & 3.8 & 2.691 & 4.267 & 3.381 \\
\hline $12 / 17 / 08$ & $16: 00$ & 7.119 & 3.776 & 2.677 & 4.248 & 3.349 \\
\hline 12/17/08 & 20:00 & 7.115 & 3.767 & 2.677 & 4.246 & 3.318 \\
\hline 12/18/08 & $0: 00$ & 7.119 & 3.762 & 2.674 & 4.248 & 3.297 \\
\hline 12/18/08 & $4: 00$ & 7.113 & 3.762 & 2.679 & 4.248 & 3.297 \\
\hline 12/18/08 & $8: 00$ & 7.119 & 3.776 & 2.681 & 4.257 & 3.321 \\
\hline 12/18/08 & $12: 00$ & 7.122 & 3.786 & 2.681 & 4.264 & 3.342 \\
\hline $12 / 18 / 08$ & $16: 00$ & 7.101 & 3.755 & 2.656 & 4.232 & 3.304 \\
\hline $12 / 18 / 08$ & $20: 00$ & 7.089 & 3.734 & 2.646 & 4.213 & 3.269 \\
\hline $12 / 19 / 08$ & $0: 00$ & 7.061 & 3.695 & 2.625 & 4.176 & 3.223 \\
\hline 12/19/08 & $4: 00$ & 7.057 & 3.706 & 2.649 & 4.188 & 3.199 \\
\hline $12 / 19 / 08$ & $8: 00$ & 7.089 & 3.727 & 2.665 & 4.223 & 3.202 \\
\hline $12 / 19 / 08$ & $12: 00$ & 7.113 & 3.739 & 2.67 & 4.248 & 3.202 \\
\hline $12 / 19 / 08$ & $16: 00$ & 7.103 & 3.718 & 2.653 & 4.225 & 3.176 \\
\hline $12 / 19 / 08$ & $20: 00$ & 7.106 & 3.72 & 2.667 & 4.239 & 3.174 \\
\hline $12 / 20 / 08$ & $0: 00$ & 7.099 & 3.711 & 2.663 & 4.227 & 3.164 \\
\hline $12 / 20 / 08$ & 4:00 & 7.08 & 3.7 & 2.663 & & 3.155 \\
\hline $12 / 20 / 08$ & $8: 00$ & 7.092 & 3.718 & 2.6 & 4.225 & 3.174 \\
\hline $12 / 20 / 08$ & $12: 00$ & 7.108 & 3.737 & 2.684 & 4.251 & 3.195 \\
\hline $12 / 20 / 08$ & $16: 00$ & 7.115 & 3.741 & 2.684 & 4.255 & 3.199 \\
\hline $12 / 20 / 08$ & $20: 00$ & 7.133 & 3.755 & 2.695 & 4.274 & 3.218 \\
\hline $12 / 21 / 08$ & 0:00 & 7.14 & 3.769 & 2.705 & 4.288 & 3.26 \\
\hline $12 / 21 / 08$ & $4: 00$ & 7.145 & 3.786 & 2.714 & 4.304 & 3.316 \\
\hline $12 / 21 / 08$ & 8:00 & & 3.797 & 2.719 & & 365 \\
\hline $12 / 21 / 08$ & $12: 00$ & 7.154 & 3.814 & 2.726 & 4.332 & 3.412 \\
\hline $12 / 21 / 08$ & $16: 00$ & 7.164 & 3.821 & 2.719 & 4.341 & 3.423 \\
\hline $12 / 21 / 08$ & 20:00 & 7.175 & 3.823 & 2.719 & 4.348 & 3.43 \\
\hline $12 / 22 / 08$ & 0:00 & 7.18 & 3.83 & 2.721 & 4.358 & 3.456 \\
\hline $12 / 22 / 08$ & $4: 00$ & 7.185 & 3.835 & 2.721 & 4.358 & 3.479 \\
\hline $12 / 22 / 08$ & $8: 00$ & & 3.839 & 2.726 & 58 & \\
\hline $12 / 22 / 08$ & $12: 00$ & 7.185 & 3.839 & 2.716 & 4.351 & 3.517 \\
\hline $12 / 22 / 08$ & $16: 00$ & 7.159 & 3.811 & 2.684 & 4.313 & 3.477 \\
\hline $12 / 22 / 08$ & 20:00 & 7.145 & 3.79 & 2.677 & 4.295 & 3.435 \\
\hline $12 / 23 / 08$ & $0: 00$ & 7.129 & 3.772 & 2.663 & 4.272 & 3.395 \\
\hline $12 / 23 / 08$ & $4: 00$ & 7.112 & 3.755 & 2.644 & 4.248 & 3.363 \\
\hline $12 / 23 / 08$ & 8:00 & & 3.744 & & & 3.337 \\
\hline $12 / 23 / 08$ & $12: 00$ & 7.103 & 3.741 & 2.644 & 4.234 & 3.316 \\
\hline $12 / 23 / 08$ & $16: 00$ & 7.099 & 3.732 & 2.642 & 4.225 & 3.288 \\
\hline $12 / 23 / 08$ & 20:00 & 7.113 & 3.739 & 2.653 & 4.241 & 3.269 \\
\hline $12 / 24 / 08$ & $0: 00$ & 7.126 & 3.739 & 2.653 & 4.248 & 3.253 \\
\hline
\end{tabular}

Table S1.4 (Cont.)

Depth to Water (ft below top of casing) in Indicated Well

Date Time PMW4 PMW5 PMW6 PMW7 PMW8

\begin{tabular}{|c|c|c|c|c|c|c|}
\hline $12 / 24 / 08$ & $4: 00$ & 7.126 & 3.739 & 2.66 & 4.258 & 3.237 \\
\hline $12 / 24 / 08$ & 8:00 & 7.14 & 3.751 & 2.672 & 4.274 & 3.246 \\
\hline $12 / 24 / 08$ & $12: 00$ & 7.154 & 3.769 & 2.691 & 4.3 & 3.272 \\
\hline $12 / 24 / 08$ & $16: 00$ & 7.164 & 3.776 & 2.695 & 4.309 & 3.286 \\
\hline $12 / 24 / 08$ & $20: 00$ & 7.173 & 3.772 & 2.7 & 4.311 & 3.279 \\
\hline $12 / 25 / 08$ & 0:00 & 7.175 & 3.781 & 2.714 & 4.323 & 3.297 \\
\hline $12 / 25 / 08$ & $4: 00$ & 7.173 & 3.786 & 2.721 & 4.325 & 3.328 \\
\hline $12 / 25 / 08$ & 8:00 & 7.168 & 3.783 & 2.716 & 4.316 & 3.349 \\
\hline $12 / 25 / 08$ & $12: 00$ & 7.161 & 3.783 & 2.712 & 4.309 & 3.351 \\
\hline $12 / 25 / 08$ & $16: 00$ & 7.131 & 3.744 & 2.679 & 4.262 & 3.302 \\
\hline $12 / 25 / 08$ & $20: 00$ & 7.119 & 3.734 & 2.672 & 4.255 & 3.267 \\
\hline $12 / 26 / 08$ & $0: 00$ & 7.11 & 3.716 & 2.658 & 4.234 & 3.232 \\
\hline $12 / 26 / 08$ & $4: 00$ & 7.103 & 3.706 & 2.66 & 4.23 & 3.206 \\
\hline $12 / 26 / 08$ & 8:00 & 7.101 & 3.702 & 2.656 & 4.22 & 3.19 \\
\hline $12 / 26 / 08$ & $12: 00$ & 7.094 & 3.674 & 2.63 & 4.202 & 3.127 \\
\hline $12 / 26 / 08$ & $16: 00$ & 6.94 & 3.207 & 2.59 & 4.139 & 2.691 \\
\hline $12 / 26 / 08$ & $20: 00$ & 6.798 & 3.093 & 2.607 & 4.122 & 2.532 \\
\hline $12 / 27 / 08$ & $0: 00$ & 6.791 & 3.132 & 2.628 & 4.15 & 2.525 \\
\hline $12 / 27 / 08$ & $4: 00$ & 6.814 & 3.275 & 2.649 & 4.171 & 2.588 \\
\hline $12 / 27 / 08$ & 8:00 & 6.851 & 3.373 & 2.658 & 4.192 & 2.677 \\
\hline $12 / 27 / 08$ & $12: 00$ & 6.877 & 3.436 & 2.667 & 4.204 & 2.766 \\
\hline $12 / 27 / 08$ & $16: 00$ & 6.898 & 3.471 & 2.665 & 4.202 & 2.826 \\
\hline $12 / 27 / 08$ & 20:00 & 6.928 & 3.508 & 2.679 & 4.22 & 2.882 \\
\hline $12 / 28 / 08$ & $0: 00$ & 6.954 & 3.534 & 2.684 & 4.23 & 2.934 \\
\hline $12 / 28 / 08$ & $4: 00$ & 6.975 & 3.557 & 2.693 & 4.244 & 2.976 \\
\hline $12 / 28 / 08$ & $8: 00$ & 6.991 & 3.585 & 2.707 & 4.253 & 3.034 \\
\hline $12 / 28 / 08$ & $12: 00$ & 7.015 & 3.618 & 2.723 & 4.272 & 3.104 \\
\hline $12 / 28 / 08$ & $16: 00$ & 7.024 & 3.62 & 2.705 & 4.262 & 3.094 \\
\hline $12 / 28 / 08$ & 20:00 & 7.038 & 3.625 & 2.702 & 4.269 & 3.073 \\
\hline $12 / 29 / 08$ & 0:00 & 7.043 & 3.627 & 2.705 & 4.267 & 3.094 \\
\hline $12 / 29 / 08$ & $4: 00$ & 7.047 & 3.634 & 2.712 & 4.267 & 3.108 \\
\hline $12 / 29 / 08$ & 8:00 & 7.064 & 3.657 & 2.735 & 4.295 & 3.143 \\
\hline $12 / 29 / 08$ & $12: 00$ & 7.085 & 3.674 & 2.744 & 4.316 & 3.178 \\
\hline $12 / 29 / 08$ & $16: 00$ & 7.089 & 3.641 & 2.712 & 4.302 & 3.073 \\
\hline $12 / 29 / 08$ & 20:00 & 7.089 & 3.629 & 2.716 & 4.3 & 3.017 \\
\hline $12 / 30 / 08$ & 0:00 & 7.073 & 3.629 & 2.709 & 4.279 & 3.052 \\
\hline $12 / 30 / 08$ & $4: 00$ & 7.052 & 3.618 & 2.695 & 4.253 & 3.066 \\
\hline $12 / 30 / 08$ & 8:00 & 7.038 & 3.618 & 2.688 & 4.234 & 3.094 \\
\hline $12 / 30 / 08$ & $12: 00$ & 7.057 & 3.655 & 2.712 & 4.262 & 3.15 \\
\hline $12 / 30 / 08$ & $16: 00$ & 7.085 & 3.648 & 2.709 & 4.279 & 3.09 \\
\hline $12 / 30 / 08$ & 20:00 & 7.108 & 3.671 & 2.742 & 4.318 & 3.066 \\
\hline $12 / 31 / 08$ & 0:00 & 7.126 & 3.688 & 2.744 & 4.339 & 3.125 \\
\hline 12/31/08 & $4: 00$ & 7.138 & 3.706 & 2.765 & 4.358 & 3.209 \\
\hline $12 / 31 / 08$ & $8: 00$ & 7.147 & 3.739 & 2.791 & 4.367 & 3.316 \\
\hline $12 / 31 / 08$ & $12: 00$ & 7.157 & 3.774 & 2.814 & 4.381 & 3.4 \\
\hline $12 / 31 / 08$ & $16: 00$ & 7.145 & 3.751 & 2.772 & 4.353 & 3.372 \\
\hline $12 / 31 / 08$ & $20: 00$ & 7.131 & 3.737 & 2.763 & 4.332 & 3.358 \\
\hline
\end{tabular}


TABLE S1.5 Wetlands discharge flow rates calculated from measurements made at the V-notch and R-notch weirs.

\begin{tabular}{|c|c|c|c|c|c|c|c|c|c|c|}
\hline \multirow[b]{3}{*}{ Date Time } & \multicolumn{4}{|c|}{ V-Notch Weir } & \multicolumn{6}{|c|}{ R-Notch Weir } \\
\hline & \multicolumn{2}{|c|}{ Measured Water Level } & \multicolumn{2}{|c|}{$\begin{array}{c}\text { Calculated Rate } \\
\left(\mathrm{ft}^{3} / \mathrm{s}=448.8 \mathrm{gpm}\right)\end{array}$} & \multicolumn{3}{|c|}{ Measured Water Level } & \multicolumn{3}{|c|}{$\begin{array}{c}\text { Calculated Rate } \\
\left(\mathrm{ft}^{3} / \mathrm{s}=448.8 \mathrm{gpm}\right)\end{array}$} \\
\hline & $\begin{array}{c}\text { (ft above } \\
\text { sensor) }\end{array}$ & $\begin{array}{c}\text { (ft above } \\
\text { notch) }\end{array}$ & $\left(\mathrm{ft}^{3} / \mathrm{s}\right)$ & (gpm) & $\begin{array}{l}\text { (ft above } \\
\text { sensor) }\end{array}$ & $\begin{array}{c}\text { (ft above } \\
\text { log) }\end{array}$ & $\begin{array}{c}\text { (m above } \\
\text { log) }\end{array}$ & $\left(\mathrm{m}^{3} / \mathrm{s}\right)$ & $\left(\mathrm{ft}^{3} / \mathrm{s}\right)$ & (gpm) \\
\hline 1/1/08 0:00 & 1.599 & 0.620 & 0.175 & 78.322 & 1.363 & 0.032 & 0.009753 & 0.002963 & 0.104644 & 46.964 \\
\hline 1/1/08 1:00 & 1.595 & 0.616 & 0.172 & 77.080 & 1.363 & 0.032 & 0.009753 & 0.002963 & 0.104644 & 46.964 \\
\hline 1/1/08 2:00 & 1.6 & 0.621 & 0.175 & 78.634 & 1.365 & 0.034 & 0.010363 & 0.003218 & 0.113667 & 51.014 \\
\hline 1/1/08 3:00 & 1.602 & 0.623 & 0.177 & 79.261 & 1.363 & 0.032 & 0.009753 & 0.002963 & 0.104644 & 46.964 \\
\hline 1/1/08 4:00 & 1.586 & 0.607 & 0.166 & 74.329 & 1.361 & 0.03 & 0.009144 & 0.002714 & 0.095873 & 43.028 \\
\hline 1/1/08 5:00 & 1.581 & 0.602 & 0.162 & 72.827 & 1.361 & 0.03 & 0.009144 & 0.002714 & 0.095873 & 43.028 \\
\hline 1/1/08 6:00 & 1.579 & 0.600 & 0.161 & 72.231 & 1.361 & 0.03 & 0.009144 & 0.002714 & 0.095873 & 43.028 \\
\hline 1/1/08 7:00 & 1.565 & 0.586 & 0.152 & 68.143 & 1.358 & 0.027 & 0.008229 & 0.002356 & 0.083206 & 37.343 \\
\hline 1/1/08 8:00 & 1.575 & 0.596 & 0.158 & 71.049 & 1.361 & 0.03 & 0.009144 & 0.002714 & 0.095873 & 43.028 \\
\hline 1/1/08 9:00 & 1.572 & 0.593 & 0.156 & 70.169 & 1.358 & 0.027 & 0.008229 & 0.002356 & 0.083206 & 37.343 \\
\hline 1/1/08 10:00 & 1.57 & 0.591 & 0.155 & 69.587 & 1.358 & 0.027 & 0.008229 & 0.002356 & 0.083206 & 37.343 \\
\hline 1/1/08 11:00 & 1.577 & 0.598 & 0.160 & 71.638 & 1.358 & 0.027 & 0.008229 & 0.002356 & 0.083206 & 37.343 \\
\hline 1/1/08 12:00 & 1.565 & 0.586 & 0.152 & 68.143 & 1.358 & 0.027 & 0.008229 & 0.002356 & 0.083206 & 37.343 \\
\hline 1/1/08 13:00 & 1.56 & 0.581 & 0.149 & 66.717 & 1.358 & 0.027 & 0.008229 & 0.002356 & 0.083206 & 37.343 \\
\hline 1/1/08 14:00 & 1.564 & 0.585 & 0.151 & 67.856 & 1.361 & 0.03 & 0.009144 & 0.002714 & 0.095873 & 43.028 \\
\hline 1/1/08 15:00 & 1.569 & 0.590 & 0.154 & 69.296 & 1.361 & 0.03 & 0.009144 & 0.002714 & 0.095873 & 43.028 \\
\hline 1/1/08 16:00 & 1.585 & 0.606 & 0.165 & 74.027 & 1.361 & 0.03 & 0.009144 & 0.002714 & 0.095873 & 43.028 \\
\hline 1/1/08 17:00 & 1.583 & 0.604 & 0.164 & 73.426 & 1.361 & 0.03 & 0.009144 & 0.002714 & 0.095873 & 43.028 \\
\hline 1/1/08 18:00 & 1.58 & 0.601 & 0.162 & 72.529 & 1.361 & 0.03 & 0.009144 & 0.002714 & 0.095873 & 43.028 \\
\hline 1/1/08 19:00 & 1.562 & 0.583 & 0.150 & 67.285 & 1.361 & 0.03 & 0.009144 & 0.002714 & 0.095873 & 43.028 \\
\hline 1/1/08 20:00 & 1.56 & 0.581 & 0.149 & 66.717 & 1.356 & 0.025 & 0.007620 & 0.002126 & 0.075100 & 33.705 \\
\hline 1/1/08 21:00 & 1.555 & 0.576 & 0.146 & 65.310 & 1.356 & 0.025 & 0.007620 & 0.002126 & 0.075100 & 33.705 \\
\hline 1/1/08 22:00 & 1.553 & 0.574 & 0.144 & 64.752 & 1.356 & 0.025 & 0.007620 & 0.002126 & 0.075100 & 33.705 \\
\hline 1/1/08 23:00 & 1.551 & 0.572 & 0.143 & 64.197 & 1.356 & 0.025 & 0.007620 & 0.002126 & 0.075100 & 33.705 \\
\hline 1/2/08 0:00 & 1.551 & 0.572 & 0.143 & 64.197 & 1.358 & 0.027 & 0.008229 & 0.002356 & 0.083206 & 37.343 \\
\hline
\end{tabular}




\begin{tabular}{|c|c|c|c|c|c|c|c|c|c|c|}
\hline \multirow[b]{3}{*}{ Date Time } & \multicolumn{4}{|c|}{ V-Notch Weir } & \multicolumn{6}{|c|}{ R-Notch Weir } \\
\hline & \multicolumn{2}{|c|}{ Measured Water Level } & \multicolumn{2}{|c|}{$\begin{array}{c}\text { Calculated Rate } \\
\left(\mathrm{ft}^{3} / \mathrm{s}=448.8 \mathrm{gpm}\right)\end{array}$} & \multicolumn{3}{|c|}{ Measured Water Level } & \multicolumn{3}{|c|}{$\begin{array}{c}\text { Calculated Rate } \\
\left(\mathrm{ft}^{3} / \mathrm{s}=448.8 \mathrm{gpm}\right)\end{array}$} \\
\hline & $\begin{array}{c}\text { (ft above } \\
\text { sensor) }\end{array}$ & $\begin{array}{c}\text { (ft above } \\
\text { notch) }\end{array}$ & $\left(\mathrm{ft}^{3} / \mathrm{s}\right)$ & (gpm) & $\begin{array}{l}\text { (ft above } \\
\text { sensor) }\end{array}$ & $\begin{array}{c}\text { (ft above } \\
\text { log) }\end{array}$ & $\begin{array}{c}\text { (m above } \\
\text { log) }\end{array}$ & $\left(\mathrm{m}^{3} / \mathrm{s}\right)$ & $\left(\mathrm{ft}^{3} / \mathrm{s}\right)$ & (gpm) \\
\hline 1/2/08 1:00 & 1.546 & 0.567 & 0.140 & 62.821 & 1.354 & 0.023 & 0.007010 & 0.001905 & 0.067276 & 30.194 \\
\hline 1/2/08 2:00 & 1.546 & 0.567 & 0.140 & 62.821 & 1.356 & 0.025 & 0.007620 & 0.002126 & 0.075100 & 33.705 \\
\hline 1/2/08 3:00 & 1.546 & 0.567 & 0.140 & 62.821 & 1.356 & 0.025 & 0.007620 & 0.002126 & 0.075100 & 33.705 \\
\hline 1/2/08 4:00 & 1.546 & 0.567 & 0.140 & 62.821 & 1.356 & 0.025 & 0.007620 & 0.002126 & 0.075100 & 33.705 \\
\hline 1/2/08 5:00 & 1.546 & 0.567 & 0.140 & 62.821 & 1.356 & 0.025 & 0.007620 & 0.002126 & 0.075100 & 33.705 \\
\hline 1/2/08 6:00 & 1.544 & 0.565 & 0.139 & 62.276 & 1.356 & 0.025 & 0.007620 & 0.002126 & 0.075100 & 33.705 \\
\hline 1/2/08 7:00 & 1.54 & 0.561 & 0.136 & 61.195 & 1.354 & 0.023 & 0.007010 & 0.001905 & 0.067276 & 30.194 \\
\hline 1/2/08 8:00 & 1.54 & 0.561 & 0.136 & 61.195 & 1.356 & 0.025 & 0.007620 & 0.002126 & 0.075100 & 33.705 \\
\hline 1/2/08 9:00 & 1.537 & 0.558 & 0.135 & 60.391 & 1.356 & 0.025 & 0.007620 & 0.002126 & 0.075100 & 33.705 \\
\hline 1/2/08 10:00 & 1.532 & 0.553 & 0.132 & 59.065 & 1.354 & 0.023 & 0.007010 & 0.001905 & 0.067276 & 30.194 \\
\hline 1/2/08 11:00 & 1.535 & 0.556 & 0.133 & 59.859 & 1.354 & 0.023 & 0.007010 & 0.001905 & 0.067276 & 30.194 \\
\hline 1/2/08 12:00 & 1.537 & 0.558 & 0.135 & 60.391 & 1.354 & 0.023 & 0.007010 & 0.001905 & 0.067276 & 30.194 \\
\hline 1/2/08 13:00 & 1.56 & 0.581 & 0.149 & 66.717 & 1.356 & 0.025 & 0.007620 & 0.002126 & 0.075100 & 33.705 \\
\hline 1/2/08 14:00 & 1.553 & 0.574 & 0.144 & 64.752 & 1.358 & 0.027 & 0.008229 & 0.002356 & 0.083206 & 37.343 \\
\hline 1/2/08 15:00 & 1.562 & 0.583 & 0.150 & 67.285 & 1.358 & 0.027 & 0.008229 & 0.002356 & 0.083206 & 37.343 \\
\hline 1/2/08 16:00 & 1.573 & 0.594 & 0.157 & 70.462 & 1.361 & 0.03 & 0.009144 & 0.002714 & 0.095873 & 43.028 \\
\hline 1/2/08 17:00 & 1.578 & 0.599 & 0.160 & 71.935 & 1.361 & 0.03 & 0.009144 & 0.002714 & 0.095873 & 43.028 \\
\hline 1/2/08 18:00 & 1.576 & 0.597 & 0.159 & 71.343 & 1.361 & 0.03 & 0.009144 & 0.002714 & 0.095873 & 43.028 \\
\hline 1/2/08 19:00 & 1.573 & 0.594 & 0.157 & 70.462 & 1.358 & 0.027 & 0.008229 & 0.002356 & 0.083206 & 37.343 \\
\hline 1/2/08 20:00 & 1.569 & 0.590 & 0.154 & 69.296 & 1.358 & 0.027 & 0.008229 & 0.002356 & 0.083206 & 37.343 \\
\hline 1/2/08 21:00 & 1.567 & 0.588 & 0.153 & 68.718 & 1.358 & 0.027 & 0.008229 & 0.002356 & 0.083206 & 37.343 \\
\hline 1/2/08 22:00 & 1.567 & 0.588 & 0.153 & 68.718 & 1.358 & 0.027 & 0.008229 & 0.002356 & 0.083206 & 37.343 \\
\hline 1/2/08 23:00 & 1.565 & 0.586 & 0.152 & 68.143 & 1.358 & 0.027 & 0.008229 & 0.002356 & 0.083206 & 37.343 \\
\hline 1/3/08 0:00 & 1.563 & 0.584 & 0.151 & 67.571 & 1.358 & 0.027 & 0.008229 & 0.002356 & 0.083206 & 37.343 \\
\hline 1/3/08 1:00 & 1.563 & 0.584 & 0.151 & 67.571 & 1.358 & 0.027 & 0.008229 & 0.002356 & 0.083206 & 37.343 \\
\hline
\end{tabular}




\begin{tabular}{|c|c|c|c|c|c|c|c|c|c|c|}
\hline \multirow[b]{3}{*}{ Date Time } & \multicolumn{4}{|c|}{ V-Notch Weir } & \multicolumn{6}{|c|}{ R-Notch Weir } \\
\hline & \multicolumn{2}{|c|}{ Measured Water Level } & \multicolumn{2}{|c|}{$\begin{array}{c}\text { Calculated Rate } \\
\left(\mathrm{ft}^{3} / \mathrm{s}=448.8 \mathrm{gpm}\right)\end{array}$} & \multicolumn{3}{|c|}{ Measured Water Level } & \multicolumn{3}{|c|}{$\begin{array}{c}\text { Calculated Rate } \\
\left(\mathrm{ft}^{3} / \mathrm{s}=448.8 \mathrm{gpm}\right)\end{array}$} \\
\hline & $\begin{array}{c}\text { (ft above } \\
\text { sensor) }\end{array}$ & $\begin{array}{c}\text { (ft above } \\
\text { notch) }\end{array}$ & $\left(\mathrm{ft}^{3} / \mathrm{s}\right)$ & (gpm) & $\begin{array}{l}\text { (ft above } \\
\text { sensor) }\end{array}$ & $\begin{array}{c}\text { (ft above } \\
\text { log) }\end{array}$ & $\begin{array}{c}\text { (m above } \\
\text { log) }\end{array}$ & $\left(\mathrm{m}^{3} / \mathrm{s}\right)$ & $\left(\mathrm{ft}^{3} / \mathrm{s}\right)$ & (gpm) \\
\hline 1/3/08 2:00 & 1.563 & 0.584 & 0.151 & 67.571 & 1.358 & 0.027 & 0.008229 & 0.002356 & 0.083206 & 37.343 \\
\hline 1/3/08 3:00 & 1.565 & 0.586 & 0.152 & 68.143 & 1.358 & 0.027 & 0.008229 & 0.002356 & 0.083206 & 37.343 \\
\hline 1/3/08 4:00 & 1.563 & 0.584 & 0.151 & 67.571 & 1.358 & 0.027 & 0.008229 & 0.002356 & 0.083206 & 37.343 \\
\hline 1/3/08 5:00 & 1.565 & 0.586 & 0.152 & 68.143 & 1.356 & 0.025 & 0.007620 & 0.002126 & 0.075100 & 33.705 \\
\hline 1/3/08 6:00 & 1.558 & 0.579 & 0.147 & 66.152 & 1.356 & 0.025 & 0.007620 & 0.002126 & 0.075100 & 33.705 \\
\hline 1/3/08 7:00 & 1.556 & 0.577 & 0.146 & 65.590 & 1.354 & 0.023 & 0.007010 & 0.001905 & 0.067276 & 30.194 \\
\hline 1/3/08 8:00 & 1.554 & 0.575 & 0.145 & 65.030 & 1.356 & 0.025 & 0.007620 & 0.002126 & 0.075100 & 33.705 \\
\hline 1/3/08 9:00 & 1.551 & 0.572 & 0.143 & 64.197 & 1.356 & 0.025 & 0.007620 & 0.002126 & 0.075100 & 33.705 \\
\hline 1/3/08 10:00 & 1.554 & 0.575 & 0.145 & 65.030 & 1.361 & 0.03 & 0.009144 & 0.002714 & 0.095873 & 43.028 \\
\hline 1/3/08 11:00 & 1.542 & 0.563 & 0.138 & 61.734 & 1.356 & 0.025 & 0.007620 & 0.002126 & 0.075100 & 33.705 \\
\hline 1/3/08 12:00 & 1.565 & 0.586 & 0.152 & 68.143 & 1.361 & 0.03 & 0.009144 & 0.002714 & 0.095873 & 43.028 \\
\hline 1/3/08 13:00 & 1.562 & 0.583 & 0.150 & 67.285 & 1.356 & 0.025 & 0.007620 & 0.002126 & 0.075100 & 33.705 \\
\hline 1/3/08 14:00 & 1.571 & 0.592 & 0.156 & 69.878 & 1.358 & 0.027 & 0.008229 & 0.002356 & 0.083206 & 37.343 \\
\hline 1/3/08 15:00 & 1.583 & 0.604 & 0.164 & 73.426 & 1.363 & 0.032 & 0.009753 & 0.002963 & 0.104644 & 46.964 \\
\hline 1/3/08 16:00 & 1.59 & 0.611 & 0.168 & 75.544 & 1.361 & 0.03 & 0.009144 & 0.002714 & 0.095873 & 43.028 \\
\hline 1/3/08 17:00 & 1.594 & 0.615 & 0.171 & 76.771 & 1.363 & 0.032 & 0.009753 & 0.002963 & 0.104644 & 46.964 \\
\hline 1/3/08 18:00 & 1.594 & 0.615 & 0.171 & 76.771 & 1.361 & 0.03 & 0.009144 & 0.002714 & 0.095873 & 43.028 \\
\hline 1/3/08 19:00 & 1.59 & 0.611 & 0.168 & 75.544 & 1.363 & 0.032 & 0.009753 & 0.002963 & 0.104644 & 46.964 \\
\hline 1/3/08 20:00 & 1.59 & 0.611 & 0.168 & 75.544 & 1.361 & 0.03 & 0.009144 & 0.002714 & 0.095873 & 43.028 \\
\hline 1/3/08 21:00 & 1.588 & 0.609 & 0.167 & 74.935 & 1.363 & 0.032 & 0.009753 & 0.002963 & 0.104644 & 46.964 \\
\hline 1/3/08 22:00 & 1.59 & 0.611 & 0.168 & 75.544 & 1.363 & 0.032 & 0.009753 & 0.002963 & 0.104644 & 46.964 \\
\hline 1/3/08 23:00 & 1.593 & 0.614 & 0.170 & 76.463 & 1.363 & 0.032 & 0.009753 & 0.002963 & 0.104644 & 46.964 \\
\hline 1/4/08 0:00 & 1.593 & 0.614 & 0.170 & 76.463 & 1.361 & 0.03 & 0.009144 & 0.002714 & 0.095873 & 43.028 \\
\hline 1/4/08 1:00 & 1.591 & 0.612 & 0.169 & 75.850 & 1.361 & 0.03 & 0.009144 & 0.002714 & 0.095873 & 43.028 \\
\hline 1/4/08 2:00 & 1.591 & 0.612 & 0.169 & 75.850 & 1.363 & 0.032 & 0.009753 & 0.002963 & 0.104644 & 46.964 \\
\hline
\end{tabular}




\begin{tabular}{|c|c|c|c|c|c|c|c|c|c|c|}
\hline \multirow[b]{3}{*}{ Date Time } & \multicolumn{4}{|c|}{ V-Notch Weir } & \multicolumn{6}{|c|}{ R-Notch Weir } \\
\hline & \multicolumn{2}{|c|}{ Measured Water Level } & \multicolumn{2}{|c|}{$\begin{array}{c}\text { Calculated Rate } \\
\left(\mathrm{ft}^{3} / \mathrm{s}=448.8 \mathrm{gpm}\right)\end{array}$} & \multicolumn{3}{|c|}{ Measured Water Level } & \multicolumn{3}{|c|}{$\begin{array}{c}\text { Calculated Rate } \\
\left(\mathrm{ft}^{3} / \mathrm{s}=448.8 \mathrm{gpm}\right)\end{array}$} \\
\hline & $\begin{array}{c}\text { (ft above } \\
\text { sensor) }\end{array}$ & $\begin{array}{c}\text { (ft above } \\
\text { notch) }\end{array}$ & $\left(\mathrm{ft}^{3} / \mathrm{s}\right)$ & (gpm) & $\begin{array}{l}\text { (ft above } \\
\text { sensor) }\end{array}$ & $\begin{array}{c}\text { (ft above } \\
\text { log) }\end{array}$ & $\begin{array}{c}\text { (m above } \\
\text { log) }\end{array}$ & $\left(\mathrm{m}^{3} / \mathrm{s}\right)$ & $\left(\mathrm{ft}^{3} / \mathrm{s}\right)$ & (gpm) \\
\hline 1/4/08 3:00 & 1.598 & 0.619 & 0.174 & 78.010 & 1.363 & 0.032 & 0.009753 & 0.002963 & 0.104644 & 46.964 \\
\hline 1/4/08 4:00 & 1.591 & 0.612 & 0.169 & 75.850 & 1.363 & 0.032 & 0.009753 & 0.002963 & 0.104644 & 46.964 \\
\hline 1/4/08 5:00 & 1.591 & 0.612 & 0.169 & 75.850 & 1.361 & 0.03 & 0.009144 & 0.002714 & 0.095873 & 43.028 \\
\hline 1/4/08 6:00 & 1.591 & 0.612 & 0.169 & 75.850 & 1.361 & 0.03 & 0.009144 & 0.002714 & 0.095873 & 43.028 \\
\hline 1/4/08 7:00 & 1.588 & 0.609 & 0.167 & 74.935 & 1.361 & 0.03 & 0.009144 & 0.002714 & 0.095873 & 43.028 \\
\hline 1/4/08 8:00 & 1.588 & 0.609 & 0.167 & 74.935 & 1.361 & 0.03 & 0.009144 & 0.002714 & 0.095873 & 43.028 \\
\hline 1/4/08 9:00 & 1.586 & 0.607 & 0.166 & 74.329 & 1.361 & 0.03 & 0.009144 & 0.002714 & 0.095873 & 43.028 \\
\hline 1/4/08 10:00 & 1.584 & 0.605 & 0.164 & 73.726 & 1.361 & 0.03 & 0.009144 & 0.002714 & 0.095873 & 43.028 \\
\hline 1/4/08 11:00 & 1.586 & 0.607 & 0.166 & 74.329 & 1.361 & 0.03 & 0.009144 & 0.002714 & 0.095873 & 43.028 \\
\hline 1/4/08 12:00 & 1.585 & 0.606 & 0.165 & 74.027 & 1.361 & 0.03 & 0.009144 & 0.002714 & 0.095873 & 43.028 \\
\hline 1/4/08 13:00 & 1.583 & 0.604 & 0.164 & 73.426 & 1.361 & 0.03 & 0.009144 & 0.002714 & 0.095873 & 43.028 \\
\hline 1/4/08 14:00 & 1.597 & 0.618 & 0.173 & 77.699 & 1.363 & 0.032 & 0.009753 & 0.002963 & 0.104644 & 46.964 \\
\hline 1/4/08 15:00 & 1.596 & 0.617 & 0.172 & 77.389 & 1.363 & 0.032 & 0.009753 & 0.002963 & 0.104644 & 46.964 \\
\hline 1/4/08 16:00 & 1.601 & 0.622 & 0.176 & 78.947 & 1.365 & 0.034 & 0.010363 & 0.003218 & 0.113667 & 51.014 \\
\hline 1/4/08 17:00 & 1.598 & 0.619 & 0.174 & 78.010 & 1.365 & 0.034 & 0.010363 & 0.003218 & 0.113667 & 51.014 \\
\hline 1/4/08 18:00 & 1.6 & 0.621 & 0.175 & 78.634 & 1.363 & 0.032 & 0.009753 & 0.002963 & 0.104644 & 46.964 \\
\hline 1/4/08 19:00 & 1.601 & 0.622 & 0.176 & 78.947 & 1.363 & 0.032 & 0.009753 & 0.002963 & 0.104644 & 46.964 \\
\hline 1/4/08 20:00 & 1.603 & 0.624 & 0.177 & 79.575 & 1.365 & 0.034 & 0.010363 & 0.003218 & 0.113667 & 51.014 \\
\hline 1/4/08 21:00 & 1.603 & 0.624 & 0.177 & 79.575 & 1.365 & 0.034 & 0.010363 & 0.003218 & 0.113667 & 51.014 \\
\hline 1/4/08 22:00 & 1.604 & 0.625 & 0.178 & 79.890 & 1.363 & 0.032 & 0.009753 & 0.002963 & 0.104644 & 46.964 \\
\hline 1/4/08 23:00 & 1.606 & 0.627 & 0.179 & 80.523 & 1.363 & 0.032 & 0.009753 & 0.002963 & 0.104644 & 46.964 \\
\hline 1/5/08 0:00 & 1.604 & 0.625 & 0.178 & 79.890 & 1.363 & 0.032 & 0.009753 & 0.002963 & 0.104644 & 46.964 \\
\hline 1/5/08 1:00 & 1.602 & 0.623 & 0.177 & 79.261 & 1.363 & 0.032 & 0.009753 & 0.002963 & 0.104644 & 46.964 \\
\hline 1/5/08 2:00 & 1.604 & 0.625 & 0.178 & 79.890 & 1.363 & 0.032 & 0.009753 & 0.002963 & 0.104644 & 46.964 \\
\hline 1/5/08 3:00 & 1.599 & 0.620 & 0.175 & 78.322 & 1.363 & 0.032 & 0.009753 & 0.002963 & 0.104644 & 46.964 \\
\hline
\end{tabular}




\begin{tabular}{|c|c|c|c|c|c|c|c|c|c|c|}
\hline \multirow[b]{3}{*}{ Date Time } & \multicolumn{4}{|c|}{ V-Notch Weir } & \multicolumn{6}{|c|}{ R-Notch Weir } \\
\hline & \multicolumn{2}{|c|}{ Measured Water Level } & \multicolumn{2}{|c|}{$\begin{array}{c}\text { Calculated Rate } \\
\left(\mathrm{ft}^{3} / \mathrm{s}=448.8 \mathrm{gpm}\right)\end{array}$} & \multicolumn{3}{|c|}{ Measured Water Level } & \multicolumn{3}{|c|}{$\begin{array}{c}\text { Calculated Rate } \\
\left(\mathrm{ft}^{3} / \mathrm{s}=448.8 \mathrm{gpm}\right)\end{array}$} \\
\hline & $\begin{array}{c}\text { (ft above } \\
\text { sensor) }\end{array}$ & $\begin{array}{c}\text { (ft above } \\
\text { notch) }\end{array}$ & $\left(\mathrm{ft}^{3} / \mathrm{s}\right)$ & (gpm) & $\begin{array}{l}\text { (ft above } \\
\text { sensor) }\end{array}$ & $\begin{array}{c}\text { (ft above } \\
\text { log) }\end{array}$ & $\begin{array}{c}\text { (m above } \\
\text { log) }\end{array}$ & $\left(\mathrm{m}^{3} / \mathrm{s}\right)$ & $\left(\mathrm{ft}^{3} / \mathrm{s}\right)$ & (gpm) \\
\hline 1/5/08 4:00 & 1.597 & 0.618 & 0.173 & 77.699 & 1.363 & 0.032 & 0.009753 & 0.002963 & 0.104644 & 46.964 \\
\hline 1/5/08 5:00 & 1.599 & 0.620 & 0.175 & 78.322 & 1.363 & 0.032 & 0.009753 & 0.002963 & 0.104644 & 46.964 \\
\hline 1/5/08 6:00 & 1.6 & 0.621 & 0.175 & 78.634 & 1.363 & 0.032 & 0.009753 & 0.002963 & 0.104644 & 46.964 \\
\hline 1/5/08 7:00 & 1.6 & 0.621 & 0.175 & 78.634 & 1.363 & 0.032 & 0.009753 & 0.002963 & 0.104644 & 46.964 \\
\hline 1/5/08 8:00 & 1.597 & 0.618 & 0.173 & 77.699 & 1.363 & 0.032 & 0.009753 & 0.002963 & 0.104644 & 46.964 \\
\hline 1/5/08 9:00 & 1.597 & 0.618 & 0.173 & 77.699 & 1.363 & 0.032 & 0.009753 & 0.002963 & 0.104644 & 46.964 \\
\hline 1/5/08 10:00 & 1.595 & 0.616 & 0.172 & 77.080 & 1.363 & 0.032 & 0.009753 & 0.002963 & 0.104644 & 46.964 \\
\hline 1/5/08 11:00 & 1.59 & 0.611 & 0.168 & 75.544 & 1.363 & 0.032 & 0.009753 & 0.002963 & 0.104644 & 46.964 \\
\hline 1/5/08 12:00 & 1.594 & 0.615 & 0.171 & 76.771 & 1.363 & 0.032 & 0.009753 & 0.002963 & 0.104644 & 46.964 \\
\hline 1/5/08 13:00 & 1.603 & 0.624 & 0.177 & 79.575 & 1.365 & 0.034 & 0.010363 & 0.003218 & 0.113667 & 51.014 \\
\hline 1/5/08 14:00 & 1.605 & 0.626 & 0.179 & 80.206 & 1.365 & 0.034 & 0.010363 & 0.003218 & 0.113667 & 51.014 \\
\hline 1/5/08 15:00 & 1.614 & 0.635 & 0.185 & 83.085 & 1.365 & 0.034 & 0.010363 & 0.003218 & 0.113667 & 51.014 \\
\hline 1/5/08 16:00 & 1.621 & 0.642 & 0.190 & 85.365 & 1.368 & 0.037 & 0.011277 & 0.003614 & 0.127660 & 57.294 \\
\hline 1/5/08 17:00 & 1.632 & 0.653 & 0.198 & 89.024 & 1.37 & 0.039 & 0.011887 & 0.003887 & 0.137285 & 61.614 \\
\hline 1/5/08 18:00 & 1.626 & 0.647 & 0.194 & 87.017 & 1.365 & 0.034 & 0.010363 & 0.003218 & 0.113667 & 51.014 \\
\hline 1/5/08 19:00 & 1.626 & 0.647 & 0.194 & 87.017 & 1.37 & 0.039 & 0.011887 & 0.003887 & 0.137285 & 61.614 \\
\hline 1/5/08 20:00 & 1.621 & 0.642 & 0.190 & 85.365 & 1.368 & 0.037 & 0.011277 & 0.003614 & 0.127660 & 57.294 \\
\hline 1/5/08 21:00 & 1.619 & 0.640 & 0.189 & 84.710 & 1.368 & 0.037 & 0.011277 & 0.003614 & 0.127660 & 57.294 \\
\hline 1/5/08 22:00 & 1.624 & 0.645 & 0.192 & 86.354 & 1.365 & 0.034 & 0.010363 & 0.003218 & 0.113667 & 51.014 \\
\hline 1/5/08 23:00 & 1.624 & 0.645 & 0.192 & 86.354 & 1.368 & 0.037 & 0.011277 & 0.003614 & 0.127660 & 57.294 \\
\hline 1/6/08 0:00 & 1.622 & 0.643 & 0.191 & 85.694 & 1.368 & 0.037 & 0.011277 & 0.003614 & 0.127660 & 57.294 \\
\hline 1/6/08 1:00 & 1.622 & 0.643 & 0.191 & 85.694 & 1.365 & 0.034 & 0.010363 & 0.003218 & 0.113667 & 51.014 \\
\hline 1/6/08 2:00 & 1.625 & 0.646 & 0.193 & 86.685 & 1.368 & 0.037 & 0.011277 & 0.003614 & 0.127660 & 57.294 \\
\hline 1/6/08 3:00 & 1.622 & 0.643 & 0.191 & 85.694 & 1.368 & 0.037 & 0.011277 & 0.003614 & 0.127660 & 57.294 \\
\hline 1/6/08 4:00 & 1.622 & 0.643 & 0.191 & 85.694 & 1.368 & 0.037 & 0.011277 & 0.003614 & 0.127660 & 57.294 \\
\hline
\end{tabular}




\begin{tabular}{|c|c|c|c|c|c|c|c|c|c|c|}
\hline \multirow[b]{3}{*}{ Date Time } & \multicolumn{4}{|c|}{ V-Notch Weir } & \multicolumn{6}{|c|}{ R-Notch Weir } \\
\hline & \multicolumn{2}{|c|}{ Measured Water Level } & \multicolumn{2}{|c|}{$\begin{array}{c}\text { Calculated Rate } \\
\left(\mathrm{ft}^{3} / \mathrm{s}=448.8 \mathrm{gpm}\right)\end{array}$} & \multicolumn{3}{|c|}{ Measured Water Level } & \multicolumn{3}{|c|}{$\begin{array}{c}\text { Calculated Rate } \\
\left(\mathrm{ft}^{3} / \mathrm{s}=448.8 \mathrm{gpm}\right)\end{array}$} \\
\hline & $\begin{array}{c}\text { (ft above } \\
\text { sensor) }\end{array}$ & $\begin{array}{c}\text { (ft above } \\
\text { notch) }\end{array}$ & $\left(\mathrm{ft}^{3} / \mathrm{s}\right)$ & (gpm) & $\begin{array}{l}\text { (ft above } \\
\text { sensor) }\end{array}$ & $\begin{array}{c}\text { (ft above } \\
\text { log) }\end{array}$ & $\begin{array}{c}\text { (m above } \\
\text { log) }\end{array}$ & $\left(\mathrm{m}^{3} / \mathrm{s}\right)$ & $\left(\mathrm{ft}^{3} / \mathrm{s}\right)$ & (gpm) \\
\hline 1/6/08 5:00 & 1.618 & 0.639 & 0.188 & 84.383 & 1.368 & 0.037 & 0.011277 & 0.003614 & 0.127660 & 57.294 \\
\hline 1/6/08 6:00 & 1.62 & 0.641 & 0.189 & 85.037 & 1.368 & 0.037 & 0.011277 & 0.003614 & 0.127660 & 57.294 \\
\hline 1/6/08 7:00 & 1.62 & 0.641 & 0.189 & 85.037 & 1.365 & 0.034 & 0.010363 & 0.003218 & 0.113667 & 51.014 \\
\hline 1/6/08 8:00 & 1.618 & 0.639 & 0.188 & 84.383 & 1.365 & 0.034 & 0.010363 & 0.003218 & 0.113667 & 51.014 \\
\hline 1/6/08 9:00 & 1.618 & 0.639 & 0.188 & 84.383 & 1.365 & 0.034 & 0.010363 & 0.003218 & 0.113667 & 51.014 \\
\hline 1/6/08 10:00 & 1.615 & 0.636 & 0.186 & 83.408 & 1.365 & 0.034 & 0.010363 & 0.003218 & 0.113667 & 51.014 \\
\hline 1/6/08 11:00 & 1.615 & 0.636 & 0.186 & 83.408 & 1.365 & 0.034 & 0.010363 & 0.003218 & 0.113667 & 51.014 \\
\hline 1/6/08 12:00 & 1.615 & 0.636 & 0.186 & 83.408 & 1.365 & 0.034 & 0.010363 & 0.003218 & 0.113667 & 51.014 \\
\hline 1/6/08 13:00 & 1.617 & 0.638 & 0.187 & 84.057 & 1.365 & 0.034 & 0.010363 & 0.003218 & 0.113667 & 51.014 \\
\hline 1/6/08 14:00 & 1.621 & 0.642 & 0.190 & 85.365 & 1.368 & 0.037 & 0.011277 & 0.003614 & 0.127660 & 57.294 \\
\hline 1/6/08 15:00 & 1.63 & 0.651 & 0.197 & 88.352 & 1.368 & 0.037 & 0.011277 & 0.003614 & 0.127660 & 57.294 \\
\hline 1/6/08 16:00 & 1.642 & 0.663 & 0.206 & 92.431 & 1.37 & 0.039 & 0.011887 & 0.003887 & 0.137285 & 61.614 \\
\hline 1/6/08 17:00 & 1.646 & 0.667 & 0.209 & 93.815 & 1.37 & 0.039 & 0.011887 & 0.003887 & 0.137285 & 61.614 \\
\hline 1/6/08 18:00 & 1.649 & 0.670 & 0.211 & 94.862 & 1.37 & 0.039 & 0.011887 & 0.003887 & 0.137285 & 61.614 \\
\hline 1/6/08 19:00 & 1.644 & 0.665 & 0.207 & 93.122 & 1.368 & 0.037 & 0.011277 & 0.003614 & 0.127660 & 57.294 \\
\hline 1/6/08 20:00 & 1.64 & 0.661 & 0.204 & 91.744 & 1.368 & 0.037 & 0.011277 & 0.003614 & 0.127660 & 57.294 \\
\hline 1/6/08 21:00 & 1.631 & 0.652 & 0.198 & 88.688 & 1.368 & 0.037 & 0.011277 & 0.003614 & 0.127660 & 57.294 \\
\hline 1/6/08 22:00 & 1.628 & 0.649 & 0.195 & 87.683 & 1.365 & 0.034 & 0.010363 & 0.003218 & 0.113667 & 51.014 \\
\hline 1/6/08 23:00 & 1.626 & 0.647 & 0.194 & 87.017 & 1.368 & 0.037 & 0.011277 & 0.003614 & 0.127660 & 57.294 \\
\hline 1/7/08 0:00 & 1.626 & 0.647 & 0.194 & 87.017 & 1.365 & 0.034 & 0.010363 & 0.003218 & 0.113667 & 51.014 \\
\hline 1/7/08 1:00 & 1.624 & 0.645 & 0.192 & 86.354 & 1.365 & 0.034 & 0.010363 & 0.003218 & 0.113667 & 51.014 \\
\hline 1/7/08 2:00 & 1.624 & 0.645 & 0.192 & 86.354 & 1.365 & 0.034 & 0.010363 & 0.003218 & 0.113667 & 51.014 \\
\hline 1/7/08 3:00 & 1.622 & 0.643 & 0.191 & 85.694 & 1.365 & 0.034 & 0.010363 & 0.003218 & 0.113667 & 51.014 \\
\hline 1/7/08 4:00 & 1.62 & 0.641 & 0.189 & 85.037 & 1.365 & 0.034 & 0.010363 & 0.003218 & 0.113667 & 51.014 \\
\hline 1/7/08 5:00 & 1.629 & 0.650 & 0.196 & 88.017 & 1.368 & 0.037 & 0.011277 & 0.003614 & 0.127660 & 57.294 \\
\hline
\end{tabular}




\begin{tabular}{|c|c|c|c|c|c|c|c|c|c|c|}
\hline \multirow[b]{3}{*}{ Date Time } & \multicolumn{4}{|c|}{ V-Notch Weir } & \multicolumn{6}{|c|}{ R-Notch Weir } \\
\hline & \multicolumn{2}{|c|}{ Measured Water Level } & \multicolumn{2}{|c|}{$\begin{array}{c}\text { Calculated Rate } \\
\left(\mathrm{ft}^{3} / \mathrm{s}=448.8 \mathrm{gpm}\right)\end{array}$} & \multicolumn{3}{|c|}{ Measured Water Level } & \multicolumn{3}{|c|}{$\begin{array}{c}\text { Calculated Rate } \\
\left(\mathrm{ft}^{3} / \mathrm{s}=448.8 \mathrm{gpm}\right)\end{array}$} \\
\hline & $\begin{array}{c}\text { (ft above } \\
\text { sensor) }\end{array}$ & $\begin{array}{c}\text { (ft above } \\
\text { notch) }\end{array}$ & $\left(\mathrm{ft}^{3} / \mathrm{s}\right)$ & (gpm) & $\begin{array}{l}\text { (ft above } \\
\text { sensor) }\end{array}$ & $\begin{array}{c}\text { (ft above } \\
\text { log) }\end{array}$ & $\begin{array}{c}\text { (m above } \\
\text { log) }\end{array}$ & $\left(\mathrm{m}^{3} / \mathrm{s}\right)$ & $\left(\mathrm{ft}^{3} / \mathrm{s}\right)$ & (gpm) \\
\hline 1/7/08 6:00 & 1.634 & 0.655 & 0.200 & 89.700 & 1.365 & 0.034 & 0.010363 & 0.003218 & 0.113667 & 51.014 \\
\hline 1/7/08 7:00 & 1.625 & 0.646 & 0.193 & 86.685 & 1.368 & 0.037 & 0.011277 & 0.003614 & 0.127660 & 57.294 \\
\hline 1/7/08 8:00 & 1.625 & 0.646 & 0.193 & 86.685 & 1.365 & 0.034 & 0.010363 & 0.003218 & 0.113667 & 51.014 \\
\hline 1/7/08 9:00 & 1.622 & 0.643 & 0.191 & 85.694 & 1.365 & 0.034 & 0.010363 & 0.003218 & 0.113667 & 51.014 \\
\hline 1/7/08 10:00 & 1.62 & 0.641 & 0.189 & 85.037 & 1.365 & 0.034 & 0.010363 & 0.003218 & 0.113667 & 51.014 \\
\hline 1/7/08 11:00 & 1.62 & 0.641 & 0.189 & 85.037 & 1.365 & 0.034 & 0.010363 & 0.003218 & 0.113667 & 51.014 \\
\hline 1/7/08 12:00 & 1.622 & 0.643 & 0.191 & 85.694 & 1.365 & 0.034 & 0.010363 & 0.003218 & 0.113667 & 51.014 \\
\hline 1/7/08 13:00 & 1.623 & 0.644 & 0.192 & 86.024 & 1.368 & 0.037 & 0.011277 & 0.003614 & 0.127660 & 57.294 \\
\hline 1/7/08 14:00 & 1.635 & 0.656 & 0.201 & 90.038 & 1.368 & 0.037 & 0.011277 & 0.003614 & 0.127660 & 57.294 \\
\hline 1/7/08 15:00 & 1.651 & 0.672 & 0.213 & 95.563 & 1.372 & 0.041 & 0.012496 & 0.004166 & 0.147141 & 66.037 \\
\hline 1/7/08 16:00 & 1.665 & 0.686 & 0.224 & 100.560 & 1.375 & 0.044 & 0.013411 & 0.004596 & 0.162345 & 72.860 \\
\hline 1/7/08 17:00 & 1.676 & 0.697 & 0.233 & 104.594 & 1.377 & 0.046 & 0.014020 & 0.004891 & 0.172754 & 77.532 \\
\hline 1/7/08 18:00 & 1.679 & 0.700 & 0.236 & 105.711 & 1.375 & 0.044 & 0.013411 & 0.004596 & 0.162345 & 72.860 \\
\hline 1/7/08 19:00 & 1.667 & 0.688 & 0.226 & 101.286 & 1.372 & 0.041 & 0.012496 & 0.004166 & 0.147141 & 66.037 \\
\hline 1/7/08 20:00 & 1.658 & 0.679 & 0.218 & 98.042 & 1.37 & 0.039 & 0.011887 & 0.003887 & 0.137285 & 61.614 \\
\hline 1/7/08 21:00 & 1.649 & 0.670 & 0.211 & 94.862 & 1.37 & 0.039 & 0.011887 & 0.003887 & 0.137285 & 61.614 \\
\hline 1/7/08 22:00 & 1.644 & 0.665 & 0.207 & 93.122 & 1.37 & 0.039 & 0.011887 & 0.003887 & 0.137285 & 61.614 \\
\hline 1/7/08 23:00 & 1.642 & 0.663 & 0.206 & 92.431 & 1.368 & 0.037 & 0.011277 & 0.003614 & 0.127660 & 57.294 \\
\hline 1/8/08 0:00 & 1.633 & 0.654 & 0.199 & 89.362 & 1.368 & 0.037 & 0.011277 & 0.003614 & 0.127660 & 57.294 \\
\hline 1/8/08 1:00 & 1.633 & 0.654 & 0.199 & 89.362 & 1.368 & 0.037 & 0.011277 & 0.003614 & 0.127660 & 57.294 \\
\hline 1/8/08 2:00 & 1.631 & 0.652 & 0.198 & 88.688 & 1.368 & 0.037 & 0.011277 & 0.003614 & 0.127660 & 57.294 \\
\hline 1/8/08 3:00 & 1.629 & 0.650 & 0.196 & 88.017 & 1.368 & 0.037 & 0.011277 & 0.003614 & 0.127660 & 57.294 \\
\hline 1/8/08 4:00 & 1.634 & 0.655 & 0.200 & 89.700 & 1.368 & 0.037 & 0.011277 & 0.003614 & 0.127660 & 57.294 \\
\hline 1/8/08 5:00 & 1.629 & 0.650 & 0.196 & 88.017 & 1.365 & 0.034 & 0.010363 & 0.003218 & 0.113667 & 51.014 \\
\hline 1/8/08 6:00 & 1.624 & 0.645 & 0.192 & 86.354 & 1.365 & 0.034 & 0.010363 & 0.003218 & 0.113667 & 51.014 \\
\hline
\end{tabular}




\begin{tabular}{|c|c|c|c|c|c|c|c|c|c|c|}
\hline \multirow[b]{3}{*}{ Date Time } & \multicolumn{4}{|c|}{ V-Notch Weir } & \multicolumn{6}{|c|}{ R-Notch Weir } \\
\hline & \multicolumn{2}{|c|}{ Measured Water Level } & \multicolumn{2}{|c|}{$\begin{array}{c}\text { Calculated Rate } \\
\left(\mathrm{ft}^{3} / \mathrm{s}=448.8 \mathrm{gpm}\right)\end{array}$} & \multicolumn{3}{|c|}{ Measured Water Level } & \multicolumn{3}{|c|}{$\begin{array}{c}\text { Calculated Rate } \\
\left(\mathrm{ft}^{3} / \mathrm{s}=448.8 \mathrm{gpm}\right)\end{array}$} \\
\hline & $\begin{array}{c}\text { (ft above } \\
\text { sensor) }\end{array}$ & $\begin{array}{c}\text { (ft above } \\
\text { notch) }\end{array}$ & $\left(\mathrm{ft}^{3} / \mathrm{s}\right)$ & (gpm) & $\begin{array}{l}\text { (ft above } \\
\text { sensor) }\end{array}$ & $\begin{array}{c}\text { (ft above } \\
\text { log) }\end{array}$ & $\begin{array}{c}\text { (m above } \\
\text { log) }\end{array}$ & $\left(\mathrm{m}^{3} / \mathrm{s}\right)$ & $\left(\mathrm{ft}^{3} / \mathrm{s}\right)$ & (gpm) \\
\hline 1/8/08 7:00 & 1.627 & 0.648 & 0.195 & 87.350 & 1.365 & 0.034 & 0.010363 & 0.003218 & 0.113667 & 51.014 \\
\hline 1/8/08 8:00 & 1.624 & 0.645 & 0.192 & 86.354 & 1.365 & 0.034 & 0.010363 & 0.003218 & 0.113667 & 51.014 \\
\hline 1/8/08 9:00 & 1.622 & 0.643 & 0.191 & 85.694 & 1.365 & 0.034 & 0.010363 & 0.003218 & 0.113667 & 51.014 \\
\hline 1/8/08 10:00 & 1.624 & 0.645 & 0.192 & 86.354 & 1.365 & 0.034 & 0.010363 & 0.003218 & 0.113667 & 51.014 \\
\hline 1/8/08 11:00 & 1.627 & 0.648 & 0.195 & 87.350 & 1.365 & 0.034 & 0.010363 & 0.003218 & 0.113667 & 51.014 \\
\hline 1/8/08 12:00 & 1.61 & 0.631 & 0.182 & 81.798 & 1.365 & 0.034 & 0.010363 & 0.003218 & 0.113667 & 51.014 \\
\hline 1/8/08 13:00 & 1.624 & 0.645 & 0.192 & 86.354 & 1.365 & 0.034 & 0.010363 & 0.003218 & 0.113667 & 51.014 \\
\hline 1/8/08 14:00 & 1.631 & 0.652 & 0.198 & 88.688 & 1.365 & 0.034 & 0.010363 & 0.003218 & 0.113667 & 51.014 \\
\hline 1/8/08 15:00 & 1.623 & 0.644 & 0.192 & 86.024 & 1.365 & 0.034 & 0.010363 & 0.003218 & 0.113667 & 51.014 \\
\hline 1/8/08 16:00 & 1.637 & 0.658 & 0.202 & 90.718 & 1.368 & 0.037 & 0.011277 & 0.003614 & 0.127660 & 57.294 \\
\hline 1/8/08 17:00 & 1.639 & 0.660 & 0.204 & 91.401 & 1.368 & 0.037 & 0.011277 & 0.003614 & 0.127660 & 57.294 \\
\hline 1/8/08 18:00 & 1.637 & 0.658 & 0.202 & 90.718 & 1.368 & 0.037 & 0.011277 & 0.003614 & 0.127660 & 57.294 \\
\hline 1/8/08 19:00 & 1.635 & 0.656 & 0.201 & 90.038 & 1.368 & 0.037 & 0.011277 & 0.003614 & 0.127660 & 57.294 \\
\hline 1/8/08 20:00 & 1.631 & 0.652 & 0.198 & 88.688 & 1.365 & 0.034 & 0.010363 & 0.003218 & 0.113667 & 51.014 \\
\hline 1/8/08 21:00 & 1.628 & 0.649 & 0.195 & 87.683 & 1.365 & 0.034 & 0.010363 & 0.003218 & 0.113667 & 51.014 \\
\hline 1/8/08 22:00 & 1.621 & 0.642 & 0.190 & 85.365 & 1.365 & 0.034 & 0.010363 & 0.003218 & 0.113667 & 51.014 \\
\hline 1/8/08 23:00 & 1.619 & 0.640 & 0.189 & 84.710 & 1.365 & 0.034 & 0.010363 & 0.003218 & 0.113667 & 51.014 \\
\hline 1/9/08 0:00 & 1.62 & 0.641 & 0.189 & 85.037 & 1.363 & 0.032 & 0.009753 & 0.002963 & 0.104644 & 46.964 \\
\hline 1/9/08 1:00 & 1.613 & 0.634 & 0.184 & 82.762 & 1.363 & 0.032 & 0.009753 & 0.002963 & 0.104644 & 46.964 \\
\hline 1/9/08 2:00 & 1.613 & 0.634 & 0.184 & 82.762 & 1.363 & 0.032 & 0.009753 & 0.002963 & 0.104644 & 46.964 \\
\hline 1/9/08 3:00 & 1.611 & 0.632 & 0.183 & 82.118 & 1.363 & 0.032 & 0.009753 & 0.002963 & 0.104644 & 46.964 \\
\hline 1/9/08 4:00 & 1.613 & 0.634 & 0.184 & 82.762 & 1.363 & 0.032 & 0.009753 & 0.002963 & 0.104644 & 46.964 \\
\hline 1/9/08 5:00 & 1.608 & 0.629 & 0.181 & 81.159 & 1.363 & 0.032 & 0.009753 & 0.002963 & 0.104644 & 46.964 \\
\hline 1/9/08 6:00 & 1.611 & 0.632 & 0.183 & 82.118 & 1.363 & 0.032 & 0.009753 & 0.002963 & 0.104644 & 46.964 \\
\hline 1/9/08 7:00 & 1.609 & 0.630 & 0.182 & 81.478 & 1.363 & 0.032 & 0.009753 & 0.002963 & 0.104644 & 46.964 \\
\hline
\end{tabular}




\begin{tabular}{|c|c|c|c|c|c|c|c|c|c|c|}
\hline \multirow[b]{3}{*}{ Date Time } & \multicolumn{4}{|c|}{ V-Notch Weir } & \multicolumn{6}{|c|}{ R-Notch Weir } \\
\hline & \multicolumn{2}{|c|}{ Measured Water Level } & \multicolumn{2}{|c|}{$\begin{array}{c}\text { Calculated Rate } \\
\left(\mathrm{ft}^{3} / \mathrm{s}=448.8 \mathrm{gpm}\right)\end{array}$} & \multicolumn{3}{|c|}{ Measured Water Level } & \multicolumn{3}{|c|}{$\begin{array}{c}\text { Calculated Rate } \\
\left(\mathrm{ft}^{3} / \mathrm{s}=448.8 \mathrm{gpm}\right)\end{array}$} \\
\hline & $\begin{array}{c}\text { (ft above } \\
\text { sensor) }\end{array}$ & $\begin{array}{c}\text { (ft above } \\
\text { notch) }\end{array}$ & $\left(\mathrm{ft}^{3} / \mathrm{s}\right)$ & (gpm) & $\begin{array}{l}\text { (ft above } \\
\text { sensor) }\end{array}$ & $\begin{array}{c}\text { (ft above } \\
\text { log) }\end{array}$ & $\begin{array}{c}\text { (m above } \\
\text { log) }\end{array}$ & $\left(\mathrm{m}^{3} / \mathrm{s}\right)$ & $\left(\mathrm{ft}^{3} / \mathrm{s}\right)$ & (gpm) \\
\hline 1/9/08 8:00 & 1.611 & 0.632 & 0.183 & 82.118 & 1.363 & 0.032 & 0.009753 & 0.002963 & 0.104644 & 46.964 \\
\hline 1/9/08 9:00 & 1.611 & 0.632 & 0.183 & 82.118 & 1.363 & 0.032 & 0.009753 & 0.002963 & 0.104644 & 46.964 \\
\hline 1/9/08 10:00 & 1.606 & 0.627 & 0.179 & 80.523 & 1.363 & 0.032 & 0.009753 & 0.002963 & 0.104644 & 46.964 \\
\hline 1/9/08 11:00 & 1.608 & 0.629 & 0.181 & 81.159 & 1.363 & 0.032 & 0.009753 & 0.002963 & 0.104644 & 46.964 \\
\hline 1/9/08 12:00 & 1.61 & 0.631 & 0.182 & 81.798 & 1.363 & 0.032 & 0.009753 & 0.002963 & 0.104644 & 46.964 \\
\hline 1/9/08 13:00 & 1.617 & 0.638 & 0.187 & 84.057 & 1.363 & 0.032 & 0.009753 & 0.002963 & 0.104644 & 46.964 \\
\hline 1/9/08 14:00 & 1.61 & 0.631 & 0.182 & 81.798 & 1.363 & 0.032 & 0.009753 & 0.002963 & 0.104644 & 46.964 \\
\hline 1/9/08 15:00 & 1.619 & 0.640 & 0.189 & 84.710 & 1.365 & 0.034 & 0.010363 & 0.003218 & 0.113667 & 51.014 \\
\hline 1/9/08 16:00 & 1.628 & 0.649 & 0.195 & 87.683 & 1.368 & 0.037 & 0.011277 & 0.003614 & 0.127660 & 57.294 \\
\hline 1/9/08 17:00 & 1.623 & 0.644 & 0.192 & 86.024 & 1.365 & 0.034 & 0.010363 & 0.003218 & 0.113667 & 51.014 \\
\hline 1/9/08 18:00 & 1.625 & 0.646 & 0.193 & 86.685 & 1.365 & 0.034 & 0.010363 & 0.003218 & 0.113667 & 51.014 \\
\hline 1/9/08 19:00 & 1.626 & 0.647 & 0.194 & 87.017 & 1.365 & 0.034 & 0.010363 & 0.003218 & 0.113667 & 51.014 \\
\hline 1/9/08 20:00 & 1.626 & 0.647 & 0.194 & 87.017 & 1.365 & 0.034 & 0.010363 & 0.003218 & 0.113667 & 51.014 \\
\hline 1/9/08 21:00 & 1.626 & 0.647 & 0.194 & 87.017 & 1.368 & 0.037 & 0.011277 & 0.003614 & 0.127660 & 57.294 \\
\hline 1/9/08 22:00 & 1.624 & 0.645 & 0.192 & 86.354 & 1.365 & 0.034 & 0.010363 & 0.003218 & 0.113667 & 51.014 \\
\hline 1/9/08 23:00 & 1.619 & 0.640 & 0.189 & 84.710 & 1.365 & 0.034 & 0.010363 & 0.003218 & 0.113667 & 51.014 \\
\hline 1/10/08 0:00 & 1.622 & 0.643 & 0.191 & 85.694 & 1.365 & 0.034 & 0.010363 & 0.003218 & 0.113667 & 51.014 \\
\hline 1/10/08 1:00 & 1.615 & 0.636 & 0.186 & 83.408 & 1.363 & 0.032 & 0.009753 & 0.002963 & 0.104644 & 46.964 \\
\hline 1/10/08 2:00 & 1.617 & 0.638 & 0.187 & 84.057 & 1.365 & 0.034 & 0.010363 & 0.003218 & 0.113667 & 51.014 \\
\hline 1/10/08 3:00 & 1.617 & 0.638 & 0.187 & 84.057 & 1.365 & 0.034 & 0.010363 & 0.003218 & 0.113667 & 51.014 \\
\hline 1/10/08 4:00 & 1.617 & 0.638 & 0.187 & 84.057 & 1.365 & 0.034 & 0.010363 & 0.003218 & 0.113667 & 51.014 \\
\hline 1/10/08 5:00 & 1.62 & 0.641 & 0.189 & 85.037 & 1.365 & 0.034 & 0.010363 & 0.003218 & 0.113667 & 51.014 \\
\hline 1/10/08 6:00 & 1.62 & 0.641 & 0.189 & 85.037 & 1.365 & 0.034 & 0.010363 & 0.003218 & 0.113667 & 51.014 \\
\hline 1/10/08 7:00 & 1.62 & 0.641 & 0.189 & 85.037 & 1.365 & 0.034 & 0.010363 & 0.003218 & 0.113667 & 51.014 \\
\hline 1/10/08 8:00 & 1.62 & 0.641 & 0.189 & 85.037 & 1.365 & 0.034 & 0.010363 & 0.003218 & 0.113667 & 51.014 \\
\hline
\end{tabular}




\begin{tabular}{|c|c|c|c|c|c|c|c|c|c|c|}
\hline \multirow[b]{3}{*}{ Date Time } & \multicolumn{4}{|c|}{ V-Notch Weir } & \multicolumn{6}{|c|}{ R-Notch Weir } \\
\hline & \multicolumn{2}{|c|}{ Measured Water Level } & \multicolumn{2}{|c|}{$\begin{array}{c}\text { Calculated Rate } \\
\left(\mathrm{ft}^{3} / \mathrm{s}=448.8 \mathrm{gpm}\right)\end{array}$} & \multicolumn{3}{|c|}{ Measured Water Level } & \multicolumn{3}{|c|}{$\begin{array}{c}\text { Calculated Rate } \\
\left(\mathrm{ft}^{3} / \mathrm{s}=448.8 \mathrm{gpm}\right)\end{array}$} \\
\hline & $\begin{array}{c}\text { (ft above } \\
\text { sensor) }\end{array}$ & $\begin{array}{c}\text { (ft above } \\
\text { notch) }\end{array}$ & $\left(\mathrm{ft}^{3} / \mathrm{s}\right)$ & (gpm) & $\begin{array}{l}\text { (ft above } \\
\text { sensor) }\end{array}$ & $\begin{array}{c}\text { (ft above } \\
\text { log) }\end{array}$ & $\begin{array}{c}\text { (m above } \\
\text { log) }\end{array}$ & $\left(\mathrm{m}^{3} / \mathrm{s}\right)$ & $\left(\mathrm{ft}^{3} / \mathrm{s}\right)$ & (gpm) \\
\hline 1/10/08 9:00 & 1.615 & 0.636 & 0.186 & 83.408 & 1.365 & 0.034 & 0.010363 & 0.003218 & 0.113667 & 51.014 \\
\hline 1/10/08 10:00 & 1.62 & 0.641 & 0.189 & 85.037 & 1.365 & 0.034 & 0.010363 & 0.003218 & 0.113667 & 51.014 \\
\hline 1/10/08 11:00 & 1.617 & 0.638 & 0.187 & 84.057 & 1.365 & 0.034 & 0.010363 & 0.003218 & 0.113667 & 51.014 \\
\hline 1/10/08 12:00 & 1.634 & 0.655 & 0.200 & 89.700 & 1.365 & 0.034 & 0.010363 & 0.003218 & 0.113667 & 51.014 \\
\hline 1/10/08 13:00 & 1.629 & 0.650 & 0.196 & 88.017 & 1.365 & 0.034 & 0.010363 & 0.003218 & 0.113667 & 51.014 \\
\hline 1/10/08 14:00 & 1.62 & 0.641 & 0.189 & 85.037 & 1.363 & 0.032 & 0.009753 & 0.002963 & 0.104644 & 46.964 \\
\hline 1/10/08 15:00 & 1.631 & 0.652 & 0.198 & 88.688 & 1.363 & 0.032 & 0.009753 & 0.002963 & 0.104644 & 46.964 \\
\hline 1/10/08 16:00 & 1.621 & 0.642 & 0.190 & 85.365 & 1.365 & 0.034 & 0.010363 & 0.003218 & 0.113667 & 51.014 \\
\hline 1/10/08 17:00 & 1.626 & 0.647 & 0.194 & 87.017 & 1.368 & 0.037 & 0.011277 & 0.003614 & 0.127660 & 57.294 \\
\hline 1/10/08 18:00 & 1.622 & 0.643 & 0.191 & 85.694 & 1.365 & 0.034 & 0.010363 & 0.003218 & 0.113667 & 51.014 \\
\hline 1/10/08 19:00 & 1.62 & 0.641 & 0.189 & 85.037 & 1.365 & 0.034 & 0.010363 & 0.003218 & 0.113667 & 51.014 \\
\hline 1/10/08 20:00 & 1.617 & 0.638 & 0.187 & 84.057 & 1.365 & 0.034 & 0.010363 & 0.003218 & 0.113667 & 51.014 \\
\hline 1/10/08 21:00 & 1.62 & 0.641 & 0.189 & 85.037 & 1.363 & 0.032 & 0.009753 & 0.002963 & 0.104644 & 46.964 \\
\hline 1/10/08 22:00 & 1.618 & 0.639 & 0.188 & 84.383 & 1.365 & 0.034 & 0.010363 & 0.003218 & 0.113667 & 51.014 \\
\hline 1/10/08 23:00 & 1.618 & 0.639 & 0.188 & 84.383 & 1.363 & 0.032 & 0.009753 & 0.002963 & 0.104644 & 46.964 \\
\hline 1/11/08 0:00 & 1.616 & 0.637 & 0.187 & 83.732 & 1.363 & 0.032 & 0.009753 & 0.002963 & 0.104644 & 46.964 \\
\hline 1/11/08 1:00 & 1.613 & 0.634 & 0.184 & 82.762 & 1.363 & 0.032 & 0.009753 & 0.002963 & 0.104644 & 46.964 \\
\hline 1/11/08 2:00 & 1.611 & 0.632 & 0.183 & 82.118 & 1.363 & 0.032 & 0.009753 & 0.002963 & 0.104644 & 46.964 \\
\hline 1/11/08 3:00 & 1.611 & 0.632 & 0.183 & 82.118 & 1.363 & 0.032 & 0.009753 & 0.002963 & 0.104644 & 46.964 \\
\hline 1/11/08 4:00 & 1.609 & 0.630 & 0.182 & 81.478 & 1.361 & 0.03 & 0.009144 & 0.002714 & 0.095873 & 43.028 \\
\hline 1/11/08 5:00 & 1.607 & 0.628 & 0.180 & 80.841 & 1.363 & 0.032 & 0.009753 & 0.002963 & 0.104644 & 46.964 \\
\hline 1/11/08 6:00 & 1.604 & 0.625 & 0.178 & 79.890 & 1.361 & 0.03 & 0.009144 & 0.002714 & 0.095873 & 43.028 \\
\hline 1/11/08 7:00 & 1.605 & 0.626 & 0.179 & 80.206 & 1.361 & 0.03 & 0.009144 & 0.002714 & 0.095873 & 43.028 \\
\hline 1/11/08 8:00 & 1.6 & 0.621 & 0.175 & 78.634 & 1.361 & 0.03 & 0.009144 & 0.002714 & 0.095873 & 43.028 \\
\hline 1/11/08 9:00 & 1.6 & 0.621 & 0.175 & 78.634 & 1.361 & 0.03 & 0.009144 & 0.002714 & 0.095873 & 43.028 \\
\hline
\end{tabular}




\begin{tabular}{|c|c|c|c|c|c|c|c|c|c|c|}
\hline \multirow[b]{3}{*}{ Date Time } & \multicolumn{4}{|c|}{ V-Notch Weir } & \multicolumn{6}{|c|}{ R-Notch Weir } \\
\hline & \multicolumn{2}{|c|}{ Measured Water Level } & \multicolumn{2}{|c|}{$\begin{array}{c}\text { Calculated Rate } \\
\left(\mathrm{ft}^{3} / \mathrm{s}=448.8 \mathrm{gpm}\right)\end{array}$} & \multicolumn{3}{|c|}{ Measured Water Level } & \multicolumn{3}{|c|}{$\begin{array}{c}\text { Calculated Rate } \\
\left(\mathrm{ft}^{3} / \mathrm{s}=448.8 \mathrm{gpm}\right)\end{array}$} \\
\hline & $\begin{array}{c}\text { (ft above } \\
\text { sensor) }\end{array}$ & $\begin{array}{c}\text { (ft above } \\
\text { notch) }\end{array}$ & $\left(\mathrm{ft}^{3} / \mathrm{s}\right)$ & (gpm) & $\begin{array}{l}\text { (ft above } \\
\text { sensor) }\end{array}$ & $\begin{array}{c}\text { (ft above } \\
\text { log) }\end{array}$ & $\begin{array}{c}\text { (m above } \\
\text { log) }\end{array}$ & $\left(\mathrm{m}^{3} / \mathrm{s}\right)$ & $\left(\mathrm{ft}^{3} / \mathrm{s}\right)$ & (gpm) \\
\hline 1/11/08 10:00 & 1.595 & 0.616 & 0.172 & 77.080 & 1.361 & 0.03 & 0.009144 & 0.002714 & 0.095873 & 43.028 \\
\hline 1/11/08 11:00 & 1.593 & 0.614 & 0.170 & 76.463 & 1.358 & 0.027 & 0.008229 & 0.002356 & 0.083206 & 37.343 \\
\hline 1/11/08 12:00 & 1.597 & 0.618 & 0.173 & 77.699 & 1.363 & 0.032 & 0.009753 & 0.002963 & 0.104644 & 46.964 \\
\hline 1/11/08 13:00 & 1.601 & 0.622 & 0.176 & 78.947 & 1.363 & 0.032 & 0.009753 & 0.002963 & 0.104644 & 46.964 \\
\hline 1/11/08 14:00 & 1.605 & 0.626 & 0.179 & 80.206 & 1.363 & 0.032 & 0.009753 & 0.002963 & 0.104644 & 46.964 \\
\hline 1/11/08 15:00 & 1.605 & 0.626 & 0.179 & 80.206 & 1.363 & 0.032 & 0.009753 & 0.002963 & 0.104644 & 46.964 \\
\hline 1/11/08 16:00 & 1.612 & 0.633 & 0.184 & 82.440 & 1.365 & 0.034 & 0.010363 & 0.003218 & 0.113667 & 51.014 \\
\hline 1/11/08 17:00 & 1.612 & 0.633 & 0.184 & 82.440 & 1.363 & 0.032 & 0.009753 & 0.002963 & 0.104644 & 46.964 \\
\hline 1/11/08 18:00 & 1.612 & 0.633 & 0.184 & 82.440 & 1.363 & 0.032 & 0.009753 & 0.002963 & 0.104644 & 46.964 \\
\hline 1/11/08 19:00 & 1.612 & 0.633 & 0.184 & 82.440 & 1.363 & 0.032 & 0.009753 & 0.002963 & 0.104644 & 46.964 \\
\hline 1/11/08 20:00 & 1.612 & 0.633 & 0.184 & 82.440 & 1.36 & 0.029 & 0.008839 & 0.002593 & 0.091584 & 41.103 \\
\hline 1/11/08 21:00 & 1.607 & 0.628 & 0.180 & 80.841 & 1.363 & 0.032 & 0.009753 & 0.002963 & 0.104644 & 46.964 \\
\hline 1/11/08 22:00 & 1.607 & 0.628 & 0.180 & 80.841 & 1.36 & 0.029 & 0.008839 & 0.002593 & 0.091584 & 41.103 \\
\hline 1/11/08 23:00 & 1.61 & 0.631 & 0.182 & 81.798 & 1.363 & 0.032 & 0.009753 & 0.002963 & 0.104644 & 46.964 \\
\hline 1/12/08 0:00 & 1.61 & 0.631 & 0.182 & 81.798 & 1.363 & 0.032 & 0.009753 & 0.002963 & 0.104644 & 46.964 \\
\hline 1/12/08 1:00 & 1.606 & 0.627 & 0.179 & 80.523 & 1.36 & 0.029 & 0.008839 & 0.002593 & 0.091584 & 41.103 \\
\hline 1/12/08 2:00 & 1.606 & 0.627 & 0.179 & 80.523 & 1.36 & 0.029 & 0.008839 & 0.002593 & 0.091584 & 41.103 \\
\hline 1/12/08 3:00 & 1.606 & 0.627 & 0.179 & 80.523 & 1.36 & 0.029 & 0.008839 & 0.002593 & 0.091584 & 41.103 \\
\hline 1/12/08 4:00 & 1.604 & 0.625 & 0.178 & 79.890 & 1.36 & 0.029 & 0.008839 & 0.002593 & 0.091584 & 41.103 \\
\hline 1/12/08 5:00 & 1.599 & 0.620 & 0.175 & 78.322 & 1.361 & 0.03 & 0.009144 & 0.002714 & 0.095873 & 43.028 \\
\hline 1/12/08 6:00 & 1.601 & 0.622 & 0.176 & 78.947 & 1.358 & 0.027 & 0.008229 & 0.002356 & 0.083206 & 37.343 \\
\hline 1/12/08 7:00 & 1.599 & 0.620 & 0.175 & 78.322 & 1.361 & 0.03 & 0.009144 & 0.002714 & 0.095873 & 43.028 \\
\hline 1/12/08 8:00 & 1.597 & 0.618 & 0.173 & 77.699 & 1.361 & 0.03 & 0.009144 & 0.002714 & 0.095873 & 43.028 \\
\hline 1/12/08 9:00 & 1.592 & 0.613 & 0.170 & 76.156 & 1.358 & 0.027 & 0.008229 & 0.002356 & 0.083206 & 37.343 \\
\hline 1/12/08 10:00 & 1.604 & 0.625 & 0.178 & 79.890 & 1.358 & 0.027 & 0.008229 & 0.002356 & 0.083206 & 37.343 \\
\hline
\end{tabular}




\begin{tabular}{|c|c|c|c|c|c|c|c|c|c|c|}
\hline \multirow[b]{3}{*}{ Date Time } & \multicolumn{4}{|c|}{ V-Notch Weir } & \multicolumn{6}{|c|}{ R-Notch Weir } \\
\hline & \multicolumn{2}{|c|}{ Measured Water Level } & \multicolumn{2}{|c|}{$\begin{array}{c}\text { Calculated Rate } \\
\left(\mathrm{ft}^{3} / \mathrm{s}=448.8 \mathrm{gpm}\right)\end{array}$} & \multicolumn{3}{|c|}{ Measured Water Level } & \multicolumn{3}{|c|}{$\begin{array}{c}\text { Calculated Rate } \\
\left(\mathrm{ft}^{3} / \mathrm{s}=448.8 \mathrm{gpm}\right)\end{array}$} \\
\hline & $\begin{array}{c}\text { (ft above } \\
\text { sensor) }\end{array}$ & $\begin{array}{c}\text { (ft above } \\
\text { notch) }\end{array}$ & $\left(\mathrm{ft}^{3} / \mathrm{s}\right)$ & (gpm) & $\begin{array}{l}\text { (ft above } \\
\text { sensor) }\end{array}$ & $\begin{array}{c}\text { (ft above } \\
\text { log) }\end{array}$ & $\begin{array}{c}\text { (m above } \\
\text { log) }\end{array}$ & $\left(\mathrm{m}^{3} / \mathrm{s}\right)$ & $\left(\mathrm{ft}^{3} / \mathrm{s}\right)$ & (gpm) \\
\hline 1/12/08 11:00 & 1.597 & 0.618 & 0.173 & 77.699 & 1.358 & 0.027 & 0.008229 & 0.002356 & 0.083206 & 37.343 \\
\hline 1/12/08 12:00 & 1.609 & 0.630 & 0.182 & 81.478 & 1.361 & 0.03 & 0.009144 & 0.002714 & 0.095873 & 43.028 \\
\hline 1/12/08 13:00 & 1.599 & 0.620 & 0.175 & 78.322 & 1.358 & 0.027 & 0.008229 & 0.002356 & 0.083206 & 37.343 \\
\hline 1/12/08 14:00 & 1.596 & 0.617 & 0.172 & 77.389 & 1.361 & 0.03 & 0.009144 & 0.002714 & 0.095873 & 43.028 \\
\hline 1/12/08 15:00 & 1.591 & 0.612 & 0.169 & 75.850 & 1.358 & 0.027 & 0.008229 & 0.002356 & 0.083206 & 37.343 \\
\hline 1/12/08 16:00 & 1.603 & 0.624 & 0.177 & 79.575 & 1.36 & 0.029 & 0.008839 & 0.002593 & 0.091584 & 41.103 \\
\hline 1/12/08 17:00 & 1.598 & 0.619 & 0.174 & 78.010 & 1.36 & 0.029 & 0.008839 & 0.002593 & 0.091584 & 41.103 \\
\hline 1/12/08 18:00 & 1.601 & 0.622 & 0.176 & 78.947 & 1.36 & 0.029 & 0.008839 & 0.002593 & 0.091584 & 41.103 \\
\hline 1/12/08 19:00 & 1.596 & 0.617 & 0.172 & 77.389 & 1.358 & 0.027 & 0.008229 & 0.002356 & 0.083206 & 37.343 \\
\hline 1/12/08 20:00 & 1.594 & 0.615 & 0.171 & 76.771 & 1.358 & 0.027 & 0.008229 & 0.002356 & 0.083206 & 37.343 \\
\hline 1/12/08 21:00 & 1.599 & 0.620 & 0.175 & 78.322 & 1.361 & 0.03 & 0.009144 & 0.002714 & 0.095873 & 43.028 \\
\hline 1/12/08 22:00 & 1.592 & 0.613 & 0.170 & 76.156 & 1.358 & 0.027 & 0.008229 & 0.002356 & 0.083206 & 37.343 \\
\hline 1/12/08 23:00 & 1.594 & 0.615 & 0.171 & 76.771 & 1.361 & 0.03 & 0.009144 & 0.002714 & 0.095873 & 43.028 \\
\hline 1/13/08 0:00 & 1.592 & 0.613 & 0.170 & 76.156 & 1.358 & 0.027 & 0.008229 & 0.002356 & 0.083206 & 37.343 \\
\hline 1/13/08 1:00 & 1.592 & 0.613 & 0.170 & 76.156 & 1.358 & 0.027 & 0.008229 & 0.002356 & 0.083206 & 37.343 \\
\hline 1/13/08 2:00 & 1.59 & 0.611 & 0.168 & 75.544 & 1.358 & 0.027 & 0.008229 & 0.002356 & 0.083206 & 37.343 \\
\hline 1/13/08 3:00 & 1.585 & 0.606 & 0.165 & 74.027 & 1.356 & 0.025 & 0.007620 & 0.002126 & 0.075100 & 33.705 \\
\hline 1/13/08 4:00 & 1.588 & 0.609 & 0.167 & 74.935 & 1.356 & 0.025 & 0.007620 & 0.002126 & 0.075100 & 33.705 \\
\hline 1/13/08 5:00 & 1.585 & 0.606 & 0.165 & 74.027 & 1.358 & 0.027 & 0.008229 & 0.002356 & 0.083206 & 37.343 \\
\hline 1/13/08 6:00 & 1.588 & 0.609 & 0.167 & 74.935 & 1.358 & 0.027 & 0.008229 & 0.002356 & 0.083206 & 37.343 \\
\hline 1/13/08 7:00 & 1.59 & 0.611 & 0.168 & 75.544 & 1.358 & 0.027 & 0.008229 & 0.002356 & 0.083206 & 37.343 \\
\hline 1/13/08 8:00 & 1.586 & 0.607 & 0.166 & 74.329 & 1.358 & 0.027 & 0.008229 & 0.002356 & 0.083206 & 37.343 \\
\hline 1/13/08 9:00 & 1.586 & 0.607 & 0.166 & 74.329 & 1.358 & 0.027 & 0.008229 & 0.002356 & 0.083206 & 37.343 \\
\hline 1/13/08 10:00 & 1.586 & 0.607 & 0.166 & 74.329 & 1.358 & 0.027 & 0.008229 & 0.002356 & 0.083206 & 37.343 \\
\hline 1/13/08 11:00 & 1.583 & 0.604 & 0.164 & 73.426 & 1.358 & 0.027 & 0.008229 & 0.002356 & 0.083206 & 37.343 \\
\hline
\end{tabular}




\begin{tabular}{|c|c|c|c|c|c|c|c|c|c|c|}
\hline \multirow[b]{3}{*}{ Date Time } & \multicolumn{4}{|c|}{ V-Notch Weir } & \multicolumn{6}{|c|}{ R-Notch Weir } \\
\hline & \multicolumn{2}{|c|}{ Measured Water Level } & \multicolumn{2}{|c|}{$\begin{array}{c}\text { Calculated Rate } \\
\left(\mathrm{ft}^{3} / \mathrm{s}=448.8 \mathrm{gpm}\right)\end{array}$} & \multicolumn{3}{|c|}{ Measured Water Level } & \multicolumn{3}{|c|}{$\begin{array}{c}\text { Calculated Rate } \\
\left(\mathrm{ft}^{3} / \mathrm{s}=448.8 \mathrm{gpm}\right)\end{array}$} \\
\hline & $\begin{array}{c}\text { (ft above } \\
\text { sensor) }\end{array}$ & $\begin{array}{c}\text { (ft above } \\
\text { notch) }\end{array}$ & $\left(\mathrm{ft}^{3} / \mathrm{s}\right)$ & (gpm) & $\begin{array}{l}\text { (ft above } \\
\text { sensor) }\end{array}$ & $\begin{array}{c}\text { (ft above } \\
\text { log) }\end{array}$ & $\begin{array}{c}\text { (m above } \\
\text { log) }\end{array}$ & $\left(\mathrm{m}^{3} / \mathrm{s}\right)$ & $\left(\mathrm{ft}^{3} / \mathrm{s}\right)$ & (gpm) \\
\hline 1/13/08 12:00 & 1.585 & 0.606 & 0.165 & 74.027 & 1.356 & 0.025 & 0.007620 & 0.002126 & 0.075100 & 33.705 \\
\hline 1/13/08 13:00 & 1.587 & 0.608 & 0.166 & 74.632 & 1.358 & 0.027 & 0.008229 & 0.002356 & 0.083206 & 37.343 \\
\hline 1/13/08 14:00 & 1.591 & 0.612 & 0.169 & 75.850 & 1.361 & 0.03 & 0.009144 & 0.002714 & 0.095873 & 43.028 \\
\hline 1/13/08 15:00 & 1.591 & 0.612 & 0.169 & 75.850 & 1.358 & 0.027 & 0.008229 & 0.002356 & 0.083206 & 37.343 \\
\hline 1/13/08 16:00 & 1.595 & 0.616 & 0.172 & 77.080 & 1.36 & 0.029 & 0.008839 & 0.002593 & 0.091584 & 41.103 \\
\hline 1/13/08 17:00 & 1.598 & 0.619 & 0.174 & 78.010 & 1.358 & 0.027 & 0.008229 & 0.002356 & 0.083206 & 37.343 \\
\hline 1/13/08 18:00 & 1.605 & 0.626 & 0.179 & 80.206 & 1.36 & 0.029 & 0.008839 & 0.002593 & 0.091584 & 41.103 \\
\hline 1/13/08 19:00 & 1.598 & 0.619 & 0.174 & 78.010 & 1.36 & 0.029 & 0.008839 & 0.002593 & 0.091584 & 41.103 \\
\hline 1/13/08 20:00 & 1.598 & 0.619 & 0.174 & 78.010 & 1.36 & 0.029 & 0.008839 & 0.002593 & 0.091584 & 41.103 \\
\hline 1/13/08 21:00 & 1.595 & 0.616 & 0.172 & 77.080 & 1.36 & 0.029 & 0.008839 & 0.002593 & 0.091584 & 41.103 \\
\hline 1/13/08 22:00 & 1.596 & 0.617 & 0.172 & 77.389 & 1.36 & 0.029 & 0.008839 & 0.002593 & 0.091584 & 41.103 \\
\hline 1/13/08 23:00 & 1.587 & 0.608 & 0.166 & 74.632 & 1.358 & 0.027 & 0.008229 & 0.002356 & 0.083206 & 37.343 \\
\hline 1/14/08 0:00 & 1.582 & 0.603 & 0.163 & 73.126 & 1.358 & 0.027 & 0.008229 & 0.002356 & 0.083206 & 37.343 \\
\hline 1/14/08 1:00 & 1.582 & 0.603 & 0.163 & 73.126 & 1.358 & 0.027 & 0.008229 & 0.002356 & 0.083206 & 37.343 \\
\hline 1/14/08 2:00 & 1.582 & 0.603 & 0.163 & 73.126 & 1.358 & 0.027 & 0.008229 & 0.002356 & 0.083206 & 37.343 \\
\hline 1/14/08 3:00 & 1.578 & 0.599 & 0.160 & 71.935 & 1.358 & 0.027 & 0.008229 & 0.002356 & 0.083206 & 37.343 \\
\hline 1/14/08 4:00 & 1.576 & 0.597 & 0.159 & 71.343 & 1.358 & 0.027 & 0.008229 & 0.002356 & 0.083206 & 37.343 \\
\hline 1/14/08 5:00 & 1.573 & 0.594 & 0.157 & 70.462 & 1.356 & 0.025 & 0.007620 & 0.002126 & 0.075100 & 33.705 \\
\hline 1/14/08 6:00 & 1.571 & 0.592 & 0.156 & 69.878 & 1.356 & 0.025 & 0.007620 & 0.002126 & 0.075100 & 33.705 \\
\hline 1/14/08 7:00 & 1.564 & 0.585 & 0.151 & 67.856 & 1.356 & 0.025 & 0.007620 & 0.002126 & 0.075100 & 33.705 \\
\hline 1/14/08 8:00 & 1.564 & 0.585 & 0.151 & 67.856 & 1.356 & 0.025 & 0.007620 & 0.002126 & 0.075100 & 33.705 \\
\hline 1/14/08 9:00 & 1.564 & 0.585 & 0.151 & 67.856 & 1.356 & 0.025 & 0.007620 & 0.002126 & 0.075100 & 33.705 \\
\hline 1/14/08 10:00 & 1.562 & 0.583 & 0.150 & 67.285 & 1.356 & 0.025 & 0.007620 & 0.002126 & 0.075100 & 33.705 \\
\hline 1/14/08 11:00 & 1.567 & 0.588 & 0.153 & 68.718 & 1.356 & 0.025 & 0.007620 & 0.002126 & 0.075100 & 33.705 \\
\hline 1/14/08 12:00 & 1.564 & 0.585 & 0.151 & 67.856 & 1.353 & 0.022 & 0.006705 & 0.001797 & 0.063473 & 28.487 \\
\hline
\end{tabular}




\begin{tabular}{|c|c|c|c|c|c|c|c|c|c|c|}
\hline \multirow[b]{3}{*}{ Date Time } & \multicolumn{4}{|c|}{ V-Notch Weir } & \multicolumn{6}{|c|}{ R-Notch Weir } \\
\hline & \multicolumn{2}{|c|}{ Measured Water Level } & \multicolumn{2}{|c|}{$\begin{array}{c}\text { Calculated Rate } \\
\left(\mathrm{ft}^{3} / \mathrm{s}=448.8 \mathrm{gpm}\right)\end{array}$} & \multicolumn{3}{|c|}{ Measured Water Level } & \multicolumn{3}{|c|}{$\begin{array}{c}\text { Calculated Rate } \\
\left(\mathrm{ft}^{3} / \mathrm{s}=448.8 \mathrm{gpm}\right)\end{array}$} \\
\hline & $\begin{array}{c}\text { (ft above } \\
\text { sensor) }\end{array}$ & $\begin{array}{c}\text { (ft above } \\
\text { notch) }\end{array}$ & $\left(\mathrm{ft}^{3} / \mathrm{s}\right)$ & (gpm) & $\begin{array}{l}\text { (ft above } \\
\text { sensor) }\end{array}$ & $\begin{array}{c}\text { (ft above } \\
\text { log) }\end{array}$ & $\begin{array}{c}\text { (m above } \\
\text { log) }\end{array}$ & $\left(\mathrm{m}^{3} / \mathrm{s}\right)$ & $\left(\mathrm{ft}^{3} / \mathrm{s}\right)$ & (gpm) \\
\hline 1/14/08 13:00 & 1.564 & 0.585 & 0.151 & 67.856 & 1.356 & 0.025 & 0.007620 & 0.002126 & 0.075100 & 33.705 \\
\hline 1/14/08 14:00 & 1.57 & 0.591 & 0.155 & 69.587 & 1.358 & 0.027 & 0.008229 & 0.002356 & 0.083206 & 37.343 \\
\hline 1/14/08 15:00 & 1.563 & 0.584 & 0.151 & 67.571 & 1.358 & 0.027 & 0.008229 & 0.002356 & 0.083206 & 37.343 \\
\hline 1/14/08 16:00 & 1.57 & 0.591 & 0.155 & 69.587 & 1.358 & 0.027 & 0.008229 & 0.002356 & 0.083206 & 37.343 \\
\hline 1/14/08 17:00 & 1.572 & 0.593 & 0.156 & 70.169 & 1.358 & 0.027 & 0.008229 & 0.002356 & 0.083206 & 37.343 \\
\hline 1/14/08 18:00 & 1.567 & 0.588 & 0.153 & 68.718 & 1.358 & 0.027 & 0.008229 & 0.002356 & 0.083206 & 37.343 \\
\hline 1/14/08 19:00 & 1.567 & 0.588 & 0.153 & 68.718 & 1.356 & 0.025 & 0.007620 & 0.002126 & 0.075100 & 33.705 \\
\hline 1/14/08 20:00 & 1.568 & 0.589 & 0.154 & 69.007 & 1.356 & 0.025 & 0.007620 & 0.002126 & 0.075100 & 33.705 \\
\hline 1/14/08 21:00 & 1.563 & 0.584 & 0.151 & 67.571 & 1.356 & 0.025 & 0.007620 & 0.002126 & 0.075100 & 33.705 \\
\hline 1/14/08 22:00 & 1.561 & 0.582 & 0.149 & 67.001 & 1.353 & 0.022 & 0.006705 & 0.001797 & 0.063473 & 28.487 \\
\hline 1/14/08 23:00 & 1.559 & 0.580 & 0.148 & 66.434 & 1.356 & 0.025 & 0.007620 & 0.002126 & 0.075100 & 33.705 \\
\hline 1/15/08 0:00 & 1.557 & 0.578 & 0.147 & 65.871 & 1.353 & 0.022 & 0.006705 & 0.001797 & 0.063473 & 28.487 \\
\hline 1/15/08 1:00 & 1.555 & 0.576 & 0.146 & 65.310 & 1.353 & 0.022 & 0.006705 & 0.001797 & 0.063473 & 28.487 \\
\hline 1/15/08 2:00 & 1.555 & 0.576 & 0.146 & 65.310 & 1.353 & 0.022 & 0.006705 & 0.001797 & 0.063473 & 28.487 \\
\hline 1/15/08 3:00 & 1.553 & 0.574 & 0.144 & 64.752 & 1.353 & 0.022 & 0.006705 & 0.001797 & 0.063473 & 28.487 \\
\hline 1/15/08 4:00 & 1.553 & 0.574 & 0.144 & 64.752 & 1.353 & 0.022 & 0.006705 & 0.001797 & 0.063473 & 28.487 \\
\hline 1/15/08 5:00 & 1.553 & 0.574 & 0.144 & 64.752 & 1.353 & 0.022 & 0.006705 & 0.001797 & 0.063473 & 28.487 \\
\hline 1/15/08 6:00 & 1.551 & 0.572 & 0.143 & 64.197 & 1.353 & 0.022 & 0.006705 & 0.001797 & 0.063473 & 28.487 \\
\hline 1/15/08 7:00 & 1.553 & 0.574 & 0.144 & 64.752 & 1.354 & 0.023 & 0.007010 & 0.001905 & 0.067276 & 30.194 \\
\hline 1/15/08 8:00 & 1.553 & 0.574 & 0.144 & 64.752 & 1.354 & 0.023 & 0.007010 & 0.001905 & 0.067276 & 30.194 \\
\hline 1/15/08 9:00 & 1.551 & 0.572 & 0.143 & 64.197 & 1.356 & 0.025 & 0.007620 & 0.002126 & 0.075100 & 33.705 \\
\hline 1/15/08 10:00 & 1.553 & 0.574 & 0.144 & 64.752 & 1.354 & 0.023 & 0.007010 & 0.001905 & 0.067276 & 30.194 \\
\hline 1/15/08 11:00 & 1.551 & 0.572 & 0.143 & 64.197 & 1.354 & 0.023 & 0.007010 & 0.001905 & 0.067276 & 30.194 \\
\hline 1/15/08 12:00 & 1.55 & 0.571 & 0.142 & 63.920 & 1.356 & 0.025 & 0.007620 & 0.002126 & 0.075100 & 33.705 \\
\hline 1/15/08 13:00 & 1.557 & 0.578 & 0.147 & 65.871 & 1.356 & 0.025 & 0.007620 & 0.002126 & 0.075100 & 33.705 \\
\hline
\end{tabular}




\begin{tabular}{|c|c|c|c|c|c|c|c|c|c|c|}
\hline \multirow[b]{3}{*}{ Date Time } & \multicolumn{4}{|c|}{ V-Notch Weir } & \multicolumn{6}{|c|}{ R-Notch Weir } \\
\hline & \multicolumn{2}{|c|}{ Measured Water Level } & \multicolumn{2}{|c|}{$\begin{array}{c}\text { Calculated Rate } \\
\left(\mathrm{ft}^{3} / \mathrm{s}=448.8 \mathrm{gpm}\right)\end{array}$} & \multicolumn{3}{|c|}{ Measured Water Level } & \multicolumn{3}{|c|}{$\begin{array}{c}\text { Calculated Rate } \\
\left(\mathrm{ft}^{3} / \mathrm{s}=448.8 \mathrm{gpm}\right)\end{array}$} \\
\hline & $\begin{array}{c}\text { (ft above } \\
\text { sensor) }\end{array}$ & $\begin{array}{c}\text { (ft above } \\
\text { notch) }\end{array}$ & $\left(\mathrm{ft}^{3} / \mathrm{s}\right)$ & (gpm) & $\begin{array}{l}\text { (ft above } \\
\text { sensor) }\end{array}$ & $\begin{array}{c}\text { (ft above } \\
\text { log) }\end{array}$ & $\begin{array}{c}\text { (m above } \\
\text { log) }\end{array}$ & $\left(\mathrm{m}^{3} / \mathrm{s}\right)$ & $\left(\mathrm{ft}^{3} / \mathrm{s}\right)$ & (gpm) \\
\hline 1/15/08 14:00 & 1.573 & 0.594 & 0.157 & 70.462 & 1.358 & 0.027 & 0.008229 & 0.002356 & 0.083206 & 37.343 \\
\hline 1/15/08 15:00 & 1.57 & 0.591 & 0.155 & 69.587 & 1.358 & 0.027 & 0.008229 & 0.002356 & 0.083206 & 37.343 \\
\hline 1/15/08 16:00 & 1.574 & 0.595 & 0.158 & 70.755 & 1.36 & 0.029 & 0.008839 & 0.002593 & 0.091584 & 41.103 \\
\hline 1/15/08 17:00 & 1.586 & 0.607 & 0.166 & 74.329 & 1.36 & 0.029 & 0.008839 & 0.002593 & 0.091584 & 41.103 \\
\hline 1/15/08 18:00 & 1.586 & 0.607 & 0.166 & 74.329 & 1.36 & 0.029 & 0.008839 & 0.002593 & 0.091584 & 41.103 \\
\hline 1/15/08 19:00 & 1.589 & 0.610 & 0.168 & 75.239 & 1.361 & 0.03 & 0.009144 & 0.002714 & 0.095873 & 43.028 \\
\hline 1/15/08 20:00 & 1.586 & 0.607 & 0.166 & 74.329 & 1.358 & 0.027 & 0.008229 & 0.002356 & 0.083206 & 37.343 \\
\hline 1/15/08 21:00 & 1.584 & 0.605 & 0.164 & 73.726 & 1.361 & 0.03 & 0.009144 & 0.002714 & 0.095873 & 43.028 \\
\hline 1/15/08 22:00 & 1.589 & 0.610 & 0.168 & 75.239 & 1.363 & 0.032 & 0.009753 & 0.002963 & 0.104644 & 46.964 \\
\hline 1/15/08 23:00 & 1.589 & 0.610 & 0.168 & 75.239 & 1.358 & 0.027 & 0.008229 & 0.002356 & 0.083206 & 37.343 \\
\hline 1/16/08 0:00 & 1.585 & 0.606 & 0.165 & 74.027 & 1.361 & 0.03 & 0.009144 & 0.002714 & 0.095873 & 43.028 \\
\hline 1/16/08 1:00 & 1.58 & 0.601 & 0.162 & 72.529 & 1.361 & 0.03 & 0.009144 & 0.002714 & 0.095873 & 43.028 \\
\hline 1/16/08 2:00 & 1.585 & 0.606 & 0.165 & 74.027 & 1.361 & 0.03 & 0.009144 & 0.002714 & 0.095873 & 43.028 \\
\hline 1/16/08 3:00 & 1.583 & 0.604 & 0.164 & 73.426 & 1.358 & 0.027 & 0.008229 & 0.002356 & 0.083206 & 37.343 \\
\hline 1/16/08 4:00 & 1.58 & 0.601 & 0.162 & 72.529 & 1.358 & 0.027 & 0.008229 & 0.002356 & 0.083206 & 37.343 \\
\hline 1/16/08 5:00 & 1.583 & 0.604 & 0.164 & 73.426 & 1.358 & 0.027 & 0.008229 & 0.002356 & 0.083206 & 37.343 \\
\hline 1/16/08 6:00 & 1.585 & 0.606 & 0.165 & 74.027 & 1.358 & 0.027 & 0.008229 & 0.002356 & 0.083206 & 37.343 \\
\hline 1/16/08 7:00 & 1.58 & 0.601 & 0.162 & 72.529 & 1.358 & 0.027 & 0.008229 & 0.002356 & 0.083206 & 37.343 \\
\hline 1/16/08 8:00 & 1.578 & 0.599 & 0.160 & 71.935 & 1.358 & 0.027 & 0.008229 & 0.002356 & 0.083206 & 37.343 \\
\hline 1/16/08 9:00 & 1.583 & 0.604 & 0.164 & 73.426 & 1.358 & 0.027 & 0.008229 & 0.002356 & 0.083206 & 37.343 \\
\hline 1/16/08 10:00 & 1.588 & 0.609 & 0.167 & 74.935 & 1.358 & 0.027 & 0.008229 & 0.002356 & 0.083206 & 37.343 \\
\hline 1/16/08 11:00 & 1.581 & 0.602 & 0.162 & 72.827 & 1.356 & 0.025 & 0.007620 & 0.002126 & 0.075100 & 33.705 \\
\hline 1/16/08 12:00 & 1.588 & 0.609 & 0.167 & 74.935 & 1.358 & 0.027 & 0.008229 & 0.002356 & 0.083206 & 37.343 \\
\hline 1/16/08 13:00 & 1.583 & 0.604 & 0.164 & 73.426 & 1.358 & 0.027 & 0.008229 & 0.002356 & 0.083206 & 37.343 \\
\hline 1/16/08 14:00 & 1.592 & 0.613 & 0.170 & 76.156 & 1.358 & 0.027 & 0.008229 & 0.002356 & 0.083206 & 37.343 \\
\hline
\end{tabular}




\begin{tabular}{|c|c|c|c|c|c|c|c|c|c|c|}
\hline \multirow[b]{3}{*}{ Date Time } & \multicolumn{4}{|c|}{ V-Notch Weir } & \multicolumn{6}{|c|}{ R-Notch Weir } \\
\hline & \multicolumn{2}{|c|}{ Measured Water Level } & \multicolumn{2}{|c|}{$\begin{array}{c}\text { Calculated Rate } \\
\left(\mathrm{ft}^{3} / \mathrm{s}=448.8 \mathrm{gpm}\right)\end{array}$} & \multicolumn{3}{|c|}{ Measured Water Level } & \multicolumn{3}{|c|}{$\begin{array}{c}\text { Calculated Rate } \\
\left(\mathrm{ft}^{3} / \mathrm{s}=448.8 \mathrm{gpm}\right)\end{array}$} \\
\hline & $\begin{array}{c}\text { (ft above } \\
\text { sensor) }\end{array}$ & $\begin{array}{c}\text { (ft above } \\
\text { notch) }\end{array}$ & $\left(\mathrm{ft}^{3} / \mathrm{s}\right)$ & (gpm) & $\begin{array}{l}\text { (ft above } \\
\text { sensor) }\end{array}$ & $\begin{array}{c}\text { (ft above } \\
\text { log) }\end{array}$ & $\begin{array}{c}\text { (m above } \\
\text { log) }\end{array}$ & $\left(\mathrm{m}^{3} / \mathrm{s}\right)$ & $\left(\mathrm{ft}^{3} / \mathrm{s}\right)$ & (gpm) \\
\hline 1/16/08 15:00 & 1.576 & 0.597 & 0.159 & 71.343 & 1.356 & 0.025 & 0.007620 & 0.002126 & 0.075100 & 33.705 \\
\hline 1/16/08 16:00 & 1.576 & 0.597 & 0.159 & 71.343 & 1.358 & 0.027 & 0.008229 & 0.002356 & 0.083206 & 37.343 \\
\hline 1/16/08 17:00 & 1.59 & 0.611 & 0.168 & 75.544 & 1.358 & 0.027 & 0.008229 & 0.002356 & 0.083206 & 37.343 \\
\hline 1/16/08 18:00 & 1.578 & 0.599 & 0.160 & 71.935 & 1.358 & 0.027 & 0.008229 & 0.002356 & 0.083206 & 37.343 \\
\hline 1/16/08 19:00 & 1.581 & 0.602 & 0.162 & 72.827 & 1.358 & 0.027 & 0.008229 & 0.002356 & 0.083206 & 37.343 \\
\hline 1/16/08 20:00 & 1.583 & 0.604 & 0.164 & 73.426 & 1.358 & 0.027 & 0.008229 & 0.002356 & 0.083206 & 37.343 \\
\hline 1/16/08 21:00 & 1.579 & 0.600 & 0.161 & 72.231 & 1.358 & 0.027 & 0.008229 & 0.002356 & 0.083206 & 37.343 \\
\hline 1/16/08 22:00 & 1.579 & 0.600 & 0.161 & 72.231 & 1.358 & 0.027 & 0.008229 & 0.002356 & 0.083206 & 37.343 \\
\hline 1/16/08 23:00 & 1.589 & 0.610 & 0.168 & 75.239 & 1.358 & 0.027 & 0.008229 & 0.002356 & 0.083206 & 37.343 \\
\hline 1/17/08 0:00 & 1.572 & 0.593 & 0.156 & 70.169 & 1.358 & 0.027 & 0.008229 & 0.002356 & 0.083206 & 37.343 \\
\hline 1/17/08 1:00 & 1.575 & 0.596 & 0.158 & 71.049 & 1.358 & 0.027 & 0.008229 & 0.002356 & 0.083206 & 37.343 \\
\hline 1/17/08 2:00 & 1.58 & 0.601 & 0.162 & 72.529 & 1.356 & 0.025 & 0.007620 & 0.002126 & 0.075100 & 33.705 \\
\hline 1/17/08 3:00 & 1.575 & 0.596 & 0.158 & 71.049 & 1.358 & 0.027 & 0.008229 & 0.002356 & 0.083206 & 37.343 \\
\hline 1/17/08 4:00 & 1.575 & 0.596 & 0.158 & 71.049 & 1.358 & 0.027 & 0.008229 & 0.002356 & 0.083206 & 37.343 \\
\hline 1/17/08 5:00 & 1.58 & 0.601 & 0.162 & 72.529 & 1.358 & 0.027 & 0.008229 & 0.002356 & 0.083206 & 37.343 \\
\hline 1/17/08 6:00 & 1.584 & 0.605 & 0.164 & 73.726 & 1.358 & 0.027 & 0.008229 & 0.002356 & 0.083206 & 37.343 \\
\hline 1/17/08 7:00 & 1.584 & 0.605 & 0.164 & 73.726 & 1.358 & 0.027 & 0.008229 & 0.002356 & 0.083206 & 37.343 \\
\hline 1/17/08 8:00 & 1.58 & 0.601 & 0.162 & 72.529 & 1.358 & 0.027 & 0.008229 & 0.002356 & 0.083206 & 37.343 \\
\hline 1/17/08 9:00 & 1.582 & 0.603 & 0.163 & 73.126 & 1.358 & 0.027 & 0.008229 & 0.002356 & 0.083206 & 37.343 \\
\hline 1/17/08 10:00 & 1.58 & 0.601 & 0.162 & 72.529 & 1.358 & 0.027 & 0.008229 & 0.002356 & 0.083206 & 37.343 \\
\hline 1/17/08 11:00 & 1.579 & 0.600 & 0.161 & 72.231 & 1.358 & 0.027 & 0.008229 & 0.002356 & 0.083206 & 37.343 \\
\hline 1/17/08 12:00 & 1.577 & 0.598 & 0.160 & 71.638 & 1.358 & 0.027 & 0.008229 & 0.002356 & 0.083206 & 37.343 \\
\hline 1/17/08 13:00 & 1.577 & 0.598 & 0.160 & 71.638 & 1.358 & 0.027 & 0.008229 & 0.002356 & 0.083206 & 37.343 \\
\hline 1/17/08 14:00 & 1.579 & 0.600 & 0.161 & 72.231 & 1.361 & 0.03 & 0.009144 & 0.002714 & 0.095873 & 43.028 \\
\hline 1/17/08 15:00 & 1.579 & 0.600 & 0.161 & 72.231 & 1.361 & 0.03 & 0.009144 & 0.002714 & 0.095873 & 43.028 \\
\hline
\end{tabular}




\begin{tabular}{|c|c|c|c|c|c|c|c|c|c|c|}
\hline \multirow[b]{3}{*}{ Date Time } & \multicolumn{4}{|c|}{ V-Notch Weir } & \multicolumn{6}{|c|}{ R-Notch Weir } \\
\hline & \multicolumn{2}{|c|}{ Measured Water Level } & \multicolumn{2}{|c|}{$\begin{array}{c}\text { Calculated Rate } \\
\left(\mathrm{ft}^{3} / \mathrm{s}=448.8 \mathrm{gpm}\right)\end{array}$} & \multicolumn{3}{|c|}{ Measured Water Level } & \multicolumn{3}{|c|}{$\begin{array}{c}\text { Calculated Rate } \\
\left(\mathrm{ft}^{3} / \mathrm{s}=448.8 \mathrm{gpm}\right)\end{array}$} \\
\hline & $\begin{array}{c}\text { (ft above } \\
\text { sensor) }\end{array}$ & $\begin{array}{c}\text { (ft above } \\
\text { notch) }\end{array}$ & $\left(\mathrm{ft}^{3} / \mathrm{s}\right)$ & (gpm) & $\begin{array}{l}\text { (ft above } \\
\text { sensor) }\end{array}$ & $\begin{array}{c}\text { (ft above } \\
\text { log) }\end{array}$ & $\begin{array}{c}\text { (m above } \\
\text { log) }\end{array}$ & $\left(\mathrm{m}^{3} / \mathrm{s}\right)$ & $\left(\mathrm{ft}^{3} / \mathrm{s}\right)$ & (gpm) \\
\hline 1/17/08 16:00 & 1.583 & 0.604 & 0.164 & 73.426 & 1.361 & 0.03 & 0.009144 & 0.002714 & 0.095873 & 43.028 \\
\hline 1/17/08 17:00 & 1.583 & 0.604 & 0.164 & 73.426 & 1.361 & 0.03 & 0.009144 & 0.002714 & 0.095873 & 43.028 \\
\hline 1/17/08 18:00 & 1.583 & 0.604 & 0.164 & 73.426 & 1.361 & 0.03 & 0.009144 & 0.002714 & 0.095873 & 43.028 \\
\hline 1/17/08 19:00 & 1.581 & 0.602 & 0.162 & 72.827 & 1.358 & 0.027 & 0.008229 & 0.002356 & 0.083206 & 37.343 \\
\hline 1/17/08 20:00 & 1.579 & 0.600 & 0.161 & 72.231 & 1.358 & 0.027 & 0.008229 & 0.002356 & 0.083206 & 37.343 \\
\hline 1/17/08 21:00 & 1.577 & 0.598 & 0.160 & 71.638 & 1.358 & 0.027 & 0.008229 & 0.002356 & 0.083206 & 37.343 \\
\hline 1/17/08 22:00 & 1.579 & 0.600 & 0.161 & 72.231 & 1.358 & 0.027 & 0.008229 & 0.002356 & 0.083206 & 37.343 \\
\hline 1/17/08 23:00 & 1.579 & 0.600 & 0.161 & 72.231 & 1.358 & 0.027 & 0.008229 & 0.002356 & 0.083206 & 37.343 \\
\hline 1/18/08 0:00 & 1.575 & 0.596 & 0.158 & 71.049 & 1.358 & 0.027 & 0.008229 & 0.002356 & 0.083206 & 37.343 \\
\hline 1/18/08 1:00 & 1.577 & 0.598 & 0.160 & 71.638 & 1.358 & 0.027 & 0.008229 & 0.002356 & 0.083206 & 37.343 \\
\hline 1/18/08 2:00 & 1.577 & 0.598 & 0.160 & 71.638 & 1.358 & 0.027 & 0.008229 & 0.002356 & 0.083206 & 37.343 \\
\hline 1/18/08 3:00 & 1.577 & 0.598 & 0.160 & 71.638 & 1.358 & 0.027 & 0.008229 & 0.002356 & 0.083206 & 37.343 \\
\hline 1/18/08 4:00 & 1.575 & 0.596 & 0.158 & 71.049 & 1.358 & 0.027 & 0.008229 & 0.002356 & 0.083206 & 37.343 \\
\hline 1/18/08 5:00 & 1.575 & 0.596 & 0.158 & 71.049 & 1.356 & 0.025 & 0.007620 & 0.002126 & 0.075100 & 33.705 \\
\hline 1/18/08 6:00 & 1.575 & 0.596 & 0.158 & 71.049 & 1.358 & 0.027 & 0.008229 & 0.002356 & 0.083206 & 37.343 \\
\hline 1/18/08 7:00 & 1.577 & 0.598 & 0.160 & 71.638 & 1.358 & 0.027 & 0.008229 & 0.002356 & 0.083206 & 37.343 \\
\hline 1/18/08 8:00 & 1.579 & 0.600 & 0.161 & 72.231 & 1.358 & 0.027 & 0.008229 & 0.002356 & 0.083206 & 37.343 \\
\hline 1/18/08 9:00 & 1.584 & 0.605 & 0.164 & 73.726 & 1.358 & 0.027 & 0.008229 & 0.002356 & 0.083206 & 37.343 \\
\hline 1/18/08 10:00 & 1.582 & 0.603 & 0.163 & 73.126 & 1.356 & 0.025 & 0.007620 & 0.002126 & 0.075100 & 33.705 \\
\hline 1/18/08 11:00 & 1.577 & 0.598 & 0.160 & 71.638 & 1.358 & 0.027 & 0.008229 & 0.002356 & 0.083206 & 37.343 \\
\hline 1/18/08 12:00 & 1.572 & 0.593 & 0.156 & 70.169 & 1.358 & 0.027 & 0.008229 & 0.002356 & 0.083206 & 37.343 \\
\hline 1/18/08 13:00 & 1.574 & 0.595 & 0.158 & 70.755 & 1.358 & 0.027 & 0.008229 & 0.002356 & 0.083206 & 37.343 \\
\hline 1/18/08 14:00 & 1.572 & 0.593 & 0.156 & 70.169 & 1.358 & 0.027 & 0.008229 & 0.002356 & 0.083206 & 37.343 \\
\hline 1/18/08 15:00 & 1.569 & 0.590 & 0.154 & 69.296 & 1.358 & 0.027 & 0.008229 & 0.002356 & 0.083206 & 37.343 \\
\hline 1/18/08 16:00 & 1.569 & 0.590 & 0.154 & 69.296 & 1.358 & 0.027 & 0.008229 & 0.002356 & 0.083206 & 37.343 \\
\hline
\end{tabular}




\begin{tabular}{|c|c|c|c|c|c|c|c|c|c|c|}
\hline \multirow[b]{3}{*}{ Date Time } & \multicolumn{4}{|c|}{ V-Notch Weir } & \multicolumn{6}{|c|}{ R-Notch Weir } \\
\hline & \multicolumn{2}{|c|}{ Measured Water Level } & \multicolumn{2}{|c|}{$\begin{array}{c}\text { Calculated Rate } \\
\left(\mathrm{ft}^{3} / \mathrm{s}=448.8 \mathrm{gpm}\right)\end{array}$} & \multicolumn{3}{|c|}{ Measured Water Level } & \multicolumn{3}{|c|}{$\begin{array}{c}\text { Calculated Rate } \\
\left(\mathrm{ft}^{3} / \mathrm{s}=448.8 \mathrm{gpm}\right)\end{array}$} \\
\hline & $\begin{array}{c}\text { (ft above } \\
\text { sensor) }\end{array}$ & $\begin{array}{c}\text { (ft above } \\
\text { notch) }\end{array}$ & $\left(\mathrm{ft}^{3} / \mathrm{s}\right)$ & (gpm) & $\begin{array}{l}\text { (ft above } \\
\text { sensor) }\end{array}$ & $\begin{array}{c}\text { (ft above } \\
\text { log) }\end{array}$ & $\begin{array}{c}\text { (m above } \\
\text { log) }\end{array}$ & $\left(\mathrm{m}^{3} / \mathrm{s}\right)$ & $\left(\mathrm{ft}^{3} / \mathrm{s}\right)$ & (gpm) \\
\hline 1/18/08 17:00 & 1.572 & 0.593 & 0.156 & 70.169 & 1.358 & 0.027 & 0.008229 & 0.002356 & 0.083206 & 37.343 \\
\hline 1/18/08 18:00 & 1.579 & 0.600 & 0.161 & 72.231 & 1.358 & 0.027 & 0.008229 & 0.002356 & 0.083206 & 37.343 \\
\hline 1/18/08 19:00 & 1.57 & 0.591 & 0.155 & 69.587 & 1.356 & 0.025 & 0.007620 & 0.002126 & 0.075100 & 33.705 \\
\hline 1/18/08 20:00 & 1.567 & 0.588 & 0.153 & 68.718 & 1.356 & 0.025 & 0.007620 & 0.002126 & 0.075100 & 33.705 \\
\hline 1/18/08 21:00 & 1.567 & 0.588 & 0.153 & 68.718 & 1.356 & 0.025 & 0.007620 & 0.002126 & 0.075100 & 33.705 \\
\hline 1/18/08 22:00 & 1.567 & 0.588 & 0.153 & 68.718 & 1.356 & 0.025 & 0.007620 & 0.002126 & 0.075100 & 33.705 \\
\hline 1/18/08 23:00 & 1.563 & 0.584 & 0.151 & 67.571 & 1.356 & 0.025 & 0.007620 & 0.002126 & 0.075100 & 33.705 \\
\hline 1/19/08 0:00 & 1.561 & 0.582 & 0.149 & 67.001 & 1.358 & 0.027 & 0.008229 & 0.002356 & 0.083206 & 37.343 \\
\hline 1/19/08 1:00 & 1.558 & 0.579 & 0.147 & 66.152 & 1.358 & 0.027 & 0.008229 & 0.002356 & 0.083206 & 37.343 \\
\hline 1/19/08 2:00 & 1.556 & 0.577 & 0.146 & 65.590 & 1.358 & 0.027 & 0.008229 & 0.002356 & 0.083206 & 37.343 \\
\hline 1/19/08 3:00 & 1.561 & 0.582 & 0.149 & 67.001 & 1.361 & 0.03 & 0.009144 & 0.002714 & 0.095873 & 43.028 \\
\hline 1/19/08 4:00 & 1.551 & 0.572 & 0.143 & 64.197 & 1.361 & 0.03 & 0.009144 & 0.002714 & 0.095873 & 43.028 \\
\hline 1/19/08 5:00 & 1.542 & 0.563 & 0.138 & 61.734 & 1.366 & 0.035 & 0.010667 & 0.003349 & 0.118271 & 53.080 \\
\hline 1/19/08 6:00 & 1.554 & 0.575 & 0.145 & 65.030 & 1.366 & 0.035 & 0.010667 & 0.003349 & 0.118271 & 53.080 \\
\hline 1/19/08 7:00 & 1.561 & 0.582 & 0.149 & 67.001 & 1.366 & 0.035 & 0.010667 & 0.003349 & 0.118271 & 53.080 \\
\hline 1/19/08 8:00 & 1.563 & 0.584 & 0.151 & 67.571 & 1.363 & 0.032 & 0.009753 & 0.002963 & 0.104644 & 46.964 \\
\hline 1/19/08 9:00 & 1.559 & 0.580 & 0.148 & 66.434 & 1.358 & 0.027 & 0.008229 & 0.002356 & 0.083206 & 37.343 \\
\hline 1/19/08 10:00 & 1.549 & 0.570 & 0.142 & 63.644 & 1.356 & 0.025 & 0.007620 & 0.002126 & 0.075100 & 33.705 \\
\hline 1/19/08 11:00 & 1.554 & 0.575 & 0.145 & 65.030 & 1.356 & 0.025 & 0.007620 & 0.002126 & 0.075100 & 33.705 \\
\hline 1/19/08 12:00 & 1.551 & 0.572 & 0.143 & 64.197 & 1.356 & 0.025 & 0.007620 & 0.002126 & 0.075100 & 33.705 \\
\hline 1/19/08 13:00 & 1.563 & 0.584 & 0.151 & 67.571 & 1.356 & 0.025 & 0.007620 & 0.002126 & 0.075100 & 33.705 \\
\hline 1/19/08 14:00 & 1.565 & 0.586 & 0.152 & 68.143 & 1.356 & 0.025 & 0.007620 & 0.002126 & 0.075100 & 33.705 \\
\hline 1/19/08 15:00 & 1.567 & 0.588 & 0.153 & 68.718 & 1.356 & 0.025 & 0.007620 & 0.002126 & 0.075100 & 33.705 \\
\hline 1/19/08 16:00 & 1.565 & 0.586 & 0.152 & 68.143 & 1.356 & 0.025 & 0.007620 & 0.002126 & 0.075100 & 33.705 \\
\hline 1/19/08 17:00 & 1.572 & 0.593 & 0.156 & 70.169 & 1.356 & 0.025 & 0.007620 & 0.002126 & 0.075100 & 33.705 \\
\hline
\end{tabular}




\begin{tabular}{|c|c|c|c|c|c|c|c|c|c|c|}
\hline \multirow[b]{3}{*}{ Date Time } & \multicolumn{4}{|c|}{ V-Notch Weir } & \multicolumn{6}{|c|}{ R-Notch Weir } \\
\hline & \multicolumn{2}{|c|}{ Measured Water Level } & \multicolumn{2}{|c|}{$\begin{array}{c}\text { Calculated Rate } \\
\left(\mathrm{ft}^{3} / \mathrm{s}=448.8 \mathrm{gpm}\right)\end{array}$} & \multicolumn{3}{|c|}{ Measured Water Level } & \multicolumn{3}{|c|}{$\begin{array}{c}\text { Calculated Rate } \\
\left(\mathrm{ft}^{3} / \mathrm{s}=448.8 \mathrm{gpm}\right)\end{array}$} \\
\hline & $\begin{array}{c}\text { (ft above } \\
\text { sensor) }\end{array}$ & $\begin{array}{c}\text { (ft above } \\
\text { notch) }\end{array}$ & $\left(\mathrm{ft}^{3} / \mathrm{s}\right)$ & (gpm) & $\begin{array}{l}\text { (ft above } \\
\text { sensor) }\end{array}$ & $\begin{array}{c}\text { (ft above } \\
\text { log) }\end{array}$ & $\begin{array}{c}\text { (m above } \\
\text { log) }\end{array}$ & $\left(\mathrm{m}^{3} / \mathrm{s}\right)$ & $\left(\mathrm{ft}^{3} / \mathrm{s}\right)$ & (gpm) \\
\hline 1/19/08 18:00 & 1.567 & 0.588 & 0.153 & 68.718 & 1.358 & 0.027 & 0.008229 & 0.002356 & 0.083206 & 37.343 \\
\hline 1/19/08 19:00 & 1.57 & 0.591 & 0.155 & 69.587 & 1.356 & 0.025 & 0.007620 & 0.002126 & 0.075100 & 33.705 \\
\hline 1/19/08 20:00 & 1.565 & 0.586 & 0.152 & 68.143 & 1.356 & 0.025 & 0.007620 & 0.002126 & 0.075100 & 33.705 \\
\hline 1/19/08 21:00 & 1.563 & 0.584 & 0.151 & 67.571 & 1.356 & 0.025 & 0.007620 & 0.002126 & 0.075100 & 33.705 \\
\hline 1/19/08 22:00 & 1.561 & 0.582 & 0.149 & 67.001 & 1.356 & 0.025 & 0.007620 & 0.002126 & 0.075100 & 33.705 \\
\hline 1/19/08 23:00 & 1.561 & 0.582 & 0.149 & 67.001 & 1.354 & 0.023 & 0.007010 & 0.001905 & 0.067276 & 30.194 \\
\hline 1/20/08 0:00 & 1.558 & 0.579 & 0.147 & 66.152 & 1.356 & 0.025 & 0.007620 & 0.002126 & 0.075100 & 33.705 \\
\hline 1/20/08 1:00 & 1.559 & 0.580 & 0.148 & 66.434 & 1.354 & 0.023 & 0.007010 & 0.001905 & 0.067276 & 30.194 \\
\hline 1/20/08 2:00 & 1.554 & 0.575 & 0.145 & 65.030 & 1.354 & 0.023 & 0.007010 & 0.001905 & 0.067276 & 30.194 \\
\hline 1/20/08 3:00 & 1.552 & 0.573 & 0.144 & 64.474 & 1.354 & 0.023 & 0.007010 & 0.001905 & 0.067276 & 30.194 \\
\hline 1/20/08 4:00 & 1.554 & 0.575 & 0.145 & 65.030 & 1.354 & 0.023 & 0.007010 & 0.001905 & 0.067276 & 30.194 \\
\hline 1/20/08 5:00 & 1.556 & 0.577 & 0.146 & 65.590 & 1.354 & 0.023 & 0.007010 & 0.001905 & 0.067276 & 30.194 \\
\hline 1/20/08 6:00 & 1.559 & 0.580 & 0.148 & 66.434 & 1.354 & 0.023 & 0.007010 & 0.001905 & 0.067276 & 30.194 \\
\hline 1/20/08 7:00 & 1.559 & 0.580 & 0.148 & 66.434 & 1.356 & 0.025 & 0.007620 & 0.002126 & 0.075100 & 33.705 \\
\hline 1/20/08 8:00 & 1.559 & 0.580 & 0.148 & 66.434 & 1.354 & 0.023 & 0.007010 & 0.001905 & 0.067276 & 30.194 \\
\hline 1/20/08 9:00 & 1.556 & 0.577 & 0.146 & 65.590 & 1.354 & 0.023 & 0.007010 & 0.001905 & 0.067276 & 30.194 \\
\hline 1/20/08 10:00 & 1.556 & 0.577 & 0.146 & 65.590 & 1.354 & 0.023 & 0.007010 & 0.001905 & 0.067276 & 30.194 \\
\hline 1/20/08 11:00 & 1.556 & 0.577 & 0.146 & 65.590 & 1.354 & 0.023 & 0.007010 & 0.001905 & 0.067276 & 30.194 \\
\hline 1/20/08 12:00 & 1.563 & 0.584 & 0.151 & 67.571 & 1.356 & 0.025 & 0.007620 & 0.002126 & 0.075100 & 33.705 \\
\hline 1/20/08 13:00 & 1.563 & 0.584 & 0.151 & 67.571 & 1.356 & 0.025 & 0.007620 & 0.002126 & 0.075100 & 33.705 \\
\hline 1/20/08 14:00 & 1.572 & 0.593 & 0.156 & 70.169 & 1.359 & 0.028 & 0.008534 & 0.002473 & 0.087362 & 39.208 \\
\hline 1/20/08 15:00 & 1.572 & 0.593 & 0.156 & 70.169 & 1.361 & 0.03 & 0.009144 & 0.002714 & 0.095873 & 43.028 \\
\hline 1/20/08 16:00 & 1.574 & 0.595 & 0.158 & 70.755 & 1.363 & 0.032 & 0.009753 & 0.002963 & 0.104644 & 46.964 \\
\hline 1/20/08 17:00 & 1.572 & 0.593 & 0.156 & 70.169 & 1.361 & 0.03 & 0.009144 & 0.002714 & 0.095873 & 43.028 \\
\hline 1/20/08 18:00 & 1.57 & 0.591 & 0.155 & 69.587 & 1.361 & 0.03 & 0.009144 & 0.002714 & 0.095873 & 43.028 \\
\hline
\end{tabular}




\begin{tabular}{|c|c|c|c|c|c|c|c|c|c|c|}
\hline \multirow[b]{3}{*}{ Date Time } & \multicolumn{4}{|c|}{ V-Notch Weir } & \multicolumn{6}{|c|}{ R-Notch Weir } \\
\hline & \multicolumn{2}{|c|}{ Measured Water Level } & \multicolumn{2}{|c|}{$\begin{array}{c}\text { Calculated Rate } \\
\left(\mathrm{ft}^{3} / \mathrm{s}=448.8 \mathrm{gpm}\right)\end{array}$} & \multicolumn{3}{|c|}{ Measured Water Level } & \multicolumn{3}{|c|}{$\begin{array}{c}\text { Calculated Rate } \\
\left(\mathrm{ft}^{3} / \mathrm{s}=448.8 \mathrm{gpm}\right)\end{array}$} \\
\hline & $\begin{array}{c}\text { (ft above } \\
\text { sensor) }\end{array}$ & $\begin{array}{c}\text { (ft above } \\
\text { notch) }\end{array}$ & $\left(\mathrm{ft}^{3} / \mathrm{s}\right)$ & (gpm) & $\begin{array}{l}\text { (ft above } \\
\text { sensor) }\end{array}$ & $\begin{array}{c}\text { (ft above } \\
\text { log) }\end{array}$ & $\begin{array}{c}\text { (m above } \\
\text { log) }\end{array}$ & $\left(\mathrm{m}^{3} / \mathrm{s}\right)$ & $\left(\mathrm{ft}^{3} / \mathrm{s}\right)$ & (gpm) \\
\hline 1/20/08 19:00 & 1.57 & 0.591 & 0.155 & 69.587 & 1.361 & 0.03 & 0.009144 & 0.002714 & 0.095873 & 43.028 \\
\hline 1/20/08 20:00 & 1.563 & 0.584 & 0.151 & 67.571 & 1.358 & 0.027 & 0.008229 & 0.002356 & 0.083206 & 37.343 \\
\hline 1/20/08 21:00 & 1.563 & 0.584 & 0.151 & 67.571 & 1.361 & 0.03 & 0.009144 & 0.002714 & 0.095873 & 43.028 \\
\hline 1/20/08 22:00 & 1.554 & 0.575 & 0.145 & 65.030 & 1.358 & 0.027 & 0.008229 & 0.002356 & 0.083206 & 37.343 \\
\hline 1/20/08 23:00 & 1.565 & 0.586 & 0.152 & 68.143 & 1.356 & 0.025 & 0.007620 & 0.002126 & 0.075100 & 33.705 \\
\hline 1/21/08 0:00 & 1.572 & 0.593 & 0.156 & 70.169 & 1.361 & 0.03 & 0.009144 & 0.002714 & 0.095873 & 43.028 \\
\hline 1/21/08 1:00 & 1.563 & 0.584 & 0.151 & 67.571 & 1.361 & 0.03 & 0.009144 & 0.002714 & 0.095873 & 43.028 \\
\hline 1/21/08 2:00 & 1.559 & 0.580 & 0.148 & 66.434 & 1.361 & 0.03 & 0.009144 & 0.002714 & 0.095873 & 43.028 \\
\hline 1/21/08 3:00 & 1.563 & 0.584 & 0.151 & 67.571 & 1.361 & 0.03 & 0.009144 & 0.002714 & 0.095873 & 43.028 \\
\hline 1/21/08 4:00 & 1.563 & 0.584 & 0.151 & 67.571 & 1.358 & 0.027 & 0.008229 & 0.002356 & 0.083206 & 37.343 \\
\hline 1/21/08 5:00 & 1.561 & 0.582 & 0.149 & 67.001 & 1.359 & 0.028 & 0.008534 & 0.002473 & 0.087362 & 39.208 \\
\hline 1/21/08 6:00 & 1.561 & 0.582 & 0.149 & 67.001 & 1.359 & 0.028 & 0.008534 & 0.002473 & 0.087362 & 39.208 \\
\hline 1/21/08 7:00 & 1.561 & 0.582 & 0.149 & 67.001 & 1.356 & 0.025 & 0.007620 & 0.002126 & 0.075100 & 33.705 \\
\hline 1/21/08 8:00 & 1.561 & 0.582 & 0.149 & 67.001 & 1.359 & 0.028 & 0.008534 & 0.002473 & 0.087362 & 39.208 \\
\hline 1/21/08 9:00 & 1.561 & 0.582 & 0.149 & 67.001 & 1.356 & 0.025 & 0.007620 & 0.002126 & 0.075100 & 33.705 \\
\hline 1/21/08 10:00 & 1.554 & 0.575 & 0.145 & 65.030 & 1.356 & 0.025 & 0.007620 & 0.002126 & 0.075100 & 33.705 \\
\hline 1/21/08 11:00 & 1.565 & 0.586 & 0.152 & 68.143 & 1.359 & 0.028 & 0.008534 & 0.002473 & 0.087362 & 39.208 \\
\hline 1/21/08 12:00 & 1.568 & 0.589 & 0.154 & 69.007 & 1.356 & 0.025 & 0.007620 & 0.002126 & 0.075100 & 33.705 \\
\hline 1/21/08 13:00 & 1.56 & 0.581 & 0.149 & 66.717 & 1.356 & 0.025 & 0.007620 & 0.002126 & 0.075100 & 33.705 \\
\hline 1/21/08 14:00 & 1.57 & 0.591 & 0.155 & 69.587 & 1.356 & 0.025 & 0.007620 & 0.002126 & 0.075100 & 33.705 \\
\hline 1/21/08 15:00 & 1.572 & 0.593 & 0.156 & 70.169 & 1.359 & 0.028 & 0.008534 & 0.002473 & 0.087362 & 39.208 \\
\hline 1/21/08 16:00 & 1.572 & 0.593 & 0.156 & 70.169 & 1.356 & 0.025 & 0.007620 & 0.002126 & 0.075100 & 33.705 \\
\hline 1/21/08 17:00 & 1.577 & 0.598 & 0.160 & 71.638 & 1.359 & 0.028 & 0.008534 & 0.002473 & 0.087362 & 39.208 \\
\hline 1/21/08 18:00 & 1.577 & 0.598 & 0.160 & 71.638 & 1.359 & 0.028 & 0.008534 & 0.002473 & 0.087362 & 39.208 \\
\hline 1/21/08 19:00 & 1.575 & 0.596 & 0.158 & 71.049 & 1.359 & 0.028 & 0.008534 & 0.002473 & 0.087362 & 39.208 \\
\hline
\end{tabular}




\begin{tabular}{|c|c|c|c|c|c|c|c|c|c|c|}
\hline \multirow[b]{3}{*}{ Date Time } & \multicolumn{4}{|c|}{ V-Notch Weir } & \multicolumn{6}{|c|}{ R-Notch Weir } \\
\hline & \multicolumn{2}{|c|}{ Measured Water Level } & \multicolumn{2}{|c|}{$\begin{array}{c}\text { Calculated Rate } \\
\left(\mathrm{ft}^{3} / \mathrm{s}=448.8 \mathrm{gpm}\right)\end{array}$} & \multicolumn{3}{|c|}{ Measured Water Level } & \multicolumn{3}{|c|}{$\begin{array}{c}\text { Calculated Rate } \\
\left(\mathrm{ft}^{3} / \mathrm{s}=448.8 \mathrm{gpm}\right)\end{array}$} \\
\hline & $\begin{array}{c}\text { (ft above } \\
\text { sensor) }\end{array}$ & $\begin{array}{c}\text { (ft above } \\
\text { notch) }\end{array}$ & $\left(\mathrm{ft}^{3} / \mathrm{s}\right)$ & (gpm) & $\begin{array}{l}\text { (ft above } \\
\text { sensor) }\end{array}$ & $\begin{array}{c}\text { (ft above } \\
\text { log) }\end{array}$ & $\begin{array}{c}\text { (m above } \\
\text { log) }\end{array}$ & $\left(\mathrm{m}^{3} / \mathrm{s}\right)$ & $\left(\mathrm{ft}^{3} / \mathrm{s}\right)$ & (gpm) \\
\hline 1/21/08 20:00 & 1.57 & 0.591 & 0.155 & 69.587 & 1.356 & 0.025 & 0.007620 & 0.002126 & 0.075100 & 33.705 \\
\hline 1/21/08 21:00 & 1.57 & 0.591 & 0.155 & 69.587 & 1.356 & 0.025 & 0.007620 & 0.002126 & 0.075100 & 33.705 \\
\hline 1/21/08 22:00 & 1.582 & 0.603 & 0.163 & 73.126 & 1.356 & 0.025 & 0.007620 & 0.002126 & 0.075100 & 33.705 \\
\hline 1/21/08 23:00 & 1.561 & 0.582 & 0.149 & 67.001 & 1.354 & 0.023 & 0.007010 & 0.001905 & 0.067276 & 30.194 \\
\hline 1/22/08 0:00 & 1.565 & 0.586 & 0.152 & 68.143 & 1.354 & 0.023 & 0.007010 & 0.001905 & 0.067276 & 30.194 \\
\hline 1/22/08 1:00 & 1.561 & 0.582 & 0.149 & 67.001 & 1.354 & 0.023 & 0.007010 & 0.001905 & 0.067276 & 30.194 \\
\hline 1/22/08 2:00 & 1.561 & 0.582 & 0.149 & 67.001 & 1.356 & 0.025 & 0.007620 & 0.002126 & 0.075100 & 33.705 \\
\hline 1/22/08 3:00 & 1.561 & 0.582 & 0.149 & 67.001 & 1.354 & 0.023 & 0.007010 & 0.001905 & 0.067276 & 30.194 \\
\hline 1/22/08 4:00 & 1.559 & 0.580 & 0.148 & 66.434 & 1.356 & 0.025 & 0.007620 & 0.002126 & 0.075100 & 33.705 \\
\hline 1/22/08 5:00 & 1.559 & 0.580 & 0.148 & 66.434 & 1.354 & 0.023 & 0.007010 & 0.001905 & 0.067276 & 30.194 \\
\hline 1/22/08 6:00 & 1.559 & 0.580 & 0.148 & 66.434 & 1.354 & 0.023 & 0.007010 & 0.001905 & 0.067276 & 30.194 \\
\hline 1/22/08 7:00 & 1.556 & 0.577 & 0.146 & 65.590 & 1.354 & 0.023 & 0.007010 & 0.001905 & 0.067276 & 30.194 \\
\hline 1/22/08 8:00 & 1.559 & 0.580 & 0.148 & 66.434 & 1.356 & 0.025 & 0.007620 & 0.002126 & 0.075100 & 33.705 \\
\hline 1/22/08 9:00 & 1.556 & 0.577 & 0.146 & 65.590 & 1.354 & 0.023 & 0.007010 & 0.001905 & 0.067276 & 30.194 \\
\hline 1/22/08 10:00 & 1.556 & 0.577 & 0.146 & 65.590 & 1.354 & 0.023 & 0.007010 & 0.001905 & 0.067276 & 30.194 \\
\hline 1/22/08 11:00 & 1.552 & 0.573 & 0.144 & 64.474 & 1.354 & 0.023 & 0.007010 & 0.001905 & 0.067276 & 30.194 \\
\hline 1/22/08 12:00 & 1.551 & 0.572 & 0.143 & 64.197 & 1.354 & 0.023 & 0.007010 & 0.001905 & 0.067276 & 30.194 \\
\hline 1/22/08 13:00 & 1.554 & 0.575 & 0.145 & 65.030 & 1.356 & 0.025 & 0.007620 & 0.002126 & 0.075100 & 33.705 \\
\hline 1/22/08 14:00 & 1.558 & 0.579 & 0.147 & 66.152 & 1.356 & 0.025 & 0.007620 & 0.002126 & 0.075100 & 33.705 \\
\hline 1/22/08 15:00 & 1.565 & 0.586 & 0.152 & 68.143 & 1.356 & 0.025 & 0.007620 & 0.002126 & 0.075100 & 33.705 \\
\hline 1/22/08 16:00 & 1.572 & 0.593 & 0.156 & 70.169 & 1.359 & 0.028 & 0.008534 & 0.002473 & 0.087362 & 39.208 \\
\hline 1/22/08 17:00 & 1.57 & 0.591 & 0.155 & 69.587 & 1.359 & 0.028 & 0.008534 & 0.002473 & 0.087362 & 39.208 \\
\hline 1/22/08 18:00 & 1.572 & 0.593 & 0.156 & 70.169 & 1.358 & 0.027 & 0.008229 & 0.002356 & 0.083206 & 37.343 \\
\hline 1/22/08 19:00 & 1.572 & 0.593 & 0.156 & 70.169 & 1.358 & 0.027 & 0.008229 & 0.002356 & 0.083206 & 37.343 \\
\hline 1/22/08 20:00 & 1.568 & 0.589 & 0.154 & 69.007 & 1.356 & 0.025 & 0.007620 & 0.002126 & 0.075100 & 33.705 \\
\hline
\end{tabular}




\begin{tabular}{|c|c|c|c|c|c|c|c|c|c|c|}
\hline \multirow[b]{3}{*}{ Date Time } & \multicolumn{4}{|c|}{ V-Notch Weir } & \multicolumn{6}{|c|}{ R-Notch Weir } \\
\hline & \multicolumn{2}{|c|}{ Measured Water Level } & \multicolumn{2}{|c|}{$\begin{array}{c}\text { Calculated Rate } \\
\left(\mathrm{ft}^{3} / \mathrm{s}=448.8 \mathrm{gpm}\right)\end{array}$} & \multicolumn{3}{|c|}{ Measured Water Level } & \multicolumn{3}{|c|}{$\begin{array}{c}\text { Calculated Rate } \\
\left(\mathrm{ft}^{3} / \mathrm{s}=448.8 \mathrm{gpm}\right)\end{array}$} \\
\hline & $\begin{array}{c}\text { (ft above } \\
\text { sensor) }\end{array}$ & $\begin{array}{c}\text { (ft above } \\
\text { notch) }\end{array}$ & $\left(\mathrm{ft}^{3} / \mathrm{s}\right)$ & (gpm) & $\begin{array}{l}\text { (ft above } \\
\text { sensor) }\end{array}$ & $\begin{array}{c}\text { (ft above } \\
\text { log) }\end{array}$ & $\begin{array}{c}\text { (m above } \\
\text { log) }\end{array}$ & $\left(\mathrm{m}^{3} / \mathrm{s}\right)$ & $\left(\mathrm{ft}^{3} / \mathrm{s}\right)$ & (gpm) \\
\hline 1/22/08 21:00 & 1.568 & 0.589 & 0.154 & 69.007 & 1.358 & 0.027 & 0.008229 & 0.002356 & 0.083206 & 37.343 \\
\hline 1/22/08 22:00 & 1.565 & 0.586 & 0.152 & 68.143 & 1.356 & 0.025 & 0.007620 & 0.002126 & 0.075100 & 33.705 \\
\hline 1/22/08 23:00 & 1.565 & 0.586 & 0.152 & 68.143 & 1.356 & 0.025 & 0.007620 & 0.002126 & 0.075100 & 33.705 \\
\hline 1/23/08 0:00 & 1.565 & 0.586 & 0.152 & 68.143 & 1.359 & 0.028 & 0.008534 & 0.002473 & 0.087362 & 39.208 \\
\hline 1/23/08 1:00 & 1.565 & 0.586 & 0.152 & 68.143 & 1.356 & 0.025 & 0.007620 & 0.002126 & 0.075100 & 33.705 \\
\hline 1/23/08 2:00 & 1.565 & 0.586 & 0.152 & 68.143 & 1.356 & 0.025 & 0.007620 & 0.002126 & 0.075100 & 33.705 \\
\hline 1/23/08 3:00 & 1.565 & 0.586 & 0.152 & 68.143 & 1.359 & 0.028 & 0.008534 & 0.002473 & 0.087362 & 39.208 \\
\hline 1/23/08 4:00 & 1.563 & 0.584 & 0.151 & 67.571 & 1.356 & 0.025 & 0.007620 & 0.002126 & 0.075100 & 33.705 \\
\hline 1/23/08 5:00 & 1.566 & 0.587 & 0.152 & 68.430 & 1.356 & 0.025 & 0.007620 & 0.002126 & 0.075100 & 33.705 \\
\hline 1/23/08 6:00 & 1.565 & 0.586 & 0.152 & 68.143 & 1.356 & 0.025 & 0.007620 & 0.002126 & 0.075100 & 33.705 \\
\hline 1/23/08 7:00 & 1.563 & 0.584 & 0.151 & 67.571 & 1.356 & 0.025 & 0.007620 & 0.002126 & 0.075100 & 33.705 \\
\hline 1/23/08 8:00 & 1.559 & 0.580 & 0.148 & 66.434 & 1.356 & 0.025 & 0.007620 & 0.002126 & 0.075100 & 33.705 \\
\hline 1/23/08 9:00 & 1.558 & 0.579 & 0.147 & 66.152 & 1.359 & 0.028 & 0.008534 & 0.002473 & 0.087362 & 39.208 \\
\hline 1/23/08 10:00 & 1.558 & 0.579 & 0.147 & 66.152 & 1.356 & 0.025 & 0.007620 & 0.002126 & 0.075100 & 33.705 \\
\hline 1/23/08 11:00 & 1.565 & 0.586 & 0.152 & 68.143 & 1.356 & 0.025 & 0.007620 & 0.002126 & 0.075100 & 33.705 \\
\hline 1/23/08 12:00 & 1.556 & 0.577 & 0.146 & 65.590 & 1.356 & 0.025 & 0.007620 & 0.002126 & 0.075100 & 33.705 \\
\hline 1/23/08 13:00 & 1.56 & 0.581 & 0.149 & 66.717 & 1.359 & 0.028 & 0.008534 & 0.002473 & 0.087362 & 39.208 \\
\hline 1/23/08 14:00 & 1.563 & 0.584 & 0.151 & 67.571 & 1.359 & 0.028 & 0.008534 & 0.002473 & 0.087362 & 39.208 \\
\hline 1/23/08 15:00 & 1.567 & 0.588 & 0.153 & 68.718 & 1.361 & 0.03 & 0.009144 & 0.002714 & 0.095873 & 43.028 \\
\hline 1/23/08 16:00 & 1.567 & 0.588 & 0.153 & 68.718 & 1.358 & 0.027 & 0.008229 & 0.002356 & 0.083206 & 37.343 \\
\hline 1/23/08 17:00 & 1.581 & 0.602 & 0.162 & 72.827 & 1.358 & 0.027 & 0.008229 & 0.002356 & 0.083206 & 37.343 \\
\hline 1/23/08 18:00 & 1.556 & 0.577 & 0.146 & 65.590 & 1.358 & 0.027 & 0.008229 & 0.002356 & 0.083206 & 37.343 \\
\hline 1/23/08 19:00 & 1.558 & 0.579 & 0.147 & 66.152 & 1.356 & 0.025 & 0.007620 & 0.002126 & 0.075100 & 33.705 \\
\hline 1/23/08 20:00 & 1.556 & 0.577 & 0.146 & 65.590 & 1.356 & 0.025 & 0.007620 & 0.002126 & 0.075100 & 33.705 \\
\hline 1/23/08 21:00 & 1.556 & 0.577 & 0.146 & 65.590 & 1.356 & 0.025 & 0.007620 & 0.002126 & 0.075100 & 33.705 \\
\hline
\end{tabular}




\begin{tabular}{|c|c|c|c|c|c|c|c|c|c|c|}
\hline \multirow[b]{3}{*}{ Date Time } & \multicolumn{4}{|c|}{ V-Notch Weir } & \multicolumn{6}{|c|}{ R-Notch Weir } \\
\hline & \multicolumn{2}{|c|}{ Measured Water Level } & \multicolumn{2}{|c|}{$\begin{array}{c}\text { Calculated Rate } \\
\left(\mathrm{ft}^{3} / \mathrm{s}=448.8 \mathrm{gpm}\right)\end{array}$} & \multicolumn{3}{|c|}{ Measured Water Level } & \multicolumn{3}{|c|}{$\begin{array}{c}\text { Calculated Rate } \\
\left(\mathrm{ft}^{3} / \mathrm{s}=448.8 \mathrm{gpm}\right)\end{array}$} \\
\hline & $\begin{array}{c}\text { (ft above } \\
\text { sensor) }\end{array}$ & $\begin{array}{c}\text { (ft above } \\
\text { notch) }\end{array}$ & $\left(\mathrm{ft}^{3} / \mathrm{s}\right)$ & (gpm) & $\begin{array}{l}\text { (ft above } \\
\text { sensor) }\end{array}$ & $\begin{array}{c}\text { (ft above } \\
\text { log) }\end{array}$ & $\begin{array}{c}\text { (m above } \\
\text { log) }\end{array}$ & $\left(\mathrm{m}^{3} / \mathrm{s}\right)$ & $\left(\mathrm{ft}^{3} / \mathrm{s}\right)$ & (gpm) \\
\hline 1/23/08 22:00 & 1.554 & 0.575 & 0.145 & 65.030 & 1.356 & 0.025 & 0.007620 & 0.002126 & 0.075100 & 33.705 \\
\hline 1/23/08 23:00 & 1.554 & 0.575 & 0.145 & 65.030 & 1.356 & 0.025 & 0.007620 & 0.002126 & 0.075100 & 33.705 \\
\hline 1/24/08 0:00 & 1.551 & 0.572 & 0.143 & 64.197 & 1.356 & 0.025 & 0.007620 & 0.002126 & 0.075100 & 33.705 \\
\hline 1/24/08 1:00 & 1.551 & 0.572 & 0.143 & 64.197 & 1.356 & 0.025 & 0.007620 & 0.002126 & 0.075100 & 33.705 \\
\hline 1/24/08 2:00 & 1.552 & 0.573 & 0.144 & 64.474 & 1.356 & 0.025 & 0.007620 & 0.002126 & 0.075100 & 33.705 \\
\hline 1/24/08 3:00 & 1.549 & 0.570 & 0.142 & 63.644 & 1.354 & 0.023 & 0.007010 & 0.001905 & 0.067276 & 30.194 \\
\hline 1/24/08 4:00 & 1.547 & 0.568 & 0.141 & 63.095 & 1.354 & 0.023 & 0.007010 & 0.001905 & 0.067276 & 30.194 \\
\hline 1/24/08 5:00 & 1.547 & 0.568 & 0.141 & 63.095 & 1.354 & 0.023 & 0.007010 & 0.001905 & 0.067276 & 30.194 \\
\hline 1/24/08 6:00 & 1.542 & 0.563 & 0.138 & 61.734 & 1.354 & 0.023 & 0.007010 & 0.001905 & 0.067276 & 30.194 \\
\hline 1/24/08 7:00 & 1.542 & 0.563 & 0.138 & 61.734 & 1.354 & 0.023 & 0.007010 & 0.001905 & 0.067276 & 30.194 \\
\hline 1/24/08 8:00 & 1.542 & 0.563 & 0.138 & 61.734 & 1.354 & 0.023 & 0.007010 & 0.001905 & 0.067276 & 30.194 \\
\hline 1/24/08 9:00 & 1.545 & 0.566 & 0.139 & 62.549 & 1.354 & 0.023 & 0.007010 & 0.001905 & 0.067276 & 30.194 \\
\hline 1/24/08 10:00 & 1.54 & 0.561 & 0.136 & 61.195 & 1.351 & 0.02 & 0.006096 & 0.001588 & 0.056092 & 25.174 \\
\hline 1/24/08 11:00 & 1.542 & 0.563 & 0.138 & 61.734 & 1.354 & 0.023 & 0.007010 & 0.001905 & 0.067276 & 30.194 \\
\hline 1/24/08 12:00 & 1.544 & 0.565 & 0.139 & 62.276 & 1.354 & 0.023 & 0.007010 & 0.001905 & 0.067276 & 30.194 \\
\hline 1/24/08 13:00 & 1.542 & 0.563 & 0.138 & 61.734 & 1.354 & 0.023 & 0.007010 & 0.001905 & 0.067276 & 30.194 \\
\hline 1/24/08 14:00 & 1.549 & 0.570 & 0.142 & 63.644 & 1.354 & 0.023 & 0.007010 & 0.001905 & 0.067276 & 30.194 \\
\hline 1/24/08 15:00 & 1.556 & 0.577 & 0.146 & 65.590 & 1.356 & 0.025 & 0.007620 & 0.002126 & 0.075100 & 33.705 \\
\hline 1/24/08 16:00 & 1.553 & 0.574 & 0.144 & 64.752 & 1.356 & 0.025 & 0.007620 & 0.002126 & 0.075100 & 33.705 \\
\hline 1/24/08 17:00 & 1.558 & 0.579 & 0.147 & 66.152 & 1.356 & 0.025 & 0.007620 & 0.002126 & 0.075100 & 33.705 \\
\hline 1/24/08 18:00 & 1.56 & 0.581 & 0.149 & 66.717 & 1.359 & 0.028 & 0.008534 & 0.002473 & 0.087362 & 39.208 \\
\hline 1/24/08 19:00 & 1.556 & 0.577 & 0.146 & 65.590 & 1.356 & 0.025 & 0.007620 & 0.002126 & 0.075100 & 33.705 \\
\hline 1/24/08 20:00 & 1.556 & 0.577 & 0.146 & 65.590 & 1.354 & 0.023 & 0.007010 & 0.001905 & 0.067276 & 30.194 \\
\hline 1/24/08 21:00 & 1.558 & 0.579 & 0.147 & 66.152 & 1.354 & 0.023 & 0.007010 & 0.001905 & 0.067276 & 30.194 \\
\hline 1/24/08 22:00 & 1.554 & 0.575 & 0.145 & 65.030 & 1.354 & 0.023 & 0.007010 & 0.001905 & 0.067276 & 30.194 \\
\hline
\end{tabular}




\begin{tabular}{|c|c|c|c|c|c|c|c|c|c|c|}
\hline \multirow[b]{3}{*}{ Date Time } & \multicolumn{4}{|c|}{ V-Notch Weir } & \multicolumn{6}{|c|}{ R-Notch Weir } \\
\hline & \multicolumn{2}{|c|}{ Measured Water Level } & \multicolumn{2}{|c|}{$\begin{array}{c}\text { Calculated Rate } \\
\left(\mathrm{ft}^{3} / \mathrm{s}=448.8 \mathrm{gpm}\right)\end{array}$} & \multicolumn{3}{|c|}{ Measured Water Level } & \multicolumn{3}{|c|}{$\begin{array}{c}\text { Calculated Rate } \\
\left(\mathrm{ft}^{3} / \mathrm{s}=448.8 \mathrm{gpm}\right)\end{array}$} \\
\hline & $\begin{array}{c}\text { (ft above } \\
\text { sensor) }\end{array}$ & $\begin{array}{c}\text { (ft above } \\
\text { notch) }\end{array}$ & $\left(\mathrm{ft}^{3} / \mathrm{s}\right)$ & (gpm) & $\begin{array}{l}\text { (ft above } \\
\text { sensor) }\end{array}$ & $\begin{array}{c}\text { (ft above } \\
\text { log) }\end{array}$ & $\begin{array}{c}\text { (m above } \\
\text { log) }\end{array}$ & $\left(\mathrm{m}^{3} / \mathrm{s}\right)$ & $\left(\mathrm{ft}^{3} / \mathrm{s}\right)$ & (gpm) \\
\hline 1/24/08 23:00 & 1.554 & 0.575 & 0.145 & 65.030 & 1.354 & 0.023 & 0.007010 & 0.001905 & 0.067276 & 30.194 \\
\hline 1/25/08 0:00 & 1.551 & 0.572 & 0.143 & 64.197 & 1.354 & 0.023 & 0.007010 & 0.001905 & 0.067276 & 30.194 \\
\hline 1/25/08 1:00 & 1.549 & 0.570 & 0.142 & 63.644 & 1.354 & 0.023 & 0.007010 & 0.001905 & 0.067276 & 30.194 \\
\hline 1/25/08 2:00 & 1.544 & 0.565 & 0.139 & 62.276 & 1.356 & 0.025 & 0.007620 & 0.002126 & 0.075100 & 33.705 \\
\hline 1/25/08 3:00 & 1.551 & 0.572 & 0.143 & 64.197 & 1.354 & 0.023 & 0.007010 & 0.001905 & 0.067276 & 30.194 \\
\hline 1/25/08 4:00 & 1.551 & 0.572 & 0.143 & 64.197 & 1.354 & 0.023 & 0.007010 & 0.001905 & 0.067276 & 30.194 \\
\hline 1/25/08 5:00 & 1.556 & 0.577 & 0.146 & 65.590 & 1.356 & 0.025 & 0.007620 & 0.002126 & 0.075100 & 33.705 \\
\hline 1/25/08 6:00 & 1.568 & 0.589 & 0.154 & 69.007 & 1.354 & 0.023 & 0.007010 & 0.001905 & 0.067276 & 30.194 \\
\hline 1/25/08 7:00 & 1.551 & 0.572 & 0.143 & 64.197 & 1.354 & 0.023 & 0.007010 & 0.001905 & 0.067276 & 30.194 \\
\hline 1/25/08 8:00 & 1.563 & 0.584 & 0.151 & 67.571 & 1.356 & 0.025 & 0.007620 & 0.002126 & 0.075100 & 33.705 \\
\hline 1/25/08 9:00 & 1.556 & 0.577 & 0.146 & 65.590 & 1.354 & 0.023 & 0.007010 & 0.001905 & 0.067276 & 30.194 \\
\hline 1/25/08 10:00 & 1.556 & 0.577 & 0.146 & 65.590 & 1.356 & 0.025 & 0.007620 & 0.002126 & 0.075100 & 33.705 \\
\hline 1/25/08 11:00 & 1.561 & 0.582 & 0.149 & 67.001 & 1.356 & 0.025 & 0.007620 & 0.002126 & 0.075100 & 33.705 \\
\hline 1/25/08 12:00 & 1.556 & 0.577 & 0.146 & 65.590 & 1.354 & 0.023 & 0.007010 & 0.001905 & 0.067276 & 30.194 \\
\hline 1/25/08 13:00 & 1.56 & 0.581 & 0.149 & 66.717 & 1.356 & 0.025 & 0.007620 & 0.002126 & 0.075100 & 33.705 \\
\hline 1/25/08 14:00 & 1.562 & 0.583 & 0.150 & 67.285 & 1.361 & 0.03 & 0.009144 & 0.002714 & 0.095873 & 43.028 \\
\hline 1/25/08 15:00 & 1.567 & 0.588 & 0.153 & 68.718 & 1.359 & 0.028 & 0.008534 & 0.002473 & 0.087362 & 39.208 \\
\hline 1/25/08 16:00 & 1.567 & 0.588 & 0.153 & 68.718 & 1.359 & 0.028 & 0.008534 & 0.002473 & 0.087362 & 39.208 \\
\hline 1/25/08 17:00 & 1.574 & 0.595 & 0.158 & 70.755 & 1.358 & 0.027 & 0.008229 & 0.002356 & 0.083206 & 37.343 \\
\hline 1/25/08 18:00 & 1.574 & 0.595 & 0.158 & 70.755 & 1.361 & 0.03 & 0.009144 & 0.002714 & 0.095873 & 43.028 \\
\hline 1/25/08 19:00 & 1.574 & 0.595 & 0.158 & 70.755 & 1.361 & 0.03 & 0.009144 & 0.002714 & 0.095873 & 43.028 \\
\hline 1/25/08 20:00 & 1.572 & 0.593 & 0.156 & 70.169 & 1.358 & 0.027 & 0.008229 & 0.002356 & 0.083206 & 37.343 \\
\hline 1/25/08 21:00 & 1.572 & 0.593 & 0.156 & 70.169 & 1.358 & 0.027 & 0.008229 & 0.002356 & 0.083206 & 37.343 \\
\hline 1/25/08 22:00 & 1.569 & 0.590 & 0.154 & 69.296 & 1.358 & 0.027 & 0.008229 & 0.002356 & 0.083206 & 37.343 \\
\hline 1/25/08 23:00 & 1.57 & 0.591 & 0.155 & 69.587 & 1.358 & 0.027 & 0.008229 & 0.002356 & 0.083206 & 37.343 \\
\hline
\end{tabular}




\begin{tabular}{|c|c|c|c|c|c|c|c|c|c|c|}
\hline \multirow[b]{3}{*}{ Date Time } & \multicolumn{4}{|c|}{ V-Notch Weir } & \multicolumn{6}{|c|}{ R-Notch Weir } \\
\hline & \multicolumn{2}{|c|}{ Measured Water Level } & \multicolumn{2}{|c|}{$\begin{array}{c}\text { Calculated Rate } \\
\left(\mathrm{ft}^{3} / \mathrm{s}=448.8 \mathrm{gpm}\right)\end{array}$} & \multicolumn{3}{|c|}{ Measured Water Level } & \multicolumn{3}{|c|}{$\begin{array}{c}\text { Calculated Rate } \\
\left(\mathrm{ft}^{3} / \mathrm{s}=448.8 \mathrm{gpm}\right)\end{array}$} \\
\hline & $\begin{array}{c}\text { (ft above } \\
\text { sensor) }\end{array}$ & $\begin{array}{c}\text { (ft above } \\
\text { notch) }\end{array}$ & $\left(\mathrm{ft}^{3} / \mathrm{s}\right)$ & (gpm) & $\begin{array}{l}\text { (ft above } \\
\text { sensor) }\end{array}$ & $\begin{array}{c}\text { (ft above } \\
\text { log) }\end{array}$ & $\begin{array}{c}\text { (m above } \\
\text { log) }\end{array}$ & $\left(\mathrm{m}^{3} / \mathrm{s}\right)$ & $\left(\mathrm{ft}^{3} / \mathrm{s}\right)$ & (gpm) \\
\hline 1/26/08 0:00 & 1.57 & 0.591 & 0.155 & 69.587 & 1.358 & 0.027 & 0.008229 & 0.002356 & 0.083206 & 37.343 \\
\hline 1/26/08 1:00 & 1.57 & 0.591 & 0.155 & 69.587 & 1.358 & 0.027 & 0.008229 & 0.002356 & 0.083206 & 37.343 \\
\hline 1/26/08 2:00 & 1.567 & 0.588 & 0.153 & 68.718 & 1.356 & 0.025 & 0.007620 & 0.002126 & 0.075100 & 33.705 \\
\hline 1/26/08 3:00 & 1.57 & 0.591 & 0.155 & 69.587 & 1.358 & 0.027 & 0.008229 & 0.002356 & 0.083206 & 37.343 \\
\hline 1/26/08 4:00 & 1.567 & 0.588 & 0.153 & 68.718 & 1.358 & 0.027 & 0.008229 & 0.002356 & 0.083206 & 37.343 \\
\hline 1/26/08 5:00 & 1.565 & 0.586 & 0.152 & 68.143 & 1.358 & 0.027 & 0.008229 & 0.002356 & 0.083206 & 37.343 \\
\hline 1/26/08 6:00 & 1.563 & 0.584 & 0.151 & 67.571 & 1.358 & 0.027 & 0.008229 & 0.002356 & 0.083206 & 37.343 \\
\hline 1/26/08 7:00 & 1.567 & 0.588 & 0.153 & 68.718 & 1.358 & 0.027 & 0.008229 & 0.002356 & 0.083206 & 37.343 \\
\hline 1/26/08 8:00 & 1.567 & 0.588 & 0.153 & 68.718 & 1.358 & 0.027 & 0.008229 & 0.002356 & 0.083206 & 37.343 \\
\hline 1/26/08 9:00 & 1.565 & 0.586 & 0.152 & 68.143 & 1.358 & 0.027 & 0.008229 & 0.002356 & 0.083206 & 37.343 \\
\hline 1/26/08 10:00 & 1.563 & 0.584 & 0.151 & 67.571 & 1.358 & 0.027 & 0.008229 & 0.002356 & 0.083206 & 37.343 \\
\hline 1/26/08 11:00 & 1.558 & 0.579 & 0.147 & 66.152 & 1.356 & 0.025 & 0.007620 & 0.002126 & 0.075100 & 33.705 \\
\hline 1/26/08 12:00 & 1.558 & 0.579 & 0.147 & 66.152 & 1.358 & 0.027 & 0.008229 & 0.002356 & 0.083206 & 37.343 \\
\hline 1/26/08 13:00 & 1.564 & 0.585 & 0.151 & 67.856 & 1.358 & 0.027 & 0.008229 & 0.002356 & 0.083206 & 37.343 \\
\hline 1/26/08 14:00 & 1.567 & 0.588 & 0.153 & 68.718 & 1.358 & 0.027 & 0.008229 & 0.002356 & 0.083206 & 37.343 \\
\hline 1/26/08 15:00 & 1.573 & 0.594 & 0.157 & 70.462 & 1.358 & 0.027 & 0.008229 & 0.002356 & 0.083206 & 37.343 \\
\hline 1/26/08 16:00 & 1.58 & 0.601 & 0.162 & 72.529 & 1.361 & 0.03 & 0.009144 & 0.002714 & 0.095873 & 43.028 \\
\hline 1/26/08 17:00 & 1.582 & 0.603 & 0.163 & 73.126 & 1.361 & 0.03 & 0.009144 & 0.002714 & 0.095873 & 43.028 \\
\hline 1/26/08 18:00 & 1.583 & 0.604 & 0.164 & 73.426 & 1.361 & 0.03 & 0.009144 & 0.002714 & 0.095873 & 43.028 \\
\hline 1/26/08 19:00 & 1.58 & 0.601 & 0.162 & 72.529 & 1.361 & 0.03 & 0.009144 & 0.002714 & 0.095873 & 43.028 \\
\hline 1/26/08 20:00 & 1.576 & 0.597 & 0.159 & 71.343 & 1.358 & 0.027 & 0.008229 & 0.002356 & 0.083206 & 37.343 \\
\hline 1/26/08 21:00 & 1.576 & 0.597 & 0.159 & 71.343 & 1.358 & 0.027 & 0.008229 & 0.002356 & 0.083206 & 37.343 \\
\hline 1/26/08 22:00 & 1.574 & 0.595 & 0.158 & 70.755 & 1.358 & 0.027 & 0.008229 & 0.002356 & 0.083206 & 37.343 \\
\hline 1/26/08 23:00 & 1.571 & 0.592 & 0.156 & 69.878 & 1.358 & 0.027 & 0.008229 & 0.002356 & 0.083206 & 37.343 \\
\hline 1/27/08 0:00 & 1.571 & 0.592 & 0.156 & 69.878 & 1.358 & 0.027 & 0.008229 & 0.002356 & 0.083206 & 37.343 \\
\hline
\end{tabular}




\begin{tabular}{|c|c|c|c|c|c|c|c|c|c|c|}
\hline \multirow[b]{3}{*}{ Date Time } & \multicolumn{4}{|c|}{ V-Notch Weir } & \multicolumn{6}{|c|}{ R-Notch Weir } \\
\hline & \multicolumn{2}{|c|}{ Measured Water Level } & \multicolumn{2}{|c|}{$\begin{array}{c}\text { Calculated Rate } \\
\left(\mathrm{ft}^{3} / \mathrm{s}=448.8 \mathrm{gpm}\right)\end{array}$} & \multicolumn{3}{|c|}{ Measured Water Level } & \multicolumn{3}{|c|}{$\begin{array}{c}\text { Calculated Rate } \\
\left(\mathrm{ft}^{3} / \mathrm{s}=448.8 \mathrm{gpm}\right)\end{array}$} \\
\hline & $\begin{array}{c}\text { (ft above } \\
\text { sensor) }\end{array}$ & $\begin{array}{c}\text { (ft above } \\
\text { notch) }\end{array}$ & $\left(\mathrm{ft}^{3} / \mathrm{s}\right)$ & (gpm) & $\begin{array}{l}\text { (ft above } \\
\text { sensor) }\end{array}$ & $\begin{array}{c}\text { (ft above } \\
\text { log) }\end{array}$ & $\begin{array}{c}\text { (m above } \\
\text { log) }\end{array}$ & $\left(\mathrm{m}^{3} / \mathrm{s}\right)$ & $\left(\mathrm{ft}^{3} / \mathrm{s}\right)$ & (gpm) \\
\hline 1/27/08 1:00 & 1.571 & 0.592 & 0.156 & 69.878 & 1.358 & 0.027 & 0.008229 & 0.002356 & 0.083206 & 37.343 \\
\hline 1/27/08 2:00 & 1.572 & 0.593 & 0.156 & 70.169 & 1.358 & 0.027 & 0.008229 & 0.002356 & 0.083206 & 37.343 \\
\hline 1/27/08 3:00 & 1.574 & 0.595 & 0.158 & 70.755 & 1.358 & 0.027 & 0.008229 & 0.002356 & 0.083206 & 37.343 \\
\hline 1/27/08 4:00 & 1.569 & 0.590 & 0.154 & 69.296 & 1.356 & 0.025 & 0.007620 & 0.002126 & 0.075100 & 33.705 \\
\hline 1/27/08 5:00 & 1.569 & 0.590 & 0.154 & 69.296 & 1.358 & 0.027 & 0.008229 & 0.002356 & 0.083206 & 37.343 \\
\hline 1/27/08 6:00 & 1.569 & 0.590 & 0.154 & 69.296 & 1.358 & 0.027 & 0.008229 & 0.002356 & 0.083206 & 37.343 \\
\hline 1/27/08 7:00 & 1.567 & 0.588 & 0.153 & 68.718 & 1.356 & 0.025 & 0.007620 & 0.002126 & 0.075100 & 33.705 \\
\hline 1/27/08 8:00 & 1.569 & 0.590 & 0.154 & 69.296 & 1.358 & 0.027 & 0.008229 & 0.002356 & 0.083206 & 37.343 \\
\hline 1/27/08 9:00 & 1.567 & 0.588 & 0.153 & 68.718 & 1.358 & 0.027 & 0.008229 & 0.002356 & 0.083206 & 37.343 \\
\hline 1/27/08 10:00 & 1.567 & 0.588 & 0.153 & 68.718 & 1.358 & 0.027 & 0.008229 & 0.002356 & 0.083206 & 37.343 \\
\hline 1/27/08 11:00 & 1.564 & 0.585 & 0.151 & 67.856 & 1.356 & 0.025 & 0.007620 & 0.002126 & 0.075100 & 33.705 \\
\hline 1/27/08 12:00 & 1.569 & 0.590 & 0.154 & 69.296 & 1.358 & 0.027 & 0.008229 & 0.002356 & 0.083206 & 37.343 \\
\hline 1/27/08 13:00 & 1.576 & 0.597 & 0.159 & 71.343 & 1.358 & 0.027 & 0.008229 & 0.002356 & 0.083206 & 37.343 \\
\hline 1/27/08 14:00 & 1.58 & 0.601 & 0.162 & 72.529 & 1.361 & 0.03 & 0.009144 & 0.002714 & 0.095873 & 43.028 \\
\hline 1/27/08 15:00 & 1.598 & 0.619 & 0.174 & 78.010 & 1.363 & 0.032 & 0.009753 & 0.002963 & 0.104644 & 46.964 \\
\hline 1/27/08 16:00 & 1.624 & 0.645 & 0.192 & 86.354 & 1.368 & 0.037 & 0.011277 & 0.003614 & 0.127660 & 57.294 \\
\hline 1/27/08 17:00 & 1.633 & 0.654 & 0.199 & 89.362 & 1.368 & 0.037 & 0.011277 & 0.003614 & 0.127660 & 57.294 \\
\hline 1/27/08 18:00 & 1.671 & 0.692 & 0.229 & 102.749 & 1.375 & 0.044 & 0.013411 & 0.004596 & 0.162345 & 72.860 \\
\hline 1/27/08 19:00 & 1.669 & 0.690 & 0.227 & 102.016 & 1.375 & 0.044 & 0.013411 & 0.004596 & 0.162345 & 72.860 \\
\hline 1/27/08 20:00 & 1.655 & 0.676 & 0.216 & 96.975 & 1.372 & 0.041 & 0.012496 & 0.004166 & 0.147141 & 66.037 \\
\hline 1/27/08 21:00 & 1.641 & 0.662 & 0.205 & 92.087 & 1.368 & 0.037 & 0.011277 & 0.003614 & 0.127660 & 57.294 \\
\hline 1/27/08 22:00 & 1.632 & 0.653 & 0.198 & 89.024 & 1.368 & 0.037 & 0.011277 & 0.003614 & 0.127660 & 57.294 \\
\hline 1/27/08 23:00 & 1.625 & 0.646 & 0.193 & 86.685 & 1.365 & 0.034 & 0.010363 & 0.003218 & 0.113667 & 51.014 \\
\hline 1/28/08 0:00 & 1.623 & 0.644 & 0.192 & 86.024 & 1.368 & 0.037 & 0.011277 & 0.003614 & 0.127660 & 57.294 \\
\hline 1/28/08 1:00 & 1.625 & 0.646 & 0.193 & 86.685 & 1.365 & 0.034 & 0.010363 & 0.003218 & 0.113667 & 51.014 \\
\hline
\end{tabular}




\begin{tabular}{|c|c|c|c|c|c|c|c|c|c|c|}
\hline \multirow[b]{3}{*}{ Date Time } & \multicolumn{4}{|c|}{ V-Notch Weir } & \multicolumn{6}{|c|}{ R-Notch Weir } \\
\hline & \multicolumn{2}{|c|}{ Measured Water Level } & \multicolumn{2}{|c|}{$\begin{array}{c}\text { Calculated Rate } \\
\left(\mathrm{ft}^{3} / \mathrm{s}=448.8 \mathrm{gpm}\right)\end{array}$} & \multicolumn{3}{|c|}{ Measured Water Level } & \multicolumn{3}{|c|}{$\begin{array}{c}\text { Calculated Rate } \\
\left(\mathrm{ft}^{3} / \mathrm{s}=448.8 \mathrm{gpm}\right)\end{array}$} \\
\hline & $\begin{array}{c}\text { (ft above } \\
\text { sensor) }\end{array}$ & $\begin{array}{c}\text { (ft above } \\
\text { notch) }\end{array}$ & $\left(\mathrm{ft}^{3} / \mathrm{s}\right)$ & (gpm) & $\begin{array}{l}\text { (ft above } \\
\text { sensor) }\end{array}$ & $\begin{array}{c}\text { (ft above } \\
\text { log) }\end{array}$ & $\begin{array}{c}\text { (m above } \\
\text { log) }\end{array}$ & $\left(\mathrm{m}^{3} / \mathrm{s}\right)$ & $\left(\mathrm{ft}^{3} / \mathrm{s}\right)$ & (gpm) \\
\hline 1/28/08 2:00 & 1.62 & 0.641 & 0.189 & 85.037 & 1.365 & 0.034 & 0.010363 & 0.003218 & 0.113667 & 51.014 \\
\hline 1/28/08 3:00 & 1.62 & 0.641 & 0.189 & 85.037 & 1.368 & 0.037 & 0.011277 & 0.003614 & 0.127660 & 57.294 \\
\hline 1/28/08 4:00 & 1.623 & 0.644 & 0.192 & 86.024 & 1.365 & 0.034 & 0.010363 & 0.003218 & 0.113667 & 51.014 \\
\hline 1/28/08 5:00 & 1.627 & 0.648 & 0.195 & 87.350 & 1.368 & 0.037 & 0.011277 & 0.003614 & 0.127660 & 57.294 \\
\hline 1/28/08 6:00 & 1.637 & 0.658 & 0.202 & 90.718 & 1.37 & 0.039 & 0.011887 & 0.003887 & 0.137285 & 61.614 \\
\hline 1/28/08 7:00 & 1.646 & 0.667 & 0.209 & 93.815 & 1.37 & 0.039 & 0.011887 & 0.003887 & 0.137285 & 61.614 \\
\hline 1/28/08 8:00 & 1.648 & 0.669 & 0.211 & 94.512 & 1.37 & 0.039 & 0.011887 & 0.003887 & 0.137285 & 61.614 \\
\hline 1/28/08 9:00 & 1.651 & 0.672 & 0.213 & 95.563 & 1.37 & 0.039 & 0.011887 & 0.003887 & 0.137285 & 61.614 \\
\hline 1/28/08 10:00 & 1.648 & 0.669 & 0.211 & 94.512 & 1.372 & 0.041 & 0.012496 & 0.004166 & 0.147141 & 66.037 \\
\hline 1/28/08 11:00 & 1.653 & 0.674 & 0.215 & 96.268 & 1.37 & 0.039 & 0.011887 & 0.003887 & 0.137285 & 61.614 \\
\hline 1/28/08 12:00 & 1.678 & 0.699 & 0.235 & 105.338 & 1.377 & 0.046 & 0.014020 & 0.004891 & 0.172754 & 77.532 \\
\hline 1/28/08 13:00 & 1.755 & 0.776 & 0.304 & 136.416 & 1.391 & 0.06 & 0.018287 & 0.007117 & 0.251378 & 112.818 \\
\hline 1/28/08 14:00 & 1.899 & 0.920 & 0.463 & 207.972 & 1.415 & 0.084 & 0.025602 & 0.011529 & 0.407202 & 182.752 \\
\hline 1/28/08 15:00 & 2.023 & 1.044 & 0.634 & 284.585 & 1.453 & 0.122 & 0.037184 & 0.019827 & 0.700289 & 314.290 \\
\hline 1/28/08 16:00 & 2.131 & 1.152 & 0.810 & 363.365 & 1.526 & 0.195 & 0.059433 & 0.039481 & 1.394471 & 625.838 \\
\hline 1/28/08 17:00 & 2.188 & 1.209 & 0.913 & 409.673 & 1.552 & 0.221 & 0.067358 & 0.047498 & 1.677606 & 752.910 \\
\hline 1/28/08 18:00 & 2.245 & 1.266 & 1.024 & 459.351 & 1.574 & 0.243 & 0.074063 & 0.054656 & 1.930452 & 866.387 \\
\hline 1/28/08 19:00 & 2.215 & 1.236 & 0.964 & 432.780 & 1.557 & 0.226 & 0.068881 & 0.049095 & 1.734028 & 778.232 \\
\hline 1/28/08 20:00 & 2.161 & 1.182 & 0.863 & 387.322 & 1.538 & 0.207 & 0.063091 & 0.043120 & 1.522979 & 683.513 \\
\hline 1/28/08 21:00 & 2.121 & 1.142 & 0.792 & 355.583 & 1.507 & 0.176 & 0.053642 & 0.033944 & 1.198890 & 538.062 \\
\hline 1/28/08 22:00 & 2.05 & 1.071 & 0.676 & 303.203 & 1.481 & 0.15 & 0.045718 & 0.026834 & 0.947757 & 425.353 \\
\hline 1/28/08 23:00 & 2.017 & 1.038 & 0.625 & 280.543 & 1.46 & 0.129 & 0.039317 & 0.021512 & 0.759800 & 340.998 \\
\hline 1/29/08 0:00 & 1.989 & 1.010 & 0.584 & 262.139 & 1.441 & 0.11 & 0.033526 & 0.017047 & 0.602114 & 270.229 \\
\hline 1/29/08 1:00 & 1.951 & 0.972 & 0.531 & 238.351 & 1.427 & 0.096 & 0.029259 & 0.013987 & 0.494010 & 221.711 \\
\hline 1/29/08 2:00 & 1.908 & 0.929 & 0.475 & 213.053 & 1.413 & 0.082 & 0.024992 & 0.011135 & 0.393285 & 176.506 \\
\hline
\end{tabular}




\begin{tabular}{|c|c|c|c|c|c|c|c|c|c|c|}
\hline \multirow[b]{3}{*}{ Date Time } & \multicolumn{4}{|c|}{ V-Notch Weir } & \multicolumn{6}{|c|}{ R-Notch Weir } \\
\hline & \multicolumn{2}{|c|}{ Measured Water Level } & \multicolumn{2}{|c|}{$\begin{array}{c}\text { Calculated Rate } \\
\left(\mathrm{ft}^{3} / \mathrm{s}=448.8 \mathrm{gpm}\right)\end{array}$} & \multicolumn{3}{|c|}{ Measured Water Level } & \multicolumn{3}{|c|}{$\begin{array}{c}\text { Calculated Rate } \\
\left(\mathrm{ft}^{3} / \mathrm{s}=448.8 \mathrm{gpm}\right)\end{array}$} \\
\hline & $\begin{array}{c}\text { (ft above } \\
\text { sensor) }\end{array}$ & $\begin{array}{c}\text { (ft above } \\
\text { notch) }\end{array}$ & $\left(\mathrm{ft}^{3} / \mathrm{s}\right)$ & (gpm) & $\begin{array}{l}\text { (ft above } \\
\text { sensor) }\end{array}$ & $\begin{array}{c}\text { (ft above } \\
\text { log) }\end{array}$ & $\begin{array}{c}\text { (m above } \\
\text { log) }\end{array}$ & $\left(\mathrm{m}^{3} / \mathrm{s}\right)$ & $\left(\mathrm{ft}^{3} / \mathrm{s}\right)$ & (gpm) \\
\hline 1/29/08 3:00 & 1.875 & 0.896 & 0.434 & 194.781 & 1.41 & 0.079 & 0.024078 & 0.010553 & 0.372715 & 167.274 \\
\hline 1/29/08 4:00 & 1.84 & 0.861 & 0.393 & 176.467 & 1.401 & 0.07 & 0.021335 & 0.008869 & 0.313263 & 140.593 \\
\hline 1/29/08 5:00 & 1.791 & 0.812 & 0.340 & 152.626 & 1.391 & 0.06 & 0.018287 & 0.007117 & 0.251378 & 112.818 \\
\hline 1/29/08 6:00 & 1.746 & 0.767 & 0.295 & 132.534 & 1.387 & 0.056 & 0.017068 & 0.006453 & 0.227923 & 102.292 \\
\hline 1/29/08 7:00 & 1.709 & 0.730 & 0.261 & 117.272 & 1.382 & 0.051 & 0.015544 & 0.005654 & 0.199703 & 89.627 \\
\hline 1/29/08 8:00 & 1.686 & 0.707 & 0.241 & 108.344 & 1.38 & 0.049 & 0.014934 & 0.005345 & 0.188768 & 84.719 \\
\hline 1/29/08 9:00 & 1.677 & 0.698 & 0.234 & 104.965 & 1.373 & 0.042 & 0.012801 & 0.004308 & 0.152154 & 68.287 \\
\hline 1/29/08 10:00 & 1.627 & 0.648 & 0.195 & 87.350 & 1.37 & 0.039 & 0.011887 & 0.003887 & 0.137285 & 61.614 \\
\hline 1/29/08 11:00 & 1.627 & 0.648 & 0.195 & 87.350 & 1.373 & 0.042 & 0.012801 & 0.004308 & 0.152154 & 68.287 \\
\hline 1/29/08 12:00 & 1.59 & 0.611 & 0.168 & 75.544 & 1.366 & 0.035 & 0.010667 & 0.003349 & 0.118271 & 53.080 \\
\hline 1/29/08 13:00 & 1.585 & 0.606 & 0.165 & 74.027 & 1.373 & 0.042 & 0.012801 & 0.004308 & 0.152154 & 68.287 \\
\hline 1/29/08 14:00 & 1.624 & 0.645 & 0.192 & 86.354 & 1.368 & 0.037 & 0.011277 & 0.003614 & 0.127660 & 57.294 \\
\hline 1/29/08 15:00 & 1.629 & 0.650 & 0.196 & 88.017 & 1.363 & 0.032 & 0.009753 & 0.002963 & 0.104644 & 46.964 \\
\hline 1/29/08 16:00 & 1.605 & 0.626 & 0.179 & 80.206 & 1.363 & 0.032 & 0.009753 & 0.002963 & 0.104644 & 46.964 \\
\hline 1/29/08 17:00 & 1.593 & 0.614 & 0.170 & 76.463 & 1.363 & 0.032 & 0.009753 & 0.002963 & 0.104644 & 46.964 \\
\hline 1/29/08 18:00 & 1.6 & 0.621 & 0.175 & 78.634 & 1.363 & 0.032 & 0.009753 & 0.002963 & 0.104644 & 46.964 \\
\hline 1/29/08 19:00 & 1.583 & 0.604 & 0.164 & 73.426 & 1.361 & 0.03 & 0.009144 & 0.002714 & 0.095873 & 43.028 \\
\hline 1/29/08 20:00 & 1.574 & 0.595 & 0.158 & 70.755 & 1.361 & 0.03 & 0.009144 & 0.002714 & 0.095873 & 43.028 \\
\hline 1/29/08 21:00 & 1.572 & 0.593 & 0.156 & 70.169 & 1.361 & 0.03 & 0.009144 & 0.002714 & 0.095873 & 43.028 \\
\hline 1/29/08 22:00 & 1.57 & 0.591 & 0.155 & 69.587 & 1.358 & 0.027 & 0.008229 & 0.002356 & 0.083206 & 37.343 \\
\hline 1/29/08 23:00 & 1.565 & 0.586 & 0.152 & 68.143 & 1.358 & 0.027 & 0.008229 & 0.002356 & 0.083206 & 37.343 \\
\hline 1/30/08 0:00 & 1.563 & 0.584 & 0.151 & 67.571 & 1.358 & 0.027 & 0.008229 & 0.002356 & 0.083206 & 37.343 \\
\hline 1/30/08 1:00 & 1.556 & 0.577 & 0.146 & 65.590 & 1.358 & 0.027 & 0.008229 & 0.002356 & 0.083206 & 37.343 \\
\hline 1/30/08 2:00 & 1.554 & 0.575 & 0.145 & 65.030 & 1.358 & 0.027 & 0.008229 & 0.002356 & 0.083206 & 37.343 \\
\hline 1/30/08 3:00 & 1.549 & 0.570 & 0.142 & 63.644 & 1.356 & 0.025 & 0.007620 & 0.002126 & 0.075100 & 33.705 \\
\hline
\end{tabular}




\begin{tabular}{|c|c|c|c|c|c|c|c|c|c|c|}
\hline \multirow[b]{3}{*}{ Date Time } & \multicolumn{4}{|c|}{ V-Notch Weir } & \multicolumn{6}{|c|}{ R-Notch Weir } \\
\hline & \multicolumn{2}{|c|}{ Measured Water Level } & \multicolumn{2}{|c|}{$\begin{array}{c}\text { Calculated Rate } \\
\left(\mathrm{ft}^{3} / \mathrm{s}=448.8 \mathrm{gpm}\right)\end{array}$} & \multicolumn{3}{|c|}{ Measured Water Level } & \multicolumn{3}{|c|}{$\begin{array}{c}\text { Calculated Rate } \\
\left(\mathrm{ft}^{3} / \mathrm{s}=448.8 \mathrm{gpm}\right)\end{array}$} \\
\hline & $\begin{array}{c}\text { (ft above } \\
\text { sensor) }\end{array}$ & $\begin{array}{c}\text { (ft above } \\
\text { notch) }\end{array}$ & $\left(\mathrm{ft}^{3} / \mathrm{s}\right)$ & (gpm) & $\begin{array}{l}\text { (ft above } \\
\text { sensor) }\end{array}$ & $\begin{array}{c}\text { (ft above } \\
\text { log) }\end{array}$ & $\begin{array}{c}\text { (m above } \\
\text { log) }\end{array}$ & $\left(\mathrm{m}^{3} / \mathrm{s}\right)$ & $\left(\mathrm{ft}^{3} / \mathrm{s}\right)$ & (gpm) \\
\hline 1/30/08 4:00 & 1.551 & 0.572 & 0.143 & 64.197 & 1.356 & 0.025 & 0.007620 & 0.002126 & 0.075100 & 33.705 \\
\hline 1/30/08 5:00 & 1.542 & 0.563 & 0.138 & 61.734 & 1.356 & 0.025 & 0.007620 & 0.002126 & 0.075100 & 33.705 \\
\hline 1/30/08 6:00 & 1.537 & 0.558 & 0.135 & 60.391 & 1.356 & 0.025 & 0.007620 & 0.002126 & 0.075100 & 33.705 \\
\hline 1/30/08 7:00 & 1.537 & 0.558 & 0.135 & 60.391 & 1.356 & 0.025 & 0.007620 & 0.002126 & 0.075100 & 33.705 \\
\hline 1/30/08 8:00 & 1.537 & 0.558 & 0.135 & 60.391 & 1.356 & 0.025 & 0.007620 & 0.002126 & 0.075100 & 33.705 \\
\hline 1/30/08 9:00 & 1.537 & 0.558 & 0.135 & 60.391 & 1.356 & 0.025 & 0.007620 & 0.002126 & 0.075100 & 33.705 \\
\hline 1/30/08 10:00 & 1.537 & 0.558 & 0.135 & 60.391 & 1.356 & 0.025 & 0.007620 & 0.002126 & 0.075100 & 33.705 \\
\hline 1/30/08 11:00 & 1.537 & 0.558 & 0.135 & 60.391 & 1.356 & 0.025 & 0.007620 & 0.002126 & 0.075100 & 33.705 \\
\hline 1/30/08 12:00 & 1.539 & 0.560 & 0.136 & 60.926 & 1.356 & 0.025 & 0.007620 & 0.002126 & 0.075100 & 33.705 \\
\hline 1/30/08 13:00 & 1.539 & 0.560 & 0.136 & 60.926 & 1.354 & 0.023 & 0.007010 & 0.001905 & 0.067276 & 30.194 \\
\hline 1/30/08 14:00 & 1.562 & 0.583 & 0.150 & 67.285 & 1.358 & 0.027 & 0.008229 & 0.002356 & 0.083206 & 37.343 \\
\hline 1/30/08 15:00 & 1.543 & 0.564 & 0.138 & 62.005 & 1.363 & 0.032 & 0.009753 & 0.002963 & 0.104644 & 46.964 \\
\hline 1/30/08 16:00 & 1.557 & 0.578 & 0.147 & 65.871 & 1.361 & 0.03 & 0.009144 & 0.002714 & 0.095873 & 43.028 \\
\hline 1/30/08 17:00 & 1.555 & 0.576 & 0.146 & 65.310 & 1.361 & 0.03 & 0.009144 & 0.002714 & 0.095873 & 43.028 \\
\hline 1/30/08 18:00 & 1.552 & 0.573 & 0.144 & 64.474 & 1.361 & 0.03 & 0.009144 & 0.002714 & 0.095873 & 43.028 \\
\hline 1/30/08 19:00 & 1.55 & 0.571 & 0.142 & 63.920 & 1.358 & 0.027 & 0.008229 & 0.002356 & 0.083206 & 37.343 \\
\hline 1/30/08 20:00 & 1.539 & 0.560 & 0.136 & 60.926 & 1.356 & 0.025 & 0.007620 & 0.002126 & 0.075100 & 33.705 \\
\hline 1/30/08 21:00 & 1.537 & 0.558 & 0.135 & 60.391 & 1.356 & 0.025 & 0.007620 & 0.002126 & 0.075100 & 33.705 \\
\hline 1/30/08 22:00 & 1.534 & 0.555 & 0.133 & 59.593 & 1.354 & 0.023 & 0.007010 & 0.001905 & 0.067276 & 30.194 \\
\hline 1/30/08 23:00 & 1.537 & 0.558 & 0.135 & 60.391 & 1.354 & 0.023 & 0.007010 & 0.001905 & 0.067276 & 30.194 \\
\hline 1/31/08 0:00 & 1.528 & 0.549 & 0.129 & 58.018 & 1.356 & 0.025 & 0.007620 & 0.002126 & 0.075100 & 33.705 \\
\hline 1/31/08 1:00 & 1.521 & 0.542 & 0.125 & 56.211 & 1.354 & 0.023 & 0.007010 & 0.001905 & 0.067276 & 30.194 \\
\hline 1/31/08 2:00 & 1.523 & 0.544 & 0.126 & 56.724 & 1.354 & 0.023 & 0.007010 & 0.001905 & 0.067276 & 30.194 \\
\hline 1/31/08 3:00 & 1.525 & 0.546 & 0.128 & 57.239 & 1.354 & 0.023 & 0.007010 & 0.001905 & 0.067276 & 30.194 \\
\hline 1/31/08 4:00 & 1.523 & 0.544 & 0.126 & 56.724 & 1.354 & 0.023 & 0.007010 & 0.001905 & 0.067276 & 30.194 \\
\hline
\end{tabular}




\begin{tabular}{|c|c|c|c|c|c|c|c|c|c|c|}
\hline \multirow[b]{3}{*}{ Date Time } & \multicolumn{4}{|c|}{ V-Notch Weir } & \multicolumn{6}{|c|}{ R-Notch Weir } \\
\hline & \multicolumn{2}{|c|}{ Measured Water Level } & \multicolumn{2}{|c|}{$\begin{array}{c}\text { Calculated Rate } \\
\left(\mathrm{ft}^{3} / \mathrm{s}=448.8 \mathrm{gpm}\right)\end{array}$} & \multicolumn{3}{|c|}{ Measured Water Level } & \multicolumn{3}{|c|}{$\begin{array}{c}\text { Calculated Rate } \\
\left(\mathrm{ft}^{3} / \mathrm{s}=448.8 \mathrm{gpm}\right)\end{array}$} \\
\hline & $\begin{array}{c}\text { (ft above } \\
\text { sensor) }\end{array}$ & $\begin{array}{c}\text { (ft above } \\
\text { notch) }\end{array}$ & $\left(\mathrm{ft}^{3} / \mathrm{s}\right)$ & (gpm) & $\begin{array}{l}\text { (ft above } \\
\text { sensor) }\end{array}$ & $\begin{array}{c}\text { (ft above } \\
\text { log) }\end{array}$ & $\begin{array}{c}\text { (m above } \\
\text { log) }\end{array}$ & $\left(\mathrm{m}^{3} / \mathrm{s}\right)$ & $\left(\mathrm{ft}^{3} / \mathrm{s}\right)$ & (gpm) \\
\hline 1/31/08 5:00 & 1.516 & 0.537 & 0.122 & 54.942 & 1.354 & 0.023 & 0.007010 & 0.001905 & 0.067276 & 30.194 \\
\hline 1/31/08 6:00 & 1.514 & 0.535 & 0.121 & 54.439 & 1.354 & 0.023 & 0.007010 & 0.001905 & 0.067276 & 30.194 \\
\hline 1/31/08 7:00 & 1.511 & 0.532 & 0.120 & 53.689 & 1.354 & 0.023 & 0.007010 & 0.001905 & 0.067276 & 30.194 \\
\hline 1/31/08 8:00 & 1.507 & 0.528 & 0.117 & 52.700 & 1.351 & 0.02 & 0.006096 & 0.001588 & 0.056092 & 25.174 \\
\hline 1/31/08 9:00 & 1.504 & 0.525 & 0.116 & 51.966 & 1.351 & 0.02 & 0.006096 & 0.001588 & 0.056092 & 25.174 \\
\hline 1/31/08 10:00 & 1.502 & 0.523 & 0.115 & 51.479 & 1.351 & 0.02 & 0.006096 & 0.001588 & 0.056092 & 25.174 \\
\hline 1/31/08 11:00 & 1.502 & 0.523 & 0.115 & 51.479 & 1.351 & 0.02 & 0.006096 & 0.001588 & 0.056092 & 25.174 \\
\hline 1/31/08 12:00 & 1.509 & 0.530 & 0.119 & 53.194 & 1.351 & 0.02 & 0.006096 & 0.001588 & 0.056092 & 25.174 \\
\hline 1/31/08 13:00 & 1.52 & 0.541 & 0.125 & 55.956 & 1.354 & 0.023 & 0.007010 & 0.001905 & 0.067276 & 30.194 \\
\hline 1/31/08 14:00 & 1.522 & 0.543 & 0.126 & 56.467 & 1.354 & 0.023 & 0.007010 & 0.001905 & 0.067276 & 30.194 \\
\hline 1/31/08 15:00 & 1.538 & 0.559 & 0.135 & 60.658 & 1.356 & 0.025 & 0.007620 & 0.002126 & 0.075100 & 33.705 \\
\hline 1/31/08 16:00 & 1.547 & 0.568 & 0.141 & 63.095 & 1.361 & 0.03 & 0.009144 & 0.002714 & 0.095873 & 43.028 \\
\hline 1/31/08 17:00 & 1.554 & 0.575 & 0.145 & 65.030 & 1.358 & 0.027 & 0.008229 & 0.002356 & 0.083206 & 37.343 \\
\hline 1/31/08 18:00 & 1.554 & 0.575 & 0.145 & 65.030 & 1.361 & 0.03 & 0.009144 & 0.002714 & 0.095873 & 43.028 \\
\hline 1/31/08 19:00 & 1.54 & 0.561 & 0.136 & 61.195 & 1.358 & 0.027 & 0.008229 & 0.002356 & 0.083206 & 37.343 \\
\hline 1/31/08 20:00 & 1.536 & 0.557 & 0.134 & 60.124 & 1.356 & 0.025 & 0.007620 & 0.002126 & 0.075100 & 33.705 \\
\hline 1/31/08 21:00 & 1.534 & 0.555 & 0.133 & 59.593 & 1.356 & 0.025 & 0.007620 & 0.002126 & 0.075100 & 33.705 \\
\hline 1/31/08 22:00 & 1.532 & 0.553 & 0.132 & 59.065 & 1.354 & 0.023 & 0.007010 & 0.001905 & 0.067276 & 30.194 \\
\hline 1/31/08 23:00 & 1.53 & 0.551 & 0.130 & 58.540 & 1.356 & 0.025 & 0.007620 & 0.002126 & 0.075100 & 33.705 \\
\hline 2/1/08 0:00 & 1.525 & 0.546 & 0.128 & 57.239 & 1.354 & 0.023 & 0.007010 & 0.001905 & 0.067276 & 30.194 \\
\hline 2/1/08 1:00 & 1.525 & 0.546 & 0.128 & 57.239 & 1.354 & 0.023 & 0.007010 & 0.001905 & 0.067276 & 30.194 \\
\hline 2/1/08 2:00 & 1.52 & 0.541 & 0.125 & 55.956 & 1.354 & 0.023 & 0.007010 & 0.001905 & 0.067276 & 30.194 \\
\hline 2/1/08 3:00 & 1.518 & 0.539 & 0.124 & 55.447 & 1.354 & 0.023 & 0.007010 & 0.001905 & 0.067276 & 30.194 \\
\hline 2/1/08 4:00 & 1.518 & 0.539 & 0.124 & 55.447 & 1.354 & 0.023 & 0.007010 & 0.001905 & 0.067276 & 30.194 \\
\hline 2/1/08 5:00 & 1.516 & 0.537 & 0.122 & 54.942 & 1.351 & 0.02 & 0.006096 & 0.001588 & 0.056092 & 25.174 \\
\hline
\end{tabular}




\begin{tabular}{|c|c|c|c|c|c|c|c|c|c|c|}
\hline \multirow[b]{3}{*}{ Date Time } & \multicolumn{4}{|c|}{ V-Notch Weir } & \multicolumn{6}{|c|}{ R-Notch Weir } \\
\hline & \multicolumn{2}{|c|}{ Measured Water Level } & \multicolumn{2}{|c|}{$\begin{array}{c}\text { Calculated Rate } \\
\left(\mathrm{ft}^{3} / \mathrm{s}=448.8 \mathrm{gpm}\right)\end{array}$} & \multicolumn{3}{|c|}{ Measured Water Level } & \multicolumn{3}{|c|}{$\begin{array}{c}\text { Calculated Rate } \\
\left(\mathrm{ft}^{3} / \mathrm{s}=448.8 \mathrm{gpm}\right)\end{array}$} \\
\hline & $\begin{array}{c}\text { (ft above } \\
\text { sensor) }\end{array}$ & $\begin{array}{c}\text { (ft above } \\
\text { notch) }\end{array}$ & $\left(\mathrm{ft}^{3} / \mathrm{s}\right)$ & (gpm) & $\begin{array}{l}\text { (ft above } \\
\text { sensor) }\end{array}$ & $\begin{array}{c}\text { (ft above } \\
\text { log) }\end{array}$ & $\begin{array}{c}\text { (m above } \\
\text { log) }\end{array}$ & $\left(\mathrm{m}^{3} / \mathrm{s}\right)$ & $\left(\mathrm{ft}^{3} / \mathrm{s}\right)$ & (gpm) \\
\hline 2/1/08 6:00 & 1.514 & 0.535 & 0.121 & 54.439 & 1.354 & 0.023 & 0.007010 & 0.001905 & 0.067276 & 30.194 \\
\hline 2/1/08 7:00 & 1.511 & 0.532 & 0.120 & 53.689 & 1.351 & 0.02 & 0.006096 & 0.001588 & 0.056092 & 25.174 \\
\hline 2/1/08 8:00 & 1.509 & 0.530 & 0.119 & 53.194 & 1.351 & 0.02 & 0.006096 & 0.001588 & 0.056092 & 25.174 \\
\hline 2/1/08 9:00 & 1.511 & 0.532 & 0.120 & 53.689 & 1.351 & 0.02 & 0.006096 & 0.001588 & 0.056092 & 25.174 \\
\hline 2/1/08 10:00 & 1.507 & 0.528 & 0.117 & 52.700 & 1.351 & 0.02 & 0.006096 & 0.001588 & 0.056092 & 25.174 \\
\hline 2/1/08 11:00 & 1.518 & 0.539 & 0.124 & 55.447 & 1.354 & 0.023 & 0.007010 & 0.001905 & 0.067276 & 30.194 \\
\hline 2/1/08 12:00 & 1.515 & 0.536 & 0.122 & 54.690 & 1.354 & 0.023 & 0.007010 & 0.001905 & 0.067276 & 30.194 \\
\hline 2/1/08 13:00 & 1.522 & 0.543 & 0.126 & 56.467 & 1.356 & 0.025 & 0.007620 & 0.002126 & 0.075100 & 33.705 \\
\hline 2/1/08 14:00 & 1.535 & 0.556 & 0.133 & 59.859 & 1.356 & 0.025 & 0.007620 & 0.002126 & 0.075100 & 33.705 \\
\hline 2/1/08 15:00 & 1.547 & 0.568 & 0.141 & 63.095 & 1.358 & 0.027 & 0.008229 & 0.002356 & 0.083206 & 37.343 \\
\hline 2/1/08 16:00 & 1.556 & 0.577 & 0.146 & 65.590 & 1.358 & 0.027 & 0.008229 & 0.002356 & 0.083206 & 37.343 \\
\hline 2/1/08 17:00 & 1.558 & 0.579 & 0.147 & 66.152 & 1.361 & 0.03 & 0.009144 & 0.002714 & 0.095873 & 43.028 \\
\hline 2/1/08 18:00 & 1.556 & 0.577 & 0.146 & 65.590 & 1.358 & 0.027 & 0.008229 & 0.002356 & 0.083206 & 37.343 \\
\hline 2/1/08 19:00 & 1.547 & 0.568 & 0.141 & 63.095 & 1.358 & 0.027 & 0.008229 & 0.002356 & 0.083206 & 37.343 \\
\hline 2/1/08 20:00 & 1.543 & 0.564 & 0.138 & 62.005 & 1.356 & 0.025 & 0.007620 & 0.002126 & 0.075100 & 33.705 \\
\hline 2/1/08 21:00 & 1.545 & 0.566 & 0.139 & 62.549 & 1.356 & 0.025 & 0.007620 & 0.002126 & 0.075100 & 33.705 \\
\hline 2/1/08 22:00 & 1.548 & 0.569 & 0.141 & 63.369 & 1.358 & 0.027 & 0.008229 & 0.002356 & 0.083206 & 37.343 \\
\hline 2/1/08 23:00 & 1.55 & 0.571 & 0.142 & 63.920 & 1.358 & 0.027 & 0.008229 & 0.002356 & 0.083206 & 37.343 \\
\hline 2/2/08 0:00 & 1.548 & 0.569 & 0.141 & 63.369 & 1.358 & 0.027 & 0.008229 & 0.002356 & 0.083206 & 37.343 \\
\hline 2/2/08 1:00 & 1.553 & 0.574 & 0.144 & 64.752 & 1.358 & 0.027 & 0.008229 & 0.002356 & 0.083206 & 37.343 \\
\hline 2/2/08 2:00 & 1.548 & 0.569 & 0.141 & 63.369 & 1.356 & 0.025 & 0.007620 & 0.002126 & 0.075100 & 33.705 \\
\hline 2/2/08 3:00 & 1.543 & 0.564 & 0.138 & 62.005 & 1.356 & 0.025 & 0.007620 & 0.002126 & 0.075100 & 33.705 \\
\hline 2/2/08 4:00 & 1.548 & 0.569 & 0.141 & 63.369 & 1.356 & 0.025 & 0.007620 & 0.002126 & 0.075100 & 33.705 \\
\hline 2/2/08 5:00 & 1.548 & 0.569 & 0.141 & 63.369 & 1.358 & 0.027 & 0.008229 & 0.002356 & 0.083206 & 37.343 \\
\hline 2/2/08 6:00 & 1.548 & 0.569 & 0.141 & 63.369 & 1.358 & 0.027 & 0.008229 & 0.002356 & 0.083206 & 37.343 \\
\hline
\end{tabular}




\begin{tabular}{|c|c|c|c|c|c|c|c|c|c|c|}
\hline \multirow[b]{3}{*}{ Date Time } & \multicolumn{4}{|c|}{ V-Notch Weir } & \multicolumn{6}{|c|}{ R-Notch Weir } \\
\hline & \multicolumn{2}{|c|}{ Measured Water Level } & \multicolumn{2}{|c|}{$\begin{array}{c}\text { Calculated Rate } \\
\left(\mathrm{ft}^{3} / \mathrm{s}=448.8 \mathrm{gpm}\right)\end{array}$} & \multicolumn{3}{|c|}{ Measured Water Level } & \multicolumn{3}{|c|}{$\begin{array}{c}\text { Calculated Rate } \\
\left(\mathrm{ft}^{3} / \mathrm{s}=448.8 \mathrm{gpm}\right)\end{array}$} \\
\hline & $\begin{array}{c}\text { (ft above } \\
\text { sensor) }\end{array}$ & $\begin{array}{c}\text { (ft above } \\
\text { notch) }\end{array}$ & $\left(\mathrm{ft}^{3} / \mathrm{s}\right)$ & (gpm) & $\begin{array}{l}\text { (ft above } \\
\text { sensor) }\end{array}$ & $\begin{array}{c}\text { (ft above } \\
\text { log) }\end{array}$ & $\begin{array}{c}\text { (m above } \\
\text { log) }\end{array}$ & $\left(\mathrm{m}^{3} / \mathrm{s}\right)$ & $\left(\mathrm{ft}^{3} / \mathrm{s}\right)$ & (gpm) \\
\hline 2/2/08 7:00 & 1.548 & 0.569 & 0.141 & 63.369 & 1.358 & 0.027 & 0.008229 & 0.002356 & 0.083206 & 37.343 \\
\hline 2/2/08 8:00 & 1.546 & 0.567 & 0.140 & 62.821 & 1.356 & 0.025 & 0.007620 & 0.002126 & 0.075100 & 33.705 \\
\hline 2/2/08 9:00 & 1.541 & 0.562 & 0.137 & 61.464 & 1.356 & 0.025 & 0.007620 & 0.002126 & 0.075100 & 33.705 \\
\hline 2/2/08 10:00 & 1.544 & 0.565 & 0.139 & 62.276 & 1.358 & 0.027 & 0.008229 & 0.002356 & 0.083206 & 37.343 \\
\hline 2/2/08 11:00 & 1.546 & 0.567 & 0.140 & 62.821 & 1.356 & 0.025 & 0.007620 & 0.002126 & 0.075100 & 33.705 \\
\hline 2/2/08 12:00 & 1.536 & 0.557 & 0.134 & 60.124 & 1.356 & 0.025 & 0.007620 & 0.002126 & 0.075100 & 33.705 \\
\hline 2/2/08 13:00 & 1.545 & 0.566 & 0.139 & 62.549 & 1.356 & 0.025 & 0.007620 & 0.002126 & 0.075100 & 33.705 \\
\hline 2/2/08 14:00 & 1.547 & 0.568 & 0.141 & 63.095 & 1.358 & 0.027 & 0.008229 & 0.002356 & 0.083206 & 37.343 \\
\hline 2/2/08 15:00 & 1.556 & 0.577 & 0.146 & 65.590 & 1.358 & 0.027 & 0.008229 & 0.002356 & 0.083206 & 37.343 \\
\hline 2/2/08 16:00 & 1.558 & 0.579 & 0.147 & 66.152 & 1.358 & 0.027 & 0.008229 & 0.002356 & 0.083206 & 37.343 \\
\hline 2/2/08 17:00 & 1.558 & 0.579 & 0.147 & 66.152 & 1.358 & 0.027 & 0.008229 & 0.002356 & 0.083206 & 37.343 \\
\hline 2/2/08 18:00 & 1.558 & 0.579 & 0.147 & 66.152 & 1.358 & 0.027 & 0.008229 & 0.002356 & 0.083206 & 37.343 \\
\hline 2/2/08 19:00 & 1.558 & 0.579 & 0.147 & 66.152 & 1.358 & 0.027 & 0.008229 & 0.002356 & 0.083206 & 37.343 \\
\hline 2/2/08 20:00 & 1.554 & 0.575 & 0.145 & 65.030 & 1.358 & 0.027 & 0.008229 & 0.002356 & 0.083206 & 37.343 \\
\hline 2/2/08 21:00 & 1.547 & 0.568 & 0.141 & 63.095 & 1.358 & 0.027 & 0.008229 & 0.002356 & 0.083206 & 37.343 \\
\hline 2/2/08 22:00 & 1.547 & 0.568 & 0.141 & 63.095 & 1.358 & 0.027 & 0.008229 & 0.002356 & 0.083206 & 37.343 \\
\hline 2/2/08 23:00 & 1.54 & 0.561 & 0.136 & 61.195 & 1.356 & 0.025 & 0.007620 & 0.002126 & 0.075100 & 33.705 \\
\hline 2/3/08 0:00 & 1.545 & 0.566 & 0.139 & 62.549 & 1.356 & 0.025 & 0.007620 & 0.002126 & 0.075100 & 33.705 \\
\hline 2/3/08 1:00 & 1.543 & 0.564 & 0.138 & 62.005 & 1.356 & 0.025 & 0.007620 & 0.002126 & 0.075100 & 33.705 \\
\hline 2/3/08 2:00 & 1.546 & 0.567 & 0.140 & 62.821 & 1.356 & 0.025 & 0.007620 & 0.002126 & 0.075100 & 33.705 \\
\hline 2/3/08 3:00 & 1.546 & 0.567 & 0.140 & 62.821 & 1.356 & 0.025 & 0.007620 & 0.002126 & 0.075100 & 33.705 \\
\hline 2/3/08 4:00 & 1.541 & 0.562 & 0.137 & 61.464 & 1.356 & 0.025 & 0.007620 & 0.002126 & 0.075100 & 33.705 \\
\hline 2/3/08 5:00 & 1.543 & 0.564 & 0.138 & 62.005 & 1.356 & 0.025 & 0.007620 & 0.002126 & 0.075100 & 33.705 \\
\hline 2/3/08 6:00 & 1.539 & 0.560 & 0.136 & 60.926 & 1.356 & 0.025 & 0.007620 & 0.002126 & 0.075100 & 33.705 \\
\hline 2/3/08 7:00 & 1.537 & 0.558 & 0.135 & 60.391 & 1.356 & 0.025 & 0.007620 & 0.002126 & 0.075100 & 33.705 \\
\hline
\end{tabular}




\begin{tabular}{|c|c|c|c|c|c|c|c|c|c|c|}
\hline \multirow[b]{3}{*}{ Date Time } & \multicolumn{4}{|c|}{ V-Notch Weir } & \multicolumn{6}{|c|}{ R-Notch Weir } \\
\hline & \multicolumn{2}{|c|}{ Measured Water Level } & \multicolumn{2}{|c|}{$\begin{array}{c}\text { Calculated Rate } \\
\left(\mathrm{ft}^{3} / \mathrm{s}=448.8 \mathrm{gpm}\right)\end{array}$} & \multicolumn{3}{|c|}{ Measured Water Level } & \multicolumn{3}{|c|}{$\begin{array}{c}\text { Calculated Rate } \\
\left(\mathrm{ft}^{3} / \mathrm{s}=448.8 \mathrm{gpm}\right)\end{array}$} \\
\hline & $\begin{array}{c}\text { (ft above } \\
\text { sensor) }\end{array}$ & $\begin{array}{c}\text { (ft above } \\
\text { notch) }\end{array}$ & $\left(\mathrm{ft}^{3} / \mathrm{s}\right)$ & (gpm) & $\begin{array}{l}\text { (ft above } \\
\text { sensor) }\end{array}$ & $\begin{array}{c}\text { (ft above } \\
\text { log) }\end{array}$ & $\begin{array}{c}\text { (m above } \\
\text { log) }\end{array}$ & $\left(\mathrm{m}^{3} / \mathrm{s}\right)$ & $\left(\mathrm{ft}^{3} / \mathrm{s}\right)$ & (gpm) \\
\hline 2/3/08 8:00 & 1.536 & 0.557 & 0.134 & 60.124 & 1.356 & 0.025 & 0.007620 & 0.002126 & 0.075100 & 33.705 \\
\hline 2/3/08 9:00 & 1.539 & 0.560 & 0.136 & 60.926 & 1.356 & 0.025 & 0.007620 & 0.002126 & 0.075100 & 33.705 \\
\hline 2/3/08 10:00 & 1.543 & 0.564 & 0.138 & 62.005 & 1.356 & 0.025 & 0.007620 & 0.002126 & 0.075100 & 33.705 \\
\hline 2/3/08 11:00 & 1.541 & 0.562 & 0.137 & 61.464 & 1.356 & 0.025 & 0.007620 & 0.002126 & 0.075100 & 33.705 \\
\hline 2/3/08 12:00 & 1.543 & 0.564 & 0.138 & 62.005 & 1.356 & 0.025 & 0.007620 & 0.002126 & 0.075100 & 33.705 \\
\hline 2/3/08 13:00 & 1.545 & 0.566 & 0.139 & 62.549 & 1.358 & 0.027 & 0.008229 & 0.002356 & 0.083206 & 37.343 \\
\hline 2/3/08 14:00 & 1.55 & 0.571 & 0.142 & 63.920 & 1.358 & 0.027 & 0.008229 & 0.002356 & 0.083206 & 37.343 \\
\hline 2/3/08 15:00 & 1.566 & 0.587 & 0.152 & 68.430 & 1.363 & 0.032 & 0.009753 & 0.002963 & 0.104644 & 46.964 \\
\hline 2/3/08 16:00 & 1.563 & 0.584 & 0.151 & 67.571 & 1.361 & 0.03 & 0.009144 & 0.002714 & 0.095873 & 43.028 \\
\hline 2/3/08 17:00 & 1.575 & 0.596 & 0.158 & 71.049 & 1.365 & 0.034 & 0.010363 & 0.003218 & 0.113667 & 51.014 \\
\hline 2/3/08 18:00 & 1.568 & 0.589 & 0.154 & 69.007 & 1.363 & 0.032 & 0.009753 & 0.002963 & 0.104644 & 46.964 \\
\hline 2/3/08 19:00 & 1.571 & 0.592 & 0.156 & 69.878 & 1.361 & 0.03 & 0.009144 & 0.002714 & 0.095873 & 43.028 \\
\hline 2/3/08 20:00 & 1.564 & 0.585 & 0.151 & 67.856 & 1.361 & 0.03 & 0.009144 & 0.002714 & 0.095873 & 43.028 \\
\hline 2/3/08 21:00 & 1.566 & 0.587 & 0.152 & 68.430 & 1.361 & 0.03 & 0.009144 & 0.002714 & 0.095873 & 43.028 \\
\hline 2/3/08 22:00 & 1.561 & 0.582 & 0.149 & 67.001 & 1.361 & 0.03 & 0.009144 & 0.002714 & 0.095873 & 43.028 \\
\hline 2/3/08 23:00 & 1.559 & 0.580 & 0.148 & 66.434 & 1.361 & 0.03 & 0.009144 & 0.002714 & 0.095873 & 43.028 \\
\hline 2/4/08 0:00 & 1.566 & 0.587 & 0.152 & 68.430 & 1.361 & 0.03 & 0.009144 & 0.002714 & 0.095873 & 43.028 \\
\hline 2/4/08 1:00 & 1.559 & 0.580 & 0.148 & 66.434 & 1.365 & 0.034 & 0.010363 & 0.003218 & 0.113667 & 51.014 \\
\hline 2/4/08 2:00 & 1.559 & 0.580 & 0.148 & 66.434 & 1.363 & 0.032 & 0.009753 & 0.002963 & 0.104644 & 46.964 \\
\hline 2/4/08 3:00 & 1.576 & 0.597 & 0.159 & 71.343 & 1.361 & 0.03 & 0.009144 & 0.002714 & 0.095873 & 43.028 \\
\hline 2/4/08 4:00 & 1.569 & 0.590 & 0.154 & 69.296 & 1.37 & 0.039 & 0.011887 & 0.003887 & 0.137285 & 61.614 \\
\hline 2/4/08 5:00 & 1.56 & 0.581 & 0.149 & 66.717 & 1.361 & 0.03 & 0.009144 & 0.002714 & 0.095873 & 43.028 \\
\hline 2/4/08 6:00 & 1.557 & 0.578 & 0.147 & 65.871 & 1.361 & 0.03 & 0.009144 & 0.002714 & 0.095873 & 43.028 \\
\hline 2/4/08 7:00 & 1.564 & 0.585 & 0.151 & 67.856 & 1.361 & 0.03 & 0.009144 & 0.002714 & 0.095873 & 43.028 \\
\hline 2/4/08 8:00 & 1.564 & 0.585 & 0.151 & 67.856 & 1.361 & 0.03 & 0.009144 & 0.002714 & 0.095873 & 43.028 \\
\hline
\end{tabular}




\begin{tabular}{|c|c|c|c|c|c|c|c|c|c|c|}
\hline \multirow[b]{3}{*}{ Date Time } & \multicolumn{4}{|c|}{ V-Notch Weir } & \multicolumn{6}{|c|}{ R-Notch Weir } \\
\hline & \multicolumn{2}{|c|}{ Measured Water Level } & \multicolumn{2}{|c|}{$\begin{array}{c}\text { Calculated Rate } \\
\left(\mathrm{ft}^{3} / \mathrm{s}=448.8 \mathrm{gpm}\right)\end{array}$} & \multicolumn{3}{|c|}{ Measured Water Level } & \multicolumn{3}{|c|}{$\begin{array}{c}\text { Calculated Rate } \\
\left(\mathrm{ft}^{3} / \mathrm{s}=448.8 \mathrm{gpm}\right)\end{array}$} \\
\hline & $\begin{array}{c}\text { (ft above } \\
\text { sensor) }\end{array}$ & $\begin{array}{c}\text { (ft above } \\
\text { notch) }\end{array}$ & $\left(\mathrm{ft}^{3} / \mathrm{s}\right)$ & (gpm) & $\begin{array}{l}\text { (ft above } \\
\text { sensor) }\end{array}$ & $\begin{array}{c}\text { (ft above } \\
\text { log) }\end{array}$ & $\begin{array}{c}\text { (m above } \\
\text { log) }\end{array}$ & $\left(\mathrm{m}^{3} / \mathrm{s}\right)$ & $\left(\mathrm{ft}^{3} / \mathrm{s}\right)$ & (gpm) \\
\hline 2/4/08 9:00 & 1.562 & 0.583 & 0.150 & 67.285 & 1.361 & 0.03 & 0.009144 & 0.002714 & 0.095873 & 43.028 \\
\hline 2/4/08 10:00 & 1.562 & 0.583 & 0.150 & 67.285 & 1.358 & 0.027 & 0.008229 & 0.002356 & 0.083206 & 37.343 \\
\hline 2/4/08 11:00 & 1.566 & 0.587 & 0.152 & 68.430 & 1.361 & 0.03 & 0.009144 & 0.002714 & 0.095873 & 43.028 \\
\hline 2/4/08 12:00 & 1.564 & 0.585 & 0.151 & 67.856 & 1.358 & 0.027 & 0.008229 & 0.002356 & 0.083206 & 37.343 \\
\hline 2/4/08 13:00 & 1.571 & 0.592 & 0.156 & 69.878 & 1.361 & 0.03 & 0.009144 & 0.002714 & 0.095873 & 43.028 \\
\hline 2/4/08 14:00 & 1.587 & 0.608 & 0.166 & 74.632 & 1.363 & 0.032 & 0.009753 & 0.002963 & 0.104644 & 46.964 \\
\hline 2/4/08 15:00 & 1.596 & 0.617 & 0.172 & 77.389 & 1.363 & 0.032 & 0.009753 & 0.002963 & 0.104644 & 46.964 \\
\hline 2/4/08 16:00 & 1.603 & 0.624 & 0.177 & 79.575 & 1.365 & 0.034 & 0.010363 & 0.003218 & 0.113667 & 51.014 \\
\hline 2/4/08 17:00 & 1.61 & 0.631 & 0.182 & 81.798 & 1.365 & 0.034 & 0.010363 & 0.003218 & 0.113667 & 51.014 \\
\hline 2/4/08 18:00 & 1.612 & 0.633 & 0.184 & 82.440 & 1.365 & 0.034 & 0.010363 & 0.003218 & 0.113667 & 51.014 \\
\hline 2/4/08 19:00 & 1.601 & 0.622 & 0.176 & 78.947 & 1.365 & 0.034 & 0.010363 & 0.003218 & 0.113667 & 51.014 \\
\hline 2/4/08 20:00 & 1.589 & 0.610 & 0.168 & 75.239 & 1.363 & 0.032 & 0.009753 & 0.002963 & 0.104644 & 46.964 \\
\hline 2/4/08 21:00 & 1.589 & 0.610 & 0.168 & 75.239 & 1.363 & 0.032 & 0.009753 & 0.002963 & 0.104644 & 46.964 \\
\hline 2/4/08 22:00 & 1.585 & 0.606 & 0.165 & 74.027 & 1.361 & 0.03 & 0.009144 & 0.002714 & 0.095873 & 43.028 \\
\hline 2/4/08 23:00 & 1.58 & 0.601 & 0.162 & 72.529 & 1.358 & 0.027 & 0.008229 & 0.002356 & 0.083206 & 37.343 \\
\hline 2/5/08 0:00 & 1.576 & 0.597 & 0.159 & 71.343 & 1.358 & 0.027 & 0.008229 & 0.002356 & 0.083206 & 37.343 \\
\hline 2/5/08 1:00 & 1.578 & 0.599 & 0.160 & 71.935 & 1.358 & 0.027 & 0.008229 & 0.002356 & 0.083206 & 37.343 \\
\hline 2/5/08 2:00 & 1.559 & 0.580 & 0.148 & 66.434 & 1.361 & 0.03 & 0.009144 & 0.002714 & 0.095873 & 43.028 \\
\hline 2/5/08 3:00 & 1.567 & 0.588 & 0.153 & 68.718 & 1.358 & 0.027 & 0.008229 & 0.002356 & 0.083206 & 37.343 \\
\hline 2/5/08 4:00 & 1.56 & 0.581 & 0.149 & 66.717 & 1.358 & 0.027 & 0.008229 & 0.002356 & 0.083206 & 37.343 \\
\hline 2/5/08 5:00 & 1.56 & 0.581 & 0.149 & 66.717 & 1.358 & 0.027 & 0.008229 & 0.002356 & 0.083206 & 37.343 \\
\hline 2/5/08 6:00 & 1.56 & 0.581 & 0.149 & 66.717 & 1.358 & 0.027 & 0.008229 & 0.002356 & 0.083206 & 37.343 \\
\hline 2/5/08 7:00 & 1.565 & 0.586 & 0.152 & 68.143 & 1.358 & 0.027 & 0.008229 & 0.002356 & 0.083206 & 37.343 \\
\hline 2/5/08 8:00 & 1.56 & 0.581 & 0.149 & 66.717 & 1.358 & 0.027 & 0.008229 & 0.002356 & 0.083206 & 37.343 \\
\hline 2/5/08 9:00 & 1.572 & 0.593 & 0.156 & 70.169 & 1.361 & 0.03 & 0.009144 & 0.002714 & 0.095873 & 43.028 \\
\hline
\end{tabular}




\begin{tabular}{|c|c|c|c|c|c|c|c|c|c|c|}
\hline \multirow[b]{3}{*}{ Date Time } & \multicolumn{4}{|c|}{ V-Notch Weir } & \multicolumn{6}{|c|}{ R-Notch Weir } \\
\hline & \multicolumn{2}{|c|}{ Measured Water Level } & \multicolumn{2}{|c|}{$\begin{array}{c}\text { Calculated Rate } \\
\left(\mathrm{ft}^{3} / \mathrm{s}=448.8 \mathrm{gpm}\right)\end{array}$} & \multicolumn{3}{|c|}{ Measured Water Level } & \multicolumn{3}{|c|}{$\begin{array}{c}\text { Calculated Rate } \\
\left(\mathrm{ft}^{3} / \mathrm{s}=448.8 \mathrm{gpm}\right)\end{array}$} \\
\hline & $\begin{array}{c}\text { (ft above } \\
\text { sensor) }\end{array}$ & $\begin{array}{c}\text { (ft above } \\
\text { notch) }\end{array}$ & $\left(\mathrm{ft}^{3} / \mathrm{s}\right)$ & (gpm) & $\begin{array}{l}\text { (ft above } \\
\text { sensor) }\end{array}$ & $\begin{array}{c}\text { (ft above } \\
\text { log) }\end{array}$ & $\begin{array}{c}\text { (m above } \\
\text { log) }\end{array}$ & $\left(\mathrm{m}^{3} / \mathrm{s}\right)$ & $\left(\mathrm{ft}^{3} / \mathrm{s}\right)$ & (gpm) \\
\hline 2/5/08 10:00 & 1.567 & 0.588 & 0.153 & 68.718 & 1.358 & 0.027 & 0.008229 & 0.002356 & 0.083206 & 37.343 \\
\hline 2/5/08 11:00 & 1.569 & 0.590 & 0.154 & 69.296 & 1.358 & 0.027 & 0.008229 & 0.002356 & 0.083206 & 37.343 \\
\hline 2/5/08 12:00 & 1.576 & 0.597 & 0.159 & 71.343 & 1.361 & 0.03 & 0.009144 & 0.002714 & 0.095873 & 43.028 \\
\hline 2/5/08 13:00 & 1.572 & 0.593 & 0.156 & 70.169 & 1.358 & 0.027 & 0.008229 & 0.002356 & 0.083206 & 37.343 \\
\hline 2/5/08 14:00 & 1.581 & 0.602 & 0.162 & 72.827 & 1.358 & 0.027 & 0.008229 & 0.002356 & 0.083206 & 37.343 \\
\hline 2/5/08 15:00 & 1.581 & 0.602 & 0.162 & 72.827 & 1.358 & 0.027 & 0.008229 & 0.002356 & 0.083206 & 37.343 \\
\hline 2/5/08 16:00 & 1.571 & 0.592 & 0.156 & 69.878 & 1.363 & 0.032 & 0.009753 & 0.002963 & 0.104644 & 46.964 \\
\hline 2/5/08 17:00 & 1.574 & 0.595 & 0.158 & 70.755 & 1.361 & 0.03 & 0.009144 & 0.002714 & 0.095873 & 43.028 \\
\hline 2/5/08 18:00 & 1.574 & 0.595 & 0.158 & 70.755 & 1.358 & 0.027 & 0.008229 & 0.002356 & 0.083206 & 37.343 \\
\hline 2/5/08 19:00 & 1.579 & 0.600 & 0.161 & 72.231 & 1.358 & 0.027 & 0.008229 & 0.002356 & 0.083206 & 37.343 \\
\hline 2/5/08 20:00 & 1.579 & 0.600 & 0.161 & 72.231 & 1.358 & 0.027 & 0.008229 & 0.002356 & 0.083206 & 37.343 \\
\hline 2/5/08 21:00 & 1.563 & 0.584 & 0.151 & 67.571 & 1.358 & 0.027 & 0.008229 & 0.002356 & 0.083206 & 37.343 \\
\hline 2/5/08 22:00 & 1.561 & 0.582 & 0.149 & 67.001 & 1.358 & 0.027 & 0.008229 & 0.002356 & 0.083206 & 37.343 \\
\hline 2/5/08 23:00 & 1.563 & 0.584 & 0.151 & 67.571 & 1.358 & 0.027 & 0.008229 & 0.002356 & 0.083206 & 37.343 \\
\hline 2/6/08 0:00 & 1.556 & 0.577 & 0.146 & 65.590 & 1.358 & 0.027 & 0.008229 & 0.002356 & 0.083206 & 37.343 \\
\hline 2/6/08 1:00 & 1.559 & 0.580 & 0.148 & 66.434 & 1.358 & 0.027 & 0.008229 & 0.002356 & 0.083206 & 37.343 \\
\hline 2/6/08 2:00 & 1.564 & 0.585 & 0.151 & 67.856 & 1.356 & 0.025 & 0.007620 & 0.002126 & 0.075100 & 33.705 \\
\hline 2/6/08 3:00 & 1.566 & 0.587 & 0.152 & 68.430 & 1.358 & 0.027 & 0.008229 & 0.002356 & 0.083206 & 37.343 \\
\hline 2/6/08 4:00 & 1.564 & 0.585 & 0.151 & 67.856 & 1.361 & 0.03 & 0.009144 & 0.002714 & 0.095873 & 43.028 \\
\hline 2/6/08 5:00 & 1.554 & 0.575 & 0.145 & 65.030 & 1.361 & 0.03 & 0.009144 & 0.002714 & 0.095873 & 43.028 \\
\hline 2/6/08 6:00 & 1.556 & 0.577 & 0.146 & 65.590 & 1.363 & 0.032 & 0.009753 & 0.002963 & 0.104644 & 46.964 \\
\hline 2/6/08 7:00 & 1.564 & 0.585 & 0.151 & 67.856 & 1.363 & 0.032 & 0.009753 & 0.002963 & 0.104644 & 46.964 \\
\hline 2/6/08 8:00 & 1.559 & 0.580 & 0.148 & 66.434 & 1.363 & 0.032 & 0.009753 & 0.002963 & 0.104644 & 46.964 \\
\hline 2/6/08 9:00 & 1.545 & 0.566 & 0.139 & 62.549 & 1.366 & 0.035 & 0.010667 & 0.003349 & 0.118271 & 53.080 \\
\hline 2/6/08 10:00 & 1.54 & 0.561 & 0.136 & 61.195 & 1.368 & 0.037 & 0.011277 & 0.003614 & 0.127660 & 57.294 \\
\hline
\end{tabular}




\begin{tabular}{|c|c|c|c|c|c|c|c|c|c|c|}
\hline \multirow[b]{3}{*}{ Date Time } & \multicolumn{4}{|c|}{ V-Notch Weir } & \multicolumn{6}{|c|}{ R-Notch Weir } \\
\hline & \multicolumn{2}{|c|}{ Measured Water Level } & \multicolumn{2}{|c|}{$\begin{array}{c}\text { Calculated Rate } \\
\left(\mathrm{ft}^{3} / \mathrm{s}=448.8 \mathrm{gpm}\right)\end{array}$} & \multicolumn{3}{|c|}{ Measured Water Level } & \multicolumn{3}{|c|}{$\begin{array}{c}\text { Calculated Rate } \\
\left(\mathrm{ft}^{3} / \mathrm{s}=448.8 \mathrm{gpm}\right)\end{array}$} \\
\hline & $\begin{array}{c}\text { (ft above } \\
\text { sensor) }\end{array}$ & $\begin{array}{c}\text { (ft above } \\
\text { notch) }\end{array}$ & $\left(\mathrm{ft}^{3} / \mathrm{s}\right)$ & (gpm) & $\begin{array}{l}\text { (ft above } \\
\text { sensor) }\end{array}$ & $\begin{array}{c}\text { (ft above } \\
\text { log) }\end{array}$ & $\begin{array}{c}\text { (m above } \\
\text { log) }\end{array}$ & $\left(\mathrm{m}^{3} / \mathrm{s}\right)$ & $\left(\mathrm{ft}^{3} / \mathrm{s}\right)$ & (gpm) \\
\hline 2/6/08 11:00 & 1.54 & 0.561 & 0.136 & 61.195 & 1.368 & 0.037 & 0.011277 & 0.003614 & 0.127660 & 57.294 \\
\hline 2/6/08 12:00 & 1.558 & 0.579 & 0.147 & 66.152 & 1.366 & 0.035 & 0.010667 & 0.003349 & 0.118271 & 53.080 \\
\hline 2/6/08 13:00 & 1.553 & 0.574 & 0.144 & 64.752 & 1.361 & 0.03 & 0.009144 & 0.002714 & 0.095873 & 43.028 \\
\hline 2/6/08 14:00 & 1.553 & 0.574 & 0.144 & 64.752 & 1.358 & 0.027 & 0.008229 & 0.002356 & 0.083206 & 37.343 \\
\hline 2/6/08 15:00 & 1.548 & 0.569 & 0.141 & 63.369 & 1.358 & 0.027 & 0.008229 & 0.002356 & 0.083206 & 37.343 \\
\hline 2/6/08 16:00 & 1.548 & 0.569 & 0.141 & 63.369 & 1.358 & 0.027 & 0.008229 & 0.002356 & 0.083206 & 37.343 \\
\hline 2/6/08 17:00 & 1.545 & 0.566 & 0.139 & 62.549 & 1.361 & 0.03 & 0.009144 & 0.002714 & 0.095873 & 43.028 \\
\hline 2/6/08 18:00 & 1.536 & 0.557 & 0.134 & 60.124 & 1.358 & 0.027 & 0.008229 & 0.002356 & 0.083206 & 37.343 \\
\hline 2/6/08 19:00 & 1.54 & 0.561 & 0.136 & 61.195 & 1.361 & 0.03 & 0.009144 & 0.002714 & 0.095873 & 43.028 \\
\hline 2/6/08 20:00 & 1.531 & 0.552 & 0.131 & 58.802 & 1.358 & 0.027 & 0.008229 & 0.002356 & 0.083206 & 37.343 \\
\hline 2/6/08 21:00 & 1.534 & 0.555 & 0.133 & 59.593 & 1.358 & 0.027 & 0.008229 & 0.002356 & 0.083206 & 37.343 \\
\hline 2/6/08 22:00 & 1.525 & 0.546 & 0.128 & 57.239 & 1.356 & 0.025 & 0.007620 & 0.002126 & 0.075100 & 33.705 \\
\hline 2/6/08 23:00 & 1.527 & 0.548 & 0.129 & 57.757 & 1.356 & 0.025 & 0.007620 & 0.002126 & 0.075100 & 33.705 \\
\hline 2/7/08 0:00 & 1.527 & 0.548 & 0.129 & 57.757 & 1.356 & 0.025 & 0.007620 & 0.002126 & 0.075100 & 33.705 \\
\hline 2/7/08 1:00 & 1.53 & 0.551 & 0.130 & 58.540 & 1.356 & 0.025 & 0.007620 & 0.002126 & 0.075100 & 33.705 \\
\hline 2/7/08 2:00 & 1.53 & 0.551 & 0.130 & 58.540 & 1.356 & 0.025 & 0.007620 & 0.002126 & 0.075100 & 33.705 \\
\hline 2/7/08 3:00 & 1.525 & 0.546 & 0.128 & 57.239 & 1.356 & 0.025 & 0.007620 & 0.002126 & 0.075100 & 33.705 \\
\hline 2/7/08 4:00 & 1.53 & 0.551 & 0.130 & 58.540 & 1.356 & 0.025 & 0.007620 & 0.002126 & 0.075100 & 33.705 \\
\hline 2/7/08 5:00 & 1.528 & 0.549 & 0.129 & 58.018 & 1.356 & 0.025 & 0.007620 & 0.002126 & 0.075100 & 33.705 \\
\hline 2/7/08 6:00 & 1.528 & 0.549 & 0.129 & 58.018 & 1.356 & 0.025 & 0.007620 & 0.002126 & 0.075100 & 33.705 \\
\hline 2/7/08 7:00 & 1.532 & 0.553 & 0.132 & 59.065 & 1.358 & 0.027 & 0.008229 & 0.002356 & 0.083206 & 37.343 \\
\hline 2/7/08 8:00 & 1.525 & 0.546 & 0.128 & 57.239 & 1.356 & 0.025 & 0.007620 & 0.002126 & 0.075100 & 33.705 \\
\hline 2/7/08 9:00 & 1.518 & 0.539 & 0.124 & 55.447 & 1.356 & 0.025 & 0.007620 & 0.002126 & 0.075100 & 33.705 \\
\hline 2/7/08 10:00 & 1.516 & 0.537 & 0.122 & 54.942 & 1.356 & 0.025 & 0.007620 & 0.002126 & 0.075100 & 33.705 \\
\hline 2/7/08 11:00 & 1.518 & 0.539 & 0.124 & 55.447 & 1.358 & 0.027 & 0.008229 & 0.002356 & 0.083206 & 37.343 \\
\hline
\end{tabular}




\begin{tabular}{|c|c|c|c|c|c|c|c|c|c|c|}
\hline \multirow[b]{3}{*}{ Date Time } & \multicolumn{4}{|c|}{ V-Notch Weir } & \multicolumn{6}{|c|}{ R-Notch Weir } \\
\hline & \multicolumn{2}{|c|}{ Measured Water Level } & \multicolumn{2}{|c|}{$\begin{array}{c}\text { Calculated Rate } \\
\left(\mathrm{ft}^{3} / \mathrm{s}=448.8 \mathrm{gpm}\right)\end{array}$} & \multicolumn{3}{|c|}{ Measured Water Level } & \multicolumn{3}{|c|}{$\begin{array}{c}\text { Calculated Rate } \\
\left(\mathrm{ft}^{3} / \mathrm{s}=448.8 \mathrm{gpm}\right)\end{array}$} \\
\hline & $\begin{array}{c}\text { (ft above } \\
\text { sensor) }\end{array}$ & $\begin{array}{c}\text { (ft above } \\
\text { notch) }\end{array}$ & $\left(\mathrm{ft}^{3} / \mathrm{s}\right)$ & (gpm) & $\begin{array}{l}\text { (ft above } \\
\text { sensor) }\end{array}$ & $\begin{array}{c}\text { (ft above } \\
\text { log) }\end{array}$ & $\begin{array}{c}\text { (m above } \\
\text { log) }\end{array}$ & $\left(\mathrm{m}^{3} / \mathrm{s}\right)$ & $\left(\mathrm{ft}^{3} / \mathrm{s}\right)$ & (gpm) \\
\hline 2/7/08 12:00 & 1.515 & 0.536 & 0.122 & 54.690 & 1.356 & 0.025 & 0.007620 & 0.002126 & 0.075100 & 33.705 \\
\hline 2/7/08 13:00 & 1.513 & 0.534 & 0.121 & 54.188 & 1.358 & 0.027 & 0.008229 & 0.002356 & 0.083206 & 37.343 \\
\hline 2/7/08 14:00 & 1.519 & 0.540 & 0.124 & 55.701 & 1.358 & 0.027 & 0.008229 & 0.002356 & 0.083206 & 37.343 \\
\hline 2/7/08 15:00 & 1.521 & 0.542 & 0.125 & 56.211 & 1.361 & 0.03 & 0.009144 & 0.002714 & 0.095873 & 43.028 \\
\hline 2/7/08 16:00 & 1.523 & 0.544 & 0.126 & 56.724 & 1.358 & 0.027 & 0.008229 & 0.002356 & 0.083206 & 37.343 \\
\hline 2/7/08 17:00 & 1.526 & 0.547 & 0.128 & 57.498 & 1.358 & 0.027 & 0.008229 & 0.002356 & 0.083206 & 37.343 \\
\hline 2/7/08 18:00 & 1.528 & 0.549 & 0.129 & 58.018 & 1.358 & 0.027 & 0.008229 & 0.002356 & 0.083206 & 37.343 \\
\hline 2/7/08 19:00 & 1.523 & 0.544 & 0.126 & 56.724 & 1.358 & 0.027 & 0.008229 & 0.002356 & 0.083206 & 37.343 \\
\hline 2/7/08 20:00 & 1.526 & 0.547 & 0.128 & 57.498 & 1.358 & 0.027 & 0.008229 & 0.002356 & 0.083206 & 37.343 \\
\hline 2/7/08 21:00 & 1.521 & 0.542 & 0.125 & 56.211 & 1.358 & 0.027 & 0.008229 & 0.002356 & 0.083206 & 37.343 \\
\hline 2/7/08 22:00 & 1.522 & 0.543 & 0.126 & 56.467 & 1.358 & 0.027 & 0.008229 & 0.002356 & 0.083206 & 37.343 \\
\hline 2/7/08 23:00 & 1.522 & 0.543 & 0.126 & 56.467 & 1.358 & 0.027 & 0.008229 & 0.002356 & 0.083206 & 37.343 \\
\hline 2/8/08 0:00 & 1.522 & 0.543 & 0.126 & 56.467 & 1.358 & 0.027 & 0.008229 & 0.002356 & 0.083206 & 37.343 \\
\hline 2/8/08 1:00 & 1.524 & 0.545 & 0.127 & 56.981 & 1.358 & 0.027 & 0.008229 & 0.002356 & 0.083206 & 37.343 \\
\hline 2/8/08 2:00 & 1.524 & 0.545 & 0.127 & 56.981 & 1.358 & 0.027 & 0.008229 & 0.002356 & 0.083206 & 37.343 \\
\hline 2/8/08 3:00 & 1.527 & 0.548 & 0.129 & 57.757 & 1.358 & 0.027 & 0.008229 & 0.002356 & 0.083206 & 37.343 \\
\hline 2/8/08 4:00 & 1.527 & 0.548 & 0.129 & 57.757 & 1.358 & 0.027 & 0.008229 & 0.002356 & 0.083206 & 37.343 \\
\hline 2/8/08 5:00 & 1.525 & 0.546 & 0.128 & 57.239 & 1.358 & 0.027 & 0.008229 & 0.002356 & 0.083206 & 37.343 \\
\hline 2/8/08 6:00 & 1.52 & 0.541 & 0.125 & 55.956 & 1.358 & 0.027 & 0.008229 & 0.002356 & 0.083206 & 37.343 \\
\hline 2/8/08 7:00 & 1.522 & 0.543 & 0.126 & 56.467 & 1.356 & 0.025 & 0.007620 & 0.002126 & 0.075100 & 33.705 \\
\hline 2/8/08 8:00 & 1.52 & 0.541 & 0.125 & 55.956 & 1.358 & 0.027 & 0.008229 & 0.002356 & 0.083206 & 37.343 \\
\hline 2/8/08 9:00 & 1.518 & 0.539 & 0.124 & 55.447 & 1.356 & 0.025 & 0.007620 & 0.002126 & 0.075100 & 33.705 \\
\hline 2/8/08 10:00 & 1.518 & 0.539 & 0.124 & 55.447 & 1.356 & 0.025 & 0.007620 & 0.002126 & 0.075100 & 33.705 \\
\hline 2/8/08 11:00 & 1.515 & 0.536 & 0.122 & 54.690 & 1.358 & 0.027 & 0.008229 & 0.002356 & 0.083206 & 37.343 \\
\hline 2/8/08 12:00 & 1.517 & 0.538 & 0.123 & 55.194 & 1.358 & 0.027 & 0.008229 & 0.002356 & 0.083206 & 37.343 \\
\hline
\end{tabular}




\begin{tabular}{|c|c|c|c|c|c|c|c|c|c|c|}
\hline \multirow[b]{3}{*}{ Date Time } & \multicolumn{4}{|c|}{ V-Notch Weir } & \multicolumn{6}{|c|}{ R-Notch Weir } \\
\hline & \multicolumn{2}{|c|}{ Measured Water Level } & \multicolumn{2}{|c|}{$\begin{array}{c}\text { Calculated Rate } \\
\left(\mathrm{ft}^{3} / \mathrm{s}=448.8 \mathrm{gpm}\right)\end{array}$} & \multicolumn{3}{|c|}{ Measured Water Level } & \multicolumn{3}{|c|}{$\begin{array}{c}\text { Calculated Rate } \\
\left(\mathrm{ft}^{3} / \mathrm{s}=448.8 \mathrm{gpm}\right)\end{array}$} \\
\hline & $\begin{array}{c}\text { (ft above } \\
\text { sensor) }\end{array}$ & $\begin{array}{c}\text { (ft above } \\
\text { notch) }\end{array}$ & $\left(\mathrm{ft}^{3} / \mathrm{s}\right)$ & (gpm) & $\begin{array}{l}\text { (ft above } \\
\text { sensor) }\end{array}$ & $\begin{array}{c}\text { (ft above } \\
\text { log) }\end{array}$ & $\begin{array}{c}\text { (m above } \\
\text { log) }\end{array}$ & $\left(\mathrm{m}^{3} / \mathrm{s}\right)$ & $\left(\mathrm{ft}^{3} / \mathrm{s}\right)$ & (gpm) \\
\hline 2/8/08 13:00 & 1.519 & 0.540 & 0.124 & 55.701 & 1.356 & 0.025 & 0.007620 & 0.002126 & 0.075100 & 33.705 \\
\hline 2/8/08 14:00 & 1.528 & 0.549 & 0.129 & 58.018 & 1.361 & 0.03 & 0.009144 & 0.002714 & 0.095873 & 43.028 \\
\hline 2/8/08 15:00 & 1.535 & 0.556 & 0.133 & 59.859 & 1.361 & 0.03 & 0.009144 & 0.002714 & 0.095873 & 43.028 \\
\hline 2/8/08 16:00 & 1.56 & 0.581 & 0.149 & 66.717 & 1.363 & 0.032 & 0.009753 & 0.002963 & 0.104644 & 46.964 \\
\hline 2/8/08 17:00 & 1.565 & 0.586 & 0.152 & 68.143 & 1.363 & 0.032 & 0.009753 & 0.002963 & 0.104644 & 46.964 \\
\hline 2/8/08 18:00 & 1.567 & 0.588 & 0.153 & 68.718 & 1.363 & 0.032 & 0.009753 & 0.002963 & 0.104644 & 46.964 \\
\hline 2/8/08 19:00 & 1.558 & 0.579 & 0.147 & 66.152 & 1.363 & 0.032 & 0.009753 & 0.002963 & 0.104644 & 46.964 \\
\hline 2/8/08 20:00 & 1.546 & 0.567 & 0.140 & 62.821 & 1.361 & 0.03 & 0.009144 & 0.002714 & 0.095873 & 43.028 \\
\hline 2/8/08 21:00 & 1.537 & 0.558 & 0.135 & 60.391 & 1.358 & 0.027 & 0.008229 & 0.002356 & 0.083206 & 37.343 \\
\hline 2/8/08 22:00 & 1.535 & 0.556 & 0.133 & 59.859 & 1.361 & 0.03 & 0.009144 & 0.002714 & 0.095873 & 43.028 \\
\hline 2/8/08 23:00 & 1.533 & 0.554 & 0.132 & 59.329 & 1.358 & 0.027 & 0.008229 & 0.002356 & 0.083206 & 37.343 \\
\hline 2/9/08 0:00 & 1.529 & 0.550 & 0.130 & 58.278 & 1.358 & 0.027 & 0.008229 & 0.002356 & 0.083206 & 37.343 \\
\hline 2/9/08 1:00 & 1.526 & 0.547 & 0.128 & 57.498 & 1.358 & 0.027 & 0.008229 & 0.002356 & 0.083206 & 37.343 \\
\hline 2/9/08 2:00 & 1.526 & 0.547 & 0.128 & 57.498 & 1.358 & 0.027 & 0.008229 & 0.002356 & 0.083206 & 37.343 \\
\hline 2/9/08 3:00 & 1.527 & 0.548 & 0.129 & 57.757 & 1.358 & 0.027 & 0.008229 & 0.002356 & 0.083206 & 37.343 \\
\hline 2/9/08 4:00 & 1.527 & 0.548 & 0.129 & 57.757 & 1.358 & 0.027 & 0.008229 & 0.002356 & 0.083206 & 37.343 \\
\hline 2/9/08 5:00 & 1.524 & 0.545 & 0.127 & 56.981 & 1.358 & 0.027 & 0.008229 & 0.002356 & 0.083206 & 37.343 \\
\hline 2/9/08 6:00 & 1.524 & 0.545 & 0.127 & 56.981 & 1.358 & 0.027 & 0.008229 & 0.002356 & 0.083206 & 37.343 \\
\hline 2/9/08 7:00 & 1.522 & 0.543 & 0.126 & 56.467 & 1.358 & 0.027 & 0.008229 & 0.002356 & 0.083206 & 37.343 \\
\hline 2/9/08 8:00 & 1.522 & 0.543 & 0.126 & 56.467 & 1.358 & 0.027 & 0.008229 & 0.002356 & 0.083206 & 37.343 \\
\hline 2/9/08 9:00 & 1.515 & 0.536 & 0.122 & 54.690 & 1.356 & 0.025 & 0.007620 & 0.002126 & 0.075100 & 33.705 \\
\hline 2/9/08 10:00 & 1.515 & 0.536 & 0.122 & 54.690 & 1.356 & 0.025 & 0.007620 & 0.002126 & 0.075100 & 33.705 \\
\hline 2/9/08 11:00 & 1.515 & 0.536 & 0.122 & 54.690 & 1.358 & 0.027 & 0.008229 & 0.002356 & 0.083206 & 37.343 \\
\hline 2/9/08 12:00 & 1.51 & 0.531 & 0.119 & 53.441 & 1.358 & 0.027 & 0.008229 & 0.002356 & 0.083206 & 37.343 \\
\hline 2/9/08 13:00 & 1.512 & 0.533 & 0.120 & 53.939 & 1.356 & 0.025 & 0.007620 & 0.002126 & 0.075100 & 33.705 \\
\hline
\end{tabular}




\begin{tabular}{|c|c|c|c|c|c|c|c|c|c|c|}
\hline \multirow[b]{3}{*}{ Date Time } & \multicolumn{4}{|c|}{ V-Notch Weir } & \multicolumn{6}{|c|}{ R-Notch Weir } \\
\hline & \multicolumn{2}{|c|}{ Measured Water Level } & \multicolumn{2}{|c|}{$\begin{array}{c}\text { Calculated Rate } \\
\left(\mathrm{ft}^{3} / \mathrm{s}=448.8 \mathrm{gpm}\right)\end{array}$} & \multicolumn{3}{|c|}{ Measured Water Level } & \multicolumn{3}{|c|}{$\begin{array}{c}\text { Calculated Rate } \\
\left(\mathrm{ft}^{3} / \mathrm{s}=448.8 \mathrm{gpm}\right)\end{array}$} \\
\hline & $\begin{array}{c}\text { (ft above } \\
\text { sensor) }\end{array}$ & $\begin{array}{c}\text { (ft above } \\
\text { notch) }\end{array}$ & $\left(\mathrm{ft}^{3} / \mathrm{s}\right)$ & (gpm) & $\begin{array}{l}\text { (ft above } \\
\text { sensor) }\end{array}$ & $\begin{array}{c}\text { (ft above } \\
\text { log) }\end{array}$ & $\begin{array}{c}\text { (m above } \\
\text { log) }\end{array}$ & $\left(\mathrm{m}^{3} / \mathrm{s}\right)$ & $\left(\mathrm{ft}^{3} / \mathrm{s}\right)$ & (gpm) \\
\hline 2/9/08 14:00 & 1.537 & 0.558 & 0.135 & 60.391 & 1.356 & 0.025 & 0.007620 & 0.002126 & 0.075100 & 33.705 \\
\hline 2/9/08 15:00 & 1.5 & 0.521 & 0.114 & 50.996 & 1.358 & 0.027 & 0.008229 & 0.002356 & 0.083206 & 37.343 \\
\hline 2/9/08 16:00 & 1.511 & 0.532 & 0.120 & 53.689 & 1.358 & 0.027 & 0.008229 & 0.002356 & 0.083206 & 37.343 \\
\hline 2/9/08 17:00 & 1.509 & 0.530 & 0.119 & 53.194 & 1.356 & 0.025 & 0.007620 & 0.002126 & 0.075100 & 33.705 \\
\hline 2/9/08 18:00 & 1.514 & 0.535 & 0.121 & 54.439 & 1.356 & 0.025 & 0.007620 & 0.002126 & 0.075100 & 33.705 \\
\hline 2/9/08 19:00 & 1.507 & 0.528 & 0.117 & 52.700 & 1.356 & 0.025 & 0.007620 & 0.002126 & 0.075100 & 33.705 \\
\hline 2/9/08 20:00 & 1.507 & 0.528 & 0.117 & 52.700 & 1.356 & 0.025 & 0.007620 & 0.002126 & 0.075100 & 33.705 \\
\hline 2/9/08 21:00 & 1.512 & 0.533 & 0.120 & 53.939 & 1.353 & 0.022 & 0.006705 & 0.001797 & 0.063473 & 28.487 \\
\hline 2/9/08 22:00 & 1.52 & 0.541 & 0.125 & 55.956 & 1.353 & 0.022 & 0.006705 & 0.001797 & 0.063473 & 28.487 \\
\hline 2/9/08 23:00 & 1.508 & 0.529 & 0.118 & 52.947 & 1.356 & 0.025 & 0.007620 & 0.002126 & 0.075100 & 33.705 \\
\hline 2/10/08 0:00 & 1.509 & 0.530 & 0.119 & 53.194 & 1.354 & 0.023 & 0.007010 & 0.001905 & 0.067276 & 30.194 \\
\hline 2/10/08 1:00 & 1.516 & 0.537 & 0.122 & 54.942 & 1.354 & 0.023 & 0.007010 & 0.001905 & 0.067276 & 30.194 \\
\hline 2/10/08 2:00 & 1.495 & 0.516 & 0.111 & 49.799 & 1.354 & 0.023 & 0.007010 & 0.001905 & 0.067276 & 30.194 \\
\hline 2/10/08 3:00 & 1.505 & 0.526 & 0.116 & 52.210 & 1.354 & 0.023 & 0.007010 & 0.001905 & 0.067276 & 30.194 \\
\hline 2/10/08 4:00 & 1.476 & 0.497 & 0.101 & 45.404 & 1.356 & 0.025 & 0.007620 & 0.002126 & 0.075100 & 33.705 \\
\hline 2/10/08 5:00 & 1.465 & 0.486 & 0.096 & 42.971 & 1.361 & 0.03 & 0.009144 & 0.002714 & 0.095873 & 43.028 \\
\hline 2/10/08 6:00 & 1.465 & 0.486 & 0.096 & 42.971 & 1.368 & 0.037 & 0.011277 & 0.003614 & 0.127660 & 57.294 \\
\hline 2/10/08 7:00 & 1.458 & 0.479 & 0.092 & 41.464 & 1.375 & 0.044 & 0.013411 & 0.004596 & 0.162345 & 72.860 \\
\hline 2/10/08 8:00 & 1.439 & 0.460 & 0.084 & 37.535 & 1.384 & 0.053 & 0.016154 & 0.005969 & 0.210842 & 94.626 \\
\hline 2/10/08 9:00 & 1.425 & 0.446 & 0.078 & 34.789 & 1.389 & 0.058 & 0.017678 & 0.006782 & 0.239555 & 107.512 \\
\hline 2/10/08 10:00 & 1.458 & 0.479 & 0.092 & 41.464 & 1.394 & 0.063 & 0.019201 & 0.007629 & 0.269465 & 120.936 \\
\hline 2/10/08 11:00 & 1.49 & 0.511 & 0.108 & 48.618 & 1.389 & 0.058 & 0.017678 & 0.006782 & 0.239555 & 107.512 \\
\hline 2/10/08 12:00 & 1.509 & 0.530 & 0.119 & 53.194 & 1.375 & 0.044 & 0.013411 & 0.004596 & 0.162345 & 72.860 \\
\hline 2/10/08 13:00 & 1.525 & 0.546 & 0.128 & 57.239 & 1.363 & 0.032 & 0.009753 & 0.002963 & 0.104644 & 46.964 \\
\hline 2/10/08 14:00 & 1.498 & 0.519 & 0.113 & 50.515 & 1.356 & 0.025 & 0.007620 & 0.002126 & 0.075100 & 33.705 \\
\hline
\end{tabular}




\begin{tabular}{|c|c|c|c|c|c|c|c|c|c|c|}
\hline \multirow[b]{3}{*}{ Date Time } & \multicolumn{4}{|c|}{ V-Notch Weir } & \multicolumn{6}{|c|}{ R-Notch Weir } \\
\hline & \multicolumn{2}{|c|}{ Measured Water Level } & \multicolumn{2}{|c|}{$\begin{array}{c}\text { Calculated Rate } \\
\left(\mathrm{ft}^{3} / \mathrm{s}=448.8 \mathrm{gpm}\right)\end{array}$} & \multicolumn{3}{|c|}{ Measured Water Level } & \multicolumn{3}{|c|}{$\begin{array}{c}\text { Calculated Rate } \\
\left(\mathrm{ft}^{3} / \mathrm{s}=448.8 \mathrm{gpm}\right)\end{array}$} \\
\hline & $\begin{array}{c}\text { (ft above } \\
\text { sensor) }\end{array}$ & $\begin{array}{c}\text { (ft above } \\
\text { notch) }\end{array}$ & $\left(\mathrm{ft}^{3} / \mathrm{s}\right)$ & (gpm) & $\begin{array}{l}\text { (ft above } \\
\text { sensor) }\end{array}$ & $\begin{array}{c}\text { (ft above } \\
\text { log) }\end{array}$ & $\begin{array}{c}\text { (m above } \\
\text { log) }\end{array}$ & $\left(\mathrm{m}^{3} / \mathrm{s}\right)$ & $\left(\mathrm{ft}^{3} / \mathrm{s}\right)$ & (gpm) \\
\hline 2/10/08 15:00 & 1.496 & 0.517 & 0.111 & 50.037 & 1.354 & 0.023 & 0.007010 & 0.001905 & 0.067276 & 30.194 \\
\hline 2/10/08 16:00 & 1.498 & 0.519 & 0.113 & 50.515 & 1.356 & 0.025 & 0.007620 & 0.002126 & 0.075100 & 33.705 \\
\hline 2/10/08 17:00 & 1.497 & 0.518 & 0.112 & 50.275 & 1.353 & 0.022 & 0.006705 & 0.001797 & 0.063473 & 28.487 \\
\hline 2/10/08 18:00 & 1.493 & 0.514 & 0.110 & 49.324 & 1.353 & 0.022 & 0.006705 & 0.001797 & 0.063473 & 28.487 \\
\hline 2/10/08 19:00 & 1.491 & 0.512 & 0.109 & 48.853 & 1.354 & 0.023 & 0.007010 & 0.001905 & 0.067276 & 30.194 \\
\hline 2/10/08 20:00 & 1.491 & 0.512 & 0.109 & 48.853 & 1.354 & 0.023 & 0.007010 & 0.001905 & 0.067276 & 30.194 \\
\hline 2/10/08 21:00 & 1.482 & 0.503 & 0.104 & 46.766 & 1.351 & 0.02 & 0.006096 & 0.001588 & 0.056092 & 25.174 \\
\hline 2/10/08 22:00 & 1.496 & 0.517 & 0.111 & 50.037 & 1.356 & 0.025 & 0.007620 & 0.002126 & 0.075100 & 33.705 \\
\hline 2/10/08 23:00 & 1.48 & 0.501 & 0.103 & 46.309 & 1.354 & 0.023 & 0.007010 & 0.001905 & 0.067276 & 30.194 \\
\hline 2/11/08 0:00 & 1.473 & 0.494 & 0.100 & 44.732 & 1.351 & 0.02 & 0.006096 & 0.001588 & 0.056092 & 25.174 \\
\hline 2/11/08 1:00 & 1.467 & 0.488 & 0.097 & 43.407 & 1.351 & 0.02 & 0.006096 & 0.001588 & 0.056092 & 25.174 \\
\hline 2/11/08 2:00 & 1.469 & 0.490 & 0.098 & 43.846 & 1.351 & 0.02 & 0.006096 & 0.001588 & 0.056092 & 25.174 \\
\hline 2/11/08 3:00 & 1.46 & 0.481 & 0.093 & 41.891 & 1.354 & 0.023 & 0.007010 & 0.001905 & 0.067276 & 30.194 \\
\hline 2/11/08 4:00 & 1.455 & 0.476 & 0.091 & 40.828 & 1.361 & 0.03 & 0.009144 & 0.002714 & 0.095873 & 43.028 \\
\hline 2/11/08 5:00 & 1.434 & 0.455 & 0.081 & 36.540 & 1.366 & 0.035 & 0.010667 & 0.003349 & 0.118271 & 53.080 \\
\hline 2/11/08 6:00 & 1.427 & 0.448 & 0.078 & 35.174 & 1.377 & 0.046 & 0.014020 & 0.004891 & 0.172754 & 77.532 \\
\hline 2/11/08 7:00 & 1.422 & 0.443 & 0.076 & 34.217 & 1.389 & 0.058 & 0.017678 & 0.006782 & 0.239555 & 107.512 \\
\hline 2/11/08 8:00 & 1.427 & 0.448 & 0.078 & 35.174 & 1.396 & 0.065 & 0.019811 & 0.007977 & 0.281754 & 126.451 \\
\hline 2/11/08 9:00 & 1.446 & 0.467 & 0.087 & 38.955 & 1.396 & 0.065 & 0.019811 & 0.007977 & 0.281754 & 126.451 \\
\hline 2/11/08 10:00 & 1.448 & 0.469 & 0.088 & 39.367 & 1.392 & 0.061 & 0.018592 & 0.007287 & 0.257360 & 115.503 \\
\hline 2/11/08 11:00 & 1.486 & 0.507 & 0.106 & 47.687 & 1.389 & 0.058 & 0.017678 & 0.006782 & 0.239555 & 107.512 \\
\hline 2/11/08 12:00 & 1.492 & 0.513 & 0.109 & 49.088 & 1.368 & 0.037 & 0.011277 & 0.003614 & 0.127660 & 57.294 \\
\hline 2/11/08 13:00 & 1.494 & 0.515 & 0.110 & 49.561 & 1.361 & 0.03 & 0.009144 & 0.002714 & 0.095873 & 43.028 \\
\hline 2/11/08 14:00 & 1.492 & 0.513 & 0.109 & 49.088 & 1.356 & 0.025 & 0.007620 & 0.002126 & 0.075100 & 33.705 \\
\hline 2/11/08 15:00 & 1.477 & 0.498 & 0.102 & 45.629 & 1.356 & 0.025 & 0.007620 & 0.002126 & 0.075100 & 33.705 \\
\hline
\end{tabular}




\begin{tabular}{|c|c|c|c|c|c|c|c|c|c|c|}
\hline \multirow[b]{3}{*}{ Date Time } & \multicolumn{4}{|c|}{ V-Notch Weir } & \multicolumn{6}{|c|}{ R-Notch Weir } \\
\hline & \multicolumn{2}{|c|}{ Measured Water Level } & \multicolumn{2}{|c|}{$\begin{array}{c}\text { Calculated Rate } \\
\left(\mathrm{ft}^{3} / \mathrm{s}=448.8 \mathrm{gpm}\right)\end{array}$} & \multicolumn{3}{|c|}{ Measured Water Level } & \multicolumn{3}{|c|}{$\begin{array}{c}\text { Calculated Rate } \\
\left(\mathrm{ft}^{3} / \mathrm{s}=448.8 \mathrm{gpm}\right)\end{array}$} \\
\hline & $\begin{array}{c}\text { (ft above } \\
\text { sensor) }\end{array}$ & $\begin{array}{c}\text { (ft above } \\
\text { notch) }\end{array}$ & $\left(\mathrm{ft}^{3} / \mathrm{s}\right)$ & (gpm) & $\begin{array}{l}\text { (ft above } \\
\text { sensor) }\end{array}$ & $\begin{array}{c}\text { (ft above } \\
\text { log) }\end{array}$ & $\begin{array}{c}\text { (m above } \\
\text { log) }\end{array}$ & $\left(\mathrm{m}^{3} / \mathrm{s}\right)$ & $\left(\mathrm{ft}^{3} / \mathrm{s}\right)$ & (gpm) \\
\hline 2/11/08 16:00 & 1.501 & 0.522 & 0.114 & 51.237 & 1.356 & 0.025 & 0.007620 & 0.002126 & 0.075100 & 33.705 \\
\hline 2/11/08 17:00 & 1.493 & 0.514 & 0.110 & 49.324 & 1.354 & 0.023 & 0.007010 & 0.001905 & 0.067276 & 30.194 \\
\hline 2/11/08 18:00 & 1.493 & 0.514 & 0.110 & 49.324 & 1.354 & 0.023 & 0.007010 & 0.001905 & 0.067276 & 30.194 \\
\hline 2/11/08 19:00 & 1.489 & 0.510 & 0.108 & 48.384 & 1.356 & 0.025 & 0.007620 & 0.002126 & 0.075100 & 33.705 \\
\hline 2/11/08 20:00 & 1.489 & 0.510 & 0.108 & 48.384 & 1.354 & 0.023 & 0.007010 & 0.001905 & 0.067276 & 30.194 \\
\hline 2/11/08 21:00 & 1.482 & 0.503 & 0.104 & 46.766 & 1.354 & 0.023 & 0.007010 & 0.001905 & 0.067276 & 30.194 \\
\hline 2/11/08 22:00 & 1.487 & 0.508 & 0.107 & 47.919 & 1.354 & 0.023 & 0.007010 & 0.001905 & 0.067276 & 30.194 \\
\hline 2/11/08 23:00 & 1.476 & 0.497 & 0.101 & 45.404 & 1.351 & 0.02 & 0.006096 & 0.001588 & 0.056092 & 25.174 \\
\hline 2/12/08 0:00 & 1.476 & 0.497 & 0.101 & 45.404 & 1.351 & 0.02 & 0.006096 & 0.001588 & 0.056092 & 25.174 \\
\hline 2/12/08 1:00 & 1.471 & 0.492 & 0.099 & 44.288 & 1.351 & 0.02 & 0.006096 & 0.001588 & 0.056092 & 25.174 \\
\hline 2/12/08 2:00 & 1.476 & 0.497 & 0.101 & 45.404 & 1.351 & 0.02 & 0.006096 & 0.001588 & 0.056092 & 25.174 \\
\hline 2/12/08 3:00 & 1.474 & 0.495 & 0.100 & 44.956 & 1.351 & 0.02 & 0.006096 & 0.001588 & 0.056092 & 25.174 \\
\hline 2/12/08 4:00 & 1.476 & 0.497 & 0.101 & 45.404 & 1.351 & 0.02 & 0.006096 & 0.001588 & 0.056092 & 25.174 \\
\hline 2/12/08 5:00 & 1.471 & 0.492 & 0.099 & 44.288 & 1.351 & 0.02 & 0.006096 & 0.001588 & 0.056092 & 25.174 \\
\hline 2/12/08 6:00 & 1.483 & 0.504 & 0.105 & 46.995 & 1.351 & 0.02 & 0.006096 & 0.001588 & 0.056092 & 25.174 \\
\hline 2/12/08 7:00 & 1.467 & 0.488 & 0.097 & 43.407 & 1.351 & 0.02 & 0.006096 & 0.001588 & 0.056092 & 25.174 \\
\hline 2/12/08 8:00 & 1.467 & 0.488 & 0.097 & 43.407 & 1.351 & 0.02 & 0.006096 & 0.001588 & 0.056092 & 25.174 \\
\hline 2/12/08 9:00 & 1.467 & 0.488 & 0.097 & 43.407 & 1.351 & 0.02 & 0.006096 & 0.001588 & 0.056092 & 25.174 \\
\hline 2/12/08 10:00 & 1.467 & 0.488 & 0.097 & 43.407 & 1.354 & 0.023 & 0.007010 & 0.001905 & 0.067276 & 30.194 \\
\hline 2/12/08 11:00 & 1.455 & 0.476 & 0.091 & 40.828 & 1.354 & 0.023 & 0.007010 & 0.001905 & 0.067276 & 30.194 \\
\hline 2/12/08 12:00 & 1.471 & 0.492 & 0.099 & 44.288 & 1.354 & 0.023 & 0.007010 & 0.001905 & 0.067276 & 30.194 \\
\hline 2/12/08 13:00 & 1.471 & 0.492 & 0.099 & 44.288 & 1.354 & 0.023 & 0.007010 & 0.001905 & 0.067276 & 30.194 \\
\hline 2/12/08 14:00 & 1.477 & 0.498 & 0.102 & 45.629 & 1.354 & 0.023 & 0.007010 & 0.001905 & 0.067276 & 30.194 \\
\hline 2/12/08 15:00 & 1.489 & 0.510 & 0.108 & 48.384 & 1.356 & 0.025 & 0.007620 & 0.002126 & 0.075100 & 33.705 \\
\hline 2/12/08 16:00 & 1.498 & 0.519 & 0.113 & 50.515 & 1.354 & 0.023 & 0.007010 & 0.001905 & 0.067276 & 30.194 \\
\hline
\end{tabular}




\begin{tabular}{|c|c|c|c|c|c|c|c|c|c|c|}
\hline \multirow[b]{3}{*}{ Date Time } & \multicolumn{4}{|c|}{ V-Notch Weir } & \multicolumn{6}{|c|}{ R-Notch Weir } \\
\hline & \multicolumn{2}{|c|}{ Measured Water Level } & \multicolumn{2}{|c|}{$\begin{array}{c}\text { Calculated Rate } \\
\left(\mathrm{ft}^{3} / \mathrm{s}=448.8 \mathrm{gpm}\right)\end{array}$} & \multicolumn{3}{|c|}{ Measured Water Level } & \multicolumn{3}{|c|}{$\begin{array}{c}\text { Calculated Rate } \\
\left(\mathrm{ft}^{3} / \mathrm{s}=448.8 \mathrm{gpm}\right)\end{array}$} \\
\hline & $\begin{array}{c}\text { (ft above } \\
\text { sensor) }\end{array}$ & $\begin{array}{c}\text { (ft above } \\
\text { notch) }\end{array}$ & $\left(\mathrm{ft}^{3} / \mathrm{s}\right)$ & (gpm) & $\begin{array}{l}\text { (ft above } \\
\text { sensor) }\end{array}$ & $\begin{array}{c}\text { (ft above } \\
\text { log) }\end{array}$ & $\begin{array}{c}\text { (m above } \\
\text { log) }\end{array}$ & $\left(\mathrm{m}^{3} / \mathrm{s}\right)$ & $\left(\mathrm{ft}^{3} / \mathrm{s}\right)$ & (gpm) \\
\hline 2/12/08 17:00 & 1.488 & 0.509 & 0.107 & 48.151 & 1.354 & 0.023 & 0.007010 & 0.001905 & 0.067276 & 30.194 \\
\hline 2/12/08 18:00 & 1.488 & 0.509 & 0.107 & 48.151 & 1.356 & 0.025 & 0.007620 & 0.002126 & 0.075100 & 33.705 \\
\hline 2/12/08 19:00 & 1.482 & 0.503 & 0.104 & 46.766 & 1.354 & 0.023 & 0.007010 & 0.001905 & 0.067276 & 30.194 \\
\hline 2/12/08 20:00 & 1.48 & 0.501 & 0.103 & 46.309 & 1.351 & 0.02 & 0.006096 & 0.001588 & 0.056092 & 25.174 \\
\hline 2/12/08 21:00 & 1.475 & 0.496 & 0.101 & 45.180 & 1.351 & 0.02 & 0.006096 & 0.001588 & 0.056092 & 25.174 \\
\hline 2/12/08 22:00 & 1.473 & 0.494 & 0.100 & 44.732 & 1.349 & 0.018 & 0.005486 & 0.001388 & 0.049021 & 22.001 \\
\hline 2/12/08 23:00 & 1.476 & 0.497 & 0.101 & 45.404 & 1.351 & 0.02 & 0.006096 & 0.001588 & 0.056092 & 25.174 \\
\hline 2/13/08 0:00 & 1.469 & 0.490 & 0.098 & 43.846 & 1.351 & 0.02 & 0.006096 & 0.001588 & 0.056092 & 25.174 \\
\hline 2/13/08 1:00 & 1.466 & 0.487 & 0.096 & 43.188 & 1.349 & 0.018 & 0.005486 & 0.001388 & 0.049021 & 22.001 \\
\hline 2/13/08 2:00 & 1.471 & 0.492 & 0.099 & 44.288 & 1.351 & 0.02 & 0.006096 & 0.001588 & 0.056092 & 25.174 \\
\hline 2/13/08 3:00 & 1.471 & 0.492 & 0.099 & 44.288 & 1.351 & 0.02 & 0.006096 & 0.001588 & 0.056092 & 25.174 \\
\hline 2/13/08 4:00 & 1.471 & 0.492 & 0.099 & 44.288 & 1.351 & 0.02 & 0.006096 & 0.001588 & 0.056092 & 25.174 \\
\hline 2/13/08 5:00 & 1.478 & 0.499 & 0.102 & 45.855 & 1.351 & 0.02 & 0.006096 & 0.001588 & 0.056092 & 25.174 \\
\hline 2/13/08 6:00 & 1.471 & 0.492 & 0.099 & 44.288 & 1.351 & 0.02 & 0.006096 & 0.001588 & 0.056092 & 25.174 \\
\hline 2/13/08 7:00 & 1.474 & 0.495 & 0.100 & 44.956 & 1.349 & 0.018 & 0.005486 & 0.001388 & 0.049021 & 22.001 \\
\hline 2/13/08 8:00 & 1.469 & 0.490 & 0.098 & 43.846 & 1.351 & 0.02 & 0.006096 & 0.001588 & 0.056092 & 25.174 \\
\hline 2/13/08 9:00 & 1.471 & 0.492 & 0.099 & 44.288 & 1.349 & 0.018 & 0.005486 & 0.001388 & 0.049021 & 22.001 \\
\hline 2/13/08 10:00 & 1.467 & 0.488 & 0.097 & 43.407 & 1.349 & 0.018 & 0.005486 & 0.001388 & 0.049021 & 22.001 \\
\hline 2/13/08 11:00 & 1.464 & 0.485 & 0.095 & 42.753 & 1.349 & 0.018 & 0.005486 & 0.001388 & 0.049021 & 22.001 \\
\hline 2/13/08 12:00 & 1.473 & 0.494 & 0.100 & 44.732 & 1.351 & 0.02 & 0.006096 & 0.001588 & 0.056092 & 25.174 \\
\hline 2/13/08 13:00 & 1.496 & 0.517 & 0.111 & 50.037 & 1.356 & 0.025 & 0.007620 & 0.002126 & 0.075100 & 33.705 \\
\hline 2/13/08 14:00 & 1.491 & 0.512 & 0.109 & 48.853 & 1.356 & 0.025 & 0.007620 & 0.002126 & 0.075100 & 33.705 \\
\hline 2/13/08 15:00 & 1.502 & 0.523 & 0.115 & 51.479 & 1.356 & 0.025 & 0.007620 & 0.002126 & 0.075100 & 33.705 \\
\hline 2/13/08 16:00 & 1.511 & 0.532 & 0.120 & 53.689 & 1.356 & 0.025 & 0.007620 & 0.002126 & 0.075100 & 33.705 \\
\hline 2/13/08 17:00 & 1.53 & 0.551 & 0.130 & 58.540 & 1.358 & 0.027 & 0.008229 & 0.002356 & 0.083206 & 37.343 \\
\hline
\end{tabular}




\begin{tabular}{|c|c|c|c|c|c|c|c|c|c|c|}
\hline \multirow[b]{3}{*}{ Date Time } & \multicolumn{4}{|c|}{ V-Notch Weir } & \multicolumn{6}{|c|}{ R-Notch Weir } \\
\hline & \multicolumn{2}{|c|}{ Measured Water Level } & \multicolumn{2}{|c|}{$\begin{array}{c}\text { Calculated Rate } \\
\left(\mathrm{ft}^{3} / \mathrm{s}=448.8 \mathrm{gpm}\right)\end{array}$} & \multicolumn{3}{|c|}{ Measured Water Level } & \multicolumn{3}{|c|}{$\begin{array}{c}\text { Calculated Rate } \\
\left(\mathrm{ft}^{3} / \mathrm{s}=448.8 \mathrm{gpm}\right)\end{array}$} \\
\hline & $\begin{array}{c}\text { (ft above } \\
\text { sensor) }\end{array}$ & $\begin{array}{c}\text { (ft above } \\
\text { notch) }\end{array}$ & $\left(\mathrm{ft}^{3} / \mathrm{s}\right)$ & (gpm) & $\begin{array}{l}\text { (ft above } \\
\text { sensor) }\end{array}$ & $\begin{array}{c}\text { (ft above } \\
\text { log) }\end{array}$ & $\begin{array}{c}\text { (m above } \\
\text { log) }\end{array}$ & $\left(\mathrm{m}^{3} / \mathrm{s}\right)$ & $\left(\mathrm{ft}^{3} / \mathrm{s}\right)$ & (gpm) \\
\hline 2/13/08 18:00 & 1.53 & 0.551 & 0.131 & 58.585 & 1.358 & 0.027 & 0.008229 & 0.002356 & 0.083206 & 37.343 \\
\hline 2/13/08 19:00 & 1.526 & 0.547 & 0.128 & 57.542 & 1.361 & 0.03 & 0.009144 & 0.002714 & 0.095873 & 43.028 \\
\hline 2/13/08 20:00 & 1.521 & 0.542 & 0.125 & 56.255 & 1.358 & 0.027 & 0.008229 & 0.002356 & 0.083206 & 37.343 \\
\hline 2/13/08 21:00 & 1.522 & 0.543 & 0.126 & 56.511 & 1.358 & 0.027 & 0.008229 & 0.002356 & 0.083206 & 37.343 \\
\hline 2/13/08 22:00 & 1.52 & 0.541 & 0.125 & 55.999 & 1.356 & 0.025 & 0.007620 & 0.002126 & 0.075100 & 33.705 \\
\hline 2/13/08 23:00 & 1.508 & 0.529 & 0.118 & 52.988 & 1.358 & 0.027 & 0.008229 & 0.002356 & 0.083206 & 37.343 \\
\hline 2/14/08 0:00 & 1.513 & 0.534 & 0.121 & 54.231 & 1.356 & 0.025 & 0.007620 & 0.002126 & 0.075100 & 33.705 \\
\hline 2/14/08 1:00 & 1.51 & 0.531 & 0.119 & 53.483 & 1.356 & 0.025 & 0.007620 & 0.002126 & 0.075100 & 33.705 \\
\hline 2/14/08 2:00 & 1.511 & 0.532 & 0.120 & 53.732 & 1.356 & 0.025 & 0.007620 & 0.002126 & 0.075100 & 33.705 \\
\hline 2/14/08 3:00 & 1.513 & 0.534 & 0.121 & 54.231 & 1.356 & 0.025 & 0.007620 & 0.002126 & 0.075100 & 33.705 \\
\hline 2/14/08 4:00 & 1.511 & 0.532 & 0.120 & 53.732 & 1.356 & 0.025 & 0.007620 & 0.002126 & 0.075100 & 33.705 \\
\hline 2/14/08 5:00 & 1.508 & 0.529 & 0.118 & 52.988 & 1.356 & 0.025 & 0.007620 & 0.002126 & 0.075100 & 33.705 \\
\hline 2/14/08 6:00 & 1.513 & 0.534 & 0.121 & 54.231 & 1.356 & 0.025 & 0.007620 & 0.002126 & 0.075100 & 33.705 \\
\hline 2/14/08 7:00 & 1.511 & 0.532 & 0.120 & 53.732 & 1.356 & 0.025 & 0.007620 & 0.002126 & 0.075100 & 33.705 \\
\hline 2/14/08 8:00 & 1.508 & 0.529 & 0.118 & 52.988 & 1.356 & 0.025 & 0.007620 & 0.002126 & 0.075100 & 33.705 \\
\hline 2/14/08 9:00 & 1.511 & 0.532 & 0.120 & 53.732 & 1.358 & 0.027 & 0.008229 & 0.002356 & 0.083206 & 37.343 \\
\hline 2/14/08 10:00 & 1.511 & 0.532 & 0.120 & 53.732 & 1.356 & 0.025 & 0.007620 & 0.002126 & 0.075100 & 33.705 \\
\hline 2/14/08 11:00 & 1.508 & 0.529 & 0.118 & 52.988 & 1.354 & 0.023 & 0.007010 & 0.001905 & 0.067276 & 30.194 \\
\hline 2/14/08 12:00 & 1.506 & 0.527 & 0.117 & 52.496 & 1.354 & 0.023 & 0.007010 & 0.001905 & 0.067276 & 30.194 \\
\hline 2/14/08 13:00 & 1.504 & 0.525 & 0.116 & 52.007 & 1.356 & 0.025 & 0.007620 & 0.002126 & 0.075100 & 33.705 \\
\hline 2/14/08 14:00 & 1.52 & 0.541 & 0.125 & 55.999 & 1.356 & 0.025 & 0.007620 & 0.002126 & 0.075100 & 33.705 \\
\hline 2/14/08 15:00 & 1.513 & 0.534 & 0.121 & 54.231 & 1.356 & 0.025 & 0.007620 & 0.002126 & 0.075100 & 33.705 \\
\hline 2/14/08 16:00 & 1.499 & 0.520 & 0.113 & 50.796 & 1.354 & 0.023 & 0.007010 & 0.001905 & 0.067276 & 30.194 \\
\hline 2/14/08 17:00 & 1.501 & 0.522 & 0.114 & 51.278 & 1.354 & 0.023 & 0.007010 & 0.001905 & 0.067276 & 30.194 \\
\hline 2/14/08 18:00 & 1.49 & 0.511 & 0.108 & 48.658 & 1.354 & 0.023 & 0.007010 & 0.001905 & 0.067276 & 30.194 \\
\hline
\end{tabular}




\begin{tabular}{|c|c|c|c|c|c|c|c|c|c|c|}
\hline \multirow[b]{3}{*}{ Date Time } & \multicolumn{4}{|c|}{ V-Notch Weir } & \multicolumn{6}{|c|}{ R-Notch Weir } \\
\hline & \multicolumn{2}{|c|}{ Measured Water Level } & \multicolumn{2}{|c|}{$\begin{array}{c}\text { Calculated Rate } \\
\left(\mathrm{ft}^{3} / \mathrm{s}=448.8 \mathrm{gpm}\right)\end{array}$} & \multicolumn{3}{|c|}{ Measured Water Level } & \multicolumn{3}{|c|}{$\begin{array}{c}\text { Calculated Rate } \\
\left(\mathrm{ft}^{3} / \mathrm{s}=448.8 \mathrm{gpm}\right)\end{array}$} \\
\hline & $\begin{array}{c}\text { (ft above } \\
\text { sensor) }\end{array}$ & $\begin{array}{c}\text { (ft above } \\
\text { notch) }\end{array}$ & $\left(\mathrm{ft}^{3} / \mathrm{s}\right)$ & (gpm) & $\begin{array}{l}\text { (ft above } \\
\text { sensor) }\end{array}$ & $\begin{array}{c}\text { (ft above } \\
\text { log) }\end{array}$ & $\begin{array}{c}\text { (m above } \\
\text { log) }\end{array}$ & $\left(\mathrm{m}^{3} / \mathrm{s}\right)$ & $\left(\mathrm{ft}^{3} / \mathrm{s}\right)$ & (gpm) \\
\hline 2/14/08 19:00 & 1.502 & 0.523 & 0.115 & 51.520 & 1.354 & 0.023 & 0.007010 & 0.001905 & 0.067276 & 30.194 \\
\hline 2/14/08 20:00 & 1.483 & 0.504 & 0.105 & 47.034 & 1.354 & 0.023 & 0.007010 & 0.001905 & 0.067276 & 30.194 \\
\hline 2/14/08 21:00 & 1.486 & 0.507 & 0.106 & 47.726 & 1.354 & 0.023 & 0.007010 & 0.001905 & 0.067276 & 30.194 \\
\hline 2/14/08 22:00 & 1.488 & 0.509 & 0.107 & 48.191 & 1.354 & 0.023 & 0.007010 & 0.001905 & 0.067276 & 30.194 \\
\hline 2/14/08 23:00 & 1.479 & 0.500 & 0.103 & 46.120 & 1.351 & 0.02 & 0.006096 & 0.001588 & 0.056092 & 25.174 \\
\hline 2/15/08 0:00 & 1.469 & 0.490 & 0.098 & 43.884 & 1.354 & 0.023 & 0.007010 & 0.001905 & 0.067276 & 30.194 \\
\hline 2/15/08 1:00 & 1.474 & 0.495 & 0.100 & 44.994 & 1.356 & 0.025 & 0.007620 & 0.002126 & 0.075100 & 33.705 \\
\hline 2/15/08 2:00 & 1.469 & 0.490 & 0.098 & 43.884 & 1.356 & 0.025 & 0.007620 & 0.002126 & 0.075100 & 33.705 \\
\hline 2/15/08 3:00 & 1.467 & 0.488 & 0.097 & 43.444 & 1.356 & 0.025 & 0.007620 & 0.002126 & 0.075100 & 33.705 \\
\hline 2/15/08 4:00 & 1.462 & 0.483 & 0.094 & 42.357 & 1.356 & 0.025 & 0.007620 & 0.002126 & 0.075100 & 33.705 \\
\hline 2/15/08 5:00 & 1.465 & 0.486 & 0.096 & 43.008 & 1.356 & 0.025 & 0.007620 & 0.002126 & 0.075100 & 33.705 \\
\hline 2/15/08 6:00 & 1.467 & 0.488 & 0.097 & 43.444 & 1.354 & 0.023 & 0.007010 & 0.001905 & 0.067276 & 30.194 \\
\hline 2/15/08 7:00 & 1.462 & 0.483 & 0.094 & 42.357 & 1.354 & 0.023 & 0.007010 & 0.001905 & 0.067276 & 30.194 \\
\hline 2/15/08 8:00 & 1.458 & 0.479 & 0.092 & 41.500 & 1.351 & 0.02 & 0.006096 & 0.001588 & 0.056092 & 25.174 \\
\hline 2/15/08 9:00 & 1.458 & 0.479 & 0.092 & 41.500 & 1.351 & 0.02 & 0.006096 & 0.001588 & 0.056092 & 25.174 \\
\hline 2/15/08 10:00 & 1.462 & 0.483 & 0.094 & 42.357 & 1.349 & 0.018 & 0.005486 & 0.001388 & 0.049021 & 22.001 \\
\hline 2/15/08 11:00 & 1.464 & 0.485 & 0.095 & 42.790 & 1.351 & 0.02 & 0.006096 & 0.001588 & 0.056092 & 25.174 \\
\hline 2/15/08 12:00 & 1.466 & 0.487 & 0.096 & 43.226 & 1.351 & 0.02 & 0.006096 & 0.001588 & 0.056092 & 25.174 \\
\hline 2/15/08 13:00 & 1.47 & 0.491 & 0.098 & 44.104 & 1.351 & 0.02 & 0.006096 & 0.001588 & 0.056092 & 25.174 \\
\hline 2/15/08 14:00 & 1.479 & 0.500 & 0.103 & 46.120 & 1.351 & 0.02 & 0.006096 & 0.001588 & 0.056092 & 25.174 \\
\hline 2/15/08 15:00 & 1.481 & 0.502 & 0.104 & 46.576 & 1.354 & 0.023 & 0.007010 & 0.001905 & 0.067276 & 30.194 \\
\hline 2/15/08 16:00 & 1.483 & 0.504 & 0.105 & 47.034 & 1.354 & 0.023 & 0.007010 & 0.001905 & 0.067276 & 30.194 \\
\hline 2/15/08 17:00 & 1.488 & 0.509 & 0.107 & 48.191 & 1.354 & 0.023 & 0.007010 & 0.001905 & 0.067276 & 30.194 \\
\hline 2/15/08 18:00 & 1.488 & 0.509 & 0.107 & 48.191 & 1.354 & 0.023 & 0.007010 & 0.001905 & 0.067276 & 30.194 \\
\hline 2/15/08 19:00 & 1.486 & 0.507 & 0.106 & 47.726 & 1.351 & 0.02 & 0.006096 & 0.001588 & 0.056092 & 25.174 \\
\hline
\end{tabular}




\begin{tabular}{|c|c|c|c|c|c|c|c|c|c|c|}
\hline \multirow[b]{3}{*}{ Date Time } & \multicolumn{4}{|c|}{ V-Notch Weir } & \multicolumn{6}{|c|}{ R-Notch Weir } \\
\hline & \multicolumn{2}{|c|}{ Measured Water Level } & \multicolumn{2}{|c|}{$\begin{array}{c}\text { Calculated Rate } \\
\left(\mathrm{ft}^{3} / \mathrm{s}=448.8 \mathrm{gpm}\right)\end{array}$} & \multicolumn{3}{|c|}{ Measured Water Level } & \multicolumn{3}{|c|}{$\begin{array}{c}\text { Calculated Rate } \\
\left(\mathrm{ft}^{3} / \mathrm{s}=448.8 \mathrm{gpm}\right)\end{array}$} \\
\hline & $\begin{array}{c}\text { (ft above } \\
\text { sensor) }\end{array}$ & $\begin{array}{c}\text { (ft above } \\
\text { notch) }\end{array}$ & $\left(\mathrm{ft}^{3} / \mathrm{s}\right)$ & (gpm) & $\begin{array}{l}\text { (ft above } \\
\text { sensor) }\end{array}$ & $\begin{array}{c}\text { (ft above } \\
\text { log) }\end{array}$ & $\begin{array}{c}\text { (m above } \\
\text { log) }\end{array}$ & $\left(\mathrm{m}^{3} / \mathrm{s}\right)$ & $\left(\mathrm{ft}^{3} / \mathrm{s}\right)$ & (gpm) \\
\hline 2/15/08 20:00 & 1.488 & 0.509 & 0.107 & 48.191 & 1.351 & 0.02 & 0.006096 & 0.001588 & 0.056092 & 25.174 \\
\hline 2/15/08 21:00 & 1.489 & 0.510 & 0.108 & 48.424 & 1.354 & 0.023 & 0.007010 & 0.001905 & 0.067276 & 30.194 \\
\hline 2/15/08 22:00 & 1.487 & 0.508 & 0.107 & 47.958 & 1.351 & 0.02 & 0.006096 & 0.001588 & 0.056092 & 25.174 \\
\hline 2/15/08 23:00 & 1.487 & 0.508 & 0.107 & 47.958 & 1.351 & 0.02 & 0.006096 & 0.001588 & 0.056092 & 25.174 \\
\hline 2/16/08 0:00 & 1.482 & 0.503 & 0.104 & 46.804 & 1.351 & 0.02 & 0.006096 & 0.001588 & 0.056092 & 25.174 \\
\hline 2/16/08 1:00 & 1.482 & 0.503 & 0.104 & 46.804 & 1.349 & 0.018 & 0.005486 & 0.001388 & 0.049021 & 22.001 \\
\hline 2/16/08 2:00 & 1.48 & 0.501 & 0.103 & 46.348 & 1.351 & 0.02 & 0.006096 & 0.001588 & 0.056092 & 25.174 \\
\hline 2/16/08 3:00 & 1.48 & 0.501 & 0.103 & 46.348 & 1.351 & 0.02 & 0.006096 & 0.001588 & 0.056092 & 25.174 \\
\hline 2/16/08 4:00 & 1.478 & 0.499 & 0.102 & 45.894 & 1.351 & 0.02 & 0.006096 & 0.001588 & 0.056092 & 25.174 \\
\hline 2/16/08 5:00 & 1.478 & 0.499 & 0.102 & 45.894 & 1.351 & 0.02 & 0.006096 & 0.001588 & 0.056092 & 25.174 \\
\hline 2/16/08 6:00 & 1.478 & 0.499 & 0.102 & 45.894 & 1.351 & 0.02 & 0.006096 & 0.001588 & 0.056092 & 25.174 \\
\hline 2/16/08 7:00 & 1.481 & 0.502 & 0.104 & 46.576 & 1.351 & 0.02 & 0.006096 & 0.001588 & 0.056092 & 25.174 \\
\hline 2/16/08 8:00 & 1.476 & 0.497 & 0.101 & 45.442 & 1.351 & 0.02 & 0.006096 & 0.001588 & 0.056092 & 25.174 \\
\hline 2/16/08 9:00 & 1.474 & 0.495 & 0.100 & 44.994 & 1.349 & 0.018 & 0.005486 & 0.001388 & 0.049021 & 22.001 \\
\hline 2/16/08 10:00 & 1.476 & 0.497 & 0.101 & 45.442 & 1.351 & 0.02 & 0.006096 & 0.001588 & 0.056092 & 25.174 \\
\hline 2/16/08 11:00 & 1.48 & 0.501 & 0.103 & 46.348 & 1.351 & 0.02 & 0.006096 & 0.001588 & 0.056092 & 25.174 \\
\hline 2/16/08 12:00 & 1.482 & 0.503 & 0.104 & 46.804 & 1.351 & 0.02 & 0.006096 & 0.001588 & 0.056092 & 25.174 \\
\hline 2/16/08 13:00 & 1.489 & 0.510 & 0.108 & 48.424 & 1.354 & 0.023 & 0.007010 & 0.001905 & 0.067276 & 30.194 \\
\hline 2/16/08 14:00 & 1.495 & 0.516 & 0.111 & 49.839 & 1.356 & 0.025 & 0.007620 & 0.002126 & 0.075100 & 33.705 \\
\hline 2/16/08 15:00 & 1.499 & 0.520 & 0.113 & 50.796 & 1.356 & 0.025 & 0.007620 & 0.002126 & 0.075100 & 33.705 \\
\hline 2/16/08 16:00 & 1.523 & 0.544 & 0.126 & 56.767 & 1.358 & 0.027 & 0.008229 & 0.002356 & 0.083206 & 37.343 \\
\hline 2/16/08 17:00 & 1.532 & 0.553 & 0.132 & 59.110 & 1.358 & 0.027 & 0.008229 & 0.002356 & 0.083206 & 37.343 \\
\hline 2/16/08 18:00 & 1.539 & 0.560 & 0.136 & 60.972 & 1.361 & 0.03 & 0.009144 & 0.002714 & 0.095873 & 43.028 \\
\hline 2/16/08 19:00 & 1.544 & 0.565 & 0.139 & 62.323 & 1.363 & 0.032 & 0.009753 & 0.002963 & 0.104644 & 46.964 \\
\hline 2/16/08 20:00 & 1.535 & 0.556 & 0.133 & 59.904 & 1.358 & 0.027 & 0.008229 & 0.002356 & 0.083206 & 37.343 \\
\hline
\end{tabular}




\begin{tabular}{|c|c|c|c|c|c|c|c|c|c|c|}
\hline \multirow[b]{3}{*}{ Date Time } & \multicolumn{4}{|c|}{ V-Notch Weir } & \multicolumn{6}{|c|}{ R-Notch Weir } \\
\hline & \multicolumn{2}{|c|}{ Measured Water Level } & \multicolumn{2}{|c|}{$\begin{array}{c}\text { Calculated Rate } \\
\left(\mathrm{ft}^{3} / \mathrm{s}=448.8 \mathrm{gpm}\right)\end{array}$} & \multicolumn{3}{|c|}{ Measured Water Level } & \multicolumn{3}{|c|}{$\begin{array}{c}\text { Calculated Rate } \\
\left(\mathrm{ft}^{3} / \mathrm{s}=448.8 \mathrm{gpm}\right)\end{array}$} \\
\hline & $\begin{array}{c}\text { (ft above } \\
\text { sensor) }\end{array}$ & $\begin{array}{c}\text { (ft above } \\
\text { notch) }\end{array}$ & $\left(\mathrm{ft}^{3} / \mathrm{s}\right)$ & (gpm) & $\begin{array}{l}\text { (ft above } \\
\text { sensor) }\end{array}$ & $\begin{array}{c}\text { (ft above } \\
\text { log) }\end{array}$ & $\begin{array}{c}\text { (m above } \\
\text { log) }\end{array}$ & $\left(\mathrm{m}^{3} / \mathrm{s}\right)$ & $\left(\mathrm{ft}^{3} / \mathrm{s}\right)$ & (gpm) \\
\hline 2/16/08 21:00 & 1.526 & 0.547 & 0.128 & 57.542 & 1.358 & 0.027 & 0.008229 & 0.002356 & 0.083206 & 37.343 \\
\hline 2/16/08 22:00 & 1.522 & 0.543 & 0.126 & 56.511 & 1.358 & 0.027 & 0.008229 & 0.002356 & 0.083206 & 37.343 \\
\hline 2/16/08 23:00 & 1.517 & 0.538 & 0.123 & 55.237 & 1.356 & 0.025 & 0.007620 & 0.002126 & 0.075100 & 33.705 \\
\hline 2/17/08 0:00 & 1.512 & 0.533 & 0.120 & 53.981 & 1.356 & 0.025 & 0.007620 & 0.002126 & 0.075100 & 33.705 \\
\hline 2/17/08 1:00 & 1.512 & 0.533 & 0.120 & 53.981 & 1.356 & 0.025 & 0.007620 & 0.002126 & 0.075100 & 33.705 \\
\hline 2/17/08 2:00 & 1.51 & 0.531 & 0.119 & 53.483 & 1.356 & 0.025 & 0.007620 & 0.002126 & 0.075100 & 33.705 \\
\hline 2/17/08 3:00 & 1.515 & 0.536 & 0.122 & 54.733 & 1.356 & 0.025 & 0.007620 & 0.002126 & 0.075100 & 33.705 \\
\hline 2/17/08 4:00 & 1.515 & 0.536 & 0.122 & 54.733 & 1.356 & 0.025 & 0.007620 & 0.002126 & 0.075100 & 33.705 \\
\hline 2/17/08 5:00 & 1.513 & 0.534 & 0.121 & 54.231 & 1.356 & 0.025 & 0.007620 & 0.002126 & 0.075100 & 33.705 \\
\hline 2/17/08 6:00 & 1.517 & 0.538 & 0.123 & 55.237 & 1.358 & 0.027 & 0.008229 & 0.002356 & 0.083206 & 37.343 \\
\hline 2/17/08 7:00 & 1.522 & 0.543 & 0.126 & 56.511 & 1.358 & 0.027 & 0.008229 & 0.002356 & 0.083206 & 37.343 \\
\hline 2/17/08 8:00 & 1.527 & 0.548 & 0.129 & 57.802 & 1.358 & 0.027 & 0.008229 & 0.002356 & 0.083206 & 37.343 \\
\hline 2/17/08 9:00 & 1.527 & 0.548 & 0.129 & 57.802 & 1.356 & 0.025 & 0.007620 & 0.002126 & 0.075100 & 33.705 \\
\hline 2/17/08 10:00 & 1.525 & 0.546 & 0.128 & 57.283 & 1.356 & 0.025 & 0.007620 & 0.002126 & 0.075100 & 33.705 \\
\hline 2/17/08 11:00 & 1.527 & 0.548 & 0.129 & 57.802 & 1.354 & 0.023 & 0.007010 & 0.001905 & 0.067276 & 30.194 \\
\hline 2/17/08 12:00 & 1.541 & 0.562 & 0.137 & 61.510 & 1.361 & 0.03 & 0.009144 & 0.002714 & 0.095873 & 43.028 \\
\hline 2/17/08 13:00 & 1.512 & 0.533 & 0.120 & 53.981 & 1.356 & 0.025 & 0.007620 & 0.002126 & 0.075100 & 33.705 \\
\hline 2/17/08 14:00 & 1.533 & 0.554 & 0.132 & 59.374 & 1.361 & 0.03 & 0.009144 & 0.002714 & 0.095873 & 43.028 \\
\hline 2/17/08 15:00 & 1.564 & 0.585 & 0.151 & 67.905 & 1.363 & 0.032 & 0.009753 & 0.002963 & 0.104644 & 46.964 \\
\hline 2/17/08 16:00 & 1.646 & 0.667 & 0.209 & 93.875 & 1.375 & 0.044 & 0.013411 & 0.004596 & 0.162345 & 72.860 \\
\hline 2/17/08 17:00 & 1.681 & 0.702 & 0.237 & 106.523 & 1.382 & 0.051 & 0.015544 & 0.005654 & 0.199703 & 89.627 \\
\hline 2/17/08 18:00 & 1.684 & 0.705 & 0.240 & 107.652 & 1.382 & 0.051 & 0.015544 & 0.005654 & 0.199703 & 89.627 \\
\hline 2/17/08 19:00 & 1.656 & 0.677 & 0.217 & 97.391 & 1.377 & 0.046 & 0.014020 & 0.004891 & 0.172754 & 77.532 \\
\hline 2/17/08 20:00 & 1.64 & 0.661 & 0.205 & 91.802 & 1.375 & 0.044 & 0.013411 & 0.004596 & 0.162345 & 72.860 \\
\hline 2/17/08 21:00 & 1.616 & 0.637 & 0.187 & 83.788 & 1.37 & 0.039 & 0.011887 & 0.003887 & 0.137285 & 61.614 \\
\hline
\end{tabular}




\begin{tabular}{|c|c|c|c|c|c|c|c|c|c|c|}
\hline \multirow[b]{3}{*}{ Date Time } & \multicolumn{4}{|c|}{ V-Notch Weir } & \multicolumn{6}{|c|}{ R-Notch Weir } \\
\hline & \multicolumn{2}{|c|}{ Measured Water Level } & \multicolumn{2}{|c|}{$\begin{array}{c}\text { Calculated Rate } \\
\left(\mathrm{ft}^{3} / \mathrm{s}=448.8 \mathrm{gpm}\right)\end{array}$} & \multicolumn{3}{|c|}{ Measured Water Level } & \multicolumn{3}{|c|}{$\begin{array}{c}\text { Calculated Rate } \\
\left(\mathrm{ft}^{3} / \mathrm{s}=448.8 \mathrm{gpm}\right)\end{array}$} \\
\hline & $\begin{array}{c}\text { (ft above } \\
\text { sensor) }\end{array}$ & $\begin{array}{c}\text { (ft above } \\
\text { notch) }\end{array}$ & $\left(\mathrm{ft}^{3} / \mathrm{s}\right)$ & (gpm) & $\begin{array}{l}\text { (ft above } \\
\text { sensor) }\end{array}$ & $\begin{array}{c}\text { (ft above } \\
\text { log) }\end{array}$ & $\begin{array}{c}\text { (m above } \\
\text { log) }\end{array}$ & $\left(\mathrm{m}^{3} / \mathrm{s}\right)$ & $\left(\mathrm{ft}^{3} / \mathrm{s}\right)$ & (gpm) \\
\hline 2/17/08 22:00 & 1.614 & 0.635 & 0.185 & 83.140 & 1.372 & 0.041 & 0.012496 & 0.004166 & 0.147141 & 66.037 \\
\hline 2/17/08 23:00 & 1.61 & 0.631 & 0.182 & 81.852 & 1.368 & 0.037 & 0.011277 & 0.003614 & 0.127660 & 57.294 \\
\hline 2/18/08 0:00 & 1.605 & 0.626 & 0.179 & 80.260 & 1.37 & 0.039 & 0.011887 & 0.003887 & 0.137285 & 61.614 \\
\hline 2/18/08 1:00 & 1.584 & 0.605 & 0.164 & 73.777 & 1.363 & 0.032 & 0.009753 & 0.002963 & 0.104644 & 46.964 \\
\hline 2/18/08 2:00 & 1.56 & 0.581 & 0.149 & 66.766 & 1.361 & 0.03 & 0.009144 & 0.002714 & 0.095873 & 43.028 \\
\hline 2/18/08 3:00 & 1.546 & 0.567 & 0.140 & 62.868 & 1.361 & 0.03 & 0.009144 & 0.002714 & 0.095873 & 43.028 \\
\hline 2/18/08 4:00 & 1.537 & 0.558 & 0.135 & 60.436 & 1.358 & 0.027 & 0.008229 & 0.002356 & 0.083206 & 37.343 \\
\hline 2/18/08 5:00 & 1.531 & 0.552 & 0.131 & 58.847 & 1.356 & 0.025 & 0.007620 & 0.002126 & 0.075100 & 33.705 \\
\hline 2/18/08 6:00 & 1.519 & 0.540 & 0.124 & 55.744 & 1.356 & 0.025 & 0.007620 & 0.002126 & 0.075100 & 33.705 \\
\hline 2/18/08 7:00 & 1.516 & 0.537 & 0.123 & 54.984 & 1.356 & 0.025 & 0.007620 & 0.002126 & 0.075100 & 33.705 \\
\hline 2/18/08 8:00 & 1.519 & 0.540 & 0.124 & 55.744 & 1.356 & 0.025 & 0.007620 & 0.002126 & 0.075100 & 33.705 \\
\hline 2/18/08 9:00 & 1.516 & 0.537 & 0.123 & 54.984 & 1.356 & 0.025 & 0.007620 & 0.002126 & 0.075100 & 33.705 \\
\hline 2/18/08 10:00 & 1.516 & 0.537 & 0.123 & 54.984 & 1.356 & 0.025 & 0.007620 & 0.002126 & 0.075100 & 33.705 \\
\hline 2/18/08 11:00 & 1.511 & 0.532 & 0.120 & 53.732 & 1.356 & 0.025 & 0.007620 & 0.002126 & 0.075100 & 33.705 \\
\hline 2/18/08 12:00 & 1.504 & 0.525 & 0.116 & 52.007 & 1.356 & 0.025 & 0.007620 & 0.002126 & 0.075100 & 33.705 \\
\hline 2/18/08 13:00 & 1.513 & 0.534 & 0.121 & 54.231 & 1.358 & 0.027 & 0.008229 & 0.002356 & 0.083206 & 37.343 \\
\hline 2/18/08 14:00 & 1.52 & 0.541 & 0.125 & 55.999 & 1.354 & 0.023 & 0.007010 & 0.001905 & 0.067276 & 30.194 \\
\hline 2/18/08 15:00 & 1.493 & 0.514 & 0.110 & 49.365 & 1.356 & 0.025 & 0.007620 & 0.002126 & 0.075100 & 33.705 \\
\hline 2/18/08 16:00 & 1.509 & 0.530 & 0.119 & 53.236 & 1.356 & 0.025 & 0.007620 & 0.002126 & 0.075100 & 33.705 \\
\hline 2/18/08 17:00 & 1.502 & 0.523 & 0.115 & 51.520 & 1.356 & 0.025 & 0.007620 & 0.002126 & 0.075100 & 33.705 \\
\hline 2/18/08 18:00 & 1.497 & 0.518 & 0.112 & 50.316 & 1.353 & 0.022 & 0.006705 & 0.001797 & 0.063473 & 28.487 \\
\hline 2/18/08 19:00 & 1.5 & 0.521 & 0.114 & 51.037 & 1.356 & 0.025 & 0.007620 & 0.002126 & 0.075100 & 33.705 \\
\hline 2/18/08 20:00 & 1.489 & 0.510 & 0.108 & 48.424 & 1.353 & 0.022 & 0.006705 & 0.001797 & 0.063473 & 28.487 \\
\hline 2/18/08 21:00 & 1.489 & 0.510 & 0.108 & 48.424 & 1.354 & 0.023 & 0.007010 & 0.001905 & 0.067276 & 30.194 \\
\hline 2/18/08 22:00 & 1.485 & 0.506 & 0.106 & 47.495 & 1.354 & 0.023 & 0.007010 & 0.001905 & 0.067276 & 30.194 \\
\hline
\end{tabular}




\begin{tabular}{|c|c|c|c|c|c|c|c|c|c|c|}
\hline \multirow[b]{3}{*}{ Date Time } & \multicolumn{4}{|c|}{ V-Notch Weir } & \multicolumn{6}{|c|}{ R-Notch Weir } \\
\hline & \multicolumn{2}{|c|}{ Measured Water Level } & \multicolumn{2}{|c|}{$\begin{array}{c}\text { Calculated Rate } \\
\left(\mathrm{ft}^{3} / \mathrm{s}=448.8 \mathrm{gpm}\right)\end{array}$} & \multicolumn{3}{|c|}{ Measured Water Level } & \multicolumn{3}{|c|}{$\begin{array}{c}\text { Calculated Rate } \\
\left(\mathrm{ft}^{3} / \mathrm{s}=448.8 \mathrm{gpm}\right)\end{array}$} \\
\hline & $\begin{array}{c}\text { (ft above } \\
\text { sensor) }\end{array}$ & $\begin{array}{c}\text { (ft above } \\
\text { notch) }\end{array}$ & $\left(\mathrm{ft}^{3} / \mathrm{s}\right)$ & (gpm) & $\begin{array}{l}\text { (ft above } \\
\text { sensor) }\end{array}$ & $\begin{array}{c}\text { (ft above } \\
\text { log) }\end{array}$ & $\begin{array}{c}\text { (m above } \\
\text { log) }\end{array}$ & $\left(\mathrm{m}^{3} / \mathrm{s}\right)$ & $\left(\mathrm{ft}^{3} / \mathrm{s}\right)$ & (gpm) \\
\hline 2/18/08 23:00 & 1.483 & 0.504 & 0.105 & 47.034 & 1.351 & 0.02 & 0.006096 & 0.001588 & 0.056092 & 25.174 \\
\hline 2/19/08 0:00 & 1.483 & 0.504 & 0.105 & 47.034 & 1.351 & 0.02 & 0.006096 & 0.001588 & 0.056092 & 25.174 \\
\hline 2/19/08 1:00 & 1.478 & 0.499 & 0.102 & 45.894 & 1.351 & 0.02 & 0.006096 & 0.001588 & 0.056092 & 25.174 \\
\hline 2/19/08 2:00 & 1.478 & 0.499 & 0.102 & 45.894 & 1.351 & 0.02 & 0.006096 & 0.001588 & 0.056092 & 25.174 \\
\hline 2/19/08 3:00 & 1.474 & 0.495 & 0.100 & 44.994 & 1.351 & 0.02 & 0.006096 & 0.001588 & 0.056092 & 25.174 \\
\hline 2/19/08 4:00 & 1.474 & 0.495 & 0.100 & 44.994 & 1.351 & 0.02 & 0.006096 & 0.001588 & 0.056092 & 25.174 \\
\hline 2/19/08 5:00 & 1.467 & 0.488 & 0.097 & 43.444 & 1.351 & 0.02 & 0.006096 & 0.001588 & 0.056092 & 25.174 \\
\hline 2/19/08 6:00 & 1.467 & 0.488 & 0.097 & 43.444 & 1.349 & 0.018 & 0.005486 & 0.001388 & 0.049021 & 22.001 \\
\hline 2/19/08 7:00 & 1.465 & 0.486 & 0.096 & 43.008 & 1.349 & 0.018 & 0.005486 & 0.001388 & 0.049021 & 22.001 \\
\hline 2/19/08 8:00 & 1.469 & 0.490 & 0.098 & 43.884 & 1.351 & 0.02 & 0.006096 & 0.001588 & 0.056092 & 25.174 \\
\hline 2/19/08 9:00 & 1.474 & 0.495 & 0.100 & 44.994 & 1.351 & 0.02 & 0.006096 & 0.001588 & 0.056092 & 25.174 \\
\hline 2/19/08 10:00 & 1.469 & 0.490 & 0.098 & 43.884 & 1.351 & 0.02 & 0.006096 & 0.001588 & 0.056092 & 25.174 \\
\hline 2/19/08 11:00 & 1.474 & 0.495 & 0.100 & 44.994 & 1.351 & 0.02 & 0.006096 & 0.001588 & 0.056092 & 25.174 \\
\hline 2/19/08 12:00 & 1.476 & 0.497 & 0.101 & 45.442 & 1.351 & 0.02 & 0.006096 & 0.001588 & 0.056092 & 25.174 \\
\hline 2/19/08 13:00 & 1.482 & 0.503 & 0.104 & 46.804 & 1.351 & 0.02 & 0.006096 & 0.001588 & 0.056092 & 25.174 \\
\hline 2/19/08 14:00 & 1.482 & 0.503 & 0.104 & 46.804 & 1.351 & 0.02 & 0.006096 & 0.001588 & 0.056092 & 25.174 \\
\hline 2/19/08 15:00 & 1.493 & 0.514 & 0.110 & 49.365 & 1.354 & 0.023 & 0.007010 & 0.001905 & 0.067276 & 30.194 \\
\hline 2/19/08 16:00 & 1.507 & 0.528 & 0.118 & 52.742 & 1.356 & 0.025 & 0.007620 & 0.002126 & 0.075100 & 33.705 \\
\hline 2/19/08 17:00 & 1.518 & 0.539 & 0.124 & 55.490 & 1.358 & 0.027 & 0.008229 & 0.002356 & 0.083206 & 37.343 \\
\hline 2/19/08 18:00 & 1.526 & 0.547 & 0.128 & 57.542 & 1.358 & 0.027 & 0.008229 & 0.002356 & 0.083206 & 37.343 \\
\hline 2/19/08 19:00 & 1.535 & 0.556 & 0.133 & 59.904 & 1.361 & 0.03 & 0.009144 & 0.002714 & 0.095873 & 43.028 \\
\hline 2/19/08 20:00 & 1.528 & 0.549 & 0.129 & 58.062 & 1.358 & 0.027 & 0.008229 & 0.002356 & 0.083206 & 37.343 \\
\hline 2/19/08 21:00 & 1.522 & 0.543 & 0.126 & 56.511 & 1.358 & 0.027 & 0.008229 & 0.002356 & 0.083206 & 37.343 \\
\hline 2/19/08 22:00 & 1.501 & 0.522 & 0.114 & 51.278 & 1.354 & 0.023 & 0.007010 & 0.001905 & 0.067276 & 30.194 \\
\hline 2/19/08 23:00 & 1.496 & 0.517 & 0.112 & 50.077 & 1.351 & 0.02 & 0.006096 & 0.001588 & 0.056092 & 25.174 \\
\hline
\end{tabular}




\begin{tabular}{|c|c|c|c|c|c|c|c|c|c|c|}
\hline \multirow[b]{3}{*}{ Date Time } & \multicolumn{4}{|c|}{ V-Notch Weir } & \multicolumn{6}{|c|}{ R-Notch Weir } \\
\hline & \multicolumn{2}{|c|}{ Measured Water Level } & \multicolumn{2}{|c|}{$\begin{array}{c}\text { Calculated Rate } \\
\left(\mathrm{ft}^{3} / \mathrm{s}=448.8 \mathrm{gpm}\right)\end{array}$} & \multicolumn{3}{|c|}{ Measured Water Level } & \multicolumn{3}{|c|}{$\begin{array}{c}\text { Calculated Rate } \\
\left(\mathrm{ft}^{3} / \mathrm{s}=448.8 \mathrm{gpm}\right)\end{array}$} \\
\hline & $\begin{array}{c}\text { (ft above } \\
\text { sensor) }\end{array}$ & $\begin{array}{c}\text { (ft above } \\
\text { notch) }\end{array}$ & $\left(\mathrm{ft}^{3} / \mathrm{s}\right)$ & (gpm) & $\begin{array}{l}\text { (ft above } \\
\text { sensor) }\end{array}$ & $\begin{array}{c}\text { (ft above } \\
\text { log) }\end{array}$ & $\begin{array}{c}\text { (m above } \\
\text { log) }\end{array}$ & $\left(\mathrm{m}^{3} / \mathrm{s}\right)$ & $\left(\mathrm{ft}^{3} / \mathrm{s}\right)$ & (gpm) \\
\hline 2/20/08 0:00 & 1.49 & 0.511 & 0.108 & 48.658 & 1.354 & 0.023 & 0.007010 & 0.001905 & 0.067276 & 30.194 \\
\hline 2/20/08 1:00 & 1.469 & 0.490 & 0.098 & 43.884 & 1.351 & 0.02 & 0.006096 & 0.001588 & 0.056092 & 25.174 \\
\hline 2/20/08 2:00 & 1.467 & 0.488 & 0.097 & 43.444 & 1.351 & 0.02 & 0.006096 & 0.001588 & 0.056092 & 25.174 \\
\hline 2/20/08 3:00 & 1.462 & 0.483 & 0.094 & 42.357 & 1.351 & 0.02 & 0.006096 & 0.001588 & 0.056092 & 25.174 \\
\hline 2/20/08 4:00 & 1.465 & 0.486 & 0.096 & 43.008 & 1.351 & 0.02 & 0.006096 & 0.001588 & 0.056092 & 25.174 \\
\hline 2/20/08 5:00 & 1.422 & 0.443 & 0.076 & 34.249 & 1.354 & 0.023 & 0.007010 & 0.001905 & 0.067276 & 30.194 \\
\hline 2/20/08 6:00 & 1.38 & 0.401 & 0.060 & 26.822 & 1.366 & 0.035 & 0.010667 & 0.003349 & 0.118271 & 53.080 \\
\hline 2/20/08 7:00 & 1.305 & 0.326 & 0.036 & 16.162 & 1.392 & 0.061 & 0.018592 & 0.007287 & 0.257360 & 115.503 \\
\hline 2/20/08 8:00 & 1.288 & 0.309 & 0.032 & 14.182 & 1.418 & 0.087 & 0.026516 & 0.012129 & 0.428377 & 192.256 \\
\hline 2/20/08 9:00 & 1.371 & 0.392 & 0.057 & 25.370 & 1.432 & 0.101 & 0.030783 & 0.015057 & 0.531798 & 238.671 \\
\hline 2/20/08 10:00 & 1.49 & 0.511 & 0.108 & 48.658 & 1.425 & 0.094 & 0.028650 & 0.013566 & 0.479156 & 215.045 \\
\hline 2/20/08 11:00 & 1.568 & 0.589 & 0.154 & 69.056 & 1.384 & 0.053 & 0.016154 & 0.005969 & 0.210842 & 94.626 \\
\hline 2/20/08 12:00 & 1.497 & 0.518 & 0.112 & 50.316 & 1.358 & 0.027 & 0.008229 & 0.002356 & 0.083206 & 37.343 \\
\hline 2/20/08 13:00 & 1.485 & 0.506 & 0.106 & 47.495 & 1.354 & 0.023 & 0.007010 & 0.001905 & 0.067276 & 30.194 \\
\hline 2/20/08 14:00 & 1.475 & 0.496 & 0.101 & 45.218 & 1.354 & 0.023 & 0.007010 & 0.001905 & 0.067276 & 30.194 \\
\hline 2/20/08 15:00 & 1.465 & 0.486 & 0.096 & 43.008 & 1.353 & 0.022 & 0.006705 & 0.001797 & 0.063473 & 28.487 \\
\hline 2/20/08 16:00 & 1.467 & 0.488 & 0.097 & 43.444 & 1.351 & 0.02 & 0.006096 & 0.001588 & 0.056092 & 25.174 \\
\hline 2/20/08 17:00 & 1.457 & 0.478 & 0.092 & 41.287 & 1.351 & 0.02 & 0.006096 & 0.001588 & 0.056092 & 25.174 \\
\hline 2/20/08 18:00 & 1.46 & 0.481 & 0.093 & 41.927 & 1.349 & 0.018 & 0.005486 & 0.001388 & 0.049021 & 22.001 \\
\hline 2/20/08 19:00 & 1.448 & 0.469 & 0.088 & 39.402 & 1.349 & 0.018 & 0.005486 & 0.001388 & 0.049021 & 22.001 \\
\hline 2/20/08 20:00 & 1.448 & 0.469 & 0.088 & 39.402 & 1.346 & 0.015 & 0.004572 & 0.001105 & 0.039030 & 17.516 \\
\hline 2/20/08 21:00 & 1.444 & 0.465 & 0.086 & 38.581 & 1.349 & 0.018 & 0.005486 & 0.001388 & 0.049021 & 22.001 \\
\hline 2/20/08 22:00 & 1.445 & 0.466 & 0.086 & 38.785 & 1.349 & 0.018 & 0.005486 & 0.001388 & 0.049021 & 22.001 \\
\hline 2/20/08 23:00 & 1.44 & 0.461 & 0.084 & 37.770 & 1.349 & 0.018 & 0.005486 & 0.001388 & 0.049021 & 22.001 \\
\hline 2/21/08 0:00 & 1.438 & 0.459 & 0.083 & 37.369 & 1.349 & 0.018 & 0.005486 & 0.001388 & 0.049021 & 22.001 \\
\hline
\end{tabular}




\begin{tabular}{|c|c|c|c|c|c|c|c|c|c|c|}
\hline \multirow[b]{3}{*}{ Date Time } & \multicolumn{4}{|c|}{ V-Notch Weir } & \multicolumn{6}{|c|}{ R-Notch Weir } \\
\hline & \multicolumn{2}{|c|}{ Measured Water Level } & \multicolumn{2}{|c|}{$\begin{array}{c}\text { Calculated Rate } \\
\left(\mathrm{ft}^{3} / \mathrm{s}=448.8 \mathrm{gpm}\right)\end{array}$} & \multicolumn{3}{|c|}{ Measured Water Level } & \multicolumn{3}{|c|}{$\begin{array}{c}\text { Calculated Rate } \\
\left(\mathrm{ft}^{3} / \mathrm{s}=448.8 \mathrm{gpm}\right)\end{array}$} \\
\hline & $\begin{array}{c}\text { (ft above } \\
\text { sensor) }\end{array}$ & $\begin{array}{c}\text { (ft above } \\
\text { notch) }\end{array}$ & $\left(\mathrm{ft}^{3} / \mathrm{s}\right)$ & (gpm) & $\begin{array}{l}\text { (ft above } \\
\text { sensor) }\end{array}$ & $\begin{array}{c}\text { (ft above } \\
\text { log) }\end{array}$ & $\begin{array}{c}\text { (m above } \\
\text { log) }\end{array}$ & $\left(\mathrm{m}^{3} / \mathrm{s}\right)$ & $\left(\mathrm{ft}^{3} / \mathrm{s}\right)$ & (gpm) \\
\hline 2/21/08 1:00 & 1.434 & 0.455 & 0.081 & 36.573 & 1.347 & 0.016 & 0.004877 & 0.001197 & 0.042276 & 18.973 \\
\hline 2/21/08 2:00 & 1.429 & 0.450 & 0.079 & 35.594 & 1.349 & 0.018 & 0.005486 & 0.001388 & 0.049021 & 22.001 \\
\hline 2/21/08 3:00 & 1.434 & 0.455 & 0.081 & 36.573 & 1.351 & 0.02 & 0.006096 & 0.001588 & 0.056092 & 25.174 \\
\hline 2/21/08 4:00 & 1.436 & 0.457 & 0.082 & 36.970 & 1.351 & 0.02 & 0.006096 & 0.001588 & 0.056092 & 25.174 \\
\hline 2/21/08 5:00 & 1.434 & 0.455 & 0.081 & 36.573 & 1.351 & 0.02 & 0.006096 & 0.001588 & 0.056092 & 25.174 \\
\hline 2/21/08 6:00 & 1.434 & 0.455 & 0.081 & 36.573 & 1.354 & 0.023 & 0.007010 & 0.001905 & 0.067276 & 30.194 \\
\hline 2/21/08 7:00 & 1.432 & 0.453 & 0.081 & 36.180 & 1.356 & 0.025 & 0.007620 & 0.002126 & 0.075100 & 33.705 \\
\hline 2/21/08 8:00 & 1.432 & 0.453 & 0.081 & 36.180 & 1.359 & 0.028 & 0.008534 & 0.002473 & 0.087362 & 39.208 \\
\hline 2/21/08 9:00 & 1.436 & 0.457 & 0.082 & 36.970 & 1.356 & 0.025 & 0.007620 & 0.002126 & 0.075100 & 33.705 \\
\hline 2/21/08 10:00 & 1.436 & 0.457 & 0.082 & 36.970 & 1.354 & 0.023 & 0.007010 & 0.001905 & 0.067276 & 30.194 \\
\hline 2/21/08 11:00 & 1.446 & 0.467 & 0.087 & 38.990 & 1.354 & 0.023 & 0.007010 & 0.001905 & 0.067276 & 30.194 \\
\hline 2/21/08 12:00 & 1.45 & 0.471 & 0.089 & 39.816 & 1.349 & 0.018 & 0.005486 & 0.001388 & 0.049021 & 22.001 \\
\hline 2/21/08 13:00 & 1.448 & 0.469 & 0.088 & 39.402 & 1.347 & 0.016 & 0.004877 & 0.001197 & 0.042276 & 18.973 \\
\hline 2/21/08 14:00 & 1.45 & 0.471 & 0.089 & 39.816 & 1.347 & 0.016 & 0.004877 & 0.001197 & 0.042276 & 18.973 \\
\hline 2/21/08 15:00 & 1.456 & 0.477 & 0.092 & 41.075 & 1.349 & 0.018 & 0.005486 & 0.001388 & 0.049021 & 22.001 \\
\hline 2/21/08 16:00 & 1.461 & 0.482 & 0.094 & 42.142 & 1.351 & 0.02 & 0.006096 & 0.001588 & 0.056092 & 25.174 \\
\hline 2/21/08 17:00 & 1.463 & 0.484 & 0.095 & 42.574 & 1.349 & 0.018 & 0.005486 & 0.001388 & 0.049021 & 22.001 \\
\hline 2/21/08 18:00 & 1.461 & 0.482 & 0.094 & 42.142 & 1.349 & 0.018 & 0.005486 & 0.001388 & 0.049021 & 22.001 \\
\hline 2/21/08 19:00 & 1.461 & 0.482 & 0.094 & 42.142 & 1.349 & 0.018 & 0.005486 & 0.001388 & 0.049021 & 22.001 \\
\hline 2/21/08 20:00 & 1.456 & 0.477 & 0.092 & 41.075 & 1.349 & 0.018 & 0.005486 & 0.001388 & 0.049021 & 22.001 \\
\hline 2/21/08 21:00 & 1.454 & 0.475 & 0.091 & 40.653 & 1.349 & 0.018 & 0.005486 & 0.001388 & 0.049021 & 22.001 \\
\hline 2/21/08 22:00 & 1.454 & 0.475 & 0.091 & 40.653 & 1.349 & 0.018 & 0.005486 & 0.001388 & 0.049021 & 22.001 \\
\hline 2/21/08 23:00 & 1.452 & 0.473 & 0.090 & 40.233 & 1.349 & 0.018 & 0.005486 & 0.001388 & 0.049021 & 22.001 \\
\hline 2/22/08 0:00 & 1.455 & 0.476 & 0.091 & 40.864 & 1.349 & 0.018 & 0.005486 & 0.001388 & 0.049021 & 22.001 \\
\hline 2/22/08 1:00 & 1.452 & 0.473 & 0.090 & 40.233 & 1.349 & 0.018 & 0.005486 & 0.001388 & 0.049021 & 22.001 \\
\hline
\end{tabular}




\begin{tabular}{|c|c|c|c|c|c|c|c|c|c|c|}
\hline \multirow[b]{3}{*}{ Date Time } & \multicolumn{4}{|c|}{ V-Notch Weir } & \multicolumn{6}{|c|}{ R-Notch Weir } \\
\hline & \multicolumn{2}{|c|}{ Measured Water Level } & \multicolumn{2}{|c|}{$\begin{array}{c}\text { Calculated Rate } \\
\left(\mathrm{ft}^{3} / \mathrm{s}=448.8 \mathrm{gpm}\right)\end{array}$} & \multicolumn{3}{|c|}{ Measured Water Level } & \multicolumn{3}{|c|}{$\begin{array}{c}\text { Calculated Rate } \\
\left(\mathrm{ft}^{3} / \mathrm{s}=448.8 \mathrm{gpm}\right)\end{array}$} \\
\hline & $\begin{array}{c}\text { (ft above } \\
\text { sensor) }\end{array}$ & $\begin{array}{c}\text { (ft above } \\
\text { notch) }\end{array}$ & $\left(\mathrm{ft}^{3} / \mathrm{s}\right)$ & (gpm) & $\begin{array}{l}\text { (ft above } \\
\text { sensor) }\end{array}$ & $\begin{array}{c}\text { (ft above } \\
\text { log) }\end{array}$ & $\begin{array}{c}\text { (m above } \\
\text { log) }\end{array}$ & $\left(\mathrm{m}^{3} / \mathrm{s}\right)$ & $\left(\mathrm{ft}^{3} / \mathrm{s}\right)$ & (gpm) \\
\hline 2/22/08 2:00 & 1.452 & 0.473 & 0.090 & 40.233 & 1.349 & 0.018 & 0.005486 & 0.001388 & 0.049021 & 22.001 \\
\hline 2/22/08 3:00 & 1.452 & 0.473 & 0.090 & 40.233 & 1.349 & 0.018 & 0.005486 & 0.001388 & 0.049021 & 22.001 \\
\hline 2/22/08 4:00 & 1.45 & 0.471 & 0.089 & 39.816 & 1.349 & 0.018 & 0.005486 & 0.001388 & 0.049021 & 22.001 \\
\hline 2/22/08 5:00 & 1.445 & 0.466 & 0.086 & 38.785 & 1.349 & 0.018 & 0.005486 & 0.001388 & 0.049021 & 22.001 \\
\hline 2/22/08 6:00 & 1.448 & 0.469 & 0.088 & 39.402 & 1.349 & 0.018 & 0.005486 & 0.001388 & 0.049021 & 22.001 \\
\hline 2/22/08 7:00 & 1.443 & 0.464 & 0.086 & 38.377 & 1.347 & 0.016 & 0.004877 & 0.001197 & 0.042276 & 18.973 \\
\hline 2/22/08 8:00 & 1.443 & 0.464 & 0.086 & 38.377 & 1.347 & 0.016 & 0.004877 & 0.001197 & 0.042276 & 18.973 \\
\hline 2/22/08 9:00 & 1.443 & 0.464 & 0.086 & 38.377 & 1.347 & 0.016 & 0.004877 & 0.001197 & 0.042276 & 18.973 \\
\hline 2/22/08 10:00 & 1.452 & 0.473 & 0.090 & 40.233 & 1.349 & 0.018 & 0.005486 & 0.001388 & 0.049021 & 22.001 \\
\hline 2/22/08 11:00 & 1.459 & 0.480 & 0.093 & 41.713 & 1.349 & 0.018 & 0.005486 & 0.001388 & 0.049021 & 22.001 \\
\hline 2/22/08 12:00 & 1.461 & 0.482 & 0.094 & 42.142 & 1.349 & 0.018 & 0.005486 & 0.001388 & 0.049021 & 22.001 \\
\hline 2/22/08 13:00 & 1.46 & 0.481 & 0.093 & 41.927 & 1.351 & 0.02 & 0.006096 & 0.001588 & 0.056092 & 25.174 \\
\hline 2/22/08 14:00 & 1.464 & 0.485 & 0.095 & 42.790 & 1.351 & 0.02 & 0.006096 & 0.001588 & 0.056092 & 25.174 \\
\hline 2/22/08 15:00 & 1.478 & 0.499 & 0.102 & 45.894 & 1.353 & 0.022 & 0.006705 & 0.001797 & 0.063473 & 28.487 \\
\hline 2/22/08 16:00 & 1.499 & 0.520 & 0.113 & 50.796 & 1.358 & 0.027 & 0.008229 & 0.002356 & 0.083206 & 37.343 \\
\hline 2/22/08 17:00 & 1.511 & 0.532 & 0.120 & 53.732 & 1.358 & 0.027 & 0.008229 & 0.002356 & 0.083206 & 37.343 \\
\hline 2/22/08 18:00 & 1.518 & 0.539 & 0.124 & 55.490 & 1.358 & 0.027 & 0.008229 & 0.002356 & 0.083206 & 37.343 \\
\hline 2/22/08 19:00 & 1.521 & 0.542 & 0.125 & 56.255 & 1.358 & 0.027 & 0.008229 & 0.002356 & 0.083206 & 37.343 \\
\hline 2/22/08 20:00 & 1.507 & 0.528 & 0.118 & 52.742 & 1.356 & 0.025 & 0.007620 & 0.002126 & 0.075100 & 33.705 \\
\hline 2/22/08 21:00 & 1.493 & 0.514 & 0.110 & 49.365 & 1.354 & 0.023 & 0.007010 & 0.001905 & 0.067276 & 30.194 \\
\hline 2/22/08 22:00 & 1.484 & 0.505 & 0.105 & 47.264 & 1.354 & 0.023 & 0.007010 & 0.001905 & 0.067276 & 30.194 \\
\hline 2/22/08 23:00 & 1.484 & 0.505 & 0.105 & 47.264 & 1.354 & 0.023 & 0.007010 & 0.001905 & 0.067276 & 30.194 \\
\hline 2/23/08 0:00 & 1.48 & 0.501 & 0.103 & 46.348 & 1.351 & 0.02 & 0.006096 & 0.001588 & 0.056092 & 25.174 \\
\hline 2/23/08 1:00 & 1.475 & 0.496 & 0.101 & 45.218 & 1.351 & 0.02 & 0.006096 & 0.001588 & 0.056092 & 25.174 \\
\hline 2/23/08 2:00 & 1.478 & 0.499 & 0.102 & 45.894 & 1.351 & 0.02 & 0.006096 & 0.001588 & 0.056092 & 25.174 \\
\hline
\end{tabular}




\begin{tabular}{|c|c|c|c|c|c|c|c|c|c|c|}
\hline \multirow[b]{3}{*}{ Date Time } & \multicolumn{4}{|c|}{ V-Notch Weir } & \multicolumn{6}{|c|}{ R-Notch Weir } \\
\hline & \multicolumn{2}{|c|}{ Measured Water Level } & \multicolumn{2}{|c|}{$\begin{array}{c}\text { Calculated Rate } \\
\left(\mathrm{ft}^{3} / \mathrm{s}=448.8 \mathrm{gpm}\right)\end{array}$} & \multicolumn{3}{|c|}{ Measured Water Level } & \multicolumn{3}{|c|}{$\begin{array}{c}\text { Calculated Rate } \\
\left(\mathrm{ft}^{3} / \mathrm{s}=448.8 \mathrm{gpm}\right)\end{array}$} \\
\hline & $\begin{array}{c}\text { (ft above } \\
\text { sensor) }\end{array}$ & $\begin{array}{c}\text { (ft above } \\
\text { notch) }\end{array}$ & $\left(\mathrm{ft}^{3} / \mathrm{s}\right)$ & (gpm) & $\begin{array}{l}\text { (ft above } \\
\text { sensor) }\end{array}$ & $\begin{array}{c}\text { (ft above } \\
\text { log) }\end{array}$ & $\begin{array}{c}\text { (m above } \\
\text { log) }\end{array}$ & $\left(\mathrm{m}^{3} / \mathrm{s}\right)$ & $\left(\mathrm{ft}^{3} / \mathrm{s}\right)$ & (gpm) \\
\hline 2/23/08 3:00 & 1.473 & 0.494 & 0.100 & 44.770 & 1.351 & 0.02 & 0.006096 & 0.001588 & 0.056092 & 25.174 \\
\hline 2/23/08 4:00 & 1.471 & 0.492 & 0.099 & 44.326 & 1.351 & 0.02 & 0.006096 & 0.001588 & 0.056092 & 25.174 \\
\hline 2/23/08 5:00 & 1.469 & 0.490 & 0.098 & 43.884 & 1.351 & 0.02 & 0.006096 & 0.001588 & 0.056092 & 25.174 \\
\hline 2/23/08 6:00 & 1.466 & 0.487 & 0.096 & 43.226 & 1.349 & 0.018 & 0.005486 & 0.001388 & 0.049021 & 22.001 \\
\hline 2/23/08 7:00 & 1.469 & 0.490 & 0.098 & 43.884 & 1.351 & 0.02 & 0.006096 & 0.001588 & 0.056092 & 25.174 \\
\hline 2/23/08 8:00 & 1.464 & 0.485 & 0.095 & 42.790 & 1.351 & 0.02 & 0.006096 & 0.001588 & 0.056092 & 25.174 \\
\hline 2/23/08 9:00 & 1.466 & 0.487 & 0.096 & 43.226 & 1.349 & 0.018 & 0.005486 & 0.001388 & 0.049021 & 22.001 \\
\hline 2/23/08 10:00 & 1.466 & 0.487 & 0.096 & 43.226 & 1.351 & 0.02 & 0.006096 & 0.001588 & 0.056092 & 25.174 \\
\hline 2/23/08 11:00 & 1.475 & 0.496 & 0.101 & 45.218 & 1.351 & 0.02 & 0.006096 & 0.001588 & 0.056092 & 25.174 \\
\hline 2/23/08 12:00 & 1.47 & 0.491 & 0.098 & 44.104 & 1.351 & 0.02 & 0.006096 & 0.001588 & 0.056092 & 25.174 \\
\hline 2/23/08 13:00 & 1.476 & 0.497 & 0.101 & 45.442 & 1.354 & 0.023 & 0.007010 & 0.001905 & 0.067276 & 30.194 \\
\hline 2/23/08 14:00 & 1.513 & 0.534 & 0.121 & 54.231 & 1.358 & 0.027 & 0.008229 & 0.002356 & 0.083206 & 37.343 \\
\hline 2/23/08 15:00 & 1.604 & 0.625 & 0.178 & 79.944 & 1.372 & 0.041 & 0.012496 & 0.004166 & 0.147141 & 66.037 \\
\hline 2/23/08 16:00 & 1.752 & 0.773 & 0.301 & 135.188 & 1.396 & 0.065 & 0.019811 & 0.007977 & 0.281754 & 126.451 \\
\hline 2/23/08 17:00 & 1.837 & 0.858 & 0.390 & 175.033 & 1.405 & 0.074 & 0.022554 & 0.009605 & 0.339261 & 152.260 \\
\hline 2/23/08 18:00 & 1.864 & 0.885 & 0.421 & 188.998 & 1.408 & 0.077 & 0.023468 & 0.010170 & 0.359208 & 161.212 \\
\hline 2/23/08 19:00 & 1.825 & 0.846 & 0.377 & 169.032 & 1.403 & 0.072 & 0.021945 & 0.009235 & 0.326176 & 146.388 \\
\hline 2/23/08 20:00 & 1.776 & 0.797 & 0.325 & 145.816 & 1.394 & 0.063 & 0.019201 & 0.007629 & 0.269465 & 120.936 \\
\hline 2/23/08 21:00 & 1.682 & 0.703 & 0.238 & 106.898 & 1.38 & 0.049 & 0.014934 & 0.005345 & 0.188768 & 84.719 \\
\hline 2/23/08 22:00 & 1.633 & 0.654 & 0.199 & 89.419 & 1.373 & 0.042 & 0.012801 & 0.004308 & 0.152154 & 68.287 \\
\hline 2/23/08 23:00 & 1.598 & 0.619 & 0.174 & 78.063 & 1.368 & 0.037 & 0.011277 & 0.003614 & 0.127660 & 57.294 \\
\hline 2/24/08 0:00 & 1.579 & 0.600 & 0.161 & 72.282 & 1.365 & 0.034 & 0.010363 & 0.003218 & 0.113667 & 51.014 \\
\hline 2/24/08 1:00 & 1.56 & 0.581 & 0.149 & 66.766 & 1.361 & 0.03 & 0.009144 & 0.002714 & 0.095873 & 43.028 \\
\hline 2/24/08 2:00 & 1.539 & 0.560 & 0.136 & 60.972 & 1.358 & 0.027 & 0.008229 & 0.002356 & 0.083206 & 37.343 \\
\hline 2/24/08 3:00 & 1.527 & 0.548 & 0.129 & 57.802 & 1.358 & 0.027 & 0.008229 & 0.002356 & 0.083206 & 37.343 \\
\hline
\end{tabular}




\begin{tabular}{|c|c|c|c|c|c|c|c|c|c|c|}
\hline \multirow[b]{3}{*}{ Date Time } & \multicolumn{4}{|c|}{ V-Notch Weir } & \multicolumn{6}{|c|}{ R-Notch Weir } \\
\hline & \multicolumn{2}{|c|}{ Measured Water Level } & \multicolumn{2}{|c|}{$\begin{array}{c}\text { Calculated Rate } \\
\left(\mathrm{ft}^{3} / \mathrm{s}=448.8 \mathrm{gpm}\right)\end{array}$} & \multicolumn{3}{|c|}{ Measured Water Level } & \multicolumn{3}{|c|}{$\begin{array}{c}\text { Calculated Rate } \\
\left(\mathrm{ft}^{3} / \mathrm{s}=448.8 \mathrm{gpm}\right)\end{array}$} \\
\hline & $\begin{array}{c}\text { (ft above } \\
\text { sensor) }\end{array}$ & $\begin{array}{c}\text { (ft above } \\
\text { notch) }\end{array}$ & $\left(\mathrm{ft}^{3} / \mathrm{s}\right)$ & (gpm) & $\begin{array}{l}\text { (ft above } \\
\text { sensor) }\end{array}$ & $\begin{array}{c}\text { (ft above } \\
\text { log) }\end{array}$ & $\begin{array}{c}\text { (m above } \\
\text { log) }\end{array}$ & $\left(\mathrm{m}^{3} / \mathrm{s}\right)$ & $\left(\mathrm{ft}^{3} / \mathrm{s}\right)$ & (gpm) \\
\hline 2/24/08 4:00 & 1.52 & 0.541 & 0.125 & 55.999 & 1.358 & 0.027 & 0.008229 & 0.002356 & 0.083206 & 37.343 \\
\hline 2/24/08 5:00 & 1.511 & 0.532 & 0.120 & 53.732 & 1.356 & 0.025 & 0.007620 & 0.002126 & 0.075100 & 33.705 \\
\hline 2/24/08 6:00 & 1.511 & 0.532 & 0.120 & 53.732 & 1.354 & 0.023 & 0.007010 & 0.001905 & 0.067276 & 30.194 \\
\hline 2/24/08 7:00 & 1.501 & 0.522 & 0.114 & 51.278 & 1.354 & 0.023 & 0.007010 & 0.001905 & 0.067276 & 30.194 \\
\hline 2/24/08 8:00 & 1.497 & 0.518 & 0.112 & 50.316 & 1.354 & 0.023 & 0.007010 & 0.001905 & 0.067276 & 30.194 \\
\hline 2/24/08 9:00 & 1.499 & 0.520 & 0.113 & 50.796 & 1.356 & 0.025 & 0.007620 & 0.002126 & 0.075100 & 33.705 \\
\hline 2/24/08 10:00 & 1.504 & 0.525 & 0.116 & 52.007 & 1.354 & 0.023 & 0.007010 & 0.001905 & 0.067276 & 30.194 \\
\hline 2/24/08 11:00 & 1.503 & 0.524 & 0.115 & 51.763 & 1.354 & 0.023 & 0.007010 & 0.001905 & 0.067276 & 30.194 \\
\hline 2/24/08 12:00 & 1.505 & 0.526 & 0.116 & 52.251 & 1.356 & 0.025 & 0.007620 & 0.002126 & 0.075100 & 33.705 \\
\hline 2/24/08 13:00 & 1.524 & 0.545 & 0.127 & 57.025 & 1.358 & 0.027 & 0.008229 & 0.002356 & 0.083206 & 37.343 \\
\hline 2/24/08 14:00 & 1.579 & 0.600 & 0.161 & 72.282 & 1.368 & 0.037 & 0.011277 & 0.003614 & 0.127660 & 57.294 \\
\hline 2/24/08 15:00 & 1.759 & 0.780 & 0.308 & 138.238 & 1.396 & 0.065 & 0.019811 & 0.007977 & 0.281754 & 126.451 \\
\hline 2/24/08 16:00 & 1.928 & 0.949 & 0.501 & 224.707 & 1.422 & 0.091 & 0.027735 & 0.012943 & 0.457162 & 205.174 \\
\hline 2/24/08 17:00 & 1.995 & 1.016 & 0.593 & 266.131 & 1.443 & 0.112 & 0.034136 & 0.017501 & 0.618132 & 277.418 \\
\hline 2/24/08 18:00 & 2.001 & 1.022 & 0.602 & 270.047 & 1.453 & 0.122 & 0.037184 & 0.019827 & 0.700289 & 314.290 \\
\hline 2/24/08 19:00 & 1.976 & 0.997 & 0.566 & 253.956 & 1.446 & 0.115 & 0.035050 & 0.018189 & 0.642419 & 288.318 \\
\hline 2/24/08 20:00 & 1.955 & 0.976 & 0.537 & 240.895 & 1.429 & 0.098 & 0.029869 & 0.014412 & 0.509013 & 228.445 \\
\hline 2/24/08 21:00 & 1.868 & 0.889 & 0.426 & 191.122 & 1.41 & 0.079 & 0.024078 & 0.010553 & 0.372715 & 167.274 \\
\hline 2/24/08 22:00 & 1.791 & 0.812 & 0.340 & 152.705 & 1.396 & 0.065 & 0.019811 & 0.007977 & 0.281754 & 126.451 \\
\hline 2/24/08 23:00 & 1.732 & 0.753 & 0.282 & 126.698 & 1.387 & 0.056 & 0.017068 & 0.006453 & 0.227923 & 102.292 \\
\hline 2/25/08 0:00 & 1.694 & 0.715 & 0.248 & 111.467 & 1.382 & 0.051 & 0.015544 & 0.005654 & 0.199703 & 89.627 \\
\hline 2/25/08 1:00 & 1.663 & 0.684 & 0.223 & 99.898 & 1.377 & 0.046 & 0.014020 & 0.004891 & 0.172754 & 77.532 \\
\hline 2/25/08 2:00 & 1.642 & 0.663 & 0.206 & 92.490 & 1.375 & 0.044 & 0.013411 & 0.004596 & 0.162345 & 72.860 \\
\hline 2/25/08 3:00 & 1.626 & 0.647 & 0.194 & 87.074 & 1.372 & 0.041 & 0.012496 & 0.004166 & 0.147141 & 66.037 \\
\hline 2/25/08 4:00 & 1.621 & 0.642 & 0.190 & 85.421 & 1.372 & 0.041 & 0.012496 & 0.004166 & 0.147141 & 66.037 \\
\hline
\end{tabular}




\begin{tabular}{|c|c|c|c|c|c|c|c|c|c|c|}
\hline \multirow[b]{3}{*}{ Date Time } & \multicolumn{4}{|c|}{ V-Notch Weir } & \multicolumn{6}{|c|}{ R-Notch Weir } \\
\hline & \multicolumn{2}{|c|}{ Measured Water Level } & \multicolumn{2}{|c|}{$\begin{array}{c}\text { Calculated Rate } \\
\left(\mathrm{ft}^{3} / \mathrm{s}=448.8 \mathrm{gpm}\right)\end{array}$} & \multicolumn{3}{|c|}{ Measured Water Level } & \multicolumn{3}{|c|}{$\begin{array}{c}\text { Calculated Rate } \\
\left(\mathrm{ft}^{3} / \mathrm{s}=448.8 \mathrm{gpm}\right)\end{array}$} \\
\hline & $\begin{array}{c}\text { (ft above } \\
\text { sensor) }\end{array}$ & $\begin{array}{c}\text { (ft above } \\
\text { notch) }\end{array}$ & $\left(\mathrm{ft}^{3} / \mathrm{s}\right)$ & (gpm) & $\begin{array}{l}\text { (ft above } \\
\text { sensor) }\end{array}$ & $\begin{array}{c}\text { (ft above } \\
\text { log) }\end{array}$ & $\begin{array}{c}\text { (m above } \\
\text { log) }\end{array}$ & $\left(\mathrm{m}^{3} / \mathrm{s}\right)$ & $\left(\mathrm{ft}^{3} / \mathrm{s}\right)$ & (gpm) \\
\hline 2/25/08 5:00 & 1.614 & 0.635 & 0.185 & 83.140 & 1.37 & 0.039 & 0.011887 & 0.003887 & 0.137285 & 61.614 \\
\hline 2/25/08 6:00 & 1.593 & 0.614 & 0.170 & 76.516 & 1.368 & 0.037 & 0.011277 & 0.003614 & 0.127660 & 57.294 \\
\hline 2/25/08 7:00 & 1.597 & 0.618 & 0.173 & 77.752 & 1.37 & 0.039 & 0.011887 & 0.003887 & 0.137285 & 61.614 \\
\hline 2/25/08 8:00 & 1.593 & 0.614 & 0.170 & 76.516 & 1.372 & 0.041 & 0.012496 & 0.004166 & 0.147141 & 66.037 \\
\hline 2/25/08 9:00 & 1.586 & 0.607 & 0.166 & 74.381 & 1.368 & 0.037 & 0.011277 & 0.003614 & 0.127660 & 57.294 \\
\hline 2/25/08 10:00 & 1.583 & 0.604 & 0.164 & 73.477 & 1.368 & 0.037 & 0.011277 & 0.003614 & 0.127660 & 57.294 \\
\hline 2/25/08 11:00 & 1.583 & 0.604 & 0.164 & 73.477 & 1.365 & 0.034 & 0.010363 & 0.003218 & 0.113667 & 51.014 \\
\hline 2/25/08 12:00 & 1.583 & 0.604 & 0.164 & 73.477 & 1.368 & 0.037 & 0.011277 & 0.003614 & 0.127660 & 57.294 \\
\hline 2/25/08 13:00 & 1.599 & 0.620 & 0.175 & 78.375 & 1.368 & 0.037 & 0.011277 & 0.003614 & 0.127660 & 57.294 \\
\hline 2/25/08 14:00 & 1.585 & 0.606 & 0.165 & 74.079 & 1.365 & 0.034 & 0.010363 & 0.003218 & 0.113667 & 51.014 \\
\hline 2/25/08 15:00 & 1.634 & 0.655 & 0.200 & 89.757 & 1.372 & 0.041 & 0.012496 & 0.004166 & 0.147141 & 66.037 \\
\hline 2/25/08 16:00 & 1.881 & 0.902 & 0.441 & 198.123 & 1.42 & 0.089 & 0.027126 & 0.012534 & 0.442691 & 198.680 \\
\hline 2/25/08 17:00 & 1.997 & 1.018 & 0.596 & 267.432 & 1.455 & 0.124 & 0.037793 & 0.020304 & 0.717127 & 321.847 \\
\hline 2/25/08 18:00 & 1.977 & 0.998 & 0.567 & 254.588 & 1.444 & 0.113 & 0.034441 & 0.017729 & 0.626193 & 281.035 \\
\hline 2/25/08 19:00 & 1.914 & 0.935 & 0.483 & 216.578 & 1.422 & 0.091 & 0.027735 & 0.012943 & 0.457162 & 205.174 \\
\hline 2/25/08 20:00 & 1.789 & 0.810 & 0.338 & 151.775 & 1.396 & 0.065 & 0.019811 & 0.007977 & 0.281754 & 126.451 \\
\hline 2/25/08 21:00 & 1.709 & 0.730 & 0.261 & 117.339 & 1.384 & 0.053 & 0.016154 & 0.005969 & 0.210842 & 94.626 \\
\hline 2/25/08 22:00 & 1.62 & 0.641 & 0.190 & 85.093 & 1.375 & 0.044 & 0.013411 & 0.004596 & 0.162345 & 72.860 \\
\hline 2/25/08 23:00 & 1.639 & 0.660 & 0.204 & 91.459 & 1.368 & 0.037 & 0.011277 & 0.003614 & 0.127660 & 57.294 \\
\hline 2/26/08 0:00 & 1.585 & 0.606 & 0.165 & 74.079 & 1.366 & 0.035 & 0.010667 & 0.003349 & 0.118271 & 53.080 \\
\hline 2/26/08 1:00 & 1.566 & 0.587 & 0.153 & 68.479 & 1.366 & 0.035 & 0.010667 & 0.003349 & 0.118271 & 53.080 \\
\hline 2/26/08 2:00 & 1.557 & 0.578 & 0.147 & 65.918 & 1.363 & 0.032 & 0.009753 & 0.002963 & 0.104644 & 46.964 \\
\hline 2/26/08 3:00 & 1.531 & 0.552 & 0.131 & 58.847 & 1.356 & 0.025 & 0.007620 & 0.002126 & 0.075100 & 33.705 \\
\hline 2/26/08 4:00 & 1.522 & 0.543 & 0.126 & 56.511 & 1.354 & 0.023 & 0.007010 & 0.001905 & 0.067276 & 30.194 \\
\hline 2/26/08 5:00 & 1.51 & 0.531 & 0.119 & 53.483 & 1.354 & 0.023 & 0.007010 & 0.001905 & 0.067276 & 30.194 \\
\hline
\end{tabular}




\begin{tabular}{|c|c|c|c|c|c|c|c|c|c|c|}
\hline \multirow[b]{3}{*}{ Date Time } & \multicolumn{4}{|c|}{ V-Notch Weir } & \multicolumn{6}{|c|}{ R-Notch Weir } \\
\hline & \multicolumn{2}{|c|}{ Measured Water Level } & \multicolumn{2}{|c|}{$\begin{array}{c}\text { Calculated Rate } \\
\left(\mathrm{ft}^{3} / \mathrm{s}=448.8 \mathrm{gpm}\right)\end{array}$} & \multicolumn{3}{|c|}{ Measured Water Level } & \multicolumn{3}{|c|}{$\begin{array}{c}\text { Calculated Rate } \\
\left(\mathrm{ft}^{3} / \mathrm{s}=448.8 \mathrm{gpm}\right)\end{array}$} \\
\hline & $\begin{array}{c}\text { (ft above } \\
\text { sensor) }\end{array}$ & $\begin{array}{c}\text { (ft above } \\
\text { notch) }\end{array}$ & $\left(\mathrm{ft}^{3} / \mathrm{s}\right)$ & (gpm) & $\begin{array}{l}\text { (ft above } \\
\text { sensor) }\end{array}$ & $\begin{array}{c}\text { (ft above } \\
\text { log) }\end{array}$ & $\begin{array}{c}\text { (m above } \\
\text { log) }\end{array}$ & $\left(\mathrm{m}^{3} / \mathrm{s}\right)$ & $\left(\mathrm{ft}^{3} / \mathrm{s}\right)$ & (gpm) \\
\hline 2/26/08 6:00 & 1.51 & 0.531 & 0.119 & 53.483 & 1.359 & 0.028 & 0.008534 & 0.002473 & 0.087362 & 39.208 \\
\hline 2/26/08 7:00 & 1.484 & 0.505 & 0.105 & 47.264 & 1.366 & 0.035 & 0.010667 & 0.003349 & 0.118271 & 53.080 \\
\hline 2/26/08 8:00 & 1.47 & 0.491 & 0.098 & 44.104 & 1.373 & 0.042 & 0.012801 & 0.004308 & 0.152154 & 68.287 \\
\hline 2/26/08 9:00 & 1.467 & 0.488 & 0.097 & 43.444 & 1.382 & 0.051 & 0.015544 & 0.005654 & 0.199703 & 89.627 \\
\hline 2/26/08 10:00 & 1.498 & 0.519 & 0.113 & 50.555 & 1.387 & 0.056 & 0.017068 & 0.006453 & 0.227923 & 102.292 \\
\hline 2/26/08 11:00 & 1.554 & 0.575 & 0.145 & 65.078 & 1.373 & 0.042 & 0.012801 & 0.004308 & 0.152154 & 68.287 \\
\hline 2/26/08 12:00 & 1.534 & 0.555 & 0.133 & 59.638 & 1.363 & 0.032 & 0.009753 & 0.002963 & 0.104644 & 46.964 \\
\hline 2/26/08 13:00 & 1.52 & 0.541 & 0.125 & 55.999 & 1.354 & 0.023 & 0.007010 & 0.001905 & 0.067276 & 30.194 \\
\hline 2/26/08 14:00 & 1.508 & 0.529 & 0.118 & 52.988 & 1.356 & 0.025 & 0.007620 & 0.002126 & 0.075100 & 33.705 \\
\hline 2/26/08 15:00 & 1.507 & 0.528 & 0.118 & 52.742 & 1.354 & 0.023 & 0.007010 & 0.001905 & 0.067276 & 30.194 \\
\hline 2/26/08 16:00 & 1.516 & 0.537 & 0.123 & 54.984 & 1.356 & 0.025 & 0.007620 & 0.002126 & 0.075100 & 33.705 \\
\hline 2/26/08 17:00 & 1.509 & 0.530 & 0.119 & 53.236 & 1.354 & 0.023 & 0.007010 & 0.001905 & 0.067276 & 30.194 \\
\hline 2/26/08 18:00 & 1.516 & 0.537 & 0.123 & 54.984 & 1.356 & 0.025 & 0.007620 & 0.002126 & 0.075100 & 33.705 \\
\hline 2/26/08 19:00 & 1.507 & 0.528 & 0.118 & 52.742 & 1.356 & 0.025 & 0.007620 & 0.002126 & 0.075100 & 33.705 \\
\hline 2/26/08 20:00 & 1.507 & 0.528 & 0.118 & 52.742 & 1.356 & 0.025 & 0.007620 & 0.002126 & 0.075100 & 33.705 \\
\hline 2/26/08 21:00 & 1.507 & 0.528 & 0.118 & 52.742 & 1.356 & 0.025 & 0.007620 & 0.002126 & 0.075100 & 33.705 \\
\hline 2/26/08 22:00 & 1.508 & 0.529 & 0.118 & 52.988 & 1.354 & 0.023 & 0.007010 & 0.001905 & 0.067276 & 30.194 \\
\hline 2/26/08 23:00 & 1.501 & 0.522 & 0.114 & 51.278 & 1.354 & 0.023 & 0.007010 & 0.001905 & 0.067276 & 30.194 \\
\hline 2/27/08 0:00 & 1.501 & 0.522 & 0.114 & 51.278 & 1.354 & 0.023 & 0.007010 & 0.001905 & 0.067276 & 30.194 \\
\hline 2/27/08 1:00 & 1.499 & 0.520 & 0.113 & 50.796 & 1.354 & 0.023 & 0.007010 & 0.001905 & 0.067276 & 30.194 \\
\hline 2/27/08 2:00 & 1.497 & 0.518 & 0.112 & 50.316 & 1.354 & 0.023 & 0.007010 & 0.001905 & 0.067276 & 30.194 \\
\hline 2/27/08 3:00 & 1.497 & 0.518 & 0.112 & 50.316 & 1.354 & 0.023 & 0.007010 & 0.001905 & 0.067276 & 30.194 \\
\hline 2/27/08 4:00 & 1.495 & 0.516 & 0.111 & 49.839 & 1.354 & 0.023 & 0.007010 & 0.001905 & 0.067276 & 30.194 \\
\hline 2/27/08 5:00 & 1.495 & 0.516 & 0.111 & 49.839 & 1.354 & 0.023 & 0.007010 & 0.001905 & 0.067276 & 30.194 \\
\hline 2/27/08 6:00 & 1.49 & 0.511 & 0.108 & 48.658 & 1.354 & 0.023 & 0.007010 & 0.001905 & 0.067276 & 30.194 \\
\hline
\end{tabular}




\begin{tabular}{|c|c|c|c|c|c|c|c|c|c|c|}
\hline \multirow[b]{3}{*}{ Date Time } & \multicolumn{4}{|c|}{ V-Notch Weir } & \multicolumn{6}{|c|}{ R-Notch Weir } \\
\hline & \multicolumn{2}{|c|}{ Measured Water Level } & \multicolumn{2}{|c|}{$\begin{array}{c}\text { Calculated Rate } \\
\left(\mathrm{ft}^{3} / \mathrm{s}=448.8 \mathrm{gpm}\right)\end{array}$} & \multicolumn{3}{|c|}{ Measured Water Level } & \multicolumn{3}{|c|}{$\begin{array}{c}\text { Calculated Rate } \\
\left(\mathrm{ft}^{3} / \mathrm{s}=448.8 \mathrm{gpm}\right)\end{array}$} \\
\hline & $\begin{array}{c}\text { (ft above } \\
\text { sensor) }\end{array}$ & $\begin{array}{c}\text { (ft above } \\
\text { notch) }\end{array}$ & $\left(\mathrm{ft}^{3} / \mathrm{s}\right)$ & (gpm) & $\begin{array}{l}\text { (ft above } \\
\text { sensor) }\end{array}$ & $\begin{array}{c}\text { (ft above } \\
\text { log) }\end{array}$ & $\begin{array}{c}\text { (m above } \\
\text { log) }\end{array}$ & $\left(\mathrm{m}^{3} / \mathrm{s}\right)$ & $\left(\mathrm{ft}^{3} / \mathrm{s}\right)$ & (gpm) \\
\hline 2/27/08 7:00 & 1.49 & 0.511 & 0.108 & 48.658 & 1.354 & 0.023 & 0.007010 & 0.001905 & 0.067276 & 30.194 \\
\hline 2/27/08 8:00 & 1.49 & 0.511 & 0.108 & 48.658 & 1.354 & 0.023 & 0.007010 & 0.001905 & 0.067276 & 30.194 \\
\hline 2/27/08 9:00 & 1.488 & 0.509 & 0.107 & 48.191 & 1.351 & 0.02 & 0.006096 & 0.001588 & 0.056092 & 25.174 \\
\hline 2/27/08 10:00 & 1.492 & 0.513 & 0.109 & 49.129 & 1.354 & 0.023 & 0.007010 & 0.001905 & 0.067276 & 30.194 \\
\hline 2/27/08 11:00 & 1.496 & 0.517 & 0.112 & 50.077 & 1.354 & 0.023 & 0.007010 & 0.001905 & 0.067276 & 30.194 \\
\hline 2/27/08 12:00 & 1.498 & 0.519 & 0.113 & 50.555 & 1.356 & 0.025 & 0.007620 & 0.002126 & 0.075100 & 33.705 \\
\hline 2/27/08 13:00 & 1.507 & 0.528 & 0.118 & 52.742 & 1.356 & 0.025 & 0.007620 & 0.002126 & 0.075100 & 33.705 \\
\hline 2/27/08 14:00 & 1.52 & 0.541 & 0.125 & 55.999 & 1.358 & 0.027 & 0.008229 & 0.002356 & 0.083206 & 37.343 \\
\hline 2/27/08 15:00 & 1.583 & 0.604 & 0.164 & 73.477 & 1.368 & 0.037 & 0.011277 & 0.003614 & 0.127660 & 57.294 \\
\hline 2/27/08 16:00 & 1.693 & 0.714 & 0.248 & 111.082 & 1.384 & 0.053 & 0.016154 & 0.005969 & 0.210842 & 94.626 \\
\hline 2/27/08 17:00 & 1.787 & 0.808 & 0.336 & 150.849 & 1.398 & 0.067 & 0.020421 & 0.008330 & 0.294224 & 132.048 \\
\hline 2/27/08 18:00 & 1.875 & 0.896 & 0.434 & 194.873 & 1.412 & 0.081 & 0.024688 & 0.010940 & 0.386387 & 173.411 \\
\hline 2/27/08 19:00 & 1.899 & 0.920 & 0.464 & 208.067 & 1.417 & 0.086 & 0.026212 & 0.011928 & 0.421279 & 189.070 \\
\hline 2/27/08 20:00 & 1.837 & 0.858 & 0.390 & 175.033 & 1.403 & 0.072 & 0.021945 & 0.009235 & 0.326176 & 146.388 \\
\hline 2/27/08 21:00 & 1.762 & 0.783 & 0.311 & 139.558 & 1.391 & 0.06 & 0.018287 & 0.007117 & 0.251378 & 112.818 \\
\hline 2/27/08 22:00 & 1.708 & 0.729 & 0.261 & 116.942 & 1.384 & 0.053 & 0.016154 & 0.005969 & 0.210842 & 94.626 \\
\hline 2/27/08 23:00 & 1.668 & 0.689 & 0.227 & 101.713 & 1.377 & 0.046 & 0.014020 & 0.004891 & 0.172754 & 77.532 \\
\hline 2/28/08 0:00 & 1.638 & 0.659 & 0.203 & 91.117 & 1.372 & 0.041 & 0.012496 & 0.004166 & 0.147141 & 66.037 \\
\hline 2/28/08 1:00 & 1.609 & 0.630 & 0.182 & 81.532 & 1.368 & 0.037 & 0.011277 & 0.003614 & 0.127660 & 57.294 \\
\hline 2/28/08 2:00 & 1.593 & 0.614 & 0.170 & 76.516 & 1.368 & 0.037 & 0.011277 & 0.003614 & 0.127660 & 57.294 \\
\hline 2/28/08 3:00 & 1.588 & 0.609 & 0.167 & 74.987 & 1.363 & 0.032 & 0.009753 & 0.002963 & 0.104644 & 46.964 \\
\hline 2/28/08 4:00 & 1.572 & 0.593 & 0.156 & 70.219 & 1.363 & 0.032 & 0.009753 & 0.002963 & 0.104644 & 46.964 \\
\hline 2/28/08 5:00 & 1.562 & 0.583 & 0.150 & 67.334 & 1.363 & 0.032 & 0.009753 & 0.002963 & 0.104644 & 46.964 \\
\hline 2/28/08 6:00 & 1.558 & 0.579 & 0.148 & 66.200 & 1.361 & 0.03 & 0.009144 & 0.002714 & 0.095873 & 43.028 \\
\hline 2/28/08 7:00 & 1.553 & 0.574 & 0.144 & 64.799 & 1.363 & 0.032 & 0.009753 & 0.002963 & 0.104644 & 46.964 \\
\hline
\end{tabular}




\begin{tabular}{|c|c|c|c|c|c|c|c|c|c|c|}
\hline \multirow[b]{3}{*}{ Date Time } & \multicolumn{4}{|c|}{ V-Notch Weir } & \multicolumn{6}{|c|}{ R-Notch Weir } \\
\hline & \multicolumn{2}{|c|}{ Measured Water Level } & \multicolumn{2}{|c|}{$\begin{array}{c}\text { Calculated Rate } \\
\left(\mathrm{ft}^{3} / \mathrm{s}=448.8 \mathrm{gpm}\right)\end{array}$} & \multicolumn{3}{|c|}{ Measured Water Level } & \multicolumn{3}{|c|}{$\begin{array}{c}\text { Calculated Rate } \\
\left(\mathrm{ft}^{3} / \mathrm{s}=448.8 \mathrm{gpm}\right)\end{array}$} \\
\hline & $\begin{array}{c}\text { (ft above } \\
\text { sensor) }\end{array}$ & $\begin{array}{c}\text { (ft above } \\
\text { notch) }\end{array}$ & $\left(\mathrm{ft}^{3} / \mathrm{s}\right)$ & (gpm) & $\begin{array}{l}\text { (ft above } \\
\text { sensor) }\end{array}$ & $\begin{array}{c}\text { (ft above } \\
\text { log) }\end{array}$ & $\begin{array}{c}\text { (m above } \\
\text { log) }\end{array}$ & $\left(\mathrm{m}^{3} / \mathrm{s}\right)$ & $\left(\mathrm{ft}^{3} / \mathrm{s}\right)$ & (gpm) \\
\hline 2/28/08 8:00 & 1.539 & 0.560 & 0.136 & 60.972 & 1.361 & 0.03 & 0.009144 & 0.002714 & 0.095873 & 43.028 \\
\hline 2/28/08 9:00 & 1.532 & 0.553 & 0.132 & 59.110 & 1.358 & 0.027 & 0.008229 & 0.002356 & 0.083206 & 37.343 \\
\hline 2/28/08 10:00 & 1.534 & 0.555 & 0.133 & 59.638 & 1.358 & 0.027 & 0.008229 & 0.002356 & 0.083206 & 37.343 \\
\hline 2/28/08 11:00 & 1.527 & 0.548 & 0.129 & 57.802 & 1.358 & 0.027 & 0.008229 & 0.002356 & 0.083206 & 37.343 \\
\hline 2/28/08 12:00 & 1.524 & 0.545 & 0.127 & 57.025 & 1.358 & 0.027 & 0.008229 & 0.002356 & 0.083206 & 37.343 \\
\hline 2/28/08 13:00 & 1.533 & 0.554 & 0.132 & 59.374 & 1.356 & 0.025 & 0.007620 & 0.002126 & 0.075100 & 33.705 \\
\hline 2/28/08 14:00 & 1.543 & 0.564 & 0.138 & 62.051 & 1.358 & 0.027 & 0.008229 & 0.002356 & 0.083206 & 37.343 \\
\hline 2/28/08 15:00 & 1.552 & 0.573 & 0.144 & 64.521 & 1.361 & 0.03 & 0.009144 & 0.002714 & 0.095873 & 43.028 \\
\hline 2/28/08 16:00 & 1.582 & 0.603 & 0.163 & 73.177 & 1.365 & 0.034 & 0.010363 & 0.003218 & 0.113667 & 51.014 \\
\hline 2/28/08 17:00 & 1.663 & 0.684 & 0.223 & 99.898 & 1.379 & 0.048 & 0.014630 & 0.005192 & 0.183377 & 82.300 \\
\hline 2/28/08 18:00 & 1.769 & 0.790 & 0.318 & 142.666 & 1.394 & 0.063 & 0.019201 & 0.007629 & 0.269465 & 120.936 \\
\hline 2/28/08 19:00 & 1.825 & 0.846 & 0.377 & 169.032 & 1.403 & 0.072 & 0.021945 & 0.009235 & 0.326176 & 146.388 \\
\hline 2/28/08 20:00 & 1.833 & 0.854 & 0.386 & 173.019 & 1.403 & 0.072 & 0.021945 & 0.009235 & 0.326176 & 146.388 \\
\hline 2/28/08 21:00 & 1.803 & 0.824 & 0.353 & 158.354 & 1.398 & 0.067 & 0.020421 & 0.008330 & 0.294224 & 132.048 \\
\hline 2/28/08 22:00 & 1.761 & 0.782 & 0.310 & 139.117 & 1.391 & 0.06 & 0.018287 & 0.007117 & 0.251378 & 112.818 \\
\hline 2/28/08 23:00 & 1.722 & 0.743 & 0.273 & 122.576 & 1.384 & 0.053 & 0.016154 & 0.005969 & 0.210842 & 94.626 \\
\hline 2/29/08 0:00 & 1.668 & 0.689 & 0.227 & 101.713 & 1.377 & 0.046 & 0.014020 & 0.004891 & 0.172754 & 77.532 \\
\hline 2/29/08 1:00 & 1.644 & 0.665 & 0.208 & 93.181 & 1.375 & 0.044 & 0.013411 & 0.004596 & 0.162345 & 72.860 \\
\hline 2/29/08 2:00 & 1.618 & 0.639 & 0.188 & 84.439 & 1.37 & 0.039 & 0.011887 & 0.003887 & 0.137285 & 61.614 \\
\hline 2/29/08 3:00 & 1.595 & 0.616 & 0.172 & 77.132 & 1.368 & 0.037 & 0.011277 & 0.003614 & 0.127660 & 57.294 \\
\hline 2/29/08 4:00 & 1.576 & 0.597 & 0.159 & 71.393 & 1.363 & 0.032 & 0.009753 & 0.002963 & 0.104644 & 46.964 \\
\hline 2/29/08 5:00 & 1.56 & 0.581 & 0.149 & 66.766 & 1.361 & 0.03 & 0.009144 & 0.002714 & 0.095873 & 43.028 \\
\hline 2/29/08 6:00 & 1.555 & 0.576 & 0.146 & 65.357 & 1.361 & 0.03 & 0.009144 & 0.002714 & 0.095873 & 43.028 \\
\hline 2/29/08 7:00 & 1.55 & 0.571 & 0.143 & 63.967 & 1.358 & 0.027 & 0.008229 & 0.002356 & 0.083206 & 37.343 \\
\hline 2/29/08 8:00 & 1.534 & 0.555 & 0.133 & 59.638 & 1.358 & 0.027 & 0.008229 & 0.002356 & 0.083206 & 37.343 \\
\hline
\end{tabular}




\begin{tabular}{|c|c|c|c|c|c|c|c|c|c|c|}
\hline \multirow[b]{3}{*}{ Date Time } & \multicolumn{4}{|c|}{ V-Notch Weir } & \multicolumn{6}{|c|}{ R-Notch Weir } \\
\hline & \multicolumn{2}{|c|}{ Measured Water Level } & \multicolumn{2}{|c|}{$\begin{array}{c}\text { Calculated Rate } \\
\left(\mathrm{ft}^{3} / \mathrm{s}=448.8 \mathrm{gpm}\right)\end{array}$} & \multicolumn{3}{|c|}{ Measured Water Level } & \multicolumn{3}{|c|}{$\begin{array}{c}\text { Calculated Rate } \\
\left(\mathrm{ft}^{3} / \mathrm{s}=448.8 \mathrm{gpm}\right)\end{array}$} \\
\hline & $\begin{array}{c}\text { (ft above } \\
\text { sensor) }\end{array}$ & $\begin{array}{c}\text { (ft above } \\
\text { notch) }\end{array}$ & $\left(\mathrm{ft}^{3} / \mathrm{s}\right)$ & (gpm) & $\begin{array}{l}\text { (ft above } \\
\text { sensor) }\end{array}$ & $\begin{array}{c}\text { (ft above } \\
\text { log) }\end{array}$ & $\begin{array}{c}\text { (m above } \\
\text { log) }\end{array}$ & $\left(\mathrm{m}^{3} / \mathrm{s}\right)$ & $\left(\mathrm{ft}^{3} / \mathrm{s}\right)$ & (gpm) \\
\hline 2/29/08 9:00 & 1.529 & 0.550 & 0.130 & 58.323 & 1.358 & 0.027 & 0.008229 & 0.002356 & 0.083206 & 37.343 \\
\hline 2/29/08 10:00 & 1.524 & 0.545 & 0.127 & 57.025 & 1.361 & 0.03 & 0.009144 & 0.002714 & 0.095873 & 43.028 \\
\hline 2/29/08 11:00 & 1.536 & 0.557 & 0.134 & 60.170 & 1.358 & 0.027 & 0.008229 & 0.002356 & 0.083206 & 37.343 \\
\hline 2/29/08 12:00 & 1.546 & 0.567 & 0.140 & 62.868 & 1.36 & 0.029 & 0.008839 & 0.002593 & 0.091584 & 41.103 \\
\hline 2/29/08 13:00 & 1.585 & 0.606 & 0.165 & 74.079 & 1.368 & 0.037 & 0.011277 & 0.003614 & 0.127660 & 57.294 \\
\hline 2/29/08 14:00 & 1.73 & 0.751 & 0.280 & 125.867 & 1.391 & 0.06 & 0.018287 & 0.007117 & 0.251378 & 112.818 \\
\hline 2/29/08 15:00 & 1.896 & 0.917 & 0.460 & 206.389 & 1.417 & 0.086 & 0.026212 & 0.011928 & 0.421279 & 189.070 \\
\hline 2/29/08 16:00 & 1.95 & 0.971 & 0.530 & 237.846 & 1.436 & 0.105 & 0.032002 & 0.015931 & 0.562690 & 252.535 \\
\hline 2/29/08 17:00 & 1.998 & 1.019 & 0.597 & 268.084 & 1.446 & 0.115 & 0.035050 & 0.018189 & 0.642419 & 288.318 \\
\hline 2/29/08 18:00 & 1.996 & 1.017 & 0.594 & 266.781 & 1.446 & 0.115 & 0.035050 & 0.018189 & 0.642419 & 288.318 \\
\hline 2/29/08 19:00 & 1.965 & 0.986 & 0.550 & 247.063 & 1.431 & 0.1 & 0.030479 & 0.014841 & 0.524166 & 235.246 \\
\hline 2/29/08 20:00 & 1.89 & 0.911 & 0.452 & 203.059 & 1.415 & 0.084 & 0.025602 & 0.011529 & 0.407202 & 182.752 \\
\hline 2/29/08 21:00 & 1.792 & 0.813 & 0.341 & 153.171 & 1.396 & 0.065 & 0.019811 & 0.007977 & 0.281754 & 126.451 \\
\hline 2/29/08 22:00 & 1.722 & 0.743 & 0.273 & 122.576 & 1.384 & 0.053 & 0.016154 & 0.005969 & 0.210842 & 94.626 \\
\hline 2/29/08 23:00 & 1.672 & 0.693 & 0.230 & 103.179 & 1.377 & 0.046 & 0.014020 & 0.004891 & 0.172754 & 77.532 \\
\hline 3/1/08 0:00 & 1.644 & 0.665 & 0.208 & 93.181 & 1.372 & 0.041 & 0.012496 & 0.004166 & 0.147141 & 66.037 \\
\hline 3/1/08 1:00 & 1.611 & 0.632 & 0.183 & 82.173 & 1.37 & 0.039 & 0.011887 & 0.003887 & 0.137285 & 61.614 \\
\hline 3/1/08 2:00 & 1.595 & 0.616 & 0.172 & 77.132 & 1.368 & 0.037 & 0.011277 & 0.003614 & 0.127660 & 57.294 \\
\hline 3/1/08 3:00 & 1.581 & 0.602 & 0.162 & 72.878 & 1.365 & 0.034 & 0.010363 & 0.003218 & 0.113667 & 51.014 \\
\hline 3/1/08 4:00 & 1.567 & 0.588 & 0.153 & 68.767 & 1.363 & 0.032 & 0.009753 & 0.002963 & 0.104644 & 46.964 \\
\hline 3/1/08 5:00 & 1.567 & 0.588 & 0.153 & 68.767 & 1.365 & 0.034 & 0.010363 & 0.003218 & 0.113667 & 51.014 \\
\hline 3/1/08 6:00 & 1.557 & 0.578 & 0.147 & 65.918 & 1.363 & 0.032 & 0.009753 & 0.002963 & 0.104644 & 46.964 \\
\hline 3/1/08 7:00 & 1.553 & 0.574 & 0.144 & 64.799 & 1.361 & 0.03 & 0.009144 & 0.002714 & 0.095873 & 43.028 \\
\hline 3/1/08 8:00 & 1.562 & 0.583 & 0.150 & 67.334 & 1.361 & 0.03 & 0.009144 & 0.002714 & 0.095873 & 43.028 \\
\hline 3/1/08 9:00 & 1.569 & 0.590 & 0.155 & 69.346 & 1.363 & 0.032 & 0.009753 & 0.002963 & 0.104644 & 46.964 \\
\hline
\end{tabular}




\begin{tabular}{|c|c|c|c|c|c|c|c|c|c|c|}
\hline \multirow[b]{3}{*}{ Date Time } & \multicolumn{4}{|c|}{ V-Notch Weir } & \multicolumn{6}{|c|}{ R-Notch Weir } \\
\hline & \multicolumn{2}{|c|}{ Measured Water Level } & \multicolumn{2}{|c|}{$\begin{array}{c}\text { Calculated Rate } \\
\left(\mathrm{ft}^{3} / \mathrm{s}=448.8 \mathrm{gpm}\right)\end{array}$} & \multicolumn{3}{|c|}{ Measured Water Level } & \multicolumn{3}{|c|}{$\begin{array}{c}\text { Calculated Rate } \\
\left(\mathrm{ft}^{3} / \mathrm{s}=448.8 \mathrm{gpm}\right)\end{array}$} \\
\hline & $\begin{array}{c}\text { (ft above } \\
\text { sensor) }\end{array}$ & $\begin{array}{c}\text { (ft above } \\
\text { notch) }\end{array}$ & $\left(\mathrm{ft}^{3} / \mathrm{s}\right)$ & (gpm) & $\begin{array}{l}\text { (ft above } \\
\text { sensor) }\end{array}$ & $\begin{array}{c}\text { (ft above } \\
\text { log) }\end{array}$ & $\begin{array}{c}\text { (m above } \\
\text { log) }\end{array}$ & $\left(\mathrm{m}^{3} / \mathrm{s}\right)$ & $\left(\mathrm{ft}^{3} / \mathrm{s}\right)$ & (gpm) \\
\hline 3/1/08 10:00 & 1.547 & 0.568 & 0.141 & 63.142 & 1.363 & 0.032 & 0.009753 & 0.002963 & 0.104644 & 46.964 \\
\hline 3/1/08 11:00 & 1.584 & 0.605 & 0.164 & 73.777 & 1.365 & 0.034 & 0.010363 & 0.003218 & 0.113667 & 51.014 \\
\hline 3/1/08 12:00 & 1.641 & 0.662 & 0.205 & 92.146 & 1.375 & 0.044 & 0.013411 & 0.004596 & 0.162345 & 72.860 \\
\hline 3/1/08 13:00 & 1.743 & 0.764 & 0.293 & 131.327 & 1.389 & 0.058 & 0.017678 & 0.006782 & 0.239555 & 107.512 \\
\hline 3/1/08 14:00 & 1.827 & 0.848 & 0.379 & 170.023 & 1.405 & 0.074 & 0.022554 & 0.009605 & 0.339261 & 152.260 \\
\hline 3/1/08 15:00 & 1.89 & 0.911 & 0.452 & 203.059 & 1.413 & 0.082 & 0.024992 & 0.011135 & 0.393285 & 176.506 \\
\hline 3/1/08 16:00 & 1.913 & 0.934 & 0.481 & 216.004 & 1.42 & 0.089 & 0.027126 & 0.012534 & 0.442691 & 198.680 \\
\hline 3/1/08 17:00 & 1.911 & 0.932 & 0.479 & 214.860 & 1.417 & 0.086 & 0.026212 & 0.011928 & 0.421279 & 189.070 \\
\hline 3/1/08 18:00 & 1.873 & 0.894 & 0.432 & 193.797 & 1.413 & 0.082 & 0.024992 & 0.011135 & 0.393285 & 176.506 \\
\hline 3/1/08 19:00 & 1.82 & 0.841 & 0.371 & 166.568 & 1.403 & 0.072 & 0.021945 & 0.009235 & 0.326176 & 146.388 \\
\hline 3/1/08 20:00 & 1.762 & 0.783 & 0.311 & 139.558 & 1.391 & 0.06 & 0.018287 & 0.007117 & 0.251378 & 112.818 \\
\hline 3/1/08 21:00 & 1.715 & 0.736 & 0.267 & 119.739 & 1.382 & 0.051 & 0.015544 & 0.005654 & 0.199703 & 89.627 \\
\hline 3/1/08 22:00 & 1.678 & 0.699 & 0.235 & 105.401 & 1.377 & 0.046 & 0.014020 & 0.004891 & 0.172754 & 77.532 \\
\hline 3/1/08 23:00 & 1.657 & 0.678 & 0.218 & 97.747 & 1.375 & 0.044 & 0.013411 & 0.004596 & 0.162345 & 72.860 \\
\hline 3/2/08 0:00 & 1.643 & 0.664 & 0.207 & 92.835 & 1.372 & 0.041 & 0.012496 & 0.004166 & 0.147141 & 66.037 \\
\hline 3/2/08 1:00 & 1.634 & 0.655 & 0.200 & 89.757 & 1.372 & 0.041 & 0.012496 & 0.004166 & 0.147141 & 66.037 \\
\hline 3/2/08 2:00 & 1.623 & 0.644 & 0.192 & 86.080 & 1.37 & 0.039 & 0.011887 & 0.003887 & 0.137285 & 61.614 \\
\hline 3/2/08 3:00 & 1.627 & 0.648 & 0.195 & 87.406 & 1.37 & 0.039 & 0.011887 & 0.003887 & 0.137285 & 61.614 \\
\hline 3/2/08 4:00 & 1.621 & 0.642 & 0.190 & 85.421 & 1.372 & 0.041 & 0.012496 & 0.004166 & 0.147141 & 66.037 \\
\hline 3/2/08 5:00 & 1.632 & 0.653 & 0.198 & 89.082 & 1.368 & 0.037 & 0.011277 & 0.003614 & 0.127660 & 57.294 \\
\hline 3/2/08 6:00 & 1.623 & 0.644 & 0.192 & 86.080 & 1.368 & 0.037 & 0.011277 & 0.003614 & 0.127660 & 57.294 \\
\hline 3/2/08 7:00 & 1.616 & 0.637 & 0.187 & 83.788 & 1.37 & 0.039 & 0.011887 & 0.003887 & 0.137285 & 61.614 \\
\hline 3/2/08 8:00 & 1.618 & 0.639 & 0.188 & 84.439 & 1.37 & 0.039 & 0.011887 & 0.003887 & 0.137285 & 61.614 \\
\hline 3/2/08 9:00 & 1.616 & 0.637 & 0.187 & 83.788 & 1.37 & 0.039 & 0.011887 & 0.003887 & 0.137285 & 61.614 \\
\hline 3/2/08 10:00 & 1.609 & 0.630 & 0.182 & 81.532 & 1.368 & 0.037 & 0.011277 & 0.003614 & 0.127660 & 57.294 \\
\hline
\end{tabular}




\begin{tabular}{|c|c|c|c|c|c|c|c|c|c|c|}
\hline \multirow[b]{3}{*}{ Date Time } & \multicolumn{4}{|c|}{ V-Notch Weir } & \multicolumn{6}{|c|}{ R-Notch Weir } \\
\hline & \multicolumn{2}{|c|}{ Measured Water Level } & \multicolumn{2}{|c|}{$\begin{array}{c}\text { Calculated Rate } \\
\left(\mathrm{ft}^{3} / \mathrm{s}=448.8 \mathrm{gpm}\right)\end{array}$} & \multicolumn{3}{|c|}{ Measured Water Level } & \multicolumn{3}{|c|}{$\begin{array}{c}\text { Calculated Rate } \\
\left(\mathrm{ft}^{3} / \mathrm{s}=448.8 \mathrm{gpm}\right)\end{array}$} \\
\hline & $\begin{array}{c}\text { (ft above } \\
\text { sensor) }\end{array}$ & $\begin{array}{c}\text { (ft above } \\
\text { notch) }\end{array}$ & $\left(\mathrm{ft}^{3} / \mathrm{s}\right)$ & (gpm) & $\begin{array}{l}\text { (ft above } \\
\text { sensor) }\end{array}$ & $\begin{array}{c}\text { (ft above } \\
\text { log) }\end{array}$ & $\begin{array}{c}\text { (m above } \\
\text { log) }\end{array}$ & $\left(\mathrm{m}^{3} / \mathrm{s}\right)$ & $\left(\mathrm{ft}^{3} / \mathrm{s}\right)$ & (gpm) \\
\hline 3/2/08 11:00 & 1.624 & 0.645 & 0.193 & 86.410 & 1.372 & 0.041 & 0.012496 & 0.004166 & 0.147141 & 66.037 \\
\hline 3/2/08 12:00 & 1.691 & 0.712 & 0.246 & 110.314 & 1.384 & 0.053 & 0.016154 & 0.005969 & 0.210842 & 94.626 \\
\hline 3/2/08 13:00 & 1.707 & 0.728 & 0.260 & 116.546 & 1.382 & 0.051 & 0.015544 & 0.005654 & 0.199703 & 89.627 \\
\hline 3/2/08 14:00 & 1.677 & 0.698 & 0.234 & 105.029 & 1.379 & 0.048 & 0.014630 & 0.005192 & 0.183377 & 82.300 \\
\hline 3/2/08 15:00 & 1.654 & 0.675 & 0.215 & 96.681 & 1.377 & 0.046 & 0.014020 & 0.004891 & 0.172754 & 77.532 \\
\hline 3/2/08 16:00 & 1.638 & 0.659 & 0.203 & 91.117 & 1.375 & 0.044 & 0.013411 & 0.004596 & 0.162345 & 72.860 \\
\hline 3/2/08 17:00 & 1.634 & 0.655 & 0.200 & 89.757 & 1.37 & 0.039 & 0.011887 & 0.003887 & 0.137285 & 61.614 \\
\hline 3/2/08 18:00 & 1.684 & 0.705 & 0.240 & 107.652 & 1.377 & 0.046 & 0.014020 & 0.004891 & 0.172754 & 77.532 \\
\hline 3/2/08 19:00 & 1.764 & 0.785 & 0.313 & 140.442 & 1.394 & 0.063 & 0.019201 & 0.007629 & 0.269465 & 120.936 \\
\hline 3/2/08 20:00 & 1.76 & 0.781 & 0.309 & 138.677 & 1.391 & 0.06 & 0.018287 & 0.007117 & 0.251378 & 112.818 \\
\hline 3/2/08 21:00 & 1.721 & 0.742 & 0.272 & 122.168 & 1.386 & 0.055 & 0.016763 & 0.006291 & 0.222180 & 99.714 \\
\hline 3/2/08 22:00 & 1.698 & 0.719 & 0.252 & 113.015 & 1.379 & 0.048 & 0.014630 & 0.005192 & 0.183377 & 82.300 \\
\hline 3/2/08 23:00 & 1.686 & 0.707 & 0.242 & 108.408 & 1.377 & 0.046 & 0.014020 & 0.004891 & 0.172754 & 77.532 \\
\hline 3/3/08 0:00 & 1.693 & 0.714 & 0.248 & 111.082 & 1.375 & 0.044 & 0.013411 & 0.004596 & 0.162345 & 72.860 \\
\hline 3/3/08 1:00 & 1.656 & 0.677 & 0.217 & 97.391 & 1.365 & 0.034 & 0.010363 & 0.003218 & 0.113667 & 51.014 \\
\hline 3/3/08 2:00 & 1.633 & 0.654 & 0.199 & 89.419 & 1.368 & 0.037 & 0.011277 & 0.003614 & 0.127660 & 57.294 \\
\hline 3/3/08 3:00 & 1.61 & 0.631 & 0.182 & 81.852 & 1.363 & 0.032 & 0.009753 & 0.002963 & 0.104644 & 46.964 \\
\hline 3/3/08 4:00 & 1.599 & 0.620 & 0.175 & 78.375 & 1.361 & 0.03 & 0.009144 & 0.002714 & 0.095873 & 43.028 \\
\hline 3/3/08 5:00 & 1.573 & 0.594 & 0.157 & 70.511 & 1.363 & 0.032 & 0.009753 & 0.002963 & 0.104644 & 46.964 \\
\hline 3/3/08 6:00 & 1.764 & 0.785 & 0.313 & 140.442 & 1.354 & 0.023 & 0.007010 & 0.001905 & 0.067276 & 30.194 \\
\hline 3/3/08 7:00 & 1.846 & 0.867 & 0.400 & 179.617 & 1.37 & 0.039 & 0.011887 & 0.003887 & 0.137285 & 61.614 \\
\hline 3/3/08 8:00 & 1.616 & 0.637 & 0.187 & 83.788 & 1.387 & 0.056 & 0.017068 & 0.006453 & 0.227923 & 102.292 \\
\hline 3/3/08 9:00 & 1.623 & 0.644 & 0.192 & 86.080 & 1.375 & 0.044 & 0.013411 & 0.004596 & 0.162345 & 72.860 \\
\hline 3/3/08 10:00 & 1.653 & 0.674 & 0.215 & 96.328 & 1.377 & 0.046 & 0.014020 & 0.004891 & 0.172754 & 77.532 \\
\hline 3/3/08 11:00 & 1.603 & 0.624 & 0.177 & 79.629 & 1.363 & 0.032 & 0.009753 & 0.002963 & 0.104644 & 46.964 \\
\hline
\end{tabular}




\begin{tabular}{|c|c|c|c|c|c|c|c|c|c|c|}
\hline \multirow[b]{3}{*}{ Date Time } & \multicolumn{4}{|c|}{ V-Notch Weir } & \multicolumn{6}{|c|}{ R-Notch Weir } \\
\hline & \multicolumn{2}{|c|}{ Measured Water Level } & \multicolumn{2}{|c|}{$\begin{array}{c}\text { Calculated Rate } \\
\left(\mathrm{ft}^{3} / \mathrm{s}=448.8 \mathrm{gpm}\right)\end{array}$} & \multicolumn{3}{|c|}{ Measured Water Level } & \multicolumn{3}{|c|}{$\begin{array}{c}\text { Calculated Rate } \\
\left(\mathrm{ft}^{3} / \mathrm{s}=448.8 \mathrm{gpm}\right)\end{array}$} \\
\hline & $\begin{array}{c}\text { (ft above } \\
\text { sensor) }\end{array}$ & $\begin{array}{c}\text { (ft above } \\
\text { notch) }\end{array}$ & $\left(\mathrm{ft}^{3} / \mathrm{s}\right)$ & (gpm) & $\begin{array}{l}\text { (ft above } \\
\text { sensor) }\end{array}$ & $\begin{array}{c}\text { (ft above } \\
\text { log) }\end{array}$ & $\begin{array}{c}\text { (m above } \\
\text { log) }\end{array}$ & $\left(\mathrm{m}^{3} / \mathrm{s}\right)$ & $\left(\mathrm{ft}^{3} / \mathrm{s}\right)$ & (gpm) \\
\hline 3/3/08 12:00 & 1.558 & 0.579 & 0.148 & 66.200 & 1.358 & 0.027 & 0.008229 & 0.002356 & 0.083206 & 37.343 \\
\hline 3/3/08 13:00 & 1.547 & 0.568 & 0.141 & 63.142 & 1.358 & 0.027 & 0.008229 & 0.002356 & 0.083206 & 37.343 \\
\hline 3/3/08 14:00 & 1.548 & 0.569 & 0.141 & 63.416 & 1.36 & 0.029 & 0.008839 & 0.002593 & 0.091584 & 41.103 \\
\hline 3/3/08 15:00 & 1.57 & 0.591 & 0.155 & 69.636 & 1.358 & 0.027 & 0.008229 & 0.002356 & 0.083206 & 37.343 \\
\hline 3/3/08 16:00 & 1.558 & 0.579 & 0.148 & 66.200 & 1.36 & 0.029 & 0.008839 & 0.002593 & 0.091584 & 41.103 \\
\hline 3/3/08 17:00 & 1.565 & 0.586 & 0.152 & 68.192 & 1.363 & 0.032 & 0.009753 & 0.002963 & 0.104644 & 46.964 \\
\hline 3/3/08 18:00 & 1.558 & 0.579 & 0.148 & 66.200 & 1.36 & 0.029 & 0.008839 & 0.002593 & 0.091584 & 41.103 \\
\hline 3/3/08 19:00 & 1.553 & 0.574 & 0.144 & 64.799 & 1.36 & 0.029 & 0.008839 & 0.002593 & 0.091584 & 41.103 \\
\hline 3/3/08 20:00 & 1.552 & 0.573 & 0.144 & 64.521 & 1.358 & 0.027 & 0.008229 & 0.002356 & 0.083206 & 37.343 \\
\hline 3/3/08 21:00 & 1.543 & 0.564 & 0.138 & 62.051 & 1.358 & 0.027 & 0.008229 & 0.002356 & 0.083206 & 37.343 \\
\hline 3/3/08 22:00 & 1.539 & 0.560 & 0.136 & 60.972 & 1.358 & 0.027 & 0.008229 & 0.002356 & 0.083206 & 37.343 \\
\hline 3/3/08 23:00 & 1.534 & 0.555 & 0.133 & 59.638 & 1.356 & 0.025 & 0.007620 & 0.002126 & 0.075100 & 33.705 \\
\hline 3/4/08 0:00 & 1.532 & 0.553 & 0.132 & 59.110 & 1.356 & 0.025 & 0.007620 & 0.002126 & 0.075100 & 33.705 \\
\hline 3/4/08 1:00 & 1.526 & 0.547 & 0.128 & 57.542 & 1.356 & 0.025 & 0.007620 & 0.002126 & 0.075100 & 33.705 \\
\hline 3/4/08 2:00 & 1.523 & 0.544 & 0.126 & 56.767 & 1.356 & 0.025 & 0.007620 & 0.002126 & 0.075100 & 33.705 \\
\hline 3/4/08 3:00 & 1.521 & 0.542 & 0.125 & 56.255 & 1.356 & 0.025 & 0.007620 & 0.002126 & 0.075100 & 33.705 \\
\hline 3/4/08 4:00 & 1.521 & 0.542 & 0.125 & 56.255 & 1.353 & 0.022 & 0.006705 & 0.001797 & 0.063473 & 28.487 \\
\hline 3/4/08 5:00 & 1.519 & 0.540 & 0.124 & 55.744 & 1.356 & 0.025 & 0.007620 & 0.002126 & 0.075100 & 33.705 \\
\hline 3/4/08 6:00 & 1.515 & 0.536 & 0.122 & 54.733 & 1.353 & 0.022 & 0.006705 & 0.001797 & 0.063473 & 28.487 \\
\hline 3/4/08 7:00 & 1.515 & 0.536 & 0.122 & 54.733 & 1.353 & 0.022 & 0.006705 & 0.001797 & 0.063473 & 28.487 \\
\hline 3/4/08 8:00 & 1.515 & 0.536 & 0.122 & 54.733 & 1.353 & 0.022 & 0.006705 & 0.001797 & 0.063473 & 28.487 \\
\hline 3/4/08 9:00 & 1.513 & 0.534 & 0.121 & 54.231 & 1.353 & 0.022 & 0.006705 & 0.001797 & 0.063473 & 28.487 \\
\hline 3/4/08 10:00 & 1.513 & 0.534 & 0.121 & 54.231 & 1.353 & 0.022 & 0.006705 & 0.001797 & 0.063473 & 28.487 \\
\hline 3/4/08 11:00 & 1.512 & 0.533 & 0.120 & 53.981 & 1.356 & 0.025 & 0.007620 & 0.002126 & 0.075100 & 33.705 \\
\hline 3/4/08 12:00 & 1.518 & 0.539 & 0.124 & 55.490 & 1.353 & 0.022 & 0.006705 & 0.001797 & 0.063473 & 28.487 \\
\hline
\end{tabular}




\begin{tabular}{|c|c|c|c|c|c|c|c|c|c|c|}
\hline \multirow[b]{3}{*}{ Date Time } & \multicolumn{4}{|c|}{ V-Notch Weir } & \multicolumn{6}{|c|}{ R-Notch Weir } \\
\hline & \multicolumn{2}{|c|}{ Measured Water Level } & \multicolumn{2}{|c|}{$\begin{array}{c}\text { Calculated Rate } \\
\left(\mathrm{ft}^{3} / \mathrm{s}=448.8 \mathrm{gpm}\right)\end{array}$} & \multicolumn{3}{|c|}{ Measured Water Level } & \multicolumn{3}{|c|}{$\begin{array}{c}\text { Calculated Rate } \\
\left(\mathrm{ft}^{3} / \mathrm{s}=448.8 \mathrm{gpm}\right)\end{array}$} \\
\hline & $\begin{array}{c}\text { (ft above } \\
\text { sensor) }\end{array}$ & $\begin{array}{c}\text { (ft above } \\
\text { notch) }\end{array}$ & $\left(\mathrm{ft}^{3} / \mathrm{s}\right)$ & (gpm) & $\begin{array}{l}\text { (ft above } \\
\text { sensor) }\end{array}$ & $\begin{array}{c}\text { (ft above } \\
\text { log) }\end{array}$ & $\begin{array}{c}\text { (m above } \\
\text { log) }\end{array}$ & $\left(\mathrm{m}^{3} / \mathrm{s}\right)$ & $\left(\mathrm{ft}^{3} / \mathrm{s}\right)$ & (gpm) \\
\hline 3/4/08 13:00 & 1.524 & 0.545 & 0.127 & 57.025 & 1.356 & 0.025 & 0.007620 & 0.002126 & 0.075100 & 33.705 \\
\hline 3/4/08 14:00 & 1.537 & 0.558 & 0.135 & 60.436 & 1.36 & 0.029 & 0.008839 & 0.002593 & 0.091584 & 41.103 \\
\hline 3/4/08 15:00 & 1.55 & 0.571 & 0.143 & 63.967 & 1.36 & 0.029 & 0.008839 & 0.002593 & 0.091584 & 41.103 \\
\hline 3/4/08 16:00 & 1.569 & 0.590 & 0.155 & 69.346 & 1.36 & 0.029 & 0.008839 & 0.002593 & 0.091584 & 41.103 \\
\hline 3/4/08 17:00 & 1.562 & 0.583 & 0.150 & 67.334 & 1.363 & 0.032 & 0.009753 & 0.002963 & 0.104644 & 46.964 \\
\hline 3/4/08 18:00 & 1.558 & 0.579 & 0.148 & 66.200 & 1.36 & 0.029 & 0.008839 & 0.002593 & 0.091584 & 41.103 \\
\hline 3/4/08 19:00 & 1.553 & 0.574 & 0.144 & 64.799 & 1.36 & 0.029 & 0.008839 & 0.002593 & 0.091584 & 41.103 \\
\hline 3/4/08 20:00 & 1.542 & 0.563 & 0.138 & 61.780 & 1.356 & 0.025 & 0.007620 & 0.002126 & 0.075100 & 33.705 \\
\hline 3/4/08 21:00 & 1.54 & 0.561 & 0.136 & 61.240 & 1.358 & 0.027 & 0.008229 & 0.002356 & 0.083206 & 37.343 \\
\hline 3/4/08 22:00 & 1.54 & 0.561 & 0.136 & 61.240 & 1.358 & 0.027 & 0.008229 & 0.002356 & 0.083206 & 37.343 \\
\hline 3/4/08 23:00 & 1.536 & 0.557 & 0.134 & 60.170 & 1.356 & 0.025 & 0.007620 & 0.002126 & 0.075100 & 33.705 \\
\hline 3/5/08 0:00 & 1.527 & 0.548 & 0.129 & 57.802 & 1.358 & 0.027 & 0.008229 & 0.002356 & 0.083206 & 37.343 \\
\hline 3/5/08 1:00 & 1.53 & 0.551 & 0.131 & 58.585 & 1.356 & 0.025 & 0.007620 & 0.002126 & 0.075100 & 33.705 \\
\hline 3/5/08 2:00 & 1.526 & 0.547 & 0.128 & 57.542 & 1.358 & 0.027 & 0.008229 & 0.002356 & 0.083206 & 37.343 \\
\hline 3/5/08 3:00 & 1.526 & 0.547 & 0.128 & 57.542 & 1.356 & 0.025 & 0.007620 & 0.002126 & 0.075100 & 33.705 \\
\hline 3/5/08 4:00 & 1.533 & 0.554 & 0.132 & 59.374 & 1.356 & 0.025 & 0.007620 & 0.002126 & 0.075100 & 33.705 \\
\hline 3/5/08 5:00 & 1.531 & 0.552 & 0.131 & 58.847 & 1.356 & 0.025 & 0.007620 & 0.002126 & 0.075100 & 33.705 \\
\hline 3/5/08 6:00 & 1.515 & 0.536 & 0.122 & 54.733 & 1.353 & 0.022 & 0.006705 & 0.001797 & 0.063473 & 28.487 \\
\hline 3/5/08 7:00 & 1.523 & 0.544 & 0.126 & 56.767 & 1.356 & 0.025 & 0.007620 & 0.002126 & 0.075100 & 33.705 \\
\hline 3/5/08 8:00 & 1.516 & 0.537 & 0.123 & 54.984 & 1.354 & 0.023 & 0.007010 & 0.001905 & 0.067276 & 30.194 \\
\hline 3/5/08 9:00 & 1.504 & 0.525 & 0.116 & 52.007 & 1.356 & 0.025 & 0.007620 & 0.002126 & 0.075100 & 33.705 \\
\hline 3/5/08 10:00 & 1.525 & 0.546 & 0.128 & 57.283 & 1.351 & 0.02 & 0.006096 & 0.001588 & 0.056092 & 25.174 \\
\hline 3/5/08 11:00 & 1.535 & 0.556 & 0.133 & 59.904 & 1.356 & 0.025 & 0.007620 & 0.002126 & 0.075100 & 33.705 \\
\hline 3/5/08 12:00 & 1.554 & 0.575 & 0.145 & 65.078 & 1.354 & 0.023 & 0.007010 & 0.001905 & 0.067276 & 30.194 \\
\hline 3/5/08 13:00 & 1.513 & 0.534 & 0.121 & 54.231 & 1.354 & 0.023 & 0.007010 & 0.001905 & 0.067276 & 30.194 \\
\hline
\end{tabular}




\begin{tabular}{|c|c|c|c|c|c|c|c|c|c|c|}
\hline \multirow[b]{3}{*}{ Date Time } & \multicolumn{4}{|c|}{ V-Notch Weir } & \multicolumn{6}{|c|}{ R-Notch Weir } \\
\hline & \multicolumn{2}{|c|}{ Measured Water Level } & \multicolumn{2}{|c|}{$\begin{array}{c}\text { Calculated Rate } \\
\left(\mathrm{ft}^{3} / \mathrm{s}=448.8 \mathrm{gpm}\right)\end{array}$} & \multicolumn{3}{|c|}{ Measured Water Level } & \multicolumn{3}{|c|}{$\begin{array}{c}\text { Calculated Rate } \\
\left(\mathrm{ft}^{3} / \mathrm{s}=448.8 \mathrm{gpm}\right)\end{array}$} \\
\hline & $\begin{array}{c}\text { (ft above } \\
\text { sensor) }\end{array}$ & $\begin{array}{c}\text { (ft above } \\
\text { notch) }\end{array}$ & $\left(\mathrm{ft}^{3} / \mathrm{s}\right)$ & (gpm) & $\begin{array}{l}\text { (ft above } \\
\text { sensor) }\end{array}$ & $\begin{array}{c}\text { (ft above } \\
\text { log) }\end{array}$ & $\begin{array}{c}\text { (m above } \\
\text { log) }\end{array}$ & $\left(\mathrm{m}^{3} / \mathrm{s}\right)$ & $\left(\mathrm{ft}^{3} / \mathrm{s}\right)$ & (gpm) \\
\hline 3/5/08 14:00 & 1.512 & 0.533 & 0.120 & 53.981 & 1.358 & 0.027 & 0.008229 & 0.002356 & 0.083206 & 37.343 \\
\hline 3/5/08 15:00 & 1.521 & 0.542 & 0.125 & 56.255 & 1.356 & 0.025 & 0.007620 & 0.002126 & 0.075100 & 33.705 \\
\hline 3/5/08 16:00 & 1.534 & 0.555 & 0.133 & 59.638 & 1.353 & 0.022 & 0.006705 & 0.001797 & 0.063473 & 28.487 \\
\hline 3/5/08 17:00 & 1.515 & 0.536 & 0.122 & 54.733 & 1.358 & 0.027 & 0.008229 & 0.002356 & 0.083206 & 37.343 \\
\hline 3/5/08 18:00 & 1.523 & 0.544 & 0.126 & 56.767 & 1.351 & 0.02 & 0.006096 & 0.001588 & 0.056092 & 25.174 \\
\hline 3/5/08 19:00 & 1.521 & 0.542 & 0.125 & 56.255 & 1.353 & 0.022 & 0.006705 & 0.001797 & 0.063473 & 28.487 \\
\hline 3/5/08 20:00 & 1.519 & 0.540 & 0.124 & 55.744 & 1.353 & 0.022 & 0.006705 & 0.001797 & 0.063473 & 28.487 \\
\hline 3/5/08 21:00 & 1.515 & 0.536 & 0.122 & 54.733 & 1.353 & 0.022 & 0.006705 & 0.001797 & 0.063473 & 28.487 \\
\hline 3/5/08 22:00 & 1.508 & 0.529 & 0.118 & 52.988 & 1.353 & 0.022 & 0.006705 & 0.001797 & 0.063473 & 28.487 \\
\hline 3/5/08 23:00 & 1.499 & 0.520 & 0.113 & 50.796 & 1.351 & 0.02 & 0.006096 & 0.001588 & 0.056092 & 25.174 \\
\hline 3/6/08 0:00 & 1.504 & 0.525 & 0.116 & 52.007 & 1.351 & 0.02 & 0.006096 & 0.001588 & 0.056092 & 25.174 \\
\hline 3/6/08 1:00 & 1.5 & 0.521 & 0.114 & 51.037 & 1.351 & 0.02 & 0.006096 & 0.001588 & 0.056092 & 25.174 \\
\hline 3/6/08 2:00 & 1.498 & 0.519 & 0.113 & 50.555 & 1.351 & 0.02 & 0.006096 & 0.001588 & 0.056092 & 25.174 \\
\hline 3/6/08 3:00 & 1.498 & 0.519 & 0.113 & 50.555 & 1.351 & 0.02 & 0.006096 & 0.001588 & 0.056092 & 25.174 \\
\hline 3/6/08 4:00 & 1.493 & 0.514 & 0.110 & 49.365 & 1.351 & 0.02 & 0.006096 & 0.001588 & 0.056092 & 25.174 \\
\hline 3/6/08 5:00 & 1.49 & 0.511 & 0.108 & 48.658 & 1.351 & 0.02 & 0.006096 & 0.001588 & 0.056092 & 25.174 \\
\hline 3/6/08 6:00 & 1.49 & 0.511 & 0.108 & 48.658 & 1.351 & 0.02 & 0.006096 & 0.001588 & 0.056092 & 25.174 \\
\hline 3/6/08 7:00 & 1.49 & 0.511 & 0.108 & 48.658 & 1.349 & 0.018 & 0.005486 & 0.001388 & 0.049021 & 22.001 \\
\hline 3/6/08 8:00 & 1.488 & 0.509 & 0.107 & 48.191 & 1.349 & 0.018 & 0.005486 & 0.001388 & 0.049021 & 22.001 \\
\hline 3/6/08 9:00 & 1.488 & 0.509 & 0.107 & 48.191 & 1.349 & 0.018 & 0.005486 & 0.001388 & 0.049021 & 22.001 \\
\hline 3/6/08 10:00 & 1.488 & 0.509 & 0.107 & 48.191 & 1.351 & 0.02 & 0.006096 & 0.001588 & 0.056092 & 25.174 \\
\hline 3/6/08 11:00 & 1.491 & 0.512 & 0.109 & 48.893 & 1.353 & 0.022 & 0.006705 & 0.001797 & 0.063473 & 28.487 \\
\hline 3/6/08 12:00 & 1.5 & 0.521 & 0.114 & 51.037 & 1.351 & 0.02 & 0.006096 & 0.001588 & 0.056092 & 25.174 \\
\hline 3/6/08 13:00 & 1.51 & 0.531 & 0.119 & 53.483 & 1.356 & 0.025 & 0.007620 & 0.002126 & 0.075100 & 33.705 \\
\hline 3/6/08 14:00 & 1.528 & 0.549 & 0.129 & 58.062 & 1.356 & 0.025 & 0.007620 & 0.002126 & 0.075100 & 33.705 \\
\hline
\end{tabular}




\begin{tabular}{|c|c|c|c|c|c|c|c|c|c|c|}
\hline \multirow[b]{3}{*}{ Date Time } & \multicolumn{4}{|c|}{ V-Notch Weir } & \multicolumn{6}{|c|}{ R-Notch Weir } \\
\hline & \multicolumn{2}{|c|}{ Measured Water Level } & \multicolumn{2}{|c|}{$\begin{array}{c}\text { Calculated Rate } \\
\left(\mathrm{ft}^{3} / \mathrm{s}=448.8 \mathrm{gpm}\right)\end{array}$} & \multicolumn{3}{|c|}{ Measured Water Level } & \multicolumn{3}{|c|}{$\begin{array}{c}\text { Calculated Rate } \\
\left(\mathrm{ft}^{3} / \mathrm{s}=448.8 \mathrm{gpm}\right)\end{array}$} \\
\hline & $\begin{array}{l}\text { (ft above } \\
\text { sensor) }\end{array}$ & $\begin{array}{l}\text { (ft above } \\
\text { notch) }\end{array}$ & $\left(\mathrm{ft}^{3} / \mathrm{s}\right)$ & (gpm) & $\begin{array}{l}\text { (ft above } \\
\text { sensor) }\end{array}$ & $\begin{array}{l}\text { (ft above } \\
\text { log) }\end{array}$ & $\begin{array}{c}\text { (m above } \\
\text { log) }\end{array}$ & $\left(\mathrm{m}^{3} / \mathrm{s}\right)$ & $\left(\mathrm{ft}^{3} / \mathrm{s}\right)$ & (gpm) \\
\hline 3/6/08 15:00 & 1.541 & 0.562 & 0.137 & 61.510 & 1.358 & 0.027 & 0.008229 & 0.002356 & 0.083206 & 37.343 \\
\hline 3/6/08 16:00 & 1.55 & 0.571 & 0.143 & 63.967 & 1.36 & 0.029 & 0.008839 & 0.002593 & 0.091584 & 41.103 \\
\hline 3/6/08 17:00 & 1.554 & 0.575 & 0.145 & 65.078 & 1.363 & 0.032 & 0.009753 & 0.002963 & 0.104644 & 46.964 \\
\hline 3/6/08 18:00 & 1.545 & 0.566 & 0.139 & 62.595 & 1.358 & 0.027 & 0.008229 & 0.002356 & 0.083206 & 37.343 \\
\hline 3/6/08 19:00 & 1.54 & 0.561 & 0.136 & 61.240 & 1.358 & 0.027 & 0.008229 & 0.002356 & 0.083206 & 37.343 \\
\hline 3/6/08 20:00 & 1.533 & 0.554 & 0.132 & 59.374 & 1.358 & 0.027 & 0.008229 & 0.002356 & 0.083206 & 37.343 \\
\hline 3/6/08 21:00 & 1.534 & 0.555 & 0.133 & 59.638 & 1.358 & 0.027 & 0.008229 & 0.002356 & 0.083206 & 37.343 \\
\hline 3/6/08 22:00 & 1.532 & 0.553 & 0.132 & 59.110 & 1.356 & 0.025 & 0.007620 & 0.002126 & 0.075100 & 33.705 \\
\hline 3/6/08 23:00 & 1.549 & 0.570 & 0.142 & 63.691 & 1.356 & 0.025 & 0.007620 & 0.002126 & 0.075100 & 33.705 \\
\hline 3/7/08 0:00 & 1.542 & 0.563 & 0.138 & 61.780 & 1.356 & 0.025 & 0.007620 & 0.002126 & 0.075100 & 33.705 \\
\hline 3/7/08 1:00 & 1.547 & 0.568 & 0.141 & 63.142 & 1.353 & 0.022 & 0.006705 & 0.001797 & 0.063473 & 28.487 \\
\hline 3/7/08 2:00 & 1.56 & 0.581 & 0.149 & 66.766 & 1.353 & 0.022 & 0.006705 & 0.001797 & 0.063473 & 28.487 \\
\hline 3/7/08 3:00 & 1.554 & 0.575 & 0.145 & 65.078 & 1.353 & 0.022 & 0.006705 & 0.001797 & 0.063473 & 28.487 \\
\hline 3/7/08 4:00 & 1.543 & 0.564 & 0.138 & 62.051 & 1.356 & 0.025 & 0.007620 & 0.002126 & 0.075100 & 33.705 \\
\hline 3/7/08 5:00 & 1.547 & 0.568 & 0.141 & 63.142 & 1.353 & 0.022 & 0.006705 & 0.001797 & 0.063473 & 28.487 \\
\hline 3/7/08 6:00 & 1.526 & 0.547 & 0.128 & 57.542 & 1.349 & 0.018 & 0.005486 & 0.001388 & 0.049021 & 22.001 \\
\hline 3/7/08 7:00 & 1.514 & 0.535 & 0.121 & 54.481 & 1.349 & 0.018 & 0.005486 & 0.001388 & 0.049021 & 22.001 \\
\hline 3/7/08 8:00 & 1.498 & 0.519 & 0.113 & 50.555 & 1.354 & 0.023 & 0.007010 & 0.001905 & 0.067276 & 30.194 \\
\hline 3/7/08 9:00 & 1.439 & 0.460 & 0.084 & 37.569 & 1.358 & 0.027 & 0.008229 & 0.002356 & 0.083206 & 37.343 \\
\hline 3/7/08 10:00 & 1.342 & 0.363 & 0.047 & 21.018 & 1.375 & 0.044 & 0.013411 & 0.004596 & 0.162345 & 72.860 \\
\hline 3/7/08 11:00 & 1.57 & 0.591 & 0.155 & 69.636 & 1.384 & 0.053 & 0.016154 & 0.005969 & 0.210842 & 94.626 \\
\hline 3/7/08 12:00 & 1.612 & 0.633 & 0.184 & 82.494 & 1.37 & 0.039 & 0.011887 & 0.003887 & 0.137285 & 61.614 \\
\hline 3/7/08 13:00 & 1.571 & 0.592 & 0.156 & 69.927 & 1.356 & 0.025 & 0.007620 & 0.002126 & 0.075100 & 33.705 \\
\hline 3/7/08 14:00 & 1.549 & 0.570 & 0.142 & 63.691 & 1.353 & 0.022 & 0.006705 & 0.001797 & 0.063473 & 28.487 \\
\hline 3/7/08 15:00 & 1.541 & 0.562 & 0.137 & 61.510 & 1.356 & 0.025 & 0.007620 & 0.002126 & 0.075100 & 33.705 \\
\hline
\end{tabular}




\begin{tabular}{|c|c|c|c|c|c|c|c|c|c|c|}
\hline \multirow[b]{3}{*}{ Date Time } & \multicolumn{4}{|c|}{ V-Notch Weir } & \multicolumn{6}{|c|}{ R-Notch Weir } \\
\hline & \multicolumn{2}{|c|}{ Measured Water Level } & \multicolumn{2}{|c|}{$\begin{array}{c}\text { Calculated Rate } \\
\left(\mathrm{ft}^{3} / \mathrm{s}=448.8 \mathrm{gpm}\right)\end{array}$} & \multicolumn{3}{|c|}{ Measured Water Level } & \multicolumn{3}{|c|}{$\begin{array}{c}\text { Calculated Rate } \\
\left(\mathrm{ft}^{3} / \mathrm{s}=448.8 \mathrm{gpm}\right)\end{array}$} \\
\hline & $\begin{array}{c}\text { (ft above } \\
\text { sensor) }\end{array}$ & $\begin{array}{c}\text { (ft above } \\
\text { notch) }\end{array}$ & $\left(\mathrm{ft}^{3} / \mathrm{s}\right)$ & (gpm) & $\begin{array}{l}\text { (ft above } \\
\text { sensor) }\end{array}$ & $\begin{array}{c}\text { (ft above } \\
\text { log) }\end{array}$ & $\begin{array}{c}\text { (m above } \\
\text { log) }\end{array}$ & $\left(\mathrm{m}^{3} / \mathrm{s}\right)$ & $\left(\mathrm{ft}^{3} / \mathrm{s}\right)$ & (gpm) \\
\hline 3/7/08 16:00 & 1.547 & 0.568 & 0.141 & 63.142 & 1.356 & 0.025 & 0.007620 & 0.002126 & 0.075100 & 33.705 \\
\hline 3/7/08 17:00 & 1.549 & 0.570 & 0.142 & 63.691 & 1.356 & 0.025 & 0.007620 & 0.002126 & 0.075100 & 33.705 \\
\hline 3/7/08 18:00 & 1.545 & 0.566 & 0.139 & 62.595 & 1.356 & 0.025 & 0.007620 & 0.002126 & 0.075100 & 33.705 \\
\hline 3/7/08 19:00 & 1.536 & 0.557 & 0.134 & 60.170 & 1.353 & 0.022 & 0.006705 & 0.001797 & 0.063473 & 28.487 \\
\hline 3/7/08 20:00 & 1.531 & 0.552 & 0.131 & 58.847 & 1.353 & 0.022 & 0.006705 & 0.001797 & 0.063473 & 28.487 \\
\hline 3/7/08 21:00 & 1.532 & 0.553 & 0.132 & 59.110 & 1.353 & 0.022 & 0.006705 & 0.001797 & 0.063473 & 28.487 \\
\hline 3/7/08 22:00 & 1.525 & 0.546 & 0.128 & 57.283 & 1.353 & 0.022 & 0.006705 & 0.001797 & 0.063473 & 28.487 \\
\hline 3/7/08 23:00 & 1.52 & 0.541 & 0.125 & 55.999 & 1.351 & 0.02 & 0.006096 & 0.001588 & 0.056092 & 25.174 \\
\hline 3/8/08 0:00 & 1.516 & 0.537 & 0.123 & 54.984 & 1.351 & 0.02 & 0.006096 & 0.001588 & 0.056092 & 25.174 \\
\hline 3/8/08 1:00 & 1.514 & 0.535 & 0.121 & 54.481 & 1.349 & 0.018 & 0.005486 & 0.001388 & 0.049021 & 22.001 \\
\hline 3/8/08 2:00 & 1.509 & 0.530 & 0.119 & 53.236 & 1.351 & 0.02 & 0.006096 & 0.001588 & 0.056092 & 25.174 \\
\hline 3/8/08 3:00 & 1.505 & 0.526 & 0.116 & 52.251 & 1.349 & 0.018 & 0.005486 & 0.001388 & 0.049021 & 22.001 \\
\hline 3/8/08 4:00 & 1.505 & 0.526 & 0.116 & 52.251 & 1.349 & 0.018 & 0.005486 & 0.001388 & 0.049021 & 22.001 \\
\hline 3/8/08 5:00 & 1.503 & 0.524 & 0.115 & 51.763 & 1.349 & 0.018 & 0.005486 & 0.001388 & 0.049021 & 22.001 \\
\hline 3/8/08 6:00 & 1.501 & 0.522 & 0.114 & 51.278 & 1.349 & 0.018 & 0.005486 & 0.001388 & 0.049021 & 22.001 \\
\hline 3/8/08 7:00 & 1.499 & 0.520 & 0.113 & 50.796 & 1.349 & 0.018 & 0.005486 & 0.001388 & 0.049021 & 22.001 \\
\hline 3/8/08 8:00 & 1.497 & 0.518 & 0.112 & 50.316 & 1.349 & 0.018 & 0.005486 & 0.001388 & 0.049021 & 22.001 \\
\hline 3/8/08 9:00 & 1.494 & 0.515 & 0.111 & 49.601 & 1.346 & 0.015 & 0.004572 & 0.001105 & 0.039030 & 17.516 \\
\hline 3/8/08 10:00 & 1.497 & 0.518 & 0.112 & 50.316 & 1.349 & 0.018 & 0.005486 & 0.001388 & 0.049021 & 22.001 \\
\hline 3/8/08 11:00 & 1.501 & 0.522 & 0.114 & 51.278 & 1.349 & 0.018 & 0.005486 & 0.001388 & 0.049021 & 22.001 \\
\hline 3/8/08 12:00 & 1.505 & 0.526 & 0.116 & 52.251 & 1.349 & 0.018 & 0.005486 & 0.001388 & 0.049021 & 22.001 \\
\hline 3/8/08 13:00 & 1.525 & 0.546 & 0.128 & 57.283 & 1.353 & 0.022 & 0.006705 & 0.001797 & 0.063473 & 28.487 \\
\hline 3/8/08 14:00 & 1.529 & 0.550 & 0.130 & 58.323 & 1.353 & 0.022 & 0.006705 & 0.001797 & 0.063473 & 28.487 \\
\hline 3/8/08 15:00 & 1.55 & 0.571 & 0.143 & 63.967 & 1.356 & 0.025 & 0.007620 & 0.002126 & 0.075100 & 33.705 \\
\hline 3/8/08 16:00 & 1.554 & 0.575 & 0.145 & 65.078 & 1.358 & 0.027 & 0.008229 & 0.002356 & 0.083206 & 37.343 \\
\hline
\end{tabular}




\begin{tabular}{|c|c|c|c|c|c|c|c|c|c|c|}
\hline \multirow[b]{3}{*}{ Date Time } & \multicolumn{4}{|c|}{ V-Notch Weir } & \multicolumn{6}{|c|}{ R-Notch Weir } \\
\hline & \multicolumn{2}{|c|}{ Measured Water Level } & \multicolumn{2}{|c|}{$\begin{array}{c}\text { Calculated Rate } \\
\left(\mathrm{ft}^{3} / \mathrm{s}=448.8 \mathrm{gpm}\right)\end{array}$} & \multicolumn{3}{|c|}{ Measured Water Level } & \multicolumn{3}{|c|}{$\begin{array}{c}\text { Calculated Rate } \\
\left(\mathrm{ft}^{3} / \mathrm{s}=448.8 \mathrm{gpm}\right)\end{array}$} \\
\hline & $\begin{array}{l}\text { (ft above } \\
\text { sensor) }\end{array}$ & $\begin{array}{l}\text { (ft above } \\
\text { notch) }\end{array}$ & $\left(\mathrm{ft}^{3} / \mathrm{s}\right)$ & (gpm) & $\begin{array}{l}\text { (ft above } \\
\text { sensor) }\end{array}$ & $\begin{array}{l}\text { (ft above } \\
\text { log) }\end{array}$ & $\begin{array}{c}\text { (m above } \\
\text { log) }\end{array}$ & $\left(\mathrm{m}^{3} / \mathrm{s}\right)$ & $\left(\mathrm{ft}^{3} / \mathrm{s}\right)$ & (gpm) \\
\hline 3/8/08 17:00 & 1.57 & 0.591 & 0.155 & 69.636 & 1.36 & 0.029 & 0.008839 & 0.002593 & 0.091584 & 41.103 \\
\hline 3/8/08 18:00 & 1.566 & 0.587 & 0.153 & 68.479 & 1.358 & 0.027 & 0.008229 & 0.002356 & 0.083206 & 37.343 \\
\hline 3/8/08 19:00 & 1.556 & 0.577 & 0.146 & 65.638 & 1.358 & 0.027 & 0.008229 & 0.002356 & 0.083206 & 37.343 \\
\hline 3/8/08 20:00 & 1.554 & 0.575 & 0.145 & 65.078 & 1.356 & 0.025 & 0.007620 & 0.002126 & 0.075100 & 33.705 \\
\hline 3/8/08 21:00 & 1.543 & 0.564 & 0.138 & 62.051 & 1.356 & 0.025 & 0.007620 & 0.002126 & 0.075100 & 33.705 \\
\hline 3/8/08 22:00 & 1.541 & 0.562 & 0.137 & 61.510 & 1.353 & 0.022 & 0.006705 & 0.001797 & 0.063473 & 28.487 \\
\hline 3/8/08 23:00 & 1.534 & 0.555 & 0.133 & 59.638 & 1.353 & 0.022 & 0.006705 & 0.001797 & 0.063473 & 28.487 \\
\hline 3/9/08 0:00 & 1.534 & 0.555 & 0.133 & 59.638 & 1.353 & 0.022 & 0.006705 & 0.001797 & 0.063473 & 28.487 \\
\hline 3/9/08 1:00 & 1.532 & 0.553 & 0.132 & 59.110 & 1.353 & 0.022 & 0.006705 & 0.001797 & 0.063473 & 28.487 \\
\hline 3/9/08 2:00 & 1.53 & 0.551 & 0.131 & 58.585 & 1.353 & 0.022 & 0.006705 & 0.001797 & 0.063473 & 28.487 \\
\hline 3/9/08 3:00 & 1.521 & 0.542 & 0.125 & 56.255 & 1.353 & 0.022 & 0.006705 & 0.001797 & 0.063473 & 28.487 \\
\hline 3/9/08 4:00 & 1.521 & 0.542 & 0.125 & 56.255 & 1.353 & 0.022 & 0.006705 & 0.001797 & 0.063473 & 28.487 \\
\hline 3/9/08 5:00 & 1.521 & 0.542 & 0.125 & 56.255 & 1.353 & 0.022 & 0.006705 & 0.001797 & 0.063473 & 28.487 \\
\hline 3/9/08 6:00 & 1.519 & 0.540 & 0.124 & 55.744 & 1.353 & 0.022 & 0.006705 & 0.001797 & 0.063473 & 28.487 \\
\hline 3/9/08 7:00 & 1.515 & 0.536 & 0.122 & 54.733 & 1.351 & 0.02 & 0.006096 & 0.001588 & 0.056092 & 25.174 \\
\hline 3/9/08 8:00 & 1.512 & 0.533 & 0.120 & 53.981 & 1.351 & 0.02 & 0.006096 & 0.001588 & 0.056092 & 25.174 \\
\hline 3/9/08 9:00 & 1.51 & 0.531 & 0.119 & 53.483 & 1.351 & 0.02 & 0.006096 & 0.001588 & 0.056092 & 25.174 \\
\hline 3/9/08 10:00 & 1.515 & 0.536 & 0.122 & 54.733 & 1.351 & 0.02 & 0.006096 & 0.001588 & 0.056092 & 25.174 \\
\hline 3/9/08 11:00 & 1.514 & 0.535 & 0.121 & 54.481 & 1.351 & 0.02 & 0.006096 & 0.001588 & 0.056092 & 25.174 \\
\hline 3/9/08 12:00 & 1.516 & 0.537 & 0.123 & 54.984 & 1.353 & 0.022 & 0.006705 & 0.001797 & 0.063473 & 28.487 \\
\hline 3/9/08 13:00 & 1.522 & 0.543 & 0.126 & 56.511 & 1.353 & 0.022 & 0.006705 & 0.001797 & 0.063473 & 28.487 \\
\hline 3/9/08 14:00 & 1.531 & 0.552 & 0.131 & 58.847 & 1.353 & 0.022 & 0.006705 & 0.001797 & 0.063473 & 28.487 \\
\hline 3/9/08 15:00 & 1.551 & 0.572 & 0.143 & 64.244 & 1.356 & 0.025 & 0.007620 & 0.002126 & 0.075100 & 33.705 \\
\hline 3/9/08 16:00 & 1.576 & 0.597 & 0.159 & 71.393 & 1.36 & 0.029 & 0.008839 & 0.002593 & 0.091584 & 41.103 \\
\hline 3/9/08 17:00 & 1.567 & 0.588 & 0.153 & 68.767 & 1.36 & 0.029 & 0.008839 & 0.002593 & 0.091584 & 41.103 \\
\hline
\end{tabular}




\begin{tabular}{|c|c|c|c|c|c|c|c|c|c|c|}
\hline \multirow[b]{3}{*}{ Date Time } & \multicolumn{4}{|c|}{ V-Notch Weir } & \multicolumn{6}{|c|}{ R-Notch Weir } \\
\hline & \multicolumn{2}{|c|}{ Measured Water Level } & \multicolumn{2}{|c|}{$\begin{array}{c}\text { Calculated Rate } \\
\left(\mathrm{ft}^{3} / \mathrm{s}=448.8 \mathrm{gpm}\right)\end{array}$} & \multicolumn{3}{|c|}{ Measured Water Level } & \multicolumn{3}{|c|}{$\begin{array}{c}\text { Calculated Rate } \\
\left(\mathrm{ft}^{3} / \mathrm{s}=448.8 \mathrm{gpm}\right)\end{array}$} \\
\hline & $\begin{array}{c}\text { (ft above } \\
\text { sensor) }\end{array}$ & $\begin{array}{c}\text { (ft above } \\
\text { notch) }\end{array}$ & $\left(\mathrm{ft}^{3} / \mathrm{s}\right)$ & (gpm) & $\begin{array}{l}\text { (ft above } \\
\text { sensor) }\end{array}$ & $\begin{array}{c}\text { (ft above } \\
\text { log) }\end{array}$ & $\begin{array}{c}\text { (m above } \\
\text { log) }\end{array}$ & $\left(\mathrm{m}^{3} / \mathrm{s}\right)$ & $\left(\mathrm{ft}^{3} / \mathrm{s}\right)$ & (gpm) \\
\hline 3/9/08 18:00 & 1.56 & 0.581 & 0.149 & 66.766 & 1.358 & 0.027 & 0.008229 & 0.002356 & 0.083206 & 37.343 \\
\hline 3/9/08 19:00 & 1.549 & 0.570 & 0.142 & 63.691 & 1.356 & 0.025 & 0.007620 & 0.002126 & 0.075100 & 33.705 \\
\hline 3/9/08 20:00 & 1.542 & 0.563 & 0.138 & 61.780 & 1.356 & 0.025 & 0.007620 & 0.002126 & 0.075100 & 33.705 \\
\hline 3/9/08 21:00 & 1.537 & 0.558 & 0.135 & 60.436 & 1.356 & 0.025 & 0.007620 & 0.002126 & 0.075100 & 33.705 \\
\hline 3/9/08 22:00 & 1.531 & 0.552 & 0.131 & 58.847 & 1.353 & 0.022 & 0.006705 & 0.001797 & 0.063473 & 28.487 \\
\hline 3/9/08 23:00 & 1.524 & 0.545 & 0.127 & 57.025 & 1.353 & 0.022 & 0.006705 & 0.001797 & 0.063473 & 28.487 \\
\hline 3/10/08 0:00 & 1.522 & 0.543 & 0.126 & 56.511 & 1.353 & 0.022 & 0.006705 & 0.001797 & 0.063473 & 28.487 \\
\hline 3/10/08 1:00 & 1.525 & 0.546 & 0.128 & 57.283 & 1.353 & 0.022 & 0.006705 & 0.001797 & 0.063473 & 28.487 \\
\hline 3/10/08 2:00 & 1.518 & 0.539 & 0.124 & 55.490 & 1.351 & 0.02 & 0.006096 & 0.001588 & 0.056092 & 25.174 \\
\hline 3/10/08 3:00 & 1.518 & 0.539 & 0.124 & 55.490 & 1.351 & 0.02 & 0.006096 & 0.001588 & 0.056092 & 25.174 \\
\hline 3/10/08 4:00 & 1.514 & 0.535 & 0.121 & 54.481 & 1.351 & 0.02 & 0.006096 & 0.001588 & 0.056092 & 25.174 \\
\hline 3/10/08 5:00 & 1.514 & 0.535 & 0.121 & 54.481 & 1.351 & 0.02 & 0.006096 & 0.001588 & 0.056092 & 25.174 \\
\hline 3/10/08 6:00 & 1.512 & 0.533 & 0.120 & 53.981 & 1.351 & 0.02 & 0.006096 & 0.001588 & 0.056092 & 25.174 \\
\hline 3/10/08 7:00 & 1.51 & 0.531 & 0.119 & 53.483 & 1.351 & 0.02 & 0.006096 & 0.001588 & 0.056092 & 25.174 \\
\hline 3/10/08 8:00 & 1.51 & 0.531 & 0.119 & 53.483 & 1.349 & 0.018 & 0.005486 & 0.001388 & 0.049021 & 22.001 \\
\hline 3/10/08 9:00 & 1.503 & 0.524 & 0.115 & 51.763 & 1.349 & 0.018 & 0.005486 & 0.001388 & 0.049021 & 22.001 \\
\hline 3/10/08 10:00 & 1.505 & 0.526 & 0.116 & 52.251 & 1.349 & 0.018 & 0.005486 & 0.001388 & 0.049021 & 22.001 \\
\hline 3/10/08 11:00 & 1.507 & 0.528 & 0.118 & 52.742 & 1.351 & 0.02 & 0.006096 & 0.001588 & 0.056092 & 25.174 \\
\hline 3/10/08 12:00 & 1.513 & 0.534 & 0.121 & 54.231 & 1.351 & 0.02 & 0.006096 & 0.001588 & 0.056092 & 25.174 \\
\hline 3/10/08 13:00 & 1.523 & 0.544 & 0.126 & 56.767 & 1.353 & 0.022 & 0.006705 & 0.001797 & 0.063473 & 28.487 \\
\hline 3/10/08 14:00 & 1.534 & 0.555 & 0.133 & 59.638 & 1.356 & 0.025 & 0.007620 & 0.002126 & 0.075100 & 33.705 \\
\hline 3/10/08 15:00 & 1.547 & 0.568 & 0.141 & 63.142 & 1.358 & 0.027 & 0.008229 & 0.002356 & 0.083206 & 37.343 \\
\hline 3/10/08 16:00 & 1.561 & 0.582 & 0.149 & 67.049 & 1.358 & 0.027 & 0.008229 & 0.002356 & 0.083206 & 37.343 \\
\hline 3/10/08 17:00 & 1.572 & 0.593 & 0.156 & 70.219 & 1.358 & 0.027 & 0.008229 & 0.002356 & 0.083206 & 37.343 \\
\hline 3/10/08 18:00 & 1.574 & 0.595 & 0.158 & 70.805 & 1.358 & 0.027 & 0.008229 & 0.002356 & 0.083206 & 37.343 \\
\hline
\end{tabular}




\begin{tabular}{|c|c|c|c|c|c|c|c|c|c|c|}
\hline \multirow[b]{3}{*}{ Date Time } & \multicolumn{4}{|c|}{ V-Notch Weir } & \multicolumn{6}{|c|}{ R-Notch Weir } \\
\hline & \multicolumn{2}{|c|}{ Measured Water Level } & \multicolumn{2}{|c|}{$\begin{array}{c}\text { Calculated Rate } \\
\left(\mathrm{ft}^{3} / \mathrm{s}=448.8 \mathrm{gpm}\right)\end{array}$} & \multicolumn{3}{|c|}{ Measured Water Level } & \multicolumn{3}{|c|}{$\begin{array}{c}\text { Calculated Rate } \\
\left(\mathrm{ft}^{3} / \mathrm{s}=448.8 \mathrm{gpm}\right)\end{array}$} \\
\hline & $\begin{array}{l}\text { (ft above } \\
\text { sensor) }\end{array}$ & $\begin{array}{l}\text { (ft above } \\
\text { notch) }\end{array}$ & $\left(\mathrm{ft}^{3} / \mathrm{s}\right)$ & (gpm) & $\begin{array}{l}\text { (ft above } \\
\text { sensor) }\end{array}$ & $\begin{array}{l}\text { (ft above } \\
\text { log) }\end{array}$ & $\begin{array}{c}\text { (m above } \\
\text { log) }\end{array}$ & $\left(\mathrm{m}^{3} / \mathrm{s}\right)$ & $\left(\mathrm{ft}^{3} / \mathrm{s}\right)$ & (gpm) \\
\hline 3/10/08 19:00 & 1.57 & 0.591 & 0.155 & 69.636 & 1.358 & 0.027 & 0.008229 & 0.002356 & 0.083206 & 37.343 \\
\hline 3/10/08 20:00 & 1.563 & 0.584 & 0.151 & 67.619 & 1.356 & 0.025 & 0.007620 & 0.002126 & 0.075100 & 33.705 \\
\hline 3/10/08 21:00 & 1.555 & 0.576 & 0.146 & 65.357 & 1.356 & 0.025 & 0.007620 & 0.002126 & 0.075100 & 33.705 \\
\hline 3/10/08 22:00 & 1.548 & 0.569 & 0.141 & 63.416 & 1.356 & 0.025 & 0.007620 & 0.002126 & 0.075100 & 33.705 \\
\hline 3/10/08 23:00 & 1.542 & 0.563 & 0.138 & 61.780 & 1.353 & 0.022 & 0.006705 & 0.001797 & 0.063473 & 28.487 \\
\hline 3/11/08 0:00 & 1.54 & 0.561 & 0.136 & 61.240 & 1.353 & 0.022 & 0.006705 & 0.001797 & 0.063473 & 28.487 \\
\hline 3/11/08 1:00 & 1.538 & 0.559 & 0.135 & 60.704 & 1.353 & 0.022 & 0.006705 & 0.001797 & 0.063473 & 28.487 \\
\hline 3/11/08 2:00 & 1.533 & 0.554 & 0.132 & 59.374 & 1.353 & 0.022 & 0.006705 & 0.001797 & 0.063473 & 28.487 \\
\hline 3/11/08 3:00 & 1.536 & 0.557 & 0.134 & 60.170 & 1.353 & 0.022 & 0.006705 & 0.001797 & 0.063473 & 28.487 \\
\hline 3/11/08 4:00 & 1.553 & 0.574 & 0.144 & 64.799 & 1.353 & 0.022 & 0.006705 & 0.001797 & 0.063473 & 28.487 \\
\hline 3/11/08 5:00 & 1.566 & 0.587 & 0.153 & 68.479 & 1.353 & 0.022 & 0.006705 & 0.001797 & 0.063473 & 28.487 \\
\hline 3/11/08 6:00 & 1.573 & 0.594 & 0.157 & 70.511 & 1.353 & 0.022 & 0.006705 & 0.001797 & 0.063473 & 28.487 \\
\hline 3/11/08 7:00 & 1.578 & 0.599 & 0.160 & 71.985 & 1.353 & 0.022 & 0.006705 & 0.001797 & 0.063473 & 28.487 \\
\hline 3/11/08 8:00 & 1.575 & 0.596 & 0.158 & 71.099 & 1.353 & 0.022 & 0.006705 & 0.001797 & 0.063473 & 28.487 \\
\hline 3/11/08 9:00 & 1.575 & 0.596 & 0.158 & 71.099 & 1.353 & 0.022 & 0.006705 & 0.001797 & 0.063473 & 28.487 \\
\hline 3/11/08 10:00 & 1.578 & 0.599 & 0.160 & 71.985 & 1.353 & 0.022 & 0.006705 & 0.001797 & 0.063473 & 28.487 \\
\hline 3/11/08 11:00 & 1.588 & 0.609 & 0.167 & 74.987 & 1.353 & 0.022 & 0.006705 & 0.001797 & 0.063473 & 28.487 \\
\hline 3/11/08 12:00 & 1.592 & 0.613 & 0.170 & 76.208 & 1.356 & 0.025 & 0.007620 & 0.002126 & 0.075100 & 33.705 \\
\hline 3/11/08 13:00 & 1.602 & 0.623 & 0.177 & 79.314 & 1.358 & 0.027 & 0.008229 & 0.002356 & 0.083206 & 37.343 \\
\hline 3/11/08 14:00 & 1.633 & 0.654 & 0.199 & 89.419 & 1.361 & 0.03 & 0.009144 & 0.002714 & 0.095873 & 43.028 \\
\hline 3/11/08 15:00 & 1.651 & 0.672 & 0.213 & 95.623 & 1.363 & 0.032 & 0.009753 & 0.002963 & 0.104644 & 46.964 \\
\hline 3/11/08 16:00 & 1.65 & 0.671 & 0.212 & 95.272 & 1.366 & 0.035 & 0.010667 & 0.003349 & 0.118271 & 53.080 \\
\hline 3/11/08 17:00 & 1.654 & 0.675 & 0.215 & 96.681 & 1.366 & 0.035 & 0.010667 & 0.003349 & 0.118271 & 53.080 \\
\hline 3/11/08 18:00 & 1.661 & 0.682 & 0.221 & 99.178 & 1.367 & 0.036 & 0.010972 & 0.003481 & 0.122936 & 55.174 \\
\hline 3/11/08 19:00 & 1.666 & 0.687 & 0.225 & 100.985 & 1.369 & 0.038 & 0.011582 & 0.003750 & 0.132444 & 59.441 \\
\hline
\end{tabular}




\begin{tabular}{|c|c|c|c|c|c|c|c|c|c|c|}
\hline \multirow[b]{3}{*}{ Date Time } & \multicolumn{4}{|c|}{ V-Notch Weir } & \multicolumn{6}{|c|}{ R-Notch Weir } \\
\hline & \multicolumn{2}{|c|}{ Measured Water Level } & \multicolumn{2}{|c|}{$\begin{array}{c}\text { Calculated Rate } \\
\left(\mathrm{ft}^{3} / \mathrm{s}=448.8 \mathrm{gpm}\right)\end{array}$} & \multicolumn{3}{|c|}{ Measured Water Level } & \multicolumn{3}{|c|}{$\begin{array}{c}\text { Calculated Rate } \\
\left(\mathrm{ft}^{3} / \mathrm{s}=448.8 \mathrm{gpm}\right)\end{array}$} \\
\hline & $\begin{array}{l}\text { (ft above } \\
\text { sensor) }\end{array}$ & $\begin{array}{l}\text { (ft above } \\
\text { notch) }\end{array}$ & $\left(\mathrm{ft}^{3} / \mathrm{s}\right)$ & (gpm) & $\begin{array}{l}\text { (ft above } \\
\text { sensor) }\end{array}$ & $\begin{array}{l}\text { (ft above } \\
\text { log) }\end{array}$ & $\begin{array}{c}\text { (m above } \\
\text { log) }\end{array}$ & $\left(\mathrm{m}^{3} / \mathrm{s}\right)$ & $\left(\mathrm{ft}^{3} / \mathrm{s}\right)$ & (gpm) \\
\hline 3/11/08 20:00 & 1.649 & 0.670 & 0.212 & 94.921 & 1.367 & 0.036 & 0.010972 & 0.003481 & 0.122936 & 55.174 \\
\hline 3/11/08 21:00 & 1.596 & 0.617 & 0.173 & 77.442 & 1.362 & 0.031 & 0.009448 & 0.002838 & 0.100226 & 44.982 \\
\hline 3/11/08 22:00 & 1.586 & 0.607 & 0.166 & 74.381 & 1.362 & 0.031 & 0.009448 & 0.002838 & 0.100226 & 44.982 \\
\hline 3/11/08 23:00 & 1.582 & 0.603 & 0.163 & 73.177 & 1.359 & 0.028 & 0.008534 & 0.002473 & 0.087362 & 39.208 \\
\hline 3/12/08 0:00 & 1.578 & 0.599 & 0.160 & 71.985 & 1.359 & 0.028 & 0.008534 & 0.002473 & 0.087362 & 39.208 \\
\hline 3/12/08 1:00 & 1.581 & 0.602 & 0.162 & 72.878 & 1.359 & 0.028 & 0.008534 & 0.002473 & 0.087362 & 39.208 \\
\hline 3/12/08 2:00 & 1.579 & 0.600 & 0.161 & 72.282 & 1.359 & 0.028 & 0.008534 & 0.002473 & 0.087362 & 39.208 \\
\hline 3/12/08 3:00 & 1.584 & 0.605 & 0.164 & 73.777 & 1.356 & 0.025 & 0.007620 & 0.002126 & 0.075100 & 33.705 \\
\hline 3/12/08 4:00 & 1.585 & 0.606 & 0.165 & 74.079 & 1.359 & 0.028 & 0.008534 & 0.002473 & 0.087362 & 39.208 \\
\hline 3/12/08 5:00 & 1.597 & 0.618 & 0.173 & 77.752 & 1.358 & 0.027 & 0.008229 & 0.002356 & 0.083206 & 37.343 \\
\hline 3/12/08 6:00 & 1.588 & 0.609 & 0.167 & 74.987 & 1.356 & 0.025 & 0.007620 & 0.002126 & 0.075100 & 33.705 \\
\hline 3/12/08 7:00 & 1.605 & 0.626 & 0.179 & 80.260 & 1.358 & 0.027 & 0.008229 & 0.002356 & 0.083206 & 37.343 \\
\hline 3/12/08 8:00 & 1.601 & 0.622 & 0.176 & 79.000 & 1.356 & 0.025 & 0.007620 & 0.002126 & 0.075100 & 33.705 \\
\hline 3/12/08 9:00 & 1.585 & 0.606 & 0.165 & 74.079 & 1.358 & 0.027 & 0.008229 & 0.002356 & 0.083206 & 37.343 \\
\hline 3/12/08 10:00 & 1.59 & 0.611 & 0.168 & 75.596 & 1.358 & 0.027 & 0.008229 & 0.002356 & 0.083206 & 37.343 \\
\hline 3/12/08 11:00 & 1.589 & 0.610 & 0.168 & 75.291 & 1.358 & 0.027 & 0.008229 & 0.002356 & 0.083206 & 37.343 \\
\hline 3/12/08 12:00 & 1.587 & 0.608 & 0.166 & 74.683 & 1.358 & 0.027 & 0.008229 & 0.002356 & 0.083206 & 37.343 \\
\hline 3/12/08 13:00 & 1.553 & 0.574 & 0.144 & 64.799 & 1.361 & 0.03 & 0.009144 & 0.002714 & 0.095873 & 43.028 \\
\hline 3/12/08 14:00 & 1.587 & 0.608 & 0.166 & 74.683 & 1.363 & 0.032 & 0.009753 & 0.002963 & 0.104644 & 46.964 \\
\hline 3/12/08 15:00 & 1.582 & 0.603 & 0.163 & 73.177 & 1.364 & 0.033 & 0.010058 & 0.003090 & 0.109124 & 48.975 \\
\hline 3/12/08 16:00 & 1.619 & 0.640 & 0.189 & 84.765 & 1.369 & 0.038 & 0.011582 & 0.003750 & 0.132444 & 59.441 \\
\hline 3/12/08 17:00 & 1.616 & 0.637 & 0.187 & 83.788 & 1.369 & 0.038 & 0.011582 & 0.003750 & 0.132444 & 59.441 \\
\hline 3/12/08 18:00 & 1.609 & 0.630 & 0.182 & 81.532 & 1.372 & 0.041 & 0.012496 & 0.004166 & 0.147141 & 66.037 \\
\hline 3/12/08 19:00 & 1.607 & 0.628 & 0.180 & 80.895 & 1.369 & 0.038 & 0.011582 & 0.003750 & 0.132444 & 59.441 \\
\hline 3/12/08 20:00 & 1.597 & 0.618 & 0.173 & 77.752 & 1.369 & 0.038 & 0.011582 & 0.003750 & 0.132444 & 59.441 \\
\hline
\end{tabular}




\begin{tabular}{|c|c|c|c|c|c|c|c|c|c|c|}
\hline \multirow[b]{3}{*}{ Date Time } & \multicolumn{4}{|c|}{ V-Notch Weir } & \multicolumn{6}{|c|}{ R-Notch Weir } \\
\hline & \multicolumn{2}{|c|}{ Measured Water Level } & \multicolumn{2}{|c|}{$\begin{array}{c}\text { Calculated Rate } \\
\left(\mathrm{ft}^{3} / \mathrm{s}=448.8 \mathrm{gpm}\right)\end{array}$} & \multicolumn{3}{|c|}{ Measured Water Level } & \multicolumn{3}{|c|}{$\begin{array}{c}\text { Calculated Rate } \\
\left(\mathrm{ft}^{3} / \mathrm{s}=448.8 \mathrm{gpm}\right)\end{array}$} \\
\hline & $\begin{array}{c}\text { (ft above } \\
\text { sensor) }\end{array}$ & $\begin{array}{c}\text { (ft above } \\
\text { notch) }\end{array}$ & $\left(\mathrm{ft}^{3} / \mathrm{s}\right)$ & (gpm) & $\begin{array}{l}\text { (ft above } \\
\text { sensor) }\end{array}$ & $\begin{array}{c}\text { (ft above } \\
\text { log) }\end{array}$ & $\begin{array}{c}\text { (m above } \\
\text { log) }\end{array}$ & $\left(\mathrm{m}^{3} / \mathrm{s}\right)$ & $\left(\mathrm{ft}^{3} / \mathrm{s}\right)$ & (gpm) \\
\hline 3/12/08 21:00 & 1.591 & 0.612 & 0.169 & 75.902 & 1.367 & 0.036 & 0.010972 & 0.003481 & 0.122936 & 55.174 \\
\hline 3/12/08 22:00 & 1.574 & 0.595 & 0.158 & 70.805 & 1.364 & 0.033 & 0.010058 & 0.003090 & 0.109124 & 48.975 \\
\hline 3/12/08 23:00 & 1.565 & 0.586 & 0.152 & 68.192 & 1.362 & 0.031 & 0.009448 & 0.002838 & 0.100226 & 44.982 \\
\hline 3/13/08 0:00 & 1.559 & 0.580 & 0.148 & 66.482 & 1.362 & 0.031 & 0.009448 & 0.002838 & 0.100226 & 44.982 \\
\hline 3/13/08 1:00 & 1.55 & 0.571 & 0.143 & 63.967 & 1.359 & 0.028 & 0.008534 & 0.002473 & 0.087362 & 39.208 \\
\hline 3/13/08 2:00 & 1.546 & 0.567 & 0.140 & 62.868 & 1.359 & 0.028 & 0.008534 & 0.002473 & 0.087362 & 39.208 \\
\hline 3/13/08 3:00 & 1.539 & 0.560 & 0.136 & 60.972 & 1.356 & 0.025 & 0.007620 & 0.002126 & 0.075100 & 33.705 \\
\hline 3/13/08 4:00 & 1.535 & 0.556 & 0.133 & 59.904 & 1.359 & 0.028 & 0.008534 & 0.002473 & 0.087362 & 39.208 \\
\hline 3/13/08 5:00 & 1.536 & 0.557 & 0.134 & 60.170 & 1.359 & 0.028 & 0.008534 & 0.002473 & 0.087362 & 39.208 \\
\hline 3/13/08 6:00 & 1.532 & 0.553 & 0.132 & 59.110 & 1.358 & 0.027 & 0.008229 & 0.002356 & 0.083206 & 37.343 \\
\hline 3/13/08 7:00 & 1.528 & 0.549 & 0.129 & 58.062 & 1.356 & 0.025 & 0.007620 & 0.002126 & 0.075100 & 33.705 \\
\hline 3/13/08 8:00 & 1.527 & 0.548 & 0.129 & 57.802 & 1.356 & 0.025 & 0.007620 & 0.002126 & 0.075100 & 33.705 \\
\hline 3/13/08 9:00 & 1.525 & 0.546 & 0.128 & 57.283 & 1.356 & 0.025 & 0.007620 & 0.002126 & 0.075100 & 33.705 \\
\hline 3/13/08 10:00 & 1.525 & 0.546 & 0.128 & 57.283 & 1.356 & 0.025 & 0.007620 & 0.002126 & 0.075100 & 33.705 \\
\hline 3/13/08 11:00 & 1.525 & 0.546 & 0.128 & 57.283 & 1.358 & 0.027 & 0.008229 & 0.002356 & 0.083206 & 37.343 \\
\hline 3/13/08 12:00 & 1.534 & 0.555 & 0.133 & 59.638 & 1.356 & 0.025 & 0.007620 & 0.002126 & 0.075100 & 33.705 \\
\hline 3/13/08 13:00 & 1.537 & 0.558 & 0.135 & 60.436 & 1.358 & 0.027 & 0.008229 & 0.002356 & 0.083206 & 37.343 \\
\hline 3/13/08 14:00 & 1.541 & 0.562 & 0.137 & 61.510 & 1.359 & 0.028 & 0.008534 & 0.002473 & 0.087362 & 39.208 \\
\hline 3/13/08 15:00 & 1.547 & 0.568 & 0.141 & 63.142 & 1.361 & 0.03 & 0.009144 & 0.002714 & 0.095873 & 43.028 \\
\hline 3/13/08 16:00 & 1.558 & 0.579 & 0.148 & 66.200 & 1.362 & 0.031 & 0.009448 & 0.002838 & 0.100226 & 44.982 \\
\hline 3/13/08 17:00 & 1.571 & 0.592 & 0.156 & 69.927 & 1.365 & 0.034 & 0.010363 & 0.003218 & 0.113667 & 51.014 \\
\hline 3/13/08 18:00 & 1.581 & 0.602 & 0.162 & 72.878 & 1.365 & 0.034 & 0.010363 & 0.003218 & 0.113667 & 51.014 \\
\hline 3/13/08 19:00 & 1.574 & 0.595 & 0.158 & 70.805 & 1.367 & 0.036 & 0.010972 & 0.003481 & 0.122936 & 55.174 \\
\hline 3/13/08 20:00 & 1.579 & 0.600 & 0.161 & 72.282 & 1.365 & 0.034 & 0.010363 & 0.003218 & 0.113667 & 51.014 \\
\hline 3/13/08 21:00 & 1.57 & 0.591 & 0.155 & 69.636 & 1.365 & 0.034 & 0.010363 & 0.003218 & 0.113667 & 51.014 \\
\hline
\end{tabular}




\begin{tabular}{|c|c|c|c|c|c|c|c|c|c|c|}
\hline \multirow[b]{3}{*}{ Date Time } & \multicolumn{4}{|c|}{ V-Notch Weir } & \multicolumn{6}{|c|}{ R-Notch Weir } \\
\hline & \multicolumn{2}{|c|}{ Measured Water Level } & \multicolumn{2}{|c|}{$\begin{array}{c}\text { Calculated Rate } \\
\left(\mathrm{ft}^{3} / \mathrm{s}=448.8 \mathrm{gpm}\right)\end{array}$} & \multicolumn{3}{|c|}{ Measured Water Level } & \multicolumn{3}{|c|}{$\begin{array}{c}\text { Calculated Rate } \\
\left(\mathrm{ft}^{3} / \mathrm{s}=448.8 \mathrm{gpm}\right)\end{array}$} \\
\hline & $\begin{array}{l}\text { (ft above } \\
\text { sensor) }\end{array}$ & $\begin{array}{l}\text { (ft above } \\
\text { notch) }\end{array}$ & $\left(\mathrm{ft}^{3} / \mathrm{s}\right)$ & (gpm) & $\begin{array}{l}\text { (ft above } \\
\text { sensor) }\end{array}$ & $\begin{array}{l}\text { (ft above } \\
\text { log) }\end{array}$ & $\begin{array}{c}\text { (m above } \\
\text { log) }\end{array}$ & $\left(\mathrm{m}^{3} / \mathrm{s}\right)$ & $\left(\mathrm{ft}^{3} / \mathrm{s}\right)$ & (gpm) \\
\hline 3/13/08 22:00 & 1.565 & 0.586 & 0.152 & 68.192 & 1.362 & 0.031 & 0.009448 & 0.002838 & 0.100226 & 44.982 \\
\hline 3/13/08 23:00 & 1.561 & 0.582 & 0.149 & 67.049 & 1.362 & 0.031 & 0.009448 & 0.002838 & 0.100226 & 44.982 \\
\hline 3/14/08 0:00 & 1.556 & 0.577 & 0.146 & 65.638 & 1.362 & 0.031 & 0.009448 & 0.002838 & 0.100226 & 44.982 \\
\hline 3/14/08 1:00 & 1.557 & 0.578 & 0.147 & 65.918 & 1.362 & 0.031 & 0.009448 & 0.002838 & 0.100226 & 44.982 \\
\hline 3/14/08 2:00 & 1.545 & 0.566 & 0.139 & 62.595 & 1.359 & 0.028 & 0.008534 & 0.002473 & 0.087362 & 39.208 \\
\hline 3/14/08 3:00 & 1.546 & 0.567 & 0.140 & 62.868 & 1.359 & 0.028 & 0.008534 & 0.002473 & 0.087362 & 39.208 \\
\hline 3/14/08 4:00 & 1.542 & 0.563 & 0.138 & 61.780 & 1.359 & 0.028 & 0.008534 & 0.002473 & 0.087362 & 39.208 \\
\hline 3/14/08 5:00 & 1.536 & 0.557 & 0.134 & 60.170 & 1.359 & 0.028 & 0.008534 & 0.002473 & 0.087362 & 39.208 \\
\hline 3/14/08 6:00 & 1.534 & 0.555 & 0.133 & 59.638 & 1.359 & 0.028 & 0.008534 & 0.002473 & 0.087362 & 39.208 \\
\hline 3/14/08 7:00 & 1.535 & 0.556 & 0.133 & 59.904 & 1.356 & 0.025 & 0.007620 & 0.002126 & 0.075100 & 33.705 \\
\hline 3/14/08 8:00 & 1.528 & 0.549 & 0.129 & 58.062 & 1.356 & 0.025 & 0.007620 & 0.002126 & 0.075100 & 33.705 \\
\hline 3/14/08 9:00 & 1.526 & 0.547 & 0.128 & 57.542 & 1.356 & 0.025 & 0.007620 & 0.002126 & 0.075100 & 33.705 \\
\hline 3/14/08 10:00 & 1.526 & 0.547 & 0.128 & 57.542 & 1.356 & 0.025 & 0.007620 & 0.002126 & 0.075100 & 33.705 \\
\hline 3/14/08 11:00 & 1.528 & 0.549 & 0.129 & 58.062 & 1.356 & 0.025 & 0.007620 & 0.002126 & 0.075100 & 33.705 \\
\hline 3/14/08 12:00 & 1.538 & 0.559 & 0.135 & 60.704 & 1.359 & 0.028 & 0.008534 & 0.002473 & 0.087362 & 39.208 \\
\hline 3/14/08 13:00 & 1.537 & 0.558 & 0.135 & 60.436 & 1.361 & 0.03 & 0.009144 & 0.002714 & 0.095873 & 43.028 \\
\hline 3/14/08 14:00 & 1.559 & 0.580 & 0.148 & 66.482 & 1.359 & 0.028 & 0.008534 & 0.002473 & 0.087362 & 39.208 \\
\hline 3/14/08 15:00 & 1.547 & 0.568 & 0.141 & 63.142 & 1.361 & 0.03 & 0.009144 & 0.002714 & 0.095873 & 43.028 \\
\hline 3/14/08 16:00 & 1.549 & 0.570 & 0.142 & 63.691 & 1.362 & 0.031 & 0.009448 & 0.002838 & 0.100226 & 44.982 \\
\hline 3/14/08 17:00 & 1.553 & 0.574 & 0.144 & 64.799 & 1.362 & 0.031 & 0.009448 & 0.002838 & 0.100226 & 44.982 \\
\hline 3/14/08 18:00 & 1.549 & 0.570 & 0.142 & 63.691 & 1.362 & 0.031 & 0.009448 & 0.002838 & 0.100226 & 44.982 \\
\hline 3/14/08 19:00 & 1.556 & 0.577 & 0.146 & 65.638 & 1.362 & 0.031 & 0.009448 & 0.002838 & 0.100226 & 44.982 \\
\hline 3/14/08 20:00 & 1.554 & 0.575 & 0.145 & 65.078 & 1.362 & 0.031 & 0.009448 & 0.002838 & 0.100226 & 44.982 \\
\hline 3/14/08 21:00 & 1.554 & 0.575 & 0.145 & 65.078 & 1.359 & 0.028 & 0.008534 & 0.002473 & 0.087362 & 39.208 \\
\hline 3/14/08 22:00 & 1.538 & 0.559 & 0.135 & 60.704 & 1.362 & 0.031 & 0.009448 & 0.002838 & 0.100226 & 44.982 \\
\hline
\end{tabular}




\begin{tabular}{|c|c|c|c|c|c|c|c|c|c|c|}
\hline \multirow[b]{3}{*}{ Date Time } & \multicolumn{4}{|c|}{ V-Notch Weir } & \multicolumn{6}{|c|}{ R-Notch Weir } \\
\hline & \multicolumn{2}{|c|}{ Measured Water Level } & \multicolumn{2}{|c|}{$\begin{array}{c}\text { Calculated Rate } \\
\left(\mathrm{ft}^{3} / \mathrm{s}=448.8 \mathrm{gpm}\right)\end{array}$} & \multicolumn{3}{|c|}{ Measured Water Level } & \multicolumn{3}{|c|}{$\begin{array}{c}\text { Calculated Rate } \\
\left(\mathrm{ft}^{3} / \mathrm{s}=448.8 \mathrm{gpm}\right)\end{array}$} \\
\hline & $\begin{array}{c}\text { (ft above } \\
\text { sensor) }\end{array}$ & $\begin{array}{c}\text { (ft above } \\
\text { notch) }\end{array}$ & $\left(\mathrm{ft}^{3} / \mathrm{s}\right)$ & (gpm) & $\begin{array}{l}\text { (ft above } \\
\text { sensor) }\end{array}$ & $\begin{array}{c}\text { (ft above } \\
\text { log) }\end{array}$ & $\begin{array}{c}\text { (m above } \\
\text { log) }\end{array}$ & $\left(\mathrm{m}^{3} / \mathrm{s}\right)$ & $\left(\mathrm{ft}^{3} / \mathrm{s}\right)$ & (gpm) \\
\hline 3/14/08 23:00 & 1.538 & 0.559 & 0.135 & 60.704 & 1.359 & 0.028 & 0.008534 & 0.002473 & 0.087362 & 39.208 \\
\hline 3/15/08 0:00 & 1.541 & 0.562 & 0.137 & 61.510 & 1.359 & 0.028 & 0.008534 & 0.002473 & 0.087362 & 39.208 \\
\hline 3/15/08 1:00 & 1.551 & 0.572 & 0.143 & 64.244 & 1.356 & 0.025 & 0.007620 & 0.002126 & 0.075100 & 33.705 \\
\hline 3/15/08 2:00 & 1.564 & 0.585 & 0.151 & 67.905 & 1.359 & 0.028 & 0.008534 & 0.002473 & 0.087362 & 39.208 \\
\hline 3/15/08 3:00 & 1.557 & 0.578 & 0.147 & 65.918 & 1.356 & 0.025 & 0.007620 & 0.002126 & 0.075100 & 33.705 \\
\hline 3/15/08 4:00 & 1.558 & 0.579 & 0.148 & 66.200 & 1.356 & 0.025 & 0.007620 & 0.002126 & 0.075100 & 33.705 \\
\hline 3/15/08 5:00 & 1.556 & 0.577 & 0.146 & 65.638 & 1.356 & 0.025 & 0.007620 & 0.002126 & 0.075100 & 33.705 \\
\hline 3/15/08 6:00 & 1.549 & 0.570 & 0.142 & 63.691 & 1.356 & 0.025 & 0.007620 & 0.002126 & 0.075100 & 33.705 \\
\hline 3/15/08 7:00 & 1.545 & 0.566 & 0.139 & 62.595 & 1.356 & 0.025 & 0.007620 & 0.002126 & 0.075100 & 33.705 \\
\hline 3/15/08 8:00 & 1.553 & 0.574 & 0.144 & 64.799 & 1.356 & 0.025 & 0.007620 & 0.002126 & 0.075100 & 33.705 \\
\hline 3/15/08 9:00 & 1.577 & 0.598 & 0.160 & 71.689 & 1.354 & 0.023 & 0.007010 & 0.001905 & 0.067276 & 30.194 \\
\hline 3/15/08 10:00 & 1.561 & 0.582 & 0.149 & 67.049 & 1.354 & 0.023 & 0.007010 & 0.001905 & 0.067276 & 30.194 \\
\hline 3/15/08 11:00 & 1.562 & 0.583 & 0.150 & 67.334 & 1.354 & 0.023 & 0.007010 & 0.001905 & 0.067276 & 30.194 \\
\hline 3/15/08 12:00 & 1.556 & 0.577 & 0.146 & 65.638 & 1.354 & 0.023 & 0.007010 & 0.001905 & 0.067276 & 30.194 \\
\hline 3/15/08 13:00 & 1.567 & 0.588 & 0.153 & 68.767 & 1.351 & 0.02 & 0.006096 & 0.001588 & 0.056092 & 25.174 \\
\hline 3/15/08 14:00 & 1.568 & 0.589 & 0.154 & 69.056 & 1.354 & 0.023 & 0.007010 & 0.001905 & 0.067276 & 30.194 \\
\hline 3/15/08 15:00 & 1.56 & 0.581 & 0.149 & 66.766 & 1.354 & 0.023 & 0.007010 & 0.001905 & 0.067276 & 30.194 \\
\hline 3/15/08 16:00 & 1.583 & 0.604 & 0.164 & 73.477 & 1.356 & 0.025 & 0.007620 & 0.002126 & 0.075100 & 33.705 \\
\hline 3/15/08 17:00 & 1.582 & 0.603 & 0.163 & 73.177 & 1.359 & 0.028 & 0.008534 & 0.002473 & 0.087362 & 39.208 \\
\hline 3/15/08 18:00 & 1.587 & 0.608 & 0.166 & 74.683 & 1.354 & 0.023 & 0.007010 & 0.001905 & 0.067276 & 30.194 \\
\hline 3/15/08 19:00 & 1.578 & 0.599 & 0.160 & 71.985 & 1.357 & 0.026 & 0.007924 & 0.002240 & 0.079119 & 35.508 \\
\hline 3/15/08 20:00 & 1.58 & 0.601 & 0.162 & 72.579 & 1.356 & 0.025 & 0.007620 & 0.002126 & 0.075100 & 33.705 \\
\hline 3/15/08 21:00 & 1.579 & 0.600 & 0.161 & 72.282 & 1.354 & 0.023 & 0.007010 & 0.001905 & 0.067276 & 30.194 \\
\hline 3/15/08 22:00 & 1.577 & 0.598 & 0.160 & 71.689 & 1.356 & 0.025 & 0.007620 & 0.002126 & 0.075100 & 33.705 \\
\hline 3/15/08 23:00 & 1.61 & 0.631 & 0.182 & 81.852 & 1.354 & 0.023 & 0.007010 & 0.001905 & 0.067276 & 30.194 \\
\hline
\end{tabular}




\begin{tabular}{|c|c|c|c|c|c|c|c|c|c|c|}
\hline \multirow[b]{3}{*}{ Date Time } & \multicolumn{4}{|c|}{ V-Notch Weir } & \multicolumn{6}{|c|}{ R-Notch Weir } \\
\hline & \multicolumn{2}{|c|}{ Measured Water Level } & \multicolumn{2}{|c|}{$\begin{array}{c}\text { Calculated Rate } \\
\left(\mathrm{ft}^{3} / \mathrm{s}=448.8 \mathrm{gpm}\right)\end{array}$} & \multicolumn{3}{|c|}{ Measured Water Level } & \multicolumn{3}{|c|}{$\begin{array}{c}\text { Calculated Rate } \\
\left(\mathrm{ft}^{3} / \mathrm{s}=448.8 \mathrm{gpm}\right)\end{array}$} \\
\hline & $\begin{array}{c}\text { (ft above } \\
\text { sensor) }\end{array}$ & $\begin{array}{c}\text { (ft above } \\
\text { notch) }\end{array}$ & $\left(\mathrm{ft}^{3} / \mathrm{s}\right)$ & (gpm) & $\begin{array}{l}\text { (ft above } \\
\text { sensor) }\end{array}$ & $\begin{array}{c}\text { (ft above } \\
\text { log) }\end{array}$ & $\begin{array}{c}\text { (m above } \\
\text { log) }\end{array}$ & $\left(\mathrm{m}^{3} / \mathrm{s}\right)$ & $\left(\mathrm{ft}^{3} / \mathrm{s}\right)$ & (gpm) \\
\hline 3/16/08 0:00 & 1.611 & 0.632 & 0.183 & 82.173 & 1.354 & 0.023 & 0.007010 & 0.001905 & 0.067276 & 30.194 \\
\hline 3/16/08 1:00 & 1.607 & 0.628 & 0.180 & 80.895 & 1.354 & 0.023 & 0.007010 & 0.001905 & 0.067276 & 30.194 \\
\hline 3/16/08 2:00 & 1.605 & 0.626 & 0.179 & 80.260 & 1.354 & 0.023 & 0.007010 & 0.001905 & 0.067276 & 30.194 \\
\hline 3/16/08 3:00 & 1.616 & 0.637 & 0.187 & 83.788 & 1.351 & 0.02 & 0.006096 & 0.001588 & 0.056092 & 25.174 \\
\hline 3/16/08 4:00 & 1.614 & 0.635 & 0.185 & 83.140 & 1.351 & 0.02 & 0.006096 & 0.001588 & 0.056092 & 25.174 \\
\hline 3/16/08 5:00 & 1.61 & 0.631 & 0.182 & 81.852 & 1.351 & 0.02 & 0.006096 & 0.001588 & 0.056092 & 25.174 \\
\hline 3/16/08 6:00 & 1.603 & 0.624 & 0.177 & 79.629 & 1.351 & 0.02 & 0.006096 & 0.001588 & 0.056092 & 25.174 \\
\hline 3/16/08 7:00 & 1.601 & 0.622 & 0.176 & 79.000 & 1.351 & 0.02 & 0.006096 & 0.001588 & 0.056092 & 25.174 \\
\hline 3/16/08 8:00 & 1.599 & 0.620 & 0.175 & 78.375 & 1.351 & 0.02 & 0.006096 & 0.001588 & 0.056092 & 25.174 \\
\hline 3/16/08 9:00 & 1.589 & 0.610 & 0.168 & 75.291 & 1.349 & 0.018 & 0.005486 & 0.001388 & 0.049021 & 22.001 \\
\hline 3/16/08 10:00 & 1.528 & 0.549 & 0.129 & 58.062 & 1.351 & 0.02 & 0.006096 & 0.001588 & 0.056092 & 25.174 \\
\hline 3/16/08 11:00 & 1.534 & 0.555 & 0.133 & 59.638 & 1.351 & 0.02 & 0.006096 & 0.001588 & 0.056092 & 25.174 \\
\hline 3/16/08 12:00 & 1.54 & 0.561 & 0.136 & 61.240 & 1.351 & 0.02 & 0.006096 & 0.001588 & 0.056092 & 25.174 \\
\hline 3/16/08 13:00 & 1.546 & 0.567 & 0.140 & 62.868 & 1.356 & 0.025 & 0.007620 & 0.002126 & 0.075100 & 33.705 \\
\hline 3/16/08 14:00 & 1.576 & 0.597 & 0.159 & 71.393 & 1.354 & 0.023 & 0.007010 & 0.001905 & 0.067276 & 30.194 \\
\hline 3/16/08 15:00 & 1.59 & 0.611 & 0.168 & 75.596 & 1.354 & 0.023 & 0.007010 & 0.001905 & 0.067276 & 30.194 \\
\hline 3/16/08 16:00 & 1.594 & 0.615 & 0.171 & 76.824 & 1.354 & 0.023 & 0.007010 & 0.001905 & 0.067276 & 30.194 \\
\hline 3/16/08 17:00 & 1.597 & 0.618 & 0.173 & 77.752 & 1.358 & 0.027 & 0.008229 & 0.002356 & 0.083206 & 37.343 \\
\hline 3/16/08 18:00 & 1.592 & 0.613 & 0.170 & 76.208 & 1.361 & 0.03 & 0.009144 & 0.002714 & 0.095873 & 43.028 \\
\hline 3/16/08 19:00 & 1.576 & 0.597 & 0.159 & 71.393 & 1.356 & 0.025 & 0.007620 & 0.002126 & 0.075100 & 33.705 \\
\hline 3/16/08 20:00 & 1.59 & 0.611 & 0.168 & 75.596 & 1.356 & 0.025 & 0.007620 & 0.002126 & 0.075100 & 33.705 \\
\hline 3/16/08 21:00 & 1.586 & 0.607 & 0.166 & 74.381 & 1.356 & 0.025 & 0.007620 & 0.002126 & 0.075100 & 33.705 \\
\hline 3/16/08 22:00 & 1.581 & 0.602 & 0.162 & 72.878 & 1.356 & 0.025 & 0.007620 & 0.002126 & 0.075100 & 33.705 \\
\hline 3/16/08 23:00 & 1.579 & 0.600 & 0.161 & 72.282 & 1.356 & 0.025 & 0.007620 & 0.002126 & 0.075100 & 33.705 \\
\hline 3/17/08 0:00 & 1.594 & 0.615 & 0.171 & 76.824 & 1.353 & 0.022 & 0.006705 & 0.001797 & 0.063473 & 28.487 \\
\hline
\end{tabular}




\begin{tabular}{|c|c|c|c|c|c|c|c|c|c|c|}
\hline \multirow[b]{3}{*}{ Date Time } & \multicolumn{4}{|c|}{ V-Notch Weir } & \multicolumn{6}{|c|}{ R-Notch Weir } \\
\hline & \multicolumn{2}{|c|}{ Measured Water Level } & \multicolumn{2}{|c|}{$\begin{array}{c}\text { Calculated Rate } \\
\left(\mathrm{ft}^{3} / \mathrm{s}=448.8 \mathrm{gpm}\right)\end{array}$} & \multicolumn{3}{|c|}{ Measured Water Level } & \multicolumn{3}{|c|}{$\begin{array}{c}\text { Calculated Rate } \\
\left(\mathrm{ft}^{3} / \mathrm{s}=448.8 \mathrm{gpm}\right)\end{array}$} \\
\hline & $\begin{array}{l}\text { (ft above } \\
\text { sensor) }\end{array}$ & $\begin{array}{c}\text { (ft above } \\
\text { notch) }\end{array}$ & $\left(\mathrm{ft}^{3} / \mathrm{s}\right)$ & (gpm) & $\begin{array}{c}\text { (ft above } \\
\text { sensor) }\end{array}$ & $\begin{array}{c}\text { (ft above } \\
\text { log) }\end{array}$ & $\begin{array}{c}\text { (m above } \\
\text { log) }\end{array}$ & $\left(\mathrm{m}^{3} / \mathrm{s}\right)$ & $\left(\mathrm{ft}^{3} / \mathrm{s}\right)$ & (gpm) \\
\hline 3/17/08 1:00 & 1.575 & 0.596 & 0.158 & 71.099 & 1.356 & 0.025 & 0.007620 & 0.002126 & 0.075100 & 33.705 \\
\hline 3/17/08 2:00 & 1.573 & 0.594 & 0.157 & 70.511 & 1.356 & 0.025 & 0.007620 & 0.002126 & 0.075100 & 33.705 \\
\hline 3/17/08 3:00 & 1.583 & 0.604 & 0.164 & 73.477 & 1.361 & 0.03 & 0.009144 & 0.002714 & 0.095873 & 43.028 \\
\hline 3/17/08 4:00 & 1.581 & 0.602 & 0.162 & 72.878 & 1.358 & 0.027 & 0.008229 & 0.002356 & 0.083206 & 37.343 \\
\hline 3/17/08 5:00 & 1.585 & 0.606 & 0.165 & 74.079 & 1.356 & 0.025 & 0.007620 & 0.002126 & 0.075100 & 33.705 \\
\hline 3/17/08 6:00 & 1.586 & 0.607 & 0.166 & 74.381 & 1.356 & 0.025 & 0.007620 & 0.002126 & 0.075100 & 33.705 \\
\hline 3/17/08 7:00 & 1.574 & 0.595 & 0.158 & 70.805 & 1.353 & 0.022 & 0.006705 & 0.001797 & 0.063473 & 28.487 \\
\hline 3/17/08 8:00 & 1.567 & 0.588 & 0.153 & 68.767 & 1.353 & 0.022 & 0.006705 & 0.001797 & 0.063473 & 28.487 \\
\hline 3/17/08 9:00 & 1.59 & 0.611 & 0.168 & 75.596 & 1.356 & 0.025 & 0.007620 & 0.002126 & 0.075100 & 33.705 \\
\hline 3/17/08 10:00 & 1.6 & 0.621 & 0.175 & 78.687 & 1.358 & 0.027 & 0.008229 & 0.002356 & 0.083206 & 37.343 \\
\hline 3/17/08 11:00 & 1.635 & 0.656 & 0.201 & 90.096 & 1.363 & 0.032 & 0.009753 & 0.002963 & 0.104644 & 46.964 \\
\hline 3/17/08 12:00 & 1.675 & 0.696 & 0.232 & 104.286 & 1.368 & 0.037 & 0.011277 & 0.003614 & 0.127660 & 57.294 \\
\hline 3/17/08 13:00 & 1.658 & 0.679 & 0.219 & 98.103 & 1.365 & 0.034 & 0.010363 & 0.003218 & 0.113667 & 51.014 \\
\hline 3/17/08 14:00 & 1.648 & 0.669 & 0.211 & 94.572 & 1.363 & 0.032 & 0.009753 & 0.002963 & 0.104644 & 46.964 \\
\hline 3/17/08 15:00 & 1.631 & 0.652 & 0.198 & 88.745 & 1.36 & 0.029 & 0.008839 & 0.002593 & 0.091584 & 41.103 \\
\hline 3/17/08 16:00 & 1.621 & 0.642 & 0.190 & 85.421 & 1.36 & 0.029 & 0.008839 & 0.002593 & 0.091584 & 41.103 \\
\hline 3/17/08 17:00 & 1.63 & 0.651 & 0.197 & 88.409 & 1.36 & 0.029 & 0.008839 & 0.002593 & 0.091584 & 41.103 \\
\hline 3/17/08 18:00 & 1.647 & 0.668 & 0.210 & 94.223 & 1.36 & 0.029 & 0.008839 & 0.002593 & 0.091584 & 41.103 \\
\hline 3/17/08 19:00 & 1.647 & 0.668 & 0.210 & 94.223 & 1.358 & 0.027 & 0.008229 & 0.002356 & 0.083206 & 37.343 \\
\hline 3/17/08 20:00 & 1.649 & 0.670 & 0.212 & 94.921 & 1.358 & 0.027 & 0.008229 & 0.002356 & 0.083206 & 37.343 \\
\hline 3/17/08 21:00 & 1.654 & 0.675 & 0.215 & 96.681 & 1.358 & 0.027 & 0.008229 & 0.002356 & 0.083206 & 37.343 \\
\hline 3/17/08 22:00 & 1.656 & 0.677 & 0.217 & 97.391 & 1.358 & 0.027 & 0.008229 & 0.002356 & 0.083206 & 37.343 \\
\hline 3/17/08 23:00 & 1.661 & 0.682 & 0.221 & 99.178 & 1.358 & 0.027 & 0.008229 & 0.002356 & 0.083206 & 37.343 \\
\hline 3/18/08 0:00 & 1.659 & 0.680 & 0.219 & 98.461 & 1.358 & 0.027 & 0.008229 & 0.002356 & 0.083206 & 37.343 \\
\hline 3/18/08 1:00 & 1.659 & 0.680 & 0.219 & 98.461 & 1.358 & 0.027 & 0.008229 & 0.002356 & 0.083206 & 37.343 \\
\hline
\end{tabular}




\begin{tabular}{|c|c|c|c|c|c|c|c|c|c|c|}
\hline \multirow[b]{3}{*}{ Date Time } & \multicolumn{4}{|c|}{ V-Notch Weir } & \multicolumn{6}{|c|}{ R-Notch Weir } \\
\hline & \multicolumn{2}{|c|}{ Measured Water Level } & \multicolumn{2}{|c|}{$\begin{array}{c}\text { Calculated Rate } \\
\left(\mathrm{ft}^{3} / \mathrm{s}=448.8 \mathrm{gpm}\right)\end{array}$} & \multicolumn{3}{|c|}{ Measured Water Level } & \multicolumn{3}{|c|}{$\begin{array}{c}\text { Calculated Rate } \\
\left(\mathrm{ft}^{3} / \mathrm{s}=448.8 \mathrm{gpm}\right)\end{array}$} \\
\hline & $\begin{array}{c}\text { (ft above } \\
\text { sensor) }\end{array}$ & $\begin{array}{c}\text { (ft above } \\
\text { notch) }\end{array}$ & $\left(\mathrm{ft}^{3} / \mathrm{s}\right)$ & (gpm) & $\begin{array}{l}\text { (ft above } \\
\text { sensor) }\end{array}$ & $\begin{array}{c}\text { (ft above } \\
\text { log) }\end{array}$ & $\begin{array}{c}\text { (m above } \\
\text { log) }\end{array}$ & $\left(\mathrm{m}^{3} / \mathrm{s}\right)$ & $\left(\mathrm{ft}^{3} / \mathrm{s}\right)$ & (gpm) \\
\hline 3/18/08 2:00 & 1.659 & 0.680 & 0.219 & 98.461 & 1.358 & 0.027 & 0.008229 & 0.002356 & 0.083206 & 37.343 \\
\hline 3/18/08 3:00 & 1.652 & 0.673 & 0.214 & 95.975 & 1.358 & 0.027 & 0.008229 & 0.002356 & 0.083206 & 37.343 \\
\hline 3/18/08 4:00 & 1.596 & 0.617 & 0.173 & 77.442 & 1.358 & 0.027 & 0.008229 & 0.002356 & 0.083206 & 37.343 \\
\hline 3/18/08 5:00 & 1.593 & 0.614 & 0.170 & 76.516 & 1.358 & 0.027 & 0.008229 & 0.002356 & 0.083206 & 37.343 \\
\hline 3/18/08 6:00 & 1.596 & 0.617 & 0.173 & 77.442 & 1.358 & 0.027 & 0.008229 & 0.002356 & 0.083206 & 37.343 \\
\hline 3/18/08 7:00 & 1.594 & 0.615 & 0.171 & 76.824 & 1.356 & 0.025 & 0.007620 & 0.002126 & 0.075100 & 33.705 \\
\hline 3/18/08 8:00 & 1.589 & 0.610 & 0.168 & 75.291 & 1.356 & 0.025 & 0.007620 & 0.002126 & 0.075100 & 33.705 \\
\hline 3/18/08 9:00 & 1.59 & 0.611 & 0.168 & 75.596 & 1.356 & 0.025 & 0.007620 & 0.002126 & 0.075100 & 33.705 \\
\hline 3/18/08 10:00 & 1.592 & 0.613 & 0.170 & 76.208 & 1.356 & 0.025 & 0.007620 & 0.002126 & 0.075100 & 33.705 \\
\hline 3/18/08 11:00 & 1.591 & 0.612 & 0.169 & 75.902 & 1.356 & 0.025 & 0.007620 & 0.002126 & 0.075100 & 33.705 \\
\hline 3/18/08 12:00 & 1.59 & 0.611 & 0.168 & 75.596 & 1.356 & 0.025 & 0.007620 & 0.002126 & 0.075100 & 33.705 \\
\hline 3/18/08 13:00 & 1.603 & 0.624 & 0.177 & 79.629 & 1.356 & 0.025 & 0.007620 & 0.002126 & 0.075100 & 33.705 \\
\hline 3/18/08 14:00 & 1.573 & 0.594 & 0.157 & 70.511 & 1.356 & 0.025 & 0.007620 & 0.002126 & 0.075100 & 33.705 \\
\hline 3/18/08 15:00 & 1.591 & 0.612 & 0.169 & 75.902 & 1.359 & 0.028 & 0.008534 & 0.002473 & 0.087362 & 39.208 \\
\hline 3/18/08 16:00 & 1.536 & 0.557 & 0.134 & 60.170 & 1.357 & 0.026 & 0.007924 & 0.002240 & 0.079119 & 35.508 \\
\hline 3/18/08 17:00 & 1.527 & 0.548 & 0.129 & 57.802 & 1.364 & 0.033 & 0.010058 & 0.003090 & 0.109124 & 48.975 \\
\hline 3/18/08 18:00 & 1.564 & 0.585 & 0.151 & 67.905 & 1.362 & 0.031 & 0.009448 & 0.002838 & 0.100226 & 44.982 \\
\hline 3/18/08 19:00 & 1.55 & 0.571 & 0.143 & 63.967 & 1.362 & 0.031 & 0.009448 & 0.002838 & 0.100226 & 44.982 \\
\hline 3/18/08 20:00 & 1.551 & 0.572 & 0.143 & 64.244 & 1.359 & 0.028 & 0.008534 & 0.002473 & 0.087362 & 39.208 \\
\hline 3/18/08 21:00 & 1.549 & 0.570 & 0.142 & 63.691 & 1.359 & 0.028 & 0.008534 & 0.002473 & 0.087362 & 39.208 \\
\hline 3/18/08 22:00 & 1.544 & 0.565 & 0.139 & 62.323 & 1.359 & 0.028 & 0.008534 & 0.002473 & 0.087362 & 39.208 \\
\hline 3/18/08 23:00 & 1.542 & 0.563 & 0.138 & 61.780 & 1.359 & 0.028 & 0.008534 & 0.002473 & 0.087362 & 39.208 \\
\hline 3/19/08 0:00 & 1.538 & 0.559 & 0.135 & 60.704 & 1.357 & 0.026 & 0.007924 & 0.002240 & 0.079119 & 35.508 \\
\hline 3/19/08 1:00 & 1.539 & 0.560 & 0.136 & 60.972 & 1.357 & 0.026 & 0.007924 & 0.002240 & 0.079119 & 35.508 \\
\hline 3/19/08 2:00 & 1.535 & 0.556 & 0.133 & 59.904 & 1.356 & 0.025 & 0.007620 & 0.002126 & 0.075100 & 33.705 \\
\hline
\end{tabular}




\begin{tabular}{|c|c|c|c|c|c|c|c|c|c|c|}
\hline \multirow[b]{3}{*}{ Date Time } & \multicolumn{4}{|c|}{ V-Notch Weir } & \multicolumn{6}{|c|}{ R-Notch Weir } \\
\hline & \multicolumn{2}{|c|}{ Measured Water Level } & \multicolumn{2}{|c|}{$\begin{array}{c}\text { Calculated Rate } \\
\left(\mathrm{ft}^{3} / \mathrm{s}=448.8 \mathrm{gpm}\right)\end{array}$} & \multicolumn{3}{|c|}{ Measured Water Level } & \multicolumn{3}{|c|}{$\begin{array}{c}\text { Calculated Rate } \\
\left(\mathrm{ft}^{3} / \mathrm{s}=448.8 \mathrm{gpm}\right)\end{array}$} \\
\hline & $\begin{array}{l}\text { (ft above } \\
\text { sensor) }\end{array}$ & $\begin{array}{c}\text { (ft above } \\
\text { notch) }\end{array}$ & $\left(\mathrm{ft}^{3} / \mathrm{s}\right)$ & (gpm) & $\begin{array}{c}\text { (ft above } \\
\text { sensor) }\end{array}$ & $\begin{array}{c}\text { (ft above } \\
\text { log) }\end{array}$ & $\begin{array}{c}\text { (m above } \\
\text { log) }\end{array}$ & $\left(\mathrm{m}^{3} / \mathrm{s}\right)$ & $\left(\mathrm{ft}^{3} / \mathrm{s}\right)$ & (gpm) \\
\hline 3/19/08 3:00 & 1.531 & 0.552 & 0.131 & 58.847 & 1.354 & 0.023 & 0.007010 & 0.001905 & 0.067276 & 30.194 \\
\hline 3/19/08 4:00 & 1.529 & 0.550 & 0.130 & 58.323 & 1.356 & 0.025 & 0.007620 & 0.002126 & 0.075100 & 33.705 \\
\hline 3/19/08 5:00 & 1.528 & 0.549 & 0.129 & 58.062 & 1.354 & 0.023 & 0.007010 & 0.001905 & 0.067276 & 30.194 \\
\hline 3/19/08 6:00 & 1.524 & 0.545 & 0.127 & 57.025 & 1.354 & 0.023 & 0.007010 & 0.001905 & 0.067276 & 30.194 \\
\hline 3/19/08 7:00 & 1.525 & 0.546 & 0.128 & 57.283 & 1.354 & 0.023 & 0.007010 & 0.001905 & 0.067276 & 30.194 \\
\hline 3/19/08 8:00 & 1.523 & 0.544 & 0.126 & 56.767 & 1.354 & 0.023 & 0.007010 & 0.001905 & 0.067276 & 30.194 \\
\hline 3/19/08 9:00 & 1.521 & 0.542 & 0.125 & 56.255 & 1.351 & 0.02 & 0.006096 & 0.001588 & 0.056092 & 25.174 \\
\hline 3/19/08 10:00 & 1.52 & 0.541 & 0.125 & 55.999 & 1.354 & 0.023 & 0.007010 & 0.001905 & 0.067276 & 30.194 \\
\hline 3/19/08 11:00 & 1.53 & 0.551 & 0.131 & 58.585 & 1.356 & 0.025 & 0.007620 & 0.002126 & 0.075100 & 33.705 \\
\hline 3/19/08 12:00 & 1.526 & 0.547 & 0.128 & 57.542 & 1.356 & 0.025 & 0.007620 & 0.002126 & 0.075100 & 33.705 \\
\hline 3/19/08 13:00 & 1.537 & 0.558 & 0.135 & 60.436 & 1.356 & 0.025 & 0.007620 & 0.002126 & 0.075100 & 33.705 \\
\hline 3/19/08 14:00 & 1.54 & 0.561 & 0.136 & 61.240 & 1.359 & 0.028 & 0.008534 & 0.002473 & 0.087362 & 39.208 \\
\hline 3/19/08 15:00 & 1.527 & 0.548 & 0.129 & 57.802 & 1.359 & 0.028 & 0.008534 & 0.002473 & 0.087362 & 39.208 \\
\hline 3/19/08 16:00 & 1.536 & 0.557 & 0.134 & 60.170 & 1.357 & 0.026 & 0.007924 & 0.002240 & 0.079119 & 35.508 \\
\hline 3/19/08 17:00 & 1.532 & 0.553 & 0.132 & 59.110 & 1.357 & 0.026 & 0.007924 & 0.002240 & 0.079119 & 35.508 \\
\hline 3/19/08 18:00 & 1.541 & 0.562 & 0.137 & 61.510 & 1.357 & 0.026 & 0.007924 & 0.002240 & 0.079119 & 35.508 \\
\hline 3/19/08 19:00 & 1.546 & 0.567 & 0.140 & 62.868 & 1.36 & 0.029 & 0.008839 & 0.002593 & 0.091584 & 41.103 \\
\hline 3/19/08 20:00 & 1.546 & 0.567 & 0.140 & 62.868 & 1.36 & 0.029 & 0.008839 & 0.002593 & 0.091584 & 41.103 \\
\hline 3/19/08 21:00 & 1.565 & 0.586 & 0.152 & 68.192 & 1.362 & 0.031 & 0.009448 & 0.002838 & 0.100226 & 44.982 \\
\hline 3/19/08 22:00 & 1.56 & 0.581 & 0.149 & 66.766 & 1.36 & 0.029 & 0.008839 & 0.002593 & 0.091584 & 41.103 \\
\hline 3/19/08 23:00 & 1.558 & 0.579 & 0.148 & 66.200 & 1.357 & 0.026 & 0.007924 & 0.002240 & 0.079119 & 35.508 \\
\hline 3/20/08 0:00 & 1.549 & 0.570 & 0.142 & 63.691 & 1.359 & 0.028 & 0.008534 & 0.002473 & 0.087362 & 39.208 \\
\hline 3/20/08 1:00 & 1.549 & 0.570 & 0.142 & 63.691 & 1.359 & 0.028 & 0.008534 & 0.002473 & 0.087362 & 39.208 \\
\hline 3/20/08 2:00 & 1.543 & 0.564 & 0.138 & 62.051 & 1.357 & 0.026 & 0.007924 & 0.002240 & 0.079119 & 35.508 \\
\hline 3/20/08 3:00 & 1.536 & 0.557 & 0.134 & 60.170 & 1.359 & 0.028 & 0.008534 & 0.002473 & 0.087362 & 39.208 \\
\hline
\end{tabular}




\begin{tabular}{|c|c|c|c|c|c|c|c|c|c|c|}
\hline \multirow[b]{3}{*}{ Date Time } & \multicolumn{4}{|c|}{ V-Notch Weir } & \multicolumn{6}{|c|}{ R-Notch Weir } \\
\hline & \multicolumn{2}{|c|}{ Measured Water Level } & \multicolumn{2}{|c|}{$\begin{array}{c}\text { Calculated Rate } \\
\left(\mathrm{ft}^{3} / \mathrm{s}=448.8 \mathrm{gpm}\right)\end{array}$} & \multicolumn{3}{|c|}{ Measured Water Level } & \multicolumn{3}{|c|}{$\begin{array}{c}\text { Calculated Rate } \\
\left(\mathrm{ft}^{3} / \mathrm{s}=448.8 \mathrm{gpm}\right)\end{array}$} \\
\hline & $\begin{array}{c}\text { (ft above } \\
\text { sensor) }\end{array}$ & $\begin{array}{c}\text { (ft above } \\
\text { notch) }\end{array}$ & $\left(\mathrm{ft}^{3} / \mathrm{s}\right)$ & (gpm) & $\begin{array}{l}\text { (ft above } \\
\text { sensor) }\end{array}$ & $\begin{array}{c}\text { (ft above } \\
\text { log) }\end{array}$ & $\begin{array}{c}\text { (m above } \\
\text { log) }\end{array}$ & $\left(\mathrm{m}^{3} / \mathrm{s}\right)$ & $\left(\mathrm{ft}^{3} / \mathrm{s}\right)$ & (gpm) \\
\hline 3/20/08 4:00 & 1.547 & 0.568 & 0.141 & 63.142 & 1.359 & 0.028 & 0.008534 & 0.002473 & 0.087362 & 39.208 \\
\hline 3/20/08 5:00 & 1.543 & 0.564 & 0.138 & 62.051 & 1.356 & 0.025 & 0.007620 & 0.002126 & 0.075100 & 33.705 \\
\hline 3/20/08 6:00 & 1.541 & 0.562 & 0.137 & 61.510 & 1.356 & 0.025 & 0.007620 & 0.002126 & 0.075100 & 33.705 \\
\hline 3/20/08 7:00 & 1.537 & 0.558 & 0.135 & 60.436 & 1.356 & 0.025 & 0.007620 & 0.002126 & 0.075100 & 33.705 \\
\hline 3/20/08 8:00 & 1.533 & 0.554 & 0.132 & 59.374 & 1.356 & 0.025 & 0.007620 & 0.002126 & 0.075100 & 33.705 \\
\hline 3/20/08 9:00 & 1.534 & 0.555 & 0.133 & 59.638 & 1.356 & 0.025 & 0.007620 & 0.002126 & 0.075100 & 33.705 \\
\hline 3/20/08 10:00 & 1.533 & 0.554 & 0.132 & 59.374 & 1.356 & 0.025 & 0.007620 & 0.002126 & 0.075100 & 33.705 \\
\hline 3/20/08 11:00 & 1.546 & 0.567 & 0.140 & 62.868 & 1.356 & 0.025 & 0.007620 & 0.002126 & 0.075100 & 33.705 \\
\hline 3/20/08 12:00 & 1.556 & 0.577 & 0.146 & 65.638 & 1.361 & 0.03 & 0.009144 & 0.002714 & 0.095873 & 43.028 \\
\hline 3/20/08 13:00 & 1.557 & 0.578 & 0.147 & 65.918 & 1.361 & 0.03 & 0.009144 & 0.002714 & 0.095873 & 43.028 \\
\hline 3/20/08 14:00 & 1.526 & 0.547 & 0.128 & 57.542 & 1.359 & 0.028 & 0.008534 & 0.002473 & 0.087362 & 39.208 \\
\hline 3/20/08 15:00 & 1.532 & 0.553 & 0.132 & 59.110 & 1.373 & 0.042 & 0.012801 & 0.004308 & 0.152154 & 68.287 \\
\hline 3/20/08 16:00 & 1.565 & 0.586 & 0.152 & 68.192 & 1.357 & 0.026 & 0.007924 & 0.002240 & 0.079119 & 35.508 \\
\hline 3/20/08 17:00 & 1.541 & 0.562 & 0.137 & 61.510 & 1.357 & 0.026 & 0.007924 & 0.002240 & 0.079119 & 35.508 \\
\hline 3/20/08 18:00 & 1.536 & 0.557 & 0.134 & 60.170 & 1.362 & 0.031 & 0.009448 & 0.002838 & 0.100226 & 44.982 \\
\hline 3/20/08 19:00 & 1.524 & 0.545 & 0.127 & 57.025 & 1.358 & 0.027 & 0.008229 & 0.002356 & 0.083206 & 37.343 \\
\hline 3/20/08 20:00 & 1.546 & 0.567 & 0.140 & 62.868 & 1.36 & 0.029 & 0.008839 & 0.002593 & 0.091584 & 41.103 \\
\hline 3/20/08 21:00 & 1.553 & 0.574 & 0.144 & 64.799 & 1.36 & 0.029 & 0.008839 & 0.002593 & 0.091584 & 41.103 \\
\hline 3/20/08 22:00 & 1.558 & 0.579 & 0.148 & 66.200 & 1.36 & 0.029 & 0.008839 & 0.002593 & 0.091584 & 41.103 \\
\hline 3/20/08 23:00 & 1.579 & 0.600 & 0.161 & 72.282 & 1.362 & 0.031 & 0.009448 & 0.002838 & 0.100226 & 44.982 \\
\hline 3/21/08 0:00 & 1.537 & 0.558 & 0.135 & 60.436 & 1.36 & 0.029 & 0.008839 & 0.002593 & 0.091584 & 41.103 \\
\hline 3/21/08 1:00 & 1.545 & 0.566 & 0.139 & 62.595 & 1.355 & 0.024 & 0.007315 & 0.002015 & 0.071152 & 31.933 \\
\hline 3/21/08 2:00 & 1.554 & 0.575 & 0.145 & 65.078 & 1.357 & 0.026 & 0.007924 & 0.002240 & 0.079119 & 35.508 \\
\hline 3/21/08 3:00 & 1.554 & 0.575 & 0.145 & 65.078 & 1.359 & 0.028 & 0.008534 & 0.002473 & 0.087362 & 39.208 \\
\hline 3/21/08 4:00 & 1.555 & 0.576 & 0.146 & 65.357 & 1.357 & 0.026 & 0.007924 & 0.002240 & 0.079119 & 35.508 \\
\hline
\end{tabular}




\begin{tabular}{|c|c|c|c|c|c|c|c|c|c|c|}
\hline \multirow[b]{3}{*}{ Date Time } & \multicolumn{4}{|c|}{ V-Notch Weir } & \multicolumn{6}{|c|}{ R-Notch Weir } \\
\hline & \multicolumn{2}{|c|}{ Measured Water Level } & \multicolumn{2}{|c|}{$\begin{array}{c}\text { Calculated Rate } \\
\left(\mathrm{ft}^{3} / \mathrm{s}=448.8 \mathrm{gpm}\right)\end{array}$} & \multicolumn{3}{|c|}{ Measured Water Level } & \multicolumn{3}{|c|}{$\begin{array}{c}\text { Calculated Rate } \\
\left(\mathrm{ft}^{3} / \mathrm{s}=448.8 \mathrm{gpm}\right)\end{array}$} \\
\hline & $\begin{array}{c}\text { (ft above } \\
\text { sensor) }\end{array}$ & $\begin{array}{c}\text { (ft above } \\
\text { notch) }\end{array}$ & $\left(\mathrm{ft}^{3} / \mathrm{s}\right)$ & (gpm) & $\begin{array}{l}\text { (ft above } \\
\text { sensor) }\end{array}$ & $\begin{array}{c}\text { (ft above } \\
\text { log) }\end{array}$ & $\begin{array}{c}\text { (m above } \\
\text { log) }\end{array}$ & $\left(\mathrm{m}^{3} / \mathrm{s}\right)$ & $\left(\mathrm{ft}^{3} / \mathrm{s}\right)$ & (gpm) \\
\hline 3/21/08 5:00 & 1.555 & 0.576 & 0.146 & 65.357 & 1.357 & 0.026 & 0.007924 & 0.002240 & 0.079119 & 35.508 \\
\hline 3/21/08 6:00 & 1.553 & 0.574 & 0.144 & 64.799 & 1.357 & 0.026 & 0.007924 & 0.002240 & 0.079119 & 35.508 \\
\hline 3/21/08 7:00 & 1.544 & 0.565 & 0.139 & 62.323 & 1.357 & 0.026 & 0.007924 & 0.002240 & 0.079119 & 35.508 \\
\hline 3/21/08 8:00 & 1.533 & 0.554 & 0.132 & 59.374 & 1.354 & 0.023 & 0.007010 & 0.001905 & 0.067276 & 30.194 \\
\hline 3/21/08 9:00 & 1.547 & 0.568 & 0.141 & 63.142 & 1.352 & 0.021 & 0.006400 & 0.001692 & 0.059744 & 26.813 \\
\hline 3/21/08 10:00 & 1.531 & 0.552 & 0.131 & 58.847 & 1.354 & 0.023 & 0.007010 & 0.001905 & 0.067276 & 30.194 \\
\hline 3/21/08 11:00 & 1.536 & 0.557 & 0.134 & 60.170 & 1.354 & 0.023 & 0.007010 & 0.001905 & 0.067276 & 30.194 \\
\hline 3/21/08 12:00 & 1.545 & 0.566 & 0.139 & 62.595 & 1.352 & 0.021 & 0.006400 & 0.001692 & 0.059744 & 26.813 \\
\hline 3/21/08 13:00 & 1.532 & 0.553 & 0.132 & 59.110 & 1.356 & 0.025 & 0.007620 & 0.002126 & 0.075100 & 33.705 \\
\hline 3/21/08 14:00 & 1.529 & 0.550 & 0.130 & 58.323 & 1.354 & 0.023 & 0.007010 & 0.001905 & 0.067276 & 30.194 \\
\hline 3/21/08 15:00 & 1.556 & 0.577 & 0.146 & 65.638 & 1.357 & 0.026 & 0.007924 & 0.002240 & 0.079119 & 35.508 \\
\hline 3/21/08 16:00 & 1.565 & 0.586 & 0.152 & 68.192 & 1.355 & 0.024 & 0.007315 & 0.002015 & 0.071152 & 31.933 \\
\hline 3/21/08 17:00 & 1.548 & 0.569 & 0.141 & 63.416 & 1.357 & 0.026 & 0.007924 & 0.002240 & 0.079119 & 35.508 \\
\hline 3/21/08 18:00 & 1.525 & 0.546 & 0.128 & 57.283 & 1.355 & 0.024 & 0.007315 & 0.002015 & 0.071152 & 31.933 \\
\hline 3/21/08 19:00 & 1.532 & 0.553 & 0.132 & 59.110 & 1.355 & 0.024 & 0.007315 & 0.002015 & 0.071152 & 31.933 \\
\hline 3/21/08 20:00 & 1.532 & 0.553 & 0.132 & 59.110 & 1.357 & 0.026 & 0.007924 & 0.002240 & 0.079119 & 35.508 \\
\hline 3/21/08 21:00 & 1.532 & 0.553 & 0.132 & 59.110 & 1.355 & 0.024 & 0.007315 & 0.002015 & 0.071152 & 31.933 \\
\hline 3/21/08 22:00 & 1.53 & 0.551 & 0.131 & 58.585 & 1.355 & 0.024 & 0.007315 & 0.002015 & 0.071152 & 31.933 \\
\hline 3/21/08 23:00 & 1.531 & 0.552 & 0.131 & 58.847 & 1.355 & 0.024 & 0.007315 & 0.002015 & 0.071152 & 31.933 \\
\hline 3/22/08 0:00 & 1.529 & 0.550 & 0.130 & 58.323 & 1.355 & 0.024 & 0.007315 & 0.002015 & 0.071152 & 31.933 \\
\hline 3/22/08 1:00 & 1.529 & 0.550 & 0.130 & 58.323 & 1.355 & 0.024 & 0.007315 & 0.002015 & 0.071152 & 31.933 \\
\hline 3/22/08 2:00 & 1.529 & 0.550 & 0.130 & 58.323 & 1.354 & 0.023 & 0.007010 & 0.001905 & 0.067276 & 30.194 \\
\hline 3/22/08 3:00 & 1.528 & 0.549 & 0.129 & 58.062 & 1.354 & 0.023 & 0.007010 & 0.001905 & 0.067276 & 30.194 \\
\hline 3/22/08 4:00 & 1.528 & 0.549 & 0.129 & 58.062 & 1.354 & 0.023 & 0.007010 & 0.001905 & 0.067276 & 30.194 \\
\hline 3/22/08 5:00 & 1.527 & 0.548 & 0.129 & 57.802 & 1.352 & 0.021 & 0.006400 & 0.001692 & 0.059744 & 26.813 \\
\hline
\end{tabular}




\begin{tabular}{|c|c|c|c|c|c|c|c|c|c|c|}
\hline \multirow[b]{3}{*}{ Date Time } & \multicolumn{4}{|c|}{ V-Notch Weir } & \multicolumn{6}{|c|}{ R-Notch Weir } \\
\hline & \multicolumn{2}{|c|}{ Measured Water Level } & \multicolumn{2}{|c|}{$\begin{array}{c}\text { Calculated Rate } \\
\left(\mathrm{ft}^{3} / \mathrm{s}=448.8 \mathrm{gpm}\right)\end{array}$} & \multicolumn{3}{|c|}{ Measured Water Level } & \multicolumn{3}{|c|}{$\begin{array}{c}\text { Calculated Rate } \\
\left(\mathrm{ft}^{3} / \mathrm{s}=448.8 \mathrm{gpm}\right)\end{array}$} \\
\hline & $\begin{array}{c}\text { (ft above } \\
\text { sensor) }\end{array}$ & $\begin{array}{c}\text { (ft above } \\
\text { notch) }\end{array}$ & $\left(\mathrm{ft}^{3} / \mathrm{s}\right)$ & (gpm) & $\begin{array}{l}\text { (ft above } \\
\text { sensor) }\end{array}$ & $\begin{array}{c}\text { (ft above } \\
\text { log) }\end{array}$ & $\begin{array}{c}\text { (m above } \\
\text { log) }\end{array}$ & $\left(\mathrm{m}^{3} / \mathrm{s}\right)$ & $\left(\mathrm{ft}^{3} / \mathrm{s}\right)$ & (gpm) \\
\hline 3/22/08 6:00 & 1.532 & 0.553 & 0.132 & 59.110 & 1.352 & 0.021 & 0.006400 & 0.001692 & 0.059744 & 26.813 \\
\hline 3/22/08 7:00 & 1.528 & 0.549 & 0.129 & 58.062 & 1.354 & 0.023 & 0.007010 & 0.001905 & 0.067276 & 30.194 \\
\hline 3/22/08 8:00 & 1.524 & 0.545 & 0.127 & 57.025 & 1.351 & 0.02 & 0.006096 & 0.001588 & 0.056092 & 25.174 \\
\hline 3/22/08 9:00 & 1.526 & 0.547 & 0.128 & 57.542 & 1.351 & 0.02 & 0.006096 & 0.001588 & 0.056092 & 25.174 \\
\hline 3/22/08 10:00 & 1.526 & 0.547 & 0.128 & 57.542 & 1.354 & 0.023 & 0.007010 & 0.001905 & 0.067276 & 30.194 \\
\hline 3/22/08 11:00 & 1.526 & 0.547 & 0.128 & 57.542 & 1.351 & 0.02 & 0.006096 & 0.001588 & 0.056092 & 25.174 \\
\hline 3/22/08 12:00 & 1.523 & 0.544 & 0.126 & 56.767 & 1.351 & 0.02 & 0.006096 & 0.001588 & 0.056092 & 25.174 \\
\hline 3/22/08 13:00 & 1.541 & 0.562 & 0.137 & 61.510 & 1.354 & 0.023 & 0.007010 & 0.001905 & 0.067276 & 30.194 \\
\hline 3/22/08 14:00 & 1.525 & 0.546 & 0.128 & 57.283 & 1.351 & 0.02 & 0.006096 & 0.001588 & 0.056092 & 25.174 \\
\hline 3/22/08 15:00 & 1.519 & 0.540 & 0.124 & 55.744 & 1.354 & 0.023 & 0.007010 & 0.001905 & 0.067276 & 30.194 \\
\hline 3/22/08 16:00 & 1.514 & 0.535 & 0.121 & 54.481 & 1.354 & 0.023 & 0.007010 & 0.001905 & 0.067276 & 30.194 \\
\hline 3/22/08 17:00 & 1.514 & 0.535 & 0.121 & 54.481 & 1.354 & 0.023 & 0.007010 & 0.001905 & 0.067276 & 30.194 \\
\hline 3/22/08 18:00 & 1.505 & 0.526 & 0.116 & 52.251 & 1.354 & 0.023 & 0.007010 & 0.001905 & 0.067276 & 30.194 \\
\hline 3/22/08 19:00 & 1.521 & 0.542 & 0.125 & 56.255 & 1.351 & 0.02 & 0.006096 & 0.001588 & 0.056092 & 25.174 \\
\hline 3/22/08 20:00 & 1.524 & 0.545 & 0.127 & 57.025 & 1.354 & 0.023 & 0.007010 & 0.001905 & 0.067276 & 30.194 \\
\hline 3/22/08 21:00 & 1.529 & 0.550 & 0.130 & 58.323 & 1.354 & 0.023 & 0.007010 & 0.001905 & 0.067276 & 30.194 \\
\hline 3/22/08 22:00 & 1.516 & 0.537 & 0.123 & 54.984 & 1.351 & 0.02 & 0.006096 & 0.001588 & 0.056092 & 25.174 \\
\hline 3/22/08 23:00 & 1.525 & 0.546 & 0.128 & 57.283 & 1.351 & 0.02 & 0.006096 & 0.001588 & 0.056092 & 25.174 \\
\hline 3/23/08 0:00 & 1.533 & 0.554 & 0.132 & 59.374 & 1.351 & 0.02 & 0.006096 & 0.001588 & 0.056092 & 25.174 \\
\hline 3/23/08 1:00 & 1.522 & 0.543 & 0.126 & 56.511 & 1.354 & 0.023 & 0.007010 & 0.001905 & 0.067276 & 30.194 \\
\hline 3/23/08 2:00 & 1.522 & 0.543 & 0.126 & 56.511 & 1.351 & 0.02 & 0.006096 & 0.001588 & 0.056092 & 25.174 \\
\hline 3/23/08 3:00 & 1.525 & 0.546 & 0.128 & 57.283 & 1.354 & 0.023 & 0.007010 & 0.001905 & 0.067276 & 30.194 \\
\hline 3/23/08 4:00 & 1.521 & 0.542 & 0.125 & 56.255 & 1.354 & 0.023 & 0.007010 & 0.001905 & 0.067276 & 30.194 \\
\hline 3/23/08 5:00 & 1.521 & 0.542 & 0.125 & 56.255 & 1.351 & 0.02 & 0.006096 & 0.001588 & 0.056092 & 25.174 \\
\hline 3/23/08 6:00 & 1.52 & 0.541 & 0.125 & 55.999 & 1.351 & 0.02 & 0.006096 & 0.001588 & 0.056092 & 25.174 \\
\hline
\end{tabular}




\begin{tabular}{|c|c|c|c|c|c|c|c|c|c|c|}
\hline \multirow[b]{3}{*}{ Date Time } & \multicolumn{4}{|c|}{ V-Notch Weir } & \multicolumn{6}{|c|}{ R-Notch Weir } \\
\hline & \multicolumn{2}{|c|}{ Measured Water Level } & \multicolumn{2}{|c|}{$\begin{array}{c}\text { Calculated Rate } \\
\left(\mathrm{ft}^{3} / \mathrm{s}=448.8 \mathrm{gpm}\right)\end{array}$} & \multicolumn{3}{|c|}{ Measured Water Level } & \multicolumn{3}{|c|}{$\begin{array}{c}\text { Calculated Rate } \\
\left(\mathrm{ft}^{3} / \mathrm{s}=448.8 \mathrm{gpm}\right)\end{array}$} \\
\hline & $\begin{array}{c}\text { (ft above } \\
\text { sensor) }\end{array}$ & $\begin{array}{c}\text { (ft above } \\
\text { notch) }\end{array}$ & $\left(\mathrm{ft}^{3} / \mathrm{s}\right)$ & (gpm) & $\begin{array}{l}\text { (ft above } \\
\text { sensor) }\end{array}$ & $\begin{array}{c}\text { (ft above } \\
\text { log) }\end{array}$ & $\begin{array}{c}\text { (m above } \\
\text { log) }\end{array}$ & $\left(\mathrm{m}^{3} / \mathrm{s}\right)$ & $\left(\mathrm{ft}^{3} / \mathrm{s}\right)$ & (gpm) \\
\hline 3/23/08 7:00 & 1.513 & 0.534 & 0.121 & 54.231 & 1.351 & 0.02 & 0.006096 & 0.001588 & 0.056092 & 25.174 \\
\hline 3/23/08 8:00 & 1.511 & 0.532 & 0.120 & 53.732 & 1.349 & 0.018 & 0.005486 & 0.001388 & 0.049021 & 22.001 \\
\hline 3/23/08 9:00 & 1.515 & 0.536 & 0.122 & 54.733 & 1.351 & 0.02 & 0.006096 & 0.001588 & 0.056092 & 25.174 \\
\hline 3/23/08 10:00 & 1.524 & 0.545 & 0.127 & 57.025 & 1.351 & 0.02 & 0.006096 & 0.001588 & 0.056092 & 25.174 \\
\hline 3/23/08 11:00 & 1.525 & 0.546 & 0.128 & 57.283 & 1.351 & 0.02 & 0.006096 & 0.001588 & 0.056092 & 25.174 \\
\hline 3/23/08 12:00 & 1.519 & 0.540 & 0.124 & 55.744 & 1.351 & 0.02 & 0.006096 & 0.001588 & 0.056092 & 25.174 \\
\hline 3/23/08 13:00 & 1.514 & 0.535 & 0.121 & 54.481 & 1.351 & 0.02 & 0.006096 & 0.001588 & 0.056092 & 25.174 \\
\hline 3/23/08 14:00 & 1.512 & 0.533 & 0.120 & 53.981 & 1.351 & 0.02 & 0.006096 & 0.001588 & 0.056092 & 25.174 \\
\hline 3/23/08 15:00 & 1.518 & 0.539 & 0.124 & 55.490 & 1.351 & 0.02 & 0.006096 & 0.001588 & 0.056092 & 25.174 \\
\hline 3/23/08 16:00 & 1.503 & 0.524 & 0.115 & 51.763 & 1.351 & 0.02 & 0.006096 & 0.001588 & 0.056092 & 25.174 \\
\hline 3/23/08 17:00 & 1.491 & 0.512 & 0.109 & 48.893 & 1.354 & 0.023 & 0.007010 & 0.001905 & 0.067276 & 30.194 \\
\hline 3/23/08 18:00 & 1.573 & 0.594 & 0.157 & 70.511 & 1.359 & 0.028 & 0.008534 & 0.002473 & 0.087362 & 39.208 \\
\hline 3/23/08 19:00 & 1.55 & 0.571 & 0.143 & 63.967 & 1.354 & 0.023 & 0.007010 & 0.001905 & 0.067276 & 30.194 \\
\hline 3/23/08 20:00 & 1.484 & 0.505 & 0.105 & 47.264 & 1.359 & 0.028 & 0.008534 & 0.002473 & 0.087362 & 39.208 \\
\hline 3/23/08 21:00 & 1.536 & 0.557 & 0.134 & 60.170 & 1.356 & 0.025 & 0.007620 & 0.002126 & 0.075100 & 33.705 \\
\hline 3/23/08 22:00 & 1.556 & 0.577 & 0.146 & 65.638 & 1.354 & 0.023 & 0.007010 & 0.001905 & 0.067276 & 30.194 \\
\hline 3/23/08 23:00 & 1.544 & 0.565 & 0.139 & 62.323 & 1.351 & 0.02 & 0.006096 & 0.001588 & 0.056092 & 25.174 \\
\hline 3/24/08 0:00 & 1.538 & 0.559 & 0.135 & 60.704 & 1.351 & 0.02 & 0.006096 & 0.001588 & 0.056092 & 25.174 \\
\hline 3/24/08 1:00 & 1.539 & 0.560 & 0.136 & 60.972 & 1.351 & 0.02 & 0.006096 & 0.001588 & 0.056092 & 25.174 \\
\hline 3/24/08 2:00 & 1.537 & 0.558 & 0.135 & 60.436 & 1.351 & 0.02 & 0.006096 & 0.001588 & 0.056092 & 25.174 \\
\hline 3/24/08 3:00 & 1.536 & 0.557 & 0.134 & 60.170 & 1.351 & 0.02 & 0.006096 & 0.001588 & 0.056092 & 25.174 \\
\hline 3/24/08 4:00 & 1.542 & 0.563 & 0.138 & 61.780 & 1.351 & 0.02 & 0.006096 & 0.001588 & 0.056092 & 25.174 \\
\hline 3/24/08 5:00 & 1.552 & 0.573 & 0.144 & 64.521 & 1.351 & 0.02 & 0.006096 & 0.001588 & 0.056092 & 25.174 \\
\hline 3/24/08 6:00 & 1.538 & 0.559 & 0.135 & 60.704 & 1.351 & 0.02 & 0.006096 & 0.001588 & 0.056092 & 25.174 \\
\hline 3/24/08 7:00 & 1.547 & 0.568 & 0.141 & 63.142 & 1.349 & 0.018 & 0.005486 & 0.001388 & 0.049021 & 22.001 \\
\hline
\end{tabular}




\begin{tabular}{|c|c|c|c|c|c|c|c|c|c|c|}
\hline \multirow[b]{3}{*}{ Date Time } & \multicolumn{4}{|c|}{ V-Notch Weir } & \multicolumn{6}{|c|}{ R-Notch Weir } \\
\hline & \multicolumn{2}{|c|}{ Measured Water Level } & \multicolumn{2}{|c|}{$\begin{array}{c}\text { Calculated Rate } \\
\left(\mathrm{ft}^{3} / \mathrm{s}=448.8 \mathrm{gpm}\right)\end{array}$} & \multicolumn{3}{|c|}{ Measured Water Level } & \multicolumn{3}{|c|}{$\begin{array}{c}\text { Calculated Rate } \\
\left(\mathrm{ft}^{3} / \mathrm{s}=448.8 \mathrm{gpm}\right)\end{array}$} \\
\hline & $\begin{array}{c}\text { (ft above } \\
\text { sensor) }\end{array}$ & $\begin{array}{c}\text { (ft above } \\
\text { notch) }\end{array}$ & $\left(\mathrm{ft}^{3} / \mathrm{s}\right)$ & (gpm) & $\begin{array}{l}\text { (ft above } \\
\text { sensor) }\end{array}$ & $\begin{array}{c}\text { (ft above } \\
\text { log) }\end{array}$ & $\begin{array}{c}\text { (m above } \\
\text { log) }\end{array}$ & $\left(\mathrm{m}^{3} / \mathrm{s}\right)$ & $\left(\mathrm{ft}^{3} / \mathrm{s}\right)$ & (gpm) \\
\hline 3/24/08 8:00 & 1.529 & 0.550 & 0.130 & 58.323 & 1.349 & 0.018 & 0.005486 & 0.001388 & 0.049021 & 22.001 \\
\hline 3/24/08 9:00 & 1.527 & 0.548 & 0.129 & 57.802 & 1.349 & 0.018 & 0.005486 & 0.001388 & 0.049021 & 22.001 \\
\hline 3/24/08 10:00 & 1.557 & 0.578 & 0.147 & 65.918 & 1.349 & 0.018 & 0.005486 & 0.001388 & 0.049021 & 22.001 \\
\hline 3/24/08 11:00 & 1.561 & 0.582 & 0.149 & 67.049 & 1.351 & 0.02 & 0.006096 & 0.001588 & 0.056092 & 25.174 \\
\hline 3/24/08 12:00 & 1.529 & 0.550 & 0.130 & 58.323 & 1.351 & 0.02 & 0.006096 & 0.001588 & 0.056092 & 25.174 \\
\hline 3/24/08 13:00 & 1.623 & 0.644 & 0.192 & 86.080 & 1.356 & 0.025 & 0.007620 & 0.002126 & 0.075100 & 33.705 \\
\hline 3/24/08 14:00 & 1.626 & 0.647 & 0.194 & 87.074 & 1.358 & 0.027 & 0.008229 & 0.002356 & 0.083206 & 37.343 \\
\hline 3/24/08 15:00 & 1.625 & 0.646 & 0.193 & 86.742 & 1.356 & 0.025 & 0.007620 & 0.002126 & 0.075100 & 33.705 \\
\hline 3/24/08 16:00 & 1.633 & 0.654 & 0.199 & 89.419 & 1.356 & 0.025 & 0.007620 & 0.002126 & 0.075100 & 33.705 \\
\hline 3/24/08 17:00 & 1.647 & 0.668 & 0.210 & 94.223 & 1.359 & 0.028 & 0.008534 & 0.002473 & 0.087362 & 39.208 \\
\hline 3/24/08 18:00 & 1.612 & 0.633 & 0.184 & 82.494 & 1.349 & 0.018 & 0.005486 & 0.001388 & 0.049021 & 22.001 \\
\hline 3/24/08 19:00 & 1.633 & 0.654 & 0.199 & 89.419 & 1.357 & 0.026 & 0.007924 & 0.002240 & 0.079119 & 35.508 \\
\hline 3/24/08 20:00 & 1.635 & 0.656 & 0.201 & 90.096 & 1.357 & 0.026 & 0.007924 & 0.002240 & 0.079119 & 35.508 \\
\hline 3/24/08 21:00 & 1.624 & 0.645 & 0.193 & 86.410 & 1.357 & 0.026 & 0.007924 & 0.002240 & 0.079119 & 35.508 \\
\hline 3/24/08 22:00 & 1.631 & 0.652 & 0.198 & 88.745 & 1.357 & 0.026 & 0.007924 & 0.002240 & 0.079119 & 35.508 \\
\hline 3/24/08 23:00 & 1.641 & 0.662 & 0.205 & 92.146 & 1.357 & 0.026 & 0.007924 & 0.002240 & 0.079119 & 35.508 \\
\hline 3/25/08 0:00 & 1.674 & 0.695 & 0.232 & 103.916 & 1.361 & 0.03 & 0.009144 & 0.002714 & 0.095873 & 43.028 \\
\hline 3/25/08 1:00 & 1.63 & 0.651 & 0.197 & 88.409 & 1.354 & 0.023 & 0.007010 & 0.001905 & 0.067276 & 30.194 \\
\hline 3/25/08 2:00 & 1.602 & 0.623 & 0.177 & 79.314 & 1.352 & 0.021 & 0.006400 & 0.001692 & 0.059744 & 26.813 \\
\hline 3/25/08 3:00 & 1.621 & 0.642 & 0.190 & 85.421 & 1.356 & 0.025 & 0.007620 & 0.002126 & 0.075100 & 33.705 \\
\hline 3/25/08 4:00 & 1.619 & 0.640 & 0.189 & 84.765 & 1.354 & 0.023 & 0.007010 & 0.001905 & 0.067276 & 30.194 \\
\hline 3/25/08 5:00 & 1.603 & 0.624 & 0.177 & 79.629 & 1.356 & 0.025 & 0.007620 & 0.002126 & 0.075100 & 33.705 \\
\hline 3/25/08 6:00 & 1.615 & 0.636 & 0.186 & 83.463 & 1.356 & 0.025 & 0.007620 & 0.002126 & 0.075100 & 33.705 \\
\hline 3/25/08 7:00 & 1.605 & 0.626 & 0.179 & 80.260 & 1.354 & 0.023 & 0.007010 & 0.001905 & 0.067276 & 30.194 \\
\hline 3/25/08 8:00 & 1.603 & 0.624 & 0.177 & 79.629 & 1.354 & 0.023 & 0.007010 & 0.001905 & 0.067276 & 30.194 \\
\hline
\end{tabular}




\begin{tabular}{|c|c|c|c|c|c|c|c|c|c|c|}
\hline \multirow[b]{3}{*}{ Date Time } & \multicolumn{4}{|c|}{ V-Notch Weir } & \multicolumn{6}{|c|}{ R-Notch Weir } \\
\hline & \multicolumn{2}{|c|}{ Measured Water Level } & \multicolumn{2}{|c|}{$\begin{array}{c}\text { Calculated Rate } \\
\left(\mathrm{ft}^{3} / \mathrm{s}=448.8 \mathrm{gpm}\right)\end{array}$} & \multicolumn{3}{|c|}{ Measured Water Level } & \multicolumn{3}{|c|}{$\begin{array}{c}\text { Calculated Rate } \\
\left(\mathrm{ft}^{3} / \mathrm{s}=448.8 \mathrm{gpm}\right)\end{array}$} \\
\hline & $\begin{array}{c}\text { (ft above } \\
\text { sensor) }\end{array}$ & $\begin{array}{c}\text { (ft above } \\
\text { notch) }\end{array}$ & $\left(\mathrm{ft}^{3} / \mathrm{s}\right)$ & (gpm) & $\begin{array}{c}\text { (ft above } \\
\text { sensor) }\end{array}$ & $\begin{array}{c}\text { (ft above } \\
\text { log) }\end{array}$ & $\begin{array}{c}\text { (m above } \\
\text { log) }\end{array}$ & $\left(\mathrm{m}^{3} / \mathrm{s}\right)$ & $\left(\mathrm{ft}^{3} / \mathrm{s}\right)$ & (gpm) \\
\hline 3/25/08 9:00 & 1.601 & 0.622 & 0.176 & 79.000 & 1.352 & 0.021 & 0.006400 & 0.001692 & 0.059744 & 26.813 \\
\hline 3/25/08 10:00 & 1.601 & 0.622 & 0.176 & 79.000 & 1.352 & 0.021 & 0.006400 & 0.001692 & 0.059744 & 26.813 \\
\hline 3/25/08 11:00 & 1.608 & 0.629 & 0.181 & 81.213 & 1.354 & 0.023 & 0.007010 & 0.001905 & 0.067276 & 30.194 \\
\hline 3/25/08 12:00 & 1.619 & 0.640 & 0.189 & 84.765 & 1.352 & 0.021 & 0.006400 & 0.001692 & 0.059744 & 26.813 \\
\hline 3/25/08 13:00 & 1.592 & 0.613 & 0.170 & 76.208 & 1.352 & 0.021 & 0.006400 & 0.001692 & 0.059744 & 26.813 \\
\hline 3/25/08 14:00 & 1.596 & 0.617 & 0.173 & 77.442 & 1.352 & 0.021 & 0.006400 & 0.001692 & 0.059744 & 26.813 \\
\hline 3/25/08 15:00 & 1.497 & 0.518 & 0.112 & 50.316 & 1.355 & 0.024 & 0.007315 & 0.002015 & 0.071152 & 31.933 \\
\hline 3/25/08 16:00 & 1.567 & 0.588 & 0.153 & 68.767 & 1.355 & 0.024 & 0.007315 & 0.002015 & 0.071152 & 31.933 \\
\hline 3/25/08 17:00 & 1.588 & 0.609 & 0.167 & 74.987 & 1.357 & 0.026 & 0.007924 & 0.002240 & 0.079119 & 35.508 \\
\hline 3/25/08 18:00 & 1.59 & 0.611 & 0.168 & 75.596 & 1.36 & 0.029 & 0.008839 & 0.002593 & 0.091584 & 41.103 \\
\hline 3/25/08 19:00 & 1.651 & 0.672 & 0.213 & 95.623 & 1.357 & 0.026 & 0.007924 & 0.002240 & 0.079119 & 35.508 \\
\hline 3/25/08 20:00 & 1.63 & 0.651 & 0.197 & 88.409 & 1.36 & 0.029 & 0.008839 & 0.002593 & 0.091584 & 41.103 \\
\hline 3/25/08 21:00 & 1.635 & 0.656 & 0.201 & 90.096 & 1.357 & 0.026 & 0.007924 & 0.002240 & 0.079119 & 35.508 \\
\hline 3/25/08 22:00 & 1.633 & 0.654 & 0.199 & 89.419 & 1.357 & 0.026 & 0.007924 & 0.002240 & 0.079119 & 35.508 \\
\hline 3/25/08 23:00 & 1.636 & 0.657 & 0.202 & 90.436 & 1.357 & 0.026 & 0.007924 & 0.002240 & 0.079119 & 35.508 \\
\hline 3/26/08 0:00 & 1.662 & 0.683 & 0.222 & 99.538 & 1.357 & 0.026 & 0.007924 & 0.002240 & 0.079119 & 35.508 \\
\hline 3/26/08 1:00 & 1.664 & 0.685 & 0.223 & 100.260 & 1.357 & 0.026 & 0.007924 & 0.002240 & 0.079119 & 35.508 \\
\hline 3/26/08 2:00 & 1.667 & 0.688 & 0.226 & 101.348 & 1.357 & 0.026 & 0.007924 & 0.002240 & 0.079119 & 35.508 \\
\hline 3/26/08 3:00 & 1.67 & 0.691 & 0.228 & 102.444 & 1.357 & 0.026 & 0.007924 & 0.002240 & 0.079119 & 35.508 \\
\hline 3/26/08 4:00 & 1.675 & 0.696 & 0.232 & 104.286 & 1.357 & 0.026 & 0.007924 & 0.002240 & 0.079119 & 35.508 \\
\hline 3/26/08 5:00 & 1.67 & 0.691 & 0.228 & 102.444 & 1.359 & 0.028 & 0.008534 & 0.002473 & 0.087362 & 39.208 \\
\hline 3/26/08 6:00 & 1.669 & 0.690 & 0.227 & 102.078 & 1.355 & 0.024 & 0.007315 & 0.002015 & 0.071152 & 31.933 \\
\hline 3/26/08 7:00 & 1.664 & 0.685 & 0.223 & 100.260 & 1.354 & 0.023 & 0.007010 & 0.001905 & 0.067276 & 30.194 \\
\hline 3/26/08 8:00 & 1.674 & 0.695 & 0.232 & 103.916 & 1.357 & 0.026 & 0.007924 & 0.002240 & 0.079119 & 35.508 \\
\hline 3/26/08 9:00 & 1.667 & 0.688 & 0.226 & 101.348 & 1.357 & 0.026 & 0.007924 & 0.002240 & 0.079119 & 35.508 \\
\hline
\end{tabular}




\begin{tabular}{|c|c|c|c|c|c|c|c|c|c|c|}
\hline \multirow[b]{3}{*}{ Date Time } & \multicolumn{4}{|c|}{ V-Notch Weir } & \multicolumn{6}{|c|}{ R-Notch Weir } \\
\hline & \multicolumn{2}{|c|}{ Measured Water Level } & \multicolumn{2}{|c|}{$\begin{array}{c}\text { Calculated Rate } \\
\left(\mathrm{ft}^{3} / \mathrm{s}=448.8 \mathrm{gpm}\right)\end{array}$} & \multicolumn{3}{|c|}{ Measured Water Level } & \multicolumn{3}{|c|}{$\begin{array}{c}\text { Calculated Rate } \\
\left(\mathrm{ft}^{3} / \mathrm{s}=448.8 \mathrm{gpm}\right)\end{array}$} \\
\hline & $\begin{array}{c}\text { (ft above } \\
\text { sensor) }\end{array}$ & $\begin{array}{c}\text { (ft above } \\
\text { notch) }\end{array}$ & $\left(\mathrm{ft}^{3} / \mathrm{s}\right)$ & (gpm) & $\begin{array}{l}\text { (ft above } \\
\text { sensor) }\end{array}$ & $\begin{array}{c}\text { (ft above } \\
\text { log) }\end{array}$ & $\begin{array}{c}\text { (m above } \\
\text { log) }\end{array}$ & $\left(\mathrm{m}^{3} / \mathrm{s}\right)$ & $\left(\mathrm{ft}^{3} / \mathrm{s}\right)$ & (gpm) \\
\hline 3/26/08 10:00 & 1.653 & 0.674 & 0.215 & 96.328 & 1.359 & 0.028 & 0.008534 & 0.002473 & 0.087362 & 39.208 \\
\hline 3/26/08 11:00 & 1.674 & 0.695 & 0.232 & 103.916 & 1.357 & 0.026 & 0.007924 & 0.002240 & 0.079119 & 35.508 \\
\hline 3/26/08 12:00 & 1.673 & 0.694 & 0.231 & 103.547 & 1.357 & 0.026 & 0.007924 & 0.002240 & 0.079119 & 35.508 \\
\hline 3/26/08 13:00 & 1.67 & 0.691 & 0.228 & 102.444 & 1.359 & 0.028 & 0.008534 & 0.002473 & 0.087362 & 39.208 \\
\hline 3/26/08 14:00 & 1.666 & 0.687 & 0.225 & 100.985 & 1.359 & 0.028 & 0.008534 & 0.002473 & 0.087362 & 39.208 \\
\hline 3/26/08 15:00 & 1.659 & 0.680 & 0.219 & 98.461 & 1.362 & 0.031 & 0.009448 & 0.002838 & 0.100226 & 44.982 \\
\hline 3/26/08 16:00 & 1.611 & 0.632 & 0.183 & 82.173 & 1.369 & 0.038 & 0.011582 & 0.003750 & 0.132444 & 59.441 \\
\hline 3/26/08 17:00 & 1.661 & 0.682 & 0.221 & 99.178 & 1.364 & 0.033 & 0.010058 & 0.003090 & 0.109124 & 48.975 \\
\hline 3/26/08 18:00 & 1.675 & 0.696 & 0.232 & 104.286 & 1.369 & 0.038 & 0.011582 & 0.003750 & 0.132444 & 59.441 \\
\hline 3/26/08 19:00 & 1.675 & 0.696 & 0.232 & 104.286 & 1.358 & 0.027 & 0.008229 & 0.002356 & 0.083206 & 37.343 \\
\hline 3/26/08 20:00 & 1.656 & 0.677 & 0.217 & 97.391 & 1.362 & 0.031 & 0.009448 & 0.002838 & 0.100226 & 44.982 \\
\hline 3/26/08 21:00 & 1.699 & 0.720 & 0.253 & 113.404 & 1.365 & 0.034 & 0.010363 & 0.003218 & 0.113667 & 51.014 \\
\hline 3/26/08 22:00 & 1.589 & 0.610 & 0.168 & 75.291 & 1.358 & 0.027 & 0.008229 & 0.002356 & 0.083206 & 37.343 \\
\hline 3/26/08 23:00 & 1.568 & 0.589 & 0.154 & 69.056 & 1.357 & 0.026 & 0.007924 & 0.002240 & 0.079119 & 35.508 \\
\hline 3/27/08 0:00 & 1.556 & 0.577 & 0.146 & 65.638 & 1.357 & 0.026 & 0.007924 & 0.002240 & 0.079119 & 35.508 \\
\hline 3/27/08 1:00 & 1.582 & 0.603 & 0.163 & 73.177 & 1.357 & 0.026 & 0.007924 & 0.002240 & 0.079119 & 35.508 \\
\hline 3/27/08 2:00 & 1.554 & 0.575 & 0.145 & 65.078 & 1.357 & 0.026 & 0.007924 & 0.002240 & 0.079119 & 35.508 \\
\hline 3/27/08 3:00 & 1.569 & 0.590 & 0.155 & 69.346 & 1.357 & 0.026 & 0.007924 & 0.002240 & 0.079119 & 35.508 \\
\hline 3/27/08 4:00 & 1.581 & 0.602 & 0.162 & 72.878 & 1.36 & 0.029 & 0.008839 & 0.002593 & 0.091584 & 41.103 \\
\hline 3/27/08 5:00 & 1.541 & 0.562 & 0.137 & 61.510 & 1.352 & 0.021 & 0.006400 & 0.001692 & 0.059744 & 26.813 \\
\hline 3/27/08 6:00 & 1.565 & 0.586 & 0.152 & 68.192 & 1.355 & 0.024 & 0.007315 & 0.002015 & 0.071152 & 31.933 \\
\hline 3/27/08 7:00 & 1.551 & 0.572 & 0.143 & 64.244 & 1.352 & 0.021 & 0.006400 & 0.001692 & 0.059744 & 26.813 \\
\hline 3/27/08 8:00 & 1.537 & 0.558 & 0.135 & 60.436 & 1.354 & 0.023 & 0.007010 & 0.001905 & 0.067276 & 30.194 \\
\hline 3/27/08 9:00 & 1.589 & 0.610 & 0.168 & 75.291 & 1.361 & 0.03 & 0.009144 & 0.002714 & 0.095873 & 43.028 \\
\hline 3/27/08 10:00 & 1.564 & 0.585 & 0.151 & 67.905 & 1.354 & 0.023 & 0.007010 & 0.001905 & 0.067276 & 30.194 \\
\hline
\end{tabular}




\begin{tabular}{|c|c|c|c|c|c|c|c|c|c|c|}
\hline \multirow[b]{3}{*}{ Date Time } & \multicolumn{4}{|c|}{ V-Notch Weir } & \multicolumn{6}{|c|}{ R-Notch Weir } \\
\hline & \multicolumn{2}{|c|}{ Measured Water Level } & \multicolumn{2}{|c|}{$\begin{array}{c}\text { Calculated Rate } \\
\left(\mathrm{ft}^{3} / \mathrm{s}=448.8 \mathrm{gpm}\right)\end{array}$} & \multicolumn{3}{|c|}{ Measured Water Level } & \multicolumn{3}{|c|}{$\begin{array}{c}\text { Calculated Rate } \\
\left(\mathrm{ft}^{3} / \mathrm{s}=448.8 \mathrm{gpm}\right)\end{array}$} \\
\hline & $\begin{array}{c}\text { (ft above } \\
\text { sensor) }\end{array}$ & $\begin{array}{c}\text { (ft above } \\
\text { notch) }\end{array}$ & $\left(\mathrm{ft}^{3} / \mathrm{s}\right)$ & (gpm) & $\begin{array}{l}\text { (ft above } \\
\text { sensor) }\end{array}$ & $\begin{array}{c}\text { (ft above } \\
\text { log) }\end{array}$ & $\begin{array}{c}\text { (m above } \\
\text { log) }\end{array}$ & $\left(\mathrm{m}^{3} / \mathrm{s}\right)$ & $\left(\mathrm{ft}^{3} / \mathrm{s}\right)$ & (gpm) \\
\hline 3/27/08 11:00 & 1.559 & 0.580 & 0.148 & 66.482 & 1.352 & 0.021 & 0.006400 & 0.001692 & 0.059744 & 26.813 \\
\hline 3/27/08 12:00 & 1.557 & 0.578 & 0.147 & 65.918 & 1.354 & 0.023 & 0.007010 & 0.001905 & 0.067276 & 30.194 \\
\hline 3/27/08 13:00 & 1.547 & 0.568 & 0.141 & 63.142 & 1.352 & 0.021 & 0.006400 & 0.001692 & 0.059744 & 26.813 \\
\hline 3/27/08 14:00 & 1.556 & 0.577 & 0.146 & 65.638 & 1.352 & 0.021 & 0.006400 & 0.001692 & 0.059744 & 26.813 \\
\hline 3/27/08 15:00 & 1.547 & 0.568 & 0.141 & 63.142 & 1.352 & 0.021 & 0.006400 & 0.001692 & 0.059744 & 26.813 \\
\hline 3/27/08 16:00 & 1.425 & 0.446 & 0.078 & 34.821 & 1.354 & 0.023 & 0.007010 & 0.001905 & 0.067276 & 30.194 \\
\hline 3/27/08 17:00 & 1.495 & 0.516 & 0.111 & 49.839 & 1.361 & 0.03 & 0.009144 & 0.002714 & 0.095873 & 43.028 \\
\hline 3/27/08 18:00 & 1.523 & 0.544 & 0.126 & 56.767 & 1.366 & 0.035 & 0.010667 & 0.003349 & 0.118271 & 53.080 \\
\hline 3/27/08 19:00 & 1.578 & 0.599 & 0.160 & 71.985 & 1.363 & 0.032 & 0.009753 & 0.002963 & 0.104644 & 46.964 \\
\hline 3/27/08 20:00 & 1.569 & 0.590 & 0.155 & 69.346 & 1.361 & 0.03 & 0.009144 & 0.002714 & 0.095873 & 43.028 \\
\hline 3/27/08 21:00 & 1.545 & 0.566 & 0.139 & 62.595 & 1.359 & 0.028 & 0.008534 & 0.002473 & 0.087362 & 39.208 \\
\hline 3/27/08 22:00 & 1.546 & 0.567 & 0.140 & 62.868 & 1.359 & 0.028 & 0.008534 & 0.002473 & 0.087362 & 39.208 \\
\hline 3/27/08 23:00 & 1.544 & 0.565 & 0.139 & 62.323 & 1.359 & 0.028 & 0.008534 & 0.002473 & 0.087362 & 39.208 \\
\hline 3/28/08 0:00 & 1.539 & 0.560 & 0.136 & 60.972 & 1.358 & 0.027 & 0.008229 & 0.002356 & 0.083206 & 37.343 \\
\hline 3/28/08 1:00 & 1.537 & 0.558 & 0.135 & 60.436 & 1.361 & 0.03 & 0.009144 & 0.002714 & 0.095873 & 43.028 \\
\hline 3/28/08 2:00 & 1.545 & 0.566 & 0.139 & 62.595 & 1.361 & 0.03 & 0.009144 & 0.002714 & 0.095873 & 43.028 \\
\hline 3/28/08 3:00 & 1.538 & 0.559 & 0.135 & 60.704 & 1.361 & 0.03 & 0.009144 & 0.002714 & 0.095873 & 43.028 \\
\hline 3/28/08 4:00 & 1.545 & 0.566 & 0.139 & 62.595 & 1.361 & 0.03 & 0.009144 & 0.002714 & 0.095873 & 43.028 \\
\hline 3/28/08 5:00 & 1.543 & 0.564 & 0.138 & 62.051 & 1.363 & 0.032 & 0.009753 & 0.002963 & 0.104644 & 46.964 \\
\hline 3/28/08 6:00 & 1.539 & 0.560 & 0.136 & 60.972 & 1.363 & 0.032 & 0.009753 & 0.002963 & 0.104644 & 46.964 \\
\hline 3/28/08 7:00 & 1.544 & 0.565 & 0.139 & 62.323 & 1.363 & 0.032 & 0.009753 & 0.002963 & 0.104644 & 46.964 \\
\hline 3/28/08 8:00 & 1.539 & 0.560 & 0.136 & 60.972 & 1.363 & 0.032 & 0.009753 & 0.002963 & 0.104644 & 46.964 \\
\hline 3/28/08 9:00 & 1.549 & 0.570 & 0.142 & 63.691 & 1.363 & 0.032 & 0.009753 & 0.002963 & 0.104644 & 46.964 \\
\hline 3/28/08 10:00 & 1.551 & 0.572 & 0.143 & 64.244 & 1.363 & 0.032 & 0.009753 & 0.002963 & 0.104644 & 46.964 \\
\hline 3/28/08 11:00 & 1.545 & 0.566 & 0.139 & 62.595 & 1.363 & 0.032 & 0.009753 & 0.002963 & 0.104644 & 46.964 \\
\hline
\end{tabular}




\begin{tabular}{|c|c|c|c|c|c|c|c|c|c|c|}
\hline \multirow[b]{3}{*}{ Date Time } & \multicolumn{4}{|c|}{ V-Notch Weir } & \multicolumn{6}{|c|}{ R-Notch Weir } \\
\hline & \multicolumn{2}{|c|}{ Measured Water Level } & \multicolumn{2}{|c|}{$\begin{array}{c}\text { Calculated Rate } \\
\left(\mathrm{ft}^{3} / \mathrm{s}=448.8 \mathrm{gpm}\right)\end{array}$} & \multicolumn{3}{|c|}{ Measured Water Level } & \multicolumn{3}{|c|}{$\begin{array}{c}\text { Calculated Rate } \\
\left(\mathrm{ft}^{3} / \mathrm{s}=448.8 \mathrm{gpm}\right)\end{array}$} \\
\hline & $\begin{array}{c}\text { (ft above } \\
\text { sensor) }\end{array}$ & $\begin{array}{c}\text { (ft above } \\
\text { notch) }\end{array}$ & $\left(\mathrm{ft}^{3} / \mathrm{s}\right)$ & (gpm) & $\begin{array}{l}\text { (ft above } \\
\text { sensor) }\end{array}$ & $\begin{array}{c}\text { (ft above } \\
\text { log) }\end{array}$ & $\begin{array}{c}\text { (m above } \\
\text { log) }\end{array}$ & $\left(\mathrm{m}^{3} / \mathrm{s}\right)$ & $\left(\mathrm{ft}^{3} / \mathrm{s}\right)$ & (gpm) \\
\hline 3/28/08 12:00 & 1.556 & 0.577 & 0.146 & 65.638 & 1.365 & 0.034 & 0.010363 & 0.003218 & 0.113667 & 51.014 \\
\hline 3/28/08 13:00 & 1.491 & 0.512 & 0.109 & 48.893 & 1.365 & 0.034 & 0.010363 & 0.003218 & 0.113667 & 51.014 \\
\hline 3/28/08 14:00 & 1.688 & 0.709 & 0.243 & 109.168 & 1.368 & 0.037 & 0.011277 & 0.003614 & 0.127660 & 57.294 \\
\hline 3/28/08 15:00 & 1.629 & 0.650 & 0.196 & 88.074 & 1.363 & 0.032 & 0.009753 & 0.002963 & 0.104644 & 46.964 \\
\hline 3/28/08 16:00 & 1.487 & 0.508 & 0.107 & 47.958 & 1.361 & 0.03 & 0.009144 & 0.002714 & 0.095873 & 43.028 \\
\hline 3/28/08 17:00 & 1.534 & 0.555 & 0.133 & 59.638 & 1.368 & 0.037 & 0.011277 & 0.003614 & 0.127660 & 57.294 \\
\hline 3/28/08 18:00 & 1.536 & 0.557 & 0.134 & 60.170 & 1.369 & 0.038 & 0.011582 & 0.003750 & 0.132444 & 59.441 \\
\hline 3/28/08 19:00 & 1.562 & 0.583 & 0.150 & 67.334 & 1.366 & 0.035 & 0.010667 & 0.003349 & 0.118271 & 53.080 \\
\hline 3/28/08 20:00 & 1.56 & 0.581 & 0.149 & 66.766 & 1.369 & 0.038 & 0.011582 & 0.003750 & 0.132444 & 59.441 \\
\hline 3/28/08 21:00 & 1.544 & 0.565 & 0.139 & 62.323 & 1.364 & 0.033 & 0.010058 & 0.003090 & 0.109124 & 48.975 \\
\hline 3/28/08 22:00 & 1.591 & 0.612 & 0.169 & 75.902 & 1.366 & 0.035 & 0.010667 & 0.003349 & 0.118271 & 53.080 \\
\hline 3/28/08 23:00 & 1.598 & 0.619 & 0.174 & 78.063 & 1.371 & 0.04 & 0.012191 & 0.004026 & 0.142185 & 63.812 \\
\hline 3/29/08 0:00 & 1.641 & 0.662 & 0.205 & 92.146 & 1.366 & 0.035 & 0.010667 & 0.003349 & 0.118271 & 53.080 \\
\hline 3/29/08 1:00 & 1.646 & 0.667 & 0.209 & 93.875 & 1.357 & 0.026 & 0.007924 & 0.002240 & 0.079119 & 35.508 \\
\hline 3/29/08 2:00 & 1.606 & 0.627 & 0.180 & 80.577 & 1.354 & 0.023 & 0.007010 & 0.001905 & 0.067276 & 30.194 \\
\hline 3/29/08 3:00 & 1.59 & 0.611 & 0.168 & 75.596 & 1.352 & 0.021 & 0.006400 & 0.001692 & 0.059744 & 26.813 \\
\hline 3/29/08 4:00 & 1.551 & 0.572 & 0.143 & 64.244 & 1.357 & 0.026 & 0.007924 & 0.002240 & 0.079119 & 35.508 \\
\hline 3/29/08 5:00 & 1.601 & 0.622 & 0.176 & 79.000 & 1.354 & 0.023 & 0.007010 & 0.001905 & 0.067276 & 30.194 \\
\hline 3/29/08 6:00 & 1.62 & 0.641 & 0.190 & 85.093 & 1.352 & 0.021 & 0.006400 & 0.001692 & 0.059744 & 26.813 \\
\hline 3/29/08 7:00 & 1.592 & 0.613 & 0.170 & 76.208 & 1.352 & 0.021 & 0.006400 & 0.001692 & 0.059744 & 26.813 \\
\hline 3/29/08 8:00 & 1.628 & 0.649 & 0.195 & 87.740 & 1.352 & 0.021 & 0.006400 & 0.001692 & 0.059744 & 26.813 \\
\hline 3/29/08 9:00 & 1.593 & 0.614 & 0.170 & 76.516 & 1.351 & 0.02 & 0.006096 & 0.001588 & 0.056092 & 25.174 \\
\hline 3/29/08 10:00 & 1.642 & 0.663 & 0.206 & 92.490 & 1.356 & 0.025 & 0.007620 & 0.002126 & 0.075100 & 33.705 \\
\hline 3/29/08 11:00 & 1.621 & 0.642 & 0.190 & 85.421 & 1.356 & 0.025 & 0.007620 & 0.002126 & 0.075100 & 33.705 \\
\hline 3/29/08 12:00 & 1.589 & 0.610 & 0.168 & 75.291 & 1.349 & 0.018 & 0.005486 & 0.001388 & 0.049021 & 22.001 \\
\hline
\end{tabular}




\begin{tabular}{|c|c|c|c|c|c|c|c|c|c|c|}
\hline \multirow[b]{3}{*}{ Date Time } & \multicolumn{4}{|c|}{ V-Notch Weir } & \multicolumn{6}{|c|}{ R-Notch Weir } \\
\hline & \multicolumn{2}{|c|}{ Measured Water Level } & \multicolumn{2}{|c|}{$\begin{array}{c}\text { Calculated Rate } \\
\left(\mathrm{ft}^{3} / \mathrm{s}=448.8 \mathrm{gpm}\right)\end{array}$} & \multicolumn{3}{|c|}{ Measured Water Level } & \multicolumn{3}{|c|}{$\begin{array}{c}\text { Calculated Rate } \\
\left(\mathrm{ft}^{3} / \mathrm{s}=448.8 \mathrm{gpm}\right)\end{array}$} \\
\hline & $\begin{array}{c}\text { (ft above } \\
\text { sensor) }\end{array}$ & $\begin{array}{c}\text { (ft above } \\
\text { notch) }\end{array}$ & $\left(\mathrm{ft}^{3} / \mathrm{s}\right)$ & (gpm) & $\begin{array}{l}\text { (ft above } \\
\text { sensor) }\end{array}$ & $\begin{array}{c}\text { (ft above } \\
\text { log) }\end{array}$ & $\begin{array}{c}\text { (m above } \\
\text { log) }\end{array}$ & $\left(\mathrm{m}^{3} / \mathrm{s}\right)$ & $\left(\mathrm{ft}^{3} / \mathrm{s}\right)$ & (gpm) \\
\hline 3/29/08 13:00 & 1.591 & 0.612 & 0.169 & 75.902 & 1.352 & 0.021 & 0.006400 & 0.001692 & 0.059744 & 26.813 \\
\hline 3/29/08 14:00 & 1.547 & 0.568 & 0.141 & 63.142 & 1.352 & 0.021 & 0.006400 & 0.001692 & 0.059744 & 26.813 \\
\hline 3/29/08 15:00 & 1.523 & 0.544 & 0.126 & 56.767 & 1.352 & 0.021 & 0.006400 & 0.001692 & 0.059744 & 26.813 \\
\hline 3/29/08 16:00 & 1.56 & 0.581 & 0.149 & 66.766 & 1.35 & 0.019 & 0.005791 & 0.001487 & 0.052517 & 23.570 \\
\hline 3/29/08 17:00 & 1.666 & 0.687 & 0.225 & 100.985 & 1.352 & 0.021 & 0.006400 & 0.001692 & 0.059744 & 26.813 \\
\hline 3/29/08 18:00 & 1.59 & 0.611 & 0.168 & 75.596 & 1.357 & 0.026 & 0.007924 & 0.002240 & 0.079119 & 35.508 \\
\hline 3/29/08 19:00 & 1.612 & 0.633 & 0.184 & 82.494 & 1.35 & 0.019 & 0.005791 & 0.001487 & 0.052517 & 23.570 \\
\hline 3/29/08 20:00 & 1.616 & 0.637 & 0.187 & 83.788 & 1.355 & 0.024 & 0.007315 & 0.002015 & 0.071152 & 31.933 \\
\hline 3/29/08 21:00 & 1.621 & 0.642 & 0.190 & 85.421 & 1.355 & 0.024 & 0.007315 & 0.002015 & 0.071152 & 31.933 \\
\hline 3/29/08 22:00 & 1.652 & 0.673 & 0.214 & 95.975 & 1.352 & 0.021 & 0.006400 & 0.001692 & 0.059744 & 26.813 \\
\hline 3/29/08 23:00 & 1.622 & 0.643 & 0.191 & 85.750 & 1.35 & 0.019 & 0.005791 & 0.001487 & 0.052517 & 23.570 \\
\hline 3/30/08 0:00 & 1.605 & 0.626 & 0.179 & 80.260 & 1.35 & 0.019 & 0.005791 & 0.001487 & 0.052517 & 23.570 \\
\hline 3/30/08 1:00 & 1.589 & 0.610 & 0.168 & 75.291 & 1.352 & 0.021 & 0.006400 & 0.001692 & 0.059744 & 26.813 \\
\hline 3/30/08 2:00 & 1.599 & 0.620 & 0.175 & 78.375 & 1.35 & 0.019 & 0.005791 & 0.001487 & 0.052517 & 23.570 \\
\hline 3/30/08 3:00 & 1.592 & 0.613 & 0.170 & 76.208 & 1.35 & 0.019 & 0.005791 & 0.001487 & 0.052517 & 23.570 \\
\hline 3/30/08 4:00 & 1.564 & 0.585 & 0.151 & 67.905 & 1.352 & 0.021 & 0.006400 & 0.001692 & 0.059744 & 26.813 \\
\hline 3/30/08 5:00 & 1.587 & 0.608 & 0.166 & 74.683 & 1.355 & 0.024 & 0.007315 & 0.002015 & 0.071152 & 31.933 \\
\hline 3/30/08 6:00 & 1.597 & 0.618 & 0.173 & 77.752 & 1.357 & 0.026 & 0.007924 & 0.002240 & 0.079119 & 35.508 \\
\hline 3/30/08 7:00 & 1.595 & 0.616 & 0.172 & 77.132 & 1.357 & 0.026 & 0.007924 & 0.002240 & 0.079119 & 35.508 \\
\hline 3/30/08 8:00 & 1.581 & 0.602 & 0.162 & 72.878 & 1.357 & 0.026 & 0.007924 & 0.002240 & 0.079119 & 35.508 \\
\hline 3/30/08 9:00 & 1.581 & 0.602 & 0.162 & 72.878 & 1.355 & 0.024 & 0.007315 & 0.002015 & 0.071152 & 31.933 \\
\hline 3/30/08 10:00 & 1.602 & 0.623 & 0.177 & 79.314 & 1.355 & 0.024 & 0.007315 & 0.002015 & 0.071152 & 31.933 \\
\hline 3/30/08 11:00 & 1.599 & 0.620 & 0.175 & 78.375 & 1.352 & 0.021 & 0.006400 & 0.001692 & 0.059744 & 26.813 \\
\hline 3/30/08 12:00 & 1.577 & 0.598 & 0.160 & 71.689 & 1.352 & 0.021 & 0.006400 & 0.001692 & 0.059744 & 26.813 \\
\hline 3/30/08 13:00 & 1.582 & 0.603 & 0.163 & 73.177 & 1.352 & 0.021 & 0.006400 & 0.001692 & 0.059744 & 26.813 \\
\hline
\end{tabular}




\begin{tabular}{|c|c|c|c|c|c|c|c|c|c|c|}
\hline \multirow[b]{3}{*}{ Date Time } & \multicolumn{4}{|c|}{ V-Notch Weir } & \multicolumn{6}{|c|}{ R-Notch Weir } \\
\hline & \multicolumn{2}{|c|}{ Measured Water Level } & \multicolumn{2}{|c|}{$\begin{array}{c}\text { Calculated Rate } \\
\left(\mathrm{ft}^{3} / \mathrm{s}=448.8 \mathrm{gpm}\right)\end{array}$} & \multicolumn{3}{|c|}{ Measured Water Level } & \multicolumn{3}{|c|}{$\begin{array}{c}\text { Calculated Rate } \\
\left(\mathrm{ft}^{3} / \mathrm{s}=448.8 \mathrm{gpm}\right)\end{array}$} \\
\hline & $\begin{array}{l}\text { (ft above } \\
\text { sensor) }\end{array}$ & $\begin{array}{l}\text { (ft above } \\
\text { notch) }\end{array}$ & $\left(\mathrm{ft}^{3} / \mathrm{s}\right)$ & (gpm) & $\begin{array}{l}\text { (ft above } \\
\text { sensor) }\end{array}$ & $\begin{array}{l}\text { (ft above } \\
\text { log) }\end{array}$ & $\begin{array}{c}\text { (m above } \\
\text { log) }\end{array}$ & $\left(\mathrm{m}^{3} / \mathrm{s}\right)$ & $\left(\mathrm{ft}^{3} / \mathrm{s}\right)$ & (gpm) \\
\hline 3/30/08 14:00 & 1.588 & 0.609 & 0.167 & 74.987 & 1.353 & 0.022 & 0.006705 & 0.001797 & 0.063473 & 28.487 \\
\hline 3/30/08 15:00 & 1.572 & 0.593 & 0.156 & 70.219 & 1.35 & 0.019 & 0.005791 & 0.001487 & 0.052517 & 23.570 \\
\hline 3/30/08 16:00 & 1.583 & 0.604 & 0.164 & 73.477 & 1.353 & 0.022 & 0.006705 & 0.001797 & 0.063473 & 28.487 \\
\hline 3/30/08 17:00 & 1.588 & 0.609 & 0.167 & 74.987 & 1.35 & 0.019 & 0.005791 & 0.001487 & 0.052517 & 23.570 \\
\hline 3/30/08 18:00 & 1.581 & 0.602 & 0.162 & 72.878 & 1.351 & 0.02 & 0.006096 & 0.001588 & 0.056092 & 25.174 \\
\hline 3/30/08 19:00 & 1.586 & 0.607 & 0.166 & 74.381 & 1.351 & 0.02 & 0.006096 & 0.001588 & 0.056092 & 25.174 \\
\hline 3/30/08 20:00 & 1.577 & 0.598 & 0.160 & 71.689 & 1.351 & 0.02 & 0.006096 & 0.001588 & 0.056092 & 25.174 \\
\hline 3/30/08 21:00 & 1.57 & 0.591 & 0.155 & 69.636 & 1.35 & 0.019 & 0.005791 & 0.001487 & 0.052517 & 23.570 \\
\hline 3/30/08 22:00 & 1.575 & 0.596 & 0.158 & 71.099 & 1.35 & 0.019 & 0.005791 & 0.001487 & 0.052517 & 23.570 \\
\hline 3/30/08 23:00 & 1.568 & 0.589 & 0.154 & 69.056 & 1.35 & 0.019 & 0.005791 & 0.001487 & 0.052517 & 23.570 \\
\hline 3/31/08 0:00 & 1.583 & 0.604 & 0.164 & 73.477 & 1.353 & 0.022 & 0.006705 & 0.001797 & 0.063473 & 28.487 \\
\hline 3/31/08 1:00 & 1.578 & 0.599 & 0.160 & 71.985 & 1.352 & 0.021 & 0.006400 & 0.001692 & 0.059744 & 26.813 \\
\hline 3/31/08 2:00 & 1.574 & 0.595 & 0.158 & 70.805 & 1.35 & 0.019 & 0.005791 & 0.001487 & 0.052517 & 23.570 \\
\hline 3/31/08 3:00 & 1.578 & 0.599 & 0.160 & 71.985 & 1.35 & 0.019 & 0.005791 & 0.001487 & 0.052517 & 23.570 \\
\hline 3/31/08 4:00 & 1.602 & 0.623 & 0.177 & 79.314 & 1.355 & 0.024 & 0.007315 & 0.002015 & 0.071152 & 31.933 \\
\hline 3/31/08 5:00 & 1.706 & 0.727 & 0.259 & 116.150 & 1.373 & 0.042 & 0.012801 & 0.004308 & 0.152154 & 68.287 \\
\hline 3/31/08 6:00 & 1.871 & 0.892 & 0.429 & 192.724 & 1.397 & 0.066 & 0.020116 & 0.008153 & 0.287966 & 129.239 \\
\hline 3/31/08 7:00 & 1.991 & 1.012 & 0.587 & 263.539 & 1.439 & 0.108 & 0.032917 & 0.016598 & 0.586238 & 263.104 \\
\hline 3/31/08 8:00 & 1.977 & 0.998 & 0.567 & 254.588 & 1.432 & 0.101 & 0.030783 & 0.015057 & 0.531798 & 238.671 \\
\hline 3/31/08 9:00 & 1.949 & 0.970 & 0.529 & 237.239 & 1.416 & 0.085 & 0.025907 & 0.011728 & 0.414220 & 185.902 \\
\hline 3/31/08 10:00 & 1.916 & 0.937 & 0.485 & 217.729 & 1.411 & 0.08 & 0.024383 & 0.010746 & 0.379531 & 170.333 \\
\hline 3/31/08 11:00 & 1.878 & 0.899 & 0.438 & 196.494 & 1.401 & 0.07 & 0.021335 & 0.008869 & 0.313263 & 140.593 \\
\hline 3/31/08 12:00 & 1.824 & 0.845 & 0.376 & 168.537 & 1.392 & 0.061 & 0.018592 & 0.007287 & 0.257360 & 115.503 \\
\hline 3/31/08 13:00 & 1.756 & 0.777 & 0.305 & 136.926 & 1.382 & 0.051 & 0.015544 & 0.005654 & 0.199703 & 89.627 \\
\hline 3/31/08 14:00 & 1.704 & 0.725 & 0.257 & 115.362 & 1.373 & 0.042 & 0.012801 & 0.004308 & 0.152154 & 68.287 \\
\hline
\end{tabular}




\begin{tabular}{|c|c|c|c|c|c|c|c|c|c|c|}
\hline \multirow[b]{3}{*}{ Date Time } & \multicolumn{4}{|c|}{ V-Notch Weir } & \multicolumn{6}{|c|}{ R-Notch Weir } \\
\hline & \multicolumn{2}{|c|}{ Measured Water Level } & \multicolumn{2}{|c|}{$\begin{array}{c}\text { Calculated Rate } \\
\left(\mathrm{ft}^{3} / \mathrm{s}=448.8 \mathrm{gpm}\right)\end{array}$} & \multicolumn{3}{|c|}{ Measured Water Level } & \multicolumn{3}{|c|}{$\begin{array}{c}\text { Calculated Rate } \\
\left(\mathrm{ft}^{3} / \mathrm{s}=448.8 \mathrm{gpm}\right)\end{array}$} \\
\hline & $\begin{array}{c}\text { (ft above } \\
\text { sensor) }\end{array}$ & $\begin{array}{c}\text { (ft above } \\
\text { notch) }\end{array}$ & $\left(\mathrm{ft}^{3} / \mathrm{s}\right)$ & (gpm) & $\begin{array}{l}\text { (ft above } \\
\text { sensor) }\end{array}$ & $\begin{array}{c}\text { (ft above } \\
\text { log) }\end{array}$ & $\begin{array}{c}\text { (m above } \\
\text { log) }\end{array}$ & $\left(\mathrm{m}^{3} / \mathrm{s}\right)$ & $\left(\mathrm{ft}^{3} / \mathrm{s}\right)$ & (gpm) \\
\hline 3/31/08 15:00 & 1.697 & 0.718 & 0.251 & 112.627 & 1.371 & 0.04 & 0.012191 & 0.004026 & 0.142185 & 63.812 \\
\hline 3/31/08 16:00 & 1.683 & 0.704 & 0.239 & 107.275 & 1.371 & 0.04 & 0.012191 & 0.004026 & 0.142185 & 63.812 \\
\hline 3/31/08 17:00 & 1.671 & 0.692 & 0.229 & 102.811 & 1.371 & 0.04 & 0.012191 & 0.004026 & 0.142185 & 63.812 \\
\hline 3/31/08 18:00 & 1.645 & 0.666 & 0.208 & 93.527 & 1.366 & 0.035 & 0.010667 & 0.003349 & 0.118271 & 53.080 \\
\hline 3/31/08 19:00 & 1.617 & 0.638 & 0.187 & 84.113 & 1.361 & 0.03 & 0.009144 & 0.002714 & 0.095873 & 43.028 \\
\hline 3/31/08 20:00 & 1.627 & 0.648 & 0.195 & 87.406 & 1.363 & 0.032 & 0.009753 & 0.002963 & 0.104644 & 46.964 \\
\hline 3/31/08 21:00 & 1.616 & 0.637 & 0.187 & 83.788 & 1.361 & 0.03 & 0.009144 & 0.002714 & 0.095873 & 43.028 \\
\hline 3/31/08 22:00 & 1.614 & 0.635 & 0.185 & 83.140 & 1.359 & 0.028 & 0.008534 & 0.002473 & 0.087362 & 39.208 \\
\hline 3/31/08 23:00 & 1.612 & 0.633 & 0.184 & 82.494 & 1.361 & 0.03 & 0.009144 & 0.002714 & 0.095873 & 43.028 \\
\hline 4/1/08 0:00 & 1.619 & 0.640 & 0.189 & 84.765 & 1.359 & 0.028 & 0.008534 & 0.002473 & 0.087362 & 39.208 \\
\hline 4/1/08 1:00 & 1.617 & 0.638 & 0.187 & 84.113 & 1.356 & 0.025 & 0.007620 & 0.002126 & 0.075100 & 33.705 \\
\hline 4/1/08 2:00 & 1.601 & 0.622 & 0.176 & 79.000 & 1.356 & 0.025 & 0.007620 & 0.002126 & 0.075100 & 33.705 \\
\hline 4/1/08 3:00 & 1.597 & 0.618 & 0.173 & 77.752 & 1.358 & 0.027 & 0.008229 & 0.002356 & 0.083206 & 37.343 \\
\hline 4/1/08 4:00 & 1.59 & 0.611 & 0.168 & 75.596 & 1.358 & 0.027 & 0.008229 & 0.002356 & 0.083206 & 37.343 \\
\hline 4/1/08 5:00 & 1.597 & 0.618 & 0.173 & 77.752 & 1.356 & 0.025 & 0.007620 & 0.002126 & 0.075100 & 33.705 \\
\hline 4/1/08 6:00 & 1.6 & 0.621 & 0.175 & 78.687 & 1.356 & 0.025 & 0.007620 & 0.002126 & 0.075100 & 33.705 \\
\hline 4/1/08 7:00 & 1.574 & 0.595 & 0.158 & 70.805 & 1.358 & 0.027 & 0.008229 & 0.002356 & 0.083206 & 37.343 \\
\hline 4/1/08 8:00 & 1.588 & 0.609 & 0.167 & 74.987 & 1.354 & 0.023 & 0.007010 & 0.001905 & 0.067276 & 30.194 \\
\hline 4/1/08 9:00 & 1.572 & 0.593 & 0.156 & 70.219 & 1.354 & 0.023 & 0.007010 & 0.001905 & 0.067276 & 30.194 \\
\hline 4/1/08 10:00 & 1.577 & 0.598 & 0.160 & 71.689 & 1.356 & 0.025 & 0.007620 & 0.002126 & 0.075100 & 33.705 \\
\hline 4/1/08 11:00 & 1.581 & 0.602 & 0.162 & 72.878 & 1.356 & 0.025 & 0.007620 & 0.002126 & 0.075100 & 33.705 \\
\hline 4/1/08 12:00 & 1.554 & 0.575 & 0.145 & 65.078 & 1.356 & 0.025 & 0.007620 & 0.002126 & 0.075100 & 33.705 \\
\hline 4/1/08 13:00 & 1.55 & 0.571 & 0.143 & 63.967 & 1.358 & 0.027 & 0.008229 & 0.002356 & 0.083206 & 37.343 \\
\hline 4/1/08 14:00 & 1.594 & 0.615 & 0.171 & 76.824 & 1.356 & 0.025 & 0.007620 & 0.002126 & 0.075100 & 33.705 \\
\hline 4/1/08 15:00 & 1.574 & 0.595 & 0.158 & 70.805 & 1.356 & 0.025 & 0.007620 & 0.002126 & 0.075100 & 33.705 \\
\hline
\end{tabular}




\begin{tabular}{|c|c|c|c|c|c|c|c|c|c|c|}
\hline \multirow[b]{3}{*}{ Date Time } & \multicolumn{4}{|c|}{ V-Notch Weir } & \multicolumn{6}{|c|}{ R-Notch Weir } \\
\hline & \multicolumn{2}{|c|}{ Measured Water Level } & \multicolumn{2}{|c|}{$\begin{array}{c}\text { Calculated Rate } \\
\left(\mathrm{ft}^{3} / \mathrm{s}=448.8 \mathrm{gpm}\right)\end{array}$} & \multicolumn{3}{|c|}{ Measured Water Level } & \multicolumn{3}{|c|}{$\begin{array}{c}\text { Calculated Rate } \\
\left(\mathrm{ft}^{3} / \mathrm{s}=448.8 \mathrm{gpm}\right)\end{array}$} \\
\hline & $\begin{array}{c}\text { (ft above } \\
\text { sensor) }\end{array}$ & $\begin{array}{c}\text { (ft above } \\
\text { notch) }\end{array}$ & $\left(\mathrm{ft}^{3} / \mathrm{s}\right)$ & (gpm) & $\begin{array}{l}\text { (ft above } \\
\text { sensor) }\end{array}$ & $\begin{array}{c}\text { (ft above } \\
\text { log) }\end{array}$ & $\begin{array}{c}\text { (m above } \\
\text { log) }\end{array}$ & $\left(\mathrm{m}^{3} / \mathrm{s}\right)$ & $\left(\mathrm{ft}^{3} / \mathrm{s}\right)$ & (gpm) \\
\hline 4/1/08 16:00 & 1.576 & 0.597 & 0.159 & 71.393 & 1.354 & 0.023 & 0.007010 & 0.001905 & 0.067276 & 30.194 \\
\hline 4/1/08 17:00 & 1.554 & 0.575 & 0.145 & 65.078 & 1.359 & 0.028 & 0.008534 & 0.002473 & 0.087362 & 39.208 \\
\hline 4/1/08 18:00 & 1.564 & 0.585 & 0.151 & 67.905 & 1.359 & 0.028 & 0.008534 & 0.002473 & 0.087362 & 39.208 \\
\hline 4/1/08 19:00 & 1.589 & 0.610 & 0.168 & 75.291 & 1.359 & 0.028 & 0.008534 & 0.002473 & 0.087362 & 39.208 \\
\hline 4/1/08 20:00 & 1.573 & 0.594 & 0.157 & 70.511 & 1.356 & 0.025 & 0.007620 & 0.002126 & 0.075100 & 33.705 \\
\hline 4/1/08 21:00 & 1.571 & 0.592 & 0.156 & 69.927 & 1.356 & 0.025 & 0.007620 & 0.002126 & 0.075100 & 33.705 \\
\hline 4/1/08 22:00 & 1.571 & 0.592 & 0.156 & 69.927 & 1.356 & 0.025 & 0.007620 & 0.002126 & 0.075100 & 33.705 \\
\hline 4/1/08 23:00 & 1.555 & 0.576 & 0.146 & 65.357 & 1.356 & 0.025 & 0.007620 & 0.002126 & 0.075100 & 33.705 \\
\hline 4/2/08 0:00 & 1.541 & 0.562 & 0.137 & 61.510 & 1.356 & 0.025 & 0.007620 & 0.002126 & 0.075100 & 33.705 \\
\hline 4/2/08 1:00 & 1.532 & 0.553 & 0.132 & 59.110 & 1.359 & 0.028 & 0.008534 & 0.002473 & 0.087362 & 39.208 \\
\hline 4/2/08 2:00 & 1.578 & 0.599 & 0.160 & 71.985 & 1.359 & 0.028 & 0.008534 & 0.002473 & 0.087362 & 39.208 \\
\hline 4/2/08 3:00 & 1.559 & 0.580 & 0.148 & 66.482 & 1.356 & 0.025 & 0.007620 & 0.002126 & 0.075100 & 33.705 \\
\hline 4/2/08 4:00 & 1.557 & 0.578 & 0.147 & 65.918 & 1.356 & 0.025 & 0.007620 & 0.002126 & 0.075100 & 33.705 \\
\hline 4/2/08 5:00 & 1.562 & 0.583 & 0.150 & 67.334 & 1.359 & 0.028 & 0.008534 & 0.002473 & 0.087362 & 39.208 \\
\hline 4/2/08 6:00 & 1.563 & 0.584 & 0.151 & 67.619 & 1.356 & 0.025 & 0.007620 & 0.002126 & 0.075100 & 33.705 \\
\hline 4/2/08 7:00 & 1.564 & 0.585 & 0.151 & 67.905 & 1.354 & 0.023 & 0.007010 & 0.001905 & 0.067276 & 30.194 \\
\hline 4/2/08 8:00 & 1.56 & 0.581 & 0.149 & 66.766 & 1.354 & 0.023 & 0.007010 & 0.001905 & 0.067276 & 30.194 \\
\hline 4/2/08 9:00 & 1.557 & 0.578 & 0.147 & 65.918 & 1.354 & 0.023 & 0.007010 & 0.001905 & 0.067276 & 30.194 \\
\hline 4/2/08 10:00 & 1.571 & 0.592 & 0.156 & 69.927 & 1.356 & 0.025 & 0.007620 & 0.002126 & 0.075100 & 33.705 \\
\hline 4/2/08 11:00 & 1.563 & 0.584 & 0.151 & 67.619 & 1.354 & 0.023 & 0.007010 & 0.001905 & 0.067276 & 30.194 \\
\hline 4/2/08 12:00 & 1.55 & 0.571 & 0.143 & 63.967 & 1.356 & 0.025 & 0.007620 & 0.002126 & 0.075100 & 33.705 \\
\hline 4/2/08 13:00 & 1.554 & 0.575 & 0.145 & 65.078 & 1.354 & 0.023 & 0.007010 & 0.001905 & 0.067276 & 30.194 \\
\hline 4/2/08 14:00 & 1.542 & 0.563 & 0.138 & 61.780 & 1.354 & 0.023 & 0.007010 & 0.001905 & 0.067276 & 30.194 \\
\hline 4/2/08 15:00 & 1.56 & 0.581 & 0.149 & 66.766 & 1.356 & 0.025 & 0.007620 & 0.002126 & 0.075100 & 33.705 \\
\hline 4/2/08 16:00 & 1.535 & 0.556 & 0.133 & 59.904 & 1.356 & 0.025 & 0.007620 & 0.002126 & 0.075100 & 33.705 \\
\hline
\end{tabular}




\begin{tabular}{|c|c|c|c|c|c|c|c|c|c|c|}
\hline \multirow[b]{3}{*}{ Date Time } & \multicolumn{4}{|c|}{ V-Notch Weir } & \multicolumn{6}{|c|}{ R-Notch Weir } \\
\hline & \multicolumn{2}{|c|}{ Measured Water Level } & \multicolumn{2}{|c|}{$\begin{array}{c}\text { Calculated Rate } \\
\left(\mathrm{ft}^{3} / \mathrm{s}=448.8 \mathrm{gpm}\right)\end{array}$} & \multicolumn{3}{|c|}{ Measured Water Level } & \multicolumn{3}{|c|}{$\begin{array}{c}\text { Calculated Rate } \\
\left(\mathrm{ft}^{3} / \mathrm{s}=448.8 \mathrm{gpm}\right)\end{array}$} \\
\hline & $\begin{array}{l}\text { (ft above } \\
\text { sensor) }\end{array}$ & $\begin{array}{c}\text { (ft above } \\
\text { notch) }\end{array}$ & $\left(\mathrm{ft}^{3} / \mathrm{s}\right)$ & (gpm) & $\begin{array}{c}\text { (ft above } \\
\text { sensor) }\end{array}$ & $\begin{array}{c}\text { (ft above } \\
\text { log) }\end{array}$ & $\begin{array}{c}\text { (m above } \\
\text { log) }\end{array}$ & $\left(\mathrm{m}^{3} / \mathrm{s}\right)$ & $\left(\mathrm{ft}^{3} / \mathrm{s}\right)$ & (gpm) \\
\hline 4/2/08 17:00 & 1.532 & 0.553 & 0.132 & 59.110 & 1.359 & 0.028 & 0.008534 & 0.002473 & 0.087362 & 39.208 \\
\hline 4/2/08 18:00 & 1.546 & 0.567 & 0.140 & 62.868 & 1.364 & 0.033 & 0.010058 & 0.003090 & 0.109124 & 48.975 \\
\hline 4/2/08 19:00 & 1.607 & 0.628 & 0.180 & 80.895 & 1.362 & 0.031 & 0.009448 & 0.002838 & 0.100226 & 44.982 \\
\hline 4/2/08 20:00 & 1.595 & 0.616 & 0.172 & 77.132 & 1.359 & 0.028 & 0.008534 & 0.002473 & 0.087362 & 39.208 \\
\hline 4/2/08 21:00 & 1.596 & 0.617 & 0.173 & 77.442 & 1.362 & 0.031 & 0.009448 & 0.002838 & 0.100226 & 44.982 \\
\hline 4/2/08 22:00 & 1.617 & 0.638 & 0.187 & 84.113 & 1.359 & 0.028 & 0.008534 & 0.002473 & 0.087362 & 39.208 \\
\hline 4/2/08 23:00 & 1.601 & 0.622 & 0.176 & 79.000 & 1.359 & 0.028 & 0.008534 & 0.002473 & 0.087362 & 39.208 \\
\hline 4/3/08 0:00 & 1.606 & 0.627 & 0.180 & 80.577 & 1.357 & 0.026 & 0.007924 & 0.002240 & 0.079119 & 35.508 \\
\hline 4/3/08 1:00 & 1.63 & 0.651 & 0.197 & 88.409 & 1.357 & 0.026 & 0.007924 & 0.002240 & 0.079119 & 35.508 \\
\hline 4/3/08 2:00 & 1.651 & 0.672 & 0.213 & 95.623 & 1.357 & 0.026 & 0.007924 & 0.002240 & 0.079119 & 35.508 \\
\hline 4/3/08 3:00 & 1.6 & 0.621 & 0.175 & 78.687 & 1.357 & 0.026 & 0.007924 & 0.002240 & 0.079119 & 35.508 \\
\hline 4/3/08 4:00 & 1.577 & 0.598 & 0.160 & 71.689 & 1.354 & 0.023 & 0.007010 & 0.001905 & 0.067276 & 30.194 \\
\hline 4/3/08 5:00 & 1.551 & 0.572 & 0.143 & 64.244 & 1.354 & 0.023 & 0.007010 & 0.001905 & 0.067276 & 30.194 \\
\hline 4/3/08 6:00 & 1.575 & 0.596 & 0.158 & 71.099 & 1.356 & 0.025 & 0.007620 & 0.002126 & 0.075100 & 33.705 \\
\hline 4/3/08 7:00 & 1.57 & 0.591 & 0.155 & 69.636 & 1.356 & 0.025 & 0.007620 & 0.002126 & 0.075100 & 33.705 \\
\hline 4/3/08 8:00 & 1.561 & 0.582 & 0.149 & 67.049 & 1.361 & 0.03 & 0.009144 & 0.002714 & 0.095873 & 43.028 \\
\hline 4/3/08 9:00 & 1.62 & 0.641 & 0.190 & 85.093 & 1.366 & 0.035 & 0.010667 & 0.003349 & 0.118271 & 53.080 \\
\hline 4/3/08 10:00 & 1.573 & 0.594 & 0.157 & 70.511 & 1.368 & 0.037 & 0.011277 & 0.003614 & 0.127660 & 57.294 \\
\hline 4/3/08 11:00 & 1.609 & 0.630 & 0.182 & 81.532 & 1.366 & 0.035 & 0.010667 & 0.003349 & 0.118271 & 53.080 \\
\hline 4/3/08 12:00 & 1.736 & 0.757 & 0.286 & 128.369 & 1.377 & 0.046 & 0.014020 & 0.004891 & 0.172754 & 77.532 \\
\hline 4/3/08 13:00 & 1.86 & 0.881 & 0.416 & 186.889 & 1.387 & 0.056 & 0.017068 & 0.006453 & 0.227923 & 102.292 \\
\hline 4/3/08 14:00 & 1.94 & 0.961 & 0.517 & 231.819 & 1.418 & 0.087 & 0.026516 & 0.012129 & 0.428377 & 192.256 \\
\hline 4/3/08 15:00 & 1.942 & 0.963 & 0.519 & 233.017 & 1.413 & 0.082 & 0.024992 & 0.011135 & 0.393285 & 176.506 \\
\hline 4/3/08 16:00 & 1.882 & 0.903 & 0.443 & 198.668 & 1.394 & 0.063 & 0.019201 & 0.007629 & 0.269465 & 120.936 \\
\hline 4/3/08 17:00 & 1.811 & 0.832 & 0.361 & 162.188 & 1.385 & 0.054 & 0.016458 & 0.006129 & 0.216486 & 97.159 \\
\hline
\end{tabular}




\begin{tabular}{|c|c|c|c|c|c|c|c|c|c|c|}
\hline \multirow[b]{3}{*}{ Date Time } & \multicolumn{4}{|c|}{ V-Notch Weir } & \multicolumn{6}{|c|}{ R-Notch Weir } \\
\hline & \multicolumn{2}{|c|}{ Measured Water Level } & \multicolumn{2}{|c|}{$\begin{array}{c}\text { Calculated Rate } \\
\left(\mathrm{ft}^{3} / \mathrm{s}=448.8 \mathrm{gpm}\right)\end{array}$} & \multicolumn{3}{|c|}{ Measured Water Level } & \multicolumn{3}{|c|}{$\begin{array}{c}\text { Calculated Rate } \\
\left(\mathrm{ft}^{3} / \mathrm{s}=448.8 \mathrm{gpm}\right)\end{array}$} \\
\hline & $\begin{array}{c}\text { (ft above } \\
\text { sensor) }\end{array}$ & $\begin{array}{c}\text { (ft above } \\
\text { notch) }\end{array}$ & $\left(\mathrm{ft}^{3} / \mathrm{s}\right)$ & (gpm) & $\begin{array}{l}\text { (ft above } \\
\text { sensor) }\end{array}$ & $\begin{array}{c}\text { (ft above } \\
\text { log) }\end{array}$ & $\begin{array}{c}\text { (m above } \\
\text { log) }\end{array}$ & $\left(\mathrm{m}^{3} / \mathrm{s}\right)$ & $\left(\mathrm{ft}^{3} / \mathrm{s}\right)$ & (gpm) \\
\hline 4/3/08 18:00 & 1.748 & 0.769 & 0.297 & 133.464 & 1.375 & 0.044 & 0.013411 & 0.004596 & 0.162345 & 72.860 \\
\hline 4/3/08 19:00 & 1.705 & 0.726 & 0.258 & 115.756 & 1.371 & 0.04 & 0.012191 & 0.004026 & 0.142185 & 63.812 \\
\hline 4/3/08 20:00 & 1.675 & 0.696 & 0.232 & 104.286 & 1.371 & 0.04 & 0.012191 & 0.004026 & 0.142185 & 63.812 \\
\hline 4/3/08 21:00 & 1.673 & 0.694 & 0.231 & 103.547 & 1.371 & 0.04 & 0.012191 & 0.004026 & 0.142185 & 63.812 \\
\hline 4/3/08 22:00 & 1.692 & 0.713 & 0.247 & 110.697 & 1.366 & 0.035 & 0.010667 & 0.003349 & 0.118271 & 53.080 \\
\hline 4/3/08 23:00 & 1.668 & 0.689 & 0.227 & 101.713 & 1.363 & 0.032 & 0.009753 & 0.002963 & 0.104644 & 46.964 \\
\hline 4/4/08 0:00 & 1.657 & 0.678 & 0.218 & 97.747 & 1.361 & 0.03 & 0.009144 & 0.002714 & 0.095873 & 43.028 \\
\hline 4/4/08 1:00 & 1.653 & 0.674 & 0.215 & 96.328 & 1.361 & 0.03 & 0.009144 & 0.002714 & 0.095873 & 43.028 \\
\hline 4/4/08 2:00 & 1.649 & 0.670 & 0.212 & 94.921 & 1.361 & 0.03 & 0.009144 & 0.002714 & 0.095873 & 43.028 \\
\hline 4/4/08 3:00 & 1.647 & 0.668 & 0.210 & 94.223 & 1.359 & 0.028 & 0.008534 & 0.002473 & 0.087362 & 39.208 \\
\hline 4/4/08 4:00 & 1.642 & 0.663 & 0.206 & 92.490 & 1.358 & 0.027 & 0.008229 & 0.002356 & 0.083206 & 37.343 \\
\hline 4/4/08 5:00 & 1.64 & 0.661 & 0.205 & 91.802 & 1.358 & 0.027 & 0.008229 & 0.002356 & 0.083206 & 37.343 \\
\hline 4/4/08 6:00 & 1.64 & 0.661 & 0.205 & 91.802 & 1.358 & 0.027 & 0.008229 & 0.002356 & 0.083206 & 37.343 \\
\hline 4/4/08 7:00 & 1.634 & 0.655 & 0.200 & 89.757 & 1.358 & 0.027 & 0.008229 & 0.002356 & 0.083206 & 37.343 \\
\hline 4/4/08 8:00 & 1.634 & 0.655 & 0.200 & 89.757 & 1.356 & 0.025 & 0.007620 & 0.002126 & 0.075100 & 33.705 \\
\hline 4/4/08 9:00 & 1.636 & 0.657 & 0.202 & 90.436 & 1.358 & 0.027 & 0.008229 & 0.002356 & 0.083206 & 37.343 \\
\hline 4/4/08 10:00 & 1.626 & 0.647 & 0.194 & 87.074 & 1.358 & 0.027 & 0.008229 & 0.002356 & 0.083206 & 37.343 \\
\hline 4/4/08 11:00 & 1.632 & 0.653 & 0.198 & 89.082 & 1.359 & 0.028 & 0.008534 & 0.002473 & 0.087362 & 39.208 \\
\hline 4/4/08 12:00 & 1.64 & 0.661 & 0.205 & 91.802 & 1.361 & 0.03 & 0.009144 & 0.002714 & 0.095873 & 43.028 \\
\hline 4/4/08 13:00 & 1.631 & 0.652 & 0.198 & 88.745 & 1.359 & 0.028 & 0.008534 & 0.002473 & 0.087362 & 39.208 \\
\hline 4/4/08 14:00 & 1.633 & 0.654 & 0.199 & 89.419 & 1.36 & 0.029 & 0.008839 & 0.002593 & 0.091584 & 41.103 \\
\hline 4/4/08 15:00 & 1.651 & 0.672 & 0.213 & 95.623 & 1.36 & 0.029 & 0.008839 & 0.002593 & 0.091584 & 41.103 \\
\hline 4/4/08 16:00 & 1.59 & 0.611 & 0.168 & 75.596 & 1.358 & 0.027 & 0.008229 & 0.002356 & 0.083206 & 37.343 \\
\hline 4/4/08 17:00 & 1.621 & 0.642 & 0.190 & 85.421 & 1.361 & 0.03 & 0.009144 & 0.002714 & 0.095873 & 43.028 \\
\hline 4/4/08 18:00 & 1.628 & 0.649 & 0.195 & 87.740 & 1.362 & 0.031 & 0.009448 & 0.002838 & 0.100226 & 44.982 \\
\hline
\end{tabular}




\begin{tabular}{|c|c|c|c|c|c|c|c|c|c|c|}
\hline \multirow[b]{3}{*}{ Date Time } & \multicolumn{4}{|c|}{ V-Notch Weir } & \multicolumn{6}{|c|}{ R-Notch Weir } \\
\hline & \multicolumn{2}{|c|}{ Measured Water Level } & \multicolumn{2}{|c|}{$\begin{array}{c}\text { Calculated Rate } \\
\left(\mathrm{ft}^{3} / \mathrm{s}=448.8 \mathrm{gpm}\right)\end{array}$} & \multicolumn{3}{|c|}{ Measured Water Level } & \multicolumn{3}{|c|}{$\begin{array}{c}\text { Calculated Rate } \\
\left(\mathrm{ft}^{3} / \mathrm{s}=448.8 \mathrm{gpm}\right)\end{array}$} \\
\hline & $\begin{array}{c}\text { (ft above } \\
\text { sensor) }\end{array}$ & $\begin{array}{c}\text { (ft above } \\
\text { notch) }\end{array}$ & $\left(\mathrm{ft}^{3} / \mathrm{s}\right)$ & (gpm) & $\begin{array}{l}\text { (ft above } \\
\text { sensor) }\end{array}$ & $\begin{array}{c}\text { (ft above } \\
\text { log) }\end{array}$ & $\begin{array}{c}\text { (m above } \\
\text { log) }\end{array}$ & $\left(\mathrm{m}^{3} / \mathrm{s}\right)$ & $\left(\mathrm{ft}^{3} / \mathrm{s}\right)$ & (gpm) \\
\hline 4/4/08 19:00 & 1.621 & 0.642 & 0.190 & 85.421 & 1.362 & 0.031 & 0.009448 & 0.002838 & 0.100226 & 44.982 \\
\hline 4/4/08 20:00 & 1.632 & 0.653 & 0.198 & 89.082 & 1.364 & 0.033 & 0.010058 & 0.003090 & 0.109124 & 48.975 \\
\hline 4/4/08 21:00 & 1.632 & 0.653 & 0.198 & 89.082 & 1.362 & 0.031 & 0.009448 & 0.002838 & 0.100226 & 44.982 \\
\hline 4/4/08 22:00 & 1.658 & 0.679 & 0.219 & 98.103 & 1.361 & 0.03 & 0.009144 & 0.002714 & 0.095873 & 43.028 \\
\hline 4/4/08 23:00 & 1.687 & 0.708 & 0.242 & 108.788 & 1.361 & 0.03 & 0.009144 & 0.002714 & 0.095873 & 43.028 \\
\hline 4/5/08 0:00 & 1.703 & 0.724 & 0.256 & 114.969 & 1.361 & 0.03 & 0.009144 & 0.002714 & 0.095873 & 43.028 \\
\hline 4/5/08 1:00 & 1.704 & 0.725 & 0.257 & 115.362 & 1.361 & 0.03 & 0.009144 & 0.002714 & 0.095873 & 43.028 \\
\hline 4/5/08 2:00 & 1.72 & 0.741 & 0.271 & 121.761 & 1.36 & 0.029 & 0.008839 & 0.002593 & 0.091584 & 41.103 \\
\hline 4/5/08 3:00 & 1.73 & 0.751 & 0.280 & 125.867 & 1.36 & 0.029 & 0.008839 & 0.002593 & 0.091584 & 41.103 \\
\hline 4/5/08 4:00 & 1.717 & 0.738 & 0.269 & 120.546 & 1.36 & 0.029 & 0.008839 & 0.002593 & 0.091584 & 41.103 \\
\hline 4/5/08 5:00 & 1.717 & 0.738 & 0.269 & 120.546 & 1.36 & 0.029 & 0.008839 & 0.002593 & 0.091584 & 41.103 \\
\hline 4/5/08 6:00 & 1.335 & 0.356 & 0.045 & 20.040 & 1.359 & 0.028 & 0.008534 & 0.002473 & 0.087362 & 39.208 \\
\hline 4/5/08 7:00 & 1.725 & 0.746 & 0.276 & 123.804 & 1.359 & 0.028 & 0.008534 & 0.002473 & 0.087362 & 39.208 \\
\hline 4/5/08 8:00 & 1.714 & 0.735 & 0.266 & 119.337 & 1.359 & 0.028 & 0.008534 & 0.002473 & 0.087362 & 39.208 \\
\hline 4/5/08 9:00 & 1.695 & 0.716 & 0.249 & 111.853 & 1.359 & 0.028 & 0.008534 & 0.002473 & 0.087362 & 39.208 \\
\hline 4/5/08 10:00 & 1.643 & 0.664 & 0.207 & 92.835 & 1.361 & 0.03 & 0.009144 & 0.002714 & 0.095873 & 43.028 \\
\hline 4/5/08 11:00 & 1.661 & 0.682 & 0.221 & 99.178 & 1.357 & 0.026 & 0.007924 & 0.002240 & 0.079119 & 35.508 \\
\hline 4/5/08 12:00 & 1.723 & 0.744 & 0.274 & 122.984 & 1.362 & 0.031 & 0.009448 & 0.002838 & 0.100226 & 44.982 \\
\hline 4/5/08 13:00 & 1.701 & 0.722 & 0.254 & 114.185 & 1.36 & 0.029 & 0.008839 & 0.002593 & 0.091584 & 41.103 \\
\hline 4/5/08 14:00 & 1.696 & 0.717 & 0.250 & 112.239 & 1.361 & 0.03 & 0.009144 & 0.002714 & 0.095873 & 43.028 \\
\hline 4/5/08 15:00 & 1.703 & 0.724 & 0.256 & 114.969 & 1.359 & 0.028 & 0.008534 & 0.002473 & 0.087362 & 39.208 \\
\hline 4/5/08 16:00 & 1.706 & 0.727 & 0.259 & 116.150 & 1.362 & 0.031 & 0.009448 & 0.002838 & 0.100226 & 44.982 \\
\hline 4/5/08 17:00 & 1.659 & 0.680 & 0.219 & 98.461 & 1.358 & 0.027 & 0.008229 & 0.002356 & 0.083206 & 37.343 \\
\hline 4/5/08 18:00 & 1.628 & 0.649 & 0.195 & 87.740 & 1.353 & 0.022 & 0.006705 & 0.001797 & 0.063473 & 28.487 \\
\hline 4/5/08 19:00 & 1.628 & 0.649 & 0.195 & 87.740 & 1.356 & 0.025 & 0.007620 & 0.002126 & 0.075100 & 33.705 \\
\hline
\end{tabular}




\begin{tabular}{|c|c|c|c|c|c|c|c|c|c|c|}
\hline \multirow[b]{3}{*}{ Date Time } & \multicolumn{4}{|c|}{ V-Notch Weir } & \multicolumn{6}{|c|}{ R-Notch Weir } \\
\hline & \multicolumn{2}{|c|}{ Measured Water Level } & \multicolumn{2}{|c|}{$\begin{array}{c}\text { Calculated Rate } \\
\left(\mathrm{ft}^{3} / \mathrm{s}=448.8 \mathrm{gpm}\right)\end{array}$} & \multicolumn{3}{|c|}{ Measured Water Level } & \multicolumn{3}{|c|}{$\begin{array}{c}\text { Calculated Rate } \\
\left(\mathrm{ft}^{3} / \mathrm{s}=448.8 \mathrm{gpm}\right)\end{array}$} \\
\hline & $\begin{array}{l}\text { (ft above } \\
\text { sensor) }\end{array}$ & $\begin{array}{c}\text { (ft above } \\
\text { notch) }\end{array}$ & $\left(\mathrm{ft}^{3} / \mathrm{s}\right)$ & (gpm) & $\begin{array}{c}\text { (ft above } \\
\text { sensor) }\end{array}$ & $\begin{array}{c}\text { (ft above } \\
\text { log) }\end{array}$ & $\begin{array}{c}\text { (m above } \\
\text { log) }\end{array}$ & $\left(\mathrm{m}^{3} / \mathrm{s}\right)$ & $\left(\mathrm{ft}^{3} / \mathrm{s}\right)$ & (gpm) \\
\hline 4/5/08 20:00 & 1.644 & 0.665 & 0.208 & 93.181 & 1.358 & 0.027 & 0.008229 & 0.002356 & 0.083206 & 37.343 \\
\hline 4/5/08 21:00 & 1.632 & 0.653 & 0.198 & 89.082 & 1.363 & 0.032 & 0.009753 & 0.002963 & 0.104644 & 46.964 \\
\hline 4/5/08 22:00 & 1.67 & 0.691 & 0.228 & 102.444 & 1.358 & 0.027 & 0.008229 & 0.002356 & 0.083206 & 37.343 \\
\hline 4/5/08 23:00 & 1.649 & 0.670 & 0.212 & 94.921 & 1.357 & 0.026 & 0.007924 & 0.002240 & 0.079119 & 35.508 \\
\hline 4/6/08 0:00 & 1.64 & 0.661 & 0.205 & 91.802 & 1.357 & 0.026 & 0.007924 & 0.002240 & 0.079119 & 35.508 \\
\hline 4/6/08 1:00 & 1.654 & 0.675 & 0.215 & 96.681 & 1.359 & 0.028 & 0.008534 & 0.002473 & 0.087362 & 39.208 \\
\hline 4/6/08 2:00 & 1.668 & 0.689 & 0.227 & 101.713 & 1.357 & 0.026 & 0.007924 & 0.002240 & 0.079119 & 35.508 \\
\hline 4/6/08 3:00 & 1.659 & 0.680 & 0.219 & 98.461 & 1.359 & 0.028 & 0.008534 & 0.002473 & 0.087362 & 39.208 \\
\hline 4/6/08 4:00 & 1.664 & 0.685 & 0.223 & 100.260 & 1.361 & 0.03 & 0.009144 & 0.002714 & 0.095873 & 43.028 \\
\hline 4/6/08 5:00 & 1.664 & 0.685 & 0.223 & 100.260 & 1.358 & 0.027 & 0.008229 & 0.002356 & 0.083206 & 37.343 \\
\hline 4/6/08 6:00 & 1.556 & 0.577 & 0.146 & 65.638 & 1.356 & 0.025 & 0.007620 & 0.002126 & 0.075100 & 33.705 \\
\hline 4/6/08 7:00 & 1.578 & 0.599 & 0.160 & 71.985 & 1.358 & 0.027 & 0.008229 & 0.002356 & 0.083206 & 37.343 \\
\hline 4/6/08 8:00 & 1.538 & 0.559 & 0.135 & 60.704 & 1.358 & 0.027 & 0.008229 & 0.002356 & 0.083206 & 37.343 \\
\hline 4/6/08 9:00 & 1.538 & 0.559 & 0.135 & 60.704 & 1.36 & 0.029 & 0.008839 & 0.002593 & 0.091584 & 41.103 \\
\hline 4/6/08 10:00 & 1.559 & 0.580 & 0.148 & 66.482 & 1.355 & 0.024 & 0.007315 & 0.002015 & 0.071152 & 31.933 \\
\hline 4/6/08 11:00 & 1.575 & 0.596 & 0.158 & 71.099 & 1.355 & 0.024 & 0.007315 & 0.002015 & 0.071152 & 31.933 \\
\hline 4/6/08 12:00 & 1.57 & 0.591 & 0.155 & 69.636 & 1.358 & 0.027 & 0.008229 & 0.002356 & 0.083206 & 37.343 \\
\hline 4/6/08 13:00 & 1.591 & 0.612 & 0.169 & 75.902 & 1.356 & 0.025 & 0.007620 & 0.002126 & 0.075100 & 33.705 \\
\hline 4/6/08 14:00 & 1.604 & 0.625 & 0.178 & 79.944 & 1.354 & 0.023 & 0.007010 & 0.001905 & 0.067276 & 30.194 \\
\hline 4/6/08 15:00 & 1.616 & 0.637 & 0.187 & 83.788 & 1.359 & 0.028 & 0.008534 & 0.002473 & 0.087362 & 39.208 \\
\hline 4/6/08 16:00 & 1.614 & 0.635 & 0.185 & 83.140 & 1.355 & 0.024 & 0.007315 & 0.002015 & 0.071152 & 31.933 \\
\hline 4/6/08 17:00 & 1.654 & 0.675 & 0.215 & 96.681 & 1.358 & 0.027 & 0.008229 & 0.002356 & 0.083206 & 37.343 \\
\hline 4/6/08 18:00 & 1.6 & 0.621 & 0.175 & 78.687 & 1.356 & 0.025 & 0.007620 & 0.002126 & 0.075100 & 33.705 \\
\hline 4/6/08 19:00 & 1.663 & 0.684 & 0.223 & 99.898 & 1.356 & 0.025 & 0.007620 & 0.002126 & 0.075100 & 33.705 \\
\hline 4/6/08 20:00 & 1.63 & 0.651 & 0.197 & 88.409 & 1.353 & 0.022 & 0.006705 & 0.001797 & 0.063473 & 28.487 \\
\hline
\end{tabular}




\begin{tabular}{|c|c|c|c|c|c|c|c|c|c|c|}
\hline \multirow[b]{3}{*}{ Date Time } & \multicolumn{4}{|c|}{ V-Notch Weir } & \multicolumn{6}{|c|}{ R-Notch Weir } \\
\hline & \multicolumn{2}{|c|}{ Measured Water Level } & \multicolumn{2}{|c|}{$\begin{array}{c}\text { Calculated Rate } \\
\left(\mathrm{ft}^{3} / \mathrm{s}=448.8 \mathrm{gpm}\right)\end{array}$} & \multicolumn{3}{|c|}{ Measured Water Level } & \multicolumn{3}{|c|}{$\begin{array}{c}\text { Calculated Rate } \\
\left(\mathrm{ft}^{3} / \mathrm{s}=448.8 \mathrm{gpm}\right)\end{array}$} \\
\hline & $\begin{array}{c}\text { (ft above } \\
\text { sensor) }\end{array}$ & $\begin{array}{c}\text { (ft above } \\
\text { notch) }\end{array}$ & $\left(\mathrm{ft}^{3} / \mathrm{s}\right)$ & (gpm) & $\begin{array}{l}\text { (ft above } \\
\text { sensor) }\end{array}$ & $\begin{array}{c}\text { (ft above } \\
\text { log) }\end{array}$ & $\begin{array}{c}\text { (m above } \\
\text { log) }\end{array}$ & $\left(\mathrm{m}^{3} / \mathrm{s}\right)$ & $\left(\mathrm{ft}^{3} / \mathrm{s}\right)$ & (gpm) \\
\hline 4/6/08 21:00 & 1.628 & 0.649 & 0.195 & 87.740 & 1.355 & 0.024 & 0.007315 & 0.002015 & 0.071152 & 31.933 \\
\hline 4/6/08 22:00 & 1.631 & 0.652 & 0.198 & 88.745 & 1.352 & 0.021 & 0.006400 & 0.001692 & 0.059744 & 26.813 \\
\hline 4/6/08 23:00 & 1.622 & 0.643 & 0.191 & 85.750 & 1.354 & 0.023 & 0.007010 & 0.001905 & 0.067276 & 30.194 \\
\hline 4/7/08 0:00 & 1.643 & 0.664 & 0.207 & 92.835 & 1.351 & 0.02 & 0.006096 & 0.001588 & 0.056092 & 25.174 \\
\hline 4/7/08 1:00 & 1.663 & 0.684 & 0.223 & 99.898 & 1.355 & 0.024 & 0.007315 & 0.002015 & 0.071152 & 31.933 \\
\hline 4/7/08 2:00 & 1.669 & 0.690 & 0.227 & 102.078 & 1.353 & 0.022 & 0.006705 & 0.001797 & 0.063473 & 28.487 \\
\hline 4/7/08 3:00 & 1.66 & 0.681 & 0.220 & 98.819 & 1.355 & 0.024 & 0.007315 & 0.002015 & 0.071152 & 31.933 \\
\hline 4/7/08 4:00 & 1.673 & 0.694 & 0.231 & 103.547 & 1.355 & 0.024 & 0.007315 & 0.002015 & 0.071152 & 31.933 \\
\hline 4/7/08 5:00 & 1.671 & 0.692 & 0.229 & 102.811 & 1.354 & 0.023 & 0.007010 & 0.001905 & 0.067276 & 30.194 \\
\hline 4/7/08 6:00 & 1.677 & 0.698 & 0.234 & 105.029 & 1.354 & 0.023 & 0.007010 & 0.001905 & 0.067276 & 30.194 \\
\hline 4/7/08 7:00 & 1.677 & 0.698 & 0.234 & 105.029 & 1.354 & 0.023 & 0.007010 & 0.001905 & 0.067276 & 30.194 \\
\hline 4/7/08 8:00 & 1.67 & 0.691 & 0.228 & 102.444 & 1.354 & 0.023 & 0.007010 & 0.001905 & 0.067276 & 30.194 \\
\hline 4/7/08 9:00 & 1.663 & 0.684 & 0.223 & 99.898 & 1.354 & 0.023 & 0.007010 & 0.001905 & 0.067276 & 30.194 \\
\hline 4/7/08 10:00 & 1.653 & 0.674 & 0.215 & 96.328 & 1.352 & 0.021 & 0.006400 & 0.001692 & 0.059744 & 26.813 \\
\hline 4/7/08 11:00 & 1.664 & 0.685 & 0.223 & 100.260 & 1.356 & 0.025 & 0.007620 & 0.002126 & 0.075100 & 33.705 \\
\hline 4/7/08 12:00 & 1.651 & 0.672 & 0.213 & 95.623 & 1.354 & 0.023 & 0.007010 & 0.001905 & 0.067276 & 30.194 \\
\hline 4/7/08 13:00 & 1.643 & 0.664 & 0.207 & 92.835 & 1.357 & 0.026 & 0.007924 & 0.002240 & 0.079119 & 35.508 \\
\hline 4/7/08 14:00 & 1.661 & 0.682 & 0.221 & 99.178 & 1.354 & 0.023 & 0.007010 & 0.001905 & 0.067276 & 30.194 \\
\hline 4/7/08 15:00 & 1.653 & 0.674 & 0.215 & 96.328 & 1.355 & 0.024 & 0.007315 & 0.002015 & 0.071152 & 31.933 \\
\hline 4/7/08 16:00 & 1.627 & 0.648 & 0.195 & 87.406 & 1.357 & 0.026 & 0.007924 & 0.002240 & 0.079119 & 35.508 \\
\hline 4/7/08 17:00 & 1.646 & 0.667 & 0.209 & 93.875 & 1.357 & 0.026 & 0.007924 & 0.002240 & 0.079119 & 35.508 \\
\hline 4/7/08 18:00 & 1.66 & 0.681 & 0.220 & 98.819 & 1.357 & 0.026 & 0.007924 & 0.002240 & 0.079119 & 35.508 \\
\hline 4/7/08 19:00 & 1.656 & 0.677 & 0.217 & 97.391 & 1.357 & 0.026 & 0.007924 & 0.002240 & 0.079119 & 35.508 \\
\hline 4/7/08 20:00 & 1.655 & 0.676 & 0.216 & 97.036 & 1.359 & 0.028 & 0.008534 & 0.002473 & 0.087362 & 39.208 \\
\hline 4/7/08 21:00 & 1.646 & 0.667 & 0.209 & 93.875 & 1.364 & 0.033 & 0.010058 & 0.003090 & 0.109124 & 48.975 \\
\hline
\end{tabular}




\begin{tabular}{|c|c|c|c|c|c|c|c|c|c|c|}
\hline \multirow[b]{3}{*}{ Date Time } & \multicolumn{4}{|c|}{ V-Notch Weir } & \multicolumn{6}{|c|}{ R-Notch Weir } \\
\hline & \multicolumn{2}{|c|}{ Measured Water Level } & \multicolumn{2}{|c|}{$\begin{array}{c}\text { Calculated Rate } \\
\left(\mathrm{ft}^{3} / \mathrm{s}=448.8 \mathrm{gpm}\right)\end{array}$} & \multicolumn{3}{|c|}{ Measured Water Level } & \multicolumn{3}{|c|}{$\begin{array}{c}\text { Calculated Rate } \\
\left(\mathrm{ft}^{3} / \mathrm{s}=448.8 \mathrm{gpm}\right)\end{array}$} \\
\hline & $\begin{array}{c}\text { (ft above } \\
\text { sensor) }\end{array}$ & $\begin{array}{c}\text { (ft above } \\
\text { notch) }\end{array}$ & $\left(\mathrm{ft}^{3} / \mathrm{s}\right)$ & (gpm) & $\begin{array}{l}\text { (ft above } \\
\text { sensor) }\end{array}$ & $\begin{array}{c}\text { (ft above } \\
\text { log) }\end{array}$ & $\begin{array}{c}\text { (m above } \\
\text { log) }\end{array}$ & $\left(\mathrm{m}^{3} / \mathrm{s}\right)$ & $\left(\mathrm{ft}^{3} / \mathrm{s}\right)$ & (gpm) \\
\hline 4/7/08 22:00 & 1.696 & 0.717 & 0.250 & 112.239 & 1.376 & 0.045 & 0.013715 & 0.004743 & 0.167523 & 75.184 \\
\hline 4/7/08 23:00 & 1.668 & 0.689 & 0.227 & 101.713 & 1.385 & 0.054 & 0.016458 & 0.006129 & 0.216486 & 97.159 \\
\hline 4/8/08 0:00 & 1.718 & 0.739 & 0.269 & 120.950 & 1.381 & 0.05 & 0.015239 & 0.005499 & 0.194210 & 87.161 \\
\hline 4/8/08 1:00 & 1.678 & 0.699 & 0.235 & 105.401 & 1.371 & 0.04 & 0.012191 & 0.004026 & 0.142185 & 63.812 \\
\hline 4/8/08 2:00 & 1.638 & 0.659 & 0.203 & 91.117 & 1.366 & 0.035 & 0.010667 & 0.003349 & 0.118271 & 53.080 \\
\hline 4/8/08 3:00 & 1.634 & 0.655 & 0.200 & 89.757 & 1.364 & 0.033 & 0.010058 & 0.003090 & 0.109124 & 48.975 \\
\hline 4/8/08 4:00 & 1.643 & 0.664 & 0.207 & 92.835 & 1.361 & 0.03 & 0.009144 & 0.002714 & 0.095873 & 43.028 \\
\hline 4/8/08 5:00 & 1.643 & 0.664 & 0.207 & 92.835 & 1.361 & 0.03 & 0.009144 & 0.002714 & 0.095873 & 43.028 \\
\hline 4/8/08 6:00 & 1.639 & 0.660 & 0.204 & 91.459 & 1.361 & 0.03 & 0.009144 & 0.002714 & 0.095873 & 43.028 \\
\hline 4/8/08 7:00 & 1.594 & 0.615 & 0.171 & 76.824 & 1.361 & 0.03 & 0.009144 & 0.002714 & 0.095873 & 43.028 \\
\hline 4/8/08 8:00 & 1.613 & 0.634 & 0.185 & 82.817 & 1.359 & 0.028 & 0.008534 & 0.002473 & 0.087362 & 39.208 \\
\hline 4/8/08 9:00 & 1.63 & 0.651 & 0.197 & 88.409 & 1.359 & 0.028 & 0.008534 & 0.002473 & 0.087362 & 39.208 \\
\hline 4/8/08 10:00 & 1.62 & 0.641 & 0.190 & 85.093 & 1.359 & 0.028 & 0.008534 & 0.002473 & 0.087362 & 39.208 \\
\hline 4/8/08 11:00 & 1.62 & 0.641 & 0.190 & 85.093 & 1.359 & 0.028 & 0.008534 & 0.002473 & 0.087362 & 39.208 \\
\hline 4/8/08 12:00 & 1.601 & 0.622 & 0.176 & 79.000 & 1.359 & 0.028 & 0.008534 & 0.002473 & 0.087362 & 39.208 \\
\hline 4/8/08 13:00 & 1.632 & 0.653 & 0.198 & 89.082 & 1.361 & 0.03 & 0.009144 & 0.002714 & 0.095873 & 43.028 \\
\hline 4/8/08 14:00 & 1.622 & 0.643 & 0.191 & 85.750 & 1.359 & 0.028 & 0.008534 & 0.002473 & 0.087362 & 39.208 \\
\hline 4/8/08 15:00 & 1.633 & 0.654 & 0.199 & 89.419 & 1.359 & 0.028 & 0.008534 & 0.002473 & 0.087362 & 39.208 \\
\hline 4/8/08 16:00 & 1.609 & 0.630 & 0.182 & 81.532 & 1.357 & 0.026 & 0.007924 & 0.002240 & 0.079119 & 35.508 \\
\hline 4/8/08 17:00 & 1.641 & 0.662 & 0.205 & 92.146 & 1.359 & 0.028 & 0.008534 & 0.002473 & 0.087362 & 39.208 \\
\hline 4/8/08 18:00 & 1.589 & 0.610 & 0.168 & 75.291 & 1.359 & 0.028 & 0.008534 & 0.002473 & 0.087362 & 39.208 \\
\hline 4/8/08 19:00 & 1.629 & 0.650 & 0.196 & 88.074 & 1.359 & 0.028 & 0.008534 & 0.002473 & 0.087362 & 39.208 \\
\hline 4/8/08 20:00 & 1.612 & 0.633 & 0.184 & 82.494 & 1.362 & 0.031 & 0.009448 & 0.002838 & 0.100226 & 44.982 \\
\hline 4/8/08 21:00 & 1.582 & 0.603 & 0.163 & 73.177 & 1.359 & 0.028 & 0.008534 & 0.002473 & 0.087362 & 39.208 \\
\hline 4/8/08 22:00 & 1.61 & 0.631 & 0.182 & 81.852 & 1.362 & 0.031 & 0.009448 & 0.002838 & 0.100226 & 44.982 \\
\hline
\end{tabular}




\begin{tabular}{|c|c|c|c|c|c|c|c|c|c|c|}
\hline \multirow[b]{3}{*}{ Date Time } & \multicolumn{4}{|c|}{ V-Notch Weir } & \multicolumn{6}{|c|}{ R-Notch Weir } \\
\hline & \multicolumn{2}{|c|}{ Measured Water Level } & \multicolumn{2}{|c|}{$\begin{array}{c}\text { Calculated Rate } \\
\left(\mathrm{ft}^{3} / \mathrm{s}=448.8 \mathrm{gpm}\right)\end{array}$} & \multicolumn{3}{|c|}{ Measured Water Level } & \multicolumn{3}{|c|}{$\begin{array}{c}\text { Calculated Rate } \\
\left(\mathrm{ft}^{3} / \mathrm{s}=448.8 \mathrm{gpm}\right)\end{array}$} \\
\hline & $\begin{array}{c}\text { (ft above } \\
\text { sensor) }\end{array}$ & $\begin{array}{c}\text { (ft above } \\
\text { notch) }\end{array}$ & $\left(\mathrm{ft}^{3} / \mathrm{s}\right)$ & (gpm) & $\begin{array}{l}\text { (ft above } \\
\text { sensor) }\end{array}$ & $\begin{array}{c}\text { (ft above } \\
\text { log) }\end{array}$ & $\begin{array}{c}\text { (m above } \\
\text { log) }\end{array}$ & $\left(\mathrm{m}^{3} / \mathrm{s}\right)$ & $\left(\mathrm{ft}^{3} / \mathrm{s}\right)$ & (gpm) \\
\hline 4/8/08 23:00 & 1.63 & 0.651 & 0.197 & 88.409 & 1.359 & 0.028 & 0.008534 & 0.002473 & 0.087362 & 39.208 \\
\hline 4/9/08 0:00 & 1.63 & 0.651 & 0.197 & 88.409 & 1.359 & 0.028 & 0.008534 & 0.002473 & 0.087362 & 39.208 \\
\hline 4/9/08 1:00 & 1.611 & 0.632 & 0.183 & 82.173 & 1.357 & 0.026 & 0.007924 & 0.002240 & 0.079119 & 35.508 \\
\hline 4/9/08 2:00 & 1.57 & 0.591 & 0.155 & 69.636 & 1.357 & 0.026 & 0.007924 & 0.002240 & 0.079119 & 35.508 \\
\hline 4/9/08 3:00 & 1.511 & 0.532 & 0.120 & 53.732 & 1.357 & 0.026 & 0.007924 & 0.002240 & 0.079119 & 35.508 \\
\hline 4/9/08 4:00 & 1.54 & 0.561 & 0.136 & 61.240 & 1.356 & 0.025 & 0.007620 & 0.002126 & 0.075100 & 33.705 \\
\hline 4/9/08 5:00 & 1.55 & 0.571 & 0.143 & 63.967 & 1.356 & 0.025 & 0.007620 & 0.002126 & 0.075100 & 33.705 \\
\hline 4/9/08 6:00 & 1.562 & 0.583 & 0.150 & 67.334 & 1.356 & 0.025 & 0.007620 & 0.002126 & 0.075100 & 33.705 \\
\hline 4/9/08 7:00 & 1.553 & 0.574 & 0.144 & 64.799 & 1.356 & 0.025 & 0.007620 & 0.002126 & 0.075100 & 33.705 \\
\hline 4/9/08 8:00 & 1.622 & 0.643 & 0.191 & 85.750 & 1.356 & 0.025 & 0.007620 & 0.002126 & 0.075100 & 33.705 \\
\hline 4/9/08 9:00 & 1.633 & 0.654 & 0.199 & 89.419 & 1.356 & 0.025 & 0.007620 & 0.002126 & 0.075100 & 33.705 \\
\hline 4/9/08 10:00 & 1.612 & 0.633 & 0.184 & 82.494 & 1.354 & 0.023 & 0.007010 & 0.001905 & 0.067276 & 30.194 \\
\hline 4/9/08 11:00 & 1.517 & 0.538 & 0.123 & 55.237 & 1.356 & 0.025 & 0.007620 & 0.002126 & 0.075100 & 33.705 \\
\hline 4/9/08 12:00 & 1.513 & 0.534 & 0.121 & 54.231 & 1.359 & 0.028 & 0.008534 & 0.002473 & 0.087362 & 39.208 \\
\hline 4/9/08 13:00 & 1.582 & 0.603 & 0.163 & 73.177 & 1.362 & 0.031 & 0.009448 & 0.002838 & 0.100226 & 44.982 \\
\hline 4/9/08 14:00 & 1.562 & 0.583 & 0.150 & 67.334 & 1.364 & 0.033 & 0.010058 & 0.003090 & 0.109124 & 48.975 \\
\hline 4/9/08 15:00 & 1.531 & 0.552 & 0.131 & 58.847 & 1.358 & 0.027 & 0.008229 & 0.002356 & 0.083206 & 37.343 \\
\hline 4/9/08 16:00 & 1.515 & 0.536 & 0.122 & 54.733 & 1.36 & 0.029 & 0.008839 & 0.002593 & 0.091584 & 41.103 \\
\hline 4/9/08 17:00 & 1.508 & 0.529 & 0.118 & 52.988 & 1.36 & 0.029 & 0.008839 & 0.002593 & 0.091584 & 41.103 \\
\hline 4/9/08 18:00 & 1.52 & 0.541 & 0.125 & 55.999 & 1.358 & 0.027 & 0.008229 & 0.002356 & 0.083206 & 37.343 \\
\hline 4/9/08 19:00 & 1.531 & 0.552 & 0.131 & 58.847 & 1.356 & 0.025 & 0.007620 & 0.002126 & 0.075100 & 33.705 \\
\hline 4/9/08 20:00 & 1.548 & 0.569 & 0.141 & 63.416 & 1.365 & 0.034 & 0.010363 & 0.003218 & 0.113667 & 51.014 \\
\hline 4/9/08 21:00 & 1.525 & 0.546 & 0.128 & 57.283 & 1.358 & 0.027 & 0.008229 & 0.002356 & 0.083206 & 37.343 \\
\hline 4/9/08 22:00 & 1.544 & 0.565 & 0.139 & 62.323 & 1.365 & 0.034 & 0.010363 & 0.003218 & 0.113667 & 51.014 \\
\hline 4/9/08 23:00 & 1.533 & 0.554 & 0.132 & 59.374 & 1.353 & 0.022 & 0.006705 & 0.001797 & 0.063473 & 28.487 \\
\hline
\end{tabular}




\begin{tabular}{|c|c|c|c|c|c|c|c|c|c|c|}
\hline \multirow[b]{3}{*}{ Date Time } & \multicolumn{4}{|c|}{ V-Notch Weir } & \multicolumn{6}{|c|}{ R-Notch Weir } \\
\hline & \multicolumn{2}{|c|}{ Measured Water Level } & \multicolumn{2}{|c|}{$\begin{array}{c}\text { Calculated Rate } \\
\left(\mathrm{ft}^{3} / \mathrm{s}=448.8 \mathrm{gpm}\right)\end{array}$} & \multicolumn{3}{|c|}{ Measured Water Level } & \multicolumn{3}{|c|}{$\begin{array}{c}\text { Calculated Rate } \\
\left(\mathrm{ft}^{3} / \mathrm{s}=448.8 \mathrm{gpm}\right)\end{array}$} \\
\hline & $\begin{array}{l}\text { (ft above } \\
\text { sensor) }\end{array}$ & $\begin{array}{c}\text { (ft above } \\
\text { notch) }\end{array}$ & $\left(\mathrm{ft}^{3} / \mathrm{s}\right)$ & (gpm) & $\begin{array}{c}\text { (ft above } \\
\text { sensor) }\end{array}$ & $\begin{array}{c}\text { (ft above } \\
\text { log) }\end{array}$ & $\begin{array}{c}\text { (m above } \\
\text { log) }\end{array}$ & $\left(\mathrm{m}^{3} / \mathrm{s}\right)$ & $\left(\mathrm{ft}^{3} / \mathrm{s}\right)$ & (gpm) \\
\hline 4/10/08 0:00 & 1.54 & 0.561 & 0.136 & 61.240 & 1.36 & 0.029 & 0.008839 & 0.002593 & 0.091584 & 41.103 \\
\hline 4/10/08 1:00 & 1.526 & 0.547 & 0.128 & 57.542 & 1.364 & 0.033 & 0.010058 & 0.003090 & 0.109124 & 48.975 \\
\hline 4/10/08 2:00 & 1.599 & 0.620 & 0.175 & 78.375 & 1.371 & 0.04 & 0.012191 & 0.004026 & 0.142185 & 63.812 \\
\hline 4/10/08 3:00 & 1.602 & 0.623 & 0.177 & 79.314 & 1.385 & 0.054 & 0.016458 & 0.006129 & 0.216486 & 97.159 \\
\hline 4/10/08 4:00 & 1.666 & 0.687 & 0.225 & 100.985 & 1.381 & 0.05 & 0.015239 & 0.005499 & 0.194210 & 87.161 \\
\hline 4/10/08 5:00 & 1.629 & 0.650 & 0.196 & 88.074 & 1.383 & 0.052 & 0.015849 & 0.005811 & 0.205247 & 92.115 \\
\hline 4/10/08 6:00 & 1.608 & 0.629 & 0.181 & 81.213 & 1.38 & 0.049 & 0.014934 & 0.005345 & 0.188768 & 84.719 \\
\hline 4/10/08 7:00 & 1.655 & 0.676 & 0.216 & 97.036 & 1.383 & 0.052 & 0.015849 & 0.005811 & 0.205247 & 92.115 \\
\hline 4/10/08 8:00 & 1.662 & 0.683 & 0.222 & 99.538 & 1.383 & 0.052 & 0.015849 & 0.005811 & 0.205247 & 92.115 \\
\hline 4/10/08 9:00 & 1.643 & 0.664 & 0.207 & 92.835 & 1.38 & 0.049 & 0.014934 & 0.005345 & 0.188768 & 84.719 \\
\hline 4/10/08 10:00 & 1.646 & 0.667 & 0.209 & 93.875 & 1.385 & 0.054 & 0.016458 & 0.006129 & 0.216486 & 97.159 \\
\hline 4/10/08 11:00 & 1.712 & 0.733 & 0.264 & 118.536 & 1.394 & 0.063 & 0.019201 & 0.007629 & 0.269465 & 120.936 \\
\hline 4/10/08 12:00 & 1.869 & 0.890 & 0.427 & 191.655 & 1.432 & 0.101 & 0.030783 & 0.015057 & 0.531798 & 238.671 \\
\hline 4/10/08 13:00 & 1.911 & 0.932 & 0.479 & 214.860 & 1.446 & 0.115 & 0.035050 & 0.018189 & 0.642419 & 288.318 \\
\hline 4/10/08 14:00 & 1.906 & 0.927 & 0.472 & 212.014 & 1.444 & 0.113 & 0.034441 & 0.017729 & 0.626193 & 281.035 \\
\hline 4/10/08 15:00 & 1.916 & 0.937 & 0.485 & 217.729 & 1.465 & 0.134 & 0.040841 & 0.022743 & 0.803289 & 360.516 \\
\hline 4/10/08 16:00 & 2.52 & 1.541 & 1.669 & 749.050 & 1.678 & 0.347 & 0.105760 & 0.092720 & 3.274853 & 1469.754 \\
\hline 4/10/08 17:00 & 2.205 & 1.226 & 0.945 & 424.279 & 1.545 & 0.214 & 0.065224 & 0.045291 & 1.599669 & 717.932 \\
\hline 4/10/08 18:00 & 2.042 & 1.063 & 0.663 & 297.731 & 1.477 & 0.146 & 0.044499 & 0.025790 & 0.910896 & 408.810 \\
\hline 4/10/08 19:00 & 1.969 & 0.990 & 0.556 & 249.556 & 1.444 & 0.113 & 0.034441 & 0.017729 & 0.626193 & 281.035 \\
\hline 4/10/08 20:00 & 1.941 & 0.962 & 0.518 & 232.417 & 1.425 & 0.094 & 0.028650 & 0.013566 & 0.479156 & 215.045 \\
\hline 4/10/08 21:00 & 1.911 & 0.932 & 0.479 & 214.860 & 1.416 & 0.085 & 0.025907 & 0.011728 & 0.414220 & 185.902 \\
\hline 4/10/08 22:00 & 1.871 & 0.892 & 0.429 & 192.724 & 1.406 & 0.075 & 0.022859 & 0.009792 & 0.345867 & 155.225 \\
\hline 4/10/08 23:00 & 1.852 & 0.873 & 0.407 & 182.712 & 1.404 & 0.073 & 0.022249 & 0.009420 & 0.332697 & 149.314 \\
\hline 4/11/08 0:00 & 1.833 & 0.854 & 0.386 & 173.019 & 1.401 & 0.07 & 0.021335 & 0.008869 & 0.313263 & 140.593 \\
\hline
\end{tabular}




\begin{tabular}{|c|c|c|c|c|c|c|c|c|c|c|}
\hline \multirow[b]{3}{*}{ Date Time } & \multicolumn{4}{|c|}{ V-Notch Weir } & \multicolumn{6}{|c|}{ R-Notch Weir } \\
\hline & \multicolumn{2}{|c|}{ Measured Water Level } & \multicolumn{2}{|c|}{$\begin{array}{c}\text { Calculated Rate } \\
\left(\mathrm{ft}^{3} / \mathrm{s}=448.8 \mathrm{gpm}\right)\end{array}$} & \multicolumn{3}{|c|}{ Measured Water Level } & \multicolumn{3}{|c|}{$\begin{array}{c}\text { Calculated Rate } \\
\left(\mathrm{ft}^{3} / \mathrm{s}=448.8 \mathrm{gpm}\right)\end{array}$} \\
\hline & $\begin{array}{c}\text { (ft above } \\
\text { sensor) }\end{array}$ & $\begin{array}{c}\text { (ft above } \\
\text { notch) }\end{array}$ & $\left(\mathrm{ft}^{3} / \mathrm{s}\right)$ & (gpm) & $\begin{array}{l}\text { (ft above } \\
\text { sensor) }\end{array}$ & $\begin{array}{c}\text { (ft above } \\
\text { log) }\end{array}$ & $\begin{array}{c}\text { (m above } \\
\text { log) }\end{array}$ & $\left(\mathrm{m}^{3} / \mathrm{s}\right)$ & $\left(\mathrm{ft}^{3} / \mathrm{s}\right)$ & (gpm) \\
\hline 4/11/08 1:00 & 1.815 & 0.836 & 0.366 & 164.126 & 1.399 & 0.068 & 0.020725 & 0.008509 & 0.300526 & 134.876 \\
\hline 4/11/08 2:00 & 1.794 & 0.815 & 0.343 & 154.105 & 1.397 & 0.066 & 0.020116 & 0.008153 & 0.287966 & 129.239 \\
\hline 4/11/08 3:00 & 1.78 & 0.801 & 0.329 & 147.634 & 1.392 & 0.061 & 0.018592 & 0.007287 & 0.257360 & 115.503 \\
\hline 4/11/08 4:00 & 1.782 & 0.803 & 0.331 & 148.549 & 1.392 & 0.061 & 0.018592 & 0.007287 & 0.257360 & 115.503 \\
\hline 4/11/08 5:00 & 1.778 & 0.799 & 0.327 & 146.723 & 1.392 & 0.061 & 0.018592 & 0.007287 & 0.257360 & 115.503 \\
\hline 4/11/08 6:00 & 1.804 & 0.825 & 0.354 & 158.830 & 1.394 & 0.063 & 0.019201 & 0.007629 & 0.269465 & 120.936 \\
\hline 4/11/08 7:00 & 1.831 & 0.852 & 0.383 & 172.017 & 1.401 & 0.07 & 0.021335 & 0.008869 & 0.313263 & 140.593 \\
\hline 4/11/08 8:00 & 1.824 & 0.845 & 0.376 & 168.537 & 1.403 & 0.072 & 0.021945 & 0.009235 & 0.326176 & 146.388 \\
\hline 4/11/08 9:00 & 1.817 & 0.838 & 0.368 & 165.100 & 1.399 & 0.068 & 0.020725 & 0.008509 & 0.300526 & 134.876 \\
\hline 4/11/08 10:00 & 1.78 & 0.801 & 0.329 & 147.634 & 1.394 & 0.063 & 0.019201 & 0.007629 & 0.269465 & 120.936 \\
\hline 4/11/08 11:00 & 1.765 & 0.786 & 0.314 & 140.885 & 1.387 & 0.056 & 0.017068 & 0.006453 & 0.227923 & 102.292 \\
\hline 4/11/08 12:00 & 1.739 & 0.760 & 0.289 & 129.632 & 1.391 & 0.06 & 0.018287 & 0.007117 & 0.251378 & 112.818 \\
\hline 4/11/08 13:00 & 1.734 & 0.755 & 0.284 & 127.532 & 1.384 & 0.053 & 0.016154 & 0.005969 & 0.210842 & 94.626 \\
\hline 4/11/08 14:00 & 1.734 & 0.755 & 0.284 & 127.532 & 1.377 & 0.046 & 0.014020 & 0.004891 & 0.172754 & 77.532 \\
\hline 4/11/08 15:00 & 1.752 & 0.773 & 0.301 & 135.188 & 1.384 & 0.053 & 0.016154 & 0.005969 & 0.210842 & 94.626 \\
\hline 4/11/08 16:00 & 1.707 & 0.728 & 0.260 & 116.546 & 1.382 & 0.051 & 0.015544 & 0.005654 & 0.199703 & 89.627 \\
\hline 4/11/08 17:00 & 1.693 & 0.714 & 0.248 & 111.082 & 1.384 & 0.053 & 0.016154 & 0.005969 & 0.210842 & 94.626 \\
\hline 4/11/08 18:00 & 1.721 & 0.742 & 0.272 & 122.168 & 1.384 & 0.053 & 0.016154 & 0.005969 & 0.210842 & 94.626 \\
\hline 4/11/08 19:00 & 1.717 & 0.738 & 0.269 & 120.546 & 1.382 & 0.051 & 0.015544 & 0.005654 & 0.199703 & 89.627 \\
\hline 4/11/08 20:00 & 1.694 & 0.715 & 0.248 & 111.467 & 1.377 & 0.046 & 0.014020 & 0.004891 & 0.172754 & 77.532 \\
\hline 4/11/08 21:00 & 1.704 & 0.725 & 0.257 & 115.362 & 1.377 & 0.046 & 0.014020 & 0.004891 & 0.172754 & 77.532 \\
\hline 4/11/08 22:00 & 1.685 & 0.706 & 0.241 & 108.030 & 1.377 & 0.046 & 0.014020 & 0.004891 & 0.172754 & 77.532 \\
\hline 4/11/08 23:00 & 1.674 & 0.695 & 0.232 & 103.916 & 1.375 & 0.044 & 0.013411 & 0.004596 & 0.162345 & 72.860 \\
\hline 4/12/08 0:00 & 1.686 & 0.707 & 0.242 & 108.408 & 1.38 & 0.049 & 0.014934 & 0.005345 & 0.188768 & 84.719 \\
\hline 4/12/08 1:00 & 1.677 & 0.698 & 0.234 & 105.029 & 1.375 & 0.044 & 0.013411 & 0.004596 & 0.162345 & 72.860 \\
\hline
\end{tabular}




\begin{tabular}{|c|c|c|c|c|c|c|c|c|c|c|}
\hline \multirow[b]{3}{*}{ Date Time } & \multicolumn{4}{|c|}{ V-Notch Weir } & \multicolumn{6}{|c|}{ R-Notch Weir } \\
\hline & \multicolumn{2}{|c|}{ Measured Water Level } & \multicolumn{2}{|c|}{$\begin{array}{c}\text { Calculated Rate } \\
\left(\mathrm{ft}^{3} / \mathrm{s}=448.8 \mathrm{gpm}\right)\end{array}$} & \multicolumn{3}{|c|}{ Measured Water Level } & \multicolumn{3}{|c|}{$\begin{array}{c}\text { Calculated Rate } \\
\left(\mathrm{ft}^{3} / \mathrm{s}=448.8 \mathrm{gpm}\right)\end{array}$} \\
\hline & $\begin{array}{c}\text { (ft above } \\
\text { sensor) }\end{array}$ & $\begin{array}{c}\text { (ft above } \\
\text { notch) }\end{array}$ & $\left(\mathrm{ft}^{3} / \mathrm{s}\right)$ & (gpm) & $\begin{array}{l}\text { (ft above } \\
\text { sensor) }\end{array}$ & $\begin{array}{c}\text { (ft above } \\
\text { log) }\end{array}$ & $\begin{array}{c}\text { (m above } \\
\text { log) }\end{array}$ & $\left(\mathrm{m}^{3} / \mathrm{s}\right)$ & $\left(\mathrm{ft}^{3} / \mathrm{s}\right)$ & (gpm) \\
\hline 4/12/08 2:00 & 1.68 & 0.701 & 0.237 & 106.148 & 1.375 & 0.044 & 0.013411 & 0.004596 & 0.162345 & 72.860 \\
\hline 4/12/08 3:00 & 1.677 & 0.698 & 0.234 & 105.029 & 1.377 & 0.046 & 0.014020 & 0.004891 & 0.172754 & 77.532 \\
\hline 4/12/08 4:00 & 1.666 & 0.687 & 0.225 & 100.985 & 1.372 & 0.041 & 0.012496 & 0.004166 & 0.147141 & 66.037 \\
\hline 4/12/08 5:00 & 1.676 & 0.697 & 0.233 & 104.657 & 1.375 & 0.044 & 0.013411 & 0.004596 & 0.162345 & 72.860 \\
\hline 4/12/08 6:00 & 1.673 & 0.694 & 0.231 & 103.547 & 1.372 & 0.041 & 0.012496 & 0.004166 & 0.147141 & 66.037 \\
\hline 4/12/08 7:00 & 1.676 & 0.697 & 0.233 & 104.657 & 1.37 & 0.039 & 0.011887 & 0.003887 & 0.137285 & 61.614 \\
\hline 4/12/08 8:00 & 1.669 & 0.690 & 0.227 & 102.078 & 1.37 & 0.039 & 0.011887 & 0.003887 & 0.137285 & 61.614 \\
\hline 4/12/08 9:00 & 1.65 & 0.671 & 0.212 & 95.272 & 1.365 & 0.034 & 0.010363 & 0.003218 & 0.113667 & 51.014 \\
\hline 4/12/08 10:00 & 1.654 & 0.675 & 0.215 & 96.681 & 1.368 & 0.037 & 0.011277 & 0.003614 & 0.127660 & 57.294 \\
\hline 4/12/08 11:00 & 1.627 & 0.648 & 0.195 & 87.406 & 1.368 & 0.037 & 0.011277 & 0.003614 & 0.127660 & 57.294 \\
\hline 4/12/08 12:00 & 1.649 & 0.670 & 0.212 & 94.921 & 1.372 & 0.041 & 0.012496 & 0.004166 & 0.147141 & 66.037 \\
\hline 4/12/08 13:00 & 1.646 & 0.667 & 0.209 & 93.875 & 1.372 & 0.041 & 0.012496 & 0.004166 & 0.147141 & 66.037 \\
\hline 4/12/08 14:00 & 1.651 & 0.672 & 0.213 & 95.623 & 1.372 & 0.041 & 0.012496 & 0.004166 & 0.147141 & 66.037 \\
\hline 4/12/08 15:00 & 1.641 & 0.662 & 0.205 & 92.146 & 1.37 & 0.039 & 0.011887 & 0.003887 & 0.137285 & 61.614 \\
\hline 4/12/08 16:00 & 1.634 & 0.655 & 0.200 & 89.757 & 1.37 & 0.039 & 0.011887 & 0.003887 & 0.137285 & 61.614 \\
\hline 4/12/08 17:00 & 1.637 & 0.658 & 0.202 & 90.776 & 1.37 & 0.039 & 0.011887 & 0.003887 & 0.137285 & 61.614 \\
\hline 4/12/08 18:00 & 1.639 & 0.660 & 0.204 & 91.459 & 1.368 & 0.037 & 0.011277 & 0.003614 & 0.127660 & 57.294 \\
\hline 4/12/08 19:00 & 1.637 & 0.658 & 0.202 & 90.776 & 1.37 & 0.039 & 0.011887 & 0.003887 & 0.137285 & 61.614 \\
\hline 4/12/08 20:00 & 1.639 & 0.660 & 0.204 & 91.459 & 1.37 & 0.039 & 0.011887 & 0.003887 & 0.137285 & 61.614 \\
\hline 4/12/08 21:00 & 1.637 & 0.658 & 0.202 & 90.776 & 1.368 & 0.037 & 0.011277 & 0.003614 & 0.127660 & 57.294 \\
\hline 4/12/08 22:00 & 1.638 & 0.659 & 0.203 & 91.117 & 1.368 & 0.037 & 0.011277 & 0.003614 & 0.127660 & 57.294 \\
\hline 4/12/08 23:00 & 1.635 & 0.656 & 0.201 & 90.096 & 1.368 & 0.037 & 0.011277 & 0.003614 & 0.127660 & 57.294 \\
\hline 4/13/08 0:00 & 1.631 & 0.652 & 0.198 & 88.745 & 1.37 & 0.039 & 0.011887 & 0.003887 & 0.137285 & 61.614 \\
\hline 4/13/08 1:00 & 1.638 & 0.659 & 0.203 & 91.117 & 1.368 & 0.037 & 0.011277 & 0.003614 & 0.127660 & 57.294 \\
\hline 4/13/08 2:00 & 1.625 & 0.646 & 0.193 & 86.742 & 1.365 & 0.034 & 0.010363 & 0.003218 & 0.113667 & 51.014 \\
\hline
\end{tabular}




\begin{tabular}{|c|c|c|c|c|c|c|c|c|c|c|}
\hline \multirow[b]{3}{*}{ Date Time } & \multicolumn{4}{|c|}{ V-Notch Weir } & \multicolumn{6}{|c|}{ R-Notch Weir } \\
\hline & \multicolumn{2}{|c|}{ Measured Water Level } & \multicolumn{2}{|c|}{$\begin{array}{c}\text { Calculated Rate } \\
\left(\mathrm{ft}^{3} / \mathrm{s}=448.8 \mathrm{gpm}\right)\end{array}$} & \multicolumn{3}{|c|}{ Measured Water Level } & \multicolumn{3}{|c|}{$\begin{array}{c}\text { Calculated Rate } \\
\left(\mathrm{ft}^{3} / \mathrm{s}=448.8 \mathrm{gpm}\right)\end{array}$} \\
\hline & $\begin{array}{c}\text { (ft above } \\
\text { sensor) }\end{array}$ & $\begin{array}{c}\text { (ft above } \\
\text { notch) }\end{array}$ & $\left(\mathrm{ft}^{3} / \mathrm{s}\right)$ & (gpm) & $\begin{array}{l}\text { (ft above } \\
\text { sensor) }\end{array}$ & $\begin{array}{c}\text { (ft above } \\
\text { log) }\end{array}$ & $\begin{array}{c}\text { (m above } \\
\text { log) }\end{array}$ & $\left(\mathrm{m}^{3} / \mathrm{s}\right)$ & $\left(\mathrm{ft}^{3} / \mathrm{s}\right)$ & (gpm) \\
\hline 4/13/08 3:00 & 1.627 & 0.648 & 0.195 & 87.406 & 1.368 & 0.037 & 0.011277 & 0.003614 & 0.127660 & 57.294 \\
\hline 4/13/08 4:00 & 1.628 & 0.649 & 0.195 & 87.740 & 1.368 & 0.037 & 0.011277 & 0.003614 & 0.127660 & 57.294 \\
\hline 4/13/08 5:00 & 1.614 & 0.635 & 0.185 & 83.140 & 1.365 & 0.034 & 0.010363 & 0.003218 & 0.113667 & 51.014 \\
\hline 4/13/08 6:00 & 1.614 & 0.635 & 0.185 & 83.140 & 1.365 & 0.034 & 0.010363 & 0.003218 & 0.113667 & 51.014 \\
\hline 4/13/08 7:00 & 1.617 & 0.638 & 0.187 & 84.113 & 1.365 & 0.034 & 0.010363 & 0.003218 & 0.113667 & 51.014 \\
\hline 4/13/08 8:00 & 1.615 & 0.636 & 0.186 & 83.463 & 1.365 & 0.034 & 0.010363 & 0.003218 & 0.113667 & 51.014 \\
\hline 4/13/08 9:00 & 1.62 & 0.641 & 0.190 & 85.093 & 1.363 & 0.032 & 0.009753 & 0.002963 & 0.104644 & 46.964 \\
\hline 4/13/08 10:00 & 1.619 & 0.640 & 0.189 & 84.765 & 1.365 & 0.034 & 0.010363 & 0.003218 & 0.113667 & 51.014 \\
\hline 4/13/08 11:00 & 1.623 & 0.644 & 0.192 & 86.080 & 1.363 & 0.032 & 0.009753 & 0.002963 & 0.104644 & 46.964 \\
\hline 4/13/08 12:00 & 1.609 & 0.630 & 0.182 & 81.532 & 1.365 & 0.034 & 0.010363 & 0.003218 & 0.113667 & 51.014 \\
\hline 4/13/08 13:00 & 1.619 & 0.640 & 0.189 & 84.765 & 1.361 & 0.03 & 0.009144 & 0.002714 & 0.095873 & 43.028 \\
\hline 4/13/08 14:00 & 1.561 & 0.582 & 0.149 & 67.049 & 1.368 & 0.037 & 0.011277 & 0.003614 & 0.127660 & 57.294 \\
\hline 4/13/08 15:00 & 1.619 & 0.640 & 0.189 & 84.765 & 1.369 & 0.038 & 0.011582 & 0.003750 & 0.132444 & 59.441 \\
\hline 4/13/08 16:00 & 1.595 & 0.616 & 0.172 & 77.132 & 1.369 & 0.038 & 0.011582 & 0.003750 & 0.132444 & 59.441 \\
\hline 4/13/08 17:00 & 1.614 & 0.635 & 0.185 & 83.140 & 1.367 & 0.036 & 0.010972 & 0.003481 & 0.122936 & 55.174 \\
\hline 4/13/08 18:00 & 1.597 & 0.618 & 0.173 & 77.752 & 1.365 & 0.034 & 0.010363 & 0.003218 & 0.113667 & 51.014 \\
\hline 4/13/08 19:00 & 1.597 & 0.618 & 0.173 & 77.752 & 1.365 & 0.034 & 0.010363 & 0.003218 & 0.113667 & 51.014 \\
\hline 4/13/08 20:00 & 1.593 & 0.614 & 0.170 & 76.516 & 1.365 & 0.034 & 0.010363 & 0.003218 & 0.113667 & 51.014 \\
\hline 4/13/08 21:00 & 1.595 & 0.616 & 0.172 & 77.132 & 1.367 & 0.036 & 0.010972 & 0.003481 & 0.122936 & 55.174 \\
\hline 4/13/08 22:00 & 1.598 & 0.619 & 0.174 & 78.063 & 1.365 & 0.034 & 0.010363 & 0.003218 & 0.113667 & 51.014 \\
\hline 4/13/08 23:00 & 1.603 & 0.624 & 0.177 & 79.629 & 1.364 & 0.033 & 0.010058 & 0.003090 & 0.109124 & 48.975 \\
\hline 4/14/08 0:00 & 1.599 & 0.620 & 0.175 & 78.375 & 1.364 & 0.033 & 0.010058 & 0.003090 & 0.109124 & 48.975 \\
\hline 4/14/08 1:00 & 1.59 & 0.611 & 0.168 & 75.596 & 1.364 & 0.033 & 0.010058 & 0.003090 & 0.109124 & 48.975 \\
\hline 4/14/08 2:00 & 1.597 & 0.618 & 0.173 & 77.752 & 1.364 & 0.033 & 0.010058 & 0.003090 & 0.109124 & 48.975 \\
\hline 4/14/08 3:00 & 1.596 & 0.617 & 0.173 & 77.442 & 1.364 & 0.033 & 0.010058 & 0.003090 & 0.109124 & 48.975 \\
\hline
\end{tabular}




\begin{tabular}{|c|c|c|c|c|c|c|c|c|c|c|}
\hline \multirow[b]{3}{*}{ Date Time } & \multicolumn{4}{|c|}{ V-Notch Weir } & \multicolumn{6}{|c|}{ R-Notch Weir } \\
\hline & \multicolumn{2}{|c|}{ Measured Water Level } & \multicolumn{2}{|c|}{$\begin{array}{c}\text { Calculated Rate } \\
\left(\mathrm{ft}^{3} / \mathrm{s}=448.8 \mathrm{gpm}\right)\end{array}$} & \multicolumn{3}{|c|}{ Measured Water Level } & \multicolumn{3}{|c|}{$\begin{array}{c}\text { Calculated Rate } \\
\left(\mathrm{ft}^{3} / \mathrm{s}=448.8 \mathrm{gpm}\right)\end{array}$} \\
\hline & $\begin{array}{c}\text { (ft above } \\
\text { sensor) }\end{array}$ & $\begin{array}{c}\text { (ft above } \\
\text { notch) }\end{array}$ & $\left(\mathrm{ft}^{3} / \mathrm{s}\right)$ & (gpm) & $\begin{array}{l}\text { (ft above } \\
\text { sensor) }\end{array}$ & $\begin{array}{c}\text { (ft above } \\
\text { log) }\end{array}$ & $\begin{array}{c}\text { (m above } \\
\text { log) }\end{array}$ & $\left(\mathrm{m}^{3} / \mathrm{s}\right)$ & $\left(\mathrm{ft}^{3} / \mathrm{s}\right)$ & (gpm) \\
\hline 4/14/08 4:00 & 1.594 & 0.615 & 0.171 & 76.824 & 1.364 & 0.033 & 0.010058 & 0.003090 & 0.109124 & 48.975 \\
\hline 4/14/08 5:00 & 1.607 & 0.628 & 0.180 & 80.895 & 1.363 & 0.032 & 0.009753 & 0.002963 & 0.104644 & 46.964 \\
\hline 4/14/08 6:00 & 1.622 & 0.643 & 0.191 & 85.750 & 1.366 & 0.035 & 0.010667 & 0.003349 & 0.118271 & 53.080 \\
\hline 4/14/08 7:00 & 1.611 & 0.632 & 0.183 & 82.173 & 1.366 & 0.035 & 0.010667 & 0.003349 & 0.118271 & 53.080 \\
\hline 4/14/08 8:00 & 1.614 & 0.635 & 0.185 & 83.140 & 1.363 & 0.032 & 0.009753 & 0.002963 & 0.104644 & 46.964 \\
\hline 4/14/08 9:00 & 1.618 & 0.639 & 0.188 & 84.439 & 1.366 & 0.035 & 0.010667 & 0.003349 & 0.118271 & 53.080 \\
\hline 4/14/08 10:00 & 1.634 & 0.655 & 0.200 & 89.757 & 1.366 & 0.035 & 0.010667 & 0.003349 & 0.118271 & 53.080 \\
\hline 4/14/08 11:00 & 1.646 & 0.667 & 0.209 & 93.875 & 1.366 & 0.035 & 0.010667 & 0.003349 & 0.118271 & 53.080 \\
\hline 4/14/08 12:00 & 1.682 & 0.703 & 0.238 & 106.898 & 1.366 & 0.035 & 0.010667 & 0.003349 & 0.118271 & 53.080 \\
\hline 4/14/08 13:00 & 1.723 & 0.744 & 0.274 & 122.984 & 1.366 & 0.035 & 0.010667 & 0.003349 & 0.118271 & 53.080 \\
\hline 4/14/08 14:00 & 1.668 & 0.689 & 0.227 & 101.713 & 1.367 & 0.036 & 0.010972 & 0.003481 & 0.122936 & 55.174 \\
\hline 4/14/08 15:00 & 1.694 & 0.715 & 0.248 & 111.467 & 1.374 & 0.043 & 0.013106 & 0.004451 & 0.157222 & 70.561 \\
\hline 4/14/08 16:00 & 1.696 & 0.717 & 0.250 & 112.239 & 1.367 & 0.036 & 0.010972 & 0.003481 & 0.122936 & 55.174 \\
\hline 4/14/08 17:00 & 1.717 & 0.738 & 0.269 & 120.546 & 1.365 & 0.034 & 0.010363 & 0.003218 & 0.113667 & 51.014 \\
\hline 4/14/08 18:00 & 1.701 & 0.722 & 0.254 & 114.185 & 1.366 & 0.035 & 0.010667 & 0.003349 & 0.118271 & 53.080 \\
\hline 4/14/08 19:00 & 1.703 & 0.724 & 0.256 & 114.969 & 1.366 & 0.035 & 0.010667 & 0.003349 & 0.118271 & 53.080 \\
\hline 4/14/08 20:00 & 1.705 & 0.726 & 0.258 & 115.756 & 1.366 & 0.035 & 0.010667 & 0.003349 & 0.118271 & 53.080 \\
\hline 4/14/08 21:00 & 1.712 & 0.733 & 0.264 & 118.536 & 1.366 & 0.035 & 0.010667 & 0.003349 & 0.118271 & 53.080 \\
\hline 4/14/08 22:00 & 1.687 & 0.708 & 0.242 & 108.788 & 1.368 & 0.037 & 0.011277 & 0.003614 & 0.127660 & 57.294 \\
\hline 4/14/08 23:00 & 1.706 & 0.727 & 0.259 & 116.150 & 1.368 & 0.037 & 0.011277 & 0.003614 & 0.127660 & 57.294 \\
\hline 4/15/08 0:00 & 1.718 & 0.739 & 0.269 & 120.950 & 1.37 & 0.039 & 0.011887 & 0.003887 & 0.137285 & 61.614 \\
\hline 4/15/08 1:00 & 1.727 & 0.748 & 0.278 & 124.627 & 1.37 & 0.039 & 0.011887 & 0.003887 & 0.137285 & 61.614 \\
\hline 4/15/08 2:00 & 1.73 & 0.751 & 0.280 & 125.867 & 1.367 & 0.036 & 0.010972 & 0.003481 & 0.122936 & 55.174 \\
\hline 4/15/08 3:00 & 1.726 & 0.747 & 0.277 & 124.215 & 1.369 & 0.038 & 0.011582 & 0.003750 & 0.132444 & 59.441 \\
\hline 4/15/08 4:00 & 1.733 & 0.754 & 0.283 & 127.114 & 1.369 & 0.038 & 0.011582 & 0.003750 & 0.132444 & 59.441 \\
\hline
\end{tabular}




\begin{tabular}{|c|c|c|c|c|c|c|c|c|c|c|}
\hline \multirow[b]{3}{*}{ Date Time } & \multicolumn{4}{|c|}{ V-Notch Weir } & \multicolumn{6}{|c|}{ R-Notch Weir } \\
\hline & \multicolumn{2}{|c|}{ Measured Water Level } & \multicolumn{2}{|c|}{$\begin{array}{c}\text { Calculated Rate } \\
\left(\mathrm{ft}^{3} / \mathrm{s}=448.8 \mathrm{gpm}\right)\end{array}$} & \multicolumn{3}{|c|}{ Measured Water Level } & \multicolumn{3}{|c|}{$\begin{array}{c}\text { Calculated Rate } \\
\left(\mathrm{ft}^{3} / \mathrm{s}=448.8 \mathrm{gpm}\right)\end{array}$} \\
\hline & $\begin{array}{c}\text { (ft above } \\
\text { sensor) }\end{array}$ & $\begin{array}{c}\text { (ft above } \\
\text { notch) }\end{array}$ & $\left(\mathrm{ft}^{3} / \mathrm{s}\right)$ & (gpm) & $\begin{array}{l}\text { (ft above } \\
\text { sensor) }\end{array}$ & $\begin{array}{c}\text { (ft above } \\
\text { log) }\end{array}$ & $\begin{array}{c}\text { (m above } \\
\text { log) }\end{array}$ & $\left(\mathrm{m}^{3} / \mathrm{s}\right)$ & $\left(\mathrm{ft}^{3} / \mathrm{s}\right)$ & (gpm) \\
\hline 4/15/08 5:00 & 1.72 & 0.741 & 0.271 & 121.761 & 1.369 & 0.038 & 0.011582 & 0.003750 & 0.132444 & 59.441 \\
\hline 4/15/08 6:00 & 1.73 & 0.751 & 0.280 & 125.867 & 1.366 & 0.035 & 0.010667 & 0.003349 & 0.118271 & 53.080 \\
\hline 4/15/08 7:00 & 1.735 & 0.756 & 0.285 & 127.950 & 1.366 & 0.035 & 0.010667 & 0.003349 & 0.118271 & 53.080 \\
\hline 4/15/08 8:00 & 1.738 & 0.759 & 0.288 & 129.210 & 1.366 & 0.035 & 0.010667 & 0.003349 & 0.118271 & 53.080 \\
\hline 4/15/08 9:00 & 1.748 & 0.769 & 0.297 & 133.464 & 1.366 & 0.035 & 0.010667 & 0.003349 & 0.118271 & 53.080 \\
\hline 4/15/08 10:00 & 1.749 & 0.770 & 0.298 & 133.893 & 1.366 & 0.035 & 0.010667 & 0.003349 & 0.118271 & 53.080 \\
\hline 4/15/08 11:00 & 1.755 & 0.776 & 0.304 & 136.490 & 1.362 & 0.031 & 0.009448 & 0.002838 & 0.100226 & 44.982 \\
\hline 4/15/08 12:00 & 1.758 & 0.779 & 0.307 & 137.800 & 1.364 & 0.033 & 0.010058 & 0.003090 & 0.109124 & 48.975 \\
\hline 4/15/08 13:00 & 1.734 & 0.755 & 0.284 & 127.532 & 1.367 & 0.036 & 0.010972 & 0.003481 & 0.122936 & 55.174 \\
\hline 4/15/08 14:00 & 1.736 & 0.757 & 0.286 & 128.369 & 1.363 & 0.032 & 0.009753 & 0.002963 & 0.104644 & 46.964 \\
\hline 4/15/08 15:00 & 1.75 & 0.771 & 0.299 & 134.324 & 1.361 & 0.03 & 0.009144 & 0.002714 & 0.095873 & 43.028 \\
\hline 4/15/08 16:00 & 1.727 & 0.748 & 0.278 & 124.627 & 1.357 & 0.026 & 0.007924 & 0.002240 & 0.079119 & 35.508 \\
\hline 4/15/08 17:00 & 1.711 & 0.732 & 0.263 & 118.136 & 1.36 & 0.029 & 0.008839 & 0.002593 & 0.091584 & 41.103 \\
\hline 4/15/08 18:00 & 1.678 & 0.699 & 0.235 & 105.401 & 1.363 & 0.032 & 0.009753 & 0.002963 & 0.104644 & 46.964 \\
\hline 4/15/08 19:00 & 1.724 & 0.745 & 0.275 & 123.394 & 1.355 & 0.024 & 0.007315 & 0.002015 & 0.071152 & 31.933 \\
\hline 4/15/08 20:00 & 1.694 & 0.715 & 0.248 & 111.467 & 1.363 & 0.032 & 0.009753 & 0.002963 & 0.104644 & 46.964 \\
\hline 4/15/08 21:00 & 1.719 & 0.740 & 0.270 & 121.355 & 1.363 & 0.032 & 0.009753 & 0.002963 & 0.104644 & 46.964 \\
\hline 4/15/08 22:00 & 1.722 & 0.743 & 0.273 & 122.576 & 1.365 & 0.034 & 0.010363 & 0.003218 & 0.113667 & 51.014 \\
\hline 4/15/08 23:00 & 1.738 & 0.759 & 0.288 & 129.210 & 1.36 & 0.029 & 0.008839 & 0.002593 & 0.091584 & 41.103 \\
\hline 4/16/08 0:00 & 1.72 & 0.741 & 0.271 & 121.761 & 1.364 & 0.033 & 0.010058 & 0.003090 & 0.109124 & 48.975 \\
\hline 4/16/08 1:00 & 1.724 & 0.745 & 0.275 & 123.394 & 1.364 & 0.033 & 0.010058 & 0.003090 & 0.109124 & 48.975 \\
\hline 4/16/08 2:00 & 1.713 & 0.734 & 0.265 & 118.936 & 1.364 & 0.033 & 0.010058 & 0.003090 & 0.109124 & 48.975 \\
\hline 4/16/08 3:00 & 1.72 & 0.741 & 0.271 & 121.761 & 1.364 & 0.033 & 0.010058 & 0.003090 & 0.109124 & 48.975 \\
\hline 4/16/08 4:00 & 1.72 & 0.741 & 0.271 & 121.761 & 1.363 & 0.032 & 0.009753 & 0.002963 & 0.104644 & 46.964 \\
\hline 4/16/08 5:00 & 1.725 & 0.746 & 0.276 & 123.804 & 1.365 & 0.034 & 0.010363 & 0.003218 & 0.113667 & 51.014 \\
\hline
\end{tabular}




\begin{tabular}{|c|c|c|c|c|c|c|c|c|c|c|}
\hline \multirow[b]{3}{*}{ Date Time } & \multicolumn{4}{|c|}{ V-Notch Weir } & \multicolumn{6}{|c|}{ R-Notch Weir } \\
\hline & \multicolumn{2}{|c|}{ Measured Water Level } & \multicolumn{2}{|c|}{$\begin{array}{c}\text { Calculated Rate } \\
\left(\mathrm{ft}^{3} / \mathrm{s}=448.8 \mathrm{gpm}\right)\end{array}$} & \multicolumn{3}{|c|}{ Measured Water Level } & \multicolumn{3}{|c|}{$\begin{array}{c}\text { Calculated Rate } \\
\left(\mathrm{ft}^{3} / \mathrm{s}=448.8 \mathrm{gpm}\right)\end{array}$} \\
\hline & $\begin{array}{c}\text { (ft above } \\
\text { sensor) }\end{array}$ & $\begin{array}{c}\text { (ft above } \\
\text { notch) }\end{array}$ & $\left(\mathrm{ft}^{3} / \mathrm{s}\right)$ & (gpm) & $\begin{array}{l}\text { (ft above } \\
\text { sensor) }\end{array}$ & $\begin{array}{c}\text { (ft above } \\
\text { log) }\end{array}$ & $\begin{array}{c}\text { (m above } \\
\text { log) }\end{array}$ & $\left(\mathrm{m}^{3} / \mathrm{s}\right)$ & $\left(\mathrm{ft}^{3} / \mathrm{s}\right)$ & (gpm) \\
\hline 4/16/08 6:00 & 1.709 & 0.730 & 0.261 & 117.339 & 1.363 & 0.032 & 0.009753 & 0.002963 & 0.104644 & 46.964 \\
\hline 4/16/08 7:00 & 1.709 & 0.730 & 0.261 & 117.339 & 1.363 & 0.032 & 0.009753 & 0.002963 & 0.104644 & 46.964 \\
\hline 4/16/08 8:00 & 1.721 & 0.742 & 0.272 & 122.168 & 1.36 & 0.029 & 0.008839 & 0.002593 & 0.091584 & 41.103 \\
\hline 4/16/08 9:00 & 1.714 & 0.735 & 0.266 & 119.337 & 1.363 & 0.032 & 0.009753 & 0.002963 & 0.104644 & 46.964 \\
\hline 4/16/08 10:00 & 1.723 & 0.744 & 0.274 & 122.984 & 1.361 & 0.03 & 0.009144 & 0.002714 & 0.095873 & 43.028 \\
\hline 4/16/08 11:00 & 1.724 & 0.745 & 0.275 & 123.394 & 1.363 & 0.032 & 0.009753 & 0.002963 & 0.104644 & 46.964 \\
\hline 4/16/08 12:00 & 1.717 & 0.738 & 0.269 & 120.546 & 1.364 & 0.033 & 0.010058 & 0.003090 & 0.109124 & 48.975 \\
\hline 4/16/08 13:00 & 1.717 & 0.738 & 0.269 & 120.546 & 1.362 & 0.031 & 0.009448 & 0.002838 & 0.100226 & 44.982 \\
\hline 4/16/08 14:00 & 1.691 & 0.712 & 0.246 & 110.314 & 1.361 & 0.03 & 0.009144 & 0.002714 & 0.095873 & 43.028 \\
\hline 4/16/08 15:00 & 1.706 & 0.727 & 0.259 & 116.150 & 1.362 & 0.031 & 0.009448 & 0.002838 & 0.100226 & 44.982 \\
\hline 4/16/08 16:00 & 1.723 & 0.744 & 0.274 & 122.984 & 1.365 & 0.034 & 0.010363 & 0.003218 & 0.113667 & 51.014 \\
\hline 4/16/08 17:00 & 1.711 & 0.732 & 0.263 & 118.136 & 1.363 & 0.032 & 0.009753 & 0.002963 & 0.104644 & 46.964 \\
\hline 4/16/08 18:00 & 1.7 & 0.721 & 0.254 & 113.794 & 1.363 & 0.032 & 0.009753 & 0.002963 & 0.104644 & 46.964 \\
\hline 4/16/08 19:00 & 1.692 & 0.713 & 0.247 & 110.697 & 1.366 & 0.035 & 0.010667 & 0.003349 & 0.118271 & 53.080 \\
\hline 4/16/08 20:00 & 1.704 & 0.725 & 0.257 & 115.362 & 1.363 & 0.032 & 0.009753 & 0.002963 & 0.104644 & 46.964 \\
\hline 4/16/08 21:00 & 1.708 & 0.729 & 0.261 & 116.942 & 1.363 & 0.032 & 0.009753 & 0.002963 & 0.104644 & 46.964 \\
\hline 4/16/08 22:00 & 1.703 & 0.724 & 0.256 & 114.969 & 1.363 & 0.032 & 0.009753 & 0.002963 & 0.104644 & 46.964 \\
\hline 4/16/08 23:00 & 1.712 & 0.733 & 0.264 & 118.536 & 1.365 & 0.034 & 0.010363 & 0.003218 & 0.113667 & 51.014 \\
\hline 4/17/08 0:00 & 1.715 & 0.736 & 0.267 & 119.739 & 1.362 & 0.031 & 0.009448 & 0.002838 & 0.100226 & 44.982 \\
\hline 4/17/08 1:00 & 1.701 & 0.722 & 0.254 & 114.185 & 1.359 & 0.028 & 0.008534 & 0.002473 & 0.087362 & 39.208 \\
\hline 4/17/08 2:00 & 1.71 & 0.731 & 0.262 & 117.737 & 1.361 & 0.03 & 0.009144 & 0.002714 & 0.095873 & 43.028 \\
\hline 4/17/08 3:00 & 1.713 & 0.734 & 0.265 & 118.936 & 1.363 & 0.032 & 0.009753 & 0.002963 & 0.104644 & 46.964 \\
\hline 4/17/08 4:00 & 1.717 & 0.738 & 0.269 & 120.546 & 1.362 & 0.031 & 0.009448 & 0.002838 & 0.100226 & 44.982 \\
\hline 4/17/08 5:00 & 1.776 & 0.797 & 0.325 & 145.816 & 1.374 & 0.043 & 0.013106 & 0.004451 & 0.157222 & 70.561 \\
\hline 4/17/08 6:00 & 2.047 & 1.068 & 0.671 & 301.218 & 1.461 & 0.13 & 0.039622 & 0.021756 & 0.768433 & 344.873 \\
\hline
\end{tabular}




\begin{tabular}{|c|c|c|c|c|c|c|c|c|c|c|}
\hline \multirow[b]{3}{*}{ Date Time } & \multicolumn{4}{|c|}{ V-Notch Weir } & \multicolumn{6}{|c|}{ R-Notch Weir } \\
\hline & \multicolumn{2}{|c|}{ Measured Water Level } & \multicolumn{2}{|c|}{$\begin{array}{c}\text { Calculated Rate } \\
\left(\mathrm{ft}^{3} / \mathrm{s}=448.8 \mathrm{gpm}\right)\end{array}$} & \multicolumn{3}{|c|}{ Measured Water Level } & \multicolumn{3}{|c|}{$\begin{array}{c}\text { Calculated Rate } \\
\left(\mathrm{ft}^{3} / \mathrm{s}=448.8 \mathrm{gpm}\right)\end{array}$} \\
\hline & $\begin{array}{c}\text { (ft above } \\
\text { sensor) }\end{array}$ & $\begin{array}{c}\text { (ft above } \\
\text { notch) }\end{array}$ & $\left(\mathrm{ft}^{3} / \mathrm{s}\right)$ & (gpm) & $\begin{array}{l}\text { (ft above } \\
\text { sensor) }\end{array}$ & $\begin{array}{c}\text { (ft above } \\
\text { log) }\end{array}$ & $\begin{array}{c}\text { (m above } \\
\text { log) }\end{array}$ & $\left(\mathrm{m}^{3} / \mathrm{s}\right)$ & $\left(\mathrm{ft}^{3} / \mathrm{s}\right)$ & (gpm) \\
\hline 4/17/08 7:00 & 2.18 & 1.201 & 0.898 & 403.114 & 1.519 & 0.188 & 0.057300 & 0.037409 & 1.321271 & 592.986 \\
\hline 4/17/08 8:00 & 2.173 & 1.194 & 0.885 & 397.303 & 1.521 & 0.19 & 0.057909 & 0.037997 & 1.342051 & 602.312 \\
\hline 4/17/08 9:00 & 2.105 & 1.126 & 0.765 & 343.470 & 1.488 & 0.157 & 0.047851 & 0.028693 & 1.013424 & 454.824 \\
\hline 4/17/08 10:00 & 2.025 & 1.046 & 0.637 & 286.055 & 1.438 & 0.107 & 0.032612 & 0.016375 & 0.578353 & 259.565 \\
\hline 4/17/08 11:00 & 1.969 & 0.990 & 0.556 & 249.556 & 1.412 & 0.081 & 0.024688 & 0.010940 & 0.386387 & 173.411 \\
\hline 4/17/08 12:00 & 1.939 & 0.960 & 0.515 & 231.221 & 1.402 & 0.071 & 0.021640 & 0.009052 & 0.319698 & 143.480 \\
\hline 4/17/08 13:00 & 1.898 & 0.919 & 0.462 & 207.507 & 1.395 & 0.064 & 0.019506 & 0.007803 & 0.275587 & 123.683 \\
\hline 4/17/08 14:00 & 1.882 & 0.903 & 0.443 & 198.668 & 1.391 & 0.06 & 0.018287 & 0.007117 & 0.251378 & 112.818 \\
\hline 4/17/08 15:00 & 1.889 & 0.910 & 0.451 & 202.507 & 1.395 & 0.064 & 0.019506 & 0.007803 & 0.275587 & 123.683 \\
\hline 4/17/08 16:00 & 1.924 & 0.945 & 0.495 & 222.366 & 1.4 & 0.069 & 0.021030 & 0.008688 & 0.306873 & 137.724 \\
\hline 4/17/08 17:00 & 1.931 & 0.952 & 0.505 & 226.473 & 1.405 & 0.074 & 0.022554 & 0.009605 & 0.339261 & 152.260 \\
\hline 4/17/08 18:00 & 1.926 & 0.947 & 0.498 & 223.535 & 1.402 & 0.071 & 0.021640 & 0.009052 & 0.319698 & 143.480 \\
\hline 4/17/08 19:00 & 1.908 & 0.929 & 0.475 & 213.149 & 1.397 & 0.066 & 0.020116 & 0.008153 & 0.287966 & 129.239 \\
\hline 4/17/08 20:00 & 1.891 & 0.912 & 0.454 & 203.611 & 1.395 & 0.064 & 0.019506 & 0.007803 & 0.275587 & 123.683 \\
\hline 4/17/08 21:00 & 1.889 & 0.910 & 0.451 & 202.507 & 1.393 & 0.062 & 0.018897 & 0.007457 & 0.263390 & 118.209 \\
\hline 4/17/08 22:00 & 1.937 & 0.958 & 0.513 & 230.028 & 1.404 & 0.073 & 0.022249 & 0.009420 & 0.332697 & 149.314 \\
\hline 4/17/08 23:00 & 2.005 & 1.026 & 0.608 & 272.676 & 1.43 & 0.099 & 0.030174 & 0.014626 & 0.516571 & 231.837 \\
\hline 4/18/08 0:00 & 2.005 & 1.026 & 0.608 & 272.676 & 1.428 & 0.097 & 0.029564 & 0.014199 & 0.501493 & 225.070 \\
\hline 4/18/08 1:00 & 1.97 & 0.991 & 0.557 & 250.182 & 1.411 & 0.08 & 0.024383 & 0.010746 & 0.379531 & 170.333 \\
\hline 4/18/08 2:00 & 1.93 & 0.951 & 0.503 & 225.883 & 1.399 & 0.068 & 0.020725 & 0.008509 & 0.300526 & 134.876 \\
\hline 4/18/08 3:00 & 1.914 & 0.935 & 0.483 & 216.578 & 1.397 & 0.066 & 0.020116 & 0.008153 & 0.287966 & 129.239 \\
\hline 4/18/08 4:00 & 1.911 & 0.932 & 0.479 & 214.860 & 1.401 & 0.07 & 0.021335 & 0.008869 & 0.313263 & 140.593 \\
\hline 4/18/08 5:00 & 1.97 & 0.991 & 0.557 & 250.182 & 1.411 & 0.08 & 0.024383 & 0.010746 & 0.379531 & 170.333 \\
\hline 4/18/08 6:00 & 2.043 & 1.064 & 0.665 & 298.426 & 1.446 & 0.115 & 0.035050 & 0.018189 & 0.642419 & 288.318 \\
\hline 4/18/08 7:00 & 2.521 & 1.542 & 1.672 & 750.259 & 1.711 & 0.38 & 0.115818 & 0.106131 & 3.748522 & 1682.337 \\
\hline
\end{tabular}




\begin{tabular}{|c|c|c|c|c|c|c|c|c|c|c|}
\hline \multirow[b]{3}{*}{ Date Time } & \multicolumn{4}{|c|}{ V-Notch Weir } & \multicolumn{6}{|c|}{ R-Notch Weir } \\
\hline & \multicolumn{2}{|c|}{ Measured Water Level } & \multicolumn{2}{|c|}{$\begin{array}{c}\text { Calculated Rate } \\
\left(\mathrm{ft}^{3} / \mathrm{s}=448.8 \mathrm{gpm}\right)\end{array}$} & \multicolumn{3}{|c|}{ Measured Water Level } & \multicolumn{3}{|c|}{$\begin{array}{c}\text { Calculated Rate } \\
\left(\mathrm{ft}^{3} / \mathrm{s}=448.8 \mathrm{gpm}\right)\end{array}$} \\
\hline & $\begin{array}{c}\text { (ft above } \\
\text { sensor) }\end{array}$ & $\begin{array}{c}\text { (ft above } \\
\text { notch) }\end{array}$ & $\left(\mathrm{ft}^{3} / \mathrm{s}\right)$ & (gpm) & $\begin{array}{l}\text { (ft above } \\
\text { sensor) }\end{array}$ & $\begin{array}{c}\text { (ft above } \\
\text { log) }\end{array}$ & $\begin{array}{c}\text { (m above } \\
\text { log) }\end{array}$ & $\left(\mathrm{m}^{3} / \mathrm{s}\right)$ & $\left(\mathrm{ft}^{3} / \mathrm{s}\right)$ & (gpm) \\
\hline 4/18/08 8:00 & 2.684 & 1.705 & 2.146 & 963.346 & 1.725 & 0.394 & 0.120085 & 0.112000 & 3.955834 & 1775.378 \\
\hline 4/18/08 9:00 & 2.383 & 1.404 & 1.324 & 594.240 & 1.628 & 0.297 & 0.090521 & 0.073589 & 2.599158 & 1166.502 \\
\hline 4/18/08 10:00 & 2.197 & 1.218 & 0.930 & 417.435 & 1.548 & 0.217 & 0.066138 & 0.046232 & 1.632919 & 732.854 \\
\hline 4/18/08 11:00 & 2.135 & 1.156 & 0.817 & 366.641 & 1.5 & 0.169 & 0.051509 & 0.031975 & 1.129354 & 506.854 \\
\hline 4/18/08 12:00 & 2.053 & 1.074 & 0.681 & 305.435 & 1.47 & 0.139 & 0.042365 & 0.023997 & 0.847577 & 380.392 \\
\hline 4/18/08 13:00 & 2.015 & 1.036 & 0.622 & 279.318 & 1.458 & 0.127 & 0.038708 & 0.021026 & 0.742632 & 333.293 \\
\hline 4/18/08 14:00 & 2.019 & 1.040 & 0.628 & 282.001 & 1.453 & 0.122 & 0.037184 & 0.019827 & 0.700289 & 314.290 \\
\hline 4/18/08 15:00 & 2.019 & 1.040 & 0.628 & 282.001 & 1.444 & 0.113 & 0.034441 & 0.017729 & 0.626193 & 281.035 \\
\hline 4/18/08 16:00 & 2.002 & 1.023 & 0.603 & 270.703 & 1.434 & 0.103 & 0.031393 & 0.015492 & 0.547171 & 245.570 \\
\hline 4/18/08 17:00 & 1.99 & 1.011 & 0.586 & 262.894 & 1.425 & 0.094 & 0.028650 & 0.013566 & 0.479156 & 215.045 \\
\hline 4/18/08 18:00 & 1.974 & 0.995 & 0.563 & 252.694 & 1.42 & 0.089 & 0.027126 & 0.012534 & 0.442691 & 198.680 \\
\hline 4/18/08 19:00 & 1.969 & 0.990 & 0.556 & 249.556 & 1.413 & 0.082 & 0.024992 & 0.011135 & 0.393285 & 176.506 \\
\hline 4/18/08 20:00 & 1.955 & 0.976 & 0.537 & 240.895 & 1.411 & 0.08 & 0.024383 & 0.010746 & 0.379531 & 170.333 \\
\hline 4/18/08 21:00 & 1.934 & 0.955 & 0.509 & 228.246 & 1.408 & 0.077 & 0.023468 & 0.010170 & 0.359208 & 161.212 \\
\hline 4/18/08 22:00 & 1.925 & 0.946 & 0.497 & 222.950 & 1.406 & 0.075 & 0.022859 & 0.009792 & 0.345867 & 155.225 \\
\hline 4/18/08 23:00 & 1.93 & 0.951 & 0.503 & 225.883 & 1.401 & 0.07 & 0.021335 & 0.008869 & 0.313263 & 140.593 \\
\hline 4/19/08 0:00 & 1.923 & 0.944 & 0.494 & 221.783 & 1.401 & 0.07 & 0.021335 & 0.008869 & 0.313263 & 140.593 \\
\hline 4/19/08 1:00 & 1.93 & 0.951 & 0.503 & 225.883 & 1.399 & 0.068 & 0.020725 & 0.008509 & 0.300526 & 134.876 \\
\hline 4/19/08 2:00 & 1.923 & 0.944 & 0.494 & 221.783 & 1.399 & 0.068 & 0.020725 & 0.008509 & 0.300526 & 134.876 \\
\hline 4/19/08 3:00 & 1.914 & 0.935 & 0.483 & 216.578 & 1.396 & 0.065 & 0.019811 & 0.007977 & 0.281754 & 126.451 \\
\hline 4/19/08 4:00 & 1.914 & 0.935 & 0.483 & 216.578 & 1.394 & 0.063 & 0.019201 & 0.007629 & 0.269465 & 120.936 \\
\hline 4/19/08 5:00 & 1.903 & 0.924 & 0.469 & 210.317 & 1.394 & 0.063 & 0.019201 & 0.007629 & 0.269465 & 120.936 \\
\hline 4/19/08 6:00 & 1.898 & 0.919 & 0.462 & 207.507 & 1.394 & 0.063 & 0.019201 & 0.007629 & 0.269465 & 120.936 \\
\hline 4/19/08 7:00 & 1.891 & 0.912 & 0.454 & 203.611 & 1.392 & 0.061 & 0.018592 & 0.007287 & 0.257360 & 115.503 \\
\hline 4/19/08 8:00 & 1.887 & 0.908 & 0.449 & 201.405 & 1.392 & 0.061 & 0.018592 & 0.007287 & 0.257360 & 115.503 \\
\hline
\end{tabular}




\begin{tabular}{|c|c|c|c|c|c|c|c|c|c|c|}
\hline \multirow[b]{3}{*}{ Date Time } & \multicolumn{4}{|c|}{ V-Notch Weir } & \multicolumn{6}{|c|}{ R-Notch Weir } \\
\hline & \multicolumn{2}{|c|}{ Measured Water Level } & \multicolumn{2}{|c|}{$\begin{array}{c}\text { Calculated Rate } \\
\left(\mathrm{ft}^{3} / \mathrm{s}=448.8 \mathrm{gpm}\right)\end{array}$} & \multicolumn{3}{|c|}{ Measured Water Level } & \multicolumn{3}{|c|}{$\begin{array}{c}\text { Calculated Rate } \\
\left(\mathrm{ft}^{3} / \mathrm{s}=448.8 \mathrm{gpm}\right)\end{array}$} \\
\hline & $\begin{array}{c}\text { (ft above } \\
\text { sensor) }\end{array}$ & $\begin{array}{c}\text { (ft above } \\
\text { notch) }\end{array}$ & $\left(\mathrm{ft}^{3} / \mathrm{s}\right)$ & (gpm) & $\begin{array}{l}\text { (ft above } \\
\text { sensor) }\end{array}$ & $\begin{array}{c}\text { (ft above } \\
\text { log) }\end{array}$ & $\begin{array}{c}\text { (m above } \\
\text { log) }\end{array}$ & $\left(\mathrm{m}^{3} / \mathrm{s}\right)$ & $\left(\mathrm{ft}^{3} / \mathrm{s}\right)$ & (gpm) \\
\hline 4/19/08 9:00 & 1.886 & 0.907 & 0.448 & 200.856 & 1.389 & 0.058 & 0.017678 & 0.006782 & 0.239555 & 107.512 \\
\hline 4/19/08 10:00 & 1.879 & 0.900 & 0.439 & 197.036 & 1.389 & 0.058 & 0.017678 & 0.006782 & 0.239555 & 107.512 \\
\hline 4/19/08 11:00 & 1.878 & 0.899 & 0.438 & 196.494 & 1.39 & 0.059 & 0.017982 & 0.006949 & 0.245443 & 110.155 \\
\hline 4/19/08 12:00 & 1.876 & 0.897 & 0.435 & 195.412 & 1.388 & 0.057 & 0.017373 & 0.006617 & 0.233715 & 104.891 \\
\hline 4/19/08 13:00 & 1.873 & 0.894 & 0.432 & 193.797 & 1.388 & 0.057 & 0.017373 & 0.006617 & 0.233715 & 104.891 \\
\hline 4/19/08 14:00 & 1.863 & 0.884 & 0.420 & 188.469 & 1.387 & 0.056 & 0.017068 & 0.006453 & 0.227923 & 102.292 \\
\hline 4/19/08 15:00 & 1.865 & 0.886 & 0.422 & 189.528 & 1.385 & 0.054 & 0.016458 & 0.006129 & 0.216486 & 97.159 \\
\hline 4/19/08 16:00 & 1.861 & 0.882 & 0.418 & 187.415 & 1.386 & 0.055 & 0.016763 & 0.006291 & 0.222180 & 99.714 \\
\hline 4/19/08 17:00 & 1.852 & 0.873 & 0.407 & 182.712 & 1.387 & 0.056 & 0.017068 & 0.006453 & 0.227923 & 102.292 \\
\hline 4/19/08 18:00 & 1.854 & 0.875 & 0.409 & 183.751 & 1.388 & 0.057 & 0.017373 & 0.006617 & 0.233715 & 104.891 \\
\hline 4/19/08 19:00 & 1.857 & 0.878 & 0.413 & 185.316 & 1.388 & 0.057 & 0.017373 & 0.006617 & 0.233715 & 104.891 \\
\hline 4/19/08 20:00 & 1.854 & 0.875 & 0.409 & 183.751 & 1.388 & 0.057 & 0.017373 & 0.006617 & 0.233715 & 104.891 \\
\hline 4/19/08 21:00 & 1.868 & 0.889 & 0.426 & 191.122 & 1.388 & 0.057 & 0.017373 & 0.006617 & 0.233715 & 104.891 \\
\hline 4/19/08 22:00 & 1.865 & 0.886 & 0.422 & 189.528 & 1.388 & 0.057 & 0.017373 & 0.006617 & 0.233715 & 104.891 \\
\hline 4/19/08 23:00 & 1.858 & 0.879 & 0.414 & 185.839 & 1.385 & 0.054 & 0.016458 & 0.006129 & 0.216486 & 97.159 \\
\hline 4/20/08 0:00 & 1.863 & 0.884 & 0.420 & 188.469 & 1.384 & 0.053 & 0.016154 & 0.005969 & 0.210842 & 94.626 \\
\hline 4/20/08 1:00 & 1.856 & 0.877 & 0.412 & 184.793 & 1.386 & 0.055 & 0.016763 & 0.006291 & 0.222180 & 99.714 \\
\hline 4/20/08 2:00 & 1.863 & 0.884 & 0.420 & 188.469 & 1.385 & 0.054 & 0.016458 & 0.006129 & 0.216486 & 97.159 \\
\hline 4/20/08 3:00 & 1.852 & 0.873 & 0.407 & 182.712 & 1.382 & 0.051 & 0.015544 & 0.005654 & 0.199703 & 89.627 \\
\hline 4/20/08 4:00 & 1.857 & 0.878 & 0.413 & 185.316 & 1.382 & 0.051 & 0.015544 & 0.005654 & 0.199703 & 89.627 \\
\hline 4/20/08 5:00 & 1.843 & 0.864 & 0.397 & 178.081 & 1.384 & 0.053 & 0.016154 & 0.005969 & 0.210842 & 94.626 \\
\hline 4/20/08 6:00 & 1.856 & 0.877 & 0.412 & 184.793 & 1.384 & 0.053 & 0.016154 & 0.005969 & 0.210842 & 94.626 \\
\hline 4/20/08 7:00 & 1.844 & 0.865 & 0.398 & 178.592 & 1.379 & 0.048 & 0.014630 & 0.005192 & 0.183377 & 82.300 \\
\hline 4/20/08 8:00 & 1.842 & 0.863 & 0.396 & 177.571 & 1.381 & 0.05 & 0.015239 & 0.005499 & 0.194210 & 87.161 \\
\hline 4/20/08 9:00 & 1.844 & 0.865 & 0.398 & 178.592 & 1.386 & 0.055 & 0.016763 & 0.006291 & 0.222180 & 99.714 \\
\hline
\end{tabular}




\begin{tabular}{|c|c|c|c|c|c|c|c|c|c|c|}
\hline \multirow[b]{3}{*}{ Date Time } & \multicolumn{4}{|c|}{ V-Notch Weir } & \multicolumn{6}{|c|}{ R-Notch Weir } \\
\hline & \multicolumn{2}{|c|}{ Measured Water Level } & \multicolumn{2}{|c|}{$\begin{array}{c}\text { Calculated Rate } \\
\left(\mathrm{ft}^{3} / \mathrm{s}=448.8 \mathrm{gpm}\right)\end{array}$} & \multicolumn{3}{|c|}{ Measured Water Level } & \multicolumn{3}{|c|}{$\begin{array}{c}\text { Calculated Rate } \\
\left(\mathrm{ft}^{3} / \mathrm{s}=448.8 \mathrm{gpm}\right)\end{array}$} \\
\hline & $\begin{array}{c}\text { (ft above } \\
\text { sensor) }\end{array}$ & $\begin{array}{c}\text { (ft above } \\
\text { notch) }\end{array}$ & $\left(\mathrm{ft}^{3} / \mathrm{s}\right)$ & (gpm) & $\begin{array}{l}\text { (ft above } \\
\text { sensor) }\end{array}$ & $\begin{array}{c}\text { (ft above } \\
\text { log) }\end{array}$ & $\begin{array}{c}\text { (m above } \\
\text { log) }\end{array}$ & $\left(\mathrm{m}^{3} / \mathrm{s}\right)$ & $\left(\mathrm{ft}^{3} / \mathrm{s}\right)$ & (gpm) \\
\hline 4/20/08 10:00 & 1.836 & 0.857 & 0.389 & 174.528 & 1.381 & 0.05 & 0.015239 & 0.005499 & 0.194210 & 87.161 \\
\hline 4/20/08 11:00 & 1.871 & 0.892 & 0.429 & 192.724 & 1.389 & 0.058 & 0.017678 & 0.006782 & 0.239555 & 107.512 \\
\hline 4/20/08 12:00 & 1.849 & 0.870 & 0.404 & 181.160 & 1.385 & 0.054 & 0.016458 & 0.006129 & 0.216486 & 97.159 \\
\hline 4/20/08 13:00 & 1.83 & 0.851 & 0.382 & 171.517 & 1.385 & 0.054 & 0.016458 & 0.006129 & 0.216486 & 97.159 \\
\hline 4/20/08 14:00 & 1.828 & 0.849 & 0.380 & 170.520 & 1.384 & 0.053 & 0.016154 & 0.005969 & 0.210842 & 94.626 \\
\hline 4/20/08 15:00 & 1.798 & 0.819 & 0.348 & 155.985 & 1.38 & 0.049 & 0.014934 & 0.005345 & 0.188768 & 84.719 \\
\hline 4/20/08 16:00 & 1.826 & 0.847 & 0.378 & 169.527 & 1.386 & 0.055 & 0.016763 & 0.006291 & 0.222180 & 99.714 \\
\hline 4/20/08 17:00 & 1.785 & 0.806 & 0.334 & 149.926 & 1.382 & 0.051 & 0.015544 & 0.005654 & 0.199703 & 89.627 \\
\hline 4/20/08 18:00 & 1.794 & 0.815 & 0.343 & 154.105 & 1.38 & 0.049 & 0.014934 & 0.005345 & 0.188768 & 84.719 \\
\hline 4/20/08 19:00 & 1.801 & 0.822 & 0.351 & 157.404 & 1.382 & 0.051 & 0.015544 & 0.005654 & 0.199703 & 89.627 \\
\hline 4/20/08 20:00 & 1.815 & 0.836 & 0.366 & 164.126 & 1.382 & 0.051 & 0.015544 & 0.005654 & 0.199703 & 89.627 \\
\hline 4/20/08 21:00 & 1.802 & 0.823 & 0.352 & 157.878 & 1.382 & 0.051 & 0.015544 & 0.005654 & 0.199703 & 89.627 \\
\hline 4/20/08 22:00 & 1.816 & 0.837 & 0.367 & 164.613 & 1.382 & 0.051 & 0.015544 & 0.005654 & 0.199703 & 89.627 \\
\hline 4/20/08 23:00 & 1.818 & 0.839 & 0.369 & 165.589 & 1.382 & 0.051 & 0.015544 & 0.005654 & 0.199703 & 89.627 \\
\hline 4/21/08 0:00 & 1.82 & 0.841 & 0.371 & 166.568 & 1.381 & 0.05 & 0.015239 & 0.005499 & 0.194210 & 87.161 \\
\hline 4/21/08 1:00 & 1.809 & 0.830 & 0.359 & 161.224 & 1.379 & 0.048 & 0.014630 & 0.005192 & 0.183377 & 82.300 \\
\hline 4/21/08 2:00 & 1.82 & 0.841 & 0.371 & 166.568 & 1.378 & 0.047 & 0.014325 & 0.005041 & 0.178040 & 79.904 \\
\hline 4/21/08 3:00 & 1.813 & 0.834 & 0.364 & 163.155 & 1.38 & 0.049 & 0.014934 & 0.005345 & 0.188768 & 84.719 \\
\hline 4/21/08 4:00 & 1.809 & 0.830 & 0.359 & 161.224 & 1.38 & 0.049 & 0.014934 & 0.005345 & 0.188768 & 84.719 \\
\hline 4/21/08 5:00 & 1.821 & 0.842 & 0.372 & 167.059 & 1.379 & 0.048 & 0.014630 & 0.005192 & 0.183377 & 82.300 \\
\hline 4/21/08 6:00 & 1.804 & 0.825 & 0.354 & 158.830 & 1.379 & 0.048 & 0.014630 & 0.005192 & 0.183377 & 82.300 \\
\hline 4/21/08 7:00 & 1.807 & 0.828 & 0.357 & 160.264 & 1.379 & 0.048 & 0.014630 & 0.005192 & 0.183377 & 82.300 \\
\hline 4/21/08 8:00 & 1.802 & 0.823 & 0.352 & 157.878 & 1.378 & 0.047 & 0.014325 & 0.005041 & 0.178040 & 79.904 \\
\hline 4/21/08 9:00 & 1.8 & 0.821 & 0.350 & 156.930 & 1.378 & 0.047 & 0.014325 & 0.005041 & 0.178040 & 79.904 \\
\hline 4/21/08 10:00 & 1.795 & 0.816 & 0.344 & 154.574 & 1.376 & 0.045 & 0.013715 & 0.004743 & 0.167523 & 75.184 \\
\hline
\end{tabular}




\begin{tabular}{|c|c|c|c|c|c|c|c|c|c|c|}
\hline \multirow[b]{3}{*}{ Date Time } & \multicolumn{4}{|c|}{ V-Notch Weir } & \multicolumn{6}{|c|}{ R-Notch Weir } \\
\hline & \multicolumn{2}{|c|}{ Measured Water Level } & \multicolumn{2}{|c|}{$\begin{array}{c}\text { Calculated Rate } \\
\left(\mathrm{ft}^{3} / \mathrm{s}=448.8 \mathrm{gpm}\right)\end{array}$} & \multicolumn{3}{|c|}{ Measured Water Level } & \multicolumn{3}{|c|}{$\begin{array}{c}\text { Calculated Rate } \\
\left(\mathrm{ft}^{3} / \mathrm{s}=448.8 \mathrm{gpm}\right)\end{array}$} \\
\hline & $\begin{array}{c}\text { (ft above } \\
\text { sensor) }\end{array}$ & $\begin{array}{c}\text { (ft above } \\
\text { notch) }\end{array}$ & $\left(\mathrm{ft}^{3} / \mathrm{s}\right)$ & (gpm) & $\begin{array}{l}\text { (ft above } \\
\text { sensor) }\end{array}$ & $\begin{array}{c}\text { (ft above } \\
\text { log) }\end{array}$ & $\begin{array}{c}\text { (m above } \\
\text { log) }\end{array}$ & $\left(\mathrm{m}^{3} / \mathrm{s}\right)$ & $\left(\mathrm{ft}^{3} / \mathrm{s}\right)$ & (gpm) \\
\hline 4/21/08 11:00 & 1.811 & 0.832 & 0.361 & 162.188 & 1.376 & 0.045 & 0.013715 & 0.004743 & 0.167523 & 75.184 \\
\hline 4/21/08 12:00 & 1.788 & 0.809 & 0.337 & 151.312 & 1.378 & 0.047 & 0.014325 & 0.005041 & 0.178040 & 79.904 \\
\hline 4/21/08 13:00 & 1.793 & 0.814 & 0.342 & 153.638 & 1.374 & 0.043 & 0.013106 & 0.004451 & 0.157222 & 70.561 \\
\hline 4/21/08 14:00 & 1.795 & 0.816 & 0.344 & 154.574 & 1.376 & 0.045 & 0.013715 & 0.004743 & 0.167523 & 75.184 \\
\hline 4/21/08 15:00 & 1.799 & 0.820 & 0.349 & 156.457 & 1.377 & 0.046 & 0.014020 & 0.004891 & 0.172754 & 77.532 \\
\hline 4/21/08 16:00 & 1.785 & 0.806 & 0.334 & 149.926 & 1.377 & 0.046 & 0.014020 & 0.004891 & 0.172754 & 77.532 \\
\hline 4/21/08 17:00 & 1.787 & 0.808 & 0.336 & 150.849 & 1.375 & 0.044 & 0.013411 & 0.004596 & 0.162345 & 72.860 \\
\hline 4/21/08 18:00 & 1.787 & 0.808 & 0.336 & 150.849 & 1.375 & 0.044 & 0.013411 & 0.004596 & 0.162345 & 72.860 \\
\hline 4/21/08 19:00 & 1.79 & 0.811 & 0.339 & 152.239 & 1.378 & 0.047 & 0.014325 & 0.005041 & 0.178040 & 79.904 \\
\hline 4/21/08 20:00 & 1.797 & 0.818 & 0.347 & 155.514 & 1.378 & 0.047 & 0.014325 & 0.005041 & 0.178040 & 79.904 \\
\hline 4/21/08 21:00 & 1.785 & 0.806 & 0.334 & 149.926 & 1.375 & 0.044 & 0.013411 & 0.004596 & 0.162345 & 72.860 \\
\hline 4/21/08 22:00 & 1.797 & 0.818 & 0.347 & 155.514 & 1.375 & 0.044 & 0.013411 & 0.004596 & 0.162345 & 72.860 \\
\hline 4/21/08 23:00 & 1.797 & 0.818 & 0.347 & 155.514 & 1.374 & 0.043 & 0.013106 & 0.004451 & 0.157222 & 70.561 \\
\hline 4/22/08 0:00 & 1.811 & 0.832 & 0.361 & 162.188 & 1.374 & 0.043 & 0.013106 & 0.004451 & 0.157222 & 70.561 \\
\hline 4/22/08 1:00 & 1.814 & 0.835 & 0.365 & 163.640 & 1.376 & 0.045 & 0.013715 & 0.004743 & 0.167523 & 75.184 \\
\hline 4/22/08 2:00 & 1.816 & 0.837 & 0.367 & 164.613 & 1.376 & 0.045 & 0.013715 & 0.004743 & 0.167523 & 75.184 \\
\hline 4/22/08 3:00 & 1.821 & 0.842 & 0.372 & 167.059 & 1.373 & 0.042 & 0.012801 & 0.004308 & 0.152154 & 68.287 \\
\hline 4/22/08 4:00 & 1.814 & 0.835 & 0.365 & 163.640 & 1.373 & 0.042 & 0.012801 & 0.004308 & 0.152154 & 68.287 \\
\hline 4/22/08 5:00 & 1.808 & 0.829 & 0.358 & 160.744 & 1.373 & 0.042 & 0.012801 & 0.004308 & 0.152154 & 68.287 \\
\hline 4/22/08 6:00 & 1.808 & 0.829 & 0.358 & 160.744 & 1.373 & 0.042 & 0.012801 & 0.004308 & 0.152154 & 68.287 \\
\hline 4/22/08 7:00 & 1.806 & 0.827 & 0.356 & 159.785 & 1.372 & 0.041 & 0.012496 & 0.004166 & 0.147141 & 66.037 \\
\hline 4/22/08 8:00 & 1.806 & 0.827 & 0.356 & 159.785 & 1.372 & 0.041 & 0.012496 & 0.004166 & 0.147141 & 66.037 \\
\hline 4/22/08 9:00 & 1.811 & 0.832 & 0.361 & 162.188 & 1.372 & 0.041 & 0.012496 & 0.004166 & 0.147141 & 66.037 \\
\hline 4/22/08 10:00 & 1.808 & 0.829 & 0.358 & 160.744 & 1.373 & 0.042 & 0.012801 & 0.004308 & 0.152154 & 68.287 \\
\hline 4/22/08 11:00 & 1.814 & 0.835 & 0.365 & 163.640 & 1.373 & 0.042 & 0.012801 & 0.004308 & 0.152154 & 68.287 \\
\hline
\end{tabular}




\begin{tabular}{|c|c|c|c|c|c|c|c|c|c|c|}
\hline \multirow[b]{3}{*}{ Date Time } & \multicolumn{4}{|c|}{ V-Notch Weir } & \multicolumn{6}{|c|}{ R-Notch Weir } \\
\hline & \multicolumn{2}{|c|}{ Measured Water Level } & \multicolumn{2}{|c|}{$\begin{array}{l}\text { Calculated Rate } \\
\left(\mathrm{ft}^{3} / \mathrm{s}=448.8 \mathrm{gpm}\right)\end{array}$} & \multicolumn{3}{|c|}{ Measured Water Level } & \multicolumn{3}{|c|}{$\begin{array}{l}\text { Calculated Rate } \\
\left(\mathrm{ft}^{3} / \mathrm{s}=448.8 \mathrm{gpm}\right)\end{array}$} \\
\hline & $\begin{array}{l}\text { (ft above } \\
\text { sensor) }\end{array}$ & $\begin{array}{c}\text { (ft above } \\
\text { notch) }\end{array}$ & $\left(\mathrm{ft}^{3} / \mathrm{s}\right)$ & (gpm) & $\begin{array}{l}\text { (ft above } \\
\text { sensor) }\end{array}$ & $\begin{array}{c}\text { (ft above } \\
\text { log) }\end{array}$ & $\begin{array}{c}\text { (m above } \\
\text { log) }\end{array}$ & $\left(\mathrm{m}^{3} / \mathrm{s}\right)$ & $\left(\mathrm{ft}^{3} / \mathrm{s}\right)$ & (gpm) \\
\hline 4/22/08 12:00 & 1.814 & 0.835 & 0.365 & 163.640 & 1.374 & 0.043 & 0.013106 & 0.004451 & 0.157222 & 70.561 \\
\hline 4/22/08 13:00 & 1.764 & 0.785 & 0.313 & 140.442 & 1.37 & 0.039 & 0.011887 & 0.003887 & 0.137285 & 61.614 \\
\hline 4/22/08 14:00 & 1.743 & 0.764 & 0.293 & 131.327 & 1.373 & 0.042 & 0.012801 & 0.004308 & 0.152154 & 68.287 \\
\hline 4/22/08 15:00 & 1.755 & 0.776 & 0.304 & 136.490 & 1.372 & 0.041 & 0.012496 & 0.004166 & 0.147141 & 66.037 \\
\hline 4/22/08 16:00 & 1.753 & 0.774 & 0.302 & 135.621 & 1.375 & 0.044 & 0.013411 & 0.004596 & 0.162345 & 72.860 \\
\hline 4/22/08 17:00 & 1.758 & 0.779 & 0.307 & 137.800 & 1.373 & 0.042 & 0.012801 & 0.004308 & 0.152154 & 68.287 \\
\hline 4/22/08 18:00 & 1.766 & 0.787 & 0.315 & 141.329 & 1.374 & 0.043 & 0.013106 & 0.004451 & 0.157222 & 70.561 \\
\hline 4/22/08 19:00 & 1.747 & 0.768 & 0.296 & 133.034 & 1.374 & 0.043 & 0.013106 & 0.004451 & 0.157222 & 70.561 \\
\hline 4/22/08 20:00 & 1.754 & 0.775 & 0.303 & 136.055 & 1.377 & 0.046 & 0.014020 & 0.004891 & 0.172754 & 77.532 \\
\hline 4/22/08 21:00 & 1.76 & 0.781 & 0.309 & 138.677 & 1.376 & 0.045 & 0.013715 & 0.004743 & 0.167523 & 75.184 \\
\hline 4/22/08 22:00 & 1.748 & 0.769 & 0.297 & 133.464 & 1.376 & 0.045 & 0.013715 & 0.004743 & 0.167523 & 75.184 \\
\hline 4/22/08 23:00 & 1.767 & 0.788 & 0.316 & 141.774 & 1.375 & 0.044 & 0.013411 & 0.004596 & 0.162345 & 72.860 \\
\hline 4/23/08 0:00 & 1.778 & 0.799 & 0.327 & 146.723 & 1.375 & 0.044 & 0.013411 & 0.004596 & 0.162345 & 72.860 \\
\hline 4/23/08 1:00 & 1.776 & 0.797 & 0.325 & 145.816 & 1.372 & 0.041 & 0.012496 & 0.004166 & 0.147141 & 66.037 \\
\hline 4/23/08 2:00 & 1.776 & 0.797 & 0.325 & 145.816 & 1.371 & 0.04 & 0.012191 & 0.004026 & 0.142185 & 63.812 \\
\hline 4/23/08 3:00 & 1.771 & 0.792 & 0.320 & 143.562 & 1.373 & 0.042 & 0.012801 & 0.004308 & 0.152154 & 68.287 \\
\hline 4/23/08 4:00 & 1.774 & 0.795 & 0.323 & 144.912 & 1.372 & 0.041 & 0.012496 & 0.004166 & 0.147141 & 66.037 \\
\hline 4/23/08 5:00 & 1.776 & 0.797 & 0.325 & 145.816 & 1.372 & 0.041 & 0.012496 & 0.004166 & 0.147141 & 66.037 \\
\hline 4/23/08 6:00 & 1.772 & 0.793 & 0.321 & 144.011 & 1.371 & 0.04 & 0.012191 & 0.004026 & 0.142185 & 63.812 \\
\hline 4/23/08 7:00 & 1.77 & 0.791 & 0.319 & 143.114 & 1.371 & 0.04 & 0.012191 & 0.004026 & 0.142185 & 63.812 \\
\hline 4/23/08 8:00 & 1.775 & 0.796 & 0.324 & 145.363 & 1.371 & 0.04 & 0.012191 & 0.004026 & 0.142185 & 63.812 \\
\hline 4/23/08 9:00 & 1.78 & 0.801 & 0.329 & 147.634 & 1.375 & 0.044 & 0.013411 & 0.004596 & 0.162345 & 72.860 \\
\hline 4/23/08 10:00 & 1.767 & 0.788 & 0.316 & 141.774 & 1.373 & 0.042 & 0.012801 & 0.004308 & 0.152154 & 68.287 \\
\hline 4/23/08 11:00 & 1.748 & 0.769 & 0.297 & 133.464 & 1.374 & 0.043 & 0.013106 & 0.004451 & 0.157222 & 70.561 \\
\hline 4/23/08 12:00 & 1.752 & 0.773 & 0.301 & 135.188 & 1.37 & 0.039 & 0.011887 & 0.003887 & 0.137285 & 61.614 \\
\hline
\end{tabular}




\begin{tabular}{|c|c|c|c|c|c|c|c|c|c|c|}
\hline \multirow[b]{3}{*}{ Date Time } & \multicolumn{4}{|c|}{ V-Notch Weir } & \multicolumn{6}{|c|}{ R-Notch Weir } \\
\hline & \multicolumn{2}{|c|}{ Measured Water Level } & \multicolumn{2}{|c|}{$\begin{array}{c}\text { Calculated Rate } \\
\left(\mathrm{ft}^{3} / \mathrm{s}=448.8 \mathrm{gpm}\right)\end{array}$} & \multicolumn{3}{|c|}{ Measured Water Level } & \multicolumn{3}{|c|}{$\begin{array}{c}\text { Calculated Rate } \\
\left(\mathrm{ft}^{3} / \mathrm{s}=448.8 \mathrm{gpm}\right)\end{array}$} \\
\hline & $\begin{array}{l}\text { (ft above } \\
\text { sensor) }\end{array}$ & $\begin{array}{l}\text { (ft above } \\
\text { notch) }\end{array}$ & $\left(\mathrm{ft}^{3} / \mathrm{s}\right)$ & (gpm) & $\begin{array}{l}\text { (ft above } \\
\text { sensor) }\end{array}$ & $\begin{array}{l}\text { (ft above } \\
\text { log) }\end{array}$ & $\begin{array}{c}\text { (m above } \\
\text { log) }\end{array}$ & $\left(\mathrm{m}^{3} / \mathrm{s}\right)$ & $\left(\mathrm{ft}^{3} / \mathrm{s}\right)$ & (gpm) \\
\hline 4/23/08 13:00 & 1.755 & 0.776 & 0.304 & 136.490 & 1.374 & 0.043 & 0.013106 & 0.004451 & 0.157222 & 70.561 \\
\hline 4/23/08 14:00 & 1.772 & 0.793 & 0.321 & 144.011 & 1.372 & 0.041 & 0.012496 & 0.004166 & 0.147141 & 66.037 \\
\hline 4/23/08 15:00 & 1.742 & 0.763 & 0.292 & 130.902 & 1.369 & 0.038 & 0.011582 & 0.003750 & 0.132444 & 59.441 \\
\hline 4/23/08 16:00 & 1.745 & 0.766 & 0.295 & 132.179 & 1.377 & 0.046 & 0.014020 & 0.004891 & 0.172754 & 77.532 \\
\hline 4/23/08 17:00 & 1.745 & 0.766 & 0.295 & 132.179 & 1.371 & 0.04 & 0.012191 & 0.004026 & 0.142185 & 63.812 \\
\hline 4/23/08 18:00 & 1.764 & 0.785 & 0.313 & 140.442 & 1.373 & 0.042 & 0.012801 & 0.004308 & 0.152154 & 68.287 \\
\hline 4/23/08 19:00 & 1.738 & 0.759 & 0.288 & 129.210 & 1.376 & 0.045 & 0.013715 & 0.004743 & 0.167523 & 75.184 \\
\hline 4/23/08 20:00 & 1.749 & 0.770 & 0.298 & 133.893 & 1.373 & 0.042 & 0.012801 & 0.004308 & 0.152154 & 68.287 \\
\hline 4/23/08 21:00 & 1.753 & 0.774 & 0.302 & 135.621 & 1.375 & 0.044 & 0.013411 & 0.004596 & 0.162345 & 72.860 \\
\hline 4/23/08 22:00 & 1.756 & 0.777 & 0.305 & 136.926 & 1.375 & 0.044 & 0.013411 & 0.004596 & 0.162345 & 72.860 \\
\hline 4/23/08 23:00 & 1.762 & 0.783 & 0.311 & 139.558 & 1.375 & 0.044 & 0.013411 & 0.004596 & 0.162345 & 72.860 \\
\hline 4/24/08 0:00 & 1.769 & 0.790 & 0.318 & 142.666 & 1.377 & 0.046 & 0.014020 & 0.004891 & 0.172754 & 77.532 \\
\hline 4/24/08 1:00 & 1.774 & 0.795 & 0.323 & 144.912 & 1.376 & 0.045 & 0.013715 & 0.004743 & 0.167523 & 75.184 \\
\hline 4/24/08 2:00 & 1.778 & 0.799 & 0.327 & 146.723 & 1.373 & 0.042 & 0.012801 & 0.004308 & 0.152154 & 68.287 \\
\hline 4/24/08 3:00 & 1.769 & 0.790 & 0.318 & 142.666 & 1.373 & 0.042 & 0.012801 & 0.004308 & 0.152154 & 68.287 \\
\hline 4/24/08 4:00 & 1.769 & 0.790 & 0.318 & 142.666 & 1.375 & 0.044 & 0.013411 & 0.004596 & 0.162345 & 72.860 \\
\hline 4/24/08 5:00 & 1.769 & 0.790 & 0.318 & 142.666 & 1.374 & 0.043 & 0.013106 & 0.004451 & 0.157222 & 70.561 \\
\hline 4/24/08 6:00 & 1.771 & 0.792 & 0.320 & 143.562 & 1.374 & 0.043 & 0.013106 & 0.004451 & 0.157222 & 70.561 \\
\hline 4/24/08 7:00 & 1.769 & 0.790 & 0.318 & 142.666 & 1.376 & 0.045 & 0.013715 & 0.004743 & 0.167523 & 75.184 \\
\hline 4/24/08 8:00 & 1.769 & 0.790 & 0.318 & 142.666 & 1.374 & 0.043 & 0.013106 & 0.004451 & 0.157222 & 70.561 \\
\hline 4/24/08 9:00 & 1.788 & 0.809 & 0.337 & 151.312 & 1.376 & 0.045 & 0.013715 & 0.004743 & 0.167523 & 75.184 \\
\hline 4/24/08 10:00 & 1.773 & 0.794 & 0.322 & 144.461 & 1.376 & 0.045 & 0.013715 & 0.004743 & 0.167523 & 75.184 \\
\hline 4/24/08 11:00 & 1.771 & 0.792 & 0.320 & 143.562 & 1.371 & 0.04 & 0.012191 & 0.004026 & 0.142185 & 63.812 \\
\hline 4/24/08 12:00 & 1.776 & 0.797 & 0.325 & 145.816 & 1.376 & 0.045 & 0.013715 & 0.004743 & 0.167523 & 75.184 \\
\hline 4/24/08 13:00 & 1.773 & 0.794 & 0.322 & 144.461 & 1.374 & 0.043 & 0.013106 & 0.004451 & 0.157222 & 70.561 \\
\hline
\end{tabular}




\begin{tabular}{|c|c|c|c|c|c|c|c|c|c|c|}
\hline \multirow[b]{3}{*}{ Date Time } & \multicolumn{4}{|c|}{ V-Notch Weir } & \multicolumn{6}{|c|}{ R-Notch Weir } \\
\hline & \multicolumn{2}{|c|}{ Measured Water Level } & \multicolumn{2}{|c|}{$\begin{array}{c}\text { Calculated Rate } \\
\left(\mathrm{ft}^{3} / \mathrm{s}=448.8 \mathrm{gpm}\right)\end{array}$} & \multicolumn{3}{|c|}{ Measured Water Level } & \multicolumn{3}{|c|}{$\begin{array}{c}\text { Calculated Rate } \\
\left(\mathrm{ft}^{3} / \mathrm{s}=448.8 \mathrm{gpm}\right)\end{array}$} \\
\hline & $\begin{array}{l}\text { (ft above } \\
\text { sensor) }\end{array}$ & $\begin{array}{l}\text { (ft above } \\
\text { notch) }\end{array}$ & $\left(\mathrm{ft}^{3} / \mathrm{s}\right)$ & (gpm) & $\begin{array}{l}\text { (ft above } \\
\text { sensor) }\end{array}$ & $\begin{array}{l}\text { (ft above } \\
\text { log) }\end{array}$ & $\begin{array}{c}\text { (m above } \\
\text { log) }\end{array}$ & $\left(\mathrm{m}^{3} / \mathrm{s}\right)$ & $\left(\mathrm{ft}^{3} / \mathrm{s}\right)$ & (gpm) \\
\hline 4/24/08 14:00 & 1.781 & 0.802 & 0.330 & 148.091 & 1.375 & 0.044 & 0.013411 & 0.004596 & 0.162345 & 72.860 \\
\hline 4/24/08 15:00 & 1.767 & 0.788 & 0.316 & 141.774 & 1.372 & 0.041 & 0.012496 & 0.004166 & 0.147141 & 66.037 \\
\hline 4/24/08 16:00 & 1.774 & 0.795 & 0.323 & 144.912 & 1.373 & 0.042 & 0.012801 & 0.004308 & 0.152154 & 68.287 \\
\hline 4/24/08 17:00 & 1.717 & 0.738 & 0.269 & 120.546 & 1.373 & 0.042 & 0.012801 & 0.004308 & 0.152154 & 68.287 \\
\hline 4/24/08 18:00 & 1.673 & 0.694 & 0.231 & 103.547 & 1.375 & 0.044 & 0.013411 & 0.004596 & 0.162345 & 72.860 \\
\hline 4/24/08 19:00 & 1.741 & 0.762 & 0.291 & 130.478 & 1.373 & 0.042 & 0.012801 & 0.004308 & 0.152154 & 68.287 \\
\hline 4/24/08 20:00 & 1.734 & 0.755 & 0.284 & 127.532 & 1.375 & 0.044 & 0.013411 & 0.004596 & 0.162345 & 72.860 \\
\hline 4/24/08 21:00 & 1.759 & 0.780 & 0.308 & 138.238 & 1.375 & 0.044 & 0.013411 & 0.004596 & 0.162345 & 72.860 \\
\hline 4/24/08 22:00 & 1.762 & 0.783 & 0.311 & 139.558 & 1.375 & 0.044 & 0.013411 & 0.004596 & 0.162345 & 72.860 \\
\hline 4/24/08 23:00 & 1.766 & 0.787 & 0.315 & 141.329 & 1.375 & 0.044 & 0.013411 & 0.004596 & 0.162345 & 72.860 \\
\hline 4/25/08 0:00 & 1.757 & 0.778 & 0.306 & 137.362 & 1.375 & 0.044 & 0.013411 & 0.004596 & 0.162345 & 72.860 \\
\hline 4/25/08 1:00 & 1.757 & 0.778 & 0.306 & 137.362 & 1.375 & 0.044 & 0.013411 & 0.004596 & 0.162345 & 72.860 \\
\hline 4/25/08 2:00 & 1.797 & 0.818 & 0.347 & 155.514 & 1.382 & 0.051 & 0.015544 & 0.005654 & 0.199703 & 89.627 \\
\hline 4/25/08 3:00 & 1.928 & 0.949 & 0.501 & 224.707 & 1.414 & 0.083 & 0.025297 & 0.011331 & 0.400223 & 179.620 \\
\hline 4/25/08 4:00 & 2.023 & 1.044 & 0.634 & 284.700 & 1.433 & 0.102 & 0.031088 & 0.015274 & 0.539466 & 242.112 \\
\hline 4/25/08 5:00 & 1.976 & 0.997 & 0.566 & 253.956 & 1.416 & 0.085 & 0.025907 & 0.011728 & 0.414220 & 185.902 \\
\hline 4/25/08 6:00 & 1.905 & 0.926 & 0.471 & 211.447 & 1.401 & 0.07 & 0.021335 & 0.008869 & 0.313263 & 140.593 \\
\hline 4/25/08 7:00 & 1.856 & 0.877 & 0.412 & 184.793 & 1.387 & 0.056 & 0.017068 & 0.006453 & 0.227923 & 102.292 \\
\hline 4/25/08 8:00 & 1.819 & 0.840 & 0.370 & 166.078 & 1.384 & 0.053 & 0.016154 & 0.005969 & 0.210842 & 94.626 \\
\hline 4/25/08 9:00 & 1.8 & 0.821 & 0.350 & 156.930 & 1.381 & 0.05 & 0.015239 & 0.005499 & 0.194210 & 87.161 \\
\hline 4/25/08 10:00 & 1.786 & 0.807 & 0.335 & 150.387 & 1.381 & 0.05 & 0.015239 & 0.005499 & 0.194210 & 87.161 \\
\hline 4/25/08 11:00 & 1.777 & 0.798 & 0.326 & 146.269 & 1.378 & 0.047 & 0.014325 & 0.005041 & 0.178040 & 79.904 \\
\hline 4/25/08 12:00 & 1.76 & 0.781 & 0.309 & 138.677 & 1.376 & 0.045 & 0.013715 & 0.004743 & 0.167523 & 75.184 \\
\hline 4/25/08 13:00 & 1.758 & 0.779 & 0.307 & 137.800 & 1.374 & 0.043 & 0.013106 & 0.004451 & 0.157222 & 70.561 \\
\hline 4/25/08 14:00 & 1.748 & 0.769 & 0.297 & 133.464 & 1.374 & 0.043 & 0.013106 & 0.004451 & 0.157222 & 70.561 \\
\hline
\end{tabular}




\begin{tabular}{|c|c|c|c|c|c|c|c|c|c|c|}
\hline \multirow[b]{3}{*}{ Date Time } & \multicolumn{4}{|c|}{ V-Notch Weir } & \multicolumn{6}{|c|}{ R-Notch Weir } \\
\hline & \multicolumn{2}{|c|}{ Measured Water Level } & \multicolumn{2}{|c|}{$\begin{array}{c}\text { Calculated Rate } \\
\left(\mathrm{ft}^{3} / \mathrm{s}=448.8 \mathrm{gpm}\right)\end{array}$} & \multicolumn{3}{|c|}{ Measured Water Level } & \multicolumn{3}{|c|}{$\begin{array}{c}\text { Calculated Rate } \\
\left(\mathrm{ft}^{3} / \mathrm{s}=448.8 \mathrm{gpm}\right)\end{array}$} \\
\hline & $\begin{array}{c}\text { (ft above } \\
\text { sensor) }\end{array}$ & $\begin{array}{c}\text { (ft above } \\
\text { notch) }\end{array}$ & $\left(\mathrm{ft}^{3} / \mathrm{s}\right)$ & (gpm) & $\begin{array}{l}\text { (ft above } \\
\text { sensor) }\end{array}$ & $\begin{array}{c}\text { (ft above } \\
\text { log) }\end{array}$ & $\begin{array}{c}\text { (m above } \\
\text { log) }\end{array}$ & $\left(\mathrm{m}^{3} / \mathrm{s}\right)$ & $\left(\mathrm{ft}^{3} / \mathrm{s}\right)$ & (gpm) \\
\hline 4/25/08 15:00 & 1.751 & 0.772 & 0.300 & 134.756 & 1.374 & 0.043 & 0.013106 & 0.004451 & 0.157222 & 70.561 \\
\hline 4/25/08 16:00 & 1.739 & 0.760 & 0.289 & 129.632 & 1.376 & 0.045 & 0.013715 & 0.004743 & 0.167523 & 75.184 \\
\hline 4/25/08 17:00 & 1.751 & 0.772 & 0.300 & 134.756 & 1.371 & 0.04 & 0.012191 & 0.004026 & 0.142185 & 63.812 \\
\hline 4/25/08 18:00 & 1.753 & 0.774 & 0.302 & 135.621 & 1.376 & 0.045 & 0.013715 & 0.004743 & 0.167523 & 75.184 \\
\hline 4/25/08 19:00 & 1.751 & 0.772 & 0.300 & 134.756 & 1.373 & 0.042 & 0.012801 & 0.004308 & 0.152154 & 68.287 \\
\hline 4/25/08 20:00 & 1.744 & 0.765 & 0.294 & 131.752 & 1.373 & 0.042 & 0.012801 & 0.004308 & 0.152154 & 68.287 \\
\hline 4/25/08 21:00 & 1.738 & 0.759 & 0.288 & 129.210 & 1.375 & 0.044 & 0.013411 & 0.004596 & 0.162345 & 72.860 \\
\hline 4/25/08 22:00 & 1.736 & 0.757 & 0.286 & 128.369 & 1.37 & 0.039 & 0.011887 & 0.003887 & 0.137285 & 61.614 \\
\hline 4/25/08 23:00 & 1.75 & 0.771 & 0.299 & 134.324 & 1.374 & 0.043 & 0.013106 & 0.004451 & 0.157222 & 70.561 \\
\hline 4/26/08 0:00 & 1.746 & 0.767 & 0.295 & 132.606 & 1.372 & 0.041 & 0.012496 & 0.004166 & 0.147141 & 66.037 \\
\hline 4/26/08 1:00 & 1.744 & 0.765 & 0.294 & 131.752 & 1.371 & 0.04 & 0.012191 & 0.004026 & 0.142185 & 63.812 \\
\hline 4/26/08 2:00 & 1.747 & 0.768 & 0.296 & 133.034 & 1.374 & 0.043 & 0.013106 & 0.004451 & 0.157222 & 70.561 \\
\hline 4/26/08 3:00 & 1.743 & 0.764 & 0.293 & 131.327 & 1.371 & 0.04 & 0.012191 & 0.004026 & 0.142185 & 63.812 \\
\hline 4/26/08 4:00 & 1.741 & 0.762 & 0.291 & 130.478 & 1.373 & 0.042 & 0.012801 & 0.004308 & 0.152154 & 68.287 \\
\hline 4/26/08 5:00 & 1.746 & 0.767 & 0.295 & 132.606 & 1.371 & 0.04 & 0.012191 & 0.004026 & 0.142185 & 63.812 \\
\hline 4/26/08 6:00 & 1.746 & 0.767 & 0.295 & 132.606 & 1.371 & 0.04 & 0.012191 & 0.004026 & 0.142185 & 63.812 \\
\hline 4/26/08 7:00 & 1.739 & 0.760 & 0.289 & 129.632 & 1.373 & 0.042 & 0.012801 & 0.004308 & 0.152154 & 68.287 \\
\hline 4/26/08 8:00 & 1.74 & 0.761 & 0.290 & 130.054 & 1.371 & 0.04 & 0.012191 & 0.004026 & 0.142185 & 63.812 \\
\hline 4/26/08 9:00 & 1.741 & 0.762 & 0.291 & 130.478 & 1.371 & 0.04 & 0.012191 & 0.004026 & 0.142185 & 63.812 \\
\hline 4/26/08 10:00 & 1.736 & 0.757 & 0.286 & 128.369 & 1.371 & 0.04 & 0.012191 & 0.004026 & 0.142185 & 63.812 \\
\hline 4/26/08 11:00 & 1.742 & 0.763 & 0.292 & 130.902 & 1.371 & 0.04 & 0.012191 & 0.004026 & 0.142185 & 63.812 \\
\hline 4/26/08 12:00 & 1.747 & 0.768 & 0.296 & 133.034 & 1.372 & 0.041 & 0.012496 & 0.004166 & 0.147141 & 66.037 \\
\hline 4/26/08 13:00 & 1.744 & 0.765 & 0.294 & 131.752 & 1.372 & 0.041 & 0.012496 & 0.004166 & 0.147141 & 66.037 \\
\hline 4/26/08 14:00 & 1.737 & 0.758 & 0.287 & 128.789 & 1.37 & 0.039 & 0.011887 & 0.003887 & 0.137285 & 61.614 \\
\hline 4/26/08 15:00 & 1.739 & 0.760 & 0.289 & 129.632 & 1.37 & 0.039 & 0.011887 & 0.003887 & 0.137285 & 61.614 \\
\hline
\end{tabular}




\begin{tabular}{|c|c|c|c|c|c|c|c|c|c|c|}
\hline \multirow[b]{3}{*}{ Date Time } & \multicolumn{4}{|c|}{ V-Notch Weir } & \multicolumn{6}{|c|}{ R-Notch Weir } \\
\hline & \multicolumn{2}{|c|}{ Measured Water Level } & \multicolumn{2}{|c|}{$\begin{array}{c}\text { Calculated Rate } \\
\left(\mathrm{ft}^{3} / \mathrm{s}=448.8 \mathrm{gpm}\right)\end{array}$} & \multicolumn{3}{|c|}{ Measured Water Level } & \multicolumn{3}{|c|}{$\begin{array}{c}\text { Calculated Rate } \\
\left(\mathrm{ft}^{3} / \mathrm{s}=448.8 \mathrm{gpm}\right)\end{array}$} \\
\hline & $\begin{array}{c}\text { (ft above } \\
\text { sensor) }\end{array}$ & $\begin{array}{c}\text { (ft above } \\
\text { notch) }\end{array}$ & $\left(\mathrm{ft}^{3} / \mathrm{s}\right)$ & (gpm) & $\begin{array}{l}\text { (ft above } \\
\text { sensor) }\end{array}$ & $\begin{array}{c}\text { (ft above } \\
\text { log) }\end{array}$ & $\begin{array}{c}\text { (m above } \\
\text { log) }\end{array}$ & $\left(\mathrm{m}^{3} / \mathrm{s}\right)$ & $\left(\mathrm{ft}^{3} / \mathrm{s}\right)$ & (gpm) \\
\hline 4/26/08 16:00 & 1.715 & 0.736 & 0.267 & 119.739 & 1.37 & 0.039 & 0.011887 & 0.003887 & 0.137285 & 61.614 \\
\hline 4/26/08 17:00 & 1.736 & 0.757 & 0.286 & 128.369 & 1.373 & 0.042 & 0.012801 & 0.004308 & 0.152154 & 68.287 \\
\hline 4/26/08 18:00 & 1.736 & 0.757 & 0.286 & 128.369 & 1.371 & 0.04 & 0.012191 & 0.004026 & 0.142185 & 63.812 \\
\hline 4/26/08 19:00 & 1.745 & 0.766 & 0.295 & 132.179 & 1.373 & 0.042 & 0.012801 & 0.004308 & 0.152154 & 68.287 \\
\hline 4/26/08 20:00 & 1.781 & 0.802 & 0.330 & 148.091 & 1.378 & 0.047 & 0.014325 & 0.005041 & 0.178040 & 79.904 \\
\hline 4/26/08 21:00 & 1.795 & 0.816 & 0.344 & 154.574 & 1.38 & 0.049 & 0.014934 & 0.005345 & 0.188768 & 84.719 \\
\hline 4/26/08 22:00 & 1.823 & 0.844 & 0.374 & 168.044 & 1.385 & 0.054 & 0.016458 & 0.006129 & 0.216486 & 97.159 \\
\hline 4/26/08 23:00 & 1.788 & 0.809 & 0.337 & 151.312 & 1.384 & 0.053 & 0.016154 & 0.005969 & 0.210842 & 94.626 \\
\hline 4/27/08 0:00 & 1.786 & 0.807 & 0.335 & 150.387 & 1.382 & 0.051 & 0.015544 & 0.005654 & 0.199703 & 89.627 \\
\hline 4/27/08 1:00 & 1.775 & 0.796 & 0.324 & 145.363 & 1.379 & 0.048 & 0.014630 & 0.005192 & 0.183377 & 82.300 \\
\hline 4/27/08 2:00 & 1.768 & 0.789 & 0.317 & 142.220 & 1.377 & 0.046 & 0.014020 & 0.004891 & 0.172754 & 77.532 \\
\hline 4/27/08 3:00 & 1.764 & 0.785 & 0.313 & 140.442 & 1.374 & 0.043 & 0.013106 & 0.004451 & 0.157222 & 70.561 \\
\hline 4/27/08 4:00 & 1.771 & 0.792 & 0.320 & 143.562 & 1.374 & 0.043 & 0.013106 & 0.004451 & 0.157222 & 70.561 \\
\hline 4/27/08 5:00 & 1.758 & 0.779 & 0.307 & 137.800 & 1.374 & 0.043 & 0.013106 & 0.004451 & 0.157222 & 70.561 \\
\hline 4/27/08 6:00 & 1.753 & 0.774 & 0.302 & 135.621 & 1.373 & 0.042 & 0.012801 & 0.004308 & 0.152154 & 68.287 \\
\hline 4/27/08 7:00 & 1.747 & 0.768 & 0.296 & 133.034 & 1.373 & 0.042 & 0.012801 & 0.004308 & 0.152154 & 68.287 \\
\hline 4/27/08 8:00 & 1.749 & 0.770 & 0.298 & 133.893 & 1.371 & 0.04 & 0.012191 & 0.004026 & 0.142185 & 63.812 \\
\hline 4/27/08 9:00 & 1.747 & 0.768 & 0.296 & 133.034 & 1.371 & 0.04 & 0.012191 & 0.004026 & 0.142185 & 63.812 \\
\hline 4/27/08 10:00 & 1.746 & 0.767 & 0.295 & 132.606 & 1.371 & 0.04 & 0.012191 & 0.004026 & 0.142185 & 63.812 \\
\hline 4/27/08 11:00 & 1.748 & 0.769 & 0.297 & 133.464 & 1.372 & 0.041 & 0.012496 & 0.004166 & 0.147141 & 66.037 \\
\hline 4/27/08 12:00 & 1.737 & 0.758 & 0.287 & 128.789 & 1.37 & 0.039 & 0.011887 & 0.003887 & 0.137285 & 61.614 \\
\hline 4/27/08 13:00 & 1.671 & 0.692 & 0.229 & 102.811 & 1.373 & 0.042 & 0.012801 & 0.004308 & 0.152154 & 68.287 \\
\hline 4/27/08 14:00 & 1.736 & 0.757 & 0.286 & 128.369 & 1.373 & 0.042 & 0.012801 & 0.004308 & 0.152154 & 68.287 \\
\hline 4/27/08 15:00 & 1.724 & 0.745 & 0.275 & 123.394 & 1.374 & 0.043 & 0.013106 & 0.004451 & 0.157222 & 70.561 \\
\hline 4/27/08 16:00 & 1.724 & 0.745 & 0.275 & 123.394 & 1.372 & 0.041 & 0.012496 & 0.004166 & 0.147141 & 66.037 \\
\hline
\end{tabular}




\begin{tabular}{|c|c|c|c|c|c|c|c|c|c|c|}
\hline \multirow[b]{3}{*}{ Date Time } & \multicolumn{4}{|c|}{ V-Notch Weir } & \multicolumn{6}{|c|}{ R-Notch Weir } \\
\hline & \multicolumn{2}{|c|}{ Measured Water Level } & \multicolumn{2}{|c|}{$\begin{array}{c}\text { Calculated Rate } \\
\left(\mathrm{ft}^{3} / \mathrm{s}=448.8 \mathrm{gpm}\right)\end{array}$} & \multicolumn{3}{|c|}{ Measured Water Level } & \multicolumn{3}{|c|}{$\begin{array}{c}\text { Calculated Rate } \\
\left(\mathrm{ft}^{3} / \mathrm{s}=448.8 \mathrm{gpm}\right)\end{array}$} \\
\hline & $\begin{array}{l}\text { (ft above } \\
\text { sensor) }\end{array}$ & $\begin{array}{l}\text { (ft above } \\
\text { notch) }\end{array}$ & $\left(\mathrm{ft}^{3} / \mathrm{s}\right)$ & (gpm) & $\begin{array}{l}\text { (ft above } \\
\text { sensor) }\end{array}$ & $\begin{array}{l}\text { (ft above } \\
\text { log) }\end{array}$ & $\begin{array}{c}\text { (m above } \\
\log )\end{array}$ & $\left(\mathrm{m}^{3} / \mathrm{s}\right)$ & $\left(\mathrm{ft}^{3} / \mathrm{s}\right)$ & (gpm) \\
\hline 4/27/08 17:00 & 1.731 & 0.752 & 0.281 & 126.282 & 1.373 & 0.042 & 0.012801 & 0.004308 & 0.152154 & 68.287 \\
\hline 4/27/08 18:00 & 1.715 & 0.736 & 0.267 & 119.739 & 1.373 & 0.042 & 0.012801 & 0.004308 & 0.152154 & 68.287 \\
\hline 4/27/08 19:00 & 1.691 & 0.712 & 0.246 & 110.314 & 1.373 & 0.042 & 0.012801 & 0.004308 & 0.152154 & 68.287 \\
\hline 4/27/08 20:00 & 1.696 & 0.717 & 0.250 & 112.239 & 1.373 & 0.042 & 0.012801 & 0.004308 & 0.152154 & 68.287 \\
\hline 4/27/08 21:00 & 1.63 & 0.651 & 0.197 & 88.409 & 1.373 & 0.042 & 0.012801 & 0.004308 & 0.152154 & 68.287 \\
\hline 4/27/08 22:00 & 1.63 & 0.651 & 0.197 & 88.409 & 1.373 & 0.042 & 0.012801 & 0.004308 & 0.152154 & 68.287 \\
\hline 4/27/08 23:00 & 1.649 & 0.670 & 0.212 & 94.921 & 1.373 & 0.042 & 0.012801 & 0.004308 & 0.152154 & 68.287 \\
\hline 4/28/08 0:00 & 1.623 & 0.644 & 0.192 & 86.080 & 1.372 & 0.041 & 0.012496 & 0.004166 & 0.147141 & 66.037 \\
\hline 4/28/08 1:00 & 1.63 & 0.651 & 0.197 & 88.409 & 1.372 & 0.041 & 0.012496 & 0.004166 & 0.147141 & 66.037 \\
\hline 4/28/08 2:00 & 1.623 & 0.644 & 0.192 & 86.080 & 1.374 & 0.043 & 0.013106 & 0.004451 & 0.157222 & 70.561 \\
\hline 4/28/08 3:00 & 1.645 & 0.666 & 0.208 & 93.527 & 1.371 & 0.04 & 0.012191 & 0.004026 & 0.142185 & 63.812 \\
\hline 4/28/08 4:00 & 1.659 & 0.680 & 0.219 & 98.461 & 1.373 & 0.042 & 0.012801 & 0.004308 & 0.152154 & 68.287 \\
\hline 4/28/08 5:00 & 1.653 & 0.674 & 0.215 & 96.328 & 1.373 & 0.042 & 0.012801 & 0.004308 & 0.152154 & 68.287 \\
\hline 4/28/08 6:00 & 1.632 & 0.653 & 0.198 & 89.082 & 1.372 & 0.041 & 0.012496 & 0.004166 & 0.147141 & 66.037 \\
\hline 4/28/08 7:00 & 1.602 & 0.623 & 0.177 & 79.314 & 1.372 & 0.041 & 0.012496 & 0.004166 & 0.147141 & 66.037 \\
\hline 4/28/08 8:00 & 1.567 & 0.588 & 0.153 & 68.767 & 1.369 & 0.038 & 0.011582 & 0.003750 & 0.132444 & 59.441 \\
\hline 4/28/08 9:00 & 1.579 & 0.600 & 0.161 & 72.282 & 1.372 & 0.041 & 0.012496 & 0.004166 & 0.147141 & 66.037 \\
\hline 4/28/08 10:00 & 1.593 & 0.614 & 0.170 & 76.516 & 1.369 & 0.038 & 0.011582 & 0.003750 & 0.132444 & 59.441 \\
\hline 4/28/08 11:00 & 1.58 & 0.601 & 0.162 & 72.579 & 1.37 & 0.039 & 0.011887 & 0.003887 & 0.137285 & 61.614 \\
\hline 4/28/08 12:00 & 1.556 & 0.577 & 0.146 & 65.638 & 1.371 & 0.04 & 0.012191 & 0.004026 & 0.142185 & 63.812 \\
\hline 4/28/08 13:00 & 1.574 & 0.595 & 0.158 & 70.805 & 1.369 & 0.038 & 0.011582 & 0.003750 & 0.132444 & 59.441 \\
\hline 4/28/08 14:00 & 1.581 & 0.602 & 0.162 & 72.878 & 1.372 & 0.041 & 0.012496 & 0.004166 & 0.147141 & 66.037 \\
\hline 4/28/08 15:00 & 1.621 & 0.642 & 0.190 & 85.421 & 1.37 & 0.039 & 0.011887 & 0.003887 & 0.137285 & 61.614 \\
\hline 4/28/08 16:00 & 1.628 & 0.649 & 0.195 & 87.740 & 1.371 & 0.04 & 0.012191 & 0.004026 & 0.142185 & 63.812 \\
\hline 4/28/08 17:00 & 1.63 & 0.651 & 0.197 & 88.409 & 1.371 & 0.04 & 0.012191 & 0.004026 & 0.142185 & 63.812 \\
\hline
\end{tabular}




\begin{tabular}{|c|c|c|c|c|c|c|c|c|c|c|}
\hline \multirow[b]{3}{*}{ Date Time } & \multicolumn{4}{|c|}{ V-Notch Weir } & \multicolumn{6}{|c|}{ R-Notch Weir } \\
\hline & \multicolumn{2}{|c|}{ Measured Water Level } & \multicolumn{2}{|c|}{$\begin{array}{c}\text { Calculated Rate } \\
\left(\mathrm{ft}^{3} / \mathrm{s}=448.8 \mathrm{gpm}\right)\end{array}$} & \multicolumn{3}{|c|}{ Measured Water Level } & \multicolumn{3}{|c|}{$\begin{array}{c}\text { Calculated Rate } \\
\left(\mathrm{ft}^{3} / \mathrm{s}=448.8 \mathrm{gpm}\right)\end{array}$} \\
\hline & $\begin{array}{c}\text { (ft above } \\
\text { sensor) }\end{array}$ & $\begin{array}{c}\text { (ft above } \\
\text { notch) }\end{array}$ & $\left(\mathrm{ft}^{3} / \mathrm{s}\right)$ & (gpm) & $\begin{array}{l}\text { (ft above } \\
\text { sensor) }\end{array}$ & $\begin{array}{c}\text { (ft above } \\
\text { log) }\end{array}$ & $\begin{array}{c}\text { (m above } \\
\text { log) }\end{array}$ & $\left(\mathrm{m}^{3} / \mathrm{s}\right)$ & $\left(\mathrm{ft}^{3} / \mathrm{s}\right)$ & (gpm) \\
\hline 4/28/08 18:00 & 1.628 & 0.649 & 0.195 & 87.740 & 1.369 & 0.038 & 0.011582 & 0.003750 & 0.132444 & 59.441 \\
\hline 4/28/08 19:00 & 1.621 & 0.642 & 0.190 & 85.421 & 1.371 & 0.04 & 0.012191 & 0.004026 & 0.142185 & 63.812 \\
\hline 4/28/08 20:00 & 1.609 & 0.630 & 0.182 & 81.532 & 1.374 & 0.043 & 0.013106 & 0.004451 & 0.157222 & 70.561 \\
\hline 4/28/08 21:00 & 1.597 & 0.618 & 0.173 & 77.752 & 1.373 & 0.042 & 0.012801 & 0.004308 & 0.152154 & 68.287 \\
\hline 4/28/08 22:00 & 1.578 & 0.599 & 0.160 & 71.985 & 1.373 & 0.042 & 0.012801 & 0.004308 & 0.152154 & 68.287 \\
\hline 4/28/08 23:00 & 1.538 & 0.559 & 0.135 & 60.704 & 1.373 & 0.042 & 0.012801 & 0.004308 & 0.152154 & 68.287 \\
\hline 4/29/08 0:00 & 1.499 & 0.520 & 0.113 & 50.796 & 1.375 & 0.044 & 0.013411 & 0.004596 & 0.162345 & 72.860 \\
\hline 4/29/08 1:00 & 1.513 & 0.534 & 0.121 & 54.231 & 1.374 & 0.043 & 0.013106 & 0.004451 & 0.157222 & 70.561 \\
\hline 4/29/08 2:00 & 1.511 & 0.532 & 0.120 & 53.732 & 1.374 & 0.043 & 0.013106 & 0.004451 & 0.157222 & 70.561 \\
\hline 4/29/08 3:00 & 1.49 & 0.511 & 0.108 & 48.658 & 1.373 & 0.042 & 0.012801 & 0.004308 & 0.152154 & 68.287 \\
\hline 4/29/08 4:00 & 1.481 & 0.502 & 0.104 & 46.576 & 1.375 & 0.044 & 0.013411 & 0.004596 & 0.162345 & 72.860 \\
\hline 4/29/08 5:00 & 1.472 & 0.493 & 0.099 & 44.548 & 1.37 & 0.039 & 0.011887 & 0.003887 & 0.137285 & 61.614 \\
\hline 4/29/08 6:00 & 1.456 & 0.477 & 0.092 & 41.075 & 1.372 & 0.041 & 0.012496 & 0.004166 & 0.147141 & 66.037 \\
\hline 4/29/08 7:00 & 1.551 & 0.572 & 0.143 & 64.244 & 1.374 & 0.043 & 0.013106 & 0.004451 & 0.157222 & 70.561 \\
\hline 4/29/08 8:00 & 1.608 & 0.629 & 0.181 & 81.213 & 1.374 & 0.043 & 0.013106 & 0.004451 & 0.157222 & 70.561 \\
\hline 4/29/08 9:00 & 1.643 & 0.664 & 0.207 & 92.835 & 1.374 & 0.043 & 0.013106 & 0.004451 & 0.157222 & 70.561 \\
\hline 4/29/08 10:00 & 1.659 & 0.680 & 0.219 & 98.461 & 1.372 & 0.041 & 0.012496 & 0.004166 & 0.147141 & 66.037 \\
\hline 4/29/08 11:00 & 1.682 & 0.703 & 0.238 & 106.898 & 1.37 & 0.039 & 0.011887 & 0.003887 & 0.137285 & 61.614 \\
\hline 4/29/08 12:00 & 1.539 & 0.560 & 0.136 & 60.972 & 1.37 & 0.039 & 0.011887 & 0.003887 & 0.137285 & 61.614 \\
\hline 4/29/08 13:00 & 1.583 & 0.604 & 0.164 & 73.477 & 1.369 & 0.038 & 0.011582 & 0.003750 & 0.132444 & 59.441 \\
\hline 4/29/08 14:00 & 1.503 & 0.524 & 0.115 & 51.763 & 1.37 & 0.039 & 0.011887 & 0.003887 & 0.137285 & 61.614 \\
\hline 4/29/08 15:00 & 1.543 & 0.564 & 0.138 & 62.051 & 1.371 & 0.04 & 0.012191 & 0.004026 & 0.142185 & 63.812 \\
\hline 4/29/08 16:00 & 1.56 & 0.581 & 0.149 & 66.766 & 1.371 & 0.04 & 0.012191 & 0.004026 & 0.142185 & 63.812 \\
\hline 4/29/08 17:00 & 1.551 & 0.572 & 0.143 & 64.244 & 1.372 & 0.041 & 0.012496 & 0.004166 & 0.147141 & 66.037 \\
\hline 4/29/08 18:00 & 1.523 & 0.544 & 0.126 & 56.767 & 1.377 & 0.046 & 0.014020 & 0.004891 & 0.172754 & 77.532 \\
\hline
\end{tabular}




\begin{tabular}{|c|c|c|c|c|c|c|c|c|c|c|}
\hline \multirow[b]{3}{*}{ Date Time } & \multicolumn{4}{|c|}{ V-Notch Weir } & \multicolumn{6}{|c|}{ R-Notch Weir } \\
\hline & \multicolumn{2}{|c|}{ Measured Water Level } & \multicolumn{2}{|c|}{$\begin{array}{c}\text { Calculated Rate } \\
\left(\mathrm{ft}^{3} / \mathrm{s}=448.8 \mathrm{gpm}\right)\end{array}$} & \multicolumn{3}{|c|}{ Measured Water Level } & \multicolumn{3}{|c|}{$\begin{array}{c}\text { Calculated Rate } \\
\left(\mathrm{ft}^{3} / \mathrm{s}=448.8 \mathrm{gpm}\right)\end{array}$} \\
\hline & $\begin{array}{l}\text { (ft above } \\
\text { sensor) }\end{array}$ & $\begin{array}{l}\text { (ft above } \\
\text { notch) }\end{array}$ & $\left(\mathrm{ft}^{3} / \mathrm{s}\right)$ & (gpm) & $\begin{array}{l}\text { (ft above } \\
\text { sensor) }\end{array}$ & $\begin{array}{l}\text { (ft above } \\
\text { log) }\end{array}$ & $\begin{array}{c}\text { (m above } \\
\text { log) }\end{array}$ & $\left(\mathrm{m}^{3} / \mathrm{s}\right)$ & $\left(\mathrm{ft}^{3} / \mathrm{s}\right)$ & (gpm) \\
\hline 4/29/08 19:00 & 1.49 & 0.511 & 0.108 & 48.658 & 1.377 & 0.046 & 0.014020 & 0.004891 & 0.172754 & 77.532 \\
\hline 4/29/08 20:00 & 1.473 & 0.494 & 0.100 & 44.770 & 1.377 & 0.046 & 0.014020 & 0.004891 & 0.172754 & 77.532 \\
\hline 4/29/08 21:00 & 1.456 & 0.477 & 0.092 & 41.075 & 1.379 & 0.048 & 0.014630 & 0.005192 & 0.183377 & 82.300 \\
\hline 4/29/08 22:00 & 1.437 & 0.458 & 0.083 & 37.169 & 1.376 & 0.045 & 0.013715 & 0.004743 & 0.167523 & 75.184 \\
\hline 4/29/08 23:00 & 1.442 & 0.463 & 0.085 & 38.174 & 1.376 & 0.045 & 0.013715 & 0.004743 & 0.167523 & 75.184 \\
\hline 4/30/08 0:00 & 1.426 & 0.447 & 0.078 & 35.014 & 1.378 & 0.047 & 0.014325 & 0.005041 & 0.178040 & 79.904 \\
\hline 4/30/08 1:00 & 1.414 & 0.435 & 0.073 & 32.750 & 1.378 & 0.047 & 0.014325 & 0.005041 & 0.178040 & 79.904 \\
\hline 4/30/08 2:00 & 1.409 & 0.430 & 0.071 & 31.833 & 1.38 & 0.049 & 0.014934 & 0.005345 & 0.188768 & 84.719 \\
\hline 4/30/08 3:00 & 1.405 & 0.426 & 0.069 & 31.111 & 1.379 & 0.048 & 0.014630 & 0.005192 & 0.183377 & 82.300 \\
\hline 4/30/08 4:00 & 1.405 & 0.426 & 0.069 & 31.111 & 1.381 & 0.05 & 0.015239 & 0.005499 & 0.194210 & 87.161 \\
\hline 4/30/08 5:00 & 1.389 & 0.410 & 0.063 & 28.322 & 1.378 & 0.047 & 0.014325 & 0.005041 & 0.178040 & 79.904 \\
\hline 4/30/08 6:00 & 1.373 & 0.394 & 0.057 & 25.689 & 1.382 & 0.051 & 0.015544 & 0.005654 & 0.199703 & 89.627 \\
\hline 4/30/08 7:00 & 1.383 & 0.404 & 0.061 & 27.317 & 1.377 & 0.046 & 0.014020 & 0.004891 & 0.172754 & 77.532 \\
\hline 4/30/08 8:00 & 1.4 & 0.421 & 0.067 & 30.223 & 1.377 & 0.046 & 0.014020 & 0.004891 & 0.172754 & 77.532 \\
\hline 4/30/08 9:00 & 1.423 & 0.444 & 0.077 & 34.439 & 1.377 & 0.046 & 0.014020 & 0.004891 & 0.172754 & 77.532 \\
\hline 4/30/08 10:00 & 1.446 & 0.467 & 0.087 & 38.990 & 1.379 & 0.048 & 0.014630 & 0.005192 & 0.183377 & 82.300 \\
\hline 4/30/08 11:00 & 1.457 & 0.478 & 0.092 & 41.287 & 1.382 & 0.051 & 0.015544 & 0.005654 & 0.199703 & 89.627 \\
\hline 4/30/08 12:00 & 1.459 & 0.480 & 0.093 & 41.713 & 1.375 & 0.044 & 0.013411 & 0.004596 & 0.162345 & 72.860 \\
\hline 4/30/08 13:00 & 1.454 & 0.475 & 0.091 & 40.653 & 1.373 & 0.042 & 0.012801 & 0.004308 & 0.152154 & 68.287 \\
\hline 4/30/08 14:00 & 1.444 & 0.465 & 0.086 & 38.581 & 1.376 & 0.045 & 0.013715 & 0.004743 & 0.167523 & 75.184 \\
\hline 4/30/08 15:00 & 1.419 & 0.440 & 0.075 & 33.682 & 1.374 & 0.043 & 0.013106 & 0.004451 & 0.157222 & 70.561 \\
\hline 4/30/08 16:00 & 1.391 & 0.412 & 0.064 & 28.662 & 1.382 & 0.051 & 0.015544 & 0.005654 & 0.199703 & 89.627 \\
\hline 4/30/08 17:00 & 1.356 & 0.377 & 0.051 & 23.058 & 1.38 & 0.049 & 0.014934 & 0.005345 & 0.188768 & 84.719 \\
\hline 4/30/08 18:00 & 1.333 & 0.354 & 0.044 & 19.766 & 1.376 & 0.045 & 0.013715 & 0.004743 & 0.167523 & 75.184 \\
\hline 4/30/08 19:00 & 1.298 & 0.319 & 0.034 & 15.328 & 1.381 & 0.05 & 0.015239 & 0.005499 & 0.194210 & 87.161 \\
\hline
\end{tabular}




\begin{tabular}{|c|c|c|c|c|c|c|c|c|c|c|}
\hline \multirow[b]{3}{*}{ Date Time } & \multicolumn{4}{|c|}{ V-Notch Weir } & \multicolumn{6}{|c|}{ R-Notch Weir } \\
\hline & \multicolumn{2}{|c|}{ Measured Water Level } & \multicolumn{2}{|c|}{$\begin{array}{c}\text { Calculated Rate } \\
\left(\mathrm{ft}^{3} / \mathrm{s}=448.8 \mathrm{gpm}\right)\end{array}$} & \multicolumn{3}{|c|}{ Measured Water Level } & \multicolumn{3}{|c|}{$\begin{array}{c}\text { Calculated Rate } \\
\left(\mathrm{ft}^{3} / \mathrm{s}=448.8 \mathrm{gpm}\right)\end{array}$} \\
\hline & $\begin{array}{l}\text { (ft above } \\
\text { sensor) }\end{array}$ & $\begin{array}{c}\text { (ft above } \\
\text { notch) }\end{array}$ & $\left(\mathrm{ft}^{3} / \mathrm{s}\right)$ & (gpm) & $\begin{array}{c}\text { (ft above } \\
\text { sensor) }\end{array}$ & $\begin{array}{c}\text { (ft above } \\
\text { log) }\end{array}$ & $\begin{array}{c}\text { (m above } \\
\text { log) }\end{array}$ & $\left(\mathrm{m}^{3} / \mathrm{s}\right)$ & $\left(\mathrm{ft}^{3} / \mathrm{s}\right)$ & (gpm) \\
\hline 4/30/08 20:00 & 1.27 & 0.291 & 0.027 & 12.253 & 1.382 & 0.051 & 0.015544 & 0.005654 & 0.199703 & 89.627 \\
\hline 4/30/08 21:00 & 1.276 & 0.297 & 0.029 & 12.878 & 1.384 & 0.053 & 0.016154 & 0.005969 & 0.210842 & 94.626 \\
\hline 4/30/08 22:00 & 1.271 & 0.292 & 0.028 & 12.356 & 1.38 & 0.049 & 0.014934 & 0.005345 & 0.188768 & 84.719 \\
\hline 4/30/08 23:00 & 1.224 & 0.245 & 0.018 & 8.069 & 1.384 & 0.053 & 0.016154 & 0.005969 & 0.210842 & 94.626 \\
\hline 5/1/08 0:00 & 1.215 & 0.236 & 0.016 & 7.370 & 1.382 & 0.051 & 0.015544 & 0.005654 & 0.199703 & 89.627 \\
\hline 5/1/08 1:00 & 1.233 & 0.254 & 0.020 & 8.806 & 1.382 & 0.051 & 0.015544 & 0.005654 & 0.199703 & 89.627 \\
\hline 5/1/08 2:00 & 1.254 & 0.275 & 0.024 & 10.679 & 1.384 & 0.053 & 0.016154 & 0.005969 & 0.210842 & 94.626 \\
\hline 5/1/08 3:00 & 1.264 & 0.285 & 0.026 & 11.648 & 1.384 & 0.053 & 0.016154 & 0.005969 & 0.210842 & 94.626 \\
\hline 5/1/08 4:00 & 1.252 & 0.273 & 0.023 & 10.491 & 1.386 & 0.055 & 0.016763 & 0.006291 & 0.222180 & 99.714 \\
\hline 5/1/08 5:00 & 1.238 & 0.259 & 0.021 & 9.232 & 1.388 & 0.057 & 0.017373 & 0.006617 & 0.233715 & 104.891 \\
\hline 5/1/08 6:00 & 1.226 & 0.247 & 0.018 & 8.229 & 1.383 & 0.052 & 0.015849 & 0.005811 & 0.205247 & 92.115 \\
\hline 5/1/08 7:00 & 1.217 & 0.238 & 0.017 & 7.522 & 1.385 & 0.054 & 0.016458 & 0.006129 & 0.216486 & 97.159 \\
\hline 5/1/08 8:00 & 1.214 & 0.235 & 0.016 & 7.295 & 1.392 & 0.061 & 0.018592 & 0.007287 & 0.257360 & 115.503 \\
\hline 5/1/08 9:00 & 1.21 & 0.231 & 0.016 & 6.998 & 1.39 & 0.059 & 0.017982 & 0.006949 & 0.245443 & 110.155 \\
\hline 5/1/08 10:00 & 1.205 & 0.226 & 0.015 & 6.638 & 1.385 & 0.054 & 0.016458 & 0.006129 & 0.216486 & 97.159 \\
\hline 5/1/08 11:00 & 1.2 & 0.221 & 0.014 & 6.289 & 1.387 & 0.056 & 0.017068 & 0.006453 & 0.227923 & 102.292 \\
\hline 5/1/08 12:00 & 1.193 & 0.214 & 0.013 & 5.819 & 1.383 & 0.052 & 0.015849 & 0.005811 & 0.205247 & 92.115 \\
\hline 5/1/08 13:00 & 1.189 & 0.210 & 0.012 & 5.560 & 1.386 & 0.055 & 0.016763 & 0.006291 & 0.222180 & 99.714 \\
\hline 5/1/08 14:00 & 1.181 & 0.202 & 0.011 & 5.064 & 1.375 & 0.044 & 0.013411 & 0.004596 & 0.162345 & 72.860 \\
\hline 5/1/08 15:00 & 1.175 & 0.196 & 0.010 & 4.710 & 1.385 & 0.054 & 0.016458 & 0.006129 & 0.216486 & 97.159 \\
\hline 5/1/08 16:00 & 1.171 & 0.192 & 0.010 & 4.482 & 1.384 & 0.053 & 0.016154 & 0.005969 & 0.210842 & 94.626 \\
\hline 5/1/08 17:00 & 1.167 & 0.188 & 0.009 & 4.261 & 1.387 & 0.056 & 0.017068 & 0.006453 & 0.227923 & 102.292 \\
\hline 5/1/08 18:00 & 1.164 & 0.185 & 0.009 & 4.100 & 1.39 & 0.059 & 0.017982 & 0.006949 & 0.245443 & 110.155 \\
\hline 5/1/08 19:00 & 1.164 & 0.185 & 0.009 & 4.100 & 1.391 & 0.06 & 0.018287 & 0.007117 & 0.251378 & 112.818 \\
\hline 5/1/08 20:00 & 1.166 & 0.187 & 0.009 & 4.207 & 1.391 & 0.06 & 0.018287 & 0.007117 & 0.251378 & 112.818 \\
\hline
\end{tabular}




\begin{tabular}{|c|c|c|c|c|c|c|c|c|c|c|}
\hline \multirow[b]{3}{*}{ Date Time } & \multicolumn{4}{|c|}{ V-Notch Weir } & \multicolumn{6}{|c|}{ R-Notch Weir } \\
\hline & \multicolumn{2}{|c|}{ Measured Water Level } & \multicolumn{2}{|c|}{$\begin{array}{c}\text { Calculated Rate } \\
\left(\mathrm{ft}^{3} / \mathrm{s}=448.8 \mathrm{gpm}\right)\end{array}$} & \multicolumn{3}{|c|}{ Measured Water Level } & \multicolumn{3}{|c|}{$\begin{array}{c}\text { Calculated Rate } \\
\left(\mathrm{ft}^{3} / \mathrm{s}=448.8 \mathrm{gpm}\right)\end{array}$} \\
\hline & $\begin{array}{c}\text { (ft above } \\
\text { sensor) }\end{array}$ & $\begin{array}{c}\text { (ft above } \\
\text { notch) }\end{array}$ & $\left(\mathrm{ft}^{3} / \mathrm{s}\right)$ & (gpm) & $\begin{array}{c}\text { (ft above } \\
\text { sensor) }\end{array}$ & $\begin{array}{c}\text { (ft above } \\
\text { log) }\end{array}$ & $\begin{array}{c}\text { (m above } \\
\text { log) }\end{array}$ & $\left(\mathrm{m}^{3} / \mathrm{s}\right)$ & $\left(\mathrm{ft}^{3} / \mathrm{s}\right)$ & (gpm) \\
\hline 5/1/08 21:00 & 1.167 & 0.188 & 0.009 & 4.261 & 1.39 & 0.059 & 0.017982 & 0.006949 & 0.245443 & 110.155 \\
\hline 5/1/08 22:00 & 1.166 & 0.187 & 0.009 & 4.207 & 1.388 & 0.057 & 0.017373 & 0.006617 & 0.233715 & 104.891 \\
\hline 5/1/08 23:00 & 1.166 & 0.187 & 0.009 & 4.207 & 1.388 & 0.057 & 0.017373 & 0.006617 & 0.233715 & 104.891 \\
\hline 5/2/08 0:00 & 1.166 & 0.187 & 0.009 & 4.207 & 1.385 & 0.054 & 0.016458 & 0.006129 & 0.216486 & 97.159 \\
\hline 5/2/08 1:00 & 1.165 & 0.186 & 0.009 & 4.153 & 1.384 & 0.053 & 0.016154 & 0.005969 & 0.210842 & 94.626 \\
\hline $5 / 2 / 082: 00$ & 1.165 & 0.186 & 0.009 & 4.153 & 1.386 & 0.055 & 0.016763 & 0.006291 & 0.222180 & 99.714 \\
\hline 5/2/08 3:00 & 1.17 & 0.191 & 0.010 & 4.426 & 1.393 & 0.062 & 0.018897 & 0.007457 & 0.263390 & 118.209 \\
\hline 5/2/08 4:00 & 1.177 & 0.198 & 0.011 & 4.826 & 1.394 & 0.063 & 0.019201 & 0.007629 & 0.269465 & 120.936 \\
\hline 5/2/08 5:00 & 1.184 & 0.205 & 0.012 & 5.247 & 1.401 & 0.07 & 0.021335 & 0.008869 & 0.313263 & 140.593 \\
\hline 5/2/08 6:00 & 1.184 & 0.205 & 0.012 & 5.247 & 1.396 & 0.065 & 0.019811 & 0.007977 & 0.281754 & 126.451 \\
\hline 5/2/08 7:00 & 1.181 & 0.202 & 0.011 & 5.064 & 1.393 & 0.062 & 0.018897 & 0.007457 & 0.263390 & 118.209 \\
\hline 5/2/08 8:00 & 1.179 & 0.200 & 0.011 & 4.944 & 1.388 & 0.057 & 0.017373 & 0.006617 & 0.233715 & 104.891 \\
\hline 5/2/08 9:00 & 1.182 & 0.203 & 0.011 & 5.124 & 1.411 & 0.08 & 0.024383 & 0.010746 & 0.379531 & 170.333 \\
\hline 5/2/08 10:00 & 1.203 & 0.224 & 0.014 & 6.497 & 1.434 & 0.103 & 0.031393 & 0.015492 & 0.547171 & 245.570 \\
\hline 5/2/08 11:00 & 1.213 & 0.234 & 0.016 & 7.220 & 1.42 & 0.089 & 0.027126 & 0.012534 & 0.442691 & 198.680 \\
\hline 5/2/08 12:00 & 1.208 & 0.229 & 0.015 & 6.853 & 1.405 & 0.074 & 0.022554 & 0.009605 & 0.339261 & 152.260 \\
\hline 5/2/08 13:00 & 1.195 & 0.216 & 0.013 & 5.951 & 1.399 & 0.068 & 0.020725 & 0.008509 & 0.300526 & 134.876 \\
\hline 5/2/08 14:00 & 1.193 & 0.214 & 0.013 & 5.819 & 1.394 & 0.063 & 0.019201 & 0.007629 & 0.269465 & 120.936 \\
\hline 5/2/08 15:00 & 1.186 & 0.207 & 0.012 & 5.371 & 1.392 & 0.061 & 0.018592 & 0.007287 & 0.257360 & 115.503 \\
\hline 5/2/08 16:00 & 1.181 & 0.202 & 0.011 & 5.064 & 1.39 & 0.059 & 0.017982 & 0.006949 & 0.245443 & 110.155 \\
\hline 5/2/08 17:00 & 1.179 & 0.200 & 0.011 & 4.944 & 1.392 & 0.061 & 0.018592 & 0.007287 & 0.257360 & 115.503 \\
\hline 5/2/08 18:00 & 1.174 & 0.195 & 0.010 & 4.652 & 1.387 & 0.056 & 0.017068 & 0.006453 & 0.227923 & 102.292 \\
\hline 5/2/08 19:00 & 1.172 & 0.193 & 0.010 & 4.538 & 1.387 & 0.056 & 0.017068 & 0.006453 & 0.227923 & 102.292 \\
\hline 5/2/08 20:00 & 1.172 & 0.193 & 0.010 & 4.538 & 1.385 & 0.054 & 0.016458 & 0.006129 & 0.216486 & 97.159 \\
\hline 5/2/08 21:00 & 1.17 & 0.191 & 0.010 & 4.426 & 1.387 & 0.056 & 0.017068 & 0.006453 & 0.227923 & 102.292 \\
\hline
\end{tabular}




\begin{tabular}{|c|c|c|c|c|c|c|c|c|c|c|}
\hline \multirow[b]{3}{*}{ Date Time } & \multicolumn{4}{|c|}{ V-Notch Weir } & \multicolumn{6}{|c|}{ R-Notch Weir } \\
\hline & \multicolumn{2}{|c|}{ Measured Water Level } & \multicolumn{2}{|c|}{$\begin{array}{c}\text { Calculated Rate } \\
\left(\mathrm{ft}^{3} / \mathrm{s}=448.8 \mathrm{gpm}\right)\end{array}$} & \multicolumn{3}{|c|}{ Measured Water Level } & \multicolumn{3}{|c|}{$\begin{array}{c}\text { Calculated Rate } \\
\left(\mathrm{ft}^{3} / \mathrm{s}=448.8 \mathrm{gpm}\right)\end{array}$} \\
\hline & $\begin{array}{c}\text { (ft above } \\
\text { sensor) }\end{array}$ & $\begin{array}{c}\text { (ft above } \\
\text { notch) }\end{array}$ & $\left(\mathrm{ft}^{3} / \mathrm{s}\right)$ & (gpm) & $\begin{array}{c}\text { (ft above } \\
\text { sensor) }\end{array}$ & $\begin{array}{c}\text { (ft above } \\
\text { log) }\end{array}$ & $\begin{array}{c}\text { (m above } \\
\text { log) }\end{array}$ & $\left(\mathrm{m}^{3} / \mathrm{s}\right)$ & $\left(\mathrm{ft}^{3} / \mathrm{s}\right)$ & (gpm) \\
\hline 5/2/08 22:00 & 1.168 & 0.189 & 0.010 & 4.316 & 1.389 & 0.058 & 0.017678 & 0.006782 & 0.239555 & 107.512 \\
\hline 5/2/08 23:00 & 1.17 & 0.191 & 0.010 & 4.426 & 1.388 & 0.057 & 0.017373 & 0.006617 & 0.233715 & 104.891 \\
\hline 5/3/08 0:00 & 1.173 & 0.194 & 0.010 & 4.595 & 1.393 & 0.062 & 0.018897 & 0.007457 & 0.263390 & 118.209 \\
\hline 5/3/08 1:00 & 1.178 & 0.199 & 0.011 & 4.885 & 1.397 & 0.066 & 0.020116 & 0.008153 & 0.287966 & 129.239 \\
\hline 5/3/08 2:00 & 1.183 & 0.204 & 0.012 & 5.185 & 1.397 & 0.066 & 0.020116 & 0.008153 & 0.287966 & 129.239 \\
\hline 5/3/08 3:00 & 1.181 & 0.202 & 0.011 & 5.064 & 1.389 & 0.058 & 0.017678 & 0.006782 & 0.239555 & 107.512 \\
\hline 5/3/08 4:00 & 1.179 & 0.200 & 0.011 & 4.944 & 1.391 & 0.06 & 0.018287 & 0.007117 & 0.251378 & 112.818 \\
\hline 5/3/08 5:00 & 1.175 & 0.196 & 0.010 & 4.710 & 1.384 & 0.053 & 0.016154 & 0.005969 & 0.210842 & 94.626 \\
\hline 5/3/08 6:00 & 1.173 & 0.194 & 0.010 & 4.595 & 1.379 & 0.048 & 0.014630 & 0.005192 & 0.183377 & 82.300 \\
\hline 5/3/08 7:00 & 1.169 & 0.190 & 0.010 & 4.371 & 1.381 & 0.05 & 0.015239 & 0.005499 & 0.194210 & 87.161 \\
\hline 5/3/08 8:00 & 1.167 & 0.188 & 0.009 & 4.261 & 1.379 & 0.048 & 0.014630 & 0.005192 & 0.183377 & 82.300 \\
\hline 5/3/08 9:00 & 1.164 & 0.185 & 0.009 & 4.100 & 1.376 & 0.045 & 0.013715 & 0.004743 & 0.167523 & 75.184 \\
\hline 5/3/08 10:00 & 1.161 & 0.182 & 0.009 & 3.942 & 1.379 & 0.048 & 0.014630 & 0.005192 & 0.183377 & 82.300 \\
\hline 5/3/08 11:00 & 1.16 & 0.181 & 0.009 & 3.891 & 1.377 & 0.046 & 0.014020 & 0.004891 & 0.172754 & 77.532 \\
\hline 5/3/08 12:00 & 1.157 & 0.178 & 0.008 & 3.738 & 1.38 & 0.049 & 0.014934 & 0.005345 & 0.188768 & 84.719 \\
\hline 5/3/08 13:00 & 1.161 & 0.182 & 0.009 & 3.942 & 1.378 & 0.047 & 0.014325 & 0.005041 & 0.178040 & 79.904 \\
\hline 5/3/08 14:00 & 1.165 & 0.186 & 0.009 & 4.153 & 1.379 & 0.048 & 0.014630 & 0.005192 & 0.183377 & 82.300 \\
\hline 5/3/08 15:00 & 1.165 & 0.186 & 0.009 & 4.153 & 1.375 & 0.044 & 0.013411 & 0.004596 & 0.162345 & 72.860 \\
\hline 5/3/08 16:00 & 1.165 & 0.186 & 0.009 & 4.153 & 1.378 & 0.047 & 0.014325 & 0.005041 & 0.178040 & 79.904 \\
\hline 5/3/08 17:00 & 1.163 & 0.184 & 0.009 & 4.047 & 1.376 & 0.045 & 0.013715 & 0.004743 & 0.167523 & 75.184 \\
\hline 5/3/08 18:00 & 1.165 & 0.186 & 0.009 & 4.153 & 1.378 & 0.047 & 0.014325 & 0.005041 & 0.178040 & 79.904 \\
\hline 5/3/08 19:00 & 1.165 & 0.186 & 0.009 & 4.153 & 1.376 & 0.045 & 0.013715 & 0.004743 & 0.167523 & 75.184 \\
\hline 5/3/08 20:00 & 1.165 & 0.186 & 0.009 & 4.153 & 1.378 & 0.047 & 0.014325 & 0.005041 & 0.178040 & 79.904 \\
\hline 5/3/08 21:00 & 1.165 & 0.186 & 0.009 & 4.153 & 1.378 & 0.047 & 0.014325 & 0.005041 & 0.178040 & 79.904 \\
\hline 5/3/08 22:00 & 1.163 & 0.184 & 0.009 & 4.047 & 1.378 & 0.047 & 0.014325 & 0.005041 & 0.178040 & 79.904 \\
\hline
\end{tabular}




\begin{tabular}{|c|c|c|c|c|c|c|c|c|c|c|}
\hline \multirow[b]{3}{*}{ Date Time } & \multicolumn{4}{|c|}{ V-Notch Weir } & \multicolumn{6}{|c|}{ R-Notch Weir } \\
\hline & \multicolumn{2}{|c|}{ Measured Water Level } & \multicolumn{2}{|c|}{$\begin{array}{c}\text { Calculated Rate } \\
\left(\mathrm{ft}^{3} / \mathrm{s}=448.8 \mathrm{gpm}\right)\end{array}$} & \multicolumn{3}{|c|}{ Measured Water Level } & \multicolumn{3}{|c|}{$\begin{array}{c}\text { Calculated Rate } \\
\left(\mathrm{ft}^{3} / \mathrm{s}=448.8 \mathrm{gpm}\right)\end{array}$} \\
\hline & $\begin{array}{c}\text { (ft above } \\
\text { sensor) }\end{array}$ & $\begin{array}{c}\text { (ft above } \\
\text { notch) }\end{array}$ & $\left(\mathrm{ft}^{3} / \mathrm{s}\right)$ & (gpm) & $\begin{array}{c}\text { (ft above } \\
\text { sensor) }\end{array}$ & $\begin{array}{c}\text { (ft above } \\
\text { log) }\end{array}$ & $\begin{array}{c}\text { (m above } \\
\text { log) }\end{array}$ & $\left(\mathrm{m}^{3} / \mathrm{s}\right)$ & $\left(\mathrm{ft}^{3} / \mathrm{s}\right)$ & (gpm) \\
\hline 5/3/08 23:00 & 1.16 & 0.181 & 0.009 & 3.891 & 1.378 & 0.047 & 0.014325 & 0.005041 & 0.178040 & 79.904 \\
\hline 5/4/08 0:00 & 1.16 & 0.181 & 0.009 & 3.891 & 1.378 & 0.047 & 0.014325 & 0.005041 & 0.178040 & 79.904 \\
\hline 5/4/08 1:00 & 1.16 & 0.181 & 0.009 & 3.891 & 1.377 & 0.046 & 0.014020 & 0.004891 & 0.172754 & 77.532 \\
\hline 5/4/08 2:00 & 1.16 & 0.181 & 0.009 & 3.891 & 1.377 & 0.046 & 0.014020 & 0.004891 & 0.172754 & 77.532 \\
\hline 5/4/08 3:00 & 1.161 & 0.182 & 0.009 & 3.942 & 1.377 & 0.046 & 0.014020 & 0.004891 & 0.172754 & 77.532 \\
\hline 5/4/08 4:00 & 1.158 & 0.179 & 0.008 & 3.788 & 1.379 & 0.048 & 0.014630 & 0.005192 & 0.183377 & 82.300 \\
\hline 5/4/08 5:00 & 1.161 & 0.182 & 0.009 & 3.942 & 1.378 & 0.047 & 0.014325 & 0.005041 & 0.178040 & 79.904 \\
\hline 5/4/08 6:00 & 1.162 & 0.183 & 0.009 & 3.994 & 1.376 & 0.045 & 0.013715 & 0.004743 & 0.167523 & 75.184 \\
\hline 5/4/08 7:00 & 1.162 & 0.183 & 0.009 & 3.994 & 1.378 & 0.047 & 0.014325 & 0.005041 & 0.178040 & 79.904 \\
\hline 5/4/08 8:00 & 1.16 & 0.181 & 0.009 & 3.891 & 1.377 & 0.046 & 0.014020 & 0.004891 & 0.172754 & 77.532 \\
\hline 5/4/08 9:00 & 1.16 & 0.181 & 0.009 & 3.891 & 1.377 & 0.046 & 0.014020 & 0.004891 & 0.172754 & 77.532 \\
\hline 5/4/08 10:00 & 1.162 & 0.183 & 0.009 & 3.994 & 1.375 & 0.044 & 0.013411 & 0.004596 & 0.162345 & 72.860 \\
\hline 5/4/08 11:00 & 1.161 & 0.182 & 0.009 & 3.942 & 1.376 & 0.045 & 0.013715 & 0.004743 & 0.167523 & 75.184 \\
\hline 5/4/08 12:00 & 1.16 & 0.181 & 0.009 & 3.891 & 1.376 & 0.045 & 0.013715 & 0.004743 & 0.167523 & 75.184 \\
\hline 5/4/08 13:00 & 1.162 & 0.183 & 0.009 & 3.994 & 1.375 & 0.044 & 0.013411 & 0.004596 & 0.162345 & 72.860 \\
\hline 5/4/08 14:00 & 1.163 & 0.184 & 0.009 & 4.047 & 1.376 & 0.045 & 0.013715 & 0.004743 & 0.167523 & 75.184 \\
\hline 5/4/08 15:00 & 1.166 & 0.187 & 0.009 & 4.207 & 1.375 & 0.044 & 0.013411 & 0.004596 & 0.162345 & 72.860 \\
\hline 5/4/08 16:00 & 1.169 & 0.190 & 0.010 & 4.371 & 1.378 & 0.047 & 0.014325 & 0.005041 & 0.178040 & 79.904 \\
\hline 5/4/08 17:00 & 1.169 & 0.190 & 0.010 & 4.371 & 1.379 & 0.048 & 0.014630 & 0.005192 & 0.183377 & 82.300 \\
\hline 5/4/08 18:00 & 1.169 & 0.190 & 0.010 & 4.371 & 1.379 & 0.048 & 0.014630 & 0.005192 & 0.183377 & 82.300 \\
\hline 5/4/08 19:00 & 1.169 & 0.190 & 0.010 & 4.371 & 1.379 & 0.048 & 0.014630 & 0.005192 & 0.183377 & 82.300 \\
\hline 5/4/08 20:00 & 1.164 & 0.185 & 0.009 & 4.100 & 1.382 & 0.051 & 0.015544 & 0.005654 & 0.199703 & 89.627 \\
\hline 5/4/08 21:00 & 1.161 & 0.182 & 0.009 & 3.942 & 1.382 & 0.051 & 0.015544 & 0.005654 & 0.199703 & 89.627 \\
\hline 5/4/08 22:00 & 1.156 & 0.177 & 0.008 & 3.688 & 1.379 & 0.048 & 0.014630 & 0.005192 & 0.183377 & 82.300 \\
\hline 5/4/08 23:00 & 1.156 & 0.177 & 0.008 & 3.688 & 1.379 & 0.048 & 0.014630 & 0.005192 & 0.183377 & 82.300 \\
\hline
\end{tabular}




\begin{tabular}{|c|c|c|c|c|c|c|c|c|c|c|}
\hline \multirow[b]{3}{*}{ Date Time } & \multicolumn{4}{|c|}{ V-Notch Weir } & \multicolumn{6}{|c|}{ R-Notch Weir } \\
\hline & \multicolumn{2}{|c|}{ Measured Water Level } & \multicolumn{2}{|c|}{$\begin{array}{c}\text { Calculated Rate } \\
\left(\mathrm{ft}^{3} / \mathrm{s}=448.8 \mathrm{gpm}\right)\end{array}$} & \multicolumn{3}{|c|}{ Measured Water Level } & \multicolumn{3}{|c|}{$\begin{array}{c}\text { Calculated Rate } \\
\left(\mathrm{ft}^{3} / \mathrm{s}=448.8 \mathrm{gpm}\right)\end{array}$} \\
\hline & $\begin{array}{l}\text { (ft above } \\
\text { sensor) }\end{array}$ & $\begin{array}{c}\text { (ft above } \\
\text { notch) }\end{array}$ & $\left(\mathrm{ft}^{3} / \mathrm{s}\right)$ & (gpm) & $\begin{array}{c}\text { (ft above } \\
\text { sensor) }\end{array}$ & $\begin{array}{c}\text { (ft above } \\
\text { log) }\end{array}$ & $\begin{array}{c}\text { (m above } \\
\text { log) }\end{array}$ & $\left(\mathrm{m}^{3} / \mathrm{s}\right)$ & $\left(\mathrm{ft}^{3} / \mathrm{s}\right)$ & (gpm) \\
\hline 5/5/08 0:00 & 1.153 & 0.174 & 0.008 & 3.540 & 1.378 & 0.047 & 0.014325 & 0.005041 & 0.178040 & 79.904 \\
\hline 5/5/08 1:00 & 1.153 & 0.174 & 0.008 & 3.540 & 1.38 & 0.049 & 0.014934 & 0.005345 & 0.188768 & 84.719 \\
\hline 5/5/08 2:00 & 1.155 & 0.176 & 0.008 & 3.638 & 1.38 & 0.049 & 0.014934 & 0.005345 & 0.188768 & 84.719 \\
\hline 5/5/08 3:00 & 1.153 & 0.174 & 0.008 & 3.540 & 1.38 & 0.049 & 0.014934 & 0.005345 & 0.188768 & 84.719 \\
\hline 5/5/08 4:00 & 1.155 & 0.176 & 0.008 & 3.638 & 1.379 & 0.048 & 0.014630 & 0.005192 & 0.183377 & 82.300 \\
\hline 5/5/08 5:00 & 1.153 & 0.174 & 0.008 & 3.540 & 1.379 & 0.048 & 0.014630 & 0.005192 & 0.183377 & 82.300 \\
\hline 5/5/08 6:00 & 1.153 & 0.174 & 0.008 & 3.540 & 1.381 & 0.05 & 0.015239 & 0.005499 & 0.194210 & 87.161 \\
\hline 5/5/08 7:00 & 1.153 & 0.174 & 0.008 & 3.540 & 1.378 & 0.047 & 0.014325 & 0.005041 & 0.178040 & 79.904 \\
\hline 5/5/08 8:00 & 1.156 & 0.177 & 0.008 & 3.688 & 1.377 & 0.046 & 0.014020 & 0.004891 & 0.172754 & 77.532 \\
\hline 5/5/08 9:00 & 1.158 & 0.179 & 0.008 & 3.788 & 1.377 & 0.046 & 0.014020 & 0.004891 & 0.172754 & 77.532 \\
\hline 5/5/08 10:00 & 1.158 & 0.179 & 0.008 & 3.788 & 1.378 & 0.047 & 0.014325 & 0.005041 & 0.178040 & 79.904 \\
\hline 5/5/08 11:00 & 1.158 & 0.179 & 0.008 & 3.788 & 1.376 & 0.045 & 0.013715 & 0.004743 & 0.167523 & 75.184 \\
\hline 5/5/08 12:00 & 1.16 & 0.181 & 0.009 & 3.891 & 1.376 & 0.045 & 0.013715 & 0.004743 & 0.167523 & 75.184 \\
\hline 5/5/08 13:00 & 1.163 & 0.184 & 0.009 & 4.047 & 1.375 & 0.044 & 0.013411 & 0.004596 & 0.162345 & 72.860 \\
\hline 5/5/08 14:00 & 1.161 & 0.182 & 0.009 & 3.942 & 1.376 & 0.045 & 0.013715 & 0.004743 & 0.167523 & 75.184 \\
\hline 5/5/08 15:00 & 1.16 & 0.181 & 0.009 & 3.891 & 1.377 & 0.046 & 0.014020 & 0.004891 & 0.172754 & 77.532 \\
\hline 5/5/08 16:00 & 1.157 & 0.178 & 0.008 & 3.738 & 1.383 & 0.052 & 0.015849 & 0.005811 & 0.205247 & 92.115 \\
\hline 5/5/08 17:00 & 1.152 & 0.173 & 0.008 & 3.492 & 1.384 & 0.053 & 0.016154 & 0.005969 & 0.210842 & 94.626 \\
\hline 5/5/08 18:00 & 1.148 & 0.169 & 0.007 & 3.302 & 1.385 & 0.054 & 0.016458 & 0.006129 & 0.216486 & 97.159 \\
\hline 5/5/08 19:00 & 1.145 & 0.166 & 0.007 & 3.164 & 1.385 & 0.054 & 0.016458 & 0.006129 & 0.216486 & 97.159 \\
\hline 5/5/08 20:00 & 1.147 & 0.168 & 0.007 & 3.255 & 1.384 & 0.053 & 0.016154 & 0.005969 & 0.210842 & 94.626 \\
\hline 5/5/08 21:00 & 1.151 & 0.172 & 0.008 & 3.444 & 1.387 & 0.056 & 0.017068 & 0.006453 & 0.227923 & 102.292 \\
\hline 5/5/08 22:00 & 1.153 & 0.174 & 0.008 & 3.540 & 1.386 & 0.055 & 0.016763 & 0.006291 & 0.222180 & 99.714 \\
\hline 5/5/08 23:00 & 1.154 & 0.175 & 0.008 & 3.589 & 1.381 & 0.05 & 0.015239 & 0.005499 & 0.194210 & 87.161 \\
\hline 5/6/08 0:00 & 1.159 & 0.180 & 0.009 & 3.839 & 1.383 & 0.052 & 0.015849 & 0.005811 & 0.205247 & 92.115 \\
\hline
\end{tabular}




\begin{tabular}{|c|c|c|c|c|c|c|c|c|c|c|}
\hline \multirow[b]{3}{*}{ Date Time } & \multicolumn{4}{|c|}{ V-Notch Weir } & \multicolumn{6}{|c|}{ R-Notch Weir } \\
\hline & \multicolumn{2}{|c|}{ Measured Water Level } & \multicolumn{2}{|c|}{$\begin{array}{c}\text { Calculated Rate } \\
\left(\mathrm{ft}^{3} / \mathrm{s}=448.8 \mathrm{gpm}\right)\end{array}$} & \multicolumn{3}{|c|}{ Measured Water Level } & \multicolumn{3}{|c|}{$\begin{array}{c}\begin{array}{c}\text { Calculated Rate } \\
\left(\mathrm{ft}^{3} / \mathrm{s}=448.8 \mathrm{gpm}\right)\end{array} \\
\end{array}$} \\
\hline & $\begin{array}{l}\text { (ft above } \\
\text { sensor) }\end{array}$ & $\begin{array}{c}\text { (ft above } \\
\text { notch) }\end{array}$ & $\left(\mathrm{ft}^{3} / \mathrm{s}\right)$ & (gpm) & $\begin{array}{l}\text { (ft above } \\
\text { sensor) }\end{array}$ & $\begin{array}{c}\text { (ft above } \\
\text { log) }\end{array}$ & $\begin{array}{c}\text { (m above } \\
\text { log) }\end{array}$ & $\left(\mathrm{m}^{3} / \mathrm{s}\right)$ & $\left(\mathrm{ft}^{3} / \mathrm{s}\right)$ & (gpm) \\
\hline 5/6/08 1:00 & 1.161 & 0.182 & 0.009 & 3.942 & 1.383 & 0.052 & 0.015849 & 0.005811 & 0.205247 & 92.115 \\
\hline 5/6/08 2:00 & 1.163 & 0.184 & 0.009 & 4.047 & 1.382 & 0.051 & 0.015544 & 0.005654 & 0.199703 & 89.627 \\
\hline 5/6/08 3:00 & 1.165 & 0.186 & 0.009 & 4.153 & 1.382 & 0.051 & 0.015544 & 0.005654 & 0.199703 & 89.627 \\
\hline 5/6/08 4:00 & 1.165 & 0.186 & 0.009 & 4.153 & 1.381 & 0.05 & 0.015239 & 0.005499 & 0.194210 & 87.161 \\
\hline 5/6/08 5:00 & 1.17 & 0.191 & 0.010 & 4.426 & 1.383 & 0.052 & 0.015849 & 0.005811 & 0.205247 & 92.115 \\
\hline 5/6/08 6:00 & 1.167 & 0.188 & 0.009 & 4.261 & 1.383 & 0.052 & 0.015849 & 0.005811 & 0.205247 & 92.115 \\
\hline 5/6/08 7:00 & 1.167 & 0.188 & 0.009 & 4.261 & 1.382 & 0.051 & 0.015544 & 0.005654 & 0.199703 & 89.627 \\
\hline 5/6/08 8:00 & 1.167 & 0.188 & 0.009 & 4.261 & 1.382 & 0.051 & 0.015544 & 0.005654 & 0.199703 & 89.627 \\
\hline 5/6/08 9:00 & 1.17 & 0.191 & 0.010 & 4.426 & 1.382 & 0.051 & 0.015544 & 0.005654 & 0.199703 & 89.627 \\
\hline 5/6/08 10:00 & 1.172 & 0.193 & 0.010 & 4.538 & 1.38 & 0.049 & 0.014934 & 0.005345 & 0.188768 & 84.719 \\
\hline 5/6/08 11:00 & 1.172 & 0.193 & 0.010 & 4.538 & 1.38 & 0.049 & 0.014934 & 0.005345 & 0.188768 & 84.719 \\
\hline 5/6/08 12:00 & 1.172 & 0.193 & 0.010 & 4.538 & 1.379 & 0.048 & 0.014630 & 0.005192 & 0.183377 & 82.300 \\
\hline 5/6/08 13:00 & 1.176 & 0.197 & 0.011 & 4.768 & 1.382 & 0.051 & 0.015544 & 0.005654 & 0.199703 & 89.627 \\
\hline 5/6/08 14:00 & 1.179 & 0.200 & 0.011 & 4.944 & 1.384 & 0.053 & 0.016154 & 0.005969 & 0.210842 & 94.626 \\
\hline 5/6/08 15:00 & 1.18 & 0.201 & 0.011 & 5.004 & 1.385 & 0.054 & 0.016458 & 0.006129 & 0.216486 & 97.159 \\
\hline 5/6/08 16:00 & 1.179 & 0.200 & 0.011 & 4.944 & 1.384 & 0.053 & 0.016154 & 0.005969 & 0.210842 & 94.626 \\
\hline 5/6/08 17:00 & 1.18 & 0.201 & 0.011 & 5.004 & 1.388 & 0.057 & 0.017373 & 0.006617 & 0.233715 & 104.891 \\
\hline 5/6/08 18:00 & 1.18 & 0.201 & 0.011 & 5.004 & 1.386 & 0.055 & 0.016763 & 0.006291 & 0.222180 & 99.714 \\
\hline 5/6/08 19:00 & 1.182 & 0.203 & 0.011 & 5.124 & 1.39 & 0.059 & 0.017982 & 0.006949 & 0.245443 & 110.155 \\
\hline 5/6/08 20:00 & 1.181 & 0.202 & 0.011 & 5.064 & 1.388 & 0.057 & 0.017373 & 0.006617 & 0.233715 & 104.891 \\
\hline 5/6/08 21:00 & 1.178 & 0.199 & 0.011 & 4.885 & 1.387 & 0.056 & 0.017068 & 0.006453 & 0.227923 & 102.292 \\
\hline 5/6/08 22:00 & 1.177 & 0.198 & 0.011 & 4.826 & 1.386 & 0.055 & 0.016763 & 0.006291 & 0.222180 & 99.714 \\
\hline 5/6/08 23:00 & 1.183 & 0.204 & 0.012 & 5.185 & 1.405 & 0.074 & 0.022554 & 0.009605 & 0.339261 & 152.260 \\
\hline 5/7/08 0:00 & 1.204 & 0.225 & 0.015 & 6.567 & 1.411 & 0.08 & 0.024383 & 0.010746 & 0.379531 & 170.333 \\
\hline 5/7/08 1:00 & 1.213 & 0.234 & 0.016 & 7.220 & 1.42 & 0.089 & 0.027126 & 0.012534 & 0.442691 & 198.680 \\
\hline
\end{tabular}




\begin{tabular}{|c|c|c|c|c|c|c|c|c|c|c|}
\hline \multirow[b]{3}{*}{ Date Time } & \multicolumn{4}{|c|}{ V-Notch Weir } & \multicolumn{6}{|c|}{ R-Notch Weir } \\
\hline & \multicolumn{2}{|c|}{ Measured Water Level } & \multicolumn{2}{|c|}{$\begin{array}{c}\text { Calculated Rate } \\
\left(\mathrm{ft}^{3} / \mathrm{s}=448.8 \mathrm{gpm}\right)\end{array}$} & \multicolumn{3}{|c|}{ Measured Water Level } & \multicolumn{3}{|c|}{$\begin{array}{c}\text { Calculated Rate } \\
\left(\mathrm{ft}^{3} / \mathrm{s}=448.8 \mathrm{gpm}\right)\end{array}$} \\
\hline & $\begin{array}{l}\text { (ft above } \\
\text { sensor) }\end{array}$ & $\begin{array}{l}\text { (ft above } \\
\text { notch) }\end{array}$ & $\left(\mathrm{ft}^{3} / \mathrm{s}\right)$ & (gpm) & $\begin{array}{l}\text { (ft above } \\
\text { sensor) }\end{array}$ & $\begin{array}{l}\text { (ft above } \\
\text { log) }\end{array}$ & $\begin{array}{c}\text { (m above } \\
\text { log) }\end{array}$ & $\left(\mathrm{m}^{3} / \mathrm{s}\right)$ & $\left(\mathrm{ft}^{3} / \mathrm{s}\right)$ & (gpm) \\
\hline 5/7/08 2:00 & 1.222 & 0.243 & 0.018 & 7.910 & 1.415 & 0.084 & 0.025602 & 0.011529 & 0.407202 & 182.752 \\
\hline 5/7/08 3:00 & 1.222 & 0.243 & 0.018 & 7.910 & 1.407 & 0.076 & 0.023164 & 0.009981 & 0.352516 & 158.209 \\
\hline 5/7/08 4:00 & 1.217 & 0.238 & 0.017 & 7.522 & 1.396 & 0.065 & 0.019811 & 0.007977 & 0.281754 & 126.451 \\
\hline 5/7/08 5:00 & 1.209 & 0.230 & 0.015 & 6.925 & 1.391 & 0.06 & 0.018287 & 0.007117 & 0.251378 & 112.818 \\
\hline 5/7/08 6:00 & 1.202 & 0.223 & 0.014 & 6.427 & 1.39 & 0.059 & 0.017982 & 0.006949 & 0.245443 & 110.155 \\
\hline 5/7/08 7:00 & 1.2 & 0.221 & 0.014 & 6.289 & 1.388 & 0.057 & 0.017373 & 0.006617 & 0.233715 & 104.891 \\
\hline 5/7/08 8:00 & 1.195 & 0.216 & 0.013 & 5.951 & 1.385 & 0.054 & 0.016458 & 0.006129 & 0.216486 & 97.159 \\
\hline 5/7/08 9:00 & 1.195 & 0.216 & 0.013 & 5.951 & 1.385 & 0.054 & 0.016458 & 0.006129 & 0.216486 & 97.159 \\
\hline 5/7/08 10:00 & 1.193 & 0.214 & 0.013 & 5.819 & 1.385 & 0.054 & 0.016458 & 0.006129 & 0.216486 & 97.159 \\
\hline 5/7/08 11:00 & 1.193 & 0.214 & 0.013 & 5.819 & 1.385 & 0.054 & 0.016458 & 0.006129 & 0.216486 & 97.159 \\
\hline 5/7/08 12:00 & 1.195 & 0.216 & 0.013 & 5.951 & 1.385 & 0.054 & 0.016458 & 0.006129 & 0.216486 & 97.159 \\
\hline 5/7/08 13:00 & 1.193 & 0.214 & 0.013 & 5.819 & 1.383 & 0.052 & 0.015849 & 0.005811 & 0.205247 & 92.115 \\
\hline 5/7/08 14:00 & 1.193 & 0.214 & 0.013 & 5.819 & 1.383 & 0.052 & 0.015849 & 0.005811 & 0.205247 & 92.115 \\
\hline 5/7/08 15:00 & 1.193 & 0.214 & 0.013 & 5.819 & 1.381 & 0.05 & 0.015239 & 0.005499 & 0.194210 & 87.161 \\
\hline 5/7/08 16:00 & 1.193 & 0.214 & 0.013 & 5.819 & 1.383 & 0.052 & 0.015849 & 0.005811 & 0.205247 & 92.115 \\
\hline 5/7/08 17:00 & 1.196 & 0.217 & 0.013 & 6.017 & 1.381 & 0.05 & 0.015239 & 0.005499 & 0.194210 & 87.161 \\
\hline 5/7/08 18:00 & 1.198 & 0.219 & 0.014 & 6.152 & 1.384 & 0.053 & 0.016154 & 0.005969 & 0.210842 & 94.626 \\
\hline 5/7/08 19:00 & 1.196 & 0.217 & 0.013 & 6.017 & 1.382 & 0.051 & 0.015544 & 0.005654 & 0.199703 & 89.627 \\
\hline 5/7/08 20:00 & 1.196 & 0.217 & 0.013 & 6.017 & 1.382 & 0.051 & 0.015544 & 0.005654 & 0.199703 & 89.627 \\
\hline 5/7/08 21:00 & 1.196 & 0.217 & 0.013 & 6.017 & 1.381 & 0.05 & 0.015239 & 0.005499 & 0.194210 & 87.161 \\
\hline 5/7/08 22:00 & 1.193 & 0.214 & 0.013 & 5.819 & 1.381 & 0.05 & 0.015239 & 0.005499 & 0.194210 & 87.161 \\
\hline 5/7/08 23:00 & 1.191 & 0.212 & 0.013 & 5.689 & 1.381 & 0.05 & 0.015239 & 0.005499 & 0.194210 & 87.161 \\
\hline 5/8/08 0:00 & 1.188 & 0.209 & 0.012 & 5.497 & 1.381 & 0.05 & 0.015239 & 0.005499 & 0.194210 & 87.161 \\
\hline 5/8/08 1:00 & 1.188 & 0.209 & 0.012 & 5.497 & 1.38 & 0.049 & 0.014934 & 0.005345 & 0.188768 & 84.719 \\
\hline 5/8/08 2:00 & 1.186 & 0.207 & 0.012 & 5.371 & 1.38 & 0.049 & 0.014934 & 0.005345 & 0.188768 & 84.719 \\
\hline
\end{tabular}




\begin{tabular}{|c|c|c|c|c|c|c|c|c|c|c|}
\hline \multirow[b]{3}{*}{ Date Time } & \multicolumn{4}{|c|}{ V-Notch Weir } & \multicolumn{6}{|c|}{ R-Notch Weir } \\
\hline & \multicolumn{2}{|c|}{ Measured Water Level } & \multicolumn{2}{|c|}{$\begin{array}{c}\text { Calculated Rate } \\
\left(\mathrm{ft}^{3} / \mathrm{s}=448.8 \mathrm{gpm}\right)\end{array}$} & \multicolumn{3}{|c|}{ Measured Water Level } & \multicolumn{3}{|c|}{$\begin{array}{c}\text { Calculated Rate } \\
\left(\mathrm{ft}^{3} / \mathrm{s}=448.8 \mathrm{gpm}\right)\end{array}$} \\
\hline & $\begin{array}{c}\text { (ft above } \\
\text { sensor) }\end{array}$ & $\begin{array}{c}\text { (ft above } \\
\text { notch) }\end{array}$ & $\left(\mathrm{ft}^{3} / \mathrm{s}\right)$ & (gpm) & $\begin{array}{c}\text { (ft above } \\
\text { sensor) }\end{array}$ & $\begin{array}{c}\text { (ft above } \\
\text { log) }\end{array}$ & $\begin{array}{c}\text { (m above } \\
\text { log) }\end{array}$ & $\left(\mathrm{m}^{3} / \mathrm{s}\right)$ & $\left(\mathrm{ft}^{3} / \mathrm{s}\right)$ & (gpm) \\
\hline 5/8/08 3:00 & 1.188 & 0.209 & 0.012 & 5.497 & 1.379 & 0.048 & 0.014630 & 0.005192 & 0.183377 & 82.300 \\
\hline 5/8/08 4:00 & 1.186 & 0.207 & 0.012 & 5.371 & 1.379 & 0.048 & 0.014630 & 0.005192 & 0.183377 & 82.300 \\
\hline 5/8/08 5:00 & 1.186 & 0.207 & 0.012 & 5.371 & 1.378 & 0.047 & 0.014325 & 0.005041 & 0.178040 & 79.904 \\
\hline 5/8/08 6:00 & 1.186 & 0.207 & 0.012 & 5.371 & 1.38 & 0.049 & 0.014934 & 0.005345 & 0.188768 & 84.719 \\
\hline 5/8/08 7:00 & 1.187 & 0.208 & 0.012 & 5.433 & 1.377 & 0.046 & 0.014020 & 0.004891 & 0.172754 & 77.532 \\
\hline 5/8/08 8:00 & 1.187 & 0.208 & 0.012 & 5.433 & 1.379 & 0.048 & 0.014630 & 0.005192 & 0.183377 & 82.300 \\
\hline 5/8/08 9:00 & 1.187 & 0.208 & 0.012 & 5.433 & 1.379 & 0.048 & 0.014630 & 0.005192 & 0.183377 & 82.300 \\
\hline 5/8/08 10:00 & 1.189 & 0.210 & 0.012 & 5.560 & 1.391 & 0.06 & 0.018287 & 0.007117 & 0.251378 & 112.818 \\
\hline 5/8/08 11:00 & 1.193 & 0.214 & 0.013 & 5.819 & 1.38 & 0.049 & 0.014934 & 0.005345 & 0.188768 & 84.719 \\
\hline 5/8/08 12:00 & 1.198 & 0.219 & 0.014 & 6.152 & 1.379 & 0.048 & 0.014630 & 0.005192 & 0.183377 & 82.300 \\
\hline 5/8/08 13:00 & 1.198 & 0.219 & 0.014 & 6.152 & 1.375 & 0.044 & 0.013411 & 0.004596 & 0.162345 & 72.860 \\
\hline 5/8/08 14:00 & 1.201 & 0.222 & 0.014 & 6.358 & 1.374 & 0.043 & 0.013106 & 0.004451 & 0.157222 & 70.561 \\
\hline 5/8/08 15:00 & 1.204 & 0.225 & 0.015 & 6.567 & 1.382 & 0.051 & 0.015544 & 0.005654 & 0.199703 & 89.627 \\
\hline 5/8/08 16:00 & 1.207 & 0.228 & 0.015 & 6.780 & 1.378 & 0.047 & 0.014325 & 0.005041 & 0.178040 & 79.904 \\
\hline 5/8/08 17:00 & 1.208 & 0.229 & 0.015 & 6.853 & 1.379 & 0.048 & 0.014630 & 0.005192 & 0.183377 & 82.300 \\
\hline 5/8/08 18:00 & 1.208 & 0.229 & 0.015 & 6.853 & 1.379 & 0.048 & 0.014630 & 0.005192 & 0.183377 & 82.300 \\
\hline 5/8/08 19:00 & 1.207 & 0.228 & 0.015 & 6.780 & 1.382 & 0.051 & 0.015544 & 0.005654 & 0.199703 & 89.627 \\
\hline 5/8/08 20:00 & 1.207 & 0.228 & 0.015 & 6.780 & 1.381 & 0.05 & 0.015239 & 0.005499 & 0.194210 & 87.161 \\
\hline 5/8/08 21:00 & 1.206 & 0.227 & 0.015 & 6.709 & 1.381 & 0.05 & 0.015239 & 0.005499 & 0.194210 & 87.161 \\
\hline 5/8/08 22:00 & 1.206 & 0.227 & 0.015 & 6.709 & 1.381 & 0.05 & 0.015239 & 0.005499 & 0.194210 & 87.161 \\
\hline 5/8/08 23:00 & 1.208 & 0.229 & 0.015 & 6.853 & 1.383 & 0.052 & 0.015849 & 0.005811 & 0.205247 & 92.115 \\
\hline 5/9/08 0:00 & 1.208 & 0.229 & 0.015 & 6.853 & 1.383 & 0.052 & 0.015849 & 0.005811 & 0.205247 & 92.115 \\
\hline 5/9/08 1:00 & 1.21 & 0.231 & 0.016 & 6.998 & 1.385 & 0.054 & 0.016458 & 0.006129 & 0.216486 & 97.159 \\
\hline 5/9/08 2:00 & 1.21 & 0.231 & 0.016 & 6.998 & 1.392 & 0.061 & 0.018592 & 0.007287 & 0.257360 & 115.503 \\
\hline 5/9/08 3:00 & 1.217 & 0.238 & 0.017 & 7.522 & 1.431 & 0.1 & 0.030479 & 0.014841 & 0.524166 & 235.246 \\
\hline
\end{tabular}




\begin{tabular}{|c|c|c|c|c|c|c|c|c|c|c|}
\hline \multirow[b]{3}{*}{ Date Time } & \multicolumn{4}{|c|}{ V-Notch Weir } & \multicolumn{6}{|c|}{ R-Notch Weir } \\
\hline & \multicolumn{2}{|c|}{ Measured Water Level } & \multicolumn{2}{|c|}{$\begin{array}{c}\text { Calculated Rate } \\
\left(\mathrm{ft}^{3} / \mathrm{s}=448.8 \mathrm{gpm}\right)\end{array}$} & \multicolumn{3}{|c|}{ Measured Water Level } & \multicolumn{3}{|c|}{$\begin{array}{c}\begin{array}{c}\text { Calculated Rate } \\
\left(\mathrm{ft}^{3} / \mathrm{s}=448.8 \mathrm{gpm}\right)\end{array} \\
\end{array}$} \\
\hline & $\begin{array}{l}\text { (ft above } \\
\text { sensor) }\end{array}$ & $\begin{array}{c}\text { (ft above } \\
\text { notch) }\end{array}$ & $\left(\mathrm{ft}^{3} / \mathrm{s}\right)$ & (gpm) & $\begin{array}{c}\text { (ft above } \\
\text { sensor) }\end{array}$ & $\begin{array}{c}\text { (ft above } \\
\text { log) }\end{array}$ & $\begin{array}{c}\text { (m above } \\
\text { log) }\end{array}$ & $\left(\mathrm{m}^{3} / \mathrm{s}\right)$ & $\left(\mathrm{ft}^{3} / \mathrm{s}\right)$ & (gpm) \\
\hline 5/9/08 4:00 & 1.242 & 0.263 & 0.021 & 9.582 & 1.472 & 0.141 & 0.042975 & 0.024505 & 0.865512 & 388.442 \\
\hline 5/9/08 5:00 & 1.275 & 0.296 & 0.028 & 12.772 & 1.481 & 0.15 & 0.045718 & 0.026834 & 0.947757 & 425.353 \\
\hline 5/9/08 6:00 & 1.285 & 0.306 & 0.031 & 13.849 & 1.454 & 0.123 & 0.037489 & 0.020065 & 0.708692 & 318.061 \\
\hline 5/9/08 7:00 & 1.254 & 0.275 & 0.024 & 10.679 & 1.428 & 0.097 & 0.029564 & 0.014199 & 0.501493 & 225.070 \\
\hline 5/9/08 8:00 & 1.224 & 0.245 & 0.018 & 8.069 & 1.411 & 0.08 & 0.024383 & 0.010746 & 0.379531 & 170.333 \\
\hline 5/9/08 9:00 & 1.205 & 0.226 & 0.015 & 6.638 & 1.404 & 0.073 & 0.022249 & 0.009420 & 0.332697 & 149.314 \\
\hline 5/9/08 10:00 & 1.191 & 0.212 & 0.013 & 5.689 & 1.399 & 0.068 & 0.020725 & 0.008509 & 0.300526 & 134.876 \\
\hline 5/9/08 11:00 & 1.186 & 0.207 & 0.012 & 5.371 & 1.397 & 0.066 & 0.020116 & 0.008153 & 0.287966 & 129.239 \\
\hline 5/9/08 12:00 & 1.184 & 0.205 & 0.012 & 5.247 & 1.395 & 0.064 & 0.019506 & 0.007803 & 0.275587 & 123.683 \\
\hline 5/9/08 13:00 & 1.191 & 0.212 & 0.013 & 5.689 & 1.393 & 0.062 & 0.018897 & 0.007457 & 0.263390 & 118.209 \\
\hline 5/9/08 14:00 & 1.2 & 0.221 & 0.014 & 6.289 & 1.393 & 0.062 & 0.018897 & 0.007457 & 0.263390 & 118.209 \\
\hline 5/9/08 15:00 & 1.262 & 0.283 & 0.026 & 11.450 & 1.392 & 0.061 & 0.018592 & 0.007287 & 0.257360 & 115.503 \\
\hline 5/9/08 16:00 & 1.285 & 0.306 & 0.031 & 13.849 & 1.392 & 0.061 & 0.018592 & 0.007287 & 0.257360 & 115.503 \\
\hline 5/9/08 17:00 & 1.267 & 0.288 & 0.027 & 11.948 & 1.39 & 0.059 & 0.017982 & 0.006949 & 0.245443 & 110.155 \\
\hline 5/9/08 18:00 & 1.262 & 0.283 & 0.026 & 11.450 & 1.391 & 0.06 & 0.018287 & 0.007117 & 0.251378 & 112.818 \\
\hline 5/9/08 19:00 & 1.248 & 0.269 & 0.023 & 10.122 & 1.391 & 0.06 & 0.018287 & 0.007117 & 0.251378 & 112.818 \\
\hline 5/9/08 20:00 & 1.231 & 0.252 & 0.019 & 8.639 & 1.391 & 0.06 & 0.018287 & 0.007117 & 0.251378 & 112.818 \\
\hline 5/9/08 21:00 & 1.215 & 0.236 & 0.016 & 7.370 & 1.391 & 0.06 & 0.018287 & 0.007117 & 0.251378 & 112.818 \\
\hline 5/9/08 22:00 & 1.2 & 0.221 & 0.014 & 6.289 & 1.391 & 0.06 & 0.018287 & 0.007117 & 0.251378 & 112.818 \\
\hline 5/9/08 23:00 & 1.189 & 0.210 & 0.012 & 5.560 & 1.39 & 0.059 & 0.017982 & 0.006949 & 0.245443 & 110.155 \\
\hline 5/10/08 0:00 & 1.181 & 0.202 & 0.011 & 5.064 & 1.39 & 0.059 & 0.017982 & 0.006949 & 0.245443 & 110.155 \\
\hline 5/10/08 1:00 & 1.179 & 0.200 & 0.011 & 4.944 & 1.39 & 0.059 & 0.017982 & 0.006949 & 0.245443 & 110.155 \\
\hline 5/10/08 2:00 & 1.177 & 0.198 & 0.011 & 4.826 & 1.389 & 0.058 & 0.017678 & 0.006782 & 0.239555 & 107.512 \\
\hline 5/10/08 3:00 & 1.177 & 0.198 & 0.011 & 4.826 & 1.394 & 0.063 & 0.019201 & 0.007629 & 0.269465 & 120.936 \\
\hline 5/10/08 4:00 & 1.181 & 0.202 & 0.011 & 5.064 & 1.405 & 0.074 & 0.022554 & 0.009605 & 0.339261 & 152.260 \\
\hline
\end{tabular}




\begin{tabular}{|c|c|c|c|c|c|c|c|c|c|c|}
\hline \multirow[b]{3}{*}{ Date Time } & \multicolumn{4}{|c|}{ V-Notch Weir } & \multicolumn{6}{|c|}{ R-Notch Weir } \\
\hline & \multicolumn{2}{|c|}{ Measured Water Level } & \multicolumn{2}{|c|}{$\begin{array}{c}\text { Calculated Rate } \\
\left(\mathrm{ft}^{3} / \mathrm{s}=448.8 \mathrm{gpm}\right)\end{array}$} & \multicolumn{3}{|c|}{ Measured Water Level } & \multicolumn{3}{|c|}{$\begin{array}{c}\begin{array}{c}\text { Calculated Rate } \\
\left(\mathrm{ft}^{3} / \mathrm{s}=448.8 \mathrm{gpm}\right)\end{array} \\
\end{array}$} \\
\hline & $\begin{array}{l}\text { (ft above } \\
\text { sensor) }\end{array}$ & $\begin{array}{c}\text { (ft above } \\
\text { notch) }\end{array}$ & $\left(\mathrm{ft}^{3} / \mathrm{s}\right)$ & (gpm) & $\begin{array}{l}\text { (ft above } \\
\text { sensor) }\end{array}$ & $\begin{array}{c}\text { (ft above } \\
\text { log) }\end{array}$ & $\begin{array}{c}\text { (m above } \\
\text { log) }\end{array}$ & $\left(\mathrm{m}^{3} / \mathrm{s}\right)$ & $\left(\mathrm{ft}^{3} / \mathrm{s}\right)$ & (gpm) \\
\hline 5/10/08 5:00 & 1.193 & 0.214 & 0.013 & 5.819 & 1.409 & 0.078 & 0.023773 & 0.010361 & 0.365940 & 164.234 \\
\hline 5/10/08 6:00 & 1.2 & 0.221 & 0.014 & 6.289 & 1.406 & 0.075 & 0.022859 & 0.009792 & 0.345867 & 155.225 \\
\hline 5/10/08 7:00 & 1.198 & 0.219 & 0.014 & 6.152 & 1.399 & 0.068 & 0.020725 & 0.008509 & 0.300526 & 134.876 \\
\hline 5/10/08 8:00 & 1.191 & 0.212 & 0.013 & 5.689 & 1.396 & 0.065 & 0.019811 & 0.007977 & 0.281754 & 126.451 \\
\hline 5/10/08 9:00 & 1.179 & 0.200 & 0.011 & 4.944 & 1.396 & 0.065 & 0.019811 & 0.007977 & 0.281754 & 126.451 \\
\hline 5/10/08 10:00 & 1.177 & 0.198 & 0.011 & 4.826 & 1.399 & 0.068 & 0.020725 & 0.008509 & 0.300526 & 134.876 \\
\hline 5/10/08 11:00 & 1.174 & 0.195 & 0.010 & 4.652 & 1.396 & 0.065 & 0.019811 & 0.007977 & 0.281754 & 126.451 \\
\hline 5/10/08 12:00 & 1.174 & 0.195 & 0.010 & 4.652 & 1.389 & 0.058 & 0.017678 & 0.006782 & 0.239555 & 107.512 \\
\hline 5/10/08 13:00 & 1.174 & 0.195 & 0.010 & 4.652 & 1.399 & 0.068 & 0.020725 & 0.008509 & 0.300526 & 134.876 \\
\hline 5/10/08 14:00 & 1.184 & 0.205 & 0.012 & 5.247 & 1.408 & 0.077 & 0.023468 & 0.010170 & 0.359208 & 161.212 \\
\hline 5/10/08 15:00 & 1.198 & 0.219 & 0.014 & 6.152 & 1.415 & 0.084 & 0.025602 & 0.011529 & 0.407202 & 182.752 \\
\hline 5/10/08 16:00 & 1.214 & 0.235 & 0.016 & 7.295 & 1.448 & 0.117 & 0.035660 & 0.018652 & 0.658783 & 295.662 \\
\hline 5/10/08 17:00 & 1.261 & 0.282 & 0.025 & 11.352 & 1.536 & 0.205 & 0.062481 & 0.042506 & 1.501300 & 673.783 \\
\hline 5/10/08 18:00 & 1.318 & 0.339 & 0.040 & 17.781 & 1.559 & 0.228 & 0.069491 & 0.049739 & 1.756770 & 788.438 \\
\hline 5/10/08 19:00 & 1.344 & 0.365 & 0.047 & 21.302 & 1.535 & 0.204 & 0.062176 & 0.042200 & 1.490499 & 668.936 \\
\hline 5/10/08 20:00 & 1.334 & 0.355 & 0.044 & 19.903 & 1.5 & 0.169 & 0.051509 & 0.031975 & 1.129354 & 506.854 \\
\hline 5/10/08 21:00 & 1.306 & 0.327 & 0.036 & 16.283 & 1.471 & 0.14 & 0.042670 & 0.024251 & 0.856529 & 384.410 \\
\hline 5/10/08 22:00 & 1.292 & 0.313 & 0.033 & 14.634 & 1.452 & 0.121 & 0.036879 & 0.019590 & 0.691921 & 310.534 \\
\hline 5/10/08 23:00 & 1.276 & 0.297 & 0.029 & 12.878 & 1.442 & 0.111 & 0.033831 & 0.017274 & 0.610106 & 273.815 \\
\hline 5/11/08 0:00 & 1.26 & 0.281 & 0.025 & 11.254 & 1.43 & 0.099 & 0.030174 & 0.014626 & 0.516571 & 231.837 \\
\hline 5/11/08 1:00 & 1.244 & 0.265 & 0.022 & 9.760 & 1.422 & 0.091 & 0.027735 & 0.012943 & 0.457162 & 205.174 \\
\hline 5/11/08 2:00 & 1.235 & 0.256 & 0.020 & 8.975 & 1.413 & 0.082 & 0.024992 & 0.011135 & 0.393285 & 176.506 \\
\hline 5/11/08 3:00 & 1.226 & 0.247 & 0.018 & 8.229 & 1.415 & 0.084 & 0.025602 & 0.011529 & 0.407202 & 182.752 \\
\hline 5/11/08 4:00 & 1.211 & 0.232 & 0.016 & 7.072 & 1.403 & 0.072 & 0.021945 & 0.009235 & 0.326176 & 146.388 \\
\hline 5/11/08 5:00 & 1.211 & 0.232 & 0.016 & 7.072 & 1.405 & 0.074 & 0.022554 & 0.009605 & 0.339261 & 152.260 \\
\hline
\end{tabular}




\begin{tabular}{|c|c|c|c|c|c|c|c|c|c|c|}
\hline \multirow[b]{3}{*}{ Date Time } & \multicolumn{4}{|c|}{ V-Notch Weir } & \multicolumn{6}{|c|}{ R-Notch Weir } \\
\hline & \multicolumn{2}{|c|}{ Measured Water Level } & \multicolumn{2}{|c|}{$\begin{array}{c}\text { Calculated Rate } \\
\left(\mathrm{ft}^{3} / \mathrm{s}=448.8 \mathrm{gpm}\right)\end{array}$} & \multicolumn{3}{|c|}{ Measured Water Level } & \multicolumn{3}{|c|}{$\begin{array}{c}\text { Calculated Rate } \\
\left(\mathrm{ft}^{3} / \mathrm{s}=448.8 \mathrm{gpm}\right)\end{array}$} \\
\hline & $\begin{array}{l}\text { (ft above } \\
\text { sensor) }\end{array}$ & $\begin{array}{c}\text { (ft above } \\
\text { notch) }\end{array}$ & $\left(\mathrm{ft}^{3} / \mathrm{s}\right)$ & $(g p m)$ & $\begin{array}{c}\text { (ft above } \\
\text { sensor) }\end{array}$ & $\begin{array}{c}\text { (ft above } \\
\text { log) }\end{array}$ & $\begin{array}{c}\text { (m above } \\
\text { log) }\end{array}$ & $\left(\mathrm{m}^{3} / \mathrm{s}\right)$ & $\left(\mathrm{ft}^{3} / \mathrm{s}\right)$ & (gpm) \\
\hline 5/11/08 6:00 & 1.204 & 0.225 & 0.015 & 6.567 & 1.405 & 0.074 & 0.022554 & 0.009605 & 0.339261 & 152.260 \\
\hline 5/11/08 7:00 & 1.198 & 0.219 & 0.014 & 6.152 & 1.402 & 0.071 & 0.021640 & 0.009052 & 0.319698 & 143.480 \\
\hline 5/11/08 8:00 & 1.198 & 0.219 & 0.014 & 6.152 & 1.402 & 0.071 & 0.021640 & 0.009052 & 0.319698 & 143.480 \\
\hline 5/11/08 9:00 & 1.195 & 0.216 & 0.013 & 5.951 & 1.397 & 0.066 & 0.020116 & 0.008153 & 0.287966 & 129.239 \\
\hline 5/11/08 10:00 & 1.191 & 0.212 & 0.013 & 5.689 & 1.398 & 0.067 & 0.020421 & 0.008330 & 0.294224 & 132.048 \\
\hline 5/11/08 11:00 & 1.19 & 0.211 & 0.013 & 5.624 & 1.398 & 0.067 & 0.020421 & 0.008330 & 0.294224 & 132.048 \\
\hline 5/11/08 12:00 & 1.189 & 0.210 & 0.012 & 5.560 & 1.399 & 0.068 & 0.020725 & 0.008509 & 0.300526 & 134.876 \\
\hline 5/11/08 13:00 & 1.188 & 0.209 & 0.012 & 5.497 & 1.395 & 0.064 & 0.019506 & 0.007803 & 0.275587 & 123.683 \\
\hline 5/11/08 14:00 & 1.191 & 0.212 & 0.013 & 5.689 & 1.396 & 0.065 & 0.019811 & 0.007977 & 0.281754 & 126.451 \\
\hline 5/11/08 15:00 & 1.193 & 0.214 & 0.013 & 5.819 & 1.395 & 0.064 & 0.019506 & 0.007803 & 0.275587 & 123.683 \\
\hline 5/11/08 16:00 & 1.196 & 0.217 & 0.013 & 6.017 & 1.396 & 0.065 & 0.019811 & 0.007977 & 0.281754 & 126.451 \\
\hline 5/11/08 17:00 & 1.201 & 0.222 & 0.014 & 6.358 & 1.397 & 0.066 & 0.020116 & 0.008153 & 0.287966 & 129.239 \\
\hline 5/11/08 18:00 & 1.204 & 0.225 & 0.015 & 6.567 & 1.396 & 0.065 & 0.019811 & 0.007977 & 0.281754 & 126.451 \\
\hline 5/11/08 19:00 & 1.206 & 0.227 & 0.015 & 6.709 & 1.397 & 0.066 & 0.020116 & 0.008153 & 0.287966 & 129.239 \\
\hline 5/11/08 20:00 & 1.208 & 0.229 & 0.015 & 6.853 & 1.397 & 0.066 & 0.020116 & 0.008153 & 0.287966 & 129.239 \\
\hline 5/11/08 21:00 & 1.208 & 0.229 & 0.015 & 6.853 & 1.397 & 0.066 & 0.020116 & 0.008153 & 0.287966 & 129.239 \\
\hline 5/11/08 22:00 & 1.205 & 0.226 & 0.015 & 6.638 & 1.397 & 0.066 & 0.020116 & 0.008153 & 0.287966 & 129.239 \\
\hline 5/11/08 23:00 & 1.205 & 0.226 & 0.015 & 6.638 & 1.394 & 0.063 & 0.019201 & 0.007629 & 0.269465 & 120.936 \\
\hline 5/12/08 0:00 & 1.205 & 0.226 & 0.015 & 6.638 & 1.396 & 0.065 & 0.019811 & 0.007977 & 0.281754 & 126.451 \\
\hline 5/12/08 1:00 & 1.202 & 0.223 & 0.014 & 6.427 & 1.395 & 0.064 & 0.019506 & 0.007803 & 0.275587 & 123.683 \\
\hline 5/12/08 2:00 & 1.202 & 0.223 & 0.014 & 6.427 & 1.395 & 0.064 & 0.019506 & 0.007803 & 0.275587 & 123.683 \\
\hline 5/12/08 3:00 & 1.205 & 0.226 & 0.015 & 6.638 & 1.394 & 0.063 & 0.019201 & 0.007629 & 0.269465 & 120.936 \\
\hline 5/12/08 4:00 & 1.205 & 0.226 & 0.015 & 6.638 & 1.393 & 0.062 & 0.018897 & 0.007457 & 0.263390 & 118.209 \\
\hline 5/12/08 5:00 & 1.208 & 0.229 & 0.015 & 6.853 & 1.395 & 0.064 & 0.019506 & 0.007803 & 0.275587 & 123.683 \\
\hline 5/12/08 6:00 & 1.215 & 0.236 & 0.016 & 7.370 & 1.395 & 0.064 & 0.019506 & 0.007803 & 0.275587 & 123.683 \\
\hline
\end{tabular}




\begin{tabular}{|c|c|c|c|c|c|c|c|c|c|c|}
\hline \multirow[b]{3}{*}{ Date Time } & \multicolumn{4}{|c|}{ V-Notch Weir } & \multicolumn{6}{|c|}{ R-Notch Weir } \\
\hline & \multicolumn{2}{|c|}{ Measured Water Level } & \multicolumn{2}{|c|}{$\begin{array}{c}\text { Calculated Rate } \\
\left(\mathrm{ft}^{3} / \mathrm{s}=448.8 \mathrm{gpm}\right)\end{array}$} & \multicolumn{3}{|c|}{ Measured Water Level } & \multicolumn{3}{|c|}{$\begin{array}{c}\text { Calculated Rate } \\
\left(\mathrm{ft}^{3} / \mathrm{s}=448.8 \mathrm{gpm}\right)\end{array}$} \\
\hline & $\begin{array}{l}\text { (ft above } \\
\text { sensor) }\end{array}$ & $\begin{array}{c}\text { (ft above } \\
\text { notch) }\end{array}$ & $\left(\mathrm{ft}^{3} / \mathrm{s}\right)$ & (gpm) & $\begin{array}{c}\text { (ft above } \\
\text { sensor) }\end{array}$ & $\begin{array}{c}\text { (ft above } \\
\text { log) }\end{array}$ & $\begin{array}{c}\text { (m above } \\
\text { log) }\end{array}$ & $\left(\mathrm{m}^{3} / \mathrm{s}\right)$ & $\left(\mathrm{ft}^{3} / \mathrm{s}\right)$ & (gpm) \\
\hline 5/12/08 7:00 & 1.22 & 0.241 & 0.017 & 7.754 & 1.392 & 0.061 & 0.018592 & 0.007287 & 0.257360 & 115.503 \\
\hline 5/12/08 8:00 & 1.247 & 0.268 & 0.022 & 10.030 & 1.392 & 0.061 & 0.018592 & 0.007287 & 0.257360 & 115.503 \\
\hline 5/12/08 9:00 & 1.263 & 0.284 & 0.026 & 11.549 & 1.392 & 0.061 & 0.018592 & 0.007287 & 0.257360 & 115.503 \\
\hline 5/12/08 10:00 & 1.276 & 0.297 & 0.029 & 12.878 & 1.395 & 0.064 & 0.019506 & 0.007803 & 0.275587 & 123.683 \\
\hline 5/12/08 11:00 & 1.292 & 0.313 & 0.033 & 14.634 & 1.395 & 0.064 & 0.019506 & 0.007803 & 0.275587 & 123.683 \\
\hline 5/12/08 12:00 & 1.301 & 0.322 & 0.035 & 15.682 & 1.394 & 0.063 & 0.019201 & 0.007629 & 0.269465 & 120.936 \\
\hline 5/12/08 13:00 & 1.306 & 0.327 & 0.036 & 16.283 & 1.388 & 0.057 & 0.017373 & 0.006617 & 0.233715 & 104.891 \\
\hline 5/12/08 14:00 & 1.311 & 0.332 & 0.038 & 16.897 & 1.389 & 0.058 & 0.017678 & 0.006782 & 0.239555 & 107.512 \\
\hline 5/12/08 15:00 & 1.31 & 0.331 & 0.037 & 16.773 & 1.388 & 0.057 & 0.017373 & 0.006617 & 0.233715 & 104.891 \\
\hline 5/12/08 16:00 & 1.315 & 0.336 & 0.039 & 17.399 & 1.392 & 0.061 & 0.018592 & 0.007287 & 0.257360 & 115.503 \\
\hline 5/12/08 17:00 & 1.316 & 0.337 & 0.039 & 17.526 & 1.39 & 0.059 & 0.017982 & 0.006949 & 0.245443 & 110.155 \\
\hline 5/12/08 18:00 & 1.316 & 0.337 & 0.039 & 17.526 & 1.393 & 0.062 & 0.018897 & 0.007457 & 0.263390 & 118.209 \\
\hline 5/12/08 19:00 & 1.313 & 0.334 & 0.038 & 17.147 & 1.393 & 0.062 & 0.018897 & 0.007457 & 0.263390 & 118.209 \\
\hline 5/12/08 20:00 & 1.308 & 0.329 & 0.037 & 16.527 & 1.39 & 0.059 & 0.017982 & 0.006949 & 0.245443 & 110.155 \\
\hline 5/12/08 21:00 & 1.303 & 0.324 & 0.035 & 15.921 & 1.392 & 0.061 & 0.018592 & 0.007287 & 0.257360 & 115.503 \\
\hline 5/12/08 22:00 & 1.297 & 0.318 & 0.034 & 15.211 & 1.394 & 0.063 & 0.019201 & 0.007629 & 0.269465 & 120.936 \\
\hline 5/12/08 23:00 & 1.292 & 0.313 & 0.033 & 14.634 & 1.394 & 0.063 & 0.019201 & 0.007629 & 0.269465 & 120.936 \\
\hline 5/13/08 0:00 & 1.287 & 0.308 & 0.031 & 14.071 & 1.393 & 0.062 & 0.018897 & 0.007457 & 0.263390 & 118.209 \\
\hline 5/13/08 1:00 & 1.285 & 0.306 & 0.031 & 13.849 & 1.391 & 0.06 & 0.018287 & 0.007117 & 0.251378 & 112.818 \\
\hline 5/13/08 2:00 & 1.282 & 0.303 & 0.030 & 13.521 & 1.392 & 0.061 & 0.018592 & 0.007287 & 0.257360 & 115.503 \\
\hline 5/13/08 3:00 & 1.278 & 0.299 & 0.029 & 13.090 & 1.394 & 0.063 & 0.019201 & 0.007629 & 0.269465 & 120.936 \\
\hline 5/13/08 4:00 & 1.275 & 0.296 & 0.028 & 12.772 & 1.391 & 0.06 & 0.018287 & 0.007117 & 0.251378 & 112.818 \\
\hline 5/13/08 5:00 & 1.273 & 0.294 & 0.028 & 12.563 & 1.391 & 0.06 & 0.018287 & 0.007117 & 0.251378 & 112.818 \\
\hline 5/13/08 6:00 & 1.273 & 0.294 & 0.028 & 12.563 & 1.39 & 0.059 & 0.017982 & 0.006949 & 0.245443 & 110.155 \\
\hline 5/13/08 7:00 & 1.273 & 0.294 & 0.028 & 12.563 & 1.39 & 0.059 & 0.017982 & 0.006949 & 0.245443 & 110.155 \\
\hline
\end{tabular}




\begin{tabular}{|c|c|c|c|c|c|c|c|c|c|c|}
\hline \multirow[b]{3}{*}{ Date Time } & \multicolumn{4}{|c|}{ V-Notch Weir } & \multicolumn{6}{|c|}{ R-Notch Weir } \\
\hline & \multicolumn{2}{|c|}{ Measured Water Level } & \multicolumn{2}{|c|}{$\begin{array}{c}\text { Calculated Rate } \\
\left(\mathrm{ft}^{3} / \mathrm{s}=448.8 \mathrm{gpm}\right)\end{array}$} & \multicolumn{3}{|c|}{ Measured Water Level } & \multicolumn{3}{|c|}{$\begin{array}{c}\text { Calculated Rate } \\
\left(\mathrm{ft}^{3} / \mathrm{s}=448.8 \mathrm{gpm}\right)\end{array}$} \\
\hline & $\begin{array}{c}\text { (ft above } \\
\text { sensor) }\end{array}$ & $\begin{array}{c}\text { (ft above } \\
\text { notch) }\end{array}$ & $\left(\mathrm{ft}^{3} / \mathrm{s}\right)$ & (gpm) & $\begin{array}{c}\text { (ft above } \\
\text { sensor) }\end{array}$ & $\begin{array}{c}\text { (ft above } \\
\text { log) }\end{array}$ & $\begin{array}{c}\text { (m above } \\
\text { log) }\end{array}$ & $\left(\mathrm{m}^{3} / \mathrm{s}\right)$ & $\left(\mathrm{ft}^{3} / \mathrm{s}\right)$ & (gpm) \\
\hline 5/13/08 8:00 & 1.276 & 0.297 & 0.029 & 12.878 & 1.39 & 0.059 & 0.017982 & 0.006949 & 0.245443 & 110.155 \\
\hline 5/13/08 9:00 & 1.276 & 0.297 & 0.029 & 12.878 & 1.389 & 0.058 & 0.017678 & 0.006782 & 0.239555 & 107.512 \\
\hline 5/13/08 10:00 & 1.276 & 0.297 & 0.029 & 12.878 & 1.389 & 0.058 & 0.017678 & 0.006782 & 0.239555 & 107.512 \\
\hline 5/13/08 11:00 & 1.275 & 0.296 & 0.028 & 12.772 & 1.389 & 0.058 & 0.017678 & 0.006782 & 0.239555 & 107.512 \\
\hline 5/13/08 12:00 & 1.278 & 0.299 & 0.029 & 13.090 & 1.39 & 0.059 & 0.017982 & 0.006949 & 0.245443 & 110.155 \\
\hline 5/13/08 13:00 & 1.28 & 0.301 & 0.030 & 13.304 & 1.388 & 0.057 & 0.017373 & 0.006617 & 0.233715 & 104.891 \\
\hline 5/13/08 14:00 & 1.282 & 0.303 & 0.030 & 13.521 & 1.388 & 0.057 & 0.017373 & 0.006617 & 0.233715 & 104.891 \\
\hline 5/13/08 15:00 & 1.282 & 0.303 & 0.030 & 13.521 & 1.386 & 0.055 & 0.016763 & 0.006291 & 0.222180 & 99.714 \\
\hline 5/13/08 16:00 & 1.285 & 0.306 & 0.031 & 13.849 & 1.386 & 0.055 & 0.016763 & 0.006291 & 0.222180 & 99.714 \\
\hline 5/13/08 17:00 & 1.285 & 0.306 & 0.031 & 13.849 & 1.386 & 0.055 & 0.016763 & 0.006291 & 0.222180 & 99.714 \\
\hline 5/13/08 18:00 & 1.285 & 0.306 & 0.031 & 13.849 & 1.389 & 0.058 & 0.017678 & 0.006782 & 0.239555 & 107.512 \\
\hline 5/13/08 19:00 & 1.285 & 0.306 & 0.031 & 13.849 & 1.389 & 0.058 & 0.017678 & 0.006782 & 0.239555 & 107.512 \\
\hline 5/13/08 20:00 & 1.282 & 0.303 & 0.030 & 13.521 & 1.386 & 0.055 & 0.016763 & 0.006291 & 0.222180 & 99.714 \\
\hline 5/13/08 21:00 & 1.28 & 0.301 & 0.030 & 13.304 & 1.389 & 0.058 & 0.017678 & 0.006782 & 0.239555 & 107.512 \\
\hline $5 / 13 / 08$ 22:00 & 1.278 & 0.299 & 0.029 & 13.090 & 1.388 & 0.057 & 0.017373 & 0.006617 & 0.233715 & 104.891 \\
\hline 5/13/08 23:00 & 1.275 & 0.296 & 0.028 & 12.772 & 1.39 & 0.059 & 0.017982 & 0.006949 & 0.245443 & 110.155 \\
\hline 5/14/08 0:00 & 1.271 & 0.292 & 0.028 & 12.356 & 1.39 & 0.059 & 0.017982 & 0.006949 & 0.245443 & 110.155 \\
\hline 5/14/08 1:00 & 1.271 & 0.292 & 0.028 & 12.356 & 1.389 & 0.058 & 0.017678 & 0.006782 & 0.239555 & 107.512 \\
\hline 5/14/08 2:00 & 1.269 & 0.290 & 0.027 & 12.151 & 1.389 & 0.058 & 0.017678 & 0.006782 & 0.239555 & 107.512 \\
\hline 5/14/08 3:00 & 1.269 & 0.290 & 0.027 & 12.151 & 1.388 & 0.057 & 0.017373 & 0.006617 & 0.233715 & 104.891 \\
\hline 5/14/08 4:00 & 1.269 & 0.290 & 0.027 & 12.151 & 1.388 & 0.057 & 0.017373 & 0.006617 & 0.233715 & 104.891 \\
\hline 5/14/08 5:00 & 1.269 & 0.290 & 0.027 & 12.151 & 1.388 & 0.057 & 0.017373 & 0.006617 & 0.233715 & 104.891 \\
\hline 5/14/08 6:00 & 1.27 & 0.291 & 0.027 & 12.253 & 1.39 & 0.059 & 0.017982 & 0.006949 & 0.245443 & 110.155 \\
\hline 5/14/08 7:00 & 1.27 & 0.291 & 0.027 & 12.253 & 1.387 & 0.056 & 0.017068 & 0.006453 & 0.227923 & 102.292 \\
\hline 5/14/08 8:00 & 1.27 & 0.291 & 0.027 & 12.253 & 1.387 & 0.056 & 0.017068 & 0.006453 & 0.227923 & 102.292 \\
\hline
\end{tabular}




\begin{tabular}{|c|c|c|c|c|c|c|c|c|c|c|}
\hline \multirow[b]{3}{*}{ Date Time } & \multicolumn{4}{|c|}{ V-Notch Weir } & \multicolumn{6}{|c|}{ R-Notch Weir } \\
\hline & \multicolumn{2}{|c|}{ Measured Water Level } & \multicolumn{2}{|c|}{$\begin{array}{c}\text { Calculated Rate } \\
\left(\mathrm{ft}^{3} / \mathrm{s}=448.8 \mathrm{gpm}\right)\end{array}$} & \multicolumn{3}{|c|}{ Measured Water Level } & \multicolumn{3}{|c|}{$\begin{array}{c}\text { Calculated Rate } \\
\left(\mathrm{ft}^{3} / \mathrm{s}=448.8 \mathrm{gpm}\right)\end{array}$} \\
\hline & $\begin{array}{c}\text { (ft above } \\
\text { sensor) }\end{array}$ & $\begin{array}{c}\text { (ft above } \\
\text { notch) }\end{array}$ & $\left(\mathrm{ft}^{3} / \mathrm{s}\right)$ & (gpm) & $\begin{array}{l}\text { (ft above } \\
\text { sensor) }\end{array}$ & $\begin{array}{c}\text { (ft above } \\
\text { log) }\end{array}$ & $\begin{array}{c}\text { (m above } \\
\text { log) }\end{array}$ & $\left(\mathrm{m}^{3} / \mathrm{s}\right)$ & $\left(\mathrm{ft}^{3} / \mathrm{s}\right)$ & (gpm) \\
\hline 5/14/08 9:00 & 1.272 & 0.293 & 0.028 & 12.459 & 1.389 & 0.058 & 0.017678 & 0.006782 & 0.239555 & 107.512 \\
\hline 5/14/08 10:00 & 1.274 & 0.295 & 0.028 & 12.668 & 1.39 & 0.059 & 0.017982 & 0.006949 & 0.245443 & 110.155 \\
\hline 5/14/08 11:00 & 1.278 & 0.299 & 0.029 & 13.090 & 1.388 & 0.057 & 0.017373 & 0.006617 & 0.233715 & 104.891 \\
\hline 5/14/08 12:00 & 1.285 & 0.306 & 0.031 & 13.849 & 1.389 & 0.058 & 0.017678 & 0.006782 & 0.239555 & 107.512 \\
\hline 5/14/08 13:00 & 1.289 & 0.310 & 0.032 & 14.295 & 1.388 & 0.057 & 0.017373 & 0.006617 & 0.233715 & 104.891 \\
\hline 5/14/08 14:00 & 1.292 & 0.313 & 0.033 & 14.634 & 1.385 & 0.054 & 0.016458 & 0.006129 & 0.216486 & 97.159 \\
\hline 5/14/08 15:00 & 1.295 & 0.316 & 0.033 & 14.979 & 1.389 & 0.058 & 0.017678 & 0.006782 & 0.239555 & 107.512 \\
\hline 5/14/08 16:00 & 1.301 & 0.322 & 0.035 & 15.682 & 1.388 & 0.057 & 0.017373 & 0.006617 & 0.233715 & 104.891 \\
\hline 5/14/08 17:00 & 1.304 & 0.325 & 0.036 & 16.041 & 1.389 & 0.058 & 0.017678 & 0.006782 & 0.239555 & 107.512 \\
\hline 5/14/08 18:00 & 1.307 & 0.328 & 0.037 & 16.405 & 1.392 & 0.061 & 0.018592 & 0.007287 & 0.257360 & 115.503 \\
\hline 5/14/08 19:00 & 1.309 & 0.330 & 0.037 & 16.650 & 1.392 & 0.061 & 0.018592 & 0.007287 & 0.257360 & 115.503 \\
\hline 5/14/08 20:00 & 1.217 & 0.238 & 0.017 & 7.522 & 1.394 & 0.063 & 0.019201 & 0.007629 & 0.269465 & 120.936 \\
\hline 5/14/08 21:00 & 1.219 & 0.240 & 0.017 & 7.676 & 1.394 & 0.063 & 0.019201 & 0.007629 & 0.269465 & 120.936 \\
\hline 5/14/08 22:00 & 1.22 & 0.241 & 0.017 & 7.754 & 1.393 & 0.062 & 0.018897 & 0.007457 & 0.263390 & 118.209 \\
\hline 5/14/08 23:00 & 1.22 & 0.241 & 0.017 & 7.754 & 1.395 & 0.064 & 0.019506 & 0.007803 & 0.275587 & 123.683 \\
\hline 5/15/08 0:00 & 1.222 & 0.243 & 0.018 & 7.910 & 1.392 & 0.061 & 0.018592 & 0.007287 & 0.257360 & 115.503 \\
\hline 5/15/08 1:00 & 1.224 & 0.245 & 0.018 & 8.069 & 1.391 & 0.06 & 0.018287 & 0.007117 & 0.251378 & 112.818 \\
\hline 5/15/08 2:00 & 1.224 & 0.245 & 0.018 & 8.069 & 1.391 & 0.06 & 0.018287 & 0.007117 & 0.251378 & 112.818 \\
\hline 5/15/08 3:00 & 1.224 & 0.245 & 0.018 & 8.069 & 1.39 & 0.059 & 0.017982 & 0.006949 & 0.245443 & 110.155 \\
\hline 5/15/08 4:00 & 1.224 & 0.245 & 0.018 & 8.069 & 1.389 & 0.058 & 0.017678 & 0.006782 & 0.239555 & 107.512 \\
\hline 5/15/08 5:00 & 1.224 & 0.245 & 0.018 & 8.069 & 1.39 & 0.059 & 0.017982 & 0.006949 & 0.245443 & 110.155 \\
\hline 5/15/08 6:00 & 1.224 & 0.245 & 0.018 & 8.069 & 1.39 & 0.059 & 0.017982 & 0.006949 & 0.245443 & 110.155 \\
\hline 5/15/08 7:00 & 1.224 & 0.245 & 0.018 & 8.069 & 1.389 & 0.058 & 0.017678 & 0.006782 & 0.239555 & 107.512 \\
\hline 5/15/08 8:00 & 1.224 & 0.245 & 0.018 & 8.069 & 1.389 & 0.058 & 0.017678 & 0.006782 & 0.239555 & 107.512 \\
\hline 5/15/08 9:00 & 1.226 & 0.247 & 0.018 & 8.229 & 1.387 & 0.056 & 0.017068 & 0.006453 & 0.227923 & 102.292 \\
\hline
\end{tabular}




\begin{tabular}{|c|c|c|c|c|c|c|c|c|c|c|}
\hline \multirow[b]{3}{*}{ Date Time } & \multicolumn{4}{|c|}{ V-Notch Weir } & \multicolumn{6}{|c|}{ R-Notch Weir } \\
\hline & \multicolumn{2}{|c|}{ Measured Water Level } & \multicolumn{2}{|c|}{$\begin{array}{c}\text { Calculated Rate } \\
\left(\mathrm{ft}^{3} / \mathrm{s}=448.8 \mathrm{gpm}\right)\end{array}$} & \multicolumn{3}{|c|}{ Measured Water Level } & \multicolumn{3}{|c|}{$\begin{array}{c}\text { Calculated Rate } \\
\left(\mathrm{ft}^{3} / \mathrm{s}=448.8 \mathrm{gpm}\right)\end{array}$} \\
\hline & $\begin{array}{l}\text { (ft above } \\
\text { sensor) }\end{array}$ & $\begin{array}{c}\text { (ft above } \\
\text { notch) }\end{array}$ & $\left(\mathrm{ft}^{3} / \mathrm{s}\right)$ & $(g p m)$ & $\begin{array}{c}\text { (ft above } \\
\text { sensor) }\end{array}$ & $\begin{array}{c}\text { (ft above } \\
\text { log) }\end{array}$ & $\begin{array}{c}\text { (m above } \\
\text { log) }\end{array}$ & $\left(\mathrm{m}^{3} / \mathrm{s}\right)$ & $\left(\mathrm{ft}^{3} / \mathrm{s}\right)$ & (gpm) \\
\hline 5/15/08 10:00 & 1.231 & 0.252 & 0.019 & 8.639 & 1.387 & 0.056 & 0.017068 & 0.006453 & 0.227923 & 102.292 \\
\hline 5/15/08 11:00 & 1.233 & 0.254 & 0.020 & 8.806 & 1.388 & 0.057 & 0.017373 & 0.006617 & 0.233715 & 104.891 \\
\hline 5/15/08 12:00 & 1.238 & 0.259 & 0.021 & 9.232 & 1.389 & 0.058 & 0.017678 & 0.006782 & 0.239555 & 107.512 \\
\hline 5/15/08 13:00 & 1.222 & 0.243 & 0.018 & 7.910 & 1.391 & 0.06 & 0.018287 & 0.007117 & 0.251378 & 112.818 \\
\hline 5/15/08 14:00 & 1.204 & 0.225 & 0.015 & 6.567 & 1.39 & 0.059 & 0.017982 & 0.006949 & 0.245443 & 110.155 \\
\hline 5/15/08 15:00 & 1.213 & 0.234 & 0.016 & 7.220 & 1.39 & 0.059 & 0.017982 & 0.006949 & 0.245443 & 110.155 \\
\hline 5/15/08 16:00 & 1.218 & 0.239 & 0.017 & 7.599 & 1.393 & 0.062 & 0.018897 & 0.007457 & 0.263390 & 118.209 \\
\hline 5/15/08 17:00 & 1.226 & 0.247 & 0.018 & 8.229 & 1.395 & 0.064 & 0.019506 & 0.007803 & 0.275587 & 123.683 \\
\hline 5/15/08 18:00 & 1.23 & 0.251 & 0.019 & 8.556 & 1.395 & 0.064 & 0.019506 & 0.007803 & 0.275587 & 123.683 \\
\hline 5/15/08 19:00 & 1.227 & 0.248 & 0.019 & 8.310 & 1.394 & 0.063 & 0.019201 & 0.007629 & 0.269465 & 120.936 \\
\hline 5/15/08 20:00 & 1.229 & 0.250 & 0.019 & 8.474 & 1.391 & 0.06 & 0.018287 & 0.007117 & 0.251378 & 112.818 \\
\hline 5/15/08 21:00 & 1.225 & 0.246 & 0.018 & 8.149 & 1.39 & 0.059 & 0.017982 & 0.006949 & 0.245443 & 110.155 \\
\hline 5/15/08 22:00 & 1.22 & 0.241 & 0.017 & 7.754 & 1.391 & 0.06 & 0.018287 & 0.007117 & 0.251378 & 112.818 \\
\hline 5/15/08 23:00 & 1.215 & 0.236 & 0.016 & 7.370 & 1.391 & 0.06 & 0.018287 & 0.007117 & 0.251378 & 112.818 \\
\hline 5/16/08 0:00 & 1.213 & 0.234 & 0.016 & 7.220 & 1.39 & 0.059 & 0.017982 & 0.006949 & 0.245443 & 110.155 \\
\hline 5/16/08 1:00 & 1.21 & 0.231 & 0.016 & 6.998 & 1.39 & 0.059 & 0.017982 & 0.006949 & 0.245443 & 110.155 \\
\hline 5/16/08 2:00 & 1.21 & 0.231 & 0.016 & 6.998 & 1.389 & 0.058 & 0.017678 & 0.006782 & 0.239555 & 107.512 \\
\hline 5/16/08 3:00 & 1.207 & 0.228 & 0.015 & 6.780 & 1.388 & 0.057 & 0.017373 & 0.006617 & 0.233715 & 104.891 \\
\hline 5/16/08 4:00 & 1.205 & 0.226 & 0.015 & 6.638 & 1.39 & 0.059 & 0.017982 & 0.006949 & 0.245443 & 110.155 \\
\hline 5/16/08 5:00 & 1.202 & 0.223 & 0.014 & 6.427 & 1.389 & 0.058 & 0.017678 & 0.006782 & 0.239555 & 107.512 \\
\hline 5/16/08 6:00 & 1.2 & 0.221 & 0.014 & 6.289 & 1.388 & 0.057 & 0.017373 & 0.006617 & 0.233715 & 104.891 \\
\hline 5/16/08 7:00 & 1.2 & 0.221 & 0.014 & 6.289 & 1.388 & 0.057 & 0.017373 & 0.006617 & 0.233715 & 104.891 \\
\hline 5/16/08 8:00 & 1.196 & 0.217 & 0.013 & 6.017 & 1.385 & 0.054 & 0.016458 & 0.006129 & 0.216486 & 97.159 \\
\hline 5/16/08 9:00 & 1.196 & 0.217 & 0.013 & 6.017 & 1.388 & 0.057 & 0.017373 & 0.006617 & 0.233715 & 104.891 \\
\hline 5/16/08 10:00 & 1.191 & 0.212 & 0.013 & 5.689 & 1.388 & 0.057 & 0.017373 & 0.006617 & 0.233715 & 104.891 \\
\hline
\end{tabular}




\begin{tabular}{|c|c|c|c|c|c|c|c|c|c|c|}
\hline \multirow[b]{3}{*}{ Date Time } & \multicolumn{4}{|c|}{ V-Notch Weir } & \multicolumn{6}{|c|}{ R-Notch Weir } \\
\hline & \multicolumn{2}{|c|}{ Measured Water Level } & \multicolumn{2}{|c|}{$\begin{array}{c}\text { Calculated Rate } \\
\left(\mathrm{ft}^{3} / \mathrm{s}=448.8 \mathrm{gpm}\right)\end{array}$} & \multicolumn{3}{|c|}{ Measured Water Level } & \multicolumn{3}{|c|}{$\begin{array}{c}\text { Calculated Rate } \\
\left(\mathrm{ft}^{3} / \mathrm{s}=448.8 \mathrm{gpm}\right)\end{array}$} \\
\hline & $\begin{array}{l}\text { (ft above } \\
\text { sensor) }\end{array}$ & $\begin{array}{c}\text { (ft above } \\
\text { notch) }\end{array}$ & $\left(\mathrm{ft}^{3} / \mathrm{s}\right)$ & (gpm) & $\begin{array}{c}\text { (ft above } \\
\text { sensor) }\end{array}$ & $\begin{array}{c}\text { (ft above } \\
\text { log) }\end{array}$ & $\begin{array}{c}\text { (m above } \\
\text { log) }\end{array}$ & $\left(\mathrm{m}^{3} / \mathrm{s}\right)$ & $\left(\mathrm{ft}^{3} / \mathrm{s}\right)$ & (gpm) \\
\hline 5/16/08 11:00 & 1.193 & 0.214 & 0.013 & 5.819 & 1.387 & 0.056 & 0.017068 & 0.006453 & 0.227923 & 102.292 \\
\hline 5/16/08 12:00 & 1.196 & 0.217 & 0.013 & 6.017 & 1.385 & 0.054 & 0.016458 & 0.006129 & 0.216486 & 97.159 \\
\hline 5/16/08 13:00 & 1.203 & 0.224 & 0.014 & 6.497 & 1.384 & 0.053 & 0.016154 & 0.005969 & 0.210842 & 94.626 \\
\hline 5/16/08 14:00 & 1.211 & 0.232 & 0.016 & 7.072 & 1.386 & 0.055 & 0.016763 & 0.006291 & 0.222180 & 99.714 \\
\hline 5/16/08 15:00 & 1.215 & 0.236 & 0.016 & 7.370 & 1.388 & 0.057 & 0.017373 & 0.006617 & 0.233715 & 104.891 \\
\hline 5/16/08 16:00 & 1.22 & 0.241 & 0.017 & 7.754 & 1.387 & 0.056 & 0.017068 & 0.006453 & 0.227923 & 102.292 \\
\hline 5/16/08 17:00 & 1.218 & 0.239 & 0.017 & 7.599 & 1.39 & 0.059 & 0.017982 & 0.006949 & 0.245443 & 110.155 \\
\hline 5/16/08 18:00 & 1.223 & 0.244 & 0.018 & 7.989 & 1.39 & 0.059 & 0.017982 & 0.006949 & 0.245443 & 110.155 \\
\hline 5/16/08 19:00 & 1.214 & 0.235 & 0.016 & 7.295 & 1.39 & 0.059 & 0.017982 & 0.006949 & 0.245443 & 110.155 \\
\hline 5/16/08 20:00 & 1.204 & 0.225 & 0.015 & 6.567 & 1.392 & 0.061 & 0.018592 & 0.007287 & 0.257360 & 115.503 \\
\hline 5/16/08 21:00 & 1.189 & 0.210 & 0.012 & 5.560 & 1.392 & 0.061 & 0.018592 & 0.007287 & 0.257360 & 115.503 \\
\hline 5/16/08 22:00 & 1.174 & 0.195 & 0.010 & 4.652 & 1.391 & 0.06 & 0.018287 & 0.007117 & 0.251378 & 112.818 \\
\hline 5/16/08 23:00 & 1.169 & 0.190 & 0.010 & 4.371 & 1.39 & 0.059 & 0.017982 & 0.006949 & 0.245443 & 110.155 \\
\hline 5/17/08 0:00 & 1.169 & 0.190 & 0.010 & 4.371 & 1.392 & 0.061 & 0.018592 & 0.007287 & 0.257360 & 115.503 \\
\hline 5/17/08 1:00 & 1.168 & 0.189 & 0.010 & 4.316 & 1.389 & 0.058 & 0.017678 & 0.006782 & 0.239555 & 107.512 \\
\hline 5/17/08 2:00 & 1.172 & 0.193 & 0.010 & 4.538 & 1.391 & 0.06 & 0.018287 & 0.007117 & 0.251378 & 112.818 \\
\hline 5/17/08 3:00 & 1.172 & 0.193 & 0.010 & 4.538 & 1.39 & 0.059 & 0.017982 & 0.006949 & 0.245443 & 110.155 \\
\hline 5/17/08 4:00 & 1.172 & 0.193 & 0.010 & 4.538 & 1.391 & 0.06 & 0.018287 & 0.007117 & 0.251378 & 112.818 \\
\hline 5/17/08 5:00 & 1.167 & 0.188 & 0.009 & 4.261 & 1.391 & 0.06 & 0.018287 & 0.007117 & 0.251378 & 112.818 \\
\hline 5/17/08 6:00 & 1.167 & 0.188 & 0.009 & 4.261 & 1.39 & 0.059 & 0.017982 & 0.006949 & 0.245443 & 110.155 \\
\hline 5/17/08 7:00 & 1.165 & 0.186 & 0.009 & 4.153 & 1.389 & 0.058 & 0.017678 & 0.006782 & 0.239555 & 107.512 \\
\hline 5/17/08 8:00 & 1.165 & 0.186 & 0.009 & 4.153 & 1.389 & 0.058 & 0.017678 & 0.006782 & 0.239555 & 107.512 \\
\hline 5/17/08 9:00 & 1.165 & 0.186 & 0.009 & 4.153 & 1.389 & 0.058 & 0.017678 & 0.006782 & 0.239555 & 107.512 \\
\hline 5/17/08 10:00 & 1.167 & 0.188 & 0.009 & 4.261 & 1.39 & 0.059 & 0.017982 & 0.006949 & 0.245443 & 110.155 \\
\hline 5/17/08 11:00 & 1.167 & 0.188 & 0.009 & 4.261 & 1.388 & 0.057 & 0.017373 & 0.006617 & 0.233715 & 104.891 \\
\hline
\end{tabular}




\begin{tabular}{|c|c|c|c|c|c|c|c|c|c|c|}
\hline \multirow[b]{3}{*}{ Date Time } & \multicolumn{4}{|c|}{ V-Notch Weir } & \multicolumn{6}{|c|}{ R-Notch Weir } \\
\hline & \multicolumn{2}{|c|}{ Measured Water Level } & \multicolumn{2}{|c|}{$\begin{array}{c}\text { Calculated Rate } \\
\left(\mathrm{ft}^{3} / \mathrm{s}=448.8 \mathrm{gpm}\right)\end{array}$} & \multicolumn{3}{|c|}{ Measured Water Level } & \multicolumn{3}{|c|}{$\begin{array}{c}\text { Calculated Rate } \\
\left(\mathrm{ft}^{3} / \mathrm{s}=448.8 \mathrm{gpm}\right)\end{array}$} \\
\hline & $\begin{array}{l}\text { (ft above } \\
\text { sensor) }\end{array}$ & $\begin{array}{l}\text { (ft above } \\
\text { notch) }\end{array}$ & $\left(\mathrm{ft}^{3} / \mathrm{s}\right)$ & (gpm) & $\begin{array}{l}\text { (ft above } \\
\text { sensor) }\end{array}$ & $\begin{array}{l}\text { (ft above } \\
\text { log) }\end{array}$ & $\begin{array}{c}\text { (m above } \\
\text { log) }\end{array}$ & $\left(\mathrm{m}^{3} / \mathrm{s}\right)$ & $\left(\mathrm{ft}^{3} / \mathrm{s}\right)$ & (gpm) \\
\hline 5/17/08 12:00 & 1.17 & 0.191 & 0.010 & 4.426 & 1.387 & 0.056 & 0.017068 & 0.006453 & 0.227923 & 102.292 \\
\hline 5/17/08 13:00 & 1.173 & 0.194 & 0.010 & 4.595 & 1.389 & 0.058 & 0.017678 & 0.006782 & 0.239555 & 107.512 \\
\hline 5/17/08 14:00 & 1.174 & 0.195 & 0.010 & 4.652 & 1.388 & 0.057 & 0.017373 & 0.006617 & 0.233715 & 104.891 \\
\hline 5/17/08 15:00 & 1.182 & 0.203 & 0.011 & 5.124 & 1.385 & 0.054 & 0.016458 & 0.006129 & 0.216486 & 97.159 \\
\hline 5/17/08 16:00 & 1.188 & 0.209 & 0.012 & 5.497 & 1.388 & 0.057 & 0.017373 & 0.006617 & 0.233715 & 104.891 \\
\hline 5/17/08 17:00 & 1.193 & 0.214 & 0.013 & 5.819 & 1.384 & 0.053 & 0.016154 & 0.005969 & 0.210842 & 94.626 \\
\hline 5/17/08 18:00 & 1.198 & 0.219 & 0.014 & 6.152 & 1.387 & 0.056 & 0.017068 & 0.006453 & 0.227923 & 102.292 \\
\hline 5/17/08 19:00 & 1.199 & 0.220 & 0.014 & 6.220 & 1.389 & 0.058 & 0.017678 & 0.006782 & 0.239555 & 107.512 \\
\hline 5/17/08 20:00 & 1.192 & 0.213 & 0.013 & 5.753 & 1.388 & 0.057 & 0.017373 & 0.006617 & 0.233715 & 104.891 \\
\hline 5/17/08 21:00 & 1.188 & 0.209 & 0.012 & 5.497 & 1.387 & 0.056 & 0.017068 & 0.006453 & 0.227923 & 102.292 \\
\hline 5/17/08 22:00 & 1.183 & 0.204 & 0.012 & 5.185 & 1.388 & 0.057 & 0.017373 & 0.006617 & 0.233715 & 104.891 \\
\hline 5/17/08 23:00 & 1.18 & 0.201 & 0.011 & 5.004 & 1.388 & 0.057 & 0.017373 & 0.006617 & 0.233715 & 104.891 \\
\hline 5/18/08 0:00 & 1.18 & 0.201 & 0.011 & 5.004 & 1.389 & 0.058 & 0.017678 & 0.006782 & 0.239555 & 107.512 \\
\hline 5/18/08 1:00 & 1.177 & 0.198 & 0.011 & 4.826 & 1.389 & 0.058 & 0.017678 & 0.006782 & 0.239555 & 107.512 \\
\hline 5/18/08 2:00 & 1.177 & 0.198 & 0.011 & 4.826 & 1.388 & 0.057 & 0.017373 & 0.006617 & 0.233715 & 104.891 \\
\hline 5/18/08 3:00 & 1.174 & 0.195 & 0.010 & 4.652 & 1.387 & 0.056 & 0.017068 & 0.006453 & 0.227923 & 102.292 \\
\hline 5/18/08 4:00 & 1.174 & 0.195 & 0.010 & 4.652 & 1.389 & 0.058 & 0.017678 & 0.006782 & 0.239555 & 107.512 \\
\hline 5/18/08 5:00 & 1.172 & 0.193 & 0.010 & 4.538 & 1.388 & 0.057 & 0.017373 & 0.006617 & 0.233715 & 104.891 \\
\hline 5/18/08 6:00 & 1.167 & 0.188 & 0.009 & 4.261 & 1.387 & 0.056 & 0.017068 & 0.006453 & 0.227923 & 102.292 \\
\hline 5/18/08 7:00 & 1.165 & 0.186 & 0.009 & 4.153 & 1.387 & 0.056 & 0.017068 & 0.006453 & 0.227923 & 102.292 \\
\hline 5/18/08 8:00 & 1.163 & 0.184 & 0.009 & 4.047 & 1.386 & 0.055 & 0.016763 & 0.006291 & 0.222180 & 99.714 \\
\hline 5/18/08 9:00 & 1.163 & 0.184 & 0.009 & 4.047 & 1.389 & 0.058 & 0.017678 & 0.006782 & 0.239555 & 107.512 \\
\hline 5/18/08 10:00 & 1.162 & 0.183 & 0.009 & 3.994 & 1.389 & 0.058 & 0.017678 & 0.006782 & 0.239555 & 107.512 \\
\hline 5/18/08 11:00 & 1.162 & 0.183 & 0.009 & 3.994 & 1.387 & 0.056 & 0.017068 & 0.006453 & 0.227923 & 102.292 \\
\hline 5/18/08 12:00 & 1.167 & 0.188 & 0.009 & 4.261 & 1.386 & 0.055 & 0.016763 & 0.006291 & 0.222180 & 99.714 \\
\hline
\end{tabular}




\begin{tabular}{|c|c|c|c|c|c|c|c|c|c|c|}
\hline \multirow[b]{3}{*}{ Date Time } & \multicolumn{4}{|c|}{ V-Notch Weir } & \multicolumn{6}{|c|}{ R-Notch Weir } \\
\hline & \multicolumn{2}{|c|}{ Measured Water Level } & \multicolumn{2}{|c|}{$\begin{array}{c}\text { Calculated Rate } \\
\left(\mathrm{ft}^{3} / \mathrm{s}=448.8 \mathrm{gpm}\right)\end{array}$} & \multicolumn{3}{|c|}{ Measured Water Level } & \multicolumn{3}{|c|}{$\begin{array}{c}\text { Calculated Rate } \\
\left(\mathrm{ft}^{3} / \mathrm{s}=448.8 \mathrm{gpm}\right)\end{array}$} \\
\hline & $\begin{array}{c}\text { (ft above } \\
\text { sensor) }\end{array}$ & $\begin{array}{c}\text { (ft above } \\
\text { notch) }\end{array}$ & $\left(\mathrm{ft}^{3} / \mathrm{s}\right)$ & (gpm) & $\begin{array}{l}\text { (ft above } \\
\text { sensor) }\end{array}$ & $\begin{array}{c}\text { (ft above } \\
\text { log) }\end{array}$ & $\begin{array}{c}\text { (m above } \\
\text { log) }\end{array}$ & $\left(\mathrm{m}^{3} / \mathrm{s}\right)$ & $\left(\mathrm{ft}^{3} / \mathrm{s}\right)$ & (gpm) \\
\hline \multirow[t]{2}{*}{ 5/18/08 13:00 } & 1.173 & 0.194 & 0.010 & 4.595 & 1.385 & 0.054 & 0.016458 & 0.006129 & 0.216486 & 97.159 \\
\hline & 2.32 & No measurer & 0.392 & 176.045 & , during rep & positioning $\mathrm{c}$ & the $V$-notch & eir at the we & ands outfall. & \\
\hline 6/9/08 14:00 & 2.303 & 0.843 & 0.373 & 167.551 & 1.414 & 0.068 & 0.020725 & 0.008509 & 0.300526 & 134.876 \\
\hline 6/9/08 15:00 & 2.288 & 0.828 & 0.357 & 160.264 & 1.411 & 0.065 & 0.019811 & 0.007977 & 0.281754 & 126.451 \\
\hline 6/9/08 16:00 & 2.272 & 0.812 & 0.340 & 152.705 & 1.413 & 0.067 & 0.020421 & 0.008330 & 0.294224 & 132.048 \\
\hline 6/9/08 17:00 & 2.263 & 0.803 & 0.331 & 148.549 & 1.411 & 0.065 & 0.019811 & 0.007977 & 0.281754 & 126.451 \\
\hline 6/9/08 18:00 & 2.254 & 0.794 & 0.322 & 144.461 & 1.412 & 0.066 & 0.020116 & 0.008153 & 0.287966 & 129.239 \\
\hline 6/9/08 19:00 & 2.248 & 0.788 & 0.316 & 141.774 & 1.41 & 0.064 & 0.019506 & 0.007803 & 0.275587 & 123.683 \\
\hline 6/9/08 20:00 & 2.256 & 0.796 & 0.324 & 145.363 & 1.412 & 0.066 & 0.020116 & 0.008153 & 0.287966 & 129.239 \\
\hline 6/9/08 21:00 & 2.262 & 0.802 & 0.330 & 148.091 & 1.412 & 0.066 & 0.020116 & 0.008153 & 0.287966 & 129.239 \\
\hline 6/9/08 22:00 & 2.268 & 0.808 & 0.336 & 150.849 & 1.413 & 0.067 & 0.020421 & 0.008330 & 0.294224 & 132.048 \\
\hline 6/9/08 23:00 & 2.274 & 0.814 & 0.342 & 153.638 & 1.414 & 0.068 & 0.020725 & 0.008509 & 0.300526 & 134.876 \\
\hline 6/10/08 0:00 & 2.282 & 0.822 & 0.351 & 157.404 & 1.413 & 0.067 & 0.020421 & 0.008330 & 0.294224 & 132.048 \\
\hline 6/10/08 1:00 & 2.289 & 0.829 & 0.358 & 160.744 & 1.414 & 0.068 & 0.020725 & 0.008509 & 0.300526 & 134.876 \\
\hline 6/10/08 2:00 & 2.291 & 0.831 & 0.360 & 161.706 & 1.413 & 0.067 & 0.020421 & 0.008330 & 0.294224 & 132.048 \\
\hline 6/10/08 3:00 & 2.291 & 0.831 & 0.360 & 161.706 & 1.413 & 0.067 & 0.020421 & 0.008330 & 0.294224 & 132.048 \\
\hline 6/10/08 4:00 & 2.293 & 0.833 & 0.362 & 162.671 & 1.414 & 0.068 & 0.020725 & 0.008509 & 0.300526 & 134.876 \\
\hline 6/10/08 5:00 & 2.293 & 0.833 & 0.362 & 162.671 & 1.413 & 0.067 & 0.020421 & 0.008330 & 0.294224 & 132.048 \\
\hline 6/10/08 6:00 & 2.286 & 0.826 & 0.355 & 159.307 & 1.412 & 0.066 & 0.020116 & 0.008153 & 0.287966 & 129.239 \\
\hline 6/10/08 7:00 & 2.288 & 0.828 & 0.357 & 160.264 & 1.412 & 0.066 & 0.020116 & 0.008153 & 0.287966 & 129.239 \\
\hline 6/10/08 8:00 & 2.288 & 0.828 & 0.357 & 160.264 & 1.414 & 0.068 & 0.020725 & 0.008509 & 0.300526 & 134.876 \\
\hline 6/10/08 9:00 & 2.288 & 0.828 & 0.357 & 160.264 & 1.414 & 0.068 & 0.020725 & 0.008509 & 0.300526 & 134.876 \\
\hline 6/10/08 10:00 & 2.286 & 0.826 & 0.355 & 159.307 & 1.412 & 0.066 & 0.020116 & 0.008153 & 0.287966 & 129.239 \\
\hline 6/10/08 11:00 & 2.272 & 0.812 & 0.340 & 152.705 & 1.41 & 0.064 & 0.019506 & 0.007803 & 0.275587 & 123.683 \\
\hline
\end{tabular}




\begin{tabular}{|c|c|c|c|c|c|c|c|c|c|c|}
\hline \multirow[b]{3}{*}{ Date Time } & \multicolumn{4}{|c|}{ V-Notch Weir } & \multicolumn{6}{|c|}{ R-Notch Weir } \\
\hline & \multicolumn{2}{|c|}{ Measured Water Level } & \multicolumn{2}{|c|}{$\begin{array}{l}\text { Calculated Rate } \\
\left(\mathrm{ft}^{3} / \mathrm{s}=448.8 \mathrm{gpm}\right)\end{array}$} & \multicolumn{3}{|c|}{ Measured Water Level } & \multicolumn{3}{|c|}{$\begin{array}{c}\text { Calculated Rate } \\
\left(\mathrm{ft}^{3} / \mathrm{s}=448.8 \mathrm{gpm}\right)\end{array}$} \\
\hline & $\begin{array}{c}\text { (ft above } \\
\text { sensor) }\end{array}$ & $\begin{array}{c}\text { (ft above } \\
\text { notch) }\end{array}$ & $\left(\mathrm{ft}^{3} / \mathrm{s}\right)$ & (gpm) & $\begin{array}{c}\text { (ft above } \\
\text { sensor) }\end{array}$ & $\begin{array}{c}\text { (ft above } \\
\text { log) }\end{array}$ & $\begin{array}{c}\text { (m above } \\
\text { log) }\end{array}$ & $\left(\mathrm{m}^{3} / \mathrm{s}\right)$ & $\left(\mathrm{ft}^{3} / \mathrm{s}\right)$ & (gpm) \\
\hline 6/10/08 12:00 & 2.262 & 0.802 & 0.330 & 148.091 & 1.409 & 0.063 & 0.019201 & 0.007629 & 0.269465 & 120.936 \\
\hline 6/10/08 13:00 & 2.24 & 0.780 & 0.308 & 138.238 & 1.406 & 0.06 & 0.018287 & 0.007117 & 0.251378 & 112.818 \\
\hline 6/10/08 14:00 & 2.215 & 0.755 & 0.284 & 127.532 & 1.405 & 0.059 & 0.017982 & 0.006949 & 0.245443 & 110.155 \\
\hline 6/10/08 15:00 & 2.205 & 0.745 & 0.275 & 123.394 & 1.402 & 0.056 & 0.017068 & 0.006453 & 0.227923 & 102.292 \\
\hline 6/10/08 16:00 & 2.189 & 0.729 & 0.261 & 116.942 & 1.403 & 0.057 & 0.017373 & 0.006617 & 0.233715 & 104.891 \\
\hline 6/10/08 17:00 & 2.175 & 0.715 & 0.248 & 111.467 & 1.401 & 0.055 & 0.016763 & 0.006291 & 0.222180 & 99.714 \\
\hline 6/10/08 18:00 & 2.174 & 0.714 & 0.248 & 111.082 & 1.402 & 0.056 & 0.017068 & 0.006453 & 0.227923 & 102.292 \\
\hline 6/10/08 19:00 & 2.171 & 0.711 & 0.245 & 109.931 & 1.402 & 0.056 & 0.017068 & 0.006453 & 0.227923 & 102.292 \\
\hline 6/10/08 20:00 & 2.18 & 0.720 & 0.253 & 113.404 & 1.404 & 0.058 & 0.017678 & 0.006782 & 0.239555 & 107.512 \\
\hline 6/10/08 21:00 & 2.188 & 0.728 & 0.260 & 116.546 & 1.403 & 0.057 & 0.017373 & 0.006617 & 0.233715 & 104.891 \\
\hline 6/10/08 22:00 & 2.199 & 0.739 & 0.269 & 120.950 & 1.405 & 0.059 & 0.017982 & 0.006949 & 0.245443 & 110.155 \\
\hline 6/10/08 23:00 & 2.21 & 0.750 & 0.280 & 125.453 & 1.406 & 0.06 & 0.018287 & 0.007117 & 0.251378 & 112.818 \\
\hline 6/11/08 0:00 & 2.221 & 0.761 & 0.290 & 130.054 & 1.408 & 0.062 & 0.018897 & 0.007457 & 0.263390 & 118.209 \\
\hline 6/11/08 1:00 & 2.228 & 0.768 & 0.296 & 133.034 & 1.407 & 0.061 & 0.018592 & 0.007287 & 0.257360 & 115.503 \\
\hline 6/11/08 2:00 & 2.232 & 0.772 & 0.300 & 134.756 & 1.409 & 0.063 & 0.019201 & 0.007629 & 0.269465 & 120.936 \\
\hline 6/11/08 3:00 & 2.239 & 0.779 & 0.307 & 137.800 & 1.408 & 0.062 & 0.018897 & 0.007457 & 0.263390 & 118.209 \\
\hline 6/11/08 4:00 & 2.418 & 0.958 & 0.513 & 230.028 & 1.438 & 0.092 & 0.028040 & 0.013150 & 0.464455 & 208.447 \\
\hline 6/11/08 5:00 & 2.415 & 0.955 & 0.509 & 228.246 & 1.438 & 0.092 & 0.028040 & 0.013150 & 0.464455 & 208.447 \\
\hline 6/11/08 6:00 & 2.366 & 0.906 & 0.446 & 200.308 & 1.431 & 0.085 & 0.025907 & 0.011728 & 0.414220 & 185.902 \\
\hline 6/11/08 7:00 & 2.342 & 0.882 & 0.418 & 187.415 & 1.425 & 0.079 & 0.024078 & 0.010553 & 0.372715 & 167.274 \\
\hline 6/11/08 8:00 & 2.312 & 0.852 & 0.383 & 172.017 & 1.416 & 0.07 & 0.021335 & 0.008869 & 0.313263 & 140.593 \\
\hline 6/11/08 9:00 & 2.307 & 0.847 & 0.378 & 169.527 & 1.418 & 0.072 & 0.021945 & 0.009235 & 0.326176 & 146.388 \\
\hline 6/11/08 10:00 & 2.289 & 0.829 & 0.358 & 160.744 & 1.414 & 0.068 & 0.020725 & 0.008509 & 0.300526 & 134.876 \\
\hline 6/11/08 11:00 & 2.284 & 0.824 & 0.353 & 158.354 & 1.416 & 0.07 & 0.021335 & 0.008869 & 0.313263 & 140.593 \\
\hline 6/11/08 12:00 & 2.271 & 0.811 & 0.339 & 152.239 & 1.412 & 0.066 & 0.020116 & 0.008153 & 0.287966 & 129.239 \\
\hline
\end{tabular}




\begin{tabular}{|c|c|c|c|c|c|c|c|c|c|c|}
\hline \multirow[b]{3}{*}{ Date Time } & \multicolumn{4}{|c|}{ V-Notch Weir } & \multicolumn{6}{|c|}{ R-Notch Weir } \\
\hline & \multicolumn{2}{|c|}{ Measured Water Level } & \multicolumn{2}{|c|}{$\begin{array}{c}\text { Calculated Rate } \\
\left(\mathrm{ft}^{3} / \mathrm{s}=448.8 \mathrm{gpm}\right)\end{array}$} & \multicolumn{3}{|c|}{ Measured Water Level } & \multicolumn{3}{|c|}{$\begin{array}{c}\text { Calculated Rate } \\
\left(\mathrm{ft}^{3} / \mathrm{s}=448.8 \mathrm{gpm}\right)\end{array}$} \\
\hline & $\begin{array}{l}\text { (ft above } \\
\text { sensor) }\end{array}$ & $\begin{array}{c}\text { (ft above } \\
\text { notch) }\end{array}$ & $\left(\mathrm{ft}^{3} / \mathrm{s}\right)$ & (gpm) & $\begin{array}{c}\text { (ft above } \\
\text { sensor) }\end{array}$ & $\begin{array}{c}\text { (ft above } \\
\text { log) }\end{array}$ & $\begin{array}{c}\text { (m above } \\
\text { log) }\end{array}$ & $\left(\mathrm{m}^{3} / \mathrm{s}\right)$ & $\left(\mathrm{ft}^{3} / \mathrm{s}\right)$ & (gpm) \\
\hline 6/11/08 13:00 & 2.274 & 0.814 & 0.342 & 153.638 & 1.413 & 0.067 & 0.020421 & 0.008330 & 0.294224 & 132.048 \\
\hline 6/11/08 14:00 & 2.253 & 0.793 & 0.321 & 144.011 & 1.409 & 0.063 & 0.019201 & 0.007629 & 0.269465 & 120.936 \\
\hline 6/11/08 15:00 & 2.236 & 0.776 & 0.304 & 136.490 & 1.41 & 0.064 & 0.019506 & 0.007803 & 0.275587 & 123.683 \\
\hline 6/11/08 16:00 & 2.22 & 0.760 & 0.289 & 129.632 & 1.407 & 0.061 & 0.018592 & 0.007287 & 0.257360 & 115.503 \\
\hline 6/11/08 17:00 & 2.217 & 0.757 & 0.286 & 128.369 & 1.408 & 0.062 & 0.018897 & 0.007457 & 0.263390 & 118.209 \\
\hline 6/11/08 18:00 & 2.196 & 0.736 & 0.267 & 119.739 & 1.406 & 0.06 & 0.018287 & 0.007117 & 0.251378 & 112.818 \\
\hline 6/11/08 19:00 & 2.197 & 0.737 & 0.268 & 120.142 & 1.406 & 0.06 & 0.018287 & 0.007117 & 0.251378 & 112.818 \\
\hline 6/11/08 20:00 & 2.604 & 1.144 & 0.796 & 357.263 & 1.51 & 0.164 & 0.049985 & 0.030593 & 1.080542 & 484.947 \\
\hline 6/11/08 21:00 & 4.117 & 2.657 & 6.480 & 2908.408 & 3.398 & 2.052 & 0.625419 & 1.320512 & 46.640266 & 20932.152 \\
\hline 6/11/08 22:00 & 3.908 & 2.448 & 5.284 & 2371.263 & 2.947 & 1.601 & 0.487961 & 0.910168 & 32.146992 & 14427.570 \\
\hline 6/11/08 23:00 & 3.34 & 1.880 & 2.737 & 1228.570 & 2.256 & 0.91 & 0.277354 & 0.390614 & 13.796431 & 6191.838 \\
\hline 6/12/08 0:00 & 3.126 & 1.666 & 2.026 & 909.444 & 1.921 & 0.575 & 0.175251 & 0.196732 & 6.948548 & 3118.508 \\
\hline 6/12/08 1:00 & 2.94 & 1.480 & 1.510 & 677.464 & 1.741 & 0.395 & 0.120390 & 0.112424 & 3.970784 & 1782.088 \\
\hline 6/12/08 2:00 & 2.895 & 1.435 & 1.398 & 627.397 & 1.68 & 0.334 & 0.101798 & 0.087605 & 3.094197 & 1388.676 \\
\hline 6/12/08 3:00 & 2.813 & 1.353 & 1.208 & 542.023 & 1.609 & 0.263 & 0.080158 & 0.061449 & 2.170368 & 974.061 \\
\hline 6/12/08 4:00 & 2.785 & 1.325 & 1.147 & 514.571 & 1.571 & 0.225 & 0.068577 & 0.048774 & 1.722693 & 773.145 \\
\hline 6/12/08 5:00 & 2.721 & 1.261 & 1.014 & 455.009 & 1.549 & 0.203 & 0.061871 & 0.041895 & 1.479724 & 664.100 \\
\hline 6/12/08 6:00 & 2.697 & 1.237 & 0.967 & 433.798 & 1.523 & 0.177 & 0.053947 & 0.034228 & 1.208936 & 542.570 \\
\hline 6/12/08 7:00 & 2.648 & 1.188 & 0.874 & 392.364 & 1.504 & 0.158 & 0.048156 & 0.028962 & 1.022924 & 459.088 \\
\hline 6/12/08 8:00 & 2.631 & 1.171 & 0.844 & 378.568 & 1.492 & 0.146 & 0.044499 & 0.025790 & 0.910896 & 408.810 \\
\hline 6/12/08 9:00 & 2.589 & 1.129 & 0.770 & 345.746 & 1.48 & 0.134 & 0.040841 & 0.022743 & 0.803289 & 360.516 \\
\hline 6/12/08 10:00 & 2.578 & 1.118 & 0.752 & 337.443 & 1.473 & 0.127 & 0.038708 & 0.021026 & 0.742632 & 333.293 \\
\hline 6/12/08 11:00 & 2.56 & 1.100 & 0.722 & 324.118 & 1.466 & 0.12 & 0.036574 & 0.019354 & 0.683586 & 306.793 \\
\hline 6/12/08 12:00 & 2.542 & 1.082 & 0.693 & 311.113 & 1.459 & 0.113 & 0.034441 & 0.017729 & 0.626193 & 281.035 \\
\hline 6/12/08 13:00 & 2.509 & 1.049 & 0.642 & 288.095 & 1.452 & 0.106 & 0.032307 & 0.016152 & 0.570504 & 256.042 \\
\hline
\end{tabular}




\begin{tabular}{|c|c|c|c|c|c|c|c|c|c|c|}
\hline \multirow[b]{3}{*}{ Date Time } & \multicolumn{4}{|c|}{ V-Notch Weir } & \multicolumn{6}{|c|}{ R-Notch Weir } \\
\hline & \multicolumn{2}{|c|}{ Measured Water Level } & \multicolumn{2}{|c|}{$\begin{array}{c}\text { Calculated Rate } \\
\left(\mathrm{ft}^{3} / \mathrm{s}=448.8 \mathrm{gpm}\right)\end{array}$} & \multicolumn{3}{|c|}{ Measured Water Level } & \multicolumn{3}{|c|}{$\begin{array}{c}\text { Calculated Rate } \\
\left(\mathrm{ft}^{3} / \mathrm{s}=448.8 \mathrm{gpm}\right)\end{array}$} \\
\hline & $\begin{array}{c}\text { (ft above } \\
\text { sensor) }\end{array}$ & $\begin{array}{c}\text { (ft above } \\
\text { notch) }\end{array}$ & $\left(\mathrm{ft}^{3} / \mathrm{s}\right)$ & (gpm) & $\begin{array}{c}\text { (ft above } \\
\text { sensor) }\end{array}$ & $\begin{array}{c}\text { (ft above } \\
\text { log) }\end{array}$ & $\begin{array}{c}\text { (m above } \\
\text { log) }\end{array}$ & $\left(\mathrm{m}^{3} / \mathrm{s}\right)$ & $\left(\mathrm{ft}^{3} / \mathrm{s}\right)$ & (gpm) \\
\hline 6/12/08 14:00 & 2.48 & 1.020 & 0.599 & 268.737 & 1.446 & 0.1 & 0.030479 & 0.014841 & 0.524166 & 235.246 \\
\hline 6/12/08 15:00 & 2.455 & 0.995 & 0.563 & 252.694 & 1.442 & 0.096 & 0.029259 & 0.013987 & 0.494010 & 221.711 \\
\hline 6/12/08 16:00 & 2.425 & 0.965 & 0.522 & 234.219 & 1.436 & 0.09 & 0.027431 & 0.012738 & 0.449907 & 201.918 \\
\hline 6/12/08 17:00 & 2.409 & 0.949 & 0.501 & 224.707 & 1.435 & 0.089 & 0.027126 & 0.012534 & 0.442691 & 198.680 \\
\hline 6/12/08 18:00 & 2.385 & 0.925 & 0.470 & 210.881 & 1.43 & 0.084 & 0.025602 & 0.011529 & 0.407202 & 182.752 \\
\hline 6/12/08 19:00 & 2.375 & 0.915 & 0.457 & 205.276 & 1.429 & 0.083 & 0.025297 & 0.011331 & 0.400223 & 179.620 \\
\hline 6/12/08 20:00 & 2.371 & 0.911 & 0.452 & 203.059 & 1.429 & 0.083 & 0.025297 & 0.011331 & 0.400223 & 179.620 \\
\hline 6/12/08 21:00 & 2.369 & 0.909 & 0.450 & 201.955 & 1.429 & 0.083 & 0.025297 & 0.011331 & 0.400223 & 179.620 \\
\hline $6 / 12 / 08$ 22:00 & 2.368 & 0.908 & 0.449 & 201.405 & 1.425 & 0.079 & 0.024078 & 0.010553 & 0.372715 & 167.274 \\
\hline 6/12/08 23:00 & 2.377 & 0.917 & 0.460 & 206.389 & 1.427 & 0.081 & 0.024688 & 0.010940 & 0.386387 & 173.411 \\
\hline 6/13/08 0:00 & 2.378 & 0.918 & 0.461 & 206.948 & 1.426 & 0.08 & 0.024383 & 0.010746 & 0.379531 & 170.333 \\
\hline 6/13/08 1:00 & 2.38 & 0.920 & 0.464 & 208.067 & 1.425 & 0.079 & 0.024078 & 0.010553 & 0.372715 & 167.274 \\
\hline 6/13/08 2:00 & 2.375 & 0.915 & 0.457 & 205.276 & 1.424 & 0.078 & 0.023773 & 0.010361 & 0.365940 & 164.234 \\
\hline 6/13/08 3:00 & 2.375 & 0.915 & 0.457 & 205.276 & 1.423 & 0.077 & 0.023468 & 0.010170 & 0.359208 & 161.212 \\
\hline 6/13/08 4:00 & 2.373 & 0.913 & 0.455 & 204.165 & 1.422 & 0.076 & 0.023164 & 0.009981 & 0.352516 & 158.209 \\
\hline 6/13/08 5:00 & 2.37 & 0.910 & 0.451 & 202.507 & 1.421 & 0.075 & 0.022859 & 0.009792 & 0.345867 & 155.225 \\
\hline 6/13/08 6:00 & 2.361 & 0.901 & 0.440 & 197.579 & 1.421 & 0.075 & 0.022859 & 0.009792 & 0.345867 & 155.225 \\
\hline 6/13/08 7:00 & 2.358 & 0.898 & 0.437 & 195.953 & 1.42 & 0.074 & 0.022554 & 0.009605 & 0.339261 & 152.260 \\
\hline 6/13/08 8:00 & 2.358 & 0.898 & 0.437 & 195.953 & 1.422 & 0.076 & 0.023164 & 0.009981 & 0.352516 & 158.209 \\
\hline 6/13/08 9:00 & 2.354 & 0.894 & 0.432 & 193.797 & 1.42 & 0.074 & 0.022554 & 0.009605 & 0.339261 & 152.260 \\
\hline 6/13/08 10:00 & 2.335 & 0.875 & 0.409 & 183.751 & 1.418 & 0.072 & 0.021945 & 0.009235 & 0.326176 & 146.388 \\
\hline 6/13/08 11:00 & 2.322 & 0.862 & 0.395 & 177.061 & 1.416 & 0.07 & 0.021335 & 0.008869 & 0.313263 & 140.593 \\
\hline 6/13/08 12:00 & 2.316 & 0.856 & 0.388 & 174.024 & 1.411 & 0.065 & 0.019811 & 0.007977 & 0.281754 & 126.451 \\
\hline 6/13/08 13:00 & 2.293 & 0.833 & 0.362 & 162.671 & 1.41 & 0.064 & 0.019506 & 0.007803 & 0.275587 & 123.683 \\
\hline 6/13/08 14:00 & 2.271 & 0.811 & 0.339 & 152.239 & 1.406 & 0.06 & 0.018287 & 0.007117 & 0.251378 & 112.818 \\
\hline
\end{tabular}




\begin{tabular}{|c|c|c|c|c|c|c|c|c|c|c|}
\hline \multirow[b]{3}{*}{ Date Time } & \multicolumn{4}{|c|}{ V-Notch Weir } & \multicolumn{6}{|c|}{ R-Notch Weir } \\
\hline & \multicolumn{2}{|c|}{ Measured Water Level } & \multicolumn{2}{|c|}{$\begin{array}{c}\text { Calculated Rate } \\
\left(\mathrm{ft}^{3} / \mathrm{s}=448.8 \mathrm{gpm}\right)\end{array}$} & \multicolumn{3}{|c|}{ Measured Water Level } & \multicolumn{3}{|c|}{$\begin{array}{c}\text { Calculated Rate } \\
\left(\mathrm{ft}^{3} / \mathrm{s}=448.8 \mathrm{gpm}\right)\end{array}$} \\
\hline & $\begin{array}{c}\text { (ft above } \\
\text { sensor) }\end{array}$ & $\begin{array}{c}\text { (ft above } \\
\text { notch) }\end{array}$ & $\left(\mathrm{ft}^{3} / \mathrm{s}\right)$ & (gpm) & $\begin{array}{c}\text { (ft above } \\
\text { sensor) }\end{array}$ & $\begin{array}{c}\text { (ft above } \\
\text { log) }\end{array}$ & $\begin{array}{c}\text { (m above } \\
\text { log) }\end{array}$ & $\left(\mathrm{m}^{3} / \mathrm{s}\right)$ & $\left(\mathrm{ft}^{3} / \mathrm{s}\right)$ & (gpm) \\
\hline 6/13/08 15:00 & 2.255 & 0.795 & 0.323 & 144.912 & 1.405 & 0.059 & 0.017982 & 0.006949 & 0.245443 & 110.155 \\
\hline 6/13/08 16:00 & 2.239 & 0.779 & 0.307 & 137.800 & 1.401 & 0.055 & 0.016763 & 0.006291 & 0.222180 & 99.714 \\
\hline 6/13/08 17:00 & 2.223 & 0.763 & 0.292 & 130.902 & 1.402 & 0.056 & 0.017068 & 0.006453 & 0.227923 & 102.292 \\
\hline 6/13/08 18:00 & 2.213 & 0.753 & 0.282 & 126.698 & 1.401 & 0.055 & 0.016763 & 0.006291 & 0.222180 & 99.714 \\
\hline 6/13/08 19:00 & 2.215 & 0.755 & 0.284 & 127.532 & 1.401 & 0.055 & 0.016763 & 0.006291 & 0.222180 & 99.714 \\
\hline 6/13/08 20:00 & 2.212 & 0.752 & 0.281 & 126.282 & 1.4 & 0.054 & 0.016458 & 0.006129 & 0.216486 & 97.159 \\
\hline 6/13/08 21:00 & 2.221 & 0.761 & 0.290 & 130.054 & 1.402 & 0.056 & 0.017068 & 0.006453 & 0.227923 & 102.292 \\
\hline 6/13/08 22:00 & 2.234 & 0.774 & 0.302 & 135.621 & 1.404 & 0.058 & 0.017678 & 0.006782 & 0.239555 & 107.512 \\
\hline 6/13/08 23:00 & 2.245 & 0.785 & 0.313 & 140.442 & 1.403 & 0.057 & 0.017373 & 0.006617 & 0.233715 & 104.891 \\
\hline 6/14/08 0:00 & 2.251 & 0.791 & 0.319 & 143.114 & 1.404 & 0.058 & 0.017678 & 0.006782 & 0.239555 & 107.512 \\
\hline 6/14/08 1:00 & 2.265 & 0.805 & 0.333 & 149.466 & 1.406 & 0.06 & 0.018287 & 0.007117 & 0.251378 & 112.818 \\
\hline 6/14/08 2:00 & 2.269 & 0.809 & 0.337 & 151.312 & 1.407 & 0.061 & 0.018592 & 0.007287 & 0.257360 & 115.503 \\
\hline 6/14/08 3:00 & 2.274 & 0.814 & 0.342 & 153.638 & 1.406 & 0.06 & 0.018287 & 0.007117 & 0.251378 & 112.818 \\
\hline 6/14/08 4:00 & 2.279 & 0.819 & 0.348 & 155.985 & 1.408 & 0.062 & 0.018897 & 0.007457 & 0.263390 & 118.209 \\
\hline 6/14/08 5:00 & 2.281 & 0.821 & 0.350 & 156.930 & 1.407 & 0.061 & 0.018592 & 0.007287 & 0.257360 & 115.503 \\
\hline 6/14/08 6:00 & 2.279 & 0.819 & 0.348 & 155.985 & 1.407 & 0.061 & 0.018592 & 0.007287 & 0.257360 & 115.503 \\
\hline 6/14/08 7:00 & 2.276 & 0.816 & 0.344 & 154.574 & 1.406 & 0.06 & 0.018287 & 0.007117 & 0.251378 & 112.818 \\
\hline 6/14/08 8:00 & 2.276 & 0.816 & 0.344 & 154.574 & 1.408 & 0.062 & 0.018897 & 0.007457 & 0.263390 & 118.209 \\
\hline 6/14/08 9:00 & 2.276 & 0.816 & 0.344 & 154.574 & 1.405 & 0.059 & 0.017982 & 0.006949 & 0.245443 & 110.155 \\
\hline 6/14/08 10:00 & 2.262 & 0.802 & 0.330 & 148.091 & 1.405 & 0.059 & 0.017982 & 0.006949 & 0.245443 & 110.155 \\
\hline 6/14/08 11:00 & 2.249 & 0.789 & 0.317 & 142.220 & 1.405 & 0.059 & 0.017982 & 0.006949 & 0.245443 & 110.155 \\
\hline 6/14/08 12:00 & 2.238 & 0.778 & 0.306 & 137.362 & 1.401 & 0.055 & 0.016763 & 0.006291 & 0.222180 & 99.714 \\
\hline 6/14/08 13:00 & 2.215 & 0.755 & 0.284 & 127.532 & 1.398 & 0.052 & 0.015849 & 0.005811 & 0.205247 & 92.115 \\
\hline 6/14/08 14:00 & 2.205 & 0.745 & 0.275 & 123.394 & 1.396 & 0.05 & 0.015239 & 0.005499 & 0.194210 & 87.161 \\
\hline 6/14/08 15:00 & 2.183 & 0.723 & 0.255 & 114.576 & 1.393 & 0.047 & 0.014325 & 0.005041 & 0.178040 & 79.904 \\
\hline
\end{tabular}




\begin{tabular}{|c|c|c|c|c|c|c|c|c|c|c|}
\hline \multirow[b]{3}{*}{ Date Time } & \multicolumn{4}{|c|}{ V-Notch Weir } & \multicolumn{6}{|c|}{ R-Notch Weir } \\
\hline & \multicolumn{2}{|c|}{ Measured Water Level } & \multicolumn{2}{|c|}{$\begin{array}{c}\text { Calculated Rate } \\
\left(\mathrm{ft}^{3} / \mathrm{s}=448.8 \mathrm{gpm}\right)\end{array}$} & \multicolumn{3}{|c|}{ Measured Water Level } & \multicolumn{3}{|c|}{$\begin{array}{c}\text { Calculated Rate } \\
\left(\mathrm{ft}^{3} / \mathrm{s}=448.8 \mathrm{gpm}\right)\end{array}$} \\
\hline & $\begin{array}{c}\text { (ft above } \\
\text { sensor) }\end{array}$ & $\begin{array}{c}\text { (ft above } \\
\text { notch) }\end{array}$ & $\left(\mathrm{ft}^{3} / \mathrm{s}\right)$ & (gpm) & $\begin{array}{c}\text { (ft above } \\
\text { sensor) }\end{array}$ & $\begin{array}{c}\text { (ft above } \\
\text { log) }\end{array}$ & $\begin{array}{c}\text { (m above } \\
\text { log) }\end{array}$ & $\left(\mathrm{m}^{3} / \mathrm{s}\right)$ & $\left(\mathrm{ft}^{3} / \mathrm{s}\right)$ & (gpm) \\
\hline 6/14/08 16:00 & 2.165 & 0.705 & 0.240 & 107.652 & 1.394 & 0.048 & 0.014630 & 0.005192 & 0.183377 & 82.300 \\
\hline 6/14/08 17:00 & 2.156 & 0.696 & 0.232 & 104.286 & 1.391 & 0.045 & 0.013715 & 0.004743 & 0.167523 & 75.184 \\
\hline 6/14/08 18:00 & 2.156 & 0.696 & 0.232 & 104.286 & 1.394 & 0.048 & 0.014630 & 0.005192 & 0.183377 & 82.300 \\
\hline 6/14/08 19:00 & 2.16 & 0.700 & 0.236 & 105.774 & 1.392 & 0.046 & 0.014020 & 0.004891 & 0.172754 & 77.532 \\
\hline 6/14/08 20:00 & 2.169 & 0.709 & 0.243 & 109.168 & 1.395 & 0.049 & 0.014934 & 0.005345 & 0.188768 & 84.719 \\
\hline 6/14/08 21:00 & 2.183 & 0.723 & 0.255 & 114.576 & 1.397 & 0.051 & 0.015544 & 0.005654 & 0.199703 & 89.627 \\
\hline 6/14/08 22:00 & 2.191 & 0.731 & 0.262 & 117.737 & 1.397 & 0.051 & 0.015544 & 0.005654 & 0.199703 & 89.627 \\
\hline 6/14/08 23:00 & 2.202 & 0.742 & 0.272 & 122.168 & 1.398 & 0.052 & 0.015849 & 0.005811 & 0.205247 & 92.115 \\
\hline 6/15/08 0:00 & 2.213 & 0.753 & 0.282 & 126.698 & 1.4 & 0.054 & 0.016458 & 0.006129 & 0.216486 & 97.159 \\
\hline 6/15/08 1:00 & 2.221 & 0.761 & 0.290 & 130.054 & 1.401 & 0.055 & 0.016763 & 0.006291 & 0.222180 & 99.714 \\
\hline 6/15/08 2:00 & 2.221 & 0.761 & 0.290 & 130.054 & 1.4 & 0.054 & 0.016458 & 0.006129 & 0.216486 & 97.159 \\
\hline 6/15/08 3:00 & 2.235 & 0.775 & 0.303 & 136.055 & 1.402 & 0.056 & 0.017068 & 0.006453 & 0.227923 & 102.292 \\
\hline 6/15/08 4:00 & 2.239 & 0.779 & 0.307 & 137.800 & 1.404 & 0.058 & 0.017678 & 0.006782 & 0.239555 & 107.512 \\
\hline 6/15/08 5:00 & 2.244 & 0.784 & 0.312 & 139.999 & 1.403 & 0.057 & 0.017373 & 0.006617 & 0.233715 & 104.891 \\
\hline 6/15/08 6:00 & 2.244 & 0.784 & 0.312 & 139.999 & 1.405 & 0.059 & 0.017982 & 0.006949 & 0.245443 & 110.155 \\
\hline 6/15/08 7:00 & 2.248 & 0.788 & 0.316 & 141.774 & 1.404 & 0.058 & 0.017678 & 0.006782 & 0.239555 & 107.512 \\
\hline 6/15/08 8:00 & 2.251 & 0.791 & 0.319 & 143.114 & 1.404 & 0.058 & 0.017678 & 0.006782 & 0.239555 & 107.512 \\
\hline 6/15/08 9:00 & 2.763 & 1.303 & 1.100 & 493.601 & 1.553 & 0.207 & 0.063091 & 0.043120 & 1.522979 & 683.513 \\
\hline 6/15/08 10:00 & 2.826 & 1.366 & 1.237 & 555.060 & 1.631 & 0.285 & 0.086864 & 0.069221 & 2.444886 & 1097.265 \\
\hline 6/15/08 11:00 & 2.86 & 1.400 & 1.315 & 590.040 & 1.648 & 0.302 & 0.092045 & 0.075435 & 2.664361 & 1195.765 \\
\hline 6/15/08 12:00 & 2.762 & 1.302 & 1.098 & 492.660 & 1.552 & 0.206 & 0.062786 & 0.042812 & 1.512127 & 678.642 \\
\hline 6/15/08 13:00 & 2.646 & 1.186 & 0.871 & 390.725 & 1.503 & 0.157 & 0.047851 & 0.028693 & 1.013424 & 454.824 \\
\hline 6/15/08 14:00 & 2.577 & 1.117 & 0.750 & 336.695 & 1.473 & 0.127 & 0.038708 & 0.021026 & 0.742632 & 333.293 \\
\hline 6/15/08 15:00 & 2.508 & 1.048 & 0.640 & 287.414 & 1.454 & 0.108 & 0.032917 & 0.016598 & 0.586238 & 263.104 \\
\hline 6/15/08 16:00 & 2.462 & 1.002 & 0.573 & 257.127 & 1.445 & 0.099 & 0.030174 & 0.014626 & 0.516571 & 231.837 \\
\hline
\end{tabular}




\begin{tabular}{|c|c|c|c|c|c|c|c|c|c|c|}
\hline \multirow[b]{3}{*}{ Date Time } & \multicolumn{4}{|c|}{ V-Notch Weir } & \multicolumn{6}{|c|}{ R-Notch Weir } \\
\hline & \multicolumn{2}{|c|}{ Measured Water Level } & \multicolumn{2}{|c|}{$\begin{array}{c}\text { Calculated Rate } \\
\left(\mathrm{ft}^{3} / \mathrm{s}=448.8 \mathrm{gpm}\right)\end{array}$} & \multicolumn{3}{|c|}{ Measured Water Level } & \multicolumn{3}{|c|}{$\begin{array}{c}\text { Calculated Rate } \\
\left(\mathrm{ft}^{3} / \mathrm{s}=448.8 \mathrm{gpm}\right)\end{array}$} \\
\hline & $\begin{array}{c}\text { (ft above } \\
\text { sensor) }\end{array}$ & $\begin{array}{c}\text { (ft above } \\
\text { notch) }\end{array}$ & $\left(\mathrm{ft}^{3} / \mathrm{s}\right)$ & (gpm) & $\begin{array}{c}\text { (ft above } \\
\text { sensor) }\end{array}$ & $\begin{array}{c}\text { (ft above } \\
\text { log) }\end{array}$ & $\begin{array}{c}\text { (m above } \\
\text { log) }\end{array}$ & $\left(\mathrm{m}^{3} / \mathrm{s}\right)$ & $\left(\mathrm{ft}^{3} / \mathrm{s}\right)$ & (gpm) \\
\hline 6/15/08 17:00 & 2.415 & 0.955 & 0.509 & 228.246 & 1.436 & 0.09 & 0.027431 & 0.012738 & 0.449907 & 201.918 \\
\hline 6/15/08 18:00 & 2.387 & 0.927 & 0.472 & 212.014 & 1.437 & 0.091 & 0.027735 & 0.012943 & 0.457162 & 205.174 \\
\hline 6/15/08 19:00 & 2.362 & 0.902 & 0.441 & 198.123 & 1.435 & 0.089 & 0.027126 & 0.012534 & 0.442691 & 198.680 \\
\hline 6/15/08 20:00 & 2.354 & 0.894 & 0.432 & 193.797 & 1.43 & 0.084 & 0.025602 & 0.011529 & 0.407202 & 182.752 \\
\hline 6/15/08 21:00 & 2.343 & 0.883 & 0.419 & 187.942 & 1.43 & 0.084 & 0.025602 & 0.011529 & 0.407202 & 182.752 \\
\hline 6/15/08 22:00 & 2.338 & 0.878 & 0.413 & 185.316 & 1.427 & 0.081 & 0.024688 & 0.010940 & 0.386387 & 173.411 \\
\hline 6/15/08 23:00 & 2.337 & 0.877 & 0.412 & 184.793 & 1.426 & 0.08 & 0.024383 & 0.010746 & 0.379531 & 170.333 \\
\hline 6/16/08 0:00 & 2.336 & 0.876 & 0.411 & 184.272 & 1.426 & 0.08 & 0.024383 & 0.010746 & 0.379531 & 170.333 \\
\hline 6/16/08 1:00 & 2.331 & 0.871 & 0.405 & 181.677 & 1.424 & 0.078 & 0.023773 & 0.010361 & 0.365940 & 164.234 \\
\hline 6/16/08 2:00 & 2.326 & 0.866 & 0.399 & 179.104 & 1.423 & 0.077 & 0.023468 & 0.010170 & 0.359208 & 161.212 \\
\hline 6/16/08 3:00 & 2.324 & 0.864 & 0.397 & 178.081 & 1.42 & 0.074 & 0.022554 & 0.009605 & 0.339261 & 152.260 \\
\hline 6/16/08 4:00 & 2.319 & 0.859 & 0.391 & 175.539 & 1.422 & 0.076 & 0.023164 & 0.009981 & 0.352516 & 158.209 \\
\hline 6/16/08 5:00 & 2.321 & 0.861 & 0.393 & 176.553 & 1.422 & 0.076 & 0.023164 & 0.009981 & 0.352516 & 158.209 \\
\hline 6/16/08 6:00 & 2.314 & 0.854 & 0.386 & 173.019 & 1.421 & 0.075 & 0.022859 & 0.009792 & 0.345867 & 155.225 \\
\hline 6/16/08 7:00 & 2.309 & 0.849 & 0.380 & 170.520 & 1.418 & 0.072 & 0.021945 & 0.009235 & 0.326176 & 146.388 \\
\hline 6/16/08 8:00 & 2.309 & 0.849 & 0.380 & 170.520 & 1.417 & 0.071 & 0.021640 & 0.009052 & 0.319698 & 143.480 \\
\hline 6/16/08 9:00 & 2.3 & 0.840 & 0.370 & 166.078 & 1.417 & 0.071 & 0.021640 & 0.009052 & 0.319698 & 143.480 \\
\hline 6/16/08 10:00 & 2.293 & 0.833 & 0.362 & 162.671 & 1.417 & 0.071 & 0.021640 & 0.009052 & 0.319698 & 143.480 \\
\hline 6/16/08 11:00 & 2.286 & 0.826 & 0.355 & 159.307 & 1.415 & 0.069 & 0.021030 & 0.008688 & 0.306873 & 137.724 \\
\hline 6/16/08 12:00 & 2.273 & 0.813 & 0.341 & 153.171 & 1.413 & 0.067 & 0.020421 & 0.008330 & 0.294224 & 132.048 \\
\hline 6/16/08 13:00 & 2.262 & 0.802 & 0.330 & 148.091 & 1.409 & 0.063 & 0.019201 & 0.007629 & 0.269465 & 120.936 \\
\hline 6/16/08 14:00 & 2.249 & 0.789 & 0.317 & 142.220 & 1.41 & 0.064 & 0.019506 & 0.007803 & 0.275587 & 123.683 \\
\hline 6/16/08 15:00 & 2.235 & 0.775 & 0.303 & 136.055 & 1.409 & 0.063 & 0.019201 & 0.007629 & 0.269465 & 120.936 \\
\hline 6/16/08 16:00 & 2.229 & 0.769 & 0.297 & 133.464 & 1.407 & 0.061 & 0.018592 & 0.007287 & 0.257360 & 115.503 \\
\hline 6/16/08 17:00 & 2.22 & 0.760 & 0.289 & 129.632 & 1.408 & 0.062 & 0.018897 & 0.007457 & 0.263390 & 118.209 \\
\hline
\end{tabular}




\begin{tabular}{|c|c|c|c|c|c|c|c|c|c|c|}
\hline \multirow[b]{3}{*}{ Date Time } & \multicolumn{4}{|c|}{ V-Notch Weir } & \multicolumn{6}{|c|}{ R-Notch Weir } \\
\hline & \multicolumn{2}{|c|}{ Measured Water Level } & \multicolumn{2}{|c|}{$\begin{array}{c}\text { Calculated Rate } \\
\left(\mathrm{ft}^{3} / \mathrm{s}=448.8 \mathrm{gpm}\right)\end{array}$} & \multicolumn{3}{|c|}{ Measured Water Level } & \multicolumn{3}{|c|}{$\begin{array}{c}\text { Calculated Rate } \\
\left(\mathrm{ft}^{3} / \mathrm{s}=448.8 \mathrm{gpm}\right)\end{array}$} \\
\hline & $\begin{array}{c}\text { (ft above } \\
\text { sensor) }\end{array}$ & $\begin{array}{c}\text { (ft above } \\
\text { notch) }\end{array}$ & $\left(\mathrm{ft}^{3} / \mathrm{s}\right)$ & (gpm) & $\begin{array}{c}\text { (ft above } \\
\text { sensor) }\end{array}$ & $\begin{array}{c}\text { (ft above } \\
\text { log) }\end{array}$ & $\begin{array}{c}\text { (m above } \\
\text { log) }\end{array}$ & $\left(\mathrm{m}^{3} / \mathrm{s}\right)$ & $\left(\mathrm{ft}^{3} / \mathrm{s}\right)$ & (gpm) \\
\hline 6/16/08 18:00 & 2.215 & 0.755 & 0.284 & 127.532 & 1.406 & 0.06 & 0.018287 & 0.007117 & 0.251378 & 112.818 \\
\hline 6/16/08 19:00 & 2.21 & 0.750 & 0.280 & 125.453 & 1.407 & 0.061 & 0.018592 & 0.007287 & 0.257360 & 115.503 \\
\hline 6/16/08 20:00 & 2.212 & 0.752 & 0.281 & 126.282 & 1.407 & 0.061 & 0.018592 & 0.007287 & 0.257360 & 115.503 \\
\hline 6/16/08 21:00 & 2.218 & 0.758 & 0.287 & 128.789 & 1.409 & 0.063 & 0.019201 & 0.007629 & 0.269465 & 120.936 \\
\hline 6/16/08 22:00 & 2.229 & 0.769 & 0.297 & 133.464 & 1.408 & 0.062 & 0.018897 & 0.007457 & 0.263390 & 118.209 \\
\hline 6/16/08 23:00 & 2.235 & 0.775 & 0.303 & 136.055 & 1.41 & 0.064 & 0.019506 & 0.007803 & 0.275587 & 123.683 \\
\hline 6/17/08 0:00 & 2.249 & 0.789 & 0.317 & 142.220 & 1.411 & 0.065 & 0.019811 & 0.007977 & 0.281754 & 126.451 \\
\hline 6/17/08 1:00 & 2.246 & 0.786 & 0.314 & 140.885 & 1.41 & 0.064 & 0.019506 & 0.007803 & 0.275587 & 123.683 \\
\hline 6/17/08 2:00 & 2.253 & 0.793 & 0.321 & 144.011 & 1.41 & 0.064 & 0.019506 & 0.007803 & 0.275587 & 123.683 \\
\hline 6/17/08 3:00 & 2.258 & 0.798 & 0.326 & 146.269 & 1.414 & 0.068 & 0.020725 & 0.008509 & 0.300526 & 134.876 \\
\hline 6/17/08 4:00 & 2.26 & 0.800 & 0.328 & 147.178 & 1.413 & 0.067 & 0.020421 & 0.008330 & 0.294224 & 132.048 \\
\hline 6/17/08 5:00 & 2.255 & 0.795 & 0.323 & 144.912 & 1.412 & 0.066 & 0.020116 & 0.008153 & 0.287966 & 129.239 \\
\hline 6/17/08 6:00 & 2.26 & 0.800 & 0.328 & 147.178 & 1.414 & 0.068 & 0.020725 & 0.008509 & 0.300526 & 134.876 \\
\hline 6/17/08 7:00 & 2.257 & 0.797 & 0.325 & 145.816 & 1.412 & 0.066 & 0.020116 & 0.008153 & 0.287966 & 129.239 \\
\hline 6/17/08 8:00 & 2.302 & 0.842 & 0.372 & 167.059 & 1.414 & 0.068 & 0.020725 & 0.008509 & 0.300526 & 134.876 \\
\hline 6/17/08 9:00 & 2.304 & 0.844 & 0.374 & 168.044 & 1.412 & 0.066 & 0.020116 & 0.008153 & 0.287966 & 129.239 \\
\hline 6/17/08 10:00 & 2.288 & 0.828 & 0.357 & 160.264 & 1.409 & 0.063 & 0.019201 & 0.007629 & 0.269465 & 120.936 \\
\hline 6/17/08 11:00 & 2.281 & 0.821 & 0.350 & 156.930 & 1.41 & 0.064 & 0.019506 & 0.007803 & 0.275587 & 123.683 \\
\hline 6/17/08 12:00 & 2.268 & 0.808 & 0.336 & 150.849 & 1.408 & 0.062 & 0.018897 & 0.007457 & 0.263390 & 118.209 \\
\hline 6/17/08 13:00 & 2.252 & 0.792 & 0.320 & 143.562 & 1.404 & 0.058 & 0.017678 & 0.006782 & 0.239555 & 107.512 \\
\hline 6/17/08 14:00 & 2.246 & 0.786 & 0.314 & 140.885 & 1.405 & 0.059 & 0.017982 & 0.006949 & 0.245443 & 110.155 \\
\hline 6/17/08 15:00 & 2.229 & 0.769 & 0.297 & 133.464 & 1.404 & 0.058 & 0.017678 & 0.006782 & 0.239555 & 107.512 \\
\hline 6/17/08 16:00 & 2.22 & 0.760 & 0.289 & 129.632 & 1.4 & 0.054 & 0.016458 & 0.006129 & 0.216486 & 97.159 \\
\hline 6/17/08 17:00 & 2.209 & 0.749 & 0.279 & 125.039 & 1.401 & 0.055 & 0.016763 & 0.006291 & 0.222180 & 99.714 \\
\hline 6/17/08 18:00 & 2.211 & 0.751 & 0.280 & 125.867 & 1.402 & 0.056 & 0.017068 & 0.006453 & 0.227923 & 102.292 \\
\hline
\end{tabular}




\begin{tabular}{|c|c|c|c|c|c|c|c|c|c|c|}
\hline \multirow[b]{3}{*}{ Date Time } & \multicolumn{4}{|c|}{ V-Notch Weir } & \multicolumn{6}{|c|}{ R-Notch Weir } \\
\hline & \multicolumn{2}{|c|}{ Measured Water Level } & \multicolumn{2}{|c|}{$\begin{array}{c}\text { Calculated Rate } \\
\left(\mathrm{ft}^{3} / \mathrm{s}=448.8 \mathrm{gpm}\right)\end{array}$} & \multicolumn{3}{|c|}{ Measured Water Level } & \multicolumn{3}{|c|}{$\begin{array}{c}\text { Calculated Rate } \\
\left(\mathrm{ft}^{3} / \mathrm{s}=448.8 \mathrm{gpm}\right)\end{array}$} \\
\hline & $\begin{array}{c}\text { (ft above } \\
\text { sensor) }\end{array}$ & $\begin{array}{c}\text { (ft above } \\
\text { notch) }\end{array}$ & $\left(\mathrm{ft}^{3} / \mathrm{s}\right)$ & (gpm) & $\begin{array}{c}\text { (ft above } \\
\text { sensor) }\end{array}$ & $\begin{array}{c}\text { (ft above } \\
\text { log) }\end{array}$ & $\begin{array}{c}\text { (m above } \\
\text { log) }\end{array}$ & $\left(\mathrm{m}^{3} / \mathrm{s}\right)$ & $\left(\mathrm{ft}^{3} / \mathrm{s}\right)$ & (gpm) \\
\hline 6/17/08 19:00 & 2.211 & 0.751 & 0.280 & 125.867 & 1.402 & 0.056 & 0.017068 & 0.006453 & 0.227923 & 102.292 \\
\hline 6/17/08 20:00 & 2.215 & 0.755 & 0.284 & 127.532 & 1.405 & 0.059 & 0.017982 & 0.006949 & 0.245443 & 110.155 \\
\hline 6/17/08 21:00 & 2.222 & 0.762 & 0.291 & 130.478 & 1.405 & 0.059 & 0.017982 & 0.006949 & 0.245443 & 110.155 \\
\hline 6/17/08 22:00 & 2.232 & 0.772 & 0.300 & 134.756 & 1.407 & 0.061 & 0.018592 & 0.007287 & 0.257360 & 115.503 \\
\hline 6/17/08 23:00 & 2.253 & 0.793 & 0.321 & 144.011 & 1.406 & 0.06 & 0.018287 & 0.007117 & 0.251378 & 112.818 \\
\hline 6/18/08 0:00 & 2.311 & 0.851 & 0.382 & 171.517 & 1.417 & 0.071 & 0.021640 & 0.009052 & 0.319698 & 143.480 \\
\hline 6/18/08 1:00 & 2.341 & 0.881 & 0.416 & 186.889 & 1.421 & 0.075 & 0.022859 & 0.009792 & 0.345867 & 155.225 \\
\hline 6/18/08 2:00 & 2.333 & 0.873 & 0.407 & 182.712 & 1.42 & 0.074 & 0.022554 & 0.009605 & 0.339261 & 152.260 \\
\hline 6/18/08 3:00 & 2.321 & 0.861 & 0.393 & 176.553 & 1.42 & 0.074 & 0.022554 & 0.009605 & 0.339261 & 152.260 \\
\hline 6/18/08 4:00 & 2.312 & 0.852 & 0.383 & 172.017 & 1.417 & 0.071 & 0.021640 & 0.009052 & 0.319698 & 143.480 \\
\hline 6/18/08 5:00 & 2.309 & 0.849 & 0.380 & 170.520 & 1.416 & 0.07 & 0.021335 & 0.008869 & 0.313263 & 140.593 \\
\hline 6/18/08 6:00 & 2.307 & 0.847 & 0.378 & 169.527 & 1.415 & 0.069 & 0.021030 & 0.008688 & 0.306873 & 137.724 \\
\hline 6/18/08 7:00 & 2.3 & 0.840 & 0.370 & 166.078 & 1.415 & 0.069 & 0.021030 & 0.008688 & 0.306873 & 137.724 \\
\hline 6/18/08 8:00 & 2.3 & 0.840 & 0.370 & 166.078 & 1.413 & 0.067 & 0.020421 & 0.008330 & 0.294224 & 132.048 \\
\hline 6/18/08 9:00 & 2.307 & 0.847 & 0.378 & 169.527 & 1.415 & 0.069 & 0.021030 & 0.008688 & 0.306873 & 137.724 \\
\hline 6/18/08 10:00 & 2.302 & 0.842 & 0.372 & 167.059 & 1.413 & 0.067 & 0.020421 & 0.008330 & 0.294224 & 132.048 \\
\hline 6/18/08 11:00 & 2.296 & 0.836 & 0.366 & 164.126 & 1.413 & 0.067 & 0.020421 & 0.008330 & 0.294224 & 132.048 \\
\hline 6/18/08 12:00 & 2.28 & 0.820 & 0.349 & 156.457 & 1.412 & 0.066 & 0.020116 & 0.008153 & 0.287966 & 129.239 \\
\hline 6/18/08 13:00 & 2.27 & 0.810 & 0.338 & 151.775 & 1.41 & 0.064 & 0.019506 & 0.007803 & 0.275587 & 123.683 \\
\hline 6/18/08 14:00 & 2.258 & 0.798 & 0.326 & 146.269 & 1.411 & 0.065 & 0.019811 & 0.007977 & 0.281754 & 126.451 \\
\hline 6/18/08 15:00 & 2.25 & 0.790 & 0.318 & 142.666 & 1.409 & 0.063 & 0.019201 & 0.007629 & 0.269465 & 120.936 \\
\hline 6/18/08 16:00 & 2.242 & 0.782 & 0.310 & 139.117 & 1.408 & 0.062 & 0.018897 & 0.007457 & 0.263390 & 118.209 \\
\hline 6/18/08 17:00 & 2.232 & 0.772 & 0.300 & 134.756 & 1.406 & 0.06 & 0.018287 & 0.007117 & 0.251378 & 112.818 \\
\hline 6/18/08 18:00 & 2.228 & 0.768 & 0.296 & 133.034 & 1.407 & 0.061 & 0.018592 & 0.007287 & 0.257360 & 115.503 \\
\hline 6/18/08 19:00 & 2.223 & 0.763 & 0.292 & 130.902 & 1.407 & 0.061 & 0.018592 & 0.007287 & 0.257360 & 115.503 \\
\hline
\end{tabular}




\begin{tabular}{|c|c|c|c|c|c|c|c|c|c|c|}
\hline \multirow[b]{3}{*}{ Date Time } & \multicolumn{4}{|c|}{ V-Notch Weir } & \multicolumn{6}{|c|}{ R-Notch Weir } \\
\hline & \multicolumn{2}{|c|}{ Measured Water Level } & \multicolumn{2}{|c|}{$\begin{array}{c}\text { Calculated Rate } \\
\left(\mathrm{ft}^{3} / \mathrm{s}=448.8 \mathrm{gpm}\right)\end{array}$} & \multicolumn{3}{|c|}{ Measured Water Level } & \multicolumn{3}{|c|}{$\begin{array}{c}\text { Calculated Rate } \\
\left(\mathrm{ft}^{3} / \mathrm{s}=448.8 \mathrm{gpm}\right)\end{array}$} \\
\hline & $\begin{array}{c}\text { (ft above } \\
\text { sensor) }\end{array}$ & $\begin{array}{c}\text { (ft above } \\
\text { notch) }\end{array}$ & $\left(\mathrm{ft}^{3} / \mathrm{s}\right)$ & (gpm) & $\begin{array}{c}\text { (ft above } \\
\text { sensor) }\end{array}$ & $\begin{array}{c}\text { (ft above } \\
\text { log) }\end{array}$ & $\begin{array}{c}\text { (m above } \\
\text { log) }\end{array}$ & $\left(\mathrm{m}^{3} / \mathrm{s}\right)$ & $\left(\mathrm{ft}^{3} / \mathrm{s}\right)$ & (gpm) \\
\hline 6/18/08 20:00 & 2.227 & 0.767 & 0.295 & 132.606 & 1.408 & 0.062 & 0.018897 & 0.007457 & 0.263390 & 118.209 \\
\hline 6/18/08 21:00 & 2.238 & 0.778 & 0.306 & 137.362 & 1.408 & 0.062 & 0.018897 & 0.007457 & 0.263390 & 118.209 \\
\hline 6/18/08 22:00 & 2.249 & 0.789 & 0.317 & 142.220 & 1.41 & 0.064 & 0.019506 & 0.007803 & 0.275587 & 123.683 \\
\hline 6/18/08 23:00 & 2.258 & 0.798 & 0.326 & 146.269 & 1.412 & 0.066 & 0.020116 & 0.008153 & 0.287966 & 129.239 \\
\hline 6/19/08 0:00 & 2.262 & 0.802 & 0.330 & 148.091 & 1.411 & 0.065 & 0.019811 & 0.007977 & 0.281754 & 126.451 \\
\hline 6/19/08 1:00 & 2.266 & 0.806 & 0.334 & 149.926 & 1.413 & 0.067 & 0.020421 & 0.008330 & 0.294224 & 132.048 \\
\hline 6/19/08 2:00 & 2.268 & 0.808 & 0.336 & 150.849 & 1.412 & 0.066 & 0.020116 & 0.008153 & 0.287966 & 129.239 \\
\hline 6/19/08 3:00 & 2.286 & 0.826 & 0.355 & 159.307 & 1.414 & 0.068 & 0.020725 & 0.008509 & 0.300526 & 134.876 \\
\hline 6/19/08 4:00 & 2.281 & 0.821 & 0.350 & 156.930 & 1.413 & 0.067 & 0.020421 & 0.008330 & 0.294224 & 132.048 \\
\hline 6/19/08 5:00 & 2.284 & 0.824 & 0.353 & 158.354 & 1.413 & 0.067 & 0.020421 & 0.008330 & 0.294224 & 132.048 \\
\hline 6/19/08 6:00 & 2.281 & 0.821 & 0.350 & 156.930 & 1.412 & 0.066 & 0.020116 & 0.008153 & 0.287966 & 129.239 \\
\hline 6/19/08 7:00 & 2.286 & 0.826 & 0.355 & 159.307 & 1.412 & 0.066 & 0.020116 & 0.008153 & 0.287966 & 129.239 \\
\hline 6/19/08 8:00 & 2.286 & 0.826 & 0.355 & 159.307 & 1.414 & 0.068 & 0.020725 & 0.008509 & 0.300526 & 134.876 \\
\hline 6/19/08 9:00 & 2.291 & 0.831 & 0.360 & 161.706 & 1.414 & 0.068 & 0.020725 & 0.008509 & 0.300526 & 134.876 \\
\hline 6/19/08 10:00 & 2.288 & 0.828 & 0.357 & 160.264 & 1.412 & 0.066 & 0.020116 & 0.008153 & 0.287966 & 129.239 \\
\hline 6/19/08 11:00 & 2.284 & 0.824 & 0.353 & 158.354 & 1.412 & 0.066 & 0.020116 & 0.008153 & 0.287966 & 129.239 \\
\hline 6/19/08 12:00 & 2.271 & 0.811 & 0.339 & 152.239 & 1.41 & 0.064 & 0.019506 & 0.007803 & 0.275587 & 123.683 \\
\hline 6/19/08 13:00 & 2.213 & 0.753 & 0.282 & 126.698 & 1.408 & 0.062 & 0.018897 & 0.007457 & 0.263390 & 118.209 \\
\hline 6/19/08 14:00 & 2.2 & 0.740 & 0.270 & 121.355 & 1.409 & 0.063 & 0.019201 & 0.007629 & 0.269465 & 120.936 \\
\hline 6/19/08 15:00 & 2.186 & 0.726 & 0.258 & 115.756 & 1.405 & 0.059 & 0.017982 & 0.006949 & 0.245443 & 110.155 \\
\hline 6/19/08 16:00 & 2.243 & 0.783 & 0.311 & 139.558 & 1.417 & 0.071 & 0.021640 & 0.009052 & 0.319698 & 143.480 \\
\hline 6/19/08 17:00 & 2.872 & 1.412 & 1.343 & 602.693 & 1.702 & 0.356 & 0.108504 & 0.096317 & 3.401911 & 1526.778 \\
\hline 6/19/08 18:00 & 2.826 & 1.366 & 1.237 & 555.060 & 1.63 & 0.284 & 0.086559 & 0.068861 & 2.432173 & 1091.559 \\
\hline 6/19/08 19:00 & 2.762 & 1.302 & 1.098 & 492.660 & 1.569 & 0.223 & 0.067967 & 0.048134 & 1.700100 & 763.005 \\
\hline 6/19/08 20:00 & 4.258 & 2.798 & 7.372 & 3308.519 & 4.122 & 2.776 & 0.846084 & 2.078311 & 73.405588 & 32944.428 \\
\hline
\end{tabular}




\begin{tabular}{|c|c|c|c|c|c|c|c|c|c|c|}
\hline \multirow[b]{3}{*}{ Date Time } & \multicolumn{4}{|c|}{ V-Notch Weir } & \multicolumn{6}{|c|}{ R-Notch Weir } \\
\hline & \multicolumn{2}{|c|}{ Measured Water Level } & \multicolumn{2}{|c|}{$\begin{array}{c}\text { Calculated Rate } \\
\left(\mathrm{ft}^{3} / \mathrm{s}=448.8 \mathrm{gpm}\right)\end{array}$} & \multicolumn{3}{|c|}{ Measured Water Level } & \multicolumn{3}{|c|}{$\begin{array}{c}\text { Calculated Rate } \\
\left(\mathrm{ft}^{3} / \mathrm{s}=448.8 \mathrm{gpm}\right)\end{array}$} \\
\hline & $\begin{array}{l}\text { (ft above } \\
\text { sensor) }\end{array}$ & $\begin{array}{c}\text { (ft above } \\
\text { notch) }\end{array}$ & $\left(\mathrm{ft}^{3} / \mathrm{s}\right)$ & (gpm) & $\begin{array}{c}\text { (ft above } \\
\text { sensor) }\end{array}$ & $\begin{array}{c}\text { (ft above } \\
\text { log) }\end{array}$ & $\begin{array}{c}\text { (m above } \\
\text { log) }\end{array}$ & $\left(\mathrm{m}^{3} / \mathrm{s}\right)$ & $\left(\mathrm{ft}^{3} / \mathrm{s}\right)$ & (gpm) \\
\hline 6/19/08 21:00 & 2.753 & 1.293 & 1.079 & 484.241 & 2.463 & 1.117 & 0.340445 & 0.530811 & 18.748145 & 8414.168 \\
\hline 6/19/08 22:00 & 2.081 & 0.621 & 0.175 & 78.687 & 2.042 & 0.696 & 0.212130 & 0.261642 & 9.241156 & 4147.431 \\
\hline 6/19/08 23:00 & 1.835 & 0.375 & 0.051 & 22.759 & 1.8 & 0.454 & 0.138372 & 0.138318 & 4.885369 & 2192.554 \\
\hline 6/20/08 0:00 & 1.788 & 0.328 & 0.037 & 16.405 & 1.685 & 0.339 & 0.103322 & 0.089561 & 3.163275 & 1419.678 \\
\hline 6/20/08 1:00 & 1.755 & 0.295 & 0.028 & 12.668 & 1.624 & 0.278 & 0.084730 & 0.066715 & 2.356360 & 1057.534 \\
\hline 6/20/08 2:00 & 1.743 & 0.283 & 0.026 & 11.450 & 1.583 & 0.237 & 0.072234 & 0.052671 & 1.860325 & 834.914 \\
\hline 6/20/08 3:00 & 1.724 & 0.264 & 0.022 & 9.671 & 1.559 & 0.213 & 0.064919 & 0.044979 & 1.588637 & 712.980 \\
\hline 6/20/08 4:00 & 1.708 & 0.248 & 0.019 & 8.310 & 1.542 & 0.196 & 0.059738 & 0.039780 & 1.405035 & 630.580 \\
\hline 6/20/08 5:00 & 1.698 & 0.238 & 0.017 & 7.522 & 1.528 & 0.182 & 0.055471 & 0.035662 & 1.259584 & 565.301 \\
\hline 6/20/08 6:00 & 1.691 & 0.231 & 0.016 & 6.998 & 1.519 & 0.173 & 0.052728 & 0.033095 & 1.168919 & 524.611 \\
\hline 6/20/08 7:00 & 1.682 & 0.222 & 0.014 & 6.358 & 1.511 & 0.165 & 0.050290 & 0.030868 & 1.090247 & 489.303 \\
\hline 6/20/08 8:00 & 1.679 & 0.219 & 0.014 & 6.152 & 1.507 & 0.161 & 0.049070 & 0.029774 & 1.051601 & 471.959 \\
\hline 6/20/08 9:00 & 1.672 & 0.212 & 0.013 & 5.689 & 1.503 & 0.157 & 0.047851 & 0.028693 & 1.013424 & 454.824 \\
\hline 6/20/08 10:00 & 1.671 & 0.211 & 0.013 & 5.624 & 1.496 & 0.15 & 0.045718 & 0.026834 & 0.947757 & 425.353 \\
\hline 6/20/08 11:00 & 1.669 & 0.209 & 0.012 & 5.497 & 1.494 & 0.148 & 0.045108 & 0.026310 & 0.929266 & 417.054 \\
\hline 6/20/08 12:00 & 1.665 & 0.205 & 0.012 & 5.247 & 1.489 & 0.143 & 0.043584 & 0.025016 & 0.883573 & 396.548 \\
\hline 6/20/08 13:00 & 1.66 & 0.200 & 0.011 & 4.944 & 1.486 & 0.14 & 0.042670 & 0.024251 & 0.856529 & 384.410 \\
\hline 6/20/08 14:00 & 1.654 & 0.194 & 0.010 & 4.595 & 1.481 & 0.135 & 0.041146 & 0.022992 & 0.812083 & 364.463 \\
\hline 6/20/08 15:00 & 1.653 & 0.193 & 0.010 & 4.538 & 1.478 & 0.132 & 0.040232 & 0.022248 & 0.785797 & 352.665 \\
\hline 6/20/08 16:00 & 1.649 & 0.189 & 0.010 & 4.316 & 1.475 & 0.129 & 0.039317 & 0.021512 & 0.759800 & 340.998 \\
\hline 6/20/08 17:00 & 1.649 & 0.189 & 0.010 & 4.316 & 1.474 & 0.128 & 0.039012 & 0.021269 & 0.751200 & 337.139 \\
\hline 6/20/08 18:00 & 2.359 & 0.899 & 0.438 & 196.494 & 1.434 & 0.088 & 0.026821 & 0.012331 & 0.435514 & 195.459 \\
\hline 6/20/08 19:00 & 2.326 & 0.866 & 0.399 & 179.104 & 1.43 & 0.084 & 0.025602 & 0.011529 & 0.407202 & 182.752 \\
\hline 6/20/08 20:00 & 2.313 & 0.853 & 0.384 & 172.517 & 1.426 & 0.08 & 0.024383 & 0.010746 & 0.379531 & 170.333 \\
\hline 6/20/08 21:00 & 2.308 & 0.848 & 0.379 & 170.023 & 1.424 & 0.078 & 0.023773 & 0.010361 & 0.365940 & 164.234 \\
\hline
\end{tabular}




\begin{tabular}{|c|c|c|c|c|c|c|c|c|c|c|}
\hline \multirow[b]{3}{*}{ Date Time } & \multicolumn{4}{|c|}{ V-Notch Weir } & \multicolumn{6}{|c|}{ R-Notch Weir } \\
\hline & \multicolumn{2}{|c|}{ Measured Water Level } & \multicolumn{2}{|c|}{$\begin{array}{l}\text { Calculated Rate } \\
\left(\mathrm{ft}^{3} / \mathrm{s}=448.8 \mathrm{gpm}\right)\end{array}$} & \multicolumn{3}{|c|}{ Measured Water Level } & \multicolumn{3}{|c|}{$\begin{array}{c}\text { Calculated Rate } \\
\left(\mathrm{ft}^{3} / \mathrm{s}=448.8 \mathrm{gpm}\right)\end{array}$} \\
\hline & $\begin{array}{c}\text { (ft above } \\
\text { sensor) }\end{array}$ & $\begin{array}{c}\text { (ft above } \\
\text { notch) }\end{array}$ & $\left(\mathrm{ft}^{3} / \mathrm{s}\right)$ & (gpm) & $\begin{array}{c}\text { (ft above } \\
\text { sensor) }\end{array}$ & $\begin{array}{c}\text { (ft above } \\
\text { log) }\end{array}$ & $\begin{array}{c}\text { (m above } \\
\text { log) }\end{array}$ & $\left(\mathrm{m}^{3} / \mathrm{s}\right)$ & $\left(\mathrm{ft}^{3} / \mathrm{s}\right)$ & (gpm) \\
\hline 6/20/08 22:00 & 2.309 & 0.849 & 0.380 & 170.520 & 1.425 & 0.079 & 0.024078 & 0.010553 & 0.372715 & 167.274 \\
\hline 6/20/08 23:00 & 2.308 & 0.848 & 0.379 & 170.023 & 1.423 & 0.077 & 0.023468 & 0.010170 & 0.359208 & 161.212 \\
\hline 6/21/08 0:00 & 2.312 & 0.852 & 0.383 & 172.017 & 1.425 & 0.079 & 0.024078 & 0.010553 & 0.372715 & 167.274 \\
\hline 6/21/08 1:00 & 2.314 & 0.854 & 0.386 & 173.019 & 1.424 & 0.078 & 0.023773 & 0.010361 & 0.365940 & 164.234 \\
\hline 6/21/08 2:00 & 2.31 & 0.850 & 0.381 & 171.018 & 1.422 & 0.076 & 0.023164 & 0.009981 & 0.352516 & 158.209 \\
\hline 6/21/08 3:00 & 2.309 & 0.849 & 0.380 & 170.520 & 1.424 & 0.078 & 0.023773 & 0.010361 & 0.365940 & 164.234 \\
\hline 6/21/08 4:00 & 2.309 & 0.849 & 0.380 & 170.520 & 1.423 & 0.077 & 0.023468 & 0.010170 & 0.359208 & 161.212 \\
\hline 6/21/08 5:00 & 2.307 & 0.847 & 0.378 & 169.527 & 1.423 & 0.077 & 0.023468 & 0.010170 & 0.359208 & 161.212 \\
\hline 6/21/08 6:00 & 2.302 & 0.842 & 0.372 & 167.059 & 1.42 & 0.074 & 0.022554 & 0.009605 & 0.339261 & 152.260 \\
\hline 6/21/08 7:00 & 2.302 & 0.842 & 0.372 & 167.059 & 1.42 & 0.074 & 0.022554 & 0.009605 & 0.339261 & 152.260 \\
\hline 6/21/08 8:00 & 2.304 & 0.844 & 0.374 & 168.044 & 1.42 & 0.074 & 0.022554 & 0.009605 & 0.339261 & 152.260 \\
\hline 6/21/08 9:00 & 2.305 & 0.845 & 0.376 & 168.537 & 1.42 & 0.074 & 0.022554 & 0.009605 & 0.339261 & 152.260 \\
\hline 6/21/08 10:00 & 2.298 & 0.838 & 0.368 & 165.100 & 1.419 & 0.073 & 0.022249 & 0.009420 & 0.332697 & 149.314 \\
\hline 6/21/08 11:00 & 2.282 & 0.822 & 0.351 & 157.404 & 1.418 & 0.072 & 0.021945 & 0.009235 & 0.326176 & 146.388 \\
\hline 6/21/08 12:00 & 2.271 & 0.811 & 0.339 & 152.239 & 1.417 & 0.071 & 0.021640 & 0.009052 & 0.319698 & 143.480 \\
\hline 6/21/08 13:00 & 2.251 & 0.791 & 0.319 & 143.114 & 1.414 & 0.068 & 0.020725 & 0.008509 & 0.300526 & 134.876 \\
\hline 6/21/08 14:00 & 2.238 & 0.778 & 0.306 & 137.362 & 1.414 & 0.068 & 0.020725 & 0.008509 & 0.300526 & 134.876 \\
\hline 6/21/08 15:00 & 2.218 & 0.758 & 0.287 & 128.789 & 1.411 & 0.065 & 0.019811 & 0.007977 & 0.281754 & 126.451 \\
\hline 6/21/08 16:00 & 2.205 & 0.745 & 0.275 & 123.394 & 1.41 & 0.064 & 0.019506 & 0.007803 & 0.275587 & 123.683 \\
\hline 6/21/08 17:00 & 2.195 & 0.735 & 0.266 & 119.337 & 1.408 & 0.062 & 0.018897 & 0.007457 & 0.263390 & 118.209 \\
\hline 6/21/08 18:00 & 2.202 & 0.742 & 0.272 & 122.168 & 1.408 & 0.062 & 0.018897 & 0.007457 & 0.263390 & 118.209 \\
\hline 6/21/08 19:00 & 2.206 & 0.746 & 0.276 & 123.804 & 1.407 & 0.061 & 0.018592 & 0.007287 & 0.257360 & 115.503 \\
\hline 6/21/08 20:00 & 2.209 & 0.749 & 0.279 & 125.039 & 1.409 & 0.063 & 0.019201 & 0.007629 & 0.269465 & 120.936 \\
\hline 6/21/08 21:00 & 2.223 & 0.763 & 0.292 & 130.902 & 1.41 & 0.064 & 0.019506 & 0.007803 & 0.275587 & 123.683 \\
\hline 6/21/08 22:00 & 2.238 & 0.778 & 0.306 & 137.362 & 1.413 & 0.067 & 0.020421 & 0.008330 & 0.294224 & 132.048 \\
\hline
\end{tabular}




\begin{tabular}{|c|c|c|c|c|c|c|c|c|c|c|}
\hline \multirow[b]{3}{*}{ Date Time } & \multicolumn{4}{|c|}{ V-Notch Weir } & \multicolumn{6}{|c|}{ R-Notch Weir } \\
\hline & \multicolumn{2}{|c|}{ Measured Water Level } & \multicolumn{2}{|c|}{$\begin{array}{c}\text { Calculated Rate } \\
\left(\mathrm{ft}^{3} / \mathrm{s}=448.8 \mathrm{gpm}\right)\end{array}$} & \multicolumn{3}{|c|}{ Measured Water Level } & \multicolumn{3}{|c|}{$\begin{array}{c}\text { Calculated Rate } \\
\left(\mathrm{ft}^{3} / \mathrm{s}=448.8 \mathrm{gpm}\right)\end{array}$} \\
\hline & $\begin{array}{c}\text { (ft above } \\
\text { sensor) }\end{array}$ & $\begin{array}{c}\text { (ft above } \\
\text { notch) }\end{array}$ & $\left(\mathrm{ft}^{3} / \mathrm{s}\right)$ & (gpm) & $\begin{array}{c}\text { (ft above } \\
\text { sensor) }\end{array}$ & $\begin{array}{c}\text { (ft above } \\
\text { log) }\end{array}$ & $\begin{array}{c}\text { (m above } \\
\text { log) }\end{array}$ & $\left(\mathrm{m}^{3} / \mathrm{s}\right)$ & $\left(\mathrm{ft}^{3} / \mathrm{s}\right)$ & (gpm) \\
\hline 6/21/08 23:00 & 2.247 & 0.787 & 0.315 & 141.329 & 1.414 & 0.068 & 0.020725 & 0.008509 & 0.300526 & 134.876 \\
\hline 6/22/08 0:00 & 2.261 & 0.801 & 0.329 & 147.634 & 1.415 & 0.069 & 0.021030 & 0.008688 & 0.306873 & 137.724 \\
\hline 6/22/08 1:00 & 2.265 & 0.805 & 0.333 & 149.466 & 1.416 & 0.07 & 0.021335 & 0.008869 & 0.313263 & 140.593 \\
\hline 6/22/08 2:00 & 2.272 & 0.812 & 0.340 & 152.705 & 1.418 & 0.072 & 0.021945 & 0.009235 & 0.326176 & 146.388 \\
\hline 6/22/08 3:00 & 2.267 & 0.807 & 0.335 & 150.387 & 1.415 & 0.069 & 0.021030 & 0.008688 & 0.306873 & 137.724 \\
\hline 6/22/08 4:00 & 2.276 & 0.816 & 0.344 & 154.574 & 1.416 & 0.07 & 0.021335 & 0.008869 & 0.313263 & 140.593 \\
\hline 6/22/08 5:00 & 2.272 & 0.812 & 0.340 & 152.705 & 1.418 & 0.072 & 0.021945 & 0.009235 & 0.326176 & 146.388 \\
\hline 6/22/08 6:00 & 2.272 & 0.812 & 0.340 & 152.705 & 1.415 & 0.069 & 0.021030 & 0.008688 & 0.306873 & 137.724 \\
\hline 6/22/08 7:00 & 2.267 & 0.807 & 0.335 & 150.387 & 1.415 & 0.069 & 0.021030 & 0.008688 & 0.306873 & 137.724 \\
\hline 6/22/08 8:00 & 2.272 & 0.812 & 0.340 & 152.705 & 1.415 & 0.069 & 0.021030 & 0.008688 & 0.306873 & 137.724 \\
\hline 6/22/08 9:00 & 2.272 & 0.812 & 0.340 & 152.705 & 1.416 & 0.07 & 0.021335 & 0.008869 & 0.313263 & 140.593 \\
\hline 6/22/08 10:00 & 2.274 & 0.814 & 0.342 & 153.638 & 1.416 & 0.07 & 0.021335 & 0.008869 & 0.313263 & 140.593 \\
\hline 6/22/08 11:00 & 2.258 & 0.798 & 0.326 & 146.269 & 1.415 & 0.069 & 0.021030 & 0.008688 & 0.306873 & 137.724 \\
\hline 6/22/08 12:00 & 2.247 & 0.787 & 0.315 & 141.329 & 1.412 & 0.066 & 0.020116 & 0.008153 & 0.287966 & 129.239 \\
\hline 6/22/08 13:00 & 2.232 & 0.772 & 0.300 & 134.756 & 1.411 & 0.065 & 0.019811 & 0.007977 & 0.281754 & 126.451 \\
\hline 6/22/08 14:00 & 2.214 & 0.754 & 0.283 & 127.114 & 1.408 & 0.062 & 0.018897 & 0.007457 & 0.263390 & 118.209 \\
\hline 6/22/08 15:00 & 2.199 & 0.739 & 0.269 & 120.950 & 1.408 & 0.062 & 0.018897 & 0.007457 & 0.263390 & 118.209 \\
\hline 6/22/08 16:00 & 2.188 & 0.728 & 0.260 & 116.546 & 1.407 & 0.061 & 0.018592 & 0.007287 & 0.257360 & 115.503 \\
\hline 6/22/08 17:00 & 2.177 & 0.717 & 0.250 & 112.239 & 1.406 & 0.06 & 0.018287 & 0.007117 & 0.251378 & 112.818 \\
\hline 6/22/08 18:00 & 2.172 & 0.712 & 0.246 & 110.314 & 1.406 & 0.06 & 0.018287 & 0.007117 & 0.251378 & 112.818 \\
\hline 6/22/08 19:00 & 2.169 & 0.709 & 0.243 & 109.168 & 1.404 & 0.058 & 0.017678 & 0.006782 & 0.239555 & 107.512 \\
\hline 6/22/08 20:00 & 2.171 & 0.711 & 0.245 & 109.931 & 1.403 & 0.057 & 0.017373 & 0.006617 & 0.233715 & 104.891 \\
\hline 6/22/08 21:00 & 2.179 & 0.719 & 0.252 & 113.015 & 1.405 & 0.059 & 0.017982 & 0.006949 & 0.245443 & 110.155 \\
\hline 6/22/08 22:00 & 2.195 & 0.735 & 0.266 & 119.337 & 1.408 & 0.062 & 0.018897 & 0.007457 & 0.263390 & 118.209 \\
\hline 6/22/08 23:00 & 2.201 & 0.741 & 0.271 & 121.761 & 1.409 & 0.063 & 0.019201 & 0.007629 & 0.269465 & 120.936 \\
\hline
\end{tabular}




\begin{tabular}{|c|c|c|c|c|c|c|c|c|c|c|}
\hline \multirow[b]{3}{*}{ Date Time } & \multicolumn{4}{|c|}{ V-Notch Weir } & \multicolumn{6}{|c|}{ R-Notch Weir } \\
\hline & \multicolumn{2}{|c|}{ Measured Water Level } & \multicolumn{2}{|c|}{$\begin{array}{c}\text { Calculated Rate } \\
\left(\mathrm{ft}^{3} / \mathrm{s}=448.8 \mathrm{gpm}\right)\end{array}$} & \multicolumn{3}{|c|}{ Measured Water Level } & \multicolumn{3}{|c|}{$\begin{array}{c}\text { Calculated Rate } \\
\left(\mathrm{ft}^{3} / \mathrm{s}=448.8 \mathrm{gpm}\right)\end{array}$} \\
\hline & $\begin{array}{c}\text { (ft above } \\
\text { sensor) }\end{array}$ & $\begin{array}{c}\text { (ft above } \\
\text { notch) }\end{array}$ & $\left(\mathrm{ft}^{3} / \mathrm{s}\right)$ & (gpm) & $\begin{array}{c}\text { (ft above } \\
\text { sensor) }\end{array}$ & $\begin{array}{c}\text { (ft above } \\
\text { log) }\end{array}$ & $\begin{array}{c}\text { (m above } \\
\text { log) }\end{array}$ & $\left(\mathrm{m}^{3} / \mathrm{s}\right)$ & $\left(\mathrm{ft}^{3} / \mathrm{s}\right)$ & (gpm) \\
\hline 6/23/08 0:00 & 2.21 & 0.750 & 0.280 & 125.453 & 1.409 & 0.063 & 0.019201 & 0.007629 & 0.269465 & 120.936 \\
\hline 6/23/08 1:00 & 2.218 & 0.758 & 0.287 & 128.789 & 1.408 & 0.062 & 0.018897 & 0.007457 & 0.263390 & 118.209 \\
\hline 6/23/08 2:00 & 2.225 & 0.765 & 0.294 & 131.752 & 1.409 & 0.063 & 0.019201 & 0.007629 & 0.269465 & 120.936 \\
\hline 6/23/08 3:00 & 2.229 & 0.769 & 0.297 & 133.464 & 1.41 & 0.064 & 0.019506 & 0.007803 & 0.275587 & 123.683 \\
\hline 6/23/08 4:00 & 2.236 & 0.776 & 0.304 & 136.490 & 1.412 & 0.066 & 0.020116 & 0.008153 & 0.287966 & 129.239 \\
\hline 6/23/08 5:00 & 2.241 & 0.781 & 0.309 & 138.677 & 1.413 & 0.067 & 0.020421 & 0.008330 & 0.294224 & 132.048 \\
\hline 6/23/08 6:00 & 2.241 & 0.781 & 0.309 & 138.677 & 1.413 & 0.067 & 0.020421 & 0.008330 & 0.294224 & 132.048 \\
\hline 6/23/08 7:00 & 2.243 & 0.783 & 0.311 & 139.558 & 1.412 & 0.066 & 0.020116 & 0.008153 & 0.287966 & 129.239 \\
\hline 6/23/08 8:00 & 2.248 & 0.788 & 0.316 & 141.774 & 1.413 & 0.067 & 0.020421 & 0.008330 & 0.294224 & 132.048 \\
\hline 6/23/08 9:00 & 2.253 & 0.793 & 0.321 & 144.011 & 1.415 & 0.069 & 0.021030 & 0.008688 & 0.306873 & 137.724 \\
\hline 6/23/08 10:00 & 2.239 & 0.779 & 0.307 & 137.800 & 1.411 & 0.065 & 0.019811 & 0.007977 & 0.281754 & 126.451 \\
\hline 6/23/08 11:00 & 2.23 & 0.770 & 0.298 & 133.893 & 1.41 & 0.064 & 0.019506 & 0.007803 & 0.275587 & 123.683 \\
\hline 6/23/08 12:00 & 2.214 & 0.754 & 0.283 & 127.114 & 1.409 & 0.063 & 0.019201 & 0.007629 & 0.269465 & 120.936 \\
\hline 6/23/08 13:00 & 2.196 & 0.736 & 0.267 & 119.739 & 1.405 & 0.059 & 0.017982 & 0.006949 & 0.245443 & 110.155 \\
\hline 6/23/08 14:00 & 2.18 & 0.720 & 0.253 & 113.404 & 1.406 & 0.06 & 0.018287 & 0.007117 & 0.251378 & 112.818 \\
\hline 6/23/08 15:00 & 2.167 & 0.707 & 0.242 & 108.408 & 1.403 & 0.057 & 0.017373 & 0.006617 & 0.233715 & 104.891 \\
\hline 6/23/08 16:00 & 2.153 & 0.693 & 0.230 & 103.179 & 1.402 & 0.056 & 0.017068 & 0.006453 & 0.227923 & 102.292 \\
\hline 6/23/08 17:00 & 2.149 & 0.689 & 0.227 & 101.713 & 1.403 & 0.057 & 0.017373 & 0.006617 & 0.233715 & 104.891 \\
\hline 6/23/08 18:00 & 2.146 & 0.686 & 0.224 & 100.622 & 1.401 & 0.055 & 0.016763 & 0.006291 & 0.222180 & 99.714 \\
\hline 6/23/08 19:00 & 2.151 & 0.691 & 0.228 & 102.444 & 1.403 & 0.057 & 0.017373 & 0.006617 & 0.233715 & 104.891 \\
\hline 6/23/08 20:00 & 2.153 & 0.693 & 0.230 & 103.179 & 1.4 & 0.054 & 0.016458 & 0.006129 & 0.216486 & 97.159 \\
\hline 6/23/08 21:00 & 2.164 & 0.704 & 0.239 & 107.275 & 1.404 & 0.058 & 0.017678 & 0.006782 & 0.239555 & 107.512 \\
\hline 6/23/08 22:00 & 2.175 & 0.715 & 0.248 & 111.467 & 1.406 & 0.06 & 0.018287 & 0.007117 & 0.251378 & 112.818 \\
\hline 6/23/08 23:00 & 2.186 & 0.726 & 0.258 & 115.756 & 1.404 & 0.058 & 0.017678 & 0.006782 & 0.239555 & 107.512 \\
\hline 6/24/08 0:00 & 2.195 & 0.735 & 0.266 & 119.337 & 1.406 & 0.06 & 0.018287 & 0.007117 & 0.251378 & 112.818 \\
\hline
\end{tabular}




\begin{tabular}{|c|c|c|c|c|c|c|c|c|c|c|}
\hline \multirow[b]{3}{*}{ Date Time } & \multicolumn{4}{|c|}{ V-Notch Weir } & \multicolumn{6}{|c|}{ R-Notch Weir } \\
\hline & \multicolumn{2}{|c|}{ Measured Water Level } & \multicolumn{2}{|c|}{$\begin{array}{c}\text { Calculated Rate } \\
\left(\mathrm{ft}^{3} / \mathrm{s}=448.8 \mathrm{gpm}\right)\end{array}$} & \multicolumn{3}{|c|}{ Measured Water Level } & \multicolumn{3}{|c|}{$\begin{array}{c}\text { Calculated Rate } \\
\left(\mathrm{ft}^{3} / \mathrm{s}=448.8 \mathrm{gpm}\right)\end{array}$} \\
\hline & $\begin{array}{c}\text { (ft above } \\
\text { sensor) }\end{array}$ & $\begin{array}{c}\text { (ft above } \\
\text { notch) }\end{array}$ & $\left(\mathrm{ft}^{3} / \mathrm{s}\right)$ & (gpm) & $\begin{array}{c}\text { (ft above } \\
\text { sensor) }\end{array}$ & $\begin{array}{c}\text { (ft above } \\
\text { log) }\end{array}$ & $\begin{array}{c}\text { (m above } \\
\text { log) }\end{array}$ & $\left(\mathrm{m}^{3} / \mathrm{s}\right)$ & $\left(\mathrm{ft}^{3} / \mathrm{s}\right)$ & (gpm) \\
\hline 6/24/08 1:00 & 2.202 & 0.742 & 0.272 & 122.168 & 1.407 & 0.061 & 0.018592 & 0.007287 & 0.257360 & 115.503 \\
\hline 6/24/08 2:00 & 2.211 & 0.751 & 0.280 & 125.867 & 1.409 & 0.063 & 0.019201 & 0.007629 & 0.269465 & 120.936 \\
\hline 6/24/08 3:00 & 2.215 & 0.755 & 0.284 & 127.532 & 1.41 & 0.064 & 0.019506 & 0.007803 & 0.275587 & 123.683 \\
\hline 6/24/08 4:00 & 2.225 & 0.765 & 0.294 & 131.752 & 1.41 & 0.064 & 0.019506 & 0.007803 & 0.275587 & 123.683 \\
\hline 6/24/08 5:00 & 2.22 & 0.760 & 0.289 & 129.632 & 1.409 & 0.063 & 0.019201 & 0.007629 & 0.269465 & 120.936 \\
\hline 6/24/08 6:00 & 2.229 & 0.769 & 0.297 & 133.464 & 1.411 & 0.065 & 0.019811 & 0.007977 & 0.281754 & 126.451 \\
\hline 6/24/08 7:00 & 2.283 & 0.823 & 0.352 & 157.878 & 1.423 & 0.077 & 0.023468 & 0.010170 & 0.359208 & 161.212 \\
\hline 6/24/08 8:00 & 2.351 & 0.891 & 0.428 & 192.189 & 1.432 & 0.086 & 0.026212 & 0.011928 & 0.421279 & 189.070 \\
\hline 6/24/08 9:00 & 2.363 & 0.903 & 0.443 & 198.668 & 1.435 & 0.089 & 0.027126 & 0.012534 & 0.442691 & 198.680 \\
\hline 6/24/08 10:00 & 2.34 & 0.880 & 0.415 & 186.363 & 1.433 & 0.087 & 0.026516 & 0.012129 & 0.428377 & 192.256 \\
\hline 6/24/08 11:00 & 2.312 & 0.852 & 0.383 & 172.017 & 1.426 & 0.08 & 0.024383 & 0.010746 & 0.379531 & 170.333 \\
\hline 6/24/08 12:00 & 2.298 & 0.838 & 0.368 & 165.100 & 1.424 & 0.078 & 0.023773 & 0.010361 & 0.365940 & 164.234 \\
\hline 6/24/08 13:00 & 2.288 & 0.828 & 0.357 & 160.264 & 1.422 & 0.076 & 0.023164 & 0.009981 & 0.352516 & 158.209 \\
\hline 6/24/08 14:00 & 2.284 & 0.824 & 0.353 & 158.354 & 1.422 & 0.076 & 0.023164 & 0.009981 & 0.352516 & 158.209 \\
\hline 6/24/08 15:00 & 2.286 & 0.826 & 0.355 & 159.307 & 1.419 & 0.073 & 0.022249 & 0.009420 & 0.332697 & 149.314 \\
\hline 6/24/08 16:00 & 2.601 & 1.141 & 0.791 & 354.942 & 1.493 & 0.147 & 0.044803 & 0.026050 & 0.920066 & 412.925 \\
\hline 6/24/08 17:00 & 2.625 & 1.165 & 0.833 & 373.770 & 1.501 & 0.155 & 0.047242 & 0.028157 & 0.994512 & 446.337 \\
\hline 6/24/08 18:00 & 2.556 & 1.096 & 0.716 & 321.200 & 1.475 & 0.129 & 0.039317 & 0.021512 & 0.759800 & 340.998 \\
\hline 6/24/08 19:00 & 2.502 & 1.042 & 0.631 & 283.348 & 1.457 & 0.111 & 0.033831 & 0.017274 & 0.610106 & 273.815 \\
\hline 6/24/08 20:00 & 2.463 & 1.003 & 0.574 & 257.764 & 1.45 & 0.104 & 0.031698 & 0.015711 & 0.554913 & 249.045 \\
\hline 6/24/08 21:00 & 2.42 & 0.960 & 0.515 & 231.221 & 1.445 & 0.099 & 0.030174 & 0.014626 & 0.516571 & 231.837 \\
\hline 6/24/08 22:00 & 2.397 & 0.937 & 0.485 & 217.729 & 1.44 & 0.094 & 0.028650 & 0.013566 & 0.479156 & 215.045 \\
\hline 6/24/08 23:00 & 2.378 & 0.918 & 0.461 & 206.948 & 1.437 & 0.091 & 0.027735 & 0.012943 & 0.457162 & 205.174 \\
\hline 6/25/08 0:00 & 2.366 & 0.906 & 0.446 & 200.308 & 1.434 & 0.088 & 0.026821 & 0.012331 & 0.435514 & 195.459 \\
\hline 6/25/08 1:00 & 2.359 & 0.899 & 0.438 & 196.494 & 1.433 & 0.087 & 0.026516 & 0.012129 & 0.428377 & 192.256 \\
\hline
\end{tabular}




\begin{tabular}{|c|c|c|c|c|c|c|c|c|c|c|}
\hline \multirow[b]{3}{*}{ Date Time } & \multicolumn{4}{|c|}{ V-Notch Weir } & \multicolumn{6}{|c|}{ R-Notch Weir } \\
\hline & \multicolumn{2}{|c|}{ Measured Water Level } & \multicolumn{2}{|c|}{$\begin{array}{c}\text { Calculated Rate } \\
\left(\mathrm{ft}^{3} / \mathrm{s}=448.8 \mathrm{gpm}\right)\end{array}$} & \multicolumn{3}{|c|}{ Measured Water Level } & \multicolumn{3}{|c|}{$\begin{array}{c}\text { Calculated Rate } \\
\left(\mathrm{ft}^{3} / \mathrm{s}=448.8 \mathrm{gpm}\right)\end{array}$} \\
\hline & $\begin{array}{c}\text { (ft above } \\
\text { sensor) }\end{array}$ & $\begin{array}{c}\text { (ft above } \\
\text { notch) }\end{array}$ & $\left(\mathrm{ft}^{3} / \mathrm{s}\right)$ & (gpm) & $\begin{array}{l}\text { (ft above } \\
\text { sensor) }\end{array}$ & $\begin{array}{c}\text { (ft above } \\
\text { log) }\end{array}$ & $\begin{array}{c}\text { (m above } \\
\text { log) }\end{array}$ & $\left(\mathrm{m}^{3} / \mathrm{s}\right)$ & $\left(\mathrm{ft}^{3} / \mathrm{s}\right)$ & (gpm) \\
\hline 6/25/08 2:00 & 2.356 & 0.896 & 0.434 & 194.873 & 1.433 & 0.087 & 0.026516 & 0.012129 & 0.428377 & 192.256 \\
\hline 6/25/08 3:00 & 2.347 & 0.887 & 0.423 & 190.058 & 1.43 & 0.084 & 0.025602 & 0.011529 & 0.407202 & 182.752 \\
\hline 6/25/08 4:00 & 2.349 & 0.889 & 0.426 & 191.122 & 1.433 & 0.087 & 0.026516 & 0.012129 & 0.428377 & 192.256 \\
\hline 6/25/08 5:00 & 2.34 & 0.880 & 0.415 & 186.363 & 1.429 & 0.083 & 0.025297 & 0.011331 & 0.400223 & 179.620 \\
\hline 6/25/08 6:00 & 2.347 & 0.887 & 0.423 & 190.058 & 1.429 & 0.083 & 0.025297 & 0.011331 & 0.400223 & 179.620 \\
\hline 6/25/08 7:00 & 2.333 & 0.873 & 0.407 & 182.712 & 1.431 & 0.085 & 0.025907 & 0.011728 & 0.414220 & 185.902 \\
\hline 6/25/08 8:00 & 2.328 & 0.868 & 0.401 & 180.130 & 1.428 & 0.082 & 0.024992 & 0.011135 & 0.393285 & 176.506 \\
\hline 6/25/08 9:00 & 2.326 & 0.866 & 0.399 & 179.104 & 1.428 & 0.082 & 0.024992 & 0.011135 & 0.393285 & 176.506 \\
\hline $6 / 25 / 08$ 10:00 & 2.329 & 0.869 & 0.403 & 180.645 & 1.429 & 0.083 & 0.025297 & 0.011331 & 0.400223 & 179.620 \\
\hline 6/25/08 11:00 & 2.32 & 0.860 & 0.392 & 176.045 & 1.43 & 0.084 & 0.025602 & 0.011529 & 0.407202 & 182.752 \\
\hline 6/25/08 12:00 & 2.316 & 0.856 & 0.388 & 174.024 & 1.426 & 0.08 & 0.024383 & 0.010746 & 0.379531 & 170.333 \\
\hline 6/25/08 13:00 & 2.301 & 0.841 & 0.371 & 166.568 & 1.425 & 0.079 & 0.024078 & 0.010553 & 0.372715 & 167.274 \\
\hline 6/25/08 14:00 & 2.286 & 0.826 & 0.355 & 159.307 & 1.424 & 0.078 & 0.023773 & 0.010361 & 0.365940 & 164.234 \\
\hline 6/25/08 15:00 & 2.265 & 0.805 & 0.333 & 149.466 & 1.423 & 0.077 & 0.023468 & 0.010170 & 0.359208 & 161.212 \\
\hline 6/25/08 16:00 & 2.252 & 0.792 & 0.320 & 143.562 & 1.419 & 0.073 & 0.022249 & 0.009420 & 0.332697 & 149.314 \\
\hline 6/25/08 17:00 & 2.239 & 0.779 & 0.307 & 137.800 & 1.418 & 0.072 & 0.021945 & 0.009235 & 0.326176 & 146.388 \\
\hline 6/25/08 18:00 & 2.222 & 0.762 & 0.291 & 130.478 & 1.417 & 0.071 & 0.021640 & 0.009052 & 0.319698 & 143.480 \\
\hline 6/25/08 19:00 & 2.213 & 0.753 & 0.282 & 126.698 & 1.415 & 0.069 & 0.021030 & 0.008688 & 0.306873 & 137.724 \\
\hline 6/25/08 20:00 & 2.208 & 0.748 & 0.278 & 124.627 & 1.415 & 0.069 & 0.021030 & 0.008688 & 0.306873 & 137.724 \\
\hline 6/25/08 21:00 & 2.216 & 0.756 & 0.285 & 127.950 & 1.417 & 0.071 & 0.021640 & 0.009052 & 0.319698 & 143.480 \\
\hline 6/25/08 22:00 & 2.22 & 0.760 & 0.289 & 129.632 & 1.417 & 0.071 & 0.021640 & 0.009052 & 0.319698 & 143.480 \\
\hline 6/25/08 23:00 & 2.228 & 0.768 & 0.296 & 133.034 & 1.416 & 0.07 & 0.021335 & 0.008869 & 0.313263 & 140.593 \\
\hline 6/26/08 0:00 & 2.237 & 0.777 & 0.305 & 136.926 & 1.417 & 0.071 & 0.021640 & 0.009052 & 0.319698 & 143.480 \\
\hline 6/26/08 1:00 & 2.243 & 0.783 & 0.311 & 139.558 & 1.418 & 0.072 & 0.021945 & 0.009235 & 0.326176 & 146.388 \\
\hline 6/26/08 2:00 & 2.254 & 0.794 & 0.322 & 144.461 & 1.417 & 0.071 & 0.021640 & 0.009052 & 0.319698 & 143.480 \\
\hline
\end{tabular}




\begin{tabular}{|c|c|c|c|c|c|c|c|c|c|c|}
\hline \multirow[b]{3}{*}{ Date Time } & \multicolumn{4}{|c|}{ V-Notch Weir } & \multicolumn{6}{|c|}{ R-Notch Weir } \\
\hline & \multicolumn{2}{|c|}{ Measured Water Level } & \multicolumn{2}{|c|}{$\begin{array}{c}\text { Calculated Rate } \\
\left(\mathrm{ft}^{3} / \mathrm{s}=448.8 \mathrm{gpm}\right)\end{array}$} & \multicolumn{3}{|c|}{ Measured Water Level } & \multicolumn{3}{|c|}{$\begin{array}{c}\text { Calculated Rate } \\
\left(\mathrm{ft}^{3} / \mathrm{s}=448.8 \mathrm{gpm}\right)\end{array}$} \\
\hline & $\begin{array}{c}\text { (ft above } \\
\text { sensor) }\end{array}$ & $\begin{array}{c}\text { (ft above } \\
\text { notch) }\end{array}$ & $\left(\mathrm{ft}^{3} / \mathrm{s}\right)$ & (gpm) & $\begin{array}{c}\text { (ft above } \\
\text { sensor) }\end{array}$ & $\begin{array}{c}\text { (ft above } \\
\text { log) }\end{array}$ & $\begin{array}{c}\text { (m above } \\
\text { log) }\end{array}$ & $\left(\mathrm{m}^{3} / \mathrm{s}\right)$ & $\left(\mathrm{ft}^{3} / \mathrm{s}\right)$ & (gpm) \\
\hline 6/26/08 3:00 & 2.254 & 0.794 & 0.322 & 144.461 & 1.419 & 0.073 & 0.022249 & 0.009420 & 0.332697 & 149.314 \\
\hline 6/26/08 4:00 & 2.256 & 0.796 & 0.324 & 145.363 & 1.418 & 0.072 & 0.021945 & 0.009235 & 0.326176 & 146.388 \\
\hline 6/26/08 5:00 & 2.258 & 0.798 & 0.326 & 146.269 & 1.417 & 0.071 & 0.021640 & 0.009052 & 0.319698 & 143.480 \\
\hline 6/26/08 6:00 & 2.263 & 0.803 & 0.331 & 148.549 & 1.419 & 0.073 & 0.022249 & 0.009420 & 0.332697 & 149.314 \\
\hline 6/26/08 7:00 & 2.26 & 0.800 & 0.328 & 147.178 & 1.419 & 0.073 & 0.022249 & 0.009420 & 0.332697 & 149.314 \\
\hline 6/26/08 8:00 & 2.26 & 0.800 & 0.328 & 147.178 & 1.419 & 0.073 & 0.022249 & 0.009420 & 0.332697 & 149.314 \\
\hline 6/26/08 9:00 & 2.265 & 0.805 & 0.333 & 149.466 & 1.417 & 0.071 & 0.021640 & 0.009052 & 0.319698 & 143.480 \\
\hline 6/26/08 10:00 & 2.254 & 0.794 & 0.322 & 144.461 & 1.42 & 0.074 & 0.022554 & 0.009605 & 0.339261 & 152.260 \\
\hline 6/26/08 11:00 & 3.37 & 1.910 & 2.848 & 1277.961 & 2.385 & 1.039 & 0.316672 & 0.476306 & 16.823041 & 7550.181 \\
\hline 6/26/08 12:00 & 3.288 & 1.828 & 2.553 & 1145.711 & 2.224 & 0.878 & 0.267601 & 0.370251 & 13.077209 & 5869.051 \\
\hline 6/26/08 13:00 & 3.069 & 1.609 & 1.858 & 833.989 & 1.879 & 0.533 & 0.162450 & 0.175685 & 6.205152 & 2784.872 \\
\hline 6/26/08 14:00 & 2.898 & 1.438 & 1.405 & 630.663 & 1.71 & 0.364 & 0.110942 & 0.099553 & 3.516199 & 1578.070 \\
\hline 6/26/08 15:00 & 2.775 & 1.315 & 1.125 & 504.974 & 1.633 & 0.287 & 0.087473 & 0.069943 & 2.470379 & 1108.706 \\
\hline 6/26/08 16:00 & 2.705 & 1.245 & 0.982 & 440.801 & 1.587 & 0.241 & 0.073453 & 0.053992 & 1.906980 & 855.853 \\
\hline 6/26/08 17:00 & 2.687 & 1.227 & 0.947 & 425.139 & 1.553 & 0.207 & 0.063091 & 0.043120 & 1.522979 & 683.513 \\
\hline 6/26/08 18:00 & 2.626 & 1.166 & 0.835 & 374.567 & 1.524 & 0.178 & 0.054252 & 0.034513 & 1.219010 & 547.092 \\
\hline 6/26/08 19:00 & 2.588 & 1.128 & 0.769 & 344.986 & 1.508 & 0.162 & 0.049375 & 0.030046 & 1.061219 & 476.275 \\
\hline 6/26/08 20:00 & 2.554 & 1.094 & 0.712 & 319.747 & 1.493 & 0.147 & 0.044803 & 0.026050 & 0.920066 & 412.925 \\
\hline 6/26/08 21:00 & 2.537 & 1.077 & 0.685 & 307.557 & 1.464 & 0.118 & 0.035965 & 0.018885 & 0.667017 & 299.357 \\
\hline 6/26/08 22:00 & 2.508 & 1.048 & 0.640 & 287.414 & 1.456 & 0.11 & 0.033526 & 0.017047 & 0.602114 & 270.229 \\
\hline 6/26/08 23:00 & 2.496 & 1.036 & 0.622 & 279.318 & 1.454 & 0.108 & 0.032917 & 0.016598 & 0.586238 & 263.104 \\
\hline 6/27/08 0:00 & 2.482 & 1.022 & 0.602 & 270.047 & 1.45 & 0.104 & 0.031698 & 0.015711 & 0.554913 & 249.045 \\
\hline 6/27/08 1:00 & 2.442 & 0.982 & 0.545 & 244.585 & 1.445 & 0.099 & 0.030174 & 0.014626 & 0.516571 & 231.837 \\
\hline 6/27/08 2:00 & 2.43 & 0.970 & 0.529 & 237.239 & 1.44 & 0.094 & 0.028650 & 0.013566 & 0.479156 & 215.045 \\
\hline 6/27/08 3:00 & 2.408 & 0.948 & 0.499 & 224.121 & 1.439 & 0.093 & 0.028345 & 0.013358 & 0.471786 & 211.738 \\
\hline
\end{tabular}




\begin{tabular}{|c|c|c|c|c|c|c|c|c|c|c|}
\hline \multirow[b]{3}{*}{ Date Time } & \multicolumn{4}{|c|}{ V-Notch Weir } & \multicolumn{6}{|c|}{ R-Notch Weir } \\
\hline & \multicolumn{2}{|c|}{ Measured Water Level } & \multicolumn{2}{|c|}{$\begin{array}{c}\text { Calculated Rate } \\
\left(\mathrm{ft}^{3} / \mathrm{s}=448.8 \mathrm{gpm}\right)\end{array}$} & \multicolumn{3}{|c|}{ Measured Water Level } & \multicolumn{3}{|c|}{$\begin{array}{c}\text { Calculated Rate } \\
\left(\mathrm{ft}^{3} / \mathrm{s}=448.8 \mathrm{gpm}\right)\end{array}$} \\
\hline & $\begin{array}{c}\text { (ft above } \\
\text { sensor) }\end{array}$ & $\begin{array}{c}\text { (ft above } \\
\text { notch) }\end{array}$ & $\left(\mathrm{ft}^{3} / \mathrm{s}\right)$ & (gpm) & $\begin{array}{c}\text { (ft above } \\
\text { sensor) }\end{array}$ & $\begin{array}{c}\text { (ft above } \\
\text { log) }\end{array}$ & $\begin{array}{c}\text { (m above } \\
\text { log) }\end{array}$ & $\left(\mathrm{m}^{3} / \mathrm{s}\right)$ & $\left(\mathrm{ft}^{3} / \mathrm{s}\right)$ & (gpm) \\
\hline 6/27/08 4:00 & 2.401 & 0.941 & 0.490 & 220.040 & 1.436 & 0.09 & 0.027431 & 0.012738 & 0.449907 & 201.918 \\
\hline 6/27/08 5:00 & 2.389 & 0.929 & 0.475 & 213.149 & 1.436 & 0.09 & 0.027431 & 0.012738 & 0.449907 & 201.918 \\
\hline 6/27/08 6:00 & 2.382 & 0.922 & 0.466 & 209.190 & 1.433 & 0.087 & 0.026516 & 0.012129 & 0.428377 & 192.256 \\
\hline 6/27/08 7:00 & 2.377 & 0.917 & 0.460 & 206.389 & 1.433 & 0.087 & 0.026516 & 0.012129 & 0.428377 & 192.256 \\
\hline 6/27/08 8:00 & 2.38 & 0.920 & 0.464 & 208.067 & 1.433 & 0.087 & 0.026516 & 0.012129 & 0.428377 & 192.256 \\
\hline 6/27/08 9:00 & 2.366 & 0.906 & 0.446 & 200.308 & 1.431 & 0.085 & 0.025907 & 0.011728 & 0.414220 & 185.902 \\
\hline 6/27/08 10:00 & 2.366 & 0.906 & 0.446 & 200.308 & 1.431 & 0.085 & 0.025907 & 0.011728 & 0.414220 & 185.902 \\
\hline 6/27/08 11:00 & 2.351 & 0.891 & 0.428 & 192.189 & 1.429 & 0.083 & 0.025297 & 0.011331 & 0.400223 & 179.620 \\
\hline 6/27/08 12:00 & 2.328 & 0.868 & 0.401 & 180.130 & 1.428 & 0.082 & 0.024992 & 0.011135 & 0.393285 & 176.506 \\
\hline 6/27/08 13:00 & 2.315 & 0.855 & 0.387 & 173.521 & 1.424 & 0.078 & 0.023773 & 0.010361 & 0.365940 & 164.234 \\
\hline 6/27/08 14:00 & 2.295 & 0.835 & 0.365 & 163.640 & 1.423 & 0.077 & 0.023468 & 0.010170 & 0.359208 & 161.212 \\
\hline 6/27/08 15:00 & 2.284 & 0.824 & 0.353 & 158.354 & 1.421 & 0.075 & 0.022859 & 0.009792 & 0.345867 & 155.225 \\
\hline 6/27/08 16:00 & 2.271 & 0.811 & 0.339 & 152.239 & 1.42 & 0.074 & 0.022554 & 0.009605 & 0.339261 & 152.260 \\
\hline 6/27/08 17:00 & 2.266 & 0.806 & 0.334 & 149.926 & 1.423 & 0.077 & 0.023468 & 0.010170 & 0.359208 & 161.212 \\
\hline 6/27/08 18:00 & 2.279 & 0.819 & 0.348 & 155.985 & 1.423 & 0.077 & 0.023468 & 0.010170 & 0.359208 & 161.212 \\
\hline 6/27/08 19:00 & 2.304 & 0.844 & 0.374 & 168.044 & 1.425 & 0.079 & 0.024078 & 0.010553 & 0.372715 & 167.274 \\
\hline 6/27/08 20:00 & 2.315 & 0.855 & 0.387 & 173.521 & 1.425 & 0.079 & 0.024078 & 0.010553 & 0.372715 & 167.274 \\
\hline 6/27/08 21:00 & 2.312 & 0.852 & 0.383 & 172.017 & 1.427 & 0.081 & 0.024688 & 0.010940 & 0.386387 & 173.411 \\
\hline 6/27/08 22:00 & 2.306 & 0.846 & 0.377 & 169.032 & 1.426 & 0.08 & 0.024383 & 0.010746 & 0.379531 & 170.333 \\
\hline 6/27/08 23:00 & 2.306 & 0.846 & 0.377 & 169.032 & 1.425 & 0.079 & 0.024078 & 0.010553 & 0.372715 & 167.274 \\
\hline 6/28/08 0:00 & 2.303 & 0.843 & 0.373 & 167.551 & 1.426 & 0.08 & 0.024383 & 0.010746 & 0.379531 & 170.333 \\
\hline 6/28/08 1:00 & 2.31 & 0.850 & 0.381 & 171.018 & 1.423 & 0.077 & 0.023468 & 0.010170 & 0.359208 & 161.212 \\
\hline 6/28/08 2:00 & 2.307 & 0.847 & 0.378 & 169.527 & 1.425 & 0.079 & 0.024078 & 0.010553 & 0.372715 & 167.274 \\
\hline 6/28/08 3:00 & 2.314 & 0.854 & 0.386 & 173.019 & 1.424 & 0.078 & 0.023773 & 0.010361 & 0.365940 & 164.234 \\
\hline 6/28/08 4:00 & 2.312 & 0.852 & 0.383 & 172.017 & 1.424 & 0.078 & 0.023773 & 0.010361 & 0.365940 & 164.234 \\
\hline
\end{tabular}




\begin{tabular}{|c|c|c|c|c|c|c|c|c|c|c|}
\hline \multirow[b]{3}{*}{ Date Time } & \multicolumn{4}{|c|}{ V-Notch Weir } & \multicolumn{6}{|c|}{ R-Notch Weir } \\
\hline & \multicolumn{2}{|c|}{ Measured Water Level } & \multicolumn{2}{|c|}{$\begin{array}{c}\text { Calculated Rate } \\
\left(\mathrm{ft}^{3} / \mathrm{s}=448.8 \mathrm{gpm}\right)\end{array}$} & \multicolumn{3}{|c|}{ Measured Water Level } & \multicolumn{3}{|c|}{$\begin{array}{c}\text { Calculated Rate } \\
\left(\mathrm{ft}^{3} / \mathrm{s}=448.8 \mathrm{gpm}\right)\end{array}$} \\
\hline & $\begin{array}{c}\text { (ft above } \\
\text { sensor) }\end{array}$ & $\begin{array}{c}\text { (ft above } \\
\text { notch) }\end{array}$ & $\left(\mathrm{ft}^{3} / \mathrm{s}\right)$ & (gpm) & $\begin{array}{l}\text { (ft above } \\
\text { sensor) }\end{array}$ & $\begin{array}{c}\text { (ft above } \\
\text { log) }\end{array}$ & $\begin{array}{c}\text { (m above } \\
\text { log) }\end{array}$ & $\left(\mathrm{m}^{3} / \mathrm{s}\right)$ & $\left(\mathrm{ft}^{3} / \mathrm{s}\right)$ & (gpm) \\
\hline 6/28/08 5:00 & 2.316 & 0.856 & 0.388 & 174.024 & 1.425 & 0.079 & 0.024078 & 0.010553 & 0.372715 & 167.274 \\
\hline 6/28/08 6:00 & 2.314 & 0.854 & 0.386 & 173.019 & 1.425 & 0.079 & 0.024078 & 0.010553 & 0.372715 & 167.274 \\
\hline 6/28/08 7:00 & 2.309 & 0.849 & 0.380 & 170.520 & 1.422 & 0.076 & 0.023164 & 0.009981 & 0.352516 & 158.209 \\
\hline 6/28/08 8:00 & 2.309 & 0.849 & 0.380 & 170.520 & 1.422 & 0.076 & 0.023164 & 0.009981 & 0.352516 & 158.209 \\
\hline 6/28/08 9:00 & 2.314 & 0.854 & 0.386 & 173.019 & 1.422 & 0.076 & 0.023164 & 0.009981 & 0.352516 & 158.209 \\
\hline 6/28/08 10:00 & 2.307 & 0.847 & 0.378 & 169.527 & 1.422 & 0.076 & 0.023164 & 0.009981 & 0.352516 & 158.209 \\
\hline 6/28/08 11:00 & 2.3 & 0.840 & 0.370 & 166.078 & 1.423 & 0.077 & 0.023468 & 0.010170 & 0.359208 & 161.212 \\
\hline 6/28/08 12:00 & 2.273 & 0.813 & 0.341 & 153.171 & 1.416 & 0.07 & 0.021335 & 0.008869 & 0.313263 & 140.593 \\
\hline 6/28/08 13:00 & 2.252 & 0.792 & 0.320 & 143.562 & 1.415 & 0.069 & 0.021030 & 0.008688 & 0.306873 & 137.724 \\
\hline 6/28/08 14:00 & 2.236 & 0.776 & 0.304 & 136.490 & 1.416 & 0.07 & 0.021335 & 0.008869 & 0.313263 & 140.593 \\
\hline 6/28/08 15:00 & 2.218 & 0.758 & 0.287 & 128.789 & 1.412 & 0.066 & 0.020116 & 0.008153 & 0.287966 & 129.239 \\
\hline 6/28/08 16:00 & 2.213 & 0.753 & 0.282 & 126.698 & 1.412 & 0.066 & 0.020116 & 0.008153 & 0.287966 & 129.239 \\
\hline 6/28/08 17:00 & 2.201 & 0.741 & 0.271 & 121.761 & 1.41 & 0.064 & 0.019506 & 0.007803 & 0.275587 & 123.683 \\
\hline 6/28/08 18:00 & 2.203 & 0.743 & 0.273 & 122.576 & 1.41 & 0.064 & 0.019506 & 0.007803 & 0.275587 & 123.683 \\
\hline 6/28/08 19:00 & 2.217 & 0.757 & 0.286 & 128.369 & 1.412 & 0.066 & 0.020116 & 0.008153 & 0.287966 & 129.239 \\
\hline 6/28/08 20:00 & 2.212 & 0.752 & 0.281 & 126.282 & 1.412 & 0.066 & 0.020116 & 0.008153 & 0.287966 & 129.239 \\
\hline 6/28/08 21:00 & 2.226 & 0.766 & 0.295 & 132.179 & 1.414 & 0.068 & 0.020725 & 0.008509 & 0.300526 & 134.876 \\
\hline 6/28/08 22:00 & 2.228 & 0.768 & 0.296 & 133.034 & 1.416 & 0.07 & 0.021335 & 0.008869 & 0.313263 & 140.593 \\
\hline 6/28/08 23:00 & 2.23 & 0.770 & 0.298 & 133.893 & 1.415 & 0.069 & 0.021030 & 0.008688 & 0.306873 & 137.724 \\
\hline 6/29/08 0:00 & 2.244 & 0.784 & 0.312 & 139.999 & 1.417 & 0.071 & 0.021640 & 0.009052 & 0.319698 & 143.480 \\
\hline 6/29/08 1:00 & 2.246 & 0.786 & 0.314 & 140.885 & 1.418 & 0.072 & 0.021945 & 0.009235 & 0.326176 & 146.388 \\
\hline 6/29/08 2:00 & 2.253 & 0.793 & 0.321 & 144.011 & 1.416 & 0.07 & 0.021335 & 0.008869 & 0.313263 & 140.593 \\
\hline 6/29/08 3:00 & 2.26 & 0.800 & 0.328 & 147.178 & 1.418 & 0.072 & 0.021945 & 0.009235 & 0.326176 & 146.388 \\
\hline 6/29/08 4:00 & 2.257 & 0.797 & 0.325 & 145.816 & 1.419 & 0.073 & 0.022249 & 0.009420 & 0.332697 & 149.314 \\
\hline 6/29/08 5:00 & 2.262 & 0.802 & 0.330 & 148.091 & 1.417 & 0.071 & 0.021640 & 0.009052 & 0.319698 & 143.480 \\
\hline
\end{tabular}




\begin{tabular}{|c|c|c|c|c|c|c|c|c|c|c|}
\hline \multirow[b]{3}{*}{ Date Time } & \multicolumn{4}{|c|}{ V-Notch Weir } & \multicolumn{6}{|c|}{ R-Notch Weir } \\
\hline & \multicolumn{2}{|c|}{ Measured Water Level } & \multicolumn{2}{|c|}{$\begin{array}{c}\text { Calculated Rate } \\
\left(\mathrm{ft}^{3} / \mathrm{s}=448.8 \mathrm{gpm}\right)\end{array}$} & \multicolumn{3}{|c|}{ Measured Water Level } & \multicolumn{3}{|c|}{$\begin{array}{c}\text { Calculated Rate } \\
\left(\mathrm{ft}^{3} / \mathrm{s}=448.8 \mathrm{gpm}\right)\end{array}$} \\
\hline & $\begin{array}{c}\text { (ft above } \\
\text { sensor) }\end{array}$ & $\begin{array}{c}\text { (ft above } \\
\text { notch) }\end{array}$ & $\left(\mathrm{ft}^{3} / \mathrm{s}\right)$ & (gpm) & $\begin{array}{c}\text { (ft above } \\
\text { sensor) }\end{array}$ & $\begin{array}{c}\text { (ft above } \\
\text { log) }\end{array}$ & $\begin{array}{c}\text { (m above } \\
\text { log) }\end{array}$ & $\left(\mathrm{m}^{3} / \mathrm{s}\right)$ & $\left(\mathrm{ft}^{3} / \mathrm{s}\right)$ & (gpm) \\
\hline 6/29/08 6:00 & 2.267 & 0.807 & 0.335 & 150.387 & 1.421 & 0.075 & 0.022859 & 0.009792 & 0.345867 & 155.225 \\
\hline 6/29/08 7:00 & 2.267 & 0.807 & 0.335 & 150.387 & 1.418 & 0.072 & 0.021945 & 0.009235 & 0.326176 & 146.388 \\
\hline 6/29/08 8:00 & 2.269 & 0.809 & 0.337 & 151.312 & 1.423 & 0.077 & 0.023468 & 0.010170 & 0.359208 & 161.212 \\
\hline 6/29/08 9:00 & 2.267 & 0.807 & 0.335 & 150.387 & 1.419 & 0.073 & 0.022249 & 0.009420 & 0.332697 & 149.314 \\
\hline 6/29/08 10:00 & 2.265 & 0.805 & 0.333 & 149.466 & 1.419 & 0.073 & 0.022249 & 0.009420 & 0.332697 & 149.314 \\
\hline 6/29/08 11:00 & 2.255 & 0.795 & 0.323 & 144.912 & 1.417 & 0.071 & 0.021640 & 0.009052 & 0.319698 & 143.480 \\
\hline 6/29/08 12:00 & 2.239 & 0.779 & 0.307 & 137.800 & 1.418 & 0.072 & 0.021945 & 0.009235 & 0.326176 & 146.388 \\
\hline 6/29/08 13:00 & 2.231 & 0.771 & 0.299 & 134.324 & 1.414 & 0.068 & 0.020725 & 0.008509 & 0.300526 & 134.876 \\
\hline 6/29/08 14:00 & 2.22 & 0.760 & 0.289 & 129.632 & 1.412 & 0.066 & 0.020116 & 0.008153 & 0.287966 & 129.239 \\
\hline 6/29/08 15:00 & 2.206 & 0.746 & 0.276 & 123.804 & 1.413 & 0.067 & 0.020421 & 0.008330 & 0.294224 & 132.048 \\
\hline 6/29/08 16:00 & 2.204 & 0.744 & 0.274 & 122.984 & 1.411 & 0.065 & 0.019811 & 0.007977 & 0.281754 & 126.451 \\
\hline 6/29/08 17:00 & 2.195 & 0.735 & 0.266 & 119.337 & 1.409 & 0.063 & 0.019201 & 0.007629 & 0.269465 & 120.936 \\
\hline 6/29/08 18:00 & 2.192 & 0.732 & 0.263 & 118.136 & 1.41 & 0.064 & 0.019506 & 0.007803 & 0.275587 & 123.683 \\
\hline 6/29/08 19:00 & 2.195 & 0.735 & 0.266 & 119.337 & 1.41 & 0.064 & 0.019506 & 0.007803 & 0.275587 & 123.683 \\
\hline 6/29/08 20:00 & 2.192 & 0.732 & 0.263 & 118.136 & 1.412 & 0.066 & 0.020116 & 0.008153 & 0.287966 & 129.239 \\
\hline 6/29/08 21:00 & 2.201 & 0.741 & 0.271 & 121.761 & 1.412 & 0.066 & 0.020116 & 0.008153 & 0.287966 & 129.239 \\
\hline 6/29/08 22:00 & 2.21 & 0.750 & 0.280 & 125.453 & 1.412 & 0.066 & 0.020116 & 0.008153 & 0.287966 & 129.239 \\
\hline 6/29/08 23:00 & 2.221 & 0.761 & 0.290 & 130.054 & 1.414 & 0.068 & 0.020725 & 0.008509 & 0.300526 & 134.876 \\
\hline 6/30/08 0:00 & 2.232 & 0.772 & 0.300 & 134.756 & 1.415 & 0.069 & 0.021030 & 0.008688 & 0.306873 & 137.724 \\
\hline 6/30/08 1:00 & 2.234 & 0.774 & 0.302 & 135.621 & 1.415 & 0.069 & 0.021030 & 0.008688 & 0.306873 & 137.724 \\
\hline 6/30/08 2:00 & 2.241 & 0.781 & 0.309 & 138.677 & 1.416 & 0.07 & 0.021335 & 0.008869 & 0.313263 & 140.593 \\
\hline 6/30/08 3:00 & 2.246 & 0.786 & 0.314 & 140.885 & 1.418 & 0.072 & 0.021945 & 0.009235 & 0.326176 & 146.388 \\
\hline 6/30/08 4:00 & 2.248 & 0.788 & 0.316 & 141.774 & 1.422 & 0.076 & 0.023164 & 0.009981 & 0.352516 & 158.209 \\
\hline 6/30/08 5:00 & 2.243 & 0.783 & 0.311 & 139.558 & 1.422 & 0.076 & 0.023164 & 0.009981 & 0.352516 & 158.209 \\
\hline 6/30/08 6:00 & 2.248 & 0.788 & 0.316 & 141.774 & 1.421 & 0.075 & 0.022859 & 0.009792 & 0.345867 & 155.225 \\
\hline
\end{tabular}




\begin{tabular}{|c|c|c|c|c|c|c|c|c|c|c|}
\hline \multirow[b]{3}{*}{ Date Time } & \multicolumn{4}{|c|}{ V-Notch Weir } & \multicolumn{6}{|c|}{ R-Notch Weir } \\
\hline & \multicolumn{2}{|c|}{ Measured Water Level } & \multicolumn{2}{|c|}{$\begin{array}{c}\text { Calculated Rate } \\
\left(\mathrm{ft}^{3} / \mathrm{s}=448.8 \mathrm{gpm}\right)\end{array}$} & \multicolumn{3}{|c|}{ Measured Water Level } & \multicolumn{3}{|c|}{$\begin{array}{c}\text { Calculated Rate } \\
\left(\mathrm{ft}^{3} / \mathrm{s}=448.8 \mathrm{gpm}\right)\end{array}$} \\
\hline & $\begin{array}{c}\text { (ft above } \\
\text { sensor) }\end{array}$ & $\begin{array}{c}\text { (ft above } \\
\text { notch) }\end{array}$ & $\left(\mathrm{ft}^{3} / \mathrm{s}\right)$ & (gpm) & $\begin{array}{c}\text { (ft above } \\
\text { sensor) }\end{array}$ & $\begin{array}{c}\text { (ft above } \\
\text { log) }\end{array}$ & $\begin{array}{c}\text { (m above } \\
\text { log) }\end{array}$ & $\left(\mathrm{m}^{3} / \mathrm{s}\right)$ & $\left(\mathrm{ft}^{3} / \mathrm{s}\right)$ & (gpm) \\
\hline 6/30/08 7:00 & 2.246 & 0.786 & 0.314 & 140.885 & 1.421 & 0.075 & 0.022859 & 0.009792 & 0.345867 & 155.225 \\
\hline 6/30/08 8:00 & 2.25 & 0.790 & 0.318 & 142.666 & 1.421 & 0.075 & 0.022859 & 0.009792 & 0.345867 & 155.225 \\
\hline 6/30/08 9:00 & 2.25 & 0.790 & 0.318 & 142.666 & 1.423 & 0.077 & 0.023468 & 0.010170 & 0.359208 & 161.212 \\
\hline 6/30/08 10:00 & 2.253 & 0.793 & 0.321 & 144.011 & 1.424 & 0.078 & 0.023773 & 0.010361 & 0.365940 & 164.234 \\
\hline 6/30/08 11:00 & 2.248 & 0.788 & 0.316 & 141.774 & 1.422 & 0.076 & 0.023164 & 0.009981 & 0.352516 & 158.209 \\
\hline 6/30/08 12:00 & 2.235 & 0.775 & 0.303 & 136.055 & 1.42 & 0.074 & 0.022554 & 0.009605 & 0.339261 & 152.260 \\
\hline 6/30/08 13:00 & 2.219 & 0.759 & 0.288 & 129.210 & 1.418 & 0.072 & 0.021945 & 0.009235 & 0.326176 & 146.388 \\
\hline 6/30/08 14:00 & 2.206 & 0.746 & 0.276 & 123.804 & 1.414 & 0.068 & 0.020725 & 0.008509 & 0.300526 & 134.876 \\
\hline 6/30/08 15:00 & 2.197 & 0.737 & 0.268 & 120.142 & 1.415 & 0.069 & 0.021030 & 0.008688 & 0.306873 & 137.724 \\
\hline 6/30/08 16:00 & 2.188 & 0.728 & 0.260 & 116.546 & 1.416 & 0.07 & 0.021335 & 0.008869 & 0.313263 & 140.593 \\
\hline 6/30/08 17:00 & 2.18 & 0.720 & 0.253 & 113.404 & 1.414 & 0.068 & 0.020725 & 0.008509 & 0.300526 & 134.876 \\
\hline 6/30/08 18:00 & 2.175 & 0.715 & 0.248 & 111.467 & 1.413 & 0.067 & 0.020421 & 0.008330 & 0.294224 & 132.048 \\
\hline 6/30/08 19:00 & 2.177 & 0.717 & 0.250 & 112.239 & 1.415 & 0.069 & 0.021030 & 0.008688 & 0.306873 & 137.724 \\
\hline 6/30/08 20:00 & 2.184 & 0.724 & 0.256 & 114.969 & 1.416 & 0.07 & 0.021335 & 0.008869 & 0.313263 & 140.593 \\
\hline 6/30/08 21:00 & 2.188 & 0.728 & 0.260 & 116.546 & 1.418 & 0.072 & 0.021945 & 0.009235 & 0.326176 & 146.388 \\
\hline 6/30/08 22:00 & 2.194 & 0.734 & 0.265 & 118.936 & 1.418 & 0.072 & 0.021945 & 0.009235 & 0.326176 & 146.388 \\
\hline 6/30/08 23:00 & 2.208 & 0.748 & 0.278 & 124.627 & 1.42 & 0.074 & 0.022554 & 0.009605 & 0.339261 & 152.260 \\
\hline 7/1/08 0:00 & 2.219 & 0.759 & 0.288 & 129.210 & 1.419 & 0.073 & 0.022249 & 0.009420 & 0.332697 & 149.314 \\
\hline 7/1/08 1:00 & 2.23 & 0.770 & 0.298 & 133.893 & 1.421 & 0.075 & 0.022859 & 0.009792 & 0.345867 & 155.225 \\
\hline 7/1/08 2:00 & 2.232 & 0.772 & 0.300 & 134.756 & 1.425 & 0.079 & 0.024078 & 0.010553 & 0.372715 & 167.274 \\
\hline 7/1/08 3:00 & 2.237 & 0.777 & 0.305 & 136.926 & 1.422 & 0.076 & 0.023164 & 0.009981 & 0.352516 & 158.209 \\
\hline 7/1/08 4:00 & 2.26 & 0.800 & 0.328 & 147.178 & 1.419 & 0.073 & 0.022249 & 0.009420 & 0.332697 & 149.314 \\
\hline 7/1/08 5:00 & 2.246 & 0.786 & 0.314 & 140.885 & 1.418 & 0.072 & 0.021945 & 0.009235 & 0.326176 & 146.388 \\
\hline 7/1/08 6:00 & 2.246 & 0.786 & 0.314 & 140.885 & 1.418 & 0.072 & 0.021945 & 0.009235 & 0.326176 & 146.388 \\
\hline 7/1/08 7:00 & 2.246 & 0.786 & 0.314 & 140.885 & 1.415 & 0.069 & 0.021030 & 0.008688 & 0.306873 & 137.724 \\
\hline
\end{tabular}




\begin{tabular}{|c|c|c|c|c|c|c|c|c|c|c|}
\hline \multirow[b]{3}{*}{ Date Time } & \multicolumn{4}{|c|}{ V-Notch Weir } & \multicolumn{6}{|c|}{ R-Notch Weir } \\
\hline & \multicolumn{2}{|c|}{ Measured Water Level } & \multicolumn{2}{|c|}{$\begin{array}{c}\text { Calculated Rate } \\
\left(\mathrm{ft}^{3} / \mathrm{s}=448.8 \mathrm{gpm}\right)\end{array}$} & \multicolumn{3}{|c|}{ Measured Water Level } & \multicolumn{3}{|c|}{$\begin{array}{c}\text { Calculated Rate } \\
\left(\mathrm{ft}^{3} / \mathrm{s}=448.8 \mathrm{gpm}\right)\end{array}$} \\
\hline & $\begin{array}{c}\text { (ft above } \\
\text { sensor) }\end{array}$ & $\begin{array}{c}\text { (ft above } \\
\text { notch) }\end{array}$ & $\left(\mathrm{ft}^{3} / \mathrm{s}\right)$ & (gpm) & $\begin{array}{l}\text { (ft above } \\
\text { sensor) }\end{array}$ & $\begin{array}{c}\text { (ft above } \\
\text { log) }\end{array}$ & $\begin{array}{c}\text { (m above } \\
\text { log) }\end{array}$ & $\left(\mathrm{m}^{3} / \mathrm{s}\right)$ & $\left(\mathrm{ft}^{3} / \mathrm{s}\right)$ & (gpm) \\
\hline 7/1/08 8:00 & 2.248 & 0.788 & 0.316 & 141.774 & 1.414 & 0.068 & 0.020725 & 0.008509 & 0.300526 & 134.876 \\
\hline 7/1/08 9:00 & 2.255 & 0.795 & 0.323 & 144.912 & 1.417 & 0.071 & 0.021640 & 0.009052 & 0.319698 & 143.480 \\
\hline 7/1/08 10:00 & 2.246 & 0.786 & 0.314 & 140.885 & 1.415 & 0.069 & 0.021030 & 0.008688 & 0.306873 & 137.724 \\
\hline 7/1/08 11:00 & 2.232 & 0.772 & 0.300 & 134.756 & 1.416 & 0.07 & 0.021335 & 0.008869 & 0.313263 & 140.593 \\
\hline 7/1/08 12:00 & 2.219 & 0.759 & 0.288 & 129.210 & 1.411 & 0.065 & 0.019811 & 0.007977 & 0.281754 & 126.451 \\
\hline 7/1/08 13:00 & 2.205 & 0.745 & 0.275 & 123.394 & 1.412 & 0.066 & 0.020116 & 0.008153 & 0.287966 & 129.239 \\
\hline 7/1/08 14:00 & 2.197 & 0.737 & 0.268 & 120.142 & 1.41 & 0.064 & 0.019506 & 0.007803 & 0.275587 & 123.683 \\
\hline 7/1/08 15:00 & 2.182 & 0.722 & 0.254 & 114.185 & 1.407 & 0.061 & 0.018592 & 0.007287 & 0.257360 & 115.503 \\
\hline 7/1/08 16:00 & 2.173 & 0.713 & 0.247 & 110.697 & 1.405 & 0.059 & 0.017982 & 0.006949 & 0.245443 & 110.155 \\
\hline 7/1/08 17:00 & 2.164 & 0.704 & 0.239 & 107.275 & 1.406 & 0.06 & 0.018287 & 0.007117 & 0.251378 & 112.818 \\
\hline 7/1/08 18:00 & 2.157 & 0.697 & 0.233 & 104.657 & 1.404 & 0.058 & 0.017678 & 0.006782 & 0.239555 & 107.512 \\
\hline 7/1/08 19:00 & 2.159 & 0.699 & 0.235 & 105.401 & 1.405 & 0.059 & 0.017982 & 0.006949 & 0.245443 & 110.155 \\
\hline 7/1/08 20:00 & 2.159 & 0.699 & 0.235 & 105.401 & 1.405 & 0.059 & 0.017982 & 0.006949 & 0.245443 & 110.155 \\
\hline 7/1/08 21:00 & 2.158 & 0.698 & 0.234 & 105.029 & 1.408 & 0.062 & 0.018897 & 0.007457 & 0.263390 & 118.209 \\
\hline 7/1/08 22:00 & 2.165 & 0.705 & 0.240 & 107.652 & 1.412 & 0.066 & 0.020116 & 0.008153 & 0.287966 & 129.239 \\
\hline 7/1/08 23:00 & 2.181 & 0.721 & 0.254 & 113.794 & 1.414 & 0.068 & 0.020725 & 0.008509 & 0.300526 & 134.876 \\
\hline 7/2/08 0:00 & 2.201 & 0.741 & 0.271 & 121.761 & 1.411 & 0.065 & 0.019811 & 0.007977 & 0.281754 & 126.451 \\
\hline 7/2/08 1:00 & 2.205 & 0.745 & 0.275 & 123.394 & 1.413 & 0.067 & 0.020421 & 0.008330 & 0.294224 & 132.048 \\
\hline 7/2/08 2:00 & 2.219 & 0.759 & 0.288 & 129.210 & 1.415 & 0.069 & 0.021030 & 0.008688 & 0.306873 & 137.724 \\
\hline 7/2/08 3:00 & 2.228 & 0.768 & 0.296 & 133.034 & 1.415 & 0.069 & 0.021030 & 0.008688 & 0.306873 & 137.724 \\
\hline 7/2/08 4:00 & 2.228 & 0.768 & 0.296 & 133.034 & 1.419 & 0.073 & 0.022249 & 0.009420 & 0.332697 & 149.314 \\
\hline 7/2/08 5:00 & 2.237 & 0.777 & 0.305 & 136.926 & 1.421 & 0.075 & 0.022859 & 0.009792 & 0.345867 & 155.225 \\
\hline 7/2/08 6:00 & 2.242 & 0.782 & 0.310 & 139.117 & 1.418 & 0.072 & 0.021945 & 0.009235 & 0.326176 & 146.388 \\
\hline 7/2/08 7:00 & 2.239 & 0.779 & 0.307 & 137.800 & 1.42 & 0.074 & 0.022554 & 0.009605 & 0.339261 & 152.260 \\
\hline 7/2/08 8:00 & 2.246 & 0.786 & 0.314 & 140.885 & 1.417 & 0.071 & 0.021640 & 0.009052 & 0.319698 & 143.480 \\
\hline
\end{tabular}




\begin{tabular}{|c|c|c|c|c|c|c|c|c|c|c|}
\hline \multirow[b]{3}{*}{ Date Time } & \multicolumn{4}{|c|}{ V-Notch Weir } & \multicolumn{6}{|c|}{ R-Notch Weir } \\
\hline & \multicolumn{2}{|c|}{ Measured Water Level } & \multicolumn{2}{|c|}{$\begin{array}{c}\text { Calculated Rate } \\
\left(\mathrm{ft}^{3} / \mathrm{s}=448.8 \mathrm{gpm}\right)\end{array}$} & \multicolumn{3}{|c|}{ Measured Water Level } & \multicolumn{3}{|c|}{$\begin{array}{c}\text { Calculated Rate } \\
\left(\mathrm{ft}^{3} / \mathrm{s}=448.8 \mathrm{gpm}\right)\end{array}$} \\
\hline & $\begin{array}{c}\text { (ft above } \\
\text { sensor) }\end{array}$ & $\begin{array}{c}\text { (ft above } \\
\text { notch) }\end{array}$ & $\left(\mathrm{ft}^{3} / \mathrm{s}\right)$ & (gpm) & $\begin{array}{l}\text { (ft above } \\
\text { sensor) }\end{array}$ & $\begin{array}{c}\text { (ft above } \\
\text { log) }\end{array}$ & $\begin{array}{c}\text { (m above } \\
\text { log) }\end{array}$ & $\left(\mathrm{m}^{3} / \mathrm{s}\right)$ & $\left(\mathrm{ft}^{3} / \mathrm{s}\right)$ & (gpm) \\
\hline 7/2/08 9:00 & 2.249 & 0.789 & 0.317 & 142.220 & 1.417 & 0.071 & 0.021640 & 0.009052 & 0.319698 & 143.480 \\
\hline 7/2/08 10:00 & 2.249 & 0.789 & 0.317 & 142.220 & 1.418 & 0.072 & 0.021945 & 0.009235 & 0.326176 & 146.388 \\
\hline 7/2/08 11:00 & 2.238 & 0.778 & 0.306 & 137.362 & 1.416 & 0.07 & 0.021335 & 0.008869 & 0.313263 & 140.593 \\
\hline 7/2/08 12:00 & 2.227 & 0.767 & 0.295 & 132.606 & 1.414 & 0.068 & 0.020725 & 0.008509 & 0.300526 & 134.876 \\
\hline 7/2/08 13:00 & 2.204 & 0.744 & 0.274 & 122.984 & 1.412 & 0.066 & 0.020116 & 0.008153 & 0.287966 & 129.239 \\
\hline 7/2/08 14:00 & 2.192 & 0.732 & 0.263 & 118.136 & 1.411 & 0.065 & 0.019811 & 0.007977 & 0.281754 & 126.451 \\
\hline 7/2/08 15:00 & 2.176 & 0.716 & 0.249 & 111.853 & 1.412 & 0.066 & 0.020116 & 0.008153 & 0.287966 & 129.239 \\
\hline 7/2/08 16:00 & 2.163 & 0.703 & 0.238 & 106.898 & 1.413 & 0.067 & 0.020421 & 0.008330 & 0.294224 & 132.048 \\
\hline 7/2/08 17:00 & 2.156 & 0.696 & 0.232 & 104.286 & 1.406 & 0.06 & 0.018287 & 0.007117 & 0.251378 & 112.818 \\
\hline 7/2/08 18:00 & 2.139 & 0.679 & 0.219 & 98.103 & 1.409 & 0.063 & 0.019201 & 0.007629 & 0.269465 & 120.936 \\
\hline 7/2/08 19:00 & 2.141 & 0.681 & 0.220 & 98.819 & 1.412 & 0.066 & 0.020116 & 0.008153 & 0.287966 & 129.239 \\
\hline 7/2/08 20:00 & 2.158 & 0.698 & 0.234 & 105.029 & 1.411 & 0.065 & 0.019811 & 0.007977 & 0.281754 & 126.451 \\
\hline 7/2/08 21:00 & 2.148 & 0.688 & 0.226 & 101.348 & 1.413 & 0.067 & 0.020421 & 0.008330 & 0.294224 & 132.048 \\
\hline 7/2/08 22:00 & 2.162 & 0.702 & 0.237 & 106.523 & 1.415 & 0.069 & 0.021030 & 0.008688 & 0.306873 & 137.724 \\
\hline 7/2/08 23:00 & 2.177 & 0.717 & 0.250 & 112.239 & 1.415 & 0.069 & 0.021030 & 0.008688 & 0.306873 & 137.724 \\
\hline 7/3/08 0:00 & 2.189 & 0.729 & 0.261 & 116.942 & 1.421 & 0.075 & 0.022859 & 0.009792 & 0.345867 & 155.225 \\
\hline 7/3/08 1:00 & 2.212 & 0.752 & 0.281 & 126.282 & 1.42 & 0.074 & 0.022554 & 0.009605 & 0.339261 & 152.260 \\
\hline 7/3/08 2:00 & 2.209 & 0.749 & 0.279 & 125.039 & 1.412 & 0.066 & 0.020116 & 0.008153 & 0.287966 & 129.239 \\
\hline 7/3/08 3:00 & 2.211 & 0.751 & 0.280 & 125.867 & 1.414 & 0.068 & 0.020725 & 0.008509 & 0.300526 & 134.876 \\
\hline 7/3/08 4:00 & 2.215 & 0.755 & 0.284 & 127.532 & 1.413 & 0.067 & 0.020421 & 0.008330 & 0.294224 & 132.048 \\
\hline 7/3/08 5:00 & 2.215 & 0.755 & 0.284 & 127.532 & 1.415 & 0.069 & 0.021030 & 0.008688 & 0.306873 & 137.724 \\
\hline 7/3/08 6:00 & 2.218 & 0.758 & 0.287 & 128.789 & 1.414 & 0.068 & 0.020725 & 0.008509 & 0.300526 & 134.876 \\
\hline 7/3/08 7:00 & 2.218 & 0.758 & 0.287 & 128.789 & 1.414 & 0.068 & 0.020725 & 0.008509 & 0.300526 & 134.876 \\
\hline 7/3/08 8:00 & 2.222 & 0.762 & 0.291 & 130.478 & 1.413 & 0.067 & 0.020421 & 0.008330 & 0.294224 & 132.048 \\
\hline 7/3/08 9:00 & 2.222 & 0.762 & 0.291 & 130.478 & 1.413 & 0.067 & 0.020421 & 0.008330 & 0.294224 & 132.048 \\
\hline
\end{tabular}




\begin{tabular}{|c|c|c|c|c|c|c|c|c|c|c|}
\hline \multirow[b]{3}{*}{ Date Time } & \multicolumn{4}{|c|}{ V-Notch Weir } & \multicolumn{6}{|c|}{ R-Notch Weir } \\
\hline & \multicolumn{2}{|c|}{ Measured Water Level } & \multicolumn{2}{|c|}{$\begin{array}{c}\text { Calculated Rate } \\
\left(\mathrm{ft}^{3} / \mathrm{s}=448.8 \mathrm{gpm}\right)\end{array}$} & \multicolumn{3}{|c|}{ Measured Water Level } & \multicolumn{3}{|c|}{$\begin{array}{c}\text { Calculated Rate } \\
\left(\mathrm{ft}^{3} / \mathrm{s}=448.8 \mathrm{gpm}\right)\end{array}$} \\
\hline & $\begin{array}{c}\text { (ft above } \\
\text { sensor) }\end{array}$ & $\begin{array}{c}\text { (ft above } \\
\text { notch) }\end{array}$ & $\left(\mathrm{ft}^{3} / \mathrm{s}\right)$ & (gpm) & $\begin{array}{l}\text { (ft above } \\
\text { sensor) }\end{array}$ & $\begin{array}{c}\text { (ft above } \\
\text { log) }\end{array}$ & $\begin{array}{c}\text { (m above } \\
\text { log) }\end{array}$ & $\left(\mathrm{m}^{3} / \mathrm{s}\right)$ & $\left(\mathrm{ft}^{3} / \mathrm{s}\right)$ & (gpm) \\
\hline 7/3/08 10:00 & 2.225 & 0.765 & 0.294 & 131.752 & 1.413 & 0.067 & 0.020421 & 0.008330 & 0.294224 & 132.048 \\
\hline 7/3/08 11:00 & 2.22 & 0.760 & 0.289 & 129.632 & 1.414 & 0.068 & 0.020725 & 0.008509 & 0.300526 & 134.876 \\
\hline 7/3/08 12:00 & 2.214 & 0.754 & 0.283 & 127.114 & 1.414 & 0.068 & 0.020725 & 0.008509 & 0.300526 & 134.876 \\
\hline 7/3/08 13:00 & 2.2 & 0.740 & 0.270 & 121.355 & 1.412 & 0.066 & 0.020116 & 0.008153 & 0.287966 & 129.239 \\
\hline 7/3/08 14:00 & 2.194 & 0.734 & 0.265 & 118.936 & 1.41 & 0.064 & 0.019506 & 0.007803 & 0.275587 & 123.683 \\
\hline 7/3/08 15:00 & 2.18 & 0.720 & 0.253 & 113.404 & 1.411 & 0.065 & 0.019811 & 0.007977 & 0.281754 & 126.451 \\
\hline 7/3/08 16:00 & 2.169 & 0.709 & 0.243 & 109.168 & 1.412 & 0.066 & 0.020116 & 0.008153 & 0.287966 & 129.239 \\
\hline 7/3/08 17:00 & 2.162 & 0.702 & 0.237 & 106.523 & 1.41 & 0.064 & 0.019506 & 0.007803 & 0.275587 & 123.683 \\
\hline 7/3/08 18:00 & 2.159 & 0.699 & 0.235 & 105.401 & 1.41 & 0.064 & 0.019506 & 0.007803 & 0.275587 & 123.683 \\
\hline 7/3/08 19:00 & 2.162 & 0.702 & 0.237 & 106.523 & 1.408 & 0.062 & 0.018897 & 0.007457 & 0.263390 & 118.209 \\
\hline 7/3/08 20:00 & 2.164 & 0.704 & 0.239 & 107.275 & 1.41 & 0.064 & 0.019506 & 0.007803 & 0.275587 & 123.683 \\
\hline 7/3/08 21:00 & 2.168 & 0.708 & 0.242 & 108.788 & 1.41 & 0.064 & 0.019506 & 0.007803 & 0.275587 & 123.683 \\
\hline 7/3/08 22:00 & 2.177 & 0.717 & 0.250 & 112.239 & 1.413 & 0.067 & 0.020421 & 0.008330 & 0.294224 & 132.048 \\
\hline 7/3/08 23:00 & 2.186 & 0.726 & 0.258 & 115.756 & 1.41 & 0.064 & 0.019506 & 0.007803 & 0.275587 & 123.683 \\
\hline 7/4/08 0:00 & 2.204 & 0.744 & 0.274 & 122.984 & 1.414 & 0.068 & 0.020725 & 0.008509 & 0.300526 & 134.876 \\
\hline 7/4/08 1:00 & 2.26 & 0.800 & 0.328 & 147.178 & 1.416 & 0.07 & 0.021335 & 0.008869 & 0.313263 & 140.593 \\
\hline 7/4/08 2:00 & 2.258 & 0.798 & 0.326 & 146.269 & 1.415 & 0.069 & 0.021030 & 0.008688 & 0.306873 & 137.724 \\
\hline 7/4/08 3:00 & 2.26 & 0.800 & 0.328 & 147.178 & 1.415 & 0.069 & 0.021030 & 0.008688 & 0.306873 & 137.724 \\
\hline 7/4/08 4:00 & 2.269 & 0.809 & 0.337 & 151.312 & 1.416 & 0.07 & 0.021335 & 0.008869 & 0.313263 & 140.593 \\
\hline 7/4/08 5:00 & 2.267 & 0.807 & 0.335 & 150.387 & 1.418 & 0.072 & 0.021945 & 0.009235 & 0.326176 & 146.388 \\
\hline 7/4/08 6:00 & 2.269 & 0.809 & 0.337 & 151.312 & 1.416 & 0.07 & 0.021335 & 0.008869 & 0.313263 & 140.593 \\
\hline 7/4/08 7:00 & 2.267 & 0.807 & 0.335 & 150.387 & 1.415 & 0.069 & 0.021030 & 0.008688 & 0.306873 & 137.724 \\
\hline 7/4/08 8:00 & 2.274 & 0.814 & 0.342 & 153.638 & 1.417 & 0.071 & 0.021640 & 0.009052 & 0.319698 & 143.480 \\
\hline 7/4/08 9:00 & 2.279 & 0.819 & 0.348 & 155.985 & 1.417 & 0.071 & 0.021640 & 0.009052 & 0.319698 & 143.480 \\
\hline 7/4/08 10:00 & 2.279 & 0.819 & 0.348 & 155.985 & 1.418 & 0.072 & 0.021945 & 0.009235 & 0.326176 & 146.388 \\
\hline
\end{tabular}




\begin{tabular}{|c|c|c|c|c|c|c|c|c|c|c|}
\hline \multirow[b]{3}{*}{ Date Time } & \multicolumn{4}{|c|}{ V-Notch Weir } & \multicolumn{6}{|c|}{ R-Notch Weir } \\
\hline & \multicolumn{2}{|c|}{ Measured Water Level } & \multicolumn{2}{|c|}{$\begin{array}{c}\text { Calculated Rate } \\
\left(\mathrm{ft}^{3} / \mathrm{s}=448.8 \mathrm{gpm}\right)\end{array}$} & \multicolumn{3}{|c|}{ Measured Water Level } & \multicolumn{3}{|c|}{$\begin{array}{c}\text { Calculated Rate } \\
\left(\mathrm{ft}^{3} / \mathrm{s}=448.8 \mathrm{gpm}\right)\end{array}$} \\
\hline & $\begin{array}{c}\text { (ft above } \\
\text { sensor) }\end{array}$ & $\begin{array}{c}\text { (ft above } \\
\text { notch) }\end{array}$ & $\left(\mathrm{ft}^{3} / \mathrm{s}\right)$ & (gpm) & $\begin{array}{l}\text { (ft above } \\
\text { sensor) }\end{array}$ & $\begin{array}{c}\text { (ft above } \\
\text { log) }\end{array}$ & $\begin{array}{c}\text { (m above } \\
\text { log) }\end{array}$ & $\left(\mathrm{m}^{3} / \mathrm{s}\right)$ & $\left(\mathrm{ft}^{3} / \mathrm{s}\right)$ & (gpm) \\
\hline 7/4/08 11:00 & 2.272 & 0.812 & 0.340 & 152.705 & 1.418 & 0.072 & 0.021945 & 0.009235 & 0.326176 & 146.388 \\
\hline 7/4/08 12:00 & 2.256 & 0.796 & 0.324 & 145.363 & 1.414 & 0.068 & 0.020725 & 0.008509 & 0.300526 & 134.876 \\
\hline 7/4/08 13:00 & 2.247 & 0.787 & 0.315 & 141.329 & 1.414 & 0.068 & 0.020725 & 0.008509 & 0.300526 & 134.876 \\
\hline 7/4/08 14:00 & 2.237 & 0.777 & 0.305 & 136.926 & 1.413 & 0.067 & 0.020421 & 0.008330 & 0.294224 & 132.048 \\
\hline 7/4/08 15:00 & 2.219 & 0.759 & 0.288 & 129.210 & 1.411 & 0.065 & 0.019811 & 0.007977 & 0.281754 & 126.451 \\
\hline 7/4/08 16:00 & 2.21 & 0.750 & 0.280 & 125.453 & 1.412 & 0.066 & 0.020116 & 0.008153 & 0.287966 & 129.239 \\
\hline 7/4/08 17:00 & 2.205 & 0.745 & 0.275 & 123.394 & 1.41 & 0.064 & 0.019506 & 0.007803 & 0.275587 & 123.683 \\
\hline 7/4/08 18:00 & 2.201 & 0.741 & 0.271 & 121.761 & 1.41 & 0.064 & 0.019506 & 0.007803 & 0.275587 & 123.683 \\
\hline 7/4/08 19:00 & 2.198 & 0.738 & 0.269 & 120.546 & 1.411 & 0.065 & 0.019811 & 0.007977 & 0.281754 & 126.451 \\
\hline 7/4/08 20:00 & 2.198 & 0.738 & 0.269 & 120.546 & 1.409 & 0.063 & 0.019201 & 0.007629 & 0.269465 & 120.936 \\
\hline 7/4/08 21:00 & 2.209 & 0.749 & 0.279 & 125.039 & 1.411 & 0.065 & 0.019811 & 0.007977 & 0.281754 & 126.451 \\
\hline 7/4/08 22:00 & 2.215 & 0.755 & 0.284 & 127.532 & 1.411 & 0.065 & 0.019811 & 0.007977 & 0.281754 & 126.451 \\
\hline 7/4/08 23:00 & 2.224 & 0.764 & 0.293 & 131.327 & 1.413 & 0.067 & 0.020421 & 0.008330 & 0.294224 & 132.048 \\
\hline 7/5/08 0:00 & 2.231 & 0.771 & 0.299 & 134.324 & 1.417 & 0.071 & 0.021640 & 0.009052 & 0.319698 & 143.480 \\
\hline 7/5/08 1:00 & 2.247 & 0.787 & 0.315 & 141.329 & 1.417 & 0.071 & 0.021640 & 0.009052 & 0.319698 & 143.480 \\
\hline 7/5/08 2:00 & 2.251 & 0.791 & 0.319 & 143.114 & 1.418 & 0.072 & 0.021945 & 0.009235 & 0.326176 & 146.388 \\
\hline 7/5/08 3:00 & 2.265 & 0.805 & 0.333 & 149.466 & 1.418 & 0.072 & 0.021945 & 0.009235 & 0.326176 & 146.388 \\
\hline 7/5/08 4:00 & 2.265 & 0.805 & 0.333 & 149.466 & 1.417 & 0.071 & 0.021640 & 0.009052 & 0.319698 & 143.480 \\
\hline 7/5/08 5:00 & 2.269 & 0.809 & 0.337 & 151.312 & 1.417 & 0.071 & 0.021640 & 0.009052 & 0.319698 & 143.480 \\
\hline 7/5/08 6:00 & 2.274 & 0.814 & 0.342 & 153.638 & 1.418 & 0.072 & 0.021945 & 0.009235 & 0.326176 & 146.388 \\
\hline 7/5/08 7:00 & 2.272 & 0.812 & 0.340 & 152.705 & 1.418 & 0.072 & 0.021945 & 0.009235 & 0.326176 & 146.388 \\
\hline 7/5/08 8:00 & 2.272 & 0.812 & 0.340 & 152.705 & 1.418 & 0.072 & 0.021945 & 0.009235 & 0.326176 & 146.388 \\
\hline 7/5/08 9:00 & 2.276 & 0.816 & 0.344 & 154.574 & 1.418 & 0.072 & 0.021945 & 0.009235 & 0.326176 & 146.388 \\
\hline 7/5/08 10:00 & 2.283 & 0.823 & 0.352 & 157.878 & 1.42 & 0.074 & 0.022554 & 0.009605 & 0.339261 & 152.260 \\
\hline 7/5/08 11:00 & 2.307 & 0.847 & 0.378 & 169.527 & 1.425 & 0.079 & 0.024078 & 0.010553 & 0.372715 & 167.274 \\
\hline
\end{tabular}




\begin{tabular}{|c|c|c|c|c|c|c|c|c|c|c|}
\hline \multirow[b]{3}{*}{ Date } & \multicolumn{4}{|c|}{ V-Notch Weir } & \multicolumn{6}{|c|}{ R-Notch Weir } \\
\hline & \multicolumn{2}{|c|}{ Measured Water Level } & \multicolumn{2}{|c|}{$\begin{array}{c}\text { Calculated Rate } \\
\left(\mathrm{ft}^{3} / \mathrm{s}=448.8 \mathrm{gpm}\right)\end{array}$} & \multicolumn{3}{|c|}{ Measured Water Level } & \multicolumn{3}{|c|}{$\begin{array}{c}\text { Calculated Rate } \\
\left(\mathrm{ft}^{3} / \mathrm{s}=448.8 \mathrm{gpm}\right)\end{array}$} \\
\hline & $\begin{array}{c}\text { (ft above } \\
\text { sensor) }\end{array}$ & $\begin{array}{c}\text { (ft above } \\
\text { notch) }\end{array}$ & $\left(\mathrm{ft}^{3} / \mathrm{s}\right)$ & (gpm) & $\begin{array}{l}\text { (ft above } \\
\text { sensor) }\end{array}$ & $\begin{array}{c}\text { (ft above } \\
\text { log) }\end{array}$ & $\begin{array}{c}\text { (m above } \\
\text { log) }\end{array}$ & $\left(\mathrm{m}^{3} / \mathrm{s}\right)$ & $\left(\mathrm{ft}^{3} / \mathrm{s}\right)$ & (gpm) \\
\hline 7/5/08 12:00 & 2.326 & 0.866 & 0.399 & 179.104 & 1.425 & 0.079 & 0.024078 & 0.010553 & 0.372715 & 167.274 \\
\hline 7/5/08 13:00 & 2.336 & 0.876 & 0.411 & 184.272 & 1.423 & 0.077 & 0.023468 & 0.010170 & 0.359208 & 161.212 \\
\hline 7/5/08 14:00 & 2.308 & 0.848 & 0.379 & 170.023 & 1.423 & 0.077 & 0.023468 & 0.010170 & 0.359208 & 161.212 \\
\hline 7/5/08 15:00 & 2.22 & 0.760 & 0.289 & 129.632 & 1.419 & 0.073 & 0.022249 & 0.009420 & 0.332697 & 149.314 \\
\hline 7/5/08 16:00 & 2.197 & 0.737 & 0.268 & 120.142 & 1.418 & 0.072 & 0.021945 & 0.009235 & 0.326176 & 146.388 \\
\hline 7/5/08 17:00 & 2.188 & 0.728 & 0.260 & 116.546 & 1.418 & 0.072 & 0.021945 & 0.009235 & 0.326176 & 146.388 \\
\hline 7/5/08 18:00 & 2.174 & 0.714 & 0.248 & 111.082 & 1.412 & 0.066 & 0.020116 & 0.008153 & 0.287966 & 129.239 \\
\hline 7/5/08 19:00 & 2.169 & 0.709 & 0.243 & 109.168 & 1.412 & 0.066 & 0.020116 & 0.008153 & 0.287966 & 129.239 \\
\hline 7/5/08 20:00 & 2.167 & 0.707 & 0.242 & 108.408 & 1.413 & 0.067 & 0.020421 & 0.008330 & 0.294224 & 132.048 \\
\hline 7/5/08 21:00 & 2.171 & 0.711 & 0.245 & 109.931 & 1.413 & 0.067 & 0.020421 & 0.008330 & 0.294224 & 132.048 \\
\hline 7/5/08 22:00 & 2.183 & 0.723 & 0.255 & 114.576 & 1.415 & 0.069 & 0.021030 & 0.008688 & 0.306873 & 137.724 \\
\hline 7/5/08 23:00 & 2.194 & 0.734 & 0.265 & 118.936 & 1.417 & 0.071 & 0.021640 & 0.009052 & 0.319698 & 143.480 \\
\hline 7/6/08 0:00 & 2.203 & 0.743 & 0.273 & 122.576 & 1.417 & 0.071 & 0.021640 & 0.009052 & 0.319698 & 143.480 \\
\hline 7/6/08 1:00 & 2.212 & 0.752 & 0.281 & 126.282 & 1.419 & 0.073 & 0.022249 & 0.009420 & 0.332697 & 149.314 \\
\hline 7/6/08 2:00 & 2.214 & 0.754 & 0.283 & 127.114 & 1.419 & 0.073 & 0.022249 & 0.009420 & 0.332697 & 149.314 \\
\hline 7/6/08 3:00 & 2.223 & 0.763 & 0.292 & 130.902 & 1.421 & 0.075 & 0.022859 & 0.009792 & 0.345867 & 155.225 \\
\hline 7/6/08 4:00 & 2.235 & 0.775 & 0.303 & 136.055 & 1.423 & 0.077 & 0.023468 & 0.010170 & 0.359208 & 161.212 \\
\hline 7/6/08 5:00 & 2.237 & 0.777 & 0.305 & 136.926 & 1.423 & 0.077 & 0.023468 & 0.010170 & 0.359208 & 161.212 \\
\hline 7/6/08 6:00 & 2.242 & 0.782 & 0.310 & 139.117 & 1.425 & 0.079 & 0.024078 & 0.010553 & 0.372715 & 167.274 \\
\hline 7/6/08 7:00 & 2.239 & 0.779 & 0.307 & 137.800 & 1.422 & 0.076 & 0.023164 & 0.009981 & 0.352516 & 158.209 \\
\hline 7/6/08 8:00 & 2.244 & 0.784 & 0.312 & 139.999 & 1.422 & 0.076 & 0.023164 & 0.009981 & 0.352516 & 158.209 \\
\hline 7/6/08 9:00 & 2.244 & 0.784 & 0.312 & 139.999 & 1.424 & 0.078 & 0.023773 & 0.010361 & 0.365940 & 164.234 \\
\hline 7/6/08 10:00 & 2.237 & 0.777 & 0.305 & 136.926 & 1.422 & 0.076 & 0.023164 & 0.009981 & 0.352516 & 158.209 \\
\hline 7/6/08 11:00 & 2.229 & 0.769 & 0.297 & 133.464 & 1.42 & 0.074 & 0.022554 & 0.009605 & 0.339261 & 152.260 \\
\hline 7/6/08 12:00 & 2.213 & 0.753 & 0.282 & 126.698 & 1.418 & 0.072 & 0.021945 & 0.009235 & 0.326176 & 146.388 \\
\hline
\end{tabular}




\begin{tabular}{|c|c|c|c|c|c|c|c|c|c|c|}
\hline \multirow[b]{3}{*}{ Date Time } & \multicolumn{4}{|c|}{ V-Notch Weir } & \multicolumn{6}{|c|}{ R-Notch Weir } \\
\hline & \multicolumn{2}{|c|}{ Measured Water Level } & \multicolumn{2}{|c|}{$\begin{array}{c}\text { Calculated Rate } \\
\left(\mathrm{ft}^{3} / \mathrm{s}=448.8 \mathrm{gpm}\right)\end{array}$} & \multicolumn{3}{|c|}{ Measured Water Level } & \multicolumn{3}{|c|}{$\begin{array}{c}\begin{array}{c}\text { Calculated Rate } \\
\left(\mathrm{ft}^{3} / \mathrm{s}=448.8 \mathrm{gpm}\right)\end{array} \\
\end{array}$} \\
\hline & $\begin{array}{l}\text { (ft above } \\
\text { sensor) }\end{array}$ & $\begin{array}{c}\text { (ft above } \\
\text { notch) }\end{array}$ & $\left(\mathrm{ft}^{3} / \mathrm{s}\right)$ & (gpm) & $\begin{array}{c}\text { (ft above } \\
\text { sensor) }\end{array}$ & $\begin{array}{c}\text { (ft above } \\
\text { log) }\end{array}$ & $\begin{array}{c}\text { (m above } \\
\text { log) }\end{array}$ & $\left(\mathrm{m}^{3} / \mathrm{s}\right)$ & $\left(\mathrm{ft}^{3} / \mathrm{s}\right)$ & (gpm) \\
\hline 7/6/08 13:00 & 2.199 & 0.739 & 0.269 & 120.950 & 1.418 & 0.072 & 0.021945 & 0.009235 & 0.326176 & 146.388 \\
\hline 7/6/08 14:00 & 2.193 & 0.733 & 0.264 & 118.536 & 1.419 & 0.073 & 0.022249 & 0.009420 & 0.332697 & 149.314 \\
\hline 7/6/08 15:00 & 2.179 & 0.719 & 0.252 & 113.015 & 1.417 & 0.071 & 0.021640 & 0.009052 & 0.319698 & 143.480 \\
\hline 7/6/08 16:00 & 2.179 & 0.719 & 0.252 & 113.015 & 1.418 & 0.072 & 0.021945 & 0.009235 & 0.326176 & 146.388 \\
\hline 7/6/08 17:00 & 2.17 & 0.710 & 0.244 & 109.549 & 1.416 & 0.07 & 0.021335 & 0.008869 & 0.313263 & 140.593 \\
\hline 7/6/08 18:00 & 2.167 & 0.707 & 0.242 & 108.408 & 1.416 & 0.07 & 0.021335 & 0.008869 & 0.313263 & 140.593 \\
\hline 7/6/08 19:00 & 2.172 & 0.712 & 0.246 & 110.314 & 1.416 & 0.07 & 0.021335 & 0.008869 & 0.313263 & 140.593 \\
\hline 7/6/08 20:00 & 2.177 & 0.717 & 0.250 & 112.239 & 1.419 & 0.073 & 0.022249 & 0.009420 & 0.332697 & 149.314 \\
\hline 7/6/08 21:00 & 2.19 & 0.730 & 0.261 & 117.339 & 1.419 & 0.073 & 0.022249 & 0.009420 & 0.332697 & 149.314 \\
\hline 7/6/08 22:00 & 2.195 & 0.735 & 0.266 & 119.337 & 1.419 & 0.073 & 0.022249 & 0.009420 & 0.332697 & 149.314 \\
\hline 7/6/08 23:00 & 2.203 & 0.743 & 0.273 & 122.576 & 1.421 & 0.075 & 0.022859 & 0.009792 & 0.345867 & 155.225 \\
\hline 7/7/08 0:00 & 2.215 & 0.755 & 0.284 & 127.532 & 1.423 & 0.077 & 0.023468 & 0.010170 & 0.359208 & 161.212 \\
\hline 7/7/08 1:00 & 2.217 & 0.757 & 0.286 & 128.369 & 1.423 & 0.077 & 0.023468 & 0.010170 & 0.359208 & 161.212 \\
\hline 7/7/08 2:00 & 2.238 & 0.778 & 0.306 & 137.362 & 1.427 & 0.081 & 0.024688 & 0.010940 & 0.386387 & 173.411 \\
\hline 7/7/08 3:00 & 2.23 & 0.770 & 0.298 & 133.893 & 1.424 & 0.078 & 0.023773 & 0.010361 & 0.365940 & 164.234 \\
\hline 7/7/08 4:00 & 2.228 & 0.768 & 0.296 & 133.034 & 1.424 & 0.078 & 0.023773 & 0.010361 & 0.365940 & 164.234 \\
\hline 7/7/08 5:00 & 2.228 & 0.768 & 0.296 & 133.034 & 1.424 & 0.078 & 0.023773 & 0.010361 & 0.365940 & 164.234 \\
\hline 7/7/08 6:00 & 2.235 & 0.775 & 0.303 & 136.055 & 1.426 & 0.08 & 0.024383 & 0.010746 & 0.379531 & 170.333 \\
\hline 7/7/08 7:00 & 2.23 & 0.770 & 0.298 & 133.893 & 1.423 & 0.077 & 0.023468 & 0.010170 & 0.359208 & 161.212 \\
\hline 7/7/08 8:00 & 2.235 & 0.775 & 0.303 & 136.055 & 1.423 & 0.077 & 0.023468 & 0.010170 & 0.359208 & 161.212 \\
\hline 7/7/08 9:00 & 2.235 & 0.775 & 0.303 & 136.055 & 1.423 & 0.077 & 0.023468 & 0.010170 & 0.359208 & 161.212 \\
\hline 7/7/08 10:00 & 2.226 & 0.766 & 0.295 & 132.179 & 1.423 & 0.077 & 0.023468 & 0.010170 & 0.359208 & 161.212 \\
\hline 7/7/08 11:00 & 2.21 & 0.750 & 0.280 & 125.453 & 1.421 & 0.075 & 0.022859 & 0.009792 & 0.345867 & 155.225 \\
\hline 7/7/08 12:00 & 2.202 & 0.742 & 0.272 & 122.168 & 1.419 & 0.073 & 0.022249 & 0.009420 & 0.332697 & 149.314 \\
\hline 7/7/08 13:00 & 2.187 & 0.727 & 0.259 & 116.150 & 1.42 & 0.074 & 0.022554 & 0.009605 & 0.339261 & 152.260 \\
\hline
\end{tabular}




\begin{tabular}{|c|c|c|c|c|c|c|c|c|c|c|}
\hline \multirow[b]{3}{*}{ Date Time } & \multicolumn{4}{|c|}{ V-Notch Weir } & \multicolumn{6}{|c|}{ R-Notch Weir } \\
\hline & \multicolumn{2}{|c|}{ Measured Water Level } & \multicolumn{2}{|c|}{$\begin{array}{c}\text { Calculated Rate } \\
\left(\mathrm{ft}^{3} / \mathrm{s}=448.8 \mathrm{gpm}\right)\end{array}$} & \multicolumn{3}{|c|}{ Measured Water Level } & \multicolumn{3}{|c|}{$\begin{array}{c}\text { Calculated Rate } \\
\left(\mathrm{ft}^{3} / \mathrm{s}=448.8 \mathrm{gpm}\right)\end{array}$} \\
\hline & $\begin{array}{c}\text { (ft above } \\
\text { sensor) }\end{array}$ & $\begin{array}{c}\text { (ft above } \\
\text { notch) }\end{array}$ & $\left(\mathrm{ft}^{3} / \mathrm{s}\right)$ & (gpm) & $\begin{array}{l}\text { (ft above } \\
\text { sensor) }\end{array}$ & $\begin{array}{c}\text { (ft above } \\
\text { log) }\end{array}$ & $\begin{array}{c}\text { (m above } \\
\text { log) }\end{array}$ & $\left(\mathrm{m}^{3} / \mathrm{s}\right)$ & $\left(\mathrm{ft}^{3} / \mathrm{s}\right)$ & (gpm) \\
\hline 7/7/08 14:00 & 2.171 & 0.711 & 0.245 & 109.931 & 1.421 & 0.075 & 0.022859 & 0.009792 & 0.345867 & 155.225 \\
\hline 7/7/08 15:00 & 2.157 & 0.697 & 0.233 & 104.657 & 1.416 & 0.07 & 0.021335 & 0.008869 & 0.313263 & 140.593 \\
\hline 7/7/08 16:00 & 2.152 & 0.692 & 0.229 & 102.811 & 1.417 & 0.071 & 0.021640 & 0.009052 & 0.319698 & 143.480 \\
\hline 7/7/08 17:00 & 2.152 & 0.692 & 0.229 & 102.811 & 1.417 & 0.071 & 0.021640 & 0.009052 & 0.319698 & 143.480 \\
\hline 7/7/08 18:00 & 2.166 & 0.706 & 0.241 & 108.030 & 1.42 & 0.074 & 0.022554 & 0.009605 & 0.339261 & 152.260 \\
\hline 7/7/08 19:00 & 2.17 & 0.710 & 0.244 & 109.549 & 1.418 & 0.072 & 0.021945 & 0.009235 & 0.326176 & 146.388 \\
\hline 7/7/08 20:00 & 2.184 & 0.724 & 0.256 & 114.969 & 1.42 & 0.074 & 0.022554 & 0.009605 & 0.339261 & 152.260 \\
\hline 7/7/08 21:00 & 2.195 & 0.735 & 0.266 & 119.337 & 1.425 & 0.079 & 0.024078 & 0.010553 & 0.372715 & 167.274 \\
\hline 7/7/08 22:00 & 2.201 & 0.741 & 0.271 & 121.761 & 1.429 & 0.083 & 0.025297 & 0.011331 & 0.400223 & 179.620 \\
\hline 7/7/08 23:00 & 2.21 & 0.750 & 0.280 & 125.453 & 1.429 & 0.083 & 0.025297 & 0.011331 & 0.400223 & 179.620 \\
\hline 7/8/08 0:00 & 2.219 & 0.759 & 0.288 & 129.210 & 1.431 & 0.085 & 0.025907 & 0.011728 & 0.414220 & 185.902 \\
\hline 7/8/08 1:00 & 2.221 & 0.761 & 0.290 & 130.054 & 1.43 & 0.084 & 0.025602 & 0.011529 & 0.407202 & 182.752 \\
\hline 7/8/08 2:00 & 2.226 & 0.766 & 0.295 & 132.179 & 1.432 & 0.086 & 0.026212 & 0.011928 & 0.421279 & 189.070 \\
\hline 7/8/08 3:00 & 2.228 & 0.768 & 0.296 & 133.034 & 1.432 & 0.086 & 0.026212 & 0.011928 & 0.421279 & 189.070 \\
\hline 7/8/08 4:00 & 2.24 & 0.780 & 0.308 & 138.238 & 1.434 & 0.088 & 0.026821 & 0.012331 & 0.435514 & 195.459 \\
\hline 7/8/08 5:00 & 2.249 & 0.789 & 0.317 & 142.220 & 1.436 & 0.09 & 0.027431 & 0.012738 & 0.449907 & 201.918 \\
\hline 7/8/08 6:00 & 2.253 & 0.793 & 0.321 & 144.011 & 1.435 & 0.089 & 0.027126 & 0.012534 & 0.442691 & 198.680 \\
\hline 7/8/08 7:00 & 2.244 & 0.784 & 0.312 & 139.999 & 1.435 & 0.089 & 0.027126 & 0.012534 & 0.442691 & 198.680 \\
\hline 7/8/08 8:00 & 2.246 & 0.786 & 0.314 & 140.885 & 1.435 & 0.089 & 0.027126 & 0.012534 & 0.442691 & 198.680 \\
\hline 7/8/08 9:00 & 2.244 & 0.784 & 0.312 & 139.999 & 1.432 & 0.086 & 0.026212 & 0.011928 & 0.421279 & 189.070 \\
\hline 7/8/08 10:00 & 2.24 & 0.780 & 0.308 & 138.238 & 1.432 & 0.086 & 0.026212 & 0.011928 & 0.421279 & 189.070 \\
\hline 7/8/08 11:00 & 2.233 & 0.773 & 0.301 & 135.188 & 1.433 & 0.087 & 0.026516 & 0.012129 & 0.428377 & 192.256 \\
\hline 7/8/08 12:00 & 2.235 & 0.775 & 0.303 & 136.055 & 1.43 & 0.084 & 0.025602 & 0.011529 & 0.407202 & 182.752 \\
\hline 7/8/08 13:00 & 2.224 & 0.764 & 0.293 & 131.327 & 1.431 & 0.085 & 0.025907 & 0.011728 & 0.414220 & 185.902 \\
\hline 7/8/08 14:00 & 2.222 & 0.762 & 0.291 & 130.478 & 1.431 & 0.085 & 0.025907 & 0.011728 & 0.414220 & 185.902 \\
\hline
\end{tabular}




\begin{tabular}{|c|c|c|c|c|c|c|c|c|c|c|}
\hline \multirow[b]{3}{*}{ Date Time } & \multicolumn{4}{|c|}{ V-Notch Weir } & \multicolumn{6}{|c|}{ R-Notch Weir } \\
\hline & \multicolumn{2}{|c|}{ Measured Water Level } & \multicolumn{2}{|c|}{$\begin{array}{c}\text { Calculated Rate } \\
\left(\mathrm{ft}^{3} / \mathrm{s}=448.8 \mathrm{gpm}\right)\end{array}$} & \multicolumn{3}{|c|}{ Measured Water Level } & \multicolumn{3}{|c|}{$\begin{array}{c}\text { Calculated Rate } \\
\left(\mathrm{ft}^{3} / \mathrm{s}=448.8 \mathrm{gpm}\right)\end{array}$} \\
\hline & $\begin{array}{l}\text { (ft above } \\
\text { sensor) }\end{array}$ & $\begin{array}{l}\text { (ft above } \\
\text { notch) }\end{array}$ & $\left(\mathrm{ft}^{3} / \mathrm{s}\right)$ & (gpm) & $\begin{array}{l}\text { (ft above } \\
\text { sensor) }\end{array}$ & $\begin{array}{l}\text { (ft above } \\
\text { log) }\end{array}$ & $\begin{array}{c}\text { (m above } \\
\text { log) }\end{array}$ & $\left(\mathrm{m}^{3} / \mathrm{s}\right)$ & $\left(\mathrm{ft}^{3} / \mathrm{s}\right)$ & (gpm) \\
\hline 7/8/08 15:00 & 2.212 & 0.752 & 0.281 & 126.282 & 1.431 & 0.085 & 0.025907 & 0.011728 & 0.414220 & 185.902 \\
\hline 7/8/08 16:00 & 2.203 & 0.743 & 0.273 & 122.576 & 1.43 & 0.084 & 0.025602 & 0.011529 & 0.407202 & 182.752 \\
\hline 7/8/08 17:00 & 2.195 & 0.735 & 0.266 & 119.337 & 1.428 & 0.082 & 0.024992 & 0.011135 & 0.393285 & 176.506 \\
\hline 7/8/08 18:00 & 2.186 & 0.726 & 0.258 & 115.756 & 1.428 & 0.082 & 0.024992 & 0.011135 & 0.393285 & 176.506 \\
\hline 7/8/08 19:00 & 2.191 & 0.731 & 0.262 & 117.737 & 1.428 & 0.082 & 0.024992 & 0.011135 & 0.393285 & 176.506 \\
\hline 7/8/08 20:00 & 2.188 & 0.728 & 0.260 & 116.546 & 1.429 & 0.083 & 0.025297 & 0.011331 & 0.400223 & 179.620 \\
\hline 7/8/08 21:00 & 2.19 & 0.730 & 0.261 & 117.339 & 1.429 & 0.083 & 0.025297 & 0.011331 & 0.400223 & 179.620 \\
\hline 7/8/08 22:00 & 2.194 & 0.734 & 0.265 & 118.936 & 1.428 & 0.082 & 0.024992 & 0.011135 & 0.393285 & 176.506 \\
\hline 7/8/08 23:00 & 2.203 & 0.743 & 0.273 & 122.576 & 1.43 & 0.084 & 0.025602 & 0.011529 & 0.407202 & 182.752 \\
\hline 7/9/08 0:00 & 2.207 & 0.747 & 0.277 & 124.215 & 1.43 & 0.084 & 0.025602 & 0.011529 & 0.407202 & 182.752 \\
\hline 7/9/08 1:00 & 2.216 & 0.756 & 0.285 & 127.950 & 1.429 & 0.083 & 0.025297 & 0.011331 & 0.400223 & 179.620 \\
\hline 7/9/08 2:00 & 2.214 & 0.754 & 0.283 & 127.114 & 1.431 & 0.085 & 0.025907 & 0.011728 & 0.414220 & 185.902 \\
\hline 7/9/08 3:00 & 2.223 & 0.763 & 0.292 & 130.902 & 1.431 & 0.085 & 0.025907 & 0.011728 & 0.414220 & 185.902 \\
\hline 7/9/08 4:00 & 2.23 & 0.770 & 0.298 & 133.893 & 1.433 & 0.087 & 0.026516 & 0.012129 & 0.428377 & 192.256 \\
\hline 7/9/08 5:00 & 2.23 & 0.770 & 0.298 & 133.893 & 1.432 & 0.086 & 0.026212 & 0.011928 & 0.421279 & 189.070 \\
\hline 7/9/08 6:00 & 2.229 & 0.769 & 0.297 & 133.464 & 1.431 & 0.085 & 0.025907 & 0.011728 & 0.414220 & 185.902 \\
\hline 7/9/08 7:00 & 2.227 & 0.767 & 0.295 & 132.606 & 1.431 & 0.085 & 0.025907 & 0.011728 & 0.414220 & 185.902 \\
\hline 7/9/08 8:00 & 2.234 & 0.774 & 0.302 & 135.621 & 1.433 & 0.087 & 0.026516 & 0.012129 & 0.428377 & 192.256 \\
\hline 7/9/08 9:00 & 2.236 & 0.776 & 0.304 & 136.490 & 1.43 & 0.084 & 0.025602 & 0.011529 & 0.407202 & 182.752 \\
\hline 7/9/08 10:00 & 2.237 & 0.777 & 0.305 & 136.926 & 1.431 & 0.085 & 0.025907 & 0.011728 & 0.414220 & 185.902 \\
\hline 7/9/08 11:00 & 2.228 & 0.768 & 0.296 & 133.034 & 1.431 & 0.085 & 0.025907 & 0.011728 & 0.414220 & 185.902 \\
\hline 7/9/08 12:00 & 2.217 & 0.757 & 0.286 & 128.369 & 1.429 & 0.083 & 0.025297 & 0.011331 & 0.400223 & 179.620 \\
\hline 7/9/08 13:00 & 2.208 & 0.748 & 0.278 & 124.627 & 1.429 & 0.083 & 0.025297 & 0.011331 & 0.400223 & 179.620 \\
\hline 7/9/08 14:00 & 2.197 & 0.737 & 0.268 & 120.142 & 1.428 & 0.082 & 0.024992 & 0.011135 & 0.393285 & 176.506 \\
\hline 7/9/08 15:00 & 2.186 & 0.726 & 0.258 & 115.756 & 1.426 & 0.08 & 0.024383 & 0.010746 & 0.379531 & 170.333 \\
\hline
\end{tabular}




\begin{tabular}{|c|c|c|c|c|c|c|c|c|c|c|}
\hline \multirow[b]{3}{*}{ Date Time } & \multicolumn{4}{|c|}{ V-Notch Weir } & \multicolumn{6}{|c|}{ R-Notch Weir } \\
\hline & \multicolumn{2}{|c|}{ Measured Water Level } & \multicolumn{2}{|c|}{$\begin{array}{c}\text { Calculated Rate } \\
\left(\mathrm{ft}^{3} / \mathrm{s}=448.8 \mathrm{gpm}\right)\end{array}$} & \multicolumn{3}{|c|}{ Measured Water Level } & \multicolumn{3}{|c|}{$\begin{array}{c}\text { Calculated Rate } \\
\left(\mathrm{ft}^{3} / \mathrm{s}=448.8 \mathrm{gpm}\right)\end{array}$} \\
\hline & $\begin{array}{c}\text { (ft above } \\
\text { sensor) }\end{array}$ & $\begin{array}{c}\text { (ft above } \\
\text { notch) }\end{array}$ & $\left(\mathrm{ft}^{3} / \mathrm{s}\right)$ & (gpm) & $\begin{array}{l}\text { (ft above } \\
\text { sensor) }\end{array}$ & $\begin{array}{c}\text { (ft above } \\
\text { log) }\end{array}$ & $\begin{array}{c}\text { (m above } \\
\text { log) }\end{array}$ & $\left(\mathrm{m}^{3} / \mathrm{s}\right)$ & $\left(\mathrm{ft}^{3} / \mathrm{s}\right)$ & (gpm) \\
\hline 7/9/08 16:00 & 2.177 & 0.717 & 0.250 & 112.239 & 1.427 & 0.081 & 0.024688 & 0.010940 & 0.386387 & 173.411 \\
\hline 7/9/08 17:00 & 2.172 & 0.712 & 0.246 & 110.314 & 1.425 & 0.079 & 0.024078 & 0.010553 & 0.372715 & 167.274 \\
\hline 7/9/08 18:00 & 2.176 & 0.716 & 0.249 & 111.853 & 1.425 & 0.079 & 0.024078 & 0.010553 & 0.372715 & 167.274 \\
\hline 7/9/08 19:00 & 2.183 & 0.723 & 0.255 & 114.576 & 1.428 & 0.082 & 0.024992 & 0.011135 & 0.393285 & 176.506 \\
\hline 7/9/08 20:00 & 2.185 & 0.725 & 0.257 & 115.362 & 1.428 & 0.082 & 0.024992 & 0.011135 & 0.393285 & 176.506 \\
\hline 7/9/08 21:00 & 2.197 & 0.737 & 0.268 & 120.142 & 1.43 & 0.084 & 0.025602 & 0.011529 & 0.407202 & 182.752 \\
\hline 7/9/08 22:00 & 2.205 & 0.745 & 0.275 & 123.394 & 1.432 & 0.086 & 0.026212 & 0.011928 & 0.421279 & 189.070 \\
\hline 7/9/08 23:00 & 2.214 & 0.754 & 0.283 & 127.114 & 1.432 & 0.086 & 0.026212 & 0.011928 & 0.421279 & 189.070 \\
\hline 7/10/08 0:00 & 2.219 & 0.759 & 0.288 & 129.210 & 1.434 & 0.088 & 0.026821 & 0.012331 & 0.435514 & 195.459 \\
\hline 7/10/08 1:00 & 2.223 & 0.763 & 0.292 & 130.902 & 1.433 & 0.087 & 0.026516 & 0.012129 & 0.428377 & 192.256 \\
\hline 7/10/08 2:00 & 2.237 & 0.777 & 0.305 & 136.926 & 1.435 & 0.089 & 0.027126 & 0.012534 & 0.442691 & 198.680 \\
\hline 7/10/08 3:00 & 2.232 & 0.772 & 0.300 & 134.756 & 1.435 & 0.089 & 0.027126 & 0.012534 & 0.442691 & 198.680 \\
\hline 7/10/08 4:00 & 2.234 & 0.774 & 0.302 & 135.621 & 1.434 & 0.088 & 0.026821 & 0.012331 & 0.435514 & 195.459 \\
\hline 7/10/08 5:00 & 2.234 & 0.774 & 0.302 & 135.621 & 1.436 & 0.09 & 0.027431 & 0.012738 & 0.449907 & 201.918 \\
\hline 7/10/08 6:00 & 2.232 & 0.772 & 0.300 & 134.756 & 1.436 & 0.09 & 0.027431 & 0.012738 & 0.449907 & 201.918 \\
\hline 7/10/08 7:00 & 2.229 & 0.769 & 0.297 & 133.464 & 1.435 & 0.089 & 0.027126 & 0.012534 & 0.442691 & 198.680 \\
\hline 7/10/08 8:00 & 2.237 & 0.777 & 0.305 & 136.926 & 1.435 & 0.089 & 0.027126 & 0.012534 & 0.442691 & 198.680 \\
\hline 7/10/08 9:00 & 2.244 & 0.784 & 0.312 & 139.999 & 1.438 & 0.092 & 0.028040 & 0.013150 & 0.464455 & 208.447 \\
\hline 7/10/08 10:00 & 2.237 & 0.777 & 0.305 & 136.926 & 1.436 & 0.09 & 0.027431 & 0.012738 & 0.449907 & 201.918 \\
\hline 7/10/08 11:00 & 2.219 & 0.759 & 0.288 & 129.210 & 1.431 & 0.085 & 0.025907 & 0.011728 & 0.414220 & 185.902 \\
\hline 7/10/08 12:00 & 2.203 & 0.743 & 0.273 & 122.576 & 1.432 & 0.086 & 0.026212 & 0.011928 & 0.421279 & 189.070 \\
\hline 7/10/08 13:00 & 2.19 & 0.730 & 0.261 & 117.339 & 1.43 & 0.084 & 0.025602 & 0.011529 & 0.407202 & 182.752 \\
\hline 7/10/08 14:00 & 2.184 & 0.724 & 0.256 & 114.969 & 1.431 & 0.085 & 0.025907 & 0.011728 & 0.414220 & 185.902 \\
\hline 7/10/08 15:00 & 2.166 & 0.706 & 0.241 & 108.030 & 1.429 & 0.083 & 0.025297 & 0.011331 & 0.400223 & 179.620 \\
\hline 7/10/08 16:00 & 2.146 & 0.686 & 0.224 & 100.622 & 1.425 & 0.079 & 0.024078 & 0.010553 & 0.372715 & 167.274 \\
\hline
\end{tabular}




\begin{tabular}{|c|c|c|c|c|c|c|c|c|c|c|}
\hline \multirow[b]{3}{*}{ Date Time } & \multicolumn{4}{|c|}{ V-Notch Weir } & \multicolumn{6}{|c|}{ R-Notch Weir } \\
\hline & \multicolumn{2}{|c|}{ Measured Water Level } & \multicolumn{2}{|c|}{$\begin{array}{c}\text { Calculated Rate } \\
\left(\mathrm{ft}^{3} / \mathrm{s}=448.8 \mathrm{gpm}\right)\end{array}$} & \multicolumn{3}{|c|}{ Measured Water Level } & \multicolumn{3}{|c|}{$\begin{array}{c}\text { Calculated Rate } \\
\left(\mathrm{ft}^{3} / \mathrm{s}=448.8 \mathrm{gpm}\right)\end{array}$} \\
\hline & $\begin{array}{l}\text { (ft above } \\
\text { sensor) }\end{array}$ & $\begin{array}{l}\text { (ft above } \\
\text { notch) }\end{array}$ & $\left(\mathrm{ft}^{3} / \mathrm{s}\right)$ & (gpm) & $\begin{array}{l}\text { (ft above } \\
\text { sensor) }\end{array}$ & $\begin{array}{l}\text { (ft above } \\
\text { log) }\end{array}$ & $\begin{array}{c}\text { (m above } \\
\text { log) }\end{array}$ & $\left(\mathrm{m}^{3} / \mathrm{s}\right)$ & $\left(\mathrm{ft}^{3} / \mathrm{s}\right)$ & (gpm) \\
\hline 7/10/08 17:00 & 2.137 & 0.677 & 0.217 & 97.391 & 1.403 & 0.057 & 0.017373 & 0.006617 & 0.233715 & 104.891 \\
\hline 7/10/08 18:00 & 2.118 & 0.658 & 0.202 & 90.776 & 1.403 & 0.057 & 0.017373 & 0.006617 & 0.233715 & 104.891 \\
\hline 7/10/08 19:00 & 2.109 & 0.649 & 0.195 & 87.740 & 1.404 & 0.058 & 0.017678 & 0.006782 & 0.239555 & 107.512 \\
\hline 7/10/08 20:00 & 2.103 & 0.643 & 0.191 & 85.750 & 1.402 & 0.056 & 0.017068 & 0.006453 & 0.227923 & 102.292 \\
\hline 7/10/08 21:00 & 2.112 & 0.652 & 0.198 & 88.745 & 1.402 & 0.056 & 0.017068 & 0.006453 & 0.227923 & 102.292 \\
\hline 7/10/08 22:00 & 2.119 & 0.659 & 0.203 & 91.117 & 1.402 & 0.056 & 0.017068 & 0.006453 & 0.227923 & 102.292 \\
\hline 7/10/08 23:00 & 2.134 & 0.674 & 0.215 & 96.328 & 1.404 & 0.058 & 0.017678 & 0.006782 & 0.239555 & 107.512 \\
\hline 7/11/08 0:00 & 2.143 & 0.683 & 0.222 & 99.538 & 1.406 & 0.06 & 0.018287 & 0.007117 & 0.251378 & 112.818 \\
\hline 7/11/08 1:00 & 2.157 & 0.697 & 0.233 & 104.657 & 1.411 & 0.065 & 0.019811 & 0.007977 & 0.281754 & 126.451 \\
\hline 7/11/08 2:00 & 2.173 & 0.713 & 0.247 & 110.697 & 1.41 & 0.064 & 0.019506 & 0.007803 & 0.275587 & 123.683 \\
\hline 7/11/08 3:00 & 2.177 & 0.717 & 0.250 & 112.239 & 1.412 & 0.066 & 0.020116 & 0.008153 & 0.287966 & 129.239 \\
\hline 7/11/08 4:00 & 2.181 & 0.721 & 0.254 & 113.794 & 1.411 & 0.065 & 0.019811 & 0.007977 & 0.281754 & 126.451 \\
\hline 7/11/08 5:00 & 2.181 & 0.721 & 0.254 & 113.794 & 1.413 & 0.067 & 0.020421 & 0.008330 & 0.294224 & 132.048 \\
\hline 7/11/08 6:00 & 2.19 & 0.730 & 0.261 & 117.339 & 1.413 & 0.067 & 0.020421 & 0.008330 & 0.294224 & 132.048 \\
\hline 7/11/08 7:00 & 2.188 & 0.728 & 0.260 & 116.546 & 1.413 & 0.067 & 0.020421 & 0.008330 & 0.294224 & 132.048 \\
\hline 7/11/08 8:00 & 2.2 & 0.740 & 0.270 & 121.355 & 1.415 & 0.069 & 0.021030 & 0.008688 & 0.306873 & 137.724 \\
\hline 7/11/08 9:00 & 2.2 & 0.740 & 0.270 & 121.355 & 1.412 & 0.066 & 0.020116 & 0.008153 & 0.287966 & 129.239 \\
\hline 7/11/08 10:00 & 2.187 & 0.727 & 0.259 & 116.150 & 1.413 & 0.067 & 0.020421 & 0.008330 & 0.294224 & 132.048 \\
\hline 7/11/08 11:00 & 2.174 & 0.714 & 0.248 & 111.082 & 1.408 & 0.062 & 0.018897 & 0.007457 & 0.263390 & 118.209 \\
\hline 7/11/08 12:00 & 2.16 & 0.700 & 0.236 & 105.774 & 1.409 & 0.063 & 0.019201 & 0.007629 & 0.269465 & 120.936 \\
\hline 7/11/08 13:00 & 2.155 & 0.695 & 0.232 & 103.916 & 1.407 & 0.061 & 0.018592 & 0.007287 & 0.257360 & 115.503 \\
\hline 7/11/08 14:00 & 2.139 & 0.679 & 0.219 & 98.103 & 1.406 & 0.06 & 0.018287 & 0.007117 & 0.251378 & 112.818 \\
\hline 7/11/08 15:00 & 2.131 & 0.671 & 0.212 & 95.272 & 1.407 & 0.061 & 0.018592 & 0.007287 & 0.257360 & 115.503 \\
\hline 7/11/08 16:00 & 2.115 & 0.655 & 0.200 & 89.757 & 1.405 & 0.059 & 0.017982 & 0.006949 & 0.245443 & 110.155 \\
\hline 7/11/08 17:00 & 2.104 & 0.644 & 0.192 & 86.080 & 1.401 & 0.055 & 0.016763 & 0.006291 & 0.222180 & 99.714 \\
\hline
\end{tabular}




\begin{tabular}{|c|c|c|c|c|c|c|c|c|c|c|}
\hline \multirow[b]{3}{*}{ Date Time } & \multicolumn{4}{|c|}{ V-Notch Weir } & \multicolumn{6}{|c|}{ R-Notch Weir } \\
\hline & \multicolumn{2}{|c|}{ Measured Water Level } & \multicolumn{2}{|c|}{$\begin{array}{c}\text { Calculated Rate } \\
\left(\mathrm{ft}^{3} / \mathrm{s}=448.8 \mathrm{gpm}\right)\end{array}$} & \multicolumn{3}{|c|}{ Measured Water Level } & \multicolumn{3}{|c|}{$\begin{array}{c}\text { Calculated Rate } \\
\left(\mathrm{ft}^{3} / \mathrm{s}=448.8 \mathrm{gpm}\right)\end{array}$} \\
\hline & $\begin{array}{c}\text { (ft above } \\
\text { sensor) }\end{array}$ & $\begin{array}{c}\text { (ft above } \\
\text { notch) }\end{array}$ & $\left(\mathrm{ft}^{3} / \mathrm{s}\right)$ & (gpm) & $\begin{array}{l}\text { (ft above } \\
\text { sensor) }\end{array}$ & $\begin{array}{c}\text { (ft above } \\
\text { log) }\end{array}$ & $\begin{array}{c}\text { (m above } \\
\text { log) }\end{array}$ & $\left(\mathrm{m}^{3} / \mathrm{s}\right)$ & $\left(\mathrm{ft}^{3} / \mathrm{s}\right)$ & (gpm) \\
\hline 7/11/08 18:00 & 2.092 & 0.632 & 0.183 & 82.173 & 1.404 & 0.058 & 0.017678 & 0.006782 & 0.239555 & 107.512 \\
\hline 7/11/08 19:00 & 2.092 & 0.632 & 0.183 & 82.173 & 1.405 & 0.059 & 0.017982 & 0.006949 & 0.245443 & 110.155 \\
\hline 7/11/08 20:00 & 2.091 & 0.631 & 0.182 & 81.852 & 1.402 & 0.056 & 0.017068 & 0.006453 & 0.227923 & 102.292 \\
\hline 7/11/08 21:00 & 2.1 & 0.640 & 0.189 & 84.765 & 1.405 & 0.059 & 0.017982 & 0.006949 & 0.245443 & 110.155 \\
\hline 7/11/08 22:00 & 2.111 & 0.651 & 0.197 & 88.409 & 1.407 & 0.061 & 0.018592 & 0.007287 & 0.257360 & 115.503 \\
\hline 7/11/08 23:00 & 2.133 & 0.673 & 0.214 & 95.975 & 1.409 & 0.063 & 0.019201 & 0.007629 & 0.269465 & 120.936 \\
\hline 7/12/08 0:00 & 2.16 & 0.700 & 0.236 & 105.774 & 1.414 & 0.068 & 0.020725 & 0.008509 & 0.300526 & 134.876 \\
\hline 7/12/08 1:00 & 2.169 & 0.709 & 0.243 & 109.168 & 1.413 & 0.067 & 0.020421 & 0.008330 & 0.294224 & 132.048 \\
\hline 7/12/08 2:00 & 2.185 & 0.725 & 0.257 & 115.362 & 1.414 & 0.068 & 0.020725 & 0.008509 & 0.300526 & 134.876 \\
\hline 7/12/08 3:00 & 2.184 & 0.724 & 0.256 & 114.969 & 1.414 & 0.068 & 0.020725 & 0.008509 & 0.300526 & 134.876 \\
\hline 7/12/08 4:00 & 2.195 & 0.735 & 0.266 & 119.337 & 1.415 & 0.069 & 0.021030 & 0.008688 & 0.306873 & 137.724 \\
\hline 7/12/08 5:00 & 2.195 & 0.735 & 0.266 & 119.337 & 1.416 & 0.07 & 0.021335 & 0.008869 & 0.313263 & 140.593 \\
\hline 7/12/08 6:00 & 2.19 & 0.730 & 0.261 & 117.339 & 1.413 & 0.067 & 0.020421 & 0.008330 & 0.294224 & 132.048 \\
\hline 7/12/08 7:00 & 2.176 & 0.716 & 0.249 & 111.853 & 1.413 & 0.067 & 0.020421 & 0.008330 & 0.294224 & 132.048 \\
\hline 7/12/08 8:00 & 2.185 & 0.725 & 0.257 & 115.362 & 1.412 & 0.066 & 0.020116 & 0.008153 & 0.287966 & 129.239 \\
\hline 7/12/08 9:00 & 2.194 & 0.734 & 0.265 & 118.936 & 1.414 & 0.068 & 0.020725 & 0.008509 & 0.300526 & 134.876 \\
\hline 7/12/08 10:00 & 2.194 & 0.734 & 0.265 & 118.936 & 1.414 & 0.068 & 0.020725 & 0.008509 & 0.300526 & 134.876 \\
\hline 7/12/08 11:00 & 2.197 & 0.737 & 0.268 & 120.142 & 1.414 & 0.068 & 0.020725 & 0.008509 & 0.300526 & 134.876 \\
\hline 7/12/08 12:00 & 2.192 & 0.732 & 0.263 & 118.136 & 1.411 & 0.065 & 0.019811 & 0.007977 & 0.281754 & 126.451 \\
\hline 7/12/08 13:00 & 2.179 & 0.719 & 0.252 & 113.015 & 1.409 & 0.063 & 0.019201 & 0.007629 & 0.269465 & 120.936 \\
\hline 7/12/08 14:00 & 2.177 & 0.717 & 0.250 & 112.239 & 1.412 & 0.066 & 0.020116 & 0.008153 & 0.287966 & 129.239 \\
\hline 7/12/08 15:00 & 2.166 & 0.706 & 0.241 & 108.030 & 1.41 & 0.064 & 0.019506 & 0.007803 & 0.275587 & 123.683 \\
\hline 7/12/08 16:00 & 2.157 & 0.697 & 0.233 & 104.657 & 1.41 & 0.064 & 0.019506 & 0.007803 & 0.275587 & 123.683 \\
\hline 7/12/08 17:00 & 2.152 & 0.692 & 0.229 & 102.811 & 1.408 & 0.062 & 0.018897 & 0.007457 & 0.263390 & 118.209 \\
\hline 7/12/08 18:00 & 2.143 & 0.683 & 0.222 & 99.538 & 1.409 & 0.063 & 0.019201 & 0.007629 & 0.269465 & 120.936 \\
\hline
\end{tabular}




\begin{tabular}{|c|c|c|c|c|c|c|c|c|c|c|}
\hline \multirow[b]{3}{*}{ Date Time } & \multicolumn{4}{|c|}{ V-Notch Weir } & \multicolumn{6}{|c|}{ R-Notch Weir } \\
\hline & \multicolumn{2}{|c|}{ Measured Water Level } & \multicolumn{2}{|c|}{$\begin{array}{c}\text { Calculated Rate } \\
\left(\mathrm{ft}^{3} / \mathrm{s}=448.8 \mathrm{gpm}\right)\end{array}$} & \multicolumn{3}{|c|}{ Measured Water Level } & \multicolumn{3}{|c|}{$\begin{array}{c}\text { Calculated Rate } \\
\left(\mathrm{ft}^{3} / \mathrm{s}=448.8 \mathrm{gpm}\right)\end{array}$} \\
\hline & $\begin{array}{c}\text { (ft above } \\
\text { sensor) }\end{array}$ & $\begin{array}{c}\text { (ft above } \\
\text { notch) }\end{array}$ & $\left(\mathrm{ft}^{3} / \mathrm{s}\right)$ & (gpm) & $\begin{array}{l}\text { (ft above } \\
\text { sensor) }\end{array}$ & $\begin{array}{c}\text { (ft above } \\
\text { log) }\end{array}$ & $\begin{array}{c}\text { (m above } \\
\text { log) }\end{array}$ & $\left(\mathrm{m}^{3} / \mathrm{s}\right)$ & $\left(\mathrm{ft}^{3} / \mathrm{s}\right)$ & (gpm) \\
\hline 7/12/08 19:00 & 2.138 & 0.678 & 0.218 & 97.747 & 1.404 & 0.058 & 0.017678 & 0.006782 & 0.239555 & 107.512 \\
\hline 7/12/08 20:00 & 2.131 & 0.671 & 0.212 & 95.272 & 1.404 & 0.058 & 0.017678 & 0.006782 & 0.239555 & 107.512 \\
\hline 7/12/08 21:00 & 2.135 & 0.675 & 0.215 & 96.681 & 1.406 & 0.06 & 0.018287 & 0.007117 & 0.251378 & 112.818 \\
\hline 7/12/08 22:00 & 2.139 & 0.679 & 0.219 & 98.103 & 1.406 & 0.06 & 0.018287 & 0.007117 & 0.251378 & 112.818 \\
\hline 7/12/08 23:00 & 2.148 & 0.688 & 0.226 & 101.348 & 1.408 & 0.062 & 0.018897 & 0.007457 & 0.263390 & 118.209 \\
\hline 7/13/08 0:00 & 2.16 & 0.700 & 0.236 & 105.774 & 1.408 & 0.062 & 0.018897 & 0.007457 & 0.263390 & 118.209 \\
\hline 7/13/08 1:00 & 2.171 & 0.711 & 0.245 & 109.931 & 1.41 & 0.064 & 0.019506 & 0.007803 & 0.275587 & 123.683 \\
\hline 7/13/08 2:00 & 2.176 & 0.716 & 0.249 & 111.853 & 1.411 & 0.065 & 0.019811 & 0.007977 & 0.281754 & 126.451 \\
\hline 7/13/08 3:00 & 2.183 & 0.723 & 0.255 & 114.576 & 1.413 & 0.067 & 0.020421 & 0.008330 & 0.294224 & 132.048 \\
\hline 7/13/08 4:00 & 2.182 & 0.722 & 0.254 & 114.185 & 1.413 & 0.067 & 0.020421 & 0.008330 & 0.294224 & 132.048 \\
\hline 7/13/08 5:00 & 2.189 & 0.729 & 0.261 & 116.942 & 1.414 & 0.068 & 0.020725 & 0.008509 & 0.300526 & 134.876 \\
\hline 7/13/08 6:00 & 2.201 & 0.741 & 0.271 & 121.761 & 1.416 & 0.07 & 0.021335 & 0.008869 & 0.313263 & 140.593 \\
\hline 7/13/08 7:00 & 2.196 & 0.736 & 0.267 & 119.739 & 1.413 & 0.067 & 0.020421 & 0.008330 & 0.294224 & 132.048 \\
\hline 7/13/08 8:00 & 2.201 & 0.741 & 0.271 & 121.761 & 1.413 & 0.067 & 0.020421 & 0.008330 & 0.294224 & 132.048 \\
\hline 7/13/08 9:00 & 2.201 & 0.741 & 0.271 & 121.761 & 1.415 & 0.069 & 0.021030 & 0.008688 & 0.306873 & 137.724 \\
\hline 7/13/08 10:00 & 2.19 & 0.730 & 0.261 & 117.339 & 1.413 & 0.067 & 0.020421 & 0.008330 & 0.294224 & 132.048 \\
\hline 7/13/08 11:00 & 2.169 & 0.709 & 0.243 & 109.168 & 1.411 & 0.065 & 0.019811 & 0.007977 & 0.281754 & 126.451 \\
\hline 7/13/08 12:00 & 2.158 & 0.698 & 0.234 & 105.029 & 1.409 & 0.063 & 0.019201 & 0.007629 & 0.269465 & 120.936 \\
\hline 7/13/08 13:00 & 2.147 & 0.687 & 0.225 & 100.985 & 1.41 & 0.064 & 0.019506 & 0.007803 & 0.275587 & 123.683 \\
\hline 7/13/08 14:00 & 2.145 & 0.685 & 0.223 & 100.260 & 1.408 & 0.062 & 0.018897 & 0.007457 & 0.263390 & 118.209 \\
\hline 7/13/08 15:00 & 2.132 & 0.672 & 0.213 & 95.623 & 1.409 & 0.063 & 0.019201 & 0.007629 & 0.269465 & 120.936 \\
\hline 7/13/08 16:00 & 2.123 & 0.663 & 0.206 & 92.490 & 1.407 & 0.061 & 0.018592 & 0.007287 & 0.257360 & 115.503 \\
\hline 7/13/08 17:00 & 2.114 & 0.654 & 0.199 & 89.419 & 1.406 & 0.06 & 0.018287 & 0.007117 & 0.251378 & 112.818 \\
\hline 7/13/08 18:00 & 2.102 & 0.642 & 0.190 & 85.421 & 1.406 & 0.06 & 0.018287 & 0.007117 & 0.251378 & 112.818 \\
\hline 7/13/08 19:00 & 2.095 & 0.635 & 0.185 & 83.140 & 1.404 & 0.058 & 0.017678 & 0.006782 & 0.239555 & 107.512 \\
\hline
\end{tabular}




\begin{tabular}{|c|c|c|c|c|c|c|c|c|c|c|}
\hline \multirow[b]{3}{*}{ Date Time } & \multicolumn{4}{|c|}{ V-Notch Weir } & \multicolumn{6}{|c|}{ R-Notch Weir } \\
\hline & \multicolumn{2}{|c|}{ Measured Water Level } & \multicolumn{2}{|c|}{$\begin{array}{c}\text { Calculated Rate } \\
\left(\mathrm{ft}^{3} / \mathrm{s}=448.8 \mathrm{gpm}\right)\end{array}$} & \multicolumn{3}{|c|}{ Measured Water Level } & \multicolumn{3}{|c|}{$\begin{array}{c}\text { Calculated Rate } \\
\left(\mathrm{ft}^{3} / \mathrm{s}=448.8 \mathrm{gpm}\right)\end{array}$} \\
\hline & $\begin{array}{c}\text { (ft above } \\
\text { sensor) }\end{array}$ & $\begin{array}{c}\text { (ft above } \\
\text { notch) }\end{array}$ & $\left(\mathrm{ft}^{3} / \mathrm{s}\right)$ & (gpm) & $\begin{array}{l}\text { (ft above } \\
\text { sensor) }\end{array}$ & $\begin{array}{c}\text { (ft above } \\
\text { log) }\end{array}$ & $\begin{array}{c}\text { (m above } \\
\text { log) }\end{array}$ & $\left(\mathrm{m}^{3} / \mathrm{s}\right)$ & $\left(\mathrm{ft}^{3} / \mathrm{s}\right)$ & (gpm) \\
\hline 7/13/08 20:00 & 2.092 & 0.632 & 0.183 & 82.173 & 1.407 & 0.061 & 0.018592 & 0.007287 & 0.257360 & 115.503 \\
\hline 7/13/08 21:00 & 2.101 & 0.641 & 0.190 & 85.093 & 1.407 & 0.061 & 0.018592 & 0.007287 & 0.257360 & 115.503 \\
\hline 7/13/08 22:00 & 2.11 & 0.650 & 0.196 & 88.074 & 1.407 & 0.061 & 0.018592 & 0.007287 & 0.257360 & 115.503 \\
\hline 7/13/08 23:00 & 2.126 & 0.666 & 0.208 & 93.527 & 1.409 & 0.063 & 0.019201 & 0.007629 & 0.269465 & 120.936 \\
\hline 7/14/08 0:00 & 2.139 & 0.679 & 0.219 & 98.103 & 1.411 & 0.065 & 0.019811 & 0.007977 & 0.281754 & 126.451 \\
\hline 7/14/08 1:00 & 2.151 & 0.691 & 0.228 & 102.444 & 1.413 & 0.067 & 0.020421 & 0.008330 & 0.294224 & 132.048 \\
\hline 7/14/08 2:00 & 2.164 & 0.704 & 0.239 & 107.275 & 1.415 & 0.069 & 0.021030 & 0.008688 & 0.306873 & 137.724 \\
\hline 7/14/08 3:00 & 2.169 & 0.709 & 0.243 & 109.168 & 1.417 & 0.071 & 0.021640 & 0.009052 & 0.319698 & 143.480 \\
\hline 7/14/08 4:00 & 2.18 & 0.720 & 0.253 & 113.404 & 1.418 & 0.072 & 0.021945 & 0.009235 & 0.326176 & 146.388 \\
\hline 7/14/08 5:00 & 2.194 & 0.734 & 0.265 & 118.936 & 1.42 & 0.074 & 0.022554 & 0.009605 & 0.339261 & 152.260 \\
\hline 7/14/08 6:00 & 2.192 & 0.732 & 0.263 & 118.136 & 1.42 & 0.074 & 0.022554 & 0.009605 & 0.339261 & 152.260 \\
\hline 7/14/08 7:00 & 2.185 & 0.725 & 0.257 & 115.362 & 1.419 & 0.073 & 0.022249 & 0.009420 & 0.332697 & 149.314 \\
\hline 7/14/08 8:00 & 2.189 & 0.729 & 0.261 & 116.942 & 1.419 & 0.073 & 0.022249 & 0.009420 & 0.332697 & 149.314 \\
\hline 7/14/08 9:00 & 2.199 & 0.739 & 0.269 & 120.950 & 1.419 & 0.073 & 0.022249 & 0.009420 & 0.332697 & 149.314 \\
\hline 7/14/08 10:00 & 2.178 & 0.718 & 0.251 & 112.627 & 1.416 & 0.07 & 0.021335 & 0.008869 & 0.313263 & 140.593 \\
\hline 7/14/08 11:00 & 2.155 & 0.695 & 0.232 & 103.916 & 1.412 & 0.066 & 0.020116 & 0.008153 & 0.287966 & 129.239 \\
\hline 7/14/08 12:00 & 2.141 & 0.681 & 0.220 & 98.819 & 1.412 & 0.066 & 0.020116 & 0.008153 & 0.287966 & 129.239 \\
\hline 7/14/08 13:00 & 2.135 & 0.675 & 0.215 & 96.681 & 1.41 & 0.064 & 0.019506 & 0.007803 & 0.275587 & 123.683 \\
\hline 7/14/08 14:00 & 2.127 & 0.667 & 0.209 & 93.875 & 1.411 & 0.065 & 0.019811 & 0.007977 & 0.281754 & 126.451 \\
\hline 7/14/08 15:00 & 2.116 & 0.656 & 0.201 & 90.096 & 1.409 & 0.063 & 0.019201 & 0.007629 & 0.269465 & 120.936 \\
\hline 7/14/08 16:00 & 2.102 & 0.642 & 0.190 & 85.421 & 1.408 & 0.062 & 0.018897 & 0.007457 & 0.263390 & 118.209 \\
\hline 7/14/08 17:00 & 2.091 & 0.631 & 0.182 & 81.852 & 1.406 & 0.06 & 0.018287 & 0.007117 & 0.251378 & 112.818 \\
\hline 7/14/08 18:00 & 2.082 & 0.622 & 0.176 & 79.000 & 1.407 & 0.061 & 0.018592 & 0.007287 & 0.257360 & 115.503 \\
\hline 7/14/08 19:00 & 2.077 & 0.617 & 0.173 & 77.442 & 1.405 & 0.059 & 0.017982 & 0.006949 & 0.245443 & 110.155 \\
\hline 7/14/08 20:00 & 2.076 & 0.616 & 0.172 & 77.132 & 1.405 & 0.059 & 0.017982 & 0.006949 & 0.245443 & 110.155 \\
\hline
\end{tabular}




\begin{tabular}{|c|c|c|c|c|c|c|c|c|c|c|}
\hline \multirow[b]{3}{*}{ Date Time } & \multicolumn{4}{|c|}{ V-Notch Weir } & \multicolumn{6}{|c|}{ R-Notch Weir } \\
\hline & \multicolumn{2}{|c|}{ Measured Water Level } & \multicolumn{2}{|c|}{$\begin{array}{c}\text { Calculated Rate } \\
\left(\mathrm{ft}^{3} / \mathrm{s}=448.8 \mathrm{gpm}\right)\end{array}$} & \multicolumn{3}{|c|}{ Measured Water Level } & \multicolumn{3}{|c|}{$\begin{array}{c}\text { Calculated Rate } \\
\left(\mathrm{ft}^{3} / \mathrm{s}=448.8 \mathrm{gpm}\right)\end{array}$} \\
\hline & $\begin{array}{l}\text { (ft above } \\
\text { sensor) }\end{array}$ & $\begin{array}{l}\text { (ft above } \\
\text { notch) }\end{array}$ & $\left(\mathrm{ft}^{3} / \mathrm{s}\right)$ & (gpm) & $\begin{array}{l}\text { (ft above } \\
\text { sensor) }\end{array}$ & $\begin{array}{l}\text { (ft above } \\
\text { log) }\end{array}$ & $\begin{array}{c}\text { (m above } \\
\text { log) }\end{array}$ & $\left(\mathrm{m}^{3} / \mathrm{s}\right)$ & $\left(\mathrm{ft}^{3} / \mathrm{s}\right)$ & (gpm) \\
\hline 7/14/08 21:00 & 2.085 & 0.625 & 0.178 & 79.944 & 1.407 & 0.061 & 0.018592 & 0.007287 & 0.257360 & 115.503 \\
\hline 7/14/08 22:00 & 2.096 & 0.636 & 0.186 & 83.463 & 1.407 & 0.061 & 0.018592 & 0.007287 & 0.257360 & 115.503 \\
\hline 7/14/08 23:00 & 2.103 & 0.643 & 0.191 & 85.750 & 1.407 & 0.061 & 0.018592 & 0.007287 & 0.257360 & 115.503 \\
\hline 7/15/08 0:00 & 2.121 & 0.661 & 0.205 & 91.802 & 1.411 & 0.065 & 0.019811 & 0.007977 & 0.281754 & 126.451 \\
\hline 7/15/08 1:00 & 2.128 & 0.668 & 0.210 & 94.223 & 1.411 & 0.065 & 0.019811 & 0.007977 & 0.281754 & 126.451 \\
\hline 7/15/08 2:00 & 2.141 & 0.681 & 0.220 & 98.819 & 1.413 & 0.067 & 0.020421 & 0.008330 & 0.294224 & 132.048 \\
\hline 7/15/08 3:00 & 2.15 & 0.690 & 0.227 & 102.078 & 1.415 & 0.069 & 0.021030 & 0.008688 & 0.306873 & 137.724 \\
\hline 7/15/08 4:00 & 2.155 & 0.695 & 0.232 & 103.916 & 1.417 & 0.071 & 0.021640 & 0.009052 & 0.319698 & 143.480 \\
\hline 7/15/08 5:00 & 2.159 & 0.699 & 0.235 & 105.401 & 1.416 & 0.07 & 0.021335 & 0.008869 & 0.313263 & 140.593 \\
\hline 7/15/08 6:00 & 2.164 & 0.704 & 0.239 & 107.275 & 1.418 & 0.072 & 0.021945 & 0.009235 & 0.326176 & 146.388 \\
\hline 7/15/08 7:00 & 2.173 & 0.713 & 0.247 & 110.697 & 1.418 & 0.072 & 0.021945 & 0.009235 & 0.326176 & 146.388 \\
\hline 7/15/08 8:00 & 2.173 & 0.713 & 0.247 & 110.697 & 1.418 & 0.072 & 0.021945 & 0.009235 & 0.326176 & 146.388 \\
\hline 7/15/08 9:00 & 2.173 & 0.713 & 0.247 & 110.697 & 1.418 & 0.072 & 0.021945 & 0.009235 & 0.326176 & 146.388 \\
\hline 7/15/08 10:00 & 2.167 & 0.707 & 0.242 & 108.408 & 1.415 & 0.069 & 0.021030 & 0.008688 & 0.306873 & 137.724 \\
\hline 7/15/08 11:00 & 2.146 & 0.686 & 0.224 & 100.622 & 1.413 & 0.067 & 0.020421 & 0.008330 & 0.294224 & 132.048 \\
\hline 7/15/08 12:00 & 2.135 & 0.675 & 0.215 & 96.681 & 1.414 & 0.068 & 0.020725 & 0.008509 & 0.300526 & 134.876 \\
\hline 7/15/08 13:00 & 2.127 & 0.667 & 0.209 & 93.875 & 1.414 & 0.068 & 0.020725 & 0.008509 & 0.300526 & 134.876 \\
\hline 7/15/08 14:00 & 2.116 & 0.656 & 0.201 & 90.096 & 1.412 & 0.066 & 0.020116 & 0.008153 & 0.287966 & 129.239 \\
\hline 7/15/08 15:00 & 2.108 & 0.648 & 0.195 & 87.406 & 1.413 & 0.067 & 0.020421 & 0.008330 & 0.294224 & 132.048 \\
\hline 7/15/08 16:00 & 2.099 & 0.639 & 0.188 & 84.439 & 1.414 & 0.068 & 0.020725 & 0.008509 & 0.300526 & 134.876 \\
\hline 7/15/08 17:00 & 2.09 & 0.630 & 0.182 & 81.532 & 1.41 & 0.064 & 0.019506 & 0.007803 & 0.275587 & 123.683 \\
\hline 7/15/08 18:00 & 2.081 & 0.621 & 0.175 & 78.687 & 1.411 & 0.065 & 0.019811 & 0.007977 & 0.281754 & 126.451 \\
\hline 7/15/08 19:00 & 2.081 & 0.621 & 0.175 & 78.687 & 1.411 & 0.065 & 0.019811 & 0.007977 & 0.281754 & 126.451 \\
\hline 7/15/08 20:00 & 2.082 & 0.622 & 0.176 & 79.000 & 1.414 & 0.068 & 0.020725 & 0.008509 & 0.300526 & 134.876 \\
\hline 7/15/08 21:00 & 2.093 & 0.633 & 0.184 & 82.494 & 1.412 & 0.066 & 0.020116 & 0.008153 & 0.287966 & 129.239 \\
\hline
\end{tabular}




\begin{tabular}{|c|c|c|c|c|c|c|c|c|c|c|}
\hline \multirow[b]{3}{*}{ Date Time } & \multicolumn{4}{|c|}{ V-Notch Weir } & \multicolumn{6}{|c|}{ R-Notch Weir } \\
\hline & \multicolumn{2}{|c|}{ Measured Water Level } & \multicolumn{2}{|c|}{$\begin{array}{c}\text { Calculated Rate } \\
\left(\mathrm{ft}^{3} / \mathrm{s}=448.8 \mathrm{gpm}\right)\end{array}$} & \multicolumn{3}{|c|}{ Measured Water Level } & \multicolumn{3}{|c|}{$\begin{array}{c}\text { Calculated Rate } \\
\left(\mathrm{ft}^{3} / \mathrm{s}=448.8 \mathrm{gpm}\right)\end{array}$} \\
\hline & $\begin{array}{c}\text { (ft above } \\
\text { sensor) }\end{array}$ & $\begin{array}{c}\text { (ft above } \\
\text { notch) }\end{array}$ & $\left(\mathrm{ft}^{3} / \mathrm{s}\right)$ & (gpm) & $\begin{array}{l}\text { (ft above } \\
\text { sensor) }\end{array}$ & $\begin{array}{c}\text { (ft above } \\
\text { log) }\end{array}$ & $\begin{array}{c}\text { (m above } \\
\text { log) }\end{array}$ & $\left(\mathrm{m}^{3} / \mathrm{s}\right)$ & $\left(\mathrm{ft}^{3} / \mathrm{s}\right)$ & (gpm) \\
\hline 7/15/08 22:00 & 2.161 & 0.701 & 0.237 & 106.148 & 1.426 & 0.08 & 0.024383 & 0.010746 & 0.379531 & 170.333 \\
\hline 7/15/08 23:00 & 2.783 & 1.323 & 1.142 & 512.643 & 1.659 & 0.313 & 0.095398 & 0.079550 & 2.809693 & 1260.990 \\
\hline 7/16/08 0:00 & 2.757 & 1.297 & 1.087 & 487.972 & 1.625 & 0.279 & 0.085035 & 0.067071 & 2.368939 & 1063.180 \\
\hline 7/16/08 1:00 & 2.757 & 1.297 & 1.087 & 487.972 & 1.626 & 0.28 & 0.085340 & 0.067428 & 2.381542 & 1068.836 \\
\hline 7/16/08 2:00 & 2.651 & 1.191 & 0.880 & 394.829 & 1.548 & 0.202 & 0.061567 & 0.041591 & 1.468975 & 659.276 \\
\hline 7/16/08 3:00 & 2.569 & 1.109 & 0.737 & 330.740 & 1.505 & 0.159 & 0.048461 & 0.029232 & 1.032453 & 463.365 \\
\hline 7/16/08 4:00 & 2.66 & 1.200 & 0.896 & 402.280 & 1.555 & 0.209 & 0.063700 & 0.043736 & 1.544762 & 693.289 \\
\hline 7/16/08 5:00 & 2.721 & 1.261 & 1.014 & 455.009 & 1.587 & 0.241 & 0.073453 & 0.053992 & 1.906980 & 855.853 \\
\hline 7/16/08 6:00 & 2.688 & 1.228 & 0.949 & 426.000 & 1.566 & 0.22 & 0.067053 & 0.047180 & 1.666397 & 747.879 \\
\hline 7/16/08 7:00 & 2.618 & 1.158 & 0.820 & 368.218 & 1.528 & 0.182 & 0.055471 & 0.035662 & 1.259584 & 565.301 \\
\hline 7/16/08 8:00 & 2.521 & 1.061 & 0.660 & 296.342 & 1.49 & 0.144 & 0.043889 & 0.025273 & 0.892650 & 400.621 \\
\hline 7/16/08 9:00 & 2.449 & 0.989 & 0.555 & 248.932 & 1.466 & 0.12 & 0.036574 & 0.019354 & 0.683586 & 306.793 \\
\hline 7/16/08 10:00 & 2.404 & 0.944 & 0.494 & 221.783 & 1.459 & 0.113 & 0.034441 & 0.017729 & 0.626193 & 281.035 \\
\hline 7/16/08 11:00 & 2.364 & 0.904 & 0.444 & 199.213 & 1.452 & 0.106 & 0.032307 & 0.016152 & 0.570504 & 256.042 \\
\hline 7/16/08 12:00 & 2.345 & 0.885 & 0.421 & 188.998 & 1.447 & 0.101 & 0.030783 & 0.015057 & 0.531798 & 238.671 \\
\hline 7/16/08 13:00 & 2.329 & 0.869 & 0.403 & 180.645 & 1.447 & 0.101 & 0.030783 & 0.015057 & 0.531798 & 238.671 \\
\hline 7/16/08 14:00 & 2.314 & 0.854 & 0.386 & 173.019 & 1.442 & 0.096 & 0.029259 & 0.013987 & 0.494010 & 221.711 \\
\hline 7/16/08 15:00 & 2.291 & 0.831 & 0.360 & 161.706 & 1.44 & 0.094 & 0.028650 & 0.013566 & 0.479156 & 215.045 \\
\hline 7/16/08 16:00 & 2.273 & 0.813 & 0.341 & 153.171 & 1.439 & 0.093 & 0.028345 & 0.013358 & 0.471786 & 211.738 \\
\hline 7/16/08 17:00 & 2.259 & 0.799 & 0.327 & 146.723 & 1.437 & 0.091 & 0.027735 & 0.012943 & 0.457162 & 205.174 \\
\hline 7/16/08 18:00 & 2.245 & 0.785 & 0.313 & 140.442 & 1.435 & 0.089 & 0.027126 & 0.012534 & 0.442691 & 198.680 \\
\hline 7/16/08 19:00 & 2.238 & 0.778 & 0.306 & 137.362 & 1.435 & 0.089 & 0.027126 & 0.012534 & 0.442691 & 198.680 \\
\hline 7/16/08 20:00 & 2.24 & 0.780 & 0.308 & 138.238 & 1.436 & 0.09 & 0.027431 & 0.012738 & 0.449907 & 201.918 \\
\hline 7/16/08 21:00 & 2.242 & 0.782 & 0.310 & 139.117 & 1.436 & 0.09 & 0.027431 & 0.012738 & 0.449907 & 201.918 \\
\hline 7/16/08 22:00 & 2.244 & 0.784 & 0.312 & 139.999 & 1.436 & 0.09 & 0.027431 & 0.012738 & 0.449907 & 201.918 \\
\hline
\end{tabular}




\begin{tabular}{|c|c|c|c|c|c|c|c|c|c|c|}
\hline \multirow[b]{3}{*}{ Date Time } & \multicolumn{4}{|c|}{ V-Notch Weir } & \multicolumn{6}{|c|}{ R-Notch Weir } \\
\hline & \multicolumn{2}{|c|}{ Measured Water Level } & \multicolumn{2}{|c|}{$\begin{array}{c}\text { Calculated Rate } \\
\left(\mathrm{ft}^{3} / \mathrm{s}=448.8 \mathrm{gpm}\right)\end{array}$} & \multicolumn{3}{|c|}{ Measured Water Level } & \multicolumn{3}{|c|}{$\begin{array}{c}\text { Calculated Rate } \\
\left(\mathrm{ft}^{3} / \mathrm{s}=448.8 \mathrm{gpm}\right)\end{array}$} \\
\hline & $\begin{array}{c}\text { (ft above } \\
\text { sensor) }\end{array}$ & $\begin{array}{c}\text { (ft above } \\
\text { notch) }\end{array}$ & $\left(\mathrm{ft}^{3} / \mathrm{s}\right)$ & (gpm) & $\begin{array}{l}\text { (ft above } \\
\text { sensor) }\end{array}$ & $\begin{array}{c}\text { (ft above } \\
\text { log) }\end{array}$ & $\begin{array}{c}\text { (m above } \\
\text { log) }\end{array}$ & $\left(\mathrm{m}^{3} / \mathrm{s}\right)$ & $\left(\mathrm{ft}^{3} / \mathrm{s}\right)$ & (gpm) \\
\hline 7/16/08 23:00 & 2.248 & 0.788 & 0.316 & 141.774 & 1.436 & 0.09 & 0.027431 & 0.012738 & 0.449907 & 201.918 \\
\hline 7/17/08 0:00 & 2.25 & 0.790 & 0.318 & 142.666 & 1.438 & 0.092 & 0.028040 & 0.013150 & 0.464455 & 208.447 \\
\hline 7/17/08 1:00 & 2.247 & 0.787 & 0.315 & 141.329 & 1.437 & 0.091 & 0.027735 & 0.012943 & 0.457162 & 205.174 \\
\hline 7/17/08 2:00 & 2.249 & 0.789 & 0.317 & 142.220 & 1.437 & 0.091 & 0.027735 & 0.012943 & 0.457162 & 205.174 \\
\hline 7/17/08 3:00 & 2.249 & 0.789 & 0.317 & 142.220 & 1.439 & 0.093 & 0.028345 & 0.013358 & 0.471786 & 211.738 \\
\hline 7/17/08 4:00 & 2.251 & 0.791 & 0.319 & 143.114 & 1.436 & 0.09 & 0.027431 & 0.012738 & 0.449907 & 201.918 \\
\hline 7/17/08 5:00 & 2.251 & 0.791 & 0.319 & 143.114 & 1.438 & 0.092 & 0.028040 & 0.013150 & 0.464455 & 208.447 \\
\hline 7/17/08 6:00 & 2.251 & 0.791 & 0.319 & 143.114 & 1.438 & 0.092 & 0.028040 & 0.013150 & 0.464455 & 208.447 \\
\hline 7/17/08 7:00 & 2.249 & 0.789 & 0.317 & 142.220 & 1.435 & 0.089 & 0.027126 & 0.012534 & 0.442691 & 198.680 \\
\hline 7/17/08 8:00 & 2.251 & 0.791 & 0.319 & 143.114 & 1.438 & 0.092 & 0.028040 & 0.013150 & 0.464455 & 208.447 \\
\hline 7/17/08 9:00 & 2.249 & 0.789 & 0.317 & 142.220 & 1.437 & 0.091 & 0.027735 & 0.012943 & 0.457162 & 205.174 \\
\hline 7/17/08 10:00 & 2.244 & 0.784 & 0.312 & 139.999 & 1.433 & 0.087 & 0.026516 & 0.012129 & 0.428377 & 192.256 \\
\hline 7/17/08 11:00 & 2.226 & 0.766 & 0.295 & 132.179 & 1.433 & 0.087 & 0.026516 & 0.012129 & 0.428377 & 192.256 \\
\hline 7/17/08 12:00 & 2.203 & 0.743 & 0.273 & 122.576 & 1.429 & 0.083 & 0.025297 & 0.011331 & 0.400223 & 179.620 \\
\hline 7/17/08 13:00 & 2.195 & 0.735 & 0.266 & 119.337 & 1.429 & 0.083 & 0.025297 & 0.011331 & 0.400223 & 179.620 \\
\hline 7/17/08 14:00 & 2.178 & 0.718 & 0.251 & 112.627 & 1.43 & 0.084 & 0.025602 & 0.011529 & 0.407202 & 182.752 \\
\hline 7/17/08 15:00 & 2.172 & 0.712 & 0.246 & 110.314 & 1.428 & 0.082 & 0.024992 & 0.011135 & 0.393285 & 176.506 \\
\hline 7/17/08 16:00 & 2.17 & 0.710 & 0.244 & 109.549 & 1.429 & 0.083 & 0.025297 & 0.011331 & 0.400223 & 179.620 \\
\hline 7/17/08 17:00 & 2.171 & 0.711 & 0.245 & 109.931 & 1.434 & 0.088 & 0.026821 & 0.012331 & 0.435514 & 195.459 \\
\hline 7/17/08 18:00 & 3.42 & 1.960 & 3.037 & 1362.888 & 2.688 & 1.342 & 0.409022 & 0.698692 & 24.677687 & 11075.346 \\
\hline 7/17/08 19:00 & 2.958 & 1.498 & 1.556 & 698.140 & 2.042 & 0.696 & 0.212130 & 0.261642 & 9.241156 & 4147.431 \\
\hline 7/17/08 20:00 & 2.726 & 1.266 & 1.024 & 459.505 & 1.715 & 0.369 & 0.112466 & 0.101594 & 3.588266 & 1610.414 \\
\hline 7/17/08 21:00 & 2.644 & 1.184 & 0.867 & 389.091 & 1.619 & 0.273 & 0.083206 & 0.064944 & 2.293797 & 1029.456 \\
\hline 7/17/08 22:00 & 2.594 & 1.134 & 0.779 & 349.560 & 1.576 & 0.23 & 0.070101 & 0.050386 & 1.779611 & 798.689 \\
\hline 7/17/08 23:00 & 2.571 & 1.111 & 0.740 & 332.223 & 1.548 & 0.202 & 0.061567 & 0.041591 & 1.468975 & 659.276 \\
\hline
\end{tabular}




\begin{tabular}{|c|c|c|c|c|c|c|c|c|c|c|}
\hline \multirow[b]{3}{*}{ Date Time } & \multicolumn{4}{|c|}{ V-Notch Weir } & \multicolumn{6}{|c|}{ R-Notch Weir } \\
\hline & \multicolumn{2}{|c|}{ Measured Water Level } & \multicolumn{2}{|c|}{$\begin{array}{c}\text { Calculated Rate } \\
\left(\mathrm{ft}^{3} / \mathrm{s}=448.8 \mathrm{gpm}\right)\end{array}$} & \multicolumn{3}{|c|}{ Measured Water Level } & \multicolumn{3}{|c|}{$\begin{array}{c}\text { Calculated Rate } \\
\left(\mathrm{ft}^{3} / \mathrm{s}=448.8 \mathrm{gpm}\right)\end{array}$} \\
\hline & $\begin{array}{c}\text { (ft above } \\
\text { sensor) }\end{array}$ & $\begin{array}{c}\text { (ft above } \\
\text { notch) }\end{array}$ & $\left(\mathrm{ft}^{3} / \mathrm{s}\right)$ & (gpm) & $\begin{array}{l}\text { (ft above } \\
\text { sensor) }\end{array}$ & $\begin{array}{c}\text { (ft above } \\
\text { log) }\end{array}$ & $\begin{array}{c}\text { (m above } \\
\text { log) }\end{array}$ & $\left(\mathrm{m}^{3} / \mathrm{s}\right)$ & $\left(\mathrm{ft}^{3} / \mathrm{s}\right)$ & (gpm) \\
\hline 7/18/08 0:00 & 2.547 & 1.087 & 0.701 & 314.693 & 1.527 & 0.181 & 0.055166 & 0.035374 & 1.249399 & 560.730 \\
\hline 7/18/08 1:00 & 2.535 & 1.075 & 0.682 & 306.141 & 1.503 & 0.157 & 0.047851 & 0.028693 & 1.013424 & 454.824 \\
\hline 7/18/08 2:00 & 2.53 & 1.070 & 0.674 & 302.620 & 1.494 & 0.148 & 0.045108 & 0.026310 & 0.929266 & 417.054 \\
\hline 7/18/08 3:00 & 2.481 & 1.021 & 0.600 & 269.392 & 1.477 & 0.131 & 0.039927 & 0.022002 & 0.777099 & 348.762 \\
\hline 7/18/08 4:00 & 2.459 & 0.999 & 0.569 & 255.221 & 1.477 & 0.131 & 0.039927 & 0.022002 & 0.777099 & 348.762 \\
\hline 7/18/08 5:00 & 2.454 & 0.994 & 0.562 & 252.065 & 1.481 & 0.135 & 0.041146 & 0.022992 & 0.812083 & 364.463 \\
\hline 7/18/08 6:00 & 2.447 & 0.987 & 0.552 & 247.685 & 1.476 & 0.13 & 0.039622 & 0.021756 & 0.768433 & 344.873 \\
\hline 7/18/08 7:00 & 2.442 & 0.982 & 0.545 & 244.585 & 1.471 & 0.125 & 0.038098 & 0.020544 & 0.725596 & 325.647 \\
\hline 7/18/08 8:00 & 2.435 & 0.975 & 0.535 & 240.284 & 1.468 & 0.122 & 0.037184 & 0.019827 & 0.700289 & 314.290 \\
\hline 7/18/08 9:00 & 2.423 & 0.963 & 0.519 & 233.017 & 1.466 & 0.12 & 0.036574 & 0.019354 & 0.683586 & 306.793 \\
\hline 7/18/08 10:00 & 2.417 & 0.957 & 0.511 & 229.433 & 1.463 & 0.117 & 0.035660 & 0.018652 & 0.658783 & 295.662 \\
\hline 7/18/08 11:00 & 2.399 & 0.939 & 0.488 & 218.882 & 1.459 & 0.113 & 0.034441 & 0.017729 & 0.626193 & 281.035 \\
\hline 7/18/08 12:00 & 2.384 & 0.924 & 0.469 & 210.317 & 1.457 & 0.111 & 0.033831 & 0.017274 & 0.610106 & 273.815 \\
\hline 7/18/08 13:00 & 2.374 & 0.914 & 0.456 & 204.720 & 1.455 & 0.109 & 0.033222 & 0.016822 & 0.594159 & 266.658 \\
\hline 7/18/08 14:00 & 2.355 & 0.895 & 0.433 & 194.334 & 1.451 & 0.105 & 0.032002 & 0.015931 & 0.562690 & 252.535 \\
\hline 7/18/08 15:00 & 2.34 & 0.880 & 0.415 & 186.363 & 1.449 & 0.103 & 0.031393 & 0.015492 & 0.547171 & 245.570 \\
\hline 7/18/08 16:00 & 2.329 & 0.869 & 0.403 & 180.645 & 1.447 & 0.101 & 0.030783 & 0.015057 & 0.531798 & 238.671 \\
\hline 7/18/08 17:00 & 2.315 & 0.855 & 0.387 & 173.521 & 1.446 & 0.1 & 0.030479 & 0.014841 & 0.524166 & 235.246 \\
\hline 7/18/08 18:00 & 2.305 & 0.845 & 0.376 & 168.537 & 1.444 & 0.098 & 0.029869 & 0.014412 & 0.509013 & 228.445 \\
\hline 7/18/08 19:00 & 2.295 & 0.835 & 0.365 & 163.640 & 1.444 & 0.098 & 0.029869 & 0.014412 & 0.509013 & 228.445 \\
\hline 7/18/08 20:00 & 2.296 & 0.836 & 0.366 & 164.126 & 1.444 & 0.098 & 0.029869 & 0.014412 & 0.509013 & 228.445 \\
\hline 7/18/08 21:00 & 2.304 & 0.844 & 0.374 & 168.044 & 1.442 & 0.096 & 0.029259 & 0.013987 & 0.494010 & 221.711 \\
\hline 7/18/08 22:00 & 2.304 & 0.844 & 0.374 & 168.044 & 1.441 & 0.095 & 0.028955 & 0.013776 & 0.486564 & 218.370 \\
\hline 7/18/08 23:00 & 2.303 & 0.843 & 0.373 & 167.551 & 1.441 & 0.095 & 0.028955 & 0.013776 & 0.486564 & 218.370 \\
\hline 7/19/08 0:00 & 2.297 & 0.837 & 0.367 & 164.613 & 1.44 & 0.094 & 0.028650 & 0.013566 & 0.479156 & 215.045 \\
\hline
\end{tabular}




\begin{tabular}{|c|c|c|c|c|c|c|c|c|c|c|}
\hline \multirow[b]{3}{*}{ Date Time } & \multicolumn{4}{|c|}{ V-Notch Weir } & \multicolumn{6}{|c|}{ R-Notch Weir } \\
\hline & \multicolumn{2}{|c|}{ Measured Water Level } & \multicolumn{2}{|c|}{$\begin{array}{c}\text { Calculated Rate } \\
\left(\mathrm{ft}^{3} / \mathrm{s}=448.8 \mathrm{gpm}\right)\end{array}$} & \multicolumn{3}{|c|}{ Measured Water Level } & \multicolumn{3}{|c|}{$\begin{array}{c}\text { Calculated Rate } \\
\left(\mathrm{ft}^{3} / \mathrm{s}=448.8 \mathrm{gpm}\right)\end{array}$} \\
\hline & $\begin{array}{c}\text { (ft above } \\
\text { sensor) }\end{array}$ & $\begin{array}{c}\text { (ft above } \\
\text { notch) }\end{array}$ & $\left(\mathrm{ft}^{3} / \mathrm{s}\right)$ & (gpm) & $\begin{array}{l}\text { (ft above } \\
\text { sensor) }\end{array}$ & $\begin{array}{c}\text { (ft above } \\
\text { log) }\end{array}$ & $\begin{array}{c}\text { (m above } \\
\text { log) }\end{array}$ & $\left(\mathrm{m}^{3} / \mathrm{s}\right)$ & $\left(\mathrm{ft}^{3} / \mathrm{s}\right)$ & (gpm) \\
\hline 7/19/08 1:00 & 2.306 & 0.846 & 0.377 & 169.032 & 1.44 & 0.094 & 0.028650 & 0.013566 & 0.479156 & 215.045 \\
\hline 7/19/08 2:00 & 2.304 & 0.844 & 0.374 & 168.044 & 1.439 & 0.093 & 0.028345 & 0.013358 & 0.471786 & 211.738 \\
\hline 7/19/08 3:00 & 2.308 & 0.848 & 0.379 & 170.023 & 1.438 & 0.092 & 0.028040 & 0.013150 & 0.464455 & 208.447 \\
\hline 7/19/08 4:00 & 2.305 & 0.845 & 0.376 & 168.537 & 1.437 & 0.091 & 0.027735 & 0.012943 & 0.457162 & 205.174 \\
\hline 7/19/08 5:00 & 2.305 & 0.845 & 0.376 & 168.537 & 1.437 & 0.091 & 0.027735 & 0.012943 & 0.457162 & 205.174 \\
\hline 7/19/08 6:00 & 2.303 & 0.843 & 0.373 & 167.551 & 1.437 & 0.091 & 0.027735 & 0.012943 & 0.457162 & 205.174 \\
\hline 7/19/08 7:00 & 2.303 & 0.843 & 0.373 & 167.551 & 1.436 & 0.09 & 0.027431 & 0.012738 & 0.449907 & 201.918 \\
\hline 7/19/08 8:00 & 2.3 & 0.840 & 0.370 & 166.078 & 1.436 & 0.09 & 0.027431 & 0.012738 & 0.449907 & 201.918 \\
\hline 7/19/08 9:00 & 2.298 & 0.838 & 0.368 & 165.100 & 1.434 & 0.088 & 0.026821 & 0.012331 & 0.435514 & 195.459 \\
\hline 7/19/08 10:00 & 2.294 & 0.834 & 0.364 & 163.155 & 1.436 & 0.09 & 0.027431 & 0.012738 & 0.449907 & 201.918 \\
\hline 7/19/08 11:00 & 2.279 & 0.819 & 0.348 & 155.985 & 1.434 & 0.088 & 0.026821 & 0.012331 & 0.435514 & 195.459 \\
\hline 7/19/08 12:00 & 2.273 & 0.813 & 0.341 & 153.171 & 1.433 & 0.087 & 0.026516 & 0.012129 & 0.428377 & 192.256 \\
\hline 7/19/08 13:00 & 2.256 & 0.796 & 0.324 & 145.363 & 1.431 & 0.085 & 0.025907 & 0.011728 & 0.414220 & 185.902 \\
\hline 7/19/08 14:00 & 2.245 & 0.785 & 0.313 & 140.442 & 1.432 & 0.086 & 0.026212 & 0.011928 & 0.421279 & 189.070 \\
\hline 7/19/08 15:00 & 2.225 & 0.765 & 0.294 & 131.752 & 1.43 & 0.084 & 0.025602 & 0.011529 & 0.407202 & 182.752 \\
\hline 7/19/08 16:00 & 2.215 & 0.755 & 0.284 & 127.532 & 1.429 & 0.083 & 0.025297 & 0.011331 & 0.400223 & 179.620 \\
\hline 7/19/08 17:00 & 2.198 & 0.738 & 0.269 & 120.546 & 1.427 & 0.081 & 0.024688 & 0.010940 & 0.386387 & 173.411 \\
\hline 7/19/08 18:00 & 2.186 & 0.726 & 0.258 & 115.756 & 1.425 & 0.079 & 0.024078 & 0.010553 & 0.372715 & 167.274 \\
\hline 7/19/08 19:00 & 2.183 & 0.723 & 0.255 & 114.576 & 1.425 & 0.079 & 0.024078 & 0.010553 & 0.372715 & 167.274 \\
\hline 7/19/08 20:00 & 2.185 & 0.725 & 0.257 & 115.362 & 1.426 & 0.08 & 0.024383 & 0.010746 & 0.379531 & 170.333 \\
\hline 7/19/08 21:00 & 2.191 & 0.731 & 0.262 & 117.737 & 1.428 & 0.082 & 0.024992 & 0.011135 & 0.393285 & 176.506 \\
\hline 7/19/08 22:00 & 2.201 & 0.741 & 0.271 & 121.761 & 1.428 & 0.082 & 0.024992 & 0.011135 & 0.393285 & 176.506 \\
\hline 7/19/08 23:00 & 2.215 & 0.755 & 0.284 & 127.532 & 1.43 & 0.084 & 0.025602 & 0.011529 & 0.407202 & 182.752 \\
\hline 7/20/08 0:00 & 2.223 & 0.763 & 0.292 & 130.902 & 1.432 & 0.086 & 0.026212 & 0.011928 & 0.421279 & 189.070 \\
\hline 7/20/08 1:00 & 2.223 & 0.763 & 0.292 & 130.902 & 1.432 & 0.086 & 0.026212 & 0.011928 & 0.421279 & 189.070 \\
\hline
\end{tabular}




\begin{tabular}{|c|c|c|c|c|c|c|c|c|c|c|}
\hline \multirow[b]{3}{*}{ Date Time } & \multicolumn{4}{|c|}{ V-Notch Weir } & \multicolumn{6}{|c|}{ R-Notch Weir } \\
\hline & \multicolumn{2}{|c|}{ Measured Water Level } & \multicolumn{2}{|c|}{$\begin{array}{c}\text { Calculated Rate } \\
\left(\mathrm{ft}^{3} / \mathrm{s}=448.8 \mathrm{gpm}\right)\end{array}$} & \multicolumn{3}{|c|}{ Measured Water Level } & \multicolumn{3}{|c|}{$\begin{array}{c}\text { Calculated Rate } \\
\left(\mathrm{ft}^{3} / \mathrm{s}=448.8 \mathrm{gpm}\right)\end{array}$} \\
\hline & $\begin{array}{l}\text { (ft above } \\
\text { sensor) }\end{array}$ & $\begin{array}{l}\text { (ft above } \\
\text { notch) }\end{array}$ & $\left(\mathrm{ft}^{3} / \mathrm{s}\right)$ & (gpm) & $\begin{array}{l}\text { (ft above } \\
\text { sensor) }\end{array}$ & $\begin{array}{l}\text { (ft above } \\
\text { log) }\end{array}$ & $\begin{array}{c}\text { (m above } \\
\text { log) }\end{array}$ & $\left(\mathrm{m}^{3} / \mathrm{s}\right)$ & $\left(\mathrm{ft}^{3} / \mathrm{s}\right)$ & (gpm) \\
\hline 7/20/08 2:00 & 2.231 & 0.771 & 0.299 & 134.324 & 1.434 & 0.088 & 0.026821 & 0.012331 & 0.435514 & 195.459 \\
\hline 7/20/08 3:00 & 2.24 & 0.780 & 0.308 & 138.238 & 1.433 & 0.087 & 0.026516 & 0.012129 & 0.428377 & 192.256 \\
\hline 7/20/08 4:00 & 2.238 & 0.778 & 0.306 & 137.362 & 1.432 & 0.086 & 0.026212 & 0.011928 & 0.421279 & 189.070 \\
\hline 7/20/08 5:00 & 2.242 & 0.782 & 0.310 & 139.117 & 1.431 & 0.085 & 0.025907 & 0.011728 & 0.414220 & 185.902 \\
\hline 7/20/08 6:00 & 2.244 & 0.784 & 0.312 & 139.999 & 1.431 & 0.085 & 0.025907 & 0.011728 & 0.414220 & 185.902 \\
\hline 7/20/08 7:00 & 2.244 & 0.784 & 0.312 & 139.999 & 1.43 & 0.084 & 0.025602 & 0.011529 & 0.407202 & 182.752 \\
\hline 7/20/08 8:00 & 2.244 & 0.784 & 0.312 & 139.999 & 1.43 & 0.084 & 0.025602 & 0.011529 & 0.407202 & 182.752 \\
\hline 7/20/08 9:00 & 2.244 & 0.784 & 0.312 & 139.999 & 1.428 & 0.082 & 0.024992 & 0.011135 & 0.393285 & 176.506 \\
\hline 7/20/08 10:00 & 2.24 & 0.780 & 0.308 & 138.238 & 1.428 & 0.082 & 0.024992 & 0.011135 & 0.393285 & 176.506 \\
\hline 7/20/08 11:00 & 2.222 & 0.762 & 0.291 & 130.478 & 1.426 & 0.08 & 0.024383 & 0.010746 & 0.379531 & 170.333 \\
\hline 7/20/08 12:00 & 2.214 & 0.754 & 0.283 & 127.114 & 1.426 & 0.08 & 0.024383 & 0.010746 & 0.379531 & 170.333 \\
\hline 7/20/08 13:00 & 2.198 & 0.738 & 0.269 & 120.546 & 1.422 & 0.076 & 0.023164 & 0.009981 & 0.352516 & 158.209 \\
\hline 7/20/08 14:00 & 2.188 & 0.728 & 0.260 & 116.546 & 1.422 & 0.076 & 0.023164 & 0.009981 & 0.352516 & 158.209 \\
\hline 7/20/08 15:00 & 2.18 & 0.720 & 0.253 & 113.404 & 1.423 & 0.077 & 0.023468 & 0.010170 & 0.359208 & 161.212 \\
\hline 7/20/08 16:00 & 2.17 & 0.710 & 0.244 & 109.549 & 1.422 & 0.076 & 0.023164 & 0.009981 & 0.352516 & 158.209 \\
\hline 7/20/08 17:00 & 2.154 & 0.694 & 0.231 & 103.547 & 1.42 & 0.074 & 0.022554 & 0.009605 & 0.339261 & 152.260 \\
\hline 7/20/08 18:00 & 2.149 & 0.689 & 0.227 & 101.713 & 1.423 & 0.077 & 0.023468 & 0.010170 & 0.359208 & 161.212 \\
\hline 7/20/08 19:00 & 2.147 & 0.687 & 0.225 & 100.985 & 1.424 & 0.078 & 0.023773 & 0.010361 & 0.365940 & 164.234 \\
\hline 7/20/08 20:00 & 2.153 & 0.693 & 0.230 & 103.179 & 1.426 & 0.08 & 0.024383 & 0.010746 & 0.379531 & 170.333 \\
\hline 7/20/08 21:00 & 2.163 & 0.703 & 0.238 & 106.898 & 1.429 & 0.083 & 0.025297 & 0.011331 & 0.400223 & 179.620 \\
\hline 7/20/08 22:00 & 2.172 & 0.712 & 0.246 & 110.314 & 1.429 & 0.083 & 0.025297 & 0.011331 & 0.400223 & 179.620 \\
\hline 7/20/08 23:00 & 2.187 & 0.727 & 0.259 & 116.150 & 1.431 & 0.085 & 0.025907 & 0.011728 & 0.414220 & 185.902 \\
\hline 7/21/08 0:00 & 2.196 & 0.736 & 0.267 & 119.739 & 1.43 & 0.084 & 0.025602 & 0.011529 & 0.407202 & 182.752 \\
\hline 7/21/08 1:00 & 2.209 & 0.749 & 0.279 & 125.039 & 1.432 & 0.086 & 0.026212 & 0.011928 & 0.421279 & 189.070 \\
\hline 7/21/08 2:00 & 2.131 & 0.671 & 0.212 & 95.272 & 1.42 & 0.074 & 0.022554 & 0.009605 & 0.339261 & 152.260 \\
\hline
\end{tabular}




\begin{tabular}{|c|c|c|c|c|c|c|c|c|c|c|}
\hline \multirow[b]{3}{*}{ Date Time } & \multicolumn{4}{|c|}{ V-Notch Weir } & \multicolumn{6}{|c|}{ R-Notch Weir } \\
\hline & \multicolumn{2}{|c|}{ Measured Water Level } & \multicolumn{2}{|c|}{$\begin{array}{c}\text { Calculated Rate } \\
\left(\mathrm{ft}^{3} / \mathrm{s}=448.8 \mathrm{gpm}\right)\end{array}$} & \multicolumn{3}{|c|}{ Measured Water Level } & \multicolumn{3}{|c|}{$\begin{array}{c}\text { Calculated Rate } \\
\left(\mathrm{ft}^{3} / \mathrm{s}=448.8 \mathrm{gpm}\right)\end{array}$} \\
\hline & $\begin{array}{c}\text { (ft above } \\
\text { sensor) }\end{array}$ & $\begin{array}{c}\text { (ft above } \\
\text { notch) }\end{array}$ & $\left(\mathrm{ft}^{3} / \mathrm{s}\right)$ & (gpm) & $\begin{array}{l}\text { (ft above } \\
\text { sensor) }\end{array}$ & $\begin{array}{c}\text { (ft above } \\
\text { log) }\end{array}$ & $\begin{array}{c}\text { (m above } \\
\text { log) }\end{array}$ & $\left(\mathrm{m}^{3} / \mathrm{s}\right)$ & $\left(\mathrm{ft}^{3} / \mathrm{s}\right)$ & (gpm) \\
\hline 7/21/08 3:00 & 2.128 & 0.668 & 0.210 & 94.223 & 1.422 & 0.076 & 0.023164 & 0.009981 & 0.352516 & 158.209 \\
\hline 7/21/08 4:00 & 2.151 & 0.691 & 0.228 & 102.444 & 1.423 & 0.077 & 0.023468 & 0.010170 & 0.359208 & 161.212 \\
\hline 7/21/08 5:00 & 2.205 & 0.745 & 0.275 & 123.394 & 1.43 & 0.084 & 0.025602 & 0.011529 & 0.407202 & 182.752 \\
\hline 7/21/08 6:00 & 2.2 & 0.740 & 0.270 & 121.355 & 1.429 & 0.083 & 0.025297 & 0.011331 & 0.400223 & 179.620 \\
\hline 7/21/08 7:00 & 2.212 & 0.752 & 0.281 & 126.282 & 1.429 & 0.083 & 0.025297 & 0.011331 & 0.400223 & 179.620 \\
\hline 7/21/08 8:00 & 2.233 & 0.773 & 0.301 & 135.188 & 1.431 & 0.085 & 0.025907 & 0.011728 & 0.414220 & 185.902 \\
\hline 7/21/08 9:00 & 2.224 & 0.764 & 0.293 & 131.327 & 1.429 & 0.083 & 0.025297 & 0.011331 & 0.400223 & 179.620 \\
\hline 7/21/08 10:00 & 2.215 & 0.755 & 0.284 & 127.532 & 1.429 & 0.083 & 0.025297 & 0.011331 & 0.400223 & 179.620 \\
\hline 7/21/08 11:00 & 2.201 & 0.741 & 0.271 & 121.761 & 1.427 & 0.081 & 0.024688 & 0.010940 & 0.386387 & 173.411 \\
\hline 7/21/08 12:00 & 2.186 & 0.726 & 0.258 & 115.756 & 1.424 & 0.078 & 0.023773 & 0.010361 & 0.365940 & 164.234 \\
\hline 7/21/08 13:00 & 2.172 & 0.712 & 0.246 & 110.314 & 1.425 & 0.079 & 0.024078 & 0.010553 & 0.372715 & 167.274 \\
\hline 7/21/08 14:00 & 2.162 & 0.702 & 0.237 & 106.523 & 1.423 & 0.077 & 0.023468 & 0.010170 & 0.359208 & 161.212 \\
\hline 7/21/08 15:00 & 2.153 & 0.693 & 0.230 & 103.179 & 1.421 & 0.075 & 0.022859 & 0.009792 & 0.345867 & 155.225 \\
\hline 7/21/08 16:00 & 2.149 & 0.689 & 0.227 & 101.713 & 1.424 & 0.078 & 0.023773 & 0.010361 & 0.365940 & 164.234 \\
\hline 7/21/08 17:00 & 2.151 & 0.691 & 0.228 & 102.444 & 1.424 & 0.078 & 0.023773 & 0.010361 & 0.365940 & 164.234 \\
\hline 7/21/08 18:00 & 2.156 & 0.696 & 0.232 & 104.286 & 1.425 & 0.079 & 0.024078 & 0.010553 & 0.372715 & 167.274 \\
\hline 7/21/08 19:00 & 2.163 & 0.703 & 0.238 & 106.898 & 1.427 & 0.081 & 0.024688 & 0.010940 & 0.386387 & 173.411 \\
\hline 7/21/08 20:00 & 2.167 & 0.707 & 0.242 & 108.408 & 1.428 & 0.082 & 0.024992 & 0.011135 & 0.393285 & 176.506 \\
\hline 7/21/08 21:00 & 2.164 & 0.704 & 0.239 & 107.275 & 1.428 & 0.082 & 0.024992 & 0.011135 & 0.393285 & 176.506 \\
\hline 7/21/08 22:00 & 2.2 & 0.740 & 0.270 & 121.355 & 1.432 & 0.086 & 0.026212 & 0.011928 & 0.421279 & 189.070 \\
\hline 7/21/08 23:00 & 2.2 & 0.740 & 0.270 & 121.355 & 1.432 & 0.086 & 0.026212 & 0.011928 & 0.421279 & 189.070 \\
\hline 7/22/08 0:00 & 2.197 & 0.737 & 0.268 & 120.142 & 1.432 & 0.086 & 0.026212 & 0.011928 & 0.421279 & 189.070 \\
\hline 7/22/08 1:00 & 2.203 & 0.743 & 0.273 & 122.576 & 1.434 & 0.088 & 0.026821 & 0.012331 & 0.435514 & 195.459 \\
\hline 7/22/08 2:00 & 2.173 & 0.713 & 0.247 & 110.697 & 1.426 & 0.08 & 0.024383 & 0.010746 & 0.379531 & 170.333 \\
\hline 7/22/08 3:00 & 1.954 & 0.494 & 0.100 & 44.770 & 1.397 & 0.051 & 0.015544 & 0.005654 & 0.199703 & 89.627 \\
\hline
\end{tabular}




\begin{tabular}{|c|c|c|c|c|c|c|c|c|c|c|}
\hline \multirow[b]{3}{*}{ Date Time } & \multicolumn{4}{|c|}{ V-Notch Weir } & \multicolumn{6}{|c|}{ R-Notch Weir } \\
\hline & \multicolumn{2}{|c|}{ Measured Water Level } & \multicolumn{2}{|c|}{$\begin{array}{c}\text { Calculated Rate } \\
\left(\mathrm{ft}^{3} / \mathrm{s}=448.8 \mathrm{gpm}\right)\end{array}$} & \multicolumn{3}{|c|}{ Measured Water Level } & \multicolumn{3}{|c|}{$\begin{array}{c}\text { Calculated Rate } \\
\left(\mathrm{ft}^{3} / \mathrm{s}=448.8 \mathrm{gpm}\right)\end{array}$} \\
\hline & $\begin{array}{c}\text { (ft above } \\
\text { sensor) }\end{array}$ & $\begin{array}{c}\text { (ft above } \\
\text { notch) }\end{array}$ & $\left(\mathrm{ft}^{3} / \mathrm{s}\right)$ & (gpm) & $\begin{array}{l}\text { (ft above } \\
\text { sensor) }\end{array}$ & $\begin{array}{c}\text { (ft above } \\
\text { log) }\end{array}$ & $\begin{array}{c}\text { (m above } \\
\text { log) }\end{array}$ & $\left(\mathrm{m}^{3} / \mathrm{s}\right)$ & $\left(\mathrm{ft}^{3} / \mathrm{s}\right)$ & (gpm) \\
\hline 7/22/08 4:00 & 2.076 & 0.616 & 0.172 & 77.132 & 1.406 & 0.06 & 0.018287 & 0.007117 & 0.251378 & 112.818 \\
\hline 7/22/08 5:00 & 2.151 & 0.691 & 0.228 & 102.444 & 1.417 & 0.071 & 0.021640 & 0.009052 & 0.319698 & 143.480 \\
\hline 7/22/08 6:00 & 2.183 & 0.723 & 0.255 & 114.576 & 1.424 & 0.078 & 0.023773 & 0.010361 & 0.365940 & 164.234 \\
\hline 7/22/08 7:00 & 2.202 & 0.742 & 0.272 & 122.168 & 1.424 & 0.078 & 0.023773 & 0.010361 & 0.365940 & 164.234 \\
\hline 7/22/08 8:00 & 2.211 & 0.751 & 0.280 & 125.867 & 1.428 & 0.082 & 0.024992 & 0.011135 & 0.393285 & 176.506 \\
\hline 7/22/08 9:00 & 2.214 & 0.754 & 0.283 & 127.114 & 1.424 & 0.078 & 0.023773 & 0.010361 & 0.365940 & 164.234 \\
\hline 7/22/08 10:00 & 2.21 & 0.750 & 0.280 & 125.453 & 1.426 & 0.08 & 0.024383 & 0.010746 & 0.379531 & 170.333 \\
\hline 7/22/08 11:00 & 2.194 & 0.734 & 0.265 & 118.936 & 1.424 & 0.078 & 0.023773 & 0.010361 & 0.365940 & 164.234 \\
\hline 7/22/08 12:00 & 2.173 & 0.713 & 0.247 & 110.697 & 1.419 & 0.073 & 0.022249 & 0.009420 & 0.332697 & 149.314 \\
\hline 7/22/08 13:00 & 2.162 & 0.702 & 0.237 & 106.523 & 1.42 & 0.074 & 0.022554 & 0.009605 & 0.339261 & 152.260 \\
\hline 7/22/08 14:00 & 2.144 & 0.684 & 0.223 & 99.898 & 1.413 & 0.067 & 0.020421 & 0.008330 & 0.294224 & 132.048 \\
\hline 7/22/08 15:00 & 2.138 & 0.678 & 0.218 & 97.747 & 1.414 & 0.068 & 0.020725 & 0.008509 & 0.300526 & 134.876 \\
\hline 7/22/08 16:00 & 2.132 & 0.672 & 0.213 & 95.623 & 1.414 & 0.068 & 0.020725 & 0.008509 & 0.300526 & 134.876 \\
\hline 7/22/08 17:00 & 2.123 & 0.663 & 0.206 & 92.490 & 1.415 & 0.069 & 0.021030 & 0.008688 & 0.306873 & 137.724 \\
\hline 7/22/08 18:00 & 2.123 & 0.663 & 0.206 & 92.490 & 1.413 & 0.067 & 0.020421 & 0.008330 & 0.294224 & 132.048 \\
\hline 7/22/08 19:00 & 2.116 & 0.656 & 0.201 & 90.096 & 1.413 & 0.067 & 0.020421 & 0.008330 & 0.294224 & 132.048 \\
\hline 7/22/08 20:00 & 2.122 & 0.662 & 0.205 & 92.146 & 1.413 & 0.067 & 0.020421 & 0.008330 & 0.294224 & 132.048 \\
\hline 7/22/08 21:00 & 2.14 & 0.680 & 0.219 & 98.461 & 1.415 & 0.069 & 0.021030 & 0.008688 & 0.306873 & 137.724 \\
\hline 7/22/08 22:00 & 2.146 & 0.686 & 0.224 & 100.622 & 1.418 & 0.072 & 0.021945 & 0.009235 & 0.326176 & 146.388 \\
\hline 7/22/08 23:00 & 2.158 & 0.698 & 0.234 & 105.029 & 1.418 & 0.072 & 0.021945 & 0.009235 & 0.326176 & 146.388 \\
\hline 7/23/08 0:00 & 2.174 & 0.714 & 0.248 & 111.082 & 1.422 & 0.076 & 0.023164 & 0.009981 & 0.352516 & 158.209 \\
\hline 7/23/08 1:00 & 2.173 & 0.713 & 0.247 & 110.697 & 1.419 & 0.073 & 0.022249 & 0.009420 & 0.332697 & 149.314 \\
\hline 7/23/08 2:00 & 2.142 & 0.682 & 0.221 & 99.178 & 1.414 & 0.068 & 0.020725 & 0.008509 & 0.300526 & 134.876 \\
\hline 7/23/08 3:00 & 2.165 & 0.705 & 0.240 & 107.652 & 1.416 & 0.07 & 0.021335 & 0.008869 & 0.313263 & 140.593 \\
\hline 7/23/08 4:00 & 2.179 & 0.719 & 0.252 & 113.015 & 1.417 & 0.071 & 0.021640 & 0.009052 & 0.319698 & 143.480 \\
\hline
\end{tabular}




\begin{tabular}{|c|c|c|c|c|c|c|c|c|c|c|}
\hline \multirow[b]{3}{*}{ Date Time } & \multicolumn{4}{|c|}{ V-Notch Weir } & \multicolumn{6}{|c|}{ R-Notch Weir } \\
\hline & \multicolumn{2}{|c|}{ Measured Water Level } & \multicolumn{2}{|c|}{$\begin{array}{c}\text { Calculated Rate } \\
\left(\mathrm{ft}^{3} / \mathrm{s}=448.8 \mathrm{gpm}\right)\end{array}$} & \multicolumn{3}{|c|}{ Measured Water Level } & \multicolumn{3}{|c|}{$\begin{array}{c}\text { Calculated Rate } \\
\left(\mathrm{ft}^{3} / \mathrm{s}=448.8 \mathrm{gpm}\right)\end{array}$} \\
\hline & $\begin{array}{c}\text { (ft above } \\
\text { sensor) }\end{array}$ & $\begin{array}{c}\text { (ft above } \\
\text { notch) }\end{array}$ & $\left(\mathrm{ft}^{3} / \mathrm{s}\right)$ & (gpm) & $\begin{array}{l}\text { (ft above } \\
\text { sensor) }\end{array}$ & $\begin{array}{c}\text { (ft above } \\
\text { log) }\end{array}$ & $\begin{array}{c}\text { (m above } \\
\text { log) }\end{array}$ & $\left(\mathrm{m}^{3} / \mathrm{s}\right)$ & $\left(\mathrm{ft}^{3} / \mathrm{s}\right)$ & (gpm) \\
\hline 7/23/08 5:00 & 2.191 & 0.731 & 0.262 & 117.737 & 1.419 & 0.073 & 0.022249 & 0.009420 & 0.332697 & 149.314 \\
\hline 7/23/08 6:00 & 2.193 & 0.733 & 0.264 & 118.536 & 1.419 & 0.073 & 0.022249 & 0.009420 & 0.332697 & 149.314 \\
\hline 7/23/08 7:00 & 2.2 & 0.740 & 0.270 & 121.355 & 1.421 & 0.075 & 0.022859 & 0.009792 & 0.345867 & 155.225 \\
\hline 7/23/08 8:00 & 2.202 & 0.742 & 0.272 & 122.168 & 1.42 & 0.074 & 0.022554 & 0.009605 & 0.339261 & 152.260 \\
\hline 7/23/08 9:00 & 2.202 & 0.742 & 0.272 & 122.168 & 1.423 & 0.077 & 0.023468 & 0.010170 & 0.359208 & 161.212 \\
\hline 7/23/08 10:00 & 2.205 & 0.745 & 0.275 & 123.394 & 1.421 & 0.075 & 0.022859 & 0.009792 & 0.345867 & 155.225 \\
\hline 7/23/08 11:00 & 2.203 & 0.743 & 0.273 & 122.576 & 1.421 & 0.075 & 0.022859 & 0.009792 & 0.345867 & 155.225 \\
\hline 7/23/08 12:00 & 2.199 & 0.739 & 0.269 & 120.950 & 1.423 & 0.077 & 0.023468 & 0.010170 & 0.359208 & 161.212 \\
\hline 7/23/08 13:00 & 2.188 & 0.728 & 0.260 & 116.546 & 1.417 & 0.071 & 0.021640 & 0.009052 & 0.319698 & 143.480 \\
\hline 7/23/08 14:00 & 2.181 & 0.721 & 0.254 & 113.794 & 1.417 & 0.071 & 0.021640 & 0.009052 & 0.319698 & 143.480 \\
\hline 7/23/08 15:00 & 2.172 & 0.712 & 0.246 & 110.314 & 1.417 & 0.071 & 0.021640 & 0.009052 & 0.319698 & 143.480 \\
\hline 7/23/08 16:00 & 2.163 & 0.703 & 0.238 & 106.898 & 1.418 & 0.072 & 0.021945 & 0.009235 & 0.326176 & 146.388 \\
\hline 7/23/08 17:00 & 2.163 & 0.703 & 0.238 & 106.898 & 1.416 & 0.07 & 0.021335 & 0.008869 & 0.313263 & 140.593 \\
\hline 7/23/08 18:00 & 2.162 & 0.702 & 0.237 & 106.523 & 1.418 & 0.072 & 0.021945 & 0.009235 & 0.326176 & 146.388 \\
\hline 7/23/08 19:00 & 2.169 & 0.709 & 0.243 & 109.168 & 1.418 & 0.072 & 0.021945 & 0.009235 & 0.326176 & 146.388 \\
\hline 7/23/08 20:00 & 2.181 & 0.721 & 0.254 & 113.794 & 1.421 & 0.075 & 0.022859 & 0.009792 & 0.345867 & 155.225 \\
\hline 7/23/08 21:00 & 2.188 & 0.728 & 0.260 & 116.546 & 1.421 & 0.075 & 0.022859 & 0.009792 & 0.345867 & 155.225 \\
\hline 7/23/08 22:00 & 2.192 & 0.732 & 0.263 & 118.136 & 1.423 & 0.077 & 0.023468 & 0.010170 & 0.359208 & 161.212 \\
\hline 7/23/08 23:00 & 2.196 & 0.736 & 0.267 & 119.739 & 1.425 & 0.079 & 0.024078 & 0.010553 & 0.372715 & 167.274 \\
\hline 7/24/08 0:00 & 2.21 & 0.750 & 0.280 & 125.453 & 1.425 & 0.079 & 0.024078 & 0.010553 & 0.372715 & 167.274 \\
\hline 7/24/08 1:00 & 2.217 & 0.757 & 0.286 & 128.369 & 1.427 & 0.081 & 0.024688 & 0.010940 & 0.386387 & 173.411 \\
\hline 7/24/08 2:00 & 2.217 & 0.757 & 0.286 & 128.369 & 1.424 & 0.078 & 0.023773 & 0.010361 & 0.365940 & 164.234 \\
\hline 7/24/08 3:00 & 2.217 & 0.757 & 0.286 & 128.369 & 1.427 & 0.081 & 0.024688 & 0.010940 & 0.386387 & 173.411 \\
\hline 7/24/08 4:00 & 2.226 & 0.766 & 0.295 & 132.179 & 1.426 & 0.08 & 0.024383 & 0.010746 & 0.379531 & 170.333 \\
\hline 7/24/08 5:00 & 2.233 & 0.773 & 0.301 & 135.188 & 1.429 & 0.083 & 0.025297 & 0.011331 & 0.400223 & 179.620 \\
\hline
\end{tabular}




\begin{tabular}{|c|c|c|c|c|c|c|c|c|c|c|}
\hline \multirow[b]{3}{*}{ Date Time } & \multicolumn{4}{|c|}{ V-Notch Weir } & \multicolumn{6}{|c|}{ R-Notch Weir } \\
\hline & \multicolumn{2}{|c|}{ Measured Water Level } & \multicolumn{2}{|c|}{$\begin{array}{c}\text { Calculated Rate } \\
\left(\mathrm{ft}^{3} / \mathrm{s}=448.8 \mathrm{gpm}\right)\end{array}$} & \multicolumn{3}{|c|}{ Measured Water Level } & \multicolumn{3}{|c|}{$\begin{array}{c}\text { Calculated Rate } \\
\left(\mathrm{ft}^{3} / \mathrm{s}=448.8 \mathrm{gpm}\right)\end{array}$} \\
\hline & $\begin{array}{c}\text { (ft above } \\
\text { sensor) }\end{array}$ & $\begin{array}{c}\text { (ft above } \\
\text { notch) }\end{array}$ & $\left(\mathrm{ft}^{3} / \mathrm{s}\right)$ & (gpm) & $\begin{array}{l}\text { (ft above } \\
\text { sensor) }\end{array}$ & $\begin{array}{c}\text { (ft above } \\
\text { log) }\end{array}$ & $\begin{array}{c}\text { (m above } \\
\text { log) }\end{array}$ & $\left(\mathrm{m}^{3} / \mathrm{s}\right)$ & $\left(\mathrm{ft}^{3} / \mathrm{s}\right)$ & (gpm) \\
\hline 7/24/08 6:00 & 2.23 & 0.770 & 0.298 & 133.893 & 1.428 & 0.082 & 0.024992 & 0.011135 & 0.393285 & 176.506 \\
\hline 7/24/08 7:00 & 2.23 & 0.770 & 0.298 & 133.893 & 1.426 & 0.08 & 0.024383 & 0.010746 & 0.379531 & 170.333 \\
\hline 7/24/08 8:00 & 2.233 & 0.773 & 0.301 & 135.188 & 1.425 & 0.079 & 0.024078 & 0.010553 & 0.372715 & 167.274 \\
\hline 7/24/08 9:00 & 2.235 & 0.775 & 0.303 & 136.055 & 1.425 & 0.079 & 0.024078 & 0.010553 & 0.372715 & 167.274 \\
\hline 7/24/08 10:00 & 2.244 & 0.784 & 0.312 & 139.999 & 1.427 & 0.081 & 0.024688 & 0.010940 & 0.386387 & 173.411 \\
\hline 7/24/08 11:00 & 2.238 & 0.778 & 0.306 & 137.362 & 1.425 & 0.079 & 0.024078 & 0.010553 & 0.372715 & 167.274 \\
\hline 7/24/08 12:00 & 2.243 & 0.783 & 0.311 & 139.558 & 1.427 & 0.081 & 0.024688 & 0.010940 & 0.386387 & 173.411 \\
\hline 7/24/08 13:00 & 2.224 & 0.764 & 0.293 & 131.327 & 1.425 & 0.079 & 0.024078 & 0.010553 & 0.372715 & 167.274 \\
\hline 7/24/08 14:00 & 2.219 & 0.759 & 0.288 & 129.210 & 1.423 & 0.077 & 0.023468 & 0.010170 & 0.359208 & 161.212 \\
\hline 7/24/08 15:00 & 2.208 & 0.748 & 0.278 & 124.627 & 1.423 & 0.077 & 0.023468 & 0.010170 & 0.359208 & 161.212 \\
\hline 7/24/08 16:00 & 2.197 & 0.737 & 0.268 & 120.142 & 1.426 & 0.08 & 0.024383 & 0.010746 & 0.379531 & 170.333 \\
\hline 7/24/08 17:00 & 2.193 & 0.733 & 0.264 & 118.536 & 1.424 & 0.078 & 0.023773 & 0.010361 & 0.365940 & 164.234 \\
\hline 7/24/08 18:00 & 2.186 & 0.726 & 0.258 & 115.756 & 1.421 & 0.075 & 0.022859 & 0.009792 & 0.345867 & 155.225 \\
\hline 7/24/08 19:00 & 2.186 & 0.726 & 0.258 & 115.756 & 1.422 & 0.076 & 0.023164 & 0.009981 & 0.352516 & 158.209 \\
\hline 7/24/08 20:00 & 2.188 & 0.728 & 0.260 & 116.546 & 1.422 & 0.076 & 0.023164 & 0.009981 & 0.352516 & 158.209 \\
\hline 7/24/08 21:00 & 2.19 & 0.730 & 0.261 & 117.339 & 1.422 & 0.076 & 0.023164 & 0.009981 & 0.352516 & 158.209 \\
\hline 7/24/08 22:00 & 2.204 & 0.744 & 0.274 & 122.984 & 1.424 & 0.078 & 0.023773 & 0.010361 & 0.365940 & 164.234 \\
\hline 7/24/08 23:00 & 2.208 & 0.748 & 0.278 & 124.627 & 1.424 & 0.078 & 0.023773 & 0.010361 & 0.365940 & 164.234 \\
\hline 7/25/08 0:00 & 2.222 & 0.762 & 0.291 & 130.478 & 1.427 & 0.081 & 0.024688 & 0.010940 & 0.386387 & 173.411 \\
\hline 7/25/08 1:00 & 2.224 & 0.764 & 0.293 & 131.327 & 1.427 & 0.081 & 0.024688 & 0.010940 & 0.386387 & 173.411 \\
\hline 7/25/08 2:00 & 2.224 & 0.764 & 0.293 & 131.327 & 1.427 & 0.081 & 0.024688 & 0.010940 & 0.386387 & 173.411 \\
\hline 7/25/08 3:00 & 2.231 & 0.771 & 0.299 & 134.324 & 1.429 & 0.083 & 0.025297 & 0.011331 & 0.400223 & 179.620 \\
\hline 7/25/08 4:00 & 2.231 & 0.771 & 0.299 & 134.324 & 1.429 & 0.083 & 0.025297 & 0.011331 & 0.400223 & 179.620 \\
\hline 7/25/08 5:00 & 2.233 & 0.773 & 0.301 & 135.188 & 1.428 & 0.082 & 0.024992 & 0.011135 & 0.393285 & 176.506 \\
\hline 7/25/08 6:00 & 2.233 & 0.773 & 0.301 & 135.188 & 1.428 & 0.082 & 0.024992 & 0.011135 & 0.393285 & 176.506 \\
\hline
\end{tabular}




\begin{tabular}{|c|c|c|c|c|c|c|c|c|c|c|}
\hline \multirow[b]{3}{*}{ Date Time } & \multicolumn{4}{|c|}{ V-Notch Weir } & \multicolumn{6}{|c|}{ R-Notch Weir } \\
\hline & \multicolumn{2}{|c|}{ Measured Water Level } & \multicolumn{2}{|c|}{$\begin{array}{c}\text { Calculated Rate } \\
\left(\mathrm{ft}^{3} / \mathrm{s}=448.8 \mathrm{gpm}\right)\end{array}$} & \multicolumn{3}{|c|}{ Measured Water Level } & \multicolumn{3}{|c|}{$\begin{array}{c}\text { Calculated Rate } \\
\left(\mathrm{ft}^{3} / \mathrm{s}=448.8 \mathrm{gpm}\right)\end{array}$} \\
\hline & $\begin{array}{c}\text { (ft above } \\
\text { sensor) }\end{array}$ & $\begin{array}{c}\text { (ft above } \\
\text { notch) }\end{array}$ & $\left(\mathrm{ft}^{3} / \mathrm{s}\right)$ & (gpm) & $\begin{array}{l}\text { (ft above } \\
\text { sensor) }\end{array}$ & $\begin{array}{c}\text { (ft above } \\
\text { log) }\end{array}$ & $\begin{array}{c}\text { (m above } \\
\text { log) }\end{array}$ & $\left(\mathrm{m}^{3} / \mathrm{s}\right)$ & $\left(\mathrm{ft}^{3} / \mathrm{s}\right)$ & (gpm) \\
\hline 7/25/08 7:00 & 2.238 & 0.778 & 0.306 & 137.362 & 1.43 & 0.084 & 0.025602 & 0.011529 & 0.407202 & 182.752 \\
\hline 7/25/08 8:00 & 2.242 & 0.782 & 0.310 & 139.117 & 1.43 & 0.084 & 0.025602 & 0.011529 & 0.407202 & 182.752 \\
\hline 7/25/08 9:00 & 2.242 & 0.782 & 0.310 & 139.117 & 1.43 & 0.084 & 0.025602 & 0.011529 & 0.407202 & 182.752 \\
\hline 7/25/08 10:00 & 2.245 & 0.785 & 0.313 & 140.442 & 1.43 & 0.084 & 0.025602 & 0.011529 & 0.407202 & 182.752 \\
\hline 7/25/08 11:00 & 2.245 & 0.785 & 0.313 & 140.442 & 1.43 & 0.084 & 0.025602 & 0.011529 & 0.407202 & 182.752 \\
\hline 7/25/08 12:00 & 2.247 & 0.787 & 0.315 & 141.329 & 1.43 & 0.084 & 0.025602 & 0.011529 & 0.407202 & 182.752 \\
\hline 7/25/08 13:00 & 2.245 & 0.785 & 0.313 & 140.442 & 1.43 & 0.084 & 0.025602 & 0.011529 & 0.407202 & 182.752 \\
\hline 7/25/08 14:00 & 2.236 & 0.776 & 0.304 & 136.490 & 1.427 & 0.081 & 0.024688 & 0.010940 & 0.386387 & 173.411 \\
\hline 7/25/08 15:00 & 2.225 & 0.765 & 0.294 & 131.752 & 1.428 & 0.082 & 0.024992 & 0.011135 & 0.393285 & 176.506 \\
\hline 7/25/08 16:00 & 2.209 & 0.749 & 0.279 & 125.039 & 1.425 & 0.079 & 0.024078 & 0.010553 & 0.372715 & 167.274 \\
\hline 7/25/08 17:00 & 2.204 & 0.744 & 0.274 & 122.984 & 1.428 & 0.082 & 0.024992 & 0.011135 & 0.393285 & 176.506 \\
\hline 7/25/08 18:00 & 2.195 & 0.735 & 0.266 & 119.337 & 1.426 & 0.08 & 0.024383 & 0.010746 & 0.379531 & 170.333 \\
\hline 7/25/08 19:00 & 2.198 & 0.738 & 0.269 & 120.546 & 1.424 & 0.078 & 0.023773 & 0.010361 & 0.365940 & 164.234 \\
\hline 7/25/08 20:00 & 2.197 & 0.737 & 0.268 & 120.142 & 1.424 & 0.078 & 0.023773 & 0.010361 & 0.365940 & 164.234 \\
\hline 7/25/08 21:00 & 2.202 & 0.742 & 0.272 & 122.168 & 1.427 & 0.081 & 0.024688 & 0.010940 & 0.386387 & 173.411 \\
\hline 7/25/08 22:00 & 2.209 & 0.749 & 0.279 & 125.039 & 1.427 & 0.081 & 0.024688 & 0.010940 & 0.386387 & 173.411 \\
\hline 7/25/08 23:00 & 2.222 & 0.762 & 0.291 & 130.478 & 1.427 & 0.081 & 0.024688 & 0.010940 & 0.386387 & 173.411 \\
\hline 7/26/08 0:00 & 2.224 & 0.764 & 0.293 & 131.327 & 1.426 & 0.08 & 0.024383 & 0.010746 & 0.379531 & 170.333 \\
\hline 7/26/08 1:00 & 2.252 & 0.792 & 0.320 & 143.562 & 1.433 & 0.087 & 0.026516 & 0.012129 & 0.428377 & 192.256 \\
\hline 7/26/08 2:00 & 2.245 & 0.785 & 0.313 & 140.442 & 1.431 & 0.085 & 0.025907 & 0.011728 & 0.414220 & 185.902 \\
\hline 7/26/08 3:00 & 2.245 & 0.785 & 0.313 & 140.442 & 1.431 & 0.085 & 0.025907 & 0.011728 & 0.414220 & 185.902 \\
\hline 7/26/08 4:00 & 2.242 & 0.782 & 0.310 & 139.117 & 1.43 & 0.084 & 0.025602 & 0.011529 & 0.407202 & 182.752 \\
\hline 7/26/08 5:00 & 2.242 & 0.782 & 0.310 & 139.117 & 1.43 & 0.084 & 0.025602 & 0.011529 & 0.407202 & 182.752 \\
\hline 7/26/08 6:00 & 2.247 & 0.787 & 0.315 & 141.329 & 1.43 & 0.084 & 0.025602 & 0.011529 & 0.407202 & 182.752 \\
\hline 7/26/08 7:00 & 2.244 & 0.784 & 0.312 & 139.999 & 1.43 & 0.084 & 0.025602 & 0.011529 & 0.407202 & 182.752 \\
\hline
\end{tabular}




\begin{tabular}{|c|c|c|c|c|c|c|c|c|c|c|}
\hline \multirow[b]{3}{*}{ Date Time } & \multicolumn{4}{|c|}{ V-Notch Weir } & \multicolumn{6}{|c|}{ R-Notch Weir } \\
\hline & \multicolumn{2}{|c|}{ Measured Water Level } & \multicolumn{2}{|c|}{$\begin{array}{c}\text { Calculated Rate } \\
\left(\mathrm{ft}^{3} / \mathrm{s}=448.8 \mathrm{gpm}\right)\end{array}$} & \multicolumn{3}{|c|}{ Measured Water Level } & \multicolumn{3}{|c|}{$\begin{array}{c}\text { Calculated Rate } \\
\left(\mathrm{ft}^{3} / \mathrm{s}=448.8 \mathrm{gpm}\right)\end{array}$} \\
\hline & $\begin{array}{c}\text { (ft above } \\
\text { sensor) }\end{array}$ & $\begin{array}{c}\text { (ft above } \\
\text { notch) }\end{array}$ & $\left(\mathrm{ft}^{3} / \mathrm{s}\right)$ & (gpm) & $\begin{array}{l}\text { (ft above } \\
\text { sensor) }\end{array}$ & $\begin{array}{c}\text { (ft above } \\
\text { log) }\end{array}$ & $\begin{array}{c}\text { (m above } \\
\text { log) }\end{array}$ & $\left(\mathrm{m}^{3} / \mathrm{s}\right)$ & $\left(\mathrm{ft}^{3} / \mathrm{s}\right)$ & (gpm) \\
\hline 7/26/08 8:00 & 2.246 & 0.786 & 0.314 & 140.885 & 1.429 & 0.083 & 0.025297 & 0.011331 & 0.400223 & 179.620 \\
\hline 7/26/08 9:00 & 2.249 & 0.789 & 0.317 & 142.220 & 1.429 & 0.083 & 0.025297 & 0.011331 & 0.400223 & 179.620 \\
\hline 7/26/08 10:00 & 2.252 & 0.792 & 0.320 & 143.562 & 1.431 & 0.085 & 0.025907 & 0.011728 & 0.414220 & 185.902 \\
\hline 7/26/08 11:00 & 2.249 & 0.789 & 0.317 & 142.220 & 1.431 & 0.085 & 0.025907 & 0.011728 & 0.414220 & 185.902 \\
\hline 7/26/08 12:00 & 2.242 & 0.782 & 0.310 & 139.117 & 1.429 & 0.083 & 0.025297 & 0.011331 & 0.400223 & 179.620 \\
\hline 7/26/08 13:00 & 2.238 & 0.778 & 0.306 & 137.362 & 1.427 & 0.081 & 0.024688 & 0.010940 & 0.386387 & 173.411 \\
\hline 7/26/08 14:00 & 2.229 & 0.769 & 0.297 & 133.464 & 1.432 & 0.086 & 0.026212 & 0.011928 & 0.421279 & 189.070 \\
\hline 7/26/08 15:00 & 2.22 & 0.760 & 0.289 & 129.632 & 1.429 & 0.083 & 0.025297 & 0.011331 & 0.400223 & 179.620 \\
\hline 7/26/08 16:00 & 2.206 & 0.746 & 0.276 & 123.804 & 1.427 & 0.081 & 0.024688 & 0.010940 & 0.386387 & 173.411 \\
\hline 7/26/08 17:00 & 2.2 & 0.740 & 0.270 & 121.355 & 1.428 & 0.082 & 0.024992 & 0.011135 & 0.393285 & 176.506 \\
\hline 7/26/08 18:00 & 2.193 & 0.733 & 0.264 & 118.536 & 1.428 & 0.082 & 0.024992 & 0.011135 & 0.393285 & 176.506 \\
\hline 7/26/08 19:00 & 2.193 & 0.733 & 0.264 & 118.536 & 1.428 & 0.082 & 0.024992 & 0.011135 & 0.393285 & 176.506 \\
\hline 7/26/08 20:00 & 2.19 & 0.730 & 0.261 & 117.339 & 1.429 & 0.083 & 0.025297 & 0.011331 & 0.400223 & 179.620 \\
\hline 7/26/08 21:00 & 2.195 & 0.735 & 0.266 & 119.337 & 1.429 & 0.083 & 0.025297 & 0.011331 & 0.400223 & 179.620 \\
\hline 7/26/08 22:00 & 2.206 & 0.746 & 0.276 & 123.804 & 1.429 & 0.083 & 0.025297 & 0.011331 & 0.400223 & 179.620 \\
\hline 7/26/08 23:00 & 2.218 & 0.758 & 0.287 & 128.789 & 1.433 & 0.087 & 0.026516 & 0.012129 & 0.428377 & 192.256 \\
\hline 7/27/08 0:00 & 2.225 & 0.765 & 0.294 & 131.752 & 1.433 & 0.087 & 0.026516 & 0.012129 & 0.428377 & 192.256 \\
\hline 7/27/08 1:00 & 2.231 & 0.771 & 0.299 & 134.324 & 1.436 & 0.09 & 0.027431 & 0.012738 & 0.449907 & 201.918 \\
\hline 7/27/08 2:00 & 2.24 & 0.780 & 0.308 & 138.238 & 1.438 & 0.092 & 0.028040 & 0.013150 & 0.464455 & 208.447 \\
\hline 7/27/08 3:00 & 2.245 & 0.785 & 0.313 & 140.442 & 1.438 & 0.092 & 0.028040 & 0.013150 & 0.464455 & 208.447 \\
\hline 7/27/08 4:00 & 2.252 & 0.792 & 0.320 & 143.562 & 1.44 & 0.094 & 0.028650 & 0.013566 & 0.479156 & 215.045 \\
\hline 7/27/08 5:00 & 2.252 & 0.792 & 0.320 & 143.562 & 1.437 & 0.091 & 0.027735 & 0.012943 & 0.457162 & 205.174 \\
\hline 7/27/08 6:00 & 2.254 & 0.794 & 0.322 & 144.461 & 1.437 & 0.091 & 0.027735 & 0.012943 & 0.457162 & 205.174 \\
\hline 7/27/08 7:00 & 2.256 & 0.796 & 0.324 & 145.363 & 1.437 & 0.091 & 0.027735 & 0.012943 & 0.457162 & 205.174 \\
\hline 7/27/08 8:00 & 2.254 & 0.794 & 0.322 & 144.461 & 1.434 & 0.088 & 0.026821 & 0.012331 & 0.435514 & 195.459 \\
\hline
\end{tabular}




\begin{tabular}{|c|c|c|c|c|c|c|c|c|c|c|}
\hline \multirow[b]{3}{*}{ Date Time } & \multicolumn{4}{|c|}{ V-Notch Weir } & \multicolumn{6}{|c|}{ R-Notch Weir } \\
\hline & \multicolumn{2}{|c|}{ Measured Water Level } & \multicolumn{2}{|c|}{$\begin{array}{c}\text { Calculated Rate } \\
\left(\mathrm{ft}^{3} / \mathrm{s}=448.8 \mathrm{gpm}\right)\end{array}$} & \multicolumn{3}{|c|}{ Measured Water Level } & \multicolumn{3}{|c|}{$\begin{array}{c}\text { Calculated Rate } \\
\left(\mathrm{ft}^{3} / \mathrm{s}=448.8 \mathrm{gpm}\right)\end{array}$} \\
\hline & $\begin{array}{c}\text { (ft above } \\
\text { sensor) }\end{array}$ & $\begin{array}{c}\text { (ft above } \\
\text { notch) }\end{array}$ & $\left(\mathrm{ft}^{3} / \mathrm{s}\right)$ & (gpm) & $\begin{array}{l}\text { (ft above } \\
\text { sensor) }\end{array}$ & $\begin{array}{c}\text { (ft above } \\
\text { log) }\end{array}$ & $\begin{array}{c}\text { (m above } \\
\text { log) }\end{array}$ & $\left(\mathrm{m}^{3} / \mathrm{s}\right)$ & $\left(\mathrm{ft}^{3} / \mathrm{s}\right)$ & (gpm) \\
\hline 7/27/08 9:00 & 2.256 & 0.796 & 0.324 & 145.363 & 1.434 & 0.088 & 0.026821 & 0.012331 & 0.435514 & 195.459 \\
\hline 7/27/08 10:00 & 2.254 & 0.794 & 0.322 & 144.461 & 1.434 & 0.088 & 0.026821 & 0.012331 & 0.435514 & 195.459 \\
\hline 7/27/08 11:00 & 2.247 & 0.787 & 0.315 & 141.329 & 1.434 & 0.088 & 0.026821 & 0.012331 & 0.435514 & 195.459 \\
\hline 7/27/08 12:00 & 2.241 & 0.781 & 0.309 & 138.677 & 1.434 & 0.088 & 0.026821 & 0.012331 & 0.435514 & 195.459 \\
\hline 7/27/08 13:00 & 2.222 & 0.762 & 0.291 & 130.478 & 1.432 & 0.086 & 0.026212 & 0.011928 & 0.421279 & 189.070 \\
\hline 7/27/08 14:00 & 2.207 & 0.747 & 0.277 & 124.215 & 1.435 & 0.089 & 0.027126 & 0.012534 & 0.442691 & 198.680 \\
\hline 7/27/08 15:00 & 2.193 & 0.733 & 0.264 & 118.536 & 1.433 & 0.087 & 0.026516 & 0.012129 & 0.428377 & 192.256 \\
\hline 7/27/08 16:00 & 2.184 & 0.724 & 0.256 & 114.969 & 1.433 & 0.087 & 0.026516 & 0.012129 & 0.428377 & 192.256 \\
\hline 7/27/08 17:00 & 2.178 & 0.718 & 0.251 & 112.627 & 1.433 & 0.087 & 0.026516 & 0.012129 & 0.428377 & 192.256 \\
\hline 7/27/08 18:00 & 2.171 & 0.711 & 0.245 & 109.931 & 1.434 & 0.088 & 0.026821 & 0.012331 & 0.435514 & 195.459 \\
\hline 7/27/08 19:00 & 2.171 & 0.711 & 0.245 & 109.931 & 1.437 & 0.091 & 0.027735 & 0.012943 & 0.457162 & 205.174 \\
\hline 7/27/08 20:00 & 2.173 & 0.713 & 0.247 & 110.697 & 1.437 & 0.091 & 0.027735 & 0.012943 & 0.457162 & 205.174 \\
\hline 7/27/08 21:00 & 2.18 & 0.720 & 0.253 & 113.404 & 1.437 & 0.091 & 0.027735 & 0.012943 & 0.457162 & 205.174 \\
\hline 7/27/08 22:00 & 2.22 & 0.760 & 0.289 & 129.632 & 1.442 & 0.096 & 0.029259 & 0.013987 & 0.494010 & 221.711 \\
\hline 7/27/08 23:00 & 2.224 & 0.764 & 0.293 & 131.327 & 1.442 & 0.096 & 0.029259 & 0.013987 & 0.494010 & 221.711 \\
\hline 7/28/08 0:00 & 2.228 & 0.768 & 0.296 & 133.034 & 1.442 & 0.096 & 0.029259 & 0.013987 & 0.494010 & 221.711 \\
\hline 7/28/08 1:00 & 2.23 & 0.770 & 0.298 & 133.893 & 1.442 & 0.096 & 0.029259 & 0.013987 & 0.494010 & 221.711 \\
\hline 7/28/08 2:00 & 2.232 & 0.772 & 0.300 & 134.756 & 1.442 & 0.096 & 0.029259 & 0.013987 & 0.494010 & 221.711 \\
\hline 7/28/08 3:00 & 2.239 & 0.779 & 0.307 & 137.800 & 1.442 & 0.096 & 0.029259 & 0.013987 & 0.494010 & 221.711 \\
\hline 7/28/08 4:00 & 2.241 & 0.781 & 0.309 & 138.677 & 1.439 & 0.093 & 0.028345 & 0.013358 & 0.471786 & 211.738 \\
\hline 7/28/08 5:00 & 2.238 & 0.778 & 0.306 & 137.362 & 1.439 & 0.093 & 0.028345 & 0.013358 & 0.471786 & 211.738 \\
\hline 7/28/08 6:00 & 2.236 & 0.776 & 0.304 & 136.490 & 1.439 & 0.093 & 0.028345 & 0.013358 & 0.471786 & 211.738 \\
\hline 7/28/08 7:00 & 2.229 & 0.769 & 0.297 & 133.464 & 1.439 & 0.093 & 0.028345 & 0.013358 & 0.471786 & 211.738 \\
\hline 7/28/08 8:00 & 2.24 & 0.780 & 0.308 & 138.238 & 1.434 & 0.088 & 0.026821 & 0.012331 & 0.435514 & 195.459 \\
\hline 7/28/08 9:00 & 2.24 & 0.780 & 0.308 & 138.238 & 1.433 & 0.087 & 0.026516 & 0.012129 & 0.428377 & 192.256 \\
\hline
\end{tabular}




\begin{tabular}{|c|c|c|c|c|c|c|c|c|c|c|}
\hline \multirow[b]{3}{*}{ Date Time } & \multicolumn{4}{|c|}{ V-Notch Weir } & \multicolumn{6}{|c|}{ R-Notch Weir } \\
\hline & \multicolumn{2}{|c|}{ Measured Water Level } & \multicolumn{2}{|c|}{$\begin{array}{c}\text { Calculated Rate } \\
\left(\mathrm{ft}^{3} / \mathrm{s}=448.8 \mathrm{gpm}\right)\end{array}$} & \multicolumn{3}{|c|}{ Measured Water Level } & \multicolumn{3}{|c|}{$\begin{array}{c}\text { Calculated Rate } \\
\left(\mathrm{ft}^{3} / \mathrm{s}=448.8 \mathrm{gpm}\right)\end{array}$} \\
\hline & $\begin{array}{c}\text { (ft above } \\
\text { sensor) }\end{array}$ & $\begin{array}{c}\text { (ft above } \\
\text { notch) }\end{array}$ & $\left(\mathrm{ft}^{3} / \mathrm{s}\right)$ & (gpm) & $\begin{array}{l}\text { (ft above } \\
\text { sensor) }\end{array}$ & $\begin{array}{c}\text { (ft above } \\
\text { log) }\end{array}$ & $\begin{array}{c}\text { (m above } \\
\text { log) }\end{array}$ & $\left(\mathrm{m}^{3} / \mathrm{s}\right)$ & $\left(\mathrm{ft}^{3} / \mathrm{s}\right)$ & (gpm) \\
\hline 7/28/08 10:00 & 2.236 & 0.776 & 0.304 & 136.490 & 1.438 & 0.092 & 0.028040 & 0.013150 & 0.464455 & 208.447 \\
\hline 7/28/08 11:00 & 2.224 & 0.764 & 0.293 & 131.327 & 1.438 & 0.092 & 0.028040 & 0.013150 & 0.464455 & 208.447 \\
\hline 7/28/08 12:00 & 2.213 & 0.753 & 0.282 & 126.698 & 1.436 & 0.09 & 0.027431 & 0.012738 & 0.449907 & 201.918 \\
\hline 7/28/08 13:00 & 2.202 & 0.742 & 0.272 & 122.168 & 1.436 & 0.09 & 0.027431 & 0.012738 & 0.449907 & 201.918 \\
\hline 7/28/08 14:00 & 2.193 & 0.733 & 0.264 & 118.536 & 1.436 & 0.09 & 0.027431 & 0.012738 & 0.449907 & 201.918 \\
\hline 7/28/08 15:00 & 2.191 & 0.731 & 0.262 & 117.737 & 1.436 & 0.09 & 0.027431 & 0.012738 & 0.449907 & 201.918 \\
\hline 7/28/08 16:00 & 2.183 & 0.723 & 0.255 & 114.576 & 1.437 & 0.091 & 0.027735 & 0.012943 & 0.457162 & 205.174 \\
\hline 7/28/08 17:00 & 2.178 & 0.718 & 0.251 & 112.627 & 1.435 & 0.089 & 0.027126 & 0.012534 & 0.442691 & 198.680 \\
\hline 7/28/08 18:00 & 2.174 & 0.714 & 0.248 & 111.082 & 1.435 & 0.089 & 0.027126 & 0.012534 & 0.442691 & 198.680 \\
\hline 7/28/08 19:00 & 2.171 & 0.711 & 0.245 & 109.931 & 1.435 & 0.089 & 0.027126 & 0.012534 & 0.442691 & 198.680 \\
\hline 7/28/08 20:00 & 2.173 & 0.713 & 0.247 & 110.697 & 1.438 & 0.092 & 0.028040 & 0.013150 & 0.464455 & 208.447 \\
\hline 7/28/08 21:00 & 2.18 & 0.720 & 0.253 & 113.404 & 1.438 & 0.092 & 0.028040 & 0.013150 & 0.464455 & 208.447 \\
\hline 7/28/08 22:00 & 2.191 & 0.731 & 0.262 & 117.737 & 1.436 & 0.09 & 0.027431 & 0.012738 & 0.449907 & 201.918 \\
\hline 7/28/08 23:00 & 2.198 & 0.738 & 0.269 & 120.546 & 1.436 & 0.09 & 0.027431 & 0.012738 & 0.449907 & 201.918 \\
\hline 7/29/08 0:00 & 2.202 & 0.742 & 0.272 & 122.168 & 1.436 & 0.09 & 0.027431 & 0.012738 & 0.449907 & 201.918 \\
\hline 7/29/08 1:00 & 2.242 & 0.782 & 0.310 & 139.117 & 1.438 & 0.092 & 0.028040 & 0.013150 & 0.464455 & 208.447 \\
\hline 7/29/08 2:00 & 2.382 & 0.922 & 0.466 & 209.190 & 1.466 & 0.12 & 0.036574 & 0.019354 & 0.683586 & 306.793 \\
\hline 7/29/08 3:00 & 2.285 & 0.825 & 0.354 & 158.830 & 1.449 & 0.103 & 0.031393 & 0.015492 & 0.547171 & 245.570 \\
\hline 7/29/08 4:00 & 2.25 & 0.790 & 0.318 & 142.666 & 1.442 & 0.096 & 0.029259 & 0.013987 & 0.494010 & 221.711 \\
\hline 7/29/08 5:00 & 2.294 & 0.834 & 0.364 & 163.155 & 1.448 & 0.102 & 0.031088 & 0.015274 & 0.539466 & 242.112 \\
\hline 7/29/08 6:00 & 2.249 & 0.789 & 0.317 & 142.220 & 1.443 & 0.097 & 0.029564 & 0.014199 & 0.501493 & 225.070 \\
\hline 7/29/08 7:00 & 2.223 & 0.763 & 0.292 & 130.902 & 1.438 & 0.092 & 0.028040 & 0.013150 & 0.464455 & 208.447 \\
\hline 7/29/08 8:00 & 2.211 & 0.751 & 0.280 & 125.867 & 1.435 & 0.089 & 0.027126 & 0.012534 & 0.442691 & 198.680 \\
\hline 7/29/08 9:00 & 2.209 & 0.749 & 0.279 & 125.039 & 1.435 & 0.089 & 0.027126 & 0.012534 & 0.442691 & 198.680 \\
\hline 7/29/08 10:00 & 2.207 & 0.747 & 0.277 & 124.215 & 1.435 & 0.089 & 0.027126 & 0.012534 & 0.442691 & 198.680 \\
\hline
\end{tabular}




\begin{tabular}{|c|c|c|c|c|c|c|c|c|c|c|}
\hline \multirow[b]{3}{*}{ Date Time } & \multicolumn{4}{|c|}{ V-Notch Weir } & \multicolumn{6}{|c|}{ R-Notch Weir } \\
\hline & \multicolumn{2}{|c|}{ Measured Water Level } & \multicolumn{2}{|c|}{$\begin{array}{c}\text { Calculated Rate } \\
\left(\mathrm{ft}^{3} / \mathrm{s}=448.8 \mathrm{gpm}\right)\end{array}$} & \multicolumn{3}{|c|}{ Measured Water Level } & \multicolumn{3}{|c|}{$\begin{array}{c}\text { Calculated Rate } \\
\left(\mathrm{ft}^{3} / \mathrm{s}=448.8 \mathrm{gpm}\right)\end{array}$} \\
\hline & $\begin{array}{c}\text { (ft above } \\
\text { sensor) }\end{array}$ & $\begin{array}{c}\text { (ft above } \\
\text { notch) }\end{array}$ & $\left(\mathrm{ft}^{3} / \mathrm{s}\right)$ & (gpm) & $\begin{array}{l}\text { (ft above } \\
\text { sensor) }\end{array}$ & $\begin{array}{c}\text { (ft above } \\
\text { log) }\end{array}$ & $\begin{array}{c}\text { (m above } \\
\text { log) }\end{array}$ & $\left(\mathrm{m}^{3} / \mathrm{s}\right)$ & $\left(\mathrm{ft}^{3} / \mathrm{s}\right)$ & (gpm) \\
\hline 7/29/08 11:00 & 2.198 & 0.738 & 0.269 & 120.546 & 1.433 & 0.087 & 0.026516 & 0.012129 & 0.428377 & 192.256 \\
\hline 7/29/08 12:00 & 2.189 & 0.729 & 0.261 & 116.942 & 1.43 & 0.084 & 0.025602 & 0.011529 & 0.407202 & 182.752 \\
\hline 7/29/08 13:00 & 2.184 & 0.724 & 0.256 & 114.969 & 1.43 & 0.084 & 0.025602 & 0.011529 & 0.407202 & 182.752 \\
\hline 7/29/08 14:00 & 2.168 & 0.708 & 0.242 & 108.788 & 1.431 & 0.085 & 0.025907 & 0.011728 & 0.414220 & 185.902 \\
\hline 7/29/08 15:00 & 2.164 & 0.704 & 0.239 & 107.275 & 1.431 & 0.085 & 0.025907 & 0.011728 & 0.414220 & 185.902 \\
\hline 7/29/08 16:00 & 2.15 & 0.690 & 0.227 & 102.078 & 1.431 & 0.085 & 0.025907 & 0.011728 & 0.414220 & 185.902 \\
\hline 7/29/08 17:00 & 2.145 & 0.685 & 0.223 & 100.260 & 1.431 & 0.085 & 0.025907 & 0.011728 & 0.414220 & 185.902 \\
\hline 7/29/08 18:00 & 2.139 & 0.679 & 0.219 & 98.103 & 1.433 & 0.087 & 0.026516 & 0.012129 & 0.428377 & 192.256 \\
\hline 7/29/08 19:00 & 2.162 & 0.702 & 0.237 & 106.523 & 1.436 & 0.09 & 0.027431 & 0.012738 & 0.449907 & 201.918 \\
\hline 7/29/08 20:00 & 2.155 & 0.695 & 0.232 & 103.916 & 1.439 & 0.093 & 0.028345 & 0.013358 & 0.471786 & 211.738 \\
\hline 7/29/08 21:00 & 2.159 & 0.699 & 0.235 & 105.401 & 1.439 & 0.093 & 0.028345 & 0.013358 & 0.471786 & 211.738 \\
\hline 7/29/08 22:00 & 2.163 & 0.703 & 0.238 & 106.898 & 1.436 & 0.09 & 0.027431 & 0.012738 & 0.449907 & 201.918 \\
\hline 7/29/08 23:00 & 2.17 & 0.710 & 0.244 & 109.549 & 1.436 & 0.09 & 0.027431 & 0.012738 & 0.449907 & 201.918 \\
\hline 7/30/08 0:00 & 2.179 & 0.719 & 0.252 & 113.015 & 1.438 & 0.092 & 0.028040 & 0.013150 & 0.464455 & 208.447 \\
\hline 7/30/08 1:00 & 2.186 & 0.726 & 0.258 & 115.756 & 1.438 & 0.092 & 0.028040 & 0.013150 & 0.464455 & 208.447 \\
\hline 7/30/08 2:00 & 2.191 & 0.731 & 0.262 & 117.737 & 1.438 & 0.092 & 0.028040 & 0.013150 & 0.464455 & 208.447 \\
\hline 7/30/08 3:00 & 2.195 & 0.735 & 0.266 & 119.337 & 1.44 & 0.094 & 0.028650 & 0.013566 & 0.479156 & 215.045 \\
\hline 7/30/08 4:00 & 2.185 & 0.725 & 0.257 & 115.362 & 1.437 & 0.091 & 0.027735 & 0.012943 & 0.457162 & 205.174 \\
\hline 7/30/08 5:00 & 2.195 & 0.735 & 0.266 & 119.337 & 1.436 & 0.09 & 0.027431 & 0.012738 & 0.449907 & 201.918 \\
\hline 7/30/08 6:00 & 2.202 & 0.742 & 0.272 & 122.168 & 1.434 & 0.088 & 0.026821 & 0.012331 & 0.435514 & 195.459 \\
\hline 7/30/08 7:00 & 2.202 & 0.742 & 0.272 & 122.168 & 1.433 & 0.087 & 0.026516 & 0.012129 & 0.428377 & 192.256 \\
\hline 7/30/08 8:00 & 2.206 & 0.746 & 0.276 & 123.804 & 1.431 & 0.085 & 0.025907 & 0.011728 & 0.414220 & 185.902 \\
\hline 7/30/08 9:00 & 2.206 & 0.746 & 0.276 & 123.804 & 1.431 & 0.085 & 0.025907 & 0.011728 & 0.414220 & 185.902 \\
\hline 7/30/08 10:00 & 2.218 & 0.758 & 0.287 & 128.789 & 1.434 & 0.088 & 0.026821 & 0.012331 & 0.435514 & 195.459 \\
\hline 7/30/08 11:00 & 2.21 & 0.750 & 0.280 & 125.453 & 1.434 & 0.088 & 0.026821 & 0.012331 & 0.435514 & 195.459 \\
\hline
\end{tabular}




\begin{tabular}{|c|c|c|c|c|c|c|c|c|c|c|}
\hline \multirow[b]{3}{*}{ Date Time } & \multicolumn{4}{|c|}{ V-Notch Weir } & \multicolumn{6}{|c|}{ R-Notch Weir } \\
\hline & \multicolumn{2}{|c|}{ Measured Water Level } & \multicolumn{2}{|c|}{$\begin{array}{c}\text { Calculated Rate } \\
\left(\mathrm{ft}^{3} / \mathrm{s}=448.8 \mathrm{gpm}\right)\end{array}$} & \multicolumn{3}{|c|}{ Measured Water Level } & \multicolumn{3}{|c|}{$\begin{array}{c}\text { Calculated Rate } \\
\left(\mathrm{ft}^{3} / \mathrm{s}=448.8 \mathrm{gpm}\right)\end{array}$} \\
\hline & $\begin{array}{c}\text { (ft above } \\
\text { sensor) }\end{array}$ & $\begin{array}{c}\text { (ft above } \\
\text { notch) }\end{array}$ & $\left(\mathrm{ft}^{3} / \mathrm{s}\right)$ & (gpm) & $\begin{array}{l}\text { (ft above } \\
\text { sensor) }\end{array}$ & $\begin{array}{c}\text { (ft above } \\
\text { log) }\end{array}$ & $\begin{array}{c}\text { (m above } \\
\text { log) }\end{array}$ & $\left(\mathrm{m}^{3} / \mathrm{s}\right)$ & $\left(\mathrm{ft}^{3} / \mathrm{s}\right)$ & (gpm) \\
\hline 7/30/08 12:00 & 2.198 & 0.738 & 0.269 & 120.546 & 1.429 & 0.083 & 0.025297 & 0.011331 & 0.400223 & 179.620 \\
\hline 7/30/08 13:00 & 2.178 & 0.718 & 0.251 & 112.627 & 1.429 & 0.083 & 0.025297 & 0.011331 & 0.400223 & 179.620 \\
\hline 7/30/08 14:00 & 2.165 & 0.705 & 0.240 & 107.652 & 1.43 & 0.084 & 0.025602 & 0.011529 & 0.407202 & 182.752 \\
\hline 7/30/08 15:00 & 2.146 & 0.686 & 0.224 & 100.622 & 1.428 & 0.082 & 0.024992 & 0.011135 & 0.393285 & 176.506 \\
\hline 7/30/08 16:00 & 2.131 & 0.671 & 0.212 & 95.272 & 1.435 & 0.089 & 0.027126 & 0.012534 & 0.442691 & 198.680 \\
\hline 7/30/08 17:00 & 2.129 & 0.669 & 0.211 & 94.572 & 1.428 & 0.082 & 0.024992 & 0.011135 & 0.393285 & 176.506 \\
\hline 7/30/08 18:00 & 2.117 & 0.657 & 0.202 & 90.436 & 1.429 & 0.083 & 0.025297 & 0.011331 & 0.400223 & 179.620 \\
\hline 7/30/08 19:00 & 2.12 & 0.660 & 0.204 & 91.459 & 1.432 & 0.086 & 0.026212 & 0.011928 & 0.421279 & 189.070 \\
\hline 7/30/08 20:00 & 2.126 & 0.666 & 0.208 & 93.527 & 1.432 & 0.086 & 0.026212 & 0.011928 & 0.421279 & 189.070 \\
\hline 7/30/08 21:00 & 2.135 & 0.675 & 0.215 & 96.681 & 1.432 & 0.086 & 0.026212 & 0.011928 & 0.421279 & 189.070 \\
\hline 7/30/08 22:00 & 2.155 & 0.695 & 0.232 & 103.916 & 1.442 & 0.096 & 0.029259 & 0.013987 & 0.494010 & 221.711 \\
\hline 7/30/08 23:00 & 2.152 & 0.692 & 0.229 & 102.811 & 1.432 & 0.086 & 0.026212 & 0.011928 & 0.421279 & 189.070 \\
\hline 7/31/08 0:00 & 2.161 & 0.701 & 0.237 & 106.148 & 1.435 & 0.089 & 0.027126 & 0.012534 & 0.442691 & 198.680 \\
\hline 7/31/08 1:00 & 2.17 & 0.710 & 0.244 & 109.549 & 1.437 & 0.091 & 0.027735 & 0.012943 & 0.457162 & 205.174 \\
\hline 7/31/08 2:00 & 2.179 & 0.719 & 0.252 & 113.015 & 1.439 & 0.093 & 0.028345 & 0.013358 & 0.471786 & 211.738 \\
\hline 7/31/08 3:00 & 2.181 & 0.721 & 0.254 & 113.794 & 1.438 & 0.092 & 0.028040 & 0.013150 & 0.464455 & 208.447 \\
\hline 7/31/08 4:00 & 2.205 & 0.745 & 0.275 & 123.394 & 1.44 & 0.094 & 0.028650 & 0.013566 & 0.479156 & 215.045 \\
\hline 7/31/08 5:00 & 2.197 & 0.737 & 0.268 & 120.142 & 1.437 & 0.091 & 0.027735 & 0.012943 & 0.457162 & 205.174 \\
\hline 7/31/08 6:00 & 2.207 & 0.747 & 0.277 & 124.215 & 1.434 & 0.088 & 0.026821 & 0.012331 & 0.435514 & 195.459 \\
\hline 7/31/08 7:00 & 2.202 & 0.742 & 0.272 & 122.168 & 1.432 & 0.086 & 0.026212 & 0.011928 & 0.421279 & 189.070 \\
\hline 7/31/08 8:00 & 2.207 & 0.747 & 0.277 & 124.215 & 1.432 & 0.086 & 0.026212 & 0.011928 & 0.421279 & 189.070 \\
\hline 7/31/08 9:00 & 2.204 & 0.744 & 0.274 & 122.984 & 1.432 & 0.086 & 0.026212 & 0.011928 & 0.421279 & 189.070 \\
\hline 7/31/08 10:00 & 2.202 & 0.742 & 0.272 & 122.168 & 1.432 & 0.086 & 0.026212 & 0.011928 & 0.421279 & 189.070 \\
\hline 7/31/08 11:00 & 2.205 & 0.745 & 0.275 & 123.394 & 1.432 & 0.086 & 0.026212 & 0.011928 & 0.421279 & 189.070 \\
\hline 7/31/08 12:00 & 2.192 & 0.732 & 0.263 & 118.136 & 1.432 & 0.086 & 0.026212 & 0.011928 & 0.421279 & 189.070 \\
\hline
\end{tabular}




\begin{tabular}{|c|c|c|c|c|c|c|c|c|c|c|}
\hline \multirow[b]{3}{*}{ Date Time } & \multicolumn{4}{|c|}{ V-Notch Weir } & \multicolumn{6}{|c|}{ R-Notch Weir } \\
\hline & \multicolumn{2}{|c|}{ Measured Water Level } & \multicolumn{2}{|c|}{$\begin{array}{c}\text { Calculated Rate } \\
\left(\mathrm{ft}^{3} / \mathrm{s}=448.8 \mathrm{gpm}\right)\end{array}$} & \multicolumn{3}{|c|}{ Measured Water Level } & \multicolumn{3}{|c|}{$\begin{array}{c}\text { Calculated Rate } \\
\left(\mathrm{ft}^{3} / \mathrm{s}=448.8 \mathrm{gpm}\right)\end{array}$} \\
\hline & $\begin{array}{c}\text { (ft above } \\
\text { sensor) }\end{array}$ & $\begin{array}{c}\text { (ft above } \\
\text { notch) }\end{array}$ & $\left(\mathrm{ft}^{3} / \mathrm{s}\right)$ & (gpm) & $\begin{array}{l}\text { (ft above } \\
\text { sensor) }\end{array}$ & $\begin{array}{c}\text { (ft above } \\
\text { log) }\end{array}$ & $\begin{array}{c}\text { (m above } \\
\text { log) }\end{array}$ & $\left(\mathrm{m}^{3} / \mathrm{s}\right)$ & $\left(\mathrm{ft}^{3} / \mathrm{s}\right)$ & (gpm) \\
\hline 7/31/08 13:00 & 2.178 & 0.718 & 0.251 & 112.627 & 1.43 & 0.084 & 0.025602 & 0.011529 & 0.407202 & 182.752 \\
\hline 7/31/08 14:00 & 2.163 & 0.703 & 0.238 & 106.898 & 1.43 & 0.084 & 0.025602 & 0.011529 & 0.407202 & 182.752 \\
\hline 7/31/08 15:00 & 2.147 & 0.687 & 0.225 & 100.985 & 1.428 & 0.082 & 0.024992 & 0.011135 & 0.393285 & 176.506 \\
\hline 7/31/08 16:00 & 2.127 & 0.667 & 0.209 & 93.875 & 1.426 & 0.08 & 0.024383 & 0.010746 & 0.379531 & 170.333 \\
\hline 7/31/08 17:00 & 2.116 & 0.656 & 0.201 & 90.096 & 1.427 & 0.081 & 0.024688 & 0.010940 & 0.386387 & 173.411 \\
\hline 7/31/08 18:00 & 2.104 & 0.644 & 0.192 & 86.080 & 1.427 & 0.081 & 0.024688 & 0.010940 & 0.386387 & 173.411 \\
\hline 7/31/08 19:00 & 2.101 & 0.641 & 0.190 & 85.093 & 1.43 & 0.084 & 0.025602 & 0.011529 & 0.407202 & 182.752 \\
\hline 7/31/08 20:00 & 2.106 & 0.646 & 0.193 & 86.742 & 1.43 & 0.084 & 0.025602 & 0.011529 & 0.407202 & 182.752 \\
\hline 7/31/08 21:00 & 2.112 & 0.652 & 0.198 & 88.745 & 1.433 & 0.087 & 0.026516 & 0.012129 & 0.428377 & 192.256 \\
\hline 7/31/08 22:00 & 2.13 & 0.670 & 0.212 & 94.921 & 1.431 & 0.085 & 0.025907 & 0.011728 & 0.414220 & 185.902 \\
\hline 7/31/08 23:00 & 2.142 & 0.682 & 0.221 & 99.178 & 1.431 & 0.085 & 0.025907 & 0.011728 & 0.414220 & 185.902 \\
\hline 8/1/08 0:00 & 2.139 & 0.679 & 0.219 & 98.103 & 1.431 & 0.085 & 0.025907 & 0.011728 & 0.414220 & 185.902 \\
\hline 8/1/08 1:00 & 2.152 & 0.692 & 0.229 & 102.811 & 1.431 & 0.085 & 0.025907 & 0.011728 & 0.414220 & 185.902 \\
\hline 8/1/08 2:00 & 2.166 & 0.706 & 0.241 & 108.030 & 1.433 & 0.087 & 0.026516 & 0.012129 & 0.428377 & 192.256 \\
\hline 8/1/08 3:00 & 2.208 & 0.748 & 0.278 & 124.627 & 1.437 & 0.091 & 0.027735 & 0.012943 & 0.457162 & 205.174 \\
\hline 8/1/08 4:00 & 1.896 & 0.436 & 0.073 & 32.935 & 1.406 & 0.06 & 0.018287 & 0.007117 & 0.251378 & 112.818 \\
\hline 8/1/08 5:00 & 2.055 & 0.595 & 0.158 & 70.805 & 1.413 & 0.067 & 0.020421 & 0.008330 & 0.294224 & 132.048 \\
\hline 8/1/08 6:00 & 2.109 & 0.649 & 0.195 & 87.740 & 1.42 & 0.074 & 0.022554 & 0.009605 & 0.339261 & 152.260 \\
\hline 8/1/08 7:00 & 2.254 & 0.794 & 0.322 & 144.461 & 1.441 & 0.095 & 0.028955 & 0.013776 & 0.486564 & 218.370 \\
\hline 8/1/08 8:00 & 2.231 & 0.771 & 0.299 & 134.324 & 1.433 & 0.087 & 0.026516 & 0.012129 & 0.428377 & 192.256 \\
\hline 8/1/08 9:00 & 2.238 & 0.778 & 0.306 & 137.362 & 1.443 & 0.097 & 0.029564 & 0.014199 & 0.501493 & 225.070 \\
\hline 8/1/08 10:00 & 2.222 & 0.762 & 0.291 & 130.478 & 1.431 & 0.085 & 0.025907 & 0.011728 & 0.414220 & 185.902 \\
\hline 8/1/08 11:00 & 2.203 & 0.743 & 0.273 & 122.576 & 1.431 & 0.085 & 0.025907 & 0.011728 & 0.414220 & 185.902 \\
\hline 8/1/08 12:00 & 2.188 & 0.728 & 0.260 & 116.546 & 1.429 & 0.083 & 0.025297 & 0.011331 & 0.400223 & 179.620 \\
\hline 8/1/08 13:00 & 2.167 & 0.707 & 0.242 & 108.408 & 1.427 & 0.081 & 0.024688 & 0.010940 & 0.386387 & 173.411 \\
\hline
\end{tabular}




\begin{tabular}{|c|c|c|c|c|c|c|c|c|c|c|}
\hline \multirow[b]{3}{*}{ Date Time } & \multicolumn{4}{|c|}{ V-Notch Weir } & \multicolumn{6}{|c|}{ R-Notch Weir } \\
\hline & \multicolumn{2}{|c|}{ Measured Water Level } & \multicolumn{2}{|c|}{$\begin{array}{c}\text { Calculated Rate } \\
\left(\mathrm{ft}^{3} / \mathrm{s}=448.8 \mathrm{gpm}\right)\end{array}$} & \multicolumn{3}{|c|}{ Measured Water Level } & \multicolumn{3}{|c|}{$\begin{array}{c}\text { Calculated Rate } \\
\left(\mathrm{ft}^{3} / \mathrm{s}=448.8 \mathrm{gpm}\right)\end{array}$} \\
\hline & $\begin{array}{c}\text { (ft above } \\
\text { sensor) }\end{array}$ & $\begin{array}{c}\text { (ft above } \\
\text { notch) }\end{array}$ & $\left(\mathrm{ft}^{3} / \mathrm{s}\right)$ & (gpm) & $\begin{array}{l}\text { (ft above } \\
\text { sensor) }\end{array}$ & $\begin{array}{c}\text { (ft above } \\
\text { log) }\end{array}$ & $\begin{array}{c}\text { (m above } \\
\text { log) }\end{array}$ & $\left(\mathrm{m}^{3} / \mathrm{s}\right)$ & $\left(\mathrm{ft}^{3} / \mathrm{s}\right)$ & (gpm) \\
\hline 8/1/08 14:00 & 2.149 & 0.689 & 0.227 & 101.713 & 1.425 & 0.079 & 0.024078 & 0.010553 & 0.372715 & 167.274 \\
\hline 8/1/08 15:00 & 2.129 & 0.669 & 0.211 & 94.572 & 1.425 & 0.079 & 0.024078 & 0.010553 & 0.372715 & 167.274 \\
\hline 8/1/08 16:00 & 2.078 & 0.618 & 0.173 & 77.752 & 1.43 & 0.084 & 0.025602 & 0.011529 & 0.407202 & 182.752 \\
\hline 8/1/08 17:00 & 2.106 & 0.646 & 0.193 & 86.742 & 1.426 & 0.08 & 0.024383 & 0.010746 & 0.379531 & 170.333 \\
\hline 8/1/08 18:00 & 2.097 & 0.637 & 0.187 & 83.788 & 1.426 & 0.08 & 0.024383 & 0.010746 & 0.379531 & 170.333 \\
\hline 8/1/08 19:00 & 2.099 & 0.639 & 0.188 & 84.439 & 1.426 & 0.08 & 0.024383 & 0.010746 & 0.379531 & 170.333 \\
\hline 8/1/08 20:00 & 2.096 & 0.636 & 0.186 & 83.463 & 1.429 & 0.083 & 0.025297 & 0.011331 & 0.400223 & 179.620 \\
\hline 8/1/08 21:00 & 2.128 & 0.668 & 0.210 & 94.223 & 1.431 & 0.085 & 0.025907 & 0.011728 & 0.414220 & 185.902 \\
\hline 8/1/08 22:00 & 2.13 & 0.670 & 0.212 & 94.921 & 1.431 & 0.085 & 0.025907 & 0.011728 & 0.414220 & 185.902 \\
\hline 8/1/08 23:00 & 2.136 & 0.676 & 0.216 & 97.036 & 1.431 & 0.085 & 0.025907 & 0.011728 & 0.414220 & 185.902 \\
\hline 8/2/08 0:00 & 2.141 & 0.681 & 0.220 & 98.819 & 1.431 & 0.085 & 0.025907 & 0.011728 & 0.414220 & 185.902 \\
\hline 8/2/08 1:00 & 2.213 & 0.753 & 0.282 & 126.698 & 1.443 & 0.097 & 0.029564 & 0.014199 & 0.501493 & 225.070 \\
\hline 8/2/08 2:00 & 2.196 & 0.736 & 0.267 & 119.739 & 1.44 & 0.094 & 0.028650 & 0.013566 & 0.479156 & 215.045 \\
\hline 8/2/08 3:00 & 2.184 & 0.724 & 0.256 & 114.969 & 1.437 & 0.091 & 0.027735 & 0.012943 & 0.457162 & 205.174 \\
\hline 8/2/08 4:00 & 2.158 & 0.698 & 0.234 & 105.029 & 1.432 & 0.086 & 0.026212 & 0.011928 & 0.421279 & 189.070 \\
\hline 8/2/08 5:00 & 2.172 & 0.712 & 0.246 & 110.314 & 1.433 & 0.087 & 0.026516 & 0.012129 & 0.428377 & 192.256 \\
\hline 8/2/08 6:00 & 2.16 & 0.700 & 0.236 & 105.774 & 1.426 & 0.08 & 0.024383 & 0.010746 & 0.379531 & 170.333 \\
\hline 8/2/08 7:00 & 2.178 & 0.718 & 0.251 & 112.627 & 1.43 & 0.084 & 0.025602 & 0.011529 & 0.407202 & 182.752 \\
\hline 8/2/08 8:00 & 2.19 & 0.730 & 0.261 & 117.339 & 1.432 & 0.086 & 0.026212 & 0.011928 & 0.421279 & 189.070 \\
\hline 8/2/08 9:00 & 2.193 & 0.733 & 0.264 & 118.536 & 1.435 & 0.089 & 0.027126 & 0.012534 & 0.442691 & 198.680 \\
\hline 8/2/08 10:00 & 2.193 & 0.733 & 0.264 & 118.536 & 1.432 & 0.086 & 0.026212 & 0.011928 & 0.421279 & 189.070 \\
\hline 8/2/08 11:00 & 2.165 & 0.705 & 0.240 & 107.652 & 1.428 & 0.082 & 0.024992 & 0.011135 & 0.393285 & 176.506 \\
\hline 8/2/08 12:00 & 2.164 & 0.704 & 0.239 & 107.275 & 1.428 & 0.082 & 0.024992 & 0.011135 & 0.393285 & 176.506 \\
\hline 8/2/08 13:00 & 2.148 & 0.688 & 0.226 & 101.348 & 1.428 & 0.082 & 0.024992 & 0.011135 & 0.393285 & 176.506 \\
\hline 8/2/08 14:00 & 2.133 & 0.673 & 0.214 & 95.975 & 1.429 & 0.083 & 0.025297 & 0.011331 & 0.400223 & 179.620 \\
\hline
\end{tabular}




\begin{tabular}{|c|c|c|c|c|c|c|c|c|c|c|}
\hline \multirow[b]{3}{*}{ Date Time } & \multicolumn{4}{|c|}{ V-Notch Weir } & \multicolumn{6}{|c|}{ R-Notch Weir } \\
\hline & \multicolumn{2}{|c|}{ Measured Water Level } & \multicolumn{2}{|c|}{$\begin{array}{c}\text { Calculated Rate } \\
\left(\mathrm{ft}^{3} / \mathrm{s}=448.8 \mathrm{gpm}\right)\end{array}$} & \multicolumn{3}{|c|}{ Measured Water Level } & \multicolumn{3}{|c|}{$\begin{array}{c}\begin{array}{c}\text { Calculated Rate } \\
\left(\mathrm{ft}^{3} / \mathrm{s}=448.8 \mathrm{gpm}\right)\end{array} \\
\end{array}$} \\
\hline & $\begin{array}{l}\text { (ft above } \\
\text { sensor) }\end{array}$ & $\begin{array}{c}\text { (ft above } \\
\text { notch) }\end{array}$ & $\left(\mathrm{ft}^{3} / \mathrm{s}\right)$ & (gpm) & $\begin{array}{c}\text { (ft above } \\
\text { sensor) }\end{array}$ & $\begin{array}{c}\text { (ft above } \\
\text { log) }\end{array}$ & $\begin{array}{c}\text { (m above } \\
\text { log) }\end{array}$ & $\left(\mathrm{m}^{3} / \mathrm{s}\right)$ & $\left(\mathrm{ft}^{3} / \mathrm{s}\right)$ & (gpm) \\
\hline 8/2/08 15:00 & 2.12 & 0.660 & 0.204 & 91.459 & 1.427 & 0.081 & 0.024688 & 0.010940 & 0.386387 & 173.411 \\
\hline 8/2/08 16:00 & 2.102 & 0.642 & 0.190 & 85.421 & 1.43 & 0.084 & 0.025602 & 0.011529 & 0.407202 & 182.752 \\
\hline 8/2/08 17:00 & 2.096 & 0.636 & 0.186 & 83.463 & 1.428 & 0.082 & 0.024992 & 0.011135 & 0.393285 & 176.506 \\
\hline 8/2/08 18:00 & 2.086 & 0.626 & 0.179 & 80.260 & 1.426 & 0.08 & 0.024383 & 0.010746 & 0.379531 & 170.333 \\
\hline 8/2/08 19:00 & 2.091 & 0.631 & 0.182 & 81.852 & 1.434 & 0.088 & 0.026821 & 0.012331 & 0.435514 & 195.459 \\
\hline 8/2/08 20:00 & 2.095 & 0.635 & 0.185 & 83.140 & 1.434 & 0.088 & 0.026821 & 0.012331 & 0.435514 & 195.459 \\
\hline 8/2/08 21:00 & 2.104 & 0.644 & 0.192 & 86.080 & 1.434 & 0.088 & 0.026821 & 0.012331 & 0.435514 & 195.459 \\
\hline 8/2/08 22:00 & 2.124 & 0.664 & 0.207 & 92.835 & 1.437 & 0.091 & 0.027735 & 0.012943 & 0.457162 & 205.174 \\
\hline 8/2/08 23:00 & 2.126 & 0.666 & 0.208 & 93.527 & 1.437 & 0.091 & 0.027735 & 0.012943 & 0.457162 & 205.174 \\
\hline 8/3/08 0:00 & 2.154 & 0.694 & 0.231 & 103.547 & 1.444 & 0.098 & 0.029869 & 0.014412 & 0.509013 & 228.445 \\
\hline 8/3/08 1:00 & 2.158 & 0.698 & 0.234 & 105.029 & 1.444 & 0.098 & 0.029869 & 0.014412 & 0.509013 & 228.445 \\
\hline 8/3/08 2:00 & 2.165 & 0.705 & 0.240 & 107.652 & 1.453 & 0.107 & 0.032612 & 0.016375 & 0.578353 & 259.565 \\
\hline 8/3/08 3:00 & 2.176 & 0.716 & 0.249 & 111.853 & 1.45 & 0.104 & 0.031698 & 0.015711 & 0.554913 & 249.045 \\
\hline 8/3/08 4:00 & 2.19 & 0.730 & 0.261 & 117.339 & 1.453 & 0.107 & 0.032612 & 0.016375 & 0.578353 & 259.565 \\
\hline 8/3/08 5:00 & 2.222 & 0.762 & 0.291 & 130.478 & 1.457 & 0.111 & 0.033831 & 0.017274 & 0.610106 & 273.815 \\
\hline 8/3/08 6:00 & 2.161 & 0.701 & 0.237 & 106.148 & 1.445 & 0.099 & 0.030174 & 0.014626 & 0.516571 & 231.837 \\
\hline 8/3/08 7:00 & 2.163 & 0.703 & 0.238 & 106.898 & 1.444 & 0.098 & 0.029869 & 0.014412 & 0.509013 & 228.445 \\
\hline 8/3/08 8:00 & 2.163 & 0.703 & 0.238 & 106.898 & 1.439 & 0.093 & 0.028345 & 0.013358 & 0.471786 & 211.738 \\
\hline 8/3/08 9:00 & 2.17 & 0.710 & 0.244 & 109.549 & 1.441 & 0.095 & 0.028955 & 0.013776 & 0.486564 & 218.370 \\
\hline 8/3/08 10:00 & 2.175 & 0.715 & 0.248 & 111.467 & 1.441 & 0.095 & 0.028955 & 0.013776 & 0.486564 & 218.370 \\
\hline 8/3/08 11:00 & 2.164 & 0.704 & 0.239 & 107.275 & 1.439 & 0.093 & 0.028345 & 0.013358 & 0.471786 & 211.738 \\
\hline 8/3/08 12:00 & 2.139 & 0.679 & 0.219 & 98.103 & 1.437 & 0.091 & 0.027735 & 0.012943 & 0.457162 & 205.174 \\
\hline 8/3/08 13:00 & 2.113 & 0.653 & 0.198 & 89.082 & 1.435 & 0.089 & 0.027126 & 0.012534 & 0.442691 & 198.680 \\
\hline 8/3/08 14:00 & 2.091 & 0.631 & 0.182 & 81.852 & 1.431 & 0.085 & 0.025907 & 0.011728 & 0.414220 & 185.902 \\
\hline 8/3/08 15:00 & 2.068 & 0.608 & 0.166 & 74.683 & 1.434 & 0.088 & 0.026821 & 0.012331 & 0.435514 & 195.459 \\
\hline
\end{tabular}




\begin{tabular}{|c|c|c|c|c|c|c|c|c|c|c|}
\hline \multirow[b]{3}{*}{ Date Time } & \multicolumn{4}{|c|}{ V-Notch Weir } & \multicolumn{6}{|c|}{ R-Notch Weir } \\
\hline & \multicolumn{2}{|c|}{ Measured Water Level } & \multicolumn{2}{|c|}{$\begin{array}{c}\text { Calculated Rate } \\
\left(\mathrm{ft}^{3} / \mathrm{s}=448.8 \mathrm{gpm}\right)\end{array}$} & \multicolumn{3}{|c|}{ Measured Water Level } & \multicolumn{3}{|c|}{$\begin{array}{c}\text { Calculated Rate } \\
\left(\mathrm{ft}^{3} / \mathrm{s}=448.8 \mathrm{gpm}\right)\end{array}$} \\
\hline & $\begin{array}{c}\text { (ft above } \\
\text { sensor) }\end{array}$ & $\begin{array}{c}\text { (ft above } \\
\text { notch) }\end{array}$ & $\left(\mathrm{ft}^{3} / \mathrm{s}\right)$ & (gpm) & $\begin{array}{l}\text { (ft above } \\
\text { sensor) }\end{array}$ & $\begin{array}{c}\text { (ft above } \\
\text { log) }\end{array}$ & $\begin{array}{c}\text { (m above } \\
\text { log) }\end{array}$ & $\left(\mathrm{m}^{3} / \mathrm{s}\right)$ & $\left(\mathrm{ft}^{3} / \mathrm{s}\right)$ & (gpm) \\
\hline 8/3/08 16:00 & 2.032 & 0.572 & 0.143 & 64.244 & 1.43 & 0.084 & 0.025602 & 0.011529 & 0.407202 & 182.752 \\
\hline 8/3/08 17:00 & 2.037 & 0.577 & 0.146 & 65.638 & 1.433 & 0.087 & 0.026516 & 0.012129 & 0.428377 & 192.256 \\
\hline 8/3/08 18:00 & 2.061 & 0.601 & 0.162 & 72.579 & 1.447 & 0.101 & 0.030783 & 0.015057 & 0.531798 & 238.671 \\
\hline 8/3/08 19:00 & 2.118 & 0.658 & 0.202 & 90.776 & 1.429 & 0.083 & 0.025297 & 0.011331 & 0.400223 & 179.620 \\
\hline 8/3/08 20:00 & 2.085 & 0.625 & 0.178 & 79.944 & 1.446 & 0.1 & 0.030479 & 0.014841 & 0.524166 & 235.246 \\
\hline 8/3/08 21:00 & 2.095 & 0.635 & 0.185 & 83.140 & 1.448 & 0.102 & 0.031088 & 0.015274 & 0.539466 & 242.112 \\
\hline 8/3/08 22:00 & 2.108 & 0.648 & 0.195 & 87.406 & 1.448 & 0.102 & 0.031088 & 0.015274 & 0.539466 & 242.112 \\
\hline 8/3/08 23:00 & 2.11 & 0.650 & 0.196 & 88.074 & 1.453 & 0.107 & 0.032612 & 0.016375 & 0.578353 & 259.565 \\
\hline 8/4/08 0:00 & 2.121 & 0.661 & 0.205 & 91.802 & 1.448 & 0.102 & 0.031088 & 0.015274 & 0.539466 & 242.112 \\
\hline 8/4/08 1:00 & 2.165 & 0.705 & 0.240 & 107.652 & 1.455 & 0.109 & 0.033222 & 0.016822 & 0.594159 & 266.658 \\
\hline 8/4/08 2:00 & 2.122 & 0.662 & 0.205 & 92.146 & 1.454 & 0.108 & 0.032917 & 0.016598 & 0.586238 & 263.104 \\
\hline 8/4/08 3:00 & 2.122 & 0.662 & 0.205 & 92.146 & 1.449 & 0.103 & 0.031393 & 0.015492 & 0.547171 & 245.570 \\
\hline 8/4/08 4:00 & 2.128 & 0.668 & 0.210 & 94.223 & 1.451 & 0.105 & 0.032002 & 0.015931 & 0.562690 & 252.535 \\
\hline 8/4/08 5:00 & 2.123 & 0.663 & 0.206 & 92.490 & 1.451 & 0.105 & 0.032002 & 0.015931 & 0.562690 & 252.535 \\
\hline 8/4/08 6:00 & 2.123 & 0.663 & 0.206 & 92.490 & 1.45 & 0.104 & 0.031698 & 0.015711 & 0.554913 & 249.045 \\
\hline 8/4/08 7:00 & 2.135 & 0.675 & 0.215 & 96.681 & 1.447 & 0.101 & 0.030783 & 0.015057 & 0.531798 & 238.671 \\
\hline 8/4/08 8:00 & 2.118 & 0.658 & 0.202 & 90.776 & 1.449 & 0.103 & 0.031393 & 0.015492 & 0.547171 & 245.570 \\
\hline 8/4/08 9:00 & 2.135 & 0.675 & 0.215 & 96.681 & 1.447 & 0.101 & 0.030783 & 0.015057 & 0.531798 & 238.671 \\
\hline 8/4/08 10:00 & 2.128 & 0.668 & 0.210 & 94.223 & 1.449 & 0.103 & 0.031393 & 0.015492 & 0.547171 & 245.570 \\
\hline 8/4/08 11:00 & 2.136 & 0.676 & 0.216 & 97.036 & 1.449 & 0.103 & 0.031393 & 0.015492 & 0.547171 & 245.570 \\
\hline 8/4/08 12:00 & 2.128 & 0.668 & 0.210 & 94.223 & 1.447 & 0.101 & 0.030783 & 0.015057 & 0.531798 & 238.671 \\
\hline 8/4/08 13:00 & 2.11 & 0.650 & 0.196 & 88.074 & 1.445 & 0.099 & 0.030174 & 0.014626 & 0.516571 & 231.837 \\
\hline 8/4/08 14:00 & 2.103 & 0.643 & 0.191 & 85.750 & 1.441 & 0.095 & 0.028955 & 0.013776 & 0.486564 & 218.370 \\
\hline 8/4/08 15:00 & 2.101 & 0.641 & 0.190 & 85.093 & 1.441 & 0.095 & 0.028955 & 0.013776 & 0.486564 & 218.370 \\
\hline 8/4/08 16:00 & 2.088 & 0.628 & 0.180 & 80.895 & 1.437 & 0.091 & 0.027735 & 0.012943 & 0.457162 & 205.174 \\
\hline
\end{tabular}




\begin{tabular}{|c|c|c|c|c|c|c|c|c|c|c|}
\hline \multirow[b]{3}{*}{ Date Time } & \multicolumn{4}{|c|}{ V-Notch Weir } & \multicolumn{6}{|c|}{ R-Notch Weir } \\
\hline & \multicolumn{2}{|c|}{ Measured Water Level } & \multicolumn{2}{|c|}{$\begin{array}{c}\text { Calculated Rate } \\
\left(\mathrm{ft}^{3} / \mathrm{s}=448.8 \mathrm{gpm}\right)\end{array}$} & \multicolumn{3}{|c|}{ Measured Water Level } & \multicolumn{3}{|c|}{$\begin{array}{c}\begin{array}{c}\text { Calculated Rate } \\
\left(\mathrm{ft}^{3} / \mathrm{s}=448.8 \mathrm{gpm}\right)\end{array} \\
\end{array}$} \\
\hline & $\begin{array}{l}\text { (ft above } \\
\text { sensor) }\end{array}$ & $\begin{array}{c}\text { (ft above } \\
\text { notch) }\end{array}$ & $\left(\mathrm{ft}^{3} / \mathrm{s}\right)$ & (gpm) & $\begin{array}{c}\text { (ft above } \\
\text { sensor) }\end{array}$ & $\begin{array}{c}\text { (ft above } \\
\text { log) }\end{array}$ & $\begin{array}{c}\text { (m above } \\
\text { log) }\end{array}$ & $\left(\mathrm{m}^{3} / \mathrm{s}\right)$ & $\left(\mathrm{ft}^{3} / \mathrm{s}\right)$ & (gpm) \\
\hline 8/4/08 17:00 & 2.082 & 0.622 & 0.176 & 79.000 & 1.428 & 0.082 & 0.024992 & 0.011135 & 0.393285 & 176.506 \\
\hline 8/4/08 18:00 & 2.058 & 0.598 & 0.160 & 71.689 & 1.431 & 0.085 & 0.025907 & 0.011728 & 0.414220 & 185.902 \\
\hline 8/4/08 19:00 & 2.046 & 0.586 & 0.152 & 68.192 & 1.431 & 0.085 & 0.025907 & 0.011728 & 0.414220 & 185.902 \\
\hline 8/4/08 20:00 & 2.048 & 0.588 & 0.153 & 68.767 & 1.431 & 0.085 & 0.025907 & 0.011728 & 0.414220 & 185.902 \\
\hline 8/4/08 21:00 & 2.099 & 0.639 & 0.188 & 84.439 & 1.434 & 0.088 & 0.026821 & 0.012331 & 0.435514 & 195.459 \\
\hline 8/4/08 22:00 & 2.079 & 0.619 & 0.174 & 78.063 & 1.441 & 0.095 & 0.028955 & 0.013776 & 0.486564 & 218.370 \\
\hline 8/4/08 23:00 & 2.086 & 0.626 & 0.179 & 80.260 & 1.441 & 0.095 & 0.028955 & 0.013776 & 0.486564 & 218.370 \\
\hline 8/5/08 0:00 & 2.102 & 0.642 & 0.190 & 85.421 & 1.44 & 0.094 & 0.028650 & 0.013566 & 0.479156 & 215.045 \\
\hline 8/5/08 1:00 & 2.113 & 0.653 & 0.198 & 89.082 & 1.438 & 0.092 & 0.028040 & 0.013150 & 0.464455 & 208.447 \\
\hline 8/5/08 2:00 & 2.117 & 0.657 & 0.202 & 90.436 & 1.44 & 0.094 & 0.028650 & 0.013566 & 0.479156 & 215.045 \\
\hline 8/5/08 3:00 & 2.122 & 0.662 & 0.205 & 92.146 & 1.437 & 0.091 & 0.027735 & 0.012943 & 0.457162 & 205.174 \\
\hline 8/5/08 4:00 & 2.14 & 0.680 & 0.219 & 98.461 & 1.437 & 0.091 & 0.027735 & 0.012943 & 0.457162 & 205.174 \\
\hline 8/5/08 5:00 & 2.13 & 0.670 & 0.212 & 94.921 & 1.436 & 0.09 & 0.027431 & 0.012738 & 0.449907 & 201.918 \\
\hline 8/5/08 6:00 & 2.125 & 0.665 & 0.208 & 93.181 & 1.436 & 0.09 & 0.027431 & 0.012738 & 0.449907 & 201.918 \\
\hline 8/5/08 7:00 & 2.128 & 0.668 & 0.210 & 94.223 & 1.435 & 0.089 & 0.027126 & 0.012534 & 0.442691 & 198.680 \\
\hline 8/5/08 8:00 & 2.125 & 0.665 & 0.208 & 93.181 & 1.435 & 0.089 & 0.027126 & 0.012534 & 0.442691 & 198.680 \\
\hline 8/5/08 9:00 & 2.132 & 0.672 & 0.213 & 95.623 & 1.435 & 0.089 & 0.027126 & 0.012534 & 0.442691 & 198.680 \\
\hline 8/5/08 10:00 & 2.128 & 0.668 & 0.210 & 94.223 & 1.438 & 0.092 & 0.028040 & 0.013150 & 0.464455 & 208.447 \\
\hline 8/5/08 11:00 & 2.128 & 0.668 & 0.210 & 94.223 & 1.438 & 0.092 & 0.028040 & 0.013150 & 0.464455 & 208.447 \\
\hline 8/5/08 12:00 & 2.119 & 0.659 & 0.203 & 91.117 & 1.433 & 0.087 & 0.026516 & 0.012129 & 0.428377 & 192.256 \\
\hline 8/5/08 13:00 & 2.106 & 0.646 & 0.193 & 86.742 & 1.433 & 0.087 & 0.026516 & 0.012129 & 0.428377 & 192.256 \\
\hline 8/5/08 14:00 & 2.094 & 0.634 & 0.185 & 82.817 & 1.431 & 0.085 & 0.025907 & 0.011728 & 0.414220 & 185.902 \\
\hline 8/5/08 15:00 & 2.094 & 0.634 & 0.185 & 82.817 & 1.431 & 0.085 & 0.025907 & 0.011728 & 0.414220 & 185.902 \\
\hline 8/5/08 16:00 & 2.088 & 0.628 & 0.180 & 80.895 & 1.429 & 0.083 & 0.025297 & 0.011331 & 0.400223 & 179.620 \\
\hline 8/5/08 17:00 & 2.09 & 0.630 & 0.182 & 81.532 & 1.434 & 0.088 & 0.026821 & 0.012331 & 0.435514 & 195.459 \\
\hline
\end{tabular}




\begin{tabular}{|c|c|c|c|c|c|c|c|c|c|c|}
\hline \multirow[b]{3}{*}{ Date Time } & \multicolumn{4}{|c|}{ V-Notch Weir } & \multicolumn{6}{|c|}{ R-Notch Weir } \\
\hline & \multicolumn{2}{|c|}{ Measured Water Level } & \multicolumn{2}{|c|}{$\begin{array}{c}\text { Calculated Rate } \\
\left(\mathrm{ft}^{3} / \mathrm{s}=448.8 \mathrm{gpm}\right)\end{array}$} & \multicolumn{3}{|c|}{ Measured Water Level } & \multicolumn{3}{|c|}{$\begin{array}{c}\text { Calculated Rate } \\
\left(\mathrm{ft}^{3} / \mathrm{s}=448.8 \mathrm{gpm}\right)\end{array}$} \\
\hline & $\begin{array}{c}\text { (ft above } \\
\text { sensor) }\end{array}$ & $\begin{array}{c}\text { (ft above } \\
\text { notch) }\end{array}$ & $\left(\mathrm{ft}^{3} / \mathrm{s}\right)$ & (gpm) & $\begin{array}{l}\text { (ft above } \\
\text { sensor) }\end{array}$ & $\begin{array}{c}\text { (ft above } \\
\text { log) }\end{array}$ & $\begin{array}{c}\text { (m above } \\
\text { log) }\end{array}$ & $\left(\mathrm{m}^{3} / \mathrm{s}\right)$ & $\left(\mathrm{ft}^{3} / \mathrm{s}\right)$ & (gpm) \\
\hline 8/5/08 18:00 & 2.09 & 0.630 & 0.182 & 81.532 & 1.434 & 0.088 & 0.026821 & 0.012331 & 0.435514 & 195.459 \\
\hline 8/5/08 19:00 & 2.083 & 0.623 & 0.177 & 79.314 & 1.432 & 0.086 & 0.026212 & 0.011928 & 0.421279 & 189.070 \\
\hline 8/5/08 20:00 & 2.083 & 0.623 & 0.177 & 79.314 & 1.434 & 0.088 & 0.026821 & 0.012331 & 0.435514 & 195.459 \\
\hline 8/5/08 21:00 & 2.089 & 0.629 & 0.181 & 81.213 & 1.436 & 0.09 & 0.027431 & 0.012738 & 0.449907 & 201.918 \\
\hline 8/5/08 22:00 & 2.1 & 0.640 & 0.189 & 84.765 & 1.434 & 0.088 & 0.026821 & 0.012331 & 0.435514 & 195.459 \\
\hline 8/5/08 23:00 & 2.098 & 0.638 & 0.187 & 84.113 & 1.436 & 0.09 & 0.027431 & 0.012738 & 0.449907 & 201.918 \\
\hline 8/6/08 0:00 & 2.102 & 0.642 & 0.190 & 85.421 & 1.435 & 0.089 & 0.027126 & 0.012534 & 0.442691 & 198.680 \\
\hline 8/6/08 1:00 & 2.109 & 0.649 & 0.195 & 87.740 & 1.435 & 0.089 & 0.027126 & 0.012534 & 0.442691 & 198.680 \\
\hline 8/6/08 2:00 & 2.115 & 0.655 & 0.200 & 89.757 & 1.434 & 0.088 & 0.026821 & 0.012331 & 0.435514 & 195.459 \\
\hline 8/6/08 3:00 & 2.122 & 0.662 & 0.205 & 92.146 & 1.436 & 0.09 & 0.027431 & 0.012738 & 0.449907 & 201.918 \\
\hline 8/6/08 4:00 & 2.129 & 0.669 & 0.211 & 94.572 & 1.435 & 0.089 & 0.027126 & 0.012534 & 0.442691 & 198.680 \\
\hline 8/6/08 5:00 & 2.143 & 0.683 & 0.222 & 99.538 & 1.435 & 0.089 & 0.027126 & 0.012534 & 0.442691 & 198.680 \\
\hline 8/6/08 6:00 & 2.138 & 0.678 & 0.218 & 97.747 & 1.437 & 0.091 & 0.027735 & 0.012943 & 0.457162 & 205.174 \\
\hline 8/6/08 7:00 & 2.147 & 0.687 & 0.225 & 100.985 & 1.434 & 0.088 & 0.026821 & 0.012331 & 0.435514 & 195.459 \\
\hline 8/6/08 8:00 & 2.15 & 0.690 & 0.227 & 102.078 & 1.434 & 0.088 & 0.026821 & 0.012331 & 0.435514 & 195.459 \\
\hline 8/6/08 9:00 & 2.152 & 0.692 & 0.229 & 102.811 & 1.436 & 0.09 & 0.027431 & 0.012738 & 0.449907 & 201.918 \\
\hline 8/6/08 10:00 & 2.117 & 0.657 & 0.202 & 90.436 & 1.434 & 0.088 & 0.026821 & 0.012331 & 0.435514 & 195.459 \\
\hline 8/6/08 11:00 & 2.122 & 0.662 & 0.205 & 92.146 & 1.439 & 0.093 & 0.028345 & 0.013358 & 0.471786 & 211.738 \\
\hline 8/6/08 12:00 & 2.115 & 0.655 & 0.200 & 89.757 & 1.436 & 0.09 & 0.027431 & 0.012738 & 0.449907 & 201.918 \\
\hline 8/6/08 13:00 & 2.104 & 0.644 & 0.192 & 86.080 & 1.434 & 0.088 & 0.026821 & 0.012331 & 0.435514 & 195.459 \\
\hline 8/6/08 14:00 & 2.106 & 0.646 & 0.193 & 86.742 & 1.432 & 0.086 & 0.026212 & 0.011928 & 0.421279 & 189.070 \\
\hline 8/6/08 15:00 & 2.091 & 0.631 & 0.182 & 81.852 & 1.43 & 0.084 & 0.025602 & 0.011529 & 0.407202 & 182.752 \\
\hline 8/6/08 16:00 & 2.093 & 0.633 & 0.184 & 82.494 & 1.432 & 0.086 & 0.026212 & 0.011928 & 0.421279 & 189.070 \\
\hline 8/6/08 17:00 & 2.096 & 0.636 & 0.186 & 83.463 & 1.435 & 0.089 & 0.027126 & 0.012534 & 0.442691 & 198.680 \\
\hline 8/6/08 18:00 & 2.082 & 0.622 & 0.176 & 79.000 & 1.43 & 0.084 & 0.025602 & 0.011529 & 0.407202 & 182.752 \\
\hline
\end{tabular}




\begin{tabular}{|c|c|c|c|c|c|c|c|c|c|c|}
\hline \multirow[b]{3}{*}{ Date Time } & \multicolumn{4}{|c|}{ V-Notch Weir } & \multicolumn{6}{|c|}{ R-Notch Weir } \\
\hline & \multicolumn{2}{|c|}{ Measured Water Level } & \multicolumn{2}{|c|}{$\begin{array}{c}\text { Calculated Rate } \\
\left(\mathrm{ft}^{3} / \mathrm{s}=448.8 \mathrm{gpm}\right)\end{array}$} & \multicolumn{3}{|c|}{ Measured Water Level } & \multicolumn{3}{|c|}{$\begin{array}{c}\begin{array}{c}\text { Calculated Rate } \\
\left(\mathrm{ft}^{3} / \mathrm{s}=448.8 \mathrm{gpm}\right)\end{array} \\
\end{array}$} \\
\hline & $\begin{array}{l}\text { (ft above } \\
\text { sensor) }\end{array}$ & $\begin{array}{c}\text { (ft above } \\
\text { notch) }\end{array}$ & $\left(\mathrm{ft}^{3} / \mathrm{s}\right)$ & (gpm) & $\begin{array}{c}\text { (ft above } \\
\text { sensor) }\end{array}$ & $\begin{array}{c}\text { (ft above } \\
\text { log) }\end{array}$ & $\begin{array}{c}\text { (m above } \\
\text { log) }\end{array}$ & $\left(\mathrm{m}^{3} / \mathrm{s}\right)$ & $\left(\mathrm{ft}^{3} / \mathrm{s}\right)$ & (gpm) \\
\hline 8/6/08 19:00 & 2.075 & 0.615 & 0.171 & 76.824 & 1.428 & 0.082 & 0.024992 & 0.011135 & 0.393285 & 176.506 \\
\hline 8/6/08 20:00 & 2.082 & 0.622 & 0.176 & 79.000 & 1.431 & 0.085 & 0.025907 & 0.011728 & 0.414220 & 185.902 \\
\hline 8/6/08 21:00 & 2.083 & 0.623 & 0.177 & 79.314 & 1.431 & 0.085 & 0.025907 & 0.011728 & 0.414220 & 185.902 \\
\hline 8/6/08 22:00 & 2.081 & 0.621 & 0.175 & 78.687 & 1.431 & 0.085 & 0.025907 & 0.011728 & 0.414220 & 185.902 \\
\hline 8/6/08 23:00 & 2.094 & 0.634 & 0.185 & 82.817 & 1.433 & 0.087 & 0.026516 & 0.012129 & 0.428377 & 192.256 \\
\hline 8/7/08 0:00 & 2.115 & 0.655 & 0.200 & 89.757 & 1.43 & 0.084 & 0.025602 & 0.011529 & 0.407202 & 182.752 \\
\hline 8/7/08 1:00 & 2.131 & 0.671 & 0.212 & 95.272 & 1.43 & 0.084 & 0.025602 & 0.011529 & 0.407202 & 182.752 \\
\hline 8/7/08 2:00 & 2.134 & 0.674 & 0.215 & 96.328 & 1.432 & 0.086 & 0.026212 & 0.011928 & 0.421279 & 189.070 \\
\hline 8/7/08 3:00 & 2.133 & 0.673 & 0.214 & 95.975 & 1.431 & 0.085 & 0.025907 & 0.011728 & 0.414220 & 185.902 \\
\hline 8/7/08 4:00 & 2.129 & 0.669 & 0.211 & 94.572 & 1.429 & 0.083 & 0.025297 & 0.011331 & 0.400223 & 179.620 \\
\hline 8/7/08 5:00 & 2.128 & 0.668 & 0.210 & 94.223 & 1.428 & 0.082 & 0.024992 & 0.011135 & 0.393285 & 176.506 \\
\hline 8/7/08 6:00 & 2.131 & 0.671 & 0.212 & 95.272 & 1.43 & 0.084 & 0.025602 & 0.011529 & 0.407202 & 182.752 \\
\hline 8/7/08 7:00 & 2.131 & 0.671 & 0.212 & 95.272 & 1.43 & 0.084 & 0.025602 & 0.011529 & 0.407202 & 182.752 \\
\hline 8/7/08 8:00 & 2.135 & 0.675 & 0.215 & 96.681 & 1.43 & 0.084 & 0.025602 & 0.011529 & 0.407202 & 182.752 \\
\hline 8/7/08 9:00 & 2.143 & 0.683 & 0.222 & 99.538 & 1.429 & 0.083 & 0.025297 & 0.011331 & 0.400223 & 179.620 \\
\hline 8/7/08 10:00 & 2.145 & 0.685 & 0.223 & 100.260 & 1.43 & 0.084 & 0.025602 & 0.011529 & 0.407202 & 182.752 \\
\hline 8/7/08 11:00 & 2.139 & 0.679 & 0.219 & 98.103 & 1.43 & 0.084 & 0.025602 & 0.011529 & 0.407202 & 182.752 \\
\hline 8/7/08 12:00 & 2.163 & 0.703 & 0.238 & 106.898 & 1.416 & 0.07 & 0.021335 & 0.008869 & 0.313263 & 140.593 \\
\hline 8/7/08 13:00 & 2.136 & 0.676 & 0.216 & 97.036 & 1.412 & 0.066 & 0.020116 & 0.008153 & 0.287966 & 129.239 \\
\hline 8/7/08 14:00 & 2.111 & 0.651 & 0.197 & 88.409 & 1.407 & 0.061 & 0.018592 & 0.007287 & 0.257360 & 115.503 \\
\hline 8/7/08 15:00 & 2.096 & 0.636 & 0.186 & 83.463 & 1.408 & 0.062 & 0.018897 & 0.007457 & 0.263390 & 118.209 \\
\hline 8/7/08 16:00 & 2.083 & 0.623 & 0.177 & 79.314 & 1.409 & 0.063 & 0.019201 & 0.007629 & 0.269465 & 120.936 \\
\hline 8/7/08 17:00 & 2.081 & 0.621 & 0.175 & 78.687 & 1.407 & 0.061 & 0.018592 & 0.007287 & 0.257360 & 115.503 \\
\hline 8/7/08 18:00 & 2.078 & 0.618 & 0.173 & 77.752 & 1.41 & 0.064 & 0.019506 & 0.007803 & 0.275587 & 123.683 \\
\hline 8/7/08 19:00 & 2.078 & 0.618 & 0.173 & 77.752 & 1.417 & 0.071 & 0.021640 & 0.009052 & 0.319698 & 143.480 \\
\hline
\end{tabular}




\begin{tabular}{|c|c|c|c|c|c|c|c|c|c|c|}
\hline \multirow[b]{3}{*}{ Date Time } & \multicolumn{4}{|c|}{ V-Notch Weir } & \multicolumn{6}{|c|}{ R-Notch Weir } \\
\hline & \multicolumn{2}{|c|}{ Measured Water Level } & \multicolumn{2}{|c|}{$\begin{array}{c}\text { Calculated Rate } \\
\left(\mathrm{ft}^{3} / \mathrm{s}=448.8 \mathrm{gpm}\right)\end{array}$} & \multicolumn{3}{|c|}{ Measured Water Level } & \multicolumn{3}{|c|}{$\begin{array}{c}\text { Calculated Rate } \\
\left(\mathrm{ft}^{3} / \mathrm{s}=448.8 \mathrm{gpm}\right)\end{array}$} \\
\hline & $\begin{array}{c}\text { (ft above } \\
\text { sensor) }\end{array}$ & $\begin{array}{c}\text { (ft above } \\
\text { notch) }\end{array}$ & $\left(\mathrm{ft}^{3} / \mathrm{s}\right)$ & (gpm) & $\begin{array}{l}\text { (ft above } \\
\text { sensor) }\end{array}$ & $\begin{array}{c}\text { (ft above } \\
\text { log) }\end{array}$ & $\begin{array}{c}\text { (m above } \\
\text { log) }\end{array}$ & $\left(\mathrm{m}^{3} / \mathrm{s}\right)$ & $\left(\mathrm{ft}^{3} / \mathrm{s}\right)$ & (gpm) \\
\hline 8/7/08 20:00 & 2.089 & 0.629 & 0.181 & 81.213 & 1.42 & 0.074 & 0.022554 & 0.009605 & 0.339261 & 152.260 \\
\hline 8/7/08 21:00 & 2.099 & 0.639 & 0.188 & 84.439 & 1.422 & 0.076 & 0.023164 & 0.009981 & 0.352516 & 158.209 \\
\hline 8/7/08 22:00 & 2.117 & 0.657 & 0.202 & 90.436 & 1.418 & 0.072 & 0.021945 & 0.009235 & 0.326176 & 146.388 \\
\hline 8/7/08 23:00 & 2.117 & 0.657 & 0.202 & 90.436 & 1.418 & 0.072 & 0.021945 & 0.009235 & 0.326176 & 146.388 \\
\hline 8/8/08 0:00 & 2.13 & 0.670 & 0.212 & 94.921 & 1.417 & 0.071 & 0.021640 & 0.009052 & 0.319698 & 143.480 \\
\hline 8/8/08 1:00 & 2.139 & 0.679 & 0.219 & 98.103 & 1.419 & 0.073 & 0.022249 & 0.009420 & 0.332697 & 149.314 \\
\hline 8/8/08 2:00 & 2.146 & 0.686 & 0.224 & 100.622 & 1.419 & 0.073 & 0.022249 & 0.009420 & 0.332697 & 149.314 \\
\hline 8/8/08 3:00 & 2.15 & 0.690 & 0.227 & 102.078 & 1.421 & 0.075 & 0.022859 & 0.009792 & 0.345867 & 155.225 \\
\hline 8/8/08 4:00 & 2.157 & 0.697 & 0.233 & 104.657 & 1.42 & 0.074 & 0.022554 & 0.009605 & 0.339261 & 152.260 \\
\hline 8/8/08 5:00 & 2.162 & 0.702 & 0.237 & 106.523 & 1.42 & 0.074 & 0.022554 & 0.009605 & 0.339261 & 152.260 \\
\hline 8/8/08 6:00 & 2.164 & 0.704 & 0.239 & 107.275 & 1.419 & 0.073 & 0.022249 & 0.009420 & 0.332697 & 149.314 \\
\hline 8/8/08 7:00 & 2.166 & 0.706 & 0.241 & 108.030 & 1.419 & 0.073 & 0.022249 & 0.009420 & 0.332697 & 149.314 \\
\hline 8/8/08 8:00 & 2.171 & 0.711 & 0.245 & 109.931 & 1.419 & 0.073 & 0.022249 & 0.009420 & 0.332697 & 149.314 \\
\hline 8/8/08 9:00 & 2.171 & 0.711 & 0.245 & 109.931 & 1.419 & 0.073 & 0.022249 & 0.009420 & 0.332697 & 149.314 \\
\hline 8/8/08 10:00 & 2.171 & 0.711 & 0.245 & 109.931 & 1.419 & 0.073 & 0.022249 & 0.009420 & 0.332697 & 149.314 \\
\hline 8/8/08 11:00 & 2.172 & 0.712 & 0.246 & 110.314 & 1.419 & 0.073 & 0.022249 & 0.009420 & 0.332697 & 149.314 \\
\hline 8/8/08 12:00 & 2.149 & 0.689 & 0.227 & 101.713 & 1.414 & 0.068 & 0.020725 & 0.008509 & 0.300526 & 134.876 \\
\hline 8/8/08 13:00 & 2.124 & 0.664 & 0.207 & 92.835 & 1.412 & 0.066 & 0.020116 & 0.008153 & 0.287966 & 129.239 \\
\hline 8/8/08 14:00 & 2.104 & 0.644 & 0.192 & 86.080 & 1.41 & 0.064 & 0.019506 & 0.007803 & 0.275587 & 123.683 \\
\hline 8/8/08 15:00 & 2.093 & 0.633 & 0.184 & 82.494 & 1.411 & 0.065 & 0.019811 & 0.007977 & 0.281754 & 126.451 \\
\hline 8/8/08 16:00 & 2.08 & 0.620 & 0.175 & 78.375 & 1.409 & 0.063 & 0.019201 & 0.007629 & 0.269465 & 120.936 \\
\hline 8/8/08 17:00 & 2.073 & 0.613 & 0.170 & 76.208 & 1.41 & 0.064 & 0.019506 & 0.007803 & 0.275587 & 123.683 \\
\hline 8/8/08 18:00 & 2.059 & 0.599 & 0.160 & 71.985 & 1.41 & 0.064 & 0.019506 & 0.007803 & 0.275587 & 123.683 \\
\hline 8/8/08 19:00 & 2.078 & 0.618 & 0.173 & 77.752 & 1.415 & 0.069 & 0.021030 & 0.008688 & 0.306873 & 137.724 \\
\hline 8/8/08 20:00 & 2.072 & 0.612 & 0.169 & 75.902 & 1.415 & 0.069 & 0.021030 & 0.008688 & 0.306873 & 137.724 \\
\hline
\end{tabular}




\begin{tabular}{|c|c|c|c|c|c|c|c|c|c|c|}
\hline \multirow[b]{3}{*}{ Date Time } & \multicolumn{4}{|c|}{ V-Notch Weir } & \multicolumn{6}{|c|}{ R-Notch Weir } \\
\hline & \multicolumn{2}{|c|}{ Measured Water Level } & \multicolumn{2}{|c|}{$\begin{array}{c}\text { Calculated Rate } \\
\left(\mathrm{ft}^{3} / \mathrm{s}=448.8 \mathrm{gpm}\right)\end{array}$} & \multicolumn{3}{|c|}{ Measured Water Level } & \multicolumn{3}{|c|}{$\begin{array}{c}\begin{array}{c}\text { Calculated Rate } \\
\left(\mathrm{ft}^{3} / \mathrm{s}=448.8 \mathrm{gpm}\right)\end{array} \\
\end{array}$} \\
\hline & $\begin{array}{l}\text { (ft above } \\
\text { sensor) }\end{array}$ & $\begin{array}{c}\text { (ft above } \\
\text { notch) }\end{array}$ & $\left(\mathrm{ft}^{3} / \mathrm{s}\right)$ & (gpm) & $\begin{array}{c}\text { (ft above } \\
\text { sensor) }\end{array}$ & $\begin{array}{c}\text { (ft above } \\
\text { log) }\end{array}$ & $\begin{array}{c}\text { (m above } \\
\text { log) }\end{array}$ & $\left(\mathrm{m}^{3} / \mathrm{s}\right)$ & $\left(\mathrm{ft}^{3} / \mathrm{s}\right)$ & (gpm) \\
\hline 8/8/08 21:00 & 2.088 & 0.628 & 0.180 & 80.895 & 1.418 & 0.072 & 0.021945 & 0.009235 & 0.326176 & 146.388 \\
\hline 8/8/08 22:00 & 2.103 & 0.643 & 0.191 & 85.750 & 1.418 & 0.072 & 0.021945 & 0.009235 & 0.326176 & 146.388 \\
\hline 8/8/08 23:00 & 2.117 & 0.657 & 0.202 & 90.436 & 1.42 & 0.074 & 0.022554 & 0.009605 & 0.339261 & 152.260 \\
\hline 8/9/08 0:00 & 2.126 & 0.666 & 0.208 & 93.527 & 1.425 & 0.079 & 0.024078 & 0.010553 & 0.372715 & 167.274 \\
\hline 8/9/08 1:00 & 2.135 & 0.675 & 0.215 & 96.681 & 1.427 & 0.081 & 0.024688 & 0.010940 & 0.386387 & 173.411 \\
\hline 8/9/08 2:00 & 2.139 & 0.679 & 0.219 & 98.103 & 1.431 & 0.085 & 0.025907 & 0.011728 & 0.414220 & 185.902 \\
\hline 8/9/08 3:00 & 2.136 & 0.676 & 0.216 & 97.036 & 1.423 & 0.077 & 0.023468 & 0.010170 & 0.359208 & 161.212 \\
\hline 8/9/08 4:00 & 2.146 & 0.686 & 0.224 & 100.622 & 1.423 & 0.077 & 0.023468 & 0.010170 & 0.359208 & 161.212 \\
\hline 8/9/08 5:00 & 2.153 & 0.693 & 0.230 & 103.179 & 1.423 & 0.077 & 0.023468 & 0.010170 & 0.359208 & 161.212 \\
\hline 8/9/08 6:00 & 2.157 & 0.697 & 0.233 & 104.657 & 1.427 & 0.081 & 0.024688 & 0.010940 & 0.386387 & 173.411 \\
\hline 8/9/08 7:00 & 2.164 & 0.704 & 0.239 & 107.275 & 1.425 & 0.079 & 0.024078 & 0.010553 & 0.372715 & 167.274 \\
\hline 8/9/08 8:00 & 2.134 & 0.674 & 0.215 & 96.328 & 1.429 & 0.083 & 0.025297 & 0.011331 & 0.400223 & 179.620 \\
\hline 8/9/08 9:00 & 2.166 & 0.706 & 0.241 & 108.030 & 1.424 & 0.078 & 0.023773 & 0.010361 & 0.365940 & 164.234 \\
\hline 8/9/08 10:00 & 2.166 & 0.706 & 0.241 & 108.030 & 1.427 & 0.081 & 0.024688 & 0.010940 & 0.386387 & 173.411 \\
\hline 8/9/08 11:00 & 2.164 & 0.704 & 0.239 & 107.275 & 1.426 & 0.08 & 0.024383 & 0.010746 & 0.379531 & 170.333 \\
\hline 8/9/08 12:00 & 2.164 & 0.704 & 0.239 & 107.275 & 1.424 & 0.078 & 0.023773 & 0.010361 & 0.365940 & 164.234 \\
\hline 8/9/08 13:00 & 2.16 & 0.700 & 0.236 & 105.774 & 1.424 & 0.078 & 0.023773 & 0.010361 & 0.365940 & 164.234 \\
\hline 8/9/08 14:00 & 2.154 & 0.694 & 0.231 & 103.547 & 1.424 & 0.078 & 0.023773 & 0.010361 & 0.365940 & 164.234 \\
\hline 8/9/08 15:00 & 2.136 & 0.676 & 0.216 & 97.036 & 1.424 & 0.078 & 0.023773 & 0.010361 & 0.365940 & 164.234 \\
\hline 8/9/08 16:00 & 2.127 & 0.667 & 0.209 & 93.875 & 1.422 & 0.076 & 0.023164 & 0.009981 & 0.352516 & 158.209 \\
\hline 8/9/08 17:00 & 2.099 & 0.639 & 0.188 & 84.439 & 1.411 & 0.065 & 0.019811 & 0.007977 & 0.281754 & 126.451 \\
\hline 8/9/08 18:00 & 2.081 & 0.621 & 0.175 & 78.687 & 1.409 & 0.063 & 0.019201 & 0.007629 & 0.269465 & 120.936 \\
\hline 8/9/08 19:00 & 2.092 & 0.632 & 0.183 & 82.173 & 1.412 & 0.066 & 0.020116 & 0.008153 & 0.287966 & 129.239 \\
\hline 8/9/08 20:00 & 2.099 & 0.639 & 0.188 & 84.439 & 1.414 & 0.068 & 0.020725 & 0.008509 & 0.300526 & 134.876 \\
\hline 8/9/08 21:00 & 2.107 & 0.647 & 0.194 & 87.074 & 1.417 & 0.071 & 0.021640 & 0.009052 & 0.319698 & 143.480 \\
\hline
\end{tabular}




\begin{tabular}{|c|c|c|c|c|c|c|c|c|c|c|}
\hline \multirow[b]{3}{*}{ Date Time } & \multicolumn{4}{|c|}{ V-Notch Weir } & \multicolumn{6}{|c|}{ R-Notch Weir } \\
\hline & \multicolumn{2}{|c|}{ Measured Water Level } & \multicolumn{2}{|c|}{$\begin{array}{c}\text { Calculated Rate } \\
\left(\mathrm{ft}^{3} / \mathrm{s}=448.8 \mathrm{gpm}\right)\end{array}$} & \multicolumn{3}{|c|}{ Measured Water Level } & \multicolumn{3}{|c|}{$\begin{array}{c}\text { Calculated Rate } \\
\left(\mathrm{ft}^{3} / \mathrm{s}=448.8 \mathrm{gpm}\right)\end{array}$} \\
\hline & $\begin{array}{c}\text { (ft above } \\
\text { sensor) }\end{array}$ & $\begin{array}{c}\text { (ft above } \\
\text { notch) }\end{array}$ & $\left(\mathrm{ft}^{3} / \mathrm{s}\right)$ & (gpm) & $\begin{array}{l}\text { (ft above } \\
\text { sensor) }\end{array}$ & $\begin{array}{c}\text { (ft above } \\
\text { log) }\end{array}$ & $\begin{array}{c}\text { (m above } \\
\text { log) }\end{array}$ & $\left(\mathrm{m}^{3} / \mathrm{s}\right)$ & $\left(\mathrm{ft}^{3} / \mathrm{s}\right)$ & (gpm) \\
\hline 8/9/08 22:00 & 2.119 & 0.659 & 0.203 & 91.117 & 1.417 & 0.071 & 0.021640 & 0.009052 & 0.319698 & 143.480 \\
\hline 8/9/08 23:00 & 2.125 & 0.665 & 0.208 & 93.181 & 1.416 & 0.07 & 0.021335 & 0.008869 & 0.313263 & 140.593 \\
\hline 8/10/08 0:00 & 2.129 & 0.669 & 0.211 & 94.572 & 1.418 & 0.072 & 0.021945 & 0.009235 & 0.326176 & 146.388 \\
\hline 8/10/08 1:00 & 2.139 & 0.679 & 0.219 & 98.103 & 1.418 & 0.072 & 0.021945 & 0.009235 & 0.326176 & 146.388 \\
\hline 8/10/08 2:00 & 2.15 & 0.690 & 0.227 & 102.078 & 1.42 & 0.074 & 0.022554 & 0.009605 & 0.339261 & 152.260 \\
\hline 8/10/08 3:00 & 2.157 & 0.697 & 0.233 & 104.657 & 1.422 & 0.076 & 0.023164 & 0.009981 & 0.352516 & 158.209 \\
\hline 8/10/08 4:00 & 2.178 & 0.718 & 0.251 & 112.627 & 1.421 & 0.075 & 0.022859 & 0.009792 & 0.345867 & 155.225 \\
\hline 8/10/08 5:00 & 2.175 & 0.715 & 0.248 & 111.467 & 1.421 & 0.075 & 0.022859 & 0.009792 & 0.345867 & 155.225 \\
\hline 8/10/08 6:00 & 2.178 & 0.718 & 0.251 & 112.627 & 1.422 & 0.076 & 0.023164 & 0.009981 & 0.352516 & 158.209 \\
\hline 8/10/08 7:00 & 2.171 & 0.711 & 0.245 & 109.931 & 1.42 & 0.074 & 0.022554 & 0.009605 & 0.339261 & 152.260 \\
\hline 8/10/08 8:00 & 2.168 & 0.708 & 0.242 & 108.788 & 1.417 & 0.071 & 0.021640 & 0.009052 & 0.319698 & 143.480 \\
\hline 8/10/08 9:00 & 2.168 & 0.708 & 0.242 & 108.788 & 1.419 & 0.073 & 0.022249 & 0.009420 & 0.332697 & 149.314 \\
\hline 8/10/08 10:00 & 2.173 & 0.713 & 0.247 & 110.697 & 1.42 & 0.074 & 0.022554 & 0.009605 & 0.339261 & 152.260 \\
\hline 8/10/08 11:00 & 2.167 & 0.707 & 0.242 & 108.408 & 1.42 & 0.074 & 0.022554 & 0.009605 & 0.339261 & 152.260 \\
\hline 8/10/08 12:00 & 2.151 & 0.691 & 0.228 & 102.444 & 1.418 & 0.072 & 0.021945 & 0.009235 & 0.326176 & 146.388 \\
\hline 8/10/08 13:00 & 2.133 & 0.673 & 0.214 & 95.975 & 1.416 & 0.07 & 0.021335 & 0.008869 & 0.313263 & 140.593 \\
\hline 8/10/08 14:00 & 2.111 & 0.651 & 0.197 & 88.409 & 1.414 & 0.068 & 0.020725 & 0.008509 & 0.300526 & 134.876 \\
\hline 8/10/08 15:00 & 2.104 & 0.644 & 0.192 & 86.080 & 1.412 & 0.066 & 0.020116 & 0.008153 & 0.287966 & 129.239 \\
\hline 8/10/08 16:00 & 2.091 & 0.631 & 0.182 & 81.852 & 1.415 & 0.069 & 0.021030 & 0.008688 & 0.306873 & 137.724 \\
\hline 8/10/08 17:00 & 2.089 & 0.629 & 0.181 & 81.213 & 1.418 & 0.072 & 0.021945 & 0.009235 & 0.326176 & 146.388 \\
\hline 8/10/08 18:00 & 2.121 & 0.661 & 0.205 & 91.802 & 1.418 & 0.072 & 0.021945 & 0.009235 & 0.326176 & 146.388 \\
\hline 8/10/08 19:00 & 2.13 & 0.670 & 0.212 & 94.921 & 1.421 & 0.075 & 0.022859 & 0.009792 & 0.345867 & 155.225 \\
\hline 8/10/08 20:00 & 2.134 & 0.674 & 0.215 & 96.328 & 1.423 & 0.077 & 0.023468 & 0.010170 & 0.359208 & 161.212 \\
\hline 8/10/08 21:00 & 2.155 & 0.695 & 0.232 & 103.916 & 1.426 & 0.08 & 0.024383 & 0.010746 & 0.379531 & 170.333 \\
\hline 8/10/08 22:00 & 2.159 & 0.699 & 0.235 & 105.401 & 1.426 & 0.08 & 0.024383 & 0.010746 & 0.379531 & 170.333 \\
\hline
\end{tabular}




\begin{tabular}{|c|c|c|c|c|c|c|c|c|c|c|}
\hline \multirow[b]{3}{*}{ Date Time } & \multicolumn{4}{|c|}{ V-Notch Weir } & \multicolumn{6}{|c|}{ R-Notch Weir } \\
\hline & \multicolumn{2}{|c|}{ Measured Water Level } & \multicolumn{2}{|c|}{$\begin{array}{c}\text { Calculated Rate } \\
\left(\mathrm{ft}^{3} / \mathrm{s}=448.8 \mathrm{gpm}\right)\end{array}$} & \multicolumn{3}{|c|}{ Measured Water Level } & \multicolumn{3}{|c|}{$\begin{array}{c}\text { Calculated Rate } \\
\left(\mathrm{ft}^{3} / \mathrm{s}=448.8 \mathrm{gpm}\right)\end{array}$} \\
\hline & $\begin{array}{c}\text { (ft above } \\
\text { sensor) }\end{array}$ & $\begin{array}{c}\text { (ft above } \\
\text { notch) }\end{array}$ & $\left(\mathrm{ft}^{3} / \mathrm{s}\right)$ & (gpm) & $\begin{array}{l}\text { (ft above } \\
\text { sensor) }\end{array}$ & $\begin{array}{c}\text { (ft above } \\
\text { log) }\end{array}$ & $\begin{array}{c}\text { (m above } \\
\text { log) }\end{array}$ & $\left(\mathrm{m}^{3} / \mathrm{s}\right)$ & $\left(\mathrm{ft}^{3} / \mathrm{s}\right)$ & (gpm) \\
\hline 8/10/08 23:00 & 2.161 & 0.701 & 0.237 & 106.148 & 1.423 & 0.077 & 0.023468 & 0.010170 & 0.359208 & 161.212 \\
\hline 8/11/08 0:00 & 2.165 & 0.705 & 0.240 & 107.652 & 1.426 & 0.08 & 0.024383 & 0.010746 & 0.379531 & 170.333 \\
\hline 8/11/08 1:00 & 2.162 & 0.702 & 0.237 & 106.523 & 1.425 & 0.079 & 0.024078 & 0.010553 & 0.372715 & 167.274 \\
\hline 8/11/08 2:00 & 2.158 & 0.698 & 0.234 & 105.029 & 1.425 & 0.079 & 0.024078 & 0.010553 & 0.372715 & 167.274 \\
\hline 8/11/08 3:00 & 2.162 & 0.702 & 0.237 & 106.523 & 1.427 & 0.081 & 0.024688 & 0.010940 & 0.386387 & 173.411 \\
\hline 8/11/08 4:00 & 2.174 & 0.714 & 0.248 & 111.082 & 1.427 & 0.081 & 0.024688 & 0.010940 & 0.386387 & 173.411 \\
\hline 8/11/08 5:00 & 2.171 & 0.711 & 0.245 & 109.931 & 1.426 & 0.08 & 0.024383 & 0.010746 & 0.379531 & 170.333 \\
\hline 8/11/08 6:00 & 2.173 & 0.713 & 0.247 & 110.697 & 1.426 & 0.08 & 0.024383 & 0.010746 & 0.379531 & 170.333 \\
\hline 8/11/08 7:00 & 2.183 & 0.723 & 0.255 & 114.576 & 1.426 & 0.08 & 0.024383 & 0.010746 & 0.379531 & 170.333 \\
\hline 8/11/08 8:00 & 2.178 & 0.718 & 0.251 & 112.627 & 1.426 & 0.08 & 0.024383 & 0.010746 & 0.379531 & 170.333 \\
\hline 8/11/08 9:00 & 2.18 & 0.720 & 0.253 & 113.404 & 1.426 & 0.08 & 0.024383 & 0.010746 & 0.379531 & 170.333 \\
\hline 8/11/08 10:00 & 2.174 & 0.714 & 0.248 & 111.082 & 1.423 & 0.077 & 0.023468 & 0.010170 & 0.359208 & 161.212 \\
\hline 8/11/08 11:00 & 2.165 & 0.705 & 0.240 & 107.652 & 1.423 & 0.077 & 0.023468 & 0.010170 & 0.359208 & 161.212 \\
\hline 8/11/08 12:00 & 2.145 & 0.685 & 0.223 & 100.260 & 1.428 & 0.082 & 0.024992 & 0.011135 & 0.393285 & 176.506 \\
\hline 8/11/08 13:00 & 2.128 & 0.668 & 0.210 & 94.223 & 1.426 & 0.08 & 0.024383 & 0.010746 & 0.379531 & 170.333 \\
\hline 8/11/08 14:00 & 2.132 & 0.672 & 0.213 & 95.623 & 1.419 & 0.073 & 0.022249 & 0.009420 & 0.332697 & 149.314 \\
\hline 8/11/08 15:00 & 2.123 & 0.663 & 0.206 & 92.490 & 1.422 & 0.076 & 0.023164 & 0.009981 & 0.352516 & 158.209 \\
\hline 8/11/08 16:00 & 2.125 & 0.665 & 0.208 & 93.181 & 1.423 & 0.077 & 0.023468 & 0.010170 & 0.359208 & 161.212 \\
\hline 8/11/08 17:00 & 2.118 & 0.658 & 0.202 & 90.776 & 1.423 & 0.077 & 0.023468 & 0.010170 & 0.359208 & 161.212 \\
\hline 8/11/08 18:00 & 2.113 & 0.653 & 0.198 & 89.082 & 1.423 & 0.077 & 0.023468 & 0.010170 & 0.359208 & 161.212 \\
\hline 8/11/08 19:00 & 2.115 & 0.655 & 0.200 & 89.757 & 1.423 & 0.077 & 0.023468 & 0.010170 & 0.359208 & 161.212 \\
\hline 8/11/08 20:00 & 2.131 & 0.671 & 0.212 & 95.272 & 1.426 & 0.08 & 0.024383 & 0.010746 & 0.379531 & 170.333 \\
\hline 8/11/08 21:00 & 2.131 & 0.671 & 0.212 & 95.272 & 1.426 & 0.08 & 0.024383 & 0.010746 & 0.379531 & 170.333 \\
\hline 8/11/08 22:00 & 2.14 & 0.680 & 0.219 & 98.461 & 1.426 & 0.08 & 0.024383 & 0.010746 & 0.379531 & 170.333 \\
\hline 8/11/08 23:00 & 2.153 & 0.693 & 0.230 & 103.179 & 1.428 & 0.082 & 0.024992 & 0.011135 & 0.393285 & 176.506 \\
\hline
\end{tabular}




\begin{tabular}{|c|c|c|c|c|c|c|c|c|c|c|}
\hline \multirow[b]{3}{*}{ Date Time } & \multicolumn{4}{|c|}{ V-Notch Weir } & \multicolumn{6}{|c|}{ R-Notch Weir } \\
\hline & \multicolumn{2}{|c|}{ Measured Water Level } & \multicolumn{2}{|c|}{$\begin{array}{c}\text { Calculated Rate } \\
\left(\mathrm{ft}^{3} / \mathrm{s}=448.8 \mathrm{gpm}\right)\end{array}$} & \multicolumn{3}{|c|}{ Measured Water Level } & \multicolumn{3}{|c|}{$\begin{array}{c}\text { Calculated Rate } \\
\left(\mathrm{ft}^{3} / \mathrm{s}=448.8 \mathrm{gpm}\right)\end{array}$} \\
\hline & $\begin{array}{c}\text { (ft above } \\
\text { sensor) }\end{array}$ & $\begin{array}{c}\text { (ft above } \\
\text { notch) }\end{array}$ & $\left(\mathrm{ft}^{3} / \mathrm{s}\right)$ & (gpm) & $\begin{array}{l}\text { (ft above } \\
\text { sensor) }\end{array}$ & $\begin{array}{c}\text { (ft above } \\
\text { log) }\end{array}$ & $\begin{array}{c}\text { (m above } \\
\text { log) }\end{array}$ & $\left(\mathrm{m}^{3} / \mathrm{s}\right)$ & $\left(\mathrm{ft}^{3} / \mathrm{s}\right)$ & (gpm) \\
\hline 8/12/08 0:00 & 2.155 & 0.695 & 0.232 & 103.916 & 1.426 & 0.08 & 0.024383 & 0.010746 & 0.379531 & 170.333 \\
\hline 8/12/08 1:00 & 2.212 & 0.752 & 0.281 & 126.282 & 1.435 & 0.089 & 0.027126 & 0.012534 & 0.442691 & 198.680 \\
\hline 8/12/08 2:00 & 2.418 & 0.958 & 0.513 & 230.028 & 1.465 & 0.119 & 0.036269 & 0.019119 & 0.675284 & 303.068 \\
\hline 8/12/08 3:00 & 2.357 & 0.897 & 0.435 & 195.412 & 1.458 & 0.112 & 0.034136 & 0.017501 & 0.618132 & 277.418 \\
\hline 8/12/08 4:00 & 2.289 & 0.829 & 0.358 & 160.744 & 1.443 & 0.097 & 0.029564 & 0.014199 & 0.501493 & 225.070 \\
\hline 8/12/08 5:00 & 2.246 & 0.786 & 0.314 & 140.885 & 1.436 & 0.09 & 0.027431 & 0.012738 & 0.449907 & 201.918 \\
\hline 8/12/08 6:00 & 2.227 & 0.767 & 0.295 & 132.606 & 1.433 & 0.087 & 0.026516 & 0.012129 & 0.428377 & 192.256 \\
\hline 8/12/08 7:00 & 2.22 & 0.760 & 0.289 & 129.632 & 1.431 & 0.085 & 0.025907 & 0.011728 & 0.414220 & 185.902 \\
\hline 8/12/08 8:00 & 2.206 & 0.746 & 0.276 & 123.804 & 1.428 & 0.082 & 0.024992 & 0.011135 & 0.393285 & 176.506 \\
\hline 8/12/08 9:00 & 2.202 & 0.742 & 0.272 & 122.168 & 1.428 & 0.082 & 0.024992 & 0.011135 & 0.393285 & 176.506 \\
\hline 8/12/08 10:00 & 2.195 & 0.735 & 0.266 & 119.337 & 1.425 & 0.079 & 0.024078 & 0.010553 & 0.372715 & 167.274 \\
\hline 8/12/08 11:00 & 2.191 & 0.731 & 0.262 & 117.737 & 1.426 & 0.08 & 0.024383 & 0.010746 & 0.379531 & 170.333 \\
\hline 8/12/08 12:00 & 2.175 & 0.715 & 0.248 & 111.467 & 1.421 & 0.075 & 0.022859 & 0.009792 & 0.345867 & 155.225 \\
\hline 8/12/08 13:00 & 2.151 & 0.691 & 0.228 & 102.444 & 1.419 & 0.073 & 0.022249 & 0.009420 & 0.332697 & 149.314 \\
\hline 8/12/08 14:00 & 2.133 & 0.673 & 0.214 & 95.975 & 1.419 & 0.073 & 0.022249 & 0.009420 & 0.332697 & 149.314 \\
\hline 8/12/08 15:00 & 2.132 & 0.672 & 0.213 & 95.623 & 1.42 & 0.074 & 0.022554 & 0.009605 & 0.339261 & 152.260 \\
\hline 8/12/08 16:00 & 2.114 & 0.654 & 0.199 & 89.419 & 1.418 & 0.072 & 0.021945 & 0.009235 & 0.326176 & 146.388 \\
\hline 8/12/08 17:00 & 2.103 & 0.643 & 0.191 & 85.750 & 1.419 & 0.073 & 0.022249 & 0.009420 & 0.332697 & 149.314 \\
\hline 8/12/08 18:00 & 2.095 & 0.635 & 0.185 & 83.140 & 1.417 & 0.071 & 0.021640 & 0.009052 & 0.319698 & 143.480 \\
\hline 8/12/08 19:00 & 2.099 & 0.639 & 0.188 & 84.439 & 1.422 & 0.076 & 0.023164 & 0.009981 & 0.352516 & 158.209 \\
\hline 8/12/08 20:00 & 2.112 & 0.652 & 0.198 & 88.745 & 1.425 & 0.079 & 0.024078 & 0.010553 & 0.372715 & 167.274 \\
\hline 8/12/08 21:00 & 2.118 & 0.658 & 0.202 & 90.776 & 1.425 & 0.079 & 0.024078 & 0.010553 & 0.372715 & 167.274 \\
\hline 8/12/08 22:00 & 2.129 & 0.669 & 0.211 & 94.572 & 1.425 & 0.079 & 0.024078 & 0.010553 & 0.372715 & 167.274 \\
\hline 8/12/08 23:00 & 2.133 & 0.673 & 0.214 & 95.975 & 1.425 & 0.079 & 0.024078 & 0.010553 & 0.372715 & 167.274 \\
\hline 8/13/08 0:00 & 2.135 & 0.675 & 0.215 & 96.681 & 1.425 & 0.079 & 0.024078 & 0.010553 & 0.372715 & 167.274 \\
\hline
\end{tabular}




\begin{tabular}{|c|c|c|c|c|c|c|c|c|c|c|}
\hline \multirow[b]{3}{*}{ Date Time } & \multicolumn{4}{|c|}{ V-Notch Weir } & \multicolumn{6}{|c|}{ R-Notch Weir } \\
\hline & \multicolumn{2}{|c|}{ Measured Water Level } & \multicolumn{2}{|c|}{$\begin{array}{c}\text { Calculated Rate } \\
\left(\mathrm{ft}^{3} / \mathrm{s}=448.8 \mathrm{gpm}\right)\end{array}$} & \multicolumn{3}{|c|}{ Measured Water Level } & \multicolumn{3}{|c|}{$\begin{array}{c}\text { Calculated Rate } \\
\left(\mathrm{ft}^{3} / \mathrm{s}=448.8 \mathrm{gpm}\right)\end{array}$} \\
\hline & $\begin{array}{c}\text { (ft above } \\
\text { sensor) }\end{array}$ & $\begin{array}{c}\text { (ft above } \\
\text { notch) }\end{array}$ & $\left(\mathrm{ft}^{3} / \mathrm{s}\right)$ & (gpm) & $\begin{array}{l}\text { (ft above } \\
\text { sensor) }\end{array}$ & $\begin{array}{c}\text { (ft above } \\
\text { log) }\end{array}$ & $\begin{array}{c}\text { (m above } \\
\text { log) }\end{array}$ & $\left(\mathrm{m}^{3} / \mathrm{s}\right)$ & $\left(\mathrm{ft}^{3} / \mathrm{s}\right)$ & (gpm) \\
\hline 8/13/08 1:00 & 2.148 & 0.688 & 0.226 & 101.348 & 1.427 & 0.081 & 0.024688 & 0.010940 & 0.386387 & 173.411 \\
\hline 8/13/08 2:00 & 2.155 & 0.695 & 0.232 & 103.916 & 1.426 & 0.08 & 0.024383 & 0.010746 & 0.379531 & 170.333 \\
\hline 8/13/08 3:00 & 2.155 & 0.695 & 0.232 & 103.916 & 1.423 & 0.077 & 0.023468 & 0.010170 & 0.359208 & 161.212 \\
\hline 8/13/08 4:00 & 2.162 & 0.702 & 0.237 & 106.523 & 1.425 & 0.079 & 0.024078 & 0.010553 & 0.372715 & 167.274 \\
\hline 8/13/08 5:00 & 2.176 & 0.716 & 0.249 & 111.853 & 1.427 & 0.081 & 0.024688 & 0.010940 & 0.386387 & 173.411 \\
\hline 8/13/08 6:00 & 2.169 & 0.709 & 0.243 & 109.168 & 1.422 & 0.076 & 0.023164 & 0.009981 & 0.352516 & 158.209 \\
\hline 8/13/08 7:00 & 2.173 & 0.713 & 0.247 & 110.697 & 1.421 & 0.075 & 0.022859 & 0.009792 & 0.345867 & 155.225 \\
\hline 8/13/08 8:00 & 2.173 & 0.713 & 0.247 & 110.697 & 1.421 & 0.075 & 0.022859 & 0.009792 & 0.345867 & 155.225 \\
\hline 8/13/08 9:00 & 2.173 & 0.713 & 0.247 & 110.697 & 1.421 & 0.075 & 0.022859 & 0.009792 & 0.345867 & 155.225 \\
\hline 8/13/08 10:00 & 2.174 & 0.714 & 0.248 & 111.082 & 1.423 & 0.077 & 0.023468 & 0.010170 & 0.359208 & 161.212 \\
\hline 8/13/08 11:00 & 2.174 & 0.714 & 0.248 & 111.082 & 1.421 & 0.075 & 0.022859 & 0.009792 & 0.345867 & 155.225 \\
\hline 8/13/08 12:00 & 2.156 & 0.696 & 0.232 & 104.286 & 1.421 & 0.075 & 0.022859 & 0.009792 & 0.345867 & 155.225 \\
\hline 8/13/08 13:00 & 2.14 & 0.680 & 0.219 & 98.461 & 1.419 & 0.073 & 0.022249 & 0.009420 & 0.332697 & 149.314 \\
\hline 8/13/08 14:00 & 2.132 & 0.672 & 0.213 & 95.623 & 1.419 & 0.073 & 0.022249 & 0.009420 & 0.332697 & 149.314 \\
\hline 8/13/08 15:00 & 2.112 & 0.652 & 0.198 & 88.745 & 1.417 & 0.071 & 0.021640 & 0.009052 & 0.319698 & 143.480 \\
\hline 8/13/08 16:00 & 2.102 & 0.642 & 0.190 & 85.421 & 1.418 & 0.072 & 0.021945 & 0.009235 & 0.326176 & 146.388 \\
\hline 8/13/08 17:00 & 2.092 & 0.632 & 0.183 & 82.173 & 1.416 & 0.07 & 0.021335 & 0.008869 & 0.313263 & 140.593 \\
\hline 8/13/08 18:00 & 2.085 & 0.625 & 0.178 & 79.944 & 1.417 & 0.071 & 0.021640 & 0.009052 & 0.319698 & 143.480 \\
\hline 8/13/08 19:00 & 2.08 & 0.620 & 0.175 & 78.375 & 1.417 & 0.071 & 0.021640 & 0.009052 & 0.319698 & 143.480 \\
\hline 8/13/08 20:00 & 2.091 & 0.631 & 0.182 & 81.852 & 1.42 & 0.074 & 0.022554 & 0.009605 & 0.339261 & 152.260 \\
\hline 8/13/08 21:00 & 2.09 & 0.630 & 0.182 & 81.532 & 1.42 & 0.074 & 0.022554 & 0.009605 & 0.339261 & 152.260 \\
\hline 8/13/08 22:00 & 2.094 & 0.634 & 0.185 & 82.817 & 1.42 & 0.074 & 0.022554 & 0.009605 & 0.339261 & 152.260 \\
\hline 8/13/08 23:00 & 2.096 & 0.636 & 0.186 & 83.463 & 1.42 & 0.074 & 0.022554 & 0.009605 & 0.339261 & 152.260 \\
\hline 8/14/08 0:00 & 2.119 & 0.659 & 0.203 & 91.117 & 1.422 & 0.076 & 0.023164 & 0.009981 & 0.352516 & 158.209 \\
\hline 8/14/08 1:00 & 2.132 & 0.672 & 0.213 & 95.623 & 1.422 & 0.076 & 0.023164 & 0.009981 & 0.352516 & 158.209 \\
\hline
\end{tabular}




\begin{tabular}{|c|c|c|c|c|c|c|c|c|c|c|}
\hline \multirow[b]{3}{*}{ Date Time } & \multicolumn{4}{|c|}{ V-Notch Weir } & \multicolumn{6}{|c|}{ R-Notch Weir } \\
\hline & \multicolumn{2}{|c|}{ Measured Water Level } & \multicolumn{2}{|c|}{$\begin{array}{l}\text { Calculated Rate } \\
\left(\mathrm{ft}^{3} / \mathrm{s}=448.8 \mathrm{gpm}\right)\end{array}$} & \multicolumn{3}{|c|}{ Measured Water Level } & \multicolumn{3}{|c|}{$\begin{array}{c}\text { Calculated Rate } \\
\left(\mathrm{ft}^{3} / \mathrm{s}=448.8 \mathrm{gpm}\right)\end{array}$} \\
\hline & $\begin{array}{c}\text { (ft above } \\
\text { sensor) }\end{array}$ & $\begin{array}{c}\text { (ft above } \\
\text { notch) }\end{array}$ & $\left(\mathrm{ft}^{3} / \mathrm{s}\right)$ & (gpm) & $\begin{array}{l}\text { (ft above } \\
\text { sensor) }\end{array}$ & $\begin{array}{c}\text { (ft above } \\
\text { log) }\end{array}$ & $\begin{array}{c}\text { (m above } \\
\text { log) }\end{array}$ & $\left(\mathrm{m}^{3} / \mathrm{s}\right)$ & $\left(\mathrm{ft}^{3} / \mathrm{s}\right)$ & (gpm) \\
\hline 8/14/08 2:00 & 2.141 & 0.681 & 0.220 & 98.819 & 1.424 & 0.078 & 0.023773 & 0.010361 & 0.365940 & 164.234 \\
\hline 8/14/08 3:00 & 2.146 & 0.686 & 0.224 & 100.622 & 1.423 & 0.077 & 0.023468 & 0.010170 & 0.359208 & 161.212 \\
\hline 8/14/08 4:00 & 2.153 & 0.693 & 0.230 & 103.179 & 1.423 & 0.077 & 0.023468 & 0.010170 & 0.359208 & 161.212 \\
\hline 8/14/08 5:00 & 2.155 & 0.695 & 0.232 & 103.916 & 1.425 & 0.079 & 0.024078 & 0.010553 & 0.372715 & 167.274 \\
\hline 8/14/08 6:00 & 2.157 & 0.697 & 0.233 & 104.657 & 1.424 & 0.078 & 0.023773 & 0.010361 & 0.365940 & 164.234 \\
\hline 8/14/08 7:00 & 2.157 & 0.697 & 0.233 & 104.657 & 1.424 & 0.078 & 0.023773 & 0.010361 & 0.365940 & 164.234 \\
\hline 8/14/08 8:00 & 2.154 & 0.694 & 0.231 & 103.547 & 1.421 & 0.075 & 0.022859 & 0.009792 & 0.345867 & 155.225 \\
\hline 8/14/08 9:00 & 2.159 & 0.699 & 0.235 & 105.401 & 1.423 & 0.077 & 0.023468 & 0.010170 & 0.359208 & 161.212 \\
\hline 8/14/08 10:00 & 2.162 & 0.702 & 0.237 & 106.523 & 1.421 & 0.075 & 0.022859 & 0.009792 & 0.345867 & 155.225 \\
\hline 8/14/08 11:00 & 2.157 & 0.697 & 0.233 & 104.657 & 1.421 & 0.075 & 0.022859 & 0.009792 & 0.345867 & 155.225 \\
\hline 8/14/08 12:00 & 2.16 & 0.700 & 0.236 & 105.774 & 1.421 & 0.075 & 0.022859 & 0.009792 & 0.345867 & 155.225 \\
\hline 8/14/08 13:00 & 2.151 & 0.691 & 0.228 & 102.444 & 1.421 & 0.075 & 0.022859 & 0.009792 & 0.345867 & 155.225 \\
\hline 8/14/08 14:00 & 2.14 & 0.680 & 0.219 & 98.461 & 1.419 & 0.073 & 0.022249 & 0.009420 & 0.332697 & 149.314 \\
\hline 8/14/08 15:00 & 2.116 & 0.656 & 0.201 & 90.096 & 1.427 & 0.081 & 0.024688 & 0.010940 & 0.386387 & 173.411 \\
\hline 8/14/08 16:00 & 2.105 & 0.645 & 0.193 & 86.410 & 1.425 & 0.079 & 0.024078 & 0.010553 & 0.372715 & 167.274 \\
\hline 8/14/08 17:00 & 2.098 & 0.638 & 0.187 & 84.113 & 1.413 & 0.067 & 0.020421 & 0.008330 & 0.294224 & 132.048 \\
\hline 8/14/08 18:00 & 2.089 & 0.629 & 0.181 & 81.213 & 1.414 & 0.068 & 0.020725 & 0.008509 & 0.300526 & 134.876 \\
\hline 8/14/08 19:00 & 2.088 & 0.628 & 0.180 & 80.895 & 1.414 & 0.068 & 0.020725 & 0.008509 & 0.300526 & 134.876 \\
\hline 8/14/08 20:00 & 2.092 & 0.632 & 0.183 & 82.173 & 1.417 & 0.071 & 0.021640 & 0.009052 & 0.319698 & 143.480 \\
\hline 8/14/08 21:00 & 2.101 & 0.641 & 0.190 & 85.093 & 1.417 & 0.071 & 0.021640 & 0.009052 & 0.319698 & 143.480 \\
\hline 8/14/08 22:00 & 2.105 & 0.645 & 0.193 & 86.410 & 1.417 & 0.071 & 0.021640 & 0.009052 & 0.319698 & 143.480 \\
\hline 8/14/08 23:00 & 2.111 & 0.651 & 0.197 & 88.409 & 1.419 & 0.073 & 0.022249 & 0.009420 & 0.332697 & 149.314 \\
\hline 8/15/08 0:00 & 2.123 & 0.663 & 0.206 & 92.490 & 1.419 & 0.073 & 0.022249 & 0.009420 & 0.332697 & 149.314 \\
\hline 8/15/08 1:00 & 2.129 & 0.669 & 0.211 & 94.572 & 1.418 & 0.072 & 0.021945 & 0.009235 & 0.326176 & 146.388 \\
\hline 8/15/08 2:00 & 2.134 & 0.674 & 0.215 & 96.328 & 1.42 & 0.074 & 0.022554 & 0.009605 & 0.339261 & 152.260 \\
\hline
\end{tabular}




\begin{tabular}{|c|c|c|c|c|c|c|c|c|c|c|}
\hline \multirow[b]{3}{*}{ Date Time } & \multicolumn{4}{|c|}{ V-Notch Weir } & \multicolumn{6}{|c|}{ R-Notch Weir } \\
\hline & \multicolumn{2}{|c|}{ Measured Water Level } & \multicolumn{2}{|c|}{$\begin{array}{c}\text { Calculated Rate } \\
\left(\mathrm{ft}^{3} / \mathrm{s}=448.8 \mathrm{gpm}\right)\end{array}$} & \multicolumn{3}{|c|}{ Measured Water Level } & \multicolumn{3}{|c|}{$\begin{array}{c}\text { Calculated Rate } \\
\left(\mathrm{ft}^{3} / \mathrm{s}=448.8 \mathrm{gpm}\right)\end{array}$} \\
\hline & $\begin{array}{l}\text { (ft above } \\
\text { sensor) }\end{array}$ & $\begin{array}{c}\text { (ft above } \\
\text { notch) }\end{array}$ & $\left(\mathrm{ft}^{3} / \mathrm{s}\right)$ & (gpm) & $\begin{array}{c}\text { (ft above } \\
\text { sensor) }\end{array}$ & $\begin{array}{c}\text { (ft above } \\
\text { log) }\end{array}$ & $\begin{array}{c}\text { (m above } \\
\text { log) }\end{array}$ & $\left(\mathrm{m}^{3} / \mathrm{s}\right)$ & $\left(\mathrm{ft}^{3} / \mathrm{s}\right)$ & (gpm) \\
\hline 8/15/08 3:00 & 2.136 & 0.676 & 0.216 & 97.036 & 1.42 & 0.074 & 0.022554 & 0.009605 & 0.339261 & 152.260 \\
\hline 8/15/08 4:00 & 2.14 & 0.680 & 0.219 & 98.461 & 1.419 & 0.073 & 0.022249 & 0.009420 & 0.332697 & 149.314 \\
\hline 8/15/08 5:00 & 2.152 & 0.692 & 0.229 & 102.811 & 1.416 & 0.07 & 0.021335 & 0.008869 & 0.313263 & 140.593 \\
\hline 8/15/08 6:00 & 2.157 & 0.697 & 0.233 & 104.657 & 1.418 & 0.072 & 0.021945 & 0.009235 & 0.326176 & 146.388 \\
\hline 8/15/08 7:00 & 2.161 & 0.701 & 0.237 & 106.148 & 1.418 & 0.072 & 0.021945 & 0.009235 & 0.326176 & 146.388 \\
\hline 8/15/08 8:00 & 2.157 & 0.697 & 0.233 & 104.657 & 1.415 & 0.069 & 0.021030 & 0.008688 & 0.306873 & 137.724 \\
\hline 8/15/08 9:00 & 2.157 & 0.697 & 0.233 & 104.657 & 1.415 & 0.069 & 0.021030 & 0.008688 & 0.306873 & 137.724 \\
\hline 8/15/08 10:00 & 2.162 & 0.702 & 0.237 & 106.523 & 1.418 & 0.072 & 0.021945 & 0.009235 & 0.326176 & 146.388 \\
\hline 8/15/08 11:00 & 2.167 & 0.707 & 0.242 & 108.408 & 1.418 & 0.072 & 0.021945 & 0.009235 & 0.326176 & 146.388 \\
\hline 8/15/08 12:00 & 2.165 & 0.705 & 0.240 & 107.652 & 1.416 & 0.07 & 0.021335 & 0.008869 & 0.313263 & 140.593 \\
\hline 8/15/08 13:00 & 2.133 & 0.673 & 0.214 & 95.975 & 1.414 & 0.068 & 0.020725 & 0.008509 & 0.300526 & 134.876 \\
\hline 8/15/08 14:00 & 2.105 & 0.645 & 0.193 & 86.410 & 1.414 & 0.068 & 0.020725 & 0.008509 & 0.300526 & 134.876 \\
\hline 8/15/08 15:00 & 2.099 & 0.639 & 0.188 & 84.439 & 1.41 & 0.064 & 0.019506 & 0.007803 & 0.275587 & 123.683 \\
\hline 8/15/08 16:00 & 2.086 & 0.626 & 0.179 & 80.260 & 1.408 & 0.062 & 0.018897 & 0.007457 & 0.263390 & 118.209 \\
\hline 8/15/08 17:00 & 2.086 & 0.626 & 0.179 & 80.260 & 1.406 & 0.06 & 0.018287 & 0.007117 & 0.251378 & 112.818 \\
\hline 8/15/08 18:00 & 2.074 & 0.614 & 0.170 & 76.516 & 1.406 & 0.06 & 0.018287 & 0.007117 & 0.251378 & 112.818 \\
\hline 8/15/08 19:00 & 2.076 & 0.616 & 0.172 & 77.132 & 1.409 & 0.063 & 0.019201 & 0.007629 & 0.269465 & 120.936 \\
\hline 8/15/08 20:00 & 2.087 & 0.627 & 0.180 & 80.577 & 1.409 & 0.063 & 0.019201 & 0.007629 & 0.269465 & 120.936 \\
\hline 8/15/08 21:00 & 2.091 & 0.631 & 0.182 & 81.852 & 1.412 & 0.066 & 0.020116 & 0.008153 & 0.287966 & 129.239 \\
\hline 8/15/08 22:00 & 2.105 & 0.645 & 0.193 & 86.410 & 1.411 & 0.065 & 0.019811 & 0.007977 & 0.281754 & 126.451 \\
\hline 8/15/08 23:00 & 2.116 & 0.656 & 0.201 & 90.096 & 1.411 & 0.065 & 0.019811 & 0.007977 & 0.281754 & 126.451 \\
\hline 8/16/08 0:00 & 2.132 & 0.672 & 0.213 & 95.623 & 1.413 & 0.067 & 0.020421 & 0.008330 & 0.294224 & 132.048 \\
\hline 8/16/08 1:00 & 2.131 & 0.671 & 0.212 & 95.272 & 1.413 & 0.067 & 0.020421 & 0.008330 & 0.294224 & 132.048 \\
\hline 8/16/08 2:00 & 2.136 & 0.676 & 0.216 & 97.036 & 1.412 & 0.066 & 0.020116 & 0.008153 & 0.287966 & 129.239 \\
\hline 8/16/08 3:00 & 2.145 & 0.685 & 0.223 & 100.260 & 1.414 & 0.068 & 0.020725 & 0.008509 & 0.300526 & 134.876 \\
\hline
\end{tabular}




\begin{tabular}{|c|c|c|c|c|c|c|c|c|c|c|}
\hline \multirow[b]{3}{*}{ Date Time } & \multicolumn{4}{|c|}{ V-Notch Weir } & \multicolumn{6}{|c|}{ R-Notch Weir } \\
\hline & \multicolumn{2}{|c|}{ Measured Water Level } & \multicolumn{2}{|c|}{$\begin{array}{c}\text { Calculated Rate } \\
\left(\mathrm{ft}^{3} / \mathrm{s}=448.8 \mathrm{gpm}\right)\end{array}$} & \multicolumn{3}{|c|}{ Measured Water Level } & \multicolumn{3}{|c|}{$\begin{array}{c}\text { Calculated Rate } \\
\left(\mathrm{ft}^{3} / \mathrm{s}=448.8 \mathrm{gpm}\right)\end{array}$} \\
\hline & $\begin{array}{c}\text { (ft above } \\
\text { sensor) }\end{array}$ & $\begin{array}{c}\text { (ft above } \\
\text { notch) }\end{array}$ & $\left(\mathrm{ft}^{3} / \mathrm{s}\right)$ & (gpm) & $\begin{array}{l}\text { (ft above } \\
\text { sensor) }\end{array}$ & $\begin{array}{c}\text { (ft above } \\
\text { log) }\end{array}$ & $\begin{array}{c}\text { (m above } \\
\text { log) }\end{array}$ & $\left(\mathrm{m}^{3} / \mathrm{s}\right)$ & $\left(\mathrm{ft}^{3} / \mathrm{s}\right)$ & (gpm) \\
\hline 8/16/08 4:00 & 2.15 & 0.690 & 0.227 & 102.078 & 1.414 & 0.068 & 0.020725 & 0.008509 & 0.300526 & 134.876 \\
\hline 8/16/08 5:00 & 2.145 & 0.685 & 0.223 & 100.260 & 1.411 & 0.065 & 0.019811 & 0.007977 & 0.281754 & 126.451 \\
\hline 8/16/08 6:00 & 2.194 & 0.734 & 0.265 & 118.936 & 1.411 & 0.065 & 0.019811 & 0.007977 & 0.281754 & 126.451 \\
\hline 8/16/08 7:00 & 2.248 & 0.788 & 0.316 & 141.774 & 1.413 & 0.067 & 0.020421 & 0.008330 & 0.294224 & 132.048 \\
\hline 8/16/08 8:00 & 2.3 & 0.840 & 0.370 & 166.078 & 1.412 & 0.066 & 0.020116 & 0.008153 & 0.287966 & 129.239 \\
\hline 8/16/08 9:00 & 2.335 & 0.875 & 0.409 & 183.751 & 1.415 & 0.069 & 0.021030 & 0.008688 & 0.306873 & 137.724 \\
\hline 8/16/08 10:00 & 2.349 & 0.889 & 0.426 & 191.122 & 1.415 & 0.069 & 0.021030 & 0.008688 & 0.306873 & 137.724 \\
\hline 8/16/08 11:00 & 2.164 & 0.704 & 0.239 & 107.275 & 1.415 & 0.069 & 0.021030 & 0.008688 & 0.306873 & 137.724 \\
\hline 8/16/08 12:00 & 2.148 & 0.688 & 0.226 & 101.348 & 1.413 & 0.067 & 0.020421 & 0.008330 & 0.294224 & 132.048 \\
\hline 8/16/08 13:00 & 2.128 & 0.668 & 0.210 & 94.223 & 1.41 & 0.064 & 0.019506 & 0.007803 & 0.275587 & 123.683 \\
\hline 8/16/08 14:00 & 2.089 & 0.629 & 0.181 & 81.213 & 1.408 & 0.062 & 0.018897 & 0.007457 & 0.263390 & 118.209 \\
\hline 8/16/08 15:00 & 2.084 & 0.624 & 0.177 & 79.629 & 1.404 & 0.058 & 0.017678 & 0.006782 & 0.239555 & 107.512 \\
\hline 8/16/08 16:00 & 2.083 & 0.623 & 0.177 & 79.314 & 1.407 & 0.061 & 0.018592 & 0.007287 & 0.257360 & 115.503 \\
\hline 8/16/08 17:00 & 2.07 & 0.610 & 0.168 & 75.291 & 1.406 & 0.06 & 0.018287 & 0.007117 & 0.251378 & 112.818 \\
\hline 8/16/08 18:00 & 2.073 & 0.613 & 0.170 & 76.208 & 1.409 & 0.063 & 0.019201 & 0.007629 & 0.269465 & 120.936 \\
\hline 8/16/08 19:00 & 2.07 & 0.610 & 0.168 & 75.291 & 1.409 & 0.063 & 0.019201 & 0.007629 & 0.269465 & 120.936 \\
\hline 8/16/08 20:00 & 2.081 & 0.621 & 0.175 & 78.687 & 1.407 & 0.061 & 0.018592 & 0.007287 & 0.257360 & 115.503 \\
\hline 8/16/08 21:00 & 2.094 & 0.634 & 0.185 & 82.817 & 1.409 & 0.063 & 0.019201 & 0.007629 & 0.269465 & 120.936 \\
\hline 8/16/08 22:00 & 2.105 & 0.645 & 0.193 & 86.410 & 1.409 & 0.063 & 0.019201 & 0.007629 & 0.269465 & 120.936 \\
\hline 8/16/08 23:00 & 2.113 & 0.653 & 0.198 & 89.082 & 1.409 & 0.063 & 0.019201 & 0.007629 & 0.269465 & 120.936 \\
\hline 8/17/08 0:00 & 2.118 & 0.658 & 0.202 & 90.776 & 1.413 & 0.067 & 0.020421 & 0.008330 & 0.294224 & 132.048 \\
\hline 8/17/08 1:00 & 2.122 & 0.662 & 0.205 & 92.146 & 1.412 & 0.066 & 0.020116 & 0.008153 & 0.287966 & 129.239 \\
\hline 8/17/08 2:00 & 2.11 & 0.650 & 0.196 & 88.074 & 1.414 & 0.068 & 0.020725 & 0.008509 & 0.300526 & 134.876 \\
\hline 8/17/08 3:00 & 2.136 & 0.676 & 0.216 & 97.036 & 1.414 & 0.068 & 0.020725 & 0.008509 & 0.300526 & 134.876 \\
\hline 8/17/08 4:00 & 2.154 & 0.694 & 0.231 & 103.547 & 1.411 & 0.065 & 0.019811 & 0.007977 & 0.281754 & 126.451 \\
\hline
\end{tabular}




\begin{tabular}{|c|c|c|c|c|c|c|c|c|c|c|}
\hline \multirow[b]{3}{*}{ Date Time } & \multicolumn{4}{|c|}{ V-Notch Weir } & \multicolumn{6}{|c|}{ R-Notch Weir } \\
\hline & \multicolumn{2}{|c|}{ Measured Water Level } & \multicolumn{2}{|c|}{$\begin{array}{c}\text { Calculated Rate } \\
\left(\mathrm{ft}^{3} / \mathrm{s}=448.8 \mathrm{gpm}\right)\end{array}$} & \multicolumn{3}{|c|}{ Measured Water Level } & \multicolumn{3}{|c|}{$\begin{array}{c}\text { Calculated Rate } \\
\left(\mathrm{ft}^{3} / \mathrm{s}=448.8 \mathrm{gpm}\right)\end{array}$} \\
\hline & $\begin{array}{c}\text { (ft above } \\
\text { sensor) }\end{array}$ & $\begin{array}{c}\text { (ft above } \\
\text { notch) }\end{array}$ & $\left(\mathrm{ft}^{3} / \mathrm{s}\right)$ & (gpm) & $\begin{array}{l}\text { (ft above } \\
\text { sensor) }\end{array}$ & $\begin{array}{c}\text { (ft above } \\
\text { log) }\end{array}$ & $\begin{array}{c}\text { (m above } \\
\text { log) }\end{array}$ & $\left(\mathrm{m}^{3} / \mathrm{s}\right)$ & $\left(\mathrm{ft}^{3} / \mathrm{s}\right)$ & (gpm) \\
\hline 8/17/08 5:00 & 2.154 & 0.694 & 0.231 & 103.547 & 1.411 & 0.065 & 0.019811 & 0.007977 & 0.281754 & 126.451 \\
\hline 8/17/08 6:00 & 2.182 & 0.722 & 0.254 & 114.185 & 1.415 & 0.069 & 0.021030 & 0.008688 & 0.306873 & 137.724 \\
\hline 8/17/08 7:00 & 2.194 & 0.734 & 0.265 & 118.936 & 1.415 & 0.069 & 0.021030 & 0.008688 & 0.306873 & 137.724 \\
\hline 8/17/08 8:00 & 2.201 & 0.741 & 0.271 & 121.761 & 1.415 & 0.069 & 0.021030 & 0.008688 & 0.306873 & 137.724 \\
\hline 8/17/08 9:00 & 2.194 & 0.734 & 0.265 & 118.936 & 1.414 & 0.068 & 0.020725 & 0.008509 & 0.300526 & 134.876 \\
\hline 8/17/08 10:00 & 2.183 & 0.723 & 0.255 & 114.576 & 1.412 & 0.066 & 0.020116 & 0.008153 & 0.287966 & 129.239 \\
\hline 8/17/08 11:00 & 2.183 & 0.723 & 0.255 & 114.576 & 1.412 & 0.066 & 0.020116 & 0.008153 & 0.287966 & 129.239 \\
\hline 8/17/08 12:00 & 2.172 & 0.712 & 0.246 & 110.314 & 1.41 & 0.064 & 0.019506 & 0.007803 & 0.275587 & 123.683 \\
\hline 8/17/08 13:00 & 2.143 & 0.683 & 0.222 & 99.538 & 1.405 & 0.059 & 0.017982 & 0.006949 & 0.245443 & 110.155 \\
\hline 8/17/08 14:00 & 2.128 & 0.668 & 0.210 & 94.223 & 1.404 & 0.058 & 0.017678 & 0.006782 & 0.239555 & 107.512 \\
\hline 8/17/08 15:00 & 2.103 & 0.643 & 0.191 & 85.750 & 1.4 & 0.054 & 0.016458 & 0.006129 & 0.216486 & 97.159 \\
\hline 8/17/08 16:00 & 2.101 & 0.641 & 0.190 & 85.093 & 1.403 & 0.057 & 0.017373 & 0.006617 & 0.233715 & 104.891 \\
\hline 8/17/08 17:00 & 2.097 & 0.637 & 0.187 & 83.788 & 1.403 & 0.057 & 0.017373 & 0.006617 & 0.233715 & 104.891 \\
\hline 8/17/08 18:00 & 2.097 & 0.637 & 0.187 & 83.788 & 1.409 & 0.063 & 0.019201 & 0.007629 & 0.269465 & 120.936 \\
\hline 8/17/08 19:00 & 2.118 & 0.658 & 0.202 & 90.776 & 1.409 & 0.063 & 0.019201 & 0.007629 & 0.269465 & 120.936 \\
\hline 8/17/08 20:00 & 2.124 & 0.664 & 0.207 & 92.835 & 1.409 & 0.063 & 0.019201 & 0.007629 & 0.269465 & 120.936 \\
\hline 8/17/08 21:00 & 2.129 & 0.669 & 0.211 & 94.572 & 1.412 & 0.066 & 0.020116 & 0.008153 & 0.287966 & 129.239 \\
\hline 8/17/08 22:00 & 2.133 & 0.673 & 0.214 & 95.975 & 1.409 & 0.063 & 0.019201 & 0.007629 & 0.269465 & 120.936 \\
\hline 8/17/08 23:00 & 2.159 & 0.699 & 0.235 & 105.401 & 1.412 & 0.066 & 0.020116 & 0.008153 & 0.287966 & 129.239 \\
\hline 8/18/08 0:00 & 2.172 & 0.712 & 0.246 & 110.314 & 1.414 & 0.068 & 0.020725 & 0.008509 & 0.300526 & 134.876 \\
\hline 8/18/08 1:00 & 2.188 & 0.728 & 0.260 & 116.546 & 1.413 & 0.067 & 0.020421 & 0.008330 & 0.294224 & 132.048 \\
\hline 8/18/08 2:00 & 2.199 & 0.739 & 0.269 & 120.950 & 1.413 & 0.067 & 0.020421 & 0.008330 & 0.294224 & 132.048 \\
\hline 8/18/08 3:00 & 2.187 & 0.727 & 0.259 & 116.150 & 1.412 & 0.066 & 0.020116 & 0.008153 & 0.287966 & 129.239 \\
\hline 8/18/08 4:00 & 2.206 & 0.746 & 0.276 & 123.804 & 1.412 & 0.066 & 0.020116 & 0.008153 & 0.287966 & 129.239 \\
\hline 8/18/08 5:00 & 2.234 & 0.774 & 0.302 & 135.621 & 1.416 & 0.07 & 0.021335 & 0.008869 & 0.313263 & 140.593 \\
\hline
\end{tabular}




\begin{tabular}{|c|c|c|c|c|c|c|c|c|c|c|}
\hline \multirow[b]{3}{*}{ Date Time } & \multicolumn{4}{|c|}{ V-Notch Weir } & \multicolumn{6}{|c|}{ R-Notch Weir } \\
\hline & \multicolumn{2}{|c|}{ Measured Water Level } & \multicolumn{2}{|c|}{$\begin{array}{c}\text { Calculated Rate } \\
\left(\mathrm{ft}^{3} / \mathrm{s}=448.8 \mathrm{gpm}\right)\end{array}$} & \multicolumn{3}{|c|}{ Measured Water Level } & \multicolumn{3}{|c|}{$\begin{array}{c}\text { Calculated Rate } \\
\left(\mathrm{ft}^{3} / \mathrm{s}=448.8 \mathrm{gpm}\right)\end{array}$} \\
\hline & $\begin{array}{c}\text { (ft above } \\
\text { sensor) }\end{array}$ & $\begin{array}{c}\text { (ft above } \\
\text { notch) }\end{array}$ & $\left(\mathrm{ft}^{3} / \mathrm{s}\right)$ & (gpm) & $\begin{array}{c}\text { (ft above } \\
\text { sensor) }\end{array}$ & $\begin{array}{c}\text { (ft above } \\
\text { log) }\end{array}$ & $\begin{array}{c}\text { (m above } \\
\text { log) }\end{array}$ & $\left(\mathrm{m}^{3} / \mathrm{s}\right)$ & $\left(\mathrm{ft}^{3} / \mathrm{s}\right)$ & (gpm) \\
\hline 8/18/08 6:00 & 2.26 & 0.800 & 0.328 & 147.178 & 1.416 & 0.07 & 0.021335 & 0.008869 & 0.313263 & 140.593 \\
\hline 8/18/08 7:00 & 2.234 & 0.774 & 0.302 & 135.621 & 1.415 & 0.069 & 0.021030 & 0.008688 & 0.306873 & 137.724 \\
\hline 8/18/08 8:00 & 2.243 & 0.783 & 0.311 & 139.558 & 1.413 & 0.067 & 0.020421 & 0.008330 & 0.294224 & 132.048 \\
\hline 8/18/08 9:00 & 2.248 & 0.788 & 0.316 & 141.774 & 1.415 & 0.069 & 0.021030 & 0.008688 & 0.306873 & 137.724 \\
\hline 8/18/08 10:00 & 2.251 & 0.791 & 0.319 & 143.114 & 1.415 & 0.069 & 0.021030 & 0.008688 & 0.306873 & 137.724 \\
\hline 8/18/08 11:00 & 2.239 & 0.779 & 0.307 & 137.800 & 1.413 & 0.067 & 0.020421 & 0.008330 & 0.294224 & 132.048 \\
\hline 8/18/08 12:00 & 2.205 & 0.745 & 0.275 & 123.394 & 1.413 & 0.067 & 0.020421 & 0.008330 & 0.294224 & 132.048 \\
\hline 8/18/08 13:00 & 2.168 & 0.708 & 0.242 & 108.788 & 1.406 & 0.06 & 0.018287 & 0.007117 & 0.251378 & 112.818 \\
\hline 8/18/08 14:00 & 2.137 & 0.677 & 0.217 & 97.391 & 1.404 & 0.058 & 0.017678 & 0.006782 & 0.239555 & 107.512 \\
\hline 8/18/08 15:00 & 2.143 & 0.683 & 0.222 & 99.538 & 1.402 & 0.056 & 0.017068 & 0.006453 & 0.227923 & 102.292 \\
\hline 8/18/08 16:00 & 2.113 & 0.653 & 0.198 & 89.082 & 1.4 & 0.054 & 0.016458 & 0.006129 & 0.216486 & 97.159 \\
\hline 8/18/08 17:00 & 2.179 & 0.719 & 0.252 & 113.015 & 1.406 & 0.06 & 0.018287 & 0.007117 & 0.251378 & 112.818 \\
\hline 8/18/08 18:00 & 2.137 & 0.677 & 0.217 & 97.391 & 1.406 & 0.06 & 0.018287 & 0.007117 & 0.251378 & 112.818 \\
\hline 8/18/08 19:00 & 2.129 & 0.669 & 0.211 & 94.572 & 1.407 & 0.061 & 0.018592 & 0.007287 & 0.257360 & 115.503 \\
\hline 8/18/08 20:00 & 2.138 & 0.678 & 0.218 & 97.747 & 1.41 & 0.064 & 0.019506 & 0.007803 & 0.275587 & 123.683 \\
\hline 8/18/08 21:00 & 2.153 & 0.693 & 0.230 & 103.179 & 1.41 & 0.064 & 0.019506 & 0.007803 & 0.275587 & 123.683 \\
\hline 8/18/08 22:00 & 2.164 & 0.704 & 0.239 & 107.275 & 1.41 & 0.064 & 0.019506 & 0.007803 & 0.275587 & 123.683 \\
\hline 8/18/08 23:00 & 2.206 & 0.746 & 0.276 & 123.804 & 1.41 & 0.064 & 0.019506 & 0.007803 & 0.275587 & 123.683 \\
\hline 8/19/08 0:00 & 2.217 & 0.757 & 0.286 & 128.369 & 1.409 & 0.063 & 0.019201 & 0.007629 & 0.269465 & 120.936 \\
\hline 8/19/08 1:00 & 2.195 & 0.735 & 0.266 & 119.337 & 1.413 & 0.067 & 0.020421 & 0.008330 & 0.294224 & 132.048 \\
\hline 8/19/08 2:00 & 2.207 & 0.747 & 0.277 & 124.215 & 1.413 & 0.067 & 0.020421 & 0.008330 & 0.294224 & 132.048 \\
\hline 8/19/08 3:00 & 2.218 & 0.758 & 0.287 & 128.789 & 1.412 & 0.066 & 0.020116 & 0.008153 & 0.287966 & 129.239 \\
\hline 8/19/08 4:00 & 2.225 & 0.765 & 0.294 & 131.752 & 1.412 & 0.066 & 0.020116 & 0.008153 & 0.287966 & 129.239 \\
\hline 8/19/08 5:00 & 2.239 & 0.779 & 0.307 & 137.800 & 1.411 & 0.065 & 0.019811 & 0.007977 & 0.281754 & 126.451 \\
\hline 8/19/08 6:00 & 2.229 & 0.769 & 0.297 & 133.464 & 1.413 & 0.067 & 0.020421 & 0.008330 & 0.294224 & 132.048 \\
\hline
\end{tabular}




\begin{tabular}{|c|c|c|c|c|c|c|c|c|c|c|}
\hline \multirow[b]{3}{*}{ Date Time } & \multicolumn{4}{|c|}{ V-Notch Weir } & \multicolumn{6}{|c|}{ R-Notch Weir } \\
\hline & \multicolumn{2}{|c|}{ Measured Water Level } & \multicolumn{2}{|c|}{$\begin{array}{c}\text { Calculated Rate } \\
\left(\mathrm{ft}^{3} / \mathrm{s}=448.8 \mathrm{gpm}\right)\end{array}$} & \multicolumn{3}{|c|}{ Measured Water Level } & \multicolumn{3}{|c|}{$\begin{array}{c}\text { Calculated Rate } \\
\left(\mathrm{ft}^{3} / \mathrm{s}=448.8 \mathrm{gpm}\right)\end{array}$} \\
\hline & $\begin{array}{l}\text { (ft above } \\
\text { sensor) }\end{array}$ & $\begin{array}{c}\text { (ft above } \\
\text { notch) }\end{array}$ & $\left(\mathrm{ft}^{3} / \mathrm{s}\right)$ & (gpm) & $\begin{array}{c}\text { (ft above } \\
\text { sensor) }\end{array}$ & $\begin{array}{c}\text { (ft above } \\
\text { log) }\end{array}$ & $\begin{array}{c}\text { (m above } \\
\text { log) }\end{array}$ & $\left(\mathrm{m}^{3} / \mathrm{s}\right)$ & $\left(\mathrm{ft}^{3} / \mathrm{s}\right)$ & (gpm) \\
\hline 8/19/08 7:00 & 2.239 & 0.779 & 0.307 & 137.800 & 1.413 & 0.067 & 0.020421 & 0.008330 & 0.294224 & 132.048 \\
\hline 8/19/08 8:00 & 2.225 & 0.765 & 0.294 & 131.752 & 1.415 & 0.069 & 0.021030 & 0.008688 & 0.306873 & 137.724 \\
\hline 8/19/08 9:00 & 2.241 & 0.781 & 0.309 & 138.677 & 1.415 & 0.069 & 0.021030 & 0.008688 & 0.306873 & 137.724 \\
\hline 8/19/08 10:00 & 2.239 & 0.779 & 0.307 & 137.800 & 1.413 & 0.067 & 0.020421 & 0.008330 & 0.294224 & 132.048 \\
\hline 8/19/08 11:00 & 2.23 & 0.770 & 0.298 & 133.893 & 1.413 & 0.067 & 0.020421 & 0.008330 & 0.294224 & 132.048 \\
\hline 8/19/08 12:00 & 2.196 & 0.736 & 0.267 & 119.739 & 1.408 & 0.062 & 0.018897 & 0.007457 & 0.263390 & 118.209 \\
\hline 8/19/08 13:00 & 2.164 & 0.704 & 0.239 & 107.275 & 1.406 & 0.06 & 0.018287 & 0.007117 & 0.251378 & 112.818 \\
\hline 8/19/08 14:00 & 2.138 & 0.678 & 0.218 & 97.747 & 1.402 & 0.056 & 0.017068 & 0.006453 & 0.227923 & 102.292 \\
\hline 8/19/08 15:00 & 2.149 & 0.689 & 0.227 & 101.713 & 1.402 & 0.056 & 0.017068 & 0.006453 & 0.227923 & 102.292 \\
\hline 8/19/08 16:00 & 2.135 & 0.675 & 0.215 & 96.681 & 1.4 & 0.054 & 0.016458 & 0.006129 & 0.216486 & 97.159 \\
\hline 8/19/08 17:00 & 2.134 & 0.674 & 0.215 & 96.328 & 1.4 & 0.054 & 0.016458 & 0.006129 & 0.216486 & 97.159 \\
\hline 8/19/08 18:00 & 2.136 & 0.676 & 0.216 & 97.036 & 1.401 & 0.055 & 0.016763 & 0.006291 & 0.222180 & 99.714 \\
\hline 8/19/08 19:00 & 2.145 & 0.685 & 0.223 & 100.260 & 1.401 & 0.055 & 0.016763 & 0.006291 & 0.222180 & 99.714 \\
\hline 8/19/08 20:00 & 2.163 & 0.703 & 0.238 & 106.898 & 1.404 & 0.058 & 0.017678 & 0.006782 & 0.239555 & 107.512 \\
\hline 8/19/08 21:00 & 2.181 & 0.721 & 0.254 & 113.794 & 1.407 & 0.061 & 0.018592 & 0.007287 & 0.257360 & 115.503 \\
\hline 8/19/08 22:00 & 2.178 & 0.718 & 0.251 & 112.627 & 1.409 & 0.063 & 0.019201 & 0.007629 & 0.269465 & 120.936 \\
\hline 8/19/08 23:00 & 2.208 & 0.748 & 0.278 & 124.627 & 1.409 & 0.063 & 0.019201 & 0.007629 & 0.269465 & 120.936 \\
\hline 8/20/08 0:00 & 2.245 & 0.785 & 0.313 & 140.442 & 1.411 & 0.065 & 0.019811 & 0.007977 & 0.281754 & 126.451 \\
\hline 8/20/08 1:00 & 2.284 & 0.824 & 0.353 & 158.354 & 1.418 & 0.072 & 0.021945 & 0.009235 & 0.326176 & 146.388 \\
\hline 8/20/08 2:00 & 2.246 & 0.786 & 0.314 & 140.885 & 1.415 & 0.069 & 0.021030 & 0.008688 & 0.306873 & 137.724 \\
\hline 8/20/08 3:00 & 2.248 & 0.788 & 0.316 & 141.774 & 1.414 & 0.068 & 0.020725 & 0.008509 & 0.300526 & 134.876 \\
\hline 8/20/08 4:00 & 2.248 & 0.788 & 0.316 & 141.774 & 1.414 & 0.068 & 0.020725 & 0.008509 & 0.300526 & 134.876 \\
\hline 8/20/08 5:00 & 2.253 & 0.793 & 0.321 & 144.011 & 1.413 & 0.067 & 0.020421 & 0.008330 & 0.294224 & 132.048 \\
\hline 8/20/08 6:00 & 2.232 & 0.772 & 0.300 & 134.756 & 1.41 & 0.064 & 0.019506 & 0.007803 & 0.275587 & 123.683 \\
\hline 8/20/08 7:00 & 2.262 & 0.802 & 0.330 & 148.091 & 1.412 & 0.066 & 0.020116 & 0.008153 & 0.287966 & 129.239 \\
\hline
\end{tabular}




\begin{tabular}{|c|c|c|c|c|c|c|c|c|c|c|}
\hline \multirow[b]{3}{*}{ Date Time } & \multicolumn{4}{|c|}{ V-Notch Weir } & \multicolumn{6}{|c|}{ R-Notch Weir } \\
\hline & \multicolumn{2}{|c|}{ Measured Water Level } & \multicolumn{2}{|c|}{$\begin{array}{c}\text { Calculated Rate } \\
\left(\mathrm{ft}^{3} / \mathrm{s}=448.8 \mathrm{gpm}\right)\end{array}$} & \multicolumn{3}{|c|}{ Measured Water Level } & \multicolumn{3}{|c|}{$\begin{array}{c}\text { Calculated Rate } \\
\left(\mathrm{ft}^{3} / \mathrm{s}=448.8 \mathrm{gpm}\right)\end{array}$} \\
\hline & $\begin{array}{c}\text { (ft above } \\
\text { sensor) }\end{array}$ & $\begin{array}{c}\text { (ft above } \\
\text { notch) }\end{array}$ & $\left(\mathrm{ft}^{3} / \mathrm{s}\right)$ & (gpm) & $\begin{array}{l}\text { (ft above } \\
\text { sensor) }\end{array}$ & $\begin{array}{c}\text { (ft above } \\
\text { log) }\end{array}$ & $\begin{array}{c}\text { (m above } \\
\text { log) }\end{array}$ & $\left(\mathrm{m}^{3} / \mathrm{s}\right)$ & $\left(\mathrm{ft}^{3} / \mathrm{s}\right)$ & (gpm) \\
\hline 8/20/08 8:00 & 2.267 & 0.807 & 0.335 & 150.387 & 1.412 & 0.066 & 0.020116 & 0.008153 & 0.287966 & 129.239 \\
\hline 8/20/08 9:00 & 2.236 & 0.776 & 0.304 & 136.490 & 1.412 & 0.066 & 0.020116 & 0.008153 & 0.287966 & 129.239 \\
\hline 8/20/08 10:00 & 2.248 & 0.788 & 0.316 & 141.774 & 1.412 & 0.066 & 0.020116 & 0.008153 & 0.287966 & 129.239 \\
\hline 8/20/08 11:00 & 2.202 & 0.742 & 0.272 & 122.168 & 1.407 & 0.061 & 0.018592 & 0.007287 & 0.257360 & 115.503 \\
\hline 8/20/08 12:00 & 2.197 & 0.737 & 0.268 & 120.142 & 1.403 & 0.057 & 0.017373 & 0.006617 & 0.233715 & 104.891 \\
\hline 8/20/08 13:00 & 2.224 & 0.764 & 0.293 & 131.327 & 1.408 & 0.062 & 0.018897 & 0.007457 & 0.263390 & 118.209 \\
\hline 8/20/08 14:00 & 2.216 & 0.756 & 0.285 & 127.950 & 1.405 & 0.059 & 0.017982 & 0.006949 & 0.245443 & 110.155 \\
\hline 8/20/08 15:00 & 2.193 & 0.733 & 0.264 & 118.536 & 1.406 & 0.06 & 0.018287 & 0.007117 & 0.251378 & 112.818 \\
\hline 8/20/08 16:00 & 2.18 & 0.720 & 0.253 & 113.404 & 1.402 & 0.056 & 0.017068 & 0.006453 & 0.227923 & 102.292 \\
\hline 8/20/08 17:00 & 2.164 & 0.704 & 0.239 & 107.275 & 1.402 & 0.056 & 0.017068 & 0.006453 & 0.227923 & 102.292 \\
\hline 8/20/08 18:00 & 2.051 & 0.591 & 0.155 & 69.636 & 1.4 & 0.054 & 0.016458 & 0.006129 & 0.216486 & 97.159 \\
\hline 8/20/08 19:00 & 2.053 & 0.593 & 0.156 & 70.219 & 1.403 & 0.057 & 0.017373 & 0.006617 & 0.233715 & 104.891 \\
\hline 8/20/08 20:00 & 2.055 & 0.595 & 0.158 & 70.805 & 1.404 & 0.058 & 0.017678 & 0.006782 & 0.239555 & 107.512 \\
\hline 8/20/08 21:00 & 2.068 & 0.608 & 0.166 & 74.683 & 1.406 & 0.06 & 0.018287 & 0.007117 & 0.251378 & 112.818 \\
\hline 8/20/08 22:00 & 2.086 & 0.626 & 0.179 & 80.260 & 1.409 & 0.063 & 0.019201 & 0.007629 & 0.269465 & 120.936 \\
\hline 8/20/08 23:00 & 2.081 & 0.621 & 0.175 & 78.687 & 1.409 & 0.063 & 0.019201 & 0.007629 & 0.269465 & 120.936 \\
\hline 8/21/08 0:00 & 2.109 & 0.649 & 0.195 & 87.740 & 1.411 & 0.065 & 0.019811 & 0.007977 & 0.281754 & 126.451 \\
\hline 8/21/08 1:00 & 2.101 & 0.641 & 0.190 & 85.093 & 1.408 & 0.062 & 0.018897 & 0.007457 & 0.263390 & 118.209 \\
\hline 8/21/08 2:00 & 2.101 & 0.641 & 0.190 & 85.093 & 1.41 & 0.064 & 0.019506 & 0.007803 & 0.275587 & 123.683 \\
\hline 8/21/08 3:00 & 2.117 & 0.657 & 0.202 & 90.436 & 1.41 & 0.064 & 0.019506 & 0.007803 & 0.275587 & 123.683 \\
\hline 8/21/08 4:00 & 2.122 & 0.662 & 0.205 & 92.146 & 1.41 & 0.064 & 0.019506 & 0.007803 & 0.275587 & 123.683 \\
\hline 8/21/08 5:00 & 2.131 & 0.671 & 0.212 & 95.272 & 1.409 & 0.063 & 0.019201 & 0.007629 & 0.269465 & 120.936 \\
\hline 8/21/08 6:00 & 2.138 & 0.678 & 0.218 & 97.747 & 1.411 & 0.065 & 0.019811 & 0.007977 & 0.281754 & 126.451 \\
\hline 8/21/08 7:00 & 2.145 & 0.685 & 0.223 & 100.260 & 1.413 & 0.067 & 0.020421 & 0.008330 & 0.294224 & 132.048 \\
\hline 8/21/08 8:00 & 2.147 & 0.687 & 0.225 & 100.985 & 1.416 & 0.07 & 0.021335 & 0.008869 & 0.313263 & 140.593 \\
\hline
\end{tabular}




\begin{tabular}{|c|c|c|c|c|c|c|c|c|c|c|}
\hline \multirow[b]{3}{*}{ Date Time } & \multicolumn{4}{|c|}{ V-Notch Weir } & \multicolumn{6}{|c|}{ R-Notch Weir } \\
\hline & \multicolumn{2}{|c|}{ Measured Water Level } & \multicolumn{2}{|c|}{$\begin{array}{c}\text { Calculated Rate } \\
\left(\mathrm{ft}^{3} / \mathrm{s}=448.8 \mathrm{gpm}\right)\end{array}$} & \multicolumn{3}{|c|}{ Measured Water Level } & \multicolumn{3}{|c|}{$\begin{array}{c}\text { Calculated Rate } \\
\left(\mathrm{ft}^{3} / \mathrm{s}=448.8 \mathrm{gpm}\right)\end{array}$} \\
\hline & $\begin{array}{c}\text { (ft above } \\
\text { sensor) }\end{array}$ & $\begin{array}{c}\text { (ft above } \\
\text { notch) }\end{array}$ & $\left(\mathrm{ft}^{3} / \mathrm{s}\right)$ & (gpm) & $\begin{array}{l}\text { (ft above } \\
\text { sensor) }\end{array}$ & $\begin{array}{c}\text { (ft above } \\
\text { log) }\end{array}$ & $\begin{array}{c}\text { (m above } \\
\text { log) }\end{array}$ & $\left(\mathrm{m}^{3} / \mathrm{s}\right)$ & $\left(\mathrm{ft}^{3} / \mathrm{s}\right)$ & (gpm) \\
\hline 8/21/08 9:00 & 2.147 & 0.687 & 0.225 & 100.985 & 1.413 & 0.067 & 0.020421 & 0.008330 & 0.294224 & 132.048 \\
\hline 8/21/08 10:00 & 2.145 & 0.685 & 0.223 & 100.260 & 1.415 & 0.069 & 0.021030 & 0.008688 & 0.306873 & 137.724 \\
\hline 8/21/08 11:00 & 2.147 & 0.687 & 0.225 & 100.985 & 1.413 & 0.067 & 0.020421 & 0.008330 & 0.294224 & 132.048 \\
\hline 8/21/08 12:00 & 2.153 & 0.693 & 0.230 & 103.179 & 1.415 & 0.069 & 0.021030 & 0.008688 & 0.306873 & 137.724 \\
\hline 8/21/08 13:00 & 2.141 & 0.681 & 0.220 & 98.819 & 1.413 & 0.067 & 0.020421 & 0.008330 & 0.294224 & 132.048 \\
\hline 8/21/08 14:00 & 2.135 & 0.675 & 0.215 & 96.681 & 1.413 & 0.067 & 0.020421 & 0.008330 & 0.294224 & 132.048 \\
\hline 8/21/08 15:00 & 2.118 & 0.658 & 0.202 & 90.776 & 1.411 & 0.065 & 0.019811 & 0.007977 & 0.281754 & 126.451 \\
\hline 8/21/08 16:00 & 2.103 & 0.643 & 0.191 & 85.750 & 1.409 & 0.063 & 0.019201 & 0.007629 & 0.269465 & 120.936 \\
\hline 8/21/08 17:00 & 2.098 & 0.638 & 0.187 & 84.113 & 1.407 & 0.061 & 0.018592 & 0.007287 & 0.257360 & 115.503 \\
\hline 8/21/08 18:00 & 2.094 & 0.634 & 0.185 & 82.817 & 1.407 & 0.061 & 0.018592 & 0.007287 & 0.257360 & 115.503 \\
\hline 8/21/08 19:00 & 2.089 & 0.629 & 0.181 & 81.213 & 1.407 & 0.061 & 0.018592 & 0.007287 & 0.257360 & 115.503 \\
\hline 8/21/08 20:00 & 2.098 & 0.638 & 0.187 & 84.113 & 1.41 & 0.064 & 0.019506 & 0.007803 & 0.275587 & 123.683 \\
\hline 8/21/08 21:00 & 2.095 & 0.635 & 0.185 & 83.140 & 1.41 & 0.064 & 0.019506 & 0.007803 & 0.275587 & 123.683 \\
\hline 8/21/08 22:00 & 2.107 & 0.647 & 0.194 & 87.074 & 1.412 & 0.066 & 0.020116 & 0.008153 & 0.287966 & 129.239 \\
\hline 8/21/08 23:00 & 2.109 & 0.649 & 0.195 & 87.740 & 1.412 & 0.066 & 0.020116 & 0.008153 & 0.287966 & 129.239 \\
\hline 8/22/08 0:00 & 2.106 & 0.646 & 0.193 & 86.742 & 1.415 & 0.069 & 0.021030 & 0.008688 & 0.306873 & 137.724 \\
\hline 8/22/08 1:00 & 2.127 & 0.667 & 0.209 & 93.875 & 1.414 & 0.068 & 0.020725 & 0.008509 & 0.300526 & 134.876 \\
\hline 8/22/08 2:00 & 2.136 & 0.676 & 0.216 & 97.036 & 1.414 & 0.068 & 0.020725 & 0.008509 & 0.300526 & 134.876 \\
\hline 8/22/08 3:00 & 2.143 & 0.683 & 0.222 & 99.538 & 1.414 & 0.068 & 0.020725 & 0.008509 & 0.300526 & 134.876 \\
\hline 8/22/08 4:00 & 2.143 & 0.683 & 0.222 & 99.538 & 1.414 & 0.068 & 0.020725 & 0.008509 & 0.300526 & 134.876 \\
\hline 8/22/08 5:00 & 2.126 & 0.666 & 0.208 & 93.527 & 1.414 & 0.068 & 0.020725 & 0.008509 & 0.300526 & 134.876 \\
\hline 8/22/08 6:00 & 2.15 & 0.690 & 0.227 & 102.078 & 1.416 & 0.07 & 0.021335 & 0.008869 & 0.313263 & 140.593 \\
\hline 8/22/08 7:00 & 2.164 & 0.704 & 0.239 & 107.275 & 1.418 & 0.072 & 0.021945 & 0.009235 & 0.326176 & 146.388 \\
\hline 8/22/08 8:00 & 2.157 & 0.697 & 0.233 & 104.657 & 1.415 & 0.069 & 0.021030 & 0.008688 & 0.306873 & 137.724 \\
\hline 8/22/08 9:00 & 2.209 & 0.749 & 0.279 & 125.039 & 1.415 & 0.069 & 0.021030 & 0.008688 & 0.306873 & 137.724 \\
\hline
\end{tabular}




\begin{tabular}{|c|c|c|c|c|c|c|c|c|c|c|}
\hline \multirow[b]{3}{*}{ Date Time } & \multicolumn{4}{|c|}{ V-Notch Weir } & \multicolumn{6}{|c|}{ R-Notch Weir } \\
\hline & \multicolumn{2}{|c|}{ Measured Water Level } & \multicolumn{2}{|c|}{$\begin{array}{c}\text { Calculated Rate } \\
\left(\mathrm{ft}^{3} / \mathrm{s}=448.8 \mathrm{gpm}\right)\end{array}$} & \multicolumn{3}{|c|}{ Measured Water Level } & \multicolumn{3}{|c|}{$\begin{array}{c}\text { Calculated Rate } \\
\left(\mathrm{ft}^{3} / \mathrm{s}=448.8 \mathrm{gpm}\right)\end{array}$} \\
\hline & $\begin{array}{c}\text { (ft above } \\
\text { sensor) }\end{array}$ & $\begin{array}{c}\text { (ft above } \\
\text { notch) }\end{array}$ & $\left(\mathrm{ft}^{3} / \mathrm{s}\right)$ & (gpm) & $\begin{array}{l}\text { (ft above } \\
\text { sensor) }\end{array}$ & $\begin{array}{c}\text { (ft above } \\
\text { log) }\end{array}$ & $\begin{array}{c}\text { (m above } \\
\text { log) }\end{array}$ & $\left(\mathrm{m}^{3} / \mathrm{s}\right)$ & $\left(\mathrm{ft}^{3} / \mathrm{s}\right)$ & (gpm) \\
\hline 8/22/08 10:00 & 2.235 & 0.775 & 0.303 & 136.055 & 1.434 & 0.088 & 0.026821 & 0.012331 & 0.435514 & 195.459 \\
\hline 8/22/08 11:00 & 2.2 & 0.740 & 0.270 & 121.355 & 1.437 & 0.091 & 0.027735 & 0.012943 & 0.457162 & 205.174 \\
\hline 8/22/08 12:00 & 2.048 & 0.588 & 0.153 & 68.767 & 1.456 & 0.11 & 0.033526 & 0.017047 & 0.602114 & 270.229 \\
\hline 8/22/08 13:00 & 2.343 & 0.883 & 0.419 & 187.942 & 1.435 & 0.089 & 0.027126 & 0.012534 & 0.442691 & 198.680 \\
\hline 8/22/08 14:00 & 2.422 & 0.962 & 0.518 & 232.417 & 1.428 & 0.082 & 0.024992 & 0.011135 & 0.393285 & 176.506 \\
\hline 8/22/08 15:00 & 2.407 & 0.947 & 0.498 & 223.535 & 1.426 & 0.08 & 0.024383 & 0.010746 & 0.379531 & 170.333 \\
\hline 8/22/08 16:00 & 2.347 & 0.887 & 0.423 & 190.058 & 1.418 & 0.072 & 0.021945 & 0.009235 & 0.326176 & 146.388 \\
\hline 8/22/08 17:00 & 2.362 & 0.902 & 0.441 & 198.123 & 1.426 & 0.08 & 0.024383 & 0.010746 & 0.379531 & 170.333 \\
\hline 8/22/08 18:00 & 2.329 & 0.869 & 0.403 & 180.645 & 1.438 & 0.092 & 0.028040 & 0.013150 & 0.464455 & 208.447 \\
\hline 8/22/08 19:00 & 2.357 & 0.897 & 0.435 & 195.412 & 1.438 & 0.092 & 0.028040 & 0.013150 & 0.464455 & 208.447 \\
\hline 8/22/08 20:00 & 2.419 & 0.959 & 0.514 & 230.624 & 1.439 & 0.093 & 0.028345 & 0.013358 & 0.471786 & 211.738 \\
\hline 8/22/08 21:00 & 2.449 & 0.989 & 0.555 & 248.932 & 1.439 & 0.093 & 0.028345 & 0.013358 & 0.471786 & 211.738 \\
\hline 8/22/08 22:00 & 2.437 & 0.977 & 0.538 & 241.508 & 1.435 & 0.089 & 0.027126 & 0.012534 & 0.442691 & 198.680 \\
\hline 8/22/08 23:00 & 2.328 & 0.868 & 0.401 & 180.130 & 1.421 & 0.075 & 0.022859 & 0.009792 & 0.345867 & 155.225 \\
\hline 8/23/08 0:00 & 2.461 & 1.001 & 0.572 & 256.490 & 1.437 & 0.091 & 0.027735 & 0.012943 & 0.457162 & 205.174 \\
\hline 8/23/08 1:00 & 2.494 & 1.034 & 0.619 & 277.982 & 1.442 & 0.096 & 0.029259 & 0.013987 & 0.494010 & 221.711 \\
\hline 8/23/08 2:00 & 2.448 & 0.988 & 0.553 & 248.308 & 1.458 & 0.112 & 0.034136 & 0.017501 & 0.618132 & 277.418 \\
\hline 8/23/08 3:00 & 2.603 & 1.143 & 0.794 & 356.488 & 1.427 & 0.081 & 0.024688 & 0.010940 & 0.386387 & 173.411 \\
\hline 8/23/08 4:00 & 2.579 & 1.119 & 0.754 & 338.193 & 1.441 & 0.095 & 0.028955 & 0.013776 & 0.486564 & 218.370 \\
\hline 8/23/08 5:00 & 2.67 & 1.210 & 0.915 & 410.658 & 1.431 & 0.085 & 0.025907 & 0.011728 & 0.414220 & 185.902 \\
\hline 8/23/08 6:00 & 2.67 & 1.210 & 0.915 & 410.658 & 1.426 & 0.08 & 0.024383 & 0.010746 & 0.379531 & 170.333 \\
\hline 8/23/08 7:00 & 2.688 & 1.228 & 0.949 & 426.000 & 1.423 & 0.077 & 0.023468 & 0.010170 & 0.359208 & 161.212 \\
\hline 8/23/08 8:00 & 2.683 & 1.223 & 0.940 & 421.705 & 1.42 & 0.074 & 0.022554 & 0.009605 & 0.339261 & 152.260 \\
\hline 8/23/08 9:00 & 2.7 & 1.240 & 0.972 & 436.416 & 1.422 & 0.076 & 0.023164 & 0.009981 & 0.352516 & 158.209 \\
\hline 8/23/08 10:00 & 2.69 & 1.230 & 0.953 & 427.726 & 1.424 & 0.078 & 0.023773 & 0.010361 & 0.365940 & 164.234 \\
\hline
\end{tabular}




\begin{tabular}{|c|c|c|c|c|c|c|c|c|c|c|}
\hline \multirow[b]{3}{*}{ Date Time } & \multicolumn{4}{|c|}{ V-Notch Weir } & \multicolumn{6}{|c|}{ R-Notch Weir } \\
\hline & \multicolumn{2}{|c|}{ Measured Water Level } & \multicolumn{2}{|c|}{$\begin{array}{c}\text { Calculated Rate } \\
\left(\mathrm{ft}^{3} / \mathrm{s}=448.8 \mathrm{gpm}\right)\end{array}$} & \multicolumn{3}{|c|}{ Measured Water Level } & \multicolumn{3}{|c|}{$\begin{array}{c}\text { Calculated Rate } \\
\left(\mathrm{ft}^{3} / \mathrm{s}=448.8 \mathrm{gpm}\right)\end{array}$} \\
\hline & $\begin{array}{c}\text { (ft above } \\
\text { sensor) }\end{array}$ & $\begin{array}{c}\text { (ft above } \\
\text { notch) }\end{array}$ & $\left(\mathrm{ft}^{3} / \mathrm{s}\right)$ & (gpm) & $\begin{array}{l}\text { (ft above } \\
\text { sensor) }\end{array}$ & $\begin{array}{c}\text { (ft above } \\
\text { log) }\end{array}$ & $\begin{array}{c}\text { (m above } \\
\text { log) }\end{array}$ & $\left(\mathrm{m}^{3} / \mathrm{s}\right)$ & $\left(\mathrm{ft}^{3} / \mathrm{s}\right)$ & (gpm) \\
\hline 8/23/08 11:00 & 2.665 & 1.205 & 0.906 & 406.456 & 1.424 & 0.078 & 0.023773 & 0.010361 & 0.365940 & 164.234 \\
\hline 8/23/08 12:00 & 2.687 & 1.227 & 0.947 & 425.139 & 1.424 & 0.078 & 0.023773 & 0.010361 & 0.365940 & 164.234 \\
\hline 8/23/08 13:00 & 2.648 & 1.188 & 0.874 & 392.364 & 1.408 & 0.062 & 0.018897 & 0.007457 & 0.263390 & 118.209 \\
\hline 8/23/08 14:00 & 2.63 & 1.170 & 0.842 & 377.766 & 1.406 & 0.06 & 0.018287 & 0.007117 & 0.251378 & 112.818 \\
\hline 8/23/08 15:00 & 2.605 & 1.145 & 0.798 & 358.039 & 1.402 & 0.056 & 0.017068 & 0.006453 & 0.227923 & 102.292 \\
\hline 8/23/08 16:00 & 2.598 & 1.138 & 0.786 & 352.629 & 1.402 & 0.056 & 0.017068 & 0.006453 & 0.227923 & 102.292 \\
\hline 8/23/08 17:00 & 2.577 & 1.117 & 0.750 & 336.695 & 1.4 & 0.054 & 0.016458 & 0.006129 & 0.216486 & 97.159 \\
\hline 8/23/08 18:00 & 2.575 & 1.115 & 0.747 & 335.200 & 1.4 & 0.054 & 0.016458 & 0.006129 & 0.216486 & 97.159 \\
\hline 8/23/08 19:00 & 2.572 & 1.112 & 0.742 & 332.966 & 1.401 & 0.055 & 0.016763 & 0.006291 & 0.222180 & 99.714 \\
\hline 8/23/08 20:00 & 2.081 & 0.621 & 0.175 & 78.687 & 1.396 & 0.05 & 0.015239 & 0.005499 & 0.194210 & 87.161 \\
\hline 8/23/08 21:00 & 2.061 & 0.601 & 0.162 & 72.579 & 1.396 & 0.05 & 0.015239 & 0.005499 & 0.194210 & 87.161 \\
\hline 8/23/08 22:00 & 2.117 & 0.657 & 0.202 & 90.436 & 1.396 & 0.05 & 0.015239 & 0.005499 & 0.194210 & 87.161 \\
\hline 8/23/08 23:00 & 2.184 & 0.724 & 0.256 & 114.969 & 1.398 & 0.052 & 0.015849 & 0.005811 & 0.205247 & 92.115 \\
\hline 8/24/08 0:00 & 2.198 & 0.738 & 0.269 & 120.546 & 1.4 & 0.054 & 0.016458 & 0.006129 & 0.216486 & 97.159 \\
\hline 8/24/08 1:00 & 2.228 & 0.768 & 0.296 & 133.034 & 1.402 & 0.056 & 0.017068 & 0.006453 & 0.227923 & 102.292 \\
\hline 8/24/08 2:00 & 2.251 & 0.791 & 0.319 & 143.114 & 1.401 & 0.055 & 0.016763 & 0.006291 & 0.222180 & 99.714 \\
\hline 8/24/08 3:00 & 2.148 & 0.688 & 0.226 & 101.348 & 1.401 & 0.055 & 0.016763 & 0.006291 & 0.222180 & 99.714 \\
\hline 8/24/08 4:00 & 2.136 & 0.676 & 0.216 & 97.036 & 1.4 & 0.054 & 0.016458 & 0.006129 & 0.216486 & 97.159 \\
\hline 8/24/08 5:00 & 2.114 & 0.654 & 0.199 & 89.419 & 1.397 & 0.051 & 0.015544 & 0.005654 & 0.199703 & 89.627 \\
\hline 8/24/08 6:00 & 2.128 & 0.668 & 0.210 & 94.223 & 1.397 & 0.051 & 0.015544 & 0.005654 & 0.199703 & 89.627 \\
\hline 8/24/08 7:00 & 2.133 & 0.673 & 0.214 & 95.975 & 1.402 & 0.056 & 0.017068 & 0.006453 & 0.227923 & 102.292 \\
\hline 8/24/08 8:00 & 2.145 & 0.685 & 0.223 & 100.260 & 1.399 & 0.053 & 0.016154 & 0.005969 & 0.210842 & 94.626 \\
\hline 8/24/08 9:00 & 2.145 & 0.685 & 0.223 & 100.260 & 1.406 & 0.06 & 0.018287 & 0.007117 & 0.251378 & 112.818 \\
\hline 8/24/08 10:00 & 2.208 & 0.748 & 0.278 & 124.627 & 1.413 & 0.067 & 0.020421 & 0.008330 & 0.294224 & 132.048 \\
\hline 8/24/08 11:00 & 2.27 & 0.810 & 0.338 & 151.775 & 1.408 & 0.062 & 0.018897 & 0.007457 & 0.263390 & 118.209 \\
\hline
\end{tabular}




\begin{tabular}{|c|c|c|c|c|c|c|c|c|c|c|}
\hline \multirow[b]{3}{*}{ Date Time } & \multicolumn{4}{|c|}{ V-Notch Weir } & \multicolumn{6}{|c|}{ R-Notch Weir } \\
\hline & \multicolumn{2}{|c|}{ Measured Water Level } & \multicolumn{2}{|c|}{$\begin{array}{c}\text { Calculated Rate } \\
\left(\mathrm{ft}^{3} / \mathrm{s}=448.8 \mathrm{gpm}\right)\end{array}$} & \multicolumn{3}{|c|}{ Measured Water Level } & \multicolumn{3}{|c|}{$\begin{array}{c}\text { Calculated Rate } \\
\left(\mathrm{ft}^{3} / \mathrm{s}=448.8 \mathrm{gpm}\right)\end{array}$} \\
\hline & $\begin{array}{c}\text { (ft above } \\
\text { sensor) }\end{array}$ & $\begin{array}{c}\text { (ft above } \\
\text { notch) }\end{array}$ & $\left(\mathrm{ft}^{3} / \mathrm{s}\right)$ & (gpm) & $\begin{array}{l}\text { (ft above } \\
\text { sensor) }\end{array}$ & $\begin{array}{c}\text { (ft above } \\
\text { log) }\end{array}$ & $\begin{array}{c}\text { (m above } \\
\text { log) }\end{array}$ & $\left(\mathrm{m}^{3} / \mathrm{s}\right)$ & $\left(\mathrm{ft}^{3} / \mathrm{s}\right)$ & (gpm) \\
\hline 8/24/08 12:00 & 2.29 & 0.830 & 0.359 & 161.224 & 1.408 & 0.062 & 0.018897 & 0.007457 & 0.263390 & 118.209 \\
\hline 8/24/08 13:00 & 2.307 & 0.847 & 0.378 & 169.527 & 1.404 & 0.058 & 0.017678 & 0.006782 & 0.239555 & 107.512 \\
\hline 8/24/08 14:00 & 2.341 & 0.881 & 0.416 & 186.889 & 1.397 & 0.051 & 0.015544 & 0.005654 & 0.199703 & 89.627 \\
\hline 8/24/08 15:00 & 2.317 & 0.857 & 0.389 & 174.528 & 1.395 & 0.049 & 0.014934 & 0.005345 & 0.188768 & 84.719 \\
\hline 8/24/08 16:00 & 2.306 & 0.846 & 0.377 & 169.032 & 1.398 & 0.052 & 0.015849 & 0.005811 & 0.205247 & 92.115 \\
\hline 8/24/08 17:00 & 2.281 & 0.821 & 0.350 & 156.930 & 1.401 & 0.055 & 0.016763 & 0.006291 & 0.222180 & 99.714 \\
\hline 8/24/08 18:00 & 2.309 & 0.849 & 0.380 & 170.520 & 1.399 & 0.053 & 0.016154 & 0.005969 & 0.210842 & 94.626 \\
\hline 8/24/08 19:00 & 2.376 & 0.916 & 0.459 & 205.832 & 1.395 & 0.049 & 0.014934 & 0.005345 & 0.188768 & 84.719 \\
\hline 8/24/08 20:00 & 2.387 & 0.927 & 0.472 & 212.014 & 1.395 & 0.049 & 0.014934 & 0.005345 & 0.188768 & 84.719 \\
\hline 8/24/08 21:00 & 2.41 & 0.950 & 0.502 & 225.295 & 1.395 & 0.049 & 0.014934 & 0.005345 & 0.188768 & 84.719 \\
\hline 8/24/08 22:00 & 2.446 & 0.986 & 0.550 & 247.063 & 1.4 & 0.054 & 0.016458 & 0.006129 & 0.216486 & 97.159 \\
\hline 8/24/08 23:00 & 2.493 & 1.033 & 0.618 & 277.315 & 1.4 & 0.054 & 0.016458 & 0.006129 & 0.216486 & 97.159 \\
\hline 8/25/08 0:00 & 2.501 & 1.041 & 0.630 & 282.674 & 1.402 & 0.056 & 0.017068 & 0.006453 & 0.227923 & 102.292 \\
\hline 8/25/08 1:00 & 2.508 & 1.048 & 0.640 & 287.414 & 1.404 & 0.058 & 0.017678 & 0.006782 & 0.239555 & 107.512 \\
\hline 8/25/08 2:00 & 2.536 & 1.076 & 0.684 & 306.849 & 1.404 & 0.058 & 0.017678 & 0.006782 & 0.239555 & 107.512 \\
\hline 8/25/08 3:00 & 2.54 & 1.080 & 0.690 & 309.687 & 1.403 & 0.057 & 0.017373 & 0.006617 & 0.233715 & 104.891 \\
\hline 8/25/08 4:00 & 2.556 & 1.096 & 0.716 & 321.200 & 1.402 & 0.056 & 0.017068 & 0.006453 & 0.227923 & 102.292 \\
\hline 8/25/08 5:00 & 2.57 & 1.110 & 0.739 & 331.481 & 1.404 & 0.058 & 0.017678 & 0.006782 & 0.239555 & 107.512 \\
\hline 8/25/08 6:00 & 2.521 & 1.061 & 0.660 & 296.342 & 1.404 & 0.058 & 0.017678 & 0.006782 & 0.239555 & 107.512 \\
\hline 8/25/08 7:00 & 2.741 & 1.281 & 1.054 & 473.151 & 1.403 & 0.057 & 0.017373 & 0.006617 & 0.233715 & 104.891 \\
\hline 8/25/08 8:00 & 2.763 & 1.303 & 1.100 & 493.601 & 1.403 & 0.057 & 0.017373 & 0.006617 & 0.233715 & 104.891 \\
\hline 8/25/08 9:00 & 2.706 & 1.246 & 0.984 & 441.681 & 1.407 & 0.061 & 0.018592 & 0.007287 & 0.257360 & 115.503 \\
\hline 8/25/08 10:00 & 2.669 & 1.209 & 0.913 & 409.816 & 1.419 & 0.073 & 0.022249 & 0.009420 & 0.332697 & 149.314 \\
\hline 8/25/08 11:00 & 2.662 & 1.202 & 0.900 & 403.948 & 1.419 & 0.073 & 0.022249 & 0.009420 & 0.332697 & 149.314 \\
\hline 8/25/08 12:00 & 2.63 & 1.170 & 0.842 & 377.766 & 1.419 & 0.073 & 0.022249 & 0.009420 & 0.332697 & 149.314 \\
\hline
\end{tabular}




\begin{tabular}{|c|c|c|c|c|c|c|c|c|c|c|}
\hline \multirow[b]{3}{*}{ Date Time } & \multicolumn{4}{|c|}{ V-Notch Weir } & \multicolumn{6}{|c|}{ R-Notch Weir } \\
\hline & \multicolumn{2}{|c|}{ Measured Water Level } & \multicolumn{2}{|c|}{$\begin{array}{c}\text { Calculated Rate } \\
\left(\mathrm{ft}^{3} / \mathrm{s}=448.8 \mathrm{gpm}\right)\end{array}$} & \multicolumn{3}{|c|}{ Measured Water Level } & \multicolumn{3}{|c|}{$\begin{array}{c}\text { Calculated Rate } \\
\left(\mathrm{ft}^{3} / \mathrm{s}=448.8 \mathrm{gpm}\right)\end{array}$} \\
\hline & $\begin{array}{c}\text { (ft above } \\
\text { sensor) }\end{array}$ & $\begin{array}{c}\text { (ft above } \\
\text { notch) }\end{array}$ & $\left(\mathrm{ft}^{3} / \mathrm{s}\right)$ & (gpm) & $\begin{array}{l}\text { (ft above } \\
\text { sensor) }\end{array}$ & $\begin{array}{c}\text { (ft above } \\
\text { log) }\end{array}$ & $\begin{array}{c}\text { (m above } \\
\text { log) }\end{array}$ & $\left(\mathrm{m}^{3} / \mathrm{s}\right)$ & $\left(\mathrm{ft}^{3} / \mathrm{s}\right)$ & (gpm) \\
\hline 8/25/08 13:00 & 2.636 & 1.176 & 0.852 & 382.595 & 1.417 & 0.071 & 0.021640 & 0.009052 & 0.319698 & 143.480 \\
\hline 8/25/08 14:00 & 2.602 & 1.142 & 0.793 & 355.714 & 1.415 & 0.069 & 0.021030 & 0.008688 & 0.306873 & 137.724 \\
\hline 8/25/08 15:00 & 2.54 & 1.080 & 0.690 & 309.687 & 1.409 & 0.063 & 0.019201 & 0.007629 & 0.269465 & 120.936 \\
\hline 8/25/08 16:00 & 2.533 & 1.073 & 0.679 & 304.730 & 1.402 & 0.056 & 0.017068 & 0.006453 & 0.227923 & 102.292 \\
\hline 8/25/08 17:00 & 2.058 & 0.598 & 0.160 & 71.689 & 1.391 & 0.045 & 0.013715 & 0.004743 & 0.167523 & 75.184 \\
\hline 8/25/08 18:00 & 2.045 & 0.585 & 0.151 & 67.905 & 1.389 & 0.043 & 0.013106 & 0.004451 & 0.157222 & 70.561 \\
\hline 8/25/08 19:00 & 2.042 & 0.582 & 0.149 & 67.049 & 1.39 & 0.044 & 0.013411 & 0.004596 & 0.162345 & 72.860 \\
\hline 8/25/08 20:00 & 2.056 & 0.596 & 0.158 & 71.099 & 1.395 & 0.049 & 0.014934 & 0.005345 & 0.188768 & 84.719 \\
\hline 8/25/08 21:00 & 2.067 & 0.607 & 0.166 & 74.381 & 1.392 & 0.046 & 0.014020 & 0.004891 & 0.172754 & 77.532 \\
\hline 8/25/08 22:00 & 2.073 & 0.613 & 0.170 & 76.208 & 1.4 & 0.054 & 0.016458 & 0.006129 & 0.216486 & 97.159 \\
\hline 8/25/08 23:00 & 2.065 & 0.605 & 0.164 & 73.777 & 1.397 & 0.051 & 0.015544 & 0.005654 & 0.199703 & 89.627 \\
\hline 8/26/08 0:00 & 2.097 & 0.637 & 0.187 & 83.788 & 1.395 & 0.049 & 0.014934 & 0.005345 & 0.188768 & 84.719 \\
\hline 8/26/08 1:00 & 2.104 & 0.644 & 0.192 & 86.080 & 1.397 & 0.051 & 0.015544 & 0.005654 & 0.199703 & 89.627 \\
\hline 8/26/08 2:00 & 2.106 & 0.646 & 0.193 & 86.742 & 1.396 & 0.05 & 0.015239 & 0.005499 & 0.194210 & 87.161 \\
\hline 8/26/08 3:00 & 2.105 & 0.645 & 0.193 & 86.410 & 1.395 & 0.049 & 0.014934 & 0.005345 & 0.188768 & 84.719 \\
\hline 8/26/08 4:00 & 2.086 & 0.626 & 0.179 & 80.260 & 1.409 & 0.063 & 0.019201 & 0.007629 & 0.269465 & 120.936 \\
\hline 8/26/08 5:00 & 2.089 & 0.629 & 0.181 & 81.213 & 1.411 & 0.065 & 0.019811 & 0.007977 & 0.281754 & 126.451 \\
\hline 8/26/08 6:00 & 2.208 & 0.748 & 0.278 & 124.627 & 1.408 & 0.062 & 0.018897 & 0.007457 & 0.263390 & 118.209 \\
\hline 8/26/08 7:00 & 2.208 & 0.748 & 0.278 & 124.627 & 1.407 & 0.061 & 0.018592 & 0.007287 & 0.257360 & 115.503 \\
\hline 8/26/08 8:00 & 2.213 & 0.753 & 0.282 & 126.698 & 1.409 & 0.063 & 0.019201 & 0.007629 & 0.269465 & 120.936 \\
\hline 8/26/08 9:00 & 2.21 & 0.750 & 0.280 & 125.453 & 1.409 & 0.063 & 0.019201 & 0.007629 & 0.269465 & 120.936 \\
\hline 8/26/08 10:00 & 2.22 & 0.760 & 0.289 & 129.632 & 1.412 & 0.066 & 0.020116 & 0.008153 & 0.287966 & 129.239 \\
\hline 8/26/08 11:00 & 2.209 & 0.749 & 0.279 & 125.039 & 1.407 & 0.061 & 0.018592 & 0.007287 & 0.257360 & 115.503 \\
\hline 8/26/08 12:00 & 2.19 & 0.730 & 0.261 & 117.339 & 1.412 & 0.066 & 0.020116 & 0.008153 & 0.287966 & 129.239 \\
\hline 8/26/08 13:00 & 2.177 & 0.717 & 0.250 & 112.239 & 1.403 & 0.057 & 0.017373 & 0.006617 & 0.233715 & 104.891 \\
\hline
\end{tabular}




\begin{tabular}{|c|c|c|c|c|c|c|c|c|c|c|}
\hline \multirow[b]{3}{*}{ Date Time } & \multicolumn{4}{|c|}{ V-Notch Weir } & \multicolumn{6}{|c|}{ R-Notch Weir } \\
\hline & \multicolumn{2}{|c|}{ Measured Water Level } & \multicolumn{2}{|c|}{$\begin{array}{c}\text { Calculated Rate } \\
\left(\mathrm{ft}^{3} / \mathrm{s}=448.8 \mathrm{gpm}\right)\end{array}$} & \multicolumn{3}{|c|}{ Measured Water Level } & \multicolumn{3}{|c|}{$\begin{array}{c}\text { Calculated Rate } \\
\left(\mathrm{ft}^{3} / \mathrm{s}=448.8 \mathrm{gpm}\right)\end{array}$} \\
\hline & $\begin{array}{c}\text { (ft above } \\
\text { sensor) }\end{array}$ & $\begin{array}{c}\text { (ft above } \\
\text { notch) }\end{array}$ & $\left(\mathrm{ft}^{3} / \mathrm{s}\right)$ & (gpm) & $\begin{array}{l}\text { (ft above } \\
\text { sensor) }\end{array}$ & $\begin{array}{c}\text { (ft above } \\
\text { log) }\end{array}$ & $\begin{array}{c}\text { (m above } \\
\text { log) }\end{array}$ & $\left(\mathrm{m}^{3} / \mathrm{s}\right)$ & $\left(\mathrm{ft}^{3} / \mathrm{s}\right)$ & (gpm) \\
\hline 8/26/08 14:00 & 2.168 & 0.708 & 0.242 & 108.788 & 1.403 & 0.057 & 0.017373 & 0.006617 & 0.233715 & 104.891 \\
\hline 8/26/08 15:00 & 2.174 & 0.714 & 0.248 & 111.082 & 1.406 & 0.06 & 0.018287 & 0.007117 & 0.251378 & 112.818 \\
\hline 8/26/08 16:00 & 2.156 & 0.696 & 0.232 & 104.286 & 1.406 & 0.06 & 0.018287 & 0.007117 & 0.251378 & 112.818 \\
\hline 8/26/08 17:00 & 2.138 & 0.678 & 0.218 & 97.747 & 1.4 & 0.054 & 0.016458 & 0.006129 & 0.216486 & 97.159 \\
\hline 8/26/08 18:00 & 2.136 & 0.676 & 0.216 & 97.036 & 1.4 & 0.054 & 0.016458 & 0.006129 & 0.216486 & 97.159 \\
\hline 8/26/08 19:00 & 2.135 & 0.675 & 0.215 & 96.681 & 1.398 & 0.052 & 0.015849 & 0.005811 & 0.205247 & 92.115 \\
\hline 8/26/08 20:00 & 2.14 & 0.680 & 0.219 & 98.461 & 1.403 & 0.057 & 0.017373 & 0.006617 & 0.233715 & 104.891 \\
\hline 8/26/08 21:00 & 2.148 & 0.688 & 0.226 & 101.348 & 1.401 & 0.055 & 0.016763 & 0.006291 & 0.222180 & 99.714 \\
\hline 8/26/08 22:00 & 2.148 & 0.688 & 0.226 & 101.348 & 1.401 & 0.055 & 0.016763 & 0.006291 & 0.222180 & 99.714 \\
\hline 8/26/08 23:00 & 2.159 & 0.699 & 0.235 & 105.401 & 1.406 & 0.06 & 0.018287 & 0.007117 & 0.251378 & 112.818 \\
\hline 8/27/08 0:00 & 2.17 & 0.710 & 0.244 & 109.549 & 1.406 & 0.06 & 0.018287 & 0.007117 & 0.251378 & 112.818 \\
\hline 8/27/08 1:00 & 2.181 & 0.721 & 0.254 & 113.794 & 1.405 & 0.059 & 0.017982 & 0.006949 & 0.245443 & 110.155 \\
\hline 8/27/08 2:00 & 2.183 & 0.723 & 0.255 & 114.576 & 1.408 & 0.062 & 0.018897 & 0.007457 & 0.263390 & 118.209 \\
\hline 8/27/08 3:00 & 2.192 & 0.732 & 0.263 & 118.136 & 1.409 & 0.063 & 0.019201 & 0.007629 & 0.269465 & 120.936 \\
\hline 8/27/08 4:00 & 2.192 & 0.732 & 0.263 & 118.136 & 1.409 & 0.063 & 0.019201 & 0.007629 & 0.269465 & 120.936 \\
\hline 8/27/08 5:00 & 2.194 & 0.734 & 0.265 & 118.936 & 1.409 & 0.063 & 0.019201 & 0.007629 & 0.269465 & 120.936 \\
\hline 8/27/08 6:00 & 2.204 & 0.744 & 0.274 & 122.984 & 1.411 & 0.065 & 0.019811 & 0.007977 & 0.281754 & 126.451 \\
\hline 8/27/08 7:00 & 2.206 & 0.746 & 0.276 & 123.804 & 1.41 & 0.064 & 0.019506 & 0.007803 & 0.275587 & 123.683 \\
\hline 8/27/08 8:00 & 2.211 & 0.751 & 0.280 & 125.867 & 1.412 & 0.066 & 0.020116 & 0.008153 & 0.287966 & 129.239 \\
\hline 8/27/08 9:00 & 2.603 & 1.143 & 0.794 & 356.488 & 1.5 & 0.154 & 0.046937 & 0.027891 & 0.985101 & 442.113 \\
\hline 8/27/08 10:00 & 2.692 & 1.232 & 0.957 & 429.456 & 1.525 & 0.179 & 0.054557 & 0.034799 & 1.229112 & 551.626 \\
\hline 8/27/08 11:00 & 2.566 & 1.106 & 0.732 & 328.524 & 1.452 & 0.106 & 0.032307 & 0.016152 & 0.570504 & 256.042 \\
\hline 8/27/08 12:00 & 2.521 & 1.061 & 0.660 & 296.342 & 1.419 & 0.073 & 0.022249 & 0.009420 & 0.332697 & 149.314 \\
\hline 8/27/08 13:00 & 2.502 & 1.042 & 0.631 & 283.348 & 1.417 & 0.071 & 0.021640 & 0.009052 & 0.319698 & 143.480 \\
\hline 8/27/08 14:00 & 2.482 & 1.022 & 0.602 & 270.047 & 1.408 & 0.062 & 0.018897 & 0.007457 & 0.263390 & 118.209 \\
\hline
\end{tabular}




\begin{tabular}{|c|c|c|c|c|c|c|c|c|c|c|}
\hline \multirow[b]{3}{*}{ Date Time } & \multicolumn{4}{|c|}{ V-Notch Weir } & \multicolumn{6}{|c|}{ R-Notch Weir } \\
\hline & \multicolumn{2}{|c|}{ Measured Water Level } & \multicolumn{2}{|c|}{$\begin{array}{c}\text { Calculated Rate } \\
\left(\mathrm{ft}^{3} / \mathrm{s}=448.8 \mathrm{gpm}\right)\end{array}$} & \multicolumn{3}{|c|}{ Measured Water Level } & \multicolumn{3}{|c|}{$\begin{array}{c}\text { Calculated Rate } \\
\left(\mathrm{ft}^{3} / \mathrm{s}=448.8 \mathrm{gpm}\right)\end{array}$} \\
\hline & $\begin{array}{c}\text { (ft above } \\
\text { sensor) }\end{array}$ & $\begin{array}{c}\text { (ft above } \\
\text { notch) }\end{array}$ & $\left(\mathrm{ft}^{3} / \mathrm{s}\right)$ & (gpm) & $\begin{array}{l}\text { (ft above } \\
\text { sensor) }\end{array}$ & $\begin{array}{c}\text { (ft above } \\
\text { log) }\end{array}$ & $\begin{array}{c}\text { (m above } \\
\text { log) }\end{array}$ & $\left(\mathrm{m}^{3} / \mathrm{s}\right)$ & $\left(\mathrm{ft}^{3} / \mathrm{s}\right)$ & (gpm) \\
\hline 8/27/08 15:00 & 2.449 & 0.989 & 0.555 & 248.932 & 1.404 & 0.058 & 0.017678 & 0.006782 & 0.239555 & 107.512 \\
\hline 8/27/08 16:00 & 2.429 & 0.969 & 0.527 & 236.633 & 1.402 & 0.056 & 0.017068 & 0.006453 & 0.227923 & 102.292 \\
\hline 8/27/08 17:00 & 2.406 & 0.946 & 0.497 & 222.950 & 1.398 & 0.052 & 0.015849 & 0.005811 & 0.205247 & 92.115 \\
\hline 8/27/08 18:00 & 2.413 & 0.953 & 0.506 & 227.063 & 1.401 & 0.055 & 0.016763 & 0.006291 & 0.222180 & 99.714 \\
\hline 8/27/08 19:00 & 2.401 & 0.941 & 0.490 & 220.040 & 1.402 & 0.056 & 0.017068 & 0.006453 & 0.227923 & 102.292 \\
\hline 8/27/08 20:00 & 2.407 & 0.947 & 0.498 & 223.535 & 1.402 & 0.056 & 0.017068 & 0.006453 & 0.227923 & 102.292 \\
\hline 8/27/08 21:00 & 2.42 & 0.960 & 0.515 & 231.221 & 1.405 & 0.059 & 0.017982 & 0.006949 & 0.245443 & 110.155 \\
\hline 8/27/08 22:00 & 2.429 & 0.969 & 0.527 & 236.633 & 1.405 & 0.059 & 0.017982 & 0.006949 & 0.245443 & 110.155 \\
\hline 8/27/08 23:00 & 2.435 & 0.975 & 0.535 & 240.284 & 1.407 & 0.061 & 0.018592 & 0.007287 & 0.257360 & 115.503 \\
\hline 8/28/08 0:00 & 2.444 & 0.984 & 0.548 & 245.822 & 1.41 & 0.064 & 0.019506 & 0.007803 & 0.275587 & 123.683 \\
\hline 8/28/08 1:00 & 2.443 & 0.983 & 0.546 & 245.203 & 1.41 & 0.064 & 0.019506 & 0.007803 & 0.275587 & 123.683 \\
\hline 8/28/08 2:00 & 2.438 & 0.978 & 0.539 & 242.121 & 1.407 & 0.061 & 0.018592 & 0.007287 & 0.257360 & 115.503 \\
\hline 8/28/08 3:00 & 2.44 & 0.980 & 0.542 & 243.351 & 1.407 & 0.061 & 0.018592 & 0.007287 & 0.257360 & 115.503 \\
\hline 8/28/08 4:00 & 2.447 & 0.987 & 0.552 & 247.685 & 1.407 & 0.061 & 0.018592 & 0.007287 & 0.257360 & 115.503 \\
\hline 8/28/08 5:00 & 2.452 & 0.992 & 0.559 & 250.809 & 1.409 & 0.063 & 0.019201 & 0.007629 & 0.269465 & 120.936 \\
\hline 8/28/08 6:00 & 2.454 & 0.994 & 0.562 & 252.065 & 1.409 & 0.063 & 0.019201 & 0.007629 & 0.269465 & 120.936 \\
\hline 8/28/08 7:00 & 2.453 & 0.993 & 0.560 & 251.436 & 1.406 & 0.06 & 0.018287 & 0.007117 & 0.251378 & 112.818 \\
\hline 8/28/08 8:00 & 2.456 & 0.996 & 0.564 & 253.324 & 1.405 & 0.059 & 0.017982 & 0.006949 & 0.245443 & 110.155 \\
\hline 8/28/08 9:00 & 2.453 & 0.993 & 0.560 & 251.436 & 1.408 & 0.062 & 0.018897 & 0.007457 & 0.263390 & 118.209 \\
\hline 8/28/08 10:00 & 2.458 & 0.998 & 0.567 & 254.588 & 1.405 & 0.059 & 0.017982 & 0.006949 & 0.245443 & 110.155 \\
\hline 8/28/08 11:00 & 2.452 & 0.992 & 0.559 & 250.809 & 1.405 & 0.059 & 0.017982 & 0.006949 & 0.245443 & 110.155 \\
\hline 8/28/08 12:00 & 2.443 & 0.983 & 0.546 & 245.203 & 1.403 & 0.057 & 0.017373 & 0.006617 & 0.233715 & 104.891 \\
\hline 8/28/08 13:00 & 2.194 & 0.734 & 0.265 & 118.936 & 1.401 & 0.055 & 0.016763 & 0.006291 & 0.222180 & 99.714 \\
\hline 8/28/08 14:00 & 2.206 & 0.746 & 0.276 & 123.804 & 1.399 & 0.053 & 0.016154 & 0.005969 & 0.210842 & 94.626 \\
\hline 8/28/08 15:00 & 2.259 & 0.799 & 0.327 & 146.723 & 1.397 & 0.051 & 0.015544 & 0.005654 & 0.199703 & 89.627 \\
\hline
\end{tabular}




\begin{tabular}{|c|c|c|c|c|c|c|c|c|c|c|}
\hline \multirow[b]{3}{*}{ Date Time } & \multicolumn{4}{|c|}{ V-Notch Weir } & \multicolumn{6}{|c|}{ R-Notch Weir } \\
\hline & \multicolumn{2}{|c|}{ Measured Water Level } & \multicolumn{2}{|c|}{$\begin{array}{c}\text { Calculated Rate } \\
\left(\mathrm{ft}^{3} / \mathrm{s}=448.8 \mathrm{gpm}\right)\end{array}$} & \multicolumn{3}{|c|}{ Measured Water Level } & \multicolumn{3}{|c|}{$\begin{array}{c}\text { Calculated Rate } \\
\left(\mathrm{ft}^{3} / \mathrm{s}=448.8 \mathrm{gpm}\right)\end{array}$} \\
\hline & $\begin{array}{c}\text { (ft above } \\
\text { sensor) }\end{array}$ & $\begin{array}{c}\text { (ft above } \\
\text { notch) }\end{array}$ & $\left(\mathrm{ft}^{3} / \mathrm{s}\right)$ & (gpm) & $\begin{array}{l}\text { (ft above } \\
\text { sensor) }\end{array}$ & $\begin{array}{c}\text { (ft above } \\
\text { log) }\end{array}$ & $\begin{array}{c}\text { (m above } \\
\text { log) }\end{array}$ & $\left(\mathrm{m}^{3} / \mathrm{s}\right)$ & $\left(\mathrm{ft}^{3} / \mathrm{s}\right)$ & (gpm) \\
\hline 8/28/08 16:00 & 2.139 & 0.679 & 0.219 & 98.103 & 1.395 & 0.049 & 0.014934 & 0.005345 & 0.188768 & 84.719 \\
\hline 8/28/08 17:00 & 2.064 & 0.604 & 0.164 & 73.477 & 1.396 & 0.05 & 0.015239 & 0.005499 & 0.194210 & 87.161 \\
\hline 8/28/08 18:00 & 2.214 & 0.754 & 0.283 & 127.114 & 1.399 & 0.053 & 0.016154 & 0.005969 & 0.210842 & 94.626 \\
\hline 8/28/08 19:00 & 2.07 & 0.610 & 0.168 & 75.291 & 1.402 & 0.056 & 0.017068 & 0.006453 & 0.227923 & 102.292 \\
\hline 8/28/08 20:00 & 2.266 & 0.806 & 0.334 & 149.926 & 1.407 & 0.061 & 0.018592 & 0.007287 & 0.257360 & 115.503 \\
\hline 8/28/08 21:00 & 2.246 & 0.786 & 0.314 & 140.885 & 1.407 & 0.061 & 0.018592 & 0.007287 & 0.257360 & 115.503 \\
\hline 8/28/08 22:00 & 2.29 & 0.830 & 0.359 & 161.224 & 1.405 & 0.059 & 0.017982 & 0.006949 & 0.245443 & 110.155 \\
\hline 8/28/08 23:00 & 2.458 & 0.998 & 0.567 & 254.588 & 1.407 & 0.061 & 0.018592 & 0.007287 & 0.257360 & 115.503 \\
\hline 8/29/08 0:00 & 2.248 & 0.788 & 0.316 & 141.774 & 1.402 & 0.056 & 0.017068 & 0.006453 & 0.227923 & 102.292 \\
\hline 8/29/08 1:00 & 2.346 & 0.886 & 0.422 & 189.528 & 1.403 & 0.057 & 0.017373 & 0.006617 & 0.233715 & 104.891 \\
\hline 8/29/08 2:00 & 2.146 & 0.686 & 0.224 & 100.622 & 1.402 & 0.056 & 0.017068 & 0.006453 & 0.227923 & 102.292 \\
\hline 8/29/08 3:00 & 2.411 & 0.951 & 0.503 & 225.883 & 1.406 & 0.06 & 0.018287 & 0.007117 & 0.251378 & 112.818 \\
\hline 8/29/08 4:00 & 2.3 & 0.840 & 0.370 & 166.078 & 1.403 & 0.057 & 0.017373 & 0.006617 & 0.233715 & 104.891 \\
\hline 8/29/08 5:00 & 2.147 & 0.687 & 0.225 & 100.985 & 1.402 & 0.056 & 0.017068 & 0.006453 & 0.227923 & 102.292 \\
\hline 8/29/08 6:00 & 2.203 & 0.743 & 0.273 & 122.576 & 1.402 & 0.056 & 0.017068 & 0.006453 & 0.227923 & 102.292 \\
\hline 8/29/08 7:00 & 2.483 & 1.023 & 0.603 & 270.703 & 1.401 & 0.055 & 0.016763 & 0.006291 & 0.222180 & 99.714 \\
\hline 8/29/08 8:00 & 2.436 & 0.976 & 0.537 & 240.895 & 1.403 & 0.057 & 0.017373 & 0.006617 & 0.233715 & 104.891 \\
\hline 8/29/08 9:00 & 2.14 & 0.680 & 0.219 & 98.461 & 1.403 & 0.057 & 0.017373 & 0.006617 & 0.233715 & 104.891 \\
\hline 8/29/08 10:00 & 2.142 & 0.682 & 0.221 & 99.178 & 1.403 & 0.057 & 0.017373 & 0.006617 & 0.233715 & 104.891 \\
\hline 8/29/08 11:00 & 2.138 & 0.678 & 0.218 & 97.747 & 1.403 & 0.057 & 0.017373 & 0.006617 & 0.233715 & 104.891 \\
\hline 8/29/08 12:00 & 2.125 & 0.665 & 0.208 & 93.181 & 1.401 & 0.055 & 0.016763 & 0.006291 & 0.222180 & 99.714 \\
\hline 8/29/08 13:00 & 2.098 & 0.638 & 0.187 & 84.113 & 1.399 & 0.053 & 0.016154 & 0.005969 & 0.210842 & 94.626 \\
\hline 8/29/08 14:00 & 2.081 & 0.621 & 0.175 & 78.687 & 1.397 & 0.051 & 0.015544 & 0.005654 & 0.199703 & 89.627 \\
\hline 8/29/08 15:00 & 2.248 & 0.788 & 0.316 & 141.774 & 1.395 & 0.049 & 0.014934 & 0.005345 & 0.188768 & 84.719 \\
\hline 8/29/08 16:00 & 2.284 & 0.824 & 0.353 & 158.354 & 1.396 & 0.05 & 0.015239 & 0.005499 & 0.194210 & 87.161 \\
\hline
\end{tabular}




\begin{tabular}{|c|c|c|c|c|c|c|c|c|c|c|}
\hline \multirow[b]{3}{*}{ Date Time } & \multicolumn{4}{|c|}{ V-Notch Weir } & \multicolumn{6}{|c|}{ R-Notch Weir } \\
\hline & \multicolumn{2}{|c|}{ Measured Water Level } & \multicolumn{2}{|c|}{$\begin{array}{c}\text { Calculated Rate } \\
\left(\mathrm{ft}^{3} / \mathrm{s}=448.8 \mathrm{gpm}\right)\end{array}$} & \multicolumn{3}{|c|}{ Measured Water Level } & \multicolumn{3}{|c|}{$\begin{array}{c}\text { Calculated Rate } \\
\left(\mathrm{ft}^{3} / \mathrm{s}=448.8 \mathrm{gpm}\right)\end{array}$} \\
\hline & $\begin{array}{c}\text { (ft above } \\
\text { sensor) }\end{array}$ & $\begin{array}{c}\text { (ft above } \\
\text { notch) }\end{array}$ & $\left(\mathrm{ft}^{3} / \mathrm{s}\right)$ & (gpm) & $\begin{array}{l}\text { (ft above } \\
\text { sensor) }\end{array}$ & $\begin{array}{c}\text { (ft above } \\
\text { log) }\end{array}$ & $\begin{array}{c}\text { (m above } \\
\text { log) }\end{array}$ & $\left(\mathrm{m}^{3} / \mathrm{s}\right)$ & $\left(\mathrm{ft}^{3} / \mathrm{s}\right)$ & (gpm) \\
\hline 8/29/08 17:00 & 2.311 & 0.851 & 0.382 & 171.517 & 1.397 & 0.051 & 0.015544 & 0.005654 & 0.199703 & 89.627 \\
\hline 8/29/08 18:00 & 2.043 & 0.583 & 0.150 & 67.334 & 1.397 & 0.051 & 0.015544 & 0.005654 & 0.199703 & 89.627 \\
\hline 8/29/08 19:00 & 2.054 & 0.594 & 0.157 & 70.511 & 1.4 & 0.054 & 0.016458 & 0.006129 & 0.216486 & 97.159 \\
\hline 8/29/08 20:00 & 2.285 & 0.825 & 0.354 & 158.830 & 1.403 & 0.057 & 0.017373 & 0.006617 & 0.233715 & 104.891 \\
\hline 8/29/08 21:00 & 2.3 & 0.840 & 0.370 & 166.078 & 1.403 & 0.057 & 0.017373 & 0.006617 & 0.233715 & 104.891 \\
\hline 8/29/08 22:00 & 2.33 & 0.870 & 0.404 & 181.160 & 1.406 & 0.06 & 0.018287 & 0.007117 & 0.251378 & 112.818 \\
\hline 8/29/08 23:00 & 2.077 & 0.617 & 0.173 & 77.442 & 1.406 & 0.06 & 0.018287 & 0.007117 & 0.251378 & 112.818 \\
\hline 8/30/08 0:00 & 2.095 & 0.635 & 0.185 & 83.140 & 1.408 & 0.062 & 0.018897 & 0.007457 & 0.263390 & 118.209 \\
\hline 8/30/08 1:00 & 2.344 & 0.884 & 0.420 & 188.469 & 1.405 & 0.059 & 0.017982 & 0.006949 & 0.245443 & 110.155 \\
\hline 8/30/08 2:00 & 2.397 & 0.937 & 0.485 & 217.729 & 1.407 & 0.061 & 0.018592 & 0.007287 & 0.257360 & 115.503 \\
\hline 8/30/08 3:00 & 2.31 & 0.850 & 0.381 & 171.018 & 1.406 & 0.06 & 0.018287 & 0.007117 & 0.251378 & 112.818 \\
\hline 8/30/08 4:00 & 2.357 & 0.897 & 0.435 & 195.412 & 1.408 & 0.062 & 0.018897 & 0.007457 & 0.263390 & 118.209 \\
\hline 8/30/08 5:00 & 2.354 & 0.894 & 0.432 & 193.797 & 1.409 & 0.063 & 0.019201 & 0.007629 & 0.269465 & 120.936 \\
\hline 8/30/08 6:00 & 2.424 & 0.964 & 0.521 & 233.617 & 1.409 & 0.063 & 0.019201 & 0.007629 & 0.269465 & 120.936 \\
\hline 8/30/08 7:00 & 2.455 & 0.995 & 0.563 & 252.694 & 1.408 & 0.062 & 0.018897 & 0.007457 & 0.263390 & 118.209 \\
\hline 8/30/08 8:00 & 2.499 & 1.039 & 0.627 & 281.329 & 1.405 & 0.059 & 0.017982 & 0.006949 & 0.245443 & 110.155 \\
\hline 8/30/08 9:00 & 2.135 & 0.675 & 0.215 & 96.681 & 1.407 & 0.061 & 0.018592 & 0.007287 & 0.257360 & 115.503 \\
\hline 8/30/08 10:00 & 2.142 & 0.682 & 0.221 & 99.178 & 1.407 & 0.061 & 0.018592 & 0.007287 & 0.257360 & 115.503 \\
\hline 8/30/08 11:00 & 2.134 & 0.674 & 0.215 & 96.328 & 1.408 & 0.062 & 0.018897 & 0.007457 & 0.263390 & 118.209 \\
\hline 8/30/08 12:00 & 2.318 & 0.858 & 0.390 & 175.033 & 1.405 & 0.059 & 0.017982 & 0.006949 & 0.245443 & 110.155 \\
\hline 8/30/08 13:00 & 2.094 & 0.634 & 0.185 & 82.817 & 1.403 & 0.057 & 0.017373 & 0.006617 & 0.233715 & 104.891 \\
\hline 8/30/08 14:00 & 2.259 & 0.799 & 0.327 & 146.723 & 1.399 & 0.053 & 0.016154 & 0.005969 & 0.210842 & 94.626 \\
\hline 8/30/08 15:00 & 2.388 & 0.928 & 0.474 & 212.581 & 1.397 & 0.051 & 0.015544 & 0.005654 & 0.199703 & 89.627 \\
\hline 8/30/08 16:00 & 2.044 & 0.584 & 0.151 & 67.619 & 1.398 & 0.052 & 0.015849 & 0.005811 & 0.205247 & 92.115 \\
\hline 8/30/08 17:00 & 2.317 & 0.857 & 0.389 & 174.528 & 1.396 & 0.05 & 0.015239 & 0.005499 & 0.194210 & 87.161 \\
\hline
\end{tabular}




\begin{tabular}{|c|c|c|c|c|c|c|c|c|c|c|}
\hline \multirow[b]{3}{*}{ Date Time } & \multicolumn{4}{|c|}{ V-Notch Weir } & \multicolumn{6}{|c|}{ R-Notch Weir } \\
\hline & \multicolumn{2}{|c|}{ Measured Water Level } & \multicolumn{2}{|c|}{$\begin{array}{c}\text { Calculated Rate } \\
\left(\mathrm{ft}^{3} / \mathrm{s}=448.8 \mathrm{gpm}\right)\end{array}$} & \multicolumn{3}{|c|}{ Measured Water Level } & \multicolumn{3}{|c|}{$\begin{array}{c}\text { Calculated Rate } \\
\left(\mathrm{ft}^{3} / \mathrm{s}=448.8 \mathrm{gpm}\right)\end{array}$} \\
\hline & $\begin{array}{c}\text { (ft above } \\
\text { sensor) }\end{array}$ & $\begin{array}{c}\text { (ft above } \\
\text { notch) }\end{array}$ & $\left(\mathrm{ft}^{3} / \mathrm{s}\right)$ & (gpm) & $\begin{array}{c}\text { (ft above } \\
\text { sensor) }\end{array}$ & $\begin{array}{c}\text { (ft above } \\
\text { log) }\end{array}$ & $\begin{array}{c}\text { (m above } \\
\text { log) }\end{array}$ & $\left(\mathrm{m}^{3} / \mathrm{s}\right)$ & $\left(\mathrm{ft}^{3} / \mathrm{s}\right)$ & (gpm) \\
\hline 8/30/08 18:00 & 2.213 & 0.753 & 0.282 & 126.698 & 1.399 & 0.053 & 0.016154 & 0.005969 & 0.210842 & 94.626 \\
\hline 8/30/08 19:00 & 2.309 & 0.849 & 0.380 & 170.520 & 1.405 & 0.059 & 0.017982 & 0.006949 & 0.245443 & 110.155 \\
\hline 8/30/08 20:00 & 2.27 & 0.810 & 0.338 & 151.775 & 1.407 & 0.061 & 0.018592 & 0.007287 & 0.257360 & 115.503 \\
\hline 8/30/08 21:00 & 2.29 & 0.830 & 0.359 & 161.224 & 1.403 & 0.057 & 0.017373 & 0.006617 & 0.233715 & 104.891 \\
\hline 8/30/08 22:00 & 2.301 & 0.841 & 0.371 & 166.568 & 1.405 & 0.059 & 0.017982 & 0.006949 & 0.245443 & 110.155 \\
\hline 8/30/08 23:00 & 2.387 & 0.927 & 0.472 & 212.014 & 1.41 & 0.064 & 0.019506 & 0.007803 & 0.275587 & 123.683 \\
\hline 8/31/08 0:00 & 2.408 & 0.948 & 0.499 & 224.121 & 1.41 & 0.064 & 0.019506 & 0.007803 & 0.275587 & 123.683 \\
\hline 8/31/08 1:00 & 2.398 & 0.938 & 0.486 & 218.305 & 1.407 & 0.061 & 0.018592 & 0.007287 & 0.257360 & 115.503 \\
\hline 8/31/08 2:00 & 2.332 & 0.872 & 0.406 & 182.194 & 1.405 & 0.059 & 0.017982 & 0.006949 & 0.245443 & 110.155 \\
\hline 8/31/08 3:00 & 2.425 & 0.965 & 0.522 & 234.219 & 1.406 & 0.06 & 0.018287 & 0.007117 & 0.251378 & 112.818 \\
\hline 8/31/08 4:00 & 2.437 & 0.977 & 0.538 & 241.508 & 1.408 & 0.062 & 0.018897 & 0.007457 & 0.263390 & 118.209 \\
\hline 8/31/08 5:00 & 2.453 & 0.993 & 0.560 & 251.436 & 1.405 & 0.059 & 0.017982 & 0.006949 & 0.245443 & 110.155 \\
\hline 8/31/08 6:00 & 2.455 & 0.995 & 0.563 & 252.694 & 1.407 & 0.061 & 0.018592 & 0.007287 & 0.257360 & 115.503 \\
\hline 8/31/08 7:00 & 2.425 & 0.965 & 0.522 & 234.219 & 1.409 & 0.063 & 0.019201 & 0.007629 & 0.269465 & 120.936 \\
\hline 8/31/08 8:00 & 2.413 & 0.953 & 0.506 & 227.063 & 1.409 & 0.063 & 0.019201 & 0.007629 & 0.269465 & 120.936 \\
\hline 8/31/08 9:00 & 2.145 & 0.685 & 0.223 & 100.260 & 1.411 & 0.065 & 0.019811 & 0.007977 & 0.281754 & 126.451 \\
\hline 8/31/08 10:00 & 2.244 & 0.784 & 0.312 & 139.999 & 1.409 & 0.063 & 0.019201 & 0.007629 & 0.269465 & 120.936 \\
\hline 8/31/08 11:00 & 2.355 & 0.895 & 0.433 & 194.334 & 1.409 & 0.063 & 0.019201 & 0.007629 & 0.269465 & 120.936 \\
\hline 8/31/08 12:00 & 2.351 & 0.891 & 0.428 & 192.189 & 1.407 & 0.061 & 0.018592 & 0.007287 & 0.257360 & 115.503 \\
\hline 8/31/08 13:00 & 2.341 & 0.881 & 0.416 & 186.889 & 1.405 & 0.059 & 0.017982 & 0.006949 & 0.245443 & 110.155 \\
\hline 8/31/08 14:00 & 2.341 & 0.881 & 0.416 & 186.889 & 1.403 & 0.057 & 0.017373 & 0.006617 & 0.233715 & 104.891 \\
\hline 8/31/08 15:00 & 2.328 & 0.868 & 0.401 & 180.130 & 1.399 & 0.053 & 0.016154 & 0.005969 & 0.210842 & 94.626 \\
\hline 8/31/08 16:00 & 2.332 & 0.872 & 0.406 & 182.194 & 1.4 & 0.054 & 0.016458 & 0.006129 & 0.216486 & 97.159 \\
\hline 8/31/08 17:00 & 2.032 & 0.572 & 0.143 & 64.244 & 1.403 & 0.057 & 0.017373 & 0.006617 & 0.233715 & 104.891 \\
\hline 8/31/08 18:00 & 2.029 & 0.569 & 0.141 & 63.416 & 1.403 & 0.057 & 0.017373 & 0.006617 & 0.233715 & 104.891 \\
\hline
\end{tabular}




\begin{tabular}{|c|c|c|c|c|c|c|c|c|c|c|}
\hline \multirow[b]{3}{*}{ Date Time } & \multicolumn{4}{|c|}{ V-Notch Weir } & \multicolumn{6}{|c|}{ R-Notch Weir } \\
\hline & \multicolumn{2}{|c|}{ Measured Water Level } & \multicolumn{2}{|c|}{$\begin{array}{c}\text { Calculated Rate } \\
\left(\mathrm{ft}^{3} / \mathrm{s}=448.8 \mathrm{gpm}\right)\end{array}$} & \multicolumn{3}{|c|}{ Measured Water Level } & \multicolumn{3}{|c|}{$\begin{array}{c}\text { Calculated Rate } \\
\left(\mathrm{ft}^{3} / \mathrm{s}=448.8 \mathrm{gpm}\right)\end{array}$} \\
\hline & $\begin{array}{c}\text { (ft above } \\
\text { sensor) }\end{array}$ & $\begin{array}{c}\text { (ft above } \\
\text { notch) }\end{array}$ & $\left(\mathrm{ft}^{3} / \mathrm{s}\right)$ & (gpm) & $\begin{array}{l}\text { (ft above } \\
\text { sensor) }\end{array}$ & $\begin{array}{c}\text { (ft above } \\
\text { log) }\end{array}$ & $\begin{array}{c}\text { (m above } \\
\text { log) }\end{array}$ & $\left(\mathrm{m}^{3} / \mathrm{s}\right)$ & $\left(\mathrm{ft}^{3} / \mathrm{s}\right)$ & (gpm) \\
\hline 8/31/08 19:00 & 2.303 & 0.843 & 0.373 & 167.551 & 1.406 & 0.06 & 0.018287 & 0.007117 & 0.251378 & 112.818 \\
\hline 8/31/08 20:00 & 2.352 & 0.892 & 0.429 & 192.724 & 1.407 & 0.061 & 0.018592 & 0.007287 & 0.257360 & 115.503 \\
\hline 8/31/08 21:00 & 2.229 & 0.769 & 0.297 & 133.464 & 1.407 & 0.061 & 0.018592 & 0.007287 & 0.257360 & 115.503 \\
\hline 8/31/08 22:00 & 2.322 & 0.862 & 0.395 & 177.061 & 1.407 & 0.061 & 0.018592 & 0.007287 & 0.257360 & 115.503 \\
\hline 8/31/08 23:00 & 2.392 & 0.932 & 0.479 & 214.860 & 1.41 & 0.064 & 0.019506 & 0.007803 & 0.275587 & 123.683 \\
\hline 9/1/08 0:00 & 2.403 & 0.943 & 0.493 & 221.201 & 1.41 & 0.064 & 0.019506 & 0.007803 & 0.275587 & 123.683 \\
\hline 9/1/08 1:00 & 2.425 & 0.965 & 0.522 & 234.219 & 1.41 & 0.064 & 0.019506 & 0.007803 & 0.275587 & 123.683 \\
\hline 9/1/08 2:00 & 2.324 & 0.864 & 0.397 & 178.081 & 1.409 & 0.063 & 0.019201 & 0.007629 & 0.269465 & 120.936 \\
\hline 9/1/08 3:00 & 2.133 & 0.673 & 0.214 & 95.975 & 1.411 & 0.065 & 0.019811 & 0.007977 & 0.281754 & 126.451 \\
\hline 9/1/08 4:00 & 2.147 & 0.687 & 0.225 & 100.985 & 1.411 & 0.065 & 0.019811 & 0.007977 & 0.281754 & 126.451 \\
\hline 9/1/08 5:00 & 2.154 & 0.694 & 0.231 & 103.547 & 1.413 & 0.067 & 0.020421 & 0.008330 & 0.294224 & 132.048 \\
\hline 9/1/08 6:00 & 2.163 & 0.703 & 0.238 & 106.898 & 1.41 & 0.064 & 0.019506 & 0.007803 & 0.275587 & 123.683 \\
\hline 9/1/08 7:00 & 2.176 & 0.716 & 0.249 & 111.853 & 1.412 & 0.066 & 0.020116 & 0.008153 & 0.287966 & 129.239 \\
\hline 9/1/08 8:00 & 2.402 & 0.942 & 0.492 & 220.620 & 1.409 & 0.063 & 0.019201 & 0.007629 & 0.269465 & 120.936 \\
\hline 9/1/08 9:00 & 2.162 & 0.702 & 0.237 & 106.523 & 1.411 & 0.065 & 0.019811 & 0.007977 & 0.281754 & 126.451 \\
\hline 9/1/08 10:00 & 2.423 & 0.963 & 0.519 & 233.017 & 1.411 & 0.065 & 0.019811 & 0.007977 & 0.281754 & 126.451 \\
\hline 9/1/08 11:00 & 2.485 & 1.025 & 0.606 & 272.017 & 1.409 & 0.063 & 0.019201 & 0.007629 & 0.269465 & 120.936 \\
\hline 9/1/08 12:00 & 2.106 & 0.646 & 0.193 & 86.742 & 1.407 & 0.061 & 0.018592 & 0.007287 & 0.257360 & 115.503 \\
\hline 9/1/08 13:00 & 2.084 & 0.624 & 0.177 & 79.629 & 1.409 & 0.063 & 0.019201 & 0.007629 & 0.269465 & 120.936 \\
\hline 9/1/08 14:00 & 2.402 & 0.942 & 0.492 & 220.620 & 1.403 & 0.057 & 0.017373 & 0.006617 & 0.233715 & 104.891 \\
\hline 9/1/08 15:00 & 2.413 & 0.953 & 0.506 & 227.063 & 1.403 & 0.057 & 0.017373 & 0.006617 & 0.233715 & 104.891 \\
\hline 9/1/08 16:00 & 2.407 & 0.947 & 0.498 & 223.535 & 1.406 & 0.06 & 0.018287 & 0.007117 & 0.251378 & 112.818 \\
\hline 9/1/08 17:00 & 2.405 & 0.945 & 0.495 & 222.366 & 1.405 & 0.059 & 0.017982 & 0.006949 & 0.245443 & 110.155 \\
\hline 9/1/08 18:00 & 2.398 & 0.938 & 0.486 & 218.305 & 1.405 & 0.059 & 0.017982 & 0.006949 & 0.245443 & 110.155 \\
\hline 9/1/08 19:00 & 2.395 & 0.935 & 0.483 & 216.578 & 1.41 & 0.064 & 0.019506 & 0.007803 & 0.275587 & 123.683 \\
\hline
\end{tabular}




\begin{tabular}{|c|c|c|c|c|c|c|c|c|c|c|}
\hline \multirow[b]{3}{*}{ Date } & \multicolumn{4}{|c|}{ V-Notch Weir } & \multicolumn{6}{|c|}{ R-Notch Weir } \\
\hline & \multicolumn{2}{|c|}{ Measured Water Level } & \multicolumn{2}{|c|}{$\begin{array}{c}\text { Calculated Rate } \\
\left(\mathrm{ft}^{3} / \mathrm{s}=448.8 \mathrm{gpm}\right)\end{array}$} & \multicolumn{3}{|c|}{ Measured Water Level } & \multicolumn{3}{|c|}{$\begin{array}{c}\text { Calculated Rate } \\
\left(\mathrm{ft}^{3} / \mathrm{s}=448.8 \mathrm{gpm}\right)\end{array}$} \\
\hline & $\begin{array}{c}\text { (ft above } \\
\text { sensor) }\end{array}$ & $\begin{array}{c}\text { (ft above } \\
\text { notch) }\end{array}$ & $\left(\mathrm{ft}^{3} / \mathrm{s}\right)$ & (gpm) & $\begin{array}{l}\text { (ft above } \\
\text { sensor) }\end{array}$ & $\begin{array}{c}\text { (ft above } \\
\text { log) }\end{array}$ & $\begin{array}{c}\text { (m above } \\
\text { log) }\end{array}$ & $\left(\mathrm{m}^{3} / \mathrm{s}\right)$ & $\left(\mathrm{ft}^{3} / \mathrm{s}\right)$ & (gpm) \\
\hline 9/1/08 20:00 & 2.378 & 0.918 & 0.461 & 206.948 & 1.415 & 0.069 & 0.021030 & 0.008688 & 0.306873 & 137.724 \\
\hline 9/1/08 21:00 & 2.21 & 0.750 & 0.280 & 125.453 & 1.423 & 0.077 & 0.023468 & 0.010170 & 0.359208 & 161.212 \\
\hline 9/1/08 22:00 & 2.108 & 0.648 & 0.195 & 87.406 & 1.413 & 0.067 & 0.020421 & 0.008330 & 0.294224 & 132.048 \\
\hline 9/1/08 23:00 & 2.126 & 0.666 & 0.208 & 93.527 & 1.413 & 0.067 & 0.020421 & 0.008330 & 0.294224 & 132.048 \\
\hline 9/2/08 0:00 & 2.133 & 0.673 & 0.214 & 95.975 & 1.413 & 0.067 & 0.020421 & 0.008330 & 0.294224 & 132.048 \\
\hline 9/2/08 1:00 & 2.466 & 1.006 & 0.579 & 259.680 & 1.415 & 0.069 & 0.021030 & 0.008688 & 0.306873 & 137.724 \\
\hline 9/2/08 2:00 & 2.357 & 0.897 & 0.435 & 195.412 & 1.415 & 0.069 & 0.021030 & 0.008688 & 0.306873 & 137.724 \\
\hline 9/2/08 3:00 & 2.159 & 0.699 & 0.235 & 105.401 & 1.417 & 0.071 & 0.021640 & 0.009052 & 0.319698 & 143.480 \\
\hline 9/2/08 4:00 & 2.199 & 0.739 & 0.269 & 120.950 & 1.419 & 0.073 & 0.022249 & 0.009420 & 0.332697 & 149.314 \\
\hline 9/2/08 5:00 & 2.222 & 0.762 & 0.291 & 130.478 & 1.421 & 0.075 & 0.022859 & 0.009792 & 0.345867 & 155.225 \\
\hline 9/2/08 6:00 & 2.196 & 0.736 & 0.267 & 119.739 & 1.408 & 0.062 & 0.018897 & 0.007457 & 0.263390 & 118.209 \\
\hline 9/2/08 7:00 & 2.391 & 0.931 & 0.477 & 214.289 & 1.408 & 0.062 & 0.018897 & 0.007457 & 0.263390 & 118.209 \\
\hline 9/2/08 8:00 & 2.13 & 0.670 & 0.212 & 94.921 & 1.405 & 0.059 & 0.017982 & 0.006949 & 0.245443 & 110.155 \\
\hline 9/2/08 9:00 & 2.303 & 0.843 & 0.373 & 167.551 & 1.405 & 0.059 & 0.017982 & 0.006949 & 0.245443 & 110.155 \\
\hline 9/2/08 10:00 & 2.216 & 0.756 & 0.285 & 127.950 & 1.405 & 0.059 & 0.017982 & 0.006949 & 0.245443 & 110.155 \\
\hline 9/2/08 11:00 & 2.106 & 0.646 & 0.193 & 86.742 & 1.402 & 0.056 & 0.017068 & 0.006453 & 0.227923 & 102.292 \\
\hline 9/2/08 12:00 & 2.102 & 0.642 & 0.190 & 85.421 & 1.402 & 0.056 & 0.017068 & 0.006453 & 0.227923 & 102.292 \\
\hline 9/2/08 13:00 & 2.108 & 0.648 & 0.195 & 87.406 & 1.402 & 0.056 & 0.017068 & 0.006453 & 0.227923 & 102.292 \\
\hline 9/2/08 14:00 & 2.113 & 0.653 & 0.198 & 89.082 & 1.402 & 0.056 & 0.017068 & 0.006453 & 0.227923 & 102.292 \\
\hline 9/2/08 15:00 & 2.125 & 0.665 & 0.208 & 93.181 & 1.406 & 0.06 & 0.018287 & 0.007117 & 0.251378 & 112.818 \\
\hline 9/2/08 16:00 & 2.15 & 0.690 & 0.227 & 102.078 & 1.409 & 0.063 & 0.019201 & 0.007629 & 0.269465 & 120.936 \\
\hline 9/2/08 17:00 & 2.143 & 0.683 & 0.222 & 99.538 & 1.408 & 0.062 & 0.018897 & 0.007457 & 0.263390 & 118.209 \\
\hline 9/2/08 18:00 & 2.139 & 0.679 & 0.219 & 98.103 & 1.406 & 0.06 & 0.018287 & 0.007117 & 0.251378 & 112.818 \\
\hline 9/2/08 19:00 & 2.127 & 0.667 & 0.209 & 93.875 & 1.406 & 0.06 & 0.018287 & 0.007117 & 0.251378 & 112.818 \\
\hline 9/2/08 20:00 & 2.113 & 0.653 & 0.198 & 89.082 & 1.405 & 0.059 & 0.017982 & 0.006949 & 0.245443 & 110.155 \\
\hline
\end{tabular}




\begin{tabular}{|c|c|c|c|c|c|c|c|c|c|c|}
\hline \multirow[b]{3}{*}{ Date Time } & \multicolumn{4}{|c|}{ V-Notch Weir } & \multicolumn{6}{|c|}{ R-Notch Weir } \\
\hline & \multicolumn{2}{|c|}{ Measured Water Level } & \multicolumn{2}{|c|}{$\begin{array}{c}\text { Calculated Rate } \\
\left(\mathrm{ft}^{3} / \mathrm{s}=448.8 \mathrm{gpm}\right)\end{array}$} & \multicolumn{3}{|c|}{ Measured Water Level } & \multicolumn{3}{|c|}{$\begin{array}{c}\text { Calculated Rate } \\
\left(\mathrm{ft}^{3} / \mathrm{s}=448.8 \mathrm{gpm}\right)\end{array}$} \\
\hline & $\begin{array}{c}\text { (ft above } \\
\text { sensor) }\end{array}$ & $\begin{array}{c}\text { (ft above } \\
\text { notch) }\end{array}$ & $\left(\mathrm{ft}^{3} / \mathrm{s}\right)$ & (gpm) & $\begin{array}{l}\text { (ft above } \\
\text { sensor) }\end{array}$ & $\begin{array}{c}\text { (ft above } \\
\text { log) }\end{array}$ & $\begin{array}{c}\text { (m above } \\
\text { log) }\end{array}$ & $\left(\mathrm{m}^{3} / \mathrm{s}\right)$ & $\left(\mathrm{ft}^{3} / \mathrm{s}\right)$ & (gpm) \\
\hline 9/2/08 21:00 & 2.12 & 0.660 & 0.204 & 91.459 & 1.405 & 0.059 & 0.017982 & 0.006949 & 0.245443 & 110.155 \\
\hline 9/2/08 22:00 & 2.122 & 0.662 & 0.205 & 92.146 & 1.405 & 0.059 & 0.017982 & 0.006949 & 0.245443 & 110.155 \\
\hline 9/2/08 23:00 & 2.122 & 0.662 & 0.205 & 92.146 & 1.402 & 0.056 & 0.017068 & 0.006453 & 0.227923 & 102.292 \\
\hline 9/3/08 0:00 & 2.124 & 0.664 & 0.207 & 92.835 & 1.404 & 0.058 & 0.017678 & 0.006782 & 0.239555 & 107.512 \\
\hline 9/3/08 1:00 & 2.124 & 0.664 & 0.207 & 92.835 & 1.404 & 0.058 & 0.017678 & 0.006782 & 0.239555 & 107.512 \\
\hline 9/3/08 2:00 & 2.117 & 0.657 & 0.202 & 90.436 & 1.403 & 0.057 & 0.017373 & 0.006617 & 0.233715 & 104.891 \\
\hline 9/3/08 3:00 & 2.119 & 0.659 & 0.203 & 91.117 & 1.403 & 0.057 & 0.017373 & 0.006617 & 0.233715 & 104.891 \\
\hline 9/3/08 4:00 & 2.126 & 0.666 & 0.208 & 93.527 & 1.405 & 0.059 & 0.017982 & 0.006949 & 0.245443 & 110.155 \\
\hline 9/3/08 5:00 & 2.119 & 0.659 & 0.203 & 91.117 & 1.404 & 0.058 & 0.017678 & 0.006782 & 0.239555 & 107.512 \\
\hline 9/3/08 6:00 & 2.121 & 0.661 & 0.205 & 91.802 & 1.404 & 0.058 & 0.017678 & 0.006782 & 0.239555 & 107.512 \\
\hline 9/3/08 7:00 & 2.123 & 0.663 & 0.206 & 92.490 & 1.404 & 0.058 & 0.017678 & 0.006782 & 0.239555 & 107.512 \\
\hline 9/3/08 8:00 & 2.123 & 0.663 & 0.206 & 92.490 & 1.403 & 0.057 & 0.017373 & 0.006617 & 0.233715 & 104.891 \\
\hline 9/3/08 9:00 & 2.128 & 0.668 & 0.210 & 94.223 & 1.403 & 0.057 & 0.017373 & 0.006617 & 0.233715 & 104.891 \\
\hline 9/3/08 10:00 & 2.123 & 0.663 & 0.206 & 92.490 & 1.406 & 0.06 & 0.018287 & 0.007117 & 0.251378 & 112.818 \\
\hline 9/3/08 11:00 & 2.126 & 0.666 & 0.208 & 93.527 & 1.403 & 0.057 & 0.017373 & 0.006617 & 0.233715 & 104.891 \\
\hline 9/3/08 12:00 & 2.114 & 0.654 & 0.199 & 89.419 & 1.401 & 0.055 & 0.016763 & 0.006291 & 0.222180 & 99.714 \\
\hline 9/3/08 13:00 & 2.105 & 0.645 & 0.193 & 86.410 & 1.399 & 0.053 & 0.016154 & 0.005969 & 0.210842 & 94.626 \\
\hline 9/3/08 14:00 & 2.09 & 0.630 & 0.182 & 81.532 & 1.397 & 0.051 & 0.015544 & 0.005654 & 0.199703 & 89.627 \\
\hline 9/3/08 15:00 & 2.072 & 0.612 & 0.169 & 75.902 & 1.4 & 0.054 & 0.016458 & 0.006129 & 0.216486 & 97.159 \\
\hline 9/3/08 16:00 & 2.072 & 0.612 & 0.169 & 75.902 & 1.398 & 0.052 & 0.015849 & 0.005811 & 0.205247 & 92.115 \\
\hline 9/3/08 17:00 & 2.064 & 0.604 & 0.164 & 73.477 & 1.398 & 0.052 & 0.015849 & 0.005811 & 0.205247 & 92.115 \\
\hline 9/3/08 18:00 & 2.059 & 0.599 & 0.160 & 71.985 & 1.396 & 0.05 & 0.015239 & 0.005499 & 0.194210 & 87.161 \\
\hline 9/3/08 19:00 & 2.058 & 0.598 & 0.160 & 71.689 & 1.399 & 0.053 & 0.016154 & 0.005969 & 0.210842 & 94.626 \\
\hline 9/3/08 20:00 & 2.06 & 0.600 & 0.161 & 72.282 & 1.399 & 0.053 & 0.016154 & 0.005969 & 0.210842 & 94.626 \\
\hline 9/3/08 21:00 & 2.076 & 0.616 & 0.172 & 77.132 & 1.402 & 0.056 & 0.017068 & 0.006453 & 0.227923 & 102.292 \\
\hline
\end{tabular}




\begin{tabular}{|c|c|c|c|c|c|c|c|c|c|c|}
\hline \multirow[b]{3}{*}{ Date Time } & \multicolumn{4}{|c|}{ V-Notch Weir } & \multicolumn{6}{|c|}{ R-Notch Weir } \\
\hline & \multicolumn{2}{|c|}{ Measured Water Level } & \multicolumn{2}{|c|}{$\begin{array}{c}\text { Calculated Rate } \\
\left(\mathrm{ft}^{3} / \mathrm{s}=448.8 \mathrm{gpm}\right)\end{array}$} & \multicolumn{3}{|c|}{ Measured Water Level } & \multicolumn{3}{|c|}{$\begin{array}{c}\text { Calculated Rate } \\
\left(\mathrm{ft}^{3} / \mathrm{s}=448.8 \mathrm{gpm}\right)\end{array}$} \\
\hline & $\begin{array}{c}\text { (ft above } \\
\text { sensor) }\end{array}$ & $\begin{array}{c}\text { (ft above } \\
\text { notch) }\end{array}$ & $\left(\mathrm{ft}^{3} / \mathrm{s}\right)$ & (gpm) & $\begin{array}{l}\text { (ft above } \\
\text { sensor) }\end{array}$ & $\begin{array}{c}\text { (ft above } \\
\text { log) }\end{array}$ & $\begin{array}{c}\text { (m above } \\
\text { log) }\end{array}$ & $\left(\mathrm{m}^{3} / \mathrm{s}\right)$ & $\left(\mathrm{ft}^{3} / \mathrm{s}\right)$ & (gpm) \\
\hline 9/3/08 22:00 & 2.092 & 0.632 & 0.183 & 82.173 & 1.402 & 0.056 & 0.017068 & 0.006453 & 0.227923 & 102.292 \\
\hline 9/3/08 23:00 & 2.099 & 0.639 & 0.188 & 84.439 & 1.404 & 0.058 & 0.017678 & 0.006782 & 0.239555 & 107.512 \\
\hline 9/4/08 0:00 & 2.108 & 0.648 & 0.195 & 87.406 & 1.404 & 0.058 & 0.017678 & 0.006782 & 0.239555 & 107.512 \\
\hline 9/4/08 1:00 & 2.143 & 0.683 & 0.222 & 99.538 & 1.406 & 0.06 & 0.018287 & 0.007117 & 0.251378 & 112.818 \\
\hline 9/4/08 2:00 & 2.159 & 0.699 & 0.235 & 105.401 & 1.405 & 0.059 & 0.017982 & 0.006949 & 0.245443 & 110.155 \\
\hline 9/4/08 3:00 & 2.112 & 0.652 & 0.198 & 88.745 & 1.405 & 0.059 & 0.017982 & 0.006949 & 0.245443 & 110.155 \\
\hline 9/4/08 4:00 & 2.112 & 0.652 & 0.198 & 88.745 & 1.402 & 0.056 & 0.017068 & 0.006453 & 0.227923 & 102.292 \\
\hline 9/4/08 5:00 & 2.119 & 0.659 & 0.203 & 91.117 & 1.407 & 0.061 & 0.018592 & 0.007287 & 0.257360 & 115.503 \\
\hline 9/4/08 6:00 & 2.128 & 0.668 & 0.210 & 94.223 & 1.406 & 0.06 & 0.018287 & 0.007117 & 0.251378 & 112.818 \\
\hline 9/4/08 7:00 & 2.131 & 0.671 & 0.212 & 95.272 & 1.406 & 0.06 & 0.018287 & 0.007117 & 0.251378 & 112.818 \\
\hline 9/4/08 8:00 & 2.135 & 0.675 & 0.215 & 96.681 & 1.403 & 0.057 & 0.017373 & 0.006617 & 0.233715 & 104.891 \\
\hline 9/4/08 9:00 & 2.133 & 0.673 & 0.214 & 95.975 & 1.408 & 0.062 & 0.018897 & 0.007457 & 0.263390 & 118.209 \\
\hline 9/4/08 10:00 & 2.14 & 0.680 & 0.219 & 98.461 & 1.406 & 0.06 & 0.018287 & 0.007117 & 0.251378 & 112.818 \\
\hline 9/4/08 11:00 & 2.135 & 0.675 & 0.215 & 96.681 & 1.406 & 0.06 & 0.018287 & 0.007117 & 0.251378 & 112.818 \\
\hline 9/4/08 12:00 & 2.126 & 0.666 & 0.208 & 93.527 & 1.406 & 0.06 & 0.018287 & 0.007117 & 0.251378 & 112.818 \\
\hline 9/4/08 13:00 & 2.11 & 0.650 & 0.196 & 88.074 & 1.404 & 0.058 & 0.017678 & 0.006782 & 0.239555 & 107.512 \\
\hline 9/4/08 14:00 & 2.092 & 0.632 & 0.183 & 82.173 & 1.402 & 0.056 & 0.017068 & 0.006453 & 0.227923 & 102.292 \\
\hline 9/4/08 15:00 & 2.081 & 0.621 & 0.175 & 78.687 & 1.402 & 0.056 & 0.017068 & 0.006453 & 0.227923 & 102.292 \\
\hline 9/4/08 16:00 & 2.073 & 0.613 & 0.170 & 76.208 & 1.4 & 0.054 & 0.016458 & 0.006129 & 0.216486 & 97.159 \\
\hline 9/4/08 17:00 & 2.062 & 0.602 & 0.162 & 72.878 & 1.4 & 0.054 & 0.016458 & 0.006129 & 0.216486 & 97.159 \\
\hline 9/4/08 18:00 & 2.052 & 0.592 & 0.156 & 69.927 & 1.398 & 0.052 & 0.015849 & 0.005811 & 0.205247 & 92.115 \\
\hline 9/4/08 19:00 & 2.054 & 0.594 & 0.157 & 70.511 & 1.401 & 0.055 & 0.016763 & 0.006291 & 0.222180 & 99.714 \\
\hline 9/4/08 20:00 & 2.063 & 0.603 & 0.163 & 73.177 & 1.401 & 0.055 & 0.016763 & 0.006291 & 0.222180 & 99.714 \\
\hline 9/4/08 21:00 & 2.067 & 0.607 & 0.166 & 74.381 & 1.402 & 0.056 & 0.017068 & 0.006453 & 0.227923 & 102.292 \\
\hline 9/4/08 22:00 & 2.083 & 0.623 & 0.177 & 79.314 & 1.402 & 0.056 & 0.017068 & 0.006453 & 0.227923 & 102.292 \\
\hline
\end{tabular}




\begin{tabular}{|c|c|c|c|c|c|c|c|c|c|c|}
\hline \multirow[b]{3}{*}{ Date Time } & \multicolumn{4}{|c|}{ V-Notch Weir } & \multicolumn{6}{|c|}{ R-Notch Weir } \\
\hline & \multicolumn{2}{|c|}{ Measured Water Level } & \multicolumn{2}{|c|}{$\begin{array}{c}\text { Calculated Rate } \\
\left(\mathrm{ft}^{3} / \mathrm{s}=448.8 \mathrm{gpm}\right)\end{array}$} & \multicolumn{3}{|c|}{ Measured Water Level } & \multicolumn{3}{|c|}{$\begin{array}{c}\text { Calculated Rate } \\
\left(\mathrm{ft}^{3} / \mathrm{s}=448.8 \mathrm{gpm}\right)\end{array}$} \\
\hline & $\begin{array}{c}\text { (ft above } \\
\text { sensor) }\end{array}$ & $\begin{array}{c}\text { (ft above } \\
\text { notch) }\end{array}$ & $\left(\mathrm{ft}^{3} / \mathrm{s}\right)$ & (gpm) & $\begin{array}{l}\text { (ft above } \\
\text { sensor) }\end{array}$ & $\begin{array}{c}\text { (ft above } \\
\text { log) }\end{array}$ & $\begin{array}{c}\text { (m above } \\
\text { log) }\end{array}$ & $\left(\mathrm{m}^{3} / \mathrm{s}\right)$ & $\left(\mathrm{ft}^{3} / \mathrm{s}\right)$ & (gpm) \\
\hline 9/4/08 23:00 & 2.091 & 0.631 & 0.182 & 81.852 & 1.406 & 0.06 & 0.018287 & 0.007117 & 0.251378 & 112.818 \\
\hline 9/5/08 0:00 & 2.103 & 0.643 & 0.191 & 85.750 & 1.406 & 0.06 & 0.018287 & 0.007117 & 0.251378 & 112.818 \\
\hline 9/5/08 1:00 & 2.11 & 0.650 & 0.196 & 88.074 & 1.405 & 0.059 & 0.017982 & 0.006949 & 0.245443 & 110.155 \\
\hline 9/5/08 2:00 & 2.114 & 0.654 & 0.199 & 89.419 & 1.407 & 0.061 & 0.018592 & 0.007287 & 0.257360 & 115.503 \\
\hline 9/5/08 3:00 & 2.114 & 0.654 & 0.199 & 89.419 & 1.406 & 0.06 & 0.018287 & 0.007117 & 0.251378 & 112.818 \\
\hline 9/5/08 4:00 & 2.116 & 0.656 & 0.201 & 90.096 & 1.406 & 0.06 & 0.018287 & 0.007117 & 0.251378 & 112.818 \\
\hline 9/5/08 5:00 & 2.116 & 0.656 & 0.201 & 90.096 & 1.403 & 0.057 & 0.017373 & 0.006617 & 0.233715 & 104.891 \\
\hline 9/5/08 6:00 & 2.123 & 0.663 & 0.206 & 92.490 & 1.405 & 0.059 & 0.017982 & 0.006949 & 0.245443 & 110.155 \\
\hline 9/5/08 7:00 & 2.126 & 0.666 & 0.208 & 93.527 & 1.405 & 0.059 & 0.017982 & 0.006949 & 0.245443 & 110.155 \\
\hline 9/5/08 8:00 & 2.126 & 0.666 & 0.208 & 93.527 & 1.407 & 0.061 & 0.018592 & 0.007287 & 0.257360 & 115.503 \\
\hline 9/5/08 9:00 & 2.128 & 0.668 & 0.210 & 94.223 & 1.407 & 0.061 & 0.018592 & 0.007287 & 0.257360 & 115.503 \\
\hline 9/5/08 10:00 & 2.133 & 0.673 & 0.214 & 95.975 & 1.407 & 0.061 & 0.018592 & 0.007287 & 0.257360 & 115.503 \\
\hline 9/5/08 11:00 & 2.135 & 0.675 & 0.215 & 96.681 & 1.407 & 0.061 & 0.018592 & 0.007287 & 0.257360 & 115.503 \\
\hline 9/5/08 12:00 & 2.133 & 0.673 & 0.214 & 95.975 & 1.407 & 0.061 & 0.018592 & 0.007287 & 0.257360 & 115.503 \\
\hline 9/5/08 13:00 & 2.126 & 0.666 & 0.208 & 93.527 & 1.407 & 0.061 & 0.018592 & 0.007287 & 0.257360 & 115.503 \\
\hline 9/5/08 14:00 & 2.119 & 0.659 & 0.203 & 91.117 & 1.405 & 0.059 & 0.017982 & 0.006949 & 0.245443 & 110.155 \\
\hline 9/5/08 15:00 & 2.112 & 0.652 & 0.198 & 88.745 & 1.405 & 0.059 & 0.017982 & 0.006949 & 0.245443 & 110.155 \\
\hline 9/5/08 16:00 & 2.112 & 0.652 & 0.198 & 88.745 & 1.405 & 0.059 & 0.017982 & 0.006949 & 0.245443 & 110.155 \\
\hline 9/5/08 17:00 & 2.121 & 0.661 & 0.205 & 91.802 & 1.407 & 0.061 & 0.018592 & 0.007287 & 0.257360 & 115.503 \\
\hline 9/5/08 18:00 & 2.119 & 0.659 & 0.203 & 91.117 & 1.405 & 0.059 & 0.017982 & 0.006949 & 0.245443 & 110.155 \\
\hline 9/5/08 19:00 & 2.117 & 0.657 & 0.202 & 90.436 & 1.407 & 0.061 & 0.018592 & 0.007287 & 0.257360 & 115.503 \\
\hline 9/5/08 20:00 & 2.117 & 0.657 & 0.202 & 90.436 & 1.407 & 0.061 & 0.018592 & 0.007287 & 0.257360 & 115.503 \\
\hline 9/5/08 21:00 & 2.121 & 0.661 & 0.205 & 91.802 & 1.407 & 0.061 & 0.018592 & 0.007287 & 0.257360 & 115.503 \\
\hline 9/5/08 22:00 & 2.116 & 0.656 & 0.201 & 90.096 & 1.405 & 0.059 & 0.017982 & 0.006949 & 0.245443 & 110.155 \\
\hline 9/5/08 23:00 & 2.123 & 0.663 & 0.206 & 92.490 & 1.407 & 0.061 & 0.018592 & 0.007287 & 0.257360 & 115.503 \\
\hline
\end{tabular}




\begin{tabular}{|c|c|c|c|c|c|c|c|c|c|c|}
\hline \multirow[b]{3}{*}{ Date Time } & \multicolumn{4}{|c|}{ V-Notch Weir } & \multicolumn{6}{|c|}{ R-Notch Weir } \\
\hline & \multicolumn{2}{|c|}{ Measured Water Level } & \multicolumn{2}{|c|}{$\begin{array}{c}\text { Calculated Rate } \\
\left(\mathrm{ft}^{3} / \mathrm{s}=448.8 \mathrm{gpm}\right)\end{array}$} & \multicolumn{3}{|c|}{ Measured Water Level } & \multicolumn{3}{|c|}{$\begin{array}{c}\text { Calculated Rate } \\
\left(\mathrm{ft}^{3} / \mathrm{s}=448.8 \mathrm{gpm}\right)\end{array}$} \\
\hline & $\begin{array}{c}\text { (ft above } \\
\text { sensor) }\end{array}$ & $\begin{array}{c}\text { (ft above } \\
\text { notch) }\end{array}$ & $\left(\mathrm{ft}^{3} / \mathrm{s}\right)$ & (gpm) & $\begin{array}{c}\text { (ft above } \\
\text { sensor) }\end{array}$ & $\begin{array}{c}\text { (ft above } \\
\text { log) }\end{array}$ & $\begin{array}{c}\text { (m above } \\
\text { log) }\end{array}$ & $\left(\mathrm{m}^{3} / \mathrm{s}\right)$ & $\left(\mathrm{ft}^{3} / \mathrm{s}\right)$ & (gpm) \\
\hline 9/6/08 0:00 & 2.135 & 0.675 & 0.215 & 96.681 & 1.407 & 0.061 & 0.018592 & 0.007287 & 0.257360 & 115.503 \\
\hline 9/6/08 1:00 & 2.128 & 0.668 & 0.210 & 94.223 & 1.406 & 0.06 & 0.018287 & 0.007117 & 0.251378 & 112.818 \\
\hline 9/6/08 2:00 & 2.135 & 0.675 & 0.215 & 96.681 & 1.406 & 0.06 & 0.018287 & 0.007117 & 0.251378 & 112.818 \\
\hline 9/6/08 3:00 & 2.133 & 0.673 & 0.214 & 95.975 & 1.406 & 0.06 & 0.018287 & 0.007117 & 0.251378 & 112.818 \\
\hline 9/6/08 4:00 & 2.135 & 0.675 & 0.215 & 96.681 & 1.408 & 0.062 & 0.018897 & 0.007457 & 0.263390 & 118.209 \\
\hline 9/6/08 5:00 & 2.14 & 0.680 & 0.219 & 98.461 & 1.408 & 0.062 & 0.018897 & 0.007457 & 0.263390 & 118.209 \\
\hline 9/6/08 6:00 & 2.15 & 0.690 & 0.227 & 102.078 & 1.408 & 0.062 & 0.018897 & 0.007457 & 0.263390 & 118.209 \\
\hline 9/6/08 7:00 & 2.159 & 0.699 & 0.235 & 105.401 & 1.407 & 0.061 & 0.018592 & 0.007287 & 0.257360 & 115.503 \\
\hline 9/6/08 8:00 & 2.159 & 0.699 & 0.235 & 105.401 & 1.407 & 0.061 & 0.018592 & 0.007287 & 0.257360 & 115.503 \\
\hline 9/6/08 9:00 & 2.154 & 0.694 & 0.231 & 103.547 & 1.407 & 0.061 & 0.018592 & 0.007287 & 0.257360 & 115.503 \\
\hline 9/6/08 10:00 & 2.154 & 0.694 & 0.231 & 103.547 & 1.407 & 0.061 & 0.018592 & 0.007287 & 0.257360 & 115.503 \\
\hline 9/6/08 11:00 & 2.156 & 0.696 & 0.232 & 104.286 & 1.407 & 0.061 & 0.018592 & 0.007287 & 0.257360 & 115.503 \\
\hline 9/6/08 12:00 & 2.168 & 0.708 & 0.242 & 108.788 & 1.407 & 0.061 & 0.018592 & 0.007287 & 0.257360 & 115.503 \\
\hline 9/6/08 13:00 & 2.159 & 0.699 & 0.235 & 105.401 & 1.407 & 0.061 & 0.018592 & 0.007287 & 0.257360 & 115.503 \\
\hline 9/6/08 14:00 & 2.154 & 0.694 & 0.231 & 103.547 & 1.405 & 0.059 & 0.017982 & 0.006949 & 0.245443 & 110.155 \\
\hline 9/6/08 15:00 & 2.154 & 0.694 & 0.231 & 103.547 & 1.408 & 0.062 & 0.018897 & 0.007457 & 0.263390 & 118.209 \\
\hline 9/6/08 16:00 & 2.149 & 0.689 & 0.227 & 101.713 & 1.408 & 0.062 & 0.018897 & 0.007457 & 0.263390 & 118.209 \\
\hline 9/6/08 17:00 & 2.152 & 0.692 & 0.229 & 102.811 & 1.408 & 0.062 & 0.018897 & 0.007457 & 0.263390 & 118.209 \\
\hline 9/6/08 18:00 & 2.154 & 0.694 & 0.231 & 103.547 & 1.408 & 0.062 & 0.018897 & 0.007457 & 0.263390 & 118.209 \\
\hline 9/6/08 19:00 & 2.154 & 0.694 & 0.231 & 103.547 & 1.408 & 0.062 & 0.018897 & 0.007457 & 0.263390 & 118.209 \\
\hline 9/6/08 20:00 & 2.159 & 0.699 & 0.235 & 105.401 & 1.408 & 0.062 & 0.018897 & 0.007457 & 0.263390 & 118.209 \\
\hline 9/6/08 21:00 & 2.215 & 0.755 & 0.284 & 127.532 & 1.42 & 0.074 & 0.022554 & 0.009605 & 0.339261 & 152.260 \\
\hline 9/6/08 22:00 & 2.506 & 1.046 & 0.637 & 286.055 & 1.472 & 0.126 & 0.038403 & 0.020784 & 0.734098 & 329.463 \\
\hline 9/6/08 23:00 & 2.518 & 1.058 & 0.656 & 294.267 & 1.467 & 0.121 & 0.036879 & 0.019590 & 0.691921 & 310.534 \\
\hline 9/7/08 0:00 & 2.347 & 0.887 & 0.423 & 190.058 & 1.439 & 0.093 & 0.028345 & 0.013358 & 0.471786 & 211.738 \\
\hline
\end{tabular}




\begin{tabular}{|c|c|c|c|c|c|c|c|c|c|c|}
\hline \multirow[b]{3}{*}{ Date Time } & \multicolumn{4}{|c|}{ V-Notch Weir } & \multicolumn{6}{|c|}{ R-Notch Weir } \\
\hline & \multicolumn{2}{|c|}{ Measured Water Level } & \multicolumn{2}{|c|}{$\begin{array}{c}\text { Calculated Rate } \\
\left(\mathrm{ft}^{3} / \mathrm{s}=448.8 \mathrm{gpm}\right)\end{array}$} & \multicolumn{3}{|c|}{ Measured Water Level } & \multicolumn{3}{|c|}{$\begin{array}{c}\text { Calculated Rate } \\
\left(\mathrm{ft}^{3} / \mathrm{s}=448.8 \mathrm{gpm}\right)\end{array}$} \\
\hline & $\begin{array}{c}\text { (ft above } \\
\text { sensor) }\end{array}$ & $\begin{array}{c}\text { (ft above } \\
\text { notch) }\end{array}$ & $\left(\mathrm{ft}^{3} / \mathrm{s}\right)$ & (gpm) & $\begin{array}{c}\text { (ft above } \\
\text { sensor) }\end{array}$ & $\begin{array}{c}\text { (ft above } \\
\text { log) }\end{array}$ & $\begin{array}{c}\text { (m above } \\
\text { log) }\end{array}$ & $\left(\mathrm{m}^{3} / \mathrm{s}\right)$ & $\left(\mathrm{ft}^{3} / \mathrm{s}\right)$ & (gpm) \\
\hline 9/7/08 1:00 & 2.269 & 0.809 & 0.337 & 151.312 & 1.424 & 0.078 & 0.023773 & 0.010361 & 0.365940 & 164.234 \\
\hline 9/7/08 2:00 & 2.232 & 0.772 & 0.300 & 134.756 & 1.417 & 0.071 & 0.021640 & 0.009052 & 0.319698 & 143.480 \\
\hline 9/7/08 3:00 & 2.215 & 0.755 & 0.284 & 127.532 & 1.414 & 0.068 & 0.020725 & 0.008509 & 0.300526 & 134.876 \\
\hline 9/7/08 4:00 & 2.203 & 0.743 & 0.273 & 122.576 & 1.414 & 0.068 & 0.020725 & 0.008509 & 0.300526 & 134.876 \\
\hline 9/7/08 5:00 & 2.203 & 0.743 & 0.273 & 122.576 & 1.412 & 0.066 & 0.020116 & 0.008153 & 0.287966 & 129.239 \\
\hline 9/7/08 6:00 & 2.192 & 0.732 & 0.263 & 118.136 & 1.412 & 0.066 & 0.020116 & 0.008153 & 0.287966 & 129.239 \\
\hline 9/7/08 7:00 & 2.187 & 0.727 & 0.259 & 116.150 & 1.412 & 0.066 & 0.020116 & 0.008153 & 0.287966 & 129.239 \\
\hline 9/7/08 8:00 & 2.192 & 0.732 & 0.263 & 118.136 & 1.411 & 0.065 & 0.019811 & 0.007977 & 0.281754 & 126.451 \\
\hline 9/7/08 9:00 & 2.185 & 0.725 & 0.257 & 115.362 & 1.412 & 0.066 & 0.020116 & 0.008153 & 0.287966 & 129.239 \\
\hline 9/7/08 10:00 & 2.185 & 0.725 & 0.257 & 115.362 & 1.411 & 0.065 & 0.019811 & 0.007977 & 0.281754 & 126.451 \\
\hline 9/7/08 11:00 & 2.185 & 0.725 & 0.257 & 115.362 & 1.412 & 0.066 & 0.020116 & 0.008153 & 0.287966 & 129.239 \\
\hline 9/7/08 12:00 & 2.185 & 0.725 & 0.257 & 115.362 & 1.409 & 0.063 & 0.019201 & 0.007629 & 0.269465 & 120.936 \\
\hline 9/7/08 13:00 & 2.182 & 0.722 & 0.254 & 114.185 & 1.409 & 0.063 & 0.019201 & 0.007629 & 0.269465 & 120.936 \\
\hline 9/7/08 14:00 & 2.176 & 0.716 & 0.249 & 111.853 & 1.412 & 0.066 & 0.020116 & 0.008153 & 0.287966 & 129.239 \\
\hline 9/7/08 15:00 & 2.171 & 0.711 & 0.245 & 109.931 & 1.41 & 0.064 & 0.019506 & 0.007803 & 0.275587 & 123.683 \\
\hline 9/7/08 16:00 & 2.155 & 0.695 & 0.232 & 103.916 & 1.41 & 0.064 & 0.019506 & 0.007803 & 0.275587 & 123.683 \\
\hline 9/7/08 17:00 & 2.153 & 0.693 & 0.230 & 103.179 & 1.408 & 0.062 & 0.018897 & 0.007457 & 0.263390 & 118.209 \\
\hline 9/7/08 18:00 & 2.137 & 0.677 & 0.217 & 97.391 & 1.406 & 0.06 & 0.018287 & 0.007117 & 0.251378 & 112.818 \\
\hline 9/7/08 19:00 & 2.137 & 0.677 & 0.217 & 97.391 & 1.408 & 0.062 & 0.018897 & 0.007457 & 0.263390 & 118.209 \\
\hline 9/7/08 20:00 & 2.143 & 0.683 & 0.222 & 99.538 & 1.408 & 0.062 & 0.018897 & 0.007457 & 0.263390 & 118.209 \\
\hline 9/7/08 21:00 & 2.148 & 0.688 & 0.226 & 101.348 & 1.409 & 0.063 & 0.019201 & 0.007629 & 0.269465 & 120.936 \\
\hline 9/7/08 22:00 & 2.155 & 0.695 & 0.232 & 103.916 & 1.409 & 0.063 & 0.019201 & 0.007629 & 0.269465 & 120.936 \\
\hline 9/7/08 23:00 & 2.159 & 0.699 & 0.235 & 105.401 & 1.409 & 0.063 & 0.019201 & 0.007629 & 0.269465 & 120.936 \\
\hline 9/8/08 0:00 & 2.161 & 0.701 & 0.237 & 106.148 & 1.411 & 0.065 & 0.019811 & 0.007977 & 0.281754 & 126.451 \\
\hline 9/8/08 1:00 & 2.164 & 0.704 & 0.239 & 107.275 & 1.409 & 0.063 & 0.019201 & 0.007629 & 0.269465 & 120.936 \\
\hline
\end{tabular}




\begin{tabular}{|c|c|c|c|c|c|c|c|c|c|c|}
\hline \multirow[b]{3}{*}{ Date Time } & \multicolumn{4}{|c|}{ V-Notch Weir } & \multicolumn{6}{|c|}{ R-Notch Weir } \\
\hline & \multicolumn{2}{|c|}{ Measured Water Level } & \multicolumn{2}{|c|}{$\begin{array}{c}\text { Calculated Rate } \\
\left(\mathrm{ft}^{3} / \mathrm{s}=448.8 \mathrm{gpm}\right)\end{array}$} & \multicolumn{3}{|c|}{ Measured Water Level } & \multicolumn{3}{|c|}{$\begin{array}{c}\text { Calculated Rate } \\
\left(\mathrm{ft}^{3} / \mathrm{s}=448.8 \mathrm{gpm}\right)\end{array}$} \\
\hline & $\begin{array}{c}\text { (ft above } \\
\text { sensor) }\end{array}$ & $\begin{array}{c}\text { (ft above } \\
\text { notch) }\end{array}$ & $\left(\mathrm{ft}^{3} / \mathrm{s}\right)$ & (gpm) & $\begin{array}{c}\text { (ft above } \\
\text { sensor) }\end{array}$ & $\begin{array}{c}\text { (ft above } \\
\text { log) }\end{array}$ & $\begin{array}{c}\text { (m above } \\
\text { log) }\end{array}$ & $\left(\mathrm{m}^{3} / \mathrm{s}\right)$ & $\left(\mathrm{ft}^{3} / \mathrm{s}\right)$ & (gpm) \\
\hline 9/8/08 2:00 & 2.168 & 0.708 & 0.242 & 108.788 & 1.411 & 0.065 & 0.019811 & 0.007977 & 0.281754 & 126.451 \\
\hline 9/8/08 3:00 & 2.173 & 0.713 & 0.247 & 110.697 & 1.411 & 0.065 & 0.019811 & 0.007977 & 0.281754 & 126.451 \\
\hline 9/8/08 4:00 & 2.166 & 0.706 & 0.241 & 108.030 & 1.411 & 0.065 & 0.019811 & 0.007977 & 0.281754 & 126.451 \\
\hline 9/8/08 5:00 & 2.173 & 0.713 & 0.247 & 110.697 & 1.411 & 0.065 & 0.019811 & 0.007977 & 0.281754 & 126.451 \\
\hline 9/8/08 6:00 & 2.185 & 0.725 & 0.257 & 115.362 & 1.41 & 0.064 & 0.019506 & 0.007803 & 0.275587 & 123.683 \\
\hline 9/8/08 7:00 & 2.222 & 0.762 & 0.291 & 130.478 & 1.42 & 0.074 & 0.022554 & 0.009605 & 0.339261 & 152.260 \\
\hline 9/8/08 8:00 & 2.307 & 0.847 & 0.378 & 169.527 & 1.434 & 0.088 & 0.026821 & 0.012331 & 0.435514 & 195.459 \\
\hline 9/8/08 9:00 & 2.49 & 1.030 & 0.613 & 275.321 & 1.464 & 0.118 & 0.035965 & 0.018885 & 0.667017 & 299.357 \\
\hline 9/8/08 10:00 & 2.471 & 1.011 & 0.586 & 262.894 & 1.462 & 0.116 & 0.035355 & 0.018420 & 0.650584 & 291.982 \\
\hline 9/8/08 11:00 & 2.368 & 0.908 & 0.449 & 201.405 & 1.443 & 0.097 & 0.029564 & 0.014199 & 0.501493 & 225.070 \\
\hline 9/8/08 12:00 & 2.3 & 0.840 & 0.370 & 166.078 & 1.431 & 0.085 & 0.025907 & 0.011728 & 0.414220 & 185.902 \\
\hline 9/8/08 13:00 & 2.267 & 0.807 & 0.335 & 150.387 & 1.426 & 0.08 & 0.024383 & 0.010746 & 0.379531 & 170.333 \\
\hline 9/8/08 14:00 & 2.243 & 0.783 & 0.311 & 139.558 & 1.421 & 0.075 & 0.022859 & 0.009792 & 0.345867 & 155.225 \\
\hline 9/8/08 15:00 & 2.218 & 0.758 & 0.287 & 128.789 & 1.419 & 0.073 & 0.022249 & 0.009420 & 0.332697 & 149.314 \\
\hline 9/8/08 16:00 & 2.192 & 0.732 & 0.263 & 118.136 & 1.417 & 0.071 & 0.021640 & 0.009052 & 0.319698 & 143.480 \\
\hline 9/8/08 17:00 & 2.178 & 0.718 & 0.251 & 112.627 & 1.412 & 0.066 & 0.020116 & 0.008153 & 0.287966 & 129.239 \\
\hline 9/8/08 18:00 & 2.164 & 0.704 & 0.239 & 107.275 & 1.412 & 0.066 & 0.020116 & 0.008153 & 0.287966 & 129.239 \\
\hline 9/8/08 19:00 & 2.162 & 0.702 & 0.237 & 106.523 & 1.412 & 0.066 & 0.020116 & 0.008153 & 0.287966 & 129.239 \\
\hline 9/8/08 20:00 & 2.161 & 0.701 & 0.237 & 106.148 & 1.412 & 0.066 & 0.020116 & 0.008153 & 0.287966 & 129.239 \\
\hline 9/8/08 21:00 & 2.166 & 0.706 & 0.241 & 108.030 & 1.412 & 0.066 & 0.020116 & 0.008153 & 0.287966 & 129.239 \\
\hline 9/8/08 22:00 & 2.166 & 0.706 & 0.241 & 108.030 & 1.413 & 0.067 & 0.020421 & 0.008330 & 0.294224 & 132.048 \\
\hline 9/8/08 23:00 & 2.166 & 0.706 & 0.241 & 108.030 & 1.412 & 0.066 & 0.020116 & 0.008153 & 0.287966 & 129.239 \\
\hline 9/9/08 0:00 & 2.17 & 0.710 & 0.244 & 109.549 & 1.412 & 0.066 & 0.020116 & 0.008153 & 0.287966 & 129.239 \\
\hline 9/9/08 1:00 & 2.175 & 0.715 & 0.248 & 111.467 & 1.412 & 0.066 & 0.020116 & 0.008153 & 0.287966 & 129.239 \\
\hline 9/9/08 2:00 & 2.173 & 0.713 & 0.247 & 110.697 & 1.414 & 0.068 & 0.020725 & 0.008509 & 0.300526 & 134.876 \\
\hline
\end{tabular}




\begin{tabular}{|c|c|c|c|c|c|c|c|c|c|c|}
\hline \multirow[b]{3}{*}{ Date Time } & \multicolumn{4}{|c|}{ V-Notch Weir } & \multicolumn{6}{|c|}{ R-Notch Weir } \\
\hline & \multicolumn{2}{|c|}{ Measured Water Level } & \multicolumn{2}{|c|}{$\begin{array}{c}\text { Calculated Rate } \\
\left(\mathrm{ft}^{3} / \mathrm{s}=448.8 \mathrm{gpm}\right)\end{array}$} & \multicolumn{3}{|c|}{ Measured Water Level } & \multicolumn{3}{|c|}{$\begin{array}{c}\text { Calculated Rate } \\
\left(\mathrm{ft}^{3} / \mathrm{s}=448.8 \mathrm{gpm}\right)\end{array}$} \\
\hline & $\begin{array}{c}\text { (ft above } \\
\text { sensor) }\end{array}$ & $\begin{array}{c}\text { (ft above } \\
\text { notch) }\end{array}$ & $\left(\mathrm{ft}^{3} / \mathrm{s}\right)$ & (gpm) & $\begin{array}{c}\text { (ft above } \\
\text { sensor) }\end{array}$ & $\begin{array}{c}\text { (ft above } \\
\text { log) }\end{array}$ & $\begin{array}{c}\text { (m above } \\
\text { log) }\end{array}$ & $\left(\mathrm{m}^{3} / \mathrm{s}\right)$ & $\left(\mathrm{ft}^{3} / \mathrm{s}\right)$ & (gpm) \\
\hline 9/9/08 3:00 & 2.166 & 0.706 & 0.241 & 108.030 & 1.411 & 0.065 & 0.019811 & 0.007977 & 0.281754 & 126.451 \\
\hline 9/9/08 4:00 & 2.164 & 0.704 & 0.239 & 107.275 & 1.411 & 0.065 & 0.019811 & 0.007977 & 0.281754 & 126.451 \\
\hline 9/9/08 5:00 & 2.18 & 0.720 & 0.253 & 113.404 & 1.411 & 0.065 & 0.019811 & 0.007977 & 0.281754 & 126.451 \\
\hline 9/9/08 6:00 & 2.18 & 0.720 & 0.253 & 113.404 & 1.411 & 0.065 & 0.019811 & 0.007977 & 0.281754 & 126.451 \\
\hline 9/9/08 7:00 & 2.178 & 0.718 & 0.251 & 112.627 & 1.41 & 0.064 & 0.019506 & 0.007803 & 0.275587 & 123.683 \\
\hline 9/9/08 8:00 & 2.171 & 0.711 & 0.245 & 109.931 & 1.41 & 0.064 & 0.019506 & 0.007803 & 0.275587 & 123.683 \\
\hline 9/9/08 9:00 & 2.176 & 0.716 & 0.249 & 111.853 & 1.408 & 0.062 & 0.018897 & 0.007457 & 0.263390 & 118.209 \\
\hline 9/9/08 10:00 & 2.178 & 0.718 & 0.251 & 112.627 & 1.41 & 0.064 & 0.019506 & 0.007803 & 0.275587 & 123.683 \\
\hline 9/9/08 11:00 & 2.175 & 0.715 & 0.248 & 111.467 & 1.408 & 0.062 & 0.018897 & 0.007457 & 0.263390 & 118.209 \\
\hline 9/9/08 12:00 & 2.166 & 0.706 & 0.241 & 108.030 & 1.411 & 0.065 & 0.019811 & 0.007977 & 0.281754 & 126.451 \\
\hline 9/9/08 13:00 & 2.157 & 0.697 & 0.233 & 104.657 & 1.406 & 0.06 & 0.018287 & 0.007117 & 0.251378 & 112.818 \\
\hline 9/9/08 14:00 & 2.134 & 0.674 & 0.215 & 96.328 & 1.404 & 0.058 & 0.017678 & 0.006782 & 0.239555 & 107.512 \\
\hline 9/9/08 15:00 & 2.132 & 0.672 & 0.213 & 95.623 & 1.404 & 0.058 & 0.017678 & 0.006782 & 0.239555 & 107.512 \\
\hline 9/9/08 16:00 & 2.109 & 0.649 & 0.195 & 87.740 & 1.404 & 0.058 & 0.017678 & 0.006782 & 0.239555 & 107.512 \\
\hline 9/9/08 17:00 & 2.109 & 0.649 & 0.195 & 87.740 & 1.402 & 0.056 & 0.017068 & 0.006453 & 0.227923 & 102.292 \\
\hline 9/9/08 18:00 & 2.102 & 0.642 & 0.190 & 85.421 & 1.403 & 0.057 & 0.017373 & 0.006617 & 0.233715 & 104.891 \\
\hline 9/9/08 19:00 & 2.106 & 0.646 & 0.193 & 86.742 & 1.403 & 0.057 & 0.017373 & 0.006617 & 0.233715 & 104.891 \\
\hline 9/9/08 20:00 & 2.103 & 0.643 & 0.191 & 85.750 & 1.403 & 0.057 & 0.017373 & 0.006617 & 0.233715 & 104.891 \\
\hline 9/9/08 21:00 & 2.117 & 0.657 & 0.202 & 90.436 & 1.403 & 0.057 & 0.017373 & 0.006617 & 0.233715 & 104.891 \\
\hline 9/9/08 22:00 & 2.117 & 0.657 & 0.202 & 90.436 & 1.406 & 0.06 & 0.018287 & 0.007117 & 0.251378 & 112.818 \\
\hline 9/9/08 23:00 & 2.112 & 0.652 & 0.198 & 88.745 & 1.403 & 0.057 & 0.017373 & 0.006617 & 0.233715 & 104.891 \\
\hline 9/10/08 0:00 & 2.154 & 0.694 & 0.231 & 103.547 & 1.41 & 0.064 & 0.019506 & 0.007803 & 0.275587 & 123.683 \\
\hline 9/10/08 1:00 & 2.149 & 0.689 & 0.227 & 101.713 & 1.41 & 0.064 & 0.019506 & 0.007803 & 0.275587 & 123.683 \\
\hline 9/10/08 2:00 & 2.159 & 0.699 & 0.235 & 105.401 & 1.41 & 0.064 & 0.019506 & 0.007803 & 0.275587 & 123.683 \\
\hline 9/10/08 3:00 & 2.156 & 0.696 & 0.232 & 104.286 & 1.408 & 0.062 & 0.018897 & 0.007457 & 0.263390 & 118.209 \\
\hline
\end{tabular}




\begin{tabular}{|c|c|c|c|c|c|c|c|c|c|c|}
\hline \multirow[b]{3}{*}{ Date Time } & \multicolumn{4}{|c|}{ V-Notch Weir } & \multicolumn{6}{|c|}{ R-Notch Weir } \\
\hline & \multicolumn{2}{|c|}{ Measured Water Level } & \multicolumn{2}{|c|}{$\begin{array}{c}\text { Calculated Rate } \\
\left(\mathrm{ft}^{3} / \mathrm{s}=448.8 \mathrm{gpm}\right)\end{array}$} & \multicolumn{3}{|c|}{ Measured Water Level } & \multicolumn{3}{|c|}{$\begin{array}{c}\text { Calculated Rate } \\
\left(\mathrm{ft}^{3} / \mathrm{s}=448.8 \mathrm{gpm}\right)\end{array}$} \\
\hline & $\begin{array}{c}\text { (ft above } \\
\text { sensor) }\end{array}$ & $\begin{array}{c}\text { (ft above } \\
\text { notch) }\end{array}$ & $\left(\mathrm{ft}^{3} / \mathrm{s}\right)$ & (gpm) & $\begin{array}{c}\text { (ft above } \\
\text { sensor) }\end{array}$ & $\begin{array}{c}\text { (ft above } \\
\text { log) }\end{array}$ & $\begin{array}{c}\text { (m above } \\
\text { log) }\end{array}$ & $\left(\mathrm{m}^{3} / \mathrm{s}\right)$ & $\left(\mathrm{ft}^{3} / \mathrm{s}\right)$ & (gpm) \\
\hline 9/10/08 4:00 & 2.182 & 0.722 & 0.254 & 114.185 & 1.407 & 0.061 & 0.018592 & 0.007287 & 0.257360 & 115.503 \\
\hline 9/10/08 5:00 & 2.156 & 0.696 & 0.232 & 104.286 & 1.407 & 0.061 & 0.018592 & 0.007287 & 0.257360 & 115.503 \\
\hline 9/10/08 6:00 & 2.159 & 0.699 & 0.235 & 105.401 & 1.407 & 0.061 & 0.018592 & 0.007287 & 0.257360 & 115.503 \\
\hline 9/10/08 7:00 & 2.161 & 0.701 & 0.237 & 106.148 & 1.407 & 0.061 & 0.018592 & 0.007287 & 0.257360 & 115.503 \\
\hline 9/10/08 8:00 & 2.161 & 0.701 & 0.237 & 106.148 & 1.407 & 0.061 & 0.018592 & 0.007287 & 0.257360 & 115.503 \\
\hline 9/10/08 9:00 & 2.159 & 0.699 & 0.235 & 105.401 & 1.409 & 0.063 & 0.019201 & 0.007629 & 0.269465 & 120.936 \\
\hline 9/10/08 10:00 & 2.166 & 0.706 & 0.241 & 108.030 & 1.406 & 0.06 & 0.018287 & 0.007117 & 0.251378 & 112.818 \\
\hline 9/10/08 11:00 & 2.163 & 0.703 & 0.238 & 106.898 & 1.407 & 0.061 & 0.018592 & 0.007287 & 0.257360 & 115.503 \\
\hline 9/10/08 12:00 & 2.159 & 0.699 & 0.235 & 105.401 & 1.407 & 0.061 & 0.018592 & 0.007287 & 0.257360 & 115.503 \\
\hline 9/10/08 13:00 & 2.148 & 0.688 & 0.226 & 101.348 & 1.407 & 0.061 & 0.018592 & 0.007287 & 0.257360 & 115.503 \\
\hline 9/10/08 14:00 & 2.132 & 0.672 & 0.213 & 95.623 & 1.405 & 0.059 & 0.017982 & 0.006949 & 0.245443 & 110.155 \\
\hline 9/10/08 15:00 & 2.117 & 0.657 & 0.202 & 90.436 & 1.403 & 0.057 & 0.017373 & 0.006617 & 0.233715 & 104.891 \\
\hline 9/10/08 16:00 & 2.112 & 0.652 & 0.198 & 88.745 & 1.401 & 0.055 & 0.016763 & 0.006291 & 0.222180 & 99.714 \\
\hline 9/10/08 17:00 & 2.107 & 0.647 & 0.194 & 87.074 & 1.403 & 0.057 & 0.017373 & 0.006617 & 0.233715 & 104.891 \\
\hline 9/10/08 18:00 & 2.109 & 0.649 & 0.195 & 87.740 & 1.404 & 0.058 & 0.017678 & 0.006782 & 0.239555 & 107.512 \\
\hline 9/10/08 19:00 & 2.118 & 0.658 & 0.202 & 90.776 & 1.406 & 0.06 & 0.018287 & 0.007117 & 0.251378 & 112.818 \\
\hline 9/10/08 20:00 & 2.127 & 0.667 & 0.209 & 93.875 & 1.409 & 0.063 & 0.019201 & 0.007629 & 0.269465 & 120.936 \\
\hline 9/10/08 21:00 & 2.148 & 0.688 & 0.226 & 101.348 & 1.409 & 0.063 & 0.019201 & 0.007629 & 0.269465 & 120.936 \\
\hline 9/10/08 22:00 & 2.143 & 0.683 & 0.222 & 99.538 & 1.409 & 0.063 & 0.019201 & 0.007629 & 0.269465 & 120.936 \\
\hline 9/10/08 23:00 & 2.148 & 0.688 & 0.226 & 101.348 & 1.412 & 0.066 & 0.020116 & 0.008153 & 0.287966 & 129.239 \\
\hline 9/11/08 0:00 & 2.16 & 0.700 & 0.236 & 105.774 & 1.409 & 0.063 & 0.019201 & 0.007629 & 0.269465 & 120.936 \\
\hline 9/11/08 1:00 & 2.157 & 0.697 & 0.233 & 104.657 & 1.412 & 0.066 & 0.020116 & 0.008153 & 0.287966 & 129.239 \\
\hline 9/11/08 2:00 & 2.164 & 0.704 & 0.239 & 107.275 & 1.412 & 0.066 & 0.020116 & 0.008153 & 0.287966 & 129.239 \\
\hline 9/11/08 3:00 & 2.173 & 0.713 & 0.247 & 110.697 & 1.412 & 0.066 & 0.020116 & 0.008153 & 0.287966 & 129.239 \\
\hline 9/11/08 4:00 & 2.173 & 0.713 & 0.247 & 110.697 & 1.412 & 0.066 & 0.020116 & 0.008153 & 0.287966 & 129.239 \\
\hline
\end{tabular}




\begin{tabular}{|c|c|c|c|c|c|c|c|c|c|c|}
\hline \multirow[b]{3}{*}{ Date Time } & \multicolumn{4}{|c|}{ V-Notch Weir } & \multicolumn{6}{|c|}{ R-Notch Weir } \\
\hline & \multicolumn{2}{|c|}{ Measured Water Level } & \multicolumn{2}{|c|}{$\begin{array}{c}\text { Calculated Rate } \\
\left(\mathrm{ft}^{3} / \mathrm{s}=448.8 \mathrm{gpm}\right)\end{array}$} & \multicolumn{3}{|c|}{ Measured Water Level } & \multicolumn{3}{|c|}{$\begin{array}{c}\text { Calculated Rate } \\
\left(\mathrm{ft}^{3} / \mathrm{s}=448.8 \mathrm{gpm}\right)\end{array}$} \\
\hline & $\begin{array}{c}\text { (ft above } \\
\text { sensor) }\end{array}$ & $\begin{array}{c}\text { (ft above } \\
\text { notch) }\end{array}$ & $\left(\mathrm{ft}^{3} / \mathrm{s}\right)$ & (gpm) & $\begin{array}{c}\text { (ft above } \\
\text { sensor) }\end{array}$ & $\begin{array}{c}\text { (ft above } \\
\text { log) }\end{array}$ & $\begin{array}{c}\text { (m above } \\
\text { log) }\end{array}$ & $\left(\mathrm{m}^{3} / \mathrm{s}\right)$ & $\left(\mathrm{ft}^{3} / \mathrm{s}\right)$ & (gpm) \\
\hline 9/11/08 5:00 & 2.18 & 0.720 & 0.253 & 113.404 & 1.412 & 0.066 & 0.020116 & 0.008153 & 0.287966 & 129.239 \\
\hline 9/11/08 6:00 & 2.197 & 0.737 & 0.268 & 120.142 & 1.414 & 0.068 & 0.020725 & 0.008509 & 0.300526 & 134.876 \\
\hline 9/11/08 7:00 & 2.192 & 0.732 & 0.263 & 118.136 & 1.412 & 0.066 & 0.020116 & 0.008153 & 0.287966 & 129.239 \\
\hline 9/11/08 8:00 & 2.206 & 0.746 & 0.276 & 123.804 & 1.412 & 0.066 & 0.020116 & 0.008153 & 0.287966 & 129.239 \\
\hline 9/11/08 9:00 & 2.3 & 0.840 & 0.370 & 166.078 & 1.426 & 0.08 & 0.024383 & 0.010746 & 0.379531 & 170.333 \\
\hline 9/11/08 10:00 & 2.514 & 1.054 & 0.650 & 291.515 & 1.466 & 0.12 & 0.036574 & 0.019354 & 0.683586 & 306.793 \\
\hline 9/11/08 11:00 & 2.39 & 0.930 & 0.476 & 213.718 & 1.445 & 0.099 & 0.030174 & 0.014626 & 0.516571 & 231.837 \\
\hline 9/11/08 12:00 & 2.31 & 0.850 & 0.381 & 171.018 & 1.428 & 0.082 & 0.024992 & 0.011135 & 0.393285 & 176.506 \\
\hline 9/11/08 13:00 & 2.259 & 0.799 & 0.327 & 146.723 & 1.424 & 0.078 & 0.023773 & 0.010361 & 0.365940 & 164.234 \\
\hline 9/11/08 14:00 & 2.231 & 0.771 & 0.299 & 134.324 & 1.419 & 0.073 & 0.022249 & 0.009420 & 0.332697 & 149.314 \\
\hline 9/11/08 15:00 & 2.219 & 0.759 & 0.288 & 129.210 & 1.419 & 0.073 & 0.022249 & 0.009420 & 0.332697 & 149.314 \\
\hline 9/11/08 16:00 & 2.234 & 0.774 & 0.302 & 135.621 & 1.422 & 0.076 & 0.023164 & 0.009981 & 0.352516 & 158.209 \\
\hline 9/11/08 17:00 & 2.271 & 0.811 & 0.339 & 152.239 & 1.427 & 0.081 & 0.024688 & 0.010940 & 0.386387 & 173.411 \\
\hline 9/11/08 18:00 & 2.325 & 0.865 & 0.398 & 178.592 & 1.436 & 0.09 & 0.027431 & 0.012738 & 0.449907 & 201.918 \\
\hline 9/11/08 19:00 & 2.59 & 1.130 & 0.772 & 346.507 & 1.512 & 0.166 & 0.050594 & 0.031143 & 1.099980 & 493.671 \\
\hline 9/11/08 20:00 & 2.596 & 1.136 & 0.782 & 351.092 & 1.543 & 0.197 & 0.060043 & 0.040080 & 1.415626 & 635.333 \\
\hline 9/11/08 21:00 & 2.5 & 1.040 & 0.628 & 282.001 & 1.479 & 0.133 & 0.040536 & 0.022495 & 0.794527 & 356.583 \\
\hline 9/11/08 22:00 & 2.394 & 0.934 & 0.481 & 216.004 & 1.448 & 0.102 & 0.031088 & 0.015274 & 0.539466 & 242.112 \\
\hline 9/11/08 23:00 & 2.319 & 0.859 & 0.391 & 175.539 & 1.437 & 0.091 & 0.027735 & 0.012943 & 0.457162 & 205.174 \\
\hline 9/12/08 0:00 & 2.272 & 0.812 & 0.340 & 152.705 & 1.429 & 0.083 & 0.025297 & 0.011331 & 0.400223 & 179.620 \\
\hline 9/12/08 1:00 & 2.251 & 0.791 & 0.319 & 143.114 & 1.427 & 0.081 & 0.024688 & 0.010940 & 0.386387 & 173.411 \\
\hline 9/12/08 2:00 & 2.239 & 0.779 & 0.307 & 137.800 & 1.425 & 0.079 & 0.024078 & 0.010553 & 0.372715 & 167.274 \\
\hline 9/12/08 3:00 & 2.23 & 0.770 & 0.298 & 133.893 & 1.422 & 0.076 & 0.023164 & 0.009981 & 0.352516 & 158.209 \\
\hline 9/12/08 4:00 & 2.222 & 0.762 & 0.291 & 130.478 & 1.422 & 0.076 & 0.023164 & 0.009981 & 0.352516 & 158.209 \\
\hline 9/12/08 5:00 & 2.218 & 0.758 & 0.287 & 128.789 & 1.422 & 0.076 & 0.023164 & 0.009981 & 0.352516 & 158.209 \\
\hline
\end{tabular}




\begin{tabular}{|c|c|c|c|c|c|c|c|c|c|c|}
\hline \multirow[b]{3}{*}{ Date Time } & \multicolumn{4}{|c|}{ V-Notch Weir } & \multicolumn{6}{|c|}{ R-Notch Weir } \\
\hline & \multicolumn{2}{|c|}{ Measured Water Level } & \multicolumn{2}{|c|}{$\begin{array}{c}\text { Calculated Rate } \\
\left(\mathrm{ft}^{3} / \mathrm{s}=448.8 \mathrm{gpm}\right)\end{array}$} & \multicolumn{3}{|c|}{ Measured Water Level } & \multicolumn{3}{|c|}{$\begin{array}{c}\text { Calculated Rate } \\
\left(\mathrm{ft}^{3} / \mathrm{s}=448.8 \mathrm{gpm}\right)\end{array}$} \\
\hline & $\begin{array}{c}\text { (ft above } \\
\text { sensor) }\end{array}$ & $\begin{array}{c}\text { (ft above } \\
\text { notch) }\end{array}$ & $\left(\mathrm{ft}^{3} / \mathrm{s}\right)$ & (gpm) & $\begin{array}{l}\text { (ft above } \\
\text { sensor) }\end{array}$ & $\begin{array}{c}\text { (ft above } \\
\text { log) }\end{array}$ & $\begin{array}{c}\text { (m above } \\
\text { log) }\end{array}$ & $\left(\mathrm{m}^{3} / \mathrm{s}\right)$ & $\left(\mathrm{ft}^{3} / \mathrm{s}\right)$ & (gpm) \\
\hline 9/12/08 6:00 & 2.211 & 0.751 & 0.280 & 125.867 & 1.42 & 0.074 & 0.022554 & 0.009605 & 0.339261 & 152.260 \\
\hline 9/12/08 7:00 & 2.211 & 0.751 & 0.280 & 125.867 & 1.422 & 0.076 & 0.023164 & 0.009981 & 0.352516 & 158.209 \\
\hline 9/12/08 8:00 & 2.211 & 0.751 & 0.280 & 125.867 & 1.419 & 0.073 & 0.022249 & 0.009420 & 0.332697 & 149.314 \\
\hline 9/12/08 9:00 & 2.218 & 0.758 & 0.287 & 128.789 & 1.422 & 0.076 & 0.023164 & 0.009981 & 0.352516 & 158.209 \\
\hline 9/12/08 10:00 & 2.244 & 0.784 & 0.312 & 139.999 & 1.424 & 0.078 & 0.023773 & 0.010361 & 0.365940 & 164.234 \\
\hline 9/12/08 11:00 & 2.272 & 0.812 & 0.340 & 152.705 & 1.429 & 0.083 & 0.025297 & 0.011331 & 0.400223 & 179.620 \\
\hline 9/12/08 12:00 & 2.291 & 0.831 & 0.360 & 161.706 & 1.433 & 0.087 & 0.026516 & 0.012129 & 0.428377 & 192.256 \\
\hline 9/12/08 13:00 & 2.295 & 0.835 & 0.365 & 163.640 & 1.433 & 0.087 & 0.026516 & 0.012129 & 0.428377 & 192.256 \\
\hline 9/12/08 14:00 & 2.474 & 1.014 & 0.590 & 264.833 & 1.464 & 0.118 & 0.035965 & 0.018885 & 0.667017 & 299.357 \\
\hline 9/12/08 15:00 & 2.558 & 1.098 & 0.719 & 322.657 & 1.502 & 0.156 & 0.047546 & 0.028425 & 1.003953 & 450.574 \\
\hline 9/12/08 16:00 & 2.519 & 1.059 & 0.657 & 294.958 & 1.485 & 0.139 & 0.042365 & 0.023997 & 0.847577 & 380.392 \\
\hline 9/12/08 17:00 & 2.467 & 1.007 & 0.580 & 260.321 & 1.464 & 0.118 & 0.035965 & 0.018885 & 0.667017 & 299.357 \\
\hline 9/12/08 18:00 & 2.389 & 0.929 & 0.475 & 213.149 & 1.45 & 0.104 & 0.031698 & 0.015711 & 0.554913 & 249.045 \\
\hline 9/12/08 19:00 & 2.357 & 0.897 & 0.435 & 195.412 & 1.445 & 0.099 & 0.030174 & 0.014626 & 0.516571 & 231.837 \\
\hline 9/12/08 20:00 & 2.338 & 0.878 & 0.413 & 185.316 & 1.441 & 0.095 & 0.028955 & 0.013776 & 0.486564 & 218.370 \\
\hline 9/12/08 21:00 & 2.319 & 0.859 & 0.391 & 175.539 & 1.438 & 0.092 & 0.028040 & 0.013150 & 0.464455 & 208.447 \\
\hline 9/12/08 22:00 & 2.307 & 0.847 & 0.378 & 169.527 & 1.436 & 0.09 & 0.027431 & 0.012738 & 0.449907 & 201.918 \\
\hline 9/12/08 23:00 & 2.298 & 0.838 & 0.368 & 165.100 & 1.433 & 0.087 & 0.026516 & 0.012129 & 0.428377 & 192.256 \\
\hline 9/13/08 0:00 & 2.276 & 0.816 & 0.344 & 154.574 & 1.431 & 0.085 & 0.025907 & 0.011728 & 0.414220 & 185.902 \\
\hline 9/13/08 1:00 & 2.281 & 0.821 & 0.350 & 156.930 & 1.431 & 0.085 & 0.025907 & 0.011728 & 0.414220 & 185.902 \\
\hline 9/13/08 2:00 & 2.288 & 0.828 & 0.357 & 160.264 & 1.433 & 0.087 & 0.026516 & 0.012129 & 0.428377 & 192.256 \\
\hline 9/13/08 3:00 & 2.279 & 0.819 & 0.348 & 155.985 & 1.433 & 0.087 & 0.026516 & 0.012129 & 0.428377 & 192.256 \\
\hline 9/13/08 4:00 & 2.272 & 0.812 & 0.340 & 152.705 & 1.431 & 0.085 & 0.025907 & 0.011728 & 0.414220 & 185.902 \\
\hline 9/13/08 5:00 & 2.265 & 0.805 & 0.333 & 149.466 & 1.431 & 0.085 & 0.025907 & 0.011728 & 0.414220 & 185.902 \\
\hline 9/13/08 6:00 & 2.255 & 0.795 & 0.323 & 144.912 & 1.429 & 0.083 & 0.025297 & 0.011331 & 0.400223 & 179.620 \\
\hline
\end{tabular}




\begin{tabular}{|c|c|c|c|c|c|c|c|c|c|c|}
\hline \multirow[b]{3}{*}{ Date Time } & \multicolumn{4}{|c|}{ V-Notch Weir } & \multicolumn{6}{|c|}{ R-Notch Weir } \\
\hline & \multicolumn{2}{|c|}{ Measured Water Level } & \multicolumn{2}{|c|}{$\begin{array}{c}\text { Calculated Rate } \\
\left(\mathrm{ft}^{3} / \mathrm{s}=448.8 \mathrm{gpm}\right)\end{array}$} & \multicolumn{3}{|c|}{ Measured Water Level } & \multicolumn{3}{|c|}{$\begin{array}{c}\text { Calculated Rate } \\
\left(\mathrm{ft}^{3} / \mathrm{s}=448.8 \mathrm{gpm}\right)\end{array}$} \\
\hline & $\begin{array}{c}\text { (ft above } \\
\text { sensor) }\end{array}$ & $\begin{array}{c}\text { (ft above } \\
\text { notch) }\end{array}$ & $\left(\mathrm{ft}^{3} / \mathrm{s}\right)$ & (gpm) & $\begin{array}{l}\text { (ft above } \\
\text { sensor) }\end{array}$ & $\begin{array}{c}\text { (ft above } \\
\text { log) }\end{array}$ & $\begin{array}{c}\text { (m above } \\
\text { log) }\end{array}$ & $\left(\mathrm{m}^{3} / \mathrm{s}\right)$ & $\left(\mathrm{ft}^{3} / \mathrm{s}\right)$ & (gpm) \\
\hline 9/13/08 7:00 & 2.246 & 0.786 & 0.314 & 140.885 & 1.428 & 0.082 & 0.024992 & 0.011135 & 0.393285 & 176.506 \\
\hline 9/13/08 8:00 & 2.243 & 0.783 & 0.311 & 139.558 & 1.426 & 0.08 & 0.024383 & 0.010746 & 0.379531 & 170.333 \\
\hline 9/13/08 9:00 & 2.236 & 0.776 & 0.304 & 136.490 & 1.426 & 0.08 & 0.024383 & 0.010746 & 0.379531 & 170.333 \\
\hline 9/13/08 10:00 & 2.234 & 0.774 & 0.302 & 135.621 & 1.424 & 0.078 & 0.023773 & 0.010361 & 0.365940 & 164.234 \\
\hline 9/13/08 11:00 & 2.23 & 0.770 & 0.298 & 133.893 & 1.426 & 0.08 & 0.024383 & 0.010746 & 0.379531 & 170.333 \\
\hline 9/13/08 12:00 & 2.223 & 0.763 & 0.292 & 130.902 & 1.426 & 0.08 & 0.024383 & 0.010746 & 0.379531 & 170.333 \\
\hline 9/13/08 13:00 & 2.21 & 0.750 & 0.280 & 125.453 & 1.424 & 0.078 & 0.023773 & 0.010361 & 0.365940 & 164.234 \\
\hline 9/13/08 14:00 & 2.199 & 0.739 & 0.269 & 120.950 & 1.422 & 0.076 & 0.023164 & 0.009981 & 0.352516 & 158.209 \\
\hline 9/13/08 15:00 & 2.177 & 0.717 & 0.250 & 112.239 & 1.422 & 0.076 & 0.023164 & 0.009981 & 0.352516 & 158.209 \\
\hline 9/13/08 16:00 & 2.163 & 0.703 & 0.238 & 106.898 & 1.42 & 0.074 & 0.022554 & 0.009605 & 0.339261 & 152.260 \\
\hline 9/13/08 17:00 & 2.149 & 0.689 & 0.227 & 101.713 & 1.418 & 0.072 & 0.021945 & 0.009235 & 0.326176 & 146.388 \\
\hline 9/13/08 18:00 & 2.144 & 0.684 & 0.223 & 99.898 & 1.418 & 0.072 & 0.021945 & 0.009235 & 0.326176 & 146.388 \\
\hline 9/13/08 19:00 & 2.137 & 0.677 & 0.217 & 97.391 & 1.418 & 0.072 & 0.021945 & 0.009235 & 0.326176 & 146.388 \\
\hline 9/13/08 20:00 & 2.145 & 0.685 & 0.223 & 100.260 & 1.419 & 0.073 & 0.022249 & 0.009420 & 0.332697 & 149.314 \\
\hline 9/13/08 21:00 & 2.149 & 0.689 & 0.227 & 101.713 & 1.419 & 0.073 & 0.022249 & 0.009420 & 0.332697 & 149.314 \\
\hline 9/13/08 22:00 & 2.158 & 0.698 & 0.234 & 105.029 & 1.419 & 0.073 & 0.022249 & 0.009420 & 0.332697 & 149.314 \\
\hline 9/13/08 23:00 & 2.162 & 0.702 & 0.237 & 106.523 & 1.421 & 0.075 & 0.022859 & 0.009792 & 0.345867 & 155.225 \\
\hline 9/14/08 0:00 & 2.169 & 0.709 & 0.243 & 109.168 & 1.426 & 0.08 & 0.024383 & 0.010746 & 0.379531 & 170.333 \\
\hline 9/14/08 1:00 & 2.169 & 0.709 & 0.243 & 109.168 & 1.426 & 0.08 & 0.024383 & 0.010746 & 0.379531 & 170.333 \\
\hline 9/14/08 2:00 & 2.171 & 0.711 & 0.245 & 109.931 & 1.426 & 0.08 & 0.024383 & 0.010746 & 0.379531 & 170.333 \\
\hline 9/14/08 3:00 & 2.175 & 0.715 & 0.248 & 111.467 & 1.425 & 0.079 & 0.024078 & 0.010553 & 0.372715 & 167.274 \\
\hline 9/14/08 4:00 & 2.175 & 0.715 & 0.248 & 111.467 & 1.425 & 0.079 & 0.024078 & 0.010553 & 0.372715 & 167.274 \\
\hline 9/14/08 5:00 & 2.168 & 0.708 & 0.242 & 108.788 & 1.42 & 0.074 & 0.022554 & 0.009605 & 0.339261 & 152.260 \\
\hline 9/14/08 6:00 & 2.173 & 0.713 & 0.247 & 110.697 & 1.42 & 0.074 & 0.022554 & 0.009605 & 0.339261 & 152.260 \\
\hline 9/14/08 7:00 & 2.17 & 0.710 & 0.244 & 109.549 & 1.419 & 0.073 & 0.022249 & 0.009420 & 0.332697 & 149.314 \\
\hline
\end{tabular}




\begin{tabular}{|c|c|c|c|c|c|c|c|c|c|c|}
\hline \multirow[b]{3}{*}{ Date Time } & \multicolumn{4}{|c|}{ V-Notch Weir } & \multicolumn{6}{|c|}{ R-Notch Weir } \\
\hline & \multicolumn{2}{|c|}{ Measured Water Level } & \multicolumn{2}{|c|}{$\begin{array}{c}\text { Calculated Rate } \\
\left(\mathrm{ft}^{3} / \mathrm{s}=448.8 \mathrm{gpm}\right)\end{array}$} & \multicolumn{3}{|c|}{ Measured Water Level } & \multicolumn{3}{|c|}{$\begin{array}{c}\text { Calculated Rate } \\
\left(\mathrm{ft}^{3} / \mathrm{s}=448.8 \mathrm{gpm}\right)\end{array}$} \\
\hline & $\begin{array}{c}\text { (ft above } \\
\text { sensor) }\end{array}$ & $\begin{array}{c}\text { (ft above } \\
\text { notch) }\end{array}$ & $\left(\mathrm{ft}^{3} / \mathrm{s}\right)$ & (gpm) & $\begin{array}{c}\text { (ft above } \\
\text { sensor) }\end{array}$ & $\begin{array}{c}\text { (ft above } \\
\text { log) }\end{array}$ & $\begin{array}{c}\text { (m above } \\
\text { log) }\end{array}$ & $\left(\mathrm{m}^{3} / \mathrm{s}\right)$ & $\left(\mathrm{ft}^{3} / \mathrm{s}\right)$ & (gpm) \\
\hline 9/14/08 8:00 & 2.166 & 0.706 & 0.241 & 108.030 & 1.416 & 0.07 & 0.021335 & 0.008869 & 0.313263 & 140.593 \\
\hline 9/14/08 9:00 & 2.163 & 0.703 & 0.238 & 106.898 & 1.418 & 0.072 & 0.021945 & 0.009235 & 0.326176 & 146.388 \\
\hline 9/14/08 10:00 & 2.163 & 0.703 & 0.238 & 106.898 & 1.415 & 0.069 & 0.021030 & 0.008688 & 0.306873 & 137.724 \\
\hline 9/14/08 11:00 & 2.161 & 0.701 & 0.237 & 106.148 & 1.415 & 0.069 & 0.021030 & 0.008688 & 0.306873 & 137.724 \\
\hline 9/14/08 12:00 & 2.152 & 0.692 & 0.229 & 102.811 & 1.42 & 0.074 & 0.022554 & 0.009605 & 0.339261 & 152.260 \\
\hline 9/14/08 13:00 & 2.14 & 0.680 & 0.219 & 98.461 & 1.416 & 0.07 & 0.021335 & 0.008869 & 0.313263 & 140.593 \\
\hline 9/14/08 14:00 & 2.133 & 0.673 & 0.214 & 95.975 & 1.418 & 0.072 & 0.021945 & 0.009235 & 0.326176 & 146.388 \\
\hline 9/14/08 15:00 & 2.138 & 0.678 & 0.218 & 97.747 & 1.413 & 0.067 & 0.020421 & 0.008330 & 0.294224 & 132.048 \\
\hline 9/14/08 16:00 & 2.126 & 0.666 & 0.208 & 93.527 & 1.416 & 0.07 & 0.021335 & 0.008869 & 0.313263 & 140.593 \\
\hline 9/14/08 17:00 & 2.121 & 0.661 & 0.205 & 91.802 & 1.413 & 0.067 & 0.020421 & 0.008330 & 0.294224 & 132.048 \\
\hline 9/14/08 18:00 & 2.126 & 0.666 & 0.208 & 93.527 & 1.413 & 0.067 & 0.020421 & 0.008330 & 0.294224 & 132.048 \\
\hline 9/14/08 19:00 & 2.117 & 0.657 & 0.202 & 90.436 & 1.413 & 0.067 & 0.020421 & 0.008330 & 0.294224 & 132.048 \\
\hline 9/14/08 20:00 & 2.119 & 0.659 & 0.203 & 91.117 & 1.413 & 0.067 & 0.020421 & 0.008330 & 0.294224 & 132.048 \\
\hline 9/14/08 21:00 & 2.121 & 0.661 & 0.205 & 91.802 & 1.413 & 0.067 & 0.020421 & 0.008330 & 0.294224 & 132.048 \\
\hline 9/14/08 22:00 & 2.126 & 0.666 & 0.208 & 93.527 & 1.413 & 0.067 & 0.020421 & 0.008330 & 0.294224 & 132.048 \\
\hline 9/14/08 23:00 & 2.133 & 0.673 & 0.214 & 95.975 & 1.413 & 0.067 & 0.020421 & 0.008330 & 0.294224 & 132.048 \\
\hline 9/15/08 0:00 & 2.142 & 0.682 & 0.221 & 99.178 & 1.415 & 0.069 & 0.021030 & 0.008688 & 0.306873 & 137.724 \\
\hline 9/15/08 1:00 & 2.142 & 0.682 & 0.221 & 99.178 & 1.412 & 0.066 & 0.020116 & 0.008153 & 0.287966 & 129.239 \\
\hline 9/15/08 2:00 & 2.145 & 0.685 & 0.223 & 100.260 & 1.411 & 0.065 & 0.019811 & 0.007977 & 0.281754 & 126.451 \\
\hline 9/15/08 3:00 & 2.152 & 0.692 & 0.229 & 102.811 & 1.414 & 0.068 & 0.020725 & 0.008509 & 0.300526 & 134.876 \\
\hline 9/15/08 4:00 & 2.155 & 0.695 & 0.232 & 103.916 & 1.411 & 0.065 & 0.019811 & 0.007977 & 0.281754 & 126.451 \\
\hline 9/15/08 5:00 & 2.157 & 0.697 & 0.233 & 104.657 & 1.411 & 0.065 & 0.019811 & 0.007977 & 0.281754 & 126.451 \\
\hline 9/15/08 6:00 & 2.155 & 0.695 & 0.232 & 103.916 & 1.41 & 0.064 & 0.019506 & 0.007803 & 0.275587 & 123.683 \\
\hline 9/15/08 7:00 & 2.157 & 0.697 & 0.233 & 104.657 & 1.41 & 0.064 & 0.019506 & 0.007803 & 0.275587 & 123.683 \\
\hline 9/15/08 8:00 & 2.158 & 0.698 & 0.234 & 105.029 & 1.41 & 0.064 & 0.019506 & 0.007803 & 0.275587 & 123.683 \\
\hline
\end{tabular}




\begin{tabular}{|c|c|c|c|c|c|c|c|c|c|c|}
\hline \multirow[b]{3}{*}{ Date Time } & \multicolumn{4}{|c|}{ V-Notch Weir } & \multicolumn{6}{|c|}{ R-Notch Weir } \\
\hline & \multicolumn{2}{|c|}{ Measured Water Level } & \multicolumn{2}{|c|}{$\begin{array}{c}\text { Calculated Rate } \\
\left(\mathrm{ft}^{3} / \mathrm{s}=448.8 \mathrm{gpm}\right)\end{array}$} & \multicolumn{3}{|c|}{ Measured Water Level } & \multicolumn{3}{|c|}{$\begin{array}{c}\text { Calculated Rate } \\
\left(\mathrm{ft}^{3} / \mathrm{s}=448.8 \mathrm{gpm}\right)\end{array}$} \\
\hline & $\begin{array}{c}\text { (ft above } \\
\text { sensor) }\end{array}$ & $\begin{array}{c}\text { (ft above } \\
\text { notch) }\end{array}$ & $\left(\mathrm{ft}^{3} / \mathrm{s}\right)$ & (gpm) & $\begin{array}{c}\text { (ft above } \\
\text { sensor) }\end{array}$ & $\begin{array}{c}\text { (ft above } \\
\text { log) }\end{array}$ & $\begin{array}{c}\text { (m above } \\
\text { log) }\end{array}$ & $\left(\mathrm{m}^{3} / \mathrm{s}\right)$ & $\left(\mathrm{ft}^{3} / \mathrm{s}\right)$ & (gpm) \\
\hline 9/15/08 9:00 & 2.16 & 0.700 & 0.236 & 105.774 & 1.413 & 0.067 & 0.020421 & 0.008330 & 0.294224 & 132.048 \\
\hline 9/15/08 10:00 & 2.162 & 0.702 & 0.237 & 106.523 & 1.413 & 0.067 & 0.020421 & 0.008330 & 0.294224 & 132.048 \\
\hline 9/15/08 11:00 & 2.161 & 0.701 & 0.237 & 106.148 & 1.413 & 0.067 & 0.020421 & 0.008330 & 0.294224 & 132.048 \\
\hline 9/15/08 12:00 & 2.142 & 0.682 & 0.221 & 99.178 & 1.41 & 0.064 & 0.019506 & 0.007803 & 0.275587 & 123.683 \\
\hline 9/15/08 13:00 & 2.157 & 0.697 & 0.233 & 104.657 & 1.408 & 0.062 & 0.018897 & 0.007457 & 0.263390 & 118.209 \\
\hline 9/15/08 14:00 & 2.15 & 0.690 & 0.227 & 102.078 & 1.408 & 0.062 & 0.018897 & 0.007457 & 0.263390 & 118.209 \\
\hline 9/15/08 15:00 & 2.132 & 0.672 & 0.213 & 95.623 & 1.406 & 0.06 & 0.018287 & 0.007117 & 0.251378 & 112.818 \\
\hline 9/15/08 16:00 & 2.123 & 0.663 & 0.206 & 92.490 & 1.404 & 0.058 & 0.017678 & 0.006782 & 0.239555 & 107.512 \\
\hline 9/15/08 17:00 & 2.114 & 0.654 & 0.199 & 89.419 & 1.405 & 0.059 & 0.017982 & 0.006949 & 0.245443 & 110.155 \\
\hline 9/15/08 18:00 & 2.109 & 0.649 & 0.195 & 87.740 & 1.405 & 0.059 & 0.017982 & 0.006949 & 0.245443 & 110.155 \\
\hline 9/15/08 19:00 & 2.116 & 0.656 & 0.201 & 90.096 & 1.405 & 0.059 & 0.017982 & 0.006949 & 0.245443 & 110.155 \\
\hline 9/15/08 20:00 & 2.12 & 0.660 & 0.204 & 91.459 & 1.408 & 0.062 & 0.018897 & 0.007457 & 0.263390 & 118.209 \\
\hline 9/15/08 21:00 & 2.134 & 0.674 & 0.215 & 96.328 & 1.408 & 0.062 & 0.018897 & 0.007457 & 0.263390 & 118.209 \\
\hline 9/15/08 22:00 & 2.15 & 0.690 & 0.227 & 102.078 & 1.408 & 0.062 & 0.018897 & 0.007457 & 0.263390 & 118.209 \\
\hline 9/15/08 23:00 & 2.161 & 0.701 & 0.237 & 106.148 & 1.408 & 0.062 & 0.018897 & 0.007457 & 0.263390 & 118.209 \\
\hline 9/16/08 0:00 & 2.168 & 0.708 & 0.242 & 108.788 & 1.411 & 0.065 & 0.019811 & 0.007977 & 0.281754 & 126.451 \\
\hline 9/16/08 1:00 & 2.189 & 0.729 & 0.261 & 116.942 & 1.411 & 0.065 & 0.019811 & 0.007977 & 0.281754 & 126.451 \\
\hline 9/16/08 2:00 & 2.192 & 0.732 & 0.263 & 118.136 & 1.41 & 0.064 & 0.019506 & 0.007803 & 0.275587 & 123.683 \\
\hline 9/16/08 3:00 & 2.194 & 0.734 & 0.265 & 118.936 & 1.41 & 0.064 & 0.019506 & 0.007803 & 0.275587 & 123.683 \\
\hline 9/16/08 4:00 & 2.199 & 0.739 & 0.269 & 120.950 & 1.41 & 0.064 & 0.019506 & 0.007803 & 0.275587 & 123.683 \\
\hline 9/16/08 5:00 & 2.199 & 0.739 & 0.269 & 120.950 & 1.409 & 0.063 & 0.019201 & 0.007629 & 0.269465 & 120.936 \\
\hline 9/16/08 6:00 & 2.211 & 0.751 & 0.280 & 125.867 & 1.409 & 0.063 & 0.019201 & 0.007629 & 0.269465 & 120.936 \\
\hline 9/16/08 7:00 & 2.213 & 0.753 & 0.282 & 126.698 & 1.411 & 0.065 & 0.019811 & 0.007977 & 0.281754 & 126.451 \\
\hline 9/16/08 8:00 & 2.213 & 0.753 & 0.282 & 126.698 & 1.411 & 0.065 & 0.019811 & 0.007977 & 0.281754 & 126.451 \\
\hline 9/16/08 9:00 & 2.22 & 0.760 & 0.289 & 129.632 & 1.411 & 0.065 & 0.019811 & 0.007977 & 0.281754 & 126.451 \\
\hline
\end{tabular}




\begin{tabular}{|c|c|c|c|c|c|c|c|c|c|c|}
\hline \multirow[b]{3}{*}{ Date Time } & \multicolumn{4}{|c|}{ V-Notch Weir } & \multicolumn{6}{|c|}{ R-Notch Weir } \\
\hline & \multicolumn{2}{|c|}{ Measured Water Level } & \multicolumn{2}{|c|}{$\begin{array}{c}\text { Calculated Rate } \\
\left(\mathrm{ft}^{3} / \mathrm{s}=448.8 \mathrm{gpm}\right)\end{array}$} & \multicolumn{3}{|c|}{ Measured Water Level } & \multicolumn{3}{|c|}{$\begin{array}{c}\text { Calculated Rate } \\
\left(\mathrm{ft}^{3} / \mathrm{s}=448.8 \mathrm{gpm}\right)\end{array}$} \\
\hline & $\begin{array}{c}\text { (ft above } \\
\text { sensor) }\end{array}$ & $\begin{array}{c}\text { (ft above } \\
\text { notch) }\end{array}$ & $\left(\mathrm{ft}^{3} / \mathrm{s}\right)$ & (gpm) & $\begin{array}{c}\text { (ft above } \\
\text { sensor) }\end{array}$ & $\begin{array}{c}\text { (ft above } \\
\text { log) }\end{array}$ & $\begin{array}{c}\text { (m above } \\
\text { log) }\end{array}$ & $\left(\mathrm{m}^{3} / \mathrm{s}\right)$ & $\left(\mathrm{ft}^{3} / \mathrm{s}\right)$ & (gpm) \\
\hline 9/16/08 10:00 & 2.222 & 0.762 & 0.291 & 130.478 & 1.409 & 0.063 & 0.019201 & 0.007629 & 0.269465 & 120.936 \\
\hline 9/16/08 11:00 & 2.217 & 0.757 & 0.286 & 128.369 & 1.409 & 0.063 & 0.019201 & 0.007629 & 0.269465 & 120.936 \\
\hline 9/16/08 12:00 & 2.208 & 0.748 & 0.278 & 124.627 & 1.409 & 0.063 & 0.019201 & 0.007629 & 0.269465 & 120.936 \\
\hline 9/16/08 13:00 & 2.195 & 0.735 & 0.266 & 119.337 & 1.407 & 0.061 & 0.018592 & 0.007287 & 0.257360 & 115.503 \\
\hline 9/16/08 14:00 & 2.18 & 0.720 & 0.253 & 113.404 & 1.405 & 0.059 & 0.017982 & 0.006949 & 0.245443 & 110.155 \\
\hline 9/16/08 15:00 & 2.167 & 0.707 & 0.242 & 108.408 & 1.405 & 0.059 & 0.017982 & 0.006949 & 0.245443 & 110.155 \\
\hline 9/16/08 16:00 & 2.158 & 0.698 & 0.234 & 105.029 & 1.403 & 0.057 & 0.017373 & 0.006617 & 0.233715 & 104.891 \\
\hline 9/16/08 17:00 & 2.152 & 0.692 & 0.229 & 102.811 & 1.404 & 0.058 & 0.017678 & 0.006782 & 0.239555 & 107.512 \\
\hline 9/16/08 18:00 & 2.149 & 0.689 & 0.227 & 101.713 & 1.404 & 0.058 & 0.017678 & 0.006782 & 0.239555 & 107.512 \\
\hline 9/16/08 19:00 & 2.155 & 0.695 & 0.232 & 103.916 & 1.407 & 0.061 & 0.018592 & 0.007287 & 0.257360 & 115.503 \\
\hline 9/16/08 20:00 & 2.163 & 0.703 & 0.238 & 106.898 & 1.407 & 0.061 & 0.018592 & 0.007287 & 0.257360 & 115.503 \\
\hline 9/16/08 21:00 & 2.179 & 0.719 & 0.252 & 113.015 & 1.41 & 0.064 & 0.019506 & 0.007803 & 0.275587 & 123.683 \\
\hline 9/16/08 22:00 & 2.193 & 0.733 & 0.264 & 118.536 & 1.412 & 0.066 & 0.020116 & 0.008153 & 0.287966 & 129.239 \\
\hline 9/16/08 23:00 & 2.213 & 0.753 & 0.282 & 126.698 & 1.412 & 0.066 & 0.020116 & 0.008153 & 0.287966 & 129.239 \\
\hline 9/17/08 0:00 & 2.216 & 0.756 & 0.285 & 127.950 & 1.412 & 0.066 & 0.020116 & 0.008153 & 0.287966 & 129.239 \\
\hline 9/17/08 1:00 & 2.218 & 0.758 & 0.287 & 128.789 & 1.412 & 0.066 & 0.020116 & 0.008153 & 0.287966 & 129.239 \\
\hline 9/17/08 2:00 & 2.229 & 0.769 & 0.297 & 133.464 & 1.414 & 0.068 & 0.020725 & 0.008509 & 0.300526 & 134.876 \\
\hline 9/17/08 3:00 & 2.232 & 0.772 & 0.300 & 134.756 & 1.412 & 0.066 & 0.020116 & 0.008153 & 0.287966 & 129.239 \\
\hline 9/17/08 4:00 & 2.243 & 0.783 & 0.311 & 139.558 & 1.412 & 0.066 & 0.020116 & 0.008153 & 0.287966 & 129.239 \\
\hline 9/17/08 5:00 & 2.239 & 0.779 & 0.307 & 137.800 & 1.414 & 0.068 & 0.020725 & 0.008509 & 0.300526 & 134.876 \\
\hline 9/17/08 6:00 & 2.241 & 0.781 & 0.309 & 138.677 & 1.413 & 0.067 & 0.020421 & 0.008330 & 0.294224 & 132.048 \\
\hline 9/17/08 7:00 & 2.25 & 0.790 & 0.318 & 142.666 & 1.413 & 0.067 & 0.020421 & 0.008330 & 0.294224 & 132.048 \\
\hline 9/17/08 8:00 & 2.25 & 0.790 & 0.318 & 142.666 & 1.413 & 0.067 & 0.020421 & 0.008330 & 0.294224 & 132.048 \\
\hline 9/17/08 9:00 & 2.255 & 0.795 & 0.323 & 144.912 & 1.41 & 0.064 & 0.019506 & 0.007803 & 0.275587 & 123.683 \\
\hline 9/17/08 10:00 & 2.264 & 0.804 & 0.332 & 149.007 & 1.413 & 0.067 & 0.020421 & 0.008330 & 0.294224 & 132.048 \\
\hline
\end{tabular}




\begin{tabular}{|c|c|c|c|c|c|c|c|c|c|c|}
\hline \multirow[b]{3}{*}{ Date Time } & \multicolumn{4}{|c|}{ V-Notch Weir } & \multicolumn{6}{|c|}{ R-Notch Weir } \\
\hline & \multicolumn{2}{|c|}{ Measured Water Level } & \multicolumn{2}{|c|}{$\begin{array}{c}\text { Calculated Rate } \\
\left(\mathrm{ft}^{3} / \mathrm{s}=448.8 \mathrm{gpm}\right)\end{array}$} & \multicolumn{3}{|c|}{ Measured Water Level } & \multicolumn{3}{|c|}{$\begin{array}{c}\text { Calculated Rate } \\
\left(\mathrm{ft}^{3} / \mathrm{s}=448.8 \mathrm{gpm}\right)\end{array}$} \\
\hline & $\begin{array}{c}\text { (ft above } \\
\text { sensor) }\end{array}$ & $\begin{array}{c}\text { (ft above } \\
\text { notch) }\end{array}$ & $\left(\mathrm{ft}^{3} / \mathrm{s}\right)$ & (gpm) & $\begin{array}{c}\text { (ft above } \\
\text { sensor) }\end{array}$ & $\begin{array}{c}\text { (ft above } \\
\text { log) }\end{array}$ & $\begin{array}{c}\text { (m above } \\
\text { log) }\end{array}$ & $\left(\mathrm{m}^{3} / \mathrm{s}\right)$ & $\left(\mathrm{ft}^{3} / \mathrm{s}\right)$ & (gpm) \\
\hline 9/17/08 11:00 & 2.26 & 0.800 & 0.328 & 147.178 & 1.41 & 0.064 & 0.019506 & 0.007803 & 0.275587 & 123.683 \\
\hline 9/17/08 12:00 & 2.242 & 0.782 & 0.310 & 139.117 & 1.41 & 0.064 & 0.019506 & 0.007803 & 0.275587 & 123.683 \\
\hline 9/17/08 13:00 & 2.229 & 0.769 & 0.297 & 133.464 & 1.406 & 0.06 & 0.018287 & 0.007117 & 0.251378 & 112.818 \\
\hline 9/17/08 14:00 & 2.209 & 0.749 & 0.279 & 125.039 & 1.406 & 0.06 & 0.018287 & 0.007117 & 0.251378 & 112.818 \\
\hline 9/17/08 15:00 & 2.196 & 0.736 & 0.267 & 119.739 & 1.404 & 0.058 & 0.017678 & 0.006782 & 0.239555 & 107.512 \\
\hline 9/17/08 16:00 & 2.187 & 0.727 & 0.259 & 116.150 & 1.405 & 0.059 & 0.017982 & 0.006949 & 0.245443 & 110.155 \\
\hline 9/17/08 17:00 & 2.183 & 0.723 & 0.255 & 114.576 & 1.405 & 0.059 & 0.017982 & 0.006949 & 0.245443 & 110.155 \\
\hline 9/17/08 18:00 & 2.182 & 0.722 & 0.254 & 114.185 & 1.403 & 0.057 & 0.017373 & 0.006617 & 0.233715 & 104.891 \\
\hline 9/17/08 19:00 & 2.188 & 0.728 & 0.260 & 116.546 & 1.406 & 0.06 & 0.018287 & 0.007117 & 0.251378 & 112.818 \\
\hline 9/17/08 20:00 & 2.194 & 0.734 & 0.265 & 118.936 & 1.409 & 0.063 & 0.019201 & 0.007629 & 0.269465 & 120.936 \\
\hline 9/17/08 21:00 & 2.205 & 0.745 & 0.275 & 123.394 & 1.411 & 0.065 & 0.019811 & 0.007977 & 0.281754 & 126.451 \\
\hline 9/17/08 22:00 & 2.221 & 0.761 & 0.290 & 130.054 & 1.414 & 0.068 & 0.020725 & 0.008509 & 0.300526 & 134.876 \\
\hline 9/17/08 23:00 & 2.23 & 0.770 & 0.298 & 133.893 & 1.414 & 0.068 & 0.020725 & 0.008509 & 0.300526 & 134.876 \\
\hline 9/18/08 0:00 & 2.232 & 0.772 & 0.300 & 134.756 & 1.413 & 0.067 & 0.020421 & 0.008330 & 0.294224 & 132.048 \\
\hline 9/18/08 1:00 & 2.237 & 0.777 & 0.305 & 136.926 & 1.413 & 0.067 & 0.020421 & 0.008330 & 0.294224 & 132.048 \\
\hline 9/18/08 2:00 & 2.246 & 0.786 & 0.314 & 140.885 & 1.413 & 0.067 & 0.020421 & 0.008330 & 0.294224 & 132.048 \\
\hline 9/18/08 3:00 & 2.255 & 0.795 & 0.323 & 144.912 & 1.413 & 0.067 & 0.020421 & 0.008330 & 0.294224 & 132.048 \\
\hline 9/18/08 4:00 & 2.26 & 0.800 & 0.328 & 147.178 & 1.412 & 0.066 & 0.020116 & 0.008153 & 0.287966 & 129.239 \\
\hline 9/18/08 5:00 & 2.267 & 0.807 & 0.335 & 150.387 & 1.414 & 0.068 & 0.020725 & 0.008509 & 0.300526 & 134.876 \\
\hline 9/18/08 6:00 & 2.267 & 0.807 & 0.335 & 150.387 & 1.414 & 0.068 & 0.020725 & 0.008509 & 0.300526 & 134.876 \\
\hline 9/18/08 7:00 & 2.267 & 0.807 & 0.335 & 150.387 & 1.413 & 0.067 & 0.020421 & 0.008330 & 0.294224 & 132.048 \\
\hline 9/18/08 8:00 & 2.269 & 0.809 & 0.337 & 151.312 & 1.411 & 0.065 & 0.019811 & 0.007977 & 0.281754 & 126.451 \\
\hline 9/18/08 9:00 & 2.269 & 0.809 & 0.337 & 151.312 & 1.411 & 0.065 & 0.019811 & 0.007977 & 0.281754 & 126.451 \\
\hline 9/18/08 10:00 & 2.271 & 0.811 & 0.339 & 152.239 & 1.413 & 0.067 & 0.020421 & 0.008330 & 0.294224 & 132.048 \\
\hline 9/18/08 11:00 & 2.269 & 0.809 & 0.337 & 151.312 & 1.413 & 0.067 & 0.020421 & 0.008330 & 0.294224 & 132.048 \\
\hline
\end{tabular}




\begin{tabular}{|c|c|c|c|c|c|c|c|c|c|c|}
\hline \multirow[b]{3}{*}{ Date Time } & \multicolumn{4}{|c|}{ V-Notch Weir } & \multicolumn{6}{|c|}{ R-Notch Weir } \\
\hline & \multicolumn{2}{|c|}{ Measured Water Level } & \multicolumn{2}{|c|}{$\begin{array}{c}\text { Calculated Rate } \\
\left(\mathrm{ft}^{3} / \mathrm{s}=448.8 \mathrm{gpm}\right)\end{array}$} & \multicolumn{3}{|c|}{ Measured Water Level } & \multicolumn{3}{|c|}{$\begin{array}{c}\text { Calculated Rate } \\
\left(\mathrm{ft}^{3} / \mathrm{s}=448.8 \mathrm{gpm}\right)\end{array}$} \\
\hline & $\begin{array}{c}\text { (ft above } \\
\text { sensor) }\end{array}$ & $\begin{array}{c}\text { (ft above } \\
\text { notch) }\end{array}$ & $\left(\mathrm{ft}^{3} / \mathrm{s}\right)$ & (gpm) & $\begin{array}{c}\text { (ft above } \\
\text { sensor) }\end{array}$ & $\begin{array}{c}\text { (ft above } \\
\text { log) }\end{array}$ & $\begin{array}{c}\text { (m above } \\
\text { log) }\end{array}$ & $\left(\mathrm{m}^{3} / \mathrm{s}\right)$ & $\left(\mathrm{ft}^{3} / \mathrm{s}\right)$ & (gpm) \\
\hline 9/18/08 12:00 & 2.26 & 0.800 & 0.328 & 147.178 & 1.408 & 0.062 & 0.018897 & 0.007457 & 0.263390 & 118.209 \\
\hline 9/18/08 13:00 & 2.247 & 0.787 & 0.315 & 141.329 & 1.409 & 0.063 & 0.019201 & 0.007629 & 0.269465 & 120.936 \\
\hline 9/18/08 14:00 & 2.232 & 0.772 & 0.300 & 134.756 & 1.409 & 0.063 & 0.019201 & 0.007629 & 0.269465 & 120.936 \\
\hline 9/18/08 15:00 & 2.221 & 0.761 & 0.290 & 130.054 & 1.404 & 0.058 & 0.017678 & 0.006782 & 0.239555 & 107.512 \\
\hline 9/18/08 16:00 & 2.21 & 0.750 & 0.280 & 125.453 & 1.405 & 0.059 & 0.017982 & 0.006949 & 0.245443 & 110.155 \\
\hline 9/18/08 17:00 & 2.045 & 0.585 & 0.151 & 67.905 & 1.405 & 0.059 & 0.017982 & 0.006949 & 0.245443 & 110.155 \\
\hline 9/18/08 18:00 & 2.05 & 0.590 & 0.155 & 69.346 & 1.408 & 0.062 & 0.018897 & 0.007457 & 0.263390 & 118.209 \\
\hline 9/18/08 19:00 & 2.054 & 0.594 & 0.157 & 70.511 & 1.408 & 0.062 & 0.018897 & 0.007457 & 0.263390 & 118.209 \\
\hline 9/18/08 20:00 & 2.065 & 0.605 & 0.164 & 73.777 & 1.411 & 0.065 & 0.019811 & 0.007977 & 0.281754 & 126.451 \\
\hline 9/18/08 21:00 & 2.073 & 0.613 & 0.170 & 76.208 & 1.411 & 0.065 & 0.019811 & 0.007977 & 0.281754 & 126.451 \\
\hline 9/18/08 22:00 & 2.085 & 0.625 & 0.178 & 79.944 & 1.413 & 0.067 & 0.020421 & 0.008330 & 0.294224 & 132.048 \\
\hline 9/18/08 23:00 & 2.096 & 0.636 & 0.186 & 83.463 & 1.413 & 0.067 & 0.020421 & 0.008330 & 0.294224 & 132.048 \\
\hline 9/19/08 0:00 & 2.103 & 0.643 & 0.191 & 85.750 & 1.413 & 0.067 & 0.020421 & 0.008330 & 0.294224 & 132.048 \\
\hline 9/19/08 1:00 & 2.1 & 0.640 & 0.189 & 84.765 & 1.413 & 0.067 & 0.020421 & 0.008330 & 0.294224 & 132.048 \\
\hline 9/19/08 2:00 & 2.112 & 0.652 & 0.198 & 88.745 & 1.416 & 0.07 & 0.021335 & 0.008869 & 0.313263 & 140.593 \\
\hline 9/19/08 3:00 & 2.114 & 0.654 & 0.199 & 89.419 & 1.415 & 0.069 & 0.021030 & 0.008688 & 0.306873 & 137.724 \\
\hline 9/19/08 4:00 & 2.114 & 0.654 & 0.199 & 89.419 & 1.415 & 0.069 & 0.021030 & 0.008688 & 0.306873 & 137.724 \\
\hline 9/19/08 5:00 & 2.119 & 0.659 & 0.203 & 91.117 & 1.414 & 0.068 & 0.020725 & 0.008509 & 0.300526 & 134.876 \\
\hline 9/19/08 6:00 & 2.123 & 0.663 & 0.206 & 92.490 & 1.414 & 0.068 & 0.020725 & 0.008509 & 0.300526 & 134.876 \\
\hline 9/19/08 7:00 & 2.128 & 0.668 & 0.210 & 94.223 & 1.416 & 0.07 & 0.021335 & 0.008869 & 0.313263 & 140.593 \\
\hline 9/19/08 8:00 & 2.126 & 0.666 & 0.208 & 93.527 & 1.414 & 0.068 & 0.020725 & 0.008509 & 0.300526 & 134.876 \\
\hline 9/19/08 9:00 & 2.131 & 0.671 & 0.212 & 95.272 & 1.416 & 0.07 & 0.021335 & 0.008869 & 0.313263 & 140.593 \\
\hline 9/19/08 10:00 & 2.128 & 0.668 & 0.210 & 94.223 & 1.416 & 0.07 & 0.021335 & 0.008869 & 0.313263 & 140.593 \\
\hline 9/19/08 11:00 & 2.133 & 0.673 & 0.214 & 95.975 & 1.413 & 0.067 & 0.020421 & 0.008330 & 0.294224 & 132.048 \\
\hline 9/19/08 12:00 & 2.119 & 0.659 & 0.203 & 91.117 & 1.414 & 0.068 & 0.020725 & 0.008509 & 0.300526 & 134.876 \\
\hline
\end{tabular}




\begin{tabular}{|c|c|c|c|c|c|c|c|c|c|c|}
\hline \multirow[b]{3}{*}{ Date Time } & \multicolumn{4}{|c|}{ V-Notch Weir } & \multicolumn{6}{|c|}{ R-Notch Weir } \\
\hline & \multicolumn{2}{|c|}{ Measured Water Level } & \multicolumn{2}{|c|}{$\begin{array}{c}\text { Calculated Rate } \\
\left(\mathrm{ft}^{3} / \mathrm{s}=448.8 \mathrm{gpm}\right)\end{array}$} & \multicolumn{3}{|c|}{ Measured Water Level } & \multicolumn{3}{|c|}{$\begin{array}{c}\text { Calculated Rate } \\
\left(\mathrm{ft}^{3} / \mathrm{s}=448.8 \mathrm{gpm}\right)\end{array}$} \\
\hline & $\begin{array}{l}\text { (ft above } \\
\text { sensor) }\end{array}$ & $\begin{array}{c}\text { (ft above } \\
\text { notch) }\end{array}$ & $\left(\mathrm{ft}^{3} / \mathrm{s}\right)$ & (gpm) & $\begin{array}{c}\text { (ft above } \\
\text { sensor) }\end{array}$ & $\begin{array}{c}\text { (ft above } \\
\text { log) }\end{array}$ & $\begin{array}{c}\text { (m above } \\
\text { log) }\end{array}$ & $\left(\mathrm{m}^{3} / \mathrm{s}\right)$ & $\left(\mathrm{ft}^{3} / \mathrm{s}\right)$ & (gpm) \\
\hline 9/19/08 13:00 & 2.099 & 0.639 & 0.188 & 84.439 & 1.414 & 0.068 & 0.020725 & 0.008509 & 0.300526 & 134.876 \\
\hline 9/19/08 14:00 & 2.09 & 0.630 & 0.182 & 81.532 & 1.412 & 0.066 & 0.020116 & 0.008153 & 0.287966 & 129.239 \\
\hline 9/19/08 15:00 & 2.081 & 0.621 & 0.175 & 78.687 & 1.409 & 0.063 & 0.019201 & 0.007629 & 0.269465 & 120.936 \\
\hline 9/19/08 16:00 & 2.068 & 0.608 & 0.166 & 74.683 & 1.41 & 0.064 & 0.019506 & 0.007803 & 0.275587 & 123.683 \\
\hline 9/19/08 17:00 & 2.064 & 0.604 & 0.164 & 73.477 & 1.408 & 0.062 & 0.018897 & 0.007457 & 0.263390 & 118.209 \\
\hline 9/19/08 18:00 & 2.059 & 0.599 & 0.160 & 71.985 & 1.408 & 0.062 & 0.018897 & 0.007457 & 0.263390 & 118.209 \\
\hline 9/19/08 19:00 & 2.061 & 0.601 & 0.162 & 72.579 & 1.411 & 0.065 & 0.019811 & 0.007977 & 0.281754 & 126.451 \\
\hline 9/19/08 20:00 & 2.069 & 0.609 & 0.167 & 74.987 & 1.411 & 0.065 & 0.019811 & 0.007977 & 0.281754 & 126.451 \\
\hline 9/19/08 21:00 & 2.08 & 0.620 & 0.175 & 78.375 & 1.413 & 0.067 & 0.020421 & 0.008330 & 0.294224 & 132.048 \\
\hline 9/19/08 22:00 & 2.089 & 0.629 & 0.181 & 81.213 & 1.414 & 0.068 & 0.020725 & 0.008509 & 0.300526 & 134.876 \\
\hline 9/19/08 23:00 & 2.096 & 0.636 & 0.186 & 83.463 & 1.414 & 0.068 & 0.020725 & 0.008509 & 0.300526 & 134.876 \\
\hline 9/20/08 0:00 & 2.107 & 0.647 & 0.194 & 87.074 & 1.416 & 0.07 & 0.021335 & 0.008869 & 0.313263 & 140.593 \\
\hline 9/20/08 1:00 & 2.112 & 0.652 & 0.198 & 88.745 & 1.416 & 0.07 & 0.021335 & 0.008869 & 0.313263 & 140.593 \\
\hline 9/20/08 2:00 & 2.117 & 0.657 & 0.202 & 90.436 & 1.416 & 0.07 & 0.021335 & 0.008869 & 0.313263 & 140.593 \\
\hline 9/20/08 3:00 & 2.119 & 0.659 & 0.203 & 91.117 & 1.415 & 0.069 & 0.021030 & 0.008688 & 0.306873 & 137.724 \\
\hline 9/20/08 4:00 & 2.112 & 0.652 & 0.198 & 88.745 & 1.415 & 0.069 & 0.021030 & 0.008688 & 0.306873 & 137.724 \\
\hline 9/20/08 5:00 & 2.116 & 0.656 & 0.201 & 90.096 & 1.414 & 0.068 & 0.020725 & 0.008509 & 0.300526 & 134.876 \\
\hline 9/20/08 6:00 & 2.126 & 0.666 & 0.208 & 93.527 & 1.416 & 0.07 & 0.021335 & 0.008869 & 0.313263 & 140.593 \\
\hline 9/20/08 7:00 & 2.123 & 0.663 & 0.206 & 92.490 & 1.416 & 0.07 & 0.021335 & 0.008869 & 0.313263 & 140.593 \\
\hline 9/20/08 8:00 & 2.126 & 0.666 & 0.208 & 93.527 & 1.413 & 0.067 & 0.020421 & 0.008330 & 0.294224 & 132.048 \\
\hline 9/20/08 9:00 & 2.128 & 0.668 & 0.210 & 94.223 & 1.413 & 0.067 & 0.020421 & 0.008330 & 0.294224 & 132.048 \\
\hline 9/20/08 10:00 & 2.126 & 0.666 & 0.208 & 93.527 & 1.416 & 0.07 & 0.021335 & 0.008869 & 0.313263 & 140.593 \\
\hline 9/20/08 11:00 & 2.126 & 0.666 & 0.208 & 93.527 & 1.416 & 0.07 & 0.021335 & 0.008869 & 0.313263 & 140.593 \\
\hline 9/20/08 12:00 & 2.117 & 0.657 & 0.202 & 90.436 & 1.413 & 0.067 & 0.020421 & 0.008330 & 0.294224 & 132.048 \\
\hline 9/20/08 13:00 & 2.104 & 0.644 & 0.192 & 86.080 & 1.411 & 0.065 & 0.019811 & 0.007977 & 0.281754 & 126.451 \\
\hline
\end{tabular}




\begin{tabular}{|c|c|c|c|c|c|c|c|c|c|c|}
\hline \multirow[b]{3}{*}{ Date Time } & \multicolumn{4}{|c|}{ V-Notch Weir } & \multicolumn{6}{|c|}{ R-Notch Weir } \\
\hline & \multicolumn{2}{|c|}{ Measured Water Level } & \multicolumn{2}{|c|}{$\begin{array}{c}\text { Calculated Rate } \\
\left(\mathrm{ft}^{3} / \mathrm{s}=448.8 \mathrm{gpm}\right)\end{array}$} & \multicolumn{3}{|c|}{ Measured Water Level } & \multicolumn{3}{|c|}{$\begin{array}{c}\text { Calculated Rate } \\
\left(\mathrm{ft}^{3} / \mathrm{s}=448.8 \mathrm{gpm}\right)\end{array}$} \\
\hline & $\begin{array}{c}\text { (ft above } \\
\text { sensor) }\end{array}$ & $\begin{array}{c}\text { (ft above } \\
\text { notch) }\end{array}$ & $\left(\mathrm{ft}^{3} / \mathrm{s}\right)$ & (gpm) & $\begin{array}{l}\text { (ft above } \\
\text { sensor) }\end{array}$ & $\begin{array}{c}\text { (ft above } \\
\text { log) }\end{array}$ & $\begin{array}{c}\text { (m above } \\
\text { log) }\end{array}$ & $\left(\mathrm{m}^{3} / \mathrm{s}\right)$ & $\left(\mathrm{ft}^{3} / \mathrm{s}\right)$ & (gpm) \\
\hline 9/20/08 14:00 & 2.086 & 0.626 & 0.179 & 80.260 & 1.411 & 0.065 & 0.019811 & 0.007977 & 0.281754 & 126.451 \\
\hline 9/20/08 15:00 & 2.07 & 0.610 & 0.168 & 75.291 & 1.409 & 0.063 & 0.019201 & 0.007629 & 0.269465 & 120.936 \\
\hline 9/20/08 16:00 & 2.059 & 0.599 & 0.160 & 71.985 & 1.41 & 0.064 & 0.019506 & 0.007803 & 0.275587 & 123.683 \\
\hline 9/20/08 17:00 & 2.052 & 0.592 & 0.156 & 69.927 & 1.408 & 0.062 & 0.018897 & 0.007457 & 0.263390 & 118.209 \\
\hline 9/20/08 18:00 & 2.05 & 0.590 & 0.155 & 69.346 & 1.408 & 0.062 & 0.018897 & 0.007457 & 0.263390 & 118.209 \\
\hline 9/20/08 19:00 & 2.051 & 0.591 & 0.155 & 69.636 & 1.411 & 0.065 & 0.019811 & 0.007977 & 0.281754 & 126.451 \\
\hline 9/20/08 20:00 & 2.058 & 0.598 & 0.160 & 71.689 & 1.413 & 0.067 & 0.020421 & 0.008330 & 0.294224 & 132.048 \\
\hline 9/20/08 21:00 & 2.073 & 0.613 & 0.170 & 76.208 & 1.413 & 0.067 & 0.020421 & 0.008330 & 0.294224 & 132.048 \\
\hline 9/20/08 22:00 & 2.085 & 0.625 & 0.178 & 79.944 & 1.416 & 0.07 & 0.021335 & 0.008869 & 0.313263 & 140.593 \\
\hline 9/20/08 23:00 & 2.091 & 0.631 & 0.182 & 81.852 & 1.416 & 0.07 & 0.021335 & 0.008869 & 0.313263 & 140.593 \\
\hline 9/21/08 0:00 & 2.094 & 0.634 & 0.185 & 82.817 & 1.416 & 0.07 & 0.021335 & 0.008869 & 0.313263 & 140.593 \\
\hline 9/21/08 1:00 & 2.1 & 0.640 & 0.189 & 84.765 & 1.416 & 0.07 & 0.021335 & 0.008869 & 0.313263 & 140.593 \\
\hline 9/21/08 2:00 & 2.105 & 0.645 & 0.193 & 86.410 & 1.416 & 0.07 & 0.021335 & 0.008869 & 0.313263 & 140.593 \\
\hline 9/21/08 3:00 & 2.109 & 0.649 & 0.195 & 87.740 & 1.413 & 0.067 & 0.020421 & 0.008330 & 0.294224 & 132.048 \\
\hline 9/21/08 4:00 & 2.119 & 0.659 & 0.203 & 91.117 & 1.415 & 0.069 & 0.021030 & 0.008688 & 0.306873 & 137.724 \\
\hline 9/21/08 5:00 & 2.121 & 0.661 & 0.205 & 91.802 & 1.413 & 0.067 & 0.020421 & 0.008330 & 0.294224 & 132.048 \\
\hline 9/21/08 6:00 & 2.116 & 0.656 & 0.201 & 90.096 & 1.412 & 0.066 & 0.020116 & 0.008153 & 0.287966 & 129.239 \\
\hline 9/21/08 7:00 & 2.121 & 0.661 & 0.205 & 91.802 & 1.414 & 0.068 & 0.020725 & 0.008509 & 0.300526 & 134.876 \\
\hline 9/21/08 8:00 & 2.119 & 0.659 & 0.203 & 91.117 & 1.412 & 0.066 & 0.020116 & 0.008153 & 0.287966 & 129.239 \\
\hline 9/21/08 9:00 & 2.121 & 0.661 & 0.205 & 91.802 & 1.414 & 0.068 & 0.020725 & 0.008509 & 0.300526 & 134.876 \\
\hline 9/21/08 10:00 & 2.121 & 0.661 & 0.205 & 91.802 & 1.414 & 0.068 & 0.020725 & 0.008509 & 0.300526 & 134.876 \\
\hline 9/21/08 11:00 & 2.121 & 0.661 & 0.205 & 91.802 & 1.414 & 0.068 & 0.020725 & 0.008509 & 0.300526 & 134.876 \\
\hline 9/21/08 12:00 & 2.117 & 0.657 & 0.202 & 90.436 & 1.412 & 0.066 & 0.020116 & 0.008153 & 0.287966 & 129.239 \\
\hline 9/21/08 13:00 & 2.104 & 0.644 & 0.192 & 86.080 & 1.412 & 0.066 & 0.020116 & 0.008153 & 0.287966 & 129.239 \\
\hline 9/21/08 14:00 & 2.088 & 0.628 & 0.180 & 80.895 & 1.41 & 0.064 & 0.019506 & 0.007803 & 0.275587 & 123.683 \\
\hline
\end{tabular}




\begin{tabular}{|c|c|c|c|c|c|c|c|c|c|c|}
\hline \multirow[b]{3}{*}{ Date Time } & \multicolumn{4}{|c|}{ V-Notch Weir } & \multicolumn{6}{|c|}{ R-Notch Weir } \\
\hline & \multicolumn{2}{|c|}{ Measured Water Level } & \multicolumn{2}{|c|}{$\begin{array}{c}\text { Calculated Rate } \\
\left(\mathrm{ft}^{3} / \mathrm{s}=448.8 \mathrm{gpm}\right)\end{array}$} & \multicolumn{3}{|c|}{ Measured Water Level } & \multicolumn{3}{|c|}{$\begin{array}{c}\text { Calculated Rate } \\
\left(\mathrm{ft}^{3} / \mathrm{s}=448.8 \mathrm{gpm}\right)\end{array}$} \\
\hline & $\begin{array}{c}\text { (ft above } \\
\text { sensor) }\end{array}$ & $\begin{array}{c}\text { (ft above } \\
\text { notch) }\end{array}$ & $\left(\mathrm{ft}^{3} / \mathrm{s}\right)$ & (gpm) & $\begin{array}{c}\text { (ft above } \\
\text { sensor) }\end{array}$ & $\begin{array}{c}\text { (ft above } \\
\text { log) }\end{array}$ & $\begin{array}{c}\text { (m above } \\
\text { log) }\end{array}$ & $\left(\mathrm{m}^{3} / \mathrm{s}\right)$ & $\left(\mathrm{ft}^{3} / \mathrm{s}\right)$ & (gpm) \\
\hline 9/21/08 15:00 & 2.084 & 0.624 & 0.177 & 79.629 & 1.412 & 0.066 & 0.020116 & 0.008153 & 0.287966 & 129.239 \\
\hline 9/21/08 16:00 & 2.066 & 0.606 & 0.165 & 74.079 & 1.405 & 0.059 & 0.017982 & 0.006949 & 0.245443 & 110.155 \\
\hline 9/21/08 17:00 & 2.059 & 0.599 & 0.160 & 71.985 & 1.408 & 0.062 & 0.018897 & 0.007457 & 0.263390 & 118.209 \\
\hline 9/21/08 18:00 & 2.059 & 0.599 & 0.160 & 71.985 & 1.408 & 0.062 & 0.018897 & 0.007457 & 0.263390 & 118.209 \\
\hline 9/21/08 19:00 & 2.06 & 0.600 & 0.161 & 72.282 & 1.409 & 0.063 & 0.019201 & 0.007629 & 0.269465 & 120.936 \\
\hline 9/21/08 20:00 & 2.065 & 0.605 & 0.164 & 73.777 & 1.409 & 0.063 & 0.019201 & 0.007629 & 0.269465 & 120.936 \\
\hline 9/21/08 21:00 & 2.076 & 0.616 & 0.172 & 77.132 & 1.411 & 0.065 & 0.019811 & 0.007977 & 0.281754 & 126.451 \\
\hline 9/21/08 22:00 & 2.087 & 0.627 & 0.180 & 80.577 & 1.412 & 0.066 & 0.020116 & 0.008153 & 0.287966 & 129.239 \\
\hline 9/21/08 23:00 & 2.096 & 0.636 & 0.186 & 83.463 & 1.414 & 0.068 & 0.020725 & 0.008509 & 0.300526 & 134.876 \\
\hline 9/22/08 0:00 & 2.098 & 0.638 & 0.187 & 84.113 & 1.416 & 0.07 & 0.021335 & 0.008869 & 0.313263 & 140.593 \\
\hline 9/22/08 1:00 & 2.108 & 0.648 & 0.195 & 87.406 & 1.414 & 0.068 & 0.020725 & 0.008509 & 0.300526 & 134.876 \\
\hline 9/22/08 2:00 & 2.11 & 0.650 & 0.196 & 88.074 & 1.411 & 0.065 & 0.019811 & 0.007977 & 0.281754 & 126.451 \\
\hline 9/22/08 3:00 & 2.112 & 0.652 & 0.198 & 88.745 & 1.414 & 0.068 & 0.020725 & 0.008509 & 0.300526 & 134.876 \\
\hline 9/22/08 4:00 & 2.114 & 0.654 & 0.199 & 89.419 & 1.413 & 0.067 & 0.020421 & 0.008330 & 0.294224 & 132.048 \\
\hline 9/22/08 5:00 & 2.133 & 0.673 & 0.214 & 95.975 & 1.416 & 0.07 & 0.021335 & 0.008869 & 0.313263 & 140.593 \\
\hline 9/22/08 6:00 & 2.147 & 0.687 & 0.225 & 100.985 & 1.418 & 0.072 & 0.021945 & 0.009235 & 0.326176 & 146.388 \\
\hline 9/22/08 7:00 & 2.164 & 0.704 & 0.239 & 107.275 & 1.42 & 0.074 & 0.022554 & 0.009605 & 0.339261 & 152.260 \\
\hline 9/22/08 8:00 & 2.196 & 0.736 & 0.267 & 119.739 & 1.422 & 0.076 & 0.023164 & 0.009981 & 0.352516 & 158.209 \\
\hline 9/22/08 9:00 & 2.46 & 1.000 & 0.570 & 255.855 & 1.467 & 0.121 & 0.036879 & 0.019590 & 0.691921 & 310.534 \\
\hline 9/22/08 10:00 & 2.401 & 0.941 & 0.490 & 220.040 & 1.455 & 0.109 & 0.033222 & 0.016822 & 0.594159 & 266.658 \\
\hline 9/22/08 11:00 & 2.288 & 0.828 & 0.357 & 160.264 & 1.436 & 0.09 & 0.027431 & 0.012738 & 0.449907 & 201.918 \\
\hline 9/22/08 12:00 & 2.22 & 0.760 & 0.289 & 129.632 & 1.424 & 0.078 & 0.023773 & 0.010361 & 0.365940 & 164.234 \\
\hline 9/22/08 13:00 & 2.186 & 0.726 & 0.258 & 115.756 & 1.422 & 0.076 & 0.023164 & 0.009981 & 0.352516 & 158.209 \\
\hline 9/22/08 14:00 & 2.156 & 0.696 & 0.232 & 104.286 & 1.417 & 0.071 & 0.021640 & 0.009052 & 0.319698 & 143.480 \\
\hline 9/22/08 15:00 & 2.133 & 0.673 & 0.214 & 95.975 & 1.413 & 0.067 & 0.020421 & 0.008330 & 0.294224 & 132.048 \\
\hline
\end{tabular}




\begin{tabular}{|c|c|c|c|c|c|c|c|c|c|c|}
\hline \multirow[b]{3}{*}{ Date Time } & \multicolumn{4}{|c|}{ V-Notch Weir } & \multicolumn{6}{|c|}{ R-Notch Weir } \\
\hline & \multicolumn{2}{|c|}{ Measured Water Level } & \multicolumn{2}{|c|}{$\begin{array}{c}\text { Calculated Rate } \\
\left(\mathrm{ft}^{3} / \mathrm{s}=448.8 \mathrm{gpm}\right)\end{array}$} & \multicolumn{3}{|c|}{ Measured Water Level } & \multicolumn{3}{|c|}{$\begin{array}{c}\text { Calculated Rate } \\
\left(\mathrm{ft}^{3} / \mathrm{s}=448.8 \mathrm{gpm}\right)\end{array}$} \\
\hline & $\begin{array}{c}\text { (ft above } \\
\text { sensor) }\end{array}$ & $\begin{array}{c}\text { (ft above } \\
\text { notch) }\end{array}$ & $\left(\mathrm{ft}^{3} / \mathrm{s}\right)$ & (gpm) & $\begin{array}{c}\text { (ft above } \\
\text { sensor) }\end{array}$ & $\begin{array}{c}\text { (ft above } \\
\text { log) }\end{array}$ & $\begin{array}{c}\text { (m above } \\
\text { log) }\end{array}$ & $\left(\mathrm{m}^{3} / \mathrm{s}\right)$ & $\left(\mathrm{ft}^{3} / \mathrm{s}\right)$ & (gpm) \\
\hline 9/22/08 16:00 & 2.12 & 0.660 & 0.204 & 91.459 & 1.413 & 0.067 & 0.020421 & 0.008330 & 0.294224 & 132.048 \\
\hline 9/22/08 17:00 & 2.113 & 0.653 & 0.198 & 89.082 & 1.411 & 0.065 & 0.019811 & 0.007977 & 0.281754 & 126.451 \\
\hline 9/22/08 18:00 & 2.106 & 0.646 & 0.193 & 86.742 & 1.411 & 0.065 & 0.019811 & 0.007977 & 0.281754 & 126.451 \\
\hline 9/22/08 19:00 & 2.1 & 0.640 & 0.189 & 84.765 & 1.409 & 0.063 & 0.019201 & 0.007629 & 0.269465 & 120.936 \\
\hline 9/22/08 20:00 & 2.109 & 0.649 & 0.195 & 87.740 & 1.409 & 0.063 & 0.019201 & 0.007629 & 0.269465 & 120.936 \\
\hline 9/22/08 21:00 & 2.113 & 0.653 & 0.198 & 89.082 & 1.412 & 0.066 & 0.020116 & 0.008153 & 0.287966 & 129.239 \\
\hline 9/22/08 22:00 & 2.113 & 0.653 & 0.198 & 89.082 & 1.412 & 0.066 & 0.020116 & 0.008153 & 0.287966 & 129.239 \\
\hline 9/22/08 23:00 & 2.115 & 0.655 & 0.200 & 89.757 & 1.412 & 0.066 & 0.020116 & 0.008153 & 0.287966 & 129.239 \\
\hline 9/23/08 0:00 & 2.131 & 0.671 & 0.212 & 95.272 & 1.414 & 0.068 & 0.020725 & 0.008509 & 0.300526 & 134.876 \\
\hline 9/23/08 1:00 & 2.129 & 0.669 & 0.211 & 94.572 & 1.414 & 0.068 & 0.020725 & 0.008509 & 0.300526 & 134.876 \\
\hline 9/23/08 2:00 & 2.136 & 0.676 & 0.216 & 97.036 & 1.414 & 0.068 & 0.020725 & 0.008509 & 0.300526 & 134.876 \\
\hline 9/23/08 3:00 & 2.143 & 0.683 & 0.222 & 99.538 & 1.414 & 0.068 & 0.020725 & 0.008509 & 0.300526 & 134.876 \\
\hline 9/23/08 4:00 & 2.145 & 0.685 & 0.223 & 100.260 & 1.416 & 0.07 & 0.021335 & 0.008869 & 0.313263 & 140.593 \\
\hline 9/23/08 5:00 & 2.152 & 0.692 & 0.229 & 102.811 & 1.414 & 0.068 & 0.020725 & 0.008509 & 0.300526 & 134.876 \\
\hline 9/23/08 6:00 & 2.154 & 0.694 & 0.231 & 103.547 & 1.413 & 0.067 & 0.020421 & 0.008330 & 0.294224 & 132.048 \\
\hline 9/23/08 7:00 & 2.164 & 0.704 & 0.239 & 107.275 & 1.413 & 0.067 & 0.020421 & 0.008330 & 0.294224 & 132.048 \\
\hline 9/23/08 8:00 & 2.128 & 0.668 & 0.210 & 94.223 & 1.415 & 0.069 & 0.021030 & 0.008688 & 0.306873 & 137.724 \\
\hline 9/23/08 9:00 & 2.133 & 0.673 & 0.214 & 95.975 & 1.415 & 0.069 & 0.021030 & 0.008688 & 0.306873 & 137.724 \\
\hline 9/23/08 10:00 & 2.131 & 0.671 & 0.212 & 95.272 & 1.415 & 0.069 & 0.021030 & 0.008688 & 0.306873 & 137.724 \\
\hline 9/23/08 11:00 & 2.124 & 0.664 & 0.207 & 92.835 & 1.413 & 0.067 & 0.020421 & 0.008330 & 0.294224 & 132.048 \\
\hline 9/23/08 12:00 & 2.106 & 0.646 & 0.193 & 86.742 & 1.413 & 0.067 & 0.020421 & 0.008330 & 0.294224 & 132.048 \\
\hline 9/23/08 13:00 & 2.102 & 0.642 & 0.190 & 85.421 & 1.411 & 0.065 & 0.019811 & 0.007977 & 0.281754 & 126.451 \\
\hline 9/23/08 14:00 & 2.091 & 0.631 & 0.182 & 81.852 & 1.411 & 0.065 & 0.019811 & 0.007977 & 0.281754 & 126.451 \\
\hline 9/23/08 15:00 & 2.086 & 0.626 & 0.179 & 80.260 & 1.411 & 0.065 & 0.019811 & 0.007977 & 0.281754 & 126.451 \\
\hline 9/23/08 16:00 & 2.102 & 0.642 & 0.190 & 85.421 & 1.411 & 0.065 & 0.019811 & 0.007977 & 0.281754 & 126.451 \\
\hline
\end{tabular}




\begin{tabular}{|c|c|c|c|c|c|c|c|c|c|c|}
\hline \multirow[b]{3}{*}{ Date Time } & \multicolumn{4}{|c|}{ V-Notch Weir } & \multicolumn{6}{|c|}{ R-Notch Weir } \\
\hline & \multicolumn{2}{|c|}{ Measured Water Level } & \multicolumn{2}{|c|}{$\begin{array}{l}\text { Calculated Rate } \\
\left(\mathrm{ft}^{3} / \mathrm{s}=448.8 \mathrm{gpm}\right)\end{array}$} & \multicolumn{3}{|c|}{ Measured Water Level } & \multicolumn{3}{|c|}{$\begin{array}{c}\text { Calculated Rate } \\
\left(\mathrm{ft}^{3} / \mathrm{s}=448.8 \mathrm{gpm}\right)\end{array}$} \\
\hline & $\begin{array}{c}\text { (ft above } \\
\text { sensor) }\end{array}$ & $\begin{array}{c}\text { (ft above } \\
\text { notch) }\end{array}$ & $\left(\mathrm{ft}^{3} / \mathrm{s}\right)$ & (gpm) & $\begin{array}{c}\text { (ft above } \\
\text { sensor) }\end{array}$ & $\begin{array}{c}\text { (ft above } \\
\text { log) }\end{array}$ & $\begin{array}{c}\text { (m above } \\
\text { log) }\end{array}$ & $\left(\mathrm{m}^{3} / \mathrm{s}\right)$ & $\left(\mathrm{ft}^{3} / \mathrm{s}\right)$ & (gpm) \\
\hline 9/23/08 17:00 & 2.133 & 0.673 & 0.214 & 95.975 & 1.418 & 0.072 & 0.021945 & 0.009235 & 0.326176 & 146.388 \\
\hline 9/23/08 18:00 & 2.144 & 0.684 & 0.223 & 99.898 & 1.421 & 0.075 & 0.022859 & 0.009792 & 0.345867 & 155.225 \\
\hline 9/23/08 19:00 & 2.153 & 0.693 & 0.230 & 103.179 & 1.419 & 0.073 & 0.022249 & 0.009420 & 0.332697 & 149.314 \\
\hline 9/23/08 20:00 & 2.151 & 0.691 & 0.228 & 102.444 & 1.419 & 0.073 & 0.022249 & 0.009420 & 0.332697 & 149.314 \\
\hline 9/23/08 21:00 & 2.148 & 0.688 & 0.226 & 101.348 & 1.419 & 0.073 & 0.022249 & 0.009420 & 0.332697 & 149.314 \\
\hline 9/23/08 22:00 & 2.138 & 0.678 & 0.218 & 97.747 & 1.417 & 0.071 & 0.021640 & 0.009052 & 0.319698 & 143.480 \\
\hline 9/23/08 23:00 & 2.141 & 0.681 & 0.220 & 98.819 & 1.416 & 0.07 & 0.021335 & 0.008869 & 0.313263 & 140.593 \\
\hline 9/24/08 0:00 & 2.143 & 0.683 & 0.222 & 99.538 & 1.416 & 0.07 & 0.021335 & 0.008869 & 0.313263 & 140.593 \\
\hline 9/24/08 1:00 & 2.14 & 0.680 & 0.219 & 98.461 & 1.416 & 0.07 & 0.021335 & 0.008869 & 0.313263 & 140.593 \\
\hline 9/24/08 2:00 & 2.135 & 0.675 & 0.215 & 96.681 & 1.416 & 0.07 & 0.021335 & 0.008869 & 0.313263 & 140.593 \\
\hline 9/24/08 3:00 & 2.135 & 0.675 & 0.215 & 96.681 & 1.414 & 0.068 & 0.020725 & 0.008509 & 0.300526 & 134.876 \\
\hline 9/24/08 4:00 & 2.135 & 0.675 & 0.215 & 96.681 & 1.416 & 0.07 & 0.021335 & 0.008869 & 0.313263 & 140.593 \\
\hline 9/24/08 5:00 & 2.14 & 0.680 & 0.219 & 98.461 & 1.416 & 0.07 & 0.021335 & 0.008869 & 0.313263 & 140.593 \\
\hline 9/24/08 6:00 & 2.145 & 0.685 & 0.223 & 100.260 & 1.415 & 0.069 & 0.021030 & 0.008688 & 0.306873 & 137.724 \\
\hline 9/24/08 7:00 & 2.142 & 0.682 & 0.221 & 99.178 & 1.415 & 0.069 & 0.021030 & 0.008688 & 0.306873 & 137.724 \\
\hline 9/24/08 8:00 & 2.142 & 0.682 & 0.221 & 99.178 & 1.415 & 0.069 & 0.021030 & 0.008688 & 0.306873 & 137.724 \\
\hline 9/24/08 9:00 & 2.147 & 0.687 & 0.225 & 100.985 & 1.417 & 0.071 & 0.021640 & 0.009052 & 0.319698 & 143.480 \\
\hline 9/24/08 10:00 & 2.145 & 0.685 & 0.223 & 100.260 & 1.417 & 0.071 & 0.021640 & 0.009052 & 0.319698 & 143.480 \\
\hline 9/24/08 11:00 & 2.147 & 0.687 & 0.225 & 100.985 & 1.417 & 0.071 & 0.021640 & 0.009052 & 0.319698 & 143.480 \\
\hline 9/24/08 12:00 & 2.136 & 0.676 & 0.216 & 97.036 & 1.415 & 0.069 & 0.021030 & 0.008688 & 0.306873 & 137.724 \\
\hline 9/24/08 13:00 & 2.132 & 0.672 & 0.213 & 95.623 & 1.412 & 0.066 & 0.020116 & 0.008153 & 0.287966 & 129.239 \\
\hline 9/24/08 14:00 & 2.128 & 0.668 & 0.210 & 94.223 & 1.415 & 0.069 & 0.021030 & 0.008688 & 0.306873 & 137.724 \\
\hline 9/24/08 15:00 & 2.117 & 0.657 & 0.202 & 90.436 & 1.415 & 0.069 & 0.021030 & 0.008688 & 0.306873 & 137.724 \\
\hline 9/24/08 16:00 & 2.106 & 0.646 & 0.193 & 86.742 & 1.415 & 0.069 & 0.021030 & 0.008688 & 0.306873 & 137.724 \\
\hline 9/24/08 17:00 & 2.097 & 0.637 & 0.187 & 83.788 & 1.409 & 0.063 & 0.019201 & 0.007629 & 0.269465 & 120.936 \\
\hline
\end{tabular}




\begin{tabular}{|c|c|c|c|c|c|c|c|c|c|c|}
\hline \multirow[b]{3}{*}{ Date Time } & \multicolumn{4}{|c|}{ V-Notch Weir } & \multicolumn{6}{|c|}{ R-Notch Weir } \\
\hline & \multicolumn{2}{|c|}{ Measured Water Level } & \multicolumn{2}{|c|}{$\begin{array}{c}\text { Calculated Rate } \\
\left(\mathrm{ft}^{3} / \mathrm{s}=448.8 \mathrm{gpm}\right)\end{array}$} & \multicolumn{3}{|c|}{ Measured Water Level } & \multicolumn{3}{|c|}{$\begin{array}{c}\text { Calculated Rate } \\
\left(\mathrm{ft}^{3} / \mathrm{s}=448.8 \mathrm{gpm}\right)\end{array}$} \\
\hline & $\begin{array}{c}\text { (ft above } \\
\text { sensor) }\end{array}$ & $\begin{array}{c}\text { (ft above } \\
\text { notch) }\end{array}$ & $\left(\mathrm{ft}^{3} / \mathrm{s}\right)$ & (gpm) & $\begin{array}{c}\text { (ft above } \\
\text { sensor) }\end{array}$ & $\begin{array}{c}\text { (ft above } \\
\text { log) }\end{array}$ & $\begin{array}{c}\text { (m above } \\
\text { log) }\end{array}$ & $\left(\mathrm{m}^{3} / \mathrm{s}\right)$ & $\left(\mathrm{ft}^{3} / \mathrm{s}\right)$ & (gpm) \\
\hline 9/24/08 18:00 & 2.085 & 0.625 & 0.178 & 79.944 & 1.409 & 0.063 & 0.019201 & 0.007629 & 0.269465 & 120.936 \\
\hline 9/24/08 19:00 & 2.079 & 0.619 & 0.174 & 78.063 & 1.407 & 0.061 & 0.018592 & 0.007287 & 0.257360 & 115.503 \\
\hline 9/24/08 20:00 & 2.083 & 0.623 & 0.177 & 79.314 & 1.407 & 0.061 & 0.018592 & 0.007287 & 0.257360 & 115.503 \\
\hline 9/24/08 21:00 & 2.09 & 0.630 & 0.182 & 81.532 & 1.409 & 0.063 & 0.019201 & 0.007629 & 0.269465 & 120.936 \\
\hline 9/24/08 22:00 & 2.099 & 0.639 & 0.188 & 84.439 & 1.409 & 0.063 & 0.019201 & 0.007629 & 0.269465 & 120.936 \\
\hline 9/24/08 23:00 & 2.106 & 0.646 & 0.193 & 86.742 & 1.41 & 0.064 & 0.019506 & 0.007803 & 0.275587 & 123.683 \\
\hline 9/25/08 0:00 & 2.115 & 0.655 & 0.200 & 89.757 & 1.412 & 0.066 & 0.020116 & 0.008153 & 0.287966 & 129.239 \\
\hline 9/25/08 1:00 & 2.122 & 0.662 & 0.205 & 92.146 & 1.412 & 0.066 & 0.020116 & 0.008153 & 0.287966 & 129.239 \\
\hline 9/25/08 2:00 & 2.119 & 0.659 & 0.203 & 91.117 & 1.412 & 0.066 & 0.020116 & 0.008153 & 0.287966 & 129.239 \\
\hline 9/25/08 3:00 & 2.124 & 0.664 & 0.207 & 92.835 & 1.412 & 0.066 & 0.020116 & 0.008153 & 0.287966 & 129.239 \\
\hline 9/25/08 4:00 & 2.126 & 0.666 & 0.208 & 93.527 & 1.414 & 0.068 & 0.020725 & 0.008509 & 0.300526 & 134.876 \\
\hline 9/25/08 5:00 & 2.131 & 0.671 & 0.212 & 95.272 & 1.411 & 0.065 & 0.019811 & 0.007977 & 0.281754 & 126.451 \\
\hline 9/25/08 6:00 & 2.128 & 0.668 & 0.210 & 94.223 & 1.413 & 0.067 & 0.020421 & 0.008330 & 0.294224 & 132.048 \\
\hline 9/25/08 7:00 & 2.128 & 0.668 & 0.210 & 94.223 & 1.413 & 0.067 & 0.020421 & 0.008330 & 0.294224 & 132.048 \\
\hline 9/25/08 8:00 & 2.135 & 0.675 & 0.215 & 96.681 & 1.413 & 0.067 & 0.020421 & 0.008330 & 0.294224 & 132.048 \\
\hline 9/25/08 9:00 & 2.138 & 0.678 & 0.218 & 97.747 & 1.413 & 0.067 & 0.020421 & 0.008330 & 0.294224 & 132.048 \\
\hline 9/25/08 10:00 & 2.14 & 0.680 & 0.219 & 98.461 & 1.413 & 0.067 & 0.020421 & 0.008330 & 0.294224 & 132.048 \\
\hline 9/25/08 11:00 & 2.131 & 0.671 & 0.212 & 95.272 & 1.41 & 0.064 & 0.019506 & 0.007803 & 0.275587 & 123.683 \\
\hline 9/25/08 12:00 & 2.115 & 0.655 & 0.200 & 89.757 & 1.41 & 0.064 & 0.019506 & 0.007803 & 0.275587 & 123.683 \\
\hline 9/25/08 13:00 & 2.109 & 0.649 & 0.195 & 87.740 & 1.408 & 0.062 & 0.018897 & 0.007457 & 0.263390 & 118.209 \\
\hline 9/25/08 14:00 & 2.096 & 0.636 & 0.186 & 83.463 & 1.411 & 0.065 & 0.019811 & 0.007977 & 0.281754 & 126.451 \\
\hline 9/25/08 15:00 & 2.087 & 0.627 & 0.180 & 80.577 & 1.409 & 0.063 & 0.019201 & 0.007629 & 0.269465 & 120.936 \\
\hline 9/25/08 16:00 & 2.076 & 0.616 & 0.172 & 77.132 & 1.406 & 0.06 & 0.018287 & 0.007117 & 0.251378 & 112.818 \\
\hline 9/25/08 17:00 & 2.072 & 0.612 & 0.169 & 75.902 & 1.404 & 0.058 & 0.017678 & 0.006782 & 0.239555 & 107.512 \\
\hline 9/25/08 18:00 & 2.069 & 0.609 & 0.167 & 74.987 & 1.405 & 0.059 & 0.017982 & 0.006949 & 0.245443 & 110.155 \\
\hline
\end{tabular}




\begin{tabular}{|c|c|c|c|c|c|c|c|c|c|c|}
\hline \multirow[b]{3}{*}{ Date Time } & \multicolumn{4}{|c|}{ V-Notch Weir } & \multicolumn{6}{|c|}{ R-Notch Weir } \\
\hline & \multicolumn{2}{|c|}{ Measured Water Level } & \multicolumn{2}{|c|}{$\begin{array}{c}\text { Calculated Rate } \\
\left(\mathrm{ft}^{3} / \mathrm{s}=448.8 \mathrm{gpm}\right)\end{array}$} & \multicolumn{3}{|c|}{ Measured Water Level } & \multicolumn{3}{|c|}{$\begin{array}{c}\text { Calculated Rate } \\
\left(\mathrm{ft}^{3} / \mathrm{s}=448.8 \mathrm{gpm}\right)\end{array}$} \\
\hline & $\begin{array}{c}\text { (ft above } \\
\text { sensor) }\end{array}$ & $\begin{array}{c}\text { (ft above } \\
\text { notch) }\end{array}$ & $\left(\mathrm{ft}^{3} / \mathrm{s}\right)$ & (gpm) & $\begin{array}{c}\text { (ft above } \\
\text { sensor) }\end{array}$ & $\begin{array}{c}\text { (ft above } \\
\text { log) }\end{array}$ & $\begin{array}{c}\text { (m above } \\
\text { log) }\end{array}$ & $\left(\mathrm{m}^{3} / \mathrm{s}\right)$ & $\left(\mathrm{ft}^{3} / \mathrm{s}\right)$ & (gpm) \\
\hline 9/25/08 19:00 & 2.071 & 0.611 & 0.168 & 75.596 & 1.407 & 0.061 & 0.018592 & 0.007287 & 0.257360 & 115.503 \\
\hline 9/25/08 20:00 & 2.07 & 0.610 & 0.168 & 75.291 & 1.408 & 0.062 & 0.018897 & 0.007457 & 0.263390 & 118.209 \\
\hline 9/25/08 21:00 & 2.076 & 0.616 & 0.172 & 77.132 & 1.408 & 0.062 & 0.018897 & 0.007457 & 0.263390 & 118.209 \\
\hline 9/25/08 22:00 & 2.085 & 0.625 & 0.178 & 79.944 & 1.41 & 0.064 & 0.019506 & 0.007803 & 0.275587 & 123.683 \\
\hline 9/25/08 23:00 & 2.094 & 0.634 & 0.185 & 82.817 & 1.41 & 0.064 & 0.019506 & 0.007803 & 0.275587 & 123.683 \\
\hline 9/26/08 0:00 & 2.096 & 0.636 & 0.186 & 83.463 & 1.41 & 0.064 & 0.019506 & 0.007803 & 0.275587 & 123.683 \\
\hline 9/26/08 1:00 & 2.105 & 0.645 & 0.193 & 86.410 & 1.413 & 0.067 & 0.020421 & 0.008330 & 0.294224 & 132.048 \\
\hline 9/26/08 2:00 & 2.11 & 0.650 & 0.196 & 88.074 & 1.412 & 0.066 & 0.020116 & 0.008153 & 0.287966 & 129.239 \\
\hline 9/26/08 3:00 & 2.114 & 0.654 & 0.199 & 89.419 & 1.412 & 0.066 & 0.020116 & 0.008153 & 0.287966 & 129.239 \\
\hline 9/26/08 4:00 & 2.119 & 0.659 & 0.203 & 91.117 & 1.414 & 0.068 & 0.020725 & 0.008509 & 0.300526 & 134.876 \\
\hline 9/26/08 5:00 & 2.126 & 0.666 & 0.208 & 93.527 & 1.414 & 0.068 & 0.020725 & 0.008509 & 0.300526 & 134.876 \\
\hline 9/26/08 6:00 & 2.124 & 0.664 & 0.207 & 92.835 & 1.414 & 0.068 & 0.020725 & 0.008509 & 0.300526 & 134.876 \\
\hline 9/26/08 7:00 & 2.128 & 0.668 & 0.210 & 94.223 & 1.413 & 0.067 & 0.020421 & 0.008330 & 0.294224 & 132.048 \\
\hline 9/26/08 8:00 & 2.128 & 0.668 & 0.210 & 94.223 & 1.413 & 0.067 & 0.020421 & 0.008330 & 0.294224 & 132.048 \\
\hline 9/26/08 9:00 & 2.126 & 0.666 & 0.208 & 93.527 & 1.411 & 0.065 & 0.019811 & 0.007977 & 0.281754 & 126.451 \\
\hline 9/26/08 10:00 & 2.128 & 0.668 & 0.210 & 94.223 & 1.413 & 0.067 & 0.020421 & 0.008330 & 0.294224 & 132.048 \\
\hline 9/26/08 11:00 & 2.126 & 0.666 & 0.208 & 93.527 & 1.413 & 0.067 & 0.020421 & 0.008330 & 0.294224 & 132.048 \\
\hline 9/26/08 12:00 & 2.113 & 0.653 & 0.198 & 89.082 & 1.413 & 0.067 & 0.020421 & 0.008330 & 0.294224 & 132.048 \\
\hline 9/26/08 13:00 & 2.095 & 0.635 & 0.185 & 83.140 & 1.411 & 0.065 & 0.019811 & 0.007977 & 0.281754 & 126.451 \\
\hline 9/26/08 14:00 & 2.086 & 0.626 & 0.179 & 80.260 & 1.409 & 0.063 & 0.019201 & 0.007629 & 0.269465 & 120.936 \\
\hline 9/26/08 15:00 & 2.07 & 0.610 & 0.168 & 75.291 & 1.406 & 0.06 & 0.018287 & 0.007117 & 0.251378 & 112.818 \\
\hline 9/26/08 16:00 & 2.062 & 0.602 & 0.162 & 72.878 & 1.407 & 0.061 & 0.018592 & 0.007287 & 0.257360 & 115.503 \\
\hline 9/26/08 17:00 & 2.052 & 0.592 & 0.156 & 69.927 & 1.405 & 0.059 & 0.017982 & 0.006949 & 0.245443 & 110.155 \\
\hline 9/26/08 18:00 & 2.05 & 0.590 & 0.155 & 69.346 & 1.403 & 0.057 & 0.017373 & 0.006617 & 0.233715 & 104.891 \\
\hline 9/26/08 19:00 & 2.052 & 0.592 & 0.156 & 69.927 & 1.403 & 0.057 & 0.017373 & 0.006617 & 0.233715 & 104.891 \\
\hline
\end{tabular}




\begin{tabular}{|c|c|c|c|c|c|c|c|c|c|c|}
\hline \multirow[b]{3}{*}{ Date Time } & \multicolumn{4}{|c|}{ V-Notch Weir } & \multicolumn{6}{|c|}{ R-Notch Weir } \\
\hline & \multicolumn{2}{|c|}{ Measured Water Level } & \multicolumn{2}{|c|}{$\begin{array}{c}\text { Calculated Rate } \\
\left(\mathrm{ft}^{3} / \mathrm{s}=448.8 \mathrm{gpm}\right)\end{array}$} & \multicolumn{3}{|c|}{ Measured Water Level } & \multicolumn{3}{|c|}{$\begin{array}{c}\text { Calculated Rate } \\
\left(\mathrm{ft}^{3} / \mathrm{s}=448.8 \mathrm{gpm}\right)\end{array}$} \\
\hline & $\begin{array}{c}\text { (ft above } \\
\text { sensor) }\end{array}$ & $\begin{array}{c}\text { (ft above } \\
\text { notch) }\end{array}$ & $\left(\mathrm{ft}^{3} / \mathrm{s}\right)$ & (gpm) & $\begin{array}{c}\text { (ft above } \\
\text { sensor) }\end{array}$ & $\begin{array}{c}\text { (ft above } \\
\text { log) }\end{array}$ & $\begin{array}{c}\text { (m above } \\
\text { log) }\end{array}$ & $\left(\mathrm{m}^{3} / \mathrm{s}\right)$ & $\left(\mathrm{ft}^{3} / \mathrm{s}\right)$ & (gpm) \\
\hline 9/26/08 20:00 & 2.058 & 0.598 & 0.160 & 71.689 & 1.403 & 0.057 & 0.017373 & 0.006617 & 0.233715 & 104.891 \\
\hline 9/26/08 21:00 & 2.067 & 0.607 & 0.166 & 74.381 & 1.406 & 0.06 & 0.018287 & 0.007117 & 0.251378 & 112.818 \\
\hline 9/26/08 22:00 & 2.076 & 0.616 & 0.172 & 77.132 & 1.406 & 0.06 & 0.018287 & 0.007117 & 0.251378 & 112.818 \\
\hline 9/26/08 23:00 & 2.085 & 0.625 & 0.178 & 79.944 & 1.406 & 0.06 & 0.018287 & 0.007117 & 0.251378 & 112.818 \\
\hline 9/27/08 0:00 & 2.092 & 0.632 & 0.183 & 82.173 & 1.408 & 0.062 & 0.018897 & 0.007457 & 0.263390 & 118.209 \\
\hline 9/27/08 1:00 & 2.096 & 0.636 & 0.186 & 83.463 & 1.408 & 0.062 & 0.018897 & 0.007457 & 0.263390 & 118.209 \\
\hline 9/27/08 2:00 & 2.103 & 0.643 & 0.191 & 85.750 & 1.41 & 0.064 & 0.019506 & 0.007803 & 0.275587 & 123.683 \\
\hline 9/27/08 3:00 & 2.107 & 0.647 & 0.194 & 87.074 & 1.41 & 0.064 & 0.019506 & 0.007803 & 0.275587 & 123.683 \\
\hline 9/27/08 4:00 & 2.11 & 0.650 & 0.196 & 88.074 & 1.41 & 0.064 & 0.019506 & 0.007803 & 0.275587 & 123.683 \\
\hline 9/27/08 5:00 & 2.112 & 0.652 & 0.198 & 88.745 & 1.41 & 0.064 & 0.019506 & 0.007803 & 0.275587 & 123.683 \\
\hline 9/27/08 6:00 & 2.114 & 0.654 & 0.199 & 89.419 & 1.414 & 0.068 & 0.020725 & 0.008509 & 0.300526 & 134.876 \\
\hline 9/27/08 7:00 & 2.119 & 0.659 & 0.203 & 91.117 & 1.414 & 0.068 & 0.020725 & 0.008509 & 0.300526 & 134.876 \\
\hline 9/27/08 8:00 & 2.116 & 0.656 & 0.201 & 90.096 & 1.413 & 0.067 & 0.020421 & 0.008330 & 0.294224 & 132.048 \\
\hline 9/27/08 9:00 & 2.121 & 0.661 & 0.205 & 91.802 & 1.413 & 0.067 & 0.020421 & 0.008330 & 0.294224 & 132.048 \\
\hline 9/27/08 10:00 & 2.126 & 0.666 & 0.208 & 93.527 & 1.416 & 0.07 & 0.021335 & 0.008869 & 0.313263 & 140.593 \\
\hline 9/27/08 11:00 & 2.128 & 0.668 & 0.210 & 94.223 & 1.413 & 0.067 & 0.020421 & 0.008330 & 0.294224 & 132.048 \\
\hline 9/27/08 12:00 & 2.124 & 0.664 & 0.207 & 92.835 & 1.409 & 0.063 & 0.019201 & 0.007629 & 0.269465 & 120.936 \\
\hline 9/27/08 13:00 & 2.113 & 0.653 & 0.198 & 89.082 & 1.406 & 0.06 & 0.018287 & 0.007117 & 0.251378 & 112.818 \\
\hline 9/27/08 14:00 & 2.102 & 0.642 & 0.190 & 85.421 & 1.406 & 0.06 & 0.018287 & 0.007117 & 0.251378 & 112.818 \\
\hline 9/27/08 15:00 & 2.095 & 0.635 & 0.185 & 83.140 & 1.406 & 0.06 & 0.018287 & 0.007117 & 0.251378 & 112.818 \\
\hline 9/27/08 16:00 & 2.091 & 0.631 & 0.182 & 81.852 & 1.404 & 0.058 & 0.017678 & 0.006782 & 0.239555 & 107.512 \\
\hline 9/27/08 17:00 & 2.082 & 0.622 & 0.176 & 79.000 & 1.404 & 0.058 & 0.017678 & 0.006782 & 0.239555 & 107.512 \\
\hline 9/27/08 18:00 & 2.072 & 0.612 & 0.169 & 75.902 & 1.405 & 0.059 & 0.017982 & 0.006949 & 0.245443 & 110.155 \\
\hline 9/27/08 19:00 & 2.07 & 0.610 & 0.168 & 75.291 & 1.403 & 0.057 & 0.017373 & 0.006617 & 0.233715 & 104.891 \\
\hline 9/27/08 20:00 & 2.071 & 0.611 & 0.168 & 75.596 & 1.403 & 0.057 & 0.017373 & 0.006617 & 0.233715 & 104.891 \\
\hline
\end{tabular}




\begin{tabular}{|c|c|c|c|c|c|c|c|c|c|c|}
\hline \multirow[b]{3}{*}{ Date Time } & \multicolumn{4}{|c|}{ V-Notch Weir } & \multicolumn{6}{|c|}{ R-Notch Weir } \\
\hline & \multicolumn{2}{|c|}{ Measured Water Level } & \multicolumn{2}{|c|}{$\begin{array}{c}\text { Calculated Rate } \\
\left(\mathrm{ft}^{3} / \mathrm{s}=448.8 \mathrm{gpm}\right)\end{array}$} & \multicolumn{3}{|c|}{ Measured Water Level } & \multicolumn{3}{|c|}{$\begin{array}{c}\text { Calculated Rate } \\
\left(\mathrm{ft}^{3} / \mathrm{s}=448.8 \mathrm{gpm}\right)\end{array}$} \\
\hline & $\begin{array}{c}\text { (ft above } \\
\text { sensor) }\end{array}$ & $\begin{array}{c}\text { (ft above } \\
\text { notch) }\end{array}$ & $\left(\mathrm{ft}^{3} / \mathrm{s}\right)$ & (gpm) & $\begin{array}{c}\text { (ft above } \\
\text { sensor) }\end{array}$ & $\begin{array}{c}\text { (ft above } \\
\text { log) }\end{array}$ & $\begin{array}{c}\text { (m above } \\
\text { log) }\end{array}$ & $\left(\mathrm{m}^{3} / \mathrm{s}\right)$ & $\left(\mathrm{ft}^{3} / \mathrm{s}\right)$ & (gpm) \\
\hline 9/27/08 21:00 & 2.078 & 0.618 & 0.173 & 77.752 & 1.405 & 0.059 & 0.017982 & 0.006949 & 0.245443 & 110.155 \\
\hline 9/27/08 22:00 & 2.085 & 0.625 & 0.178 & 79.944 & 1.405 & 0.059 & 0.017982 & 0.006949 & 0.245443 & 110.155 \\
\hline 9/27/08 23:00 & 2.087 & 0.627 & 0.180 & 80.577 & 1.408 & 0.062 & 0.018897 & 0.007457 & 0.263390 & 118.209 \\
\hline 9/28/08 0:00 & 2.098 & 0.638 & 0.187 & 84.113 & 1.408 & 0.062 & 0.018897 & 0.007457 & 0.263390 & 118.209 \\
\hline 9/28/08 1:00 & 2.1 & 0.640 & 0.189 & 84.765 & 1.407 & 0.061 & 0.018592 & 0.007287 & 0.257360 & 115.503 \\
\hline 9/28/08 2:00 & 2.109 & 0.649 & 0.195 & 87.740 & 1.407 & 0.061 & 0.018592 & 0.007287 & 0.257360 & 115.503 \\
\hline 9/28/08 3:00 & 2.109 & 0.649 & 0.195 & 87.740 & 1.407 & 0.061 & 0.018592 & 0.007287 & 0.257360 & 115.503 \\
\hline 9/28/08 4:00 & 2.116 & 0.656 & 0.201 & 90.096 & 1.409 & 0.063 & 0.019201 & 0.007629 & 0.269465 & 120.936 \\
\hline 9/28/08 5:00 & 2.119 & 0.659 & 0.203 & 91.117 & 1.408 & 0.062 & 0.018897 & 0.007457 & 0.263390 & 118.209 \\
\hline 9/28/08 6:00 & 2.128 & 0.668 & 0.210 & 94.223 & 1.408 & 0.062 & 0.018897 & 0.007457 & 0.263390 & 118.209 \\
\hline 9/28/08 7:00 & 2.123 & 0.663 & 0.206 & 92.490 & 1.408 & 0.062 & 0.018897 & 0.007457 & 0.263390 & 118.209 \\
\hline 9/28/08 8:00 & 2.124 & 0.664 & 0.207 & 92.835 & 1.407 & 0.061 & 0.018592 & 0.007287 & 0.257360 & 115.503 \\
\hline 9/28/08 9:00 & 2.126 & 0.666 & 0.208 & 93.527 & 1.407 & 0.061 & 0.018592 & 0.007287 & 0.257360 & 115.503 \\
\hline 9/28/08 10:00 & 2.128 & 0.668 & 0.210 & 94.223 & 1.41 & 0.064 & 0.019506 & 0.007803 & 0.275587 & 123.683 \\
\hline 9/28/08 11:00 & 2.123 & 0.663 & 0.206 & 92.490 & 1.407 & 0.061 & 0.018592 & 0.007287 & 0.257360 & 115.503 \\
\hline 9/28/08 12:00 & 2.121 & 0.661 & 0.205 & 91.802 & 1.407 & 0.061 & 0.018592 & 0.007287 & 0.257360 & 115.503 \\
\hline 9/28/08 13:00 & 2.115 & 0.655 & 0.200 & 89.757 & 1.407 & 0.061 & 0.018592 & 0.007287 & 0.257360 & 115.503 \\
\hline 9/28/08 14:00 & 2.106 & 0.646 & 0.193 & 86.742 & 1.405 & 0.059 & 0.017982 & 0.006949 & 0.245443 & 110.155 \\
\hline 9/28/08 15:00 & 2.097 & 0.637 & 0.187 & 83.788 & 1.405 & 0.059 & 0.017982 & 0.006949 & 0.245443 & 110.155 \\
\hline 9/28/08 16:00 & 2.084 & 0.624 & 0.177 & 79.629 & 1.403 & 0.057 & 0.017373 & 0.006617 & 0.233715 & 104.891 \\
\hline 9/28/08 17:00 & 2.079 & 0.619 & 0.174 & 78.063 & 1.401 & 0.055 & 0.016763 & 0.006291 & 0.222180 & 99.714 \\
\hline 9/28/08 18:00 & 2.082 & 0.622 & 0.176 & 79.000 & 1.404 & 0.058 & 0.017678 & 0.006782 & 0.239555 & 107.512 \\
\hline 9/28/08 19:00 & 2.077 & 0.617 & 0.173 & 77.442 & 1.404 & 0.058 & 0.017678 & 0.006782 & 0.239555 & 107.512 \\
\hline 9/28/08 20:00 & 2.081 & 0.621 & 0.175 & 78.687 & 1.402 & 0.056 & 0.017068 & 0.006453 & 0.227923 & 102.292 \\
\hline 9/28/08 21:00 & 2.085 & 0.625 & 0.178 & 79.944 & 1.404 & 0.058 & 0.017678 & 0.006782 & 0.239555 & 107.512 \\
\hline
\end{tabular}




\begin{tabular}{|c|c|c|c|c|c|c|c|c|c|c|}
\hline \multirow[b]{3}{*}{ Date Time } & \multicolumn{4}{|c|}{ V-Notch Weir } & \multicolumn{6}{|c|}{ R-Notch Weir } \\
\hline & \multicolumn{2}{|c|}{ Measured Water Level } & \multicolumn{2}{|c|}{$\begin{array}{c}\text { Calculated Rate } \\
\left(\mathrm{ft}^{3} / \mathrm{s}=448.8 \mathrm{gpm}\right)\end{array}$} & \multicolumn{3}{|c|}{ Measured Water Level } & \multicolumn{3}{|c|}{$\begin{array}{c}\text { Calculated Rate } \\
\left(\mathrm{ft}^{3} / \mathrm{s}=448.8 \mathrm{gpm}\right)\end{array}$} \\
\hline & $\begin{array}{c}\text { (ft above } \\
\text { sensor) }\end{array}$ & $\begin{array}{c}\text { (ft above } \\
\text { notch) }\end{array}$ & $\left(\mathrm{ft}^{3} / \mathrm{s}\right)$ & (gpm) & $\begin{array}{c}\text { (ft above } \\
\text { sensor) }\end{array}$ & $\begin{array}{c}\text { (ft above } \\
\text { log) }\end{array}$ & $\begin{array}{c}\text { (m above } \\
\text { log) }\end{array}$ & $\left(\mathrm{m}^{3} / \mathrm{s}\right)$ & $\left(\mathrm{ft}^{3} / \mathrm{s}\right)$ & (gpm) \\
\hline 9/28/08 22:00 & 2.089 & 0.629 & 0.181 & 81.213 & 1.407 & 0.061 & 0.018592 & 0.007287 & 0.257360 & 115.503 \\
\hline 9/28/08 23:00 & 2.085 & 0.625 & 0.178 & 79.944 & 1.404 & 0.058 & 0.017678 & 0.006782 & 0.239555 & 107.512 \\
\hline 9/29/08 0:00 & 2.108 & 0.648 & 0.195 & 87.406 & 1.409 & 0.063 & 0.019201 & 0.007629 & 0.269465 & 120.936 \\
\hline 9/29/08 1:00 & 2.112 & 0.652 & 0.198 & 88.745 & 1.409 & 0.063 & 0.019201 & 0.007629 & 0.269465 & 120.936 \\
\hline 9/29/08 2:00 & 2.124 & 0.664 & 0.207 & 92.835 & 1.409 & 0.063 & 0.019201 & 0.007629 & 0.269465 & 120.936 \\
\hline 9/29/08 3:00 & 2.136 & 0.676 & 0.216 & 97.036 & 1.412 & 0.066 & 0.020116 & 0.008153 & 0.287966 & 129.239 \\
\hline 9/29/08 4:00 & 2.138 & 0.678 & 0.218 & 97.747 & 1.412 & 0.066 & 0.020116 & 0.008153 & 0.287966 & 129.239 \\
\hline 9/29/08 5:00 & 2.138 & 0.678 & 0.218 & 97.747 & 1.411 & 0.065 & 0.019811 & 0.007977 & 0.281754 & 126.451 \\
\hline 9/29/08 6:00 & 2.145 & 0.685 & 0.223 & 100.260 & 1.414 & 0.068 & 0.020725 & 0.008509 & 0.300526 & 134.876 \\
\hline 9/29/08 7:00 & 2.138 & 0.678 & 0.218 & 97.747 & 1.411 & 0.065 & 0.019811 & 0.007977 & 0.281754 & 126.451 \\
\hline 9/29/08 8:00 & 2.133 & 0.673 & 0.214 & 95.975 & 1.411 & 0.065 & 0.019811 & 0.007977 & 0.281754 & 126.451 \\
\hline 9/29/08 9:00 & 2.128 & 0.668 & 0.210 & 94.223 & 1.409 & 0.063 & 0.019201 & 0.007629 & 0.269465 & 120.936 \\
\hline 9/29/08 10:00 & 2.131 & 0.671 & 0.212 & 95.272 & 1.409 & 0.063 & 0.019201 & 0.007629 & 0.269465 & 120.936 \\
\hline 9/29/08 11:00 & 2.126 & 0.666 & 0.208 & 93.527 & 1.409 & 0.063 & 0.019201 & 0.007629 & 0.269465 & 120.936 \\
\hline 9/29/08 12:00 & 2.124 & 0.664 & 0.207 & 92.835 & 1.406 & 0.06 & 0.018287 & 0.007117 & 0.251378 & 112.818 \\
\hline 9/29/08 13:00 & 2.105 & 0.645 & 0.193 & 86.410 & 1.406 & 0.06 & 0.018287 & 0.007117 & 0.251378 & 112.818 \\
\hline 9/29/08 14:00 & 2.103 & 0.643 & 0.191 & 85.750 & 1.404 & 0.058 & 0.017678 & 0.006782 & 0.239555 & 107.512 \\
\hline 9/29/08 15:00 & 2.097 & 0.637 & 0.187 & 83.788 & 1.406 & 0.06 & 0.018287 & 0.007117 & 0.251378 & 112.818 \\
\hline 9/29/08 16:00 & 2.085 & 0.625 & 0.178 & 79.944 & 1.402 & 0.056 & 0.017068 & 0.006453 & 0.227923 & 102.292 \\
\hline 9/29/08 17:00 & 2.073 & 0.613 & 0.170 & 76.208 & 1.402 & 0.056 & 0.017068 & 0.006453 & 0.227923 & 102.292 \\
\hline 9/29/08 18:00 & 2.071 & 0.611 & 0.168 & 75.596 & 1.402 & 0.056 & 0.017068 & 0.006453 & 0.227923 & 102.292 \\
\hline 9/29/08 19:00 & 2.066 & 0.606 & 0.165 & 74.079 & 1.402 & 0.056 & 0.017068 & 0.006453 & 0.227923 & 102.292 \\
\hline 9/29/08 20:00 & 2.068 & 0.608 & 0.166 & 74.683 & 1.402 & 0.056 & 0.017068 & 0.006453 & 0.227923 & 102.292 \\
\hline 9/29/08 21:00 & 2.074 & 0.614 & 0.170 & 76.516 & 1.402 & 0.056 & 0.017068 & 0.006453 & 0.227923 & 102.292 \\
\hline 9/29/08 22:00 & 2.081 & 0.621 & 0.175 & 78.687 & 1.404 & 0.058 & 0.017678 & 0.006782 & 0.239555 & 107.512 \\
\hline
\end{tabular}




\begin{tabular}{|c|c|c|c|c|c|c|c|c|c|c|}
\hline \multirow[b]{3}{*}{ Date Time } & \multicolumn{4}{|c|}{ V-Notch Weir } & \multicolumn{6}{|c|}{ R-Notch Weir } \\
\hline & \multicolumn{2}{|c|}{ Measured Water Level } & \multicolumn{2}{|c|}{$\begin{array}{c}\text { Calculated Rate } \\
\left(\mathrm{ft}^{3} / \mathrm{s}=448.8 \mathrm{gpm}\right)\end{array}$} & \multicolumn{3}{|c|}{ Measured Water Level } & \multicolumn{3}{|c|}{$\begin{array}{c}\text { Calculated Rate } \\
\left(\mathrm{ft}^{3} / \mathrm{s}=448.8 \mathrm{gpm}\right)\end{array}$} \\
\hline & $\begin{array}{c}\text { (ft above } \\
\text { sensor) }\end{array}$ & $\begin{array}{c}\text { (ft above } \\
\text { notch) }\end{array}$ & $\left(\mathrm{ft}^{3} / \mathrm{s}\right)$ & (gpm) & $\begin{array}{c}\text { (ft above } \\
\text { sensor) }\end{array}$ & $\begin{array}{c}\text { (ft above } \\
\text { log) }\end{array}$ & $\begin{array}{c}\text { (m above } \\
\text { log) }\end{array}$ & $\left(\mathrm{m}^{3} / \mathrm{s}\right)$ & $\left(\mathrm{ft}^{3} / \mathrm{s}\right)$ & (gpm) \\
\hline 9/29/08 23:00 & 2.088 & 0.628 & 0.180 & 80.895 & 1.404 & 0.058 & 0.017678 & 0.006782 & 0.239555 & 107.512 \\
\hline 9/30/08 0:00 & 2.095 & 0.635 & 0.185 & 83.140 & 1.403 & 0.057 & 0.017373 & 0.006617 & 0.233715 & 104.891 \\
\hline 9/30/08 1:00 & 2.102 & 0.642 & 0.190 & 85.421 & 1.405 & 0.059 & 0.017982 & 0.006949 & 0.245443 & 110.155 \\
\hline 9/30/08 2:00 & 2.107 & 0.647 & 0.194 & 87.074 & 1.405 & 0.059 & 0.017982 & 0.006949 & 0.245443 & 110.155 \\
\hline 9/30/08 3:00 & 2.11 & 0.650 & 0.196 & 88.074 & 1.405 & 0.059 & 0.017982 & 0.006949 & 0.245443 & 110.155 \\
\hline 9/30/08 4:00 & 2.112 & 0.652 & 0.198 & 88.745 & 1.404 & 0.058 & 0.017678 & 0.006782 & 0.239555 & 107.512 \\
\hline 9/30/08 5:00 & 2.114 & 0.654 & 0.199 & 89.419 & 1.404 & 0.058 & 0.017678 & 0.006782 & 0.239555 & 107.512 \\
\hline 9/30/08 6:00 & 2.119 & 0.659 & 0.203 & 91.117 & 1.404 & 0.058 & 0.017678 & 0.006782 & 0.239555 & 107.512 \\
\hline 9/30/08 7:00 & 2.119 & 0.659 & 0.203 & 91.117 & 1.406 & 0.06 & 0.018287 & 0.007117 & 0.251378 & 112.818 \\
\hline 9/30/08 8:00 & 2.117 & 0.657 & 0.202 & 90.436 & 1.403 & 0.057 & 0.017373 & 0.006617 & 0.233715 & 104.891 \\
\hline 9/30/08 9:00 & 2.122 & 0.662 & 0.205 & 92.146 & 1.405 & 0.059 & 0.017982 & 0.006949 & 0.245443 & 110.155 \\
\hline 9/30/08 10:00 & 2.126 & 0.666 & 0.208 & 93.527 & 1.405 & 0.059 & 0.017982 & 0.006949 & 0.245443 & 110.155 \\
\hline 9/30/08 11:00 & 2.126 & 0.666 & 0.208 & 93.527 & 1.407 & 0.061 & 0.018592 & 0.007287 & 0.257360 & 115.503 \\
\hline 9/30/08 12:00 & 2.121 & 0.661 & 0.205 & 91.802 & 1.405 & 0.059 & 0.017982 & 0.006949 & 0.245443 & 110.155 \\
\hline 9/30/08 13:00 & 2.112 & 0.652 & 0.198 & 88.745 & 1.403 & 0.057 & 0.017373 & 0.006617 & 0.233715 & 104.891 \\
\hline 9/30/08 14:00 & 2.109 & 0.649 & 0.195 & 87.740 & 1.403 & 0.057 & 0.017373 & 0.006617 & 0.233715 & 104.891 \\
\hline 9/30/08 15:00 & 2.098 & 0.638 & 0.187 & 84.113 & 1.403 & 0.057 & 0.017373 & 0.006617 & 0.233715 & 104.891 \\
\hline 9/30/08 16:00 & 2.091 & 0.631 & 0.182 & 81.852 & 1.401 & 0.055 & 0.016763 & 0.006291 & 0.222180 & 99.714 \\
\hline 9/30/08 17:00 & 2.08 & 0.620 & 0.175 & 78.375 & 1.401 & 0.055 & 0.016763 & 0.006291 & 0.222180 & 99.714 \\
\hline 9/30/08 18:00 & 2.075 & 0.615 & 0.171 & 76.824 & 1.399 & 0.053 & 0.016154 & 0.005969 & 0.210842 & 94.626 \\
\hline 9/30/08 19:00 & 2.068 & 0.608 & 0.166 & 74.683 & 1.401 & 0.055 & 0.016763 & 0.006291 & 0.222180 & 99.714 \\
\hline 9/30/08 20:00 & 2.07 & 0.610 & 0.168 & 75.291 & 1.399 & 0.053 & 0.016154 & 0.005969 & 0.210842 & 94.626 \\
\hline 9/30/08 21:00 & 2.079 & 0.619 & 0.174 & 78.063 & 1.401 & 0.055 & 0.016763 & 0.006291 & 0.222180 & 99.714 \\
\hline 9/30/08 22:00 & 2.086 & 0.626 & 0.179 & 80.260 & 1.401 & 0.055 & 0.016763 & 0.006291 & 0.222180 & 99.714 \\
\hline 9/30/08 23:00 & 2.093 & 0.633 & 0.184 & 82.494 & 1.404 & 0.058 & 0.017678 & 0.006782 & 0.239555 & 107.512 \\
\hline
\end{tabular}




\begin{tabular}{|c|c|c|c|c|c|c|c|c|c|c|}
\hline \multirow[b]{3}{*}{ Date Time } & \multicolumn{4}{|c|}{ V-Notch Weir } & \multicolumn{6}{|c|}{ R-Notch Weir } \\
\hline & \multicolumn{2}{|c|}{ Measured Water Level } & \multicolumn{2}{|c|}{$\begin{array}{c}\text { Calculated Rate } \\
\left(\mathrm{ft}^{3} / \mathrm{s}=448.8 \mathrm{gpm}\right)\end{array}$} & \multicolumn{3}{|c|}{ Measured Water Level } & \multicolumn{3}{|c|}{$\begin{array}{c}\text { Calculated Rate } \\
\left(\mathrm{ft}^{3} / \mathrm{s}=448.8 \mathrm{gpm}\right)\end{array}$} \\
\hline & $\begin{array}{l}\text { (ft above } \\
\text { sensor) }\end{array}$ & $\begin{array}{l}\text { (ft above } \\
\text { notch) }\end{array}$ & $\left(\mathrm{ft}^{3} / \mathrm{s}\right)$ & (gpm) & $\begin{array}{l}\text { (ft above } \\
\text { sensor) }\end{array}$ & $\begin{array}{l}\text { (ft above } \\
\text { log) }\end{array}$ & $\begin{array}{c}\text { (m above } \\
\log )\end{array}$ & $\left(\mathrm{m}^{3} / \mathrm{s}\right)$ & $\left(\mathrm{ft}^{3} / \mathrm{s}\right)$ & (gpm) \\
\hline 10/1/08 0:00 & 2.1 & 0.640 & 0.189 & 84.765 & 1.401 & 0.055 & 0.016763 & 0.006291 & 0.222180 & 99.714 \\
\hline 10/1/08 1:00 & 2.105 & 0.645 & 0.193 & 86.410 & 1.404 & 0.058 & 0.017678 & 0.006782 & 0.239555 & 107.512 \\
\hline 10/1/08 2:00 & 2.109 & 0.649 & 0.195 & 87.740 & 1.403 & 0.057 & 0.017373 & 0.006617 & 0.233715 & 104.891 \\
\hline 10/1/08 3:00 & 2.109 & 0.649 & 0.195 & 87.740 & 1.406 & 0.06 & 0.018287 & 0.007117 & 0.251378 & 112.818 \\
\hline 10/1/08 4:00 & 2.117 & 0.657 & 0.202 & 90.436 & 1.405 & 0.059 & 0.017982 & 0.006949 & 0.245443 & 110.155 \\
\hline 10/1/08 5:00 & 2.119 & 0.659 & 0.203 & 91.117 & 1.405 & 0.059 & 0.017982 & 0.006949 & 0.245443 & 110.155 \\
\hline 10/1/08 6:00 & 2.114 & 0.654 & 0.199 & 89.419 & 1.405 & 0.059 & 0.017982 & 0.006949 & 0.245443 & 110.155 \\
\hline 10/1/08 7:00 & 2.117 & 0.657 & 0.202 & 90.436 & 1.405 & 0.059 & 0.017982 & 0.006949 & 0.245443 & 110.155 \\
\hline 10/1/08 8:00 & 2.115 & 0.655 & 0.200 & 89.757 & 1.402 & 0.056 & 0.017068 & 0.006453 & 0.227923 & 102.292 \\
\hline 10/1/08 9:00 & 2.119 & 0.659 & 0.203 & 91.117 & 1.404 & 0.058 & 0.017678 & 0.006782 & 0.239555 & 107.512 \\
\hline 10/1/08 10:00 & 2.122 & 0.662 & 0.205 & 92.146 & 1.407 & 0.061 & 0.018592 & 0.007287 & 0.257360 & 115.503 \\
\hline 10/1/08 11:00 & 2.119 & 0.659 & 0.203 & 91.117 & 1.407 & 0.061 & 0.018592 & 0.007287 & 0.257360 & 115.503 \\
\hline 10/1/08 12:00 & 2.109 & 0.649 & 0.195 & 87.740 & 1.404 & 0.058 & 0.017678 & 0.006782 & 0.239555 & 107.512 \\
\hline 10/1/08 13:00 & 2.105 & 0.645 & 0.193 & 86.410 & 1.405 & 0.059 & 0.017982 & 0.006949 & 0.245443 & 110.155 \\
\hline 10/1/08 14:00 & 2.091 & 0.631 & 0.182 & 81.852 & 1.405 & 0.059 & 0.017982 & 0.006949 & 0.245443 & 110.155 \\
\hline 10/1/08 15:00 & 2.086 & 0.626 & 0.179 & 80.260 & 1.402 & 0.056 & 0.017068 & 0.006453 & 0.227923 & 102.292 \\
\hline 10/1/08 16:00 & 2.082 & 0.622 & 0.176 & 79.000 & 1.402 & 0.056 & 0.017068 & 0.006453 & 0.227923 & 102.292 \\
\hline 10/1/08 17:00 & 2.079 & 0.619 & 0.174 & 78.063 & 1.4 & 0.054 & 0.016458 & 0.006129 & 0.216486 & 97.159 \\
\hline 10/1/08 18:00 & 2.077 & 0.617 & 0.173 & 77.442 & 1.4 & 0.054 & 0.016458 & 0.006129 & 0.216486 & 97.159 \\
\hline 10/1/08 19:00 & 2.07 & 0.610 & 0.168 & 75.291 & 1.401 & 0.055 & 0.016763 & 0.006291 & 0.222180 & 99.714 \\
\hline 10/1/08 20:00 & 2.077 & 0.617 & 0.173 & 77.442 & 1.401 & 0.055 & 0.016763 & 0.006291 & 0.222180 & 99.714 \\
\hline 10/1/08 21:00 & 2.076 & 0.616 & 0.172 & 77.132 & 1.401 & 0.055 & 0.016763 & 0.006291 & 0.222180 & 99.714 \\
\hline 10/1/08 22:00 & 2.086 & 0.626 & 0.179 & 80.260 & 1.403 & 0.057 & 0.017373 & 0.006617 & 0.233715 & 104.891 \\
\hline 10/1/08 23:00 & 2.098 & 0.638 & 0.187 & 84.113 & 1.405 & 0.059 & 0.017982 & 0.006949 & 0.245443 & 110.155 \\
\hline 10/2/08 0:00 & 2.1 & 0.640 & 0.189 & 84.765 & 1.405 & 0.059 & 0.017982 & 0.006949 & 0.245443 & 110.155 \\
\hline
\end{tabular}




\begin{tabular}{|c|c|c|c|c|c|c|c|c|c|c|}
\hline \multirow[b]{3}{*}{ Date Time } & \multicolumn{4}{|c|}{ V-Notch Weir } & \multicolumn{6}{|c|}{ R-Notch Weir } \\
\hline & \multicolumn{2}{|c|}{ Measured Water Level } & \multicolumn{2}{|c|}{$\begin{array}{c}\text { Calculated Rate } \\
\left(\mathrm{ft}^{3} / \mathrm{s}=448.8 \mathrm{gpm}\right)\end{array}$} & \multicolumn{3}{|c|}{ Measured Water Level } & \multicolumn{3}{|c|}{$\begin{array}{c}\text { Calculated Rate } \\
\left(\mathrm{ft}^{3} / \mathrm{s}=448.8 \mathrm{gpm}\right)\end{array}$} \\
\hline & $\begin{array}{c}\text { (ft above } \\
\text { sensor) }\end{array}$ & $\begin{array}{c}\text { (ft above } \\
\text { notch) }\end{array}$ & $\left(\mathrm{ft}^{3} / \mathrm{s}\right)$ & (gpm) & $\begin{array}{l}\text { (ft above } \\
\text { sensor) }\end{array}$ & $\begin{array}{c}\text { (ft above } \\
\text { log) }\end{array}$ & $\begin{array}{c}\text { (m above } \\
\text { log) }\end{array}$ & $\left(\mathrm{m}^{3} / \mathrm{s}\right)$ & $\left(\mathrm{ft}^{3} / \mathrm{s}\right)$ & (gpm) \\
\hline 10/2/08 1:00 & 2.105 & 0.645 & 0.193 & 86.410 & 1.405 & 0.059 & 0.017982 & 0.006949 & 0.245443 & 110.155 \\
\hline 10/2/08 2:00 & 2.107 & 0.647 & 0.194 & 87.074 & 1.405 & 0.059 & 0.017982 & 0.006949 & 0.245443 & 110.155 \\
\hline 10/2/08 3:00 & 2.11 & 0.650 & 0.196 & 88.074 & 1.407 & 0.061 & 0.018592 & 0.007287 & 0.257360 & 115.503 \\
\hline 10/2/08 4:00 & 2.122 & 0.662 & 0.205 & 92.146 & 1.407 & 0.061 & 0.018592 & 0.007287 & 0.257360 & 115.503 \\
\hline 10/2/08 5:00 & 2.124 & 0.664 & 0.207 & 92.835 & 1.406 & 0.06 & 0.018287 & 0.007117 & 0.251378 & 112.818 \\
\hline 10/2/08 6:00 & 2.124 & 0.664 & 0.207 & 92.835 & 1.406 & 0.06 & 0.018287 & 0.007117 & 0.251378 & 112.818 \\
\hline 10/2/08 7:00 & 2.122 & 0.662 & 0.205 & 92.146 & 1.406 & 0.06 & 0.018287 & 0.007117 & 0.251378 & 112.818 \\
\hline 10/2/08 8:00 & 2.122 & 0.662 & 0.205 & 92.146 & 1.406 & 0.06 & 0.018287 & 0.007117 & 0.251378 & 112.818 \\
\hline 10/2/08 9:00 & 2.125 & 0.665 & 0.208 & 93.181 & 1.406 & 0.06 & 0.018287 & 0.007117 & 0.251378 & 112.818 \\
\hline 10/2/08 10:00 & 2.129 & 0.669 & 0.211 & 94.572 & 1.408 & 0.062 & 0.018897 & 0.007457 & 0.263390 & 118.209 \\
\hline 10/2/08 11:00 & 2.129 & 0.669 & 0.211 & 94.572 & 1.408 & 0.062 & 0.018897 & 0.007457 & 0.263390 & 118.209 \\
\hline 10/2/08 12:00 & 2.124 & 0.664 & 0.207 & 92.835 & 1.406 & 0.06 & 0.018287 & 0.007117 & 0.251378 & 112.818 \\
\hline 10/2/08 13:00 & 2.116 & 0.656 & 0.201 & 90.096 & 1.406 & 0.06 & 0.018287 & 0.007117 & 0.251378 & 112.818 \\
\hline 10/2/08 14:00 & 2.105 & 0.645 & 0.193 & 86.410 & 1.403 & 0.057 & 0.017373 & 0.006617 & 0.233715 & 104.891 \\
\hline 10/2/08 15:00 & 2.095 & 0.635 & 0.185 & 83.140 & 1.403 & 0.057 & 0.017373 & 0.006617 & 0.233715 & 104.891 \\
\hline 10/2/08 16:00 & 2.091 & 0.631 & 0.182 & 81.852 & 1.404 & 0.058 & 0.017678 & 0.006782 & 0.239555 & 107.512 \\
\hline 10/2/08 17:00 & 2.133 & 0.673 & 0.214 & 95.975 & 1.404 & 0.058 & 0.017678 & 0.006782 & 0.239555 & 107.512 \\
\hline 10/2/08 18:00 & 2.129 & 0.669 & 0.211 & 94.572 & 1.402 & 0.056 & 0.017068 & 0.006453 & 0.227923 & 102.292 \\
\hline 10/2/08 19:00 & 2.131 & 0.671 & 0.212 & 95.272 & 1.404 & 0.058 & 0.017678 & 0.006782 & 0.239555 & 107.512 \\
\hline 10/2/08 20:00 & 2.131 & 0.671 & 0.212 & 95.272 & 1.402 & 0.056 & 0.017068 & 0.006453 & 0.227923 & 102.292 \\
\hline 10/2/08 21:00 & 2.135 & 0.675 & 0.215 & 96.681 & 1.404 & 0.058 & 0.017678 & 0.006782 & 0.239555 & 107.512 \\
\hline 10/2/08 22:00 & 2.142 & 0.682 & 0.221 & 99.178 & 1.404 & 0.058 & 0.017678 & 0.006782 & 0.239555 & 107.512 \\
\hline 10/2/08 23:00 & 2.145 & 0.685 & 0.223 & 100.260 & 1.404 & 0.058 & 0.017678 & 0.006782 & 0.239555 & 107.512 \\
\hline 10/3/08 0:00 & 2.149 & 0.689 & 0.227 & 101.713 & 1.407 & 0.061 & 0.018592 & 0.007287 & 0.257360 & 115.503 \\
\hline 10/3/08 1:00 & 2.154 & 0.694 & 0.231 & 103.547 & 1.407 & 0.061 & 0.018592 & 0.007287 & 0.257360 & 115.503 \\
\hline
\end{tabular}




\begin{tabular}{|c|c|c|c|c|c|c|c|c|c|c|}
\hline \multirow[b]{3}{*}{ Date Time } & \multicolumn{4}{|c|}{ V-Notch Weir } & \multicolumn{6}{|c|}{ R-Notch Weir } \\
\hline & \multicolumn{2}{|c|}{ Measured Water Level } & \multicolumn{2}{|c|}{$\begin{array}{c}\text { Calculated Rate } \\
\left(\mathrm{ft}^{3} / \mathrm{s}=448.8 \mathrm{gpm}\right)\end{array}$} & \multicolumn{3}{|c|}{ Measured Water Level } & \multicolumn{3}{|c|}{$\begin{array}{c}\text { Calculated Rate } \\
\left(\mathrm{ft}^{3} / \mathrm{s}=448.8 \mathrm{gpm}\right)\end{array}$} \\
\hline & $\begin{array}{l}\text { (ft above } \\
\text { sensor) }\end{array}$ & $\begin{array}{l}\text { (ft above } \\
\text { notch) }\end{array}$ & $\left(\mathrm{ft}^{3} / \mathrm{s}\right)$ & (gpm) & $\begin{array}{l}\text { (ft above } \\
\text { sensor) }\end{array}$ & $\begin{array}{l}\text { (ft above } \\
\text { log) }\end{array}$ & $\begin{array}{c}\text { (m above } \\
\text { log) }\end{array}$ & $\left(\mathrm{m}^{3} / \mathrm{s}\right)$ & $\left(\mathrm{ft}^{3} / \mathrm{s}\right)$ & (gpm) \\
\hline 10/3/08 2:00 & 2.154 & 0.694 & 0.231 & 103.547 & 1.409 & 0.063 & 0.019201 & 0.007629 & 0.269465 & 120.936 \\
\hline 10/3/08 3:00 & 2.168 & 0.708 & 0.242 & 108.788 & 1.407 & 0.061 & 0.018592 & 0.007287 & 0.257360 & 115.503 \\
\hline 10/3/08 4:00 & 2.168 & 0.708 & 0.242 & 108.788 & 1.404 & 0.058 & 0.017678 & 0.006782 & 0.239555 & 107.512 \\
\hline 10/3/08 5:00 & 2.161 & 0.701 & 0.237 & 106.148 & 1.399 & 0.053 & 0.016154 & 0.005969 & 0.210842 & 94.626 \\
\hline 10/3/08 6:00 & 2.159 & 0.699 & 0.235 & 105.401 & 1.401 & 0.055 & 0.016763 & 0.006291 & 0.222180 & 99.714 \\
\hline 10/3/08 7:00 & 2.155 & 0.695 & 0.232 & 103.916 & 1.41 & 0.064 & 0.019506 & 0.007803 & 0.275587 & 123.683 \\
\hline 10/3/08 8:00 & 2.157 & 0.697 & 0.233 & 104.657 & 1.41 & 0.064 & 0.019506 & 0.007803 & 0.275587 & 123.683 \\
\hline 10/3/08 9:00 & 2.159 & 0.699 & 0.235 & 105.401 & 1.41 & 0.064 & 0.019506 & 0.007803 & 0.275587 & 123.683 \\
\hline 10/3/08 10:00 & 2.162 & 0.702 & 0.237 & 106.523 & 1.413 & 0.067 & 0.020421 & 0.008330 & 0.294224 & 132.048 \\
\hline 10/3/08 11:00 & 2.159 & 0.699 & 0.235 & 105.401 & 1.413 & 0.067 & 0.020421 & 0.008330 & 0.294224 & 132.048 \\
\hline 10/3/08 12:00 & 2.279 & 0.819 & 0.348 & 155.985 & 1.396 & 0.05 & 0.015239 & 0.005499 & 0.194210 & 87.161 \\
\hline 10/3/08 13:00 & 2.161 & 0.701 & 0.237 & 106.148 & 1.396 & 0.05 & 0.015239 & 0.005499 & 0.194210 & 87.161 \\
\hline 10/3/08 14:00 & 2.152 & 0.692 & 0.229 & 102.811 & 1.394 & 0.048 & 0.014630 & 0.005192 & 0.183377 & 82.300 \\
\hline 10/3/08 15:00 & 2.141 & 0.681 & 0.220 & 98.819 & 1.394 & 0.048 & 0.014630 & 0.005192 & 0.183377 & 82.300 \\
\hline 10/3/08 16:00 & 2.134 & 0.674 & 0.215 & 96.328 & 1.392 & 0.046 & 0.014020 & 0.004891 & 0.172754 & 77.532 \\
\hline 10/3/08 17:00 & 2.132 & 0.672 & 0.213 & 95.623 & 1.392 & 0.046 & 0.014020 & 0.004891 & 0.172754 & 77.532 \\
\hline 10/3/08 18:00 & 2.073 & 0.613 & 0.170 & 76.208 & 1.39 & 0.044 & 0.013411 & 0.004596 & 0.162345 & 72.860 \\
\hline 10/3/08 19:00 & 2.07 & 0.610 & 0.168 & 75.291 & 1.393 & 0.047 & 0.014325 & 0.005041 & 0.178040 & 79.904 \\
\hline 10/3/08 20:00 & 2.07 & 0.610 & 0.168 & 75.291 & 1.391 & 0.045 & 0.013715 & 0.004743 & 0.167523 & 75.184 \\
\hline 10/3/08 21:00 & 2.077 & 0.617 & 0.173 & 77.442 & 1.393 & 0.047 & 0.014325 & 0.005041 & 0.178040 & 79.904 \\
\hline 10/3/08 22:00 & 2.081 & 0.621 & 0.175 & 78.687 & 1.393 & 0.047 & 0.014325 & 0.005041 & 0.178040 & 79.904 \\
\hline 10/3/08 23:00 & 2.088 & 0.628 & 0.180 & 80.895 & 1.393 & 0.047 & 0.014325 & 0.005041 & 0.178040 & 79.904 \\
\hline 10/4/08 0:00 & 2.093 & 0.633 & 0.184 & 82.494 & 1.396 & 0.05 & 0.015239 & 0.005499 & 0.194210 & 87.161 \\
\hline 10/4/08 1:00 & 2.095 & 0.635 & 0.185 & 83.140 & 1.396 & 0.05 & 0.015239 & 0.005499 & 0.194210 & 87.161 \\
\hline 10/4/08 2:00 & 2.102 & 0.642 & 0.190 & 85.421 & 1.398 & 0.052 & 0.015849 & 0.005811 & 0.205247 & 92.115 \\
\hline
\end{tabular}




\begin{tabular}{|c|c|c|c|c|c|c|c|c|c|c|}
\hline \multirow[b]{3}{*}{ Date Time } & \multicolumn{4}{|c|}{ V-Notch Weir } & \multicolumn{6}{|c|}{ R-Notch Weir } \\
\hline & \multicolumn{2}{|c|}{ Measured Water Level } & \multicolumn{2}{|c|}{$\begin{array}{c}\text { Calculated Rate } \\
\left(\mathrm{ft}^{3} / \mathrm{s}=448.8 \mathrm{gpm}\right)\end{array}$} & \multicolumn{3}{|c|}{ Measured Water Level } & \multicolumn{3}{|c|}{$\begin{array}{c}\text { Calculated Rate } \\
\left(\mathrm{ft}^{3} / \mathrm{s}=448.8 \mathrm{gpm}\right)\end{array}$} \\
\hline & $\begin{array}{l}\text { (ft above } \\
\text { sensor) }\end{array}$ & $\begin{array}{l}\text { (ft above } \\
\text { notch) }\end{array}$ & $\left(\mathrm{ft}^{3} / \mathrm{s}\right)$ & (gpm) & $\begin{array}{l}\text { (ft above } \\
\text { sensor) }\end{array}$ & $\begin{array}{l}\text { (ft above } \\
\text { log) }\end{array}$ & $\begin{array}{c}\text { (m above } \\
\log )\end{array}$ & $\left(\mathrm{m}^{3} / \mathrm{s}\right)$ & $\left(\mathrm{ft}^{3} / \mathrm{s}\right)$ & (gpm) \\
\hline 10/4/08 3:00 & 2.1 & 0.640 & 0.189 & 84.765 & 1.395 & 0.049 & 0.014934 & 0.005345 & 0.188768 & 84.719 \\
\hline 10/4/08 4:00 & 2.102 & 0.642 & 0.190 & 85.421 & 1.398 & 0.052 & 0.015849 & 0.005811 & 0.205247 & 92.115 \\
\hline 10/4/08 5:00 & 2.121 & 0.661 & 0.205 & 91.802 & 1.397 & 0.051 & 0.015544 & 0.005654 & 0.199703 & 89.627 \\
\hline 10/4/08 6:00 & 2.128 & 0.668 & 0.210 & 94.223 & 1.397 & 0.051 & 0.015544 & 0.005654 & 0.199703 & 89.627 \\
\hline 10/4/08 7:00 & 2.145 & 0.685 & 0.223 & 100.260 & 1.404 & 0.058 & 0.017678 & 0.006782 & 0.239555 & 107.512 \\
\hline 10/4/08 8:00 & 2.138 & 0.678 & 0.218 & 97.747 & 1.399 & 0.053 & 0.016154 & 0.005969 & 0.210842 & 94.626 \\
\hline 10/4/08 9:00 & 2.14 & 0.680 & 0.219 & 98.461 & 1.402 & 0.056 & 0.017068 & 0.006453 & 0.227923 & 102.292 \\
\hline 10/4/08 10:00 & 2.14 & 0.680 & 0.219 & 98.461 & 1.399 & 0.053 & 0.016154 & 0.005969 & 0.210842 & 94.626 \\
\hline 10/4/08 11:00 & 2.14 & 0.680 & 0.219 & 98.461 & 1.397 & 0.051 & 0.015544 & 0.005654 & 0.199703 & 89.627 \\
\hline 10/4/08 12:00 & 2.135 & 0.675 & 0.215 & 96.681 & 1.397 & 0.051 & 0.015544 & 0.005654 & 0.199703 & 89.627 \\
\hline 10/4/08 13:00 & 2.124 & 0.664 & 0.207 & 92.835 & 1.392 & 0.046 & 0.014020 & 0.004891 & 0.172754 & 77.532 \\
\hline 10/4/08 14:00 & 2.114 & 0.654 & 0.199 & 89.419 & 1.395 & 0.049 & 0.014934 & 0.005345 & 0.188768 & 84.719 \\
\hline 10/4/08 15:00 & 2.105 & 0.645 & 0.193 & 86.410 & 1.397 & 0.051 & 0.015544 & 0.005654 & 0.199703 & 89.627 \\
\hline 10/4/08 16:00 & 2.091 & 0.631 & 0.182 & 81.852 & 1.393 & 0.047 & 0.014325 & 0.005041 & 0.178040 & 79.904 \\
\hline 10/4/08 17:00 & 2.087 & 0.627 & 0.180 & 80.577 & 1.393 & 0.047 & 0.014325 & 0.005041 & 0.178040 & 79.904 \\
\hline 10/4/08 18:00 & 2.08 & 0.620 & 0.175 & 78.375 & 1.393 & 0.047 & 0.014325 & 0.005041 & 0.178040 & 79.904 \\
\hline 10/4/08 19:00 & 2.084 & 0.624 & 0.177 & 79.629 & 1.391 & 0.045 & 0.013715 & 0.004743 & 0.167523 & 75.184 \\
\hline 10/4/08 20:00 & 2.082 & 0.622 & 0.176 & 79.000 & 1.393 & 0.047 & 0.014325 & 0.005041 & 0.178040 & 79.904 \\
\hline 10/4/08 21:00 & 2.086 & 0.626 & 0.179 & 80.260 & 1.394 & 0.048 & 0.014630 & 0.005192 & 0.183377 & 82.300 \\
\hline 10/4/08 22:00 & 2.096 & 0.636 & 0.186 & 83.463 & 1.396 & 0.05 & 0.015239 & 0.005499 & 0.194210 & 87.161 \\
\hline 10/4/08 23:00 & 2.1 & 0.640 & 0.189 & 84.765 & 1.396 & 0.05 & 0.015239 & 0.005499 & 0.194210 & 87.161 \\
\hline 10/5/08 0:00 & 2.107 & 0.647 & 0.194 & 87.074 & 1.396 & 0.05 & 0.015239 & 0.005499 & 0.194210 & 87.161 \\
\hline 10/5/08 1:00 & 2.107 & 0.647 & 0.194 & 87.074 & 1.396 & 0.05 & 0.015239 & 0.005499 & 0.194210 & 87.161 \\
\hline 10/5/08 2:00 & 2.112 & 0.652 & 0.198 & 88.745 & 1.396 & 0.05 & 0.015239 & 0.005499 & 0.194210 & 87.161 \\
\hline 10/5/08 3:00 & 2.126 & 0.666 & 0.208 & 93.527 & 1.398 & 0.052 & 0.015849 & 0.005811 & 0.205247 & 92.115 \\
\hline
\end{tabular}




\begin{tabular}{|c|c|c|c|c|c|c|c|c|c|c|}
\hline \multirow[b]{3}{*}{ Date Time } & \multicolumn{4}{|c|}{ V-Notch Weir } & \multicolumn{6}{|c|}{ R-Notch Weir } \\
\hline & \multicolumn{2}{|c|}{ Measured Water Level } & \multicolumn{2}{|c|}{$\begin{array}{c}\text { Calculated Rate } \\
\left(\mathrm{ft}^{3} / \mathrm{s}=448.8 \mathrm{gpm}\right)\end{array}$} & \multicolumn{3}{|c|}{ Measured Water Level } & \multicolumn{3}{|c|}{$\begin{array}{c}\text { Calculated Rate } \\
\left(\mathrm{ft}^{3} / \mathrm{s}=448.8 \mathrm{gpm}\right)\end{array}$} \\
\hline & $\begin{array}{c}\text { (ft above } \\
\text { sensor) }\end{array}$ & $\begin{array}{c}\text { (ft above } \\
\text { notch) }\end{array}$ & $\left(\mathrm{ft}^{3} / \mathrm{s}\right)$ & (gpm) & $\begin{array}{l}\text { (ft above } \\
\text { sensor) }\end{array}$ & $\begin{array}{c}\text { (ft above } \\
\text { log) }\end{array}$ & $\begin{array}{c}\text { (m above } \\
\text { log) }\end{array}$ & $\left(\mathrm{m}^{3} / \mathrm{s}\right)$ & $\left(\mathrm{ft}^{3} / \mathrm{s}\right)$ & (gpm) \\
\hline 10/5/08 4:00 & 2.131 & 0.671 & 0.212 & 95.272 & 1.398 & 0.052 & 0.015849 & 0.005811 & 0.205247 & 92.115 \\
\hline 10/5/08 5:00 & 2.098 & 0.638 & 0.187 & 84.113 & 1.41 & 0.064 & 0.019506 & 0.007803 & 0.275587 & 123.683 \\
\hline 10/5/08 6:00 & 2.098 & 0.638 & 0.187 & 84.113 & 1.401 & 0.055 & 0.016763 & 0.006291 & 0.222180 & 99.714 \\
\hline 10/5/08 7:00 & 2.102 & 0.642 & 0.190 & 85.421 & 1.401 & 0.055 & 0.016763 & 0.006291 & 0.222180 & 99.714 \\
\hline 10/5/08 8:00 & 2.1 & 0.640 & 0.189 & 84.765 & 1.4 & 0.054 & 0.016458 & 0.006129 & 0.216486 & 97.159 \\
\hline 10/5/08 9:00 & 2.102 & 0.642 & 0.190 & 85.421 & 1.4 & 0.054 & 0.016458 & 0.006129 & 0.216486 & 97.159 \\
\hline 10/5/08 10:00 & 2.114 & 0.654 & 0.199 & 89.419 & 1.4 & 0.054 & 0.016458 & 0.006129 & 0.216486 & 97.159 \\
\hline 10/5/08 11:00 & 2.107 & 0.647 & 0.194 & 87.074 & 1.403 & 0.057 & 0.017373 & 0.006617 & 0.233715 & 104.891 \\
\hline 10/5/08 12:00 & 2.107 & 0.647 & 0.194 & 87.074 & 1.4 & 0.054 & 0.016458 & 0.006129 & 0.216486 & 97.159 \\
\hline 10/5/08 13:00 & 2.084 & 0.624 & 0.177 & 79.629 & 1.398 & 0.052 & 0.015849 & 0.005811 & 0.205247 & 92.115 \\
\hline 10/5/08 14:00 & 2.075 & 0.615 & 0.171 & 76.824 & 1.396 & 0.05 & 0.015239 & 0.005499 & 0.194210 & 87.161 \\
\hline 10/5/08 15:00 & 2.059 & 0.599 & 0.160 & 71.985 & 1.396 & 0.05 & 0.015239 & 0.005499 & 0.194210 & 87.161 \\
\hline 10/5/08 16:00 & 2.055 & 0.595 & 0.158 & 70.805 & 1.396 & 0.05 & 0.015239 & 0.005499 & 0.194210 & 87.161 \\
\hline 10/5/08 17:00 & 2.047 & 0.587 & 0.153 & 68.479 & 1.397 & 0.051 & 0.015544 & 0.005654 & 0.199703 & 89.627 \\
\hline 10/5/08 18:00 & 2.043 & 0.583 & 0.150 & 67.334 & 1.394 & 0.048 & 0.014630 & 0.005192 & 0.183377 & 82.300 \\
\hline 10/5/08 19:00 & 2.043 & 0.583 & 0.150 & 67.334 & 1.395 & 0.049 & 0.014934 & 0.005345 & 0.188768 & 84.719 \\
\hline 10/5/08 20:00 & 2.042 & 0.582 & 0.149 & 67.049 & 1.395 & 0.049 & 0.014934 & 0.005345 & 0.188768 & 84.719 \\
\hline 10/5/08 21:00 & 2.047 & 0.587 & 0.153 & 68.479 & 1.397 & 0.051 & 0.015544 & 0.005654 & 0.199703 & 89.627 \\
\hline 10/5/08 22:00 & 2.051 & 0.591 & 0.155 & 69.636 & 1.397 & 0.051 & 0.015544 & 0.005654 & 0.199703 & 89.627 \\
\hline 10/5/08 23:00 & 2.051 & 0.591 & 0.155 & 69.636 & 1.397 & 0.051 & 0.015544 & 0.005654 & 0.199703 & 89.627 \\
\hline 10/6/08 0:00 & 2.051 & 0.591 & 0.155 & 69.636 & 1.4 & 0.054 & 0.016458 & 0.006129 & 0.216486 & 97.159 \\
\hline 10/6/08 1:00 & 2.06 & 0.600 & 0.161 & 72.282 & 1.4 & 0.054 & 0.016458 & 0.006129 & 0.216486 & 97.159 \\
\hline 10/6/08 2:00 & 2.058 & 0.598 & 0.160 & 71.689 & 1.402 & 0.056 & 0.017068 & 0.006453 & 0.227923 & 102.292 \\
\hline 10/6/08 3:00 & 2.058 & 0.598 & 0.160 & 71.689 & 1.404 & 0.058 & 0.017678 & 0.006782 & 0.239555 & 107.512 \\
\hline 10/6/08 4:00 & 2.051 & 0.591 & 0.155 & 69.636 & 1.404 & 0.058 & 0.017678 & 0.006782 & 0.239555 & 107.512 \\
\hline
\end{tabular}




\begin{tabular}{|c|c|c|c|c|c|c|c|c|c|c|}
\hline \multirow[b]{3}{*}{ Date } & \multicolumn{4}{|c|}{ V-Notch Weir } & \multicolumn{6}{|c|}{ R-Notch Weir } \\
\hline & \multicolumn{2}{|c|}{ Measured Water Level } & \multicolumn{2}{|c|}{$\begin{array}{c}\text { Calculated Rate } \\
\left(\mathrm{ft}^{3} / \mathrm{s}=448.8 \mathrm{gpm}\right)\end{array}$} & \multicolumn{3}{|c|}{ Measured Water Level } & \multicolumn{3}{|c|}{$\begin{array}{c}\text { Calculated Rate } \\
\left(\mathrm{ft}^{3} / \mathrm{s}=448.8 \mathrm{gpm}\right)\end{array}$} \\
\hline & $\begin{array}{c}\text { (ft above } \\
\text { sensor) }\end{array}$ & $\begin{array}{c}\text { (ft above } \\
\text { notch) }\end{array}$ & $\left(\mathrm{ft}^{3} / \mathrm{s}\right)$ & (gpm) & $\begin{array}{l}\text { (ft above } \\
\text { sensor) }\end{array}$ & $\begin{array}{c}\text { (ft above } \\
\text { log) }\end{array}$ & $\begin{array}{c}\text { (m above } \\
\text { log) }\end{array}$ & $\left(\mathrm{m}^{3} / \mathrm{s}\right)$ & $\left(\mathrm{ft}^{3} / \mathrm{s}\right)$ & (gpm) \\
\hline 10/6/08 5:00 & 2.048 & 0.588 & 0.153 & 68.767 & 1.404 & 0.058 & 0.017678 & 0.006782 & 0.239555 & 107.512 \\
\hline 10/6/08 6:00 & 2.046 & 0.586 & 0.152 & 68.192 & 1.404 & 0.058 & 0.017678 & 0.006782 & 0.239555 & 107.512 \\
\hline 10/6/08 7:00 & 2.027 & 0.567 & 0.140 & 62.868 & 1.404 & 0.058 & 0.017678 & 0.006782 & 0.239555 & 107.512 \\
\hline 10/6/08 8:00 & 2.03 & 0.570 & 0.142 & 63.691 & 1.404 & 0.058 & 0.017678 & 0.006782 & 0.239555 & 107.512 \\
\hline 10/6/08 9:00 & 2.03 & 0.570 & 0.142 & 63.691 & 1.404 & 0.058 & 0.017678 & 0.006782 & 0.239555 & 107.512 \\
\hline 10/6/08 10:00 & 2.044 & 0.584 & 0.151 & 67.619 & 1.404 & 0.058 & 0.017678 & 0.006782 & 0.239555 & 107.512 \\
\hline 10/6/08 11:00 & 2.039 & 0.579 & 0.148 & 66.200 & 1.406 & 0.06 & 0.018287 & 0.007117 & 0.251378 & 112.818 \\
\hline 10/6/08 12:00 & 2.034 & 0.574 & 0.144 & 64.799 & 1.406 & 0.06 & 0.018287 & 0.007117 & 0.251378 & 112.818 \\
\hline 10/6/08 13:00 & 2.03 & 0.570 & 0.142 & 63.691 & 1.406 & 0.06 & 0.018287 & 0.007117 & 0.251378 & 112.818 \\
\hline 10/6/08 14:00 & 2.014 & 0.554 & 0.132 & 59.374 & 1.404 & 0.058 & 0.017678 & 0.006782 & 0.239555 & 107.512 \\
\hline 10/6/08 15:00 & 2.002 & 0.542 & 0.125 & 56.255 & 1.402 & 0.056 & 0.017068 & 0.006453 & 0.227923 & 102.292 \\
\hline 10/6/08 16:00 & 2.188 & 0.728 & 0.260 & 116.546 & 1.421 & 0.075 & 0.022859 & 0.009792 & 0.345867 & 155.225 \\
\hline 10/6/08 17:00 & 2.462 & 1.002 & 0.573 & 257.127 & 1.621 & 0.275 & 0.083816 & 0.065650 & 2.318754 & 1040.657 \\
\hline 10/6/08 18:00 & 2.495 & 1.035 & 0.621 & 278.649 & 1.532 & 0.186 & 0.056690 & 0.036823 & 1.300599 & 583.709 \\
\hline 10/6/08 19:00 & 2.441 & 0.981 & 0.544 & 243.967 & 1.478 & 0.132 & 0.040232 & 0.022248 & 0.785797 & 352.665 \\
\hline 10/6/08 20:00 & 2.406 & 0.946 & 0.497 & 222.950 & 1.449 & 0.103 & 0.031393 & 0.015492 & 0.547171 & 245.570 \\
\hline 10/6/08 21:00 & 2.377 & 0.917 & 0.460 & 206.389 & 1.433 & 0.087 & 0.026516 & 0.012129 & 0.428377 & 192.256 \\
\hline 10/6/08 22:00 & 2.344 & 0.884 & 0.420 & 188.469 & 1.426 & 0.08 & 0.024383 & 0.010746 & 0.379531 & 170.333 \\
\hline 10/6/08 23:00 & 2.328 & 0.868 & 0.401 & 180.130 & 1.421 & 0.075 & 0.022859 & 0.009792 & 0.345867 & 155.225 \\
\hline 10/7/08 0:00 & 2.312 & 0.852 & 0.383 & 172.017 & 1.418 & 0.072 & 0.021945 & 0.009235 & 0.326176 & 146.388 \\
\hline 10/7/08 1:00 & 2.314 & 0.854 & 0.386 & 173.019 & 1.421 & 0.075 & 0.022859 & 0.009792 & 0.345867 & 155.225 \\
\hline 10/7/08 2:00 & 2.422 & 0.962 & 0.518 & 232.417 & 1.482 & 0.136 & 0.041451 & 0.023242 & 0.820909 & 368.424 \\
\hline 10/7/08 3:00 & 2.488 & 1.028 & 0.611 & 273.997 & 1.565 & 0.219 & 0.066748 & 0.046864 & 1.655212 & 742.859 \\
\hline 10/7/08 4:00 & 2.506 & 1.046 & 0.637 & 286.055 & 1.56 & 0.214 & 0.065224 & 0.045291 & 1.599669 & 717.932 \\
\hline 10/7/08 5:00 & 2.443 & 0.983 & 0.546 & 245.203 & 1.489 & 0.143 & 0.043584 & 0.025016 & 0.883573 & 396.548 \\
\hline
\end{tabular}




\begin{tabular}{|c|c|c|c|c|c|c|c|c|c|c|}
\hline \multirow[b]{3}{*}{ Date Time } & \multicolumn{4}{|c|}{ V-Notch Weir } & \multicolumn{6}{|c|}{ R-Notch Weir } \\
\hline & \multicolumn{2}{|c|}{ Measured Water Level } & \multicolumn{2}{|c|}{$\begin{array}{c}\text { Calculated Rate } \\
\left(\mathrm{ft}^{3} / \mathrm{s}=448.8 \mathrm{gpm}\right)\end{array}$} & \multicolumn{3}{|c|}{ Measured Water Level } & \multicolumn{3}{|c|}{$\begin{array}{c}\text { Calculated Rate } \\
\left(\mathrm{ft}^{3} / \mathrm{s}=448.8 \mathrm{gpm}\right)\end{array}$} \\
\hline & $\begin{array}{c}\text { (ft above } \\
\text { sensor) }\end{array}$ & $\begin{array}{c}\text { (ft above } \\
\text { notch) }\end{array}$ & $\left(\mathrm{ft}^{3} / \mathrm{s}\right)$ & (gpm) & $\begin{array}{l}\text { (ft above } \\
\text { sensor) }\end{array}$ & $\begin{array}{c}\text { (ft above } \\
\text { log) }\end{array}$ & $\begin{array}{c}\text { (m above } \\
\text { log) }\end{array}$ & $\left(\mathrm{m}^{3} / \mathrm{s}\right)$ & $\left(\mathrm{ft}^{3} / \mathrm{s}\right)$ & (gpm) \\
\hline 10/7/08 6:00 & 2.412 & 0.952 & 0.505 & 226.473 & 1.456 & 0.11 & 0.033526 & 0.017047 & 0.602114 & 270.229 \\
\hline 10/7/08 7:00 & 2.384 & 0.924 & 0.469 & 210.317 & 1.439 & 0.093 & 0.028345 & 0.013358 & 0.471786 & 211.738 \\
\hline 10/7/08 8:00 & 2.375 & 0.915 & 0.457 & 205.276 & 1.432 & 0.086 & 0.026212 & 0.011928 & 0.421279 & 189.070 \\
\hline 10/7/08 9:00 & 2.349 & 0.889 & 0.426 & 191.122 & 1.427 & 0.081 & 0.024688 & 0.010940 & 0.386387 & 173.411 \\
\hline 10/7/08 10:00 & 2.257 & 0.797 & 0.325 & 145.816 & 1.424 & 0.078 & 0.023773 & 0.010361 & 0.365940 & 164.234 \\
\hline 10/7/08 11:00 & 2.239 & 0.779 & 0.307 & 137.800 & 1.422 & 0.076 & 0.023164 & 0.009981 & 0.352516 & 158.209 \\
\hline 10/7/08 12:00 & 2.217 & 0.757 & 0.286 & 128.369 & 1.417 & 0.071 & 0.021640 & 0.009052 & 0.319698 & 143.480 \\
\hline 10/7/08 13:00 & 2.201 & 0.741 & 0.271 & 121.761 & 1.417 & 0.071 & 0.021640 & 0.009052 & 0.319698 & 143.480 \\
\hline 10/7/08 14:00 & 2.197 & 0.737 & 0.268 & 120.142 & 1.412 & 0.066 & 0.020116 & 0.008153 & 0.287966 & 129.239 \\
\hline 10/7/08 15:00 & 2.169 & 0.709 & 0.243 & 109.168 & 1.415 & 0.069 & 0.021030 & 0.008688 & 0.306873 & 137.724 \\
\hline 10/7/08 16:00 & 2.157 & 0.697 & 0.233 & 104.657 & 1.413 & 0.067 & 0.020421 & 0.008330 & 0.294224 & 132.048 \\
\hline 10/7/08 17:00 & 2.162 & 0.702 & 0.237 & 106.523 & 1.413 & 0.067 & 0.020421 & 0.008330 & 0.294224 & 132.048 \\
\hline 10/7/08 18:00 & 2.152 & 0.692 & 0.229 & 102.811 & 1.413 & 0.067 & 0.020421 & 0.008330 & 0.294224 & 132.048 \\
\hline 10/7/08 19:00 & 2.15 & 0.690 & 0.227 & 102.078 & 1.413 & 0.067 & 0.020421 & 0.008330 & 0.294224 & 132.048 \\
\hline 10/7/08 20:00 & 2.154 & 0.694 & 0.231 & 103.547 & 1.413 & 0.067 & 0.020421 & 0.008330 & 0.294224 & 132.048 \\
\hline 10/7/08 21:00 & 2.152 & 0.692 & 0.229 & 102.811 & 1.413 & 0.067 & 0.020421 & 0.008330 & 0.294224 & 132.048 \\
\hline 10/7/08 22:00 & 2.156 & 0.696 & 0.232 & 104.286 & 1.413 & 0.067 & 0.020421 & 0.008330 & 0.294224 & 132.048 \\
\hline 10/7/08 23:00 & 2.152 & 0.692 & 0.229 & 102.811 & 1.415 & 0.069 & 0.021030 & 0.008688 & 0.306873 & 137.724 \\
\hline 10/8/08 0:00 & 2.159 & 0.699 & 0.235 & 105.401 & 1.415 & 0.069 & 0.021030 & 0.008688 & 0.306873 & 137.724 \\
\hline 10/8/08 1:00 & 2.161 & 0.701 & 0.237 & 106.148 & 1.414 & 0.068 & 0.020725 & 0.008509 & 0.300526 & 134.876 \\
\hline 10/8/08 2:00 & 2.159 & 0.699 & 0.235 & 105.401 & 1.414 & 0.068 & 0.020725 & 0.008509 & 0.300526 & 134.876 \\
\hline 10/8/08 3:00 & 2.159 & 0.699 & 0.235 & 105.401 & 1.414 & 0.068 & 0.020725 & 0.008509 & 0.300526 & 134.876 \\
\hline 10/8/08 4:00 & 2.159 & 0.699 & 0.235 & 105.401 & 1.413 & 0.067 & 0.020421 & 0.008330 & 0.294224 & 132.048 \\
\hline 10/8/08 5:00 & 2.157 & 0.697 & 0.233 & 104.657 & 1.413 & 0.067 & 0.020421 & 0.008330 & 0.294224 & 132.048 \\
\hline 10/8/08 6:00 & 2.155 & 0.695 & 0.232 & 103.916 & 1.413 & 0.067 & 0.020421 & 0.008330 & 0.294224 & 132.048 \\
\hline
\end{tabular}




\begin{tabular}{|c|c|c|c|c|c|c|c|c|c|c|}
\hline \multirow[b]{3}{*}{ Date Time } & \multicolumn{4}{|c|}{ V-Notch Weir } & \multicolumn{6}{|c|}{ R-Notch Weir } \\
\hline & \multicolumn{2}{|c|}{ Measured Water Level } & \multicolumn{2}{|c|}{$\begin{array}{c}\text { Calculated Rate } \\
\left(\mathrm{ft}^{3} / \mathrm{s}=448.8 \mathrm{gpm}\right)\end{array}$} & \multicolumn{3}{|c|}{ Measured Water Level } & \multicolumn{3}{|c|}{$\begin{array}{c}\text { Calculated Rate } \\
\left(\mathrm{ft}^{3} / \mathrm{s}=448.8 \mathrm{gpm}\right)\end{array}$} \\
\hline & $\begin{array}{c}\text { (ft above } \\
\text { sensor) }\end{array}$ & $\begin{array}{c}\text { (ft above } \\
\text { notch) }\end{array}$ & $\left(\mathrm{ft}^{3} / \mathrm{s}\right)$ & (gpm) & $\begin{array}{l}\text { (ft above } \\
\text { sensor) }\end{array}$ & $\begin{array}{c}\text { (ft above } \\
\text { log) }\end{array}$ & $\begin{array}{c}\text { (m above } \\
\text { log) }\end{array}$ & $\left(\mathrm{m}^{3} / \mathrm{s}\right)$ & $\left(\mathrm{ft}^{3} / \mathrm{s}\right)$ & (gpm) \\
\hline 10/8/08 7:00 & 2.16 & 0.700 & 0.236 & 105.774 & 1.415 & 0.069 & 0.021030 & 0.008688 & 0.306873 & 137.724 \\
\hline 10/8/08 8:00 & 2.158 & 0.698 & 0.234 & 105.029 & 1.413 & 0.067 & 0.020421 & 0.008330 & 0.294224 & 132.048 \\
\hline 10/8/08 9:00 & 2.16 & 0.700 & 0.236 & 105.774 & 1.412 & 0.066 & 0.020116 & 0.008153 & 0.287966 & 129.239 \\
\hline 10/8/08 10:00 & 2.157 & 0.697 & 0.233 & 104.657 & 1.415 & 0.069 & 0.021030 & 0.008688 & 0.306873 & 137.724 \\
\hline 10/8/08 11:00 & 2.15 & 0.690 & 0.227 & 102.078 & 1.413 & 0.067 & 0.020421 & 0.008330 & 0.294224 & 132.048 \\
\hline 10/8/08 12:00 & 2.135 & 0.675 & 0.215 & 96.681 & 1.413 & 0.067 & 0.020421 & 0.008330 & 0.294224 & 132.048 \\
\hline 10/8/08 13:00 & 2.121 & 0.661 & 0.205 & 91.802 & 1.41 & 0.064 & 0.019506 & 0.007803 & 0.275587 & 123.683 \\
\hline 10/8/08 14:00 & 2.121 & 0.661 & 0.205 & 91.802 & 1.41 & 0.064 & 0.019506 & 0.007803 & 0.275587 & 123.683 \\
\hline 10/8/08 15:00 & 2.128 & 0.668 & 0.210 & 94.223 & 1.411 & 0.065 & 0.019811 & 0.007977 & 0.281754 & 126.451 \\
\hline 10/8/08 16:00 & 2.119 & 0.659 & 0.203 & 91.117 & 1.411 & 0.065 & 0.019811 & 0.007977 & 0.281754 & 126.451 \\
\hline 10/8/08 17:00 & 2.122 & 0.662 & 0.205 & 92.146 & 1.413 & 0.067 & 0.020421 & 0.008330 & 0.294224 & 132.048 \\
\hline 10/8/08 18:00 & 2.112 & 0.652 & 0.198 & 88.745 & 1.411 & 0.065 & 0.019811 & 0.007977 & 0.281754 & 126.451 \\
\hline 10/8/08 19:00 & 2.112 & 0.652 & 0.198 & 88.745 & 1.414 & 0.068 & 0.020725 & 0.008509 & 0.300526 & 134.876 \\
\hline 10/8/08 20:00 & 2.114 & 0.654 & 0.199 & 89.419 & 1.414 & 0.068 & 0.020725 & 0.008509 & 0.300526 & 134.876 \\
\hline 10/8/08 21:00 & 2.121 & 0.661 & 0.205 & 91.802 & 1.414 & 0.068 & 0.020725 & 0.008509 & 0.300526 & 134.876 \\
\hline 10/8/08 22:00 & 2.128 & 0.668 & 0.210 & 94.223 & 1.414 & 0.068 & 0.020725 & 0.008509 & 0.300526 & 134.876 \\
\hline 10/8/08 23:00 & 2.131 & 0.671 & 0.212 & 95.272 & 1.416 & 0.07 & 0.021335 & 0.008869 & 0.313263 & 140.593 \\
\hline 10/9/08 0:00 & 2.133 & 0.673 & 0.214 & 95.975 & 1.414 & 0.068 & 0.020725 & 0.008509 & 0.300526 & 134.876 \\
\hline 10/9/08 1:00 & 2.133 & 0.673 & 0.214 & 95.975 & 1.414 & 0.068 & 0.020725 & 0.008509 & 0.300526 & 134.876 \\
\hline 10/9/08 2:00 & 2.14 & 0.680 & 0.219 & 98.461 & 1.416 & 0.07 & 0.021335 & 0.008869 & 0.313263 & 140.593 \\
\hline 10/9/08 3:00 & 2.136 & 0.676 & 0.216 & 97.036 & 1.416 & 0.07 & 0.021335 & 0.008869 & 0.313263 & 140.593 \\
\hline 10/9/08 4:00 & 2.143 & 0.683 & 0.222 & 99.538 & 1.415 & 0.069 & 0.021030 & 0.008688 & 0.306873 & 137.724 \\
\hline 10/9/08 5:00 & 2.143 & 0.683 & 0.222 & 99.538 & 1.415 & 0.069 & 0.021030 & 0.008688 & 0.306873 & 137.724 \\
\hline 10/9/08 6:00 & 2.141 & 0.681 & 0.220 & 98.819 & 1.415 & 0.069 & 0.021030 & 0.008688 & 0.306873 & 137.724 \\
\hline 10/9/08 7:00 & 2.143 & 0.683 & 0.222 & 99.538 & 1.415 & 0.069 & 0.021030 & 0.008688 & 0.306873 & 137.724 \\
\hline
\end{tabular}




\begin{tabular}{|c|c|c|c|c|c|c|c|c|c|c|}
\hline \multirow[b]{3}{*}{ Date Time } & \multicolumn{4}{|c|}{ V-Notch Weir } & \multicolumn{6}{|c|}{ R-Notch Weir } \\
\hline & \multicolumn{2}{|c|}{ Measured Water Level } & \multicolumn{2}{|c|}{$\begin{array}{c}\text { Calculated Rate } \\
\left(\mathrm{ft}^{3} / \mathrm{s}=448.8 \mathrm{gpm}\right)\end{array}$} & \multicolumn{3}{|c|}{ Measured Water Level } & \multicolumn{3}{|c|}{$\begin{array}{c}\text { Calculated Rate } \\
\left(\mathrm{ft}^{3} / \mathrm{s}=448.8 \mathrm{gpm}\right)\end{array}$} \\
\hline & $\begin{array}{c}\text { (ft above } \\
\text { sensor) }\end{array}$ & $\begin{array}{c}\text { (ft above } \\
\text { notch) }\end{array}$ & $\left(\mathrm{ft}^{3} / \mathrm{s}\right)$ & (gpm) & $\begin{array}{l}\text { (ft above } \\
\text { sensor) }\end{array}$ & $\begin{array}{c}\text { (ft above } \\
\text { log) }\end{array}$ & $\begin{array}{c}\text { (m above } \\
\text { log) }\end{array}$ & $\left(\mathrm{m}^{3} / \mathrm{s}\right)$ & $\left(\mathrm{ft}^{3} / \mathrm{s}\right)$ & (gpm) \\
\hline 10/9/08 8:00 & 2.146 & 0.686 & 0.224 & 100.622 & 1.412 & 0.066 & 0.020116 & 0.008153 & 0.287966 & 129.239 \\
\hline 10/9/08 9:00 & 2.146 & 0.686 & 0.224 & 100.622 & 1.412 & 0.066 & 0.020116 & 0.008153 & 0.287966 & 129.239 \\
\hline 10/9/08 10:00 & 2.15 & 0.690 & 0.227 & 102.078 & 1.414 & 0.068 & 0.020725 & 0.008509 & 0.300526 & 134.876 \\
\hline 10/9/08 11:00 & 2.143 & 0.683 & 0.222 & 99.538 & 1.414 & 0.068 & 0.020725 & 0.008509 & 0.300526 & 134.876 \\
\hline 10/9/08 12:00 & 2.131 & 0.671 & 0.212 & 95.272 & 1.414 & 0.068 & 0.020725 & 0.008509 & 0.300526 & 134.876 \\
\hline 10/9/08 13:00 & 2.114 & 0.654 & 0.199 & 89.419 & 1.412 & 0.066 & 0.020116 & 0.008153 & 0.287966 & 129.239 \\
\hline 10/9/08 14:00 & 2.107 & 0.647 & 0.194 & 87.074 & 1.412 & 0.066 & 0.020116 & 0.008153 & 0.287966 & 129.239 \\
\hline 10/9/08 15:00 & 2.107 & 0.647 & 0.194 & 87.074 & 1.413 & 0.067 & 0.020421 & 0.008330 & 0.294224 & 132.048 \\
\hline 10/9/08 16:00 & 2.1 & 0.640 & 0.189 & 84.765 & 1.411 & 0.065 & 0.019811 & 0.007977 & 0.281754 & 126.451 \\
\hline 10/9/08 17:00 & 2.098 & 0.638 & 0.187 & 84.113 & 1.411 & 0.065 & 0.019811 & 0.007977 & 0.281754 & 126.451 \\
\hline 10/9/08 18:00 & 2.095 & 0.635 & 0.185 & 83.140 & 1.411 & 0.065 & 0.019811 & 0.007977 & 0.281754 & 126.451 \\
\hline 10/9/08 19:00 & 2.095 & 0.635 & 0.185 & 83.140 & 1.411 & 0.065 & 0.019811 & 0.007977 & 0.281754 & 126.451 \\
\hline 10/9/08 20:00 & 2.093 & 0.633 & 0.184 & 82.494 & 1.411 & 0.065 & 0.019811 & 0.007977 & 0.281754 & 126.451 \\
\hline 10/9/08 21:00 & 2.098 & 0.638 & 0.187 & 84.113 & 1.413 & 0.067 & 0.020421 & 0.008330 & 0.294224 & 132.048 \\
\hline 10/9/08 22:00 & 2.107 & 0.647 & 0.194 & 87.074 & 1.413 & 0.067 & 0.020421 & 0.008330 & 0.294224 & 132.048 \\
\hline 10/9/08 23:00 & 2.116 & 0.656 & 0.201 & 90.096 & 1.416 & 0.07 & 0.021335 & 0.008869 & 0.313263 & 140.593 \\
\hline 10/10/08 0:00 & 2.121 & 0.661 & 0.205 & 91.802 & 1.416 & 0.07 & 0.021335 & 0.008869 & 0.313263 & 140.593 \\
\hline 10/10/08 1:00 & 2.121 & 0.661 & 0.205 & 91.802 & 1.415 & 0.069 & 0.021030 & 0.008688 & 0.306873 & 137.724 \\
\hline 10/10/08 2:00 & 2.121 & 0.661 & 0.205 & 91.802 & 1.418 & 0.072 & 0.021945 & 0.009235 & 0.326176 & 146.388 \\
\hline 10/10/08 3:00 & 2.117 & 0.657 & 0.202 & 90.436 & 1.415 & 0.069 & 0.021030 & 0.008688 & 0.306873 & 137.724 \\
\hline 10/10/08 4:00 & 2.136 & 0.676 & 0.216 & 97.036 & 1.417 & 0.071 & 0.021640 & 0.009052 & 0.319698 & 143.480 \\
\hline 10/10/08 5:00 & 2.133 & 0.673 & 0.214 & 95.975 & 1.42 & 0.074 & 0.022554 & 0.009605 & 0.339261 & 152.260 \\
\hline 10/10/08 6:00 & 2.131 & 0.671 & 0.212 & 95.272 & 1.417 & 0.071 & 0.021640 & 0.009052 & 0.319698 & 143.480 \\
\hline 10/10/08 7:00 & 2.136 & 0.676 & 0.216 & 97.036 & 1.417 & 0.071 & 0.021640 & 0.009052 & 0.319698 & 143.480 \\
\hline 10/10/08 8:00 & 2.133 & 0.673 & 0.214 & 95.975 & 1.417 & 0.071 & 0.021640 & 0.009052 & 0.319698 & 143.480 \\
\hline
\end{tabular}




\begin{tabular}{|c|c|c|c|c|c|c|c|c|c|c|}
\hline \multirow[b]{3}{*}{ Date Time } & \multicolumn{4}{|c|}{ V-Notch Weir } & \multicolumn{6}{|c|}{ R-Notch Weir } \\
\hline & \multicolumn{2}{|c|}{ Measured Water Level } & \multicolumn{2}{|c|}{$\begin{array}{c}\text { Calculated Rate } \\
\left(\mathrm{ft}^{3} / \mathrm{s}=448.8 \mathrm{gpm}\right)\end{array}$} & \multicolumn{3}{|c|}{ Measured Water Level } & \multicolumn{3}{|c|}{$\begin{array}{c}\text { Calculated Rate } \\
\left(\mathrm{ft}^{3} / \mathrm{s}=448.8 \mathrm{gpm}\right)\end{array}$} \\
\hline & $\begin{array}{c}\text { (ft above } \\
\text { sensor) }\end{array}$ & $\begin{array}{c}\text { (ft above } \\
\text { notch) }\end{array}$ & $\left(\mathrm{ft}^{3} / \mathrm{s}\right)$ & (gpm) & $\begin{array}{l}\text { (ft above } \\
\text { sensor) }\end{array}$ & $\begin{array}{c}\text { (ft above } \\
\text { log) }\end{array}$ & $\begin{array}{c}\text { (m above } \\
\text { log) }\end{array}$ & $\left(\mathrm{m}^{3} / \mathrm{s}\right)$ & $\left(\mathrm{ft}^{3} / \mathrm{s}\right)$ & (gpm) \\
\hline 10/10/08 9:00 & 2.136 & 0.676 & 0.216 & 97.036 & 1.414 & 0.068 & 0.020725 & 0.008509 & 0.300526 & 134.876 \\
\hline 10/10/08 10:00 & 2.133 & 0.673 & 0.214 & 95.975 & 1.417 & 0.071 & 0.021640 & 0.009052 & 0.319698 & 143.480 \\
\hline 10/10/08 11:00 & 2.126 & 0.666 & 0.208 & 93.527 & 1.417 & 0.071 & 0.021640 & 0.009052 & 0.319698 & 143.480 \\
\hline 10/10/08 12:00 & 2.114 & 0.654 & 0.199 & 89.419 & 1.415 & 0.069 & 0.021030 & 0.008688 & 0.306873 & 137.724 \\
\hline 10/10/08 13:00 & 2.102 & 0.642 & 0.190 & 85.421 & 1.412 & 0.066 & 0.020116 & 0.008153 & 0.287966 & 129.239 \\
\hline 10/10/08 14:00 & 2.098 & 0.638 & 0.187 & 84.113 & 1.415 & 0.069 & 0.021030 & 0.008688 & 0.306873 & 137.724 \\
\hline 10/10/08 15:00 & 2.089 & 0.629 & 0.181 & 81.213 & 1.415 & 0.069 & 0.021030 & 0.008688 & 0.306873 & 137.724 \\
\hline 10/10/08 16:00 & 2.084 & 0.624 & 0.177 & 79.629 & 1.416 & 0.07 & 0.021335 & 0.008869 & 0.313263 & 140.593 \\
\hline 10/10/08 17:00 & 2.087 & 0.627 & 0.180 & 80.577 & 1.413 & 0.067 & 0.020421 & 0.008330 & 0.294224 & 132.048 \\
\hline 10/10/08 18:00 & 2.084 & 0.624 & 0.177 & 79.629 & 1.414 & 0.068 & 0.020725 & 0.008509 & 0.300526 & 134.876 \\
\hline 10/10/08 19:00 & 2.077 & 0.617 & 0.173 & 77.442 & 1.411 & 0.065 & 0.019811 & 0.007977 & 0.281754 & 126.451 \\
\hline 10/10/08 20:00 & 2.084 & 0.624 & 0.177 & 79.629 & 1.414 & 0.068 & 0.020725 & 0.008509 & 0.300526 & 134.876 \\
\hline 10/10/08 21:00 & 2.093 & 0.633 & 0.184 & 82.494 & 1.414 & 0.068 & 0.020725 & 0.008509 & 0.300526 & 134.876 \\
\hline 10/10/08 22:00 & 2.1 & 0.640 & 0.189 & 84.765 & 1.414 & 0.068 & 0.020725 & 0.008509 & 0.300526 & 134.876 \\
\hline 10/10/08 23:00 & 2.102 & 0.642 & 0.190 & 85.421 & 1.416 & 0.07 & 0.021335 & 0.008869 & 0.313263 & 140.593 \\
\hline 10/11/08 0:00 & 2.107 & 0.647 & 0.194 & 87.074 & 1.416 & 0.07 & 0.021335 & 0.008869 & 0.313263 & 140.593 \\
\hline 10/11/08 1:00 & 2.114 & 0.654 & 0.199 & 89.419 & 1.419 & 0.073 & 0.022249 & 0.009420 & 0.332697 & 149.314 \\
\hline 10/11/08 2:00 & 2.109 & 0.649 & 0.195 & 87.740 & 1.416 & 0.07 & 0.021335 & 0.008869 & 0.313263 & 140.593 \\
\hline 10/11/08 3:00 & 2.112 & 0.652 & 0.198 & 88.745 & 1.418 & 0.072 & 0.021945 & 0.009235 & 0.326176 & 146.388 \\
\hline 10/11/08 4:00 & 2.112 & 0.652 & 0.198 & 88.745 & 1.416 & 0.07 & 0.021335 & 0.008869 & 0.313263 & 140.593 \\
\hline 10/11/08 5:00 & 2.116 & 0.656 & 0.201 & 90.096 & 1.418 & 0.072 & 0.021945 & 0.009235 & 0.326176 & 146.388 \\
\hline 10/11/08 6:00 & 2.117 & 0.657 & 0.202 & 90.436 & 1.418 & 0.072 & 0.021945 & 0.009235 & 0.326176 & 146.388 \\
\hline 10/11/08 7:00 & 2.124 & 0.664 & 0.207 & 92.835 & 1.42 & 0.074 & 0.022554 & 0.009605 & 0.339261 & 152.260 \\
\hline 10/11/08 8:00 & 2.126 & 0.666 & 0.208 & 93.527 & 1.418 & 0.072 & 0.021945 & 0.009235 & 0.326176 & 146.388 \\
\hline 10/11/08 9:00 & 2.126 & 0.666 & 0.208 & 93.527 & 1.416 & 0.07 & 0.021335 & 0.008869 & 0.313263 & 140.593 \\
\hline
\end{tabular}




\begin{tabular}{|c|c|c|c|c|c|c|c|c|c|c|}
\hline \multirow[b]{3}{*}{ Date Time } & \multicolumn{4}{|c|}{ V-Notch Weir } & \multicolumn{6}{|c|}{ R-Notch Weir } \\
\hline & \multicolumn{2}{|c|}{ Measured Water Level } & \multicolumn{2}{|c|}{$\begin{array}{c}\text { Calculated Rate } \\
\left(\mathrm{ft}^{3} / \mathrm{s}=448.8 \mathrm{gpm}\right)\end{array}$} & \multicolumn{3}{|c|}{ Measured Water Level } & \multicolumn{3}{|c|}{$\begin{array}{c}\text { Calculated Rate } \\
\left(\mathrm{ft}^{3} / \mathrm{s}=448.8 \mathrm{gpm}\right)\end{array}$} \\
\hline & $\begin{array}{c}\text { (ft above } \\
\text { sensor) }\end{array}$ & $\begin{array}{c}\text { (ft above } \\
\text { notch) }\end{array}$ & $\left(\mathrm{ft}^{3} / \mathrm{s}\right)$ & (gpm) & $\begin{array}{l}\text { (ft above } \\
\text { sensor) }\end{array}$ & $\begin{array}{c}\text { (ft above } \\
\text { log) }\end{array}$ & $\begin{array}{c}\text { (m above } \\
\text { log) }\end{array}$ & $\left(\mathrm{m}^{3} / \mathrm{s}\right)$ & $\left(\mathrm{ft}^{3} / \mathrm{s}\right)$ & (gpm) \\
\hline 10/11/08 10:00 & 2.126 & 0.666 & 0.208 & 93.527 & 1.418 & 0.072 & 0.021945 & 0.009235 & 0.326176 & 146.388 \\
\hline 10/11/08 11:00 & 2.126 & 0.666 & 0.208 & 93.527 & 1.416 & 0.07 & 0.021335 & 0.008869 & 0.313263 & 140.593 \\
\hline 10/11/08 12:00 & 2.116 & 0.656 & 0.201 & 90.096 & 1.416 & 0.07 & 0.021335 & 0.008869 & 0.313263 & 140.593 \\
\hline 10/11/08 13:00 & 2.105 & 0.645 & 0.193 & 86.410 & 1.416 & 0.07 & 0.021335 & 0.008869 & 0.313263 & 140.593 \\
\hline 10/11/08 14:00 & 2.101 & 0.641 & 0.190 & 85.093 & 1.418 & 0.072 & 0.021945 & 0.009235 & 0.326176 & 146.388 \\
\hline 10/11/08 15:00 & 2.094 & 0.634 & 0.185 & 82.817 & 1.416 & 0.07 & 0.021335 & 0.008869 & 0.313263 & 140.593 \\
\hline 10/11/08 16:00 & 2.09 & 0.630 & 0.182 & 81.532 & 1.416 & 0.07 & 0.021335 & 0.008869 & 0.313263 & 140.593 \\
\hline 10/11/08 17:00 & 2.085 & 0.625 & 0.178 & 79.944 & 1.419 & 0.073 & 0.022249 & 0.009420 & 0.332697 & 149.314 \\
\hline 10/11/08 18:00 & 2.092 & 0.632 & 0.183 & 82.173 & 1.417 & 0.071 & 0.021640 & 0.009052 & 0.319698 & 143.480 \\
\hline 10/11/08 19:00 & 2.087 & 0.627 & 0.180 & 80.577 & 1.415 & 0.069 & 0.021030 & 0.008688 & 0.306873 & 137.724 \\
\hline 10/11/08 20:00 & 2.091 & 0.631 & 0.182 & 81.852 & 1.42 & 0.074 & 0.022554 & 0.009605 & 0.339261 & 152.260 \\
\hline 10/11/08 21:00 & 2.101 & 0.641 & 0.190 & 85.093 & 1.42 & 0.074 & 0.022554 & 0.009605 & 0.339261 & 152.260 \\
\hline 10/11/08 22:00 & 2.108 & 0.648 & 0.195 & 87.406 & 1.425 & 0.079 & 0.024078 & 0.010553 & 0.372715 & 167.274 \\
\hline 10/11/08 23:00 & 2.107 & 0.647 & 0.194 & 87.074 & 1.427 & 0.081 & 0.024688 & 0.010940 & 0.386387 & 173.411 \\
\hline 10/12/08 0:00 & 2.112 & 0.652 & 0.198 & 88.745 & 1.427 & 0.081 & 0.024688 & 0.010940 & 0.386387 & 173.411 \\
\hline 10/12/08 1:00 & 2.117 & 0.657 & 0.202 & 90.436 & 1.427 & 0.081 & 0.024688 & 0.010940 & 0.386387 & 173.411 \\
\hline 10/12/08 2:00 & 2.112 & 0.652 & 0.198 & 88.745 & 1.427 & 0.081 & 0.024688 & 0.010940 & 0.386387 & 173.411 \\
\hline 10/12/08 3:00 & 2.114 & 0.654 & 0.199 & 89.419 & 1.427 & 0.081 & 0.024688 & 0.010940 & 0.386387 & 173.411 \\
\hline 10/12/08 4:00 & 2.112 & 0.652 & 0.198 & 88.745 & 1.427 & 0.081 & 0.024688 & 0.010940 & 0.386387 & 173.411 \\
\hline 10/12/08 5:00 & 2.114 & 0.654 & 0.199 & 89.419 & 1.427 & 0.081 & 0.024688 & 0.010940 & 0.386387 & 173.411 \\
\hline 10/12/08 6:00 & 2.116 & 0.656 & 0.201 & 90.096 & 1.427 & 0.081 & 0.024688 & 0.010940 & 0.386387 & 173.411 \\
\hline 10/12/08 7:00 & 2.128 & 0.668 & 0.210 & 94.223 & 1.427 & 0.081 & 0.024688 & 0.010940 & 0.386387 & 173.411 \\
\hline 10/12/08 8:00 & 2.121 & 0.661 & 0.205 & 91.802 & 1.427 & 0.081 & 0.024688 & 0.010940 & 0.386387 & 173.411 \\
\hline 10/12/08 9:00 & 2.119 & 0.659 & 0.203 & 91.117 & 1.424 & 0.078 & 0.023773 & 0.010361 & 0.365940 & 164.234 \\
\hline 10/12/08 10:00 & 2.131 & 0.671 & 0.212 & 95.272 & 1.429 & 0.083 & 0.025297 & 0.011331 & 0.400223 & 179.620 \\
\hline
\end{tabular}




\begin{tabular}{|c|c|c|c|c|c|c|c|c|c|c|}
\hline \multirow[b]{3}{*}{ Date Time } & \multicolumn{4}{|c|}{ V-Notch Weir } & \multicolumn{6}{|c|}{ R-Notch Weir } \\
\hline & \multicolumn{2}{|c|}{ Measured Water Level } & \multicolumn{2}{|c|}{$\begin{array}{c}\text { Calculated Rate } \\
\left(\mathrm{ft}^{3} / \mathrm{s}=448.8 \mathrm{gpm}\right)\end{array}$} & \multicolumn{3}{|c|}{ Measured Water Level } & \multicolumn{3}{|c|}{$\begin{array}{c}\text { Calculated Rate } \\
\left(\mathrm{ft}^{3} / \mathrm{s}=448.8 \mathrm{gpm}\right)\end{array}$} \\
\hline & $\begin{array}{c}\text { (ft above } \\
\text { sensor) }\end{array}$ & $\begin{array}{c}\text { (ft above } \\
\text { notch) }\end{array}$ & $\left(\mathrm{ft}^{3} / \mathrm{s}\right)$ & (gpm) & $\begin{array}{l}\text { (ft above } \\
\text { sensor) }\end{array}$ & $\begin{array}{c}\text { (ft above } \\
\text { log) }\end{array}$ & $\begin{array}{c}\text { (m above } \\
\text { log) }\end{array}$ & $\left(\mathrm{m}^{3} / \mathrm{s}\right)$ & $\left(\mathrm{ft}^{3} / \mathrm{s}\right)$ & (gpm) \\
\hline 10/12/08 11:00 & 2.126 & 0.666 & 0.208 & 93.527 & 1.417 & 0.071 & 0.021640 & 0.009052 & 0.319698 & 143.480 \\
\hline 10/12/08 12:00 & 2.117 & 0.657 & 0.202 & 90.436 & 1.417 & 0.071 & 0.021640 & 0.009052 & 0.319698 & 143.480 \\
\hline 10/12/08 13:00 & 2.105 & 0.645 & 0.193 & 86.410 & 1.417 & 0.071 & 0.021640 & 0.009052 & 0.319698 & 143.480 \\
\hline 10/12/08 14:00 & 2.099 & 0.639 & 0.188 & 84.439 & 1.413 & 0.067 & 0.020421 & 0.008330 & 0.294224 & 132.048 \\
\hline 10/12/08 15:00 & 2.087 & 0.627 & 0.180 & 80.577 & 1.418 & 0.072 & 0.021945 & 0.009235 & 0.326176 & 146.388 \\
\hline 10/12/08 16:00 & 2.09 & 0.630 & 0.182 & 81.532 & 1.418 & 0.072 & 0.021945 & 0.009235 & 0.326176 & 146.388 \\
\hline 10/12/08 17:00 & 2.08 & 0.620 & 0.175 & 78.375 & 1.418 & 0.072 & 0.021945 & 0.009235 & 0.326176 & 146.388 \\
\hline 10/12/08 18:00 & 2.08 & 0.620 & 0.175 & 78.375 & 1.418 & 0.072 & 0.021945 & 0.009235 & 0.326176 & 146.388 \\
\hline 10/12/08 19:00 & 2.082 & 0.622 & 0.176 & 79.000 & 1.416 & 0.07 & 0.021335 & 0.008869 & 0.313263 & 140.593 \\
\hline 10/12/08 20:00 & 2.087 & 0.627 & 0.180 & 80.577 & 1.418 & 0.072 & 0.021945 & 0.009235 & 0.326176 & 146.388 \\
\hline 10/12/08 21:00 & 2.089 & 0.629 & 0.181 & 81.213 & 1.421 & 0.075 & 0.022859 & 0.009792 & 0.345867 & 155.225 \\
\hline 10/12/08 22:00 & 2.101 & 0.641 & 0.190 & 85.093 & 1.416 & 0.07 & 0.021335 & 0.008869 & 0.313263 & 140.593 \\
\hline 10/12/08 23:00 & 2.103 & 0.643 & 0.191 & 85.750 & 1.421 & 0.075 & 0.022859 & 0.009792 & 0.345867 & 155.225 \\
\hline 10/13/08 0:00 & 2.107 & 0.647 & 0.194 & 87.074 & 1.421 & 0.075 & 0.022859 & 0.009792 & 0.345867 & 155.225 \\
\hline 10/13/08 1:00 & 2.107 & 0.647 & 0.194 & 87.074 & 1.421 & 0.075 & 0.022859 & 0.009792 & 0.345867 & 155.225 \\
\hline 10/13/08 2:00 & 2.107 & 0.647 & 0.194 & 87.074 & 1.421 & 0.075 & 0.022859 & 0.009792 & 0.345867 & 155.225 \\
\hline 10/13/08 3:00 & 2.114 & 0.654 & 0.199 & 89.419 & 1.421 & 0.075 & 0.022859 & 0.009792 & 0.345867 & 155.225 \\
\hline 10/13/08 4:00 & 2.133 & 0.673 & 0.214 & 95.975 & 1.426 & 0.08 & 0.024383 & 0.010746 & 0.379531 & 170.333 \\
\hline 10/13/08 5:00 & 2.215 & 0.755 & 0.284 & 127.532 & 1.437 & 0.091 & 0.027735 & 0.012943 & 0.457162 & 205.174 \\
\hline 10/13/08 6:00 & 2.603 & 1.143 & 0.794 & 356.488 & 1.522 & 0.176 & 0.053642 & 0.033944 & 1.198890 & 538.062 \\
\hline 10/13/08 7:00 & 2.612 & 1.152 & 0.810 & 363.499 & 1.586 & 0.24 & 0.073148 & 0.053661 & 1.895280 & 850.602 \\
\hline 10/13/08 8:00 & 2.6 & 1.140 & 0.789 & 354.170 & 1.565 & 0.219 & 0.066748 & 0.046864 & 1.655212 & 742.859 \\
\hline 10/13/08 9:00 & 2.575 & 1.115 & 0.747 & 335.200 & 1.546 & 0.2 & 0.060957 & 0.040984 & 1.447556 & 649.663 \\
\hline 10/13/08 10:00 & 2.591 & 1.131 & 0.774 & 347.268 & 1.548 & 0.202 & 0.061567 & 0.041591 & 1.468975 & 659.276 \\
\hline 10/13/08 11:00 & 2.61 & 1.150 & 0.806 & 361.934 & 1.559 & 0.213 & 0.064919 & 0.044979 & 1.588637 & 712.980 \\
\hline
\end{tabular}




\begin{tabular}{|c|c|c|c|c|c|c|c|c|c|c|}
\hline \multirow[b]{3}{*}{ Date Time } & \multicolumn{4}{|c|}{ V-Notch Weir } & \multicolumn{6}{|c|}{ R-Notch Weir } \\
\hline & \multicolumn{2}{|c|}{ Measured Water Level } & \multicolumn{2}{|c|}{$\begin{array}{c}\text { Calculated Rate } \\
\left(\mathrm{ft}^{3} / \mathrm{s}=448.8 \mathrm{gpm}\right)\end{array}$} & \multicolumn{3}{|c|}{ Measured Water Level } & \multicolumn{3}{|c|}{$\begin{array}{c}\text { Calculated Rate } \\
\left(\mathrm{ft}^{3} / \mathrm{s}=448.8 \mathrm{gpm}\right)\end{array}$} \\
\hline & $\begin{array}{c}\text { (ft above } \\
\text { sensor) }\end{array}$ & $\begin{array}{c}\text { (ft above } \\
\text { notch) }\end{array}$ & $\left(\mathrm{ft}^{3} / \mathrm{s}\right)$ & (gpm) & $\begin{array}{l}\text { (ft above } \\
\text { sensor) }\end{array}$ & $\begin{array}{c}\text { (ft above } \\
\text { log) }\end{array}$ & $\begin{array}{c}\text { (m above } \\
\text { log) }\end{array}$ & $\left(\mathrm{m}^{3} / \mathrm{s}\right)$ & $\left(\mathrm{ft}^{3} / \mathrm{s}\right)$ & (gpm) \\
\hline 10/13/08 12:00 & 2.568 & 1.108 & 0.735 & 330.000 & 1.533 & 0.187 & 0.056995 & 0.037116 & 1.310922 & 588.342 \\
\hline 10/13/08 13:00 & 2.537 & 1.077 & 0.685 & 307.557 & 1.512 & 0.166 & 0.050594 & 0.031143 & 1.099980 & 493.671 \\
\hline 10/13/08 14:00 & 2.516 & 1.056 & 0.653 & 292.889 & 1.504 & 0.158 & 0.048156 & 0.028962 & 1.022924 & 459.088 \\
\hline 10/13/08 15:00 & 2.469 & 1.009 & 0.583 & 261.605 & 1.483 & 0.137 & 0.041756 & 0.023493 & 0.829766 & 372.399 \\
\hline 10/13/08 16:00 & 2.417 & 0.957 & 0.511 & 229.433 & 1.464 & 0.118 & 0.035965 & 0.018885 & 0.667017 & 299.357 \\
\hline 10/13/08 17:00 & 2.37 & 0.910 & 0.451 & 202.507 & 1.452 & 0.106 & 0.032307 & 0.016152 & 0.570504 & 256.042 \\
\hline 10/13/08 18:00 & 2.328 & 0.868 & 0.401 & 180.130 & 1.447 & 0.101 & 0.030783 & 0.015057 & 0.531798 & 238.671 \\
\hline 10/13/08 19:00 & 2.302 & 0.842 & 0.372 & 167.059 & 1.442 & 0.096 & 0.029259 & 0.013987 & 0.494010 & 221.711 \\
\hline 10/13/08 20:00 & 2.281 & 0.821 & 0.350 & 156.930 & 1.437 & 0.091 & 0.027735 & 0.012943 & 0.457162 & 205.174 \\
\hline 10/13/08 21:00 & 2.262 & 0.802 & 0.330 & 148.091 & 1.435 & 0.089 & 0.027126 & 0.012534 & 0.442691 & 198.680 \\
\hline 10/13/08 22:00 & 2.255 & 0.795 & 0.323 & 144.912 & 1.432 & 0.086 & 0.026212 & 0.011928 & 0.421279 & 189.070 \\
\hline 10/13/08 23:00 & 2.248 & 0.788 & 0.316 & 141.774 & 1.432 & 0.086 & 0.026212 & 0.011928 & 0.421279 & 189.070 \\
\hline 10/14/08 0:00 & 2.246 & 0.786 & 0.314 & 140.885 & 1.432 & 0.086 & 0.026212 & 0.011928 & 0.421279 & 189.070 \\
\hline 10/14/08 1:00 & 2.242 & 0.782 & 0.310 & 139.117 & 1.429 & 0.083 & 0.025297 & 0.011331 & 0.400223 & 179.620 \\
\hline 10/14/08 2:00 & 2.235 & 0.775 & 0.303 & 136.055 & 1.429 & 0.083 & 0.025297 & 0.011331 & 0.400223 & 179.620 \\
\hline 10/14/08 3:00 & 2.23 & 0.770 & 0.298 & 133.893 & 1.429 & 0.083 & 0.025297 & 0.011331 & 0.400223 & 179.620 \\
\hline 10/14/08 4:00 & 2.228 & 0.768 & 0.296 & 133.034 & 1.429 & 0.083 & 0.025297 & 0.011331 & 0.400223 & 179.620 \\
\hline 10/14/08 5:00 & 2.223 & 0.763 & 0.292 & 130.902 & 1.426 & 0.08 & 0.024383 & 0.010746 & 0.379531 & 170.333 \\
\hline 10/14/08 6:00 & 2.228 & 0.768 & 0.296 & 133.034 & 1.429 & 0.083 & 0.025297 & 0.011331 & 0.400223 & 179.620 \\
\hline 10/14/08 7:00 & 2.221 & 0.761 & 0.290 & 130.054 & 1.426 & 0.08 & 0.024383 & 0.010746 & 0.379531 & 170.333 \\
\hline 10/14/08 8:00 & 2.219 & 0.759 & 0.288 & 129.210 & 1.426 & 0.08 & 0.024383 & 0.010746 & 0.379531 & 170.333 \\
\hline 10/14/08 9:00 & 2.218 & 0.758 & 0.287 & 128.789 & 1.423 & 0.077 & 0.023468 & 0.010170 & 0.359208 & 161.212 \\
\hline 10/14/08 10:00 & 2.216 & 0.756 & 0.285 & 127.950 & 1.426 & 0.08 & 0.024383 & 0.010746 & 0.379531 & 170.333 \\
\hline 10/14/08 11:00 & 2.216 & 0.756 & 0.285 & 127.950 & 1.426 & 0.08 & 0.024383 & 0.010746 & 0.379531 & 170.333 \\
\hline 10/14/08 12:00 & 2.213 & 0.753 & 0.282 & 126.698 & 1.426 & 0.08 & 0.024383 & 0.010746 & 0.379531 & 170.333 \\
\hline
\end{tabular}




\begin{tabular}{|c|c|c|c|c|c|c|c|c|c|c|}
\hline \multirow[b]{3}{*}{ Date Time } & \multicolumn{4}{|c|}{ V-Notch Weir } & \multicolumn{6}{|c|}{ R-Notch Weir } \\
\hline & \multicolumn{2}{|c|}{ Measured Water Level } & \multicolumn{2}{|c|}{$\begin{array}{c}\text { Calculated Rate } \\
\left(\mathrm{ft}^{3} / \mathrm{s}=448.8 \mathrm{gpm}\right)\end{array}$} & \multicolumn{3}{|c|}{ Measured Water Level } & \multicolumn{3}{|c|}{$\begin{array}{c}\text { Calculated Rate } \\
\left(\mathrm{ft}^{3} / \mathrm{s}=448.8 \mathrm{gpm}\right)\end{array}$} \\
\hline & $\begin{array}{c}\text { (ft above } \\
\text { sensor) }\end{array}$ & $\begin{array}{c}\text { (ft above } \\
\text { notch) }\end{array}$ & $\left(\mathrm{ft}^{3} / \mathrm{s}\right)$ & (gpm) & $\begin{array}{l}\text { (ft above } \\
\text { sensor) }\end{array}$ & $\begin{array}{c}\text { (ft above } \\
\text { log) }\end{array}$ & $\begin{array}{c}\text { (m above } \\
\text { log) }\end{array}$ & $\left(\mathrm{m}^{3} / \mathrm{s}\right)$ & $\left(\mathrm{ft}^{3} / \mathrm{s}\right)$ & (gpm) \\
\hline 10/14/08 13:00 & 2.211 & 0.751 & 0.280 & 125.867 & 1.426 & 0.08 & 0.024383 & 0.010746 & 0.379531 & 170.333 \\
\hline 10/14/08 14:00 & 2.211 & 0.751 & 0.280 & 125.867 & 1.426 & 0.08 & 0.024383 & 0.010746 & 0.379531 & 170.333 \\
\hline 10/14/08 15:00 & 2.215 & 0.755 & 0.284 & 127.532 & 1.426 & 0.08 & 0.024383 & 0.010746 & 0.379531 & 170.333 \\
\hline 10/14/08 16:00 & 2.227 & 0.767 & 0.295 & 132.606 & 1.428 & 0.082 & 0.024992 & 0.011135 & 0.393285 & 176.506 \\
\hline 10/14/08 17:00 & 2.251 & 0.791 & 0.319 & 143.114 & 1.433 & 0.087 & 0.026516 & 0.012129 & 0.428377 & 192.256 \\
\hline 10/14/08 18:00 & 2.335 & 0.875 & 0.409 & 183.751 & 1.447 & 0.101 & 0.030783 & 0.015057 & 0.531798 & 238.671 \\
\hline 10/14/08 19:00 & 2.427 & 0.967 & 0.525 & 235.424 & 1.478 & 0.132 & 0.040232 & 0.022248 & 0.785797 & 352.665 \\
\hline 10/14/08 20:00 & 2.509 & 1.049 & 0.642 & 288.095 & 1.508 & 0.162 & 0.049375 & 0.030046 & 1.061219 & 476.275 \\
\hline 10/14/08 21:00 & 2.528 & 1.068 & 0.671 & 301.218 & 1.527 & 0.181 & 0.055166 & 0.035374 & 1.249399 & 560.730 \\
\hline 10/14/08 22:00 & 2.512 & 1.052 & 0.646 & 290.144 & 1.518 & 0.172 & 0.052423 & 0.032814 & 1.158986 & 520.153 \\
\hline 10/14/08 23:00 & 2.467 & 1.007 & 0.580 & 260.321 & 1.494 & 0.148 & 0.045108 & 0.026310 & 0.929266 & 417.054 \\
\hline 10/15/08 0:00 & 2.437 & 0.977 & 0.538 & 241.508 & 1.477 & 0.131 & 0.039927 & 0.022002 & 0.777099 & 348.762 \\
\hline 10/15/08 1:00 & 2.409 & 0.949 & 0.501 & 224.707 & 1.463 & 0.117 & 0.035660 & 0.018652 & 0.658783 & 295.662 \\
\hline 10/15/08 2:00 & 2.43 & 0.970 & 0.529 & 237.239 & 1.47 & 0.124 & 0.037793 & 0.020304 & 0.717127 & 321.847 \\
\hline 10/15/08 3:00 & 2.566 & 1.106 & 0.732 & 328.524 & 1.543 & 0.197 & 0.060043 & 0.040080 & 1.415626 & 635.333 \\
\hline 10/15/08 4:00 & 2.522 & 1.062 & 0.662 & 297.036 & 1.638 & 0.292 & 0.088997 & 0.071758 & 2.534495 & 1137.482 \\
\hline 10/15/08 5:00 & 2.515 & 1.055 & 0.651 & 292.201 & 1.647 & 0.301 & 0.091740 & 0.075065 & 2.651278 & 1189.893 \\
\hline 10/15/08 6:00 & 2.496 & 1.036 & 0.622 & 279.318 & 1.588 & 0.242 & 0.073758 & 0.054324 & 1.918704 & 861.115 \\
\hline 10/15/08 7:00 & 2.47 & 1.010 & 0.584 & 262.249 & 1.531 & 0.185 & 0.056385 & 0.036532 & 1.290305 & 579.089 \\
\hline 10/15/08 8:00 & 2.444 & 0.984 & 0.548 & 245.822 & 1.496 & 0.15 & 0.045718 & 0.026834 & 0.947757 & 425.353 \\
\hline 10/15/08 9:00 & 2.411 & 0.951 & 0.503 & 225.883 & 1.477 & 0.131 & 0.039927 & 0.022002 & 0.777099 & 348.762 \\
\hline 10/15/08 10:00 & 2.385 & 0.925 & 0.470 & 210.881 & 1.465 & 0.119 & 0.036269 & 0.019119 & 0.675284 & 303.068 \\
\hline 10/15/08 11:00 & 2.369 & 0.909 & 0.450 & 201.955 & 1.456 & 0.11 & 0.033526 & 0.017047 & 0.602114 & 270.229 \\
\hline 10/15/08 12:00 & 2.354 & 0.894 & 0.432 & 193.797 & 1.449 & 0.103 & 0.031393 & 0.015492 & 0.547171 & 245.570 \\
\hline 10/15/08 13:00 & 2.328 & 0.868 & 0.401 & 180.130 & 1.446 & 0.1 & 0.030479 & 0.014841 & 0.524166 & 235.246 \\
\hline
\end{tabular}




\begin{tabular}{|c|c|c|c|c|c|c|c|c|c|c|}
\hline \multirow[b]{3}{*}{ Date Time } & \multicolumn{4}{|c|}{ V-Notch Weir } & \multicolumn{6}{|c|}{ R-Notch Weir } \\
\hline & \multicolumn{2}{|c|}{ Measured Water Level } & \multicolumn{2}{|c|}{$\begin{array}{c}\text { Calculated Rate } \\
\left(\mathrm{ft}^{3} / \mathrm{s}=448.8 \mathrm{gpm}\right)\end{array}$} & \multicolumn{3}{|c|}{ Measured Water Level } & \multicolumn{3}{|c|}{$\begin{array}{c}\text { Calculated Rate } \\
\left(\mathrm{ft}^{3} / \mathrm{s}=448.8 \mathrm{gpm}\right)\end{array}$} \\
\hline & $\begin{array}{c}\text { (ft above } \\
\text { sensor) }\end{array}$ & $\begin{array}{c}\text { (ft above } \\
\text { notch) }\end{array}$ & $\left(\mathrm{ft}^{3} / \mathrm{s}\right)$ & (gpm) & $\begin{array}{c}\text { (ft above } \\
\text { sensor) }\end{array}$ & $\begin{array}{c}\text { (ft above } \\
\text { log) }\end{array}$ & $\begin{array}{c}\text { (m above } \\
\text { log) }\end{array}$ & $\left(\mathrm{m}^{3} / \mathrm{s}\right)$ & $\left(\mathrm{ft}^{3} / \mathrm{s}\right)$ & (gpm) \\
\hline 10/15/08 14:00 & 2.314 & 0.854 & 0.386 & 173.019 & 1.444 & 0.098 & 0.029869 & 0.014412 & 0.509013 & 228.445 \\
\hline 10/15/08 15:00 & 2.297 & 0.837 & 0.367 & 164.613 & 1.442 & 0.096 & 0.029259 & 0.013987 & 0.494010 & 221.711 \\
\hline 10/15/08 16:00 & 2.286 & 0.826 & 0.355 & 159.307 & 1.439 & 0.093 & 0.028345 & 0.013358 & 0.471786 & 211.738 \\
\hline 10/15/08 17:00 & 2.269 & 0.809 & 0.337 & 151.312 & 1.437 & 0.091 & 0.027735 & 0.012943 & 0.457162 & 205.174 \\
\hline 10/15/08 18:00 & 2.262 & 0.802 & 0.330 & 148.091 & 1.437 & 0.091 & 0.027735 & 0.012943 & 0.457162 & 205.174 \\
\hline 10/15/08 19:00 & 2.253 & 0.793 & 0.321 & 144.011 & 1.435 & 0.089 & 0.027126 & 0.012534 & 0.442691 & 198.680 \\
\hline 10/15/08 20:00 & 2.251 & 0.791 & 0.319 & 143.114 & 1.435 & 0.089 & 0.027126 & 0.012534 & 0.442691 & 198.680 \\
\hline 10/15/08 21:00 & 2.249 & 0.789 & 0.317 & 142.220 & 1.435 & 0.089 & 0.027126 & 0.012534 & 0.442691 & 198.680 \\
\hline 10/15/08 22:00 & 2.251 & 0.791 & 0.319 & 143.114 & 1.435 & 0.089 & 0.027126 & 0.012534 & 0.442691 & 198.680 \\
\hline 10/15/08 23:00 & 2.249 & 0.789 & 0.317 & 142.220 & 1.432 & 0.086 & 0.026212 & 0.011928 & 0.421279 & 189.070 \\
\hline 10/16/08 0:00 & 2.247 & 0.787 & 0.315 & 141.329 & 1.432 & 0.086 & 0.026212 & 0.011928 & 0.421279 & 189.070 \\
\hline 10/16/08 1:00 & 2.245 & 0.785 & 0.313 & 140.442 & 1.432 & 0.086 & 0.026212 & 0.011928 & 0.421279 & 189.070 \\
\hline 10/16/08 2:00 & 2.247 & 0.787 & 0.315 & 141.329 & 1.434 & 0.088 & 0.026821 & 0.012331 & 0.435514 & 195.459 \\
\hline 10/16/08 3:00 & 2.241 & 0.781 & 0.309 & 138.677 & 1.432 & 0.086 & 0.026212 & 0.011928 & 0.421279 & 189.070 \\
\hline 10/16/08 4:00 & 2.241 & 0.781 & 0.309 & 138.677 & 1.432 & 0.086 & 0.026212 & 0.011928 & 0.421279 & 189.070 \\
\hline 10/16/08 5:00 & 2.241 & 0.781 & 0.309 & 138.677 & 1.432 & 0.086 & 0.026212 & 0.011928 & 0.421279 & 189.070 \\
\hline 10/16/08 6:00 & 2.239 & 0.779 & 0.307 & 137.800 & 1.429 & 0.083 & 0.025297 & 0.011331 & 0.400223 & 179.620 \\
\hline 10/16/08 7:00 & 2.234 & 0.774 & 0.302 & 135.621 & 1.431 & 0.085 & 0.025907 & 0.011728 & 0.414220 & 185.902 \\
\hline 10/16/08 8:00 & 2.234 & 0.774 & 0.302 & 135.621 & 1.431 & 0.085 & 0.025907 & 0.011728 & 0.414220 & 185.902 \\
\hline 10/16/08 9:00 & 2.232 & 0.772 & 0.300 & 134.756 & 1.429 & 0.083 & 0.025297 & 0.011331 & 0.400223 & 179.620 \\
\hline 10/16/08 10:00 & 2.231 & 0.771 & 0.299 & 134.324 & 1.429 & 0.083 & 0.025297 & 0.011331 & 0.400223 & 179.620 \\
\hline 10/16/08 11:00 & 2.228 & 0.768 & 0.296 & 133.034 & 1.431 & 0.085 & 0.025907 & 0.011728 & 0.414220 & 185.902 \\
\hline 10/16/08 12:00 & 2.221 & 0.761 & 0.290 & 130.054 & 1.431 & 0.085 & 0.025907 & 0.011728 & 0.414220 & 185.902 \\
\hline 10/16/08 13:00 & 2.211 & 0.751 & 0.280 & 125.867 & 1.429 & 0.083 & 0.025297 & 0.011331 & 0.400223 & 179.620 \\
\hline 10/16/08 14:00 & 2.206 & 0.746 & 0.276 & 123.804 & 1.431 & 0.085 & 0.025907 & 0.011728 & 0.414220 & 185.902 \\
\hline
\end{tabular}




\begin{tabular}{|c|c|c|c|c|c|c|c|c|c|c|}
\hline \multirow[b]{3}{*}{ Date Time } & \multicolumn{4}{|c|}{ V-Notch Weir } & \multicolumn{6}{|c|}{ R-Notch Weir } \\
\hline & \multicolumn{2}{|c|}{ Measured Water Level } & \multicolumn{2}{|c|}{$\begin{array}{c}\text { Calculated Rate } \\
\left(\mathrm{ft}^{3} / \mathrm{s}=448.8 \mathrm{gpm}\right)\end{array}$} & \multicolumn{3}{|c|}{ Measured Water Level } & \multicolumn{3}{|c|}{$\begin{array}{c}\text { Calculated Rate } \\
\left(\mathrm{ft}^{3} / \mathrm{s}=448.8 \mathrm{gpm}\right)\end{array}$} \\
\hline & $\begin{array}{c}\text { (ft above } \\
\text { sensor) }\end{array}$ & $\begin{array}{c}\text { (ft above } \\
\text { notch) }\end{array}$ & $\left(\mathrm{ft}^{3} / \mathrm{s}\right)$ & (gpm) & $\begin{array}{l}\text { (ft above } \\
\text { sensor) }\end{array}$ & $\begin{array}{c}\text { (ft above } \\
\text { log) }\end{array}$ & $\begin{array}{c}\text { (m above } \\
\text { log) }\end{array}$ & $\left(\mathrm{m}^{3} / \mathrm{s}\right)$ & $\left(\mathrm{ft}^{3} / \mathrm{s}\right)$ & (gpm) \\
\hline 10/16/08 15:00 & 2.201 & 0.741 & 0.271 & 121.761 & 1.431 & 0.085 & 0.025907 & 0.011728 & 0.414220 & 185.902 \\
\hline 10/16/08 16:00 & 2.201 & 0.741 & 0.271 & 121.761 & 1.431 & 0.085 & 0.025907 & 0.011728 & 0.414220 & 185.902 \\
\hline 10/16/08 17:00 & 2.196 & 0.736 & 0.267 & 119.739 & 1.431 & 0.085 & 0.025907 & 0.011728 & 0.414220 & 185.902 \\
\hline 10/16/08 18:00 & 2.196 & 0.736 & 0.267 & 119.739 & 1.429 & 0.083 & 0.025297 & 0.011331 & 0.400223 & 179.620 \\
\hline 10/16/08 19:00 & 2.199 & 0.739 & 0.269 & 120.950 & 1.432 & 0.086 & 0.026212 & 0.011928 & 0.421279 & 189.070 \\
\hline 10/16/08 20:00 & 2.199 & 0.739 & 0.269 & 120.950 & 1.429 & 0.083 & 0.025297 & 0.011331 & 0.400223 & 179.620 \\
\hline 10/16/08 21:00 & 2.204 & 0.744 & 0.274 & 122.984 & 1.429 & 0.083 & 0.025297 & 0.011331 & 0.400223 & 179.620 \\
\hline 10/16/08 22:00 & 2.204 & 0.744 & 0.274 & 122.984 & 1.429 & 0.083 & 0.025297 & 0.011331 & 0.400223 & 179.620 \\
\hline 10/16/08 23:00 & 2.206 & 0.746 & 0.276 & 123.804 & 1.429 & 0.083 & 0.025297 & 0.011331 & 0.400223 & 179.620 \\
\hline 10/17/08 0:00 & 2.202 & 0.742 & 0.272 & 122.168 & 1.429 & 0.083 & 0.025297 & 0.011331 & 0.400223 & 179.620 \\
\hline 10/17/08 1:00 & 2.204 & 0.744 & 0.274 & 122.984 & 1.429 & 0.083 & 0.025297 & 0.011331 & 0.400223 & 179.620 \\
\hline 10/17/08 2:00 & 2.204 & 0.744 & 0.274 & 122.984 & 1.432 & 0.086 & 0.026212 & 0.011928 & 0.421279 & 189.070 \\
\hline 10/17/08 3:00 & 2.207 & 0.747 & 0.277 & 124.215 & 1.429 & 0.083 & 0.025297 & 0.011331 & 0.400223 & 179.620 \\
\hline 10/17/08 4:00 & 2.207 & 0.747 & 0.277 & 124.215 & 1.431 & 0.085 & 0.025907 & 0.011728 & 0.414220 & 185.902 \\
\hline 10/17/08 5:00 & 2.209 & 0.749 & 0.279 & 125.039 & 1.429 & 0.083 & 0.025297 & 0.011331 & 0.400223 & 179.620 \\
\hline 10/17/08 6:00 & 2.244 & 0.784 & 0.312 & 139.999 & 1.436 & 0.09 & 0.027431 & 0.012738 & 0.449907 & 201.918 \\
\hline 10/17/08 7:00 & 2.266 & 0.806 & 0.334 & 149.926 & 1.438 & 0.092 & 0.028040 & 0.013150 & 0.464455 & 208.447 \\
\hline 10/17/08 8:00 & 2.266 & 0.806 & 0.334 & 149.926 & 1.438 & 0.092 & 0.028040 & 0.013150 & 0.464455 & 208.447 \\
\hline 10/17/08 9:00 & 2.247 & 0.787 & 0.315 & 141.329 & 1.436 & 0.09 & 0.027431 & 0.012738 & 0.449907 & 201.918 \\
\hline 10/17/08 10:00 & 2.233 & 0.773 & 0.301 & 135.188 & 1.433 & 0.087 & 0.026516 & 0.012129 & 0.428377 & 192.256 \\
\hline 10/17/08 11:00 & 2.225 & 0.765 & 0.294 & 131.752 & 1.433 & 0.087 & 0.026516 & 0.012129 & 0.428377 & 192.256 \\
\hline 10/17/08 12:00 & 2.211 & 0.751 & 0.280 & 125.867 & 1.433 & 0.087 & 0.026516 & 0.012129 & 0.428377 & 192.256 \\
\hline 10/17/08 13:00 & 2.208 & 0.748 & 0.278 & 124.627 & 1.431 & 0.085 & 0.025907 & 0.011728 & 0.414220 & 185.902 \\
\hline 10/17/08 14:00 & 2.201 & 0.741 & 0.271 & 121.761 & 1.431 & 0.085 & 0.025907 & 0.011728 & 0.414220 & 185.902 \\
\hline 10/17/08 15:00 & 2.199 & 0.739 & 0.269 & 120.950 & 1.429 & 0.083 & 0.025297 & 0.011331 & 0.400223 & 179.620 \\
\hline
\end{tabular}




\begin{tabular}{|c|c|c|c|c|c|c|c|c|c|c|}
\hline \multirow[b]{3}{*}{ Date Time } & \multicolumn{4}{|c|}{ V-Notch Weir } & \multicolumn{6}{|c|}{ R-Notch Weir } \\
\hline & \multicolumn{2}{|c|}{ Measured Water Level } & \multicolumn{2}{|c|}{$\begin{array}{c}\text { Calculated Rate } \\
\left(\mathrm{ft}^{3} / \mathrm{s}=448.8 \mathrm{gpm}\right)\end{array}$} & \multicolumn{3}{|c|}{ Measured Water Level } & \multicolumn{3}{|c|}{$\begin{array}{c}\text { Calculated Rate } \\
\left(\mathrm{ft}^{3} / \mathrm{s}=448.8 \mathrm{gpm}\right)\end{array}$} \\
\hline & $\begin{array}{l}\text { (ft above } \\
\text { sensor) }\end{array}$ & $\begin{array}{c}\text { (ft above } \\
\text { notch) }\end{array}$ & $\left(\mathrm{ft}^{3} / \mathrm{s}\right)$ & (gpm) & $\begin{array}{c}\text { (ft above } \\
\text { sensor) }\end{array}$ & $\begin{array}{c}\text { (ft above } \\
\text { log) }\end{array}$ & $\begin{array}{c}\text { (m above } \\
\text { log) }\end{array}$ & $\left(\mathrm{m}^{3} / \mathrm{s}\right)$ & $\left(\mathrm{ft}^{3} / \mathrm{s}\right)$ & (gpm) \\
\hline 10/17/08 16:00 & 2.196 & 0.736 & 0.267 & 119.739 & 1.429 & 0.083 & 0.025297 & 0.011331 & 0.400223 & 179.620 \\
\hline 10/17/08 17:00 & 2.196 & 0.736 & 0.267 & 119.739 & 1.429 & 0.083 & 0.025297 & 0.011331 & 0.400223 & 179.620 \\
\hline 10/17/08 18:00 & 2.194 & 0.734 & 0.265 & 118.936 & 1.429 & 0.083 & 0.025297 & 0.011331 & 0.400223 & 179.620 \\
\hline 10/17/08 19:00 & 2.194 & 0.734 & 0.265 & 118.936 & 1.429 & 0.083 & 0.025297 & 0.011331 & 0.400223 & 179.620 \\
\hline 10/17/08 20:00 & 2.194 & 0.734 & 0.265 & 118.936 & 1.43 & 0.084 & 0.025602 & 0.011529 & 0.407202 & 182.752 \\
\hline 10/17/08 21:00 & 2.194 & 0.734 & 0.265 & 118.936 & 1.427 & 0.081 & 0.024688 & 0.010940 & 0.386387 & 173.411 \\
\hline 10/17/08 22:00 & 2.194 & 0.734 & 0.265 & 118.936 & 1.43 & 0.084 & 0.025602 & 0.011529 & 0.407202 & 182.752 \\
\hline 10/17/08 23:00 & 2.197 & 0.737 & 0.268 & 120.142 & 1.429 & 0.083 & 0.025297 & 0.011331 & 0.400223 & 179.620 \\
\hline 10/18/08 0:00 & 2.195 & 0.735 & 0.266 & 119.337 & 1.429 & 0.083 & 0.025297 & 0.011331 & 0.400223 & 179.620 \\
\hline 10/18/08 1:00 & 2.195 & 0.735 & 0.266 & 119.337 & 1.429 & 0.083 & 0.025297 & 0.011331 & 0.400223 & 179.620 \\
\hline 10/18/08 2:00 & 2.198 & 0.738 & 0.269 & 120.546 & 1.427 & 0.081 & 0.024688 & 0.010940 & 0.386387 & 173.411 \\
\hline 10/18/08 3:00 & 2.2 & 0.740 & 0.270 & 121.355 & 1.427 & 0.081 & 0.024688 & 0.010940 & 0.386387 & 173.411 \\
\hline 10/18/08 4:00 & 2.196 & 0.736 & 0.267 & 119.739 & 1.427 & 0.081 & 0.024688 & 0.010940 & 0.386387 & 173.411 \\
\hline 10/18/08 5:00 & 2.196 & 0.736 & 0.267 & 119.739 & 1.427 & 0.081 & 0.024688 & 0.010940 & 0.386387 & 173.411 \\
\hline 10/18/08 6:00 & 2.196 & 0.736 & 0.267 & 119.739 & 1.426 & 0.08 & 0.024383 & 0.010746 & 0.379531 & 170.333 \\
\hline 10/18/08 7:00 & 2.196 & 0.736 & 0.267 & 119.739 & 1.424 & 0.078 & 0.023773 & 0.010361 & 0.365940 & 164.234 \\
\hline 10/18/08 8:00 & 2.196 & 0.736 & 0.267 & 119.739 & 1.426 & 0.08 & 0.024383 & 0.010746 & 0.379531 & 170.333 \\
\hline 10/18/08 9:00 & 2.198 & 0.738 & 0.269 & 120.546 & 1.426 & 0.08 & 0.024383 & 0.010746 & 0.379531 & 170.333 \\
\hline 10/18/08 10:00 & 2.195 & 0.735 & 0.266 & 119.337 & 1.426 & 0.08 & 0.024383 & 0.010746 & 0.379531 & 170.333 \\
\hline 10/18/08 11:00 & 2.195 & 0.735 & 0.266 & 119.337 & 1.426 & 0.08 & 0.024383 & 0.010746 & 0.379531 & 170.333 \\
\hline 10/18/08 12:00 & 2.193 & 0.733 & 0.264 & 118.536 & 1.426 & 0.08 & 0.024383 & 0.010746 & 0.379531 & 170.333 \\
\hline 10/18/08 13:00 & 2.188 & 0.728 & 0.260 & 116.546 & 1.428 & 0.082 & 0.024992 & 0.011135 & 0.393285 & 176.506 \\
\hline 10/18/08 14:00 & 2.185 & 0.725 & 0.257 & 115.362 & 1.431 & 0.085 & 0.025907 & 0.011728 & 0.414220 & 185.902 \\
\hline 10/18/08 15:00 & 2.182 & 0.722 & 0.254 & 114.185 & 1.431 & 0.085 & 0.025907 & 0.011728 & 0.414220 & 185.902 \\
\hline 10/18/08 16:00 & 2.185 & 0.725 & 0.257 & 115.362 & 1.431 & 0.085 & 0.025907 & 0.011728 & 0.414220 & 185.902 \\
\hline
\end{tabular}




\begin{tabular}{|c|c|c|c|c|c|c|c|c|c|c|}
\hline \multirow[b]{3}{*}{ Date Time } & \multicolumn{4}{|c|}{ V-Notch Weir } & \multicolumn{6}{|c|}{ R-Notch Weir } \\
\hline & \multicolumn{2}{|c|}{ Measured Water Level } & \multicolumn{2}{|c|}{$\begin{array}{c}\text { Calculated Rate } \\
\left(\mathrm{ft}^{3} / \mathrm{s}=448.8 \mathrm{gpm}\right)\end{array}$} & \multicolumn{3}{|c|}{ Measured Water Level } & \multicolumn{3}{|c|}{$\begin{array}{c}\text { Calculated Rate } \\
\left(\mathrm{ft}^{3} / \mathrm{s}=448.8 \mathrm{gpm}\right)\end{array}$} \\
\hline & $\begin{array}{c}\text { (ft above } \\
\text { sensor) }\end{array}$ & $\begin{array}{c}\text { (ft above } \\
\text { notch) }\end{array}$ & $\left(\mathrm{ft}^{3} / \mathrm{s}\right)$ & (gpm) & $\begin{array}{l}\text { (ft above } \\
\text { sensor) }\end{array}$ & $\begin{array}{c}\text { (ft above } \\
\text { log) }\end{array}$ & $\begin{array}{c}\text { (m above } \\
\text { log) }\end{array}$ & $\left(\mathrm{m}^{3} / \mathrm{s}\right)$ & $\left(\mathrm{ft}^{3} / \mathrm{s}\right)$ & (gpm) \\
\hline 10/18/08 17:00 & 2.178 & 0.718 & 0.251 & 112.627 & 1.431 & 0.085 & 0.025907 & 0.011728 & 0.414220 & 185.902 \\
\hline 10/18/08 18:00 & 2.175 & 0.715 & 0.248 & 111.467 & 1.429 & 0.083 & 0.025297 & 0.011331 & 0.400223 & 179.620 \\
\hline 10/18/08 19:00 & 2.173 & 0.713 & 0.247 & 110.697 & 1.431 & 0.085 & 0.025907 & 0.011728 & 0.414220 & 185.902 \\
\hline 10/18/08 20:00 & 2.175 & 0.715 & 0.248 & 111.467 & 1.431 & 0.085 & 0.025907 & 0.011728 & 0.414220 & 185.902 \\
\hline 10/18/08 21:00 & 2.178 & 0.718 & 0.251 & 112.627 & 1.431 & 0.085 & 0.025907 & 0.011728 & 0.414220 & 185.902 \\
\hline 10/18/08 22:00 & 2.183 & 0.723 & 0.255 & 114.576 & 1.431 & 0.085 & 0.025907 & 0.011728 & 0.414220 & 185.902 \\
\hline 10/18/08 23:00 & 2.185 & 0.725 & 0.257 & 115.362 & 1.431 & 0.085 & 0.025907 & 0.011728 & 0.414220 & 185.902 \\
\hline 10/19/08 0:00 & 2.187 & 0.727 & 0.259 & 116.150 & 1.429 & 0.083 & 0.025297 & 0.011331 & 0.400223 & 179.620 \\
\hline 10/19/08 1:00 & 2.185 & 0.725 & 0.257 & 115.362 & 1.431 & 0.085 & 0.025907 & 0.011728 & 0.414220 & 185.902 \\
\hline 10/19/08 2:00 & 2.19 & 0.730 & 0.261 & 117.339 & 1.431 & 0.085 & 0.025907 & 0.011728 & 0.414220 & 185.902 \\
\hline 10/19/08 3:00 & 2.188 & 0.728 & 0.260 & 116.546 & 1.431 & 0.085 & 0.025907 & 0.011728 & 0.414220 & 185.902 \\
\hline 10/19/08 4:00 & 2.183 & 0.723 & 0.255 & 114.576 & 1.431 & 0.085 & 0.025907 & 0.011728 & 0.414220 & 185.902 \\
\hline 10/19/08 5:00 & 2.188 & 0.728 & 0.260 & 116.546 & 1.434 & 0.088 & 0.026821 & 0.012331 & 0.435514 & 195.459 \\
\hline 10/19/08 6:00 & 2.186 & 0.726 & 0.258 & 115.756 & 1.429 & 0.083 & 0.025297 & 0.011331 & 0.400223 & 179.620 \\
\hline 10/19/08 7:00 & 2.188 & 0.728 & 0.260 & 116.546 & 1.431 & 0.085 & 0.025907 & 0.011728 & 0.414220 & 185.902 \\
\hline 10/19/08 8:00 & 2.183 & 0.723 & 0.255 & 114.576 & 1.431 & 0.085 & 0.025907 & 0.011728 & 0.414220 & 185.902 \\
\hline 10/19/08 9:00 & 2.183 & 0.723 & 0.255 & 114.576 & 1.429 & 0.083 & 0.025297 & 0.011331 & 0.400223 & 179.620 \\
\hline 10/19/08 10:00 & 2.181 & 0.721 & 0.254 & 113.794 & 1.429 & 0.083 & 0.025297 & 0.011331 & 0.400223 & 179.620 \\
\hline 10/19/08 11:00 & 2.178 & 0.718 & 0.251 & 112.627 & 1.429 & 0.083 & 0.025297 & 0.011331 & 0.400223 & 179.620 \\
\hline 10/19/08 12:00 & 2.159 & 0.699 & 0.235 & 105.401 & 1.429 & 0.083 & 0.025297 & 0.011331 & 0.400223 & 179.620 \\
\hline 10/19/08 13:00 & 2.147 & 0.687 & 0.225 & 100.985 & 1.429 & 0.083 & 0.025297 & 0.011331 & 0.400223 & 179.620 \\
\hline 10/19/08 14:00 & 2.145 & 0.685 & 0.223 & 100.260 & 1.431 & 0.085 & 0.025907 & 0.011728 & 0.414220 & 185.902 \\
\hline 10/19/08 15:00 & 2.142 & 0.682 & 0.221 & 99.178 & 1.429 & 0.083 & 0.025297 & 0.011331 & 0.400223 & 179.620 \\
\hline 10/19/08 16:00 & 2.138 & 0.678 & 0.218 & 97.747 & 1.429 & 0.083 & 0.025297 & 0.011331 & 0.400223 & 179.620 \\
\hline 10/19/08 17:00 & 2.135 & 0.675 & 0.215 & 96.681 & 1.427 & 0.081 & 0.024688 & 0.010940 & 0.386387 & 173.411 \\
\hline
\end{tabular}




\begin{tabular}{|c|c|c|c|c|c|c|c|c|c|c|}
\hline \multirow[b]{3}{*}{ Date Time } & \multicolumn{4}{|c|}{ V-Notch Weir } & \multicolumn{6}{|c|}{ R-Notch Weir } \\
\hline & \multicolumn{2}{|c|}{ Measured Water Level } & \multicolumn{2}{|c|}{$\begin{array}{c}\text { Calculated Rate } \\
\left(\mathrm{ft}^{3} / \mathrm{s}=448.8 \mathrm{gpm}\right)\end{array}$} & \multicolumn{3}{|c|}{ Measured Water Level } & \multicolumn{3}{|c|}{$\begin{array}{c}\text { Calculated Rate } \\
\left(\mathrm{ft}^{3} / \mathrm{s}=448.8 \mathrm{gpm}\right)\end{array}$} \\
\hline & $\begin{array}{c}\text { (ft above } \\
\text { sensor) }\end{array}$ & $\begin{array}{c}\text { (ft above } \\
\text { notch) }\end{array}$ & $\left(\mathrm{ft}^{3} / \mathrm{s}\right)$ & (gpm) & $\begin{array}{l}\text { (ft above } \\
\text { sensor) }\end{array}$ & $\begin{array}{c}\text { (ft above } \\
\text { log) }\end{array}$ & $\begin{array}{c}\text { (m above } \\
\text { log) }\end{array}$ & $\left(\mathrm{m}^{3} / \mathrm{s}\right)$ & $\left(\mathrm{ft}^{3} / \mathrm{s}\right)$ & (gpm) \\
\hline 10/19/08 18:00 & 2.135 & 0.675 & 0.215 & 96.681 & 1.425 & 0.079 & 0.024078 & 0.010553 & 0.372715 & 167.274 \\
\hline 10/19/08 19:00 & 2.14 & 0.680 & 0.219 & 98.461 & 1.425 & 0.079 & 0.024078 & 0.010553 & 0.372715 & 167.274 \\
\hline 10/19/08 20:00 & 2.147 & 0.687 & 0.225 & 100.985 & 1.428 & 0.082 & 0.024992 & 0.011135 & 0.393285 & 176.506 \\
\hline 10/19/08 21:00 & 2.152 & 0.692 & 0.229 & 102.811 & 1.428 & 0.082 & 0.024992 & 0.011135 & 0.393285 & 176.506 \\
\hline 10/19/08 22:00 & 2.159 & 0.699 & 0.235 & 105.401 & 1.43 & 0.084 & 0.025602 & 0.011529 & 0.407202 & 182.752 \\
\hline 10/19/08 23:00 & 2.161 & 0.701 & 0.237 & 106.148 & 1.43 & 0.084 & 0.025602 & 0.011529 & 0.407202 & 182.752 \\
\hline 10/20/08 0:00 & 2.156 & 0.696 & 0.232 & 104.286 & 1.43 & 0.084 & 0.025602 & 0.011529 & 0.407202 & 182.752 \\
\hline 10/20/08 1:00 & 2.163 & 0.703 & 0.238 & 106.898 & 1.433 & 0.087 & 0.026516 & 0.012129 & 0.428377 & 192.256 \\
\hline 10/20/08 2:00 & 2.156 & 0.696 & 0.232 & 104.286 & 1.433 & 0.087 & 0.026516 & 0.012129 & 0.428377 & 192.256 \\
\hline 10/20/08 3:00 & 2.161 & 0.701 & 0.237 & 106.148 & 1.433 & 0.087 & 0.026516 & 0.012129 & 0.428377 & 192.256 \\
\hline 10/20/08 4:00 & 2.161 & 0.701 & 0.237 & 106.148 & 1.433 & 0.087 & 0.026516 & 0.012129 & 0.428377 & 192.256 \\
\hline 10/20/08 5:00 & 2.166 & 0.706 & 0.241 & 108.030 & 1.433 & 0.087 & 0.026516 & 0.012129 & 0.428377 & 192.256 \\
\hline 10/20/08 6:00 & 2.164 & 0.704 & 0.239 & 107.275 & 1.43 & 0.084 & 0.025602 & 0.011529 & 0.407202 & 182.752 \\
\hline 10/20/08 7:00 & 2.161 & 0.701 & 0.237 & 106.148 & 1.43 & 0.084 & 0.025602 & 0.011529 & 0.407202 & 182.752 \\
\hline 10/20/08 8:00 & 2.169 & 0.709 & 0.243 & 109.168 & 1.43 & 0.084 & 0.025602 & 0.011529 & 0.407202 & 182.752 \\
\hline 10/20/08 9:00 & 2.166 & 0.706 & 0.241 & 108.030 & 1.428 & 0.082 & 0.024992 & 0.011135 & 0.393285 & 176.506 \\
\hline 10/20/08 10:00 & 2.166 & 0.706 & 0.241 & 108.030 & 1.428 & 0.082 & 0.024992 & 0.011135 & 0.393285 & 176.506 \\
\hline 10/20/08 11:00 & 2.168 & 0.708 & 0.242 & 108.788 & 1.43 & 0.084 & 0.025602 & 0.011529 & 0.407202 & 182.752 \\
\hline 10/20/08 12:00 & 2.147 & 0.687 & 0.225 & 100.985 & 1.428 & 0.082 & 0.024992 & 0.011135 & 0.393285 & 176.506 \\
\hline 10/20/08 13:00 & 2.135 & 0.675 & 0.215 & 96.681 & 1.428 & 0.082 & 0.024992 & 0.011135 & 0.393285 & 176.506 \\
\hline 10/20/08 14:00 & 2.142 & 0.682 & 0.221 & 99.178 & 1.43 & 0.084 & 0.025602 & 0.011529 & 0.407202 & 182.752 \\
\hline 10/20/08 15:00 & 2.135 & 0.675 & 0.215 & 96.681 & 1.428 & 0.082 & 0.024992 & 0.011135 & 0.393285 & 176.506 \\
\hline 10/20/08 16:00 & 2.133 & 0.673 & 0.214 & 95.975 & 1.425 & 0.079 & 0.024078 & 0.010553 & 0.372715 & 167.274 \\
\hline 10/20/08 17:00 & 2.185 & 0.725 & 0.257 & 115.362 & 1.426 & 0.08 & 0.024383 & 0.010746 & 0.379531 & 170.333 \\
\hline 10/20/08 18:00 & 2.182 & 0.722 & 0.254 & 114.185 & 1.428 & 0.082 & 0.024992 & 0.011135 & 0.393285 & 176.506 \\
\hline
\end{tabular}




\begin{tabular}{|c|c|c|c|c|c|c|c|c|c|c|}
\hline \multirow[b]{3}{*}{ Date Time } & \multicolumn{4}{|c|}{ V-Notch Weir } & \multicolumn{6}{|c|}{ R-Notch Weir } \\
\hline & \multicolumn{2}{|c|}{ Measured Water Level } & \multicolumn{2}{|c|}{$\begin{array}{c}\text { Calculated Rate } \\
\left(\mathrm{ft}^{3} / \mathrm{s}=448.8 \mathrm{gpm}\right)\end{array}$} & \multicolumn{3}{|c|}{ Measured Water Level } & \multicolumn{3}{|c|}{$\begin{array}{c}\text { Calculated Rate } \\
\left(\mathrm{ft}^{3} / \mathrm{s}=448.8 \mathrm{gpm}\right)\end{array}$} \\
\hline & $\begin{array}{c}\text { (ft above } \\
\text { sensor) }\end{array}$ & $\begin{array}{c}\text { (ft above } \\
\text { notch) }\end{array}$ & $\left(\mathrm{ft}^{3} / \mathrm{s}\right)$ & (gpm) & $\begin{array}{c}\text { (ft above } \\
\text { sensor) }\end{array}$ & $\begin{array}{c}\text { (ft above } \\
\text { log) }\end{array}$ & $\begin{array}{c}\text { (m above } \\
\text { log) }\end{array}$ & $\left(\mathrm{m}^{3} / \mathrm{s}\right)$ & $\left(\mathrm{ft}^{3} / \mathrm{s}\right)$ & (gpm) \\
\hline 10/20/08 19:00 & 2.187 & 0.727 & 0.259 & 116.150 & 1.426 & 0.08 & 0.024383 & 0.010746 & 0.379531 & 170.333 \\
\hline 10/20/08 20:00 & 2.192 & 0.732 & 0.263 & 118.136 & 1.426 & 0.08 & 0.024383 & 0.010746 & 0.379531 & 170.333 \\
\hline 10/20/08 21:00 & 2.197 & 0.737 & 0.268 & 120.142 & 1.428 & 0.082 & 0.024992 & 0.011135 & 0.393285 & 176.506 \\
\hline 10/20/08 22:00 & 2.199 & 0.739 & 0.269 & 120.950 & 1.428 & 0.082 & 0.024992 & 0.011135 & 0.393285 & 176.506 \\
\hline 10/20/08 23:00 & 2.201 & 0.741 & 0.271 & 121.761 & 1.428 & 0.082 & 0.024992 & 0.011135 & 0.393285 & 176.506 \\
\hline 10/21/08 0:00 & 2.202 & 0.742 & 0.272 & 122.168 & 1.428 & 0.082 & 0.024992 & 0.011135 & 0.393285 & 176.506 \\
\hline 10/21/08 1:00 & 2.204 & 0.744 & 0.274 & 122.984 & 1.426 & 0.08 & 0.024383 & 0.010746 & 0.379531 & 170.333 \\
\hline 10/21/08 2:00 & 2.2 & 0.740 & 0.270 & 121.355 & 1.425 & 0.079 & 0.024078 & 0.010553 & 0.372715 & 167.274 \\
\hline 10/21/08 3:00 & 2.202 & 0.742 & 0.272 & 122.168 & 1.428 & 0.082 & 0.024992 & 0.011135 & 0.393285 & 176.506 \\
\hline 10/21/08 4:00 & 2.202 & 0.742 & 0.272 & 122.168 & 1.427 & 0.081 & 0.024688 & 0.010940 & 0.386387 & 173.411 \\
\hline 10/21/08 5:00 & 2.205 & 0.745 & 0.275 & 123.394 & 1.427 & 0.081 & 0.024688 & 0.010940 & 0.386387 & 173.411 \\
\hline 10/21/08 6:00 & 2.202 & 0.742 & 0.272 & 122.168 & 1.427 & 0.081 & 0.024688 & 0.010940 & 0.386387 & 173.411 \\
\hline 10/21/08 7:00 & 2.205 & 0.745 & 0.275 & 123.394 & 1.427 & 0.081 & 0.024688 & 0.010940 & 0.386387 & 173.411 \\
\hline 10/21/08 8:00 & 2.207 & 0.747 & 0.277 & 124.215 & 1.429 & 0.083 & 0.025297 & 0.011331 & 0.400223 & 179.620 \\
\hline 10/21/08 9:00 & 2.21 & 0.750 & 0.280 & 125.453 & 1.429 & 0.083 & 0.025297 & 0.011331 & 0.400223 & 179.620 \\
\hline 10/21/08 10:00 & 2.212 & 0.752 & 0.281 & 126.282 & 1.427 & 0.081 & 0.024688 & 0.010940 & 0.386387 & 173.411 \\
\hline 10/21/08 11:00 & 2.207 & 0.747 & 0.277 & 124.215 & 1.429 & 0.083 & 0.025297 & 0.011331 & 0.400223 & 179.620 \\
\hline 10/21/08 12:00 & 2.205 & 0.745 & 0.275 & 123.394 & 1.429 & 0.083 & 0.025297 & 0.011331 & 0.400223 & 179.620 \\
\hline 10/21/08 13:00 & 2.207 & 0.747 & 0.277 & 124.215 & 1.434 & 0.088 & 0.026821 & 0.012331 & 0.435514 & 195.459 \\
\hline 10/21/08 14:00 & 2.214 & 0.754 & 0.283 & 127.114 & 1.431 & 0.085 & 0.025907 & 0.011728 & 0.414220 & 185.902 \\
\hline 10/21/08 15:00 & 2.226 & 0.766 & 0.295 & 132.179 & 1.434 & 0.088 & 0.026821 & 0.012331 & 0.435514 & 195.459 \\
\hline 10/21/08 16:00 & 2.287 & 0.827 & 0.356 & 159.785 & 1.448 & 0.102 & 0.031088 & 0.015274 & 0.539466 & 242.112 \\
\hline 10/21/08 17:00 & 2.324 & 0.864 & 0.397 & 178.081 & 1.464 & 0.118 & 0.035965 & 0.018885 & 0.667017 & 299.357 \\
\hline 10/21/08 18:00 & 2.329 & 0.869 & 0.403 & 180.645 & 1.464 & 0.118 & 0.035965 & 0.018885 & 0.667017 & 299.357 \\
\hline 10/21/08 19:00 & 2.32 & 0.860 & 0.392 & 176.045 & 1.462 & 0.116 & 0.035355 & 0.018420 & 0.650584 & 291.982 \\
\hline
\end{tabular}




\begin{tabular}{|c|c|c|c|c|c|c|c|c|c|c|}
\hline \multirow[b]{3}{*}{ Date Time } & \multicolumn{4}{|c|}{ V-Notch Weir } & \multicolumn{6}{|c|}{ R-Notch Weir } \\
\hline & \multicolumn{2}{|c|}{ Measured Water Level } & \multicolumn{2}{|c|}{$\begin{array}{c}\text { Calculated Rate } \\
\left(\mathrm{ft}^{3} / \mathrm{s}=448.8 \mathrm{gpm}\right)\end{array}$} & \multicolumn{3}{|c|}{ Measured Water Level } & \multicolumn{3}{|c|}{$\begin{array}{c}\text { Calculated Rate } \\
\left(\mathrm{ft}^{3} / \mathrm{s}=448.8 \mathrm{gpm}\right)\end{array}$} \\
\hline & $\begin{array}{c}\text { (ft above } \\
\text { sensor) }\end{array}$ & $\begin{array}{c}\text { (ft above } \\
\text { notch) }\end{array}$ & $\left(\mathrm{ft}^{3} / \mathrm{s}\right)$ & (gpm) & $\begin{array}{l}\text { (ft above } \\
\text { sensor) }\end{array}$ & $\begin{array}{c}\text { (ft above } \\
\text { log) }\end{array}$ & $\begin{array}{c}\text { (m above } \\
\text { log) }\end{array}$ & $\left(\mathrm{m}^{3} / \mathrm{s}\right)$ & $\left(\mathrm{ft}^{3} / \mathrm{s}\right)$ & (gpm) \\
\hline 10/21/08 20:00 & 2.31 & 0.850 & 0.381 & 171.018 & 1.455 & 0.109 & 0.033222 & 0.016822 & 0.594159 & 266.658 \\
\hline 10/21/08 21:00 & 2.294 & 0.834 & 0.364 & 163.155 & 1.45 & 0.104 & 0.031698 & 0.015711 & 0.554913 & 249.045 \\
\hline 10/21/08 22:00 & 2.282 & 0.822 & 0.351 & 157.404 & 1.448 & 0.102 & 0.031088 & 0.015274 & 0.539466 & 242.112 \\
\hline 10/21/08 23:00 & 2.27 & 0.810 & 0.338 & 151.775 & 1.443 & 0.097 & 0.029564 & 0.014199 & 0.501493 & 225.070 \\
\hline 10/22/08 0:00 & 2.263 & 0.803 & 0.331 & 148.549 & 1.443 & 0.097 & 0.029564 & 0.014199 & 0.501493 & 225.070 \\
\hline 10/22/08 1:00 & 2.265 & 0.805 & 0.333 & 149.466 & 1.443 & 0.097 & 0.029564 & 0.014199 & 0.501493 & 225.070 \\
\hline 10/22/08 2:00 & 2.289 & 0.829 & 0.358 & 160.744 & 1.483 & 0.137 & 0.041756 & 0.023493 & 0.829766 & 372.399 \\
\hline 10/22/08 3:00 & 2.388 & 0.928 & 0.474 & 212.581 & 1.646 & 0.3 & 0.091436 & 0.074695 & 2.638215 & 1184.031 \\
\hline 10/22/08 4:00 & 2.414 & 0.954 & 0.507 & 227.654 & 1.594 & 0.248 & 0.075587 & 0.056330 & 1.989550 & 892.910 \\
\hline 10/22/08 5:00 & 2.477 & 1.017 & 0.594 & 266.781 & 1.783 & 0.437 & 0.133191 & 0.130674 & 4.615393 & 2071.389 \\
\hline 10/22/08 6:00 & 2.44 & 0.980 & 0.542 & 243.351 & 1.735 & 0.389 & 0.118561 & 0.109892 & 3.881367 & 1741.958 \\
\hline 10/22/08 7:00 & 2.442 & 0.982 & 0.545 & 244.585 & 1.754 & 0.408 & 0.124352 & 0.117974 & 4.166839 & 1870.077 \\
\hline 10/22/08 8:00 & 2.402 & 0.942 & 0.492 & 220.620 & 1.65 & 0.304 & 0.092655 & 0.076178 & 2.690593 & 1207.538 \\
\hline 10/22/08 9:00 & 2.371 & 0.911 & 0.452 & 203.059 & 1.57 & 0.224 & 0.068272 & 0.048454 & 1.711384 & 768.069 \\
\hline 10/22/08 10:00 & 2.346 & 0.886 & 0.422 & 189.528 & 1.523 & 0.177 & 0.053947 & 0.034228 & 1.208936 & 542.570 \\
\hline 10/22/08 11:00 & 2.345 & 0.885 & 0.421 & 188.998 & 1.513 & 0.167 & 0.050899 & 0.031420 & 1.109743 & 498.053 \\
\hline 10/22/08 12:00 & 2.355 & 0.895 & 0.433 & 194.334 & 1.509 & 0.163 & 0.049680 & 0.030319 & 1.070866 & 480.605 \\
\hline 10/22/08 13:00 & 2.357 & 0.897 & 0.435 & 195.412 & 1.501 & 0.155 & 0.047242 & 0.028157 & 0.994512 & 446.337 \\
\hline 10/22/08 14:00 & 2.354 & 0.894 & 0.432 & 193.797 & 1.501 & 0.155 & 0.047242 & 0.028157 & 0.994512 & 446.337 \\
\hline 10/22/08 15:00 & 2.34 & 0.880 & 0.415 & 186.363 & 1.49 & 0.144 & 0.043889 & 0.025273 & 0.892650 & 400.621 \\
\hline 10/22/08 16:00 & 2.328 & 0.868 & 0.401 & 180.130 & 1.48 & 0.134 & 0.040841 & 0.022743 & 0.803289 & 360.516 \\
\hline 10/22/08 17:00 & 2.321 & 0.861 & 0.393 & 176.553 & 1.473 & 0.127 & 0.038708 & 0.021026 & 0.742632 & 333.293 \\
\hline 10/22/08 18:00 & 2.314 & 0.854 & 0.386 & 173.019 & 1.468 & 0.122 & 0.037184 & 0.019827 & 0.700289 & 314.290 \\
\hline 10/22/08 19:00 & 2.314 & 0.854 & 0.386 & 173.019 & 1.471 & 0.125 & 0.038098 & 0.020544 & 0.725596 & 325.647 \\
\hline 10/22/08 20:00 & 2.333 & 0.873 & 0.407 & 182.712 & 1.478 & 0.132 & 0.040232 & 0.022248 & 0.785797 & 352.665 \\
\hline
\end{tabular}




\begin{tabular}{|c|c|c|c|c|c|c|c|c|c|c|}
\hline \multirow[b]{3}{*}{ Date Time } & \multicolumn{4}{|c|}{ V-Notch Weir } & \multicolumn{6}{|c|}{ R-Notch Weir } \\
\hline & \multicolumn{2}{|c|}{ Measured Water Level } & \multicolumn{2}{|c|}{$\begin{array}{c}\text { Calculated Rate } \\
\left(\mathrm{ft}^{3} / \mathrm{s}=448.8 \mathrm{gpm}\right)\end{array}$} & \multicolumn{3}{|c|}{ Measured Water Level } & \multicolumn{3}{|c|}{$\begin{array}{c}\text { Calculated Rate } \\
\left(\mathrm{ft}^{3} / \mathrm{s}=448.8 \mathrm{gpm}\right)\end{array}$} \\
\hline & $\begin{array}{c}\text { (ft above } \\
\text { sensor) }\end{array}$ & $\begin{array}{c}\text { (ft above } \\
\text { notch) }\end{array}$ & $\left(\mathrm{ft}^{3} / \mathrm{s}\right)$ & (gpm) & $\begin{array}{c}\text { (ft above } \\
\text { sensor) }\end{array}$ & $\begin{array}{c}\text { (ft above } \\
\text { log) }\end{array}$ & $\begin{array}{c}\text { (m above } \\
\text { log) }\end{array}$ & $\left(\mathrm{m}^{3} / \mathrm{s}\right)$ & $\left(\mathrm{ft}^{3} / \mathrm{s}\right)$ & (gpm) \\
\hline 10/22/08 21:00 & 2.385 & 0.925 & 0.470 & 210.881 & 1.539 & 0.193 & 0.058824 & 0.038885 & 1.373422 & 616.392 \\
\hline 10/22/08 22:00 & 2.39 & 0.930 & 0.476 & 213.718 & 1.535 & 0.189 & 0.057604 & 0.037703 & 1.331647 & 597.643 \\
\hline 10/22/08 23:00 & 2.381 & 0.921 & 0.465 & 208.628 & 1.518 & 0.172 & 0.052423 & 0.032814 & 1.158986 & 520.153 \\
\hline 10/23/08 0:00 & 2.359 & 0.899 & 0.438 & 196.494 & 1.506 & 0.16 & 0.048766 & 0.029502 & 1.042013 & 467.655 \\
\hline 10/23/08 1:00 & 2.345 & 0.885 & 0.421 & 188.998 & 1.499 & 0.153 & 0.046632 & 0.027625 & 0.975720 & 437.903 \\
\hline 10/23/08 2:00 & 2.334 & 0.874 & 0.408 & 183.231 & 1.487 & 0.141 & 0.042975 & 0.024505 & 0.865512 & 388.442 \\
\hline 10/23/08 3:00 & 2.334 & 0.874 & 0.408 & 183.231 & 1.483 & 0.137 & 0.041756 & 0.023493 & 0.829766 & 372.399 \\
\hline 10/23/08 4:00 & 2.322 & 0.862 & 0.395 & 177.061 & 1.478 & 0.132 & 0.040232 & 0.022248 & 0.785797 & 352.665 \\
\hline 10/23/08 5:00 & 2.32 & 0.860 & 0.392 & 176.045 & 1.473 & 0.127 & 0.038708 & 0.021026 & 0.742632 & 333.293 \\
\hline 10/23/08 6:00 & 2.316 & 0.856 & 0.388 & 174.024 & 1.471 & 0.125 & 0.038098 & 0.020544 & 0.725596 & 325.647 \\
\hline 10/23/08 7:00 & 2.316 & 0.856 & 0.388 & 174.024 & 1.466 & 0.12 & 0.036574 & 0.019354 & 0.683586 & 306.793 \\
\hline 10/23/08 8:00 & 2.316 & 0.856 & 0.388 & 174.024 & 1.463 & 0.117 & 0.035660 & 0.018652 & 0.658783 & 295.662 \\
\hline 10/23/08 9:00 & 2.313 & 0.853 & 0.384 & 172.517 & 1.463 & 0.117 & 0.035660 & 0.018652 & 0.658783 & 295.662 \\
\hline 10/23/08 10:00 & 2.316 & 0.856 & 0.388 & 174.024 & 1.461 & 0.115 & 0.035050 & 0.018189 & 0.642419 & 288.318 \\
\hline 10/23/08 11:00 & 2.308 & 0.848 & 0.379 & 170.023 & 1.459 & 0.113 & 0.034441 & 0.017729 & 0.626193 & 281.035 \\
\hline 10/23/08 12:00 & 2.308 & 0.848 & 0.379 & 170.023 & 1.461 & 0.115 & 0.035050 & 0.018189 & 0.642419 & 288.318 \\
\hline 10/23/08 13:00 & 2.306 & 0.846 & 0.377 & 169.032 & 1.461 & 0.115 & 0.035050 & 0.018189 & 0.642419 & 288.318 \\
\hline 10/23/08 14:00 & 2.301 & 0.841 & 0.371 & 166.568 & 1.461 & 0.115 & 0.035050 & 0.018189 & 0.642419 & 288.318 \\
\hline 10/23/08 15:00 & 2.286 & 0.826 & 0.355 & 159.307 & 1.461 & 0.115 & 0.035050 & 0.018189 & 0.642419 & 288.318 \\
\hline 10/23/08 16:00 & 2.282 & 0.822 & 0.351 & 157.404 & 1.454 & 0.108 & 0.032917 & 0.016598 & 0.586238 & 263.104 \\
\hline 10/23/08 17:00 & 2.284 & 0.824 & 0.353 & 158.354 & 1.458 & 0.112 & 0.034136 & 0.017501 & 0.618132 & 277.418 \\
\hline 10/23/08 18:00 & 2.286 & 0.826 & 0.355 & 159.307 & 1.459 & 0.113 & 0.034441 & 0.017729 & 0.626193 & 281.035 \\
\hline 10/23/08 19:00 & 2.298 & 0.838 & 0.368 & 165.100 & 1.461 & 0.115 & 0.035050 & 0.018189 & 0.642419 & 288.318 \\
\hline 10/23/08 20:00 & 2.303 & 0.843 & 0.373 & 167.551 & 1.463 & 0.117 & 0.035660 & 0.018652 & 0.658783 & 295.662 \\
\hline 10/23/08 21:00 & 2.301 & 0.841 & 0.371 & 166.568 & 1.461 & 0.115 & 0.035050 & 0.018189 & 0.642419 & 288.318 \\
\hline
\end{tabular}




\begin{tabular}{|c|c|c|c|c|c|c|c|c|c|c|}
\hline \multirow[b]{3}{*}{ Date Time } & \multicolumn{4}{|c|}{ V-Notch Weir } & \multicolumn{6}{|c|}{ R-Notch Weir } \\
\hline & \multicolumn{2}{|c|}{ Measured Water Level } & \multicolumn{2}{|c|}{$\begin{array}{c}\text { Calculated Rate } \\
\left(\mathrm{ft}^{3} / \mathrm{s}=448.8 \mathrm{gpm}\right)\end{array}$} & \multicolumn{3}{|c|}{ Measured Water Level } & \multicolumn{3}{|c|}{$\begin{array}{c}\text { Calculated Rate } \\
\left(\mathrm{ft}^{3} / \mathrm{s}=448.8 \mathrm{gpm}\right)\end{array}$} \\
\hline & $\begin{array}{c}\text { (ft above } \\
\text { sensor) }\end{array}$ & $\begin{array}{c}\text { (ft above } \\
\text { notch) }\end{array}$ & $\left(\mathrm{ft}^{3} / \mathrm{s}\right)$ & (gpm) & $\begin{array}{c}\text { (ft above } \\
\text { sensor) }\end{array}$ & $\begin{array}{c}\text { (ft above } \\
\text { log) }\end{array}$ & $\begin{array}{c}\text { (m above } \\
\text { log) }\end{array}$ & $\left(\mathrm{m}^{3} / \mathrm{s}\right)$ & $\left(\mathrm{ft}^{3} / \mathrm{s}\right)$ & (gpm) \\
\hline 10/23/08 22:00 & 2.282 & 0.822 & 0.351 & 157.404 & 1.458 & 0.112 & 0.034136 & 0.017501 & 0.618132 & 277.418 \\
\hline 10/23/08 23:00 & 2.285 & 0.825 & 0.354 & 158.830 & 1.456 & 0.11 & 0.033526 & 0.017047 & 0.602114 & 270.229 \\
\hline 10/24/08 0:00 & 2.287 & 0.827 & 0.356 & 159.785 & 1.456 & 0.11 & 0.033526 & 0.017047 & 0.602114 & 270.229 \\
\hline 10/24/08 1:00 & 2.289 & 0.829 & 0.358 & 160.744 & 1.458 & 0.112 & 0.034136 & 0.017501 & 0.618132 & 277.418 \\
\hline 10/24/08 2:00 & 2.294 & 0.834 & 0.364 & 163.155 & 1.461 & 0.115 & 0.035050 & 0.018189 & 0.642419 & 288.318 \\
\hline 10/24/08 3:00 & 2.299 & 0.839 & 0.369 & 165.589 & 1.463 & 0.117 & 0.035660 & 0.018652 & 0.658783 & 295.662 \\
\hline 10/24/08 4:00 & 2.294 & 0.834 & 0.364 & 163.155 & 1.458 & 0.112 & 0.034136 & 0.017501 & 0.618132 & 277.418 \\
\hline 10/24/08 5:00 & 2.294 & 0.834 & 0.364 & 163.155 & 1.458 & 0.112 & 0.034136 & 0.017501 & 0.618132 & 277.418 \\
\hline 10/24/08 6:00 & 2.301 & 0.841 & 0.371 & 166.568 & 1.456 & 0.11 & 0.033526 & 0.017047 & 0.602114 & 270.229 \\
\hline 10/24/08 7:00 & 2.301 & 0.841 & 0.371 & 166.568 & 1.458 & 0.112 & 0.034136 & 0.017501 & 0.618132 & 277.418 \\
\hline 10/24/08 8:00 & 2.297 & 0.837 & 0.367 & 164.613 & 1.456 & 0.11 & 0.033526 & 0.017047 & 0.602114 & 270.229 \\
\hline 10/24/08 9:00 & 2.304 & 0.844 & 0.374 & 168.044 & 1.456 & 0.11 & 0.033526 & 0.017047 & 0.602114 & 270.229 \\
\hline 10/24/08 10:00 & 2.311 & 0.851 & 0.382 & 171.517 & 1.458 & 0.112 & 0.034136 & 0.017501 & 0.618132 & 277.418 \\
\hline 10/24/08 11:00 & 2.327 & 0.867 & 0.400 & 179.617 & 1.463 & 0.117 & 0.035660 & 0.018652 & 0.658783 & 295.662 \\
\hline 10/24/08 12:00 & 2.325 & 0.865 & 0.398 & 178.592 & 1.465 & 0.119 & 0.036269 & 0.019119 & 0.675284 & 303.068 \\
\hline 10/24/08 13:00 & 2.315 & 0.855 & 0.387 & 173.521 & 1.46 & 0.114 & 0.034746 & 0.017958 & 0.634289 & 284.669 \\
\hline 10/24/08 14:00 & 2.308 & 0.848 & 0.379 & 170.023 & 1.458 & 0.112 & 0.034136 & 0.017501 & 0.618132 & 277.418 \\
\hline 10/24/08 15:00 & 2.298 & 0.838 & 0.368 & 165.100 & 1.458 & 0.112 & 0.034136 & 0.017501 & 0.618132 & 277.418 \\
\hline 10/24/08 16:00 & 2.291 & 0.831 & 0.360 & 161.706 & 1.456 & 0.11 & 0.033526 & 0.017047 & 0.602114 & 270.229 \\
\hline 10/24/08 17:00 & 2.289 & 0.829 & 0.358 & 160.744 & 1.456 & 0.11 & 0.033526 & 0.017047 & 0.602114 & 270.229 \\
\hline 10/24/08 18:00 & 2.289 & 0.829 & 0.358 & 160.744 & 1.456 & 0.11 & 0.033526 & 0.017047 & 0.602114 & 270.229 \\
\hline 10/24/08 19:00 & 2.289 & 0.829 & 0.358 & 160.744 & 1.453 & 0.107 & 0.032612 & 0.016375 & 0.578353 & 259.565 \\
\hline 10/24/08 20:00 & 2.291 & 0.831 & 0.360 & 161.706 & 1.454 & 0.108 & 0.032917 & 0.016598 & 0.586238 & 263.104 \\
\hline 10/24/08 21:00 & 2.294 & 0.834 & 0.364 & 163.155 & 1.456 & 0.11 & 0.033526 & 0.017047 & 0.602114 & 270.229 \\
\hline 10/24/08 22:00 & 2.294 & 0.834 & 0.364 & 163.155 & 1.454 & 0.108 & 0.032917 & 0.016598 & 0.586238 & 263.104 \\
\hline
\end{tabular}




\begin{tabular}{|c|c|c|c|c|c|c|c|c|c|c|}
\hline \multirow[b]{3}{*}{ Date Time } & \multicolumn{4}{|c|}{ V-Notch Weir } & \multicolumn{6}{|c|}{ R-Notch Weir } \\
\hline & \multicolumn{2}{|c|}{ Measured Water Level } & \multicolumn{2}{|c|}{$\begin{array}{c}\text { Calculated Rate } \\
\left(\mathrm{ft}^{3} / \mathrm{s}=448.8 \mathrm{gpm}\right)\end{array}$} & \multicolumn{3}{|c|}{ Measured Water Level } & \multicolumn{3}{|c|}{$\begin{array}{c}\text { Calculated Rate } \\
\left(\mathrm{ft}^{3} / \mathrm{s}=448.8 \mathrm{gpm}\right)\end{array}$} \\
\hline & $\begin{array}{c}\text { (ft above } \\
\text { sensor) }\end{array}$ & $\begin{array}{c}\text { (ft above } \\
\text { notch) }\end{array}$ & $\left(\mathrm{ft}^{3} / \mathrm{s}\right)$ & (gpm) & $\begin{array}{c}\text { (ft above } \\
\text { sensor) }\end{array}$ & $\begin{array}{c}\text { (ft above } \\
\text { log) }\end{array}$ & $\begin{array}{c}\text { (m above } \\
\text { log) }\end{array}$ & $\left(\mathrm{m}^{3} / \mathrm{s}\right)$ & $\left(\mathrm{ft}^{3} / \mathrm{s}\right)$ & (gpm) \\
\hline 10/24/08 23:00 & 2.292 & 0.832 & 0.361 & 162.188 & 1.454 & 0.108 & 0.032917 & 0.016598 & 0.586238 & 263.104 \\
\hline 10/25/08 0:00 & 2.294 & 0.834 & 0.364 & 163.155 & 1.442 & 0.096 & 0.029259 & 0.013987 & 0.494010 & 221.711 \\
\hline 10/25/08 1:00 & 2.292 & 0.832 & 0.361 & 162.188 & 1.449 & 0.103 & 0.031393 & 0.015492 & 0.547171 & 245.570 \\
\hline 10/25/08 2:00 & 2.292 & 0.832 & 0.361 & 162.188 & 1.451 & 0.105 & 0.032002 & 0.015931 & 0.562690 & 252.535 \\
\hline 10/25/08 3:00 & 2.292 & 0.832 & 0.361 & 162.188 & 1.451 & 0.105 & 0.032002 & 0.015931 & 0.562690 & 252.535 \\
\hline 10/25/08 4:00 & 2.295 & 0.835 & 0.365 & 163.640 & 1.453 & 0.107 & 0.032612 & 0.016375 & 0.578353 & 259.565 \\
\hline 10/25/08 5:00 & 2.293 & 0.833 & 0.362 & 162.671 & 1.451 & 0.105 & 0.032002 & 0.015931 & 0.562690 & 252.535 \\
\hline 10/25/08 6:00 & 2.3 & 0.840 & 0.370 & 166.078 & 1.451 & 0.105 & 0.032002 & 0.015931 & 0.562690 & 252.535 \\
\hline 10/25/08 7:00 & 2.305 & 0.845 & 0.376 & 168.537 & 1.451 & 0.105 & 0.032002 & 0.015931 & 0.562690 & 252.535 \\
\hline 10/25/08 8:00 & 2.303 & 0.843 & 0.373 & 167.551 & 1.451 & 0.105 & 0.032002 & 0.015931 & 0.562690 & 252.535 \\
\hline 10/25/08 9:00 & 2.3 & 0.840 & 0.370 & 166.078 & 1.439 & 0.093 & 0.028345 & 0.013358 & 0.471786 & 211.738 \\
\hline 10/25/08 10:00 & 2.293 & 0.833 & 0.362 & 162.671 & 1.444 & 0.098 & 0.029869 & 0.014412 & 0.509013 & 228.445 \\
\hline 10/25/08 11:00 & 2.287 & 0.827 & 0.356 & 159.785 & 1.446 & 0.1 & 0.030479 & 0.014841 & 0.524166 & 235.246 \\
\hline 10/25/08 12:00 & 2.268 & 0.808 & 0.336 & 150.849 & 1.448 & 0.102 & 0.031088 & 0.015274 & 0.539466 & 242.112 \\
\hline 10/25/08 13:00 & 2.26 & 0.800 & 0.328 & 147.178 & 1.451 & 0.105 & 0.032002 & 0.015931 & 0.562690 & 252.535 \\
\hline 10/25/08 14:00 & 2.25 & 0.790 & 0.318 & 142.666 & 1.451 & 0.105 & 0.032002 & 0.015931 & 0.562690 & 252.535 \\
\hline 10/25/08 15:00 & 2.234 & 0.774 & 0.302 & 135.621 & 1.444 & 0.098 & 0.029869 & 0.014412 & 0.509013 & 228.445 \\
\hline 10/25/08 16:00 & 2.232 & 0.772 & 0.300 & 134.756 & 1.442 & 0.096 & 0.029259 & 0.013987 & 0.494010 & 221.711 \\
\hline 10/25/08 17:00 & 2.234 & 0.774 & 0.302 & 135.621 & 1.444 & 0.098 & 0.029869 & 0.014412 & 0.509013 & 228.445 \\
\hline 10/25/08 18:00 & 2.229 & 0.769 & 0.297 & 133.464 & 1.444 & 0.098 & 0.029869 & 0.014412 & 0.509013 & 228.445 \\
\hline 10/25/08 19:00 & 2.25 & 0.790 & 0.318 & 142.666 & 1.447 & 0.101 & 0.030783 & 0.015057 & 0.531798 & 238.671 \\
\hline 10/25/08 20:00 & 2.269 & 0.809 & 0.337 & 151.312 & 1.447 & 0.101 & 0.030783 & 0.015057 & 0.531798 & 238.671 \\
\hline 10/25/08 21:00 & 2.281 & 0.821 & 0.350 & 156.930 & 1.449 & 0.103 & 0.031393 & 0.015492 & 0.547171 & 245.570 \\
\hline 10/25/08 22:00 & 2.279 & 0.819 & 0.348 & 155.985 & 1.449 & 0.103 & 0.031393 & 0.015492 & 0.547171 & 245.570 \\
\hline 10/25/08 23:00 & 2.279 & 0.819 & 0.348 & 155.985 & 1.449 & 0.103 & 0.031393 & 0.015492 & 0.547171 & 245.570 \\
\hline
\end{tabular}




\begin{tabular}{|c|c|c|c|c|c|c|c|c|c|c|}
\hline \multirow[b]{3}{*}{ Date Time } & \multicolumn{4}{|c|}{ V-Notch Weir } & \multicolumn{6}{|c|}{ R-Notch Weir } \\
\hline & \multicolumn{2}{|c|}{ Measured Water Level } & \multicolumn{2}{|c|}{$\begin{array}{c}\text { Calculated Rate } \\
\left(\mathrm{ft}^{3} / \mathrm{s}=448.8 \mathrm{gpm}\right)\end{array}$} & \multicolumn{3}{|c|}{ Measured Water Level } & \multicolumn{3}{|c|}{$\begin{array}{c}\text { Calculated Rate } \\
\left(\mathrm{ft}^{3} / \mathrm{s}=448.8 \mathrm{gpm}\right)\end{array}$} \\
\hline & $\begin{array}{c}\text { (ft above } \\
\text { sensor) }\end{array}$ & $\begin{array}{c}\text { (ft above } \\
\text { notch) }\end{array}$ & $\left(\mathrm{ft}^{3} / \mathrm{s}\right)$ & (gpm) & $\begin{array}{c}\text { (ft above } \\
\text { sensor) }\end{array}$ & $\begin{array}{c}\text { (ft above } \\
\text { log) }\end{array}$ & $\begin{array}{c}\text { (m above } \\
\text { log) }\end{array}$ & $\left(\mathrm{m}^{3} / \mathrm{s}\right)$ & $\left(\mathrm{ft}^{3} / \mathrm{s}\right)$ & (gpm) \\
\hline 10/26/08 0:00 & 2.282 & 0.822 & 0.351 & 157.404 & 1.451 & 0.105 & 0.032002 & 0.015931 & 0.562690 & 252.535 \\
\hline 10/26/08 1:00 & 2.28 & 0.820 & 0.349 & 156.457 & 1.444 & 0.098 & 0.029869 & 0.014412 & 0.509013 & 228.445 \\
\hline 10/26/08 2:00 & 2.282 & 0.822 & 0.351 & 157.404 & 1.442 & 0.096 & 0.029259 & 0.013987 & 0.494010 & 221.711 \\
\hline 10/26/08 3:00 & 2.282 & 0.822 & 0.351 & 157.404 & 1.442 & 0.096 & 0.029259 & 0.013987 & 0.494010 & 221.711 \\
\hline 10/26/08 4:00 & 2.289 & 0.829 & 0.358 & 160.744 & 1.442 & 0.096 & 0.029259 & 0.013987 & 0.494010 & 221.711 \\
\hline 10/26/08 5:00 & 2.296 & 0.836 & 0.366 & 164.126 & 1.44 & 0.094 & 0.028650 & 0.013566 & 0.479156 & 215.045 \\
\hline 10/26/08 6:00 & 2.292 & 0.832 & 0.361 & 162.188 & 1.44 & 0.094 & 0.028650 & 0.013566 & 0.479156 & 215.045 \\
\hline 10/26/08 7:00 & 2.29 & 0.830 & 0.359 & 161.224 & 1.437 & 0.091 & 0.027735 & 0.012943 & 0.457162 & 205.174 \\
\hline 10/26/08 8:00 & 2.285 & 0.825 & 0.354 & 158.830 & 1.437 & 0.091 & 0.027735 & 0.012943 & 0.457162 & 205.174 \\
\hline 10/26/08 9:00 & 2.295 & 0.835 & 0.365 & 163.640 & 1.435 & 0.089 & 0.027126 & 0.012534 & 0.442691 & 198.680 \\
\hline 10/26/08 10:00 & 2.288 & 0.828 & 0.357 & 160.264 & 1.437 & 0.091 & 0.027735 & 0.012943 & 0.457162 & 205.174 \\
\hline 10/26/08 11:00 & 2.283 & 0.823 & 0.352 & 157.878 & 1.435 & 0.089 & 0.027126 & 0.012534 & 0.442691 & 198.680 \\
\hline 10/26/08 12:00 & 2.28 & 0.820 & 0.349 & 156.457 & 1.437 & 0.091 & 0.027735 & 0.012943 & 0.457162 & 205.174 \\
\hline 10/26/08 13:00 & 2.268 & 0.808 & 0.336 & 150.849 & 1.437 & 0.091 & 0.027735 & 0.012943 & 0.457162 & 205.174 \\
\hline 10/26/08 14:00 & 2.289 & 0.829 & 0.358 & 160.744 & 1.435 & 0.089 & 0.027126 & 0.012534 & 0.442691 & 198.680 \\
\hline 10/26/08 15:00 & 2.277 & 0.817 & 0.345 & 155.043 & 1.437 & 0.091 & 0.027735 & 0.012943 & 0.457162 & 205.174 \\
\hline 10/26/08 16:00 & 2.272 & 0.812 & 0.340 & 152.705 & 1.428 & 0.082 & 0.024992 & 0.011135 & 0.393285 & 176.506 \\
\hline 10/26/08 17:00 & 2.259 & 0.799 & 0.327 & 146.723 & 1.432 & 0.086 & 0.026212 & 0.011928 & 0.421279 & 189.070 \\
\hline 10/26/08 18:00 & 2.269 & 0.809 & 0.337 & 151.312 & 1.437 & 0.091 & 0.027735 & 0.012943 & 0.457162 & 205.174 \\
\hline 10/26/08 19:00 & 2.271 & 0.811 & 0.339 & 152.239 & 1.437 & 0.091 & 0.027735 & 0.012943 & 0.457162 & 205.174 \\
\hline 10/26/08 20:00 & 2.282 & 0.822 & 0.351 & 157.404 & 1.432 & 0.086 & 0.026212 & 0.011928 & 0.421279 & 189.070 \\
\hline 10/26/08 21:00 & 2.282 & 0.822 & 0.351 & 157.404 & 1.434 & 0.088 & 0.026821 & 0.012331 & 0.435514 & 195.459 \\
\hline 10/26/08 22:00 & 2.283 & 0.823 & 0.352 & 157.878 & 1.434 & 0.088 & 0.026821 & 0.012331 & 0.435514 & 195.459 \\
\hline 10/26/08 23:00 & 2.288 & 0.828 & 0.357 & 160.264 & 1.434 & 0.088 & 0.026821 & 0.012331 & 0.435514 & 195.459 \\
\hline 10/27/08 0:00 & 2.293 & 0.833 & 0.362 & 162.671 & 1.434 & 0.088 & 0.026821 & 0.012331 & 0.435514 & 195.459 \\
\hline
\end{tabular}




\begin{tabular}{|c|c|c|c|c|c|c|c|c|c|c|}
\hline \multirow[b]{3}{*}{ Date Time } & \multicolumn{4}{|c|}{ V-Notch Weir } & \multicolumn{6}{|c|}{ R-Notch Weir } \\
\hline & \multicolumn{2}{|c|}{ Measured Water Level } & \multicolumn{2}{|c|}{$\begin{array}{c}\text { Calculated Rate } \\
\left(\mathrm{ft}^{3} / \mathrm{s}=448.8 \mathrm{gpm}\right)\end{array}$} & \multicolumn{3}{|c|}{ Measured Water Level } & \multicolumn{3}{|c|}{$\begin{array}{c}\text { Calculated Rate } \\
\left(\mathrm{ft}^{3} / \mathrm{s}=448.8 \mathrm{gpm}\right)\end{array}$} \\
\hline & $\begin{array}{c}\text { (ft above } \\
\text { sensor) }\end{array}$ & $\begin{array}{c}\text { (ft above } \\
\text { notch) }\end{array}$ & $\left(\mathrm{ft}^{3} / \mathrm{s}\right)$ & (gpm) & $\begin{array}{l}\text { (ft above } \\
\text { sensor) }\end{array}$ & $\begin{array}{c}\text { (ft above } \\
\text { log) }\end{array}$ & $\begin{array}{c}\text { (m above } \\
\text { log) }\end{array}$ & $\left(\mathrm{m}^{3} / \mathrm{s}\right)$ & $\left(\mathrm{ft}^{3} / \mathrm{s}\right)$ & (gpm) \\
\hline 10/27/08 1:00 & 2.314 & 0.854 & 0.386 & 173.019 & 1.436 & 0.09 & 0.027431 & 0.012738 & 0.449907 & 201.918 \\
\hline 10/27/08 2:00 & 2.31 & 0.850 & 0.381 & 171.018 & 1.436 & 0.09 & 0.027431 & 0.012738 & 0.449907 & 201.918 \\
\hline 10/27/08 3:00 & 2.312 & 0.852 & 0.383 & 172.017 & 1.436 & 0.09 & 0.027431 & 0.012738 & 0.449907 & 201.918 \\
\hline 10/27/08 4:00 & 2.331 & 0.871 & 0.405 & 181.677 & 1.436 & 0.09 & 0.027431 & 0.012738 & 0.449907 & 201.918 \\
\hline 10/27/08 5:00 & 2.334 & 0.874 & 0.408 & 183.231 & 1.436 & 0.09 & 0.027431 & 0.012738 & 0.449907 & 201.918 \\
\hline 10/27/08 6:00 & 2.322 & 0.862 & 0.395 & 177.061 & 1.436 & 0.09 & 0.027431 & 0.012738 & 0.449907 & 201.918 \\
\hline 10/27/08 7:00 & 2.327 & 0.867 & 0.400 & 179.617 & 1.436 & 0.09 & 0.027431 & 0.012738 & 0.449907 & 201.918 \\
\hline 10/27/08 8:00 & 2.327 & 0.867 & 0.400 & 179.617 & 1.433 & 0.087 & 0.026516 & 0.012129 & 0.428377 & 192.256 \\
\hline 10/27/08 9:00 & 2.33 & 0.870 & 0.404 & 181.160 & 1.433 & 0.087 & 0.026516 & 0.012129 & 0.428377 & 192.256 \\
\hline 10/27/08 10:00 & 2.325 & 0.865 & 0.398 & 178.592 & 1.433 & 0.087 & 0.026516 & 0.012129 & 0.428377 & 192.256 \\
\hline 10/27/08 11:00 & 2.326 & 0.866 & 0.399 & 179.104 & 1.435 & 0.089 & 0.027126 & 0.012534 & 0.442691 & 198.680 \\
\hline 10/27/08 12:00 & 2.323 & 0.863 & 0.396 & 177.571 & 1.435 & 0.089 & 0.027126 & 0.012534 & 0.442691 & 198.680 \\
\hline 10/27/08 13:00 & 2.329 & 0.869 & 0.403 & 180.645 & 1.438 & 0.092 & 0.028040 & 0.013150 & 0.464455 & 208.447 \\
\hline 10/27/08 14:00 & 2.328 & 0.868 & 0.401 & 180.130 & 1.438 & 0.092 & 0.028040 & 0.013150 & 0.464455 & 208.447 \\
\hline 10/27/08 15:00 & 2.325 & 0.865 & 0.398 & 178.592 & 1.443 & 0.097 & 0.029564 & 0.014199 & 0.501493 & 225.070 \\
\hline 10/27/08 16:00 & 2.323 & 0.863 & 0.396 & 177.571 & 1.44 & 0.094 & 0.028650 & 0.013566 & 0.479156 & 215.045 \\
\hline 10/27/08 17:00 & 2.32 & 0.860 & 0.392 & 176.045 & 1.44 & 0.094 & 0.028650 & 0.013566 & 0.479156 & 215.045 \\
\hline 10/27/08 18:00 & 2.316 & 0.856 & 0.388 & 174.024 & 1.438 & 0.092 & 0.028040 & 0.013150 & 0.464455 & 208.447 \\
\hline 10/27/08 19:00 & 2.323 & 0.863 & 0.396 & 177.571 & 1.44 & 0.094 & 0.028650 & 0.013566 & 0.479156 & 215.045 \\
\hline 10/27/08 20:00 & 2.328 & 0.868 & 0.401 & 180.130 & 1.44 & 0.094 & 0.028650 & 0.013566 & 0.479156 & 215.045 \\
\hline 10/27/08 21:00 & 2.329 & 0.869 & 0.403 & 180.645 & 1.44 & 0.094 & 0.028650 & 0.013566 & 0.479156 & 215.045 \\
\hline 10/27/08 22:00 & 2.327 & 0.867 & 0.400 & 179.617 & 1.44 & 0.094 & 0.028650 & 0.013566 & 0.479156 & 215.045 \\
\hline 10/27/08 23:00 & 2.332 & 0.872 & 0.406 & 182.194 & 1.438 & 0.092 & 0.028040 & 0.013150 & 0.464455 & 208.447 \\
\hline 10/28/08 0:00 & 2.33 & 0.870 & 0.404 & 181.160 & 1.44 & 0.094 & 0.028650 & 0.013566 & 0.479156 & 215.045 \\
\hline 10/28/08 1:00 & 2.333 & 0.873 & 0.407 & 182.712 & 1.438 & 0.092 & 0.028040 & 0.013150 & 0.464455 & 208.447 \\
\hline
\end{tabular}




\begin{tabular}{|c|c|c|c|c|c|c|c|c|c|c|}
\hline \multirow[b]{3}{*}{ Date } & \multicolumn{4}{|c|}{ V-Notch Weir } & \multicolumn{6}{|c|}{ R-Notch Weir } \\
\hline & \multicolumn{2}{|c|}{ Measured Water Level } & \multicolumn{2}{|c|}{$\begin{array}{c}\text { Calculated Rate } \\
\left(\mathrm{ft}^{3} / \mathrm{s}=448.8 \mathrm{gpm}\right)\end{array}$} & \multicolumn{3}{|c|}{ Measured Water Level } & \multicolumn{3}{|c|}{$\begin{array}{c}\text { Calculated Rate } \\
\left(\mathrm{ft}^{3} / \mathrm{s}=448.8 \mathrm{gpm}\right)\end{array}$} \\
\hline & $\begin{array}{c}\text { (ft above } \\
\text { sensor) }\end{array}$ & $\begin{array}{c}\text { (ft above } \\
\text { notch) }\end{array}$ & $\left(\mathrm{ft}^{3} / \mathrm{s}\right)$ & (gpm) & $\begin{array}{c}\text { (ft above } \\
\text { sensor) }\end{array}$ & $\begin{array}{c}\text { (ft above } \\
\text { log) }\end{array}$ & $\begin{array}{c}\text { (m above } \\
\text { log) }\end{array}$ & $\left(\mathrm{m}^{3} / \mathrm{s}\right)$ & $\left(\mathrm{ft}^{3} / \mathrm{s}\right)$ & (gpm) \\
\hline 10/28/08 2:00 & 2.333 & 0.873 & 0.407 & 182.712 & 1.438 & 0.092 & 0.028040 & 0.013150 & 0.464455 & 208.447 \\
\hline 10/28/08 3:00 & 2.333 & 0.873 & 0.407 & 182.712 & 1.44 & 0.094 & 0.028650 & 0.013566 & 0.479156 & 215.045 \\
\hline 10/28/08 4:00 & 2.341 & 0.881 & 0.416 & 186.889 & 1.438 & 0.092 & 0.028040 & 0.013150 & 0.464455 & 208.447 \\
\hline 10/28/08 5:00 & 2.334 & 0.874 & 0.408 & 183.231 & 1.44 & 0.094 & 0.028650 & 0.013566 & 0.479156 & 215.045 \\
\hline 10/28/08 6:00 & 2.332 & 0.872 & 0.406 & 182.194 & 1.44 & 0.094 & 0.028650 & 0.013566 & 0.479156 & 215.045 \\
\hline 10/28/08 7:00 & 2.332 & 0.872 & 0.406 & 182.194 & 1.437 & 0.091 & 0.027735 & 0.012943 & 0.457162 & 205.174 \\
\hline 10/28/08 8:00 & 2.339 & 0.879 & 0.414 & 185.839 & 1.437 & 0.091 & 0.027735 & 0.012943 & 0.457162 & 205.174 \\
\hline 10/28/08 9:00 & 2.339 & 0.879 & 0.414 & 185.839 & 1.44 & 0.094 & 0.028650 & 0.013566 & 0.479156 & 215.045 \\
\hline 10/28/08 10:00 & 2.334 & 0.874 & 0.408 & 183.231 & 1.437 & 0.091 & 0.027735 & 0.012943 & 0.457162 & 205.174 \\
\hline 10/28/08 11:00 & 2.335 & 0.875 & 0.409 & 183.751 & 1.44 & 0.094 & 0.028650 & 0.013566 & 0.479156 & 215.045 \\
\hline 10/28/08 12:00 & 2.334 & 0.874 & 0.408 & 183.231 & 1.442 & 0.096 & 0.029259 & 0.013987 & 0.494010 & 221.711 \\
\hline 10/28/08 13:00 & 2.33 & 0.870 & 0.404 & 181.160 & 1.444 & 0.098 & 0.029869 & 0.014412 & 0.509013 & 228.445 \\
\hline 10/28/08 14:00 & 2.33 & 0.870 & 0.404 & 181.160 & 1.444 & 0.098 & 0.029869 & 0.014412 & 0.509013 & 228.445 \\
\hline 10/28/08 15:00 & 2.334 & 0.874 & 0.408 & 183.231 & 1.449 & 0.103 & 0.031393 & 0.015492 & 0.547171 & 245.570 \\
\hline 10/28/08 16:00 & 2.331 & 0.871 & 0.405 & 181.677 & 1.447 & 0.101 & 0.030783 & 0.015057 & 0.531798 & 238.671 \\
\hline 10/28/08 17:00 & 2.331 & 0.871 & 0.405 & 181.677 & 1.449 & 0.103 & 0.031393 & 0.015492 & 0.547171 & 245.570 \\
\hline 10/28/08 18:00 & 2.327 & 0.867 & 0.400 & 179.617 & 1.44 & 0.094 & 0.028650 & 0.013566 & 0.479156 & 215.045 \\
\hline 10/28/08 19:00 & 2.327 & 0.867 & 0.400 & 179.617 & 1.44 & 0.094 & 0.028650 & 0.013566 & 0.479156 & 215.045 \\
\hline 10/28/08 20:00 & 2.33 & 0.870 & 0.404 & 181.160 & 1.443 & 0.097 & 0.029564 & 0.014199 & 0.501493 & 225.070 \\
\hline 10/28/08 21:00 & 2.328 & 0.868 & 0.401 & 180.130 & 1.443 & 0.097 & 0.029564 & 0.014199 & 0.501493 & 225.070 \\
\hline 10/28/08 22:00 & 2.333 & 0.873 & 0.407 & 182.712 & 1.443 & 0.097 & 0.029564 & 0.014199 & 0.501493 & 225.070 \\
\hline 10/28/08 23:00 & 2.338 & 0.878 & 0.413 & 185.316 & 1.445 & 0.099 & 0.030174 & 0.014626 & 0.516571 & 231.837 \\
\hline 10/29/08 0:00 & 2.34 & 0.880 & 0.415 & 186.363 & 1.445 & 0.099 & 0.030174 & 0.014626 & 0.516571 & 231.837 \\
\hline 10/29/08 1:00 & 2.34 & 0.880 & 0.415 & 186.363 & 1.438 & 0.092 & 0.028040 & 0.013150 & 0.464455 & 208.447 \\
\hline 10/29/08 2:00 & 2.341 & 0.881 & 0.416 & 186.889 & 1.44 & 0.094 & 0.028650 & 0.013566 & 0.479156 & 215.045 \\
\hline
\end{tabular}




\begin{tabular}{|c|c|c|c|c|c|c|c|c|c|c|}
\hline \multirow[b]{3}{*}{ Date Time } & \multicolumn{4}{|c|}{ V-Notch Weir } & \multicolumn{6}{|c|}{ R-Notch Weir } \\
\hline & \multicolumn{2}{|c|}{ Measured Water Level } & \multicolumn{2}{|c|}{$\begin{array}{c}\text { Calculated Rate } \\
\left(\mathrm{ft}^{3} / \mathrm{s}=448.8 \mathrm{gpm}\right)\end{array}$} & \multicolumn{3}{|c|}{ Measured Water Level } & \multicolumn{3}{|c|}{$\begin{array}{c}\text { Calculated Rate } \\
\left(\mathrm{ft}^{3} / \mathrm{s}=448.8 \mathrm{gpm}\right)\end{array}$} \\
\hline & $\begin{array}{l}\text { (ft above } \\
\text { sensor) }\end{array}$ & $\begin{array}{l}\text { (ft above } \\
\text { notch) }\end{array}$ & $\left(\mathrm{ft}^{3} / \mathrm{s}\right)$ & (gpm) & $\begin{array}{l}\text { (ft above } \\
\text { sensor) }\end{array}$ & $\begin{array}{l}\text { (ft above } \\
\text { log) }\end{array}$ & $\begin{array}{c}\text { (m above } \\
\text { log) }\end{array}$ & $\left(\mathrm{m}^{3} / \mathrm{s}\right)$ & $\left(\mathrm{ft}^{3} / \mathrm{s}\right)$ & (gpm) \\
\hline 10/29/08 3:00 & 2.343 & 0.883 & 0.419 & 187.942 & 1.44 & 0.094 & 0.028650 & 0.013566 & 0.479156 & 215.045 \\
\hline 10/29/08 4:00 & 2.355 & 0.895 & 0.433 & 194.334 & 1.445 & 0.099 & 0.030174 & 0.014626 & 0.516571 & 231.837 \\
\hline 10/29/08 5:00 & 2.344 & 0.884 & 0.420 & 188.469 & 1.443 & 0.097 & 0.029564 & 0.014199 & 0.501493 & 225.070 \\
\hline 10/29/08 6:00 & 2.344 & 0.884 & 0.420 & 188.469 & 1.445 & 0.099 & 0.030174 & 0.014626 & 0.516571 & 231.837 \\
\hline 10/29/08 7:00 & 2.344 & 0.884 & 0.420 & 188.469 & 1.445 & 0.099 & 0.030174 & 0.014626 & 0.516571 & 231.837 \\
\hline 10/29/08 8:00 & 2.349 & 0.889 & 0.426 & 191.122 & 1.445 & 0.099 & 0.030174 & 0.014626 & 0.516571 & 231.837 \\
\hline 10/29/08 9:00 & 2.349 & 0.889 & 0.426 & 191.122 & 1.442 & 0.096 & 0.029259 & 0.013987 & 0.494010 & 221.711 \\
\hline 10/29/08 10:00 & 2.344 & 0.884 & 0.420 & 188.469 & 1.442 & 0.096 & 0.029259 & 0.013987 & 0.494010 & 221.711 \\
\hline 10/29/08 11:00 & 2.345 & 0.885 & 0.421 & 188.998 & 1.442 & 0.096 & 0.029259 & 0.013987 & 0.494010 & 221.711 \\
\hline 10/29/08 12:00 & 2.334 & 0.874 & 0.408 & 183.231 & 1.442 & 0.096 & 0.029259 & 0.013987 & 0.494010 & 221.711 \\
\hline 10/29/08 13:00 & 2.326 & 0.866 & 0.399 & 179.104 & 1.442 & 0.096 & 0.029259 & 0.013987 & 0.494010 & 221.711 \\
\hline 10/29/08 14:00 & 2.321 & 0.861 & 0.393 & 176.553 & 1.445 & 0.099 & 0.030174 & 0.014626 & 0.516571 & 231.837 \\
\hline 10/29/08 15:00 & 2.323 & 0.863 & 0.396 & 177.571 & 1.447 & 0.101 & 0.030783 & 0.015057 & 0.531798 & 238.671 \\
\hline 10/29/08 16:00 & 2.326 & 0.866 & 0.399 & 179.104 & 1.447 & 0.101 & 0.030783 & 0.015057 & 0.531798 & 238.671 \\
\hline 10/29/08 17:00 & 2.323 & 0.863 & 0.396 & 177.571 & 1.448 & 0.102 & 0.031088 & 0.015274 & 0.539466 & 242.112 \\
\hline 10/29/08 18:00 & 2.319 & 0.859 & 0.391 & 175.539 & 1.45 & 0.104 & 0.031698 & 0.015711 & 0.554913 & 249.045 \\
\hline 10/29/08 19:00 & 2.324 & 0.864 & 0.397 & 178.081 & 1.448 & 0.102 & 0.031088 & 0.015274 & 0.539466 & 242.112 \\
\hline 10/29/08 20:00 & 2.331 & 0.871 & 0.405 & 181.677 & 1.438 & 0.092 & 0.028040 & 0.013150 & 0.464455 & 208.447 \\
\hline 10/29/08 21:00 & 2.331 & 0.871 & 0.405 & 181.677 & 1.443 & 0.097 & 0.029564 & 0.014199 & 0.501493 & 225.070 \\
\hline 10/29/08 22:00 & 2.331 & 0.871 & 0.405 & 181.677 & 1.443 & 0.097 & 0.029564 & 0.014199 & 0.501493 & 225.070 \\
\hline 10/29/08 23:00 & 2.336 & 0.876 & 0.411 & 184.272 & 1.446 & 0.1 & 0.030479 & 0.014841 & 0.524166 & 235.246 \\
\hline 10/30/08 0:00 & 2.341 & 0.881 & 0.416 & 186.889 & 1.446 & 0.1 & 0.030479 & 0.014841 & 0.524166 & 235.246 \\
\hline 10/30/08 1:00 & 2.339 & 0.879 & 0.414 & 185.839 & 1.448 & 0.102 & 0.031088 & 0.015274 & 0.539466 & 242.112 \\
\hline 10/30/08 2:00 & 2.344 & 0.884 & 0.420 & 188.469 & 1.448 & 0.102 & 0.031088 & 0.015274 & 0.539466 & 242.112 \\
\hline 10/30/08 3:00 & 2.349 & 0.889 & 0.426 & 191.122 & 1.446 & 0.1 & 0.030479 & 0.014841 & 0.524166 & 235.246 \\
\hline
\end{tabular}




\begin{tabular}{|c|c|c|c|c|c|c|c|c|c|c|}
\hline \multirow[b]{3}{*}{ Date Time } & \multicolumn{4}{|c|}{ V-Notch Weir } & \multicolumn{6}{|c|}{ R-Notch Weir } \\
\hline & \multicolumn{2}{|c|}{ Measured Water Level } & \multicolumn{2}{|c|}{$\begin{array}{c}\text { Calculated Rate } \\
\left(\mathrm{ft}^{3} / \mathrm{s}=448.8 \mathrm{gpm}\right)\end{array}$} & \multicolumn{3}{|c|}{ Measured Water Level } & \multicolumn{3}{|c|}{$\begin{array}{c}\text { Calculated Rate } \\
\left(\mathrm{ft}^{3} / \mathrm{s}=448.8 \mathrm{gpm}\right)\end{array}$} \\
\hline & $\begin{array}{c}\text { (ft above } \\
\text { sensor) }\end{array}$ & $\begin{array}{c}\text { (ft above } \\
\text { notch) }\end{array}$ & $\left(\mathrm{ft}^{3} / \mathrm{s}\right)$ & (gpm) & $\begin{array}{l}\text { (ft above } \\
\text { sensor) }\end{array}$ & $\begin{array}{c}\text { (ft above } \\
\text { log) }\end{array}$ & $\begin{array}{c}\text { (m above } \\
\text { log) }\end{array}$ & $\left(\mathrm{m}^{3} / \mathrm{s}\right)$ & $\left(\mathrm{ft}^{3} / \mathrm{s}\right)$ & (gpm) \\
\hline 10/30/08 4:00 & 2.347 & 0.887 & 0.423 & 190.058 & 1.446 & 0.1 & 0.030479 & 0.014841 & 0.524166 & 235.246 \\
\hline 10/30/08 5:00 & 2.349 & 0.889 & 0.426 & 191.122 & 1.446 & 0.1 & 0.030479 & 0.014841 & 0.524166 & 235.246 \\
\hline 10/30/08 6:00 & 2.349 & 0.889 & 0.426 & 191.122 & 1.443 & 0.097 & 0.029564 & 0.014199 & 0.501493 & 225.070 \\
\hline 10/30/08 7:00 & 2.345 & 0.885 & 0.421 & 188.998 & 1.438 & 0.092 & 0.028040 & 0.013150 & 0.464455 & 208.447 \\
\hline 10/30/08 8:00 & 2.345 & 0.885 & 0.421 & 188.998 & 1.441 & 0.095 & 0.028955 & 0.013776 & 0.486564 & 218.370 \\
\hline 10/30/08 9:00 & 2.352 & 0.892 & 0.429 & 192.724 & 1.443 & 0.097 & 0.029564 & 0.014199 & 0.501493 & 225.070 \\
\hline 10/30/08 10:00 & 2.342 & 0.882 & 0.418 & 187.415 & 1.441 & 0.095 & 0.028955 & 0.013776 & 0.486564 & 218.370 \\
\hline 10/30/08 11:00 & 2.351 & 0.891 & 0.428 & 192.189 & 1.443 & 0.097 & 0.029564 & 0.014199 & 0.501493 & 225.070 \\
\hline 10/30/08 12:00 & 2.338 & 0.878 & 0.413 & 185.316 & 1.443 & 0.097 & 0.029564 & 0.014199 & 0.501493 & 225.070 \\
\hline 10/30/08 13:00 & 2.321 & 0.861 & 0.393 & 176.553 & 1.445 & 0.099 & 0.030174 & 0.014626 & 0.516571 & 231.837 \\
\hline 10/30/08 14:00 & 2.314 & 0.854 & 0.386 & 173.019 & 1.445 & 0.099 & 0.030174 & 0.014626 & 0.516571 & 231.837 \\
\hline 10/30/08 15:00 & 2.304 & 0.844 & 0.374 & 168.044 & 1.45 & 0.104 & 0.031698 & 0.015711 & 0.554913 & 249.045 \\
\hline 10/30/08 16:00 & 2.297 & 0.837 & 0.367 & 164.613 & 1.45 & 0.104 & 0.031698 & 0.015711 & 0.554913 & 249.045 \\
\hline 10/30/08 17:00 & 2.29 & 0.830 & 0.359 & 161.224 & 1.439 & 0.093 & 0.028345 & 0.013358 & 0.471786 & 211.738 \\
\hline 10/30/08 18:00 & 2.293 & 0.833 & 0.362 & 162.671 & 1.441 & 0.095 & 0.028955 & 0.013776 & 0.486564 & 218.370 \\
\hline 10/30/08 19:00 & 2.3 & 0.840 & 0.370 & 166.078 & 1.444 & 0.098 & 0.029869 & 0.014412 & 0.509013 & 228.445 \\
\hline 10/30/08 20:00 & 2.307 & 0.847 & 0.378 & 169.527 & 1.444 & 0.098 & 0.029869 & 0.014412 & 0.509013 & 228.445 \\
\hline 10/30/08 21:00 & 2.312 & 0.852 & 0.383 & 172.017 & 1.446 & 0.1 & 0.030479 & 0.014841 & 0.524166 & 235.246 \\
\hline 10/30/08 22:00 & 2.319 & 0.859 & 0.391 & 175.539 & 1.446 & 0.1 & 0.030479 & 0.014841 & 0.524166 & 235.246 \\
\hline 10/30/08 23:00 & 2.319 & 0.859 & 0.391 & 175.539 & 1.442 & 0.096 & 0.029259 & 0.013987 & 0.494010 & 221.711 \\
\hline 10/31/08 0:00 & 2.319 & 0.859 & 0.391 & 175.539 & 1.442 & 0.096 & 0.029259 & 0.013987 & 0.494010 & 221.711 \\
\hline 10/31/08 1:00 & 2.326 & 0.866 & 0.399 & 179.104 & 1.444 & 0.098 & 0.029869 & 0.014412 & 0.509013 & 228.445 \\
\hline 10/31/08 2:00 & 2.336 & 0.876 & 0.411 & 184.272 & 1.442 & 0.096 & 0.029259 & 0.013987 & 0.494010 & 221.711 \\
\hline 10/31/08 3:00 & 2.336 & 0.876 & 0.411 & 184.272 & 1.442 & 0.096 & 0.029259 & 0.013987 & 0.494010 & 221.711 \\
\hline 10/31/08 4:00 & 2.334 & 0.874 & 0.408 & 183.231 & 1.44 & 0.094 & 0.028650 & 0.013566 & 0.479156 & 215.045 \\
\hline
\end{tabular}




\begin{tabular}{|c|c|c|c|c|c|c|c|c|c|c|}
\hline \multirow[b]{3}{*}{ Date Time } & \multicolumn{4}{|c|}{ V-Notch Weir } & \multicolumn{6}{|c|}{ R-Notch Weir } \\
\hline & \multicolumn{2}{|c|}{ Measured Water Level } & \multicolumn{2}{|c|}{$\begin{array}{c}\text { Calculated Rate } \\
\left(\mathrm{ft}^{3} / \mathrm{s}=448.8 \mathrm{gpm}\right)\end{array}$} & \multicolumn{3}{|c|}{ Measured Water Level } & \multicolumn{3}{|c|}{$\begin{array}{c}\text { Calculated Rate } \\
\left(\mathrm{ft}^{3} / \mathrm{s}=448.8 \mathrm{gpm}\right)\end{array}$} \\
\hline & $\begin{array}{c}\text { (ft above } \\
\text { sensor) }\end{array}$ & $\begin{array}{c}\text { (ft above } \\
\text { notch) }\end{array}$ & $\left(\mathrm{ft}^{3} / \mathrm{s}\right)$ & (gpm) & $\begin{array}{c}\text { (ft above } \\
\text { sensor) }\end{array}$ & $\begin{array}{c}\text { (ft above } \\
\text { log) }\end{array}$ & $\begin{array}{c}\text { (m above } \\
\text { log) }\end{array}$ & $\left(\mathrm{m}^{3} / \mathrm{s}\right)$ & $\left(\mathrm{ft}^{3} / \mathrm{s}\right)$ & (gpm) \\
\hline 10/31/08 5:00 & 2.339 & 0.879 & 0.414 & 185.839 & 1.442 & 0.096 & 0.029259 & 0.013987 & 0.494010 & 221.711 \\
\hline 10/31/08 6:00 & 2.341 & 0.881 & 0.416 & 186.889 & 1.442 & 0.096 & 0.029259 & 0.013987 & 0.494010 & 221.711 \\
\hline 10/31/08 7:00 & 2.339 & 0.879 & 0.414 & 185.839 & 1.439 & 0.093 & 0.028345 & 0.013358 & 0.471786 & 211.738 \\
\hline 10/31/08 8:00 & 2.342 & 0.882 & 0.418 & 187.415 & 1.439 & 0.093 & 0.028345 & 0.013358 & 0.471786 & 211.738 \\
\hline 10/31/08 9:00 & 2.344 & 0.884 & 0.420 & 188.469 & 1.444 & 0.098 & 0.029869 & 0.014412 & 0.509013 & 228.445 \\
\hline 10/31/08 10:00 & 2.341 & 0.881 & 0.416 & 186.889 & 1.441 & 0.095 & 0.028955 & 0.013776 & 0.486564 & 218.370 \\
\hline 10/31/08 11:00 & 2.343 & 0.883 & 0.419 & 187.942 & 1.441 & 0.095 & 0.028955 & 0.013776 & 0.486564 & 218.370 \\
\hline 10/31/08 12:00 & 2.331 & 0.871 & 0.405 & 181.677 & 1.439 & 0.093 & 0.028345 & 0.013358 & 0.471786 & 211.738 \\
\hline 10/31/08 13:00 & 2.321 & 0.861 & 0.393 & 176.553 & 1.442 & 0.096 & 0.029259 & 0.013987 & 0.494010 & 221.711 \\
\hline 10/31/08 14:00 & 2.323 & 0.863 & 0.396 & 177.571 & 1.446 & 0.1 & 0.030479 & 0.014841 & 0.524166 & 235.246 \\
\hline 10/31/08 15:00 & 2.321 & 0.861 & 0.393 & 176.553 & 1.444 & 0.098 & 0.029869 & 0.014412 & 0.509013 & 228.445 \\
\hline 10/31/08 16:00 & 2.323 & 0.863 & 0.396 & 177.571 & 1.447 & 0.101 & 0.030783 & 0.015057 & 0.531798 & 238.671 \\
\hline 10/31/08 17:00 & 2.326 & 0.866 & 0.399 & 179.104 & 1.447 & 0.101 & 0.030783 & 0.015057 & 0.531798 & 238.671 \\
\hline 10/31/08 18:00 & 2.323 & 0.863 & 0.396 & 177.571 & 1.444 & 0.098 & 0.029869 & 0.014412 & 0.509013 & 228.445 \\
\hline 10/31/08 19:00 & 2.33 & 0.870 & 0.404 & 181.160 & 1.444 & 0.098 & 0.029869 & 0.014412 & 0.509013 & 228.445 \\
\hline 10/31/08 20:00 & 2.335 & 0.875 & 0.409 & 183.751 & 1.445 & 0.099 & 0.030174 & 0.014626 & 0.516571 & 231.837 \\
\hline 10/31/08 21:00 & 2.333 & 0.873 & 0.407 & 182.712 & 1.442 & 0.096 & 0.029259 & 0.013987 & 0.494010 & 221.711 \\
\hline 10/31/08 22:00 & 2.333 & 0.873 & 0.407 & 182.712 & 1.438 & 0.092 & 0.028040 & 0.013150 & 0.464455 & 208.447 \\
\hline 10/31/08 23:00 & 2.338 & 0.878 & 0.413 & 185.316 & 1.44 & 0.094 & 0.028650 & 0.013566 & 0.479156 & 215.045 \\
\hline 11/1/08 0:00 & 2.341 & 0.881 & 0.416 & 186.889 & 1.44 & 0.094 & 0.028650 & 0.013566 & 0.479156 & 215.045 \\
\hline 11/1/08 1:00 & 2.341 & 0.881 & 0.416 & 186.889 & 1.44 & 0.094 & 0.028650 & 0.013566 & 0.479156 & 215.045 \\
\hline 11/1/08 2:00 & 2.341 & 0.881 & 0.416 & 186.889 & 1.44 & 0.094 & 0.028650 & 0.013566 & 0.479156 & 215.045 \\
\hline 11/1/08 3:00 & 2.342 & 0.882 & 0.418 & 187.415 & 1.44 & 0.094 & 0.028650 & 0.013566 & 0.479156 & 215.045 \\
\hline 11/1/08 4:00 & 2.342 & 0.882 & 0.418 & 187.415 & 1.442 & 0.096 & 0.029259 & 0.013987 & 0.494010 & 221.711 \\
\hline 11/1/08 5:00 & 2.342 & 0.882 & 0.418 & 187.415 & 1.442 & 0.096 & 0.029259 & 0.013987 & 0.494010 & 221.711 \\
\hline
\end{tabular}




\begin{tabular}{|c|c|c|c|c|c|c|c|c|c|c|}
\hline \multirow[b]{3}{*}{ Date Time } & \multicolumn{4}{|c|}{ V-Notch Weir } & \multicolumn{6}{|c|}{ R-Notch Weir } \\
\hline & \multicolumn{2}{|c|}{ Measured Water Level } & \multicolumn{2}{|c|}{$\begin{array}{c}\text { Calculated Rate } \\
\left(\mathrm{ft}^{3} / \mathrm{s}=448.8 \mathrm{gpm}\right)\end{array}$} & \multicolumn{3}{|c|}{ Measured Water Level } & \multicolumn{3}{|c|}{$\begin{array}{c}\text { Calculated Rate } \\
\left(\mathrm{ft}^{3} / \mathrm{s}=448.8 \mathrm{gpm}\right)\end{array}$} \\
\hline & $\begin{array}{l}\text { (ft above } \\
\text { sensor) }\end{array}$ & $\begin{array}{l}\text { (ft above } \\
\text { notch) }\end{array}$ & $\left(\mathrm{ft}^{3} / \mathrm{s}\right)$ & (gpm) & $\begin{array}{l}\text { (ft above } \\
\text { sensor) }\end{array}$ & $\begin{array}{l}\text { (ft above } \\
\text { log) }\end{array}$ & $\begin{array}{c}\text { (m above } \\
\text { log) }\end{array}$ & $\left(\mathrm{m}^{3} / \mathrm{s}\right)$ & $\left(\mathrm{ft}^{3} / \mathrm{s}\right)$ & (gpm) \\
\hline 11/1/08 6:00 & 2.345 & 0.885 & 0.421 & 188.998 & 1.442 & 0.096 & 0.029259 & 0.013987 & 0.494010 & 221.711 \\
\hline 11/1/08 7:00 & 2.345 & 0.885 & 0.421 & 188.998 & 1.442 & 0.096 & 0.029259 & 0.013987 & 0.494010 & 221.711 \\
\hline 11/1/08 8:00 & 2.345 & 0.885 & 0.421 & 188.998 & 1.442 & 0.096 & 0.029259 & 0.013987 & 0.494010 & 221.711 \\
\hline 11/1/08 9:00 & 2.35 & 0.890 & 0.427 & 191.655 & 1.442 & 0.096 & 0.029259 & 0.013987 & 0.494010 & 221.711 \\
\hline 11/1/08 10:00 & 2.347 & 0.887 & 0.423 & 190.058 & 1.442 & 0.096 & 0.029259 & 0.013987 & 0.494010 & 221.711 \\
\hline 11/1/08 11:00 & 2.347 & 0.887 & 0.423 & 190.058 & 1.441 & 0.095 & 0.028955 & 0.013776 & 0.486564 & 218.370 \\
\hline 11/1/08 12:00 & 2.339 & 0.879 & 0.414 & 185.839 & 1.444 & 0.098 & 0.029869 & 0.014412 & 0.509013 & 228.445 \\
\hline 11/1/08 13:00 & 2.326 & 0.866 & 0.399 & 179.104 & 1.444 & 0.098 & 0.029869 & 0.014412 & 0.509013 & 228.445 \\
\hline 11/1/08 14:00 & 2.328 & 0.868 & 0.401 & 180.130 & 1.446 & 0.1 & 0.030479 & 0.014841 & 0.524166 & 235.246 \\
\hline 11/1/08 15:00 & 2.326 & 0.866 & 0.399 & 179.104 & 1.449 & 0.103 & 0.031393 & 0.015492 & 0.547171 & 245.570 \\
\hline 11/1/08 16:00 & 2.333 & 0.873 & 0.407 & 182.712 & 1.446 & 0.1 & 0.030479 & 0.014841 & 0.524166 & 235.246 \\
\hline 11/1/08 17:00 & 2.335 & 0.875 & 0.409 & 183.751 & 1.449 & 0.103 & 0.031393 & 0.015492 & 0.547171 & 245.570 \\
\hline 11/1/08 18:00 & 2.34 & 0.880 & 0.415 & 186.363 & 1.447 & 0.101 & 0.030783 & 0.015057 & 0.531798 & 238.671 \\
\hline 11/1/08 19:00 & 2.342 & 0.882 & 0.418 & 187.415 & 1.447 & 0.101 & 0.030783 & 0.015057 & 0.531798 & 238.671 \\
\hline 11/1/08 20:00 & 2.338 & 0.878 & 0.413 & 185.316 & 1.442 & 0.096 & 0.029259 & 0.013987 & 0.494010 & 221.711 \\
\hline 11/1/08 21:00 & 2.335 & 0.875 & 0.409 & 183.751 & 1.442 & 0.096 & 0.029259 & 0.013987 & 0.494010 & 221.711 \\
\hline 11/1/08 22:00 & 2.343 & 0.883 & 0.419 & 187.942 & 1.444 & 0.098 & 0.029869 & 0.014412 & 0.509013 & 228.445 \\
\hline 11/1/08 23:00 & 2.347 & 0.887 & 0.423 & 190.058 & 1.442 & 0.096 & 0.029259 & 0.013987 & 0.494010 & 221.711 \\
\hline 11/2/08 0:00 & 2.345 & 0.885 & 0.421 & 188.998 & 1.444 & 0.098 & 0.029869 & 0.014412 & 0.509013 & 228.445 \\
\hline 11/2/08 1:00 & 2.343 & 0.883 & 0.419 & 187.942 & 1.447 & 0.101 & 0.030783 & 0.015057 & 0.531798 & 238.671 \\
\hline 11/2/08 2:00 & 2.357 & 0.897 & 0.435 & 195.412 & 1.447 & 0.101 & 0.030783 & 0.015057 & 0.531798 & 238.671 \\
\hline 11/2/08 3:00 & 2.355 & 0.895 & 0.433 & 194.334 & 1.447 & 0.101 & 0.030783 & 0.015057 & 0.531798 & 238.671 \\
\hline 11/2/08 4:00 & 2.353 & 0.893 & 0.431 & 193.260 & 1.447 & 0.101 & 0.030783 & 0.015057 & 0.531798 & 238.671 \\
\hline 11/2/08 5:00 & 2.362 & 0.902 & 0.441 & 198.123 & 1.444 & 0.098 & 0.029869 & 0.014412 & 0.509013 & 228.445 \\
\hline 11/2/08 6:00 & 2.358 & 0.898 & 0.437 & 195.953 & 1.444 & 0.098 & 0.029869 & 0.014412 & 0.509013 & 228.445 \\
\hline
\end{tabular}




\begin{tabular}{|c|c|c|c|c|c|c|c|c|c|c|}
\hline \multirow[b]{3}{*}{ Date Time } & \multicolumn{4}{|c|}{ V-Notch Weir } & \multicolumn{6}{|c|}{ R-Notch Weir } \\
\hline & \multicolumn{2}{|c|}{ Measured Water Level } & \multicolumn{2}{|c|}{$\begin{array}{c}\text { Calculated Rate } \\
\left(\mathrm{ft}^{3} / \mathrm{s}=448.8 \mathrm{gpm}\right)\end{array}$} & \multicolumn{3}{|c|}{ Measured Water Level } & \multicolumn{3}{|c|}{$\begin{array}{c}\text { Calculated Rate } \\
\left(\mathrm{ft}^{3} / \mathrm{s}=448.8 \mathrm{gpm}\right)\end{array}$} \\
\hline & $\begin{array}{c}\text { (ft above } \\
\text { sensor) }\end{array}$ & $\begin{array}{c}\text { (ft above } \\
\text { notch) }\end{array}$ & $\left(\mathrm{ft}^{3} / \mathrm{s}\right)$ & (gpm) & $\begin{array}{l}\text { (ft above } \\
\text { sensor) }\end{array}$ & $\begin{array}{c}\text { (ft above } \\
\text { log) }\end{array}$ & $\begin{array}{c}\text { (m above } \\
\text { log) }\end{array}$ & $\left(\mathrm{m}^{3} / \mathrm{s}\right)$ & $\left(\mathrm{ft}^{3} / \mathrm{s}\right)$ & (gpm) \\
\hline 11/2/08 7:00 & 2.355 & 0.895 & 0.433 & 194.334 & 1.447 & 0.101 & 0.030783 & 0.015057 & 0.531798 & 238.671 \\
\hline 11/2/08 8:00 & 2.36 & 0.900 & 0.439 & 197.036 & 1.447 & 0.101 & 0.030783 & 0.015057 & 0.531798 & 238.671 \\
\hline 11/2/08 9:00 & 2.36 & 0.900 & 0.439 & 197.036 & 1.444 & 0.098 & 0.029869 & 0.014412 & 0.509013 & 228.445 \\
\hline 11/2/08 10:00 & 2.379 & 0.919 & 0.462 & 207.507 & 1.444 & 0.098 & 0.029869 & 0.014412 & 0.509013 & 228.445 \\
\hline 11/2/08 11:00 & 2.348 & 0.888 & 0.425 & 190.590 & 1.447 & 0.101 & 0.030783 & 0.015057 & 0.531798 & 238.671 \\
\hline 11/2/08 12:00 & 2.34 & 0.880 & 0.415 & 186.363 & 1.444 & 0.098 & 0.029869 & 0.014412 & 0.509013 & 228.445 \\
\hline 11/2/08 13:00 & 2.326 & 0.866 & 0.399 & 179.104 & 1.447 & 0.101 & 0.030783 & 0.015057 & 0.531798 & 238.671 \\
\hline 11/2/08 14:00 & 2.33 & 0.870 & 0.404 & 181.160 & 1.449 & 0.103 & 0.031393 & 0.015492 & 0.547171 & 245.570 \\
\hline 11/2/08 15:00 & 2.323 & 0.863 & 0.396 & 177.571 & 1.437 & 0.091 & 0.027735 & 0.012943 & 0.457162 & 205.174 \\
\hline 11/2/08 16:00 & 2.319 & 0.859 & 0.391 & 175.539 & 1.44 & 0.094 & 0.028650 & 0.013566 & 0.479156 & 215.045 \\
\hline 11/2/08 17:00 & 2.318 & 0.858 & 0.390 & 175.033 & 1.445 & 0.099 & 0.030174 & 0.014626 & 0.516571 & 231.837 \\
\hline 11/2/08 18:00 & 2.328 & 0.868 & 0.401 & 180.130 & 1.447 & 0.101 & 0.030783 & 0.015057 & 0.531798 & 238.671 \\
\hline 11/2/08 19:00 & 2.335 & 0.875 & 0.409 & 183.751 & 1.445 & 0.099 & 0.030174 & 0.014626 & 0.516571 & 231.837 \\
\hline 11/2/08 20:00 & 2.33 & 0.870 & 0.404 & 181.160 & 1.447 & 0.101 & 0.030783 & 0.015057 & 0.531798 & 238.671 \\
\hline 11/2/08 21:00 & 2.342 & 0.882 & 0.418 & 187.415 & 1.447 & 0.101 & 0.030783 & 0.015057 & 0.531798 & 238.671 \\
\hline 11/2/08 22:00 & 2.349 & 0.889 & 0.426 & 191.122 & 1.44 & 0.094 & 0.028650 & 0.013566 & 0.479156 & 215.045 \\
\hline 11/2/08 23:00 & 2.345 & 0.885 & 0.421 & 188.998 & 1.44 & 0.094 & 0.028650 & 0.013566 & 0.479156 & 215.045 \\
\hline 11/3/08 0:00 & 2.347 & 0.887 & 0.423 & 190.058 & 1.443 & 0.097 & 0.029564 & 0.014199 & 0.501493 & 225.070 \\
\hline 11/3/08 1:00 & 2.349 & 0.889 & 0.426 & 191.122 & 1.443 & 0.097 & 0.029564 & 0.014199 & 0.501493 & 225.070 \\
\hline 11/3/08 2:00 & 2.35 & 0.890 & 0.427 & 191.655 & 1.443 & 0.097 & 0.029564 & 0.014199 & 0.501493 & 225.070 \\
\hline 11/3/08 3:00 & 2.357 & 0.897 & 0.435 & 195.412 & 1.445 & 0.099 & 0.030174 & 0.014626 & 0.516571 & 231.837 \\
\hline 11/3/08 4:00 & 2.359 & 0.899 & 0.438 & 196.494 & 1.448 & 0.102 & 0.031088 & 0.015274 & 0.539466 & 242.112 \\
\hline 11/3/08 5:00 & 2.359 & 0.899 & 0.438 & 196.494 & 1.448 & 0.102 & 0.031088 & 0.015274 & 0.539466 & 242.112 \\
\hline 11/3/08 6:00 & 2.359 & 0.899 & 0.438 & 196.494 & 1.445 & 0.099 & 0.030174 & 0.014626 & 0.516571 & 231.837 \\
\hline 11/3/08 7:00 & 2.359 & 0.899 & 0.438 & 196.494 & 1.447 & 0.101 & 0.030783 & 0.015057 & 0.531798 & 238.671 \\
\hline
\end{tabular}




\begin{tabular}{|c|c|c|c|c|c|c|c|c|c|c|}
\hline \multirow[b]{3}{*}{ Date } & \multicolumn{4}{|c|}{ V-Notch Weir } & \multicolumn{6}{|c|}{ R-Notch Weir } \\
\hline & \multicolumn{2}{|c|}{ Measured Water Level } & \multicolumn{2}{|c|}{$\begin{array}{c}\text { Calculated Rate } \\
\left(\mathrm{ft}^{3} / \mathrm{s}=448.8 \mathrm{gpm}\right)\end{array}$} & \multicolumn{3}{|c|}{ Measured Water Level } & \multicolumn{3}{|c|}{$\begin{array}{c}\text { Calculated Rate } \\
\left(\mathrm{ft}^{3} / \mathrm{s}=448.8 \mathrm{gpm}\right)\end{array}$} \\
\hline & $\begin{array}{c}\text { (ft above } \\
\text { sensor) }\end{array}$ & $\begin{array}{c}\text { (ft above } \\
\text { notch) }\end{array}$ & $\left(\mathrm{ft}^{3} / \mathrm{s}\right)$ & (gpm) & $\begin{array}{l}\text { (ft above } \\
\text { sensor) }\end{array}$ & $\begin{array}{c}\text { (ft above } \\
\text { log) }\end{array}$ & $\begin{array}{c}\text { (m above } \\
\text { log) }\end{array}$ & $\left(\mathrm{m}^{3} / \mathrm{s}\right)$ & $\left(\mathrm{ft}^{3} / \mathrm{s}\right)$ & (gpm) \\
\hline 11/3/08 8:00 & 2.362 & 0.902 & 0.441 & 198.123 & 1.447 & 0.101 & 0.030783 & 0.015057 & 0.531798 & 238.671 \\
\hline 11/3/08 9:00 & 2.357 & 0.897 & 0.435 & 195.412 & 1.447 & 0.101 & 0.030783 & 0.015057 & 0.531798 & 238.671 \\
\hline 11/3/08 10:00 & 2.357 & 0.897 & 0.435 & 195.412 & 1.447 & 0.101 & 0.030783 & 0.015057 & 0.531798 & 238.671 \\
\hline 11/3/08 11:00 & 2.349 & 0.889 & 0.426 & 191.122 & 1.45 & 0.104 & 0.031698 & 0.015711 & 0.554913 & 249.045 \\
\hline 11/3/08 12:00 & 2.328 & 0.868 & 0.401 & 180.130 & 1.447 & 0.101 & 0.030783 & 0.015057 & 0.531798 & 238.671 \\
\hline 11/3/08 13:00 & 2.208 & 0.748 & 0.278 & 124.627 & 1.447 & 0.101 & 0.030783 & 0.015057 & 0.531798 & 238.671 \\
\hline 11/3/08 14:00 & 2.213 & 0.753 & 0.282 & 126.698 & 1.45 & 0.104 & 0.031698 & 0.015711 & 0.554913 & 249.045 \\
\hline 11/3/08 15:00 & 2.206 & 0.746 & 0.276 & 123.804 & 1.438 & 0.092 & 0.028040 & 0.013150 & 0.464455 & 208.447 \\
\hline 11/3/08 16:00 & 2.208 & 0.748 & 0.278 & 124.627 & 1.441 & 0.095 & 0.028955 & 0.013776 & 0.486564 & 218.370 \\
\hline 11/3/08 17:00 & 2.199 & 0.739 & 0.269 & 120.950 & 1.446 & 0.1 & 0.030479 & 0.014841 & 0.524166 & 235.246 \\
\hline 11/3/08 18:00 & 2.206 & 0.746 & 0.276 & 123.804 & 1.446 & 0.1 & 0.030479 & 0.014841 & 0.524166 & 235.246 \\
\hline 11/3/08 19:00 & 2.203 & 0.743 & 0.273 & 122.576 & 1.446 & 0.1 & 0.030479 & 0.014841 & 0.524166 & 235.246 \\
\hline 11/3/08 20:00 & 2.206 & 0.746 & 0.276 & 123.804 & 1.446 & 0.1 & 0.030479 & 0.014841 & 0.524166 & 235.246 \\
\hline 11/3/08 21:00 & 2.206 & 0.746 & 0.276 & 123.804 & 1.446 & 0.1 & 0.030479 & 0.014841 & 0.524166 & 235.246 \\
\hline 11/3/08 22:00 & 2.206 & 0.746 & 0.276 & 123.804 & 1.446 & 0.1 & 0.030479 & 0.014841 & 0.524166 & 235.246 \\
\hline 11/3/08 23:00 & 2.206 & 0.746 & 0.276 & 123.804 & 1.446 & 0.1 & 0.030479 & 0.014841 & 0.524166 & 235.246 \\
\hline 11/4/08 0:00 & 2.208 & 0.748 & 0.278 & 124.627 & 1.446 & 0.1 & 0.030479 & 0.014841 & 0.524166 & 235.246 \\
\hline 11/4/08 1:00 & 2.208 & 0.748 & 0.278 & 124.627 & 1.446 & 0.1 & 0.030479 & 0.014841 & 0.524166 & 235.246 \\
\hline 11/4/08 2:00 & 2.206 & 0.746 & 0.276 & 123.804 & 1.437 & 0.091 & 0.027735 & 0.012943 & 0.457162 & 205.174 \\
\hline 11/4/08 3:00 & 2.206 & 0.746 & 0.276 & 123.804 & 1.441 & 0.095 & 0.028955 & 0.013776 & 0.486564 & 218.370 \\
\hline 11/4/08 4:00 & 2.208 & 0.748 & 0.278 & 124.627 & 1.444 & 0.098 & 0.029869 & 0.014412 & 0.509013 & 228.445 \\
\hline 11/4/08 5:00 & 2.208 & 0.748 & 0.278 & 124.627 & 1.444 & 0.098 & 0.029869 & 0.014412 & 0.509013 & 228.445 \\
\hline 11/4/08 6:00 & 2.211 & 0.751 & 0.280 & 125.867 & 1.444 & 0.098 & 0.029869 & 0.014412 & 0.509013 & 228.445 \\
\hline 11/4/08 7:00 & 2.209 & 0.749 & 0.279 & 125.039 & 1.446 & 0.1 & 0.030479 & 0.014841 & 0.524166 & 235.246 \\
\hline 11/4/08 8:00 & 2.209 & 0.749 & 0.279 & 125.039 & 1.446 & 0.1 & 0.030479 & 0.014841 & 0.524166 & 235.246 \\
\hline
\end{tabular}




\begin{tabular}{|c|c|c|c|c|c|c|c|c|c|c|}
\hline \multirow[b]{3}{*}{ Date Time } & \multicolumn{4}{|c|}{ V-Notch Weir } & \multicolumn{6}{|c|}{ R-Notch Weir } \\
\hline & \multicolumn{2}{|c|}{ Measured Water Level } & \multicolumn{2}{|c|}{$\begin{array}{c}\text { Calculated Rate } \\
\left(\mathrm{ft}^{3} / \mathrm{s}=448.8 \mathrm{gpm}\right)\end{array}$} & \multicolumn{3}{|c|}{ Measured Water Level } & \multicolumn{3}{|c|}{$\begin{array}{c}\text { Calculated Rate } \\
\left(\mathrm{ft}^{3} / \mathrm{s}=448.8 \mathrm{gpm}\right)\end{array}$} \\
\hline & $\begin{array}{l}\text { (ft above } \\
\text { sensor) }\end{array}$ & $\begin{array}{l}\text { (ft above } \\
\text { notch) }\end{array}$ & $\left(\mathrm{ft}^{3} / \mathrm{s}\right)$ & (gpm) & $\begin{array}{l}\text { (ft above } \\
\text { sensor) }\end{array}$ & $\begin{array}{l}\text { (ft above } \\
\text { log) }\end{array}$ & $\begin{array}{c}\text { (m above } \\
\log )\end{array}$ & $\left(\mathrm{m}^{3} / \mathrm{s}\right)$ & $\left(\mathrm{ft}^{3} / \mathrm{s}\right)$ & (gpm) \\
\hline 11/4/08 9:00 & 2.209 & 0.749 & 0.279 & 125.039 & 1.446 & 0.1 & 0.030479 & 0.014841 & 0.524166 & 235.246 \\
\hline 11/4/08 10:00 & 2.209 & 0.749 & 0.279 & 125.039 & 1.446 & 0.1 & 0.030479 & 0.014841 & 0.524166 & 235.246 \\
\hline 11/4/08 11:00 & 2.211 & 0.751 & 0.280 & 125.867 & 1.451 & 0.105 & 0.032002 & 0.015931 & 0.562690 & 252.535 \\
\hline 11/4/08 12:00 & 2.203 & 0.743 & 0.273 & 122.576 & 1.448 & 0.102 & 0.031088 & 0.015274 & 0.539466 & 242.112 \\
\hline 11/4/08 13:00 & 2.203 & 0.743 & 0.273 & 122.576 & 1.451 & 0.105 & 0.032002 & 0.015931 & 0.562690 & 252.535 \\
\hline 11/4/08 14:00 & 2.201 & 0.741 & 0.271 & 121.761 & 1.444 & 0.098 & 0.029869 & 0.014412 & 0.509013 & 228.445 \\
\hline 11/4/08 15:00 & 2.201 & 0.741 & 0.271 & 121.761 & 1.441 & 0.095 & 0.028955 & 0.013776 & 0.486564 & 218.370 \\
\hline 11/4/08 16:00 & 2.199 & 0.739 & 0.269 & 120.950 & 1.446 & 0.1 & 0.030479 & 0.014841 & 0.524166 & 235.246 \\
\hline 11/4/08 17:00 & 2.189 & 0.729 & 0.261 & 116.942 & 1.446 & 0.1 & 0.030479 & 0.014841 & 0.524166 & 235.246 \\
\hline 11/4/08 18:00 & 2.215 & 0.755 & 0.284 & 127.532 & 1.451 & 0.105 & 0.032002 & 0.015931 & 0.562690 & 252.535 \\
\hline 11/4/08 19:00 & 2.213 & 0.753 & 0.282 & 126.698 & 1.451 & 0.105 & 0.032002 & 0.015931 & 0.562690 & 252.535 \\
\hline 11/4/08 20:00 & 2.222 & 0.762 & 0.291 & 130.478 & 1.451 & 0.105 & 0.032002 & 0.015931 & 0.562690 & 252.535 \\
\hline 11/4/08 21:00 & 2.215 & 0.755 & 0.284 & 127.532 & 1.452 & 0.106 & 0.032307 & 0.016152 & 0.570504 & 256.042 \\
\hline 11/4/08 22:00 & 2.213 & 0.753 & 0.282 & 126.698 & 1.449 & 0.103 & 0.031393 & 0.015492 & 0.547171 & 245.570 \\
\hline 11/4/08 23:00 & 2.208 & 0.748 & 0.278 & 124.627 & 1.452 & 0.106 & 0.032307 & 0.016152 & 0.570504 & 256.042 \\
\hline 11/5/08 0:00 & 2.213 & 0.753 & 0.282 & 126.698 & 1.454 & 0.108 & 0.032917 & 0.016598 & 0.586238 & 263.104 \\
\hline 11/5/08 1:00 & 2.211 & 0.751 & 0.280 & 125.867 & 1.452 & 0.106 & 0.032307 & 0.016152 & 0.570504 & 256.042 \\
\hline 11/5/08 2:00 & 2.211 & 0.751 & 0.280 & 125.867 & 1.442 & 0.096 & 0.029259 & 0.013987 & 0.494010 & 221.711 \\
\hline 11/5/08 3:00 & 2.215 & 0.755 & 0.284 & 127.532 & 1.447 & 0.101 & 0.030783 & 0.015057 & 0.531798 & 238.671 \\
\hline 11/5/08 4:00 & 2.213 & 0.753 & 0.282 & 126.698 & 1.447 & 0.101 & 0.030783 & 0.015057 & 0.531798 & 238.671 \\
\hline 11/5/08 5:00 & 2.208 & 0.748 & 0.278 & 124.627 & 1.454 & 0.108 & 0.032917 & 0.016598 & 0.586238 & 263.104 \\
\hline 11/5/08 6:00 & 2.208 & 0.748 & 0.278 & 124.627 & 1.447 & 0.101 & 0.030783 & 0.015057 & 0.531798 & 238.671 \\
\hline 11/5/08 7:00 & 2.215 & 0.755 & 0.284 & 127.532 & 1.447 & 0.101 & 0.030783 & 0.015057 & 0.531798 & 238.671 \\
\hline 11/5/08 8:00 & 2.218 & 0.758 & 0.287 & 128.789 & 1.447 & 0.101 & 0.030783 & 0.015057 & 0.531798 & 238.671 \\
\hline 11/5/08 9:00 & 2.218 & 0.758 & 0.287 & 128.789 & 1.447 & 0.101 & 0.030783 & 0.015057 & 0.531798 & 238.671 \\
\hline
\end{tabular}




\begin{tabular}{|c|c|c|c|c|c|c|c|c|c|c|}
\hline \multirow[b]{3}{*}{ Date Time } & \multicolumn{4}{|c|}{ V-Notch Weir } & \multicolumn{6}{|c|}{ R-Notch Weir } \\
\hline & \multicolumn{2}{|c|}{ Measured Water Level } & \multicolumn{2}{|c|}{$\begin{array}{c}\text { Calculated Rate } \\
\left(\mathrm{ft}^{3} / \mathrm{s}=448.8 \mathrm{gpm}\right)\end{array}$} & \multicolumn{3}{|c|}{ Measured Water Level } & \multicolumn{3}{|c|}{$\begin{array}{c}\text { Calculated Rate } \\
\left(\mathrm{ft}^{3} / \mathrm{s}=448.8 \mathrm{gpm}\right)\end{array}$} \\
\hline & $\begin{array}{c}\text { (ft above } \\
\text { sensor) }\end{array}$ & $\begin{array}{c}\text { (ft above } \\
\text { notch) }\end{array}$ & $\left(\mathrm{ft}^{3} / \mathrm{s}\right)$ & (gpm) & $\begin{array}{l}\text { (ft above } \\
\text { sensor) }\end{array}$ & $\begin{array}{c}\text { (ft above } \\
\text { log) }\end{array}$ & $\begin{array}{c}\text { (m above } \\
\text { log) }\end{array}$ & $\left(\mathrm{m}^{3} / \mathrm{s}\right)$ & $\left(\mathrm{ft}^{3} / \mathrm{s}\right)$ & (gpm) \\
\hline 11/5/08 10:00 & 2.215 & 0.755 & 0.284 & 127.532 & 1.449 & 0.103 & 0.031393 & 0.015492 & 0.547171 & 245.570 \\
\hline 11/5/08 11:00 & 2.213 & 0.753 & 0.282 & 126.698 & 1.446 & 0.1 & 0.030479 & 0.014841 & 0.524166 & 235.246 \\
\hline 11/5/08 12:00 & 2.213 & 0.753 & 0.282 & 126.698 & 1.446 & 0.1 & 0.030479 & 0.014841 & 0.524166 & 235.246 \\
\hline 11/5/08 13:00 & 2.156 & 0.696 & 0.232 & 104.286 & 1.444 & 0.098 & 0.029869 & 0.014412 & 0.509013 & 228.445 \\
\hline 11/5/08 14:00 & 2.149 & 0.689 & 0.227 & 101.713 & 1.444 & 0.098 & 0.029869 & 0.014412 & 0.509013 & 228.445 \\
\hline 11/5/08 15:00 & 2.156 & 0.696 & 0.232 & 104.286 & 1.444 & 0.098 & 0.029869 & 0.014412 & 0.509013 & 228.445 \\
\hline 11/5/08 16:00 & 2.163 & 0.703 & 0.238 & 106.898 & 1.447 & 0.101 & 0.030783 & 0.015057 & 0.531798 & 238.671 \\
\hline 11/5/08 17:00 & 2.161 & 0.701 & 0.237 & 106.148 & 1.447 & 0.101 & 0.030783 & 0.015057 & 0.531798 & 238.671 \\
\hline 11/5/08 18:00 & 2.159 & 0.699 & 0.235 & 105.401 & 1.444 & 0.098 & 0.029869 & 0.014412 & 0.509013 & 228.445 \\
\hline 11/5/08 19:00 & 2.194 & 0.734 & 0.265 & 118.936 & 1.449 & 0.103 & 0.031393 & 0.015492 & 0.547171 & 245.570 \\
\hline 11/5/08 20:00 & 2.234 & 0.774 & 0.302 & 135.621 & 1.452 & 0.106 & 0.032307 & 0.016152 & 0.570504 & 256.042 \\
\hline 11/5/08 21:00 & 2.21 & 0.750 & 0.280 & 125.453 & 1.447 & 0.101 & 0.030783 & 0.015057 & 0.531798 & 238.671 \\
\hline 11/5/08 22:00 & 2.189 & 0.729 & 0.261 & 116.942 & 1.447 & 0.101 & 0.030783 & 0.015057 & 0.531798 & 238.671 \\
\hline 11/5/08 23:00 & 2.18 & 0.720 & 0.253 & 113.404 & 1.445 & 0.099 & 0.030174 & 0.014626 & 0.516571 & 231.837 \\
\hline 11/6/08 0:00 & 2.164 & 0.704 & 0.239 & 107.275 & 1.442 & 0.096 & 0.029259 & 0.013987 & 0.494010 & 221.711 \\
\hline 11/6/08 1:00 & 2.168 & 0.708 & 0.242 & 108.788 & 1.442 & 0.096 & 0.029259 & 0.013987 & 0.494010 & 221.711 \\
\hline 11/6/08 2:00 & 2.162 & 0.702 & 0.237 & 106.523 & 1.44 & 0.094 & 0.028650 & 0.013566 & 0.479156 & 215.045 \\
\hline 11/6/08 3:00 & 2.152 & 0.692 & 0.229 & 102.811 & 1.44 & 0.094 & 0.028650 & 0.013566 & 0.479156 & 215.045 \\
\hline 11/6/08 4:00 & 2.155 & 0.695 & 0.232 & 103.916 & 1.442 & 0.096 & 0.029259 & 0.013987 & 0.494010 & 221.711 \\
\hline 11/6/08 5:00 & 2.16 & 0.700 & 0.236 & 105.774 & 1.44 & 0.094 & 0.028650 & 0.013566 & 0.479156 & 215.045 \\
\hline 11/6/08 6:00 & 2.16 & 0.700 & 0.236 & 105.774 & 1.439 & 0.093 & 0.028345 & 0.013358 & 0.471786 & 211.738 \\
\hline 11/6/08 7:00 & 2.162 & 0.702 & 0.237 & 106.523 & 1.442 & 0.096 & 0.029259 & 0.013987 & 0.494010 & 221.711 \\
\hline 11/6/08 8:00 & 2.16 & 0.700 & 0.236 & 105.774 & 1.441 & 0.095 & 0.028955 & 0.013776 & 0.486564 & 218.370 \\
\hline 11/6/08 9:00 & 2.163 & 0.703 & 0.238 & 106.898 & 1.441 & 0.095 & 0.028955 & 0.013776 & 0.486564 & 218.370 \\
\hline 11/6/08 10:00 & 2.156 & 0.696 & 0.232 & 104.286 & 1.439 & 0.093 & 0.028345 & 0.013358 & 0.471786 & 211.738 \\
\hline
\end{tabular}




\begin{tabular}{|c|c|c|c|c|c|c|c|c|c|c|}
\hline \multirow[b]{3}{*}{ Date Time } & \multicolumn{4}{|c|}{ V-Notch Weir } & \multicolumn{6}{|c|}{ R-Notch Weir } \\
\hline & \multicolumn{2}{|c|}{ Measured Water Level } & \multicolumn{2}{|c|}{$\begin{array}{c}\text { Calculated Rate } \\
\left(\mathrm{ft}^{3} / \mathrm{s}=448.8 \mathrm{gpm}\right)\end{array}$} & \multicolumn{3}{|c|}{ Measured Water Level } & \multicolumn{3}{|c|}{$\begin{array}{c}\text { Calculated Rate } \\
\left(\mathrm{ft}^{3} / \mathrm{s}=448.8 \mathrm{gpm}\right)\end{array}$} \\
\hline & $\begin{array}{c}\text { (ft above } \\
\text { sensor) }\end{array}$ & $\begin{array}{c}\text { (ft above } \\
\text { notch) }\end{array}$ & $\left(\mathrm{ft}^{3} / \mathrm{s}\right)$ & (gpm) & $\begin{array}{l}\text { (ft above } \\
\text { sensor) }\end{array}$ & $\begin{array}{c}\text { (ft above } \\
\text { log) }\end{array}$ & $\begin{array}{c}\text { (m above } \\
\text { log) }\end{array}$ & $\left(\mathrm{m}^{3} / \mathrm{s}\right)$ & $\left(\mathrm{ft}^{3} / \mathrm{s}\right)$ & (gpm) \\
\hline 11/6/08 11:00 & 2.153 & 0.693 & 0.230 & 103.179 & 1.439 & 0.093 & 0.028345 & 0.013358 & 0.471786 & 211.738 \\
\hline 11/6/08 12:00 & 2.16 & 0.700 & 0.236 & 105.774 & 1.436 & 0.09 & 0.027431 & 0.012738 & 0.449907 & 201.918 \\
\hline 11/6/08 13:00 & 2.156 & 0.696 & 0.232 & 104.286 & 1.436 & 0.09 & 0.027431 & 0.012738 & 0.449907 & 201.918 \\
\hline 11/6/08 14:00 & 2.167 & 0.707 & 0.242 & 108.408 & 1.434 & 0.088 & 0.026821 & 0.012331 & 0.435514 & 195.459 \\
\hline 11/6/08 15:00 & 2.158 & 0.698 & 0.234 & 105.029 & 1.441 & 0.095 & 0.028955 & 0.013776 & 0.486564 & 218.370 \\
\hline 11/6/08 16:00 & 2.158 & 0.698 & 0.234 & 105.029 & 1.436 & 0.09 & 0.027431 & 0.012738 & 0.449907 & 201.918 \\
\hline 11/6/08 17:00 & 2.17 & 0.710 & 0.244 & 109.549 & 1.431 & 0.085 & 0.025907 & 0.011728 & 0.414220 & 185.902 \\
\hline 11/6/08 18:00 & 2.163 & 0.703 & 0.238 & 106.898 & 1.429 & 0.083 & 0.025297 & 0.011331 & 0.400223 & 179.620 \\
\hline 11/6/08 19:00 & 2.164 & 0.704 & 0.239 & 107.275 & 1.429 & 0.083 & 0.025297 & 0.011331 & 0.400223 & 179.620 \\
\hline 11/6/08 20:00 & 2.166 & 0.706 & 0.241 & 108.030 & 1.431 & 0.085 & 0.025907 & 0.011728 & 0.414220 & 185.902 \\
\hline 11/6/08 21:00 & 2.157 & 0.697 & 0.233 & 104.657 & 1.426 & 0.08 & 0.024383 & 0.010746 & 0.379531 & 170.333 \\
\hline 11/6/08 22:00 & 2.155 & 0.695 & 0.232 & 103.916 & 1.428 & 0.082 & 0.024992 & 0.011135 & 0.393285 & 176.506 \\
\hline 11/6/08 23:00 & 2.148 & 0.688 & 0.226 & 101.348 & 1.43 & 0.084 & 0.025602 & 0.011529 & 0.407202 & 182.752 \\
\hline 11/7/08 0:00 & 2.151 & 0.691 & 0.228 & 102.444 & 1.43 & 0.084 & 0.025602 & 0.011529 & 0.407202 & 182.752 \\
\hline 11/7/08 1:00 & 2.149 & 0.689 & 0.227 & 101.713 & 1.43 & 0.084 & 0.025602 & 0.011529 & 0.407202 & 182.752 \\
\hline 11/7/08 2:00 & 2.154 & 0.694 & 0.231 & 103.547 & 1.43 & 0.084 & 0.025602 & 0.011529 & 0.407202 & 182.752 \\
\hline 11/7/08 3:00 & 2.145 & 0.685 & 0.223 & 100.260 & 1.43 & 0.084 & 0.025602 & 0.011529 & 0.407202 & 182.752 \\
\hline 11/7/08 4:00 & 2.178 & 0.718 & 0.251 & 112.627 & 1.434 & 0.088 & 0.026821 & 0.012331 & 0.435514 & 195.459 \\
\hline 11/7/08 5:00 & 2.171 & 0.711 & 0.245 & 109.931 & 1.434 & 0.088 & 0.026821 & 0.012331 & 0.435514 & 195.459 \\
\hline 11/7/08 6:00 & 2.171 & 0.711 & 0.245 & 109.931 & 1.432 & 0.086 & 0.026212 & 0.011928 & 0.421279 & 189.070 \\
\hline 11/7/08 7:00 & 2.155 & 0.695 & 0.232 & 103.916 & 1.429 & 0.083 & 0.025297 & 0.011331 & 0.400223 & 179.620 \\
\hline 11/7/08 8:00 & 2.155 & 0.695 & 0.232 & 103.916 & 1.432 & 0.086 & 0.026212 & 0.011928 & 0.421279 & 189.070 \\
\hline 11/7/08 9:00 & 2.16 & 0.700 & 0.236 & 105.774 & 1.432 & 0.086 & 0.026212 & 0.011928 & 0.421279 & 189.070 \\
\hline 11/7/08 10:00 & 2.157 & 0.697 & 0.233 & 104.657 & 1.431 & 0.085 & 0.025907 & 0.011728 & 0.414220 & 185.902 \\
\hline 11/7/08 11:00 & 2.167 & 0.707 & 0.242 & 108.408 & 1.427 & 0.081 & 0.024688 & 0.010940 & 0.386387 & 173.411 \\
\hline
\end{tabular}




\begin{tabular}{|c|c|c|c|c|c|c|c|c|c|c|}
\hline \multirow[b]{3}{*}{ Date Time } & \multicolumn{4}{|c|}{ V-Notch Weir } & \multicolumn{6}{|c|}{ R-Notch Weir } \\
\hline & \multicolumn{2}{|c|}{ Measured Water Level } & \multicolumn{2}{|c|}{$\begin{array}{c}\text { Calculated Rate } \\
\left(\mathrm{ft}^{3} / \mathrm{s}=448.8 \mathrm{gpm}\right)\end{array}$} & \multicolumn{3}{|c|}{ Measured Water Level } & \multicolumn{3}{|c|}{$\begin{array}{c}\text { Calculated Rate } \\
\left(\mathrm{ft}^{3} / \mathrm{s}=448.8 \mathrm{gpm}\right)\end{array}$} \\
\hline & $\begin{array}{c}\text { (ft above } \\
\text { sensor) }\end{array}$ & $\begin{array}{c}\text { (ft above } \\
\text { notch) }\end{array}$ & $\left(\mathrm{ft}^{3} / \mathrm{s}\right)$ & (gpm) & $\begin{array}{l}\text { (ft above } \\
\text { sensor) }\end{array}$ & $\begin{array}{c}\text { (ft above } \\
\text { log) }\end{array}$ & $\begin{array}{c}\text { (m above } \\
\text { log) }\end{array}$ & $\left(\mathrm{m}^{3} / \mathrm{s}\right)$ & $\left(\mathrm{ft}^{3} / \mathrm{s}\right)$ & (gpm) \\
\hline 11/7/08 12:00 & 2.162 & 0.702 & 0.237 & 106.523 & 1.434 & 0.088 & 0.026821 & 0.012331 & 0.435514 & 195.459 \\
\hline 11/7/08 13:00 & 2.152 & 0.692 & 0.229 & 102.811 & 1.431 & 0.085 & 0.025907 & 0.011728 & 0.414220 & 185.902 \\
\hline 11/7/08 14:00 & 2.156 & 0.696 & 0.232 & 104.286 & 1.426 & 0.08 & 0.024383 & 0.010746 & 0.379531 & 170.333 \\
\hline 11/7/08 15:00 & 2.154 & 0.694 & 0.231 & 103.547 & 1.429 & 0.083 & 0.025297 & 0.011331 & 0.400223 & 179.620 \\
\hline 11/7/08 16:00 & 2.158 & 0.698 & 0.234 & 105.029 & 1.429 & 0.083 & 0.025297 & 0.011331 & 0.400223 & 179.620 \\
\hline 11/7/08 17:00 & 2.149 & 0.689 & 0.227 & 101.713 & 1.429 & 0.083 & 0.025297 & 0.011331 & 0.400223 & 179.620 \\
\hline 11/7/08 18:00 & 2.154 & 0.694 & 0.231 & 103.547 & 1.429 & 0.083 & 0.025297 & 0.011331 & 0.400223 & 179.620 \\
\hline 11/7/08 19:00 & 2.154 & 0.694 & 0.231 & 103.547 & 1.426 & 0.08 & 0.024383 & 0.010746 & 0.379531 & 170.333 \\
\hline 11/7/08 20:00 & 2.159 & 0.699 & 0.235 & 105.401 & 1.428 & 0.082 & 0.024992 & 0.011135 & 0.393285 & 176.506 \\
\hline 11/7/08 21:00 & 2.152 & 0.692 & 0.229 & 102.811 & 1.428 & 0.082 & 0.024992 & 0.011135 & 0.393285 & 176.506 \\
\hline 11/7/08 22:00 & 2.15 & 0.690 & 0.227 & 102.078 & 1.428 & 0.082 & 0.024992 & 0.011135 & 0.393285 & 176.506 \\
\hline 11/7/08 23:00 & 2.155 & 0.695 & 0.232 & 103.916 & 1.431 & 0.085 & 0.025907 & 0.011728 & 0.414220 & 185.902 \\
\hline 11/8/08 0:00 & 2.146 & 0.686 & 0.224 & 100.622 & 1.428 & 0.082 & 0.024992 & 0.011135 & 0.393285 & 176.506 \\
\hline 11/8/08 1:00 & 2.151 & 0.691 & 0.228 & 102.444 & 1.431 & 0.085 & 0.025907 & 0.011728 & 0.414220 & 185.902 \\
\hline 11/8/08 2:00 & 2.153 & 0.693 & 0.230 & 103.179 & 1.428 & 0.082 & 0.024992 & 0.011135 & 0.393285 & 176.506 \\
\hline 11/8/08 3:00 & 2.156 & 0.696 & 0.232 & 104.286 & 1.426 & 0.08 & 0.024383 & 0.010746 & 0.379531 & 170.333 \\
\hline 11/8/08 4:00 & 2.153 & 0.693 & 0.230 & 103.179 & 1.428 & 0.082 & 0.024992 & 0.011135 & 0.393285 & 176.506 \\
\hline 11/8/08 5:00 & 2.161 & 0.701 & 0.237 & 106.148 & 1.426 & 0.08 & 0.024383 & 0.010746 & 0.379531 & 170.333 \\
\hline 11/8/08 6:00 & 2.151 & 0.691 & 0.228 & 102.444 & 1.428 & 0.082 & 0.024992 & 0.011135 & 0.393285 & 176.506 \\
\hline 11/8/08 7:00 & 2.161 & 0.701 & 0.237 & 106.148 & 1.426 & 0.08 & 0.024383 & 0.010746 & 0.379531 & 170.333 \\
\hline 11/8/08 8:00 & 2.154 & 0.694 & 0.231 & 103.547 & 1.428 & 0.082 & 0.024992 & 0.011135 & 0.393285 & 176.506 \\
\hline 11/8/08 9:00 & 2.149 & 0.689 & 0.227 & 101.713 & 1.425 & 0.079 & 0.024078 & 0.010553 & 0.372715 & 167.274 \\
\hline 11/8/08 10:00 & 2.157 & 0.697 & 0.233 & 104.657 & 1.425 & 0.079 & 0.024078 & 0.010553 & 0.372715 & 167.274 \\
\hline 11/8/08 11:00 & 2.149 & 0.689 & 0.227 & 101.713 & 1.428 & 0.082 & 0.024992 & 0.011135 & 0.393285 & 176.506 \\
\hline 11/8/08 12:00 & 2.156 & 0.696 & 0.232 & 104.286 & 1.425 & 0.079 & 0.024078 & 0.010553 & 0.372715 & 167.274 \\
\hline
\end{tabular}




\begin{tabular}{|c|c|c|c|c|c|c|c|c|c|c|}
\hline \multirow[b]{3}{*}{ Date Time } & \multicolumn{4}{|c|}{ V-Notch Weir } & \multicolumn{6}{|c|}{ R-Notch Weir } \\
\hline & \multicolumn{2}{|c|}{ Measured Water Level } & \multicolumn{2}{|c|}{$\begin{array}{c}\text { Calculated Rate } \\
\left(\mathrm{ft}^{3} / \mathrm{s}=448.8 \mathrm{gpm}\right)\end{array}$} & \multicolumn{3}{|c|}{ Measured Water Level } & \multicolumn{3}{|c|}{$\begin{array}{c}\text { Calculated Rate } \\
\left(\mathrm{ft}^{3} / \mathrm{s}=448.8 \mathrm{gpm}\right)\end{array}$} \\
\hline & $\begin{array}{c}\text { (ft above } \\
\text { sensor) }\end{array}$ & $\begin{array}{c}\text { (ft above } \\
\text { notch) }\end{array}$ & $\left(\mathrm{ft}^{3} / \mathrm{s}\right)$ & (gpm) & $\begin{array}{l}\text { (ft above } \\
\text { sensor) }\end{array}$ & $\begin{array}{c}\text { (ft above } \\
\text { log) }\end{array}$ & $\begin{array}{c}\text { (m above } \\
\text { log) }\end{array}$ & $\left(\mathrm{m}^{3} / \mathrm{s}\right)$ & $\left(\mathrm{ft}^{3} / \mathrm{s}\right)$ & (gpm) \\
\hline 11/8/08 13:00 & 2.153 & 0.693 & 0.230 & 103.179 & 1.425 & 0.079 & 0.024078 & 0.010553 & 0.372715 & 167.274 \\
\hline 11/8/08 14:00 & 2.153 & 0.693 & 0.230 & 103.179 & 1.425 & 0.079 & 0.024078 & 0.010553 & 0.372715 & 167.274 \\
\hline 11/8/08 15:00 & 2.155 & 0.695 & 0.232 & 103.916 & 1.425 & 0.079 & 0.024078 & 0.010553 & 0.372715 & 167.274 \\
\hline 11/8/08 16:00 & 2.153 & 0.693 & 0.230 & 103.179 & 1.425 & 0.079 & 0.024078 & 0.010553 & 0.372715 & 167.274 \\
\hline 11/8/08 17:00 & 2.15 & 0.690 & 0.227 & 102.078 & 1.423 & 0.077 & 0.023468 & 0.010170 & 0.359208 & 161.212 \\
\hline 11/8/08 18:00 & 2.15 & 0.690 & 0.227 & 102.078 & 1.425 & 0.079 & 0.024078 & 0.010553 & 0.372715 & 167.274 \\
\hline 11/8/08 19:00 & 2.158 & 0.698 & 0.234 & 105.029 & 1.423 & 0.077 & 0.023468 & 0.010170 & 0.359208 & 161.212 \\
\hline 11/8/08 20:00 & 2.153 & 0.693 & 0.230 & 103.179 & 1.425 & 0.079 & 0.024078 & 0.010553 & 0.372715 & 167.274 \\
\hline 11/8/08 21:00 & 2.151 & 0.691 & 0.228 & 102.444 & 1.425 & 0.079 & 0.024078 & 0.010553 & 0.372715 & 167.274 \\
\hline 11/8/08 22:00 & 2.151 & 0.691 & 0.228 & 102.444 & 1.428 & 0.082 & 0.024992 & 0.011135 & 0.393285 & 176.506 \\
\hline 11/8/08 23:00 & 2.154 & 0.694 & 0.231 & 103.547 & 1.425 & 0.079 & 0.024078 & 0.010553 & 0.372715 & 167.274 \\
\hline 11/9/08 0:00 & 2.152 & 0.692 & 0.229 & 102.811 & 1.425 & 0.079 & 0.024078 & 0.010553 & 0.372715 & 167.274 \\
\hline 11/9/08 1:00 & 2.152 & 0.692 & 0.229 & 102.811 & 1.425 & 0.079 & 0.024078 & 0.010553 & 0.372715 & 167.274 \\
\hline 11/9/08 2:00 & 2.154 & 0.694 & 0.231 & 103.547 & 1.425 & 0.079 & 0.024078 & 0.010553 & 0.372715 & 167.274 \\
\hline 11/9/08 3:00 & 2.152 & 0.692 & 0.229 & 102.811 & 1.427 & 0.081 & 0.024688 & 0.010940 & 0.386387 & 173.411 \\
\hline 11/9/08 4:00 & 2.152 & 0.692 & 0.229 & 102.811 & 1.427 & 0.081 & 0.024688 & 0.010940 & 0.386387 & 173.411 \\
\hline 11/9/08 5:00 & 2.15 & 0.690 & 0.227 & 102.078 & 1.427 & 0.081 & 0.024688 & 0.010940 & 0.386387 & 173.411 \\
\hline 11/9/08 6:00 & 2.145 & 0.685 & 0.223 & 100.260 & 1.427 & 0.081 & 0.024688 & 0.010940 & 0.386387 & 173.411 \\
\hline 11/9/08 7:00 & 2.148 & 0.688 & 0.226 & 101.348 & 1.427 & 0.081 & 0.024688 & 0.010940 & 0.386387 & 173.411 \\
\hline 11/9/08 8:00 & 2.148 & 0.688 & 0.226 & 101.348 & 1.427 & 0.081 & 0.024688 & 0.010940 & 0.386387 & 173.411 \\
\hline 11/9/08 9:00 & 2.148 & 0.688 & 0.226 & 101.348 & 1.425 & 0.079 & 0.024078 & 0.010553 & 0.372715 & 167.274 \\
\hline 11/9/08 10:00 & 2.145 & 0.685 & 0.223 & 100.260 & 1.427 & 0.081 & 0.024688 & 0.010940 & 0.386387 & 173.411 \\
\hline 11/9/08 11:00 & 2.149 & 0.689 & 0.227 & 101.713 & 1.427 & 0.081 & 0.024688 & 0.010940 & 0.386387 & 173.411 \\
\hline 11/9/08 12:00 & 2.144 & 0.684 & 0.223 & 99.898 & 1.427 & 0.081 & 0.024688 & 0.010940 & 0.386387 & 173.411 \\
\hline 11/9/08 13:00 & 2.145 & 0.685 & 0.223 & 100.260 & 1.43 & 0.084 & 0.025602 & 0.011529 & 0.407202 & 182.752 \\
\hline
\end{tabular}




\begin{tabular}{|c|c|c|c|c|c|c|c|c|c|c|}
\hline \multirow[b]{3}{*}{ Date Time } & \multicolumn{4}{|c|}{ V-Notch Weir } & \multicolumn{6}{|c|}{ R-Notch Weir } \\
\hline & \multicolumn{2}{|c|}{ Measured Water Level } & \multicolumn{2}{|c|}{$\begin{array}{c}\text { Calculated Rate } \\
\left(\mathrm{ft}^{3} / \mathrm{s}=448.8 \mathrm{gpm}\right)\end{array}$} & \multicolumn{3}{|c|}{ Measured Water Level } & \multicolumn{3}{|c|}{$\begin{array}{c}\text { Calculated Rate } \\
\left(\mathrm{ft}^{3} / \mathrm{s}=448.8 \mathrm{gpm}\right)\end{array}$} \\
\hline & $\begin{array}{c}\text { (ft above } \\
\text { sensor) }\end{array}$ & $\begin{array}{c}\text { (ft above } \\
\text { notch) }\end{array}$ & $\left(\mathrm{ft}^{3} / \mathrm{s}\right)$ & (gpm) & $\begin{array}{l}\text { (ft above } \\
\text { sensor) }\end{array}$ & $\begin{array}{c}\text { (ft above } \\
\text { log) }\end{array}$ & $\begin{array}{c}\text { (m above } \\
\text { log) }\end{array}$ & $\left(\mathrm{m}^{3} / \mathrm{s}\right)$ & $\left(\mathrm{ft}^{3} / \mathrm{s}\right)$ & (gpm) \\
\hline 11/9/08 14:00 & 2.146 & 0.686 & 0.224 & 100.622 & 1.43 & 0.084 & 0.025602 & 0.011529 & 0.407202 & 182.752 \\
\hline 11/9/08 15:00 & 2.143 & 0.683 & 0.222 & 99.538 & 1.432 & 0.086 & 0.026212 & 0.011928 & 0.421279 & 189.070 \\
\hline 11/9/08 16:00 & 2.15 & 0.690 & 0.227 & 102.078 & 1.432 & 0.086 & 0.026212 & 0.011928 & 0.421279 & 189.070 \\
\hline 11/9/08 17:00 & 2.147 & 0.687 & 0.225 & 100.985 & 1.43 & 0.084 & 0.025602 & 0.011529 & 0.407202 & 182.752 \\
\hline 11/9/08 18:00 & 2.15 & 0.690 & 0.227 & 102.078 & 1.427 & 0.081 & 0.024688 & 0.010940 & 0.386387 & 173.411 \\
\hline 11/9/08 19:00 & 2.15 & 0.690 & 0.227 & 102.078 & 1.427 & 0.081 & 0.024688 & 0.010940 & 0.386387 & 173.411 \\
\hline 11/9/08 20:00 & 2.149 & 0.689 & 0.227 & 101.713 & 1.427 & 0.081 & 0.024688 & 0.010940 & 0.386387 & 173.411 \\
\hline 11/9/08 21:00 & 2.149 & 0.689 & 0.227 & 101.713 & 1.427 & 0.081 & 0.024688 & 0.010940 & 0.386387 & 173.411 \\
\hline 11/9/08 22:00 & 2.149 & 0.689 & 0.227 & 101.713 & 1.427 & 0.081 & 0.024688 & 0.010940 & 0.386387 & 173.411 \\
\hline 11/9/08 23:00 & 2.149 & 0.689 & 0.227 & 101.713 & 1.425 & 0.079 & 0.024078 & 0.010553 & 0.372715 & 167.274 \\
\hline 11/10/08 0:00 & 2.147 & 0.687 & 0.225 & 100.985 & 1.425 & 0.079 & 0.024078 & 0.010553 & 0.372715 & 167.274 \\
\hline 11/10/08 1:00 & 2.152 & 0.692 & 0.229 & 102.811 & 1.425 & 0.079 & 0.024078 & 0.010553 & 0.372715 & 167.274 \\
\hline 11/10/08 2:00 & 2.15 & 0.690 & 0.227 & 102.078 & 1.425 & 0.079 & 0.024078 & 0.010553 & 0.372715 & 167.274 \\
\hline 11/10/08 3:00 & 2.148 & 0.688 & 0.226 & 101.348 & 1.427 & 0.081 & 0.024688 & 0.010940 & 0.386387 & 173.411 \\
\hline 11/10/08 4:00 & 2.144 & 0.684 & 0.223 & 99.898 & 1.425 & 0.079 & 0.024078 & 0.010553 & 0.372715 & 167.274 \\
\hline 11/10/08 5:00 & 2.151 & 0.691 & 0.228 & 102.444 & 1.427 & 0.081 & 0.024688 & 0.010940 & 0.386387 & 173.411 \\
\hline 11/10/08 6:00 & 2.151 & 0.691 & 0.228 & 102.444 & 1.427 & 0.081 & 0.024688 & 0.010940 & 0.386387 & 173.411 \\
\hline 11/10/08 7:00 & 2.146 & 0.686 & 0.224 & 100.622 & 1.427 & 0.081 & 0.024688 & 0.010940 & 0.386387 & 173.411 \\
\hline 11/10/08 8:00 & 2.153 & 0.693 & 0.230 & 103.179 & 1.427 & 0.081 & 0.024688 & 0.010940 & 0.386387 & 173.411 \\
\hline 11/10/08 9:00 & 2.149 & 0.689 & 0.227 & 101.713 & 1.427 & 0.081 & 0.024688 & 0.010940 & 0.386387 & 173.411 \\
\hline 11/10/08 10:00 & 2.146 & 0.686 & 0.224 & 100.622 & 1.427 & 0.081 & 0.024688 & 0.010940 & 0.386387 & 173.411 \\
\hline 11/10/08 11:00 & 2.148 & 0.688 & 0.226 & 101.348 & 1.43 & 0.084 & 0.025602 & 0.011529 & 0.407202 & 182.752 \\
\hline 11/10/08 12:00 & 2.148 & 0.688 & 0.226 & 101.348 & 1.427 & 0.081 & 0.024688 & 0.010940 & 0.386387 & 173.411 \\
\hline 11/10/08 13:00 & 2.15 & 0.690 & 0.227 & 102.078 & 1.43 & 0.084 & 0.025602 & 0.011529 & 0.407202 & 182.752 \\
\hline 11/10/08 14:00 & 2.15 & 0.690 & 0.227 & 102.078 & 1.427 & 0.081 & 0.024688 & 0.010940 & 0.386387 & 173.411 \\
\hline
\end{tabular}




\begin{tabular}{|c|c|c|c|c|c|c|c|c|c|c|}
\hline \multirow[b]{3}{*}{ Date Time } & \multicolumn{4}{|c|}{ V-Notch Weir } & \multicolumn{6}{|c|}{ R-Notch Weir } \\
\hline & \multicolumn{2}{|c|}{ Measured Water Level } & \multicolumn{2}{|c|}{$\begin{array}{c}\text { Calculated Rate } \\
\left(\mathrm{ft}^{3} / \mathrm{s}=448.8 \mathrm{gpm}\right)\end{array}$} & \multicolumn{3}{|c|}{ Measured Water Level } & \multicolumn{3}{|c|}{$\begin{array}{c}\text { Calculated Rate } \\
\left(\mathrm{ft}^{3} / \mathrm{s}=448.8 \mathrm{gpm}\right)\end{array}$} \\
\hline & $\begin{array}{c}\text { (ft above } \\
\text { sensor) }\end{array}$ & $\begin{array}{c}\text { (ft above } \\
\text { notch) }\end{array}$ & $\left(\mathrm{ft}^{3} / \mathrm{s}\right)$ & (gpm) & $\begin{array}{l}\text { (ft above } \\
\text { sensor) }\end{array}$ & $\begin{array}{c}\text { (ft above } \\
\text { log) }\end{array}$ & $\begin{array}{c}\text { (m above } \\
\text { log) }\end{array}$ & $\left(\mathrm{m}^{3} / \mathrm{s}\right)$ & $\left(\mathrm{ft}^{3} / \mathrm{s}\right)$ & (gpm) \\
\hline 11/10/08 15:00 & 2.154 & 0.694 & 0.231 & 103.547 & 1.43 & 0.084 & 0.025602 & 0.011529 & 0.407202 & 182.752 \\
\hline 11/10/08 16:00 & 2.18 & 0.720 & 0.253 & 113.404 & 1.432 & 0.086 & 0.026212 & 0.011928 & 0.421279 & 189.070 \\
\hline 11/10/08 17:00 & 2.216 & 0.756 & 0.285 & 127.950 & 1.439 & 0.093 & 0.028345 & 0.013358 & 0.471786 & 211.738 \\
\hline 11/10/08 18:00 & 2.268 & 0.808 & 0.336 & 150.849 & 1.448 & 0.102 & 0.031088 & 0.015274 & 0.539466 & 242.112 \\
\hline 11/10/08 19:00 & 2.315 & 0.855 & 0.387 & 173.521 & 1.456 & 0.11 & 0.033526 & 0.017047 & 0.602114 & 270.229 \\
\hline 11/10/08 20:00 & 2.291 & 0.831 & 0.360 & 161.706 & 1.451 & 0.105 & 0.032002 & 0.015931 & 0.562690 & 252.535 \\
\hline 11/10/08 21:00 & 2.256 & 0.796 & 0.324 & 145.363 & 1.444 & 0.098 & 0.029869 & 0.014412 & 0.509013 & 228.445 \\
\hline 11/10/08 22:00 & 2.232 & 0.772 & 0.300 & 134.756 & 1.441 & 0.095 & 0.028955 & 0.013776 & 0.486564 & 218.370 \\
\hline 11/10/08 23:00 & 2.223 & 0.763 & 0.292 & 130.902 & 1.441 & 0.095 & 0.028955 & 0.013776 & 0.486564 & 218.370 \\
\hline 11/11/08 0:00 & 2.225 & 0.765 & 0.294 & 131.752 & 1.441 & 0.095 & 0.028955 & 0.013776 & 0.486564 & 218.370 \\
\hline 11/11/08 1:00 & 2.23 & 0.770 & 0.298 & 133.893 & 1.439 & 0.093 & 0.028345 & 0.013358 & 0.471786 & 211.738 \\
\hline 11/11/08 2:00 & 2.242 & 0.782 & 0.310 & 139.117 & 1.441 & 0.095 & 0.028955 & 0.013776 & 0.486564 & 218.370 \\
\hline 11/11/08 3:00 & 2.246 & 0.786 & 0.314 & 140.885 & 1.444 & 0.098 & 0.029869 & 0.014412 & 0.509013 & 228.445 \\
\hline 11/11/08 4:00 & 2.23 & 0.770 & 0.298 & 133.893 & 1.441 & 0.095 & 0.028955 & 0.013776 & 0.486564 & 218.370 \\
\hline 11/11/08 5:00 & 2.223 & 0.763 & 0.292 & 130.902 & 1.441 & 0.095 & 0.028955 & 0.013776 & 0.486564 & 218.370 \\
\hline 11/11/08 6:00 & 2.218 & 0.758 & 0.287 & 128.789 & 1.437 & 0.091 & 0.027735 & 0.012943 & 0.457162 & 205.174 \\
\hline 11/11/08 7:00 & 2.218 & 0.758 & 0.287 & 128.789 & 1.439 & 0.093 & 0.028345 & 0.013358 & 0.471786 & 211.738 \\
\hline 11/11/08 8:00 & 2.229 & 0.769 & 0.297 & 133.464 & 1.439 & 0.093 & 0.028345 & 0.013358 & 0.471786 & 211.738 \\
\hline 11/11/08 9:00 & 2.22 & 0.760 & 0.289 & 129.632 & 1.439 & 0.093 & 0.028345 & 0.013358 & 0.471786 & 211.738 \\
\hline 11/11/08 10:00 & 2.213 & 0.753 & 0.282 & 126.698 & 1.437 & 0.091 & 0.027735 & 0.012943 & 0.457162 & 205.174 \\
\hline 11/11/08 11:00 & 2.205 & 0.745 & 0.275 & 123.394 & 1.437 & 0.091 & 0.027735 & 0.012943 & 0.457162 & 205.174 \\
\hline 11/11/08 12:00 & 2.219 & 0.759 & 0.288 & 129.210 & 1.437 & 0.091 & 0.027735 & 0.012943 & 0.457162 & 205.174 \\
\hline 11/11/08 13:00 & 2.245 & 0.785 & 0.313 & 140.442 & 1.441 & 0.095 & 0.028955 & 0.013776 & 0.486564 & 218.370 \\
\hline 11/11/08 14:00 & 2.27 & 0.810 & 0.338 & 151.775 & 1.446 & 0.1 & 0.030479 & 0.014841 & 0.524166 & 235.246 \\
\hline 11/11/08 15:00 & 2.301 & 0.841 & 0.371 & 166.568 & 1.451 & 0.105 & 0.032002 & 0.015931 & 0.562690 & 252.535 \\
\hline
\end{tabular}




\begin{tabular}{|c|c|c|c|c|c|c|c|c|c|c|}
\hline \multirow[b]{3}{*}{ Date Time } & \multicolumn{4}{|c|}{ V-Notch Weir } & \multicolumn{6}{|c|}{ R-Notch Weir } \\
\hline & \multicolumn{2}{|c|}{ Measured Water Level } & \multicolumn{2}{|c|}{$\begin{array}{c}\text { Calculated Rate } \\
\left(\mathrm{ft}^{3} / \mathrm{s}=448.8 \mathrm{gpm}\right)\end{array}$} & \multicolumn{3}{|c|}{ Measured Water Level } & \multicolumn{3}{|c|}{$\begin{array}{c}\text { Calculated Rate } \\
\left(\mathrm{ft}^{3} / \mathrm{s}=448.8 \mathrm{gpm}\right)\end{array}$} \\
\hline & $\begin{array}{c}\text { (ft above } \\
\text { sensor) }\end{array}$ & $\begin{array}{c}\text { (ft above } \\
\text { notch) }\end{array}$ & $\left(\mathrm{ft}^{3} / \mathrm{s}\right)$ & (gpm) & $\begin{array}{l}\text { (ft above } \\
\text { sensor) }\end{array}$ & $\begin{array}{c}\text { (ft above } \\
\text { log) }\end{array}$ & $\begin{array}{c}\text { (m above } \\
\text { log) }\end{array}$ & $\left(\mathrm{m}^{3} / \mathrm{s}\right)$ & $\left(\mathrm{ft}^{3} / \mathrm{s}\right)$ & (gpm) \\
\hline 11/11/08 16:00 & 2.352 & 0.892 & 0.429 & 192.724 & 1.458 & 0.112 & 0.034136 & 0.017501 & 0.618132 & 277.418 \\
\hline 11/11/08 17:00 & 2.373 & 0.913 & 0.455 & 204.165 & 1.46 & 0.114 & 0.034746 & 0.017958 & 0.634289 & 284.669 \\
\hline 11/11/08 18:00 & 2.42 & 0.960 & 0.515 & 231.221 & 1.467 & 0.121 & 0.036879 & 0.019590 & 0.691921 & 310.534 \\
\hline 11/11/08 19:00 & 2.486 & 1.026 & 0.608 & 272.676 & 1.479 & 0.133 & 0.040536 & 0.022495 & 0.794527 & 356.583 \\
\hline 11/11/08 20:00 & 2.451 & 0.991 & 0.557 & 250.182 & 1.47 & 0.124 & 0.037793 & 0.020304 & 0.717127 & 321.847 \\
\hline 11/11/08 21:00 & 2.373 & 0.913 & 0.455 & 204.165 & 1.458 & 0.112 & 0.034136 & 0.017501 & 0.618132 & 277.418 \\
\hline 11/11/08 22:00 & 2.319 & 0.859 & 0.391 & 175.539 & 1.448 & 0.102 & 0.031088 & 0.015274 & 0.539466 & 242.112 \\
\hline 11/11/08 23:00 & 2.291 & 0.831 & 0.360 & 161.706 & 1.441 & 0.095 & 0.028955 & 0.013776 & 0.486564 & 218.370 \\
\hline 11/12/08 0:00 & 2.266 & 0.806 & 0.334 & 149.926 & 1.439 & 0.093 & 0.028345 & 0.013358 & 0.471786 & 211.738 \\
\hline 11/12/08 1:00 & 2.249 & 0.789 & 0.317 & 142.220 & 1.437 & 0.091 & 0.027735 & 0.012943 & 0.457162 & 205.174 \\
\hline 11/12/08 2:00 & 2.238 & 0.778 & 0.306 & 137.362 & 1.437 & 0.091 & 0.027735 & 0.012943 & 0.457162 & 205.174 \\
\hline 11/12/08 3:00 & 2.228 & 0.768 & 0.296 & 133.034 & 1.434 & 0.088 & 0.026821 & 0.012331 & 0.435514 & 195.459 \\
\hline 11/12/08 4:00 & 2.226 & 0.766 & 0.295 & 132.179 & 1.434 & 0.088 & 0.026821 & 0.012331 & 0.435514 & 195.459 \\
\hline 11/12/08 5:00 & 2.219 & 0.759 & 0.288 & 129.210 & 1.434 & 0.088 & 0.026821 & 0.012331 & 0.435514 & 195.459 \\
\hline 11/12/08 6:00 & 2.217 & 0.757 & 0.286 & 128.369 & 1.434 & 0.088 & 0.026821 & 0.012331 & 0.435514 & 195.459 \\
\hline 11/12/08 7:00 & 2.21 & 0.750 & 0.280 & 125.453 & 1.432 & 0.086 & 0.026212 & 0.011928 & 0.421279 & 189.070 \\
\hline 11/12/08 8:00 & 2.208 & 0.748 & 0.278 & 124.627 & 1.432 & 0.086 & 0.026212 & 0.011928 & 0.421279 & 189.070 \\
\hline 11/12/08 9:00 & 2.203 & 0.743 & 0.273 & 122.576 & 1.43 & 0.084 & 0.025602 & 0.011529 & 0.407202 & 182.752 \\
\hline 11/12/08 10:00 & 2.2 & 0.740 & 0.270 & 121.355 & 1.43 & 0.084 & 0.025602 & 0.011529 & 0.407202 & 182.752 \\
\hline 11/12/08 11:00 & 2.198 & 0.738 & 0.269 & 120.546 & 1.43 & 0.084 & 0.025602 & 0.011529 & 0.407202 & 182.752 \\
\hline 11/12/08 12:00 & 2.198 & 0.738 & 0.269 & 120.546 & 1.43 & 0.084 & 0.025602 & 0.011529 & 0.407202 & 182.752 \\
\hline 11/12/08 13:00 & 2.193 & 0.733 & 0.264 & 118.536 & 1.43 & 0.084 & 0.025602 & 0.011529 & 0.407202 & 182.752 \\
\hline 11/12/08 14:00 & 2.195 & 0.735 & 0.266 & 119.337 & 1.43 & 0.084 & 0.025602 & 0.011529 & 0.407202 & 182.752 \\
\hline 11/12/08 15:00 & 2.197 & 0.737 & 0.268 & 120.142 & 1.432 & 0.086 & 0.026212 & 0.011928 & 0.421279 & 189.070 \\
\hline 11/12/08 16:00 & 2.192 & 0.732 & 0.263 & 118.136 & 1.432 & 0.086 & 0.026212 & 0.011928 & 0.421279 & 189.070 \\
\hline
\end{tabular}




\begin{tabular}{|c|c|c|c|c|c|c|c|c|c|c|}
\hline \multirow[b]{3}{*}{ Date Time } & \multicolumn{4}{|c|}{ V-Notch Weir } & \multicolumn{6}{|c|}{ R-Notch Weir } \\
\hline & \multicolumn{2}{|c|}{ Measured Water Level } & \multicolumn{2}{|c|}{$\begin{array}{c}\text { Calculated Rate } \\
\left(\mathrm{ft}^{3} / \mathrm{s}=448.8 \mathrm{gpm}\right)\end{array}$} & \multicolumn{3}{|c|}{ Measured Water Level } & \multicolumn{3}{|c|}{$\begin{array}{c}\text { Calculated Rate } \\
\left(\mathrm{ft}^{3} / \mathrm{s}=448.8 \mathrm{gpm}\right)\end{array}$} \\
\hline & $\begin{array}{c}\text { (ft above } \\
\text { sensor) }\end{array}$ & $\begin{array}{c}\text { (ft above } \\
\text { notch) }\end{array}$ & $\left(\mathrm{ft}^{3} / \mathrm{s}\right)$ & (gpm) & $\begin{array}{l}\text { (ft above } \\
\text { sensor) }\end{array}$ & $\begin{array}{c}\text { (ft above } \\
\text { log) }\end{array}$ & $\begin{array}{c}\text { (m above } \\
\text { log) }\end{array}$ & $\left(\mathrm{m}^{3} / \mathrm{s}\right)$ & $\left(\mathrm{ft}^{3} / \mathrm{s}\right)$ & (gpm) \\
\hline 11/12/08 17:00 & 2.192 & 0.732 & 0.263 & 118.136 & 1.432 & 0.086 & 0.026212 & 0.011928 & 0.421279 & 189.070 \\
\hline 11/12/08 18:00 & 2.192 & 0.732 & 0.263 & 118.136 & 1.432 & 0.086 & 0.026212 & 0.011928 & 0.421279 & 189.070 \\
\hline 11/12/08 19:00 & 2.192 & 0.732 & 0.263 & 118.136 & 1.434 & 0.088 & 0.026821 & 0.012331 & 0.435514 & 195.459 \\
\hline 11/12/08 20:00 & 2.192 & 0.732 & 0.263 & 118.136 & 1.434 & 0.088 & 0.026821 & 0.012331 & 0.435514 & 195.459 \\
\hline 11/12/08 21:00 & 2.19 & 0.730 & 0.261 & 117.339 & 1.432 & 0.086 & 0.026212 & 0.011928 & 0.421279 & 189.070 \\
\hline 11/12/08 22:00 & 2.191 & 0.731 & 0.262 & 117.737 & 1.432 & 0.086 & 0.026212 & 0.011928 & 0.421279 & 189.070 \\
\hline 11/12/08 23:00 & 2.189 & 0.729 & 0.261 & 116.942 & 1.434 & 0.088 & 0.026821 & 0.012331 & 0.435514 & 195.459 \\
\hline 11/13/08 0:00 & 2.189 & 0.729 & 0.261 & 116.942 & 1.434 & 0.088 & 0.026821 & 0.012331 & 0.435514 & 195.459 \\
\hline 11/13/08 1:00 & 2.163 & 0.703 & 0.238 & 106.898 & 1.427 & 0.081 & 0.024688 & 0.010940 & 0.386387 & 173.411 \\
\hline 11/13/08 2:00 & 2.187 & 0.727 & 0.259 & 116.150 & 1.432 & 0.086 & 0.026212 & 0.011928 & 0.421279 & 189.070 \\
\hline 11/13/08 3:00 & 2.199 & 0.739 & 0.269 & 120.950 & 1.432 & 0.086 & 0.026212 & 0.011928 & 0.421279 & 189.070 \\
\hline 11/13/08 4:00 & 2.196 & 0.736 & 0.267 & 119.739 & 1.432 & 0.086 & 0.026212 & 0.011928 & 0.421279 & 189.070 \\
\hline 11/13/08 5:00 & 2.192 & 0.732 & 0.263 & 118.136 & 1.432 & 0.086 & 0.026212 & 0.011928 & 0.421279 & 189.070 \\
\hline 11/13/08 6:00 & 2.215 & 0.755 & 0.284 & 127.532 & 1.434 & 0.088 & 0.026821 & 0.012331 & 0.435514 & 195.459 \\
\hline 11/13/08 7:00 & 2.201 & 0.741 & 0.271 & 121.761 & 1.432 & 0.086 & 0.026212 & 0.011928 & 0.421279 & 189.070 \\
\hline 11/13/08 8:00 & 2.193 & 0.733 & 0.264 & 118.536 & 1.432 & 0.086 & 0.026212 & 0.011928 & 0.421279 & 189.070 \\
\hline 11/13/08 9:00 & 2.193 & 0.733 & 0.264 & 118.536 & 1.432 & 0.086 & 0.026212 & 0.011928 & 0.421279 & 189.070 \\
\hline 11/13/08 10:00 & 2.193 & 0.733 & 0.264 & 118.536 & 1.432 & 0.086 & 0.026212 & 0.011928 & 0.421279 & 189.070 \\
\hline 11/13/08 11:00 & 2.186 & 0.726 & 0.258 & 115.756 & 1.432 & 0.086 & 0.026212 & 0.011928 & 0.421279 & 189.070 \\
\hline 11/13/08 12:00 & 2.187 & 0.727 & 0.259 & 116.150 & 1.432 & 0.086 & 0.026212 & 0.011928 & 0.421279 & 189.070 \\
\hline 11/13/08 13:00 & 2.192 & 0.732 & 0.263 & 118.136 & 1.432 & 0.086 & 0.026212 & 0.011928 & 0.421279 & 189.070 \\
\hline 11/13/08 14:00 & 2.187 & 0.727 & 0.259 & 116.150 & 1.434 & 0.088 & 0.026821 & 0.012331 & 0.435514 & 195.459 \\
\hline 11/13/08 15:00 & 2.191 & 0.731 & 0.262 & 117.737 & 1.432 & 0.086 & 0.026212 & 0.011928 & 0.421279 & 189.070 \\
\hline 11/13/08 16:00 & 2.191 & 0.731 & 0.262 & 117.737 & 1.432 & 0.086 & 0.026212 & 0.011928 & 0.421279 & 189.070 \\
\hline 11/13/08 17:00 & 2.193 & 0.733 & 0.264 & 118.536 & 1.432 & 0.086 & 0.026212 & 0.011928 & 0.421279 & 189.070 \\
\hline
\end{tabular}




\begin{tabular}{|c|c|c|c|c|c|c|c|c|c|c|}
\hline \multirow[b]{3}{*}{ Date Time } & \multicolumn{4}{|c|}{ V-Notch Weir } & \multicolumn{6}{|c|}{ R-Notch Weir } \\
\hline & \multicolumn{2}{|c|}{ Measured Water Level } & \multicolumn{2}{|c|}{$\begin{array}{c}\text { Calculated Rate } \\
\left(\mathrm{ft}^{3} / \mathrm{s}=448.8 \mathrm{gpm}\right)\end{array}$} & \multicolumn{3}{|c|}{ Measured Water Level } & \multicolumn{3}{|c|}{$\begin{array}{c}\text { Calculated Rate } \\
\left(\mathrm{ft}^{3} / \mathrm{s}=448.8 \mathrm{gpm}\right)\end{array}$} \\
\hline & $\begin{array}{c}\text { (ft above } \\
\text { sensor) }\end{array}$ & $\begin{array}{c}\text { (ft above } \\
\text { notch) }\end{array}$ & $\left(\mathrm{ft}^{3} / \mathrm{s}\right)$ & (gpm) & $\begin{array}{l}\text { (ft above } \\
\text { sensor) }\end{array}$ & $\begin{array}{c}\text { (ft above } \\
\text { log) }\end{array}$ & $\begin{array}{c}\text { (m above } \\
\text { log) }\end{array}$ & $\left(\mathrm{m}^{3} / \mathrm{s}\right)$ & $\left(\mathrm{ft}^{3} / \mathrm{s}\right)$ & (gpm) \\
\hline 11/13/08 18:00 & 2.205 & 0.745 & 0.275 & 123.394 & 1.432 & 0.086 & 0.026212 & 0.011928 & 0.421279 & 189.070 \\
\hline 11/13/08 19:00 & 2.228 & 0.768 & 0.296 & 133.034 & 1.437 & 0.091 & 0.027735 & 0.012943 & 0.457162 & 205.174 \\
\hline 11/13/08 20:00 & 2.24 & 0.780 & 0.308 & 138.238 & 1.451 & 0.105 & 0.032002 & 0.015931 & 0.562690 & 252.535 \\
\hline 11/13/08 21:00 & 2.231 & 0.771 & 0.299 & 134.324 & 1.451 & 0.105 & 0.032002 & 0.015931 & 0.562690 & 252.535 \\
\hline 11/13/08 22:00 & 2.221 & 0.761 & 0.290 & 130.054 & 1.449 & 0.103 & 0.031393 & 0.015492 & 0.547171 & 245.570 \\
\hline 11/13/08 23:00 & 2.212 & 0.752 & 0.281 & 126.282 & 1.444 & 0.098 & 0.029869 & 0.014412 & 0.509013 & 228.445 \\
\hline 11/14/08 0:00 & 2.201 & 0.741 & 0.271 & 121.761 & 1.444 & 0.098 & 0.029869 & 0.014412 & 0.509013 & 228.445 \\
\hline 11/14/08 1:00 & 2.196 & 0.736 & 0.267 & 119.739 & 1.442 & 0.096 & 0.029259 & 0.013987 & 0.494010 & 221.711 \\
\hline 11/14/08 2:00 & 2.196 & 0.736 & 0.267 & 119.739 & 1.442 & 0.096 & 0.029259 & 0.013987 & 0.494010 & 221.711 \\
\hline 11/14/08 3:00 & 2.189 & 0.729 & 0.261 & 116.942 & 1.439 & 0.093 & 0.028345 & 0.013358 & 0.471786 & 211.738 \\
\hline 11/14/08 4:00 & 2.185 & 0.725 & 0.257 & 115.362 & 1.44 & 0.094 & 0.028650 & 0.013566 & 0.479156 & 215.045 \\
\hline 11/14/08 5:00 & 2.183 & 0.723 & 0.255 & 114.576 & 1.437 & 0.091 & 0.027735 & 0.012943 & 0.457162 & 205.174 \\
\hline 11/14/08 6:00 & 2.183 & 0.723 & 0.255 & 114.576 & 1.44 & 0.094 & 0.028650 & 0.013566 & 0.479156 & 215.045 \\
\hline 11/14/08 7:00 & 2.178 & 0.718 & 0.251 & 112.627 & 1.437 & 0.091 & 0.027735 & 0.012943 & 0.457162 & 205.174 \\
\hline 11/14/08 8:00 & 2.176 & 0.716 & 0.249 & 111.853 & 1.435 & 0.089 & 0.027126 & 0.012534 & 0.442691 & 198.680 \\
\hline 11/14/08 9:00 & 2.176 & 0.716 & 0.249 & 111.853 & 1.432 & 0.086 & 0.026212 & 0.011928 & 0.421279 & 189.070 \\
\hline 11/14/08 10:00 & 2.174 & 0.714 & 0.248 & 111.082 & 1.432 & 0.086 & 0.026212 & 0.011928 & 0.421279 & 189.070 \\
\hline 11/14/08 11:00 & 2.169 & 0.709 & 0.243 & 109.168 & 1.435 & 0.089 & 0.027126 & 0.012534 & 0.442691 & 198.680 \\
\hline 11/14/08 12:00 & 2.183 & 0.723 & 0.255 & 114.576 & 1.432 & 0.086 & 0.026212 & 0.011928 & 0.421279 & 189.070 \\
\hline 11/14/08 13:00 & 2.168 & 0.708 & 0.242 & 108.788 & 1.435 & 0.089 & 0.027126 & 0.012534 & 0.442691 & 198.680 \\
\hline 11/14/08 14:00 & 2.168 & 0.708 & 0.242 & 108.788 & 1.43 & 0.084 & 0.025602 & 0.011529 & 0.407202 & 182.752 \\
\hline 11/14/08 15:00 & 2.165 & 0.705 & 0.240 & 107.652 & 1.435 & 0.089 & 0.027126 & 0.012534 & 0.442691 & 198.680 \\
\hline 11/14/08 16:00 & 2.161 & 0.701 & 0.237 & 106.148 & 1.432 & 0.086 & 0.026212 & 0.011928 & 0.421279 & 189.070 \\
\hline 11/14/08 17:00 & 2.163 & 0.703 & 0.238 & 106.898 & 1.432 & 0.086 & 0.026212 & 0.011928 & 0.421279 & 189.070 \\
\hline 11/14/08 18:00 & 2.159 & 0.699 & 0.235 & 105.401 & 1.432 & 0.086 & 0.026212 & 0.011928 & 0.421279 & 189.070 \\
\hline
\end{tabular}




\begin{tabular}{|c|c|c|c|c|c|c|c|c|c|c|}
\hline \multirow[b]{3}{*}{ Date Time } & \multicolumn{4}{|c|}{ V-Notch Weir } & \multicolumn{6}{|c|}{ R-Notch Weir } \\
\hline & \multicolumn{2}{|c|}{ Measured Water Level } & \multicolumn{2}{|c|}{$\begin{array}{c}\text { Calculated Rate } \\
\left(\mathrm{ft}^{3} / \mathrm{s}=448.8 \mathrm{gpm}\right)\end{array}$} & \multicolumn{3}{|c|}{ Measured Water Level } & \multicolumn{3}{|c|}{$\begin{array}{c}\text { Calculated Rate } \\
\left(\mathrm{ft}^{3} / \mathrm{s}=448.8 \mathrm{gpm}\right)\end{array}$} \\
\hline & $\begin{array}{c}\text { (ft above } \\
\text { sensor) }\end{array}$ & $\begin{array}{c}\text { (ft above } \\
\text { notch) }\end{array}$ & $\left(\mathrm{ft}^{3} / \mathrm{s}\right)$ & (gpm) & $\begin{array}{l}\text { (ft above } \\
\text { sensor) }\end{array}$ & $\begin{array}{c}\text { (ft above } \\
\text { log) }\end{array}$ & $\begin{array}{c}\text { (m above } \\
\text { log) }\end{array}$ & $\left(\mathrm{m}^{3} / \mathrm{s}\right)$ & $\left(\mathrm{ft}^{3} / \mathrm{s}\right)$ & (gpm) \\
\hline 11/14/08 19:00 & 2.166 & 0.706 & 0.241 & 108.030 & 1.432 & 0.086 & 0.026212 & 0.011928 & 0.421279 & 189.070 \\
\hline 11/14/08 20:00 & 2.166 & 0.706 & 0.241 & 108.030 & 1.432 & 0.086 & 0.026212 & 0.011928 & 0.421279 & 189.070 \\
\hline 11/14/08 21:00 & 2.162 & 0.702 & 0.237 & 106.523 & 1.43 & 0.084 & 0.025602 & 0.011529 & 0.407202 & 182.752 \\
\hline 11/14/08 22:00 & 2.181 & 0.721 & 0.254 & 113.794 & 1.43 & 0.084 & 0.025602 & 0.011529 & 0.407202 & 182.752 \\
\hline 11/14/08 23:00 & 2.179 & 0.719 & 0.252 & 113.015 & 1.43 & 0.084 & 0.025602 & 0.011529 & 0.407202 & 182.752 \\
\hline 11/15/08 0:00 & 2.173 & 0.713 & 0.247 & 110.697 & 1.432 & 0.086 & 0.026212 & 0.011928 & 0.421279 & 189.070 \\
\hline 11/15/08 1:00 & 2.164 & 0.704 & 0.239 & 107.275 & 1.428 & 0.082 & 0.024992 & 0.011135 & 0.393285 & 176.506 \\
\hline 11/15/08 2:00 & 2.167 & 0.707 & 0.242 & 108.408 & 1.428 & 0.082 & 0.024992 & 0.011135 & 0.393285 & 176.506 \\
\hline 11/15/08 3:00 & 2.163 & 0.703 & 0.238 & 106.898 & 1.428 & 0.082 & 0.024992 & 0.011135 & 0.393285 & 176.506 \\
\hline 11/15/08 4:00 & 2.156 & 0.696 & 0.232 & 104.286 & 1.428 & 0.082 & 0.024992 & 0.011135 & 0.393285 & 176.506 \\
\hline 11/15/08 5:00 & 2.152 & 0.692 & 0.229 & 102.811 & 1.428 & 0.082 & 0.024992 & 0.011135 & 0.393285 & 176.506 \\
\hline 11/15/08 6:00 & 2.157 & 0.697 & 0.233 & 104.657 & 1.427 & 0.081 & 0.024688 & 0.010940 & 0.386387 & 173.411 \\
\hline 11/15/08 7:00 & 2.157 & 0.697 & 0.233 & 104.657 & 1.43 & 0.084 & 0.025602 & 0.011529 & 0.407202 & 182.752 \\
\hline 11/15/08 8:00 & 2.16 & 0.700 & 0.236 & 105.774 & 1.427 & 0.081 & 0.024688 & 0.010940 & 0.386387 & 173.411 \\
\hline 11/15/08 9:00 & 2.162 & 0.702 & 0.237 & 106.523 & 1.427 & 0.081 & 0.024688 & 0.010940 & 0.386387 & 173.411 \\
\hline 11/15/08 10:00 & 2.16 & 0.700 & 0.236 & 105.774 & 1.427 & 0.081 & 0.024688 & 0.010940 & 0.386387 & 173.411 \\
\hline 11/15/08 11:00 & 2.159 & 0.699 & 0.235 & 105.401 & 1.427 & 0.081 & 0.024688 & 0.010940 & 0.386387 & 173.411 \\
\hline 11/15/08 12:00 & 2.161 & 0.701 & 0.237 & 106.148 & 1.427 & 0.081 & 0.024688 & 0.010940 & 0.386387 & 173.411 \\
\hline 11/15/08 13:00 & 2.158 & 0.698 & 0.234 & 105.029 & 1.43 & 0.084 & 0.025602 & 0.011529 & 0.407202 & 182.752 \\
\hline 11/15/08 14:00 & 2.167 & 0.707 & 0.242 & 108.408 & 1.43 & 0.084 & 0.025602 & 0.011529 & 0.407202 & 182.752 \\
\hline 11/15/08 15:00 & 2.162 & 0.702 & 0.237 & 106.523 & 1.43 & 0.084 & 0.025602 & 0.011529 & 0.407202 & 182.752 \\
\hline 11/15/08 16:00 & 2.164 & 0.704 & 0.239 & 107.275 & 1.432 & 0.086 & 0.026212 & 0.011928 & 0.421279 & 189.070 \\
\hline 11/15/08 17:00 & 2.168 & 0.708 & 0.242 & 108.788 & 1.43 & 0.084 & 0.025602 & 0.011529 & 0.407202 & 182.752 \\
\hline 11/15/08 18:00 & 2.161 & 0.701 & 0.237 & 106.148 & 1.43 & 0.084 & 0.025602 & 0.011529 & 0.407202 & 182.752 \\
\hline 11/15/08 19:00 & 2.162 & 0.702 & 0.237 & 106.523 & 1.43 & 0.084 & 0.025602 & 0.011529 & 0.407202 & 182.752 \\
\hline
\end{tabular}




\begin{tabular}{|c|c|c|c|c|c|c|c|c|c|c|}
\hline \multirow[b]{3}{*}{ Date Time } & \multicolumn{4}{|c|}{ V-Notch Weir } & \multicolumn{6}{|c|}{ R-Notch Weir } \\
\hline & \multicolumn{2}{|c|}{ Measured Water Level } & \multicolumn{2}{|c|}{$\begin{array}{c}\text { Calculated Rate } \\
\left(\mathrm{ft}^{3} / \mathrm{s}=448.8 \mathrm{gpm}\right)\end{array}$} & \multicolumn{3}{|c|}{ Measured Water Level } & \multicolumn{3}{|c|}{$\begin{array}{c}\text { Calculated Rate } \\
\left(\mathrm{ft}^{3} / \mathrm{s}=448.8 \mathrm{gpm}\right)\end{array}$} \\
\hline & $\begin{array}{c}\text { (ft above } \\
\text { sensor) }\end{array}$ & $\begin{array}{c}\text { (ft above } \\
\text { notch) }\end{array}$ & $\left(\mathrm{ft}^{3} / \mathrm{s}\right)$ & (gpm) & $\begin{array}{l}\text { (ft above } \\
\text { sensor) }\end{array}$ & $\begin{array}{c}\text { (ft above } \\
\text { log) }\end{array}$ & $\begin{array}{c}\text { (m above } \\
\text { log) }\end{array}$ & $\left(\mathrm{m}^{3} / \mathrm{s}\right)$ & $\left(\mathrm{ft}^{3} / \mathrm{s}\right)$ & (gpm) \\
\hline 11/15/08 20:00 & 2.162 & 0.702 & 0.237 & 106.523 & 1.432 & 0.086 & 0.026212 & 0.011928 & 0.421279 & 189.070 \\
\hline 11/15/08 21:00 & 2.16 & 0.700 & 0.236 & 105.774 & 1.432 & 0.086 & 0.026212 & 0.011928 & 0.421279 & 189.070 \\
\hline 11/15/08 22:00 & 2.153 & 0.693 & 0.230 & 103.179 & 1.429 & 0.083 & 0.025297 & 0.011331 & 0.400223 & 179.620 \\
\hline 11/15/08 23:00 & 2.153 & 0.693 & 0.230 & 103.179 & 1.432 & 0.086 & 0.026212 & 0.011928 & 0.421279 & 189.070 \\
\hline 11/16/08 0:00 & 2.156 & 0.696 & 0.232 & 104.286 & 1.434 & 0.088 & 0.026821 & 0.012331 & 0.435514 & 195.459 \\
\hline 11/16/08 1:00 & 2.158 & 0.698 & 0.234 & 105.029 & 1.434 & 0.088 & 0.026821 & 0.012331 & 0.435514 & 195.459 \\
\hline 11/16/08 2:00 & 2.159 & 0.699 & 0.235 & 105.401 & 1.434 & 0.088 & 0.026821 & 0.012331 & 0.435514 & 195.459 \\
\hline 11/16/08 3:00 & 2.166 & 0.706 & 0.241 & 108.030 & 1.434 & 0.088 & 0.026821 & 0.012331 & 0.435514 & 195.459 \\
\hline 11/16/08 4:00 & 2.164 & 0.704 & 0.239 & 107.275 & 1.434 & 0.088 & 0.026821 & 0.012331 & 0.435514 & 195.459 \\
\hline 11/16/08 5:00 & 2.161 & 0.701 & 0.237 & 106.148 & 1.434 & 0.088 & 0.026821 & 0.012331 & 0.435514 & 195.459 \\
\hline 11/16/08 6:00 & 2.164 & 0.704 & 0.239 & 107.275 & 1.432 & 0.086 & 0.026212 & 0.011928 & 0.421279 & 189.070 \\
\hline 11/16/08 7:00 & 2.159 & 0.699 & 0.235 & 105.401 & 1.432 & 0.086 & 0.026212 & 0.011928 & 0.421279 & 189.070 \\
\hline 11/16/08 8:00 & 2.159 & 0.699 & 0.235 & 105.401 & 1.432 & 0.086 & 0.026212 & 0.011928 & 0.421279 & 189.070 \\
\hline 11/16/08 9:00 & 2.161 & 0.701 & 0.237 & 106.148 & 1.429 & 0.083 & 0.025297 & 0.011331 & 0.400223 & 179.620 \\
\hline 11/16/08 10:00 & 2.159 & 0.699 & 0.235 & 105.401 & 1.429 & 0.083 & 0.025297 & 0.011331 & 0.400223 & 179.620 \\
\hline 11/16/08 11:00 & 2.16 & 0.700 & 0.236 & 105.774 & 1.427 & 0.081 & 0.024688 & 0.010940 & 0.386387 & 173.411 \\
\hline 11/16/08 12:00 & 2.164 & 0.704 & 0.239 & 107.275 & 1.427 & 0.081 & 0.024688 & 0.010940 & 0.386387 & 173.411 \\
\hline 11/16/08 13:00 & 2.168 & 0.708 & 0.242 & 108.788 & 1.429 & 0.083 & 0.025297 & 0.011331 & 0.400223 & 179.620 \\
\hline 11/16/08 14:00 & 2.16 & 0.700 & 0.236 & 105.774 & 1.429 & 0.083 & 0.025297 & 0.011331 & 0.400223 & 179.620 \\
\hline 11/16/08 15:00 & 2.15 & 0.690 & 0.227 & 102.078 & 1.432 & 0.086 & 0.026212 & 0.011928 & 0.421279 & 189.070 \\
\hline 11/16/08 16:00 & 2.157 & 0.697 & 0.233 & 104.657 & 1.42 & 0.074 & 0.022554 & 0.009605 & 0.339261 & 152.260 \\
\hline 11/16/08 17:00 & 2.154 & 0.694 & 0.231 & 103.547 & 1.42 & 0.074 & 0.022554 & 0.009605 & 0.339261 & 152.260 \\
\hline 11/16/08 18:00 & 2.152 & 0.692 & 0.229 & 102.811 & 1.42 & 0.074 & 0.022554 & 0.009605 & 0.339261 & 152.260 \\
\hline 11/16/08 19:00 & 2.154 & 0.694 & 0.231 & 103.547 & 1.42 & 0.074 & 0.022554 & 0.009605 & 0.339261 & 152.260 \\
\hline 11/16/08 20:00 & 2.152 & 0.692 & 0.229 & 102.811 & 1.42 & 0.074 & 0.022554 & 0.009605 & 0.339261 & 152.260 \\
\hline
\end{tabular}




\begin{tabular}{|c|c|c|c|c|c|c|c|c|c|c|}
\hline \multirow[b]{3}{*}{ Date Time } & \multicolumn{4}{|c|}{ V-Notch Weir } & \multicolumn{6}{|c|}{ R-Notch Weir } \\
\hline & \multicolumn{2}{|c|}{ Measured Water Level } & \multicolumn{2}{|c|}{$\begin{array}{c}\text { Calculated Rate } \\
\left(\mathrm{ft}^{3} / \mathrm{s}=448.8 \mathrm{gpm}\right)\end{array}$} & \multicolumn{3}{|c|}{ Measured Water Level } & \multicolumn{3}{|c|}{$\begin{array}{c}\text { Calculated Rate } \\
\left(\mathrm{ft}^{3} / \mathrm{s}=448.8 \mathrm{gpm}\right)\end{array}$} \\
\hline & $\begin{array}{c}\text { (ft above } \\
\text { sensor) }\end{array}$ & $\begin{array}{c}\text { (ft above } \\
\text { notch) }\end{array}$ & $\left(\mathrm{ft}^{3} / \mathrm{s}\right)$ & (gpm) & $\begin{array}{l}\text { (ft above } \\
\text { sensor) }\end{array}$ & $\begin{array}{c}\text { (ft above } \\
\text { log) }\end{array}$ & $\begin{array}{c}\text { (m above } \\
\text { log) }\end{array}$ & $\left(\mathrm{m}^{3} / \mathrm{s}\right)$ & $\left(\mathrm{ft}^{3} / \mathrm{s}\right)$ & (gpm) \\
\hline 11/16/08 21:00 & 2.155 & 0.695 & 0.232 & 103.916 & 1.42 & 0.074 & 0.022554 & 0.009605 & 0.339261 & 152.260 \\
\hline 11/16/08 22:00 & 2.157 & 0.697 & 0.233 & 104.657 & 1.42 & 0.074 & 0.022554 & 0.009605 & 0.339261 & 152.260 \\
\hline 11/16/08 23:00 & 2.157 & 0.697 & 0.233 & 104.657 & 1.422 & 0.076 & 0.023164 & 0.009981 & 0.352516 & 158.209 \\
\hline 11/17/08 0:00 & 2.153 & 0.693 & 0.230 & 103.179 & 1.42 & 0.074 & 0.022554 & 0.009605 & 0.339261 & 152.260 \\
\hline 11/17/08 1:00 & 2.153 & 0.693 & 0.230 & 103.179 & 1.42 & 0.074 & 0.022554 & 0.009605 & 0.339261 & 152.260 \\
\hline 11/17/08 2:00 & 2.153 & 0.693 & 0.230 & 103.179 & 1.422 & 0.076 & 0.023164 & 0.009981 & 0.352516 & 158.209 \\
\hline 11/17/08 3:00 & 2.153 & 0.693 & 0.230 & 103.179 & 1.42 & 0.074 & 0.022554 & 0.009605 & 0.339261 & 152.260 \\
\hline 11/17/08 4:00 & 2.151 & 0.691 & 0.228 & 102.444 & 1.42 & 0.074 & 0.022554 & 0.009605 & 0.339261 & 152.260 \\
\hline 11/17/08 5:00 & 2.153 & 0.693 & 0.230 & 103.179 & 1.423 & 0.077 & 0.023468 & 0.010170 & 0.359208 & 161.212 \\
\hline 11/17/08 6:00 & 2.153 & 0.693 & 0.230 & 103.179 & 1.423 & 0.077 & 0.023468 & 0.010170 & 0.359208 & 161.212 \\
\hline 11/17/08 7:00 & 2.149 & 0.689 & 0.227 & 101.713 & 1.423 & 0.077 & 0.023468 & 0.010170 & 0.359208 & 161.212 \\
\hline 11/17/08 8:00 & 2.145 & 0.685 & 0.223 & 100.260 & 1.42 & 0.074 & 0.022554 & 0.009605 & 0.339261 & 152.260 \\
\hline 11/17/08 9:00 & 2.145 & 0.685 & 0.223 & 100.260 & 1.42 & 0.074 & 0.022554 & 0.009605 & 0.339261 & 152.260 \\
\hline 11/17/08 10:00 & 2.147 & 0.687 & 0.225 & 100.985 & 1.42 & 0.074 & 0.022554 & 0.009605 & 0.339261 & 152.260 \\
\hline 11/17/08 11:00 & 2.147 & 0.687 & 0.225 & 100.985 & 1.42 & 0.074 & 0.022554 & 0.009605 & 0.339261 & 152.260 \\
\hline 11/17/08 12:00 & 2.146 & 0.686 & 0.224 & 100.622 & 1.422 & 0.076 & 0.023164 & 0.009981 & 0.352516 & 158.209 \\
\hline 11/17/08 13:00 & 2.142 & 0.682 & 0.221 & 99.178 & 1.422 & 0.076 & 0.023164 & 0.009981 & 0.352516 & 158.209 \\
\hline 11/17/08 14:00 & 2.144 & 0.684 & 0.223 & 99.898 & 1.422 & 0.076 & 0.023164 & 0.009981 & 0.352516 & 158.209 \\
\hline 11/17/08 15:00 & 2.141 & 0.681 & 0.220 & 98.819 & 1.422 & 0.076 & 0.023164 & 0.009981 & 0.352516 & 158.209 \\
\hline 11/17/08 16:00 & 2.141 & 0.681 & 0.220 & 98.819 & 1.422 & 0.076 & 0.023164 & 0.009981 & 0.352516 & 158.209 \\
\hline 11/17/08 17:00 & 2.143 & 0.683 & 0.222 & 99.538 & 1.422 & 0.076 & 0.023164 & 0.009981 & 0.352516 & 158.209 \\
\hline 11/17/08 18:00 & 2.143 & 0.683 & 0.222 & 99.538 & 1.422 & 0.076 & 0.023164 & 0.009981 & 0.352516 & 158.209 \\
\hline 11/17/08 19:00 & 2.144 & 0.684 & 0.223 & 99.898 & 1.423 & 0.077 & 0.023468 & 0.010170 & 0.359208 & 161.212 \\
\hline 11/17/08 20:00 & 2.144 & 0.684 & 0.223 & 99.898 & 1.423 & 0.077 & 0.023468 & 0.010170 & 0.359208 & 161.212 \\
\hline 11/17/08 21:00 & 2.142 & 0.682 & 0.221 & 99.178 & 1.423 & 0.077 & 0.023468 & 0.010170 & 0.359208 & 161.212 \\
\hline
\end{tabular}




\begin{tabular}{|c|c|c|c|c|c|c|c|c|c|c|}
\hline \multirow[b]{3}{*}{ Date Time } & \multicolumn{4}{|c|}{ V-Notch Weir } & \multicolumn{6}{|c|}{ R-Notch Weir } \\
\hline & \multicolumn{2}{|c|}{ Measured Water Level } & \multicolumn{2}{|c|}{$\begin{array}{c}\text { Calculated Rate } \\
\left(\mathrm{ft}^{3} / \mathrm{s}=448.8 \mathrm{gpm}\right)\end{array}$} & \multicolumn{3}{|c|}{ Measured Water Level } & \multicolumn{3}{|c|}{$\begin{array}{c}\text { Calculated Rate } \\
\left(\mathrm{ft}^{3} / \mathrm{s}=448.8 \mathrm{gpm}\right)\end{array}$} \\
\hline & $\begin{array}{c}\text { (ft above } \\
\text { sensor) }\end{array}$ & $\begin{array}{c}\text { (ft above } \\
\text { notch) }\end{array}$ & $\left(\mathrm{ft}^{3} / \mathrm{s}\right)$ & (gpm) & $\begin{array}{c}\text { (ft above } \\
\text { sensor) }\end{array}$ & $\begin{array}{c}\text { (ft above } \\
\text { log) }\end{array}$ & $\begin{array}{c}\text { (m above } \\
\text { log) }\end{array}$ & $\left(\mathrm{m}^{3} / \mathrm{s}\right)$ & $\left(\mathrm{ft}^{3} / \mathrm{s}\right)$ & (gpm) \\
\hline 11/17/08 22:00 & 2.147 & 0.687 & 0.225 & 100.985 & 1.423 & 0.077 & 0.023468 & 0.010170 & 0.359208 & 161.212 \\
\hline 11/17/08 23:00 & 2.15 & 0.690 & 0.227 & 102.078 & 1.423 & 0.077 & 0.023468 & 0.010170 & 0.359208 & 161.212 \\
\hline 11/18/08 0:00 & 2.148 & 0.688 & 0.226 & 101.348 & 1.422 & 0.076 & 0.023164 & 0.009981 & 0.352516 & 158.209 \\
\hline 11/18/08 1:00 & 2.148 & 0.688 & 0.226 & 101.348 & 1.422 & 0.076 & 0.023164 & 0.009981 & 0.352516 & 158.209 \\
\hline 11/18/08 2:00 & 2.148 & 0.688 & 0.226 & 101.348 & 1.425 & 0.079 & 0.024078 & 0.010553 & 0.372715 & 167.274 \\
\hline 11/18/08 3:00 & 2.151 & 0.691 & 0.228 & 102.444 & 1.425 & 0.079 & 0.024078 & 0.010553 & 0.372715 & 167.274 \\
\hline 11/18/08 4:00 & 2.146 & 0.686 & 0.224 & 100.622 & 1.425 & 0.079 & 0.024078 & 0.010553 & 0.372715 & 167.274 \\
\hline 11/18/08 5:00 & 2.142 & 0.682 & 0.221 & 99.178 & 1.422 & 0.076 & 0.023164 & 0.009981 & 0.352516 & 158.209 \\
\hline 11/18/08 6:00 & 2.137 & 0.677 & 0.217 & 97.391 & 1.422 & 0.076 & 0.023164 & 0.009981 & 0.352516 & 158.209 \\
\hline 11/18/08 7:00 & 2.144 & 0.684 & 0.223 & 99.898 & 1.425 & 0.079 & 0.024078 & 0.010553 & 0.372715 & 167.274 \\
\hline 11/18/08 8:00 & 2.142 & 0.682 & 0.221 & 99.178 & 1.425 & 0.079 & 0.024078 & 0.010553 & 0.372715 & 167.274 \\
\hline 11/18/08 9:00 & 2.142 & 0.682 & 0.221 & 99.178 & 1.422 & 0.076 & 0.023164 & 0.009981 & 0.352516 & 158.209 \\
\hline 11/18/08 10:00 & 2.142 & 0.682 & 0.221 & 99.178 & 1.425 & 0.079 & 0.024078 & 0.010553 & 0.372715 & 167.274 \\
\hline 11/18/08 11:00 & 2.144 & 0.684 & 0.223 & 99.898 & 1.425 & 0.079 & 0.024078 & 0.010553 & 0.372715 & 167.274 \\
\hline 11/18/08 12:00 & 2.141 & 0.681 & 0.220 & 98.819 & 1.425 & 0.079 & 0.024078 & 0.010553 & 0.372715 & 167.274 \\
\hline 11/18/08 13:00 & 2.147 & 0.687 & 0.225 & 100.985 & 1.427 & 0.081 & 0.024688 & 0.010940 & 0.386387 & 173.411 \\
\hline 11/18/08 14:00 & 2.148 & 0.688 & 0.226 & 101.348 & 1.427 & 0.081 & 0.024688 & 0.010940 & 0.386387 & 173.411 \\
\hline 11/18/08 15:00 & 2.145 & 0.685 & 0.223 & 100.260 & 1.429 & 0.083 & 0.025297 & 0.011331 & 0.400223 & 179.620 \\
\hline 11/18/08 16:00 & 2.145 & 0.685 & 0.223 & 100.260 & 1.427 & 0.081 & 0.024688 & 0.010940 & 0.386387 & 173.411 \\
\hline 11/18/08 17:00 & 2.147 & 0.687 & 0.225 & 100.985 & 1.432 & 0.086 & 0.026212 & 0.011928 & 0.421279 & 189.070 \\
\hline 11/18/08 18:00 & 2.145 & 0.685 & 0.223 & 100.260 & 1.432 & 0.086 & 0.026212 & 0.011928 & 0.421279 & 189.070 \\
\hline 11/18/08 19:00 & 2.148 & 0.688 & 0.226 & 101.348 & 1.432 & 0.086 & 0.026212 & 0.011928 & 0.421279 & 189.070 \\
\hline 11/18/08 20:00 & 2.151 & 0.691 & 0.228 & 102.444 & 1.432 & 0.086 & 0.026212 & 0.011928 & 0.421279 & 189.070 \\
\hline 11/18/08 21:00 & 2.154 & 0.694 & 0.231 & 103.547 & 1.434 & 0.088 & 0.026821 & 0.012331 & 0.435514 & 195.459 \\
\hline 11/18/08 22:00 & 2.154 & 0.694 & 0.231 & 103.547 & 1.434 & 0.088 & 0.026821 & 0.012331 & 0.435514 & 195.459 \\
\hline
\end{tabular}




\begin{tabular}{|c|c|c|c|c|c|c|c|c|c|c|}
\hline \multirow[b]{3}{*}{ Date Time } & \multicolumn{4}{|c|}{ V-Notch Weir } & \multicolumn{6}{|c|}{ R-Notch Weir } \\
\hline & \multicolumn{2}{|c|}{ Measured Water Level } & \multicolumn{2}{|c|}{$\begin{array}{c}\text { Calculated Rate } \\
\left(\mathrm{ft}^{3} / \mathrm{s}=448.8 \mathrm{gpm}\right)\end{array}$} & \multicolumn{3}{|c|}{ Measured Water Level } & \multicolumn{3}{|c|}{$\begin{array}{c}\text { Calculated Rate } \\
\left(\mathrm{ft}^{3} / \mathrm{s}=448.8 \mathrm{gpm}\right)\end{array}$} \\
\hline & $\begin{array}{c}\text { (ft above } \\
\text { sensor) }\end{array}$ & $\begin{array}{c}\text { (ft above } \\
\text { notch) }\end{array}$ & $\left(\mathrm{ft}^{3} / \mathrm{s}\right)$ & (gpm) & $\begin{array}{l}\text { (ft above } \\
\text { sensor) }\end{array}$ & $\begin{array}{c}\text { (ft above } \\
\text { log) }\end{array}$ & $\begin{array}{c}\text { (m above } \\
\text { log) }\end{array}$ & $\left(\mathrm{m}^{3} / \mathrm{s}\right)$ & $\left(\mathrm{ft}^{3} / \mathrm{s}\right)$ & (gpm) \\
\hline 11/18/08 23:00 & 2.154 & 0.694 & 0.231 & 103.547 & 1.437 & 0.091 & 0.027735 & 0.012943 & 0.457162 & 205.174 \\
\hline 11/19/08 0:00 & 2.157 & 0.697 & 0.233 & 104.657 & 1.439 & 0.093 & 0.028345 & 0.013358 & 0.471786 & 211.738 \\
\hline 11/19/08 1:00 & 2.152 & 0.692 & 0.229 & 102.811 & 1.439 & 0.093 & 0.028345 & 0.013358 & 0.471786 & 211.738 \\
\hline 11/19/08 2:00 & 2.155 & 0.695 & 0.232 & 103.916 & 1.439 & 0.093 & 0.028345 & 0.013358 & 0.471786 & 211.738 \\
\hline 11/19/08 3:00 & 2.155 & 0.695 & 0.232 & 103.916 & 1.441 & 0.095 & 0.028955 & 0.013776 & 0.486564 & 218.370 \\
\hline 11/19/08 4:00 & 2.155 & 0.695 & 0.232 & 103.916 & 1.439 & 0.093 & 0.028345 & 0.013358 & 0.471786 & 211.738 \\
\hline 11/19/08 5:00 & 2.148 & 0.688 & 0.226 & 101.348 & 1.439 & 0.093 & 0.028345 & 0.013358 & 0.471786 & 211.738 \\
\hline 11/19/08 6:00 & 2.151 & 0.691 & 0.228 & 102.444 & 1.439 & 0.093 & 0.028345 & 0.013358 & 0.471786 & 211.738 \\
\hline 11/19/08 7:00 & 2.146 & 0.686 & 0.224 & 100.622 & 1.437 & 0.091 & 0.027735 & 0.012943 & 0.457162 & 205.174 \\
\hline 11/19/08 8:00 & 2.142 & 0.682 & 0.221 & 99.178 & 1.437 & 0.091 & 0.027735 & 0.012943 & 0.457162 & 205.174 \\
\hline 11/19/08 9:00 & 2.139 & 0.679 & 0.219 & 98.103 & 1.434 & 0.088 & 0.026821 & 0.012331 & 0.435514 & 195.459 \\
\hline 11/19/08 10:00 & 2.137 & 0.677 & 0.217 & 97.391 & 1.432 & 0.086 & 0.026212 & 0.011928 & 0.421279 & 189.070 \\
\hline 11/19/08 11:00 & 2.138 & 0.678 & 0.218 & 97.747 & 1.434 & 0.088 & 0.026821 & 0.012331 & 0.435514 & 195.459 \\
\hline 11/19/08 12:00 & 2.137 & 0.677 & 0.217 & 97.391 & 1.432 & 0.086 & 0.026212 & 0.011928 & 0.421279 & 189.070 \\
\hline 11/19/08 13:00 & 2.141 & 0.681 & 0.220 & 98.819 & 1.432 & 0.086 & 0.026212 & 0.011928 & 0.421279 & 189.070 \\
\hline 11/19/08 14:00 & 2.137 & 0.677 & 0.217 & 97.391 & 1.432 & 0.086 & 0.026212 & 0.011928 & 0.421279 & 189.070 \\
\hline 11/19/08 15:00 & 2.134 & 0.674 & 0.215 & 96.328 & 1.432 & 0.086 & 0.026212 & 0.011928 & 0.421279 & 189.070 \\
\hline 11/19/08 16:00 & 2.137 & 0.677 & 0.217 & 97.391 & 1.432 & 0.086 & 0.026212 & 0.011928 & 0.421279 & 189.070 \\
\hline 11/19/08 17:00 & 2.141 & 0.681 & 0.220 & 98.819 & 1.432 & 0.086 & 0.026212 & 0.011928 & 0.421279 & 189.070 \\
\hline 11/19/08 18:00 & 2.144 & 0.684 & 0.223 & 99.898 & 1.432 & 0.086 & 0.026212 & 0.011928 & 0.421279 & 189.070 \\
\hline 11/19/08 19:00 & 2.145 & 0.685 & 0.223 & 100.260 & 1.432 & 0.086 & 0.026212 & 0.011928 & 0.421279 & 189.070 \\
\hline 11/19/08 20:00 & 2.145 & 0.685 & 0.223 & 100.260 & 1.432 & 0.086 & 0.026212 & 0.011928 & 0.421279 & 189.070 \\
\hline 11/19/08 21:00 & 2.141 & 0.681 & 0.220 & 98.819 & 1.43 & 0.084 & 0.025602 & 0.011529 & 0.407202 & 182.752 \\
\hline 11/19/08 22:00 & 2.132 & 0.672 & 0.213 & 95.623 & 1.43 & 0.084 & 0.025602 & 0.011529 & 0.407202 & 182.752 \\
\hline 11/19/08 23:00 & 2.128 & 0.668 & 0.210 & 94.223 & 1.43 & 0.084 & 0.025602 & 0.011529 & 0.407202 & 182.752 \\
\hline
\end{tabular}




\begin{tabular}{|c|c|c|c|c|c|c|c|c|c|c|}
\hline \multirow[b]{3}{*}{ Date Time } & \multicolumn{4}{|c|}{ V-Notch Weir } & \multicolumn{6}{|c|}{ R-Notch Weir } \\
\hline & \multicolumn{2}{|c|}{ Measured Water Level } & \multicolumn{2}{|c|}{$\begin{array}{c}\text { Calculated Rate } \\
\left(\mathrm{ft}^{3} / \mathrm{s}=448.8 \mathrm{gpm}\right)\end{array}$} & \multicolumn{3}{|c|}{ Measured Water Level } & \multicolumn{3}{|c|}{$\begin{array}{c}\text { Calculated Rate } \\
\left(\mathrm{ft}^{3} / \mathrm{s}=448.8 \mathrm{gpm}\right)\end{array}$} \\
\hline & $\begin{array}{c}\text { (ft above } \\
\text { sensor) }\end{array}$ & $\begin{array}{c}\text { (ft above } \\
\text { notch) }\end{array}$ & $\left(\mathrm{ft}^{3} / \mathrm{s}\right)$ & (gpm) & $\begin{array}{l}\text { (ft above } \\
\text { sensor) }\end{array}$ & $\begin{array}{c}\text { (ft above } \\
\text { log) }\end{array}$ & $\begin{array}{c}\text { (m above } \\
\text { log) }\end{array}$ & $\left(\mathrm{m}^{3} / \mathrm{s}\right)$ & $\left(\mathrm{ft}^{3} / \mathrm{s}\right)$ & (gpm) \\
\hline 11/20/08 0:00 & 2.128 & 0.668 & 0.210 & 94.223 & 1.43 & 0.084 & 0.025602 & 0.011529 & 0.407202 & 182.752 \\
\hline 11/20/08 1:00 & 2.129 & 0.669 & 0.211 & 94.572 & 1.43 & 0.084 & 0.025602 & 0.011529 & 0.407202 & 182.752 \\
\hline 11/20/08 2:00 & 2.129 & 0.669 & 0.211 & 94.572 & 1.43 & 0.084 & 0.025602 & 0.011529 & 0.407202 & 182.752 \\
\hline 11/20/08 3:00 & 2.12 & 0.660 & 0.204 & 91.459 & 1.427 & 0.081 & 0.024688 & 0.010940 & 0.386387 & 173.411 \\
\hline 11/20/08 4:00 & 2.125 & 0.665 & 0.208 & 93.181 & 1.43 & 0.084 & 0.025602 & 0.011529 & 0.407202 & 182.752 \\
\hline 11/20/08 5:00 & 2.12 & 0.660 & 0.204 & 91.459 & 1.427 & 0.081 & 0.024688 & 0.010940 & 0.386387 & 173.411 \\
\hline 11/20/08 6:00 & 2.125 & 0.665 & 0.208 & 93.181 & 1.427 & 0.081 & 0.024688 & 0.010940 & 0.386387 & 173.411 \\
\hline 11/20/08 7:00 & 2.114 & 0.654 & 0.199 & 89.419 & 1.427 & 0.081 & 0.024688 & 0.010940 & 0.386387 & 173.411 \\
\hline 11/20/08 8:00 & 2.109 & 0.649 & 0.195 & 87.740 & 1.427 & 0.081 & 0.024688 & 0.010940 & 0.386387 & 173.411 \\
\hline 11/20/08 9:00 & 2.112 & 0.652 & 0.198 & 88.745 & 1.425 & 0.079 & 0.024078 & 0.010553 & 0.372715 & 167.274 \\
\hline 11/20/08 10:00 & 2.102 & 0.642 & 0.190 & 85.421 & 1.425 & 0.079 & 0.024078 & 0.010553 & 0.372715 & 167.274 \\
\hline 11/20/08 11:00 & 2.09 & 0.630 & 0.182 & 81.532 & 1.427 & 0.081 & 0.024688 & 0.010940 & 0.386387 & 173.411 \\
\hline 11/20/08 12:00 & 2.097 & 0.637 & 0.187 & 83.788 & 1.422 & 0.076 & 0.023164 & 0.009981 & 0.352516 & 158.209 \\
\hline 11/20/08 13:00 & 2.087 & 0.627 & 0.180 & 80.577 & 1.427 & 0.081 & 0.024688 & 0.010940 & 0.386387 & 173.411 \\
\hline 11/20/08 14:00 & 2.086 & 0.626 & 0.179 & 80.260 & 1.425 & 0.079 & 0.024078 & 0.010553 & 0.372715 & 167.274 \\
\hline 11/20/08 15:00 & 2.084 & 0.624 & 0.177 & 79.629 & 1.425 & 0.079 & 0.024078 & 0.010553 & 0.372715 & 167.274 \\
\hline 11/20/08 16:00 & 2.079 & 0.619 & 0.174 & 78.063 & 1.425 & 0.079 & 0.024078 & 0.010553 & 0.372715 & 167.274 \\
\hline 11/20/08 17:00 & 2.079 & 0.619 & 0.174 & 78.063 & 1.425 & 0.079 & 0.024078 & 0.010553 & 0.372715 & 167.274 \\
\hline 11/20/08 18:00 & 2.07 & 0.610 & 0.168 & 75.291 & 1.425 & 0.079 & 0.024078 & 0.010553 & 0.372715 & 167.274 \\
\hline 11/20/08 19:00 & 2.066 & 0.606 & 0.165 & 74.079 & 1.425 & 0.079 & 0.024078 & 0.010553 & 0.372715 & 167.274 \\
\hline 11/20/08 20:00 & 2.067 & 0.607 & 0.166 & 74.381 & 1.425 & 0.079 & 0.024078 & 0.010553 & 0.372715 & 167.274 \\
\hline 11/20/08 21:00 & 2.065 & 0.605 & 0.164 & 73.777 & 1.425 & 0.079 & 0.024078 & 0.010553 & 0.372715 & 167.274 \\
\hline 11/20/08 22:00 & 2.059 & 0.599 & 0.160 & 71.985 & 1.425 & 0.079 & 0.024078 & 0.010553 & 0.372715 & 167.274 \\
\hline 11/20/08 23:00 & 2.059 & 0.599 & 0.160 & 71.985 & 1.425 & 0.079 & 0.024078 & 0.010553 & 0.372715 & 167.274 \\
\hline 11/21/08 0:00 & 2.055 & 0.595 & 0.158 & 70.805 & 1.425 & 0.079 & 0.024078 & 0.010553 & 0.372715 & 167.274 \\
\hline
\end{tabular}




\begin{tabular}{|c|c|c|c|c|c|c|c|c|c|c|}
\hline \multirow[b]{3}{*}{ Date Time } & \multicolumn{4}{|c|}{ V-Notch Weir } & \multicolumn{6}{|c|}{ R-Notch Weir } \\
\hline & \multicolumn{2}{|c|}{ Measured Water Level } & \multicolumn{2}{|c|}{$\begin{array}{c}\text { Calculated Rate } \\
\left(\mathrm{ft}^{3} / \mathrm{s}=448.8 \mathrm{gpm}\right)\end{array}$} & \multicolumn{3}{|c|}{ Measured Water Level } & \multicolumn{3}{|c|}{$\begin{array}{c}\text { Calculated Rate } \\
\left(\mathrm{ft}^{3} / \mathrm{s}=448.8 \mathrm{gpm}\right)\end{array}$} \\
\hline & $\begin{array}{c}\text { (ft above } \\
\text { sensor) }\end{array}$ & $\begin{array}{c}\text { (ft above } \\
\text { notch) }\end{array}$ & $\left(\mathrm{ft}^{3} / \mathrm{s}\right)$ & (gpm) & $\begin{array}{l}\text { (ft above } \\
\text { sensor) }\end{array}$ & $\begin{array}{c}\text { (ft above } \\
\text { log) }\end{array}$ & $\begin{array}{c}\text { (m above } \\
\text { log) }\end{array}$ & $\left(\mathrm{m}^{3} / \mathrm{s}\right)$ & $\left(\mathrm{ft}^{3} / \mathrm{s}\right)$ & (gpm) \\
\hline 11/21/08 1:00 & 2.065 & 0.605 & 0.164 & 73.777 & 1.425 & 0.079 & 0.024078 & 0.010553 & 0.372715 & 167.274 \\
\hline 11/21/08 2:00 & 2.072 & 0.612 & 0.169 & 75.902 & 1.425 & 0.079 & 0.024078 & 0.010553 & 0.372715 & 167.274 \\
\hline 11/21/08 3:00 & 2.06 & 0.600 & 0.161 & 72.282 & 1.425 & 0.079 & 0.024078 & 0.010553 & 0.372715 & 167.274 \\
\hline 11/21/08 4:00 & 2.058 & 0.598 & 0.160 & 71.689 & 1.425 & 0.079 & 0.024078 & 0.010553 & 0.372715 & 167.274 \\
\hline 11/21/08 5:00 & 2.055 & 0.595 & 0.158 & 70.805 & 1.422 & 0.076 & 0.023164 & 0.009981 & 0.352516 & 158.209 \\
\hline 11/21/08 6:00 & 2.055 & 0.595 & 0.158 & 70.805 & 1.422 & 0.076 & 0.023164 & 0.009981 & 0.352516 & 158.209 \\
\hline 11/21/08 7:00 & 2.055 & 0.595 & 0.158 & 70.805 & 1.422 & 0.076 & 0.023164 & 0.009981 & 0.352516 & 158.209 \\
\hline 11/21/08 8:00 & 2.051 & 0.591 & 0.155 & 69.636 & 1.422 & 0.076 & 0.023164 & 0.009981 & 0.352516 & 158.209 \\
\hline 11/21/08 9:00 & 2.051 & 0.591 & 0.155 & 69.636 & 1.42 & 0.074 & 0.022554 & 0.009605 & 0.339261 & 152.260 \\
\hline 11/21/08 10:00 & 2.051 & 0.591 & 0.155 & 69.636 & 1.422 & 0.076 & 0.023164 & 0.009981 & 0.352516 & 158.209 \\
\hline 11/21/08 11:00 & 2.05 & 0.590 & 0.155 & 69.346 & 1.422 & 0.076 & 0.023164 & 0.009981 & 0.352516 & 158.209 \\
\hline 11/21/08 12:00 & 2.066 & 0.606 & 0.165 & 74.079 & 1.424 & 0.078 & 0.023773 & 0.010361 & 0.365940 & 164.234 \\
\hline 11/21/08 13:00 & 2.067 & 0.607 & 0.166 & 74.381 & 1.424 & 0.078 & 0.023773 & 0.010361 & 0.365940 & 164.234 \\
\hline 11/21/08 14:00 & 2.08 & 0.620 & 0.175 & 78.375 & 1.427 & 0.081 & 0.024688 & 0.010940 & 0.386387 & 173.411 \\
\hline 11/21/08 15:00 & 2.089 & 0.629 & 0.181 & 81.213 & 1.429 & 0.083 & 0.025297 & 0.011331 & 0.400223 & 179.620 \\
\hline 11/21/08 16:00 & 2.096 & 0.636 & 0.186 & 83.463 & 1.431 & 0.085 & 0.025907 & 0.011728 & 0.414220 & 185.902 \\
\hline 11/21/08 17:00 & 2.099 & 0.639 & 0.188 & 84.439 & 1.429 & 0.083 & 0.025297 & 0.011331 & 0.400223 & 179.620 \\
\hline 11/21/08 18:00 & 2.101 & 0.641 & 0.190 & 85.093 & 1.431 & 0.085 & 0.025907 & 0.011728 & 0.414220 & 185.902 \\
\hline 11/21/08 19:00 & 2.109 & 0.649 & 0.195 & 87.740 & 1.431 & 0.085 & 0.025907 & 0.011728 & 0.414220 & 185.902 \\
\hline 11/21/08 20:00 & 2.107 & 0.647 & 0.194 & 87.074 & 1.431 & 0.085 & 0.025907 & 0.011728 & 0.414220 & 185.902 \\
\hline 11/21/08 21:00 & 2.105 & 0.645 & 0.193 & 86.410 & 1.431 & 0.085 & 0.025907 & 0.011728 & 0.414220 & 185.902 \\
\hline 11/21/08 22:00 & 2.105 & 0.645 & 0.193 & 86.410 & 1.431 & 0.085 & 0.025907 & 0.011728 & 0.414220 & 185.902 \\
\hline 11/21/08 23:00 & 2.107 & 0.647 & 0.194 & 87.074 & 1.431 & 0.085 & 0.025907 & 0.011728 & 0.414220 & 185.902 \\
\hline 11/22/08 0:00 & 2.11 & 0.650 & 0.196 & 88.074 & 1.431 & 0.085 & 0.025907 & 0.011728 & 0.414220 & 185.902 \\
\hline 11/22/08 1:00 & 2.105 & 0.645 & 0.193 & 86.410 & 1.431 & 0.085 & 0.025907 & 0.011728 & 0.414220 & 185.902 \\
\hline
\end{tabular}




\begin{tabular}{|c|c|c|c|c|c|c|c|c|c|c|}
\hline \multirow[b]{3}{*}{ Date Time } & \multicolumn{4}{|c|}{ V-Notch Weir } & \multicolumn{6}{|c|}{ R-Notch Weir } \\
\hline & \multicolumn{2}{|c|}{ Measured Water Level } & \multicolumn{2}{|c|}{$\begin{array}{c}\text { Calculated Rate } \\
\left(\mathrm{ft}^{3} / \mathrm{s}=448.8 \mathrm{gpm}\right)\end{array}$} & \multicolumn{3}{|c|}{ Measured Water Level } & \multicolumn{3}{|c|}{$\begin{array}{c}\text { Calculated Rate } \\
\left(\mathrm{ft}^{3} / \mathrm{s}=448.8 \mathrm{gpm}\right)\end{array}$} \\
\hline & $\begin{array}{c}\text { (ft above } \\
\text { sensor) }\end{array}$ & $\begin{array}{c}\text { (ft above } \\
\text { notch) }\end{array}$ & $\left(\mathrm{ft}^{3} / \mathrm{s}\right)$ & (gpm) & $\begin{array}{l}\text { (ft above } \\
\text { sensor) }\end{array}$ & $\begin{array}{c}\text { (ft above } \\
\text { log) }\end{array}$ & $\begin{array}{c}\text { (m above } \\
\text { log) }\end{array}$ & $\left(\mathrm{m}^{3} / \mathrm{s}\right)$ & $\left(\mathrm{ft}^{3} / \mathrm{s}\right)$ & (gpm) \\
\hline 11/22/08 2:00 & 2.108 & 0.648 & 0.195 & 87.406 & 1.434 & 0.088 & 0.026821 & 0.012331 & 0.435514 & 195.459 \\
\hline 11/22/08 3:00 & 2.113 & 0.653 & 0.198 & 89.082 & 1.434 & 0.088 & 0.026821 & 0.012331 & 0.435514 & 195.459 \\
\hline 11/22/08 4:00 & 2.108 & 0.648 & 0.195 & 87.406 & 1.434 & 0.088 & 0.026821 & 0.012331 & 0.435514 & 195.459 \\
\hline 11/22/08 5:00 & 2.108 & 0.648 & 0.195 & 87.406 & 1.434 & 0.088 & 0.026821 & 0.012331 & 0.435514 & 195.459 \\
\hline 11/22/08 6:00 & 2.111 & 0.651 & 0.197 & 88.409 & 1.434 & 0.088 & 0.026821 & 0.012331 & 0.435514 & 195.459 \\
\hline 11/22/08 7:00 & 2.111 & 0.651 & 0.197 & 88.409 & 1.434 & 0.088 & 0.026821 & 0.012331 & 0.435514 & 195.459 \\
\hline 11/22/08 8:00 & 2.111 & 0.651 & 0.197 & 88.409 & 1.434 & 0.088 & 0.026821 & 0.012331 & 0.435514 & 195.459 \\
\hline 11/22/08 9:00 & 2.108 & 0.648 & 0.195 & 87.406 & 1.436 & 0.09 & 0.027431 & 0.012738 & 0.449907 & 201.918 \\
\hline 11/22/08 10:00 & 2.108 & 0.648 & 0.195 & 87.406 & 1.434 & 0.088 & 0.026821 & 0.012331 & 0.435514 & 195.459 \\
\hline 11/22/08 11:00 & 2.11 & 0.650 & 0.196 & 88.074 & 1.434 & 0.088 & 0.026821 & 0.012331 & 0.435514 & 195.459 \\
\hline 11/22/08 12:00 & 2.112 & 0.652 & 0.198 & 88.745 & 1.434 & 0.088 & 0.026821 & 0.012331 & 0.435514 & 195.459 \\
\hline 11/22/08 13:00 & 2.114 & 0.654 & 0.199 & 89.419 & 1.434 & 0.088 & 0.026821 & 0.012331 & 0.435514 & 195.459 \\
\hline 11/22/08 14:00 & 2.117 & 0.657 & 0.202 & 90.436 & 1.434 & 0.088 & 0.026821 & 0.012331 & 0.435514 & 195.459 \\
\hline 11/22/08 15:00 & 2.119 & 0.659 & 0.203 & 91.117 & 1.436 & 0.09 & 0.027431 & 0.012738 & 0.449907 & 201.918 \\
\hline 11/22/08 16:00 & 2.121 & 0.661 & 0.205 & 91.802 & 1.434 & 0.088 & 0.026821 & 0.012331 & 0.435514 & 195.459 \\
\hline 11/22/08 17:00 & 2.125 & 0.665 & 0.208 & 93.181 & 1.434 & 0.088 & 0.026821 & 0.012331 & 0.435514 & 195.459 \\
\hline 11/22/08 18:00 & 2.126 & 0.666 & 0.208 & 93.527 & 1.434 & 0.088 & 0.026821 & 0.012331 & 0.435514 & 195.459 \\
\hline 11/22/08 19:00 & 2.124 & 0.664 & 0.207 & 92.835 & 1.434 & 0.088 & 0.026821 & 0.012331 & 0.435514 & 195.459 \\
\hline 11/22/08 20:00 & 2.117 & 0.657 & 0.202 & 90.436 & 1.431 & 0.085 & 0.025907 & 0.011728 & 0.414220 & 185.902 \\
\hline 11/22/08 21:00 & 2.115 & 0.655 & 0.200 & 89.757 & 1.431 & 0.085 & 0.025907 & 0.011728 & 0.414220 & 185.902 \\
\hline 11/22/08 22:00 & 2.116 & 0.656 & 0.201 & 90.096 & 1.431 & 0.085 & 0.025907 & 0.011728 & 0.414220 & 185.902 \\
\hline 11/22/08 23:00 & 2.116 & 0.656 & 0.201 & 90.096 & 1.431 & 0.085 & 0.025907 & 0.011728 & 0.414220 & 185.902 \\
\hline 11/23/08 0:00 & 2.114 & 0.654 & 0.199 & 89.419 & 1.431 & 0.085 & 0.025907 & 0.011728 & 0.414220 & 185.902 \\
\hline 11/23/08 1:00 & 2.119 & 0.659 & 0.203 & 91.117 & 1.431 & 0.085 & 0.025907 & 0.011728 & 0.414220 & 185.902 \\
\hline 11/23/08 2:00 & 2.115 & 0.655 & 0.200 & 89.757 & 1.431 & 0.085 & 0.025907 & 0.011728 & 0.414220 & 185.902 \\
\hline
\end{tabular}




\begin{tabular}{|c|c|c|c|c|c|c|c|c|c|c|}
\hline \multirow[b]{3}{*}{ Date Time } & \multicolumn{4}{|c|}{ V-Notch Weir } & \multicolumn{6}{|c|}{ R-Notch Weir } \\
\hline & \multicolumn{2}{|c|}{ Measured Water Level } & \multicolumn{2}{|c|}{$\begin{array}{c}\text { Calculated Rate } \\
\left(\mathrm{ft}^{3} / \mathrm{s}=448.8 \mathrm{gpm}\right)\end{array}$} & \multicolumn{3}{|c|}{ Measured Water Level } & \multicolumn{3}{|c|}{$\begin{array}{c}\text { Calculated Rate } \\
\left(\mathrm{ft}^{3} / \mathrm{s}=448.8 \mathrm{gpm}\right)\end{array}$} \\
\hline & $\begin{array}{c}\text { (ft above } \\
\text { sensor) }\end{array}$ & $\begin{array}{c}\text { (ft above } \\
\text { notch) }\end{array}$ & $\left(\mathrm{ft}^{3} / \mathrm{s}\right)$ & (gpm) & $\begin{array}{l}\text { (ft above } \\
\text { sensor) }\end{array}$ & $\begin{array}{c}\text { (ft above } \\
\text { log) }\end{array}$ & $\begin{array}{c}\text { (m above } \\
\text { log) }\end{array}$ & $\left(\mathrm{m}^{3} / \mathrm{s}\right)$ & $\left(\mathrm{ft}^{3} / \mathrm{s}\right)$ & (gpm) \\
\hline 11/23/08 3:00 & 2.117 & 0.657 & 0.202 & 90.436 & 1.434 & 0.088 & 0.026821 & 0.012331 & 0.435514 & 195.459 \\
\hline 11/23/08 4:00 & 2.119 & 0.659 & 0.203 & 91.117 & 1.434 & 0.088 & 0.026821 & 0.012331 & 0.435514 & 195.459 \\
\hline 11/23/08 5:00 & 2.117 & 0.657 & 0.202 & 90.436 & 1.434 & 0.088 & 0.026821 & 0.012331 & 0.435514 & 195.459 \\
\hline 11/23/08 6:00 & 2.12 & 0.660 & 0.204 & 91.459 & 1.434 & 0.088 & 0.026821 & 0.012331 & 0.435514 & 195.459 \\
\hline 11/23/08 7:00 & 2.12 & 0.660 & 0.204 & 91.459 & 1.434 & 0.088 & 0.026821 & 0.012331 & 0.435514 & 195.459 \\
\hline 11/23/08 8:00 & 2.115 & 0.655 & 0.200 & 89.757 & 1.434 & 0.088 & 0.026821 & 0.012331 & 0.435514 & 195.459 \\
\hline 11/23/08 9:00 & 2.11 & 0.650 & 0.196 & 88.074 & 1.436 & 0.09 & 0.027431 & 0.012738 & 0.449907 & 201.918 \\
\hline 11/23/08 10:00 & 2.117 & 0.657 & 0.202 & 90.436 & 1.434 & 0.088 & 0.026821 & 0.012331 & 0.435514 & 195.459 \\
\hline 11/23/08 11:00 & 2.121 & 0.661 & 0.205 & 91.802 & 1.434 & 0.088 & 0.026821 & 0.012331 & 0.435514 & 195.459 \\
\hline 11/23/08 12:00 & 2.12 & 0.660 & 0.204 & 91.459 & 1.434 & 0.088 & 0.026821 & 0.012331 & 0.435514 & 195.459 \\
\hline 11/23/08 13:00 & 2.125 & 0.665 & 0.208 & 93.181 & 1.439 & 0.093 & 0.028345 & 0.013358 & 0.471786 & 211.738 \\
\hline 11/23/08 14:00 & 2.127 & 0.667 & 0.209 & 93.875 & 1.439 & 0.093 & 0.028345 & 0.013358 & 0.471786 & 211.738 \\
\hline 11/23/08 15:00 & 2.129 & 0.669 & 0.211 & 94.572 & 1.439 & 0.093 & 0.028345 & 0.013358 & 0.471786 & 211.738 \\
\hline 11/23/08 16:00 & 2.128 & 0.668 & 0.210 & 94.223 & 1.436 & 0.09 & 0.027431 & 0.012738 & 0.449907 & 201.918 \\
\hline 11/23/08 17:00 & 2.124 & 0.664 & 0.207 & 92.835 & 1.436 & 0.09 & 0.027431 & 0.012738 & 0.449907 & 201.918 \\
\hline 11/23/08 18:00 & 2.129 & 0.669 & 0.211 & 94.572 & 1.436 & 0.09 & 0.027431 & 0.012738 & 0.449907 & 201.918 \\
\hline 11/23/08 19:00 & 2.136 & 0.676 & 0.216 & 97.036 & 1.434 & 0.088 & 0.026821 & 0.012331 & 0.435514 & 195.459 \\
\hline 11/23/08 20:00 & 2.13 & 0.670 & 0.212 & 94.921 & 1.431 & 0.085 & 0.025907 & 0.011728 & 0.414220 & 185.902 \\
\hline 11/23/08 21:00 & 2.123 & 0.663 & 0.206 & 92.490 & 1.432 & 0.086 & 0.026212 & 0.011928 & 0.421279 & 189.070 \\
\hline 11/23/08 22:00 & 2.128 & 0.668 & 0.210 & 94.223 & 1.429 & 0.083 & 0.025297 & 0.011331 & 0.400223 & 179.620 \\
\hline 11/23/08 23:00 & 2.119 & 0.659 & 0.203 & 91.117 & 1.432 & 0.086 & 0.026212 & 0.011928 & 0.421279 & 189.070 \\
\hline 11/24/08 0:00 & 2.122 & 0.662 & 0.205 & 92.146 & 1.432 & 0.086 & 0.026212 & 0.011928 & 0.421279 & 189.070 \\
\hline 11/24/08 1:00 & 2.116 & 0.656 & 0.201 & 90.096 & 1.429 & 0.083 & 0.025297 & 0.011331 & 0.400223 & 179.620 \\
\hline 11/24/08 2:00 & 2.118 & 0.658 & 0.202 & 90.776 & 1.429 & 0.083 & 0.025297 & 0.011331 & 0.400223 & 179.620 \\
\hline 11/24/08 3:00 & 2.119 & 0.659 & 0.203 & 91.117 & 1.429 & 0.083 & 0.025297 & 0.011331 & 0.400223 & 179.620 \\
\hline
\end{tabular}




\begin{tabular}{|c|c|c|c|c|c|c|c|c|c|c|}
\hline \multirow[b]{3}{*}{ Date Time } & \multicolumn{4}{|c|}{ V-Notch Weir } & \multicolumn{6}{|c|}{ R-Notch Weir } \\
\hline & \multicolumn{2}{|c|}{ Measured Water Level } & \multicolumn{2}{|c|}{$\begin{array}{c}\text { Calculated Rate } \\
\left(\mathrm{ft}^{3} / \mathrm{s}=448.8 \mathrm{gpm}\right)\end{array}$} & \multicolumn{3}{|c|}{ Measured Water Level } & \multicolumn{3}{|c|}{$\begin{array}{c}\text { Calculated Rate } \\
\left(\mathrm{ft}^{3} / \mathrm{s}=448.8 \mathrm{gpm}\right)\end{array}$} \\
\hline & $\begin{array}{c}\text { (ft above } \\
\text { sensor) }\end{array}$ & $\begin{array}{c}\text { (ft above } \\
\text { notch) }\end{array}$ & $\left(\mathrm{ft}^{3} / \mathrm{s}\right)$ & (gpm) & $\begin{array}{l}\text { (ft above } \\
\text { sensor) }\end{array}$ & $\begin{array}{c}\text { (ft above } \\
\text { log) }\end{array}$ & $\begin{array}{c}\text { (m above } \\
\text { log) }\end{array}$ & $\left(\mathrm{m}^{3} / \mathrm{s}\right)$ & $\left(\mathrm{ft}^{3} / \mathrm{s}\right)$ & (gpm) \\
\hline 11/24/08 4:00 & 2.119 & 0.659 & 0.203 & 91.117 & 1.429 & 0.083 & 0.025297 & 0.011331 & 0.400223 & 179.620 \\
\hline 11/24/08 5:00 & 2.117 & 0.657 & 0.202 & 90.436 & 1.429 & 0.083 & 0.025297 & 0.011331 & 0.400223 & 179.620 \\
\hline 11/24/08 6:00 & 2.119 & 0.659 & 0.203 & 91.117 & 1.429 & 0.083 & 0.025297 & 0.011331 & 0.400223 & 179.620 \\
\hline 11/24/08 7:00 & 2.115 & 0.655 & 0.200 & 89.757 & 1.427 & 0.081 & 0.024688 & 0.010940 & 0.386387 & 173.411 \\
\hline 11/24/08 8:00 & 2.113 & 0.653 & 0.198 & 89.082 & 1.427 & 0.081 & 0.024688 & 0.010940 & 0.386387 & 173.411 \\
\hline 11/24/08 9:00 & 2.115 & 0.655 & 0.200 & 89.757 & 1.427 & 0.081 & 0.024688 & 0.010940 & 0.386387 & 173.411 \\
\hline 11/24/08 10:00 & 2.115 & 0.655 & 0.200 & 89.757 & 1.429 & 0.083 & 0.025297 & 0.011331 & 0.400223 & 179.620 \\
\hline 11/24/08 11:00 & 2.114 & 0.654 & 0.199 & 89.419 & 1.429 & 0.083 & 0.025297 & 0.011331 & 0.400223 & 179.620 \\
\hline 11/24/08 12:00 & 2.118 & 0.658 & 0.202 & 90.776 & 1.429 & 0.083 & 0.025297 & 0.011331 & 0.400223 & 179.620 \\
\hline 11/24/08 13:00 & 2.121 & 0.661 & 0.205 & 91.802 & 1.429 & 0.083 & 0.025297 & 0.011331 & 0.400223 & 179.620 \\
\hline 11/24/08 14:00 & 2.125 & 0.665 & 0.208 & 93.181 & 1.431 & 0.085 & 0.025907 & 0.011728 & 0.414220 & 185.902 \\
\hline 11/24/08 15:00 & 2.127 & 0.667 & 0.209 & 93.875 & 1.431 & 0.085 & 0.025907 & 0.011728 & 0.414220 & 185.902 \\
\hline 11/24/08 16:00 & 2.127 & 0.667 & 0.209 & 93.875 & 1.431 & 0.085 & 0.025907 & 0.011728 & 0.414220 & 185.902 \\
\hline 11/24/08 17:00 & 2.12 & 0.660 & 0.204 & 91.459 & 1.429 & 0.083 & 0.025297 & 0.011331 & 0.400223 & 179.620 \\
\hline 11/24/08 18:00 & 2.125 & 0.665 & 0.208 & 93.181 & 1.432 & 0.086 & 0.026212 & 0.011928 & 0.421279 & 189.070 \\
\hline 11/24/08 19:00 & 2.125 & 0.665 & 0.208 & 93.181 & 1.432 & 0.086 & 0.026212 & 0.011928 & 0.421279 & 189.070 \\
\hline 11/24/08 20:00 & 2.123 & 0.663 & 0.206 & 92.490 & 1.432 & 0.086 & 0.026212 & 0.011928 & 0.421279 & 189.070 \\
\hline 11/24/08 21:00 & 2.124 & 0.664 & 0.207 & 92.835 & 1.429 & 0.083 & 0.025297 & 0.011331 & 0.400223 & 179.620 \\
\hline 11/24/08 22:00 & 2.124 & 0.664 & 0.207 & 92.835 & 1.429 & 0.083 & 0.025297 & 0.011331 & 0.400223 & 179.620 \\
\hline 11/24/08 23:00 & 2.125 & 0.665 & 0.208 & 93.181 & 1.429 & 0.083 & 0.025297 & 0.011331 & 0.400223 & 179.620 \\
\hline 11/25/08 0:00 & 2.123 & 0.663 & 0.206 & 92.490 & 1.429 & 0.083 & 0.025297 & 0.011331 & 0.400223 & 179.620 \\
\hline 11/25/08 1:00 & 2.121 & 0.661 & 0.205 & 91.802 & 1.429 & 0.083 & 0.025297 & 0.011331 & 0.400223 & 179.620 \\
\hline 11/25/08 2:00 & 2.119 & 0.659 & 0.203 & 91.117 & 1.427 & 0.081 & 0.024688 & 0.010940 & 0.386387 & 173.411 \\
\hline 11/25/08 3:00 & 2.117 & 0.657 & 0.202 & 90.436 & 1.429 & 0.083 & 0.025297 & 0.011331 & 0.400223 & 179.620 \\
\hline 11/25/08 4:00 & 2.117 & 0.657 & 0.202 & 90.436 & 1.427 & 0.081 & 0.024688 & 0.010940 & 0.386387 & 173.411 \\
\hline
\end{tabular}




\begin{tabular}{|c|c|c|c|c|c|c|c|c|c|c|}
\hline \multirow[b]{3}{*}{ Date Time } & \multicolumn{4}{|c|}{ V-Notch Weir } & \multicolumn{6}{|c|}{ R-Notch Weir } \\
\hline & \multicolumn{2}{|c|}{ Measured Water Level } & \multicolumn{2}{|c|}{$\begin{array}{c}\text { Calculated Rate } \\
\left(\mathrm{ft}^{3} / \mathrm{s}=448.8 \mathrm{gpm}\right)\end{array}$} & \multicolumn{3}{|c|}{ Measured Water Level } & \multicolumn{3}{|c|}{$\begin{array}{c}\text { Calculated Rate } \\
\left(\mathrm{ft}^{3} / \mathrm{s}=448.8 \mathrm{gpm}\right)\end{array}$} \\
\hline & $\begin{array}{c}\text { (ft above } \\
\text { sensor) }\end{array}$ & $\begin{array}{c}\text { (ft above } \\
\text { notch) }\end{array}$ & $\left(\mathrm{ft}^{3} / \mathrm{s}\right)$ & (gpm) & $\begin{array}{l}\text { (ft above } \\
\text { sensor) }\end{array}$ & $\begin{array}{c}\text { (ft above } \\
\text { log) }\end{array}$ & $\begin{array}{c}\text { (m above } \\
\text { log) }\end{array}$ & $\left(\mathrm{m}^{3} / \mathrm{s}\right)$ & $\left(\mathrm{ft}^{3} / \mathrm{s}\right)$ & (gpm) \\
\hline 11/25/08 5:00 & 2.11 & 0.650 & 0.196 & 88.074 & 1.427 & 0.081 & 0.024688 & 0.010940 & 0.386387 & 173.411 \\
\hline 11/25/08 6:00 & 2.115 & 0.655 & 0.200 & 89.757 & 1.427 & 0.081 & 0.024688 & 0.010940 & 0.386387 & 173.411 \\
\hline 11/25/08 7:00 & 2.115 & 0.655 & 0.200 & 89.757 & 1.427 & 0.081 & 0.024688 & 0.010940 & 0.386387 & 173.411 \\
\hline 11/25/08 8:00 & 2.111 & 0.651 & 0.197 & 88.409 & 1.427 & 0.081 & 0.024688 & 0.010940 & 0.386387 & 173.411 \\
\hline 11/25/08 9:00 & 2.113 & 0.653 & 0.198 & 89.082 & 1.424 & 0.078 & 0.023773 & 0.010361 & 0.365940 & 164.234 \\
\hline 11/25/08 10:00 & 2.113 & 0.653 & 0.198 & 89.082 & 1.427 & 0.081 & 0.024688 & 0.010940 & 0.386387 & 173.411 \\
\hline 11/25/08 11:00 & 2.115 & 0.655 & 0.200 & 89.757 & 1.427 & 0.081 & 0.024688 & 0.010940 & 0.386387 & 173.411 \\
\hline 11/25/08 12:00 & 2.116 & 0.656 & 0.201 & 90.096 & 1.429 & 0.083 & 0.025297 & 0.011331 & 0.400223 & 179.620 \\
\hline 11/25/08 13:00 & 2.127 & 0.667 & 0.209 & 93.875 & 1.429 & 0.083 & 0.025297 & 0.011331 & 0.400223 & 179.620 \\
\hline 11/25/08 14:00 & 2.13 & 0.670 & 0.212 & 94.921 & 1.431 & 0.085 & 0.025907 & 0.011728 & 0.414220 & 185.902 \\
\hline 11/25/08 15:00 & 2.132 & 0.672 & 0.213 & 95.623 & 1.434 & 0.088 & 0.026821 & 0.012331 & 0.435514 & 195.459 \\
\hline 11/25/08 16:00 & 2.131 & 0.671 & 0.212 & 95.272 & 1.431 & 0.085 & 0.025907 & 0.011728 & 0.414220 & 185.902 \\
\hline 11/25/08 17:00 & 2.129 & 0.669 & 0.211 & 94.572 & 1.431 & 0.085 & 0.025907 & 0.011728 & 0.414220 & 185.902 \\
\hline 11/25/08 18:00 & 2.132 & 0.672 & 0.213 & 95.623 & 1.434 & 0.088 & 0.026821 & 0.012331 & 0.435514 & 195.459 \\
\hline 11/25/08 19:00 & 2.13 & 0.670 & 0.212 & 94.921 & 1.434 & 0.088 & 0.026821 & 0.012331 & 0.435514 & 195.459 \\
\hline 11/25/08 20:00 & 2.128 & 0.668 & 0.210 & 94.223 & 1.432 & 0.086 & 0.026212 & 0.011928 & 0.421279 & 189.070 \\
\hline 11/25/08 21:00 & 2.129 & 0.669 & 0.211 & 94.572 & 1.432 & 0.086 & 0.026212 & 0.011928 & 0.421279 & 189.070 \\
\hline 11/25/08 22:00 & 2.129 & 0.669 & 0.211 & 94.572 & 1.432 & 0.086 & 0.026212 & 0.011928 & 0.421279 & 189.070 \\
\hline 11/25/08 23:00 & 2.13 & 0.670 & 0.212 & 94.921 & 1.432 & 0.086 & 0.026212 & 0.011928 & 0.421279 & 189.070 \\
\hline 11/26/08 0:00 & 2.13 & 0.670 & 0.212 & 94.921 & 1.432 & 0.086 & 0.026212 & 0.011928 & 0.421279 & 189.070 \\
\hline 11/26/08 1:00 & 2.126 & 0.666 & 0.208 & 93.527 & 1.432 & 0.086 & 0.026212 & 0.011928 & 0.421279 & 189.070 \\
\hline 11/26/08 2:00 & 2.126 & 0.666 & 0.208 & 93.527 & 1.432 & 0.086 & 0.026212 & 0.011928 & 0.421279 & 189.070 \\
\hline 11/26/08 3:00 & 2.124 & 0.664 & 0.207 & 92.835 & 1.429 & 0.083 & 0.025297 & 0.011331 & 0.400223 & 179.620 \\
\hline 11/26/08 4:00 & 2.122 & 0.662 & 0.205 & 92.146 & 1.429 & 0.083 & 0.025297 & 0.011331 & 0.400223 & 179.620 \\
\hline 11/26/08 5:00 & 2.119 & 0.659 & 0.203 & 91.117 & 1.432 & 0.086 & 0.026212 & 0.011928 & 0.421279 & 189.070 \\
\hline
\end{tabular}




\begin{tabular}{|c|c|c|c|c|c|c|c|c|c|c|}
\hline \multirow[b]{3}{*}{ Date Time } & \multicolumn{4}{|c|}{ V-Notch Weir } & \multicolumn{6}{|c|}{ R-Notch Weir } \\
\hline & \multicolumn{2}{|c|}{ Measured Water Level } & \multicolumn{2}{|c|}{$\begin{array}{c}\text { Calculated Rate } \\
\left(\mathrm{ft}^{3} / \mathrm{s}=448.8 \mathrm{gpm}\right)\end{array}$} & \multicolumn{3}{|c|}{ Measured Water Level } & \multicolumn{3}{|c|}{$\begin{array}{c}\text { Calculated Rate } \\
\left(\mathrm{ft}^{3} / \mathrm{s}=448.8 \mathrm{gpm}\right)\end{array}$} \\
\hline & $\begin{array}{c}\text { (ft above } \\
\text { sensor) }\end{array}$ & $\begin{array}{c}\text { (ft above } \\
\text { notch) }\end{array}$ & $\left(\mathrm{ft}^{3} / \mathrm{s}\right)$ & (gpm) & $\begin{array}{l}\text { (ft above } \\
\text { sensor) }\end{array}$ & $\begin{array}{c}\text { (ft above } \\
\text { log) }\end{array}$ & $\begin{array}{c}\text { (m above } \\
\text { log) }\end{array}$ & $\left(\mathrm{m}^{3} / \mathrm{s}\right)$ & $\left(\mathrm{ft}^{3} / \mathrm{s}\right)$ & (gpm) \\
\hline 11/26/08 6:00 & 2.124 & 0.664 & 0.207 & 92.835 & 1.432 & 0.086 & 0.026212 & 0.011928 & 0.421279 & 189.070 \\
\hline 11/26/08 7:00 & 2.129 & 0.669 & 0.211 & 94.572 & 1.429 & 0.083 & 0.025297 & 0.011331 & 0.400223 & 179.620 \\
\hline 11/26/08 8:00 & 2.124 & 0.664 & 0.207 & 92.835 & 1.429 & 0.083 & 0.025297 & 0.011331 & 0.400223 & 179.620 \\
\hline 11/26/08 9:00 & 2.12 & 0.660 & 0.204 & 91.459 & 1.429 & 0.083 & 0.025297 & 0.011331 & 0.400223 & 179.620 \\
\hline 11/26/08 10:00 & 2.122 & 0.662 & 0.205 & 92.146 & 1.429 & 0.083 & 0.025297 & 0.011331 & 0.400223 & 179.620 \\
\hline 11/26/08 11:00 & 2.121 & 0.661 & 0.205 & 91.802 & 1.429 & 0.083 & 0.025297 & 0.011331 & 0.400223 & 179.620 \\
\hline 11/26/08 12:00 & 2.12 & 0.660 & 0.204 & 91.459 & 1.429 & 0.083 & 0.025297 & 0.011331 & 0.400223 & 179.620 \\
\hline 11/26/08 13:00 & 2.121 & 0.661 & 0.205 & 91.802 & 1.431 & 0.085 & 0.025907 & 0.011728 & 0.414220 & 185.902 \\
\hline 11/26/08 14:00 & 2.125 & 0.665 & 0.208 & 93.181 & 1.431 & 0.085 & 0.025907 & 0.011728 & 0.414220 & 185.902 \\
\hline 11/26/08 15:00 & 2.12 & 0.660 & 0.204 & 91.459 & 1.431 & 0.085 & 0.025907 & 0.011728 & 0.414220 & 185.902 \\
\hline 11/26/08 16:00 & 2.126 & 0.666 & 0.208 & 93.527 & 1.431 & 0.085 & 0.025907 & 0.011728 & 0.414220 & 185.902 \\
\hline 11/26/08 17:00 & 2.124 & 0.664 & 0.207 & 92.835 & 1.431 & 0.085 & 0.025907 & 0.011728 & 0.414220 & 185.902 \\
\hline 11/26/08 18:00 & 2.129 & 0.669 & 0.211 & 94.572 & 1.432 & 0.086 & 0.026212 & 0.011928 & 0.421279 & 189.070 \\
\hline 11/26/08 19:00 & 2.127 & 0.667 & 0.209 & 93.875 & 1.432 & 0.086 & 0.026212 & 0.011928 & 0.421279 & 189.070 \\
\hline 11/26/08 20:00 & 2.128 & 0.668 & 0.210 & 94.223 & 1.432 & 0.086 & 0.026212 & 0.011928 & 0.421279 & 189.070 \\
\hline 11/26/08 21:00 & 2.128 & 0.668 & 0.210 & 94.223 & 1.432 & 0.086 & 0.026212 & 0.011928 & 0.421279 & 189.070 \\
\hline 11/26/08 22:00 & 2.129 & 0.669 & 0.211 & 94.572 & 1.432 & 0.086 & 0.026212 & 0.011928 & 0.421279 & 189.070 \\
\hline 11/26/08 23:00 & 2.127 & 0.667 & 0.209 & 93.875 & 1.432 & 0.086 & 0.026212 & 0.011928 & 0.421279 & 189.070 \\
\hline 11/27/08 0:00 & 2.125 & 0.665 & 0.208 & 93.181 & 1.429 & 0.083 & 0.025297 & 0.011331 & 0.400223 & 179.620 \\
\hline 11/27/08 1:00 & 2.123 & 0.663 & 0.206 & 92.490 & 1.429 & 0.083 & 0.025297 & 0.011331 & 0.400223 & 179.620 \\
\hline 11/27/08 2:00 & 2.123 & 0.663 & 0.206 & 92.490 & 1.429 & 0.083 & 0.025297 & 0.011331 & 0.400223 & 179.620 \\
\hline 11/27/08 3:00 & 2.121 & 0.661 & 0.205 & 91.802 & 1.429 & 0.083 & 0.025297 & 0.011331 & 0.400223 & 179.620 \\
\hline 11/27/08 4:00 & 2.121 & 0.661 & 0.205 & 91.802 & 1.429 & 0.083 & 0.025297 & 0.011331 & 0.400223 & 179.620 \\
\hline 11/27/08 5:00 & 2.121 & 0.661 & 0.205 & 91.802 & 1.429 & 0.083 & 0.025297 & 0.011331 & 0.400223 & 179.620 \\
\hline 11/27/08 6:00 & 2.122 & 0.662 & 0.205 & 92.146 & 1.429 & 0.083 & 0.025297 & 0.011331 & 0.400223 & 179.620 \\
\hline
\end{tabular}




\begin{tabular}{|c|c|c|c|c|c|c|c|c|c|c|}
\hline \multirow[b]{3}{*}{ Date Time } & \multicolumn{4}{|c|}{ V-Notch Weir } & \multicolumn{6}{|c|}{ R-Notch Weir } \\
\hline & \multicolumn{2}{|c|}{ Measured Water Level } & \multicolumn{2}{|c|}{$\begin{array}{c}\text { Calculated Rate } \\
\left(\mathrm{ft}^{3} / \mathrm{s}=448.8 \mathrm{gpm}\right)\end{array}$} & \multicolumn{3}{|c|}{ Measured Water Level } & \multicolumn{3}{|c|}{$\begin{array}{c}\text { Calculated Rate } \\
\left(\mathrm{ft}^{3} / \mathrm{s}=448.8 \mathrm{gpm}\right)\end{array}$} \\
\hline & $\begin{array}{c}\text { (ft above } \\
\text { sensor) }\end{array}$ & $\begin{array}{c}\text { (ft above } \\
\text { notch) }\end{array}$ & $\left(\mathrm{ft}^{3} / \mathrm{s}\right)$ & (gpm) & $\begin{array}{l}\text { (ft above } \\
\text { sensor) }\end{array}$ & $\begin{array}{c}\text { (ft above } \\
\text { log) }\end{array}$ & $\begin{array}{c}\text { (m above } \\
\text { log) }\end{array}$ & $\left(\mathrm{m}^{3} / \mathrm{s}\right)$ & $\left(\mathrm{ft}^{3} / \mathrm{s}\right)$ & (gpm) \\
\hline 11/27/08 7:00 & 2.119 & 0.659 & 0.203 & 91.117 & 1.429 & 0.083 & 0.025297 & 0.011331 & 0.400223 & 179.620 \\
\hline 11/27/08 8:00 & 2.119 & 0.659 & 0.203 & 91.117 & 1.429 & 0.083 & 0.025297 & 0.011331 & 0.400223 & 179.620 \\
\hline 11/27/08 9:00 & 2.12 & 0.660 & 0.204 & 91.459 & 1.427 & 0.081 & 0.024688 & 0.010940 & 0.386387 & 173.411 \\
\hline 11/27/08 10:00 & 2.115 & 0.655 & 0.200 & 89.757 & 1.427 & 0.081 & 0.024688 & 0.010940 & 0.386387 & 173.411 \\
\hline 11/27/08 11:00 & 2.117 & 0.657 & 0.202 & 90.436 & 1.429 & 0.083 & 0.025297 & 0.011331 & 0.400223 & 179.620 \\
\hline 11/27/08 12:00 & 2.12 & 0.660 & 0.204 & 91.459 & 1.427 & 0.081 & 0.024688 & 0.010940 & 0.386387 & 173.411 \\
\hline 11/27/08 13:00 & 2.126 & 0.666 & 0.208 & 93.527 & 1.429 & 0.083 & 0.025297 & 0.011331 & 0.400223 & 179.620 \\
\hline 11/27/08 14:00 & 2.123 & 0.663 & 0.206 & 92.490 & 1.429 & 0.083 & 0.025297 & 0.011331 & 0.400223 & 179.620 \\
\hline 11/27/08 15:00 & 2.116 & 0.656 & 0.201 & 90.096 & 1.429 & 0.083 & 0.025297 & 0.011331 & 0.400223 & 179.620 \\
\hline 11/27/08 16:00 & 2.118 & 0.658 & 0.202 & 90.776 & 1.429 & 0.083 & 0.025297 & 0.011331 & 0.400223 & 179.620 \\
\hline 11/27/08 17:00 & 2.123 & 0.663 & 0.206 & 92.490 & 1.429 & 0.083 & 0.025297 & 0.011331 & 0.400223 & 179.620 \\
\hline 11/27/08 18:00 & 2.123 & 0.663 & 0.206 & 92.490 & 1.429 & 0.083 & 0.025297 & 0.011331 & 0.400223 & 179.620 \\
\hline 11/27/08 19:00 & 2.123 & 0.663 & 0.206 & 92.490 & 1.429 & 0.083 & 0.025297 & 0.011331 & 0.400223 & 179.620 \\
\hline 11/27/08 20:00 & 2.123 & 0.663 & 0.206 & 92.490 & 1.429 & 0.083 & 0.025297 & 0.011331 & 0.400223 & 179.620 \\
\hline 11/27/08 21:00 & 2.124 & 0.664 & 0.207 & 92.835 & 1.429 & 0.083 & 0.025297 & 0.011331 & 0.400223 & 179.620 \\
\hline 11/27/08 22:00 & 2.129 & 0.669 & 0.211 & 94.572 & 1.432 & 0.086 & 0.026212 & 0.011928 & 0.421279 & 189.070 \\
\hline 11/27/08 23:00 & 2.124 & 0.664 & 0.207 & 92.835 & 1.429 & 0.083 & 0.025297 & 0.011331 & 0.400223 & 179.620 \\
\hline 11/28/08 0:00 & 2.129 & 0.669 & 0.211 & 94.572 & 1.432 & 0.086 & 0.026212 & 0.011928 & 0.421279 & 189.070 \\
\hline 11/28/08 1:00 & 2.132 & 0.672 & 0.213 & 95.623 & 1.432 & 0.086 & 0.026212 & 0.011928 & 0.421279 & 189.070 \\
\hline 11/28/08 2:00 & 2.13 & 0.670 & 0.212 & 94.921 & 1.432 & 0.086 & 0.026212 & 0.011928 & 0.421279 & 189.070 \\
\hline 11/28/08 3:00 & 2.125 & 0.665 & 0.208 & 93.181 & 1.432 & 0.086 & 0.026212 & 0.011928 & 0.421279 & 189.070 \\
\hline 11/28/08 4:00 & 2.128 & 0.668 & 0.210 & 94.223 & 1.432 & 0.086 & 0.026212 & 0.011928 & 0.421279 & 189.070 \\
\hline 11/28/08 5:00 & 2.124 & 0.664 & 0.207 & 92.835 & 1.429 & 0.083 & 0.025297 & 0.011331 & 0.400223 & 179.620 \\
\hline 11/28/08 6:00 & 2.121 & 0.661 & 0.205 & 91.802 & 1.429 & 0.083 & 0.025297 & 0.011331 & 0.400223 & 179.620 \\
\hline 11/28/08 7:00 & 2.122 & 0.662 & 0.205 & 92.146 & 1.429 & 0.083 & 0.025297 & 0.011331 & 0.400223 & 179.620 \\
\hline
\end{tabular}




\begin{tabular}{|c|c|c|c|c|c|c|c|c|c|c|}
\hline \multirow[b]{3}{*}{ Date Time } & \multicolumn{4}{|c|}{ V-Notch Weir } & \multicolumn{6}{|c|}{ R-Notch Weir } \\
\hline & \multicolumn{2}{|c|}{ Measured Water Level } & \multicolumn{2}{|c|}{$\begin{array}{c}\text { Calculated Rate } \\
\left(\mathrm{ft}^{3} / \mathrm{s}=448.8 \mathrm{gpm}\right)\end{array}$} & \multicolumn{3}{|c|}{ Measured Water Level } & \multicolumn{3}{|c|}{$\begin{array}{c}\text { Calculated Rate } \\
\left(\mathrm{ft}^{3} / \mathrm{s}=448.8 \mathrm{gpm}\right)\end{array}$} \\
\hline & $\begin{array}{c}\text { (ft above } \\
\text { sensor) }\end{array}$ & $\begin{array}{c}\text { (ft above } \\
\text { notch) }\end{array}$ & $\left(\mathrm{ft}^{3} / \mathrm{s}\right)$ & (gpm) & $\begin{array}{l}\text { (ft above } \\
\text { sensor) }\end{array}$ & $\begin{array}{c}\text { (ft above } \\
\text { log) }\end{array}$ & $\begin{array}{c}\text { (m above } \\
\text { log) }\end{array}$ & $\left(\mathrm{m}^{3} / \mathrm{s}\right)$ & $\left(\mathrm{ft}^{3} / \mathrm{s}\right)$ & (gpm) \\
\hline 11/28/08 8:00 & 2.124 & 0.664 & 0.207 & 92.835 & 1.429 & 0.083 & 0.025297 & 0.011331 & 0.400223 & 179.620 \\
\hline 11/28/08 9:00 & 2.124 & 0.664 & 0.207 & 92.835 & 1.429 & 0.083 & 0.025297 & 0.011331 & 0.400223 & 179.620 \\
\hline 11/28/08 10:00 & 2.122 & 0.662 & 0.205 & 92.146 & 1.429 & 0.083 & 0.025297 & 0.011331 & 0.400223 & 179.620 \\
\hline 11/28/08 11:00 & 2.119 & 0.659 & 0.203 & 91.117 & 1.429 & 0.083 & 0.025297 & 0.011331 & 0.400223 & 179.620 \\
\hline 11/28/08 12:00 & 2.123 & 0.663 & 0.206 & 92.490 & 1.429 & 0.083 & 0.025297 & 0.011331 & 0.400223 & 179.620 \\
\hline 11/28/08 13:00 & 2.12 & 0.660 & 0.204 & 91.459 & 1.431 & 0.085 & 0.025907 & 0.011728 & 0.414220 & 185.902 \\
\hline 11/28/08 14:00 & 2.128 & 0.668 & 0.210 & 94.223 & 1.431 & 0.085 & 0.025907 & 0.011728 & 0.414220 & 185.902 \\
\hline 11/28/08 15:00 & 2.13 & 0.670 & 0.212 & 94.921 & 1.431 & 0.085 & 0.025907 & 0.011728 & 0.414220 & 185.902 \\
\hline 11/28/08 16:00 & 2.129 & 0.669 & 0.211 & 94.572 & 1.431 & 0.085 & 0.025907 & 0.011728 & 0.414220 & 185.902 \\
\hline 11/28/08 17:00 & 2.132 & 0.672 & 0.213 & 95.623 & 1.431 & 0.085 & 0.025907 & 0.011728 & 0.414220 & 185.902 \\
\hline 11/28/08 18:00 & 2.132 & 0.672 & 0.213 & 95.623 & 1.434 & 0.088 & 0.026821 & 0.012331 & 0.435514 & 195.459 \\
\hline 11/28/08 19:00 & 2.133 & 0.673 & 0.214 & 95.975 & 1.432 & 0.086 & 0.026212 & 0.011928 & 0.421279 & 189.070 \\
\hline 11/28/08 20:00 & 2.131 & 0.671 & 0.212 & 95.272 & 1.432 & 0.086 & 0.026212 & 0.011928 & 0.421279 & 189.070 \\
\hline 11/28/08 21:00 & 2.133 & 0.673 & 0.214 & 95.975 & 1.432 & 0.086 & 0.026212 & 0.011928 & 0.421279 & 189.070 \\
\hline 11/28/08 22:00 & 2.133 & 0.673 & 0.214 & 95.975 & 1.432 & 0.086 & 0.026212 & 0.011928 & 0.421279 & 189.070 \\
\hline 11/28/08 23:00 & 2.131 & 0.671 & 0.212 & 95.272 & 1.432 & 0.086 & 0.026212 & 0.011928 & 0.421279 & 189.070 \\
\hline 11/29/08 0:00 & 2.131 & 0.671 & 0.212 & 95.272 & 1.432 & 0.086 & 0.026212 & 0.011928 & 0.421279 & 189.070 \\
\hline 11/29/08 1:00 & 2.131 & 0.671 & 0.212 & 95.272 & 1.432 & 0.086 & 0.026212 & 0.011928 & 0.421279 & 189.070 \\
\hline 11/29/08 2:00 & 2.141 & 0.681 & 0.220 & 98.819 & 1.434 & 0.088 & 0.026821 & 0.012331 & 0.435514 & 195.459 \\
\hline 11/29/08 3:00 & 2.143 & 0.683 & 0.222 & 99.538 & 1.434 & 0.088 & 0.026821 & 0.012331 & 0.435514 & 195.459 \\
\hline 11/29/08 4:00 & 2.146 & 0.686 & 0.224 & 100.622 & 1.434 & 0.088 & 0.026821 & 0.012331 & 0.435514 & 195.459 \\
\hline 11/29/08 5:00 & 2.153 & 0.693 & 0.230 & 103.179 & 1.436 & 0.09 & 0.027431 & 0.012738 & 0.449907 & 201.918 \\
\hline 11/29/08 6:00 & 2.15 & 0.690 & 0.227 & 102.078 & 1.436 & 0.09 & 0.027431 & 0.012738 & 0.449907 & 201.918 \\
\hline 11/29/08 7:00 & 2.153 & 0.693 & 0.230 & 103.179 & 1.436 & 0.09 & 0.027431 & 0.012738 & 0.449907 & 201.918 \\
\hline 11/29/08 8:00 & 2.15 & 0.690 & 0.227 & 102.078 & 1.434 & 0.088 & 0.026821 & 0.012331 & 0.435514 & 195.459 \\
\hline
\end{tabular}




\begin{tabular}{|c|c|c|c|c|c|c|c|c|c|c|}
\hline \multirow[b]{3}{*}{ Date Time } & \multicolumn{4}{|c|}{ V-Notch Weir } & \multicolumn{6}{|c|}{ R-Notch Weir } \\
\hline & \multicolumn{2}{|c|}{ Measured Water Level } & \multicolumn{2}{|c|}{$\begin{array}{c}\text { Calculated Rate } \\
\left(\mathrm{ft}^{3} / \mathrm{s}=448.8 \mathrm{gpm}\right)\end{array}$} & \multicolumn{3}{|c|}{ Measured Water Level } & \multicolumn{3}{|c|}{$\begin{array}{c}\text { Calculated Rate } \\
\left(\mathrm{ft}^{3} / \mathrm{s}=448.8 \mathrm{gpm}\right)\end{array}$} \\
\hline & $\begin{array}{c}\text { (ft above } \\
\text { sensor) }\end{array}$ & $\begin{array}{c}\text { (ft above } \\
\text { notch) }\end{array}$ & $\left(\mathrm{ft}^{3} / \mathrm{s}\right)$ & (gpm) & $\begin{array}{l}\text { (ft above } \\
\text { sensor) }\end{array}$ & $\begin{array}{c}\text { (ft above } \\
\text { log) }\end{array}$ & $\begin{array}{c}\text { (m above } \\
\text { log) }\end{array}$ & $\left(\mathrm{m}^{3} / \mathrm{s}\right)$ & $\left(\mathrm{ft}^{3} / \mathrm{s}\right)$ & (gpm) \\
\hline 11/29/08 9:00 & 2.148 & 0.688 & 0.226 & 101.348 & 1.434 & 0.088 & 0.026821 & 0.012331 & 0.435514 & 195.459 \\
\hline 11/29/08 10:00 & 2.146 & 0.686 & 0.224 & 100.622 & 1.434 & 0.088 & 0.026821 & 0.012331 & 0.435514 & 195.459 \\
\hline 11/29/08 11:00 & 2.141 & 0.681 & 0.220 & 98.819 & 1.434 & 0.088 & 0.026821 & 0.012331 & 0.435514 & 195.459 \\
\hline 11/29/08 12:00 & 2.148 & 0.688 & 0.226 & 101.348 & 1.434 & 0.088 & 0.026821 & 0.012331 & 0.435514 & 195.459 \\
\hline 11/29/08 13:00 & 2.152 & 0.692 & 0.229 & 102.811 & 1.434 & 0.088 & 0.026821 & 0.012331 & 0.435514 & 195.459 \\
\hline 11/29/08 14:00 & 2.154 & 0.694 & 0.231 & 103.547 & 1.436 & 0.09 & 0.027431 & 0.012738 & 0.449907 & 201.918 \\
\hline 11/29/08 15:00 & 2.152 & 0.692 & 0.229 & 102.811 & 1.436 & 0.09 & 0.027431 & 0.012738 & 0.449907 & 201.918 \\
\hline 11/29/08 16:00 & 2.154 & 0.694 & 0.231 & 103.547 & 1.436 & 0.09 & 0.027431 & 0.012738 & 0.449907 & 201.918 \\
\hline 11/29/08 17:00 & 2.152 & 0.692 & 0.229 & 102.811 & 1.434 & 0.088 & 0.026821 & 0.012331 & 0.435514 & 195.459 \\
\hline 11/29/08 18:00 & 2.154 & 0.694 & 0.231 & 103.547 & 1.434 & 0.088 & 0.026821 & 0.012331 & 0.435514 & 195.459 \\
\hline 11/29/08 19:00 & 2.145 & 0.685 & 0.223 & 100.260 & 1.434 & 0.088 & 0.026821 & 0.012331 & 0.435514 & 195.459 \\
\hline 11/29/08 20:00 & 2.155 & 0.695 & 0.232 & 103.916 & 1.434 & 0.088 & 0.026821 & 0.012331 & 0.435514 & 195.459 \\
\hline 11/29/08 21:00 & 2.143 & 0.683 & 0.222 & 99.538 & 1.432 & 0.086 & 0.026212 & 0.011928 & 0.421279 & 189.070 \\
\hline 11/29/08 22:00 & 2.141 & 0.681 & 0.220 & 98.819 & 1.432 & 0.086 & 0.026212 & 0.011928 & 0.421279 & 189.070 \\
\hline 11/29/08 23:00 & 2.139 & 0.679 & 0.219 & 98.103 & 1.429 & 0.083 & 0.025297 & 0.011331 & 0.400223 & 179.620 \\
\hline 11/30/08 0:00 & 2.137 & 0.677 & 0.217 & 97.391 & 1.436 & 0.09 & 0.027431 & 0.012738 & 0.449907 & 201.918 \\
\hline 11/30/08 1:00 & 2.142 & 0.682 & 0.221 & 99.178 & 1.436 & 0.09 & 0.027431 & 0.012738 & 0.449907 & 201.918 \\
\hline 11/30/08 2:00 & 2.142 & 0.682 & 0.221 & 99.178 & 1.436 & 0.09 & 0.027431 & 0.012738 & 0.449907 & 201.918 \\
\hline 11/30/08 3:00 & 2.14 & 0.680 & 0.219 & 98.461 & 1.436 & 0.09 & 0.027431 & 0.012738 & 0.449907 & 201.918 \\
\hline 11/30/08 4:00 & 2.143 & 0.683 & 0.222 & 99.538 & 1.436 & 0.09 & 0.027431 & 0.012738 & 0.449907 & 201.918 \\
\hline 11/30/08 5:00 & 2.133 & 0.673 & 0.214 & 95.975 & 1.436 & 0.09 & 0.027431 & 0.012738 & 0.449907 & 201.918 \\
\hline 11/30/08 6:00 & 2.136 & 0.676 & 0.216 & 97.036 & 1.436 & 0.09 & 0.027431 & 0.012738 & 0.449907 & 201.918 \\
\hline 11/30/08 7:00 & 2.136 & 0.676 & 0.216 & 97.036 & 1.436 & 0.09 & 0.027431 & 0.012738 & 0.449907 & 201.918 \\
\hline 11/30/08 8:00 & 2.129 & 0.669 & 0.211 & 94.572 & 1.436 & 0.09 & 0.027431 & 0.012738 & 0.449907 & 201.918 \\
\hline 11/30/08 9:00 & 2.135 & 0.675 & 0.215 & 96.681 & 1.434 & 0.088 & 0.026821 & 0.012331 & 0.435514 & 195.459 \\
\hline
\end{tabular}




\begin{tabular}{|c|c|c|c|c|c|c|c|c|c|c|}
\hline \multirow[b]{3}{*}{ Date Time } & \multicolumn{4}{|c|}{ V-Notch Weir } & \multicolumn{6}{|c|}{ R-Notch Weir } \\
\hline & \multicolumn{2}{|c|}{ Measured Water Level } & \multicolumn{2}{|c|}{$\begin{array}{c}\text { Calculated Rate } \\
\left(\mathrm{ft}^{3} / \mathrm{s}=448.8 \mathrm{gpm}\right)\end{array}$} & \multicolumn{3}{|c|}{ Measured Water Level } & \multicolumn{3}{|c|}{$\begin{array}{c}\text { Calculated Rate } \\
\left(\mathrm{ft}^{3} / \mathrm{s}=448.8 \mathrm{gpm}\right)\end{array}$} \\
\hline & $\begin{array}{c}\text { (ft above } \\
\text { sensor) }\end{array}$ & $\begin{array}{c}\text { (ft above } \\
\text { notch) }\end{array}$ & $\left(\mathrm{ft}^{3} / \mathrm{s}\right)$ & (gpm) & $\begin{array}{l}\text { (ft above } \\
\text { sensor) }\end{array}$ & $\begin{array}{c}\text { (ft above } \\
\text { log) }\end{array}$ & $\begin{array}{c}\text { (m above } \\
\text { log) }\end{array}$ & $\left(\mathrm{m}^{3} / \mathrm{s}\right)$ & $\left(\mathrm{ft}^{3} / \mathrm{s}\right)$ & (gpm) \\
\hline 11/30/08 10:00 & 2.137 & 0.677 & 0.217 & 97.391 & 1.434 & 0.088 & 0.026821 & 0.012331 & 0.435514 & 195.459 \\
\hline 11/30/08 11:00 & 2.13 & 0.670 & 0.212 & 94.921 & 1.434 & 0.088 & 0.026821 & 0.012331 & 0.435514 & 195.459 \\
\hline 11/30/08 12:00 & 2.134 & 0.674 & 0.215 & 96.328 & 1.434 & 0.088 & 0.026821 & 0.012331 & 0.435514 & 195.459 \\
\hline 11/30/08 13:00 & 2.133 & 0.673 & 0.214 & 95.975 & 1.436 & 0.09 & 0.027431 & 0.012738 & 0.449907 & 201.918 \\
\hline 11/30/08 14:00 & 2.13 & 0.670 & 0.212 & 94.921 & 1.436 & 0.09 & 0.027431 & 0.012738 & 0.449907 & 201.918 \\
\hline 11/30/08 15:00 & 2.125 & 0.665 & 0.208 & 93.181 & 1.434 & 0.088 & 0.026821 & 0.012331 & 0.435514 & 195.459 \\
\hline 11/30/08 16:00 & 2.132 & 0.672 & 0.213 & 95.623 & 1.434 & 0.088 & 0.026821 & 0.012331 & 0.435514 & 195.459 \\
\hline 11/30/08 17:00 & 2.122 & 0.662 & 0.205 & 92.146 & 1.436 & 0.09 & 0.027431 & 0.012738 & 0.449907 & 201.918 \\
\hline 11/30/08 18:00 & 2.136 & 0.676 & 0.216 & 97.036 & 1.436 & 0.09 & 0.027431 & 0.012738 & 0.449907 & 201.918 \\
\hline 11/30/08 19:00 & 2.13 & 0.670 & 0.212 & 94.921 & 1.434 & 0.088 & 0.026821 & 0.012331 & 0.435514 & 195.459 \\
\hline 11/30/08 20:00 & 2.132 & 0.672 & 0.213 & 95.623 & 1.436 & 0.09 & 0.027431 & 0.012738 & 0.449907 & 201.918 \\
\hline 11/30/08 21:00 & 2.14 & 0.680 & 0.219 & 98.461 & 1.436 & 0.09 & 0.027431 & 0.012738 & 0.449907 & 201.918 \\
\hline 11/30/08 22:00 & 2.133 & 0.673 & 0.214 & 95.975 & 1.436 & 0.09 & 0.027431 & 0.012738 & 0.449907 & 201.918 \\
\hline 11/30/08 23:00 & 2.138 & 0.678 & 0.218 & 97.747 & 1.436 & 0.09 & 0.027431 & 0.012738 & 0.449907 & 201.918 \\
\hline 12/1/08 0:00 & 2.138 & 0.678 & 0.218 & 97.747 & 1.436 & 0.09 & 0.027431 & 0.012738 & 0.449907 & 201.918 \\
\hline 12/1/08 1:00 & 2.138 & 0.678 & 0.218 & 97.747 & 1.434 & 0.088 & 0.026821 & 0.012331 & 0.435514 & 195.459 \\
\hline 12/1/08 2:00 & 2.129 & 0.669 & 0.211 & 94.572 & 1.434 & 0.088 & 0.026821 & 0.012331 & 0.435514 & 195.459 \\
\hline 12/1/08 3:00 & 2.127 & 0.667 & 0.209 & 93.875 & 1.434 & 0.088 & 0.026821 & 0.012331 & 0.435514 & 195.459 \\
\hline 12/1/08 4:00 & 2.129 & 0.669 & 0.211 & 94.572 & 1.431 & 0.085 & 0.025907 & 0.011728 & 0.414220 & 185.902 \\
\hline 12/1/08 5:00 & 2.129 & 0.669 & 0.211 & 94.572 & 1.431 & 0.085 & 0.025907 & 0.011728 & 0.414220 & 185.902 \\
\hline 12/1/08 6:00 & 2.132 & 0.672 & 0.213 & 95.623 & 1.429 & 0.083 & 0.025297 & 0.011331 & 0.400223 & 179.620 \\
\hline 12/1/08 7:00 & 2.121 & 0.661 & 0.205 & 91.802 & 1.431 & 0.085 & 0.025907 & 0.011728 & 0.414220 & 185.902 \\
\hline 12/1/08 8:00 & 2.121 & 0.661 & 0.205 & 91.802 & 1.431 & 0.085 & 0.025907 & 0.011728 & 0.414220 & 185.902 \\
\hline 12/1/08 9:00 & 2.114 & 0.654 & 0.199 & 89.419 & 1.431 & 0.085 & 0.025907 & 0.011728 & 0.414220 & 185.902 \\
\hline 12/1/08 10:00 & 2.116 & 0.656 & 0.201 & 90.096 & 1.429 & 0.083 & 0.025297 & 0.011331 & 0.400223 & 179.620 \\
\hline
\end{tabular}




\begin{tabular}{|c|c|c|c|c|c|c|c|c|c|c|}
\hline \multirow[b]{3}{*}{ Date } & \multicolumn{4}{|c|}{ V-Notch Weir } & \multicolumn{6}{|c|}{ R-Notch Weir } \\
\hline & \multicolumn{2}{|c|}{ Measured Water Level } & \multicolumn{2}{|c|}{$\begin{array}{c}\text { Calculated Rate } \\
\left(\mathrm{ft}^{3} / \mathrm{s}=448.8 \mathrm{gpm}\right)\end{array}$} & \multicolumn{3}{|c|}{ Measured Water Level } & \multicolumn{3}{|c|}{$\begin{array}{c}\text { Calculated Rate } \\
\left(\mathrm{ft}^{3} / \mathrm{s}=448.8 \mathrm{gpm}\right)\end{array}$} \\
\hline & $\begin{array}{c}\text { (ft above } \\
\text { sensor) }\end{array}$ & $\begin{array}{c}\text { (ft above } \\
\text { notch) }\end{array}$ & $\left(\mathrm{ft}^{3} / \mathrm{s}\right)$ & (gpm) & $\begin{array}{l}\text { (ft above } \\
\text { sensor) }\end{array}$ & $\begin{array}{c}\text { (ft above } \\
\text { log) }\end{array}$ & $\begin{array}{c}\text { (m above } \\
\text { log) }\end{array}$ & $\left(\mathrm{m}^{3} / \mathrm{s}\right)$ & $\left(\mathrm{ft}^{3} / \mathrm{s}\right)$ & (gpm) \\
\hline 12/1/08 11:00 & 2.114 & 0.654 & 0.199 & 89.419 & 1.431 & 0.085 & 0.025907 & 0.011728 & 0.414220 & 185.902 \\
\hline 12/1/08 12:00 & 2.111 & 0.651 & 0.197 & 88.409 & 1.431 & 0.085 & 0.025907 & 0.011728 & 0.414220 & 185.902 \\
\hline 12/1/08 13:00 & 2.117 & 0.657 & 0.202 & 90.436 & 1.431 & 0.085 & 0.025907 & 0.011728 & 0.414220 & 185.902 \\
\hline 12/1/08 14:00 & 2.122 & 0.662 & 0.205 & 92.146 & 1.431 & 0.085 & 0.025907 & 0.011728 & 0.414220 & 185.902 \\
\hline 12/1/08 15:00 & 2.117 & 0.657 & 0.202 & 90.436 & 1.429 & 0.083 & 0.025297 & 0.011331 & 0.400223 & 179.620 \\
\hline 12/1/08 16:00 & 2.119 & 0.659 & 0.203 & 91.117 & 1.431 & 0.085 & 0.025907 & 0.011728 & 0.414220 & 185.902 \\
\hline 12/1/08 17:00 & 2.116 & 0.656 & 0.201 & 90.096 & 1.431 & 0.085 & 0.025907 & 0.011728 & 0.414220 & 185.902 \\
\hline 12/1/08 18:00 & 2.121 & 0.661 & 0.205 & 91.802 & 1.431 & 0.085 & 0.025907 & 0.011728 & 0.414220 & 185.902 \\
\hline 12/1/08 19:00 & 2.121 & 0.661 & 0.205 & 91.802 & 1.431 & 0.085 & 0.025907 & 0.011728 & 0.414220 & 185.902 \\
\hline 12/1/08 20:00 & 2.122 & 0.662 & 0.205 & 92.146 & 1.431 & 0.085 & 0.025907 & 0.011728 & 0.414220 & 185.902 \\
\hline 12/1/08 21:00 & 2.12 & 0.660 & 0.204 & 91.459 & 1.431 & 0.085 & 0.025907 & 0.011728 & 0.414220 & 185.902 \\
\hline 12/1/08 22:00 & 2.122 & 0.662 & 0.205 & 92.146 & 1.431 & 0.085 & 0.025907 & 0.011728 & 0.414220 & 185.902 \\
\hline 12/1/08 23:00 & 2.115 & 0.655 & 0.200 & 89.757 & 1.431 & 0.085 & 0.025907 & 0.011728 & 0.414220 & 185.902 \\
\hline 12/2/08 0:00 & 2.116 & 0.656 & 0.201 & 90.096 & 1.429 & 0.083 & 0.025297 & 0.011331 & 0.400223 & 179.620 \\
\hline 12/2/08 1:00 & 2.111 & 0.651 & 0.197 & 88.409 & 1.429 & 0.083 & 0.025297 & 0.011331 & 0.400223 & 179.620 \\
\hline 12/2/08 2:00 & 2.114 & 0.654 & 0.199 & 89.419 & 1.429 & 0.083 & 0.025297 & 0.011331 & 0.400223 & 179.620 \\
\hline 12/2/08 3:00 & 2.111 & 0.651 & 0.197 & 88.409 & 1.429 & 0.083 & 0.025297 & 0.011331 & 0.400223 & 179.620 \\
\hline 12/2/08 4:00 & 2.116 & 0.656 & 0.201 & 90.096 & 1.431 & 0.085 & 0.025907 & 0.011728 & 0.414220 & 185.902 \\
\hline 12/2/08 5:00 & 2.116 & 0.656 & 0.201 & 90.096 & 1.429 & 0.083 & 0.025297 & 0.011331 & 0.400223 & 179.620 \\
\hline 12/2/08 6:00 & 2.119 & 0.659 & 0.203 & 91.117 & 1.431 & 0.085 & 0.025907 & 0.011728 & 0.414220 & 185.902 \\
\hline 12/2/08 7:00 & 2.123 & 0.663 & 0.206 & 92.490 & 1.431 & 0.085 & 0.025907 & 0.011728 & 0.414220 & 185.902 \\
\hline 12/2/08 8:00 & 2.121 & 0.661 & 0.205 & 91.802 & 1.431 & 0.085 & 0.025907 & 0.011728 & 0.414220 & 185.902 \\
\hline 12/2/08 9:00 & 2.118 & 0.658 & 0.202 & 90.776 & 1.431 & 0.085 & 0.025907 & 0.011728 & 0.414220 & 185.902 \\
\hline 12/2/08 10:00 & 2.125 & 0.665 & 0.208 & 93.181 & 1.431 & 0.085 & 0.025907 & 0.011728 & 0.414220 & 185.902 \\
\hline 12/2/08 11:00 & 2.12 & 0.660 & 0.204 & 91.459 & 1.431 & 0.085 & 0.025907 & 0.011728 & 0.414220 & 185.902 \\
\hline
\end{tabular}




\begin{tabular}{|c|c|c|c|c|c|c|c|c|c|c|}
\hline \multirow[b]{3}{*}{ Date Time } & \multicolumn{4}{|c|}{ V-Notch Weir } & \multicolumn{6}{|c|}{ R-Notch Weir } \\
\hline & \multicolumn{2}{|c|}{ Measured Water Level } & \multicolumn{2}{|c|}{$\begin{array}{c}\text { Calculated Rate } \\
\left(\mathrm{ft}^{3} / \mathrm{s}=448.8 \mathrm{gpm}\right)\end{array}$} & \multicolumn{3}{|c|}{ Measured Water Level } & \multicolumn{3}{|c|}{$\begin{array}{c}\text { Calculated Rate } \\
\left(\mathrm{ft}^{3} / \mathrm{s}=448.8 \mathrm{gpm}\right)\end{array}$} \\
\hline & $\begin{array}{c}\text { (ft above } \\
\text { sensor) }\end{array}$ & $\begin{array}{c}\text { (ft above } \\
\text { notch) }\end{array}$ & $\left(\mathrm{ft}^{3} / \mathrm{s}\right)$ & (gpm) & $\begin{array}{l}\text { (ft above } \\
\text { sensor) }\end{array}$ & $\begin{array}{c}\text { (ft above } \\
\text { log) }\end{array}$ & $\begin{array}{c}\text { (m above } \\
\text { log) }\end{array}$ & $\left(\mathrm{m}^{3} / \mathrm{s}\right)$ & $\left(\mathrm{ft}^{3} / \mathrm{s}\right)$ & (gpm) \\
\hline 12/2/08 12:00 & 2.124 & 0.664 & 0.207 & 92.835 & 1.434 & 0.088 & 0.026821 & 0.012331 & 0.435514 & 195.459 \\
\hline 12/2/08 13:00 & 2.127 & 0.667 & 0.209 & 93.875 & 1.436 & 0.09 & 0.027431 & 0.012738 & 0.449907 & 201.918 \\
\hline 12/2/08 14:00 & 2.133 & 0.673 & 0.214 & 95.975 & 1.434 & 0.088 & 0.026821 & 0.012331 & 0.435514 & 195.459 \\
\hline 12/2/08 15:00 & 2.133 & 0.673 & 0.214 & 95.975 & 1.436 & 0.09 & 0.027431 & 0.012738 & 0.449907 & 201.918 \\
\hline 12/2/08 16:00 & 2.139 & 0.679 & 0.219 & 98.103 & 1.436 & 0.09 & 0.027431 & 0.012738 & 0.449907 & 201.918 \\
\hline 12/2/08 17:00 & 2.144 & 0.684 & 0.223 & 99.898 & 1.436 & 0.09 & 0.027431 & 0.012738 & 0.449907 & 201.918 \\
\hline 12/2/08 18:00 & 2.139 & 0.679 & 0.219 & 98.103 & 1.436 & 0.09 & 0.027431 & 0.012738 & 0.449907 & 201.918 \\
\hline 12/2/08 19:00 & 2.14 & 0.680 & 0.219 & 98.461 & 1.436 & 0.09 & 0.027431 & 0.012738 & 0.449907 & 201.918 \\
\hline 12/2/08 20:00 & 2.14 & 0.680 & 0.219 & 98.461 & 1.434 & 0.088 & 0.026821 & 0.012331 & 0.435514 & 195.459 \\
\hline 12/2/08 21:00 & 2.138 & 0.678 & 0.218 & 97.747 & 1.436 & 0.09 & 0.027431 & 0.012738 & 0.449907 & 201.918 \\
\hline 12/2/08 22:00 & 2.136 & 0.676 & 0.216 & 97.036 & 1.434 & 0.088 & 0.026821 & 0.012331 & 0.435514 & 195.459 \\
\hline 12/2/08 23:00 & 2.139 & 0.679 & 0.219 & 98.103 & 1.436 & 0.09 & 0.027431 & 0.012738 & 0.449907 & 201.918 \\
\hline 12/3/08 0:00 & 2.146 & 0.686 & 0.224 & 100.622 & 1.436 & 0.09 & 0.027431 & 0.012738 & 0.449907 & 201.918 \\
\hline 12/3/08 1:00 & 2.137 & 0.677 & 0.217 & 97.391 & 1.434 & 0.088 & 0.026821 & 0.012331 & 0.435514 & 195.459 \\
\hline 12/3/08 2:00 & 2.137 & 0.677 & 0.217 & 97.391 & 1.434 & 0.088 & 0.026821 & 0.012331 & 0.435514 & 195.459 \\
\hline 12/3/08 3:00 & 2.135 & 0.675 & 0.215 & 96.681 & 1.431 & 0.085 & 0.025907 & 0.011728 & 0.414220 & 185.902 \\
\hline 12/3/08 4:00 & 2.128 & 0.668 & 0.210 & 94.223 & 1.429 & 0.083 & 0.025297 & 0.011331 & 0.400223 & 179.620 \\
\hline 12/3/08 5:00 & 2.131 & 0.671 & 0.212 & 95.272 & 1.431 & 0.085 & 0.025907 & 0.011728 & 0.414220 & 185.902 \\
\hline 12/3/08 6:00 & 2.138 & 0.678 & 0.218 & 97.747 & 1.434 & 0.088 & 0.026821 & 0.012331 & 0.435514 & 195.459 \\
\hline 12/3/08 7:00 & 2.134 & 0.674 & 0.215 & 96.328 & 1.431 & 0.085 & 0.025907 & 0.011728 & 0.414220 & 185.902 \\
\hline 12/3/08 8:00 & 2.137 & 0.677 & 0.217 & 97.391 & 1.429 & 0.083 & 0.025297 & 0.011331 & 0.400223 & 179.620 \\
\hline 12/3/08 9:00 & 2.133 & 0.673 & 0.214 & 95.975 & 1.427 & 0.081 & 0.024688 & 0.010940 & 0.386387 & 173.411 \\
\hline 12/3/08 10:00 & 2.126 & 0.666 & 0.208 & 93.527 & 1.427 & 0.081 & 0.024688 & 0.010940 & 0.386387 & 173.411 \\
\hline 12/3/08 11:00 & 2.117 & 0.657 & 0.202 & 90.436 & 1.429 & 0.083 & 0.025297 & 0.011331 & 0.400223 & 179.620 \\
\hline 12/3/08 12:00 & 2.121 & 0.661 & 0.205 & 91.802 & 1.427 & 0.081 & 0.024688 & 0.010940 & 0.386387 & 173.411 \\
\hline
\end{tabular}




\begin{tabular}{|c|c|c|c|c|c|c|c|c|c|c|}
\hline \multirow[b]{3}{*}{ Date Time } & \multicolumn{4}{|c|}{ V-Notch Weir } & \multicolumn{6}{|c|}{ R-Notch Weir } \\
\hline & \multicolumn{2}{|c|}{ Measured Water Level } & \multicolumn{2}{|c|}{$\begin{array}{c}\text { Calculated Rate } \\
\left(\mathrm{ft}^{3} / \mathrm{s}=448.8 \mathrm{gpm}\right)\end{array}$} & \multicolumn{3}{|c|}{ Measured Water Level } & \multicolumn{3}{|c|}{$\begin{array}{c}\text { Calculated Rate } \\
\left(\mathrm{ft}^{3} / \mathrm{s}=448.8 \mathrm{gpm}\right)\end{array}$} \\
\hline & $\begin{array}{c}\text { (ft above } \\
\text { sensor) }\end{array}$ & $\begin{array}{c}\text { (ft above } \\
\text { notch) }\end{array}$ & $\left(\mathrm{ft}^{3} / \mathrm{s}\right)$ & (gpm) & $\begin{array}{l}\text { (ft above } \\
\text { sensor) }\end{array}$ & $\begin{array}{c}\text { (ft above } \\
\text { log) }\end{array}$ & $\begin{array}{c}\text { (m above } \\
\text { log) }\end{array}$ & $\left(\mathrm{m}^{3} / \mathrm{s}\right)$ & $\left(\mathrm{ft}^{3} / \mathrm{s}\right)$ & (gpm) \\
\hline 12/3/08 13:00 & 2.111 & 0.651 & 0.197 & 88.409 & 1.429 & 0.083 & 0.025297 & 0.011331 & 0.400223 & 179.620 \\
\hline 12/3/08 14:00 & 2.108 & 0.648 & 0.195 & 87.406 & 1.427 & 0.081 & 0.024688 & 0.010940 & 0.386387 & 173.411 \\
\hline 12/3/08 15:00 & 2.11 & 0.650 & 0.196 & 88.074 & 1.429 & 0.083 & 0.025297 & 0.011331 & 0.400223 & 179.620 \\
\hline 12/3/08 16:00 & 2.11 & 0.650 & 0.196 & 88.074 & 1.427 & 0.081 & 0.024688 & 0.010940 & 0.386387 & 173.411 \\
\hline 12/3/08 17:00 & 2.115 & 0.655 & 0.200 & 89.757 & 1.427 & 0.081 & 0.024688 & 0.010940 & 0.386387 & 173.411 \\
\hline 12/3/08 18:00 & 2.115 & 0.655 & 0.200 & 89.757 & 1.427 & 0.081 & 0.024688 & 0.010940 & 0.386387 & 173.411 \\
\hline 12/3/08 19:00 & 2.115 & 0.655 & 0.200 & 89.757 & 1.427 & 0.081 & 0.024688 & 0.010940 & 0.386387 & 173.411 \\
\hline 12/3/08 20:00 & 2.113 & 0.653 & 0.198 & 89.082 & 1.427 & 0.081 & 0.024688 & 0.010940 & 0.386387 & 173.411 \\
\hline 12/3/08 21:00 & 2.111 & 0.651 & 0.197 & 88.409 & 1.427 & 0.081 & 0.024688 & 0.010940 & 0.386387 & 173.411 \\
\hline 12/3/08 22:00 & 2.109 & 0.649 & 0.195 & 87.740 & 1.424 & 0.078 & 0.023773 & 0.010361 & 0.365940 & 164.234 \\
\hline 12/3/08 23:00 & 2.105 & 0.645 & 0.193 & 86.410 & 1.424 & 0.078 & 0.023773 & 0.010361 & 0.365940 & 164.234 \\
\hline 12/4/08 0:00 & 2.105 & 0.645 & 0.193 & 86.410 & 1.424 & 0.078 & 0.023773 & 0.010361 & 0.365940 & 164.234 \\
\hline 12/4/08 1:00 & 2.1 & 0.640 & 0.189 & 84.765 & 1.424 & 0.078 & 0.023773 & 0.010361 & 0.365940 & 164.234 \\
\hline 12/4/08 2:00 & 2.103 & 0.643 & 0.191 & 85.750 & 1.422 & 0.076 & 0.023164 & 0.009981 & 0.352516 & 158.209 \\
\hline 12/4/08 3:00 & 2.098 & 0.638 & 0.187 & 84.113 & 1.424 & 0.078 & 0.023773 & 0.010361 & 0.365940 & 164.234 \\
\hline 12/4/08 4:00 & 2.096 & 0.636 & 0.186 & 83.463 & 1.424 & 0.078 & 0.023773 & 0.010361 & 0.365940 & 164.234 \\
\hline 12/4/08 5:00 & 2.096 & 0.636 & 0.186 & 83.463 & 1.424 & 0.078 & 0.023773 & 0.010361 & 0.365940 & 164.234 \\
\hline 12/4/08 6:00 & 2.099 & 0.639 & 0.188 & 84.439 & 1.422 & 0.076 & 0.023164 & 0.009981 & 0.352516 & 158.209 \\
\hline 12/4/08 7:00 & 2.096 & 0.636 & 0.186 & 83.463 & 1.422 & 0.076 & 0.023164 & 0.009981 & 0.352516 & 158.209 \\
\hline 12/4/08 8:00 & 2.094 & 0.634 & 0.185 & 82.817 & 1.422 & 0.076 & 0.023164 & 0.009981 & 0.352516 & 158.209 \\
\hline 12/4/08 9:00 & 2.092 & 0.632 & 0.183 & 82.173 & 1.422 & 0.076 & 0.023164 & 0.009981 & 0.352516 & 158.209 \\
\hline 12/4/08 10:00 & 2.087 & 0.627 & 0.180 & 80.577 & 1.422 & 0.076 & 0.023164 & 0.009981 & 0.352516 & 158.209 \\
\hline 12/4/08 11:00 & 2.094 & 0.634 & 0.185 & 82.817 & 1.422 & 0.076 & 0.023164 & 0.009981 & 0.352516 & 158.209 \\
\hline 12/4/08 12:00 & 2.087 & 0.627 & 0.180 & 80.577 & 1.422 & 0.076 & 0.023164 & 0.009981 & 0.352516 & 158.209 \\
\hline 12/4/08 13:00 & 2.088 & 0.628 & 0.180 & 80.895 & 1.42 & 0.074 & 0.022554 & 0.009605 & 0.339261 & 152.260 \\
\hline
\end{tabular}




\begin{tabular}{|c|c|c|c|c|c|c|c|c|c|c|}
\hline \multirow[b]{3}{*}{ Date Time } & \multicolumn{4}{|c|}{ V-Notch Weir } & \multicolumn{6}{|c|}{ R-Notch Weir } \\
\hline & \multicolumn{2}{|c|}{ Measured Water Level } & \multicolumn{2}{|c|}{$\begin{array}{c}\text { Calculated Rate } \\
\left(\mathrm{ft}^{3} / \mathrm{s}=448.8 \mathrm{gpm}\right)\end{array}$} & \multicolumn{3}{|c|}{ Measured Water Level } & \multicolumn{3}{|c|}{$\begin{array}{c}\text { Calculated Rate } \\
\left(\mathrm{ft}^{3} / \mathrm{s}=448.8 \mathrm{gpm}\right)\end{array}$} \\
\hline & $\begin{array}{c}\text { (ft above } \\
\text { sensor) }\end{array}$ & $\begin{array}{c}\text { (ft above } \\
\text { notch) }\end{array}$ & $\left(\mathrm{ft}^{3} / \mathrm{s}\right)$ & (gpm) & $\begin{array}{l}\text { (ft above } \\
\text { sensor) }\end{array}$ & $\begin{array}{c}\text { (ft above } \\
\text { log) }\end{array}$ & $\begin{array}{c}\text { (m above } \\
\text { log) }\end{array}$ & $\left(\mathrm{m}^{3} / \mathrm{s}\right)$ & $\left(\mathrm{ft}^{3} / \mathrm{s}\right)$ & (gpm) \\
\hline 12/4/08 14:00 & 2.097 & 0.637 & 0.187 & 83.788 & 1.422 & 0.076 & 0.023164 & 0.009981 & 0.352516 & 158.209 \\
\hline 12/4/08 15:00 & 2.095 & 0.635 & 0.185 & 83.140 & 1.422 & 0.076 & 0.023164 & 0.009981 & 0.352516 & 158.209 \\
\hline 12/4/08 16:00 & 2.097 & 0.637 & 0.187 & 83.788 & 1.422 & 0.076 & 0.023164 & 0.009981 & 0.352516 & 158.209 \\
\hline 12/4/08 17:00 & 2.099 & 0.639 & 0.188 & 84.439 & 1.422 & 0.076 & 0.023164 & 0.009981 & 0.352516 & 158.209 \\
\hline 12/4/08 18:00 & 2.102 & 0.642 & 0.190 & 85.421 & 1.422 & 0.076 & 0.023164 & 0.009981 & 0.352516 & 158.209 \\
\hline 12/4/08 19:00 & 2.1 & 0.640 & 0.189 & 84.765 & 1.422 & 0.076 & 0.023164 & 0.009981 & 0.352516 & 158.209 \\
\hline 12/4/08 20:00 & 2.1 & 0.640 & 0.189 & 84.765 & 1.422 & 0.076 & 0.023164 & 0.009981 & 0.352516 & 158.209 \\
\hline 12/4/08 21:00 & 2.098 & 0.638 & 0.187 & 84.113 & 1.422 & 0.076 & 0.023164 & 0.009981 & 0.352516 & 158.209 \\
\hline 12/4/08 22:00 & 2.093 & 0.633 & 0.184 & 82.494 & 1.422 & 0.076 & 0.023164 & 0.009981 & 0.352516 & 158.209 \\
\hline 12/4/08 23:00 & 2.091 & 0.631 & 0.182 & 81.852 & 1.42 & 0.074 & 0.022554 & 0.009605 & 0.339261 & 152.260 \\
\hline 12/5/08 0:00 & 2.084 & 0.624 & 0.177 & 79.629 & 1.42 & 0.074 & 0.022554 & 0.009605 & 0.339261 & 152.260 \\
\hline 12/5/08 1:00 & 2.082 & 0.622 & 0.176 & 79.000 & 1.42 & 0.074 & 0.022554 & 0.009605 & 0.339261 & 152.260 \\
\hline 12/5/08 2:00 & 2.085 & 0.625 & 0.178 & 79.944 & 1.42 & 0.074 & 0.022554 & 0.009605 & 0.339261 & 152.260 \\
\hline 12/5/08 3:00 & 2.082 & 0.622 & 0.176 & 79.000 & 1.42 & 0.074 & 0.022554 & 0.009605 & 0.339261 & 152.260 \\
\hline 12/5/08 4:00 & 2.085 & 0.625 & 0.178 & 79.944 & 1.42 & 0.074 & 0.022554 & 0.009605 & 0.339261 & 152.260 \\
\hline 12/5/08 5:00 & 2.078 & 0.618 & 0.173 & 77.752 & 1.42 & 0.074 & 0.022554 & 0.009605 & 0.339261 & 152.260 \\
\hline 12/5/08 6:00 & 2.078 & 0.618 & 0.173 & 77.752 & 1.42 & 0.074 & 0.022554 & 0.009605 & 0.339261 & 152.260 \\
\hline 12/5/08 7:00 & 2.08 & 0.620 & 0.175 & 78.375 & 1.42 & 0.074 & 0.022554 & 0.009605 & 0.339261 & 152.260 \\
\hline 12/5/08 8:00 & 2.078 & 0.618 & 0.173 & 77.752 & 1.417 & 0.071 & 0.021640 & 0.009052 & 0.319698 & 143.480 \\
\hline 12/5/08 9:00 & 2.078 & 0.618 & 0.173 & 77.752 & 1.417 & 0.071 & 0.021640 & 0.009052 & 0.319698 & 143.480 \\
\hline 12/5/08 10:00 & 2.08 & 0.620 & 0.175 & 78.375 & 1.42 & 0.074 & 0.022554 & 0.009605 & 0.339261 & 152.260 \\
\hline 12/5/08 11:00 & 2.084 & 0.624 & 0.177 & 79.629 & 1.422 & 0.076 & 0.023164 & 0.009981 & 0.352516 & 158.209 \\
\hline 12/5/08 12:00 & 2.088 & 0.628 & 0.180 & 80.895 & 1.422 & 0.076 & 0.023164 & 0.009981 & 0.352516 & 158.209 \\
\hline 12/5/08 13:00 & 2.09 & 0.630 & 0.182 & 81.532 & 1.424 & 0.078 & 0.023773 & 0.010361 & 0.365940 & 164.234 \\
\hline 12/5/08 14:00 & 2.092 & 0.632 & 0.183 & 82.173 & 1.424 & 0.078 & 0.023773 & 0.010361 & 0.365940 & 164.234 \\
\hline
\end{tabular}




\begin{tabular}{|c|c|c|c|c|c|c|c|c|c|c|}
\hline \multirow[b]{3}{*}{ Date Time } & \multicolumn{4}{|c|}{ V-Notch Weir } & \multicolumn{6}{|c|}{ R-Notch Weir } \\
\hline & \multicolumn{2}{|c|}{ Measured Water Level } & \multicolumn{2}{|c|}{$\begin{array}{c}\text { Calculated Rate } \\
\left(\mathrm{ft}^{3} / \mathrm{s}=448.8 \mathrm{gpm}\right)\end{array}$} & \multicolumn{3}{|c|}{ Measured Water Level } & \multicolumn{3}{|c|}{$\begin{array}{c}\text { Calculated Rate } \\
\left(\mathrm{ft}^{3} / \mathrm{s}=448.8 \mathrm{gpm}\right)\end{array}$} \\
\hline & $\begin{array}{c}\text { (ft above } \\
\text { sensor) }\end{array}$ & $\begin{array}{c}\text { (ft above } \\
\text { notch) }\end{array}$ & $\left(\mathrm{ft}^{3} / \mathrm{s}\right)$ & (gpm) & $\begin{array}{l}\text { (ft above } \\
\text { sensor) }\end{array}$ & $\begin{array}{c}\text { (ft above } \\
\text { log) }\end{array}$ & $\begin{array}{c}\text { (m above } \\
\text { log) }\end{array}$ & $\left(\mathrm{m}^{3} / \mathrm{s}\right)$ & $\left(\mathrm{ft}^{3} / \mathrm{s}\right)$ & (gpm) \\
\hline 12/5/08 15:00 & 2.106 & 0.646 & 0.193 & 86.742 & 1.427 & 0.081 & 0.024688 & 0.010940 & 0.386387 & 173.411 \\
\hline 12/5/08 16:00 & 2.117 & 0.657 & 0.202 & 90.436 & 1.429 & 0.083 & 0.025297 & 0.011331 & 0.400223 & 179.620 \\
\hline 12/5/08 17:00 & 2.125 & 0.665 & 0.208 & 93.181 & 1.429 & 0.083 & 0.025297 & 0.011331 & 0.400223 & 179.620 \\
\hline 12/5/08 18:00 & 2.13 & 0.670 & 0.212 & 94.921 & 1.429 & 0.083 & 0.025297 & 0.011331 & 0.400223 & 179.620 \\
\hline 12/5/08 19:00 & 2.135 & 0.675 & 0.215 & 96.681 & 1.431 & 0.085 & 0.025907 & 0.011728 & 0.414220 & 185.902 \\
\hline 12/5/08 20:00 & 2.137 & 0.677 & 0.217 & 97.391 & 1.431 & 0.085 & 0.025907 & 0.011728 & 0.414220 & 185.902 \\
\hline 12/5/08 21:00 & 2.137 & 0.677 & 0.217 & 97.391 & 1.431 & 0.085 & 0.025907 & 0.011728 & 0.414220 & 185.902 \\
\hline 12/5/08 22:00 & 2.142 & 0.682 & 0.221 & 99.178 & 1.431 & 0.085 & 0.025907 & 0.011728 & 0.414220 & 185.902 \\
\hline 12/5/08 23:00 & 2.142 & 0.682 & 0.221 & 99.178 & 1.431 & 0.085 & 0.025907 & 0.011728 & 0.414220 & 185.902 \\
\hline 12/6/08 0:00 & 2.146 & 0.686 & 0.224 & 100.622 & 1.431 & 0.085 & 0.025907 & 0.011728 & 0.414220 & 185.902 \\
\hline 12/6/08 1:00 & 2.144 & 0.684 & 0.223 & 99.898 & 1.431 & 0.085 & 0.025907 & 0.011728 & 0.414220 & 185.902 \\
\hline 12/6/08 2:00 & 2.148 & 0.688 & 0.226 & 101.348 & 1.431 & 0.085 & 0.025907 & 0.011728 & 0.414220 & 185.902 \\
\hline 12/6/08 3:00 & 2.146 & 0.686 & 0.224 & 100.622 & 1.431 & 0.085 & 0.025907 & 0.011728 & 0.414220 & 185.902 \\
\hline 12/6/08 4:00 & 2.148 & 0.688 & 0.226 & 101.348 & 1.431 & 0.085 & 0.025907 & 0.011728 & 0.414220 & 185.902 \\
\hline 12/6/08 5:00 & 2.141 & 0.681 & 0.220 & 98.819 & 1.434 & 0.088 & 0.026821 & 0.012331 & 0.435514 & 195.459 \\
\hline 12/6/08 6:00 & 2.146 & 0.686 & 0.224 & 100.622 & 1.431 & 0.085 & 0.025907 & 0.011728 & 0.414220 & 185.902 \\
\hline 12/6/08 7:00 & 2.144 & 0.684 & 0.223 & 99.898 & 1.431 & 0.085 & 0.025907 & 0.011728 & 0.414220 & 185.902 \\
\hline 12/6/08 8:00 & 2.149 & 0.689 & 0.227 & 101.713 & 1.431 & 0.085 & 0.025907 & 0.011728 & 0.414220 & 185.902 \\
\hline 12/6/08 9:00 & 2.142 & 0.682 & 0.221 & 99.178 & 1.431 & 0.085 & 0.025907 & 0.011728 & 0.414220 & 185.902 \\
\hline 12/6/08 10:00 & 2.132 & 0.672 & 0.213 & 95.623 & 1.429 & 0.083 & 0.025297 & 0.011331 & 0.400223 & 179.620 \\
\hline 12/6/08 11:00 & 2.132 & 0.672 & 0.213 & 95.623 & 1.429 & 0.083 & 0.025297 & 0.011331 & 0.400223 & 179.620 \\
\hline 12/6/08 12:00 & 2.143 & 0.683 & 0.222 & 99.538 & 1.429 & 0.083 & 0.025297 & 0.011331 & 0.400223 & 179.620 \\
\hline 12/6/08 13:00 & 2.136 & 0.676 & 0.216 & 97.036 & 1.429 & 0.083 & 0.025297 & 0.011331 & 0.400223 & 179.620 \\
\hline 12/6/08 14:00 & 2.138 & 0.678 & 0.218 & 97.747 & 1.429 & 0.083 & 0.025297 & 0.011331 & 0.400223 & 179.620 \\
\hline 12/6/08 15:00 & 2.129 & 0.669 & 0.211 & 94.572 & 1.427 & 0.081 & 0.024688 & 0.010940 & 0.386387 & 173.411 \\
\hline
\end{tabular}




\begin{tabular}{|c|c|c|c|c|c|c|c|c|c|c|}
\hline \multirow[b]{3}{*}{ Date Time } & \multicolumn{4}{|c|}{ V-Notch Weir } & \multicolumn{6}{|c|}{ R-Notch Weir } \\
\hline & \multicolumn{2}{|c|}{ Measured Water Level } & \multicolumn{2}{|c|}{$\begin{array}{c}\text { Calculated Rate } \\
\left(\mathrm{ft}^{3} / \mathrm{s}=448.8 \mathrm{gpm}\right)\end{array}$} & \multicolumn{3}{|c|}{ Measured Water Level } & \multicolumn{3}{|c|}{$\begin{array}{c}\text { Calculated Rate } \\
\left(\mathrm{ft}^{3} / \mathrm{s}=448.8 \mathrm{gpm}\right)\end{array}$} \\
\hline & $\begin{array}{c}\text { (ft above } \\
\text { sensor) }\end{array}$ & $\begin{array}{c}\text { (ft above } \\
\text { notch) }\end{array}$ & $\left(\mathrm{ft}^{3} / \mathrm{s}\right)$ & (gpm) & $\begin{array}{l}\text { (ft above } \\
\text { sensor) }\end{array}$ & $\begin{array}{c}\text { (ft above } \\
\text { log) }\end{array}$ & $\begin{array}{c}\text { (m above } \\
\text { log) }\end{array}$ & $\left(\mathrm{m}^{3} / \mathrm{s}\right)$ & $\left(\mathrm{ft}^{3} / \mathrm{s}\right)$ & (gpm) \\
\hline 12/6/08 16:00 & 2.133 & 0.673 & 0.214 & 95.975 & 1.427 & 0.081 & 0.024688 & 0.010940 & 0.386387 & 173.411 \\
\hline 12/6/08 17:00 & 2.131 & 0.671 & 0.212 & 95.272 & 1.429 & 0.083 & 0.025297 & 0.011331 & 0.400223 & 179.620 \\
\hline 12/6/08 18:00 & 2.132 & 0.672 & 0.213 & 95.623 & 1.429 & 0.083 & 0.025297 & 0.011331 & 0.400223 & 179.620 \\
\hline 12/6/08 19:00 & 2.13 & 0.670 & 0.212 & 94.921 & 1.429 & 0.083 & 0.025297 & 0.011331 & 0.400223 & 179.620 \\
\hline 12/6/08 20:00 & 2.126 & 0.666 & 0.208 & 93.527 & 1.427 & 0.081 & 0.024688 & 0.010940 & 0.386387 & 173.411 \\
\hline 12/6/08 21:00 & 2.124 & 0.664 & 0.207 & 92.835 & 1.427 & 0.081 & 0.024688 & 0.010940 & 0.386387 & 173.411 \\
\hline 12/6/08 22:00 & 2.121 & 0.661 & 0.205 & 91.802 & 1.424 & 0.078 & 0.023773 & 0.010361 & 0.365940 & 164.234 \\
\hline 12/6/08 23:00 & 2.114 & 0.654 & 0.199 & 89.419 & 1.424 & 0.078 & 0.023773 & 0.010361 & 0.365940 & 164.234 \\
\hline 12/7/08 0:00 & 2.112 & 0.652 & 0.198 & 88.745 & 1.424 & 0.078 & 0.023773 & 0.010361 & 0.365940 & 164.234 \\
\hline 12/7/08 1:00 & 2.11 & 0.650 & 0.196 & 88.074 & 1.424 & 0.078 & 0.023773 & 0.010361 & 0.365940 & 164.234 \\
\hline 12/7/08 2:00 & 2.11 & 0.650 & 0.196 & 88.074 & 1.422 & 0.076 & 0.023164 & 0.009981 & 0.352516 & 158.209 \\
\hline 12/7/08 3:00 & 2.107 & 0.647 & 0.194 & 87.074 & 1.424 & 0.078 & 0.023773 & 0.010361 & 0.365940 & 164.234 \\
\hline 12/7/08 4:00 & 2.112 & 0.652 & 0.198 & 88.745 & 1.427 & 0.081 & 0.024688 & 0.010940 & 0.386387 & 173.411 \\
\hline 12/7/08 5:00 & 2.103 & 0.643 & 0.191 & 85.750 & 1.424 & 0.078 & 0.023773 & 0.010361 & 0.365940 & 164.234 \\
\hline 12/7/08 6:00 & 2.105 & 0.645 & 0.193 & 86.410 & 1.422 & 0.076 & 0.023164 & 0.009981 & 0.352516 & 158.209 \\
\hline 12/7/08 7:00 & 2.105 & 0.645 & 0.193 & 86.410 & 1.424 & 0.078 & 0.023773 & 0.010361 & 0.365940 & 164.234 \\
\hline 12/7/08 8:00 & 2.103 & 0.643 & 0.191 & 85.750 & 1.422 & 0.076 & 0.023164 & 0.009981 & 0.352516 & 158.209 \\
\hline 12/7/08 9:00 & 2.101 & 0.641 & 0.190 & 85.093 & 1.424 & 0.078 & 0.023773 & 0.010361 & 0.365940 & 164.234 \\
\hline 12/7/08 10:00 & 2.108 & 0.648 & 0.195 & 87.406 & 1.422 & 0.076 & 0.023164 & 0.009981 & 0.352516 & 158.209 \\
\hline 12/7/08 11:00 & 2.101 & 0.641 & 0.190 & 85.093 & 1.424 & 0.078 & 0.023773 & 0.010361 & 0.365940 & 164.234 \\
\hline 12/7/08 12:00 & 2.1 & 0.640 & 0.189 & 84.765 & 1.424 & 0.078 & 0.023773 & 0.010361 & 0.365940 & 164.234 \\
\hline 12/7/08 13:00 & 2.104 & 0.644 & 0.192 & 86.080 & 1.424 & 0.078 & 0.023773 & 0.010361 & 0.365940 & 164.234 \\
\hline 12/7/08 14:00 & 2.105 & 0.645 & 0.193 & 86.410 & 1.424 & 0.078 & 0.023773 & 0.010361 & 0.365940 & 164.234 \\
\hline 12/7/08 15:00 & 2.109 & 0.649 & 0.195 & 87.740 & 1.427 & 0.081 & 0.024688 & 0.010940 & 0.386387 & 173.411 \\
\hline 12/7/08 16:00 & 2.118 & 0.658 & 0.202 & 90.776 & 1.427 & 0.081 & 0.024688 & 0.010940 & 0.386387 & 173.411 \\
\hline
\end{tabular}




\begin{tabular}{|c|c|c|c|c|c|c|c|c|c|c|}
\hline \multirow[b]{3}{*}{ Date Time } & \multicolumn{4}{|c|}{ V-Notch Weir } & \multicolumn{6}{|c|}{ R-Notch Weir } \\
\hline & \multicolumn{2}{|c|}{ Measured Water Level } & \multicolumn{2}{|c|}{$\begin{array}{c}\text { Calculated Rate } \\
\left(\mathrm{ft}^{3} / \mathrm{s}=448.8 \mathrm{gpm}\right)\end{array}$} & \multicolumn{3}{|c|}{ Measured Water Level } & \multicolumn{3}{|c|}{$\begin{array}{c}\text { Calculated Rate } \\
\left(\mathrm{ft}^{3} / \mathrm{s}=448.8 \mathrm{gpm}\right)\end{array}$} \\
\hline & $\begin{array}{c}\text { (ft above } \\
\text { sensor) }\end{array}$ & $\begin{array}{c}\text { (ft above } \\
\text { notch) }\end{array}$ & $\left(\mathrm{ft}^{3} / \mathrm{s}\right)$ & (gpm) & $\begin{array}{l}\text { (ft above } \\
\text { sensor) }\end{array}$ & $\begin{array}{c}\text { (ft above } \\
\text { log) }\end{array}$ & $\begin{array}{c}\text { (m above } \\
\text { log) }\end{array}$ & $\left(\mathrm{m}^{3} / \mathrm{s}\right)$ & $\left(\mathrm{ft}^{3} / \mathrm{s}\right)$ & (gpm) \\
\hline 12/7/08 17:00 & 2.126 & 0.666 & 0.208 & 93.527 & 1.429 & 0.083 & 0.025297 & 0.011331 & 0.400223 & 179.620 \\
\hline 12/7/08 18:00 & 2.131 & 0.671 & 0.212 & 95.272 & 1.429 & 0.083 & 0.025297 & 0.011331 & 0.400223 & 179.620 \\
\hline 12/7/08 19:00 & 2.133 & 0.673 & 0.214 & 95.975 & 1.429 & 0.083 & 0.025297 & 0.011331 & 0.400223 & 179.620 \\
\hline 12/7/08 20:00 & 2.133 & 0.673 & 0.214 & 95.975 & 1.429 & 0.083 & 0.025297 & 0.011331 & 0.400223 & 179.620 \\
\hline 12/7/08 21:00 & 2.131 & 0.671 & 0.212 & 95.272 & 1.429 & 0.083 & 0.025297 & 0.011331 & 0.400223 & 179.620 \\
\hline 12/7/08 22:00 & 2.131 & 0.671 & 0.212 & 95.272 & 1.429 & 0.083 & 0.025297 & 0.011331 & 0.400223 & 179.620 \\
\hline 12/7/08 23:00 & 2.136 & 0.676 & 0.216 & 97.036 & 1.429 & 0.083 & 0.025297 & 0.011331 & 0.400223 & 179.620 \\
\hline 12/8/08 0:00 & 2.134 & 0.674 & 0.215 & 96.328 & 1.431 & 0.085 & 0.025907 & 0.011728 & 0.414220 & 185.902 \\
\hline 12/8/08 1:00 & 2.136 & 0.676 & 0.216 & 97.036 & 1.431 & 0.085 & 0.025907 & 0.011728 & 0.414220 & 185.902 \\
\hline 12/8/08 2:00 & 2.136 & 0.676 & 0.216 & 97.036 & 1.429 & 0.083 & 0.025297 & 0.011331 & 0.400223 & 179.620 \\
\hline 12/8/08 3:00 & 2.136 & 0.676 & 0.216 & 97.036 & 1.431 & 0.085 & 0.025907 & 0.011728 & 0.414220 & 185.902 \\
\hline 12/8/08 4:00 & 2.136 & 0.676 & 0.216 & 97.036 & 1.431 & 0.085 & 0.025907 & 0.011728 & 0.414220 & 185.902 \\
\hline 12/8/08 5:00 & 2.134 & 0.674 & 0.215 & 96.328 & 1.431 & 0.085 & 0.025907 & 0.011728 & 0.414220 & 185.902 \\
\hline 12/8/08 6:00 & 2.136 & 0.676 & 0.216 & 97.036 & 1.431 & 0.085 & 0.025907 & 0.011728 & 0.414220 & 185.902 \\
\hline 12/8/08 7:00 & 2.134 & 0.674 & 0.215 & 96.328 & 1.429 & 0.083 & 0.025297 & 0.011331 & 0.400223 & 179.620 \\
\hline 12/8/08 8:00 & 2.136 & 0.676 & 0.216 & 97.036 & 1.429 & 0.083 & 0.025297 & 0.011331 & 0.400223 & 179.620 \\
\hline 12/8/08 9:00 & 2.136 & 0.676 & 0.216 & 97.036 & 1.429 & 0.083 & 0.025297 & 0.011331 & 0.400223 & 179.620 \\
\hline 12/8/08 10:00 & 2.138 & 0.678 & 0.218 & 97.747 & 1.429 & 0.083 & 0.025297 & 0.011331 & 0.400223 & 179.620 \\
\hline 12/8/08 11:00 & 2.14 & 0.680 & 0.219 & 98.461 & 1.431 & 0.085 & 0.025907 & 0.011728 & 0.414220 & 185.902 \\
\hline 12/8/08 12:00 & 2.14 & 0.680 & 0.219 & 98.461 & 1.431 & 0.085 & 0.025907 & 0.011728 & 0.414220 & 185.902 \\
\hline 12/8/08 13:00 & 2.146 & 0.686 & 0.224 & 100.622 & 1.431 & 0.085 & 0.025907 & 0.011728 & 0.414220 & 185.902 \\
\hline 12/8/08 14:00 & 2.148 & 0.688 & 0.226 & 101.348 & 1.431 & 0.085 & 0.025907 & 0.011728 & 0.414220 & 185.902 \\
\hline 12/8/08 15:00 & 2.155 & 0.695 & 0.232 & 103.916 & 1.434 & 0.088 & 0.026821 & 0.012331 & 0.435514 & 195.459 \\
\hline 12/8/08 16:00 & 2.159 & 0.699 & 0.235 & 105.401 & 1.436 & 0.09 & 0.027431 & 0.012738 & 0.449907 & 201.918 \\
\hline 12/8/08 17:00 & 2.162 & 0.702 & 0.237 & 106.523 & 1.434 & 0.088 & 0.026821 & 0.012331 & 0.435514 & 195.459 \\
\hline
\end{tabular}




\begin{tabular}{|c|c|c|c|c|c|c|c|c|c|c|}
\hline \multirow[b]{3}{*}{ Date Time } & \multicolumn{4}{|c|}{ V-Notch Weir } & \multicolumn{6}{|c|}{ R-Notch Weir } \\
\hline & \multicolumn{2}{|c|}{ Measured Water Level } & \multicolumn{2}{|c|}{$\begin{array}{c}\text { Calculated Rate } \\
\left(\mathrm{ft}^{3} / \mathrm{s}=448.8 \mathrm{gpm}\right)\end{array}$} & \multicolumn{3}{|c|}{ Measured Water Level } & \multicolumn{3}{|c|}{$\begin{array}{c}\text { Calculated Rate } \\
\left(\mathrm{ft}^{3} / \mathrm{s}=448.8 \mathrm{gpm}\right)\end{array}$} \\
\hline & $\begin{array}{l}\text { (ft above } \\
\text { sensor) }\end{array}$ & $\begin{array}{l}\text { (ft above } \\
\text { notch) }\end{array}$ & $\left(\mathrm{ft}^{3} / \mathrm{s}\right)$ & (gpm) & $\begin{array}{l}\text { (ft above } \\
\text { sensor) }\end{array}$ & $\begin{array}{l}\text { (ft above } \\
\text { log) }\end{array}$ & $\begin{array}{c}\text { (m above } \\
\text { log) }\end{array}$ & $\left(\mathrm{m}^{3} / \mathrm{s}\right)$ & $\left(\mathrm{ft}^{3} / \mathrm{s}\right)$ & (gpm) \\
\hline 12/8/08 18:00 & 2.162 & 0.702 & 0.237 & 106.523 & 1.434 & 0.088 & 0.026821 & 0.012331 & 0.435514 & 195.459 \\
\hline 12/8/08 19:00 & 2.162 & 0.702 & 0.237 & 106.523 & 1.434 & 0.088 & 0.026821 & 0.012331 & 0.435514 & 195.459 \\
\hline 12/8/08 20:00 & 2.158 & 0.698 & 0.234 & 105.029 & 1.434 & 0.088 & 0.026821 & 0.012331 & 0.435514 & 195.459 \\
\hline 12/8/08 21:00 & 2.151 & 0.691 & 0.228 & 102.444 & 1.431 & 0.085 & 0.025907 & 0.011728 & 0.414220 & 185.902 \\
\hline 12/8/08 22:00 & 2.151 & 0.691 & 0.228 & 102.444 & 1.431 & 0.085 & 0.025907 & 0.011728 & 0.414220 & 185.902 \\
\hline 12/8/08 23:00 & 2.144 & 0.684 & 0.223 & 99.898 & 1.431 & 0.085 & 0.025907 & 0.011728 & 0.414220 & 185.902 \\
\hline 12/9/08 0:00 & 2.147 & 0.687 & 0.225 & 100.985 & 1.429 & 0.083 & 0.025297 & 0.011331 & 0.400223 & 179.620 \\
\hline 12/9/08 1:00 & 2.15 & 0.690 & 0.227 & 102.078 & 1.431 & 0.085 & 0.025907 & 0.011728 & 0.414220 & 185.902 \\
\hline 12/9/08 2:00 & 2.143 & 0.683 & 0.222 & 99.538 & 1.427 & 0.081 & 0.024688 & 0.010940 & 0.386387 & 173.411 \\
\hline 12/9/08 3:00 & 2.141 & 0.681 & 0.220 & 98.819 & 1.427 & 0.081 & 0.024688 & 0.010940 & 0.386387 & 173.411 \\
\hline 12/9/08 4:00 & 2.133 & 0.673 & 0.214 & 95.975 & 1.429 & 0.083 & 0.025297 & 0.011331 & 0.400223 & 179.620 \\
\hline 12/9/08 5:00 & 2.131 & 0.671 & 0.212 & 95.272 & 1.427 & 0.081 & 0.024688 & 0.010940 & 0.386387 & 173.411 \\
\hline 12/9/08 6:00 & 2.131 & 0.671 & 0.212 & 95.272 & 1.427 & 0.081 & 0.024688 & 0.010940 & 0.386387 & 173.411 \\
\hline 12/9/08 7:00 & 2.125 & 0.665 & 0.208 & 93.181 & 1.429 & 0.083 & 0.025297 & 0.011331 & 0.400223 & 179.620 \\
\hline 12/9/08 8:00 & 2.127 & 0.667 & 0.209 & 93.875 & 1.424 & 0.078 & 0.023773 & 0.010361 & 0.365940 & 164.234 \\
\hline 12/9/08 9:00 & 2.127 & 0.667 & 0.209 & 93.875 & 1.429 & 0.083 & 0.025297 & 0.011331 & 0.400223 & 179.620 \\
\hline 12/9/08 10:00 & 2.132 & 0.672 & 0.213 & 95.623 & 1.424 & 0.078 & 0.023773 & 0.010361 & 0.365940 & 164.234 \\
\hline 12/9/08 11:00 & 2.104 & 0.644 & 0.192 & 86.080 & 1.422 & 0.076 & 0.023164 & 0.009981 & 0.352516 & 158.209 \\
\hline 12/9/08 12:00 & 2.097 & 0.637 & 0.187 & 83.788 & 1.422 & 0.076 & 0.023164 & 0.009981 & 0.352516 & 158.209 \\
\hline 12/9/08 13:00 & 2.09 & 0.630 & 0.182 & 81.532 & 1.422 & 0.076 & 0.023164 & 0.009981 & 0.352516 & 158.209 \\
\hline 12/9/08 14:00 & 2.088 & 0.628 & 0.180 & 80.895 & 1.422 & 0.076 & 0.023164 & 0.009981 & 0.352516 & 158.209 \\
\hline 12/9/08 15:00 & 2.09 & 0.630 & 0.182 & 81.532 & 1.422 & 0.076 & 0.023164 & 0.009981 & 0.352516 & 158.209 \\
\hline 12/9/08 16:00 & 2.083 & 0.623 & 0.177 & 79.314 & 1.422 & 0.076 & 0.023164 & 0.009981 & 0.352516 & 158.209 \\
\hline 12/9/08 17:00 & 2.064 & 0.604 & 0.164 & 73.477 & 1.424 & 0.078 & 0.023773 & 0.010361 & 0.365940 & 164.234 \\
\hline 12/9/08 18:00 & 2.06 & 0.600 & 0.161 & 72.282 & 1.422 & 0.076 & 0.023164 & 0.009981 & 0.352516 & 158.209 \\
\hline
\end{tabular}




\begin{tabular}{|c|c|c|c|c|c|c|c|c|c|c|}
\hline \multirow[b]{3}{*}{ Date Time } & \multicolumn{4}{|c|}{ V-Notch Weir } & \multicolumn{6}{|c|}{ R-Notch Weir } \\
\hline & \multicolumn{2}{|c|}{ Measured Water Level } & \multicolumn{2}{|c|}{$\begin{array}{c}\text { Calculated Rate } \\
\left(\mathrm{ft}^{3} / \mathrm{s}=448.8 \mathrm{gpm}\right)\end{array}$} & \multicolumn{3}{|c|}{ Measured Water Level } & \multicolumn{3}{|c|}{$\begin{array}{c}\text { Calculated Rate } \\
\left(\mathrm{ft}^{3} / \mathrm{s}=448.8 \mathrm{gpm}\right)\end{array}$} \\
\hline & $\begin{array}{c}\text { (ft above } \\
\text { sensor) }\end{array}$ & $\begin{array}{c}\text { (ft above } \\
\text { notch) }\end{array}$ & $\left(\mathrm{ft}^{3} / \mathrm{s}\right)$ & (gpm) & $\begin{array}{l}\text { (ft above } \\
\text { sensor) }\end{array}$ & $\begin{array}{c}\text { (ft above } \\
\text { log) }\end{array}$ & $\begin{array}{c}\text { (m above } \\
\text { log) }\end{array}$ & $\left(\mathrm{m}^{3} / \mathrm{s}\right)$ & $\left(\mathrm{ft}^{3} / \mathrm{s}\right)$ & (gpm) \\
\hline 12/9/08 19:00 & 2.048 & 0.588 & 0.153 & 68.767 & 1.42 & 0.074 & 0.022554 & 0.009605 & 0.339261 & 152.260 \\
\hline 12/9/08 20:00 & 2.039 & 0.579 & 0.148 & 66.200 & 1.42 & 0.074 & 0.022554 & 0.009605 & 0.339261 & 152.260 \\
\hline 12/9/08 21:00 & 2.037 & 0.577 & 0.146 & 65.638 & 1.417 & 0.071 & 0.021640 & 0.009052 & 0.319698 & 143.480 \\
\hline 12/9/08 22:00 & 2.025 & 0.565 & 0.139 & 62.323 & 1.42 & 0.074 & 0.022554 & 0.009605 & 0.339261 & 152.260 \\
\hline 12/9/08 23:00 & 2.02 & 0.560 & 0.136 & 60.972 & 1.417 & 0.071 & 0.021640 & 0.009052 & 0.319698 & 143.480 \\
\hline 12/10/08 0:00 & 2.013 & 0.553 & 0.132 & 59.110 & 1.417 & 0.071 & 0.021640 & 0.009052 & 0.319698 & 143.480 \\
\hline 12/10/08 1:00 & 2.01 & 0.550 & 0.130 & 58.323 & 1.415 & 0.069 & 0.021030 & 0.008688 & 0.306873 & 137.724 \\
\hline 12/10/08 2:00 & 2.005 & 0.545 & 0.127 & 57.025 & 1.417 & 0.071 & 0.021640 & 0.009052 & 0.319698 & 143.480 \\
\hline 12/10/08 3:00 & 2.001 & 0.541 & 0.125 & 55.999 & 1.417 & 0.071 & 0.021640 & 0.009052 & 0.319698 & 143.480 \\
\hline 12/10/08 4:00 & 1.994 & 0.534 & 0.121 & 54.231 & 1.415 & 0.069 & 0.021030 & 0.008688 & 0.306873 & 137.724 \\
\hline 12/10/08 5:00 & 1.989 & 0.529 & 0.118 & 52.988 & 1.415 & 0.069 & 0.021030 & 0.008688 & 0.306873 & 137.724 \\
\hline 12/10/08 6:00 & 1.986 & 0.526 & 0.116 & 52.251 & 1.415 & 0.069 & 0.021030 & 0.008688 & 0.306873 & 137.724 \\
\hline 12/10/08 7:00 & 1.982 & 0.522 & 0.114 & 51.278 & 1.415 & 0.069 & 0.021030 & 0.008688 & 0.306873 & 137.724 \\
\hline 12/10/08 8:00 & 1.979 & 0.519 & 0.113 & 50.555 & 1.415 & 0.069 & 0.021030 & 0.008688 & 0.306873 & 137.724 \\
\hline 12/10/08 9:00 & 1.972 & 0.512 & 0.109 & 48.893 & 1.413 & 0.067 & 0.020421 & 0.008330 & 0.294224 & 132.048 \\
\hline 12/10/08 10:00 & 1.977 & 0.517 & 0.112 & 50.077 & 1.413 & 0.067 & 0.020421 & 0.008330 & 0.294224 & 132.048 \\
\hline 12/10/08 11:00 & 1.988 & 0.528 & 0.118 & 52.742 & 1.413 & 0.067 & 0.020421 & 0.008330 & 0.294224 & 132.048 \\
\hline 12/10/08 12:00 & 1.98 & 0.520 & 0.113 & 50.796 & 1.417 & 0.071 & 0.021640 & 0.009052 & 0.319698 & 143.480 \\
\hline 12/10/08 13:00 & 1.989 & 0.529 & 0.118 & 52.988 & 1.417 & 0.071 & 0.021640 & 0.009052 & 0.319698 & 143.480 \\
\hline 12/10/08 14:00 & 1.998 & 0.538 & 0.123 & 55.237 & 1.42 & 0.074 & 0.022554 & 0.009605 & 0.339261 & 152.260 \\
\hline 12/10/08 15:00 & 2.007 & 0.547 & 0.128 & 57.542 & 1.422 & 0.076 & 0.023164 & 0.009981 & 0.352516 & 158.209 \\
\hline 12/10/08 16:00 & 2.009 & 0.549 & 0.129 & 58.062 & 1.424 & 0.078 & 0.023773 & 0.010361 & 0.365940 & 164.234 \\
\hline 12/10/08 17:00 & 2.014 & 0.554 & 0.132 & 59.374 & 1.417 & 0.071 & 0.021640 & 0.009052 & 0.319698 & 143.480 \\
\hline 12/10/08 18:00 & 2.012 & 0.552 & 0.131 & 58.847 & 1.42 & 0.074 & 0.022554 & 0.009605 & 0.339261 & 152.260 \\
\hline 12/10/08 19:00 & 2.008 & 0.548 & 0.129 & 57.802 & 1.417 & 0.071 & 0.021640 & 0.009052 & 0.319698 & 143.480 \\
\hline
\end{tabular}




\begin{tabular}{|c|c|c|c|c|c|c|c|c|c|c|}
\hline \multirow[b]{3}{*}{ Date Time } & \multicolumn{4}{|c|}{ V-Notch Weir } & \multicolumn{6}{|c|}{ R-Notch Weir } \\
\hline & \multicolumn{2}{|c|}{ Measured Water Level } & \multicolumn{2}{|c|}{$\begin{array}{c}\text { Calculated Rate } \\
\left(\mathrm{ft}^{3} / \mathrm{s}=448.8 \mathrm{gpm}\right)\end{array}$} & \multicolumn{3}{|c|}{ Measured Water Level } & \multicolumn{3}{|c|}{$\begin{array}{c}\text { Calculated Rate } \\
\left(\mathrm{ft}^{3} / \mathrm{s}=448.8 \mathrm{gpm}\right)\end{array}$} \\
\hline & $\begin{array}{c}\text { (ft above } \\
\text { sensor) }\end{array}$ & $\begin{array}{c}\text { (ft above } \\
\text { notch) }\end{array}$ & $\left(\mathrm{ft}^{3} / \mathrm{s}\right)$ & (gpm) & $\begin{array}{l}\text { (ft above } \\
\text { sensor) }\end{array}$ & $\begin{array}{c}\text { (ft above } \\
\text { log) }\end{array}$ & $\begin{array}{c}\text { (m above } \\
\text { log) }\end{array}$ & $\left(\mathrm{m}^{3} / \mathrm{s}\right)$ & $\left(\mathrm{ft}^{3} / \mathrm{s}\right)$ & (gpm) \\
\hline 12/10/08 20:00 & 2.006 & 0.546 & 0.128 & 57.283 & 1.417 & 0.071 & 0.021640 & 0.009052 & 0.319698 & 143.480 \\
\hline 12/10/08 21:00 & 2.003 & 0.543 & 0.126 & 56.511 & 1.417 & 0.071 & 0.021640 & 0.009052 & 0.319698 & 143.480 \\
\hline 12/10/08 22:00 & 2.001 & 0.541 & 0.125 & 55.999 & 1.417 & 0.071 & 0.021640 & 0.009052 & 0.319698 & 143.480 \\
\hline 12/10/08 23:00 & 1.997 & 0.537 & 0.123 & 54.984 & 1.415 & 0.069 & 0.021030 & 0.008688 & 0.306873 & 137.724 \\
\hline 12/11/08 0:00 & 1.994 & 0.534 & 0.121 & 54.231 & 1.415 & 0.069 & 0.021030 & 0.008688 & 0.306873 & 137.724 \\
\hline 12/11/08 1:00 & 1.994 & 0.534 & 0.121 & 54.231 & 1.415 & 0.069 & 0.021030 & 0.008688 & 0.306873 & 137.724 \\
\hline 12/11/08 2:00 & 1.987 & 0.527 & 0.117 & 52.496 & 1.415 & 0.069 & 0.021030 & 0.008688 & 0.306873 & 137.724 \\
\hline 12/11/08 3:00 & 1.985 & 0.525 & 0.116 & 52.007 & 1.415 & 0.069 & 0.021030 & 0.008688 & 0.306873 & 137.724 \\
\hline 12/11/08 4:00 & 1.987 & 0.527 & 0.117 & 52.496 & 1.417 & 0.071 & 0.021640 & 0.009052 & 0.319698 & 143.480 \\
\hline 12/11/08 5:00 & 1.983 & 0.523 & 0.115 & 51.520 & 1.415 & 0.069 & 0.021030 & 0.008688 & 0.306873 & 137.724 \\
\hline 12/11/08 6:00 & 1.978 & 0.518 & 0.112 & 50.316 & 1.415 & 0.069 & 0.021030 & 0.008688 & 0.306873 & 137.724 \\
\hline 12/11/08 7:00 & 1.978 & 0.518 & 0.112 & 50.316 & 1.415 & 0.069 & 0.021030 & 0.008688 & 0.306873 & 137.724 \\
\hline 12/11/08 8:00 & 1.978 & 0.518 & 0.112 & 50.316 & 1.415 & 0.069 & 0.021030 & 0.008688 & 0.306873 & 137.724 \\
\hline 12/11/08 9:00 & 1.97 & 0.510 & 0.108 & 48.424 & 1.413 & 0.067 & 0.020421 & 0.008330 & 0.294224 & 132.048 \\
\hline 12/11/08 10:00 & 1.97 & 0.510 & 0.108 & 48.424 & 1.415 & 0.069 & 0.021030 & 0.008688 & 0.306873 & 137.724 \\
\hline 12/11/08 11:00 & 1.968 & 0.508 & 0.107 & 47.958 & 1.415 & 0.069 & 0.021030 & 0.008688 & 0.306873 & 137.724 \\
\hline 12/11/08 12:00 & 1.974 & 0.514 & 0.110 & 49.365 & 1.413 & 0.067 & 0.020421 & 0.008330 & 0.294224 & 132.048 \\
\hline 12/11/08 13:00 & 1.973 & 0.513 & 0.109 & 49.129 & 1.417 & 0.071 & 0.021640 & 0.009052 & 0.319698 & 143.480 \\
\hline 12/11/08 14:00 & 1.979 & 0.519 & 0.113 & 50.555 & 1.417 & 0.071 & 0.021640 & 0.009052 & 0.319698 & 143.480 \\
\hline 12/11/08 15:00 & 1.991 & 0.531 & 0.119 & 53.483 & 1.42 & 0.074 & 0.022554 & 0.009605 & 0.339261 & 152.260 \\
\hline 12/11/08 16:00 & 1.988 & 0.528 & 0.118 & 52.742 & 1.42 & 0.074 & 0.022554 & 0.009605 & 0.339261 & 152.260 \\
\hline 12/11/08 17:00 & 1.998 & 0.538 & 0.123 & 55.237 & 1.422 & 0.076 & 0.023164 & 0.009981 & 0.352516 & 158.209 \\
\hline 12/11/08 18:00 & 1.994 & 0.534 & 0.121 & 54.231 & 1.422 & 0.076 & 0.023164 & 0.009981 & 0.352516 & 158.209 \\
\hline 12/11/08 19:00 & 1.989 & 0.529 & 0.118 & 52.988 & 1.422 & 0.076 & 0.023164 & 0.009981 & 0.352516 & 158.209 \\
\hline 12/11/08 20:00 & 1.98 & 0.520 & 0.113 & 50.796 & 1.422 & 0.076 & 0.023164 & 0.009981 & 0.352516 & 158.209 \\
\hline
\end{tabular}




\begin{tabular}{|c|c|c|c|c|c|c|c|c|c|c|}
\hline \multirow[b]{3}{*}{ Date Time } & \multicolumn{4}{|c|}{ V-Notch Weir } & \multicolumn{6}{|c|}{ R-Notch Weir } \\
\hline & \multicolumn{2}{|c|}{ Measured Water Level } & \multicolumn{2}{|c|}{$\begin{array}{c}\text { Calculated Rate } \\
\left(\mathrm{ft}^{3} / \mathrm{s}=448.8 \mathrm{gpm}\right)\end{array}$} & \multicolumn{3}{|c|}{ Measured Water Level } & \multicolumn{3}{|c|}{$\begin{array}{c}\text { Calculated Rate } \\
\left(\mathrm{ft}^{3} / \mathrm{s}=448.8 \mathrm{gpm}\right)\end{array}$} \\
\hline & $\begin{array}{c}\text { (ft above } \\
\text { sensor) }\end{array}$ & $\begin{array}{c}\text { (ft above } \\
\text { notch) }\end{array}$ & $\left(\mathrm{ft}^{3} / \mathrm{s}\right)$ & (gpm) & $\begin{array}{l}\text { (ft above } \\
\text { sensor) }\end{array}$ & $\begin{array}{c}\text { (ft above } \\
\text { log) }\end{array}$ & $\begin{array}{c}\text { (m above } \\
\text { log) }\end{array}$ & $\left(\mathrm{m}^{3} / \mathrm{s}\right)$ & $\left(\mathrm{ft}^{3} / \mathrm{s}\right)$ & (gpm) \\
\hline 12/11/08 21:00 & 1.98 & 0.520 & 0.113 & 50.796 & 1.42 & 0.074 & 0.022554 & 0.009605 & 0.339261 & 152.260 \\
\hline 12/11/08 22:00 & 1.978 & 0.518 & 0.112 & 50.316 & 1.42 & 0.074 & 0.022554 & 0.009605 & 0.339261 & 152.260 \\
\hline 12/11/08 23:00 & 1.973 & 0.513 & 0.109 & 49.129 & 1.42 & 0.074 & 0.022554 & 0.009605 & 0.339261 & 152.260 \\
\hline 12/12/08 0:00 & 1.974 & 0.514 & 0.110 & 49.365 & 1.422 & 0.076 & 0.023164 & 0.009981 & 0.352516 & 158.209 \\
\hline 12/12/08 1:00 & 1.972 & 0.512 & 0.109 & 48.893 & 1.42 & 0.074 & 0.022554 & 0.009605 & 0.339261 & 152.260 \\
\hline 12/12/08 2:00 & 1.965 & 0.505 & 0.105 & 47.264 & 1.42 & 0.074 & 0.022554 & 0.009605 & 0.339261 & 152.260 \\
\hline 12/12/08 3:00 & 1.96 & 0.500 & 0.103 & 46.120 & 1.42 & 0.074 & 0.022554 & 0.009605 & 0.339261 & 152.260 \\
\hline 12/12/08 4:00 & 1.958 & 0.498 & 0.102 & 45.668 & 1.417 & 0.071 & 0.021640 & 0.009052 & 0.319698 & 143.480 \\
\hline 12/12/08 5:00 & 1.956 & 0.496 & 0.101 & 45.218 & 1.417 & 0.071 & 0.021640 & 0.009052 & 0.319698 & 143.480 \\
\hline 12/12/08 6:00 & 1.954 & 0.494 & 0.100 & 44.770 & 1.417 & 0.071 & 0.021640 & 0.009052 & 0.319698 & 143.480 \\
\hline 12/12/08 7:00 & 1.952 & 0.492 & 0.099 & 44.326 & 1.417 & 0.071 & 0.021640 & 0.009052 & 0.319698 & 143.480 \\
\hline 12/12/08 8:00 & 1.943 & 0.483 & 0.094 & 42.357 & 1.417 & 0.071 & 0.021640 & 0.009052 & 0.319698 & 143.480 \\
\hline 12/12/08 9:00 & 1.943 & 0.483 & 0.094 & 42.357 & 1.417 & 0.071 & 0.021640 & 0.009052 & 0.319698 & 143.480 \\
\hline 12/12/08 10:00 & 1.945 & 0.485 & 0.095 & 42.790 & 1.417 & 0.071 & 0.021640 & 0.009052 & 0.319698 & 143.480 \\
\hline 12/12/08 11:00 & 1.94 & 0.480 & 0.093 & 41.713 & 1.417 & 0.071 & 0.021640 & 0.009052 & 0.319698 & 143.480 \\
\hline 12/12/08 12:00 & 1.937 & 0.477 & 0.092 & 41.075 & 1.417 & 0.071 & 0.021640 & 0.009052 & 0.319698 & 143.480 \\
\hline 12/12/08 13:00 & 1.933 & 0.473 & 0.090 & 40.233 & 1.42 & 0.074 & 0.022554 & 0.009605 & 0.339261 & 152.260 \\
\hline 12/12/08 14:00 & 1.939 & 0.479 & 0.092 & 41.500 & 1.422 & 0.076 & 0.023164 & 0.009981 & 0.352516 & 158.209 \\
\hline 12/12/08 15:00 & 1.943 & 0.483 & 0.094 & 42.357 & 1.422 & 0.076 & 0.023164 & 0.009981 & 0.352516 & 158.209 \\
\hline 12/12/08 16:00 & 1.955 & 0.495 & 0.100 & 44.994 & 1.424 & 0.078 & 0.023773 & 0.010361 & 0.365940 & 164.234 \\
\hline 12/12/08 17:00 & 1.958 & 0.498 & 0.102 & 45.668 & 1.424 & 0.078 & 0.023773 & 0.010361 & 0.365940 & 164.234 \\
\hline 12/12/08 18:00 & 1.958 & 0.498 & 0.102 & 45.668 & 1.422 & 0.076 & 0.023164 & 0.009981 & 0.352516 & 158.209 \\
\hline 12/12/08 19:00 & 1.954 & 0.494 & 0.100 & 44.770 & 1.424 & 0.078 & 0.023773 & 0.010361 & 0.365940 & 164.234 \\
\hline 12/12/08 20:00 & 1.954 & 0.494 & 0.100 & 44.770 & 1.422 & 0.076 & 0.023164 & 0.009981 & 0.352516 & 158.209 \\
\hline 12/12/08 21:00 & 1.952 & 0.492 & 0.099 & 44.326 & 1.424 & 0.078 & 0.023773 & 0.010361 & 0.365940 & 164.234 \\
\hline
\end{tabular}




\begin{tabular}{|c|c|c|c|c|c|c|c|c|c|c|}
\hline \multirow[b]{3}{*}{ Date Time } & \multicolumn{4}{|c|}{ V-Notch Weir } & \multicolumn{6}{|c|}{ R-Notch Weir } \\
\hline & \multicolumn{2}{|c|}{ Measured Water Level } & \multicolumn{2}{|c|}{$\begin{array}{c}\text { Calculated Rate } \\
\left(\mathrm{ft}^{3} / \mathrm{s}=448.8 \mathrm{gpm}\right)\end{array}$} & \multicolumn{3}{|c|}{ Measured Water Level } & \multicolumn{3}{|c|}{$\begin{array}{c}\text { Calculated Rate } \\
\left(\mathrm{ft}^{3} / \mathrm{s}=448.8 \mathrm{gpm}\right)\end{array}$} \\
\hline & $\begin{array}{c}\text { (ft above } \\
\text { sensor) }\end{array}$ & $\begin{array}{c}\text { (ft above } \\
\text { notch) }\end{array}$ & $\left(\mathrm{ft}^{3} / \mathrm{s}\right)$ & (gpm) & $\begin{array}{l}\text { (ft above } \\
\text { sensor) }\end{array}$ & $\begin{array}{c}\text { (ft above } \\
\text { log) }\end{array}$ & $\begin{array}{c}\text { (m above } \\
\text { log) }\end{array}$ & $\left(\mathrm{m}^{3} / \mathrm{s}\right)$ & $\left(\mathrm{ft}^{3} / \mathrm{s}\right)$ & (gpm) \\
\hline 12/12/08 22:00 & 1.95 & 0.490 & 0.098 & 43.884 & 1.422 & 0.076 & 0.023164 & 0.009981 & 0.352516 & 158.209 \\
\hline 12/12/08 23:00 & 1.948 & 0.488 & 0.097 & 43.444 & 1.422 & 0.076 & 0.023164 & 0.009981 & 0.352516 & 158.209 \\
\hline 12/13/08 0:00 & 1.946 & 0.486 & 0.096 & 43.008 & 1.422 & 0.076 & 0.023164 & 0.009981 & 0.352516 & 158.209 \\
\hline 12/13/08 1:00 & 1.949 & 0.489 & 0.097 & 43.664 & 1.422 & 0.076 & 0.023164 & 0.009981 & 0.352516 & 158.209 \\
\hline 12/13/08 2:00 & 1.946 & 0.486 & 0.096 & 43.008 & 1.424 & 0.078 & 0.023773 & 0.010361 & 0.365940 & 164.234 \\
\hline 12/13/08 3:00 & 1.944 & 0.484 & 0.095 & 42.574 & 1.424 & 0.078 & 0.023773 & 0.010361 & 0.365940 & 164.234 \\
\hline 12/13/08 4:00 & 1.944 & 0.484 & 0.095 & 42.574 & 1.424 & 0.078 & 0.023773 & 0.010361 & 0.365940 & 164.234 \\
\hline 12/13/08 5:00 & 1.944 & 0.484 & 0.095 & 42.574 & 1.424 & 0.078 & 0.023773 & 0.010361 & 0.365940 & 164.234 \\
\hline 12/13/08 6:00 & 1.942 & 0.482 & 0.094 & 42.142 & 1.424 & 0.078 & 0.023773 & 0.010361 & 0.365940 & 164.234 \\
\hline 12/13/08 7:00 & 1.942 & 0.482 & 0.094 & 42.142 & 1.427 & 0.081 & 0.024688 & 0.010940 & 0.386387 & 173.411 \\
\hline 12/13/08 8:00 & 1.94 & 0.480 & 0.093 & 41.713 & 1.427 & 0.081 & 0.024688 & 0.010940 & 0.386387 & 173.411 \\
\hline 12/13/08 9:00 & 1.937 & 0.477 & 0.092 & 41.075 & 1.424 & 0.078 & 0.023773 & 0.010361 & 0.365940 & 164.234 \\
\hline 12/13/08 10:00 & 1.947 & 0.487 & 0.096 & 43.226 & 1.417 & 0.071 & 0.021640 & 0.009052 & 0.319698 & 143.480 \\
\hline 12/13/08 11:00 & 1.943 & 0.483 & 0.094 & 42.357 & 1.417 & 0.071 & 0.021640 & 0.009052 & 0.319698 & 143.480 \\
\hline 12/13/08 12:00 & 1.941 & 0.481 & 0.093 & 41.927 & 1.415 & 0.069 & 0.021030 & 0.008688 & 0.306873 & 137.724 \\
\hline 12/13/08 13:00 & 1.939 & 0.479 & 0.092 & 41.500 & 1.424 & 0.078 & 0.023773 & 0.010361 & 0.365940 & 164.234 \\
\hline 12/13/08 14:00 & 1.94 & 0.480 & 0.093 & 41.713 & 1.422 & 0.076 & 0.023164 & 0.009981 & 0.352516 & 158.209 \\
\hline 12/13/08 15:00 & 1.961 & 0.501 & 0.103 & 46.348 & 1.422 & 0.076 & 0.023164 & 0.009981 & 0.352516 & 158.209 \\
\hline 12/13/08 16:00 & 1.967 & 0.507 & 0.106 & 47.726 & 1.422 & 0.076 & 0.023164 & 0.009981 & 0.352516 & 158.209 \\
\hline 12/13/08 17:00 & 1.965 & 0.505 & 0.105 & 47.264 & 1.422 & 0.076 & 0.023164 & 0.009981 & 0.352516 & 158.209 \\
\hline 12/13/08 18:00 & 1.965 & 0.505 & 0.105 & 47.264 & 1.422 & 0.076 & 0.023164 & 0.009981 & 0.352516 & 158.209 \\
\hline 12/13/08 19:00 & 1.957 & 0.497 & 0.101 & 45.442 & 1.422 & 0.076 & 0.023164 & 0.009981 & 0.352516 & 158.209 \\
\hline 12/13/08 20:00 & 1.952 & 0.492 & 0.099 & 44.326 & 1.422 & 0.076 & 0.023164 & 0.009981 & 0.352516 & 158.209 \\
\hline 12/13/08 21:00 & 1.948 & 0.488 & 0.097 & 43.444 & 1.42 & 0.074 & 0.022554 & 0.009605 & 0.339261 & 152.260 \\
\hline 12/13/08 22:00 & 1.943 & 0.483 & 0.094 & 42.357 & 1.42 & 0.074 & 0.022554 & 0.009605 & 0.339261 & 152.260 \\
\hline
\end{tabular}




\begin{tabular}{|c|c|c|c|c|c|c|c|c|c|c|}
\hline \multirow[b]{3}{*}{ Date Time } & \multicolumn{4}{|c|}{ V-Notch Weir } & \multicolumn{6}{|c|}{ R-Notch Weir } \\
\hline & \multicolumn{2}{|c|}{ Measured Water Level } & \multicolumn{2}{|c|}{$\begin{array}{c}\text { Calculated Rate } \\
\left(\mathrm{ft}^{3} / \mathrm{s}=448.8 \mathrm{gpm}\right)\end{array}$} & \multicolumn{3}{|c|}{ Measured Water Level } & \multicolumn{3}{|c|}{$\begin{array}{c}\text { Calculated Rate } \\
\left(\mathrm{ft}^{3} / \mathrm{s}=448.8 \mathrm{gpm}\right)\end{array}$} \\
\hline & $\begin{array}{c}\text { (ft above } \\
\text { sensor) }\end{array}$ & $\begin{array}{c}\text { (ft above } \\
\text { notch) }\end{array}$ & $\left(\mathrm{ft}^{3} / \mathrm{s}\right)$ & (gpm) & $\begin{array}{l}\text { (ft above } \\
\text { sensor) }\end{array}$ & $\begin{array}{c}\text { (ft above } \\
\text { log) }\end{array}$ & $\begin{array}{c}\text { (m above } \\
\text { log) }\end{array}$ & $\left(\mathrm{m}^{3} / \mathrm{s}\right)$ & $\left(\mathrm{ft}^{3} / \mathrm{s}\right)$ & (gpm) \\
\hline 12/13/08 23:00 & 1.944 & 0.484 & 0.095 & 42.574 & 1.422 & 0.076 & 0.023164 & 0.009981 & 0.352516 & 158.209 \\
\hline 12/14/08 0:00 & 1.941 & 0.481 & 0.093 & 41.927 & 1.42 & 0.074 & 0.022554 & 0.009605 & 0.339261 & 152.260 \\
\hline 12/14/08 1:00 & 1.941 & 0.481 & 0.093 & 41.927 & 1.42 & 0.074 & 0.022554 & 0.009605 & 0.339261 & 152.260 \\
\hline 12/14/08 2:00 & 1.946 & 0.486 & 0.096 & 43.008 & 1.422 & 0.076 & 0.023164 & 0.009981 & 0.352516 & 158.209 \\
\hline 12/14/08 3:00 & 1.946 & 0.486 & 0.096 & 43.008 & 1.422 & 0.076 & 0.023164 & 0.009981 & 0.352516 & 158.209 \\
\hline 12/14/08 4:00 & 1.946 & 0.486 & 0.096 & 43.008 & 1.422 & 0.076 & 0.023164 & 0.009981 & 0.352516 & 158.209 \\
\hline 12/14/08 5:00 & 1.943 & 0.483 & 0.094 & 42.357 & 1.422 & 0.076 & 0.023164 & 0.009981 & 0.352516 & 158.209 \\
\hline 12/14/08 6:00 & 1.939 & 0.479 & 0.092 & 41.500 & 1.422 & 0.076 & 0.023164 & 0.009981 & 0.352516 & 158.209 \\
\hline 12/14/08 7:00 & 1.932 & 0.472 & 0.089 & 40.024 & 1.42 & 0.074 & 0.022554 & 0.009605 & 0.339261 & 152.260 \\
\hline 12/14/08 8:00 & 1.924 & 0.464 & 0.086 & 38.377 & 1.417 & 0.071 & 0.021640 & 0.009052 & 0.319698 & 143.480 \\
\hline 12/14/08 9:00 & 1.904 & 0.444 & 0.077 & 34.439 & 1.412 & 0.066 & 0.020116 & 0.008153 & 0.287966 & 129.239 \\
\hline 12/14/08 10:00 & 1.91 & 0.450 & 0.079 & 35.594 & 1.412 & 0.066 & 0.020116 & 0.008153 & 0.287966 & 129.239 \\
\hline 12/14/08 11:00 & 1.897 & 0.437 & 0.074 & 33.121 & 1.41 & 0.064 & 0.019506 & 0.007803 & 0.275587 & 123.683 \\
\hline 12/14/08 12:00 & 1.883 & 0.423 & 0.068 & 30.576 & 1.41 & 0.064 & 0.019506 & 0.007803 & 0.275587 & 123.683 \\
\hline 12/14/08 13:00 & 1.874 & 0.414 & 0.065 & 29.005 & 1.41 & 0.064 & 0.019506 & 0.007803 & 0.275587 & 123.683 \\
\hline 12/14/08 14:00 & 1.869 & 0.409 & 0.063 & 28.153 & 1.408 & 0.062 & 0.018897 & 0.007457 & 0.263390 & 118.209 \\
\hline 12/14/08 15:00 & 1.86 & 0.400 & 0.059 & 26.658 & 1.408 & 0.062 & 0.018897 & 0.007457 & 0.263390 & 118.209 \\
\hline 12/14/08 16:00 & 1.851 & 0.391 & 0.056 & 25.212 & 1.408 & 0.062 & 0.018897 & 0.007457 & 0.263390 & 118.209 \\
\hline 12/14/08 17:00 & 1.837 & 0.377 & 0.051 & 23.058 & 1.405 & 0.059 & 0.017982 & 0.006949 & 0.245443 & 110.155 \\
\hline 12/14/08 18:00 & 1.825 & 0.365 & 0.047 & 21.302 & 1.405 & 0.059 & 0.017982 & 0.006949 & 0.245443 & 110.155 \\
\hline 12/14/08 19:00 & 1.799 & 0.339 & 0.040 & 17.781 & 1.415 & 0.069 & 0.021030 & 0.008688 & 0.306873 & 137.724 \\
\hline 12/14/08 20:00 & 1.757 & 0.297 & 0.029 & 12.878 & 1.441 & 0.095 & 0.028955 & 0.013776 & 0.486564 & 218.370 \\
\hline 12/14/08 21:00 & 1.707 & 0.247 & 0.018 & 8.229 & 1.472 & 0.126 & 0.038403 & 0.020784 & 0.734098 & 329.463 \\
\hline 12/14/08 22:00 & 1.689 & 0.229 & 0.015 & 6.853 & 1.495 & 0.149 & 0.045413 & 0.026571 & 0.938496 & 421.197 \\
\hline 12/14/08 23:00 & 1.7 & 0.240 & 0.017 & 7.676 & 1.519 & 0.173 & 0.052728 & 0.033095 & 1.168919 & 524.611 \\
\hline
\end{tabular}




\begin{tabular}{|c|c|c|c|c|c|c|c|c|c|c|}
\hline \multirow[b]{3}{*}{ Date Time } & \multicolumn{4}{|c|}{ V-Notch Weir } & \multicolumn{6}{|c|}{ R-Notch Weir } \\
\hline & \multicolumn{2}{|c|}{ Measured Water Level } & \multicolumn{2}{|c|}{$\begin{array}{c}\text { Calculated Rate } \\
\left(\mathrm{ft}^{3} / \mathrm{s}=448.8 \mathrm{gpm}\right)\end{array}$} & \multicolumn{3}{|c|}{ Measured Water Level } & \multicolumn{3}{|c|}{$\begin{array}{c}\text { Calculated Rate } \\
\left(\mathrm{ft}^{3} / \mathrm{s}=448.8 \mathrm{gpm}\right)\end{array}$} \\
\hline & $\begin{array}{c}\text { (ft above } \\
\text { sensor) }\end{array}$ & $\begin{array}{c}\text { (ft above } \\
\text { notch) }\end{array}$ & $\left(\mathrm{ft}^{3} / \mathrm{s}\right)$ & (gpm) & $\begin{array}{l}\text { (ft above } \\
\text { sensor) }\end{array}$ & $\begin{array}{c}\text { (ft above } \\
\text { log) }\end{array}$ & $\begin{array}{c}\text { (m above } \\
\text { log) }\end{array}$ & $\left(\mathrm{m}^{3} / \mathrm{s}\right)$ & $\left(\mathrm{ft}^{3} / \mathrm{s}\right)$ & (gpm) \\
\hline 12/15/08 0:00 & 1.754 & 0.294 & 0.028 & 12.563 & 1.524 & 0.178 & 0.054252 & 0.034513 & 1.219010 & 547.092 \\
\hline 12/15/08 1:00 & 1.724 & 0.264 & 0.022 & 9.671 & 1.535 & 0.189 & 0.057604 & 0.037703 & 1.331647 & 597.643 \\
\hline 12/15/08 2:00 & 1.726 & 0.266 & 0.022 & 9.850 & 1.543 & 0.197 & 0.060043 & 0.040080 & 1.415626 & 635.333 \\
\hline 12/15/08 3:00 & 1.79 & 0.330 & 0.037 & 16.650 & 1.526 & 0.18 & 0.054861 & 0.035086 & 1.239242 & 556.172 \\
\hline 12/15/08 4:00 & 1.764 & 0.304 & 0.030 & 13.630 & 1.524 & 0.178 & 0.054252 & 0.034513 & 1.219010 & 547.092 \\
\hline 12/15/08 5:00 & 1.75 & 0.290 & 0.027 & 12.151 & 1.517 & 0.171 & 0.052118 & 0.032534 & 1.149080 & 515.707 \\
\hline 12/15/08 6:00 & 1.752 & 0.292 & 0.028 & 12.356 & 1.519 & 0.173 & 0.052728 & 0.033095 & 1.168919 & 524.611 \\
\hline 12/15/08 7:00 & 1.759 & 0.299 & 0.029 & 13.090 & 1.512 & 0.166 & 0.050594 & 0.031143 & 1.099980 & 493.671 \\
\hline 12/15/08 8:00 & 1.768 & 0.308 & 0.031 & 14.071 & 1.503 & 0.157 & 0.047851 & 0.028693 & 1.013424 & 454.824 \\
\hline 12/15/08 9:00 & 1.773 & 0.313 & 0.033 & 14.634 & 1.495 & 0.149 & 0.045413 & 0.026571 & 0.938496 & 421.197 \\
\hline 12/15/08 10:00 & 1.78 & 0.320 & 0.034 & 15.445 & 1.486 & 0.14 & 0.042670 & 0.024251 & 0.856529 & 384.410 \\
\hline 12/15/08 11:00 & 1.78 & 0.320 & 0.034 & 15.445 & 1.479 & 0.133 & 0.040536 & 0.022495 & 0.794527 & 356.583 \\
\hline 12/15/08 12:00 & 1.796 & 0.336 & 0.039 & 17.399 & 1.47 & 0.124 & 0.037793 & 0.020304 & 0.717127 & 321.847 \\
\hline 12/15/08 13:00 & 1.812 & 0.352 & 0.043 & 19.494 & 1.451 & 0.105 & 0.032002 & 0.015931 & 0.562690 & 252.535 \\
\hline 12/15/08 14:00 & 1.81 & 0.350 & 0.043 & 19.224 & 1.439 & 0.093 & 0.028345 & 0.013358 & 0.471786 & 211.738 \\
\hline 12/15/08 15:00 & 1.805 & 0.345 & 0.041 & 18.560 & 1.429 & 0.083 & 0.025297 & 0.011331 & 0.400223 & 179.620 \\
\hline 12/15/08 16:00 & 1.808 & 0.348 & 0.042 & 18.957 & 1.425 & 0.079 & 0.024078 & 0.010553 & 0.372715 & 167.274 \\
\hline 12/15/08 17:00 & 1.801 & 0.341 & 0.040 & 18.038 & 1.42 & 0.074 & 0.022554 & 0.009605 & 0.339261 & 152.260 \\
\hline 12/15/08 18:00 & 1.796 & 0.336 & 0.039 & 17.399 & 1.415 & 0.069 & 0.021030 & 0.008688 & 0.306873 & 137.724 \\
\hline 12/15/08 19:00 & 1.791 & 0.331 & 0.037 & 16.773 & 1.415 & 0.069 & 0.021030 & 0.008688 & 0.306873 & 137.724 \\
\hline 12/15/08 20:00 & 1.792 & 0.332 & 0.038 & 16.897 & 1.413 & 0.067 & 0.020421 & 0.008330 & 0.294224 & 132.048 \\
\hline 12/15/08 21:00 & 1.787 & 0.327 & 0.036 & 16.283 & 1.41 & 0.064 & 0.019506 & 0.007803 & 0.275587 & 123.683 \\
\hline 12/15/08 22:00 & 1.785 & 0.325 & 0.036 & 16.041 & 1.408 & 0.062 & 0.018897 & 0.007457 & 0.263390 & 118.209 \\
\hline 12/15/08 23:00 & 1.78 & 0.320 & 0.034 & 15.445 & 1.406 & 0.06 & 0.018287 & 0.007117 & 0.251378 & 112.818 \\
\hline 12/16/08 0:00 & 1.78 & 0.320 & 0.034 & 15.445 & 1.406 & 0.06 & 0.018287 & 0.007117 & 0.251378 & 112.818 \\
\hline
\end{tabular}




\begin{tabular}{|c|c|c|c|c|c|c|c|c|c|c|}
\hline \multirow[b]{3}{*}{ Date Time } & \multicolumn{4}{|c|}{ V-Notch Weir } & \multicolumn{6}{|c|}{ R-Notch Weir } \\
\hline & \multicolumn{2}{|c|}{ Measured Water Level } & \multicolumn{2}{|c|}{$\begin{array}{c}\text { Calculated Rate } \\
\left(\mathrm{ft}^{3} / \mathrm{s}=448.8 \mathrm{gpm}\right)\end{array}$} & \multicolumn{3}{|c|}{ Measured Water Level } & \multicolumn{3}{|c|}{$\begin{array}{c}\text { Calculated Rate } \\
\left(\mathrm{ft}^{3} / \mathrm{s}=448.8 \mathrm{gpm}\right)\end{array}$} \\
\hline & $\begin{array}{c}\text { (ft above } \\
\text { sensor) }\end{array}$ & $\begin{array}{c}\text { (ft above } \\
\text { notch) }\end{array}$ & $\left(\mathrm{ft}^{3} / \mathrm{s}\right)$ & (gpm) & $\begin{array}{l}\text { (ft above } \\
\text { sensor) }\end{array}$ & $\begin{array}{c}\text { (ft above } \\
\text { log) }\end{array}$ & $\begin{array}{c}\text { (m above } \\
\text { log) }\end{array}$ & $\left(\mathrm{m}^{3} / \mathrm{s}\right)$ & $\left(\mathrm{ft}^{3} / \mathrm{s}\right)$ & (gpm) \\
\hline 12/16/08 1:00 & 1.78 & 0.320 & 0.034 & 15.445 & 1.406 & 0.06 & 0.018287 & 0.007117 & 0.251378 & 112.818 \\
\hline 12/16/08 2:00 & 1.778 & 0.318 & 0.034 & 15.211 & 1.403 & 0.057 & 0.017373 & 0.006617 & 0.233715 & 104.891 \\
\hline 12/16/08 3:00 & 1.778 & 0.318 & 0.034 & 15.211 & 1.406 & 0.06 & 0.018287 & 0.007117 & 0.251378 & 112.818 \\
\hline 12/16/08 4:00 & 1.778 & 0.318 & 0.034 & 15.211 & 1.403 & 0.057 & 0.017373 & 0.006617 & 0.233715 & 104.891 \\
\hline 12/16/08 5:00 & 1.778 & 0.318 & 0.034 & 15.211 & 1.403 & 0.057 & 0.017373 & 0.006617 & 0.233715 & 104.891 \\
\hline 12/16/08 6:00 & 1.775 & 0.315 & 0.033 & 14.863 & 1.403 & 0.057 & 0.017373 & 0.006617 & 0.233715 & 104.891 \\
\hline 12/16/08 7:00 & 1.775 & 0.315 & 0.033 & 14.863 & 1.403 & 0.057 & 0.017373 & 0.006617 & 0.233715 & 104.891 \\
\hline 12/16/08 8:00 & 1.775 & 0.315 & 0.033 & 14.863 & 1.403 & 0.057 & 0.017373 & 0.006617 & 0.233715 & 104.891 \\
\hline 12/16/08 9:00 & 1.78 & 0.320 & 0.034 & 15.445 & 1.406 & 0.06 & 0.018287 & 0.007117 & 0.251378 & 112.818 \\
\hline 12/16/08 10:00 & 1.771 & 0.311 & 0.032 & 14.407 & 1.403 & 0.057 & 0.017373 & 0.006617 & 0.233715 & 104.891 \\
\hline 12/16/08 11:00 & 1.778 & 0.318 & 0.034 & 15.211 & 1.406 & 0.06 & 0.018287 & 0.007117 & 0.251378 & 112.818 \\
\hline 12/16/08 12:00 & 1.787 & 0.327 & 0.036 & 16.283 & 1.406 & 0.06 & 0.018287 & 0.007117 & 0.251378 & 112.818 \\
\hline 12/16/08 13:00 & 1.792 & 0.332 & 0.038 & 16.897 & 1.403 & 0.057 & 0.017373 & 0.006617 & 0.233715 & 104.891 \\
\hline 12/16/08 14:00 & 1.799 & 0.339 & 0.040 & 17.781 & 1.408 & 0.062 & 0.018897 & 0.007457 & 0.263390 & 118.209 \\
\hline 12/16/08 15:00 & 1.803 & 0.343 & 0.041 & 18.298 & 1.406 & 0.06 & 0.018287 & 0.007117 & 0.251378 & 112.818 \\
\hline 12/16/08 16:00 & 1.803 & 0.343 & 0.041 & 18.298 & 1.408 & 0.062 & 0.018897 & 0.007457 & 0.263390 & 118.209 \\
\hline 12/16/08 17:00 & 1.805 & 0.345 & 0.041 & 18.560 & 1.408 & 0.062 & 0.018897 & 0.007457 & 0.263390 & 118.209 \\
\hline 12/16/08 18:00 & 1.807 & 0.347 & 0.042 & 18.824 & 1.408 & 0.062 & 0.018897 & 0.007457 & 0.263390 & 118.209 \\
\hline 12/16/08 19:00 & 1.81 & 0.350 & 0.043 & 19.224 & 1.408 & 0.062 & 0.018897 & 0.007457 & 0.263390 & 118.209 \\
\hline 12/16/08 20:00 & 1.807 & 0.347 & 0.042 & 18.824 & 1.408 & 0.062 & 0.018897 & 0.007457 & 0.263390 & 118.209 \\
\hline 12/16/08 21:00 & 1.81 & 0.350 & 0.043 & 19.224 & 1.411 & 0.065 & 0.019811 & 0.007977 & 0.281754 & 126.451 \\
\hline 12/16/08 22:00 & 1.807 & 0.347 & 0.042 & 18.824 & 1.408 & 0.062 & 0.018897 & 0.007457 & 0.263390 & 118.209 \\
\hline 12/16/08 23:00 & 1.807 & 0.347 & 0.042 & 18.824 & 1.408 & 0.062 & 0.018897 & 0.007457 & 0.263390 & 118.209 \\
\hline 12/17/08 0:00 & 1.807 & 0.347 & 0.042 & 18.824 & 1.411 & 0.065 & 0.019811 & 0.007977 & 0.281754 & 126.451 \\
\hline 12/17/08 1:00 & 1.81 & 0.350 & 0.043 & 19.224 & 1.408 & 0.062 & 0.018897 & 0.007457 & 0.263390 & 118.209 \\
\hline
\end{tabular}




\begin{tabular}{|c|c|c|c|c|c|c|c|c|c|c|}
\hline \multirow[b]{3}{*}{ Date Time } & \multicolumn{4}{|c|}{ V-Notch Weir } & \multicolumn{6}{|c|}{ R-Notch Weir } \\
\hline & \multicolumn{2}{|c|}{ Measured Water Level } & \multicolumn{2}{|c|}{$\begin{array}{c}\text { Calculated Rate } \\
\left(\mathrm{ft}^{3} / \mathrm{s}=448.8 \mathrm{gpm}\right)\end{array}$} & \multicolumn{3}{|c|}{ Measured Water Level } & \multicolumn{3}{|c|}{$\begin{array}{c}\text { Calculated Rate } \\
\left(\mathrm{ft}^{3} / \mathrm{s}=448.8 \mathrm{gpm}\right)\end{array}$} \\
\hline & $\begin{array}{c}\text { (ft above } \\
\text { sensor) }\end{array}$ & $\begin{array}{c}\text { (ft above } \\
\text { notch) }\end{array}$ & $\left(\mathrm{ft}^{3} / \mathrm{s}\right)$ & (gpm) & $\begin{array}{l}\text { (ft above } \\
\text { sensor) }\end{array}$ & $\begin{array}{c}\text { (ft above } \\
\text { log) }\end{array}$ & $\begin{array}{c}\text { (m above } \\
\text { log) }\end{array}$ & $\left(\mathrm{m}^{3} / \mathrm{s}\right)$ & $\left(\mathrm{ft}^{3} / \mathrm{s}\right)$ & (gpm) \\
\hline 12/17/08 2:00 & 1.805 & 0.345 & 0.041 & 18.560 & 1.408 & 0.062 & 0.018897 & 0.007457 & 0.263390 & 118.209 \\
\hline 12/17/08 3:00 & 1.803 & 0.343 & 0.041 & 18.298 & 1.408 & 0.062 & 0.018897 & 0.007457 & 0.263390 & 118.209 \\
\hline 12/17/08 4:00 & 1.803 & 0.343 & 0.041 & 18.298 & 1.408 & 0.062 & 0.018897 & 0.007457 & 0.263390 & 118.209 \\
\hline 12/17/08 5:00 & 1.798 & 0.338 & 0.039 & 17.653 & 1.408 & 0.062 & 0.018897 & 0.007457 & 0.263390 & 118.209 \\
\hline 12/17/08 6:00 & 1.796 & 0.336 & 0.039 & 17.399 & 1.408 & 0.062 & 0.018897 & 0.007457 & 0.263390 & 118.209 \\
\hline 12/17/08 7:00 & 1.793 & 0.333 & 0.038 & 17.022 & 1.408 & 0.062 & 0.018897 & 0.007457 & 0.263390 & 118.209 \\
\hline 12/17/08 8:00 & 1.796 & 0.336 & 0.039 & 17.399 & 1.408 & 0.062 & 0.018897 & 0.007457 & 0.263390 & 118.209 \\
\hline 12/17/08 9:00 & 1.793 & 0.333 & 0.038 & 17.022 & 1.406 & 0.06 & 0.018287 & 0.007117 & 0.251378 & 112.818 \\
\hline 12/17/08 10:00 & 1.793 & 0.333 & 0.038 & 17.022 & 1.406 & 0.06 & 0.018287 & 0.007117 & 0.251378 & 112.818 \\
\hline 12/17/08 11:00 & 1.793 & 0.333 & 0.038 & 17.022 & 1.408 & 0.062 & 0.018897 & 0.007457 & 0.263390 & 118.209 \\
\hline 12/17/08 12:00 & 1.793 & 0.333 & 0.038 & 17.022 & 1.406 & 0.06 & 0.018287 & 0.007117 & 0.251378 & 112.818 \\
\hline 12/17/08 13:00 & 1.797 & 0.337 & 0.039 & 17.526 & 1.406 & 0.06 & 0.018287 & 0.007117 & 0.251378 & 112.818 \\
\hline 12/17/08 14:00 & 1.799 & 0.339 & 0.040 & 17.781 & 1.408 & 0.062 & 0.018897 & 0.007457 & 0.263390 & 118.209 \\
\hline 12/17/08 15:00 & 1.801 & 0.341 & 0.040 & 18.038 & 1.408 & 0.062 & 0.018897 & 0.007457 & 0.263390 & 118.209 \\
\hline 12/17/08 16:00 & 1.808 & 0.348 & 0.042 & 18.957 & 1.411 & 0.065 & 0.019811 & 0.007977 & 0.281754 & 126.451 \\
\hline 12/17/08 17:00 & 1.808 & 0.348 & 0.042 & 18.957 & 1.411 & 0.065 & 0.019811 & 0.007977 & 0.281754 & 126.451 \\
\hline 12/17/08 18:00 & 1.797 & 0.337 & 0.039 & 17.526 & 1.406 & 0.06 & 0.018287 & 0.007117 & 0.251378 & 112.818 \\
\hline 12/17/08 19:00 & 1.792 & 0.332 & 0.038 & 16.897 & 1.406 & 0.06 & 0.018287 & 0.007117 & 0.251378 & 112.818 \\
\hline 12/17/08 20:00 & 1.792 & 0.332 & 0.038 & 16.897 & 1.406 & 0.06 & 0.018287 & 0.007117 & 0.251378 & 112.818 \\
\hline 12/17/08 21:00 & 1.79 & 0.330 & 0.037 & 16.650 & 1.406 & 0.06 & 0.018287 & 0.007117 & 0.251378 & 112.818 \\
\hline 12/17/08 22:00 & 1.788 & 0.328 & 0.037 & 16.405 & 1.406 & 0.06 & 0.018287 & 0.007117 & 0.251378 & 112.818 \\
\hline 12/17/08 23:00 & 1.785 & 0.325 & 0.036 & 16.041 & 1.403 & 0.057 & 0.017373 & 0.006617 & 0.233715 & 104.891 \\
\hline 12/18/08 0:00 & 1.783 & 0.323 & 0.035 & 15.801 & 1.403 & 0.057 & 0.017373 & 0.006617 & 0.233715 & 104.891 \\
\hline 12/18/08 1:00 & 1.783 & 0.323 & 0.035 & 15.801 & 1.403 & 0.057 & 0.017373 & 0.006617 & 0.233715 & 104.891 \\
\hline 12/18/08 2:00 & 1.781 & 0.321 & 0.035 & 15.563 & 1.403 & 0.057 & 0.017373 & 0.006617 & 0.233715 & 104.891 \\
\hline
\end{tabular}




\begin{tabular}{|c|c|c|c|c|c|c|c|c|c|c|}
\hline \multirow[b]{3}{*}{ Date Time } & \multicolumn{4}{|c|}{ V-Notch Weir } & \multicolumn{6}{|c|}{ R-Notch Weir } \\
\hline & \multicolumn{2}{|c|}{ Measured Water Level } & \multicolumn{2}{|c|}{$\begin{array}{c}\text { Calculated Rate } \\
\left(\mathrm{ft}^{3} / \mathrm{s}=448.8 \mathrm{gpm}\right)\end{array}$} & \multicolumn{3}{|c|}{ Measured Water Level } & \multicolumn{3}{|c|}{$\begin{array}{c}\text { Calculated Rate } \\
\left(\mathrm{ft}^{3} / \mathrm{s}=448.8 \mathrm{gpm}\right)\end{array}$} \\
\hline & $\begin{array}{c}\text { (ft above } \\
\text { sensor) }\end{array}$ & $\begin{array}{c}\text { (ft above } \\
\text { notch) }\end{array}$ & $\left(\mathrm{ft}^{3} / \mathrm{s}\right)$ & (gpm) & $\begin{array}{l}\text { (ft above } \\
\text { sensor) }\end{array}$ & $\begin{array}{c}\text { (ft above } \\
\text { log) }\end{array}$ & $\begin{array}{c}\text { (m above } \\
\text { log) }\end{array}$ & $\left(\mathrm{m}^{3} / \mathrm{s}\right)$ & $\left(\mathrm{ft}^{3} / \mathrm{s}\right)$ & (gpm) \\
\hline 12/18/08 3:00 & 1.781 & 0.321 & 0.035 & 15.563 & 1.403 & 0.057 & 0.017373 & 0.006617 & 0.233715 & 104.891 \\
\hline 12/18/08 4:00 & 1.783 & 0.323 & 0.035 & 15.801 & 1.403 & 0.057 & 0.017373 & 0.006617 & 0.233715 & 104.891 \\
\hline 12/18/08 5:00 & 1.783 & 0.323 & 0.035 & 15.801 & 1.406 & 0.06 & 0.018287 & 0.007117 & 0.251378 & 112.818 \\
\hline 12/18/08 6:00 & 1.779 & 0.319 & 0.034 & 15.328 & 1.403 & 0.057 & 0.017373 & 0.006617 & 0.233715 & 104.891 \\
\hline 12/18/08 7:00 & 1.781 & 0.321 & 0.035 & 15.563 & 1.403 & 0.057 & 0.017373 & 0.006617 & 0.233715 & 104.891 \\
\hline 12/18/08 8:00 & 1.779 & 0.319 & 0.034 & 15.328 & 1.403 & 0.057 & 0.017373 & 0.006617 & 0.233715 & 104.891 \\
\hline 12/18/08 9:00 & 1.779 & 0.319 & 0.034 & 15.328 & 1.403 & 0.057 & 0.017373 & 0.006617 & 0.233715 & 104.891 \\
\hline 12/18/08 10:00 & 1.779 & 0.319 & 0.034 & 15.328 & 1.403 & 0.057 & 0.017373 & 0.006617 & 0.233715 & 104.891 \\
\hline 12/18/08 11:00 & 1.779 & 0.319 & 0.034 & 15.328 & 1.403 & 0.057 & 0.017373 & 0.006617 & 0.233715 & 104.891 \\
\hline 12/18/08 12:00 & 1.778 & 0.318 & 0.034 & 15.211 & 1.406 & 0.06 & 0.018287 & 0.007117 & 0.251378 & 112.818 \\
\hline 12/18/08 13:00 & 1.782 & 0.322 & 0.035 & 15.682 & 1.406 & 0.06 & 0.018287 & 0.007117 & 0.251378 & 112.818 \\
\hline 12/18/08 14:00 & 1.785 & 0.325 & 0.036 & 16.041 & 1.406 & 0.06 & 0.018287 & 0.007117 & 0.251378 & 112.818 \\
\hline 12/18/08 15:00 & 1.792 & 0.332 & 0.038 & 16.897 & 1.411 & 0.065 & 0.019811 & 0.007977 & 0.281754 & 126.451 \\
\hline 12/18/08 16:00 & 1.792 & 0.332 & 0.038 & 16.897 & 1.411 & 0.065 & 0.019811 & 0.007977 & 0.281754 & 126.451 \\
\hline 12/18/08 17:00 & 1.796 & 0.336 & 0.039 & 17.399 & 1.408 & 0.062 & 0.018897 & 0.007457 & 0.263390 & 118.209 \\
\hline 12/18/08 18:00 & 1.799 & 0.339 & 0.040 & 17.781 & 1.408 & 0.062 & 0.018897 & 0.007457 & 0.263390 & 118.209 \\
\hline 12/18/08 19:00 & 1.797 & 0.337 & 0.039 & 17.526 & 1.408 & 0.062 & 0.018897 & 0.007457 & 0.263390 & 118.209 \\
\hline 12/18/08 20:00 & 1.804 & 0.344 & 0.041 & 18.429 & 1.411 & 0.065 & 0.019811 & 0.007977 & 0.281754 & 126.451 \\
\hline 12/18/08 21:00 & 1.819 & 0.359 & 0.046 & 20.456 & 1.413 & 0.067 & 0.020421 & 0.008330 & 0.294224 & 132.048 \\
\hline 12/18/08 22:00 & 1.84 & 0.380 & 0.052 & 23.510 & 1.418 & 0.072 & 0.021945 & 0.009235 & 0.326176 & 146.388 \\
\hline 12/18/08 23:00 & 1.84 & 0.380 & 0.052 & 23.510 & 1.415 & 0.069 & 0.021030 & 0.008688 & 0.306873 & 137.724 \\
\hline 12/19/08 0:00 & 1.833 & 0.373 & 0.050 & 22.463 & 1.413 & 0.067 & 0.020421 & 0.008330 & 0.294224 & 132.048 \\
\hline 12/19/08 1:00 & 1.819 & 0.359 & 0.046 & 20.456 & 1.411 & 0.065 & 0.019811 & 0.007977 & 0.281754 & 126.451 \\
\hline 12/19/08 2:00 & 1.819 & 0.359 & 0.046 & 20.456 & 1.408 & 0.062 & 0.018897 & 0.007457 & 0.263390 & 118.209 \\
\hline 12/19/08 3:00 & 1.814 & 0.354 & 0.044 & 19.766 & 1.408 & 0.062 & 0.018897 & 0.007457 & 0.263390 & 118.209 \\
\hline
\end{tabular}




\begin{tabular}{|c|c|c|c|c|c|c|c|c|c|c|}
\hline \multirow[b]{3}{*}{ Date Time } & \multicolumn{4}{|c|}{ V-Notch Weir } & \multicolumn{6}{|c|}{ R-Notch Weir } \\
\hline & \multicolumn{2}{|c|}{ Measured Water Level } & \multicolumn{2}{|c|}{$\begin{array}{c}\text { Calculated Rate } \\
\left(\mathrm{ft}^{3} / \mathrm{s}=448.8 \mathrm{gpm}\right)\end{array}$} & \multicolumn{3}{|c|}{ Measured Water Level } & \multicolumn{3}{|c|}{$\begin{array}{c}\text { Calculated Rate } \\
\left(\mathrm{ft}^{3} / \mathrm{s}=448.8 \mathrm{gpm}\right)\end{array}$} \\
\hline & $\begin{array}{c}\text { (ft above } \\
\text { sensor) }\end{array}$ & $\begin{array}{c}\text { (ft above } \\
\text { notch) }\end{array}$ & $\left(\mathrm{ft}^{3} / \mathrm{s}\right)$ & (gpm) & $\begin{array}{l}\text { (ft above } \\
\text { sensor) }\end{array}$ & $\begin{array}{c}\text { (ft above } \\
\text { log) }\end{array}$ & $\begin{array}{c}\text { (m above } \\
\text { log) }\end{array}$ & $\left(\mathrm{m}^{3} / \mathrm{s}\right)$ & $\left(\mathrm{ft}^{3} / \mathrm{s}\right)$ & (gpm) \\
\hline 12/19/08 4:00 & 1.814 & 0.354 & 0.044 & 19.766 & 1.408 & 0.062 & 0.018897 & 0.007457 & 0.263390 & 118.209 \\
\hline 12/19/08 5:00 & 1.812 & 0.352 & 0.043 & 19.494 & 1.408 & 0.062 & 0.018897 & 0.007457 & 0.263390 & 118.209 \\
\hline 12/19/08 6:00 & 1.802 & 0.342 & 0.040 & 18.168 & 1.408 & 0.062 & 0.018897 & 0.007457 & 0.263390 & 118.209 \\
\hline 12/19/08 7:00 & 1.802 & 0.342 & 0.040 & 18.168 & 1.406 & 0.06 & 0.018287 & 0.007117 & 0.251378 & 112.818 \\
\hline 12/19/08 8:00 & 1.8 & 0.340 & 0.040 & 17.909 & 1.406 & 0.06 & 0.018287 & 0.007117 & 0.251378 & 112.818 \\
\hline 12/19/08 9:00 & 1.798 & 0.338 & 0.039 & 17.653 & 1.406 & 0.06 & 0.018287 & 0.007117 & 0.251378 & 112.818 \\
\hline 12/19/08 10:00 & 1.793 & 0.333 & 0.038 & 17.022 & 1.403 & 0.057 & 0.017373 & 0.006617 & 0.233715 & 104.891 \\
\hline 12/19/08 11:00 & 1.79 & 0.330 & 0.037 & 16.650 & 1.403 & 0.057 & 0.017373 & 0.006617 & 0.233715 & 104.891 \\
\hline 12/19/08 12:00 & 1.787 & 0.327 & 0.036 & 16.283 & 1.403 & 0.057 & 0.017373 & 0.006617 & 0.233715 & 104.891 \\
\hline 12/19/08 13:00 & 1.792 & 0.332 & 0.038 & 16.897 & 1.403 & 0.057 & 0.017373 & 0.006617 & 0.233715 & 104.891 \\
\hline 12/19/08 14:00 & 1.789 & 0.329 & 0.037 & 16.527 & 1.403 & 0.057 & 0.017373 & 0.006617 & 0.233715 & 104.891 \\
\hline 12/19/08 15:00 & 1.793 & 0.333 & 0.038 & 17.022 & 1.403 & 0.057 & 0.017373 & 0.006617 & 0.233715 & 104.891 \\
\hline 12/19/08 16:00 & 1.791 & 0.331 & 0.037 & 16.773 & 1.403 & 0.057 & 0.017373 & 0.006617 & 0.233715 & 104.891 \\
\hline 12/19/08 17:00 & 1.799 & 0.339 & 0.040 & 17.781 & 1.411 & 0.065 & 0.019811 & 0.007977 & 0.281754 & 126.451 \\
\hline 12/19/08 18:00 & 1.797 & 0.337 & 0.039 & 17.526 & 1.406 & 0.06 & 0.018287 & 0.007117 & 0.251378 & 112.818 \\
\hline 12/19/08 19:00 & 1.797 & 0.337 & 0.039 & 17.526 & 1.406 & 0.06 & 0.018287 & 0.007117 & 0.251378 & 112.818 \\
\hline 12/19/08 20:00 & 1.794 & 0.334 & 0.038 & 17.147 & 1.406 & 0.06 & 0.018287 & 0.007117 & 0.251378 & 112.818 \\
\hline 12/19/08 21:00 & 1.792 & 0.332 & 0.038 & 16.897 & 1.406 & 0.06 & 0.018287 & 0.007117 & 0.251378 & 112.818 \\
\hline 12/19/08 22:00 & 1.792 & 0.332 & 0.038 & 16.897 & 1.406 & 0.06 & 0.018287 & 0.007117 & 0.251378 & 112.818 \\
\hline 12/19/08 23:00 & 1.792 & 0.332 & 0.038 & 16.897 & 1.406 & 0.06 & 0.018287 & 0.007117 & 0.251378 & 112.818 \\
\hline 12/20/08 0:00 & 1.79 & 0.330 & 0.037 & 16.650 & 1.406 & 0.06 & 0.018287 & 0.007117 & 0.251378 & 112.818 \\
\hline 12/20/08 1:00 & 1.792 & 0.332 & 0.038 & 16.897 & 1.408 & 0.062 & 0.018897 & 0.007457 & 0.263390 & 118.209 \\
\hline 12/20/08 2:00 & 1.787 & 0.327 & 0.036 & 16.283 & 1.408 & 0.062 & 0.018897 & 0.007457 & 0.263390 & 118.209 \\
\hline 12/20/08 3:00 & 1.792 & 0.332 & 0.038 & 16.897 & 1.408 & 0.062 & 0.018897 & 0.007457 & 0.263390 & 118.209 \\
\hline 12/20/08 4:00 & 1.79 & 0.330 & 0.037 & 16.650 & 1.41 & 0.064 & 0.019506 & 0.007803 & 0.275587 & 123.683 \\
\hline
\end{tabular}




\begin{tabular}{|c|c|c|c|c|c|c|c|c|c|c|}
\hline \multirow[b]{3}{*}{ Date Time } & \multicolumn{4}{|c|}{ V-Notch Weir } & \multicolumn{6}{|c|}{ R-Notch Weir } \\
\hline & \multicolumn{2}{|c|}{ Measured Water Level } & \multicolumn{2}{|c|}{$\begin{array}{c}\text { Calculated Rate } \\
\left(\mathrm{ft}^{3} / \mathrm{s}=448.8 \mathrm{gpm}\right)\end{array}$} & \multicolumn{3}{|c|}{ Measured Water Level } & \multicolumn{3}{|c|}{$\begin{array}{c}\text { Calculated Rate } \\
\left(\mathrm{ft}^{3} / \mathrm{s}=448.8 \mathrm{gpm}\right)\end{array}$} \\
\hline & $\begin{array}{c}\text { (ft above } \\
\text { sensor) }\end{array}$ & $\begin{array}{c}\text { (ft above } \\
\text { notch) }\end{array}$ & $\left(\mathrm{ft}^{3} / \mathrm{s}\right)$ & (gpm) & $\begin{array}{l}\text { (ft above } \\
\text { sensor) }\end{array}$ & $\begin{array}{c}\text { (ft above } \\
\text { log) }\end{array}$ & $\begin{array}{c}\text { (m above } \\
\text { log) }\end{array}$ & $\left(\mathrm{m}^{3} / \mathrm{s}\right)$ & $\left(\mathrm{ft}^{3} / \mathrm{s}\right)$ & (gpm) \\
\hline 12/20/08 5:00 & 1.785 & 0.325 & 0.036 & 16.041 & 1.408 & 0.062 & 0.018897 & 0.007457 & 0.263390 & 118.209 \\
\hline 12/20/08 6:00 & 1.785 & 0.325 & 0.036 & 16.041 & 1.408 & 0.062 & 0.018897 & 0.007457 & 0.263390 & 118.209 \\
\hline 12/20/08 7:00 & 1.787 & 0.327 & 0.036 & 16.283 & 1.408 & 0.062 & 0.018897 & 0.007457 & 0.263390 & 118.209 \\
\hline 12/20/08 8:00 & 1.787 & 0.327 & 0.036 & 16.283 & 1.408 & 0.062 & 0.018897 & 0.007457 & 0.263390 & 118.209 \\
\hline 12/20/08 9:00 & 1.785 & 0.325 & 0.036 & 16.041 & 1.408 & 0.062 & 0.018897 & 0.007457 & 0.263390 & 118.209 \\
\hline 12/20/08 10:00 & 1.783 & 0.323 & 0.035 & 15.801 & 1.408 & 0.062 & 0.018897 & 0.007457 & 0.263390 & 118.209 \\
\hline 12/20/08 11:00 & 1.788 & 0.328 & 0.037 & 16.405 & 1.408 & 0.062 & 0.018897 & 0.007457 & 0.263390 & 118.209 \\
\hline 12/20/08 12:00 & 1.791 & 0.331 & 0.037 & 16.773 & 1.406 & 0.06 & 0.018287 & 0.007117 & 0.251378 & 112.818 \\
\hline 12/20/08 13:00 & 1.774 & 0.314 & 0.033 & 14.748 & 1.406 & 0.06 & 0.018287 & 0.007117 & 0.251378 & 112.818 \\
\hline 12/20/08 14:00 & 1.767 & 0.307 & 0.031 & 13.960 & 1.403 & 0.057 & 0.017373 & 0.006617 & 0.233715 & 104.891 \\
\hline 12/20/08 15:00 & 1.776 & 0.316 & 0.033 & 14.979 & 1.406 & 0.06 & 0.018287 & 0.007117 & 0.251378 & 112.818 \\
\hline 12/20/08 16:00 & 1.774 & 0.314 & 0.033 & 14.748 & 1.408 & 0.062 & 0.018897 & 0.007457 & 0.263390 & 118.209 \\
\hline 12/20/08 17:00 & 1.77 & 0.310 & 0.032 & 14.295 & 1.403 & 0.057 & 0.017373 & 0.006617 & 0.233715 & 104.891 \\
\hline 12/20/08 18:00 & 1.765 & 0.305 & 0.031 & 13.739 & 1.403 & 0.057 & 0.017373 & 0.006617 & 0.233715 & 104.891 \\
\hline 12/20/08 19:00 & 1.763 & 0.303 & 0.030 & 13.521 & 1.403 & 0.057 & 0.017373 & 0.006617 & 0.233715 & 104.891 \\
\hline 12/20/08 20:00 & 1.756 & 0.296 & 0.028 & 12.772 & 1.403 & 0.057 & 0.017373 & 0.006617 & 0.233715 & 104.891 \\
\hline 12/20/08 21:00 & 1.756 & 0.296 & 0.028 & 12.772 & 1.403 & 0.057 & 0.017373 & 0.006617 & 0.233715 & 104.891 \\
\hline 12/20/08 22:00 & 1.754 & 0.294 & 0.028 & 12.563 & 1.401 & 0.055 & 0.016763 & 0.006291 & 0.222180 & 99.714 \\
\hline 12/20/08 23:00 & 1.754 & 0.294 & 0.028 & 12.563 & 1.403 & 0.057 & 0.017373 & 0.006617 & 0.233715 & 104.891 \\
\hline 12/21/08 0:00 & 1.751 & 0.291 & 0.027 & 12.253 & 1.401 & 0.055 & 0.016763 & 0.006291 & 0.222180 & 99.714 \\
\hline 12/21/08 1:00 & 1.749 & 0.289 & 0.027 & 12.049 & 1.401 & 0.055 & 0.016763 & 0.006291 & 0.222180 & 99.714 \\
\hline 12/21/08 2:00 & 1.749 & 0.289 & 0.027 & 12.049 & 1.403 & 0.057 & 0.017373 & 0.006617 & 0.233715 & 104.891 \\
\hline 12/21/08 3:00 & 1.747 & 0.287 & 0.026 & 11.848 & 1.401 & 0.055 & 0.016763 & 0.006291 & 0.222180 & 99.714 \\
\hline 12/21/08 4:00 & 1.742 & 0.282 & 0.025 & 11.352 & 1.401 & 0.055 & 0.016763 & 0.006291 & 0.222180 & 99.714 \\
\hline 12/21/08 5:00 & 1.74 & 0.280 & 0.025 & 11.157 & 1.401 & 0.055 & 0.016763 & 0.006291 & 0.222180 & 99.714 \\
\hline
\end{tabular}




\begin{tabular}{|c|c|c|c|c|c|c|c|c|c|c|}
\hline \multirow[b]{3}{*}{ Date Time } & \multicolumn{4}{|c|}{ V-Notch Weir } & \multicolumn{6}{|c|}{ R-Notch Weir } \\
\hline & \multicolumn{2}{|c|}{ Measured Water Level } & \multicolumn{2}{|c|}{$\begin{array}{c}\text { Calculated Rate } \\
\left(\mathrm{ft}^{3} / \mathrm{s}=448.8 \mathrm{gpm}\right)\end{array}$} & \multicolumn{3}{|c|}{ Measured Water Level } & \multicolumn{3}{|c|}{$\begin{array}{c}\text { Calculated Rate } \\
\left(\mathrm{ft}^{3} / \mathrm{s}=448.8 \mathrm{gpm}\right)\end{array}$} \\
\hline & $\begin{array}{c}\text { (ft above } \\
\text { sensor) }\end{array}$ & $\begin{array}{c}\text { (ft above } \\
\text { notch) }\end{array}$ & $\left(\mathrm{ft}^{3} / \mathrm{s}\right)$ & (gpm) & $\begin{array}{l}\text { (ft above } \\
\text { sensor) }\end{array}$ & $\begin{array}{c}\text { (ft above } \\
\text { log) }\end{array}$ & $\begin{array}{c}\text { (m above } \\
\text { log) }\end{array}$ & $\left(\mathrm{m}^{3} / \mathrm{s}\right)$ & $\left(\mathrm{ft}^{3} / \mathrm{s}\right)$ & (gpm) \\
\hline 12/21/08 6:00 & 1.738 & 0.278 & 0.024 & 10.964 & 1.401 & 0.055 & 0.016763 & 0.006291 & 0.222180 & 99.714 \\
\hline 12/21/08 7:00 & 1.738 & 0.278 & 0.024 & 10.964 & 1.401 & 0.055 & 0.016763 & 0.006291 & 0.222180 & 99.714 \\
\hline 12/21/08 8:00 & 1.738 & 0.278 & 0.024 & 10.964 & 1.401 & 0.055 & 0.016763 & 0.006291 & 0.222180 & 99.714 \\
\hline 12/21/08 9:00 & 1.738 & 0.278 & 0.024 & 10.964 & 1.401 & 0.055 & 0.016763 & 0.006291 & 0.222180 & 99.714 \\
\hline 12/21/08 10:00 & 1.733 & 0.273 & 0.023 & 10.491 & 1.401 & 0.055 & 0.016763 & 0.006291 & 0.222180 & 99.714 \\
\hline 12/21/08 11:00 & 1.74 & 0.280 & 0.025 & 11.157 & 1.404 & 0.058 & 0.017678 & 0.006782 & 0.239555 & 107.512 \\
\hline 12/21/08 12:00 & 1.733 & 0.273 & 0.023 & 10.491 & 1.401 & 0.055 & 0.016763 & 0.006291 & 0.222180 & 99.714 \\
\hline 12/21/08 13:00 & 1.735 & 0.275 & 0.024 & 10.679 & 1.404 & 0.058 & 0.017678 & 0.006782 & 0.239555 & 107.512 \\
\hline 12/21/08 14:00 & 1.742 & 0.282 & 0.025 & 11.352 & 1.404 & 0.058 & 0.017678 & 0.006782 & 0.239555 & 107.512 \\
\hline 12/21/08 15:00 & 1.735 & 0.275 & 0.024 & 10.679 & 1.401 & 0.055 & 0.016763 & 0.006291 & 0.222180 & 99.714 \\
\hline 12/21/08 16:00 & 1.73 & 0.270 & 0.023 & 10.213 & 1.401 & 0.055 & 0.016763 & 0.006291 & 0.222180 & 99.714 \\
\hline 12/21/08 17:00 & 1.732 & 0.272 & 0.023 & 10.398 & 1.399 & 0.053 & 0.016154 & 0.005969 & 0.210842 & 94.626 \\
\hline 12/21/08 18:00 & 1.723 & 0.263 & 0.021 & 9.582 & 1.397 & 0.051 & 0.015544 & 0.005654 & 0.199703 & 89.627 \\
\hline 12/21/08 19:00 & 1.721 & 0.261 & 0.021 & 9.406 & 1.397 & 0.051 & 0.015544 & 0.005654 & 0.199703 & 89.627 \\
\hline 12/21/08 20:00 & 1.723 & 0.263 & 0.021 & 9.582 & 1.399 & 0.053 & 0.016154 & 0.005969 & 0.210842 & 94.626 \\
\hline 12/21/08 21:00 & 1.719 & 0.259 & 0.021 & 9.232 & 1.394 & 0.048 & 0.014630 & 0.005192 & 0.183377 & 82.300 \\
\hline 12/21/08 22:00 & 1.719 & 0.259 & 0.021 & 9.232 & 1.394 & 0.048 & 0.014630 & 0.005192 & 0.183377 & 82.300 \\
\hline 12/21/08 23:00 & 1.721 & 0.261 & 0.021 & 9.406 & 1.397 & 0.051 & 0.015544 & 0.005654 & 0.199703 & 89.627 \\
\hline 12/22/08 0:00 & 1.721 & 0.261 & 0.021 & 9.406 & 1.397 & 0.051 & 0.015544 & 0.005654 & 0.199703 & 89.627 \\
\hline 12/22/08 1:00 & 1.719 & 0.259 & 0.021 & 9.232 & 1.394 & 0.048 & 0.014630 & 0.005192 & 0.183377 & 82.300 \\
\hline 12/22/08 2:00 & 1.719 & 0.259 & 0.021 & 9.232 & 1.397 & 0.051 & 0.015544 & 0.005654 & 0.199703 & 89.627 \\
\hline 12/22/08 3:00 & 1.719 & 0.259 & 0.021 & 9.232 & 1.394 & 0.048 & 0.014630 & 0.005192 & 0.183377 & 82.300 \\
\hline 12/22/08 4:00 & 1.716 & 0.256 & 0.020 & 8.975 & 1.394 & 0.048 & 0.014630 & 0.005192 & 0.183377 & 82.300 \\
\hline 12/22/08 5:00 & 1.719 & 0.259 & 0.021 & 9.232 & 1.394 & 0.048 & 0.014630 & 0.005192 & 0.183377 & 82.300 \\
\hline 12/22/08 6:00 & 1.719 & 0.259 & 0.021 & 9.232 & 1.394 & 0.048 & 0.014630 & 0.005192 & 0.183377 & 82.300 \\
\hline
\end{tabular}




\begin{tabular}{|c|c|c|c|c|c|c|c|c|c|c|}
\hline \multirow[b]{3}{*}{ Date Time } & \multicolumn{4}{|c|}{ V-Notch Weir } & \multicolumn{6}{|c|}{ R-Notch Weir } \\
\hline & \multicolumn{2}{|c|}{ Measured Water Level } & \multicolumn{2}{|c|}{$\begin{array}{c}\text { Calculated Rate } \\
\left(\mathrm{ft}^{3} / \mathrm{s}=448.8 \mathrm{gpm}\right)\end{array}$} & \multicolumn{3}{|c|}{ Measured Water Level } & \multicolumn{3}{|c|}{$\begin{array}{c}\text { Calculated Rate } \\
\left(\mathrm{ft}^{3} / \mathrm{s}=448.8 \mathrm{gpm}\right)\end{array}$} \\
\hline & $\begin{array}{c}\text { (ft above } \\
\text { sensor) }\end{array}$ & $\begin{array}{c}\text { (ft above } \\
\text { notch) }\end{array}$ & $\left(\mathrm{ft}^{3} / \mathrm{s}\right)$ & (gpm) & $\begin{array}{l}\text { (ft above } \\
\text { sensor) }\end{array}$ & $\begin{array}{c}\text { (ft above } \\
\text { log) }\end{array}$ & $\begin{array}{c}\text { (m above } \\
\text { log) }\end{array}$ & $\left(\mathrm{m}^{3} / \mathrm{s}\right)$ & $\left(\mathrm{ft}^{3} / \mathrm{s}\right)$ & (gpm) \\
\hline 12/22/08 7:00 & 1.714 & 0.254 & 0.020 & 8.806 & 1.394 & 0.048 & 0.014630 & 0.005192 & 0.183377 & 82.300 \\
\hline 12/22/08 8:00 & 1.717 & 0.257 & 0.020 & 9.060 & 1.397 & 0.051 & 0.015544 & 0.005654 & 0.199703 & 89.627 \\
\hline 12/22/08 9:00 & 1.717 & 0.257 & 0.020 & 9.060 & 1.394 & 0.048 & 0.014630 & 0.005192 & 0.183377 & 82.300 \\
\hline 12/22/08 10:00 & 1.719 & 0.259 & 0.021 & 9.232 & 1.392 & 0.046 & 0.014020 & 0.004891 & 0.172754 & 77.532 \\
\hline 12/22/08 11:00 & 1.716 & 0.256 & 0.020 & 8.975 & 1.378 & 0.032 & 0.009753 & 0.002963 & 0.104644 & 46.964 \\
\hline 12/22/08 12:00 & 1.721 & 0.261 & 0.021 & 9.406 & 1.378 & 0.032 & 0.009753 & 0.002963 & 0.104644 & 46.964 \\
\hline 12/22/08 13:00 & 1.721 & 0.261 & 0.021 & 9.406 & 1.378 & 0.032 & 0.009753 & 0.002963 & 0.104644 & 46.964 \\
\hline 12/22/08 14:00 & 1.723 & 0.263 & 0.021 & 9.582 & 1.385 & 0.039 & 0.011887 & 0.003887 & 0.137285 & 61.614 \\
\hline 12/22/08 15:00 & 1.725 & 0.265 & 0.022 & 9.760 & 1.385 & 0.039 & 0.011887 & 0.003887 & 0.137285 & 61.614 \\
\hline 12/22/08 16:00 & 1.73 & 0.270 & 0.023 & 10.213 & 1.385 & 0.039 & 0.011887 & 0.003887 & 0.137285 & 61.614 \\
\hline 12/22/08 17:00 & 1.727 & 0.267 & 0.022 & 9.940 & 1.375 & 0.029 & 0.008839 & 0.002593 & 0.091584 & 41.103 \\
\hline 12/22/08 18:00 & 1.73 & 0.270 & 0.023 & 10.213 & 1.387 & 0.041 & 0.012496 & 0.004166 & 0.147141 & 66.037 \\
\hline 12/22/08 19:00 & 1.73 & 0.270 & 0.023 & 10.213 & 1.387 & 0.041 & 0.012496 & 0.004166 & 0.147141 & 66.037 \\
\hline 12/22/08 20:00 & 1.732 & 0.272 & 0.023 & 10.398 & 1.389 & 0.043 & 0.013106 & 0.004451 & 0.157222 & 70.561 \\
\hline 12/22/08 21:00 & 1.735 & 0.275 & 0.024 & 10.679 & 1.385 & 0.039 & 0.011887 & 0.003887 & 0.137285 & 61.614 \\
\hline 12/22/08 22:00 & 1.742 & 0.282 & 0.025 & 11.352 & 1.387 & 0.041 & 0.012496 & 0.004166 & 0.147141 & 66.037 \\
\hline 12/22/08 23:00 & 1.737 & 0.277 & 0.024 & 10.869 & 1.404 & 0.058 & 0.017678 & 0.006782 & 0.239555 & 107.512 \\
\hline 12/23/08 0:00 & 1.739 & 0.279 & 0.025 & 11.060 & 1.387 & 0.041 & 0.012496 & 0.004166 & 0.147141 & 66.037 \\
\hline 12/23/08 1:00 & 1.737 & 0.277 & 0.024 & 10.869 & 1.378 & 0.032 & 0.009753 & 0.002963 & 0.104644 & 46.964 \\
\hline 12/23/08 2:00 & 1.742 & 0.282 & 0.025 & 11.352 & 1.394 & 0.048 & 0.014630 & 0.005192 & 0.183377 & 82.300 \\
\hline 12/23/08 3:00 & 1.742 & 0.282 & 0.025 & 11.352 & 1.397 & 0.051 & 0.015544 & 0.005654 & 0.199703 & 89.627 \\
\hline 12/23/08 4:00 & 1.741 & 0.281 & 0.025 & 11.254 & 1.397 & 0.051 & 0.015544 & 0.005654 & 0.199703 & 89.627 \\
\hline 12/23/08 5:00 & 1.739 & 0.279 & 0.025 & 11.060 & 1.401 & 0.055 & 0.016763 & 0.006291 & 0.222180 & 99.714 \\
\hline 12/23/08 6:00 & 1.744 & 0.284 & 0.026 & 11.549 & 1.399 & 0.053 & 0.016154 & 0.005969 & 0.210842 & 94.626 \\
\hline 12/23/08 7:00 & 1.744 & 0.284 & 0.026 & 11.549 & 1.394 & 0.048 & 0.014630 & 0.005192 & 0.183377 & 82.300 \\
\hline
\end{tabular}




\begin{tabular}{|c|c|c|c|c|c|c|c|c|c|c|}
\hline \multirow[b]{3}{*}{ Date Time } & \multicolumn{4}{|c|}{ V-Notch Weir } & \multicolumn{6}{|c|}{ R-Notch Weir } \\
\hline & \multicolumn{2}{|c|}{ Measured Water Level } & \multicolumn{2}{|c|}{$\begin{array}{c}\text { Calculated Rate } \\
\left(\mathrm{ft}^{3} / \mathrm{s}=448.8 \mathrm{gpm}\right)\end{array}$} & \multicolumn{3}{|c|}{ Measured Water Level } & \multicolumn{3}{|c|}{$\begin{array}{c}\text { Calculated Rate } \\
\left(\mathrm{ft}^{3} / \mathrm{s}=448.8 \mathrm{gpm}\right)\end{array}$} \\
\hline & $\begin{array}{c}\text { (ft above } \\
\text { sensor) }\end{array}$ & $\begin{array}{c}\text { (ft above } \\
\text { notch) }\end{array}$ & $\left(\mathrm{ft}^{3} / \mathrm{s}\right)$ & (gpm) & $\begin{array}{l}\text { (ft above } \\
\text { sensor) }\end{array}$ & $\begin{array}{c}\text { (ft above } \\
\text { log) }\end{array}$ & $\begin{array}{c}\text { (m above } \\
\text { log) }\end{array}$ & $\left(\mathrm{m}^{3} / \mathrm{s}\right)$ & $\left(\mathrm{ft}^{3} / \mathrm{s}\right)$ & (gpm) \\
\hline 12/23/08 8:00 & 1.744 & 0.284 & 0.026 & 11.549 & 1.394 & 0.048 & 0.014630 & 0.005192 & 0.183377 & 82.300 \\
\hline 12/23/08 9:00 & 1.744 & 0.284 & 0.026 & 11.549 & 1.387 & 0.041 & 0.012496 & 0.004166 & 0.147141 & 66.037 \\
\hline 12/23/08 10:00 & 1.741 & 0.281 & 0.025 & 11.254 & 1.394 & 0.048 & 0.014630 & 0.005192 & 0.183377 & 82.300 \\
\hline 12/23/08 11:00 & 1.738 & 0.278 & 0.024 & 10.964 & 1.397 & 0.051 & 0.015544 & 0.005654 & 0.199703 & 89.627 \\
\hline 12/23/08 12:00 & 1.743 & 0.283 & 0.026 & 11.450 & 1.399 & 0.053 & 0.016154 & 0.005969 & 0.210842 & 94.626 \\
\hline 12/23/08 13:00 & 1.738 & 0.278 & 0.024 & 10.964 & 1.397 & 0.051 & 0.015544 & 0.005654 & 0.199703 & 89.627 \\
\hline 12/23/08 14:00 & 1.747 & 0.287 & 0.026 & 11.848 & 1.401 & 0.055 & 0.016763 & 0.006291 & 0.222180 & 99.714 \\
\hline 12/23/08 15:00 & 1.747 & 0.287 & 0.026 & 11.848 & 1.399 & 0.053 & 0.016154 & 0.005969 & 0.210842 & 94.626 \\
\hline 12/23/08 16:00 & 1.744 & 0.284 & 0.026 & 11.549 & 1.394 & 0.048 & 0.014630 & 0.005192 & 0.183377 & 82.300 \\
\hline 12/23/08 17:00 & 1.747 & 0.287 & 0.026 & 11.848 & 1.399 & 0.053 & 0.016154 & 0.005969 & 0.210842 & 94.626 \\
\hline 12/23/08 18:00 & 1.747 & 0.287 & 0.026 & 11.848 & 1.401 & 0.055 & 0.016763 & 0.006291 & 0.222180 & 99.714 \\
\hline 12/23/08 19:00 & 1.75 & 0.290 & 0.027 & 12.151 & 1.401 & 0.055 & 0.016763 & 0.006291 & 0.222180 & 99.714 \\
\hline 12/23/08 20:00 & 1.745 & 0.285 & 0.026 & 11.648 & 1.401 & 0.055 & 0.016763 & 0.006291 & 0.222180 & 99.714 \\
\hline 12/23/08 21:00 & 1.745 & 0.285 & 0.026 & 11.648 & 1.401 & 0.055 & 0.016763 & 0.006291 & 0.222180 & 99.714 \\
\hline 12/23/08 22:00 & 1.743 & 0.283 & 0.026 & 11.450 & 1.401 & 0.055 & 0.016763 & 0.006291 & 0.222180 & 99.714 \\
\hline 12/23/08 23:00 & 1.743 & 0.283 & 0.026 & 11.450 & 1.401 & 0.055 & 0.016763 & 0.006291 & 0.222180 & 99.714 \\
\hline 12/24/08 0:00 & 1.741 & 0.281 & 0.025 & 11.254 & 1.404 & 0.058 & 0.017678 & 0.006782 & 0.239555 & 107.512 \\
\hline 12/24/08 1:00 & 1.739 & 0.279 & 0.025 & 11.060 & 1.401 & 0.055 & 0.016763 & 0.006291 & 0.222180 & 99.714 \\
\hline 12/24/08 2:00 & 1.734 & 0.274 & 0.024 & 10.585 & 1.406 & 0.06 & 0.018287 & 0.007117 & 0.251378 & 112.818 \\
\hline 12/24/08 3:00 & 1.741 & 0.281 & 0.025 & 11.254 & 1.401 & 0.055 & 0.016763 & 0.006291 & 0.222180 & 99.714 \\
\hline 12/24/08 4:00 & 1.734 & 0.274 & 0.024 & 10.585 & 1.404 & 0.058 & 0.017678 & 0.006782 & 0.239555 & 107.512 \\
\hline 12/24/08 5:00 & 1.737 & 0.277 & 0.024 & 10.869 & 1.401 & 0.055 & 0.016763 & 0.006291 & 0.222180 & 99.714 \\
\hline 12/24/08 6:00 & 1.734 & 0.274 & 0.024 & 10.585 & 1.401 & 0.055 & 0.016763 & 0.006291 & 0.222180 & 99.714 \\
\hline 12/24/08 7:00 & 1.73 & 0.270 & 0.023 & 10.213 & 1.399 & 0.053 & 0.016154 & 0.005969 & 0.210842 & 94.626 \\
\hline 12/24/08 8:00 & 1.725 & 0.265 & 0.022 & 9.760 & 1.399 & 0.053 & 0.016154 & 0.005969 & 0.210842 & 94.626 \\
\hline
\end{tabular}




\begin{tabular}{|c|c|c|c|c|c|c|c|c|c|c|}
\hline \multirow[b]{3}{*}{ Date Time } & \multicolumn{4}{|c|}{ V-Notch Weir } & \multicolumn{6}{|c|}{ R-Notch Weir } \\
\hline & \multicolumn{2}{|c|}{ Measured Water Level } & \multicolumn{2}{|c|}{$\begin{array}{c}\text { Calculated Rate } \\
\left(\mathrm{ft}^{3} / \mathrm{s}=448.8 \mathrm{gpm}\right)\end{array}$} & \multicolumn{3}{|c|}{ Measured Water Level } & \multicolumn{3}{|c|}{$\begin{array}{c}\text { Calculated Rate } \\
\left(\mathrm{ft}^{3} / \mathrm{s}=448.8 \mathrm{gpm}\right)\end{array}$} \\
\hline & $\begin{array}{c}\text { (ft above } \\
\text { sensor) }\end{array}$ & $\begin{array}{c}\text { (ft above } \\
\text { notch) }\end{array}$ & $\left(\mathrm{ft}^{3} / \mathrm{s}\right)$ & (gpm) & $\begin{array}{l}\text { (ft above } \\
\text { sensor) }\end{array}$ & $\begin{array}{c}\text { (ft above } \\
\text { log) }\end{array}$ & $\begin{array}{c}\text { (m above } \\
\text { log) }\end{array}$ & $\left(\mathrm{m}^{3} / \mathrm{s}\right)$ & $\left(\mathrm{ft}^{3} / \mathrm{s}\right)$ & (gpm) \\
\hline 12/24/08 9:00 & 1.725 & 0.265 & 0.022 & 9.760 & 1.399 & 0.053 & 0.016154 & 0.005969 & 0.210842 & 94.626 \\
\hline 12/24/08 10:00 & 1.725 & 0.265 & 0.022 & 9.760 & 1.397 & 0.051 & 0.015544 & 0.005654 & 0.199703 & 89.627 \\
\hline 12/24/08 11:00 & 1.72 & 0.260 & 0.021 & 9.319 & 1.394 & 0.048 & 0.014630 & 0.005192 & 0.183377 & 82.300 \\
\hline 12/24/08 12:00 & 1.727 & 0.267 & 0.022 & 9.940 & 1.394 & 0.048 & 0.014630 & 0.005192 & 0.183377 & 82.300 \\
\hline 12/24/08 13:00 & 1.724 & 0.264 & 0.022 & 9.671 & 1.394 & 0.048 & 0.014630 & 0.005192 & 0.183377 & 82.300 \\
\hline 12/24/08 14:00 & 1.724 & 0.264 & 0.022 & 9.671 & 1.389 & 0.043 & 0.013106 & 0.004451 & 0.157222 & 70.561 \\
\hline 12/24/08 15:00 & 1.719 & 0.259 & 0.021 & 9.232 & 1.397 & 0.051 & 0.015544 & 0.005654 & 0.199703 & 89.627 \\
\hline 12/24/08 16:00 & 1.719 & 0.259 & 0.021 & 9.232 & 1.389 & 0.043 & 0.013106 & 0.004451 & 0.157222 & 70.561 \\
\hline 12/24/08 17:00 & 1.717 & 0.257 & 0.020 & 9.060 & 1.392 & 0.046 & 0.014020 & 0.004891 & 0.172754 & 77.532 \\
\hline 12/24/08 18:00 & 1.719 & 0.259 & 0.021 & 9.232 & 1.387 & 0.041 & 0.012496 & 0.004166 & 0.147141 & 66.037 \\
\hline 12/24/08 19:00 & 1.717 & 0.257 & 0.020 & 9.060 & 1.392 & 0.046 & 0.014020 & 0.004891 & 0.172754 & 77.532 \\
\hline 12/24/08 20:00 & 1.715 & 0.255 & 0.020 & 8.890 & 1.394 & 0.048 & 0.014630 & 0.005192 & 0.183377 & 82.300 \\
\hline 12/24/08 21:00 & 1.715 & 0.255 & 0.020 & 8.890 & 1.397 & 0.051 & 0.015544 & 0.005654 & 0.199703 & 89.627 \\
\hline 12/24/08 22:00 & 1.715 & 0.255 & 0.020 & 8.890 & 1.394 & 0.048 & 0.014630 & 0.005192 & 0.183377 & 82.300 \\
\hline 12/24/08 23:00 & 1.713 & 0.253 & 0.019 & 8.722 & 1.397 & 0.051 & 0.015544 & 0.005654 & 0.199703 & 89.627 \\
\hline 12/25/08 0:00 & 1.713 & 0.253 & 0.019 & 8.722 & 1.397 & 0.051 & 0.015544 & 0.005654 & 0.199703 & 89.627 \\
\hline 12/25/08 1:00 & 1.713 & 0.253 & 0.019 & 8.722 & 1.397 & 0.051 & 0.015544 & 0.005654 & 0.199703 & 89.627 \\
\hline 12/25/08 2:00 & 1.715 & 0.255 & 0.020 & 8.890 & 1.397 & 0.051 & 0.015544 & 0.005654 & 0.199703 & 89.627 \\
\hline 12/25/08 3:00 & 1.711 & 0.251 & 0.019 & 8.556 & 1.401 & 0.055 & 0.016763 & 0.006291 & 0.222180 & 99.714 \\
\hline 12/25/08 4:00 & 1.711 & 0.251 & 0.019 & 8.556 & 1.401 & 0.055 & 0.016763 & 0.006291 & 0.222180 & 99.714 \\
\hline 12/25/08 5:00 & 1.713 & 0.253 & 0.019 & 8.722 & 1.401 & 0.055 & 0.016763 & 0.006291 & 0.222180 & 99.714 \\
\hline 12/25/08 6:00 & 1.711 & 0.251 & 0.019 & 8.556 & 1.404 & 0.058 & 0.017678 & 0.006782 & 0.239555 & 107.512 \\
\hline 12/25/08 7:00 & 1.713 & 0.253 & 0.019 & 8.722 & 1.406 & 0.06 & 0.018287 & 0.007117 & 0.251378 & 112.818 \\
\hline 12/25/08 8:00 & 1.713 & 0.253 & 0.019 & 8.722 & 1.411 & 0.065 & 0.019811 & 0.007977 & 0.281754 & 126.451 \\
\hline 12/25/08 9:00 & 1.711 & 0.251 & 0.019 & 8.556 & 1.397 & 0.051 & 0.015544 & 0.005654 & 0.199703 & 89.627 \\
\hline
\end{tabular}




\begin{tabular}{|c|c|c|c|c|c|c|c|c|c|c|}
\hline \multirow[b]{3}{*}{ Date Time } & \multicolumn{4}{|c|}{ V-Notch Weir } & \multicolumn{6}{|c|}{ R-Notch Weir } \\
\hline & \multicolumn{2}{|c|}{ Measured Water Level } & \multicolumn{2}{|c|}{$\begin{array}{c}\text { Calculated Rate } \\
\left(\mathrm{ft}^{3} / \mathrm{s}=448.8 \mathrm{gpm}\right)\end{array}$} & \multicolumn{3}{|c|}{ Measured Water Level } & \multicolumn{3}{|c|}{$\begin{array}{c}\text { Calculated Rate } \\
\left(\mathrm{ft}^{3} / \mathrm{s}=448.8 \mathrm{gpm}\right)\end{array}$} \\
\hline & $\begin{array}{c}\text { (ft above } \\
\text { sensor) }\end{array}$ & $\begin{array}{c}\text { (ft above } \\
\text { notch) }\end{array}$ & $\left(\mathrm{ft}^{3} / \mathrm{s}\right)$ & (gpm) & $\begin{array}{c}\text { (ft above } \\
\text { sensor) }\end{array}$ & $\begin{array}{c}\text { (ft above } \\
\text { log) }\end{array}$ & $\begin{array}{c}\text { (m above } \\
\text { log) }\end{array}$ & $\left(\mathrm{m}^{3} / \mathrm{s}\right)$ & $\left(\mathrm{ft}^{3} / \mathrm{s}\right)$ & (gpm) \\
\hline 12/25/08 10:00 & 1.711 & 0.251 & 0.019 & 8.556 & 1.406 & 0.06 & 0.018287 & 0.007117 & 0.251378 & 112.818 \\
\hline 12/25/08 11:00 & 1.713 & 0.253 & 0.019 & 8.722 & 1.406 & 0.06 & 0.018287 & 0.007117 & 0.251378 & 112.818 \\
\hline 12/25/08 12:00 & 1.71 & 0.250 & 0.019 & 8.474 & 1.411 & 0.065 & 0.019811 & 0.007977 & 0.281754 & 126.451 \\
\hline 12/25/08 13:00 & 1.719 & 0.259 & 0.021 & 9.232 & 1.415 & 0.069 & 0.021030 & 0.008688 & 0.306873 & 137.724 \\
\hline 12/25/08 14:00 & 1.719 & 0.259 & 0.021 & 9.232 & 1.415 & 0.069 & 0.021030 & 0.008688 & 0.306873 & 137.724 \\
\hline 12/25/08 15:00 & 1.726 & 0.266 & 0.022 & 9.850 & 1.418 & 0.072 & 0.021945 & 0.009235 & 0.326176 & 146.388 \\
\hline 12/25/08 16:00 & 1.721 & 0.261 & 0.021 & 9.406 & 1.418 & 0.072 & 0.021945 & 0.009235 & 0.326176 & 146.388 \\
\hline 12/25/08 17:00 & 1.726 & 0.266 & 0.022 & 9.850 & 1.413 & 0.067 & 0.020421 & 0.008330 & 0.294224 & 132.048 \\
\hline 12/25/08 18:00 & 1.724 & 0.264 & 0.022 & 9.671 & 1.413 & 0.067 & 0.020421 & 0.008330 & 0.294224 & 132.048 \\
\hline 12/25/08 19:00 & 1.726 & 0.266 & 0.022 & 9.850 & 1.411 & 0.065 & 0.019811 & 0.007977 & 0.281754 & 126.451 \\
\hline 12/25/08 20:00 & 1.728 & 0.268 & 0.022 & 10.030 & 1.413 & 0.067 & 0.020421 & 0.008330 & 0.294224 & 132.048 \\
\hline 12/25/08 21:00 & 1.731 & 0.271 & 0.023 & 10.305 & 1.413 & 0.067 & 0.020421 & 0.008330 & 0.294224 & 132.048 \\
\hline 12/25/08 22:00 & 1.731 & 0.271 & 0.023 & 10.305 & 1.415 & 0.069 & 0.021030 & 0.008688 & 0.306873 & 137.724 \\
\hline 12/25/08 23:00 & 1.731 & 0.271 & 0.023 & 10.305 & 1.413 & 0.067 & 0.020421 & 0.008330 & 0.294224 & 132.048 \\
\hline 12/26/08 0:00 & 1.733 & 0.273 & 0.023 & 10.491 & 1.413 & 0.067 & 0.020421 & 0.008330 & 0.294224 & 132.048 \\
\hline 12/26/08 1:00 & 1.735 & 0.275 & 0.024 & 10.679 & 1.413 & 0.067 & 0.020421 & 0.008330 & 0.294224 & 132.048 \\
\hline 12/26/08 2:00 & 1.735 & 0.275 & 0.024 & 10.679 & 1.413 & 0.067 & 0.020421 & 0.008330 & 0.294224 & 132.048 \\
\hline 12/26/08 3:00 & 1.733 & 0.273 & 0.023 & 10.491 & 1.413 & 0.067 & 0.020421 & 0.008330 & 0.294224 & 132.048 \\
\hline 12/26/08 4:00 & 1.737 & 0.277 & 0.024 & 10.869 & 1.413 & 0.067 & 0.020421 & 0.008330 & 0.294224 & 132.048 \\
\hline 12/26/08 5:00 & 1.74 & 0.280 & 0.025 & 11.157 & 1.413 & 0.067 & 0.020421 & 0.008330 & 0.294224 & 132.048 \\
\hline 12/26/08 6:00 & 1.742 & 0.282 & 0.025 & 11.352 & 1.415 & 0.069 & 0.021030 & 0.008688 & 0.306873 & 137.724 \\
\hline 12/26/08 7:00 & 1.744 & 0.284 & 0.026 & 11.549 & 1.413 & 0.067 & 0.020421 & 0.008330 & 0.294224 & 132.048 \\
\hline 12/26/08 8:00 & 1.749 & 0.289 & 0.027 & 12.049 & 1.413 & 0.067 & 0.020421 & 0.008330 & 0.294224 & 132.048 \\
\hline 12/26/08 9:00 & 1.753 & 0.293 & 0.028 & 12.459 & 1.415 & 0.069 & 0.021030 & 0.008688 & 0.306873 & 137.724 \\
\hline 12/26/08 10:00 & 1.765 & 0.305 & 0.031 & 13.739 & 1.415 & 0.069 & 0.021030 & 0.008688 & 0.306873 & 137.724 \\
\hline
\end{tabular}




\begin{tabular}{|c|c|c|c|c|c|c|c|c|c|c|}
\hline \multirow[b]{3}{*}{ Date Time } & \multicolumn{4}{|c|}{ V-Notch Weir } & \multicolumn{6}{|c|}{ R-Notch Weir } \\
\hline & \multicolumn{2}{|c|}{ Measured Water Level } & \multicolumn{2}{|c|}{$\begin{array}{c}\text { Calculated Rate } \\
\left(\mathrm{ft}^{3} / \mathrm{s}=448.8 \mathrm{gpm}\right)\end{array}$} & \multicolumn{3}{|c|}{ Measured Water Level } & \multicolumn{3}{|c|}{$\begin{array}{c}\text { Calculated Rate } \\
\left(\mathrm{ft}^{3} / \mathrm{s}=448.8 \mathrm{gpm}\right)\end{array}$} \\
\hline & $\begin{array}{c}\text { (ft above } \\
\text { sensor) }\end{array}$ & $\begin{array}{c}\text { (ft above } \\
\text { notch) }\end{array}$ & $\left(\mathrm{ft}^{3} / \mathrm{s}\right)$ & (gpm) & $\begin{array}{l}\text { (ft above } \\
\text { sensor) }\end{array}$ & $\begin{array}{c}\text { (ft above } \\
\text { log) }\end{array}$ & $\begin{array}{c}\text { (m above } \\
\text { log) }\end{array}$ & $\left(\mathrm{m}^{3} / \mathrm{s}\right)$ & $\left(\mathrm{ft}^{3} / \mathrm{s}\right)$ & (gpm) \\
\hline 12/26/08 11:00 & 1.786 & 0.326 & 0.036 & 16.162 & 1.418 & 0.072 & 0.021945 & 0.009235 & 0.326176 & 146.388 \\
\hline 12/26/08 12:00 & 1.809 & 0.349 & 0.043 & 19.090 & 1.422 & 0.076 & 0.023164 & 0.009981 & 0.352516 & 158.209 \\
\hline 12/26/08 13:00 & 1.832 & 0.372 & 0.050 & 22.316 & 1.427 & 0.081 & 0.024688 & 0.010940 & 0.386387 & 173.411 \\
\hline 12/26/08 14:00 & 1.872 & 0.412 & 0.064 & 28.662 & 1.434 & 0.088 & 0.026821 & 0.012331 & 0.435514 & 195.459 \\
\hline 12/26/08 15:00 & 1.923 & 0.463 & 0.085 & 38.174 & 1.444 & 0.098 & 0.029869 & 0.014412 & 0.509013 & 228.445 \\
\hline 12/26/08 16:00 & 2.145 & 0.685 & 0.223 & 100.260 & 1.489 & 0.143 & 0.043584 & 0.025016 & 0.883573 & 396.548 \\
\hline 12/26/08 17:00 & 2.177 & 0.717 & 0.250 & 112.239 & 1.503 & 0.157 & 0.047851 & 0.028693 & 1.013424 & 454.824 \\
\hline 12/26/08 18:00 & 2.14 & 0.680 & 0.219 & 98.461 & 1.491 & 0.145 & 0.044194 & 0.025531 & 0.901758 & 404.709 \\
\hline 12/26/08 19:00 & 2.178 & 0.718 & 0.251 & 112.627 & 1.508 & 0.162 & 0.049375 & 0.030046 & 1.061219 & 476.275 \\
\hline 12/26/08 20:00 & 2.174 & 0.714 & 0.248 & 111.082 & 1.503 & 0.157 & 0.047851 & 0.028693 & 1.013424 & 454.824 \\
\hline 12/26/08 21:00 & 2.157 & 0.697 & 0.233 & 104.657 & 1.493 & 0.147 & 0.044803 & 0.026050 & 0.920066 & 412.925 \\
\hline 12/26/08 22:00 & 2.143 & 0.683 & 0.222 & 99.538 & 1.489 & 0.143 & 0.043584 & 0.025016 & 0.883573 & 396.548 \\
\hline 12/26/08 23:00 & 2.114 & 0.654 & 0.199 & 89.419 & 1.479 & 0.133 & 0.040536 & 0.022495 & 0.794527 & 356.583 \\
\hline 12/27/08 0:00 & 2.065 & 0.605 & 0.164 & 73.777 & 1.465 & 0.119 & 0.036269 & 0.019119 & 0.675284 & 303.068 \\
\hline 12/27/08 1:00 & 1.997 & 0.537 & 0.123 & 54.984 & 1.451 & 0.105 & 0.032002 & 0.015931 & 0.562690 & 252.535 \\
\hline 12/27/08 2:00 & 1.925 & 0.465 & 0.086 & 38.581 & 1.439 & 0.093 & 0.028345 & 0.013358 & 0.471786 & 211.738 \\
\hline 12/27/08 3:00 & 1.885 & 0.425 & 0.069 & 30.933 & 1.434 & 0.088 & 0.026821 & 0.012331 & 0.435514 & 195.459 \\
\hline 12/27/08 4:00 & 1.855 & 0.395 & 0.058 & 25.849 & 1.429 & 0.083 & 0.025297 & 0.011331 & 0.400223 & 179.620 \\
\hline 12/27/08 5:00 & 1.824 & 0.364 & 0.047 & 21.160 & 1.425 & 0.079 & 0.024078 & 0.010553 & 0.372715 & 167.274 \\
\hline 12/27/08 6:00 & 1.803 & 0.343 & 0.041 & 18.298 & 1.422 & 0.076 & 0.023164 & 0.009981 & 0.352516 & 158.209 \\
\hline 12/27/08 7:00 & 1.786 & 0.326 & 0.036 & 16.162 & 1.42 & 0.074 & 0.022554 & 0.009605 & 0.339261 & 152.260 \\
\hline 12/27/08 8:00 & 1.777 & 0.317 & 0.034 & 15.094 & 1.418 & 0.072 & 0.021945 & 0.009235 & 0.326176 & 146.388 \\
\hline 12/27/08 9:00 & 1.765 & 0.305 & 0.031 & 13.739 & 1.415 & 0.069 & 0.021030 & 0.008688 & 0.306873 & 137.724 \\
\hline 12/27/08 10:00 & 1.76 & 0.300 & 0.029 & 13.197 & 1.415 & 0.069 & 0.021030 & 0.008688 & 0.306873 & 137.724 \\
\hline 12/27/08 11:00 & 1.756 & 0.296 & 0.028 & 12.772 & 1.415 & 0.069 & 0.021030 & 0.008688 & 0.306873 & 137.724 \\
\hline
\end{tabular}




\begin{tabular}{|c|c|c|c|c|c|c|c|c|c|c|}
\hline \multirow[b]{3}{*}{ Date Time } & \multicolumn{4}{|c|}{ V-Notch Weir } & \multicolumn{6}{|c|}{ R-Notch Weir } \\
\hline & \multicolumn{2}{|c|}{ Measured Water Level } & \multicolumn{2}{|c|}{$\begin{array}{c}\text { Calculated Rate } \\
\left(\mathrm{ft}^{3} / \mathrm{s}=448.8 \mathrm{gpm}\right)\end{array}$} & \multicolumn{3}{|c|}{ Measured Water Level } & \multicolumn{3}{|c|}{$\begin{array}{c}\text { Calculated Rate } \\
\left(\mathrm{ft}^{3} / \mathrm{s}=448.8 \mathrm{gpm}\right)\end{array}$} \\
\hline & $\begin{array}{c}\text { (ft above } \\
\text { sensor) }\end{array}$ & $\begin{array}{c}\text { (ft above } \\
\text { notch) }\end{array}$ & $\left(\mathrm{ft}^{3} / \mathrm{s}\right)$ & (gpm) & $\begin{array}{l}\text { (ft above } \\
\text { sensor) }\end{array}$ & $\begin{array}{c}\text { (ft above } \\
\text { log) }\end{array}$ & $\begin{array}{c}\text { (m above } \\
\text { log) }\end{array}$ & $\left(\mathrm{m}^{3} / \mathrm{s}\right)$ & $\left(\mathrm{ft}^{3} / \mathrm{s}\right)$ & (gpm) \\
\hline 12/27/08 12:00 & 1.748 & 0.288 & 0.027 & 11.948 & 1.415 & 0.069 & 0.021030 & 0.008688 & 0.306873 & 137.724 \\
\hline 12/27/08 13:00 & 1.736 & 0.276 & 0.024 & 10.773 & 1.415 & 0.069 & 0.021030 & 0.008688 & 0.306873 & 137.724 \\
\hline 12/27/08 14:00 & 1.742 & 0.282 & 0.025 & 11.352 & 1.415 & 0.069 & 0.021030 & 0.008688 & 0.306873 & 137.724 \\
\hline 12/27/08 15:00 & 1.74 & 0.280 & 0.025 & 11.157 & 1.415 & 0.069 & 0.021030 & 0.008688 & 0.306873 & 137.724 \\
\hline 12/27/08 16:00 & 1.737 & 0.277 & 0.024 & 10.869 & 1.413 & 0.067 & 0.020421 & 0.008330 & 0.294224 & 132.048 \\
\hline 12/27/08 17:00 & 1.735 & 0.275 & 0.024 & 10.679 & 1.413 & 0.067 & 0.020421 & 0.008330 & 0.294224 & 132.048 \\
\hline 12/27/08 18:00 & 1.733 & 0.273 & 0.023 & 10.491 & 1.413 & 0.067 & 0.020421 & 0.008330 & 0.294224 & 132.048 \\
\hline 12/27/08 19:00 & 1.729 & 0.269 & 0.023 & 10.122 & 1.413 & 0.067 & 0.020421 & 0.008330 & 0.294224 & 132.048 \\
\hline 12/27/08 20:00 & 1.724 & 0.264 & 0.022 & 9.671 & 1.413 & 0.067 & 0.020421 & 0.008330 & 0.294224 & 132.048 \\
\hline 12/27/08 21:00 & 1.722 & 0.262 & 0.021 & 9.494 & 1.411 & 0.065 & 0.019811 & 0.007977 & 0.281754 & 126.451 \\
\hline 12/27/08 22:00 & 1.715 & 0.255 & 0.020 & 8.890 & 1.411 & 0.065 & 0.019811 & 0.007977 & 0.281754 & 126.451 \\
\hline 12/27/08 23:00 & 1.715 & 0.255 & 0.020 & 8.890 & 1.411 & 0.065 & 0.019811 & 0.007977 & 0.281754 & 126.451 \\
\hline 12/28/08 0:00 & 1.706 & 0.246 & 0.018 & 8.149 & 1.411 & 0.065 & 0.019811 & 0.007977 & 0.281754 & 126.451 \\
\hline 12/28/08 1:00 & 1.708 & 0.248 & 0.019 & 8.310 & 1.411 & 0.065 & 0.019811 & 0.007977 & 0.281754 & 126.451 \\
\hline 12/28/08 2:00 & 1.708 & 0.248 & 0.019 & 8.310 & 1.408 & 0.062 & 0.018897 & 0.007457 & 0.263390 & 118.209 \\
\hline 12/28/08 3:00 & 1.706 & 0.246 & 0.018 & 8.149 & 1.411 & 0.065 & 0.019811 & 0.007977 & 0.281754 & 126.451 \\
\hline 12/28/08 4:00 & 1.704 & 0.244 & 0.018 & 7.989 & 1.411 & 0.065 & 0.019811 & 0.007977 & 0.281754 & 126.451 \\
\hline 12/28/08 5:00 & 1.701 & 0.241 & 0.017 & 7.754 & 1.408 & 0.062 & 0.018897 & 0.007457 & 0.263390 & 118.209 \\
\hline 12/28/08 6:00 & 1.699 & 0.239 & 0.017 & 7.599 & 1.411 & 0.065 & 0.019811 & 0.007977 & 0.281754 & 126.451 \\
\hline 12/28/08 7:00 & 1.701 & 0.241 & 0.017 & 7.754 & 1.408 & 0.062 & 0.018897 & 0.007457 & 0.263390 & 118.209 \\
\hline 12/28/08 8:00 & 1.694 & 0.234 & 0.016 & 7.220 & 1.408 & 0.062 & 0.018897 & 0.007457 & 0.263390 & 118.209 \\
\hline 12/28/08 9:00 & 1.692 & 0.232 & 0.016 & 7.072 & 1.411 & 0.065 & 0.019811 & 0.007977 & 0.281754 & 126.451 \\
\hline 12/28/08 10:00 & 1.701 & 0.241 & 0.017 & 7.754 & 1.408 & 0.062 & 0.018897 & 0.007457 & 0.263390 & 118.209 \\
\hline 12/28/08 11:00 & 1.707 & 0.247 & 0.018 & 8.229 & 1.411 & 0.065 & 0.019811 & 0.007977 & 0.281754 & 126.451 \\
\hline 12/28/08 12:00 & 1.693 & 0.233 & 0.016 & 7.145 & 1.408 & 0.062 & 0.018897 & 0.007457 & 0.263390 & 118.209 \\
\hline
\end{tabular}




\begin{tabular}{|c|c|c|c|c|c|c|c|c|c|c|}
\hline \multirow[b]{3}{*}{ Date Time } & \multicolumn{4}{|c|}{ V-Notch Weir } & \multicolumn{6}{|c|}{ R-Notch Weir } \\
\hline & \multicolumn{2}{|c|}{ Measured Water Level } & \multicolumn{2}{|c|}{$\begin{array}{c}\text { Calculated Rate } \\
\left(\mathrm{ft}^{3} / \mathrm{s}=448.8 \mathrm{gpm}\right)\end{array}$} & \multicolumn{3}{|c|}{ Measured Water Level } & \multicolumn{3}{|c|}{$\begin{array}{c}\text { Calculated Rate } \\
\left(\mathrm{ft}^{3} / \mathrm{s}=448.8 \mathrm{gpm}\right)\end{array}$} \\
\hline & $\begin{array}{c}\text { (ft above } \\
\text { sensor) }\end{array}$ & $\begin{array}{c}\text { (ft above } \\
\text { notch) }\end{array}$ & $\left(\mathrm{ft}^{3} / \mathrm{s}\right)$ & (gpm) & $\begin{array}{l}\text { (ft above } \\
\text { sensor) }\end{array}$ & $\begin{array}{c}\text { (ft above } \\
\text { log) }\end{array}$ & $\begin{array}{c}\text { (m above } \\
\text { log) }\end{array}$ & $\left(\mathrm{m}^{3} / \mathrm{s}\right)$ & $\left(\mathrm{ft}^{3} / \mathrm{s}\right)$ & (gpm) \\
\hline 12/28/08 13:00 & 1.689 & 0.229 & 0.015 & 6.853 & 1.408 & 0.062 & 0.018897 & 0.007457 & 0.263390 & 118.209 \\
\hline 12/28/08 14:00 & 1.702 & 0.242 & 0.017 & 7.832 & 1.411 & 0.065 & 0.019811 & 0.007977 & 0.281754 & 126.451 \\
\hline 12/28/08 15:00 & 1.709 & 0.249 & 0.019 & 8.392 & 1.413 & 0.067 & 0.020421 & 0.008330 & 0.294224 & 132.048 \\
\hline 12/28/08 16:00 & 1.706 & 0.246 & 0.018 & 8.149 & 1.415 & 0.069 & 0.021030 & 0.008688 & 0.306873 & 137.724 \\
\hline 12/28/08 17:00 & 1.711 & 0.251 & 0.019 & 8.556 & 1.413 & 0.067 & 0.020421 & 0.008330 & 0.294224 & 132.048 \\
\hline 12/28/08 18:00 & 1.712 & 0.252 & 0.019 & 8.639 & 1.415 & 0.069 & 0.021030 & 0.008688 & 0.306873 & 137.724 \\
\hline 12/28/08 19:00 & 1.708 & 0.248 & 0.019 & 8.310 & 1.413 & 0.067 & 0.020421 & 0.008330 & 0.294224 & 132.048 \\
\hline 12/28/08 20:00 & 1.704 & 0.244 & 0.018 & 7.989 & 1.413 & 0.067 & 0.020421 & 0.008330 & 0.294224 & 132.048 \\
\hline 12/28/08 21:00 & 1.706 & 0.246 & 0.018 & 8.149 & 1.41 & 0.064 & 0.019506 & 0.007803 & 0.275587 & 123.683 \\
\hline 12/28/08 22:00 & 1.702 & 0.242 & 0.017 & 7.832 & 1.413 & 0.067 & 0.020421 & 0.008330 & 0.294224 & 132.048 \\
\hline 12/28/08 23:00 & 1.707 & 0.247 & 0.018 & 8.229 & 1.413 & 0.067 & 0.020421 & 0.008330 & 0.294224 & 132.048 \\
\hline 12/29/08 0:00 & 1.704 & 0.244 & 0.018 & 7.989 & 1.413 & 0.067 & 0.020421 & 0.008330 & 0.294224 & 132.048 \\
\hline 12/29/08 1:00 & 1.702 & 0.242 & 0.017 & 7.832 & 1.413 & 0.067 & 0.020421 & 0.008330 & 0.294224 & 132.048 \\
\hline 12/29/08 2:00 & 1.7 & 0.240 & 0.017 & 7.676 & 1.413 & 0.067 & 0.020421 & 0.008330 & 0.294224 & 132.048 \\
\hline 12/29/08 3:00 & 1.702 & 0.242 & 0.017 & 7.832 & 1.413 & 0.067 & 0.020421 & 0.008330 & 0.294224 & 132.048 \\
\hline 12/29/08 4:00 & 1.695 & 0.235 & 0.016 & 7.295 & 1.41 & 0.064 & 0.019506 & 0.007803 & 0.275587 & 123.683 \\
\hline 12/29/08 5:00 & 1.698 & 0.238 & 0.017 & 7.522 & 1.413 & 0.067 & 0.020421 & 0.008330 & 0.294224 & 132.048 \\
\hline 12/29/08 6:00 & 1.7 & 0.240 & 0.017 & 7.676 & 1.41 & 0.064 & 0.019506 & 0.007803 & 0.275587 & 123.683 \\
\hline 12/29/08 7:00 & 1.695 & 0.235 & 0.016 & 7.295 & 1.41 & 0.064 & 0.019506 & 0.007803 & 0.275587 & 123.683 \\
\hline 12/29/08 8:00 & 1.693 & 0.233 & 0.016 & 7.145 & 1.41 & 0.064 & 0.019506 & 0.007803 & 0.275587 & 123.683 \\
\hline 12/29/08 9:00 & 1.693 & 0.233 & 0.016 & 7.145 & 1.41 & 0.064 & 0.019506 & 0.007803 & 0.275587 & 123.683 \\
\hline 12/29/08 10:00 & 1.695 & 0.235 & 0.016 & 7.295 & 1.41 & 0.064 & 0.019506 & 0.007803 & 0.275587 & 123.683 \\
\hline 12/29/08 11:00 & 1.69 & 0.230 & 0.015 & 6.925 & 1.41 & 0.064 & 0.019506 & 0.007803 & 0.275587 & 123.683 \\
\hline 12/29/08 12:00 & 1.693 & 0.233 & 0.016 & 7.145 & 1.41 & 0.064 & 0.019506 & 0.007803 & 0.275587 & 123.683 \\
\hline 12/29/08 13:00 & 1.702 & 0.242 & 0.017 & 7.832 & 1.41 & 0.064 & 0.019506 & 0.007803 & 0.275587 & 123.683 \\
\hline
\end{tabular}




\begin{tabular}{|c|c|c|c|c|c|c|c|c|c|c|}
\hline \multirow[b]{3}{*}{ Date Time } & \multicolumn{4}{|c|}{ V-Notch Weir } & \multicolumn{6}{|c|}{ R-Notch Weir } \\
\hline & \multicolumn{2}{|c|}{ Measured Water Level } & \multicolumn{2}{|c|}{$\begin{array}{c}\text { Calculated Rate } \\
\left(\mathrm{ft}^{3} / \mathrm{s}=448.8 \mathrm{gpm}\right)\end{array}$} & \multicolumn{3}{|c|}{ Measured Water Level } & \multicolumn{3}{|c|}{$\begin{array}{c}\text { Calculated Rate } \\
\left(\mathrm{ft}^{3} / \mathrm{s}=448.8 \mathrm{gpm}\right)\end{array}$} \\
\hline & $\begin{array}{c}\text { (ft above } \\
\text { sensor) }\end{array}$ & $\begin{array}{c}\text { (ft above } \\
\text { notch) }\end{array}$ & $\left(\mathrm{ft}^{3} / \mathrm{s}\right)$ & (gpm) & $\begin{array}{l}\text { (ft above } \\
\text { sensor) }\end{array}$ & $\begin{array}{c}\text { (ft above } \\
\text { log) }\end{array}$ & $\begin{array}{c}\text { (m above } \\
\text { log) }\end{array}$ & $\left(\mathrm{m}^{3} / \mathrm{s}\right)$ & $\left(\mathrm{ft}^{3} / \mathrm{s}\right)$ & (gpm) \\
\hline 12/29/08 14:00 & 1.713 & 0.253 & 0.019 & 8.722 & 1.415 & 0.069 & 0.021030 & 0.008688 & 0.306873 & 137.724 \\
\hline 12/29/08 15:00 & 1.722 & 0.262 & 0.021 & 9.494 & 1.418 & 0.072 & 0.021945 & 0.009235 & 0.326176 & 146.388 \\
\hline 12/29/08 16:00 & 1.731 & 0.271 & 0.023 & 10.305 & 1.42 & 0.074 & 0.022554 & 0.009605 & 0.339261 & 152.260 \\
\hline 12/29/08 17:00 & 1.734 & 0.274 & 0.024 & 10.585 & 1.42 & 0.074 & 0.022554 & 0.009605 & 0.339261 & 152.260 \\
\hline 12/29/08 18:00 & 1.73 & 0.270 & 0.023 & 10.213 & 1.42 & 0.074 & 0.022554 & 0.009605 & 0.339261 & 152.260 \\
\hline 12/29/08 19:00 & 1.726 & 0.266 & 0.022 & 9.850 & 1.417 & 0.071 & 0.021640 & 0.009052 & 0.319698 & 143.480 \\
\hline 12/29/08 20:00 & 1.717 & 0.257 & 0.020 & 9.060 & 1.417 & 0.071 & 0.021640 & 0.009052 & 0.319698 & 143.480 \\
\hline 12/29/08 21:00 & 1.715 & 0.255 & 0.020 & 8.890 & 1.417 & 0.071 & 0.021640 & 0.009052 & 0.319698 & 143.480 \\
\hline 12/29/08 22:00 & 1.713 & 0.253 & 0.019 & 8.722 & 1.417 & 0.071 & 0.021640 & 0.009052 & 0.319698 & 143.480 \\
\hline 12/29/08 23:00 & 1.716 & 0.256 & 0.020 & 8.975 & 1.42 & 0.074 & 0.022554 & 0.009605 & 0.339261 & 152.260 \\
\hline 12/30/08 0:00 & 1.707 & 0.247 & 0.018 & 8.229 & 1.417 & 0.071 & 0.021640 & 0.009052 & 0.319698 & 143.480 \\
\hline 12/30/08 1:00 & 1.707 & 0.247 & 0.018 & 8.229 & 1.417 & 0.071 & 0.021640 & 0.009052 & 0.319698 & 143.480 \\
\hline 12/30/08 2:00 & 1.709 & 0.249 & 0.019 & 8.392 & 1.42 & 0.074 & 0.022554 & 0.009605 & 0.339261 & 152.260 \\
\hline 12/30/08 3:00 & 1.707 & 0.247 & 0.018 & 8.229 & 1.42 & 0.074 & 0.022554 & 0.009605 & 0.339261 & 152.260 \\
\hline 12/30/08 4:00 & 1.702 & 0.242 & 0.017 & 7.832 & 1.42 & 0.074 & 0.022554 & 0.009605 & 0.339261 & 152.260 \\
\hline 12/30/08 5:00 & 1.705 & 0.245 & 0.018 & 8.069 & 1.424 & 0.078 & 0.023773 & 0.010361 & 0.365940 & 164.234 \\
\hline 12/30/08 6:00 & 1.696 & 0.236 & 0.016 & 7.370 & 1.42 & 0.074 & 0.022554 & 0.009605 & 0.339261 & 152.260 \\
\hline 12/30/08 7:00 & 1.698 & 0.238 & 0.017 & 7.522 & 1.422 & 0.076 & 0.023164 & 0.009981 & 0.352516 & 158.209 \\
\hline 12/30/08 8:00 & 1.698 & 0.238 & 0.017 & 7.522 & 1.422 & 0.076 & 0.023164 & 0.009981 & 0.352516 & 158.209 \\
\hline 12/30/08 9:00 & 1.698 & 0.238 & 0.017 & 7.522 & 1.422 & 0.076 & 0.023164 & 0.009981 & 0.352516 & 158.209 \\
\hline 12/30/08 10:00 & 1.703 & 0.243 & 0.018 & 7.910 & 1.417 & 0.071 & 0.021640 & 0.009052 & 0.319698 & 143.480 \\
\hline 12/30/08 11:00 & 1.697 & 0.237 & 0.017 & 7.446 & 1.417 & 0.071 & 0.021640 & 0.009052 & 0.319698 & 143.480 \\
\hline 12/30/08 12:00 & 1.696 & 0.236 & 0.016 & 7.370 & 1.415 & 0.069 & 0.021030 & 0.008688 & 0.306873 & 137.724 \\
\hline 12/30/08 13:00 & 1.714 & 0.254 & 0.020 & 8.806 & 1.42 & 0.074 & 0.022554 & 0.009605 & 0.339261 & 152.260 \\
\hline 12/30/08 14:00 & 1.714 & 0.254 & 0.020 & 8.806 & 1.422 & 0.076 & 0.023164 & 0.009981 & 0.352516 & 158.209 \\
\hline
\end{tabular}




\begin{tabular}{|c|c|c|c|c|c|c|c|c|c|c|}
\hline \multirow[b]{3}{*}{ Date Time } & \multicolumn{4}{|c|}{ V-Notch Weir } & \multicolumn{6}{|c|}{ R-Notch Weir } \\
\hline & \multicolumn{2}{|c|}{ Measured Water Level } & \multicolumn{2}{|c|}{$\begin{array}{c}\text { Calculated Rate } \\
\left(\mathrm{ft}^{3} / \mathrm{s}=448.8 \mathrm{gpm}\right)\end{array}$} & \multicolumn{3}{|c|}{ Measured Water Level } & \multicolumn{3}{|c|}{$\begin{array}{c}\text { Calculated Rate } \\
\left(\mathrm{ft}^{3} / \mathrm{s}=448.8 \mathrm{gpm}\right)\end{array}$} \\
\hline & $\begin{array}{c}\text { (ft above } \\
\text { sensor) }\end{array}$ & $\begin{array}{c}\text { (ft above } \\
\text { notch) }\end{array}$ & $\left(\mathrm{ft}^{3} / \mathrm{s}\right)$ & (gpm) & $\begin{array}{c}\text { (ft above } \\
\text { sensor) }\end{array}$ & $\begin{array}{c}\text { (ft above } \\
\text { log) }\end{array}$ & $\begin{array}{c}\text { (m above } \\
\text { log) }\end{array}$ & $\left(\mathrm{m}^{3} / \mathrm{s}\right)$ & $\left(\mathrm{ft}^{3} / \mathrm{s}\right)$ & (gpm) \\
\hline 12/30/08 15:00 & 1.723 & 0.263 & 0.021 & 9.582 & 1.417 & 0.071 & 0.021640 & 0.009052 & 0.319698 & 143.480 \\
\hline 12/30/08 16:00 & 1.735 & 0.275 & 0.024 & 10.679 & 1.424 & 0.078 & 0.023773 & 0.010361 & 0.365940 & 164.234 \\
\hline 12/30/08 17:00 & 1.748 & 0.288 & 0.027 & 11.948 & 1.422 & 0.076 & 0.023164 & 0.009981 & 0.352516 & 158.209 \\
\hline 12/30/08 18:00 & 1.727 & 0.267 & 0.022 & 9.940 & 1.42 & 0.074 & 0.022554 & 0.009605 & 0.339261 & 152.260 \\
\hline 12/30/08 19:00 & 1.731 & 0.271 & 0.023 & 10.305 & 1.415 & 0.069 & 0.021030 & 0.008688 & 0.306873 & 137.724 \\
\hline 12/30/08 20:00 & 1.729 & 0.269 & 0.023 & 10.122 & 1.413 & 0.067 & 0.020421 & 0.008330 & 0.294224 & 132.048 \\
\hline 12/30/08 21:00 & 1.712 & 0.252 & 0.019 & 8.639 & 1.415 & 0.069 & 0.021030 & 0.008688 & 0.306873 & 137.724 \\
\hline 12/30/08 22:00 & 1.698 & 0.238 & 0.017 & 7.522 & 1.413 & 0.067 & 0.020421 & 0.008330 & 0.294224 & 132.048 \\
\hline 12/30/08 23:00 & 1.682 & 0.222 & 0.014 & 6.358 & 1.408 & 0.062 & 0.018897 & 0.007457 & 0.263390 & 118.209 \\
\hline 12/31/08 0:00 & 1.672 & 0.212 & 0.013 & 5.689 & 1.408 & 0.062 & 0.018897 & 0.007457 & 0.263390 & 118.209 \\
\hline 12/31/08 1:00 & 1.682 & 0.222 & 0.014 & 6.358 & 1.41 & 0.064 & 0.019506 & 0.007803 & 0.275587 & 123.683 \\
\hline 12/31/08 2:00 & 1.679 & 0.219 & 0.014 & 6.152 & 1.41 & 0.064 & 0.019506 & 0.007803 & 0.275587 & 123.683 \\
\hline 12/31/08 3:00 & 1.672 & 0.212 & 0.013 & 5.689 & 1.415 & 0.069 & 0.021030 & 0.008688 & 0.306873 & 137.724 \\
\hline 12/31/08 4:00 & 1.663 & 0.203 & 0.011 & 5.124 & 1.413 & 0.067 & 0.020421 & 0.008330 & 0.294224 & 132.048 \\
\hline 12/31/08 5:00 & 1.665 & 0.205 & 0.012 & 5.247 & 1.413 & 0.067 & 0.020421 & 0.008330 & 0.294224 & 132.048 \\
\hline 12/31/08 6:00 & 1.66 & 0.200 & 0.011 & 4.944 & 1.41 & 0.064 & 0.019506 & 0.007803 & 0.275587 & 123.683 \\
\hline 12/31/08 7:00 & 1.653 & 0.193 & 0.010 & 4.538 & 1.408 & 0.062 & 0.018897 & 0.007457 & 0.263390 & 118.209 \\
\hline 12/31/08 8:00 & 1.65 & 0.190 & 0.010 & 4.371 & 1.408 & 0.062 & 0.018897 & 0.007457 & 0.263390 & 118.209 \\
\hline 12/31/08 9:00 & 1.646 & 0.186 & 0.009 & 4.153 & 1.408 & 0.062 & 0.018897 & 0.007457 & 0.263390 & 118.209 \\
\hline 12/31/08 10:00 & 1.648 & 0.188 & 0.009 & 4.261 & 1.408 & 0.062 & 0.018897 & 0.007457 & 0.263390 & 118.209 \\
\hline 12/31/08 11:00 & 1.657 & 0.197 & 0.011 & 4.768 & 1.41 & 0.064 & 0.019506 & 0.007803 & 0.275587 & 123.683 \\
\hline 12/31/08 12:00 & 1.651 & 0.191 & 0.010 & 4.426 & 1.41 & 0.064 & 0.019506 & 0.007803 & 0.275587 & 123.683 \\
\hline 12/31/08 13:00 & 1.643 & 0.183 & 0.009 & 3.994 & 1.413 & 0.067 & 0.020421 & 0.008330 & 0.294224 & 132.048 \\
\hline 12/31/08 14:00 & 1.64 & 0.180 & 0.009 & 3.839 & 1.413 & 0.067 & 0.020421 & 0.008330 & 0.294224 & 132.048 \\
\hline 12/31/08 15:00 & 1.649 & 0.189 & 0.010 & 4.316 & 1.413 & 0.067 & 0.020421 & 0.008330 & 0.294224 & 132.048 \\
\hline
\end{tabular}




\begin{tabular}{|c|c|c|c|c|c|c|c|c|c|c|}
\hline \multirow[b]{3}{*}{ Date } & \multicolumn{4}{|c|}{ V-Notch Weir } & \multicolumn{6}{|c|}{ R-Notch Weir } \\
\hline & \multicolumn{2}{|c|}{ Measured Water Level } & \multicolumn{2}{|c|}{$\begin{array}{c}\text { Calculated Rate } \\
\left(\mathrm{ft}^{3} / \mathrm{s}=448.8 \mathrm{gpm}\right)\end{array}$} & \multicolumn{3}{|c|}{ Measured Water Level } & \multicolumn{3}{|c|}{$\begin{array}{c}\text { Calculated Rate } \\
\left(\mathrm{ft}^{3} / \mathrm{s}=448.8 \mathrm{gpm}\right)\end{array}$} \\
\hline & $\begin{array}{l}\text { (ft above } \\
\text { sensor) }\end{array}$ & $\begin{array}{l}\text { (ft above } \\
\text { notch) }\end{array}$ & $\left(\mathrm{ft}^{3} / \mathrm{s}\right)$ & (gpm) & $\begin{array}{l}\text { (ft above } \\
\text { sensor) }\end{array}$ & $\begin{array}{l}\text { (ft above } \\
\text { log) }\end{array}$ & $\begin{array}{c}\text { (m above } \\
\text { log) }\end{array}$ & $\left(\mathrm{m}^{3} / \mathrm{s}\right)$ & $\left(\mathrm{ft}^{3} / \mathrm{s}\right)$ & (gpm) \\
\hline 12/31/08 16:00 & 1.652 & 0.192 & 0.010 & 4.482 & 1.418 & 0.072 & 0.021945 & 0.009235 & 0.326176 & 146.388 \\
\hline 12/31/08 17:00 & 1.652 & 0.192 & 0.010 & 4.482 & 1.415 & 0.069 & 0.021030 & 0.008688 & 0.306873 & 137.724 \\
\hline 12/31/08 18:00 & 1.652 & 0.192 & 0.010 & 4.482 & 1.415 & 0.069 & 0.021030 & 0.008688 & 0.306873 & 137.724 \\
\hline 12/31/08 19:00 & 1.651 & 0.191 & 0.010 & 4.426 & 1.417 & 0.071 & 0.021640 & 0.009052 & 0.319698 & 143.480 \\
\hline 12/31/08 20:00 & 1.651 & 0.191 & 0.010 & 4.426 & 1.417 & 0.071 & 0.021640 & 0.009052 & 0.319698 & 143.480 \\
\hline 12/31/08 21:00 & 1.654 & 0.194 & 0.010 & 4.595 & 1.417 & 0.071 & 0.021640 & 0.009052 & 0.319698 & 143.480 \\
\hline 12/31/08 22:00 & 1.652 & 0.192 & 0.010 & 4.482 & 1.42 & 0.074 & 0.022554 & 0.009605 & 0.339261 & 152.260 \\
\hline 12/31/08 23:00 & 1.647 & 0.187 & 0.009 & 4.207 & 1.417 & 0.071 & 0.021640 & 0.009052 & 0.319698 & 143.480 \\
\hline
\end{tabular}




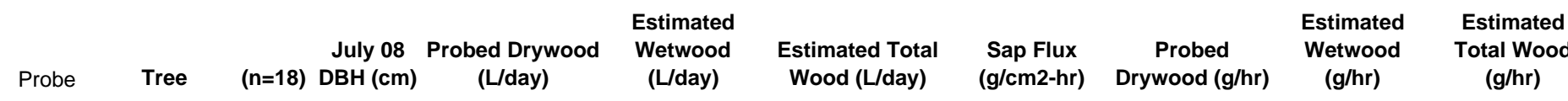
Murdock, NE Mean Daily Sapflow Summer 2008 (JD 204-239)

$\begin{array}{llllcccccc}1 & \text { W506EC } & 10.9 & 19.2 & 7.1 & 26.3 & 13.13 & 1577.7 & 295.3 & 1096.5 \\ 2 & \text { W610WN1 } & 11.9 & 37.8 & 13.9 & 51.7 & 24.67 & 1577.7 & 581.3 & 2159.1 \\ 3 & \text { W609EC2 } & 6.4 & 12.0 & 4.4 & 16.5 & 24.28 & 502.9 & 185.3 & 688.2 \\ 4 & \text { W608EC2 } & 10.2 & 28.3 & 10.4 & 38.8 & 22.31 & 1182.7 & 435.8 & 1618.6 \\ 5 & \text { W608EC2 } & 10.2 & 21.3 & 7.9 & 29.2 & 16.81 & 891.5 & 328.5 & 1220.0 \\ 6 & \text { W709EC } & 11.4 & 15.4 & 5.7 & 21.0 & 9.56 & 641.7 & 236.4 & 878.1 \\ 7 & \text { W607EC1 } & 10.7 & 14.2 & 5.3 & 19.5 & 10.14 & 595.1 & 219.3 & 814.4 \\ 8 & \text { W505EC1 } & 9.1 & 24.3 & 9.0 & 33.3 & 24.43 & 1015.5 & 374.2 & 1389.7 \\ 9 & \text { W505EC1 } & 9.1 & 21.0 & 7.7 & 28.7 & 21.10 & 877.1 & 323.2 & 1200.3 \\ 10 & \text { W504WN1 } & 10.7 & 33.5 & 12.3 & 45.8 & 22.92 & 1398.8 & 515.4 & 1914.2 \\ 11 & \text { W504WN2 } & 10.9 & 28.8 & 10.6 & 39.4 & 18.98 & 1203.7 & 443.5 & 1647.2 \\ 12 & \text { W605WN1 } & 13.7 & 60.1 & 22.1 & 82.2 & 24.67 & 2510.1 & 924.9 & 3435.0 \\ 13 & \text { W605WN1 } & 13.7 & 75.0 & 27.6 & 102.7 & 30.79 & 3132.9 & 1154.4 & 4287.3 \\ 14 & \text { W606EC1 } & 9.7 & 13.1 & 4.8 & 17.9 & 11.48 & 546.7 & 201.5 & 748.2 \\ 15 & \text { W706EC2 } & 9.4 & 13.1 & 4.8 & 17.9 & 12.29 & 547.7 & 201.8 & 749.5 \\ 16 & \text { W707EC2 } & 11.2 & 18.1 & 6.7 & 24.8 & 11.73 & 757.9 & 279.3 & 1037.2 \\ 17 & \text { W810EC1 } & 10.0 & 27.5 & 10.1 & 37.6 & 18.81 & 1147.7 & 422.9 & 1570.6 \\ 18 & \text { W809EC1 } & 10.9 & 18.0 & 6.6 & 24.6 & 10.59 & 750.5 & 276.5 & 1027.0 \\ 19 & \text { W708EC1 } & 7.1 & 6.4 & 2.3 & 8.7 & 10.29 & 265.7 & 97.9 & 363.5 \\ 20 & \text { W707GA } & 2.5 & 0.4 & 0.2 & 0.7 & 6.53 & 17.4 & 9.8 & 27.2 \\ 21 & \text { W708EC2 } & 11.2 & 45.9 & 16.9 & 62.9 & 29.69 & 2547.0 & 938.5 & 3485.5\end{array}$

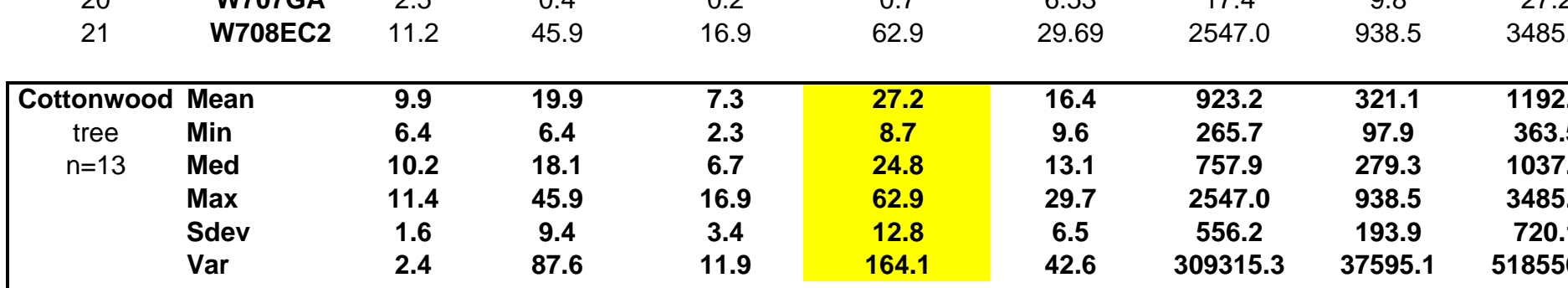

$\mathrm{n}=810 \quad$ Dly Cuml Stand Use

\begin{tabular}{|c|c|c|c|c|}
\hline \multicolumn{5}{|c|}{ Dly Cuml Stand Use } \\
\hline \multirow[t]{2}{*}{ Mean } & $\mathbf{L}$ & 16087.6 & 5927.8 & 22015.3 \\
\hline & $\mathbf{m m}$ & 2.9 & 1.1 & 4.0 \\
\hline \multirow[t]{2}{*}{ Min } & $\mathbf{L}$ & 5152.0 & 1898.4 & 7050.4 \\
\hline & $\mathbf{m m}$ & 0.9 & 0.3 & 1.3 \\
\hline \multirow[t]{2}{*}{ Max } & $\mathbf{L}$ & 37213.4 & 13712.1 & 50925.5 \\
\hline & $\mathbf{m m}$ & 6.8 & 2.5 & 9.3 \\
\hline
\end{tabular}

Ann Cuml Stand Use, 180d

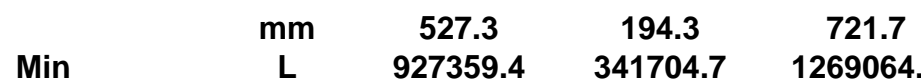

$\begin{array}{ccccc} & \mathrm{mm} & 168.9 & 62.2 & 231.1\end{array}$

$\begin{array}{llll}\text { L } & 6698420.3 & 2468170.7 & 9166591.1\end{array}$

$\begin{array}{lllll}\mathrm{mm} & 1219.8 & 449.5 & 1669.3 & \text { Poplar }\end{array}$

\begin{tabular}{|c|c|c|c|c|c|c|c|c|c|}
\hline Willow & Mean & 11.8 & 47.0 & 17.3 & 64.4 & 24.4 & 1964.6 & 723.9 & 2688.5 \\
\hline tree & Min & 10.7 & 28.8 & 10.6 & 39.4 & 19.0 & 1203.7 & 443.5 & 1647.2 \\
\hline \multirow[t]{4}{*}{$n=4$} & Med & 11.4 & 37.8 & 13.9 & 51.7 & 24.7 & 1577.7 & 581.3 & 2159.1 \\
\hline & $\operatorname{Max}$ & 13.7 & 75.0 & 27.6 & 102.7 & 30.8 & 3132.9 & 1154.4 & 4287.3 \\
\hline & Sdev & 1.4 & 19.7 & 7.3 & 27.0 & 4.3 & 823.3 & 303.4 & 1126.7 \\
\hline & Var & 1.9 & 388.6 & 52.8 & 727.7 & 18.1 & 677861.3 & 92033.6 & 1269438.8 \\
\hline \multirow[t]{14}{*}{$n=1015$} & \multicolumn{9}{|c|}{ Dly Cuml Stand Use } \\
\hline & \multirow[t]{2}{*}{ Mean } & $\mathbf{L}$ & 47744.6 & 17592.5 & 65337.1 & & & & \\
\hline & & $\mathbf{m m}$ & 8.7 & 3.2 & 11.9 & & & & \\
\hline & \multirow[t]{2}{*}{ Min } & $\mathbf{L}$ & 29251.4 & 10778.3 & 40029.7 & & & & \\
\hline & & $\mathbf{m m}$ & 5.3 & 2.0 & 7.3 & & & & \\
\hline & \multirow[t]{2}{*}{$\operatorname{Max}$} & $\mathbf{L}$ & 76136.6 & 28054.1 & 104190.7 & & & & \\
\hline & & $\mathrm{mm}$ & 13.9 & 5.1 & 19.0 & & & & \\
\hline & \multicolumn{9}{|c|}{ Ann Cuml Stand Use, 180d } \\
\hline & \multirow[t]{2}{*}{ Mean } & L & 8594031.9 & 3166647.8 & 11760679.8 & & & & \\
\hline & & $\mathbf{m m}$ & 1565.0 & 576.7 & 2141.7 & & & & \\
\hline & \multirow[t]{2}{*}{ Min } & $\mathbf{L}$ & 5265248.5 & 1940089.1 & 7205337.6 & & & & \\
\hline & & $\mathbf{m m}$ & 958.8 & 353.3 & 1312.2 & & & & \\
\hline & \multirow[t]{2}{*}{$\operatorname{Max}$} & $\mathbf{L}$ & 13704593.6 & 5049739.4 & 18754333.0 & & & & \\
\hline & & $\mathrm{mm}$ & 2495.7 & 919.6 & 3415.3 & & & & Willow \\
\hline All & Mean & 9.9 & 25.4 & 9.4 & 34.8 & 17.87 & 1128.0 & 402.2 & 1493.2 \\
\hline tree & Min & 2.5 & 0.4 & 0.2 & 0.7 & 6.53 & 17.4 & 9.8 & 27.2 \\
\hline \multirow[t]{4}{*}{$\mathrm{n}=18$} & Med & 10.7 & 21.0 & 7.7 & 28.7 & 18.81 & 891.5 & 323.2 & 1200.3 \\
\hline & $\operatorname{Max}$ & 13.7 & 75.0 & 27.6 & 102.7 & 30.79 & 3132.9 & 1154.4 & 4287.3 \\
\hline & Sdev & 2.5 & 17.6 & 6.5 & 24.1 & 7.22 & 787.5 & 288.5 & 1072.1 \\
\hline & Var & 6.1 & 310.3 & 42.1 & 580.9 & 52.10 & 620224.1 & 83231.9 & 1149374.2 \\
\hline \multirow[t]{14}{*}{$n=1825$} & \multicolumn{5}{|c|}{ Dly Cuml Stand Use } & & & & \\
\hline & \multirow[t]{2}{*}{ Mean } & $\mathbf{L}$ & 46366.2 & 17091.6 & 63457.8 & & & & \\
\hline & & $\mathbf{m m}$ & 8.4 & 3.1 & 11.6 & & & & \\
\hline & \multirow[t]{2}{*}{ Min } & $\mathbf{L}$ & 760.0 & 427.5 & 1187.4 & & & & \\
\hline & & $\mathbf{m m}$ & 0.1 & 0.1 & 0.2 & & & & \\
\hline & \multirow[t]{2}{*}{$\operatorname{Max}$} & $\mathbf{L}$ & 136895.9 & 50442.1 & 187338.0 & & & & \\
\hline & & $\mathrm{mm}$ & 24.9 & 9.2 & 34.1 & & & & \\
\hline & \multicolumn{9}{|c|}{ Ann Cuml Stand Use, 180d } \\
\hline & \multirow[t]{2}{*}{ Mean } & $\mathbf{L}$ & 8345921.5 & 3076490.3 & 11422411.8 & & & & \\
\hline & & $\mathbf{m m}$ & 1519.9 & 560.3 & 2080.1 & & & & \\
\hline & \multirow[t]{2}{*}{ Min } & $\mathbf{L}$ & 136791.5 & 76945.2 & 213736.7 & & & & \\
\hline & & $\mathbf{m m}$ & 24.9 & 14.0 & 38.9 & & & & \\
\hline & \multirow[t]{2}{*}{$\operatorname{Max}$} & $\mathbf{L}$ & 24641264.4 & 9079580.7 & 33720845.0 & & & & \\
\hline & & $\mathbf{m m}$ & 4487.38 & 1653.47 & 6140.85 & & & & \\
\hline
\end{tabular}




\begin{tabular}{|c|c|c|c|c|c|c|c|c|c|c|c|c|c|}
\hline Tree ID & TS & RN & JD & HR & $\begin{array}{c}\text { Raw } \\
\text { TC_dTA }\end{array}$ & $\begin{array}{c}\text { Fixed } \\
\text { TC_dT } \Delta\end{array}$ & dTM & $\mathbf{K}$ & $\begin{array}{l}\text { Sap } \\
\text { Velocity } \\
(\mathrm{cm} / \mathrm{s})\end{array}$ & $\begin{array}{c}\text { Probed } \\
\text { Drywood F } \\
(\mathrm{g} / \mathrm{hr})\end{array}$ & $\begin{array}{c}\text { Extrapolated } \\
\text { Wetwood F } \\
(\mathrm{g} / \mathrm{hr})\end{array}$ & $\begin{array}{c}\text { Extrapolated } \\
\text { Total Wood F } \\
(\mathrm{g} / \mathrm{hr})\end{array}$ & $\begin{array}{l}\text { Relative } \\
\text { Humidity, } \\
\text { @10m (\%) }\end{array}$ \\
\hline & $7 / 22 / 2008$ 13:00 & 0 & 204 & 1300 & 5.238 & 5.238 & 9.74 & 0.8595 & $9.88 \mathrm{E}-03$ & 2164.8498 & 797.6834 & 2962.5332 & \\
\hline \multirow[t]{2}{*}{ W506EC } & $7 / 22 / 200814: 00$ & 1 & 204 & 1400 & 5.821 & 5.821 & & 0.6733 & 7.31E-03 & 1602.7472 & 590.5652 & 2193.3124 & \\
\hline & $7 / 22 / 200815: 00$ & 2 & 204 & 1500 & 5.775 & 5.775 & & 0.6866 & 7.49E-03 & 1641.8943 & 604.9897 & 2246.8841 & \\
\hline \multirow[t]{2}{*}{ Probe \# } & $7 / 22 / 200816: 00$ & 3 & 204 & 1600 & 5.794 & 5.794 & & 0.6810 & 7.42E-03 & 1625.6280 & 598.9961 & 2224.6241 & \\
\hline & $7 / 22 / 2008$ 17:00 & 4 & 204 & 1700 & 5.868 & 5.868 & & 0.6599 & 7.13E-03 & 1563.5632 & 576.1270 & 2139.6902 & \\
\hline \multirow[t]{4}{*}{1} & $7 / 22 / 2008$ 18:00 & 5 & 204 & 1800 & 5.913 & 5.913 & & 0.6472 & $6.97 \mathrm{E}-03$ & 1526.7981 & 562.5801 & 2089.3782 & \\
\hline & $7 / 22 / 2008$ 19:00 & 6 & 204 & 1900 & 6.106 & 6.106 & & 0.5952 & $6.28 \mathrm{E}-03$ & 1377.0368 & 507.3975 & 1884.4344 & \\
\hline & $7 / 22 / 200820: 00$ & 7 & 204 & 2000 & 6.664 & 6.664 & & 0.4616 & 4.59E-03 & 1007.0980 & 371.0860 & 1378.1840 & \\
\hline & $7 / 22 / 2008$ 21:00 & 8 & 204 & 2100 & 8.19 & 8.19 & & 0.1893 & 1.53E-03 & 336.0657 & 123.8303 & 459.8960 & \\
\hline \multirow[t]{2}{*}{ DBH (cm) } & $7 / 22 / 200822: 00$ & 9 & 204 & 2200 & 9.36 & 9.36 & & 0.0406 & $2.30 \mathrm{E}-04$ & 50.5186 & 18.6146 & 69.1332 & \\
\hline & $7 / 22 / 200823: 00$ & 10 & 204 & 2300 & 9.74 & 9.74 & & 0.0000 & $0.00 \mathrm{E}+00$ & 0.0000 & 0.0000 & 0.0000 & \\
\hline \multirow[t]{2}{*}{10.9} & $7 / 23 / 20080: 00$ & 11 & 205 & 0 & 10.01 & 10.01 & 10.09 & -0.0270 & $0.00 \mathrm{E}+00$ & 0.0000 & 0.0000 & 0.0000 & \\
\hline & 7/23/2008 1:00 & 12 & 205 & 100 & 10.06 & 10.06 & & -0.0318 & $0.00 \mathrm{E}+00$ & 0.0000 & 0.0000 & 0.0000 & \\
\hline Day Count & 7/23/2008 2:00 & 13 & 205 & 200 & NAN & 10.075 & & -0.0333 & $0.00 \mathrm{E}+00$ & 0.0000 & 0.0000 & 0.0000 & \\
\hline \multirow[t]{27}{*}{35} & 7/23/2008 3:00 & 14 & 205 & 300 & 10.09 & 10.09 & & -0.0347 & $0.00 \mathrm{E}+00$ & 0.0000 & 0.0000 & 0.0000 & \\
\hline & 7/23/2008 4:00 & 15 & 205 & 400 & 10.05 & 10.05 & & -0.0308 & $0.00 \mathrm{E}+00$ & 0.0000 & 0.0000 & 0.0000 & \\
\hline & $7 / 23 / 20085: 00$ & 16 & 205 & 500 & 9.98 & 9.98 & & -0.0240 & $0.00 \mathrm{E}+00$ & 0.0000 & 0.0000 & 0.0000 & \\
\hline & 7/23/2008 6:00 & 17 & 205 & 600 & 9.84 & 9.84 & & -0.0102 & $0.00 \mathrm{E}+00$ & 0.0000 & 0.0000 & 0.0000 & \\
\hline & 7/23/2008 7:00 & 18 & 205 & 700 & 9.45 & 9.45 & & 0.0307 & 1.63E-04 & 35.7959 & 13.1897 & 48.9857 & \\
\hline & 7/23/2008 8:00 & 19 & 205 & 800 & 8.34 & 8.34 & & 0.1679 & 1.32E-03 & 289.9389 & 106.8340 & 396.7729 & \\
\hline & 7/23/2008 9:00 & 20 & 205 & 900 & 6.96 & 6.96 & & 0.3994 & 3.85E-03 & 842.8407 & 310.5620 & 1153.4027 & \\
\hline & $7 / 23 / 200810: 00$ & 21 & 205 & 1000 & 6.002 & 6.002 & & 0.6228 & $6.64 \mathrm{E}-03$ & 1456.1796 & 536.5593 & 1992.7389 & \\
\hline & $7 / 23 / 2008$ 11:00 & 22 & 205 & 1100 & 6.019 & 6.019 & & 0.6182 & $6.58 \mathrm{E}-03$ & 1442.9986 & 531.7025 & 1974.7012 & \\
\hline & $7 / 23 / 2008$ 12:00 & 23 & 205 & 1200 & 6.002 & 6.002 & & 0.6228 & $6.64 \mathrm{E}-03$ & 1456.1796 & 536.5593 & 1992.7389 & \\
\hline & $7 / 23 / 2008$ 13:00 & 24 & 205 & 1300 & 5.99 & 5.99 & & 0.6260 & 6.69E-03 & 1465.5425 & 540.0093 & 2005.5517 & \\
\hline & $7 / 23 / 200814: 00$ & 25 & 205 & 1400 & NAN & 5.9625 & & 0.6335 & $6.78 \mathrm{E}-03$ & 1487.1839 & 547.9835 & 2035.1675 & \\
\hline & $7 / 23 / 2008$ 15:00 & 26 & 205 & 1500 & 5.935 & 5.935 & & 0.6411 & $6.88 \mathrm{E}-03$ & 1509.0861 & 556.0538 & 2065.1399 & \\
\hline & $7 / 23 / 2008$ 16:00 & 27 & 205 & 1600 & 6.092 & 6.092 & & 0.5988 & $6.33 \mathrm{E}-03$ & 1387.4853 & 511.2475 & 1898.7328 & \\
\hline & $7 / 23 / 2008$ 17:00 & 28 & 205 & 1700 & 6.475 & 6.475 & & 0.5042 & $5.12 \mathrm{E}-03$ & 1122.8775 & 413.7473 & 1536.6248 & \\
\hline & $7 / 23 / 2008$ 18:00 & 29 & 205 & 1800 & 7.193 & 7.193 & & 0.3541 & 3.32E-03 & 726.6808 & 267.7605 & 994.4413 & \\
\hline & $7 / 23 / 2008$ 19:00 & 30 & 205 & 1900 & 7.094 & 7.094 & & 0.3730 & 3.53E-03 & 774.7104 & 285.4580 & 1060.1684 & \\
\hline & $7 / 23 / 200820: 00$ & 31 & 205 & 2000 & 7.614 & 7.614 & & 0.2792 & $2.47 \mathrm{E}-03$ & 542.4294 & 199.8693 & 742.2987 & \\
\hline & $7 / 23 / 2008$ 21:00 & 32 & 205 & 2100 & 8.56 & 8.56 & & 0.1379 & 1.04E-03 & 227.5044 & 83.8287 & 311.3331 & \\
\hline & $7 / 23 / 200822: 00$ & 33 & 205 & 2200 & 9.1 & 9.1 & & 0.0703 & 4.53E-04 & 99.3587 & 36.6108 & 135.9695 & \\
\hline & $7 / 23 / 200823: 00$ & 34 & 205 & 2300 & 9.21 & 9.21 & & 0.0575 & 3.54E-04 & 77.6173 & 28.5997 & 106.2169 & \\
\hline & $7 / 24 / 20080: 00$ & 35 & 206 & 0 & 9.24 & 9.24 & 10.04 & 0.0541 & $3.28 E-04$ & 71.9562 & 26.5137 & 98.4699 & \\
\hline & 7/24/2008 1:00 & 36 & 206 & 100 & 9.37 & 9.37 & & 0.0395 & 2.23E-04 & 48.8229 & 17.9898 & 66.8126 & \\
\hline & $7 / 24 / 2008$ 2:00 & 37 & 206 & 200 & 9.47 & 9.47 & & 0.0285 & 1.49E-04 & 32.6964 & 12.0477 & 44.7441 & \\
\hline & $7 / 24 / 20083: 00$ & 38 & 206 & 300 & 9.62 & 9.62 & & 0.0125 & $5.39 \mathrm{E}-05$ & 11.8185 & 4.3548 & 16.1733 & \\
\hline & $7 / 24 / 20084: 00$ & 39 & 206 & 400 & 9.7 & 9.7 & & 0.0041 & 1.38E-05 & 3.0255 & 1.1148 & 4.1403 & \\
\hline & $7 / 24 / 20085: 00$ & 40 & 206 & 500 & 9.75 & 9.75 & & -0.0010 & $0.00 \mathrm{E}+00$ & 0.0000 & 0.0000 & 0.0000 & \\
\hline
\end{tabular}




\begin{tabular}{|c|c|c|c|c|c|c|c|c|c|c|c|c|c|}
\hline Tree ID & TS & RN & JD & HR & $\begin{array}{c}\text { Raw } \\
\text { TC_dTA }\end{array}$ & $\begin{array}{c}\text { Fixed } \\
\text { TC_dT } \Delta\end{array}$ & dTM & $\mathbf{K}$ & $\begin{array}{l}\text { Sap } \\
\text { Velocity } \\
\text { (cm/s) }\end{array}$ & $\begin{array}{l}\text { Probed } \\
\text { Drywood F } \\
\text { (g/hr) }\end{array}$ & $\begin{array}{c}\text { Extrapolated } \\
\text { Wetwood F } \\
(\mathrm{g} / \mathrm{hr})\end{array}$ & $\begin{array}{c}\text { Extrapolated } \\
\text { Total Wood F } \\
(\mathrm{g} / \mathrm{hr})\end{array}$ & $\begin{array}{l}\text { Relative } \\
\text { Humidity, } \\
\text { @10m (\%) }\end{array}$ \\
\hline & $7 / 24 / 20086: 00$ & 41 & 206 & 600 & NAN & 9.76 & & -0.0020 & $0.00 \mathrm{E}+00$ & 0.0000 & 0.0000 & 0.0000 & \\
\hline & 7/24/2008 7:00 & 42 & 206 & 700 & 9.77 & 9.77 & & -0.0031 & $0.00 \mathrm{E}+00$ & 0.0000 & 0.0000 & 0.0000 & \\
\hline & 7/24/2008 8:00 & 43 & 206 & 800 & 9.47 & 9.47 & & 0.0285 & $1.49 \mathrm{E}-04$ & 32.6964 & 12.0477 & 44.7441 & \\
\hline & 7/24/2008 9:00 & 44 & 206 & 900 & 8.96 & 8.96 & & 0.0871 & $5.89 \mathrm{E}-04$ & 129.1980 & 47.6056 & 176.8036 & \\
\hline & $7 / 24 / 200810: 00$ & 45 & 206 & 1000 & 8.93 & 8.93 & & 0.0907 & $6.20 \mathrm{E}-04$ & 135.9018 & 50.0758 & 185.9776 & \\
\hline & $7 / 24 / 200811: 00$ & 46 & 206 & 1100 & NAN & 7.495 & & 0.2995 & $2.70 \mathrm{E}-03$ & 591.4006 & 217.9137 & 809.3143 & \\
\hline & $7 / 24 / 200812: 00$ & 47 & 206 & 1200 & 6.06 & 6.06 & & 0.6073 & 6.44E-03 & 1411.6050 & 520.1349 & 1931.7399 & \\
\hline & $7 / 24 / 200813: 00$ & 48 & 206 & 1300 & 6.619 & 6.619 & & 0.4715 & $4.72 \mathrm{E}-03$ & 1033.8524 & 380.9442 & 1414.7966 & \\
\hline & 7/24/2008 14:00 & 49 & 206 & 1400 & 6.44 & 6.44 & & 0.5124 & $5.23 \mathrm{E}-03$ & 1145.3296 & 422.0203 & 1567.3499 & \\
\hline & $7 / 24 / 200815: 00$ & 50 & 206 & 1500 & 6.101 & 6.101 & & 0.5965 & $6.30 \mathrm{E}-03$ & 1380.7612 & 508.7699 & 1889.5311 & \\
\hline & $7 / 24 / 200816: 00$ & 51 & 206 & 1600 & 6.063 & 6.063 & & 0.6065 & $6.43 \mathrm{E}-03$ & 1409.3297 & 519.2965 & 1928.6262 & \\
\hline & $7 / 24 / 200817: 00$ & 52 & 206 & 1700 & 6.238 & 6.238 & & 0.5614 & $5.85 \mathrm{E}-03$ & 1281.5355 & 472.2081 & 1753.7437 & \\
\hline & $7 / 24 / 200818: 00$ & 53 & 206 & 1800 & 6.18 & 6.18 & & 0.5761 & 6.03E-03 & 1322.8374 & 487.4266 & 1810.2641 & \\
\hline & 7/24/2008 19:00 & 54 & 206 & 1900 & 6.485 & 6.485 & & 0.5019 & $5.09 \mathrm{E}-03$ & 1116.5223 & 411.4056 & 1527.9280 & \\
\hline & $7 / 24 / 200820: 00$ & 55 & 206 & 2000 & 7.469 & 7.469 & & 0.3041 & $2.75 \mathrm{E}-03$ & 602.4147 & 221.9721 & 824.3867 & \\
\hline & 7/24/2008 21:00 & 56 & 206 & 2100 & 8.97 & 8.97 & & 0.0858 & 5.79E-04 & 126.9875 & 46.7912 & 173.7786 & \\
\hline & 7/24/2008 22:00 & 57 & 206 & 2200 & 9.86 & 9.86 & & -0.0122 & $0.00 \mathrm{E}+00$ & 0.0000 & 0.0000 & 0.0000 & \\
\hline & 7/24/2008 23:00 & 58 & 206 & 2300 & 10.04 & 10.04 & & -0.0299 & $0.00 \mathrm{E}+00$ & 0.0000 & 0.0000 & 0.0000 & \\
\hline & 7/25/2008 0:00 & 59 & 207 & 0 & 10.05 & 10.05 & 10.05 & -0.0308 & $0.00 \mathrm{E}+00$ & 0.0000 & 0.0000 & 0.0000 & \\
\hline & 7/25/2008 1:00 & 60 & 207 & 100 & 9.98 & 9.98 & & -0.0240 & $0.00 \mathrm{E}+00$ & 0.0000 & 0.0000 & 0.0000 & \\
\hline & $7 / 25 / 20082: 00$ & 61 & 207 & 200 & 9.87 & 9.87 & & -0.0132 & $0.00 \mathrm{E}+00$ & 0.0000 & 0.0000 & 0.0000 & \\
\hline & 7/25/2008 3:00 & 62 & 207 & 300 & 9.83 & 9.83 & & -0.0092 & $0.00 \mathrm{E}+00$ & 0.0000 & 0.0000 & 0.0000 & \\
\hline & 7/25/2008 4:00 & 63 & 207 & 400 & 9.87 & 9.87 & & -0.0132 & $0.00 \mathrm{E}+00$ & 0.0000 & 0.0000 & 0.0000 & \\
\hline & 7/25/2008 5:00 & 64 & 207 & 500 & 9.87 & 9.87 & & -0.0132 & $0.00 \mathrm{E}+00$ & 0.0000 & 0.0000 & 0.0000 & \\
\hline & 7/25/2008 6:00 & 65 & 207 & 600 & 9.89 & 9.89 & & -0.0152 & $0.00 \mathrm{E}+00$ & 0.0000 & 0.0000 & 0.0000 & \\
\hline & 7/25/2008 7:00 & 66 & 207 & 700 & 9.86 & 9.86 & & -0.0122 & $0.00 \mathrm{E}+00$ & 0.0000 & 0.0000 & 0.0000 & \\
\hline & 7/25/2008 8:00 & 67 & 207 & 800 & 9.83 & 9.83 & & -0.0092 & $0.00 \mathrm{E}+00$ & 0.0000 & 0.0000 & 0.0000 & \\
\hline & 7/25/2008 9:00 & 68 & 207 & 900 & 9.77 & 9.77 & & -0.0031 & $0.00 \mathrm{E}+00$ & 0.0000 & 0.0000 & 0.0000 & \\
\hline & $7 / 25 / 200810: 00$ & 69 & 207 & 1000 & NAN & 8.995 & & 0.0828 & $5.54 \mathrm{E}-04$ & 121.5143 & 44.7744 & 166.2887 & \\
\hline & 7/25/2008 11:00 & 70 & 207 & 1100 & 8.22 & 8.22 & & 0.1849 & $1.49 \mathrm{E}-03$ & 326.6033 & 120.3437 & 446.9470 & \\
\hline & $7 / 25 / 200812: 00$ & 71 & 207 & 1200 & 6.85 & 6.85 & & 0.4219 & 4.11E-03 & 901.5887 & 332.2089 & 1233.7977 & \\
\hline & $7 / 25 / 2008$ 13:00 & 72 & 207 & 1300 & 6.291 & 6.291 & & 0.5482 & 5.68E-03 & 1244.6714 & 458.6248 & 1703.2962 & \\
\hline & $7 / 25 / 200814: 00$ & 73 & 207 & 1400 & 5.944 & 5.944 & & 0.6386 & $6.85 \mathrm{E}-03$ & 1501.8892 & 553.4020 & 2055.2911 & \\
\hline & 7/25/2008 15:00 & 74 & 207 & 1500 & 6.007 & 6.007 & & 0.6214 & 6.63E-03 & 1452.2928 & 535.1271 & 1987.4199 & \\
\hline & 7/25/2008 16:00 & 75 & 207 & 1600 & NAN & 6.007 & & 0.6214 & $6.63 \mathrm{E}-03$ & 1452.2928 & 535.1271 & 1987.4199 & \\
\hline & $7 / 25 / 200817: 00$ & 76 & 207 & 1700 & NAN & 6.125 & & 0.5902 & $6.22 \mathrm{E}-03$ & 1362.9567 & 502.2094 & 1865.1661 & \\
\hline & $7 / 25 / 200818: 00$ & 77 & 207 & 1800 & 6.125 & 6.125 & & 0.5902 & $6.22 \mathrm{E}-03$ & 1362.9567 & 502.2094 & 1865.1661 & \\
\hline & 7/25/2008 19:00 & 78 & 207 & 1900 & 6.238 & 6.238 & & 0.5614 & 5.85E-03 & 1281.5355 & 472.2081 & 1753.7437 & \\
\hline & $7 / 25 / 200820: 00$ & 79 & 207 & 2000 & 7.079 & 7.079 & & 0.3759 & 3.57E-03 & 782.1556 & 288.2013 & 1070.3569 & \\
\hline & $7 / 25 / 200821: 00$ & 80 & 207 & 2100 & 8.68 & 8.68 & & 0.1221 & 8.94E-04 & 195.9801 & 72.2129 & 268.1931 & \\
\hline & $7 / 25 / 200822: 00$ & 81 & 207 & 2200 & 9.82 & 9.82 & & -0.0081 & $0.00 \mathrm{E}+00$ & 0.0000 & 0.0000 & 0.0000 & \\
\hline
\end{tabular}




\begin{tabular}{|c|c|c|c|c|c|c|c|c|c|c|c|c|c|}
\hline Tree ID & TS & RN & JD & HR & $\begin{array}{c}\text { Raw } \\
\text { TC_dTA }\end{array}$ & $\begin{array}{c}\text { Fixed } \\
\text { TC_dT } \Delta\end{array}$ & dTM & $\mathbf{K}$ & $\begin{array}{l}\text { Sap } \\
\text { Velocity } \\
(\mathrm{cm} / \mathrm{s})\end{array}$ & $\begin{array}{c}\text { Probed } \\
\text { Drywood F } \\
(\mathrm{g} / \mathrm{hr})\end{array}$ & $\begin{array}{c}\text { Extrapolated } \\
\text { Wetwood F } \\
(\mathrm{g} / \mathrm{hr})\end{array}$ & $\begin{array}{c}\text { Extrapolated } \\
\text { Total Wood F } \\
(\mathrm{g} / \mathrm{hr})\end{array}$ & $\begin{array}{l}\text { Relative } \\
\text { Humidity, } \\
@ 10 \mathrm{~m}(\%)\end{array}$ \\
\hline & 7/25/2008 23:00 & 82 & 207 & 2300 & 9.97 & 9.97 & & -0.0231 & $0.00 \mathrm{E}+00$ & 0.0000 & 0.0000 & 0.0000 & \\
\hline & $7 / 26 / 20080: 00$ & 83 & 208 & 0 & 9.96 & 9.96 & 9.96 & -0.0221 & $0.00 \mathrm{E}+00$ & 0.0000 & 0.0000 & 0.0000 & \\
\hline & 7/26/2008 1:00 & 84 & 208 & 100 & 9.93 & 9.93 & & -0.0191 & $0.00 E+00$ & 0.0000 & 0.0000 & 0.0000 & \\
\hline & $7 / 26 / 20082: 00$ & 85 & 208 & 200 & 9.88 & 9.88 & & -0.0142 & $0.00 \mathrm{E}+00$ & 0.0000 & 0.0000 & 0.0000 & \\
\hline & $7 / 26 / 20083: 00$ & 86 & 208 & 300 & 9.85 & 9.85 & & -0.0112 & $0.00 \mathrm{E}+00$ & 0.0000 & 0.0000 & 0.0000 & \\
\hline & 7/26/2008 4:00 & 87 & 208 & 400 & 9.81 & 9.81 & & -0.0071 & $0.00 \mathrm{E}+00$ & 0.0000 & 0.0000 & 0.0000 & \\
\hline & 7/26/2008 5:00 & 88 & 208 & 500 & 9.76 & 9.76 & & -0.0020 & $0.00 \mathrm{E}+00$ & 0.0000 & 0.0000 & 0.0000 & \\
\hline & 7/26/2008 6:00 & 89 & 208 & 600 & 9.71 & 9.71 & & 0.0031 & 9.67E-06 & 2.1205 & 0.7814 & 2.9019 & \\
\hline & 7/26/2008 7:00 & 90 & 208 & 700 & 9.65 & 9.65 & & 0.0093 & 3.77E-05 & 8.2622 & 3.0444 & 11.3066 & \\
\hline & 7/26/2008 8:00 & 91 & 208 & 800 & 9.6 & 9.6 & & 0.0146 & 6.54E-05 & 14.3247 & 5.2782 & 19.6030 & \\
\hline & 7/26/2008 9:00 & 92 & 208 & 900 & 7.936 & 7.936 & & 0.2273 & 1.92E-03 & 421.1103 & 155.1668 & 576.2770 & \\
\hline & $7 / 26 / 2008$ 10:00 & 93 & 208 & 1000 & 6.175 & 6.175 & & 0.5773 & $6.05 \mathrm{E}-03$ & 1326.4458 & 488.7562 & 1815.2021 & \\
\hline & $7 / 26 / 2008$ 11:00 & 94 & 208 & 1100 & 6.327 & 6.327 & & 0.5394 & $5.57 \mathrm{E}-03$ & 1220.0976 & 449.5700 & 1669.6676 & \\
\hline & $7 / 26 / 2008$ 12:00 & 95 & 208 & 1200 & 6.688 & 6.688 & & 0.4563 & 4.53E-03 & 993.0296 & 365.9022 & 1358.9318 & \\
\hline & $7 / 26 / 2008$ 13:00 & 96 & 208 & 1300 & 6.138 & 6.138 & & 0.5868 & $6.17 \mathrm{E}-03$ & 1353.3887 & 498.6839 & 1852.0726 & \\
\hline & $7 / 26 / 200814: 00$ & 97 & 208 & 1400 & 6.069 & 6.069 & & 0.6049 & $6.41 \mathrm{E}-03$ & 1404.7878 & 517.6229 & 1922.4107 & \\
\hline & $7 / 26 / 2008$ 15:00 & 98 & 208 & 1500 & 6.011 & 6.011 & & 0.6204 & $6.61 \mathrm{E}-03$ & 1449.1893 & 533.9836 & 1983.1730 & \\
\hline & $7 / 26 / 2008$ 16:00 & 99 & 208 & 1600 & 6.066 & 6.066 & & 0.6057 & $6.42 \mathrm{E}-03$ & 1407.0572 & 518.4592 & 1925.5164 & \\
\hline & $7 / 26 / 200817: 00$ & 100 & 208 & 1700 & NAN & 6.082 & & 0.6014 & 6.36E-03 & 1394.9871 & 514.0117 & 1908.9988 & \\
\hline & $7 / 26 / 2008$ 18:00 & 101 & 208 & 1800 & 6.098 & 6.098 & & 0.5972 & $6.31 \mathrm{E}-03$ & 1382.9997 & 509.5947 & 1892.5944 & \\
\hline & $7 / 26 / 2008$ 19:00 & 102 & 208 & 1900 & 6.232 & 6.232 & & 0.5629 & 5.87E-03 & 1285.7611 & 473.7651 & 1759.5263 & \\
\hline & $7 / 26 / 200820: 00$ & 103 & 208 & 2000 & 6.802 & 6.802 & & 0.4319 & 4.23E-03 & 928.0565 & 341.9615 & 1270.0180 & \\
\hline & $7 / 26 / 200821: 00$ & 104 & 208 & 2100 & 8.41 & 8.41 & & 0.1581 & 1.23E-03 & 269.4113 & 99.2701 & 368.6815 & \\
\hline & $7 / 26 / 200822: 00$ & 105 & 208 & 2200 & 9.52 & 9.52 & & 0.0231 & $1.15 E-04$ & 25.2463 & 9.3025 & 34.5489 & \\
\hline & $7 / 26 / 2008$ 23:00 & 106 & 208 & 2300 & 9.79 & 9.79 & & -0.0051 & $0.00 \mathrm{E}+00$ & 0.0000 & 0.0000 & 0.0000 & \\
\hline & $7 / 27 / 20080: 00$ & 107 & 209 & 0 & 9.81 & 9.81 & 9.87 & -0.0071 & $0.00 \mathrm{E}+00$ & 0.0000 & 0.0000 & 0.0000 & \\
\hline & 7/27/2008 1:00 & 108 & 209 & 100 & 9.84 & 9.84 & & -0.0102 & $0.00 \mathrm{E}+00$ & 0.0000 & 0.0000 & 0.0000 & \\
\hline & 7/27/2008 2:00 & 109 & 209 & 200 & 9.84 & 9.84 & & -0.0102 & $0.00 \mathrm{E}+00$ & 0.0000 & 0.0000 & 0.0000 & \\
\hline & 7/27/2008 3:00 & 110 & 209 & 300 & 9.87 & 9.87 & & -0.0132 & $0.00 \mathrm{E}+00$ & 0.0000 & 0.0000 & 0.0000 & \\
\hline & 7/27/2008 4:00 & 111 & 209 & 400 & 9.87 & 9.87 & & -0.0132 & $0.00 E+00$ & 0.0000 & 0.0000 & 0.0000 & \\
\hline & 7/27/2008 5:00 & 112 & 209 & 500 & 9.83 & 9.83 & & -0.0092 & $0.00 \mathrm{E}+00$ & 0.0000 & 0.0000 & 0.0000 & \\
\hline & 7/27/2008 6:00 & 113 & 209 & 600 & 9.76 & 9.76 & & -0.0020 & $0.00 \mathrm{E}+00$ & 0.0000 & 0.0000 & 0.0000 & \\
\hline & 7/27/2008 7:00 & 114 & 209 & 700 & 9.68 & 9.68 & & 0.0062 & $2.28 \mathrm{E}-05$ & 4.9965 & 1.8411 & 6.8376 & \\
\hline & 7/27/2008 8:00 & 115 & 209 & 800 & 9.35 & 9.35 & & 0.0417 & 2.38E-04 & 52.2288 & 19.2448 & 71.4735 & \\
\hline & 7/27/2008 9:00 & 116 & 209 & 900 & NAN & 7.5565 & & 0.2890 & $2.58 \mathrm{E}-03$ & 565.8003 & 208.4807 & 774.2810 & \\
\hline & $7 / 27 / 2008$ 10:00 & 117 & 209 & 1000 & 5.763 & 5.763 & & 0.6901 & 7.54E-03 & 1652.2388 & 608.8014 & 2261.0402 & \\
\hline & $7 / 27 / 2008$ 11:00 & 118 & 209 & 1100 & 5.719 & 5.719 & & 0.7031 & 7.71E-03 & 1690.6454 & 622.9531 & 2313.5985 & \\
\hline & $7 / 27 / 200812: 00$ & 119 & 209 & 1200 & 5.828 & 5.828 & & 0.6712 & 7.29E-03 & 1596.8597 & 588.3958 & 2185.2555 & \\
\hline & $7 / 27 / 2008$ 13:00 & 120 & 209 & 1300 & 5.961 & 5.961 & & 0.6340 & $6.79 \mathrm{E}-03$ & 1488.3718 & 548.4212 & 2036.7931 & \\
\hline & $7 / 27 / 2008$ 14:00 & 121 & 209 & 1400 & 5.813 & 5.813 & & 0.6756 & 7.34E-03 & 1609.4982 & 593.0527 & 2202.5510 & \\
\hline & $7 / 27 / 200815: 00$ & 122 & 209 & 1500 & 5.817 & 5.817 & & 0.6744 & 7.33E-03 & 1606.1197 & 591.8079 & 2197.9276 & \\
\hline
\end{tabular}




\begin{tabular}{|c|c|c|c|c|c|c|c|c|c|c|c|c|c|}
\hline Tree ID & TS & RN & JD & HR & $\begin{array}{c}\text { Raw } \\
\text { TC_dTA }\end{array}$ & $\begin{array}{c}\text { Fixed } \\
\text { TC_dT } \Delta\end{array}$ & dTM & $\mathbf{K}$ & $\begin{array}{l}\text { Sap } \\
\text { Velocity } \\
\text { (cm/s) }\end{array}$ & $\begin{array}{l}\text { Probed } \\
\text { Drywood F } \\
\text { (g/hr) }\end{array}$ & $\begin{array}{c}\text { Extrapolated } \\
\text { Wetwood F } \\
(\mathrm{g} / \mathrm{hr})\end{array}$ & $\begin{array}{c}\text { Extrapolated } \\
\text { Total Wood F } \\
(\mathrm{g} / \mathrm{hr})\end{array}$ & $\begin{array}{l}\text { Relative } \\
\text { Humidity, } \\
@ 10 \mathrm{~m}(\%)\end{array}$ \\
\hline & $7 / 27 / 200816: 00$ & 123 & 209 & 1600 & $\begin{array}{l}5.845 \\
\end{array}$ & 5.845 & & 0.6664 & $7.22 \mathrm{E}-03$ & 1582.6369 & 583.1551 & 2165.7920 & \\
\hline & $7 / 27 / 200817: 00$ & 124 & 209 & 1700 & 5.919 & 5.919 & & 0.6455 & $6.94 \mathrm{E}-03$ & 1521.9506 & 560.7940 & 2082.7446 & \\
\hline & $7 / 27 / 2008$ 18:00 & 125 & 209 & 1800 & 6.004 & 6.004 & & 0.6223 & $6.64 \mathrm{E}-03$ & 1454.6239 & 535.9861 & 1990.6099 & \\
\hline & $7 / 27 / 2008$ 19:00 & 126 & 209 & 1900 & 6.169 & 6.169 & & 0.5789 & 6.07E-03 & 1330.7861 & 490.3555 & 1821.1416 & \\
\hline & $7 / 27 / 200820: 00$ & 127 & 209 & 2000 & 6.644 & 6.644 & & 0.4660 & 4.65E-03 & 1018.9279 & 375.4449 & 1394.3728 & \\
\hline & 7/27/2008 21:00 & 128 & 209 & 2100 & NAN & 7.797 & & 0.2492 & $2.15 \mathrm{E}-03$ & 471.5476 & 173.7514 & 645.2990 & \\
\hline & 7/27/2008 22:00 & 129 & 209 & 2200 & 8.95 & 8.95 & & 0.0883 & 6.00E-04 & 131.4205 & 48.4246 & 179.8451 & \\
\hline & $7 / 27 / 200823: 00$ & 130 & 209 & 2300 & 9.16 & 9.16 & & 0.0633 & 3.98E-04 & 87.3102 & 32.1712 & 119.4814 & \\
\hline & $7 / 28 / 20080: 00$ & 131 & 210 & 0 & 9.33 & 9.33 & 9.76 & 0.0439 & $2.54 \mathrm{E}-04$ & 55.6918 & 20.5208 & 76.2125 & \\
\hline & 7/28/2008 1:00 & 132 & 210 & 100 & 9.49 & 9.49 & & 0.0263 & $1.35 \mathrm{E}-04$ & 29.6639 & 10.9303 & 40.5941 & \\
\hline & 7/28/2008 2:00 & 133 & 210 & 200 & 9.59 & 9.59 & & 0.0156 & 7.12E-05 & 15.6145 & 5.7535 & 21.3680 & \\
\hline & $7 / 28 / 20083: 00$ & 134 & 210 & 300 & 9.76 & 9.76 & & -0.0020 & $0.00 \mathrm{E}+00$ & 0.0000 & 0.0000 & 0.0000 & \\
\hline & 7/28/2008 4:00 & 135 & 210 & 400 & NAN & 9.76 & & -0.0020 & $0.00 \mathrm{E}+00$ & 0.0000 & 0.0000 & 0.0000 & \\
\hline & 7/28/2008 5:00 & 136 & 210 & 500 & 9.76 & 9.76 & & -0.0020 & $0.00 \mathrm{E}+00$ & 0.0000 & 0.0000 & 0.0000 & \\
\hline & $7 / 28 / 20086: 00$ & 137 & 210 & 600 & 9.73 & 9.73 & & 0.0010 & $2.50 \mathrm{E}-06$ & 0.5470 & 0.2016 & 0.7486 & \\
\hline & $7 / 28 / 20087: 00$ & 138 & 210 & 700 & 9.56 & 9.56 & & 0.0188 & 8.95E-05 & 19.6189 & 7.2290 & 26.8479 & \\
\hline & 7/28/2008 8:00 & 139 & 210 & 800 & 7.795 & 7.795 & & 0.2495 & $2.15 \mathrm{E}-03$ & 472.2943 & 174.0266 & 646.3209 & \\
\hline & 7/28/20089:00 & 140 & 210 & 900 & 6.26 & 6.26 & & 0.5559 & $5.78 \mathrm{E}-03$ & 1266.1331 & 466.5328 & 1732.6659 & \\
\hline & 7/28/2008 10:00 & 141 & 210 & 1000 & 5.836 & 5.836 & & 0.6690 & 7.25E-03 & 1590.1533 & 585.9247 & 2176.0780 & \\
\hline & 7/28/2008 11:00 & 142 & 210 & 1100 & 5.855 & 5.855 & & 0.6635 & $7.18 \mathrm{E}-03$ & 1574.3202 & 580.0907 & 2154.4109 & \\
\hline & $7 / 28 / 200812: 00$ & 143 & 210 & 1200 & 5.943 & 5.943 & & 0.6389 & $6.86 \mathrm{E}-03$ & 1502.6874 & 553.6961 & 2056.3835 & \\
\hline & $7 / 28 / 2008$ 13:00 & 144 & 210 & 1300 & 5.954 & 5.954 & & 0.6359 & $6.82 \mathrm{E}-03$ & 1493.9257 & 550.4676 & 2044.3933 & \\
\hline & $7 / 28 / 200814: 00$ & 145 & 210 & 1400 & 5.92 & 5.92 & & 0.6453 & 6.94E-03 & 1521.1439 & 560.4968 & 2081.6407 & \\
\hline & $7 / 28 / 200815: 00$ & 146 & 210 & 1500 & 5.864 & 5.864 & & 0.6610 & 7.15E-03 & 1566.8665 & 577.3442 & 2144.2106 & \\
\hline & $7 / 28 / 200816: 00$ & 147 & 210 & 1600 & 5.899 & 5.899 & & 0.6511 & 7.02E-03 & 1538.1584 & 566.7661 & 2104.9245 & \\
\hline & $7 / 28 / 200817: 00$ & 148 & 210 & 1700 & 5.916 & 5.916 & & 0.6464 & $6.95 \mathrm{E}-03$ & 1524.3727 & 561.6865 & 2086.0592 & \\
\hline & $7 / 28 / 2008$ 18:00 & 149 & 210 & 1800 & 6.026 & 6.026 & & 0.6163 & $6.56 \mathrm{E}-03$ & 1437.5993 & 529.7130 & 1967.3123 & \\
\hline & $7 / 28 / 2008$ 19:00 & 150 & 210 & 1900 & 6.22 & 6.22 & & 0.5659 & $5.90 \mathrm{E}-03$ & 1294.2447 & 476.8911 & 1771.1357 & \\
\hline & $7 / 28 / 200820: 00$ & 151 & 210 & 2000 & 6.794 & 6.794 & & 0.4336 & $4.25 \mathrm{E}-03$ & 932.5182 & 343.6055 & 1276.1236 & \\
\hline & $7 / 28 / 200821: 00$ & 152 & 210 & 2100 & 8.41 & 8.41 & & 0.1581 & $1.23 \mathrm{E}-03$ & 269.4113 & 99.2701 & 368.6815 & \\
\hline & $7 / 28 / 200822: 00$ & 153 & 210 & 2200 & 8.94 & 8.94 & & 0.0895 & $6.10 \mathrm{E}-04$ & 133.6551 & 49.2480 & 182.9031 & \\
\hline & 7/28/2008 23:00 & 154 & 210 & 2300 & 9.19 & 9.19 & & 0.0598 & $3.72 \mathrm{E}-04$ & 81.4561 & 30.0142 & 111.4702 & \\
\hline & $7 / 29 / 20080: 00$ & 155 & 211 & 0 & 9.45 & 9.45 & 9.95 & 0.0307 & $1.63 \mathrm{E}-04$ & 35.7959 & 13.1897 & 48.9857 & \\
\hline & $7 / 29 / 2008$ 1:00 & 156 & 211 & 100 & 9.64 & 9.64 & & 0.0104 & 4.30E-05 & 9.4185 & 3.4704 & 12.8889 & \\
\hline & 7/29/2008 2:00 & 157 & 211 & 200 & 9.82 & 9.82 & & -0.0081 & $0.00 \mathrm{E}+00$ & 0.0000 & 0.0000 & 0.0000 & \\
\hline & 7/29/2008 3:00 & 158 & 211 & 300 & 9.91 & 9.91 & & -0.0172 & $0.00 \mathrm{E}+00$ & 0.0000 & 0.0000 & 0.0000 & \\
\hline & 7/29/2008 4:00 & 159 & 211 & 400 & 9.95 & 9.95 & & -0.0211 & $0.00 \mathrm{E}+00$ & 0.0000 & 0.0000 & 0.0000 & \\
\hline & $7 / 29 / 2008$ 5:00 & 160 & 211 & 500 & 9.9 & 9.9 & & -0.0162 & $0.00 \mathrm{E}+00$ & 0.0000 & 0.0000 & 0.0000 & \\
\hline & $7 / 29 / 20086: 00$ & 161 & 211 & 600 & 9.84 & 9.84 & & -0.0102 & $0.00 \mathrm{E}+00$ & 0.0000 & 0.0000 & 0.0000 & \\
\hline & 7/29/2008 7:00 & 162 & 211 & 700 & 9.74 & 9.74 & & 0.0000 & $0.00 \mathrm{E}+00$ & 0.0000 & 0.0000 & 0.0000 & \\
\hline & $7 / 29 / 20088: 00$ & 163 & 211 & 800 & 9.02 & 9.02 & & 0.0798 & 5.30E-04 & 116.1169 & 42.7857 & 158.9025 & \\
\hline
\end{tabular}




\begin{tabular}{|c|c|c|c|c|c|c|c|c|c|c|c|c|c|}
\hline Tree ID & TS & RN & JD & HR & $\begin{array}{c}\text { Raw } \\
\text { TC_dTA }\end{array}$ & $\begin{array}{c}\text { Fixed } \\
\text { TC_dT } \Delta\end{array}$ & dTM & $\mathrm{K}$ & $\begin{array}{c}\text { Sap } \\
\text { Velocity } \\
(\mathrm{cm} / \mathrm{s})\end{array}$ & $\begin{array}{c}\text { Probed } \\
\text { Drywood F } \\
(\mathrm{g} / \mathrm{hr})\end{array}$ & $\begin{array}{c}\text { Extrapolated } \\
\text { Wetwood F } \\
(\mathrm{g} / \mathrm{hr})\end{array}$ & $\begin{array}{c}\text { Extrapolated } \\
\text { Total Wood F } \\
(\mathbf{g} / \mathrm{hr})\end{array}$ & $\begin{array}{l}\text { Relative } \\
\text { Humidity, } \\
\text { @10m (\%) }\end{array}$ \\
\hline & $7 / 29 / 20089: 00$ & 164 & 211 & 900 & 7.082 & 7.082 & & 0.3753 & 3.56E-03 & 780.6629 & 287.6513 & 1068.3143 & \\
\hline & $7 / 29 / 2008$ 10:00 & 165 & 211 & 1000 & 6.107 & 6.107 & & 0.5949 & $6.28 \mathrm{E}-03$ & 1376.2929 & 507.1234 & 1883.4163 & \\
\hline & 7/29/2008 11:00 & 166 & 211 & 1100 & 6.448 & 6.448 & & 0.5105 & $5.20 \mathrm{E}-03$ & 1140.1688 & 420.1187 & 1560.2875 & \\
\hline & $7 / 29 / 2008$ 12:00 & 167 & 211 & 1200 & 5.94 & 5.94 & & 0.6397 & 6.87E-03 & 1505.0843 & 554.5793 & 2059.6636 & \\
\hline & $7 / 29 / 2008$ 13:00 & 168 & 211 & 1300 & 6.03 & 6.03 & & 0.6153 & 6.54E-03 & 1434.5213 & 528.5789 & 1963.1002 & \\
\hline & 7/29/2008 14:00 & 169 & 211 & 1400 & 5.904 & 5.904 & & 0.6497 & 7.00E-03 & 1534.0932 & 565.2682 & 2099.3613 & \\
\hline & $7 / 29 / 2008$ 15:00 & 170 & 211 & 1500 & NAN & 6.0525 & & 0.6093 & 6.47E-03 & 1417.3063 & 522.2357 & 1939.5420 & \\
\hline & $7 / 29 / 2008$ 16:00 & 171 & 211 & 1600 & 6.201 & 6.201 & & 0.5707 & 5.97E-03 & 1307.7656 & 481.8731 & 1789.6387 & \\
\hline & $7 / 29 / 2008$ 17:00 & 172 & 211 & 1700 & NAN & 6.289 & & 0.5487 & $5.68 \mathrm{E}-03$ & 1246.0476 & 459.1319 & 1705.1795 & \\
\hline & $7 / 29 / 2008$ 18:00 & 173 & 211 & 1800 & 6.377 & 6.377 & & 0.5274 & 5.41E-03 & 1186.5780 & 437.2191 & 1623.7971 & \\
\hline & $7 / 29 / 2008$ 19:00 & 174 & 211 & 1900 & 6.466 & 6.466 & & 0.5063 & $5.15 E-03$ & 1128.6197 & 415.8631 & 1544.4829 & \\
\hline & $7 / 29 / 200820: 00$ & 175 & 211 & 2000 & 7.426 & 7.426 & & 0.3116 & 2.83E-03 & 620.8837 & 228.7774 & 849.6611 & \\
\hline & 7/29/2008 21:00 & 176 & 211 & 2100 & 8.96 & 8.96 & & 0.0871 & 5.89E-04 & 129.1980 & 47.6056 & 176.8036 & \\
\hline & $7 / 29 / 200822: 00$ & 177 & 211 & 2200 & 9.63 & 9.63 & & 0.0114 & 4.84E-05 & 10.6045 & 3.9074 & 14.5119 & \\
\hline & $7 / 29 / 200823: 00$ & 178 & 211 & 2300 & 9.76 & 9.76 & & -0.0020 & $0.00 \mathrm{E}+00$ & 0.0000 & 0.0000 & 0.0000 & \\
\hline & $7 / 30 / 20080: 00$ & 179 & 212 & 0 & 9.74 & 9.74 & 9.74 & 0.0000 & $0.00 \mathrm{E}+00$ & 0.0000 & 0.0000 & 0.0000 & \\
\hline & 7/30/2008 1:00 & 180 & 212 & 100 & 9.68 & 9.68 & & 0.0062 & $2.28 \mathrm{E}-05$ & 4.9965 & 1.8411 & 6.8376 & \\
\hline & 7/30/2008 2:00 & 181 & 212 & 200 & 9.58 & 9.58 & & 0.0167 & 7.72E-05 & 16.9274 & 6.2372 & 23.1646 & \\
\hline & 7/30/2008 3:00 & 182 & 212 & 300 & 9.43 & 9.43 & & 0.0329 & 1.78E-04 & 38.9602 & 14.3557 & 53.3158 & \\
\hline & 7/30/2008 4:00 & 183 & 212 & 400 & 9.3 & 9.3 & & 0.0473 & $2.78 \mathrm{E}-04$ & 60.9910 & 22.4734 & 83.4644 & \\
\hline & 7/30/2008 5:00 & 184 & 212 & 500 & 9.25 & 9.25 & & 0.0530 & $3.20 \mathrm{E}-04$ & 70.0954 & 25.8281 & 95.9234 & \\
\hline & 7/30/2008 6:00 & 185 & 212 & 600 & 9.22 & 9.22 & & 0.0564 & 3.45E-04 & 75.7172 & 27.8996 & 103.6168 & \\
\hline & 7/30/2008 7:00 & 186 & 212 & 700 & 9.15 & 9.15 & & 0.0645 & 4.07E-04 & 89.2869 & 32.8996 & 122.1865 & \\
\hline & 7/30/2008 8:00 & 187 & 212 & 800 & 9.11 & 9.11 & & 0.0692 & 4.44E-04 & 97.3194 & 35.8593 & 133.1787 & \\
\hline & 7/30/2008 9:00 & 188 & 212 & 900 & 7.369 & 7.369 & & 0.3218 & $2.95 \mathrm{E}-03$ & 645.8611 & 237.9808 & 883.8419 & \\
\hline & $7 / 30 / 2008$ 10:00 & 189 & 212 & 1000 & 5.389 & 5.389 & & 0.8074 & $9.14 \mathrm{E}-03$ & 2004.4486 & 738.5803 & 2743.0289 & \\
\hline & $7 / 30 / 2008$ 11:00 & 190 & 212 & 1100 & 5.46 & 5.46 & & 0.7839 & 8.82E-03 & 1932.8648 & 712.2038 & 2645.0685 & \\
\hline & $7 / 30 / 2008$ 12:00 & 191 & 212 & 1200 & 5.642 & 5.642 & & 0.7263 & 8.03E-03 & 1759.7067 & 648.4001 & 2408.1068 & \\
\hline & $7 / 30 / 2008$ 13:00 & 192 & 212 & 1300 & 5.756 & 5.756 & & 0.6921 & 7.57E-03 & 1658.2986 & 611.0342 & 2269.3329 & \\
\hline & $7 / 30 / 2008$ 14:00 & 193 & 212 & 1400 & 5.743 & 5.743 & & 0.6960 & 7.62E-03 & 1669.6029 & 615.1995 & 2284.8024 & \\
\hline & $7 / 30 / 2008$ 15:00 & 194 & 212 & 1500 & 5.852 & 5.852 & & 0.6644 & 7.19E-03 & 1576.8114 & 581.0086 & 2157.8199 & \\
\hline & $7 / 30 / 2008$ 16:00 & 195 & 212 & 1600 & 5.751 & 5.751 & & 0.6936 & 7.59E-03 & 1662.6387 & 612.6334 & 2275.2721 & \\
\hline & $7 / 30 / 2008$ 17:00 & 196 & 212 & 1700 & 5.845 & 5.845 & & 0.6664 & 7.22E-03 & 1582.6369 & 583.1551 & 2165.7920 & \\
\hline & $7 / 30 / 2008$ 18:00 & 197 & 212 & 1800 & 6.001 & 6.001 & & 0.6231 & $6.65 \mathrm{E}-03$ & 1456.9580 & 536.8461 & 1993.8041 & \\
\hline & $7 / 30 / 2008$ 19:00 & 198 & 212 & 1900 & 6.17 & 6.17 & & 0.5786 & $6.07 \mathrm{E}-03$ & 1330.0619 & 490.0887 & 1820.1506 & \\
\hline & $7 / 30 / 200820: 00$ & 199 & 212 & 2000 & 6.775 & 6.775 & & 0.4376 & 4.30E-03 & 943.1731 & 347.5315 & 1290.7046 & \\
\hline & $7 / 30 / 2008$ 21:00 & 200 & 212 & 2100 & 8.4 & 8.4 & & 0.1595 & $1.24 \mathrm{E}-03$ & 272.3056 & 100.3366 & 372.6422 & \\
\hline & $7 / 30 / 200822: 00$ & 201 & 212 & 2200 & 9.41 & 9.41 & & 0.0351 & $1.92 \mathrm{E}-04$ & 42.1871 & 15.5447 & 57.7319 & \\
\hline & $7 / 30 / 200823: 00$ & 202 & 212 & 2300 & 9.63 & 9.63 & & 0.0114 & 4.84E-05 & 10.6045 & 3.9074 & 14.5119 & \\
\hline & $7 / 31 / 20080: 00$ & 203 & 213 & 0 & 9.75 & 9.75 & 9.77 & -0.0010 & $0.00 \mathrm{E}+00$ & 0.0000 & 0.0000 & 0.0000 & \\
\hline & $7 / 31 / 20081: 00$ & 204 & 213 & 100 & 9.77 & 9.77 & & -0.0031 & $0.00 \mathrm{E}+00$ & 0.0000 & 0.0000 & 0.0000 & \\
\hline
\end{tabular}




\begin{tabular}{|c|c|c|c|c|c|c|c|c|c|c|c|c|c|}
\hline Tree ID & TS & RN & JD & HR & $\begin{array}{c}\text { Raw } \\
\text { TC_dTA }\end{array}$ & $\begin{array}{c}\text { Fixed } \\
\text { TC_dT } \Delta\end{array}$ & dTM & $\mathrm{K}$ & $\begin{array}{c}\text { Sap } \\
\text { Velocity } \\
\text { (cm/s) }\end{array}$ & $\begin{array}{l}\text { Probed } \\
\text { Drywood F } \\
\text { (g/hr) }\end{array}$ & $\begin{array}{c}\text { Extrapolated } \\
\text { Wetwood F } \\
(\mathrm{g} / \mathrm{hr})\end{array}$ & $\begin{array}{c}\text { Extrapolated } \\
\text { Total Wood F } \\
(\mathrm{g} / \mathrm{hr})\end{array}$ & $\begin{array}{l}\text { Relative } \\
\text { Humidity, } \\
@ 10 \mathrm{~m}(\%)\end{array}$ \\
\hline & $7 / 31 / 20082: 00$ & 205 & 213 & 200 & 9.75 & 9.75 & & -0.0010 & $0.00 \mathrm{E}+00$ & 0.0000 & 0.0000 & 0.0000 & \\
\hline & 7/31/2008 3:00 & 206 & 213 & 300 & 9.74 & 9.74 & & 0.0000 & $0.00 \mathrm{E}+00$ & 0.0000 & 0.0000 & 0.0000 & \\
\hline & 7/31/2008 4:00 & 207 & 213 & 400 & 9.69 & 9.69 & & 0.0052 & 1.82E-05 & 3.9870 & 1.4691 & 5.4561 & \\
\hline & 7/31/2008 5:00 & 208 & 213 & 500 & 9.63 & 9.63 & & 0.0114 & 4.84E-05 & 10.6045 & 3.9074 & 14.5119 & \\
\hline & $7 / 31 / 20086: 00$ & 209 & 213 & 600 & 9.57 & 9.57 & & 0.0178 & 8.33E-05 & 18.2624 & 6.7292 & 24.9916 & \\
\hline & 7/31/2008 7:00 & 210 & 213 & 700 & 9.5 & 9.5 & & 0.0253 & 1.29E-04 & 28.1735 & 10.3811 & 38.5546 & \\
\hline & $7 / 31 / 20088: 00$ & 211 & 213 & 800 & 9.4 & 9.4 & & 0.0362 & 2.00E-04 & 43.8236 & 16.1477 & 59.9714 & \\
\hline & 7/31/2008 9:00 & 212 & 213 & 900 & 7.417 & 7.417 & & 0.3132 & 2.85E-03 & 624.7898 & 230.2166 & 855.0064 & \\
\hline & $7 / 31 / 200810: 00$ & 213 & 213 & 1000 & 5.567 & 5.567 & & 0.7496 & 8.35E-03 & 1829.3237 & 674.0520 & 2503.3757 & \\
\hline & 7/31/2008 11:00 & 214 & 213 & 1100 & 5.742 & 5.742 & & 0.6963 & $7.62 \mathrm{E}-03$ & 1670.4752 & 615.5209 & 2285.9961 & \\
\hline & $7 / 31 / 200812: 00$ & 215 & 213 & 1200 & 5.683 & 5.683 & & 0.7139 & 7.86E-03 & 1722.6363 & 634.7408 & 2357.3771 & \\
\hline & $7 / 31 / 2008$ 13:00 & 216 & 213 & 1300 & 5.973 & 5.973 & & 0.6307 & 6.75E-03 & 1478.8903 & 544.9275 & 2023.8178 & \\
\hline & $7 / 31 / 200814: 00$ & 217 & 213 & 1400 & 5.723 & 5.723 & & 0.7019 & 7.70E-03 & 1687.1226 & 621.6550 & 2308.7777 & \\
\hline & $7 / 31 / 200815: 00$ & 218 & 213 & 1500 & NAN & 5.7765 & & 0.6861 & $7.48 \mathrm{E}-03$ & 1640.6051 & 604.5147 & 2245.1199 & \\
\hline & $7 / 31 / 200816: 00$ & 219 & 213 & 1600 & 5.83 & 5.83 & & 0.6707 & $7.28 \mathrm{E}-03$ & 1595.1809 & 587.7772 & 2182.9581 & \\
\hline & $7 / 31 / 200817: 00$ & 220 & 213 & 1700 & 5.888 & 5.888 & & 0.6542 & $7.06 \mathrm{E}-03$ & 1547.1335 & 570.0731 & 2117.2066 & \\
\hline & $7 / 31 / 200818: 00$ & 221 & 213 & 1800 & 5.986 & 5.986 & & 0.6271 & $6.70 \mathrm{E}-03$ & 1468.6743 & 541.1632 & 2009.8375 & \\
\hline & $7 / 31 / 2008$ 19:00 & 222 & 213 & 1900 & 6.247 & 6.247 & & 0.5591 & $5.82 \mathrm{E}-03$ & 1275.2172 & 469.8800 & 1745.0972 & \\
\hline & $7 / 31 / 200820: 00$ & 223 & 213 & 2000 & 7.178 & 7.178 & & 0.3569 & 3.35E-03 & 733.8359 & 270.3969 & 1004.2329 & \\
\hline & 7/31/2008 21:00 & 224 & 213 & 2100 & 8.13 & 8.13 & & 0.1980 & 1.62E-03 & 355.3526 & 130.9370 & 486.2896 & \\
\hline & $7 / 31 / 200822: 00$ & 225 & 213 & 2200 & 8.86 & 8.86 & & 0.0993 & $6.93 \mathrm{E}-04$ & 151.9657 & 55.9949 & 207.9606 & \\
\hline & 7/31/2008 23:00 & 226 & 213 & 2300 & 8.77 & 8.77 & & 0.1106 & 7.91E-04 & 173.4850 & 63.9241 & 237.4091 & \\
\hline & $8 / 1 / 20080: 00$ & 227 & 214 & 0 & 9.13 & 9.13 & 9.81 & 0.0668 & $4.26 \mathrm{E}-04$ & 93.2781 & 34.3702 & 127.6484 & \\
\hline & 8/1/2008 1:00 & 228 & 214 & 100 & 9.58 & 9.58 & & 0.0167 & 7.72E-05 & 16.9274 & 6.2372 & 23.1646 & \\
\hline & $8 / 1 / 20082: 00$ & 229 & 214 & 200 & 9.72 & 9.72 & & 0.0021 & 5.87E-06 & 1.2857 & 0.4737 & 1.7594 & \\
\hline & 8/1/2008 3:00 & 230 & 214 & 300 & 9.74 & 9.74 & & 0.0000 & $0.00 \mathrm{E}+00$ & 0.0000 & 0.0000 & 0.0000 & \\
\hline & 8/1/2008 4:00 & 231 & 214 & 400 & 9.81 & 9.81 & & -0.0071 & $0.00 \mathrm{E}+00$ & 0.0000 & 0.0000 & 0.0000 & \\
\hline & 8/1/2008 5:00 & 232 & 214 & 500 & 9.8 & 9.8 & & -0.0061 & $0.00 \mathrm{E}+00$ & 0.0000 & 0.0000 & 0.0000 & \\
\hline & $8 / 1 / 20086: 00$ & 233 & 214 & 600 & 9.73 & 9.73 & & 0.0010 & $2.50 \mathrm{E}-06$ & 0.5470 & 0.2016 & 0.7486 & \\
\hline & $8 / 1 / 20087: 00$ & 234 & 214 & 700 & 9.64 & 9.64 & & 0.0104 & $4.30 \mathrm{E}-05$ & 9.4185 & 3.4704 & 12.8889 & \\
\hline & $8 / 1 / 20088: 00$ & 235 & 214 & 800 & 9.45 & 9.45 & & 0.0307 & $1.63 \mathrm{E}-04$ & 35.7959 & 13.1897 & 48.9857 & \\
\hline & 8/1/2008 9:00 & 236 & 214 & 900 & 7.796 & 7.796 & & 0.2494 & $2.15 \mathrm{E}-03$ & 471.9209 & 173.8890 & 645.8099 & \\
\hline & 8/1/2008 10:00 & 237 & 214 & 1000 & 5.737 & 5.737 & & 0.6978 & 7.64E-03 & 1674.8424 & 617.1301 & 2291.9725 & \\
\hline & 8/1/2008 11:00 & 238 & 214 & 1100 & 5.742 & 5.742 & & 0.6963 & $7.62 \mathrm{E}-03$ & 1670.4752 & 615.5209 & 2285.9961 & \\
\hline & $8 / 1 / 2008$ 12:00 & 239 & 214 & 1200 & 5.856 & 5.856 & & 0.6633 & 7.18E-03 & 1573.4905 & 579.7850 & 2153.2755 & \\
\hline & $8 / 1 / 200813: 00$ & 240 & 214 & 1300 & 5.868 & 5.868 & & 0.6599 & 7.13E-03 & 1563.5632 & 576.1270 & 2139.6902 & \\
\hline & $8 / 1 / 2008$ 14:00 & 241 & 214 & 1400 & 5.818 & 5.818 & & 0.6741 & 7.32E-03 & 1605.2761 & 591.4970 & 2196.7730 & \\
\hline & $8 / 1 / 2008$ 15:00 & 242 & 214 & 1500 & 5.804 & 5.804 & & 0.6782 & 7.38E-03 & 1617.1217 & 595.8618 & 2212.9835 & \\
\hline & $8 / 1 / 2008$ 16:00 & 243 & 214 & 1600 & 5.852 & 5.852 & & 0.6644 & 7.19E-03 & 1576.8114 & 581.0086 & 2157.8199 & \\
\hline & $8 / 1 / 2008$ 17:00 & 244 & 214 & 1700 & 5.931 & 5.931 & & 0.6422 & $6.90 \mathrm{E}-03$ & 1512.2938 & 557.2357 & 2069.5295 & \\
\hline & 8/1/2008 18:00 & 245 & 214 & 1800 & 5.993 & 5.993 & & 0.6252 & 6.68E-03 & 1463.1972 & 539.1451 & 2002.3423 & \\
\hline
\end{tabular}




\begin{tabular}{|c|c|c|c|c|c|c|c|c|c|c|c|c|c|}
\hline Tree ID & TS & RN & JD & HR & $\begin{array}{c}\text { Raw } \\
\text { TC_dTA }\end{array}$ & $\begin{array}{c}\text { Fixed } \\
\text { TC_dT } \Delta\end{array}$ & dTM & $\mathbf{K}$ & $\begin{array}{c}\text { Sap } \\
\text { Velocity } \\
(\mathrm{cm} / \mathrm{s})\end{array}$ & $\begin{array}{c}\text { Probed } \\
\text { Drywood F } \\
(\mathrm{g} / \mathrm{hr})\end{array}$ & $\begin{array}{c}\text { Extrapolated } \\
\text { Wetwood F } \\
(\mathrm{g} / \mathrm{hr})\end{array}$ & $\begin{array}{c}\text { Extrapolated } \\
\text { Total Wood F } \\
(\mathrm{g} / \mathrm{hr})\end{array}$ & $\begin{array}{l}\text { Relative } \\
\text { Humidity, } \\
\text { @10m (\%) }\end{array}$ \\
\hline & 8/1/2008 19:00 & 246 & 214 & 1900 & \begin{tabular}{|r|}
6.159 \\
\end{tabular} & 6.159 & & 0.5814 & $6.10 \mathrm{E}-03$ & 1338.0446 & 493.0301 & 1831.0747 & \\
\hline & $8 / 1 / 200820: 00$ & 247 & 214 & 2000 & 7.015 & 7.015 & & 0.3885 & $3.72 \mathrm{E}-03$ & 814.4313 & 300.0939 & 1114.5252 & \\
\hline & 8/1/2008 21:00 & 248 & 214 & 2100 & 8.84 & 8.84 & & 0.1018 & 7.15E-04 & 156.6636 & 57.7259 & 214.3895 & \\
\hline & 8/1/2008 22:00 & 249 & 214 & 2200 & 9.6 & 9.6 & & 0.0146 & $6.54 \mathrm{E}-05$ & 14.3247 & 5.2782 & 19.6030 & \\
\hline & 8/1/2008 23:00 & 250 & 214 & 2300 & 9.72 & 9.72 & & 0.0021 & $5.87 \mathrm{E}-06$ & 1.2857 & 0.4737 & 1.7594 & \\
\hline & $8 / 2 / 20080: 00$ & 251 & 215 & 0 & 9.73 & 9.73 & 9.88 & 0.0010 & $2.50 \mathrm{E}-06$ & 0.5470 & 0.2016 & 0.7486 & \\
\hline & 8/2/2008 1:00 & 252 & 215 & 100 & 9.82 & 9.82 & & -0.0081 & $0.00 \mathrm{E}+00$ & 0.0000 & 0.0000 & 0.0000 & \\
\hline & 8/2/2008 2:00 & 253 & 215 & 200 & 9.88 & 9.88 & & -0.0142 & $0.00 \mathrm{E}+00$ & 0.0000 & 0.0000 & 0.0000 & \\
\hline & 8/2/2008 3:00 & 254 & 215 & 300 & 9.88 & 9.88 & & -0.0142 & $0.00 \mathrm{E}+00$ & 0.0000 & 0.0000 & 0.0000 & \\
\hline & 8/2/2008 4:00 & 255 & 215 & 400 & 9.78 & 9.78 & & -0.0041 & $0.00 \mathrm{E}+00$ & 0.0000 & 0.0000 & 0.0000 & \\
\hline & 8/2/2008 5:00 & 256 & 215 & 500 & 9.75 & 9.75 & & -0.0010 & $0.00 \mathrm{E}+00$ & 0.0000 & 0.0000 & 0.0000 & \\
\hline & 8/2/2008 6:00 & 257 & 215 & 600 & 9.74 & 9.74 & & 0.0000 & $0.00 \mathrm{E}+00$ & 0.0000 & 0.0000 & 0.0000 & \\
\hline & 8/2/2008 7:00 & 258 & 215 & 700 & 9.56 & 9.56 & & 0.0188 & 8.95E-05 & 19.6189 & 7.2290 & 26.8479 & \\
\hline & $8 / 2 / 20088: 00$ & 259 & 215 & 800 & 8.02 & 8.02 & & 0.2145 & 1.79E-03 & 391.9902 & 144.4368 & 536.4270 & \\
\hline & $8 / 2 / 20089: 00$ & 260 & 215 & 900 & 6.114 & 6.114 & & 0.5931 & $6.26 \mathrm{E}-03$ & 1371.0944 & 505.2079 & 1876.3023 & \\
\hline & 8/2/2008 10:00 & 261 & 215 & 1000 & 6.065 & 6.065 & & 0.6059 & $6.42 \mathrm{E}-03$ & 1407.8144 & 518.7382 & 1926.5526 & \\
\hline & 8/2/2008 11:00 & 262 & 215 & 1100 & 6.007 & 6.007 & & 0.6214 & $6.63 \mathrm{E}-03$ & 1452.2928 & 535.1271 & 1987.4199 & \\
\hline & $8 / 2 / 200812: 00$ & 263 & 215 & 1200 & 5.843 & 5.843 & & 0.6670 & 7.23E-03 & 1584.3046 & 583.7696 & 2168.0742 & \\
\hline & $8 / 2 / 2008$ 13:00 & 264 & 215 & 1300 & 5.968 & 5.968 & & 0.6320 & 6.76E-03 & 1482.8349 & 546.3810 & 2029.2159 & \\
\hline & 8/2/2008 14:00 & 265 & 215 & 1400 & 5.985 & 5.985 & & 0.6274 & $6.70 \mathrm{E}-03$ & 1469.4581 & 541.4520 & 2010.9101 & \\
\hline & $8 / 2 / 2008$ 15:00 & 266 & 215 & 1500 & 5.995 & 5.995 & & 0.6247 & $6.67 \mathrm{E}-03$ & 1461.6353 & 538.5696 & 2000.2049 & \\
\hline & 8/2/2008 16:00 & 267 & 215 & 1600 & 6.013 & 6.013 & & 0.6198 & $6.60 \mathrm{E}-03$ & 1447.6397 & 533.4126 & 1981.0523 & \\
\hline & $8 / 2 / 2008$ 17:00 & 268 & 215 & 1700 & 6.074 & 6.074 & & 0.6036 & 6.39E-03 & 1401.0118 & 516.2316 & 1917.2434 & \\
\hline & $8 / 2 / 2008$ 18:00 & 269 & 215 & 1800 & 6.171 & 6.171 & & 0.5784 & $6.06 \mathrm{E}-03$ & 1329.3381 & 489.8220 & 1819.1601 & \\
\hline & 8/2/2008 19:00 & 270 & 215 & 1900 & 6.309 & 6.309 & & 0.5438 & $5.62 \mathrm{E}-03$ & 1232.3380 & 454.0803 & 1686.4182 & \\
\hline & $8 / 2 / 200820: 00$ & 271 & 215 & 2000 & 6.929 & 6.929 & & 0.4057 & 3.92E-03 & 859.1333 & 316.5653 & 1175.6987 & \\
\hline & 8/2/2008 21:00 & 272 & 215 & 2100 & 8.22 & 8.22 & & 0.1849 & 1.49E-03 & 326.6033 & 120.3437 & 446.9470 & \\
\hline & $8 / 2 / 200822: 00$ & 273 & 215 & 2200 & 9.18 & 9.18 & & 0.0610 & 3.80E-04 & 83.3947 & 30.7285 & 114.1232 & \\
\hline & $8 / 2 / 2008$ 23:00 & 274 & 215 & 2300 & 9.5 & 9.5 & & 0.0253 & $1.29 \mathrm{E}-04$ & 28.1735 & 10.3811 & 38.5546 & \\
\hline & 8/3/2008 0:00 & 275 & 216 & 0 & 9.42 & 9.42 & 9.85 & 0.0340 & 1.85E-04 & 40.5659 & 14.9473 & 55.5133 & \\
\hline & 8/3/2008 1:00 & 276 & 216 & 100 & 9.2 & 9.2 & & 0.0587 & 3.63E-04 & 79.5302 & 29.3045 & 108.8348 & \\
\hline & $8 / 3 / 20082: 00$ & 277 & 216 & 200 & 9.34 & 9.34 & & 0.0428 & $2.46 \mathrm{E}-04$ & 53.9532 & 19.8802 & 73.8334 & \\
\hline & 8/3/2008 3:00 & 278 & 216 & 300 & 9.6 & 9.6 & & 0.0146 & $6.54 \mathrm{E}-05$ & 14.3247 & 5.2782 & 19.6030 & \\
\hline & $8 / 3 / 20084: 00$ & 279 & 216 & 400 & 9.76 & 9.76 & & -0.0020 & $0.00 \mathrm{E}+00$ & 0.0000 & 0.0000 & 0.0000 & \\
\hline & 8/3/2008 5:00 & 280 & 216 & 500 & 9.85 & 9.85 & & -0.0112 & $0.00 \mathrm{E}+00$ & 0.0000 & 0.0000 & 0.0000 & \\
\hline & $8 / 3 / 20086: 00$ & 281 & 216 & 600 & 9.82 & 9.82 & & -0.0081 & $0.00 \mathrm{E}+00$ & 0.0000 & 0.0000 & 0.0000 & \\
\hline & $8 / 3 / 20087: 00$ & 282 & 216 & 700 & 9.74 & 9.74 & & 0.0000 & $0.00 \mathrm{E}+00$ & 0.0000 & 0.0000 & 0.0000 & \\
\hline & $8 / 3 / 20088: 00$ & 283 & 216 & 800 & 8.37 & 8.37 & & 0.1637 & 1.28E-03 & 281.0647 & 103.5641 & 384.6288 & \\
\hline & $8 / 3 / 20089: 00$ & 284 & 216 & 900 & 6.103 & 6.103 & & 0.5959 & $6.29 \mathrm{E}-03$ & 1379.2705 & 508.2206 & 1887.4911 & \\
\hline & $8 / 3 / 2008$ 10:00 & 285 & 216 & 1000 & 5.798 & 5.798 & & 0.6799 & 7.40E-03 & 1622.2210 & 597.7407 & 2219.9617 & \\
\hline & 8/3/2008 11:00 & 286 & 216 & 1100 & 5.821 & 5.821 & & 0.6733 & 7.31E-03 & 1602.7472 & 590.5652 & 2193.3124 & \\
\hline
\end{tabular}




\begin{tabular}{|c|c|c|c|c|c|c|c|c|c|c|c|c|c|}
\hline Tree ID & TS & RN & JD & HR & $\begin{array}{c}\text { Raw } \\
\text { TC_dTA }\end{array}$ & $\begin{array}{c}\text { Fixed } \\
\text { TC_dT } \Delta\end{array}$ & dTM & $\mathrm{K}$ & $\begin{array}{l}\text { Sap } \\
\text { Velocity } \\
(\mathrm{cm} / \mathrm{s})\end{array}$ & $\begin{array}{l}\text { Probed } \\
\text { Drywood F } \\
(\mathrm{g} / \mathrm{hr})\end{array}$ & $\begin{array}{c}\text { Extrapolated } \\
\text { Wetwood F } \\
(\mathrm{g} / \mathrm{hr})\end{array}$ & $\begin{array}{c}\text { Extrapolated } \\
\text { Total Wood F } \\
(\mathrm{g} / \mathrm{hr})\end{array}$ & $\begin{array}{l}\text { Relative } \\
\text { Humidity, } \\
@ 10 \mathrm{~m}(\%)\end{array}$ \\
\hline & 8/3/2008 12:00 & 287 & 216 & 1200 & 5.922 & 5.922 & & 0.6447 & 6.93E-03 & 1519.5316 & 559.9027 & 2079.4343 & \\
\hline & 8/3/2008 13:00 & 288 & 216 & 1300 & 5.965 & 5.965 & & 0.6329 & $6.78 \mathrm{E}-03$ & 1485.2058 & 547.2546 & 2032.4605 & \\
\hline & 8/3/2008 14:00 & 289 & 216 & 1400 & NAN & 6.0155 & & 0.6192 & $6.60 \mathrm{E}-03$ & 1445.7044 & 532.6995 & 1978.4040 & \\
\hline & $8 / 3 / 2008$ 15:00 & 290 & 216 & 1500 & 6.066 & 6.066 & & 0.6057 & 6.42E-03 & 1407.0572 & 518.4592 & 1925.5164 & \\
\hline & $8 / 3 / 2008$ 16:00 & 291 & 216 & 1600 & 6.099 & 6.099 & & 0.5970 & 6.31E-03 & 1382.2532 & 509.3196 & 1891.5728 & \\
\hline & 8/3/2008 17:00 & 292 & 216 & 1700 & 6.137 & 6.137 & & 0.5871 & $6.18 \mathrm{E}-03$ & 1354.1228 & 498.9544 & 1853.0772 & \\
\hline & 8/3/2008 18:00 & 293 & 216 & 1800 & 6.189 & 6.189 & & 0.5738 & $6.01 E-03$ & 1316.3616 & 485.0405 & 1801.4021 & \\
\hline & 8/3/2008 19:00 & 294 & 216 & 1900 & 6.273 & 6.273 & & 0.5527 & $5.74 \mathrm{E}-03$ & 1257.0989 & 463.2039 & 1720.3028 & \\
\hline & $8 / 3 / 200820: 00$ & 295 & 216 & 2000 & NAN & 6.273 & & 0.5527 & $5.74 \mathrm{E}-03$ & 1257.0989 & 463.2039 & 1720.3028 & \\
\hline & 8/3/2008 21:00 & 296 & 216 & 2100 & NAN & 8.26 & & 0.1792 & 1.43E-03 & 314.1724 & 115.7633 & 429.9357 & \\
\hline & 8/3/2008 22:00 & 297 & 216 & 2200 & 8.26 & 8.26 & & 0.1792 & 1.43E-03 & 314.1724 & 115.7633 & 429.9357 & \\
\hline & 8/3/2008 23:00 & 298 & 216 & 2300 & 8.41 & 8.41 & & 0.1581 & $1.23 \mathrm{E}-03$ & 269.4113 & 99.2701 & 368.6815 & \\
\hline & $8 / 4 / 20080: 00$ & 299 & 217 & 0 & 8.44 & 8.44 & 9.41 & 0.1540 & 1.19E-03 & 260.8045 & 96.0988 & 356.9033 & \\
\hline & $8 / 4 / 2008$ 1:00 & 300 & 217 & 100 & 8.47 & 8.47 & & 0.1499 & 1.15E-03 & 252.3110 & 92.9692 & 345.2802 & \\
\hline & $8 / 4 / 20082: 00$ & 301 & 217 & 200 & 8.12 & 8.12 & & 0.1995 & 1.64E-03 & 358.6145 & 132.1389 & 490.7534 & \\
\hline & $8 / 4 / 20083: 00$ & 302 & 217 & 300 & 7.898 & 7.898 & & 0.2332 & 1.98E-03 & 434.6166 & 160.1434 & 594.7600 & \\
\hline & $8 / 4 / 20084: 00$ & 303 & 217 & 400 & 7.672 & 7.672 & & 0.2696 & 2.37E-03 & 519.3960 & 191.3821 & 710.7781 & \\
\hline & $8 / 4 / 20085: 00$ & 304 & 217 & 500 & 7.819 & 7.819 & & 0.2457 & $2.11 \mathrm{E}-03$ & 463.3737 & 170.7396 & 634.1133 & \\
\hline & 8/4/2008 6:00 & 305 & 217 & 600 & 8.3 & 8.3 & & 0.1735 & 1.38E-03 & 301.9517 & 111.2603 & 413.2120 & \\
\hline & 8/4/2008 7:00 & 306 & 217 & 700 & 8.38 & 8.38 & & 0.1623 & $1.27 \mathrm{E}-03$ & 278.1322 & 102.4835 & 380.6158 & \\
\hline & $8 / 4 / 2008$ 8:00 & 307 & 217 & 800 & 8.09 & 8.09 & & 0.2040 & $1.68 \mathrm{E}-03$ & 368.4821 & 135.7748 & 504.2570 & \\
\hline & $8 / 4 / 20089: 00$ & 308 & 217 & 900 & 7.515 & 7.515 & & 0.2961 & $2.66 \mathrm{E}-03$ & 583.0060 & 214.8205 & 797.8265 & \\
\hline & 8/4/2008 10:00 & 309 & 217 & 1000 & 6.449 & 6.449 & & 0.5103 & $5.20 \mathrm{E}-03$ & 1139.5250 & 419.8814 & 1559.4064 & \\
\hline & 8/4/2008 11:00 & 310 & 217 & 1100 & 5.974 & 5.974 & & 0.6304 & $6.74 \mathrm{E}-03$ & 1478.1024 & 544.6372 & 2022.7396 & \\
\hline & 8/4/2008 12:00 & 311 & 217 & 1200 & 6.082 & 6.082 & & 0.6014 & $6.36 \mathrm{E}-03$ & 1394.9871 & 514.0117 & 1908.9988 & \\
\hline & 8/4/2008 13:00 & 312 & 217 & 1300 & 6.107 & 6.107 & & 0.5949 & $6.28 \mathrm{E}-03$ & 1376.2929 & 507.1234 & 1883.4163 & \\
\hline & $8 / 4 / 2008$ 14:00 & 313 & 217 & 1400 & 6.381 & 6.381 & & 0.5264 & $5.40 \mathrm{E}-03$ & 1183.9267 & 436.2421 & 1620.1688 & \\
\hline & $8 / 4 / 2008$ 15:00 & 314 & 217 & 1500 & 6.664 & 6.664 & & 0.4616 & 4.59E-03 & 1007.0980 & 371.0860 & 1378.1840 & \\
\hline & $8 / 4 / 2008$ 16:00 & 315 & 217 & 1600 & 6.174 & 6.174 & & 0.5776 & $6.05 \mathrm{E}-03$ & 1327.1684 & 489.0225 & 1816.1909 & \\
\hline & 8/4/2008 17:00 & 316 & 217 & 1700 & 6.141 & 6.141 & & 0.5861 & $6.16 \mathrm{E}-03$ & 1351.1882 & 497.8731 & 1849.0613 & \\
\hline & 8/4/2008 18:00 & 317 & 217 & 1800 & 6.231 & 6.231 & & 0.5632 & 5.87E-03 & 1286.4665 & 474.0250 & 1760.4915 & \\
\hline & 8/4/2008 19:00 & 318 & 217 & 1900 & 6.45 & 6.45 & & 0.5101 & $5.20 \mathrm{E}-03$ & 1138.8813 & 419.6442 & 1558.5256 & \\
\hline & 8/4/2008 20:00 & 319 & 217 & 2000 & NAN & 7.565 & & 0.2875 & $2.57 \mathrm{E}-03$ & 562.3114 & 207.1952 & 769.5066 & \\
\hline & 8/4/2008 21:00 & 320 & 217 & 2100 & 8.68 & 8.68 & & 0.1221 & 8.94E-04 & 195.9801 & 72.2129 & 268.1931 & \\
\hline & 8/4/2008 22:00 & 321 & 217 & 2200 & 9.28 & 9.28 & & 0.0496 & $2.95 \mathrm{E}-04$ & 64.5924 & 23.8004 & 88.3928 & \\
\hline & $8 / 4 / 200823: 00$ & 322 & 217 & 2300 & 9.41 & 9.41 & & 0.0351 & 1.92E-04 & 42.1871 & 15.5447 & 57.7319 & \\
\hline & 8/5/2008 0:00 & 323 & 218 & 0 & 9.58 & 9.58 & 9.83 & 0.0167 & 7.72E-05 & 16.9274 & 6.2372 & 23.1646 & \\
\hline & 8/5/2008 1:00 & 324 & 218 & 100 & 9.69 & 9.69 & & 0.0052 & 1.82E-05 & 3.9870 & 1.4691 & 5.4561 & \\
\hline & $8 / 5 / 20082: 00$ & 325 & 218 & 200 & 9.8 & 9.8 & & -0.0061 & $0.00 \mathrm{E}+00$ & 0.0000 & 0.0000 & 0.0000 & \\
\hline & 8/5/2008 3:00 & 326 & 218 & 300 & 9.72 & 9.72 & & 0.0021 & 5.87E-06 & 1.2857 & 0.4737 & 1.7594 & \\
\hline & 8/5/2008 4:00 & 327 & 218 & 400 & 9.62 & 9.62 & & 0.0125 & 5.39E-05 & 11.8185 & 4.3548 & 16.1733 & \\
\hline
\end{tabular}




\begin{tabular}{|c|c|c|c|c|c|c|c|c|c|c|c|c|c|}
\hline Tree ID & TS & RN & JD & HR & $\begin{array}{c}\text { Raw } \\
\text { TC_dTA }\end{array}$ & $\begin{array}{c}\text { Fixed } \\
\text { TC_dT } \Delta\end{array}$ & dTM & $\mathbf{K}$ & $\begin{array}{c}\text { Sap } \\
\text { Velocity } \\
(\mathrm{cm} / \mathrm{s})\end{array}$ & $\begin{array}{c}\text { Probed } \\
\text { Drywood F } \\
(\mathrm{g} / \mathrm{hr})\end{array}$ & $\begin{array}{c}\text { Extrapolated } \\
\text { Wetwood F } \\
(\mathrm{g} / \mathrm{hr})\end{array}$ & $\begin{array}{c}\text { Extrapolated } \\
\text { Total Wood F } \\
(\mathrm{g} / \mathrm{hr})\end{array}$ & $\begin{array}{l}\text { Relative } \\
\text { Humidity, } \\
\text { @10m (\%) }\end{array}$ \\
\hline & 8/5/2008 5:00 & 328 & 218 & 500 & $\begin{array}{r}9.69 \\
\end{array}$ & $\begin{array}{r}9.69 \\
\end{array}$ & & 0.0052 & 1.82E-05 & 3.9870 & 1.4691 & 5.4561 & \\
\hline & 8/5/2008 6:00 & 329 & 218 & 600 & 9.78 & 9.78 & & -0.0041 & $0.00 \mathrm{E}+00$ & 0.0000 & 0.0000 & 0.0000 & \\
\hline & 8/5/2008 7:00 & 330 & 218 & 700 & 9.79 & 9.79 & & -0.0051 & $0.00 E+00$ & 0.0000 & 0.0000 & 0.0000 & \\
\hline & 8/5/2008 8:00 & 331 & 218 & 800 & 9.58 & 9.58 & & 0.0167 & 7.72E-05 & 16.9274 & 6.2372 & 23.1646 & \\
\hline & 8/5/2008 9:00 & 332 & 218 & 900 & 7.88 & 7.88 & & 0.2360 & $2.01 \mathrm{E}-03$ & 441.0877 & 162.5279 & 603.6156 & \\
\hline & 8/5/2008 10:00 & 333 & 218 & 1000 & 6.298 & 6.298 & & 0.5465 & $5.66 \mathrm{E}-03$ & 1239.8640 & 456.8534 & 1696.7173 & \\
\hline & 8/5/2008 11:00 & 334 & 218 & 1100 & 6.739 & 6.739 & & 0.4453 & 4.40E-03 & 963.5888 & 355.0541 & 1318.6430 & \\
\hline & 8/5/2008 12:00 & 335 & 218 & 1200 & 6.416 & 6.416 & & 0.5181 & $5.30 \mathrm{E}-03$ & 1160.9155 & 427.7632 & 1588.6787 & \\
\hline & 8/5/2008 13:00 & 336 & 218 & 1300 & 6.112 & 6.112 & & 0.5936 & $6.26 \mathrm{E}-03$ & 1372.5781 & 505.7546 & 1878.3327 & \\
\hline & $8 / 5 / 2008$ 14:00 & 337 & 218 & 1400 & 6.661 & 6.661 & & 0.4622 & $4.60 \mathrm{E}-03$ & 1008.8663 & 371.7375 & 1380.6038 & \\
\hline & 8/5/2008 15:00 & 338 & 218 & 1500 & 6.977 & 6.977 & & 0.3960 & 3.80E-03 & 833.9922 & 307.3016 & 1141.2938 & \\
\hline & 8/5/2008 16:00 & 339 & 218 & 1600 & 6.844 & 6.844 & & 0.4231 & 4.13E-03 & 904.8691 & 333.4176 & 1238.2867 & \\
\hline & 8/5/2008 17:00 & 340 & 218 & 1700 & 6.396 & 6.396 & & 0.5228 & $5.36 \mathrm{E}-03$ & 1174.0235 & 432.5931 & 1606.6166 & \\
\hline & 8/5/2008 18:00 & 341 & 218 & 1800 & NAN & 6.3935 & & 0.5234 & $5.36 \mathrm{E}-03$ & 1175.6697 & 433.1997 & 1608.8694 & \\
\hline & 8/5/2008 19:00 & 342 & 218 & 1900 & 6.391 & 6.391 & & 0.5240 & 5.37E-03 & 1177.3177 & 433.8069 & 1611.1246 & \\
\hline & $8 / 5 / 200820: 00$ & 343 & 218 & 2000 & 7.266 & 7.266 & & 0.3405 & $3.16 \mathrm{E}-03$ & 692.4664 & 255.1535 & 947.6199 & \\
\hline & 8/5/2008 21:00 & 344 & 218 & 2100 & 9.18 & 9.18 & & 0.0610 & 3.80E-04 & 83.3947 & 30.7285 & 114.1232 & \\
\hline & 8/5/2008 22:00 & 345 & 218 & 2200 & 9.76 & 9.76 & & -0.0020 & $0.00 \mathrm{E}+00$ & 0.0000 & 0.0000 & 0.0000 & \\
\hline & $8 / 5 / 2008$ 23:00 & 346 & 218 & 2300 & 9.83 & 9.83 & & -0.0092 & $0.00 \mathrm{E}+00$ & 0.0000 & 0.0000 & 0.0000 & \\
\hline & $8 / 6 / 20080: 00$ & 347 & 219 & 0 & 9.8 & 9.8 & 9.80 & -0.0061 & $0.00 \mathrm{E}+00$ & 0.0000 & 0.0000 & 0.0000 & \\
\hline & 8/6/2008 1:00 & 348 & 219 & 100 & 9.75 & 9.75 & & -0.0010 & $0.00 \mathrm{E}+00$ & 0.0000 & 0.0000 & 0.0000 & \\
\hline & $8 / 6 / 20082: 00$ & 349 & 219 & 200 & 9.74 & 9.74 & & 0.0000 & $0.00 \mathrm{E}+00$ & 0.0000 & 0.0000 & 0.0000 & \\
\hline & 8/6/2008 3:00 & 350 & 219 & 300 & 9.69 & 9.69 & & 0.0052 & 1.82E-05 & 3.9870 & 1.4691 & 5.4561 & \\
\hline & 8/6/2008 4:00 & 351 & 219 & 400 & NAN & 9.62 & & 0.0125 & $5.39 \mathrm{E}-05$ & 11.8185 & 4.3548 & 16.1733 & \\
\hline & 8/6/2008 5:00 & 352 & 219 & 500 & 9.55 & 9.55 & & 0.0199 & 9.58E-05 & 20.9962 & 7.7365 & 28.7326 & \\
\hline & 8/6/2008 6:00 & 353 & 219 & 600 & 9.49 & 9.49 & & 0.0263 & 1.35E-04 & 29.6639 & 10.9303 & 40.5941 & \\
\hline & 8/6/2008 7:00 & 354 & 219 & 700 & 9.45 & 9.45 & & 0.0307 & 1.63E-04 & 35.7959 & 13.1897 & 48.9857 & \\
\hline & 8/6/2008 8:00 & 355 & 219 & 800 & 9.37 & 9.37 & & 0.0395 & 2.23E-04 & 48.8229 & 17.9898 & 66.8126 & \\
\hline & 8/6/2008 9:00 & 356 & 219 & 900 & 9.07 & 9.07 & & 0.0739 & $4.82 E-04$ & 105.5509 & 38.8924 & 144.4433 & \\
\hline & $8 / 6 / 2008$ 10:00 & 357 & 219 & 1000 & 6.187 & 6.187 & & 0.5743 & 6.01E-03 & 1317.7985 & 485.5700 & 1803.3685 & \\
\hline & 8/6/2008 11:00 & 358 & 219 & 1100 & 5.556 & 5.556 & & 0.7531 & 8.39E-03 & 1839.7354 & 677.8884 & 2517.6238 & \\
\hline & 8/6/2008 12:00 & 359 & 219 & 1200 & 6.373 & 6.373 & & 0.5283 & $5.43 \mathrm{E}-03$ & 1189.2338 & 438.1977 & 1627.4315 & \\
\hline & 8/6/2008 13:00 & 360 & 219 & 1300 & $\begin{array}{r}6.873 \\
\end{array}$ & 6.873 & & 0.4171 & 4.06E-03 & 889.0878 & 327.6027 & 1216.6905 & \\
\hline & 8/6/2008 14:00 & 361 & 219 & 1400 & NAN & 6.873 & & 0.4171 & 4.06E-03 & 889.0878 & 327.6027 & 1216.6905 & \\
\hline & 8/6/2008 15:00 & 362 & 219 & 1500 & NAN & 6.115 & & 0.5928 & $6.25 \mathrm{E}-03$ & 1370.3530 & 504.9347 & 1875.2877 & \\
\hline & 8/6/2008 16:00 & 363 & 219 & 1600 & 6.115 & 6.115 & & 0.5928 & $6.25 \mathrm{E}-03$ & 1370.3530 & 504.9347 & 1875.2877 & \\
\hline & 8/6/2008 17:00 & 364 & 219 & 1700 & 6.065 & 6.065 & & 0.6059 & $6.42 \mathrm{E}-03$ & 1407.8144 & 518.7382 & 1926.5526 & \\
\hline & 8/6/2008 18:00 & 365 & 219 & 1800 & NAN & 6.306 & & 0.5446 & 5.63E-03 & 1234.3871 & 454.8353 & 1689.2223 & \\
\hline & 8/6/2008 19:00 & 366 & 219 & 1900 & 6.547 & 6.547 & & 0.4877 & $4.92 \mathrm{E}-03$ & 1077.7030 & 397.1018 & 1474.8048 & \\
\hline & $8 / 6 / 200820: 00$ & 367 & 219 & 2000 & 6.971 & 6.971 & & 0.3972 & 3.82E-03 & 837.1083 & 308.4498 & 1145.5581 & \\
\hline & 8/6/2008 21:00 & 368 & 219 & 2100 & 8.63 & 8.63 & & 0.1286 & 9.53E-04 & 208.9015 & 76.9741 & 285.8756 & \\
\hline
\end{tabular}




\begin{tabular}{|c|c|c|c|c|c|c|c|c|c|c|c|c|c|}
\hline Tree ID & TS & RN & JD & HR & $\begin{array}{c}\text { Raw } \\
\text { TC_dTA }\end{array}$ & $\begin{array}{c}\text { Fixed } \\
\text { TC_dT } \Delta\end{array}$ & dTM & $\mathrm{K}$ & $\begin{array}{l}\text { Sap } \\
\text { Velocity } \\
(\mathrm{cm} / \mathrm{s})\end{array}$ & $\begin{array}{l}\text { Probed } \\
\text { Drywood F } \\
\text { (g/hr) }\end{array}$ & $\begin{array}{c}\text { Extrapolated } \\
\text { Wetwood F } \\
(\mathrm{g} / \mathrm{hr})\end{array}$ & $\begin{array}{c}\text { Extrapolated } \\
\text { Total Wood F } \\
(\mathrm{g} / \mathrm{hr})\end{array}$ & $\begin{array}{l}\text { Relative } \\
\text { Humidity, } \\
@ 10 \mathrm{~m}(\%)\end{array}$ \\
\hline & 8/6/2008 22:00 & 369 & 219 & 2200 & 9.57 & 9.57 & & 0.0178 & 8.33E-05 & 18.2624 & 6.7292 & 24.9916 & \\
\hline & 8/6/2008 23:00 & 370 & 219 & 2300 & 9.69 & 9.69 & & 0.0052 & 1.82E-05 & 3.9870 & 1.4691 & 5.4561 & \\
\hline & $8 / 7 / 20080: 00$ & 371 & 220 & 0 & 9.72 & 9.72 & 9.72 & 0.0021 & 5.87E-06 & 1.2857 & 0.4737 & 1.7594 & \\
\hline & $8 / 7 / 2008$ 1:00 & 372 & 220 & 100 & 9.66 & 9.66 & & 0.0083 & $3.26 \mathrm{E}-05$ & 7.1380 & 2.6301 & 9.7681 & \\
\hline & 8/7/2008 2:00 & 373 & 220 & 200 & 9.58 & 9.58 & & 0.0167 & 7.72E-05 & 16.9274 & 6.2372 & 23.1646 & \\
\hline & 8/7/2008 3:00 & 374 & 220 & 300 & NAN & 9.505 & & 0.0247 & $1.25 E-04$ & 27.4349 & 10.1090 & 37.5439 & \\
\hline & 8/7/2008 4:00 & 375 & 220 & 400 & 9.43 & 9.43 & & 0.0329 & $1.78 \mathrm{E}-04$ & 38.9602 & 14.3557 & 53.3158 & \\
\hline & $8 / 7 / 20085: 00$ & 376 & 220 & 500 & 9.35 & 9.35 & & 0.0417 & $2.38 \mathrm{E}-04$ & 52.2288 & 19.2448 & 71.4735 & \\
\hline & 8/7/2008 6:00 & 377 & 220 & 600 & 9.28 & 9.28 & & 0.0496 & 2.95E-04 & 64.5924 & 23.8004 & 88.3928 & \\
\hline & 8/7/2008 7:00 & 378 & 220 & 700 & 9.23 & 9.23 & & 0.0553 & 3.37E-04 & 73.8302 & 27.2042 & 101.0344 & \\
\hline & 8/7/2008 8:00 & 379 & 220 & 800 & 9.18 & 9.18 & & 0.0610 & $3.80 \mathrm{E}-04$ & 83.3947 & 30.7285 & 114.1232 & \\
\hline & $8 / 7 / 20089: 00$ & 380 & 220 & 900 & 7.889 & 7.889 & & 0.2346 & $2.00 \mathrm{E}-03$ & 437.8462 & 161.3334 & 599.1797 & \\
\hline & 8/7/2008 10:00 & 381 & 220 & 1000 & 5.443 & 5.443 & & 0.7895 & 8.90E-03 & 1949.7902 & 718.4403 & 2668.2306 & \\
\hline & 8/7/2008 11:00 & 382 & 220 & 1100 & 5.289 & 5.289 & & 0.8416 & $9.62 \mathrm{E}-03$ & 2109.3894 & 777.2479 & 2886.6374 & \\
\hline & 8/7/2008 12:00 & 383 & 220 & 1200 & 5.669 & 5.669 & & 0.7181 & 7.92E-03 & 1735.2176 & 639.3766 & 2374.5942 & \\
\hline & 8/7/2008 13:00 & 384 & 220 & 1300 & 5.783 & 5.783 & & 0.6842 & 7.46E-03 & 1635.0286 & 602.4599 & 2237.4885 & \\
\hline & 8/7/2008 14:00 & 385 & 220 & 1400 & 5.827 & 5.827 & & 0.6715 & $7.29 \mathrm{E}-03$ & 1597.6996 & 588.7053 & 2186.4049 & \\
\hline & $8 / 7 / 2008$ 15:00 & 386 & 220 & 1500 & 5.902 & 5.902 & & 0.6503 & 7.01E-03 & 1535.7182 & 565.8670 & 2101.5851 & \\
\hline & 8/7/2008 16:00 & 387 & 220 & 1600 & NAN & 5.919 & & 0.6455 & 6.94E-03 & 1521.9506 & 560.7940 & 2082.7446 & \\
\hline & 8/7/2008 17:00 & 388 & 220 & 1700 & 5.936 & 5.936 & & 0.6408 & $6.88 \mathrm{E}-03$ & 1508.2850 & 555.7586 & 2064.0436 & \\
\hline & 8/7/2008 18:00 & 389 & 220 & 1800 & 6.03 & 6.03 & & 0.6153 & $6.54 \mathrm{E}-03$ & 1434.5213 & 528.5789 & 1963.1002 & \\
\hline & 8/7/2008 19:00 & 390 & 220 & 1900 & 6.259 & 6.259 & & 0.5562 & $5.78 \mathrm{E}-03$ & 1266.8301 & 466.7896 & 1733.6198 & \\
\hline & 8/7/2008 20:00 & 391 & 220 & 2000 & 6.808 & 6.808 & & 0.4307 & $4.22 \mathrm{E}-03$ & 924.7197 & 340.7320 & 1265.4517 & \\
\hline & 8/7/2008 21:00 & 392 & 220 & 2100 & 8.51 & 8.51 & & 0.1445 & $1.10 \mathrm{E}-03$ & 241.1616 & 88.8610 & 330.0226 & \\
\hline & 8/7/2008 22:00 & 393 & 220 & 2200 & 9.32 & 9.32 & & 0.0451 & 2.62E-04 & 57.4443 & 21.1665 & 78.6109 & \\
\hline & 8/7/2008 23:00 & 394 & 220 & 2300 & 9.59 & 9.59 & & 0.0156 & $7.12 \mathrm{E}-05$ & 15.6145 & 5.7535 & 21.3680 & \\
\hline & 8/8/2008 0:00 & 395 & 221 & 0 & 9.69 & 9.69 & 9.69 & 0.0052 & 1.82E-05 & 3.9870 & 1.4691 & 5.4561 & \\
\hline & $8 / 8 / 20081: 00$ & 396 & 221 & 100 & 9.66 & 9.66 & & 0.0083 & $3.26 \mathrm{E}-05$ & 7.1380 & 2.6301 & 9.7681 & \\
\hline & $8 / 8 / 20082: 00$ & 397 & 221 & 200 & NAN & 9.655 & & 0.0088 & $3.51 \mathrm{E}-05$ & 7.6960 & 2.8357 & 10.5317 & \\
\hline & 8/8/2008 3:00 & 398 & 221 & 300 & 9.65 & 9.65 & & 0.0093 & 3.77E-05 & 8.2622 & 3.0444 & 11.3066 & \\
\hline & 8/8/2008 4:00 & 399 & 221 & 400 & 9.57 & 9.57 & & 0.0178 & 8.33E-05 & 18.2624 & 6.7292 & 24.9916 & \\
\hline & 8/8/2008 5:00 & 400 & 221 & 500 & 9.49 & 9.49 & & 0.0263 & 1.35E-04 & 29.6639 & 10.9303 & 40.5941 & \\
\hline & 8/8/2008 6:00 & 401 & 221 & 600 & 9.4 & 9.4 & & 0.0362 & 2.00E-04 & 43.8236 & 16.1477 & 59.9714 & \\
\hline & 8/8/2008 7:00 & 402 & 221 & 700 & 9.33 & 9.33 & & 0.0439 & 2.54E-04 & 55.6918 & 20.5208 & 76.2125 & \\
\hline & 8/8/2008 8:00 & 403 & 221 & 800 & 9.08 & 9.08 & & 0.0727 & 4.72E-04 & 103.4745 & 38.1273 & 141.6018 & \\
\hline & 8/8/2008 9:00 & 404 & 221 & 900 & 6.751 & 6.751 & & 0.4427 & $4.36 \mathrm{E}-03$ & 956.7503 & 352.5343 & 1309.2846 & \\
\hline & 8/8/2008 10:00 & 405 & 221 & 1000 & 5.394 & 5.394 & & 0.8057 & $9.12 \mathrm{E}-03$ & 1999.3297 & 736.6941 & 2736.0238 & \\
\hline & 8/8/2008 11:00 & 406 & 221 & 1100 & 5.519 & 5.519 & & 0.7648 & 8.55E-03 & 1875.1434 & 690.9352 & 2566.0785 & \\
\hline & 8/8/2008 12:00 & 407 & 221 & 1200 & 5.699 & 5.699 & & 0.7091 & 7.79E-03 & 1708.3544 & 629.4783 & 2337.8327 & \\
\hline & 8/8/2008 13:00 & 408 & 221 & 1300 & 5.795 & 5.795 & & 0.6808 & 7.41E-03 & 1624.7757 & 598.6820 & 2223.4577 & \\
\hline & 8/8/2008 14:00 & 409 & 221 & 1400 & 5.825 & 5.825 & & 0.6721 & 7.30E-03 & 1599.3807 & 589.3247 & 2188.7054 & \\
\hline
\end{tabular}




\begin{tabular}{|c|c|c|c|c|c|c|c|c|c|c|c|c|c|}
\hline Tree ID & TS & RN & JD & HR & $\begin{array}{c}\text { Raw } \\
\text { TC_dTA }\end{array}$ & $\begin{array}{c}\text { Fixed } \\
\text { TC_dT } \Delta\end{array}$ & dTM & $\mathrm{K}$ & $\begin{array}{l}\text { Sap } \\
\text { Velocity } \\
(\mathrm{cm} / \mathrm{s})\end{array}$ & $\begin{array}{l}\text { Probed } \\
\text { Drywood F } \\
(\mathrm{g} / \mathrm{hr})\end{array}$ & $\begin{array}{c}\text { Extrapolated } \\
\text { Wetwood F } \\
(\mathrm{g} / \mathrm{hr})\end{array}$ & $\begin{array}{c}\text { Extrapolated } \\
\text { Total Wood F } \\
(\mathrm{g} / \mathrm{hr})\end{array}$ & $\begin{array}{l}\text { Relative } \\
\text { Humidity, } \\
@ 10 \mathrm{~m}(\%)\end{array}$ \\
\hline & 8/8/2008 15:00 & 410 & 221 & 1500 & 5.868 & 5.868 & & 0.6599 & 7.13E-03 & 1563.5632 & 576.1270 & 2139.6902 & \\
\hline & 8/8/2008 16:00 & 411 & 221 & 1600 & 5.879 & 5.879 & & 0.6567 & 7.09E-03 & 1554.5090 & 572.7908 & 2127.2998 & \\
\hline & 8/8/2008 17:00 & 412 & 221 & 1700 & 6.021 & 6.021 & & 0.6177 & $6.58 \mathrm{E}-03$ & 1441.4543 & 531.1335 & 1972.5878 & \\
\hline & 8/8/2008 18:00 & 413 & 221 & 1800 & 6.254 & 6.254 & & 0.5574 & $5.80 \mathrm{E}-03$ & 1270.3196 & 468.0754 & 1738.3950 & \\
\hline & $8 / 8 / 2008$ 19:00 & 414 & 221 & 1900 & 6.173 & 6.173 & & 0.5778 & $6.06 \mathrm{E}-03$ & 1327.8914 & 489.2889 & 1817.1802 & \\
\hline & 8/8/2008 20:00 & 415 & 221 & 2000 & 6.911 & 6.911 & & 0.4093 & $3.96 \mathrm{E}-03$ & 868.6877 & 320.0858 & 1188.7735 & \\
\hline & 8/8/2008 21:00 & 416 & 221 & 2100 & NAN & 7.9605 & & 0.2235 & 1.88E-03 & 412.5130 & 151.9989 & 564.5118 & \\
\hline & 8/8/2008 22:00 & 417 & 221 & 2200 & 9.01 & 9.01 & & 0.0810 & $5.40 \mathrm{E}-04$ & 118.2667 & 43.5778 & 161.8445 & \\
\hline & 8/8/2008 23:00 & 418 & 221 & 2300 & NAN & 9.15 & & 0.0645 & 4.07E-04 & 89.2869 & 32.8996 & 122.1865 & \\
\hline & 8/9/2008 0:00 & 419 & 222 & 0 & 9.29 & 9.29 & 9.59 & 0.0484 & 2.86E-04 & 62.7850 & 23.1344 & 85.9194 & \\
\hline & 8/9/2008 1:00 & 420 & 222 & 100 & 9.26 & 9.26 & & 0.0518 & $3.11 E-04$ & 68.2477 & 25.1473 & 93.3950 & \\
\hline & $8 / 9 / 20082: 00$ & 421 & 222 & 200 & 9.31 & 9.31 & & 0.0462 & $2.70 \mathrm{E}-04$ & 59.2108 & 21.8174 & 81.0282 & \\
\hline & 8/9/2008 3:00 & 422 & 222 & 300 & 9.3 & 9.3 & & 0.0473 & $2.78 \mathrm{E}-04$ & 60.9910 & 22.4734 & 83.4644 & \\
\hline & 8/9/2008 4:00 & 423 & 222 & 400 & 9.24 & 9.24 & & 0.0541 & $3.28 \mathrm{E}-04$ & 71.9562 & 26.5137 & 98.4699 & \\
\hline & 8/9/2008 5:00 & 424 & 222 & 500 & 9.12 & 9.12 & & 0.0680 & 4.35E-04 & 95.2925 & 35.1125 & 130.4050 & \\
\hline & 8/9/2008 6:00 & 425 & 222 & 600 & 9.12 & 9.12 & & 0.0680 & $4.35 \mathrm{E}-04$ & 95.2925 & 35.1125 & 130.4050 & \\
\hline & 8/9/2008 7:00 & 426 & 222 & 700 & 8.71 & 8.71 & & 0.1183 & 8.59E-04 & 188.3730 & 69.4099 & 257.7829 & \\
\hline & 8/9/2008 8:00 & 427 & 222 & 800 & 7.865 & 7.865 & & 0.2384 & $2.04 \mathrm{E}-03$ & 446.5168 & 164.5283 & 611.0450 & \\
\hline & 8/9/2008 9:00 & 428 & 222 & 900 & 8.51 & 8.51 & & 0.1445 & 1.10E-03 & 241.1616 & 88.8610 & 330.0226 & \\
\hline & 8/9/2008 10:00 & 429 & 222 & 1000 & 8.07 & 8.07 & & 0.2069 & $1.71 \mathrm{E}-03$ & 375.1293 & 138.2241 & 513.3534 & \\
\hline & 8/9/2008 11:00 & 430 & 222 & 1100 & 7.312 & 7.312 & & 0.3321 & $3.06 \mathrm{E}-03$ & 671.4148 & 247.3966 & 918.8114 & \\
\hline & 8/9/2008 12:00 & 431 & 222 & 1200 & 6.403 & 6.403 & & 0.5212 & 5.34E-03 & 1169.4233 & 430.8981 & 1600.3213 & \\
\hline & 8/9/2008 13:00 & 432 & 222 & 1300 & 6.19 & 6.19 & & 0.5735 & $6.00 \mathrm{E}-03$ & 1315.6436 & 484.7759 & 1800.4195 & \\
\hline & $8 / 9 / 2008$ 14:00 & 433 & 222 & 1400 & 5.816 & 5.816 & & 0.6747 & 7.33E-03 & 1606.9638 & 592.1189 & 2199.0827 & \\
\hline & 8/9/2008 15:00 & 434 & 222 & 1500 & 5.852 & 5.852 & & 0.6644 & 7.19E-03 & 1576.8114 & 581.0086 & 2157.8199 & \\
\hline & 8/9/2008 16:00 & 435 & 222 & 1600 & 5.918 & 5.918 & & 0.6458 & $6.95 \mathrm{E}-03$ & 1522.7576 & 561.0914 & 2083.8490 & \\
\hline & 8/9/2008 17:00 & 436 & 222 & 1700 & 6.075 & 6.075 & & 0.6033 & $6.39 \mathrm{E}-03$ & 1400.2576 & 515.9537 & 1916.2113 & \\
\hline & $8 / 9 / 2008$ 18:00 & 437 & 222 & 1800 & 6.069 & 6.069 & & 0.6049 & $6.41 \mathrm{E}-03$ & 1404.7878 & 517.6229 & 1922.4107 & \\
\hline & $8 / 9 / 2008$ 19:00 & 438 & 222 & 1900 & 6.282 & 6.282 & & 0.5505 & 5.71E-03 & 1250.8733 & 460.9100 & 1711.7834 & \\
\hline & 8/9/2008 20:00 & 439 & 222 & 2000 & 6.933 & 6.933 & & 0.4049 & $3.91 \mathrm{E}-03$ & 857.0196 & 315.7865 & 1172.8061 & \\
\hline & 8/9/2008 21:00 & 440 & 222 & 2100 & 8.79 & 8.79 & & 0.1081 & 7.69E-04 & 168.6187 & 62.1310 & 230.7498 & \\
\hline & 8/9/2008 22:00 & 441 & 222 & 2200 & 9.55 & 9.55 & & 0.0199 & $9.58 \mathrm{E}-05$ & 20.9962 & 7.7365 & 28.7326 & \\
\hline & 8/9/2008 23:00 & 442 & 222 & 2300 & 9.59 & 9.59 & & 0.0156 & 7.12E-05 & 15.6145 & 5.7535 & 21.3680 & \\
\hline & 8/10/2008 0:00 & 443 & 223 & 0 & 9.54 & 9.54 & 9.54 & 0.0210 & 1.02E-04 & 22.3935 & 8.2514 & 30.6449 & \\
\hline & 8/10/2008 1:00 & 444 & 223 & 100 & 9.5 & 9.5 & & 0.0253 & 1.29E-04 & 28.1735 & 10.3811 & 38.5546 & \\
\hline & $8 / 10 / 2008$ 2:00 & 445 & 223 & 200 & 9.43 & 9.43 & & 0.0329 & $1.78 \mathrm{E}-04$ & 38.9602 & 14.3557 & 53.3158 & \\
\hline & 8/10/2008 3:00 & 446 & 223 & 300 & 9.35 & 9.35 & & 0.0417 & $2.38 \mathrm{E}-04$ & 52.2288 & 19.2448 & 71.4735 & \\
\hline & 8/10/2008 4:00 & 447 & 223 & 400 & NAN & 9.245 & & 0.0535 & $3.24 \mathrm{E}-04$ & 71.0241 & 26.1703 & 97.1944 & \\
\hline & 8/10/2008 5:00 & 448 & 223 & 500 & 9.14 & 9.14 & & 0.0656 & $4.16 \mathrm{E}-04$ & 91.2762 & 33.6326 & 124.9089 & \\
\hline & 8/10/2008 6:00 & 449 & 223 & 600 & 9.03 & 9.03 & & 0.0786 & $5.20 \mathrm{E}-04$ & 113.9792 & 41.9980 & 155.9772 & \\
\hline & 8/10/2008 7:00 & 450 & 223 & 700 & 8.97 & 8.97 & & 0.0858 & $5.79 \mathrm{E}-04$ & 126.9875 & 46.7912 & 173.7786 & \\
\hline
\end{tabular}




\begin{tabular}{|c|c|c|c|c|c|c|c|c|c|c|c|c|c|}
\hline Tree ID & TS & RN & JD & HR & $\begin{array}{c}\text { Raw } \\
\text { TC_dTA }\end{array}$ & $\begin{array}{c}\text { Fixed } \\
\text { TC_dT } \Delta\end{array}$ & dTM & $\mathbf{K}$ & $\begin{array}{l}\text { Sap } \\
\text { Velocity } \\
(\mathrm{cm} / \mathrm{s})\end{array}$ & $\begin{array}{l}\text { Probed } \\
\text { Drywood F } \\
\text { (g/hr) }\end{array}$ & $\begin{array}{c}\text { Extrapolated } \\
\text { Wetwood F } \\
(\mathrm{g} / \mathrm{hr})\end{array}$ & $\begin{array}{c}\text { Extrapolated } \\
\text { Total Wood F } \\
(\mathrm{g} / \mathrm{hr})\end{array}$ & $\begin{array}{l}\text { Relative } \\
\text { Humidity, } \\
@ 10 \mathrm{~m}(\%)\end{array}$ \\
\hline & 8/10/2008 8:00 & 451 & 223 & 800 & 8.92 & 8.92 & & 0.0919 & $6.30 \mathrm{E}-04$ & 138.1605 & 50.9081 & 189.0686 & \\
\hline & 8/10/2008 9:00 & 452 & 223 & 900 & 7.631 & 7.631 & & 0.2764 & $2.44 \mathrm{E}-03$ & 535.6225 & 197.3611 & 732.9837 & \\
\hline & $8 / 10 / 2008$ 10:00 & 453 & 223 & 1000 & 5.185 & 5.185 & & 0.8785 & 1.01E-02 & 2223.9335 & 819.4541 & 3043.3876 & \\
\hline & 8/10/2008 11:00 & 454 & 223 & 1100 & NAN & 5.336 & & 0.8253 & $9.40 \mathrm{E}-03$ & 2059.4521 & 758.8475 & 2818.2996 & \\
\hline & $8 / 10 / 2008$ 12:00 & 455 & 223 & 1200 & 5.487 & 5.487 & & 0.7751 & 8.70E-03 & 1906.2551 & 702.3989 & 2608.6540 & \\
\hline & 8/10/2008 13:00 & 456 & 223 & 1300 & 5.63 & 5.63 & & 0.7300 & 8.08E-03 & 1770.6869 & 652.4460 & 2423.1329 & \\
\hline & $8 / 10 / 2008$ 14:00 & 457 & 223 & 1400 & 5.68 & 5.68 & & 0.7148 & 7.87E-03 & 1725.3256 & 635.7317 & 2361.0574 & \\
\hline & 8/10/2008 15:00 & 458 & 223 & 1500 & 5.863 & 5.863 & & 0.6613 & 7.15E-03 & 1567.6932 & 577.6488 & 2145.3420 & \\
\hline & 8/10/2008 16:00 & 459 & 223 & 1600 & 5.775 & 5.775 & & 0.6866 & 7.49E-03 & 1641.8943 & 604.9897 & 2246.8841 & \\
\hline & $8 / 10 / 2008$ 17:00 & 460 & 223 & 1700 & 6.034 & 6.034 & & 0.6142 & 6.53E-03 & 1431.4486 & 527.4467 & 1958.8953 & \\
\hline & 8/10/2008 18:00 & 461 & 223 & 1800 & 8.63 & 8.63 & & 0.1286 & 9.53E-04 & 208.9015 & 76.9741 & 285.8756 & \\
\hline & $8 / 10 / 2008$ 19:00 & 462 & 223 & 1900 & 9.17 & \begin{tabular}{|c|}
9.17 \\
\end{tabular} & & 0.0622 & $3.89 \mathrm{E}-04$ & 85.3461 & 31.4475 & 116.7936 & \\
\hline & 8/10/2008 20:00 & 463 & 223 & 2000 & 8.85 & 8.85 & & 0.1006 & 7.04E-04 & 154.3086 & 56.8582 & 211.1668 & \\
\hline & 8/10/2008 21:00 & 464 & 223 & 2100 & 8.91 & 8.91 & & 0.0932 & $6.41 \mathrm{E}-04$ & 140.4313 & 51.7448 & 192.1761 & \\
\hline & $8 / 10 / 200822: 00$ & 465 & 223 & 2200 & 9.03 & 9.03 & & 0.0786 & $5.20 \mathrm{E}-04$ & 113.9792 & 41.9980 & 155.9772 & \\
\hline & 8/10/2008 23:00 & 466 & 223 & 2300 & 8.82 & 8.82 & & 0.1043 & 7.36E-04 & 161.4096 & 59.4747 & 220.8843 & \\
\hline & 8/11/2008 0:00 & 467 & 224 & 0 & 8.75 & 8.75 & 9.17 & 0.1131 & 8.14E-04 & 178.3994 & 65.7349 & 244.1343 & \\
\hline & 8/11/2008 1:00 & 468 & 224 & 100 & 8.97 & 8.97 & & 0.0858 & 5.79E-04 & 126.9875 & 46.7912 & 173.7786 & \\
\hline & 8/11/2008 2:00 & 469 & 224 & 200 & 9.17 & 9.17 & & 0.0622 & 3.89E-04 & 85.3461 & 31.4475 & 116.7936 & \\
\hline & 8/11/2008 3:00 & 470 & 224 & 300 & 9.1 & 9.1 & & 0.0703 & 4.53E-04 & 99.3587 & 36.6108 & 135.9695 & \\
\hline & 8/11/2008 4:00 & 471 & 224 & 400 & 9.06 & 9.06 & & 0.0751 & $4.91 \mathrm{E}-04$ & 107.6396 & 39.6620 & 147.3016 & \\
\hline & 8/11/2008 5:00 & 472 & 224 & 500 & 8.96 & 8.96 & & 0.0871 & 5.89E-04 & 129.1980 & 47.6056 & 176.8036 & \\
\hline & 8/11/2008 6:00 & 473 & 224 & 600 & 8.91 & 8.91 & & 0.0932 & $6.41 E-04$ & 140.4313 & 51.7448 & 192.1761 & \\
\hline & $8 / 11 / 20087: 00$ & 474 & 224 & 700 & 8.92 & 8.92 & & 0.0919 & $6.30 \mathrm{E}-04$ & 138.1605 & 50.9081 & 189.0686 & \\
\hline & 8/11/2008 8:00 & 475 & 224 & 800 & 8.86 & 8.86 & & 0.0993 & 6.93E-04 & 151.9657 & 55.9949 & 207.9606 & \\
\hline & 8/11/2008 9:00 & 476 & 224 & 900 & 7.761 & 7.761 & & 0.2550 & $2.21 E-03$ & 485.0812 & 178.7381 & 663.8193 & \\
\hline & $8 / 11 / 2008$ 10:00 & 477 & 224 & 1000 & 5.682 & 5.682 & & 0.7142 & 7.86E-03 & 1723.5324 & 635.0709 & 2358.6033 & \\
\hline & 8/11/2008 11:00 & 478 & 224 & 1100 & NAN & 5.559 & & 0.7521 & 8.38E-03 & 1836.8907 & 676.8402 & 2513.7308 & \\
\hline & $8 / 11 / 200812: 00$ & 479 & 224 & 1200 & 5.436 & 5.436 & & 0.7918 & 8.93E-03 & 1956.7984 & 721.0226 & 2677.8210 & \\
\hline & 8/11/2008 13:00 & 480 & 224 & 1300 & 5.836 & 5.836 & & 0.6690 & $7.25 \mathrm{E}-03$ & 1590.1533 & 585.9247 & 2176.0780 & \\
\hline & $8 / 11 / 200814: 00$ & 481 & 224 & 1400 & 5.598 & 5.598 & & 0.7399 & 8.21E-03 & 1800.2610 & 663.3432 & 2463.6041 & \\
\hline & $8 / 11 / 2008$ 15:00 & 482 & 224 & 1500 & 5.636 & 5.636 & & 0.7282 & 8.05E-03 & 1765.1894 & 650.4203 & 2415.6097 & \\
\hline & 8/11/2008 16:00 & 483 & 224 & 1600 & 5.862 & 5.862 & & 0.6615 & 7.16E-03 & 1568.5203 & 577.9536 & 2146.4739 & \\
\hline & $8 / 11 / 200817: 00$ & 484 & 224 & 1700 & 5.919 & 5.919 & & 0.6455 & $6.94 \mathrm{E}-03$ & 1521.9506 & 560.7940 & 2082.7446 & \\
\hline & $8 / 11 / 200818: 00$ & 485 & 224 & 1800 & NAN & 6.1685 & & 0.5790 & $6.07 \mathrm{E}-03$ & 1331.1483 & 490.4890 & 1821.6373 & \\
\hline & 8/11/2008 19:00 & 486 & 224 & 1900 & 6.418 & 6.418 & & 0.5176 & 5.29E-03 & 1159.6107 & 427.2824 & 1586.8932 & \\
\hline & 8/11/2008 20:00 & 487 & 224 & 2000 & 7.142 & 7.142 & & 0.3638 & 3.43E-03 & 751.1850 & 276.7896 & 1027.9745 & \\
\hline & 8/11/2008 21:00 & 488 & 224 & 2100 & 8.17 & 8.17 & & 0.1922 & 1.56E-03 & 342.4408 & 126.1793 & 468.6201 & \\
\hline & $8 / 11 / 200822: 00$ & 489 & 224 & 2200 & 8.72 & 8.72 & & 0.1170 & 8.48E-04 & 185.8615 & 68.4845 & 254.3459 & \\
\hline & $8 / 11 / 200823: 00$ & 490 & 224 & 2300 & 8.92 & 8.92 & & 0.0919 & 6.30E-04 & 138.1605 & 50.9081 & 189.0686 & \\
\hline & 8/12/2008 0:00 & 491 & 225 & 0 & 8.99 & 8.99 & 9.04 & 0.0834 & $5.59 \mathrm{E}-04$ & 122.6029 & 45.1755 & 167.7784 & \\
\hline
\end{tabular}




\begin{tabular}{|c|c|c|c|c|c|c|c|c|c|c|c|c|c|}
\hline Tree ID & TS & RN & JD & HR & $\begin{array}{c}\text { Raw } \\
\text { TC_dTA }\end{array}$ & $\begin{array}{c}\text { Fixed } \\
\text { TC_dT } \Delta\end{array}$ & dTM & $\mathbf{K}$ & $\begin{array}{c}\text { Sap } \\
\text { Velocity } \\
(\mathrm{cm} / \mathrm{s})\end{array}$ & $\begin{array}{c}\text { Probed } \\
\text { Drywood F } \\
(\mathrm{g} / \mathrm{hr})\end{array}$ & $\begin{array}{c}\text { Extrapolated } \\
\text { Wetwood F } \\
(\mathrm{g} / \mathrm{hr})\end{array}$ & $\begin{array}{c}\text { Extrapolated } \\
\text { Total Wood F } \\
(\mathrm{g} / \mathrm{hr})\end{array}$ & $\begin{array}{l}\text { Relative } \\
\text { Humidity, } \\
@ 10 \mathrm{~m}(\%)\end{array}$ \\
\hline & $8 / 12 / 2008$ 1:00 & 492 & 225 & 100 & 8.98 & 8.98 & & 0.0846 & $5.69 \mathrm{E}-04$ & 124.7891 & 45.9811 & 170.7702 & \\
\hline & 8/12/2008 2:00 & 493 & 225 & 200 & 8.31 & 8.31 & & 0.1721 & $1.36 \mathrm{E}-03$ & 298.9291 & 110.1466 & 409.0756 & \\
\hline & $8 / 12 / 20083: 00$ & 494 & 225 & 300 & 8.56 & 8.56 & & 0.1379 & 1.04E-03 & 227.5044 & 83.8287 & 311.3331 & \\
\hline & $8 / 12 / 2008$ 4:00 & 495 & 225 & 400 & 8.62 & 8.62 & & 0.1299 & $9.65 \mathrm{E}-04$ & 211.5224 & 77.9398 & 289.4621 & \\
\hline & 8/12/2008 5:00 & 496 & 225 & 500 & 8.64 & 8.64 & & 0.1273 & $9.41 \mathrm{E}-04$ & 206.2929 & 76.0129 & 282.3058 & \\
\hline & 8/12/2008 6:00 & 497 & 225 & 600 & 8.61 & 8.61 & & 0.1312 & 9.77E-04 & 214.1554 & 78.9100 & 293.0654 & \\
\hline & $8 / 12 / 20087: 00$ & 498 & 225 & 700 & 8.59 & 8.59 & & 0.1339 & 1.00E-03 & 219.4582 & 80.8639 & 300.3221 & \\
\hline & 8/12/2008 8:00 & 499 & 225 & 800 & 8.42 & 8.42 & & 0.1568 & $1.22 \mathrm{E}-03$ & 266.5297 & 98.2084 & 364.7381 & \\
\hline & 8/12/2008 9:00 & 500 & 225 & 900 & 7.059 & 7.059 & & 0.3798 & $3.61 \mathrm{E}-03$ & 792.1526 & 291.8849 & 1084.0375 & \\
\hline & $8 / 12 / 2008$ 10:00 & 501 & 225 & 1000 & 4.98 & 4.98 & & 0.9558 & 1.13E-02 & 2467.3069 & 909.1300 & 3376.4368 & \\
\hline & $8 / 12 / 2008$ 11:00 & 502 & 225 & 1100 & 4.551 & 4.551 & & 1.1402 & $1.40 \mathrm{E}-02$ & 3065.6094 & 1129.5868 & 4195.1962 & \\
\hline & $8 / 12 / 2008$ 12:00 & 503 & 225 & 1200 & 4.92 & 4.92 & & 0.9797 & 1.16E-02 & 2543.3152 & 937.1368 & 3480.4521 & \\
\hline & 8/12/2008 13:00 & 504 & 225 & 1300 & 5.093 & 5.093 & & 0.9124 & $1.06 \mathrm{E}-02$ & 2330.1469 & 858.5905 & 3188.7374 & \\
\hline & $8 / 12 / 2008$ 14:00 & 505 & 225 & 1400 & 5.18 & 5.18 & & 0.8803 & $1.02 \mathrm{E}-02$ & 2229.5854 & 821.5366 & 3051.1220 & \\
\hline & $8 / 12 / 200815: 00$ & 506 & 225 & 1500 & 5.247 & 5.247 & & 0.8563 & 9.83E-03 & 2154.9646 & 794.0410 & 2949.0056 & \\
\hline & $8 / 12 / 2008$ 16:00 & 507 & 225 & 1600 & 5.335 & 5.335 & & 0.8257 & $9.40 \mathrm{E}-03$ & 2060.5031 & 759.2347 & 2819.7379 & \\
\hline & $8 / 12 / 200817: 00$ & 508 & 225 & 1700 & 5.448 & 5.448 & & 0.7878 & 8.87E-03 & 1944.7983 & 716.6009 & 2661.3993 & \\
\hline & 8/12/2008 18:00 & 509 & 225 & 1800 & 5.568 & 5.568 & & 0.7493 & 8.34E-03 & 1828.3798 & 673.7041 & 2502.0840 & \\
\hline & 8/12/2008 19:00 & 510 & 225 & 1900 & 5.829 & 5.829 & & 0.6710 & 7.28E-03 & 1596.0201 & 588.0864 & 2184.1065 & \\
\hline & $8 / 12 / 200820: 00$ & 511 & 225 & 2000 & 6.519 & 6.519 & & 0.4941 & 5.00E-03 & 1095.1110 & 403.5162 & 1498.6271 & \\
\hline & $8 / 12 / 2008$ 21:00 & 512 & 225 & 2100 & 8.39 & 8.39 & & 0.1609 & $1.26 \mathrm{E}-03$ & 275.2125 & 101.4077 & 376.6203 & \\
\hline & $8 / 12 / 200822: 00$ & 513 & 225 & 2200 & 9.03 & 9.03 & & 0.0786 & $5.20 \mathrm{E}-04$ & 113.9792 & 41.9980 & 155.9772 & \\
\hline & $8 / 12 / 2008$ 23:00 & 514 & 225 & 2300 & 9.04 & 9.04 & & 0.0774 & $5.10 \mathrm{E}-04$ & 111.8538 & 41.2148 & 153.0686 & \\
\hline & 8/13/2008 0:00 & 515 & 226 & 0 & 8.93 & 8.93 & 8.93 & 0.0907 & $6.20 \mathrm{E}-04$ & 135.9018 & 50.0758 & 185.9776 & \\
\hline & 8/13/2008 1:00 & 516 & 226 & 100 & 8.81 & 8.81 & & 0.1056 & 7.47E-04 & 163.8006 & 60.3557 & 224.1563 & \\
\hline & 8/13/2008 2:00 & 517 & 226 & 200 & 8.74 & 8.74 & & 0.1144 & $8.25 E-04$ & 180.8747 & 66.6470 & 247.5217 & \\
\hline & 8/13/2008 3:00 & 518 & 226 & 300 & 8.69 & 8.69 & & 0.1208 & 8.82E-04 & 193.4323 & 71.2741 & 264.7064 & \\
\hline & 8/13/2008 4:00 & 519 & 226 & 400 & 8.6 & 8.6 & & 0.1326 & $9.89 \mathrm{E}-04$ & 216.8007 & 79.8847 & 296.6853 & \\
\hline & $8 / 13 / 20085: 00$ & 520 & 226 & 500 & 8.58 & 8.58 & & 0.1352 & $1.01 \mathrm{E}-03$ & 222.1280 & 81.8476 & 303.9756 & \\
\hline & 8/13/2008 6:00 & 521 & 226 & 600 & 8.52 & 8.52 & & 0.1432 & 1.09E-03 & 238.4054 & 87.8454 & 326.2508 & \\
\hline & 8/13/2008 7:00 & 522 & 226 & 700 & 8.43 & 8.43 & & 0.1554 & 1.20E-03 & 263.6608 & 97.1512 & 360.8120 & \\
\hline & 8/13/2008 8:00 & 523 & 226 & 800 & 8.25 & 8.25 & & 0.1806 & 1.45E-03 & 317.2604 & 116.9011 & 434.1615 & \\
\hline & 8/13/2008 9:00 & 524 & 226 & 900 & 6.453 & 6.453 & & 0.5094 & $5.19 \mathrm{E}-03$ & 1136.9521 & 418.9334 & 1555.8854 & \\
\hline & $8 / 13 / 2008$ 10:00 & 525 & 226 & 1000 & 4.768 & 4.768 & & 1.0428 & 1.25E-02 & 2746.4786 & 1011.9965 & 3758.4751 & \\
\hline & 8/13/2008 11:00 & 526 & 226 & 1100 & 4.867 & 4.867 & & 1.0012 & 1.19E-02 & 2612.3839 & 962.5866 & 3574.9705 & \\
\hline & $8 / 13 / 2008$ 12:00 & 527 & 226 & 1200 & 4.672 & 4.672 & & 1.0848 & 1.32E-02 & 2883.1963 & 1062.3730 & 3945.5692 & \\
\hline
\end{tabular}




\begin{tabular}{|c|c|c|c|c|c|c|c|c|c|c|c|c|c|}
\hline Tree ID & TS & RN & JD & HR & $\begin{array}{c}\text { Raw } \\
\text { TC_dTA }\end{array}$ & $\begin{array}{c}\text { Fixed } \\
\text { TC_dT } \Delta\end{array}$ & dTM & $\mathrm{K}$ & $\begin{array}{l}\text { Sap } \\
\text { Velocity } \\
\text { (cm/s) }\end{array}$ & $\begin{array}{c}\text { Probed } \\
\text { Drywood F } \\
\text { (g/hr) }\end{array}$ & $\begin{array}{c}\text { Extrapolated } \\
\text { Wetwood F } \\
(\mathrm{g} / \mathrm{hr})\end{array}$ & $\begin{array}{l}\text { Extrapolated } \\
\text { Total Wood F } \\
\text { (g/hr) }\end{array}$ & $\begin{array}{c}\text { Relative } \\
\text { Humidity, } \\
\text { @10m (\%) }\end{array}$ \\
\hline & $7 / 22 / 2008$ 13:00 & 0 & 204 & 1300 & 5.509 & 5.509 & 9.63 & 0.7480 & 8.32E-03 & 2274.6113 & 838.1273 & 3112.7387 & \\
\hline \multirow[t]{2}{*}{ W610WN1 } & $7 / 22 / 2008$ 14:00 & 1 & 204 & 1400 & 6.088 & 6.088 & & 0.5818 & $6.11 \mathrm{E}-03$ & 1669.3071 & 615.0905 & 2284.3977 & \\
\hline & 7/22/2008 15:00 & 2 & 204 & 1500 & 6.026 & 6.026 & & 0.5981 & $6.32 \mathrm{E}-03$ & 1726.9739 & 636.3391 & 2363.3130 & \\
\hline \multirow[t]{2}{*}{ Probe \# } & $7 / 22 / 2008$ 16:00 & 3 & 204 & 1600 & 6.054 & 6.054 & & 0.5907 & $6.22 \mathrm{E}-03$ & 1700.7390 & 626.6723 & 2327.4113 & \\
\hline & 7/22/2008 17:00 & 4 & 204 & 1700 & 6.092 & 6.092 & & 0.5808 & $6.10 \mathrm{E}-03$ & 1665.6395 & 613.7391 & 2279.3787 & \\
\hline \multirow[t]{4}{*}{2} & 7/22/2008 18:00 & 5 & 204 & 1800 & 6.174 & 6.174 & & 0.5598 & 5.83E-03 & 1591.8286 & 586.5420 & 2178.3706 & \\
\hline & $7 / 22 / 2008$ 19:00 & 6 & 204 & 1900 & 6.399 & 6.399 & & 0.5049 & $5.13 E-03$ & 1402.0689 & 516.6211 & 1918.6900 & \\
\hline & $7 / 22 / 200820: 00$ & 7 & 204 & 2000 & 6.859 & 6.859 & & 0.4040 & $3.90 \mathrm{E}-03$ & 1065.4868 & 392.6005 & 1458.0873 & \\
\hline & 7/22/2008 21:00 & 8 & 204 & 2100 & 7.985 & 7.985 & & 0.2060 & 1.70E-03 & 465.0497 & 171.3571 & 636.4068 & \\
\hline \multirow[t]{2}{*}{$\mathrm{DBH}(\mathrm{cm})$} & $7 / 22 / 2008$ 22:00 & 9 & 204 & 2200 & 9.14 & 9.14 & & 0.0536 & $3.25 \mathrm{E}-04$ & 88.6760 & 32.6745 & 121.3505 & \\
\hline & 7/22/2008 23:00 & 10 & 204 & 2300 & 9.63 & 9.63 & & 0.0000 & $0.00 \mathrm{E}+00$ & 0.0000 & 0.0000 & 0.0000 & \\
\hline \multirow[t]{2}{*}{11.9} & 7/23/2008 0:00 & 11 & 205 & 0 & 9.92 & 9.92 & 10.12 & -0.0292 & $0.00 \mathrm{E}+00$ & 0.0000 & 0.0000 & 0.0000 & \\
\hline & 7/23/2008 1:00 & 12 & 205 & 100 & 10.03 & 10.03 & & -0.0399 & $0.00 \mathrm{E}+00$ & 0.0000 & 0.0000 & 0.0000 & \\
\hline Day Count & $7 / 23 / 2008$ 2:00 & 13 & 205 & 200 & NAN & 10.075 & & -0.0442 & $0.00 \mathrm{E}+00$ & 0.0000 & 0.0000 & 0.0000 & \\
\hline \multirow[t]{27}{*}{35} & 7/23/2008 3:00 & 14 & 205 & 300 & 10.12 & 10.12 & & -0.0484 & $0.00 \mathrm{E}+00$ & 0.0000 & 0.0000 & 0.0000 & \\
\hline & 7/23/2008 4:00 & 15 & 205 & 400 & 10.11 & 10.11 & & -0.0475 & $0.00 \mathrm{E}+00$ & 0.0000 & 0.0000 & 0.0000 & \\
\hline & 7/23/2008 5:00 & 16 & 205 & 500 & 10.06 & 10.06 & & -0.0427 & $0.00 \mathrm{E}+00$ & 0.0000 & 0.0000 & 0.0000 & \\
\hline & 7/23/2008 6:00 & 17 & 205 & 600 & 9.98 & 9.98 & & -0.0351 & $0.00 \mathrm{E}+00$ & 0.0000 & 0.0000 & 0.0000 & \\
\hline & 7/23/2008 7:00 & 18 & 205 & 700 & 9.79 & 9.79 & & -0.0163 & $0.00 \mathrm{E}+00$ & 0.0000 & 0.0000 & 0.0000 & \\
\hline & 7/23/2008 8:00 & 19 & 205 & 800 & 9.21 & 9.21 & & 0.0456 & $2.66 \mathrm{E}-04$ & 72.6634 & 26.7743 & 99.4378 & \\
\hline & 7/23/20089:00 & 20 & 205 & 900 & 7.972 & 7.972 & & 0.2080 & 1.72E-03 & 470.5208 & 173.3730 & 643.8938 & \\
\hline & $7 / 23 / 2008$ 10:00 & 21 & 205 & 1000 & 6.767 & 6.767 & & 0.4231 & 4.13E-03 & 1127.7918 & 415.5581 & 1543.3499 & \\
\hline & $7 / 23 / 2008$ 11:00 & 22 & 205 & 1100 & 6.493 & 6.493 & & 0.4831 & 4.86E-03 & 1327.9712 & 489.3183 & 1817.2895 & \\
\hline & $7 / 23 / 2008$ 12:00 & 23 & 205 & 1200 & 6.38 & 6.38 & & 0.5094 & $5.19 \mathrm{E}-03$ & 1417.4042 & 522.2717 & 1939.6760 & \\
\hline & $7 / 23 / 2008$ 13:00 & 24 & 205 & 1300 & 6.298 & 6.298 & & 0.5291 & $5.43 \mathrm{E}-03$ & 1485.0153 & 547.1844 & 2032.1998 & \\
\hline & $7 / 23 / 2008$ 14:00 & 25 & 205 & 1400 & NAN & 6.2655 & & 0.5370 & $5.54 \mathrm{E}-03$ & 1512.4681 & 557.3000 & 2069.7681 & \\
\hline & 7/23/2008 15:00 & 26 & 205 & 1500 & 6.233 & 6.233 & & 0.5450 & 5.64E-03 & 1540.3025 & 567.5561 & 2107.8586 & \\
\hline & 7/23/2008 16:00 & 27 & 205 & 1600 & 6.383 & 6.383 & & 0.5087 & $5.18 \mathrm{E}-03$ & 1414.9747 & 521.3765 & 1936.3512 & \\
\hline & $7 / 23 / 2008$ 17:00 & 28 & 205 & 1700 & 6.732 & 6.732 & & 0.4305 & $4.22 \mathrm{E}-03$ & 1152.1188 & 424.5219 & 1576.6407 & \\
\hline & $7 / 23 / 2008$ 18:00 & 29 & 205 & 1800 & 7.288 & 7.288 & & 0.3214 & $2.94 \mathrm{E}-03$ & 803.8780 & 296.2054 & 1100.0833 & \\
\hline & $7 / 23 / 2008$ 19:00 & 30 & 205 & 1900 & 7.522 & 7.522 & & 0.2802 & $2.49 \mathrm{E}-03$ & 679.2317 & 250.2769 & 929.5086 & \\
\hline & $7 / 23 / 200820: 00$ & 31 & 205 & 2000 & 7.868 & 7.868 & & 0.2239 & 1.89E-03 & 515.3756 & 189.9007 & 705.2763 & \\
\hline & $7 / 23 / 2008$ 21:00 & 32 & 205 & 2100 & 8.37 & 8.37 & & 0.1505 & $1.16 \mathrm{E}-03$ & 316.0681 & 116.4618 & 432.5299 & \\
\hline & $7 / 23 / 2008$ 22:00 & 33 & 205 & 2200 & 8.99 & 8.99 & & 0.0712 & $4.60 \mathrm{E}-04$ & 125.7271 & 46.3267 & 172.0538 & \\
\hline & $7 / 23 / 200823: 00$ & 34 & 205 & 2300 & 9.24 & 9.24 & & 0.0422 & $2.42 \mathrm{E}-04$ & 66.0629 & 24.3422 & 90.4052 & \\
\hline & $7 / 24 / 20080: 00$ & 35 & 206 & 0 & 9.3 & 9.3 & 9.73 & 0.0355 & $1.95 \mathrm{E}-04$ & 53.3565 & 19.6603 & 73.0169 & \\
\hline & 7/24/2008 1:00 & 36 & 206 & 100 & 9.39 & 9.39 & & 0.0256 & $1.30 \mathrm{E}-04$ & 35.6277 & 13.1278 & 48.7555 & \\
\hline & 7/24/2008 2:00 & 37 & 206 & 200 & 9.47 & 9.47 & & 0.0169 & $7.83 \mathrm{E}-05$ & 21.4035 & 7.8866 & 29.2900 & \\
\hline & 7/24/2008 3:00 & 38 & 206 & 300 & 9.58 & 9.58 & & 0.0052 & $1.84 \mathrm{E}-05$ & 5.0405 & 1.8573 & 6.8977 & \\
\hline & 7/24/2008 4:00 & 39 & 206 & 400 & 9.64 & 9.64 & & -0.0010 & $0.00 \mathrm{E}+00$ & 0.0000 & 0.0000 & 0.0000 & \\
\hline & 7/24/2008 5:00 & 40 & 206 & 500 & 9.71 & 9.71 & & -0.0082 & $0.00 \mathrm{E}+00$ & 0.0000 & 0.0000 & 0.0000 & \\
\hline
\end{tabular}




\begin{tabular}{|c|c|c|c|c|c|c|c|c|c|c|c|c|c|}
\hline \multirow[t]{42}{*}{ Tree ID } & TS & RN & JD & HR & $\begin{array}{c}\text { Raw } \\
\text { TC_dTA }\end{array}$ & $\begin{array}{c}\text { Fixed } \\
\text { TC_dT } \Delta\end{array}$ & dTM & $\mathrm{K}$ & $\begin{array}{l}\text { Sap } \\
\text { Velocity } \\
\text { (cm/s) }\end{array}$ & $\begin{array}{l}\text { Probed } \\
\text { Drywood F } \\
\text { (g/hr) }\end{array}$ & $\begin{array}{c}\text { Extrapolated } \\
\text { Wetwood F } \\
(\mathrm{g} / \mathrm{hr})\end{array}$ & $\begin{array}{c}\text { Extrapolated } \\
\text { Total Wood F } \\
(\mathrm{g} / \mathrm{hr})\end{array}$ & $\begin{array}{c}\text { Relative } \\
\text { Humidity, } \\
\text { @10m (\%) }\end{array}$ \\
\hline & 7/24/2008 6:00 & 41 & 206 & 600 & NAN & 9.72 & & -0.0093 & $0.00 \mathrm{E}+00$ & 0.0000 & 0.0000 & 0.0000 & \\
\hline & 7/24/2008 7:00 & 42 & 206 & 700 & 9.73 & 9.73 & & -0.0103 & $0.00 \mathrm{E}+00$ & 0.0000 & 0.0000 & 0.0000 & \\
\hline & 7/24/2008 8:00 & 43 & 206 & 800 & 9.59 & 9.59 & & 0.0042 & $1.40 \mathrm{E}-05$ & 3.8249 & 1.4094 & 5.2342 & \\
\hline & 7/24/20089:00 & 44 & 206 & 900 & 9.28 & 9.28 & & 0.0377 & 2.10E-04 & 57.5169 & 21.1933 & 78.7102 & \\
\hline & $7 / 24 / 2008$ 10:00 & 45 & 206 & 1000 & 9.33 & 9.33 & & 0.0322 & 1.73E-04 & 47.2619 & 17.4146 & 64.6766 & \\
\hline & 7/24/2008 11:00 & 46 & 206 & 1100 & NAN & 7.9205 & & 0.2158 & $1.80 \mathrm{E}-03$ & 492.4895 & 181.4679 & 673.9574 & \\
\hline & $7 / 24 / 2008$ 12:00 & 47 & 206 & 1200 & 6.511 & 6.511 & & 0.4790 & 4.81E-03 & 1314.1114 & 484.2114 & 1798.3227 & \\
\hline & 7/24/2008 13:00 & 48 & 206 & 1300 & 6.729 & 6.729 & & 0.4311 & $4.22 \mathrm{E}-03$ & 1154.2203 & 425.2962 & 1579.5165 & \\
\hline & $7 / 24 / 200814: 00$ & 49 & 206 & 1400 & 6.668 & 6.668 & & 0.4442 & 4.38E-03 & 1197.5184 & 441.2503 & 1638.7687 & \\
\hline & $7 / 24 / 200815: 00$ & 50 & 206 & 1500 & 6.339 & 6.339 & & 0.5192 & 5.31E-03 & 1450.9172 & 534.6203 & 1985.5375 & \\
\hline & $7 / 24 / 2008$ 16:00 & 51 & 206 & 1600 & 6.256 & 6.256 & & 0.5393 & 5.56E-03 & 1520.5646 & 560.2833 & 2080.8479 & \\
\hline & 7/24/2008 17:00 & 52 & 206 & 1700 & 6.421 & 6.421 & & 0.4998 & $5.07 E-03$ & 1384.4644 & 510.1344 & 1894.5988 & \\
\hline & $7 / 24 / 2008$ 18:00 & 53 & 206 & 1800 & 6.388 & 6.388 & & 0.5075 & $5.16 \mathrm{E}-03$ & 1410.9323 & 519.8870 & 1930.8193 & \\
\hline & $7 / 24 / 2008$ 19:00 & 54 & 206 & 1900 & 6.711 & 6.711 & & 0.4350 & 4.27E-03 & 1166.8839 & 429.9624 & 1596.8463 & \\
\hline & $7 / 24 / 200820: 00$ & 55 & 206 & 2000 & 7.411 & 7.411 & & 0.2994 & 2.70E-03 & 736.8869 & 271.5211 & 1008.4081 & \\
\hline & $7 / 24 / 2008$ 21:00 & 56 & 206 & 2100 & 8.53 & 8.53 & & 0.1290 & 9.56E-04 & 261.2496 & 96.2628 & 357.5124 & \\
\hline & 7/24/2008 22:00 & 57 & 206 & 2200 & 9.37 & 9.37 & & 0.0277 & 1.44E-04 & 39.4203 & 14.5252 & 53.9456 & \\
\hline & $7 / 24 / 200823: 00$ & 58 & 206 & 2300 & 9.56 & 9.56 & & 0.0073 & 2.80E-05 & 7.6467 & 2.8176 & 10.4642 & \\
\hline & $7 / 25 / 20080: 00$ & 59 & 207 & 0 & 9.52 & 9.52 & 9.52 & 0.0116 & 4.91E-05 & 13.4076 & 4.9403 & 18.3479 & \\
\hline & 7/25/2008 1:00 & 60 & 207 & 100 & 9.4 & 9.4 & & 0.0245 & 1.24E-04 & 33.7650 & 12.4414 & 46.2063 & \\
\hline & 7/25/2008 2:00 & 61 & 207 & 200 & 9.25 & 9.25 & & 0.0411 & $2.34 \mathrm{E}-04$ & 63.8988 & 23.5448 & 87.4436 & \\
\hline & 7/25/2008 3:00 & 62 & 207 & 300 & 9.08 & 9.08 & & 0.0606 & 3.77E-04 & 103.0582 & 37.9739 & 141.0320 & \\
\hline & 7/25/2008 4:00 & 63 & 207 & 400 & 8.92 & 8.92 & & 0.0796 & $5.28 E-04$ & 144.2446 & 53.1499 & 197.3944 & \\
\hline & 7/25/2008 5:00 & 64 & 207 & 500 & 8.79 & 8.79 & & 0.0956 & 6.61E-04 & 180.6496 & 66.5641 & 247.2137 & \\
\hline & 7/25/2008 6:00 & 65 & 207 & 600 & 8.72 & 8.72 & & 0.1044 & 7.37E-04 & 201.3278 & 74.1834 & 275.5112 & \\
\hline & 7/25/2008 7:00 & 66 & 207 & 700 & 8.6 & 8.6 & & 0.1198 & 8.73E-04 & 238.5254 & 87.8896 & 326.4150 & \\
\hline & 7/25/2008 8:00 & 67 & 207 & 800 & 8.5 & 8.5 & & 0.1329 & 9.93E-04 & 271.2217 & 99.9372 & 371.1589 & \\
\hline & 7/25/2008 9:00 & 68 & 207 & 900 & 8.4 & 8.4 & & 0.1464 & 1.12E-03 & 305.4815 & 112.5610 & 418.0425 & \\
\hline & $7 / 25 / 2008$ 10:00 & 69 & 207 & 1000 & NAN & 8.225 & & 0.1708 & $1.35 \mathrm{E}-03$ & 369.2809 & 136.0691 & 505.3500 & \\
\hline & 7/25/2008 11:00 & 70 & 207 & 1100 & 8.05 & 8.05 & & 0.1963 & 1.60E-03 & 438.1388 & 161.4412 & 599.5800 & \\
\hline & $7 / 25 / 2008$ 12:00 & 71 & 207 & 1200 & 6.911 & 6.911 & & 0.3934 & 3.77E-03 & 1031.2939 & 380.0015 & 1411.2954 & \\
\hline & $7 / 25 / 2008$ 13:00 & 72 & 207 & 1300 & 6.004 & 6.004 & & 0.6039 & 6.40E-03 & 1747.8119 & 644.0173 & 2391.8292 & \\
\hline & $7 / 25 / 200814: 00$ & 73 & 207 & 1400 & 5.582 & 5.582 & & 0.7252 & 8.01E-03 & 2189.3457 & 806.7094 & 2996.0551 & \\
\hline & $7 / 25 / 200815: 00$ & 74 & 207 & 1500 & 5.686 & 5.686 & & 0.6936 & 7.59E-03 & 2072.6724 & 763.7188 & 2836.3911 & \\
\hline & $7 / 25 / 2008$ 16:00 & 75 & 207 & 1600 & NAN & 5.686 & & 0.6936 & 7.59E-03 & 2072.6724 & 763.7188 & 2836.3911 & \\
\hline & $7 / 25 / 200817: 00$ & 76 & 207 & 1700 & NAN & 6.062 & & 0.5886 & $6.20 \mathrm{E}-03$ & 1693.3017 & 623.9318 & 2317.2335 & \\
\hline & 7/25/2008 18:00 & 77 & 207 & 1800 & 6.062 & 6.062 & & 0.5886 & $6.20 \mathrm{E}-03$ & 1693.3017 & 623.9318 & 2317.2335 & \\
\hline & $7 / 25 / 2008$ 19:00 & 78 & 207 & 1900 & 6.192 & 6.192 & & 0.5552 & 5.77E-03 & 1575.9709 & 580.6989 & 2156.6698 & \\
\hline & 7/25/2008 20:00 & 79 & 207 & 2000 & 6.833 & 6.833 & & 0.4093 & $3.96 \mathrm{E}-03$ & 1082.8576 & 399.0011 & 1481.8587 & \\
\hline & $7 / 25 / 2008$ 21:00 & 80 & 207 & 2100 & 7.906 & 7.906 & & 0.2181 & 1.83E-03 & 498.7606 & 183.7786 & 682.5391 & \\
\hline & $7 / 25 / 200822: 00$ & 81 & 207 & 2200 & 8.65 & 8.65 & & 0.1133 & 8.15E-04 & 222.7573 & 82.0795 & 304.8369 & \\
\hline
\end{tabular}




\begin{tabular}{|c|c|c|c|c|c|c|c|c|c|c|c|c|c|}
\hline Tree ID & TS & RN & JD & HR & $\begin{array}{c}\text { Raw } \\
\text { TC_dTA }\end{array}$ & $\begin{array}{c}\text { Fixed } \\
\text { TC_dT } \Delta\end{array}$ & dTM & $\mathbf{K}$ & $\begin{array}{l}\text { Sap } \\
\text { Velocity } \\
(\mathrm{cm} / \mathrm{s})\end{array}$ & $\begin{array}{l}\text { Probed } \\
\text { Drywood F } \\
(\mathrm{g} / \mathrm{hr})\end{array}$ & $\begin{array}{l}\text { Extrapolated } \\
\text { Wetwood F } \\
(\mathrm{g} / \mathrm{hr})\end{array}$ & $\begin{array}{c}\text { Extrapolated } \\
\text { Total Wood F } \\
(\mathrm{g} / \mathrm{hr})\end{array}$ & $\begin{array}{l}\text { Relative } \\
\text { Humidity, } \\
\text { @10m (\%) }\end{array}$ \\
\hline & 7/25/2008 23:00 & 82 & 207 & 2300 & \begin{tabular}{|l|} 
\\
\end{tabular} & 8.57 & & 0.1237 & $9.08 \mathrm{E}-04$ & 248.1714 & 91.4438 & 339.6152 & \\
\hline & $7 / 26 / 20080: 00$ & 83 & 208 & 0 & 8.41 & 8.41 & 8.41 & 0.1451 & 1.11E-03 & 301.9845 & 111.2724 & 413.2569 & \\
\hline & 7/26/2008 1:00 & 84 & 208 & 100 & 8.3 & 8.3 & & 0.1602 & $1.25 \mathrm{E}-03$ & 341.3311 & 125.7704 & 467.1015 & \\
\hline & $7 / 26 / 20082: 00$ & 85 & 208 & 200 & 8.21 & 8.21 & & 0.1730 & 1.37E-03 & 374.9817 & 138.1697 & 513.1514 & \\
\hline & 7/26/2008 3:00 & 86 & 208 & 300 & 8.13 & 8.13 & & 0.1845 & 1.49E-03 & 406.0191 & 149.6061 & 555.6252 & \\
\hline & $7 / 26 / 20084: 00$ & 87 & 208 & 400 & 8.08 & 8.08 & & 0.1918 & 1.56E-03 & 425.9656 & 156.9558 & 582.9213 & \\
\hline & $7 / 26 / 20085: 00$ & 88 & 208 & 500 & 8.02 & 8.02 & & 0.2007 & $1.65 \mathrm{E}-03$ & 450.4676 & 165.9841 & 616.4517 & \\
\hline & 7/26/2008 6:00 & 89 & 208 & 600 & 7.955 & 7.955 & & 0.2106 & 1.75E-03 & 477.7203 & 176.0259 & 653.7462 & \\
\hline & $7 / 26 / 20087: 00$ & 90 & 208 & 700 & 7.874 & 7.874 & & 0.2230 & 1.88E-03 & 512.7347 & 188.9276 & 701.6623 & \\
\hline & $7 / 26 / 20088: 00$ & 91 & 208 & 800 & 7.816 & 7.816 & & 0.2321 & 1.97E-03 & 538.5401 & 198.4362 & 736.9763 & \\
\hline & 7/26/2008 9:00 & 92 & 208 & 900 & 7.661 & 7.661 & & 0.2570 & 2.23E-03 & 610.6054 & 224.9901 & 835.5955 & \\
\hline & $7 / 26 / 200810: 00$ & 93 & 208 & 1000 & 6.021 & 6.021 & & 0.5994 & $6.34 \mathrm{E}-03$ & 1731.6923 & 638.0777 & 2369.7700 & \\
\hline & 7/26/2008 11:00 & 94 & 208 & 1100 & 5.32 & 5.32 & & 0.8102 & $9.18 \mathrm{E}-03$ & 2509.2496 & 924.5846 & 3433.8342 & \\
\hline & $7 / 26 / 200812: 00$ & 95 & 208 & 1200 & 5.603 & 5.603 & & 0.7187 & 7.92E-03 & 2165.3403 & 797.8642 & 2963.2045 & \\
\hline & 7/26/2008 13:00 & 96 & 208 & 1300 & 5.143 & 5.143 & & 0.8724 & 1.01E-02 & 2748.8430 & 1012.8678 & 3761.7108 & \\
\hline & 7/26/2008 14:00 & 97 & 208 & 1400 & 5.071 & 5.071 & & 0.8990 & 1.04E-02 & 2852.3170 & 1050.9949 & 3903.3119 & \\
\hline & 7/26/2008 15:00 & 98 & 208 & 1500 & 5.049 & 5.049 & & 0.9073 & 1.06E-02 & 2884.6682 & 1062.9153 & 3947.5836 & \\
\hline & 7/26/2008 16:00 & 99 & 208 & 1600 & 5.161 & 5.161 & & 0.8659 & 9.97E-03 & 2723.5361 & 1003.5429 & 3727.0789 & \\
\hline & $7 / 26 / 200817: 00$ & 100 & 208 & 1700 & NAN & 5.235 & & 0.8395 & $9.59 \mathrm{E}-03$ & 2621.7755 & 966.0471 & 3587.8226 & \\
\hline & $7 / 26 / 200818: 00$ & 101 & 208 & 1800 & 5.309 & 5.309 & & 0.8139 & $9.24 \mathrm{E}-03$ & 2523.5571 & 929.8565 & 3453.4136 & \\
\hline & 7/26/2008 19:00 & 102 & 208 & 1900 & 5.484 & 5.484 & & 0.7560 & 8.43E-03 & 2304.4764 & 849.1317 & 3153.6082 & \\
\hline & $7 / 26 / 200820: 00$ & 103 & 208 & 2000 & 5.873 & 5.873 & & 0.6397 & 6.87E-03 & 1876.1278 & 691.2979 & 2567.4256 & \\
\hline & 7/26/2008 21:00 & 104 & 208 & 2100 & 6.747 & 6.747 & & 0.4273 & 4.18E-03 & 1141.6501 & 420.6645 & 1562.3146 & \\
\hline & $7 / 26 / 200822: 00$ & 105 & 208 & 2200 & 7.614 & 7.614 & & 0.2648 & $2.32 E-03$ & 633.3765 & 233.3806 & 866.7571 & \\
\hline & $7 / 26 / 200823: 00$ & 106 & 208 & 2300 & 7.92 & 7.92 & & 0.2159 & 1.80E-03 & 492.7051 & 181.5473 & 674.2525 & \\
\hline & $7 / 27 / 20080: 00$ & 107 & 209 & 0 & 7.961 & 7.961 & 8.00 & 0.2096 & $1.74 \mathrm{E}-03$ & 475.1735 & 175.0874 & 650.2609 & \\
\hline & $7 / 27 / 20081: 00$ & 108 & 209 & 100 & 7.987 & 7.987 & & 0.2057 & 1.70E-03 & 464.2106 & 171.0480 & 635.2586 & \\
\hline & $7 / 27 / 20082: 00$ & 109 & 209 & 200 & 7.996 & 7.996 & & 0.2044 & 1.69E-03 & 460.4436 & 169.6599 & 630.1035 & \\
\hline & 7/27/2008 3:00 & 110 & 209 & 300 & 8 & 8 & & 0.2038 & 1.68E-03 & 458.7739 & 169.0447 & 627.8186 & \\
\hline & 7/27/2008 4:00 & 111 & 209 & 400 & 7.982 & 7.982 & & 0.2065 & 1.71E-03 & 466.3096 & 171.8214 & 638.1310 & \\
\hline & 7/27/2008 5:00 & 112 & 209 & 500 & 7.927 & 7.927 & & 0.2148 & 1.79E-03 & 489.6906 & 180.4366 & 670.1272 & \\
\hline & $7 / 27 / 20086: 00$ & 113 & 209 & 600 & 7.857 & 7.857 & & 0.2257 & $1.90 \mathrm{E}-03$ & 520.2343 & 191.6910 & 711.9253 & \\
\hline & 7/27/2008 7:00 & 114 & 209 & 700 & 7.772 & 7.772 & & 0.2391 & $2.04 \mathrm{E}-03$ & 558.5332 & 205.8030 & 764.3362 & \\
\hline & $7 / 27 / 20088: 00$ & 115 & 209 & 800 & 7.739 & 7.739 & & 0.2443 & $2.10 \mathrm{E}-03$ & 573.7673 & 211.4164 & 785.1837 & \\
\hline & 7/27/2008 9:00 & 116 & 209 & 900 & NAN & 6.381 & & 0.5092 & 5.18E-03 & 1416.5940 & 521.9732 & 1938.5673 & \\
\hline & $7 / 27 / 200810: 00$ & 117 & 209 & 1000 & 5.023 & 5.023 & & 0.9172 & 1.07E-02 & 2923.3560 & 1077.1707 & 4000.5267 & \\
\hline & 7/27/2008 11:00 & 118 & 209 & 1100 & 4.839 & 4.839 & & 0.9901 & 1.18E-02 & 3211.9591 & 1183.5124 & 4395.4715 & \\
\hline & $7 / 27 / 200812: 00$ & 119 & 209 & 1200 & 4.795 & 4.795 & & 1.0083 & 1.20E-02 & 3285.0414 & 1210.4411 & 4495.4825 & \\
\hline & $7 / 27 / 200813: 00$ & 120 & 209 & 1300 & 4.836 & 4.836 & & 0.9913 & 1.18E-02 & 3216.8900 & 1185.3293 & 4402.2193 & \\
\hline & $7 / 27 / 200814: 00$ & 121 & 209 & 1400 & 4.687 & 4.687 & & 1.0546 & 1.27E-02 & 3471.6051 & 1279.1843 & 4750.7894 & \\
\hline & $7 / 27 / 200815: 00$ & 122 & 209 & 1500 & 4.7 & 4.7 & & 1.0489 & 1.26E-02 & 3448.5907 & 1270.7042 & 4719.2949 & \\
\hline
\end{tabular}




\begin{tabular}{|c|c|c|c|c|c|c|c|c|c|c|c|c|c|}
\hline Tree ID & TS & RN & JD & HR & $\begin{array}{c}\text { Raw } \\
\text { TC_dT } \Delta\end{array}$ & $\begin{array}{c}\text { Fixed } \\
\text { TC_dT } \Delta\end{array}$ & dTM & $\mathbf{K}$ & $\begin{array}{l}\text { Sap } \\
\text { Velocity } \\
\text { (cm/s) }\end{array}$ & $\begin{array}{c}\text { Probed } \\
\text { Drywood F } \\
\text { (g/hr) }\end{array}$ & $\begin{array}{c}\text { Extrapolated } \\
\text { Wetwood F } \\
(\mathrm{g} / \mathrm{hr})\end{array}$ & $\begin{array}{c}\text { Extrapolated } \\
\text { Total Wood F } \\
(\mathrm{g} / \mathrm{hr})\end{array}$ & $\begin{array}{l}\text { Relative } \\
\text { Humidity, } \\
@ 10 \mathrm{~m}(\%)\end{array}$ \\
\hline & $7 / 27 / 2008$ 16:00 & 123 & 209 & 1600 & 4.774 & 4.774 & & 1.0172 & 1.22E-02 & 3320.5067 & 1223.5090 & 4544.0157 & \\
\hline & 7/27/2008 17:00 & 124 & 209 & 1700 & 4.829 & 4.829 & & 0.9942 & $1.18 \mathrm{E}-02$ & 3228.4248 & 1189.5795 & 4418.0043 & \\
\hline & $7 / 27 / 2008$ 18:00 & 125 & 209 & 1800 & 4.943 & 4.943 & & 0.9482 & $1.11 \mathrm{E}-02$ & 3045.5716 & 1122.2035 & 4167.7751 & \\
\hline & $7 / 27 / 2008$ 19:00 & 126 & 209 & 1900 & 5.087 & 5.087 & & 0.8931 & $1.04 \mathrm{E}-02$ & 2829.0073 & 1042.4059 & 3871.4133 & \\
\hline & $7 / 27 / 200820: 00$ & 127 & 209 & 2000 & 5.424 & 5.424 & & 0.7754 & 8.70E-03 & 2377.5798 & 876.0682 & 3253.6480 & \\
\hline & $7 / 27 / 2008$ 21:00 & 128 & 209 & 2100 & NAN & 6.303 & & 0.5278 & 5.42E-03 & 1480.8253 & 545.6405 & 2026.4658 & \\
\hline & $7 / 27 / 200822: 00$ & 129 & 209 & 2200 & 7.182 & 7.182 & & 0.3409 & $3.16 \mathrm{E}-03$ & 864.3474 & 318.4866 & 1182.8340 & \\
\hline & $7 / 27 / 200823: 00$ & 130 & 209 & 2300 & 7.532 & 7.532 & & 0.2785 & 2.47E-03 & 674.1639 & 248.4096 & 922.5735 & \\
\hline & $7 / 28 / 20080: 00$ & 131 & 210 & 0 & 7.658 & 7.658 & 7.81 & 0.2575 & $2.24 \mathrm{E}-03$ & 612.0459 & 225.5209 & 837.5667 & \\
\hline & 7/28/2008 1:00 & 132 & 210 & 100 & 7.734 & 7.734 & & 0.2452 & 2.11E-03 & 576.0936 & 212.2735 & 788.3671 & \\
\hline & 7/28/2008 2:00 & 133 & 210 & 200 & 7.771 & 7.771 & & 0.2392 & 2.05E-03 & 558.9918 & 205.9720 & 764.9638 & \\
\hline & 7/28/2008 3:00 & 134 & 210 & 300 & 7.806 & 7.806 & & 0.2337 & 1.99E-03 & 543.0522 & 200.0988 & 743.1510 & \\
\hline & 7/28/2008 4:00 & 135 & 210 & 400 & NAN & 7.7835 & & 0.2372 & $2.02 E-03$ & 553.2727 & 203.8647 & 757.1374 & \\
\hline & 7/28/2008 5:00 & 136 & 210 & 500 & 7.761 & 7.761 & & 0.2408 & 2.06E-03 & 563.5883 & 207.6657 & 771.2540 & \\
\hline & 7/28/2008 6:00 & 137 & 210 & 600 & 7.715 & 7.715 & & 0.2482 & $2.14 \mathrm{E}-03$ & 584.9770 & 215.5468 & 800.5238 & \\
\hline & 7/28/2008 7:00 & 138 & 210 & 700 & 7.638 & 7.638 & & 0.2608 & $2.28 \mathrm{E}-03$ & 621.6941 & 229.0760 & 850.7701 & \\
\hline & 7/28/2008 8:00 & 139 & 210 & 800 & 6.798 & 6.798 & & 0.4166 & 4.05E-03 & 1106.5350 & 407.7256 & 1514.2605 & \\
\hline & 7/28/2008 9:00 & 140 & 210 & 900 & 5.181 & 5.181 & & 0.8587 & 9.87E-03 & 2695.6745 & 993.2767 & 3688.9512 & \\
\hline & $7 / 28 / 2008$ 10:00 & 141 & 210 & 1000 & 4.524 & 4.524 & & 1.1286 & 1.38E-02 & 3773.9724 & 1390.5978 & 5164.5702 & \\
\hline & $7 / 28 / 2008$ 11:00 & 142 & 210 & 1100 & 4.494 & 4.494 & & 1.1429 & 1.40E-02 & 3832.5484 & 1412.1813 & 5244.7297 & \\
\hline & $7 / 28 / 200812: 00$ & 143 & 210 & 1200 & 4.503 & 4.503 & & 1.1386 & $1.40 \mathrm{E}-02$ & 3814.8759 & 1405.6695 & 5220.5453 & \\
\hline & $7 / 28 / 2008$ 13:00 & 144 & 210 & 1300 & 4.54 & 4.54 & & 1.1211 & 1.37E-02 & 3743.1170 & 1379.2284 & 5122.3454 & \\
\hline & $7 / 28 / 2008$ 14:00 & 145 & 210 & 1400 & 4.501 & 4.501 & & 1.1395 & 1.40E-02 & 3818.7957 & 1407.1138 & 5225.9095 & \\
\hline & $7 / 28 / 200815: 00$ & 146 & 210 & 1500 & 4.51 & 4.51 & & 1.1353 & 1.39E-02 & 3801.1899 & 1400.6266 & 5201.8164 & \\
\hline & $7 / 28 / 2008$ 16:00 & 147 & 210 & 1600 & 4.633 & 4.633 & & 1.0786 & 1.31E-02 & 3568.8992 & 1315.0343 & 4883.9335 & \\
\hline & $7 / 28 / 200817: 00$ & 148 & 210 & 1700 & 4.652 & 4.652 & & 1.0701 & 1.29E-02 & 3534.3508 & 1302.3043 & 4836.6551 & \\
\hline & $7 / 28 / 2008$ 18:00 & 149 & 210 & 1800 & 4.825 & 4.825 & & 0.9959 & 1.18E-02 & 3235.0346 & 1192.0150 & 4427.0496 & \\
\hline & $7 / 28 / 200819: 00$ & 150 & 210 & 1900 & 5.032 & 5.032 & & 0.9138 & 1.06E-02 & 2909.9079 & 1072.2154 & 3982.1233 & \\
\hline & $7 / 28 / 200820: 00$ & 151 & 210 & 2000 & 5.452 & 5.452 & & 0.7663 & 8.58E-03 & 2343.2113 & 863.4044 & 3206.6157 & \\
\hline & 7/28/2008 21:00 & 152 & 210 & 2100 & 6.58 & 6.58 & & 0.4635 & $4.62 E-03$ & 1261.9339 & 464.9855 & 1726.9193 & \\
\hline & $7 / 28 / 200822: 00$ & 153 & 210 & 2200 & 7.08 & 7.08 & & 0.3602 & 3.39E-03 & 925.0380 & 340.8493 & 1265.8873 & \\
\hline & $7 / 28 / 200823: 00$ & 154 & 210 & 2300 & 7.355 & 7.355 & & 0.3093 & 2.81E-03 & 766.9736 & 282.6072 & 1049.5807 & \\
\hline & $7 / 29 / 2008$ 0:00 & 155 & 211 & 0 & 7.529 & 7.529 & 7.74 & 0.2791 & 2.47E-03 & 675.6821 & 248.9690 & 924.6511 & \\
\hline & 7/29/2008 1:00 & 156 & 211 & 100 & 7.656 & 7.656 & & 0.2578 & $2.24 \mathrm{E}-03$ & 613.0071 & 225.8751 & 838.8822 & \\
\hline & 7/29/2008 2:00 & 157 & 211 & 200 & 7.726 & 7.726 & & 0.2464 & $2.12 \mathrm{E}-03$ & 579.8255 & 213.6486 & 793.4742 & \\
\hline & 7/29/2008 3:00 & 158 & 211 & 300 & 7.736 & 7.736 & & 0.2448 & $2.10 \mathrm{E}-03$ & 575.1625 & 211.9304 & 787.0929 & \\
\hline & 7/29/2008 4:00 & 159 & 211 & 400 & 7.721 & 7.721 & & 0.2472 & 2.13E-03 & 582.1642 & 214.5104 & 796.6746 & \\
\hline & 7/29/2008 5:00 & 160 & 211 & 500 & 7.684 & 7.684 & & 0.2533 & $2.19 \mathrm{E}-03$ & 599.6203 & 220.9424 & 820.5628 & \\
\hline & 7/29/2008 6:00 & 161 & 211 & 600 & 7.652 & 7.652 & & 0.2585 & $2.25 \mathrm{E}-03$ & 614.9321 & 226.5844 & 841.5164 & \\
\hline & 7/29/2008 7:00 & 162 & 211 & 700 & 7.622 & 7.622 & & 0.2634 & 2.30E-03 & 629.4697 & 231.9411 & 861.4107 & \\
\hline & 7/29/2008 8:00 & 163 & 211 & 800 & 7.402 & 7.402 & & 0.3010 & 2.71E-03 & 741.6764 & 273.2859 & 1014.9623 & \\
\hline
\end{tabular}




\begin{tabular}{|c|c|c|c|c|c|c|c|c|c|c|c|c|c|}
\hline \multirow[t]{41}{*}{ Tree ID } & TS & RN & JD & HR & $\begin{array}{c}\text { Raw } \\
\text { TC_dTA }\end{array}$ & $\begin{array}{c}\text { Fixed } \\
\text { TC_dT } \Delta\end{array}$ & dTM & $\mathbf{K}$ & $\begin{array}{l}\text { Sap } \\
\text { Velocity } \\
(\mathrm{cm} / \mathrm{s})\end{array}$ & $\begin{array}{c}\text { Probed } \\
\text { Drywood F } \\
(\mathrm{g} / \mathrm{hr})\end{array}$ & $\begin{array}{c}\text { Extrapolated } \\
\text { Wetwood F } \\
(\mathrm{g} / \mathrm{hr})\end{array}$ & $\begin{array}{c}\text { Extrapolated } \\
\text { Total Wood F } \\
(\mathrm{g} / \mathrm{hr})\end{array}$ & $\begin{array}{l}\text { Relative } \\
\text { Humidity, } \\
@ 10 \mathrm{~m}(\%)\end{array}$ \\
\hline & 7/29/2008 9:00 & 164 & 211 & 900 & 5.932 & 5.932 & & 0.6234 & $6.65 \mathrm{E}-03$ & 1817.4238 & 669.6672 & 2487.0910 & \\
\hline & $7 / 29 / 2008$ 10:00 & 165 & 211 & 1000 & 4.516 & 4.516 & & 1.1324 & $1.39 \mathrm{E}-02$ & 3789.5001 & 1396.3192 & 5185.8193 & \\
\hline & $7 / 29 / 2008$ 11:00 & 166 & 211 & 1100 & 4.736 & 4.736 & & 1.0334 & $1.24 \mathrm{E}-02$ & 3385.6660 & 1247.5183 & 4633.1842 & \\
\hline & $7 / 29 / 2008$ 12:00 & 167 & 211 & 1200 & 4.248 & 4.248 & & 1.2669 & $1.59 \mathrm{E}-02$ & 4351.0705 & 1603.2414 & 5954.3119 & \\
\hline & 7/29/2008 13:00 & 168 & 211 & 1300 & 4.268 & 4.268 & & 1.2563 & 1.58E-02 & 4306.2042 & 1586.7095 & 5892.9136 & \\
\hline & $7 / 29 / 2008$ 14:00 & 169 & 211 & 1400 & 4.184 & 4.184 & & 1.3016 & $1.65 \mathrm{E}-02$ & 4498.1277 & 1657.4277 & 6155.5554 & \\
\hline & $7 / 29 / 2008$ 15:00 & 170 & 211 & 1500 & NAN & 4.38 & & 1.1986 & $1.49 \mathrm{E}-02$ & 4064.0687 & 1497.4897 & 5561.5583 & \\
\hline & $7 / 29 / 2008$ 16:00 & 171 & 211 & 1600 & 4.576 & 4.576 & & 1.1045 & 1.34E-02 & 3674.6523 & 1354.0012 & 5028.6536 & \\
\hline & 7/29/2008 17:00 & 172 & 211 & 1700 & NAN & 4.6405 & & 1.0752 & 1.30E-02 & 3555.2203 & 1309.9941 & 4865.2144 & \\
\hline & 7/29/2008 18:00 & 173 & 211 & 1800 & 4.705 & 4.705 & & 1.0468 & 1.26E-02 & 3439.7806 & 1267.4579 & 4707.2384 & \\
\hline & $7 / 29 / 2008$ 19:00 & 174 & 211 & 1900 & 4.734 & 4.734 & & 1.0342 & 1.24E-02 & 3389.1310 & 1248.7950 & 4637.9260 & \\
\hline & $7 / 29 / 200820: 00$ & 175 & 211 & 2000 & 5.405 & 5.405 & & 0.7817 & 8.79E-03 & 2401.1580 & 884.7561 & 3285.9141 & \\
\hline & 7/29/2008 21:00 & 176 & 211 & 2100 & 6.554 & 6.554 & & 0.4693 & 4.69E-03 & 1281.4195 & 472.1654 & 1753.5848 & \\
\hline & $7 / 29 / 2008$ 22:00 & 177 & 211 & 2200 & 7.315 & 7.315 & & 0.3165 & 2.89E-03 & 788.8855 & 290.6811 & 1079.5666 & \\
\hline & $7 / 29 / 2008$ 23:00 & 178 & 211 & 2300 & 7.507 & 7.507 & & 0.2828 & $2.51 E-03$ & 686.8721 & 253.0921 & 939.9642 & \\
\hline & $7 / 30 / 20080: 00$ & 179 & 212 & 0 & 7.529 & 7.529 & 7.53 & 0.2791 & 2.47E-03 & 675.6821 & 248.9690 & 924.6511 & \\
\hline & 7/30/2008 1:00 & 180 & 212 & 100 & 7.526 & 7.526 & & 0.2796 & 2.48E-03 & 677.2021 & 249.5291 & 926.7312 & \\
\hline & 7/30/2008 2:00 & 181 & 212 & 200 & 7.504 & 7.504 & & 0.2833 & $2.52 \mathrm{E}-03$ & 688.4057 & 253.6572 & 942.0630 & \\
\hline & 7/30/2008 3:00 & 182 & 212 & 300 & 7.485 & 7.485 & & 0.2866 & 2.56E-03 & 698.1624 & 257.2523 & 955.4147 & \\
\hline & 7/30/2008 4:00 & 183 & 212 & 400 & 7.47 & 7.47 & & 0.2892 & $2.58 \mathrm{E}-03$ & 705.9183 & 260.1101 & 966.0285 & \\
\hline & 7/30/2008 5:00 & 184 & 212 & 500 & 7.447 & 7.447 & & 0.2931 & 2.63E-03 & 717.9028 & 264.5261 & 982.4289 & \\
\hline & 7/30/2008 6:00 & 185 & 212 & 600 & 7.426 & 7.426 & & 0.2968 & 2.67E-03 & 728.9432 & 268.5941 & 997.5373 & \\
\hline & 7/30/2008 7:00 & 186 & 212 & 700 & 7.402 & 7.402 & & 0.3010 & 2.71E-03 & 741.6764 & 273.2859 & 1014.9623 & \\
\hline & $7 / 30 / 20088: 00$ & 187 & 212 & 800 & 7.384 & 7.384 & & 0.3042 & $2.75 E-03$ & 751.3078 & 276.8348 & 1028.1426 & \\
\hline & 7/30/2008 9:00 & 188 & 212 & 900 & 7.351 & 7.351 & & 0.3100 & 2.81E-03 & 769.1488 & 283.4087 & 1052.5575 & \\
\hline & $7 / 30 / 2008$ 10:00 & 189 & 212 & 1000 & 5.207 & 5.207 & & 0.8494 & 9.73E-03 & 2659.8541 & 980.0779 & 3639.9320 & \\
\hline & $7 / 30 / 2008$ 11:00 & 190 & 212 & 1100 & 4.115 & 4.115 & & 1.3402 & 1.71E-02 & 4662.8648 & 1718.1284 & 6380.9932 & \\
\hline & $7 / 30 / 2008$ 12:00 & 191 & 212 & 1200 & 4.063 & 4.063 & & 1.3702 & 1.75E-02 & 4791.4708 & 1765.5160 & 6556.9868 & \\
\hline & 7/30/2008 13:00 & 192 & 212 & 1300 & 4.144 & 4.144 & & 1.3238 & 1.68E-02 & 4592.8234 & 1692.3203 & 6285.1437 & \\
\hline & $7 / 30 / 2008$ 14:00 & 193 & 212 & 1400 & 4.201 & 4.201 & & 1.2923 & 1.63E-02 & 4458.5387 & 1642.8403 & 6101.3789 & \\
\hline & 7/30/2008 15:00 & 194 & 212 & 1500 & 4.343 & 4.343 & & 1.2174 & $1.52 \mathrm{E}-02$ & 4142.3896 & 1526.3486 & 5668.7383 & \\
\hline & $7 / 30 / 2008$ 16:00 & 195 & 212 & 1600 & 4.299 & 4.299 & & 1.2401 & 1.55E-02 & 4237.6563 & 1561.4516 & 5799.1079 & \\
\hline & $7 / 30 / 2008$ 17:00 & 196 & 212 & 1700 & 4.399 & 4.399 & & 1.1891 & 1.47E-02 & 4024.4694 & 1482.8985 & 5507.3679 & \\
\hline & $7 / 30 / 2008$ 18:00 & 197 & 212 & 1800 & 4.567 & 4.567 & & 1.1086 & $1.35 \mathrm{E}-02$ & 3691.6451 & 1360.2626 & 5051.9077 & \\
\hline & 7/30/2008 19:00 & 198 & 212 & 1900 & 4.636 & 4.636 & & 1.0772 & 1.30E-02 & 3563.4212 & 1313.0158 & 4876.4370 & \\
\hline & $7 / 30 / 200820: 00$ & 199 & 212 & 2000 & 5.114 & 5.114 & & 0.8831 & 1.02E-02 & 2790.0835 & 1028.0637 & 3818.1472 & \\
\hline & 7/30/2008 21:00 & 200 & 212 & 2100 & 6.331 & 6.331 & & 0.5211 & 5.33E-03 & 1457.5242 & 537.0548 & 1994.5790 & \\
\hline & $7 / 30 / 2008$ 22:00 & 201 & 212 & 2200 & 7.19 & 7.19 & & 0.3394 & 3.15E-03 & 859.6926 & 316.7714 & 1176.4640 & \\
\hline & 7/30/2008 23:00 & 202 & 212 & 2300 & 7.429 & 7.429 & & 0.2963 & $2.66 \mathrm{E}-03$ & 727.3603 & 268.0108 & 995.3711 & \\
\hline & $7 / 31 / 20080: 00$ & 203 & 213 & 0 & 7.49 & 7.49 & 7.52 & 0.2857 & 2.55E-03 & 695.5876 & 256.3035 & 951.8911 & \\
\hline & 7/31/2008 1:00 & 204 & 213 & 100 & 7.512 & 7.512 & & 0.2819 & $2.50 \mathrm{E}-03$ & 684.3201 & 252.1518 & 936.4719 & \\
\hline
\end{tabular}




\begin{tabular}{|c|c|c|c|c|c|c|c|c|c|c|c|c|c|}
\hline \multirow[t]{42}{*}{ Tree ID } & TS & RN & JD & HR & $\begin{array}{c}\text { Raw } \\
\text { TC_dTA }\end{array}$ & $\begin{array}{c}\text { Fixed } \\
\text { TC_dT } \Delta\end{array}$ & dTM & $\mathbf{K}$ & $\begin{array}{c}\text { Sap } \\
\text { Velocity } \\
(\mathrm{cm} / \mathrm{s})\end{array}$ & $\begin{array}{c}\text { Probed } \\
\text { Drywood F } \\
\text { (g/hr) }\end{array}$ & $\begin{array}{c}\text { Extrapolated } \\
\text { Wetwood F } \\
(\mathrm{g} / \mathrm{hr})\end{array}$ & $\begin{array}{c}\text { Extrapolated } \\
\text { Total Wood F } \\
(\mathrm{g} / \mathrm{hr})\end{array}$ & $\begin{array}{l}\text { Relative } \\
\text { Humidity, } \\
\text { @10m (\%) }\end{array}$ \\
\hline & 7/31/2008 2:00 & 205 & 213 & 200 & 7.521 & 7.521 & & 0.2804 & 2.49E-03 & 679.7396 & 250.4640 & $\begin{array}{r}930.2037 \\
\end{array}$ & \\
\hline & 7/31/2008 3:00 & 206 & 213 & 300 & 7.523 & 7.523 & & 0.2801 & 2.48E-03 & 678.7240 & 250.0898 & 928.8138 & \\
\hline & 7/31/2008 4:00 & 207 & 213 & 400 & 7.511 & 7.511 & & 0.2821 & $2.51 \mathrm{E}-03$ & 684.8301 & 252.3397 & 937.1698 & \\
\hline & 7/31/2008 5:00 & 208 & 213 & 500 & 7.505 & 7.505 & & 0.2831 & $2.52 \mathrm{E}-03$ & 687.8943 & 253.4688 & 941.3631 & \\
\hline & 7/31/2008 6:00 & 209 & 213 & 600 & 7.496 & 7.496 & & 0.2847 & $2.53 \mathrm{E}-03$ & 692.5046 & 255.1676 & 947.6722 & \\
\hline & 7/31/2008 7:00 & 210 & 213 & 700 & 7.483 & 7.483 & & 0.2869 & $2.56 \mathrm{E}-03$ & 699.1938 & 257.6323 & 956.8261 & \\
\hline & 7/31/2008 8:00 & 211 & 213 & 800 & 7.494 & 7.494 & & 0.2850 & $2.54 \mathrm{E}-03$ & 693.5314 & 255.5459 & 949.0774 & \\
\hline & 7/31/2008 9:00 & 212 & 213 & 900 & 7.217 & 7.217 & & 0.3343 & $3.09 \mathrm{E}-03$ & 844.0934 & 311.0236 & 1155.1170 & \\
\hline & $7 / 31 / 2008$ 10:00 & 213 & 213 & 1000 & 4.589 & 4.589 & & 1.0985 & $1.34 \mathrm{E}-02$ & 3650.2507 & 1345.0099 & 4995.2606 & \\
\hline & 7/31/2008 11:00 & 214 & 213 & 1100 & 4.176 & 4.176 & & 1.3060 & 1.65E-02 & 4516.8922 & 1664.3418 & 6181.2341 & \\
\hline & 7/31/2008 12:00 & 215 & 213 & 1200 & 4.101 & 4.101 & & 1.3482 & $1.72 \mathrm{E}-02$ & 4697.1044 & 1730.7447 & 6427.8491 & \\
\hline & $7 / 31 / 2008$ 13:00 & 216 & 213 & 1300 & 4.373 & 4.373 & & 1.2021 & 1.49E-02 & 4078.7631 & 1502.9041 & 5581.6672 & \\
\hline & 7/31/2008 14:00 & 217 & 213 & 1400 & 4.222 & 4.222 & & 1.2809 & 1.61E-02 & 4410.1644 & 1625.0158 & 6035.1802 & \\
\hline & 7/31/2008 15:00 & 218 & 213 & 1500 & NAN & 4.322 & & 1.2281 & 1.53E-02 & 4187.5651 & 1542.9945 & 5730.5596 & \\
\hline & $7 / 31 / 2008$ 16:00 & 219 & 213 & 1600 & 4.422 & 4.422 & & 1.1777 & 1.46E-02 & 3977.0849 & 1465.4387 & 5442.5237 & \\
\hline & 7/31/2008 17:00 & 220 & 213 & 1700 & 4.457 & 4.457 & & 1.1606 & 1.43E-02 & 3906.1154 & 1439.2886 & 5345.4040 & \\
\hline & $7 / 31 / 2008$ 18:00 & 221 & 213 & 1800 & 4.624 & 4.624 & & 1.0826 & $1.31 E-02$ & 3585.3854 & 1321.1090 & 4906.4944 & \\
\hline & 7/31/2008 19:00 & 222 & 213 & 1900 & 4.885 & 4.885 & & 0.9713 & 1.15E-02 & 3137.2856 & 1155.9974 & 4293.2831 & \\
\hline & $7 / 31 / 200820: 00$ & 223 & 213 & 2000 & 5.476 & 5.476 & & 0.7586 & 8.47E-03 & 2314.1064 & 852.6801 & 3166.7865 & \\
\hline & $7 / 31 / 200821: 00$ & 224 & 213 & 2100 & 6.137 & 6.137 & & 0.5692 & 5.95E-03 & 1624.8117 & 598.6953 & 2223.5070 & \\
\hline & $7 / 31 / 200822: 00$ & 225 & 213 & 2200 & 6.913 & 6.913 & & 0.3930 & 3.77E-03 & 1029.9932 & 379.5222 & 1409.5154 & \\
\hline & 7/31/2008 23:00 & 226 & 213 & 2300 & 7.006 & 7.006 & & 0.3745 & $3.55 E-03$ & 970.6671 & 357.6623 & 1328.3293 & \\
\hline & 8/1/2008 0:00 & 227 & 214 & 0 & 7.172 & 7.172 & 7.64 & 0.3427 & $3.18 \mathrm{E}-03$ & 870.1872 & 320.6384 & 1190.8255 & \\
\hline & 8/1/2008 1:00 & 228 & 214 & 100 & 7.413 & 7.413 & & 0.2991 & 2.69E-03 & 735.8250 & 271.1298 & 1006.9548 & \\
\hline & 8/1/2008 2:00 & 229 & 214 & 200 & 7.511 & 7.511 & & 0.2821 & 2.51E-03 & 684.8301 & 252.3397 & 937.1698 & \\
\hline & 8/1/2008 3:00 & 230 & 214 & 300 & 7.526 & 7.526 & & 0.2796 & 2.48E-03 & 677.2021 & 249.5291 & 926.7312 & \\
\hline & 8/1/2008 4:00 & 231 & 214 & 400 & 7.534 & 7.534 & & 0.2782 & 2.46E-03 & 673.1529 & 248.0370 & 921.1899 & \\
\hline & 8/1/2008 5:00 & 232 & 214 & 500 & 7.515 & 7.515 & & 0.2814 & $2.50 \mathrm{E}-03$ & 682.7914 & 251.5885 & 934.3800 & \\
\hline & 8/1/2008 6:00 & 233 & 214 & 600 & 7.472 & 7.472 & & 0.2888 & 2.58E-03 & 704.8815 & 259.7281 & 964.6096 & \\
\hline & 8/1/2008 7:00 & 234 & 214 & 700 & 7.439 & 7.439 & & 0.2945 & 2.64E-03 & 722.0976 & 266.0717 & 988.1693 & \\
\hline & 8/1/2008 8:00 & 235 & 214 & 800 & 7.427 & 7.427 & & 0.2966 & 2.67E-03 & 728.4153 & 268.3996 & 996.8150 & \\
\hline & 8/1/20089:00 & 236 & 214 & 900 & 6.837 & 6.837 & & 0.4085 & $3.95 \mathrm{E}-03$ & 1080.1731 & 398.0120 & 1478.1851 & \\
\hline & 8/1/2008 10:00 & 237 & 214 & 1000 & 4.6 & 4.6 & & 1.0935 & 1.33E-02 & 3629.7345 & 1337.4503 & 4967.1849 & \\
\hline & 8/1/2008 11:00 & 238 & 214 & 1100 & 4.399 & 4.399 & & 1.1891 & $1.47 \mathrm{E}-02$ & 4024.4694 & 1482.8985 & 5507.3679 & \\
\hline & 8/1/2008 12:00 & 239 & 214 & 1200 & 4.444 & 4.444 & & 1.1670 & 1.44E-02 & 3932.3171 & 1448.9431 & 5381.2602 & \\
\hline & 8/1/2008 13:00 & 240 & 214 & 1300 & 4.449 & 4.449 & & 1.1645 & 1.44E-02 & 3922.2175 & 1445.2217 & 5367.4392 & \\
\hline & 8/1/2008 14:00 & 241 & 214 & 1400 & 4.434 & 4.434 & & 1.1719 & $1.45 \mathrm{E}-02$ & 3952.5993 & 1456.4165 & 5409.0157 & \\
\hline & 8/1/2008 15:00 & 242 & 214 & 1500 & 4.45 & 4.45 & & 1.1640 & $1.43 \mathrm{E}-02$ & 3920.2009 & 1444.4786 & 5364.6795 & \\
\hline & 8/1/2008 16:00 & 243 & 214 & 1600 & 4.56 & 4.56 & & 1.1118 & 1.36E-02 & 3704.9183 & 1365.1534 & 5070.0717 & \\
\hline & 8/1/2008 17:00 & 244 & 214 & 1700 & 4.648 & 4.648 & & 1.0719 & 1.30E-02 & 3541.5955 & 1304.9737 & 4846.5692 & \\
\hline & $8 / 1 / 2008$ 18:00 & 245 & 214 & 1800 & 4.772 & 4.772 & & 1.0180 & 1.22E-02 & 3323.9044 & 1224.7609 & 4548.6653 & \\
\hline
\end{tabular}




\begin{tabular}{|c|c|c|c|c|c|c|c|c|c|c|c|c|c|}
\hline Tree ID & TS & RN & JD & HR & $\begin{array}{c}\text { Raw } \\
\text { TC_dTA }\end{array}$ & $\begin{array}{c}\text { Fixed } \\
\text { TC_dT } \Delta\end{array}$ & dTM & $\mathbf{K}$ & $\begin{array}{c}\text { Sap } \\
\text { Velocity } \\
(\mathrm{cm} / \mathrm{s})\end{array}$ & $\begin{array}{c}\text { Probed } \\
\text { Drywood F } \\
\text { (g/hr) }\end{array}$ & $\begin{array}{c}\text { Extrapolated } \\
\text { Wetwood F } \\
(\mathrm{g} / \mathrm{hr})\end{array}$ & $\begin{array}{c}\text { Extrapolated } \\
\text { Total Wood F } \\
(\mathrm{g} / \mathrm{hr})\end{array}$ & $\begin{array}{l}\text { Relative } \\
\text { Humidity, } \\
\text { @10m (\%) }\end{array}$ \\
\hline & 8/1/2008 19:00 & 246 & 214 & 1900 & 5.011 & 5.011 & & 0.9218 & $1.08 \mathrm{E}-02$ & 2941.3802 & 1083.8120 & \begin{tabular}{|r|}
4025.1922 \\
\end{tabular} & \\
\hline & 8/1/2008 20:00 & 247 & 214 & 2000 & 5.813 & 5.813 & & 0.6566 & 7.09E-03 & 1937.4154 & \begin{tabular}{|l|}
713.8805 \\
\end{tabular} & 2651.2959 & \\
\hline & 8/1/2008 21:00 & 248 & 214 & 2100 & 6.973 & 6.973 & & 0.3810 & $3.63 \mathrm{E}-03$ & 991.4617 & 365.3245 & 1356.7862 & \\
\hline & 8/1/2008 22:00 & 249 & 214 & 2200 & 7.517 & 7.517 & & 0.2811 & $2.50 \mathrm{E}-03$ & 681.7733 & 251.2134 & 932.9867 & \\
\hline & 8/1/2008 23:00 & 250 & 214 & 2300 & 7.637 & 7.637 & & 0.2610 & $2.28 \mathrm{E}-03$ & 622.1786 & 229.2545 & 851.4331 & \\
\hline & 8/2/2008 0:00 & 251 & 215 & 0 & 7.675 & 7.675 & 7.70 & 0.2547 & $2.21 \mathrm{E}-03$ & 603.9065 & 222.5218 & 826.4283 & \\
\hline & 8/2/2008 1:00 & 252 & 215 & 100 & 7.703 & 7.703 & & 0.2502 & $2.16 \mathrm{E}-03$ & 590.6234 & 217.6273 & 808.2507 & \\
\hline & 8/2/2008 2:00 & 253 & 215 & 200 & 7.695 & 7.695 & & 0.2515 & $2.18 \mathrm{E}-03$ & 594.4030 & 219.0200 & 813.4231 & \\
\hline & 8/2/2008 3:00 & 254 & 215 & 300 & 7.681 & 7.681 & & 0.2537 & $2.20 \mathrm{E}-03$ & 601.0473 & 221.4682 & 822.5156 & \\
\hline & 8/2/2008 4:00 & 255 & 215 & 400 & 7.673 & 7.673 & & 0.2551 & 2.21E-03 & 604.8612 & 222.8735 & 827.7347 & \\
\hline & 8/2/2008 5:00 & 256 & 215 & 500 & 7.677 & 7.677 & & 0.2544 & $2.21 \mathrm{E}-03$ & 602.9527 & 222.1703 & 825.1230 & \\
\hline & 8/2/2008 6:00 & 257 & 215 & 600 & 7.672 & 7.672 & & 0.2552 & $2.22 E-03$ & 605.3388 & 223.0495 & 828.3883 & \\
\hline & 8/2/2008 7:00 & 258 & 215 & 700 & 7.618 & 7.618 & & 0.2641 & $2.31 \mathrm{E}-03$ & 631.4215 & 232.6602 & 864.0817 & \\
\hline & 8/2/2008 8:00 & 259 & 215 & 800 & 7.042 & 7.042 & & 0.3675 & 3.47E-03 & 948.2979 & 349.4199 & 1297.7177 & \\
\hline & 8/2/20089:00 & 260 & 215 & 900 & 5.168 & 5.168 & & 0.8634 & 9.93E-03 & 2713.7539 & 999.9384 & 3713.6923 & \\
\hline & 8/2/2008 10:00 & 261 & 215 & 1000 & 4.646 & 4.646 & & 1.0728 & 1.30E-02 & 3545.2235 & 1306.3105 & 4851.5340 & \\
\hline & 8/2/2008 11:00 & 262 & 215 & 1100 & 4.566 & 4.566 & & 1.1091 & 1.35E-02 & 3693.5383 & 1360.9601 & 5054.4984 & \\
\hline & 8/2/2008 12:00 & 263 & 215 & 1200 & 4.508 & 4.508 & & 1.1362 & 1.39E-02 & 3805.0949 & 1402.0655 & 5207.1603 & \\
\hline & 8/2/2008 13:00 & 264 & 215 & 1300 & 4.555 & 4.555 & & 1.1142 & 1.36E-02 & 3714.4297 & 1368.6580 & 5083.0877 & \\
\hline & 8/2/2008 14:00 & 265 & 215 & 1400 & 4.611 & 4.611 & & 1.0885 & 1.32E-02 & 3609.3378 & 1329.9348 & 4939.2726 & \\
\hline & 8/2/2008 15:00 & 266 & 215 & 1500 & 4.647 & 4.647 & & 1.0723 & 1.30E-02 & 3543.4090 & 1305.6419 & 4849.0509 & \\
\hline & 8/2/2008 16:00 & 267 & 215 & 1600 & 4.705 & 4.705 & & 1.0468 & $1.26 \mathrm{E}-02$ & 3439.7806 & 1267.4579 & 4707.2384 & \\
\hline & 8/2/2008 17:00 & 268 & 215 & 1700 & 4.765 & 4.765 & & 1.0210 & $1.22 \mathrm{E}-02$ & 3335.8238 & 1229.1529 & 4564.9767 & \\
\hline & 8/2/2008 18:00 & 269 & 215 & 1800 & 4.903 & 4.903 & & 0.9641 & $1.14 \mathrm{E}-02$ & 3108.5356 & 1145.4039 & 4253.9395 & \\
\hline & 8/2/2008 19:00 & 270 & 215 & 1900 & 5.086 & 5.086 & & 0.8934 & 1.04E-02 & 2830.4588 & 1042.9408 & 3873.3996 & \\
\hline & 8/2/2008 20:00 & 271 & 215 & 2000 & 5.631 & 5.631 & & 0.7102 & 7.81E-03 & 2133.6882 & 786.2013 & 2919.8895 & \\
\hline & 8/2/2008 21:00 & 272 & 215 & 2100 & 6.276 & 6.276 & & 0.5344 & 5.50E-03 & 1503.5573 & 554.0166 & 2057.5740 & \\
\hline & 8/2/2008 22:00 & 273 & 215 & 2200 & 7.083 & 7.083 & & 0.3596 & 3.38E-03 & 923.2170 & 340.1783 & 1263.3952 & \\
\hline & 8/2/2008 23:00 & 274 & 215 & 2300 & 7.442 & 7.442 & & 0.2940 & 2.64E-03 & 720.5230 & 265.4915 & 986.0145 & \\
\hline & 8/3/2008 0:00 & 275 & 216 & 0 & 7.521 & 7.521 & 7.76 & 0.2804 & $2.49 \mathrm{E}-03$ & 679.7396 & 250.4640 & 930.2037 & \\
\hline & 8/3/2008 1:00 & 276 & 216 & 100 & 7.5 & 7.5 & & 0.2840 & 2.53E-03 & 690.4535 & 254.4118 & 944.8653 & \\
\hline & $8 / 3 / 2008$ 2:00 & 277 & 216 & 200 & 7.567 & 7.567 & & 0.2726 & $2.40 \mathrm{E}-03$ & 656.5881 & 241.9334 & 898.5215 & \\
\hline & 8/3/2008 3:00 & 278 & 216 & 300 & 7.652 & 7.652 & & 0.2585 & $2.25 \mathrm{E}-03$ & 614.9321 & 226.5844 & 841.5164 & \\
\hline & 8/3/2008 4:00 & 279 & 216 & 400 & 7.726 & 7.726 & & 0.2464 & 2.12E-03 & 579.8255 & 213.6486 & 793.4742 & \\
\hline & $8 / 3 / 20085: 00$ & 280 & 216 & 500 & 7.753 & 7.753 & & 0.2421 & 2.08E-03 & 567.2791 & 209.0256 & 776.3047 & \\
\hline & 8/3/2008 6:00 & 281 & 216 & 600 & 7.763 & 7.763 & & 0.2405 & 2.06E-03 & 562.6675 & 207.3264 & 769.9939 & \\
\hline & 8/3/2008 7:00 & 282 & 216 & 700 & 7.764 & 7.764 & & 0.2403 & 2.06E-03 & 562.2073 & 207.1569 & 769.3642 & \\
\hline & 8/3/2008 8:00 & 283 & 216 & 800 & 7.479 & 7.479 & & 0.2876 & $2.57 \mathrm{E}-03$ & 701.2591 & 258.3933 & 959.6525 & \\
\hline & 8/3/2008 9:00 & 284 & 216 & 900 & 5.477 & 5.477 & & 0.7583 & $8.46 \mathrm{E}-03$ & 2312.9007 & 852.2358 & 3165.1365 & \\
\hline & $8 / 3 / 2008$ 10:00 & 285 & 216 & 1000 & 4.629 & 4.629 & & 1.0804 & 1.31E-02 & 3576.2167 & 1317.7306 & 4893.9473 & \\
\hline & 8/3/2008 11:00 & 286 & 216 & 1100 & 4.694 & 4.694 & & 1.0516 & 1.27E-02 & 3459.1933 & 1274.6109 & 4733.8043 & \\
\hline
\end{tabular}




\begin{tabular}{|c|c|c|c|c|c|c|c|c|c|c|c|c|c|}
\hline Tree ID & TS & RN & JD & HR & $\begin{array}{c}\text { Raw } \\
\text { TC_dTA }\end{array}$ & $\begin{array}{c}\text { Fixed } \\
\text { TC_dT } \Delta\end{array}$ & dTM & $\mathbf{K}$ & $\begin{array}{l}\text { Sap } \\
\text { Velocity } \\
(\mathrm{cm} / \mathrm{s})\end{array}$ & $\begin{array}{c}\text { Probed } \\
\text { Drywood F } \\
(\mathrm{g} / \mathrm{hr})\end{array}$ & $\begin{array}{c}\text { Extrapolated } \\
\text { Wetwood F } \\
(\mathrm{g} / \mathrm{hr})\end{array}$ & $\begin{array}{c}\text { Extrapolated } \\
\text { Total Wood F } \\
(\mathrm{g} / \mathrm{hr})\end{array}$ & $\begin{array}{l}\text { Relative } \\
\text { Humidity, } \\
@ 10 \mathrm{~m}(\%)\end{array}$ \\
\hline & 8/3/2008 12:00 & 287 & 216 & 1200 & 4.72 & 4.72 & & 1.0403 & 1.25E-02 & 3413.4872 & 1257.7696 & 4671.2568 & \\
\hline & 8/3/2008 13:00 & 288 & 216 & 1300 & 4.723 & 4.723 & & 1.0390 & $1.25 \mathrm{E}-02$ & 3408.2531 & 1255.8410 & 4664.0941 & \\
\hline & $8 / 3 / 2008$ 14:00 & 289 & 216 & 1400 & NAN & 4.749 & & 1.0278 & 1.23E-02 & 3363.2305 & 1239.2515 & 4602.4820 & \\
\hline & 8/3/2008 15:00 & 290 & 216 & 1500 & 4.775 & 4.775 & & 1.0168 & $1.21 \mathrm{E}-02$ & 3318.8092 & 1222.8835 & 4541.6927 & \\
\hline & $8 / 3 / 2008$ 16:00 & 291 & 216 & 1600 & 4.832 & 4.832 & & 0.9930 & 1.18E-02 & 3223.4763 & 1187.7561 & 4411.2324 & \\
\hline & 8/3/2008 17:00 & 292 & 216 & 1700 & 4.886 & 4.886 & & 0.9709 & 1.15E-02 & 3135.6816 & 1155.4064 & 4291.0879 & \\
\hline & $8 / 3 / 2008$ 18:00 & 293 & 216 & 1800 & 5.006 & 5.006 & & 0.9237 & 1.08E-02 & 2948.9219 & 1086.5909 & 4035.5129 & \\
\hline & 8/3/2008 19:00 & 294 & 216 & 1900 & 5.174 & 5.174 & & 0.8612 & $9.90 \mathrm{E}-03$ & 2705.3954 & 996.8586 & 3702.2540 & \\
\hline & $8 / 3 / 200820: 00$ & 295 & 216 & 2000 & NAN & 5.174 & & 0.8612 & $9.90 \mathrm{E}-03$ & 2705.3954 & 996.8586 & 3702.2540 & \\
\hline & $8 / 3 / 200821: 00$ & 296 & 216 & 2100 & NAN & 6.813 & & 0.4135 & $4.01 E-03$ & 1096.3459 & 403.9712 & 1500.3172 & \\
\hline & $8 / 3 / 200822: 00$ & 297 & 216 & 2200 & 6.813 & 6.813 & & 0.4135 & 4.01E-03 & 1096.3459 & 403.9712 & 1500.3172 & \\
\hline & $8 / 3 / 200823: 00$ & 298 & 216 & 2300 & 6.96 & 6.96 & & 0.3836 & 3.66E-03 & 999.7305 & 368.3713 & 1368.1018 & \\
\hline & 8/4/2008 0:00 & 299 & 217 & 0 & 6.975 & 6.975 & 7.55 & 0.3806 & $3.62 \mathrm{E}-03$ & 990.1935 & 364.8572 & 1355.0507 & \\
\hline & 8/4/2008 1:00 & 300 & 217 & 100 & 6.982 & 6.982 & & 0.3793 & 3.61E-03 & 985.7628 & 363.2246 & 1348.9873 & \\
\hline & $8 / 4 / 20082: 00$ & 301 & 217 & 200 & 6.754 & 6.754 & & 0.4258 & $4.16 \mathrm{E}-03$ & 1136.7868 & 418.8725 & 1555.6593 & \\
\hline & 8/4/2008 3:00 & 302 & 217 & 300 & 6.545 & 6.545 & & 0.4714 & $4.71 \mathrm{E}-03$ & 1288.2136 & 474.6688 & 1762.8825 & \\
\hline & $8 / 4 / 20084: 00$ & 303 & 217 & 400 & 6.321 & 6.321 & & 0.5235 & 5.36E-03 & 1465.8144 & 540.1095 & 2005.9238 & \\
\hline & 8/4/2008 5:00 & 304 & 217 & 500 & 6.322 & 6.322 & & 0.5233 & $5.36 \mathrm{E}-03$ & 1464.9838 & 539.8034 & 2004.7872 & \\
\hline & $8 / 4 / 20086: 00$ & 305 & 217 & 600 & 6.616 & 6.616 & & 0.4556 & 4.52E-03 & 1235.2983 & 455.1711 & 1690.4694 & \\
\hline & $8 / 4 / 20087: 00$ & 306 & 217 & 700 & 6.651 & 6.651 & & 0.4479 & 4.43E-03 & 1209.7803 & 445.7684 & 1655.5488 & \\
\hline & 8/4/2008 8:00 & 307 & 217 & 800 & 6.551 & 6.551 & & 0.4700 & $4.70 \mathrm{E}-03$ & 1283.6814 & 472.9988 & 1756.6802 & \\
\hline & 8/4/2008 9:00 & 308 & 217 & 900 & 6.371 & 6.371 & & 0.5115 & 5.21E-03 & 1424.7112 & 524.9642 & 1949.6754 & \\
\hline & $8 / 4 / 2008$ 10:00 & 309 & 217 & 1000 & 5.465 & 5.465 & & 0.7621 & 8.52E-03 & 2327.4060 & 857.5806 & 3184.9865 & \\
\hline & 8/4/2008 11:00 & 310 & 217 & 1100 & 4.972 & 4.972 & & 0.9368 & 1.10E-02 & 3000.7051 & 1105.6715 & 4106.3766 & \\
\hline & 8/4/2008 12:00 & 311 & 217 & 1200 & 4.914 & 4.914 & & 0.9597 & 1.13E-02 & 3091.0942 & 1138.9772 & 4230.0714 & \\
\hline & 8/4/2008 13:00 & 312 & 217 & 1300 & 4.957 & 4.957 & & 0.9427 & 1.11E-02 & 3023.8308 & 1114.1926 & 4138.0234 & \\
\hline & $8 / 4 / 2008$ 14:00 & 313 & 217 & 1400 & 5.263 & 5.263 & & 0.8298 & 9.46E-03 & 2584.2040 & 952.2031 & 3536.4071 & \\
\hline & $8 / 4 / 2008$ 15:00 & 314 & 217 & 1500 & 5.489 & 5.489 & & 0.7544 & $8.41 \mathrm{E}-03$ & 2298.4758 & 846.9207 & 3145.3965 & \\
\hline & $8 / 4 / 2008$ 16:00 & 315 & 217 & 1600 & 5.031 & 5.031 & & 0.9141 & 1.07E-02 & 2911.3992 & 1072.7649 & 3984.1641 & \\
\hline & $8 / 4 / 200817: 00$ & 316 & 217 & 1700 & 5.034 & 5.034 & & 0.9130 & 1.06E-02 & 2906.9275 & 1071.1172 & 3978.0448 & \\
\hline & 8/4/2008 18:00 & 317 & 217 & 1800 & 5.17 & 5.17 & & 0.8627 & 9.92E-03 & 2710.9650 & 998.9108 & 3709.8759 & \\
\hline & 8/4/2008 19:00 & 318 & 217 & 1900 & 5.381 & 5.381 & & 0.7896 & 8.90E-03 & 2431.2420 & 895.8411 & 3327.0831 & \\
\hline & $8 / 4 / 200820: 00$ & 319 & 217 & 2000 & NAN & 6.1255 & & 0.5721 & 5.98E-03 & 1635.1704 & 602.5122 & 2237.6825 & \\
\hline & $8 / 4 / 200821: 00$ & 320 & 217 & 2100 & 6.87 & 6.87 & & 0.4017 & 3.87E-03 & 1058.1930 & 389.9130 & 1448.1060 & \\
\hline & $8 / 4 / 200822: 00$ & 321 & 217 & 2200 & 7.35 & 7.35 & & 0.3102 & $2.82 \mathrm{E}-03$ & 769.6932 & 283.6093 & 1053.3025 & \\
\hline & 8/4/2008 23:00 & 322 & 217 & 2300 & 7.548 & 7.548 & & 0.2758 & 2.44E-03 & 666.0982 & 245.4376 & 911.5358 & \\
\hline & 8/5/2008 0:00 & 323 & 218 & 0 & 7.708 & 7.708 & 7.86 & 0.2494 & $2.15 E-03$ & 588.2673 & 216.7592 & 805.0265 & \\
\hline & $8 / 5 / 20081: 00$ & 324 & 218 & 100 & 7.795 & 7.795 & & 0.2354 & $2.01 \mathrm{E}-03$ & 548.0371 & 201.9355 & 749.9726 & \\
\hline & $8 / 5 / 2008$ 2:00 & 325 & 218 & 200 & 7.846 & 7.846 & & 0.2274 & 1.92E-03 & 525.1152 & 193.4895 & 718.6046 & \\
\hline & $8 / 5 / 2008$ 3:00 & 326 & 218 & 300 & 7.858 & 7.858 & & 0.2255 & 1.90E-03 & 519.7916 & 191.5279 & 711.3196 & \\
\hline & 8/5/2008 4:00 & 327 & 218 & 400 & 7.841 & 7.841 & & 0.2282 & 1.93E-03 & 527.3411 & 194.3097 & 721.6508 & \\
\hline
\end{tabular}




\begin{tabular}{|c|c|c|c|c|c|c|c|c|c|c|c|c|c|}
\hline \multirow[t]{42}{*}{ Tree ID } & TS & RN & JD & HR & $\begin{array}{c}\text { Raw } \\
\text { TC_dTA }\end{array}$ & $\begin{array}{c}\text { Fixed } \\
\text { TC_dT } \Delta\end{array}$ & dTM & $\mathbf{K}$ & $\begin{array}{c}\text { Sap } \\
\text { Velocity } \\
(\mathrm{cm} / \mathrm{s})\end{array}$ & $\begin{array}{c}\text { Probed } \\
\text { Drywood F } \\
\text { (g/hr) }\end{array}$ & $\begin{array}{c}\text { Extrapolated } \\
\text { Wetwood F } \\
(\mathrm{g} / \mathrm{hr})\end{array}$ & $\begin{array}{c}\text { Extrapolated } \\
\text { Total Wood F } \\
(\mathrm{g} / \mathrm{hr})\end{array}$ & $\begin{array}{l}\text { Relative } \\
\text { Humidity, } \\
\text { @10m (\%) }\end{array}$ \\
\hline & 8/5/2008 5:00 & 328 & 218 & 500 & \begin{tabular}{|l|} 
\\
\end{tabular} & 7.85 & & 0.2268 & 1.92E-03 & 523.3377 & 192.8345 & 716.1723 & \\
\hline & 8/5/2008 6:00 & 329 & 218 & 600 & 7.862 & 7.862 & & 0.2249 & $1.90 \mathrm{E}-03$ & 518.0230 & 190.8762 & 708.8992 & \\
\hline & 8/5/2008 7:00 & 330 & 218 & 700 & 7.848 & 7.848 & & 0.2271 & $1.92 \mathrm{E}-03$ & 524.2261 & 193.1619 & 717.3880 & \\
\hline & 8/5/2008 8:00 & 331 & 218 & 800 & 7.769 & 7.769 & & 0.2395 & $2.05 E-03$ & 559.9096 & 206.3102 & 766.2198 & \\
\hline & 8/5/2008 9:00 & 332 & 218 & 900 & 6.9 & 6.9 & & 0.3957 & $3.80 \mathrm{E}-03$ & 1038.4666 & 382.6444 & 1421.1110 & \\
\hline & 8/5/2008 10:00 & 333 & 218 & 1000 & 5.296 & 5.296 & & 0.8184 & $9.30 \mathrm{E}-03$ & 2540.5623 & 936.1224 & 3476.6847 & \\
\hline & 8/5/2008 11:00 & 334 & 218 & 1100 & 5.368 & 5.368 & & 0.7940 & 8.96E-03 & 2447.6793 & 901.8978 & 3349.5771 & \\
\hline & 8/5/2008 12:00 & 335 & 218 & 1200 & 5.167 & 5.167 & & 0.8638 & $9.94 \mathrm{E}-03$ & 2715.1493 & 1000.4526 & 3715.6019 & \\
\hline & 8/5/2008 13:00 & 336 & 218 & 1300 & 4.857 & 4.857 & & 0.9827 & $1.16 \mathrm{E}-02$ & 3182.5313 & 1172.6691 & 4355.2004 & \\
\hline & $8 / 5 / 2008$ 14:00 & 337 & 218 & 1400 & 5.323 & 5.323 & & 0.8091 & 9.17E-03 & 2505.3605 & 923.1516 & 3428.5121 & \\
\hline & 8/5/2008 15:00 & 338 & 218 & 1500 & 5.603 & 5.603 & & 0.7187 & 7.92E-03 & 2165.3403 & 797.8642 & 2963.2045 & \\
\hline & 8/5/2008 16:00 & 339 & 218 & 1600 & 5.456 & 5.456 & & 0.7650 & 8.56E-03 & 2338.3380 & 861.6087 & 3199.9467 & \\
\hline & 8/5/2008 17:00 & 340 & 218 & 1700 & 5.1 & 5.1 & & 0.8882 & 1.03E-02 & 2810.2022 & 1035.4768 & \begin{tabular}{|l|}
3845.6789 \\
\end{tabular} & \\
\hline & 8/5/2008 18:00 & 341 & 218 & 1800 & NAN & 5.1595 & & 0.8665 & 9.98E-03 & 2725.6366 & 1004.3168 & 3729.9534 & \\
\hline & 8/5/2008 19:00 & 342 & 218 & 1900 & 5.219 & 5.219 & & 0.8452 & 9.67E-03 & 2643.4720 & 974.0416 & 3617.5137 & \\
\hline & 8/5/2008 20:00 & 343 & 218 & 2000 & 6.064 & 6.064 & & 0.5881 & 6.19E-03 & 1691.4463 & 623.2482 & 2314.6945 & \\
\hline & 8/5/2008 21:00 & 344 & 218 & 2100 & 7.349 & 7.349 & & 0.3104 & $2.82 \mathrm{E}-03$ & 770.2378 & 283.8100 & 1054.0477 & \\
\hline & 8/5/2008 22:00 & 345 & 218 & 2200 & 7.73 & 7.73 & & 0.2458 & 2.12E-03 & 577.9580 & 212.9605 & 790.9185 & \\
\hline & 8/5/2008 23:00 & 346 & 218 & 2300 & 7.783 & 7.783 & & 0.2373 & 2.03E-03 & 553.5009 & 203.9488 & 757.4497 & \\
\hline & $8 / 6 / 20080: 00$ & 347 & 219 & 0 & 7.771 & 7.771 & 7.77 & 0.2392 & $2.05 E-03$ & 558.9918 & 205.9720 & 764.9638 & \\
\hline & 8/6/2008 1:00 & 348 & 219 & 100 & 7.719 & 7.719 & & 0.2476 & 2.13E-03 & 583.1011 & 214.8556 & 797.9566 & \\
\hline & 8/6/2008 2:00 & 349 & 219 & 200 & 7.667 & 7.667 & & 0.2560 & $2.22 \mathrm{E}-03$ & 607.7298 & 223.9305 & 831.6603 & \\
\hline & 8/6/2008 3:00 & 350 & 219 & 300 & 7.628 & 7.628 & & 0.2625 & $2.29 \mathrm{E}-03$ & 626.5479 & 230.8645 & 857.4124 & \\
\hline & 8/6/2008 4:00 & 351 & 219 & 400 & NAN & 7.591 & & 0.2686 & $2.36 \mathrm{E}-03$ & 644.6799 & 237.5456 & 882.2254 & \\
\hline & 8/6/2008 5:00 & 352 & 219 & 500 & 7.554 & 7.554 & & 0.2748 & 2.43E-03 & 663.0871 & 244.3281 & 907.4152 & \\
\hline & 8/6/2008 6:00 & 353 & 219 & 600 & 7.519 & 7.519 & & 0.2808 & $2.49 \mathrm{E}-03$ & 680.7561 & 250.8386 & 931.5946 & \\
\hline & $8 / 6 / 20087: 00$ & 354 & 219 & 700 & 7.499 & 7.499 & & 0.2842 & 2.53E-03 & 690.9660 & 254.6006 & 945.5666 & \\
\hline & 8/6/2008 8:00 & 355 & 219 & 800 & 7.464 & 7.464 & & 0.2902 & $2.59 \mathrm{E}-03$ & 709.0340 & 261.2581 & 970.2921 & \\
\hline & 8/6/2008 9:00 & 356 & 219 & 900 & 7.513 & 7.513 & & 0.2818 & 2.50E-03 & 683.8103 & 251.9640 & 935.7743 & \\
\hline & 8/6/2008 10:00 & 357 & 219 & 1000 & 6.331 & 6.331 & & 0.5211 & 5.33E-03 & 1457.5242 & 537.0548 & 1994.5790 & \\
\hline & 8/6/2008 11:00 & 358 & 219 & 1100 & 4.393 & 4.393 & & 1.1921 & 1.48E-02 & 4036.9296 & 1487.4897 & 5524.4193 & \\
\hline & 8/6/2008 12:00 & 359 & 219 & 1200 & 4.8 & 4.8 & & 1.0063 & 1.20E-02 & 3276.6535 & 1207.3504 & 4484.0039 & \\
\hline & 8/6/2008 13:00 & 360 & 219 & 1300 & 5.295 & 5.295 & & 0.8187 & 9.30E-03 & 2541.8747 & 936.6060 & 3478.4808 & \\
\hline & $8 / 6 / 2008$ 14:00 & 361 & 219 & 1400 & NAN & 5.295 & & 0.8187 & $9.30 \mathrm{E}-03$ & 2541.8747 & 936.6060 & 3478.4808 & \\
\hline & 8/6/2008 15:00 & 362 & 219 & 1500 & NAN & 4.702 & & 1.0481 & $1.26 \mathrm{E}-02$ & 3445.0639 & 1269.4047 & 4714.4686 & \\
\hline & 8/6/2008 16:00 & 363 & 219 & 1600 & 4.702 & 4.702 & & 1.0481 & 1.26E-02 & 3445.0639 & 1269.4047 & 4714.4686 & \\
\hline & 8/6/2008 17:00 & 364 & 219 & 1700 & 4.732 & 4.732 & & 1.0351 & 1.24E-02 & 3392.5996 & 1250.0731 & 4642.6728 & \\
\hline & 8/6/2008 18:00 & 365 & 219 & 1800 & NAN & 5.03 & & 0.9145 & 1.07E-02 & 2912.8912 & 1073.3147 & 3986.2059 & \\
\hline & 8/6/2008 19:00 & 366 & 219 & 1900 & 5.328 & 5.328 & & 0.8074 & $9.15 \mathrm{E}-03$ & 2498.8908 & 920.7677 & 3419.6585 & \\
\hline & 8/6/2008 20:00 & 367 & 219 & 2000 & 5.701 & 5.701 & & 0.6892 & 7.53E-03 & 2056.2931 & 757.6835 & 2813.9765 & \\
\hline & 8/6/2008 21:00 & 368 & 219 & 2100 & 6.794 & 6.794 & & 0.4174 & 4.06E-03 & 1109.2626 & 408.7306 & 1517.9933 & \\
\hline
\end{tabular}




\begin{tabular}{|c|c|c|c|c|c|c|c|c|c|c|c|c|c|}
\hline \multirow[t]{42}{*}{ Tree ID } & TS & RN & JD & HR & $\begin{array}{c}\text { Raw } \\
\text { TC_dTA }\end{array}$ & $\begin{array}{c}\text { Fixed } \\
\text { TC_dT } \Delta\end{array}$ & dTM & $\mathbf{K}$ & $\begin{array}{c}\text { Sap } \\
\text { Velocity } \\
(\mathrm{cm} / \mathrm{s})\end{array}$ & $\begin{array}{c}\text { Probed } \\
\text { Drywood F } \\
\text { (g/hr) }\end{array}$ & $\begin{array}{c}\text { Extrapolated } \\
\text { Wetwood F } \\
(\mathrm{g} / \mathrm{hr})\end{array}$ & $\begin{array}{c}\text { Extrapolated } \\
\text { Total Wood F } \\
(\mathrm{g} / \mathrm{hr})\end{array}$ & $\begin{array}{l}\text { Relative } \\
\text { Humidity, } \\
\text { @10m (\%) }\end{array}$ \\
\hline & 8/6/2008 22:00 & 369 & 219 & 2200 & 7.516 & 7.516 & & 0.2813 & $2.50 \mathrm{E}-03$ & 682.2823 & 251.4009 & $\begin{array}{r}933.6832 \\
\end{array}$ & \\
\hline & 8/6/2008 23:00 & 370 & 219 & 2300 & 7.68 & 7.68 & & 0.2539 & 2.20E-03 & 601.5234 & 221.6437 & 823.1670 & \\
\hline & 8/7/2008 0:00 & 371 & 220 & 0 & 7.704 & 7.704 & 7.70 & 0.2500 & $2.16 \mathrm{E}-03$ & 590.1518 & 217.4536 & 807.6053 & \\
\hline & 8/7/2008 1:00 & 372 & 220 & 100 & 7.678 & 7.678 & & 0.2542 & $2.20 \mathrm{E}-03$ & 602.4761 & 221.9947 & 824.4708 & \\
\hline & 8/7/2008 2:00 & 373 & 220 & 200 & 7.644 & 7.644 & & 0.2598 & $2.26 \mathrm{E}-03$ & 618.7914 & 228.0064 & 846.7978 & \\
\hline & 8/7/2008 3:00 & 374 & 220 & 300 & NAN & 7.6035 & & 0.2665 & 2.34E-03 & 638.5236 & 235.2772 & 873.8008 & \\
\hline & 8/7/2008 4:00 & 375 & 220 & 400 & \begin{tabular}{|r|}
7.563 \\
\end{tabular} & 7.563 & & 0.2733 & $2.41 \mathrm{E}-03$ & 658.5841 & 242.6689 & 901.2530 & \\
\hline & 8/7/2008 5:00 & 376 & 220 & 500 & 7.53 & 7.53 & & 0.2789 & $2.47 \mathrm{E}-03$ & 675.1758 & 248.7824 & 923.9583 & \\
\hline & 8/7/2008 6:00 & 377 & 220 & 600 & 7.498 & 7.498 & & 0.2843 & $2.53 \mathrm{E}-03$ & 691.4786 & 254.7895 & 946.2682 & \\
\hline & 8/7/2008 7:00 & 378 & 220 & 700 & 7.473 & 7.473 & & 0.2886 & $2.58 \mathrm{E}-03$ & 704.3634 & 259.5372 & 963.9006 & \\
\hline & 8/7/2008 8:00 & 379 & 220 & 800 & 7.446 & 7.446 & & 0.2933 & 2.63E-03 & 718.4264 & 264.7190 & 983.1454 & \\
\hline & 8/7/2008 9:00 & 380 & 220 & 900 & 7.468 & 7.468 & & 0.2895 & 2.59E-03 & 706.9560 & 260.4925 & 967.4485 & \\
\hline & 8/7/2008 10:00 & 381 & 220 & 1000 & 5.548 & 5.548 & & 0.7358 & $8.16 \mathrm{E}-03$ & 2228.7033 & 821.2116 & \begin{tabular}{|l|}
3049.9149 \\
\end{tabular} & \\
\hline & 8/7/2008 11:00 & 382 & 220 & 1100 & 4.307 & 4.307 & & 1.2359 & 1.54E-02 & 4220.1599 & 1555.0047 & 5775.1646 & \\
\hline & 8/7/2008 12:00 & 383 & 220 & 1200 & 4.359 & 4.359 & & 1.2092 & 1.50E-02 & 4108.3235 & 1513.7963 & 5622.1197 & \\
\hline & 8/7/2008 13:00 & 384 & 220 & 1300 & 4.438 & 4.438 & & 1.1699 & 1.44E-02 & 3944.4731 & 1453.4222 & 5397.8953 & \\
\hline & 8/7/2008 14:00 & 385 & 220 & 1400 & 4.522 & 4.522 & & 1.1296 & 1.38E-02 & 3777.8481 & 1392.0258 & 5169.8739 & \\
\hline & 8/7/2008 15:00 & 386 & 220 & 1500 & 4.611 & 4.611 & & 1.0885 & 1.32E-02 & 3609.3378 & 1329.9348 & 4939.2726 & \\
\hline & 8/7/2008 16:00 & 387 & 220 & 1600 & NAN & 4.676 & & 1.0595 & $1.28 \mathrm{E}-02$ & 3491.2013 & 1286.4049 & 4777.6062 & \\
\hline & 8/7/2008 17:00 & 388 & 220 & 1700 & 4.741 & 4.741 & & 1.0312 & 1.24E-02 & 3377.0191 & 1244.3321 & 4621.3512 & \\
\hline & 8/7/2008 18:00 & 389 & 220 & 1800 & 4.936 & 4.936 & & 0.9510 & 1.12E-02 & 3056.4992 & 1126.2300 & 4182.7292 & \\
\hline & 8/7/2008 19:00 & 390 & 220 & 1900 & 5.236 & 5.236 & & 0.8392 & 9.59E-03 & 2620.4250 & 965.5495 & 3585.9745 & \\
\hline & 8/7/2008 20:00 & 391 & 220 & 2000 & 5.801 & 5.801 & & 0.6601 & 7.14E-03 & 1949.8698 & 718.4696 & 2668.3395 & \\
\hline & 8/7/2008 21:00 & 392 & 220 & 2100 & 6.837 & 6.837 & & 0.4085 & $3.95 E-03$ & 1080.1731 & 398.0120 & 1478.1851 & \\
\hline & 8/7/2008 22:00 & 393 & 220 & 2200 & 7.448 & 7.448 & & 0.2930 & 2.63E-03 & 717.3794 & 264.3332 & 981.7126 & \\
\hline & 8/7/2008 23:00 & 394 & 220 & 2300 & 7.674 & 7.674 & & 0.2549 & 2.21E-03 & 604.3838 & 222.6976 & 827.0814 & \\
\hline & 8/8/2008 0:00 & 395 & 221 & 0 & 7.757 & 7.757 & 7.76 & 0.2415 & 2.07E-03 & 565.4322 & 208.3451 & 773.7773 & \\
\hline & 8/8/2008 1:00 & 396 & 221 & 100 & 7.756 & 7.756 & & 0.2416 & 2.07E-03 & 565.8936 & 208.5151 & 774.4088 & \\
\hline & 8/8/2008 2:00 & 397 & 221 & 200 & NAN & 7.7135 & & 0.2485 & 2.14E-03 & 585.6813 & 215.8063 & 801.4876 & \\
\hline & 8/8/2008 3:00 & 398 & 221 & 300 & 7.671 & 7.671 & & 0.2554 & $2.22 \mathrm{E}-03$ & 605.8166 & 223.2256 & 829.0422 & \\
\hline & 8/8/2008 4:00 & 399 & 221 & 400 & 7.631 & 7.631 & & 0.2620 & 2.29E-03 & 625.0897 & 230.3272 & 855.4169 & \\
\hline & 8/8/2008 5:00 & 400 & 221 & 500 & 7.593 & 7.593 & & 0.2683 & 2.36E-03 & 643.6928 & 237.1818 & 880.8746 & \\
\hline & 8/8/2008 6:00 & 401 & 221 & 600 & 7.549 & 7.549 & & 0.2757 & $2.44 \mathrm{E}-03$ & 665.5959 & 245.2525 & 910.8483 & \\
\hline & $8 / 8 / 20087: 00$ & 402 & 221 & 700 & 7.527 & 7.527 & & 0.2794 & $2.48 \mathrm{E}-03$ & 676.6953 & 249.3423 & 926.0375 & \\
\hline & 8/8/2008 8:00 & 403 & 221 & 800 & 7.518 & 7.518 & & 0.2809 & $2.49 \mathrm{E}-03$ & 681.2646 & 251.0259 & 932.2905 & \\
\hline & 8/8/2008 9:00 & 404 & 221 & 900 & 7.284 & 7.284 & & 0.3221 & 2.95E-03 & 806.1130 & 297.0289 & 1103.1420 & \\
\hline & 8/8/2008 10:00 & 405 & 221 & 1000 & 4.814 & 4.814 & & 1.0004 & 1.19E-02 & 3253.2814 & 1198.7384 & 4452.0198 & \\
\hline & 8/8/2008 11:00 & 406 & 221 & 1100 & 4.581 & 4.581 & & 1.1022 & $1.34 \mathrm{E}-02$ & 3665.2471 & 1350.5357 & 5015.7827 & \\
\hline & 8/8/2008 12:00 & 407 & 221 & 1200 & 4.485 & 4.485 & & 1.1472 & $1.41 \mathrm{E}-02$ & 3850.3073 & 1418.7249 & 5269.0322 & \\
\hline & 8/8/2008 13:00 & 408 & 221 & 1300 & 4.525 & 4.525 & & 1.1282 & 1.38E-02 & 3772.0362 & 1389.8843 & 5161.9205 & \\
\hline & 8/8/2008 14:00 & 409 & 221 & 1400 & 4.603 & 4.603 & & 1.0921 & 1.33E-02 & 3624.1600 & 1335.3963 & 4959.5563 & \\
\hline
\end{tabular}




\begin{tabular}{|c|c|c|c|c|c|c|c|c|c|c|c|c|c|}
\hline Tree ID & TS & RN & JD & HR & $\begin{array}{c}\text { Raw } \\
\text { TC_dTA }\end{array}$ & $\begin{array}{c}\text { Fixed } \\
\text { TC_dT } \Delta\end{array}$ & dTM & $\mathbf{K}$ & $\begin{array}{c}\text { Sap } \\
\text { Velocity } \\
(\mathrm{cm} / \mathrm{s})\end{array}$ & $\begin{array}{c}\text { Probed } \\
\text { Drywood F } \\
\text { (g/hr) }\end{array}$ & $\begin{array}{c}\text { Extrapolated } \\
\text { Wetwood F } \\
(\mathrm{g} / \mathrm{hr})\end{array}$ & $\begin{array}{c}\text { Extrapolated } \\
\text { Total Wood F } \\
(\mathrm{g} / \mathrm{hr})\end{array}$ & $\begin{array}{l}\text { Relative } \\
\text { Humidity, } \\
\text { @10m (\%) }\end{array}$ \\
\hline & 8/8/2008 15:00 & 410 & 221 & 1500 & 4.688 & 4.688 & & 1.0542 & 1.27E-02 & 3469.8292 & 1278.5299 & \begin{tabular}{|r|}
4748.3591 \\
\end{tabular} & \\
\hline & 8/8/2008 16:00 & 411 & 221 & 1600 & 4.742 & 4.742 & & 1.0308 & 1.24E-02 & 3375.2924 & 1243.6959 & 4618.9883 & \\
\hline & 8/8/2008 17:00 & 412 & 221 & 1700 & 4.916 & 4.916 & & 0.9589 & 1.13E-02 & 3087.9333 & 1137.8125 & 4225.7459 & \\
\hline & 8/8/2008 18:00 & 413 & 221 & 1800 & 5.225 & 5.225 & & 0.8431 & $9.64 \mathrm{E}-03$ & 2635.3163 & 971.0365 & 3606.3528 & \\
\hline & 8/8/2008 19:00 & 414 & 221 & 1900 & 5.225 & 5.225 & & 0.8431 & 9.64E-03 & 2635.3163 & 971.0365 & 3606.3528 & \\
\hline & 8/8/2008 20:00 & 415 & 221 & 2000 & 5.903 & 5.903 & & 0.6314 & $6.76 \mathrm{E}-03$ & 1846.0879 & 680.2291 & 2526.3170 & \\
\hline & 8/8/2008 21:00 & 416 & 221 & \multicolumn{2}{|c|}{2100 NAN } & 6.609 & & 0.4571 & 4.54E-03 & 1240.4464 & 457.0680 & 1697.5144 & \\
\hline & 8/8/2008 22:00 & 417 & 221 & 2200 & \begin{tabular}{|r|}
7.315 \\
\end{tabular} & 7.315 & & 0.3165 & $2.89 \mathrm{E}-03$ & 788.8855 & 290.6811 & 1079.5666 & \\
\hline & 8/8/2008 23:00 & 418 & 221 & \multicolumn{2}{|c|}{2300 NAN } & 7.489 & & 0.2859 & $2.55 \mathrm{E}-03$ & 696.1021 & 256.4931 & 952.5952 & \\
\hline & 8/9/2008 0:00 & 419 & 222 & 0 & 7.663 & 7.663 & 7.71 & 0.2567 & 2.23E-03 & 609.6461 & 224.6366 & 834.2827 & \\
\hline & 8/9/2008 1:00 & 420 & 222 & 100 & 7.69 & 7.69 & & 0.2523 & 2.18E-03 & 596.7716 & 219.8928 & 816.6644 & \\
\hline & 8/9/2008 2:00 & 421 & 222 & 200 & 7.714 & 7.714 & & 0.2484 & 2.14E-03 & 585.4465 & 215.7198 & 801.1663 & \\
\hline & 8/9/2008 3:00 & 422 & 222 & 300 & 7.714 & 7.714 & & 0.2484 & $2.14 \mathrm{E}-03$ & 585.4465 & 215.7198 & 801.1663 & \\
\hline & 8/9/2008 4:00 & 423 & 222 & 400 & 7.692 & 7.692 & & 0.2520 & $2.18 \mathrm{E}-03$ & 595.8236 & 219.5435 & 815.3671 & \\
\hline & 8/9/2008 5:00 & 424 & 222 & 500 & 7.634 & 7.634 & & 0.2615 & $2.28 \mathrm{E}-03$ & 623.6333 & 229.7905 & 853.4238 & \\
\hline & 8/9/2008 6:00 & 425 & 222 & 600 & 7.588 & 7.588 & & 0.2691 & 2.36E-03 & 646.1620 & 238.0917 & 884.2537 & \\
\hline & 8/9/2008 7:00 & 426 & 222 & 700 & 7.421 & 7.421 & & 0.2977 & $2.68 E-03$ & 731.5857 & 269.5678 & 1001.1536 & \\
\hline & 8/9/2008 8:00 & 427 & 222 & 800 & 6.991 & 6.991 & & 0.3775 & 3.59E-03 & 980.0846 & 361.1323 & 1341.2169 & \\
\hline & 8/9/2008 9:00 & 428 & 222 & 900 & 7.095 & 7.095 & & 0.3573 & 3.35E-03 & 915.9548 & 337.5024 & 1253.4572 & \\
\hline & 8/9/2008 10:00 & 429 & 222 & 1000 & 7.19 & 7.19 & & 0.3394 & $3.15 E-03$ & 859.6926 & 316.7714 & 1176.4640 & \\
\hline & 8/9/2008 11:00 & 430 & 222 & 1100 & 6.659 & 6.659 & & 0.4462 & 4.41E-03 & 1203.9993 & 443.6383 & 1647.6376 & \\
\hline & 8/9/2008 12:00 & 431 & 222 & 1200 & 5.637 & 5.637 & & 0.7084 & 7.78E-03 & 2126.9578 & 783.7214 & 2910.6792 & \\
\hline & 8/9/2008 13:00 & 432 & 222 & 1300 & 5.21 & 5.21 & & 0.8484 & $9.72 \mathrm{E}-03$ & 2655.7497 & 978.5656 & 3634.3153 & \\
\hline & 8/9/2008 14:00 & 433 & 222 & 1400 & 4.692 & 4.692 & & 1.0524 & $1.27 \mathrm{E}-02$ & 3462.7349 & 1275.9159 & 4738.6508 & \\
\hline & 8/9/2008 15:00 & 434 & 222 & 1500 & 4.675 & 4.675 & & 1.0599 & 1.28E-02 & 3492.9884 & 1287.0634 & 4780.0518 & \\
\hline & 8/9/2008 16:00 & 435 & 222 & 1600 & 4.86 & 4.86 & & 0.9815 & 1.16E-02 & 3177.6528 & 1170.8715 & 4348.5243 & \\
\hline & 8/9/2008 17:00 & 436 & 222 & 1700 & 5.068 & 5.068 & & 0.9002 & 1.05E-02 & 2856.7080 & 1052.6128 & 3909.3208 & \\
\hline & 8/9/2008 18:00 & 437 & 222 & 1800 & 5.16 & 5.16 & & 0.8663 & 9.97E-03 & 2724.9362 & 1004.0588 & 3728.9950 & \\
\hline & 8/9/2008 19:00 & 438 & 222 & 1900 & 5.433 & 5.433 & & 0.7725 & 8.66E-03 & 2366.4839 & 871.9797 & 3238.4636 & \\
\hline & 8/9/2008 20:00 & 439 & 222 & 2000 & 6.027 & 6.027 & & 0.5978 & $6.32 \mathrm{E}-03$ & 1726.0314 & 635.9918 & 2362.0232 & \\
\hline & 8/9/2008 21:00 & 440 & 222 & 2100 & 7.135 & 7.135 & & 0.3497 & $3.26 \mathrm{E}-03$ & 892.0012 & 328.6762 & 1220.6773 & \\
\hline & 8/9/2008 22:00 & 441 & 222 & 2200 & 7.646 & 7.646 & & 0.2595 & $2.26 \mathrm{E}-03$ & 617.8254 & 227.6505 & 845.4758 & \\
\hline & 8/9/2008 23:00 & 442 & 222 & 2300 & 7.713 & 7.713 & & 0.2485 & $2.14 \mathrm{E}-03$ & 585.9161 & 215.8929 & 801.8090 & \\
\hline & 8/10/2008 0:00 & 443 & 223 & 0 & 7.694 & 7.694 & 7.69 & 0.2516 & $2.18 \mathrm{E}-03$ & 594.8764 & 219.1944 & 814.0708 & \\
\hline & 8/10/2008 1:00 & 444 & 223 & 100 & 7.655 & 7.655 & & 0.2580 & $2.25 \mathrm{E}-03$ & 613.4881 & 226.0523 & 839.5404 & \\
\hline & 8/10/2008 2:00 & 445 & 223 & 200 & 7.614 & 7.614 & & 0.2648 & $2.32 \mathrm{E}-03$ & 633.3765 & 233.3806 & 866.7571 & \\
\hline & 8/10/2008 3:00 & 446 & 223 & 300 & 7.575 & 7.575 & & 0.2713 & 2.39E-03 & 652.6057 & 240.4660 & 893.0718 & \\
\hline & 8/10/2008 4:00 & 447 & 223 & \multicolumn{2}{|c|}{400 NAN } & 7.535 & & 0.2780 & $2.46 \mathrm{E}-03$ & 672.6476 & 247.8509 & 920.4985 & \\
\hline & 8/10/2008 5:00 & 448 & 223 & 500 & 7.495 & 7.495 & & 0.2849 & $2.54 \mathrm{E}-03$ & 693.0179 & 255.3567 & 948.3746 & \\
\hline & 8/10/2008 6:00 & 449 & 223 & 600 & 7.459 & 7.459 & & 0.2911 & $2.60 \mathrm{E}-03$ & 711.6361 & 262.2170 & 973.8531 & \\
\hline & 8/10/2008 7:00 & 450 & 223 & 700 & 7.441 & 7.441 & & 0.2942 & $2.64 \mathrm{E}-03$ & 721.0476 & 265.6848 & 986.7325 & \\
\hline
\end{tabular}




\begin{tabular}{|c|c|c|c|c|c|c|c|c|c|c|c|c|c|}
\hline \multirow[t]{42}{*}{ Tree ID } & TS & RN & JD & HR & $\begin{array}{c}\text { Raw } \\
\text { TC_dTA }\end{array}$ & $\begin{array}{c}\text { Fixed } \\
\text { TC_dT } \Delta\end{array}$ & dTM & $\mathbf{K}$ & $\begin{array}{c}\text { Sap } \\
\text { Velocity } \\
(\mathrm{cm} / \mathrm{s})\end{array}$ & $\begin{array}{c}\text { Probed } \\
\text { Drywood F } \\
\text { (g/hr) }\end{array}$ & $\begin{array}{c}\text { Extrapolated } \\
\text { Wetwood F } \\
(\mathrm{g} / \mathrm{hr})\end{array}$ & $\begin{array}{c}\text { Extrapolated } \\
\text { Total Wood F } \\
(\mathrm{g} / \mathrm{hr})\end{array}$ & $\begin{array}{l}\text { Relative } \\
\text { Humidity, } \\
\text { @10m (\%) }\end{array}$ \\
\hline & 8/10/2008 8:00 & 451 & 223 & 800 & 7.412 & 7.412 & & 0.2992 & 2.69E-03 & 736.3558 & 271.3255 & 1007.6813 & \\
\hline & $8 / 10 / 20089: 00$ & 452 & 223 & 900 & 7.466 & 7.466 & & 0.2898 & 2.59E-03 & 707.9946 & 260.8752 & 968.8698 & \\
\hline & 8/10/2008 10:00 & 453 & 223 & 1000 & 5.888 & 5.888 & & 0.6355 & $6.81 \mathrm{E}-03$ & 1861.0582 & 685.7452 & 2546.8034 & \\
\hline & 8/10/2008 11:00 & 454 & 223 & 1100 & NAN & 5.172 & & 0.8619 & 9.91E-03 & 2708.1789 & 997.8842 & 3706.0631 & \\
\hline & $8 / 10 / 2008$ 12:00 & 455 & 223 & 1200 & 4.456 & 4.456 & & 1.1611 & 1.43E-02 & 3908.1243 & 1440.0288 & 5348.1531 & \\
\hline & 8/10/2008 13:00 & 456 & 223 & 1300 & 4.519 & 4.519 & & 1.1310 & 1.38E-02 & 3783.6694 & 1394.1708 & 5177.8402 & \\
\hline & 8/10/2008 14:00 & 457 & 223 & 1400 & 4.55 & 4.55 & & 1.1165 & 1.36E-02 & 3723.9665 & 1372.1720 & 5096.1385 & \\
\hline & 8/10/2008 15:00 & 458 & 223 & 1500 & 4.777 & 4.777 & & 1.0159 & $1.21 \mathrm{E}-02$ & 3315.4168 & 1221.6335 & 4537.0503 & \\
\hline & 8/10/2008 16:00 & 459 & 223 & 1600 & 4.724 & 4.724 & & 1.0385 & $1.25 \mathrm{E}-02$ & 3406.5102 & 1255.1988 & 4661.7090 & \\
\hline & 8/10/2008 17:00 & 460 & 223 & 1700 & 4.991 & 4.991 & & 0.9295 & 1.09E-02 & 2971.6596 & 1094.9691 & 4066.6288 & \\
\hline & 8/10/2008 18:00 & 461 & 223 & 1800 & 6.846 & 6.846 & & 0.4067 & 3.93E-03 & 1074.1491 & 395.7923 & 1469.9414 & \\
\hline & 8/10/2008 19:00 & 462 & 223 & 1900 & 7.432 & 7.432 & & 0.2957 & $2.66 \mathrm{E}-03$ & 725.7792 & 267.4283 & 993.2075 & \\
\hline & 8/10/2008 20:00 & 463 & 223 & 2000 & 7.521 & 7.521 & & 0.2804 & $2.49 \mathrm{E}-03$ & 679.7396 & 250.4640 & 930.2037 & \\
\hline & 8/10/2008 21:00 & 464 & 223 & 2100 & 7.601 & 7.601 & & 0.2669 & 2.34E-03 & 639.7524 & 235.7299 & 875.4823 & \\
\hline & $8 / 10 / 2008$ 22:00 & 465 & 223 & 2200 & 7.621 & 7.621 & & 0.2636 & 2.31E-03 & 629.9573 & 232.1207 & 862.0781 & \\
\hline & 8/10/2008 23:00 & 466 & 223 & 2300 & 7.588 & 7.588 & & 0.2691 & $2.36 \mathrm{E}-03$ & 646.1620 & 238.0917 & 884.2537 & \\
\hline & $8 / 11 / 20080: 00$ & 467 & 224 & 0 & 7.571 & 7.571 & 7.69 & 0.2720 & $2.40 \mathrm{E}-03$ & 654.5953 & 241.1991 & 895.7944 & \\
\hline & 8/11/2008 1:00 & 468 & 224 & 100 & 7.616 & 7.616 & & 0.2644 & 2.31E-03 & 632.3986 & 233.0203 & 865.4189 & \\
\hline & 8/11/2008 2:00 & 469 & 224 & 200 & 7.654 & 7.654 & & 0.2582 & $2.25 E-03$ & 613.9692 & 226.2296 & 840.1988 & \\
\hline & 8/11/2008 3:00 & 470 & 224 & 300 & 7.664 & 7.664 & & 0.2565 & 2.23E-03 & 609.1667 & 224.4600 & 833.6267 & \\
\hline & 8/11/2008 4:00 & 471 & 224 & 400 & 7.669 & 7.669 & & 0.2557 & $2.22 \mathrm{E}-03$ & 606.7728 & 223.5779 & 830.3507 & \\
\hline & 8/11/2008 5:00 & 472 & 224 & 500 & 7.675 & 7.675 & & 0.2547 & $2.21 \mathrm{E}-03$ & 603.9065 & 222.5218 & 826.4283 & \\
\hline & 8/11/2008 6:00 & 473 & 224 & 600 & 7.688 & 7.688 & & 0.2526 & $2.19 \mathrm{E}-03$ & 597.7204 & 220.2424 & 817.9628 & \\
\hline & 8/11/2008 7:00 & 474 & 224 & 700 & 7.681 & 7.681 & & 0.2537 & $2.20 \mathrm{E}-03$ & 601.0473 & 221.4682 & 822.5156 & \\
\hline & 8/11/2008 8:00 & 475 & 224 & 800 & 7.663 & 7.663 & & 0.2567 & 2.23E-03 & 609.6461 & 224.6366 & 834.2827 & \\
\hline & 8/11/2008 9:00 & 476 & 224 & 900 & 7.4 & 7.4 & & 0.3014 & $2.72 \mathrm{E}-03$ & 742.7431 & 273.6790 & 1016.4220 & \\
\hline & 8/11/2008 10:00 & 477 & 224 & 1000 & 5.815 & 5.815 & & 0.6561 & 7.08E-03 & 1935.3461 & 713.1181 & 2648.4642 & \\
\hline & 8/11/2008 11:00 & 478 & 224 & 1100 & NAN & 5.2185 & & 0.8454 & 9.68E-03 & 2644.1527 & 974.2925 & 3618.4452 & \\
\hline & $8 / 11 / 2008$ 12:00 & 479 & 224 & 1200 & 4.622 & 4.622 & & 1.0835 & 1.31E-02 & 3589.0597 & 1322.4629 & 4911.5226 & \\
\hline & $8 / 11 / 2008$ 13:00 & 480 & 224 & 1300 & 4.931 & 4.931 & & 0.9530 & $1.12 \mathrm{E}-02$ & 3064.3282 & 1129.1147 & 4193.4429 & \\
\hline & $8 / 11 / 2008$ 14:00 & 481 & 224 & 1400 & 4.725 & 4.725 & & 1.0381 & 1.25E-02 & 3404.7682 & 1254.5569 & 4659.3251 & \\
\hline & $8 / 11 / 2008$ 15:00 & 482 & 224 & 1500 & 4.758 & 4.758 & & 1.0240 & 1.23E-02 & 3347.7864 & 1233.5608 & 4581.3472 & \\
\hline & $8 / 11 / 2008$ 16:00 & 483 & 224 & 1600 & 5.087 & 5.087 & & 0.8931 & 1.04E-02 & 2829.0073 & 1042.4059 & 3871.4133 & \\
\hline & $8 / 11 / 2008$ 17:00 & 484 & 224 & 1700 & 5.099 & 5.099 & & 0.8886 & $1.03 \mathrm{E}-02$ & 2811.6445 & 1036.0082 & 3847.6527 & \\
\hline & 8/11/2008 18:00 & 485 & 224 & 1800 & NAN & 5.2925 & & 0.8196 & 9.31E-03 & 2545.1586 & 937.8160 & 3482.9746 & \\
\hline & $8 / 11 / 2008$ 19:00 & 486 & 224 & 1900 & 5.486 & 5.486 & & 0.7554 & $8.42 E-03$ & 2302.0745 & 848.2467 & 3150.3212 & \\
\hline & $8 / 11 / 2008$ 20:00 & 487 & 224 & 2000 & 6.087 & 6.087 & & 0.5821 & 6.11E-03 & 1670.2250 & 615.4288 & 2285.6538 & \\
\hline & 8/11/2008 21:00 & 488 & 224 & 2100 & 6.841 & 6.841 & & 0.4077 & $3.94 \mathrm{E}-03$ & 1077.4930 & 397.0245 & 1474.5175 & \\
\hline & 8/11/2008 22:00 & 489 & 224 & 2200 & 7.356 & 7.356 & & 0.3091 & $2.80 \mathrm{E}-03$ & 766.4303 & 282.4070 & 1048.8373 & \\
\hline & 8/11/2008 23:00 & 490 & 224 & 2300 & 7.566 & 7.566 & & 0.2728 & $2.40 \mathrm{E}-03$ & 657.0868 & 242.1171 & 899.2039 & \\
\hline & 8/12/2008 0:00 & 491 & 225 & 0 & 7.647 & 7.647 & 7.65 & 0.2593 & $2.26 \mathrm{E}-03$ & 617.3427 & 227.4726 & 844.8153 & \\
\hline
\end{tabular}




\begin{tabular}{|c|c|c|c|c|c|c|c|c|c|c|c|c|c|}
\hline \multirow[t]{37}{*}{ Tree ID } & TS & RN & JD & HR & $\begin{array}{c}\text { Raw } \\
\text { TC_dTA }\end{array}$ & $\begin{array}{c}\text { Fixed } \\
\text { TC_dTA }\end{array}$ & dTM & $\mathbf{K}$ & $\begin{array}{l}\text { Sap } \\
\text { Velocity } \\
(\mathrm{cm} / \mathrm{s})\end{array}$ & $\begin{array}{l}\text { Probed } \\
\text { Drywood F } \\
(\mathbf{g} / \mathrm{hr})\end{array}$ & $\begin{array}{c}\text { Extrapolated } \\
\text { Wetwood F } \\
(\mathrm{g} / \mathrm{hr})\end{array}$ & $\begin{array}{c}\text { Extrapolated } \\
\text { Total Wood F } \\
(\mathrm{g} / \mathrm{hr})\end{array}$ & $\begin{array}{c}\text { Relative } \\
\text { Humidity, } \\
\text { @10m (\%) }\end{array}$ \\
\hline & 8/12/2008 1:00 & 492 & 225 & 100 & 7.641 & 7.641 & & 0.2603 & $2.27 \mathrm{E}-03$ & 620.2419 & 228.5409 & 848.7828 & \\
\hline & 8/12/2008 2:00 & 493 & 225 & 200 & 7.382 & 7.382 & & 0.3045 & $2.75 \mathrm{E}-03$ & 752.3823 & 277.2307 & 1029.6130 & \\
\hline & 8/12/2008 3:00 & 494 & 225 & 300 & 7.459 & 7.459 & & 0.2911 & $2.60 \mathrm{E}-03$ & 711.6361 & 262.2170 & 973.8531 & \\
\hline & $8 / 12 / 2008$ 4:00 & 495 & 225 & 400 & 7.444 & 7.444 & & 0.2937 & 2.63E-03 & 719.4743 & 265.1051 & 984.5794 & \\
\hline & 8/12/2008 5:00 & 496 & 225 & 500 & 7.417 & 7.417 & & 0.2984 & 2.69E-03 & 733.7036 & 270.3482 & 1004.0518 & \\
\hline & 8/12/2008 6:00 & 497 & 225 & 600 & 7.404 & 7.404 & & 0.3006 & 2.71E-03 & 740.6105 & 272.8932 & 1013.5037 & \\
\hline & 8/12/2008 7:00 & 498 & 225 & 700 & 7.396 & 7.396 & & 0.3021 & 2.73E-03 & 744.8790 & 274.4660 & 1019.3450 & \\
\hline & 8/12/2008 8:00 & 499 & 225 & 800 & 7.393 & 7.393 & & 0.3026 & 2.73E-03 & 746.4833 & 275.0571 & 1021.5404 & \\
\hline & 8/12/2008 9:00 & 500 & 225 & 900 & 7.396 & 7.396 & & 0.3021 & 2.73E-03 & 744.8790 & 274.4660 & 1019.3450 & \\
\hline & $8 / 12 / 2008$ 10:00 & 501 & 225 & 1000 & 6.059 & 6.059 & & 0.5894 & $6.21 E-03$ & 1696.0877 & 624.9584 & 2321.0461 & \\
\hline & 8/12/2008 11:00 & 502 & 225 & 1100 & 4.631 & 4.631 & & 1.0795 & 1.31E-02 & 3572.5560 & 1316.3817 & 4888.9378 & \\
\hline & $8 / 12 / 2008$ 12:00 & 503 & 225 & 1200 & 4.555 & 4.555 & & 1.1142 & 1.36E-02 & 3714.4297 & 1368.6580 & 5083.0877 & \\
\hline & 8/12/2008 13:00 & 504 & 225 & 1300 & 4.54 & 4.54 & & 1.1211 & 1.37E-02 & 3743.1170 & 1379.2284 & 5122.3454 & \\
\hline & $8 / 12 / 2008$ 14:00 & 505 & 225 & 1400 & 4.567 & 4.567 & & 1.1086 & 1.35E-02 & 3691.6451 & 1360.2626 & 5051.9077 & \\
\hline & 8/12/2008 15:00 & 506 & 225 & 1500 & 4.615 & 4.615 & & 1.0867 & 1.32E-02 & 3601.9503 & 1327.2127 & 4929.1630 & \\
\hline & $8 / 12 / 2008$ 16:00 & 507 & 225 & 1600 & 4.691 & 4.691 & & 1.0529 & 1.27E-02 & 3464.5071 & 1276.5689 & 4741.0760 & \\
\hline & $8 / 12 / 200817: 00$ & 508 & 225 & 1700 & 4.8 & 4.8 & & 1.0063 & 1.20E-02 & 3276.6535 & 1207.3504 & 4484.0039 & \\
\hline & $8 / 12 / 200818: 00$ & 509 & 225 & 1800 & 4.984 & 4.984 & & 0.9322 & 1.09E-02 & 2982.3287 & 1098.9003 & 4081.2290 & \\
\hline & 8/12/2008 19:00 & 510 & 225 & 1900 & 5.235 & 5.235 & & 0.8395 & 9.59E-03 & 2621.7755 & 966.0471 & 3587.8226 & \\
\hline & $8 / 12 / 200820: 00$ & 511 & 225 & 2000 & 5.814 & 5.814 & & 0.6563 & 7.09E-03 & 1936.3805 & 713.4992 & 2649.8797 & \\
\hline & $8 / 12 / 200821: 00$ & 512 & 225 & 2100 & 6.99 & 6.99 & & 0.3777 & $3.59 \mathrm{E}-03$ & 980.7145 & 361.3644 & 1342.0789 & \\
\hline & $8 / 12 / 200822: 00$ & 513 & 225 & 2200 & 7.531 & 7.531 & & 0.2787 & 2.47E-03 & 674.6698 & 248.5960 & 923.2658 & \\
\hline & $8 / 12 / 200823: 00$ & 514 & 225 & 2300 & 7.615 & 7.615 & & 0.2646 & $2.32 \mathrm{E}-03$ & 632.8875 & 233.2004 & 866.0879 & \\
\hline & $8 / 13 / 20080: 00$ & 515 & 226 & 0 & 7.58 & 7.58 & 7.62 & 0.2704 & 2.38E-03 & 650.1234 & 239.5513 & 889.6747 & \\
\hline & 8/13/2008 1:00 & 516 & 226 & 100 & 7.547 & 7.547 & & 0.2760 & 2.44E-03 & 666.6008 & 245.6228 & 912.2236 & \\
\hline & 8/13/2008 2:00 & 517 & 226 & 200 & 7.572 & 7.572 & & 0.2718 & $2.39 E-03$ & 654.0976 & 241.0157 & 895.1133 & \\
\hline & 8/13/2008 3:00 & 518 & 226 & 300 & 7.595 & 7.595 & & 0.2679 & 2.35E-03 & 642.7065 & 236.8184 & 879.5249 & \\
\hline & 8/13/2008 4:00 & 519 & 226 & 400 & 7.59 & 7.59 & & 0.2688 & 2.36E-03 & 645.1737 & 237.7275 & 882.9013 & \\
\hline & 8/13/2008 5:00 & 520 & 226 & 500 & 7.622 & 7.622 & & 0.2634 & $2.30 \mathrm{E}-03$ & 629.4697 & 231.9411 & 861.4107 & \\
\hline & 8/13/2008 6:00 & 521 & 226 & 600 & 7.568 & 7.568 & & 0.2725 & $2.40 \mathrm{E}-03$ & 656.0896 & 241.7497 & 897.8393 & \\
\hline & 8/13/2008 7:00 & 522 & 226 & 700 & 7.518 & 7.518 & & 0.2809 & 2.49E-03 & 681.2646 & 251.0259 & 932.2905 & \\
\hline & 8/13/2008 8:00 & 523 & 226 & 800 & 7.527 & 7.527 & & 0.2794 & 2.48E-03 & 676.6953 & 249.3423 & 926.0375 & \\
\hline & 8/13/2008 9:00 & 524 & 226 & 900 & 7.099 & 7.099 & & 0.3565 & 3.34E-03 & 913.5419 & 336.6133 & 1250.1552 & \\
\hline & $8 / 13 / 200810: 00$ & 525 & 226 & 1000 & 4.831 & 4.831 & & 0.9934 & 1.18E-02 & 3225.1249 & 1188.3636 & 4413.4886 & \\
\hline & 8/13/2008 11:00 & 526 & 226 & 1100 & 4.749 & 4.749 & & 1.0278 & 1.23E-02 & 3363.2305 & 1239.2515 & 4602.4820 & \\
\hline & 8/13/2008 12:00 & 527 & 226 & 1200 & 4.427 & 4.427 & & 1.1753 & 1.45E-02 & 3966.8630 & 1461.6723 & 5428.5353 & \\
\hline
\end{tabular}




\begin{tabular}{|c|c|c|c|c|c|c|c|c|c|c|c|c|c|}
\hline Tree ID & TS & RN & JD & HR & $\begin{array}{c}\text { Raw } \\
\text { TC_dTA }\end{array}$ & $\begin{array}{c}\text { Fixed } \\
\text { TC_dT } \Delta\end{array}$ & dTM & $\mathbf{K}$ & $\begin{array}{l}\text { Sap } \\
\text { Velocity } \\
(\mathrm{cm} / \mathrm{s})\end{array}$ & $\begin{array}{l}\text { Probed } \\
\text { Drywood F } \\
\text { (g/hr) }\end{array}$ & $\begin{array}{c}\text { Extrapolated } \\
\text { Wetwood F } \\
(\mathrm{g} / \mathrm{hr})\end{array}$ & $\begin{array}{c}\text { Extrapolated } \\
\text { Total Wood F } \\
\text { (g/hr) }\end{array}$ & $\begin{array}{l}\text { Relative } \\
\text { Humidity, } \\
@ 10 m(\%)\end{array}$ \\
\hline & 7/22/2008 13:00 & 0 & 204 & 1300 & 5.093 & 5.093 & 9.56 & 0.8771 & 1.01E-02 & 753.1672 & 277.5200 & 1030.6872 & \\
\hline \multirow[t]{2}{*}{ W609EC2 } & $7 / 22 / 2008$ 14:00 & 1 & 204 & 1400 & 5.599 & 5.599 & & 0.7074 & 7.77E-03 & 578.0696 & 213.0016 & 791.0713 & \\
\hline & $7 / 22 / 2008$ 15:00 & 2 & 204 & 1500 & 5.55 & 5.55 & & 0.7225 & $7.98 \mathrm{E}-03$ & 593.2701 & 218.6026 & 811.8726 & \\
\hline \multirow[t]{2}{*}{ Probe \# } & 7/22/2008 16:00 & 3 & 204 & 1600 & 5.521 & 5.521 & & 0.7316 & $8.10 \mathrm{E}-03$ & 602.4287 & 221.9772 & 824.4059 & \\
\hline & 7/22/2008 17:00 & 4 & 204 & 1700 & 5.555 & 5.555 & & 0.7210 & 7.96E-03 & 591.7033 & 218.0253 & 809.7286 & \\
\hline \multirow[t]{4}{*}{3} & $7 / 22 / 2008$ 18:00 & 5 & 204 & 1800 & 5.635 & 5.635 & & 0.6965 & $7.62 \mathrm{E}-03$ & 567.1169 & 208.9659 & 776.0828 & \\
\hline & $7 / 22 / 2008$ 19:00 & 6 & 204 & 1900 & 5.782 & 5.782 & & 0.6534 & 7.05E-03 & 524.2009 & 193.1526 & 717.3534 & \\
\hline & 7/22/2008 20:00 & 7 & 204 & 2000 & 6.242 & 6.242 & & 0.5316 & $5.47 \mathrm{E}-03$ & 406.5946 & 149.8182 & 556.4128 & \\
\hline & 7/22/2008 21:00 & 8 & 204 & 2100 & 7.865 & 7.865 & & 0.2155 & $1.80 \mathrm{E}-03$ & 133.8163 & 49.3074 & 183.1237 & \\
\hline \multirow[t]{2}{*}{$\mathrm{DBH}(\mathrm{cm})$} & $7 / 22 / 2008$ 22:00 & 9 & 204 & 2200 & 9.18 & 9.18 & & 0.0414 & $2.36 \mathrm{E}-04$ & 17.5574 & 6.4694 & 24.0268 & \\
\hline & 7/22/2008 23:00 & 10 & 204 & 2300 & 9.56 & 9.56 & & 0.0000 & $0.00 \mathrm{E}+00$ & 0.0000 & 0.0000 & 0.0000 & \\
\hline \multirow[t]{2}{*}{6.4} & 7/23/2008 0:00 & 11 & 205 & 0 & 9.85 & 9.85 & 10.01 & -0.0294 & $0.00 \mathrm{E}+00$ & 0.0000 & 0.0000 & 0.0000 & \\
\hline & 7/23/2008 1:00 & 12 & 205 & 100 & 9.95 & 9.95 & & -0.0392 & $0.00 \mathrm{E}+00$ & 0.0000 & 0.0000 & 0.0000 & \\
\hline Day Count & $7 / 23 / 20082: 00$ & 13 & 205 & 200 & 9.97 & 9.97 & & -0.0411 & $0.00 \mathrm{E}+00$ & 0.0000 & 0.0000 & 0.0000 & \\
\hline \multirow[t]{27}{*}{35} & 7/23/2008 3:00 & 14 & 205 & 300 & 10.01 & 10.01 & & -0.0450 & $0.00 \mathrm{E}+00$ & 0.0000 & 0.0000 & 0.0000 & \\
\hline & 7/23/2008 4:00 & 15 & 205 & 400 & 9.98 & 9.98 & & -0.0421 & $0.00 \mathrm{E}+00$ & 0.0000 & 0.0000 & 0.0000 & \\
\hline & 7/23/2008 5:00 & 16 & 205 & 500 & 9.92 & 9.92 & & -0.0363 & $0.00 E+00$ & 0.0000 & 0.0000 & 0.0000 & \\
\hline & 7/23/2008 6:00 & 17 & 205 & 600 & 9.81 & 9.81 & & -0.0255 & $0.00 \mathrm{E}+00$ & 0.0000 & 0.0000 & 0.0000 & \\
\hline & 7/23/2008 7:00 & 18 & 205 & 700 & 9.51 & 9.51 & & 0.0053 & 1.86E-05 & 1.3845 & 0.5102 & 1.8947 & \\
\hline & 7/23/2008 8:00 & 19 & 205 & 800 & 8.67 & 8.67 & & 0.1027 & $7.22 \mathrm{E}-04$ & 53.7037 & 19.7882 & 73.4919 & \\
\hline & 7/23/2008 9:00 & 20 & 205 & 900 & 7.259 & 7.259 & & 0.3170 & $2.89 \mathrm{E}-03$ & 215.1723 & 79.2847 & 294.4570 & \\
\hline & 7/23/2008 10:00 & 21 & 205 & 1000 & 5.928 & 5.928 & & 0.6127 & $6.51 \mathrm{E}-03$ & 484.2793 & 178.4427 & 662.7220 & \\
\hline & $7 / 23 / 2008$ 11:00 & 22 & 205 & 1100 & 5.993 & 5.993 & & 0.5952 & $6.28 \mathrm{E}-03$ & 467.3168 & 172.1925 & 639.5093 & \\
\hline & $7 / 23 / 2008$ 12:00 & 23 & 205 & 1200 & 5.918 & 5.918 & & 0.6154 & $6.55 E-03$ & 486.9322 & 179.4202 & 666.3524 & \\
\hline & $7 / 23 / 2008$ 13:00 & 24 & 205 & 1300 & 5.81 & 5.81 & & 0.6454 & 6.94E-03 & 516.3427 & 190.2571 & 706.5998 & \\
\hline & $7 / 23 / 2008$ 14:00 & 25 & 205 & 1400 & 5.812 & 5.812 & & 0.6449 & $6.93 \mathrm{E}-03$ & 515.7852 & 190.0517 & 705.8368 & \\
\hline & $7 / 23 / 2008$ 15:00 & 26 & 205 & 1500 & 5.811 & 5.811 & & 0.6452 & $6.94 \mathrm{E}-03$ & 516.0639 & 190.1544 & 706.2182 & \\
\hline & 7/23/2008 16:00 & 27 & 205 & 1600 & 5.985 & 5.985 & & 0.5973 & $6.31 \mathrm{E}-03$ & 469.3785 & 172.9522 & 642.3307 & \\
\hline & 7/23/2008 17:00 & 28 & 205 & 1700 & 6.431 & 6.431 & & 0.4865 & 4.90E-03 & 364.6362 & 134.3577 & 498.9939 & \\
\hline & $7 / 23 / 2008$ 18:00 & 29 & 205 & 1800 & 7.164 & 7.164 & & 0.3345 & 3.09E-03 & 229.8572 & 84.6956 & 314.5528 & \\
\hline & $7 / 23 / 2008$ 19:00 & 30 & 205 & 1900 & 7.102 & 7.102 & & 0.3461 & $3.22 \mathrm{E}-03$ & 239.7525 & 88.3417 & 328.0942 & \\
\hline & $7 / 23 / 200820: 00$ & 31 & 205 & 2000 & 7.557 & 7.557 & & 0.2651 & $2.32 \mathrm{E}-03$ & 172.6345 & 63.6107 & 236.2452 & \\
\hline & $7 / 23 / 200821: 00$ & 32 & 205 & 2100 & 8.47 & 8.47 & & 0.1287 & 9.54E-04 & 70.9339 & 26.1371 & 97.0710 & \\
\hline & $7 / 23 / 200822: 00$ & 33 & 205 & 2200 & 9.06 & 9.06 & & 0.0552 & $3.36 \mathrm{E}-04$ & 25.0158 & 9.2176 & 34.2333 & \\
\hline & $7 / 23 / 2008$ 23:00 & 34 & 205 & 2300 & 9.17 & 9.17 & & 0.0425 & 2.44E-04 & 18.1522 & 6.6886 & 24.8408 & \\
\hline & $7 / 24 / 20080: 00$ & 35 & 206 & 0 & 9.2 & 9.2 & 9.66 & 0.0391 & 2.20E-04 & 16.3829 & 6.0366 & 22.4196 & \\
\hline & 7/24/2008 1:00 & 36 & 206 & 100 & 9.29 & 9.29 & & 0.0291 & $1.53 E-04$ & 11.3602 & 4.1859 & 15.5462 & \\
\hline & 7/24/2008 2:00 & 37 & 206 & 200 & 9.37 & 9.37 & & 0.0203 & $9.81 \mathrm{E}-05$ & 7.2936 & 2.6875 & \begin{tabular}{|c|}
9.9810 \\
\end{tabular} & \\
\hline & 7/24/2008 3:00 & 38 & 206 & 300 & 9.48 & 9.48 & & 0.0084 & 3.33E-05 & 2.4789 & 0.9134 & 3.3923 & \\
\hline & 7/24/2008 4:00 & 39 & 206 & 400 & 9.55 & 9.55 & & 0.0010 & 2.55E-06 & 0.1899 & 0.0700 & 0.2599 & \\
\hline & 7/24/2008 5:00 & 40 & 206 & 500 & 9.61 & 9.61 & & -0.0052 & $0.00 \mathrm{E}+00$ & 0.0000 & 0.0000 & 0.0000 & \\
\hline
\end{tabular}




\begin{tabular}{|c|c|c|c|c|c|c|c|c|c|c|c|c|c|}
\hline Tree ID & TS & RN & JD & HR & $\begin{array}{c}\text { Raw } \\
\text { TC_dTA }\end{array}$ & $\begin{array}{c}\text { Fixed } \\
\text { TC_dT } \Delta\end{array}$ & dTM & $\mathbf{K}$ & $\begin{array}{l}\text { Sap } \\
\text { Velocity } \\
(\mathrm{cm} / \mathrm{s})\end{array}$ & $\begin{array}{c}\text { Probed } \\
\text { Drywood F } \\
(\mathrm{g} / \mathrm{hr})\end{array}$ & $\begin{array}{c}\text { Extrapolated } \\
\text { Wetwood F } \\
(\mathrm{g} / \mathrm{hr})\end{array}$ & $\begin{array}{c}\text { Extrapolated } \\
\text { Total Wood F } \\
(\mathrm{g} / \mathrm{hr})\end{array}$ & $\begin{array}{l}\text { Relative } \\
\text { Humidity, } \\
@ 10 \mathrm{~m}(\%)\end{array}$ \\
\hline & 7/24/2008 6:00 & 41 & 206 & 600 & ) $\quad 9.64$ & 9.64 & & -0.0083 & $0.00 \mathrm{E}+00$ & 0.0000 & 0.0000 & 0.0000 & \\
\hline & 7/24/2008 7:00 & 42 & 206 & 700 & 9.66 & 9.66 & & -0.0104 & $0.00 \mathrm{E}+00$ & 0.0000 & 0.0000 & 0.0000 & \\
\hline & 7/24/2008 8:00 & 43 & 206 & 800 & 9.53 & 9.53 & & 0.0031 & $9.90 \mathrm{E}-06$ & 0.7363 & 0.2713 & 1.0077 & \\
\hline & 7/24/2008 9:00 & 44 & 206 & 900 & 9.17 & 9.17 & & 0.0425 & $2.44 \mathrm{E}-04$ & 18.1522 & 6.6886 & 24.8408 & \\
\hline & $7 / 24 / 200810: 00$ & 45 & 206 & 1000 & 9.13 & 9.13 & & 0.0471 & 2.77E-04 & 20.5810 & 7.5835 & 28.1645 & \\
\hline & $7 / 24 / 2008$ 11:00 & 46 & 206 & 1100 & 7.082 & 7.082 & & 0.3499 & 3.27E-03 & 242.9982 & 89.5377 & 332.5360 & \\
\hline & $7 / 24 / 2008$ 12:00 & 47 & 206 & 1200 & 5.974 & 5.974 & & 0.6003 & $6.35 E-03$ & 472.2252 & 174.0011 & 646.2263 & \\
\hline & $7 / 24 / 2008$ 13:00 & 48 & 206 & 1300 & 6.558 & 6.558 & & 0.4578 & 4.55E-03 & 338.2621 & 124.6396 & 462.9017 & \\
\hline & $7 / 24 / 2008$ 14:00 & 49 & 206 & 1400 & 6.354 & 6.354 & & 0.5046 & 5.13E-03 & 381.3259 & 140.5074 & 521.8333 & \\
\hline & $7 / 24 / 200815: 00$ & 50 & 206 & 1500 & 5.952 & 5.952 & & 0.6062 & $6.43 \mathrm{E}-03$ & 477.9599 & 176.1141 & 654.0740 & \\
\hline & $7 / 24 / 200816: 00$ & 51 & 206 & 1600 & 5.936 & 5.936 & & 0.6105 & $6.48 \mathrm{E}-03$ & 482.1654 & 177.6638 & 659.8292 & \\
\hline & $7 / 24 / 200817: 00$ & 52 & 206 & 1700 & 6.145 & 6.145 & & 0.5557 & $5.77 \mathrm{E}-03$ & 429.4770 & 158.2496 & 587.7266 & \\
\hline & $7 / 24 / 2008$ 18:00 & 53 & 206 & 1800 & 6.074 & 6.074 & & 0.5739 & 6.01E-03 & 446.8419 & 164.6481 & 611.4900 & \\
\hline & $7 / 24 / 2008$ 19:00 & 54 & 206 & 1900 & 6.392 & 6.392 & & 0.4956 & 5.02E-03 & 373.0217 & 137.4475 & 510.4692 & \\
\hline & $7 / 24 / 2008$ 20:00 & 55 & 206 & 2000 & 7.443 & 7.443 & & 0.2844 & 2.53E-03 & 188.2985 & 69.3825 & 257.6810 & \\
\hline & 7/24/2008 21:00 & 56 & 206 & 2100 & 8.75 & 8.75 & & 0.0926 & $6.36 \mathrm{E}-04$ & 47.2868 & 17.4238 & \begin{tabular}{|l|}
64.7105 \\
\end{tabular} & \\
\hline & $7 / 24 / 200822: 00$ & 57 & 206 & 2200 & 9.51 & 9.51 & & 0.0053 & 1.86E-05 & 1.3845 & 0.5102 & 1.8947 & \\
\hline & $7 / 24 / 2008$ 23:00 & 58 & 206 & 2300 & 9.62 & 9.62 & & -0.0062 & $0.00 \mathrm{E}+00$ & 0.0000 & 0.0000 & 0.0000 & \\
\hline & $7 / 25 / 20080: 00$ & 59 & 207 & 0 & 9.6 & 9.6 & 9.60 & -0.0042 & $0.00 E+00$ & 0.0000 & 0.0000 & 0.0000 & \\
\hline & 7/25/2008 1:00 & 60 & 207 & 100 & 9.5 & 9.5 & & 0.0063 & 2.33E-05 & 1.7351 & 0.6393 & 2.3745 & \\
\hline & 7/25/2008 2:00 & 61 & 207 & 200 & 9.4 & 9.4 & & 0.0170 & 7.90E-05 & 5.8797 & 2.1665 & 8.0462 & \\
\hline & 7/25/2008 3:00 & 62 & 207 & 300 & 9.32 & 9.32 & & 0.0258 & $1.32 \mathrm{E}-04$ & 9.7880 & 3.6066 & 13.3946 & \\
\hline & 7/25/2008 4:00 & 63 & 207 & 400 & 9.27 & 9.27 & & 0.0313 & 1.67E-04 & 12.4378 & 4.5830 & 17.0207 & \\
\hline & 7/25/2008 5:00 & 64 & 207 & 500 & 9.28 & 9.28 & & 0.0302 & 1.60E-04 & 11.8961 & 4.3834 & 16.2795 & \\
\hline & 7/25/2008 6:00 & 65 & 207 & 600 & 9.31 & 9.31 & & 0.0269 & 1.39E-04 & 10.3061 & 3.7975 & 14.1036 & \\
\hline & 7/25/2008 7:00 & 66 & 207 & 700 & 9.27 & 9.27 & & 0.0313 & 1.67E-04 & 12.4378 & 4.5830 & 17.0207 & \\
\hline & 7/25/2008 8:00 & 67 & 207 & 800 & 9.21 & 9.21 & & 0.0380 & $2.12 \mathrm{E}-04$ & 15.8034 & 5.8231 & 21.6265 & \\
\hline & 7/25/2008 9:00 & 68 & 207 & 900 & 9.11 & 9.11 & & 0.0494 & 2.93E-04 & 21.8245 & 8.0417 & 29.8662 & \\
\hline & $7 / 25 / 2008$ 10:00 & 69 & 207 & 1000 & 9.03 & 9.03 & & 0.0587 & 3.63E-04 & 26.9860 & 9.9435 & 36.9295 & \\
\hline & $7 / 25 / 2008$ 11:00 & 70 & 207 & 1100 & 8.28 & 8.28 & & 0.1546 & 1.20E-03 & 88.8969 & 32.7559 & 121.6528 & \\
\hline & $7 / 25 / 2008$ 12:00 & 71 & 207 & 1200 & 6.846 & 6.846 & & 0.3964 & 3.81E-03 & 283.3722 & 104.4143 & 387.7866 & \\
\hline & $7 / 25 / 2008$ 13:00 & 72 & 207 & 1300 & 6.219 & 6.219 & & 0.5372 & 5.54E-03 & 411.9346 & 151.7858 & 563.7204 & \\
\hline & $7 / 25 / 200814: 00$ & 73 & 207 & 1400 & 5.761 & 5.761 & & 0.6594 & 7.13E-03 & 530.1593 & 195.3481 & 725.5074 & \\
\hline & $7 / 25 / 2008$ 15:00 & 74 & 207 & 1500 & 5.88 & 5.88 & & 0.6259 & 6.68E-03 & 497.1204 & 183.1742 & 680.2946 & \\
\hline & $7 / 25 / 2008$ 16:00 & 75 & 207 & 1600 & 6.057 & 6.057 & & 0.5783 & $6.06 \mathrm{E}-03$ & 451.0795 & 166.2095 & 617.2891 & \\
\hline & $7 / 25 / 200817: 00$ & 76 & 207 & 1700 & 5.879 & 5.879 & & 0.6261 & $6.69 \mathrm{E}-03$ & 497.3908 & 183.2739 & 680.6647 & \\
\hline & $7 / 25 / 2008$ 18:00 & 77 & 207 & 1800 & 5.989 & 5.989 & & 0.5963 & $6.30 \mathrm{E}-03$ & 468.3468 & 172.5720 & 640.9188 & \\
\hline & $7 / 25 / 2008$ 19:00 & 78 & 207 & 1900 & 6.073 & 6.073 & & 0.5742 & 6.01E-03 & 447.0903 & 164.7396 & 611.8300 & \\
\hline & $7 / 25 / 200820: 00$ & 79 & 207 & 2000 & 6.949 & 6.949 & & 0.3757 & 3.57E-03 & 265.2707 & 97.7444 & 363.0151 & \\
\hline & $7 / 25 / 200821: 00$ & 80 & 207 & 2100 & 8.46 & 8.46 & & 0.1300 & $9.66 \mathrm{E}-04$ & 71.8403 & 26.4710 & 98.3113 & \\
\hline & $7 / 25 / 200822: 00$ & 81 & 207 & 2200 & 9.46 & 9.46 & & 0.0106 & $4.40 \mathrm{E}-05$ & 3.2710 & 1.2053 & 4.4763 & \\
\hline
\end{tabular}




\begin{tabular}{|c|c|c|c|c|c|c|c|c|c|c|c|c|c|}
\hline Tree ID & TS & RN & JD & HR & $\begin{array}{c}\text { Raw } \\
\text { TC_dTA }\end{array}$ & $\begin{array}{c}\text { Fixed } \\
\text { TC_dT } \Delta\end{array}$ & dTM & K & $\begin{array}{l}\text { Sap } \\
\text { Velocity } \\
(\mathrm{cm} / \mathrm{s})\end{array}$ & $\begin{array}{c}\text { Probed } \\
\text { Drywood F } \\
(\mathrm{g} / \mathrm{hr})\end{array}$ & $\begin{array}{c}\text { Extrapolated } \\
\text { Wetwood F } \\
(\mathrm{g} / \mathrm{hr})\end{array}$ & $\begin{array}{c}\text { Extrapolated } \\
\text { Total Wood F } \\
(\mathrm{g} / \mathrm{hr})\end{array}$ & $\begin{array}{l}\text { Relative } \\
\text { Humidity, } \\
@ 10 \mathrm{~m}(\%)\end{array}$ \\
\hline & 7/25/2008 23:00 & 82 & 207 & 2300 & $\begin{array}{r}9.6 \\
\end{array}$ & 9.6 & & -0.0042 & $0.00 \mathrm{E}+00$ & 0.0000 & 0.0000 & 0.0000 & \\
\hline & $7 / 26 / 20080: 00$ & 83 & 208 & 0 & 9.61 & 9.61 & 9.61 & -0.0052 & $0.00 \mathrm{E}+00$ & 0.0000 & 0.0000 & 0.0000 & \\
\hline & 7/26/2008 1:00 & 84 & 208 & 100 & 9.61 & 9.61 & & -0.0052 & $0.00 E+00$ & 0.0000 & 0.0000 & 0.0000 & \\
\hline & $7 / 26 / 20082: 00$ & 85 & 208 & 200 & 9.59 & 9.59 & & -0.0031 & $0.00 \mathrm{E}+00$ & 0.0000 & 0.0000 & 0.0000 & \\
\hline & 7/26/2008 3:00 & 86 & 208 & 300 & 9.57 & 9.57 & & -0.0010 & $0.00 \mathrm{E}+00$ & 0.0000 & 0.0000 & 0.0000 & \\
\hline & 7/26/2008 4:00 & 87 & 208 & 400 & 9.56 & 9.56 & & 0.0000 & $0.00 \mathrm{E}+00$ & 0.0000 & 0.0000 & 0.0000 & \\
\hline & 7/26/2008 5:00 & 88 & 208 & 500 & 9.53 & 9.53 & & 0.0031 & $9.90 \mathrm{E}-06$ & 0.7363 & 0.2713 & 1.0077 & \\
\hline & 7/26/2008 6:00 & 89 & 208 & 600 & 9.49 & 9.49 & & 0.0074 & 2.82E-05 & 2.1004 & 0.7739 & 2.8744 & \\
\hline & 7/26/2008 7:00 & 90 & 208 & 700 & 9.46 & 9.46 & & 0.0106 & 4.40E-05 & 3.2710 & 1.2053 & 4.4763 & \\
\hline & 7/26/2008 8:00 & 91 & 208 & 800 & 9.44 & 9.44 & & 0.0127 & 5.52E-05 & 4.1048 & 1.5125 & 5.6172 & \\
\hline & 7/26/2008 9:00 & 92 & 208 & 900 & 8.82 & 8.82 & & 0.0839 & 5.63E-04 & 41.8947 & 15.4370 & 57.3316 & \\
\hline & $7 / 26 / 2008$ 10:00 & 93 & 208 & 1000 & 6.438 & 6.438 & & 0.4849 & 4.88E-03 & 363.1456 & 133.8085 & 496.9541 & \\
\hline & $7 / 26 / 2008$ 11:00 & 94 & 208 & 1100 & 6.636 & 6.636 & & 0.4406 & 4.34E-03 & 322.7437 & 118.9216 & 441.6653 & \\
\hline & $7 / 26 / 2008$ 12:00 & 95 & 208 & 1200 & 6.992 & 6.992 & & 0.3673 & 3.47E-03 & 257.9369 & 95.0422 & 352.9791 & \\
\hline & $7 / 26 / 2008$ 13:00 & 96 & 208 & 1300 & 6.221 & 6.221 & & 0.5367 & 5.53E-03 & 411.4682 & 151.6139 & 563.0821 & \\
\hline & $7 / 26 / 200814: 00$ & 97 & 208 & 1400 & 6.096 & 6.096 & & 0.5682 & 5.93E-03 & 441.4042 & 162.6444 & 604.0486 & \\
\hline & $7 / 26 / 2008$ 15:00 & 98 & 208 & 1500 & 6.022 & 6.022 & & 0.5875 & 6.18E-03 & 459.9032 & 169.4608 & 629.3640 & \\
\hline & $7 / 26 / 200816: 00$ & 99 & 208 & 1600 & 6.058 & 6.058 & & 0.5781 & $6.06 \mathrm{E}-03$ & 450.8294 & 166.1174 & 616.9468 & \\
\hline & $7 / 26 / 200817: 00$ & 100 & 208 & 1700 & 5.832 & 5.832 & & 0.6392 & $6.86 \mathrm{E}-03$ & 510.2369 & 188.0073 & 698.2442 & \\
\hline & $7 / 26 / 2008$ 18:00 & 101 & 208 & 1800 & 6.068 & 6.068 & & 0.5755 & $6.03 E-03$ & 448.3340 & 165.1979 & 613.5319 & \\
\hline & $7 / 26 / 2008$ 19:00 & 102 & 208 & 1900 & 6.14 & 6.14 & & 0.5570 & $5.79 \mathrm{E}-03$ & 430.6825 & 158.6938 & 589.3764 & \\
\hline & $7 / 26 / 200820: 00$ & 103 & 208 & 2000 & 6.677 & 6.677 & & 0.4318 & 4.23E-03 & 314.7860 & 115.9894 & 430.7754 & \\
\hline & $7 / 26 / 200821: 00$ & 104 & 208 & 2100 & 8.23 & 8.23 & & 0.1616 & 1.26E-03 & 93.8881 & 34.5950 & 128.4831 & \\
\hline & $7 / 26 / 200822: 00$ & 105 & 208 & 2200 & 9.16 & 9.16 & & 0.0437 & $2.52 E-04$ & 18.7521 & 6.9096 & 25.6616 & \\
\hline & $7 / 26 / 2008$ 23:00 & 106 & 208 & 2300 & 9.34 & 9.34 & & 0.0236 & 1.18E-04 & 8.7706 & 3.2317 & 12.0024 & \\
\hline & $7 / 27 / 20080: 00$ & 107 & 209 & 0 & 9.36 & 9.36 & 9.36 & 0.0214 & 1.05E-04 & 7.7792 & 2.8664 & 10.6456 & \\
\hline & 7/27/2008 1:00 & 108 & 209 & 100 & 9.35 & 9.35 & & 0.0225 & 1.11E-04 & 8.2716 & 3.0478 & 11.3194 & \\
\hline & 7/27/2008 2:00 & 109 & 209 & 200 & 9.33 & 9.33 & & 0.0247 & $1.25 \mathrm{E}-04$ & 9.2762 & 3.4180 & 12.6942 & \\
\hline & 7/27/2008 3:00 & 110 & 209 & 300 & 9.32 & 9.32 & & 0.0258 & $1.32 \mathrm{E}-04$ & 9.7880 & 3.6066 & 13.3946 & \\
\hline & 7/27/2008 4:00 & 111 & 209 & 400 & 9.3 & 9.3 & & 0.0280 & 1.46E-04 & 10.8302 & 3.9906 & 14.8208 & \\
\hline & 7/27/20085:00 & 112 & 209 & 500 & 9.24 & 9.24 & & 0.0346 & 1.90E-04 & 14.0963 & 5.1941 & 19.2903 & \\
\hline & 7/27/2008 6:00 & 113 & 209 & 600 & 9.16 & 9.16 & & 0.0437 & $2.52 \mathrm{E}-04$ & 18.7521 & 6.9096 & 25.6616 & \\
\hline & 7/27/2008 7:00 & 114 & 209 & 700 & 9.08 & 9.08 & & 0.0529 & 3.19E-04 & 23.7252 & 8.7421 & 32.4673 & \\
\hline & 7/27/2008 8:00 & 115 & 209 & 800 & 8.99 & 8.99 & & 0.0634 & 3.99E-04 & 29.6763 & 10.9349 & 40.6112 & \\
\hline & $7 / 27 / 20089: 00$ & 116 & 209 & 900 & 6.997 & 6.997 & & 0.3663 & 3.46E-03 & 257.0925 & 94.7310 & 351.8235 & \\
\hline & $7 / 27 / 2008$ 10:00 & 117 & 209 & 1000 & 5.366 & 5.366 & & 0.7816 & 8.79E-03 & 653.5247 & 240.8046 & 894.3293 & \\
\hline & $7 / 27 / 2008$ 11:00 & 118 & 209 & 1100 & 5.673 & 5.673 & & 0.6852 & 7.47E-03 & 555.7486 & 204.7770 & 760.5255 & \\
\hline & $7 / 27 / 2008$ 12:00 & 119 & 209 & 1200 & 5.608 & 5.608 & & 0.7047 & 7.73E-03 & 575.3146 & 211.9865 & 787.3011 & \\
\hline & $7 / 27 / 200813: 00$ & 120 & 209 & 1300 & 5.619 & 5.619 & & 0.7014 & 7.69E-03 & 571.9626 & 210.7514 & 782.7140 & \\
\hline & $7 / 27 / 200814: 00$ & 121 & 209 & 1400 & 5.515 & 5.515 & & 0.7335 & 8.12E-03 & 604.3389 & 222.6811 & 827.0200 & \\
\hline & 7/27/2008 15:00 & 122 & 209 & 1500 & 5.589 & 5.589 & & 0.7105 & 7.81E-03 & 581.1441 & 214.1345 & 795.2786 & \\
\hline
\end{tabular}




\begin{tabular}{|c|c|c|c|c|c|c|c|c|c|c|c|c|c|}
\hline Tree ID & TS & RN & JD & HR & $\begin{array}{c}\text { Raw } \\
\text { TC_dTA }\end{array}$ & $\begin{array}{c}\text { Fixed } \\
\text { TC_dT } \Delta\end{array}$ & dTM & $\mathbf{K}$ & $\begin{array}{l}\text { Sap } \\
\text { Velocity } \\
(\mathrm{cm} / \mathrm{s})\end{array}$ & $\begin{array}{c}\text { Probed } \\
\text { Drywood F } \\
(\mathrm{g} / \mathrm{hr})\end{array}$ & $\begin{array}{c}\text { Extrapolated } \\
\text { Wetwood F } \\
(\mathrm{g} / \mathrm{hr})\end{array}$ & $\begin{array}{c}\text { Extrapolated } \\
\text { Total Wood F } \\
(\mathrm{g} / \mathrm{hr})\end{array}$ & $\begin{array}{l}\text { Relative } \\
\text { Humidity, } \\
@ 10 \mathrm{~m}(\%)\end{array}$ \\
\hline & 7/27/2008 16:00 & 123 & 209 & 1600 & 5.6 & 5.6 & & 0.7071 & 7.77E-03 & 577.7630 & 212.8886 & 790.6516 & \\
\hline & $7 / 27 / 2008$ 17:00 & 124 & 209 & 1700 & 5.616 & 5.616 & & 0.7023 & 7.70E-03 & 572.8751 & 211.0876 & 783.9627 & \\
\hline & $7 / 27 / 2008$ 18:00 & 125 & 209 & 1800 & 5.779 & 5.779 & & 0.6543 & 7.06E-03 & 525.0486 & 193.4650 & 718.5136 & \\
\hline & $7 / 27 / 2008$ 19:00 & 126 & 209 & 1900 & 5.926 & 5.926 & & 0.6132 & $6.52 \mathrm{E}-03$ & 484.8090 & 178.6378 & 663.4468 & \\
\hline & $7 / 27 / 200820: 00$ & 127 & 209 & 2000 & 6.421 & 6.421 & & 0.4889 & 4.93E-03 & 366.7732 & 135.1451 & 501.9183 & \\
\hline & $7 / 27 / 2008$ 21:00 & 128 & 209 & 2100 & 7.823 & 7.823 & & 0.2220 & 1.87E-03 & 138.8217 & 51.1517 & 189.9734 & \\
\hline & $7 / 27 / 2008$ 22:00 & 129 & 209 & 2200 & 8.82 & 8.82 & & 0.0839 & $5.63 E-04$ & 41.8947 & 15.4370 & 57.3316 & \\
\hline & $7 / 27 / 200823: 00$ & 130 & 209 & 2300 & 8.96 & 8.96 & & 0.0670 & 4.27E-04 & 31.7409 & 11.6956 & 43.4365 & \\
\hline & $7 / 28 / 20080: 00$ & 131 & 210 & 0 & 9.04 & 9.04 & 9.16 & 0.0575 & 3.54E-04 & 26.3247 & 9.6999 & 36.0246 & \\
\hline & 7/28/2008 1:00 & 132 & 210 & 100 & 9.16 & 9.16 & & 0.0437 & 2.52E-04 & 18.7521 & 6.9096 & 25.6616 & \\
\hline & $7 / 28 / 20082: 00$ & 133 & 210 & 200 & 9.16 & 9.16 & & 0.0437 & $2.52 E-04$ & 18.7521 & 6.9096 & 25.6616 & \\
\hline & 7/28/2008 3:00 & 134 & 210 & 300 & 8.86 & 8.86 & & 0.0790 & $5.23 E-04$ & 38.9073 & 14.3362 & 53.2435 & \\
\hline & 7/28/2008 4:00 & 135 & 210 & 400 & 8.69 & 8.69 & & 0.1001 & 7.00E-04 & 52.0740 & 19.1877 & 71.2618 & \\
\hline & 7/28/2008 5:00 & 136 & 210 & 500 & 8.56 & 8.56 & & 0.1168 & 8.47E-04 & 62.9696 & 23.2025 & 86.1721 & \\
\hline & 7/28/2008 6:00 & 137 & 210 & 600 & 8.45 & 8.45 & & 0.1314 & $9.78 E-04$ & 72.7509 & 26.8066 & 99.5575 & \\
\hline & 7/28/2008 7:00 & 138 & 210 & 700 & 8.32 & 8.32 & & 0.1490 & $1.14 \mathrm{E}-03$ & 84.9839 & 31.3141 & 116.2980 & \\
\hline & 7/28/2008 8:00 & 139 & 210 & 800 & 6.818 & 6.818 & & 0.4022 & 3.88E-03 & 288.4268 & 106.2768 & 394.7036 & \\
\hline & $7 / 28 / 20089: 00$ & 140 & 210 & 900 & 5.14 & 5.14 & & 0.8599 & $9.88 \mathrm{E}-03$ & 735.0648 & 270.8497 & 1005.9145 & \\
\hline & $7 / 28 / 2008$ 10:00 & 141 & 210 & 1000 & 4.906 & 4.906 & & 0.9486 & 1.12E-02 & 829.4974 & 305.6454 & 1135.1428 & \\
\hline & $7 / 28 / 2008$ 11:00 & 142 & 210 & 1100 & 5.157 & 5.157 & & 0.8538 & $9.80 \mathrm{E}-03$ & 728.6184 & 268.4744 & 997.0929 & \\
\hline & $7 / 28 / 200812: 00$ & 143 & 210 & 1200 & 5.303 & 5.303 & & 0.8028 & $9.08 \mathrm{E}-03$ & 675.3779 & 248.8569 & 924.2348 & \\
\hline & 7/28/2008 13:00 & 144 & 210 & 1300 & 5.339 & 5.339 & & 0.7906 & 8.91E-03 & 662.8107 & 244.2262 & 907.0370 & \\
\hline & $7 / 28 / 2008$ 14:00 & 145 & 210 & 1400 & 5.422 & 5.422 & & 0.7632 & 8.53E-03 & 634.6366 & 233.8449 & 868.4815 & \\
\hline & $7 / 28 / 2008$ 15:00 & 146 & 210 & 1500 & 5.408 & 5.408 & & 0.7678 & $8.60 \mathrm{E}-03$ & 639.3122 & 235.5677 & 874.8800 & \\
\hline & $7 / 28 / 2008$ 16:00 & 147 & 210 & 1600 & 5.491 & 5.491 & & 0.7410 & 8.23E-03 & 612.0329 & 225.5161 & 837.5491 & \\
\hline & 7/28/2008 17:00 & 148 & 210 & 1700 & 5.501 & 5.501 & & 0.7379 & 8.19E-03 & 608.8167 & 224.3310 & 833.1478 & \\
\hline & $7 / 28 / 2008$ 18:00 & 149 & 210 & 1800 & 5.695 & 5.695 & & 0.6787 & 7.38E-03 & 549.2558 & 202.3846 & 751.6404 & \\
\hline & $7 / 28 / 2008$ 19:00 & 150 & 210 & 1900 & 5.921 & 5.921 & & 0.6146 & $6.54 \mathrm{E}-03$ & 486.1351 & 179.1265 & 665.2616 & \\
\hline & $7 / 28 / 200820: 00$ & 151 & 210 & 2000 & 6.658 & 6.658 & & 0.4359 & $4.28 \mathrm{E}-03$ & 318.4569 & 117.3420 & 435.7989 & \\
\hline & $7 / 28 / 200821: 00$ & 152 & 210 & 2100 & 8.26 & 8.26 & & 0.1574 & $1.22 \mathrm{E}-03$ & 90.8800 & 33.4866 & 124.3666 & \\
\hline & $7 / 28 / 2008$ 22:00 & 153 & 210 & 2200 & 8.71 & 8.71 & & 0.0976 & 6.78E-04 & 50.4613 & 18.5935 & 69.0549 & \\
\hline & $7 / 28 / 200823: 00$ & 154 & 210 & 2300 & 8.89 & 8.89 & & 0.0754 & 4.94E-04 & 36.7119 & 13.5272 & 50.2391 & \\
\hline & $7 / 29 / 20080: 00$ & 155 & 211 & 0 & 9.14 & 9.14 & 9.18 & 0.0460 & 2.68E-04 & 19.9665 & 7.3571 & 27.3235 & \\
\hline & 7/29/2008 1:00 & 156 & 211 & 100 & 9.18 & 9.18 & & 0.0414 & 2.36E-04 & 17.5574 & 6.4694 & 24.0268 & \\
\hline & 7/29/2008 2:00 & 157 & 211 & 200 & 9.13 & 9.13 & & 0.0471 & 2.77E-04 & 20.5810 & 7.5835 & 28.1645 & \\
\hline & 7/29/2008 3:00 & 158 & 211 & 300 & 9.09 & 9.09 & & 0.0517 & 3.10E-04 & 23.0870 & 8.5069 & 31.5938 & \\
\hline & 7/29/2008 4:00 & 159 & 211 & 400 & 8.98 & 8.98 & & 0.0646 & 4.08E-04 & 30.3601 & 11.1868 & 41.5469 & \\
\hline & 7/29/2008 5:00 & 160 & 211 & 500 & 8.61 & 8.61 & & 0.1103 & 7.89E-04 & 58.6942 & 21.6271 & 80.3213 & \\
\hline & $7 / 29 / 20086: 00$ & 161 & 211 & 600 & 8.41 & 8.41 & & 0.1367 & 1.03E-03 & 76.4366 & 28.1646 & 104.6013 & \\
\hline & 7/29/2008 7:00 & 162 & 211 & 700 & 8.28 & 8.28 & & 0.1546 & 1.20E-03 & 88.8969 & 32.7559 & 121.6528 & \\
\hline & 7/29/2008 8:00 & 163 & 211 & 800 & 7.758 & 7.758 & & 0.2323 & 1.97E-03 & 146.7434 & 54.0706 & 200.8140 & \\
\hline
\end{tabular}




\begin{tabular}{|c|c|c|c|c|c|c|c|c|c|c|c|c|c|}
\hline Tree ID & TS & RN & JD & HR & $\begin{array}{c}\text { Raw } \\
\text { TC_dTA }\end{array}$ & $\begin{array}{c}\text { Fixed } \\
\text { TC_dT } \Delta\end{array}$ & dTM & $\mathbf{K}$ & $\begin{array}{l}\text { Sap } \\
\text { Velocity } \\
(\mathrm{cm} / \mathrm{s})\end{array}$ & $\begin{array}{c}\text { Probed } \\
\text { Drywood F } \\
(\mathrm{g} / \mathrm{hr})\end{array}$ & $\begin{array}{c}\text { Extrapolated } \\
\text { Wetwood F } \\
(\mathrm{g} / \mathrm{hr})\end{array}$ & $\begin{array}{c}\text { Extrapolated } \\
\text { Total Wood F } \\
(\mathrm{g} / \mathrm{hr})\end{array}$ & $\begin{array}{l}\text { Relative } \\
\text { Humidity, } \\
@ 10 \mathrm{~m}(\%)\end{array}$ \\
\hline & 7/29/2008 9:00 & 164 & 211 & 900 & 5.964 & 5.964 & & 0.6030 & $6.38 \mathrm{E}-03$ & 474.8250 & 174.9590 & 649.7840 & \\
\hline & $7 / 29 / 2008$ 10:00 & 165 & 211 & 1000 & 5.047 & 5.047 & & 0.8942 & $1.04 \mathrm{E}-02$ & 771.2927 & 284.1987 & 1055.4913 & \\
\hline & $7 / 29 / 2008$ 11:00 & 166 & 211 & 1100 & 5.557 & 5.557 & & 0.7204 & 7.95E-03 & 591.0776 & 217.7947 & 808.8723 & \\
\hline & $7 / 29 / 2008$ 12:00 & 167 & 211 & 1200 & 5.036 & 5.036 & & 0.8983 & 1.04E-02 & 775.6882 & 285.8183 & 1061.5064 & \\
\hline & 7/29/2008 13:00 & 168 & 211 & 1300 & 5.237 & 5.237 & & 0.8255 & 9.40E-03 & 698.9842 & 257.5551 & 956.5393 & \\
\hline & $7 / 29 / 2008$ 14:00 & 169 & 211 & 1400 & 5.202 & 5.202 & & 0.8378 & 9.57E-03 & 711.8086 & 262.2805 & 974.0892 & \\
\hline & $7 / 29 / 2008$ 15:00 & 170 & 211 & 1500 & 5.547 & 5.547 & & 0.7235 & 7.99E-03 & 594.2119 & 218.9496 & 813.1614 & \\
\hline & $7 / 29 / 2008$ 16:00 & 171 & 211 & 1600 & 5.745 & 5.745 & & 0.6641 & 7.19E-03 & 534.7369 & 197.0348 & 731.7717 & \\
\hline & 7/29/2008 17:00 & 172 & 211 & 1700 & 6.003 & 6.003 & & 0.5925 & $6.25 \mathrm{E}-03$ & 464.7498 & 171.2466 & 635.9964 & \\
\hline & 7/29/2008 18:00 & 173 & 211 & 1800 & 6.006 & 6.006 & & 0.5917 & 6.24E-03 & 463.9819 & 170.9637 & 634.9455 & \\
\hline & $7 / 29 / 2008$ 19:00 & 174 & 211 & 1900 & 6.142 & 6.142 & & 0.5565 & $5.78 \mathrm{E}-03$ & 430.2000 & 158.5160 & 588.7160 & \\
\hline & $7 / 29 / 200820: 00$ & 175 & 211 & 2000 & 7.132 & 7.132 & & 0.3404 & 3.16E-03 & 234.9332 & 86.5660 & 321.4991 & \\
\hline & 7/29/2008 21:00 & 176 & 211 & 2100 & 8.49 & 8.49 & & 0.1260 & $9.29 \mathrm{E}-04$ & 69.1341 & 25.4739 & 94.6080 & \\
\hline & $7 / 29 / 2008$ 22:00 & 177 & 211 & 2200 & 8.66 & 8.66 & & 0.1039 & 7.33E-04 & 54.5248 & 20.0908 & 74.6156 & \\
\hline & $7 / 29 / 2008$ 23:00 & 178 & 211 & 2300 & 8.44 & 8.44 & & 0.1327 & $9.90 \mathrm{E}-04$ & 73.6659 & 27.1437 & 100.8096 & \\
\hline & $7 / 30 / 20080: 00$ & 179 & 212 & 0 & 8.37 & 8.37 & 8.87 & 0.1422 & 1.08E-03 & 80.1917 & 29.5483 & 109.7400 & \\
\hline & 7/30/2008 1:00 & 180 & 212 & 100 & 8.35 & 8.35 & & 0.1449 & 1.10E-03 & 82.0955 & 30.2498 & 112.3452 & \\
\hline & 7/30/2008 2:00 & 181 & 212 & 200 & 8.26 & 8.26 & & 0.1574 & $1.22 \mathrm{E}-03$ & 90.8800 & 33.4866 & 124.3666 & \\
\hline & 7/30/2008 3:00 & 182 & 212 & 300 & 8.19 & 8.19 & & 0.1673 & 1.32E-03 & 97.9618 & 36.0961 & 134.0579 & \\
\hline & 7/30/2008 4:00 & 183 & 212 & 400 & 8.11 & 8.11 & & 0.1788 & 1.43E-03 & 106.3275 & 39.1786 & 145.5061 & \\
\hline & 7/30/2008 5:00 & 184 & 212 & 500 & 8.15 & 8.15 & & 0.1730 & 1.37E-03 & 102.1081 & 37.6238 & 139.7319 & \\
\hline & 7/30/2008 6:00 & 185 & 212 & 600 & 8.07 & 8.07 & & 0.1846 & 1.49E-03 & 110.6209 & 40.7606 & 151.3815 & \\
\hline & 7/30/2008 7:00 & 186 & 212 & 700 & 7.949 & 7.949 & & 0.2027 & 1.67E-03 & 124.0670 & 45.7150 & 169.7820 & \\
\hline & $7 / 30 / 20088: 00$ & 187 & 212 & 800 & 7.913 & 7.913 & & 0.2081 & $1.72 \mathrm{E}-03$ & 128.2030 & 47.2390 & 175.4420 & \\
\hline & 7/30/2008 9:00 & 188 & 212 & 900 & 7.353 & 7.353 & & 0.3001 & $2.70 \mathrm{E}-03$ & 201.1913 & 74.1331 & 275.3244 & \\
\hline & $7 / 30 / 2008$ 10:00 & 189 & 212 & 1000 & 4.615 & 4.615 & & 1.0715 & 1.30E-02 & 963.6731 & 355.0852 & 1318.7582 & \\
\hline & 7/30/2008 11:00 & 190 & 212 & 1100 & 4.653 & 4.653 & & 1.0546 & 1.27E-02 & 944.9777 & 348.1965 & 1293.1742 & \\
\hline & $7 / 30 / 2008$ 12:00 & 191 & 212 & 1200 & 4.794 & 4.794 & & 0.9942 & $1.18 \mathrm{E}-02$ & 878.7690 & 323.8005 & 1202.5695 & \\
\hline & 7/30/2008 13:00 & 192 & 212 & 1300 & 4.753 & 4.753 & & 1.0114 & 1.21E-02 & 897.5240 & 330.7112 & 1228.2351 & \\
\hline & $7 / 30 / 2008$ 14:00 & 193 & 212 & 1400 & 4.865 & 4.865 & & 0.9651 & 1.14E-02 & 847.2095 & 312.1718 & 1159.3813 & \\
\hline & 7/30/2008 15:00 & 194 & 212 & 1500 & 5.077 & 5.077 & & 0.8830 & 1.02E-02 & 759.4253 & 279.8259 & 1039.2512 & \\
\hline & $7 / 30 / 2008$ 16:00 & 195 & 212 & 1600 & 4.968 & 4.968 & & 0.9243 & 1.08E-02 & 803.3988 & 296.0288 & 1099.4276 & \\
\hline & $7 / 30 / 2008$ 17:00 & 196 & 212 & 1700 & 5.07 & 5.07 & & 0.8856 & 1.02E-02 & 762.1787 & 280.8404 & 1043.0192 & \\
\hline & 7/30/2008 18:00 & 197 & 212 & 1800 & 5.394 & 5.394 & & 0.7723 & 8.66E-03 & 644.0186 & 237.3019 & 881.3205 & \\
\hline & $7 / 30 / 2008$ 19:00 & 198 & 212 & 1900 & 5.566 & 5.566 & & 0.7176 & 7.91E-03 & 588.2691 & 216.7598 & 805.0289 & \\
\hline & $7 / 30 / 200820: 00$ & 199 & 212 & 2000 & 6.264 & 6.264 & & 0.5262 & 5.40E-03 & 401.5357 & 147.9541 & 549.4897 & \\
\hline & 7/30/2008 21:00 & 200 & 212 & 2100 & 7.865 & 7.865 & & 0.2155 & 1.80E-03 & 133.8163 & 49.3074 & 183.1237 & \\
\hline & $7 / 30 / 2008$ 22:00 & 201 & 212 & 2200 & 8.77 & 8.77 & & 0.0901 & $6.15 E-04$ & 45.7249 & 16.8483 & 62.5732 & \\
\hline & $7 / 30 / 200823: 00$ & 202 & 212 & 2300 & 8.87 & 8.87 & & 0.0778 & 5.13E-04 & 38.1712 & 14.0650 & 52.2361 & \\
\hline & $7 / 31 / 20080: 00$ & 203 & 213 & 0 & 8.73 & 8.73 & 8.73 & 0.0951 & 6.57E-04 & 48.8656 & 18.0055 & 66.8711 & \\
\hline & 7/31/2008 1:00 & 204 & 213 & 100 & 8.56 & 8.56 & & 0.1168 & 8.47E-04 & 62.9696 & 23.2025 & 86.1721 & \\
\hline
\end{tabular}




\begin{tabular}{|c|c|c|c|c|c|c|c|c|c|c|c|c|c|}
\hline Tree ID & TS & RN & JD & HR & $\begin{array}{c}\text { Raw } \\
\text { TC_dTA }\end{array}$ & $\begin{array}{c}\text { Fixed } \\
\text { TC_dT } \Delta\end{array}$ & dTM & $\mathbf{K}$ & $\begin{array}{l}\text { Sap } \\
\text { Velocity } \\
(\mathrm{cm} / \mathrm{s})\end{array}$ & $\begin{array}{c}\text { Probed } \\
\text { Drywood F } \\
(\mathrm{g} / \mathrm{hr})\end{array}$ & $\begin{array}{c}\text { Extrapolated } \\
\text { Wetwood F } \\
(\mathrm{g} / \mathrm{hr})\end{array}$ & $\begin{array}{c}\text { Extrapolated } \\
\text { Total Wood F } \\
(\mathrm{g} / \mathrm{hr})\end{array}$ & $\begin{array}{l}\text { Relative } \\
\text { Humidity, } \\
@ 10 \mathrm{~m}(\%)\end{array}$ \\
\hline & 7/31/2008 2:00 & 205 & 213 & 200 & 8.45 & 8.45 & & 0.1314 & $9.78 \mathrm{E}-04$ & 72.7509 & 26.8066 & $\begin{array}{r}99.5575 \\
\end{array}$ & \\
\hline & 7/31/2008 3:00 & 206 & 213 & 300 & 8.43 & 8.43 & & 0.1340 & $1.00 \mathrm{E}-03$ & 74.5851 & 27.4824 & 102.0675 & \\
\hline & 7/31/2008 4:00 & 207 & 213 & 400 & 8.35 & 8.35 & & 0.1449 & $1.10 \mathrm{E}-03$ & 82.0955 & 30.2498 & 112.3452 & \\
\hline & 7/31/2008 5:00 & 208 & 213 & 500 & 8.26 & 8.26 & & 0.1574 & $1.22 \mathrm{E}-03$ & 90.8800 & 33.4866 & 124.3666 & \\
\hline & 7/31/2008 6:00 & 209 & 213 & 600 & 8.2 & 8.2 & & 0.1659 & 1.30E-03 & 96.9366 & 35.7183 & 132.6549 & \\
\hline & 7/31/2008 7:00 & 210 & 213 & 700 & 8.13 & 8.13 & & 0.1759 & 1.40E-03 & 104.2086 & 38.3978 & 142.6064 & \\
\hline & $7 / 31 / 2008$ 8:00 & 211 & 213 & 800 & 8.08 & 8.08 & & 0.1832 & 1.47E-03 & 109.5406 & 40.3625 & 149.9031 & \\
\hline & $7 / 31 / 20089: 00$ & 212 & 213 & 900 & 7.043 & 7.043 & & 0.3574 & 3.35E-03 & 249.4043 & 91.8981 & 341.3024 & \\
\hline & $7 / 31 / 2008$ 10:00 & 213 & 213 & 1000 & 4.582 & 4.582 & & 1.0864 & 1.32E-02 & 980.2168 & 361.1810 & 1341.3979 & \\
\hline & 7/31/2008 11:00 & 214 & 213 & 1100 & 4.778 & 4.778 & & 1.0008 & 1.19E-02 & 886.0409 & 326.4800 & 1212.5209 & \\
\hline & $7 / 31 / 2008$ 12:00 & 215 & 213 & 1200 & 4.675 & 4.675 & & 1.0449 & $1.26 \mathrm{E}-02$ & 934.3240 & 344.2709 & 1278.5949 & \\
\hline & 7/31/2008 13:00 & 216 & 213 & 1300 & 4.964 & 4.964 & & 0.9259 & $1.08 \mathrm{E}-02$ & 805.0582 & 296.6402 & 1101.6984 & \\
\hline & 7/31/2008 14:00 & 217 & 213 & 1400 & 4.793 & 4.793 & & 0.9946 & 1.18E-02 & 879.2217 & 323.9673 & 1203.1891 & \\
\hline & 7/31/2008 15:00 & 218 & 213 & 1500 & 4.891 & 4.891 & & 0.9546 & 1.12E-02 & 835.9349 & 308.0174 & 1143.9523 & \\
\hline & $7 / 31 / 2008$ 16:00 & 219 & 213 & 1600 & 5.01 & 5.01 & & 0.9082 & 1.06E-02 & 786.1731 & 289.6816 & 1075.8547 & \\
\hline & 7/31/2008 17:00 & 220 & 213 & 1700 & 5.07 & 5.07 & & 0.8856 & 1.02E-02 & 762.1787 & 280.8404 & 1043.0192 & \\
\hline & $7 / 31 / 2008$ 18:00 & 221 & 213 & 1800 & 5.343 & 5.343 & & 0.7893 & 8.89E-03 & 661.4275 & 243.7166 & 905.1441 & \\
\hline & $7 / 31 / 2008$ 19:00 & 222 & 213 & 1900 & 5.706 & 5.706 & & 0.6754 & 7.34E-03 & 546.0335 & 201.1973 & 747.2308 & \\
\hline & $7 / 31 / 200820: 00$ & 223 & 213 & 2000 & 6.721 & 6.721 & & 0.4224 & 4.12E-03 & 306.3951 & 112.8976 & 419.2927 & \\
\hline & $7 / 31 / 200821: 00$ & 224 & 213 & 2100 & 7.651 & 7.651 & & 0.2495 & $2.15 E-03$ & 160.2586 & 59.0506 & 219.3092 & \\
\hline & $7 / 31 / 200822: 00$ & 225 & 213 & 2200 & 8.39 & 8.39 & & 0.1395 & 1.05E-03 & 78.3055 & 28.8533 & 107.1588 & \\
\hline & 7/31/2008 23:00 & 226 & 213 & 2300 & 8.25 & 8.25 & & 0.1588 & 1.24E-03 & 91.8782 & 33.8544 & 125.7326 & \\
\hline & $8 / 1 / 20080: 00$ & 227 & 214 & 0 & 8.29 & 8.29 & 8.98 & 0.1532 & 1.18E-03 & 87.9120 & 32.3930 & 120.3050 & \\
\hline & 8/1/2008 1:00 & 228 & 214 & 100 & 8.44 & 8.44 & & 0.1327 & 9.90E-04 & 73.6659 & 27.1437 & 100.8096 & \\
\hline & 8/1/2008 2:00 & 229 & 214 & 200 & 8.47 & 8.47 & & 0.1287 & 9.54E-04 & 70.9339 & 26.1371 & 97.0710 & \\
\hline & $8 / 1 / 2008$ 3:00 & 230 & 214 & 300 & 8.45 & 8.45 & & 0.1314 & $9.78 E-04$ & 72.7509 & 26.8066 & 99.5575 & \\
\hline & 8/1/2008 4:00 & 231 & 214 & 400 & 8.45 & 8.45 & & 0.1314 & $9.78 \mathrm{E}-04$ & 72.7509 & 26.8066 & 99.5575 & \\
\hline & 8/1/2008 5:00 & 232 & 214 & 500 & 8.39 & 8.39 & & 0.1395 & $1.05 \mathrm{E}-03$ & 78.3055 & 28.8533 & 107.1588 & \\
\hline & 8/1/2008 6:00 & 233 & 214 & 600 & 8.31 & 8.31 & & 0.1504 & 1.16E-03 & 85.9555 & 31.6721 & 117.6276 & \\
\hline & 8/1/2008 7:00 & 234 & 214 & 700 & 8.22 & 8.22 & & 0.1630 & $1.28 \mathrm{E}-03$ & 94.8998 & 34.9678 & 129.8675 & \\
\hline & 8/1/2008 8:00 & 235 & 214 & 800 & 8.19 & 8.19 & & 0.1673 & 1.32E-03 & 97.9618 & 36.0961 & 134.0579 & \\
\hline & 8/1/2008 9:00 & 236 & 214 & 900 & 7.147 & 7.147 & & 0.3376 & 3.13E-03 & 232.5456 & 85.6862 & 318.2318 & \\
\hline & 8/1/2008 10:00 & 237 & 214 & 1000 & 4.76 & 4.76 & & 1.0084 & $1.20 \mathrm{E}-02$ & 894.2937 & 329.5209 & 1223.8147 & \\
\hline & 8/1/2008 11:00 & 238 & 214 & 1100 & 4.781 & 4.781 & & 0.9996 & 1.19E-02 & 884.6728 & 325.9759 & 1210.6487 & \\
\hline & 8/1/2008 12:00 & 239 & 214 & 1200 & 4.914 & 4.914 & & 0.9455 & 1.11E-02 & 826.0840 & 304.3876 & 1130.4716 & \\
\hline & 8/1/2008 13:00 & 240 & 214 & 1300 & 4.839 & 4.839 & & 0.9756 & 1.15E-02 & 858.6340 & 316.3813 & 1175.0153 & \\
\hline & 8/1/2008 14:00 & 241 & 214 & 1400 & 4.905 & 4.905 & & 0.9490 & 1.12E-02 & 829.9251 & 305.8030 & 1135.7281 & \\
\hline & 8/1/2008 15:00 & 242 & 214 & 1500 & 4.935 & 4.935 & & 0.9372 & 1.10E-02 & 817.1889 & 301.1100 & 1118.2989 & \\
\hline & 8/1/2008 16:00 & 243 & 214 & 1600 & 5.034 & 5.034 & & 0.8991 & 1.04E-02 & 776.4899 & 286.1137 & 1062.6036 & \\
\hline & 8/1/2008 17:00 & 244 & 214 & 1700 & 5.159 & 5.159 & & 0.8531 & 9.79E-03 & 727.8635 & 268.1963 & 996.0598 & \\
\hline & 8/1/2008 18:00 & 245 & 214 & 1800 & 5.359 & 5.359 & & 0.7839 & 8.82E-03 & 655.9208 & 241.6875 & 897.6084 & \\
\hline
\end{tabular}




\begin{tabular}{|c|c|c|c|c|c|c|c|c|c|c|c|c|c|}
\hline Tree ID & TS & RN & JD & HR & $\begin{array}{c}\text { Raw } \\
\text { TC_dTA }\end{array}$ & $\begin{array}{c}\text { Fixed } \\
\text { TC_dT } \Delta\end{array}$ & dTM & $\mathbf{K}$ & $\begin{array}{l}\text { Sap } \\
\text { Velocity } \\
(\mathrm{cm} / \mathrm{s})\end{array}$ & $\begin{array}{c}\text { Probed } \\
\text { Drywood F } \\
(\mathrm{g} / \mathrm{hr})\end{array}$ & $\begin{array}{c}\text { Extrapolated } \\
\text { Wetwood F } \\
(\mathrm{g} / \mathrm{hr})\end{array}$ & $\begin{array}{c}\text { Extrapolated } \\
\text { Total Wood F } \\
(\mathrm{g} / \mathrm{hr})\end{array}$ & $\begin{array}{l}\text { Relative } \\
\text { Humidity, } \\
@ 10 \mathrm{~m}(\%)\end{array}$ \\
\hline & 8/1/2008 19:00 & 246 & 214 & 1900 & \begin{tabular}{|r|}
5.613 \\
\end{tabular} & 5.613 & & 0.7032 & 7.71E-03 & 573.7889 & 211.4243 & $\begin{array}{r}785.2132 \\
\end{array}$ & \\
\hline & 8/1/2008 20:00 & 247 & 214 & 2000 & 6.598 & 6.598 & & 0.4489 & 4.44E-03 & 330.2410 & 121.6841 & 451.9251 & \\
\hline & 8/1/2008 21:00 & 248 & 214 & 2100 & 8.28 & 8.28 & & 0.1546 & $1.20 \mathrm{E}-03$ & 88.8969 & 32.7559 & 121.6528 & \\
\hline & 8/1/2008 22:00 & 249 & 214 & 2200 & 8.98 & 8.98 & & 0.0646 & $4.08 \mathrm{E}-04$ & 30.3601 & 11.1868 & 41.5469 & \\
\hline & 8/1/2008 23:00 & 250 & 214 & 2300 & 8.93 & 8.93 & & 0.0705 & 4.55E-04 & 33.8452 & 12.4709 & 46.3161 & \\
\hline & 8/2/2008 0:00 & 251 & 215 & 0 & 8.86 & 8.86 & 8.86 & 0.0790 & 5.23E-04 & 38.9073 & 14.3362 & 53.2435 & \\
\hline & 8/2/2008 1:00 & 252 & 215 & 100 & 8.76 & 8.76 & & 0.0913 & $6.25 E-04$ & 46.5037 & 17.1352 & 63.6390 & \\
\hline & 8/2/2008 2:00 & 253 & 215 & 200 & 8.69 & 8.69 & & 0.1001 & 7.00E-04 & 52.0740 & 19.1877 & 71.2618 & \\
\hline & 8/2/2008 3:00 & 254 & 215 & 300 & 8.61 & 8.61 & & 0.1103 & 7.89E-04 & 58.6942 & 21.6271 & 80.3213 & \\
\hline & 8/2/2008 4:00 & 255 & 215 & 400 & 8.51 & 8.51 & & 0.1234 & 9.05E-04 & 67.3515 & 24.8170 & 92.1685 & \\
\hline & 8/2/2008 5:00 & 256 & 215 & 500 & 8.46 & 8.46 & & 0.1300 & 9.66E-04 & 71.8403 & 26.4710 & 98.3113 & \\
\hline & 8/2/2008 6:00 & 257 & 215 & 600 & 8.51 & 8.51 & & 0.1234 & $9.05 E-04$ & 67.3515 & 24.8170 & 92.1685 & \\
\hline & 8/2/2008 7:00 & 258 & 215 & 700 & 8.27 & 8.27 & & 0.1560 & $1.21 \mathrm{E}-03$ & 89.8862 & 33.1204 & 123.0066 & \\
\hline & 8/2/2008 8:00 & 259 & 215 & 800 & 7.149 & 7.149 & & 0.3372 & 3.12E-03 & 232.2283 & 85.5693 & 317.7976 & \\
\hline & 8/2/2008 9:00 & 260 & 215 & 900 & 5.163 & 5.163 & & 0.8516 & 9.77E-03 & 726.3559 & 267.6408 & 993.9967 & \\
\hline & 8/2/2008 10:00 & 261 & 215 & 1000 & 5.031 & 5.031 & & 0.9002 & 1.05E-02 & 777.6940 & 286.5574 & 1064.2514 & \\
\hline & 8/2/2008 11:00 & 262 & 215 & 1100 & 5.038 & 5.038 & & 0.8976 & 1.04E-02 & 774.8872 & 285.5231 & 1060.4104 & \\
\hline & $8 / 2 / 200812: 00$ & 263 & 215 & 1200 & 4.968 & 4.968 & & 0.9243 & 1.08E-02 & 803.3988 & 296.0288 & 1099.4276 & \\
\hline & 8/2/2008 13:00 & 264 & 215 & 1300 & 4.977 & 4.977 & & 0.9208 & 1.08E-02 & 799.6772 & 294.6575 & 1094.3347 & \\
\hline & 8/2/2008 14:00 & 265 & 215 & 1400 & 5.147 & 5.147 & & 0.8574 & $9.85 E-03$ & 732.4039 & 269.8693 & 1002.2732 & \\
\hline & $8 / 2 / 2008$ 15:00 & 266 & 215 & 1500 & 5.251 & 5.251 & & 0.8206 & $9.33 \mathrm{E}-03$ & 693.9144 & 255.6870 & 949.6015 & \\
\hline & 8/2/2008 16:00 & 267 & 215 & 1600 & 5.36 & 5.36 & & 0.7836 & 8.81E-03 & 655.5780 & 241.5612 & 897.1393 & \\
\hline & 8/2/2008 17:00 & 268 & 215 & 1700 & 5.449 & 5.449 & & 0.7545 & 8.41E-03 & 625.7052 & 230.5539 & 856.2591 & \\
\hline & $8 / 2 / 200818: 00$ & 269 & 215 & 1800 & 5.709 & 5.709 & & 0.6745 & 7.33E-03 & 545.1575 & 200.8745 & 746.0320 & \\
\hline & 8/2/2008 19:00 & 270 & 215 & 1900 & 5.892 & 5.892 & & 0.6225 & $6.64 \mathrm{E}-03$ & 493.8846 & 181.9819 & 675.8665 & \\
\hline & 8/2/2008 20:00 & 271 & 215 & 2000 & 6.558 & 6.558 & & 0.4578 & $4.55 E-03$ & 338.2621 & 124.6396 & 462.9017 & \\
\hline & 8/2/2008 21:00 & 272 & 215 & 2100 & 7.727 & 7.727 & & 0.2372 & $2.02 E-03$ & 150.5976 & 55.4908 & 206.0883 & \\
\hline & 8/2/2008 22:00 & 273 & 215 & 2200 & 8.44 & 8.44 & & 0.1327 & 9.90E-04 & 73.6659 & 27.1437 & 100.8096 & \\
\hline & 8/2/2008 23:00 & 274 & 215 & 2300 & 8.26 & 8.26 & & 0.1574 & $1.22 \mathrm{E}-03$ & 90.8800 & 33.4866 & 124.3666 & \\
\hline & 8/3/2008 0:00 & 275 & 216 & 0 & 8.09 & 8.09 & 8.30 & 0.1817 & 1.46E-03 & 108.4649 & 39.9661 & 148.4311 & \\
\hline & 8/3/2008 1:00 & 276 & 216 & 100 & 7.902 & 7.902 & & 0.2098 & 1.74E-03 & 129.4794 & 47.7093 & 177.1887 & \\
\hline & 8/3/2008 2:00 & 277 & 216 & 200 & 8.05 & 8.05 & & 0.1876 & 1.52E-03 & 112.7956 & 41.5619 & 154.3575 & \\
\hline & 8/3/2008 3:00 & 278 & 216 & 300 & 8.21 & 8.21 & & 0.1644 & 1.29E-03 & 95.9159 & 35.3422 & 131.2582 & \\
\hline & 8/3/2008 4:00 & 279 & 216 & 400 & 8.28 & 8.28 & & 0.1546 & $1.20 \mathrm{E}-03$ & 88.8969 & 32.7559 & 121.6528 & \\
\hline & 8/3/2008 5:00 & 280 & 216 & 500 & 8.3 & 8.3 & & 0.1518 & 1.17E-03 & 86.9315 & 32.0317 & 118.9633 & \\
\hline & 8/3/2008 6:00 & 281 & 216 & 600 & 8.27 & 8.27 & & 0.1560 & 1.21E-03 & 89.8862 & 33.1204 & 123.0066 & \\
\hline & 8/3/2008 7:00 & 282 & 216 & 700 & 8.22 & 8.22 & & 0.1630 & $1.28 \mathrm{E}-03$ & 94.8998 & 34.9678 & 129.8675 & \\
\hline & 8/3/2008 8:00 & 283 & 216 & 800 & 7.273 & 7.273 & & 0.3145 & $2.86 \mathrm{E}-03$ & 213.0559 & 78.5048 & 291.5608 & \\
\hline & 8/3/2008 9:00 & 284 & 216 & 900 & 4.959 & 4.959 & & 0.9278 & 1.09E-02 & 807.1371 & 297.4063 & 1104.5434 & \\
\hline & 8/3/2008 10:00 & 285 & 216 & 1000 & 4.724 & 4.724 & & 1.0237 & $1.22 \mathrm{E}-02$ & 911.0318 & 335.6884 & 1246.7202 & \\
\hline & 8/3/2008 11:00 & 286 & 216 & 1100 & 4.819 & 4.819 & & 0.9838 & 1.17E-02 & 867.5256 & 319.6577 & 1187.1833 & \\
\hline
\end{tabular}




\begin{tabular}{|c|c|c|c|c|c|c|c|c|c|c|c|c|c|}
\hline Tree ID & TS & RN & JD & HR & $\begin{array}{c}\text { Raw } \\
\text { TC_dTA }\end{array}$ & $\begin{array}{c}\text { Fixed } \\
\text { TC_dT } \Delta\end{array}$ & dTM & $\mathbf{K}$ & $\begin{array}{l}\text { Sap } \\
\text { Velocity } \\
(\mathrm{cm} / \mathrm{s})\end{array}$ & $\begin{array}{c}\text { Probed } \\
\text { Drywood F } \\
(\mathrm{g} / \mathrm{hr})\end{array}$ & $\begin{array}{c}\text { Extrapolated } \\
\text { Wetwood F } \\
(\mathrm{g} / \mathrm{hr})\end{array}$ & $\begin{array}{c}\text { Extrapolated } \\
\text { Total Wood F } \\
(\mathrm{g} / \mathrm{hr})\end{array}$ & $\begin{array}{l}\text { Relative } \\
\text { Humidity, } \\
@ 10 \mathrm{~m}(\%)\end{array}$ \\
\hline & 8/3/2008 12:00 & 287 & 216 & 1200 & 4.841 & 4.841 & & 0.9748 & 1.15E-02 & 857.7498 & 316.0556 & 1173.8053 & \\
\hline & 8/3/2008 13:00 & 288 & 216 & 1300 & 4.886 & 4.886 & & 0.9566 & 1.13E-02 & 838.0916 & 308.8121 & 1146.9036 & \\
\hline & $8 / 3 / 2008$ 14:00 & 289 & 216 & 1400 & 5.059 & 5.059 & & 0.8897 & $1.03 E-02$ & 766.5247 & 282.4418 & 1048.9665 & \\
\hline & 8/3/2008 15:00 & 290 & 216 & 1500 & 5.22 & 5.22 & & 0.8314 & $9.48 \mathrm{E}-03$ & 705.1863 & 259.8404 & 965.0267 & \\
\hline & 8/3/2008 16:00 & 291 & 216 & 1600 & 5.351 & 5.351 & & 0.7866 & 8.86E-03 & 658.6690 & 242.7001 & 901.3691 & \\
\hline & 8/3/2008 17:00 & 292 & 216 & 1700 & 5.437 & 5.437 & & 0.7583 & 8.47E-03 & 629.6608 & 232.0115 & 861.6723 & \\
\hline & 8/3/2008 18:00 & 293 & 216 & 1800 & 5.678 & 5.678 & & 0.6837 & $7.45 E-03$ & 554.2673 & 204.2312 & 758.4984 & \\
\hline & 8/3/2008 19:00 & 294 & 216 & 1900 & 5.777 & 5.777 & & 0.6548 & 7.07E-03 & 525.6145 & 193.6735 & 719.2879 & \\
\hline & 8/3/2008 20:00 & 295 & 216 & 2000 & 6.155 & 6.155 & & 0.5532 & 5.74E-03 & 427.0737 & 157.3641 & 584.4378 & \\
\hline & 8/3/2008 21:00 & 296 & 216 & 2100 & 7.129 & 7.129 & & 0.3410 & 3.16E-03 & 235.4125 & 86.7426 & 322.1550 & \\
\hline & 8/3/2008 22:00 & 297 & 216 & 2200 & 7.835 & 7.835 & & 0.2202 & 1.85E-03 & 137.3826 & 50.6214 & 188.0041 & \\
\hline & 8/3/2008 23:00 & 298 & 216 & 2300 & 7.981 & 7.981 & & 0.1978 & $1.62 \mathrm{E}-03$ & 120.4432 & 44.3798 & 164.8230 & \\
\hline & 8/4/2008 0:00 & 299 & 217 & 0 & 7.895 & 7.895 & 8.64 & 0.2109 & 1.75E-03 & 130.2947 & 48.0098 & 178.3045 & \\
\hline & 8/4/2008 1:00 & 300 & 217 & 100 & 7.822 & 7.822 & & 0.2222 & 1.87E-03 & 138.9420 & 51.1960 & 190.1380 & \\
\hline & $8 / 4 / 2008$ 2:00 & 301 & 217 & 200 & 7.445 & 7.445 & & 0.2841 & 2.53E-03 & 188.0174 & 69.2789 & 257.2963 & \\
\hline & 8/4/2008 3:00 & 302 & 217 & 300 & 7.223 & 7.223 & & 0.3235 & $2.97 \mathrm{E}-03$ & 220.6703 & 81.3105 & 301.9808 & \\
\hline & 8/4/2008 4:00 & 303 & 217 & 400 & 7.025 & 7.025 & & 0.3609 & 3.39E-03 & 252.3955 & 93.0003 & 345.3958 & \\
\hline & $8 / 4 / 20085: 00$ & 304 & 217 & 500 & 7.159 & 7.159 & & 0.3354 & $3.10 \mathrm{E}-03$ & 230.6460 & 84.9863 & 315.6322 & \\
\hline & 8/4/2008 6:00 & 305 & 217 & 600 & 7.526 & 7.526 & & 0.2703 & 2.38E-03 & 176.8218 & 65.1536 & 241.9755 & \\
\hline & 8/4/2008 7:00 & 306 & 217 & 700 & 7.484 & 7.484 & & 0.2774 & $2.45 E-03$ & 182.5806 & 67.2756 & 249.8561 & \\
\hline & 8/4/2008 8:00 & 307 & 217 & 800 & 7.263 & 7.263 & & 0.3163 & $2.88 \mathrm{E}-03$ & 214.5664 & 79.0614 & 293.6278 & \\
\hline & 8/4/2008 9:00 & 308 & 217 & 900 & 6.862 & 6.862 & & 0.3932 & 3.77E-03 & 280.5099 & 103.3596 & 383.8695 & \\
\hline & 8/4/2008 10:00 & 309 & 217 & 1000 & 5.789 & 5.789 & & 0.6514 & 7.02E-03 & 522.2271 & 192.4253 & 714.6524 & \\
\hline & 8/4/2008 11:00 & 310 & 217 & 1100 & 5.287 & 5.287 & & 0.8082 & $9.16 \mathrm{E}-03$ & 681.0326 & 250.9405 & 931.9731 & \\
\hline & 8/4/2008 12:00 & 311 & 217 & 1200 & 5.315 & 5.315 & & 0.7987 & $9.02 \mathrm{E}-03$ & 671.1650 & 247.3045 & 918.4695 & \\
\hline & 8/4/2008 13:00 & 312 & 217 & 1300 & 5.39 & 5.39 & & 0.7737 & 8.68E-03 & 645.3690 & 237.7995 & 883.1685 & \\
\hline & 8/4/2008 14:00 & 313 & 217 & 1400 & 5.866 & 5.866 & & 0.6297 & 6.73E-03 & 500.9172 & 184.5733 & 685.4905 & \\
\hline & 8/4/2008 15:00 & 314 & 217 & 1500 & 6.201 & 6.201 & & 0.5417 & $5.59 \mathrm{E}-03$ & 416.1506 & 153.3392 & 569.4898 & \\
\hline & 8/4/2008 16:00 & 315 & 217 & 1600 & 5.671 & 5.671 & & 0.6858 & 7.48E-03 & 556.3420 & 204.9957 & 761.3377 & \\
\hline & 8/4/2008 17:00 & 316 & 217 & 1700 & 5.631 & 5.631 & & 0.6977 & 7.64E-03 & 568.3250 & 209.4110 & 777.7361 & \\
\hline & 8/4/2008 18:00 & 317 & 217 & 1800 & 5.775 & 5.775 & & 0.6554 & 7.07E-03 & 526.1808 & 193.8821 & 720.0629 & \\
\hline & 8/4/2008 19:00 & 318 & 217 & 1900 & 5.999 & 5.999 & & 0.5936 & $6.26 \mathrm{E}-03$ & 465.7752 & 171.6245 & 637.3997 & \\
\hline & 8/4/2008 20:00 & 319 & 217 & 2000 & 6.686 & 6.686 & & 0.4299 & $4.21 E-03$ & 313.0573 & 115.3524 & 428.4097 & \\
\hline & 8/4/2008 21:00 & 320 & 217 & 2100 & 8.18 & 8.18 & & 0.1687 & 1.33E-03 & 98.9916 & 36.4755 & 135.4671 & \\
\hline & 8/4/2008 22:00 & 321 & 217 & 2200 & 8.64 & 8.64 & & 0.1065 & 7.55E-04 & 56.1799 & 20.7006 & 76.8805 & \\
\hline & 8/4/2008 23:00 & 322 & 217 & 2300 & 8.55 & 8.55 & & 0.1181 & 8.58E-04 & 63.8375 & 23.5222 & 87.3597 & \\
\hline & 8/5/2008 0:00 & 323 & 218 & 0 & 8.51 & 8.51 & 8.51 & 0.1234 & $9.05 \mathrm{E}-04$ & 67.3515 & 24.8170 & 92.1685 & \\
\hline & 8/5/2008 1:00 & 324 & 218 & 100 & 8.46 & 8.46 & & 0.1300 & $9.66 \mathrm{E}-04$ & 71.8403 & 26.4710 & 98.3113 & \\
\hline & 8/5/2008 2:00 & 325 & 218 & 200 & 8.43 & 8.43 & & 0.1340 & $1.00 \mathrm{E}-03$ & 74.5851 & 27.4824 & 102.0675 & \\
\hline & 8/5/2008 3:00 & 326 & 218 & 300 & 8.28 & 8.28 & & 0.1546 & 1.20E-03 & 88.8969 & 32.7559 & 121.6528 & \\
\hline & 8/5/2008 4:00 & 327 & 218 & 400 & 8.17 & 8.17 & & 0.1701 & 1.34E-03 & 100.0259 & 36.8566 & 136.8824 & \\
\hline
\end{tabular}




\begin{tabular}{|c|c|c|c|c|c|c|c|c|c|c|c|c|c|}
\hline Tree ID & TS & RN & JD & HR & $\begin{array}{c}\text { Raw } \\
\text { TC_dTA }\end{array}$ & $\begin{array}{c}\text { Fixed } \\
\text { TC_dT } \Delta\end{array}$ & dTM & $\mathbf{K}$ & $\begin{array}{l}\text { Sap } \\
\text { Velocity } \\
(\mathrm{cm} / \mathrm{s})\end{array}$ & $\begin{array}{c}\text { Probed } \\
\text { Drywood F } \\
(\mathrm{g} / \mathrm{hr})\end{array}$ & $\begin{array}{c}\text { Extrapolated } \\
\text { Wetwood F } \\
(\mathrm{g} / \mathrm{hr})\end{array}$ & $\begin{array}{c}\text { Extrapolated } \\
\text { Total Wood F } \\
(\mathrm{g} / \mathrm{hr})\end{array}$ & $\begin{array}{l}\text { Relative } \\
\text { Humidity, } \\
@ 10 \mathrm{~m}(\%)\end{array}$ \\
\hline & 8/5/2008 5:00 & 328 & 218 & 500 & 8.17 & 8.17 & & 0.1701 & 1.34E-03 & 100.0259 & 36.8566 & \begin{tabular}{|r|}
136.8824 \\
\end{tabular} & \\
\hline & 8/5/2008 6:00 & 329 & 218 & 600 & 8.21 & 8.21 & & 0.1644 & $1.29 \mathrm{E}-03$ & 95.9159 & 35.3422 & 131.2582 & \\
\hline & 8/5/2008 7:00 & 330 & 218 & 700 & 8.18 & 8.18 & & 0.1687 & $1.33 E-03$ & 98.9916 & 36.4755 & 135.4671 & \\
\hline & 8/5/2008 8:00 & 331 & 218 & 800 & 8.07 & 8.07 & & 0.1846 & 1.49E-03 & 110.6209 & 40.7606 & 151.3815 & \\
\hline & 8/5/2008 9:00 & 332 & 218 & 900 & 6.882 & 6.882 & & 0.3891 & 3.72E-03 & 276.9583 & 102.0510 & 379.0093 & \\
\hline & 8/5/2008 10:00 & 333 & 218 & 1000 & 5.239 & 5.239 & & 0.8248 & 9.39E-03 & 698.2579 & 257.2875 & 955.5453 & \\
\hline & 8/5/2008 11:00 & 334 & 218 & 1100 & 5.602 & 5.602 & & 0.7065 & 7.76E-03 & 577.1500 & 212.6628 & 789.8128 & \\
\hline & 8/5/2008 12:00 & 335 & 218 & 1200 & 5.308 & 5.308 & & 0.8011 & 9.06E-03 & 673.6196 & 248.2090 & 921.8286 & \\
\hline & 8/5/2008 13:00 & 336 & 218 & 1300 & 4.89 & 4.89 & & 0.9550 & 1.12E-02 & 836.3658 & 308.1762 & 1144.5420 & \\
\hline & 8/5/2008 14:00 & 337 & 218 & 1400 & 5.647 & 5.647 & & 0.6929 & 7.58E-03 & 563.5057 & 207.6353 & 771.1410 & \\
\hline & 8/5/2008 15:00 & 338 & 218 & 1500 & 5.951 & 5.951 & & 0.6065 & 6.43E-03 & 478.2218 & 176.2107 & 654.4325 & \\
\hline & 8/5/2008 16:00 & 339 & 218 & 1600 & 5.74 & 5.74 & & 0.6655 & $7.21 \mathrm{E}-03$ & 536.1741 & 197.5644 & 733.7385 & \\
\hline & 8/5/2008 17:00 & 340 & 218 & 1700 & 5.36 & 5.36 & & 0.7836 & 8.81E-03 & 655.5780 & 241.5612 & 897.1393 & \\
\hline & 8/5/2008 18:00 & 341 & 218 & 1800 & 5.219 & 5.219 & & 0.8318 & 9.49E-03 & 705.5527 & 259.9754 & 965.5281 & \\
\hline & 8/5/2008 19:00 & 342 & 218 & 1900 & 5.411 & 5.411 & & 0.7668 & $8.58 \mathrm{E}-03$ & 638.3077 & 235.1976 & 873.5053 & \\
\hline & 8/5/2008 20:00 & 343 & 218 & 2000 & 6.344 & 6.344 & & 0.5069 & 5.16E-03 & 383.5336 & 141.3208 & 524.8544 & \\
\hline & 8/5/2008 21:00 & 344 & 218 & 2100 & 7.905 & 7.905 & & 0.2094 & 1.74E-03 & 129.1307 & 47.5809 & 176.7116 & \\
\hline & $8 / 5 / 200822: 00$ & 345 & 218 & 2200 & 8.18 & 8.18 & & 0.1687 & 1.33E-03 & 98.9916 & 36.4755 & 135.4671 & \\
\hline & 8/5/2008 23:00 & 346 & 218 & 2300 & 8.11 & 8.11 & & 0.1788 & 1.43E-03 & 106.3275 & 39.1786 & 145.5061 & \\
\hline & 8/6/2008 0:00 & 347 & 219 & 0 & 7.982 & 7.982 & 7.98 & 0.1977 & $1.62 E-03$ & 120.3307 & 44.3383 & 164.6691 & \\
\hline & 8/6/2008 1:00 & 348 & 219 & 100 & 7.863 & 7.863 & & 0.2158 & 1.80E-03 & 134.0527 & 49.3945 & 183.4472 & \\
\hline & $8 / 6 / 2008$ 2:00 & 349 & 219 & 200 & 7.796 & 7.796 & & 0.2263 & 1.91E-03 & 142.0862 & 52.3546 & 194.4408 & \\
\hline & 8/6/2008 3:00 & 350 & 219 & 300 & 7.72 & 7.72 & & 0.2383 & 2.04E-03 & 151.4747 & 55.8140 & 207.2887 & \\
\hline & 8/6/2008 4:00 & 351 & 219 & 400 & 7.652 & 7.652 & & 0.2493 & $2.15 E-03$ & 160.1295 & 59.0030 & 219.1325 & \\
\hline & 8/6/2008 5:00 & 352 & 219 & 500 & 7.583 & 7.583 & & 0.2607 & 2.27E-03 & 169.1633 & 62.3317 & 231.4951 & \\
\hline & 8/6/2008 6:00 & 353 & 219 & 600 & 7.539 & 7.539 & & 0.2681 & 2.35E-03 & 175.0593 & 64.5042 & 239.5636 & \\
\hline & 8/6/2008 7:00 & 354 & 219 & 700 & 7.52 & 7.52 & & 0.2713 & 2.39E-03 & 177.6384 & 65.4545 & 243.0930 & \\
\hline & 8/6/2008 8:00 & 355 & 219 & 800 & 7.482 & 7.482 & & 0.2777 & $2.46 \mathrm{E}-03$ & 182.8573 & 67.3775 & 250.2348 & \\
\hline & 8/6/2008 9:00 & 356 & 219 & 900 & 7.589 & 7.589 & & 0.2597 & $2.26 \mathrm{E}-03$ & 168.3676 & 62.0385 & 230.4061 & \\
\hline & 8/6/2008 10:00 & 357 & 219 & 1000 & 5.799 & 5.799 & & 0.6486 & $6.98 \mathrm{E}-03$ & 519.4181 & 191.3903 & 710.8084 & \\
\hline & 8/6/2008 11:00 & 358 & 219 & 1100 & 4.148 & 4.148 & & 1.3047 & 1.65E-02 & 1228.0338 & 452.4943 & 1680.5281 & \\
\hline & 8/6/2008 12:00 & 359 & 219 & 1200 & 5.05 & 5.05 & & 0.8931 & 1.04E-02 & 770.0980 & 283.7585 & 1053.8565 & \\
\hline & 8/6/2008 13:00 & 360 & 219 & 1300 & 5.49 & 5.49 & & 0.7413 & 8.23E-03 & 612.3554 & 225.6349 & 837.9903 & \\
\hline & $8 / 6 / 2008$ 14:00 & 361 & 219 & 1400 & 4.906 & 4.906 & & 0.9486 & 1.12E-02 & 829.4974 & 305.6454 & 1135.1428 & \\
\hline & 8/6/2008 15:00 & 362 & 219 & 1500 & 4.517 & 4.517 & & 1.1164 & 1.36E-02 & 1013.6687 & 373.5071 & 1387.1758 & \\
\hline & 8/6/2008 16:00 & 363 & 219 & 1600 & 4.661 & 4.661 & & 1.0511 & 1.27E-02 & 941.0894 & 346.7637 & 1287.8531 & \\
\hline & 8/6/2008 17:00 & 364 & 219 & 1700 & 4.551 & 4.551 & & 1.1006 & 1.34E-02 & 996.0253 & 367.0060 & 1363.0314 & \\
\hline & 8/6/2008 18:00 & 365 & 219 & 1800 & 5.194 & 5.194 & & 0.8406 & 9.61E-03 & 714.7704 & 263.3719 & 978.1423 & \\
\hline & 8/6/2008 19:00 & 366 & 219 & 1900 & 5.149 & 5.149 & & 0.8567 & $9.84 \mathrm{E}-03$ & 731.6454 & 269.5898 & 1001.2351 & \\
\hline & $8 / 6 / 200820: 00$ & 367 & 219 & 2000 & 5.574 & 5.574 & & 0.7151 & 7.88E-03 & 585.7823 & 215.8435 & 801.6259 & \\
\hline & 8/6/2008 21:00 & 368 & 219 & 2100 & 7.018 & 7.018 & & 0.3622 & $3.41 E-03$ & 253.5647 & 93.4311 & 346.9959 & \\
\hline
\end{tabular}




\begin{tabular}{|c|c|c|c|c|c|c|c|c|c|c|c|c|c|}
\hline Tree ID & TS & RN & JD & HR & $\begin{array}{c}\text { Raw } \\
\text { TC_dTA }\end{array}$ & $\begin{array}{c}\text { Fixed } \\
\text { TC_dT } \Delta\end{array}$ & dTM & $\mathbf{K}$ & $\begin{array}{l}\text { Sap } \\
\text { Velocity } \\
(\mathrm{cm} / \mathrm{s})\end{array}$ & $\begin{array}{c}\text { Probed } \\
\text { Drywood F } \\
(\mathrm{g} / \mathrm{hr})\end{array}$ & $\begin{array}{c}\text { Extrapolated } \\
\text { Wetwood F } \\
(\mathrm{g} / \mathrm{hr})\end{array}$ & $\begin{array}{c}\text { Extrapolated } \\
\text { Total Wood F } \\
(\mathrm{g} / \mathrm{hr})\end{array}$ & $\begin{array}{l}\text { Relative } \\
\text { Humidity, } \\
@ 10 \mathrm{~m}(\%)\end{array}$ \\
\hline & 8/6/2008 22:00 & 369 & 219 & 2200 & \begin{tabular}{|r|}
7.719 \\
\end{tabular} & 7.719 & & 0.2385 & 2.04E-03 & 151.6003 & 55.8602 & 207.4605 & \\
\hline & 8/6/2008 23:00 & 370 & 219 & 2300 & 7.767 & 7.767 & & 0.2308 & 1.96E-03 & 145.6337 & 53.6617 & 199.2954 & \\
\hline & $8 / 7 / 20080: 00$ & 371 & 220 & 0 & 7.735 & 7.735 & 7.74 & 0.2359 & $2.01 \mathrm{E}-03$ & 149.5982 & 55.1225 & 204.7207 & \\
\hline & 8/7/2008 1:00 & 372 & 220 & 100 & 7.653 & 7.653 & & 0.2492 & 2.15E-03 & 160.0005 & 58.9555 & 218.9559 & \\
\hline & 8/7/2008 2:00 & 373 & 220 & 200 & 7.574 & 7.574 & & 0.2622 & 2.29E-03 & 170.3607 & 62.7729 & 233.1336 & \\
\hline & 8/7/2008 3:00 & 374 & 220 & 300 & 7.521 & 7.521 & & 0.2711 & $2.39 \mathrm{E}-03$ & 177.5022 & 65.4043 & 242.9065 & \\
\hline & 8/7/2008 4:00 & 375 & 220 & 400 & 7.462 & 7.462 & & 0.2812 & $2.50 \mathrm{E}-03$ & 185.6368 & 68.4017 & 254.0385 & \\
\hline & 8/7/2008 5:00 & 376 & 220 & 500 & 7.425 & 7.425 & & 0.2875 & 2.57E-03 & 190.8393 & 70.3187 & 261.1580 & \\
\hline & 8/7/2008 6:00 & 377 & 220 & 600 & 7.388 & 7.388 & & 0.2940 & $2.64 \mathrm{E}-03$ & 196.1211 & 72.2648 & 268.3859 & \\
\hline & 8/7/2008 7:00 & 378 & 220 & 700 & 7.353 & 7.353 & & 0.3001 & 2.70E-03 & 201.1913 & 74.1331 & 275.3244 & \\
\hline & 8/7/2008 8:00 & 379 & 220 & 800 & 7.358 & 7.358 & & 0.2993 & 2.70E-03 & 200.4625 & 73.8645 & 274.3271 & \\
\hline & 8/7/2008 9:00 & 380 & 220 & 900 & 7.123 & 7.123 & & 0.3421 & $3.18 \mathrm{E}-03$ & 236.3728 & 87.0964 & 323.4692 & \\
\hline & 8/7/2008 10:00 & 381 & 220 & 1000 & 4.836 & 4.836 & & 0.9768 & 1.16E-02 & 859.9619 & 316.8707 & 1176.8326 & \\
\hline & 8/7/2008 11:00 & 382 & 220 & 1100 & 4.118 & 4.118 & & 1.3215 & 1.68E-02 & 1247.5164 & 459.6731 & 1707.1894 & \\
\hline & 8/7/2008 12:00 & 383 & 220 & 1200 & 4.232 & 4.232 & & 1.2590 & 1.58E-02 & 1175.2472 & 433.0440 & 1608.2912 & \\
\hline & 8/7/2008 13:00 & 384 & 220 & 1300 & 4.138 & 4.138 & & 1.3103 & 1.66E-02 & 1234.4902 & 454.8733 & 1689.3635 & \\
\hline & $8 / 7 / 2008$ 14:00 & 385 & 220 & 1400 & 4.241 & 4.241 & & 1.2542 & 1.57E-02 & 1169.7409 & 431.0151 & 1600.7559 & \\
\hline & 8/7/2008 15:00 & 386 & 220 & 1500 & 4.375 & 4.375 & & 1.1851 & 1.47E-02 & 1090.9833 & 401.9953 & 1492.9786 & \\
\hline & 8/7/2008 16:00 & 387 & 220 & 1600 & 4.353 & 4.353 & & 1.1962 & $1.48 \mathrm{E}-02$ & 1103.5114 & 406.6115 & 1510.1229 & \\
\hline & 8/7/2008 17:00 & 388 & 220 & 1700 & 4.321 & 4.321 & & 1.2125 & $1.51 E-02$ & 1122.0105 & 413.4279 & 1535.4384 & \\
\hline & 8/7/2008 18:00 & 389 & 220 & 1800 & 4.51 & 4.51 & & 1.1197 & 1.37E-02 & 1017.3415 & 374.8604 & 1392.2019 & \\
\hline & 8/7/2008 19:00 & 390 & 220 & 1900 & 4.751 & 4.751 & & 1.0122 & 1.21E-02 & 898.4490 & 331.0520 & 1229.5010 & \\
\hline & 8/7/2008 20:00 & 391 & 220 & 2000 & 5.311 & 5.311 & & 0.8000 & 9.04E-03 & 672.5666 & 247.8210 & 920.3877 & \\
\hline & 8/7/2008 21:00 & 392 & 220 & 2100 & 6.74 & 6.74 & & 0.4184 & 4.07E-03 & 302.8187 & 111.5798 & 414.3985 & \\
\hline & 8/7/2008 22:00 & 393 & 220 & 2200 & 7.427 & 7.427 & & 0.2872 & $2.56 \mathrm{E}-03$ & 190.5561 & 70.2143 & 260.7704 & \\
\hline & 8/7/2008 23:00 & 394 & 220 & 2300 & 7.62 & 7.62 & & 0.2546 & 2.21E-03 & 164.2872 & 60.5350 & 224.8222 & \\
\hline & 8/8/2008 0:00 & 395 & 221 & 0 & 7.697 & 7.697 & 7.73 & 0.2420 & 2.08E-03 & 154.3749 & 56.8826 & 211.2575 & \\
\hline & 8/8/2008 1:00 & 396 & 221 & 100 & 7.717 & 7.717 & & 0.2388 & $2.04 \mathrm{E}-03$ & 151.8515 & 55.9528 & 207.8043 & \\
\hline & 8/8/2008 2:00 & 397 & 221 & 200 & 7.731 & 7.731 & & 0.2366 & 2.02E-03 & 150.0975 & 55.3065 & 205.4040 & \\
\hline & 8/8/2008 3:00 & 398 & 221 & 300 & 7.618 & 7.618 & & 0.2549 & $2.21 \mathrm{E}-03$ & 164.5489 & 60.6314 & 225.1803 & \\
\hline & 8/8/2008 4:00 & 399 & 221 & 400 & 7.539 & 7.539 & & 0.2681 & 2.35E-03 & 175.0593 & 64.5042 & 239.5636 & \\
\hline & 8/8/2008 5:00 & 400 & 221 & 500 & 7.48 & 7.48 & & 0.2781 & 2.46E-03 & 183.1342 & 67.4796 & 250.6138 & \\
\hline & 8/8/2008 6:00 & 401 & 221 & 600 & 7.424 & 7.424 & & 0.2877 & 2.57E-03 & 190.9810 & 70.3709 & 261.3519 & \\
\hline & 8/8/2008 7:00 & 402 & 221 & 700 & 7.392 & 7.392 & & 0.2933 & 2.63E-03 & 195.5462 & 72.0530 & 267.5992 & \\
\hline & 8/8/2008 8:00 & 403 & 221 & 800 & 7.38 & 7.38 & & 0.2954 & 2.65E-03 & 197.2736 & 72.6895 & 269.9631 & \\
\hline & 8/8/20089:00 & 404 & 221 & 900 & 6.142 & 6.142 & & 0.5565 & 5.78E-03 & 430.2000 & 158.5160 & 588.7160 & \\
\hline & 8/8/2008 10:00 & 405 & 221 & 1000 & 4.178 & 4.178 & & 1.2882 & 1.63E-02 & 1208.8876 & 445.4395 & 1654.3271 & \\
\hline & 8/8/2008 11:00 & 406 & 221 & 1100 & 4.192 & 4.192 & & 1.2805 & 1.61E-02 & 1200.0656 & 442.1889 & 1642.2545 & \\
\hline & $8 / 8 / 200812: 00$ & 407 & 221 & 1200 & 4.154 & 4.154 & & 1.3014 & $1.65 \mathrm{E}-02$ & 1224.1779 & 451.0735 & 1675.2514 & \\
\hline & 8/8/2008 13:00 & 408 & 221 & 1300 & 4.019 & 4.019 & & 1.3787 & 1.77E-02 & 1314.2989 & 484.2804 & 1798.5793 & \\
\hline & 8/8/2008 14:00 & 409 & 221 & 1400 & 4.079 & 4.079 & & 1.3437 & $1.71 \mathrm{E}-02$ & 1273.3600 & 469.1957 & 1742.5556 & \\
\hline
\end{tabular}




\begin{tabular}{|c|c|c|c|c|c|c|c|c|c|c|c|c|c|}
\hline Tree ID & TS & RN & JD & HR & $\begin{array}{c}\text { Raw } \\
\text { TC_dT } \Delta\end{array}$ & $\begin{array}{c}\text { Fixed } \\
\text { TC_dT } \Delta\end{array}$ & dTM & K & $\begin{array}{l}\text { Sap } \\
\text { Velocity } \\
\text { (cm/s) }\end{array}$ & $\begin{array}{c}\text { Probed } \\
\text { Drywood F } \\
\text { (g/hr) }\end{array}$ & $\begin{array}{c}\text { Extrapolated } \\
\text { Wetwood F } \\
(\mathrm{g} / \mathrm{hr})\end{array}$ & $\begin{array}{c}\text { Extrapolated } \\
\text { Total Wood F } \\
(\mathrm{g} / \mathrm{hr})\end{array}$ & $\begin{array}{l}\text { Relative } \\
\text { Humidity, } \\
@ 10 \mathrm{~m}(\%)\end{array}$ \\
\hline & 8/8/2008 15:00 & 410 & 221 & 1500 & 4.162 & 4.162 & & 1.2970 & 1.64E-02 & 1219.0575 & 449.1868 & 1668.2444 & \\
\hline & $8 / 8 / 2008$ 16:00 & 411 & 221 & 1600 & 4.166 & 4.166 & & 1.2948 & $1.64 \mathrm{E}-02$ & 1216.5062 & 448.2467 & 1664.7530 & \\
\hline & 8/8/2008 17:00 & 412 & 221 & 1700 & 4.29 & 4.29 & & 1.2284 & $1.53 \mathrm{E}-02$ & 1140.2506 & 420.1488 & 1560.3994 & \\
\hline & 8/8/2008 18:00 & 413 & 221 & 1800 & 4.694 & 4.694 & & 1.0366 & $1.24 \mathrm{E}-02$ & 925.2215 & 340.9169 & 1266.1384 & \\
\hline & 8/8/2008 19:00 & 414 & 221 & 1900 & 4.588 & 4.588 & & 1.0837 & 1.31E-02 & 977.1872 & 360.0647 & 1337.2519 & \\
\hline & 8/8/2008 20:00 & 415 & 221 & 2000 & 5.283 & 5.283 & & 0.8096 & $9.18 E-03$ & 682.4530 & 251.4639 & 933.9169 & \\
\hline & 8/8/2008 21:00 & 416 & 221 & 2100 & 6.581 & 6.581 & & 0.4527 & 4.49E-03 & 333.6337 & 122.9342 & 456.5678 & \\
\hline & $8 / 8 / 200822: 00$ & 417 & 221 & 2200 & 7.176 & 7.176 & & 0.3322 & 3.06E-03 & 227.9707 & 84.0005 & 311.9712 & \\
\hline & 8/8/2008 23:00 & 418 & 221 & 2300 & 7.346 & 7.346 & & 0.3014 & $2.72 \mathrm{E}-03$ & 202.2141 & 74.5099 & 276.7240 & \\
\hline & 8/9/2008 0:00 & 419 & 222 & 0 & 7.332 & 7.332 & 7.41 & 0.3039 & $2.75 \mathrm{E}-03$ & 204.2684 & 75.2669 & 279.5353 & \\
\hline & 8/9/2008 1:00 & 420 & 222 & 100 & 7.301 & 7.301 & & 0.3094 & 2.81E-03 & 208.8592 & 76.9585 & 285.8177 & \\
\hline & $8 / 9 / 2008$ 2:00 & 421 & 222 & 200 & 7.352 & 7.352 & & 0.3003 & $2.71 E-03$ & 201.3372 & 74.1868 & 275.5241 & \\
\hline & 8/9/2008 3:00 & 422 & 222 & 300 & 7.37 & 7.37 & & 0.2972 & 2.67E-03 & 198.7196 & 73.2223 & 271.9419 & \\
\hline & 8/9/2008 4:00 & 423 & 222 & 400 & 7.349 & 7.349 & & 0.3009 & 2.71E-03 & 201.7754 & 74.3483 & 276.1237 & \\
\hline & $8 / 9 / 20085: 00$ & 424 & 222 & 500 & 7.229 & 7.229 & & 0.3225 & 2.95E-03 & 219.7484 & 80.9708 & 300.7192 & \\
\hline & 8/9/2008 6:00 & 425 & 222 & 600 & 7.199 & 7.199 & & 0.3280 & 3.02E-03 & 224.3807 & 82.6777 & 307.0584 & \\
\hline & 8/9/2008 7:00 & 426 & 222 & 700 & 6.837 & 6.837 & & 0.3983 & 3.83E-03 & 284.9906 & 105.0106 & 390.0012 & \\
\hline & $8 / 9 / 20088: 00$ & 427 & 222 & 800 & 6.168 & 6.168 & & 0.5499 & 5.70E-03 & 423.9648 & 156.2186 & 580.1834 & \\
\hline & 8/9/2008 9:00 & 428 & 222 & 900 & 6.761 & 6.761 & & 0.4140 & 4.02E-03 & 298.8983 & 110.1352 & 409.0336 & \\
\hline & 8/9/2008 10:00 & 429 & 222 & 1000 & 6.579 & 6.579 & & 0.4531 & 4.49E-03 & 334.0344 & 123.0818 & 457.1162 & \\
\hline & 8/9/2008 11:00 & 430 & 222 & 1100 & 5.894 & 5.894 & & 0.6220 & $6.63 \mathrm{E}-03$ & 493.3469 & 181.7838 & 675.1308 & \\
\hline & 8/9/2008 12:00 & 431 & 222 & 1200 & 4.977 & 4.977 & & 0.9208 & 1.08E-02 & 799.6772 & 294.6575 & 1094.3347 & \\
\hline & 8/9/2008 13:00 & 432 & 222 & 1300 & 4.665 & 4.665 & & 1.0493 & $1.26 \mathrm{E}-02$ & 939.1513 & 346.0496 & 1285.2009 & \\
\hline & 8/9/2008 14:00 & 433 & 222 & 1400 & 4.137 & 4.137 & & 1.3109 & 1.66E-02 & 1235.1379 & 455.1120 & 1690.2499 & \\
\hline & 8/9/2008 15:00 & 434 & 222 & 1500 & 4.084 & 4.084 & & 1.3408 & 1.71E-02 & 1270.0135 & 467.9626 & 1737.9761 & \\
\hline & $8 / 9 / 2008$ 16:00 & 435 & 222 & 1600 & 4.144 & 4.144 & & 1.3069 & 1.65E-02 & 1230.6119 & 453.4443 & 1684.0561 & \\
\hline & 8/9/2008 17:00 & 436 & 222 & 1700 & 4.216 & 4.216 & & 1.2676 & 1.59E-02 & 1185.1064 & 436.6768 & 1621.7832 & \\
\hline & $8 / 9 / 200818: 00$ & 437 & 222 & 1800 & 4.293 & 4.293 & & 1.2269 & 1.53E-02 & 1138.4715 & 419.4932 & 1557.9648 & \\
\hline & 8/9/2008 19:00 & 438 & 222 & 1900 & 4.478 & 4.478 & & 1.1349 & 1.39E-02 & 1034.3096 & 381.1126 & 1415.4222 & \\
\hline & $8 / 9 / 200820: 00$ & 439 & 222 & 2000 & 5.183 & 5.183 & & 0.8445 & 9.66E-03 & 718.8615 & 264.8793 & 983.7408 & \\
\hline & 8/9/2008 21:00 & 440 & 222 & 2100 & 6.819 & 6.819 & & 0.4020 & 3.88E-03 & 288.2453 & 106.2099 & 394.4552 & \\
\hline & 8/9/2008 22:00 & 441 & 222 & 2200 & 7.404 & 7.404 & & 0.2912 & 2.61E-03 & 193.8273 & 71.4196 & 265.2469 & \\
\hline & 8/9/2008 23:00 & 442 & 222 & 2300 & 7.409 & 7.409 & & 0.2903 & $2.60 \mathrm{E}-03$ & 193.1135 & 71.1566 & 264.2702 & \\
\hline & 8/10/2008 0:00 & 443 & 223 & 0 & 7.373 & 7.373 & 7.37 & 0.2966 & 2.67E-03 & 198.2851 & 73.0622 & 271.3474 & \\
\hline & 8/10/2008 1:00 & 444 & 223 & 100 & 7.344 & 7.344 & & 0.3017 & 2.72E-03 & 202.5068 & 74.6178 & 277.1246 & \\
\hline & 8/10/2008 2:00 & 445 & 223 & 200 & 7.297 & 7.297 & & 0.3101 & 2.82E-03 & 209.4558 & 77.1783 & 286.6341 & \\
\hline & 8/10/2008 3:00 & 446 & 223 & 300 & 7.26 & 7.26 & & 0.3168 & 2.89E-03 & 215.0207 & 79.2288 & 294.2496 & \\
\hline & $8 / 10 / 20084: 00$ & 447 & 223 & 400 & 7.239 & 7.239 & & 0.3206 & $2.93 \mathrm{E}-03$ & 218.2168 & 80.4065 & 298.6232 & \\
\hline & 8/10/2008 5:00 & 448 & 223 & 500 & 7.192 & 7.192 & & 0.3293 & 3.03E-03 & 225.4698 & 83.0790 & 308.5487 & \\
\hline & 8/10/2008 6:00 & 449 & 223 & 600 & 7.163 & 7.163 & & 0.3346 & 3.09E-03 & 230.0148 & 84.7537 & 314.7685 & \\
\hline & $8 / 10 / 2008$ 7:00 & 450 & 223 & 700 & 7.154 & 7.154 & & 0.3363 & 3.11E-03 & 231.4363 & 85.2775 & 316.7138 & \\
\hline
\end{tabular}




\begin{tabular}{|c|c|c|c|c|c|c|c|c|c|c|c|c|c|}
\hline Tree ID & TS & RN & JD & HR & $\begin{array}{c}\text { Raw } \\
\text { TC_dTA }\end{array}$ & $\begin{array}{c}\text { Fixed } \\
\text { TC_dT } \Delta\end{array}$ & dTM & $\mathbf{K}$ & $\begin{array}{l}\text { Sap } \\
\text { Velocity } \\
(\mathrm{cm} / \mathrm{s})\end{array}$ & $\begin{array}{c}\text { Probed } \\
\text { Drywood F } \\
(\mathrm{g} / \mathrm{hr})\end{array}$ & $\begin{array}{c}\text { Extrapolated } \\
\text { Wetwood F } \\
(\mathrm{g} / \mathrm{hr})\end{array}$ & $\begin{array}{c}\text { Extrapolated } \\
\text { Total Wood F } \\
(\mathrm{g} / \mathrm{hr})\end{array}$ & $\begin{array}{l}\text { Relative } \\
\text { Humidity, } \\
@ 10 \mathrm{~m}(\%)\end{array}$ \\
\hline & 8/10/2008 8:00 & 451 & 223 & 800 & 7.165 & 7.165 & & 0.3343 & 3.09E-03 & 229.6997 & 84.6376 & $\begin{array}{r}314.3372 \\
\end{array}$ & \\
\hline & 8/10/2008 9:00 & 452 & 223 & 900 & 7.026 & 7.026 & & 0.3607 & 3.39E-03 & 252.2288 & 92.9389 & 345.1676 & \\
\hline & $8 / 10 / 2008$ 10:00 & 453 & 223 & 1000 & 4.909 & 4.909 & & 0.9474 & $1.11 \mathrm{E}-02$ & 828.2158 & 305.1731 & 1133.3889 & \\
\hline & 8/10/2008 11:00 & 454 & 223 & 1100 & 3.902 & 3.902 & & 1.4500 & $1.88 \mathrm{E}-02$ & 1398.4915 & 515.3030 & 1913.7945 & \\
\hline & $8 / 10 / 200812: 00$ & 455 & 223 & 1200 & 4.169 & 4.169 & & 1.2931 & 1.63E-02 & 1214.5966 & 447.5431 & 1662.1397 & \\
\hline & 8/10/2008 13:00 & 456 & 223 & 1300 & 3.993 & 3.993 & & 1.3942 & 1.79E-02 & 1332.4983 & 490.9864 & 1823.4847 & \\
\hline & $8 / 10 / 2008$ 14:00 & 457 & 223 & 1400 & 4.059 & 4.059 & & 1.3553 & 1.73E-02 & 1286.8449 & 474.1645 & 1761.0093 & \\
\hline & $8 / 10 / 2008$ 15:00 & 458 & 223 & 1500 & 4.245 & 4.245 & & 1.2521 & 1.57E-02 & 1167.3026 & 430.1167 & 1597.4193 & \\
\hline & 8/10/2008 16:00 & 459 & 223 & 1600 & 4.041 & 4.041 & & 1.3658 & 1.75E-02 & 1299.1185 & 478.6869 & 1777.8054 & \\
\hline & 8/10/2008 17:00 & 460 & 223 & 1700 & 4.242 & 4.242 & & 1.2537 & 1.57E-02 & 1169.1308 & 430.7903 & 1599.9211 & \\
\hline & 8/10/2008 18:00 & 461 & 223 & 1800 & 6.601 & 6.601 & & 0.4483 & 4.43E-03 & 329.6448 & 121.4644 & 451.1092 & \\
\hline & $8 / 10 / 2008$ 19:00 & 462 & 223 & 1900 & 7.144 & 7.144 & & 0.3382 & $3.13 E-03$ & 233.0219 & 85.8617 & 318.8836 & \\
\hline & 8/10/2008 20:00 & 463 & 223 & 2000 & 7.047 & 7.047 & & 0.3566 & 3.34E-03 & 248.7425 & 91.6543 & 340.3969 & \\
\hline & 8/10/2008 21:00 & 464 & 223 & 2100 & 6.993 & 6.993 & & 0.3671 & 3.47E-03 & 257.7679 & 94.9799 & 352.7478 & \\
\hline & $8 / 10 / 2008$ 22:00 & 465 & 223 & 2200 & 7.086 & 7.086 & & 0.3491 & $3.26 \mathrm{E}-03$ & 242.3470 & 89.2977 & 331.6447 & \\
\hline & 8/10/2008 23:00 & 466 & 223 & 2300 & 7.027 & 7.027 & & 0.3605 & $3.39 \mathrm{E}-03$ & 252.0621 & 92.8775 & 344.9395 & \\
\hline & $8 / 11 / 20080: 00$ & 467 & 224 & 0 & 7.057 & 7.057 & 7.43 & 0.3547 & 3.32E-03 & 247.0929 & 91.0465 & 338.1394 & \\
\hline & 8/11/2008 1:00 & 468 & 224 & 100 & 7.238 & 7.238 & & 0.3208 & 2.94E-03 & 218.3697 & 80.4628 & 298.8325 & \\
\hline & 8/11/2008 2:00 & 469 & 224 & 200 & 7.392 & 7.392 & & 0.2933 & 2.63E-03 & 195.5462 & 72.0530 & 267.5992 & \\
\hline & 8/11/2008 3:00 & 470 & 224 & 300 & 7.42 & 7.42 & & 0.2884 & $2.58 \mathrm{E}-03$ & 191.5484 & 70.5799 & 262.1283 & \\
\hline & 8/11/2008 4:00 & 471 & 224 & 400 & 7.432 & 7.432 & & 0.2863 & $2.55 \mathrm{E}-03$ & 189.8490 & 69.9538 & 259.8028 & \\
\hline & 8/11/2008 5:00 & 472 & 224 & 500 & 7.404 & 7.404 & & 0.2912 & 2.61E-03 & 193.8273 & 71.4196 & 265.2469 & \\
\hline & 8/11/2008 6:00 & 473 & 224 & 600 & 7.397 & 7.397 & & 0.2924 & $2.62 E-03$ & 194.8290 & 71.7887 & 266.6177 & \\
\hline & 8/11/2008 7:00 & 474 & 224 & 700 & 7.414 & 7.414 & & 0.2895 & $2.59 \mathrm{E}-03$ & 192.4012 & 70.8942 & 263.2954 & \\
\hline & 8/11/2008 8:00 & 475 & 224 & 800 & 7.421 & 7.421 & & 0.2882 & 2.57E-03 & 191.4065 & 70.5276 & 261.9341 & \\
\hline & 8/11/2008 9:00 & 476 & 224 & 900 & 6.893 & 6.893 & & 0.3869 & 3.70E-03 & 275.0174 & 101.3358 & 376.3532 & \\
\hline & 8/11/2008 10:00 & 477 & 224 & 1000 & 4.955 & 4.955 & & 0.9294 & 1.09E-02 & 808.8041 & 298.0205 & 1106.8245 & \\
\hline & 8/11/2008 11:00 & 478 & 224 & 1100 & 4.077 & 4.077 & & 1.3449 & 1.71E-02 & 1274.7013 & 469.6899 & 1744.3912 & \\
\hline & $8 / 11 / 2008$ 12:00 & 479 & 224 & 1200 & 4.171 & 4.171 & & 1.2920 & 1.63E-02 & 1213.3254 & 447.0747 & 1660.4001 & \\
\hline & 8/11/2008 13:00 & 480 & 224 & 1300 & 4.509 & 4.509 & & 1.1202 & 1.37E-02 & 1017.8673 & 375.0541 & 1392.9214 & \\
\hline & 8/11/2008 14:00 & 481 & 224 & 1400 & 4.165 & 4.165 & & 1.2953 & 1.64E-02 & 1217.1435 & 448.4816 & 1665.6251 & \\
\hline & 8/11/2008 15:00 & 482 & 224 & 1500 & 4.111 & 4.111 & & 1.3255 & 1.68E-02 & 1252.1116 & 461.3663 & 1713.4778 & \\
\hline & $8 / 11 / 2008$ 16:00 & 483 & 224 & 1600 & 4.339 & 4.339 & & 1.2033 & 1.49E-02 & 1111.5641 & 409.5787 & 1521.1428 & \\
\hline & $8 / 11 / 2008$ 17:00 & 484 & 224 & 1700 & 4.389 & 4.389 & & 1.1782 & $1.46 \mathrm{E}-02$ & 1083.0902 & 399.0868 & 1482.1770 & \\
\hline & 8/11/2008 18:00 & 485 & 224 & 1800 & 4.713 & 4.713 & & 1.0284 & 1.23E-02 & 916.2089 & 337.5960 & 1253.8050 & \\
\hline & $8 / 11 / 2008$ 19:00 & 486 & 224 & 1900 & 4.868 & 4.868 & & 0.9638 & 1.14E-02 & 845.9010 & 311.6896 & 1157.5906 & \\
\hline & 8/11/2008 20:00 & 487 & 224 & 2000 & 5.613 & 5.613 & & 0.7032 & 7.71E-03 & 573.7889 & 211.4243 & 785.2132 & \\
\hline & 8/11/2008 21:00 & 488 & 224 & 2100 & 6.46 & 6.46 & & 0.4799 & 4.82E-03 & 358.4895 & 132.0928 & 490.5823 & \\
\hline & $8 / 11 / 200822: 00$ & 489 & 224 & 2200 & 6.959 & 6.959 & & 0.3738 & $3.54 \mathrm{E}-03$ & 263.5536 & 97.1118 & 360.6654 & \\
\hline & 8/11/2008 23:00 & 490 & 224 & 2300 & 7.19 & 7.19 & & 0.3296 & 3.04E-03 & 225.7815 & 83.1938 & 308.9753 & \\
\hline & 8/12/2008 0:00 & 491 & 225 & 0 & 7.326 & 7.326 & 7.34 & 0.3049 & $2.76 \mathrm{E}-03$ & 205.1524 & 75.5926 & 280.7450 & \\
\hline
\end{tabular}




\begin{tabular}{|c|c|c|c|c|c|c|c|c|c|c|c|c|c|}
\hline Tree ID & TS & RN & JD & HR & $\begin{array}{c}\text { Raw } \\
\text { TC_dTA }\end{array}$ & $\begin{array}{c}\text { Fixed } \\
\text { TC_dT } \Delta\end{array}$ & dTM & $\mathbf{K}$ & $\begin{array}{l}\text { Sap } \\
\text { Velocity } \\
(\mathrm{cm} / \mathrm{s})\end{array}$ & $\begin{array}{c}\text { Probed } \\
\text { Drywood F } \\
(\mathrm{g} / \mathrm{hr})\end{array}$ & $\begin{array}{c}\text { Extrapolated } \\
\text { Wetwood F } \\
(\mathrm{g} / \mathrm{hr})\end{array}$ & $\begin{array}{c}\text { Extrapolated } \\
\text { Total Wood F } \\
(\mathrm{g} / \mathrm{hr})\end{array}$ & $\begin{array}{c}\text { Relative } \\
\text { Humidity, } \\
\text { @10m (\%) }\end{array}$ \\
\hline & 8/12/2008 1:00 & 492 & 225 & 100 & $\begin{array}{l}7.339 \\
\end{array}$ & 7.339 & & 0.3026 & $2.73 \mathrm{E}-03$ & 203.2397 & 74.8879 & 278.1276 & \\
\hline & $8 / 12 / 20082: 00$ & 493 & 225 & 200 & 6.888 & 6.888 & & 0.3879 & 3.71E-03 & 275.8985 & 101.6605 & 377.5590 & \\
\hline & 8/12/2008 3:00 & 494 & 225 & 300 & 6.634 & 6.634 & & 0.4411 & 4.34E-03 & 323.1354 & 119.0659 & 442.2012 & \\
\hline & $8 / 12 / 2008$ 4:00 & 495 & 225 & 400 & 6.673 & 6.673 & & 0.4326 & $4.24 \mathrm{E}-03$ & 315.5565 & 116.2733 & 431.8297 & \\
\hline & 8/12/2008 5:00 & 496 & 225 & 500 & 6.838 & 6.838 & & 0.3981 & 3.83E-03 & 284.8105 & 104.9443 & 389.7547 & \\
\hline & 8/12/2008 6:00 & 497 & 225 & 600 & 6.959 & 6.959 & & 0.3738 & $3.54 \mathrm{E}-03$ & 263.5536 & 97.1118 & 360.6654 & \\
\hline & $8 / 12 / 20087: 00$ & 498 & 225 & 700 & 7.085 & 7.085 & & 0.3493 & $3.26 \mathrm{E}-03$ & 242.5097 & 89.3577 & 331.8674 & \\
\hline & 8/12/2008 8:00 & 499 & 225 & 800 & 7.072 & 7.072 & & 0.3518 & $3.29 \mathrm{E}-03$ & 244.6311 & 90.1394 & 334.7705 & \\
\hline & 8/12/2008 9:00 & 500 & 225 & 900 & 6.727 & 6.727 & & 0.4211 & 4.10E-03 & 305.2627 & 112.4803 & 417.7430 & \\
\hline & $8 / 12 / 2008$ 10:00 & 501 & 225 & 1000 & 5.001 & 5.001 & & 0.9116 & 1.06E-02 & 789.8341 & 291.0306 & 1080.8647 & \\
\hline & 8/12/2008 11:00 & 502 & 225 & 1100 & 4.138 & 4.138 & & 1.3103 & 1.66E-02 & 1234.4902 & 454.8733 & 1689.3635 & \\
\hline & $8 / 12 / 2008$ 12:00 & 503 & 225 & 1200 & 4.206 & 4.206 & & 1.2729 & 1.60E-02 & 1191.3144 & 438.9643 & 1630.2787 & \\
\hline & 8/12/2008 13:00 & 504 & 225 & 1300 & 3.948 & 3.948 & & 1.4215 & 1.83E-02 & 1364.6773 & 502.8434 & 1867.5207 & \\
\hline & $8 / 12 / 200814: 00$ & 505 & 225 & 1400 & 4.038 & 4.038 & & 1.3675 & 1.75E-02 & 1301.1768 & 479.4454 & 1780.6222 & \\
\hline & $8 / 12 / 2008$ 15:00 & 506 & 225 & 1500 & 4.104 & 4.104 & & 1.3294 & 1.69E-02 & 1256.7256 & 463.0664 & 1719.7920 & \\
\hline & $8 / 12 / 2008$ 16:00 & 507 & 225 & 1600 & 4.072 & 4.072 & & 1.3477 & 1.72E-02 & 1278.0616 & 470.9281 & 1748.9897 & \\
\hline & $8 / 12 / 200817: 00$ & 508 & 225 & 1700 & 4.022 & 4.022 & & 1.3769 & 1.76E-02 & 1312.2171 & 483.5134 & 1795.7304 & \\
\hline & $8 / 12 / 200818: 00$ & 509 & 225 & 1800 & 4.204 & 4.204 & & 1.2740 & 1.60E-02 & 1192.5603 & 439.4234 & 1631.9836 & \\
\hline & 8/12/2008 19:00 & 510 & 225 & 1900 & 4.389 & 4.389 & & 1.1782 & 1.46E-02 & 1083.0902 & 399.0868 & 1482.1770 & \\
\hline & $8 / 12 / 200820: 00$ & 511 & 225 & 2000 & 5.087 & 5.087 & & 0.8793 & 1.02E-02 & 755.5083 & 278.3826 & 1033.8908 & \\
\hline & $8 / 12 / 200821: 00$ & 512 & 225 & 2100 & 6.65 & 6.65 & & 0.4376 & $4.30 \mathrm{E}-03$ & 320.0112 & 117.9147 & 437.9259 & \\
\hline & $8 / 12 / 200822: 00$ & 513 & 225 & 2200 & 7.178 & 7.178 & & 0.3318 & 3.06E-03 & 227.6572 & 83.8850 & 311.5422 & \\
\hline & $8 / 12 / 2008$ 23:00 & 514 & 225 & 2300 & 7.203 & 7.203 & & 0.3272 & 3.01E-03 & 223.7598 & 82.4489 & 306.2087 & \\
\hline & $8 / 13 / 20080: 00$ & 515 & 226 & 0 & 7.162 & 7.162 & 7.37 & 0.3348 & 3.09E-03 & 230.1725 & 84.8118 & 314.9843 & \\
\hline & 8/13/2008 1:00 & 516 & 226 & 100 & 7.176 & 7.176 & & 0.3322 & $3.06 \mathrm{E}-03$ & 227.9707 & 84.0005 & 311.9712 & \\
\hline & 8/13/2008 2:00 & 517 & 226 & 200 & 7.249 & 7.249 & & 0.3188 & 2.91E-03 & 216.6914 & 79.8444 & 296.5359 & \\
\hline & 8/13/2008 3:00 & 518 & 226 & 300 & 7.297 & 7.297 & & 0.3101 & $2.82 \mathrm{E}-03$ & 209.4558 & 77.1783 & 286.6341 & \\
\hline & 8/13/2008 4:00 & 519 & 226 & 400 & 7.306 & 7.306 & & 0.3085 & 2.80E-03 & 208.1148 & 76.6842 & 284.7990 & \\
\hline & 8/13/2008 5:00 & 520 & 226 & 500 & 7.37 & 7.37 & & 0.2972 & 2.67E-03 & 198.7196 & 73.2223 & 271.9419 & \\
\hline & 8/13/2008 6:00 & 521 & 226 & 600 & 7.296 & 7.296 & & 0.3103 & $2.82 \mathrm{E}-03$ & 209.6051 & 77.2333 & 286.8384 & \\
\hline & 8/13/2008 7:00 & 522 & 226 & 700 & 7.236 & 7.236 & & 0.3212 & $2.94 \mathrm{E}-03$ & 218.6756 & 80.5755 & 299.2511 & \\
\hline & 8/13/2008 8:00 & 523 & 226 & 800 & 7.238 & 7.238 & & 0.3208 & 2.94E-03 & 218.3697 & 80.4628 & 298.8325 & \\
\hline & 8/13/2008 9:00 & 524 & 226 & 900 & 6.264 & 6.264 & & 0.5262 & 5.40E-03 & 401.5357 & 147.9541 & 549.4897 & \\
\hline & $8 / 13 / 200810: 00$ & 525 & 226 & 1000 & 4.506 & 4.506 & & 1.1216 & 1.37E-02 & 1019.4464 & 375.6360 & 1395.0824 & \\
\hline & 8/13/2008 11:00 & 526 & 226 & 1100 & 4.469 & 4.469 & & 1.1392 & 1.40E-02 & 1039.1352 & 382.8907 & 1422.0259 & \\
\hline & 8/13/2008 12:00 & 527 & 226 & 1200 & 4.024 & 4.024 & & 1.3757 & 1.76E-02 & 1310.8313 & 483.0027 & 1793.8340 & \\
\hline
\end{tabular}




\begin{tabular}{|c|c|c|c|c|c|c|c|c|c|c|c|c|c|}
\hline Tree ID & TS & RN & JD & HR & $\begin{array}{c}\text { Raw } \\
\text { TC_dTA }\end{array}$ & $\begin{array}{c}\text { Fixed } \\
\text { TC_dT } \Delta\end{array}$ & dTM & $\mathbf{K}$ & $\begin{array}{l}\text { Sap } \\
\text { Velocity } \\
(\mathrm{cm} / \mathrm{s})\end{array}$ & $\begin{array}{c}\text { Probed } \\
\text { Drywood F } \\
(\mathrm{g} / \mathrm{hr})\end{array}$ & $\begin{array}{c}\text { Extrapolated } \\
\text { Wetwood F } \\
(\mathrm{g} / \mathrm{hr})\end{array}$ & $\begin{array}{c}\text { Extrapolated } \\
\text { Total Wood F } \\
(\mathrm{g} / \mathrm{hr})\end{array}$ & $\begin{array}{l}\text { Relative } \\
\text { Humidity, } \\
@ 10 \mathrm{~m}(\%)\end{array}$ \\
\hline & 7/22/2008 13:00 & 0 & 204 & 1300 & 5.484 & 5.484 & 9.80 & 0.7870 & 8.86E-03 & 1687.3401 & 621.7352 & 2309.0753 & \\
\hline W608EC2 & $7 / 22 / 2008$ 14:00 & 1 & 204 & 1400 & 5.982 & 5.982 & & 0.6382 & $6.85 \mathrm{E}-03$ & 1303.7324 & 480.3870 & 1784.1193 & \\
\hline $24 " \mathrm{~N}$ & $7 / 22 / 2008$ 15:00 & 2 & 204 & 1500 & 5.902 & 5.902 & & 0.6605 & $7.14 \mathrm{E}-03$ & 1359.7925 & 501.0435 & 1860.8359 & \\
\hline \multirow[t]{2}{*}{ Probe \# } & 7/22/2008 16:00 & 3 & 204 & 1600 & 5.914 & 5.914 & & 0.6571 & $7.10 \mathrm{E}-03$ & 1351.2583 & 497.8989 & 1849.1573 & \\
\hline & 7/22/2008 17:00 & 4 & 204 & 1700 & 5.968 & 5.968 & & 0.6421 & $6.90 \mathrm{E}-03$ & 1313.4026 & 483.9502 & 1797.3528 & \\
\hline \multirow[t]{4}{*}{4} & $7 / 22 / 2008$ 18:00 & 5 & 204 & 1800 & 5.903 & 5.903 & & 0.6602 & 7.14E-03 & 1359.0796 & 500.7808 & 1859.8604 & \\
\hline & 7/22/2008 19:00 & 6 & 204 & 1900 & 6.182 & 6.182 & & 0.5852 & $6.15 E-03$ & 1171.7672 & 431.7617 & 1603.5290 & \\
\hline & 7/22/2008 20:00 & 7 & 204 & 2000 & 6.594 & 6.594 & & 0.4862 & $4.90 \mathrm{E}-03$ & 932.6423 & 343.6512 & 1276.2935 & \\
\hline & 7/22/2008 21:00 & 8 & 204 & 2100 & 8.06 & 8.06 & & 0.2159 & $1.80 \mathrm{E}-03$ & 343.2923 & 126.4931 & 469.7854 & \\
\hline \multirow[t]{2}{*}{$\mathrm{DBH}(\mathrm{cm})$} & $7 / 22 / 2008$ 22:00 & 9 & 204 & 2200 & 9.41 & 9.41 & & 0.0414 & $2.36 \mathrm{E}-04$ & 45.0151 & 16.5867 & 61.6018 & \\
\hline & 7/22/2008 23:00 & 10 & 204 & 2300 & 9.8 & 9.8 & & 0.0000 & $0.00 \mathrm{E}+00$ & 0.0000 & 0.0000 & 0.0000 & \\
\hline \multirow[t]{2}{*}{10.2} & 7/23/2008 0:00 & 11 & 205 & 0 & 10.05 & 10.05 & 10.09 & -0.0249 & $0.00 \mathrm{E}+00$ & 0.0000 & 0.0000 & 0.0000 & \\
\hline & 7/23/2008 1:00 & 12 & 205 & 100 & 10.09 & 10.09 & & -0.0287 & $0.00 \mathrm{E}+00$ & 0.0000 & 0.0000 & 0.0000 & \\
\hline Day Count & $7 / 23 / 20082: 00$ & 13 & 205 & 200 & 10.02 & 10.02 & & -0.0220 & $0.00 \mathrm{E}+00$ & 0.0000 & 0.0000 & 0.0000 & \\
\hline \multirow[t]{27}{*}{35} & 7/23/2008 3:00 & 14 & 205 & 300 & 9.96 & 9.96 & & -0.0161 & $0.00 \mathrm{E}+00$ & 0.0000 & 0.0000 & 0.0000 & \\
\hline & 7/23/2008 4:00 & 15 & 205 & 400 & 9.86 & 9.86 & & -0.0061 & $0.00 \mathrm{E}+00$ & 0.0000 & 0.0000 & 0.0000 & \\
\hline & 7/23/2008 5:00 & 16 & 205 & 500 & 9.71 & 9.71 & & 0.0093 & 3.74E-05 & 7.1228 & 2.6245 & 9.7474 & \\
\hline & 7/23/2008 6:00 & 17 & 205 & 600 & 9.55 & 9.55 & & 0.0262 & 1.34E-04 & 25.5697 & 9.4217 & 34.9914 & \\
\hline & 7/23/2008 7:00 & 18 & 205 & 700 & 9.23 & 9.23 & & 0.0618 & 3.86E-04 & 73.5470 & 27.0999 & 100.6469 & \\
\hline & 7/23/2008 8:00 & 19 & 205 & 800 & 8.32 & 8.32 & & 0.1779 & $1.42 \mathrm{E}-03$ & 270.4996 & 99.6712 & 370.1708 & \\
\hline & $7 / 23 / 20089: 00$ & 20 & 205 & 900 & 6.921 & 6.921 & & 0.4160 & $4.04 \mathrm{E}-03$ & 769.7062 & 283.6141 & 1053.3203 & \\
\hline & 7/23/2008 10:00 & 21 & 205 & 1000 & 6.169 & 6.169 & & 0.5886 & $6.20 \mathrm{E}-03$ & 1180.0062 & 434.7975 & 1614.8037 & \\
\hline & 7/23/2008 11:00 & 22 & 205 & 1100 & 6.281 & 6.281 & & 0.5603 & 5.83E-03 & 1110.4909 & 409.1832 & 1519.6742 & \\
\hline & $7 / 23 / 2008$ 12:00 & 23 & 205 & 1200 & 6.152 & 6.152 & & 0.5930 & $6.25 E-03$ & 1190.8491 & 438.7929 & 1629.6420 & \\
\hline & $7 / 23 / 2008$ 13:00 & 24 & 205 & 1300 & 6.107 & 6.107 & & 0.6047 & $6.41 E-03$ & 1219.9334 & 449.5095 & 1669.4429 & \\
\hline & $7 / 23 / 200814: 00$ & 25 & 205 & 1400 & 6.085 & 6.085 & & 0.6105 & $6.48 \mathrm{E}-03$ & 1234.3573 & 454.8243 & 1689.1816 & \\
\hline & 7/23/2008 15:00 & 26 & 205 & 1500 & 6.045 & 6.045 & & 0.6212 & $6.62 \mathrm{E}-03$ & 1260.9339 & 464.6170 & 1725.5510 & \\
\hline & 7/23/2008 16:00 & 27 & 205 & 1600 & 6.212 & 6.212 & & 0.5776 & $6.05 E-03$ & 1152.9269 & 424.8196 & 1577.7466 & \\
\hline & 7/23/2008 17:00 & 28 & 205 & 1700 & 6.59 & 6.59 & & 0.4871 & 4.91E-03 & 934.7729 & 344.4363 & 1279.2092 & \\
\hline & $7 / 23 / 2008$ 18:00 & 29 & 205 & 1800 & 7.193 & 7.193 & & 0.3624 & $3.41 \mathrm{E}-03$ & 649.6204 & 239.3660 & 888.9864 & \\
\hline & $7 / 23 / 2008$ 19:00 & 30 & 205 & 1900 & 7.074 & 7.074 & & 0.3854 & $3.68 E-03$ & 700.5530 & 258.1332 & 958.6861 & \\
\hline & $7 / 23 / 200820: 00$ & 31 & 205 & 2000 & 7.487 & 7.487 & & 0.3089 & 2.80E-03 & 533.6710 & 196.6421 & 730.3131 & \\
\hline & $7 / 23 / 200821: 00$ & 32 & 205 & 2100 & 8.28 & 8.28 & & 0.1836 & $1.48 \mathrm{E}-03$ & 281.1904 & 103.6104 & 384.8008 & \\
\hline & $7 / 23 / 200822: 00$ & 33 & 205 & 2200 & 8.95 & 8.95 & & 0.0950 & $6.56 \mathrm{E}-04$ & 124.9300 & 46.0330 & 170.9631 & \\
\hline & $7 / 23 / 2008$ 23:00 & 34 & 205 & 2300 & 9.01 & 9.01 & & 0.0877 & 5.95E-04 & 113.2294 & 41.7217 & 154.9511 & \\
\hline & $7 / 24 / 20080: 00$ & 35 & 206 & 0 & 8.92 & 8.92 & 9.30 & 0.0987 & 6.88E-04 & 130.9198 & 48.2401 & 179.1599 & \\
\hline & 7/24/2008 1:00 & 36 & 206 & 100 & 8.92 & 8.92 & & 0.0987 & 6.88E-04 & 130.9198 & 48.2401 & 179.1599 & \\
\hline & 7/24/2008 2:00 & 37 & 206 & 200 & 8.95 & 8.95 & & 0.0950 & $6.56 \mathrm{E}-04$ & 124.9300 & 46.0330 & 170.9631 & \\
\hline & 7/24/2008 3:00 & 38 & 206 & 300 & 9.03 & 9.03 & & 0.0853 & $5.75 E-04$ & 109.4120 & 40.3151 & 149.7271 & \\
\hline & 7/24/2008 4:00 & 39 & 206 & 400 & 9.04 & 9.04 & & 0.0841 & 5.65E-04 & 107.5188 & 39.6175 & 147.1364 & \\
\hline & 7/24/2008 5:00 & 40 & 206 & 500 & 9.12 & 9.12 & & 0.0746 & $4.87 \mathrm{E}-04$ & 92.7494 & 34.1754 & 126.9248 & \\
\hline
\end{tabular}




\begin{tabular}{|c|c|c|c|c|c|c|c|c|c|c|c|c|c|}
\hline Tree ID & TS & RN & JD & HR & $\begin{array}{c}\text { Raw } \\
\text { TC_dTA }\end{array}$ & $\begin{array}{c}\text { Fixed } \\
\text { TC_dT } \Delta\end{array}$ & dTM & $\mathbf{K}$ & $\begin{array}{l}\text { Sap } \\
\text { Velocity } \\
\text { (cm/s) }\end{array}$ & $\begin{array}{l}\text { Probed } \\
\text { Drywood F } \\
\text { (g/hr) }\end{array}$ & $\begin{array}{c}\text { Extrapolated } \\
\text { Wetwood F } \\
(\mathrm{g} / \mathrm{hr})\end{array}$ & $\begin{array}{c}\text { Extrapolated } \\
\text { Total Wood F } \\
(\mathrm{g} / \mathrm{hr})\end{array}$ & $\begin{array}{c}\text { Relative } \\
\text { Humidity, } \\
\text { @10m (\%) }\end{array}$ \\
\hline & 7/24/2008 6:00 & 41 & 206 & 600 & 9.13 & 9.13 & & 0.0734 & 4.78E-04 & 90.9505 & 33.5126 & 124.4630 & \\
\hline & 7/24/2008 7:00 & 42 & 206 & 700 & 9.08 & 9.08 & & 0.0793 & 5.25E-04 & 100.0504 & 36.8656 & 136.9161 & \\
\hline & 7/24/2008 8:00 & 43 & 206 & 800 & 8.79 & 8.79 & & 0.1149 & 8.29E-04 & 157.9486 & 58.1994 & 216.1479 & \\
\hline & 7/24/20089:00 & 44 & 206 & 900 & 8.11 & 8.11 & & 0.2084 & 1.73E-03 & 328.6777 & 121.1081 & 449.7857 & \\
\hline & $7 / 24 / 2008$ 10:00 & 45 & 206 & 1000 & 7.987 & 7.987 & & 0.2270 & 1.92E-03 & 365.1739 & 134.5558 & 499.7297 & \\
\hline & $7 / 24 / 200811: 00$ & 46 & 206 & 1100 & 6.066 & 6.066 & & 0.6156 & $6.55 \mathrm{E}-03$ & 1246.9242 & 459.4549 & 1706.3791 & \\
\hline & $7 / 24 / 2008$ 12:00 & 47 & 206 & 1200 & 5.588 & 5.588 & & 0.7538 & $8.40 \mathrm{E}-03$ & 1599.9960 & 589.5514 & 2189.5474 & \\
\hline & 7/24/2008 13:00 & 48 & 206 & 1300 & 6.06 & 6.06 & & 0.6172 & $6.57 \mathrm{E}-03$ & 1250.9141 & 460.9250 & 1711.8391 & \\
\hline & $7 / 24 / 200814: 00$ & 49 & 206 & 1400 & 5.932 & 5.932 & & 0.6521 & 7.03E-03 & 1338.5407 & 493.2129 & 1831.7536 & \\
\hline & $7 / 24 / 200815: 00$ & 50 & 206 & 1500 & 5.677 & 5.677 & & 0.7263 & 8.03E-03 & 1528.4583 & 563.1919 & 2091.6502 & \\
\hline & $7 / 24 / 2008$ 16:00 & 51 & 206 & 1600 & 5.718 & 5.718 & & 0.7139 & 7.86E-03 & 1496.4543 & 551.3994 & 2047.8537 & \\
\hline & $7 / 24 / 200817: 00$ & 52 & 206 & 1700 & 5.928 & 5.928 & & 0.6532 & 7.04E-03 & 1341.3582 & 494.2510 & 1835.6092 & \\
\hline & $7 / 24 / 2008$ 18:00 & 53 & 206 & 1800 & 5.828 & 5.828 & & 0.6815 & $7.42 \mathrm{E}-03$ & 1413.4230 & 520.8048 & 1934.2278 & \\
\hline & $7 / 24 / 2008$ 19:00 & 54 & 206 & 1900 & 6.134 & 6.134 & & 0.5977 & 6.31E-03 & 1202.4158 & 443.0548 & 1645.4707 & \\
\hline & $7 / 24 / 200820: 00$ & 55 & 206 & 2000 & 7.105 & 7.105 & & 0.3793 & 3.61E-03 & 687.0507 & 253.1580 & 940.2087 & \\
\hline & $7 / 24 / 2008$ 21:00 & 56 & 206 & 2100 & 8.25 & 8.25 & & 0.1879 & 1.52E-03 & 289.3276 & 106.6087 & 395.9363 & \\
\hline & 7/24/2008 22:00 & 57 & 206 & 2200 & 9.13 & 9.13 & & 0.0734 & $4.78 \mathrm{E}-04$ & 90.9505 & 33.5126 & 124.4630 & \\
\hline & $7 / 24 / 200823: 00$ & 58 & 206 & 2300 & 9.3 & 9.3 & & 0.0538 & $3.26 \mathrm{E}-04$ & 62.0120 & 22.8496 & 84.8616 & \\
\hline & $7 / 25 / 20080: 00$ & 59 & 207 & 0 & 9.31 & 9.31 & 9.31 & 0.0526 & 3.17E-04 & 60.4089 & 22.2589 & 82.6678 & \\
\hline & 7/25/2008 1:00 & 60 & 207 & 100 & 9.23 & 9.23 & & 0.0618 & 3.86E-04 & 73.5470 & 27.0999 & 100.6469 & \\
\hline & 7/25/2008 2:00 & 61 & 207 & 200 & 9.1 & 9.1 & & 0.0769 & $5.06 \mathrm{E}-04$ & 96.3789 & 35.5128 & 131.8917 & \\
\hline & 7/25/2008 3:00 & 62 & 207 & 300 & 8.98 & 8.98 & & 0.0913 & $6.25 \mathrm{E}-04$ & 119.0332 & 43.8602 & 162.8934 & \\
\hline & 7/25/2008 4:00 & 63 & 207 & 400 & 8.86 & 8.86 & & 0.1061 & 7.52E-04 & 143.1778 & 52.7568 & 195.9347 & \\
\hline & 7/25/2008 5:00 & 64 & 207 & 500 & 8.73 & 8.73 & & 0.1226 & 8.98E-04 & 171.0130 & 63.0133 & 234.0263 & \\
\hline & 7/25/2008 6:00 & 65 & 207 & 600 & 8.65 & 8.65 & & 0.1329 & 9.93E-04 & 189.0160 & 69.6468 & 258.6628 & \\
\hline & 7/25/2008 7:00 & 66 & 207 & 700 & 8.58 & 8.58 & & 0.1422 & 1.08E-03 & 205.3206 & 75.6546 & 280.9751 & \\
\hline & 7/25/2008 8:00 & 67 & 207 & 800 & 8.49 & 8.49 & & 0.1543 & 1.19E-03 & 227.0502 & 83.6613 & 310.7116 & \\
\hline & 7/25/2008 9:00 & 68 & 207 & 900 & 8.37 & 8.37 & & 0.1708 & 1.35E-03 & 257.3890 & 94.8403 & 352.2293 & \\
\hline & $7 / 25 / 2008$ 10:00 & 69 & 207 & 1000 & 8.19 & 8.19 & & 0.1966 & $1.61 \mathrm{E}-03$ & 305.9120 & 112.7196 & 418.6316 & \\
\hline & 7/25/2008 11:00 & 70 & 207 & 1100 & 7.218 & 7.218 & & 0.3577 & 3.36E-03 & 639.2243 & 235.5354 & 874.7597 & \\
\hline & $7 / 25 / 2008$ 12:00 & 71 & 207 & 1200 & 5.627 & 5.627 & & 0.7416 & 8.24E-03 & 1568.2938 & 577.8701 & 2146.1639 & \\
\hline & $7 / 25 / 200813: 00$ & 72 & 207 & 1300 & 5.192 & 5.192 & & 0.8875 & 1.03E-02 & 1956.3800 & 720.8684 & 2677.2484 & \\
\hline & $7 / 25 / 200814: 00$ & 73 & 207 & 1400 & 4.997 & 4.997 & & 0.9612 & 1.13E-02 & 2158.1277 & 795.2065 & 2953.3342 & \\
\hline & $7 / 25 / 200815: 00$ & 74 & 207 & 1500 & 5.046 & 5.046 & & 0.9421 & 1.11E-02 & 2105.6110 & 775.8557 & 2881.4666 & \\
\hline & $7 / 25 / 2008$ 16:00 & 75 & 207 & 1600 & 5.195 & 5.195 & & 0.8864 & 1.03E-02 & 1953.4226 & 719.7787 & 2673.2014 & \\
\hline & $7 / 25 / 200817: 00$ & 76 & 207 & 1700 & 5.149 & 5.149 & & 0.9033 & 1.05E-02 & 1999.2404 & 736.6612 & 2735.9016 & \\
\hline & 7/25/2008 18:00 & 77 & 207 & 1800 & 5.204 & 5.204 & & 0.8832 & $1.02 \mathrm{E}-02$ & 1944.5761 & 716.5191 & 2661.0952 & \\
\hline & $7 / 25 / 2008$ 19:00 & 78 & 207 & 1900 & 5.37 & 5.37 & & 0.8250 & $9.39 E-03$ & 1788.0143 & 658.8306 & 2446.8450 & \\
\hline & 7/25/2008 20:00 & 79 & 207 & 2000 & 6.184 & 6.184 & & 0.5847 & $6.15 \mathrm{E}-03$ & 1170.5037 & 431.2962 & 1601.7999 & \\
\hline & $7 / 25 / 2008$ 21:00 & 80 & 207 & 2100 & 7.391 & 7.391 & & 0.3259 & 2.99E-03 & 570.0511 & 210.0471 & 780.0982 & \\
\hline & $7 / 25 / 200822: 00$ & 81 & 207 & 2200 & 8.53 & 8.53 & & 0.1489 & $1.14 \mathrm{E}-03$ & 217.2854 & 80.0633 & 297.3486 & \\
\hline
\end{tabular}




\begin{tabular}{|c|c|c|c|c|c|c|c|c|c|c|c|c|c|}
\hline Tree ID & TS & RN & JD & HR & $\begin{array}{c}\text { Raw } \\
\text { TC_dT } \Delta\end{array}$ & $\begin{array}{c}\text { Fixed } \\
\text { TC_dT } \Delta\end{array}$ & dTM & K & $\begin{array}{l}\text { Sap } \\
\text { Velocity } \\
\text { (cm/s) }\end{array}$ & $\begin{array}{c}\text { Probed } \\
\text { Drywood F } \\
\text { (g/hr) }\end{array}$ & $\begin{array}{c}\text { Extrapolated } \\
\text { Wetwood F } \\
\text { (g/hr) }\end{array}$ & $\begin{array}{c}\text { Extrapolated } \\
\text { Total Wood F } \\
(\mathrm{g} / \mathrm{hr})\end{array}$ & $\begin{array}{l}\text { Relative } \\
\text { Humidity, } \\
@ 10 \mathrm{~m}(\%)\end{array}$ \\
\hline & $7 / 25 / 200823: 00$ & 82 & 207 & 2300 & 8.84 & 8.84 & & 0.1086 & 7.74E-04 & 147.3464 & 54.2928 & 201.6392 & \\
\hline & 7/26/2008 0:00 & 83 & 208 & 0 & 8.9 & 8.9 & 8.90 & 0.1011 & 7.09E-04 & 134.9646 & 49.7305 & 184.6950 & \\
\hline & 7/26/2008 1:00 & 84 & 208 & 100 & 8.89 & 8.89 & & 0.1024 & 7.19E-04 & 137.0024 & 50.4814 & 187.4838 & \\
\hline & 7/26/2008 2:00 & 85 & 208 & 200 & 8.85 & 8.85 & & 0.1073 & 7.63E-04 & 145.2570 & 53.5229 & 198.7799 & \\
\hline & 7/26/2008 3:00 & 86 & 208 & 300 & 8.8 & 8.8 & & 0.1136 & 8.18E-04 & 155.8074 & 57.4105 & 213.2179 & \\
\hline & 7/26/2008 4:00 & 87 & 208 & 400 & 8.74 & 8.74 & & 0.1213 & 8.87E-04 & 168.8097 & 62.2014 & 231.0111 & \\
\hline & 7/26/2008 5:00 & 88 & 208 & 500 & 8.67 & 8.67 & & 0.1303 & 9.69E-04 & 184.4524 & 67.9653 & 252.4177 & \\
\hline & 7/26/2008 6:00 & 89 & 208 & 600 & 8.62 & 8.62 & & 0.1369 & 1.03E-03 & 195.9402 & 72.1982 & 268.1385 & \\
\hline & 7/26/2008 7:00 & 90 & 208 & 700 & 8.51 & 8.51 & & 0.1516 & 1.17E-03 & 222.1463 & 81.8544 & 304.0006 & \\
\hline & 7/26/2008 8:00 & 91 & 208 & 800 & 8.3 & 8.3 & & 0.1807 & 1.45E-03 & 275.8224 & 101.6324 & 377.4549 & \\
\hline & 7/26/2008 9:00 & 92 & 208 & 900 & 6.881 & 6.881 & & 0.4242 & $4.14 \mathrm{E}-03$ & 788.4977 & 290.5382 & 1079.0359 & \\
\hline & $7 / 26 / 200810: 00$ & 93 & 208 & 1000 & 5.052 & 5.052 & & 0.9398 & 1.10E-02 & 2099.2669 & 773.5181 & 2872.7850 & \\
\hline & $7 / 26 / 2008$ 11:00 & 94 & 208 & 1100 & 5.198 & 5.198 & & 0.8853 & 1.02E-02 & 1950.4696 & 718.6906 & 2669.1602 & \\
\hline & $7 / 26 / 2008$ 12:00 & 95 & 208 & 1200 & 5.428 & 5.428 & & 0.8055 & $9.12 \mathrm{E}-03$ & 1736.1289 & 639.7124 & 2375.8413 & \\
\hline & $7 / 26 / 200813: 00$ & 96 & 208 & 1300 & 4.887 & 4.887 & & 1.0053 & 1.20E-02 & 2280.7784 & 840.3997 & 3121.1781 & \\
\hline & $7 / 26 / 200814: 00$ & 97 & 208 & 1400 & 4.902 & 4.902 & & 0.9992 & 1.19E-02 & 2263.6534 & 834.0896 & 3097.7430 & \\
\hline & $7 / 26 / 2008$ 15:00 & 98 & 208 & 1500 & 4.91 & 4.91 & & 0.9959 & 1.18E-02 & 2254.5727 & 830.7437 & 3085.3163 & \\
\hline & $7 / 26 / 200816: 00$ & 99 & 208 & 1600 & 4.958 & 4.958 & & 0.9766 & 1.16E-02 & 2200.8454 & 810.9467 & 3011.7921 & \\
\hline & $7 / 26 / 200817: 00$ & 100 & 208 & 1700 & 4.896 & 4.896 & & 1.0016 & 1.19E-02 & 2270.4879 & 836.6080 & 3107.0959 & \\
\hline & $7 / 26 / 2008$ 18:00 & 101 & 208 & 1800 & 5.086 & 5.086 & & 0.9269 & 1.08E-02 & 2063.6670 & 760.4006 & 2824.0676 & \\
\hline & $7 / 26 / 2008$ 19:00 & 102 & 208 & 1900 & 5.179 & 5.179 & & 0.8923 & 1.03E-02 & 1969.2444 & 725.6086 & 2694.8529 & \\
\hline & $7 / 26 / 200820: 00$ & 103 & 208 & 2000 & 5.708 & 5.708 & & 0.7169 & 7.90E-03 & 1504.2061 & 554.2557 & 2058.4618 & \\
\hline & $7 / 26 / 200821: 00$ & 104 & 208 & 2100 & 7.073 & 7.073 & & 0.3856 & $3.68 E-03$ & 700.9913 & 258.2947 & 959.2860 & \\
\hline & $7 / 26 / 200822: 00$ & 105 & 208 & 2200 & 8.17 & 8.17 & & 0.1995 & $1.64 \mathrm{E}-03$ & 311.5329 & 114.7907 & 426.3236 & \\
\hline & $7 / 26 / 2008$ 23:00 & 106 & 208 & 2300 & 8.52 & 8.52 & & 0.1502 & 1.15E-03 & 219.7104 & 80.9568 & 300.6673 & \\
\hline & $7 / 27 / 20080: 00$ & 107 & 209 & 0 & 8.61 & 8.61 & 8.75 & 0.1382 & 1.04E-03 & 198.2694 & 73.0565 & 271.3259 & \\
\hline & 7/27/2008 1:00 & 108 & 209 & 100 & 8.63 & 8.63 & & 0.1356 & 1.02E-03 & 193.6216 & 71.3439 & 264.9655 & \\
\hline & 7/27/2008 2:00 & 109 & 209 & 200 & 8.62 & 8.62 & & 0.1369 & 1.03E-03 & 195.9402 & 72.1982 & 268.1385 & \\
\hline & 7/27/2008 3:00 & 110 & 209 & 300 & 8.61 & 8.61 & & 0.1382 & 1.04E-03 & 198.2694 & 73.0565 & 271.3259 & \\
\hline & 7/27/2008 4:00 & 111 & 209 & 400 & 8.64 & 8.64 & & 0.1343 & 1.00E-03 & 191.3135 & 70.4934 & 261.8070 & \\
\hline & 7/27/2008 5:00 & 112 & 209 & 500 & 8.73 & 8.73 & & 0.1226 & 8.98E-04 & 171.0130 & 63.0133 & 234.0263 & \\
\hline & 7/27/2008 6:00 & 113 & 209 & 600 & 8.75 & 8.75 & & 0.1200 & 8.75E-04 & 166.6167 & 61.3933 & 228.0100 & \\
\hline & 7/27/2008 7:00 & 114 & 209 & 700 & 8.72 & 8.72 & & 0.1239 & $9.10 \mathrm{E}-04$ & 173.2268 & 63.8290 & 237.0558 & \\
\hline & 7/27/2008 8:00 & 115 & 209 & 800 & 8.36 & 8.36 & & 0.1722 & 1.37E-03 & 259.9888 & 95.7982 & 355.7870 & \\
\hline & $7 / 27 / 20089: 00$ & 116 & 209 & 900 & 5.981 & 5.981 & & 0.6385 & 6.85E-03 & 1304.4211 & 480.6408 & 1785.0619 & \\
\hline & $7 / 27 / 2008$ 10:00 & 117 & 209 & 1000 & 4.732 & 4.732 & & 1.0710 & 1.29E-02 & 2465.5859 & 908.4958 & 3374.0818 & \\
\hline & $7 / 27 / 2008$ 11:00 & 118 & 209 & 1100 & 4.807 & 4.807 & & 1.0387 & $1.25 \mathrm{E}-02$ & 2374.3372 & 874.8734 & 3249.2105 & \\
\hline & $7 / 27 / 200812: 00$ & 119 & 209 & 1200 & 4.696 & 4.696 & & 1.0869 & 1.32E-02 & 2510.6554 & 925.1026 & 3435.7580 & \\
\hline & 7/27/2008 13:00 & 120 & 209 & 1300 & 4.791 & 4.791 & & 1.0455 & $1.26 \mathrm{E}-02$ & 2393.5100 & 881.9380 & 3275.4480 & \\
\hline & $7 / 27 / 200814: 00$ & 121 & 209 & 1400 & 4.694 & 4.694 & & 1.0878 & 1.32E-02 & 2513.1840 & 926.0343 & 3439.2184 & \\
\hline & $7 / 27 / 2008$ 15:00 & 122 & 209 & 1500 & 4.677 & 4.677 & & 1.0954 & 1.33E-02 & 2534.7842 & 933.9934 & 3468.7776 & \\
\hline
\end{tabular}




\begin{tabular}{|c|c|c|c|c|c|c|c|c|c|c|c|c|c|}
\hline Tree ID & TS & RN & JD & HR & $\begin{array}{c}\text { Raw } \\
\text { TC_dTA }\end{array}$ & $\begin{array}{c}\text { Fixed } \\
\text { TC_dT } \Delta\end{array}$ & dTM & $\mathbf{K}$ & $\begin{array}{l}\text { Sap } \\
\text { Velocity } \\
\text { (cm/s) }\end{array}$ & $\begin{array}{l}\text { Probed } \\
\text { Drywood F } \\
\text { (g/hr) }\end{array}$ & $\begin{array}{c}\text { Extrapolated } \\
\text { Wetwood F } \\
(\mathrm{g} / \mathrm{hr})\end{array}$ & $\begin{array}{c}\text { Extrapolated } \\
\text { Total Wood F } \\
(\mathrm{g} / \mathrm{hr})\end{array}$ & $\begin{array}{l}\text { Relative } \\
\text { Humidity, } \\
@ 10 \mathrm{~m}(\%)\end{array}$ \\
\hline & 7/27/2008 16:00 & 123 & 209 & 1600 & 4.77 & 4.77 & & 1.0545 & 1.27E-02 & 2418.9139 & 891.2986 & 3310.2125 & \\
\hline & $7 / 27 / 2008$ 17:00 & 124 & 209 & 1700 & 4.812 & 4.812 & & 1.0366 & $1.24 \mathrm{E}-02$ & 2368.3777 & 872.6775 & 3241.0552 & \\
\hline & $7 / 27 / 2008$ 18:00 & 125 & 209 & 1800 & 4.953 & 4.953 & & 0.9786 & 1.16E-02 & 2206.3821 & 812.9869 & 3019.3690 & \\
\hline & 7/27/2008 19:00 & 126 & 209 & 1900 & 5.101 & 5.101 & & 0.9212 & $1.08 \mathrm{E}-02$ & 2048.1481 & 754.6823 & 2802.8303 & \\
\hline & $7 / 27 / 200820: 00$ & 127 & 209 & 2000 & 5.513 & 5.513 & & 0.7776 & $8.73 \mathrm{E}-03$ & 1662.5651 & 612.6063 & 2275.1714 & \\
\hline & $7 / 27 / 2008$ 21:00 & 128 & 209 & 2100 & 6.552 & 6.552 & & 0.4957 & $5.02 E-03$ & 955.1892 & 351.9591 & 1307.1483 & \\
\hline & 7/27/2008 22:00 & 129 & 209 & 2200 & 7.558 & 7.558 & & 0.2966 & 2.67E-03 & 507.6448 & 187.0522 & 694.6969 & \\
\hline & $7 / 27 / 2008$ 23:00 & 130 & 209 & 2300 & 7.825 & 7.825 & & 0.2524 & 2.19E-03 & 416.1120 & 153.3250 & 569.4370 & \\
\hline & $7 / 28 / 20080: 00$ & 131 & 210 & 0 & 7.94 & 7.94 & 8.57 & 0.2343 & 1.99E-03 & 379.6101 & 139.8751 & 519.4852 & \\
\hline & 7/28/2008 1:00 & 132 & 210 & 100 & 8.11 & 8.11 & & 0.2084 & $1.73 \mathrm{E}-03$ & 328.6777 & 121.1081 & 449.7857 & \\
\hline & 7/28/2008 2:00 & 133 & 210 & 200 & 8.23 & 8.23 & & 0.1908 & 1.55E-03 & 294.8096 & 108.6287 & 403.4383 & \\
\hline & 7/28/2008 3:00 & 134 & 210 & 300 & 8.42 & 8.42 & & 0.1639 & $1.28 \mathrm{E}-03$ & 244.5560 & 90.1117 & 334.6677 & \\
\hline & 7/28/2008 4:00 & 135 & 210 & 400 & 8.51 & 8.51 & & 0.1516 & 1.17E-03 & 222.1463 & 81.8544 & 304.0006 & \\
\hline & 7/28/2008 5:00 & 136 & 210 & 500 & 8.57 & 8.57 & & 0.1435 & 1.09E-03 & 207.6922 & 76.5285 & 284.2206 & \\
\hline & 7/28/2008 6:00 & 137 & 210 & 600 & 8.5 & 8.5 & & 0.1529 & 1.18E-03 & 224.5928 & 82.7559 & 307.3487 & \\
\hline & 7/28/2008 7:00 & 138 & 210 & 700 & 8.34 & 8.34 & & 0.1751 & 1.39E-03 & 265.2219 & 97.7264 & 362.9483 & \\
\hline & 7/28/2008 8:00 & 139 & 210 & 800 & 6.67 & 6.67 & & 0.4693 & $4.69 \mathrm{E}-03$ & 892.8172 & 328.9768 & 1221.7940 & \\
\hline & $7 / 28 / 20089: 00$ & 140 & 210 & 900 & 5.016 & 5.016 & & 0.9537 & $1.12 \mathrm{E}-02$ & 2137.6134 & 787.6476 & 2925.2610 & \\
\hline & $7 / 28 / 2008$ 10:00 & 141 & 210 & 1000 & 4.782 & 4.782 & & 1.0494 & $1.26 \mathrm{E}-02$ & 2404.3639 & 885.9373 & 3290.3013 & \\
\hline & 7/28/2008 11:00 & 142 & 210 & 1100 & 4.872 & 4.872 & & 1.0115 & $1.21 \mathrm{E}-02$ & 2298.0333 & 846.7576 & 3144.7909 & \\
\hline & $7 / 28 / 200812: 00$ & 143 & 210 & 1200 & 4.895 & 4.895 & & 1.0020 & 1.19E-02 & 2271.6290 & 837.0284 & 3108.6574 & \\
\hline & $7 / 28 / 2008$ 13:00 & 144 & 210 & 1300 & 4.903 & 4.903 & & 0.9988 & 1.19E-02 & 2262.5163 & 833.6707 & 3096.1870 & \\
\hline & $7 / 28 / 2008$ 14:00 & 145 & 210 & 1400 & 4.916 & 4.916 & & 0.9935 & 1.18E-02 & 2247.7860 & 828.2430 & 3076.0290 & \\
\hline & $7 / 28 / 2008$ 15:00 & 146 & 210 & 1500 & 4.905 & 4.905 & & 0.9980 & 1.19E-02 & 2260.2438 & 832.8333 & 3093.0772 & \\
\hline & $7 / 28 / 2008$ 16:00 & 147 & 210 & 1600 & 4.962 & 4.962 & & 0.9750 & 1.15E-02 & 2196.4259 & 809.3183 & 3005.7442 & \\
\hline & $7 / 28 / 200817: 00$ & 148 & 210 & 1700 & 4.944 & 4.944 & & 0.9822 & 1.16E-02 & 2216.3830 & 816.6719 & 3033.0549 & \\
\hline & $7 / 28 / 2008$ 18:00 & 149 & 210 & 1800 & 5.068 & 5.068 & & 0.9337 & $1.09 \mathrm{E}-02$ & 2082.4403 & 767.3180 & 2849.7582 & \\
\hline & $7 / 28 / 2008$ 19:00 & 150 & 210 & 1900 & 5.241 & 5.241 & & 0.8699 & $1.00 \mathrm{E}-02$ & 1908.6047 & 703.2647 & 2611.8693 & \\
\hline & $7 / 28 / 200820: 00$ & 151 & 210 & 2000 & 5.812 & 5.812 & & 0.6862 & $7.48 \mathrm{E}-03$ & 1425.2502 & 525.1628 & 1950.4130 & \\
\hline & $7 / 28 / 2008$ 21:00 & 152 & 210 & 2100 & 7.214 & 7.214 & & 0.3585 & 3.37E-03 & 640.8808 & 236.1457 & 877.0265 & \\
\hline & $7 / 28 / 2008$ 22:00 & 153 & 210 & 2200 & 7.835 & 7.835 & & 0.2508 & 2.17E-03 & 412.8703 & 152.1306 & 565.0009 & \\
\hline & $7 / 28 / 2008$ 23:00 & 154 & 210 & 2300 & 7.979 & 7.979 & & 0.2282 & $1.93 \mathrm{E}-03$ & 367.6117 & 135.4541 & 503.0658 & \\
\hline & $7 / 29 / 20080: 00$ & 155 & 211 & 0 & 8.16 & 8.16 & 8.78 & 0.2010 & $1.65 \mathrm{E}-03$ & 314.3609 & 115.8327 & 430.1937 & \\
\hline & 7/29/2008 1:00 & 156 & 211 & 100 & 8.39 & 8.39 & & 0.1681 & $1.32 \mathrm{E}-03$ & 252.2227 & 92.9366 & 345.1593 & \\
\hline & 7/29/2008 2:00 & 157 & 211 & 200 & 8.58 & 8.58 & & 0.1422 & 1.08E-03 & 205.3206 & 75.6546 & 280.9751 & \\
\hline & 7/29/2008 3:00 & 158 & 211 & 300 & 8.65 & 8.65 & & 0.1329 & $9.93 \mathrm{E}-04$ & 189.0160 & 69.6468 & 258.6628 & \\
\hline & 7/29/2008 4:00 & 159 & 211 & 400 & 8.75 & 8.75 & & 0.1200 & 8.75E-04 & 166.6167 & 61.3933 & 228.0100 & \\
\hline & 7/29/2008 5:00 & 160 & 211 & 500 & 8.78 & 8.78 & & 0.1162 & 8.41E-04 & 160.1000 & 58.9921 & 219.0922 & \\
\hline & $7 / 29 / 20086: 00$ & 161 & 211 & 600 & 8.72 & 8.72 & & 0.1239 & $9.10 \mathrm{E}-04$ & 173.2268 & 63.8290 & 237.0558 & \\
\hline & 7/29/2008 7:00 & 162 & 211 & 700 & 8.55 & 8.55 & & 0.1462 & $1.12 \mathrm{E}-03$ & 212.4674 & 78.2880 & 290.7554 & \\
\hline & 7/29/2008 8:00 & 163 & 211 & 800 & 7.764 & 7.764 & & 0.2622 & 2.29E-03 & 436.1707 & 160.7161 & 596.8868 & \\
\hline
\end{tabular}




\begin{tabular}{|c|c|c|c|c|c|c|c|c|c|c|c|c|c|}
\hline Tree ID & TS & RN & JD & HR & $\begin{array}{c}\text { Raw } \\
\text { TC_dTA }\end{array}$ & $\begin{array}{c}\text { Fixed } \\
\text { TC_dT } \Delta\end{array}$ & dTM & $\mathbf{K}$ & $\begin{array}{c}\text { Sap } \\
\text { Velocity } \\
(\mathrm{cm} / \mathrm{s})\end{array}$ & $\begin{array}{c}\text { Probed } \\
\text { Drywood F } \\
(\mathrm{g} / \mathrm{hr})\end{array}$ & $\begin{array}{c}\text { Extrapolated } \\
\text { Wetwood F } \\
(\mathrm{g} / \mathrm{hr})\end{array}$ & $\begin{array}{c}\text { Extrapolated } \\
\text { Total Wood F } \\
(\mathbf{g} / \mathrm{hr})\end{array}$ & $\begin{array}{c}\text { Relative } \\
\text { Humidity, } \\
@ 10 \mathrm{~m}(\%)\end{array}$ \\
\hline & 7/29/2008 9:00 & 164 & 211 & 900 & 5.688 & 5.688 & & 0.7229 & $7.98 \mathrm{E}-03$ & 1519.8141 & 560.0068 & 2079.8209 & \\
\hline & $7 / 29 / 2008$ 10:00 & 165 & 211 & 1000 & 5.039 & 5.039 & & 0.9448 & $1.11 \mathrm{E}-02$ & 2113.0360 & 778.5916 & 2891.6276 & \\
\hline & $7 / 29 / 2008$ 11:00 & 166 & 211 & 1100 & 5.411 & 5.411 & & 0.8111 & 9.20E-03 & 1751.1919 & 645.2627 & 2396.4545 & \\
\hline & $7 / 29 / 2008$ 12:00 & 167 & 211 & 1200 & 4.909 & 4.909 & & 0.9963 & $1.18 \mathrm{E}-02$ & 2255.7058 & 831.1612 & 3086.8669 & \\
\hline & 7/29/2008 13:00 & 168 & 211 & 1300 & 5.034 & 5.034 & & 0.9468 & 1.11E-02 & 2118.3553 & 780.5516 & 2898.9069 & \\
\hline & $7 / 29 / 2008$ 14:00 & 169 & 211 & 1400 & 4.906 & 4.906 & & 0.9976 & 1.19E-02 & 2259.1085 & 832.4150 & 3091.5234 & \\
\hline & 7/29/2008 15:00 & 170 & 211 & 1500 & 5.131 & 5.131 & & 0.9100 & $1.06 \mathrm{E}-02$ & 2017.4476 & 743.3701 & 2760.8176 & \\
\hline & 7/29/2008 16:00 & 171 & 211 & 1600 & 5.218 & 5.218 & & 0.8781 & $1.01 \mathrm{E}-02$ & 1930.8905 & 711.4763 & 2642.3668 & \\
\hline & $7 / 29 / 2008$ 17:00 & 172 & 211 & 1700 & 5.409 & 5.409 & & 0.8118 & $9.21 \mathrm{E}-03$ & 1752.9718 & 645.9185 & 2398.8903 & \\
\hline & $7 / 29 / 2008$ 18:00 & 173 & 211 & 1800 & 5.373 & 5.373 & & 0.8239 & 9.38E-03 & 1785.2960 & 657.8290 & 2443.1251 & \\
\hline & $7 / 29 / 2008$ 19:00 & 174 & 211 & 1900 & 5.447 & 5.447 & & 0.7992 & 9.03E-03 & 1719.4338 & 633.5608 & 2352.9946 & \\
\hline & $7 / 29 / 200820: 00$ & 175 & 211 & 2000 & 6.295 & 6.295 & & 0.5568 & 5.79E-03 & 1102.0303 & 406.0657 & 1508.0961 & \\
\hline & $7 / 29 / 200821: 00$ & 176 & 211 & 2100 & 7.677 & 7.677 & & 0.2765 & $2.45 \mathrm{E}-03$ & 465.6408 & 171.5749 & 637.2157 & \\
\hline & $7 / 29 / 2008$ 22:00 & 177 & 211 & 2200 & 8.43 & 8.43 & & 0.1625 & 1.27E-03 & 242.0224 & 89.1781 & 331.2006 & \\
\hline & 7/29/2008 23:00 & 178 & 211 & 2300 & 8.74 & 8.74 & & 0.1213 & 8.87E-04 & 168.8097 & 62.2014 & 231.0111 & \\
\hline & $7 / 30 / 20080: 00$ & 179 & 212 & 0 & 8.86 & 8.86 & 8.88 & 0.1061 & 7.52E-04 & 143.1778 & 52.7568 & 195.9347 & \\
\hline & 7/30/2008 1:00 & 180 & 212 & 100 & 8.87 & 8.87 & & 0.1048 & $7.41 \mathrm{E}-04$ & 141.1091 & 51.9945 & 193.1036 & \\
\hline & 7/30/2008 2:00 & 181 & 212 & 200 & 8.88 & 8.88 & & 0.1036 & 7.30E-04 & 139.0506 & 51.2360 & 190.2866 & \\
\hline & 7/30/2008 3:00 & 182 & 212 & 300 & 8.81 & 8.81 & & 0.1124 & 8.07E-04 & 153.6767 & 56.6253 & 210.3020 & \\
\hline & 7/30/2008 4:00 & 183 & 212 & 400 & 8.73 & 8.73 & & 0.1226 & $8.98 \mathrm{E}-04$ & 171.0130 & 63.0133 & 234.0263 & \\
\hline & 7/30/2008 5:00 & 184 & 212 & 500 & 8.7 & 8.7 & & 0.1264 & 9.33E-04 & 177.6857 & 65.4720 & 243.1577 & \\
\hline & 7/30/2008 6:00 & 185 & 212 & 600 & 8.68 & 8.68 & & 0.1290 & 9.57E-04 & 182.1864 & 67.1303 & 249.3167 & \\
\hline & 7/30/2008 7:00 & 186 & 212 & 700 & 8.68 & 8.68 & & 0.1290 & 9.57E-04 & 182.1864 & 67.1303 & 249.3167 & \\
\hline & $7 / 30 / 20088: 00$ & 187 & 212 & 800 & 8.51 & 8.51 & & 0.1516 & 1.17E-03 & 222.1463 & 81.8544 & 304.0006 & \\
\hline & 7/30/2008 9:00 & 188 & 212 & 900 & 7.046 & 7.046 & & 0.3909 & $3.74 \mathrm{E}-03$ & 712.8934 & 262.6802 & 975.5736 & \\
\hline & $7 / 30 / 2008$ 10:00 & 189 & 212 & 1000 & 4.827 & 4.827 & & 1.0302 & 1.23E-02 & 2350.5902 & 866.1233 & 3216.7135 & \\
\hline & 7/30/2008 11:00 & 190 & 212 & 1100 & 4.809 & 4.809 & & 1.0378 & 1.25E-02 & 2371.9516 & 873.9943 & 3245.9459 & \\
\hline & $7 / 30 / 2008$ 12:00 & 191 & 212 & 1200 & 4.808 & 4.808 & & 1.0383 & $1.25 \mathrm{E}-02$ & 2373.1441 & 874.4337 & 3247.5778 & \\
\hline & $7 / 30 / 2008$ 13:00 & 192 & 212 & 1300 & 4.835 & 4.835 & & 1.0269 & $1.23 \mathrm{E}-02$ & 2341.1589 & 862.6481 & 3203.8070 & \\
\hline & $7 / 30 / 2008$ 14:00 & 193 & 212 & 1400 & 4.841 & 4.841 & & 1.0244 & 1.23E-02 & 2334.1105 & 860.0510 & 3194.1615 & \\
\hline & $7 / 30 / 2008$ 15:00 & 194 & 212 & 1500 & 4.959 & 4.959 & & 0.9762 & $1.16 \mathrm{E}-02$ & 2199.7397 & 810.5393 & 3010.2790 & \\
\hline & $7 / 30 / 2008$ 16:00 & 195 & 212 & 1600 & 4.868 & 4.868 & & 1.0131 & $1.21 \mathrm{E}-02$ & 2302.6567 & 848.4612 & 3151.1179 & \\
\hline & $7 / 30 / 2008$ 17:00 & 196 & 212 & 1700 & 4.898 & 4.898 & & 1.0008 & 1.19E-02 & 2268.2074 & 835.7677 & 3103.9751 & \\
\hline & 7/30/2008 18:00 & 197 & 212 & 1800 & 5.082 & 5.082 & & 0.9284 & $1.09 \mathrm{E}-02$ & 2067.8246 & 761.9325 & 2829.7571 & \\
\hline & $7 / 30 / 2008$ 19:00 & 198 & 212 & 1900 & 5.183 & 5.183 & & 0.8908 & 1.03E-02 & 1965.2775 & 724.1469 & 2689.4244 & \\
\hline & $7 / 30 / 200820: 00$ & 199 & 212 & 2000 & 5.754 & 5.754 & & 0.7032 & 7.71E-03 & 1468.8326 & 541.2216 & 2010.0542 & \\
\hline & 7/30/2008 21:00 & 200 & 212 & 2100 & 7.113 & 7.113 & & 0.3778 & $3.59 \mathrm{E}-03$ & 683.5934 & 251.8840 & 935.4774 & \\
\hline & $7 / 30 / 200822: 00$ & 201 & 212 & 2200 & 8.12 & 8.12 & & 0.2069 & 1.71E-03 & 325.7906 & 120.0443 & 445.8349 & \\
\hline & $7 / 30 / 2008$ 23:00 & 202 & 212 & 2300 & 8.47 & 8.47 & & 0.1570 & 1.22E-03 & 231.9975 & 85.4842 & 317.4817 & \\
\hline & $7 / 31 / 20080: 00$ & 203 & 213 & 0 & 8.63 & 8.63 & 8.73 & 0.1356 & $1.02 \mathrm{E}-03$ & 193.6216 & 71.3439 & 264.9655 & \\
\hline & 7/31/2008 1:00 & 204 & 213 & 100 & 8.72 & 8.72 & & 0.1239 & $9.10 \mathrm{E}-04$ & 173.2268 & 63.8290 & 237.0558 & \\
\hline
\end{tabular}




\begin{tabular}{|c|c|c|c|c|c|c|c|c|c|c|c|c|c|}
\hline Tree ID & TS & RN & JD & HR & $\begin{array}{c}\text { Raw } \\
\text { TC_dTA }\end{array}$ & $\begin{array}{c}\text { Fixed } \\
\text { TC_dT } \Delta\end{array}$ & dTM & $\mathbf{K}$ & $\begin{array}{l}\text { Sap } \\
\text { Velocity } \\
(\mathrm{cm} / \mathrm{s})\end{array}$ & $\begin{array}{c}\text { Probed } \\
\text { Drywood F } \\
(\mathrm{g} / \mathrm{hr})\end{array}$ & $\begin{array}{c}\text { Extrapolated } \\
\text { Wetwood F } \\
(\mathrm{g} / \mathrm{hr})\end{array}$ & $\begin{array}{c}\text { Extrapolated } \\
\text { Total Wood F } \\
(\mathrm{g} / \mathrm{hr})\end{array}$ & $\begin{array}{l}\text { Relative } \\
\text { Humidity, } \\
@ 10 \mathrm{~m}(\%)\end{array}$ \\
\hline & $7 / 31 / 20082: 00$ & 205 & 213 & 200 & ) 8.73 & 8.73 & & 0.1226 & 8.98E-04 & 171.0130 & 63.0133 & 234.0263 & \\
\hline & 7/31/2008 3:00 & 206 & 213 & 300 & 8.7 & 8.7 & & 0.1264 & 9.33E-04 & 177.6857 & 65.4720 & 243.1577 & \\
\hline & 7/31/2008 4:00 & 207 & 213 & 400 & 8.71 & 8.71 & & 0.1251 & $9.21 \mathrm{E}-04$ & 175.4511 & 64.6486 & 240.0996 & \\
\hline & 7/31/2008 5:00 & 208 & 213 & 500 & 8.72 & 8.72 & & 0.1239 & $9.10 \mathrm{E}-04$ & 173.2268 & 63.8290 & 237.0558 & \\
\hline & 7/31/2008 6:00 & 209 & 213 & 600 & 8.66 & 8.66 & & 0.1316 & 9.81E-04 & 186.7290 & 68.8041 & 255.5331 & \\
\hline & 7/31/2008 7:00 & 210 & 213 & 700 & 8.58 & 8.58 & & 0.1422 & 1.08E-03 & 205.3206 & 75.6546 & 280.9751 & \\
\hline & 7/31/2008 8:00 & 211 & 213 & 800 & 8.4 & 8.4 & & 0.1667 & 1.31E-03 & 249.6561 & 91.9909 & 341.6470 & \\
\hline & 7/31/2008 9:00 & 212 & 213 & 900 & 6.656 & 6.656 & & 0.4724 & 4.73E-03 & 900.0606 & 331.6458 & 1231.7065 & \\
\hline & $7 / 31 / 2008$ 10:00 & 213 & 213 & 1000 & 4.747 & 4.747 & & 1.0645 & $1.29 \mathrm{E}-02$ & 2447.0535 & 901.6672 & 3348.7207 & \\
\hline & 7/31/2008 11:00 & 214 & 213 & 1100 & 4.884 & 4.884 & & 1.0066 & $1.20 \mathrm{E}-02$ & 2284.2190 & 841.6675 & 3125.8864 & \\
\hline & $7 / 31 / 200812: 00$ & 215 & 213 & 1200 & 4.689 & 4.689 & & 1.0900 & 1.32E-02 & 2519.5172 & 928.3679 & 3447.8851 & \\
\hline & $7 / 31 / 200813: 00$ & 216 & 213 & 1300 & 4.92 & 4.92 & & 0.9919 & 1.18E-02 & 2243.2729 & 826.5800 & 3069.8529 & \\
\hline & $7 / 31 / 200814: 00$ & 217 & 213 & 1400 & 4.717 & 4.717 & & 1.0776 & 1.30E-02 & 2484.2627 & 915.3777 & 3399.6403 & \\
\hline & 7/31/2008 15:00 & 218 & 213 & 1500 & 4.766 & 4.766 & & 1.0562 & $1.27 \mathrm{E}-02$ & 2423.7839 & 893.0930 & 3316.8769 & \\
\hline & $7 / 31 / 200816: 00$ & 219 & 213 & 1600 & 4.886 & 4.886 & & 1.0057 & 1.20E-02 & 2281.9247 & 840.8221 & 3122.7468 & \\
\hline & $7 / 31 / 200817: 00$ & 220 & 213 & 1700 & 4.869 & 4.869 & & 1.0127 & $1.21 \mathrm{E}-02$ & 2301.5000 & 848.0350 & 3149.5350 & \\
\hline & 7/31/2008 18:00 & 221 & 213 & 1800 & 5.013 & 5.013 & & 0.9549 & 1.12E-02 & 2140.8397 & 788.8364 & 2929.6761 & \\
\hline & $7 / 31 / 2008$ 19:00 & 222 & 213 & 1900 & 5.232 & 5.232 & & 0.8731 & $1.01 E-02$ & 1917.2961 & 706.4672 & 2623.7633 & \\
\hline & $7 / 31 / 200820: 00$ & 223 & 213 & 2000 & 6.064 & 6.064 & & 0.6161 & $6.56 \mathrm{E}-03$ & 1248.2531 & 459.9445 & 1708.1976 & \\
\hline & $7 / 31 / 200821: 00$ & 224 & 213 & 2100 & 6.938 & 6.938 & & 0.4125 & 4.00E-03 & 761.8110 & 280.7049 & 1042.5159 & \\
\hline & $7 / 31 / 200822: 00$ & 225 & 213 & 2200 & 7.602 & 7.602 & & 0.2891 & $2.58 \mathrm{E}-03$ & 491.8812 & 181.2437 & 673.1250 & \\
\hline & $7 / 31 / 2008$ 23:00 & 226 & 213 & 2300 & 7.447 & 7.447 & & 0.3160 & 2.88E-03 & 548.6608 & 202.1653 & 750.8261 & \\
\hline & $8 / 1 / 20080: 00$ & 227 & 214 & 0 & 7.744 & 7.744 & 8.74 & 0.2655 & 2.33E-03 & 442.8549 & 163.1790 & 606.0339 & \\
\hline & 8/1/2008 1:00 & 228 & 214 & 100 & 8.26 & 8.26 & & 0.1864 & $1.51 \mathrm{E}-03$ & 286.6038 & 105.6051 & 392.2088 & \\
\hline & 8/1/2008 2:00 & 229 & 214 & 200 & 8.53 & 8.53 & & 0.1489 & 1.14E-03 & 217.2854 & 80.0633 & 297.3486 & \\
\hline & 8/1/2008 3:00 & 230 & 214 & 300 & 8.54 & 8.54 & & 0.1475 & 1.13E-03 & 214.8710 & 79.1736 & 294.0447 & \\
\hline & $8 / 1 / 20084: 00$ & 231 & 214 & 400 & 8.63 & 8.63 & & 0.1356 & 1.02E-03 & 193.6216 & 71.3439 & 264.9655 & \\
\hline & $8 / 1 / 20085: 00$ & 232 & 214 & 500 & 8.71 & 8.71 & & 0.1251 & $9.21 \mathrm{E}-04$ & 175.4511 & 64.6486 & 240.0996 & \\
\hline & 8/1/2008 6:00 & 233 & 214 & 600 & 8.74 & 8.74 & & 0.1213 & 8.87E-04 & 168.8097 & 62.2014 & 231.0111 & \\
\hline & $8 / 1 / 20087: 00$ & 234 & 214 & 700 & 8.7 & 8.7 & & 0.1264 & 9.33E-04 & 177.6857 & 65.4720 & 243.1577 & \\
\hline & $8 / 1 / 20088: 00$ & 235 & 214 & 800 & 8.52 & 8.52 & & 0.1502 & 1.15E-03 & 219.7104 & 80.9568 & 300.6673 & \\
\hline & 8/1/2008 9:00 & 236 & 214 & 900 & 6.816 & 6.816 & & 0.4378 & $4.30 \mathrm{E}-03$ & 819.6884 & 302.0310 & 1121.7194 & \\
\hline & $8 / 1 / 2008$ 10:00 & 237 & 214 & 1000 & 4.812 & 4.812 & & 1.0366 & 1.24E-02 & 2368.3777 & 872.6775 & 3241.0552 & \\
\hline & 8/1/2008 11:00 & 238 & 214 & 1100 & 4.948 & 4.948 & & 0.9806 & 1.16E-02 & 2211.9326 & 815.0321 & 3026.9647 & \\
\hline & 8/1/2008 12:00 & 239 & 214 & 1200 & 4.901 & 4.901 & & 0.9996 & 1.19E-02 & 2264.7910 & 834.5088 & 3099.2999 & \\
\hline & 8/1/2008 13:00 & 240 & 214 & 1300 & 4.843 & 4.843 & & 1.0235 & 1.22E-02 & 2331.7658 & 859.1871 & 3190.9529 & \\
\hline & $8 / 1 / 2008$ 14:00 & 241 & 214 & 1400 & 4.85 & 4.85 & & 1.0206 & 1.22E-02 & 2323.5781 & 856.1701 & 3179.7482 & \\
\hline & $8 / 1 / 2008$ 15:00 & 242 & 214 & 1500 & 4.83 & 4.83 & & 1.0290 & 1.23E-02 & 2347.0489 & 864.8185 & 3211.8674 & \\
\hline & $8 / 1 / 200816: 00$ & 243 & 214 & 1600 & 4.925 & 4.925 & & 0.9898 & $1.18 \mathrm{E}-02$ & 2237.6442 & 824.5060 & 3062.1502 & \\
\hline & 8/1/2008 17:00 & 244 & 214 & 1700 & 4.956 & 4.956 & & 0.9774 & 1.16E-02 & 2203.0584 & 811.7622 & 3014.8206 & \\
\hline & 8/1/2008 18:00 & 245 & 214 & 1800 & 5.041 & 5.041 & & 0.9441 & 1.11E-02 & 2110.9120 & 777.8089 & 2888.7209 & \\
\hline
\end{tabular}




\begin{tabular}{|c|c|c|c|c|c|c|c|c|c|c|c|c|c|}
\hline Tree ID & TS & RN & JD & HR & $\begin{array}{c}\text { Raw } \\
\text { TC_dTA }\end{array}$ & $\begin{array}{c}\text { Fixed } \\
\text { TC_dT } \Delta\end{array}$ & dTM & $\mathbf{K}$ & $\begin{array}{l}\text { Sap } \\
\text { Velocity } \\
\text { (cm/s) }\end{array}$ & $\begin{array}{l}\text { Probed } \\
\text { Drywood F } \\
\text { (g/hr) }\end{array}$ & $\begin{array}{c}\text { Extrapolated } \\
\text { Wetwood F } \\
(\mathrm{g} / \mathrm{hr})\end{array}$ & $\begin{array}{c}\text { Extrapolated } \\
\text { Total Wood F } \\
(\mathrm{g} / \mathrm{hr})\end{array}$ & $\begin{array}{l}\text { Relative } \\
\text { Humidity, } \\
@ 10 \mathrm{~m}(\%)\end{array}$ \\
\hline & 8/1/2008 19:00 & 246 & 214 & 1900 & 5.245 & 5.245 & & 0.8684 & $1.00 \mathrm{E}-02$ & 1904.7538 & 701.8457 & 2606.5995 & \\
\hline & 8/1/2008 20:00 & 247 & 214 & 2000 & 6.011 & 6.011 & & 0.6303 & $6.74 \mathrm{E}-03$ & 1283.8867 & 473.0745 & 1756.9612 & \\
\hline & 8/1/2008 21:00 & 248 & 214 & 2100 & 7.551 & 7.551 & & 0.2978 & $2.68 \mathrm{E}-03$ & 510.1782 & 187.9856 & 698.1638 & \\
\hline & 8/1/2008 22:00 & 249 & 214 & 2200 & 8.49 & 8.49 & & 0.1543 & 1.19E-03 & 227.0502 & 83.6613 & 310.7116 & \\
\hline & 8/1/2008 23:00 & 250 & 214 & 2300 & 8.72 & 8.72 & & 0.1239 & $9.10 \mathrm{E}-04$ & 173.2268 & 63.8290 & 237.0558 & \\
\hline & $8 / 2 / 20080: 00$ & 251 & 215 & 0 & 8.72 & 8.72 & 8.84 & 0.1239 & $9.10 \mathrm{E}-04$ & 173.2268 & 63.8290 & 237.0558 & \\
\hline & 8/2/2008 1:00 & 252 & 215 & 100 & 8.75 & 8.75 & & 0.1200 & 8.75E-04 & 166.6167 & 61.3933 & 228.0100 & \\
\hline & 8/2/2008 2:00 & 253 & 215 & 200 & 8.84 & 8.84 & & 0.1086 & 7.74E-04 & 147.3464 & 54.2928 & 201.6392 & \\
\hline & 8/2/2008 3:00 & 254 & 215 & 300 & 8.82 & 8.82 & & 0.1111 & 7.96E-04 & 151.5562 & 55.8440 & 207.4003 & \\
\hline & 8/2/2008 4:00 & 255 & 215 & 400 & 8.68 & 8.68 & & 0.1290 & 9.57E-04 & 182.1864 & 67.1303 & 249.3167 & \\
\hline & 8/2/2008 5:00 & 256 & 215 & 500 & 8.56 & 8.56 & & 0.1449 & 1.10E-03 & 210.0745 & 77.4063 & 287.4807 & \\
\hline & 8/2/2008 6:00 & 257 & 215 & 600 & 8.52 & 8.52 & & 0.1502 & 1.15E-03 & 219.7104 & 80.9568 & 300.6673 & \\
\hline & 8/2/2008 7:00 & 258 & 215 & 700 & 8.4 & 8.4 & & 0.1667 & $1.31 \mathrm{E}-03$ & 249.6561 & 91.9909 & 341.6470 & \\
\hline & 8/2/2008 8:00 & 259 & 215 & 800 & 6.966 & 6.966 & & 0.4068 & 3.93E-03 & 748.9243 & 275.9566 & 1024.8808 & \\
\hline & 8/2/2008 9:00 & 260 & 215 & 900 & 5.091 & 5.091 & & 0.9250 & $1.08 \mathrm{E}-02$ & 2058.4814 & 758.4898 & 2816.9713 & \\
\hline & 8/2/2008 10:00 & 261 & 215 & 1000 & 5.132 & 5.132 & & 0.9096 & $1.06 \mathrm{E}-02$ & 2016.4319 & 742.9958 & 2759.4277 & \\
\hline & 8/2/2008 11:00 & 262 & 215 & 1100 & 5.048 & 5.048 & & 0.9414 & $1.10 \mathrm{E}-02$ & 2103.4942 & 775.0757 & 2878.5699 & \\
\hline & 8/2/2008 12:00 & 263 & 215 & 1200 & 5 & 5 & & 0.9600 & 1.13E-02 & 2154.8758 & 794.0083 & 2948.8841 & \\
\hline & 8/2/2008 13:00 & 264 & 215 & 1300 & 4.983 & 4.983 & & 0.9667 & 1.14E-02 & 2173.3672 & 800.8219 & 2974.1891 & \\
\hline & $8 / 2 / 200814: 00$ & 265 & 215 & 1400 & 5.009 & 5.009 & & 0.9565 & 1.13E-02 & 2145.1489 & 790.4242 & 2935.5731 & \\
\hline & 8/2/2008 15:00 & 266 & 215 & 1500 & 4.993 & 4.993 & & 0.9627 & $1.14 \mathrm{E}-02$ & 2162.4711 & 796.8069 & 2959.2780 & \\
\hline & 8/2/2008 16:00 & 267 & 215 & 1600 & 5.046 & 5.046 & & 0.9421 & 1.11E-02 & 2105.6110 & 775.8557 & 2881.4666 & \\
\hline & 8/2/2008 17:00 & 268 & 215 & 1700 & 5.038 & 5.038 & & 0.9452 & 1.11E-02 & 2114.0989 & 778.9832 & 2893.0821 & \\
\hline & 8/2/2008 18:00 & 269 & 215 & 1800 & 5.199 & 5.199 & & 0.8850 & $1.02 \mathrm{E}-02$ & 1949.4861 & 718.3283 & 2667.8144 & \\
\hline & 8/2/2008 19:00 & 270 & 215 & 1900 & 5.336 & 5.336 & & 0.8366 & 9.55E-03 & 1819.0898 & 670.2811 & 2489.3709 & \\
\hline & 8/2/2008 20:00 & 271 & 215 & 2000 & 5.948 & 5.948 & & 0.6476 & $6.97 \mathrm{E}-03$ & 1327.3196 & 489.0782 & 1816.3978 & \\
\hline & 8/2/2008 21:00 & 272 & 215 & 2100 & 7.03 & 7.03 & & 0.3940 & $3.78 \mathrm{E}-03$ & 720.0074 & 265.3015 & 985.3089 & \\
\hline & 8/2/2008 22:00 & 273 & 215 & 2200 & 7.872 & 7.872 & & 0.2449 & $2.11 \mathrm{E}-03$ & 400.9889 & 147.7526 & 548.7415 & \\
\hline & 8/2/2008 23:00 & 274 & 215 & 2300 & 8.23 & 8.23 & & 0.1908 & $1.55 \mathrm{E}-03$ & 294.8096 & 108.6287 & 403.4383 & \\
\hline & $8 / 3 / 20080: 00$ & 275 & 216 & 0 & 8.32 & 8.32 & 8.84 & 0.1779 & 1.42E-03 & 270.4996 & 99.6712 & 370.1708 & \\
\hline & 8/3/2008 1:00 & 276 & 216 & 100 & 8.28 & 8.28 & & 0.1836 & $1.48 \mathrm{E}-03$ & 281.1904 & 103.6104 & 384.8008 & \\
\hline & 8/3/2008 2:00 & 277 & 216 & 200 & 8.46 & 8.46 & & 0.1584 & $1.23 \mathrm{E}-03$ & 234.4874 & 86.4017 & 320.8891 & \\
\hline & 8/3/2008 3:00 & 278 & 216 & 300 & 8.62 & 8.62 & & 0.1369 & $1.03 E-03$ & 195.9402 & 72.1982 & 268.1385 & \\
\hline & 8/3/2008 4:00 & 279 & 216 & 400 & 8.76 & 8.76 & & 0.1187 & 8.64E-04 & 164.4341 & 60.5891 & 225.0232 & \\
\hline & 8/3/2008 5:00 & 280 & 216 & 500 & 8.84 & 8.84 & & 0.1086 & 7.74E-04 & 147.3464 & 54.2928 & 201.6392 & \\
\hline & 8/3/2008 6:00 & 281 & 216 & 600 & 8.81 & 8.81 & & 0.1124 & 8.07E-04 & 153.6767 & 56.6253 & 210.3020 & \\
\hline & 8/3/2008 7:00 & 282 & 216 & 700 & 8.62 & 8.62 & & 0.1369 & 1.03E-03 & 195.9402 & 72.1982 & 268.1385 & \\
\hline & 8/3/2008 8:00 & 283 & 216 & 800 & 7.062 & 7.062 & & 0.3877 & 3.71E-03 & 705.8248 & 260.0757 & 965.9004 & \\
\hline & 8/3/2008 9:00 & 284 & 216 & 900 & 5.061 & 5.061 & & 0.9364 & $1.10 \mathrm{E}-02$ & 2089.7857 & 770.0245 & 2859.8102 & \\
\hline & 8/3/2008 10:00 & 285 & 216 & 1000 & 4.951 & 4.951 & & 0.9794 & $1.16 \mathrm{E}-02$ & 2208.6006 & 813.8043 & 3022.4050 & \\
\hline & 8/3/2008 11:00 & 286 & 216 & 1100 & 4.945 & 4.945 & & 0.9818 & 1.16E-02 & 2215.2696 & 816.2616 & 3031.5312 & \\
\hline
\end{tabular}




\begin{tabular}{|c|c|c|c|c|c|c|c|c|c|c|c|c|c|}
\hline Tree ID & TS & RN & JD & HR & $\begin{array}{c}\text { Raw } \\
\text { TC_dTA }\end{array}$ & $\begin{array}{c}\text { Fixed } \\
\text { TC_dT } \Delta\end{array}$ & dTM & $\mathbf{K}$ & $\begin{array}{c}\text { Sap } \\
\text { Velocity } \\
(\mathrm{cm} / \mathrm{s})\end{array}$ & $\begin{array}{l}\text { Probed } \\
\text { Drywood F } \\
\text { (g/hr) }\end{array}$ & $\begin{array}{c}\text { Extrapolated } \\
\text { Wetwood F } \\
(\mathrm{g} / \mathrm{hr})\end{array}$ & $\begin{array}{c}\text { Extrapolated } \\
\text { Total Wood F } \\
(\mathrm{g} / \mathrm{hr})\end{array}$ & $\begin{array}{l}\text { Relative } \\
\text { Humidity, } \\
@ 10 \mathrm{~m}(\%)\end{array}$ \\
\hline & 8/3/2008 12:00 & 287 & 216 & 1200 & 4.932 & 4.932 & & 0.9870 & 1.17E-02 & 2229.7876 & 821.6111 & 3051.3988 & \\
\hline & 8/3/2008 13:00 & 288 & 216 & 1300 & 4.937 & 4.937 & & 0.9850 & 1.17E-02 & 2224.1926 & 819.5495 & 3043.7421 & \\
\hline & 8/3/2008 14:00 & 289 & 216 & 1400 & 4.975 & 4.975 & & 0.9698 & 1.15E-02 & 2182.1232 & 804.0482 & 2986.1713 & \\
\hline & 8/3/2008 15:00 & 290 & 216 & 1500 & 5.061 & 5.061 & & 0.9364 & $1.10 \mathrm{E}-02$ & 2089.7857 & 770.0245 & 2859.8102 & \\
\hline & 8/3/2008 16:00 & 291 & 216 & 1600 & 5.141 & 5.141 & & 0.9062 & $1.05 \mathrm{E}-02$ & 2007.3129 & 739.6357 & 2746.9486 & \\
\hline & 8/3/2008 17:00 & 292 & 216 & 1700 & 5.092 & 5.092 & & 0.9246 & $1.08 \mathrm{E}-02$ & 2057.4458 & 758.1082 & 2815.5541 & \\
\hline & 8/3/2008 18:00 & 293 & 216 & 1800 & 5.246 & 5.246 & & 0.8681 & $1.00 \mathrm{E}-02$ & 1903.7922 & 701.4914 & 2605.2836 & \\
\hline & 8/3/2008 19:00 & 294 & 216 & 1900 & 5.309 & 5.309 & & 0.8459 & $9.68 \mathrm{E}-03$ & 1844.1234 & 679.5052 & 2523.6286 & \\
\hline & 8/3/2008 20:00 & 295 & 216 & 2000 & 5.729 & 5.729 & & 0.7106 & 7.81E-03 & 1487.9673 & 548.2721 & 2036.2394 & \\
\hline & 8/3/2008 21:00 & 296 & 216 & 2100 & 6.629 & 6.629 & & 0.4784 & $4.80 \mathrm{E}-03$ & 914.1478 & 336.8366 & 1250.9843 & \\
\hline & 8/3/2008 22:00 & 297 & 216 & 2200 & 7.277 . & 7.277 & & 0.3467 & 3.23E-03 & 615.0963 & 226.6449 & 841.7412 & \\
\hline & 8/3/2008 23:00 & 298 & 216 & 2300 & 7.332 & 7.332 & & 0.3366 & $3.11 \mathrm{E}-03$ & 593.1088 & 218.5431 & 811.6520 & \\
\hline & 8/4/2008 0:00 & 299 & 217 & 0 & 7.321 & 7.321 & 8.34 & 0.3386 & $3.14 \mathrm{E}-03$ & 597.4679 & 220.1493 & 817.6172 & \\
\hline & 8/4/2008 1:00 & 300 & 217 & 100 & 7.344 & 7.344 & & 0.3344 & 3.09E-03 & 588.3752 & 216.7989 & 805.1741 & \\
\hline & 8/4/2008 2:00 & 301 & 217 & 200 & 7.008 & 7.008 & & 0.3984 & 3.83E-03 & 729.8639 & 268.9334 & 998.7973 & \\
\hline & 8/4/2008 3:00 & 302 & 217 & 300 & 6.792 & 6.792 & & 0.4429 & 4.37E-03 & 831.4138 & 306.3515 & 1137.7653 & \\
\hline & 8/4/2008 4:00 & 303 & 217 & 400 & 6.593 & 6.593 & & 0.4864 & $4.90 \mathrm{E}-03$ & 933.1746 & 343.8474 & 1277.0220 & \\
\hline & 8/4/2008 5:00 & 304 & 217 & 500 & 6.794 & 6.794 & & 0.4424 & $4.36 \mathrm{E}-03$ & 830.4323 & 305.9899 & 1136.4222 & \\
\hline & 8/4/2008 6:00 & 305 & 217 & 600 & 7.252 & 7.252 & & 0.3514 & $3.28 \mathrm{E}-03$ & 625.2509 & 230.3865 & 855.6374 & \\
\hline & 8/4/2008 7:00 & 306 & 217 & 700 & 7.255 & 7.255 & & 0.3508 & $3.28 \mathrm{E}-03$ & 624.0270 & 229.9356 & 853.9625 & \\
\hline & 8/4/2008 8:00 & 307 & 217 & 800 & 6.867 . & 6.867 & & 0.4271 & $4.18 \mathrm{E}-03$ & 795.1467 & 292.9882 & 1088.1349 & \\
\hline & 8/4/2008 9:00 & 308 & 217 & 900 & 6.327 & 6.327 & & 0.5489 & 5.69E-03 & 1082.8777 & 399.0086 & 1481.8862 & \\
\hline & 8/4/2008 10:00 & 309 & 217 & 1000 & 5.631 & 5.631 & & 0.7404 & $8.22 \mathrm{E}-03$ & 1565.0738 & 576.6836 & 2141.7575 & \\
\hline & 8/4/2008 11:00 & 310 & 217 & 1100 & 5.372 & 5.372 & & 0.8243 & $9.38 \mathrm{E}-03$ & 1786.2017 & 658.1628 & 2444.3645 & \\
\hline & 8/4/2008 12:00 & 311 & 217 & 1200 & 5.164 & 5.164 & & 0.8978 & 1.04E-02 & 1984.1882 & 731.1149 & 2715.3031 & \\
\hline & 8/4/2008 13:00 & 312 & 217 & 1300 & 5.119 & 5.119 & & 0.9144 & $1.07 \mathrm{E}-02$ & 2029.6742 & 747.8752 & 2777.5493 & \\
\hline & 8/4/2008 14:00 & 313 & 217 & 1400 & 5.458 & 5.458 & & 0.7955 & $8.98 \mathrm{E}-03$ & 1709.8351 & 630.0239 & 2339.8591 & \\
\hline & 8/4/2008 15:00 & 314 & 217 & 1500 & 5.783 & 5.783 & & 0.6946 & $7.60 \mathrm{E}-03$ & 1446.9014 & 533.1406 & 1980.0419 & \\
\hline & 8/4/2008 16:00 & 315 & 217 & 1600 & 5.277 & 5.277 & & 0.8571 & $9.84 \mathrm{E}-03$ & 1874.2088 & 690.5908 & 2564.7995 & \\
\hline & 8/4/2008 17:00 & 316 & 217 & 1700 & 5.175 & 5.175 & & 0.8937 & 1.04E-02 & 1973.2188 & 727.0731 & 2700.2919 & \\
\hline & 8/4/2008 18:00 & 317 & 217 & 1800 & 5.323 & 5.323 & & 0.8411 & 9.62E-03 & 1831.1033 & 674.7077 & 2505.8110 & \\
\hline & 8/4/2008 19:00 & 318 & 217 & 1900 & 5.523 & 5.523 & & 0.7744 & 8.69E-03 & 1654.0982 & 609.4865 & 2263.5847 & \\
\hline & 8/4/2008 20:00 & 319 & 217 & 2000 & 6.163 & 6.163 & & 0.5901 & $6.22 \mathrm{E}-03$ & 1183.8241 & 436.2044 & 1620.0285 & \\
\hline & 8/4/2008 21:00 & 320 & 217 & 2100 & 7.533 & 7.533 & & 0.3009 & $2.71 \mathrm{E}-03$ & 516.7252 & 190.3980 & 707.1232 & \\
\hline & 8/4/2008 22:00 & 321 & 217 & 2200 & 8.16 & 8.16 & & 0.2010 & 1.65E-03 & 314.3609 & 115.8327 & 430.1937 & \\
\hline & 8/4/2008 23:00 & 322 & 217 & 2300 & 8.34 & 8.34 & & 0.1751 & $1.39 \mathrm{E}-03$ & 265.2219 & 97.7264 & 362.9483 & \\
\hline & 8/5/2008 0:00 & 323 & 218 & 0 & 8.57 & 8.57 & 9.06 & 0.1435 & 1.09E-03 & 207.6922 & 76.5285 & 284.2206 & \\
\hline & 8/5/2008 1:00 & 324 & 218 & 100 & 8.71 & 8.71 & & 0.1251 & 9.21E-04 & 175.4511 & 64.6486 & 240.0996 & \\
\hline & 8/5/2008 2:00 & 325 & 218 & 200 & 8.78 & 8.78 & & 0.1162 & $8.41 \mathrm{E}-04$ & 160.1000 & 58.9921 & 219.0922 & \\
\hline & 8/5/2008 3:00 & 326 & 218 & 300 & 8.74 & 8.74 & & 0.1213 & 8.87E-04 & 168.8097 & 62.2014 & 231.0111 & \\
\hline & 8/5/2008 4:00 & 327 & 218 & 400 & 8.52 & 8.52 & & 0.1502 & 1.15E-03 & 219.7104 & 80.9568 & 300.6673 & \\
\hline
\end{tabular}




\begin{tabular}{|c|c|c|c|c|c|c|c|c|c|c|c|c|c|}
\hline Tree ID & TS & RN & JD & HR & $\begin{array}{c}\text { Raw } \\
\text { TC_dTA }\end{array}$ & $\begin{array}{c}\text { Fixed } \\
\text { TC_dT } \Delta\end{array}$ & dTM & $\mathbf{K}$ & $\begin{array}{c}\text { Sap } \\
\text { Velocity } \\
(\mathrm{cm} / \mathrm{s})\end{array}$ & $\begin{array}{l}\text { Probed } \\
\text { Drywood F } \\
\text { (g/hr) }\end{array}$ & $\begin{array}{c}\text { Extrapolated } \\
\text { Wetwood F } \\
(\mathrm{g} / \mathrm{hr})\end{array}$ & $\begin{array}{c}\text { Extrapolated } \\
\text { Total Wood F } \\
(\mathrm{g} / \mathrm{hr})\end{array}$ & $\begin{array}{l}\text { Relative } \\
\text { Humidity, } \\
@ 10 \mathrm{~m}(\%)\end{array}$ \\
\hline & 8/5/2008 5:00 & 328 & 218 & 500 & 8.43 & 8.43 & & 0.1625 & $1.27 \mathrm{E}-03$ & 242.0224 & 89.1781 & 331.2006 & \\
\hline & 8/5/2008 6:00 & 329 & 218 & 600 & 8.52 & 8.52 & & 0.1502 & 1.15E-03 & 219.7104 & 80.9568 & 300.6673 & \\
\hline & 8/5/2008 7:00 & 330 & 218 & 700 & 8.57 & 8.57 & & 0.1435 & 1.09E-03 & 207.6922 & 76.5285 & 284.2206 & \\
\hline & 8/5/2008 8:00 & 331 & 218 & 800 & 8.39 & 8.39 & & 0.1681 & 1.32E-03 & 252.2227 & 92.9366 & 345.1593 & \\
\hline & 8/5/2008 9:00 & 332 & 218 & 900 & 6.78 & 6.78 & & 0.4454 & 4.40E-03 & 837.3194 & 308.5275 & 1145.8469 & \\
\hline & 8/5/2008 10:00 & 333 & 218 & 1000 & 5.47 & 5.47 & & 0.7916 & 8.92E-03 & 1699.4193 & 626.1860 & 2325.6053 & \\
\hline & 8/5/2008 11:00 & 334 & 218 & 1100 & 5.83 & 5.83 & & 0.6810 & 7.42E-03 & 1411.9505 & 520.2622 & 1932.2127 & \\
\hline & 8/5/2008 12:00 & 335 & 218 & 1200 & 5.564 & 5.564 & & 0.7613 & 8.51E-03 & 1619.7858 & 596.8434 & 2216.6291 & \\
\hline & 8/5/2008 13:00 & 336 & 218 & 1300 & 5.229 & 5.229 & & 0.8742 & 1.01E-02 & 1920.2015 & 707.5378 & 2627.7393 & \\
\hline & 8/5/2008 14:00 & 337 & 218 & 1400 & 5.713 & 5.713 & & 0.7154 & $7.88 \mathrm{E}-03$ & 1500.3259 & 552.8259 & 2053.1518 & \\
\hline & 8/5/2008 15:00 & 338 & 218 & 1500 & 6.073 & 6.073 & & 0.6137 & $6.52 \mathrm{E}-03$ & 1242.2824 & 457.7445 & 1700.0269 & \\
\hline & 8/5/2008 16:00 & 339 & 218 & 1600 & 5.848 & 5.848 & & 0.6758 & 7.35E-03 & 1398.7559 & 515.4004 & 1914.1563 & \\
\hline & 8/5/2008 17:00 & 340 & 218 & 1700 & 5.538 & 5.538 & & 0.7696 & $8.62 \mathrm{E}-03$ & 1641.4703 & 604.8335 & 2246.3037 & \\
\hline & 8/5/2008 18:00 & 341 & 218 & 1800 & 5.296 & 5.296 & & 0.8505 & 9.75E-03 & 1856.2907 & 683.9885 & 2540.2792 & \\
\hline & 8/5/2008 19:00 & 342 & 218 & 1900 & 5.389 & 5.389 & & 0.8185 & 9.30E-03 & 1770.8627 & 652.5108 & 2423.3735 & \\
\hline & 8/5/2008 20:00 & 343 & 218 & 2000 & 6.294 & 6.294 & & 0.5570 & 5.79E-03 & 1102.6330 & 406.2878 & 1508.9208 & \\
\hline & 8/5/2008 21:00 & 344 & 218 & 2100 & 7.963 & 7.963 & & 0.2307 & $1.96 \mathrm{E}-03$ & 372.5112 & 137.2594 & 509.7706 & \\
\hline & 8/5/2008 22:00 & 345 & 218 & 2200 & 8.8 & 8.8 & & 0.1136 & $8.18 E-04$ & 155.8074 & 57.4105 & 213.2179 & \\
\hline & 8/5/2008 23:00 & 346 & 218 & 2300 & 9.06 & 9.06 & & 0.0817 & $5.45 \mathrm{E}-04$ & 103.7638 & 38.2339 & 141.9977 & \\
\hline & 8/6/2008 0:00 & 347 & 219 & 0 & 9.2 & 9.2 & 9.24 & 0.0652 & $4.13 \mathrm{E}-04$ & 78.6553 & 28.9821 & 107.6374 & \\
\hline & 8/6/2008 1:00 & 348 & 219 & 100 & 9.24 & 9.24 & & 0.0606 & $3.77 \mathrm{E}-04$ & 71.8660 & 26.4805 & 98.3465 & \\
\hline & 8/6/2008 2:00 & 349 & 219 & 200 & 9.22 & 9.22 & & 0.0629 & 3.95E-04 & 75.2389 & 27.7233 & 102.9622 & \\
\hline & 8/6/2008 3:00 & 350 & 219 & 300 & 9.17 & 9.17 & & 0.0687 & $4.40 \mathrm{E}-04$ & 83.8606 & 30.9002 & 114.7607 & \\
\hline & 8/6/2008 4:00 & 351 & 219 & 400 & 9.11 & 9.11 & & 0.0757 & $4.97 \mathrm{E}-04$ & 94.5589 & 34.8422 & 129.4011 & \\
\hline & 8/6/2008 5:00 & 352 & 219 & 500 & 9.05 & 9.05 & & 0.0829 & $5.55 \mathrm{E}-04$ & 105.6361 & 38.9238 & 144.5599 & \\
\hline & 8/6/2008 6:00 & 353 & 219 & 600 & 8.94 & 8.94 & & 0.0962 & $6.67 \mathrm{E}-04$ & 126.9163 & 46.7649 & 173.6812 & \\
\hline & 8/6/2008 7:00 & 354 & 219 & 700 & 8.83 & 8.83 & & 0.1099 & $7.85 \mathrm{E}-04$ & 149.4461 & 55.0665 & 204.5127 & \\
\hline & 8/6/2008 8:00 & 355 & 219 & 800 & 8.65 & 8.65 & & 0.1329 & 9.93E-04 & 189.0160 & 69.6468 & 258.6628 & \\
\hline & 8/6/2008 9:00 & 356 & 219 & 900 & 8.06 & 8.06 & & 0.2159 & $1.80 \mathrm{E}-03$ & 343.2923 & 126.4931 & 469.7854 & \\
\hline & 8/6/2008 10:00 & 357 & 219 & 1000 & 5.566 & 5.566 & & 0.7607 & 8.50E-03 & 1618.1284 & 596.2327 & 2214.3610 & \\
\hline & 8/6/2008 11:00 & 358 & 219 & 1100 & 4.75 & 4.75 & & 1.0632 & $1.28 \mathrm{E}-02$ & 2443.3642 & 900.3078 & 3343.6720 & \\
\hline & 8/6/2008 12:00 & 359 & 219 & 1200 & 5.405 & 5.405 & & 0.8131 & $9.22 \mathrm{E}-03$ & 1756.5367 & 647.2321 & 2403.7688 & \\
\hline & 8/6/2008 13:00 & 360 & 219 & 1300 & 5.759 & 5.759 & & 0.7017 & 7.69E-03 & 1465.0312 & 539.8209 & 2004.8521 & \\
\hline & 8/6/2008 14:00 & 361 & 219 & 1400 & 5.265 & 5.265 & & 0.8613 & $9.90 \mathrm{E}-03$ & 1885.6088 & 694.7913 & 2580.4001 & \\
\hline & $8 / 6 / 2008$ 15:00 & 362 & 219 & 1500 & 5.054 & 5.054 & & 0.9391 & 1.10E-02 & 2097.1564 & 772.7404 & 2869.8968 & \\
\hline & 8/6/2008 16:00 & 363 & 219 & 1600 & 5.142 & 5.142 & & 0.9059 & $1.05 \mathrm{E}-02$ & 2006.3021 & 739.2633 & 2745.5654 & \\
\hline & 8/6/2008 17:00 & 364 & 219 & 1700 & 5.071 & 5.071 & & 0.9326 & 1.09E-02 & 2079.2999 & 766.1608 & 2845.4608 & \\
\hline & 8/6/2008 18:00 & 365 & 219 & 1800 & 5.612 & 5.612 & & 0.7463 & 8.30E-03 & 1580.4207 & 582.3385 & 2162.7593 & \\
\hline & 8/6/2008 19:00 & 366 & 219 & 1900 & 5.606 & 5.606 & & 0.7481 & 8.33E-03 & 1585.2946 & 584.1344 & 2169.4290 & \\
\hline & 8/6/2008 20:00 & 367 & 219 & 2000 & 6.008 & 6.008 & & 0.6312 & $6.75 \mathrm{E}-03$ & 1285.9282 & 473.8267 & 1759.7549 & \\
\hline & 8/6/2008 21:00 & 368 & 219 & 2100 & 7.511 & 7.511 & & 0.3048 & 2.76E-03 & 524.7910 & 193.3700 & 718.1610 & \\
\hline
\end{tabular}




\begin{tabular}{|c|c|c|c|c|c|c|c|c|c|c|c|c|c|}
\hline Tree ID & TS & RN & JD & HR & $\begin{array}{c}\text { Raw } \\
\text { TC_dTA }\end{array}$ & $\begin{array}{c}\text { Fixed } \\
\text { TC_dT } \Delta\end{array}$ & dTM & $\mathbf{K}$ & $\begin{array}{l}\text { Sap } \\
\text { Velocity } \\
\text { (cm/s) }\end{array}$ & $\begin{array}{l}\text { Probed } \\
\text { Drywood F } \\
\text { (g/hr) }\end{array}$ & $\begin{array}{c}\text { Extrapolated } \\
\text { Wetwood F } \\
(\mathrm{g} / \mathrm{hr})\end{array}$ & $\begin{array}{c}\text { Extrapolated } \\
\text { Total Wood F } \\
(\mathrm{g} / \mathrm{hr})\end{array}$ & $\begin{array}{l}\text { Relative } \\
\text { Humidity, } \\
@ 10 \mathrm{~m}(\%)\end{array}$ \\
\hline & 8/6/2008 22:00 & 369 & 219 & 2200 & 8.63 & 8.63 & & 0.1356 & $1.02 \mathrm{E}-03$ & 193.6216 & 71.3439 & 264.9655 & \\
\hline & 8/6/2008 23:00 & 370 & 219 & 2300 & 8.97 & 8.97 & & 0.0925 & $6.35 \mathrm{E}-04$ & 120.9885 & 44.5807 & 165.5692 & \\
\hline & 8/7/2008 0:00 & 371 & 220 & 0 & 9.09 & 9.09 & 9.12 & 0.0781 & $5.16 \mathrm{E}-04$ & 98.2094 & 36.1873 & 134.3967 & \\
\hline & 8/7/2008 1:00 & 372 & 220 & 100 & 9.12 & 9.12 & & 0.0746 & 4.87E-04 & 92.7494 & 34.1754 & 126.9248 & \\
\hline & 8/7/2008 2:00 & 373 & 220 & 200 & 9.12 & 9.12 & & 0.0746 & 4.87E-04 & 92.7494 & 34.1754 & 126.9248 & \\
\hline & 8/7/2008 3:00 & 374 & 220 & 300 & 9.07 & 9.07 & & 0.0805 & $5.35 E-04$ & 101.9019 & 37.5478 & 139.4497 & \\
\hline & 8/7/2008 4:00 & 375 & 220 & 400 & 9.02 & 9.02 & & 0.0865 & $5.85 \mathrm{E}-04$ & 111.3155 & 41.0165 & 152.3320 & \\
\hline & 8/7/2008 5:00 & 376 & 220 & 500 & 8.94 & 8.94 & & 0.0962 & $6.67 \mathrm{E}-04$ & 126.9163 & 46.7649 & 173.6812 & \\
\hline & 8/7/2008 6:00 & 377 & 220 & 600 & 8.87 & 8.87 & & 0.1048 & 7.41E-04 & 141.1091 & 51.9945 & 193.1036 & \\
\hline & 8/7/2008 7:00 & 378 & 220 & 700 & 8.8 & 8.8 & & 0.1136 & $8.18 \mathrm{E}-04$ & 155.8074 & 57.4105 & 213.2179 & \\
\hline & 8/7/2008 8:00 & 379 & 220 & 800 & 8.56 & 8.56 & & 0.1449 & 1.10E-03 & 210.0745 & 77.4063 & 287.4807 & \\
\hline & 8/7/20089:00 & 380 & 220 & 900 & 7.279 & 7.279 & & 0.3463 & 3.23E-03 & 614.2883 & 226.3472 & 840.6354 & \\
\hline & 8/7/2008 10:00 & 381 & 220 & 1000 & 4.877 & 4.877 & & 1.0094 & $1.20 \mathrm{E}-02$ & 2292.2672 & 844.6330 & 3136.9002 & \\
\hline & 8/7/2008 11:00 & 382 & 220 & 1100 & 4.658 & 4.658 & & 1.1039 & $1.34 \mathrm{E}-02$ & 2559.1537 & 942.9728 & 3502.1265 & \\
\hline & 8/7/2008 12:00 & 383 & 220 & 1200 & 4.684 & 4.684 & & 1.0922 & 1.33E-02 & 2525.8668 & 930.7076 & 3456.5744 & \\
\hline & 8/7/2008 13:00 & 384 & 220 & 1300 & 4.7 & 4.7 & & 1.0851 & 1.32E-02 & 2505.6060 & 923.2420 & 3428.8480 & \\
\hline & 8/7/2008 14:00 & 385 & 220 & 1400 & 4.752 & 4.752 & & 1.0623 & $1.28 \mathrm{E}-02$ & 2440.9078 & 899.4027 & 3340.3105 & \\
\hline & 8/7/2008 15:00 & 386 & 220 & 1500 & 4.798 & 4.798 & & 1.0425 & $1.25 \mathrm{E}-02$ & 2385.1026 & 878.8401 & 3263.9427 & \\
\hline & 8/7/2008 16:00 & 387 & 220 & 1600 & 4.8 & 4.8 & & 1.0417 & $1.25 \mathrm{E}-02$ & 2382.7060 & 877.9570 & 3260.6630 & \\
\hline & 8/7/2008 17:00 & 388 & 220 & 1700 & 4.784 & 4.784 & & 1.0485 & $1.26 \mathrm{E}-02$ & 2401.9476 & 885.0470 & 3286.9946 & \\
\hline & 8/7/2008 18:00 & 389 & 220 & 1800 & 4.949 & 4.949 & & 0.9802 & $1.16 \mathrm{E}-02$ & 2210.8214 & 814.6226 & 3025.4440 & \\
\hline & 8/7/2008 19:00 & 390 & 220 & 1900 & 5.161 & 5.161 & & 0.8989 & 1.04E-02 & 1987.1899 & 732.2210 & 2719.4109 & \\
\hline & 8/7/2008 20:00 & 391 & 220 & 2000 & 5.8 & 5.8 & & 0.6897 & 7.53E-03 & 1434.1756 & 528.4515 & 1962.6271 & \\
\hline & 8/7/2008 21:00 & 392 & 220 & 2100 & 7.304 & 7.304 & & 0.3417 & 3.17E-03 & 604.2423 & 222.6455 & 826.8878 & \\
\hline & 8/7/2008 22:00 & 393 & 220 & 2200 & 8.23 & 8.23 & & 0.1908 & $1.55 \mathrm{E}-03$ & 294.8096 & 108.6287 & 403.4383 & \\
\hline & 8/7/2008 23:00 & 394 & 220 & 2300 & 8.61 & 8.61 & & 0.1382 & $1.04 \mathrm{E}-03$ & 198.2694 & 73.0565 & 271.3259 & \\
\hline & $8 / 8 / 20080: 00$ & 395 & 221 & 0 & 8.82 & 8.82 & 8.87 & 0.1111 & 7.96E-04 & 151.5562 & 55.8440 & 207.4003 & \\
\hline & 8/8/2008 1:00 & 396 & 221 & 100 & 8.83 & 8.83 & & 0.1099 & $7.85 \mathrm{E}-04$ & 149.4461 & 55.0665 & 204.5127 & \\
\hline & 8/8/2008 2:00 & 397 & 221 & 200 & 8.85 & 8.85 & & 0.1073 & $7.63 \mathrm{E}-04$ & 145.2570 & 53.5229 & 198.7799 & \\
\hline & 8/8/2008 3:00 & 398 & 221 & 300 & 8.87 & 8.87 & & 0.1048 & 7.41E-04 & 141.1091 & 51.9945 & 193.1036 & \\
\hline & 8/8/2008 4:00 & 399 & 221 & 400 & 8.87 & 8.87 & & 0.1048 & $7.41 \mathrm{E}-04$ & 141.1091 & 51.9945 & 193.1036 & \\
\hline & 8/8/2008 5:00 & 400 & 221 & 500 & 8.86 & 8.86 & & 0.1061 & $7.52 \mathrm{E}-04$ & 143.1778 & 52.7568 & 195.9347 & \\
\hline & 8/8/2008 6:00 & 401 & 221 & 600 & 8.77 & 8.77 & & 0.1174 & $8.52 E-04$ & 162.2619 & 59.7887 & 222.0506 & \\
\hline & 8/8/2008 7:00 & 402 & 221 & 700 & 8.7 & 8.7 & & 0.1264 & 9.33E-04 & 177.6857 & 65.4720 & 243.1577 & \\
\hline & 8/8/2008 8:00 & 403 & 221 & 800 & 8.46 & 8.46 & & 0.1584 & 1.23E-03 & 234.4874 & 86.4017 & 320.8891 & \\
\hline & 8/8/2008 9:00 & 404 & 221 & 900 & 6.232 & 6.232 & & 0.5725 & $5.99 \mathrm{E}-03$ & 1140.4991 & 420.2404 & 1560.7395 & \\
\hline & 8/8/2008 10:00 & 405 & 221 & 1000 & 4.691 & 4.691 & & 1.0891 & $1.32 \mathrm{E}-02$ & 2516.9819 & 927.4338 & 3444.4157 & \\
\hline & 8/8/2008 11:00 & 406 & 221 & 1100 & 4.884 & 4.884 & & 1.0066 & $1.20 \mathrm{E}-02$ & 2284.2190 & 841.6675 & 3125.8864 & \\
\hline & 8/8/2008 12:00 & 407 & 221 & 1200 & 4.76 & 4.76 & & 1.0588 & $1.28 \mathrm{E}-02$ & 2431.1076 & 895.7916 & 3326.8992 & \\
\hline & 8/8/2008 13:00 & 408 & 221 & 1300 & 4.73 & 4.73 & & 1.0719 & $1.30 \mathrm{E}-02$ & 2468.0678 & 909.4103 & 3377.4781 & \\
\hline & 8/8/2008 14:00 & 409 & 221 & 1400 & 4.723 & 4.723 & & 1.0750 & 1.30E-02 & 2476.7745 & 912.6185 & 3389.3931 & \\
\hline
\end{tabular}




\begin{tabular}{|c|c|c|c|c|c|c|c|c|c|c|c|c|c|}
\hline Tree ID & TS & RN & JD & HR & $\begin{array}{c}\text { Raw } \\
\text { TC_dTA }\end{array}$ & $\begin{array}{c}\text { Fixed } \\
\text { TC_dT } \Delta\end{array}$ & dTM & $\mathbf{K}$ & $\begin{array}{c}\text { Sap } \\
\text { Velocity } \\
(\mathrm{cm} / \mathrm{s})\end{array}$ & $\begin{array}{c}\text { Probed } \\
\text { Drywood F } \\
(\mathrm{g} / \mathrm{hr})\end{array}$ & $\begin{array}{c}\text { Extrapolated } \\
\text { Wetwood F } \\
(\mathrm{g} / \mathrm{hr})\end{array}$ & $\begin{array}{c}\text { Extrapolated } \\
\text { Total Wood F } \\
(\mathbf{g} / \mathrm{hr})\end{array}$ & $\begin{array}{l}\text { Relative } \\
\text { Humidity, } \\
@ 10 \mathrm{~m}(\%)\end{array}$ \\
\hline & 8/8/2008 15:00 & 410 & 221 & 1500 & 4.752 & 4.752 & & 1.0623 & $1.28 \mathrm{E}-02$ & 2440.9078 & 899.4027 & 3340.3105 & \\
\hline & 8/8/2008 16:00 & 411 & 221 & 1600 & 4.791 & 4.791 & & 1.0455 & $1.26 \mathrm{E}-02$ & 2393.5100 & 881.9380 & 3275.4480 & \\
\hline & 8/8/2008 17:00 & 412 & 221 & 1700 & 4.913 & 4.913 & & 0.9947 & 1.18E-02 & 2251.1768 & 829.4924 & 3080.6692 & \\
\hline & 8/8/2008 18:00 & 413 & 221 & 1800 & 5.222 & 5.222 & & 0.8767 & $1.01 \mathrm{E}-02$ & 1926.9971 & 710.0417 & 2637.0388 & \\
\hline & 8/8/2008 19:00 & 414 & 221 & 1900 & 5.188 & 5.188 & & 0.8890 & 1.03E-02 & 1960.3297 & 722.3238 & 2682.6535 & \\
\hline & $8 / 8 / 200820: 00$ & 415 & 221 & 2000 & 5.96 & 5.96 & & 0.6443 & $6.93 \mathrm{E}-03$ & 1318.9549 & 485.9961 & 1804.9509 & \\
\hline & 8/8/2008 21:00 & 416 & 221 & 2100 & 7.331 & 7.331 & & 0.3368 & 3.12E-03 & 593.5043 & 218.6889 & 812.1932 & \\
\hline & 8/8/2008 22:00 & 417 & 221 & 2200 & 8.07 & 8.07 & & 0.2144 & 1.79E-03 & 340.3453 & 125.4072 & 465.7525 & \\
\hline & 8/8/2008 23:00 & 418 & 221 & 2300 & 8.25 & 8.25 & & 0.1879 & $1.52 \mathrm{E}-03$ & 289.3276 & 106.6087 & 395.9363 & \\
\hline & 8/9/2008 0:00 & 419 & 222 & 0 & 8.2 & 8.2 & 9.01 & 0.1951 & $1.59 \mathrm{E}-03$ & 303.1190 & 111.6904 & 414.8095 & \\
\hline & 8/9/2008 1:00 & 420 & 222 & 100 & 8.05 & 8.05 & & 0.2174 & 1.82E-03 & 346.2513 & 127.5834 & 473.8348 & \\
\hline & 8/9/2008 2:00 & 421 & 222 & 200 & 8.01 & 8.01 & & 0.2235 & $1.88 \mathrm{E}-03$ & 358.2093 & 131.9896 & 490.1988 & \\
\hline & 8/9/2008 3:00 & 422 & 222 & 300 & 7.98 & 7.98 & & 0.2281 & $1.93 \mathrm{E}-03$ & 367.3065 & 135.3417 & 502.6482 & \\
\hline & 8/9/2008 4:00 & 423 & 222 & 400 & 7.878 & 7.878 & & 0.2440 & $2.10 \mathrm{E}-03$ & 399.0788 & 147.0488 & 546.1276 & \\
\hline & 8/9/2008 5:00 & 424 & 222 & 500 & 7.747 & 7.747 & & 0.2650 & $2.32 \mathrm{E}-03$ & 441.8489 & 162.8083 & 604.6572 & \\
\hline & 8/9/2008 6:00 & 425 & 222 & 600 & 7.719 & 7.719 & & 0.2696 & $2.37 \mathrm{E}-03$ & 451.2858 & 166.2855 & 617.5713 & \\
\hline & 8/9/2008 7:00 & 426 & 222 & 700 & 7.379 & 7.379 & & 0.3281 & $3.02 \mathrm{E}-03$ & 574.6971 & 211.7590 & 786.4561 & \\
\hline & 8/9/2008 8:00 & 427 & 222 & 800 & 6.517 & 6.517 & & 0.5038 & $5.12 E-03$ & 974.2784 & 358.9929 & 1333.2713 & \\
\hline & 8/9/2008 9:00 & 428 & 222 & 900 & 7.127 & 7.127 & & 0.3751 & 3.56E-03 & 677.5695 & 249.6644 & 927.2339 & \\
\hline & $8 / 9 / 200810: 00$ & 429 & 222 & 1000 & 6.831 & 6.831 & & 0.4346 & $4.27 \mathrm{E}-03$ & 812.4176 & 299.3520 & 1111.7696 & \\
\hline & 8/9/2008 11:00 & 430 & 222 & 1100 & 6.043 & 6.043 & & 0.6217 & $6.63 \mathrm{E}-03$ & 1262.2748 & 465.1111 & 1727.3859 & \\
\hline & 8/9/2008 12:00 & 431 & 222 & 1200 & 5.402 & 5.402 & & 0.8141 & 9.24E-03 & 1759.2147 & 648.2188 & 2407.4335 & \\
\hline & 8/9/2008 13:00 & 432 & 222 & 1300 & 5.338 & 5.338 & & 0.8359 & 9.54E-03 & 1817.2481 & 669.6024 & 2486.8505 & \\
\hline & 8/9/2008 14:00 & 433 & 222 & 1400 & 5.007 & 5.007 & & 0.9573 & $1.13 \mathrm{E}-02$ & 2147.3067 & 791.2193 & 2938.5260 & \\
\hline & 8/9/2008 15:00 & 434 & 222 & 1500 & 4.972 & 4.972 & & 0.9710 & 1.15E-02 & 2185.4156 & 805.2613 & 2990.6769 & \\
\hline & 8/9/2008 16:00 & 435 & 222 & 1600 & 5.029 & 5.029 & & 0.9487 & 1.12E-02 & 2123.6877 & 782.5164 & 2906.2041 & \\
\hline & 8/9/2008 17:00 & 436 & 222 & 1700 & 5.107 & 5.107 & & 0.9189 & $1.07 \mathrm{E}-02$ & 2041.9721 & 752.4066 & 2794.3788 & \\
\hline & 8/9/2008 18:00 & 437 & 222 & 1800 & 5.182 & 5.182 & & 0.8912 & $1.03 \mathrm{E}-02$ & 1966.2685 & 724.5121 & 2690.7806 & \\
\hline & 8/9/2008 19:00 & 438 & 222 & 1900 & 5.337 & 5.337 & & 0.8362 & $9.55 \mathrm{E}-03$ & 1818.1688 & 669.9417 & 2488.1104 & \\
\hline & 8/9/2008 20:00 & 439 & 222 & 2000 & 6.048 & 6.048 & & 0.6204 & $6.61 E-03$ & 1258.9248 & 463.8767 & 1722.8015 & \\
\hline & 8/9/2008 21:00 & 440 & 222 & 2100 & 7.8 & 7.8 & & 0.2564 & 2.23E-03 & 424.2734 & 156.3323 & 580.6057 & \\
\hline & 8/9/2008 22:00 & 441 & 222 & 2200 & 8.75 & 8.75 & & 0.1200 & $8.75 \mathrm{E}-04$ & 166.6167 & 61.3933 & 228.0100 & \\
\hline & 8/9/2008 23:00 & 442 & 222 & 2300 & 9.01 & 9.01 & & 0.0877 & $5.95 E-04$ & 113.2294 & 41.7217 & 154.9511 & \\
\hline & 8/10/2008 0:00 & 443 & 223 & 0 & 9.12 & 9.12 & 9.15 & 0.0746 & 4.87E-04 & 92.7494 & 34.1754 & 126.9248 & \\
\hline & 8/10/2008 1:00 & 444 & 223 & 100 & 9.15 & 9.15 & & 0.0710 & 4.59E-04 & 87.3843 & 32.1985 & 119.5828 & \\
\hline & 8/10/2008 2:00 & 445 & 223 & 200 & 9.14 & 9.14 & & 0.0722 & $4.68 \mathrm{E}-04$ & 89.1621 & 32.8536 & 122.0157 & \\
\hline & 8/10/2008 3:00 & 446 & 223 & 300 & 9.1 & 9.1 & & 0.0769 & $5.06 \mathrm{E}-04$ & 96.3789 & 35.5128 & 131.8917 & \\
\hline & 8/10/2008 4:00 & 447 & 223 & 400 & 9.04 & 9.04 & & 0.0841 & $5.65 \mathrm{E}-04$ & 107.5188 & 39.6175 & 147.1364 & \\
\hline & 8/10/2008 5:00 & 448 & 223 & 500 & 8.98 & 8.98 & & 0.0913 & $6.25 \mathrm{E}-04$ & 119.0332 & 43.8602 & 162.8934 & \\
\hline & 8/10/2008 6:00 & 449 & 223 & 600 & 8.91 & 8.91 & & 0.0999 & $6.98 \mathrm{E}-04$ & 132.9370 & 48.9834 & 181.9204 & \\
\hline & 8/10/2008 7:00 & 450 & 223 & 700 & 8.84 & 8.84 & & 0.1086 & 7.74E-04 & 147.3464 & 54.2928 & 201.6392 & \\
\hline
\end{tabular}




\begin{tabular}{|c|c|c|c|c|c|c|c|c|c|c|c|c|c|}
\hline Tree ID & TS & RN & JD & HR & $\begin{array}{c}\text { Raw } \\
\text { TC_dTA }\end{array}$ & $\begin{array}{c}\text { Fixed } \\
\text { TC_dT } \Delta\end{array}$ & dTM & $\mathbf{K}$ & $\begin{array}{l}\text { Sap } \\
\text { Velocity } \\
\text { (cm/s) }\end{array}$ & $\begin{array}{l}\text { Probed } \\
\text { Drywood F } \\
\text { (g/hr) }\end{array}$ & $\begin{array}{c}\text { Extrapolated } \\
\text { Wetwood F } \\
(\mathrm{g} / \mathrm{hr})\end{array}$ & $\begin{array}{c}\text { Extrapolated } \\
\text { Total Wood F } \\
(\mathrm{g} / \mathrm{hr})\end{array}$ & $\begin{array}{c}\text { Relative } \\
\text { Humidity, } \\
\text { @10m (\%) }\end{array}$ \\
\hline & 8/10/2008 8:00 & 451 & 223 & 800 & 8.65 & 8.65 & & 0.1329 & 9.93E-04 & 189.0160 & 69.6468 & 258.6628 & \\
\hline & 8/10/2008 9:00 & 452 & 223 & 900 & 7.368 & 7.368 & & 0.3301 & 3.04E-03 & 578.9754 & 213.3354 & 792.3108 & \\
\hline & $8 / 10 / 2008$ 10:00 & 453 & 223 & 1000 & 4.9 & 4.9 & & 1.0000 & 1.19E-02 & 2265.9293 & 834.9282 & 3100.8575 & \\
\hline & 8/10/2008 11:00 & 454 & 223 & 1100 & 4.635 & 4.635 & & 1.1143 & $1.36 \mathrm{E}-02$ & 2588.9800 & 953.9629 & 3542.9429 & \\
\hline & $8 / 10 / 2008$ 12:00 & 455 & 223 & 1200 & 4.722 & 4.722 & & 1.0754 & $1.30 \mathrm{E}-02$ & 2478.0209 & 913.0778 & 3391.0987 & \\
\hline & 8/10/2008 13:00 & 456 & 223 & 1300 & 4.707 & 4.707 & & 1.0820 & $1.31 \mathrm{E}-02$ & 2496.7947 & 919.9954 & 3416.7901 & \\
\hline & 8/10/2008 14:00 & 457 & 223 & 1400 & 4.717 & 4.717 & & 1.0776 & $1.30 \mathrm{E}-02$ & 2484.2627 & 915.3777 & 3399.6403 & \\
\hline & 8/10/2008 15:00 & 458 & 223 & 1500 & 4.891 & 4.891 & & 1.0037 & $1.20 \mathrm{E}-02$ & 2276.1991 & 838.7124 & 3114.9115 & \\
\hline & $8 / 10 / 2008$ 16:00 & 459 & 223 & 1600 & 4.794 & 4.794 & & 1.0442 & $1.26 \mathrm{E}-02$ & 2389.9032 & 880.6090 & 3270.5121 & \\
\hline & $8 / 10 / 2008$ 17:00 & 460 & 223 & 1700 & 4.982 & 4.982 & & 0.9671 & 1.14E-02 & 2174.4598 & 801.2244 & 2975.6843 & \\
\hline & $8 / 10 / 2008$ 18:00 & 461 & 223 & 1800 & 7.472 & 7.472 & & 0.3116 & 2.83E-03 & 539.2642 & 198.7030 & 737.9672 & \\
\hline & $8 / 10 / 2008$ 19:00 & 462 & 223 & 1900 & 8.07 & 8.07 & & 0.2144 & 1.79E-03 & 340.3453 & 125.4072 & 465.7525 & \\
\hline & $8 / 10 / 200820: 00$ & 463 & 223 & 2000 & 7.821 & 7.821 & & 0.2530 & $2.19 \mathrm{E}-03$ & 417.4123 & 153.8041 & 571.2165 & \\
\hline & $8 / 10 / 200821: 00$ & 464 & 223 & 2100 & 7.844 & 7.844 & & 0.2494 & $2.15 \mathrm{E}-03$ & 409.9639 & 151.0596 & 561.0236 & \\
\hline & $8 / 10 / 2008$ 22:00 & 465 & 223 & 2200 & 8.15 & 8.15 & & 0.2025 & 1.67E-03 & 317.2006 & 116.8791 & 434.0798 & \\
\hline & $8 / 10 / 200823: 00$ & 466 & 223 & 2300 & 8.14 & 8.14 & & 0.2039 & 1.68E-03 & 320.0522 & 117.9298 & 437.9820 & \\
\hline & $8 / 11 / 20080: 00$ & 467 & 224 & 0 & 8.08 & 8.08 & 8.34 & 0.2129 & $1.77 \mathrm{E}-03$ & 337.4104 & 124.3258 & 461.7362 & \\
\hline & 8/11/2008 1:00 & 468 & 224 & 100 & 8.11 & 8.11 & & 0.2084 & 1.73E-03 & 328.6777 & 121.1081 & 449.7857 & \\
\hline & 8/11/2008 2:00 & 469 & 224 & 200 & 8.32 & 8.32 & & 0.1779 & 1.42E-03 & 270.4996 & 99.6712 & 370.1708 & \\
\hline & 8/11/2008 3:00 & 470 & 224 & 300 & 8.34 & 8.34 & & 0.1751 & 1.39E-03 & 265.2219 & 97.7264 & 362.9483 & \\
\hline & 8/11/2008 4:00 & 471 & 224 & 400 & 8.32 & 8.32 & & 0.1779 & $1.42 \mathrm{E}-03$ & 270.4996 & 99.6712 & 370.1708 & \\
\hline & 8/11/2008 5:00 & 472 & 224 & 500 & 8.27 & 8.27 & & 0.1850 & 1.49E-03 & 283.8914 & 104.6056 & 388.4970 & \\
\hline & 8/11/2008 6:00 & 473 & 224 & 600 & 8.29 & 8.29 & & 0.1821 & 1.46E-03 & 278.5007 & 102.6193 & 381.1201 & \\
\hline & 8/11/2008 7:00 & 474 & 224 & 700 & 8.32 & 8.32 & & 0.1779 & $1.42 \mathrm{E}-03$ & 270.4996 & 99.6712 & 370.1708 & \\
\hline & 8/11/2008 8:00 & 475 & 224 & 800 & 8.24 & 8.24 & & 0.1893 & 1.53E-03 & 292.0629 & 107.6166 & 399.6794 & \\
\hline & $8 / 11 / 20089: 00$ & 476 & 224 & 900 & 7.168 & 7.168 & & 0.3672 & $3.47 \mathrm{E}-03$ & 660.1207 & 243.2350 & 903.3557 & \\
\hline & $8 / 11 / 2008$ 10:00 & 477 & 224 & 1000 & 4.963 & 4.963 & & 0.9746 & 1.15E-02 & 2195.3224 & 808.9117 & 3004.2341 & \\
\hline & 8/11/2008 11:00 & 478 & 224 & 1100 & 4.765 & 4.765 & & 1.0567 & $1.27 \mathrm{E}-02$ & 2425.0029 & 893.5422 & 3318.5451 & \\
\hline & $8 / 11 / 2008$ 12:00 & 479 & 224 & 1200 & 4.799 & 4.799 & & 1.0421 & $1.25 \mathrm{E}-02$ & 2383.9040 & 878.3985 & 3262.3025 & \\
\hline & 8/11/2008 13:00 & 480 & 224 & 1300 & 5.018 & 5.018 & & 0.9530 & $1.12 \mathrm{E}-02$ & 2135.4652 & 786.8561 & 2922.3212 & \\
\hline & $8 / 11 / 2008$ 14:00 & 481 & 224 & 1400 & 4.765 & 4.765 & & 1.0567 & $1.27 \mathrm{E}-02$ & 2425.0029 & 893.5422 & 3318.5451 & \\
\hline & $8 / 11 / 200815: 00$ & 482 & 224 & 1500 & 4.743 & 4.743 & & 1.0662 & $1.29 \mathrm{E}-02$ & 2451.9814 & 903.4830 & 3355.4644 & \\
\hline & $8 / 11 / 200816: 00$ & 483 & 224 & 1600 & 5.003 & 5.003 & & 0.9588 & $1.13 \mathrm{E}-02$ & 2151.6287 & 792.8118 & 2944.4405 & \\
\hline & $8 / 11 / 2008$ 17:00 & 484 & 224 & 1700 & 5.077 & 5.077 & & 0.9303 & $1.09 \mathrm{E}-02$ & 2073.0330 & 763.8517 & 2836.8847 & \\
\hline & 8/11/2008 18:00 & 485 & 224 & 1800 & 5.432 & 5.432 & & 0.8041 & $9.10 \mathrm{E}-03$ & 1732.6020 & 638.4128 & 2371.0148 & \\
\hline & $8 / 11 / 2008$ 19:00 & 486 & 224 & 1900 & 5.535 & 5.535 & & 0.7706 & $8.63 \mathrm{E}-03$ & 1643.9889 & 605.7615 & 2249.7504 & \\
\hline & $8 / 11 / 2008$ 20:00 & 487 & 224 & 2000 & 6.26 & 6.26 & & 0.5655 & $5.90 \mathrm{E}-03$ & 1123.2757 & 413.8940 & 1537.1697 & \\
\hline & 8/11/2008 21:00 & 488 & 224 & 2100 & 7.203 & 7.203 & & 0.3605 & 3.39E-03 & 645.4496 & 237.8292 & 883.2787 & \\
\hline & $8 / 11 / 2008$ 22:00 & 489 & 224 & 2200 & 7.848 & 7.848 & & 0.2487 & $2.15 \mathrm{E}-03$ & 408.6756 & 150.5849 & 559.2605 & \\
\hline & 8/11/2008 23:00 & 490 & 224 & 2300 & 8.13 & 8.13 & & 0.2054 & 1.70E-03 & 322.9155 & 118.9848 & 441.9003 & \\
\hline & 8/12/2008 0:00 & 491 & 225 & 0 & 8.24 & 8.24 & 8.68 & 0.1893 & 1.53E-03 & 292.0629 & 107.6166 & 399.6794 & \\
\hline
\end{tabular}




\begin{tabular}{|c|c|c|c|c|c|c|c|c|c|c|c|c|c|}
\hline Tree ID & TS & RN & JD & HR & $\begin{array}{c}\text { Raw } \\
\text { TC_dTA }\end{array}$ & $\begin{array}{c}\text { Fixed } \\
\text { TC_dT } \Delta\end{array}$ & dTM & $\mathbf{K}$ & $\begin{array}{l}\text { Sap } \\
\text { Velocity } \\
(\mathrm{cm} / \mathrm{s})\end{array}$ & $\begin{array}{c}\text { Probed } \\
\text { Drywood F } \\
(\mathrm{g} / \mathrm{hr})\end{array}$ & $\begin{array}{c}\text { Extrapolated } \\
\text { Wetwood F } \\
(\mathrm{g} / \mathrm{hr})\end{array}$ & $\begin{array}{c}\text { Extrapolated } \\
\text { Total Wood F } \\
(\mathrm{g} / \mathrm{hr})\end{array}$ & $\begin{array}{l}\text { Relative } \\
\text { Humidity, } \\
@ 10 \mathrm{~m}(\%)\end{array}$ \\
\hline & 8/12/2008 1:00 & 492 & 225 & 100 & 8.19 & 8.19 & & 0.1966 & 1.61E-03 & 305.9120 & 112.7196 & 418.6316 & \\
\hline & 8/12/2008 2:00 & 493 & 225 & 200 & 8.57 & 8.57 & & 0.1435 & 1.09E-03 & 207.6922 & 76.5285 & 284.2206 & \\
\hline & 8/12/2008 3:00 & 494 & 225 & 300 & 8.66 & 8.66 & & 0.1316 & $9.81 E-04$ & 186.7290 & 68.8041 & 255.5331 & \\
\hline & 8/12/2008 4:00 & 495 & 225 & 400 & 8.68 & 8.68 & & 0.1290 & 9.57E-04 & 182.1864 & 67.1303 & 249.3167 & \\
\hline & 8/12/2008 5:00 & 496 & 225 & 500 & 8.6 & 8.6 & & 0.1395 & 1.05E-03 & 200.6092 & 73.9186 & 274.5278 & \\
\hline & 8/12/2008 6:00 & 497 & 225 & 600 & 8.43 & 8.43 & & 0.1625 & 1.27E-03 & 242.0224 & 89.1781 & 331.2006 & \\
\hline & 8/12/2008 7:00 & 498 & 225 & 700 & 8.32 & 8.32 & & 0.1779 & 1.42E-03 & 270.4996 & 99.6712 & 370.1708 & \\
\hline & 8/12/2008 8:00 & 499 & 225 & 800 & 8.25 & 8.25 & & 0.1879 & 1.52E-03 & 289.3276 & 106.6087 & 395.9363 & \\
\hline & 8/12/2008 9:00 & 500 & 225 & 900 & 7.249 & 7.249 & & 0.3519 & 3.29E-03 & 626.4762 & 230.8380 & 857.3143 & \\
\hline & $8 / 12 / 2008$ 10:00 & 501 & 225 & 1000 & 5.073 & 5.073 & & 0.9318 & 1.09E-02 & 2077.2089 & 765.3903 & 2842.5993 & \\
\hline & $8 / 12 / 2008$ 11:00 & 502 & 225 & 1100 & 4.611 & 4.611 & & 1.1254 & 1.38E-02 & 2620.4903 & 965.5735 & 3586.0638 & \\
\hline & $8 / 12 / 2008$ 12:00 & 503 & 225 & 1200 & 4.619 & 4.619 & & 1.1217 & 1.37E-02 & 2609.9425 & 961.6870 & 3571.6295 & \\
\hline & 8/12/2008 13:00 & 504 & 225 & 1300 & 4.568 & 4.568 & & 1.1454 & 1.41E-02 & 2677.9564 & 986.7481 & 3664.7046 & \\
\hline & $8 / 12 / 2008$ 14:00 & 505 & 225 & 1400 & 4.593 & 4.593 & & 1.1337 & 1.39E-02 & 2644.3865 & 974.3786 & 3618.7651 & \\
\hline & $8 / 12 / 2008$ 15:00 & 506 & 225 & 1500 & 4.564 & 4.564 & & 1.1472 & 1.41E-02 & 2683.3692 & 988.7426 & 3672.1117 & \\
\hline & $8 / 12 / 2008$ 16:00 & 507 & 225 & 1600 & 4.594 & 4.594 & & 1.1332 & 1.39E-02 & 2643.0530 & 973.8872 & 3616.9402 & \\
\hline & $8 / 12 / 2008$ 17:00 & 508 & 225 & 1700 & 4.607 & 4.607 & & 1.1272 & 1.38E-02 & 2625.7809 & 967.5230 & 3593.3039 & \\
\hline & $8 / 12 / 2008$ 18:00 & 509 & 225 & 1800 & 4.734 & 4.734 & & 1.0701 & 1.29E-02 & 2463.1066 & 907.5823 & 3370.6889 & \\
\hline & $8 / 12 / 2008$ 19:00 & 510 & 225 & 1900 & 4.921 & 4.921 & & 0.9915 & 1.18E-02 & 2242.1460 & 826.1648 & 3068.3108 & \\
\hline & $8 / 12 / 200820: 00$ & 511 & 225 & 2000 & 5.677 & 5.677 & & 0.7263 & 8.03E-03 & 1528.4583 & 563.1919 & 2091.6502 & \\
\hline & $8 / 12 / 2008$ 21:00 & 512 & 225 & 2100 & 7.283 & 7.283 & & 0.3456 & $3.22 \mathrm{E}-03$ & 612.6742 & 225.7524 & 838.4266 & \\
\hline & $8 / 12 / 2008$ 22:00 & 513 & 225 & 2200 & 8.23 & 8.23 & & 0.1908 & 1.55E-03 & 294.8096 & 108.6287 & 403.4383 & \\
\hline & $8 / 12 / 2008$ 23:00 & 514 & 225 & 2300 & 8.51 & 8.51 & & 0.1516 & 1.17E-03 & 222.1463 & 81.8544 & 304.0006 & \\
\hline & 8/13/2008 0:00 & 515 & 226 & 0 & 8.61 & 8.61 & 8.63 & 0.1382 & 1.04E-03 & 198.2694 & 73.0565 & 271.3259 & \\
\hline & 8/13/2008 1:00 & 516 & 226 & 100 & 8.63 & 8.63 & & 0.1356 & $1.02 \mathrm{E}-03$ & 193.6216 & 71.3439 & 264.9655 & \\
\hline & 8/13/2008 2:00 & 517 & 226 & 200 & 8.59 & 8.59 & & 0.1409 & 1.07E-03 & 202.9596 & 74.7846 & 277.7442 & \\
\hline & 8/13/2008 3:00 & 518 & 226 & 300 & 8.6 & 8.6 & & 0.1395 & 1.05E-03 & 200.6092 & 73.9186 & 274.5278 & \\
\hline & 8/13/2008 4:00 & 519 & 226 & 400 & 8.5 & 8.5 & & 0.1529 & $1.18 \mathrm{E}-03$ & 224.5928 & 82.7559 & 307.3487 & \\
\hline & 8/13/2008 5:00 & 520 & 226 & 500 & 8.41 & 8.41 & & 0.1653 & $1.30 \mathrm{E}-03$ & 247.1005 & 91.0493 & 338.1498 & \\
\hline & 8/13/2008 6:00 & 521 & 226 & 600 & 8.48 & 8.48 & & 0.1557 & $1.21 \mathrm{E}-03$ & 229.5184 & 84.5708 & 314.0892 & \\
\hline & $8 / 13 / 2008$ 7:00 & 522 & 226 & 700 & 8.48 & 8.48 & & 0.1557 & $1.21 \mathrm{E}-03$ & 229.5184 & 84.5708 & 314.0892 & \\
\hline & 8/13/2008 8:00 & 523 & 226 & 800 & 8.23 & 8.23 & & 0.1908 & $1.55 E-03$ & 294.8096 & 108.6287 & 403.4383 & \\
\hline & 8/13/2008 9:00 & 524 & 226 & 900 & 6.105 & 6.105 & & 0.6052 & 6.41E-03 & 1221.2390 & 449.9906 & 1671.2296 & \\
\hline & $8 / 13 / 2008$ 10:00 & 525 & 226 & 1000 & 4.596 & 4.596 & & 1.1323 & $1.39 \mathrm{E}-02$ & 2640.3880 & 972.9053 & 3613.2932 & \\
\hline & 8/13/2008 11:00 & 526 & 226 & 1100 & 4.701 & 4.701 & & 1.0847 & 1.32E-02 & 2504.3452 & 922.7775 & 3427.1228 & \\
\hline & $8 / 13 / 2008$ 12:00 & 527 & 226 & 1200 & 4.42 & 4.42 & & 1.2172 & $1.52 \mathrm{E}-02$ & 2886.1873 & 1063.4751 & 3949.6624 & \\
\hline
\end{tabular}




\begin{tabular}{|c|c|c|c|c|c|c|c|c|c|c|c|c|c|}
\hline Tree ID & TS & RN & JD & HR & $\begin{array}{c}\text { Raw } \\
\text { TC_dTA }\end{array}$ & $\begin{array}{c}\text { Fixed } \\
\text { TC_dT } \Delta\end{array}$ & dTM & $\mathbf{K}$ & $\begin{array}{c}\text { Sap } \\
\text { Velocity } \\
(\mathbf{c m} / \mathbf{s})\end{array}$ & $\begin{array}{c}\text { Probed } \\
\text { Drywood F } \\
(\mathrm{g} / \mathrm{hr})\end{array}$ & $\begin{array}{c}\text { Extrapolated } \\
\text { Wetwood F } \\
(\mathrm{g} / \mathrm{hr})\end{array}$ & $\begin{array}{c}\text { Extrapolated } \\
\text { Total Wood F } \\
(\mathrm{g} / \mathrm{hr})\end{array}$ & $\begin{array}{c}\text { Relative } \\
\text { Humidity, } \\
@ 10 \mathrm{~m}(\%)\end{array}$ \\
\hline & $7 / 22 / 2008$ 13:00 & 0 & 204 & 1300 & 4.461 & 4.461 & 8.63 & 0.9345 & 1.09E-02 & 2084.7527 & 768.1700 & 2852.9227 & \\
\hline W608EC2 & $7 / 22 / 2008$ 14:00 & 1 & 204 & 1400 & 4.928 & 4.928 & & 0.7512 & 8.37E-03 & 1593.3601 & 587.1063 & 2180.4664 & \\
\hline $30 " \mathrm{~S}$ & 7/22/2008 15:00 & 2 & 204 & 1500 & 4.937 & 4.937 & & 0.7480 & $8.32 \mathrm{E}-03$ & 1585.0288 & 584.0365 & 2169.0653 & \\
\hline \multirow[t]{2}{*}{ Probe \# } & $7 / 22 / 2008$ 16:00 & 3 & 204 & 1600 & 4.894 & 4.894 & & 0.7634 & $8.53 E-03$ & 1625.1852 & 598.8329 & 2224.0181 & \\
\hline & 7/22/2008 17:00 & 4 & 204 & 1700 & 4.913 & 4.913 & & 0.7566 & $8.44 \mathrm{E}-03$ & 1607.3318 & 592.2544 & 2199.5862 & \\
\hline \multirow[t]{4}{*}{5} & $7 / 22 / 2008$ 18:00 & 5 & 204 & 1800 & 5.101 & 5.101 & & 0.6918 & $7.56 \mathrm{E}-03$ & 1439.7326 & 530.4991 & 1970.2317 & \\
\hline & $7 / 22 / 2008$ 19:00 & 6 & 204 & 1900 & 5.308 & 5.308 & & 0.6258 & $6.68 \mathrm{E}-03$ & 1272.6217 & 468.9237 & 1741.5454 & \\
\hline & $7 / 22 / 200820: 00$ & 7 & 204 & 2000 & 5.757 & 5.757 & & 0.4990 & $5.06 \mathrm{E}-03$ & 963.0658 & 354.8614 & 1317.9272 & \\
\hline & 7/22/2008 21:00 & 8 & 204 & 2100 & 7.195 & 7.195 & & 0.1994 & $1.64 \mathrm{E}-03$ & 311.4054 & 114.7437 & 426.1491 & \\
\hline \multirow[t]{2}{*}{$\mathrm{DBH}(\mathrm{cm})$} & $7 / 22 / 2008$ 22:00 & 9 & 204 & 2200 & 8.31 & 8.31 & & 0.0385 & $2.16 \mathrm{E}-04$ & 41.1204 & 15.1516 & 56.2720 & \\
\hline & 7/22/2008 23:00 & 10 & 204 & 2300 & 8.63 & 8.63 & & 0.0000 & $0.00 \mathrm{E}+00$ & 0.0000 & 0.0000 & 0.0000 & \\
\hline \multirow[t]{2}{*}{10.2} & 7/23/2008 0:00 & 11 & 205 & 0 & 8.84 & 8.84 & 8.97 & -0.0238 & $0.00 \mathrm{E}+00$ & 0.0000 & 0.0000 & 0.0000 & \\
\hline & 7/23/2008 1:00 & 12 & 205 & 100 & 8.92 & 8.92 & & -0.0325 & $0.00 \mathrm{E}+00$ & 0.0000 & 0.0000 & 0.0000 & \\
\hline Day Count & $7 / 23 / 2008$ 2:00 & 13 & 205 & 200 & 8.95 & 8.95 & & -0.0358 & $0.00 \mathrm{E}+00$ & 0.0000 & 0.0000 & 0.0000 & \\
\hline \multirow[t]{27}{*}{35} & 7/23/2008 3:00 & 14 & 205 & 300 & 8.97 & 8.97 & & -0.0379 & $0.00 \mathrm{E}+00$ & 0.0000 & 0.0000 & 0.0000 & \\
\hline & 7/23/2008 4:00 & 15 & 205 & 400 & 8.96 & 8.96 & & -0.0368 & $0.00 \mathrm{E}+00$ & 0.0000 & 0.0000 & 0.0000 & \\
\hline & 7/23/2008 5:00 & 16 & 205 & 500 & 8.93 & 8.93 & & -0.0336 & $0.00 \mathrm{E}+00$ & 0.0000 & 0.0000 & 0.0000 & \\
\hline & 7/23/2008 6:00 & 17 & 205 & 600 & 8.86 & 8.86 & & -0.0260 & $0.00 \mathrm{E}+00$ & 0.0000 & 0.0000 & 0.0000 & \\
\hline & 7/23/2008 7:00 & 18 & 205 & 700 & 8.69 & 8.69 & & -0.0069 & $0.00 \mathrm{E}+00$ & 0.0000 & 0.0000 & 0.0000 & \\
\hline & 7/23/2008 8:00 & 19 & 205 & 800 & 8.06 & 8.06 & & 0.0707 & $4.56 \mathrm{E}-04$ & 86.9020 & 32.0208 & 118.9229 & \\
\hline & 7/23/20089:00 & 20 & 205 & 900 & 6.681 & 6.681 & & 0.2917 & 2.61E-03 & 497.3073 & 183.2431 & 680.5505 & \\
\hline & $7 / 23 / 2008$ 10:00 & 21 & 205 & 1000 & 5.517 & 5.517 & & 0.5643 & 5.88E-03 & 1120.2462 & 412.7777 & 1533.0239 & \\
\hline & $7 / 23 / 2008$ 11:00 & 22 & 205 & 1100 & 5.5 & 5.5 & & 0.5691 & 5.95E-03 & 1132.0744 & 417.1361 & 1549.2105 & \\
\hline & $7 / 23 / 2008$ 12:00 & 23 & 205 & 1200 & 5.308 & 5.308 & & 0.6258 & $6.68 \mathrm{E}-03$ & 1272.6217 & 468.9237 & 1741.5454 & \\
\hline & $7 / 23 / 2008$ 13:00 & 24 & 205 & 1300 & 5.141 & 5.141 & & 0.6787 & 7.38E-03 & 1406.0852 & 518.1010 & 1924.1862 & \\
\hline & 7/23/2008 14:00 & 25 & 205 & 1400 & 5.153 & 5.153 & & 0.6748 & 7.33E-03 & 1396.1217 & 514.4298 & 1910.5515 & \\
\hline & 7/23/2008 15:00 & 26 & 205 & 1500 & 5.154 & 5.154 & & 0.6744 & 7.33E-03 & 1395.2941 & 514.1248 & 1909.4190 & \\
\hline & 7/23/2008 16:00 & 27 & 205 & 1600 & 5.336 & 5.336 & & 0.6173 & $6.57 \mathrm{E}-03$ & 1251.2999 & 461.0672 & 1712.3671 & \\
\hline & $7 / 23 / 2008$ 17:00 & 28 & 205 & 1700 & 5.824 & 5.824 & & 0.4818 & 4.84E-03 & 922.2630 & 339.8268 & 1262.0897 & \\
\hline & $7 / 23 / 2008$ 18:00 & 29 & 205 & 1800 & 6.576 & 6.576 & & 0.3123 & $2.84 \mathrm{E}-03$ & 540.9368 & 199.3193 & 740.2561 & \\
\hline & $7 / 23 / 2008$ 19:00 & 30 & 205 & 1900 & 6.524 & 6.524 & & 0.3228 & $2.96 \mathrm{E}-03$ & 563.3223 & 207.5677 & 770.8899 & \\
\hline & $7 / 23 / 200820: 00$ & 31 & 205 & 2000 & 6.909 & 6.909 & & 0.2491 & $2.15 \mathrm{E}-03$ & 409.4232 & 150.8604 & 560.2837 & \\
\hline & $7 / 23 / 2008$ 21:00 & 32 & 205 & 2100 & 7.757 & 7.757 & & 0.1125 & $8.09 E-04$ & 153.9649 & 56.7315 & 210.6965 & \\
\hline & $7 / 23 / 2008$ 22:00 & 33 & 205 & 2200 & 8.22 & 8.22 & & 0.0499 & $2.97 \mathrm{E}-04$ & 56.5426 & 20.8343 & 77.3769 & \\
\hline & $7 / 23 / 200823: 00$ & 34 & 205 & 2300 & 8.32 & 8.32 & & 0.0373 & 2.07E-04 & 39.4858 & 14.5493 & 54.0351 & \\
\hline & $7 / 24 / 20080: 00$ & 35 & 206 & 0 & 8.36 & 8.36 & 8.75 & 0.0323 & $1.74 \mathrm{E}-04$ & 33.1146 & 12.2017 & 45.3163 & \\
\hline & 7/24/2008 1:00 & 36 & 206 & 100 & 8.43 & 8.43 & & 0.0237 & $1.19 \mathrm{E}-04$ & 22.6527 & 8.3469 & 30.9996 & \\
\hline & 7/24/2008 2:00 & 37 & 206 & 200 & 8.5 & 8.5 & & 0.0153 & $6.93 \mathrm{E}-05$ & 13.1946 & 4.8618 & 18.0564 & \\
\hline & 7/24/2008 3:00 & 38 & 206 & 300 & 8.57 & 8.57 & & 0.0070 & $2.65 \mathrm{E}-05$ & 5.0426 & 1.8580 & 6.9006 & \\
\hline & 7/24/2008 4:00 & 39 & 206 & 400 & 8.65 & 8.65 & & -0.0023 & $0.00 \mathrm{E}+00$ & 0.0000 & 0.0000 & 0.0000 & \\
\hline & 7/24/2008 5:00 & 40 & 206 & 500 & 8.7 & 8.7 & & -0.0080 & $0.00 \mathrm{E}+00$ & 0.0000 & 0.0000 & 0.0000 & \\
\hline
\end{tabular}




\begin{tabular}{|c|c|c|c|c|c|c|c|c|c|c|c|c|c|}
\hline Tree ID & TS & RN & JD & HR & $\begin{array}{c}\text { Raw } \\
\text { TC_dT } \Delta\end{array}$ & $\begin{array}{c}\text { Fixed } \\
\text { TC_dT } \Delta\end{array}$ & dTM & $\mathbf{K}$ & $\begin{array}{l}\text { Sap } \\
\text { Velocity } \\
(\mathbf{c m} / \mathbf{s})\end{array}$ & $\begin{array}{l}\text { Probed } \\
\text { Drywood F } \\
\text { (g/hr) }\end{array}$ & $\begin{array}{c}\text { Extrapolated } \\
\text { Wetwood F } \\
(\mathrm{g} / \mathrm{hr})\end{array}$ & $\begin{array}{c}\text { Extrapolated } \\
\text { Total Wood F } \\
(\mathrm{g} / \mathrm{hr})\end{array}$ & $\begin{array}{l}\text { Relative } \\
\text { Humidity, } \\
@ 10 m(\%)\end{array}$ \\
\hline & 7/24/2008 6:00 & 41 & 206 & 600 & 8.74 & 8.74 & & -0.0126 & $0.00 \mathrm{E}+00$ & 0.0000 & 0.0000 & 0.0000 & \\
\hline & 7/24/2008 7:00 & 42 & 206 & 700 & 8.75 & 8.75 & & -0.0137 & $0.00 \mathrm{E}+00$ & 0.0000 & 0.0000 & 0.0000 & \\
\hline & 7/24/2008 8:00 & 43 & 206 & 800 & 8.65 & 8.65 & & -0.0023 & $0.00 \mathrm{E}+00$ & 0.0000 & 0.0000 & 0.0000 & \\
\hline & 7/24/2008 9:00 & 44 & 206 & 900 & 8.34 & 8.34 & & 0.0348 & 1.90E-04 & 36.2663 & 13.3631 & 49.6293 & \\
\hline & $7 / 24 / 2008$ 10:00 & 45 & 206 & 1000 & 8.32 & 8.32 & & 0.0373 & 2.07E-04 & 39.4858 & 14.5493 & 54.0351 & \\
\hline & $7 / 24 / 2008$ 11:00 & 46 & 206 & 1100 & 6.423 & 6.423 & & 0.3436 & 3.19E-03 & 608.3334 & 224.1529 & 832.4863 & \\
\hline & 7/24/2008 12:00 & 47 & 206 & 1200 & 5.353 & 5.353 & & 0.6122 & $6.50 \mathrm{E}-03$ & 1238.4961 & 456.3494 & 1694.8455 & \\
\hline & $7 / 24 / 2008$ 13:00 & 48 & 206 & 1300 & 5.807 & 5.807 & & 0.4861 & 4.90E-03 & 932.4955 & 343.5971 & 1276.0926 & \\
\hline & $7 / 24 / 200814: 00$ & 49 & 206 & 1400 & 5.677 & 5.677 & & 0.5202 & $5.32 \mathrm{E}-03$ & 1013.4919 & 373.4419 & 1386.9339 & \\
\hline & $7 / 24 / 200815: 00$ & 50 & 206 & 1500 & 5.229 & 5.229 & & 0.6504 & 7.01E-03 & 1334.3838 & 491.6812 & 1826.0650 & \\
\hline & 7/24/2008 16:00 & 51 & 206 & 1600 & 5.16 & 5.16 & & 0.6725 & 7.30E-03 & 1390.3372 & 512.2983 & 1902.6356 & \\
\hline & 7/24/2008 17:00 & 52 & 206 & 1700 & 5.417 & 5.417 & & 0.5931 & $6.26 \mathrm{E}-03$ & 1191.2319 & 438.9339 & 1630.1658 & \\
\hline & $7 / 24 / 200818: 00$ & 53 & 206 & 1800 & 5.331 & 5.331 & & 0.6188 & $6.59 \mathrm{E}-03$ & 1255.0860 & 462.4623 & 1717.5483 & \\
\hline & $7 / 24 / 200819: 00$ & 54 & 206 & 1900 & 5.683 & 5.683 & & 0.5186 & 5.30E-03 & 1009.6438 & 372.0240 & 1381.6679 & \\
\hline & $7 / 24 / 200820: 00$ & 55 & 206 & 2000 & 6.695 & 6.695 & & 0.2890 & $2.58 \mathrm{E}-03$ & 491.6450 & 181.1567 & 672.8018 & \\
\hline & $7 / 24 / 200821: 00$ & 56 & 206 & 2100 & 7.913 & 7.913 & & 0.0906 & 6.19E-04 & 117.9051 & 43.4445 & 161.3496 & \\
\hline & $7 / 24 / 200822: 00$ & 57 & 206 & 2200 & 8.59 & 8.59 & & 0.0047 & $1.60 \mathrm{E}-05$ & 3.0524 & 1.1247 & 4.1771 & \\
\hline & $7 / 24 / 2008$ 23:00 & 58 & 206 & 2300 & 8.71 & 8.71 & & -0.0092 & $0.00 \mathrm{E}+00$ & 0.0000 & 0.0000 & 0.0000 & \\
\hline & $7 / 25 / 20080: 00$ & 59 & 207 & 0 & 8.7 & 8.7 & 8.70 & -0.0080 & $0.00 \mathrm{E}+00$ & 0.0000 & 0.0000 & 0.0000 & \\
\hline & 7/25/2008 1:00 & 60 & 207 & 100 & 8.64 & 8.64 & & -0.0012 & $0.00 \mathrm{E}+00$ & 0.0000 & 0.0000 & 0.0000 & \\
\hline & 7/25/2008 2:00 & 61 & 207 & 200 & 8.57 & 8.57 & & 0.0070 & $2.65 \mathrm{E}-05$ & 5.0426 & 1.8580 & 6.9006 & \\
\hline & 7/25/2008 3:00 & 62 & 207 & 300 & 8.53 & 8.53 & & 0.0117 & $5.00 \mathrm{E}-05$ & 9.5115 & 3.5047 & 13.0162 & \\
\hline & 7/25/2008 4:00 & 63 & 207 & 400 & 8.53 & 8.53 & & 0.0117 & 5.00E-05 & 9.5115 & 3.5047 & 13.0162 & \\
\hline & 7/25/2008 5:00 & 64 & 207 & 500 & 8.55 & 8.55 & & 0.0094 & $3.78 E-05$ & 7.2061 & 2.6552 & 9.8613 & \\
\hline & 7/25/2008 6:00 & 65 & 207 & 600 & 8.57 & 8.57 & & 0.0070 & 2.65E-05 & 5.0426 & 1.8580 & 6.9006 & \\
\hline & 7/25/2008 7:00 & 66 & 207 & 700 & 8.53 & 8.53 & & 0.0117 & $5.00 \mathrm{E}-05$ & 9.5115 & 3.5047 & 13.0162 & \\
\hline & 7/25/2008 8:00 & 67 & 207 & 800 & 8.5 & 8.5 & & 0.0153 & $6.93 E-05$ & 13.1946 & 4.8618 & 18.0564 & \\
\hline & 7/25/2008 9:00 & 68 & 207 & 900 & 8.44 & 8.44 & & 0.0225 & $1.12 \mathrm{E}-04$ & 21.2356 & 7.8247 & 29.0603 & \\
\hline & $7 / 25 / 200810: 00$ & 69 & 207 & 1000 & 8.38 & 8.38 & & 0.0298 & $1.58 \mathrm{E}-04$ & 30.0329 & 11.0662 & 41.0991 & \\
\hline & 7/25/2008 11:00 & 70 & 207 & 1100 & 7.582 & 7.582 & & 0.1382 & 1.04E-03 & 198.2884 & 73.0634 & 271.3518 & \\
\hline & $7 / 25 / 2008$ 12:00 & 71 & 207 & 1200 & 6.385 & 6.385 & & 0.3516 & $3.29 E-03$ & 625.8073 & 230.5916 & 856.3988 & \\
\hline & $7 / 25 / 2008$ 13:00 & 72 & 207 & 1300 & 5.724 & 5.724 & & 0.5077 & 5.17E-03 & 983.6373 & 362.4414 & 1346.0787 & \\
\hline & $7 / 25 / 200814: 00$ & 73 & 207 & 1400 & 5.202 & 5.202 & & 0.6590 & $7.12 \mathrm{E}-03$ & 1356.0506 & 499.6647 & 1855.7153 & \\
\hline & $7 / 25 / 200815: 00$ & 74 & 207 & 1500 & 5.206 & 5.206 & & 0.6577 & $7.10 \mathrm{E}-03$ & 1352.8224 & 498.4752 & 1851.2976 & \\
\hline & $7 / 25 / 200816: 00$ & 75 & 207 & 1600 & 5.375 & 5.375 & & 0.6056 & $6.42 E-03$ & 1222.0831 & 450.3016 & 1672.3847 & \\
\hline & $7 / 25 / 2008$ 17:00 & 76 & 207 & 1700 & 5.108 & 5.108 & & 0.6895 & 7.53E-03 & 1433.7954 & 528.3114 & 1962.1069 & \\
\hline & $7 / 25 / 2008$ 18:00 & 77 & 207 & 1800 & 5.271 & 5.271 & & 0.6373 & 6.83E-03 & 1301.2495 & 479.4721 & 1780.7216 & \\
\hline & $7 / 25 / 2008$ 19:00 & 78 & 207 & 1900 & 5.416 & 5.416 & & 0.5934 & 6.26E-03 & 1191.9592 & 439.2019 & 1631.1611 & \\
\hline & $7 / 25 / 200820: 00$ & 79 & 207 & 2000 & 6.244 & 6.244 & & 0.3821 & 3.64E-03 & 693.3361 & 255.4740 & 948.8101 & \\
\hline & $7 / 25 / 200821: 00$ & 80 & 207 & 2100 & 7.617 & 7.617 & & 0.1330 & 9.93E-04 & 189.0930 & 69.6752 & 258.7682 & \\
\hline & $7 / 25 / 200822: 00$ & 81 & 207 & 2200 & 8.49 & 8.49 & & 0.0165 & $7.60 \mathrm{E}-05$ & 14.4759 & 5.3339 & 19.8098 & \\
\hline
\end{tabular}




\begin{tabular}{|c|c|c|c|c|c|c|c|c|c|c|c|c|c|}
\hline Tree ID & TS & RN & JD & HR & $\begin{array}{c}\text { Raw } \\
\text { TC_dTA }\end{array}$ & $\begin{array}{c}\text { Fixed } \\
\text { TC_dT } \Delta\end{array}$ & dTM & $\mathbf{K}$ & $\begin{array}{l}\text { Sap } \\
\text { Velocity } \\
(\mathrm{cm} / \mathrm{s})\end{array}$ & $\begin{array}{l}\text { Probed } \\
\text { Drywood F } \\
\text { (g/hr) }\end{array}$ & $\begin{array}{l}\text { Extrapolated } \\
\text { Wetwood F } \\
(\mathrm{g} / \mathrm{hr})\end{array}$ & $\begin{array}{c}\text { Extrapolated } \\
\text { Total Wood F } \\
\text { (g/hr) }\end{array}$ & $\begin{array}{l}\text { Relative } \\
\text { Humidity, } \\
\text { @10m (\%) }\end{array}$ \\
\hline & $7 / 25 / 2008$ 23:00 & 82 & 207 & 2300 & 8.61 & 8.61 & & 0.0023 & $6.81 \mathrm{E}-06$ & 1.2967 & 0.4778 & 1.7744 & \\
\hline & $7 / 26 / 20080: 00$ & 83 & 208 & 0 & 8.62 & 8.62 & 8.62 & 0.0012 & $2.90 \mathrm{E}-06$ & 0.5516 & 0.2033 & 0.7549 & \\
\hline & 7/26/2008 1:00 & 84 & 208 & 100 & 8.61 & 8.61 & & 0.0023 & $6.81 \mathrm{E}-06$ & 1.2967 & 0.4778 & 1.7744 & \\
\hline & 7/26/2008 2:00 & 85 & 208 & 200 & 8.58 & 8.58 & & 0.0058 & $2.11 \mathrm{E}-05$ & 4.0231 & 1.4824 & 5.5054 & \\
\hline & 7/26/2008 3:00 & 86 & 208 & 300 & 8.55 & 8.55 & & 0.0094 & 3.78E-05 & 7.2061 & 2.6552 & 9.8613 & \\
\hline & 7/26/2008 4:00 & 87 & 208 & 400 & 8.52 & 8.52 & & 0.0129 & 5.63E-05 & 10.7110 & 3.9467 & 14.6577 & \\
\hline & 7/26/2008 5:00 & 88 & 208 & 500 & 8.49 & 8.49 & & 0.0165 & $7.60 \mathrm{E}-05$ & 14.4759 & 5.3339 & 19.8098 & \\
\hline & 7/26/2008 6:00 & 89 & 208 & 600 & 8.44 & 8.44 & & 0.0225 & $1.12 \mathrm{E}-04$ & 21.2356 & 7.8247 & 29.0603 & \\
\hline & 7/26/2008 7:00 & 90 & 208 & 700 & 8.37 & 8.37 & & 0.0311 & 1.66E-04 & 31.5648 & 11.6307 & 43.1955 & \\
\hline & 7/26/2008 8:00 & 91 & 208 & 800 & 8.37 & 8.37 & & 0.0311 & 1.66E-04 & 31.5648 & 11.6307 & 43.1955 & \\
\hline & 7/26/2008 9:00 & 92 & 208 & 900 & 7.336 & 7.336 & & 0.1764 & $1.41 \mathrm{E}-03$ & 267.7053 & 98.6415 & 366.3469 & \\
\hline & 7/26/2008 10:00 & 93 & 208 & 1000 & 5.443 & 5.443 & & 0.5855 & $6.16 \mathrm{E}-03$ & 1172.4455 & 432.0117 & 1604.4572 & \\
\hline & 7/26/2008 11:00 & 94 & 208 & 1100 & 5.551 & 5.551 & & 0.5547 & $5.76 \mathrm{E}-03$ & 1096.8764 & 404.1667 & 1501.0431 & \\
\hline & 7/26/2008 12:00 & 95 & 208 & 1200 & 5.96 & 5.96 & & 0.4480 & 4.43E-03 & 843.2446 & 310.7108 & 1153.9554 & \\
\hline & $7 / 26 / 2008$ 13:00 & 96 & 208 & 1300 & 5.408 & 5.408 & & 0.5958 & 6.29E-03 & 1197.7901 & 441.3504 & 1639.1405 & \\
\hline & $7 / 26 / 2008$ 14:00 & 97 & 208 & 1400 & 5.275 & 5.275 & & 0.6360 & 6.82E-03 & 1298.1294 & 478.3225 & 1776.4519 & \\
\hline & $7 / 26 / 2008$ 15:00 & 98 & 208 & 1500 & 5.198 & 5.198 & & 0.6603 & $7.14 \mathrm{E}-03$ & 1359.2852 & 500.8566 & 1860.1418 & \\
\hline & $7 / 26 / 2008$ 16:00 & 99 & 208 & 1600 & 5.288 & 5.288 & & 0.6320 & $6.76 \mathrm{E}-03$ & 1288.0316 & 474.6017 & 1762.6333 & \\
\hline & $7 / 26 / 2008$ 17:00 & 100 & 208 & 1700 & 5.019 & 5.019 & & 0.7195 & 7.93E-03 & 1510.8662 & 556.7097 & 2067.5760 & \\
\hline & $7 / 26 / 2008$ 18:00 & 101 & 208 & 1800 & 5.297 & 5.297 & & 0.6292 & $6.73 \mathrm{E}-03$ & 1281.0784 & 472.0397 & 1753.1181 & \\
\hline & $7 / 26 / 2008$ 19:00 & 102 & 208 & 1900 & 5.455 & 5.455 & & 0.5820 & $6.11 \mathrm{E}-03$ & 1163.8541 & 428.8460 & 1592.7001 & \\
\hline & $7 / 26 / 200820: 00$ & 103 & 208 & 2000 & 6.035 & 6.035 & & 0.4300 & 4.21E-03 & 801.7440 & 295.4191 & 1097.1631 & \\
\hline & $7 / 26 / 200821: 00$ & 104 & 208 & 2100 & 7.48 & 7.48 & & 0.1537 & 1.19E-03 & 226.0438 & 83.2905 & 309.3342 & \\
\hline & $7 / 26 / 200822: 00$ & 105 & 208 & 2200 & 8.36 & 8.36 & & 0.0323 & 1.74E-04 & 33.1146 & 12.2017 & 45.3163 & \\
\hline & $7 / 26 / 200823: 00$ & 106 & 208 & 2300 & 8.54 & 8.54 & & 0.0105 & 4.38E-05 & 8.3425 & 3.0740 & 11.4164 & \\
\hline & 7/27/2008 0:00 & 107 & 209 & 0 & 8.57 & 8.57 & 8.57 & 0.0070 & $2.65 \mathrm{E}-05$ & 5.0426 & 1.8580 & 6.9006 & \\
\hline & 7/27/2008 1:00 & 108 & 209 & 100 & 8.53 & 8.53 & & 0.0117 & $5.00 \mathrm{E}-05$ & 9.5115 & 3.5047 & 13.0162 & \\
\hline & $7 / 27 / 20082: 00$ & 109 & 209 & 200 & 8.47 & 8.47 & & 0.0189 & 8.99E-05 & 17.1117 & 6.3052 & 23.4169 & \\
\hline & 7/27/2008 3:00 & 110 & 209 & 300 & 8.41 & 8.41 & & 0.0262 & 1.34E-04 & 25.5473 & 9.4134 & 34.9607 & \\
\hline & 7/27/2008 4:00 & 111 & 209 & 400 & 8.39 & 8.39 & & 0.0286 & $1.50 \mathrm{E}-04$ & 28.5191 & 10.5084 & 39.0275 & \\
\hline & 7/27/2008 5:00 & 112 & 209 & 500 & 8.38 & 8.38 & & 0.0298 & $1.58 \mathrm{E}-04$ & 30.0329 & 11.0662 & 41.0991 & \\
\hline & 7/27/2008 6:00 & 113 & 209 & 600 & 8.43 & 8.43 & & 0.0237 & 1.19E-04 & 22.6527 & 8.3469 & 30.9996 & \\
\hline & 7/27/2008 7:00 & 114 & 209 & 700 & 8.43 & 8.43 & & 0.0237 & 1.19E-04 & 22.6527 & 8.3469 & 30.9996 & \\
\hline & 7/27/2008 8:00 & 115 & 209 & 800 & 8.33 & 8.33 & & 0.0360 & 1.99E-04 & 37.8677 & 13.9531 & 51.8208 & \\
\hline & 7/27/2008 9:00 & 116 & 209 & 900 & 6.598 & 6.598 & & 0.3080 & 2.79E-03 & 531.6231 & 195.8875 & 727.5106 & \\
\hline & $7 / 27 / 2008$ 10:00 & 117 & 209 & 1000 & 5.19 & 5.19 & & 0.6628 & 7.17E-03 & 1365.7738 & 503.2474 & 1869.0212 & \\
\hline & $7 / 27 / 2008$ 11:00 & 118 & 209 & 1100 & 5.243 & 5.243 & & 0.6460 & 6.95E-03 & 1323.2626 & 487.5833 & 1810.8460 & \\
\hline & $7 / 27 / 200812: 00$ & 119 & 209 & 1200 & 5.116 & 5.116 & & 0.6869 & 7.49E-03 & 1427.0356 & 525.8206 & 1952.8563 & \\
\hline & $7 / 27 / 2008$ 13:00 & 120 & 209 & 1300 & 5.091 & 5.091 & & 0.6951 & 7.61E-03 & 1448.2506 & 533.6377 & 1981.8883 & \\
\hline & $7 / 27 / 2008$ 14:00 & 121 & 209 & 1400 & 4.933 & 4.933 & & 0.7494 & 8.34E-03 & 1588.7269 & 585.3991 & 2174.1260 & \\
\hline & $7 / 27 / 200815: 00$ & 122 & 209 & 1500 & 4.934 & 4.934 & & 0.7491 & 8.34E-03 & 1587.8016 & 585.0582 & 2172.8598 & \\
\hline
\end{tabular}




\begin{tabular}{|c|c|c|c|c|c|c|c|c|c|c|c|c|c|}
\hline Tree ID & TS & RN & JD & HR & $\begin{array}{c}\text { Raw } \\
\text { TC_dTA }\end{array}$ & $\begin{array}{c}\text { Fixed } \\
\text { TC_dT } \Delta\end{array}$ & dTM & $\mathbf{K}$ & $\begin{array}{c}\text { Sap } \\
\text { Velocity } \\
(\mathrm{cm} / \mathrm{s})\end{array}$ & $\begin{array}{l}\text { Probed } \\
\text { Drywood F } \\
\text { (g/hr) }\end{array}$ & $\begin{array}{c}\text { Extrapolated } \\
\text { Wetwood F } \\
(\mathrm{g} / \mathrm{hr})\end{array}$ & $\begin{array}{c}\text { Extrapolated } \\
\text { Total Wood F } \\
(\mathrm{g} / \mathrm{hr})\end{array}$ & $\begin{array}{l}\text { Relative } \\
\text { Humidity, } \\
\text { @10m (\%) }\end{array}$ \\
\hline & 7/27/2008 16:00 & 123 & 209 & 1600 & 4.956 & 4.956 & & 0.7413 & 8.23E-03 & 1567.5667 & 577.6022 & 2145.1688 & \\
\hline & 7/27/2008 17:00 & 124 & 209 & 1700 & 4.969 & 4.969 & & 0.7368 & 8.17E-03 & 1555.7166 & 573.2358 & 2128.9523 & \\
\hline & $7 / 27 / 2008$ 18:00 & 125 & 209 & 1800 & 5.126 & 5.126 & & 0.6836 & 7.45E-03 & 1418.6240 & 522.7212 & 1941.3451 & \\
\hline & 7/27/2008 19:00 & 126 & 209 & 1900 & 5.31 & 5.31 & & 0.6252 & $6.68 \mathrm{E}-03$ & 1271.0890 & 468.3589 & 1739.4479 & \\
\hline & $7 / 27 / 200820: 00$ & 127 & 209 & 2000 & 5.771 & 5.771 & & 0.4954 & 5.01E-03 & 954.4340 & 351.6808 & 1306.1148 & \\
\hline & 7/27/2008 21:00 & 128 & 209 & 2100 & 7.052 & 7.052 & & 0.2238 & 1.88E-03 & 358.7927 & 132.2046 & 490.9973 & \\
\hline & 7/27/2008 22:00 & 129 & 209 & 2200 & 8.05 & 8.05 & & 0.0720 & 4.67E-04 & 88.9184 & 32.7638 & 121.6822 & \\
\hline & 7/27/2008 23:00 & 130 & 209 & 2300 & 8.23 & 8.23 & & 0.0486 & $2.88 \mathrm{E}-04$ & 54.7678 & 20.1803 & 74.9481 & \\
\hline & 7/28/2008 0:00 & 131 & 210 & 0 & 8.3 & 8.3 & 8.67 & 0.0398 & $2.25 \mathrm{E}-04$ & 42.7712 & 15.7599 & 58.5312 & \\
\hline & 7/28/2008 1:00 & 132 & 210 & 100 & 8.41 & 8.41 & & 0.0262 & 1.34E-04 & 25.5473 & 9.4134 & 34.9607 & \\
\hline & 7/28/2008 2:00 & 133 & 210 & 200 & 8.44 & 8.44 & & 0.0225 & 1.12E-04 & 21.2356 & 7.8247 & 29.0603 & \\
\hline & 7/28/2008 3:00 & 134 & 210 & 300 & 8.56 & 8.56 & & 0.0082 & 3.21E-05 & 6.1050 & 2.2495 & 8.3545 & \\
\hline & 7/28/2008 4:00 & 135 & 210 & 400 & 8.63 & 8.63 & & 0.0000 & $0.00 E+00$ & 0.0000 & 0.0000 & 0.0000 & \\
\hline & 7/28/2008 5:00 & 136 & 210 & 500 & 8.67 & 8.67 & & -0.0046 & $0.00 E+00$ & 0.0000 & 0.0000 & 0.0000 & \\
\hline & 7/28/2008 6:00 & 137 & 210 & 600 & 8.66 & 8.66 & & -0.0035 & $0.00 \mathrm{E}+00$ & 0.0000 & 0.0000 & 0.0000 & \\
\hline & 7/28/2008 7:00 & 138 & 210 & 700 & 8.58 & 8.58 & & 0.0058 & $2.11 \mathrm{E}-05$ & 4.0231 & 1.4824 & 5.5054 & \\
\hline & 7/28/2008 8:00 & 139 & 210 & 800 & 6.925 & 6.925 & & 0.2462 & $2.12 \mathrm{E}-03$ & 403.5918 & 148.7117 & 552.3035 & \\
\hline & 7/28/2008 9:00 & 140 & 210 & 900 & 5.408 & 5.408 & & 0.5958 & $6.29 E-03$ & 1197.7901 & 441.3504 & 1639.1405 & \\
\hline & $7 / 28 / 2008$ 10:00 & 141 & 210 & 1000 & 5.09 & 5.09 & & 0.6955 & $7.61 \mathrm{E}-03$ & 1449.1047 & 533.9524 & 1983.0572 & \\
\hline & 7/28/2008 11:00 & 142 & 210 & 1100 & 5.269 & 5.269 & & 0.6379 & 6.84E-03 & 1302.8118 & 480.0478 & 1782.8596 & \\
\hline & 7/28/2008 12:00 & 143 & 210 & 1200 & 5.22 & 5.22 & & 0.6533 & 7.05E-03 & 1341.5739 & 494.3305 & 1835.9044 & \\
\hline & 7/28/2008 13:00 & 144 & 210 & 1300 & 5.098 & 5.098 & & 0.6928 & 7.57E-03 & 1442.2835 & 531.4390 & 1973.7225 & \\
\hline & 7/28/2008 14:00 & 145 & 210 & 1400 & 5.009 & 5.009 & & 0.7229 & $7.98 \mathrm{E}-03$ & 1519.7451 & 559.9813 & 2079.7264 & \\
\hline & $7 / 28 / 2008$ 15:00 & 146 & 210 & 1500 & 4.997 & 4.997 & & 0.7270 & 8.04E-03 & 1530.4595 & 563.9293 & 2094.3888 & \\
\hline & 7/28/2008 16:00 & 147 & 210 & 1600 & 5.003 & 5.003 & & 0.7250 & 8.01E-03 & 1525.0941 & 561.9523 & 2087.0464 & \\
\hline & 7/28/2008 17:00 & 148 & 210 & 1700 & 4.985 & 4.985 & & 0.7312 & 8.09E-03 & 1541.2397 & 567.9015 & 2109.1412 & \\
\hline & 7/28/2008 18:00 & 149 & 210 & 1800 & 5.15 & 5.15 & & 0.6757 & 7.35E-03 & 1398.6070 & 515.3455 & 1913.9525 & \\
\hline & $7 / 28 / 2008$ 19:00 & 150 & 210 & 1900 & 5.401 & 5.401 & & 0.5979 & 6.32E-03 & 1202.9107 & 443.2372 & 1646.1479 & \\
\hline & $7 / 28 / 200820: 00$ & 151 & 210 & 2000 & 6.063 & 6.063 & & 0.4234 & 4.13E-03 & 786.6131 & 289.8438 & 1076.4569 & \\
\hline & 7/28/2008 21:00 & 152 & 210 & 2100 & 7.485 & 7.485 & & 0.1530 & 1.18E-03 & 224.6497 & 82.7768 & 307.4265 & \\
\hline & $7 / 28 / 2008$ 22:00 & 153 & 210 & 2200 & 8.02 & 8.02 & & 0.0761 & 4.99E-04 & 95.0493 & 35.0229 & 130.0721 & \\
\hline & 7/28/2008 23:00 & 154 & 210 & 2300 & 8.2 & 8.2 & & 0.0524 & 3.16E-04 & 60.1369 & 22.1587 & 82.2956 & \\
\hline & $7 / 29 / 20080: 00$ & 155 & 211 & 0 & 8.38 & 8.38 & 8.58 & 0.0298 & 1.58E-04 & 30.0329 & 11.0662 & 41.0991 & \\
\hline & 7/29/2008 1:00 & 156 & 211 & 100 & 8.48 & 8.48 & & 0.0177 & 8.29E-05 & 15.7819 & 5.8152 & 21.5971 & \\
\hline & 7/29/2008 2:00 & 157 & 211 & 200 & 8.56 & 8.56 & & 0.0082 & 3.21E-05 & 6.1050 & 2.2495 & 8.3545 & \\
\hline & 7/29/2008 3:00 & 158 & 211 & 300 & 8.56 & 8.56 & & 0.0082 & 3.21E-05 & 6.1050 & 2.2495 & 8.3545 & \\
\hline & 7/29/2008 4:00 & 159 & 211 & 400 & 8.58 & 8.58 & & 0.0058 & 2.11E-05 & 4.0231 & 1.4824 & 5.5054 & \\
\hline & 7/29/2008 5:00 & 160 & 211 & 500 & 8.49 & 8.49 & & 0.0165 & $7.60 \mathrm{E}-05$ & 14.4759 & 5.3339 & 19.8098 & \\
\hline & $7 / 29 / 20086: 00$ & 161 & 211 & 600 & 8.41 & 8.41 & & 0.0262 & $1.34 \mathrm{E}-04$ & 25.5473 & 9.4134 & 34.9607 & \\
\hline & 7/29/2008 7:00 & 162 & 211 & 700 & 8.36 & 8.36 & & 0.0323 & 1.74E-04 & 33.1146 & 12.2017 & 45.3163 & \\
\hline & $7 / 29 / 20088: 00$ & 163 & 211 & 800 & 8.12 & 8.12 & & 0.0628 & $3.94 \mathrm{E}-04$ & 75.0934 & 27.6697 & 102.7631 & \\
\hline
\end{tabular}




\begin{tabular}{|c|c|c|c|c|c|c|c|c|c|c|c|c|c|}
\hline Tree ID & TS & RN & JD & HR & $\begin{array}{c}\text { Raw } \\
\text { TC_dTA }\end{array}$ & $\begin{array}{c}\text { Fixed } \\
\text { TC_dT } \Delta\end{array}$ & dTM & $\mathbf{K}$ & $\begin{array}{c}\text { Sap } \\
\text { Velocity } \\
(\mathrm{cm} / \mathrm{s})\end{array}$ & $\begin{array}{c}\text { Probed } \\
\text { Drywood F } \\
(\mathrm{g} / \mathrm{hr})\end{array}$ & $\begin{array}{c}\text { Extrapolated } \\
\text { Wetwood F } \\
(\mathrm{g} / \mathrm{hr})\end{array}$ & $\begin{array}{c}\text { Extrapolated } \\
\text { Total Wood F } \\
(\mathrm{g} / \mathrm{hr})\end{array}$ & $\begin{array}{c}\text { Relative } \\
\text { Humidity, } \\
@ 10 \mathrm{~m}(\%)\end{array}$ \\
\hline & 7/29/2008 9:00 & 164 & 211 & 900 & 6.779 & 6.779 & & 0.2730 & $2.41 \mathrm{E}-03$ & 458.4149 & 168.9124 & 627.3273 & \\
\hline & $7 / 29 / 2008$ 10:00 & 165 & 211 & 1000 & 5.658 & 5.658 & & 0.5253 & $5.39 \mathrm{E}-03$ & 1025.7496 & 377.9585 & 1403.7081 & \\
\hline & $7 / 29 / 2008$ 11:00 & 166 & 211 & 1100 & 5.946 & 5.946 & & 0.4514 & 4.47E-03 & 851.1513 & 313.6242 & 1164.7755 & \\
\hline & $7 / 29 / 2008$ 12:00 & 167 & 211 & 1200 & 5.352 & 5.352 & & 0.6125 & $6.51 \mathrm{E}-03$ & 1239.2463 & 456.6258 & 1695.8721 & \\
\hline & $7 / 29 / 2008$ 13:00 & 168 & 211 & 1300 & 5.362 & 5.362 & & 0.6095 & $6.47 \mathrm{E}-03$ & 1231.7604 & 453.8675 & 1685.6279 & \\
\hline & $7 / 29 / 2008$ 14:00 & 169 & 211 & 1400 & 5.132 & 5.132 & & 0.6816 & 7.42E-03 & 1413.5972 & 520.8689 & 1934.4661 & \\
\hline & 7/29/2008 15:00 & 170 & 211 & 1500 & 5.288 & 5.288 & & 0.6320 & $6.76 \mathrm{E}-03$ & 1288.0316 & 474.6017 & 1762.6333 & \\
\hline & $7 / 29 / 200816: 00$ & 171 & 211 & 1600 & 5.463 & 5.463 & & 0.5797 & $6.08 \mathrm{E}-03$ & 1158.1540 & 426.7457 & 1584.8996 & \\
\hline & $7 / 29 / 2008$ 17:00 & 172 & 211 & 1700 & 5.628 & 5.628 & & 0.5334 & $5.49 \mathrm{E}-03$ & 1045.3291 & 385.1730 & 1430.5020 & \\
\hline & $7 / 29 / 2008$ 18:00 & 173 & 211 & 1800 & 5.621 & 5.621 & & 0.5353 & $5.51 E-03$ & 1049.9377 & 386.8711 & 1436.8089 & \\
\hline & $7 / 29 / 2008$ 19:00 & 174 & 211 & 1900 & 5.686 & 5.686 & & 0.5178 & 5.29E-03 & 1007.7239 & 371.3166 & 1379.0405 & \\
\hline & $7 / 29 / 200820: 00$ & 175 & 211 & 2000 & 6.58 & 6.58 & & 0.3116 & $2.83 E-03$ & 539.2365 & 198.6928 & 737.9293 & \\
\hline & $7 / 29 / 200821: 00$ & 176 & 211 & 2100 & 7.873 & 7.873 & & 0.0962 & $6.66 \mathrm{E}-04$ & 126.8425 & 46.7377 & 173.5802 & \\
\hline & $7 / 29 / 200822: 00$ & 177. & 211 & 2200 & 8.38 & 8.38 & & 0.0298 & 1.58E-04 & 30.0329 & 11.0662 & 41.0991 & \\
\hline & 7/29/2008 23:00 & 178 & 211 & 2300 & 8.44 & 8.44 & & 0.0225 & 1.12E-04 & 21.2356 & 7.8247 & 29.0603 & \\
\hline & $7 / 30 / 20080: 00$ & 179 & 212 & 0 & 8.36 & 8.36 & 8.42 & 0.0323 & $1.74 \mathrm{E}-04$ & 33.1146 & 12.2017 & 45.3163 & \\
\hline & 7/30/2008 1:00 & 180 & 212 & 100 & 8.24 & 8.24 & & 0.0473 & $2.78 \mathrm{E}-04$ & 53.0079 & 19.5318 & 72.5397 & \\
\hline & 7/30/2008 2:00 & 181 & 212 & 200 & 8.2 & 8.2 & & 0.0524 & $3.16 \mathrm{E}-04$ & 60.1369 & 22.1587 & 82.2956 & \\
\hline & 7/30/2008 3:00 & 182 & 212 & 300 & 8.19 & 8.19 & & 0.0537 & $3.25 \mathrm{E}-04$ & 61.9561 & 22.8290 & 84.7851 & \\
\hline & 7/30/2008 4:00 & 183 & 212 & 400 & 8.13 & 8.13 & & 0.0615 & $3.84 \mathrm{E}-04$ & 73.1740 & 26.9625 & 100.1365 & \\
\hline & 7/30/2008 5:00 & 184 & 212 & 500 & 8.05 & 8.05 & & 0.0720 & 4.67E-04 & 88.9184 & 32.7638 & 121.6822 & \\
\hline & 7/30/2008 6:00 & 185 & 212 & 600 & 8.01 & 8.01 & & 0.0774 & $5.10 \mathrm{E}-04$ & 97.1200 & 35.7859 & 132.9059 & \\
\hline & 7/30/2008 7:00 & 186 & 212 & 700 & 8.01 & 8.01 & & 0.0774 & 5.10E-04 & 97.1200 & 35.7859 & 132.9059 & \\
\hline & 7/30/2008 8:00 & 187 & 212 & 800 & 8.08 & 8.08 & & 0.0681 & 4.35E-04 & 82.9105 & 30.5501 & 113.4606 & \\
\hline & 7/30/2008 9:00 & 188 & 212 & 900 & 7.605 & 7.605 & & 0.1348 & 1.01E-03 & 192.2270 & 70.8300 & 263.0569 & \\
\hline & $7 / 30 / 200810: 00$ & 189 & 212 & 1000 & 5.58 & 5.58 & & 0.5466 & $5.66 \mathrm{E}-03$ & 1077.2407 & 396.9315 & 1474.1722 & \\
\hline & 7/30/2008 11:00 & 190 & 212 & 1100 & 5.321 & 5.321 & & 0.6219 & $6.63 \mathrm{E}-03$ & 1262.6860 & 465.2626 & 1727.9486 & \\
\hline & $7 / 30 / 2008$ 12:00 & 191 & 212 & 1200 & 5.209 & 5.209 & & 0.6567 & 7.09E-03 & 1350.4054 & 497.5846 & 1847.9901 & \\
\hline & $7 / 30 / 2008$ 13:00 & 192 & 212 & 1300 & 5.09 & 5.09 & & 0.6955 & $7.61 \mathrm{E}-03$ & 1449.1047 & 533.9524 & 1983.0572 & \\
\hline & $7 / 30 / 2008$ 14:00 & 193 & 212 & 1400 & 5.055 & 5.055 & & 0.7072 & 7.77E-03 & 1479.2733 & 545.0687 & 2024.3419 & \\
\hline & $7 / 30 / 2008$ 15:00 & 194 & 212 & 1500 & 5.187 & 5.187 & & 0.6638 & 7.19E-03 & 1368.2137 & 504.1465 & 1872.3601 & \\
\hline & $7 / 30 / 2008$ 16:00 & 195 & 212 & 1600 & 5.063 & 5.063 & & 0.7045 & $7.73 \mathrm{E}-03$ & 1472.3305 & 542.5105 & 2014.8410 & \\
\hline & $7 / 30 / 2008$ 17:00 & 196 & 212 & 1700 & 5.038 & 5.038 & & 0.7130 & $7.85 E-03$ & 1494.1203 & 550.5394 & 2044.6597 & \\
\hline & 7/30/2008 18:00 & 197 & 212 & 1800 & 5.252 & 5.252 & & 0.6432 & $6.91 \mathrm{E}-03$ & 1316.1538 & 484.9639 & 1801.1177 & \\
\hline & 7/30/2008 19:00 & 198 & 212 & 1900 & 5.409 & 5.409 & & 0.5955 & $6.29 \mathrm{E}-03$ & 1197.0600 & 441.0814 & 1638.1414 & \\
\hline & $7 / 30 / 200820: 00$ & 199 & 212 & 2000 & 5.99 & 5.99 & & 0.4407 & $4.34 \mathrm{E}-03$ & 826.4724 & 304.5308 & 1131.0032 & \\
\hline & 7/30/2008 21:00 & 200 & 212 & 2100 & 7.428 & 7.428 & & 0.1618 & 1.26E-03 & 240.7495 & 88.7091 & 329.4586 & \\
\hline & $7 / 30 / 200822: 00$ & 201 & 212 & 2200 & 8.24 & 8.24 & & 0.0473 & $2.78 \mathrm{E}-04$ & 53.0079 & 19.5318 & 72.5397 & \\
\hline & $7 / 30 / 2008$ 23:00 & 202 & 212 & 2300 & 8.42 & 8.42 & & 0.0249 & $1.27 \mathrm{E}-04$ & 24.0901 & 8.8765 & 32.9666 & \\
\hline & $7 / 31 / 20080: 00$ & 203 & 213 & 0 & 8.45 & 8.45 & 8.45 & 0.0213 & $1.04 \mathrm{E}-04$ & 19.8393 & 7.3102 & 27.1495 & \\
\hline & 7/31/2008 1:00 & 204 & 213 & 100 & 8.41 & 8.41 & & 0.0262 & 1.34E-04 & 25.5473 & 9.4134 & 34.9607 & \\
\hline
\end{tabular}




\begin{tabular}{|c|c|c|c|c|c|c|c|c|c|c|c|c|c|}
\hline Tree ID & TS & RN & JD & HR & $\begin{array}{c}\text { Raw } \\
\text { TC_dTA }\end{array}$ & $\begin{array}{c}\text { Fixed } \\
\text { TC_dT } \Delta\end{array}$ & dTM & $\mathbf{K}$ & $\begin{array}{l}\text { Sap } \\
\text { Velocity } \\
(\mathrm{cm} / \mathrm{s})\end{array}$ & $\begin{array}{c}\text { Probed } \\
\text { Drywood F } \\
(\mathrm{g} / \mathrm{hr})\end{array}$ & $\begin{array}{c}\text { Extrapolated } \\
\text { Wetwood F } \\
(\mathrm{g} / \mathrm{hr})\end{array}$ & $\begin{array}{c}\text { Extrapolated } \\
\text { Total Wood F } \\
(\mathrm{g} / \mathrm{hr})\end{array}$ & $\begin{array}{l}\text { Relative } \\
\text { Humidity, } \\
@ 10 \mathrm{~m}(\%)\end{array}$ \\
\hline & 7/31/2008 2:00 & 205 & 213 & 200 & 8.36 & 8.36 & & 0.0323 & 1.74E-04 & 33.1146 & 12.2017 & $\begin{array}{r}45.3163 \\
\end{array}$ & \\
\hline & 7/31/2008 3:00 & 206 & 213 & 300 & 8.32 & 8.32 & & 0.0373 & 2.07E-04 & 39.4858 & 14.5493 & 54.0351 & \\
\hline & 7/31/2008 4:00 & 207 & 213 & 400 & 8.25 & 8.25 & & 0.0461 & 2.69E-04 & 51.2631 & 18.8890 & 70.1521 & \\
\hline & 7/31/2008 5:00 & 208 & 213 & 500 & 8.18 & 8.18 & & 0.0550 & 3.35E-04 & 63.7899 & 23.5047 & 87.2945 & \\
\hline & 7/31/2008 6:00 & 209 & 213 & 600 & 8.13 & 8.13 & & 0.0615 & 3.84E-04 & 73.1740 & 26.9625 & 100.1365 & \\
\hline & 7/31/2008 7:00 & 210 & 213 & 700 & 8.08 & 8.08 & & 0.0681 & 4.35E-04 & 82.9105 & 30.5501 & 113.4606 & \\
\hline & $7 / 31 / 2008$ 8:00 & 211 & 213 & 800 & 8.07 & 8.07 & & 0.0694 & $4.46 \mathrm{E}-04$ & 84.8994 & 31.2829 & 116.1823 & \\
\hline & $7 / 31 / 20089: 00$ & 212 & 213 & 900 & 7.326 & 7.326 & & 0.1780 & 1.42E-03 & 270.7085 & 99.7481 & 370.4566 & \\
\hline & $7 / 31 / 2008$ 10:00 & 213 & 213 & 1000 & 5.508 & 5.508 & & 0.5668 & 5.92E-03 & 1126.4962 & 415.0807 & 1541.5768 & \\
\hline & 7/31/2008 11:00 & 214 & 213 & 1100 & 5.406 & 5.406 & & 0.5964 & 6.30E-03 & 1199.2514 & 441.8888 & 1641.1402 & \\
\hline & $7 / 31 / 2008$ 12:00 & 215 & 213 & 1200 & 5.113 & 5.113 & & 0.6879 & 7.51E-03 & 1429.5674 & 526.7535 & 1956.3209 & \\
\hline & 7/31/2008 13:00 & 216 & 213 & 1300 & 5.289 & 5.289 & & 0.6317 & $6.76 \mathrm{E}-03$ & 1287.2575 & 474.3165 & 1761.5740 & \\
\hline & 7/31/2008 14:00 & 217 & 213 & 1400 & 4.931 & 4.931 & & 0.7502 & 8.35E-03 & 1590.5787 & 586.0814 & 2176.6602 & \\
\hline & 7/31/2008 15:00 & 218 & 213 & 1500 & 4.892 & 4.892 & & 0.7641 & 8.54E-03 & 1627.0747 & 599.5292 & 2226.6039 & \\
\hline & $7 / 31 / 200816: 00$ & 219 & 213 & 1600 & 5.012 & 5.012 & & 0.7219 & 7.97E-03 & 1517.0767 & 558.9981 & 2076.0748 & \\
\hline & 7/31/2008 17:00 & 220 & 213 & 1700 & 4.989 & 4.989 & & 0.7298 & 8.08E-03 & 1537.6390 & 566.5747 & 2104.2137 & \\
\hline & $7 / 31 / 2008$ 18:00 & 221 & 213 & 1800 & 5.184 & 5.184 & & 0.6647 & 7.20E-03 & 1370.6572 & 505.0468 & 1875.7040 & \\
\hline & $7 / 31 / 2008$ 19:00 & 222 & 213 & 1900 & 5.456 & 5.456 & & 0.5817 & $6.11 \mathrm{E}-03$ & 1163.1404 & 428.5830 & 1591.7234 & \\
\hline & $7 / 31 / 200820: 00$ & 223 & 213 & 2000 & 6.354 & 6.354 & & 0.3582 & $3.36 \mathrm{E}-03$ & 640.2864 & 235.9267 & 876.2131 & \\
\hline & $7 / 31 / 200821: 00$ & 224 & 213 & 2100 & 7.256 & 7.256 & & 0.1894 & 1.53E-03 & 292.1391 & 107.6447 & 399.7837 & \\
\hline & $7 / 31 / 200822: 00$ & 225 & 213 & 2200 & 7.927 & 7.927 & & 0.0887 & $6.03 E-04$ & 114.8274 & 42.3105 & 157.1379 & \\
\hline & 7/31/2008 23:00 & 226 & 213 & 2300 & 7.941 & 7.941 & & 0.0868 & 5.87E-04 & 111.7758 & 41.1861 & 152.9619 & \\
\hline & $8 / 1 / 20080: 00$ & 227 & 214 & 0 & 8.17 & 8.17 & 8.62 & 0.0563 & 3.45E-04 & 65.6381 & 24.1857 & 89.8238 & \\
\hline & 8/1/2008 1:00 & 228 & 214 & 100 & 8.45 & 8.45 & & 0.0213 & 1.04E-04 & 19.8393 & 7.3102 & 27.1495 & \\
\hline & 8/1/2008 2:00 & 229 & 214 & 200 & 8.56 & 8.56 & & 0.0082 & $3.21 \mathrm{E}-05$ & 6.1050 & 2.2495 & 8.3545 & \\
\hline & $8 / 1 / 2008$ 3:00 & 230 & 214 & 300 & 8.6 & 8.6 & & 0.0035 & 1.12E-05 & 2.1390 & 0.7882 & 2.9272 & \\
\hline & 8/1/2008 4:00 & 231 & 214 & 400 & 8.62 & 8.62 & & 0.0012 & 2.90E-06 & 0.5516 & 0.2033 & 0.7549 & \\
\hline & 8/1/2008 5:00 & 232 & 214 & 500 & 8.59 & 8.59 & & 0.0047 & 1.60E-05 & 3.0524 & 1.1247 & 4.1771 & \\
\hline & 8/1/2008 6:00 & 233 & 214 & 600 & 8.53 & 8.53 & & 0.0117 & 5.00E-05 & 9.5115 & 3.5047 & 13.0162 & \\
\hline & 8/1/2008 7:00 & 234 & 214 & 700 & 8.47 & 8.47 & & 0.0189 & 8.99E-05 & 17.1117 & 6.3052 & 23.4169 & \\
\hline & 8/1/2008 8:00 & 235 & 214 & 800 & 8.48 & 8.48 & & 0.0177 & 8.29E-05 & 15.7819 & 5.8152 & 21.5971 & \\
\hline & 8/1/2008 9:00 & 236 & 214 & 900 & 7.597 & 7.597 & & 0.1360 & 1.02E-03 & 194.3271 & 71.6038 & 265.9309 & \\
\hline & 8/1/2008 10:00 & 237 & 214 & 1000 & 5.457 & 5.457 & & 0.5815 & $6.10 \mathrm{E}-03$ & 1162.4270 & 428.3201 & 1590.7471 & \\
\hline & 8/1/2008 11:00 & 238 & 214 & 1100 & 5.432 & 5.432 & & 0.5887 & $6.20 \mathrm{E}-03$ & 1180.3648 & 434.9297 & 1615.2945 & \\
\hline & 8/1/2008 12:00 & 239 & 214 & 1200 & 5.287 & 5.287 & & 0.6323 & 6.77E-03 & 1288.8061 & 474.8871 & 1763.6931 & \\
\hline & 8/1/2008 13:00 & 240 & 214 & 1300 & 5.065 & 5.065 & & 0.7038 & 7.72E-03 & 1470.5992 & 541.8725 & 2012.4718 & \\
\hline & 8/1/2008 14:00 & 241 & 214 & 1400 & 5.005 & 5.005 & & 0.7243 & 8.00E-03 & 1523.3093 & 561.2946 & 2084.6039 & \\
\hline & 8/1/2008 15:00 & 242 & 214 & 1500 & 4.99 & 4.99 & & 0.7295 & 8.07E-03 & 1536.7399 & 566.2434 & 2102.9834 & \\
\hline & $8 / 1 / 200816: 00$ & 243 & 214 & 1600 & 5.039 & 5.039 & & 0.7126 & $7.84 \mathrm{E}-03$ & 1493.2434 & 550.2162 & 2043.4597 & \\
\hline & 8/1/2008 17:00 & 244 & 214 & 1700 & 5.054 & 5.054 & & 0.7076 & 7.77E-03 & 1480.1431 & 545.3892 & 2025.5323 & \\
\hline & 8/1/2008 18:00 & 245 & 214 & 1800 & 5.222 & 5.222 & & 0.6526 & 7.04E-03 & 1339.9733 & 493.7407 & 1833.7141 & \\
\hline
\end{tabular}




\begin{tabular}{|c|c|c|c|c|c|c|c|c|c|c|c|c|c|}
\hline Tree ID & TS & RN & JD & HR & $\begin{array}{c}\text { Raw } \\
\text { TC_dTA }\end{array}$ & $\begin{array}{c}\text { Fixed } \\
\text { TC_dT } \Delta\end{array}$ & dTM & $\mathbf{K}$ & $\begin{array}{c}\text { Sap } \\
\text { Velocity } \\
(\mathrm{cm} / \mathrm{s})\end{array}$ & $\begin{array}{c}\text { Probed } \\
\text { Drywood F } \\
\text { (g/hr) }\end{array}$ & $\begin{array}{c}\text { Extrapolated } \\
\text { Wetwood F } \\
(\mathrm{g} / \mathrm{hr})\end{array}$ & $\begin{array}{c}\text { Extrapolated } \\
\text { Total Wood F } \\
(\mathrm{g} / \mathrm{hr})\end{array}$ & $\begin{array}{l}\text { Relative } \\
\text { Humidity, } \\
\text { @10m (\%) }\end{array}$ \\
\hline & 8/1/2008 19:00 & 246 & 214 & 1900 & \begin{tabular}{|l|} 
\\
5.437 \\
\end{tabular} & 5.437 & & 0.5873 & $6.18 \mathrm{E}-03$ & 1176.7599 & 433.6014 & 1610.3614 & \\
\hline & 8/1/2008 20:00 & 247 & 214 & 2000 & 6.306 & 6.306 & & 0.3685 & 3.48E-03 & 663.1105 & 244.3367 & 907.4472 & \\
\hline & 8/1/2008 21:00 & 248 & 214 & 2100 & 7.77 & 7.77 & & 0.1107 & 7.92E-04 & 150.8362 & 55.5787 & 206.4149 & \\
\hline & 8/1/2008 22:00 & 249 & 214 & 2200 & 8.42 & 8.42 & & 0.0249 & 1.27E-04 & 24.0901 & 8.8765 & 32.9666 & \\
\hline & 8/1/2008 23:00 & 250 & 214 & 2300 & 8.56 & 8.56 & & 0.0082 & $3.21 \mathrm{E}-05$ & 6.1050 & 2.2495 & 8.3545 & \\
\hline & 8/2/2008 0:00 & 251 & 215 & 0 & 8.64 & 8.64 & 8.76 & -0.0012 & $0.00 \mathrm{E}+00$ & 0.0000 & 0.0000 & 0.0000 & \\
\hline & 8/2/2008 1:00 & 252 & 215 & 100 & 8.72 & 8.72 & & -0.0103 & $0.00 \mathrm{E}+00$ & 0.0000 & 0.0000 & 0.0000 & \\
\hline & 8/2/2008 2:00 & 253 & 215 & 200 & 8.76 & 8.76 & & -0.0148 & $0.00 \mathrm{E}+00$ & 0.0000 & 0.0000 & 0.0000 & \\
\hline & 8/2/2008 3:00 & 254 & 215 & 300 & 8.76 & 8.76 & & -0.0148 & $0.00 \mathrm{E}+00$ & 0.0000 & 0.0000 & 0.0000 & \\
\hline & 8/2/2008 4:00 & 255 & 215 & 400 & 8.74 & 8.74 & & -0.0126 & $0.00 \mathrm{E}+00$ & 0.0000 & 0.0000 & 0.0000 & \\
\hline & 8/2/2008 5:00 & 256 & 215 & 500 & 8.74 & 8.74 & & -0.0126 & $0.00 \mathrm{E}+00$ & 0.0000 & 0.0000 & 0.0000 & \\
\hline & 8/2/2008 6:00 & 257 & 215 & 600 & 8.72 & 8.72 & & -0.0103 & $0.00 \mathrm{E}+00$ & 0.0000 & 0.0000 & 0.0000 & \\
\hline & 8/2/2008 7:00 & 258 & 215 & 700 & 8.64 & 8.64 & & -0.0012 & $0.00 E+00$ & 0.0000 & 0.0000 & 0.0000 & \\
\hline & 8/2/2008 8:00 & 259 & 215 & 800 & 7.817 & 7.817 & & 0.1040 & 7.34E-04 & 139.7126 & 51.4800 & 191.1925 & \\
\hline & 8/2/2008 9:00 & 260 & 215 & 900 & 5.923 & 5.923 & & 0.4570 & 4.54E-03 & 864.2522 & 318.4515 & 1182.7038 & \\
\hline & 8/2/2008 10:00 & 261 & 215 & 1000 & 5.555 & 5.555 & & 0.5536 & 5.75E-03 & 1094.1519 & 403.1628 & 1497.3146 & \\
\hline & 8/2/2008 11:00 & 262 & 215 & 1100 & 5.405 & 5.405 & & 0.5967 & $6.30 \mathrm{E}-03$ & 1199.9825 & 442.1582 & 1642.1408 & \\
\hline & 8/2/2008 12:00 & 263 & 215 & 1200 & 5.227 & 5.227 & & 0.6510 & 7.02E-03 & 1335.9788 & 492.2689 & 1828.2477 & \\
\hline & 8/2/2008 13:00 & 264 & 215 & 1300 & 5.1 & 5.1 & & 0.6922 & 7.57E-03 & 1440.5825 & 530.8122 & 1971.3947 & \\
\hline & 8/2/2008 14:00 & 265 & 215 & 1400 & 5.062 & 5.062 & & 0.7049 & 7.74E-03 & 1473.1968 & 542.8297 & 2016.0265 & \\
\hline & 8/2/2008 15:00 & 266 & 215 & 1500 & 5.069 & 5.069 & & 0.7025 & 7.71E-03 & 1467.1419 & 540.5986 & 2007.7404 & \\
\hline & 8/2/2008 16:00 & 267 & 215 & 1600 & 5.081 & 5.081 & & 0.6985 & 7.65E-03 & 1456.8115 & 536.7922 & 1993.6037 & \\
\hline & 8/2/2008 17:00 & 268 & 215 & 1700 & 5.154 & 5.154 & & 0.6744 & 7.33E-03 & 1395.2941 & 514.1248 & 1909.4190 & \\
\hline & 8/2/2008 18:00 & 269 & 215 & 1800 & 5.279 & 5.279 & & 0.6348 & $6.80 \mathrm{E}-03$ & 1295.0155 & 477.1751 & 1772.1906 & \\
\hline & 8/2/2008 19:00 & 270 & 215 & 1900 & 5.519 & 5.519 & & 0.5637 & 5.88E-03 & 1118.8610 & 412.2673 & 1531.1283 & \\
\hline & 8/2/2008 20:00 & 271 & 215 & 2000 & 6.177 & 6.177 & & 0.3971 & 3.82E-03 & 726.9705 & 267.8672 & 994.8378 & \\
\hline & 8/2/2008 21:00 & 272 & 215 & 2100 & 7.323 & 7.323 & & 0.1785 & 1.43E-03 & 271.6123 & 100.0811 & 371.6934 & \\
\hline & 8/2/2008 22:00 & 273 & 215 & 2200 & 8.14 & 8.14 & & 0.0602 & 3.74E-04 & 71.2687 & 26.2604 & 97.5292 & \\
\hline & 8/2/2008 23:00 & 274 & 215 & 2300 & 8.35 & 8.35 & & 0.0335 & 1.82E-04 & 34.6818 & 12.7792 & 47.4610 & \\
\hline & 8/3/2008 0:00 & 275 & 216 & 0 & 8.31 & 8.31 & 8.74 & 0.0385 & 2.16E-04 & 41.1204 & 15.1516 & 56.2720 & \\
\hline & 8/3/2008 1:00 & 276 & 216 & 100 & 8.23 & 8.23 & & 0.0486 & 2.88E-04 & 54.7678 & 20.1803 & 74.9481 & \\
\hline & 8/3/2008 2:00 & 277 & 216 & 200 & 8.38 & 8.38 & & 0.0298 & $1.58 \mathrm{E}-04$ & 30.0329 & 11.0662 & 41.0991 & \\
\hline & 8/3/2008 3:00 & 278 & 216 & 300 & 8.55 & 8.55 & & 0.0094 & $3.78 E-05$ & 7.2061 & 2.6552 & 9.8613 & \\
\hline & 8/3/2008 4:00 & 279 & 216 & 400 & 8.66 & 8.66 & & -0.0035 & $0.00 \mathrm{E}+00$ & 0.0000 & 0.0000 & 0.0000 & \\
\hline & $8 / 3 / 20085: 00$ & 280 & 216 & 500 & 8.69 & 8.69 & & -0.0069 & $0.00 \mathrm{E}+00$ & 0.0000 & 0.0000 & 0.0000 & \\
\hline & 8/3/2008 6:00 & 281 & 216 & 600 & 8.7 & 8.7 & & -0.0080 & $0.00 \mathrm{E}+00$ & 0.0000 & 0.0000 & 0.0000 & \\
\hline & 8/3/2008 7:00 & 282 & 216 & 700 & 8.74 & 8.74 & & -0.0126 & $0.00 \mathrm{E}+00$ & 0.0000 & 0.0000 & 0.0000 & \\
\hline & 8/3/2008 8:00 & 283 & 216 & 800 & 8.01 & 8.01 & & 0.0774 & $5.10 \mathrm{E}-04$ & 97.1200 & 35.7859 & 132.9059 & \\
\hline & 8/3/2008 9:00 & 284 & 216 & 900 & 5.8 & 5.8 & & 0.4879 & $4.92 \mathrm{E}-03$ & 936.7325 & 345.1584 & 1281.8909 & \\
\hline & 8/3/2008 10:00 & 285 & 216 & 1000 & 5.278 & 5.278 & & 0.6351 & 6.81E-03 & 1295.7934 & 477.4617 & 1773.2552 & \\
\hline & 8/3/2008 11:00 & 286 & 216 & 1100 & 5.289 & 5.289 & & 0.6317 & $6.76 \mathrm{E}-03$ & 1287.2575 & 474.3165 & 1761.5740 & \\
\hline
\end{tabular}




\begin{tabular}{|c|c|c|c|c|c|c|c|c|c|c|c|c|c|}
\hline Tree ID & TS & RN & JD & HR & $\begin{array}{c}\text { Raw } \\
\text { TC_dT } \Delta\end{array}$ & $\begin{array}{c}\text { Fixed } \\
\text { TC_dT } \Delta\end{array}$ & dTM & K & $\begin{array}{l}\text { Sap } \\
\text { Velocity } \\
\text { (cm/s) }\end{array}$ & $\begin{array}{c}\text { Probed } \\
\text { Drywood F } \\
\text { (g/hr) }\end{array}$ & $\begin{array}{c}\text { Extrapolated } \\
\text { Wetwood F } \\
\text { (g/hr) }\end{array}$ & $\begin{array}{c}\text { Extrapolated } \\
\text { Total Wood F } \\
(\mathrm{g} / \mathrm{hr})\end{array}$ & $\begin{array}{l}\text { Relative } \\
\text { Humidity, } \\
@ 10 \mathrm{~m}(\%)\end{array}$ \\
\hline & 8/3/2008 12:00 & 287 & 216 & 1200 & 5.105 & 5.105 & & 0.6905 & 7.54E-03 & 1436.3374 & 529.2480 & 1965.5854 & \\
\hline & 8/3/2008 13:00 & 288 & 216 & 1300 & 5.071 & 5.071 & & 0.7018 & 7.70E-03 & 1465.4158 & 539.9626 & 2005.3784 & \\
\hline & 8/3/2008 14:00 & 289 & 216 & 1400 & 5.013 & 5.013 & & 0.7215 & 7.96E-03 & 1516.1881 & 558.6707 & 2074.8588 & \\
\hline & 8/3/2008 15:00 & 290 & 216 & 1500 & 5.025 & 5.025 & & 0.7174 & 7.91E-03 & 1505.5606 & 554.7548 & 2060.3154 & \\
\hline & $8 / 3 / 2008$ 16:00 & 291 & 216 & 1600 & 5.118 & 5.118 & & 0.6862 & 7.49E-03 & 1425.3499 & 525.1995 & 1950.5494 & \\
\hline & 8/3/2008 17:00 & 292 & 216 & 1700 & 5.099 & 5.099 & & 0.6925 & 7.57E-03 & 1441.4328 & 531.1255 & 1972.5583 & \\
\hline & $8 / 3 / 2008$ 18:00 & 293 & 216 & 1800 & 5.249 & 5.249 & & 0.6441 & $6.92 E-03$ & 1318.5199 & 485.8358 & 1804.3557 & \\
\hline & 8/3/2008 19:00 & 294 & 216 & 1900 & 5.416 & 5.416 & & 0.5934 & $6.26 \mathrm{E}-03$ & 1191.9592 & 439.2019 & 1631.1611 & \\
\hline & $8 / 3 / 200820: 00$ & 295 & 216 & 2000 & 5.804 & 5.804 & & 0.4869 & $4.91 E-03$ & 934.3097 & 344.2656 & 1278.5753 & \\
\hline & $8 / 3 / 200821: 00$ & 296 & 216 & 2100 & 6.721 & 6.721 & & 0.2840 & 2.53E-03 & 481.2240 & 177.3169 & 658.5409 & \\
\hline & $8 / 3 / 200822: 00$ & 297 & 216 & 2200 & 7.419 & 7.419 & & 0.1632 & $1.28 \mathrm{E}-03$ & 243.3334 & 89.6612 & 332.9945 & \\
\hline & $8 / 3 / 200823: 00$ & 298 & 216 & 2300 & 7.576 & 7.576 & & 0.1391 & 1.05E-03 & 199.8815 & 73.6504 & 273.5319 & \\
\hline & 8/4/2008 0:00 & 299 & 217 & 0 & 7.617 & 7.617 & 8.29 & 0.1330 & 9.93E-04 & 189.0930 & 69.6752 & 258.7682 & \\
\hline & 8/4/2008 1:00 & 300 & 217 & 100 & 7.634 & 7.634 & & 0.1305 & 9.70E-04 & 184.6867 & 68.0516 & 252.7384 & \\
\hline & $8 / 4 / 20082: 00$ & 301 & 217 & 200 & 7.35 & 7.35 & & 0.1741 & 1.38E-03 & 263.5252 & 97.1013 & 360.6264 & \\
\hline & 8/4/2008 3:00 & 302 & 217 & 300 & 7.144 & 7.144 & & 0.2080 & $1.72 \mathrm{E}-03$ & 327.9439 & 120.8377 & 448.7816 & \\
\hline & $8 / 4 / 20084: 00$ & 303 & 217 & 400 & 6.948 & 6.948 & & 0.2421 & 2.08E-03 & 395.2835 & 145.6503 & 540.9339 & \\
\hline & 8/4/2008 5:00 & 304 & 217 & 500 & 7.104 & 7.104 & & 0.2148 & 1.79E-03 & 341.1944 & 125.7201 & 466.9144 & \\
\hline & $8 / 4 / 20086: 00$ & 305 & 217 & 600 & 7.534 & 7.534 & & 0.1455 & 1.11E-03 & 211.1712 & 77.8104 & 288.9815 & \\
\hline & $8 / 4 / 20087: 00$ & 306 & 217 & 700 & 7.694 & 7.694 & & 0.1217 & 8.90E-04 & 169.4469 & 62.4362 & 231.8831 & \\
\hline & 8/4/2008 8:00 & 307 & 217 & 800 & 7.572 & 7.572 & & 0.1397 & $1.06 \mathrm{E}-03$ & 200.9463 & 74.0428 & 274.9891 & \\
\hline & 8/4/2008 9:00 & 308 & 217 & 900 & 7.135 & 7.135 & & 0.2095 & 1.74E-03 & 330.9037 & 121.9283 & 452.8320 & \\
\hline & $8 / 4 / 2008$ 10:00 & 309 & 217 & 1000 & 6.018 & 6.018 & & 0.4340 & $4.26 \mathrm{E}-03$ & 811.0258 & 298.8391 & 1109.8650 & \\
\hline & 8/4/2008 11:00 & 310 & 217 & 1100 & 5.529 & 5.529 & & 0.5609 & $5.84 \mathrm{E}-03$ & 1111.9546 & 409.7226 & 1521.6772 & \\
\hline & 8/4/2008 12:00 & 311 & 217 & 1200 & 5.259 & 5.259 & & 0.6410 & 6.88E-03 & 1310.6465 & 482.9347 & 1793.5811 & \\
\hline & 8/4/2008 13:00 & 312 & 217 & 1300 & 5.205 & 5.205 & & 0.6580 & 7.11E-03 & 1353.6288 & 498.7724 & 1852.4012 & \\
\hline & 8/4/2008 14:00 & 313 & 217 & 1400 & 5.557 & 5.557 & & 0.5530 & 5.74E-03 & 1092.7916 & 402.6615 & 1495.4531 & \\
\hline & $8 / 4 / 2008$ 15:00 & 314 & 217 & 1500 & 5.983 & 5.983 & & 0.4424 & 4.36E-03 & 830.3652 & 305.9651 & 1136.3304 & \\
\hline & $8 / 4 / 2008$ 16:00 & 315 & 217 & 1600 & 5.311 & 5.311 & & 0.6249 & 6.67E-03 & 1270.3233 & 468.0767 & 1738.4000 & \\
\hline & $8 / 4 / 200817: 00$ & 316 & 217 & 1700 & 5.133 & 5.133 & & 0.6813 & 7.42E-03 & 1412.7608 & 520.5608 & 1933.3216 & \\
\hline & 8/4/2008 18:00 & 317 & 217 & 1800 & 5.395 & 5.395 & & 0.5996 & 6.34E-03 & 1207.3137 & 444.8596 & 1652.1732 & \\
\hline & 8/4/2008 19:00 & 318 & 217 & 1900 & 5.678 & 5.678 & & 0.5199 & 5.32E-03 & 1012.8498 & 373.2053 & 1386.0552 & \\
\hline & $8 / 4 / 200820: 00$ & 319 & 217 & 2000 & 6.305 & 6.305 & & 0.3688 & 3.48E-03 & 663.5913 & 244.5139 & 908.1051 & \\
\hline & $8 / 4 / 200821: 00$ & 320 & 217 & 2100 & 7.676 & 7.676 & & 0.1243 & 9.14E-04 & 173.9680 & 64.1021 & 238.0700 & \\
\hline & 8/4/2008 22:00 & 321 & 217 & 2200 & 8.19 & 8.19 & & 0.0537 & 3.25E-04 & 61.9561 & 22.8290 & 84.7851 & \\
\hline & 8/4/2008 23:00 & 322 & 217 & 2300 & 8.29 & 8.29 & & 0.0410 & 2.33E-04 & 44.4382 & 16.3742 & 60.8123 & \\
\hline & 8/5/2008 0:00 & 323 & 218 & 0 & 8.43 & 8.43 & 8.61 & 0.0237 & 1.19E-04 & 22.6527 & 8.3469 & 30.9996 & \\
\hline & $8 / 5 / 20081: 00$ & 324 & 218 & 100 & 8.52 & 8.52 & & 0.0129 & 5.63E-05 & 10.7110 & 3.9467 & 14.6577 & \\
\hline & $8 / 5 / 2008$ 2:00 & 325 & 218 & 200 & 8.59 & 8.59 & & 0.0047 & 1.60E-05 & 3.0524 & 1.1247 & 4.1771 & \\
\hline & $8 / 5 / 2008$ 3:00 & 326 & 218 & 300 & 8.58 & 8.58 & & 0.0058 & 2.11E-05 & 4.0231 & 1.4824 & 5.5054 & \\
\hline & 8/5/2008 4:00 & 327 & 218 & 400 & 8.54 & 8.54 & & 0.0105 & 4.38E-05 & 8.3425 & 3.0740 & 11.4164 & \\
\hline
\end{tabular}




\begin{tabular}{|c|c|c|c|c|c|c|c|c|c|c|c|c|c|}
\hline Tree ID & TS & RN & JD & HR & $\begin{array}{c}\text { Raw } \\
\text { TC_dTA }\end{array}$ & $\begin{array}{c}\text { Fixed } \\
\text { TC_dT } \Delta\end{array}$ & dTM & $\mathbf{K}$ & $\begin{array}{c}\text { Sap } \\
\text { Velocity } \\
(\mathrm{cm} / \mathrm{s})\end{array}$ & $\begin{array}{l}\text { Probed } \\
\text { Drywood F } \\
\text { (g/hr) }\end{array}$ & $\begin{array}{c}\text { Extrapolated } \\
\text { Wetwood F } \\
(\mathrm{g} / \mathrm{hr})\end{array}$ & $\begin{array}{c}\text { Extrapolated } \\
\text { Total Wood F } \\
(\mathrm{g} / \mathrm{hr})\end{array}$ & $\begin{array}{l}\text { Relative } \\
\text { Humidity, } \\
@ 10 \mathrm{~m}(\%)\end{array}$ \\
\hline & 8/5/2008 5:00 & 328 & 218 & 500 & 8.57 & 8.57 & & 0.0070 & $2.65 \mathrm{E}-05$ & 5.0426 & 1.8580 & 6.9006 & \\
\hline & 8/5/2008 6:00 & 329 & 218 & 600 & 8.61 & 8.61 & & 0.0023 & $6.81 \mathrm{E}-06$ & 1.2967 & 0.4778 & 1.7744 & \\
\hline & 8/5/2008 7:00 & 330 & 218 & 700 & 8.61 & 8.61 & & 0.0023 & $6.81 E-06$ & 1.2967 & 0.4778 & 1.7744 & \\
\hline & 8/5/2008 8:00 & 331 & 218 & 800 & 8.55 & 8.55 & & 0.0094 & $3.78 \mathrm{E}-05$ & 7.2061 & 2.6552 & 9.8613 & \\
\hline & 8/5/2008 9:00 & 332 & 218 & 900 & 7.611 & 7.611 & & 0.1339 & 1.00E-03 & 190.6576 & 70.2517 & 260.9092 & \\
\hline & 8/5/2008 10:00 & 333 & 218 & 1000 & 5.885 & 5.885 & & 0.4664 & $4.65 E-03$ & 886.2048 & 326.5404 & 1212.7451 & \\
\hline & 8/5/2008 11:00 & 334 & 218 & 1100 & 6.167 & 6.167 & & 0.3994 & 3.84E-03 & 732.0791 & 269.7496 & 1001.8287 & \\
\hline & 8/5/2008 12:00 & 335 & 218 & 1200 & 5.843 & 5.843 & & 0.4770 & 4.78E-03 & 910.9220 & 335.6479 & 1246.5699 & \\
\hline & 8/5/2008 13:00 & 336 & 218 & 1300 & 5.273 & 5.273 & & 0.6366 & $6.83 \mathrm{E}-03$ & 1299.6887 & 478.8970 & 1778.5857 & \\
\hline & 8/5/2008 14:00 & 337 & 218 & 1400 & 5.983 & 5.983 & & 0.4424 & $4.36 \mathrm{E}-03$ & 830.3652 & 305.9651 & 1136.3304 & \\
\hline & 8/5/2008 15:00 & 338 & 218 & 1500 & 6.348 & 6.348 & & 0.3595 & 3.38E-03 & 643.1124 & 236.9680 & 880.0803 & \\
\hline & 8/5/2008 16:00 & 339 & 218 & 1600 & 6.174 & 6.174 & & 0.3978 & 3.83E-03 & 728.5007 & 268.4310 & 996.9317 & \\
\hline & 8/5/2008 17:00 & 340 & 218 & 1700 & 5.621 & 5.621 & & 0.5353 & $5.51 \mathrm{E}-03$ & 1049.9377 & 386.8711 & 1436.8089 & \\
\hline & 8/5/2008 18:00 & 341 & 218 & 1800 & 5.442 & 5.442 & & 0.5858 & $6.16 \mathrm{E}-03$ & 1173.1637 & 432.2763 & 1605.4401 & \\
\hline & 8/5/2008 19:00 & 342 & 218 & 1900 & 5.615 & 5.615 & & 0.5370 & 5.53E-03 & 1053.9002 & 388.3312 & 1442.2314 & \\
\hline & 8/5/2008 20:00 & 343 & 218 & 2000 & 6.499 & 6.499 & & 0.3279 & 3.02E-03 & 574.2731 & 211.6027 & 785.8759 & \\
\hline & 8/5/2008 21:00 & 344 & 218 & 2100 & 8.03 & 8.03 & & 0.0747 & $4.88 \mathrm{E}-04$ & 92.9920 & 34.2648 & 127.2568 & \\
\hline & 8/5/2008 22:00 & 345 & 218 & 2200 & 8.51 & 8.51 & & 0.0141 & $6.27 E-05$ & 11.9392 & 4.3992 & 16.3384 & \\
\hline & 8/5/2008 23:00 & 346 & 218 & 2300 & 8.57 & 8.57 & & 0.0070 & $2.65 \mathrm{E}-05$ & 5.0426 & 1.8580 & 6.9006 & \\
\hline & 8/6/2008 0:00 & 347 & 219 & 0 & 8.53 & 8.53 & 8.53 & 0.0117 & $5.00 \mathrm{E}-05$ & 9.5115 & 3.5047 & 13.0162 & \\
\hline & 8/6/2008 1:00 & 348 & 219 & 100 & 8.48 & 8.48 & & 0.0177 & $8.29 \mathrm{E}-05$ & 15.7819 & 5.8152 & 21.5971 & \\
\hline & 8/6/2008 2:00 & 349 & 219 & 200 & 8.47 & 8.47 & & 0.0189 & 8.99E-05 & 17.1117 & 6.3052 & 23.4169 & \\
\hline & 8/6/2008 3:00 & 350 & 219 & 300 & 8.42 & 8.42 & & 0.0249 & 1.27E-04 & 24.0901 & 8.8765 & 32.9666 & \\
\hline & 8/6/2008 4:00 & 351 & 219 & 400 & 8.34 & 8.34 & & 0.0348 & $1.90 \mathrm{E}-04$ & 36.2663 & 13.3631 & 49.6293 & \\
\hline & 8/6/2008 5:00 & 352 & 219 & 500 & 8.27 & 8.27 & & 0.0435 & $2.51 \mathrm{E}-04$ & 47.8195 & 17.6201 & 65.4396 & \\
\hline & 8/6/2008 6:00 & 353 & 219 & 600 & 8.2 & 8.2 & & 0.0524 & $3.16 \mathrm{E}-04$ & 60.1369 & 22.1587 & 82.2956 & \\
\hline & 8/6/2008 7:00 & 354 & 219 & 700 & 8.14 & 8.14 & & 0.0602 & $3.74 \mathrm{E}-04$ & 71.2687 & 26.2604 & 97.5292 & \\
\hline & 8/6/2008 8:00 & 355 & 219 & 800 & 8.05 & 8.05 & & 0.0720 & $4.67 \mathrm{E}-04$ & 88.9184 & 32.7638 & 121.6822 & \\
\hline & 8/6/2008 9:00 & 356 & 219 & 900 & 8.05 & 8.05 & & 0.0720 & $4.67 \mathrm{E}-04$ & 88.9184 & 32.7638 & 121.6822 & \\
\hline & 8/6/2008 10:00 & 357 & 219 & 1000 & 6.106 & 6.106 & & 0.4134 & 4.01E-03 & 763.7509 & 281.4197 & 1045.1706 & \\
\hline & 8/6/2008 11:00 & 358 & 219 & 1100 & 4.964 & 4.964 & & 0.7385 & 8.19E-03 & 1560.2650 & 574.9117 & 2135.1767 & \\
\hline & 8/6/2008 12:00 & 359 & 219 & 1200 & 5.591 & 5.591 & & 0.5436 & $5.62 \mathrm{E}-03$ & 1069.8633 & 394.2131 & 1464.0764 & \\
\hline & 8/6/2008 13:00 & 360 & 219 & 1300 & 6.083 & 6.083 & & 0.4187 & $4.07 E-03$ & 775.9236 & 285.9050 & 1061.8286 & \\
\hline & 8/6/2008 14:00 & 361 & 219 & 1400 & 5.582 & 5.582 & & 0.5460 & $5.65 \mathrm{E}-03$ & 1075.8965 & 396.4362 & 1472.3327 & \\
\hline & $8 / 6 / 2008$ 15:00 & 362 & 219 & 1500 & 5.012 & 5.012 & & 0.7219 & 7.97E-03 & 1517.0767 & 558.9981 & 2076.0748 & \\
\hline & 8/6/2008 16:00 & 363 & 219 & 1600 & 5.262 & 5.262 & & 0.6401 & $6.87 \mathrm{E}-03$ & 1308.2920 & 482.0671 & 1790.3591 & \\
\hline & 8/6/2008 17:00 & 364 & 219 & 1700 & 5.18 & 5.18 & & 0.6660 & 7.22E-03 & 1373.9209 & 506.2494 & 1880.1703 & \\
\hline & 8/6/2008 18:00 & 365 & 219 & 1800 & 5.775 & 5.775 & & 0.4944 & $5.00 \mathrm{E}-03$ & 951.9781 & 350.7759 & 1302.7541 & \\
\hline & 8/6/2008 19:00 & 366 & 219 & 1900 & 5.866 & 5.866 & & 0.4712 & 4.71E-03 & 897.3267 & 330.6385 & 1227.9652 & \\
\hline & 8/6/2008 20:00 & 367 & 219 & 2000 & 6.255 & 6.255 & & 0.3797 & $3.61 \mathrm{E}-03$ & 687.9113 & 253.4751 & 941.3863 & \\
\hline & 8/6/2008 21:00 & 368 & 219 & 2100 & 7.628 & 7.628 & & 0.1314 & $9.78 \mathrm{E}-04$ & 186.2374 & 68.6230 & 254.8604 & \\
\hline
\end{tabular}




\begin{tabular}{|c|c|c|c|c|c|c|c|c|c|c|c|c|c|}
\hline Tree ID & TS & RN & JD & HR & $\begin{array}{c}\text { Raw } \\
\text { TC_dTA }\end{array}$ & $\begin{array}{c}\text { Fixed } \\
\text { TC_dT } \Delta\end{array}$ & dTM & $\mathbf{K}$ & $\begin{array}{c}\text { Sap } \\
\text { Velocity } \\
(\mathrm{cm} / \mathrm{s})\end{array}$ & $\begin{array}{c}\text { Probed } \\
\text { Drywood F } \\
\text { (g/hr) }\end{array}$ & $\begin{array}{c}\text { Extrapolated } \\
\text { Wetwood F } \\
(\mathrm{g} / \mathrm{hr})\end{array}$ & $\begin{array}{c}\text { Extrapolated } \\
\text { Total Wood F } \\
(\mathrm{g} / \mathrm{hr})\end{array}$ & $\begin{array}{l}\text { Relative } \\
\text { Humidity, } \\
\text { @10m (\%) }\end{array}$ \\
\hline & 8/6/2008 22:00 & 369 & 219 & 2200 & 8.36 & 8.36 & & 0.0323 & 1.74E-04 & 33.1146 & 12.2017 & 45.3163 & \\
\hline & 8/6/2008 23:00 & 370 & 219 & 2300 & 8.46 & 8.46 & & 0.0201 & 9.70E-05 & 18.4644 & 6.8036 & 25.2680 & \\
\hline & 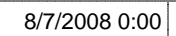 & 371 & 220 & 0 & 8.47 & 8.47 & 8.47 & 0.0189 & 8.99E-05 & 17.1117 & 6.3052 & 23.4169 & \\
\hline & 8/7/2008 1:00 & 372 & 220 & 100 & 8.39 & 8.39 & & 0.0286 & $1.50 \mathrm{E}-04$ & 28.5191 & 10.5084 & 39.0275 & \\
\hline & 8/7/2008 2:00 & 373 & 220 & 200 & 8.31 & 8.31 & & 0.0385 & $2.16 \mathrm{E}-04$ & 41.1204 & 15.1516 & 56.2720 & \\
\hline & 8/7/2008 3:00 & 374 & 220 & 300 & 8.22 & 8.22 & & 0.0499 & 2.97E-04 & 56.5426 & 20.8343 & 77.3769 & \\
\hline & 8/7/2008 4:00 & 375 & 220 & 400 & 8.14 & 8.14 & & 0.0602 & $3.74 \mathrm{E}-04$ & 71.2687 & 26.2604 & 97.5292 & \\
\hline & 8/7/2008 5:00 & 376 & 220 & 500 & 8.05 & 8.05 & & 0.0720 & $4.67 \mathrm{E}-04$ & 88.9184 & 32.7638 & 121.6822 & \\
\hline & 8/7/2008 6:00 & 377 & 220 & 600 & 7.968 & 7.968 & & 0.0831 & $5.56 \mathrm{E}-04$ & 105.9648 & 39.0449 & 145.0097 & \\
\hline & 8/7/2008 7:00 & 378 & 220 & 700 & 7.886 & 7.886 & & 0.0943 & 6.51E-04 & 123.9144 & 45.6588 & 169.5733 & \\
\hline & 8/7/2008 8:00 & 379 & 220 & 800 & 7.823 & 7.823 & & 0.1032 & 7.26E-04 & 138.3137 & 50.9645 & 189.2782 & \\
\hline & 8/7/2008 9:00 & 380 & 220 & 900 & 7.484 & 7.484 & & 0.1531 & $1.18 \mathrm{E}-03$ & 224.9282 & 82.8794 & 307.8076 & \\
\hline & 8/7/2008 10:00 & 381 & 220 & 1000 & 5.226 & 5.226 & & 0.6514 & 7.02E-03 & 1336.7769 & 492.5630 & 1829.3399 & \\
\hline & 8/7/2008 11:00 & 382 & 220 & 1100 & 4.712 & 4.712 & & 0.8315 & 9.48E-03 & 1805.4812 & 665.2667 & 2470.7478 & \\
\hline & 8/7/2008 12:00 & 383 & 220 & 1200 & 4.665 & 4.665 & & 0.8499 & $9.74 \mathrm{E}-03$ & 1854.9292 & 683.4868 & 2538.4160 & \\
\hline & 8/7/2008 13:00 & 384 & 220 & 1300 & 4.535 & 4.535 & & 0.9030 & 1.05E-02 & 1998.4085 & 736.3547 & 2734.7632 & \\
\hline & 8/7/2008 14:00 & 385 & 220 & 1400 & 4.549 & 4.549 & & 0.8971 & 1.04E-02 & 1982.4649 & 730.4800 & 2712.9449 & \\
\hline & 8/7/2008 15:00 & 386 & 220 & 1500 & 4.814 & 4.814 & & 0.7927 & 8.94E-03 & 1702.3202 & 627.2549 & 2329.5751 & \\
\hline & 8/7/2008 16:00 & 387 & 220 & 1600 & 4.794 & 4.794 & & 0.8002 & 9.04E-03 & 1722.1129 & 634.5479 & 2356.6609 & \\
\hline & 8/7/2008 17:00 & 388 & 220 & 1700 & 4.77 & 4.77 & & 0.8092 & 9.17E-03 & 1746.1405 & 643.4014 & 2389.5419 & \\
\hline & 8/7/2008 18:00 & 389 & 220 & 1800 & 5.078 & 5.078 & & 0.6995 & $7.66 \mathrm{E}-03$ & 1459.3883 & 537.7416 & 1997.1299 & \\
\hline & 8/7/2008 19:00 & 390 & 220 & 1900 & 5.376 & 5.376 & & 0.6053 & 6.41E-03 & 1221.3412 & 450.0283 & 1671.3695 & \\
\hline & 8/7/2008 20:00 & 391 & 220 & 2000 & 5.985 & 5.985 & & 0.4419 & 4.35E-03 & 829.2517 & 305.5549 & 1134.8066 & \\
\hline & 8/7/2008 21:00 & 392 & 220 & 2100 & 7.43 & 7.43 & & 0.1615 & $1.26 \mathrm{E}-03$ & 240.1769 & 88.4981 & 328.6750 & \\
\hline & 8/7/2008 22:00 & 393 & 220 & 2200 & 8.15 & 8.15 & & 0.0589 & 3.64E-04 & 69.3776 & 25.5636 & 94.9412 & \\
\hline & 8/7/2008 23:00 & 394 & 220 & 2300 & 8.37 & 8.37 & & 0.0311 & 1.66E-04 & 31.5648 & 11.6307 & 43.1955 & \\
\hline & 8/8/2008 0:00 & 395 & 221 & 0 & 8.46 & 8.46 & 8.49 & 0.0201 & 9.70E-05 & 18.4644 & 6.8036 & 25.2680 & \\
\hline & 8/8/2008 1:00 & 396 & 221 & 100 & 8.49 & 8.49 & & 0.0165 & 7.60E-05 & 14.4759 & 5.3339 & 19.8098 & \\
\hline & 8/8/2008 2:00 & 397 & 221 & 200 & 8.47 & 8.47 & & 0.0189 & 8.99E-05 & 17.1117 & 6.3052 & 23.4169 & \\
\hline & 8/8/2008 3:00 & 398 & 221 & 300 & 8.35 & 8.35 & & 0.0335 & 1.82E-04 & 34.6818 & 12.7792 & 47.4610 & \\
\hline & 8/8/2008 4:00 & 399 & 221 & 400 & 8.24 & 8.24 & & 0.0473 & 2.78E-04 & 53.0079 & 19.5318 & 72.5397 & \\
\hline & $8 / 8 / 20085: 00$ & 400 & 221 & 500 & 8.11 & 8.11 & & 0.0641 & 4.05E-04 & 77.0268 & 28.3821 & 105.4089 & \\
\hline & 8/8/2008 6:00 & 401 & 221 & 600 & 7.987 & 7.987 & & 0.0805 & $5.35 E-04$ & 101.9342 & 37.5597 & 139.4939 & \\
\hline & $8 / 8 / 20087: 00$ & 402 & 221 & 700 & 7.883 & 7.883 & & 0.0948 & $6.54 \mathrm{E}-04$ & 124.5882 & 45.9071 & 170.4952 & \\
\hline & 8/8/2008 8:00 & 403 & 221 & 800 & 7.797 & 7.797 & & 0.1068 & 7.58E-04 & 144.4101 & 53.2109 & 197.6210 & \\
\hline & 8/8/2008 9:00 & 404 & 221 & 900 & 6.601 & 6.601 & & 0.3074 & $2.79 E-03$ & 530.3603 & 195.4221 & 725.7824 & \\
\hline & 8/8/2008 10:00 & 405 & 221 & 1000 & 4.782 & 4.782 & & 0.8047 & $9.11 \mathrm{E}-03$ & 1734.0888 & 638.9607 & 2373.0495 & \\
\hline & 8/8/2008 11:00 & 406 & 221 & 1100 & 4.715 & 4.715 & & 0.8303 & 9.47E-03 & 1802.3668 & 664.1191 & 2466.4859 & \\
\hline & 8/8/2008 12:00 & 407 & 221 & 1200 & 4.489 & 4.489 & & 0.9225 & $1.08 \mathrm{E}-02$ & 2051.6663 & 755.9786 & 2807.6449 & \\
\hline & 8/8/2008 13:00 & 408 & 221 & 1300 & 4.41 & 4.41 & & 0.9569 & 1.13E-02 & 2146.3576 & 790.8696 & 2937.2271 & \\
\hline & $8 / 8 / 2008$ 14:00 & 409 & 221 & 1400 & 4.446 & 4.446 & & 0.9411 & 1.10E-02 & 2102.6902 & 774.7795 & 2877.4697 & \\
\hline
\end{tabular}




\begin{tabular}{|c|c|c|c|c|c|c|c|c|c|c|c|c|c|}
\hline Tree ID & TS & RN & JD & HR & $\begin{array}{c}\text { Raw } \\
\text { TC_dTA }\end{array}$ & $\begin{array}{c}\text { Fixed } \\
\text { TC_dT } \Delta\end{array}$ & dTM & $\mathbf{K}$ & $\begin{array}{c}\text { Sap } \\
\text { Velocity } \\
(\mathrm{cm} / \mathrm{s})\end{array}$ & $\begin{array}{c}\text { Probed } \\
\text { Drywood F } \\
\text { (g/hr) }\end{array}$ & $\begin{array}{c}\text { Extrapolated } \\
\text { Wetwood F } \\
(\mathrm{g} / \mathrm{hr})\end{array}$ & $\begin{array}{c}\text { Extrapolated } \\
\text { Total Wood F } \\
(\mathrm{g} / \mathrm{hr})\end{array}$ & $\begin{array}{l}\text { Relative } \\
\text { Humidity, } \\
\text { @10m (\%) }\end{array}$ \\
\hline & 8/8/2008 15:00 & 410 & 221 & 1500 & 4.599 & 4.599 & & 0.8765 & 1.01E-02 & 1926.5081 & 709.8615 & 2636.3697 & \\
\hline & 8/8/2008 16:00 & 411 & 221 & 1600 & 4.641 & 4.641 & & 0.8595 & 9.88E-03 & 1880.6637 & 692.9692 & 2573.6329 & \\
\hline & 8/8/2008 17:00 & 412 & 221 & 1700 & 4.834 & 4.834 & & 0.7853 & $8.84 \mathrm{E}-03$ & 1682.7338 & 620.0379 & 2302.7717 & \\
\hline & 8/8/2008 18:00 & 413 & 221 & 1800 & 5.257 & 5.257 & & 0.6416 & $6.89 \mathrm{E}-03$ & 1312.2181 & 483.5137 & 1795.7318 & \\
\hline & 8/8/2008 19:00 & 414 & 221 & 1900 & 5.275 & 5.275 & & 0.6360 & $6.82 \mathrm{E}-03$ & 1298.1294 & 478.3225 & 1776.4519 & \\
\hline & 8/8/2008 20:00 & 415 & 221 & 2000 & 6.043 & 6.043 & & 0.4281 & 4.19E-03 & 797.4011 & 293.8188 & 1091.2199 & \\
\hline & 8/8/2008 21:00 & 416 & 221 & 2100 & 7.347 & 7.347 & & 0.1746 & 1.39E-03 & 264.4185 & 97.4304 & 361.8490 & \\
\hline & 8/8/2008 22:00 & 417 & 221 & 2200 & 7.97 & 7.97 & & 0.0828 & $5.54 \mathrm{E}-04$ & 105.5382 & 38.8877 & 144.4259 & \\
\hline & 8/8/2008 23:00 & 418 & 221 & 2300 & 8.14 & 8.14 & & 0.0602 & $3.74 \mathrm{E}-04$ & 71.2687 & 26.2604 & 97.5292 & \\
\hline & 8/9/2008 0:00 & 419 & 222 & 0 & 8.17 & 8.17 & 8.17 & 0.0563 & $3.45 E-04$ & 65.6381 & 24.1857 & 89.8238 & \\
\hline & 8/9/2008 1:00 & 420 & 222 & 100 & 8.15 & 8.15 & & 0.0589 & 3.64E-04 & 69.3776 & 25.5636 & 94.9412 & \\
\hline & 8/9/2008 2:00 & 421 & 222 & 200 & 8.15 & 8.15 & & 0.0589 & $3.64 \mathrm{E}-04$ & 69.3776 & 25.5636 & 94.9412 & \\
\hline & 8/9/2008 3:00 & 422 & 222 & 300 & 8.1 & 8.1 & & 0.0654 & 4.15E-04 & 78.9741 & 29.0996 & 108.0738 & \\
\hline & 8/9/2008 4:00 & 423 & 222 & 400 & 8.03 & 8.03 & & 0.0747 & 4.88E-04 & 92.9920 & 34.2648 & 127.2568 & \\
\hline & 8/9/2008 5:00 & 424 & 222 & 500 & 7.88 & 7.88 & & 0.0952 & $6.58 \mathrm{E}-04$ & 125.2631 & 46.1557 & 171.4188 & \\
\hline & 8/9/2008 6:00 & 425 & 222 & 600 & 7.791 & 7.791 & & 0.1077 & 7.66E-04 & 145.8297 & 53.7340 & 199.5637 & \\
\hline & 8/9/2008 7:00 & 426 & 222 & 700 & 7.48 & 7.48 & & 0.1537 & 1.19E-03 & 226.0438 & 83.2905 & 309.3342 & \\
\hline & 8/9/2008 8:00 & 427 & 222 & 800 & 6.85 & 6.85 & & 0.2599 & 2.27E-03 & 431.2988 & 158.9209 & 590.2198 & \\
\hline & 8/9/2008 9:00 & 428 & 222 & 900 & 7.191 & 7.191 & & 0.2001 & 1.64E-03 & 312.6883 & 115.2164 & 427.9047 & \\
\hline & 8/9/2008 10:00 & 429 & 222 & 1000 & 6.979 & 6.979 & & 0.2366 & $2.02 E-03$ & 384.2232 & 141.5749 & 525.7981 & \\
\hline & 8/9/2008 11:00 & 430 & 222 & 1100 & 6.399 & 6.399 & & 0.3486 & $3.25 \mathrm{E}-03$ & 619.3346 & 228.2066 & 847.5411 & \\
\hline & 8/9/2008 12:00 & 431 & 222 & 1200 & 5.558 & 5.558 & & 0.5527 & $5.74 \mathrm{E}-03$ & 1092.1119 & 402.4111 & 1494.5230 & \\
\hline & 8/9/2008 13:00 & 432 & 222 & 1300 & 5.23 & 5.23 & & 0.6501 & 7.00E-03 & 1333.5869 & 491.3875 & 1824.9744 & \\
\hline & 8/9/2008 14:00 & 433 & 222 & 1400 & 4.627 & 4.627 & & 0.8651 & $9.96 \mathrm{E}-03$ & 1895.8297 & 698.5574 & 2594.3872 & \\
\hline & 8/9/2008 15:00 & 434 & 222 & 1500 & 4.511 & 4.511 & & 0.9131 & 1.06E-02 & 2026.0268 & 746.5313 & 2772.5581 & \\
\hline & 8/9/2008 16:00 & 435 & 222 & 1600 & 4.564 & 4.564 & & 0.8909 & 1.03E-02 & 1965.5175 & 724.2353 & 2689.7528 & \\
\hline & 8/9/2008 17:00 & 436 & 222 & 1700 & 4.968 & 4.968 & & 0.7371 & 8.17E-03 & 1556.6253 & 573.5706 & 2130.1959 & \\
\hline & 8/9/2008 18:00 & 437 & 222 & 1800 & 5.048 & 5.048 & & 0.7096 & 7.80E-03 & 1485.3713 & 547.3156 & 2032.6869 & \\
\hline & 8/9/2008 19:00 & 438 & 222 & 1900 & 5.4 & 5.4 & & 0.5981 & 6.32E-03 & 1203.6437 & 443.5073 & 1647.1509 & \\
\hline & 8/9/2008 20:00 & 439 & 222 & 2000 & 6.079 & 6.079 & & 0.4196 & 4.09E-03 & 778.0537 & 286.6899 & 1064.7435 & \\
\hline & 8/9/2008 21:00 & 440 & 222 & 2100 & 7.604 & 7.604 & & 0.1349 & 1.01E-03 & 192.4890 & 70.9265 & 263.4155 & \\
\hline & 8/9/2008 22:00 & 441 & 222 & 2200 & 8.15 & 8.15 & & 0.0589 & 3.64E-04 & 69.3776 & 25.5636 & 94.9412 & \\
\hline & 8/9/2008 23:00 & 442 & 222 & 2300 & 8.12 & 8.12 & & 0.0628 & $3.94 \mathrm{E}-04$ & 75.0934 & 27.6697 & 102.7631 & \\
\hline & $8 / 10 / 20080: 00$ & 443 & 223 & 0 & 8.05 & 8.05 & 8.05 & 0.0720 & 4.67E-04 & 88.9184 & 32.7638 & 121.6822 & \\
\hline & 8/10/2008 1:00 & 444 & 223 & 100 & 7.978 & 7.978 & & 0.0817 & 5.45E-04 & 103.8373 & 38.2610 & 142.0984 & \\
\hline & 8/10/2008 2:00 & 445 & 223 & 200 & 7.893 & 7.893 & & 0.0934 & 6.43E-04 & 122.3471 & 45.0813 & 167.4285 & \\
\hline & 8/10/2008 3:00 & 446 & 223 & 300 & 7.814 & 7.814 & & 0.1044 & 7.37E-04 & 140.4138 & 51.7383 & 192.1521 & \\
\hline & 8/10/2008 4:00 & 447 & 223 & 400 & 7.738 & 7.738 & & 0.1153 & 8.33E-04 & 158.5782 & 58.4314 & 217.0096 & \\
\hline & 8/10/2008 5:00 & 448 & 223 & 500 & 7.668 & 7.668 & & 0.1255 & $9.24 \mathrm{E}-04$ & 175.9913 & 64.8476 & 240.8389 & \\
\hline & $8 / 10 / 20086: 00$ & 449 & 223 & 600 & 7.603 & 7.603 & & 0.1351 & 1.01E-03 & 192.7512 & 71.0231 & 263.7743 & \\
\hline & $8 / 10 / 20087: 00$ & 450 & 223 & 700 & 7.545 & 7.545 & & 0.1438 & 1.09E-03 & 208.1909 & 76.7122 & 284.9032 & \\
\hline
\end{tabular}




\begin{tabular}{|c|c|c|c|c|c|c|c|c|c|c|c|c|c|}
\hline \multirow[t]{42}{*}{ Tree ID } & TS & RN & JD & HR & $\begin{array}{c}\text { Raw } \\
\text { TC_dT } \Delta\end{array}$ & $\begin{array}{c}\text { Fixed } \\
\text { TC_dT } \Delta\end{array}$ & dTM & $\mathrm{K}$ & $\begin{array}{l}\text { Sap } \\
\text { Velocity } \\
(\mathrm{cm} / \mathrm{s})\end{array}$ & $\begin{array}{l}\text { Probed } \\
\text { Drywood F } \\
(\mathrm{g} / \mathrm{hr})\end{array}$ & $\begin{array}{c}\text { Extrapolated } \\
\text { Wetwood F } \\
(\mathrm{g} / \mathrm{hr})\end{array}$ & $\begin{array}{c}\text { Extrapolated } \\
\text { Total Wood F } \\
(\mathrm{g} / \mathrm{hr})\end{array}$ & $\begin{array}{l}\text { Relative } \\
\text { Humidity, } \\
@ 10 \mathrm{~m}(\%)\end{array}$ \\
\hline & 8/10/2008 8:00 & 451 & 223 & 800 & 7.517 & 7.517 & & 0.1481 & 1.13E-03 & 215.8097 & 79.5195 & 295.3293 & \\
\hline & 8/10/2008 9:00 & 452 & 223 & 900 & 7.281 & 7.281 & & 0.1853 & 1.49E-03 & 284.4028 & 104.7941 & 389.1969 & \\
\hline & $8 / 10 / 2008$ 10:00 & 453 & 223 & 1000 & 5.062 & 5.062 & & 0.7049 & 7.74E-03 & 1473.1968 & 542.8297 & 2016.0265 & \\
\hline & 8/10/2008 11:00 & 454 & 223 & 1100 & 4.442 & 4.442 & & 0.9428 & 1.11E-02 & 2107.4989 & 776.5513 & 2884.0503 & \\
\hline & 8/10/2008 12:00 & 455 & 223 & 1200 & 4.351 & 4.351 & & 0.9835 & 1.17E-02 & 2219.8598 & 817.9530 & 3037.8128 & \\
\hline & 8/10/2008 13:00 & 456 & 223 & 1300 & 4.177 & 4.177 & & 1.0661 & 1.29E-02 & 2451.6228 & 903.3508 & 3354.9736 & \\
\hline & $8 / 10 / 2008$ 14:00 & 457 & 223 & 1400 & 4.173 & 4.173 & & 1.0681 & $1.29 \mathrm{E}-02$ & 2457.2303 & 905.4171 & 3362.6474 & \\
\hline & 8/10/2008 15:00 & 458 & 223 & 1500 & 4.606 & 4.606 & & 0.8736 & 1.01E-02 & 1918.7949 & 707.0194 & 2625.8143 & \\
\hline & $8 / 10 / 2008$ 16:00 & 459 & 223 & 1600 & 4.429 & 4.429 & & 0.9485 & 1.12E-02 & 2123.2015 & 782.3373 & 2905.5387 & \\
\hline & 8/10/2008 17:00 & 460 & 223 & 1700 & \begin{tabular}{|l|}
4.82 \\
\end{tabular} & 4.82 & & 0.7905 & 8.91E-03 & 1696.4228 & 625.0819 & 2321.5046 & \\
\hline & 8/10/2008 18:00 & 461 & 223 & 1800 & 6.993 & 6.993 & & 0.2341 & 1.99E-03 & 379.2796 & 139.7534 & 519.0330 & \\
\hline & $8 / 10 / 2008$ 19:00 & 462 & 223 & 1900 & 7.457 & 7.457 & & 0.1573 & 1.22E-03 & 232.5015 & 85.6700 & 318.1714 & \\
\hline & 8/10/2008 20:00 & 463 & 223 & 2000 & 7.399 & 7.399 & & 0.1664 & 1.31E-03 & 249.1162 & 91.7920 & 340.9082 & \\
\hline & 8/10/2008 21:00 & 464 & 223 & 2100 & 7.382 & 7.382 & & 0.1691 & 1.33E-03 & 254.0764 & 93.6197 & 347.6960 & \\
\hline & 8/10/2008 22:00 & 465 & 223 & 2200 & 7.474 & 7.474 & & 0.1547 & $1.20 \mathrm{E}-03$ & 227.7213 & 83.9086 & 311.6298 & \\
\hline & 8/10/2008 23:00 & 466 & 223 & 2300 & 7.472 & 7.472 & & 0.1550 & 1.20E-03 & 228.2815 & 84.1150 & 312.3966 & \\
\hline & $8 / 11 / 20080: 00$ & 467 & 224 & 0 & 7.501 & 7.501 & 7.68 & 0.1505 & 1.16E-03 & 220.2120 & 81.1416 & 301.3536 & \\
\hline & 8/11/2008 1:00 & 468 & 224 & 100 & 7.607 & 7.607 & & 0.1345 & 1.01E-03 & 191.7033 & 70.6370 & 262.3403 & \\
\hline & 8/11/2008 2:00 & 469 & 224 & 200 & 7.675 & 7.675 & & 0.1244 & $9.15 E-04$ & 174.2204 & 64.1951 & 238.4155 & \\
\hline & 8/11/2008 3:00 & 470 & 224 & 300 & 7.667 & 7.667 & & 0.1256 & $9.26 \mathrm{E}-04$ & 176.2448 & 64.9410 & 241.1858 & \\
\hline & 8/11/2008 4:00 & 471 & 224 & 400 & 7.634 & 7.634 & & 0.1305 & 9.70E-04 & 184.6867 & 68.0516 & 252.7384 & \\
\hline & 8/11/2008 5:00 & 472 & 224 & 500 & 7.609 & 7.609 & & 0.1342 & $1.00 \mathrm{E}-03$ & 191.1801 & 70.4443 & 261.6244 & \\
\hline & 8/11/2008 6:00 & 473 & 224 & 600 & 7.58 & 7.58 & & 0.1385 & $1.04 \mathrm{E}-03$ & 198.8189 & 73.2589 & 272.0778 & \\
\hline & 8/11/2008 7:00 & 474 & 224 & 700 & 7.533 & 7.533 & & 0.1456 & 1.11E-03 & 211.4429 & 77.9105 & 289.3534 & \\
\hline & 8/11/2008 8:00 & 475 & 224 & 800 & 7.514 & 7.514 & & 0.1485 & 1.14E-03 & 216.6325 & 79.8227 & 296.4551 & \\
\hline & 8/11/2008 9:00 & 476 & 224 & 900 & 7.127 & 7.127 & & 0.2109 & 1.75E-03 & 333.5452 & 122.9016 & 456.4467 & \\
\hline & 8/11/2008 10:00 & 477 & 224 & 1000 & 5.288 & 5.288 & & 0.6320 & 6.76E-03 & 1288.0316 & 474.6017 & 1762.6333 & \\
\hline & 8/11/2008 11:00 & 478 & 224 & 1100 & 4.411 & 4.411 & & 0.9565 & 1.13E-02 & 2145.1327 & 790.4182 & 2935.5509 & \\
\hline & $8 / 11 / 2008$ 12:00 & 479 & 224 & 1200 & 4.402 & 4.402 & & 0.9605 & 1.13E-02 & 2156.1815 & 794.4894 & 2950.6709 & \\
\hline & 8/11/2008 13:00 & 480 & 224 & 1300 & 4.737 & 4.737 & & 0.8218 & $9.35 \mathrm{E}-03$ & 1779.6794 & 655.7595 & 2435.4389 & \\
\hline & 8/11/2008 14:00 & 481 & 224 & 1400 & 4.431 & 4.431 & & 0.9476 & 1.11E-02 & 2120.7783 & 781.4444 & 2902.2226 & \\
\hline & $8 / 11 / 2008$ 15:00 & 482 & 224. & 1500 & 4.379 & 4.379 & & 0.9708 & 1.15E-02 & 2184.6725 & 804.9875 & 2989.6600 & \\
\hline & 8/11/2008 16:00 & 483 & 224 & 1600 & 4.702 & 4.702 & & 0.8354 & 9.54E-03 & 1815.8983 & 669.1051 & 2485.0034 & \\
\hline & 8/11/2008 17:00 & 484 & 224 & 1700 & 4.777 & 4.777 & & 0.8066 & $9.13 \mathrm{E}-03$ & 1739.1011 & 640.8076 & 2379.9087 & \\
\hline & 8/11/2008 18:00 & 485 & 224 & 1800 & 5.1 & 5.1 & & 0.6922 & 7.57E-03 & 1440.5825 & 530.8122 & 1971.3947 & \\
\hline & $8 / 11 / 2008$ 19:00 & 486 & 224 & 1900 & 5.277 & 5.277 & & 0.6354 & 6.81E-03 & 1296.5717 & 477.7485 & 1774.3202 & \\
\hline & 8/11/2008 20:00 & 487 & 224 & 2000 & 5.916 & 5.916 & & 0.4588 & $4.56 \mathrm{E}-03$ & 868.2672 & 319.9309 & 1188.1981 & \\
\hline & 8/11/2008 21:00 & 488 & 224 & 2100 & 6.747 & 6.747 & & 0.2791 & $2.47 \mathrm{E}-03$ & 470.9251 & 173.5220 & 644.4471 & \\
\hline & 8/11/2008 22:00 & 489 & 224 & 2200 & 7.18 & 7.18 & & 0.2019 & 1.66E-03 & 316.2286 & 116.5209 & 432.7496 & \\
\hline & 8/11/2008 23:00 & 490 & 224 & 2300 & 7.338 & 7.338 & & 0.1761 & $1.40 \mathrm{E}-03$ & 267.1064 & 98.4209 & 365.5273 & \\
\hline & 8/12/2008 0:00 & 491 & 225 & 0 & 7.417 & 7.417 & 7.42 & 0.1635 & $1.28 \mathrm{E}-03$ & 243.9091 & 89.8733 & 333.7824 & \\
\hline
\end{tabular}




\begin{tabular}{|c|c|c|c|c|c|c|c|c|c|c|c|c|c|}
\hline Tree ID & TS & RN & JD & HR & $\begin{array}{c}\text { Raw } \\
\text { TC_dTA }\end{array}$ & $\begin{array}{c}\text { Fixed } \\
\text { TC_dT } \Delta\end{array}$ & dTM & $\mathbf{K}$ & $\begin{array}{l}\text { Sap } \\
\text { Velocity } \\
(\mathrm{cm} / \mathrm{s})\end{array}$ & $\begin{array}{c}\text { Probed } \\
\text { Drywood F } \\
(\mathrm{g} / \mathrm{hr})\end{array}$ & $\begin{array}{c}\text { Extrapolated } \\
\text { Wetwood F } \\
(\mathrm{g} / \mathrm{hr})\end{array}$ & $\begin{array}{c}\text { Extrapolated } \\
\text { Total Wood F } \\
(\mathrm{g} / \mathrm{hr})\end{array}$ & $\begin{array}{l}\text { Relative } \\
\text { Humidity, } \\
@ 10 \mathrm{~m}(\%)\end{array}$ \\
\hline & 8/12/2008 1:00 & 492 & 225 & 100 & 7.403 & 7.403 & & 0.1657 & $1.30 \mathrm{E}-03$ & 247.9551 & 91.3642 & \begin{tabular}{|r|}
339.3193 \\
\end{tabular} & \\
\hline & 8/12/2008 2:00 & 493 & 225 & 200 & 6.754 & 6.754 & & 0.2778 & $2.46 \mathrm{E}-03$ & 468.1729 & 172.5079 & 640.6809 & \\
\hline & 8/12/2008 3:00 & 494 & 225 & 300 & 6.706 & 6.706 & & 0.2869 & $2.56 \mathrm{E}-03$ & 487.2212 & 179.5267 & 666.7478 & \\
\hline & 8/12/2008 4:00 & 495 & 225 & 400 & 6.683 & 6.683 & & 0.2913 & 2.61E-03 & 496.4962 & 182.9443 & 679.4405 & \\
\hline & 8/12/2008 5:00 & 496 & 225 & 500 & 6.688 & 6.688 & & 0.2904 & 2.60E-03 & 494.4717 & 182.1983 & 676.6700 & \\
\hline & 8/12/2008 6:00 & 497 & 225 & 600 & 6.789 & 6.789 & & 0.2712 & 2.39E-03 & 454.5426 & 167.4856 & 622.0282 & \\
\hline & 8/12/2008 7:00 & 498 & 225 & 700 & 6.947 & 6.947 & & 0.2423 & 2.08E-03 & 395.6429 & 145.7828 & 541.4257 & \\
\hline & 8/12/2008 8:00 & 499 & 225 & 800 & 7.041 & 7.041 & & 0.2257 & 1.90E-03 & 362.5701 & 133.5964 & 496.1665 & \\
\hline & 8/12/2008 9:00 & 500 & 225 & 900 & 6.902 & 6.902 & & 0.2504 & $2.16 \mathrm{E}-03$ & 411.9880 & 151.8054 & 563.7934 & \\
\hline & $8 / 12 / 2008$ 10:00 & 501 & 225 & 1000 & 5.326 & 5.326 & & 0.6204 & 6.61E-03 & 1258.8814 & 463.8607 & 1722.7421 & \\
\hline & $8 / 12 / 2008$ 11:00 & 502 & 225 & 1100 & 4.6 & 4.6 & & 0.8761 & 1.01E-02 & 1925.4044 & 709.4549 & 2634.8593 & \\
\hline & $8 / 12 / 2008$ 12:00 & 503 & 225 & 1200 & 4.38 & 4.38 & & 0.9703 & 1.15E-02 & 2183.4261 & 804.5282 & 2987.9543 & \\
\hline & $8 / 12 / 2008$ 13:00 & 504 & 225 & 1300 & 4.058 & 4.058 & & 1.1267 & $1.38 \mathrm{E}-02$ & 2624.2487 & 966.9584 & 3591.2070 & \\
\hline & $8 / 12 / 2008$ 14:00 & 505 & 225 & 1400 & 3.962 & 3.962 & & 1.1782 & 1.46E-02 & 2772.7691 & 1021.6838 & 3794.4529 & \\
\hline & $8 / 12 / 2008$ 15:00 & 506 & 225 & 1500 & 4.071 & 4.071 & & 1.1199 & 1.37E-02 & 2604.7903 & 959.7885 & 3564.5788 & \\
\hline & $8 / 12 / 2008$ 16:00 & 507 & 225 & 1600 & 4.158 & 4.158 & & 1.0755 & 1.30E-02 & 2478.3764 & 913.2087 & 3391.5851 & \\
\hline & $8 / 12 / 2008$ 17:00 & 508 & 225 & 1700 & 4.352 & 4.352 & & 0.9830 & 1.17E-02 & 2218.5935 & 817.4864 & 3036.0799 & \\
\hline & $8 / 12 / 2008$ 18:00 & 509 & 225 & 1800 & 4.523 & 4.523 & & 0.9080 & 1.06E-02 & 2012.1721 & 741.4262 & 2753.5983 & \\
\hline & $8 / 12 / 2008$ 19:00 & 510 & 225 & 1900 & 4.896 & 4.896 & & 0.7627 & 8.53E-03 & 1623.2976 & 598.1374 & 2221.4350 & \\
\hline & $8 / 12 / 200820: 00$ & 511 & 225 & 2000 & 5.494 & 5.494 & & 0.5708 & 5.97E-03 & 1136.2721 & 418.6828 & 1554.9549 & \\
\hline & $8 / 12 / 2008$ 21:00 & 512 & 225 & 2100 & 6.889 & 6.889 & & 0.2527 & $2.19 \mathrm{E}-03$ & 416.7728 & 153.5685 & 570.3413 & \\
\hline & $8 / 12 / 2008$ 22:00 & 513 & 225 & 2200 & 7.384 & 7.384 & & 0.1687 & 1.33E-03 & 253.4907 & 93.4039 & 346.8945 & \\
\hline & $8 / 12 / 2008$ 23:00 & 514 & 225 & 2300 & 7.423 & 7.423 & & 0.1626 & 1.27E-03 & 242.1836 & 89.2375 & 331.4211 & \\
\hline & 8/13/2008 0:00 & 515 & 226 & 0 & 7.382 & 7.382 & 7.41 & 0.1691 & 1.33E-03 & 254.0764 & 93.6197 & 347.6960 & \\
\hline & 8/13/2008 1:00 & 516 & 226 & 100 & 7.373 & 7.373 & & 0.1705 & $1.35 \mathrm{E}-03$ & 256.7190 & 94.5934 & 351.3124 & \\
\hline & 8/13/2008 2:00 & 517 & 226 & 200 & 7.345 & 7.345 & & 0.1749 & 1.39E-03 & 265.0148 & 97.6502 & 362.6650 & \\
\hline & 8/13/2008 3:00 & 518 & 226 & 300 & 7.405 & 7.405 & & 0.1654 & 1.30E-03 & 247.3754 & 91.1506 & 338.5260 & \\
\hline & 8/13/2008 4:00 & 519 & 226 & 400 & 7.379 & 7.379 & & 0.1695 & 1.34E-03 & 254.9560 & 93.9438 & 348.8997 & \\
\hline & $8 / 13 / 20085: 00$ & 520 & 226 & 500 & 7.384 & 7.384 & & 0.1687 & $1.33 E-03$ & 253.4907 & 93.4039 & 346.8945 & \\
\hline & 8/13/2008 6:00 & 521 & 226 & 600 & 7.387 & 7.387 & & 0.1683 & 1.33E-03 & 252.6132 & 93.0805 & 345.6938 & \\
\hline & $8 / 13 / 2008$ 7:00 & 522 & 226 & 700 & 7.321 & 7.321 & & 0.1788 & $1.43 E-03$ & 272.2155 & 100.3034 & 372.5189 & \\
\hline & 8/13/2008 8:00 & 523 & 226 & 800 & 7.33 & 7.33 & & 0.1774 & $1.42 \mathrm{E}-03$ & 269.5055 & 99.3048 & 368.8103 & \\
\hline & 8/13/2008 9:00 & 524 & 226 & 900 & 6.52 & 6.52 & & 0.3236 & $2.97 \mathrm{E}-03$ & 565.0661 & 208.2102 & 773.2763 & \\
\hline & $8 / 13 / 2008$ 10:00 & 525 & 226 & 1000 & 4.786 & 4.786 & & 0.8032 & $9.09 \mathrm{E}-03$ & 1730.0884 & 637.4867 & 2367.5751 & \\
\hline & 8/13/2008 11:00 & 526 & 226 & 1100 & 4.664 & 4.664 & & 0.8503 & 9.75E-03 & 1855.9949 & 683.8795 & 2539.8743 & \\
\hline & $8 / 13 / 2008$ 12:00 & 527 & 226 & 1200 & 4.195 & 4.195 & & 1.0572 & 1.27E-02 & 2426.5506 & 894.1125 & 3320.6631 & \\
\hline
\end{tabular}




\begin{tabular}{|c|c|c|c|c|c|c|c|c|c|c|c|c|c|}
\hline Tree ID & TS & RN & JD & HR & $\begin{array}{c}\text { Raw } \\
\text { TC_dTA }\end{array}$ & $\begin{array}{c}\text { Fixed } \\
\text { TC_dT } \Delta\end{array}$ & dTM & $\mathbf{K}$ & $\begin{array}{l}\text { Sap } \\
\text { Velocity } \\
(\mathrm{cm} / \mathrm{s})\end{array}$ & $\begin{array}{l}\text { Probed } \\
\text { Drywood F } \\
\text { (g/hr) }\end{array}$ & $\begin{array}{c}\text { Extrapolated } \\
\text { Wetwood F } \\
(\mathrm{g} / \mathrm{hr})\end{array}$ & $\begin{array}{c}\text { Extrapolated } \\
\text { Total Wood F } \\
\text { (g/hr) }\end{array}$ & $\begin{array}{l}\text { Relative } \\
\text { Humidity, } \\
@ 10 \mathrm{~m}(\%)\end{array}$ \\
\hline & $7 / 22 / 2008$ 13:00 & 0 & 204 & 1300 & 5.14 & 5.14 & 9.91 & 0.9280 & 1.09E-02 & 2615.8445 & 963.8617 & 3579.7062 & \\
\hline \multirow[t]{2}{*}{ W709EC } & $7 / 22 / 2008$ 14:00 & 1 & 204 & 1400 & 5.595 & 5.595 & & 0.7712 & $8.64 \mathrm{E}-03$ & 2082.9116 & 767.4916 & 2850.4033 & \\
\hline & $7 / 22 / 2008$ 15:00 & 2 & 204 & 1500 & 5.56 & 5.56 & & 0.7824 & $8.80 \mathrm{E}-03$ & 2120.0427 & 781.1733 & 2901.2160 & \\
\hline \multirow[t]{2}{*}{ Probe \# } & 7/22/2008 16:00 & 3 & 204 & 1600 & 5.567 & 5.567 & & 0.7801 & $8.77 \mathrm{E}-03$ & 2112.5692 & 778.4196 & 2890.9888 & \\
\hline & 7/22/2008 17:00 & 4 & 204 & 1700 & 5.598 & 5.598 & & 0.7703 & 8.63E-03 & 2079.7563 & 766.3290 & 2846.0853 & \\
\hline \multirow[t]{4}{*}{6} & $7 / 22 / 2008$ 18:00 & 5 & 204 & 1800 & 5.629 & 5.629 & & 0.7605 & $8.50 \mathrm{E}-03$ & 2047.4000 & 754.4066 & 2801.8067 & \\
\hline & $7 / 22 / 2008$ 19:00 & 6 & 204 & 1900 & 5.814 & 5.814 & & 0.7045 & 7.73E-03 & 1863.3641 & 686.5948 & 2549.9590 & \\
\hline & 7/22/2008 20:00 & 7 & 204 & 2000 & 6.284 & 6.284 & & 0.5770 & $6.05 E-03$ & 1457.3984 & 537.0084 & 1994.4068 & \\
\hline & 7/22/2008 21:00 & 8 & 204 & 2100 & 8.08 & 8.08 & & 0.2265 & 1.91E-03 & 460.8985 & 169.8275 & 630.7260 & \\
\hline \multirow[t]{2}{*}{$\mathrm{DBH}(\mathrm{cm})$} & $7 / 22 / 2008$ 22:00 & 9 & 204 & 2200 & 9.51 & 9.51 & & 0.0421 & $2.41 \mathrm{E}-04$ & 58.0159 & 21.3772 & 79.3931 & \\
\hline & 7/22/2008 23:00 & 10 & 204 & 2300 & 9.91 & 9.91 & & 0.0000 & $0.00 \mathrm{E}+00$ & 0.0000 & 0.0000 & 0.0000 & \\
\hline \multirow[t]{2}{*}{11.4} & 7/23/2008 0:00 & 11 & 205 & 0 & 10.2 & 10.2 & 10.37 & -0.0284 & $0.00 \mathrm{E}+00$ & 0.0000 & 0.0000 & 0.0000 & \\
\hline & 7/23/2008 1:00 & 12 & 205 & 100 & 10.3 & 10.3 & & -0.0379 & $0.00 \mathrm{E}+00$ & 0.0000 & 0.0000 & 0.0000 & \\
\hline Day Count & $7 / 23 / 20082: 00$ & 13 & 205 & 200 & 10.31 & 10.31 & & -0.0388 & $0.00 \mathrm{E}+00$ & 0.0000 & 0.0000 & 0.0000 & \\
\hline \multirow[t]{27}{*}{35} & 7/23/2008 3:00 & 14 & 205 & 300 & 10.37 & 10.37 & & -0.0444 & $0.00 \mathrm{E}+00$ & 0.0000 & 0.0000 & 0.0000 & \\
\hline & 7/23/2008 4:00 & 15 & 205 & 400 & 10.36 & 10.36 & & -0.0434 & $0.00 \mathrm{E}+00$ & 0.0000 & 0.0000 & 0.0000 & \\
\hline & 7/23/2008 5:00 & 16 & 205 & 500 & 10.34 & 10.34 & & -0.0416 & $0.00 E+00$ & 0.0000 & 0.0000 & 0.0000 & \\
\hline & 7/23/2008 6:00 & 17 & 205 & 600 & 10.22 & 10.22 & & -0.0303 & $0.00 \mathrm{E}+00$ & 0.0000 & 0.0000 & 0.0000 & \\
\hline & 7/23/2008 7:00 & 18 & 205 & 700 & 9.84 & 9.84 & & 0.0071 & $2.70 \mathrm{E}-05$ & 6.5086 & 2.3982 & 8.9069 & \\
\hline & 7/23/2008 8:00 & 19 & 205 & 800 & 8.62 & 8.62 & & 0.1497 & 1.15E-03 & 276.7438 & 101.9719 & 378.7157 & \\
\hline & 7/23/2008 9:00 & 20 & 205 & 900 & 7.004 & 7.004 & & 0.4149 & 4.03E-03 & 971.0625 & 357.8080 & 1328.8705 & \\
\hline & 7/23/2008 10:00 & 21 & 205 & 1000 & 5.798 & 5.798 & & 0.7092 & 7.80E-03 & 1878.6907 & 692.2422 & 2570.9329 & \\
\hline & $7 / 23 / 2008$ 11:00 & 22 & 205 & 1100 & 5.816 & 5.816 & & 0.7039 & 7.72E-03 & 1861.4559 & 685.8917 & 2547.3476 & \\
\hline & $7 / 23 / 2008$ 12:00 & 23 & 205 & 1200 & 5.83 & 5.83 & & 0.6998 & 7.67E-03 & 1848.1451 & 680.9871 & 2529.1322 & \\
\hline & $7 / 23 / 2008$ 13:00 & 24 & 205 & 1300 & 5.739 & 5.739 & & 0.7268 & 8.03E-03 & 1936.1530 & 713.4154 & 2649.5684 & \\
\hline & $7 / 23 / 200814: 00$ & 25 & 205 & 1400 & 5.736 & 5.736 & & 0.7277 & 8.05E-03 & 1939.1151 & 714.5069 & 2653.6220 & \\
\hline & 7/23/2008 15:00 & 26 & 205 & 1500 & 5.707 & 5.707 & & 0.7365 & 8.17E-03 & 1967.9539 & 725.1331 & 2693.0869 & \\
\hline & 7/23/2008 16:00 & 27 & 205 & 1600 & 5.85 & 5.85 & & 0.6940 & 7.59E-03 & 1829.2711 & 674.0326 & 2503.3036 & \\
\hline & 7/23/2008 17:00 & 28 & 205 & 1700 & 6.294 & 6.294 & & 0.5745 & $6.02 \mathrm{E}-03$ & 1449.6120 & 534.1393 & 1983.7513 & \\
\hline & $7 / 23 / 2008$ 18:00 & 29 & 205 & 1800 & 7.053 & 7.053 & & 0.4051 & $3.91 \mathrm{E}-03$ & 942.8197 & 347.4013 & 1290.2210 & \\
\hline & $7 / 23 / 2008$ 19:00 & 30 & 205 & 1900 & 6.969 & 6.969 & & 0.4220 & 4.11E-03 & 991.5758 & 365.3665 & 1356.9423 & \\
\hline & $7 / 23 / 200820: 00$ & 31 & 205 & 2000 & 7.514 & 7.514 & & 0.3189 & 2.91E-03 & 702.2669 & 258.7647 & 961.0316 & \\
\hline & $7 / 23 / 200821: 00$ & 32 & 205 & 2100 & 8.63 & 8.63 & & 0.1483 & 1.14E-03 & 273.7144 & 100.8557 & 374.5700 & \\
\hline & $7 / 23 / 200822: 00$ & 33 & 205 & 2200 & 9.33 & 9.33 & & 0.0622 & $3.89 \mathrm{E}-04$ & 93.8441 & 34.5788 & 128.4229 & \\
\hline & $7 / 23 / 2008$ 23:00 & 34 & 205 & 2300 & 9.42 & 9.42 & & 0.0520 & 3.13E-04 & 75.3574 & 27.7670 & 103.1244 & \\
\hline & $7 / 24 / 20080: 00$ & 35 & 206 & 0 & 9.42 & 9.42 & 10.14 & 0.0520 & 3.13E-04 & 75.3574 & 27.7670 & 103.1244 & \\
\hline & 7/24/2008 1:00 & 36 & 206 & 100 & 9.51 & 9.51 & & 0.0421 & $2.41 E-04$ & 58.0159 & 21.3772 & 79.3931 & \\
\hline & 7/24/2008 2:00 & 37 & 206 & 200 & 9.64 & 9.64 & & 0.0280 & $1.46 \mathrm{E}-04$ & 35.1691 & 12.9588 & 48.1279 & \\
\hline & 7/24/2008 3:00 & 38 & 206 & 300 & 9.77 & 9.77 & & 0.0143 & $6.40 \mathrm{E}-05$ & 15.4126 & 5.6791 & 21.0916 & \\
\hline & 7/24/2008 4:00 & 39 & 206 & 400 & 9.86 & 9.86 & & 0.0051 & 1.78E-05 & 4.2906 & 1.5810 & 5.8716 & \\
\hline & 7/24/2008 5:00 & 40 & 206 & 500 & 9.95 & 9.95 & & -0.0040 & $0.00 \mathrm{E}+00$ & 0.0000 & 0.0000 & 0.0000 & \\
\hline
\end{tabular}




\begin{tabular}{|c|c|c|c|c|c|c|c|c|c|c|c|c|c|}
\hline Tree ID & TS & RN & JD & HR & $\begin{array}{c}\text { Raw } \\
\text { TC_dTA }\end{array}$ & $\begin{array}{c}\text { Fixed } \\
\text { TC_dT } \Delta\end{array}$ & dTM & $\mathbf{K}$ & $\begin{array}{l}\text { Sap } \\
\text { Velocity } \\
(\mathrm{cm} / \mathrm{s})\end{array}$ & $\begin{array}{c}\text { Probed } \\
\text { Drywood F } \\
(\mathrm{g} / \mathrm{hr})\end{array}$ & $\begin{array}{c}\text { Extrapolated } \\
\text { Wetwood F } \\
(\mathrm{g} / \mathrm{hr})\end{array}$ & $\begin{array}{c}\text { Extrapolated } \\
\text { Total Wood F } \\
(\mathrm{g} / \mathrm{hr})\end{array}$ & $\begin{array}{l}\text { Relative } \\
\text { Humidity, } \\
@ 10 \mathrm{~m}(\%)\end{array}$ \\
\hline & 7/24/2008 6:00 & 41 & 206 & 600 & ) 9.98 & 9.98 & & -0.0070 & $0.00 \mathrm{E}+00$ & 0.0000 & 0.0000 & 0.0000 & \\
\hline & 7/24/2008 7:00 & 42 & 206 & 700 & 9.98 & 9.98 & & -0.0070 & $0.00 \mathrm{E}+00$ & 0.0000 & 0.0000 & 0.0000 & \\
\hline & 7/24/2008 8:00 & 43 & 206 & 800 & 9.63 & 9.63 & & 0.0291 & 1.53E-04 & 36.8264 & 13.5694 & 50.3958 & \\
\hline & 7/24/2008 9:00 & 44 & 206 & 900 & 9 & 9 & & 0.1011 & 7.09E-04 & 170.7886 & 62.9306 & 233.7191 & \\
\hline & $7 / 24 / 200810: 00$ & 45 & 206 & 1000 & 9.1 & 9.1 & & 0.0890 & 6.06E-04 & 145.9878 & 53.7922 & 199.7800 & \\
\hline & $7 / 24 / 2008$ 11:00 & 46 & 206 & 1100 & 6.748 & 6.748 & & 0.4686 & 4.68E-03 & 1127.9502 & 415.6165 & 1543.5667 & \\
\hline & $7 / 24 / 2008$ 12:00 & 47 & 206 & 1200 & 5.909 & 5.909 & & 0.6771 & 7.36E-03 & 1774.5456 & 653.8678 & 2428.4134 & \\
\hline & $7 / 24 / 2008$ 13:00 & 48 & 206 & 1300 & 6.301 & 6.301 & & 0.5728 & 5.99E-03 & 1444.1808 & 532.1381 & 1976.3190 & \\
\hline & $7 / 24 / 2008$ 14:00 & 49 & 206 & 1400 & 6.13 & 6.13 & & 0.6166 & $6.56 \mathrm{E}-03$ & 1581.5392 & 582.7506 & 2164.2898 & \\
\hline & $7 / 24 / 200815: 00$ & 50 & 206 & 1500 & 5.794 & 5.794 & & 0.7104 & 7.81E-03 & 1882.5393 & 693.6603 & 2576.1996 & \\
\hline & $7 / 24 / 200816: 00$ & 51 & 206 & 1600 & 5.74 & 5.74 & & 0.7265 & 8.03E-03 & 1935.1665 & 713.0519 & 2648.2184 & \\
\hline & 7/24/2008 17:00 & 52 & 206 & 1700 & 5.907 & 5.907 & & 0.6777 & 7.37E-03 & 1776.3777 & 654.5429 & 2430.9206 & \\
\hline & $7 / 24 / 2008$ 18:00 & 53 & 206 & 1800 & 5.825 & 5.825 & & 0.7013 & 7.69E-03 & 1852.8895 & 682.7353 & 2535.6248 & \\
\hline & $7 / 24 / 2008$ 19:00 & 54 & 206 & 1900 & 6.144 & 6.144 & & 0.6130 & $6.51 E-03$ & 1569.9168 & 578.4681 & 2148.3849 & \\
\hline & $7 / 24 / 2008$ 20:00 & 55 & 206 & 2000 & 7.242 & 7.242 & & 0.3684 & $3.48 E-03$ & 838.8809 & 309.1029 & 1147.9838 & \\
\hline & 7/24/2008 21:00 & 56 & 206 & 2100 & 8.87 & 8.87 & & 0.1172 & $8.50 \mathrm{E}-04$ & 204.9394 & 75.5141 & 280.4535 & \\
\hline & $7 / 24 / 200822: 00$ & 57 & 206 & 2200 & 9.94 & 9.94 & & -0.0030 & $0.00 \mathrm{E}+00$ & 0.0000 & 0.0000 & 0.0000 & \\
\hline & $7 / 24 / 2008$ 23:00 & 58 & 206 & 2300 & 10.14 & 10.14 & & -0.0227 & $0.00 \mathrm{E}+00$ & 0.0000 & 0.0000 & 0.0000 & \\
\hline & $7 / 25 / 20080: 00$ & 59 & 207 & 0 & 10.16 & 10.16 & 10.19 & -0.0246 & $0.00 \mathrm{E}+00$ & 0.0000 & 0.0000 & 0.0000 & \\
\hline & 7/25/2008 1:00 & 60 & 207 & 100 & 10.1 & 10.1 & & -0.0188 & $0.00 \mathrm{E}+00$ & 0.0000 & 0.0000 & 0.0000 & \\
\hline & 7/25/2008 2:00 & 61 & 207 & 200 & 10.02 & 10.02 & & -0.0110 & $0.00 \mathrm{E}+00$ & 0.0000 & 0.0000 & 0.0000 & \\
\hline & 7/25/2008 3:00 & 62 & 207 & 300 & 9.97 & 9.97 & & -0.0060 & $0.00 \mathrm{E}+00$ & 0.0000 & 0.0000 & 0.0000 & \\
\hline & 7/25/2008 4:00 & 63 & 207 & 400 & 9.98 & 9.98 & & -0.0070 & $0.00 \mathrm{E}+00$ & 0.0000 & 0.0000 & 0.0000 & \\
\hline & 7/25/2008 5:00 & 64 & 207 & 500 & 10.04 & 10.04 & & -0.0129 & $0.00 \mathrm{E}+00$ & 0.0000 & 0.0000 & 0.0000 & \\
\hline & 7/25/2008 6:00 & 65 & 207 & 600 & 10.14 & 10.14 & & -0.0227 & $0.00 \mathrm{E}+00$ & 0.0000 & 0.0000 & 0.0000 & \\
\hline & 7/25/2008 7:00 & 66 & 207 & 700 & 10.12 & 10.12 & & -0.0208 & $0.00 \mathrm{E}+00$ & 0.0000 & 0.0000 & 0.0000 & \\
\hline & 7/25/2008 8:00 & 67 & 207 & 800 & 10.1 & 10.1 & & -0.0188 & $0.00 \mathrm{E}+00$ & 0.0000 & 0.0000 & 0.0000 & \\
\hline & 7/25/20089:00 & 68 & 207 & 900 & 9.98 & 9.98 & & -0.0070 & $0.00 \mathrm{E}+00$ & 0.0000 & 0.0000 & 0.0000 & \\
\hline & $7 / 25 / 2008$ 10:00 & 69 & 207 & 1000 & 9.85 & 9.85 & & 0.0061 & 2.23E-05 & 5.3769 & 1.9812 & 7.3582 & \\
\hline & $7 / 25 / 2008$ 11:00 & 70 & 207 & 1100 & 8.76 & 8.76 & & 0.1313 & 9.77E-04 & 235.5309 & 86.7862 & 322.3171 & \\
\hline & $7 / 25 / 2008$ 12:00 & 71 & 207 & 1200 & 7.088 & 7.088 & & 0.3981 & 3.83E-03 & 922.9802 & 340.0910 & 1263.0712 & \\
\hline & $7 / 25 / 2008$ 13:00 & 72 & 207 & 1300 & 6.451 & 6.451 & & 0.5362 & 5.53E-03 & 1331.5229 & 490.6270 & 1822.1499 & \\
\hline & $7 / 25 / 200814: 00$ & 73 & 207 & 1400 & 5.987 & 5.987 & & 0.6553 & 7.07E-03 & 1704.3192 & 627.9915 & 2332.3106 & \\
\hline & $7 / 25 / 2008$ 15:00 & 74 & 207 & 1500 & 5.937 & 5.937 & & 0.6692 & 7.26E-03 & 1749.0624 & 644.4780 & 2393.5404 & \\
\hline & $7 / 25 / 2008$ 16:00 & 75 & 207 & 1600 & 6.012 & 6.012 & & 0.6484 & $6.98 \mathrm{E}-03$ & 1682.3073 & 619.8807 & 2302.1881 & \\
\hline & $7 / 25 / 200817: 00$ & 76 & 207 & 1700 & 5.801 & 5.801 & & 0.7083 & $7.78 \mathrm{E}-03$ & 1875.8087 & 691.1803 & 2566.9891 & \\
\hline & $7 / 25 / 2008$ 18:00 & 77 & 207 & 1800 & 5.837 & 5.837 & & 0.6978 & 7.64E-03 & 1841.5203 & 678.5460 & 2520.0664 & \\
\hline & $7 / 25 / 2008$ 19:00 & 78 & 207 & 1900 & 5.929 & 5.929 & & 0.6714 & 7.29E-03 & 1756.3117 & 647.1492 & 2403.4609 & \\
\hline & $7 / 25 / 200820: 00$ & 79 & 207 & 2000 & 6.815 & 6.815 & & 0.4541 & $4.50 \mathrm{E}-03$ & 1085.3209 & 399.9088 & 1485.2298 & \\
\hline & $7 / 25 / 200821: 00$ & 80 & 207 & 2100 & 8.63 & 8.63 & & 0.1483 & $1.14 \mathrm{E}-03$ & 273.7144 & 100.8557 & 374.5700 & \\
\hline & 7/25/2008 22:00 & 81 & 207 & 2200 & 9.95 & 9.95 & & -0.0040 & $0.00 \mathrm{E}+00$ & 0.0000 & 0.0000 & 0.0000 & \\
\hline
\end{tabular}




\begin{tabular}{|c|c|c|c|c|c|c|c|c|c|c|c|c|c|}
\hline Tree ID & TS & RN & JD & HR & $\begin{array}{c}\text { Raw } \\
\text { TC_dTA }\end{array}$ & $\begin{array}{c}\text { Fixed } \\
\text { TC_dT } \Delta\end{array}$ & dTM & K & $\begin{array}{l}\text { Sap } \\
\text { Velocity } \\
(\mathrm{cm} / \mathrm{s})\end{array}$ & $\begin{array}{c}\text { Probed } \\
\text { Drywood F } \\
(\mathrm{g} / \mathrm{hr})\end{array}$ & $\begin{array}{c}\text { Extrapolated } \\
\text { Wetwood F } \\
(\mathrm{g} / \mathrm{hr})\end{array}$ & $\begin{array}{c}\text { Extrapolated } \\
\text { Total Wood F } \\
(\mathrm{g} / \mathrm{hr})\end{array}$ & $\begin{array}{l}\text { Relative } \\
\text { Humidity, } \\
@ 10 \mathrm{~m}(\%)\end{array}$ \\
\hline & 7/25/2008 23:00 & 82 & 207 & 2300 & \begin{tabular}{|r|}
10.19 \\
\end{tabular} & 10.19 & & -0.0275 & $0.00 \mathrm{E}+00$ & 0.0000 & 0.0000 & 0.0000 & \\
\hline & $7 / 26 / 20080: 00$ & 83 & 208 & 0 & 10.26 & 10.26 & 10.26 & -0.0341 & $0.00 \mathrm{E}+00$ & 0.0000 & 0.0000 & 0.0000 & \\
\hline & 7/26/2008 1:00 & 84 & 208 & 100 & 10.26 & 10.26 & & -0.0341 & $0.00 E+00$ & 0.0000 & 0.0000 & 0.0000 & \\
\hline & $7 / 26 / 20082: 00$ & 85 & 208 & 200 & 10.23 & 10.23 & & -0.0313 & $0.00 \mathrm{E}+00$ & 0.0000 & 0.0000 & 0.0000 & \\
\hline & 7/26/2008 3:00 & 86 & 208 & 300 & 10.19 & 10.19 & & -0.0275 & $0.00 \mathrm{E}+00$ & 0.0000 & 0.0000 & 0.0000 & \\
\hline & 7/26/2008 4:00 & 87 & 208 & 400 & 10.14 & 10.14 & & -0.0227 & $0.00 \mathrm{E}+00$ & 0.0000 & 0.0000 & 0.0000 & \\
\hline & 7/26/2008 5:00 & 88 & 208 & 500 & 10.08 & 10.08 & & -0.0169 & $0.00 \mathrm{E}+00$ & 0.0000 & 0.0000 & 0.0000 & \\
\hline & 7/26/2008 6:00 & 89 & 208 & 600 & 10.01 & 10.01 & & -0.0100 & $0.00 \mathrm{E}+00$ & 0.0000 & 0.0000 & 0.0000 & \\
\hline & 7/26/2008 7:00 & 90 & 208 & 700 & 9.94 & 9.94 & & -0.0030 & $0.00 \mathrm{E}+00$ & 0.0000 & 0.0000 & 0.0000 & \\
\hline & 7/26/2008 8:00 & 91 & 208 & 800 & 9.86 & 9.86 & & 0.0051 & 1.78E-05 & 4.2906 & 1.5810 & 5.8716 & \\
\hline & 7/26/2008 9:00 & 92 & 208 & 900 & 8.33 & 8.33 & & 0.1897 & 1.54E-03 & 370.4966 & 136.5171 & 507.0137 & \\
\hline & $7 / 26 / 2008$ 10:00 & 93 & 208 & 1000 & 6.187 & 6.187 & & 0.6017 & 6.37E-03 & 1534.6477 & 565.4725 & 2100.1202 & \\
\hline & 7/26/2008 11:00 & 94 & 208 & 1100 & 6.365 & 6.365 & & 0.5570 & 5.79E-03 & 1395.2536 & 514.1099 & 1909.3635 & \\
\hline & $7 / 26 / 2008$ 12:00 & 95 & 208 & 1200 & 6.707 & 6.707 & & 0.4776 & 4.79E-03 & 1154.6109 & 425.4401 & 1580.0510 & \\
\hline & $7 / 26 / 2008$ 13:00 & 96 & 208 & 1300 & 6.029 & 6.029 & & 0.6437 & 6.92E-03 & 1667.4740 & 614.4151 & 2281.8891 & \\
\hline & $7 / 26 / 200814: 00$ & 97 & 208 & 1400 & 5.948 & 5.948 & & 0.6661 & $7.22 E-03$ & 1739.1356 & 640.8203 & 2379.9559 & \\
\hline & $7 / 26 / 2008$ 15:00 & 98 & 208 & 1500 & 5.902 & 5.902 & & 0.6791 & 7.39E-03 & 1780.9650 & 656.2332 & 2437.1982 & \\
\hline & $7 / 26 / 200816: 00$ & 99 & 208 & 1600 & 5.967 & 5.967 & & 0.6608 & 7.15E-03 & 1722.1004 & 634.5433 & 2356.6437 & \\
\hline & $7 / 26 / 200817: 00$ & 100 & 208 & 1700 & 5.786 & 5.786 & & 0.7128 & 7.84E-03 & 1890.2568 & 696.5040 & 2586.7608 & \\
\hline & $7 / 26 / 2008$ 18:00 & 101 & 208 & 1800 & 5.935 & 5.935 & & 0.6698 & 7.27E-03 & 1750.8724 & 645.1449 & 2396.0173 & \\
\hline & $7 / 26 / 2008$ 19:00 & 102 & 208 & 1900 & 6.069 & 6.069 & & 0.6329 & $6.78 \mathrm{E}-03$ & 1632.9961 & 601.7110 & 2234.7071 & \\
\hline & $7 / 26 / 200820: 00$ & 103 & 208 & 2000 & 6.68 & 6.68 & & 0.4835 & 4.86E-03 & 1172.4110 & 431.9989 & 1604.4099 & \\
\hline & $7 / 26 / 200821: 00$ & 104 & 208 & 2100 & 8.6 & 8.6 & & 0.1523 & 1.17E-03 & 282.8426 & 104.2192 & 387.0617 & \\
\hline & $7 / 26 / 200822: 00$ & 105 & 208 & 2200 & 9.83 & 9.83 & & 0.0081 & 3.19E-05 & 7.6811 & 2.8302 & 10.5113 & \\
\hline & $7 / 26 / 2008$ 23:00 & 106 & 208 & 2300 & 10.1 & 10.1 & & -0.0188 & $0.00 \mathrm{E}+00$ & 0.0000 & 0.0000 & 0.0000 & \\
\hline & $7 / 27 / 20080: 00$ & 107 & 209 & 0 & 10.16 & 10.16 & 10.26 & -0.0246 & $0.00 \mathrm{E}+00$ & 0.0000 & 0.0000 & 0.0000 & \\
\hline & 7/27/2008 1:00 & 108 & 209 & 100 & 10.23 & 10.23 & & -0.0313 & $0.00 \mathrm{E}+00$ & 0.0000 & 0.0000 & 0.0000 & \\
\hline & 7/27/2008 2:00 & 109 & 209 & 200 & 10.25 & 10.25 & & -0.0332 & $0.00 \mathrm{E}+00$ & 0.0000 & 0.0000 & 0.0000 & \\
\hline & 7/27/2008 3:00 & 110 & 209 & 300 & 10.26 & 10.26 & & -0.0341 & $0.00 \mathrm{E}+00$ & 0.0000 & 0.0000 & 0.0000 & \\
\hline & 7/27/2008 4:00 & 111 & 209 & 400 & 10.25 & 10.25 & & -0.0332 & $0.00 \mathrm{E}+00$ & 0.0000 & 0.0000 & 0.0000 & \\
\hline & 7/27/20085:00 & 112 & 209 & 500 & 10.22 & 10.22 & & -0.0303 & $0.00 \mathrm{E}+00$ & 0.0000 & 0.0000 & 0.0000 & \\
\hline & 7/27/2008 6:00 & 113 & 209 & 600 & 10.18 & 10.18 & & -0.0265 & $0.00 \mathrm{E}+00$ & 0.0000 & 0.0000 & 0.0000 & \\
\hline & 7/27/2008 7:00 & 114 & 209 & 700 & 10.11 & 10.11 & & -0.0198 & $0.00 \mathrm{E}+00$ & 0.0000 & 0.0000 & 0.0000 & \\
\hline & 7/27/2008 8:00 & 115 & 209 & 800 & 9.9 & 9.9 & & 0.0010 & 2.44E-06 & 0.5887 & 0.2169 & 0.8057 & \\
\hline & $7 / 27 / 20089: 00$ & 116 & 209 & 900 & 7.373 & 7.373 & & 0.3441 & $3.20 \mathrm{E}-03$ & 771.2583 & 284.1860 & 1055.4442 & \\
\hline & $7 / 27 / 2008$ 10:00 & 117 & 209 & 1000 & 5.826 & 5.826 & & 0.7010 & 7.68E-03 & 1851.9398 & 682.3853 & 2534.3251 & \\
\hline & $7 / 27 / 2008$ 11:00 & 118 & 209 & 1100 & 5.797 & 5.797 & & 0.7095 & 7.80E-03 & 1879.6522 & 692.5965 & 2572.2487 & \\
\hline & $7 / 27 / 2008$ 12:00 & 119 & 209 & 1200 & 5.817 & 5.817 & & 0.7036 & 7.72E-03 & 1860.5024 & 685.5404 & 2546.0428 & \\
\hline & $7 / 27 / 200813: 00$ & 120 & 209 & 1300 & 5.872 & 5.872 & & 0.6877 & 7.51E-03 & 1808.6999 & 666.4527 & 2475.1525 & \\
\hline & $7 / 27 / 2008$ 14:00 & 121 & 209 & 1400 & 5.77 & 5.77 & & 0.7175 & $7.91 \mathrm{E}-03$ & 1905.7740 & 702.2216 & 2607.9956 & \\
\hline & 7/27/2008 15:00 & 122 & 209 & 1500 & 5.748 & 5.748 & & 0.7241 & 8.00E-03 & 1927.2902 & 710.1497 & 2637.4399 & \\
\hline
\end{tabular}




\begin{tabular}{|c|c|c|c|c|c|c|c|c|c|c|c|c|c|}
\hline Tree ID & TS & RN & JD & HR & $\begin{array}{c}\text { Raw } \\
\text { TC_dTA }\end{array}$ & $\begin{array}{c}\text { Fixed } \\
\text { TC_dT } \Delta\end{array}$ & dTM & $\mathbf{K}$ & $\begin{array}{c}\text { Sap } \\
\text { Velocity } \\
(\mathrm{cm} / \mathrm{s})\end{array}$ & $\begin{array}{c}\text { Probed } \\
\text { Drywood F } \\
(\mathrm{g} / \mathrm{hr})\end{array}$ & $\begin{array}{c}\text { Extrapolated } \\
\text { Wetwood F } \\
(\mathrm{g} / \mathrm{hr})\end{array}$ & $\begin{array}{c}\text { Extrapolated } \\
\text { Total Wood F } \\
(\mathbf{g} / \mathrm{hr})\end{array}$ & $\begin{array}{c}\text { Relative } \\
\text { Humidity, } \\
@ 10 \mathrm{~m}(\%)\end{array}$ \\
\hline & $7 / 27 / 2008$ 16:00 & 123 & 209 & 1600 & 5.767 & 5.767 & & 0.7184 & 7.92E-03 & 1908.6957 & 703.2982 & 2611.9938 & \\
\hline & $7 / 27 / 2008$ 17:00 & 124 & 209 & 1700 & 5.794 & 5.794 & & 0.7104 & 7.81E-03 & 1882.5393 & 693.6603 & 2576.1996 & \\
\hline & $7 / 27 / 2008$ 18:00 & 125 & 209 & 1800 & 5.866 & 5.866 & & 0.6894 & 7.53E-03 & 1814.2906 & 668.5127 & 2482.8032 & \\
\hline & 7/27/2008 19:00 & 126 & 209 & 1900 & 6.012 & 6.012 & & 0.6484 & $6.98 \mathrm{E}-03$ & 1682.3073 & 619.8807 & 2302.1881 & \\
\hline & $7 / 27 / 200820: 00$ & 127 & 209 & 2000 & 6.452 & 6.452 & & 0.5360 & $5.52 \mathrm{E}-03$ & 1330.7951 & 490.3588 & 1821.1540 & \\
\hline & $7 / 27 / 2008$ 21:00 & 128 & 209 & 2100 & 7.832 & 7.832 & & 0.2653 & $2.32 \mathrm{E}-03$ & 560.0358 & 206.3567 & 766.3925 & \\
\hline & 7/27/2008 22:00 & 129 & 209 & 2200 & 9.25 & 9.25 & & 0.0714 & $4.61 \mathrm{E}-04$ & 111.1961 & 40.9725 & 152.1686 & \\
\hline & $7 / 27 / 200823: 00$ & 130 & 209 & 2300 & 9.52 & 9.52 & & 0.0410 & $2.33 \mathrm{E}-04$ & 56.1629 & 20.6944 & 76.8573 & \\
\hline & $7 / 28 / 20080: 00$ & 131 & 210 & 0 & 9.62 & 9.62 & 10.22 & 0.0301 & $1.60 \mathrm{E}-04$ & 38.5012 & 14.1866 & 52.6878 & \\
\hline & 7/28/2008 1:00 & 132 & 210 & 100 & 9.82 & 9.82 & & 0.0092 & 3.69E-05 & 8.8907 & 3.2760 & 12.1666 & \\
\hline & 7/28/2008 2:00 & 133 & 210 & 200 & 9.92 & 9.92 & & -0.0010 & $0.00 \mathrm{E}+00$ & 0.0000 & 0.0000 & 0.0000 & \\
\hline & 7/28/2008 3:00 & 134 & 210 & 300 & 10.07 & 10.07 & & -0.0159 & $0.00 \mathrm{E}+00$ & 0.0000 & 0.0000 & 0.0000 & \\
\hline & 7/28/2008 4:00 & 135 & 210 & 400 & 10.11 & 10.11 & & -0.0198 & $0.00 \mathrm{E}+00$ & 0.0000 & 0.0000 & 0.0000 & \\
\hline & 7/28/2008 5:00 & 136 & 210 & 500 & 10.21 & 10.21 & & -0.0294 & $0.00 E+00$ & 0.0000 & 0.0000 & 0.0000 & \\
\hline & 7/28/2008 6:00 & 137 & 210 & 600 & 10.22 & 10.22 & & -0.0303 & $0.00 \mathrm{E}+00$ & 0.0000 & 0.0000 & 0.0000 & \\
\hline & 7/28/2008 7:00 & 138 & 210 & 700 & 10.08 & 10.08 & & -0.0169 & $0.00 \mathrm{E}+00$ & 0.0000 & 0.0000 & 0.0000 & \\
\hline & 7/28/2008 8:00 & 139 & 210 & 800 & 7.961 & 7.961 & & 0.2448 & $2.10 \mathrm{E}-03$ & 507.2460 & 186.9052 & 694.1512 & \\
\hline & 7/28/2008 9:00 & 140 & 210 & 900 & 6.416 & 6.416 & & 0.5446 & $5.63 \mathrm{E}-03$ & 1357.1863 & 500.0832 & 1857.2695 & \\
\hline & $7 / 28 / 2008$ 10:00 & 141 & 210 & 1000 & 6.047 & 6.047 & & 0.6388 & $6.85 \mathrm{E}-03$ & 1651.8858 & 608.6713 & 2260.5570 & \\
\hline & 7/28/2008 11:00 & 142 & 210 & 1100 & 5.976 & 5.976 & & 0.6583 & $7.11 \mathrm{E}-03$ & 1714.0799 & 631.5880 & 2345.6678 & \\
\hline & $7 / 28 / 200812: 00$ & 143 & 210 & 1200 & 6.119 & 6.119 & & 0.6195 & $6.60 \mathrm{E}-03$ & 1590.7197 & 586.1334 & 2176.8531 & \\
\hline & $7 / 28 / 2008$ 13:00 & 144 & 210 & 1300 & 5.978 & 5.978 & & 0.6577 & 7.11E-03 & 1712.3017 & 630.9328 & 2343.2345 & \\
\hline & $7 / 28 / 2008$ 14:00 & 145 & 210 & 1400 & 5.953 & 5.953 & & 0.6647 & 7.20E-03 & 1734.6391 & 639.1635 & 2373.8025 & \\
\hline & $7 / 28 / 2008$ 15:00 & 146 & 210 & 1500 & 5.869 & 5.869 & & 0.6885 & $7.52 \mathrm{E}-03$ & 1811.4934 & 667.4820 & 2478.9754 & \\
\hline & $7 / 28 / 2008$ 16:00 & 147 & 210 & 1600 & 5.873 & 5.873 & & 0.6874 & 7.50E-03 & 1807.7695 & 666.1098 & 2473.8793 & \\
\hline & $7 / 28 / 2008$ 17:00 & 148 & 210 & 1700 & 5.863 & 5.863 & & 0.6903 & 7.54E-03 & 1817.0914 & 669.5447 & 2486.6361 & \\
\hline & $7 / 28 / 2008$ 18:00 & 149 & 210 & 1800 & 5.906 & 5.906 & & 0.6780 & 7.37E-03 & 1777.2944 & 654.8807 & 2432.1750 & \\
\hline & $7 / 28 / 2008$ 19:00 & 150 & 210 & 1900 & 6.073 & 6.073 & & 0.6318 & $6.76 \mathrm{E}-03$ & 1629.5807 & 600.4525 & 2230.0332 & \\
\hline & $7 / 28 / 200820: 00$ & 151 & 210 & 2000 & 6.723 & 6.723 & & 0.4740 & 4.75E-03 & 1144.1541 & 421.5871 & 1565.7412 & \\
\hline & 7/28/2008 21:00 & 152 & 210 & 2100 & 8.44 & 8.44 & & 0.1742 & $1.38 \mathrm{E}-03$ & 333.5735 & 122.9120 & 456.4855 & \\
\hline & $7 / 28 / 2008$ 22:00 & 153 & 210 & 2200 & 9.14 & 9.14 & & 0.0842 & $5.66 \mathrm{E}-04$ & 136.4259 & 50.2689 & 186.6948 & \\
\hline & $7 / 28 / 2008$ 23:00 & 154 & 210 & 2300 & 9.42 & 9.42 & & 0.0520 & $3.13 \mathrm{E}-04$ & 75.3574 & 27.7670 & 103.1244 & \\
\hline & $7 / 29 / 20080: 00$ & 155 & 211 & 0 & 9.71 & 9.71 & 10.31 & 0.0206 & $1.00 \mathrm{E}-04$ & 24.0909 & 8.8768 & 32.9676 & \\
\hline & 7/29/2008 1:00 & 156 & 211 & 100 & 9.95 & 9.95 & & -0.0040 & $0.00 \mathrm{E}+00$ & 0.0000 & 0.0000 & 0.0000 & \\
\hline & 7/29/2008 2:00 & 157 & 211 & 200 & 10.13 & 10.13 & & -0.0217 & $0.00 \mathrm{E}+00$ & 0.0000 & 0.0000 & 0.0000 & \\
\hline & 7/29/2008 3:00 & 158 & 211 & 300 & 10.23 & 10.23 & & -0.0313 & $0.00 \mathrm{E}+00$ & 0.0000 & 0.0000 & 0.0000 & \\
\hline & 7/29/2008 4:00 & 159 & 211 & 400 & 10.31 & 10.31 & & -0.0388 & $0.00 E+00$ & 0.0000 & 0.0000 & 0.0000 & \\
\hline & 7/29/2008 5:00 & 160 & 211 & 500 & 10.29 & 10.29 & & -0.0369 & $0.00 \mathrm{E}+00$ & 0.0000 & 0.0000 & 0.0000 & \\
\hline & $7 / 29 / 20086: 00$ & 161 & 211 & 600 & 10.26 & 10.26 & & -0.0341 & $0.00 \mathrm{E}+00$ & 0.0000 & 0.0000 & 0.0000 & \\
\hline & 7/29/2008 7:00 & 162 & 211 & 700 & 10.15 & 10.15 & & -0.0236 & $0.00 \mathrm{E}+00$ & 0.0000 & 0.0000 & 0.0000 & \\
\hline & 7/29/2008 8:00 & 163 & 211 & 800 & 9.27 & 9.27 & & 0.0690 & 4.43E-04 & 106.7785 & 39.3447 & 146.1232 & \\
\hline
\end{tabular}




\begin{tabular}{|c|c|c|c|c|c|c|c|c|c|c|c|c|c|}
\hline Tree ID & TS & RN & JD & HR & $\begin{array}{c}\text { Raw } \\
\text { TC_dTA }\end{array}$ & $\begin{array}{c}\text { Fixed } \\
\text { TC_dT } \Delta\end{array}$ & dTM & $\mathbf{K}$ & $\begin{array}{l}\text { Sap } \\
\text { Velocity } \\
(\mathrm{cm} / \mathrm{s})\end{array}$ & $\begin{array}{c}\text { Probed } \\
\text { Drywood F } \\
(\mathrm{g} / \mathrm{hr})\end{array}$ & $\begin{array}{c}\text { Extrapolated } \\
\text { Wetwood F } \\
(\mathrm{g} / \mathrm{hr})\end{array}$ & $\begin{array}{c}\text { Extrapolated } \\
\text { Total Wood F } \\
(\mathrm{g} / \mathrm{hr})\end{array}$ & $\begin{array}{l}\text { Relative } \\
\text { Humidity, } \\
@ 10 \mathrm{~m}(\%)\end{array}$ \\
\hline & 7/29/2008 9:00 & 164 & 211 & 900 & 7.187 & 7.187 & & 0.3789 & 3.60E-03 & 868.3302 & 319.9541 & 1188.2843 & \\
\hline & 7/29/2008 10:00 & 165 & 211 & 1000 & 6.369 & 6.369 & & 0.5560 & $5.78 \mathrm{E}-03$ & 1392.2387 & 512.9990 & 1905.2377 & \\
\hline & $7 / 29 / 2008$ 11:00 & 166 & 211 & 1100 & 6.702 & 6.702 & & 0.4787 & $4.80 \mathrm{E}-03$ & 1157.8925 & 426.6493 & 1584.5418 & \\
\hline & $7 / 29 / 200812: 00$ & 167 & 211 & 1200 & 6.126 & 6.126 & & 0.6177 & $6.58 \mathrm{E}-03$ & 1584.8726 & 583.9789 & 2168.8515 & \\
\hline & $7 / 29 / 2008$ 13:00 & 168 & 211 & 1300 & 6.172 & 6.172 & & 0.6056 & 6.42E-03 & 1546.8780 & 569.9790 & 2116.8570 & \\
\hline & $7 / 29 / 2008$ 14:00 & 169 & 211 & 1400 & 5.986 & 5.986 & & 0.6555 & 7.08E-03 & 1705.2046 & 628.3177 & 2333.5223 & \\
\hline & $7 / 29 / 2008$ 15:00 & 170 & 211 & 1500 & 6.115 & 6.115 & & 0.6206 & 6.61E-03 & 1594.0688 & 587.3674 & 2181.4362 & \\
\hline & $7 / 29 / 2008$ 16:00 & 171 & 211 & 1600 & 6.23 & 6.23 & & 0.5907 & $6.22 \mathrm{E}-03$ & 1500.0139 & 552.7110 & 2052.7248 & \\
\hline & $7 / 29 / 2008$ 17:00 & 172 & 211 & 1700 & 6.433 & 6.433 & & 0.5405 & $5.58 \mathrm{E}-03$ & 1344.6749 & 495.4731 & 1840.1480 & \\
\hline & $7 / 29 / 2008$ 18:00 & 173 & 211 & 1800 & 6.413 & 6.413 & & 0.5453 & 5.64E-03 & 1359.4033 & 500.9001 & 1860.3035 & \\
\hline & $7 / 29 / 2008$ 19:00 & 174 & 211 & 1900 & 6.454 & 6.454 & & 0.5355 & 5.52E-03 & 1329.3404 & 489.8228 & 1819.1632 & \\
\hline & $7 / 29 / 200820: 00$ & 175 & 211 & 2000 & 7.465 & 7.465 & & 0.3275 & 3.01E-03 & 725.8100 & 267.4396 & 993.2497 & \\
\hline & $7 / 29 / 200821: 00$ & 176 & 211 & 2100 & 9.11 & 9.11 & & 0.0878 & 5.96E-04 & 143.5780 & 52.9043 & 196.4823 & \\
\hline & $7 / 29 / 200822: 00$ & 177 & 211 & 2200 & 9.89 & 9.89 & & 0.0020 & 5.74E-06 & 1.3837 & 0.5098 & 1.8935 & \\
\hline & $7 / 29 / 200823: 00$ & 178 & 211 & 2300 & 10.08 & 10.08 & & -0.0169 & $0.00 \mathrm{E}+00$ & 0.0000 & 0.0000 & 0.0000 & \\
\hline & $7 / 30 / 20080: 00$ & 179 & 212 & 0 & 10.11 & 10.11 & 10.11 & -0.0198 & $0.00 \mathrm{E}+00$ & 0.0000 & 0.0000 & 0.0000 & \\
\hline & 7/30/2008 1:00 & 180 & 212 & 100 & 10.1 & 10.1 & & -0.0188 & $0.00 \mathrm{E}+00$ & 0.0000 & 0.0000 & 0.0000 & \\
\hline & 7/30/2008 2:00 & 181 & 212 & 200 & 10.07 & 10.07 & & -0.0159 & $0.00 E+00$ & 0.0000 & 0.0000 & 0.0000 & \\
\hline & 7/30/2008 3:00 & 182 & 212 & 300 & 10.05 & 10.05 & & -0.0139 & $0.00 \mathrm{E}+00$ & 0.0000 & 0.0000 & 0.0000 & \\
\hline & 7/30/2008 4:00 & 183 & 212 & 400 & 9.99 & 9.99 & & -0.0080 & $0.00 E+00$ & 0.0000 & 0.0000 & 0.0000 & \\
\hline & 7/30/2008 5:00 & 184 & 212 & 500 & 9.92 & 9.92 & & -0.0010 & $0.00 \mathrm{E}+00$ & 0.0000 & 0.0000 & 0.0000 & \\
\hline & 7/30/2008 6:00 & 185 & 212 & 600 & 9.85 & 9.85 & & 0.0061 & 2.23E-05 & 5.3769 & 1.9812 & 7.3582 & \\
\hline & 7/30/2008 7:00 & 186 & 212 & 700 & 9.76 & 9.76 & & 0.0154 & 6.97E-05 & 16.7999 & 6.1903 & 22.9902 & \\
\hline & 7/30/2008 8:00 & 187 & 212 & 800 & 9.72 & 9.72 & & 0.0195 & $9.37 \mathrm{E}-05$ & 22.5881 & 8.3231 & 30.9112 & \\
\hline & 7/30/2008 9:00 & 188 & 212 & 900 & 8.22 & 8.22 & & 0.2056 & 1.70E-03 & 409.1407 & 150.7563 & 559.8971 & \\
\hline & $7 / 30 / 2008$ 10:00 & 189 & 212 & 1000 & 5.836 & 5.836 & & 0.6981 & $7.65 E-03$ & 1842.4655 & 678.8943 & 2521.3598 & \\
\hline & 7/30/2008 11:00 & 190 & 212 & 1100 & 5.842 & 5.842 & & 0.6963 & $7.62 E-03$ & 1836.8008 & 676.8070 & 2513.6079 & \\
\hline & $7 / 30 / 200812: 00$ & 191 & 212 & 1200 & 5.952 & 5.952 & & 0.6650 & $7.20 \mathrm{E}-03$ & 1735.5376 & 639.4945 & 2375.0322 & \\
\hline & 7/30/2008 13:00 & 192 & 212 & 1300 & 5.936 & 5.936 & & 0.6695 & 7.26E-03 & 1749.9672 & 644.8114 & 2394.7786 & \\
\hline & $7 / 30 / 2008$ 14:00 & 193 & 212 & 1400 & 5.944 & 5.944 & & 0.6672 & 7.23E-03 & 1742.7399 & 642.1484 & 2384.8883 & \\
\hline & $7 / 30 / 2008$ 15:00 & 194 & 212 & 1500 & 6.045 & 6.045 & & 0.6394 & 6.86E-03 & 1653.6118 & 609.3073 & 2262.9191 & \\
\hline & $7 / 30 / 2008$ 16:00 & 195 & 212 & 1600 & 5.914 & 5.914 & & 0.6757 & 7.34E-03 & 1769.9722 & 652.1827 & 2422.1549 & \\
\hline & 7/30/2008 17:00 & 196 & 212 & 1700 & 5.976 & 5.976 & & 0.6583 & 7.11E-03 & 1714.0799 & 631.5880 & 2345.6678 & \\
\hline & $7 / 30 / 200818: 00$ & 197 & 212 & 1800 & 6.073 & 6.073 & & 0.6318 & $6.76 \mathrm{E}-03$ & 1629.5807 & 600.4525 & 2230.0332 & \\
\hline & $7 / 30 / 2008$ 19:00 & 198 & 212 & 1900 & 6.201 & 6.201 & & 0.5981 & 6.32E-03 & 1523.3025 & 561.2921 & 2084.5947 & \\
\hline & $7 / 30 / 200820: 00$ & 199 & 212 & 2000 & 6.812 & 6.812 & & 0.4548 & 4.51E-03 & 1087.2052 & 400.6031 & 1487.8084 & \\
\hline & $7 / 30 / 2008$ 21:00 & 200 & 212 & 2100 & 8.46 & 8.46 & & 0.1714 & 1.36E-03 & 327.0412 & 120.5051 & 447.5463 & \\
\hline & $7 / 30 / 200822: 00$ & 201 & 212 & 2200 & 9.54 & 9.54 & & 0.0388 & 2.18E-04 & 52.5029 & 19.3458 & 71.8487 & \\
\hline & $7 / 30 / 200823: 00$ & 202 & 212 & 2300 & 9.87 & 9.87 & & 0.0041 & $1.35 \mathrm{E}-05$ & 3.2560 & 1.1997 & 4.4557 & \\
\hline & $7 / 31 / 20080: 00$ & 203 & 213 & 0 & 10.04 & 10.04 & 10.09 & -0.0129 & $0.00 \mathrm{E}+00$ & 0.0000 & 0.0000 & 0.0000 & \\
\hline & 7/31/2008 1:00 & 204 & 213 & 100 & 10.09 & 10.09 & & -0.0178 & $0.00 \mathrm{E}+00$ & 0.0000 & 0.0000 & 0.0000 & \\
\hline
\end{tabular}




\begin{tabular}{|c|c|c|c|c|c|c|c|c|c|c|c|c|c|}
\hline Tree ID & TS & RN & JD & HR & $\begin{array}{c}\text { Raw } \\
\text { TC_dTA }\end{array}$ & $\begin{array}{c}\text { Fixed } \\
\text { TC_dT } \Delta\end{array}$ & dTM & $\mathbf{K}$ & $\begin{array}{c}\text { Sap } \\
\text { Velocity } \\
(\mathrm{cm} / \mathrm{s})\end{array}$ & $\begin{array}{l}\text { Probed } \\
\text { Drywood F } \\
\text { (g/hr) }\end{array}$ & $\begin{array}{c}\text { Extrapolated } \\
\text { Wetwood F } \\
(\mathrm{g} / \mathrm{hr})\end{array}$ & $\begin{array}{c}\text { Extrapolated } \\
\text { Total Wood F } \\
(\mathbf{g} / \mathrm{hr})\end{array}$ & $\begin{array}{l}\text { Relative } \\
\text { Humidity, } \\
@ 10 \mathrm{~m}(\%)\end{array}$ \\
\hline & 7/31/2008 2:00 & 205 & 213 & 200 & 10.09 & 10.09 & & -0.0178 & $0.00 \mathrm{E}+00$ & 0.0000 & 0.0000 & 0.0000 & \\
\hline & 7/31/2008 3:00 & 206 & 213 & 300 & 10.09 & 10.09 & & -0.0178 & $0.00 \mathrm{E}+00$ & 0.0000 & 0.0000 & 0.0000 & \\
\hline & 7/31/2008 4:00 & 207 & 213 & 400 & 10.09 & 10.09 & & -0.0178 & $0.00 E+00$ & 0.0000 & 0.0000 & 0.0000 & \\
\hline & 7/31/2008 5:00 & 208 & 213 & 500 & 10.05 & 10.05 & & -0.0139 & $0.00 \mathrm{E}+00$ & 0.0000 & 0.0000 & 0.0000 & \\
\hline & 7/31/2008 6:00 & 209 & 213 & 600 & 10.02 & 10.02 & & -0.0110 & $0.00 E+00$ & 0.0000 & 0.0000 & 0.0000 & \\
\hline & 7/31/2008 7:00 & 210 & 213 & 700 & 9.95 & 9.95 & & -0.0040 & $0.00 E+00$ & 0.0000 & 0.0000 & 0.0000 & \\
\hline & 7/31/2008 8:00 & 211 & 213 & 800 & 9.85 & 9.85 & & 0.0061 & 2.23E-05 & 5.3769 & 1.9812 & 7.3582 & \\
\hline & 7/31/2008 9:00 & 212 & 213 & 900 & 7.935 & 7.935 & & 0.2489 & $2.15 \mathrm{E}-03$ & 517.6691 & 190.7458 & 708.4149 & \\
\hline & $7 / 31 / 2008$ 10:00 & 213 & 213 & 1000 & 6.044 & 6.044 & & 0.6396 & $6.87 \mathrm{E}-03$ & 1654.4754 & 609.6255 & 2264.1009 & \\
\hline & $7 / 31 / 2008$ 11:00 & 214 & 213 & 1100 & 6.111 & 6.111 & & 0.6217 & $6.63 \mathrm{E}-03$ & 1597.4235 & 588.6035 & 2186.0271 & \\
\hline & $7 / 31 / 2008$ 12:00 & 215 & 213 & 1200 & 5.966 & 5.966 & & 0.6611 & 7.15E-03 & 1722.9935 & 634.8724 & 2357.8659 & \\
\hline & $7 / 31 / 2008$ 13:00 & 216 & 213 & 1300 & 6.162 & 6.162 & & 0.6082 & $6.45 \mathrm{E}-03$ & 1555.0748 & 572.9993 & 2128.0740 & \\
\hline & $7 / 31 / 2008$ 14:00 & 217 & 213 & 1400 & 5.947 & 5.947 & & 0.6664 & $7.22 \mathrm{E}-03$ & 1740.0361 & 641.1521 & 2381.1882 & \\
\hline & $7 / 31 / 2008$ 15:00 & 218 & 213 & 1500 & 5.971 & 5.971 & & 0.6597 & 7.13E-03 & 1718.5319 & 633.2284 & 2351.7603 & \\
\hline & $7 / 31 / 2008$ 16:00 & 219 & 213 & 1600 & 6.016 & 6.016 & & 0.6473 & 6.97E-03 & 1678.8074 & 618.5911 & 2297.3985 & \\
\hline & 7/31/2008 17:00 & 220 & 213 & 1700 & 6.032 & 6.032 & & 0.6429 & 6.91E-03 & 1664.8676 & 613.4547 & 2278.3223 & \\
\hline & $7 / 31 / 2008$ 18:00 & 221 & 213 & 1800 & 6.102 & 6.102 & & 0.6241 & $6.66 \mathrm{E}-03$ & 1604.9926 & 591.3925 & 2196.3852 & \\
\hline & $7 / 31 / 2008$ 19:00 & 222 & 213 & 1900 & 6.339 & 6.339 & & 0.5633 & $5.87 \mathrm{E}-03$ & 1414.9730 & 521.3759 & 1936.3489 & \\
\hline & $7 / 31 / 200820: 00$ & 223 & 213 & 2000 & 7.115 & 7.115 & & 0.3928 & 3.77E-03 & 907.8625 & 334.5206 & 1242.3832 & \\
\hline & 7/31/2008 21:00 & 224 & 213 & 2100 & 8.18 & 8.18 & & 0.2115 & 1.76E-03 & 423.6301 & 156.0952 & 579.7253 & \\
\hline & $7 / 31 / 200822: 00$ & 225 & 213 & 2200 & 9.05 & 9.05 & & 0.0950 & $6.57 \mathrm{E}-04$ & 158.2285 & 58.3025 & 216.5310 & \\
\hline & $7 / 31 / 2008$ 23:00 & 226 & 213 & 2300 & 9.12 & 9.12 & & 0.0866 & $5.86 \mathrm{E}-04$ & 141.1811 & 52.0211 & 193.2022 & \\
\hline & 8/1/2008 0:00 & 227 & 214 & 0 & 9.44 & 9.44 & 10.20 & 0.0498 & $2.96 \mathrm{E}-04$ & 71.4025 & 26.3097 & 97.7122 & \\
\hline & 8/1/2008 1:00 & 228 & 214 & 100 & 9.88 & 9.88 & & 0.0030 & $9.47 E-06$ & 2.2821 & 0.8409 & 3.1230 & \\
\hline & 8/1/2008 2:00 & 229 & 214 & 200 & 10.09 & 10.09 & & -0.0178 & $0.00 E+00$ & 0.0000 & 0.0000 & 0.0000 & \\
\hline & 8/1/2008 3:00 & 230 & 214 & 300 & 10.13 & 10.13 & & -0.0217 & $0.00 E+00$ & 0.0000 & 0.0000 & 0.0000 & \\
\hline & 8/1/2008 4:00 & 231 & 214 & 400 & 10.19 & 10.19 & & -0.0275 & $0.00 \mathrm{E}+00$ & 0.0000 & 0.0000 & 0.0000 & \\
\hline & 8/1/2008 5:00 & 232 & 214 & 500 & 10.2 & 10.2 & & -0.0284 & $0.00 \mathrm{E}+00$ & 0.0000 & 0.0000 & 0.0000 & \\
\hline & 8/1/2008 6:00 & 233 & 214 & 600 & 10.13 & 10.13 & & -0.0217 & $0.00 \mathrm{E}+00$ & 0.0000 & 0.0000 & 0.0000 & \\
\hline & 8/1/2008 7:00 & 234 & 214 & 700 & 10.01 & 10.01 & & -0.0100 & $0.00 E+00$ & 0.0000 & 0.0000 & 0.0000 & \\
\hline & 8/1/2008 8:00 & 235 & 214 & 800 & 9.93 & 9.93 & & -0.0020 & $0.00 \mathrm{E}+00$ & 0.0000 & 0.0000 & 0.0000 & \\
\hline & 8/1/2008 9:00 & 236 & 214 & 900 & 8.39 & 8.39 & & 0.1812 & 1.45E-03 & 350.1466 & 129.0187 & 479.1653 & \\
\hline & 8/1/2008 10:00 & 237 & 214 & 1000 & 6.14 & 6.14 & & 0.6140 & $6.53 \mathrm{E}-03$ & 1573.2304 & 579.6891 & 2152.9195 & \\
\hline & 8/1/2008 11:00 & 238 & 214 & 1100 & 6.117 & 6.117 & & 0.6201 & $6.61 \mathrm{E}-03$ & 1592.3935 & 586.7501 & 2179.1437 & \\
\hline & 8/1/2008 12:00 & 239 & 214 & 1200 & 6.161 & 6.161 & & 0.6085 & $6.46 \mathrm{E}-03$ & 1555.8964 & 573.3020 & 2129.1984 & \\
\hline & 8/1/2008 13:00 & 240 & 214 & 1300 & 6.05 & 6.05 & & 0.6380 & $6.84 \mathrm{E}-03$ & 1649.2994 & 607.7183 & 2257.0177 & \\
\hline & 8/1/2008 14:00 & 241 & 214 & 1400 & 6.007 & 6.007 & & 0.6497 & 7.00E-03 & 1686.6908 & 621.4959 & 2308.1867 & \\
\hline & 8/1/2008 15:00 & 242 & 214 & 1500 & 5.95 & 5.95 & & 0.6655 & 7.21E-03 & 1737.3359 & 640.1571 & 2377.4930 & \\
\hline & 8/1/2008 16:00 & 243 & 214 & 1600 & 5.995 & 5.995 & & 0.6530 & 7.04E-03 & 1697.2496 & 625.3865 & 2322.6361 & \\
\hline & 8/1/2008 17:00 & 244 & 214 & 1700 & 6.051 & 6.051 & & 0.6377 & $6.84 \mathrm{E}-03$ & 1648.4380 & 607.4009 & 2255.8389 & \\
\hline & 8/1/2008 18:00 & 245 & 214 & 1800 & 6.067 & 6.067 & & 0.6334 & $6.78 \mathrm{E}-03$ & 1634.7060 & 602.3410 & 2237.0470 & \\
\hline
\end{tabular}




\begin{tabular}{|c|c|c|c|c|c|c|c|c|c|c|c|c|c|}
\hline Tree ID & TS & RN & JD & HR & $\begin{array}{c}\text { Raw } \\
\text { TC_dTA }\end{array}$ & $\begin{array}{c}\text { Fixed } \\
\text { TC_dT } \Delta\end{array}$ & dTM & $\mathbf{K}$ & $\begin{array}{l}\text { Sap } \\
\text { Velocity } \\
\text { (cm/s) }\end{array}$ & $\begin{array}{l}\text { Probed } \\
\text { Drywood F } \\
\text { (g/hr) }\end{array}$ & $\begin{array}{c}\text { Extrapolated } \\
\text { Wetwood F } \\
(\mathrm{g} / \mathrm{hr})\end{array}$ & $\begin{array}{c}\text { Extrapolated } \\
\text { Total Wood F } \\
(\mathrm{g} / \mathrm{hr})\end{array}$ & $\begin{array}{l}\text { Relative } \\
\text { Humidity, } \\
@ 10 \mathrm{~m}(\%)\end{array}$ \\
\hline & 8/1/2008 19:00 & 246 & 214 & 1900 & 6.248 & 6.248 & & 0.5861 & $6.16 \mathrm{E}-03$ & 1485.7012 & 547.4372 & 2033.1384 & \\
\hline & 8/1/2008 20:00 & 247 & 214 & 2000 & 7.101 & 7.101 & & 0.3956 & $3.80 \mathrm{E}-03$ & 915.6811 & 337.4015 & 1253.0827 & \\
\hline & 8/1/2008 21:00 & 248 & 214 & 2100 & 9 & 9 & & 0.1011 & 7.09E-04 & 170.7886 & 62.9306 & 233.7191 & \\
\hline & 8/1/2008 22:00 & 249 & 214 & 2200 & 9.84 & 9.84 & & 0.0071 & 2.70E-05 & 6.5086 & 2.3982 & 8.9069 & \\
\hline & 8/1/2008 23:00 & 250 & 214 & 2300 & 10 & 10 & & -0.0090 & $0.00 \mathrm{E}+00$ & 0.0000 & 0.0000 & 0.0000 & \\
\hline & $8 / 2 / 20080: 00$ & 251 & 215 & 0 & 10.07 & 10.07 & 10.31 & -0.0159 & $0.00 E+00$ & 0.0000 & 0.0000 & 0.0000 & \\
\hline & 8/2/2008 1:00 & 252 & 215 & 100 & 10.19 & 10.19 & & -0.0275 & $0.00 \mathrm{E}+00$ & 0.0000 & 0.0000 & 0.0000 & \\
\hline & 8/2/2008 2:00 & 253 & 215 & 200 & 10.3 & 10.3 & & -0.0379 & $0.00 \mathrm{E}+00$ & 0.0000 & 0.0000 & 0.0000 & \\
\hline & 8/2/2008 3:00 & 254 & 215 & 300 & 10.31 & 10.31 & & -0.0388 & $0.00 \mathrm{E}+00$ & 0.0000 & 0.0000 & 0.0000 & \\
\hline & 8/2/2008 4:00 & 255 & 215 & 400 & 10.25 & 10.25 & & -0.0332 & $0.00 E+00$ & 0.0000 & 0.0000 & 0.0000 & \\
\hline & 8/2/2008 5:00 & 256 & 215 & 500 & 10.22 & 10.22 & & -0.0303 & $0.00 \mathrm{E}+00$ & 0.0000 & 0.0000 & 0.0000 & \\
\hline & 8/2/2008 6:00 & 257 & 215 & 600 & 10.24 & 10.24 & & -0.0322 & $0.00 \mathrm{E}+00$ & 0.0000 & 0.0000 & 0.0000 & \\
\hline & 8/2/2008 7:00 & 258 & 215 & 700 & 10.15 & 10.15 & & -0.0236 & $0.00 \mathrm{E}+00$ & 0.0000 & 0.0000 & 0.0000 & \\
\hline & 8/2/2008 8:00 & 259 & 215 & 800 & 8.62 & 8.62 & & 0.1497 & 1.15E-03 & 276.7438 & 101.9719 & 378.7157 & \\
\hline & 8/2/2008 9:00 & 260 & 215 & 900 & 6.494 & 6.494 & & 0.5260 & $5.40 \mathrm{E}-03$ & 1300.4967 & 479.1948 & 1779.6915 & \\
\hline & 8/2/2008 10:00 & 261 & 215 & 1000 & 6.38 & 6.38 & & 0.5533 & 5.74E-03 & 1383.9736 & 509.9535 & 1893.9271 & \\
\hline & 8/2/2008 11:00 & 262 & 215 & 1100 & 6.257 & 6.257 & & 0.5838 & $6.14 \mathrm{E}-03$ & 1478.5854 & 544.8152 & 2023.4006 & \\
\hline & 8/2/2008 12:00 & 263 & 215 & 1200 & 6.163 & 6.163 & & 0.6080 & $6.45 E-03$ & 1554.2535 & 572.6967 & 2126.9502 & \\
\hline & 8/2/2008 13:00 & 264 & 215 & 1300 & 6.126 & 6.126 & & 0.6177 & $6.58 \mathrm{E}-03$ & 1584.8726 & 583.9789 & 2168.8515 & \\
\hline & $8 / 2 / 200814: 00$ & 265 & 215 & 1400 & 6.143 & 6.143 & & 0.6132 & $6.52 \mathrm{E}-03$ & 1570.7446 & 578.7732 & 2149.5178 & \\
\hline & 8/2/2008 15:00 & 266 & 215 & 1500 & 6.117 & 6.117 & & 0.6201 & $6.61 \mathrm{E}-03$ & 1592.3935 & 586.7501 & 2179.1437 & \\
\hline & 8/2/2008 16:00 & 267 & 215 & 1600 & 6.142 & 6.142 & & 0.6135 & $6.52 \mathrm{E}-03$ & 1571.5729 & 579.0783 & 2150.6512 & \\
\hline & 8/2/2008 17:00 & 268 & 215 & 1700 & 6.19 & 6.19 & & 0.6010 & $6.36 \mathrm{E}-03$ & 1532.2110 & 564.5746 & 2096.7856 & \\
\hline & 8/2/2008 18:00 & 269 & 215 & 1800 & 6.263 & 6.263 & & 0.5823 & $6.12 \mathrm{E}-03$ & 1473.8564 & 543.0727 & 2016.9291 & \\
\hline & 8/2/2008 19:00 & 270 & 215 & 1900 & 6.412 & 6.412 & & 0.5455 & $5.64 \mathrm{E}-03$ & 1360.1430 & 501.1726 & 1861.3156 & \\
\hline & 8/2/2008 20:00 & 271 & 215 & 2000 & 6.99 & 6.99 & & 0.4177 & $4.06 \mathrm{E}-03$ & 979.2335 & 360.8187 & 1340.0523 & \\
\hline & 8/2/2008 21:00 & 272 & 215 & 2100 & 8.24 & 8.24 & & 0.2027 & 1.67E-03 & 401.9842 & 148.1193 & 550.1035 & \\
\hline & 8/2/2008 22:00 & 273 & 215 & 2200 & 9.34 & 9.34 & & 0.0610 & $3.81 \mathrm{E}-04$ & 91.7353 & 33.8017 & 125.5370 & \\
\hline & 8/2/2008 23:00 & 274 & 215 & 2300 & 9.72 & 9.72 & & 0.0195 & 9.37E-05 & 22.5881 & 8.3231 & 30.9112 & \\
\hline & $8 / 3 / 20080: 00$ & 275 & 216 & 0 & 9.69 & 9.69 & 10.33 & 0.0227 & 1.13E-04 & 27.1587 & 10.0072 & 37.1659 & \\
\hline & 8/3/2008 1:00 & 276 & 216 & 100 & 9.55 & 9.55 & & 0.0377 & $2.10 \mathrm{E}-04$ & 50.6962 & 18.6801 & 69.3763 & \\
\hline & 8/3/2008 2:00 & 277 & 216 & 200 & 9.82 & 9.82 & & 0.0092 & 3.69E-05 & 8.8907 & 3.2760 & 12.1666 & \\
\hline & 8/3/2008 3:00 & 278 & 216 & 300 & 10.09 & 10.09 & & -0.0178 & $0.00 E+00$ & 0.0000 & 0.0000 & 0.0000 & \\
\hline & 8/3/2008 4:00 & 279 & 216 & 400 & 10.24 & 10.24 & & -0.0322 & $0.00 \mathrm{E}+00$ & 0.0000 & 0.0000 & 0.0000 & \\
\hline & 8/3/2008 5:00 & 280 & 216 & 500 & 10.33 & 10.33 & & -0.0407 & $0.00 \mathrm{E}+00$ & 0.0000 & 0.0000 & 0.0000 & \\
\hline & 8/3/2008 6:00 & 281 & 216 & 600 & 10.25 & 10.25 & & -0.0332 & $0.00 \mathrm{E}+00$ & 0.0000 & 0.0000 & 0.0000 & \\
\hline & 8/3/2008 7:00 & 282 & 216 & 700 & 10.04 & 10.04 & & -0.0129 & $0.00 E+00$ & 0.0000 & 0.0000 & 0.0000 & \\
\hline & 8/3/2008 8:00 & 283 & 216 & 800 & 8.62 & 8.62 & & 0.1497 & 1.15E-03 & 276.7438 & 101.9719 & 378.7157 & \\
\hline & 8/3/2008 9:00 & 284 & 216 & 900 & 6.452 & 6.452 & & 0.5360 & $5.52 \mathrm{E}-03$ & 1330.7951 & 490.3588 & 1821.1540 & \\
\hline & 8/3/2008 10:00 & 285 & 216 & 1000 & 6.212 & 6.212 & & 0.5953 & $6.28 \mathrm{E}-03$ & 1514.4354 & 558.0249 & 2072.4602 & \\
\hline & 8/3/2008 11:00 & 286 & 216 & 1100 & 6.165 & 6.165 & & 0.6075 & 6.44E-03 & 1552.6121 & 572.0919 & 2124.7039 & \\
\hline
\end{tabular}




\begin{tabular}{|c|c|c|c|c|c|c|c|c|c|c|c|c|c|}
\hline Tree ID & TS & RN & JD & HR & $\begin{array}{c}\text { Raw } \\
\text { TC_dTA }\end{array}$ & $\begin{array}{c}\text { Fixed } \\
\text { TC_dT } \Delta\end{array}$ & dTM & $\mathbf{K}$ & $\begin{array}{c}\text { Sap } \\
\text { Velocity } \\
(\mathrm{cm} / \mathrm{s})\end{array}$ & $\begin{array}{c}\text { Probed } \\
\text { Drywood F } \\
(\mathrm{g} / \mathrm{hr})\end{array}$ & $\begin{array}{c}\text { Extrapolated } \\
\text { Wetwood F } \\
(\mathrm{g} / \mathrm{hr})\end{array}$ & $\begin{array}{c}\text { Extrapolated } \\
\text { Total Wood F } \\
(\mathrm{g} / \mathrm{hr})\end{array}$ & $\begin{array}{l}\text { Relative } \\
\text { Humidity, } \\
\text { @10m (\%) }\end{array}$ \\
\hline & 8/3/2008 12:00 & 287 & 216 & 1200 & 6.122 & 6.122 & & 0.6188 & $6.59 \mathrm{E}-03$ & 1588.2117 & 585.2093 & 2173.4209 & \\
\hline & 8/3/2008 13:00 & 288 & 216 & 1300 & 6.078 & 6.078 & & 0.6305 & $6.74 \mathrm{E}-03$ & 1625.3196 & 598.8824 & 2224.2020 & \\
\hline & 8/3/2008 14:00 & 289 & 216 & 1400 & 6.121 & 6.121 & & 0.6190 & $6.59 \mathrm{E}-03$ & 1589.0473 & 585.5172 & 2174.5645 & \\
\hline & 8/3/2008 15:00 & 290 & 216 & 1500 & 6.202 & 6.202 & & 0.5979 & 6.32E-03 & 1522.4947 & 560.9945 & 2083.4892 & \\
\hline & $8 / 3 / 2008$ 16:00 & 291 & 216 & 1600 & 6.214 & 6.214 & & 0.5948 & $6.28 \mathrm{E}-03$ & 1512.8276 & 557.4324 & 2070.2600 & \\
\hline & 8/3/2008 17:00 & 292 & 216 & 1700 & 6.225 & 6.225 & & 0.5920 & $6.24 \mathrm{E}-03$ & 1504.0089 & 554.1830 & 2058.1919 & \\
\hline & $8 / 3 / 200818: 00$ & 293 & 216 & 1800 & 6.229 & 6.229 & & 0.5909 & 6.23E-03 & 1500.8122 & 553.0051 & 2053.8173 & \\
\hline & 8/3/2008 19:00 & 294 & 216 & 1900 & 6.299 & 6.299 & & 0.5733 & 6.00E-03 & 1445.7310 & 532.7093 & 1978.4403 & \\
\hline & 8/3/2008 20:00 & 295 & 216 & 2000 & 6.575 & 6.575 & & 0.5072 & $5.16 \mathrm{E}-03$ & 1243.5198 & 458.2004 & 1701.7202 & \\
\hline & 8/3/2008 21:00 & 296 & 216 & 2100 & 7.597 & 7.597 & & 0.3045 & $2.75 \mathrm{E}-03$ & 663.4087 & 244.4466 & 907.8552 & \\
\hline & $8 / 3 / 200822: 00$ & 297 & 216 & 2200 & 8.39 & 8.39 & & 0.1812 & 1.45E-03 & 350.1466 & 129.0187 & 479.1653 & \\
\hline & $8 / 3 / 2008$ 23:00 & 298 & 216 & 2300 & 8.57 & 8.57 & & 0.1564 & 1.21E-03 & 292.0909 & 107.6269 & 399.7178 & \\
\hline & $8 / 4 / 20080: 00$ & 299 & 217 & 0 & 8.65 & 8.65 & 9.71 & 0.1457 & 1.11E-03 & 267.6953 & 98.6378 & 366.3331 & \\
\hline & 8/4/2008 1:00 & 300 & 217 & 100 & 8.74 & 8.74 & & 0.1339 & 1.00E-03 & 241.2613 & 88.8977 & 330.1590 & \\
\hline & $8 / 4 / 20082: 00$ & 301 & 217 & 200 & 8.42 & 8.42 & & 0.1770 & 1.41E-03 & 340.1610 & 125.3393 & 465.5003 & \\
\hline & 8/4/2008 3:00 & 302 & 217 & 300 & 8.21 & 8.21 & & 0.2071 & 1.71E-03 & 412.7410 & 152.0829 & 564.8239 & \\
\hline & $8 / 4 / 20084: 00$ & 303 & 217 & 400 & 8.02 & 8.02 & & 0.2357 & 2.01E-03 & 483.9909 & 178.3364 & 662.3273 & \\
\hline & 8/4/2008 5:00 & 304 & 217 & 500 & 8.2 & 8.2 & & 0.2085 & 1.73E-03 & 416.3559 & 153.4149 & 569.7708 & \\
\hline & 8/4/2008 6:00 & 305 & 217 & 600 & 8.69 & 8.69 & & 0.1404 & 1.06E-03 & 255.8156 & 94.2605 & 350.0761 & \\
\hline & 8/4/2008 7:00 & 306 & 217 & 700 & 8.7 & 8.7 & & 0.1391 & 1.05E-03 & 252.8785 & 93.1783 & 346.0568 & \\
\hline & $8 / 4 / 20088: 00$ & 307 & 217 & 800 & 8.28 & 8.28 & & 0.1969 & $1.61 \mathrm{E}-03$ & 387.8457 & 142.9097 & 530.7554 & \\
\hline & 8/4/2008 9:00 & 308 & 217 & 900 & 7.804 & 7.804 & & 0.2699 & 2.37E-03 & 571.8552 & 210.7118 & 782.5670 & \\
\hline & 8/4/2008 10:00 & 309 & 217 & 1000 & 6.833 & 6.833 & & 0.4503 & $4.46 \mathrm{E}-03$ & 1074.0628 & 395.7605 & 1469.8234 & \\
\hline & 8/4/2008 11:00 & 310 & 217 & 1100 & 6.389 & 6.389 & & 0.5511 & $5.71 \mathrm{E}-03$ & 1377.2392 & 507.4721 & 1884.7114 & \\
\hline & 8/4/2008 12:00 & 311 & 217 & 1200 & 6.249 & 6.249 & & 0.5859 & $6.16 \mathrm{E}-03$ & 1484.9092 & 547.1453 & 2032.0546 & \\
\hline & 8/4/2008 13:00 & 312 & 217 & 1300 & 6.158 & 6.158 & & 0.6093 & $6.47 \mathrm{E}-03$ & 1558.3632 & 574.2110 & 2132.5742 & \\
\hline & $8 / 4 / 2008$ 14:00 & 313 & 217 & 1400 & 6.462 & 6.462 & & 0.5336 & 5.49E-03 & 1323.5336 & 487.6832 & 1811.2168 & \\
\hline & $8 / 4 / 2008$ 15:00 & 314 & 217 & 1500 & 6.773 & 6.773 & & 0.4632 & $4.61 \mathrm{E}-03$ & 1111.9090 & 409.7058 & 1521.6148 & \\
\hline & 8/4/2008 16:00 & 315 & 217 & 1600 & 6.284 & 6.284 & & 0.5770 & $6.05 \mathrm{E}-03$ & 1457.3984 & 537.0084 & 1994.4068 & \\
\hline & $8 / 4 / 2008$ 17:00 & 316 & 217 & 1700 & 6.196 & 6.196 & & 0.5994 & $6.34 \mathrm{E}-03$ & 1527.3467 & 562.7823 & 2090.1290 & \\
\hline & 8/4/2008 18:00 & 317 & 217 & 1800 & 6.259 & 6.259 & & 0.5833 & $6.13 \mathrm{E}-03$ & 1477.0077 & 544.2339 & 2021.2416 & \\
\hline & 8/4/2008 19:00 & 318 & 217 & 1900 & 6.495 & 6.495 & & 0.5258 & 5.39E-03 & 1299.7817 & 478.9313 & 1778.7130 & \\
\hline & 8/4/2008 20:00 & 319 & 217 & 2000 & 7.132 & 7.132 & & 0.3895 & 3.73E-03 & 898.4267 & 331.0438 & 1229.4704 & \\
\hline & 8/4/2008 21:00 & 320 & 217 & 2100 & 8.87 & 8.87 & & 0.1172 & 8.50E-04 & 204.9394 & 75.5141 & 280.4535 & \\
\hline & 8/4/2008 22:00 & 321 & 217 & 2200 & 9.57 & 9.57 & & 0.0355 & 1.96E-04 & 47.1302 & 17.3661 & 64.4963 & \\
\hline & $8 / 4 / 2008$ 23:00 & 322 & 217 & 2300 & 9.71 & 9.71 & & 0.0206 & 1.00E-04 & 24.0909 & 8.8768 & 32.9676 & \\
\hline & $8 / 5 / 20080: 00$ & 323 & 218 & 0 & 9.9 & 9.9 & 10.23 & 0.0010 & $2.44 \mathrm{E}-06$ & 0.5887 & 0.2169 & 0.8057 & \\
\hline & 8/5/2008 1:00 & 324 & 218 & 100 & 10 & 10 & & -0.0090 & $0.00 E+00$ & 0.0000 & 0.0000 & 0.0000 & \\
\hline & $8 / 5 / 20082: 00$ & 325 & 218 & 200 & 10.08 & 10.08 & & -0.0169 & $0.00 \mathrm{E}+00$ & 0.0000 & 0.0000 & 0.0000 & \\
\hline & 8/5/2008 3:00 & 326 & 218 & 300 & 10.07 & 10.07 & & -0.0159 & $0.00 \mathrm{E}+00$ & 0.0000 & 0.0000 & 0.0000 & \\
\hline & 8/5/2008 4:00 & 327 & 218 & 400 & 10.01 & 10.01 & & -0.0100 & $0.00 \mathrm{E}+00$ & 0.0000 & 0.0000 & 0.0000 & \\
\hline
\end{tabular}




\begin{tabular}{|c|c|c|c|c|c|c|c|c|c|c|c|c|c|}
\hline Tree ID & TS & RN & JD & HR & $\begin{array}{c}\text { Raw } \\
\text { TC_dTA }\end{array}$ & $\begin{array}{c}\text { Fixed } \\
\text { TC_dT } \Delta\end{array}$ & dTM & $\mathbf{K}$ & $\begin{array}{c}\text { Sap } \\
\text { Velocity } \\
(\mathrm{cm} / \mathrm{s})\end{array}$ & $\begin{array}{l}\text { Probed } \\
\text { Drywood F } \\
\text { (g/hr) }\end{array}$ & $\begin{array}{c}\text { Extrapolated } \\
\text { Wetwood F } \\
(\mathrm{g} / \mathrm{hr})\end{array}$ & $\begin{array}{c}\text { Extrapolated } \\
\text { Total Wood F } \\
(\mathbf{g} / \mathrm{hr})\end{array}$ & $\begin{array}{c}\text { Relative } \\
\text { Humidity, } \\
\text { @10m (\%) }\end{array}$ \\
\hline & 8/5/2008 5:00 & 328 & 218 & 500 & 10.06 & 10.06 & & -0.0149 & $0.00 \mathrm{E}+00$ & 0.0000 & 0.0000 & 0.0000 & \\
\hline & 8/5/2008 6:00 & 329 & 218 & 600 & 10.15 & 10.15 & & -0.0236 & $0.00 \mathrm{E}+00$ & 0.0000 & 0.0000 & 0.0000 & \\
\hline & 8/5/2008 7:00 & 330 & 218 & 700 & 10.18 & 10.18 & & -0.0265 & $0.00 E+00$ & 0.0000 & 0.0000 & 0.0000 & \\
\hline & 8/5/2008 8:00 & 331 & 218 & 800 & 10.05 & 10.05 & & -0.0139 & $0.00 \mathrm{E}+00$ & 0.0000 & 0.0000 & 0.0000 & \\
\hline & 8/5/2008 9:00 & 332 & 218 & 900 & 8.4 & 8.4 & & 0.1798 & 1.44E-03 & 346.8041 & 127.7871 & 474.5912 & \\
\hline & 8/5/2008 10:00 & 333 & 218 & 1000 & 6.754 & 6.754 & & 0.4673 & $4.66 \mathrm{E}-03$ & 1124.0856 & 414.1924 & 1538.2780 & \\
\hline & 8/5/2008 11:00 & 334 & 218 & 1100 & 7.061 & 7.061 & & 0.4035 & 3.89E-03 & 938.2606 & 345.7214 & 1283.9821 & \\
\hline & 8/5/2008 12:00 & 335 & 218 & 1200 & 6.732 & 6.732 & & 0.4721 & 4.72E-03 & 1138.3018 & 419.4307 & 1557.7326 & \\
\hline & 8/5/2008 13:00 & 336 & 218 & 1300 & 6.336 & 6.336 & & 0.5641 & $5.88 \mathrm{E}-03$ & 1417.2621 & 522.2194 & 1939.4815 & \\
\hline & 8/5/2008 14:00 & 337 & 218 & 1400 & 6.816 & 6.816 & & 0.4539 & $4.50 \mathrm{E}-03$ & 1084.6934 & 399.6776 & 1484.3709 & \\
\hline & 8/5/2008 15:00 & 338 & 218 & 1500 & 7.204 & 7.204 & & 0.3756 & 3.57E-03 & 859.1593 & 316.5749 & 1175.7342 & \\
\hline & 8/5/2008 16:00 & 339 & 218 & 1600 & 7.032 & 7.032 & & 0.4093 & 3.96E-03 & 954.8564 & 351.8365 & 1306.6929 & \\
\hline & 8/5/2008 17:00 & 340 & 218 & 1700 & 6.562 & 6.562 & & 0.5102 & $5.20 \mathrm{E}-03$ & 1252.5374 & 461.5231 & 1714.0605 & \\
\hline & 8/5/2008 18:00 & 341 & 218 & 1800 & 6.325 & 6.325 & & 0.5668 & 5.92E-03 & 1425.6799 & 525.3211 & 1951.0010 & \\
\hline & 8/5/2008 19:00 & 342 & 218 & 1900 & 6.472 & 6.472 & & 0.5312 & $5.46 \mathrm{E}-03$ & 1316.3019 & 485.0185 & 1801.3204 & \\
\hline & 8/5/2008 20:00 & 343 & 218 & 2000 & 7.399 & 7.399 & & 0.3394 & 3.15E-03 & 758.2470 & 279.3917 & 1037.6387 & \\
\hline & 8/5/2008 21:00 & 344 & 218 & 2100 & 9.39 & 9.39 & & 0.0554 & $3.38 \mathrm{E}-04$ & 81.3955 & 29.9918 & 111.3873 & \\
\hline & 8/5/2008 22:00 & 345 & 218 & 2200 & 10.11 & 10.11 & & -0.0198 & $0.00 E+00$ & 0.0000 & 0.0000 & 0.0000 & \\
\hline & 8/5/2008 23:00 & 346 & 218 & 2300 & 10.23 & 10.23 & & -0.0313 & $0.00 \mathrm{E}+00$ & 0.0000 & 0.0000 & 0.0000 & \\
\hline & 8/6/2008 0:00 & 347 & 219 & 0 & 10.27 & 10.27 & 10.28 & -0.0351 & $0.00 E+00$ & 0.0000 & 0.0000 & 0.0000 & \\
\hline & 8/6/2008 1:00 & 348 & 219 & 100 & 10.27 & 10.27 & & -0.0351 & $0.00 E+00$ & 0.0000 & 0.0000 & 0.0000 & \\
\hline & 8/6/2008 2:00 & 349 & 219 & 200 & 10.28 & 10.28 & & -0.0360 & $0.00 \mathrm{E}+00$ & 0.0000 & 0.0000 & 0.0000 & \\
\hline & 8/6/2008 3:00 & 350 & 219 & 300 & 10.26 & 10.26 & & -0.0341 & $0.00 \mathrm{E}+00$ & 0.0000 & 0.0000 & 0.0000 & \\
\hline & 8/6/2008 4:00 & 351 & 219 & 400 & 10.23 & 10.23 & & -0.0313 & $0.00 E+00$ & 0.0000 & 0.0000 & 0.0000 & \\
\hline & 8/6/2008 5:00 & 352 & 219 & 500 & 10.22 & 10.22 & & -0.0303 & $0.00 E+00$ & 0.0000 & 0.0000 & 0.0000 & \\
\hline & 8/6/2008 6:00 & 353 & 219 & 600 & 10.2 & 10.2 & & -0.0284 & $0.00 E+00$ & 0.0000 & 0.0000 & 0.0000 & \\
\hline & 8/6/2008 7:00 & 354 & 219 & 700 & 10.17 & 10.17 & & -0.0256 & $0.00 \mathrm{E}+00$ & 0.0000 & 0.0000 & 0.0000 & \\
\hline & 8/6/2008 8:00 & 355 & 219 & 800 & 10.15 & 10.15 & & -0.0236 & $0.00 \mathrm{E}+00$ & 0.0000 & 0.0000 & 0.0000 & \\
\hline & 8/6/2008 9:00 & 356 & 219 & 900 & 9.94 & 9.94 & & -0.0030 & $0.00 \mathrm{E}+00$ & 0.0000 & 0.0000 & 0.0000 & \\
\hline & 8/6/2008 10:00 & 357 & 219 & 1000 & 7.171 & 7.171 & & 0.3820 & $3.64 \mathrm{E}-03$ & 877.0181 & 323.1553 & 1200.1734 & \\
\hline & 8/6/2008 11:00 & 358 & 219 & 1100 & 6.216 & 6.216 & & 0.5943 & 6.27E-03 & 1511.2211 & 556.8405 & 2068.0616 & \\
\hline & 8/6/2008 12:00 & 359 & 219 & 1200 & 6.841 & 6.841 & & 0.4486 & $4.44 \mathrm{E}-03$ & 1069.0853 & 393.9265 & 1463.0117 & \\
\hline & 8/6/2008 13:00 & 360 & 219 & 1300 & 7.245 & 7.245 & & 0.3678 & $3.47 \mathrm{E}-03$ & 837.2929 & 308.5178 & 1145.8107 & \\
\hline & 8/6/2008 14:00 & 361 & 219 & 1400 & 6.678 & 6.678 & & 0.4840 & 4.87E-03 & 1173.7373 & 432.4876 & 1606.2249 & \\
\hline & $8 / 6 / 2008$ 15:00 & 362 & 219 & 1500 & 6.362 & 6.362 & & 0.5577 & $5.80 \mathrm{E}-03$ & 1397.5180 & 514.9443 & 1912.4623 & \\
\hline & 8/6/2008 16:00 & 363 & 219 & 1600 & 6.407 & 6.407 & & 0.5467 & $5.66 \mathrm{E}-03$ & 1363.8457 & 502.5370 & 1866.3827 & \\
\hline & 8/6/2008 17:00 & 364 & 219 & 1700 & 6.304 & 6.304 & & 0.5720 & $5.98 \mathrm{E}-03$ & 1441.8581 & 531.2823 & 1973.1403 & \\
\hline & 8/6/2008 18:00 & 365 & 219 & 1800 & 6.813 & 6.813 & & 0.4546 & $4.51 \mathrm{E}-03$ & 1086.5769 & 400.3716 & 1486.9485 & \\
\hline & 8/6/2008 19:00 & 366 & 219 & 1900 & 6.783 & 6.783 & & 0.4610 & 4.59E-03 & 1105.5377 & 407.3581 & 1512.8958 & \\
\hline & 8/6/2008 20:00 & 367 & 219 & 2000 & 7.22 & 7.22 & & 0.3726 & $3.53 \mathrm{E}-03$ & 850.5839 & 313.4151 & 1163.9990 & \\
\hline & 8/6/2008 21:00 & 368 & 219 & 2100 & 8.88 & 8.88 & & 0.1160 & 8.39E-04 & 202.2356 & 74.5179 & 276.7535 & \\
\hline
\end{tabular}




\begin{tabular}{|c|c|c|c|c|c|c|c|c|c|c|c|c|c|}
\hline Tree ID & TS & RN & JD & HR & $\begin{array}{c}\text { Raw } \\
\text { TC_dTA }\end{array}$ & $\begin{array}{c}\text { Fixed } \\
\text { TC_dT } \Delta\end{array}$ & dTM & $\mathrm{K}$ & $\begin{array}{l}\text { Sap } \\
\text { Velocity } \\
(\mathrm{cm} / \mathrm{s})\end{array}$ & $\begin{array}{c}\text { Probed } \\
\text { Drywood F } \\
(\mathrm{g} / \mathrm{hr})\end{array}$ & $\begin{array}{c}\text { Extrapolated } \\
\text { Wetwood F } \\
(\mathrm{g} / \mathrm{hr})\end{array}$ & $\begin{array}{c}\text { Extrapolated } \\
\text { Total Wood F } \\
(\mathrm{g} / \mathrm{hr})\end{array}$ & $\begin{array}{l}\text { Relative } \\
\text { Humidity, } \\
@ 10 \mathrm{~m}(\%)\end{array}$ \\
\hline & 8/6/2008 22:00 & 369 & 219 & 2200 & ) $\quad 10.01$ & 10.01 & & -0.0100 & $0.00 \mathrm{E}+00$ & 0.0000 & 0.0000 & 0.0000 & \\
\hline & 8/6/2008 23:00 & 370 & 219 & 2300 & 10.24 & 10.24 & & -0.0322 & $0.00 \mathrm{E}+00$ & 0.0000 & 0.0000 & 0.0000 & \\
\hline & 8/7/2008 0:00 & 371 & 220 & 0 & 10.33 & 10.33 & 10.34 & -0.0407 & $0.00 E+00$ & 0.0000 & 0.0000 & 0.0000 & \\
\hline & 8/7/2008 1:00 & 372 & 220 & 100 & 10.34 & 10.34 & & -0.0416 & $0.00 \mathrm{E}+00$ & 0.0000 & 0.0000 & 0.0000 & \\
\hline & 8/7/2008 2:00 & 373 & 220 & 200 & 10.34 & 10.34 & & -0.0416 & $0.00 E+00$ & 0.0000 & 0.0000 & 0.0000 & \\
\hline & 8/7/2008 3:00 & 374 & 220 & 300 & 10.31 & 10.31 & & -0.0388 & $0.00 E+00$ & 0.0000 & 0.0000 & 0.0000 & \\
\hline & 8/7/2008 4:00 & 375 & 220 & 400 & 10.3 & 10.3 & & -0.0379 & $0.00 \mathrm{E}+00$ & 0.0000 & 0.0000 & 0.0000 & \\
\hline & 8/7/2008 5:00 & 376 & 220 & 500 & 10.28 & 10.28 & & -0.0360 & $0.00 \mathrm{E}+00$ & 0.0000 & 0.0000 & 0.0000 & \\
\hline & 8/7/2008 6:00 & 377 & 220 & 600 & 10.24 & 10.24 & & -0.0322 & $0.00 E+00$ & 0.0000 & 0.0000 & 0.0000 & \\
\hline & 8/7/2008 7:00 & 378 & 220 & 700 & 10.19 & 10.19 & & -0.0275 & $0.00 \mathrm{E}+00$ & 0.0000 & 0.0000 & 0.0000 & \\
\hline & 8/7/2008 8:00 & 379 & 220 & 800 & 10.15 & 10.15 & & -0.0236 & $0.00 \mathrm{E}+00$ & 0.0000 & 0.0000 & 0.0000 & \\
\hline & $8 / 7 / 20089: 00$ & 380 & 220 & 900 & 9.13 & 9.13 & & 0.0854 & 5.76E-04 & 138.7971 & 51.1426 & 189.9397 & \\
\hline & 8/7/2008 10:00 & 381 & 220 & 1000 & 6.528 & 6.528 & & 0.5181 & 5.30E-03 & 1276.3499 & 470.2974 & 1746.6472 & \\
\hline & 8/7/2008 11:00 & 382 & 220 & 1100 & 6.121 & 6.121 & & 0.6190 & 6.59E-03 & 1589.0473 & 585.5172 & 2174.5645 & \\
\hline & 8/7/2008 12:00 & 383 & 220 & 1200 & 6.202 & 6.202 & & 0.5979 & $6.32 \mathrm{E}-03$ & 1522.4947 & 560.9945 & 2083.4892 & \\
\hline & 8/7/2008 13:00 & 384 & 220 & 1300 & 6.178 & 6.178 & & 0.6041 & $6.40 \mathrm{E}-03$ & 1541.9766 & 568.1730 & 2110.1496 & \\
\hline & 8/7/2008 14:00 & 385 & 220 & 1400 & 6.221 & 6.221 & & 0.5930 & $6.25 \mathrm{E}-03$ & 1507.2109 & 555.3629 & 2062.5738 & \\
\hline & $8 / 7 / 2008$ 15:00 & 386 & 220 & 1500 & 6.235 & 6.235 & & 0.5894 & $6.21 E-03$ & 1496.0272 & 551.2420 & 2047.2693 & \\
\hline & $8 / 7 / 200816: 00$ & 387 & 220 & 1600 & 6.182 & 6.182 & & 0.6030 & $6.38 \mathrm{E}-03$ & 1538.7159 & 566.9715 & 2105.6874 & \\
\hline & 8/7/2008 17:00 & 388 & 220 & 1700 & 6.177 & 6.177 & & 0.6043 & $6.40 \mathrm{E}-03$ & 1542.7926 & 568.4737 & 2111.2663 & \\
\hline & 8/7/2008 18:00 & 389 & 220 & 1800 & 6.227 & 6.227 & & 0.5915 & $6.23 E-03$ & 1502.4099 & 553.5938 & 2056.0037 & \\
\hline & 8/7/2008 19:00 & 390 & 220 & 1900 & 6.467 & 6.467 & & 0.5324 & 5.48E-03 & 1319.9140 & 486.3495 & 1806.2635 & \\
\hline & $8 / 7 / 200820: 00$ & 391 & 220 & 2000 & 7.006 & 7.006 & & 0.4145 & $4.02 E-03$ & 969.8990 & 357.3792 & 1327.2782 & \\
\hline & 8/7/2008 21:00 & 392 & 220 & 2100 & 8.68 & 8.68 & & 0.1417 & 1.07E-03 & 258.7658 & 95.3476 & 354.1133 & \\
\hline & 8/7/2008 22:00 & 393 & 220 & 2200 & 9.65 & 9.65 & & 0.0269 & 1.39E-04 & 33.5298 & 12.3547 & 45.8845 & \\
\hline & $8 / 7 / 200823: 00$ & 394 & 220 & 2300 & 10.03 & 10.03 & & -0.0120 & $0.00 \mathrm{E}+00$ & 0.0000 & 0.0000 & 0.0000 & \\
\hline & 8/8/2008 0:00 & 395 & 221 & 0 & 10.22 & 10.22 & 10.36 & -0.0303 & $0.00 \mathrm{E}+00$ & 0.0000 & 0.0000 & 0.0000 & \\
\hline & $8 / 8 / 20081: 00$ & 396 & 221 & 100 & 10.26 & 10.26 & & -0.0341 & $0.00 \mathrm{E}+00$ & 0.0000 & 0.0000 & 0.0000 & \\
\hline & 8/8/2008 2:00 & 397 & 221 & 200 & 10.36 & 10.36 & & -0.0434 & $0.00 \mathrm{E}+00$ & 0.0000 & 0.0000 & 0.0000 & \\
\hline & $8 / 8 / 20083: 00$ & 398 & 221 & 300 & 10.36 & 10.36 & & -0.0434 & $0.00 E+00$ & 0.0000 & 0.0000 & 0.0000 & \\
\hline & $8 / 8 / 20084: 00$ & 399 & 221 & 400 & 10.34 & 10.34 & & -0.0416 & $0.00 \mathrm{E}+00$ & 0.0000 & 0.0000 & 0.0000 & \\
\hline & 8/8/2008 5:00 & 400 & 221 & 500 & 10.32 & 10.32 & & -0.0397 & $0.00 \mathrm{E}+00$ & 0.0000 & 0.0000 & 0.0000 & \\
\hline & 8/8/2008 6:00 & 401 & 221 & 600 & 10.29 & 10.29 & & -0.0369 & $0.00 \mathrm{E}+00$ & 0.0000 & 0.0000 & 0.0000 & \\
\hline & 8/8/2008 7:00 & 402 & 221 & 700 & 10.26 & 10.26 & & -0.0341 & $0.00 \mathrm{E}+00$ & 0.0000 & 0.0000 & 0.0000 & \\
\hline & 8/8/2008 8:00 & 403 & 221 & 800 & 10.17 & 10.17 & & -0.0256 & $0.00 \mathrm{E}+00$ & 0.0000 & 0.0000 & 0.0000 & \\
\hline & 8/8/2008 9:00 & 404 & 221 & 900 & 7.927 & 7.927 & & 0.2502 & $2.16 \mathrm{E}-03$ & 520.8980 & 191.9356 & 712.8335 & \\
\hline & 8/8/2008 10:00 & 405 & 221 & 1000 & 6.362 & 6.362 & & 0.5577 & 5.80E-03 & 1397.5180 & 514.9443 & 1912.4623 & \\
\hline & 8/8/2008 11:00 & 406 & 221 & 1100 & 6.377 & 6.377 & & 0.5540 & $5.75 \mathrm{E}-03$ & 1386.2240 & 510.7827 & 1897.0067 & \\
\hline & 8/8/2008 12:00 & 407 & 221 & 1200 & 6.286 & 6.286 & & 0.5765 & 6.04E-03 & 1455.8385 & 536.4336 & 1992.2721 & \\
\hline & 8/8/2008 13:00 & 408 & 221 & 1300 & 6.211 & 6.211 & & 0.5956 & $6.29 \mathrm{E}-03$ & 1515.2398 & 558.3213 & 2073.5610 & \\
\hline & $8 / 8 / 2008$ 14:00 & 409 & 221 & 1400 & 6.231 & 6.231 & & 0.5904 & $6.22 \mathrm{E}-03$ & 1499.2159 & 552.4169 & 2051.6328 & \\
\hline
\end{tabular}




\begin{tabular}{|c|c|c|c|c|c|c|c|c|c|c|c|c|c|}
\hline Tree ID & TS & RN & JD & HR & $\begin{array}{c}\text { Raw } \\
\text { TC_dTA }\end{array}$ & $\begin{array}{c}\text { Fixed } \\
\text { TC_dT } \Delta\end{array}$ & dTM & $\mathbf{K}$ & $\begin{array}{l}\text { Sap } \\
\text { Velocity } \\
(\mathrm{cm} / \mathrm{s})\end{array}$ & $\begin{array}{c}\text { Probed } \\
\text { Drywood F } \\
(\mathrm{g} / \mathrm{hr})\end{array}$ & $\begin{array}{c}\text { Extrapolated } \\
\text { Wetwood F } \\
(\mathrm{g} / \mathrm{hr})\end{array}$ & $\begin{array}{c}\text { Extrapolated } \\
\text { Total Wood F } \\
(\mathrm{g} / \mathrm{hr})\end{array}$ & $\begin{array}{l}\text { Relative } \\
\text { Humidity, } \\
@ 10 \mathrm{~m}(\%)\end{array}$ \\
\hline & $8 / 8 / 2008$ 15:00 & 410 & 221 & 1500 & ) 6.194 & 6.194 & & 0.5999 & $6.34 \mathrm{E}-03$ & 1528.9667 & 563.3792 & 2092.3460 & \\
\hline & $8 / 8 / 2008$ 16:00 & 411 & 221 & 1600 & 6.159 & 6.159 & & 0.6090 & $6.46 \mathrm{E}-03$ & 1557.5406 & 573.9079 & 2131.4484 & \\
\hline & 8/8/2008 17:00 & 412 & 221 & 1700 & 6.278 & 6.278 & & 0.5785 & $6.07 E-03$ & 1462.0859 & 538.7356 & 2000.8215 & \\
\hline & 8/8/2008 18:00 & 413 & 221 & 1800 & 6.464 & 6.464 & & 0.5331 & $5.49 \mathrm{E}-03$ & 1322.0849 & 487.1494 & 1809.2342 & \\
\hline & 8/8/2008 19:00 & 414 & 221 & 1900 & 6.422 & 6.422 & & 0.5431 & 5.61E-03 & 1352.7604 & 498.4524 & 1851.2128 & \\
\hline & $8 / 8 / 200820: 00$ & 415 & 221 & 2000 & 7.121 & 7.121 & & 0.3917 & $3.75 E-03$ & 904.5250 & 333.2908 & 1237.8158 & \\
\hline & $8 / 8 / 200821: 00$ & 416 & 221 & 2100 & 8.6 & 8.6 & & 0.1523 & 1.17E-03 & 282.8426 & 104.2192 & 387.0617 & \\
\hline & $8 / 8 / 200822: 00$ & 417 & 221 & 2200 & 9.45 & 9.45 & & 0.0487 & 2.88E-04 & 69.4464 & 25.5890 & 95.0354 & \\
\hline & 8/8/2008 23:00 & 418 & 221 & 2300 & 9.66 & 9.66 & & 0.0259 & 1.32E-04 & 31.9087 & 11.7574 & 43.6661 & \\
\hline & 8/9/2008 0:00 & 419 & 222 & 0 & 9.67 & 9.67 & 10.20 & 0.0248 & 1.26E-04 & 30.3062 & 11.1669 & 41.4731 & \\
\hline & 8/9/2008 1:00 & 420 & 222 & 100 & 9.67 & 9.67 & & 0.0248 & 1.26E-04 & 30.3062 & 11.1669 & 41.4731 & \\
\hline & $8 / 9 / 2008$ 2:00 & 421 & 222 & 200 & 9.73 & 9.73 & & 0.0185 & 8.76E-05 & 21.1069 & 7.7773 & 28.8842 & \\
\hline & 8/9/2008 3:00 & 422 & 222 & 300 & 9.78 & 9.78 & & 0.0133 & 5.83E-05 & 14.0510 & 5.1774 & 19.2284 & \\
\hline & 8/9/2008 4:00 & 423 & 222 & 400 & 9.78 & 9.78 & & 0.0133 & 5.83E-05 & 14.0510 & 5.1774 & 19.2284 & \\
\hline & $8 / 9 / 20085: 00$ & 424 & 222 & 500 & 9.69 & 9.69 & & 0.0227 & 1.13E-04 & 27.1587 & 10.0072 & 37.1659 & \\
\hline & 8/9/2008 6:00 & 425 & 222 & 600 & 9.7 & 9.7 & & 0.0216 & 1.06E-04 & 25.6146 & 9.4382 & 35.0528 & \\
\hline & 8/9/2008 7:00 & 426 & 222 & 700 & 9.31 & 9.31 & & 0.0644 & 4.07E-04 & 98.1021 & 36.1478 & 134.2499 & \\
\hline & 8/9/2008 8:00 & 427 & 222 & 800 & 8.33 & 8.33 & & 0.1897 & 1.54E-03 & 370.4966 & 136.5171 & 507.0137 & \\
\hline & 8/9/2008 9:00 & 428 & 222 & 900 & 9.07 & 9.07 & & 0.0926 & 6.36E-04 & 153.2938 & 56.4843 & 209.7781 & \\
\hline & 8/9/2008 10:00 & 429 & 222 & 1000 & 8.75 & 8.75 & & 0.1326 & 9.89E-04 & 238.3896 & 87.8395 & 326.2291 & \\
\hline & 8/9/2008 11:00 & 430 & 222 & 1100 & 7.943 & 7.943 & & 0.2476 & $2.13 E-03$ & 514.4505 & 189.5599 & 704.0103 & \\
\hline & 8/9/2008 12:00 & 431 & 222 & 1200 & 7.081 & 7.081 & & 0.3995 & 3.85E-03 & 926.9261 & 341.5450 & 1268.4711 & \\
\hline & 8/9/2008 13:00 & 432 & 222 & 1300 & 6.836 & 6.836 & & 0.4497 & $4.45 E-03$ & 1072.1944 & 395.0721 & 1467.2664 & \\
\hline & $8 / 9 / 2008$ 14:00 & 433 & 222 & 1400 & 6.412 & 6.412 & & 0.5455 & $5.64 \mathrm{E}-03$ & 1360.1430 & 501.1726 & 1861.3156 & \\
\hline & 8/9/2008 15:00 & 434 & 222 & 1500 & 6.332 & 6.332 & & 0.5651 & 5.89E-03 & 1420.3187 & 523.3456 & 1943.6643 & \\
\hline & $8 / 9 / 2008$ 16:00 & 435 & 222 & 1600 & 6.321 & 6.321 & & 0.5678 & 5.93E-03 & 1428.7506 & 526.4525 & 1955.2031 & \\
\hline & 8/9/2008 17:00 & 436 & 222 & 1700 & 6.438 & 6.438 & & 0.5393 & 5.56E-03 & 1341.0117 & 494.1234 & 1835.1351 & \\
\hline & $8 / 9 / 200818: 00$ & 437 & 222 & 1800 & 6.429 & 6.429 & & 0.5415 & 5.59E-03 & 1347.6108 & 496.5549 & 1844.1657 & \\
\hline & 8/9/2008 19:00 & 438 & 222 & 1900 & 6.613 & 6.613 & & 0.4986 & 5.05E-03 & 1217.4335 & 448.5884 & 1666.0219 & \\
\hline & $8 / 9 / 200820: 00$ & 439 & 222 & 2000 & 7.279 & 7.279 & & 0.3615 & $3.40 \mathrm{E}-03$ & 819.4262 & 301.9345 & 1121.3607 & \\
\hline & 8/9/2008 21:00 & 440 & 222 & 2100 & 9.2 & 9.2 & & 0.0772 & 5.08E-04 & 122.4694 & 45.1264 & 167.5958 & \\
\hline & 8/9/2008 22:00 & 441 & 222 & 2200 & 10.08 & 10.08 & & -0.0169 & $0.00 \mathrm{E}+00$ & 0.0000 & 0.0000 & 0.0000 & \\
\hline & 8/9/2008 23:00 & 442 & 222 & 2300 & 10.2 & 10.2 & & -0.0284 & $0.00 \mathrm{E}+00$ & 0.0000 & 0.0000 & 0.0000 & \\
\hline & 8/10/2008 0:00 & 443 & 223 & 0 & 10.24 & 10.24 & 10.28 & -0.0322 & $0.00 \mathrm{E}+00$ & 0.0000 & 0.0000 & 0.0000 & \\
\hline & 8/10/2008 1:00 & 444 & 223 & 100 & 10.28 & 10.28 & & -0.0360 & $0.00 \mathrm{E}+00$ & 0.0000 & 0.0000 & 0.0000 & \\
\hline & 8/10/2008 2:00 & 445 & 223 & 200 & 10.28 & 10.28 & & -0.0360 & $0.00 \mathrm{E}+00$ & 0.0000 & 0.0000 & 0.0000 & \\
\hline & 8/10/2008 3:00 & 446 & 223 & 300 & 10.28 & 10.28 & & -0.0360 & $0.00 E+00$ & 0.0000 & 0.0000 & 0.0000 & \\
\hline & $8 / 10 / 20084: 00$ & 447 & 223 & 400 & 10.26 & 10.26 & & -0.0341 & $0.00 \mathrm{E}+00$ & 0.0000 & 0.0000 & 0.0000 & \\
\hline & 8/10/2008 5:00 & 448 & 223 & 500 & 10.25 & 10.25 & & -0.0332 & $0.00 \mathrm{E}+00$ & 0.0000 & 0.0000 & 0.0000 & \\
\hline & 8/10/2008 6:00 & 449 & 223 & 600 & 10.24 & 10.24 & & -0.0322 & $0.00 E+00$ & 0.0000 & 0.0000 & 0.0000 & \\
\hline & $8 / 10 / 2008$ 7:00 & 450 & 223 & 700 & 10.22 & 10.22 & & -0.0303 & $0.00 \mathrm{E}+00$ & 0.0000 & 0.0000 & 0.0000 & \\
\hline
\end{tabular}




\begin{tabular}{|c|c|c|c|c|c|c|c|c|c|c|c|c|c|}
\hline Tree ID & TS & RN & JD & HR & $\begin{array}{c}\text { Raw } \\
\text { TC_dTA }\end{array}$ & $\begin{array}{c}\text { Fixed } \\
\text { TC_dT } \Delta\end{array}$ & dTM & $\mathbf{K}$ & $\begin{array}{c}\text { Sap } \\
\text { Velocity } \\
(\mathrm{cm} / \mathrm{s})\end{array}$ & $\begin{array}{l}\text { Probed } \\
\text { Drywood F } \\
\text { (g/hr) }\end{array}$ & $\begin{array}{c}\text { Extrapolated } \\
\text { Wetwood F } \\
(\mathrm{g} / \mathrm{hr})\end{array}$ & $\begin{array}{c}\text { Extrapolated } \\
\text { Total Wood F } \\
(\mathrm{g} / \mathrm{hr})\end{array}$ & $\begin{array}{l}\text { Relative } \\
\text { Humidity, } \\
@ 10 \mathrm{~m}(\%)\end{array}$ \\
\hline & 8/10/2008 8:00 & 451 & 223 & 800 & 10.22 & 10.22 & & -0.0303 & $0.00 \mathrm{E}+00$ & 0.0000 & 0.0000 & 0.0000 & \\
\hline & 8/10/2008 9:00 & 452 & 223 & 900 & 9.24 & 9.24 & & 0.0725 & 4.71E-04 & 113.4246 & 41.7936 & 155.2182 & \\
\hline & $8 / 10 / 2008$ 10:00 & 453 & 223 & 1000 & 6.739 & 6.739 & & 0.4705 & 4.70E-03 & 1133.7648 & 417.7590 & 1551.5237 & \\
\hline & 8/10/2008 11:00 & 454 & 223 & 1100 & 6.297 & 6.297 & & 0.5738 & $6.01 \mathrm{E}-03$ & 1447.2824 & 533.2810 & 1980.5634 & \\
\hline & 8/10/2008 12:00 & 455 & 223 & 1200 & 6.411 & 6.411 & & 0.5458 & $5.65 \mathrm{E}-03$ & 1360.8829 & 501.4453 & 1862.3282 & \\
\hline & 8/10/2008 13:00 & 456 & 223 & 1300 & 6.336 & 6.336 & & 0.5641 & $5.88 \mathrm{E}-03$ & 1417.2621 & 522.2194 & 1939.4815 & \\
\hline & 8/10/2008 14:00 & 457 & 223 & 1400 & 6.345 & 6.345 & & 0.5619 & $5.85 \mathrm{E}-03$ & 1410.4034 & 519.6921 & 1930.0955 & \\
\hline & 8/10/2008 15:00 & 458 & 223 & 1500 & 6.492 & 6.492 & & 0.5265 & $5.40 \mathrm{E}-03$ & 1301.9276 & 479.7220 & 1781.6497 & \\
\hline & $8 / 10 / 2008$ 16:00 & 459 & 223 & 1600 & 6.323 & 6.323 & & 0.5673 & 5.92E-03 & 1427.2146 & 525.8866 & 1953.1012 & \\
\hline & $8 / 10 / 2008$ 17:00 & 460 & 223 & 1700 & 6.563 & 6.563 & & 0.5100 & $5.19 \mathrm{E}-03$ & 1251.8420 & 461.2669 & 1713.1089 & \\
\hline & $8 / 10 / 2008$ 18:00 & 461 & 223 & 1800 & 9.12 & 9.12 & & 0.0866 & $5.86 \mathrm{E}-04$ & 141.1811 & 52.0211 & 193.2022 & \\
\hline & $8 / 10 / 2008$ 19:00 & 462 & 223 & 1900 & 9.85 & 9.85 & & 0.0061 & 2.23E-05 & 5.3769 & 1.9812 & 7.3582 & \\
\hline & $8 / 10 / 200820: 00$ & 463 & 223 & 2000 & 9.63 & 9.63 & & 0.0291 & $1.53 \mathrm{E}-04$ & 36.8264 & 13.5694 & 50.3958 & \\
\hline & $8 / 10 / 200821: 00$ & 464 & 223 & 2100 & 9.66 & 9.66 & & 0.0259 & $1.32 \mathrm{E}-04$ & 31.9087 & 11.7574 & 43.6661 & \\
\hline & $8 / 10 / 2008$ 22:00 & 465 & 223 & 2200 & 9.87 & 9.87 & & 0.0041 & 1.35E-05 & 3.2560 & 1.1997 & 4.4557 & \\
\hline & $8 / 10 / 200823: 00$ & 466 & 223 & 2300 & 9.84 & 9.84 & & 0.0071 & 2.70E-05 & 6.5086 & 2.3982 & 8.9069 & \\
\hline & $8 / 11 / 20080: 00$ & 467 & 224 & 0 & 9.87 & 9.87 & 10.33 & 0.0041 & $1.35 \mathrm{E}-05$ & 3.2560 & 1.1997 & 4.4557 & \\
\hline & 8/11/2008 1:00 & 468 & 224 & 100 & 10.07 & 10.07 & & -0.0159 & $0.00 E+00$ & 0.0000 & 0.0000 & 0.0000 & \\
\hline & 8/11/2008 2:00 & 469 & 224 & 200 & 10.26 & 10.26 & & -0.0341 & $0.00 \mathrm{E}+00$ & 0.0000 & 0.0000 & 0.0000 & \\
\hline & 8/11/2008 3:00 & 470 & 224 & 300 & 10.31 & 10.31 & & -0.0388 & $0.00 \mathrm{E}+00$ & 0.0000 & 0.0000 & 0.0000 & \\
\hline & 8/11/2008 4:00 & 471 & 224 & 400 & 10.32 & 10.32 & & -0.0397 & $0.00 \mathrm{E}+00$ & 0.0000 & 0.0000 & 0.0000 & \\
\hline & 8/11/2008 5:00 & 472 & 224 & 500 & 10.28 & 10.28 & & -0.0360 & $0.00 \mathrm{E}+00$ & 0.0000 & 0.0000 & 0.0000 & \\
\hline & 8/11/2008 6:00 & 473 & 224 & 600 & 10.3 & 10.3 & & -0.0379 & $0.00 \mathrm{E}+00$ & 0.0000 & 0.0000 & 0.0000 & \\
\hline & 8/11/2008 7:00 & 474 & 224 & 700 & 10.33 & 10.33 & & -0.0407 & $0.00 E+00$ & 0.0000 & 0.0000 & 0.0000 & \\
\hline & 8/11/2008 8:00 & 475 & 224 & 800 & 10.3 & 10.3 & & -0.0379 & $0.00 \mathrm{E}+00$ & 0.0000 & 0.0000 & 0.0000 & \\
\hline & $8 / 11 / 20089: 00$ & 476 & 224 & 900 & 9.3 & 9.3 & & 0.0656 & 4.16E-04 & 100.2513 & 36.9396 & 137.1909 & \\
\hline & $8 / 11 / 2008$ 10:00 & 477 & 224 & 1000 & 7.015 & 7.015 & & 0.4127 & $4.00 \mathrm{E}-03$ & 964.6743 & 355.4541 & 1320.1284 & \\
\hline & 8/11/2008 11:00 & 478 & 224 & 1100 & 6.478 & 6.478 & & 0.5298 & 5.44E-03 & 1311.9772 & 483.4250 & 1795.4022 & \\
\hline & $8 / 11 / 2008$ 12:00 & 479 & 224 & 1200 & 6.572 & 6.572 & & 0.5079 & $5.17 \mathrm{E}-03$ & 1245.5965 & 458.9656 & 1704.5621 & \\
\hline & 8/11/2008 13:00 & 480 & 224 & 1300 & 6.755 & 6.755 & & 0.4671 & $4.66 \mathrm{E}-03$ & 1123.4424 & 413.9554 & 1537.3978 & \\
\hline & $8 / 11 / 2008$ 14:00 & 481 & 224 & 1400 & 6.485 & 6.485 & & 0.5281 & 5.42E-03 & 1306.9452 & 481.5708 & 1788.5160 & \\
\hline & $8 / 11 / 200815: 00$ & 482 & 224 & 1500 & 6.435 & 6.435 & & 0.5400 & $5.57 \mathrm{E}-03$ & 1343.2087 & 494.9329 & 1838.1416 & \\
\hline & $8 / 11 / 200816: 00$ & 483 & 224 & 1600 & 6.593 & 6.593 & & 0.5031 & $5.11 \mathrm{E}-03$ & 1231.1127 & 453.6288 & 1684.7415 & \\
\hline & $8 / 11 / 2008$ 17:00 & 484 & 224 & 1700 & 6.663 & 6.663 & & 0.4873 & 4.91E-03 & 1183.7188 & 436.1655 & 1619.8844 & \\
\hline & 8/11/2008 18:00 & 485 & 224 & 1800 & 6.931 & 6.931 & & 0.4298 & 4.21E-03 & 1014.1739 & 373.6932 & 1387.8671 & \\
\hline & $8 / 11 / 2008$ 19:00 & 486 & 224 & 1900 & 7.087 & 7.087 & & 0.3983 & $3.83 E-03$ & 923.5432 & 340.2985 & 1263.8417 & \\
\hline & $8 / 11 / 2008$ 20:00 & 487 & 224 & 2000 & 7.888 & 7.888 & & 0.2563 & 2.23E-03 & 536.7867 & 197.7901 & 734.5768 & \\
\hline & 8/11/2008 21:00 & 488 & 224 & 2100 & 8.99 & 8.99 & & 0.1023 & 7.19E-04 & 173.3389 & 63.8703 & 237.2092 & \\
\hline & $8 / 11 / 200822: 00$ & 489 & 224 & 2200 & 9.6 & 9.6 & & 0.0323 & 1.74E-04 & 41.9027 & 15.4399 & 57.3426 & \\
\hline & 8/11/2008 23:00 & 490 & 224 & 2300 & 9.87 & 9.87 & & 0.0041 & 1.35E-05 & 3.2560 & 1.1997 & 4.4557 & \\
\hline & 8/12/2008 0:00 & 491 & 225 & 0 & 10.03 & 10.03 & 10.14 & -0.0120 & $0.00 E+00$ & 0.0000 & 0.0000 & 0.0000 & \\
\hline
\end{tabular}




\begin{tabular}{|c|c|c|c|c|c|c|c|c|c|c|c|c|c|}
\hline Tree ID & TS & RN & JD & HR & $\begin{array}{c}\text { Raw } \\
\text { TC_dTA }\end{array}$ & $\begin{array}{c}\text { Fixed } \\
\text { TC_dT } \Delta\end{array}$ & dTM & $\mathrm{K}$ & $\begin{array}{l}\text { Sap } \\
\text { Velocity } \\
\text { (cm/s) }\end{array}$ & $\begin{array}{c}\text { Probed } \\
\text { Drywood F } \\
\text { (g/hr) }\end{array}$ & $\begin{array}{c}\text { Extrapolated } \\
\text { Wetwood F } \\
(\mathrm{g} / \mathrm{hr})\end{array}$ & $\begin{array}{c}\text { Extrapolated } \\
\text { Total Wood F } \\
(\mathrm{g} / \mathrm{hr})\end{array}$ & $\begin{array}{c}\text { Relative } \\
\text { Humidity, } \\
@ 10 \mathrm{~m}(\%)\end{array}$ \\
\hline & 8/12/2008 1:00 & 492 & 225 & 100 & $\begin{array}{r}9.97 \\
\end{array}$ & 9.97 & & -0.0060 & $0.00 \mathrm{E}+00$ & 0.0000 & 0.0000 & 0.0000 & \\
\hline & 8/12/2008 2:00 & 493 & 225 & 200 & 9.21 & 9.21 & & 0.0760 & 4.99E-04 & 120.1887 & 44.2860 & 164.4746 & \\
\hline & $8 / 12 / 20083: 00$ & 494 & 225 & 300 & 9.64 & 9.64 & & 0.0280 & $1.46 \mathrm{E}-04$ & 35.1691 & 12.9588 & 48.1279 & \\
\hline & 8/12/2008 4:00 & 495 & 225 & 400 & 9.92 & 9.92 & & -0.0010 & $0.00 \mathrm{E}+00$ & 0.0000 & 0.0000 & 0.0000 & \\
\hline & $8 / 12 / 20085: 00$ & 496 & 225 & 500 & 10 & 10 & & -0.0090 & $0.00 \mathrm{E}+00$ & 0.0000 & 0.0000 & 0.0000 & \\
\hline & $8 / 12 / 20086: 00$ & 497 & 225 & 600 & 10.06 & 10.06 & & -0.0149 & $0.00 \mathrm{E}+00$ & 0.0000 & 0.0000 & 0.0000 & \\
\hline & 8/12/2008 7:00 & 498 & 225 & 700 & 10.1 & 10.1 & & -0.0188 & $0.00 \mathrm{E}+00$ & 0.0000 & 0.0000 & 0.0000 & \\
\hline & 8/12/2008 8:00 & 499 & 225 & 800 & 10.07 & 10.07 & & -0.0159 & $0.00 \mathrm{E}+00$ & 0.0000 & 0.0000 & 0.0000 & \\
\hline & 8/12/2008 9:00 & 500 & 225 & 900 & 9.25 & 9.25 & & 0.0714 & 4.61E-04 & 111.1961 & 40.9725 & 152.1686 & \\
\hline & $8 / 12 / 200810: 00$ & 501 & 225 & 1000 & 7.097 & 7.097 & & 0.3964 & 3.81E-03 & 917.9230 & 338.2276 & 1256.1506 & \\
\hline & 8/12/2008 11:00 & 502 & 225 & 1100 & 6.467 & 6.467 & & 0.5324 & $5.48 \mathrm{E}-03$ & 1319.9140 & 486.3495 & 1806.2635 & \\
\hline & 8/12/2008 12:00 & 503 & 225 & 1200 & 6.471 & 6.471 & & 0.5314 & $5.46 \mathrm{E}-03$ & 1317.0238 & 485.2845 & 1802.3082 & \\
\hline & $8 / 12 / 200813: 00$ & 504 & 225 & 1300 & 6.38 & 6.38 & & 0.5533 & $5.74 \mathrm{E}-03$ & 1383.9736 & 509.9535 & 1893.9271 & \\
\hline & $8 / 12 / 2008$ 14:00 & 505 & 225 & 1400 & 6.401 & 6.401 & & 0.5482 & $5.68 \mathrm{E}-03$ & 1368.2991 & 504.1779 & 1872.4771 & \\
\hline & $8 / 12 / 200815: 00$ & 506 & 225 & 1500 & 6.382 & 6.382 & & 0.5528 & $5.74 \mathrm{E}-03$ & 1382.4749 & 509.4013 & 1891.8762 & \\
\hline & $8 / 12 / 200816: 00$ & 507 & 225 & 1600 & 6.368 & 6.368 & & 0.5562 & $5.78 \mathrm{E}-03$ & 1392.9919 & 513.2765 & 1906.2685 & \\
\hline & $8 / 12 / 200817: 00$ & 508 & 225 & 1700 & 6.436 & 6.436 & & 0.5398 & 5.57E-03 & 1342.4761 & 494.6629 & 1837.1390 & \\
\hline & 8/12/2008 18:00 & 509 & 225 & 1800 & 6.493 & 6.493 & & 0.5263 & 5.40E-03 & 1301.2120 & 479.4583 & 1780.6704 & \\
\hline & $8 / 12 / 2008$ 19:00 & 510 & 225 & 1900 & 6.695 & 6.695 & & 0.4802 & $4.82 \mathrm{E}-03$ & 1162.4980 & 428.3463 & 1590.8443 & \\
\hline & $8 / 12 / 200820: 00$ & 511 & 225 & 2000 & 7.323 & 7.323 & & 0.3533 & $3.31 \mathrm{E}-03$ & 796.6574 & 293.5448 & 1090.2022 & \\
\hline & $8 / 12 / 200821: 00$ & 512 & 225 & 2100 & 9.16 & 9.16 & & 0.0819 & 5.47E-04 & 131.7220 & 48.5357 & 180.2577 & \\
\hline & $8 / 12 / 200822: 00$ & 513 & 225 & 2200 & 9.97 & 9.97 & & -0.0060 & $0.00 \mathrm{E}+00$ & 0.0000 & 0.0000 & 0.0000 & \\
\hline & 8/12/2008 23:00 & 514 & 225 & 2300 & 10.14 & 10.14 & & -0.0227 & $0.00 \mathrm{E}+00$ & 0.0000 & 0.0000 & 0.0000 & \\
\hline & 8/13/2008 0:00 & 515 & 226 & 0 & 10.19 & 10.19 & 10.30 & -0.0275 & $0.00 \mathrm{E}+00$ & 0.0000 & 0.0000 & 0.0000 & \\
\hline & 8/13/2008 1:00 & 516 & 226 & 100 & 10.2 & 10.2 & & -0.0284 & $0.00 \mathrm{E}+00$ & 0.0000 & 0.0000 & 0.0000 & \\
\hline & $8 / 13 / 20082: 00$ & 517 & 226 & 200 & 10.22 & 10.22 & & -0.0303 & $0.00 \mathrm{E}+00$ & 0.0000 & 0.0000 & 0.0000 & \\
\hline & 8/13/2008 3:00 & 518 & 226 & 300 & 10.27 & 10.27 & & -0.0351 & $0.00 \mathrm{E}+00$ & 0.0000 & 0.0000 & 0.0000 & \\
\hline & $8 / 13 / 20084: 00$ & 519 & 226 & 400 & 10.27 & 10.27 & & -0.0351 & $0.00 \mathrm{E}+00$ & 0.0000 & 0.0000 & 0.0000 & \\
\hline & 8/13/2008 5:00 & 520 & 226 & 500 & 10.29 & 10.29 & & -0.0369 & $0.00 \mathrm{E}+00$ & 0.0000 & 0.0000 & 0.0000 & \\
\hline & 8/13/2008 6:00 & 521 & 226 & 600 & 10.3 & 10.3 & & -0.0379 & $0.00 \mathrm{E}+00$ & 0.0000 & 0.0000 & 0.0000 & \\
\hline & $8 / 13 / 20087: 00$ & 522 & 226 & 700 & 10.28 & 10.28 & & -0.0360 & $0.00 \mathrm{E}+00$ & 0.0000 & 0.0000 & 0.0000 & \\
\hline & 8/13/2008 8:00 & 523 & 226 & 800 & 10.13 & 10.13 & & -0.0217 & $0.00 \mathrm{E}+00$ & 0.0000 & 0.0000 & 0.0000 & \\
\hline & 8/13/2008 9:00 & 524 & 226 & 900 & 8.27 & 8.27 & & 0.1983 & $1.62 \mathrm{E}-03$ & 391.3586 & 144.2041 & 535.5627 & \\
\hline & $8 / 13 / 2008$ 10:00 & 525 & 226 & 1000 & 6.759 & 6.759 & & 0.4662 & 4.65E-03 & 1120.8722 & 413.0084 & 1533.8806 & \\
\hline & 8/13/2008 11:00 & 526 & 226 & 1100 & 6.688 & 6.688 & & 0.4818 & 4.84E-03 & 1167.1165 & 430.0481 & 1597.1646 & \\
\hline & $8 / 13 / 2008$ 12:00 & 527 & 226 & 1200 & 6.42 & 6.42 & & 0.5436 & $5.62 \mathrm{E}-03$ & 1354.2345 & 498.9956 & 1853.2301 & \\
\hline
\end{tabular}




\begin{tabular}{|c|c|c|c|c|c|c|c|c|c|c|c|c|c|}
\hline Tree ID & TS & RN & JD & HR & $\begin{array}{c}\text { Raw } \\
\text { TC_dTA }\end{array}$ & $\begin{array}{c}\text { Fixed } \\
\text { TC_dTA }\end{array}$ & dTM & $\mathbf{K}$ & $\begin{array}{l}\text { Sap } \\
\text { Velocity } \\
(\mathrm{cm} / \mathrm{s})\end{array}$ & $\begin{array}{c}\text { Probed } \\
\text { Drywood F } \\
(\mathrm{g} / \mathrm{hr})\end{array}$ & $\begin{array}{c}\text { Extrapolated } \\
\text { Wetwood F } \\
(\mathrm{g} / \mathrm{hr})\end{array}$ & $\begin{array}{c}\text { Extrapolated } \\
\text { Total Wood F } \\
(\mathrm{g} / \mathrm{hr})\end{array}$ & $\begin{array}{l}\text { Relative } \\
\text { Humidity, } \\
@ 10 \mathrm{~m}(\%)\end{array}$ \\
\hline & $7 / 22 / 200813: 00$ & 0 & 204 & 1300 & ) $\quad 5.165$ & 5.165 & 9.31 & 0.8025 & $9.08 \mathrm{E}-03$ & 1913.0652 & 704.9082 & 2617.9734 & \\
\hline \multirow[t]{2}{*}{ W607EC1 } & $7 / 22 / 200814: 00$ & 1 & 204 & 1400 & 5.631 & 5.631 & & 0.6533 & 7.05E-03 & 1485.2146 & 547.2579 & 2032.4725 & \\
\hline & $7 / 22 / 2008$ 15:00 & 2 & 204 & 1500 & 5.539 & 5.539 & & 0.6808 & 7.41E-03 & 1562.4302 & 575.7095 & 2138.1397 & \\
\hline \multirow[t]{2}{*}{ Probe \# } & $7 / 22 / 200816: 00$ & 3 & 204 & 1600 & 5.524 & 5.524 & & 0.6854 & 7.47E-03 & 1575.3342 & 580.4643 & 2155.7984 & \\
\hline & $7 / 22 / 200817: 00$ & 4 & 204 & 1700 & 5.694 & 5.694 & & 0.6351 & 6.80E-03 & 1434.1908 & 528.4571 & 1962.6478 & \\
\hline \multirow[t]{4}{*}{7} & $7 / 22 / 2008$ 18:00 & 5 & 204 & 1800 & 5.852 & 5.852 & & 0.5909 & $6.23 \mathrm{E}-03$ & 1312.4676 & 483.6057 & 1796.0732 & \\
\hline & $7 / 22 / 2008$ 19:00 & 6 & 204 & 1900 & 5.828 & 5.828 & & 0.5975 & $6.31 \mathrm{E}-03$ & 1330.4032 & 490.2144 & 1820.6176 & \\
\hline & $7 / 22 / 200820: 00$ & 7 & 204 & 2000 & 6.301 & 6.301 & & 0.4775 & 4.79E-03 & 1009.7440 & 372.0609 & 1381.8049 & \\
\hline & 7/22/2008 21:00 & 8 & 204 & 2100 & 7.784 & 7.784 & & 0.1960 & $1.60 \mathrm{E}-03$ & 337.4672 & 124.3467 & 461.8139 & \\
\hline \multirow[t]{2}{*}{$\mathrm{DBH}(\mathrm{cm})$} & $7 / 22 / 200822: 00$ & 9 & 204 & 2200 & 9 & 9 & & 0.0344 & $1.88 \mathrm{E}-04$ & 39.6771 & 14.6199 & 54.2970 & \\
\hline & 7/22/2008 23:00 & 10 & 204 & 2300 & 9.31 & 9.31 & & 0.0000 & $0.00 \mathrm{E}+00$ & 0.0000 & 0.0000 & 0.0000 & \\
\hline \multirow[t]{2}{*}{10.7} & $7 / 23 / 20080: 00$ & 11 & 205 & 0 & 9.53 & 9.53 & 9.70 & -0.0231 & $0.00 \mathrm{E}+00$ & 0.0000 & 0.0000 & 0.0000 & \\
\hline & 7/23/2008 1:00 & 12 & 205 & 100 & 9.61 & 9.61 & & -0.0312 & $0.00 \mathrm{E}+00$ & 0.0000 & 0.0000 & 0.0000 & \\
\hline Day Count & 7/23/2008 2:00 & 13 & 205 & 200 & 9.64 & 9.64 & & -0.0342 & $0.00 \mathrm{E}+00$ & 0.0000 & 0.0000 & 0.0000 & \\
\hline \multirow[t]{27}{*}{35} & 7/23/2008 3:00 & 14 & 205 & 300 & 9.69 & 9.69 & & -0.0392 & $0.00 \mathrm{E}+00$ & 0.0000 & 0.0000 & 0.0000 & \\
\hline & 7/23/2008 4:00 & 15 & 205 & 400 & 9.7 & 9.7 & & -0.0402 & $0.00 \mathrm{E}+00$ & 0.0000 & 0.0000 & 0.0000 & \\
\hline & 7/23/2008 5:00 & 16 & 205 & 500 & 9.69 & 9.69 & & -0.0392 & $0.00 \mathrm{E}+00$ & 0.0000 & 0.0000 & 0.0000 & \\
\hline & 7/23/2008 6:00 & 17 & 205 & 600 & 9.62 & 9.62 & & -0.0322 & $0.00 \mathrm{E}+00$ & 0.0000 & 0.0000 & 0.0000 & \\
\hline & 7/23/2008 7:00 & 18 & 205 & 700 & 9.42 & 9.42 & & -0.0117 & $0.00 E+00$ & 0.0000 & 0.0000 & 0.0000 & \\
\hline & 7/23/2008 8:00 & 19 & 205 & 800 & 8.59 & 8.59 & & 0.0838 & 5.63E-04 & 118.5707 & 43.6898 & 162.2605 & \\
\hline & $7 / 23 / 20089: 00$ & 20 & 205 & 900 & 7.068 & 7.068 & & 0.3172 & 2.90E-03 & 610.2321 & 224.8526 & 835.0846 & \\
\hline & $7 / 23 / 200810: 00$ & 21 & 205 & 1000 & 5.94 & 5.94 & & 0.5673 & 5.92E-03 & 1248.3257 & 459.9713 & 1708.2970 & \\
\hline & $7 / 23 / 2008$ 11:00 & 22 & 205 & 1100 & 5.9 & 5.9 & & 0.5780 & 6.06E-03 & 1277.1692 & 470.5993 & 1747.7685 & \\
\hline & $7 / 23 / 2008$ 12:00 & 23 & 205 & 1200 & 5.837 & 5.837 & & 0.5950 & $6.28 \mathrm{E}-03$ & 1323.6547 & 487.7278 & 1811.3825 & \\
\hline & $7 / 23 / 2008$ 13:00 & 24 & 205 & 1300 & 5.765 & 5.765 & & 0.6149 & $6.54 \mathrm{E}-03$ & 1378.4159 & 507.9057 & 1886.3216 & \\
\hline & $7 / 23 / 200814: 00$ & 25 & 205 & 1400 & 5.731 & 5.731 & & 0.6245 & 6.67E-03 & 1404.9008 & 517.6646 & 1922.5653 & \\
\hline & $7 / 23 / 200815: 00$ & 26 & 205 & 1500 & 5.643 & 5.643 & & 0.6498 & 7.00E-03 & 1475.3820 & 543.6349 & 2019.0169 & \\
\hline & 7/23/2008 16:00 & 27 & 205 & 1600 & 5.83 & 5.83 & & 0.5969 & $6.31 \mathrm{E}-03$ & 1328.9012 & 489.6610 & 1818.5622 & \\
\hline & $7 / 23 / 200817: 00$ & 28 & 205 & 1700 & 6.292 & 6.292 & & 0.4797 & 4.82E-03 & 1015.2479 & 374.0890 & 1389.3369 & \\
\hline & $7 / 23 / 2008$ 18:00 & 29 & 205 & 1800 & 7.057 & 7.057 & & 0.3193 & 2.92E-03 & 615.0980 & 226.6455 & 841.7435 & \\
\hline & $7 / 23 / 2008$ 19:00 & 30 & 205 & 1900 & 7.014 & 7.014 & & 0.3273 & 3.01E-03 & 634.3357 & 233.7340 & 868.0698 & \\
\hline & $7 / 23 / 200820: 00$ & 31 & 205 & 2000 & 7.387 & 7.387 & & 0.2603 & $2.27 \mathrm{E}-03$ & 478.4548 & 176.2965 & 654.7513 & \\
\hline & $7 / 23 / 200821: 00$ & 32 & 205 & 2100 & 8.28 & 8.28 & & 0.1244 & 9.15E-04 & 192.7764 & 71.0324 & 263.8089 & \\
\hline & $7 / 23 / 2008$ 22:00 & 33 & 205 & 2200 & 8.8 & 8.8 & & 0.0580 & 3.57E-04 & 75.2848 & 27.7402 & 103.0250 & \\
\hline & $7 / 23 / 2008$ 23:00 & 34 & 205 & 2300 & 8.93 & 8.93 & & 0.0426 & 2.44E-04 & 51.4709 & 18.9655 & 70.4365 & \\
\hline & $7 / 24 / 20080: 00$ & 35 & 206 & 0 & 8.96 & 8.96 & 9.31 & 0.0391 & $2.20 \mathrm{E}-04$ & 46.3237 & 17.0689 & 63.3926 & \\
\hline & 7/24/2008 1:00 & 36 & 206 & 100 & 9.03 & 9.03 & & 0.0310 & $1.65 \mathrm{E}-04$ & 34.8615 & 12.8455 & 47.7070 & \\
\hline & 7/24/2008 2:00 & 37 & 206 & 200 & 9.09 & 9.09 & & 0.0242 & 1.22E-04 & 25.6967 & 9.4685 & 35.1651 & \\
\hline & 7/24/2008 3:00 & 38 & 206 & 300 & 9.16 & 9.16 & & 0.0164 & $7.54 \mathrm{E}-05$ & 15.8863 & 5.8536 & 21.7399 & \\
\hline & 7/24/2008 4:00 & 39 & 206 & 400 & 9.2 & 9.2 & & 0.0120 & $5.12 E-05$ & 10.7864 & 3.9745 & 14.7609 & \\
\hline & 7/24/2008 5:00 & 40 & 206 & 500 & 9.25 & 9.25 & & 0.0065 & $2.41 E-05$ & 5.0808 & 1.8721 & 6.9529 & \\
\hline
\end{tabular}




\begin{tabular}{|c|c|c|c|c|c|c|c|c|c|c|c|c|c|}
\hline Tree ID & TS & RN & JD & HR & $\begin{array}{c}\text { Raw } \\
\text { TC_dT } \Delta\end{array}$ & $\begin{array}{c}\text { Fixed } \\
\text { TC_dT } \Delta\end{array}$ & dTM & $\mathbf{K}$ & $\begin{array}{l}\text { Sap } \\
\text { Velocity } \\
(\mathbf{c m} / \mathbf{s})\end{array}$ & $\begin{array}{l}\text { Probed } \\
\text { Drywood F } \\
\text { (g/hr) }\end{array}$ & $\begin{array}{c}\text { Extrapolated } \\
\text { Wetwood F } \\
(\mathrm{g} / \mathrm{hr})\end{array}$ & $\begin{array}{c}\text { Extrapolated } \\
\text { Total Wood F } \\
(\mathrm{g} / \mathrm{hr})\end{array}$ & $\begin{array}{l}\text { Relative } \\
\text { Humidity, } \\
@ 10 m(\%)\end{array}$ \\
\hline & 7/24/2008 6:00 & 41 & 206 & 600 & 9.27 & 9.27 & & 0.0043 & 1.46E-05 & 3.0761 & 1.1335 & 4.2096 & \\
\hline & 7/24/2008 7:00 & 42 & 206 & 700 & 9.31 & 9.31 & & 0.0000 & $0.00 \mathrm{E}+00$ & 0.0000 & 0.0000 & 0.0000 & \\
\hline & 7/24/2008 8:00 & 43 & 206 & 800 & 9.21 & 9.21 & & 0.0109 & $4.55 E-05$ & 9.5795 & 3.5298 & 13.1093 & \\
\hline & 7/24/2008 9:00 & 44 & 206 & 900 & 8.74 & 8.74 & & 0.0652 & 4.13E-04 & 87.0619 & 32.0797 & 119.1416 & \\
\hline & $7 / 24 / 2008$ 10:00 & 45 & 206 & 1000 & 8.66 & 8.66 & & 0.0751 & 4.91E-04 & 103.5043 & 38.1383 & 141.6426 & \\
\hline & $7 / 24 / 2008$ 11:00 & 46 & 206 & 1100 & 6.744 & 6.744 & & 0.3805 & $3.62 E-03$ & 763.3858 & 281.2852 & 1044.6709 & \\
\hline & 7/24/2008 12:00 & 47 & 206 & 1200 & 5.704 & 5.704 & & 0.6322 & $6.77 \mathrm{E}-03$ & 1426.2259 & 525.5223 & 1951.7481 & \\
\hline & $7 / 24 / 2008$ 13:00 & 48 & 206 & 1300 & 6.187 & 6.187 & & 0.5048 & 5.13E-03 & 1081.0673 & 398.3415 & 1479.4087 & \\
\hline & $7 / 24 / 200814: 00$ & 49 & 206 & 1400 & 6.078 & 6.078 & & 0.5318 & $5.47 \mathrm{E}-03$ & 1152.6473 & 424.7166 & 1577.3639 & \\
\hline & $7 / 24 / 200815: 00$ & 50 & 206 & 1500 & 5.656 & 5.656 & & 0.6460 & $6.95 \mathrm{E}-03$ & 1464.7909 & 539.7323 & 2004.5232 & \\
\hline & 7/24/2008 16:00 & 51 & 206 & 1600 & 5.609 & 5.609 & & 0.6598 & 7.13E-03 & 1503.3823 & 553.9521 & 2057.3345 & \\
\hline & 7/24/2008 17:00 & 52 & 206 & 1700 & 5.816 & 5.816 & & 0.6008 & $6.36 \mathrm{E}-03$ & 1339.4438 & 493.5456 & 1832.9894 & \\
\hline & $7 / 24 / 200818: 00$ & 53 & 206 & 1800 & 5.781 & 5.781 & & 0.6104 & $6.48 \mathrm{E}-03$ & 1366.0927 & 503.3649 & 1869.4576 & \\
\hline & $7 / 24 / 200819: 00$ & 54 & 206 & 1900 & 6.094 & 6.094 & & 0.5277 & 5.42E-03 & 1141.9255 & 420.7659 & 1562.6914 & \\
\hline & $7 / 24 / 200820: 00$ & 55 & 206 & 2000 & 7.061 & 7.061 & & 0.3185 & 2.91E-03 & 613.3260 & 225.9926 & 839.3185 & \\
\hline & $7 / 24 / 200821: 00$ & 56 & 206 & 2100 & 8.33 & 8.33 & & 0.1176 & 8.54E-04 & 179.9832 & 66.3185 & 246.3017 & \\
\hline & $7 / 24 / 200822: 00$ & 57 & 206 & 2200 & 9.09 & 9.09 & & 0.0242 & $1.22 \mathrm{E}-04$ & 25.6967 & 9.4685 & 35.1651 & \\
\hline & $7 / 24 / 2008$ 23:00 & 58 & 206 & 2300 & 9.25 & 9.25 & & 0.0065 & $2.41 E-05$ & 5.0808 & 1.8721 & 6.9529 & \\
\hline & $7 / 25 / 20080: 00$ & 59 & 207 & 0 & 9.28 & 9.28 & 9.28 & 0.0032 & 1.02E-05 & 2.1559 & 0.7944 & 2.9503 & \\
\hline & 7/25/2008 1:00 & 60 & 207 & 100 & 9.21 & 9.21 & & 0.0109 & 4.55E-05 & 9.5795 & 3.5298 & 13.1093 & \\
\hline & 7/25/2008 2:00 & 61 & 207 & 200 & 9.1 & 9.1 & & 0.0231 & 1.15E-04 & 24.2336 & 8.9294 & 33.1630 & \\
\hline & 7/25/2008 3:00 & 62 & 207 & 300 & 9.04 & 9.04 & & 0.0299 & $1.58 \mathrm{E}-04$ & 33.2899 & 12.2663 & 45.5562 & \\
\hline & 7/25/2008 4:00 & 63 & 207 & 400 & 9.03 & 9.03 & & 0.0310 & 1.65E-04 & 34.8615 & 12.8455 & 47.7070 & \\
\hline & 7/25/2008 5:00 & 64 & 207 & 500 & 9.06 & 9.06 & & 0.0276 & 1.43E-04 & 30.1985 & 11.1273 & 41.3258 & \\
\hline & 7/25/2008 6:00 & 65 & 207 & 600 & 9.11 & 9.11 & & 0.0220 & 1.08E-04 & 22.7902 & 8.3975 & 31.1877 & \\
\hline & 7/25/2008 7:00 & 66 & 207 & 700 & 9.12 & 9.12 & & 0.0208 & 1.01E-04 & 21.3668 & 7.8730 & 29.2398 & \\
\hline & 7/25/2008 8:00 & 67 & 207 & 800 & 9.09 & 9.09 & & 0.0242 & $1.22 \mathrm{E}-04$ & 25.6967 & 9.4685 & 35.1651 & \\
\hline & 7/25/2008 9:00 & 68 & 207 & 900 & 8.91 & 8.91 & & 0.0449 & $2.61 \mathrm{E}-04$ & 54.9773 & 20.2575 & 75.2348 & \\
\hline & $7 / 25 / 200810: 00$ & 69 & 207 & 1000 & 8.89 & 8.89 & & 0.0472 & $2.78 \mathrm{E}-04$ & 58.5421 & 21.5710 & 80.1132 & \\
\hline & 7/25/2008 11:00 & 70 & 207 & 1100 & 8.37 & 8.37 & & 0.1123 & 8.06E-04 & 169.9777 & 62.6318 & 232.6095 & \\
\hline & $7 / 25 / 2008$ 12:00 & 71 & 207 & 1200 & 6.828 & 6.828 & & 0.3635 & $3.42 \mathrm{E}-03$ & 721.6595 & 265.9103 & 987.5697 & \\
\hline & $7 / 25 / 2008$ 13:00 & 72 & 207 & 1300 & 6.134 & 6.134 & & 0.5178 & 5.29E-03 & 1115.4470 & 411.0094 & 1526.4564 & \\
\hline & $7 / 25 / 200814: 00$ & 73 & 207 & 1400 & 5.695 & 5.695 & & 0.6348 & $6.80 \mathrm{E}-03$ & 1433.3926 & 528.1630 & 1961.5556 & \\
\hline & $7 / 25 / 2008$ 15:00 & 74 & 207 & 1500 & 5.714 & 5.714 & & 0.6293 & $6.73 E-03$ & 1418.2971 & 522.6008 & 1940.8979 & \\
\hline & $7 / 25 / 200816: 00$ & 75 & 207 & 1600 & 5.786 & 5.786 & & 0.6091 & 6.46E-03 & 1362.2599 & 501.9527 & 1864.2126 & \\
\hline & $7 / 25 / 200817: 00$ & 76 & 207 & 1700 & 5.625 & 5.625 & & 0.6551 & 7.07E-03 & 1490.1513 & 549.0769 & 2039.2281 & \\
\hline & $7 / 25 / 200818: 00$ & 77 & 207 & 1800 & 5.781 & 5.781 & & 0.6104 & $6.48 \mathrm{E}-03$ & 1366.0927 & 503.3649 & 1869.4576 & \\
\hline & $7 / 25 / 2008$ 19:00 & 78 & 207 & 1900 & 5.708 & 5.708 & & 0.6310 & 6.75E-03 & 1423.0501 & 524.3521 & 1947.4021 & \\
\hline & $7 / 25 / 200820: 00$ & 79 & 207 & 2000 & 6.511 & 6.511 & & 0.4299 & $4.21 E-03$ & 887.1701 & 326.8961 & 1214.0661 & \\
\hline & $7 / 25 / 200821: 00$ & 80 & 207 & 2100 & 7.928 & 7.928 & & 0.1743 & 1.39E-03 & 292.0395 & 107.6080 & 399.6475 & \\
\hline & 7/25/2008 22:00 & 81 & 207 & 2200 & 8.94 & 8.94 & & 0.0414 & $2.36 \mathrm{E}-04$ & 49.7401 & 18.3278 & 68.0678 & \\
\hline
\end{tabular}




\begin{tabular}{|c|c|c|c|c|c|c|c|c|c|c|c|c|c|}
\hline Tree ID & TS & RN & JD & HR & $\begin{array}{c}\text { Raw } \\
\text { TC_dTA }\end{array}$ & $\begin{array}{c}\text { Fixed } \\
\text { TC_dT } \Delta\end{array}$ & dTM & $\mathbf{K}$ & $\begin{array}{l}\text { Sap } \\
\text { Velocity } \\
(\mathrm{cm} / \mathrm{s})\end{array}$ & $\begin{array}{c}\text { Probed } \\
\text { Drywood F } \\
(\mathrm{g} / \mathrm{hr})\end{array}$ & $\begin{array}{c}\text { Extrapolated } \\
\text { Wetwood F } \\
(\mathrm{g} / \mathrm{hr})\end{array}$ & $\begin{array}{c}\text { Extrapolated } \\
\text { Total Wood F } \\
(\mathrm{g} / \mathrm{hr})\end{array}$ & $\begin{array}{l}\text { Relative } \\
\text { Humidity, } \\
@ 10 \mathrm{~m}(\%)\end{array}$ \\
\hline & $7 / 25 / 200823: 00$ & 82 & 207 & 2300 & 9.15 & 9.15 & & 0.0175 & 8.17E-05 & 17.2230 & 6.3462 & 23.5692 & \\
\hline & 7/26/2008 0:00 & 83 & 208 & 0 & 9.2 & 9.2 & 9.20 & 0.0120 & $5.12 \mathrm{E}-05$ & 10.7864 & 3.9745 & 14.7609 & \\
\hline & 7/26/2008 1:00 & 84 & 208 & 100 & 9.2 & 9.2 & & 0.0120 & $5.12 E-05$ & 10.7864 & 3.9745 & 14.7609 & \\
\hline & 7/26/2008 2:00 & 85 & 208 & 200 & 9.18 & 9.18 & & 0.0142 & $6.30 \mathrm{E}-05$ & 13.2847 & 4.8950 & 18.1797 & \\
\hline & 7/26/2008 3:00 & 86 & 208 & 300 & 9.16 & 9.16 & & 0.0164 & 7.54E-05 & 15.8863 & 5.8536 & 21.7399 & \\
\hline & 7/26/2008 4:00 & 87 & 208 & 400 & 9.13 & 9.13 & & 0.0197 & 9.47E-05 & 19.9640 & 7.3561 & 27.3201 & \\
\hline & 7/26/2008 5:00 & 88 & 208 & 500 & 9.1 & 9.1 & & 0.0231 & 1.15E-04 & 24.2336 & 8.9294 & 33.1630 & \\
\hline & 7/26/2008 6:00 & 89 & 208 & 600 & 9.06 & 9.06 & & 0.0276 & 1.43E-04 & 30.1985 & 11.1273 & 41.3258 & \\
\hline & 7/26/2008 7:00 & 90 & 208 & 700 & 9.02 & 9.02 & & 0.0322 & 1.73E-04 & 36.4501 & 13.4308 & 49.8810 & \\
\hline & 7/26/2008 8:00 & 91 & 208 & 800 & 8.98 & 8.98 & & 0.0367 & 2.04E-04 & 42.9689 & 15.8328 & 58.8017 & \\
\hline & 7/26/2008 9:00 & 92 & 208 & 900 & 8.09 & 8.09 & & 0.1508 & 1.16E-03 & 244.3267 & 90.0272 & 334.3539 & \\
\hline & $7 / 26 / 200810: 00$ & 93 & 208 & 1000 & 6.137 & 6.137 & & 0.5170 & $5.28 \mathrm{E}-03$ & 1113.4797 & 410.2845 & 1523.7642 & \\
\hline & $7 / 26 / 2008$ 11:00 & 94 & 208 & 1100 & 6.071 & 6.071 & & 0.5335 & 5.49E-03 & 1157.3618 & 426.4538 & 1583.8156 & \\
\hline & $7 / 26 / 2008$ 12:00 & 95 & 208 & 1200 & 6.402 & 6.402 & & 0.4542 & 4.50E-03 & 949.4164 & 349.8320 & 1299.2485 & \\
\hline & $7 / 26 / 200813: 00$ & 96 & 208 & 1300 & 5.863 & 5.863 & & 0.5879 & 6.19E-03 & 1304.3113 & 480.6003 & 1784.9116 & \\
\hline & $7 / 26 / 200814: 00$ & 97 & 208 & 1400 & 5.758 & 5.758 & & 0.6169 & $6.57 \mathrm{E}-03$ & 1383.8354 & 509.9026 & 1893.7380 & \\
\hline & $7 / 26 / 2008$ 15:00 & 98 & 208 & 1500 & 5.66 & 5.66 & & 0.6449 & 6.93E-03 & 1461.5448 & 538.5362 & 2000.0810 & \\
\hline & 7/26/2008 16:00 & 99 & 208 & 1600 & 5.707 & 5.707 & & 0.6313 & $6.76 \mathrm{E}-03$ & 1423.8435 & 524.6444 & 1948.4879 & \\
\hline & $7 / 26 / 200817: 00$ & 100 & 208 & 1700 & 5.554 & 5.554 & & 0.6763 & 7.35E-03 & 1549.6156 & 570.9878 & 2120.6034 & \\
\hline & $7 / 26 / 2008$ 18:00 & 101 & 208 & 1800 & 5.879 & 5.879 & & 0.5836 & $6.13 E-03$ & 1292.5191 & 476.2553 & 1768.7744 & \\
\hline & $7 / 26 / 2008$ 19:00 & 102 & 208 & 1900 & 5.878 & 5.878 & & 0.5839 & $6.14 \mathrm{E}-03$ & 1293.2537 & 476.5259 & 1769.7796 & \\
\hline & $7 / 26 / 200820: 00$ & 103 & 208 & 2000 & 6.342 & 6.342 & & 0.4680 & 4.67E-03 & 984.9387 & 362.9209 & 1347.8596 & \\
\hline & $7 / 26 / 200821: 00$ & 104 & 208 & 2100 & 7.844 & 7.844 & & 0.1869 & 1.51E-03 & 318.1865 & 117.2424 & 435.4289 & \\
\hline & $7 / 26 / 200822: 00$ & 105 & 208 & 2200 & 8.86 & 8.86 & & 0.0508 & 3.04E-04 & 63.9971 & 23.5810 & 87.5782 & \\
\hline & $7 / 26 / 2008$ 23:00 & 106 & 208 & 2300 & 9.07 & 9.07 & & 0.0265 & 1.36E-04 & 28.6795 & 10.5676 & 39.2471 & \\
\hline & $7 / 27 / 20080: 00$ & 107 & 209 & 0 & 9.14 & 9.14 & 9.22 & 0.0186 & 8.82E-05 & 18.5825 & 6.8471 & 25.4296 & \\
\hline & 7/27/2008 1:00 & 108 & 209 & 100 & 9.17 & 9.17 & & 0.0153 & 6.91E-05 & 14.5732 & 5.3698 & 19.9429 & \\
\hline & 7/27/2008 2:00 & 109 & 209 & 200 & 9.19 & 9.19 & & 0.0131 & 5.70E-05 & 12.0220 & 4.4298 & 16.4518 & \\
\hline & 7/27/2008 3:00 & 110 & 209 & 300 & 9.2 & 9.2 & & 0.0120 & 5.12E-05 & 10.7864 & 3.9745 & 14.7609 & \\
\hline & 7/27/2008 4:00 & 111 & 209 & 400 & 9.22 & 9.22 & & 0.0098 & 3.99E-05 & 8.4030 & 3.0963 & 11.4993 & \\
\hline & 7/27/2008 5:00 & 112 & 209 & 500 & 9.21 & 9.21 & & 0.0109 & 4.55E-05 & 9.5795 & 3.5298 & 13.1093 & \\
\hline & 7/27/2008 6:00 & 113 & 209 & 600 & 9.18 & 9.18 & & 0.0142 & 6.30E-05 & 13.2847 & 4.8950 & 18.1797 & \\
\hline & 7/27/2008 7:00 & 114 & 209 & 700 & 9.14 & 9.14 & & 0.0186 & 8.82E-05 & 18.5825 & 6.8471 & 25.4296 & \\
\hline & 7/27/2008 8:00 & 115 & 209 & 800 & 9.01 & 9.01 & & 0.0333 & 1.81E-04 & 38.0554 & 14.0223 & 52.0777 & \\
\hline & 7/27/20089:00 & 116 & 209 & 900 & 7.154 & 7.154 & & 0.3014 & 2.72E-03 & 572.9524 & 211.1161 & 784.0684 & \\
\hline & $7 / 27 / 2008$ 10:00 & 117 & 209 & 1000 & 5.687 & 5.687 & & 0.6371 & 6.83E-03 & 1439.7878 & 530.5194 & 1970.3073 & \\
\hline & $7 / 27 / 2008$ 11:00 & 118 & 209 & 1100 & 5.574 & 5.574 & & 0.6703 & 7.27E-03 & 1532.6675 & 564.7429 & 2097.4104 & \\
\hline & $7 / 27 / 200812: 00$ & 119 & 209 & 1200 & 5.556 & 5.556 & & 0.6757 & 7.34E-03 & 1547.9138 & 570.3607 & 2118.2744 & \\
\hline & 7/27/2008 13:00 & 120 & 209 & 1300 & 5.617 & 5.617 & & 0.6575 & 7.10E-03 & 1496.7546 & 551.5100 & 2048.2647 & \\
\hline & $7 / 27 / 200814: 00$ & 121 & 209 & 1400 & 5.411 & 5.411 & & 0.7206 & 7.95E-03 & 1675.5038 & 617.3738 & 2292.8776 & \\
\hline & $7 / 27 / 2008$ 15:00 & 122 & 209 & 1500 & 5.368 & 5.368 & & 0.7344 & 8.14E-03 & 1715.0413 & 631.9423 & 2346.9836 & \\
\hline
\end{tabular}




\begin{tabular}{|c|c|c|c|c|c|c|c|c|c|c|c|c|c|}
\hline Tree ID & TS & RN & JD & HR & $\begin{array}{c}\text { Raw } \\
\text { TC_dT } \Delta\end{array}$ & $\begin{array}{c}\text { Fixed } \\
\text { TC_dT } \Delta\end{array}$ & dTM & $\mathbf{K}$ & $\begin{array}{l}\text { Sap } \\
\text { Velocity } \\
\text { (cm/s) }\end{array}$ & $\begin{array}{c}\text { Probed } \\
\text { Drywood F } \\
\text { (g/hr) }\end{array}$ & $\begin{array}{c}\text { Extrapolated } \\
\text { Wetwood F } \\
(\mathrm{g} / \mathrm{hr})\end{array}$ & $\begin{array}{c}\text { Extrapolated } \\
\text { Total Wood F } \\
(\mathrm{g} / \mathrm{hr})\end{array}$ & $\begin{array}{l}\text { Relative } \\
\text { Humidity, } \\
@ 10 \mathrm{~m}(\%)\end{array}$ \\
\hline & $7 / 27 / 2008$ 16:00 & 123 & 209 & 1600 & 5.349 & 5.349 & & 0.7405 & 8.22E-03 & 1732.7696 & 638.4746 & 2371.2442 & \\
\hline & 7/27/2008 17:00 & 124 & 209 & 1700 & 5.408 & 5.408 & & 0.7215 & 7.96E-03 & 1678.2362 & 618.3807 & 2296.6169 & \\
\hline & $7 / 27 / 200818: 00$ & 125 & 209 & 1800 & 5.634 & 5.634 & & 0.6525 & 7.04E-03 & 1482.7514 & 546.3502 & 2029.1016 & \\
\hline & $7 / 27 / 2008$ 19:00 & 126 & 209 & 1900 & 5.645 & 5.645 & & 0.6492 & 6.99E-03 & 1473.7485 & 543.0330 & 2016.7815 & \\
\hline & $7 / 27 / 200820: 00$ & 127 & 209 & 2000 & 6.121 & 6.121 & & 0.5210 & 5.33E-03 & 1124.0018 & 414.1616 & 1538.1633 & \\
\hline & $7 / 27 / 2008$ 21:00 & 128 & 209 & 2100 & 7.419 & 7.419 & & 0.2549 & 2.21E-03 & 466.1855 & 171.7756 & 637.9611 & \\
\hline & $7 / 27 / 200822: 00$ & 129 & 209 & 2200 & 8.5 & 8.5 & & 0.0953 & 6.59E-04 & 138.8599 & 51.1658 & 190.0257 & \\
\hline & $7 / 27 / 200823: 00$ & 130 & 209 & 2300 & 8.75 & 8.75 & & 0.0640 & 4.04E-04 & 85.0656 & 31.3442 & 116.4098 & \\
\hline & $7 / 28 / 20080: 00$ & 131 & 210 & 0 & 8.82 & 8.82 & 9.27 & 0.0556 & 3.39E-04 & 71.4671 & 26.3335 & 97.8006 & \\
\hline & 7/28/2008 1:00 & 132 & 210 & 100 & 8.95 & 8.95 & & 0.0402 & 2.28E-04 & 48.0243 & 17.6955 & 65.7198 & \\
\hline & 7/28/2008 2:00 & 133 & 210 & 200 & 9.02 & 9.02 & & 0.0322 & 1.73E-04 & 36.4501 & 13.4308 & 49.8810 & \\
\hline & 7/28/2008 3:00 & 134 & 210 & 300 & 9.1 & 9.1 & & 0.0231 & 1.15E-04 & 24.2336 & 8.9294 & 33.1630 & \\
\hline & 7/28/2008 4:00 & 135 & 210 & 400 & 9.14 & 9.14 & & 0.0186 & 8.82E-05 & 18.5825 & 6.8471 & 25.4296 & \\
\hline & 7/28/2008 5:00 & 136 & 210 & 500 & 9.22 & 9.22 & & 0.0098 & 3.99E-05 & 8.4030 & 3.0963 & 11.4993 & \\
\hline & 7/28/2008 6:00 & 137 & 210 & 600 & 9.27 & 9.27 & & 0.0043 & 1.46E-05 & 3.0761 & 1.1335 & 4.2096 & \\
\hline & 7/28/2008 7:00 & 138 & 210 & 700 & 9.22 & 9.22 & & 0.0098 & 3.99E-05 & 8.4030 & 3.0963 & 11.4993 & \\
\hline & 7/28/2008 8:00 & 139 & 210 & 800 & 7.836 & 7.836 & & 0.1881 & 1.52E-03 & 320.7279 & 118.1788 & 438.9067 & \\
\hline & 7/28/20089:00 & 140 & 210 & 900 & 6.105 & 6.105 & & 0.5250 & 5.38E-03 & 1134.5977 & 418.0658 & 1552.6635 & \\
\hline & $7 / 28 / 2008$ 10:00 & 141 & 210 & 1000 & 5.63 & 5.63 & & 0.6536 & 7.05E-03 & 1486.0364 & 547.5607 & 2033.5971 & \\
\hline & $7 / 28 / 2008$ 11:00 & 142 & 210 & 1100 & 5.646 & 5.646 & & 0.6490 & 6.99E-03 & 1472.9323 & 542.7322 & 2015.6645 & \\
\hline & $7 / 28 / 200812: 00$ & 143 & 210 & 1200 & 5.677 & 5.677 & & 0.6400 & 6.87E-03 & 1447.8147 & 533.4771 & 1981.2918 & \\
\hline & $7 / 28 / 2008$ 13:00 & 144 & 210 & 1300 & 5.572 & 5.572 & & 0.6709 & $7.28 \mathrm{E}-03$ & 1534.3553 & 565.3648 & 2099.7200 & \\
\hline & $7 / 28 / 2008$ 14:00 & 145 & 210 & 1400 & 5.522 & 5.522 & & 0.6860 & 7.48E-03 & 1577.0615 & 581.1007 & 2158.1623 & \\
\hline & $7 / 28 / 200815: 00$ & 146 & 210 & 1500 & 5.406 & 5.406 & & 0.7222 & 7.97E-03 & 1680.0600 & 619.0527 & 2299.1127 & \\
\hline & $7 / 28 / 2008$ 16:00 & 147 & 210 & 1600 & 5.384 & 5.384 & & 0.7292 & 8.07E-03 & 1700.2357 & 626.4868 & 2326.7225 & \\
\hline & $7 / 28 / 2008$ 17:00 & 148 & 210 & 1700 & 5.396 & 5.396 & & 0.7254 & 8.01E-03 & 1689.2048 & 622.4222 & 2311.6270 & \\
\hline & $7 / 28 / 2008$ 18:00 & 149 & 210 & 1800 & 5.6 & 5.6 & & 0.6625 & 7.17E-03 & 1510.8678 & 556.7103 & 2067.5780 & \\
\hline & $7 / 28 / 200819: 00$ & 150 & 210 & 1900 & 5.672 & 5.672 & & 0.6414 & 6.89E-03 & 1451.8419 & 534.9610 & 1986.8029 & \\
\hline & $7 / 28 / 200820: 00$ & 151 & 210 & 2000 & 6.315 & 6.315 & & 0.4743 & 4.75E-03 & 1001.2246 & 368.9218 & 1370.1464 & \\
\hline & 7/28/2008 21:00 & 152 & 210 & 2100 & 7.788 & 7.788 & & 0.1954 & 1.59E-03 & 336.1660 & 123.8673 & 460.0332 & \\
\hline & $7 / 28 / 200822: 00$ & 153 & 210 & 2200 & 8.5 & 8.5 & & 0.0953 & 6.59E-04 & 138.8599 & 51.1658 & 190.0257 & \\
\hline & $7 / 28 / 200823: 00$ & 154 & 210 & 2300 & 8.7 & 8.7 & & 0.0701 & $4.52 \mathrm{E}-04$ & 95.1786 & 35.0705 & 130.2492 & \\
\hline & $7 / 29 / 2008$ 0:00 & 155 & 211 & 0 & 8.9 & 8.9 & 9.28 & 0.0461 & 2.69E-04 & 56.7524 & 20.9116 & 77.6640 & \\
\hline & 7/29/2008 1:00 & 156 & 211 & 100 & 9.05 & 9.05 & & 0.0287 & 1.51E-04 & 31.7354 & 11.6936 & 43.4290 & \\
\hline & 7/29/2008 2:00 & 157 & 211 & 200 & 9.17 & 9.17 & & 0.0153 & 6.91E-05 & 14.5732 & 5.3698 & 19.9429 & \\
\hline & 7/29/2008 3:00 & 158 & 211 & 300 & 9.23 & 9.23 & & 0.0087 & 3.44E-05 & 7.2592 & 2.6748 & 9.9340 & \\
\hline & 7/29/2008 4:00 & 159 & 211 & 400 & 9.28 & 9.28 & & 0.0032 & 1.02E-05 & 2.1559 & 0.7944 & 2.9503 & \\
\hline & 7/29/2008 5:00 & 160 & 211 & 500 & 9.27 & 9.27 & & 0.0043 & 1.46E-05 & 3.0761 & 1.1335 & 4.2096 & \\
\hline & 7/29/2008 6:00 & 161 & 211 & 600 & 9.25 & 9.25 & & 0.0065 & 2.41E-05 & 5.0808 & 1.8721 & 6.9529 & \\
\hline & 7/29/2008 7:00 & 162 & 211 & 700 & 9.19 & 9.19 & & 0.0131 & 5.70E-05 & 12.0220 & 4.4298 & 16.4518 & \\
\hline & 7/29/2008 8:00 & 163 & 211 & 800 & 8.74 & 8.74 & & 0.0652 & 4.13E-04 & 87.0619 & 32.0797 & 119.1416 & \\
\hline
\end{tabular}




\begin{tabular}{|c|c|c|c|c|c|c|c|c|c|c|c|c|c|}
\hline Tree ID & TS & RN & JD & HR & $\begin{array}{c}\text { Raw } \\
\text { TC_dTA }\end{array}$ & $\begin{array}{c}\text { Fixed } \\
\text { TC_dT } \Delta\end{array}$ & dTM & $\mathbf{K}$ & $\begin{array}{l}\text { Sap } \\
\text { Velocity } \\
(\mathrm{cm} / \mathrm{s})\end{array}$ & $\begin{array}{c}\text { Probed } \\
\text { Drywood F } \\
(\mathrm{g} / \mathrm{hr})\end{array}$ & $\begin{array}{c}\text { Extrapolated } \\
\text { Wetwood F } \\
(\mathrm{g} / \mathrm{hr})\end{array}$ & $\begin{array}{c}\text { Extrapolated } \\
\text { Total Wood F } \\
(\mathrm{g} / \mathrm{hr})\end{array}$ & $\begin{array}{l}\text { Relative } \\
\text { Humidity, } \\
@ 10 \mathrm{~m}(\%)\end{array}$ \\
\hline & 7/29/2008 9:00 & 164 & 211 & 900 & ) 7.002 & 7.002 & & 0.3296 & $3.04 \mathrm{E}-03$ & 639.7665 & 235.7351 & 875.5016 & \\
\hline & 7/29/2008 10:00 & 165 & 211 & 1000 & 5.934 & 5.934 & & 0.5689 & $5.94 \mathrm{E}-03$ & 1252.6196 & 461.5535 & 1714.1731 & \\
\hline & $7 / 29 / 2008$ 11:00 & 166 & 211 & 1100 & 6.253 & 6.253 & & 0.4889 & 4.93E-03 & 1039.3467 & 382.9687 & 1422.3154 & \\
\hline & $7 / 29 / 200812: 00$ & 167 & 211 & 1200 & 5.761 & 5.761 & & 0.6160 & $6.55 \mathrm{E}-03$ & 1381.5106 & 509.0460 & 1890.5566 & \\
\hline & $7 / 29 / 2008$ 13:00 & 168 & 211 & 1300 & 5.749 & 5.749 & & 0.6194 & 6.60E-03 & 1390.8286 & 512.4794 & 1903.3079 & \\
\hline & $7 / 29 / 2008$ 14:00 & 169 & 211 & 1400 & 5.484 & 5.484 & & 0.6977 & 7.64E-03 & 1610.1885 & 593.3071 & 2203.4956 & \\
\hline & $7 / 29 / 2008$ 15:00 & 170 & 211 & 1500 & 5.649 & 5.649 & & 0.6481 & $6.98 E-03$ & 1470.4860 & 541.8308 & 2012.3168 & \\
\hline & $7 / 29 / 2008$ 16:00 & 171 & 211 & 1600 & 5.791 & 5.791 & & 0.6077 & $6.45 \mathrm{E}-03$ & 1358.4358 & 500.5436 & 1858.9794 & \\
\hline & $7 / 29 / 2008$ 17:00 & 172 & 211 & 1700 & 5.953 & 5.953 & & 0.5639 & 5.88E-03 & 1239.0615 & 456.5577 & 1695.6192 & \\
\hline & $7 / 29 / 2008$ 18:00 & 173 & 211 & 1800 & 5.925 & 5.925 & & 0.5713 & 5.97E-03 & 1259.0819 & 463.9346 & 1723.0166 & \\
\hline & $7 / 29 / 2008$ 19:00 & 174 & 211 & 1900 & 5.908 & 5.908 & & 0.5758 & 6.03E-03 & 1271.3594 & 468.4585 & 1739.8179 & \\
\hline & $7 / 29 / 200820: 00$ & 175 & 211 & 2000 & 6.928 & 6.928 & & 0.3438 & $3.20 \mathrm{E}-03$ & 673.8661 & 248.2998 & 922.1660 & \\
\hline & $7 / 29 / 200821: 00$ & 176 & 211 & 2100 & 8.38 & 8.38 & & 0.1110 & 7.95E-04 & 167.5081 & 61.7218 & 229.2299 & \\
\hline & $7 / 29 / 2008$ 22:00 & 177 & 211 & 2200 & 8.99 & 8.99 & & 0.0356 & 1.96E-04 & 41.3150 & 15.2234 & 56.5384 & \\
\hline & $7 / 29 / 200823: 00$ & 178 & 211 & 2300 & 9.15 & 9.15 & & 0.0175 & 8.17E-05 & 17.2230 & 6.3462 & 23.5692 & \\
\hline & $7 / 30 / 20080: 00$ & 179 & 212 & 0 & 9.19 & 9.19 & 9.20 & 0.0131 & 5.70E-05 & 12.0220 & 4.4298 & 16.4518 & \\
\hline & 7/30/2008 1:00 & 180 & 212 & 100 & 9.2 & 9.2 & & 0.0120 & 5.12E-05 & 10.7864 & 3.9745 & 14.7609 & \\
\hline & 7/30/2008 2:00 & 181 & 212 & 200 & 9.19 & 9.19 & & 0.0131 & 5.70E-05 & 12.0220 & 4.4298 & 16.4518 & \\
\hline & 7/30/2008 3:00 & 182 & 212 & 300 & 9.17 & 9.17 & & 0.0153 & 6.91E-05 & 14.5732 & 5.3698 & 19.9429 & \\
\hline & 7/30/2008 4:00 & 183 & 212 & 400 & 9.13 & 9.13 & & 0.0197 & 9.47E-05 & 19.9640 & 7.3561 & 27.3201 & \\
\hline & 7/30/2008 5:00 & 184 & 212 & 500 & 9.08 & 9.08 & & 0.0253 & $1.29 \mathrm{E}-04$ & 27.1788 & 10.0146 & 37.1934 & \\
\hline & 7/30/2008 6:00 & 185 & 212 & 600 & 9.04 & 9.04 & & 0.0299 & 1.58E-04 & 33.2899 & 12.2663 & 45.5562 & \\
\hline & 7/30/2008 7:00 & 186 & 212 & 700 & 9.01 & 9.01 & & 0.0333 & 1.81E-04 & 38.0554 & 14.0223 & 52.0777 & \\
\hline & $7 / 30 / 20088: 00$ & 187 & 212 & 800 & 8.97 & 8.97 & & 0.0379 & $2.12 \mathrm{E}-04$ & 44.6385 & 16.4480 & 61.0865 & \\
\hline & 7/30/2008 9:00 & 188 & 212 & 900 & 8.12 & 8.12 & & 0.1466 & 1.12E-03 & 235.8748 & 86.9129 & 322.7877 & \\
\hline & $7 / 30 / 2008$ 10:00 & 189 & 212 & 1000 & 5.894 & 5.894 & & 0.5796 & $6.08 E-03$ & 1281.5402 & 472.2099 & 1753.7501 & \\
\hline & 7/30/2008 11:00 & 190 & 212 & 1100 & 5.549 & 5.549 & & 0.6778 & 7.37E-03 & 1553.8772 & 572.5580 & 2126.4353 & \\
\hline & $7 / 30 / 200812: 00$ & 191 & 212 & 1200 & 5.716 & 5.716 & & 0.6288 & $6.72 \mathrm{E}-03$ & 1416.7157 & 522.0180 & 1938.7338 & \\
\hline & 7/30/2008 13:00 & 192 & 212 & 1300 & 5.731 & 5.731 & & 0.6245 & 6.67E-03 & 1404.9008 & 517.6646 & 1922.5653 & \\
\hline & $7 / 30 / 2008$ 14:00 & 193 & 212 & 1400 & 5.634 & 5.634 & & 0.6525 & 7.04E-03 & 1482.7514 & 546.3502 & 2029.1016 & \\
\hline & $7 / 30 / 2008$ 15:00 & 194 & 212 & 1500 & 5.72 & 5.72 & & 0.6276 & $6.71 \mathrm{E}-03$ & 1413.5572 & 520.8542 & 1934.4114 & \\
\hline & $7 / 30 / 2008$ 16:00 & 195 & 212 & 1600 & 5.536 & 5.536 & & 0.6817 & 7.43E-03 & 1565.0038 & 576.6578 & 2141.6616 & \\
\hline & 7/30/2008 17:00 & 196 & 212 & 1700 & 5.591 & 5.591 & & 0.6652 & $7.20 \mathrm{E}-03$ & 1518.3843 & 559.4799 & 2077.8642 & \\
\hline & $7 / 30 / 200818: 00$ & 197 & 212 & 1800 & 5.93 & 5.93 & & 0.5700 & $5.96 \mathrm{E}-03$ & 1255.4886 & 462.6106 & 1718.0991 & \\
\hline & $7 / 30 / 2008$ 19:00 & 198 & 212 & 1900 & 5.897 & 5.897 & & 0.5788 & 6.07E-03 & 1279.3533 & 471.4040 & 1750.7573 & \\
\hline & $7 / 30 / 200820: 00$ & 199 & 212 & 2000 & 6.428 & 6.428 & & 0.4484 & 4.43E-03 & 934.3047 & 344.2638 & 1278.5684 & \\
\hline & $7 / 30 / 2008$ 21:00 & 200 & 212 & 2100 & 7.893 & 7.893 & & 0.1795 & 1.44E-03 & 302.8154 & 111.5785 & 414.3939 & \\
\hline & $7 / 30 / 200822: 00$ & 201 & 212 & 2200 & 8.79 & 8.79 & & 0.0592 & 3.66E-04 & 77.2141 & 28.4511 & 105.6652 & \\
\hline & $7 / 30 / 200823: 00$ & 202 & 212 & 2300 & 9.05 & 9.05 & & 0.0287 & 1.51E-04 & 31.7354 & 11.6936 & 43.4290 & \\
\hline & $7 / 31 / 20080: 00$ & 203 & 213 & 0 & 9.17 & 9.17 & 9.24 & 0.0153 & 6.91E-05 & 14.5732 & 5.3698 & 19.9429 & \\
\hline & 7/31/2008 1:00 & 204 & 213 & 100 & 9.22 & 9.22 & & 0.0098 & 3.99E-05 & 8.4030 & 3.0963 & 11.4993 & \\
\hline
\end{tabular}




\begin{tabular}{|c|c|c|c|c|c|c|c|c|c|c|c|c|c|}
\hline Tree ID & TS & RN & JD & HR & $\begin{array}{c}\text { Raw } \\
\text { TC_dTA }\end{array}$ & $\begin{array}{c}\text { Fixed } \\
\text { TC_dT } \Delta\end{array}$ & dTM & $\mathbf{K}$ & $\begin{array}{l}\text { Sap } \\
\text { Velocity } \\
(\mathrm{cm} / \mathrm{s})\end{array}$ & $\begin{array}{c}\text { Probed } \\
\text { Drywood F } \\
(\mathrm{g} / \mathrm{hr})\end{array}$ & $\begin{array}{c}\text { Extrapolated } \\
\text { Wetwood F } \\
(\mathrm{g} / \mathrm{hr})\end{array}$ & $\begin{array}{c}\text { Extrapolated } \\
\text { Total Wood F } \\
(\mathrm{g} / \mathrm{hr})\end{array}$ & $\begin{array}{l}\text { Relative } \\
\text { Humidity, } \\
@ 10 \mathrm{~m}(\%)\end{array}$ \\
\hline & 7/31/2008 2:00 & 205 & 213 & 200 & 9.24 & 9.24 & & 0.0076 & 2.92E-05 & 6.1506 & 2.2663 & 8.4170 & \\
\hline & 7/31/2008 3:00 & 206 & 213 & 300 & 9.24 & 9.24 & & 0.0076 & $2.92 \mathrm{E}-05$ & 6.1506 & 2.2663 & 8.4170 & \\
\hline & 7/31/2008 4:00 & 207 & 213 & 400 & 9.23 & 9.23 & & 0.0087 & $3.44 \mathrm{E}-05$ & 7.2592 & 2.6748 & 9.9340 & \\
\hline & 7/31/2008 5:00 & 208 & 213 & 500 & 9.21 & 9.21 & & 0.0109 & 4.55E-05 & 9.5795 & 3.5298 & 13.1093 & \\
\hline & 7/31/2008 6:00 & 209 & 213 & 600 & 9.17 & 9.17 & & 0.0153 & 6.91E-05 & 14.5732 & 5.3698 & 19.9429 & \\
\hline & 7/31/2008 7:00 & 210 & 213 & 700 & 9.12 & 9.12 & & 0.0208 & 1.01E-04 & 21.3668 & 7.8730 & 29.2398 & \\
\hline & 7/31/2008 8:00 & 211 & 213 & 800 & 9.04 & 9.04 & & 0.0299 & 1.58E-04 & 33.2899 & 12.2663 & 45.5562 & \\
\hline & 7/31/2008 9:00 & 212 & 213 & 900 & 7.804 & 7.804 & & 0.1930 & 1.57E-03 & 330.9837 & 121.9578 & 452.9415 & \\
\hline & $7 / 31 / 2008$ 10:00 & 213 & 213 & 1000 & 5.798 & 5.798 & & 0.6057 & $6.42 E-03$ & 1353.0964 & 498.5762 & 1851.6726 & \\
\hline & 7/31/2008 11:00 & 214 & 213 & 1100 & 5.678 & 5.678 & & 0.6397 & 6.87E-03 & 1447.0103 & 533.1807 & 1980.1911 & \\
\hline & $7 / 31 / 200812: 00$ & 215 & 213 & 1200 & 5.59 & 5.59 & & 0.6655 & $7.21 \mathrm{E}-03$ & 1519.2213 & 559.7884 & 2079.0097 & \\
\hline & $7 / 31 / 200813: 00$ & 216 & 213 & 1300 & 5.798 & 5.798 & & 0.6057 & $6.42 \mathrm{E}-03$ & 1353.0964 & 498.5762 & 1851.6726 & \\
\hline & $7 / 31 / 200814: 00$ & 217 & 213 & 1400 & 5.474 & 5.474 & & 0.7008 & 7.68E-03 & 1619.0042 & 596.5554 & 2215.5596 & \\
\hline & 7/31/2008 15:00 & 218 & 213 & 1500 & 5.475 & 5.475 & & 0.7005 & 7.68E-03 & 1618.1208 & 596.2299 & 2214.3507 & \\
\hline & $7 / 31 / 200816: 00$ & 219 & 213 & 1600 & 5.498 & 5.498 & & 0.6933 & 7.58E-03 & 1597.9155 & 588.7848 & 2186.7003 & \\
\hline & 7/31/2008 17:00 & 220 & 213 & 1700 & 5.499 & 5.499 & & 0.6930 & $7.58 \mathrm{E}-03$ & 1597.0419 & 588.4629 & 2185.5049 & \\
\hline & $7 / 31 / 200818: 00$ & 221 & 213 & 1800 & 5.795 & 5.795 & & 0.6066 & 6.43E-03 & 1355.3827 & 499.4186 & 1854.8013 & \\
\hline & $7 / 31 / 2008$ 19:00 & 222 & 213 & 1900 & 5.936 & 5.936 & & 0.5684 & 5.94E-03 & 1251.1870 & 461.0256 & 1712.2126 & \\
\hline & $7 / 31 / 200820: 00$ & 223 & 213 & 2000 & 6.791 & 6.791 & & 0.3709 & 3.51E-03 & 739.8575 & 272.6157 & 1012.4732 & \\
\hline & $7 / 31 / 200821: 00$ & 224 & 213 & 2100 & 7.725 & 7.725 & & 0.2052 & 1.69E-03 & 356.9272 & 131.5172 & 488.4443 & \\
\hline & $7 / 31 / 200822: 00$ & 225 & 213 & 2200 & 8.43 & 8.43 & & 0.1044 & 7.37E-04 & 155.3499 & 57.2419 & 212.5918 & \\
\hline & $7 / 31 / 2008$ 23:00 & 226 & 213 & 2300 & 8.51 & 8.51 & & 0.0940 & $6.48 \mathrm{E}-04$ & 136.5548 & 50.3164 & 186.8713 & \\
\hline & $8 / 1 / 20080: 00$ & 227 & 214 & 0 & 8.71 & 8.71 & 9.18 & 0.0689 & $4.42 E-04$ & 93.1298 & 34.3156 & 127.4453 & \\
\hline & 8/1/2008 1:00 & 228 & 214 & 100 & 9.03 & 9.03 & & 0.0310 & 1.65E-04 & 34.8615 & 12.8455 & 47.7070 & \\
\hline & 8/1/2008 2:00 & 229 & 214 & 200 & 9.16 & 9.16 & & 0.0164 & 7.54E-05 & 15.8863 & 5.8536 & 21.7399 & \\
\hline & 8/1/2008 3:00 & 230 & 214 & 300 & 9.17 & 9.17 & & 0.0153 & 6.91E-05 & 14.5732 & 5.3698 & 19.9429 & \\
\hline & 8/1/2008 4:00 & 231 & 214 & 400 & 9.18 & 9.18 & & 0.0142 & 6.30E-05 & 13.2847 & 4.8950 & 18.1797 & \\
\hline & $8 / 1 / 20085: 00$ & 232 & 214 & 500 & 9.17 & 9.17 & & 0.0153 & $6.91 \mathrm{E}-05$ & 14.5732 & 5.3698 & 19.9429 & \\
\hline & 8/1/2008 6:00 & 233 & 214 & 600 & 9.08 & 9.08 & & 0.0253 & 1.29E-04 & 27.1788 & 10.0146 & 37.1934 & \\
\hline & $8 / 1 / 20087: 00$ & 234 & 214 & 700 & 9.04 & 9.04 & & 0.0299 & 1.58E-04 & 33.2899 & 12.2663 & 45.5562 & \\
\hline & $8 / 1 / 20088: 00$ & 235 & 214 & 800 & 9.01 & 9.01 & & 0.0333 & 1.81E-04 & 38.0554 & 14.0223 & 52.0777 & \\
\hline & 8/1/2008 9:00 & 236 & 214 & 900 & 8.02 & 8.02 & & 0.1608 & 1.26E-03 & 264.5111 & 97.4646 & 361.9757 & \\
\hline & $8 / 1 / 2008$ 10:00 & 237 & 214 & 1000 & 5.818 & 5.818 & & 0.6002 & $6.35 \mathrm{E}-03$ & 1337.9336 & 492.9892 & 1830.9228 & \\
\hline & 8/1/2008 11:00 & 238 & 214 & 1100 & 5.743 & 5.743 & & 0.6211 & $6.62 \mathrm{E}-03$ & 1395.5065 & 514.2031 & 1909.7096 & \\
\hline & 8/1/2008 12:00 & 239 & 214 & 1200 & 5.757 & 5.757 & & 0.6172 & 6.57E-03 & 1384.6110 & 510.1884 & 1894.7994 & \\
\hline & 8/1/2008 13:00 & 240 & 214 & 1300 & 5.63 & 5.63 & & 0.6536 & 7.05E-03 & 1486.0364 & 547.5607 & 2033.5971 & \\
\hline & $8 / 1 / 2008$ 14:00 & 241 & 214 & 1400 & 5.53 & 5.53 & & 0.6835 & 7.45E-03 & 1570.1618 & 578.5584 & 2148.7202 & \\
\hline & $8 / 1 / 2008$ 15:00 & 242 & 214 & 1500 & 5.439 & 5.439 & & 0.7117 & 7.83E-03 & 1650.1864 & 608.0451 & 2258.2315 & \\
\hline & $8 / 1 / 2008$ 16:00 & 243 & 214 & 1600 & 5.468 & 5.468 & & 0.7026 & 7.71E-03 & 1624.3135 & 598.5117 & 2222.8252 & \\
\hline & 8/1/2008 17:00 & 244 & 214 & 1700 & 5.632 & 5.632 & & 0.6531 & 7.04E-03 & 1484.3932 & 546.9552 & 2031.3484 & \\
\hline & 8/1/2008 18:00 & 245 & 214 & 1800 & 5.86 & 5.86 & & 0.5887 & $6.20 \mathrm{E}-03$ & 1306.5318 & 481.4185 & 1787.9503 & \\
\hline
\end{tabular}




\begin{tabular}{|c|c|c|c|c|c|c|c|c|c|c|c|c|c|}
\hline Tree ID & TS & RN & JD & HR & $\begin{array}{c}\text { Raw } \\
\text { TC_dT } \Delta\end{array}$ & $\begin{array}{c}\text { Fixed } \\
\text { TC_dT } \Delta\end{array}$ & dTM & K & $\begin{array}{l}\text { Sap } \\
\text { Velocity } \\
\text { (cm/s) }\end{array}$ & $\begin{array}{c}\text { Probed } \\
\text { Drywood F } \\
\text { (g/hr) }\end{array}$ & $\begin{array}{c}\text { Extrapolated } \\
\text { Wetwood F } \\
(\mathrm{g} / \mathrm{hr})\end{array}$ & $\begin{array}{c}\text { Extrapolated } \\
\text { Total Wood F } \\
(\mathrm{g} / \mathrm{hr})\end{array}$ & $\begin{array}{l}\text { Relative } \\
\text { Humidity, } \\
@ 10 \mathrm{~m}(\%)\end{array}$ \\
\hline & 8/1/2008 19:00 & 246 & 214 & 1900 & 5.81 & 5.81 & & 0.6024 & 6.38E-03 & 1343.9824 & 495.2179 & 1839.2003 & \\
\hline & $8 / 1 / 200820: 00$ & 247 & 214 & 2000 & 6.572 & 6.572 & & 0.4166 & 4.05E-03 & 853.5747 & 314.5171 & 1168.0918 & \\
\hline & 8/1/2008 21:00 & 248 & 214 & 2100 & 8.13 & 8.13 & & 0.1451 & $1.11 \mathrm{E}-03$ & 233.0837 & 85.8845 & 318.9682 & \\
\hline & 8/1/2008 22:00 & 249 & 214 & 2200 & 8.89 & 8.89 & & 0.0472 & 2.78E-04 & 58.5421 & 21.5710 & 80.1132 & \\
\hline & 8/1/2008 23:00 & 250 & 214 & 2300 & 9.08 & 9.08 & & 0.0253 & 1.29E-04 & 27.1788 & 10.0146 & 37.1934 & \\
\hline & 8/2/2008 0:00 & 251 & 215 & 0 & 9.16 & 9.16 & 9.35 & 0.0164 & 7.54E-05 & 15.8863 & 5.8536 & 21.7399 & \\
\hline & 8/2/2008 1:00 & 252 & 215 & 100 & 9.25 & 9.25 & & 0.0065 & 2.41E-05 & 5.0808 & 1.8721 & 6.9529 & \\
\hline & $8 / 2 / 2008$ 2:00 & 253 & 215 & 200 & 9.32 & 9.32 & & -0.0011 & $0.00 \mathrm{E}+00$ & 0.0000 & 0.0000 & 0.0000 & \\
\hline & 8/2/2008 3:00 & 254 & 215 & 300 & 9.35 & 9.35 & & -0.0043 & $0.00 \mathrm{E}+00$ & 0.0000 & 0.0000 & 0.0000 & \\
\hline & 8/2/2008 4:00 & 255 & 215 & 400 & 9.34 & 9.34 & & -0.0032 & $0.00 \mathrm{E}+00$ & 0.0000 & 0.0000 & 0.0000 & \\
\hline & 8/2/2008 5:00 & 256 & 215 & 500 & 9.34 & 9.34 & & -0.0032 & $0.00 \mathrm{E}+00$ & 0.0000 & 0.0000 & 0.0000 & \\
\hline & $8 / 2 / 20086: 00$ & 257 & 215 & 600 & 9.34 & 9.34 & & -0.0032 & $0.00 \mathrm{E}+00$ & 0.0000 & 0.0000 & 0.0000 & \\
\hline & $8 / 2 / 20087: 00$ & 258 & 215 & 700 & 9.29 & 9.29 & & 0.0022 & $6.20 \mathrm{E}-06$ & 1.3070 & 0.4816 & 1.7886 & \\
\hline & $8 / 2 / 2008$ 8:00 & 259 & 215 & 800 & 8.27 & 8.27 & & 0.1258 & 9.27E-04 & 195.3734 & 71.9893 & 267.3627 & \\
\hline & 8/2/2008 9:00 & 260 & 215 & 900 & 6.184 & 6.184 & & 0.5055 & 5.14E-03 & 1082.9922 & 399.0507 & 1482.0429 & \\
\hline & $8 / 2 / 2008$ 10:00 & 261 & 215 & 1000 & 5.822 & 5.822 & & 0.5991 & 6.33E-03 & 1334.9174 & 491.8778 & 1826.7952 & \\
\hline & 8/2/2008 11:00 & 262 & 215 & 1100 & 5.757 & 5.757 & & 0.6172 & 6.57E-03 & 1384.6110 & 510.1884 & 1894.7994 & \\
\hline & $8 / 2 / 200812: 00$ & 263 & 215 & 1200 & 5.709 & 5.709 & & 0.6308 & $6.75 E-03$ & 1422.2570 & 524.0598 & 1946.3168 & \\
\hline & 8/2/2008 13:00 & 264 & 215 & 1300 & 5.664 & 5.664 & & 0.6437 & $6.92 \mathrm{E}-03$ & 1458.3045 & 537.3423 & 1995.6468 & \\
\hline & 8/2/2008 14:00 & 265 & 215 & 1400 & 5.625 & 5.625 & & 0.6551 & 7.07E-03 & 1490.1513 & 549.0769 & 2039.2281 & \\
\hline & 8/2/2008 15:00 & 266 & 215 & 1500 & 5.576 & 5.576 & & 0.6697 & $7.26 \mathrm{E}-03$ & 1530.9813 & 564.1215 & 2095.1028 & \\
\hline & $8 / 2 / 2008$ 16:00 & 267 & 215 & 1600 & 5.576 & 5.576 & & 0.6697 & 7.26E-03 & 1530.9813 & 564.1215 & 2095.1028 & \\
\hline & $8 / 2 / 2008$ 17:00 & 268 & 215 & 1700 & 5.59 & 5.59 & & 0.6655 & $7.21 E-03$ & 1519.2213 & 559.7884 & 2079.0097 & \\
\hline & $8 / 2 / 2008$ 18:00 & 269 & 215 & 1800 & 5.889 & 5.889 & & 0.5809 & $6.10 \mathrm{E}-03$ & 1285.1917 & 473.5553 & 1758.7470 & \\
\hline & 8/2/2008 19:00 & 270 & 215 & 1900 & 6.05 & 6.05 & & 0.5388 & $5.56 \mathrm{E}-03$ & 1171.5926 & 431.6974 & 1603.2900 & \\
\hline & $8 / 2 / 200820: 00$ & 271 & 215 & 2000 & 6.658 & 6.658 & & 0.3983 & 3.83E-03 & 807.6616 & 297.5995 & 1105.2611 & \\
\hline & 8/2/2008 21:00 & 272 & 215 & 2100 & 7.79 & 7.79 & & 0.1951 & 1.59E-03 & 335.5162 & 123.6278 & 459.1440 & \\
\hline & $8 / 2 / 200822: 00$ & 273 & 215 & 2200 & 8.65 & 8.65 & & 0.0763 & 5.01E-04 & 105.6181 & 38.9172 & 144.5353 & \\
\hline & 8/2/2008 23:00 & 274 & 215 & 2300 & 8.93 & 8.93 & & 0.0426 & 2.44E-04 & 51.4709 & 18.9655 & 70.4365 & \\
\hline & 8/3/2008 0:00 & 275 & 216 & 0 & 8.95 & 8.95 & 9.37 & 0.0402 & $2.28 \mathrm{E}-04$ & 48.0243 & 17.6955 & 65.7198 & \\
\hline & $8 / 3 / 2008$ 1:00 & 276 & 216 & 100 & 8.86 & 8.86 & & 0.0508 & 3.04E-04 & 63.9971 & 23.5810 & 87.5782 & \\
\hline & $8 / 3 / 2008$ 2:00 & 277 & 216 & 200 & 9 & 9 & & 0.0344 & 1.88E-04 & 39.6771 & 14.6199 & 54.2970 & \\
\hline & 8/3/2008 3:00 & 278 & 216 & 300 & 9.18 & 9.18 & & 0.0142 & $6.30 \mathrm{E}-05$ & 13.2847 & 4.8950 & 18.1797 & \\
\hline & 8/3/2008 4:00 & 279 & 216 & 400 & 9.3 & 9.3 & & 0.0011 & 2.64E-06 & 0.5561 & 0.2049 & 0.7610 & \\
\hline & $8 / 3 / 20085: 00$ & 280 & 216 & 500 & 9.37 & 9.37 & & -0.0064 & $0.00 \mathrm{E}+00$ & 0.0000 & 0.0000 & 0.0000 & \\
\hline & 8/3/2008 6:00 & 281 & 216 & 600 & 9.37 & 9.37 & & -0.0064 & $0.00 \mathrm{E}+00$ & 0.0000 & 0.0000 & 0.0000 & \\
\hline & 8/3/2008 7:00 & 282 & 216 & 700 & 9.32 & 9.32 & & -0.0011 & $0.00 E+00$ & 0.0000 & 0.0000 & 0.0000 & \\
\hline & $8 / 3 / 20088: 00$ & 283 & 216 & 800 & 8.25 & 8.25 & & 0.1285 & $9.52 \mathrm{E}-04$ & 200.6057 & 73.9173 & 274.5230 & \\
\hline & 8/3/2008 9:00 & 284 & 216 & 900 & 6.023 & 6.023 & & 0.5457 & $5.65 \mathrm{E}-03$ & 1190.0835 & 438.5107 & 1628.5942 & \\
\hline & 8/3/2008 10:00 & 285 & 216 & 1000 & 5.631 & 5.631 & & 0.6533 & 7.05E-03 & 1485.2146 & 547.2579 & 2032.4725 & \\
\hline & 8/3/2008 11:00 & 286 & 216 & 1100 & 5.6 & 5.6 & & 0.6625 & 7.17E-03 & 1510.8678 & 556.7103 & 2067.5780 & \\
\hline
\end{tabular}




\begin{tabular}{|c|c|c|c|c|c|c|c|c|c|c|c|c|c|}
\hline Tree ID & TS & RN & JD & HR & $\begin{array}{c}\text { Raw } \\
\text { TC_dTA }\end{array}$ & $\begin{array}{c}\text { Fixed } \\
\text { TC_dT } \Delta\end{array}$ & dTM & $\mathbf{K}$ & $\begin{array}{c}\text { Sap } \\
\text { Velocity } \\
(\mathrm{cm} / \mathrm{s})\end{array}$ & $\begin{array}{l}\text { Probed } \\
\text { Drywood F } \\
\text { (g/hr) }\end{array}$ & $\begin{array}{c}\text { Extrapolated } \\
\text { Wetwood F } \\
(\mathrm{g} / \mathrm{hr})\end{array}$ & $\begin{array}{c}\text { Extrapolated } \\
\text { Total Wood F } \\
(\mathbf{g} / \mathrm{hr})\end{array}$ & $\begin{array}{c}\text { Relative } \\
\text { Humidity, } \\
\text { @10m (\%) }\end{array}$ \\
\hline & 8/3/2008 12:00 & 287 & 216 & 1200 & 5.56 & 5.56 & & 0.6745 & 7.33E-03 & 1544.5147 & 569.1082 & 2113.6229 & \\
\hline & 8/3/2008 13:00 & 288 & 216 & 1300 & 5.536 & 5.536 & & 0.6817 & 7.43E-03 & 1565.0038 & 576.6578 & 2141.6616 & \\
\hline & 8/3/2008 14:00 & 289 & 216 & 1400 & 5.534 & 5.534 & & 0.6823 & 7.43E-03 & 1566.7215 & 577.2908 & 2144.0123 & \\
\hline & 8/3/2008 15:00 & 290 & 216 & 1500 & 5.592 & 5.592 & & 0.6649 & $7.20 \mathrm{E}-03$ & 1517.5475 & 559.1716 & 2076.7191 & \\
\hline & 8/3/2008 16:00 & 291 & 216 & 1600 & 5.6 & 5.6 & & 0.6625 & 7.17E-03 & 1510.8678 & 556.7103 & 2067.5780 & \\
\hline & 8/3/2008 17:00 & 292 & 216 & 1700 & 5.572 & 5.572 & & 0.6709 & $7.28 \mathrm{E}-03$ & 1534.3553 & 565.3648 & 2099.7200 & \\
\hline & 8/3/2008 18:00 & 293 & 216 & 1800 & 5.847 & 5.847 & & 0.5923 & $6.24 \mathrm{E}-03$ & 1316.1882 & 484.9766 & 1801.1649 & \\
\hline & 8/3/2008 19:00 & 294 & 216 & 1900 & 5.863 & 5.863 & & 0.5879 & $6.19 \mathrm{E}-03$ & 1304.3113 & 480.6003 & 1784.9116 & \\
\hline & 8/3/2008 20:00 & 295 & 216 & 2000 & 6.189 & 6.189 & & 0.5043 & $5.12 \mathrm{E}-03$ & 1079.7854 & 397.8691 & 1477.6545 & \\
\hline & 8/3/2008 21:00 & 296 & 216 & 2100 & 7.154 & 7.154 & & 0.3014 & $2.72 \mathrm{E}-03$ & 572.9524 & 211.1161 & 784.0684 & \\
\hline & 8/3/2008 22:00 & 297 & 216 & 2200 & 7.915 & 7.915 & & 0.1762 & 1.40E-03 & 296.0223 & 109.0755 & 405.0978 & \\
\hline & 8/3/2008 23:00 & 298 & 216 & 2300 & 8.05 & 8.05 & & 0.1565 & $1.21 \mathrm{E}-03$ & 255.7809 & 94.2477 & 350.0286 & \\
\hline & 8/4/2008 0:00 & 299 & 217 & 0 & 8.07 & 8.07 & 8.85 & 0.1537 & 1.19E-03 & 250.0273 & 92.1277 & 342.1550 & \\
\hline & 8/4/2008 1:00 & 300 & 217 & 100 & 8.1 & 8.1 & & 0.1494 & 1.15E-03 & 241.4962 & 88.9843 & 330.4805 & \\
\hline & 8/4/2008 2:00 & 301 & 217 & 200 & 7.838 & 7.838 & & 0.1878 & $1.52 \mathrm{E}-03$ & 320.0917 & 117.9444 & 438.0361 & \\
\hline & 8/4/2008 3:00 & 302 & 217 & 300 & 7.618 & 7.618 & & 0.2221 & 1.87E-03 & 393.5150 & 144.9987 & 538.5136 & \\
\hline & 8/4/2008 4:00 & 303 & 217 & 400 & 7.423 & 7.423 & & 0.2542 & $2.20 \mathrm{E}-03$ & 464.6635 & 171.2148 & 635.8783 & \\
\hline & 8/4/2008 5:00 & 304 & 217 & 500 & 7.59 & 7.59 & & 0.2266 & 1.91E-03 & 403.3709 & 148.6303 & 552.0012 & \\
\hline & 8/4/2008 6:00 & 305 & 217 & 600 & 8.1 & 8.1 & & 0.1494 & 1.15E-03 & 241.4962 & 88.9843 & 330.4805 & \\
\hline & 8/4/2008 7:00 & 306 & 217 & 700 & 8.25 & 8.25 & & 0.1285 & $9.52 \mathrm{E}-04$ & 200.6057 & 73.9173 & 274.5230 & \\
\hline & 8/4/2008 8:00 & 307 & 217 & 800 & 7.955 & 7.955 & & 0.1703 & $1.35 \mathrm{E}-03$ & 283.8415 & 104.5872 & 388.4287 & \\
\hline & 8/4/2008 9:00 & 308 & 217 & 900 & 7.462 & 7.462 & & 0.2477 & 2.13E-03 & 449.9580 & 165.7963 & 615.7542 & \\
\hline & 8/4/2008 10:00 & 309 & 217 & 1000 & 6.426 & 6.426 & & 0.4488 & 4.44E-03 & 935.4612 & 344.6899 & 1280.1511 & \\
\hline & 8/4/2008 11:00 & 310 & 217 & 1100 & 5.823 & 5.823 & & 0.5988 & $6.33 \mathrm{E}-03$ & 1334.1642 & 491.6002 & 1825.7644 & \\
\hline & 8/4/2008 12:00 & 311 & 217 & 1200 & 5.644 & 5.644 & & 0.6495 & 7.00E-03 & 1474.5651 & 543.3338 & 2017.8989 & \\
\hline & 8/4/2008 13:00 & 312 & 217 & 1300 & 5.566 & 5.566 & & 0.6727 & 7.30E-03 & 1539.4280 & 567.2339 & 2106.6618 & \\
\hline & 8/4/2008 14:00 & 313 & 217 & 1400 & 5.923 & 5.923 & & 0.5718 & $5.98 \mathrm{E}-03$ & 1260.5215 & 464.4651 & 1724.9866 & \\
\hline & 8/4/2008 15:00 & 314 & 217 & 1500 & 6.303 & 6.303 & & 0.4771 & $4.79 \mathrm{E}-03$ & 1008.5238 & 371.6113 & 1380.1351 & \\
\hline & 8/4/2008 16:00 & 315 & 217 & 1600 & 5.642 & 5.642 & & 0.6501 & $7.00 \mathrm{E}-03$ & 1476.1993 & 543.9360 & 2020.1354 & \\
\hline & 8/4/2008 17:00 & 316 & 217 & 1700 & 5.654 & 5.654 & & 0.6466 & $6.96 \mathrm{E}-03$ & 1466.4162 & 540.3312 & 2006.7474 & \\
\hline & 8/4/2008 18:00 & 317 & 217 & 1800 & 5.986 & 5.986 & & 0.5553 & $5.77 \mathrm{E}-03$ & 1215.7829 & 447.9802 & 1663.7632 & \\
\hline & 8/4/2008 19:00 & 318 & 217 & 1900 & 6.111 & 6.111 & & 0.5235 & $5.36 \mathrm{E}-03$ & 1130.6155 & 416.5985 & 1547.2140 & \\
\hline & 8/4/2008 20:00 & 319 & 217 & 2000 & 6.677 & 6.677 & & 0.3943 & $3.78 \mathrm{E}-03$ & 797.7411 & 293.9441 & 1091.6852 & \\
\hline & 8/4/2008 21:00 & 320 & 217 & 2100 & 8.15 & 8.15 & & 0.1423 & $1.08 \mathrm{E}-03$ & 227.5409 & 83.8421 & 311.3830 & \\
\hline & 8/4/2008 22:00 & 321 & 217 & 2200 & 8.72 & 8.72 & & 0.0677 & 4.32E-04 & 91.0940 & 33.5654 & 124.6594 & \\
\hline & 8/4/2008 23:00 & 322 & 217 & 2300 & 8.85 & 8.85 & & 0.0520 & $3.12 \mathrm{E}-04$ & 65.8437 & 24.2615 & 90.1052 & \\
\hline & 8/5/2008 0:00 & 323 & 218 & 0 & 9.03 & 9.03 & 9.20 & 0.0310 & 1.65E-04 & 34.8615 & 12.8455 & 47.7070 & \\
\hline & 8/5/2008 1:00 & 324 & 218 & 100 & 9.11 & 9.11 & & 0.0220 & 1.08E-04 & 22.7902 & 8.3975 & 31.1877 & \\
\hline & 8/5/2008 2:00 & 325 & 218 & 200 & 9.16 & 9.16 & & 0.0164 & 7.54E-05 & 15.8863 & 5.8536 & 21.7399 & \\
\hline & 8/5/2008 3:00 & 326 & 218 & 300 & 9.17 & 9.17 & & 0.0153 & $6.91 \mathrm{E}-05$ & 14.5732 & 5.3698 & 19.9429 & \\
\hline & 8/5/2008 4:00 & 327 & 218 & 400 & 9.11 & 9.11 & & 0.0220 & 1.08E-04 & 22.7902 & 8.3975 & 31.1877 & \\
\hline
\end{tabular}




\begin{tabular}{|c|c|c|c|c|c|c|c|c|c|c|c|c|c|}
\hline Tree ID & TS & RN & JD & HR & $\begin{array}{c}\text { Raw } \\
\text { TC_dTA }\end{array}$ & $\begin{array}{c}\text { Fixed } \\
\text { TC_dT } \Delta\end{array}$ & dTM & $\mathbf{K}$ & $\begin{array}{l}\text { Sap } \\
\text { Velocity } \\
\text { (cm/s) }\end{array}$ & $\begin{array}{l}\text { Probed } \\
\text { Drywood F } \\
\text { (g/hr) }\end{array}$ & $\begin{array}{c}\text { Extrapolated } \\
\text { Wetwood F } \\
(\mathrm{g} / \mathrm{hr})\end{array}$ & $\begin{array}{c}\text { Extrapolated } \\
\text { Total Wood F } \\
(\mathbf{g} / \mathrm{hr})\end{array}$ & $\begin{array}{l}\text { Relative } \\
\text { Humidity, } \\
@ 10 \mathrm{~m}(\%)\end{array}$ \\
\hline & 8/5/2008 5:00 & 328 & 218 & 500 & 9.13 & 9.13 & & 0.0197 & 9.47E-05 & 19.9640 & 7.3561 & 27.3201 & \\
\hline & 8/5/2008 6:00 & 329 & 218 & 600 & 9.14 & 9.14 & & 0.0186 & 8.82E-05 & 18.5825 & 6.8471 & 25.4296 & \\
\hline & 8/5/2008 7:00 & 330 & 218 & 700 & 9.15 & 9.15 & & 0.0175 & 8.17E-05 & 17.2230 & 6.3462 & 23.5692 & \\
\hline & 8/5/2008 8:00 & 331 & 218 & 800 & 9.09 & 9.09 & & 0.0242 & $1.22 \mathrm{E}-04$ & 25.6967 & 9.4685 & 35.1651 & \\
\hline & 8/5/2008 9:00 & 332 & 218 & 900 & 8.03 & 8.03 & & 0.1594 & $1.24 \mathrm{E}-03$ & 261.5877 & 96.3874 & 357.9750 & \\
\hline & 8/5/2008 10:00 & 333 & 218 & 1000 & 6.249 & 6.249 & & 0.4898 & 4.94E-03 & 1041.8415 & 383.8879 & 1425.7294 & \\
\hline & 8/5/2008 11:00 & 334 & 218 & 1100 & 6.406 & 6.406 & & 0.4533 & 4.49E-03 & 947.0806 & 348.9713 & 1296.0519 & \\
\hline & 8/5/2008 12:00 & 335 & 218 & 1200 & 6.173 & 6.173 & & 0.5082 & $5.17 \mathrm{E}-03$ & 1090.0718 & 401.6594 & 1491.7311 & \\
\hline & 8/5/2008 13:00 & 336 & 218 & 1300 & 5.764 & 5.764 & & 0.6152 & $6.54 \mathrm{E}-03$ & 1379.1890 & 508.1906 & 1887.3796 & \\
\hline & 8/5/2008 14:00 & 337 & 218 & 1400 & 6.291 & 6.291 & & 0.4799 & $4.82 \mathrm{E}-03$ & 1015.8608 & 374.3148 & 1390.1756 & \\
\hline & 8/5/2008 15:00 & 338 & 218 & 1500 & 6.677 & 6.677 & & 0.3943 & $3.78 \mathrm{E}-03$ & 797.7411 & 293.9441 & 1091.6852 & \\
\hline & 8/5/2008 16:00 & 339 & 218 & 1600 & 6.544 & 6.544 & & 0.4227 & 4.12E-03 & 868.8875 & 320.1595 & 1189.0470 & \\
\hline & 8/5/2008 17:00 & 340 & 218 & 1700 & 5.975 & 5.975 & & 0.5582 & $5.81 \mathrm{E}-03$ & 1223.5047 & 450.8255 & 1674.3302 & \\
\hline & 8/5/2008 18:00 & 341 & 218 & 1800 & 5.827 & 5.827 & & 0.5977 & $6.32 \mathrm{E}-03$ & 1331.1547 & 490.4913 & 1821.6461 & \\
\hline & 8/5/2008 19:00 & 342 & 218 & 1900 & 5.95 & 5.95 & & 0.5647 & 5.89E-03 & 1241.1947 & 457.3437 & 1698.5383 & \\
\hline & 8/5/2008 20:00 & 343 & 218 & 2000 & 6.847 & 6.847 & & 0.3597 & 3.38E-03 & 712.4238 & 262.5072 & 974.9310 & \\
\hline & 8/5/2008 21:00 & 344 & 218 & 2100 & 8.44 & 8.44 & & 0.1031 & $7.26 \mathrm{E}-04$ & 152.9563 & 56.3599 & 209.3162 & \\
\hline & 8/5/2008 22:00 & 345 & 218 & 2200 & 9.06 & 9.06 & & 0.0276 & 1.43E-04 & 30.1985 & 11.1273 & 41.3258 & \\
\hline & 8/5/2008 23:00 & 346 & 218 & 2300 & 9.2 & 9.2 & & 0.0120 & $5.12 \mathrm{E}-05$ & 10.7864 & 3.9745 & 14.7609 & \\
\hline & 8/6/2008 0:00 & 347 & 219 & 0 & 9.24 & 9.24 & 9.24 & 0.0076 & $2.92 \mathrm{E}-05$ & 6.1506 & 2.2663 & 8.4170 & \\
\hline & 8/6/2008 1:00 & 348 & 219 & 100 & 9.23 & 9.23 & & 0.0087 & $3.44 \mathrm{E}-05$ & 7.2592 & 2.6748 & 9.9340 & \\
\hline & 8/6/2008 2:00 & 349 & 219 & 200 & 9.22 & 9.22 & & 0.0098 & 3.99E-05 & 8.4030 & 3.0963 & 11.4993 & \\
\hline & 8/6/2008 3:00 & 350 & 219 & 300 & 9.2 & 9.2 & & 0.0120 & 5.12E-05 & 10.7864 & 3.9745 & 14.7609 & \\
\hline & 8/6/2008 4:00 & 351 & 219 & 400 & 9.17 & 9.17 & & 0.0153 & $6.91 \mathrm{E}-05$ & 14.5732 & 5.3698 & 19.9429 & \\
\hline & 8/6/2008 5:00 & 352 & 219 & 500 & 9.14 & 9.14 & & 0.0186 & 8.82E-05 & 18.5825 & 6.8471 & 25.4296 & \\
\hline & 8/6/2008 6:00 & 353 & 219 & 600 & 9.11 & 9.11 & & 0.0220 & $1.08 \mathrm{E}-04$ & 22.7902 & 8.3975 & 31.1877 & \\
\hline & 8/6/2008 7:00 & 354 & 219 & 700 & 9.06 & 9.06 & & 0.0276 & $1.43 \mathrm{E}-04$ & 30.1985 & 11.1273 & 41.3258 & \\
\hline & 8/6/2008 8:00 & 355 & 219 & 800 & 9.01 & 9.01 & & 0.0333 & $1.81 \mathrm{E}-04$ & 38.0554 & 14.0223 & 52.0777 & \\
\hline & 8/6/2008 9:00 & 356 & 219 & 900 & 8.9 & 8.9 & & 0.0461 & $2.69 \mathrm{E}-04$ & 56.7524 & 20.9116 & 77.6640 & \\
\hline & 8/6/2008 10:00 & 357 & 219 & 1000 & 6.777 & 6.777 & & 0.3738 & $3.54 \mathrm{E}-03$ & 746.8174 & 275.1802 & 1021.9976 & \\
\hline & 8/6/2008 11:00 & 358 & 219 & 1100 & 5.495 & 5.495 & & 0.6943 & 7.59E-03 & 1600.5387 & 589.7514 & 2190.2901 & \\
\hline & 8/6/2008 12:00 & 359 & 219 & 1200 & 6.104 & 6.104 & & 0.5252 & $5.39 \mathrm{E}-03$ & 1135.2624 & 418.3108 & 1553.5731 & \\
\hline & 8/6/2008 13:00 & 360 & 219 & 1300 & 6.677 & 6.677 & & 0.3943 & $3.78 \mathrm{E}-03$ & 797.7411 & 293.9441 & 1091.6852 & \\
\hline & 8/6/2008 14:00 & 361 & 219 & 1400 & 6.175 & 6.175 & & 0.5077 & $5.17 \mathrm{E}-03$ & 1088.7820 & 401.1841 & 1489.9662 & \\
\hline & 8/6/2008 15:00 & 362 & 219 & 1500 & 5.727 & 5.727 & & 0.6256 & $6.68 \mathrm{E}-03$ & 1408.0436 & 518.8226 & 1926.8662 & \\
\hline & 8/6/2008 16:00 & 363 & 219 & 1600 & 5.844 & 5.844 & & 0.5931 & $6.26 \mathrm{E}-03$ & 1318.4247 & 485.8007 & 1804.2254 & \\
\hline & 8/6/2008 17:00 & 364 & 219 & 1700 & 5.725 & 5.725 & & 0.6262 & $6.69 \mathrm{E}-03$ & 1409.6171 & 519.4024 & 1929.0195 & \\
\hline & 8/6/2008 18:00 & 365 & 219 & 1800 & 6.339 & 6.339 & & 0.4687 & 4.68E-03 & 986.7389 & 363.5842 & 1350.3231 & \\
\hline & 8/6/2008 19:00 & 366 & 219 & 1900 & 6.359 & 6.359 & & 0.4641 & $4.62 \mathrm{E}-03$ & 974.7811 & 359.1781 & 1333.9592 & \\
\hline & 8/6/2008 20:00 & 367 & 219 & 2000 & 6.632 & 6.632 & & 0.4038 & $3.90 \mathrm{E}-03$ & 821.3666 & 302.6494 & 1124.0160 & \\
\hline & 8/6/2008 21:00 & 368 & 219 & 2100 & 8.12 & 8.12 & & 0.1466 & 1.12E-03 & 235.8748 & 86.9129 & 322.7877 & \\
\hline
\end{tabular}




\begin{tabular}{|c|c|c|c|c|c|c|c|c|c|c|c|c|c|}
\hline Tree ID & TS & RN & JD & HR & $\begin{array}{c}\text { Raw } \\
\text { TC_dTA }\end{array}$ & $\begin{array}{c}\text { Fixed } \\
\text { TC_dT } \Delta\end{array}$ & dTM & $\mathbf{K}$ & $\begin{array}{l}\text { Sap } \\
\text { Velocity } \\
(\mathrm{cm} / \mathrm{s})\end{array}$ & $\begin{array}{c}\text { Probed } \\
\text { Drywood F } \\
(\mathrm{g} / \mathrm{hr})\end{array}$ & $\begin{array}{c}\text { Extrapolated } \\
\text { Wetwood F } \\
(\mathrm{g} / \mathrm{hr})\end{array}$ & $\begin{array}{c}\text { Extrapolated } \\
\text { Total Wood F } \\
(\mathrm{g} / \mathrm{hr})\end{array}$ & $\begin{array}{l}\text { Relative } \\
\text { Humidity, } \\
@ 10 \mathrm{~m}(\%)\end{array}$ \\
\hline & 8/6/2008 22:00 & 369 & 219 & 2200 & 9.01 & 9.01 & & 0.0333 & 1.81E-04 & 38.0554 & 14.0223 & 52.0777 & \\
\hline & 8/6/2008 23:00 & 370 & 219 & 2300 & 9.21 & 9.21 & & 0.0109 & 4.55E-05 & 9.5795 & 3.5298 & 13.1093 & \\
\hline & $8 / 7 / 20080: 00$ & 371 & 220 & 0 & 9.27 & 9.27 & 9.27 & 0.0043 & $1.46 \mathrm{E}-05$ & 3.0761 & 1.1335 & 4.2096 & \\
\hline & 8/7/2008 1:00 & 372 & 220 & 100 & 9.27 & 9.27 & & 0.0043 & 1.46E-05 & 3.0761 & 1.1335 & 4.2096 & \\
\hline & 8/7/2008 2:00 & 373 & 220 & 200 & 9.25 & 9.25 & & 0.0065 & 2.41E-05 & 5.0808 & 1.8721 & 6.9529 & \\
\hline & 8/7/2008 3:00 & 374 & 220 & 300 & 9.21 & 9.21 & & 0.0109 & $4.55 E-05$ & 9.5795 & 3.5298 & 13.1093 & \\
\hline & 8/7/2008 4:00 & 375 & 220 & 400 & 9.19 & 9.19 & & 0.0131 & 5.70E-05 & 12.0220 & 4.4298 & 16.4518 & \\
\hline & 8/7/2008 5:00 & 376 & 220 & 500 & 9.15 & 9.15 & & 0.0175 & 8.17E-05 & 17.2230 & 6.3462 & 23.5692 & \\
\hline & 8/7/2008 6:00 & 377 & 220 & 600 & 9.1 & 9.1 & & 0.0231 & 1.15E-04 & 24.2336 & 8.9294 & 33.1630 & \\
\hline & 8/7/2008 7:00 & 378 & 220 & 700 & 9.06 & 9.06 & & 0.0276 & 1.43E-04 & 30.1985 & 11.1273 & 41.3258 & \\
\hline & 8/7/2008 8:00 & 379 & 220 & 800 & 9.01 & 9.01 & & 0.0333 & 1.81E-04 & 38.0554 & 14.0223 & 52.0777 & \\
\hline & 8/7/2008 9:00 & 380 & 220 & 900 & 8.43 & 8.43 & & 0.1044 & 7.37E-04 & 155.3499 & 57.2419 & 212.5918 & \\
\hline & 8/7/2008 10:00 & 381 & 220 & 1000 & 6.254 & 6.254 & & 0.4886 & 4.93E-03 & 1038.7237 & 382.7391 & 1421.4629 & \\
\hline & 8/7/2008 11:00 & 382 & 220 & 1100 & 5.403 & 5.403 & & 0.7231 & 7.98E-03 & 1682.7989 & 620.0619 & 2302.8608 & \\
\hline & 8/7/2008 12:00 & 383 & 220 & 1200 & 5.548 & 5.548 & & 0.6781 & 7.38E-03 & 1554.7308 & 572.8725 & 2127.6033 & \\
\hline & 8/7/2008 13:00 & 384 & 220 & 1300 & 5.611 & 5.611 & & 0.6592 & 7.13E-03 & 1501.7231 & 553.3408 & 2055.0639 & \\
\hline & 8/7/2008 14:00 & 385 & 220 & 1400 & 5.616 & 5.616 & & 0.6578 & 7.11E-03 & 1497.5818 & 551.8148 & 2049.3966 & \\
\hline & 8/7/2008 15:00 & 386 & 220 & 1500 & 5.603 & 5.603 & & 0.6616 & $7.16 \mathrm{E}-03$ & 1508.3692 & 555.7896 & 2064.1588 & \\
\hline & 8/7/2008 16:00 & 387 & 220 & 1600 & 5.533 & 5.533 & & 0.6826 & 7.44E-03 & 1567.5810 & 577.6074 & 2145.1884 & \\
\hline & 8/7/2008 17:00 & 388 & 220 & 1700 & 5.57 & 5.57 & & 0.6715 & 7.29E-03 & 1536.0446 & 565.9872 & 2102.0318 & \\
\hline & 8/7/2008 18:00 & 389 & 220 & 1800 & 5.916 & 5.916 & & 0.5737 & $6.00 \mathrm{E}-03$ & 1265.5702 & 466.3254 & 1731.8955 & \\
\hline & 8/7/2008 19:00 & 390 & 220 & 1900 & 6.066 & 6.066 & & 0.5348 & 5.51E-03 & 1160.7382 & 427.6979 & 1588.4361 & \\
\hline & $8 / 7 / 200820: 00$ & 391 & 220 & 2000 & 6.468 & 6.468 & & 0.4394 & $4.32 E-03$ & 911.3810 & 335.8171 & 1247.1981 & \\
\hline & 8/7/2008 21:00 & 392 & 220 & 2100 & 7.937 & 7.937 & & 0.1730 & 1.37E-03 & 289.2958 & 106.5970 & 395.8927 & \\
\hline & 8/7/2008 22:00 & 393 & 220 & 2200 & 8.76 & 8.76 & & 0.0628 & 3.94E-04 & 83.0827 & 30.6135 & 113.6962 & \\
\hline & $8 / 7 / 200823: 00$ & 394 & 220 & 2300 & 9.06 & 9.06 & & 0.0276 & 1.43E-04 & 30.1985 & 11.1273 & 41.3258 & \\
\hline & 8/8/2008 0:00 & 395 & 221 & 0 & 9.21 & 9.21 & 9.32 & 0.0109 & 4.55E-05 & 9.5795 & 3.5298 & 13.1093 & \\
\hline & $8 / 8 / 20081: 00$ & 396 & 221 & 100 & 9.26 & 9.26 & & 0.0054 & 1.92E-05 & 4.0540 & 1.4938 & 5.5477 & \\
\hline & 8/8/2008 2:00 & 397 & 221 & 200 & 9.32 & 9.32 & & -0.0011 & $0.00 \mathrm{E}+00$ & 0.0000 & 0.0000 & 0.0000 & \\
\hline & $8 / 8 / 20083: 00$ & 398 & 221 & 300 & 9.3 & 9.3 & & 0.0011 & $2.64 \mathrm{E}-06$ & 0.5561 & 0.2049 & 0.7610 & \\
\hline & $8 / 8 / 20084: 00$ & 399 & 221 & 400 & 9.26 & 9.26 & & 0.0054 & 1.92E-05 & 4.0540 & 1.4938 & 5.5477 & \\
\hline & 8/8/2008 5:00 & 400 & 221 & 500 & 9.21 & 9.21 & & 0.0109 & $4.55 \mathrm{E}-05$ & 9.5795 & 3.5298 & 13.1093 & \\
\hline & 8/8/2008 6:00 & 401 & 221 & 600 & 9.16 & 9.16 & & 0.0164 & 7.54E-05 & 15.8863 & 5.8536 & 21.7399 & \\
\hline & 8/8/2008 7:00 & 402 & 221 & 700 & 9.11 & 9.11 & & 0.0220 & 1.08E-04 & 22.7902 & 8.3975 & 31.1877 & \\
\hline & 8/8/2008 8:00 & 403 & 221 & 800 & 9.02 & 9.02 & & 0.0322 & 1.73E-04 & 36.4501 & 13.4308 & 49.8810 & \\
\hline & 8/8/2008 9:00 & 404 & 221 & 900 & 7.451 & 7.451 & & 0.2495 & $2.15 E-03$ & 454.0811 & 167.3155 & 621.3966 & \\
\hline & 8/8/2008 10:00 & 405 & 221 & 1000 & 5.753 & 5.753 & & 0.6183 & $6.58 \mathrm{E}-03$ & 1387.7170 & 511.3329 & 1899.0498 & \\
\hline & 8/8/2008 11:00 & 406 & 221 & 1100 & 5.556 & 5.556 & & 0.6757 & 7.34E-03 & 1547.9138 & 570.3607 & 2118.2744 & \\
\hline & $8 / 8 / 200812: 00$ & 407 & 221 & 1200 & 5.574 & 5.574 & & 0.6703 & 7.27E-03 & 1532.6675 & 564.7429 & 2097.4104 & \\
\hline & 8/8/2008 13:00 & 408 & 221 & 1300 & 5.588 & 5.588 & & 0.6661 & 7.22E-03 & 1520.8967 & 560.4057 & 2081.3024 & \\
\hline & $8 / 8 / 2008$ 14:00 & 409 & 221 & 1400 & 5.527 & 5.527 & & 0.6845 & 7.46E-03 & 1572.7462 & 579.5107 & 2152.2568 & \\
\hline
\end{tabular}




\begin{tabular}{|c|c|c|c|c|c|c|c|c|c|c|c|c|c|}
\hline \multirow[t]{42}{*}{ Tree ID } & TS & RN & JD & HR & $\begin{array}{c}\text { Raw } \\
\text { TC_dTA }\end{array}$ & $\begin{array}{c}\text { Fixed } \\
\text { TC_dT } \Delta\end{array}$ & dTM & $\mathrm{K}$ & $\begin{array}{l}\text { Sap } \\
\text { Velocity } \\
\text { (cm/s) }\end{array}$ & $\begin{array}{l}\text { Probed } \\
\text { Drywood F } \\
(\mathrm{g} / \mathrm{hr})\end{array}$ & $\begin{array}{c}\text { Extrapolated } \\
\text { Wetwood F } \\
(\mathrm{g} / \mathrm{hr})\end{array}$ & $\begin{array}{c}\text { Extrapolated } \\
\text { Total Wood F } \\
(\mathrm{g} / \mathrm{hr})\end{array}$ & $\begin{array}{l}\text { Relative } \\
\text { Humidity, } \\
@ 10 m(\%)\end{array}$ \\
\hline & 8/8/2008 15:00 & 410 & 221 & 1500 & 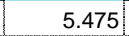 & 5.475 & & 0.7005 & $7.68 \mathrm{E}-03$ & 1618.1208 & 596.2299 & 2214.3507 & \\
\hline & 8/8/2008 16:00 & 411 & 221 & 1600 & 5.415 & 5.415 & & 0.7193 & 7.93E-03 & 1671.8666 & 616.0336 & 2287.9002 & \\
\hline & 8/8/2008 17:00 & 412 & 221 & 1700 & 5.539 & 5.539 & & 0.6808 & $7.41 \mathrm{E}-03$ & 1562.4302 & 575.7095 & 2138.1397 & \\
\hline & 8/8/2008 18:00 & 413 & 221 & 1800 & 5.993 & 5.993 & & 0.5535 & $5.75 \mathrm{E}-03$ & 1210.8886 & 446.1768 & 1657.0654 & \\
\hline & 8/8/2008 19:00 & 414 & 221 & 1900 & 5.95 & 5.95 & & 0.5647 & $5.89 \mathrm{E}-03$ & 1241.1947 & 457.3437 & 1698.5383 & \\
\hline & 8/8/2008 20:00 & 415 & 221 & 2000 & 6.587 & 6.587 & & 0.4134 & 4.01E-03 & 845.4458 & 311.5219 & 1156.9677 & \\
\hline & 8/8/2008 21:00 & 416 & 221 & 2100 & 7.927 & 7.927 & & 0.1745 & 1.39E-03 & 292.3450 & 107.7205 & 400.0656 & \\
\hline & 8/8/2008 22:00 & 417 & 221 & 2200 & 8.63 & 8.63 & & 0.0788 & 5.21E-04 & 109.8843 & 40.4891 & 150.3735 & \\
\hline & 8/8/2008 23:00 & 418 & 221 & 2300 & 8.84 & 8.84 & & 0.0532 & 3.21E-04 & 67.7043 & 24.9470 & 92.6514 & \\
\hline & $8 / 9 / 20080: 00$ & 419 & 222 & 0 & 8.95 & 8.95 & 9.17 & 0.0402 & $2.28 E-04$ & 48.0243 & 17.6955 & 65.7198 & \\
\hline & 8/9/2008 1:00 & 420 & 222 & 100 & 8.98 & 8.98 & & 0.0367 & 2.04E-04 & 42.9689 & 15.8328 & 58.8017 & \\
\hline & 8/9/2008 2:00 & 421 & 222 & 200 & 9.01 & 9.01 & & 0.0333 & $1.81 \mathrm{E}-04$ & 38.0554 & 14.0223 & 52.0777 & \\
\hline & 8/9/2008 3:00 & 422 & 222 & 300 & 9.01 & 9.01 & & 0.0333 & $1.81 \mathrm{E}-04$ & 38.0554 & 14.0223 & 52.0777 & \\
\hline & 8/9/2008 4:00 & 423 & 222 & 400 & 9 & 9 & & 0.0344 & 1.88E-04 & 39.6771 & 14.6199 & 54.2970 & \\
\hline & 8/9/2008 5:00 & 424 & 222 & 500 & 8.9 & 8.9 & & 0.0461 & 2.69E-04 & 56.7524 & 20.9116 & 77.6640 & \\
\hline & 8/9/2008 6:00 & 425 & 222 & 600 & 8.84 & 8.84 & & 0.0532 & $3.21 \mathrm{E}-04$ & 67.7043 & 24.9470 & 92.6514 & \\
\hline & $8 / 9 / 20087: 00$ & 426 & 222 & 700 & 8.57 & 8.57 & & 0.0863 & 5.84E-04 & 122.9905 & 45.3184 & 168.3088 & \\
\hline & 8/9/2008 8:00 & 427 & 222 & 800 & 7.761 & 7.761 & & 0.1996 & 1.64E-03 & 344.9938 & 127.1201 & 472.1138 & \\
\hline & 8/9/2008 9:00 & 428 & 222 & 900 & 8.13 & 8.13 & & 0.1451 & 1.11E-03 & 233.0837 & 85.8845 & 318.9682 & \\
\hline & 8/9/2008 10:00 & 429 & 222 & 1000 & 7.991 & 7.991 & & 0.1651 & $1.30 \mathrm{E}-03$ & 273.0650 & 100.6164 & 373.6814 & \\
\hline & 8/9/2008 11:00 & 430 & 222 & 1100 & 7.246 & 7.246 & & 0.2848 & $2.54 \mathrm{E}-03$ & 534.5314 & 196.9591 & 731.4905 & \\
\hline & 8/9/2008 12:00 & 431 & 222 & 1200 & 6.426 & 6.426 & & 0.4488 & 4.44E-03 & 935.4612 & 344.6899 & 1280.1511 & \\
\hline & 8/9/2008 13:00 & 432 & 222 & 1300 & 6.15 & 6.15 & & 0.5138 & $5.24 \mathrm{E}-03$ & 1104.9844 & 407.1542 & 1512.1387 & \\
\hline & 8/9/2008 14:00 & 433 & 222 & 1400 & 5.677 & 5.677 & & 0.6400 & 6.87E-03 & 1447.8147 & 533.4771 & 1981.2918 & \\
\hline & 8/9/2008 15:00 & 434 & 222 & 1500 & 5.598 & 5.598 & & 0.6631 & $7.18 \mathrm{E}-03$ & 1512.5354 & 557.3248 & 2069.8602 & \\
\hline & 8/9/2008 16:00 & 435 & 222 & 1600 & 5.642 & 5.642 & & 0.6501 & 7.00E-03 & 1476.1993 & 543.9360 & 2020.1354 & \\
\hline & 8/9/2008 17:00 & 436 & 222 & 1700 & 5.71 & 5.71 & & 0.6305 & 6.74E-03 & 1421.4643 & 523.7678 & 1945.2321 & \\
\hline & 8/9/2008 18:00 & 437 & 222 & 1800 & 5.95 & 5.95 & & 0.5647 & 5.89E-03 & 1241.1947 & 457.3437 & 1698.5383 & \\
\hline & 8/9/2008 19:00 & 438 & 222 & 1900 & 6.249 & 6.249 & & 0.4898 & 4.94E-03 & 1041.8415 & 383.8879 & 1425.7294 & \\
\hline & 8/9/2008 20:00 & 439 & 222 & 2000 & 6.765 & 6.765 & & 0.3762 & 3.57E-03 & 752.8156 & 277.3904 & 1030.2060 & \\
\hline & 8/9/2008 21:00 & 440 & 222 & 2100 & 8.32 & 8.32 & & 0.1190 & 8.66E-04 & 182.5163 & 67.2519 & 249.7682 & \\
\hline & 8/9/2008 22:00 & 441 & 222 & 2200 & 9.05 & 9.05 & & 0.0287 & 1.51E-04 & 31.7354 & 11.6936 & 43.4290 & \\
\hline & 8/9/2008 23:00 & 442 & 222 & 2300 & 9.17 & 9.17 & & 0.0153 & 6.91E-05 & 14.5732 & 5.3698 & 19.9429 & \\
\hline & 8/10/2008 0:00 & 443 & 223 & 0 & 9.21 & 9.21 & 9.23 & 0.0109 & 4.55E-05 & 9.5795 & 3.5298 & 13.1093 & \\
\hline & 8/10/2008 1:00 & 444 & 223 & 100 & 9.23 & 9.23 & & 0.0087 & 3.44E-05 & 7.2592 & 2.6748 & 9.9340 & \\
\hline & 8/10/2008 2:00 & 445 & 223 & 200 & 9.23 & 9.23 & & 0.0087 & 3.44E-05 & 7.2592 & 2.6748 & 9.9340 & \\
\hline & 8/10/2008 3:00 & 446 & 223 & 300 & 9.2 & 9.2 & & 0.0120 & $5.12 \mathrm{E}-05$ & 10.7864 & 3.9745 & 14.7609 & \\
\hline & 8/10/2008 4:00 & 447 & 223 & 400 & 9.17 & 9.17 & & 0.0153 & 6.91E-05 & 14.5732 & 5.3698 & 19.9429 & \\
\hline & 8/10/2008 5:00 & 448 & 223 & 500 & 9.15 & 9.15 & & 0.0175 & 8.17E-05 & 17.2230 & 6.3462 & 23.5692 & \\
\hline & 8/10/2008 6:00 & 449 & 223 & 600 & 9.12 & 9.12 & & 0.0208 & 1.01E-04 & 21.3668 & 7.8730 & 29.2398 & \\
\hline & 8/10/2008 7:00 & 450 & 223 & 700 & 9.1 & 9.1 & & 0.0231 & 1.15E-04 & 24.2336 & 8.9294 & 33.1630 & \\
\hline
\end{tabular}




\begin{tabular}{|c|c|c|c|c|c|c|c|c|c|c|c|c|c|}
\hline Tree ID & TS & RN & JD & HR & $\begin{array}{c}\text { Raw } \\
\text { TC_dTA }\end{array}$ & $\begin{array}{c}\text { Fixed } \\
\text { TC_dT } \Delta\end{array}$ & dTM & $\mathbf{K}$ & $\begin{array}{l}\text { Sap } \\
\text { Velocity } \\
(\mathrm{cm} / \mathrm{s})\end{array}$ & $\begin{array}{c}\text { Probed } \\
\text { Drywood F } \\
(\mathrm{g} / \mathrm{hr})\end{array}$ & $\begin{array}{c}\text { Extrapolated } \\
\text { Wetwood F } \\
(\mathrm{g} / \mathrm{hr})\end{array}$ & $\begin{array}{c}\text { Extrapolated } \\
\text { Total Wood F } \\
(\mathrm{g} / \mathrm{hr})\end{array}$ & $\begin{array}{l}\text { Relative } \\
\text { Humidity, } \\
@ 10 \mathrm{~m}(\%)\end{array}$ \\
\hline & 8/10/2008 8:00 & 451 & 223 & 800 & 9.09 & 9.09 & & 0.0242 & 1.22E-04 & 25.6967 & 9.4685 & 35.1651 & \\
\hline & 8/10/2008 9:00 & 452 & 223 & 900 & 8.49 & 8.49 & & 0.0966 & $6.70 \mathrm{E}-04$ & 141.1777 & 52.0198 & 193.1975 & \\
\hline & $8 / 10 / 2008$ 10:00 & 453 & 223 & 1000 & 6.325 & 6.325 & & 0.4719 & $4.72 E-03$ & 995.1707 & 366.6911 & 1361.8619 & \\
\hline & 8/10/2008 11:00 & 454 & 223 & 1100 & 5.451 & 5.451 & & 0.7079 & 7.78E-03 & 1639.4376 & 604.0845 & 2243.5222 & \\
\hline & $8 / 10 / 2008$ 12:00 & 455 & 223 & 1200 & 5.616 & 5.616 & & 0.6578 & 7.11E-03 & 1497.5818 & 551.8148 & 2049.3966 & \\
\hline & 8/10/2008 13:00 & 456 & 223 & 1300 & 5.695 & 5.695 & & 0.6348 & 6.80E-03 & 1433.3926 & 528.1630 & 1961.5556 & \\
\hline & $8 / 10 / 2008$ 14:00 & 457 & 223 & 1400 & 5.662 & 5.662 & & 0.6443 & 6.93E-03 & 1459.9239 & 537.9390 & 1997.8629 & \\
\hline & 8/10/2008 15:00 & 458 & 223 & 1500 & 5.811 & 5.811 & & 0.6021 & 6.37E-03 & 1343.2251 & 494.9389 & 1838.1640 & \\
\hline & 8/10/2008 16:00 & 459 & 223 & 1600 & 5.603 & 5.603 & & 0.6616 & 7.16E-03 & 1508.3692 & 555.7896 & 2064.1588 & \\
\hline & 8/10/2008 17:00 & 460 & 223 & 1700 & 5.801 & 5.801 & & 0.6049 & $6.41 E-03$ & 1350.8133 & 497.7349 & 1848.5482 & \\
\hline & 8/10/2008 18:00 & 461 & 223 & 1800 & 8.14 & 8.14 & & 0.1437 & 1.09E-03 & 230.3058 & 84.8609 & 315.1666 & \\
\hline & $8 / 10 / 2008$ 19:00 & 462 & 223 & 1900 & 8.8 & 8.8 & & 0.0580 & 3.57E-04 & 75.2848 & 27.7402 & 103.0250 & \\
\hline & 8/10/2008 20:00 & 463 & 223 & 2000 & 8.81 & 8.81 & & 0.0568 & $3.48 E-04$ & 73.3691 & 27.0344 & 100.4035 & \\
\hline & 8/10/2008 21:00 & 464 & 223 & 2100 & 8.85 & 8.85 & & 0.0520 & 3.12E-04 & 65.8437 & 24.2615 & 90.1052 & \\
\hline & $8 / 10 / 200822: 00$ & 465 & 223 & 2200 & 8.97 & 8.97 & & 0.0379 & $2.12 E-04$ & 44.6385 & 16.4480 & 61.0865 & \\
\hline & 8/10/2008 23:00 & 466 & 223 & 2300 & 8.96 & 8.96 & & 0.0391 & $2.20 \mathrm{E}-04$ & 46.3237 & 17.0689 & 63.3926 & \\
\hline & 8/11/2008 0:00 & 467 & 224 & 0 & 8.99 & 8.99 & 9.30 & 0.0356 & 1.96E-04 & 41.3150 & 15.2234 & 56.5384 & \\
\hline & 8/11/2008 1:00 & 468 & 224 & 100 & 9.11 & 9.11 & & 0.0220 & 1.08E-04 & 22.7902 & 8.3975 & 31.1877 & \\
\hline & 8/11/2008 2:00 & 469 & 224 & 200 & 9.24 & 9.24 & & 0.0076 & 2.92E-05 & 6.1506 & 2.2663 & 8.4170 & \\
\hline & 8/11/2008 3:00 & 470 & 224 & 300 & 9.29 & 9.29 & & 0.0022 & 6.20E-06 & 1.3070 & 0.4816 & 1.7886 & \\
\hline & 8/11/2008 4:00 & 471 & 224 & 400 & 9.3 & 9.3 & & 0.0011 & $2.64 \mathrm{E}-06$ & 0.5561 & 0.2049 & 0.7610 & \\
\hline & 8/11/2008 5:00 & 472 & 224 & 500 & 9.29 & 9.29 & & 0.0022 & $6.20 \mathrm{E}-06$ & 1.3070 & 0.4816 & 1.7886 & \\
\hline & 8/11/2008 6:00 & 473 & 224 & 600 & 9.29 & 9.29 & & 0.0022 & $6.20 \mathrm{E}-06$ & 1.3070 & 0.4816 & 1.7886 & \\
\hline & 8/11/2008 7:00 & 474 & 224 & 700 & 9.3 & 9.3 & & 0.0011 & 2.64E-06 & 0.5561 & 0.2049 & 0.7610 & \\
\hline & 8/11/2008 8:00 & 475 & 224 & 800 & 9.28 & 9.28 & & 0.0032 & 1.02E-05 & 2.1559 & 0.7944 & 2.9503 & \\
\hline & 8/11/2008 9:00 & 476 & 224 & 900 & 8.69 & 8.69 & & 0.0713 & 4.61E-04 & 97.2406 & 35.8303 & 133.0708 & \\
\hline & 8/11/2008 10:00 & 477 & 224 & 1000 & 6.532 & 6.532 & & 0.4253 & 4.15E-03 & 875.5061 & 322.5982 & 1198.1043 & \\
\hline & 8/11/2008 11:00 & 478 & 224 & 1100 & 5.698 & 5.698 & & 0.6339 & $6.79 \mathrm{E}-03$ & 1431.0005 & 527.2815 & 1958.2820 & \\
\hline & $8 / 11 / 2008$ 12:00 & 479 & 224 & 1200 & 5.786 & 5.786 & & 0.6091 & 6.46E-03 & 1362.2599 & 501.9527 & 1864.2126 & \\
\hline & 8/11/2008 13:00 & 480 & 224 & 1300 & 6.077 & 6.077 & & 0.5320 & 5.47E-03 & 1153.3199 & 424.9645 & 1578.2844 & \\
\hline & 8/11/2008 14:00 & 481 & 224 & 1400 & 5.757 & 5.757 & & 0.6172 & 6.57E-03 & 1384.6110 & 510.1884 & 1894.7994 & \\
\hline & 8/11/2008 15:00 & 482 & 224 & 1500 & 5.672 & 5.672 & & 0.6414 & 6.89E-03 & 1451.8419 & 534.9610 & 1986.8029 & \\
\hline & 8/11/2008 16:00 & 483 & 224 & 1600 & 5.919 & 5.919 & & 0.5729 & 5.99E-03 & 1263.4045 & 465.5274 & 1728.9319 & \\
\hline & 8/11/2008 17:00 & 484 & 224 & 1700 & 5.987 & 5.987 & & 0.5550 & 5.77E-03 & 1215.0828 & 447.7223 & 1662.8051 & \\
\hline & 8/11/2008 18:00 & 485 & 224 & 1800 & 6.354 & 6.354 & & 0.4652 & 4.64E-03 & 977.7609 & 360.2761 & 1338.0370 & \\
\hline & 8/11/2008 19:00 & 486 & 224 & 1900 & 6.556 & 6.556 & & 0.4201 & 4.09E-03 & 862.3026 & 317.7331 & 1180.0357 & \\
\hline & 8/11/2008 20:00 & 487 & 224 & 2000 & 7.258 & 7.258 & & 0.2827 & 2.51E-03 & 529.6284 & 195.1525 & 724.7809 & \\
\hline & $8 / 11 / 200821: 00$ & 488 & 224 & 2100 & 8.29 & 8.29 & & 0.1230 & 9.02E-04 & 190.1922 & 70.0802 & 260.2725 & \\
\hline & $8 / 11 / 200822: 00$ & 489 & 224 & 2200 & 8.79 & 8.79 & & 0.0592 & 3.66E-04 & 77.2141 & 28.4511 & 105.6652 & \\
\hline & 8/11/2008 23:00 & 490 & 224 & 2300 & 9.01 & 9.01 & & 0.0333 & 1.81E-04 & 38.0554 & 14.0223 & 52.0777 & \\
\hline & 8/12/2008 0:00 & 491 & 225 & 0 & 9.13 & 9.13 & 9.19 & 0.0197 & 9.47E-05 & 19.9640 & 7.3561 & 27.3201 & \\
\hline
\end{tabular}




\begin{tabular}{|c|c|c|c|c|c|c|c|c|c|c|c|c|c|}
\hline Tree ID & TS & RN & JD & HR & $\begin{array}{c}\text { Raw } \\
\text { TC_dTA }\end{array}$ & $\begin{array}{c}\text { Fixed } \\
\text { TC_dT } \Delta\end{array}$ & dTM & $\mathrm{K}$ & $\begin{array}{l}\text { Sap } \\
\text { Velocity } \\
\text { (cm/s) }\end{array}$ & $\begin{array}{c}\text { Probed } \\
\text { Drywood F } \\
\text { (g/hr) }\end{array}$ & $\begin{array}{c}\text { Extrapolated } \\
\text { Wetwood F } \\
(\mathrm{g} / \mathrm{hr})\end{array}$ & $\begin{array}{c}\text { Extrapolated } \\
\text { Total Wood F } \\
(\mathrm{g} / \mathrm{hr})\end{array}$ & $\begin{array}{c}\text { Relative } \\
\text { Humidity, } \\
@ 10 \mathrm{~m}(\%)\end{array}$ \\
\hline & 8/12/2008 1:00 & 492 & 225 & 100 & $\quad 9.09$ & 9.09 & & 0.0242 & 1.22E-04 & 25.6967 & 9.4685 & 35.1651 & \\
\hline & 8/12/2008 2:00 & 493 & 225 & 200 & 8.19 & 8.19 & & 0.1368 & 1.03E-03 & 216.6115 & 79.8150 & 296.4265 & \\
\hline & $8 / 12 / 20083: 00$ & 494 & 225 & 300 & 8.63 & 8.63 & & 0.0788 & $5.21 \mathrm{E}-04$ & 109.8843 & 40.4891 & 150.3735 & \\
\hline & 8/12/2008 4:00 & 495 & 225 & 400 & 8.92 & 8.92 & & 0.0437 & $2.52 \mathrm{E}-04$ & 53.2167 & 19.6088 & 72.8255 & \\
\hline & $8 / 12 / 20085: 00$ & 496 & 225 & 500 & 8.98 & 8.98 & & 0.0367 & 2.04E-04 & 42.9689 & 15.8328 & 58.8017 & \\
\hline & 8/12/2008 6:00 & 497 & 225 & 600 & 9.04 & 9.04 & & 0.0299 & $1.58 \mathrm{E}-04$ & 33.2899 & 12.2663 & 45.5562 & \\
\hline & 8/12/2008 7:00 & 498 & 225 & 700 & 9.05 & 9.05 & & 0.0287 & 1.51E-04 & 31.7354 & 11.6936 & 43.4290 & \\
\hline & 8/12/2008 8:00 & 499 & 225 & 800 & 9.02 & 9.02 & & 0.0322 & 1.73E-04 & 36.4501 & 13.4308 & 49.8810 & \\
\hline & 8/12/2008 9:00 & 500 & 225 & 900 & 8.56 & 8.56 & & 0.0876 & 5.94E-04 & 125.2195 & 46.1397 & 171.3592 & \\
\hline & $8 / 12 / 200810: 00$ & 501 & 225 & 1000 & 6.726 & 6.726 & & 0.3842 & 3.67E-03 & 772.5205 & 284.6511 & 1057.1716 & \\
\hline & 8/12/2008 11:00 & 502 & 225 & 1100 & 5.844 & 5.844 & & 0.5931 & $6.26 \mathrm{E}-03$ & 1318.4247 & 485.8007 & 1804.2254 & \\
\hline & 8/12/2008 12:00 & 503 & 225 & 1200 & 5.725 & 5.725 & & 0.6262 & 6.69E-03 & 1409.6171 & 519.4024 & 1929.0195 & \\
\hline & $8 / 12 / 200813: 00$ & 504 & 225 & 1300 & 5.72 & 5.72 & & 0.6276 & 6.71E-03 & 1413.5572 & 520.8542 & 1934.4114 & \\
\hline & $8 / 12 / 200814: 00$ & 505 & 225 & 1400 & 5.718 & 5.718 & & 0.6282 & 6.71E-03 & 1415.1357 & 521.4359 & 1936.5716 & \\
\hline & $8 / 12 / 200815: 00$ & 506 & 225 & 1500 & 5.644 & 5.644 & & 0.6495 & $7.00 \mathrm{E}-03$ & 1474.5651 & 543.3338 & 2017.8989 & \\
\hline & $8 / 12 / 200816: 00$ & 507 & 225 & 1600 & 5.639 & 5.639 & & 0.6510 & 7.02E-03 & 1478.6535 & 544.8403 & 2023.4939 & \\
\hline & $8 / 12 / 200817: 00$ & 508 & 225 & 1700 & 5.708 & 5.708 & & 0.6310 & $6.75 E-03$ & 1423.0501 & 524.3521 & 1947.4021 & \\
\hline & 8/12/2008 18:00 & 509 & 225 & 1800 & 6.044 & 6.044 & & 0.5404 & 5.58E-03 & 1175.6827 & 433.2045 & 1608.8872 & \\
\hline & $8 / 12 / 2008$ 19:00 & 510 & 225 & 1900 & 6.356 & 6.356 & & 0.4648 & $4.63 E-03$ & 976.5682 & 359.8366 & 1336.4048 & \\
\hline & $8 / 12 / 200820: 00$ & 511 & 225 & 2000 & 6.851 & 6.851 & & 0.3589 & $3.37 E-03$ & 710.4888 & 261.7942 & 972.2830 & \\
\hline & $8 / 12 / 200821: 00$ & 512 & 225 & 2100 & 8.4 & 8.4 & & 0.1083 & 7.72E-04 & 162.6068 & 59.9158 & 222.5227 & \\
\hline & $8 / 12 / 200822: 00$ & 513 & 225 & 2200 & 9.05 & 9.05 & & 0.0287 & 1.51E-04 & 31.7354 & 11.6936 & 43.4290 & \\
\hline & 8/12/2008 23:00 & 514 & 225 & 2300 & 9.19 & 9.19 & & 0.0131 & 5.70E-05 & 12.0220 & 4.4298 & 16.4518 & \\
\hline & 8/13/2008 0:00 & 515 & 226 & 0 & 9.23 & 9.23 & 9.34 & 0.0087 & $3.44 \mathrm{E}-05$ & 7.2592 & 2.6748 & 9.9340 & \\
\hline & 8/13/2008 1:00 & 516 & 226 & 100 & 9.26 & 9.26 & & 0.0054 & 1.92E-05 & 4.0540 & 1.4938 & 5.5477 & \\
\hline & $8 / 13 / 20082: 00$ & 517 & 226 & 200 & 9.3 & 9.3 & & 0.0011 & 2.64E-06 & 0.5561 & 0.2049 & 0.7610 & \\
\hline & 8/13/2008 3:00 & 518 & 226 & 300 & 9.32 & 9.32 & & -0.0011 & $0.00 \mathrm{E}+00$ & 0.0000 & 0.0000 & 0.0000 & \\
\hline & $8 / 13 / 20084: 00$ & 519 & 226 & 400 & 9.32 & 9.32 & & -0.0011 & $0.00 \mathrm{E}+00$ & 0.0000 & 0.0000 & 0.0000 & \\
\hline & 8/13/2008 5:00 & 520 & 226 & 500 & 9.34 & 9.34 & & -0.0032 & $0.00 \mathrm{E}+00$ & 0.0000 & 0.0000 & 0.0000 & \\
\hline & 8/13/2008 6:00 & 521 & 226 & 600 & 9.31 & 9.31 & & 0.0000 & $0.00 \mathrm{E}+00$ & 0.0000 & 0.0000 & 0.0000 & \\
\hline & $8 / 13 / 20087: 00$ & 522 & 226 & 700 & 9.28 & 9.28 & & 0.0032 & 1.02E-05 & 2.1559 & 0.7944 & 2.9503 & \\
\hline & 8/13/2008 8:00 & 523 & 226 & 800 & 9.15 & 9.15 & & 0.0175 & 8.17E-05 & 17.2230 & 6.3462 & 23.5692 & \\
\hline & 8/13/2008 9:00 & 524 & 226 & 900 & 7.838 & 7.838 & & 0.1878 & $1.52 \mathrm{E}-03$ & 320.0917 & 117.9444 & 438.0361 & \\
\hline & $8 / 13 / 2008$ 10:00 & 525 & 226 & 1000 & 6.17 & 6.17 & & 0.5089 & 5.18E-03 & 1092.0084 & 402.3730 & 1494.3814 & \\
\hline & 8/13/2008 11:00 & 526 & 226 & 1100 & 5.96 & 5.96 & & 0.5621 & 5.86E-03 & 1234.0951 & 454.7277 & 1688.8229 & \\
\hline & $8 / 13 / 2008$ 12:00 & 527 & 226 & 1200 & 5.731 & 5.731 & & 0.6245 & 6.67E-03 & 1404.9008 & 517.6646 & 1922.5653 & \\
\hline
\end{tabular}




\begin{tabular}{|c|c|c|c|c|c|c|c|c|c|c|c|c|c|}
\hline Tree ID & TS & RN & JD & HR & $\begin{array}{c}\text { Raw } \\
\text { TC_dTA }\end{array}$ & $\begin{array}{c}\text { Fixed } \\
\text { TC_dT } \Delta\end{array}$ & dTM & $\mathbf{K}$ & $\begin{array}{c}\text { Sap } \\
\text { Velocity } \\
\text { (cm/s) }\end{array}$ & $\begin{array}{c}\text { Probed } \\
\text { Drywood F } \\
(\mathrm{g} / \mathrm{hr})\end{array}$ & $\begin{array}{c}\text { Extrapolated } \\
\text { Wetwood F } \\
(\mathrm{g} / \mathrm{hr})\end{array}$ & $\begin{array}{c}\text { Extrapolated } \\
\text { Total Wood F } \\
(\mathrm{g} / \mathrm{hr})\end{array}$ & $\begin{array}{c}\text { Relative } \\
\text { Humidity, } \\
@ 10 \mathrm{~m}(\%)\end{array}$ \\
\hline & $7 / 22 / 2008$ 13:00 & 0 & 204 & 1300 & 4.885 & 4.885 & 9.73 & 0.9918 & 1.18E-02 & 1758.5151 & 647.9610 & 2406.4761 & \\
\hline W505EC1 & $7 / 22 / 2008$ 14:00 & 1 & 204 & 1400 & 5.262 & 5.262 & & 0.8491 & 9.73E-03 & 1452.4271 & 535.1766 & 1987.6038 & \\
\hline $30 " \mathrm{~N}$ & 7/22/2008 15:00 & 2 & 204 & 1500 & 5.131 & 5.131 & & 0.8963 & 1.04E-02 & 1552.4646 & 572.0375 & 2124.5021 & \\
\hline \multirow[t]{2}{*}{ Probe \# } & 7/22/2008 16:00 & 3 & 204 & 1600 & 5.075 & 5.075 & & 0.9172 & 1.07E-02 & 1597.1992 & 588.5209 & 2185.7201 & \\
\hline & 7/22/2008 17:00 & 4 & 204 & 1700 & 5.066 & 5.066 & & 0.9206 & 1.07E-02 & 1604.5035 & 591.2123 & 2195.7157 & \\
\hline \multirow[t]{4}{*}{8} & 7/22/2008 18:00 & 5 & 204 & 1800 & 5.183 & 5.183 & & 0.8773 & 1.01E-02 & 1511.9996 & 557.1274 & 2069.1270 & \\
\hline & 7/22/2008 19:00 & 6 & 204 & 1900 & 5.242 & 5.242 & & 0.8562 & 9.83E-03 & 1467.2968 & 540.6557 & 2007.9524 & \\
\hline & $7 / 22 / 200820: 00$ & 7 & 204 & 2000 & 5.889 & 5.889 & & 0.6522 & 7.03E-03 & 1049.7153 & 386.7892 & 1436.5045 & \\
\hline & 7/22/2008 21:00 & 8 & 204 & 2100 & 7.765 & 7.765 & & 0.2531 & $2.19 \mathrm{E}-03$ & 327.2705 & 120.5896 & 447.8601 & \\
\hline \multirow[t]{2}{*}{$\mathrm{DBH}(\mathrm{cm})$} & $7 / 22 / 200822: 00$ & 9 & 204 & 2200 & 9.26 & 9.26 & & 0.0508 & $3.03 E-04$ & 45.2893 & 16.6878 & 61.9771 & \\
\hline & 7/22/2008 23:00 & 10 & 204 & 2300 & 9.73 & 9.73 & & 0.0000 & $0.00 \mathrm{E}+00$ & 0.0000 & 0.0000 & 0.0000 & \\
\hline \multirow[t]{2}{*}{9.1} & 7/23/2008 0:00 & 11 & 205 & 0 & 9.94 & 9.94 & 9.98 & -0.0211 & $0.00 \mathrm{E}+00$ & 0.0000 & 0.0000 & 0.0000 & \\
\hline & 7/23/2008 1:00 & 12 & 205 & 100 & 9.98 & 9.98 & & -0.0251 & $0.00 \mathrm{E}+00$ & 0.0000 & 0.0000 & 0.0000 & \\
\hline Day Count & 7/23/2008 2:00 & 13 & 205 & 200 & 9.92 & 9.92 & & -0.0192 & $0.00 \mathrm{E}+00$ & 0.0000 & 0.0000 & 0.0000 & \\
\hline \multirow[t]{27}{*}{35} & 7/23/2008 3:00 & 14 & 205 & 300 & 9.82 & 9.82 & & -0.0092 & $0.00 \mathrm{E}+00$ & 0.0000 & 0.0000 & 0.0000 & \\
\hline & 7/23/2008 4:00 & 15 & 205 & 400 & 9.71 & 9.71 & & 0.0021 & $5.87 \mathrm{E}-06$ & 0.8767 & 0.3230 & 1.1997 & \\
\hline & 7/23/2008 5:00 & 16 & 205 & 500 & 9.54 & 9.54 & & 0.0199 & 9.59E-05 & 14.3174 & 5.2755 & 19.5929 & \\
\hline & 7/23/2008 6:00 & 17 & 205 & 600 & 9.34 & 9.34 & & 0.0418 & $2.39 \mathrm{E}-04$ & 35.6160 & 13.1235 & 48.7395 & \\
\hline & 7/23/2008 7:00 & 18 & 205 & 700 & 9.02 & 9.02 & & 0.0787 & $5.21 E-04$ & 77.7287 & 28.6407 & 106.3695 & \\
\hline & 7/23/2008 8:00 & 19 & 205 & 800 & 8.14 & 8.14 & & 0.1953 & $1.59 \mathrm{E}-03$ & 237.9485 & 87.6770 & 325.6255 & \\
\hline & 7/23/20089:00 & 20 & 205 & 900 & 6.523 & 6.523 & & 0.4916 & 4.97E-03 & 741.2502 & 273.1289 & 1014.3791 & \\
\hline & $7 / 23 / 2008$ 10:00 & 21 & 205 & 1000 & 5.486 & 5.486 & & 0.7736 & $8.68 \mathrm{E}-03$ & 1295.1180 & 477.2128 & 1772.3308 & \\
\hline & 7/23/2008 11:00 & 22 & 205 & 1100 & 5.465 & 5.465 & & 0.7804 & 8.77E-03 & 1309.1776 & 482.3934 & 1791.5710 & \\
\hline & $7 / 23 / 200812: 00$ & 23 & 205 & 1200 & 5.371 & 5.371 & & 0.8116 & $9.20 \mathrm{E}-03$ & 1373.8176 & 506.2114 & 1880.0290 & \\
\hline & 7/23/2008 13:00 & 24 & 205 & 1300 & 5.338 & 5.338 & & 0.8228 & $9.36 \mathrm{E}-03$ & 1397.1919 & 514.8241 & 1912.0159 & \\
\hline & $7 / 23 / 2008$ 14:00 & 25 & 205 & 1400 & 5.301 & 5.301 & & 0.8355 & 9.54E-03 & 1423.8347 & 524.6412 & 1948.4759 & \\
\hline & 7/23/2008 15:00 & 26 & 205 & 1500 & 5.22 & 5.22 & & 0.8640 & $9.94 \mathrm{E}-03$ & 1483.8181 & 546.7433 & 2030.5614 & \\
\hline & 7/23/2008 16:00 & 27 & 205 & 1600 & 5.445 & 5.445 & & 0.7870 & 8.86E-03 & 1322.6953 & 487.3743 & 1810.0696 & \\
\hline & $7 / 23 / 2008$ 17:00 & 28 & 205 & 1700 & 5.963 & 5.963 & & 0.6317 & $6.76 \mathrm{E}-03$ & 1009.2416 & 371.8758 & 1381.1175 & \\
\hline & $7 / 23 / 2008$ 18:00 & 29 & 205 & 1800 & 6.846 & 6.846 & & 0.4213 & 4.11E-03 & 612.8767 & 225.8270 & 838.7037 & \\
\hline & $7 / 23 / 2008$ 19:00 & 30 & 205 & 1900 & 6.715 & 6.715 & & 0.4490 & 4.44E-03 & 662.9042 & 244.2607 & 907.1649 & \\
\hline & $7 / 23 / 200820: 00$ & 31 & 205 & 2000 & 7.29 & 7.29 & & 0.3347 & 3.09E-03 & 461.7437 & 170.1390 & 631.8827 & \\
\hline & $7 / 23 / 2008$ 21:00 & 32 & 205 & 2100 & 8.21 & 8.21 & & 0.1851 & $1.49 \mathrm{E}-03$ & 222.7587 & 82.0800 & 304.8388 & \\
\hline & $7 / 23 / 2008$ 22:00 & 33 & 205 & 2200 & 8.89 & 8.89 & & 0.0945 & $6.52 \mathrm{E}-04$ & 97.3266 & 35.8620 & 133.1886 & \\
\hline & $7 / 23 / 2008$ 23:00 & 34 & 205 & 2300 & 9.06 & 9.06 & & 0.0740 & $4.82 \mathrm{E}-04$ & 71.9805 & 26.5227 & 98.5032 & \\
\hline & $7 / 24 / 20080: 00$ & 35 & 206 & 0 & 9 & 9 & 9.56 & 0.0811 & 5.40E-04 & 80.6528 & 29.7182 & 110.3710 & \\
\hline & 7/24/2008 1:00 & 36 & 206 & 100 & 9.02 & 9.02 & & 0.0787 & $5.21 E-04$ & 77.7287 & 28.6407 & 106.3695 & \\
\hline & 7/24/2008 2:00 & 37 & 206 & 200 & 9.03 & 9.03 & & 0.0775 & $5.11 E-04$ & 76.2791 & 28.1066 & 104.3858 & \\
\hline & 7/24/2008 3:00 & 38 & 206 & 300 & 9.12 & 9.12 & & 0.0669 & $4.26 \mathrm{E}-04$ & 63.6105 & 23.4386 & 87.0492 & \\
\hline & 7/24/2008 4:00 & 39 & 206 & 400 & 9.19 & 9.19 & & 0.0588 & $3.63 E-04$ & 54.2347 & 19.9839 & 74.2186 & \\
\hline & 7/24/2008 5:00 & 40 & 206 & 500 & 9.29 & 9.29 & & 0.0474 & $2.79 E-04$ & 41.5915 & 15.3252 & 56.9168 & \\
\hline
\end{tabular}




\begin{tabular}{|c|c|c|c|c|c|c|c|c|c|c|c|c|c|}
\hline Tree ID & TS & RN & JD & HR & $\begin{array}{c}\text { Raw } \\
\text { TC_dTA }\end{array}$ & $\begin{array}{c}\text { Fixed } \\
\text { TC_dT } \Delta\end{array}$ & dTM & $\mathbf{K}$ & $\begin{array}{l}\text { Sap } \\
\text { Velocity } \\
\text { (cm/s) }\end{array}$ & $\begin{array}{l}\text { Probed } \\
\text { Drywood F } \\
\text { (g/hr) }\end{array}$ & $\begin{array}{c}\text { Extrapolated } \\
\text { Wetwood F } \\
(\mathrm{g} / \mathrm{hr})\end{array}$ & $\begin{array}{c}\text { Extrapolated } \\
\text { Total Wood F } \\
(\mathrm{g} / \mathrm{hr})\end{array}$ & $\begin{array}{c}\text { Relative } \\
\text { Humidity, } \\
\text { @10m (\%) }\end{array}$ \\
\hline & 7/24/2008 6:00 & 41 & 206 & 600 & $\begin{array}{r}9.35 \\
\end{array}$ & 9.35 & & 0.0406 & 2.31E-04 & 34.4498 & 12.6937 & 47.1435 & \\
\hline & 7/24/2008 7:00 & 42 & 206 & 700 & 9.32 & 9.32 & & 0.0440 & 2.54E-04 & 37.9776 & 13.9936 & 51.9712 & \\
\hline & 7/24/2008 8:00 & 43 & 206 & 800 & 9.05 & 9.05 & & 0.0751 & 4.92E-04 & 73.4050 & 27.0476 & 100.4526 & \\
\hline & 7/24/20089:00 & 44 & 206 & 900 & 8.38 & 8.38 & & 0.1611 & 1.26E-03 & 187.7021 & 69.1627 & 256.8648 & \\
\hline & $7 / 24 / 2008$ 10:00 & 45 & 206 & 1000 & 8.21 & 8.21 & & 0.1851 & 1.49E-03 & 222.7587 & 82.0800 & 304.8388 & \\
\hline & 7/24/2008 11:00 & 46 & 206 & 1100 & 5.93 & 5.93 & & 0.6408 & $6.88 \mathrm{E}-03$ & 1027.1291 & 378.4668 & 1405.5959 & \\
\hline & $7 / 24 / 2008$ 12:00 & 47 & 206 & 1200 & 5.172 & 5.172 & & 0.8813 & $1.02 \mathrm{E}-02$ & 1520.4750 & 560.2503 & 2080.7253 & \\
\hline & 7/24/2008 13:00 & 48 & 206 & 1300 & 5.77 & 5.77 & & 0.6863 & 7.49E-03 & 1117.6275 & 411.8128 & 1529.4403 & \\
\hline & $7 / 24 / 200814: 00$ & 49 & 206 & 1400 & 5.536 & 5.536 & & 0.7576 & 8.46E-03 & 1262.1848 & 465.0780 & 1727.2628 & \\
\hline & $7 / 24 / 200815: 00$ & 50 & 206 & 1500 & 5.093 & 5.093 & & 0.9105 & 1.06E-02 & 1582.6869 & 583.1735 & 2165.8604 & \\
\hline & $7 / 24 / 2008$ 16:00 & 51 & 206 & 1600 & 5.045 & 5.045 & & 0.9286 & 1.09E-02 & 1621.6725 & 597.5386 & 2219.2110 & \\
\hline & $7 / 24 / 200817: 00$ & 52 & 206 & 1700 & 5.332 & 5.332 & & 0.8248 & $9.39 \mathrm{E}-03$ & 1401.4808 & 516.4044 & 1917.8852 & \\
\hline & $7 / 24 / 2008$ 18:00 & 53 & 206 & 1800 & 5.061 & 5.061 & & 0.9225 & $1.08 \mathrm{E}-02$ & 1608.5753 & 592.7126 & 2201.2879 & \\
\hline & $7 / 24 / 2008$ 19:00 & 54 & 206 & 1900 & 5.334 & 5.334 & & 0.8241 & 9.38E-03 & 1400.0498 & 515.8771 & 1915.9270 & \\
\hline & $7 / 24 / 200820: 00$ & 55 & 206 & 2000 & 6.741 & 6.741 & & 0.4434 & 4.37E-03 & 652.7614 & 240.5234 & 893.2848 & \\
\hline & $7 / 24 / 2008$ 21:00 & 56 & 206 & 2100 & 8.31 & 8.31 & & 0.1709 & 1.35E-03 & 201.8273 & 74.3674 & 276.1947 & \\
\hline & $7 / 24 / 200822: 00$ & 57 & 206 & 2200 & 9.28 & 9.28 & & 0.0485 & $2.87 E-04$ & 42.8149 & 15.7760 & 58.5909 & \\
\hline & $7 / 24 / 200823: 00$ & 58 & 206 & 2300 & 9.56 & 9.56 & & 0.0178 & 8.34E-05 & 12.4532 & 4.5886 & 17.0418 & \\
\hline & $7 / 25 / 20080: 00$ & 59 & 207 & 0 & 9.62 & 9.62 & 9.62 & 0.0114 & 4.84E-05 & 7.2312 & 2.6645 & 9.8956 & \\
\hline & 7/25/2008 1:00 & 60 & 207 & 100 & 9.58 & 9.58 & & 0.0157 & 7.13E-05 & 10.6475 & 3.9233 & 14.5708 & \\
\hline & 7/25/2008 2:00 & 61 & 207 & 200 & 9.5 & 9.5 & & 0.0242 & $1.22 \mathrm{E}-04$ & 18.2075 & 6.7089 & 24.9165 & \\
\hline & 7/25/2008 3:00 & 62 & 207 & 300 & 9.42 & 9.42 & & 0.0329 & $1.78 \mathrm{E}-04$ & 26.5675 & 9.7894 & 36.3569 & \\
\hline & 7/25/2008 4:00 & 63 & 207 & 400 & 9.32 & 9.32 & & 0.0440 & $2.54 \mathrm{E}-04$ & 37.9776 & 13.9936 & 51.9712 & \\
\hline & 7/25/2008 5:00 & 64 & 207 & 500 & 9.19 & 9.19 & & 0.0588 & 3.63E-04 & 54.2347 & 19.9839 & 74.2186 & \\
\hline & 7/25/2008 6:00 & 65 & 207 & 600 & 8.99 & 8.99 & & 0.0823 & $5.50 \mathrm{E}-04$ & 82.1273 & 30.2615 & 112.3889 & \\
\hline & 7/25/2008 7:00 & 66 & 207 & 700 & 8.87 & 8.87 & & 0.0970 & 6.73E-04 & 100.4651 & 37.0184 & 137.4836 & \\
\hline & 7/25/2008 8:00 & 67 & 207 & 800 & 8.77 & 8.77 & & 0.1095 & 7.81E-04 & 116.6501 & 42.9821 & 159.6322 & \\
\hline & 7/25/2008 9:00 & 68 & 207 & 900 & 8.64 & 8.64 & & 0.1262 & 9.31E-04 & 138.9202 & 51.1880 & 190.1082 & \\
\hline & $7 / 25 / 2008$ 10:00 & 69 & 207 & 1000 & 8.47 & 8.47 & & 0.1488 & 1.14E-03 & 170.1662 & 62.7013 & 232.8675 & \\
\hline & 7/25/2008 11:00 & 70 & 207 & 1100 & 7.277 & 7.277 & & 0.3371 & 3.12E-03 & 465.7963 & 171.6322 & 637.4285 & \\
\hline & $7 / 25 / 2008$ 12:00 & 71 & 207 & 1200 & 5.567 & 5.567 & & 0.7478 & 8.32E-03 & 1242.1422 & 457.6928 & 1699.8350 & \\
\hline & $7 / 25 / 2008$ 13:00 & 72 & 207 & 1300 & 5.019 & 5.019 & & 0.9386 & 1.10E-02 & 1643.1764 & 605.4622 & 2248.6386 & \\
\hline & $7 / 25 / 200814: 00$ & 73 & 207 & 1400 & 4.665 & 4.665 & & 1.0857 & 1.32E-02 & 1965.7245 & 724.3116 & 2690.0362 & \\
\hline & $7 / 25 / 200815: 00$ & 74 & 207 & 1500 & 4.646 & 4.646 & & 1.0943 & 1.33E-02 & 1984.7520 & 731.3227 & 2716.0747 & \\
\hline & $7 / 25 / 2008$ 16:00 & 75 & 207 & 1600 & 4.815 & 4.815 & & 1.0208 & 1.22E-02 & 1821.9278 & 671.3268 & 2493.2545 & \\
\hline & $7 / 25 / 200817: 00$ & 76 & 207 & 1700 & 4.578 & 4.578 & & 1.1254 & $1.38 \mathrm{E}-02$ & 2054.4336 & 756.9983 & 2811.4320 & \\
\hline & 7/25/2008 18:00 & 77 & 207 & 1800 & 4.756 & 4.756 & & 1.0458 & 1.26E-02 & 1877.1624 & 691.6791 & 2568.8414 & \\
\hline & $7 / 25 / 2008$ 19:00 & 78 & 207 & 1900 & 4.723 & 4.723 & & 1.0601 & $1.28 \mathrm{E}-02$ & 1908.7957 & 703.3350 & 2612.1308 & \\
\hline & 7/25/2008 20:00 & 79 & 207 & 2000 & 5.82 & 5.82 & & 0.6718 & $7.29 \mathrm{E}-03$ & 1088.6571 & 401.1381 & 1489.7953 & \\
\hline & $7 / 25 / 2008$ 21:00 & 80 & 207 & 2100 & 7.533 & 7.533 & & 0.2917 & 2.61E-03 & 389.7508 & 143.6117 & 533.3624 & \\
\hline & $7 / 25 / 200822: 00$ & 81 & 207 & 2200 & 8.91 & 8.91 & & 0.0920 & $6.31 \mathrm{E}-04$ & 94.2209 & 34.7176 & 128.9385 & \\
\hline
\end{tabular}




\begin{tabular}{|c|c|c|c|c|c|c|c|c|c|c|c|c|c|}
\hline Tree ID & TS & RN & JD & HR & $\begin{array}{c}\text { Raw } \\
\text { TC_dTA }\end{array}$ & $\begin{array}{c}\text { Fixed } \\
\text { TC_dT } \Delta\end{array}$ & dTM & $\mathbf{K}$ & $\begin{array}{c}\text { Sap } \\
\text { Velocity } \\
(\mathrm{cm} / \mathrm{s})\end{array}$ & $\begin{array}{l}\text { Probed } \\
\text { Drywood F } \\
\text { (g/hr) }\end{array}$ & $\begin{array}{c}\text { Extrapolated } \\
\text { Wetwood F } \\
(\mathrm{g} / \mathrm{hr})\end{array}$ & $\begin{array}{c}\text { Extrapolated } \\
\text { Total Wood F } \\
(\mathrm{g} / \mathrm{hr})\end{array}$ & $\begin{array}{l}\text { Relative } \\
\text { Humidity, } \\
\text { @10m (\%) }\end{array}$ \\
\hline & $7 / 25 / 200823: 00$ & 82 & 207 & 2300 & $\begin{array}{r}9.24 \\
\end{array}$ & 9.24 & & 0.0530 & $3.20 \mathrm{E}-04$ & 47.8003 & 17.6130 & $\begin{array}{r}65.4133 \\
\end{array}$ & \\
\hline & 7/26/2008 0:00 & 83 & 208 & 0 & 9.31 & 9.31 & 9.31 & 0.0451 & $2.62 \mathrm{E}-04$ & 39.1728 & 14.4340 & 53.6068 & \\
\hline & 7/26/2008 1:00 & 84 & 208 & 100 & 9.26 & 9.26 & & 0.0508 & 3.03E-04 & 45.2893 & 16.6878 & 61.9771 & \\
\hline & 7/26/2008 2:00 & 85 & 208 & 200 & 9.19 & 9.19 & & 0.0588 & $3.63 \mathrm{E}-04$ & 54.2347 & 19.9839 & 74.2186 & \\
\hline & 7/26/2008 3:00 & 86 & 208 & 300 & 9.1 & 9.1 & & 0.0692 & 4.45E-04 & 66.3667 & 24.4542 & 90.8208 & \\
\hline & 7/26/2008 4:00 & 87 & 208 & 400 & 9.01 & 9.01 & & 0.0799 & 5.30E-04 & 79.1866 & 29.1779 & 108.3646 & \\
\hline & 7/26/2008 5:00 & 88 & 208 & 500 & 8.92 & 8.92 & & 0.0908 & $6.21 \mathrm{E}-04$ & 92.6803 & 34.1500 & 126.8303 & \\
\hline & 7/26/2008 6:00 & 89 & 208 & 600 & 8.81 & 8.81 & & 0.1044 & 7.37E-04 & 110.0777 & 40.5604 & 150.6380 & \\
\hline & 7/26/2008 7:00 & 90 & 208 & 700 & 8.67 & 8.67 & & 0.1223 & 8.95E-04 & 133.6570 & 49.2487 & 182.9057 & \\
\hline & 7/26/2008 8:00 & 91 & 208 & 800 & 8.51 & 8.51 & & 0.1434 & 1.09E-03 & 162.5951 & 59.9115 & 222.5066 & \\
\hline & 7/26/2008 9:00 & 92 & 208 & 900 & 6.949 & 6.949 & & 0.4002 & 3.85E-03 & 575.3694 & 212.0067 & 787.3761 & \\
\hline & 7/26/2008 10:00 & 93 & 208 & 1000 & 4.803 & 4.803 & & 1.0258 & $1.23 \mathrm{E}-02$ & 1833.0271 & 675.4165 & 2508.4436 & \\
\hline & 7/26/2008 11:00 & 94 & 208 & 1100 & 4.875 & 4.875 & & 0.9959 & 1.18E-02 & 1767.4369 & 651.2485 & 2418.6854 & \\
\hline & $7 / 26 / 2008$ 12:00 & 95 & 208 & 1200 & 5.244 & 5.244 & & 0.8555 & $9.82 \mathrm{E}-03$ & 1465.8034 & 540.1054 & 2005.9088 & \\
\hline & 7/26/2008 13:00 & 96 & 208 & 1300 & 4.597 & 4.597 & & 1.1166 & 1.36E-02 & 2034.7106 & 749.7310 & 2784.4416 & \\
\hline & 7/26/2008 14:00 & 97 & 208 & 1400 & 4.555 & 4.555 & & 1.1361 & $1.39 \mathrm{E}-02$ & 2078.5773 & 765.8946 & 2844.4719 & \\
\hline & $7 / 26 / 2008$ 15:00 & 98 & 208 & 1500 & 4.483 & 4.483 & & 1.1704 & $1.44 \mathrm{E}-02$ & 2156.1111 & 794.4635 & 2950.5746 & \\
\hline & $7 / 26 / 2008$ 16:00 & 99 & 208 & 1600 & 4.619 & 4.619 & & 1.1065 & 1.35E-02 & 2012.1203 & 741.4071 & 2753.5273 & \\
\hline & 7/26/2008 17:00 & 100 & 208 & 1700 & 4.32 & 4.32 & & 1.2523 & 1.57E-02 & 2343.2956 & 863.4355 & 3206.7310 & \\
\hline & $7 / 26 / 2008$ 18:00 & 101 & 208 & 1800 & 4.552 & 4.552 & & 1.1375 & 1.39E-02 & 2081.7484 & 767.0630 & 2848.8114 & \\
\hline & 7/26/2008 19:00 & 102 & 208 & 1900 & 4.636 & 4.636 & & 1.0988 & 1.34E-02 & 1994.8430 & 735.0409 & 2729.8840 & \\
\hline & 7/26/2008 20:00 & 103 & 208 & 2000 & 5.313 & 5.313 & & 0.8314 & $9.48 \mathrm{E}-03$ & 1415.1427 & 521.4384 & 1936.5812 & \\
\hline & 7/26/2008 21:00 & 104 & 208 & 2100 & 7.19 & 7.19 & & 0.3533 & $3.31 \mathrm{E}-03$ & 493.4677 & 181.8283 & 675.2961 & \\
\hline & $7 / 26 / 200822: 00$ & 105 & 208 & 2200 & 8.4 & 8.4 & & 0.1583 & $1.23 \mathrm{E}-03$ & 183.7449 & 67.7046 & 251.4495 & \\
\hline & 7/26/2008 23:00 & 106 & 208 & 2300 & 8.67 & 8.67 & & 0.1223 & 8.95E-04 & 133.6570 & 49.2487 & 182.9057 & \\
\hline & $7 / 27 / 20080: 00$ & 107 & 209 & 0 & 8.71 & 8.71 & 8.71 & 0.1171 & 8.49E-04 & 126.7553 & 46.7056 & 173.4609 & \\
\hline & 7/27/2008 1:00 & 108 & 209 & 100 & 8.68 & 8.68 & & 0.1210 & 8.84E-04 & 131.9192 & 48.6083 & 180.5275 & \\
\hline & 7/27/2008 2:00 & 109 & 209 & 200 & 8.64 & 8.64 & & 0.1262 & 9.31E-04 & 138.9202 & 51.1880 & 190.1082 & \\
\hline & 7/27/2008 3:00 & 110 & 209 & 300 & 8.58 & 8.58 & & 0.1340 & 1.00E-03 & 149.6711 & 55.1494 & 204.8205 & \\
\hline & 7/27/2008 4:00 & 111 & 209 & 400 & 8.55 & 8.55 & & 0.1380 & 1.04E-03 & 155.1594 & 57.1717 & 212.3311 & \\
\hline & 7/27/2008 5:00 & 112 & 209 & 500 & 8.55 & 8.55 & & 0.1380 & 1.04E-03 & 155.1594 & 57.1717 & 212.3311 & \\
\hline & 7/27/2008 6:00 & 113 & 209 & 600 & 8.53 & 8.53 & & 0.1407 & 1.06E-03 & 158.8604 & 58.5354 & 217.3958 & \\
\hline & 7/27/2008 7:00 & 114 & 209 & 700 & 8.48 & 8.48 & & 0.1474 & 1.13E-03 & 168.2607 & 61.9991 & 230.2598 & \\
\hline & 7/27/2008 8:00 & 115 & 209 & 800 & 8.26 & 8.26 & & 0.1780 & $1.42 \mathrm{E}-03$ & 212.1812 & 78.1825 & 290.3637 & \\
\hline & 7/27/2008 9:00 & 116 & 209 & 900 & 5.667 & 5.667 & & 0.7170 & 7.90E-03 & 1179.3817 & 434.5674 & 1613.9491 & \\
\hline & $7 / 27 / 2008$ 10:00 & 117 & 209 & 1000 & 4.097 & 4.097 & & 1.3749 & 1.76E-02 & 2628.7953 & 968.6337 & 3597.4290 & \\
\hline & 7/27/2008 11:00 & 118 & 209 & 1100 & 3.934 & 3.934 & & 1.4733 & 1.92E-02 & 2862.2771 & 1054.6649 & 3916.9419 & \\
\hline & 7/27/2008 12:00 & 119 & 209 & 1200 & 3.913 & 3.913 & & 1.4866 & $1.94 \mathrm{E}-02$ & 2894.0542 & 1066.3738 & 3960.4280 & \\
\hline & 7/27/2008 13:00 & 120 & 209 & 1300 & 4.018 & 4.018 & & 1.4216 & $1.83 \mathrm{E}-02$ & 2739.1245 & 1009.2868 & 3748.4113 & \\
\hline & 7/27/2008 14:00 & 121 & 209 & 1400 & 3.891 & 3.891 & & 1.5006 & 1.96E-02 & 2927.7839 & 1078.8022 & 4006.5861 & \\
\hline & 7/27/2008 15:00 & 122 & 209 & 1500 & 3.853 & 3.853 & & 1.5253 & $2.00 \mathrm{E}-02$ & 2987.1278 & 1100.6687 & 4087.7965 & \\
\hline
\end{tabular}




\begin{tabular}{|c|c|c|c|c|c|c|c|c|c|c|c|c|c|}
\hline Tree ID & TS & RN & JD & HR & $\begin{array}{c}\text { Raw } \\
\text { TC_dTA }\end{array}$ & $\begin{array}{c}\text { Fixed } \\
\text { TC_dT } \Delta\end{array}$ & dTM & $\mathbf{K}$ & $\begin{array}{c}\text { Sap } \\
\text { Velocity } \\
(\mathbf{c m} / \mathbf{s})\end{array}$ & $\begin{array}{c}\text { Probed } \\
\text { Drywood F } \\
(\mathrm{g} / \mathrm{hr})\end{array}$ & $\begin{array}{c}\text { Extrapolated } \\
\text { Wetwood F } \\
(\mathrm{g} / \mathrm{hr})\end{array}$ & $\begin{array}{c}\text { Extrapolated } \\
\text { Total Wood F } \\
(\mathrm{g} / \mathrm{hr})\end{array}$ & $\begin{array}{c}\text { Relative } \\
\text { Humidity, } \\
@ 10 \mathrm{~m}(\%)\end{array}$ \\
\hline & 7/27/2008 16:00 & 123 & 209 & 1600 & 3.958 & 3.958 & & 1.4583 & 1.89E-02 & 2826.4528 & 1041.4647 & 3867.9175 & \\
\hline & 7/27/2008 17:00 & 124 & 209 & 1700 & 3.937 & 3.937 & & 1.4714 & 1.91E-02 & 2857.7705 & 1053.0043 & 3910.7748 & \\
\hline & 7/27/2008 18:00 & 125 & 209 & 1800 & 4.043 & 4.043 & & 1.4066 & 1.81E-02 & 2703.6513 & 996.2159 & 3699.8673 & \\
\hline & 7/27/2008 19:00 & 126 & 209 & 1900 & 4.247 & 4.247 & & 1.2910 & 1.63E-02 & 2432.7861 & 896.4101 & 3329.1962 & \\
\hline & $7 / 27 / 200820: 00$ & 127 & 209 & 2000 & 4.856 & 4.856 & & 1.0037 & $1.20 \mathrm{E}-02$ & 1784.5132 & 657.5406 & 2442.0538 & \\
\hline & $7 / 27 / 2008$ 21:00 & 128 & 209 & 2100 & 6.334 & 6.334 & & 0.5362 & $5.52 \mathrm{E}-03$ & 824.7024 & 303.8786 & 1128.5810 & \\
\hline & $7 / 27 / 2008 \quad 22: 00$ & 129 & 209 & 2200 & 7.51 & 7.51 & & 0.2956 & 2.65E-03 & 396.2684 & 146.0133 & 542.2817 & \\
\hline & $7 / 27 / 2008$ 23:00 & 130 & 209 & 2300 & 7.806 & 7.806 & & 0.2465 & $2.12 \mathrm{E}-03$ & 316.8244 & 116.7405 & 433.5649 & \\
\hline & $7 / 28 / 20080: 00$ & 131 & 210 & 0 & 7.981 & 7.981 & 8.43 & 0.2191 & 1.84E-03 & 274.1470 & 101.0151 & 375.1621 & \\
\hline & 7/28/2008 1:00 & 132 & 210 & 100 & 8.12 & 8.12 & & 0.1983 & 1.62E-03 & 242.3711 & 89.3066 & 331.6778 & \\
\hline & 7/28/2008 2:00 & 133 & 210 & 200 & 8.19 & 8.19 & & 0.1880 & $1.52 \mathrm{E}-03$ & 227.0530 & 83.6624 & 310.7154 & \\
\hline & 7/28/2008 3:00 & 134 & 210 & 300 & 8.32 & 8.32 & & 0.1695 & 1.34E-03 & 199.7831 & 73.6142 & 273.3972 & \\
\hline & 7/28/2008 4:00 & 135 & 210 & 400 & 8.39 & 8.39 & & 0.1597 & 1.24E-03 & 185.7192 & 68.4321 & 254.1512 & \\
\hline & 7/28/2008 5:00 & 136 & 210 & 500 & 8.43 & 8.43 & & 0.1542 & 1.19E-03 & 177.8739 & 65.5413 & 243.4152 & \\
\hline & 7/28/2008 6:00 & 137 & 210 & 600 & 8.37 & 8.37 & & 0.1625 & 1.27E-03 & 189.6938 & 69.8966 & 259.5904 & \\
\hline & 7/28/2008 7:00 & 138 & 210 & 700 & 8.18 & 8.18 & & 0.1895 & $1.54 \mathrm{E}-03$ & 229.2138 & 84.4585 & 313.6723 & \\
\hline & 7/28/2008 8:00 & 139 & 210 & 800 & 6.573 & 6.573 & & 0.4803 & $4.82 \mathrm{E}-03$ & 720.2474 & 265.3900 & 985.6374 & \\
\hline & $7 / 28 / 20089: 00$ & 140 & 210 & 900 & 4.545 & 4.545 & & 1.1408 & $1.40 \mathrm{E}-02$ & 2089.1674 & 769.7967 & 2858.9641 & \\
\hline & $7 / 28 / 2008$ 10:00 & 141 & 210 & 1000 & 4.016 & 4.016 & & 1.4228 & $1.84 \mathrm{E}-02$ & 2741.9853 & 1010.3409 & 3752.3261 & \\
\hline & 7/28/2008 11:00 & 142 & 210 & 1100 & 3.949 & 3.949 & & 1.4639 & 1.90E-02 & 2839.8260 & 1046.3923 & 3886.2183 & \\
\hline & $7 / 28 / 2008$ 12:00 & 143 & 210 & 1200 & 3.983 & 3.983 & & 1.4429 & $1.87 \mathrm{E}-02$ & 2789.6837 & 1027.9163 & 3817.6000 & \\
\hline & 7/28/2008 13:00 & 144 & 210 & 1300 & 3.958 & 3.958 & & 1.4583 & 1.89E-02 & 2826.4528 & 1041.4647 & 3867.9175 & \\
\hline & $7 / 28 / 2008$ 14:00 & 145 & 210 & 1400 & 3.873 & 3.873 & & 1.5123 & $1.98 \mathrm{E}-02$ & 2955.7212 & 1089.0963 & 4044.8175 & \\
\hline & $7 / 28 / 2008$ 15:00 & 146 & 210 & 1500 & 3.75 & 3.75 & & 1.5947 & $2.11 E-02$ & 3155.2110 & 1162.6024 & 4317.8133 & \\
\hline & 7/28/2008 16:00 & 147 & 210 & 1600 & 3.833 & 3.833 & & 1.5385 & 2.02E-02 & 3018.9253 & 1112.3851 & 4131.3104 & \\
\hline & $7 / 28 / 2008$ 17:00 & 148 & 210 & 1700 & 3.728 & 3.728 & & 1.6100 & $2.14 \mathrm{E}-02$ & 3192.5467 & 1176.3595 & 4368.9062 & \\
\hline & $7 / 28 / 2008$ 18:00 & 149 & 210 & 1800 & 3.812 & 3.812 & & 1.5525 & 2.05E-02 & 3052.7406 & 1124.8451 & 4177.5856 & \\
\hline & $7 / 28 / 2008$ 19:00 & 150 & 210 & 1900 & 4.016 & 4.016 & & 1.4228 & 1.84E-02 & 2741.9853 & 1010.3409 & 3752.3261 & \\
\hline & $7 / 28 / 200820: 00$ & 151 & 210 & 2000 & 4.761 & 4.761 & & 1.0437 & $1.25 \mathrm{E}-02$ & 1872.4163 & 689.9303 & 2562.3466 & \\
\hline & $7 / 28 / 200821: 00$ & 152 & 210 & 2100 & 6.776 & 6.776 & & 0.4360 & $4.28 \mathrm{E}-03$ & 639.2766 & 235.5546 & 874.8312 & \\
\hline & $7 / 28 / 2008 \quad 22: 00$ & 153 & 210 & 2200 & 7.662 & 7.662 & & 0.2699 & 2.37E-03 & 354.2903 & 130.5455 & 484.8358 & \\
\hline & $7 / 28 / 2008$ 23:00 & 154 & 210 & 2300 & 7.911 & 7.911 & & 0.2299 & 1.95E-03 & 290.8526 & 107.1706 & 398.0232 & \\
\hline & $7 / 29 / 20080: 00$ & 155 & 211 & 0 & 8.11 & 8.11 & 8.50 & 0.1998 & $1.64 \mathrm{E}-03$ & 244.5964 & 90.1266 & 334.7230 & \\
\hline & 7/29/2008 1:00 & 156 & 211 & 100 & 8.28 & 8.28 & & 0.1751 & $1.39 \mathrm{E}-03$ & 208.0130 & 76.6467 & 284.6596 & \\
\hline & 7/29/2008 2:00 & 157 & 211 & 200 & 8.4 & 8.4 & & 0.1583 & 1.23E-03 & 183.7449 & 67.7046 & 251.4495 & \\
\hline & 7/29/2008 3:00 & 158 & 211 & 300 & 8.45 & 8.45 & & 0.1515 & 1.17E-03 & 174.0029 & 64.1150 & 238.1179 & \\
\hline & 7/29/2008 4:00 & 159 & 211 & 400 & 8.5 & 8.5 & & 0.1447 & 1.10E-03 & 164.4751 & 60.6042 & 225.0794 & \\
\hline & 7/29/2008 5:00 & 160 & 211 & 500 & 8.48 & 8.48 & & 0.1474 & 1.13E-03 & 168.2607 & 61.9991 & 230.2598 & \\
\hline & 7/29/2008 6:00 & 161 & 211 & 600 & 8.43 & 8.43 & & 0.1542 & 1.19E-03 & 177.8739 & 65.5413 & 243.4152 & \\
\hline & 7/29/2008 7:00 & 162 & 211 & 700 & 8.33 & 8.33 & & 0.1681 & $1.32 E-03$ & 197.7477 & 72.8642 & 270.6118 & \\
\hline & 7/29/2008 8:00 & 163 & 211 & 800 & 7.649 & 7.649 & & 0.2721 & $2.40 \mathrm{E}-03$ & 357.7810 & 131.8318 & 489.6128 & \\
\hline
\end{tabular}




\begin{tabular}{|c|c|c|c|c|c|c|c|c|c|c|c|c|c|}
\hline Tree ID & TS & RN & JD & HR & $\begin{array}{c}\text { Raw } \\
\text { TC_dTA }\end{array}$ & $\begin{array}{c}\text { Fixed } \\
\text { TC_dT } \Delta\end{array}$ & dTM & $\mathbf{K}$ & $\begin{array}{l}\text { Sap } \\
\text { Velocity } \\
(\mathrm{cm} / \mathrm{s})\end{array}$ & $\begin{array}{c}\text { Probed } \\
\text { Drywood F } \\
(\mathrm{g} / \mathrm{hr})\end{array}$ & $\begin{array}{c}\text { Extrapolated } \\
\text { Wetwood F } \\
(\mathrm{g} / \mathrm{hr})\end{array}$ & $\begin{array}{c}\text { Extrapolated } \\
\text { Total Wood F } \\
(\mathrm{g} / \mathrm{hr})\end{array}$ & $\begin{array}{l}\text { Relative } \\
\text { Humidity, } \\
@ 10 \mathrm{~m}(\%)\end{array}$ \\
\hline & 7/29/2008 9:00 & 164 & 211 & 900 & \begin{tabular}{|r|}
5.261 \\
\end{tabular} & 5.261 & & 0.8495 & $9.73 \mathrm{E}-03$ & 1453.1672 & 535.4494 & \begin{tabular}{|r|}
1988.6166 \\
\end{tabular} & \\
\hline & 7/29/2008 10:00 & 165 & 211 & 1000 & 4.217 & 4.217 & & 1.3073 & $1.66 \mathrm{E}-02$ & 2470.6483 & 910.3612 & 3381.0094 & \\
\hline & $7 / 29 / 2008$ 11:00 & 166 & 211 & 1100 & 4.576 & 4.576 & & 1.1263 & $1.38 \mathrm{E}-02$ & 2056.5213 & 757.7676 & 2814.2889 & \\
\hline & $7 / 29 / 2008$ 12:00 & 167 & 211 & 1200 & 3.867 & 3.867 & & 1.5162 & 1.99E-02 & 2965.1025 & 1092.5530 & 4057.6556 & \\
\hline & 7/29/2008 13:00 & 168 & 211 & 1300 & 3.922 & 3.922 & & 1.4809 & 1.93E-02 & 2880.3857 & 1061.3373 & 3941.7230 & \\
\hline & $7 / 29 / 2008$ 14:00 & 169 & 211 & 1400 & 3.716 & 3.716 & & 1.6184 & $2.15 \mathrm{E}-02$ & 3213.1331 & 1183.9450 & 4397.0781 & \\
\hline & $7 / 29 / 2008$ 15:00 & 170 & 211 & 1500 & 3.99 & 3.99 & & 1.4386 & $1.86 \mathrm{E}-02$ & 2779.4869 & 1024.1591 & 3803.6461 & \\
\hline & $7 / 29 / 2008$ 16:00 & 171 & 211 & 1600 & 4.103 & 4.103 & & 1.3714 & 1.76E-02 & 2620.6236 & 965.6227 & 3586.2463 & \\
\hline & 7/29/2008 17:00 & 172 & 211 & 1700 & 4.298 & 4.298 & & 1.2638 & 1.59E-02 & 2369.8794 & 873.2308 & 3243.1102 & \\
\hline & 7/29/2008 18:00 & 173 & 211 & 1800 & 4.178 & 4.178 & & 1.3289 & 1.69E-02 & 2520.8492 & 928.8587 & 3449.7080 & \\
\hline & $7 / 29 / 2008$ 19:00 & 174 & 211 & 1900 & 4.202 & 4.202 & & 1.3156 & 1.67E-02 & 2489.8237 & 917.4267 & 3407.2504 & \\
\hline & $7 / 29 / 200820: 00$ & 175 & 211 & 2000 & 5.434 & 5.434 & & 0.7906 & 8.91E-03 & 1330.1836 & 490.1335 & 1820.3171 & \\
\hline & 7/29/2008 21:00 & 176 & 211 & 2100 & 7.413 & 7.413 & & 0.3126 & 2.84E-03 & 424.4271 & 156.3889 & 580.8159 & \\
\hline & $7 / 29 / 2008$ 22:00 & 177 & 211 & 2200 & 8.22 & 8.22 & & 0.1837 & 1.48E-03 & 220.6252 & 81.2939 & 301.9191 & \\
\hline & $7 / 29 / 2008$ 23:00 & 178 & 211 & 2300 & 8.47 & 8.47 & & 0.1488 & 1.14E-03 & 170.1662 & 62.7013 & 232.8675 & \\
\hline & $7 / 30 / 20080: 00$ & 179 & 212 & 0 & 8.56 & 8.56 & 8.58 & 0.1367 & 1.03E-03 & 153.3216 & 56.4945 & 209.8161 & \\
\hline & 7/30/2008 1:00 & 180 & 212 & 100 & 8.58 & 8.58 & & 0.1340 & 1.00E-03 & 149.6711 & 55.1494 & 204.8205 & \\
\hline & 7/30/2008 2:00 & 181 & 212 & 200 & 8.58 & 8.58 & & 0.1340 & 1.00E-03 & 149.6711 & 55.1494 & 204.8205 & \\
\hline & 7/30/2008 3:00 & 182 & 212 & 300 & 8.52 & 8.52 & & 0.1420 & $1.08 \mathrm{E}-03$ & 160.7235 & 59.2219 & 219.9454 & \\
\hline & 7/30/2008 4:00 & 183 & 212 & 400 & 8.46 & 8.46 & & 0.1501 & 1.15E-03 & 172.0803 & 63.4065 & 235.4868 & \\
\hline & 7/30/2008 5:00 & 184 & 212 & 500 & 8.4 & 8.4 & & 0.1583 & 1.23E-03 & 183.7449 & 67.7046 & 251.4495 & \\
\hline & 7/30/2008 6:00 & 185 & 212 & 600 & 8.35 & 8.35 & & 0.1653 & 1.30E-03 & 193.7032 & 71.3739 & 265.0772 & \\
\hline & 7/30/2008 7:00 & 186 & 212 & 700 & 8.32 & 8.32 & & 0.1695 & 1.34E-03 & 199.7831 & 73.6142 & 273.3972 & \\
\hline & $7 / 30 / 20088: 00$ & 187 & 212 & 800 & 8.24 & 8.24 & & 0.1808 & 1.45E-03 & 216.3852 & 79.7316 & 296.1168 & \\
\hline & 7/30/2008 9:00 & 188 & 212 & 900 & 6.678 & 6.678 & & 0.4570 & 4.54E-03 & 677.5254 & 249.6482 & 927.1735 & \\
\hline & $7 / 30 / 2008$ 10:00 & 189 & 212 & 1000 & 4.053 & 4.053 & & 1.4007 & 1.80E-02 & 2689.6087 & 991.0416 & 3680.6503 & \\
\hline & 7/30/2008 11:00 & 190 & 212 & 1100 & 3.727 & 3.727 & & 1.6107 & 2.14E-02 & 3194.2562 & 1176.9894 & 4371.2457 & \\
\hline & $7 / 30 / 2008$ 12:00 & 191 & 212 & 1200 & 3.774 & 3.774 & & 1.5782 & $2.09 \mathrm{E}-02$ & 3115.0701 & 1147.8116 & 4262.8817 & \\
\hline & 7/30/2008 13:00 & 192 & 212 & 1300 & 3.792 & 3.792 & & 1.5659 & 2.07E-02 & 3085.3604 & 1136.8645 & 4222.2249 & \\
\hline & $7 / 30 / 2008$ 14:00 & 193 & 212 & 1400 & 3.715 & 3.715 & & 1.6191 & $2.15 E-02$ & 3214.8558 & 1184.5797 & 4399.4355 & \\
\hline & 7/30/2008 15:00 & 194 & 212 & 1500 & 3.768 & 3.768 & & 1.5823 & 2.09E-02 & 3125.0483 & 1151.4883 & 4276.5367 & \\
\hline & $7 / 30 / 2008$ 16:00 & 195 & 212 & 1600 & 3.65 & 3.65 & & 1.6658 & 2.23E-02 & 3329.2356 & 1226.7253 & 4555.9609 & \\
\hline & $7 / 30 / 2008$ 17:00 & 196 & 212 & 1700 & 3.596 & 3.596 & & 1.7058 & $2.30 \mathrm{E}-02$ & 3427.9958 & 1263.1156 & 4691.1114 & \\
\hline & $7 / 30 / 2008$ 18:00 & 197 & 212 & 1800 & 3.776 & 3.776 & & 1.5768 & $2.08 \mathrm{E}-02$ & 3111.7524 & 1146.5892 & 4258.3415 & \\
\hline & 7/30/2008 19:00 & 198 & 212 & 1900 & 3.963 & 3.963 & & 1.4552 & 1.89E-02 & 2819.0546 & 1038.7387 & 3857.7933 & \\
\hline & $7 / 30 / 200820: 00$ & 199 & 212 & 2000 & 4.788 & 4.788 & & 1.0322 & 1.24E-02 & 1846.9973 & 680.5641 & 2527.5614 & \\
\hline & 7/30/2008 21:00 & 200 & 212 & 2100 & 6.706 & 6.706 & & 0.4509 & $4.46 \mathrm{E}-03$ & 666.4404 & 245.5637 & 912.0040 & \\
\hline & $7 / 30 / 2008$ 22:00 & 201 & 212 & 2200 & 7.923 & 7.923 & & 0.2281 & 1.93E-03 & 287.9546 & 106.1028 & 394.0574 & \\
\hline & 7/30/2008 23:00 & 202 & 212 & 2300 & 8.33 & 8.33 & & 0.1681 & $1.32 \mathrm{E}-03$ & 197.7477 & 72.8642 & 270.6118 & \\
\hline & $7 / 31 / 20080: 00$ & 203 & 213 & 0 & 8.5 & 8.5 & 8.57 & 0.1447 & 1.10E-03 & 164.4751 & 60.6042 & 225.0794 & \\
\hline & 7/31/2008 1:00 & 204 & 213 & 100 & 8.57 & 8.57 & & 0.1354 & $1.01 \mathrm{E}-03$ & 151.4921 & 55.8204 & 207.3125 & \\
\hline
\end{tabular}




\begin{tabular}{|c|c|c|c|c|c|c|c|c|c|c|c|c|c|}
\hline Tree ID & TS & RN & JD & HR & $\begin{array}{c}\text { Raw } \\
\text { TC_dTA }\end{array}$ & $\begin{array}{c}\text { Fixed } \\
\text { TC_dT } \Delta\end{array}$ & dTM & $\mathbf{K}$ & $\begin{array}{c}\text { Sap } \\
\text { Velocity } \\
(\mathrm{cm} / \mathrm{s})\end{array}$ & $\begin{array}{c}\text { Probed } \\
\text { Drywood F } \\
\text { (g/hr) }\end{array}$ & $\begin{array}{c}\text { Extrapolated } \\
\text { Wetwood F } \\
(\mathrm{g} / \mathrm{hr})\end{array}$ & $\begin{array}{c}\text { Extrapolated } \\
\text { Total Wood F } \\
(\mathrm{g} / \mathrm{hr})\end{array}$ & $\begin{array}{l}\text { Relative } \\
\text { Humidity, } \\
\text { @10m (\%) }\end{array}$ \\
\hline & 7/31/2008 2:00 & 205 & 213 & 200 & 8.57 & 8.57 & & 0.1354 & $1.01 \mathrm{E}-03$ & 151.4921 & 55.8204 & 207.3125 & \\
\hline & 7/31/2008 3:00 & 206 & 213 & 300 & 8.54 & 8.54 & & 0.1393 & 1.05E-03 & 157.0057 & 57.8520 & 214.8577 & \\
\hline & 7/31/2008 4:00 & 207 & 213 & 400 & 8.51 & 8.51 & & 0.1434 & $1.09 \mathrm{E}-03$ & 162.5951 & 59.9115 & 222.5066 & \\
\hline & 7/31/20085:00 & 208 & 213 & 500 & 8.48 & 8.48 & & 0.1474 & $1.13 \mathrm{E}-03$ & 168.2607 & 61.9991 & 230.2598 & \\
\hline & 7/31/2008 6:00 & 209 & 213 & 600 & 8.43 & 8.43 & & 0.1542 & 1.19E-03 & 177.8739 & 65.5413 & 243.4152 & \\
\hline & 7/31/2008 7:00 & 210 & 213 & 700 & 8.37 & 8.37 & & 0.1625 & $1.27 \mathrm{E}-03$ & 189.6938 & 69.8966 & 259.5904 & \\
\hline & 7/31/2008 8:00 & 211 & 213 & 800 & 8.26 & 8.26 & & 0.1780 & $1.42 \mathrm{E}-03$ & 212.1812 & 78.1825 & 290.3637 & \\
\hline & 7/31/2008 9:00 & 212 & 213 & 900 & 6.334 & 6.334 & & 0.5362 & $5.52 \mathrm{E}-03$ & 824.7024 & 303.8786 & 1128.5810 & \\
\hline & $7 / 31 / 2008$ 10:00 & 213 & 213 & 1000 & 4.127 & 4.127 & & 1.3576 & $1.73 \mathrm{E}-02$ & 2588.2218 & 953.6835 & 3541.9053 & \\
\hline & 7/31/2008 11:00 & 214 & 213 & 1100 & 4.031 & 4.031 & & 1.4138 & 1.82E-02 & 2720.6127 & 1002.4657 & 3723.0784 & \\
\hline & 7/31/2008 12:00 & 215 & 213 & 1200 & 3.758 & 3.758 & & 1.5891 & 2.10E-02 & 3141.7630 & 1157.6472 & 4299.4101 & \\
\hline & $7 / 31 / 2008$ 13:00 & 216 & 213 & 1300 & 4.012 & 4.012 & & 1.4252 & 1.84E-02 & 2747.7169 & 1012.4528 & 3760.1697 & \\
\hline & 7/31/2008 14:00 & 217 & 213 & 1400 & 3.672 & 3.672 & & 1.6498 & $2.20 \mathrm{E}-02$ & 3289.9847 & 1212.2625 & 4502.2472 & \\
\hline & 7/31/2008 15:00 & 218 & 213 & 1500 & 3.65 & 3.65 & & 1.6658 & 2.23E-02 & 3329.2356 & 1226.7253 & 4555.9609 & \\
\hline & $7 / 31 / 2008$ 16:00 & 219 & 213 & 1600 & 3.782 & 3.782 & & 1.5727 & 2.08E-02 & 3101.8243 & 1142.9309 & 4244.7552 & \\
\hline & 7/31/2008 17:00 & 220 & 213 & 1700 & 3.698 & 3.698 & & 1.6312 & 2.17E-02 & 3244.3102 & 1195.4328 & 4439.7430 & \\
\hline & $7 / 31 / 2008$ 18:00 & 221 & 213 & 1800 & 3.81 & 3.81 & & 1.5538 & 2.05E-02 & 3055.9842 & 1126.0402 & 4182.0245 & \\
\hline & 7/31/2008 19:00 & 222 & 213 & 1900 & 4.147 & 4.147 & & 1.3463 & 1.72E-02 & 2561.5639 & 943.8609 & 3505.4247 & \\
\hline & $7 / 31 / 200820: 00$ & 223 & 213 & 2000 & 5.461 & 5.461 & & 0.7817 & 8.79E-03 & 1311.8711 & 483.3859 & 1795.2571 & \\
\hline & $7 / 31 / 200821: 00$ & 224 & 213 & 2100 & 6.684 & 6.684 & & 0.4557 & $4.52 \mathrm{E}-03$ & 675.1393 & 248.7690 & 923.9082 & \\
\hline & $7 / 31 / 200822: 00$ & 225 & 213 & 2200 & 7.562 & 7.562 & & 0.2867 & $2.56 \mathrm{E}-03$ & 381.6181 & 140.6150 & 522.2331 & \\
\hline & 7/31/2008 23:00 & 226 & 213 & 2300 & 7.474 & 7.474 & & 0.3018 & $2.72 \mathrm{E}-03$ & 406.5915 & 149.8170 & 556.4085 & \\
\hline & 8/1/2008 0:00 & 227 & 214 & 0 & 7.735 & 7.735 & 8.83 & 0.2579 & $2.24 \mathrm{E}-03$ & 335.0247 & 123.4467 & 458.4714 & \\
\hline & 8/1/2008 1:00 & 228 & 214 & 100 & 8.22 & 8.22 & & 0.1837 & $1.48 \mathrm{E}-03$ & 220.6252 & 81.2939 & 301.9191 & \\
\hline & 8/1/2008 2:00 & 229 & 214 & 200 & 8.46 & 8.46 & & 0.1501 & 1.15E-03 & 172.0803 & 63.4065 & 235.4868 & \\
\hline & 8/1/2008 3:00 & 230 & 214 & 300 & 8.5 & 8.5 & & 0.1447 & 1.10E-03 & 164.4751 & 60.6042 & 225.0794 & \\
\hline & 8/1/2008 4:00 & 231 & 214 & 400 & 8.55 & 8.55 & & 0.1380 & 1.04E-03 & 155.1594 & 57.1717 & 212.3311 & \\
\hline & 8/1/2008 5:00 & 232 & 214 & 500 & 8.58 & 8.58 & & 0.1340 & 1.00E-03 & 149.6711 & 55.1494 & 204.8205 & \\
\hline & 8/1/2008 6:00 & 233 & 214 & 600 & 8.57 & 8.57 & & 0.1354 & 1.01E-03 & 151.4921 & 55.8204 & 207.3125 & \\
\hline & 8/1/2008 7:00 & 234 & 214 & 700 & 8.53 & 8.53 & & 0.1407 & 1.06E-03 & 158.8604 & 58.5354 & 217.3958 & \\
\hline & 8/1/2008 8:00 & 235 & 214 & 800 & 8.41 & 8.41 & & 0.1570 & 1.22E-03 & 181.7793 & 66.9803 & 248.7596 & \\
\hline & 8/1/2008 9:00 & 236 & 214 & 900 & 6.494 & 6.494 & & 0.4983 & 5.05E-03 & 753.6325 & 277.6914 & 1031.3238 & \\
\hline & 8/1/2008 10:00 & 237 & 214 & 1000 & 4.223 & 4.223 & & 1.3040 & $1.65 E-02$ & 2463.0240 & 907.5518 & 3370.5758 & \\
\hline & 8/1/2008 11:00 & 238 & 214 & 1100 & 4.139 & 4.139 & & 1.3508 & $1.72 \mathrm{E}-02$ & 2572.1899 & 947.7763 & 3519.9662 & \\
\hline & 8/1/2008 12:00 & 239 & 214 & 1200 & 4.054 & 4.054 & & 1.4001 & 1.80E-02 & 2688.2090 & 990.5259 & 3678.7348 & \\
\hline & 8/1/2008 13:00 & 240 & 214 & 1300 & 3.96 & 3.96 & & 1.4571 & 1.89E-02 & 2823.4909 & 1040.3733 & 3863.8641 & \\
\hline & 8/1/2008 14:00 & 241 & 214 & 1400 & 3.89 & 3.89 & & 1.5013 & 1.96E-02 & 2929.3279 & 1079.3711 & 4008.6990 & \\
\hline & 8/1/2008 15:00 & 242 & 214 & 1500 & 3.807 & 3.807 & & 1.5558 & $2.05 E-02$ & 3060.8573 & 1127.8358 & 4188.6931 & \\
\hline & 8/1/2008 16:00 & 243 & 214 & 1600 & 3.912 & 3.912 & & 1.4872 & $1.94 \mathrm{E}-02$ & 2895.5775 & 1066.9351 & 3962.5126 & \\
\hline & 8/1/2008 17:00 & 244 & 214 & 1700 & 3.953 & 3.953 & & 1.4614 & 1.90E-02 & 2833.8734 & 1044.1989 & 3878.0723 & \\
\hline & 8/1/2008 18:00 & 245 & 214 & 1800 & 4.025 & 4.025 & & 1.4174 & 1.83E-02 & 2729.1389 & 1005.6073 & 3734.7462 & \\
\hline
\end{tabular}




\begin{tabular}{|c|c|c|c|c|c|c|c|c|c|c|c|c|c|}
\hline Tree ID & TS & RN & JD & HR & $\begin{array}{c}\text { Raw } \\
\text { TC_dT } \Delta\end{array}$ & $\begin{array}{c}\text { Fixed } \\
\text { TC_dT } \Delta\end{array}$ & dTM & $\mathbf{K}$ & $\begin{array}{l}\text { Sap } \\
\text { Velocity } \\
\text { (cm/s) }\end{array}$ & $\begin{array}{c}\text { Probed } \\
\text { Drywood F } \\
\text { (g/hr) }\end{array}$ & $\begin{array}{c}\text { Extrapolated } \\
\text { Wetwood F } \\
\text { (g/hr) }\end{array}$ & $\begin{array}{c}\text { Extrapolated } \\
\text { Total Wood F } \\
(\mathrm{g} / \mathrm{hr})\end{array}$ & $\begin{array}{l}\text { Relative } \\
\text { Humidity, } \\
@ 10 \mathrm{~m}(\%)\end{array}$ \\
\hline & 8/1/2008 19:00 & 246 & 214 & 1900 & ) $\quad 4.231$ & 4.231 & & 1.2997 & 1.64E-02 & 2452.8988 & 903.8210 & \begin{tabular}{|r|}
3356.7198 \\
\end{tabular} & \\
\hline & $8 / 1 / 200820: 00$ & 247 & 214 & 2000 & 5.169 & 5.169 & & 0.8824 & $1.02 \mathrm{E}-02$ & 1522.7943 & 561.1049 & 2083.8992 & \\
\hline & 8/1/2008 21:00 & 248 & 214 & 2100 & 7.352 & 7.352 & & 0.3234 & $2.97 \mathrm{E}-03$ & 442.7039 & 163.1234 & 605.8273 & \\
\hline & 8/1/2008 22:00 & 249 & 214 & 2200 & 8.48 & 8.48 & & 0.1474 & 1.13E-03 & 168.2607 & 61.9991 & 230.2598 & \\
\hline & 8/1/2008 23:00 & 250 & 214 & 2300 & 8.83 & 8.83 & & 0.1019 & 7.16E-04 & 106.8407 & 39.3676 & 146.2083 & \\
\hline & 8/2/2008 0:00 & 251 & 215 & 0 & 8.93 & 8.93 & 8.94 & 0.0896 & 6.11E-04 & 91.1480 & 33.5854 & 124.7334 & \\
\hline & 8/2/2008 1:00 & 252 & 215 & 100 & 8.94 & 8.94 & & 0.0884 & $6.00 \mathrm{E}-04$ & 89.6240 & 33.0238 & 122.6478 & \\
\hline & $8 / 2 / 2008$ 2:00 & 253 & 215 & 200 & 8.94 & 8.94 & & 0.0884 & $6.00 \mathrm{E}-04$ & 89.6240 & 33.0238 & 122.6478 & \\
\hline & $8 / 2 / 20083: 00$ & 254 & 215 & 300 & 8.91 & 8.91 & & 0.0920 & $6.31 E-04$ & 94.2209 & 34.7176 & 128.9385 & \\
\hline & 8/2/2008 4:00 & 255 & 215 & 400 & 8.77 & 8.77 & & 0.1095 & 7.81E-04 & 116.6501 & 42.9821 & 159.6322 & \\
\hline & 8/2/2008 5:00 & 256 & 215 & 500 & 8.65 & 8.65 & & 0.1249 & $9.19 E-04$ & 137.1575 & 50.5385 & 187.6960 & \\
\hline & $8 / 2 / 20086: 00$ & 257 & 215 & 600 & 8.57 & 8.57 & & 0.1354 & 1.01E-03 & 151.4921 & 55.8204 & 207.3125 & \\
\hline & $8 / 2 / 20087: 00$ & 258 & 215 & 700 & 8.42 & 8.42 & & 0.1556 & 1.20E-03 & 179.8223 & 66.2592 & 246.0815 & \\
\hline & $8 / 2 / 2008$ 8:00 & 259 & 215 & 800 & 6.936 & 6.936 & & 0.4028 & 3.89E-03 & 580.0176 & 213.7194 & 793.7370 & \\
\hline & 8/2/2008 9:00 & 260 & 215 & 900 & 4.704 & 4.704 & & 1.0685 & 1.29E-02 & 1927.2557 & 710.1370 & 2637.3928 & \\
\hline & 8/2/2008 10:00 & 261 & 215 & 1000 & 4.501 & 4.501 & & 1.1617 & 1.43E-02 & 2136.4449 & 787.2170 & 2923.6619 & \\
\hline & 8/2/2008 11:00 & 262 & 215 & 1100 & 4.381 & 4.381 & & 1.2210 & 1.52E-02 & 2271.2693 & 836.8959 & 3108.1652 & \\
\hline & $8 / 2 / 2008$ 12:00 & 263 & 215 & 1200 & 4.209 & 4.209 & & 1.3117 & 1.66E-02 & 2480.8547 & 914.1219 & 3394.9766 & \\
\hline & 8/2/2008 13:00 & 264 & 215 & 1300 & 4.178 & 4.178 & & 1.3289 & 1.69E-02 & 2520.8492 & 928.8587 & 3449.7080 & \\
\hline & 8/2/2008 14:00 & 265 & 215 & 1400 & 4.158 & 4.158 & & 1.3401 & 1.71E-02 & 2547.0332 & 938.5068 & 3485.5400 & \\
\hline & 8/2/2008 15:00 & 266 & 215 & 1500 & 4.077 & 4.077 & & 1.3866 & $1.78 \mathrm{E}-02$ & 2656.2427 & 978.7472 & 3634.9899 & \\
\hline & $8 / 2 / 2008$ 16:00 & 267 & 215 & 1600 & 4.157 & 4.157 & & 1.3406 & 1.71E-02 & 2548.3504 & 938.9921 & 3487.3425 & \\
\hline & $8 / 2 / 2008$ 17:00 & 268 & 215 & 1700 & 4.112 & 4.112 & & 1.3662 & 1.75E-02 & 2608.4197 & 961.1259 & 3569.5456 & \\
\hline & $8 / 2 / 2008$ 18:00 & 269 & 215 & 1800 & 4.209 & 4.209 & & 1.3117 & 1.66E-02 & 2480.8547 & 914.1219 & 3394.9766 & \\
\hline & 8/2/2008 19:00 & 270 & 215 & 1900 & 4.526 & 4.526 & & 1.1498 & 1.41E-02 & 2109.4456 & 777.2686 & 2886.7142 & \\
\hline & $8 / 2 / 200820: 00$ & 271 & 215 & 2000 & 5.331 & 5.331 & & 0.8252 & 9.39E-03 & 1402.1968 & 516.6683 & 1918.8651 & \\
\hline & 8/2/2008 21:00 & 272 & 215 & 2100 & 6.985 & 6.985 & & 0.3930 & 3.77E-03 & 562.6243 & 207.3105 & 769.9348 & \\
\hline & 8/2/2008 22:00 & 273 & 215 & 2200 & 8.13 & 8.13 & & 0.1968 & 1.61E-03 & 240.1552 & 88.4901 & 328.6453 & \\
\hline & 8/2/2008 23:00 & 274 & 215 & 2300 & 8.54 & 8.54 & & 0.1393 & 1.05E-03 & 157.0057 & 57.8520 & 214.8577 & \\
\hline & 8/3/2008 0:00 & 275 & 216 & 0 & 8.61 & 8.61 & 9.03 & 0.1301 & 9.66E-04 & 144.2581 & 53.1549 & 197.4130 & \\
\hline & $8 / 3 / 2008$ 1:00 & 276 & 216 & 100 & 8.59 & 8.59 & & 0.1327 & 9.90E-04 & 147.8584 & 54.4815 & 202.3399 & \\
\hline & $8 / 3 / 2008$ 2:00 & 277 & 216 & 200 & 8.68 & 8.68 & & 0.1210 & 8.84E-04 & 131.9192 & 48.6083 & 180.5275 & \\
\hline & 8/3/2008 3:00 & 278 & 216 & 300 & 8.84 & 8.84 & & 0.1007 & 7.05E-04 & 105.2345 & 38.7758 & 144.0103 & \\
\hline & 8/3/2008 4:00 & 279 & 216 & 400 & 8.97 & 8.97 & & 0.0847 & $5.70 \mathrm{E}-04$ & 85.1012 & 31.3573 & 116.4585 & \\
\hline & $8 / 3 / 20085: 00$ & 280 & 216 & 500 & 9.03 & 9.03 & & 0.0775 & 5.11E-04 & 76.2791 & 28.1066 & 104.3858 & \\
\hline & 8/3/2008 6:00 & 281 & 216 & 600 & 9.03 & 9.03 & & 0.0775 & 5.11E-04 & 76.2791 & 28.1066 & 104.3858 & \\
\hline & 8/3/2008 7:00 & 282 & 216 & 700 & 8.92 & 8.92 & & 0.0908 & $6.21 \mathrm{E}-04$ & 92.6803 & 34.1500 & 126.8303 & \\
\hline & $8 / 3 / 20088: 00$ & 283 & 216 & 800 & 7.397 & 7.397 & & 0.3154 & $2.88 \mathrm{E}-03$ & 429.1779 & 158.1394 & 587.3173 & \\
\hline & 8/3/2008 9:00 & 284 & 216 & 900 & 4.77 & 4.77 & & 1.0398 & 1.25E-02 & 1863.9041 & 686.7938 & 2550.6978 & \\
\hline & 8/3/2008 10:00 & 285 & 216 & 1000 & 4.376 & 4.376 & & 1.2235 & 1.53E-02 & 2277.0818 & 839.0376 & 3116.1194 & \\
\hline & 8/3/2008 11:00 & 286 & 216 & 1100 & 4.177 & 4.177 & & 1.3294 & 1.69E-02 & 2522.1513 & 929.3385 & 3451.4898 & \\
\hline
\end{tabular}




\begin{tabular}{|c|c|c|c|c|c|c|c|c|c|c|c|c|c|}
\hline Tree ID & TS & RN & JD & HR & $\begin{array}{c}\text { Raw } \\
\text { TC_dTA }\end{array}$ & $\begin{array}{c}\text { Fixed } \\
\text { TC_dT } \Delta\end{array}$ & dTM & $\mathbf{K}$ & $\begin{array}{l}\text { Sap } \\
\text { Velocity } \\
(\mathrm{cm} / \mathrm{s})\end{array}$ & $\begin{array}{c}\text { Probed } \\
\text { Drywood F } \\
(\mathrm{g} / \mathrm{hr})\end{array}$ & $\begin{array}{c}\text { Extrapolated } \\
\text { Wetwood F } \\
(\mathrm{g} / \mathrm{hr})\end{array}$ & $\begin{array}{c}\text { Extrapolated } \\
\text { Total Wood F } \\
(\mathrm{g} / \mathrm{hr})\end{array}$ & $\begin{array}{l}\text { Relative } \\
\text { Humidity, } \\
@ 10 \mathrm{~m}(\%)\end{array}$ \\
\hline & 8/3/2008 12:00 & 287 & 216 & 1200 & ) $\quad 4.107$ & 4.107 & & 1.3691 & 1.75E-02 & 2615.1917 & 963.6212 & 3578.8129 & \\
\hline & 8/3/2008 13:00 & 288 & 216 & 1300 & 4.082 & 4.082 & & 1.3836 & 1.77E-02 & 2649.3506 & 976.2077 & 3625.5583 & \\
\hline & $8 / 3 / 200814: 00$ & 289 & 216 & 1400 & 4.075 & 4.075 & & 1.3877 & $1.78 \mathrm{E}-02$ & 2659.0052 & 979.7651 & 3638.7703 & \\
\hline & 8/3/2008 15:00 & 290 & 216 & 1500 & 4.112 & 4.112 & & 1.3662 & 1.75E-02 & 2608.4197 & 961.1259 & 3569.5456 & \\
\hline & $8 / 3 / 2008$ 16:00 & 291 & 216 & 1600 & 4.278 & 4.278 & & 1.2744 & 1.60E-02 & 2394.3332 & 882.2413 & 3276.5745 & \\
\hline & 8/3/2008 17:00 & 292 & 216 & 1700 & 4.202 & 4.202 & & 1.3156 & 1.67E-02 & 2489.8237 & 917.4267 & 3407.2504 & \\
\hline & 8/3/2008 18:00 & 293 & 216 & 1800 & 4.223 & 4.223 & & 1.3040 & 1.65E-02 & 2463.0240 & 907.5518 & 3370.5758 & \\
\hline & 8/3/2008 19:00 & 294 & 216 & 1900 & 4.444 & 4.444 & & 1.1895 & 1.47E-02 & 2199.3858 & 810.4089 & 3009.7947 & \\
\hline & $8 / 3 / 200820: 00$ & 295 & 216 & 2000 & 5.048 & 5.048 & & 0.9275 & 1.08E-02 & 1619.2089 & 596.6308 & 2215.8397 & \\
\hline & 8/3/2008 21:00 & 296 & 216 & 2100 & 6.332 & 6.332 & & 0.5366 & 5.53E-03 & 825.6212 & 304.2171 & 1129.8384 & \\
\hline & $8 / 3 / 200822: 00$ & 297 & 216 & 2200 & 7.277 & 7.277 & & 0.3371 & 3.12E-03 & 465.7963 & 171.6322 & 637.4285 & \\
\hline & $8 / 3 / 200823: 00$ & 298 & 216 & 2300 & 7.407 & 7.407 & & 0.3136 & $2.86 \mathrm{E}-03$ & 426.2050 & 157.0440 & 583.2491 & \\
\hline & $8 / 4 / 20080: 00$ & 299 & 217 & 0 & 7.385 & 7.385 & 8.60 & 0.3175 & $2.90 \mathrm{E}-03$ & 432.7610 & 159.4597 & 592.2207 & \\
\hline & 8/4/2008 1:00 & 300 & 217 & 100 & 7.4 & 7.4 & & 0.3149 & 2.87E-03 & 428.2848 & 157.8103 & 586.0951 & \\
\hline & $8 / 4 / 20082: 00$ & 301 & 217 & 200 & 7.028 & 7.028 & & 0.3845 & 3.67E-03 & 547.6417 & 201.7898 & 749.4315 & \\
\hline & $8 / 4 / 20083: 00$ & 302 & 217 & 300 & 6.705 & 6.705 & & 0.4512 & 4.47E-03 & 666.8341 & 245.7087 & 912.5428 & \\
\hline & 8/4/2008 4:00 & 303 & 217 & 400 & 6.436 & 6.436 & & 0.5118 & 5.22E-03 & 778.8488 & 286.9828 & 1065.8316 & \\
\hline & 8/4/2008 5:00 & 304 & 217 & 500 & 6.634 & 6.634 & & 0.4667 & $4.66 \mathrm{E}-03$ & 695.2037 & 256.1621 & 951.3659 & \\
\hline & $8 / 4 / 20086: 00$ & 305 & 217 & 600 & 7.295 & 7.295 & & 0.3338 & $3.08 \mathrm{E}-03$ & 460.1907 & 169.5667 & 629.7574 & \\
\hline & $8 / 4 / 20087: 00$ & 306 & 217 & 700 & 7.436 & 7.436 & & 0.3085 & 2.80E-03 & 417.6509 & 153.8921 & 571.5430 & \\
\hline & 8/4/2008 8:00 & 307 & 217 & 800 & 7.027 & 7.027 & & 0.3847 & 3.67E-03 & 547.9872 & 201.9171 & 749.9043 & \\
\hline & 8/4/2008 9:00 & 308 & 217 & 900 & 6.22 & 6.22 & & 0.5643 & 5.88E-03 & 878.3323 & 323.6396 & 1201.9718 & \\
\hline & $8 / 4 / 2008$ 10:00 & 309 & 217 & 1000 & 5.188 & 5.188 & & 0.8755 & 1.01E-02 & 1508.1620 & 555.7133 & 2063.8753 & \\
\hline & 8/4/2008 11:00 & 310 & 217 & 1100 & 4.686 & 4.686 & & 1.0764 & 1.30E-02 & 1944.9133 & 716.6433 & 2661.5566 & \\
\hline & 8/4/2008 12:00 & 311 & 217 & 1200 & 4.376 & 4.376 & & 1.2235 & 1.53E-02 & 2277.0818 & 839.0376 & 3116.1194 & \\
\hline & 8/4/2008 13:00 & 312 & 217 & 1300 & 4.311 & 4.311 & & 1.2570 & 1.58E-02 & 2354.1312 & 867.4281 & 3221.5593 & \\
\hline & 8/4/2008 14:00 & 313 & 217 & 1400 & 4.746 & 4.746 & & 1.0501 & 1.26E-02 & 1886.6913 & 695.1902 & 2581.8815 & \\
\hline & $8 / 4 / 2008$ 15:00 & 314 & 217 & 1500 & 5.207 & 5.207 & & 0.8686 & 1.00E-02 & 1493.6628 & 550.3708 & 2044.0335 & \\
\hline & $8 / 4 / 2008$ 16:00 & 315 & 217 & 1600 & 4.48 & 4.48 & & 1.1719 & 1.45E-02 & 2159.4075 & 795.6781 & 2955.0856 & \\
\hline & $8 / 4 / 200817: 00$ & 316 & 217 & 1700 & 4.294 & 4.294 & & 1.2660 & 1.59E-02 & 2374.7482 & 875.0248 & 3249.7730 & \\
\hline & 8/4/2008 18:00 & 317 & 217 & 1800 & 4.449 & 4.449 & & 1.1870 & 1.47E-02 & 2193.7863 & 808.3457 & 3002.1319 & \\
\hline & 8/4/2008 19:00 & 318 & 217 & 1900 & 4.783 & 4.783 & & 1.0343 & 1.24E-02 & 1851.6780 & 682.2888 & 2533.9668 & \\
\hline & $8 / 4 / 200820: 00$ & 319 & 217 & 2000 & 5.625 & 5.625 & & 0.7298 & 8.07E-03 & 1205.3951 & 444.1526 & 1649.5477 & \\
\hline & $8 / 4 / 200821: 00$ & 320 & 217 & 2100 & 7.515 & 7.515 & & 0.2947 & $2.65 E-03$ & 394.8464 & 145.4893 & 540.3357 & \\
\hline & $8 / 4 / 200822: 00$ & 321 & 217 & 2200 & 8.33 & 8.33 & & 0.1681 & 1.32E-03 & 197.7477 & 72.8642 & 270.6118 & \\
\hline & 8/4/2008 23:00 & 322 & 217 & 2300 & 8.6 & 8.6 & & 0.1314 & 9.78E-04 & 146.0541 & 53.8166 & 199.8707 & \\
\hline & 8/5/2008 0:00 & 323 & 218 & 0 & 8.85 & 8.85 & 9.28 & 0.0994 & 6.94E-04 & 103.6365 & 38.1870 & 141.8235 & \\
\hline & $8 / 5 / 20081: 00$ & 324 & 218 & 100 & 8.98 & 8.98 & & 0.0835 & 5.60E-04 & 83.6101 & 30.8079 & 114.4180 & \\
\hline & $8 / 5 / 20082: 00$ & 325 & 218 & 200 & 9.05 & 9.05 & & 0.0751 & 4.92E-04 & 73.4050 & 27.0476 & 100.4526 & \\
\hline & $8 / 5 / 2008$ 3:00 & 326 & 218 & 300 & 9.01 & 9.01 & & 0.0799 & 5.30E-04 & 79.1866 & 29.1779 & 108.3646 & \\
\hline & 8/5/2008 4:00 & 327 & 218 & 400 & 8.82 & 8.82 & & 0.1032 & 7.27E-04 & 108.4551 & 39.9625 & 148.4176 & \\
\hline
\end{tabular}




\begin{tabular}{|c|c|c|c|c|c|c|c|c|c|c|c|c|c|}
\hline Tree ID & TS & RN & JD & HR & $\begin{array}{c}\text { Raw } \\
\text { TC_dTA }\end{array}$ & $\begin{array}{c}\text { Fixed } \\
\text { TC_dT } \Delta\end{array}$ & dTM & $\mathbf{K}$ & $\begin{array}{l}\text { Sap } \\
\text { Velocity } \\
(\mathrm{cm} / \mathrm{s})\end{array}$ & $\begin{array}{c}\text { Probed } \\
\text { Drywood F } \\
(\mathrm{g} / \mathrm{hr})\end{array}$ & $\begin{array}{c}\text { Extrapolated } \\
\text { Wetwood F } \\
(\mathrm{g} / \mathrm{hr})\end{array}$ & $\begin{array}{c}\text { Extrapolated } \\
\text { Total Wood F } \\
(\mathrm{g} / \mathrm{hr})\end{array}$ & $\begin{array}{l}\text { Relative } \\
\text { Humidity, } \\
@ 10 \mathrm{~m}(\%)\end{array}$ \\
\hline & 8/5/2008 5:00 & 328 & 218 & 500 & 8.72 & 8.72 & & 0.1158 & 8.38E-04 & 125.0505 & 46.0774 & 171.1280 & \\
\hline & 8/5/2008 6:00 & 329 & 218 & 600 & 8.77 & 8.77 & & 0.1095 & 7.81E-04 & 116.6501 & 42.9821 & 159.6322 & \\
\hline & 8/5/2008 7:00 & 330 & 218 & 700 & 8.81 & 8.81 & & 0.1044 & 7.37E-04 & 110.0777 & 40.5604 & 150.6380 & \\
\hline & 8/5/2008 8:00 & 331 & 218 & 800 & 8.65 & 8.65 & & 0.1249 & $9.19 \mathrm{E}-04$ & 137.1575 & 50.5385 & 187.6960 & \\
\hline & 8/5/2008 9:00 & 332 & 218 & 900 & 6.819 & 6.819 & & 0.4269 & 4.17E-03 & 622.9706 & 229.5463 & 852.5169 & \\
\hline & 8/5/2008 10:00 & 333 & 218 & 1000 & 5.141 & 5.141 & & 0.8926 & 1.03E-02 & 1544.6037 & 569.1410 & 2113.7447 & \\
\hline & 8/5/2008 11:00 & 334 & 218 & 1100 & 5.426 & 5.426 & & 0.7932 & 8.95E-03 & 1335.6537 & 492.1491 & 1827.8028 & \\
\hline & $8 / 5 / 200812: 00$ & 335 & 218 & 1200 & 5.11 & 5.11 & & 0.9041 & 1.05E-02 & 1569.0973 & 578.1662 & 2147.2635 & \\
\hline & 8/5/2008 13:00 & 336 & 218 & 1300 & 4.629 & 4.629 & & 1.1020 & 1.34E-02 & 2001.9384 & 737.6554 & 2739.5938 & \\
\hline & 8/5/2008 14:00 & 337 & 218 & 1400 & 5.242 & 5.242 & & 0.8562 & 9.83E-03 & 1467.2968 & 540.6557 & 2007.9524 & \\
\hline & 8/5/2008 15:00 & 338 & 218 & 1500 & 5.706 & 5.706 & & 0.7052 & 7.74E-03 & 1155.6632 & 425.8279 & 1581.4911 & \\
\hline & 8/5/2008 16:00 & 339 & 218 & 1600 & 5.435 & 5.435 & & 0.7902 & $8.91 \mathrm{E}-03$ & 1329.5012 & 489.8821 & 1819.3833 & \\
\hline & 8/5/2008 17:00 & 340 & 218 & 1700 & 4.984 & 4.984 & & 0.9522 & 1.12E-02 & 1672.5635 & 616.2904 & 2288.8539 & \\
\hline & 8/5/2008 18:00 & 341 & 218 & 1800 & 4.702 & 4.702 & & 1.0693 & 1.29E-02 & 1929.2095 & 710.8569 & 2640.0665 & \\
\hline & 8/5/2008 19:00 & 342 & 218 & 1900 & 4.761 & 4.761 & & 1.0437 & $1.25 \mathrm{E}-02$ & 1872.4163 & 689.9303 & 2562.3466 & \\
\hline & 8/5/2008 20:00 & 343 & 218 & 2000 & 5.766 & 5.766 & & 0.6875 & 7.50E-03 & 1119.9731 & 412.6771 & 1532.6502 & \\
\hline & 8/5/2008 21:00 & 344 & 218 & 2100 & 7.981 & 7.981 & & 0.2191 & 1.84E-03 & 274.1470 & 101.0151 & 375.1621 & \\
\hline & $8 / 5 / 200822: 00$ & 345 & 218 & 2200 & 8.94 & 8.94 & & 0.0884 & 6.00E-04 & 89.6240 & 33.0238 & 122.6478 & \\
\hline & 8/5/2008 23:00 & 346 & 218 & 2300 & 9.28 & 9.28 & & 0.0485 & 2.87E-04 & 42.8149 & 15.7760 & 58.5909 & \\
\hline & 8/6/2008 0:00 & 347 & 219 & 0 & 9.41 & 9.41 & 9.41 & 0.0340 & $1.85 E-04$ & 27.6626 & 10.1928 & 37.8554 & \\
\hline & 8/6/2008 1:00 & 348 & 219 & 100 & 9.41 & 9.41 & & 0.0340 & 1.85E-04 & 27.6626 & 10.1928 & 37.8554 & \\
\hline & $8 / 6 / 2008$ 2:00 & 349 & 219 & 200 & 9.4 & 9.4 & & 0.0351 & 1.93E-04 & 28.7682 & 10.6002 & 39.3684 & \\
\hline & 8/6/2008 3:00 & 350 & 219 & 300 & 9.35 & 9.35 & & 0.0406 & 2.31E-04 & 34.4498 & 12.6937 & 47.1435 & \\
\hline & 8/6/2008 4:00 & 351 & 219 & 400 & 9.29 & 9.29 & & 0.0474 & $2.79 \mathrm{E}-04$ & 41.5915 & 15.3252 & 56.9168 & \\
\hline & 8/6/2008 5:00 & 352 & 219 & 500 & 9.2 & 9.2 & & 0.0576 & $3.55 E-04$ & 52.9301 & 19.5032 & 72.4333 & \\
\hline & 8/6/2008 6:00 & 353 & 219 & 600 & 9.09 & 9.09 & & 0.0704 & 4.54E-04 & 67.7575 & 24.9666 & 92.7241 & \\
\hline & 8/6/2008 7:00 & 354 & 219 & 700 & 8.99 & 8.99 & & 0.0823 & 5.50E-04 & 82.1273 & 30.2615 & 112.3889 & \\
\hline & 8/6/2008 8:00 & 355 & 219 & 800 & 8.83 & 8.83 & & 0.1019 & 7.16E-04 & 106.8407 & 39.3676 & 146.2083 & \\
\hline & 8/6/2008 9:00 & 356 & 219 & 900 & 8.36 & 8.36 & & 0.1639 & 1.28E-03 & 191.6941 & 70.6336 & 262.3278 & \\
\hline & 8/6/2008 10:00 & 357 & 219 & 1000 & 5.259 & 5.259 & & 0.8502 & $9.74 \mathrm{E}-03$ & 1454.6486 & 535.9952 & 1990.6437 & \\
\hline & 8/6/2008 11:00 & 358 & 219 & 1100 & 4.35 & 4.35 & & 1.2368 & 1.55E-02 & 2307.5676 & 850.2708 & 3157.8384 & \\
\hline & 8/6/2008 12:00 & 359 & 219 & 1200 & 5.076 & 5.076 & & 0.9169 & 1.07E-02 & 1596.3896 & 588.2226 & 2184.6122 & \\
\hline & 8/6/2008 13:00 & 360 & 219 & 1300 & 5.632 & 5.632 & & 0.7276 & 8.05E-03 & 1201.0251 & 442.5424 & 1643.5675 & \\
\hline & $8 / 6 / 2008$ 14:00 & 361 & 219 & 1400 & 4.987 & 4.987 & & 0.9511 & 1.12E-02 & 1670.0246 & 615.3549 & 2285.3795 & \\
\hline & 8/6/2008 15:00 & 362 & 219 & 1500 & 4.546 & 4.546 & & 1.1403 & 1.40E-02 & 2088.1059 & 769.4055 & 2857.5114 & \\
\hline & 8/6/2008 16:00 & 363 & 219 & 1600 & 4.659 & 4.659 & & 1.0884 & 1.32E-02 & 1971.7127 & 726.5181 & 2698.2308 & \\
\hline & 8/6/2008 17:00 & 364 & 219 & 1700 & 4.486 & 4.486 & & 1.1690 & 1.44E-02 & 2152.8201 & 793.2508 & 2946.0709 & \\
\hline & 8/6/2008 18:00 & 365 & 219 & 1800 & 5.124 & 5.124 & & 0.8989 & 1.04E-02 & 1557.9900 & 574.0735 & 2132.0635 & \\
\hline & 8/6/2008 19:00 & 366 & 219 & 1900 & 5.065 & 5.065 & & 0.9210 & $1.08 \mathrm{E}-02$ & 1605.3170 & 591.5121 & 2196.8291 & \\
\hline & $8 / 6 / 200820: 00$ & 367 & 219 & 2000 & 5.507 & 5.507 & & 0.7668 & 8.58E-03 & 1281.1938 & 472.0822 & 1753.2760 & \\
\hline & 8/6/2008 21:00 & 368 & 219 & 2100 & 7.479 & 7.479 & & 0.3010 & $2.71 \mathrm{E}-03$ & 405.1488 & 149.2854 & 554.4342 & \\
\hline
\end{tabular}




\begin{tabular}{|c|c|c|c|c|c|c|c|c|c|c|c|c|c|}
\hline Tree ID & TS & RN & JD & HR & $\begin{array}{c}\text { Raw } \\
\text { TC_dTA }\end{array}$ & $\begin{array}{c}\text { Fixed } \\
\text { TC_dT } \Delta\end{array}$ & dTM & $\mathbf{K}$ & $\begin{array}{l}\text { Sap } \\
\text { Velocity } \\
(\mathrm{cm} / \mathrm{s})\end{array}$ & $\begin{array}{c}\text { Probed } \\
\text { Drywood F } \\
(\mathrm{g} / \mathrm{hr})\end{array}$ & $\begin{array}{c}\text { Extrapolated } \\
\text { Wetwood F } \\
(\mathrm{g} / \mathrm{hr})\end{array}$ & $\begin{array}{c}\text { Extrapolated } \\
\text { Total Wood F } \\
(\mathrm{g} / \mathrm{hr})\end{array}$ & $\begin{array}{l}\text { Relative } \\
\text { Humidity, } \\
@ 10 \mathrm{~m}(\%)\end{array}$ \\
\hline & 8/6/2008 22:00 & 369 & 219 & 2200 & $\begin{array}{r}8.79 \\
\end{array}$ & 8.79 & & 0.1069 & 7.59E-04 & 113.3475 & 41.7652 & 155.1127 & \\
\hline & 8/6/2008 23:00 & 370 & 219 & 2300 & 9.15 & 9.15 & & 0.0634 & 3.99E-04 & 59.5405 & 21.9389 & 81.4794 & \\
\hline & $8 / 7 / 20080: 00$ & 371 & 220 & 0 & 9.31 & 9.31 & 9.33 & 0.0451 & $2.62 E-04$ & 39.1728 & 14.4340 & 53.6068 & \\
\hline & 8/7/2008 1:00 & 372 & 220 & 100 & 9.33 & 9.33 & & 0.0429 & $2.46 \mathrm{E}-04$ & 36.7920 & 13.5568 & 50.3488 & \\
\hline & 8/7/2008 2:00 & 373 & 220 & 200 & 9.29 & 9.29 & & 0.0474 & 2.79E-04 & 41.5915 & 15.3252 & 56.9168 & \\
\hline & 8/7/2008 3:00 & 374 & 220 & 300 & 9.23 & 9.23 & & 0.0542 & $3.29 E-04$ & 49.0694 & 18.0806 & 67.1500 & \\
\hline & 8/7/2008 4:00 & 375 & 220 & 400 & 9.13 & 9.13 & & 0.0657 & 4.17E-04 & 62.2453 & 22.9356 & 85.1808 & \\
\hline & 8/7/2008 5:00 & 376 & 220 & 500 & 9.04 & 9.04 & & 0.0763 & 5.01E-04 & 74.8379 & 27.5756 & 102.4135 & \\
\hline & 8/7/2008 6:00 & 377 & 220 & 600 & 8.96 & 8.96 & & 0.0859 & 5.80E-04 & 86.6006 & 31.9098 & 118.5103 & \\
\hline & 8/7/2008 7:00 & 378 & 220 & 700 & 8.87 & 8.87 & & 0.0970 & 6.73E-04 & 100.4651 & 37.0184 & 137.4836 & \\
\hline & 8/7/2008 8:00 & 379 & 220 & 800 & 8.69 & 8.69 & & 0.1197 & 8.72E-04 & 130.1896 & 47.9711 & 178.1607 & \\
\hline & 8/7/2008 9:00 & 380 & 220 & 900 & 7.437 & 7.437 & & 0.3083 & $2.80 \mathrm{E}-03$ & 417.3577 & 153.7840 & 571.1418 & \\
\hline & 8/7/2008 10:00 & 381 & 220 & 1000 & 4.575 & 4.575 & & 1.1268 & 1.38E-02 & 2057.5660 & 758.1525 & 2815.7186 & \\
\hline & 8/7/2008 11:00 & 382 & 220 & 1100 & 4.248 & 4.248 & & 1.2905 & 1.63E-02 & 2431.5351 & 895.9491 & 3327.4843 & \\
\hline & 8/7/2008 12:00 & 383 & 220 & 1200 & 4.224 & 4.224 & & 1.3035 & 1.65E-02 & 2461.7558 & 907.0846 & 3368.8404 & \\
\hline & 8/7/2008 13:00 & 384 & 220 & 1300 & 4.232 & 4.232 & & 1.2991 & $1.64 \mathrm{E}-02$ & 2451.6364 & 903.3558 & 3354.9922 & \\
\hline & $8 / 7 / 2008$ 14:00 & 385 & 220 & 1400 & 4.213 & 4.213 & & 1.3095 & 1.66E-02 & 2475.7456 & 912.2394 & 3387.9850 & \\
\hline & 8/7/2008 15:00 & 386 & 220 & 1500 & 4.276 & 4.276 & & 1.2755 & 1.61E-02 & 2396.7937 & 883.1479 & 3279.9417 & \\
\hline & 8/7/2008 16:00 & 387 & 220 & 1600 & 4.316 & 4.316 & & 1.2544 & 1.57E-02 & 2348.1047 & 865.2075 & 3213.3121 & \\
\hline & 8/7/2008 17:00 & 388 & 220 & 1700 & 4.159 & 4.159 & & 1.3395 & $1.71 E-02$ & 2545.7168 & 938.0217 & 3483.7385 & \\
\hline & 8/7/2008 18:00 & 389 & 220 & 1800 & 4.289 & 4.289 & & 1.2686 & $1.59 \mathrm{E}-02$ & 2380.8496 & 877.2730 & 3258.1225 & \\
\hline & 8/7/2008 19:00 & 390 & 220 & 1900 & 4.599 & 4.599 & & 1.1157 & 1.36E-02 & 2032.6460 & 748.9702 & 2781.6163 & \\
\hline & 8/7/2008 20:00 & 391 & 220 & 2000 & 5.36 & 5.36 & & 0.8153 & $9.26 \mathrm{E}-03$ & 1381.5688 & 509.0675 & 1890.6363 & \\
\hline & 8/7/2008 21:00 & 392 & 220 & 2100 & 7.279 & 7.279 & & 0.3367 & $3.12 E-03$ & 465.1715 & 171.4020 & 636.5735 & \\
\hline & 8/7/2008 22:00 & 393 & 220 & 2200 & 8.42 & 8.42 & & 0.1556 & 1.20E-03 & 179.8223 & 66.2592 & 246.0815 & \\
\hline & 8/7/2008 23:00 & 394 & 220 & 2300 & 8.92 & 8.92 & & 0.0908 & 6.21E-04 & 92.6803 & 34.1500 & 126.8303 & \\
\hline & 8/8/2008 0:00 & 395 & 221 & 0 & 9.16 & 9.16 & 9.27 & 0.0622 & 3.90E-04 & 58.2010 & 21.4454 & 79.6463 & \\
\hline & 8/8/2008 1:00 & 396 & 221 & 100 & 9.19 & 9.19 & & 0.0588 & 3.63E-04 & 54.2347 & 19.9839 & 74.2186 & \\
\hline & 8/8/2008 2:00 & 397 & 221 & 200 & 9.27 & 9.27 & & 0.0496 & 2.95E-04 & 44.0475 & 16.2302 & 60.2777 & \\
\hline & 8/8/2008 3:00 & 398 & 221 & 300 & 9.23 & 9.23 & & 0.0542 & $3.29 E-04$ & 49.0694 & 18.0806 & 67.1500 & \\
\hline & 8/8/2008 4:00 & 399 & 221 & 400 & 9.18 & 9.18 & & 0.0599 & 3.72E-04 & 55.5481 & 20.4678 & 76.0159 & \\
\hline & 8/8/2008 5:00 & 400 & 221 & 500 & 9.14 & 9.14 & & 0.0646 & 4.08E-04 & 60.8886 & 22.4356 & 83.3242 & \\
\hline & 8/8/2008 6:00 & 401 & 221 & 600 & 9.01 & 9.01 & & 0.0799 & 5.30E-04 & 79.1866 & 29.1779 & 108.3646 & \\
\hline & $8 / 8 / 20087: 00$ & 402 & 221 & 700 & 8.94 & 8.94 & & 0.0884 & $6.00 \mathrm{E}-04$ & 89.6240 & 33.0238 & 122.6478 & \\
\hline & 8/8/2008 8:00 & 403 & 221 & 800 & 8.71 & 8.71 & & 0.1171 & 8.49E-04 & 126.7553 & 46.7056 & 173.4609 & \\
\hline & 8/8/2008 9:00 & 404 & 221 & 900 & 6.061 & 6.061 & & 0.6053 & 6.41E-03 & 957.6084 & 352.8505 & 1310.4589 & \\
\hline & 8/8/2008 10:00 & 405 & 221 & 1000 & 4.58 & 4.58 & & 1.1245 & 1.37E-02 & 2052.3481 & 756.2299 & 2808.5780 & \\
\hline & 8/8/2008 11:00 & 406 & 221 & 1100 & 4.538 & 4.538 & & 1.1441 & 1.40E-02 & 2096.6143 & 772.5407 & 2869.1549 & \\
\hline & $8 / 8 / 200812: 00$ & 407 & 221 & 1200 & 4.317 & 4.317 & & 1.2539 & $1.57 \mathrm{E}-02$ & 2346.9014 & 864.7641 & 3211.6655 & \\
\hline & 8/8/2008 13:00 & 408 & 221 & 1300 & 4.26 & 4.26 & & 1.2840 & 1.62E-02 & 2416.5785 & 890.4381 & 3307.0166 & \\
\hline & 8/8/2008 14:00 & 409 & 221 & 1400 & 4.186 & 4.186 & & 1.3244 & $1.68 \mathrm{E}-02$ & 2510.4598 & 925.0306 & 3435.4904 & \\
\hline
\end{tabular}




\begin{tabular}{|c|c|c|c|c|c|c|c|c|c|c|c|c|c|}
\hline Tree ID & TS & RN & JD & HR & $\begin{array}{c}\text { Raw } \\
\text { TC_dTA }\end{array}$ & $\begin{array}{c}\text { Fixed } \\
\text { TC_dT } \Delta\end{array}$ & dTM & $\mathbf{K}$ & $\begin{array}{c}\text { Sap } \\
\text { Velocity } \\
(\mathbf{c m} / \mathbf{s})\end{array}$ & $\begin{array}{c}\text { Probed } \\
\text { Drywood F } \\
(\mathrm{g} / \mathrm{hr})\end{array}$ & $\begin{array}{c}\text { Extrapolated } \\
\text { Wetwood F } \\
(\mathrm{g} / \mathrm{hr})\end{array}$ & $\begin{array}{c}\text { Extrapolated } \\
\text { Total Wood F } \\
(\mathrm{g} / \mathrm{hr})\end{array}$ & $\begin{array}{c}\text { Relative } \\
\text { Humidity, } \\
@ 10 \mathrm{~m}(\%)\end{array}$ \\
\hline & 8/8/2008 15:00 & 410 & 221 & 1500 & \begin{tabular}{|r|}
4.16 \\
\end{tabular} & 4.16 & & 1.3389 & 1.70E-02 & 2544.4012 & 937.5369 & 3481.9381 & \\
\hline & 8/8/2008 16:00 & 411 & 221 & 1600 & 4.167 & 4.167 & & 1.3350 & 1.70E-02 & 2535.2130 & 934.1514 & 3469.3644 & \\
\hline & 8/8/2008 17:00 & 412 & 221 & 1700 & 4.192 & 4.192 & & 1.3211 & 1.68E-02 & 2502.6991 & 922.1709 & 3424.8700 & \\
\hline & 8/8/2008 18:00 & 413 & 221 & 1800 & 4.578 & 4.578 & & 1.1254 & 1.38E-02 & 2054.4336 & 756.9983 & 2811.4320 & \\
\hline & 8/8/2008 19:00 & 414 & 221 & 1900 & 4.551 & 4.551 & & 1.1380 & $1.40 \mathrm{E}-02$ & 2082.8066 & 767.4529 & 2850.2595 & \\
\hline & $8 / 8 / 200820: 00$ & 415 & 221 & 2000 & 5.486 & 5.486 & & 0.7736 & $8.68 \mathrm{E}-03$ & 1295.1180 & 477.2128 & 1772.3308 & \\
\hline & 8/8/2008 21:00 & 416 & 221 & 2100 & 7.236 & 7.236 & & 0.3447 & $3.21 \mathrm{E}-03$ & 478.7167 & 176.3930 & 655.1097 & \\
\hline & 8/8/2008 22:00 & 417 & 221 & 2200 & 8.13 & 8.13 & & 0.1968 & 1.61E-03 & 240.1552 & 88.4901 & 328.6453 & \\
\hline & 8/8/2008 23:00 & 418 & 221 & 2300 & 8.39 & 8.39 & & 0.1597 & 1.24E-03 & 185.7192 & 68.4321 & 254.1512 & \\
\hline & 8/9/2008 0:00 & 419 & 222 & 0 & 8.5 & 8.5 & 9.24 & 0.1447 & 1.10E-03 & 164.4751 & 60.6042 & 225.0794 & \\
\hline & 8/9/2008 1:00 & 420 & 222 & 100 & 8.46 & 8.46 & & 0.1501 & 1.15E-03 & 172.0803 & 63.4065 & 235.4868 & \\
\hline & 8/9/2008 2:00 & 421 & 222 & 200 & 8.46 & 8.46 & & 0.1501 & 1.15E-03 & 172.0803 & 63.4065 & 235.4868 & \\
\hline & 8/9/2008 3:00 & 422 & 222 & 300 & 8.43 & 8.43 & & 0.1542 & 1.19E-03 & 177.8739 & 65.5413 & 243.4152 & \\
\hline & 8/9/2008 4:00 & 423 & 222 & 400 & 8.3 & 8.3 & & 0.1723 & 1.37E-03 & 203.8803 & 75.1239 & 279.0042 & \\
\hline & 8/9/2008 5:00 & 424 & 222 & 500 & 8.15 & 8.15 & & 0.1939 & $1.58 \mathrm{E}-03$ & 235.7510 & 86.8673 & 322.6183 & \\
\hline & 8/9/2008 6:00 & 425 & 222 & 600 & 8.13 & 8.13 & & 0.1968 & $1.61 \mathrm{E}-03$ & 240.1552 & 88.4901 & 328.6453 & \\
\hline & 8/9/2008 7:00 & 426 & 222 & 700 & 7.823 & 7.823 & & 0.2438 & $2.09 E-03$ & 312.5438 & 115.1632 & 427.7070 & \\
\hline & 8/9/2008 8:00 & 427 & 222 & 800 & 7.02 & 7.02 & & 0.3860 & $3.69 E-03$ & 550.4095 & 202.8097 & 753.2192 & \\
\hline & 8/9/20089:00 & 428 & 222 & 900 & 7.502 & 7.502 & & 0.2970 & $2.67 \mathrm{E}-03$ & 398.5496 & 146.8538 & 545.4034 & \\
\hline & 8/9/2008 10:00 & 429 & 222 & 1000 & 7.256 & 7.256 & & 0.3410 & 3.16E-03 & 472.3875 & 174.0609 & 646.4484 & \\
\hline & 8/9/2008 11:00 & 430 & 222 & 1100 & 6.302 & 6.302 & & 0.5440 & $5.62 \mathrm{E}-03$ & 839.4968 & 309.3299 & 1148.8267 & \\
\hline & 8/9/2008 12:00 & 431 & 222 & 1200 & 5.385 & 5.385 & & 0.8069 & $9.14 \mathrm{E}-03$ & 1364.0100 & 502.5975 & 1866.6075 & \\
\hline & 8/9/2008 13:00 & 432 & 222 & 1300 & 5.166 & 5.166 & & 0.8835 & $1.02 \mathrm{E}-02$ & 1525.1169 & 561.9607 & 2087.0776 & \\
\hline & 8/9/2008 14:00 & 433 & 222 & 1400 & 4.628 & 4.628 & & 1.1024 & $1.34 \mathrm{E}-02$ & 2002.9542 & 738.0297 & 2740.9839 & \\
\hline & 8/9/2008 15:00 & 434 & 222 & 1500 & 4.421 & 4.421 & & 1.2009 & 1.49E-02 & 2225.3414 & 819.9728 & 3045.3142 & \\
\hline & 8/9/2008 16:00 & 435 & 222 & 1600 & 4.505 & 4.505 & & 1.1598 & 1.43E-02 & 2132.1005 & 785.6163 & 2917.7168 & \\
\hline & 8/9/2008 17:00 & 436 & 222 & 1700 & 4.561 & 4.561 & & 1.1333 & 1.39E-02 & 2072.2504 & 763.5633 & 2835.8136 & \\
\hline & 8/9/2008 18:00 & 437 & 222 & 1800 & 4.523 & 4.523 & & 1.1512 & 1.42E-02 & 2112.6663 & 778.4554 & 2891.1217 & \\
\hline & 8/9/2008 19:00 & 438 & 222 & 1900 & 4.879 & 4.879 & & 0.9943 & $1.18 \mathrm{E}-02$ & 1763.8628 & 649.9315 & 2413.7943 & \\
\hline & 8/9/2008 20:00 & 439 & 222 & 2000 & 5.715 & 5.715 & & 0.7025 & $7.71 \mathrm{E}-03$ & 1150.2485 & 423.8327 & 1574.0812 & \\
\hline & 8/9/2008 21:00 & 440 & 222 & 2100 & 7.812 & 7.812 & & 0.2455 & $2.11 E-03$ & 315.3102 & 116.1825 & 431.4928 & \\
\hline & 8/9/2008 22:00 & 441 & 222 & 2200 & 8.96 & 8.96 & & 0.0859 & $5.80 \mathrm{E}-04$ & 86.6006 & 31.9098 & 118.5103 & \\
\hline & 8/9/2008 23:00 & 442 & 222 & 2300 & 9.24 & 9.24 & & 0.0530 & $3.20 \mathrm{E}-04$ & 47.8003 & 17.6130 & 65.4133 & \\
\hline & 8/10/2008 0:00 & 443 & 223 & 0 & 9.38 & 9.38 & 9.44 & 0.0373 & $2.08 \mathrm{E}-04$ & 31.0104 & 11.4264 & 42.4369 & \\
\hline & 8/10/2008 1:00 & 444 & 223 & 100 & 9.44 & 9.44 & & 0.0307 & 1.64E-04 & 24.4097 & 8.9943 & 33.4040 & \\
\hline & 8/10/2008 2:00 & 445 & 223 & 200 & 9.42 & 9.42 & & 0.0329 & $1.78 \mathrm{E}-04$ & 26.5675 & 9.7894 & 36.3569 & \\
\hline & 8/10/2008 3:00 & 446 & 223 & 300 & 9.37 & 9.37 & & 0.0384 & $2.15 E-04$ & 32.1469 & 11.8452 & 43.9920 & \\
\hline & 8/10/2008 4:00 & 447 & 223 & 400 & 9.33 & 9.33 & & 0.0429 & $2.46 \mathrm{E}-04$ & 36.7920 & 13.5568 & 50.3488 & \\
\hline & 8/10/2008 5:00 & 448 & 223 & 500 & 9.25 & 9.25 & & 0.0519 & $3.12 \mathrm{E}-04$ & 46.5403 & 17.1487 & 63.6890 & \\
\hline & 8/10/2008 6:00 & 449 & 223 & 600 & 9.16 & 9.16 & & 0.0622 & 3.90E-04 & 58.2010 & 21.4454 & 79.6463 & \\
\hline & 8/10/2008 7:00 & 450 & 223 & 700 & 9.09 & 9.09 & & 0.0704 & 4.54E-04 & 67.7575 & 24.9666 & 92.7241 & \\
\hline
\end{tabular}




\begin{tabular}{|c|c|c|c|c|c|c|c|c|c|c|c|c|c|}
\hline Tree ID & TS & RN & JD & HR & $\begin{array}{c}\text { Raw } \\
\text { TC_dTA }\end{array}$ & $\begin{array}{c}\text { Fixed } \\
\text { TC_dT } \Delta\end{array}$ & dTM & $\mathbf{K}$ & $\begin{array}{c}\text { Sap } \\
\text { Velocity } \\
(\mathrm{cm} / \mathrm{s})\end{array}$ & $\begin{array}{c}\text { Probed } \\
\text { Drywood F } \\
\text { (g/hr) }\end{array}$ & $\begin{array}{c}\text { Extrapolated } \\
\text { Wetwood F } \\
(\mathrm{g} / \mathrm{hr})\end{array}$ & $\begin{array}{c}\text { Extrapolated } \\
\text { Total Wood F } \\
(\mathrm{g} / \mathrm{hr})\end{array}$ & $\begin{array}{l}\text { Relative } \\
\text { Humidity, } \\
\text { @10m (\%) }\end{array}$ \\
\hline & 8/10/2008 8:00 & 451 & 223 & 800 & 8.95 & 8.95 & & 0.0872 & 5.90E-04 & 88.1081 & 32.4653 & 120.5734 & \\
\hline & $8 / 10 / 20089: 00$ & 452 & 223 & 900 & 7.494 & 7.494 & & 0.2984 & 2.69E-03 & 400.8381 & 147.6970 & 548.5351 & \\
\hline & 8/10/2008 10:00 & 453 & 223 & 1000 & 4.745 & 4.745 & & 1.0506 & $1.26 \mathrm{E}-02$ & 1887.6469 & 695.5423 & 2583.1893 & \\
\hline & 8/10/2008 11:00 & 454 & 223 & 1100 & 4.416 & 4.416 & & 1.2034 & 1.49E-02 & 2231.0273 & 822.0679 & 3053.0952 & \\
\hline & $8 / 10 / 2008$ 12:00 & 455 & 223 & 1200 & 4.403 & 4.403 & & 1.2099 & $1.50 \mathrm{E}-02$ & 2245.8839 & 827.5421 & 3073.4260 & \\
\hline & 8/10/2008 13:00 & 456 & 223 & 1300 & 4.372 & 4.372 & & 1.2255 & 1.53E-02 & 2281.7434 & 840.7553 & 3122.4987 & \\
\hline & 8/10/2008 14:00 & 457 & 223 & 1400 & 4.325 & 4.325 & & 1.2497 & $1.57 \mathrm{E}-02$ & 2337.2993 & 861.2260 & 3198.5253 & \\
\hline & 8/10/2008 15:00 & 458 & 223 & 1500 & 4.497 & 4.497 & & 1.1637 & 1.43E-02 & 2140.7986 & 788.8213 & 2929.6199 & \\
\hline & 8/10/2008 16:00 & 459 & 223 & 1600 & 4.349 & 4.349 & & 1.2373 & $1.55 \mathrm{E}-02$ & 2308.7490 & 850.7060 & 3159.4550 & \\
\hline & 8/10/2008 17:00 & 460 & 223 & 1700 & 4.523 & 4.523 & & 1.1512 & 1.42E-02 & 2112.6663 & 778.4554 & 2891.1217 & \\
\hline & 8/10/2008 18:00 & 461 & 223 & 1800 & 7.664 & 7.664 & & 0.2696 & 2.37E-03 & 353.7549 & 130.3483 & 484.1031 & \\
\hline & $8 / 10 / 2008$ 19:00 & 462 & 223 & 1900 & 8.56 & 8.56 & & 0.1367 & 1.03E-03 & 153.3216 & 56.4945 & 209.8161 & \\
\hline & 8/10/2008 20:00 & 463 & 223 & 2000 & 8.27 & 8.27 & & 0.1765 & $1.41 E-03$ & 210.0926 & 77.4130 & 287.5056 & \\
\hline & 8/10/2008 21:00 & 464 & 223 & 2100 & 8.24 & 8.24 & & 0.1808 & 1.45E-03 & 216.3852 & 79.7316 & 296.1168 & \\
\hline & $8 / 10 / 2008$ 22:00 & 465 & 223 & 2200 & 8.56 & 8.56 & & 0.1367 & 1.03E-03 & 153.3216 & 56.4945 & 209.8161 & \\
\hline & 8/10/2008 23:00 & 466 & 223 & 2300 & 8.57 & 8.57 & & 0.1354 & 1.01E-03 & 151.4921 & 55.8204 & 207.3125 & \\
\hline & $8 / 11 / 20080: 00$ & 467 & 224 & 0 & 8.52 & 8.52 & 8.86 & 0.1420 & 1.08E-03 & 160.7235 & 59.2219 & 219.9454 & \\
\hline & 8/11/2008 1:00 & 468 & 224 & 100 & 8.58 & 8.58 & & 0.1340 & 1.00E-03 & 149.6711 & 55.1494 & 204.8205 & \\
\hline & 8/11/2008 2:00 & 469 & 224 & 200 & 8.82 & 8.82 & & 0.1032 & 7.27E-04 & 108.4551 & 39.9625 & 148.4176 & \\
\hline & 8/11/2008 3:00 & 470 & 224 & 300 & 8.86 & 8.86 & & 0.0982 & 6.84E-04 & 102.0467 & 37.6012 & 139.6479 & \\
\hline & 8/11/2008 4:00 & 471 & 224 & 400 & 8.81 & 8.81 & & 0.1044 & 7.37E-04 & 110.0777 & 40.5604 & 150.6380 & \\
\hline & 8/11/2008 5:00 & 472 & 224 & 500 & 8.75 & 8.75 & & 0.1120 & 8.04E-04 & 119.9856 & 44.2112 & \begin{tabular}{|l|}
164.1968 \\
\end{tabular} & \\
\hline & 8/11/2008 6:00 & 473 & 224 & 600 & 8.76 & 8.76 & & 0.1107 & 7.93E-04 & 118.3137 & 43.5951 & 161.9089 & \\
\hline & 8/11/2008 7:00 & 474 & 224 & 700 & 8.78 & 8.78 & & 0.1082 & 7.70E-04 & 114.9947 & 42.3722 & 157.3668 & \\
\hline & 8/11/2008 8:00 & 475 & 224 & 800 & 8.71 & 8.71 & & 0.1171 & 8.49E-04 & 126.7553 & 46.7056 & 173.4609 & \\
\hline & 8/11/2008 9:00 & 476 & 224 & 900 & 7.648 & 7.648 & & 0.2722 & 2.40E-03 & 358.0503 & 131.9310 & 489.9813 & \\
\hline & 8/11/2008 10:00 & 477 & 224 & 1000 & 5.175 & 5.175 & & 0.8802 & 1.02E-02 & 1518.1591 & 559.3969 & 2077.5560 & \\
\hline & 8/11/2008 11:00 & 478 & 224 & 1100 & 4.675 & 4.675 & & 1.0813 & 1.31E-02 & 1955.7859 & 720.6495 & 2676.4355 & \\
\hline & $8 / 11 / 2008$ 12:00 & 479 & 224 & 1200 & 4.628 & 4.628 & & 1.1024 & 1.34E-02 & 2002.9542 & 738.0297 & 2740.9839 & \\
\hline & 8/11/2008 13:00 & 480 & 224 & 1300 & 4.98 & 4.98 & & 0.9538 & 1.12E-02 & 1675.9546 & 617.5399 & 2293.4945 & \\
\hline & $8 / 11 / 2008$ 14:00 & 481 & 224 & 1400 & 4.603 & 4.603 & & 1.1138 & 1.36E-02 & 2028.5235 & 747.4512 & 2775.9747 & \\
\hline & $8 / 11 / 2008$ 15:00 & 482 & 224 & 1500 & 4.534 & 4.534 & & 1.1460 & 1.41E-02 & 2100.8822 & 774.1133 & 2874.9955 & \\
\hline & $8 / 11 / 2008$ 16:00 & 483 & 224 & 1600 & 4.873 & 4.873 & & 0.9967 & 1.19E-02 & 1769.2267 & 651.9080 & 2421.1347 & \\
\hline & $8 / 11 / 2008$ 17:00 & 484 & 224 & 1700 & 4.86 & 4.86 & & 1.0021 & $1.19 \mathrm{E}-02$ & 1780.9045 & 656.2109 & 2437.1154 & \\
\hline & 8/11/2008 18:00 & 485 & 224 & 1800 & 5.311 & 5.311 & & 0.8320 & 9.49E-03 & 1416.5880 & 521.9710 & 1938.5590 & \\
\hline & $8 / 11 / 2008$ 19:00 & 486 & 224 & 1900 & 5.5 & 5.5 & & 0.7691 & 8.61E-03 & 1285.8202 & 473.7869 & 1759.6071 & \\
\hline & $8 / 11 / 2008$ 20:00 & 487 & 224 & 2000 & 6.409 & 6.409 & & 0.5182 & 5.30E-03 & 790.7968 & 291.3853 & 1082.1821 & \\
\hline & 8/11/2008 21:00 & 488 & 224 & 2100 & 7.616 & 7.616 & & 0.2776 & $2.46 \mathrm{E}-03$ & 366.7247 & 135.1272 & 501.8519 & \\
\hline & 8/11/2008 22:00 & 489 & 224 & 2200 & 8.38 & 8.38 & & 0.1611 & $1.26 \mathrm{E}-03$ & 187.7021 & 69.1627 & 256.8648 & \\
\hline & 8/11/2008 23:00 & 490 & 224 & 2300 & 8.75 & 8.75 & & 0.1120 & 8.04E-04 & 119.9856 & 44.2112 & 164.1968 & \\
\hline & 8/12/2008 0:00 & 491 & 225 & 0 & 8.91 & 8.91 & 9.01 & 0.0920 & $6.31 \mathrm{E}-04$ & 94.2209 & 34.7176 & 128.9385 & \\
\hline
\end{tabular}




\begin{tabular}{|c|c|c|c|c|c|c|c|c|c|c|c|c|c|}
\hline Tree ID & TS & RN & JD & HR & $\begin{array}{c}\text { Raw } \\
\text { TC_dTA }\end{array}$ & $\begin{array}{c}\text { Fixed } \\
\text { TC_dT } \Delta\end{array}$ & dTM & $\mathbf{K}$ & $\begin{array}{c}\text { Sap } \\
\text { Velocity } \\
(\mathrm{cm} / \mathrm{s})\end{array}$ & $\begin{array}{c}\text { Probed } \\
\text { Drywood F } \\
\text { (g/hr) }\end{array}$ & $\begin{array}{c}\text { Extrapolated } \\
\text { Wetwood F } \\
(\mathrm{g} / \mathrm{hr})\end{array}$ & $\begin{array}{c}\text { Extrapolated } \\
\text { Total Wood F } \\
(\mathrm{g} / \mathrm{hr})\end{array}$ & $\begin{array}{c}\text { Relative } \\
\text { Humidity, } \\
@ 10 \mathrm{~m}(\%)\end{array}$ \\
\hline & 8/12/2008 1:00 & 492 & 225 & 100 & 8.86 & 8.86 & & 0.0982 & $6.84 \mathrm{E}-04$ & 102.0467 & 37.6012 & \begin{tabular}{|r|}
139.6479 \\
\end{tabular} & \\
\hline & $8 / 12 / 20082: 00$ & 493 & 225 & 200 & 8.72 & 8.72 & & 0.1158 & $8.38 \mathrm{E}-04$ & 125.0505 & 46.0774 & 171.1280 & \\
\hline & 8/12/2008 3:00 & 494 & 225 & 300 & 8.96 & 8.96 & & 0.0859 & $5.80 \mathrm{E}-04$ & 86.6006 & 31.9098 & 118.5103 & \\
\hline & 8/12/2008 4:00 & 495 & 225 & 400 & 8.93 & 8.93 & & 0.0896 & $6.11 \mathrm{E}-04$ & 91.1480 & 33.5854 & 124.7334 & \\
\hline & $8 / 12 / 20085: 00$ & 496 & 225 & 500 & 8.84 & 8.84 & & 0.1007 & 7.05E-04 & 105.2345 & 38.7758 & 144.0103 & \\
\hline & $8 / 12 / 20086: 00$ & 497 & 225 & 600 & 8.79 & 8.79 & & 0.1069 & $7.59 \mathrm{E}-04$ & 113.3475 & 41.7652 & 155.1127 & \\
\hline & $8 / 12 / 20087: 00$ & 498 & 225 & 700 & 8.78 & 8.78 & & 0.1082 & 7.70E-04 & 114.9947 & 42.3722 & 157.3668 & \\
\hline & 8/12/2008 8:00 & 499 & 225 & 800 & 8.68 & 8.68 & & 0.1210 & 8.84E-04 & 131.9192 & 48.6083 & 180.5275 & \\
\hline & 8/12/2008 9:00 & 500 & 225 & 900 & 7.667 & 7.667 & & 0.2691 & $2.36 \mathrm{E}-03$ & 352.9526 & 130.0526 & 483.0052 & \\
\hline & $8 / 12 / 200810: 00$ & 501 & 225 & 1000 & 5.302 & 5.302 & & 0.8352 & $9.53 \mathrm{E}-03$ & 1423.1085 & 524.3736 & 1947.4821 & \\
\hline & 8/12/2008 11:00 & 502 & 225 & 1100 & 4.626 & 4.626 & & 1.1033 & $1.34 \mathrm{E}-02$ & 2004.9873 & 738.7788 & 2743.7662 & \\
\hline & $8 / 12 / 200812: 00$ & 503 & 225 & 1200 & 4.56 & 4.56 & & 1.1338 & 1.39E-02 & 2073.3035 & 763.9513 & 2837.2548 & \\
\hline & 8/12/2008 13:00 & 504 & 225 & 1300 & 4.532 & 4.532 & & 1.1470 & $1.41 \mathrm{E}-02$ & 2103.0196 & 774.9008 & 2877.9204 & \\
\hline & $8 / 12 / 200814: 00$ & 505 & 225 & 1400 & 4.499 & 4.499 & & 1.1627 & 1.43E-02 & 2138.6206 & 788.0187 & 2926.6393 & \\
\hline & $8 / 12 / 200815: 00$ & 506 & 225 & 1500 & 4.421 & 4.421 & & 1.2009 & 1.49E-02 & 2225.3414 & 819.9728 & 3045.3142 & \\
\hline & 8/12/2008 16:00 & 507 & 225 & 1600 & 4.516 & 4.516 & & 1.1546 & 1.42E-02 & 2120.2017 & 781.2319 & 2901.4336 & \\
\hline & $8 / 12 / 200817: 00$ & 508 & 225 & 1700 & 4.412 & 4.412 & & 1.2053 & $1.50 \mathrm{E}-02$ & 2235.5873 & 823.7481 & 3059.3354 & \\
\hline & $8 / 12 / 2008$ 18:00 & 509 & 225 & 1800 & 4.527 & 4.527 & & 1.1493 & $1.41 E-02$ & 2108.3731 & 776.8734 & 2885.2466 & \\
\hline & 8/12/2008 19:00 & 510 & 225 & 1900 & 4.851 & 4.851 & & 1.0058 & 1.20E-02 & 1789.0343 & 659.2065 & 2448.2408 & \\
\hline & $8 / 12 / 200820: 00$ & 511 & 225 & 2000 & 5.628 & 5.628 & & 0.7289 & 8.06E-03 & 1203.5205 & 443.4619 & 1646.9824 & \\
\hline & $8 / 12 / 200821: 00$ & 512 & 225 & 2100 & 7.779 & 7.779 & & 0.2508 & $2.17 \mathrm{E}-03$ & 323.6840 & 119.2680 & 442.9521 & \\
\hline & $8 / 12 / 200822: 00$ & 513 & 225 & 2200 & 8.77 & 8.77 & & 0.1095 & $7.81 \mathrm{E}-04$ & 116.6501 & 42.9821 & 159.6322 & \\
\hline & $8 / 12 / 200823: 00$ & 514 & 225 & 2300 & 9.01 & 9.01 & & 0.0799 & $5.30 \mathrm{E}-04$ & 79.1866 & 29.1779 & 108.3646 & \\
\hline & $8 / 13 / 20080: 00$ & 515 & 226 & 0 & 9.09 & 9.09 & 9.15 & 0.0704 & $4.54 \mathrm{E}-04$ & 67.7575 & 24.9666 & 92.7241 & \\
\hline & 8/13/2008 1:00 & 516 & 226 & 100 & 9.11 & 9.11 & & 0.0681 & 4.35E-04 & 64.9844 & 23.9448 & 88.9292 & \\
\hline & 8/13/2008 2:00 & 517 & 226 & 200 & 9.12 & 9.12 & & 0.0669 & $4.26 \mathrm{E}-04$ & 63.6105 & 23.4386 & 87.0492 & \\
\hline & 8/13/2008 3:00 & 518 & 226 & 300 & 9.15 & 9.15 & & 0.0634 & 3.99E-04 & 59.5405 & 21.9389 & 81.4794 & \\
\hline & $8 / 13 / 20084: 00$ & 519 & 226 & 400 & 9.05 & 9.05 & & 0.0751 & 4.92E-04 & 73.4050 & 27.0476 & 100.4526 & \\
\hline & $8 / 13 / 20085: 00$ & 520 & 226 & 500 & 9 & 9 & & 0.0811 & $5.40 \mathrm{E}-04$ & 80.6528 & 29.7182 & 110.3710 & \\
\hline & $8 / 13 / 20086: 00$ & 521 & 226 & 600 & 8.98 & 8.98 & & 0.0835 & $5.60 \mathrm{E}-04$ & 83.6101 & 30.8079 & 114.4180 & \\
\hline & 8/13/2008 7:00 & 522 & 226 & 700 & 8.9 & 8.9 & & 0.0933 & $6.42 \mathrm{E}-04$ & 95.7696 & 35.2883 & 131.0579 & \\
\hline & 8/13/2008 8:00 & 523 & 226 & 800 & 8.69 & 8.69 & & 0.1197 & $8.72 \mathrm{E}-04$ & 130.1896 & 47.9711 & 178.1607 & \\
\hline & 8/13/2008 9:00 & 524 & 226 & 900 & 6.413 & 6.413 & & 0.5172 & $5.29 \mathrm{E}-03$ & 789.0182 & 290.7300 & 1079.7482 & \\
\hline & $8 / 13 / 2008$ 10:00 & 525 & 226 & 1000 & 4.803 & 4.803 & & 1.0258 & $1.23 \mathrm{E}-02$ & 1833.0271 & 675.4165 & 2508.4436 & \\
\hline & 8/13/2008 11:00 & 526 & 226 & 1100 & 4.769 & 4.769 & & 1.0403 & 1.25E-02 & 1864.8479 & 687.1416 & 2551.9895 & \\
\hline & $8 / 13 / 2008$ 12:00 & 527 & 226 & 1200 & 4.401 & 4.401 & & 1.2109 & $1.51 \mathrm{E}-02$ & 2248.1790 & 828.3878 & 3076.5667 & \\
\hline
\end{tabular}




\begin{tabular}{|c|c|c|c|c|c|c|c|c|c|c|c|c|c|}
\hline Tree ID & TS & RN & JD & HR & $\begin{array}{c}\text { Raw } \\
\text { TC_dTA }\end{array}$ & $\begin{array}{c}\text { Fixed } \\
\text { TC_dT } \Delta\end{array}$ & dTM & $\mathbf{K}$ & $\begin{array}{c}\text { Sap } \\
\text { Velocity } \\
(\mathbf{c m} / \mathbf{s})\end{array}$ & $\begin{array}{c}\text { Probed } \\
\text { Drywood F } \\
(\mathrm{g} / \mathrm{hr})\end{array}$ & $\begin{array}{c}\text { Extrapolated } \\
\text { Wetwood F } \\
(\mathrm{g} / \mathrm{hr})\end{array}$ & $\begin{array}{c}\text { Extrapolated } \\
\text { Total Wood F } \\
(\mathrm{g} / \mathrm{hr})\end{array}$ & $\begin{array}{c}\text { Relative } \\
\text { Humidity, } \\
@ 10 \mathrm{~m}(\%)\end{array}$ \\
\hline & $7 / 22 / 2008$ 13:00 & 0 & 204 & 1300 & 5.306 & 5.306 & 9.94 & 0.8734 & 1.01E-02 & 1503.6444 & 554.0487 & 2057.6930 & \\
\hline W505EC1 & $7 / 22 / 2008$ 14:00 & 1 & 204 & 1400 & 5.703 & 5.703 & & 0.7429 & $8.25 \mathrm{E}-03$ & 1232.2177 & 454.0360 & 1686.2537 & \\
\hline $36 " \mathrm{~N}$ & 7/22/2008 15:00 & 2 & 204 & 1500 & 5.521 & 5.521 & & 0.8004 & $9.05 \mathrm{E}-03$ & 1350.5533 & 497.6391 & 1848.1925 & \\
\hline \multirow[t]{2}{*}{ Probe \# } & 7/22/2008 16:00 & 3 & 204 & 1600 & 5.366 & 5.366 & & 0.8524 & $9.78 \mathrm{E}-03$ & 1459.3731 & 537.7360 & 1997.1091 & \\
\hline & 7/22/2008 17:00 & 4 & 204 & 1700 & 5.618 & 5.618 & & 0.7693 & 8.62E-03 & 1286.2772 & 473.9553 & 1760.2324 & \\
\hline \multirow[t]{4}{*}{9} & 7/22/2008 18:00 & 5 & 204 & 1800 & 5.512 & 5.512 & & 0.8033 & 9.09E-03 & 1356.6620 & 499.8900 & 1856.5520 & \\
\hline & 7/22/2008 19:00 & 6 & 204 & 1900 & 5.81 & 5.81 & & 0.7108 & 7.82E-03 & 1167.0123 & 430.0097 & 1597.0220 & \\
\hline & $7 / 22 / 200820: 00$ & 7 & 204 & 2000 & 6.301 & 6.301 & & 0.5775 & $6.05 \mathrm{E}-03$ & 903.7277 & 332.9970 & 1236.7247 & \\
\hline & 7/22/2008 21:00 & 8 & 204 & 2100 & 8.29 & 8.29 & & 0.1990 & $1.63 \mathrm{E}-03$ & 243.5139 & 89.7277 & 333.2417 & \\
\hline \multirow[t]{2}{*}{$\mathrm{DBH}(\mathrm{cm})$} & $7 / 22 / 200822: 00$ & 9 & 204 & 2200 & 9.63 & 9.63 & & 0.0322 & $1.73 \mathrm{E}-04$ & 25.8562 & 9.5272 & 35.3834 & \\
\hline & 7/22/2008 23:00 & 10 & 204 & 2300 & 9.94 & 9.94 & & 0.0000 & $0.00 \mathrm{E}+00$ & 0.0000 & 0.0000 & 0.0000 & \\
\hline \multirow[t]{2}{*}{9.1} & $7 / 23 / 20080: 00$ & 11 & 205 & 0 & 10.14 & 10.14 & 10.30 & -0.0197 & $0.00 \mathrm{E}+00$ & 0.0000 & 0.0000 & 0.0000 & \\
\hline & 7/23/2008 1:00 & 12 & 205 & 100 & 10.22 & 10.22 & & -0.0274 & $0.00 \mathrm{E}+00$ & 0.0000 & 0.0000 & 0.0000 & \\
\hline Day Count & 7/23/2008 2:00 & 13 & 205 & 200 & 10.24 & 10.24 & & -0.0293 & $0.00 \mathrm{E}+00$ & 0.0000 & 0.0000 & 0.0000 & \\
\hline \multirow[t]{27}{*}{35} & 7/23/2008 3:00 & 14 & 205 & 300 & 10.29 & 10.29 & & -0.0340 & $0.00 \mathrm{E}+00$ & 0.0000 & 0.0000 & 0.0000 & \\
\hline & 7/23/2008 4:00 & 15 & 205 & 400 & 10.3 & 10.3 & & -0.0350 & $0.00 \mathrm{E}+00$ & 0.0000 & 0.0000 & 0.0000 & \\
\hline & 7/23/2008 5:00 & 16 & 205 & 500 & 10.27 & 10.27 & & -0.0321 & $0.00 \mathrm{E}+00$ & 0.0000 & 0.0000 & 0.0000 & \\
\hline & 7/23/2008 6:00 & 17 & 205 & 600 & 10.19 & 10.19 & & -0.0245 & $0.00 \mathrm{E}+00$ & 0.0000 & 0.0000 & 0.0000 & \\
\hline & 7/23/2008 7:00 & 18 & 205 & 700 & 9.96 & 9.96 & & -0.0020 & $0.00 \mathrm{E}+00$ & 0.0000 & 0.0000 & 0.0000 & \\
\hline & 7/23/2008 8:00 & 19 & 205 & 800 & 9.1 & 9.1 & & 0.0923 & 6.34E-04 & 94.5692 & 34.8460 & 129.4151 & \\
\hline & 7/23/20089:00 & 20 & 205 & 900 & 7.496 & 7.496 & & 0.3260 & 2.99E-03 & 447.0737 & 164.7335 & 611.8072 & \\
\hline & $7 / 23 / 2008$ 10:00 & 21 & 205 & 1000 & 6.183 & 6.183 & & 0.6076 & $6.44 \mathrm{E}-03$ & 962.0662 & 354.4931 & 1316.5593 & \\
\hline & $7 / 23 / 2008$ 11:00 & 22 & 205 & 1100 & 6.023 & 6.023 & & 0.6503 & 7.01E-03 & 1045.9669 & 385.4080 & 1431.3749 & \\
\hline & $7 / 23 / 200812: 00$ & 23 & 205 & 1200 & 5.913 & 5.913 & & 0.6810 & $7.42 \mathrm{E}-03$ & 1107.0790 & 407.9260 & 1515.0051 & \\
\hline & 7/23/2008 13:00 & 24 & 205 & 1300 & 5.87 & 5.87 & & 0.6934 & 7.58E-03 & 1131.7720 & 417.0247 & 1548.7967 & \\
\hline & $7 / 23 / 2008$ 14:00 & 25 & 205 & 1400 & 5.841 & 5.841 & & 0.7018 & 7.69E-03 & 1148.6891 & 423.2581 & 1571.9472 & \\
\hline & 7/23/2008 15:00 & 26 & 205 & 1500 & 5.75 & 5.75 & & 0.7287 & 8.06E-03 & 1203.1952 & 443.3420 & 1646.5372 & \\
\hline & 7/23/2008 16:00 & 27 & 205 & 1600 & 5.939 & 5.939 & & 0.6737 & 7.32E-03 & 1092.3709 & 402.5065 & 1494.8774 & \\
\hline & $7 / 23 / 2008$ 17:00 & 28 & 205 & 1700 & 6.506 & 6.506 & & 0.5278 & $5.42 \mathrm{E}-03$ & 808.9511 & 298.0747 & 1107.0258 & \\
\hline & $7 / 23 / 2008$ 18:00 & 29 & 205 & 1800 & 7.415 & 7.415 & & 0.3405 & $3.16 \mathrm{E}-03$ & 471.6487 & 173.7887 & 645.4374 & \\
\hline & $7 / 23 / 2008$ 19:00 & 30 & 205 & 1900 & 7.356 & 7.356 & & 0.3513 & $3.28 \mathrm{E}-03$ & 490.0471 & 180.5679 & 670.6151 & \\
\hline & $7 / 23 / 200820: 00$ & 31 & 205 & 2000 & 7.927 & 7.927 & & 0.2539 & $2.20 \mathrm{E}-03$ & 328.6778 & 121.1081 & 449.7859 & \\
\hline & $7 / 23 / 2008$ 21:00 & 32 & 205 & 2100 & 8.87 & 8.87 & & 0.1206 & $8.81 \mathrm{E}-04$ & 131.4677 & 48.4420 & 179.9097 & \\
\hline & $7 / 23 / 2008$ 22:00 & 33 & 205 & 2200 & 9.35 & 9.35 & & 0.0631 & 3.97E-04 & 59.2095 & 21.8170 & 81.0265 & \\
\hline & $7 / 23 / 2008$ 23:00 & 34 & 205 & 2300 & 9.45 & 9.45 & & 0.0519 & $3.11 \mathrm{E}-04$ & 46.4961 & 17.1324 & 63.6285 & \\
\hline & $7 / 24 / 20080: 00$ & 35 & 206 & 0 & 9.5 & 9.5 & 10.01 & 0.0463 & $2.71 \mathrm{E}-04$ & 40.4626 & 14.9093 & 55.3719 & \\
\hline & 7/24/2008 1:00 & 36 & 206 & 100 & 9.64 & 9.64 & & 0.0311 & $1.66 \mathrm{E}-04$ & 24.8016 & 9.1386 & 33.9402 & \\
\hline & 7/24/2008 2:00 & 37 & 206 & 200 & 9.75 & 9.75 & & 0.0195 & $9.34 \mathrm{E}-05$ & 13.9387 & 5.1360 & 19.0747 & \\
\hline & 7/24/2008 3:00 & 38 & 206 & 300 & 9.86 & 9.86 & & 0.0081 & $3.18 \mathrm{E}-05$ & 4.7401 & 1.7466 & 6.4866 & \\
\hline & 7/24/2008 4:00 & 39 & 206 & 400 & 9.92 & 9.92 & & 0.0020 & $5.72 \mathrm{E}-06$ & 0.8539 & 0.3146 & 1.1685 & \\
\hline & 7/24/2008 5:00 & 40 & 206 & 500 & 9.97 & 9.97 & & -0.0030 & $0.00 \mathrm{E}+00$ & 0.0000 & 0.0000 & 0.0000 & \\
\hline
\end{tabular}




\begin{tabular}{|c|c|c|c|c|c|c|c|c|c|c|c|c|c|}
\hline Tree ID & TS & RN & JD & HR & $\begin{array}{c}\text { Raw } \\
\text { TC_dT } \Delta\end{array}$ & $\begin{array}{c}\text { Fixed } \\
\text { TC_dT } \Delta\end{array}$ & dTM & $\mathbf{K}$ & $\begin{array}{l}\text { Sap } \\
\text { Velocity } \\
(\mathbf{c m} / \mathbf{s})\end{array}$ & $\begin{array}{l}\text { Probed } \\
\text { Drywood F } \\
\text { (g/hr) }\end{array}$ & $\begin{array}{c}\text { Extrapolated } \\
\text { Wetwood F } \\
(\mathrm{g} / \mathrm{hr})\end{array}$ & $\begin{array}{c}\text { Extrapolated } \\
\text { Total Wood F } \\
(\mathrm{g} / \mathrm{hr})\end{array}$ & $\begin{array}{l}\text { Relative } \\
\text { Humidity, } \\
\text { @10m (\%) }\end{array}$ \\
\hline & 7/24/2008 6:00 & 41 & 206 & 600 & 9.98 & 9.98 & & -0.0040 & $0.00 \mathrm{E}+00$ & 0.0000 & 0.0000 & 0.0000 & \\
\hline & 7/24/2008 7:00 & 42 & 206 & 700 & 9.95 & 9.95 & & -0.0010 & $0.00 \mathrm{E}+00$ & 0.0000 & 0.0000 & 0.0000 & \\
\hline & 7/24/2008 8:00 & 43 & 206 & 800 & 9.72 & 9.72 & & 0.0226 & $1.12 E-04$ & 16.7590 & 6.1752 & 22.9341 & \\
\hline & 7/24/2008 9:00 & 44 & 206 & 900 & 9.27 & 9.27 & & 0.0723 & 4.69E-04 & 69.9785 & 25.7850 & 95.7635 & \\
\hline & $7 / 24 / 2008$ 10:00 & 45 & 206 & 1000 & 9.24 & 9.24 & & 0.0758 & 4.97E-04 & 74.1507 & 27.3223 & 101.4730 & \\
\hline & $7 / 24 / 2008$ 11:00 & 46 & 206 & 1100 & 7.042 & 7.042 & & 0.4115 & 3.99E-03 & 595.4853 & 219.4188 & 814.9041 & \\
\hline & 7/24/2008 12:00 & 47 & 206 & 1200 & 5.871 & 5.871 & & 0.6931 & $7.58 \mathrm{E}-03$ & 1131.1925 & 416.8111 & 1548.0036 & \\
\hline & $7 / 24 / 2008$ 13:00 & 48 & 206 & 1300 & 6.433 & 6.433 & & 0.5452 & 5.64E-03 & 841.7838 & 310.1726 & 1151.9564 & \\
\hline & $7 / 24 / 200814: 00$ & 49 & 206 & 1400 & 6.298 & 6.298 & & 0.5783 & $6.06 \mathrm{E}-03$ & 905.1754 & 333.5305 & 1238.7059 & \\
\hline & $7 / 24 / 200815: 00$ & 50 & 206 & 1500 & 5.775 & 5.775 & & 0.7212 & 7.96E-03 & 1188.0024 & 437.7439 & 1625.7463 & \\
\hline & 7/24/2008 16:00 & 51 & 206 & 1600 & 5.583 & 5.583 & & 0.7804 & 8.77E-03 & 1309.1445 & 482.3812 & 1791.5257 & \\
\hline & $7 / 24 / 200817: 00$ & 52 & 206 & 1700 & 6.041 & 6.041 & & 0.6454 & 6.94E-03 & 1036.2396 & 381.8238 & 1418.0634 & \\
\hline & $7 / 24 / 200818: 00$ & 53 & 206 & 1800 & 5.641 & 5.641 & & 0.7621 & $8.52 \mathrm{E}-03$ & 1271.4454 & 468.4902 & 1739.9355 & \\
\hline & $7 / 24 / 200819: 00$ & 54 & 206 & 1900 & 6.102 & 6.102 & & 0.6290 & $6.72 \mathrm{E}-03$ & 1003.8265 & 369.8805 & 1373.7071 & \\
\hline & $7 / 24 / 200820: 00$ & 55 & 206 & 2000 & 7.38 & 7.38 & & 0.3469 & 3.23E-03 & 482.5116 & 177.7913 & 660.3029 & \\
\hline & $7 / 24 / 200821: 00$ & 56 & 206 & 2100 & 8.95 & 8.95 & & 0.1106 & 7.92E-04 & 118.1611 & 43.5389 & 161.7000 & \\
\hline & $7 / 24 / 200822: 00$ & 57 & 206 & 2200 & 9.84 & 9.84 & & 0.0102 & $4.19 \mathrm{E}-05$ & 6.2541 & 2.3045 & 8.5586 & \\
\hline & $7 / 24 / 2008$ 23:00 & 58 & 206 & 2300 & 10.01 & 10.01 & & -0.0070 & $0.00 \mathrm{E}+00$ & 0.0000 & 0.0000 & 0.0000 & \\
\hline & $7 / 25 / 20080: 00$ & 59 & 207 & 0 & 10.02 & 10.02 & 10.02 & -0.0080 & $0.00 \mathrm{E}+00$ & 0.0000 & 0.0000 & 0.0000 & \\
\hline & 7/25/2008 1:00 & 60 & 207 & 100 & 9.97 & 9.97 & & -0.0030 & $0.00 \mathrm{E}+00$ & 0.0000 & 0.0000 & 0.0000 & \\
\hline & 7/25/2008 2:00 & 61 & 207 & 200 & 9.93 & 9.93 & & 0.0010 & 2.43E-06 & 0.3633 & 0.1339 & 0.4972 & \\
\hline & 7/25/2008 3:00 & 62 & 207 & 300 & 9.9 & 9.9 & & 0.0040 & 1.35E-05 & 2.0093 & 0.7404 & 2.7497 & \\
\hline & 7/25/2008 4:00 & 63 & 207 & 400 & 9.91 & 9.91 & & 0.0030 & 9.43E-06 & 1.4084 & 0.5189 & 1.9273 & \\
\hline & 7/25/2008 5:00 & 64 & 207 & 500 & 9.89 & 9.89 & & 0.0051 & 1.77E-05 & 2.6478 & 0.9756 & 3.6235 & \\
\hline & 7/25/2008 6:00 & 65 & 207 & 600 & 9.87 & 9.87 & & 0.0071 & 2.69E-05 & 4.0165 & 1.4800 & 5.4965 & \\
\hline & 7/25/2008 7:00 & 66 & 207 & 700 & 9.85 & 9.85 & & 0.0091 & $3.68 \mathrm{E}-05$ & 5.4865 & 2.0216 & 7.5081 & \\
\hline & 7/25/2008 8:00 & 67 & 207 & 800 & 9.82 & 9.82 & & 0.0122 & $5.26 \mathrm{E}-05$ & 7.8474 & 2.8915 & 10.7389 & \\
\hline & 7/25/2008 9:00 & 68 & 207 & 900 & 9.8 & 9.8 & & 0.0143 & $6.37 \mathrm{E}-05$ & 9.5110 & 3.5045 & 13.0155 & \\
\hline & $7 / 25 / 200810: 00$ & 69 & 207 & 1000 & 9.71 & 9.71 & & 0.0237 & $1.19 \mathrm{E}-04$ & 17.7240 & 6.5308 & 24.2548 & \\
\hline & 7/25/2008 11:00 & 70 & 207 & 1100 & 8.56 & 8.56 & & 0.1612 & 1.26E-03 & 187.8701 & 69.2246 & 257.0947 & \\
\hline & $7 / 25 / 2008$ 12:00 & 71 & 207 & 1200 & 7.218 & 7.218 & & 0.3771 & $3.58 \mathrm{E}-03$ & 534.7833 & 197.0519 & 731.8352 & \\
\hline & $7 / 25 / 2008$ 13:00 & 72 & 207 & 1300 & 6.33 & 6.33 & & 0.5703 & 5.96E-03 & 889.8261 & 327.8747 & 1217.7008 & \\
\hline & $7 / 25 / 200814: 00$ & 73 & 207 & 1400 & 5.712 & 5.712 & & 0.7402 & 8.22E-03 & 1226.6132 & 451.9708 & 1678.5840 & \\
\hline & $7 / 25 / 2008$ 15:00 & 74 & 207 & 1500 & 5.652 & 5.652 & & 0.7587 & 8.47E-03 & 1264.4059 & 465.8964 & 1730.3023 & \\
\hline & $7 / 25 / 200816: 00$ & 75 & 207 & 1600 & 5.798 & 5.798 & & 0.7144 & 7.87E-03 & 1174.1724 & 432.6480 & 1606.8204 & \\
\hline & $7 / 25 / 2008$ 17:00 & 76 & 207 & 1700 & 5.569 & 5.569 & & 0.7849 & 8.83E-03 & 1318.3932 & 485.7891 & 1804.1823 & \\
\hline & $7 / 25 / 2008$ 18:00 & 77 & 207 & 1800 & 5.612 & 5.612 & & 0.7712 & 8.64E-03 & 1290.1717 & 475.3903 & 1765.5619 & \\
\hline & $7 / 25 / 2008$ 19:00 & 78 & 207 & 1900 & 5.674 & 5.674 & & 0.7519 & 8.38E-03 & 1250.4307 & 460.7469 & 1711.1776 & \\
\hline & $7 / 25 / 200820: 00$ & 79 & 207 & 2000 & 6.745 & 6.745 & & 0.4737 & 4.74E-03 & 708.0576 & 260.8984 & 968.9560 & \\
\hline & $7 / 25 / 200821: 00$ & 80 & 207 & 2100 & 8.47 & 8.47 & & 0.1736 & 1.38E-03 & 205.7240 & 75.8032 & 281.5272 & \\
\hline & 7/25/2008 22:00 & 81 & 207 & 2200 & 9.72 & 9.72 & & 0.0226 & $1.12 \mathrm{E}-04$ & 16.7590 & 6.1752 & 22.9341 & \\
\hline
\end{tabular}




\begin{tabular}{|c|c|c|c|c|c|c|c|c|c|c|c|c|c|}
\hline Tree ID & TS & RN & JD & HR & $\begin{array}{c}\text { Raw } \\
\text { TC_dTA }\end{array}$ & $\begin{array}{c}\text { Fixed } \\
\text { TC_dT } \Delta\end{array}$ & dTM & $\mathbf{K}$ & $\begin{array}{l}\text { Sap } \\
\text { Velocity } \\
(\mathrm{cm} / \mathrm{s})\end{array}$ & $\begin{array}{c}\text { Probed } \\
\text { Drywood F } \\
(\mathrm{g} / \mathrm{hr})\end{array}$ & $\begin{array}{c}\text { Extrapolated } \\
\text { Wetwood F } \\
(\mathrm{g} / \mathrm{hr})\end{array}$ & $\begin{array}{c}\text { Extrapolated } \\
\text { Total Wood F } \\
(\mathrm{g} / \mathrm{hr})\end{array}$ & $\begin{array}{l}\text { Relative } \\
\text { Humidity, } \\
@ 10 \mathrm{~m}(\%)\end{array}$ \\
\hline & 7/25/2008 23:00 & 82 & 207 & 2300 & $\begin{array}{r}9.86 \\
\end{array}$ & 9.86 & & 0.0081 & 3.18E-05 & 4.7401 & 1.7466 & 6.4866 & \\
\hline & 7/26/2008 0:00 & 83 & 208 & 0 & 9.82 & 9.82 & 9.82 & 0.0122 & $5.26 \mathrm{E}-05$ & 7.8474 & 2.8915 & 10.7389 & \\
\hline & 7/26/2008 1:00 & 84 & 208 & 100 & 9.76 & 9.76 & & 0.0184 & $8.73 \mathrm{E}-05$ & 13.0248 & 4.7992 & 17.8240 & \\
\hline & $7 / 26 / 20082: 00$ & 85 & 208 & 200 & 9.69 & 9.69 & & 0.0258 & 1.32E-04 & 19.6898 & 7.2551 & 26.9449 & \\
\hline & 7/26/2008 3:00 & 86 & 208 & 300 & 9.61 & 9.61 & & 0.0343 & 1.88E-04 & 27.9963 & 10.3158 & 38.3120 & \\
\hline & 7/26/2008 4:00 & 87 & 208 & 400 & 9.54 & 9.54 & & 0.0419 & $2.40 \mathrm{E}-04$ & 35.7976 & 13.1904 & 48.9879 & \\
\hline & 7/26/2008 5:00 & 88 & 208 & 500 & 9.48 & 9.48 & & 0.0485 & 2.87E-04 & 42.8495 & 15.7888 & 58.6383 & \\
\hline & 7/26/2008 6:00 & 89 & 208 & 600 & 9.41 & 9.41 & & 0.0563 & 3.45E-04 & 51.4798 & 18.9688 & 70.4485 & \\
\hline & 7/26/2008 7:00 & 90 & 208 & 700 & 9.33 & 9.33 & & 0.0654 & 4.14E-04 & 61.8527 & 22.7909 & 84.6436 & \\
\hline & 7/26/2008 8:00 & 91 & 208 & 800 & 9.27 & 9.27 & & 0.0723 & 4.69E-04 & 69.9785 & 25.7850 & 95.7635 & \\
\hline & 7/26/2008 9:00 & 92 & 208 & 900 & 8.03 & 8.03 & & 0.2379 & 2.03E-03 & 303.2417 & 111.7357 & 414.9774 & \\
\hline & $7 / 26 / 2008$ 10:00 & 93 & 208 & 1000 & 6.395 & 6.395 & & 0.5543 & $5.76 \mathrm{E}-03$ & 859.2699 & 316.6157 & 1175.8856 & \\
\hline & $7 / 26 / 2008$ 11:00 & 94 & 208 & 1100 & 6.186 & 6.186 & & 0.6069 & $6.43 E-03$ & 960.5469 & 353.9333 & 1314.4802 & \\
\hline & $7 / 26 / 2008$ 12:00 & 95 & 208 & 1200 & 6.402 & 6.402 & & 0.5526 & 5.73E-03 & 856.0281 & 315.4212 & 1171.4493 & \\
\hline & $7 / 26 / 2008$ 13:00 & 96 & 208 & 1300 & 5.772 & 5.772 & & 0.7221 & 7.97E-03 & 1189.8167 & 438.4124 & 1628.2291 & \\
\hline & $7 / 26 / 200814: 00$ & 97 & 208 & 1400 & 5.68 & 5.68 & & 0.7500 & 8.35E-03 & 1246.6431 & 459.3513 & 1705.9944 & \\
\hline & $7 / 26 / 2008$ 15:00 & 98 & 208 & 1500 & 5.563 & 5.563 & & 0.7868 & 8.86E-03 & 1322.3749 & 487.2562 & 1809.6312 & \\
\hline & $7 / 26 / 200816: 00$ & 99 & 208 & 1600 & 5.638 & 5.638 & & 0.7630 & 8.53E-03 & 1273.3713 & 469.1998 & 1742.5711 & \\
\hline & $7 / 26 / 2008$ 17:00 & 100 & 208 & 1700 & 5.414 & 5.414 & & 0.8360 & 9.54E-03 & 1424.8376 & 525.0107 & 1949.8484 & \\
\hline & $7 / 26 / 2008$ 18:00 & 101 & 208 & 1800 & 5.489 & 5.489 & & 0.8109 & 9.19E-03 & 1372.3879 & 505.6845 & 1878.0724 & \\
\hline & $7 / 26 / 2008$ 19:00 & 102 & 208 & 1900 & 5.696 & 5.696 & & 0.7451 & 8.28E-03 & 1236.5924 & 455.6479 & 1692.2403 & \\
\hline & $7 / 26 / 200820: 00$ & 103 & 208 & 2000 & 6.293 & 6.293 & & 0.5795 & 6.08E-03 & 907.5923 & 334.4210 & 1242.0133 & \\
\hline & $7 / 26 / 200821: 00$ & 104 & 208 & 2100 & 8.02 & 8.02 & & 0.2394 & 2.05E-03 & 305.6658 & 112.6289 & 418.2947 & \\
\hline & $7 / 26 / 200822: 00$ & 105 & 208 & 2200 & 9.13 & 9.13 & & 0.0887 & 6.03E-04 & 90.0632 & 33.1856 & 123.2488 & \\
\hline & $7 / 26 / 2008$ 23:00 & 106 & 208 & 2300 & 9.32 & 9.32 & & 0.0665 & 4.23E-04 & 63.1866 & 23.2824 & 86.4690 & \\
\hline & $7 / 27 / 20080: 00$ & 107 & 209 & 0 & 9.36 & 9.36 & 9.36 & 0.0620 & 3.88E-04 & 57.9003 & 21.3346 & 79.2349 & \\
\hline & 7/27/2008 1:00 & 108 & 209 & 100 & 9.33 & 9.33 & & 0.0654 & 4.14E-04 & 61.8527 & 22.7909 & 84.6436 & \\
\hline & 7/27/2008 2:00 & 109 & 209 & 200 & 9.21 & 9.21 & & 0.0793 & 5.25E-04 & 78.3950 & 28.8863 & 107.2813 & \\
\hline & 7/27/2008 3:00 & 110 & 209 & 300 & 9.04 & 9.04 & & 0.0996 & 6.95E-04 & 103.7937 & 38.2449 & 142.0386 & \\
\hline & 7/27/2008 4:00 & 111 & 209 & 400 & 8.89 & 8.89 & & 0.1181 & 8.58E-04 & 128.0937 & 47.1987 & 175.2924 & \\
\hline & 7/27/20085:00 & 112 & 209 & 500 & 8.8 & 8.8 & & 0.1295 & $9.61 \mathrm{E}-04$ & 143.5269 & 52.8855 & 196.4124 & \\
\hline & 7/27/2008 6:00 & 113 & 209 & 600 & 8.7 & 8.7 & & 0.1425 & 1.08E-03 & 161.4342 & 59.4838 & 220.9180 & \\
\hline & 7/27/2008 7:00 & 114 & 209 & 700 & 8.59 & 8.59 & & 0.1572 & 1.22E-03 & 182.0694 & 67.0872 & 249.1566 & \\
\hline & 7/27/2008 8:00 & 115 & 209 & 800 & 8.39 & 8.39 & & 0.1847 & $1.49 \mathrm{E}-03$ & 222.1719 & 81.8638 & 304.0357 & \\
\hline & $7 / 27 / 20089: 00$ & 116 & 209 & 900 & 6.368 & 6.368 & & 0.5609 & 5.84E-03 & 871.8624 & 321.2556 & 1193.1181 & \\
\hline & $7 / 27 / 2008$ 10:00 & 117 & 209 & 1000 & 5.073 & 5.073 & & 0.9594 & 1.13E-02 & 1688.0271 & 621.9883 & 2310.0154 & \\
\hline & $7 / 27 / 2008$ 11:00 & 118 & 209 & 1100 & 4.886 & 4.886 & & 1.0344 & 1.24E-02 & 1851.8892 & 682.3667 & 2534.2559 & \\
\hline & $7 / 27 / 2008$ 12:00 & 119 & 209 & 1200 & 4.968 & 4.968 & & 1.0008 & 1.19E-02 & 1778.1648 & 655.2014 & 2433.3662 & \\
\hline & $7 / 27 / 200813: 00$ & 120 & 209 & 1300 & 5.146 & 5.146 & & 0.9316 & 1.09E-02 & 1628.0274 & 599.8802 & 2227.9076 & \\
\hline & $7 / 27 / 2008$ 14:00 & 121 & 209 & 1400 & 4.992 & 4.992 & & 0.9912 & $1.18 \mathrm{E}-02$ & 1757.1494 & 647.4578 & 2404.6072 & \\
\hline & 7/27/2008 15:00 & 122 & 209 & 1500 & 4.927 & 4.927 & & 1.0175 & 1.22E-02 & 1814.6500 & 668.6451 & 2483.2951 & \\
\hline
\end{tabular}




\begin{tabular}{|c|c|c|c|c|c|c|c|c|c|c|c|c|c|}
\hline Tree ID & TS & RN & JD & HR & $\begin{array}{c}\text { Raw } \\
\text { TC_dT } \Delta\end{array}$ & $\begin{array}{c}\text { Fixed } \\
\text { TC_dT } \Delta\end{array}$ & dTM & $\mathbf{K}$ & $\begin{array}{l}\text { Sap } \\
\text { Velocity } \\
\text { (cm/s) }\end{array}$ & $\begin{array}{c}\text { Probed } \\
\text { Drywood F } \\
\text { (g/hr) }\end{array}$ & $\begin{array}{c}\text { Extrapolated } \\
\text { Wetwood F } \\
(\mathrm{g} / \mathrm{hr})\end{array}$ & $\begin{array}{c}\text { Extrapolated } \\
\text { Total Wood F } \\
(\mathrm{g} / \mathrm{hr})\end{array}$ & $\begin{array}{l}\text { Relative } \\
\text { Humidity, } \\
@ 10 \mathrm{~m}(\%)\end{array}$ \\
\hline & $7 / 27 / 200816: 00$ & 123 & 209 & 1600 & ) $\quad 4.965$ & 4.965 & & 1.0020 & 1.19E-02 & 1780.8093 & 656.1758 & 2436.9851 & \\
\hline & 7/27/2008 17:00 & 124 & 209 & 1700 & 5.174 & 5.174 & & 0.9211 & $1.08 \mathrm{E}-02$ & 1605.5692 & 591.6050 & 2197.1742 & \\
\hline & $7 / 27 / 200818: 00$ & 125 & 209 & 1800 & 5.112 & 5.112 & & 0.9444 & $1.11 \mathrm{E}-02$ & 1655.7086 & 610.0799 & 2265.7885 & \\
\hline & $7 / 27 / 2008$ 19:00 & 126 & 209 & 1900 & 5.408 & 5.408 & & 0.8380 & 9.57E-03 & 1429.1126 & 526.5859 & 1955.6985 & \\
\hline & $7 / 27 / 200820: 00$ & 127 & 209 & 2000 & 5.863 & 5.863 & & 0.6954 & 7.61E-03 & 1135.8358 & 418.5221 & 1554.3579 & \\
\hline & $7 / 27 / 2008$ 21:00 & 128 & 209 & 2100 & 7.294 & 7.294 & & 0.3628 & $3.42 E-03$ & 509.8460 & 187.8632 & 697.7092 & \\
\hline & $7 / 27 / 200822: 00$ & 129 & 209 & 2200 & 8.43 & 8.43 & & 0.1791 & 1.43E-03 & 213.8792 & 78.8082 & 292.6874 & \\
\hline & $7 / 27 / 200823: 00$ & 130 & 209 & 2300 & 8.65 & 8.65 & & 0.1491 & 1.14E-03 & 170.6911 & 62.8946 & 233.5857 & \\
\hline & $7 / 28 / 20080: 00$ & 131 & 210 & 0 & 8.74 & 8.74 & 8.88 & 0.1373 & 1.03E-03 & 154.1748 & 56.8089 & 210.9836 & \\
\hline & 7/28/2008 1:00 & 132 & 210 & 100 & 8.83 & 8.83 & & 0.1257 & 9.27E-04 & 138.3110 & 50.9635 & 189.2745 & \\
\hline & 7/28/2008 2:00 & 133 & 210 & 200 & 8.84 & 8.84 & & 0.1244 & $9.15 E-04$ & 136.5883 & 50.3288 & 186.9170 & \\
\hline & 7/28/2008 3:00 & 134 & 210 & 300 & 8.88 & 8.88 & & 0.1194 & 8.69E-04 & 129.7767 & 47.8189 & 177.5957 & \\
\hline & 7/28/2008 4:00 & 135 & 210 & 400 & 8.84 & 8.84 & & 0.1244 & $9.15 E-04$ & 136.5883 & 50.3288 & 186.9170 & \\
\hline & 7/28/2008 5:00 & 136 & 210 & 500 & 8.85 & 8.85 & & 0.1232 & 9.04E-04 & 134.8735 & 49.6969 & 184.5704 & \\
\hline & 7/28/2008 6:00 & 137 & 210 & 600 & 8.85 & 8.85 & & 0.1232 & 9.04E-04 & 134.8735 & 49.6969 & 184.5704 & \\
\hline & 7/28/2008 7:00 & 138 & 210 & 700 & 8.74 & 8.74 & & 0.1373 & 1.03E-03 & 154.1748 & 56.8089 & 210.9836 & \\
\hline & 7/28/2008 8:00 & 139 & 210 & 800 & 7.282 & 7.282 & & 0.3650 & 3.44E-03 & 513.7340 & 189.2959 & 703.0299 & \\
\hline & 7/28/20089:00 & 140 & 210 & 900 & 5.703 & 5.703 & & 0.7429 & $8.25 E-03$ & 1232.2177 & 454.0360 & 1686.2537 & \\
\hline & $7 / 28 / 2008$ 10:00 & 141 & 210 & 1000 & 4.993 & 4.993 & & 0.9908 & 1.18E-02 & 1756.2791 & 647.1372 & 2403.4163 & \\
\hline & $7 / 28 / 2008$ 11:00 & 142 & 210 & 1100 & 5.03 & 5.03 & & 0.9761 & 1.16E-02 & 1724.3795 & 635.3831 & 2359.7626 & \\
\hline & $7 / 28 / 200812: 00$ & 143 & 210 & 1200 & 5.122 & 5.122 & & 0.9406 & $1.10 \mathrm{E}-02$ & 1647.5198 & 607.0626 & 2254.5824 & \\
\hline & $7 / 28 / 2008$ 13:00 & 144 & 210 & 1300 & 5.055 & 5.055 & & 0.9664 & 1.14E-02 & 1703.1515 & 627.5612 & 2330.7126 & \\
\hline & $7 / 28 / 2008$ 14:00 & 145 & 210 & 1400 & 4.984 & 4.984 & & 0.9944 & 1.18E-02 & 1764.1268 & 650.0288 & 2414.1556 & \\
\hline & $7 / 28 / 200815: 00$ & 146 & 210 & 1500 & 4.814 & 4.814 & & 1.0648 & $1.29 \mathrm{E}-02$ & 1919.1733 & 707.1589 & 2626.3322 & \\
\hline & $7 / 28 / 2008$ 16:00 & 147 & 210 & 1600 & 4.931 & 4.931 & & 1.0158 & $1.21 \mathrm{E}-02$ & 1811.0576 & 667.3214 & 2478.3790 & \\
\hline & $7 / 28 / 200817: 00$ & 148 & 210 & 1700 & 5.006 & 5.006 & & 0.9856 & 1.17E-02 & 1745.0049 & 642.9829 & 2387.9878 & \\
\hline & $7 / 28 / 2008$ 18:00 & 149 & 210 & 1800 & 4.972 & 4.972 & & 0.9992 & 1.19E-02 & 1774.6449 & 653.9044 & 2428.5493 & \\
\hline & $7 / 28 / 200819: 00$ & 150 & 210 & 1900 & 5.315 & 5.315 & & 0.8702 & 1.00E-02 & 1496.9240 & 551.5724 & 2048.4965 & \\
\hline & $7 / 28 / 200820: 00$ & 151 & 210 & 2000 & 5.779 & 5.779 & & 0.7200 & 7.94E-03 & 1185.5871 & 436.8539 & 1622.4410 & \\
\hline & 7/28/2008 21:00 & 152 & 210 & 2100 & 7.619 & 7.619 & & 0.3046 & $2.75 \mathrm{E}-03$ & 411.2173 & 151.5215 & 562.7388 & \\
\hline & $7 / 28 / 200822: 00$ & 153 & 210 & 2200 & 8.33 & 8.33 & & 0.1933 & 1.57E-03 & 234.8715 & 86.5432 & 321.4148 & \\
\hline & $7 / 28 / 200823: 00$ & 154 & 210 & 2300 & 8.5 & 8.5 & & 0.1694 & 1.34E-03 & 199.6969 & 73.5824 & 273.2793 & \\
\hline & $7 / 29 / 2008$ 0:00 & 155 & 211 & 0 & 8.71 & 8.71 & 8.94 & 0.1412 & 1.07E-03 & 159.6072 & 58.8106 & 218.4178 & \\
\hline & 7/29/2008 1:00 & 156 & 211 & 100 & 8.86 & 8.86 & & 0.1219 & 8.92E-04 & 133.1666 & 49.0680 & 182.2346 & \\
\hline & 7/29/2008 2:00 & 157 & 211 & 200 & 8.94 & 8.94 & & 0.1119 & 8.03E-04 & 119.7968 & 44.1416 & 163.9384 & \\
\hline & 7/29/2008 3:00 & 158 & 211 & 300 & 8.93 & 8.93 & & 0.1131 & 8.14E-04 & 121.4404 & 44.7472 & 166.1876 & \\
\hline & 7/29/2008 4:00 & 159 & 211 & 400 & 8.93 & 8.93 & & 0.1131 & 8.14E-04 & 121.4404 & 44.7472 & 166.1876 & \\
\hline & 7/29/2008 5:00 & 160 & 211 & 500 & 8.85 & 8.85 & & 0.1232 & 9.04E-04 & 134.8735 & 49.6969 & 184.5704 & \\
\hline & 7/29/2008 6:00 & 161 & 211 & 600 & 8.74 & 8.74 & & 0.1373 & 1.03E-03 & 154.1748 & 56.8089 & 210.9836 & \\
\hline & 7/29/2008 7:00 & 162 & 211 & 700 & 8.64 & 8.64 & & 0.1505 & 1.16E-03 & 172.5669 & 63.5858 & 236.1528 & \\
\hline & 7/29/2008 8:00 & 163 & 211 & 800 & 8.12 & 8.12 & & 0.2241 & 1.89E-03 & 281.8553 & 103.8554 & 385.7106 & \\
\hline
\end{tabular}




\begin{tabular}{|c|c|c|c|c|c|c|c|c|c|c|c|c|c|}
\hline Tree ID & TS & RN & JD & HR & $\begin{array}{c}\text { Raw } \\
\text { TC_dTA }\end{array}$ & $\begin{array}{c}\text { Fixed } \\
\text { TC_dT } \Delta\end{array}$ & dTM & $\mathbf{K}$ & $\begin{array}{l}\text { Sap } \\
\text { Velocity } \\
(\mathrm{cm} / \mathrm{s})\end{array}$ & $\begin{array}{c}\text { Probed } \\
\text { Drywood F } \\
(\mathrm{g} / \mathrm{hr})\end{array}$ & $\begin{array}{c}\text { Extrapolated } \\
\text { Wetwood F } \\
(\mathrm{g} / \mathrm{hr})\end{array}$ & $\begin{array}{c}\text { Extrapolated } \\
\text { Total Wood F } \\
(\mathrm{g} / \mathrm{hr})\end{array}$ & $\begin{array}{l}\text { Relative } \\
\text { Humidity, } \\
@ 10 \mathrm{~m}(\%)\end{array}$ \\
\hline & 7/29/2008 9:00 & 164 & 211 & 900 & 6.454 & 6.454 & & 0.5401 & $5.58 \mathrm{E}-03$ & 832.2375 & 306.6550 & 1138.8926 & \\
\hline & $7 / 29 / 2008$ 10:00 & 165 & 211 & 1000 & 5.318 & 5.318 & & 0.8691 & $1.00 \mathrm{E}-02$ & 1494.6902 & 550.7494 & 2045.4396 & \\
\hline & $7 / 29 / 2008$ 11:00 & 166 & 211 & 1100 & 5.477 & 5.477 & & 0.8149 & $9.25 \mathrm{E}-03$ & 1380.6586 & 508.7321 & 1889.3907 & \\
\hline & $7 / 29 / 2008$ 12:00 & 167 & 211 & 1200 & 4.89 & 4.89 & & 1.0327 & $1.24 \mathrm{E}-02$ & 1848.2223 & 681.0155 & 2529.2379 & \\
\hline & 7/29/2008 13:00 & 168 & 211 & 1300 & 4.915 & 4.915 & & 1.0224 & 1.22E-02 & 1825.4703 & 672.6321 & 2498.1024 & \\
\hline & $7 / 29 / 2008$ 14:00 & 169 & 211 & 1400 & 4.745 & 4.745 & & 1.0948 & 1.33E-02 & 1986.0070 & 731.7851 & 2717.7921 & \\
\hline & $7 / 29 / 2008$ 15:00 & 170 & 211 & 1500 & 4.93 & 4.93 & & 1.0162 & $1.21 \mathrm{E}-02$ & 1811.9550 & 667.6521 & 2479.6071 & \\
\hline & $7 / 29 / 2008$ 16:00 & 171 & 211 & 1600 & 5.103 & 5.103 & & 0.9479 & 1.11E-02 & 1663.1125 & 612.8080 & 2275.9205 & \\
\hline & 7/29/2008 17:00 & 172 & 211 & 1700 & 5.248 & 5.248 & & 0.8941 & 1.04E-02 & 1547.6439 & 570.2612 & 2117.9051 & \\
\hline & 7/29/2008 18:00 & 173 & 211 & 1800 & 5.132 & 5.132 & & 0.9369 & 1.10E-02 & 1639.3705 & 604.0598 & 2243.4303 & \\
\hline & $7 / 29 / 2008$ 19:00 & 174 & 211 & 1900 & 5.134 & 5.134 & & 0.9361 & 1.10E-02 & 1637.7454 & 603.4610 & 2241.2063 & \\
\hline & $7 / 29 / 200820: 00$ & 175 & 211 & 2000 & 6.333 & 6.333 & & 0.5696 & 5.95E-03 & 888.3976 & 327.3484 & 1215.7459 & \\
\hline & 7/29/2008 21:00 & 176 & 211 & 2100 & 7.983 & 7.983 & & 0.2451 & $2.11 \mathrm{E}-03$ & 314.7194 & 115.9648 & 430.6843 & \\
\hline & $7 / 29 / 2008$ 22:00 & 177 & 211 & 2200 & 8.63 & 8.63 & & 0.1518 & 1.17E-03 & 174.4510 & 64.2801 & 238.7310 & \\
\hline & $7 / 29 / 2008$ 23:00 & 178 & 211 & 2300 & 8.76 & 8.76 & & 0.1347 & 1.01E-03 & 150.5934 & 55.4892 & 206.0826 & \\
\hline & $7 / 30 / 20080: 00$ & 179 & 212 & 0 & 8.71 & 8.71 & 8.71 & 0.1412 & 1.07E-03 & 159.6072 & 58.8106 & 218.4178 & \\
\hline & 7/30/2008 1:00 & 180 & 212 & 100 & 8.59 & 8.59 & & 0.1572 & 1.22E-03 & 182.0694 & 67.0872 & 249.1566 & \\
\hline & 7/30/2008 2:00 & 181 & 212 & 200 & 8.55 & 8.55 & & 0.1626 & 1.27E-03 & 189.8203 & 69.9432 & 259.7635 & \\
\hline & 7/30/2008 3:00 & 182 & 212 & 300 & 8.52 & 8.52 & & 0.1667 & 1.31E-03 & 195.7211 & 72.1174 & 267.8385 & \\
\hline & 7/30/2008 4:00 & 183 & 212 & 400 & 8.46 & 8.46 & & 0.1749 & 1.39E-03 & 207.7500 & 76.5498 & 284.2997 & \\
\hline & 7/30/2008 5:00 & 184 & 212 & 500 & 8.39 & 8.39 & & 0.1847 & $1.49 \mathrm{E}-03$ & 222.1719 & 81.8638 & 304.0357 & \\
\hline & 7/30/2008 6:00 & 185 & 212 & 600 & 8.35 & 8.35 & & 0.1904 & 1.54E-03 & 230.6033 & 84.9705 & 315.5738 & \\
\hline & 7/30/2008 7:00 & 186 & 212 & 700 & 8.35 & 8.35 & & 0.1904 & 1.54E-03 & 230.6033 & 84.9705 & 315.5738 & \\
\hline & $7 / 30 / 20088: 00$ & 187 & 212 & 800 & 8.35 & 8.35 & & 0.1904 & $1.54 \mathrm{E}-03$ & 230.6033 & 84.9705 & 315.5738 & \\
\hline & 7/30/2008 9:00 & 188 & 212 & 900 & 7.117 & 7.117 & & 0.3967 & $3.81 E-03$ & 569.1009 & 209.6969 & 778.7978 & \\
\hline & $7 / 30 / 2008$ 10:00 & 189 & 212 & 1000 & 5.228 & 5.228 & & 0.9013 & 1.05E-02 & 1563.0985 & 575.9558 & 2139.0543 & \\
\hline & 7/30/2008 11:00 & 190 & 212 & 1100 & 4.761 & 4.761 & & 1.0878 & 1.32E-02 & 1970.2983 & 725.9969 & 2696.2953 & \\
\hline & $7 / 30 / 2008$ 12:00 & 191 & 212 & 1200 & 4.775 & 4.775 & & 1.0817 & $1.31 \mathrm{E}-02$ & 1956.6587 & 720.9711 & 2677.6298 & \\
\hline & 7/30/2008 13:00 & 192 & 212 & 1300 & 4.803 & 4.803 & & 1.0695 & 1.29E-02 & 1929.6708 & 711.0269 & 2640.6976 & \\
\hline & $7 / 30 / 2008$ 14:00 & 193 & 212 & 1400 & 4.718 & 4.718 & & 1.1068 & 1.35E-02 & 2012.8105 & 741.6614 & 2754.4720 & \\
\hline & 7/30/2008 15:00 & 194 & 212 & 1500 & 4.653 & 4.653 & & 1.1363 & 1.39E-02 & 2078.8972 & 766.0124 & 2844.9096 & \\
\hline & $7 / 30 / 2008$ 16:00 & 195 & 212 & 1600 & 4.562 & 4.562 & & 1.1789 & 1.46E-02 & 2175.2831 & 801.5278 & 2976.8109 & \\
\hline & $7 / 30 / 2008$ 17:00 & 196 & 212 & 1700 & 4.768 & 4.768 & & 1.0847 & 1.32E-02 & 1963.4663 & 723.4795 & 2686.9458 & \\
\hline & $7 / 30 / 2008$ 18:00 & 197 & 212 & 1800 & 4.746 & 4.746 & & 1.0944 & 1.33E-02 & 1985.0214 & 731.4220 & 2716.4433 & \\
\hline & 7/30/2008 19:00 & 198 & 212 & 1900 & 5.121 & 5.121 & & 0.9410 & 1.10E-02 & 1648.3369 & 607.3636 & 2255.7005 & \\
\hline & $7 / 30 / 200820: 00$ & 199 & 212 & 2000 & 5.763 & 5.763 & & 0.7248 & 8.01E-03 & 1195.2739 & 440.4233 & 1635.6972 & \\
\hline & 7/30/2008 21:00 & 200 & 212 & 2100 & 7.531 & 7.531 & & 0.3199 & 2.93E-03 & 436.6940 & 160.9089 & 597.6029 & \\
\hline & $7 / 30 / 2008$ 22:00 & 201 & 212 & 2200 & 8.47 & 8.47 & & 0.1736 & 1.38E-03 & 205.7240 & 75.8032 & 281.5272 & \\
\hline & 7/30/2008 23:00 & 202 & 212 & 2300 & 8.67 & 8.67 & & 0.1465 & $1.12 \mathrm{E}-03$ & 166.9639 & 61.5213 & 228.4852 & \\
\hline & $7 / 31 / 20080: 00$ & 203 & 213 & 0 & 8.67 & 8.67 & 8.67 & 0.1465 & 1.12E-03 & 166.9639 & 61.5213 & 228.4852 & \\
\hline & 7/31/2008 1:00 & 204 & 213 & 100 & 8.62 & 8.62 & & 0.1531 & $1.18 \mathrm{E}-03$ & 176.3432 & 64.9773 & 241.3205 & \\
\hline
\end{tabular}




\begin{tabular}{|c|c|c|c|c|c|c|c|c|c|c|c|c|c|}
\hline Tree ID & TS & RN & JD & HR & $\begin{array}{c}\text { Raw } \\
\text { TC_dTA }\end{array}$ & $\begin{array}{c}\text { Fixed } \\
\text { TC_dT } \Delta\end{array}$ & dTM & $\mathbf{K}$ & $\begin{array}{c}\text { Sap } \\
\text { Velocity } \\
(\mathrm{cm} / \mathrm{s})\end{array}$ & $\begin{array}{c}\text { Probed } \\
\text { Drywood F } \\
\text { (g/hr) }\end{array}$ & $\begin{array}{c}\text { Extrapolated } \\
\text { Wetwood F } \\
(\mathrm{g} / \mathrm{hr})\end{array}$ & $\begin{array}{c}\text { Extrapolated } \\
\text { Total Wood F } \\
(\mathrm{g} / \mathrm{hr})\end{array}$ & $\begin{array}{l}\text { Relative } \\
\text { Humidity, } \\
\text { @10m (\%) }\end{array}$ \\
\hline & 7/31/2008 2:00 & 205 & 213 & 200 & 8.54 & 8.54 & & 0.1639 & $1.28 \mathrm{E}-03$ & 191.7788 & 70.6649 & 262.4437 & \\
\hline & 7/31/2008 3:00 & 206 & 213 & 300 & 8.47 & 8.47 & & 0.1736 & 1.38E-03 & 205.7240 & 75.8032 & 281.5272 & \\
\hline & 7/31/2008 4:00 & 207 & 213 & 400 & 8.38 & 8.38 & & 0.1862 & $1.50 \mathrm{E}-03$ & 224.2667 & 82.6357 & 306.9023 & \\
\hline & 7/31/20085:00 & 208 & 213 & 500 & 8.31 & 8.31 & & 0.1961 & $1.60 \mathrm{E}-03$ & 239.1750 & 88.1290 & 327.3040 & \\
\hline & 7/31/2008 6:00 & 209 & 213 & 600 & 8.24 & 8.24 & & 0.2063 & 1.70E-03 & 254.5177 & 93.7823 & 348.3000 & \\
\hline & 7/31/2008 7:00 & 210 & 213 & 700 & 8.16 & 8.16 & & 0.2181 & 1.83E-03 & 272.5952 & 100.4433 & 373.0385 & \\
\hline & 7/31/2008 8:00 & 211 & 213 & 800 & 8.1 & 8.1 & & 0.2272 & $1.92 \mathrm{E}-03$ & 286.5414 & 105.5821 & 392.1235 & \\
\hline & 7/31/2008 9:00 & 212 & 213 & 900 & 6.599 & 6.599 & & 0.5063 & $5.15 \mathrm{E}-03$ & 768.5216 & 283.1776 & 1051.6992 & \\
\hline & $7 / 31 / 2008$ 10:00 & 213 & 213 & 1000 & 4.977 & 4.977 & & 0.9972 & 1.19E-02 & 1770.2548 & 652.2868 & 2422.5415 & \\
\hline & 7/31/2008 11:00 & 214 & 213 & 1100 & 4.684 & 4.684 & & 1.1221 & 1.37E-02 & 2047.1002 & 754.2962 & 2801.3963 & \\
\hline & 7/31/2008 12:00 & 215 & 213 & 1200 & 4.503 & 4.503 & & 1.2074 & $1.50 \mathrm{E}-02$ & 2240.3103 & 825.4884 & 3065.7987 & \\
\hline & $7 / 31 / 2008$ 13:00 & 216 & 213 & 1300 & 4.76 & 4.76 & & 1.0882 & 1.32E-02 & 1971.2763 & 726.3573 & 2697.6337 & \\
\hline & $7 / 31 / 2008$ 14:00 & 217 & 213 & 1400 & 4.448 & 4.448 & & 1.2347 & $1.54 \mathrm{E}-02$ & 2302.8156 & 848.5198 & 3151.3354 & \\
\hline & 7/31/2008 15:00 & 218 & 213 & 1500 & 4.383 & 4.383 & & 1.2679 & 1.59E-02 & 2379.1377 & 876.6422 & 3255.7799 & \\
\hline & $7 / 31 / 2008$ 16:00 & 219 & 213 & 1600 & 4.561 & 4.561 & & 1.1793 & 1.46E-02 & 2176.3683 & 801.9277 & 2978.2959 & \\
\hline & 7/31/2008 17:00 & 220 & 213 & 1700 & 4.656 & 4.656 & & 1.1349 & 1.39E-02 & 2075.7975 & 764.8703 & 2840.6678 & \\
\hline & $7 / 31 / 2008$ 18:00 & 221 & 213 & 1800 & 4.66 & 4.66 & & 1.1330 & 1.39E-02 & 2071.6722 & 763.3502 & 2835.0224 & \\
\hline & 7/31/2008 19:00 & 222 & 213 & 1900 & 5.138 & 5.138 & & 0.9346 & 1.09E-02 & 1634.4998 & 602.2651 & 2236.7649 & \\
\hline & $7 / 31 / 200820: 00$ & 223 & 213 & 2000 & 6.125 & 6.125 & & 0.6229 & $6.64 \mathrm{E}-03$ & 991.8224 & 365.4574 & 1357.2798 & \\
\hline & 7/31/2008 21:00 & 224 & 213 & 2100 & 7.358 & 7.358 & & 0.3509 & $3.28 E-03$ & 489.4165 & 180.3356 & 669.7520 & \\
\hline & $7 / 31 / 200822: 00$ & 225 & 213 & 2200 & 8.04 & 8.04 & & 0.2363 & 2.02E-03 & 300.8273 & 110.8460 & 411.6733 & \\
\hline & 7/31/2008 23:00 & 226 & 213 & 2300 & 7.963 & 7.963 & & 0.2483 & $2.14 \mathrm{E}-03$ & 319.6690 & 117.7886 & 437.4576 & \\
\hline & 8/1/2008 0:00 & 227 & 214 & 0 & 8.25 & 8.25 & 8.72 & 0.2048 & 1.69E-03 & 252.2990 & 92.9647 & 345.2637 & \\
\hline & 8/1/2008 1:00 & 228 & 214 & 100 & 8.62 & 8.62 & & 0.1531 & $1.18 \mathrm{E}-03$ & 176.3432 & 64.9773 & 241.3205 & \\
\hline & 8/1/2008 2:00 & 229 & 214 & 200 & 8.72 & 8.72 & & 0.1399 & 1.06E-03 & 157.7883 & 58.1404 & 215.9287 & \\
\hline & 8/1/2008 3:00 & 230 & 214 & 300 & 8.71 & 8.71 & & 0.1412 & 1.07E-03 & 159.6072 & 58.8106 & 218.4178 & \\
\hline & 8/1/2008 4:00 & 231 & 214 & 400 & 8.72 & 8.72 & & 0.1399 & 1.06E-03 & 157.7883 & 58.1404 & 215.9287 & \\
\hline & 8/1/2008 5:00 & 232 & 214 & 500 & 8.68 & 8.68 & & 0.1452 & 1.11E-03 & 165.1126 & 60.8391 & 225.9517 & \\
\hline & 8/1/2008 6:00 & 233 & 214 & 600 & 8.6 & 8.6 & & 0.1558 & $1.21 E-03$ & 180.1524 & 66.3809 & 246.5333 & \\
\hline & 8/1/2008 7:00 & 234 & 214 & 700 & 8.51 & 8.51 & & 0.1680 & 1.32E-03 & 197.7048 & 72.8484 & 270.5531 & \\
\hline & 8/1/2008 8:00 & 235 & 214 & 800 & 8.37 & 8.37 & & 0.1876 & $1.52 \mathrm{E}-03$ & 226.3702 & 83.4107 & 309.7809 & \\
\hline & 8/1/2008 9:00 & 236 & 214 & 900 & 6.844 & 6.844 & & 0.4524 & 4.48E-03 & 669.0385 & 246.5210 & 915.5595 & \\
\hline & 8/1/2008 10:00 & 237 & 214 & 1000 & 4.903 & 4.903 & & 1.0273 & 1.23E-02 & 1836.3557 & 676.6430 & 2512.9988 & \\
\hline & 8/1/2008 11:00 & 238 & 214 & 1100 & 4.693 & 4.693 & & 1.1180 & 1.37E-02 & 2037.9645 & 750.9299 & 2788.8945 & \\
\hline & 8/1/2008 12:00 & 239 & 214 & 1200 & 4.713 & 4.713 & & 1.1091 & 1.35E-02 & 2017.8153 & 743.5056 & 2761.3209 & \\
\hline & 8/1/2008 13:00 & 240 & 214 & 1300 & 4.662 & 4.662 & & 1.1321 & 1.39E-02 & 2069.6127 & 762.5914 & 2832.2041 & \\
\hline & 8/1/2008 14:00 & 241 & 214 & 1400 & 4.596 & 4.596 & & 1.1628 & 1.43E-02 & 2138.7279 & 788.0583 & 2926.7861 & \\
\hline & 8/1/2008 15:00 & 242 & 214 & 1500 & 4.442 & 4.442 & & 1.2377 & $1.55 \mathrm{E}-02$ & 2309.7478 & 851.0741 & 3160.8219 & \\
\hline & 8/1/2008 16:00 & 243 & 214 & 1600 & 4.522 & 4.522 & & 1.1981 & $1.49 \mathrm{E}-02$ & 2219.1447 & 817.6895 & 3036.8342 & \\
\hline & 8/1/2008 17:00 & 244 & 214 & 1700 & 4.819 & 4.819 & & 1.0627 & $1.28 \mathrm{E}-02$ & 1914.4212 & 705.4079 & 2619.8290 & \\
\hline & 8/1/2008 18:00 & 245 & 214 & 1800 & 4.736 & 4.736 & & 1.0988 & 1.34E-02 & 1994.9000 & 735.0620 & 2729.9620 & \\
\hline
\end{tabular}




\begin{tabular}{|c|c|c|c|c|c|c|c|c|c|c|c|c|c|}
\hline Tree ID & TS & RN & JD & HR & $\begin{array}{c}\text { Raw } \\
\text { TC_dTA }\end{array}$ & $\begin{array}{c}\text { Fixed } \\
\text { TC_dT } \Delta\end{array}$ & dTM & $\mathbf{K}$ & $\begin{array}{c}\text { Sap } \\
\text { Velocity } \\
(\mathrm{cm} / \mathrm{s})\end{array}$ & $\begin{array}{c}\text { Probed } \\
\text { Drywood F } \\
\text { (g/hr) }\end{array}$ & $\begin{array}{c}\text { Extrapolated } \\
\text { Wetwood F } \\
(\mathrm{g} / \mathrm{hr})\end{array}$ & $\begin{array}{c}\text { Extrapolated } \\
\text { Total Wood F } \\
(\mathrm{g} / \mathrm{hr})\end{array}$ & $\begin{array}{l}\text { Relative } \\
\text { Humidity, } \\
\text { @10m (\%) }\end{array}$ \\
\hline & 8/1/2008 19:00 & 246 & 214 & 1900 & 5.058 & 5.058 & & 0.9652 & $1.14 \mathrm{E}-02$ & 1700.6215 & 626.6290 & 2327.2504 & \\
\hline & 8/1/2008 20:00 & 247 & 214 & 2000 & 5.889 & 5.889 & & 0.6879 & 7.51E-03 & 1120.8041 & 412.9833 & 1533.7874 & \\
\hline & 8/1/2008 21:00 & 248 & 214 & 2100 & 7.752 & 7.752 & & 0.2822 & $2.51 \mathrm{E}-03$ & 374.3447 & 137.9350 & 512.2796 & \\
\hline & 8/1/2008 22:00 & 249 & 214 & 2200 & 8.61 & 8.61 & & 0.1545 & 1.19E-03 & 178.2437 & 65.6776 & 243.9213 & \\
\hline & 8/1/2008 23:00 & 250 & 214 & 2300 & 8.72 & 8.72 & & 0.1399 & 1.06E-03 & 157.7883 & 58.1404 & 215.9287 & \\
\hline & 8/2/2008 0:00 & 251 & 215 & 0 & 8.7 & 8.7 & 8.75 & 0.1425 & 1.08E-03 & 161.4342 & 59.4838 & 220.9180 & \\
\hline & 8/2/2008 1:00 & 252 & 215 & 100 & 8.73 & 8.73 & & 0.1386 & $1.04 \mathrm{E}-03$ & 155.9775 & 57.4731 & 213.4506 & \\
\hline & 8/2/2008 2:00 & 253 & 215 & 200 & 8.74 & 8.74 & & 0.1373 & 1.03E-03 & 154.1748 & 56.8089 & 210.9836 & \\
\hline & 8/2/2008 3:00 & 254 & 215 & 300 & 8.75 & 8.75 & & 0.1360 & $1.02 \mathrm{E}-03$ & 152.3801 & 56.1476 & 208.5276 & \\
\hline & 8/2/2008 4:00 & 255 & 215 & 400 & 8.71 & 8.71 & & 0.1412 & 1.07E-03 & 159.6072 & 58.8106 & 218.4178 & \\
\hline & 8/2/2008 5:00 & 256 & 215 & 500 & 8.7 & 8.7 & & 0.1425 & 1.08E-03 & 161.4342 & 59.4838 & 220.9180 & \\
\hline & 8/2/2008 6:00 & 257 & 215 & 600 & 8.7 & 8.7 & & 0.1425 & 1.08E-03 & 161.4342 & 59.4838 & 220.9180 & \\
\hline & 8/2/2008 7:00 & 258 & 215 & 700 & 8.59 & 8.59 & & 0.1572 & 1.22E-03 & 182.0694 & 67.0872 & 249.1566 & \\
\hline & 8/2/2008 8:00 & 259 & 215 & 800 & 7.312 & 7.312 & & 0.3594 & 3.38E-03 & 504.0481 & 185.7269 & 689.7750 & \\
\hline & 8/2/20089:00 & 260 & 215 & 900 & 5.404 & 5.404 & & 0.8394 & 9.59E-03 & 1431.9692 & 527.6385 & 1959.6077 & \\
\hline & 8/2/2008 10:00 & 261 & 215 & 1000 & 4.917 & 4.917 & & 1.0216 & 1.22E-02 & 1823.6624 & 671.9659 & 2495.6284 & \\
\hline & 8/2/2008 11:00 & 262 & 215 & 1100 & 4.791 & 4.791 & & 1.0747 & 1.30E-02 & 1941.1898 & 715.2713 & 2656.4611 & \\
\hline & 8/2/2008 12:00 & 263 & 215 & 1200 & 4.728 & 4.728 & & 1.1024 & 1.34E-02 & 2002.8397 & 737.9875 & 2740.8272 & \\
\hline & 8/2/2008 13:00 & 264 & 215 & 1300 & 4.733 & 4.733 & & 1.1001 & 1.34E-02 & 1997.8736 & 736.1576 & 2734.0312 & \\
\hline & 8/2/2008 14:00 & 265 & 215 & 1400 & 4.729 & 4.729 & & 1.1019 & 1.34E-02 & 2001.8455 & 737.6211 & 2739.4666 & \\
\hline & $8 / 2 / 200815: 00$ & 266 & 215 & 1500 & 4.582 & 4.582 & & 1.1694 & 1.44E-02 & 2153.7003 & 793.5752 & 2947.2754 & \\
\hline & $8 / 2 / 2008$ 16:00 & 267 & 215 & 1600 & 4.652 & 4.652 & & 1.1367 & 1.39E-02 & 2079.9315 & 766.3935 & 2846.3250 & \\
\hline & $8 / 2 / 2008$ 17:00 & 268 & 215 & 1700 & 4.899 & 4.899 & & 1.0290 & 1.23E-02 & 1839.9988 & 677.9854 & 2517.9841 & \\
\hline & 8/2/2008 18:00 & 269 & 215 & 1800 & 4.811 & 4.811 & & 1.0661 & 1.29E-02 & 1922.0304 & 708.2116 & 2630.2421 & \\
\hline & 8/2/2008 19:00 & 270 & 215 & 1900 & 5.253 & 5.253 & & 0.8923 & 1.03E-02 & 1543.8031 & 568.8460 & 2112.6491 & \\
\hline & 8/2/2008 20:00 & 271 & 215 & 2000 & 5.949 & 5.949 & & 0.6709 & 7.28E-03 & 1086.7579 & 400.4383 & 1487.1962 & \\
\hline & 8/2/2008 21:00 & 272 & 215 & 2100 & 7.434 & 7.434 & & 0.3371 & $3.12 E-03$ & 465.8139 & 171.6387 & 637.4526 & \\
\hline & 8/2/2008 22:00 & 273 & 215 & 2200 & 8.37 & 8.37 & & 0.1876 & $1.52 E-03$ & 226.3702 & 83.4107 & 309.7809 & \\
\hline & 8/2/2008 23:00 & 274 & 215 & 2300 & 8.59 & 8.59 & & 0.1572 & $1.22 \mathrm{E}-03$ & 182.0694 & 67.0872 & 249.1566 & \\
\hline & 8/3/2008 0:00 & 275 & 216 & 0 & 8.47 & 8.47 & 8.74 & 0.1736 & $1.38 \mathrm{E}-03$ & 205.7240 & 75.8032 & 281.5272 & \\
\hline & 8/3/2008 1:00 & 276 & 216 & 100 & 8.27 & 8.27 & & 0.2019 & 1.66E-03 & 247.8885 & 91.3396 & 339.2282 & \\
\hline & $8 / 3 / 2008$ 2:00 & 277 & 216 & 200 & 8.37 & 8.37 & & 0.1876 & $1.52 E-03$ & 226.3702 & 83.4107 & 309.7809 & \\
\hline & 8/3/2008 3:00 & 278 & 216 & 300 & 8.6 & 8.6 & & 0.1558 & $1.21 \mathrm{E}-03$ & 180.1524 & 66.3809 & 246.5333 & \\
\hline & 8/3/2008 4:00 & 279 & 216 & 400 & 8.73 & 8.73 & & 0.1386 & $1.04 \mathrm{E}-03$ & 155.9775 & 57.4731 & 213.4506 & \\
\hline & $8 / 3 / 20085: 00$ & 280 & 216 & 500 & 8.74 & 8.74 & & 0.1373 & 1.03E-03 & 154.1748 & 56.8089 & 210.9836 & \\
\hline & 8/3/2008 6:00 & 281 & 216 & 600 & 8.69 & 8.69 & & 0.1438 & 1.09E-03 & 163.2693 & 60.1599 & 223.4293 & \\
\hline & 8/3/2008 7:00 & 282 & 216 & 700 & 8.61 & 8.61 & & 0.1545 & 1.19E-03 & 178.2437 & 65.6776 & 243.9213 & \\
\hline & 8/3/2008 8:00 & 283 & 216 & 800 & 7.44 & 7.44 & & 0.3360 & 3.11E-03 & 463.9804 & 170.9631 & 634.9435 & \\
\hline & 8/3/2008 9:00 & 284 & 216 & 900 & 5.392 & 5.392 & & 0.8435 & 9.65E-03 & 1440.5708 & 530.8079 & 1971.3787 & \\
\hline & $8 / 3 / 2008$ 10:00 & 285 & 216 & 1000 & 4.657 & 4.657 & & 1.1344 & 1.39E-02 & 2074.7654 & 764.4900 & 2839.2553 & \\
\hline & 8/3/2008 11:00 & 286 & 216 & 1100 & 4.638 & 4.638 & & 1.1432 & 1.40E-02 & 2094.4688 & 771.7501 & 2866.2189 & \\
\hline
\end{tabular}




\begin{tabular}{|c|c|c|c|c|c|c|c|c|c|c|c|c|c|}
\hline Tree ID & TS & RN & JD & HR & $\begin{array}{c}\text { Raw } \\
\text { TC_dTA }\end{array}$ & $\begin{array}{c}\text { Fixed } \\
\text { TC_dT } \Delta\end{array}$ & dTM & $\mathbf{K}$ & $\begin{array}{l}\text { Sap } \\
\text { Velocity } \\
(\mathrm{cm} / \mathrm{s})\end{array}$ & $\begin{array}{c}\text { Probed } \\
\text { Drywood F } \\
(\mathrm{g} / \mathrm{hr})\end{array}$ & $\begin{array}{c}\text { Extrapolated } \\
\text { Wetwood F } \\
(\mathrm{g} / \mathrm{hr})\end{array}$ & $\begin{array}{c}\text { Extrapolated } \\
\text { Total Wood F } \\
(\mathrm{g} / \mathrm{hr})\end{array}$ & $\begin{array}{l}\text { Relative } \\
\text { Humidity, } \\
@ 10 \mathrm{~m}(\%)\end{array}$ \\
\hline & 8/3/2008 12:00 & 287 & 216 & 1200 & 4.677 & 4.677 & & 1.1253 & 1.38E-02 & 2054.2353 & 756.9252 & 2811.1605 & \\
\hline & 8/3/2008 13:00 & 288 & 216 & 1300 & 4.699 & 4.699 & & 1.1153 & 1.36E-02 & 2031.8978 & 748.6945 & 2780.5923 & \\
\hline & $8 / 3 / 2008$ 14:00 & 289 & 216 & 1400 & 4.71 & 4.71 & & 1.1104 & $1.35 \mathrm{E}-02$ & 2020.8244 & 744.6143 & 2765.4387 & \\
\hline & 8/3/2008 15:00 & 290 & 216 & 1500 & 4.681 & 4.681 & & 1.1235 & 1.37E-02 & 2050.1549 & 755.4217 & 2805.5766 & \\
\hline & 8/3/2008 16:00 & 291 & 216 & 1600 & 4.839 & 4.839 & & 1.0541 & 1.27E-02 & 1895.5326 & 698.4480 & 2593.9806 & \\
\hline & 8/3/2008 17:00 & 292 & 216 & 1700 & 4.993 & 4.993 & & 0.9908 & 1.18E-02 & 1756.2791 & 647.1372 & 2403.4163 & \\
\hline & 8/3/2008 18:00 & 293 & 216 & 1800 & 4.853 & 4.853 & & 1.0482 & 1.26E-02 & 1882.4241 & 693.6179 & 2576.0419 & \\
\hline & 8/3/2008 19:00 & 294 & 216 & 1900 & 5.207 & 5.207 & & 0.9090 & 1.06E-02 & 1579.4848 & 581.9937 & 2161.4785 & \\
\hline & 8/3/2008 20:00 & 295 & 216 & 2000 & 5.673 & 5.673 & & 0.7522 & 8.38E-03 & 1251.0630 & 460.9799 & 1712.0429 & \\
\hline & 8/3/2008 21:00 & 296 & 216 & 2100 & 6.86 & 6.86 & & 0.4490 & 4.44E-03 & 662.8766 & 244.2505 & 907.1271 & \\
\hline & 8/3/2008 22:00 & 297 & 216 & 2200 & 7.651 & 7.651 & & 0.2992 & 2.69E-03 & 402.1690 & 148.1874 & 550.3564 & \\
\hline & 8/3/2008 23:00 & 298 & 216 & 2300 & 7.807 & 7.807 & & 0.2732 & $2.41 E-03$ & 359.6511 & 132.5209 & 492.1720 & \\
\hline & 8/4/2008 0:00 & 299 & 217 & 0 & 7.889 & 7.889 & 8.64 & 0.2600 & $2.27 \mathrm{E}-03$ & 338.3276 & 124.6638 & 462.9914 & \\
\hline & 8/4/2008 1:00 & 300 & 217 & 100 & 7.913 & 7.913 & & 0.2562 & 2.23E-03 & 332.2161 & 122.4119 & 454.6280 & \\
\hline & $8 / 4 / 2008$ 2:00 & 301 & 217 & 200 & 7.545 & 7.545 & & 0.3174 & 2.90E-03 & 432.5819 & 159.3937 & 591.9755 & \\
\hline & 8/4/2008 3:00 & 302 & 217 & 300 & 7.323 & 7.323 & & 0.3574 & 3.35E-03 & 500.5252 & 184.4288 & 684.9540 & \\
\hline & 8/4/2008 4:00 & 303 & 217 & 400 & 7.057 & 7.057 & & 0.4085 & 3.95E-03 & 590.1455 & 217.4513 & 807.5968 & \\
\hline & $8 / 4 / 20085: 00$ & 304 & 217 & 500 & 7.12 & 7.12 & & 0.3961 & $3.81 E-03$ & 568.0617 & 209.3140 & 777.3757 & \\
\hline & 8/4/2008 6:00 & 305 & 217 & 600 & 7.501 & 7.501 & & 0.3252 & 2.98E-03 & 445.5821 & 164.1839 & 609.7660 & \\
\hline & 8/4/2008 7:00 & 306 & 217 & 700 & 7.653 & 7.653 & & 0.2988 & 2.69E-03 & 401.6072 & 147.9805 & 549.5877 & \\
\hline & 8/4/2008 8:00 & 307 & 217 & 800 & 7.436 & 7.436 & & 0.3367 & $3.12 \mathrm{E}-03$ & 465.2023 & 171.4133 & 636.6156 & \\
\hline & 8/4/2008 9:00 & 308 & 217 & 900 & 6.957 & 6.957 & & 0.4288 & $4.20 \mathrm{E}-03$ & 626.3519 & 230.7922 & 857.1441 & \\
\hline & 8/4/2008 10:00 & 309 & 217 & 1000 & 5.811 & 5.811 & & 0.7105 & 7.81E-03 & 1166.4173 & 429.7904 & 1596.2078 & \\
\hline & 8/4/2008 11:00 & 310 & 217 & 1100 & 5.224 & 5.224 & & 0.9028 & 1.05E-02 & 1566.2071 & 577.1012 & 2143.3083 & \\
\hline & 8/4/2008 12:00 & 311 & 217 & 1200 & 5.079 & 5.079 & & 0.9571 & 1.13E-02 & 1683.0150 & 620.1415 & 2303.1565 & \\
\hline & 8/4/2008 13:00 & 312 & 217 & 1300 & 5.076 & 5.076 & & 0.9582 & 1.13E-02 & 1685.5192 & 621.0642 & 2306.5834 & \\
\hline & 8/4/2008 14:00 & 313 & 217 & 1400 & 5.434 & 5.434 & & 0.8292 & $9.45 E-03$ & 1410.6732 & 519.7916 & 1930.4648 & \\
\hline & 8/4/2008 15:00 & 314 & 217 & 1500 & 5.831 & 5.831 & & 0.7047 & 7.73E-03 & 1154.5726 & 425.4260 & 1579.9986 & \\
\hline & 8/4/2008 16:00 & 315 & 217 & 1600 & 5.054 & 5.054 & & 0.9668 & 1.14E-02 & 1703.9956 & 627.8722 & 2331.8678 & \\
\hline & 8/4/2008 17:00 & 316 & 217 & 1700 & 5.063 & 5.063 & & 0.9633 & 1.14E-02 & 1696.4131 & 625.0783 & 2321.4914 & \\
\hline & 8/4/2008 18:00 & 317 & 217 & 1800 & 5.044 & 5.044 & & 0.9707 & 1.15E-02 & 1712.4598 & 630.9910 & 2343.4508 & \\
\hline & 8/4/2008 19:00 & 318 & 217 & 1900 & 5.505 & 5.505 & & 0.8056 & $9.12 \mathrm{E}-03$ & 1361.4306 & 501.6471 & 1863.0778 & \\
\hline & 8/4/2008 20:00 & 319 & 217 & 2000 & 6.2 & 6.2 & & 0.6032 & 6.39E-03 & 953.4820 & 351.3301 & 1304.8121 & \\
\hline & 8/4/2008 21:00 & 320 & 217 & 2100 & 7.911 & 7.911 & & 0.2565 & 2.23E-03 & 332.7232 & 122.5987 & 455.3219 & \\
\hline & 8/4/2008 22:00 & 321 & 217 & 2200 & 8.52 & 8.52 & & 0.1667 & 1.31E-03 & 195.7211 & 72.1174 & 267.8385 & \\
\hline & 8/4/2008 23:00 & 322 & 217 & 2300 & 8.64 & 8.64 & & 0.1505 & 1.16E-03 & 172.5669 & 63.5858 & 236.1528 & \\
\hline & 8/5/2008 0:00 & 323 & 218 & 0 & 8.78 & 8.78 & 8.88 & 0.1321 & $9.85 \mathrm{E}-04$ & 147.0442 & 54.1814 & 201.2256 & \\
\hline & 8/5/2008 1:00 & 324 & 218 & 100 & 8.86 & 8.86 & & 0.1219 & 8.92E-04 & 133.1666 & 49.0680 & 182.2346 & \\
\hline & 8/5/2008 2:00 & 325 & 218 & 200 & 8.88 & 8.88 & & 0.1194 & $8.69 \mathrm{E}-04$ & 129.7767 & 47.8189 & 177.5957 & \\
\hline & 8/5/2008 3:00 & 326 & 218 & 300 & 8.82 & 8.82 & & 0.1270 & 9.38E-04 & 140.0417 & 51.6012 & 191.6429 & \\
\hline & 8/5/2008 4:00 & 327 & 218 & 400 & 8.72 & 8.72 & & 0.1399 & $1.06 \mathrm{E}-03$ & 157.7883 & 58.1404 & 215.9287 & \\
\hline
\end{tabular}




\begin{tabular}{|c|c|c|c|c|c|c|c|c|c|c|c|c|c|}
\hline Tree ID & TS & RN & JD & HR & $\begin{array}{c}\text { Raw } \\
\text { TC_dTA }\end{array}$ & $\begin{array}{c}\text { Fixed } \\
\text { TC_dT } \Delta\end{array}$ & dTM & $\mathbf{K}$ & $\begin{array}{c}\text { Sap } \\
\text { Velocity } \\
(\mathbf{c m} / \mathbf{s})\end{array}$ & $\begin{array}{c}\text { Probed } \\
\text { Drywood F } \\
(\mathrm{g} / \mathrm{hr})\end{array}$ & $\begin{array}{c}\text { Extrapolated } \\
\text { Wetwood F } \\
(\mathrm{g} / \mathrm{hr})\end{array}$ & $\begin{array}{c}\text { Extrapolated } \\
\text { Total Wood F } \\
(\mathrm{g} / \mathrm{hr})\end{array}$ & $\begin{array}{c}\text { Relative } \\
\text { Humidity, } \\
@ 10 \mathrm{~m}(\%)\end{array}$ \\
\hline & 8/5/2008 5:00 & 328 & 218 & 500 & \begin{tabular}{|r|} 
\\
\end{tabular} & 8.73 & & 0.1386 & 1.04E-03 & 155.9775 & 57.4731 & 213.4506 & \\
\hline & 8/5/2008 6:00 & 329 & 218 & 600 & 8.81 & 8.81 & & 0.1283 & 9.50E-04 & 141.7803 & 52.2419 & 194.0222 & \\
\hline & 8/5/2008 7:00 & 330 & 218 & 700 & 8.83 & 8.83 & & 0.1257 & 9.27E-04 & 138.3110 & 50.9635 & 189.2745 & \\
\hline & 8/5/2008 8:00 & 331 & 218 & 800 & 8.69 & 8.69 & & 0.1438 & 1.09E-03 & 163.2693 & 60.1599 & 223.4293 & \\
\hline & 8/5/20089:00 & 332 & 218 & 900 & 7.236 & 7.236 & & 0.3737 & $3.54 \mathrm{E}-03$ & 528.8095 & 194.8507 & 723.6602 & \\
\hline & $8 / 5 / 200810: 00$ & 333 & 218 & 1000 & 5.677 & 5.677 & & 0.7509 & $8.36 \mathrm{E}-03$ & 1248.5357 & 460.0486 & 1708.5843 & \\
\hline & 8/5/2008 11:00 & 334 & 218 & 1100 & 5.772 & 5.772 & & 0.7221 & 7.97E-03 & 1189.8167 & 438.4124 & 1628.2291 & \\
\hline & 8/5/2008 12:00 & 335 & 218 & 1200 & 5.443 & 5.443 & & 0.8262 & 9.41E-03 & 1404.3418 & 517.4586 & 1921.8004 & \\
\hline & 8/5/2008 13:00 & 336 & 218 & 1300 & 5.07 & 5.07 & & 0.9606 & 1.13E-02 & 1690.5386 & 622.9137 & 2313.4523 & \\
\hline & $8 / 5 / 2008$ 14:00 & 337 & 218 & 1400 & 5.691 & 5.691 & & 0.7466 & 8.30E-03 & 1239.7255 & 456.8024 & 1696.5279 & \\
\hline & 8/5/2008 15:00 & 338 & 218 & 1500 & 6.095 & 6.095 & & 0.6308 & $6.75 \mathrm{E}-03$ & 1007.5033 & 371.2353 & 1378.7387 & \\
\hline & 8/5/2008 16:00 & 339 & 218 & 1600 & 5.897 & 5.897 & & 0.6856 & 7.48E-03 & 1116.2131 & 411.2917 & 1527.5048 & \\
\hline & 8/5/2008 17:00 & 340 & 218 & 1700 & 5.351 & 5.351 & & 0.8576 & 9.85E-03 & 1470.3247 & 541.7714 & 2012.0960 & \\
\hline & 8/5/2008 18:00 & 341 & 218 & 1800 & 5.176 & 5.176 & & 0.9204 & 1.07E-02 & 1603.9766 & 591.0182 & 2194.9947 & \\
\hline & 8/5/2008 19:00 & 342 & 218 & 1900 & 5.294 & 5.294 & & 0.8776 & 1.01E-02 & 1512.6492 & 557.3667 & 2070.0158 & \\
\hline & 8/5/2008 20:00 & 343 & 218 & 2000 & 6.171 & 6.171 & & 0.6108 & $6.49 \mathrm{E}-03$ & 968.1629 & 356.7395 & 1324.9024 & \\
\hline & 8/5/2008 21:00 & 344 & 218 & 2100 & 8.05 & 8.05 & & 0.2348 & 2.00E-03 & 298.4224 & 109.9599 & 408.3823 & \\
\hline & 8/5/2008 22:00 & 345 & 218 & 2200 & 8.69 & 8.69 & & 0.1438 & 1.09E-03 & 163.2693 & 60.1599 & 223.4293 & \\
\hline & 8/5/2008 23:00 & 346 & 218 & 2300 & 8.76 & 8.76 & & 0.1347 & 1.01E-03 & 150.5934 & 55.4892 & 206.0826 & \\
\hline & 8/6/2008 0:00 & 347 & 219 & 0 & 8.73 & 8.73 & 8.73 & 0.1386 & 1.04E-03 & 155.9775 & 57.4731 & 213.4506 & \\
\hline & 8/6/2008 1:00 & 348 & 219 & 100 & 8.65 & 8.65 & & 0.1491 & $1.14 \mathrm{E}-03$ & 170.6911 & 62.8946 & 233.5857 & \\
\hline & 8/6/2008 2:00 & 349 & 219 & 200 & 8.59 & 8.59 & & 0.1572 & $1.22 \mathrm{E}-03$ & 182.0694 & 67.0872 & 249.1566 & \\
\hline & 8/6/2008 3:00 & 350 & 219 & 300 & 8.51 & 8.51 & & 0.1680 & $1.32 \mathrm{E}-03$ & 197.7048 & 72.8484 & 270.5531 & \\
\hline & 8/6/2008 4:00 & 351 & 219 & 400 & 8.43 & 8.43 & & 0.1791 & $1.43 \mathrm{E}-03$ & 213.8792 & 78.8082 & 292.6874 & \\
\hline & 8/6/2008 5:00 & 352 & 219 & 500 & 8.36 & 8.36 & & 0.1890 & 1.53E-03 & 228.4824 & 84.1890 & 312.6714 & \\
\hline & 8/6/2008 6:00 & 353 & 219 & 600 & 8.29 & 8.29 & & 0.1990 & 1.63E-03 & 243.5139 & 89.7277 & 333.2417 & \\
\hline & 8/6/2008 7:00 & 354 & 219 & 700 & 8.23 & 8.23 & & 0.2078 & 1.72E-03 & 256.7454 & 94.6031 & 351.3486 & \\
\hline & 8/6/2008 8:00 & 355 & 219 & 800 & 8.19 & 8.19 & & 0.2137 & 1.78E-03 & 265.7474 & 97.9201 & 363.6675 & \\
\hline & 8/6/2008 9:00 & 356 & 219 & 900 & 8.08 & 8.08 & & 0.2302 & $1.95 E-03$ & 291.2653 & 107.3227 & 398.5880 & \\
\hline & 8/6/2008 10:00 & 357 & 219 & 1000 & 5.655 & 5.655 & & 0.7577 & $8.46 \mathrm{E}-03$ & 1262.4921 & 465.1912 & 1727.6832 & \\
\hline & 8/6/2008 11:00 & 358 & 219 & 1100 & 4.687 & 4.687 & & 1.1208 & 1.37E-02 & 2044.0502 & 753.1723 & 2797.2225 & \\
\hline & 8/6/2008 12:00 & 359 & 219 & 1200 & 5.197 & 5.197 & & 0.9126 & 1.06E-02 & 1587.3458 & 584.8902 & 2172.2360 & \\
\hline & 8/6/2008 13:00 & 360 & 219 & 1300 & 5.8 & 5.8 & & 0.7138 & 7.86E-03 & 1172.9765 & 432.2073 & 1605.1838 & \\
\hline & $8 / 6 / 200814: 00$ & 361 & 219 & 1400 & 5.276 & 5.276 & & 0.8840 & $1.02 \mathrm{E}-02$ & 1526.2522 & 562.3790 & 2088.6312 & \\
\hline & 8/6/2008 15:00 & 362 & 219 & 1500 & 4.672 & 4.672 & & 1.1276 & 1.38E-02 & 2059.3477 & 758.8090 & 2818.1567 & \\
\hline & 8/6/2008 16:00 & 363 & 219 & 1600 & 4.95 & 4.95 & & 1.0081 & $1.20 \mathrm{E}-02$ & 1794.0912 & 661.0698 & 2455.1609 & \\
\hline & 8/6/2008 17:00 & 364 & 219 & 1700 & 4.85 & 4.85 & & 1.0495 & $1.26 \mathrm{E}-02$ & 1885.2252 & 694.6500 & 2579.8752 & \\
\hline & 8/6/2008 18:00 & 365 & 219 & 1800 & 5.406 & 5.406 & & 0.8387 & $9.58 \mathrm{E}-03$ & 1430.5402 & 527.1120 & 1957.6522 & \\
\hline & 8/6/2008 19:00 & 366 & 219 & 1900 & 5.418 & 5.418 & & 0.8346 & 9.53E-03 & 1421.9943 & 523.9630 & 1945.9573 & \\
\hline & $8 / 6 / 200820: 00$ & 367 & 219 & 2000 & 5.717 & 5.717 & & 0.7387 & 8.20E-03 & 1223.5092 & 450.8271 & 1674.3363 & \\
\hline & 8/6/2008 21:00 & 368 & 219 & 2100 & 7.467 & 7.467 & & 0.3312 & 3.05E-03 & 455.7826 & 167.9425 & 623.7250 & \\
\hline
\end{tabular}




\begin{tabular}{|c|c|c|c|c|c|c|c|c|c|c|c|c|c|}
\hline Tree ID & TS & RN & JD & HR & $\begin{array}{c}\text { Raw } \\
\text { TC_dTA }\end{array}$ & $\begin{array}{c}\text { Fixed } \\
\text { TC_dT } \Delta\end{array}$ & dTM & $\mathbf{K}$ & $\begin{array}{c}\text { Sap } \\
\text { Velocity } \\
(\mathrm{cm} / \mathrm{s})\end{array}$ & $\begin{array}{c}\text { Probed } \\
\text { Drywood F } \\
\text { (g/hr) }\end{array}$ & $\begin{array}{c}\text { Extrapolated } \\
\text { Wetwood F } \\
(\mathrm{g} / \mathrm{hr})\end{array}$ & $\begin{array}{c}\text { Extrapolated } \\
\text { Total Wood F } \\
(\mathrm{g} / \mathrm{hr})\end{array}$ & $\begin{array}{l}\text { Relative } \\
\text { Humidity, } \\
\text { @10m (\%) }\end{array}$ \\
\hline & 8/6/2008 22:00 & 369 & 219 & 2200 & \begin{tabular}{|r|} 
\\
\end{tabular} & 8.5 & & 0.1694 & 1.34E-03 & 199.6969 & 73.5824 & 273.2793 & \\
\hline & 8/6/2008 23:00 & 370 & 219 & 2300 & 8.64 & 8.64 & & 0.1505 & 1.16E-03 & 172.5669 & 63.5858 & 236.1528 & \\
\hline & 8/7/2008 0:00 & 371 & 220 & 0 & 8.65 & 8.65 & 8.65 & 0.1491 & $1.14 \mathrm{E}-03$ & 170.6911 & 62.8946 & 233.5857 & \\
\hline & 8/7/2008 1:00 & 372 & 220 & 100 & 8.58 & 8.58 & & 0.1585 & $1.23 \mathrm{E}-03$ & 183.9947 & 67.7966 & 251.7913 & \\
\hline & 8/7/2008 2:00 & 373 & 220 & 200 & 8.48 & 8.48 & & 0.1722 & 1.36E-03 & 203.7065 & 75.0598 & 278.7663 & \\
\hline & 8/7/2008 3:00 & 374 & 220 & 300 & 8.38 & 8.38 & & 0.1862 & $1.50 \mathrm{E}-03$ & 224.2667 & 82.6357 & 306.9023 & \\
\hline & 8/7/2008 4:00 & 375 & 220 & 400 & 8.23 & 8.23 & & 0.2078 & $1.72 \mathrm{E}-03$ & 256.7454 & 94.6031 & 351.3486 & \\
\hline & 8/7/2008 5:00 & 376 & 220 & 500 & 8.15 & 8.15 & & 0.2196 & $1.84 \mathrm{E}-03$ & 274.8962 & 101.2912 & 376.1874 & \\
\hline & 8/7/2008 6:00 & 377 & 220 & 600 & 8.09 & 8.09 & & 0.2287 & $1.94 \mathrm{E}-03$ & 288.8986 & 106.4506 & 395.3493 & \\
\hline & 8/7/2008 7:00 & 378 & 220 & 700 & 8.04 & 8.04 & & 0.2363 & 2.02E-03 & 300.8273 & 110.8460 & 411.6733 & \\
\hline & 8/7/2008 8:00 & 379 & 220 & 800 & 7.998 & 7.998 & & 0.2428 & 2.08E-03 & 311.0330 & 114.6065 & 425.6395 & \\
\hline & 8/7/2008 9:00 & 380 & 220 & 900 & 7.194 & 7.194 & & 0.3817 & 3.64E-03 & 542.8146 & 200.0112 & 742.8257 & \\
\hline & 8/7/2008 10:00 & 381 & 220 & 1000 & 5.039 & 5.039 & & 0.9726 & 1.15E-02 & 1716.7075 & 632.5562 & 2349.2636 & \\
\hline & 8/7/2008 11:00 & 382 & 220 & 1100 & 4.435 & 4.435 & & 1.2413 & 1.55E-02 & 2317.8640 & 854.0647 & 3171.9286 & \\
\hline & 8/7/2008 12:00 & 383 & 220 & 1200 & 4.484 & 4.484 & & 1.2168 & $1.52 \mathrm{E}-02$ & 2261.6933 & 833.3674 & 3095.0607 & \\
\hline & 8/7/2008 13:00 & 384 & 220 & 1300 & 4.545 & 4.545 & & 1.1870 & 1.47E-02 & 2193.8099 & 808.3544 & 3002.1643 & \\
\hline & 8/7/2008 14:00 & 385 & 220 & 1400 & 4.543 & 4.543 & & 1.1880 & 1.47E-02 & 2196.0006 & 809.1616 & 3005.1622 & \\
\hline & 8/7/2008 15:00 & 386 & 220 & 1500 & 4.446 & 4.446 & & 1.2357 & 1.54E-02 & 2305.1239 & 849.3703 & 3154.4941 & \\
\hline & 8/7/2008 16:00 & 387 & 220 & 1600 & 4.503 & 4.503 & & 1.2074 & $1.50 \mathrm{E}-02$ & 2240.3103 & 825.4884 & 3065.7987 & \\
\hline & 8/7/2008 17:00 & 388 & 220 & 1700 & 4.611 & 4.611 & & 1.1557 & $1.42 \mathrm{E}-02$ & 2122.8085 & 782.1925 & 2905.0010 & \\
\hline & 8/7/2008 18:00 & 389 & 220 & 1800 & 4.599 & 4.599 & & 1.1613 & 1.43E-02 & 2135.5339 & 786.8814 & 2922.4153 & \\
\hline & 8/7/2008 19:00 & 390 & 220 & 1900 & 5.085 & 5.085 & & 0.9548 & $1.12 \mathrm{E}-02$ & 1678.0176 & 618.3001 & 2296.3177 & \\
\hline & 8/7/2008 20:00 & 391 & 220 & 2000 & 5.724 & 5.724 & & 0.7365 & 8.17E-03 & 1219.1752 & 449.2302 & 1668.4054 & \\
\hline & 8/7/2008 21:00 & 392 & 220 & 2100 & 7.447 & 7.447 & & 0.3348 & 3.09E-03 & 461.8467 & 170.1769 & 632.0236 & \\
\hline & 8/7/2008 22:00 & 393 & 220 & 2200 & 8.23 & 8.23 & & 0.2078 & 1.72E-03 & 256.7454 & 94.6031 & 351.3486 & \\
\hline & 8/7/2008 23:00 & 394 & 220 & 2300 & 8.52 & 8.52 & & 0.1667 & 1.31E-03 & 195.7211 & 72.1174 & 267.8385 & \\
\hline & 8/8/2008 0:00 & 395 & 221 & 0 & 8.64 & 8.64 & 8.70 & 0.1505 & 1.16E-03 & 172.5669 & 63.5858 & 236.1528 & \\
\hline & 8/8/2008 1:00 & 396 & 221 & 100 & 8.62 & 8.62 & & 0.1531 & 1.18E-03 & 176.3432 & 64.9773 & 241.3205 & \\
\hline & 8/8/2008 2:00 & 397 & 221 & 200 & 8.7 & 8.7 & & 0.1425 & 1.08E-03 & 161.4342 & 59.4838 & 220.9180 & \\
\hline & 8/8/2008 3:00 & 398 & 221 & 300 & 8.64 & 8.64 & & 0.1505 & 1.16E-03 & 172.5669 & 63.5858 & 236.1528 & \\
\hline & 8/8/2008 4:00 & 399 & 221 & 400 & 8.56 & 8.56 & & 0.1612 & 1.26E-03 & 187.8701 & 69.2246 & 257.0947 & \\
\hline & $8 / 8 / 20085: 00$ & 400 & 221 & 500 & 8.47 & 8.47 & & 0.1736 & 1.38E-03 & 205.7240 & 75.8032 & 281.5272 & \\
\hline & 8/8/2008 6:00 & 401 & 221 & 600 & 8.38 & 8.38 & & 0.1862 & $1.50 \mathrm{E}-03$ & 224.2667 & 82.6357 & 306.9023 & \\
\hline & $8 / 8 / 20087: 00$ & 402 & 221 & 700 & 8.31 & 8.31 & & 0.1961 & $1.60 \mathrm{E}-03$ & 239.1750 & 88.1290 & 327.3040 & \\
\hline & 8/8/2008 8:00 & 403 & 221 & 800 & 8.16 & 8.16 & & 0.2181 & 1.83E-03 & 272.5952 & 100.4433 & 373.0385 & \\
\hline & 8/8/2008 9:00 & 404 & 221 & 900 & 6.277 & 6.277 & & 0.5836 & 6.13E-03 & 915.3604 & 337.2834 & 1252.6437 & \\
\hline & 8/8/2008 10:00 & 405 & 221 & 1000 & 4.995 & 4.995 & & 0.9900 & 1.18E-02 & 1754.5399 & 646.4963 & 2401.0362 & \\
\hline & 8/8/2008 11:00 & 406 & 221 & 1100 & 4.749 & 4.749 & & 1.0931 & 1.33E-02 & 1982.0677 & 730.3336 & 2712.4013 & \\
\hline & 8/8/2008 12:00 & 407 & 221 & 1200 & 4.689 & 4.689 & & 1.1199 & 1.37E-02 & 2042.0195 & 752.4241 & 2794.4436 & \\
\hline & 8/8/2008 13:00 & 408 & 221 & 1300 & 4.711 & 4.711 & & 1.1100 & 1.35E-02 & 2019.8208 & 744.2445 & 2764.0654 & \\
\hline & 8/8/2008 14:00 & 409 & 221 & 1400 & 4.671 & 4.671 & & 1.1280 & 1.38E-02 & 2060.3718 & 759.1864 & 2819.5582 & \\
\hline
\end{tabular}




\begin{tabular}{|c|c|c|c|c|c|c|c|c|c|c|c|c|c|}
\hline Tree ID & TS & RN & JD & HR & $\begin{array}{c}\text { Raw } \\
\text { TC_dTA }\end{array}$ & $\begin{array}{c}\text { Fixed } \\
\text { TC_dT } \Delta\end{array}$ & dTM & $\mathbf{K}$ & $\begin{array}{c}\text { Sap } \\
\text { Velocity } \\
(\mathbf{c m} / \mathbf{s})\end{array}$ & $\begin{array}{c}\text { Probed } \\
\text { Drywood F } \\
(\mathrm{g} / \mathrm{hr})\end{array}$ & $\begin{array}{c}\text { Extrapolated } \\
\text { Wetwood F } \\
(\mathrm{g} / \mathrm{hr})\end{array}$ & $\begin{array}{c}\text { Extrapolated } \\
\text { Total Wood F } \\
(\mathrm{g} / \mathrm{hr})\end{array}$ & $\begin{array}{c}\text { Relative } \\
\text { Humidity, } \\
@ 10 \mathrm{~m}(\%)\end{array}$ \\
\hline & 8/8/2008 15:00 & 410 & 221 & 1500 & \begin{tabular}{|l|}
4.565 \\
\end{tabular} & 4.565 & & 1.1774 & 1.46E-02 & 2172.0310 & 800.3295 & 2972.3605 & \\
\hline & 8/8/2008 16:00 & 411 & 221 & 1600 & 4.636 & 4.636 & & 1.1441 & 1.40E-02 & 2096.5542 & 772.5185 & 2869.0728 & \\
\hline & 8/8/2008 17:00 & 412 & 221 & 1700 & 4.803 & 4.803 & & 1.0695 & 1.29E-02 & 1929.6708 & 711.0269 & 2640.6976 & \\
\hline & 8/8/2008 18:00 & 413 & 221 & 1800 & 4.988 & 4.988 & & 0.9928 & 1.18E-02 & 1760.6346 & 648.7420 & 2409.3767 & \\
\hline & 8/8/2008 19:00 & 414 & 221 & 1900 & 5.114 & 5.114 & & 0.9437 & $1.11 E-02$ & 1654.0677 & 609.4752 & 2263.5429 & \\
\hline & $8 / 8 / 200820: 00$ & 415 & 221 & 2000 & 5.912 & 5.912 & & 0.6813 & $7.42 \mathrm{E}-03$ & 1107.6480 & 408.1357 & 1515.7837 & \\
\hline & 8/8/2008 21:00 & 416 & 221 & 2100 & 7.431 & 7.431 & & 0.3376 & $3.13 \mathrm{E}-03$ & 466.7323 & 171.9771 & 638.7094 & \\
\hline & 8/8/2008 22:00 & 417 & 221 & 2200 & 7.983 & 7.983 & & 0.2451 & $2.11 E-03$ & 314.7194 & 115.9648 & 430.6843 & \\
\hline & 8/8/2008 23:00 & 418 & 221 & 2300 & 8.12 & 8.12 & & 0.2241 & 1.89E-03 & 281.8553 & 103.8554 & 385.7106 & \\
\hline & 8/9/2008 0:00 & 419 & 222 & 0 & 8.28 & 8.28 & 8.64 & 0.2005 & 1.65E-03 & 245.6968 & 90.5320 & 336.2288 & \\
\hline & 8/9/2008 1:00 & 420 & 222 & 100 & 8.35 & 8.35 & & 0.1904 & $1.54 \mathrm{E}-03$ & 230.6033 & 84.9705 & 315.5738 & \\
\hline & 8/9/2008 2:00 & 421 & 222 & 200 & 8.45 & 8.45 & & 0.1763 & $1.41 E-03$ & 209.7845 & 77.2994 & 287.0839 & \\
\hline & 8/9/2008 3:00 & 422 & 222 & 300 & 8.47 & 8.47 & & 0.1736 & 1.38E-03 & 205.7240 & 75.8032 & 281.5272 & \\
\hline & 8/9/2008 4:00 & 423 & 222 & 400 & 8.43 & 8.43 & & 0.1791 & $1.43 \mathrm{E}-03$ & 213.8792 & 78.8082 & 292.6874 & \\
\hline & 8/9/2008 5:00 & 424 & 222 & 500 & 8.33 & 8.33 & & 0.1933 & $1.57 \mathrm{E}-03$ & 234.8715 & 86.5432 & 321.4148 & \\
\hline & 8/9/2008 6:00 & 425 & 222 & 600 & 8.29 & 8.29 & & 0.1990 & $1.63 \mathrm{E}-03$ & 243.5139 & 89.7277 & 333.2417 & \\
\hline & 8/9/2008 7:00 & 426 & 222 & 700 & 7.957 & 7.957 & & 0.2492 & $2.15 E-03$ & 321.1615 & 118.3386 & 439.5001 & \\
\hline & 8/9/2008 8:00 & 427 & 222 & 800 & 7.288 & 7.288 & & 0.3639 & $3.43 E-03$ & 511.7877 & 188.5787 & 700.3664 & \\
\hline & 8/9/20089:00 & 428 & 222 & 900 & 7.755 & 7.755 & & 0.2818 & $2.50 \mathrm{E}-03$ & 373.5350 & 137.6366 & 511.1716 & \\
\hline & 8/9/2008 10:00 & 429 & 222 & 1000 & 7.406 & 7.406 & & 0.3422 & 3.18E-03 & 474.4278 & 174.8127 & 649.2405 & \\
\hline & 8/9/2008 11:00 & 430 & 222 & 1100 & 6.636 & 6.636 & & 0.4979 & 5.04E-03 & 752.8583 & 277.4061 & 1030.2645 & \\
\hline & 8/9/2008 12:00 & 431 & 222 & 1200 & 5.768 & 5.768 & & 0.7233 & 7.99E-03 & 1192.2394 & 439.3051 & 1631.5446 & \\
\hline & 8/9/2008 13:00 & 432 & 222 & 1300 & 5.411 & 5.411 & & 0.8370 & $9.56 \mathrm{E}-03$ & 1426.9736 & 525.7978 & 1952.7714 & \\
\hline & 8/9/2008 14:00 & 433 & 222 & 1400 & 4.866 & 4.866 & & 1.0427 & $1.25 \mathrm{E}-02$ & 1870.3345 & 689.1632 & 2559.4978 & \\
\hline & 8/9/2008 15:00 & 434 & 222 & 1500 & 4.686 & 4.686 & & 1.1212 & 1.37E-02 & 2045.0663 & 753.5467 & 2798.6131 & \\
\hline & 8/9/2008 16:00 & 435 & 222 & 1600 & 4.739 & 4.739 & & 1.0975 & 1.33E-02 & 1991.9311 & 733.9680 & 2725.8991 & \\
\hline & 8/9/2008 17:00 & 436 & 222 & 1700 & 5.074 & 5.074 & & 0.9590 & 1.13E-02 & 1687.1907 & 621.6801 & 2308.8708 & \\
\hline & 8/9/2008 18:00 & 437 & 222 & 1800 & 4.805 & 4.805 & & 1.0687 & 1.29E-02 & 1927.7578 & 710.3220 & 2638.0798 & \\
\hline & 8/9/2008 19:00 & 438 & 222 & 1900 & 5.333 & 5.333 & & 0.8639 & $9.94 \mathrm{E}-03$ & 1483.5683 & 546.6512 & 2030.2195 & \\
\hline & 8/9/2008 20:00 & 439 & 222 & 2000 & 5.995 & 5.995 & & 0.6580 & $7.11 \mathrm{E}-03$ & 1061.2486 & 391.0389 & 1452.2874 & \\
\hline & 8/9/2008 21:00 & 440 & 222 & 2100 & 7.838 & 7.838 & & 0.2682 & 2.35E-03 & 351.5086 & 129.5206 & 481.0291 & \\
\hline & 8/9/2008 22:00 & 441 & 222 & 2200 & 8.62 & 8.62 & & 0.1531 & $1.18 \mathrm{E}-03$ & 176.3432 & 64.9773 & 241.3205 & \\
\hline & 8/9/2008 23:00 & 442 & 222 & 2300 & 8.64 & 8.64 & & 0.1505 & 1.16E-03 & 172.5669 & 63.5858 & 236.1528 & \\
\hline & 8/10/2008 0:00 & 443 & 223 & 0 & 8.59 & 8.59 & 8.59 & 0.1572 & $1.22 \mathrm{E}-03$ & 182.0694 & 67.0872 & 249.1566 & \\
\hline & 8/10/2008 1:00 & 444 & 223 & 100 & 8.54 & 8.54 & & 0.1639 & 1.28E-03 & 191.7788 & 70.6649 & 262.4437 & \\
\hline & 8/10/2008 2:00 & 445 & 223 & 200 & 8.45 & 8.45 & & 0.1763 & $1.41 \mathrm{E}-03$ & 209.7845 & 77.2994 & 287.0839 & \\
\hline & 8/10/2008 3:00 & 446 & 223 & 300 & 8.38 & 8.38 & & 0.1862 & $1.50 \mathrm{E}-03$ & 224.2667 & 82.6357 & 306.9023 & \\
\hline & 8/10/2008 4:00 & 447 & 223 & 400 & 8.32 & 8.32 & & 0.1947 & $1.59 \mathrm{E}-03$ & 237.0189 & 87.3345 & 324.3533 & \\
\hline & 8/10/2008 5:00 & 448 & 223 & 500 & 8.2 & 8.2 & & 0.2122 & 1.77E-03 & 263.4832 & 97.0858 & 360.5691 & \\
\hline & 8/10/2008 6:00 & 449 & 223 & 600 & 8.14 & 8.14 & & 0.2211 & 1.86E-03 & 277.2066 & 102.1425 & 379.3491 & \\
\hline & 8/10/2008 7:00 & 450 & 223 & 700 & 8.1 & 8.1 & & 0.2272 & 1.92E-03 & 286.5414 & 105.5821 & 392.1235 & \\
\hline
\end{tabular}




\begin{tabular}{|c|c|c|c|c|c|c|c|c|c|c|c|c|c|}
\hline Tree ID & TS & RN & JD & HR & $\begin{array}{c}\text { Raw } \\
\text { TC_dTA }\end{array}$ & $\begin{array}{c}\text { Fixed } \\
\text { TC_dT } \Delta\end{array}$ & dTM & $\mathbf{K}$ & $\begin{array}{l}\text { Sap } \\
\text { Velocity } \\
(\mathrm{cm} / \mathrm{s})\end{array}$ & $\begin{array}{c}\text { Probed } \\
\text { Drywood F } \\
(\mathrm{g} / \mathrm{hr})\end{array}$ & $\begin{array}{c}\text { Extrapolated } \\
\text { Wetwood F } \\
(\mathrm{g} / \mathrm{hr})\end{array}$ & $\begin{array}{c}\text { Extrapolated } \\
\text { Total Wood F } \\
(\mathrm{g} / \mathrm{hr})\end{array}$ & $\begin{array}{l}\text { Relative } \\
\text { Humidity, } \\
@ 10 \mathrm{~m}(\%)\end{array}$ \\
\hline & 8/10/2008 8:00 & 451 & 223 & 800 & 8.06 & 8.06 & & 0.2333 & $1.98 \mathrm{E}-03$ & 296.0272 & 109.0773 & 405.1045 & \\
\hline & 8/10/2008 9:00 & 452 & 223 & 900 & 7.148 & 7.148 & & 0.3906 & $3.74 \mathrm{E}-03$ & 558.4219 & 205.7620 & 764.1839 & \\
\hline & $8 / 10 / 2008$ 10:00 & 453 & 223 & 1000 & 5.189 & 5.189 & & 0.9156 & $1.07 E-02$ & 1593.6616 & 587.2174 & 2180.8790 & \\
\hline & 8/10/2008 11:00 & 454 & 223 & 1100 & 4.584 & 4.584 & & 1.1684 & $1.44 \mathrm{E}-02$ & 2151.5545 & 792.7845 & 2944.3391 & \\
\hline & $8 / 10 / 200812: 00$ & 455 & 223 & 1200 & 4.648 & 4.648 & & 1.1386 & 1.40E-02 & 2084.0741 & 767.9200 & 2851.9941 & \\
\hline & 8/10/2008 13:00 & 456 & 223 & 1300 & 4.7 & 4.7 & & 1.1149 & 1.36E-02 & 2030.8885 & 748.3226 & 2779.2112 & \\
\hline & $8 / 10 / 2008$ 14:00 & 457 & 223 & 1400 & 4.685 & 4.685 & & 1.1217 & 1.37E-02 & 2046.0830 & 753.9214 & 2800.0043 & \\
\hline & $8 / 10 / 2008$ 15:00 & 458 & 223 & 1500 & 4.742 & 4.742 & & 1.0962 & 1.33E-02 & 1988.9667 & 732.8757 & 2721.8424 & \\
\hline & 8/10/2008 16:00 & 459 & 223 & 1600 & 4.646 & 4.646 & & 1.1395 & 1.40E-02 & 2086.1487 & 768.6844 & 2854.8331 & \\
\hline & 8/10/2008 17:00 & 460 & 223 & 1700 & 5.044 & 5.044 & & 0.9707 & 1.15E-02 & 1712.4598 & 630.9910 & 2343.4508 & \\
\hline & 8/10/2008 18:00 & 461 & 223 & 1800 & 7.675 & 7.675 & & 0.2951 & 2.65E-03 & 395.4569 & 145.7142 & 541.1712 & \\
\hline & $8 / 10 / 2008$ 19:00 & 462 & 223 & 1900 & 8.28 & 8.28 & & 0.2005 & $1.65 \mathrm{E}-03$ & 245.6968 & 90.5320 & 336.2288 & \\
\hline & 8/10/2008 20:00 & 463 & 223 & 2000 & 8.08 & 8.08 & & 0.2302 & 1.95E-03 & 291.2653 & 107.3227 & 398.5880 & \\
\hline & 8/10/2008 21:00 & 464 & 223 & 2100 & 8.09 & 8.09 & & 0.2287 & 1.94E-03 & 288.8986 & 106.4506 & 395.3493 & \\
\hline & $8 / 10 / 2008$ 22:00 & 465 & 223 & 2200 & 8.28 & 8.28 & & 0.2005 & 1.65E-03 & 245.6968 & 90.5320 & 336.2288 & \\
\hline & $8 / 10 / 2008$ 23:00 & 466 & 223 & 2300 & 8.2 & 8.2 & & 0.2122 & 1.77E-03 & 263.4832 & 97.0858 & 360.5691 & \\
\hline & $8 / 11 / 20080: 00$ & 467 & 224 & 0 & 8.15 & 8.15 & 8.57 & 0.2196 & 1.84E-03 & 274.8962 & 101.2912 & 376.1874 & \\
\hline & 8/11/2008 1:00 & 468 & 224 & 100 & 8.33 & 8.33 & & 0.1933 & $1.57 \mathrm{E}-03$ & 234.8715 & 86.5432 & 321.4148 & \\
\hline & 8/11/2008 2:00 & 469 & 224 & 200 & 8.53 & 8.53 & & 0.1653 & 1.30E-03 & 193.7458 & 71.3896 & 265.1354 & \\
\hline & 8/11/2008 3:00 & 470 & 224 & 300 & 8.57 & 8.57 & & 0.1599 & $1.25 \mathrm{E}-03$ & 185.9282 & 68.5091 & 254.4373 & \\
\hline & 8/11/2008 4:00 & 471 & 224 & 400 & 8.56 & 8.56 & & 0.1612 & $1.26 \mathrm{E}-03$ & 187.8701 & 69.2246 & 257.0947 & \\
\hline & 8/11/2008 5:00 & 472 & 224 & 500 & 8.51 & 8.51 & & 0.1680 & 1.32E-03 & 197.7048 & 72.8484 & 270.5531 & \\
\hline & 8/11/2008 6:00 & 473 & 224 & 600 & 8.52 & 8.52 & & 0.1667 & 1.31E-03 & 195.7211 & 72.1174 & 267.8385 & \\
\hline & 8/11/2008 7:00 & 474 & 224 & 700 & 8.51 & 8.51 & & 0.1680 & $1.32 \mathrm{E}-03$ & 197.7048 & 72.8484 & 270.5531 & \\
\hline & 8/11/2008 8:00 & 475 & 224 & 800 & 8.46 & 8.46 & & 0.1749 & 1.39E-03 & 207.7500 & 76.5498 & 284.2997 & \\
\hline & 8/11/2008 9:00 & 476 & 224 & 900 & 7.653 & 7.653 & & 0.2988 & 2.69E-03 & 401.6072 & 147.9805 & 549.5877 & \\
\hline & $8 / 11 / 2008$ 10:00 & 477 & 224 & 1000 & 5.719 & 5.719 & & 0.7381 & 8.19E-03 & 1222.2696 & 450.3704 & 1672.6399 & \\
\hline & 8/11/2008 11:00 & 478 & 224 & 1100 & 4.872 & 4.872 & & 1.0402 & $1.25 \mathrm{E}-02$ & 1864.7814 & 687.1171 & 2551.8985 & \\
\hline & $8 / 11 / 2008$ 12:00 & 479 & 224 & 1200 & 4.909 & 4.909 & & 1.0249 & 1.23E-02 & 1830.9049 & 674.6345 & 2505.5394 & \\
\hline & 8/11/2008 13:00 & 480 & 224 & 1300 & 5.221 & 5.221 & & 0.9038 & $1.05 E-02$ & 1568.5424 & 577.9617 & 2146.5041 & \\
\hline & 8/11/2008 14:00 & 481 & 224 & 1400 & 4.931 & 4.931 & & 1.0158 & 1.21E-02 & 1811.0576 & 667.3214 & 2478.3790 & \\
\hline & 8/11/2008 15:00 & 482 & 224 & 1500 & 4.891 & 4.891 & & 1.0323 & 1.24E-02 & 1847.3068 & 680.6782 & 2527.9849 & \\
\hline & $8 / 11 / 2008$ 16:00 & 483 & 224 & 1600 & 5.202 & 5.202 & & 0.9108 & 1.06E-02 & 1583.4106 & 583.4402 & 2166.8508 & \\
\hline & $8 / 11 / 2008$ 17:00 & 484 & 224 & 1700 & 5.215 & 5.215 & & 0.9060 & $1.05 \mathrm{E}-02$ & 1573.2231 & 579.6864 & 2152.9095 & \\
\hline & 8/11/2008 18:00 & 485 & 224 & 1800 & 5.572 & 5.572 & & 0.7839 & 8.82E-03 & 1316.4064 & 485.0570 & 1801.4634 & \\
\hline & $8 / 11 / 2008$ 19:00 & 486 & 224 & 1900 & 5.808 & 5.808 & & 0.7114 & 7.83E-03 & 1168.2030 & 430.4484 & 1598.6515 & \\
\hline & 8/11/2008 20:00 & 487 & 224 & 2000 & 6.419 & 6.419 & & 0.5485 & $5.68 \mathrm{E}-03$ & 848.1941 & 312.5346 & 1160.7287 & \\
\hline & 8/11/2008 21:00 & 488 & 224 & 2100 & 7.469 & 7.469 & & 0.3308 & $3.05 E-03$ & 455.1788 & 167.7200 & 622.8987 & \\
\hline & $8 / 11 / 200822: 00$ & 489 & 224 & 2200 & 8.03 & 8.03 & & 0.2379 & 2.03E-03 & 303.2417 & 111.7357 & 414.9774 & \\
\hline & 8/11/2008 23:00 & 490 & 224 & 2300 & 8.26 & 8.26 & & 0.2034 & 1.68E-03 & 250.0892 & 92.1505 & 342.2398 & \\
\hline & 8/12/2008 0:00 & 491 & 225 & 0 & 8.38 & 8.38 & 8.42 & 0.1862 & $1.50 \mathrm{E}-03$ & 224.2667 & 82.6357 & 306.9023 & \\
\hline
\end{tabular}




\begin{tabular}{|c|c|c|c|c|c|c|c|c|c|c|c|c|c|}
\hline Tree ID & TS & RN & JD & HR & $\begin{array}{c}\text { Raw } \\
\text { TC_dTA }\end{array}$ & $\begin{array}{c}\text { Fixed } \\
\text { TC_dT } \Delta\end{array}$ & dTM & $\mathbf{K}$ & $\begin{array}{l}\text { Sap } \\
\text { Velocity } \\
(\mathbf{c m} / \mathbf{s})\end{array}$ & $\begin{array}{c}\text { Probed } \\
\text { Drywood F } \\
\text { (g/hr) }\end{array}$ & $\begin{array}{l}\text { Extrapolated } \\
\text { Wetwood F } \\
(\mathrm{g} / \mathrm{hr})\end{array}$ & $\begin{array}{c}\text { Extrapolated } \\
\text { Total Wood F } \\
(\mathrm{g} / \mathrm{hr})\end{array}$ & $\begin{array}{l}\text { Relative } \\
\text { Humidity, } \\
@ 10 m(\%)\end{array}$ \\
\hline & 8/12/2008 1:00 & 492 & 225 & 100 & 8.36 & 8.36 & & 0.1890 & $1.53 \mathrm{E}-03$ & 228.4824 & 84.1890 & 312.6714 & \\
\hline & $8 / 12 / 2008$ 2:00 & 493 & 225 & 200 & 8.04 & 8.04 & & 0.2363 & $2.02 \mathrm{E}-03$ & 300.8273 & 110.8460 & 411.6733 & \\
\hline & 8/12/2008 3:00 & 494 & 225 & 300 & 8.09 & 8.09 & & 0.2287 & $1.94 \mathrm{E}-03$ & 288.8986 & 106.4506 & 395.3493 & \\
\hline & $8 / 12 / 2008$ 4:00 & 495 & 225 & 400 & 7.981 & 7.981 & & 0.2455 & $2.11 \mathrm{E}-03$ & 315.2126 & 116.1466 & 431.3592 & \\
\hline & 8/12/2008 5:00 & 496 & 225 & 500 & 7.893 & 7.893 & & 0.2593 & $2.26 \mathrm{E}-03$ & 337.3050 & 124.2870 & 461.5920 & \\
\hline & 8/12/2008 6:00 & 497 & 225 & 600 & 7.88 & 7.88 & & 0.2614 & $2.28 \mathrm{E}-03$ & 340.6344 & 125.5138 & 466.1482 & \\
\hline & $8 / 12 / 20087: 00$ & 498 & 225 & 700 & 7.98 & 7.98 & & 0.2456 & 2.11E-03 & 315.4593 & 116.2375 & 431.6968 & \\
\hline & 8/12/2008 8:00 & 499 & 225 & 800 & 7.971 & 7.971 & & 0.2470 & 2.13E-03 & 317.6844 & 117.0574 & 434.7418 & \\
\hline & 8/12/2008 9:00 & 500 & 225 & 900 & 7.259 & 7.259 & & 0.3693 & $3.49 E-03$ & 521.2376 & 192.0607 & 713.2984 & \\
\hline & $8 / 12 / 2008$ 10:00 & 501 & 225 & 1000 & 5.635 & 5.635 & & 0.7640 & 8.54E-03 & 1275.2997 & 469.9104 & 1745.2102 & \\
\hline & $8 / 12 / 2008$ 11:00 & 502 & 225 & 1100 & 4.901 & 4.901 & & 1.0282 & 1.23E-02 & 1838.1763 & 677.3139 & 2515.4902 & \\
\hline & $8 / 12 / 2008$ 12:00 & 503 & 225 & 1200 & 4.747 & 4.747 & & 1.0940 & 1.33E-02 & 1984.0363 & 731.0590 & 2715.0953 & \\
\hline & 8/12/2008 13:00 & 504 & 225 & 1300 & 4.743 & 4.743 & & 1.0957 & 1.33E-02 & 1987.9796 & 732.5120 & 2720.4916 & \\
\hline & $8 / 12 / 200814: 00$ & 505 & 225 & 1400 & 4.724 & 4.724 & & 1.1041 & 1.34E-02 & 2006.8219 & 739.4548 & 2746.2767 & \\
\hline & $8 / 12 / 200815: 00$ & 506 & 225 & 1500 & 4.515 & 4.515 & & 1.2016 & 1.49E-02 & 2226.9175 & 820.5535 & 3047.4710 & \\
\hline & $8 / 12 / 2008$ 16:00 & 507 & 225 & 1600 & 4.571 & 4.571 & & 1.1746 & 1.45E-02 & 2165.5424 & 797.9387 & 2963.4811 & \\
\hline & $8 / 12 / 2008$ 17:00 & 508 & 225 & 1700 & 4.805 & 4.805 & & 1.0687 & 1.29E-02 & 1927.7578 & 710.3220 & 2638.0798 & \\
\hline & 8/12/2008 18:00 & 509 & 225 & 1800 & 4.639 & 4.639 & & 1.1427 & $1.40 \mathrm{E}-02$ & 2093.4268 & 771.3662 & 2864.7930 & \\
\hline & 8/12/2008 19:00 & 510 & 225 & 1900 & 5.16 & 5.16 & & 0.9264 & 1.08E-02 & 1616.7605 & 595.7287 & 2212.4892 & \\
\hline & $8 / 12 / 200820: 00$ & 511 & 225 & 2000 & 5.855 & 5.855 & & 0.6977 & 7.64E-03 & 1140.4954 & 420.2390 & 1560.7344 & \\
\hline & $8 / 12 / 200821: 00$ & 512 & 225 & 2100 & 7.724 & 7.724 & & 0.2869 & $2.56 \mathrm{E}-03$ & 381.9480 & 140.7366 & 522.6846 & \\
\hline & $8 / 12 / 200822: 00$ & 513 & 225 & 2200 & 8.38 & 8.38 & & 0.1862 & 1.50E-03 & 224.2667 & 82.6357 & 306.9023 & \\
\hline & $8 / 12 / 2008$ 23:00 & 514 & 225 & 2300 & 8.42 & 8.42 & & 0.1805 & 1.45E-03 & 215.9395 & 79.5673 & 295.5068 & \\
\hline & $8 / 13 / 20080: 00$ & 515 & 226 & 0 & 8.36 & 8.36 & 8.36 & 0.1890 & $1.53 \mathrm{E}-03$ & 228.4824 & 84.1890 & 312.6714 & \\
\hline & 8/13/2008 1:00 & 516 & 226 & 100 & 8.31 & 8.31 & & 0.1961 & 1.60E-03 & 239.1750 & 88.1290 & 327.3040 & \\
\hline & $8 / 13 / 2008$ 2:00 & 517 & 226 & 200 & 8.26 & 8.26 & & 0.2034 & $1.68 \mathrm{E}-03$ & 250.0892 & 92.1505 & 342.2398 & \\
\hline & 8/13/2008 3:00 & 518 & 226 & 300 & 8.27 & 8.27 & & 0.2019 & 1.66E-03 & 247.8885 & 91.3396 & 339.2282 & \\
\hline & $8 / 13 / 20084: 00$ & 519 & 226 & 400 & 8.2 & 8.2 & & 0.2122 & 1.77E-03 & 263.4832 & 97.0858 & 360.5691 & \\
\hline & 8/13/2008 5:00 & 520 & 226 & 500 & 8.18 & 8.18 & & 0.2152 & 1.80E-03 & 268.0208 & 98.7578 & 366.7786 & \\
\hline & 8/13/2008 6:00 & 521 & 226 & 600 & 8.19 & 8.19 & & 0.2137 & 1.78E-03 & 265.7474 & 97.9201 & 363.6675 & \\
\hline & 8/13/2008 7:00 & 522 & 226 & 700 & 8.14 & 8.14 & & 0.2211 & 1.86E-03 & 277.2066 & 102.1425 & 379.3491 & \\
\hline & 8/13/2008 8:00 & 523 & 226 & 800 & 8.02 & 8.02 & & 0.2394 & 2.05E-03 & 305.6658 & 112.6289 & 418.2947 & \\
\hline & 8/13/2008 9:00 & 524 & 226 & 900 & 6.482 & 6.482 & & 0.5335 & 5.49E-03 & 819.6369 & 302.0121 & 1121.6489 & \\
\hline & $8 / 13 / 200810: 00$ & 525 & 226 & 1000 & 5.262 & 5.262 & & 0.8890 & 1.03E-02 & 1536.9126 & 566.3071 & 2103.2197 & \\
\hline & 8/13/2008 11:00 & 526 & 226 & 1100 & 4.946 & 4.946 & & 1.0097 & $1.20 \mathrm{E}-02$ & 1797.6497 & 662.3810 & 2460.0307 & \\
\hline & 8/13/2008 12:00 & 527 & 226 & 1200 & 4.639 & 4.639 & & 1.1427 & $1.40 \mathrm{E}-02$ & 2093.4268 & 771.3662 & 2864.7930 & \\
\hline
\end{tabular}




\begin{tabular}{|c|c|c|c|c|c|c|c|c|c|c|c|c|c|}
\hline Tree ID & TS & RN & JD & HR & $\begin{array}{c}\text { Raw } \\
\text { TC_dTA }\end{array}$ & $\begin{array}{c}\text { Fixed } \\
\text { TC_dT } \Delta\end{array}$ & dTM & $\mathbf{K}$ & $\begin{array}{c}\text { Sap } \\
\text { Velocity } \\
(\mathrm{cm} / \mathrm{s})\end{array}$ & $\begin{array}{c}\text { Probed } \\
\text { Drywood F } \\
(\mathrm{g} / \mathrm{hr})\end{array}$ & $\begin{array}{c}\text { Extrapolated } \\
\text { Wetwood F } \\
(\mathrm{g} / \mathrm{hr})\end{array}$ & $\begin{array}{c}\text { Extrapolated } \\
\text { Total Wood F } \\
(\mathrm{g} / \mathrm{hr})\end{array}$ & $\begin{array}{c}\text { Relative } \\
\text { Humidity, } \\
@ 10 \mathrm{~m}(\%)\end{array}$ \\
\hline & $7 / 22 / 2008$ 13:00 & 0 & 204 & 1300 & 4.546 & 4.546 & 8.70 & 0.9138 & 1.06E-02 & 2334.3650 & 860.1448 & 3194.5097 & \\
\hline \multirow[t]{2}{*}{ W504WN1 } & $7 / 22 / 200814: 00$ & 1 & 204 & 1400 & 4.922 & 4.922 & & 0.7676 & $8.59 \mathrm{E}-03$ & 1883.4820 & 694.0077 & 2577.4897 & \\
\hline & $7 / 22 / 2008$ 15:00 & 2 & 204 & 1500 & 4.885 & 4.885 & & 0.7810 & $8.78 \mathrm{E}-03$ & 1924.0034 & 708.9386 & 2632.9420 & \\
\hline \multirow[t]{2}{*}{ Probe \# } & $7 / 22 / 2008$ 16:00 & 3 & 204 & 1600 & 4.915 & 4.915 & & 0.7701 & $8.63 E-03$ & 1891.0890 & 696.8107 & 2587.8997 & \\
\hline & 7/22/2008 17:00 & 4 & 204 & 1700 & 5.007 & 5.007 & & 0.7376 & 8.18E-03 & 1793.2558 & 660.7620 & 2454.0178 & \\
\hline \multirow[t]{4}{*}{10} & $7 / 22 / 2008$ 18:00 & 5 & 204 & 1800 & 5.026 & 5.026 & & 0.7310 & $8.09 E-03$ & 1773.6166 & 653.5255 & 2427.1421 & \\
\hline & 7/22/2008 19:00 & 6 & 204 & 1900 & 5.247 & 5.247 & & 0.6581 & 7.11E-03 & 1558.4318 & 574.2362 & 2132.6680 & \\
\hline & $7 / 22 / 200820: 00$ & 7 & 204 & 2000 & 5.635 & 5.635 & & 0.5439 & 5.62E-03 & 1232.6047 & 454.1786 & 1686.7833 & \\
\hline & 7/22/2008 21:00 & 8 & 204 & 2100 & 6.873 & 6.873 & & 0.2658 & 2.33E-03 & 510.5653 & 188.1283 & 698.6936 & \\
\hline \multirow[t]{2}{*}{$\mathrm{DBH}(\mathrm{cm})$} & $7 / 22 / 2008$ 22:00 & 9 & 204 & 2200 & 8.24 & 8.24 & & 0.0558 & $3.41 \mathrm{E}-04$ & 74.7699 & 27.5505 & 102.3204 & \\
\hline & $7 / 22 / 2008$ 23:00 & 10 & 204 & 2300 & 8.7 & 8.7 & & 0.0000 & $0.00 E+00$ & 0.0000 & 0.0000 & 0.0000 & \\
\hline \multirow[t]{2}{*}{10.7} & $7 / 23 / 20080: 00$ & 11 & 205 & 0 & 8.95 & 8.95 & 9.10 & -0.0279 & $0.00 \mathrm{E}+00$ & 0.0000 & 0.0000 & 0.0000 & \\
\hline & 7/23/2008 1:00 & 12 & 205 & 100 & 9.03 & 9.03 & & -0.0365 & $0.00 \mathrm{E}+00$ & 0.0000 & 0.0000 & 0.0000 & \\
\hline Day Count & 7/23/2008 2:00 & 13 & 205 & 200 & 9.06 & 9.06 & & -0.0397 & $0.00 \mathrm{E}+00$ & 0.0000 & 0.0000 & 0.0000 & \\
\hline \multirow[t]{27}{*}{35} & 7/23/2008 3:00 & 14 & 205 & 300 & 9.1 & 9.1 & & -0.0440 & $0.00 \mathrm{E}+00$ & 0.0000 & 0.0000 & 0.0000 & \\
\hline & 7/23/2008 4:00 & 15 & 205 & 400 & 9.1 & 9.1 & & -0.0440 & $0.00 \mathrm{E}+00$ & 0.0000 & 0.0000 & 0.0000 & \\
\hline & 7/23/2008 5:00 & 16 & 205 & 500 & 9.05 & 9.05 & & -0.0387 & $0.00 \mathrm{E}+00$ & 0.0000 & 0.0000 & 0.0000 & \\
\hline & 7/23/2008 6:00 & 17 & 205 & 600 & 8.93 & 8.93 & & -0.0258 & $0.00 \mathrm{E}+00$ & 0.0000 & 0.0000 & 0.0000 & \\
\hline & 7/23/2008 7:00 & 18 & 205 & 700 & 8.59 & 8.59 & & 0.0128 & 5.57E-05 & 12.2064 & 4.4977 & 16.7041 & \\
\hline & $7 / 23 / 20088: 00$ & 19 & 205 & 800 & 7.782 & 7.782 & & 0.1180 & 8.57E-04 & 187.8038 & 69.2002 & 257.0040 & \\
\hline & 7/23/2008 9:00 & 20 & 205 & 900 & 6.774 & 6.774 & & 0.2843 & 2.53E-03 & 554.6510 & 204.3726 & 759.0235 & \\
\hline & $7 / 23 / 2008$ 10:00 & 21 & 205 & 1000 & 5.926 & 5.926 & & 0.4681 & 4.67E-03 & 1024.6435 & 377.5510 & 1402.1945 & \\
\hline & 7/23/2008 11:00 & 22 & 205 & 1100 & 5.593 & 5.593 & & 0.5555 & 5.77E-03 & 1265.0266 & 466.1251 & 1731.1516 & \\
\hline & 7/23/2008 12:00 & 23 & 205 & 1200 & 5.328 & 5.328 & & 0.6329 & $6.78 \mathrm{E}-03$ & 1485.2767 & 547.2808 & 2032.5575 & \\
\hline & 7/23/2008 13:00 & 24 & 205 & 1300 & 5.188 & 5.188 & & 0.6769 & 7.36E-03 & 1613.5817 & 594.5574 & 2208.1391 & \\
\hline & $7 / 23 / 2008$ 14:00 & 25 & 205 & 1400 & 5.161 & 5.161 & & 0.6857 & $7.48 \mathrm{E}-03$ & 1639.3622 & 604.0567 & 2243.4190 & \\
\hline & $7 / 23 / 2008$ 15:00 & 26 & 205 & 1500 & 5.133 & 5.133 & & 0.6949 & 7.60E-03 & 1666.4659 & 614.0436 & 2280.5095 & \\
\hline & $7 / 23 / 2008$ 16:00 & 27 & 205 & 1600 & 5.289 & 5.289 & & 0.6449 & $6.94 \mathrm{E}-03$ & 1520.1375 & 560.1259 & 2080.2634 & \\
\hline & 7/23/2008 17:00 & 28 & 205 & 1700 & 5.607 & 5.607 & & 0.5516 & 5.72E-03 & 1254.1477 & 462.1165 & 1716.2643 & \\
\hline & $7 / 23 / 2008$ 18:00 & 29 & 205 & 1800 & 6.101 & 6.101 & & 0.4260 & 4.16E-03 & 912.3809 & 336.1855 & 1248.5665 & \\
\hline & $7 / 23 / 2008$ 19:00 & 30 & 205 & 1900 & 6.324 & 6.324 & & 0.3757 & 3.57E-03 & 781.6716 & 288.0230 & 1069.6945 & \\
\hline & $7 / 23 / 200820: 00$ & 31 & 205 & 2000 & 6.717 & 6.717 & & 0.2952 & 2.65E-03 & 580.9379 & 214.0585 & 794.9965 & \\
\hline & $7 / 23 / 2008$ 21:00 & 32 & 205 & 2100 & 7.36 & 7.36 & & 0.1821 & $1.46 \mathrm{E}-03$ & 320.4186 & 118.0648 & 438.4835 & \\
\hline & $7 / 23 / 200822: 00$ & 33 & 205 & 2200 & 8.1 & 8.1 & & 0.0741 & $4.83 \mathrm{E}-04$ & 105.9101 & 39.0248 & 144.9349 & \\
\hline & $7 / 23 / 2008$ 23:00 & 34 & 205 & 2300 & 8.39 & 8.39 & & 0.0369 & 2.05E-04 & 44.9877 & 16.5766 & 61.5643 & \\
\hline & $7 / 24 / 20080: 00$ & 35 & 206 & 0 & 8.46 & 8.46 & 8.78 & 0.0284 & $1.48 \mathrm{E}-04$ & 32.4957 & 11.9737 & 44.4694 & \\
\hline & 7/24/2008 1:00 & 36 & 206 & 100 & 8.57 & 8.57 & & 0.0152 & $6.86 \mathrm{E}-05$ & 15.0364 & 5.5405 & 20.5768 & \\
\hline & 7/24/2008 2:00 & 37 & 206 & 200 & 8.62 & 8.62 & & 0.0093 & $3.75 E-05$ & 8.2124 & 3.0260 & 11.2385 & \\
\hline & 7/24/2008 3:00 & 38 & 206 & 300 & 8.72 & 8.72 & & -0.0023 & $0.00 \mathrm{E}+00$ & 0.0000 & 0.0000 & 0.0000 & \\
\hline & 7/24/2008 4:00 & 39 & 206 & 400 & 8.74 & 8.74 & & -0.0046 & $0.00 \mathrm{E}+00$ & 0.0000 & 0.0000 & 0.0000 & \\
\hline & $7 / 24 / 20085: 00$ & 40 & 206 & 500 & 8.77 & 8.77 & & -0.0080 & $0.00 \mathrm{E}+00$ & 0.0000 & 0.0000 & 0.0000 & \\
\hline
\end{tabular}




\begin{tabular}{|c|c|c|c|c|c|c|c|c|c|c|c|c|c|}
\hline \multirow[t]{42}{*}{ Tree ID } & TS & RN & JD & HR & $\begin{array}{c}\text { Raw } \\
\text { TC_dTA }\end{array}$ & $\begin{array}{c}\text { Fixed } \\
\text { TC_dT } \Delta\end{array}$ & dTM & $\mathbf{K}$ & $\begin{array}{l}\text { Sap } \\
\text { Velocity } \\
\text { (cm/s) }\end{array}$ & $\begin{array}{l}\text { Probed } \\
\text { Drywood F } \\
\text { (g/hr) }\end{array}$ & $\begin{array}{c}\text { Extrapolated } \\
\text { Wetwood F } \\
(\mathrm{g} / \mathrm{hr})\end{array}$ & $\begin{array}{c}\text { Extrapolated } \\
\text { Total Wood F } \\
(\mathrm{g} / \mathrm{hr})\end{array}$ & $\begin{array}{c}\text { Relative } \\
\text { Humidity, } \\
@ 10 \mathrm{~m}(\%)\end{array}$ \\
\hline & 7/24/2008 6:00 & 41 & 206 & 600 & 8.78 & 8.78 & & -0.0091 & $0.00 \mathrm{E}+00$ & 0.0000 & 0.0000 & 0.0000 & \\
\hline & 7/24/2008 7:00 & 42 & 206 & 700 & 8.71 & 8.71 & & -0.0011 & $0.00 \mathrm{E}+00$ & 0.0000 & 0.0000 & 0.0000 & \\
\hline & 7/24/2008 8:00 & 43 & 206 & 800 & 8.33 & 8.33 & & 0.0444 & $2.57 \mathrm{E}-04$ & 56.4314 & 20.7933 & 77.2247 & \\
\hline & $7 / 24 / 20089: 00$ & 44 & 206 & 900 & 7.99 & 7.99 & & 0.0889 & $6.05 E-04$ & 132.5081 & 48.8253 & 181.3334 & \\
\hline & $7 / 24 / 2008$ 10:00 & 45 & 206 & 1000 & 8.08 & 8.08 & & 0.0767 & 5.05E-04 & 110.6087 & 40.7560 & 151.3647 & \\
\hline & $7 / 24 / 2008$ 11:00 & 46 & 206 & 1100 & 6.651 & 6.651 & & 0.3081 & $2.79 \mathrm{E}-03$ & 612.2272 & 225.5877 & 837.8150 & \\
\hline & $7 / 24 / 2008$ 12:00 & 47 & 206 & 1200 & 5.563 & 5.563 & & 0.5639 & 5.88E-03 & 1288.5826 & 474.8047 & 1763.3873 & \\
\hline & 7/24/2008 13:00 & 48 & 206 & 1300 & 5.741 & 5.741 & & 0.5154 & 5.26E-03 & 1153.5704 & 425.0567 & 1578.6272 & \\
\hline & $7 / 24 / 2008$ 14:00 & 49 & 206 & 1400 & 5.61 & 5.61 & & 0.5508 & 5.71E-03 & 1251.8259 & 461.2610 & 1713.0869 & \\
\hline & 7/24/2008 15:00 & 50 & 206 & 1500 & 5.269 & 5.269 & & 0.6512 & $7.02 \mathrm{E}-03$ & 1538.2744 & 566.8089 & 2105.0833 & \\
\hline & $7 / 24 / 2008$ 16:00 & 51 & 206 & 1600 & 5.185 & 5.185 & & 0.6779 & $7.37 \mathrm{E}-03$ & 1616.4292 & 595.6066 & 2212.0358 & \\
\hline & $7 / 24 / 2008$ 17:00 & 52 & 206 & 1700 & 5.392 & 5.392 & & 0.6135 & $6.52 \mathrm{E}-03$ & 1429.4842 & 526.7229 & 1956.2070 & \\
\hline & 7/24/2008 18:00 & 53 & 206 & 1800 & 5.256 & 5.256 & & 0.6553 & 7.07E-03 & 1550.1593 & 571.1881 & 2121.3473 & \\
\hline & $7 / 24 / 2008$ 19:00 & 54 & 206 & 1900 & 5.648 & 5.648 & & 0.5404 & 5.58E-03 & 1222.6989 & 450.5286 & 1673.2275 & \\
\hline & $7 / 24 / 200820: 00$ & 55 & 206 & 2000 & 6.32 & 6.32 & & 0.3766 & $3.58 \mathrm{E}-03$ & 783.9021 & 288.8449 & 1072.7470 & \\
\hline & $7 / 24 / 200821: 00$ & 56 & 206 & 2100 & 7.563 & 7.563 & & 0.1503 & 1.15E-03 & 253.1320 & 93.2717 & 346.4037 & \\
\hline & $7 / 24 / 200822: 00$ & 57 & 206 & 2200 & 8.55 & 8.55 & & 0.0175 & 8.20E-05 & 17.9844 & 6.6267 & 24.6111 & \\
\hline & $7 / 24 / 200823: 00$ & 58 & 206 & 2300 & 8.77 & 8.77 & & -0.0080 & $0.00 \mathrm{E}+00$ & 0.0000 & 0.0000 & 0.0000 & \\
\hline & $7 / 25 / 20080: 00$ & 59 & 207 & 0 & 8.8 & 8.8 & 8.80 & -0.0114 & $0.00 \mathrm{E}+00$ & 0.0000 & 0.0000 & 0.0000 & \\
\hline & 7/25/2008 1:00 & 60 & 207 & 100 & 8.77 & 8.77 & & -0.0080 & $0.00 \mathrm{E}+00$ & 0.0000 & 0.0000 & 0.0000 & \\
\hline & 7/25/2008 2:00 & 61 & 207 & 200 & 8.72 & 8.72 & & -0.0023 & $0.00 E+00$ & 0.0000 & 0.0000 & 0.0000 & \\
\hline & 7/25/2008 3:00 & 62 & 207 & 300 & 8.66 & 8.66 & & 0.0046 & 1.59E-05 & 3.4788 & 1.2818 & 4.7606 & \\
\hline & 7/25/2008 4:00 & 63 & 207 & 400 & 8.63 & 8.63 & & 0.0081 & 3.17E-05 & 6.9577 & 2.5637 & 9.5214 & \\
\hline & 7/25/2008 5:00 & 64 & 207 & 500 & 8.64 & 8.64 & & 0.0069 & 2.62E-05 & 5.7469 & 2.1176 & 7.8645 & \\
\hline & 7/25/2008 6:00 & 65 & 207 & 600 & 8.68 & 8.68 & & 0.0023 & $6.74 \mathrm{E}-06$ & 1.4778 & 0.5445 & 2.0224 & \\
\hline & 7/25/2008 7:00 & 66 & 207 & 700 & 8.68 & 8.68 & & 0.0023 & $6.74 \mathrm{E}-06$ & 1.4778 & 0.5445 & 2.0224 & \\
\hline & 7/25/2008 8:00 & 67 & 207 & 800 & 8.69 & 8.69 & & 0.0012 & 2.87E-06 & 0.6287 & 0.2317 & 0.8604 & \\
\hline & $7 / 25 / 20089: 00$ & 68 & 207 & 900 & 8.68 & 8.68 & & 0.0023 & $6.74 \mathrm{E}-06$ & 1.4778 & 0.5445 & 2.0224 & \\
\hline & $7 / 25 / 2008$ 10:00 & 69 & 207 & 1000 & 8.58 & 8.58 & & 0.0140 & $6.21 E-05$ & 13.6059 & 5.0134 & 18.6193 & \\
\hline & 7/25/2008 11:00 & 70 & 207 & 1100 & 7.749 & 7.749 & & 0.1227 & $9.00 \mathrm{E}-04$ & 197.1774 & 72.6541 & 269.8315 & \\
\hline & $7 / 25 / 2008$ 12:00 & 71 & 207 & 1200 & 6.581 & 6.581 & & 0.3220 & 2.95E-03 & 646.4400 & 238.1941 & 884.6341 & \\
\hline & $7 / 25 / 2008$ 13:00 & 72 & 207 & 1300 & 5.927 & 5.927 & & 0.4679 & 4.67E-03 & 1023.9761 & 377.3051 & 1401.2812 & \\
\hline & $7 / 25 / 2008$ 14:00 & 73 & 207 & 1400 & 5.331 & 5.331 & & 0.6320 & $6.76 \mathrm{E}-03$ & 1482.6225 & 546.3028 & 2028.9253 & \\
\hline & $7 / 25 / 2008$ 15:00 & 74 & 207 & 1500 & 5.2 & 5.2 & & 0.6731 & 7.31E-03 & 1602.2341 & 590.3761 & 2192.6102 & \\
\hline & $7 / 25 / 200816: 00$ & 75 & 207 & 1600 & 5.285 & 5.285 & & 0.6462 & $6.95 \mathrm{E}-03$ & 1523.7507 & 561.4573 & 2085.2079 & \\
\hline & $7 / 25 / 2008$ 17:00 & 76 & 207 & 1700 & 5.096 & 5.096 & & 0.7072 & 7.77E-03 & 1702.8680 & 627.4567 & 2330.3247 & \\
\hline & $7 / 25 / 2008$ 18:00 & 77 & 207 & 1800 & 5.148 & 5.148 & & 0.6900 & $7.54 \mathrm{E}-03$ & 1651.8990 & 608.6762 & 2260.5752 & \\
\hline & $7 / 25 / 2008$ 19:00 & 78 & 207 & 1900 & 5.266 & 5.266 & & 0.6521 & 7.03E-03 & 1541.0104 & 567.8170 & 2108.8273 & \\
\hline & $7 / 25 / 200820: 00$ & 79 & 207 & 2000 & 5.976 & 5.976 & & 0.4558 & $4.52 \mathrm{E}-03$ & 991.6464 & 365.3925 & 1357.0389 & \\
\hline & $7 / 25 / 2008$ 21:00 & 80 & 207 & 2100 & 7.349 & 7.349 & & 0.1838 & 1.48E-03 & 324.2561 & 119.4788 & 443.7349 & \\
\hline & $7 / 25 / 200822: 00$ & 81 & 207 & 2200 & 8.39 & 8.39 & & 0.0369 & 2.05E-04 & 44.9877 & 16.5766 & 61.5643 & \\
\hline
\end{tabular}




\begin{tabular}{|c|c|c|c|c|c|c|c|c|c|c|c|c|c|}
\hline Tree ID & TS & RN & JD & HR & $\begin{array}{c}\text { Raw } \\
\text { TC_dTA }\end{array}$ & $\begin{array}{c}\text { Fixed } \\
\text { TC_dT } \Delta\end{array}$ & dTM & $\mathbf{K}$ & $\begin{array}{l}\text { Sap } \\
\text { Velocity } \\
(\mathrm{cm} / \mathrm{s})\end{array}$ & $\begin{array}{c}\text { Probed } \\
\text { Drywood F } \\
(\mathrm{g} / \mathrm{hr})\end{array}$ & $\begin{array}{c}\text { Extrapolated } \\
\text { Wetwood F } \\
(\mathrm{g} / \mathrm{hr})\end{array}$ & $\begin{array}{c}\text { Extrapolated } \\
\text { Total Wood F } \\
(\mathrm{g} / \mathrm{hr})\end{array}$ & $\begin{array}{l}\text { Relative } \\
\text { Humidity, } \\
@ 10 \mathrm{~m}(\%)\end{array}$ \\
\hline & 7/25/2008 23:00 & 82 & 207 & 2300 & $\begin{array}{r}8.49 \\
\end{array}$ & 8.49 & & 0.0247 & 1.25E-04 & 27.4502 & 10.1146 & $\begin{array}{r}37.5647 \\
\end{array}$ & \\
\hline & 7/26/2008 0:00 & 83 & 208 & 0 & 8.42 & 8.42 & 8.42 & 0.0333 & $1.80 \mathrm{E}-04$ & 39.5158 & 14.5604 & 54.0762 & \\
\hline & 7/26/2008 1:00 & 84 & 208 & 100 & 8.31 & 8.31 & & 0.0469 & $2.75 \mathrm{E}-04$ & 60.3879 & 22.2512 & 82.6391 & \\
\hline & $7 / 26 / 20082: 00$ & 85 & 208 & 200 & 8.21 & 8.21 & & 0.0597 & 3.70E-04 & 81.1808 & 29.9127 & 111.0935 & \\
\hline & 7/26/2008 3:00 & 86 & 208 & 300 & 8.11 & 8.11 & & 0.0727 & 4.73E-04 & 103.5840 & 38.1676 & 141.7516 & \\
\hline & 7/26/2008 4:00 & 87 & 208 & 400 & 8.02 & 8.02 & & 0.0848 & 5.71E-04 & 125.0714 & 46.0851 & 171.1566 & \\
\hline & 7/26/2008 5:00 & 88 & 208 & 500 & 7.931 & 7.931 & & 0.0970 & $6.73 E-04$ & 147.5302 & 54.3605 & 201.8907 & \\
\hline & 7/26/2008 6:00 & 89 & 208 & 600 & 7.833 & 7.833 & & 0.1107 & 7.92E-04 & 173.6418 & 63.9819 & 237.6237 & \\
\hline & 7/26/2008 7:00 & 90 & 208 & 700 & 7.757 & 7.757 & & 0.1216 & 8.89E-04 & 194.8898 & 71.8112 & 266.7010 & \\
\hline & 7/26/2008 8:00 & 91 & 208 & 800 & 7.707 & 7.707 & & 0.1288 & 9.55E-04 & 209.3471 & 77.1383 & 286.4854 & \\
\hline & 7/26/2008 9:00 & 92 & 208 & 900 & 7.305 & 7.305 & & 0.1910 & $1.55 E-03$ & 339.8073 & 125.2090 & 465.0163 & \\
\hline & $7 / 26 / 2008$ 10:00 & 93 & 208 & 1000 & 5.378 & 5.378 & & 0.6177 & 6.58E-03 & 1441.5412 & 531.1655 & 1972.7068 & \\
\hline & $7 / 26 / 2008$ 11:00 & 94 & 208 & 1100 & 5.158 & 5.158 & & 0.6867 & 7.49E-03 & 1642.2481 & 605.1201 & 2247.3682 & \\
\hline & $7 / 26 / 2008$ 12:00 & 95 & 208 & 1200 & 5.377 & 5.377 & & 0.6180 & 6.58E-03 & 1442.4056 & 531.4840 & 1973.8896 & \\
\hline & $7 / 26 / 2008$ 13:00 & 96 & 208 & 1300 & 4.89 & 4.89 & & 0.7791 & 8.75E-03 & 1918.4822 & 706.9042 & 2625.3864 & \\
\hline & $7 / 26 / 200814: 00$ & 97 & 208 & 1400 & 4.797 & 4.797 & & 0.8136 & 9.23E-03 & 2023.5606 & 745.6225 & 2769.1832 & \\
\hline & $7 / 26 / 2008$ 15:00 & 98 & 208 & 1500 & 4.739 & 4.739 & & 0.8358 & 9.54E-03 & 2091.7306 & 770.7412 & 2862.4718 & \\
\hline & $7 / 26 / 200816: 00$ & 99 & 208 & 1600 & 4.856 & 4.856 & & 0.7916 & 8.92E-03 & 1956.3097 & 720.8426 & 2677.1523 & \\
\hline & $7 / 26 / 200817: 00$ & 100 & 208 & 1700 & 4.67 & 4.67 & & 0.8630 & 9.93E-03 & 2175.6036 & 801.6459 & 2977.2495 & \\
\hline & $7 / 26 / 2008$ 18:00 & 101 & 208 & 1800 & 4.813 & 4.813 & & 0.8076 & $9.15 E-03$ & 2005.1179 & 738.8269 & 2743.9448 & \\
\hline & $7 / 26 / 2008$ 19:00 & 102 & 208 & 1900 & 4.98 & 4.98 & & 0.7470 & 8.31E-03 & 1821.4924 & 671.1663 & 2492.6588 & \\
\hline & $7 / 26 / 200820: 00$ & 103 & 208 & 2000 & 5.486 & 5.486 & & 0.5859 & 6.16E-03 & 1350.6036 & 497.6577 & 1848.2613 & \\
\hline & $7 / 26 / 200821: 00$ & 104 & 208 & 2100 & 6.678 & 6.678 & & 0.3028 & 2.73E-03 & 599.3151 & 220.8300 & 820.1450 & \\
\hline & $7 / 26 / 200822: 00$ & 105 & 208 & 2200 & 7.658 & 7.658 & & 0.1361 & 1.02E-03 & 223.8863 & 82.4955 & 306.3818 & \\
\hline & $7 / 26 / 2008$ 23:00 & 106 & 208 & 2300 & 7.828 & 7.828 & & 0.1114 & 7.98E-04 & 175.0129 & 64.4871 & 239.5000 & \\
\hline & $7 / 27 / 20080: 00$ & 107 & 209 & 0 & 7.787 & 7.787 & 7.79 & 0.1172 & 8.50E-04 & 186.3980 & 68.6822 & 255.0801 & \\
\hline & 7/27/2008 1:00 & 108 & 209 & 100 & 7.738 & 7.738 & & 0.1243 & 9.14E-04 & 200.3387 & 73.8189 & 274.1576 & \\
\hline & 7/27/2008 2:00 & 109 & 209 & 200 & 7.691 & 7.691 & & 0.1312 & 9.77E-04 & 214.0541 & 78.8726 & 292.9267 & \\
\hline & 7/27/2008 3:00 & 110 & 209 & 300 & 7.663 & 7.663 & & 0.1353 & 1.01E-03 & 222.3858 & 81.9426 & 304.3284 & \\
\hline & 7/27/2008 4:00 & 111 & 209 & 400 & 7.625 & 7.625 & & 0.1410 & 1.07E-03 & 233.8864 & 86.1802 & 320.0666 & \\
\hline & 7/27/20085:00 & 112 & 209 & 500 & 7.57 & 7.57 & & 0.1493 & 1.14E-03 & 250.9290 & 92.4600 & 343.3890 & \\
\hline & 7/27/2008 6:00 & 113 & 209 & 600 & 7.513 & 7.513 & & 0.1580 & 1.23E-03 & 269.0921 & 99.1525 & 368.2446 & \\
\hline & 7/27/2008 7:00 & 114 & 209 & 700 & 7.443 & 7.443 & & 0.1689 & 1.33E-03 & 292.1045 & 107.6319 & 399.7364 & \\
\hline & 7/27/2008 8:00 & 115 & 209 & 800 & 7.395 & 7.395 & & 0.1765 & 1.41E-03 & 308.3415 & 113.6148 & 421.9563 & \\
\hline & $7 / 27 / 20089: 00$ & 116 & 209 & 900 & 5.955 & 5.955 & & 0.4610 & 4.59E-03 & 1005.4130 & 370.4651 & 1375.8781 & \\
\hline & $7 / 27 / 2008$ 10:00 & 117 & 209 & 1000 & 4.596 & 4.596 & & 0.8930 & 1.04E-02 & 2269.0642 & 836.0834 & 3105.1475 & \\
\hline & $7 / 27 / 2008$ 11:00 & 118 & 209 & 1100 & 4.265 & 4.265 & & 1.0399 & $1.25 \mathrm{E}-02$ & 2736.9953 & 1008.5022 & 3745.4975 & \\
\hline & $7 / 27 / 2008$ 12:00 & 119 & 209 & 1200 & 4.094 & 4.094 & & 1.1251 & 1.38E-02 & 3015.6165 & 1111.1659 & 4126.7824 & \\
\hline & $7 / 27 / 200813: 00$ & 120 & 209 & 1300 & 4.132 & 4.132 & & 1.1055 & $1.35 \mathrm{E}-02$ & 2951.2623 & 1087.4533 & 4038.7156 & \\
\hline & $7 / 27 / 200814: 00$ & 121 & 209 & 1400 & 4.03 & 4.03 & & 1.1588 & 1.43E-02 & 3127.3530 & 1152.3375 & 4279.6905 & \\
\hline & 7/27/2008 15:00 & 122 & 209 & 1500 & 4.06 & 4.06 & & 1.1429 & 1.40E-02 & 3074.4428 & 1132.8417 & 4207.2845 & \\
\hline
\end{tabular}




\begin{tabular}{|c|c|c|c|c|c|c|c|c|c|c|c|c|c|}
\hline Tree ID & TS & RN & JD & HR & $\begin{array}{c}\text { Raw } \\
\text { TC_dTA }\end{array}$ & $\begin{array}{c}\text { Fixed } \\
\text { TC_dT } \Delta\end{array}$ & dTM & $\mathbf{K}$ & $\begin{array}{l}\text { Sap } \\
\text { Velocity } \\
(\mathrm{cm} / \mathrm{s})\end{array}$ & $\begin{array}{c}\text { Probed } \\
\text { Drywood F } \\
(\mathrm{g} / \mathrm{hr})\end{array}$ & $\begin{array}{c}\text { Extrapolated } \\
\text { Wetwood F } \\
(\mathrm{g} / \mathrm{hr})\end{array}$ & $\begin{array}{c}\text { Extrapolated } \\
\text { Total Wood F } \\
(\mathrm{g} / \mathrm{hr})\end{array}$ & $\begin{array}{l}\text { Relative } \\
\text { Humidity, } \\
@ 10 \mathrm{~m}(\%)\end{array}$ \\
\hline & 7/27/2008 16:00 & 123 & 209 & 1600 & 4.191 & 4.191 & & 1.0759 & 1.30E-02 & 2854.1583 & 1051.6733 & \begin{tabular}{|r|}
3905.8317 \\
\end{tabular} & \\
\hline & $7 / 27 / 2008$ 17:00 & 124 & 209 & 1700 & 4.269 & 4.269 & & 1.0379 & $1.25 \mathrm{E}-02$ & 2730.8037 & 1006.2208 & 3737.0245 & \\
\hline & $7 / 27 / 2008$ 18:00 & 125 & 209 & 1800 & 4.324 & 4.324 & & 1.0120 & $1.21 \mathrm{E}-02$ & 2647.0932 & 975.3759 & 3622.4691 & \\
\hline & $7 / 27 / 2008$ 19:00 & 126 & 209 & 1900 & 4.571 & 4.571 & & 0.9033 & 1.05E-02 & 2301.4925 & 848.0323 & 3149.5248 & \\
\hline & $7 / 27 / 200820: 00$ & 127 & 209 & 2000 & 5.145 & 5.145 & & 0.6910 & 7.55E-03 & 1654.8037 & 609.7465 & 2264.5502 & \\
\hline & $7 / 27 / 2008$ 21:00 & 128 & 209 & 2100 & 6.166 & 6.166 & & 0.4110 & $3.98 \mathrm{E}-03$ & 872.9109 & 321.6420 & 1194.5529 & \\
\hline & $7 / 27 / 2008$ 22:00 & 129 & 209 & 2200 & 6.899 & 6.899 & & 0.2611 & $2.28 \mathrm{E}-03$ & 499.3097 & 183.9809 & 683.2906 & \\
\hline & $7 / 27 / 200823: 00$ & 130 & 209 & 2300 & 7.21 & 7.21 & & 0.2067 & $1.71 \mathrm{E}-03$ & 374.5004 & 137.9924 & 512.4928 & \\
\hline & $7 / 28 / 20080: 00$ & 131 & 210 & 0 & 7.342 & 7.342 & 7.44 & 0.1850 & 1.49E-03 & 326.7085 & 120.3825 & 447.0910 & \\
\hline & 7/28/2008 1:00 & 132 & 210 & 100 & 7.404 & 7.404 & & 0.1750 & 1.39E-03 & 305.2685 & 112.4824 & 417.7509 & \\
\hline & $7 / 28 / 20082: 00$ & 133 & 210 & 200 & 7.41 & 7.41 & & 0.1741 & 1.38E-03 & 303.2271 & 111.7303 & 414.9574 & \\
\hline & 7/28/2008 3:00 & 134 & 210 & 300 & 7.436 & 7.436 & & 0.1700 & 1.34E-03 & 294.4491 & 108.4958 & 402.9449 & \\
\hline & 7/28/2008 4:00 & 135 & 210 & 400 & 7.428 & 7.428 & & 0.1712 & 1.36E-03 & 297.1383 & 109.4867 & 406.6250 & \\
\hline & 7/28/2008 5:00 & 136 & 210 & 500 & 7.391 & 7.391 & & 0.1771 & 1.41E-03 & 309.7115 & 114.1196 & 423.8311 & \\
\hline & 7/28/2008 6:00 & 137 & 210 & 600 & 7.37 & 7.37 & & 0.1805 & 1.45E-03 & 316.9474 & 116.7858 & 433.7332 & \\
\hline & 7/28/2008 7:00 & 138 & 210 & 700 & 7.204 & 7.204 & & 0.2077 & 1.72E-03 & 376.7436 & 138.8189 & 515.5625 & \\
\hline & 7/28/2008 8:00 & 139 & 210 & 800 & 5.813 & 5.813 & & 0.4966 & 5.03E-03 & 1102.0760 & 406.0826 & 1508.1585 & \\
\hline & $7 / 28 / 20089: 00$ & 140 & 210 & 900 & 4.764 & 4.764 & & 0.8262 & $9.41 \mathrm{E}-03$ & 2062.0914 & 759.8200 & 2821.9114 & \\
\hline & $7 / 28 / 2008$ 10:00 & 141 & 210 & 1000 & 4.207 & 4.207 & & 1.0680 & 1.29E-02 & 2828.3979 & 1042.1814 & 3870.5792 & \\
\hline & $7 / 28 / 2008$ 11:00 & 142 & 210 & 1100 & 4.017 & 4.017 & & 1.1658 & $1.44 \mathrm{E}-02$ & 3150.5793 & 1160.8957 & 4311.4750 & \\
\hline & $7 / 28 / 200812: 00$ & 143 & 210 & 1200 & 3.938 & 3.938 & & 1.2092 & $1.50 \mathrm{E}-02$ & 3295.7379 & 1214.3824 & 4510.1204 & \\
\hline & 7/28/2008 13:00 & 144 & 210 & 1300 & 4 & 4 & & 1.1750 & 1.45E-02 & 3181.2291 & 1172.1893 & 4353.4184 & \\
\hline & $7 / 28 / 2008$ 14:00 & 145 & 210 & 1400 & 3.987 & 3.987 & & 1.1821 & 1.46E-02 & 3204.8814 & 1180.9045 & 4385.7859 & \\
\hline & $7 / 28 / 2008$ 15:00 & 146 & 210 & 1500 & 3.96 & 3.96 & & 1.1970 & $1.48 \mathrm{E}-02$ & 3254.6081 & 1199.2273 & 4453.8353 & \\
\hline & $7 / 28 / 2008$ 16:00 & 147 & 210 & 1600 & 4.119 & 4.119 & & 1.1122 & 1.36E-02 & 2973.1153 & 1095.5055 & 4068.6208 & \\
\hline & 7/28/2008 17:00 & 148 & 210 & 1700 & 4.165 & 4.165 & & 1.0888 & 1.32E-02 & 2896.5358 & 1067.2882 & 3963.8240 & \\
\hline & $7 / 28 / 2008$ 18:00 & 149 & 210 & 1800 & 4.272 & 4.272 & & 1.0365 & 1.24E-02 & 2726.1694 & 1004.5132 & 3730.6826 & \\
\hline & $7 / 28 / 2008$ 19:00 & 150 & 210 & 1900 & 4.676 & 4.676 & & 0.8606 & $9.89 \mathrm{E}-03$ & 2168.1872 & 798.9132 & 2967.1004 & \\
\hline & $7 / 28 / 200820: 00$ & 151 & 210 & 2000 & 5.187 & 5.187 & & 0.6773 & 7.37E-03 & 1614.5304 & 594.9069 & 2209.4374 & \\
\hline & $7 / 28 / 200821: 00$ & 152 & 210 & 2100 & 6.479 & 6.479 & & 0.3428 & $3.19 E-03$ & 698.2538 & 257.2860 & 955.5397 & \\
\hline & $7 / 28 / 2008$ 22:00 & 153 & 210 & 2200 & 6.943 & 6.943 & & 0.2531 & 2.19E-03 & 480.5602 & 177.0723 & 657.6325 & \\
\hline & $7 / 28 / 200823: 00$ & 154 & 210 & 2300 & 7.187 & 7.187 & & 0.2105 & 1.75E-03 & 383.1333 & 141.1733 & 524.3066 & \\
\hline & $7 / 29 / 20080: 00$ & 155 & 211 & 0 & 7.334 & 7.334 & 7.42 & 0.1863 & 1.50E-03 & 329.5213 & 121.4189 & 450.9402 & \\
\hline & 7/29/2008 1:00 & 156 & 211 & 100 & 7.404 & 7.404 & & 0.1750 & 1.39E-03 & 305.2685 & 112.4824 & 417.7509 & \\
\hline & 7/29/2008 2:00 & 157 & 211 & 200 & 7.42 & 7.42 & & 0.1725 & 1.37E-03 & 299.8379 & 110.4815 & 410.3194 & \\
\hline & 7/29/2008 3:00 & 158 & 211 & 300 & 7.42 & 7.42 & & 0.1725 & 1.37E-03 & 299.8379 & 110.4815 & 410.3194 & \\
\hline & 7/29/2008 4:00 & 159 & 211 & 400 & 7.4 & 7.4 & & 0.1757 & 1.40E-03 & 306.6326 & 112.9851 & 419.6177 & \\
\hline & 7/29/2008 5:00 & 160 & 211 & 500 & 7.357 & 7.357 & & 0.1825 & 1.47E-03 & 321.4632 & 118.4497 & 439.9129 & \\
\hline & $7 / 29 / 20086: 00$ & 161 & 211 & 600 & 7.337 & 7.337 & & 0.1858 & $1.50 \mathrm{E}-03$ & 328.4653 & 121.0298 & 449.4950 & \\
\hline & 7/29/2008 7:00 & 162 & 211 & 700 & 7.314 & 7.314 & & 0.1895 & 1.54E-03 & 336.6000 & 124.0272 & 460.6272 & \\
\hline & 7/29/2008 8:00 & 163 & 211 & 800 & 6.823 & 6.823 & & 0.2751 & 2.43E-03 & 532.5853 & 196.2420 & 728.8273 & \\
\hline
\end{tabular}




\begin{tabular}{|c|c|c|c|c|c|c|c|c|c|c|c|c|c|}
\hline Tree ID & TS & RN & JD & HR & $\begin{array}{c}\text { Raw } \\
\text { TC_dTA }\end{array}$ & $\begin{array}{c}\text { Fixed } \\
\text { TC_dT } \Delta\end{array}$ & dTM & $\mathbf{K}$ & $\begin{array}{l}\text { Sap } \\
\text { Velocity } \\
(\mathrm{cm} / \mathrm{s})\end{array}$ & $\begin{array}{c}\text { Probed } \\
\text { Drywood F } \\
(\mathrm{g} / \mathrm{hr})\end{array}$ & $\begin{array}{c}\text { Extrapolated } \\
\text { Wetwood F } \\
(\mathrm{g} / \mathrm{hr})\end{array}$ & $\begin{array}{c}\text { Extrapolated } \\
\text { Total Wood F } \\
(\mathrm{g} / \mathrm{hr})\end{array}$ & $\begin{array}{l}\text { Relative } \\
\text { Humidity, } \\
@ 10 \mathrm{~m}(\%)\end{array}$ \\
\hline & 7/29/2008 9:00 & 164 & 211 & 900 & \begin{tabular}{|r|}
5.392 \\
\end{tabular} & 5.392 & & 0.6135 & $6.52 \mathrm{E}-03$ & 1429.4842 & 526.7229 & \begin{tabular}{|l|}
1956.2070 \\
\end{tabular} & \\
\hline & $7 / 29 / 2008$ 10:00 & 165 & 211 & 1000 & 4.428 & 4.428 & & 0.9648 & $1.14 \mathrm{E}-02$ & 2495.7658 & 919.6162 & 3415.3820 & \\
\hline & $7 / 29 / 2008$ 11:00 & 166 & 211 & 1100 & 4.557 & 4.557 & & 0.9092 & $1.06 \mathrm{E}-02$ & 2319.8459 & 854.7949 & 3174.6408 & \\
\hline & $7 / 29 / 2008$ 12:00 & 167 & 211 & 1200 & 4.04 & 4.04 & & 1.1535 & $1.42 \mathrm{E}-02$ & 3109.6101 & 1145.7998 & 4255.4099 & \\
\hline & 7/29/2008 13:00 & 168 & 211 & 1300 & 4.001 & 4.001 & & 1.1745 & 1.45E-02 & 3179.4174 & 1171.5217 & 4350.9391 & \\
\hline & $7 / 29 / 2008$ 14:00 & 169 & 211 & 1400 & 3.926 & 3.926 & & 1.2160 & $1.51 \mathrm{E}-02$ & 3318.4079 & 1222.7356 & 4541.1436 & \\
\hline & $7 / 29 / 2008$ 15:00 & 170 & 211 & 1500 & 4.102 & 4.102 & & 1.1209 & 1.37E-02 & 3001.9474 & 1106.1293 & 4108.0767 & \\
\hline & $7 / 29 / 2008$ 16:00 & 171 & 211 & 1600 & 4.255 & 4.255 & & 1.0447 & 1.26E-02 & 2752.5367 & 1014.2287 & 3766.7654 & \\
\hline & 7/29/2008 17:00 & 172 & 211 & 1700 & 4.432 & 4.432 & & 0.9630 & 1.14E-02 & 2490.1201 & 917.5360 & 3407.6560 & \\
\hline & 7/29/2008 18:00 & 173 & 211 & 1800 & 4.401 & 4.401 & & 0.9768 & 1.16E-02 & 2534.2060 & 933.7803 & 3467.9864 & \\
\hline & $7 / 29 / 2008$ 19:00 & 174 & 211 & 1900 & 4.432 & 4.432 & & 0.9630 & 1.14E-02 & 2490.1201 & 917.5360 & 3407.6560 & \\
\hline & $7 / 29 / 200820: 00$ & 175 & 211 & 2000 & 5.122 & 5.122 & & 0.6986 & $7.65 \mathrm{E}-03$ & 1677.2178 & 618.0054 & 2295.2232 & \\
\hline & 7/29/2008 21:00 & 176 & 211 & 2100 & 6.247 & 6.247 & & 0.3927 & 3.77E-03 & 825.3238 & 304.1075 & 1129.4314 & \\
\hline & $7 / 29 / 2008$ 22:00 & 177 & 211 & 2200 & 7.031 & 7.031 & & 0.2374 & 2.03E-03 & 444.1647 & 163.6616 & 607.8263 & \\
\hline & 7/29/2008 23:00 & 178 & 211 & 2300 & 7.168 & 7.168 & & 0.2137 & 1.78E-03 & 390.3345 & 143.8268 & 534.1613 & \\
\hline & $7 / 30 / 20080: 00$ & 179 & 212 & 0 & 7.161 & 7.161 & 7.19 & 0.2149 & 1.79E-03 & 393.0036 & 144.8102 & 537.8138 & \\
\hline & 7/30/2008 1:00 & 180 & 212 & 100 & 7.163 & 7.163 & & 0.2146 & 1.79E-03 & 392.2401 & 144.5289 & 536.7690 & \\
\hline & 7/30/2008 2:00 & 181 & 212 & 200 & 7.154 & 7.154 & & 0.2161 & 1.81E-03 & 395.6813 & 145.7969 & 541.4781 & \\
\hline & 7/30/2008 3:00 & 182 & 212 & 300 & 7.154 & 7.154 & & 0.2161 & 1.81E-03 & 395.6813 & 145.7969 & 541.4781 & \\
\hline & 7/30/2008 4:00 & 183 & 212 & 400 & 7.147 & 7.147 & & 0.2173 & $1.82 \mathrm{E}-03$ & 398.3676 & 146.7867 & 545.1543 & \\
\hline & 7/30/2008 5:00 & 184 & 212 & 500 & 7.129 & 7.129 & & 0.2204 & $1.85 \mathrm{E}-03$ & 405.3153 & 149.3468 & 554.6620 & \\
\hline & 7/30/2008 6:00 & 185 & 212 & 600 & 7.111 & 7.111 & & 0.2235 & 1.88E-03 & 412.3207 & 151.9281 & 564.2488 & \\
\hline & 7/30/2008 7:00 & 186 & 212 & 700 & 7.093 & 7.093 & & 0.2266 & 1.91E-03 & 419.3843 & 154.5308 & 573.9151 & \\
\hline & $7 / 30 / 20088: 00$ & 187 & 212 & 800 & 7.095 & 7.095 & & 0.2262 & 1.91E-03 & 418.5966 & 154.2405 & 572.8371 & \\
\hline & 7/30/2008 9:00 & 188 & 212 & 900 & 6.839 & 6.839 & & 0.2721 & $2.40 \mathrm{E}-03$ & 525.4849 & 193.6257 & 719.1106 & \\
\hline & $7 / 30 / 2008$ 10:00 & 189 & 212 & 1000 & 4.677 & 4.677 & & 0.8602 & 9.89E-03 & 2166.9535 & 798.4586 & 2965.4121 & \\
\hline & 7/30/2008 11:00 & 190 & 212 & 1100 & 4.067 & 4.067 & & 1.1392 & 1.40E-02 & 3062.2336 & 1128.3429 & 4190.5765 & \\
\hline & $7 / 30 / 2008$ 12:00 & 191 & 212 & 1200 & 3.817 & 3.817 & & 1.2793 & $1.61 \mathrm{E}-02$ & 3532.2523 & 1301.5310 & 4833.7832 & \\
\hline & 7/30/2008 13:00 & 192 & 212 & 1300 & 3.745 & 3.745 & & 1.3231 & 1.68E-02 & 3681.7804 & 1356.6277 & 5038.4081 & \\
\hline & $7 / 30 / 2008$ 14:00 & 193 & 212 & 1400 & 3.692 & 3.692 & & 1.3564 & 1.73E-02 & 3796.3476 & 1398.8423 & 5195.1899 & \\
\hline & 7/30/2008 15:00 & 194 & 212 & 1500 & 3.807 & 3.807 & & 1.2853 & 1.62E-02 & 3552.6130 & 1309.0333 & 4861.6463 & \\
\hline & $7 / 30 / 2008$ 16:00 & 195 & 212 & 1600 & 3.811 & 3.811 & & 1.2829 & 1.62E-02 & 3544.4532 & 1306.0267 & 4850.4799 & \\
\hline & $7 / 30 / 2008$ 17:00 & 196 & 212 & 1700 & 3.907 & 3.907 & & 1.2268 & 1.53E-02 & 3354.6471 & 1236.0887 & 4590.7358 & \\
\hline & $7 / 30 / 2008$ 18:00 & 197 & 212 & 1800 & 4.02 & 4.02 & & 1.1642 & 1.43E-02 & 3145.2032 & 1158.9148 & 4304.1180 & \\
\hline & 7/30/2008 19:00 & 198 & 212 & 1900 & 4.262 & 4.262 & & 1.0413 & 1.25E-02 & 2741.6483 & 1010.2167 & 3751.8650 & \\
\hline & $7 / 30 / 200820: 00$ & 199 & 212 & 2000 & 4.872 & 4.872 & & 0.7857 & 8.84E-03 & 1938.4255 & 714.2527 & 2652.6782 & \\
\hline & 7/30/2008 21:00 & 200 & 212 & 2100 & 6.123 & 6.123 & & 0.4209 & $4.10 \mathrm{E}-03$ & 898.8913 & 331.2150 & 1230.1063 & \\
\hline & $7 / 30 / 2008$ 22:00 & 201 & 212 & 2200 & 6.982 & 6.982 & & 0.2461 & 2.12E-03 & 464.2508 & 171.0628 & 635.3135 & \\
\hline & 7/30/2008 23:00 & 202 & 212 & 2300 & 7.188 & 7.188 & & 0.2104 & $1.75 \mathrm{E}-03$ & 382.7560 & 141.0343 & 523.7904 & \\
\hline & $7 / 31 / 20080: 00$ & 203 & 213 & 0 & 7.247 & 7.247 & 7.25 & 0.2005 & 1.65E-03 & 360.8049 & 132.9460 & 493.7510 & \\
\hline & 7/31/2008 1:00 & 204 & 213 & 100 & 7.243 & 7.243 & & 0.2012 & $1.65 \mathrm{E}-03$ & 362.2742 & 133.4874 & 495.7616 & \\
\hline
\end{tabular}




\begin{tabular}{|c|c|c|c|c|c|c|c|c|c|c|c|c|c|}
\hline Tree ID & TS & RN & JD & HR & $\begin{array}{c}\text { Raw } \\
\text { TC_dTA }\end{array}$ & $\begin{array}{c}\text { Fixed } \\
\text { TC_dT } \Delta\end{array}$ & dTM & $\mathbf{K}$ & $\begin{array}{l}\text { Sap } \\
\text { Velocity } \\
(\mathrm{cm} / \mathrm{s})\end{array}$ & $\begin{array}{c}\text { Probed } \\
\text { Drywood F } \\
(\mathrm{g} / \mathrm{hr})\end{array}$ & $\begin{array}{c}\text { Extrapolated } \\
\text { Wetwood F } \\
(\mathrm{g} / \mathrm{hr})\end{array}$ & $\begin{array}{c}\text { Extrapolated } \\
\text { Total Wood F } \\
(\mathrm{g} / \mathrm{hr})\end{array}$ & $\begin{array}{l}\text { Relative } \\
\text { Humidity, } \\
@ 10 \mathrm{~m}(\%)\end{array}$ \\
\hline & 7/31/2008 2:00 & 205 & 213 & 200 & $\begin{array}{r}7.235 \\
\end{array}$ & 7.235 & & 0.2025 & 1.67E-03 & 365.2209 & 134.5731 & \begin{tabular}{|r|}
499.7940 \\
\end{tabular} & \\
\hline & 7/31/2008 3:00 & 206 & 213 & 300 & 7.236 & 7.236 & & 0.2023 & $1.66 \mathrm{E}-03$ & 364.8520 & 134.4372 & 499.2892 & \\
\hline & 7/31/2008 4:00 & 207 & 213 & 400 & 7.222 & 7.222 & & 0.2047 & $1.69 \mathrm{E}-03$ & 370.0328 & 136.3462 & 506.3790 & \\
\hline & 7/31/2008 5:00 & 208 & 213 & 500 & 7.196 & 7.196 & & 0.2090 & 1.73E-03 & 379.7443 & 139.9246 & 519.6688 & \\
\hline & 7/31/2008 6:00 & 209 & 213 & 600 & 7.179 & 7.179 & & 0.2119 & 1.76E-03 & 386.1577 & 142.2877 & 528.4454 & \\
\hline & 7/31/2008 7:00 & 210 & 213 & 700 & 7.162 & 7.162 & & 0.2147 & 1.79E-03 & 392.6217 & 144.6696 & 537.2913 & \\
\hline & $7 / 31 / 2008$ 8:00 & 211 & 213 & 800 & 7.172 & 7.172 & & 0.2131 & 1.77E-03 & 388.8132 & 143.2662 & 532.0794 & \\
\hline & $7 / 31 / 20089: 00$ & 212 & 213 & 900 & 6.485 & 6.485 & & 0.3416 & 3.17E-03 & 695.1399 & 256.1386 & 951.2785 & \\
\hline & $7 / 31 / 2008$ 10:00 & 213 & 213 & 1000 & 4.501 & 4.501 & & 0.9329 & 1.09E-02 & 2394.6801 & 882.3691 & 3277.0493 & \\
\hline & 7/31/2008 11:00 & 214 & 213 & 1100 & 4.067 & 4.067 & & 1.1392 & 1.40E-02 & 3062.2336 & 1128.3429 & 4190.5765 & \\
\hline & $7 / 31 / 2008$ 12:00 & 215 & 213 & 1200 & 3.723 & 3.723 & & 1.3368 & 1.70E-02 & 3728.8608 & 1373.9754 & 5102.8362 & \\
\hline & $7 / 31 / 2008$ 13:00 & 216 & 213 & 1300 & 3.846 & 3.846 & & 1.2621 & 1.58E-02 & 3473.9273 & 1280.0400 & 4753.9673 & \\
\hline & 7/31/2008 14:00 & 217 & 213 & 1400 & 3.626 & 3.626 & & 1.3993 & 1.80E-02 & 3944.6559 & 1453.4896 & 5398.1456 & \\
\hline & 7/31/2008 15:00 & 218 & 213 & 1500 & 3.623 & 3.623 & & 1.4013 & 1.80E-02 & 3951.5514 & 1456.0304 & 5407.5817 & \\
\hline & $7 / 31 / 2008$ 16:00 & 219 & 213 & 1600 & 3.76 & 3.76 & & 1.3138 & 1.67E-02 & 3650.0597 & 1344.9396 & 4994.9993 & \\
\hline & 7/31/2008 17:00 & 220 & 213 & 1700 & 3.814 & 3.814 & & 1.2811 & 1.61E-02 & 3538.3470 & 1303.7767 & 4842.1237 & \\
\hline & $7 / 31 / 2008$ 18:00 & 221 & 213 & 1800 & 3.873 & 3.873 & & 1.2463 & 1.56E-02 & 3420.5711 & 1260.3798 & 4680.9508 & \\
\hline & $7 / 31 / 2008$ 19:00 & 222 & 213 & 1900 & 4.331 & 4.331 & & 1.0088 & $1.20 \mathrm{E}-02$ & 2636.6263 & 971.5192 & 3608.1454 & \\
\hline & $7 / 31 / 200820: 00$ & 223 & 213 & 2000 & 5.184 & 5.184 & & 0.6782 & 7.38E-03 & 1617.3793 & 595.9567 & 2213.3360 & \\
\hline & $7 / 31 / 200821: 00$ & 224 & 213 & 2100 & 5.992 & 5.992 & & 0.4519 & 4.48E-03 & 981.2461 & 361.5603 & 1342.8064 & \\
\hline & $7 / 31 / 200822: 00$ & 225 & 213 & 2200 & 6.795 & 6.795 & & 0.2804 & $2.49 \mathrm{E}-03$ & 545.1347 & 200.8661 & 746.0008 & \\
\hline & 7/31/2008 23:00 & 226 & 213 & 2300 & 6.841 & 6.841 & & 0.2717 & 2.39E-03 & 524.6009 & 193.3000 & 717.9009 & \\
\hline & $8 / 1 / 20080: 00$ & 227 & 214 & 0 & 7.026 & 7.026 & 7.39 & 0.2383 & 2.04E-03 & 446.1938 & 164.4093 & 610.6031 & \\
\hline & 8/1/2008 1:00 & 228 & 214 & 100 & 7.246 & 7.246 & & 0.2007 & 1.65E-03 & 361.1720 & 133.0813 & 494.2532 & \\
\hline & 8/1/2008 2:00 & 229 & 214 & 200 & 7.318 & 7.318 & & 0.1888 & 1.53E-03 & 335.1789 & 123.5036 & 458.6825 & \\
\hline & $8 / 1 / 2008$ 3:00 & 230 & 214 & 300 & 7.333 & 7.333 & & 0.1864 & 1.50E-03 & 329.8736 & 121.5487 & 451.4224 & \\
\hline & 8/1/2008 4:00 & 231 & 214 & 400 & 7.353 & 7.353 & & 0.1832 & 1.47E-03 & 322.8583 & 118.9638 & 441.8221 & \\
\hline & $8 / 1 / 20085: 00$ & 232 & 214 & 500 & 7.335 & 7.335 & & 0.1861 & $1.50 \mathrm{E}-03$ & 329.1691 & 121.2891 & 450.4582 & \\
\hline & 8/1/2008 6:00 & 233 & 214 & 600 & 7.298 & 7.298 & & 0.1921 & 1.56E-03 & 342.3113 & 126.1316 & 468.4429 & \\
\hline & 8/1/2008 7:00 & 234 & 214 & 700 & 7.266 & 7.266 & & 0.1974 & 1.61E-03 & 353.8635 & 130.3883 & 484.2517 & \\
\hline & 8/1/2008 8:00 & 235 & 214 & 800 & 7.215 & 7.215 & & 0.2058 & 1.70E-03 & 372.6359 & 137.3054 & 509.9412 & \\
\hline & 8/1/2008 9:00 & 236 & 214 & 900 & 5.987 & 5.987 & & 0.4531 & 4.49E-03 & 984.4880 & 362.7549 & 1347.2429 & \\
\hline & 8/1/2008 10:00 & 237 & 214 & 1000 & 4.323 & 4.323 & & 1.0125 & 1.21E-02 & 2648.5918 & 975.9281 & 3624.5200 & \\
\hline & 8/1/2008 11:00 & 238 & 214 & 1100 & 4.006 & 4.006 & & 1.1717 & $1.45 \mathrm{E}-02$ & 3170.3755 & 1168.1900 & 4338.5655 & \\
\hline & 8/1/2008 12:00 & 239 & 214 & 1200 & 3.861 & 3.861 & & 1.2533 & 1.57E-02 & 3444.1737 & 1269.0766 & 4713.2503 & \\
\hline & 8/1/2008 13:00 & 240 & 214 & 1300 & 3.764 & 3.764 & & 1.3114 & 1.66E-02 & 3641.6522 & 1341.8417 & 4983.4939 & \\
\hline & 8/1/2008 14:00 & 241 & 214 & 1400 & 3.733 & 3.733 & & 1.3306 & 1.69E-02 & 3707.3779 & 1366.0596 & 5073.4375 & \\
\hline & 8/1/2008 15:00 & 242 & 214 & 1500 & 3.747 & 3.747 & & 1.3219 & 1.68E-02 & 3677.5333 & 1355.0628 & 5032.5961 & \\
\hline & $8 / 1 / 200816: 00$ & 243 & 214 & 1600 & 3.897 & 3.897 & & 1.2325 & $1.54 \mathrm{E}-02$ & 3373.8922 & 1243.1800 & 4617.0721 & \\
\hline & 8/1/2008 17:00 & 244 & 214 & 1700 & 3.992 & 3.992 & & 1.1794 & 1.46E-02 & 3195.7622 & 1177.5443 & 4373.3066 & \\
\hline & 8/1/2008 18:00 & 245 & 214 & 1800 & 4.052 & 4.052 & & 1.1471 & $1.41 \mathrm{E}-02$ & 3088.4591 & 1138.0063 & 4226.4653 & \\
\hline
\end{tabular}




\begin{tabular}{|c|c|c|c|c|c|c|c|c|c|c|c|c|c|}
\hline Tree ID & TS & RN & JD & HR & $\begin{array}{c}\text { Raw } \\
\text { TC_dTA }\end{array}$ & $\begin{array}{c}\text { Fixed } \\
\text { TC_dT } \Delta\end{array}$ & dTM & $\mathbf{K}$ & $\begin{array}{l}\text { Sap } \\
\text { Velocity } \\
(\mathrm{cm} / \mathrm{s})\end{array}$ & $\begin{array}{c}\text { Probed } \\
\text { Drywood F } \\
(\mathrm{g} / \mathrm{hr})\end{array}$ & $\begin{array}{c}\text { Extrapolated } \\
\text { Wetwood F } \\
(\mathrm{g} / \mathrm{hr})\end{array}$ & $\begin{array}{c}\text { Extrapolated } \\
\text { Total Wood F } \\
(\mathrm{g} / \mathrm{hr})\end{array}$ & $\begin{array}{l}\text { Relative } \\
\text { Humidity, } \\
@ 10 \mathrm{~m}(\%)\end{array}$ \\
\hline & 8/1/2008 19:00 & 246 & 214 & 1900 & \begin{tabular}{|r|}
4.293 \\
\end{tabular} & 4.293 & & 1.0266 & 1.23E-02 & 2693.9515 & 992.6418 & 3686.5933 & \\
\hline & 8/1/2008 20:00 & 247 & 214 & 2000 & 5.236 & 5.236 & & 0.6616 & $7.16 \mathrm{E}-03$ & 1568.5926 & 577.9802 & 2146.5728 & \\
\hline & 8/1/2008 21:00 & 248 & 214 & 2100 & 6.71 & 6.71 & & 0.2966 & $2.67 \mathrm{E}-03$ & 584.2128 & 215.2652 & 799.4780 & \\
\hline & 8/1/2008 22:00 & 249 & 214 & 2200 & 7.329 & 7.329 & & 0.1871 & $1.51 \mathrm{E}-03$ & 331.2847 & 122.0687 & 453.3534 & \\
\hline & 8/1/2008 23:00 & 250 & 214 & 2300 & 7.388 & 7.388 & & 0.1776 & 1.42E-03 & 310.7408 & 114.4988 & 425.2396 & \\
\hline & 8/2/2008 0:00 & 251 & 215 & 0 & 7.381 & 7.381 & 7.44 & 0.1787 & 1.43E-03 & 313.1482 & 115.3859 & 428.5340 & \\
\hline & 8/2/2008 1:00 & 252 & 215 & 100 & 7.422 & 7.422 & & 0.1722 & 1.36E-03 & 299.1621 & 110.2324 & 409.3945 & \\
\hline & 8/2/2008 2:00 & 253 & 215 & 200 & 7.438 & 7.438 & & 0.1697 & 1.34E-03 & 293.7784 & 108.2487 & 402.0271 & \\
\hline & 8/2/2008 3:00 & 254 & 215 & 300 & 7.439 & 7.439 & & 0.1695 & 1.34E-03 & 293.4433 & 108.1252 & 401.5685 & \\
\hline & 8/2/2008 4:00 & 255 & 215 & 400 & 7.409 & 7.409 & & 0.1742 & 1.38E-03 & 303.5669 & 111.8555 & 415.4224 & \\
\hline & 8/2/2008 5:00 & 256 & 215 & 500 & 7.379 & 7.379 & & 0.1790 & 1.43E-03 & 313.8374 & 115.6399 & 429.4773 & \\
\hline & 8/2/2008 6:00 & 257 & 215 & 600 & 7.359 & 7.359 & & 0.1822 & 1.46E-03 & 320.7667 & 118.1931 & 438.9597 & \\
\hline & 8/2/2008 7:00 & 258 & 215 & 700 & 7.216 & 7.216 & & 0.2057 & 1.70E-03 & 372.2635 & 137.1681 & 509.4316 & \\
\hline & 8/2/2008 8:00 & 259 & 215 & 800 & 6.148 & 6.148 & & 0.4151 & 4.03E-03 & 883.7248 & 325.6266 & 1209.3514 & \\
\hline & 8/2/2008 9:00 & 260 & 215 & 900 & 4.675 & 4.675 & & 0.8610 & $9.90 \mathrm{E}-03$ & 2169.4216 & 799.3680 & 2968.7897 & \\
\hline & 8/2/2008 10:00 & 261 & 215 & 1000 & 4.24 & 4.24 & & 1.0519 & 1.27E-02 & 2776.0173 & 1022.8807 & 3798.8980 & \\
\hline & 8/2/2008 11:00 & 262 & 215 & 1100 & 4.046 & 4.046 & & 1.1503 & 1.41E-02 & 3099.0155 & 1141.8960 & 4240.9115 & \\
\hline & $8 / 2 / 200812: 00$ & 263 & 215 & 1200 & 3.864 & 3.864 & & 1.2516 & 1.57E-02 & 3438.2564 & 1266.8963 & 4705.1527 & \\
\hline & 8/2/2008 13:00 & 264 & 215 & 1300 & 3.823 & 3.823 & & 1.2757 & 1.61E-02 & 3520.0974 & 1297.0523 & 4817.1497 & \\
\hline & 8/2/2008 14:00 & 265 & 215 & 1400 & 3.84 & 3.84 & & 1.2656 & $1.59 E-02$ & 3485.9074 & 1284.4543 & 4770.3616 & \\
\hline & $8 / 2 / 2008$ 15:00 & 266 & 215 & 1500 & 3.857 & 3.857 & & 1.2556 & 1.57E-02 & 3452.0807 & 1271.9901 & 4724.0708 & \\
\hline & 8/2/2008 16:00 & 267 & 215 & 1600 & 3.947 & 3.947 & & 1.2042 & 1.50E-02 & 3278.8449 & 1208.1579 & 4487.0028 & \\
\hline & 8/2/2008 17:00 & 268 & 215 & 1700 & 4.01 & 4.01 & & 1.1696 & 1.44E-02 & 3163.1616 & 1165.5319 & 4328.6935 & \\
\hline & $8 / 2 / 200818: 00$ & 269 & 215 & 1800 & 4.086 & 4.086 & & 1.1292 & 1.38E-02 & 3029.3508 & 1116.2266 & 4145.5773 & \\
\hline & 8/2/2008 19:00 & 270 & 215 & 1900 & 4.371 & 4.371 & & 0.9904 & 1.18E-02 & 2577.6057 & 949.7718 & 3527.3775 & \\
\hline & 8/2/2008 20:00 & 271 & 215 & 2000 & 5.083 & 5.083 & & 0.7116 & 7.83E-03 & 1715.8191 & 632.2288 & 2348.0479 & \\
\hline & 8/2/2008 21:00 & 272 & 215 & 2100 & 6.113 & 6.113 & & 0.4232 & 4.13E-03 & 905.0062 & 333.4682 & 1238.4744 & \\
\hline & 8/2/2008 22:00 & 273 & 215 & 2200 & 7.033 & 7.033 & & 0.2370 & $2.02 \mathrm{E}-03$ & 443.3543 & 163.3630 & 606.7174 & \\
\hline & 8/2/2008 23:00 & 274 & 215 & 2300 & 7.276 & 7.276 & & 0.1957 & 1.60E-03 & 350.2347 & 129.0512 & 479.2859 & \\
\hline & 8/3/2008 0:00 & 275 & 216 & 0 & 7.278 & 7.278 & 7.42 & 0.1954 & 1.59E-03 & 349.5110 & 128.7845 & 478.2956 & \\
\hline & 8/3/2008 1:00 & 276 & 216 & 100 & 7.213 & 7.213 & & 0.2062 & 1.70E-03 & 373.3812 & 137.5800 & 510.9612 & \\
\hline & 8/3/2008 2:00 & 277 & 216 & 200 & 7.234 & 7.234 & & 0.2027 & 1.67E-03 & 365.5900 & 134.7092 & 500.2992 & \\
\hline & 8/3/2008 3:00 & 278 & 216 & 300 & 7.341 & 7.341 & & 0.1851 & 1.49E-03 & 327.0595 & 120.5118 & 447.5713 & \\
\hline & 8/3/2008 4:00 & 279 & 216 & 400 & 7.407 & 7.407 & & 0.1746 & 1.39E-03 & 304.2471 & 112.1061 & 416.3531 & \\
\hline & 8/3/2008 5:00 & 280 & 216 & 500 & 7.415 & 7.415 & & 0.1733 & 1.38E-03 & 301.5305 & 111.1051 & 412.6356 & \\
\hline & 8/3/2008 6:00 & 281 & 216 & 600 & 7.413 & 7.413 & & 0.1736 & 1.38E-03 & 302.2087 & 111.3550 & 413.5637 & \\
\hline & 8/3/2008 7:00 & 282 & 216 & 700 & 7.363 & 7.363 & & 0.1816 & 1.46E-03 & 319.3755 & 117.6805 & 437.0560 & \\
\hline & 8/3/2008 8:00 & 283 & 216 & 800 & 6.588 & 6.588 & & 0.3206 & 2.93E-03 & 642.9702 & 236.9156 & 879.8858 & \\
\hline & 8/3/2008 9:00 & 284 & 216 & 900 & 4.751 & 4.751 & & 0.8312 & $9.48 \mathrm{E}-03$ & 2077.4550 & 765.4810 & 2842.9360 & \\
\hline & 8/3/2008 10:00 & 285 & 216 & 1000 & 4.001 & 4.001 & & 1.1745 & $1.45 \mathrm{E}-02$ & 3179.4174 & 1171.5217 & 4350.9391 & \\
\hline & 8/3/2008 11:00 & 286 & 216 & 1100 & 3.788 & 3.788 & & 1.2967 & 1.64E-02 & 3591.6557 & 1323.4194 & 4915.0751 & \\
\hline
\end{tabular}




\begin{tabular}{|c|c|c|c|c|c|c|c|c|c|c|c|c|c|}
\hline Tree ID & TS & RN & JD & HR & $\begin{array}{c}\text { Raw } \\
\text { TC_dTA }\end{array}$ & $\begin{array}{c}\text { Fixed } \\
\text { TC_dT } \Delta\end{array}$ & dTM & $\mathbf{K}$ & $\begin{array}{l}\text { Sap } \\
\text { Velocity } \\
(\mathrm{cm} / \mathrm{s})\end{array}$ & $\begin{array}{c}\text { Probed } \\
\text { Drywood F } \\
(\mathrm{g} / \mathrm{hr})\end{array}$ & $\begin{array}{c}\text { Extrapolated } \\
\text { Wetwood F } \\
(\mathrm{g} / \mathrm{hr})\end{array}$ & $\begin{array}{c}\text { Extrapolated } \\
\text { Total Wood F } \\
(\mathrm{g} / \mathrm{hr})\end{array}$ & $\begin{array}{l}\text { Relative } \\
\text { Humidity, } \\
@ 10 \mathrm{~m}(\%)\end{array}$ \\
\hline & 8/3/2008 12:00 & 287 & 216 & 1200 & 3.758 & 3.758 & & 1.3151 & 1.67E-02 & 3654.2715 & 1346.4915 & 5000.7630 & \\
\hline & 8/3/2008 13:00 & 288 & 216 & 1300 & 3.78 & 3.78 & & 1.3016 & $1.65 \mathrm{E}-02$ & 3608.2363 & 1329.5289 & 4937.7651 & \\
\hline & $8 / 3 / 2008$ 14:00 & 289 & 216 & 1400 & 3.803 & 3.803 & & 1.2877 & $1.62 \mathrm{E}-02$ & 3560.7935 & 1312.0476 & 4872.8410 & \\
\hline & 8/3/2008 15:00 & 290 & 216 & 1500 & 3.862 & 3.862 & & 1.2527 & 1.57E-02 & 3442.2001 & 1268.3494 & 4710.5495 & \\
\hline & 8/3/2008 16:00 & 291 & 216 & 1600 & 3.967 & 3.967 & & 1.1931 & 1.48E-02 & 3241.6371 & 1194.4479 & 4436.0850 & \\
\hline & 8/3/2008 17:00 & 292 & 216 & 1700 & 4.047 & 4.047 & & 1.1497 & 1.41E-02 & 3097.2534 & 1141.2467 & 4238.5002 & \\
\hline & 8/3/2008 18:00 & 293 & 216 & 1800 & 4.139 & 4.139 & & 1.1020 & 1.34E-02 & 2939.5646 & 1083.1430 & 4022.7077 & \\
\hline & 8/3/2008 19:00 & 294 & 216 & 1900 & 4.371 & 4.371 & & 0.9904 & 1.18E-02 & 2577.6057 & 949.7718 & 3527.3775 & \\
\hline & 8/3/2008 20:00 & 295 & 216 & 2000 & 4.881 & 4.881 & & 0.7824 & 8.80E-03 & 1928.4306 & 710.5699 & 2639.0005 & \\
\hline & 8/3/2008 21:00 & 296 & 216 & 2100 & 5.708 & 5.708 & & 0.5242 & 5.37E-03 & 1177.7559 & 433.9684 & 1611.7243 & \\
\hline & 8/3/2008 22:00 & 297 & 216 & 2200 & 6.606 & 6.606 & & 0.3170 & 2.89E-03 & 634.0978 & 233.6464 & 867.7442 & \\
\hline & 8/3/2008 23:00 & 298 & 216 & 2300 & 6.944 & 6.944 & & 0.2529 & $2.19 E-03$ & 480.1384 & 176.9169 & 657.0553 & \\
\hline & 8/4/2008 0:00 & 299 & 217 & 0 & 7.013 & 7.013 & 7.42 & 0.2406 & 2.06E-03 & 451.4913 & 166.3613 & 617.8525 & \\
\hline & 8/4/2008 1:00 & 300 & 217 & 100 & 7.025 & 7.025 & & 0.2384 & 2.04E-03 & 446.6002 & 164.5590 & 611.1593 & \\
\hline & $8 / 4 / 2008$ 2:00 & 301 & 217 & 200 & 6.763 & 6.763 & & 0.2864 & $2.55 E-03$ & 559.6717 & 206.2225 & 765.8942 & \\
\hline & 8/4/2008 3:00 & 302 & 217 & 300 & 6.531 & 6.531 & & 0.3321 & 3.06E-03 & 671.5430 & 247.4438 & 918.9869 & \\
\hline & 8/4/2008 4:00 & 303 & 217 & 400 & 6.272 & 6.272 & & 0.3871 & 3.70E-03 & 810.9846 & 298.8240 & 1109.8086 & \\
\hline & $8 / 4 / 20085: 00$ & 304 & 217 & 500 & 6.25 & 6.25 & & 0.3920 & 3.76E-03 & 823.5946 & 303.4704 & 1127.0649 & \\
\hline & 8/4/2008 6:00 & 305 & 217 & 600 & 6.488 & 6.488 & & 0.3409 & $3.16 \mathrm{E}-03$ & 693.5861 & 255.5661 & 949.1521 & \\
\hline & 8/4/2008 7:00 & 306 & 217 & 700 & 6.387 & 6.387 & & 0.3621 & $3.41 E-03$ & 747.0644 & 275.2712 & 1022.3356 & \\
\hline & 8/4/2008 8:00 & 307 & 217 & 800 & 6.175 & 6.175 & & 0.4089 & 3.96E-03 & 867.5370 & 319.6618 & 1187.1988 & \\
\hline & 8/4/2008 9:00 & 308 & 217 & 900 & 5.805 & 5.805 & & 0.4987 & 5.05E-03 & 1107.7128 & 408.1596 & 1515.8724 & \\
\hline & 8/4/2008 10:00 & 309 & 217 & 1000 & 5.014 & 5.014 & & 0.7351 & 8.15E-03 & 1785.9983 & 658.0878 & 2444.0861 & \\
\hline & 8/4/2008 11:00 & 310 & 217 & 1100 & 4.45 & 4.45 & & 0.9551 & 1.12E-02 & 2464.8695 & 908.2319 & 3373.1014 & \\
\hline & 8/4/2008 12:00 & 311 & 217 & 1200 & 4.12 & 4.12 & & 1.1117 & 1.36E-02 & 2971.4283 & 1094.8839 & 4066.3122 & \\
\hline & 8/4/2008 13:00 & 312 & 217 & 1300 & 4.089 & 4.089 & & 1.1277 & 1.38E-02 & 3024.1927 & 1114.3260 & 4138.5187 & \\
\hline & 8/4/2008 14:00 & 313 & 217 & 1400 & 4.403 & 4.403 & & 0.9759 & 1.15E-02 & 2531.3387 & 932.7238 & 3464.0624 & \\
\hline & 8/4/2008 15:00 & 314 & 217 & 1500 & 4.712 & 4.712 & & 0.8463 & $9.69 \mathrm{E}-03$ & 2124.1844 & 782.6994 & 2906.8839 & \\
\hline & 8/4/2008 16:00 & 315 & 217 & 1600 & 4.244 & 4.244 & & 1.0500 & 1.26E-02 & 2769.7359 & 1020.5662 & 3790.3021 & \\
\hline & 8/4/2008 17:00 & 316 & 217 & 1700 & 4.224 & 4.224 & & 1.0597 & $1.28 \mathrm{E}-02$ & 2801.2888 & 1032.1925 & 3833.4813 & \\
\hline & 8/4/2008 18:00 & 317 & 217 & 1800 & 4.327 & 4.327 & & 1.0106 & 1.21E-02 & 2642.6023 & 973.7211 & 3616.3234 & \\
\hline & 8/4/2008 19:00 & 318 & 217 & 1900 & 4.633 & 4.633 & & 0.8778 & 1.01E-02 & 2221.8685 & 818.6931 & 3040.5616 & \\
\hline & 8/4/2008 20:00 & 319 & 217 & 2000 & 5.276 & 5.276 & & 0.6490 & 6.99E-03 & 1531.9063 & 564.4624 & 2096.3686 & \\
\hline & 8/4/2008 21:00 & 320 & 217 & 2100 & 6.529 & 6.529 & & 0.3325 & 3.07E-03 & 672.5589 & 247.8182 & 920.3771 & \\
\hline & 8/4/2008 22:00 & 321 & 217 & 2200 & 7.265 & 7.265 & & 0.1975 & 1.62E-03 & 354.2273 & 130.5223 & 484.7496 & \\
\hline & 8/4/2008 23:00 & 322 & 217 & 2300 & 7.423 & 7.423 & & 0.1720 & 1.36E-03 & 298.8244 & 110.1080 & 408.9324 & \\
\hline & 8/5/2008 0:00 & 323 & 218 & 0 & 7.503 & 7.503 & 7.55 & 0.1595 & 1.24E-03 & 272.3316 & 100.3462 & 372.6778 & \\
\hline & 8/5/2008 1:00 & 324 & 218 & 100 & 7.539 & 7.539 & & 0.1540 & 1.19E-03 & 260.7436 & 96.0763 & 356.8199 & \\
\hline & 8/5/2008 2:00 & 325 & 218 & 200 & 7.554 & 7.554 & & 0.1517 & $1.17 \mathrm{E}-03$ & 255.9757 & 94.3195 & 350.2952 & \\
\hline & 8/5/2008 3:00 & 326 & 218 & 300 & 7.508 & 7.508 & & 0.1588 & 1.24E-03 & 270.7098 & 99.7486 & 370.4584 & \\
\hline & 8/5/2008 4:00 & 327 & 218 & 400 & 7.446 & 7.446 & & 0.1684 & 1.33E-03 & 291.1021 & 107.2626 & 398.3647 & \\
\hline
\end{tabular}




\begin{tabular}{|c|c|c|c|c|c|c|c|c|c|c|c|c|c|}
\hline Tree ID & TS & RN & JD & HR & $\begin{array}{c}\text { Raw } \\
\text { TC_dTA }\end{array}$ & $\begin{array}{c}\text { Fixed } \\
\text { TC_dT } \Delta\end{array}$ & dTM & $\mathbf{K}$ & $\begin{array}{l}\text { Sap } \\
\text { Velocity } \\
(\mathrm{cm} / \mathrm{s})\end{array}$ & $\begin{array}{c}\text { Probed } \\
\text { Drywood F } \\
(\mathrm{g} / \mathrm{hr})\end{array}$ & $\begin{array}{c}\text { Extrapolated } \\
\text { Wetwood F } \\
(\mathrm{g} / \mathrm{hr})\end{array}$ & $\begin{array}{c}\text { Extrapolated } \\
\text { Total Wood F } \\
(\mathrm{g} / \mathrm{hr})\end{array}$ & $\begin{array}{l}\text { Relative } \\
\text { Humidity, } \\
@ 10 \mathrm{~m}(\%)\end{array}$ \\
\hline & 8/5/2008 5:00 & 328 & 218 & 500 & 7.44 & 7.44 & & 0.1694 & 1.34E-03 & 293.1083 & 108.0018 & \begin{tabular}{|r|}
401.1101 \\
\end{tabular} & \\
\hline & 8/5/2008 6:00 & 329 & 218 & 600 & 7.454 & 7.454 & & 0.1672 & $1.32 \mathrm{E}-03$ & 288.4362 & 106.2802 & 394.7164 & \\
\hline & 8/5/2008 7:00 & 330 & 218 & 700 & 7.415 & 7.415 & & 0.1733 & $1.38 E-03$ & 301.5305 & 111.1051 & 412.6356 & \\
\hline & 8/5/2008 8:00 & 331 & 218 & 800 & 7.158 & 7.158 & & 0.2154 & $1.80 \mathrm{E}-03$ & 394.1501 & 145.2327 & 539.3828 & \\
\hline & 8/5/2008 9:00 & 332 & 218 & 900 & 5.896 & 5.896 & & 0.4756 & 4.77E-03 & 1044.8088 & 384.9813 & 1429.7901 & \\
\hline & 8/5/2008 10:00 & 333 & 218 & 1000 & 4.686 & 4.686 & & 0.8566 & 9.84E-03 & 2155.8794 & 794.3781 & 2950.2575 & \\
\hline & 8/5/2008 11:00 & 334 & 218 & 1100 & 4.673 & 4.673 & & 0.8618 & 9.91E-03 & 2171.8924 & 800.2784 & 2972.1709 & \\
\hline & 8/5/2008 12:00 & 335 & 218 & 1200 & 4.371 & 4.371 & & 0.9904 & 1.18E-02 & 2577.6057 & 949.7718 & 3527.3775 & \\
\hline & 8/5/2008 13:00 & 336 & 218 & 1300 & 3.979 & 3.979 & & 1.1865 & 1.47E-02 & 3219.5299 & 1186.3020 & 4405.8320 & \\
\hline & 8/5/2008 14:00 & 337 & 218 & 1400 & 4.406 & 4.406 & & 0.9746 & 1.15E-02 & 2527.0436 & 931.1412 & 3458.1848 & \\
\hline & 8/5/2008 15:00 & 338 & 218 & 1500 & 4.756 & 4.756 & & 0.8293 & $9.45 E-03$ & 2071.5335 & 763.2991 & 2834.8326 & \\
\hline & 8/5/2008 16:00 & 339 & 218 & 1600 & 4.695 & 4.695 & & 0.8530 & $9.79 \mathrm{E}-03$ & 2144.8583 & 790.3172 & 2935.1755 & \\
\hline & 8/5/2008 17:00 & 340 & 218 & 1700 & 4.319 & 4.319 & & 1.0144 & $1.21 \mathrm{E}-02$ & 2654.5951 & 978.1401 & 3632.7352 & \\
\hline & 8/5/2008 18:00 & 341 & 218 & 1800 & 4.154 & 4.154 & & 1.0944 & 1.33E-02 & 2914.6601 & 1073.9664 & 3988.6265 & \\
\hline & 8/5/2008 19:00 & 342 & 218 & 1900 & 4.343 & 4.343 & & 1.0032 & 1.19E-02 & 2618.7795 & 964.9432 & 3583.7227 & \\
\hline & 8/5/2008 20:00 & 343 & 218 & 2000 & 5.345 & 5.345 & & 0.6277 & $6.71 \mathrm{E}-03$ & 1470.2873 & 541.7576 & 2012.0449 & \\
\hline & 8/5/2008 21:00 & 344 & 218 & 2100 & 6.931 & 6.931 & & 0.2552 & 2.22E-03 & 485.6367 & 178.9428 & 664.5796 & \\
\hline & $8 / 5 / 200822: 00$ & 345 & 218 & 2200 & 7.352 & 7.352 & & 0.1834 & 1.47E-03 & 323.2075 & 119.0924 & 442.2999 & \\
\hline & 8/5/2008 23:00 & 346 & 218 & 2300 & 7.411 & 7.411 & & 0.1739 & $1.38 \mathrm{E}-03$ & 302.8875 & 111.6051 & 414.4926 & \\
\hline & 8/6/2008 0:00 & 347 & 219 & 0 & 7.38 & 7.38 & 7.38 & 0.1789 & 1.43E-03 & 313.4927 & 115.5128 & 429.0056 & \\
\hline & 8/6/2008 1:00 & 348 & 219 & 100 & 7.321 & 7.321 & & 0.1884 & $1.52 \mathrm{E}-03$ & 334.1148 & 123.1115 & 457.2263 & \\
\hline & $8 / 6 / 2008$ 2:00 & 349 & 219 & 200 & 7.278 & 7.278 & & 0.1954 & 1.59E-03 & 349.5110 & 128.7845 & 478.2956 & \\
\hline & 8/6/2008 3:00 & 350 & 219 & 300 & 7.244 & 7.244 & & 0.2010 & 1.65E-03 & 361.9066 & 133.3519 & 495.2586 & \\
\hline & 8/6/2008 4:00 & 351 & 219 & 400 & 7.225 & 7.225 & & 0.2042 & $1.68 \mathrm{E}-03$ & 368.9198 & 135.9361 & 504.8558 & \\
\hline & 8/6/2008 5:00 & 352 & 219 & 500 & 7.187 & 7.187 & & 0.2105 & 1.75E-03 & 383.1333 & 141.1733 & 524.3066 & \\
\hline & 8/6/2008 6:00 & 353 & 219 & 600 & 7.164 & 7.164 & & 0.2144 & 1.79E-03 & 391.8586 & 144.3884 & 536.2470 & \\
\hline & 8/6/2008 7:00 & 354 & 219 & 700 & 7.156 & 7.156 & & 0.2158 & 1.80E-03 & 394.9153 & 145.5147 & 540.4300 & \\
\hline & 8/6/2008 8:00 & 355 & 219 & 800 & 7.147 & 7.147 & & 0.2173 & 1.82E-03 & 398.3676 & 146.7867 & 545.1543 & \\
\hline & 8/6/2008 9:00 & 356 & 219 & 900 & 7.185 & 7.185 & & 0.2109 & 1.75E-03 & 383.8883 & 141.4516 & 525.3399 & \\
\hline & 8/6/2008 10:00 & 357 & 219 & 1000 & 4.971 & 4.971 & & 0.7502 & 8.35E-03 & 1830.9913 & 674.6664 & 2505.6577 & \\
\hline & 8/6/2008 11:00 & 358 & 219 & 1100 & 3.873 & 3.873 & & 1.2463 & 1.56E-02 & 3420.5711 & 1260.3798 & 4680.9508 & \\
\hline & 8/6/2008 12:00 & 359 & 219 & 1200 & 4.213 & 4.213 & & 1.0650 & 1.29E-02 & 2818.7994 & 1038.6446 & 3857.4440 & \\
\hline & 8/6/2008 13:00 & 360 & 219 & 1300 & 4.767 & 4.767 & & 0.8250 & 9.39E-03 & 2058.5609 & 758.5191 & 2817.0801 & \\
\hline & $8 / 6 / 2008$ 14:00 & 361 & 219 & 1400 & 4.364 & 4.364 & & 0.9936 & 1.18E-02 & 2587.8381 & 953.5422 & 3541.3803 & \\
\hline & 8/6/2008 15:00 & 362 & 219 & 1500 & 3.85 & 3.85 & & 1.2597 & 1.58E-02 & 3465.9656 & 1277.1063 & 4743.0720 & \\
\hline & 8/6/2008 16:00 & 363 & 219 & 1600 & 3.937 & 3.937 & & 1.2098 & 1.50E-02 & 3297.6207 & 1215.0762 & 4512.6969 & \\
\hline & 8/6/2008 17:00 & 364 & 219 & 1700 & 3.874 & 3.874 & & 1.2457 & $1.56 \mathrm{E}-02$ & 3418.6122 & 1259.6580 & 4678.2701 & \\
\hline & 8/6/2008 18:00 & 365 & 219 & 1800 & 4.346 & 4.346 & & 1.0018 & 1.19E-02 & 2614.3368 & 963.3062 & 3577.6430 & \\
\hline & 8/6/2008 19:00 & 366 & 219 & 1900 & 4.471 & 4.471 & & 0.9459 & $1.11 \mathrm{E}-02$ & 2435.7279 & 897.4940 & 3333.2220 & \\
\hline & $8 / 6 / 200820: 00$ & 367 & 219 & 2000 & 5.052 & 5.052 & & 0.7221 & 7.97E-03 & 1747.0465 & 643.7352 & 2390.7817 & \\
\hline & 8/6/2008 21:00 & 368 & 219 & 2100 & 6.333 & 6.333 & & 0.3738 & $3.54 \mathrm{E}-03$ & 776.6675 & 286.1791 & 1062.8466 & \\
\hline
\end{tabular}




\begin{tabular}{|c|c|c|c|c|c|c|c|c|c|c|c|c|c|}
\hline Tree ID & TS & RN & JD & HR & $\begin{array}{c}\text { Raw } \\
\text { TC_dTA }\end{array}$ & $\begin{array}{c}\text { Fixed } \\
\text { TC_dT } \Delta\end{array}$ & dTM & $\mathbf{K}$ & $\begin{array}{l}\text { Sap } \\
\text { Velocity } \\
(\mathrm{cm} / \mathrm{s})\end{array}$ & $\begin{array}{c}\text { Probed } \\
\text { Drywood F } \\
(\mathrm{g} / \mathrm{hr})\end{array}$ & $\begin{array}{c}\text { Extrapolated } \\
\text { Wetwood F } \\
(\mathrm{g} / \mathrm{hr})\end{array}$ & $\begin{array}{c}\text { Extrapolated } \\
\text { Total Wood F } \\
(\mathrm{g} / \mathrm{hr})\end{array}$ & $\begin{array}{l}\text { Relative } \\
\text { Humidity, } \\
@ 10 \mathrm{~m}(\%)\end{array}$ \\
\hline & 8/6/2008 22:00 & 369 & 219 & 2200 & \begin{tabular}{|r|}
7.122 \\
\end{tabular} & 7.122 & & 0.2216 & 1.86E-03 & 408.0328 & 150.3481 & $\begin{array}{l}558.3808 \\
\end{array}$ & \\
\hline & 8/6/2008 23:00 & 370 & 219 & 2300 & 7.226 & 7.226 & & 0.2040 & $1.68 \mathrm{E}-03$ & 368.5491 & 135.7995 & 504.3486 & \\
\hline & $8 / 7 / 20080: 00$ & 371 & 220 & 0 & 7.231 & 7.231 & 7.33 & 0.2032 & $1.67 \mathrm{E}-03$ & 366.6984 & 135.1176 & 501.8159 & \\
\hline & 8/7/2008 1:00 & 372 & 220 & 100 & 7.209 & 7.209 & & 0.2068 & 1.71E-03 & 374.8739 & 138.1300 & 513.0038 & \\
\hline & 8/7/2008 2:00 & 373 & 220 & 200 & 7.192 & 7.192 & & 0.2097 & 1.74E-03 & 381.2488 & 140.4789 & 521.7277 & \\
\hline & 8/7/2008 3:00 & 374 & 220 & 300 & 7.182 & 7.182 & & 0.2114 & 1.76E-03 & 385.0222 & 141.8694 & 526.8916 & \\
\hline & 8/7/2008 4:00 & 375 & 220 & 400 & 7.155 & 7.155 & & 0.2159 & 1.80E-03 & 395.2982 & 145.6557 & 540.9539 & \\
\hline & 8/7/2008 5:00 & 376 & 220 & 500 & 7.145 & 7.145 & & 0.2176 & 1.82E-03 & 399.1367 & 147.0701 & 546.2069 & \\
\hline & 8/7/2008 6:00 & 377 & 220 & 600 & 7.129 & 7.129 & & 0.2204 & 1.85E-03 & 405.3153 & 149.3468 & 554.6620 & \\
\hline & 8/7/2008 7:00 & 378 & 220 & 700 & 7.117 & 7.117 & & 0.2224 & 1.87E-03 & 409.9792 & 151.0653 & 561.0444 & \\
\hline & 8/7/2008 8:00 & 379 & 220 & 800 & 7.118 & 7.118 & & 0.2223 & 1.87E-03 & 409.5895 & 150.9217 & 560.5112 & \\
\hline & 8/7/2008 9:00 & 380 & 220 & 900 & 6.865 & 6.865 & & 0.2673 & $2.35 E-03$ & 514.0553 & 189.4142 & 703.4695 & \\
\hline & 8/7/2008 10:00 & 381 & 220 & 1000 & 4.461 & 4.461 & & 0.9502 & 1.12E-02 & 2449.5625 & 902.5917 & 3352.1542 & \\
\hline & 8/7/2008 11:00 & 382 & 220 & 1100 & 3.858 & 3.858 & & 1.2551 & 1.57E-02 & 3450.1021 & 1271.2611 & 4721.3632 & \\
\hline & 8/7/2008 12:00 & 383 & 220 & 1200 & 3.659 & 3.659 & & 1.3777 & 1.77E-02 & 3869.6997 & 1425.8704 & 5295.5701 & \\
\hline & 8/7/2008 13:00 & 384 & 220 & 1300 & 3.618 & 3.618 & & 1.4046 & 1.81E-02 & 3963.0742 & 1460.2762 & 5423.3503 & \\
\hline & $8 / 7 / 2008$ 14:00 & 385 & 220 & 1400 & 3.623 & 3.623 & & 1.4013 & 1.80E-02 & 3951.5514 & 1456.0304 & 5407.5817 & \\
\hline & 8/7/2008 15:00 & 386 & 220 & 1500 & 3.699 & 3.699 & & 1.3520 & 1.72E-02 & 3780.9898 & 1393.1835 & 5174.1733 & \\
\hline & 8/7/2008 16:00 & 387 & 220 & 1600 & 3.756 & 3.756 & & 1.3163 & 1.67E-02 & 3658.4888 & 1348.0454 & 5006.5342 & \\
\hline & 8/7/2008 17:00 & 388 & 220 & 1700 & 3.793 & 3.793 & & 1.2937 & 1.63E-02 & 3581.3356 & 1319.6168 & 4900.9524 & \\
\hline & 8/7/2008 18:00 & 389 & 220 & 1800 & 3.874 & 3.874 & & 1.2457 & 1.56E-02 & 3418.6122 & 1259.6580 & 4678.2701 & \\
\hline & 8/7/2008 19:00 & 390 & 220 & 1900 & 4.317 & 4.317 & & 1.0153 & 1.21E-02 & 2657.6018 & 979.2480 & 3636.8499 & \\
\hline & 8/7/2008 20:00 & 391 & 220 & 2000 & 5.136 & 5.136 & & 0.6939 & 7.59E-03 & 1663.5438 & 612.9669 & 2276.5107 & \\
\hline & 8/7/2008 21:00 & 392 & 220 & 2100 & 6.534 & 6.534 & & 0.3315 & 3.06E-03 & 670.0209 & 246.8830 & 916.9039 & \\
\hline & 8/7/2008 22:00 & 393 & 220 & 2200 & 7.166 & 7.166 & & 0.2141 & 1.78E-03 & 391.0962 & 144.1074 & 535.2036 & \\
\hline & 8/7/2008 23:00 & 394 & 220 & 2300 & 7.334 & 7.334 & & 0.1863 & 1.50E-03 & 329.5213 & 121.4189 & 450.9402 & \\
\hline & 8/8/2008 0:00 & 395 & 221 & 0 & 7.356 & 7.356 & 7.36 & 0.1827 & 1.47E-03 & 321.8117 & 118.5782 & 440.3899 & \\
\hline & 8/8/2008 1:00 & 396 & 221 & 100 & 7.354 & 7.354 & & 0.1830 & $1.47 \mathrm{E}-03$ & 322.5093 & 118.8352 & 441.3445 & \\
\hline & 8/8/2008 2:00 & 397 & 221 & 200 & 7.341 & 7.341 & & 0.1851 & 1.49E-03 & 327.0595 & 120.5118 & 447.5713 & \\
\hline & 8/8/2008 3:00 & 398 & 221 & 300 & 7.277 & 7.277 & & 0.1955 & $1.60 \mathrm{E}-03$ & 349.8728 & 128.9178 & 478.7906 & \\
\hline & 8/8/2008 4:00 & 399 & 221 & 400 & 7.247 & 7.247 & & 0.2005 & 1.65E-03 & 360.8049 & 132.9460 & 493.7510 & \\
\hline & 8/8/2008 5:00 & 400 & 221 & 500 & 7.23 & 7.23 & & 0.2033 & 1.67E-03 & 367.0682 & 135.2538 & 502.3220 & \\
\hline & 8/8/2008 6:00 & 401 & 221 & 600 & 7.21 & 7.21 & & 0.2067 & 1.71E-03 & 374.5004 & 137.9924 & 512.4928 & \\
\hline & $8 / 8 / 20087: 00$ & 402 & 221 & 700 & 7.205 & 7.205 & & 0.2075 & $1.72 \mathrm{E}-03$ & 376.3693 & 138.6810 & 515.0503 & \\
\hline & 8/8/2008 8:00 & 403 & 221 & 800 & 7.185 & 7.185 & & 0.2109 & 1.75E-03 & 383.8883 & 141.4516 & 525.3399 & \\
\hline & 8/8/20089:00 & 404 & 221 & 900 & 5.728 & 5.728 & & 0.5189 & 5.31E-03 & 1163.0535 & 428.5510 & 1591.6045 & \\
\hline & 8/8/2008 10:00 & 405 & 221 & 1000 & 4.366 & 4.366 & & 0.9927 & 1.18E-02 & 2584.9105 & 952.4634 & 3537.3739 & \\
\hline & 8/8/2008 11:00 & 406 & 221 & 1100 & 4.063 & 4.063 & & 1.1413 & 1.40E-02 & 3069.2040 & 1130.9113 & 4200.1154 & \\
\hline & $8 / 8 / 200812: 00$ & 407 & 221 & 1200 & 3.771 & 3.771 & & 1.3071 & $1.65 \mathrm{E}-02$ & 3626.9907 & 1336.4393 & 4963.4301 & \\
\hline & 8/8/2008 13:00 & 408 & 221 & 1300 & 3.672 & 3.672 & & 1.3693 & 1.75E-02 & 3840.6145 & 1415.1534 & 5255.7679 & \\
\hline & 8/8/2008 14:00 & 409 & 221 & 1400 & 3.663 & 3.663 & & 1.3751 & $1.76 \mathrm{E}-02$ & 3860.7240 & 1422.5632 & 5283.2872 & \\
\hline
\end{tabular}




\begin{tabular}{|c|c|c|c|c|c|c|c|c|c|c|c|c|c|}
\hline Tree ID & TS & RN & JD & HR & $\begin{array}{c}\text { Raw } \\
\text { TC_dTA }\end{array}$ & $\begin{array}{c}\text { Fixed } \\
\text { TC_dT } \Delta\end{array}$ & dTM & $\mathbf{K}$ & $\begin{array}{l}\text { Sap } \\
\text { Velocity } \\
(\mathrm{cm} / \mathrm{s})\end{array}$ & $\begin{array}{c}\text { Probed } \\
\text { Drywood F } \\
(\mathrm{g} / \mathrm{hr})\end{array}$ & $\begin{array}{c}\text { Extrapolated } \\
\text { Wetwood F } \\
(\mathrm{g} / \mathrm{hr})\end{array}$ & $\begin{array}{c}\text { Extrapolated } \\
\text { Total Wood F } \\
(\mathrm{g} / \mathrm{hr})\end{array}$ & $\begin{array}{l}\text { Relative } \\
\text { Humidity, } \\
@ 10 \mathrm{~m}(\%)\end{array}$ \\
\hline & 8/8/2008 15:00 & 410 & 221 & 1500 & 3.71 & 3.71 & & 1.3450 & 1.71E-02 & 3756.9968 & 1384.3427 & $\begin{array}{r}5141.3395 \\
\end{array}$ & \\
\hline & 8/8/2008 16:00 & 411 & 221 & 1600 & 3.766 & 3.766 & & 1.3101 & $1.66 \mathrm{E}-02$ & 3637.4565 & 1340.2957 & 4977.7522 & \\
\hline & 8/8/2008 17:00 & 412 & 221 & 1700 & 3.944 & 3.944 & & 1.2059 & $1.50 \mathrm{E}-02$ & 3284.4656 & 1210.2289 & 4494.6945 & \\
\hline & 8/8/2008 18:00 & 413 & 221 & 1800 & 4.191 & 4.191 & & 1.0759 & 1.30E-02 & 2854.1583 & 1051.6733 & 3905.8317 & \\
\hline & 8/8/2008 19:00 & 414 & 221 & 1900 & 4.236 & 4.236 & & 1.0538 & 1.27E-02 & 2782.3133 & 1025.2006 & 3807.5138 & \\
\hline & 8/8/2008 20:00 & 415 & 221 & 2000 & 5.024 & 5.024 & & 0.7317 & 8.10E-03 & 1775.6750 & 654.2840 & 2429.9590 & \\
\hline & 8/8/2008 21:00 & 416 & 221 & 2100 & 6.195 & 6.195 & & 0.4044 & $3.90 \mathrm{E}-03$ & 855.6729 & 315.2903 & 1170.9632 & \\
\hline & 8/8/2008 22:00 & 417 & 221 & 2200 & 7.036 & 7.036 & & 0.2365 & 2.02E-03 & 442.1402 & 162.9156 & 605.0558 & \\
\hline & 8/8/2008 23:00 & 418 & 221 & 2300 & 7.259 & 7.259 & & 0.1985 & 1.63E-03 & 356.4137 & 131.3279 & 487.7416 & \\
\hline & 8/9/2008 0:00 & 419 & 222 & 0 & 7.297 & 7.297 & 7.30 & 0.1923 & 1.56E-03 & 342.6696 & 126.2637 & 468.9333 & \\
\hline & 8/9/2008 1:00 & 420 & 222 & 100 & 7.259 & 7.259 & & 0.1985 & 1.63E-03 & 356.4137 & 131.3279 & 487.7416 & \\
\hline & 8/9/2008 2:00 & 421 & 222 & 200 & 7.269 & 7.269 & & 0.1969 & $1.61 \mathrm{E}-03$ & 352.7731 & 129.9865 & 482.7595 & \\
\hline & 8/9/2008 3:00 & 422 & 222 & 300 & 7.254 & 7.254 & & 0.1993 & 1.63E-03 & 358.2404 & 132.0010 & 490.2414 & \\
\hline & 8/9/2008 4:00 & 423 & 222 & 400 & 7.197 & 7.197 & & 0.2088 & 1.73E-03 & 379.3686 & 139.7861 & 519.1547 & \\
\hline & $8 / 9 / 20085: 00$ & 424 & 222 & 500 & 7.101 & 7.101 & & 0.2252 & 1.90E-03 & 416.2378 & 153.3714 & 569.6091 & \\
\hline & 8/9/2008 6:00 & 425 & 222 & 600 & 7.051 & 7.051 & & 0.2339 & 1.99E-03 & 436.0942 & 160.6879 & 596.7821 & \\
\hline & 8/9/2008 7:00 & 426 & 222 & 700 & 6.76 & 6.76 & & 0.2870 & 2.56E-03 & 561.0452 & 206.7287 & 767.7739 & \\
\hline & 8/9/2008 8:00 & 427 & 222 & 800 & 6.162 & 6.162 & & 0.4119 & 3.99E-03 & 875.3064 & 322.5246 & 1197.8311 & \\
\hline & 8/9/2008 9:00 & 428 & 222 & 900 & 6.53 & 6.53 & & 0.3323 & 3.07E-03 & 672.0509 & 247.6310 & 919.6818 & \\
\hline & 8/9/2008 10:00 & 429 & 222 & 1000 & 6.688 & 6.688 & & 0.3008 & $2.71 E-03$ & 594.5723 & 219.0824 & 813.6547 & \\
\hline & 8/9/2008 11:00 & 430 & 222 & 1100 & 5.936 & 5.936 & & 0.4656 & 4.64E-03 & 1017.9834 & 375.0969 & 1393.0803 & \\
\hline & 8/9/2008 12:00 & 431 & 222 & 1200 & 4.992 & 4.992 & & 0.7428 & 8.25E-03 & 1808.8949 & 666.5245 & 2475.4194 & \\
\hline & 8/9/2008 13:00 & 432 & 222 & 1300 & 4.63 & 4.63 & & 0.8790 & 1.02E-02 & 2225.6601 & 820.0903 & 3045.7504 & \\
\hline & 8/9/2008 14:00 & 433 & 222 & 1400 & 4.008 & 4.008 & & 1.1707 & 1.44E-02 & 3166.7663 & 1166.8602 & 4333.6265 & \\
\hline & 8/9/2008 15:00 & 434 & 222 & 1500 & 3.753 & 3.753 & & 1.3181 & 1.67E-02 & 3664.8247 & 1350.3800 & 5015.2048 & \\
\hline & 8/9/2008 16:00 & 435 & 222 & 1600 & 3.815 & 3.815 & & 1.2805 & 1.61E-02 & 3536.3141 & 1303.0277 & 4839.3418 & \\
\hline & 8/9/2008 17:00 & 436 & 222 & 1700 & 4.029 & 4.029 & & 1.1593 & 1.43E-02 & 3129.1331 & 1152.9935 & 4282.1266 & \\
\hline & 8/9/2008 18:00 & 437 & 222 & 1800 & 4.031 & 4.031 & & 1.1583 & 1.43E-02 & 3125.5739 & 1151.6820 & 4277.2558 & \\
\hline & 8/9/2008 19:00 & 438 & 222 & 1900 & 4.348 & 4.348 & & 1.0009 & 1.19E-02 & 2611.3792 & 962.2164 & 3573.5955 & \\
\hline & 8/9/2008 20:00 & 439 & 222 & 2000 & 5.199 & 5.199 & & 0.6734 & $7.31 E-03$ & 1603.1772 & 590.7236 & 2193.9008 & \\
\hline & 8/9/2008 21:00 & 440 & 222 & 2100 & 6.677 & 6.677 & & 0.3030 & 2.74E-03 & 599.7905 & 221.0051 & 820.7957 & \\
\hline & 8/9/2008 22:00 & 441 & 222 & 2200 & 7.268 & 7.268 & & 0.1970 & 1.61E-03 & 353.1363 & 130.1204 & 483.2567 & \\
\hline & 8/9/2008 23:00 & 442 & 222 & 2300 & 7.248 & 7.248 & & 0.2003 & 1.64E-03 & 360.4381 & 132.8108 & 493.2489 & \\
\hline & 8/10/2008 0:00 & 443 & 223 & 0 & 7.232 & 7.232 & 7.31 & 0.2030 & 1.67E-03 & 366.3287 & 134.9814 & 501.3101 & \\
\hline & 8/10/2008 1:00 & 444 & 223 & 100 & 7.244 & 7.244 & & 0.2010 & 1.65E-03 & 361.9066 & 133.3519 & 495.2586 & \\
\hline & 8/10/2008 2:00 & 445 & 223 & 200 & 7.229 & 7.229 & & 0.2035 & 1.68E-03 & 367.4381 & 135.3901 & 502.8283 & \\
\hline & 8/10/2008 3:00 & 446 & 223 & 300 & 7.216 & 7.216 & & 0.2057 & 1.70E-03 & 372.2635 & 137.1681 & 509.4316 & \\
\hline & 8/10/2008 4:00 & 447 & 223 & 400 & 7.216 & 7.216 & & 0.2057 & 1.70E-03 & 372.2635 & 137.1681 & 509.4316 & \\
\hline & $8 / 10 / 20085: 00$ & 448 & 223 & 500 & 7.192 & 7.192 & & 0.2097 & $1.74 \mathrm{E}-03$ & 381.2488 & 140.4789 & 521.7277 & \\
\hline & 8/10/2008 6:00 & 449 & 223 & 600 & 7.172 & 7.172 & & 0.2131 & 1.77E-03 & 388.8132 & 143.2662 & 532.0794 & \\
\hline & 8/10/2008 7:00 & 450 & 223 & 700 & 7.17 & 7.17 & & 0.2134 & 1.78E-03 & 389.5735 & 143.5464 & 533.1199 & \\
\hline
\end{tabular}




\begin{tabular}{|c|c|c|c|c|c|c|c|c|c|c|c|c|c|}
\hline Tree ID & TS & RN & JD & HR & $\begin{array}{c}\text { Raw } \\
\text { TC_dTA }\end{array}$ & $\begin{array}{c}\text { Fixed } \\
\text { TC_dT } \Delta\end{array}$ & dTM & $\mathbf{K}$ & $\begin{array}{l}\text { Sap } \\
\text { Velocity } \\
(\mathrm{cm} / \mathrm{s})\end{array}$ & $\begin{array}{c}\text { Probed } \\
\text { Drywood F } \\
(\mathrm{g} / \mathrm{hr})\end{array}$ & $\begin{array}{c}\text { Extrapolated } \\
\text { Wetwood F } \\
(\mathrm{g} / \mathrm{hr})\end{array}$ & $\begin{array}{c}\text { Extrapolated } \\
\text { Total Wood F } \\
(\mathrm{g} / \mathrm{hr})\end{array}$ & $\begin{array}{l}\text { Relative } \\
\text { Humidity, } \\
@ 10 \mathrm{~m}(\%)\end{array}$ \\
\hline & 8/10/2008 8:00 & 451 & 223 & 800 & $\begin{array}{l}7.166 \\
\end{array}$ & 7.166 & & 0.2141 & $1.78 \mathrm{E}-03$ & 391.0962 & 144.1074 & 535.2036 & \\
\hline & 8/10/2008 9:00 & 452 & 223 & 900 & 6.904 & 6.904 & & 0.2601 & $2.27 \mathrm{E}-03$ & 497.1602 & 183.1889 & 680.3491 & \\
\hline & $8 / 10 / 2008$ 10:00 & 453 & 223 & 1000 & 4.574 & 4.574 & & 0.9021 & $1.05 E-02$ & 2297.5778 & 846.5898 & 3144.1676 & \\
\hline & 8/10/2008 11:00 & 454 & 223 & 1100 & 3.87 & 3.87 & & 1.2481 & $1.56 \mathrm{E}-02$ & 3426.4552 & 1262.5479 & 4689.0030 & \\
\hline & $8 / 10 / 200812: 00$ & 455 & 223 & 1200 & 3.755 & 3.755 & & 1.3169 & 1.67E-02 & 3660.5994 & 1348.8231 & 5009.4225 & \\
\hline & 8/10/2008 13:00 & 456 & 223 & 1300 & 3.709 & 3.709 & & 1.3456 & 1.71E-02 & 3759.1709 & 1385.1438 & 5144.3147 & \\
\hline & $8 / 10 / 2008$ 14:00 & 457 & 223 & 1400 & 3.702 & 3.702 & & 1.3501 & $1.72 \mathrm{E}-02$ & 3774.4293 & 1390.7661 & 5165.1954 & \\
\hline & $8 / 10 / 2008$ 15:00 & 458 & 223 & 1500 & 3.867 & 3.867 & & 1.2498 & 1.57E-02 & 3432.3503 & 1264.7200 & 4697.0703 & \\
\hline & 8/10/2008 16:00 & 459 & 223 & 1600 & 3.832 & 3.832 & & 1.2704 & 1.60E-02 & 3501.9512 & 1290.3660 & 4792.3172 & \\
\hline & 8/10/2008 17:00 & 460 & 223 & 1700 & 4.084 & 4.084 & & 1.1303 & 1.38E-02 & 3032.7946 & 1117.4955 & 4150.2901 & \\
\hline & 8/10/2008 18:00 & 461 & 223 & 1800 & 6.586 & 6.586 & & 0.3210 & 2.94E-03 & 643.9605 & 237.2805 & 881.2410 & \\
\hline & $8 / 10 / 2008$ 19:00 & 462 & 223 & 1900 & 7.307 & 7.307 & & 0.1906 & $1.55 \mathrm{E}-03$ & 339.0934 & 124.9459 & 464.0393 & \\
\hline & 8/10/2008 20:00 & 463 & 223 & 2000 & 7.268 & 7.268 & & 0.1970 & $1.61 \mathrm{E}-03$ & 353.1363 & 130.1204 & 483.2567 & \\
\hline & 8/10/2008 21:00 & 464 & 223 & 2100 & 7.158 & 7.158 & & 0.2154 & 1.80E-03 & 394.1501 & 145.2327 & 539.3828 & \\
\hline & $8 / 10 / 2008$ 22:00 & 465 & 223 & 2200 & 7.218 & 7.218 & & 0.2053 & 1.69E-03 & 371.5192 & 136.8939 & 508.4131 & \\
\hline & 8/10/2008 23:00 & 466 & 223 & 2300 & 7.121 & 7.121 & & 0.2217 & $1.86 \mathrm{E}-03$ & 408.4217 & 150.4914 & 558.9130 & \\
\hline & $8 / 11 / 20080: 00$ & 467 & 224 & 0 & 7.088 & 7.088 & 7.30 & 0.2274 & 1.92E-03 & 421.3568 & 155.2576 & 576.6144 & \\
\hline & 8/11/2008 1:00 & 468 & 224 & 100 & 7.199 & 7.199 & & 0.2085 & $1.73 \mathrm{E}-03$ & 378.6177 & 139.5095 & 518.1272 & \\
\hline & 8/11/2008 2:00 & 469 & 224 & 200 & 7.295 & 7.295 & & 0.1926 & 1.57E-03 & 343.3869 & 126.5280 & 469.9149 & \\
\hline & 8/11/2008 3:00 & 470 & 224 & 300 & 7.293 & 7.293 & & 0.1929 & $1.57 E-03$ & 344.1049 & 126.7925 & 470.8974 & \\
\hline & 8/11/2008 4:00 & 471 & 224 & 400 & 7.287 & 7.287 & & 0.1939 & $1.58 \mathrm{E}-03$ & 346.2628 & 127.5876 & 473.8504 & \\
\hline & 8/11/2008 5:00 & 472 & 224 & 500 & 7.245 & 7.245 & & 0.2008 & 1.65E-03 & 361.5392 & 133.2166 & 494.7558 & \\
\hline & 8/11/2008 6:00 & 473 & 224 & 600 & 7.233 & 7.233 & & 0.2028 & 1.67E-03 & 365.9593 & 134.8452 & 500.8045 & \\
\hline & 8/11/2008 7:00 & 474 & 224 & 700 & 7.247 & 7.247 & & 0.2005 & 1.65E-03 & 360.8049 & 132.9460 & 493.7510 & \\
\hline & 8/11/2008 8:00 & 475 & 224 & 800 & 7.194 & 7.194 & & 0.2093 & 1.74E-03 & 380.4962 & 140.2016 & 520.6978 & \\
\hline & 8/11/2008 9:00 & 476 & 224 & 900 & 6.511 & 6.511 & & 0.3362 & 3.11E-03 & 681.7428 & 251.2022 & 932.9449 & \\
\hline & $8 / 11 / 2008$ 10:00 & 477 & 224 & 1000 & 5.012 & 5.012 & & 0.7358 & 8.16E-03 & 1788.0692 & 658.8509 & 2446.9201 & \\
\hline & 8/11/2008 11:00 & 478 & 224 & 1100 & 4.211 & 4.211 & & 1.0660 & 1.29E-02 & 2821.9952 & 1039.8222 & 3861.8173 & \\
\hline & $8 / 11 / 2008$ 12:00 & 479 & 224 & 1200 & 3.999 & 3.999 & & 1.1755 & 1.45E-02 & 3183.0419 & 1172.8572 & 4355.8991 & \\
\hline & 8/11/2008 13:00 & 480 & 224 & 1300 & 4.247 & 4.247 & & 1.0485 & 1.26E-02 & 2765.0344 & 1018.8338 & 3783.8682 & \\
\hline & 8/11/2008 14:00 & 481 & 224 & 1400 & 3.999 & 3.999 & & 1.1755 & 1.45E-02 & 3183.0419 & 1172.8572 & 4355.8991 & \\
\hline & 8/11/2008 15:00 & 482 & 224 & 1500 & 3.957 & 3.957 & & 1.1986 & 1.49E-02 & 3260.1841 & 1201.2819 & 4461.4659 & \\
\hline & $8 / 11 / 2008$ 16:00 & 483 & 224 & 1600 & 4.252 & 4.252 & & 1.0461 & 1.26E-02 & 2757.2165 & 1015.9531 & 3773.1697 & \\
\hline & 8/11/2008 17:00 & 484 & 224 & 1700 & 4.266 & 4.266 & & 1.0394 & 1.25E-02 & 2735.4461 & 1007.9314 & 3743.3774 & \\
\hline & 8/11/2008 18:00 & 485 & 224 & 1800 & 4.604 & 4.604 & & 0.8897 & 1.03E-02 & 2258.7796 & 832.2938 & 3091.0734 & \\
\hline & $8 / 11 / 2008$ 19:00 & 486 & 224 & 1900 & 4.772 & 4.772 & & 0.8231 & 9.36E-03 & 2052.6892 & 756.3555 & 2809.0447 & \\
\hline & 8/11/2008 20:00 & 487 & 224 & 2000 & 5.472 & 5.472 & & 0.5899 & $6.21 \mathrm{E}-03$ & 1362.1272 & 501.9038 & 1864.0310 & \\
\hline & 8/11/2008 21:00 & 488 & 224 & 2100 & 6.365 & 6.365 & & 0.3668 & $3.46 \mathrm{E}-03$ & 759.0382 & 279.6832 & 1038.7215 & \\
\hline & $8 / 11 / 200822: 00$ & 489 & 224 & 2200 & 6.976 & 6.976 & & 0.2471 & 2.13E-03 & 466.7412 & 171.9804 & 638.7216 & \\
\hline & 8/11/2008 23:00 & 490 & 224 & 2300 & 7.16 & 7.16 & & 0.2151 & 1.79E-03 & 393.3856 & 144.9510 & 538.3366 & \\
\hline & 8/12/2008 0:00 & 491 & 225 & 0 & 7.228 & 7.228 & 7.34 & 0.2037 & $1.68 \mathrm{E}-03$ & 367.8083 & 135.5265 & 503.3348 & \\
\hline
\end{tabular}




\begin{tabular}{|c|c|c|c|c|c|c|c|c|c|c|c|c|c|}
\hline Tree ID & TS & RN & JD & HR & $\begin{array}{c}\text { Raw } \\
\text { TC_dTA }\end{array}$ & $\begin{array}{c}\text { Fixed } \\
\text { TC_dT } \Delta\end{array}$ & dTM & $\mathbf{K}$ & $\begin{array}{l}\text { Sap } \\
\text { Velocity } \\
(\mathrm{cm} / \mathrm{s})\end{array}$ & $\begin{array}{c}\text { Probed } \\
\text { Drywood F } \\
(\mathrm{g} / \mathrm{hr})\end{array}$ & $\begin{array}{c}\text { Extrapolated } \\
\text { Wetwood F } \\
(\mathrm{g} / \mathrm{hr})\end{array}$ & $\begin{array}{c}\text { Extrapolated } \\
\text { Total Wood F } \\
(\mathrm{g} / \mathrm{hr})\end{array}$ & $\begin{array}{l}\text { Relative } \\
\text { Humidity, } \\
@ 10 \mathrm{~m}(\%)\end{array}$ \\
\hline & 8/12/2008 1:00 & 492 & 225 & 100 & 7.228 & 7.228 & & 0.2037 & 1.68E-03 & 367.8083 & 135.5265 & 503.3348 & \\
\hline & 8/12/2008 2:00 & 493 & 225 & 200 & 6.451 & 6.451 & & 0.3486 & $3.25 \mathrm{E}-03$ & 712.8965 & 262.6814 & 975.5778 & \\
\hline & 8/12/2008 3:00 & 494 & 225 & 300 & 6.458 & 6.458 & & 0.3472 & $3.24 \mathrm{E}-03$ & 709.2185 & 261.3262 & 970.5447 & \\
\hline & 8/12/2008 4:00 & 495 & 225 & 400 & 6.667 & 6.667 & & 0.3049 & 2.76E-03 & 604.5567 & 222.7614 & 827.3181 & \\
\hline & 8/12/2008 5:00 & 496 & 225 & 500 & 6.663 & 6.663 & & 0.3057 & 2.77E-03 & 606.4692 & 223.4660 & 829.9352 & \\
\hline & 8/12/2008 6:00 & 497 & 225 & 600 & 6.67 & 6.67 & & 0.3043 & $2.75 E-03$ & 603.1246 & 222.2337 & 825.3583 & \\
\hline & 8/12/2008 7:00 & 498 & 225 & 700 & 6.815 & 6.815 & & 0.2766 & 2.45E-03 & 536.1547 & 197.5572 & 733.7120 & \\
\hline & 8/12/2008 8:00 & 499 & 225 & 800 & 6.947 & 6.947 & & 0.2523 & $2.18 E-03$ & 478.8742 & 176.4510 & 655.3252 & \\
\hline & 8/12/2008 9:00 & 500 & 225 & 900 & 6.697 & 6.697 & & 0.2991 & 2.69E-03 & 590.3220 & 217.5163 & 807.8382 & \\
\hline & $8 / 12 / 2008$ 10:00 & 501 & 225 & 1000 & 5.282 & 5.282 & & 0.6471 & 6.96E-03 & 1526.4652 & 562.4575 & 2088.9227 & \\
\hline & $8 / 12 / 2008$ 11:00 & 502 & 225 & 1100 & 4.331 & 4.331 & & 1.0088 & 1.20E-02 & 2636.6263 & 971.5192 & 3608.1454 & \\
\hline & $8 / 12 / 2008$ 12:00 & 503 & 225 & 1200 & 4 & 4 & & 1.1750 & 1.45E-02 & 3181.2291 & 1172.1893 & 4353.4184 & \\
\hline & 8/12/2008 13:00 & 504 & 225 & 1300 & 3.843 & 3.843 & & 1.2639 & 1.59E-02 & 3479.9117 & 1282.2450 & 4762.1567 & \\
\hline & $8 / 12 / 2008$ 14:00 & 505 & 225 & 1400 & 3.783 & 3.783 & & 1.2998 & 1.64E-02 & 3602.0087 & 1327.2342 & 4929.2428 & \\
\hline & $8 / 12 / 2008$ 15:00 & 506 & 225 & 1500 & 3.785 & 3.785 & & 1.2985 & 1.64E-02 & 3597.8635 & 1325.7068 & 4923.5703 & \\
\hline & $8 / 12 / 2008$ 16:00 & 507 & 225 & 1600 & 3.859 & 3.859 & & 1.2545 & 1.57E-02 & 3448.1247 & 1270.5325 & 4718.6572 & \\
\hline & $8 / 12 / 2008$ 17:00 & 508 & 225 & 1700 & 3.951 & 3.951 & & 1.2020 & 1.49E-02 & 3271.3669 & 1205.4024 & 4476.7693 & \\
\hline & $8 / 12 / 2008$ 18:00 & 509 & 225 & 1800 & 4.019 & 4.019 & & 1.1647 & 1.44E-02 & 3146.9941 & 1159.5747 & 4306.5688 & \\
\hline & $8 / 12 / 2008$ 19:00 & 510 & 225 & 1900 & 4.323 & 4.323 & & 1.0125 & $1.21 \mathrm{E}-02$ & 2648.5918 & 975.9281 & 3624.5200 & \\
\hline & $8 / 12 / 200820: 00$ & 511 & 225 & 2000 & 5.311 & 5.311 & & 0.6381 & 6.84E-03 & 1500.3908 & 552.8499 & 2053.2407 & \\
\hline & $8 / 12 / 2008$ 21:00 & 512 & 225 & 2100 & 6.849 & 6.849 & & 0.2703 & $2.38 \mathrm{E}-03$ & 521.0730 & 192.0001 & 713.0731 & \\
\hline & $8 / 12 / 2008$ 22:00 & 513 & 225 & 2200 & 7.341 & 7.341 & & 0.1851 & 1.49E-03 & 327.0595 & 120.5118 & 447.5713 & \\
\hline & $8 / 12 / 2008$ 23:00 & 514 & 225 & 2300 & 7.315 & 7.315 & & 0.1893 & 1.53E-03 & 336.2445 & 123.8962 & 460.1407 & \\
\hline & 8/13/2008 0:00 & 515 & 226 & 0 & 7.258 & 7.258 & 7.33 & 0.1987 & 1.63E-03 & 356.7787 & 131.4624 & 488.2411 & \\
\hline & 8/13/2008 1:00 & 516 & 226 & 100 & 7.225 & 7.225 & & 0.2042 & $1.68 \mathrm{E}-03$ & 368.9198 & 135.9361 & 504.8558 & \\
\hline & 8/13/2008 2:00 & 517 & 226 & 200 & 7.232 & 7.232 & & 0.2030 & 1.67E-03 & 366.3287 & 134.9814 & 501.3101 & \\
\hline & 8/13/2008 3:00 & 518 & 226 & 300 & 7.237 & 7.237 & & 0.2022 & 1.66E-03 & 364.4832 & 134.3013 & 498.7845 & \\
\hline & 8/13/2008 4:00 & 519 & 226 & 400 & 7.174 & 7.174 & & 0.2127 & 1.77E-03 & 388.0536 & 142.9863 & 531.0399 & \\
\hline & $8 / 13 / 20085: 00$ & 520 & 226 & 500 & 7.158 & 7.158 & & 0.2154 & $1.80 \mathrm{E}-03$ & 394.1501 & 145.2327 & 539.3828 & \\
\hline & 8/13/2008 6:00 & 521 & 226 & 600 & 7.208 & 7.208 & & 0.2070 & 1.71E-03 & 375.2475 & 138.2676 & 513.5151 & \\
\hline & $8 / 13 / 2008$ 7:00 & 522 & 226 & 700 & 7.193 & 7.193 & & 0.2095 & 1.74E-03 & 380.8724 & 140.3403 & 521.2126 & \\
\hline & 8/13/2008 8:00 & 523 & 226 & 800 & 7.171 & 7.171 & & 0.2132 & $1.78 \mathrm{E}-03$ & 389.1933 & 143.4063 & 532.5995 & \\
\hline & 8/13/2008 9:00 & 524 & 226 & 900 & 6.121 & 6.121 & & 0.4213 & 4.11E-03 & 900.1121 & 331.6648 & 1231.7769 & \\
\hline & $8 / 13 / 2008$ 10:00 & 525 & 226 & 1000 & 4.484 & 4.484 & & 0.9402 & $1.10 \mathrm{E}-02$ & 2417.8570 & 890.9091 & 3308.7662 & \\
\hline & 8/13/2008 11:00 & 526 & 226 & 1100 & 4.311 & 4.311 & & 1.0181 & 1.22E-02 & 2666.6426 & 982.5793 & 3649.2219 & \\
\hline & $8 / 13 / 2008$ 12:00 & 527 & 226 & 1200 & 3.864 & 3.864 & & 1.2516 & 1.57E-02 & 3438.2564 & 1266.8963 & 4705.1527 & \\
\hline
\end{tabular}




\begin{tabular}{|c|c|c|c|c|c|c|c|c|c|c|c|c|c|}
\hline Tree ID & TS & RN & JD & HR & $\begin{array}{c}\text { Raw } \\
\text { TC_dTA }\end{array}$ & $\begin{array}{c}\text { Fixed } \\
\text { TC_dTA }\end{array}$ & dTM & $\mathbf{K}$ & $\begin{array}{l}\text { Sap } \\
\text { Velocity } \\
(\mathrm{cm} / \mathrm{s})\end{array}$ & $\begin{array}{l}\text { Probed } \\
\text { Drywood F } \\
\text { (g/hr) }\end{array}$ & $\begin{array}{c}\text { Extrapolated } \\
\text { Wetwood F } \\
(\mathrm{g} / \mathrm{hr})\end{array}$ & $\begin{array}{c}\text { Extrapolated } \\
\text { Total Wood F } \\
\text { (g/hr) }\end{array}$ & $\begin{array}{l}\text { Relative } \\
\text { Humidity, } \\
@ 10 m(\%)\end{array}$ \\
\hline & 7/22/2008 13:00 & 0 & 204 & 1300 & 4.452 & 4.452 & 8.53 & 0.9160 & 1.07E-02 & 2433.1653 & 896.5498 & 3329.7151 & \\
\hline \multirow[t]{2}{*}{ W504WN2 } & $7 / 22 / 2008$ 14:00 & 1 & 204 & 1400 & 4.826 & 4.826 & & 0.7675 & $8.59 \mathrm{E}-03$ & 1957.1336 & 721.1461 & 2678.2797 & \\
\hline & $7 / 22 / 2008$ 15:00 & 2 & 204 & 1500 & 4.806 & 4.806 & & 0.7749 & $8.69 \mathrm{E}-03$ & 1980.2479 & 729.6631 & 2709.9110 & \\
\hline \multirow[t]{2}{*}{ Probe \# } & 7/22/2008 16:00 & 3 & 204 & 1600 & 4.837 & 4.837 & & 0.7635 & $8.54 \mathrm{E}-03$ & 1944.5237 & 716.4998 & 2661.0235 & \\
\hline & 7/22/2008 17:00 & 4 & 204 & 1700 & 4.8 & 4.8 & & 0.7771 & 8.72E-03 & 1987.2298 & 732.2357 & 2719.4655 & \\
\hline \multirow[t]{4}{*}{11} & $7 / 22 / 2008$ 18:00 & 5 & 204 & 1800 & 4.806 & 4.806 & & 0.7749 & 8.69E-03 & 1980.2479 & 729.6631 & 2709.9110 & \\
\hline & $7 / 22 / 2008$ 19:00 & 6 & 204 & 1900 & 5.011 & 5.011 & & 0.7023 & 7.70E-03 & 1754.3557 & 646.4284 & 2400.7842 & \\
\hline & 7/22/2008 20:00 & 7 & 204 & 2000 & 5.266 & 5.266 & & 0.6198 & $6.60 \mathrm{E}-03$ & 1504.4092 & 554.3305 & 2058.7397 & \\
\hline & 7/22/2008 21:00 & 8 & 204 & 2100 & 6.608 & 6.608 & & 0.2909 & $2.60 \mathrm{E}-03$ & 592.7570 & 218.4135 & 811.1705 & \\
\hline \multirow[t]{2}{*}{$\mathrm{DBH}(\mathrm{cm})$} & $7 / 22 / 2008$ 22:00 & 9 & 204 & 2200 & 8.01 & 8.01 & & 0.0649 & 4.11E-04 & 93.5645 & 34.4758 & 128.0402 & \\
\hline & 7/22/2008 23:00 & 10 & 204 & 2300 & 8.53 & 8.53 & & 0.0000 & $0.00 \mathrm{E}+00$ & 0.0000 & 0.0000 & 0.0000 & \\
\hline \multirow[t]{2}{*}{10.9} & 7/23/2008 0:00 & 11 & 205 & 0 & 8.89 & 8.89 & 9.06 & -0.0405 & $0.00 \mathrm{E}+00$ & 0.0000 & 0.0000 & 0.0000 & \\
\hline & 7/23/2008 1:00 & 12 & 205 & 100 & 9 & 9 & & -0.0522 & $0.00 \mathrm{E}+00$ & 0.0000 & 0.0000 & 0.0000 & \\
\hline Day Count & $7 / 23 / 20082: 00$ & 13 & 205 & 200 & 9.02 & 9.02 & & -0.0543 & $0.00 \mathrm{E}+00$ & 0.0000 & 0.0000 & 0.0000 & \\
\hline \multirow[t]{27}{*}{35} & 7/23/2008 3:00 & 14 & 205 & 300 & 9.06 & 9.06 & & -0.0585 & $0.00 \mathrm{E}+00$ & 0.0000 & 0.0000 & 0.0000 & \\
\hline & 7/23/2008 4:00 & 15 & 205 & 400 & 9.03 & 9.03 & & -0.0554 & $0.00 \mathrm{E}+00$ & 0.0000 & 0.0000 & 0.0000 & \\
\hline & 7/23/2008 5:00 & 16 & 205 & 500 & 8.89 & 8.89 & & -0.0405 & $0.00 E+00$ & 0.0000 & 0.0000 & 0.0000 & \\
\hline & 7/23/2008 6:00 & 17 & 205 & 600 & 8.64 & 8.64 & & -0.0127 & $0.00 \mathrm{E}+00$ & 0.0000 & 0.0000 & 0.0000 & \\
\hline & 7/23/2008 7:00 & 18 & 205 & 700 & 8.08 & 8.08 & & 0.0557 & $3.40 \mathrm{E}-04$ & 77.4754 & 28.5474 & 106.0228 & \\
\hline & 7/23/2008 8:00 & 19 & 205 & 800 & 7.032 & 7.032 & & 0.2130 & 1.77E-03 & 404.0019 & 148.8628 & 552.8648 & \\
\hline & 7/23/2008 9:00 & 20 & 205 & 900 & 6 & 6 & & 0.4217 & 4.11E-03 & 936.3100 & 345.0027 & 1281.3127 & \\
\hline & 7/23/2008 10:00 & 21 & 205 & 1000 & 5.224 & 5.224 & & 0.6328 & $6.78 \mathrm{E}-03$ & 1543.4138 & 568.7026 & 2112.1164 & \\
\hline & $7 / 23 / 2008$ 11:00 & 22 & 205 & 1100 & 5.162 & 5.162 & & 0.6525 & 7.03E-03 & 1602.5019 & 590.4748 & 2192.9767 & \\
\hline & $7 / 23 / 2008$ 12:00 & 23 & 205 & 1200 & 5.074 & 5.074 & & 0.6811 & $7.42 \mathrm{E}-03$ & 1689.5861 & 622.5627 & 2312.1488 & \\
\hline & $7 / 23 / 2008$ 13:00 & 24 & 205 & 1300 & 5.017 & 5.017 & & 0.7002 & 7.67E-03 & 1748.0973 & 644.1224 & 2392.2197 & \\
\hline & $7 / 23 / 2008$ 14:00 & 25 & 205 & 1400 & 4.995 & 4.995 & & 0.7077 & $7.78 \mathrm{E}-03$ & 1771.1391 & 652.6126 & 2423.7517 & \\
\hline & 7/23/2008 15:00 & 26 & 205 & 1500 & 4.964 & 4.964 & & 0.7184 & 7.92E-03 & 1804.0509 & 664.7397 & 2468.7906 & \\
\hline & 7/23/2008 16:00 & 27 & 205 & 1600 & 5.089 & 5.089 & & 0.6762 & 7.35E-03 & 1674.4677 & 616.9920 & 2291.4597 & \\
\hline & 7/23/2008 17:00 & 28 & 205 & 1700 & 5.33 & 5.33 & & 0.6004 & $6.35 \mathrm{E}-03$ & 1446.5082 & 532.9957 & 1979.5039 & \\
\hline & $7 / 23 / 2008$ 18:00 & 29 & 205 & 1800 & 5.765 & 5.765 & & 0.4796 & 4.82E-03 & 1097.1481 & 404.2668 & 1501.4148 & \\
\hline & $7 / 23 / 2008$ 19:00 & 30 & 205 & 1900 & 5.922 & 5.922 & & 0.4404 & 4.34E-03 & 987.7534 & 363.9580 & 1351.7114 & \\
\hline & $7 / 23 / 200820: 00$ & 31 & 205 & 2000 & 6.315 & 6.315 & & 0.3508 & $3.28 E-03$ & 746.4108 & 275.0304 & 1021.4413 & \\
\hline & $7 / 23 / 200821: 00$ & 32 & 205 & 2100 & 6.919 & 6.919 & & 0.2328 & $1.98 \mathrm{E}-03$ & 450.7376 & 166.0835 & 616.8211 & \\
\hline & $7 / 23 / 200822: 00$ & 33 & 205 & 2200 & 7.763 & 7.763 & & 0.0988 & $6.89 E-04$ & 156.9059 & 57.8152 & 214.7210 & \\
\hline & $7 / 23 / 2008$ 23:00 & 34 & 205 & 2300 & 8.06 & 8.06 & & 0.0583 & 3.60E-04 & 81.9854 & 30.2092 & 112.1946 & \\
\hline & $7 / 24 / 20080: 00$ & 35 & 206 & 0 & 8.12 & 8.12 & 8.92 & 0.0505 & 3.01E-04 & 68.6682 & 25.3022 & 93.9705 & \\
\hline & 7/24/2008 1:00 & 36 & 206 & 100 & 8.25 & 8.25 & & 0.0339 & 1.85E-04 & 42.1094 & 15.5161 & 57.6255 & \\
\hline & 7/24/2008 2:00 & 37 & 206 & 200 & 8.32 & 8.32 & & 0.0252 & $1.28 \mathrm{E}-04$ & 29.2457 & 10.7762 & 40.0219 & \\
\hline & 7/24/2008 3:00 & 38 & 206 & 300 & 8.46 & 8.46 & & 0.0083 & $3.25 \mathrm{E}-05$ & 7.4098 & 2.7303 & 10.1401 & \\
\hline & 7/24/2008 4:00 & 39 & 206 & 400 & 8.51 & 8.51 & & 0.0024 & 6.91E-06 & 1.5736 & 0.5798 & 2.1535 & \\
\hline & 7/24/2008 5:00 & 40 & 206 & 500 & 8.56 & 8.56 & & -0.0035 & $0.00 \mathrm{E}+00$ & 0.0000 & 0.0000 & 0.0000 & \\
\hline
\end{tabular}




\begin{tabular}{|c|c|c|c|c|c|c|c|c|c|c|c|c|c|}
\hline Tree ID & TS & RN & JD & HR & $\begin{array}{c}\text { Raw } \\
\text { TC_dTA }\end{array}$ & $\begin{array}{c}\text { Fixed } \\
\text { TC_dT } \Delta\end{array}$ & dTM & $\mathrm{K}$ & $\begin{array}{l}\text { Sap } \\
\text { Velocity } \\
(\mathrm{cm} / \mathrm{s})\end{array}$ & $\begin{array}{l}\text { Probed } \\
\text { Drywood F } \\
\text { (g/hr) }\end{array}$ & $\begin{array}{c}\text { Extrapolated } \\
\text { Wetwood F } \\
(\mathrm{g} / \mathrm{hr})\end{array}$ & $\begin{array}{c}\text { Extrapolated } \\
\text { Total Wood F } \\
(\mathrm{g} / \mathrm{hr})\end{array}$ & $\begin{array}{l}\text { Relative } \\
\text { Humidity, } \\
@ 10 \mathrm{~m}(\%)\end{array}$ \\
\hline & 7/24/2008 6:00 & 41 & 206 & 600 & 8.58 & 8.58 & & -0.0058 & $0.00 E+00$ & 0.0000 & 0.0000 & 0.0000 & \\
\hline & 7/24/2008 7:00 & 42 & 206 & 700 & 8.44 & 8.44 & & 0.0107 & $4.45 \mathrm{E}-05$ & 10.1258 & 3.7310 & 13.8568 & \\
\hline & 7/24/2008 8:00 & 43 & 206 & 800 & 7.851 & 7.851 & & 0.0865 & $5.85 \mathrm{E}-04$ & 133.1869 & 49.0755 & 182.2624 & \\
\hline & 7/24/2008 9:00 & 44 & 206 & 900 & 7.405 & 7.405 & & 0.1519 & 1.17E-03 & 266.4808 & 98.1903 & 364.6711 & \\
\hline & $7 / 24 / 2008$ 10:00 & 45 & 206 & 1000 & 7.412 & 7.412 & & 0.1508 & 1.16E-03 & 264.1337 & 97.3255 & 361.4592 & \\
\hline & 7/24/2008 11:00 & 46 & 206 & 1100 & 5.971 & 5.971 & & 0.4286 & $4.19 E-03$ & 955.2192 & 351.9702 & 1307.1894 & \\
\hline & 7/24/2008 12:00 & 47 & 206 & 1200 & 5.139 & 5.139 & & 0.6599 & 7.13E-03 & 1624.8917 & 598.7248 & 2223.6164 & \\
\hline & 7/24/2008 13:00 & 48 & 206 & 1300 & 5.44 & 5.44 & & 0.5680 & $5.93 \mathrm{E}-03$ & 1351.1362 & 497.8539 & 1848.9901 & \\
\hline & 7/24/2008 14:00 & 49 & 206 & 1400 & 5.315 & 5.315 & & 0.6049 & $6.41 \mathrm{E}-03$ & 1459.9156 & 537.9359 & \begin{tabular}{|l|}
1997.8515 \\
\end{tabular} & \\
\hline & $7 / 24 / 2008$ 15:00 & 50 & 206 & 1500 & 5.064 & 5.064 & & 0.6844 & 7.46E-03 & 1699.7290 & 626.3001 & 2326.0292 & \\
\hline & 7/24/2008 16:00 & 51 & 206 & 1600 & 5.046 & 5.046 & & 0.6904 & 7.54E-03 & 1718.1165 & 633.0754 & 2351.1919 & \\
\hline & 7/24/2008 17:00 & 52 & 206 & 1700 & 5.169 & 5.169 & & 0.6502 & 7.01E-03 & 1595.7387 & 587.9827 & 2183.7214 & \\
\hline & $7 / 24 / 2008$ 18:00 & 53 & 206 & 1800 & 5.061 & 5.061 & & 0.6854 & $7.48 \mathrm{E}-03$ & 1702.7820 & 627.4250 & 2330.2070 & \\
\hline & 7/24/2008 19:00 & 54 & 206 & 1900 & 5.335 & 5.335 & & 0.5989 & $6.33 \mathrm{E}-03$ & 1442.0610 & 531.3570 & 1973.4181 & \\
\hline & $7 / 24 / 200820: 00$ & 55 & 206 & 2000 & 5.991 & 5.991 & & 0.4238 & 4.14E-03 & 942.1512 & 347.1550 & 1289.3062 & \\
\hline & $7 / 24 / 200821: 00$ & 56 & 206 & 2100 & 7.309 & 7.309 & & 0.1671 & 1.31E-03 & 299.5162 & 110.3629 & 409.8791 & \\
\hline & $7 / 24 / 200822: 00$ & 57 & 206 & 2200 & 8.54 & 8.54 & & -0.0012 & $0.00 E+00$ & 0.0000 & 0.0000 & 0.0000 & \\
\hline & $7 / 24 / 200823: 00$ & 58 & 206 & 2300 & 8.92 & 8.92 & & -0.0437 & $0.00 \mathrm{E}+00$ & 0.0000 & 0.0000 & 0.0000 & \\
\hline & 7/25/2008 0:00 & 59 & 207 & 0 & 9.05 & 9.05 & 9.09 & -0.0575 & $0.00 \mathrm{E}+00$ & 0.0000 & 0.0000 & 0.0000 & \\
\hline & 7/25/2008 1:00 & 60 & 207 & 100 & 9.09 & 9.09 & & -0.0616 & $0.00 \mathrm{E}+00$ & 0.0000 & 0.0000 & 0.0000 & \\
\hline & 7/25/2008 2:00 & 61 & 207 & 200 & 9.05 & 9.05 & & -0.0575 & $0.00 \mathrm{E}+00$ & 0.0000 & 0.0000 & 0.0000 & \\
\hline & 7/25/2008 3:00 & 62 & 207 & 300 & 8.99 & 8.99 & & -0.0512 & $0.00 \mathrm{E}+00$ & 0.0000 & 0.0000 & 0.0000 & \\
\hline & 7/25/2008 4:00 & 63 & 207 & 400 & 8.97 & 8.97 & & -0.0491 & $0.00 E+00$ & 0.0000 & 0.0000 & 0.0000 & \\
\hline & 7/25/2008 5:00 & 64 & 207 & 500 & 9 & 9 & & -0.0522 & $0.00 \mathrm{E}+00$ & 0.0000 & 0.0000 & 0.0000 & \\
\hline & 7/25/2008 6:00 & 65 & 207 & 600 & 9.05 & 9.05 & & -0.0575 & $0.00 \mathrm{E}+00$ & 0.0000 & 0.0000 & 0.0000 & \\
\hline & 7/25/2008 7:00 & 66 & 207 & 700 & \begin{tabular}{|c|}
9.06 \\
\end{tabular} & 9.06 & & -0.0585 & $0.00 \mathrm{E}+00$ & 0.0000 & 0.0000 & 0.0000 & \\
\hline & 7/25/2008 8:00 & 67 & 207 & 800 & 9.03 & 9.03 & & -0.0554 & $0.00 \mathrm{E}+00$ & 0.0000 & 0.0000 & 0.0000 & \\
\hline & 7/25/2008 9:00 & 68 & 207 & 900 & 8.96 & 8.96 & & -0.0480 & $0.00 \mathrm{E}+00$ & 0.0000 & 0.0000 & 0.0000 & \\
\hline & 7/25/2008 10:00 & 69 & 207 & 1000 & 8.71 & 8.71 & & -0.0207 & $0.00 \mathrm{E}+00$ & 0.0000 & 0.0000 & 0.0000 & \\
\hline & 7/25/2008 11:00 & 70 & 207 & 1100 & 7.384 & 7.384 & & 0.1552 & $1.20 \mathrm{E}-03$ & 273.5720 & 100.8032 & 374.3753 & \\
\hline & $7 / 25 / 2008$ 12:00 & 71 & 207 & 1200 & 6.068 & 6.068 & & 0.4057 & $3.92 E-03$ & 892.9538 & 329.0272 & 1221.9809 & \\
\hline & $7 / 25 / 2008$ 13:00 & 72 & 207 & 1300 & 5.507 & 5.507 & & 0.5489 & 5.69E-03 & 1295.4939 & 477.3514 & 1772.8453 & \\
\hline & $7 / 25 / 200814: 00$ & 73 & 207 & 1400 & 5.09 & 5.09 & & 0.6758 & $7.35 E-03$ & 1673.4638 & 616.6222 & 2290.0860 & \\
\hline & $7 / 25 / 200815: 00$ & 74 & 207 & 1500 & 5.047 & 5.047 & & 0.6901 & 7.54E-03 & 1717.0906 & 632.6973 & 2349.7879 & \\
\hline & 7/25/2008 16:00 & 75 & 207 & 1600 & 5.078 & 5.078 & & 0.6798 & 7.40E-03 & 1685.5433 & 621.0731 & 2306.6164 & \\
\hline & $7 / 25 / 2008$ 17:00 & 76 & 207 & 1700 & 4.896 & 4.896 & & 0.7422 & 8.24E-03 & 1878.1123 & 692.0291 & 2570.1413 & \\
\hline & $7 / 25 / 2008$ 18:00 & 77 & 207 & 1800 & 4.926 & 4.926 & & 0.7316 & $8.10 \mathrm{E}-03$ & 1845.1171 & 679.8713 & 2524.9884 & \\
\hline & 7/25/2008 19:00 & 78 & 207 & 1900 & 5.034 & 5.034 & & 0.6945 & $7.60 \mathrm{E}-03$ & 1730.4687 & 637.6268 & 2368.0954 & \\
\hline & $7 / 25 / 200820: 00$ & 79 & 207 & 2000 & 5.627 & 5.627 & & 0.5159 & $5.27 \mathrm{E}-03$ & 1200.2074 & 442.2411 & 1642.4485 & \\
\hline & $7 / 25 / 2008$ 21:00 & 80 & 207 & 2100 & 7.215 & 7.215 & & 0.1823 & 1.46E-03 & 333.4196 & 122.8553 & 456.2749 & \\
\hline & $7 / 25 / 200822: 00$ & 81 & 207 & 2200 & 8.63 & 8.63 & & -0.0116 & $0.00 \mathrm{E}+00$ & 0.0000 & 0.0000 & 0.0000 & \\
\hline
\end{tabular}




\begin{tabular}{|c|c|c|c|c|c|c|c|c|c|c|c|c|c|}
\hline Tree ID & TS & RN & JD & HR & $\begin{array}{c}\text { Raw } \\
\text { TC_dTA }\end{array}$ & $\begin{array}{c}\text { Fixed } \\
\text { TC_dT } \Delta\end{array}$ & dTM & K & $\begin{array}{l}\text { Sap } \\
\text { Velocity } \\
(\mathrm{cm} / \mathrm{s})\end{array}$ & $\begin{array}{c}\text { Probed } \\
\text { Drywood F } \\
(\mathrm{g} / \mathrm{hr})\end{array}$ & $\begin{array}{c}\text { Extrapolated } \\
\text { Wetwood F } \\
(\mathrm{g} / \mathrm{hr})\end{array}$ & $\begin{array}{c}\text { Extrapolated } \\
\text { Total Wood F } \\
(\mathrm{g} / \mathrm{hr})\end{array}$ & $\begin{array}{l}\text { Relative } \\
\text { Humidity, } \\
@ 10 \mathrm{~m}(\%)\end{array}$ \\
\hline & 7/25/2008 23:00 & 82 & 207 & 2300 & \begin{tabular}{|r|}
8.9 \\
\end{tabular} & 8.9 & & -0.0416 & $0.00 \mathrm{E}+00$ & 0.0000 & 0.0000 & 0.0000 & \\
\hline & 7/26/2008 0:00 & 83 & 208 & 0 & 8.93 & 8.93 & 8.93 & -0.0448 & $0.00 \mathrm{E}+00$ & 0.0000 & 0.0000 & 0.0000 & \\
\hline & 7/26/2008 1:00 & 84 & 208 & 100 & 8.93 & 8.93 & & -0.0448 & $0.00 \mathrm{E}+00$ & 0.0000 & 0.0000 & 0.0000 & \\
\hline & $7 / 26 / 20082: 00$ & 85 & 208 & 200 & 8.9 & 8.9 & & -0.0416 & $0.00 \mathrm{E}+00$ & 0.0000 & 0.0000 & 0.0000 & \\
\hline & 7/26/2008 3:00 & 86 & 208 & 300 & 8.86 & 8.86 & & -0.0372 & $0.00 \mathrm{E}+00$ & 0.0000 & 0.0000 & 0.0000 & \\
\hline & 7/26/2008 4:00 & 87 & 208 & 400 & 8.81 & 8.81 & & -0.0318 & $0.00 \mathrm{E}+00$ & 0.0000 & 0.0000 & 0.0000 & \\
\hline & 7/26/2008 5:00 & 88 & 208 & 500 & 8.75 & 8.75 & & -0.0251 & $0.00 \mathrm{E}+00$ & 0.0000 & 0.0000 & 0.0000 & \\
\hline & 7/26/2008 6:00 & 89 & 208 & 600 & 8.69 & 8.69 & & -0.0184 & $0.00 \mathrm{E}+00$ & 0.0000 & 0.0000 & 0.0000 & \\
\hline & 7/26/2008 7:00 & 90 & 208 & 700 & 8.61 & 8.61 & & -0.0093 & $0.00 \mathrm{E}+00$ & 0.0000 & 0.0000 & 0.0000 & \\
\hline & 7/26/2008 8:00 & 91 & 208 & 800 & 8.53 & 8.53 & & 0.0000 & $0.00 E+00$ & 0.0000 & 0.0000 & 0.0000 & \\
\hline & 7/26/2008 9:00 & 92 & 208 & 900 & 7.207 & 7.207 & & 0.1836 & 1.48E-03 & 336.3774 & 123.9452 & 460.3226 & \\
\hline & $7 / 26 / 2008$ 10:00 & 93 & 208 & 1000 & 5.234 & 5.234 & & 0.6297 & $6.73 E-03$ & 1534.0532 & 565.2535 & 2099.3066 & \\
\hline & $7 / 26 / 2008$ 11:00 & 94 & 208 & 1100 & 5.241 & 5.241 & & 0.6276 & $6.71 \mathrm{E}-03$ & 1527.5283 & 562.8492 & 2090.3776 & \\
\hline & $7 / 26 / 2008$ 12:00 & 95 & 208 & 1200 & 5.451 & 5.451 & & 0.5649 & 5.89E-03 & 1341.8767 & 494.4421 & 1836.3188 & \\
\hline & $7 / 26 / 2008$ 13:00 & 96 & 208 & 1300 & 5.001 & 5.001 & & 0.7057 & $7.75 E-03$ & 1764.8292 & 650.2876 & 2415.1168 & \\
\hline & $7 / 26 / 200814: 00$ & 97 & 208 & 1400 & 4.95 & 4.95 & & 0.7232 & 7.99E-03 & 1819.0871 & 670.2800 & 2489.3671 & \\
\hline & $7 / 26 / 2008$ 15:00 & 98 & 208 & 1500 & 4.884 & 4.884 & & 0.7465 & 8.30E-03 & 1891.4548 & 696.9454 & 2588.4002 & \\
\hline & $7 / 26 / 200816: 00$ & 99 & 208 & 1600 & 4.945 & 4.945 & & 0.7250 & 8.01E-03 & 1824.4834 & 672.2684 & 2496.7519 & \\
\hline & $7 / 26 / 200817: 00$ & 100 & 208 & 1700 & 4.741 & 4.741 & & 0.7992 & 9.03E-03 & 2057.0761 & 757.9720 & 2815.0481 & \\
\hline & $7 / 26 / 2008$ 18:00 & 101 & 208 & 1800 & 4.912 & 4.912 & & 0.7366 & 8.17E-03 & 1860.4510 & 685.5214 & 2545.9725 & \\
\hline & $7 / 26 / 2008$ 19:00 & 102 & 208 & 1900 & 4.995 & 4.995 & & 0.7077 & $7.78 \mathrm{E}-03$ & 1771.1391 & 652.6126 & 2423.7517 & \\
\hline & $7 / 26 / 200820: 00$ & 103 & 208 & 2000 & 5.466 & 5.466 & & 0.5606 & 5.84E-03 & 1329.3295 & 489.8188 & 1819.1483 & \\
\hline & $7 / 26 / 200821: 00$ & 104 & 208 & 2100 & 6.743 & 6.743 & & 0.2650 & 2.32E-03 & 528.6029 & 194.7746 & 723.3775 & \\
\hline & $7 / 26 / 200822: 00$ & 105 & 208 & 2200 & 8.13 & 8.13 & & 0.0492 & $2.92 \mathrm{E}-04$ & 66.5115 & 24.5075 & 91.0191 & \\
\hline & $7 / 26 / 2008$ 23:00 & 106 & 208 & 2300 & 8.59 & 8.59 & & -0.0070 & $0.00 \mathrm{E}+00$ & 0.0000 & 0.0000 & 0.0000 & \\
\hline & $7 / 27 / 20080: 00$ & 107 & 209 & 0 & 8.7 & 8.7 & 8.75 & -0.0195 & $0.00 \mathrm{E}+00$ & 0.0000 & 0.0000 & 0.0000 & \\
\hline & 7/27/2008 1:00 & 108 & 209 & 100 & 8.75 & 8.75 & & -0.0251 & $0.00 \mathrm{E}+00$ & 0.0000 & 0.0000 & 0.0000 & \\
\hline & 7/27/2008 2:00 & 109 & 209 & 200 & 8.74 & 8.74 & & -0.0240 & $0.00 \mathrm{E}+00$ & 0.0000 & 0.0000 & 0.0000 & \\
\hline & 7/27/2008 3:00 & 110 & 209 & 300 & 8.74 & 8.74 & & -0.0240 & $0.00 \mathrm{E}+00$ & 0.0000 & 0.0000 & 0.0000 & \\
\hline & 7/27/2008 4:00 & 111 & 209 & 400 & 8.72 & 8.72 & & -0.0218 & $0.00 E+00$ & 0.0000 & 0.0000 & 0.0000 & \\
\hline & 7/27/20085:00 & 112 & 209 & 500 & 8.67 & 8.67 & & -0.0161 & $0.00 \mathrm{E}+00$ & 0.0000 & 0.0000 & 0.0000 & \\
\hline & 7/27/2008 6:00 & 113 & 209 & 600 & 8.61 & 8.61 & & -0.0093 & $0.00 \mathrm{E}+00$ & 0.0000 & 0.0000 & 0.0000 & \\
\hline & 7/27/2008 7:00 & 114 & 209 & 700 & 8.52 & 8.52 & & 0.0012 & $2.94 \mathrm{E}-06$ & 0.6694 & 0.2467 & 0.9161 & \\
\hline & 7/27/2008 8:00 & 115 & 209 & 800 & 8.2 & 8.2 & & 0.0402 & $2.28 \mathrm{E}-04$ & 51.9360 & 19.1369 & 71.0729 & \\
\hline & $7 / 27 / 20089: 00$ & 116 & 209 & 900 & 5.787 & 5.787 & & 0.4740 & 4.75E-03 & 1081.3299 & 398.4382 & 1479.7681 & \\
\hline & $7 / 27 / 2008$ 10:00 & 117 & 209 & 1000 & 4.791 & 4.791 & & 0.7804 & 8.77E-03 & 1997.7440 & 736.1099 & 2733.8539 & \\
\hline & $7 / 27 / 2008$ 11:00 & 118 & 209 & 1100 & 4.72 & 4.72 & & 0.8072 & $9.14 \mathrm{E}-03$ & 2082.4689 & 767.3285 & 2849.7974 & \\
\hline & $7 / 27 / 2008$ 12:00 & 119 & 209 & 1200 & 4.715 & 4.715 & & 0.8091 & 9.17E-03 & 2088.5568 & 769.5717 & 2858.1285 & \\
\hline & $7 / 27 / 200813: 00$ & 120 & 209 & 1300 & 4.727 & 4.727 & & 0.8045 & $9.10 \mathrm{E}-03$ & 2073.9731 & 764.1980 & 2838.1711 & \\
\hline & $7 / 27 / 2008$ 14:00 & 121 & 209 & 1400 & 4.618 & 4.618 & & 0.8471 & 9.70E-03 & 2209.9512 & 814.3020 & 3024.2531 & \\
\hline & 7/27/2008 15:00 & 122 & 209 & 1500 & 4.585 & 4.585 & & 0.8604 & 9.89E-03 & 2252.7221 & 830.0618 & 3082.7839 & \\
\hline
\end{tabular}




\begin{tabular}{|c|c|c|c|c|c|c|c|c|c|c|c|c|c|}
\hline Tree ID & TS & RN & JD & HR & $\begin{array}{c}\text { Raw } \\
\text { TC_dTA }\end{array}$ & $\begin{array}{c}\text { Fixed } \\
\text { TC_dT } \Delta\end{array}$ & dTM & $\mathbf{K}$ & $\begin{array}{l}\text { Sap } \\
\text { Velocity } \\
(\mathrm{cm} / \mathrm{s})\end{array}$ & $\begin{array}{c}\text { Probed } \\
\text { Drywood F } \\
(\mathrm{g} / \mathrm{hr})\end{array}$ & $\begin{array}{c}\text { Extrapolated } \\
\text { Wetwood F } \\
(\mathrm{g} / \mathrm{hr})\end{array}$ & $\begin{array}{c}\text { Extrapolated } \\
\text { Total Wood F } \\
(\mathrm{g} / \mathrm{hr})\end{array}$ & $\begin{array}{l}\text { Relative } \\
\text { Humidity, } \\
@ 10 \mathrm{~m}(\%)\end{array}$ \\
\hline & 7/27/2008 16:00 & 123 & 209 & 1600 & \begin{tabular}{|r|}
4.629 \\
\end{tabular} & 4.629 & & 0.8427 & $9.64 \mathrm{E}-03$ & 2195.8636 & 809.1111 & \begin{tabular}{|l|}
3004.9747 \\
\end{tabular} & \\
\hline & 7/27/2008 17:00 & 124 & 209 & 1700 & 4.571 & 4.571 & & 0.8661 & 9.97E-03 & 2271.1009 & 836.8338 & 3107.9347 & \\
\hline & $7 / 27 / 2008$ 18:00 & 125 & 209 & 1800 & 4.656 & 4.656 & & 0.8320 & $9.49 \mathrm{E}-03$ & 2161.6382 & 796.5000 & 2958.1382 & \\
\hline & $7 / 27 / 2008$ 19:00 & 126 & 209 & 1900 & 4.802 & 4.802 & & 0.7763 & 8.71E-03 & 1984.9001 & 731.3772 & 2716.2773 & \\
\hline & $7 / 27 / 200820: 00$ & 127 & 209 & 2000 & 5.362 & 5.362 & & 0.5908 & 6.23E-03 & 1418.2335 & 522.5773 & 1940.8107 & \\
\hline & $7 / 27 / 2008$ 21:00 & 128 & 209 & 2100 & 6.464 & 6.464 & & 0.3196 & $2.92 \mathrm{E}-03$ & 665.7033 & 245.2921 & 910.9954 & \\
\hline & $7 / 27 / 2008$ 22:00 & 129 & 209 & 2200 & 7.389 & 7.389 & & 0.1544 & 1.19E-03 & 271.8768 & 100.1786 & 372.0554 & \\
\hline & $7 / 27 / 200823: 00$ & 130 & 209 & 2300 & 7.91 & 7.91 & & 0.0784 & 5.18E-04 & 117.9944 & 43.4775 & 161.4719 & \\
\hline & $7 / 28 / 20080: 00$ & 131 & 210 & 0 & 8.21 & 8.21 & 8.58 & 0.0390 & 2.19E-04 & 49.9305 & 18.3979 & 68.3284 & \\
\hline & 7/28/2008 1:00 & 132 & 210 & 100 & 8.38 & 8.38 & & 0.0179 & 8.41E-05 & 19.1574 & 7.0590 & 26.2164 & \\
\hline & 7/28/2008 2:00 & 133 & 210 & 200 & 8.46 & 8.46 & & 0.0083 & $3.25 E-05$ & 7.4098 & 2.7303 & 10.1401 & \\
\hline & 7/28/2008 3:00 & 134 & 210 & 300 & 8.58 & 8.58 & & -0.0058 & $0.00 \mathrm{E}+00$ & 0.0000 & 0.0000 & 0.0000 & \\
\hline & 7/28/2008 4:00 & 135 & 210 & 400 & 8.57 & 8.57 & & -0.0047 & $0.00 \mathrm{E}+00$ & 0.0000 & 0.0000 & 0.0000 & \\
\hline & 7/28/2008 5:00 & 136 & 210 & 500 & 8.5 & 8.5 & & 0.0035 & 1.14E-05 & 2.5960 & 0.9565 & 3.5525 & \\
\hline & 7/28/2008 6:00 & 137 & 210 & 600 & 8.43 & 8.43 & & 0.0119 & 5.07E-05 & 11.5449 & 4.2539 & 15.7988 & \\
\hline & 7/28/2008 7:00 & 138 & 210 & 700 & 8 & 8 & & 0.0662 & $4.21 E-04$ & 95.9317 & 35.3480 & 131.2798 & \\
\hline & 7/28/2008 8:00 & 139 & 210 & 800 & 6.031 & 6.031 & & 0.4144 & 4.02E-03 & 916.3755 & 337.6574 & 1254.0329 & \\
\hline & $7 / 28 / 20089: 00$ & 140 & 210 & 900 & 5.095 & 5.095 & & 0.6742 & 7.32E-03 & 1668.4523 & 614.7756 & 2283.2279 & \\
\hline & $7 / 28 / 2008$ 10:00 & 141 & 210 & 1000 & 4.67 & 4.67 & & 0.8266 & 9.41E-03 & 2144.0868 & 790.0329 & 2934.1197 & \\
\hline & $7 / 28 / 2008$ 11:00 & 142 & 210 & 1100 & 4.612 & 4.612 & & 0.8495 & $9.74 \mathrm{E}-03$ & 2217.6708 & 817.1464 & 3034.8172 & \\
\hline & $7 / 28 / 200812: 00$ & 143 & 210 & 1200 & 4.581 & 4.581 & & 0.8620 & $9.91 \mathrm{E}-03$ & 2257.9589 & 831.9914 & 3089.9502 & \\
\hline & 7/28/2008 13:00 & 144 & 210 & 1300 & 4.617 & 4.617 & & 0.8475 & 9.71E-03 & 2211.2360 & 814.7754 & 3026.0114 & \\
\hline & $7 / 28 / 2008$ 14:00 & 145 & 210 & 1400 & 4.574 & 4.574 & & 0.8649 & 9.95E-03 & 2267.1507 & 835.3783 & 3102.5290 & \\
\hline & $7 / 28 / 2008$ 15:00 & 146 & 210 & 1500 & 4.486 & 4.486 & & 0.9015 & 1.05E-02 & 2385.7682 & 879.0854 & 3264.8535 & \\
\hline & $7 / 28 / 2008$ 16:00 & 147 & 210 & 1600 & 4.554 & 4.554 & & 0.8731 & $1.01 \mathrm{E}-02$ & 2293.6078 & 845.1270 & 3138.7347 & \\
\hline & 7/28/2008 17:00 & 148 & 210 & 1700 & 4.485 & 4.485 & & 0.9019 & 1.05E-02 & 2387.1495 & 879.5943 & 3266.7438 & \\
\hline & $7 / 28 / 2008$ 18:00 & 149 & 210 & 1800 & 4.633 & 4.633 & & 0.8411 & $9.62 \mathrm{E}-03$ & 2190.7616 & 807.2312 & 2997.9928 & \\
\hline & $7 / 28 / 2008$ 19:00 & 150 & 210 & 1900 & 4.883 & 4.883 & & 0.7469 & 8.31E-03 & 1892.5704 & 697.3565 & 2589.9269 & \\
\hline & $7 / 28 / 200820: 00$ & 151 & 210 & 2000 & 5.389 & 5.389 & & 0.5829 & $6.12 \mathrm{E}-03$ & 1394.7184 & 513.9127 & 1908.6311 & \\
\hline & $7 / 28 / 200821: 00$ & 152 & 210 & 2100 & 6.825 & 6.825 & & 0.2498 & $2.16 \mathrm{E}-03$ & 491.5354 & 181.1163 & 672.6518 & \\
\hline & $7 / 28 / 2008$ 22:00 & 153 & 210 & 2200 & 7.535 & 7.535 & & 0.1321 & 9.84E-04 & 224.2401 & 82.6259 & 306.8659 & \\
\hline & $7 / 28 / 200823: 00$ & 154 & 210 & 2300 & 7.93 & 7.93 & & 0.0757 & 4.96E-04 & 112.9747 & 41.6279 & 154.6026 & \\
\hline & $7 / 29 / 20080: 00$ & 155 & 211 & 0 & 8.18 & 8.18 & 8.43 & 0.0428 & $2.46 \mathrm{E}-04$ & 56.0055 & 20.6364 & 76.6419 & \\
\hline & 7/29/2008 1:00 & 156 & 211 & 100 & 8.32 & 8.32 & & 0.0252 & $1.28 \mathrm{E}-04$ & 29.2457 & 10.7762 & 40.0219 & \\
\hline & 7/29/2008 2:00 & 157 & 211 & 200 & 8.41 & 8.41 & & 0.0143 & 6.36E-05 & 14.4921 & 5.3399 & 19.8320 & \\
\hline & 7/29/2008 3:00 & 158 & 211 & 300 & 8.43 & 8.43 & & 0.0119 & 5.07E-05 & 11.5449 & 4.2539 & 15.7988 & \\
\hline & 7/29/2008 4:00 & 159 & 211 & 400 & 8.42 & 8.42 & & 0.0131 & 5.71E-05 & 13.0010 & 4.7905 & 17.7915 & \\
\hline & 7/29/2008 5:00 & 160 & 211 & 500 & 8.33 & 8.33 & & 0.0240 & 1.21E-04 & 27.5002 & 10.1330 & 37.6333 & \\
\hline & $7 / 29 / 20086: 00$ & 161 & 211 & 600 & 8.24 & 8.24 & & 0.0352 & 1.93E-04 & 44.0340 & 16.2252 & 60.2592 & \\
\hline & 7/29/2008 7:00 & 162 & 211 & 700 & 8.05 & 8.05 & & 0.0596 & 3.70E-04 & 84.2666 & 31.0498 & 115.3164 & \\
\hline & 7/29/2008 8:00 & 163 & 211 & 800 & 7.038 & 7.038 & & 0.2120 & 1.76E-03 & 401.5890 & 147.9737 & 549.5628 & \\
\hline
\end{tabular}




\begin{tabular}{|c|c|c|c|c|c|c|c|c|c|c|c|c|c|}
\hline Tree ID & TS & RN & JD & HR & $\begin{array}{c}\text { Raw } \\
\text { TC_dTA }\end{array}$ & $\begin{array}{c}\text { Fixed } \\
\text { TC_dT } \Delta\end{array}$ & dTM & $\mathbf{K}$ & $\begin{array}{l}\text { Sap } \\
\text { Velocity } \\
(\mathrm{cm} / \mathrm{s})\end{array}$ & $\begin{array}{c}\text { Probed } \\
\text { Drywood F } \\
(\mathrm{g} / \mathrm{hr})\end{array}$ & $\begin{array}{c}\text { Extrapolated } \\
\text { Wetwood F } \\
(\mathrm{g} / \mathrm{hr})\end{array}$ & $\begin{array}{c}\text { Extrapolated } \\
\text { Total Wood F } \\
(\mathrm{g} / \mathrm{hr})\end{array}$ & $\begin{array}{l}\text { Relative } \\
\text { Humidity, } \\
@ 10 \mathrm{~m}(\%)\end{array}$ \\
\hline & 7/29/2008 9:00 & 164 & 211 & 900 & \begin{tabular}{|r|}
5.372 \\
\end{tabular} & 5.372 & & 0.5879 & $6.19 \mathrm{E}-03$ & 1409.4880 & 519.3548 & \begin{tabular}{|r|}
1928.8429 \\
\end{tabular} & \\
\hline & $7 / 29 / 2008$ 10:00 & 165 & 211 & 1000 & 4.716 & 4.716 & & 0.8087 & $9.16 \mathrm{E}-03$ & 2087.3379 & 769.1226 & 2856.4605 & \\
\hline & $7 / 29 / 2008$ 11:00 & 166 & 211 & 1100 & 4.978 & 4.978 & & 0.7135 & $7.85 E-03$ & 1789.1227 & 659.2390 & 2448.3617 & \\
\hline & $7 / 29 / 2008$ 12:00 & 167 & 211 & 1200 & 4.553 & 4.553 & & 0.8735 & 1.01E-02 & 2294.9383 & 845.6172 & 3140.5555 & \\
\hline & 7/29/2008 13:00 & 168 & 211 & 1300 & 4.606 & 4.606 & & 0.8519 & 9.77E-03 & 2225.4155 & 820.0001 & 3045.4157 & \\
\hline & $7 / 29 / 2008$ 14:00 & 169 & 211 & 1400 & 4.552 & 4.552 & & 0.8739 & 1.01E-02 & 2296.2695 & 846.1077 & 3142.3772 & \\
\hline & $7 / 29 / 2008$ 15:00 & 170 & 211 & 1500 & 4.73 & 4.73 & & 0.8034 & $9.09 \mathrm{E}-03$ & 2070.3417 & 762.8600 & 2833.2016 & \\
\hline & $7 / 29 / 2008$ 16:00 & 171 & 211 & 1600 & 4.806 & 4.806 & & 0.7749 & 8.69E-03 & 1980.2479 & 729.6631 & 2709.9110 & \\
\hline & 7/29/2008 17:00 & 172 & 211 & 1700 & 4.936 & 4.936 & & 0.7281 & 8.05E-03 & 1834.2320 & 675.8605 & 2510.0926 & \\
\hline & 7/29/2008 18:00 & 173 & 211 & 1800 & 4.908 & 4.908 & & 0.7380 & 8.19E-03 & 1864.8526 & 687.1433 & 2551.9959 & \\
\hline & $7 / 29 / 2008$ 19:00 & 174 & 211 & 1900 & 4.912 & 4.912 & & 0.7366 & 8.17E-03 & 1860.4510 & 685.5214 & 2545.9725 & \\
\hline & $7 / 29 / 200820: 00$ & 175 & 211 & 2000 & 5.621 & 5.621 & & 0.5175 & $5.29 E-03$ & 1204.8431 & 443.9492 & 1648.7923 & \\
\hline & 7/29/2008 21:00 & 176 & 211 & 2100 & 6.94 & 6.94 & & 0.2291 & 1.94E-03 & 441.8642 & 162.8140 & 604.6782 & \\
\hline & $7 / 29 / 2008$ 22:00 & 177 & 211 & 2200 & 7.96 & 7.96 & & 0.0716 & 4.63E-04 & 105.5700 & 38.8994 & 144.4694 & \\
\hline & $7 / 29 / 2008$ 23:00 & 178 & 211 & 2300 & 8.16 & 8.16 & & 0.0453 & 2.64E-04 & 60.1517 & 22.1641 & 82.3159 & \\
\hline & $7 / 30 / 20080: 00$ & 179 & 212 & 0 & 8.14 & 8.14 & 8.14 & 0.0479 & $2.83 \mathrm{E}-04$ & 64.3731 & 23.7196 & 88.0927 & \\
\hline & 7/30/2008 1:00 & 180 & 212 & 100 & 8.09 & 8.09 & & 0.0544 & 3.30E-04 & 75.2468 & 27.7262 & 102.9730 & \\
\hline & 7/30/2008 2:00 & 181 & 212 & 200 & 8.03 & 8.03 & & 0.0623 & $3.90 \mathrm{E}-04$ & 88.8812 & 32.7501 & 121.6313 & \\
\hline & 7/30/2008 3:00 & 182 & 212 & 300 & 7.97 & 7.97 & & 0.0703 & 4.53E-04 & 103.1352 & 38.0023 & 141.1374 & \\
\hline & 7/30/2008 4:00 & 183 & 212 & 400 & 7.917 & 7.917 & & 0.0774 & 5.10E-04 & 116.2300 & 42.8273 & 159.0573 & \\
\hline & 7/30/2008 5:00 & 184 & 212 & 500 & 7.865 & 7.865 & & 0.0846 & $5.69 \mathrm{E}-04$ & 129.5301 & 47.7280 & 177.2582 & \\
\hline & 7/30/2008 6:00 & 185 & 212 & 600 & 7.818 & 7.818 & & 0.0911 & $6.23 E-04$ & 141.9334 & 52.2983 & 194.2317 & \\
\hline & 7/30/2008 7:00 & 186 & 212 & 700 & 7.773 & 7.773 & & 0.0974 & 6.77E-04 & 154.1469 & 56.7986 & 210.9455 & \\
\hline & $7 / 30 / 20088: 00$ & 187 & 212 & 800 & 7.718 & 7.718 & & 0.1052 & 7.44E-04 & 169.5226 & 62.4641 & 231.9867 & \\
\hline & 7/30/2008 9:00 & 188 & 212 & 900 & 6.315 & 6.315 & & 0.3508 & $3.28 \mathrm{E}-03$ & 746.4108 & 275.0304 & 1021.4413 & \\
\hline & $7 / 30 / 2008$ 10:00 & 189 & 212 & 1000 & 4.279 & 4.279 & & 0.9935 & 1.18E-02 & 2688.8878 & 990.7760 & 3679.6638 & \\
\hline & 7/30/2008 11:00 & 190 & 212 & 1100 & 4.116 & 4.116 & & 1.0724 & 1.30E-02 & 2954.2817 & 1088.5658 & 4042.8475 & \\
\hline & $7 / 30 / 2008$ 12:00 & 191 & 212 & 1200 & 4.122 & 4.122 & & 1.0694 & 1.29E-02 & 2944.0551 & 1084.7977 & 4028.8528 & \\
\hline & 7/30/2008 13:00 & 192 & 212 & 1300 & 4.135 & 4.135 & & 1.0629 & 1.28E-02 & 2922.0221 & 1076.6792 & 3998.7013 & \\
\hline & $7 / 30 / 2008$ 14:00 & 193 & 212 & 1400 & 4.166 & 4.166 & & 1.0475 & $1.26 \mathrm{E}-02$ & 2870.1605 & 1057.5697 & 3927.7302 & \\
\hline & 7/30/2008 15:00 & 194 & 212 & 1500 & 4.301 & 4.301 & & 0.9833 & 1.17E-02 & 2654.9545 & 978.2726 & 3633.2271 & \\
\hline & $7 / 30 / 2008$ 16:00 & 195 & 212 & 1600 & 4.293 & 4.293 & & 0.9870 & 1.17E-02 & 2667.2443 & 982.8010 & 3650.0453 & \\
\hline & $7 / 30 / 2008$ 17:00 & 196 & 212 & 1700 & 4.313 & 4.313 & & 0.9777 & 1.16E-02 & 2636.6252 & 971.5188 & 3608.1440 & \\
\hline & 7/30/2008 18:00 & 197 & 212 & 1800 & 4.5 & 4.5 & & 0.8956 & 1.04E-02 & 2366.5102 & 871.9894 & 3238.4996 & \\
\hline & $7 / 30 / 2008$ 19:00 & 198 & 212 & 1900 & 4.648 & 4.648 & & 0.8352 & 9.53E-03 & 2171.7271 & 800.2175 & 2971.9446 & \\
\hline & $7 / 30 / 200820: 00$ & 199 & 212 & 2000 & 5.289 & 5.289 & & 0.6128 & 6.51E-03 & 1483.3905 & 546.5858 & 2029.9763 & \\
\hline & 7/30/2008 21:00 & 200 & 212 & 2100 & 6.686 & 6.686 & & 0.2758 & 2.44E-03 & 555.2063 & 204.5772 & 759.7834 & \\
\hline & $7 / 30 / 2008$ 22:00 & 201 & 212 & 2200 & 7.798 & 7.798 & & 0.0939 & 6.47E-04 & 147.3209 & 54.2834 & 201.6043 & \\
\hline & $7 / 30 / 200823: 00$ & 202 & 212 & 2300 & 8.09 & 8.09 & & 0.0544 & 3.30E-04 & 75.2468 & 27.7262 & 102.9730 & \\
\hline & $7 / 31 / 20080: 00$ & 203 & 213 & 0 & 8.18 & 8.18 & 8.21 & 0.0428 & 2.46E-04 & 56.0055 & 20.6364 & 76.6419 & \\
\hline & 7/31/2008 1:00 & 204 & 213 & 100 & 8.21 & 8.21 & & 0.0390 & 2.19E-04 & 49.9305 & 18.3979 & 68.3284 & \\
\hline
\end{tabular}




\begin{tabular}{|c|c|c|c|c|c|c|c|c|c|c|c|c|c|}
\hline Tree ID & TS & RN & JD & HR & $\begin{array}{c}\text { Raw } \\
\text { TC_dTA }\end{array}$ & $\begin{array}{c}\text { Fixed } \\
\text { TC_dT } \Delta\end{array}$ & dTM & $\mathbf{K}$ & $\begin{array}{l}\text { Sap } \\
\text { Velocity } \\
(\mathrm{cm} / \mathrm{s})\end{array}$ & $\begin{array}{c}\text { Probed } \\
\text { Drywood F } \\
(\mathrm{g} / \mathrm{hr})\end{array}$ & $\begin{array}{c}\text { Extrapolated } \\
\text { Wetwood F } \\
(\mathrm{g} / \mathrm{hr})\end{array}$ & $\begin{array}{c}\text { Extrapolated } \\
\text { Total Wood F } \\
(\mathrm{g} / \mathrm{hr})\end{array}$ & $\begin{array}{l}\text { Relative } \\
\text { Humidity, } \\
@ 10 \mathrm{~m}(\%)\end{array}$ \\
\hline & 7/31/2008 2:00 & 205 & 213 & 200 & 8.16 & 8.16 & & 0.0453 & 2.64E-04 & 60.1517 & 22.1641 & $\begin{array}{r}82.3159 \\
\end{array}$ & \\
\hline & 7/31/2008 3:00 & 206 & 213 & 300 & 8.11 & 8.11 & & 0.0518 & $3.11 \mathrm{E}-04$ & 70.8431 & 26.1036 & 96.9467 & \\
\hline & 7/31/2008 4:00 & 207 & 213 & 400 & 8.04 & 8.04 & & 0.0609 & $3.80 E-04$ & 86.5653 & 31.8968 & 118.4620 & \\
\hline & 7/31/2008 5:00 & 208 & 213 & 500 & 7.968 & 7.968 & & 0.0705 & 4.55E-04 & 103.6208 & 38.1812 & 141.8020 & \\
\hline & 7/31/2008 6:00 & 209 & 213 & 600 & 7.899 & 7.899 & & 0.0799 & 5.30E-04 & 120.7834 & 44.5051 & 165.2886 & \\
\hline & 7/31/2008 7:00 & 210 & 213 & 700 & 7.823 & 7.823 & & 0.0904 & 6.17E-04 & 140.5967 & 51.8058 & 192.4025 & \\
\hline & $7 / 31 / 2008$ 8:00 & 211 & 213 & 800 & 7.723 & 7.723 & & 0.1045 & 7.38E-04 & 168.1044 & 61.9415 & 230.0460 & \\
\hline & $7 / 31 / 20089: 00$ & 212 & 213 & 900 & 5.996 & 5.996 & & 0.4226 & 4.12E-03 & 938.9031 & 345.9581 & 1284.8612 & \\
\hline & $7 / 31 / 2008$ 10:00 & 213 & 213 & 1000 & 4.215 & 4.215 & & 1.0237 & 1.22E-02 & 2790.0888 & 1028.0656 & 3818.1544 & \\
\hline & 7/31/2008 11:00 & 214 & 213 & 1100 & 4.186 & 4.186 & & 1.0377 & 1.25E-02 & 2837.2003 & 1045.4248 & 3882.6251 & \\
\hline & $7 / 31 / 2008$ 12:00 & 215 & 213 & 1200 & 4.045 & 4.045 & & 1.1088 & 1.35E-02 & 3078.1186 & 1134.1961 & 4212.3147 & \\
\hline & $7 / 31 / 2008$ 13:00 & 216 & 213 & 1300 & 4.223 & 4.223 & & 1.0199 & 1.22E-02 & 2777.2322 & 1023.3283 & 3800.5606 & \\
\hline & 7/31/2008 14:00 & 217 & 213 & 1400 & 4.082 & 4.082 & & 1.0897 & 1.32E-02 & 3012.9273 & 1110.1750 & 4123.1024 & \\
\hline & 7/31/2008 15:00 & 218 & 213 & 1500 & 4.083 & 4.083 & & 1.0892 & 1.32E-02 & 3011.1854 & 1109.5332 & 4120.7186 & \\
\hline & $7 / 31 / 2008$ 16:00 & 219 & 213 & 1600 & 4.195 & 4.195 & & 1.0334 & 1.24E-02 & 2822.4939 & 1040.0059 & 3862.4999 & \\
\hline & 7/31/2008 17:00 & 220 & 213 & 1700 & 4.184 & 4.184 & & 1.0387 & $1.25 \mathrm{E}-02$ & 2840.4790 & 1046.6329 & 3887.1118 & \\
\hline & $7 / 31 / 2008$ 18:00 & 221 & 213 & 1800 & 4.231 & 4.231 & & 1.0161 & 1.21E-02 & 2764.4354 & 1018.6131 & 3783.0484 & \\
\hline & $7 / 31 / 2008$ 19:00 & 222 & 213 & 1900 & 4.619 & 4.619 & & 0.8467 & $9.70 \mathrm{E}-03$ & 2208.6670 & 813.8288 & 3022.4958 & \\
\hline & $7 / 31 / 200820: 00$ & 223 & 213 & 2000 & 5.473 & 5.473 & & 0.5586 & 5.81E-03 & 1323.5052 & 487.6727 & 1811.1779 & \\
\hline & $7 / 31 / 200821: 00$ & 224 & 213 & 2100 & 6.371 & 6.371 & & 0.3389 & 3.14E-03 & 715.4313 & 263.6154 & 979.0466 & \\
\hline & $7 / 31 / 200822: 00$ & 225 & 213 & 2200 & 7.368 & 7.368 & & 0.1577 & $1.22 \mathrm{E}-03$ & 279.0255 & 102.8127 & 381.8381 & \\
\hline & 7/31/2008 23:00 & 226 & 213 & 2300 & 7.286 & 7.286 & & 0.1707 & 1.35E-03 & 307.6679 & 113.3666 & 421.0345 & \\
\hline & $8 / 1 / 20080: 00$ & 227 & 214 & 0 & 7.601 & 7.601 & 8.15 & 0.1222 & 8.95E-04 & 203.8722 & 75.1209 & 278.9931 & \\
\hline & 8/1/2008 1:00 & 228 & 214 & 100 & 7.999 & 7.999 & & 0.0664 & $4.22 \mathrm{E}-04$ & 96.1694 & 35.4356 & 131.6050 & \\
\hline & 8/1/2008 2:00 & 229 & 214 & 200 & 8.15 & 8.15 & & 0.0466 & 2.73E-04 & 62.2531 & 22.9384 & 85.1916 & \\
\hline & $8 / 1 / 2008$ 3:00 & 230 & 214 & 300 & 8.11 & 8.11 & & 0.0518 & 3.11E-04 & 70.8431 & 26.1036 & 96.9467 & \\
\hline & 8/1/2008 4:00 & 231 & 214 & 400 & 8.13 & 8.13 & & 0.0492 & 2.92E-04 & 66.5115 & 24.5075 & 91.0191 & \\
\hline & 8/1/2008 5:00 & 232 & 214 & 500 & 8.13 & 8.13 & & 0.0492 & $2.92 \mathrm{E}-04$ & 66.5115 & 24.5075 & 91.0191 & \\
\hline & 8/1/2008 6:00 & 233 & 214 & 600 & 8.06 & 8.06 & & 0.0583 & 3.60E-04 & 81.9854 & 30.2092 & 112.1946 & \\
\hline & 8/1/2008 7:00 & 234 & 214 & 700 & 8 & 8 & & 0.0662 & $4.21 E-04$ & 95.9317 & 35.3480 & 131.2798 & \\
\hline & 8/1/2008 8:00 & 235 & 214 & 800 & 7.593 & 7.593 & & 0.1234 & $9.06 \mathrm{E}-04$ & 206.3028 & 76.0165 & 282.3192 & \\
\hline & 8/1/2008 9:00 & 236 & 214 & 900 & 5.77 & 5.77 & & 0.4783 & $4.80 \mathrm{E}-03$ & 1093.5386 & 402.9368 & 1496.4754 & \\
\hline & 8/1/2008 10:00 & 237 & 214 & 1000 & 4.343 & 4.343 & & 0.9641 & 1.14E-02 & 2591.3480 & 954.8355 & 3546.1835 & \\
\hline & 8/1/2008 11:00 & 238 & 214 & 1100 & 4.311 & 4.311 & & 0.9787 & $1.16 \mathrm{E}-02$ & 2639.6714 & 972.6412 & 3612.3126 & \\
\hline & 8/1/2008 12:00 & 239 & 214 & 1200 & 4.319 & 4.319 & & 0.9750 & 1.15E-02 & 2627.5076 & 968.1592 & 3595.6669 & \\
\hline & 8/1/2008 13:00 & 240 & 214 & 1300 & 4.259 & 4.259 & & 1.0028 & 1.19E-02 & 2720.1113 & 1002.2810 & 3722.3923 & \\
\hline & 8/1/2008 14:00 & 241 & 214 & 1400 & 4.231 & 4.231 & & 1.0161 & $1.21 \mathrm{E}-02$ & 2764.4354 & 1018.6131 & 3783.0484 & \\
\hline & 8/1/2008 15:00 & 242 & 214 & 1500 & 4.209 & 4.209 & & 1.0266 & 1.23E-02 & 2799.7706 & 1031.6331 & 3831.4037 & \\
\hline & $8 / 1 / 200816: 00$ & 243 & 214 & 1600 & 4.292 & 4.292 & & 0.9874 & $1.17 \mathrm{E}-02$ & 2668.7845 & 983.3685 & 3652.1530 & \\
\hline & 8/1/2008 17:00 & 244 & 214 & 1700 & 4.302 & 4.302 & & 0.9828 & 1.16E-02 & 2653.4223 & 977.7080 & 3631.1303 & \\
\hline & 8/1/2008 18:00 & 245 & 214 & 1800 & 4.373 & 4.373 & & 0.9506 & 1.12E-02 & 2546.8370 & 938.4345 & 3485.2715 & \\
\hline
\end{tabular}




\begin{tabular}{|c|c|c|c|c|c|c|c|c|c|c|c|c|c|}
\hline Tree ID & TS & RN & JD & HR & $\begin{array}{c}\text { Raw } \\
\text { TC_dTA }\end{array}$ & $\begin{array}{c}\text { Fixed } \\
\text { TC_dT } \Delta\end{array}$ & dTM & $\mathbf{K}$ & $\begin{array}{l}\text { Sap } \\
\text { Velocity } \\
(\mathrm{cm} / \mathrm{s})\end{array}$ & $\begin{array}{c}\text { Probed } \\
\text { Drywood F } \\
(\mathrm{g} / \mathrm{hr})\end{array}$ & $\begin{array}{c}\text { Extrapolated } \\
\text { Wetwood F } \\
(\mathrm{g} / \mathrm{hr})\end{array}$ & $\begin{array}{c}\text { Extrapolated } \\
\text { Total Wood F } \\
(\mathrm{g} / \mathrm{hr})\end{array}$ & $\begin{array}{l}\text { Relative } \\
\text { Humidity, } \\
@ 10 \mathrm{~m}(\%)\end{array}$ \\
\hline & 8/1/2008 19:00 & 246 & 214 & 1900 & \begin{tabular}{|r|}
4.559 \\
\end{tabular} & 4.559 & & 0.8710 & 1.00E-02 & 2286.9663 & 842.6798 & 3129.6461 & \\
\hline & 8/1/2008 20:00 & 247 & 214 & 2000 & 5.38 & 5.38 & & 0.5855 & $6.16 \mathrm{E}-03$ & 1402.5224 & 516.7882 & 1919.3106 & \\
\hline & 8/1/2008 21:00 & 248 & 214 & 2100 & 7.039 & 7.039 & & 0.2118 & $1.76 \mathrm{E}-03$ & 401.1876 & 147.8258 & 549.0134 & \\
\hline & 8/1/2008 22:00 & 249 & 214 & 2200 & 7.893 & 7.893 & & 0.0807 & 5.37E-04 & 122.3131 & 45.0688 & 167.3819 & \\
\hline & 8/1/2008 23:00 & 250 & 214 & 2300 & 8.09 & 8.09 & & 0.0544 & 3.30E-04 & 75.2468 & 27.7262 & 102.9730 & \\
\hline & 8/2/2008 0:00 & 251 & 215 & 0 & 8.13 & 8.13 & 8.26 & 0.0492 & $2.92 \mathrm{E}-04$ & 66.5115 & 24.5075 & 91.0191 & \\
\hline & 8/2/2008 1:00 & 252 & 215 & 100 & 8.22 & 8.22 & & 0.0377 & $2.10 \mathrm{E}-04$ & 47.9448 & 17.6662 & 65.6110 & \\
\hline & 8/2/2008 2:00 & 253 & 215 & 200 & 8.26 & 8.26 & & 0.0327 & 1.77E-04 & 40.2058 & 14.8147 & 55.0205 & \\
\hline & 8/2/2008 3:00 & 254 & 215 & 300 & 8.26 & 8.26 & & 0.0327 & 1.77E-04 & 40.2058 & 14.8147 & 55.0205 & \\
\hline & 8/2/2008 4:00 & 255 & 215 & 400 & 8.14 & 8.14 & & 0.0479 & 2.83E-04 & 64.3731 & 23.7196 & 88.0927 & \\
\hline & 8/2/2008 5:00 & 256 & 215 & 500 & 8.06 & 8.06 & & 0.0583 & 3.60E-04 & 81.9854 & 30.2092 & 112.1946 & \\
\hline & 8/2/2008 6:00 & 257 & 215 & 600 & 7.986 & 7.986 & & 0.0681 & 4.36E-04 & 99.2744 & 36.5797 & 135.8541 & \\
\hline & 8/2/2008 7:00 & 258 & 215 & 700 & 7.631 & 7.631 & & 0.1178 & 8.55E-04 & 194.8511 & 71.7969 & 266.6480 & \\
\hline & 8/2/2008 8:00 & 259 & 215 & 800 & 6.053 & 6.053 & & 0.4092 & 3.96E-03 & 902.4008 & 332.5082 & 1234.9090 & \\
\hline & 8/2/2008 9:00 & 260 & 215 & 900 & 4.647 & 4.647 & & 0.8356 & $9.54 \mathrm{E}-03$ & 2172.9913 & 800.6833 & 2973.6746 & \\
\hline & 8/2/2008 10:00 & 261 & 215 & 1000 & 4.498 & 4.498 & & 0.8964 & 1.04E-02 & 2369.2522 & 872.9997 & 3242.2520 & \\
\hline & 8/2/2008 11:00 & 262 & 215 & 1100 & 4.446 & 4.446 & & 0.9186 & 1.07E-02 & 2441.6230 & 899.6662 & 3341.2893 & \\
\hline & $8 / 2 / 200812: 00$ & 263 & 215 & 1200 & 4.397 & 4.397 & & 0.9400 & 1.10E-02 & 2511.7684 & 925.5127 & 3437.2811 & \\
\hline & 8/2/2008 13:00 & 264 & 215 & 1300 & 4.385 & 4.385 & & 0.9453 & 1.11E-02 & 2529.2433 & 931.9517 & 3461.1950 & \\
\hline & 8/2/2008 14:00 & 265 & 215 & 1400 & 4.392 & 4.392 & & 0.9422 & $1.11 E-02$ & 2519.0352 & 928.1903 & 3447.2256 & \\
\hline & $8 / 2 / 2008$ 15:00 & 266 & 215 & 1500 & 4.373 & 4.373 & & 0.9506 & 1.12E-02 & 2546.8370 & 938.4345 & 3485.2715 & \\
\hline & 8/2/2008 16:00 & 267 & 215 & 1600 & 4.404 & 4.404 & & 0.9369 & 1.10E-02 & 2501.6291 & 921.7767 & 3423.4058 & \\
\hline & 8/2/2008 17:00 & 268 & 215 & 1700 & 4.388 & 4.388 & & 0.9439 & 1.11E-02 & 2524.8635 & 930.3379 & 3455.2013 & \\
\hline & $8 / 2 / 200818: 00$ & 269 & 215 & 1800 & 4.453 & 4.453 & & 0.9156 & 1.07E-02 & 2431.7584 & 896.0314 & 3327.7898 & \\
\hline & 8/2/2008 19:00 & 270 & 215 & 1900 & 4.658 & 4.658 & & 0.8313 & $9.48 \mathrm{E}-03$ & 2159.1227 & 795.5732 & 2954.6959 & \\
\hline & 8/2/2008 20:00 & 271 & 215 & 2000 & 5.269 & 5.269 & & 0.6189 & 6.59E-03 & 1501.6541 & 553.3153 & 2054.9694 & \\
\hline & 8/2/2008 21:00 & 272 & 215 & 2100 & 6.346 & 6.346 & & 0.3442 & $3.20 \mathrm{E}-03$ & 729.1634 & 268.6753 & 997.8387 & \\
\hline & 8/2/2008 22:00 & 273 & 215 & 2200 & 7.526 & 7.526 & & 0.1334 & 9.97E-04 & 227.0733 & 83.6698 & 310.7432 & \\
\hline & 8/2/2008 23:00 & 274 & 215 & 2300 & 7.974 & 7.974 & & 0.0697 & 4.49E-04 & 102.1659 & 37.6451 & 139.8111 & \\
\hline & 8/3/2008 0:00 & 275 & 216 & 0 & 7.993 & 7.993 & 8.34 & 0.0672 & $4.28 E-04$ & 97.5989 & 35.9623 & 133.5613 & \\
\hline & 8/3/2008 1:00 & 276 & 216 & 100 & 7.855 & 7.855 & & 0.0859 & 5.80E-04 & 132.1388 & 48.6893 & 180.8281 & \\
\hline & 8/3/2008 2:00 & 277 & 216 & 200 & 7.872 & 7.872 & & 0.0836 & 5.61E-04 & 127.7138 & 47.0588 & 174.7726 & \\
\hline & 8/3/2008 3:00 & 278 & 216 & 300 & 8.08 & 8.08 & & 0.0557 & $3.40 \mathrm{E}-04$ & 77.4754 & 28.5474 & 106.0228 & \\
\hline & 8/3/2008 4:00 & 279 & 216 & 400 & 8.26 & 8.26 & & 0.0327 & 1.77E-04 & 40.2058 & 14.8147 & 55.0205 & \\
\hline & 8/3/2008 5:00 & 280 & 216 & 500 & 8.34 & 8.34 & & 0.0228 & 1.13E-04 & 25.7794 & 9.4989 & 35.2783 & \\
\hline & 8/3/2008 6:00 & 281 & 216 & 600 & 8.33 & 8.33 & & 0.0240 & 1.21E-04 & 27.5002 & 10.1330 & 37.6333 & \\
\hline & 8/3/2008 7:00 & 282 & 216 & 700 & 8.21 & 8.21 & & 0.0390 & 2.19E-04 & 49.9305 & 18.3979 & 68.3284 & \\
\hline & 8/3/2008 8:00 & 283 & 216 & 800 & 6.895 & 6.895 & & 0.2371 & 2.02E-03 & 460.9854 & 169.8595 & 630.8449 & \\
\hline & 8/3/2008 9:00 & 284 & 216 & 900 & 4.877 & 4.877 & & 0.7490 & $8.34 \mathrm{E}-03$ & 1899.2765 & 699.8275 & 2599.1039 & \\
\hline & $8 / 3 / 2008$ 10:00 & 285 & 216 & 1000 & 4.446 & 4.446 & & 0.9186 & 1.07E-02 & 2441.6230 & 899.6662 & 3341.2893 & \\
\hline & 8/3/2008 11:00 & 286 & 216 & 1100 & 4.357 & 4.357 & & 0.9578 & 1.13E-02 & 2570.4819 & 947.1469 & 3517.6289 & \\
\hline
\end{tabular}




\begin{tabular}{|c|c|c|c|c|c|c|c|c|c|c|c|c|c|}
\hline Tree ID & TS & RN & JD & HR & $\begin{array}{c}\text { Raw } \\
\text { TC_dTA }\end{array}$ & $\begin{array}{c}\text { Fixed } \\
\text { TC_dT } \Delta\end{array}$ & dTM & $\mathbf{K}$ & $\begin{array}{l}\text { Sap } \\
\text { Velocity } \\
(\mathrm{cm} / \mathrm{s})\end{array}$ & $\begin{array}{c}\text { Probed } \\
\text { Drywood F } \\
(\mathrm{g} / \mathrm{hr})\end{array}$ & $\begin{array}{c}\text { Extrapolated } \\
\text { Wetwood F } \\
(\mathrm{g} / \mathrm{hr})\end{array}$ & $\begin{array}{c}\text { Extrapolated } \\
\text { Total Wood F } \\
(\mathrm{g} / \mathrm{hr})\end{array}$ & $\begin{array}{l}\text { Relative } \\
\text { Humidity, } \\
@ 10 \mathrm{~m}(\%)\end{array}$ \\
\hline & 8/3/2008 12:00 & 287 & 216 & 1200 & 4.341 & 4.341 & & 0.9650 & 1.14E-02 & 2594.3425 & 955.9388 & 3550.2813 & \\
\hline & 8/3/2008 13:00 & 288 & 216 & 1300 & 4.316 & 4.316 & & 0.9764 & 1.16E-02 & 2632.0625 & 969.8376 & 3601.9001 & \\
\hline & $8 / 3 / 2008$ 14:00 & 289 & 216 & 1400 & 4.283 & 4.283 & & 0.9916 & $1.18 \mathrm{E}-02$ & 2682.6861 & 988.4909 & 3671.1770 & \\
\hline & 8/3/2008 15:00 & 290 & 216 & 1500 & 4.264 & 4.264 & & 1.0005 & 1.19E-02 & 2712.2716 & 999.3923 & 3711.6639 & \\
\hline & 8/3/2008 16:00 & 291 & 216 & 1600 & 4.291 & 4.291 & & 0.9879 & 1.17E-02 & 2670.3256 & 983.9364 & 3654.2620 & \\
\hline & 8/3/2008 17:00 & 292 & 216 & 1700 & 4.306 & 4.306 & & 0.9810 & 1.16E-02 & 2647.3020 & 975.4529 & 3622.7549 & \\
\hline & 8/3/2008 18:00 & 293 & 216 & 1800 & 4.399 & 4.399 & & 0.9391 & $1.10 \mathrm{E}-02$ & 2508.8673 & 924.4438 & 3433.3111 & \\
\hline & 8/3/2008 19:00 & 294 & 216 & 1900 & 4.595 & 4.595 & & 0.8564 & 9.83E-03 & 2239.6801 & 825.2562 & 3064.9362 & \\
\hline & 8/3/2008 20:00 & 295 & 216 & 2000 & 5.038 & 5.038 & & 0.6931 & 7.58E-03 & 1726.3429 & 636.1065 & 2362.4495 & \\
\hline & 8/3/2008 21:00 & 296 & 216 & 2100 & 5.882 & 5.882 & & 0.4502 & 4.46E-03 & 1014.8672 & 373.9487 & 1388.8159 & \\
\hline & 8/3/2008 22:00 & 297 & 216 & 2200 & 6.756 & 6.756 & & 0.2626 & 2.29E-03 & 522.6325 & 192.5747 & 715.2072 & \\
\hline & 8/3/2008 23:00 & 298 & 216 & 2300 & 7.098 & 7.098 & & 0.2017 & 1.66E-03 & 377.8330 & 139.2204 & 517.0534 & \\
\hline & 8/4/2008 0:00 & 299 & 217 & 0 & 7.169 & 7.169 & 7.81 & 0.1898 & 1.54E-03 & 350.5842 & 129.1800 & 479.7641 & \\
\hline & 8/4/2008 1:00 & 300 & 217 & 100 & 7.204 & 7.204 & & 0.1841 & 1.48E-03 & 337.4895 & 124.3550 & 461.8445 & \\
\hline & 8/4/2008 2:00 & 301 & 217 & 200 & 6.913 & 6.913 & & 0.2339 & 1.99E-03 & 453.2888 & 167.0236 & 620.3124 & \\
\hline & 8/4/2008 3:00 & 302 & 217 & 300 & 6.657 & 6.657 & & 0.2814 & $2.50 \mathrm{E}-03$ & 569.0109 & 209.6638 & 778.6747 & \\
\hline & 8/4/2008 4:00 & 303 & 217 & 400 & 6.364 & 6.364 & & 0.3404 & 3.16E-03 & 719.2605 & 265.0263 & 984.2868 & \\
\hline & $8 / 4 / 20085: 00$ & 304 & 217 & 500 & 6.398 & 6.398 & & 0.3332 & $3.08 \mathrm{E}-03$ & 700.7758 & 258.2152 & 958.9910 & \\
\hline & 8/4/2008 6:00 & 305 & 217 & 600 & 6.786 & 6.786 & & 0.2570 & 2.23E-03 & 508.9903 & 187.5480 & 696.5383 & \\
\hline & 8/4/2008 7:00 & 306 & 217 & 700 & 6.707 & 6.707 & & 0.2718 & 2.39E-03 & 545.3239 & 200.9358 & 746.2597 & \\
\hline & 8/4/2008 8:00 & 307 & 217 & 800 & 6.343 & 6.343 & & 0.3448 & $3.21 \mathrm{E}-03$ & 730.8219 & 269.2864 & 1000.1082 & \\
\hline & 8/4/2008 9:00 & 308 & 217 & 900 & 5.867 & 5.867 & & 0.4539 & 4.50E-03 & 1025.1660 & 377.7435 & 1402.9095 & \\
\hline & 8/4/2008 10:00 & 309 & 217 & 1000 & 5.108 & 5.108 & & 0.6699 & 7.27E-03 & 1655.4815 & 609.9962 & 2265.4777 & \\
\hline & 8/4/2008 11:00 & 310 & 217 & 1100 & 4.776 & 4.776 & & 0.7860 & 8.85E-03 & 2015.3791 & 742.6079 & 2757.9870 & \\
\hline & 8/4/2008 12:00 & 311 & 217 & 1200 & 4.634 & 4.634 & & 0.8407 & $9.61 \mathrm{E}-03$ & 2189.4878 & 806.7618 & 2996.2496 & \\
\hline & 8/4/2008 13:00 & 312 & 217 & 1300 & 4.531 & 4.531 & & 0.8826 & 1.02E-02 & 2324.3941 & 856.4708 & 3180.8649 & \\
\hline & 8/4/2008 14:00 & 313 & 217 & 1400 & 4.724 & 4.724 & & 0.8057 & $9.12 \mathrm{E}-03$ & 2077.6103 & 765.5382 & 2843.1485 & \\
\hline & 8/4/2008 15:00 & 314 & 217 & 1500 & 4.96 & 4.96 & & 0.7198 & 7.94E-03 & 1808.3359 & 666.3186 & 2474.6545 & \\
\hline & 8/4/2008 16:00 & 315 & 217 & 1600 & 4.583 & 4.583 & & 0.8612 & 9.90E-03 & 2255.3390 & 831.0261 & 3086.3651 & \\
\hline & 8/4/2008 17:00 & 316 & 217 & 1700 & 4.495 & 4.495 & & 0.8977 & 1.04E-02 & 2373.3709 & 874.5173 & 3247.8883 & \\
\hline & 8/4/2008 18:00 & 317 & 217 & 1800 & 4.586 & 4.586 & & 0.8600 & 9.88E-03 & 2251.4147 & 829.5800 & 3080.9947 & \\
\hline & 8/4/2008 19:00 & 318 & 217 & 1900 & 4.797 & 4.797 & & 0.7782 & 8.74E-03 & 1990.7290 & 733.5250 & 2724.2540 & \\
\hline & 8/4/2008 20:00 & 319 & 217 & 2000 & 5.326 & 5.326 & & 0.6016 & 6.37E-03 & 1450.0739 & 534.3095 & 1984.3834 & \\
\hline & 8/4/2008 21:00 & 320 & 217 & 2100 & 6.637 & 6.637 & & 0.2852 & $2.54 \mathrm{E}-03$ & 578.6388 & 213.2114 & 791.8502 & \\
\hline & 8/4/2008 22:00 & 321 & 217 & 2200 & 7.522 & 7.522 & & 0.1340 & 1.00E-03 & 228.3369 & 84.1354 & 312.4723 & \\
\hline & 8/4/2008 23:00 & 322 & 217 & 2300 & 7.806 & 7.806 & & 0.0927 & 6.37E-04 & 145.1580 & 53.4865 & 198.6445 & \\
\hline & 8/5/2008 0:00 & 323 & 218 & 0 & 8.05 & 8.05 & 8.24 & 0.0596 & 3.70E-04 & 84.2666 & 31.0498 & 115.3164 & \\
\hline & 8/5/2008 1:00 & 324 & 218 & 100 & 8.17 & 8.17 & & 0.0441 & $2.55 E-04$ & 58.0692 & 21.3968 & 79.4659 & \\
\hline & 8/5/2008 2:00 & 325 & 218 & 200 & 8.24 & 8.24 & & 0.0352 & 1.93E-04 & 44.0340 & 16.2252 & 60.2592 & \\
\hline & 8/5/2008 3:00 & 326 & 218 & 300 & 8.2 & 8.2 & & 0.0402 & 2.28E-04 & 51.9360 & 19.1369 & 71.0729 & \\
\hline & 8/5/2008 4:00 & 327 & 218 & 400 & 8.06 & 8.06 & & 0.0583 & $3.60 \mathrm{E}-04$ & 81.9854 & 30.2092 & 112.1946 & \\
\hline
\end{tabular}




\begin{tabular}{|c|c|c|c|c|c|c|c|c|c|c|c|c|c|}
\hline Tree ID & TS & RN & JD & HR & $\begin{array}{c}\text { Raw } \\
\text { TC_dTA }\end{array}$ & $\begin{array}{c}\text { Fixed } \\
\text { TC_dT } \Delta\end{array}$ & dTM & $\mathbf{K}$ & $\begin{array}{l}\text { Sap } \\
\text { Velocity } \\
(\mathrm{cm} / \mathrm{s})\end{array}$ & $\begin{array}{c}\text { Probed } \\
\text { Drywood F } \\
(\mathrm{g} / \mathrm{hr})\end{array}$ & $\begin{array}{c}\text { Extrapolated } \\
\text { Wetwood F } \\
(\mathrm{g} / \mathrm{hr})\end{array}$ & $\begin{array}{c}\text { Extrapolated } \\
\text { Total Wood F } \\
(\mathrm{g} / \mathrm{hr})\end{array}$ & $\begin{array}{l}\text { Relative } \\
\text { Humidity, } \\
@ 10 \mathrm{~m}(\%)\end{array}$ \\
\hline & 8/5/2008 5:00 & 328 & 218 & 500 & \begin{tabular}{|r|}
8.03 \\
\end{tabular} & 8.03 & & 0.0623 & 3.90E-04 & 88.8812 & 32.7501 & 121.6313 & \\
\hline & 8/5/2008 6:00 & 329 & 218 & 600 & 8.07 & 8.07 & & 0.0570 & $3.50 \mathrm{E}-04$ & 79.7216 & 29.3751 & 109.0967 & \\
\hline & 8/5/2008 7:00 & 330 & 218 & 700 & 8.03 & 8.03 & & 0.0623 & $3.90 E-04$ & 88.8812 & 32.7501 & 121.6313 & \\
\hline & 8/5/2008 8:00 & 331 & 218 & 800 & 7.483 & 7.483 & & 0.1399 & 1.06E-03 & 240.7962 & 88.7263 & 329.5225 & \\
\hline & 8/5/2008 9:00 & 332 & 218 & 900 & 5.854 & 5.854 & & 0.4571 & 4.54E-03 & 1034.1501 & 381.0539 & 1415.2040 & \\
\hline & 8/5/2008 10:00 & 333 & 218 & 1000 & 4.876 & 4.876 & & 0.7494 & 8.34E-03 & 1900.3962 & 700.2401 & 2600.6362 & \\
\hline & 8/5/2008 11:00 & 334 & 218 & 1100 & 5.018 & 5.018 & & 0.6999 & 7.67E-03 & 1747.0561 & 643.7387 & 2390.7948 & \\
\hline & 8/5/2008 12:00 & 335 & 218 & 1200 & 4.777 & 4.777 & & 0.7856 & 8.84E-03 & 2014.1991 & 742.1731 & 2756.3721 & \\
\hline & 8/5/2008 13:00 & 336 & 218 & 1300 & 4.507 & 4.507 & & 0.8926 & 1.03E-02 & 2356.9371 & 868.4619 & 3225.3990 & \\
\hline & 8/5/2008 14:00 & 337 & 218 & 1400 & 4.789 & 4.789 & & 0.7812 & 8.78E-03 & 2000.0873 & 736.9733 & 2737.0606 & \\
\hline & 8/5/2008 15:00 & 338 & 218 & 1500 & 5.088 & 5.088 & & 0.6765 & 7.36E-03 & 1675.4720 & 617.3621 & 2292.8341 & \\
\hline & 8/5/2008 16:00 & 339 & 218 & 1600 & 4.968 & 4.968 & & 0.7170 & 7.90E-03 & 1799.7748 & 663.1640 & 2462.9388 & \\
\hline & 8/5/2008 17:00 & 340 & 218 & 1700 & 4.7 & 4.7 & & 0.8149 & $9.25 E-03$ & 2106.9184 & 776.3374 & 2883.2558 & \\
\hline & 8/5/2008 18:00 & 341 & 218 & 1800 & 4.554 & 4.554 & & 0.8731 & 1.01E-02 & 2293.6078 & 845.1270 & 3138.7347 & \\
\hline & 8/5/2008 19:00 & 342 & 218 & 1900 & 4.651 & 4.651 & & 0.8340 & $9.52 E-03$ & 2167.9386 & 798.8216 & 2966.7602 & \\
\hline & 8/5/2008 20:00 & 343 & 218 & 2000 & 5.483 & 5.483 & & 0.5557 & $5.77 \mathrm{E}-03$ & 1315.2188 & 484.6194 & 1799.8382 & \\
\hline & 8/5/2008 21:00 & 344 & 218 & 2100 & 7.304 & 7.304 & & 0.1679 & 1.32E-03 & 301.2804 & 111.0130 & 412.2934 & \\
\hline & $8 / 5 / 200822: 00$ & 345 & 218 & 2200 & 8 & 8 & & 0.0662 & 4.21E-04 & 95.9317 & 35.3480 & 131.2798 & \\
\hline & 8/5/2008 23:00 & 346 & 218 & 2300 & 8.24 & 8.24 & & 0.0352 & 1.93E-04 & 44.0340 & 16.2252 & 60.2592 & \\
\hline & 8/6/2008 0:00 & 347 & 219 & 0 & 8.34 & 8.34 & 8.34 & 0.0228 & 1.13E-04 & 25.7794 & 9.4989 & 35.2783 & \\
\hline & 8/6/2008 1:00 & 348 & 219 & 100 & 8.3 & 8.3 & & 0.0277 & $1.44 \mathrm{E}-04$ & 32.8083 & 12.0889 & 44.8973 & \\
\hline & $8 / 6 / 2008$ 2:00 & 349 & 219 & 200 & 8.21 & 8.21 & & 0.0390 & 2.19E-04 & 49.9305 & 18.3979 & 68.3284 & \\
\hline & 8/6/2008 3:00 & 350 & 219 & 300 & 8.12 & 8.12 & & 0.0505 & 3.01E-04 & 68.6682 & 25.3022 & 93.9705 & \\
\hline & 8/6/2008 4:00 & 351 & 219 & 400 & 8.02 & 8.02 & & 0.0636 & 4.00E-04 & 91.2143 & 33.6098 & 124.8240 & \\
\hline & 8/6/2008 5:00 & 352 & 219 & 500 & 7.916 & 7.916 & & 0.0776 & 5.11E-04 & 116.4815 & 42.9200 & 159.4016 & \\
\hline & 8/6/2008 6:00 & 353 & 219 & 600 & 7.812 & 7.812 & & 0.0919 & 6.30E-04 & 143.5428 & 52.8913 & 196.4341 & \\
\hline & 8/6/2008 7:00 & 354 & 219 & 700 & 7.744 & 7.744 & & 0.1015 & 7.12E-04 & 162.1927 & 59.7632 & 221.9559 & \\
\hline & 8/6/2008 8:00 & 355 & 219 & 800 & 7.677 & 7.677 & & 0.1111 & 7.96E-04 & 181.3051 & 66.8056 & 248.1107 & \\
\hline & 8/6/2008 9:00 & 356 & 219 & 900 & 7.342 & 7.342 & & 0.1618 & 1.26E-03 & 287.9811 & 106.1126 & 394.0937 & \\
\hline & 8/6/2008 10:00 & 357 & 219 & 1000 & 4.491 & 4.491 & & 0.8994 & 1.04E-02 & 2378.8732 & 876.5448 & 3255.4180 & \\
\hline & 8/6/2008 11:00 & 358 & 219 & 1100 & 3.811 & 3.811 & & 1.2383 & $1.55 \mathrm{E}-02$ & 3526.4104 & 1299.3784 & 4825.7889 & \\
\hline & 8/6/2008 12:00 & 359 & 219 & 1200 & 4.184 & 4.184 & & 1.0387 & 1.25E-02 & 2840.4790 & 1046.6329 & 3887.1118 & \\
\hline & 8/6/2008 13:00 & 360 & 219 & 1300 & 4.773 & 4.773 & & 0.7871 & 8.86E-03 & 2018.9229 & 743.9137 & 2762.8366 & \\
\hline & $8 / 6 / 2008$ 14:00 & 361 & 219 & 1400 & 4.369 & 4.369 & & 0.9524 & 1.12E-02 & 2552.7282 & 940.6052 & 3493.3334 & \\
\hline & 8/6/2008 15:00 & 362 & 219 & 1500 & 4.009 & 4.009 & & 1.1277 & 1.38E-02 & 3142.9594 & 1158.0880 & 4301.0475 & \\
\hline & 8/6/2008 16:00 & 363 & 219 & 1600 & 4.13 & 4.13 & & 1.0654 & 1.29E-02 & 2930.4763 & 1079.7943 & 4010.2705 & \\
\hline & 8/6/2008 17:00 & 364 & 219 & 1700 & 4.092 & 4.092 & & 1.0846 & 1.32E-02 & 2995.5549 & 1103.7738 & 4099.3288 & \\
\hline & 8/6/2008 18:00 & 365 & 219 & 1800 & 4.519 & 4.519 & & 0.8876 & 1.03E-02 & 2340.6118 & 862.4465 & 3203.0583 & \\
\hline & 8/6/2008 19:00 & 366 & 219 & 1900 & 4.582 & 4.582 & & 0.8616 & $9.91 \mathrm{E}-03$ & 2256.6486 & 831.5086 & 3088.1572 & \\
\hline & $8 / 6 / 200820: 00$ & 367 & 219 & 2000 & 5.024 & 5.024 & & 0.6979 & 7.64E-03 & 1740.8199 & 641.4409 & 2382.2608 & \\
\hline & 8/6/2008 21:00 & 368 & 219 & 2100 & 6.573 & 6.573 & & 0.2977 & 2.68E-03 & 610.0476 & 224.7846 & 834.8322 & \\
\hline
\end{tabular}




\begin{tabular}{|c|c|c|c|c|c|c|c|c|c|c|c|c|c|}
\hline Tree ID & TS & RN & JD & HR & $\begin{array}{c}\text { Raw } \\
\text { TC_dTA }\end{array}$ & $\begin{array}{c}\text { Fixed } \\
\text { TC_dT } \Delta\end{array}$ & dTM & $\mathbf{K}$ & $\begin{array}{l}\text { Sap } \\
\text { Velocity } \\
(\mathrm{cm} / \mathrm{s})\end{array}$ & $\begin{array}{c}\text { Probed } \\
\text { Drywood F } \\
(\mathrm{g} / \mathrm{hr})\end{array}$ & $\begin{array}{c}\text { Extrapolated } \\
\text { Wetwood F } \\
(\mathrm{g} / \mathrm{hr})\end{array}$ & $\begin{array}{c}\text { Extrapolated } \\
\text { Total Wood F } \\
(\mathrm{g} / \mathrm{hr})\end{array}$ & $\begin{array}{l}\text { Relative } \\
\text { Humidity, } \\
@ 10 \mathrm{~m}(\%)\end{array}$ \\
\hline & 8/6/2008 22:00 & 369 & 219 & 2200 & \begin{tabular}{|r|}
7.753 \\
\end{tabular} & 7.753 & & 0.1002 & 7.01E-04 & 159.6811 & 58.8378 & \begin{tabular}{|r|}
218.5189 \\
\end{tabular} & \\
\hline & 8/6/2008 23:00 & 370 & 219 & 2300 & 7.957 & 7.957 & & 0.0720 & 4.67E-04 & 106.3037 & 39.1698 & 145.4735 & \\
\hline & $8 / 7 / 20080: 00$ & 371 & 220 & 0 & 7.964 & 7.964 & 7.96 & 0.0711 & $4.59 E-04$ & 104.5940 & 38.5398 & 143.1339 & \\
\hline & 8/7/2008 1:00 & 372 & 220 & 100 & 7.917 & 7.917 & & 0.0774 & $5.10 \mathrm{E}-04$ & 116.2300 & 42.8273 & 159.0573 & \\
\hline & 8/7/2008 2:00 & 373 & 220 & 200 & 7.86 & 7.86 & & 0.0852 & 5.74E-04 & 130.8324 & 48.2079 & 179.0403 & \\
\hline & 8/7/2008 3:00 & 374 & 220 & 300 & 7.799 & 7.799 & & 0.0937 & $6.46 \mathrm{E}-04$ & 147.0499 & 54.1836 & 201.2335 & \\
\hline & 8/7/2008 4:00 & 375 & 220 & 400 & 7.749 & 7.749 & & 0.1008 & 7.06E-04 & 160.7957 & 59.2485 & 220.0442 & \\
\hline & 8/7/2008 5:00 & 376 & 220 & 500 & 7.7 & 7.7 & & 0.1078 & 7.67E-04 & 174.6616 & 64.3577 & 239.0193 & \\
\hline & 8/7/2008 6:00 & 377 & 220 & 600 & 7.65 & 7.65 & & 0.1150 & 8.31E-04 & 189.2141 & 69.7198 & 258.9339 & \\
\hline & 8/7/2008 7:00 & 378 & 220 & 700 & 7.613 & 7.613 & & 0.1205 & 8.79E-04 & 200.2460 & 73.7848 & 274.0308 & \\
\hline & 8/7/2008 8:00 & 379 & 220 & 800 & 7.561 & 7.561 & & 0.1282 & $9.49 \mathrm{E}-04$ & 216.1305 & 79.6377 & 295.7682 & \\
\hline & 8/7/2008 9:00 & 380 & 220 & 900 & 6.32 & 6.32 & & 0.3497 & $3.26 \mathrm{E}-03$ & 743.6124 & 273.9993 & 1017.6117 & \\
\hline & 8/7/2008 10:00 & 381 & 220 & 1000 & 3.918 & 3.918 & & 1.1771 & 1.45E-02 & 3313.3544 & 1220.8736 & 4534.2280 & \\
\hline & 8/7/2008 11:00 & 382 & 220 & 1100 & 3.695 & 3.695 & & 1.3085 & 1.66E-02 & 3774.3413 & 1390.7337 & 5165.0749 & \\
\hline & 8/7/2008 12:00 & 383 & 220 & 1200 & 3.722 & 3.722 & & 1.2918 & 1.63E-02 & 3714.9675 & 1368.8562 & 5083.8237 & \\
\hline & 8/7/2008 13:00 & 384 & 220 & 1300 & 3.727 & 3.727 & & 1.2887 & 1.63E-02 & 3704.0860 & 1364.8467 & 5068.9327 & \\
\hline & $8 / 7 / 2008$ 14:00 & 385 & 220 & 1400 & 3.767 & 3.767 & & 1.2644 & 1.59E-02 & 3618.2858 & 1333.2318 & 4951.5176 & \\
\hline & 8/7/2008 15:00 & 386 & 220 & 1500 & 3.826 & 3.826 & & 1.2295 & 1.53E-02 & 3495.6721 & 1288.0523 & 4783.7244 & \\
\hline & 8/7/2008 16:00 & 387 & 220 & 1600 & 3.879 & 3.879 & & 1.1990 & 1.49E-02 & 3389.3622 & 1248.8802 & 4638.2423 & \\
\hline & 8/7/2008 17:00 & 388 & 220 & 1700 & 3.865 & 3.865 & & 1.2070 & $1.50 \mathrm{E}-02$ & 3417.1011 & 1259.1012 & 4676.2023 & \\
\hline & 8/7/2008 18:00 & 389 & 220 & 1800 & 4.027 & 4.027 & & 1.1182 & 1.37E-02 & 3110.3624 & 1146.0770 & 4256.4394 & \\
\hline & 8/7/2008 19:00 & 390 & 220 & 1900 & 4.315 & 4.315 & & 0.9768 & 1.16E-02 & 2633.5825 & 970.3977 & 3603.9802 & \\
\hline & 8/7/2008 20:00 & 391 & 220 & 2000 & 5.164 & 5.164 & & 0.6518 & 7.03E-03 & 1600.5671 & 589.7619 & 2190.3290 & \\
\hline & 8/7/2008 21:00 & 392 & 220 & 2100 & 6.676 & 6.676 & & 0.2777 & $2.46 \mathrm{E}-03$ & 559.9458 & 206.3235 & 766.2693 & \\
\hline & 8/7/2008 22:00 & 393 & 220 & 2200 & 7.505 & 7.505 & & 0.1366 & 1.03E-03 & 233.7367 & 86.1251 & 319.8618 & \\
\hline & 8/7/2008 23:00 & 394 & 220 & 2300 & 7.88 & 7.88 & & 0.0825 & 5.52E-04 & 125.6478 & 46.2975 & 171.9454 & \\
\hline & 8/8/2008 0:00 & 395 & 221 & 0 & 8.03 & 8.03 & 8.10 & 0.0623 & 3.90E-04 & 88.8812 & 32.7501 & 121.6313 & \\
\hline & 8/8/2008 1:00 & 396 & 221 & 100 & 8.07 & 8.07 & & 0.0570 & $3.50 \mathrm{E}-04$ & 79.7216 & 29.3751 & 109.0967 & \\
\hline & 8/8/2008 2:00 & 397 & 221 & 200 & 8.1 & 8.1 & & 0.0531 & 3.21E-04 & 73.0360 & 26.9116 & 99.9476 & \\
\hline & 8/8/2008 3:00 & 398 & 221 & 300 & 8.03 & 8.03 & & 0.0623 & $3.90 \mathrm{E}-04$ & 88.8812 & 32.7501 & 121.6313 & \\
\hline & 8/8/2008 4:00 & 399 & 221 & 400 & 7.968 & 7.968 & & 0.0705 & $4.55 E-04$ & 103.6208 & 38.1812 & 141.8020 & \\
\hline & 8/8/2008 5:00 & 400 & 221 & 500 & 7.895 & 7.895 & & 0.0804 & 5.35E-04 & 121.8026 & 44.8807 & 166.6832 & \\
\hline & 8/8/2008 6:00 & 401 & 221 & 600 & 7.82 & 7.82 & & 0.0908 & $6.21 E-04$ & 141.3982 & 52.1011 & 193.4993 & \\
\hline & $8 / 8 / 20087: 00$ & 402 & 221 & 700 & 7.77 & 7.77 & & 0.0978 & $6.80 \mathrm{E}-04$ & 154.9729 & 57.1029 & 212.0758 & \\
\hline & 8/8/2008 8:00 & 403 & 221 & 800 & 7.416 & 7.416 & & 0.1502 & 1.15E-03 & 262.7963 & 96.8327 & 359.6290 & \\
\hline & 8/8/20089:00 & 404 & 221 & 900 & 4.997 & 4.997 & & 0.7070 & 7.77E-03 & 1769.0336 & 651.8368 & 2420.8705 & \\
\hline & 8/8/2008 10:00 & 405 & 221 & 1000 & 4.028 & 4.028 & & 1.1177 & 1.36E-02 & 3108.5618 & 1145.4135 & 4253.9754 & \\
\hline & 8/8/2008 11:00 & 406 & 221 & 1100 & 4.026 & 4.026 & & 1.1187 & 1.37E-02 & 3112.1640 & 1146.7408 & 4258.9049 & \\
\hline & $8 / 8 / 200812: 00$ & 407 & 221 & 1200 & 3.946 & 3.946 & & 1.1617 & $1.43 \mathrm{E}-02$ & 3259.9071 & 1201.1798 & 4461.0870 & \\
\hline & 8/8/2008 13:00 & 408 & 221 & 1300 & 3.894 & 3.894 & & 1.1905 & 1.48E-02 & 3359.9099 & 1238.0279 & 4597.9378 & \\
\hline & 8/8/2008 14:00 & 409 & 221 & 1400 & 3.889 & 3.889 & & 1.1934 & 1.48E-02 & 3369.6967 & 1241.6341 & 4611.3308 & \\
\hline
\end{tabular}




\begin{tabular}{|c|c|c|c|c|c|c|c|c|c|c|c|c|c|}
\hline Tree ID & TS & RN & JD & HR & $\begin{array}{c}\text { Raw } \\
\text { TC_dTA }\end{array}$ & $\begin{array}{c}\text { Fixed } \\
\text { TC_dT } \Delta\end{array}$ & dTM & $\mathbf{K}$ & $\begin{array}{l}\text { Sap } \\
\text { Velocity } \\
(\mathrm{cm} / \mathrm{s})\end{array}$ & $\begin{array}{c}\text { Probed } \\
\text { Drywood F } \\
(\mathrm{g} / \mathrm{hr})\end{array}$ & $\begin{array}{c}\text { Extrapolated } \\
\text { Wetwood F } \\
(\mathrm{g} / \mathrm{hr})\end{array}$ & $\begin{array}{c}\text { Extrapolated } \\
\text { Total Wood F } \\
(\mathrm{g} / \mathrm{hr})\end{array}$ & $\begin{array}{l}\text { Relative } \\
\text { Humidity, } \\
@ 10 \mathrm{~m}(\%)\end{array}$ \\
\hline & 8/8/2008 15:00 & 410 & 221 & 1500 & 3.941 & 3.941 & & 1.1644 & 1.44E-02 & 3269.3837 & 1204.6717 & 4474.0553 & \\
\hline & 8/8/2008 16:00 & 411 & 221 & 1600 & 3.981 & 3.981 & & 1.1427 & $1.40 \mathrm{E}-02$ & 3194.3804 & 1177.0351 & 4371.4155 & \\
\hline & 8/8/2008 17:00 & 412 & 221 & 1700 & 4.095 & 4.095 & & 1.0830 & $1.31 \mathrm{E}-02$ & 2990.3634 & 1101.8609 & 4092.2243 & \\
\hline & 8/8/2008 18:00 & 413 & 221 & 1800 & 4.33 & 4.33 & & 0.9700 & 1.15E-02 & 2610.8731 & 962.0299 & 3572.9029 & \\
\hline & 8/8/2008 19:00 & 414 & 221 & 1900 & 4.369 & 4.369 & & 0.9524 & 1.12E-02 & 2552.7282 & 940.6052 & 3493.3334 & \\
\hline & 8/8/2008 20:00 & 415 & 221 & 2000 & 5.043 & 5.043 & & 0.6915 & 7.56E-03 & 1721.1975 & 634.2106 & 2355.4081 & \\
\hline & 8/8/2008 21:00 & 416 & 221 & 2100 & 6.245 & 6.245 & & 0.3659 & $3.45 E-03$ & 786.2687 & 289.7169 & 1075.9855 & \\
\hline & 8/8/2008 22:00 & 417 & 221 & 2200 & 7.169 & 7.169 & & 0.1898 & 1.54E-03 & 350.5842 & 129.1800 & 479.7641 & \\
\hline & 8/8/2008 23:00 & 418 & 221 & 2300 & 7.466 & 7.466 & & 0.1425 & 1.08E-03 & 246.3068 & 90.7568 & 337.0635 & \\
\hline & 8/9/2008 0:00 & 419 & 222 & 0 & 7.59 & 7.59 & 8.06 & 0.1238 & $9.10 \mathrm{E}-04$ & 207.2169 & 76.3533 & 283.5703 & \\
\hline & 8/9/2008 1:00 & 420 & 222 & 100 & 7.599 & 7.599 & & 0.1225 & 8.98E-04 & 204.4788 & 75.3444 & 279.8233 & \\
\hline & 8/9/2008 2:00 & 421 & 222 & 200 & 7.656 & 7.656 & & 0.1142 & $8.23 E-04$ & 187.4462 & 69.0684 & 256.5146 & \\
\hline & 8/9/2008 3:00 & 422 & 222 & 300 & 7.653 & 7.653 & & 0.1146 & 8.27E-04 & 188.3294 & 69.3938 & 257.7233 & \\
\hline & 8/9/2008 4:00 & 423 & 222 & 400 & 7.555 & 7.555 & & 0.1291 & 9.57E-04 & 217.9920 & 80.3236 & 298.3156 & \\
\hline & $8 / 9 / 20085: 00$ & 424 & 222 & 500 & 7.412 & 7.412 & & 0.1508 & 1.16E-03 & 264.1337 & 97.3255 & 361.4592 & \\
\hline & 8/9/2008 6:00 & 425 & 222 & 600 & 7.35 & 7.35 & & 0.1605 & 1.25E-03 & 285.2131 & 105.0926 & 390.3058 & \\
\hline & 8/9/2008 7:00 & 426 & 222 & 700 & 6.896 & 6.896 & & 0.2369 & 2.02E-03 & 460.5561 & 169.7014 & 630.2575 & \\
\hline & 8/9/2008 8:00 & 427 & 222 & 800 & 6.149 & 6.149 & & 0.3872 & 3.70E-03 & 843.0532 & 310.6403 & 1153.6934 & \\
\hline & 8/9/2008 9:00 & 428 & 222 & 900 & 6.729 & 6.729 & & 0.2676 & 2.35E-03 & 535.0727 & 197.1585 & 732.2312 & \\
\hline & 8/9/2008 10:00 & 429 & 222 & 1000 & 6.869 & 6.869 & & 0.2418 & 2.07E-03 & 472.2170 & 173.9981 & 646.2150 & \\
\hline & 8/9/2008 11:00 & 430 & 222 & 1100 & 5.877 & 5.877 & & 0.4514 & 4.47E-03 & 1018.2921 & 375.2107 & 1393.5028 & \\
\hline & 8/9/2008 12:00 & 431 & 222 & 1200 & 4.95 & 4.95 & & 0.7232 & 7.99E-03 & 1819.0871 & 670.2800 & 2489.3671 & \\
\hline & 8/9/2008 13:00 & 432 & 222 & 1300 & 4.669 & 4.669 & & 0.8269 & 9.42E-03 & 2145.3361 & 790.4932 & 2935.8294 & \\
\hline & 8/9/2008 14:00 & 433 & 222 & 1400 & 4.264 & 4.264 & & 1.0005 & 1.19E-02 & 2712.2716 & 999.3923 & 3711.6639 & \\
\hline & 8/9/2008 15:00 & 434 & 222 & 1500 & 4.195 & 4.195 & & 1.0334 & 1.24E-02 & 2822.4939 & 1040.0059 & 3862.4999 & \\
\hline & 8/9/2008 16:00 & 435 & 222 & 1600 & 4.229 & 4.229 & & 1.0170 & 1.21E-02 & 2767.6290 & 1019.7898 & 3787.4188 & \\
\hline & 8/9/2008 17:00 & 436 & 222 & 1700 & 4.325 & 4.325 & & 0.9723 & 1.15E-02 & 2618.4213 & 964.8112 & 3583.2325 & \\
\hline & 8/9/2008 18:00 & 437 & 222 & 1800 & 4.325 & 4.325 & & 0.9723 & $1.15 \mathrm{E}-02$ & 2618.4213 & 964.8112 & 3583.2325 & \\
\hline & 8/9/2008 19:00 & 438 & 222 & 1900 & 4.559 & 4.559 & & 0.8710 & 1.00E-02 & 2286.9663 & 842.6798 & 3129.6461 & \\
\hline & 8/9/2008 20:00 & 439 & 222 & 2000 & 5.241 & 5.241 & & 0.6276 & $6.71 E-03$ & 1527.5283 & 562.8492 & 2090.3776 & \\
\hline & 8/9/2008 21:00 & 440 & 222 & 2100 & 6.904 & 6.904 & & 0.2355 & 2.01E-03 & 457.1290 & 168.4386 & 625.5676 & \\
\hline & 8/9/2008 22:00 & 441 & 222 & 2200 & 7.929 & 7.929 & & 0.0758 & 4.97E-04 & 113.2242 & 41.7198 & 154.9439 & \\
\hline & 8/9/2008 23:00 & 442 & 222 & 2300 & 8.06 & 8.06 & & 0.0583 & 3.60E-04 & 81.9854 & 30.2092 & 112.1946 & \\
\hline & 8/10/2008 0:00 & 443 & 223 & 0 & 8.05 & 8.05 & 8.06 & 0.0596 & $3.70 \mathrm{E}-04$ & 84.2666 & 31.0498 & 115.3164 & \\
\hline & 8/10/2008 1:00 & 444 & 223 & 100 & 8.06 & 8.06 & & 0.0583 & 3.60E-04 & 81.9854 & 30.2092 & 112.1946 & \\
\hline & 8/10/2008 2:00 & 445 & 223 & 200 & 8.02 & 8.02 & & 0.0636 & 4.00E-04 & 91.2143 & 33.6098 & 124.8240 & \\
\hline & 8/10/2008 3:00 & 446 & 223 & 300 & 7.952 & 7.952 & & 0.0727 & 4.72E-04 & 107.5299 & 39.6216 & 147.1515 & \\
\hline & 8/10/2008 4:00 & 447 & 223 & 400 & 7.885 & 7.885 & & 0.0818 & 5.46E-04 & 124.3620 & 45.8237 & 170.1857 & \\
\hline & $8 / 10 / 20085: 00$ & 448 & 223 & 500 & 7.803 & 7.803 & & 0.0932 & $6.41 \mathrm{E}-04$ & 145.9679 & 53.7849 & 199.7527 & \\
\hline & 8/10/2008 6:00 & 449 & 223 & 600 & 7.72 & 7.72 & & 0.1049 & 7.42E-04 & 168.9548 & 62.2549 & 231.2097 & \\
\hline & 8/10/2008 7:00 & 450 & 223 & 700 & 7.671 & 7.671 & & 0.1120 & 8.04E-04 & 183.0523 & 67.4494 & 250.5017 & \\
\hline
\end{tabular}




\begin{tabular}{|c|c|c|c|c|c|c|c|c|c|c|c|c|c|}
\hline Tree ID & TS & RN & JD & HR & $\begin{array}{c}\text { Raw } \\
\text { TC_dTA }\end{array}$ & $\begin{array}{c}\text { Fixed } \\
\text { TC_dT } \Delta\end{array}$ & dTM & $\mathbf{K}$ & $\begin{array}{l}\text { Sap } \\
\text { Velocity } \\
(\mathrm{cm} / \mathrm{s})\end{array}$ & $\begin{array}{c}\text { Probed } \\
\text { Drywood F } \\
(\mathrm{g} / \mathrm{hr})\end{array}$ & $\begin{array}{c}\text { Extrapolated } \\
\text { Wetwood F } \\
(\mathrm{g} / \mathrm{hr})\end{array}$ & $\begin{array}{c}\text { Extrapolated } \\
\text { Total Wood F } \\
(\mathrm{g} / \mathrm{hr})\end{array}$ & $\begin{array}{l}\text { Relative } \\
\text { Humidity, } \\
@ 10 \mathrm{~m}(\%)\end{array}$ \\
\hline & 8/10/2008 8:00 & 451 & 223 & 800 & \begin{tabular}{|r|}
7.61 \\
\end{tabular} & 7.61 & & 0.1209 & 8.83E-04 & 201.1503 & 74.1180 & 275.2683 & \\
\hline & 8/10/2008 9:00 & 452 & 223 & 900 & 6.306 & 6.306 & & 0.3527 & $3.30 \mathrm{E}-03$ & 751.4641 & 276.8924 & 1028.3566 & \\
\hline & $8 / 10 / 2008$ 10:00 & 453 & 223 & 1000 & 3.978 & 3.978 & & 1.1443 & $1.40 \mathrm{E}-02$ & 3199.9420 & 1179.0845 & 4379.0265 & \\
\hline & 8/10/2008 11:00 & 454 & 223 & 1100 & 3.727 & 3.727 & & 1.2887 & 1.63E-02 & 3704.0860 & 1364.8467 & 5068.9327 & \\
\hline & $8 / 10 / 200812: 00$ & 455 & 223 & 1200 & 3.817 & 3.817 & & 1.2347 & 1.54E-02 & 3514.0800 & 1294.8351 & 4808.9151 & \\
\hline & 8/10/2008 13:00 & 456 & 223 & 1300 & 3.82 & 3.82 & & 1.2330 & $1.54 \mathrm{E}-02$ & 3507.9324 & 1292.5698 & 4800.5023 & \\
\hline & $8 / 10 / 2008$ 14:00 & 457 & 223 & 1400 & 3.84 & 3.84 & & 1.2214 & $1.52 \mathrm{E}-02$ & 3467.2449 & 1277.5777 & 4744.8225 & \\
\hline & $8 / 10 / 2008$ 15:00 & 458 & 223 & 1500 & 3.987 & 3.987 & & 1.1395 & 1.40E-02 & 3183.2876 & 1172.9478 & 4356.2353 & \\
\hline & 8/10/2008 16:00 & 459 & 223 & 1600 & 3.965 & 3.965 & & 1.1513 & 1.42E-02 & 3224.1610 & 1188.0084 & 4412.1694 & \\
\hline & 8/10/2008 17:00 & 460 & 223 & 1700 & 4.149 & 4.149 & & 1.0559 & 1.27E-02 & 2898.4832 & 1068.0057 & 3966.4889 & \\
\hline & 8/10/2008 18:00 & 461 & 223 & 1800 & 6.575 & 6.575 & & 0.2973 & 2.67E-03 & 609.0521 & 224.4178 & 833.4699 & \\
\hline & $8 / 10 / 2008$ 19:00 & 462 & 223 & 1900 & 7.301 & 7.301 & & 0.1683 & 1.33E-03 & 302.3411 & 111.4038 & 413.7449 & \\
\hline & 8/10/2008 20:00 & 463 & 223 & 2000 & 7.195 & 7.195 & & 0.1855 & $1.50 \mathrm{E}-03$ & 340.8356 & 125.5879 & 466.4235 & \\
\hline & 8/10/2008 21:00 & 464 & 223 & 2100 & 7.134 & 7.134 & & 0.1957 & 1.60E-03 & 363.9011 & 134.0868 & 497.9880 & \\
\hline & $8 / 10 / 2008$ 22:00 & 465 & 223 & 2200 & 7.438 & 7.438 & & 0.1468 & 1.12E-03 & 255.4889 & 94.1401 & 349.6290 & \\
\hline & $8 / 10 / 2008$ 23:00 & 466 & 223 & 2300 & 7.317 & 7.317 & & 0.1658 & 1.30E-03 & 296.7025 & 109.3261 & 406.0286 & \\
\hline & $8 / 11 / 20080: 00$ & 467 & 224 & 0 & 7.3 & 7.3 & 7.75 & 0.1685 & 1.33E-03 & 302.6950 & 111.5342 & 414.2292 & \\
\hline & 8/11/2008 1:00 & 468 & 224 & 100 & 7.52 & 7.52 & & 0.1343 & $1.01 E-03$ & 228.9697 & 84.3686 & 313.3382 & \\
\hline & 8/11/2008 2:00 & 469 & 224 & 200 & 7.749 & 7.749 & & 0.1008 & 7.06E-04 & 160.7957 & 59.2485 & 220.0442 & \\
\hline & 8/11/2008 3:00 & 470 & 224 & 300 & 7.737 & 7.737 & & 0.1025 & 7.21E-04 & 164.1553 & 60.4864 & 224.6417 & \\
\hline & 8/11/2008 4:00 & 471 & 224 & 400 & 7.7 & 7.7 & & 0.1078 & 7.67E-04 & 174.6616 & 64.3577 & 239.0193 & \\
\hline & 8/11/2008 5:00 & 472 & 224 & 500 & 7.58 & 7.58 & & 0.1253 & 9.23E-04 & 210.2749 & 77.4801 & 287.7550 & \\
\hline & 8/11/2008 6:00 & 473 & 224 & 600 & 7.543 & 7.543 & & 0.1308 & 9.73E-04 & 221.7329 & 81.7020 & 303.4349 & \\
\hline & 8/11/2008 7:00 & 474 & 224 & 700 & 7.539 & 7.539 & & 0.1314 & 9.79E-04 & 222.9851 & 82.1635 & 305.1486 & \\
\hline & 8/11/2008 8:00 & 475 & 224 & 800 & 7.349 & 7.349 & & 0.1607 & $1.25 \mathrm{E}-03$ & 285.5585 & 105.2199 & 390.7784 & \\
\hline & 8/11/2008 9:00 & 476 & 224 & 900 & 6.196 & 6.196 & & 0.3767 & $3.58 E-03$ & 814.9397 & 300.2813 & 1115.2210 & \\
\hline & 8/11/2008 10:00 & 477 & 224 & 1000 & 4.594 & 4.594 & & 0.8568 & 9.84E-03 & 2240.9811 & 825.7356 & 3066.7166 & \\
\hline & 8/11/2008 11:00 & 478 & 224 & 1100 & 4.08 & 4.08 & & 1.0907 & $1.32 \mathrm{E}-02$ & 3016.4143 & 1111.4599 & 4127.8742 & \\
\hline & $8 / 11 / 2008$ 12:00 & 479 & 224 & 1200 & 4.128 & 4.128 & & 1.0664 & 1.29E-02 & 2933.8650 & 1081.0429 & 4014.9079 & \\
\hline & 8/11/2008 13:00 & 480 & 224 & 1300 & 4.361 & 4.361 & & 0.9560 & 1.13E-02 & 2564.5506 & 944.9614 & 3509.5120 & \\
\hline & 8/11/2008 14:00 & 481 & 224 & 1400 & 4.157 & 4.157 & & 1.0520 & 1.27E-02 & 2885.1195 & 1063.0816 & 3948.2011 & \\
\hline & 8/11/2008 15:00 & 482 & 224 & 1500 & 4.172 & 4.172 & & 1.0446 & 1.26E-02 & 2860.2317 & 1053.9112 & 3914.1430 & \\
\hline & $8 / 11 / 2008$ 16:00 & 483 & 224 & 1600 & 4.41 & 4.41 & & 0.9342 & 1.09E-02 & 2492.9700 & 918.5861 & 3411.5561 & \\
\hline & 8/11/2008 17:00 & 484 & 224 & 1700 & 4.375 & 4.375 & & 0.9497 & 1.12E-02 & 2543.8964 & 937.3510 & 3481.2474 & \\
\hline & 8/11/2008 18:00 & 485 & 224 & 1800 & 4.665 & 4.665 & & 0.8285 & 9.44E-03 & 2150.3400 & 792.3370 & 2942.6770 & \\
\hline & $8 / 11 / 2008$ 19:00 & 486 & 224 & 1900 & 4.778 & 4.778 & & 0.7853 & 8.84E-03 & 2013.0197 & 741.7385 & 2754.7582 & \\
\hline & 8/11/2008 20:00 & 487 & 224 & 2000 & 5.469 & 5.469 & & 0.5597 & 5.82E-03 & 1326.8309 & 488.8981 & 1815.7291 & \\
\hline & 8/11/2008 21:00 & 488 & 224 & 2100 & 6.434 & 6.434 & & 0.3258 & 2.99E-03 & 681.5143 & 251.1180 & 932.6322 & \\
\hline & $8 / 11 / 200822: 00$ & 489 & 224 & 2200 & 7.213 & 7.213 & & 0.1826 & $1.47 \mathrm{E}-03$ & 334.1580 & 123.1274 & 457.2854 & \\
\hline & 8/11/2008 23:00 & 490 & 224 & 2300 & 7.524 & 7.524 & & 0.1337 & 1.00E-03 & 227.7048 & 83.9025 & 311.6073 & \\
\hline & 8/12/2008 0:00 & 491 & 225 & 0 & 7.667 & 7.667 & 7.76 & 0.1126 & 8.09E-04 & 184.2204 & 67.8798 & 252.1003 & \\
\hline
\end{tabular}




\begin{tabular}{|c|c|c|c|c|c|c|c|c|c|c|c|c|c|}
\hline Tree ID & TS & RN & JD & HR & $\begin{array}{c}\text { Raw } \\
\text { TC_dTA }\end{array}$ & $\begin{array}{c}\text { Fixed } \\
\text { TC_dT } \Delta\end{array}$ & dTM & $\mathbf{K}$ & $\begin{array}{l}\text { Sap } \\
\text { Velocity } \\
(\mathrm{cm} / \mathrm{s})\end{array}$ & $\begin{array}{c}\text { Probed } \\
\text { Drywood F } \\
(\mathrm{g} / \mathrm{hr})\end{array}$ & $\begin{array}{c}\text { Extrapolated } \\
\text { Wetwood F } \\
(\mathrm{g} / \mathrm{hr})\end{array}$ & $\begin{array}{c}\text { Extrapolated } \\
\text { Total Wood F } \\
(\mathrm{g} / \mathrm{hr})\end{array}$ & $\begin{array}{l}\text { Relative } \\
\text { Humidity, } \\
@ 10 \mathrm{~m}(\%)\end{array}$ \\
\hline & 8/12/2008 1:00 & 492 & 225 & 100 & 7.696 & 7.696 & & 0.1084 & $7.72 \mathrm{E}-04$ & 175.8108 & 64.7811 & 240.5919 & \\
\hline & 8/12/2008 2:00 & 493 & 225 & 200 & 7.624 & 7.624 & & 0.1188 & $8.65 \mathrm{E}-04$ & 196.9428 & 72.5676 & 269.5105 & \\
\hline & 8/12/2008 3:00 & 494 & 225 & 300 & 7.746 & 7.746 & & 0.1012 & $7.10 \mathrm{E}-04$ & 161.6334 & 59.5572 & 221.1906 & \\
\hline & 8/12/2008 4:00 & 495 & 225 & 400 & 7.735 & 7.735 & & 0.1028 & 7.23E-04 & 164.7175 & 60.6936 & 225.4111 & \\
\hline & 8/12/2008 5:00 & 496 & 225 & 500 & 7.664 & 7.664 & & 0.1130 & 8.13E-04 & 185.0982 & 68.2033 & 253.3015 & \\
\hline & 8/12/2008 6:00 & 497 & 225 & 600 & 7.577 & 7.577 & & 0.1258 & $9.27 E-04$ & 211.1955 & 77.8193 & 289.0148 & \\
\hline & 8/12/2008 7:00 & 498 & 225 & 700 & 7.489 & 7.489 & & 0.1390 & $1.05 E-03$ & 238.8628 & 88.0139 & 326.8768 & \\
\hline & 8/12/2008 8:00 & 499 & 225 & 800 & 7.375 & 7.375 & & 0.1566 & 1.21E-03 & 276.6342 & 101.9316 & 378.5657 & \\
\hline & 8/12/2008 9:00 & 500 & 225 & 900 & 6.198 & 6.198 & & 0.3763 & 3.57E-03 & 813.7568 & 299.8454 & 1113.6022 & \\
\hline & $8 / 12 / 2008$ 10:00 & 501 & 225 & 1000 & 4.465 & 4.465 & & 0.9104 & 1.06E-02 & 2414.9369 & 889.8332 & 3304.7701 & \\
\hline & $8 / 12 / 2008$ 11:00 & 502 & 225 & 1100 & 3.88 & 3.88 & & 1.1985 & 1.49E-02 & 3387.3901 & 1248.1535 & 4635.5436 & \\
\hline & $8 / 12 / 2008$ 12:00 & 503 & 225 & 1200 & 3.823 & 3.823 & & 1.2312 & 1.54E-02 & 3501.7964 & 1290.3089 & 4792.1054 & \\
\hline & 8/12/2008 13:00 & 504 & 225 & 1300 & 3.795 & 3.795 & & 1.2477 & 1.56E-02 & 3559.5220 & 1311.5791 & 4871.1011 & \\
\hline & $8 / 12 / 2008$ 14:00 & 505 & 225 & 1400 & 3.804 & 3.804 & & 1.2424 & 1.55E-02 & 3540.8553 & 1304.7010 & 4845.5563 & \\
\hline & $8 / 12 / 2008$ 15:00 & 506 & 225 & 1500 & 3.819 & 3.819 & & 1.2336 & 1.54E-02 & 3509.9803 & 1293.3244 & 4803.3048 & \\
\hline & $8 / 12 / 2008$ 16:00 & 507 & 225 & 1600 & 3.875 & 3.875 & & 1.2013 & 1.49E-02 & 3397.2628 & 1251.7913 & 4649.0541 & \\
\hline & $8 / 12 / 2008$ 17:00 & 508 & 225 & 1700 & 3.913 & 3.913 & & 1.1799 & 1.46E-02 & 3322.9963 & 1224.4263 & 4547.4227 & \\
\hline & $8 / 12 / 2008$ 18:00 & 509 & 225 & 1800 & 4.009 & 4.009 & & 1.1277 & 1.38E-02 & 3142.9594 & 1158.0880 & 4301.0475 & \\
\hline & $8 / 12 / 2008$ 19:00 & 510 & 225 & 1900 & 4.215 & 4.215 & & 1.0237 & $1.22 \mathrm{E}-02$ & 2790.0888 & 1028.0656 & 3818.1544 & \\
\hline & $8 / 12 / 200820: 00$ & 511 & 225 & 2000 & 5.024 & 5.024 & & 0.6979 & 7.64E-03 & 1740.8199 & 641.4409 & 2382.2608 & \\
\hline & $8 / 12 / 2008$ 21:00 & 512 & 225 & 2100 & 6.849 & 6.849 & & 0.2454 & $2.11 \mathrm{E}-03$ & 480.9493 & 177.2157 & 658.1650 & \\
\hline & $8 / 12 / 2008$ 22:00 & 513 & 225 & 2200 & 7.635 & 7.635 & & 0.1172 & 8.50E-04 & 193.6595 & 71.3578 & 265.0173 & \\
\hline & $8 / 12 / 2008$ 23:00 & 514 & 225 & 2300 & 7.763 & 7.763 & & 0.0988 & 6.89E-04 & 156.9059 & 57.8152 & 214.7210 & \\
\hline & 8/13/2008 0:00 & 515 & 226 & 0 & 7.771 & 7.771 & 7.77 & 0.0977 & $6.79 E-04$ & 154.6974 & 57.0014 & 211.6988 & \\
\hline & 8/13/2008 1:00 & 516 & 226 & 100 & 7.739 & 7.739 & & 0.1022 & $7.18 \mathrm{E}-04$ & 163.5937 & 60.2795 & 223.8732 & \\
\hline & 8/13/2008 2:00 & 517 & 226 & 200 & 7.701 & 7.701 & & 0.1076 & 7.66E-04 & 174.3748 & 64.2520 & 238.6267 & \\
\hline & 8/13/2008 3:00 & 518 & 226 & 300 & 7.699 & 7.699 & & 0.1079 & 7.68E-04 & 174.9487 & 64.4634 & 239.4121 & \\
\hline & 8/13/2008 4:00 & 519 & 226 & 400 & 7.63 & 7.63 & & 0.1180 & 8.57E-04 & 195.1494 & 71.9068 & 267.0563 & \\
\hline & $8 / 13 / 20085: 00$ & 520 & 226 & 500 & 7.612 & 7.612 & & 0.1206 & $8.80 \mathrm{E}-04$ & 200.5473 & 73.8958 & 274.4431 & \\
\hline & 8/13/2008 6:00 & 521 & 226 & 600 & 7.609 & 7.609 & & 0.1210 & 8.84E-04 & 201.4521 & 74.2292 & 275.6813 & \\
\hline & $8 / 13 / 2008$ 7:00 & 522 & 226 & 700 & 7.554 & 7.554 & & 0.1292 & 9.58E-04 & 218.3028 & 80.4382 & 298.7410 & \\
\hline & 8/13/2008 8:00 & 523 & 226 & 800 & 7.408 & 7.408 & & 0.1515 & 1.17E-03 & 265.4739 & 97.8193 & 363.2932 & \\
\hline & 8/13/2008 9:00 & 524 & 226 & 900 & 5.427 & 5.427 & & 0.5718 & $5.98 \mathrm{E}-03$ & 1362.1430 & 501.9096 & 1864.0526 & \\
\hline & 8/13/2008 10:00 & 525 & 226 & 1000 & 3.946 & 3.946 & & 1.1617 & 1.43E-02 & 3259.9071 & 1201.1798 & 4461.0870 & \\
\hline & 8/13/2008 11:00 & 526 & 226 & 1100 & 3.936 & 3.936 & & 1.1672 & 1.44E-02 & 3278.8895 & 1208.1743 & 4487.0637 & \\
\hline & $8 / 13 / 2008$ 12:00 & 527 & 226 & 1200 & 3.746 & 3.746 & & 1.2771 & 1.61E-02 & 3663.0554 & 1349.7281 & 5012.7835 & \\
\hline
\end{tabular}




\begin{tabular}{|c|c|c|c|c|c|c|c|c|c|c|c|c|c|}
\hline Tree ID & TS & RN & JD & HR & $\begin{array}{c}\text { Raw } \\
\text { TC_dTA }\end{array}$ & $\begin{array}{c}\text { Fixed } \\
\text { TC_dTA }\end{array}$ & dTM & $\mathbf{K}$ & $\begin{array}{l}\text { Sap } \\
\text { Velocity } \\
(\mathrm{cm} / \mathrm{s})\end{array}$ & $\begin{array}{c}\text { Probed } \\
\text { Drywood F } \\
\text { (g/hr) }\end{array}$ & $\begin{array}{c}\text { Extrapolated } \\
\text { Wetwood F } \\
(\mathrm{g} / \mathrm{hr})\end{array}$ & $\begin{array}{c}\text { Extrapolated } \\
\text { Total Wood F } \\
(\mathrm{g} / \mathrm{hr})\end{array}$ & $\begin{array}{c}\text { Relative } \\
\text { Humidity, } \\
@ 10 \mathrm{~m}(\%)\end{array}$ \\
\hline & 7/22/2008 13:00 & 0 & 204 & 1300 & 4.232 & 4.232 & 7.97 & 0.8842 & 1.02E-02 & 3737.8358 & 1377.2825 & 5115.1182 & \\
\hline W605WN1 & $7 / 22 / 2008$ 14:00 & 1 & 204 & 1400 & 4.548 & 4.548 & & 0.7533 & $8.40 \mathrm{E}-03$ & 3068.6929 & 1130.7230 & 4199.4158 & \\
\hline $30 " \mathrm{NE}$ & $7 / 22 / 2008$ 15:00 & 2 & 204 & 1500 & 4.533 & 4.533 & & 0.7591 & $8.48 \mathrm{E}-03$ & 3097.8128 & 1141.4529 & 4239.2657 & \\
\hline \multirow[t]{2}{*}{ Probe \# } & 7/22/2008 16:00 & 3 & 204 & 1600 & 4.569 & 4.569 & & 0.7452 & $8.29 \mathrm{E}-03$ & 3028.3320 & 1115.8512 & 4144.1832 & \\
\hline & 7/22/2008 17:00 & 4 & 204 & 1700 & 4.538 & 4.538 & & 0.7572 & $8.45 \mathrm{E}-03$ & 3088.0791 & 1137.8662 & 4225.9453 & \\
\hline \multirow[t]{4}{*}{12} & $7 / 22 / 2008$ 18:00 & 5 & 204 & 1800 & 4.608 & 4.608 & & 0.7305 & $8.08 \mathrm{E}-03$ & 2954.6142 & 1088.6884 & 4043.3026 & \\
\hline & 7/22/2008 19:00 & 6 & 204 & 1900 & 4.802 & 4.802 & & 0.6606 & 7.14E-03 & 2610.4636 & 961.8790 & 3572.3425 & \\
\hline & $7 / 22 / 200820: 00$ & 7 & 204 & 2000 & 5.247 & 5.247 & & 0.5197 & 5.32E-03 & 1943.2338 & 716.0244 & 2659.2582 & \\
\hline & $7 / 22 / 2008$ 21:00 & 8 & 204 & 2100 & 6.397 & 6.397 & & 0.2465 & 2.12E-03 & 775.8559 & 285.8801 & 1061.7360 & \\
\hline \multirow[t]{2}{*}{$\mathrm{DBH}(\mathrm{cm})$} & $7 / 22 / 200822: 00$ & 9 & 204 & 2200 & 7.502 & 7.502 & & 0.0629 & $3.95 E-04$ & 144.4398 & 53.2218 & 197.6616 & \\
\hline & 7/22/2008 23:00 & 10 & 204 & 2300 & 7.974 & 7.974 & & 0.0000 & $0.00 \mathrm{E}+00$ & 0.0000 & 0.0000 & 0.0000 & \\
\hline \multirow[t]{2}{*}{13.7} & $7 / 23 / 20080: 00$ & 11 & 205 & 0 & 8.24 & 8.24 & 8.43 & -0.0323 & $0.00 \mathrm{E}+00$ & 0.0000 & 0.0000 & 0.0000 & \\
\hline & 7/23/2008 1:00 & 12 & 205 & 100 & 8.34 & 8.34 & & -0.0439 & $0.00 \mathrm{E}+00$ & 0.0000 & 0.0000 & 0.0000 & \\
\hline Day Count & 7/23/2008 2:00 & 13 & 205 & 200 & 8.39 & 8.39 & & -0.0496 & $0.00 \mathrm{E}+00$ & 0.0000 & 0.0000 & 0.0000 & \\
\hline \multirow[t]{27}{*}{35} & 7/23/2008 3:00 & 14 & 205 & 300 & 8.43 & 8.43 & & -0.0541 & $0.00 \mathrm{E}+00$ & 0.0000 & 0.0000 & 0.0000 & \\
\hline & 7/23/2008 4:00 & 15 & 205 & 400 & 8.41 & 8.41 & & -0.0518 & $0.00 \mathrm{E}+00$ & 0.0000 & 0.0000 & 0.0000 & \\
\hline & 7/23/2008 5:00 & 16 & 205 & 500 & 8.35 & 8.35 & & -0.0450 & $0.00 E+00$ & 0.0000 & 0.0000 & 0.0000 & \\
\hline & 7/23/2008 6:00 & 17 & 205 & 600 & 8.21 & 8.21 & & -0.0287 & $0.00 E+00$ & 0.0000 & 0.0000 & 0.0000 & \\
\hline & 7/23/2008 7:00 & 18 & 205 & 700 & 7.913 & 7.913 & & 0.0077 & $2.98 \mathrm{E}-05$ & 10.8966 & 4.0151 & 14.9116 & \\
\hline & 7/23/2008 8:00 & 19 & 205 & 800 & 7.246 & 7.246 & & 0.1005 & 7.03E-04 & 256.9862 & 94.6919 & 351.6781 & \\
\hline & $7 / 23 / 20089: 00$ & 20 & 205 & 900 & 6.28 & 6.28 & & 0.2697 & 2.37E-03 & 866.7847 & 319.3847 & 1186.1694 & \\
\hline & 7/23/2008 10:00 & 21 & 205 & 1000 & 5.362 & 5.362 & & 0.4871 & $4.91 \mathrm{E}-03$ & 1794.3197 & 661.1540 & 2455.4738 & \\
\hline & 7/23/2008 11:00 & 22 & 205 & 1100 & 5.102 & 5.102 & & 0.5629 & 5.87E-03 & 2143.8960 & 789.9626 & 2933.8585 & \\
\hline & $7 / 23 / 2008$ 12:00 & 23 & 205 & 1200 & 4.919 & 4.919 & & 0.6211 & $6.62 \mathrm{E}-03$ & 2419.6670 & 891.5761 & 3311.2431 & \\
\hline & $7 / 23 / 2008$ 13:00 & 24 & 205 & 1300 & 4.801 & 4.801 & & 0.6609 & 7.15E-03 & 2612.1463 & 962.4990 & 3574.6453 & \\
\hline & $7 / 23 / 200814: 00$ & 25 & 205 & 1400 & 4.772 & 4.772 & & 0.6710 & $7.28 \mathrm{E}-03$ & 2661.3414 & 980.6260 & 3641.9673 & \\
\hline & 7/23/2008 15:00 & 26 & 205 & 1500 & 4.736 & 4.736 & & 0.6837 & 7.45E-03 & 2723.4924 & 1003.5268 & 3727.0192 & \\
\hline & $7 / 23 / 2008$ 16:00 & 27 & 205 & 1600 & 4.876 & 4.876 & & 0.6354 & 6.81E-03 & 2488.4102 & 916.9059 & 3405.3161 & \\
\hline & 7/23/2008 17:00 & 28 & 205 & 1700 & 5.207 & 5.207 & & 0.5314 & 5.46E-03 & 1997.1061 & 735.8748 & 2732.9809 & \\
\hline & $7 / 23 / 2008$ 18:00 & 29 & 205 & 1800 & 5.691 & 5.691 & & 0.4012 & 3.87E-03 & 1412.8329 & 520.5873 & 1933.4202 & \\
\hline & $7 / 23 / 2008$ 19:00 & 30 & 205 & 1900 & 5.844 & 5.844 & & 0.3645 & 3.44E-03 & 1255.5158 & 462.6206 & 1718.1365 & \\
\hline & $7 / 23 / 200820: 00$ & 31 & 205 & 2000 & 6.152 & 6.152 & & 0.2962 & $2.66 \mathrm{E}-03$ & 972.4407 & 358.3158 & 1330.7564 & \\
\hline & $7 / 23 / 200821: 00$ & 32 & 205 & 2100 & 6.668 & 6.668 & & 0.1959 & $1.60 \mathrm{E}-03$ & 584.5136 & 215.3761 & 799.8897 & \\
\hline & $7 / 23 / 200822: 00$ & 33 & 205 & 2200 & 7.317 & 7.317 & & 0.0898 & $6.12 \mathrm{E}-04$ & 223.7877 & 82.4592 & 306.2468 & \\
\hline & $7 / 23 / 2008$ 23:00 & 34 & 205 & 2300 & 7.567 & 7.567 & & 0.0538 & 3.26E-04 & 119.0866 & 43.8799 & 162.9665 & \\
\hline & $7 / 24 / 20080: 00$ & 35 & 206 & 0 & 7.617 & 7.617 & 8.11 & 0.0469 & $2.75 E-04$ & 100.5231 & 37.0398 & 137.5628 & \\
\hline & 7/24/2008 1:00 & 36 & 206 & 100 & 7.693 & 7.693 & & 0.0365 & 2.02E-04 & 73.9573 & 27.2511 & 101.2083 & \\
\hline & 7/24/2008 2:00 & 37 & 206 & 200 & 7.765 & 7.765 & & 0.0269 & $1.39 \mathrm{E}-04$ & 50.7859 & 18.7131 & 69.4990 & \\
\hline & 7/24/2008 3:00 & 38 & 206 & 300 & 7.872 & 7.872 & & 0.0130 & 5.65E-05 & 20.6497 & 7.6088 & 28.2585 & \\
\hline & 7/24/2008 4:00 & 39 & 206 & 400 & 7.933 & 7.933 & & 0.0052 & 1.82E-05 & 6.6610 & 2.4544 & 9.1153 & \\
\hline & $7 / 24 / 20085: 00$ & 40 & 206 & 500 & 8.04 & 8.04 & & -0.0082 & $0.00 \mathrm{E}+00$ & 0.0000 & 0.0000 & 0.0000 & \\
\hline
\end{tabular}




\begin{tabular}{|c|c|c|c|c|c|c|c|c|c|c|c|c|c|}
\hline Tree ID & TS & RN & JD & HR & $\begin{array}{c}\text { Raw } \\
\text { TC_dTA }\end{array}$ & $\begin{array}{c}\text { Fixed } \\
\text { TC_dT } \Delta\end{array}$ & dTM & $\mathrm{K}$ & $\begin{array}{l}\text { Sap } \\
\text { Velocity } \\
(\mathrm{cm} / \mathrm{s})\end{array}$ & $\begin{array}{l}\text { Probed } \\
\text { Drywood F } \\
\text { (g/hr) }\end{array}$ & $\begin{array}{c}\text { Extrapolated } \\
\text { Wetwood F } \\
(\mathrm{g} / \mathrm{hr})\end{array}$ & $\begin{array}{c}\text { Extrapolated } \\
\text { Total Wood F } \\
(\mathrm{g} / \mathrm{hr})\end{array}$ & $\begin{array}{l}\text { Relative } \\
\text { Humidity, } \\
@ 10 \mathrm{~m}(\%)\end{array}$ \\
\hline & 7/24/2008 6:00 & 41 & 206 & 600 & 8.11 & 8.11 & & -0.0168 & $0.00 E+00$ & 0.0000 & 0.0000 & 0.0000 & \\
\hline & 7/24/2008 7:00 & 42 & 206 & 700 & 8.08 & 8.08 & & -0.0131 & $0.00 \mathrm{E}+00$ & 0.0000 & 0.0000 & 0.0000 & \\
\hline & 7/24/2008 8:00 & 43 & 206 & 800 & 7.8 & 7.8 & & 0.0223 & $1.10 \mathrm{E}-04$ & 40.3046 & 14.8511 & 55.1557 & \\
\hline & 7/24/2008 9:00 & 44 & 206 & 900 & 7.439 & 7.439 & & 0.0719 & 4.66E-04 & 170.2849 & 62.7450 & 233.0299 & \\
\hline & $7 / 24 / 2008$ 10:00 & 45 & 206 & 1000 & 7.502 & 7.502 & & 0.0629 & 3.95E-04 & 144.4398 & 53.2218 & 197.6616 & \\
\hline & 7/24/2008 11:00 & 46 & 206 & 1100 & 6.099 & 6.099 & & 0.3074 & $2.79 \mathrm{E}-03$ & 1018.1654 & 375.1640 & 1393.3294 & \\
\hline & 7/24/2008 12:00 & 47 & 206 & 1200 & 5.093 & 5.093 & & 0.5657 & $5.90 \mathrm{E}-03$ & 2156.8519 & 794.7364 & 2951.5883 & \\
\hline & 7/24/2008 13:00 & 48 & 206 & 1300 & 5.311 & 5.311 & & 0.5014 & $5.09 \mathrm{E}-03$ & 1859.2896 & 685.0935 & 2544.3831 & \\
\hline & 7/24/2008 14:00 & 49 & 206 & 1400 & 5.167 & 5.167 & & 0.5433 & 5.61E-03 & 2052.0929 & 756.1359 & 2808.2288 & \\
\hline & $7 / 24 / 2008$ 15:00 & 50 & 206 & 1500 & 4.853 & 4.853 & & 0.6431 & 6.91E-03 & 2525.8300 & 930.6940 & 3456.5241 & \\
\hline & 7/24/2008 16:00 & 51 & 206 & 1600 & 4.771 & 4.771 & & 0.6713 & 7.29E-03 & 2663.0515 & 981.2561 & 3644.3076 & \\
\hline & 7/24/2008 17:00 & 52 & 206 & 1700 & 4.911 & 4.911 & & 0.6237 & 6.66E-03 & 2432.3380 & 896.2450 & 3328.5830 & \\
\hline & $7 / 24 / 2008$ 18:00 & 53 & 206 & 1800 & 4.832 & 4.832 & & 0.6502 & 7.01E-03 & 2560.3995 & 943.4318 & 3503.8313 & \\
\hline & 7/24/2008 19:00 & 54 & 206 & 1900 & 5.188 & 5.188 & & 0.5370 & $5.54 \mathrm{E}-03$ & 2023.0842 & 745.4470 & 2768.5312 & \\
\hline & $7 / 24 / 200820: 00$ & 55 & 206 & 2000 & 5.809 & 5.809 & & 0.3727 & 3.53E-03 & 1290.4674 & 475.4993 & 1765.9667 & \\
\hline & $7 / 24 / 200821: 00$ & 56 & 206 & 2100 & 6.918 & 6.918 & & 0.1526 & 1.18E-03 & 430.0524 & 158.4617 & 588.5141 & \\
\hline & $7 / 24 / 2008$ 22:00 & 57 & 206 & 2200 & 7.821 & 7.821 & & 0.0196 & 9.38E-05 & 34.2891 & 12.6345 & 46.9237 & \\
\hline & $7 / 24 / 200823: 00$ & 58 & 206 & 2300 & 8.1 & 8.1 & & -0.0156 & $0.00 \mathrm{E}+00$ & 0.0000 & 0.0000 & 0.0000 & \\
\hline & 7/25/2008 0:00 & 59 & 207 & 0 & 8.2 & 8.2 & 8.28 & -0.0276 & $0.00 \mathrm{E}+00$ & 0.0000 & 0.0000 & 0.0000 & \\
\hline & 7/25/2008 1:00 & 60 & 207 & 100 & 8.22 & 8.22 & & -0.0299 & $0.00 \mathrm{E}+00$ & 0.0000 & 0.0000 & 0.0000 & \\
\hline & 7/25/2008 2:00 & 61 & 207 & 200 & 8.21 & 8.21 & & -0.0287 & $0.00 \mathrm{E}+00$ & 0.0000 & 0.0000 & 0.0000 & \\
\hline & 7/25/2008 3:00 & 62 & 207 & 300 & 8.2 & 8.2 & & -0.0276 & $0.00 \mathrm{E}+00$ & 0.0000 & 0.0000 & 0.0000 & \\
\hline & 7/25/2008 4:00 & 63 & 207 & 400 & 8.21 & 8.21 & & -0.0287 & $0.00 E+00$ & 0.0000 & 0.0000 & 0.0000 & \\
\hline & 7/25/2008 5:00 & 64 & 207 & 500 & 8.24 & 8.24 & & -0.0323 & $0.00 \mathrm{E}+00$ & 0.0000 & 0.0000 & 0.0000 & \\
\hline & 7/25/2008 6:00 & 65 & 207 & 600 & 8.28 & 8.28 & & -0.0370 & $0.00 \mathrm{E}+00$ & 0.0000 & 0.0000 & 0.0000 & \\
\hline & 7/25/2008 7:00 & 66 & 207 & 700 & 8.28 & 8.28 & & -0.0370 & $0.00 \mathrm{E}+00$ & 0.0000 & 0.0000 & 0.0000 & \\
\hline & 7/25/2008 8:00 & 67 & 207 & 800 & 8.26 & 8.26 & & -0.0346 & $0.00 \mathrm{E}+00$ & 0.0000 & 0.0000 & 0.0000 & \\
\hline & 7/25/2008 9:00 & 68 & 207 & 900 & 8.22 & 8.22 & & -0.0299 & $0.00 \mathrm{E}+00$ & 0.0000 & 0.0000 & 0.0000 & \\
\hline & 7/25/2008 10:00 & 69 & 207 & 1000 & 8.09 & 8.09 & & -0.0143 & $0.00 \mathrm{E}+00$ & 0.0000 & 0.0000 & 0.0000 & \\
\hline & 7/25/2008 11:00 & 70 & 207 & 1100 & 7.409 & 7.409 & & 0.0763 & 5.01E-04 & 183.0226 & 67.4384 & 250.4611 & \\
\hline & $7 / 25 / 2008$ 12:00 & 71 & 207 & 1200 & 6.228 & 6.228 & & 0.2803 & 2.49E-03 & 908.9092 & 334.9063 & 1243.8155 & \\
\hline & $7 / 25 / 2008$ 13:00 & 72 & 207 & 1300 & 5.513 & 5.513 & & 0.4464 & 4.41E-03 & 1611.4504 & 593.7720 & 2205.2224 & \\
\hline & $7 / 25 / 200814: 00$ & 73 & 207 & 1400 & 5.033 & 5.033 & & 0.5843 & $6.14 \mathrm{E}-03$ & 2244.7893 & 827.1388 & 3071.9281 & \\
\hline & $7 / 25 / 200815: 00$ & 74 & 207 & 1500 & 4.923 & 4.923 & & 0.6197 & $6.60 \mathrm{E}-03$ & 2413.3516 & 889.2490 & 3302.6006 & \\
\hline & 7/25/2008 16:00 & 75 & 207 & 1600 & 4.954 & 4.954 & & 0.6096 & 6.47E-03 & 2364.8569 & 871.3802 & 3236.2371 & \\
\hline & $7 / 25 / 2008$ 17:00 & 76 & 207 & 1700 & 4.703 & 4.703 & & 0.6955 & 7.61E-03 & 2781.5400 & 1024.9156 & 3806.4557 & \\
\hline & 7/25/2008 18:00 & 77 & 207 & 1800 & 4.778 & 4.778 & & 0.6689 & $7.25 E-03$ & 2651.0999 & 976.8523 & 3627.9522 & \\
\hline & 7/25/2008 19:00 & 78 & 207 & 1900 & 4.904 & 4.904 & & 0.6260 & $6.69 \mathrm{E}-03$ & 2443.4693 & 900.3465 & 3343.8158 & \\
\hline & $7 / 25 / 200820: 00$ & 79 & 207 & 2000 & 5.472 & 5.472 & & 0.4572 & $4.54 \mathrm{E}-03$ & 1659.7436 & 611.5667 & 2271.3103 & \\
\hline & $7 / 25 / 2008$ 21:00 & 80 & 207 & 2100 & 6.704 & 6.704 & & 0.1894 & 1.54E-03 & 561.0123 & 206.7165 & 767.7288 & \\
\hline & $7 / 25 / 200822: 00$ & 81 & 207 & 2200 & 7.761 & 7.761 & & 0.0274 & $1.42 \mathrm{E}-04$ & 52.0180 & 19.1671 & 71.1851 & \\
\hline
\end{tabular}




\begin{tabular}{|c|c|c|c|c|c|c|c|c|c|c|c|c|c|}
\hline Tree ID & TS & RN & JD & HR & $\begin{array}{c}\text { Raw } \\
\text { TC_dTA }\end{array}$ & $\begin{array}{c}\text { Fixed } \\
\text { TC_dT } \Delta\end{array}$ & dTM & K & $\begin{array}{l}\text { Sap } \\
\text { Velocity } \\
(\mathrm{cm} / \mathrm{s})\end{array}$ & $\begin{array}{c}\text { Probed } \\
\text { Drywood F } \\
(\mathrm{g} / \mathrm{hr})\end{array}$ & $\begin{array}{c}\text { Extrapolated } \\
\text { Wetwood F } \\
(\mathrm{g} / \mathrm{hr})\end{array}$ & $\begin{array}{c}\text { Extrapolated } \\
\text { Total Wood F } \\
(\mathrm{g} / \mathrm{hr})\end{array}$ & $\begin{array}{l}\text { Relative } \\
\text { Humidity, } \\
@ 10 \mathrm{~m}(\%)\end{array}$ \\
\hline & 7/25/2008 23:00 & 82 & 207 & 2300 & 8.05 & 8.05 & & -0.0094 & $0.00 \mathrm{E}+00$ & 0.0000 & 0.0000 & 0.0000 & \\
\hline & $7 / 26 / 20080: 00$ & 83 & 208 & 0 & 8.1 & 8.1 & 8.10 & -0.0156 & $0.00 \mathrm{E}+00$ & 0.0000 & 0.0000 & 0.0000 & \\
\hline & 7/26/2008 1:00 & 84 & 208 & 100 & 8.1 & 8.1 & & -0.0156 & $0.00 E+00$ & 0.0000 & 0.0000 & 0.0000 & \\
\hline & $7 / 26 / 20082: 00$ & 85 & 208 & 200 & 8.08 & 8.08 & & -0.0131 & $0.00 \mathrm{E}+00$ & 0.0000 & 0.0000 & 0.0000 & \\
\hline & 7/26/2008 3:00 & 86 & 208 & 300 & 8.04 & 8.04 & & -0.0082 & $0.00 \mathrm{E}+00$ & 0.0000 & 0.0000 & 0.0000 & \\
\hline & 7/26/2008 4:00 & 87 & 208 & 400 & 7.986 & 7.986 & & -0.0015 & $0.00 \mathrm{E}+00$ & 0.0000 & 0.0000 & 0.0000 & \\
\hline & 7/26/2008 5:00 & 88 & 208 & 500 & 7.934 & 7.934 & & 0.0050 & 1.77E-05 & 6.4605 & 2.3805 & 8.8410 & \\
\hline & 7/26/2008 6:00 & 89 & 208 & 600 & 7.859 & 7.859 & & 0.0146 & $6.56 \mathrm{E}-05$ & 23.9844 & 8.8375 & 32.8220 & \\
\hline & 7/26/2008 7:00 & 90 & 208 & 700 & 7.778 & 7.778 & & 0.0252 & 1.28E-04 & 46.8291 & 17.2552 & 64.0843 & \\
\hline & 7/26/2008 8:00 & 91 & 208 & 800 & 7.694 & 7.694 & & 0.0364 & 2.01E-04 & 73.6216 & 27.1274 & 100.7490 & \\
\hline & 7/26/2008 9:00 & 92 & 208 & 900 & 7.005 & 7.005 & & 0.1383 & 1.04E-03 & 380.9556 & 140.3709 & 521.3265 & \\
\hline & $7 / 26 / 2008$ 10:00 & 93 & 208 & 1000 & 5.433 & 5.433 & & 0.4677 & 4.67E-03 & 1706.6090 & 628.8352 & 2335.4442 & \\
\hline & $7 / 26 / 2008$ 11:00 & 94 & 208 & 1100 & 5.154 & 5.154 & & 0.5471 & $5.66 \mathrm{E}-03$ & 2070.2083 & 762.8108 & 2833.0191 & \\
\hline & $7 / 26 / 2008$ 12:00 & 95 & 208 & 1200 & 5.419 & 5.419 & & 0.4715 & 4.72E-03 & 1723.6572 & 635.1169 & 2358.7741 & \\
\hline & $7 / 26 / 2008$ 13:00 & 96 & 208 & 1300 & 4.944 & 4.944 & & 0.6129 & 6.51E-03 & 2380.4138 & 877.1124 & 3257.5262 & \\
\hline & $7 / 26 / 200814: 00$ & 97 & 208 & 1400 & 4.847 & 4.847 & & 0.6451 & $6.94 \mathrm{E}-03$ & 2535.6675 & 934.3188 & 3469.9863 & \\
\hline & $7 / 26 / 2008$ 15:00 & 98 & 208 & 1500 & 4.759 & 4.759 & & 0.6756 & 7.34E-03 & 2683.6453 & 988.8443 & 3672.4896 & \\
\hline & $7 / 26 / 200816: 00$ & 99 & 208 & 1600 & 4.811 & 4.811 & & 0.6575 & 7.10E-03 & 2595.3596 & 956.3136 & 3551.6733 & \\
\hline & $7 / 26 / 200817: 00$ & 100 & 208 & 1700 & 4.573 & 4.573 & & 0.7437 & 8.27E-03 & 3020.6976 & 1113.0382 & 4133.7357 & \\
\hline & $7 / 26 / 2008$ 18:00 & 101 & 208 & 1800 & 4.754 & 4.754 & & 0.6773 & 7.37E-03 & 2692.2656 & 992.0206 & 3684.2862 & \\
\hline & $7 / 26 / 2008$ 19:00 & 102 & 208 & 1900 & 4.901 & 4.901 & & 0.6270 & $6.70 \mathrm{E}-03$ & 2448.2525 & 902.1090 & 3350.3615 & \\
\hline & $7 / 26 / 200820: 00$ & 103 & 208 & 2000 & 5.308 & 5.308 & & 0.5023 & 5.10E-03 & 1863.1638 & 686.5210 & 2549.6849 & \\
\hline & $7 / 26 / 200821: 00$ & 104 & 208 & 2100 & 6.5 & 6.5 & & 0.2268 & 1.92E-03 & 700.0535 & 257.9491 & 958.0026 & \\
\hline & $7 / 26 / 200822: 00$ & 105 & 208 & 2200 & 7.505 & 7.505 & & 0.0625 & 3.92E-04 & 143.2400 & 52.7797 & 196.0197 & \\
\hline & $7 / 26 / 2008$ 23:00 & 106 & 208 & 2300 & 7.822 & 7.822 & & 0.0194 & $9.31 \mathrm{E}-05$ & 34.0081 & 12.5310 & 46.5391 & \\
\hline & $7 / 27 / 20080: 00$ & 107 & 209 & 0 & 7.869 & 7.869 & 7.87 & 0.0133 & 5.86E-05 & 21.4099 & 7.8889 & 29.2988 & \\
\hline & 7/27/2008 1:00 & 108 & 209 & 100 & 7.822 & 7.822 & & 0.0194 & 9.31E-05 & 34.0081 & 12.5310 & 46.5391 & \\
\hline & 7/27/2008 2:00 & 109 & 209 & 200 & 7.761 & 7.761 & & 0.0274 & 1.42E-04 & 52.0180 & 19.1671 & 71.1851 & \\
\hline & 7/27/2008 3:00 & 110 & 209 & 300 & 7.725 & 7.725 & & 0.0322 & 1.73E-04 & 63.4053 & 23.3630 & 86.7683 & \\
\hline & 7/27/2008 4:00 & 111 & 209 & 400 & 7.685 & 7.685 & & 0.0376 & $2.10 \mathrm{E}-04$ & 76.6558 & 28.2454 & 104.9012 & \\
\hline & 7/27/20085:00 & 112 & 209 & 500 & 7.615 & 7.615 & & 0.0471 & 2.77E-04 & 101.2495 & 37.3075 & 138.5569 & \\
\hline & 7/27/2008 6:00 & 113 & 209 & 600 & 7.556 & 7.556 & & 0.0553 & 3.37E-04 & 123.2815 & 45.4256 & 168.7071 & \\
\hline & 7/27/2008 7:00 & 114 & 209 & 700 & 7.487 & 7.487 & & 0.0650 & $4.12 \mathrm{E}-04$ & 150.4812 & 55.4479 & 205.9292 & \\
\hline & 7/27/2008 8:00 & 115 & 209 & 800 & 7.388 & 7.388 & & 0.0793 & $5.26 \mathrm{E}-04$ & 192.1023 & 70.7840 & 262.8863 & \\
\hline & $7 / 27 / 20089: 00$ & 116 & 209 & 900 & 6.211 & 6.211 & & 0.2839 & 2.53E-03 & 922.9155 & 340.0672 & 1262.9826 & \\
\hline & $7 / 27 / 2008$ 10:00 & 117 & 209 & 1000 & 4.671 & 4.671 & & 0.7071 & 7.77E-03 & 2838.8345 & 1046.0270 & 3884.8614 & \\
\hline & $7 / 27 / 2008$ 11:00 & 118 & 209 & 1100 & 4.46 & 4.46 & & 0.7879 & 8.87E-03 & 3243.0819 & 1194.9802 & 4438.0621 & \\
\hline & $7 / 27 / 2008$ 12:00 & 119 & 209 & 1200 & 4.41 & 4.41 & & 0.8082 & $9.16 \mathrm{E}-03$ & 3346.0973 & 1232.9384 & 4579.0357 & \\
\hline & $7 / 27 / 200813: 00$ & 120 & 209 & 1300 & 4.476 & 4.476 & & 0.7815 & $8.79 \mathrm{E}-03$ & 3210.7291 & 1183.0592 & 4393.7882 & \\
\hline & $7 / 27 / 200814: 00$ & 121 & 209 & 1400 & 4.372 & 4.372 & & 0.8239 & 9.38E-03 & 3426.3774 & 1262.5192 & 4688.8967 & \\
\hline & 7/27/2008 15:00 & 122 & 209 & 1500 & 4.357 & 4.357 & & 0.8302 & $9.46 \mathrm{E}-03$ & 3458.5519 & 1274.3746 & 4732.9264 & \\
\hline
\end{tabular}




\begin{tabular}{|c|c|c|c|c|c|c|c|c|c|c|c|c|c|}
\hline Tree ID & TS & RN & JD & HR & $\begin{array}{c}\text { Raw } \\
\text { TC_dTA }\end{array}$ & $\begin{array}{c}\text { Fixed } \\
\text { TC_dT } \Delta\end{array}$ & dTM & $\mathbf{K}$ & $\begin{array}{l}\text { Sap } \\
\text { Velocity } \\
(\mathrm{cm} / \mathrm{s})\end{array}$ & $\begin{array}{c}\text { Probed } \\
\text { Drywood F } \\
(\mathrm{g} / \mathrm{hr})\end{array}$ & $\begin{array}{c}\text { Extrapolated } \\
\text { Wetwood F } \\
(\mathrm{g} / \mathrm{hr})\end{array}$ & $\begin{array}{c}\text { Extrapolated } \\
\text { Total Wood F } \\
(\mathrm{g} / \mathrm{hr})\end{array}$ & $\begin{array}{l}\text { Relative } \\
\text { Humidity, } \\
@ 10 \mathrm{~m}(\%)\end{array}$ \\
\hline & 7/27/2008 16:00 & 123 & 209 & 1600 & \begin{tabular}{|l|}
4.387 \\
\end{tabular} & 4.387 & & 0.8176 & $9.29 \mathrm{E}-03$ & 3394.4790 & 1250.7656 & 4645.2447 & \\
\hline & 7/27/2008 17:00 & 124 & 209 & 1700 & 4.352 & 4.352 & & 0.8323 & $9.49 \mathrm{E}-03$ & 3469.3386 & 1278.3491 & 4747.6877 & \\
\hline & $7 / 27 / 2008$ 18:00 & 125 & 209 & 1800 & 4.453 & 4.453 & & 0.7907 & $8.91 \mathrm{E}-03$ & 3257.3286 & 1200.2297 & 4457.5583 & \\
\hline & $7 / 27 / 2008$ 19:00 & 126 & 209 & 1900 & 4.602 & 4.602 & & 0.7327 & 8.12E-03 & 2965.8520 & 1092.8292 & 4058.6812 & \\
\hline & $7 / 27 / 200820: 00$ & 127 & 209 & 2000 & 5.045 & 5.045 & & 0.5806 & 6.09E-03 & 2226.9815 & 820.5771 & 3047.5586 & \\
\hline & $7 / 27 / 2008$ 21:00 & 128 & 209 & 2100 & 6.09 & 6.09 & & 0.3094 & 2.81E-03 & 1026.0484 & 378.0686 & 1404.1171 & \\
\hline & $7 / 27 / 2008$ 22:00 & 129 & 209 & 2200 & 6.925 & 6.925 & & 0.1515 & 1.17E-03 & 426.0152 & 156.9740 & 582.9892 & \\
\hline & $7 / 27 / 200823: 00$ & 130 & 209 & 2300 & 7.19 & 7.19 & & 0.1090 & 7.78E-04 & 284.2346 & 104.7321 & 388.9667 & \\
\hline & $7 / 28 / 20080: 00$ & 131 & 210 & 0 & 7.291 & 7.291 & 7.56 & 0.0937 & $6.45 \mathrm{E}-04$ & 235.7698 & 86.8742 & 322.6440 & \\
\hline & 7/28/2008 1:00 & 132 & 210 & 100 & 7.403 & 7.403 & & 0.0771 & 5.08E-04 & 185.6031 & 68.3893 & 253.9924 & \\
\hline & $7 / 28 / 20082: 00$ & 133 & 210 & 200 & 7.414 & 7.414 & & 0.0755 & $4.95 E-04$ & 180.8806 & 66.6492 & 247.5297 & \\
\hline & 7/28/2008 3:00 & 134 & 210 & 300 & 7.513 & 7.513 & & 0.0614 & 3.83E-04 & 140.0544 & 51.6059 & 191.6603 & \\
\hline & 7/28/2008 4:00 & 135 & 210 & 400 & 7.528 & 7.528 & & 0.0592 & 3.67E-04 & 134.1361 & 49.4252 & 183.5613 & \\
\hline & 7/28/2008 5:00 & 136 & 210 & 500 & 7.555 & 7.555 & & 0.0555 & 3.38E-04 & 123.6648 & 45.5668 & 169.2316 & \\
\hline & 7/28/2008 6:00 & 137 & 210 & 600 & 7.54 & 7.54 & & 0.0576 & 3.54E-04 & 129.4532 & 47.6997 & 177.1529 & \\
\hline & 7/28/2008 7:00 & 138 & 210 & 700 & 7.29 & 7.29 & & 0.0938 & 6.46E-04 & 236.2347 & 87.0455 & 323.2802 & \\
\hline & 7/28/2008 8:00 & 139 & 210 & 800 & 6.059 & 6.059 & & 0.3161 & 2.88E-03 & 1053.4680 & 388.1720 & 1441.6400 & \\
\hline & $7 / 28 / 20089: 00$ & 140 & 210 & 900 & 5.064 & 5.064 & & 0.5746 & $6.02 \mathrm{E}-03$ & 2199.0125 & 810.2714 & 3009.2838 & \\
\hline & $7 / 28 / 2008$ 10:00 & 141 & 210 & 1000 & 4.557 & 4.557 & & 0.7498 & 8.35E-03 & 3051.3375 & 1124.3281 & 4175.6656 & \\
\hline & $7 / 28 / 2008$ 11:00 & 142 & 210 & 1100 & 4.427 & 4.427 & & 0.8012 & 9.06E-03 & 3310.7430 & 1219.9113 & 4530.6543 & \\
\hline & $7 / 28 / 200812: 00$ & 143 & 210 & 1200 & 4.392 & 4.392 & & 0.8156 & $9.26 \mathrm{E}-03$ & 3383.9071 & 1246.8701 & 4630.7772 & \\
\hline & 7/28/2008 13:00 & 144 & 210 & 1300 & 4.439 & 4.439 & & 0.7964 & 8.99E-03 & 3285.9922 & 1210.7914 & 4496.7836 & \\
\hline & $7 / 28 / 2008$ 14:00 & 145 & 210 & 1400 & 4.404 & 4.404 & & 0.8106 & $9.19 \mathrm{E}-03$ & 3358.6574 & 1237.5664 & 4596.2237 & \\
\hline & $7 / 28 / 2008$ 15:00 & 146 & 210 & 1500 & 4.329 & 4.329 & & 0.8420 & $9.63 \mathrm{E}-03$ & 3519.3601 & 1296.7806 & 4816.1407 & \\
\hline & $7 / 28 / 2008$ 16:00 & 147 & 210 & 1600 & 4.37 & 4.37 & & 0.8247 & $9.39 \mathrm{E}-03$ & 3430.6513 & 1264.0940 & 4694.7454 & \\
\hline & 7/28/2008 17:00 & 148 & 210 & 1700 & 4.332 & 4.332 & & 0.8407 & 9.61E-03 & 3512.7978 & 1294.3626 & 4807.1604 & \\
\hline & $7 / 28 / 2008$ 18:00 & 149 & 210 & 1800 & 4.479 & 4.479 & & 0.7803 & 8.77E-03 & 3204.6954 & 1180.8359 & 4385.5313 & \\
\hline & $7 / 28 / 2008$ 19:00 & 150 & 210 & 1900 & 4.717 & 4.717 & & 0.6905 & $7.54 \mathrm{E}-03$ & 2756.7865 & 1015.7947 & 3772.5812 & \\
\hline & $7 / 28 / 200820: 00$ & 151 & 210 & 2000 & 5.154 & 5.154 & & 0.5471 & $5.66 \mathrm{E}-03$ & 2070.2083 & 762.8108 & 2833.0191 & \\
\hline & $7 / 28 / 200821: 00$ & 152 & 210 & 2100 & 6.35 & 6.35 & & 0.2557 & $2.22 \mathrm{E}-03$ & 811.7534 & 299.1072 & 1110.8606 & \\
\hline & $7 / 28 / 2008$ 22:00 & 153 & 210 & 2200 & 6.908 & 6.908 & & 0.1543 & 1.19E-03 & 435.8465 & 160.5966 & 596.4431 & \\
\hline & $7 / 28 / 200823: 00$ & 154 & 210 & 2300 & 7.152 & 7.152 & & 0.1149 & 8.30E-04 & 303.2593 & 111.7421 & 415.0014 & \\
\hline & $7 / 29 / 20080: 00$ & 155 & 211 & 0 & 7.293 & 7.293 & 7.36 & 0.0934 & 6.43E-04 & 234.8409 & 86.5320 & 321.3728 & \\
\hline & 7/29/2008 1:00 & 156 & 211 & 100 & 7.333 & 7.333 & & 0.0874 & 5.92E-04 & 216.5148 & 79.7793 & 296.2942 & \\
\hline & 7/29/2008 2:00 & 157 & 211 & 200 & 7.364 & 7.364 & & 0.0828 & 5.54E-04 & 202.6428 & 74.6679 & 277.3107 & \\
\hline & 7/29/2008 3:00 & 158 & 211 & 300 & 7.346 & 7.346 & & 0.0855 & 5.76E-04 & 210.6623 & 77.6229 & 288.2851 & \\
\hline & 7/29/2008 4:00 & 159 & 211 & 400 & 7.316 & 7.316 & & 0.0899 & 6.14E-04 & 224.2448 & 82.6276 & 306.8724 & \\
\hline & 7/29/2008 5:00 & 160 & 211 & 500 & 7.205 & 7.205 & & 0.1067 & 7.57E-04 & 276.8440 & 102.0089 & 378.8528 & \\
\hline & $7 / 29 / 20086: 00$ & 161 & 211 & 600 & 7.11 & 7.11 & & 0.1215 & $8.89 \mathrm{E}-04$ & 324.7911 & 119.6760 & 444.4670 & \\
\hline & 7/29/2008 7:00 & 162 & 211 & 700 & 6.94 & 6.94 & & 0.1490 & 1.14E-03 & 417.4154 & 153.8053 & 571.2207 & \\
\hline & 7/29/2008 8:00 & 163 & 211 & 800 & 6.309 & 6.309 & & 0.2639 & $2.31 E-03$ & 843.7556 & 310.8991 & 1154.6547 & \\
\hline
\end{tabular}




\begin{tabular}{|c|c|c|c|c|c|c|c|c|c|c|c|c|c|}
\hline Tree ID & TS & RN & JD & HR & $\begin{array}{c}\text { Raw } \\
\text { TC_dTA }\end{array}$ & $\begin{array}{c}\text { Fixed } \\
\text { TC_dT } \Delta\end{array}$ & dTM & $\mathbf{K}$ & $\begin{array}{l}\text { Sap } \\
\text { Velocity } \\
(\mathrm{cm} / \mathrm{s})\end{array}$ & $\begin{array}{c}\text { Probed } \\
\text { Drywood F } \\
(\mathrm{g} / \mathrm{hr})\end{array}$ & $\begin{array}{c}\text { Extrapolated } \\
\text { Wetwood F } \\
(\mathrm{g} / \mathrm{hr})\end{array}$ & $\begin{array}{c}\text { Extrapolated } \\
\text { Total Wood F } \\
(\mathrm{g} / \mathrm{hr})\end{array}$ & $\begin{array}{l}\text { Relative } \\
\text { Humidity, } \\
@ 10 \mathrm{~m}(\%)\end{array}$ \\
\hline & 7/29/2008 9:00 & 164 & 211 & 900 & \begin{tabular}{|l|}
5.008 \\
\end{tabular} & 5.008 & & 0.5923 & $6.24 \mathrm{E}-03$ & 2282.2492 & 840.9417 & \begin{tabular}{|r|}
3123.1909 \\
\end{tabular} & \\
\hline & 7/29/2008 10:00 & 165 & 211 & 1000 & 4.205 & 4.205 & & 0.8963 & $1.04 \mathrm{E}-02$ & 3800.8926 & 1400.5170 & 5201.4096 & \\
\hline & $7 / 29 / 2008$ 11:00 & 166 & 211 & 1100 & 4.47 & 4.47 & & 0.7839 & 8.82E-03 & 3222.8271 & 1187.5169 & 4410.3440 & \\
\hline & $7 / 29 / 2008$ 12:00 & 167 & 211 & 1200 & 4.093 & 4.093 & & 0.9482 & $1.11 \mathrm{E}-02$ & 4073.5533 & 1500.9845 & 5574.5378 & \\
\hline & 7/29/2008 13:00 & 168 & 211 & 1300 & 4.176 & 4.176 & & 0.9095 & 1.06E-02 & 3869.7522 & 1425.8898 & 5295.6420 & \\
\hline & $7 / 29 / 2008$ 14:00 & 169 & 211 & 1400 & 4.093 & 4.093 & & 0.9482 & 1.11E-02 & 4073.5533 & 1500.9845 & 5574.5378 & \\
\hline & $7 / 29 / 2008$ 15:00 & 170 & 211 & 1500 & 4.32 & 4.32 & & 0.8458 & $9.68 \mathrm{E}-03$ & 3539.1156 & 1304.0599 & 4843.1755 & \\
\hline & $7 / 29 / 2008$ 16:00 & 171 & 211 & 1600 & 4.489 & 4.489 & & 0.7763 & 8.71E-03 & 3184.6567 & 1173.4523 & 4358.1090 & \\
\hline & 7/29/2008 17:00 & 172 & 211 & 1700 & 4.751 & 4.751 & & 0.6784 & 7.38E-03 & 2697.4489 & 993.9305 & 3691.3794 & \\
\hline & 7/29/2008 18:00 & 173 & 211 & 1800 & 4.745 & 4.745 & & 0.6805 & 7.41E-03 & 2707.8409 & 997.7597 & 3705.6006 & \\
\hline & $7 / 29 / 2008$ 19:00 & 174 & 211 & 1900 & 4.771 & 4.771 & & 0.6713 & 7.29E-03 & 2663.0515 & 981.2561 & 3644.3076 & \\
\hline & $7 / 29 / 200820: 00$ & 175 & 211 & 2000 & 5.302 & 5.302 & & 0.5040 & 5.12E-03 & 1870.9300 & 689.3826 & 2560.3126 & \\
\hline & 7/29/2008 21:00 & 176 & 211 & 2100 & 6.353 & 6.353 & & 0.2552 & $2.21 \mathrm{E}-03$ & 809.4371 & 298.2537 & 1107.6908 & \\
\hline & $7 / 29 / 2008$ 22:00 & 177 & 211 & 2200 & 7.046 & 7.046 & & 0.1317 & 9.81E-04 & 358.6256 & 132.1430 & 490.7686 & \\
\hline & $7 / 29 / 2008$ 23:00 & 178 & 211 & 2300 & 7.131 & 7.131 & & 0.1182 & 8.59E-04 & 313.9588 & 115.6846 & 429.6434 & \\
\hline & $7 / 30 / 20080: 00$ & 179 & 212 & 0 & 7.065 & 7.065 & 7.07 & 0.1287 & 9.53E-04 & 348.4514 & 128.3941 & 476.8455 & \\
\hline & 7/30/2008 1:00 & 180 & 212 & 100 & 7.028 & 7.028 & & 0.1346 & 1.01E-03 & 368.3658 & 135.7319 & 504.0977 & \\
\hline & 7/30/2008 2:00 & 181 & 212 & 200 & 6.999 & 6.999 & & 0.1393 & 1.05E-03 & 384.2665 & 141.5909 & 525.8574 & \\
\hline & 7/30/2008 3:00 & 182 & 212 & 300 & 6.97 & 6.97 & & 0.1440 & 1.10E-03 & 400.4256 & 147.5450 & 547.9706 & \\
\hline & 7/30/2008 4:00 & 183 & 212 & 400 & 6.933 & 6.933 & & 0.1502 & 1.15E-03 & 421.4199 & 155.2808 & 576.7007 & \\
\hline & 7/30/2008 5:00 & 184 & 212 & 500 & 6.887 & 6.887 & & 0.1578 & 1.23E-03 & 448.1163 & 165.1177 & 613.2339 & \\
\hline & 7/30/2008 6:00 & 185 & 212 & 600 & 6.842 & 6.842 & & 0.1654 & 1.30E-03 & 474.8777 & 174.9784 & 649.8561 & \\
\hline & 7/30/2008 7:00 & 186 & 212 & 700 & 6.809 & 6.809 & & 0.1711 & 1.35E-03 & 494.9129 & 182.3608 & 677.2738 & \\
\hline & $7 / 30 / 20088: 00$ & 187 & 212 & 800 & 6.799 & 6.799 & & 0.1728 & 1.37E-03 & 501.0533 & 184.6234 & 685.6767 & \\
\hline & 7/30/2008 9:00 & 188 & 212 & 900 & 6.252 & 6.252 & & 0.2754 & 2.43E-03 & 889.3334 & 327.6932 & 1217.0266 & \\
\hline & $7 / 30 / 2008$ 10:00 & 189 & 212 & 1000 & 4.269 & 4.269 & & 0.8679 & 1.00E-02 & 3653.0360 & 1346.0363 & 4999.0723 & \\
\hline & 7/30/2008 11:00 & 190 & 212 & 1100 & 3.642 & 3.642 & & 1.1895 & 1.47E-02 & 5384.6958 & 1984.1019 & 7368.7978 & \\
\hline & $7 / 30 / 2008$ 12:00 & 191 & 212 & 1200 & 3.653 & 3.653 & & 1.1829 & 1.46E-02 & 5347.9785 & 1970.5727 & 7318.5511 & \\
\hline & 7/30/2008 13:00 & 192 & 212 & 1300 & 3.658 & 3.658 & & 1.1799 & 1.46E-02 & 5331.3773 & 1964.4556 & 7295.8329 & \\
\hline & $7 / 30 / 2008$ 14:00 & 193 & 212 & 1400 & 3.656 & 3.656 & & 1.1811 & 1.46E-02 & 5338.0111 & 1966.9000 & 7304.9111 & \\
\hline & 7/30/2008 15:00 & 194 & 212 & 1500 & 3.83 & 3.83 & & 1.0820 & 1.31E-02 & 4792.1807 & 1765.7775 & 6557.9582 & \\
\hline & $7 / 30 / 2008$ 16:00 & 195 & 212 & 1600 & 3.811 & 3.811 & & 1.0924 & 1.33E-02 & 4848.8362 & 1786.6534 & 6635.4896 & \\
\hline & $7 / 30 / 2008$ 17:00 & 196 & 212 & 1700 & 3.878 & 3.878 & & 1.0562 & 1.27E-02 & 4652.0680 & 1714.1501 & 6366.2181 & \\
\hline & $7 / 30 / 2008$ 18:00 & 197 & 212 & 1800 & 4.093 & 4.093 & & 0.9482 & $1.11 \mathrm{E}-02$ & 4073.5533 & 1500.9845 & 5574.5378 & \\
\hline & $7 / 30 / 2008$ 19:00 & 198 & 212 & 1900 & 4.258 & 4.258 & & 0.8727 & 1.01E-02 & 3678.0548 & 1355.2549 & 5033.3097 & \\
\hline & $7 / 30 / 200820: 00$ & 199 & 212 & 2000 & 4.779 & 4.779 & & 0.6685 & 7.25E-03 & 2649.3962 & 976.2245 & 3625.6207 & \\
\hline & 7/30/2008 21:00 & 200 & 212 & 2100 & 5.933 & 5.933 & & 0.3440 & $3.20 \mathrm{E}-03$ & 1169.2925 & 430.8499 & 1600.1424 & \\
\hline & $7 / 30 / 2008$ 22:00 & 201 & 212 & 2200 & 6.726 & 6.726 & & 0.1855 & 1.50E-03 & 546.8631 & 201.5030 & 748.3661 & \\
\hline & 7/30/2008 23:00 & 202 & 212 & 2300 & 6.991 & 6.991 & & 0.1406 & 1.06E-03 & 388.6983 & 143.2239 & 531.9222 & \\
\hline & $7 / 31 / 20080: 00$ & 203 & 213 & 0 & 7.08 & 7.08 & 7.08 & 0.1263 & 9.32E-04 & 340.4965 & 125.4630 & 465.9595 & \\
\hline & 7/31/2008 1:00 & 204 & 213 & 100 & 7.072 & 7.072 & & 0.1275 & 9.43E-04 & 344.7307 & 127.0231 & 471.7537 & \\
\hline
\end{tabular}




\begin{tabular}{|c|c|c|c|c|c|c|c|c|c|c|c|c|c|}
\hline Tree ID & TS & RN & JD & HR & $\begin{array}{c}\text { Raw } \\
\text { TC_dTA }\end{array}$ & $\begin{array}{c}\text { Fixed } \\
\text { TC_dT } \Delta\end{array}$ & dTM & $\mathbf{K}$ & $\begin{array}{l}\text { Sap } \\
\text { Velocity } \\
(\mathrm{cm} / \mathrm{s})\end{array}$ & $\begin{array}{c}\text { Probed } \\
\text { Drywood F } \\
(\mathrm{g} / \mathrm{hr})\end{array}$ & $\begin{array}{c}\text { Extrapolated } \\
\text { Wetwood F } \\
(\mathrm{g} / \mathrm{hr})\end{array}$ & $\begin{array}{c}\text { Extrapolated } \\
\text { Total Wood F } \\
(\mathrm{g} / \mathrm{hr})\end{array}$ & $\begin{array}{l}\text { Relative } \\
\text { Humidity, } \\
@ 10 \mathrm{~m}(\%)\end{array}$ \\
\hline & 7/31/2008 2:00 & 205 & 213 & 200 & \begin{tabular}{|l|}
7.067 \\
\end{tabular} & 7.067 & & 0.1283 & 9.51E-04 & 347.3868 & 128.0018 & 475.3886 & \\
\hline & 7/31/2008 3:00 & 206 & 213 & 300 & 7.059 & 7.059 & & 0.1296 & $9.62 \mathrm{E}-04$ & 351.6525 & 129.5736 & 481.2261 & \\
\hline & 7/31/2008 4:00 & 207 & 213 & 400 & 7.022 & 7.022 & & 0.1356 & $1.02 \mathrm{E}-03$ & 371.6345 & 136.9364 & 508.5708 & \\
\hline & 7/31/2008 5:00 & 208 & 213 & 500 & 6.973 & 6.973 & & 0.1436 & 1.09E-03 & 398.7419 & 146.9247 & 545.6666 & \\
\hline & 7/31/2008 6:00 & 209 & 213 & 600 & 6.934 & 6.934 & & 0.1500 & 1.15E-03 & 420.8469 & 155.0697 & 575.9166 & \\
\hline & 7/31/2008 7:00 & 210 & 213 & 700 & 6.889 & 6.889 & & 0.1575 & $1.22 \mathrm{E}-03$ & 446.9417 & 164.6849 & 611.6266 & \\
\hline & $7 / 31 / 2008$ 8:00 & 211 & 213 & 800 & 6.828 & 6.828 & & 0.1678 & 1.32E-03 & 483.3349 & 178.0947 & 661.4295 & \\
\hline & $7 / 31 / 20089: 00$ & 212 & 213 & 900 & 5.881 & 5.881 & & 0.3559 & 3.34E-03 & 1219.2133 & 449.2442 & 1668.4575 & \\
\hline & $7 / 31 / 2008$ 10:00 & 213 & 213 & 1000 & 4.015 & 4.015 & & 0.9861 & 1.17E-02 & 4274.6248 & 1575.0734 & 5849.6983 & \\
\hline & 7/31/2008 11:00 & 214 & 213 & 1100 & 3.769 & 3.769 & & 1.1157 & 1.36E-02 & 4976.5542 & 1833.7137 & 6810.2679 & \\
\hline & $7 / 31 / 2008$ 12:00 & 215 & 213 & 1200 & 3.573 & 3.573 & & 1.2317 & $1.54 \mathrm{E}-02$ & 5621.2805 & 2071.2764 & 7692.5569 & \\
\hline & $7 / 31 / 2008$ 13:00 & 216 & 213 & 1300 & 3.715 & 3.715 & & 1.1464 & 1.41E-02 & 5145.9500 & 1896.1311 & 7042.0811 & \\
\hline & 7/31/2008 14:00 & 217 & 213 & 1400 & 3.542 & 3.542 & & 1.2513 & 1.57E-02 & 5731.2122 & 2111.7830 & 7842.9951 & \\
\hline & 7/31/2008 15:00 & 218 & 213 & 1500 & 3.585 & 3.585 & & 1.2243 & 1.53E-02 & 5579.3427 & 2055.8236 & 7635.1663 & \\
\hline & $7 / 31 / 2008$ 16:00 & 219 & 213 & 1600 & 3.739 & 3.739 & & 1.1327 & 1.39E-02 & 5069.9274 & 1868.1190 & 6938.0464 & \\
\hline & 7/31/2008 17:00 & 220 & 213 & 1700 & 3.798 & 3.798 & & 1.0995 & 1.34E-02 & 4887.9997 & 1801.0840 & 6689.0837 & \\
\hline & $7 / 31 / 2008$ 18:00 & 221 & 213 & 1800 & 3.985 & 3.985 & & 1.0010 & 1.19E-02 & 4354.5524 & 1604.5244 & 5959.0768 & \\
\hline & $7 / 31 / 2008$ 19:00 & 222 & 213 & 1900 & 4.346 & 4.346 & & 0.8348 & 9.53E-03 & 3482.3237 & 1283.1338 & 4765.4575 & \\
\hline & $7 / 31 / 200820: 00$ & 223 & 213 & 2000 & 5.11 & 5.11 & & 0.5605 & 5.83E-03 & 2132.4302 & 785.7377 & 2918.1679 & \\
\hline & $7 / 31 / 200821: 00$ & 224 & 213 & 2100 & 5.754 & 5.754 & & 0.3858 & $3.68 E-03$ & 1346.6191 & 496.1895 & 1842.8087 & \\
\hline & $7 / 31 / 200822: 00$ & 225 & 213 & 2200 & 6.445 & 6.445 & & 0.2372 & $2.02 \mathrm{E}-03$ & 740.0471 & 272.6856 & 1012.7327 & \\
\hline & 7/31/2008 23:00 & 226 & 213 & 2300 & 6.488 & 6.488 & & 0.2290 & 1.94E-03 & 708.6860 & 261.1299 & 969.8160 & \\
\hline & $8 / 1 / 20080: 00$ & 227 & 214 & 0 & 6.659 & 6.659 & 7.00 & 0.1975 & 1.62E-03 & 590.4570 & 217.5660 & 808.0230 & \\
\hline & 8/1/2008 1:00 & 228 & 214 & 100 & 6.889 & 6.889 & & 0.1575 & $1.22 \mathrm{E}-03$ & 446.9417 & 164.6849 & 611.6266 & \\
\hline & 8/1/2008 2:00 & 229 & 214 & 200 & 6.97 & 6.97 & & 0.1440 & 1.10E-03 & 400.4256 & 147.5450 & 547.9706 & \\
\hline & $8 / 1 / 2008$ 3:00 & 230 & 214 & 300 & 6.974 & 6.974 & & 0.1434 & 1.09E-03 & 398.1813 & 146.7181 & 544.8994 & \\
\hline & 8/1/2008 4:00 & 231 & 214 & 400 & 6.998 & 6.998 & & 0.1395 & 1.05E-03 & 384.8194 & 141.7946 & 526.6140 & \\
\hline & 8/1/2008 5:00 & 232 & 214 & 500 & 6.984 & 6.984 & & 0.1418 & $1.07 \mathrm{E}-03$ & 392.5923 & 144.6587 & 537.2510 & \\
\hline & 8/1/2008 6:00 & 233 & 214 & 600 & 6.945 & 6.945 & & 0.1482 & 1.13E-03 & 414.5643 & 152.7548 & 567.3191 & \\
\hline & 8/1/2008 7:00 & 234 & 214 & 700 & 6.91 & 6.91 & & 0.1540 & 1.19E-03 & 434.6852 & 160.1687 & 594.8539 & \\
\hline & 8/1/2008 8:00 & 235 & 214 & 800 & 6.829 & 6.829 & & 0.1677 & 1.32E-03 & 482.7287 & 177.8713 & 660.6000 & \\
\hline & 8/1/2008 9:00 & 236 & 214 & 900 & 5.752 & 5.752 & & 0.3863 & 3.69E-03 & 1348.6898 & 496.9525 & 1845.6422 & \\
\hline & 8/1/2008 10:00 & 237 & 214 & 1000 & 3.988 & 3.988 & & 0.9995 & 1.19E-02 & 4346.4930 & 1601.5547 & 5948.0477 & \\
\hline & 8/1/2008 11:00 & 238 & 214 & 1100 & 3.774 & 3.774 & & 1.1129 & $1.36 \mathrm{E}-02$ & 4961.1677 & 1828.0443 & 6789.2120 & \\
\hline & 8/1/2008 12:00 & 239 & 214 & 1200 & 3.711 & 3.711 & & 1.1487 & 1.41E-02 & 5158.7368 & 1900.8427 & 7059.5795 & \\
\hline & 8/1/2008 13:00 & 240 & 214 & 1300 & 3.638 & 3.638 & & 1.1919 & 1.48E-02 & 5398.1144 & 1989.0463 & 7387.1607 & \\
\hline & 8/1/2008 14:00 & 241 & 214 & 1400 & 3.63 & 3.63 & & 1.1967 & 1.48E-02 & 5425.0591 & 1998.9746 & 7424.0337 & \\
\hline & 8/1/2008 15:00 & 242 & 214 & 1500 & 3.654 & 3.654 & & 1.1823 & 1.46E-02 & 5344.6538 & 1969.3476 & 7314.0014 & \\
\hline & $8 / 1 / 200816: 00$ & 243 & 214 & 1600 & 3.787 & 3.787 & & 1.1056 & $1.35 \mathrm{E}-02$ & 4921.3946 & 1813.3891 & 6734.7837 & \\
\hline & 8/1/2008 17:00 & 244 & 214 & 1700 & 3.886 & 3.886 & & 1.0520 & 1.27E-02 & 4629.1273 & 1705.6972 & 6334.8245 & \\
\hline & 8/1/2008 18:00 & 245 & 214 & 1800 & 4.01 & 4.01 & & 0.9885 & 1.17E-02 & 4287.8438 & 1579.9442 & 5867.7881 & \\
\hline
\end{tabular}




\begin{tabular}{|c|c|c|c|c|c|c|c|c|c|c|c|c|c|}
\hline Tree ID & TS & RN & JD & HR & $\begin{array}{c}\text { Raw } \\
\text { TC_dTA }\end{array}$ & $\begin{array}{c}\text { Fixed } \\
\text { TC_dT } \Delta\end{array}$ & dTM & $\mathbf{K}$ & $\begin{array}{l}\text { Sap } \\
\text { Velocity } \\
(\mathrm{cm} / \mathrm{s})\end{array}$ & $\begin{array}{c}\text { Probed } \\
\text { Drywood F } \\
(\mathrm{g} / \mathrm{hr})\end{array}$ & $\begin{array}{c}\text { Extrapolated } \\
\text { Wetwood F } \\
(\mathrm{g} / \mathrm{hr})\end{array}$ & $\begin{array}{c}\text { Extrapolated } \\
\text { Total Wood F } \\
(\mathrm{g} / \mathrm{hr})\end{array}$ & $\begin{array}{l}\text { Relative } \\
\text { Humidity, } \\
@ 10 \mathrm{~m}(\%)\end{array}$ \\
\hline & 8/1/2008 19:00 & 246 & 214 & 1900 & 4.265 & 4.265 & & 0.8696 & 1.00E-02 & 3662.1151 & 1349.3816 & 5011.4968 & \\
\hline & 8/1/2008 20:00 & 247 & 214 & 2000 & 4.934 & 4.934 & & 0.6161 & $6.56 \mathrm{E}-03$ & 2396.0529 & 882.8750 & 3278.9278 & \\
\hline & 8/1/2008 21:00 & 248 & 214 & 2100 & 6.133 & 6.133 & & 0.3002 & $2.71 E-03$ & 988.6964 & 364.3055 & 1353.0019 & \\
\hline & 8/1/2008 22:00 & 249 & 214 & 2200 & 6.79 & 6.79 & & 0.1744 & 1.39E-03 & 506.6073 & 186.6699 & 693.2771 & \\
\hline & 8/1/2008 23:00 & 250 & 214 & 2300 & 6.98 & 6.98 & & 0.1424 & 1.08E-03 & 394.8242 & 145.4811 & 540.3053 & \\
\hline & 8/2/2008 0:00 & 251 & 215 & 0 & 7.002 & 7.002 & 7.03 & 0.1388 & 1.05E-03 & 382.6097 & 140.9804 & 523.5900 & \\
\hline & 8/2/2008 1:00 & 252 & 215 & 100 & 7.032 & 7.032 & & 0.1340 & $1.00 \mathrm{E}-03$ & 366.1928 & 134.9313 & 501.1240 & \\
\hline & $8 / 2 / 20082: 00$ & 253 & 215 & 200 & 7.033 & 7.033 & & 0.1338 & $1.00 \mathrm{E}-03$ & 365.6503 & 134.7314 & 500.3816 & \\
\hline & 8/2/2008 3:00 & 254 & 215 & 300 & 7.01 & 7.01 & & 0.1375 & 1.03E-03 & 378.2049 & 139.3574 & 517.5622 & \\
\hline & 8/2/2008 4:00 & 255 & 215 & 400 & 6.931 & 6.931 & & 0.1505 & 1.16E-03 & 422.5668 & 155.7034 & 578.2703 & \\
\hline & 8/2/2008 5:00 & 256 & 215 & 500 & 6.881 & 6.881 & & 0.1588 & 1.24E-03 & 451.6474 & 166.4188 & 618.0662 & \\
\hline & 8/2/2008 6:00 & 257 & 215 & 600 & 6.837 & 6.837 & & 0.1663 & 1.31E-03 & 477.8909 & 176.0887 & 653.9796 & \\
\hline & 8/2/2008 7:00 & 258 & 215 & 700 & 6.667 & 6.667 & & 0.1960 & 1.60E-03 & 585.1727 & 215.6189 & 800.7916 & \\
\hline & 8/2/2008 8:00 & 259 & 215 & 800 & 5.742 & 5.742 & & 0.3887 & $3.72 E-03$ & 1359.0734 & 500.7786 & 1859.8520 & \\
\hline & 8/2/2008 9:00 & 260 & 215 & 900 & 4.279 & 4.279 & & 0.8635 & 9.93E-03 & 3630.4310 & 1337.7070 & 4968.1380 & \\
\hline & 8/2/2008 10:00 & 261 & 215 & 1000 & 3.966 & 3.966 & & 1.0106 & $1.21 \mathrm{E}-02$ & 4405.9442 & 1623.4608 & 6029.4050 & \\
\hline & 8/2/2008 11:00 & 262 & 215 & 1100 & 3.809 & 3.809 & & 1.0935 & 1.33E-02 & 4854.8401 & 1788.8657 & 6643.7058 & \\
\hline & $8 / 2 / 200812: 00$ & 263 & 215 & 1200 & 3.653 & 3.653 & & 1.1829 & 1.46E-02 & 5347.9785 & 1970.5727 & 7318.5511 & \\
\hline & 8/2/2008 13:00 & 264 & 215 & 1300 & 3.626 & 3.626 & & 1.1991 & 1.49E-02 & 5438.5855 & 2003.9587 & 7442.5442 & \\
\hline & 8/2/2008 14:00 & 265 & 215 & 1400 & 3.646 & 3.646 & & 1.1871 & $1.47 E-02$ & 5371.3130 & 1979.1707 & 7350.4837 & \\
\hline & $8 / 2 / 2008$ 15:00 & 266 & 215 & 1500 & 3.656 & 3.656 & & 1.1811 & 1.46E-02 & 5338.0111 & 1966.9000 & 7304.9111 & \\
\hline & 8/2/2008 16:00 & 267 & 215 & 1600 & 3.7 & 3.7 & & 1.1551 & 1.42E-02 & 5194.0740 & 1913.8634 & 7107.9374 & \\
\hline & 8/2/2008 17:00 & 268 & 215 & 1700 & 3.733 & 3.733 & & 1.1361 & 1.39E-02 & 5088.8216 & 1875.0810 & 6963.9025 & \\
\hline & $8 / 2 / 200818: 00$ & 269 & 215 & 1800 & 3.914 & 3.914 & & 1.0373 & $1.24 \mathrm{E}-02$ & 4549.7390 & 1676.4449 & 6226.1839 & \\
\hline & 8/2/2008 19:00 & 270 & 215 & 1900 & 4.137 & 4.137 & & 0.9275 & 1.08E-02 & 3964.2514 & 1460.7100 & 5424.9614 & \\
\hline & 8/2/2008 20:00 & 271 & 215 & 2000 & 4.659 & 4.659 & & 0.7115 & 7.83E-03 & 2860.5798 & 1054.0395 & 3914.6193 & \\
\hline & 8/2/2008 21:00 & 272 & 215 & 2100 & 5.472 & 5.472 & & 0.4572 & 4.54E-03 & 1659.7436 & 611.5667 & 2271.3103 & \\
\hline & 8/2/2008 22:00 & 273 & 215 & 2200 & 6.249 & 6.249 & & 0.2760 & $2.44 \mathrm{E}-03$ & 891.7678 & 328.5902 & 1220.3580 & \\
\hline & 8/2/2008 23:00 & 274 & 215 & 2300 & 6.524 & 6.524 & & 0.2223 & 1.87E-03 & 682.9431 & 251.6444 & 934.5875 & \\
\hline & 8/3/2008 0:00 & 275 & 216 & 0 & 6.503 & 6.503 & 6.83 & 0.2262 & 1.91E-03 & 697.9034 & 257.1569 & 955.0603 & \\
\hline & 8/3/2008 1:00 & 276 & 216 & 100 & 6.474 & 6.474 & & 0.2317 & 1.97E-03 & 718.8229 & 264.8651 & 983.6880 & \\
\hline & 8/3/2008 2:00 & 277 & 216 & 200 & 6.625 & 6.625 & & 0.2036 & 1.68E-03 & 613.1575 & 225.9305 & 839.0880 & \\
\hline & 8/3/2008 3:00 & 278 & 216 & 300 & 6.747 & 6.747 & & 0.1819 & 1.46E-03 & 533.5063 & 196.5814 & 730.0876 & \\
\hline & 8/3/2008 4:00 & 279 & 216 & 400 & 6.805 & 6.805 & & 0.1718 & $1.36 \mathrm{E}-03$ & 497.3652 & 183.2644 & 680.6296 & \\
\hline & 8/3/2008 5:00 & 280 & 216 & 500 & 6.832 & 6.832 & & 0.1672 & 1.32E-03 & 480.9121 & 177.2020 & 658.1141 & \\
\hline & 8/3/2008 6:00 & 281 & 216 & 600 & 6.798 & 6.798 & & 0.1730 & 1.37E-03 & 501.6691 & 184.8503 & 686.5194 & \\
\hline & 8/3/2008 7:00 & 282 & 216 & 700 & 6.664 & 6.664 & & 0.1966 & 1.61E-03 & 587.1517 & 216.3481 & 803.4999 & \\
\hline & 8/3/2008 8:00 & 283 & 216 & 800 & 5.91 & 5.91 & & 0.3492 & $3.26 \mathrm{E}-03$ & 1191.2162 & 438.9281 & 1630.1444 & \\
\hline & 8/3/2008 9:00 & 284 & 216 & 900 & 4.212 & 4.212 & & 0.8932 & 1.04E-02 & 3784.4478 & 1394.4576 & 5178.9055 & \\
\hline & 8/3/2008 10:00 & 285 & 216 & 1000 & 3.63 & 3.63 & & 1.1967 & $1.48 \mathrm{E}-02$ & 5425.0591 & 1998.9746 & 7424.0337 & \\
\hline & 8/3/2008 11:00 & 286 & 216 & 1100 & 3.442 & 3.442 & & 1.3167 & 1.67E-02 & 6102.1918 & 2248.4781 & 8350.6699 & \\
\hline
\end{tabular}




\begin{tabular}{|c|c|c|c|c|c|c|c|c|c|c|c|c|c|}
\hline Tree ID & TS & RN & JD & HR & $\begin{array}{c}\text { Raw } \\
\text { TC_dTA }\end{array}$ & $\begin{array}{c}\text { Fixed } \\
\text { TC_dT } \Delta\end{array}$ & dTM & $\mathbf{K}$ & $\begin{array}{l}\text { Sap } \\
\text { Velocity } \\
(\mathrm{cm} / \mathrm{s})\end{array}$ & $\begin{array}{c}\text { Probed } \\
\text { Drywood F } \\
(\mathrm{g} / \mathrm{hr})\end{array}$ & $\begin{array}{c}\text { Extrapolated } \\
\text { Wetwood F } \\
(\mathrm{g} / \mathrm{hr})\end{array}$ & $\begin{array}{c}\text { Extrapolated } \\
\text { Total Wood F } \\
(\mathrm{g} / \mathrm{hr})\end{array}$ & $\begin{array}{l}\text { Relative } \\
\text { Humidity, } \\
@ 10 \mathrm{~m}(\%)\end{array}$ \\
\hline & 8/3/2008 12:00 & 287 & 216 & 1200 & 3.443 & 3.443 & & 1.3160 & 1.67E-02 & 6098.3533 & 2247.0637 & 8345.4170 & \\
\hline & 8/3/2008 13:00 & 288 & 216 & 1300 & 3.457 & 3.457 & & 1.3066 & $1.65 \mathrm{E}-02$ & 6044.8940 & 2227.3655 & 8272.2595 & \\
\hline & $8 / 3 / 2008$ 14:00 & 289 & 216 & 1400 & 3.476 & 3.476 & & 1.2940 & $1.63 \mathrm{E}-02$ & 5973.1705 & 2200.9375 & 8174.1081 & \\
\hline & 8/3/2008 15:00 & 290 & 216 & 1500 & 3.534 & 3.534 & & 1.2564 & $1.58 \mathrm{E}-02$ & 5759.9602 & 2122.3758 & 7882.3360 & \\
\hline & 8/3/2008 16:00 & 291 & 216 & 1600 & 3.605 & 3.605 & & 1.2119 & 1.51E-02 & 5510.1964 & 2030.3452 & 7540.5415 & \\
\hline & 8/3/2008 17:00 & 292 & 216 & 1700 & 3.69 & 3.69 & & 1.1610 & 1.43E-02 & 5226.4211 & 1925.7823 & 7152.2034 & \\
\hline & 8/3/2008 18:00 & 293 & 216 & 1800 & 3.869 & 3.869 & & 1.0610 & $1.28 \mathrm{E}-02$ & 4678.0152 & 1723.7109 & 6401.7261 & \\
\hline & 8/3/2008 19:00 & 294 & 216 & 1900 & 4.092 & 4.092 & & 0.9487 & 1.12E-02 & 4076.0713 & 1501.9123 & 5577.9836 & \\
\hline & 8/3/2008 20:00 & 295 & 216 & 2000 & 4.506 & 4.506 & & 0.7696 & 8.62E-03 & 3150.8489 & 1160.9951 & 4311.8439 & \\
\hline & 8/3/2008 21:00 & 296 & 216 & 2100 & 5.299 & 5.299 & & 0.5048 & 5.13E-03 & 1874.8220 & 690.8167 & 2565.6387 & \\
\hline & 8/3/2008 22:00 & 297 & 216 & 2200 & 5.833 & 5.833 & & 0.3670 & 3.47E-03 & 1266.4360 & 466.6444 & 1733.0804 & \\
\hline & 8/3/2008 23:00 & 298 & 216 & 2300 & 5.968 & 5.968 & & 0.3361 & 3.11E-03 & 1136.4001 & 418.7300 & 1555.1301 & \\
\hline & 8/4/2008 0:00 & 299 & 217 & 0 & 5.983 & 5.983 & 6.65 & 0.3328 & 3.07E-03 & 1122.4748 & 413.5989 & 1536.0737 & \\
\hline & 8/4/2008 1:00 & 300 & 217 & 100 & 6.003 & 6.003 & & 0.3283 & $3.02 E-03$ & 1104.0658 & 406.8158 & 1510.8816 & \\
\hline & $8 / 4 / 2008$ 2:00 & 301 & 217 & 200 & 5.83 & 5.83 & & 0.3678 & 3.47E-03 & 1269.4245 & 467.7456 & 1737.1701 & \\
\hline & 8/4/2008 3:00 & 302 & 217 & 300 & 5.679 & 5.679 & & 0.4041 & $3.90 \mathrm{E}-03$ & 1425.6798 & 525.3210 & 1951.0008 & \\
\hline & 8/4/2008 4:00 & 303 & 217 & 400 & 5.526 & 5.526 & & 0.4430 & 4.37E-03 & 1596.3430 & 588.2054 & 2184.5485 & \\
\hline & $8 / 4 / 20085: 00$ & 304 & 217 & 500 & 5.608 & 5.608 & & 0.4219 & 4.11E-03 & 1503.2688 & 553.9103 & 2057.1792 & \\
\hline & 8/4/2008 6:00 & 305 & 217 & 600 & 5.91 & 5.91 & & 0.3492 & $3.26 \mathrm{E}-03$ & 1191.2162 & 438.9281 & 1630.1444 & \\
\hline & 8/4/2008 7:00 & 306 & 217 & 700 & 5.882 & 5.882 & & 0.3557 & 3.33E-03 & 1218.2412 & 448.8860 & 1667.1273 & \\
\hline & 8/4/2008 8:00 & 307 & 217 & 800 & 5.681 & 5.681 & & 0.4036 & $3.89 \mathrm{E}-03$ & 1423.5333 & 524.5301 & 1948.0635 & \\
\hline & 8/4/2008 9:00 & 308 & 217 & 900 & 5.364 & 5.364 & & 0.4866 & 4.90E-03 & 1791.8059 & 660.2277 & 2452.0336 & \\
\hline & 8/4/2008 10:00 & 309 & 217 & 1000 & 4.627 & 4.627 & & 0.7234 & 7.99E-03 & 2919.2727 & 1075.6661 & 3994.9387 & \\
\hline & 8/4/2008 11:00 & 310 & 217 & 1100 & 4.09 & 4.09 & & 0.9496 & 1.12E-02 & 4081.1118 & 1503.7696 & 5584.8814 & \\
\hline & 8/4/2008 12:00 & 311 & 217 & 1200 & 3.877 & 3.877 & & 1.0567 & 1.27E-02 & 4654.9437 & 1715.2098 & 6370.1535 & \\
\hline & 8/4/2008 13:00 & 312 & 217 & 1300 & 3.824 & 3.824 & & 1.0853 & 1.32E-02 & 4809.9976 & 1772.3425 & 6582.3401 & \\
\hline & 8/4/2008 14:00 & 313 & 217 & 1400 & 4.118 & 4.118 & & 0.9364 & 1.10E-02 & 4011.0951 & 1477.9705 & 5489.0655 & \\
\hline & 8/4/2008 15:00 & 314 & 217 & 1500 & 4.435 & 4.435 & & 0.7980 & $9.01 \mathrm{E}-03$ & 3294.2237 & 1213.8245 & 4508.0482 & \\
\hline & 8/4/2008 16:00 & 315 & 217 & 1600 & 4 & 4 & & 0.9935 & 1.18E-02 & 4314.4040 & 1589.7309 & 5904.1349 & \\
\hline & 8/4/2008 17:00 & 316 & 217 & 1700 & 3.935 & 3.935 & & 1.0264 & 1.23E-02 & 4491.1060 & 1654.8404 & 6145.9464 & \\
\hline & 8/4/2008 18:00 & 317 & 217 & 1800 & 4.085 & 4.085 & & 0.9520 & 1.12E-02 & 4093.7399 & 1508.4227 & 5602.1626 & \\
\hline & 8/4/2008 19:00 & 318 & 217 & 1900 & 4.364 & 4.364 & & 0.8272 & $9.42 \mathrm{E}-03$ & 3443.5026 & 1268.8293 & 4712.3319 & \\
\hline & 8/4/2008 20:00 & 319 & 217 & 2000 & 4.904 & 4.904 & & 0.6260 & 6.69E-03 & 2443.4693 & 900.3465 & 3343.8158 & \\
\hline & 8/4/2008 21:00 & 320 & 217 & 2100 & 5.95 & 5.95 & & 0.3402 & $3.16 \mathrm{E}-03$ & 1153.2459 & 424.9372 & 1578.1831 & \\
\hline & 8/4/2008 22:00 & 321 & 217 & 2200 & 6.476 & 6.476 & & 0.2313 & 1.96E-03 & 717.3705 & 264.3299 & 981.7004 & \\
\hline & 8/4/2008 23:00 & 322 & 217 & 2300 & 6.645 & 6.645 & & 0.2000 & 1.64E-03 & 599.7567 & 220.9927 & 820.7494 & \\
\hline & 8/5/2008 0:00 & 323 & 218 & 0 & 6.809 & 6.809 & 6.90 & 0.1711 & 1.35E-03 & 494.9129 & 182.3608 & 677.2738 & \\
\hline & 8/5/2008 1:00 & 324 & 218 & 100 & 6.88 & 6.88 & & 0.1590 & 1.24E-03 & 452.2370 & 166.6360 & 618.8731 & \\
\hline & 8/5/2008 2:00 & 325 & 218 & 200 & 6.896 & 6.896 & & 0.1563 & $1.21 \mathrm{E}-03$ & 442.8408 & 163.1738 & 606.0146 & \\
\hline & 8/5/2008 3:00 & 326 & 218 & 300 & 6.836 & 6.836 & & 0.1665 & 1.31E-03 & 478.4945 & 176.3111 & 654.8057 & \\
\hline & 8/5/2008 4:00 & 327 & 218 & 400 & 6.736 & 6.736 & & 0.1838 & 1.48E-03 & 540.4846 & 199.1527 & 739.6373 & \\
\hline
\end{tabular}




\begin{tabular}{|c|c|c|c|c|c|c|c|c|c|c|c|c|c|}
\hline Tree ID & TS & RN & JD & HR & $\begin{array}{c}\text { Raw } \\
\text { TC_dTA }\end{array}$ & $\begin{array}{c}\text { Fixed } \\
\text { TC_dT } \Delta\end{array}$ & dTM & $\mathbf{K}$ & $\begin{array}{l}\text { Sap } \\
\text { Velocity } \\
(\mathrm{cm} / \mathrm{s})\end{array}$ & $\begin{array}{c}\text { Probed } \\
\text { Drywood F } \\
(\mathrm{g} / \mathrm{hr})\end{array}$ & $\begin{array}{c}\text { Extrapolated } \\
\text { Wetwood F } \\
(\mathrm{g} / \mathrm{hr})\end{array}$ & $\begin{array}{c}\text { Extrapolated } \\
\text { Total Wood F } \\
(\mathrm{g} / \mathrm{hr})\end{array}$ & $\begin{array}{l}\text { Relative } \\
\text { Humidity, } \\
@ 10 \mathrm{~m}(\%)\end{array}$ \\
\hline & 8/5/20085:00 & 328 & 218 & 500 & 6.69 & 6.69 & & 0.1919 & 1.56E-03 & 570.1002 & 210.0651 & 780.1653 & \\
\hline & 8/5/2008 6:00 & 329 & 218 & 600 & 6.695 & 6.695 & & 0.1910 & $1.55 \mathrm{E}-03$ & 566.8470 & 208.8664 & 775.7134 & \\
\hline & $8 / 5 / 20087: 00$ & 330 & 218 & 700 & 6.689 & 6.689 & & 0.1921 & $1.56 \mathrm{E}-03$ & 570.7518 & 210.3052 & 781.0570 & \\
\hline & 8/5/2008 8:00 & 331 & 218 & 800 & 6.52 & 6.52 & & 0.2230 & $1.88 \mathrm{E}-03$ & 685.7805 & 252.6899 & 938.4705 & \\
\hline & 8/5/2008 9:00 & 332 & 218 & 900 & 5.403 & 5.403 & & 0.4758 & 4.77E-03 & 1743.2881 & 642.3504 & 2385.6385 & \\
\hline & $8 / 5 / 2008$ 10:00 & 333 & 218 & 1000 & 4.117 & 4.117 & & 0.9368 & 1.10E-02 & 4013.5754 & 1478.8844 & 5492.4598 & \\
\hline & 8/5/2008 11:00 & 334 & 218 & 1100 & 4.215 & 4.215 & & 0.8918 & 1.03E-02 & 3777.4209 & 1391.8684 & 5169.2893 & \\
\hline & $8 / 5 / 2008$ 12:00 & 335 & 218 & 1200 & 3.942 & 3.942 & & 1.0228 & $1.22 \mathrm{E}-02$ & 4471.7320 & 1647.7016 & 6119.4337 & \\
\hline & 8/5/2008 13:00 & 336 & 218 & 1300 & 3.564 & 3.564 & & 1.2374 & $1.55 \mathrm{E}-02$ & 5652.9581 & 2082.9487 & 7735.9068 & \\
\hline & $8 / 5 / 2008$ 14:00 & 337 & 218 & 1400 & 3.992 & 3.992 & & 0.9975 & 1.19E-02 & 4335.7703 & 1597.6037 & 5933.3740 & \\
\hline & $8 / 5 / 2008$ 15:00 & 338 & 218 & 1500 & 4.275 & 4.275 & & 0.8653 & 9.96E-03 & 3639.4572 & 1341.0328 & 4980.4900 & \\
\hline & $8 / 5 / 2008$ 16:00 & 339 & 218 & 1600 & 4.155 & 4.155 & & 0.9191 & 1.07E-02 & 3920.3628 & 1444.5383 & 5364.9011 & \\
\hline & $8 / 5 / 200817: 00$ & 340 & 218 & 1700 & 3.86 & 3.86 & & 1.0658 & 1.29E-02 & 4704.1107 & 1733.3263 & 6437.4370 & \\
\hline & $8 / 5 / 2008$ 18:00 & 341 & 218 & 1800 & 3.666 & 3.666 & & 1.1751 & 1.45E-02 & 5304.9296 & 1954.7104 & 7259.6400 & \\
\hline & $8 / 5 / 2008$ 19:00 & 342 & 218 & 1900 & 3.885 & 3.885 & & 1.0525 & 1.27E-02 & 4631.9886 & 1706.7514 & 6338.7400 & \\
\hline & $8 / 5 / 200820: 00$ & 343 & 218 & 2000 & 4.564 & 4.564 & & 0.7472 & 8.31E-03 & 3037.8990 & 1119.3764 & 4157.2753 & \\
\hline & $8 / 5 / 200821: 00$ & 344 & 218 & 2100 & 5.911 & 5.911 & & 0.3490 & 3.26E-03 & 1190.2579 & 438.5750 & 1628.8329 & \\
\hline & $8 / 5 / 200822: 00$ & 345 & 218 & 2200 & 6.523 & 6.523 & & 0.2224 & 1.87E-03 & 683.6519 & 251.9056 & 935.5575 & \\
\hline & 8/5/2008 23:00 & 346 & 218 & 2300 & 6.722 & 6.722 & & 0.1863 & $1.50 \mathrm{E}-03$ & 549.4238 & 202.4465 & 751.8702 & \\
\hline & $8 / 6 / 20080: 00$ & 347 & 219 & 0 & 6.814 & 6.814 & 6.82 & 0.1702 & $1.35 E-03$ & 491.8548 & 181.2340 & 673.0888 & \\
\hline & 8/6/2008 1:00 & 348 & 219 & 100 & 6.824 & 6.824 & & 0.1685 & 1.33E-03 & 485.7627 & 178.9893 & 664.7520 & \\
\hline & $8 / 6 / 2008$ 2:00 & 349 & 219 & 200 & 6.821 & 6.821 & & 0.1690 & 1.33E-03 & 487.5870 & 179.6614 & 667.2484 & \\
\hline & $8 / 6 / 2008$ 3:00 & 350 & 219 & 300 & 6.809 & 6.809 & & 0.1711 & 1.35E-03 & 494.9129 & 182.3608 & 677.2738 & \\
\hline & 8/6/2008 4:00 & 351 & 219 & 400 & 6.8 & 6.8 & & 0.1726 & 1.37E-03 & 500.4378 & 184.3966 & 684.8344 & \\
\hline & 8/6/2008 5:00 & 352 & 219 & 500 & 6.774 & 6.774 & & 0.1771 & $1.41 \mathrm{E}-03$ & 516.5458 & 190.3319 & 706.8777 & \\
\hline & 8/6/2008 6:00 & 353 & 219 & 600 & 6.739 & 6.739 & & 0.1833 & 1.47E-03 & 538.5775 & 198.4499 & 737.0274 & \\
\hline & $8 / 6 / 20087: 00$ & 354 & 219 & 700 & 6.717 & 6.717 & & 0.1871 & 1.51E-03 & 552.6320 & 203.6286 & 756.2606 & \\
\hline & 8/6/2008 8:00 & 355 & 219 & 800 & 6.686 & 6.686 & & 0.1926 & $1.57 \mathrm{E}-03$ & 572.7087 & 211.0263 & 783.7350 & \\
\hline & 8/6/2008 9:00 & 356 & 219 & 900 & 6.58 & 6.58 & & 0.2119 & 1.76E-03 & 643.8113 & 237.2255 & 881.0368 & \\
\hline & 8/6/2008 10:00 & 357 & 219 & 1000 & 4.479 & 4.479 & & 0.7803 & 8.77E-03 & 3204.6954 & 1180.8359 & 4385.5313 & \\
\hline & 8/6/2008 11:00 & 358 & 219 & 1100 & 3.179 & 3.179 & & 1.5083 & 1.97E-02 & 7213.3716 & 2657.9151 & 9871.2867 & \\
\hline & 8/6/2008 12:00 & 359 & 219 & 1200 & 3.63 & 3.63 & & 1.1967 & 1.48E-02 & 5425.0591 & 1998.9746 & 7424.0337 & \\
\hline & 8/6/2008 13:00 & 360 & 219 & 1300 & 4.022 & 4.022 & & 0.9826 & 1.16E-02 & 4256.1863 & 1568.2794 & 5824.4657 & \\
\hline & $8 / 6 / 2008$ 14:00 & 361 & 219 & 1400 & 3.66 & 3.66 & & 1.1787 & $1.46 \mathrm{E}-02$ & 5324.7522 & 1962.0145 & 7286.7667 & \\
\hline & 8/6/2008 15:00 & 362 & 219 & 1500 & 3.275 & 3.275 & & 1.4348 & 1.86E-02 & 6782.9834 & 2499.3297 & 9282.3131 & \\
\hline & $8 / 6 / 2008$ 16:00 & 363 & 219 & 1600 & 3.324 & 3.324 & & 1.3989 & 1.80E-02 & 6574.7166 & 2422.5896 & 8997.3062 & \\
\hline & $8 / 6 / 2008$ 17:00 & 364 & 219 & 1700 & 3.308 & 3.308 & & 1.4105 & 1.82E-02 & 6641.9102 & 2447.3484 & 9089.2586 & \\
\hline & 8/6/2008 18:00 & 365 & 219 & 1800 & 3.767 & 3.767 & & 1.1168 & 1.36E-02 & 4982.7227 & 1835.9867 & 6818.7094 & \\
\hline & $8 / 6 / 2008$ 19:00 & 366 & 219 & 1900 & 3.878 & 3.878 & & 1.0562 & $1.27 \mathrm{E}-02$ & 4652.0680 & 1714.1501 & 6366.2181 & \\
\hline & $8 / 6 / 200820: 00$ & 367 & 219 & 2000 & 4.209 & 4.209 & & 0.8945 & 1.04E-02 & 3791.4872 & 1397.0514 & 5188.5387 & \\
\hline & 8/6/2008 21:00 & 368 & 219 & 2100 & 5.335 & 5.335 & & 0.4947 & $5.00 \mathrm{E}-03$ & 1828.5068 & 673.7509 & 2502.2577 & \\
\hline
\end{tabular}




\begin{tabular}{|c|c|c|c|c|c|c|c|c|c|c|c|c|c|}
\hline Tree ID & TS & RN & JD & HR & $\begin{array}{c}\text { Raw } \\
\text { TC_dTA }\end{array}$ & $\begin{array}{c}\text { Fixed } \\
\text { TC_dT } \Delta\end{array}$ & dTM & $\mathbf{K}$ & $\begin{array}{l}\text { Sap } \\
\text { Velocity } \\
(\mathrm{cm} / \mathrm{s})\end{array}$ & $\begin{array}{c}\text { Probed } \\
\text { Drywood F } \\
(\mathrm{g} / \mathrm{hr})\end{array}$ & $\begin{array}{c}\text { Extrapolated } \\
\text { Wetwood F } \\
(\mathrm{g} / \mathrm{hr})\end{array}$ & $\begin{array}{c}\text { Extrapolated } \\
\text { Total Wood F } \\
(\mathrm{g} / \mathrm{hr})\end{array}$ & $\begin{array}{l}\text { Relative } \\
\text { Humidity, } \\
@ 10 \mathrm{~m}(\%)\end{array}$ \\
\hline & 8/6/2008 22:00 & 369 & 219 & 2200 & 6.297 & 6.297 & & 0.2663 & 2.33E-03 & 853.2451 & 314.3957 & 1167.6408 & \\
\hline & 8/6/2008 23:00 & 370 & 219 & 2300 & 6.574 & 6.574 & & 0.2130 & 1.77E-03 & 647.9514 & 238.7510 & 886.7024 & \\
\hline & $8 / 7 / 20080: 00$ & 371 & 220 & 0 & 6.691 & 6.691 & 6.74 & 0.1918 & $1.56 \mathrm{E}-03$ & 569.4489 & 209.8251 & 779.2740 & \\
\hline & 8/7/2008 1:00 & 372 & 220 & 100 & 6.725 & 6.725 & & 0.1857 & $1.50 \mathrm{E}-03$ & 547.5028 & 201.7387 & 749.2414 & \\
\hline & 8/7/2008 2:00 & 373 & 220 & 200 & 6.737 & 6.737 & & 0.1836 & 1.48E-03 & 539.8486 & 198.9183 & 738.7669 & \\
\hline & 8/7/2008 3:00 & 374 & 220 & 300 & 6.733 & 6.733 & & 0.1843 & 1.48E-03 & 542.3947 & 199.8565 & 742.2512 & \\
\hline & 8/7/2008 4:00 & 375 & 220 & 400 & 6.723 & 6.723 & & 0.1861 & $1.50 \mathrm{E}-03$ & 548.7831 & 202.2104 & 750.9935 & \\
\hline & 8/7/2008 5:00 & 376 & 220 & 500 & 6.714 & 6.714 & & 0.1877 & 1.52E-03 & 554.5609 & 204.3394 & 758.9003 & \\
\hline & 8/7/2008 6:00 & 377 & 220 & 600 & 6.704 & 6.704 & & 0.1894 & 1.54E-03 & 561.0123 & 206.7165 & 767.7288 & \\
\hline & 8/7/2008 7:00 & 378 & 220 & 700 & 6.69 & 6.69 & & 0.1919 & 1.56E-03 & 570.1002 & 210.0651 & 780.1653 & \\
\hline & 8/7/2008 8:00 & 379 & 220 & 800 & 6.648 & 6.648 & & 0.1995 & 1.64E-03 & 597.7583 & 220.2563 & 818.0146 & \\
\hline & 8/7/2008 9:00 & 380 & 220 & 900 & 6.248 & 6.248 & & 0.2762 & $2.44 \mathrm{E}-03$ & 892.5801 & 328.8895 & 1221.4695 & \\
\hline & 8/7/2008 10:00 & 381 & 220 & 1000 & 4.029 & 4.029 & & 0.9792 & 1.16E-02 & 4237.8267 & 1561.5144 & 5799.3411 & \\
\hline & 8/7/2008 11:00 & 382 & 220 & 1100 & 3.054 & 3.054 & & 1.6110 & $2.14 \mathrm{E}-02$ & 7822.4434 & 2882.3401 & 10704.7835 & \\
\hline & 8/7/2008 12:00 & 383 & 220 & 1200 & 2.969 & 2.969 & & 1.6858 & $2.26 \mathrm{E}-02$ & 8271.6174 & 3047.8475 & 11319.4649 & \\
\hline & 8/7/2008 13:00 & 384 & 220 & 1300 & 2.927 & 2.927 & & 1.7243 & $2.33 \mathrm{E}-02$ & 8505.0093 & 3133.8456 & 11638.8549 & \\
\hline & $8 / 7 / 2008$ 14:00 & 385 & 220 & 1400 & 2.993 & 2.993 & & 1.6642 & 2.23E-02 & 8141.7256 & 2999.9862 & 11141.7118 & \\
\hline & 8/7/2008 15:00 & 386 & 220 & 1500 & 3.159 & 3.159 & & 1.5242 & $2.00 \mathrm{E}-02$ & 7306.9748 & 2692.4051 & 9999.3800 & \\
\hline & 8/7/2008 16:00 & 387 & 220 & 1600 & 3.18 & 3.18 & & 1.5075 & 1.97E-02 & 7208.7283 & 2656.2042 & 9864.9324 & \\
\hline & 8/7/2008 17:00 & 388 & 220 & 1700 & 3.196 & 3.196 & & 1.4950 & $1.95 E-02$ & 7134.9057 & 2629.0027 & 9763.9084 & \\
\hline & 8/7/2008 18:00 & 389 & 220 & 1800 & 3.335 & 3.335 & & 1.3910 & $1.79 \mathrm{E}-02$ & 6528.9688 & 2405.7328 & 8934.7016 & \\
\hline & 8/7/2008 19:00 & 390 & 220 & 1900 & 3.674 & 3.674 & & 1.1704 & 1.44E-02 & 5278.6217 & 1945.0167 & 7223.6384 & \\
\hline & 8/7/2008 20:00 & 391 & 220 & 2000 & 4.12 & 4.12 & & 0.9354 & 1.10E-02 & 4006.1389 & 1476.1443 & 5482.2832 & \\
\hline & 8/7/2008 21:00 & 392 & 220 & 2100 & 5.32 & 5.32 & & 0.4989 & $5.06 \mathrm{E}-03$ & 1847.7022 & 680.8239 & 2528.5261 & \\
\hline & 8/7/2008 22:00 & 393 & 220 & 2200 & 6.074 & 6.074 & & 0.3128 & $2.85 E-03$ & 1040.1486 & 383.2641 & 1423.4127 & \\
\hline & 8/7/2008 23:00 & 394 & 220 & 2300 & 6.448 & 6.448 & & 0.2367 & $2.02 E-03$ & 737.8373 & 271.8713 & 1009.7086 & \\
\hline & 8/8/2008 0:00 & 395 & 221 & 0 & 6.616 & 6.616 & 6.72 & 0.2053 & 1.69E-03 & 619.2325 & 228.1689 & 847.4014 & \\
\hline & 8/8/2008 1:00 & 396 & 221 & 100 & 6.665 & 6.665 & & 0.1964 & $1.60 \mathrm{E}-03$ & 586.4917 & 216.1049 & 802.5966 & \\
\hline & 8/8/2008 2:00 & 397 & 221 & 200 & 6.715 & 6.715 & & 0.1875 & $1.52 \mathrm{E}-03$ & 553.9176 & 204.1023 & 758.0200 & \\
\hline & 8/8/2008 3:00 & 398 & 221 & 300 & 6.703 & 6.703 & & 0.1896 & $1.54 \mathrm{E}-03$ & 561.6593 & 206.9549 & 768.6142 & \\
\hline & 8/8/2008 4:00 & 399 & 221 & 400 & 6.699 & 6.699 & & 0.1903 & 1.54E-03 & 564.2505 & 207.9097 & 772.1601 & \\
\hline & 8/8/2008 5:00 & 400 & 221 & 500 & 6.689 & 6.689 & & 0.1921 & 1.56E-03 & 570.7518 & 210.3052 & 781.0570 & \\
\hline & 8/8/2008 6:00 & 401 & 221 & 600 & 6.668 & 6.668 & & 0.1959 & 1.60E-03 & 584.5136 & 215.3761 & 799.8897 & \\
\hline & $8 / 8 / 20087: 00$ & 402 & 221 & 700 & 6.663 & 6.663 & & 0.1968 & 1.61E-03 & 587.8121 & 216.5915 & 804.4036 & \\
\hline & 8/8/2008 8:00 & 403 & 221 & 800 & 6.623 & 6.623 & & 0.2040 & 1.68E-03 & 614.5051 & 226.4270 & 840.9322 & \\
\hline & 8/8/2008 9:00 & 404 & 221 & 900 & 5.454 & 5.454 & & 0.4620 & 4.60E-03 & 1681.2601 & 619.4949 & 2300.7549 & \\
\hline & 8/8/2008 10:00 & 405 & 221 & 1000 & 3.57 & 3.57 & & 1.2336 & 1.54E-02 & 5631.8182 & 2075.1593 & 7706.9775 & \\
\hline & 8/8/2008 11:00 & 406 & 221 & 1100 & 3.271 & 3.271 & & 1.4378 & 1.86E-02 & 6800.3147 & 2505.7158 & 9306.0306 & \\
\hline & $8 / 8 / 200812: 00$ & 407 & 221 & 1200 & 3.089 & 3.089 & & 1.5814 & $2.09 \mathrm{E}-02$ & 7645.9878 & 2817.3215 & 10463.3093 & \\
\hline & 8/8/2008 13:00 & 408 & 221 & 1300 & 3.106 & 3.106 & & 1.5673 & 2.07E-02 & 7561.9836 & 2786.3684 & 10348.3519 & \\
\hline & 8/8/2008 14:00 & 409 & 221 & 1400 & 3.116 & 3.116 & & 1.5591 & $2.06 \mathrm{E}-02$ & 7513.0780 & 2768.3481 & 10281.4262 & \\
\hline
\end{tabular}




\begin{tabular}{|c|c|c|c|c|c|c|c|c|c|c|c|c|c|}
\hline Tree ID & TS & RN & JD & HR & $\begin{array}{c}\text { Raw } \\
\text { TC_dTA }\end{array}$ & $\begin{array}{c}\text { Fixed } \\
\text { TC_dT } \Delta\end{array}$ & dTM & $\mathbf{K}$ & $\begin{array}{l}\text { Sap } \\
\text { Velocity } \\
(\mathrm{cm} / \mathrm{s})\end{array}$ & $\begin{array}{c}\text { Probed } \\
\text { Drywood F } \\
(\mathrm{g} / \mathrm{hr})\end{array}$ & $\begin{array}{c}\text { Extrapolated } \\
\text { Wetwood F } \\
(\mathrm{g} / \mathrm{hr})\end{array}$ & $\begin{array}{c}\text { Extrapolated } \\
\text { Total Wood F } \\
(\mathrm{g} / \mathrm{hr})\end{array}$ & $\begin{array}{l}\text { Relative } \\
\text { Humidity, } \\
@ 10 \mathrm{~m}(\%)\end{array}$ \\
\hline & 8/8/2008 15:00 & 410 & 221 & 1500 & 3.155 & 3.155 & & 1.5274 & $2.00 \mathrm{E}-02$ & 7325.8652 & 2699.3657 & 10025.2309 & \\
\hline & 8/8/2008 16:00 & 411 & 221 & 1600 & 3.168 & 3.168 & & 1.5170 & 1.99E-02 & 7264.6791 & 2676.8204 & 9941.4995 & \\
\hline & 8/8/2008 17:00 & 412 & 221 & 1700 & 3.314 & 3.314 & & 1.4062 & $1.81 \mathrm{E}-02$ & 6616.6216 & 2438.0303 & 9054.6518 & \\
\hline & 8/8/2008 18:00 & 413 & 221 & 1800 & 3.603 & 3.603 & & 1.2132 & $1.51 E-02$ & 5517.0692 & 2032.8776 & 7549.9468 & \\
\hline & 8/8/2008 19:00 & 414 & 221 & 1900 & 3.65 & 3.65 & & 1.1847 & 1.47E-02 & 5357.9657 & 1974.2526 & 7332.2183 & \\
\hline & 8/8/2008 20:00 & 415 & 221 & 2000 & 4.235 & 4.235 & & 0.8829 & 1.02E-02 & 3730.8912 & 1374.7236 & 5105.6148 & \\
\hline & 8/8/2008 21:00 & 416 & 221 & 2100 & 5.191 & 5.191 & & 0.5361 & $5.52 E-03$ & 2018.9655 & 743.9294 & 2762.8949 & \\
\hline & 8/8/2008 22:00 & 417 & 221 & 2200 & 5.898 & 5.898 & & 0.3520 & 3.29E-03 & 1202.7530 & 443.1791 & 1645.9321 & \\
\hline & 8/8/2008 23:00 & 418 & 221 & 2300 & 6.171 & 6.171 & & 0.2922 & $2.62 \mathrm{E}-03$ & 956.3354 & 352.3814 & 1308.7168 & \\
\hline & 8/9/2008 0:00 & 419 & 222 & 0 & 6.22 & 6.22 & 6.64 & 0.2820 & 2.50E-03 & 915.4858 & 337.3296 & 1252.8154 & \\
\hline & 8/9/2008 1:00 & 420 & 222 & 100 & 6.209 & 6.209 & & 0.2843 & 2.53E-03 & 924.5709 & 340.6772 & 1265.2481 & \\
\hline & 8/9/2008 2:00 & 421 & 222 & 200 & 6.22 & 6.22 & & 0.2820 & $2.50 \mathrm{E}-03$ & 915.4858 & 337.3296 & 1252.8154 & \\
\hline & 8/9/2008 3:00 & 422 & 222 & 300 & 6.221 & 6.221 & & 0.2818 & $2.50 \mathrm{E}-03$ & 914.6623 & 337.0261 & 1251.6885 & \\
\hline & 8/9/2008 4:00 & 423 & 222 & 400 & 6.202 & 6.202 & & 0.2857 & $2.55 E-03$ & 930.3779 & 342.8169 & 1273.1948 & \\
\hline & 8/9/2008 5:00 & 424 & 222 & 500 & 6.112 & 6.112 & & 0.3046 & $2.75 \mathrm{E}-03$ & 1006.8400 & 370.9909 & 1377.8309 & \\
\hline & 8/9/2008 6:00 & 425 & 222 & 600 & 6.08 & 6.08 & & 0.3115 & $2.83 \mathrm{E}-03$ & 1034.8481 & 381.3111 & 1416.1592 & \\
\hline & 8/9/2008 7:00 & 426 & 222 & 700 & 5.862 & 5.862 & & 0.3603 & 3.39E-03 & 1237.7729 & 456.0829 & 1693.8558 & \\
\hline & 8/9/2008 8:00 & 427 & 222 & 800 & 5.374 & 5.374 & & 0.4838 & 4.87E-03 & 1779.2744 & 655.6103 & 2434.8847 & \\
\hline & 8/9/2008 9:00 & 428 & 222 & 900 & 5.66 & 5.66 & & 0.4088 & 3.96E-03 & 1446.1769 & 532.8736 & 1979.0506 & \\
\hline & 8/9/2008 10:00 & 429 & 222 & 1000 & 5.657 & 5.657 & & 0.4096 & 3.97E-03 & 1449.4309 & 534.0726 & 1983.5036 & \\
\hline & 8/9/2008 11:00 & 430 & 222 & 1100 & 5.108 & 5.108 & & 0.5611 & $5.84 \mathrm{E}-03$ & 2135.2922 & 786.7923 & 2922.0845 & \\
\hline & 8/9/2008 12:00 & 431 & 222 & 1200 & 4.308 & 4.308 & & 0.8510 & 9.76E-03 & 3565.6171 & 1313.8250 & 4879.4420 & \\
\hline & 8/9/2008 13:00 & 432 & 222 & 1300 & 3.99 & 3.99 & & 0.9985 & 1.19E-02 & 4341.1283 & 1599.5780 & 5940.7064 & \\
\hline & 8/9/2008 14:00 & 433 & 222 & 1400 & 3.465 & 3.465 & & 1.3013 & 1.65E-02 & 6014.5792 & 2216.1954 & 8230.7746 & \\
\hline & 8/9/2008 15:00 & 434 & 222 & 1500 & 3.338 & 3.338 & & 1.3889 & 1.78E-02 & 6516.5547 & 2401.1586 & 8917.7134 & \\
\hline & 8/9/2008 16:00 & 435 & 222 & 1600 & 3.334 & 3.334 & & 1.3917 & 1.79E-02 & 6533.1127 & 2407.2597 & 8940.3724 & \\
\hline & 8/9/2008 17:00 & 436 & 222 & 1700 & 3.526 & 3.526 & & 1.2615 & 1.58E-02 & 5788.8659 & 2133.0267 & 7921.8925 & \\
\hline & 8/9/2008 18:00 & 437 & 222 & 1800 & 3.62 & 3.62 & & 1.2028 & 1.49E-02 & 5458.9431 & 2011.4598 & 7470.4029 & \\
\hline & 8/9/2008 19:00 & 438 & 222 & 1900 & 3.846 & 3.846 & & 1.0733 & 1.30E-02 & 4745.0009 & 1748.3932 & 6493.3940 & \\
\hline & 8/9/2008 20:00 & 439 & 222 & 2000 & 4.401 & 4.401 & & 0.8119 & $9.21 E-03$ & 3364.9535 & 1239.8863 & 4604.8399 & \\
\hline & 8/9/2008 21:00 & 440 & 222 & 2100 & 5.75 & 5.75 & & 0.3868 & 3.70E-03 & 1350.7624 & 497.7162 & 1848.4786 & \\
\hline & 8/9/2008 22:00 & 441 & 222 & 2200 & 6.492 & 6.492 & & 0.2283 & 1.93E-03 & 705.8028 & 260.0675 & 965.8703 & \\
\hline & 8/9/2008 23:00 & 442 & 222 & 2300 & 6.637 & 6.637 & & 0.2014 & 1.66E-03 & 605.1006 & 222.9618 & 828.0624 & \\
\hline & 8/10/2008 0:00 & 443 & 223 & 0 & 6.692 & 6.692 & 6.73 & 0.1916 & $1.56 \mathrm{E}-03$ & 568.7979 & 209.5853 & 778.3832 & \\
\hline & 8/10/2008 1:00 & 444 & 223 & 100 & 6.724 & 6.724 & & 0.1859 & 1.50E-03 & 548.1428 & 201.9745 & 750.1172 & \\
\hline & 8/10/2008 2:00 & 445 & 223 & 200 & 6.725 & 6.725 & & 0.1857 & 1.50E-03 & 547.5028 & 201.7387 & 749.2414 & \\
\hline & 8/10/2008 3:00 & 446 & 223 & 300 & 6.718 & 6.718 & & 0.1870 & $1.51 \mathrm{E}-03$ & 551.9897 & 203.3920 & 755.3817 & \\
\hline & 8/10/2008 4:00 & 447 & 223 & 400 & 6.716 & 6.716 & & 0.1873 & 1.51E-03 & 553.2747 & 203.8654 & 757.1401 & \\
\hline & $8 / 10 / 20085: 00$ & 448 & 223 & 500 & 6.704 & 6.704 & & 0.1894 & $1.54 \mathrm{E}-03$ & 561.0123 & 206.7165 & 767.7288 & \\
\hline & 8/10/2008 6:00 & 449 & 223 & 600 & 6.681 & 6.681 & & 0.1935 & 1.58E-03 & 575.9769 & 212.2305 & 788.2075 & \\
\hline & 8/10/2008 7:00 & 450 & 223 & 700 & 6.674 & 6.674 & & 0.1948 & 1.59E-03 & 580.5666 & 213.9217 & 794.4882 & \\
\hline
\end{tabular}




\begin{tabular}{|c|c|c|c|c|c|c|c|c|c|c|c|c|c|}
\hline Tree ID & TS & RN & JD & HR & $\begin{array}{c}\text { Raw } \\
\text { TC_dTA }\end{array}$ & $\begin{array}{c}\text { Fixed } \\
\text { TC_dT } \Delta\end{array}$ & dTM & $\mathbf{K}$ & $\begin{array}{l}\text { Sap } \\
\text { Velocity } \\
(\mathrm{cm} / \mathrm{s})\end{array}$ & $\begin{array}{c}\text { Probed } \\
\text { Drywood F } \\
(\mathrm{g} / \mathrm{hr})\end{array}$ & $\begin{array}{c}\text { Extrapolated } \\
\text { Wetwood F } \\
(\mathrm{g} / \mathrm{hr})\end{array}$ & $\begin{array}{c}\text { Extrapolated } \\
\text { Total Wood F } \\
(\mathrm{g} / \mathrm{hr})\end{array}$ & $\begin{array}{l}\text { Relative } \\
\text { Humidity, } \\
@ 10 \mathrm{~m}(\%)\end{array}$ \\
\hline & 8/10/2008 8:00 & 451 & 223 & 800 & \begin{tabular}{|r|}
6.643 \\
\end{tabular} & 6.643 & & 0.2004 & 1.64E-03 & 601.0906 & 221.4842 & \begin{tabular}{|l|}
822.5748 \\
\end{tabular} & \\
\hline & 8/10/2008 9:00 & 452 & 223 & 900 & 6.179 & 6.179 & & 0.2905 & $2.60 \mathrm{E}-03$ & 949.5989 & 349.8992 & 1299.4982 & \\
\hline & $8 / 10 / 2008$ 10:00 & 453 & 223 & 1000 & 4.107 & 4.107 & & 0.9416 & $1.10 \mathrm{E}-02$ & 4038.4607 & 1488.0539 & 5526.5146 & \\
\hline & 8/10/2008 11:00 & 454 & 223 & 1100 & 3.072 & 3.072 & & 1.5957 & $2.12 \mathrm{E}-02$ & 7731.0983 & 2848.6822 & 10579.7804 & \\
\hline & $8 / 10 / 200812: 00$ & 455 & 223 & 1200 & 3.054 & 3.054 & & 1.6110 & 2.14E-02 & 7822.4434 & 2882.3401 & 10704.7835 & \\
\hline & 8/10/2008 13:00 & 456 & 223 & 1300 & 3.016 & 3.016 & & 1.6439 & 2.19E-02 & 8019.5411 & 2954.9649 & 10974.5060 & \\
\hline & $8 / 10 / 2008$ 14:00 & 457 & 223 & 1400 & 3.083 & 3.083 & & 1.5864 & $2.10 \mathrm{E}-02$ & 7675.8995 & 2828.3430 & 10504.2425 & \\
\hline & $8 / 10 / 2008$ 15:00 & 458 & 223 & 1500 & 3.333 & 3.333 & & 1.3924 & 1.79E-02 & 6537.2596 & 2408.7878 & 8946.0474 & \\
\hline & 8/10/2008 16:00 & 459 & 223 & 1600 & 3.258 & 3.258 & & 1.4475 & 1.88E-02 & 6856.9934 & 2526.6002 & 9383.5936 & \\
\hline & 8/10/2008 17:00 & 460 & 223 & 1700 & 3.481 & 3.481 & & 1.2907 & 1.63E-02 & 5954.4526 & 2194.0405 & 8148.4931 & \\
\hline & 8/10/2008 18:00 & 461 & 223 & 1800 & 5.439 & 5.439 & & 0.4661 & 4.65E-03 & 1699.3392 & 626.1565 & 2325.4957 & \\
\hline & $8 / 10 / 2008$ 19:00 & 462 & 223 & 1900 & 6.09 & 6.09 & & 0.3094 & $2.81 E-03$ & 1026.0484 & 378.0686 & 1404.1171 & \\
\hline & 8/10/2008 20:00 & 463 & 223 & 2000 & 6.061 & 6.061 & & 0.3156 & 2.88E-03 & 1051.6865 & 387.5155 & 1439.2020 & \\
\hline & 8/10/2008 21:00 & 464 & 223 & 2100 & 6.015 & 6.015 & & 0.3257 & 2.99E-03 & 1093.1065 & 402.7776 & 1495.8841 & \\
\hline & $8 / 10 / 2008$ 22:00 & 465 & 223 & 2200 & 6.155 & 6.155 & & 0.2955 & $2.65 E-03$ & 969.8878 & 357.3751 & 1327.2629 & \\
\hline & 8/10/2008 23:00 & 466 & 223 & 2300 & 6.186 & 6.186 & & 0.2890 & $2.58 \mathrm{E}-03$ & 943.7261 & 347.7353 & 1291.4614 & \\
\hline & $8 / 11 / 20080: 00$ & 467 & 224 & 0 & 6.214 & 6.214 & 6.50 & 0.2832 & 2.52E-03 & 920.4353 & 339.1533 & 1259.5886 & \\
\hline & 8/11/2008 1:00 & 468 & 224 & 100 & 6.313 & 6.313 & & 0.2631 & 2.30E-03 & 840.6049 & 309.7382 & 1150.3431 & \\
\hline & 8/11/2008 2:00 & 469 & 224 & 200 & 6.417 & 6.417 & & 0.2426 & 2.08E-03 & 760.8319 & 280.3442 & 1041.1760 & \\
\hline & 8/11/2008 3:00 & 470 & 224 & 300 & 6.463 & 6.463 & & 0.2338 & 1.99E-03 & 726.8374 & 267.8182 & 994.6556 & \\
\hline & 8/11/2008 4:00 & 471 & 224 & 400 & 6.475 & 6.475 & & 0.2315 & 1.96E-03 & 718.0965 & 264.5974 & 982.6939 & \\
\hline & 8/11/2008 5:00 & 472 & 224 & 500 & 6.465 & 6.465 & & 0.2334 & 1.98E-03 & 725.3770 & 267.2801 & 992.6570 & \\
\hline & 8/11/2008 6:00 & 473 & 224 & 600 & 6.492 & 6.492 & & 0.2283 & 1.93E-03 & 705.8028 & 260.0675 & 965.8703 & \\
\hline & 8/11/2008 7:00 & 474 & 224 & 700 & 6.5 & 6.5 & & 0.2268 & 1.92E-03 & 700.0535 & 257.9491 & 958.0026 & \\
\hline & 8/11/2008 8:00 & 475 & 224 & 800 & 6.451 & 6.451 & & 0.2361 & 2.01E-03 & 735.6307 & 271.0583 & 1006.6890 & \\
\hline & 8/11/2008 9:00 & 476 & 224 & 900 & 5.806 & 5.806 & & 0.3734 & 3.54E-03 & 1293.4913 & 476.6135 & 1770.1048 & \\
\hline & 8/11/2008 10:00 & 477 & 224 & 1000 & 4.305 & 4.305 & & 0.8523 & 9.77E-03 & 3572.2714 & 1316.2769 & 4888.5482 & \\
\hline & 8/11/2008 11:00 & 478 & 224 & 1100 & 3.521 & 3.521 & & 1.2647 & 1.59E-02 & 5807.0124 & 2139.7132 & 7946.7256 & \\
\hline & $8 / 11 / 2008$ 12:00 & 479 & 224 & 1200 & 3.382 & 3.382 & & 1.3578 & 1.73E-02 & 6337.5117 & 2335.1865 & 8672.6982 & \\
\hline & 8/11/2008 13:00 & 480 & 224 & 1300 & 3.594 & 3.594 & & 1.2187 & $1.52 E-02$ & 5548.1116 & 2044.3158 & 7592.4274 & \\
\hline & 8/11/2008 14:00 & 481 & 224 & 1400 & 3.309 & 3.309 & & 1.4098 & 1.82E-02 & 6637.6878 & 2445.7926 & 9083.4804 & \\
\hline & 8/11/2008 15:00 & 482 & 224 & 1500 & 3.338 & 3.338 & & 1.3889 & 1.78E-02 & 6516.5547 & 2401.1586 & 8917.7134 & \\
\hline & $8 / 11 / 2008$ 16:00 & 483 & 224 & 1600 & 3.65 & 3.65 & & 1.1847 & 1.47E-02 & 5357.9657 & 1974.2526 & 7332.2183 & \\
\hline & $8 / 11 / 2008$ 17:00 & 484 & 224 & 1700 & 3.708 & 3.708 & & 1.1505 & $1.41 \mathrm{E}-02$ & 5168.3490 & 1904.3845 & 7072.7334 & \\
\hline & 8/11/2008 18:00 & 485 & 224 & 1800 & 4.006 & 4.006 & & 0.9905 & 1.18E-02 & 4298.4483 & 1583.8517 & 5882.3000 & \\
\hline & $8 / 11 / 2008$ 19:00 & 486 & 224 & 1900 & 4.157 & 4.157 & & 0.9182 & 1.07E-02 & 3915.5154 & 1442.7522 & 5358.2675 & \\
\hline & 8/11/2008 20:00 & 487 & 224 & 2000 & 4.709 & 4.709 & & 0.6934 & 7.58E-03 & 2770.9083 & 1020.9981 & 3791.9064 & \\
\hline & 8/11/2008 21:00 & 488 & 224 & 2100 & 5.356 & 5.356 & & 0.4888 & 4.93E-03 & 1801.8766 & 663.9385 & 2465.8151 & \\
\hline & $8 / 11 / 200822: 00$ & 489 & 224 & 2200 & 5.922 & 5.922 & & 0.3465 & $3.23 \mathrm{E}-03$ & 1179.7470 & 434.7021 & 1614.4491 & \\
\hline & 8/11/2008 23:00 & 490 & 224 & 2300 & 6.143 & 6.143 & & 0.2981 & 2.68E-03 & 980.1219 & 361.1461 & 1341.2680 & \\
\hline & 8/12/2008 0:00 & 491 & 225 & 0 & 6.256 & 6.256 & 6.49 & 0.2746 & $2.42 \mathrm{E}-03$ & 886.0932 & 326.4993 & 1212.5924 & \\
\hline
\end{tabular}




\begin{tabular}{|c|c|c|c|c|c|c|c|c|c|c|c|c|c|}
\hline Tree ID & TS & RN & JD & HR & $\begin{array}{c}\text { Raw } \\
\text { TC_dTA }\end{array}$ & $\begin{array}{c}\text { Fixed } \\
\text { TC_dT } \Delta\end{array}$ & dTM & $\mathbf{K}$ & $\begin{array}{l}\text { Sap } \\
\text { Velocity } \\
(\mathrm{cm} / \mathrm{s})\end{array}$ & $\begin{array}{c}\text { Probed } \\
\text { Drywood F } \\
(\mathrm{g} / \mathrm{hr})\end{array}$ & $\begin{array}{c}\text { Extrapolated } \\
\text { Wetwood F } \\
(\mathrm{g} / \mathrm{hr})\end{array}$ & $\begin{array}{c}\text { Extrapolated } \\
\text { Total Wood F } \\
(\mathrm{g} / \mathrm{hr})\end{array}$ & $\begin{array}{l}\text { Relative } \\
\text { Humidity, } \\
@ 10 \mathrm{~m}(\%)\end{array}$ \\
\hline & 8/12/2008 1:00 & 492 & 225 & 100 & 6.254 & 6.254 & & 0.2750 & $2.43 \mathrm{E}-03$ & 887.7125 & 327.0959 & \begin{tabular}{|l|}
1214.8084 \\
\end{tabular} & \\
\hline & 8/12/2008 2:00 & 493 & 225 & 200 & 6.207 & 6.207 & & 0.2847 & 2.53E-03 & 926.2280 & 341.2878 & 1267.5158 & \\
\hline & 8/12/2008 3:00 & 494 & 225 & 300 & 6.412 & 6.412 & & 0.2436 & 2.09E-03 & 764.5739 & 281.7230 & 1046.2969 & \\
\hline & 8/12/2008 4:00 & 495 & 225 & 400 & 6.434 & 6.434 & & 0.2394 & 2.05E-03 & 748.1781 & 275.6816 & 1023.8597 & \\
\hline & 8/12/2008 5:00 & 496 & 225 & 500 & 6.463 & 6.463 & & 0.2338 & 1.99E-03 & 726.8374 & 267.8182 & 994.6556 & \\
\hline & 8/12/2008 6:00 & 497 & 225 & 600 & 6.474 & 6.474 & & 0.2317 & 1.97E-03 & 718.8229 & 264.8651 & 983.6880 & \\
\hline & 8/12/2008 7:00 & 498 & 225 & 700 & 6.483 & 6.483 & & 0.2300 & 1.95E-03 & 712.2982 & 262.4609 & 974.7592 & \\
\hline & 8/12/2008 8:00 & 499 & 225 & 800 & 6.489 & 6.489 & & 0.2288 & 1.94E-03 & 707.9647 & 260.8641 & 968.8288 & \\
\hline & 8/12/2008 9:00 & 500 & 225 & 900 & 6.178 & 6.178 & & 0.2907 & $2.60 \mathrm{E}-03$ & 950.4395 & 350.2090 & 1300.6485 & \\
\hline & $8 / 12 / 2008$ 10:00 & 501 & 225 & 1000 & 4.735 & 4.735 & & 0.6841 & 7.46E-03 & 2725.2362 & 1004.1693 & 3729.4055 & \\
\hline & $8 / 12 / 2008$ 11:00 & 502 & 225 & 1100 & 3.474 & 3.474 & & 1.2953 & 1.64E-02 & 5980.6759 & 2203.7030 & 8184.3789 & \\
\hline & $8 / 12 / 2008$ 12:00 & 503 & 225 & 1200 & 3.216 & 3.216 & & 1.4795 & 1.93E-02 & 7043.8582 & 2595.4544 & 9639.3126 & \\
\hline & 8/12/2008 13:00 & 504 & 225 & 1300 & 3.123 & 3.123 & & 1.5533 & $2.05 E-02$ & 7479.0657 & 2755.8156 & 10234.8813 & \\
\hline & $8 / 12 / 2008$ 14:00 & 505 & 225 & 1400 & 3.12 & 3.12 & & 1.5558 & 2.05E-02 & 7493.6202 & 2761.1785 & 10254.7987 & \\
\hline & $8 / 12 / 2008$ 15:00 & 506 & 225 & 1500 & 3.194 & 3.194 & & 1.4966 & 1.95E-02 & 7144.0853 & 2632.3851 & 9776.4704 & \\
\hline & $8 / 12 / 2008$ 16:00 & 507 & 225 & 1600 & 3.258 & 3.258 & & 1.4475 & 1.88E-02 & 6856.9934 & 2526.6002 & 9383.5936 & \\
\hline & $8 / 12 / 2008$ 17:00 & 508 & 225 & 1700 & 3.367 & 3.367 & & 1.3683 & 1.75E-02 & 6397.9186 & 2357.4447 & 8755.3632 & \\
\hline & $8 / 12 / 2008$ 18:00 & 509 & 225 & 1800 & 3.461 & 3.461 & & 1.3040 & $1.65 \mathrm{E}-02$ & 6029.7155 & 2221.7727 & 8251.4882 & \\
\hline & $8 / 12 / 2008$ 19:00 & 510 & 225 & 1900 & 3.74 & 3.74 & & 1.1321 & 1.39E-02 & 5066.7855 & 1866.9613 & 6933.7469 & \\
\hline & $8 / 12 / 200820: 00$ & 511 & 225 & 2000 & 4.342 & 4.342 & & 0.8365 & $9.55 E-03$ & 3491.0054 & 1286.3328 & 4777.3382 & \\
\hline & $8 / 12 / 2008$ 21:00 & 512 & 225 & 2100 & 5.71 & 5.71 & & 0.3965 & 3.81E-03 & 1392.6468 & 513.1494 & 1905.7962 & \\
\hline & $8 / 12 / 2008$ 22:00 & 513 & 225 & 2200 & 6.295 & 6.295 & & 0.2667 & 2.34E-03 & 854.8321 & 314.9805 & 1169.8126 & \\
\hline & $8 / 12 / 2008$ 23:00 & 514 & 225 & 2300 & 6.417 & 6.417 & & 0.2426 & 2.08E-03 & 760.8319 & 280.3442 & 1041.1760 & \\
\hline & 8/13/2008 0:00 & 515 & 226 & 0 & 6.464 & 6.464 & 6.56 & 0.2336 & 1.99E-03 & 726.1070 & 267.5491 & 993.6561 & \\
\hline & 8/13/2008 1:00 & 516 & 226 & 100 & 6.477 & 6.477 & & 0.2311 & $1.96 \mathrm{E}-03$ & 716.6448 & 264.0625 & 980.7073 & \\
\hline & 8/13/2008 2:00 & 517 & 226 & 200 & 6.497 & 6.497 & & 0.2273 & 1.92E-03 & 702.2068 & 258.7425 & 960.9493 & \\
\hline & 8/13/2008 3:00 & 518 & 226 & 300 & 6.543 & 6.543 & & 0.2187 & 1.83E-03 & 669.5425 & 246.7067 & 916.2492 & \\
\hline & 8/13/2008 4:00 & 519 & 226 & 400 & 6.538 & 6.538 & & 0.2196 & 1.84E-03 & 673.0566 & 248.0015 & 921.0581 & \\
\hline & 8/13/2008 5:00 & 520 & 226 & 500 & 6.551 & 6.551 & & 0.2172 & $1.82 \mathrm{E}-03$ & 663.9383 & 244.6417 & 908.5801 & \\
\hline & 8/13/2008 6:00 & 521 & 226 & 600 & 6.559 & 6.559 & & 0.2157 & 1.80E-03 & 658.3566 & 242.5850 & 900.9417 & \\
\hline & $8 / 13 / 2008$ 7:00 & 522 & 226 & 700 & 6.548 & 6.548 & & 0.2178 & $1.82 \mathrm{E}-03$ & 666.0373 & 245.4151 & 911.4524 & \\
\hline & 8/13/2008 8:00 & 523 & 226 & 800 & 6.496 & 6.496 & & 0.2275 & $1.92 \mathrm{E}-03$ & 702.9253 & 259.0073 & 961.9325 & \\
\hline & 8/13/2008 9:00 & 524 & 226 & 900 & 5.449 & 5.449 & & 0.4634 & $4.62 \mathrm{E}-03$ & 1687.2714 & 621.7098 & 2308.9812 & \\
\hline & $8 / 13 / 2008$ 10:00 & 525 & 226 & 1000 & 3.862 & 3.862 & & 1.0647 & $1.29 \mathrm{E}-02$ & 4698.2988 & 1731.1848 & 6429.4836 & \\
\hline & 8/13/2008 11:00 & 526 & 226 & 1100 & 3.624 & 3.624 & & 1.2003 & 1.49E-02 & 5445.3623 & 2006.4557 & 7451.8180 & \\
\hline & $8 / 13 / 2008$ 12:00 & 527 & 226 & 1200 & 3.198 & 3.198 & & 1.4934 & 1.95E-02 & 7125.7397 & 2625.6254 & 9751.3651 & \\
\hline
\end{tabular}




\begin{tabular}{|c|c|c|c|c|c|c|c|c|c|c|c|c|c|}
\hline Tree ID & TS & RN & JD & HR & $\begin{array}{c}\text { Raw } \\
\text { TC_dTA }\end{array}$ & $\begin{array}{c}\text { Fixed } \\
\text { TC_dT } \Delta\end{array}$ & dTM & $\mathbf{K}$ & $\begin{array}{c}\text { Sap } \\
\text { Velocity } \\
(\mathrm{cm} / \mathrm{s})\end{array}$ & $\begin{array}{c}\text { Probed } \\
\text { Drywood F } \\
(\mathrm{g} / \mathrm{hr})\end{array}$ & $\begin{array}{c}\text { Extrapolated } \\
\text { Wetwood F } \\
(\mathrm{g} / \mathrm{hr})\end{array}$ & $\begin{array}{c}\text { Extrapolated } \\
\text { Total Wood F } \\
(\mathrm{g} / \mathrm{hr})\end{array}$ & $\begin{array}{c}\text { Relative } \\
\text { Humidity, } \\
@ 10 \mathrm{~m}(\%)\end{array}$ \\
\hline & $7 / 22 / 2008$ 13:00 & 0 & 204 & 1300 & 4.058 & 4.058 & 7.70 & 0.8970 & 1.04E-02 & 3804.4409 & 1401.8245 & 5206.2654 & \\
\hline W605WN1 & $7 / 22 / 2008$ 14:00 & 1. & 204 & 1400 & 4.543 & 4.543 & & 0.6945 & 7.60E-03 & 2776.4284 & 1023.0321 & 3799.4605 & \\
\hline $36 " \mathrm{~N}$ & $7 / 22 / 2008$ 15:00 & 2 & 204 & 1500 & 4.52 & 4.52 & & 0.7031 & $7.71 \mathrm{E}-03$ & 2818.9229 & 1038.6901 & 3857.6130 & \\
\hline \multirow[t]{2}{*}{ Probe \# } & $7 / 22 / 2008$ 16:00 & 3 & 204 & 1600 & 4.476 & 4.476 & & 0.7198 & 7.94E-03 & 2901.7769 & 1069.2194 & 3970.9962 & \\
\hline & 7/22/2008 17:00 & 4 & 204 & 1700 & 4.471 & 4.471 & & 0.7218 & 7.97E-03 & 2911.3240 & 1072.7372 & 3984.0612 & \\
\hline \multirow[t]{4}{*}{13} & $7 / 22 / 2008$ 18:00 & 5 & 204 & 1800 & 4.54 & 4.54 & & 0.6956 & $7.61 E-03$ & 2781.9399 & 1025.0630 & 3807.0028 & \\
\hline & 7/22/2008 19:00 & 6 & 204 & 1900 & 4.691 & 4.691 & & 0.6410 & 6.88E-03 & 2515.7163 & 926.9674 & 3442.6837 & \\
\hline & $7 / 22 / 200820: 00$ & 7 & 204 & 2000 & 5.005 & 5.005 & & 0.5381 & 5.55E-03 & 2027.9708 & 747.2475 & 2775.2183 & \\
\hline & 7/22/2008 21:00 & 8 & 204 & 2100 & 6.045 & 6.045 & & 0.2734 & $2.41 \mathrm{E}-03$ & 881.4592 & 324.7918 & 1206.2509 & \\
\hline \multirow[t]{2}{*}{$\mathrm{DBH}(\mathrm{cm})$} & $7 / 22 / 2008$ 22:00 & 9 & 204 & 2200 & 7.209 & 7.209 & & 0.0678 & 4.34E-04 & 158.4536 & 58.3855 & 216.8391 & \\
\hline & $7 / 22 / 2008$ 23:00 & 10 & 204 & 2300 & 7.698 & 7.698 & & 0.0000 & $0.00 E+00$ & 0.0000 & 0.0000 & 0.0000 & \\
\hline \multirow[t]{2}{*}{13.7} & $7 / 23 / 20080: 00$ & 11 & 205 & 0 & 8.01 & 8.01 & 8.18 & -0.0390 & $0.00 \mathrm{E}+00$ & 0.0000 & 0.0000 & 0.0000 & \\
\hline & 7/23/2008 1:00 & 12 & 205 & 100 & 8.13 & 8.13 & & -0.0531 & $0.00 \mathrm{E}+00$ & 0.0000 & 0.0000 & 0.0000 & \\
\hline Day Count & 7/23/2008 2:00 & 13 & 205 & 200 & 8.16 & 8.16 & & -0.0566 & $0.00 \mathrm{E}+00$ & 0.0000 & 0.0000 & 0.0000 & \\
\hline \multirow{27}{*}{35} & 7/23/2008 3:00 & 14 & 205 & 300 & 8.18 & 8.18 & & -0.0589 & $0.00 \mathrm{E}+00$ & 0.0000 & 0.0000 & 0.0000 & \\
\hline & 7/23/2008 4:00 & 15 & 205 & 400 & 8.17 & 8.17 & & -0.0578 & $0.00 \mathrm{E}+00$ & 0.0000 & 0.0000 & 0.0000 & \\
\hline & 7/23/2008 5:00 & 16 & 205 & 500 & 8.09 & 8.09 & & -0.0485 & $0.00 \mathrm{E}+00$ & 0.0000 & 0.0000 & 0.0000 & \\
\hline & 7/23/2008 6:00 & 17 & 205 & 600 & 7.932 & 7.932 & & -0.0295 & $0.00 \mathrm{E}+00$ & 0.0000 & 0.0000 & 0.0000 & \\
\hline & 7/23/2008 7:00 & 18 & 205 & 700 & 7.582 & 7.582 & & 0.0153 & $6.93 \mathrm{E}-05$ & 25.3362 & 9.3356 & 34.6718 & \\
\hline & 7/23/2008 8:00 & 19 & 205 & 800 & 6.871 & 6.871 & & 0.1204 & 8.78E-04 & 320.9851 & 118.2736 & 439.2587 & \\
\hline & 7/23/2008 9:00 & 20 & 205 & 900 & 5.888 & 5.888 & & 0.3074 & 2.79E-03 & 1018.0735 & 375.1301 & 1393.2036 & \\
\hline & $7 / 23 / 2008$ 10:00 & 21 & 205 & 1000 & 5.031 & 5.031 & & 0.5301 & $5.45 \mathrm{E}-03$ & 1991.1548 & 733.6819 & 2724.8368 & \\
\hline & 7/23/2008 11:00 & 22 & 205 & 1100 & 4.858 & 4.858 & & 0.5846 & $6.15 E-03$ & 2246.0160 & 827.5908 & 3073.6068 & \\
\hline & 7/23/2008 12:00 & 23 & 205 & 1200 & 4.709 & 4.709 & & 0.6347 & $6.80 \mathrm{E}-03$ & 2485.4461 & 915.8137 & 3401.2598 & \\
\hline & 7/23/2008 13:00 & 24 & 205 & 1300 & 4.618 & 4.618 & & 0.6670 & 7.23E-03 & 2641.6198 & 973.3591 & 3614.9789 & \\
\hline & $7 / 23 / 2008$ 14:00 & 25 & 205 & 1400 & 4.6 & 4.6 & & 0.6735 & 7.31E-03 & 2673.4588 & 985.0909 & 3658.5497 & \\
\hline & $7 / 23 / 2008$ 15:00 & 26 & 205 & 1500 & 4.581 & 4.581 & & 0.6804 & 7.41E-03 & 2707.4164 & 997.6032 & 3705.0196 & \\
\hline & $7 / 23 / 2008$ 16:00 & 27 & 205 & 1600 & 4.672 & 4.672 & & 0.6477 & 6.97E-03 & 2547.9965 & 938.8617 & 3486.8582 & \\
\hline & 7/23/2008 17:00 & 28 & 205 & 1700 & 4.942 & 4.942 & & 0.5577 & 5.80E-03 & 2119.3203 & 780.9072 & 2900.2275 & \\
\hline & $7 / 23 / 2008$ 18:00 & 29 & 205 & 1800 & 5.356 & 5.356 & & 0.4373 & $4.30 \mathrm{E}-03$ & 1570.9627 & 578.8535 & 2149.8163 & \\
\hline & $7 / 23 / 2008$ 19:00 & 30 & 205 & 1900 & 5.484 & 5.484 & & 0.4037 & 3.90E-03 & 1423.9405 & 524.6802 & 1948.6207 & \\
\hline & $7 / 23 / 200820: 00$ & 31 & 205 & 2000 & 5.796 & 5.796 & & 0.3282 & 3.02E-03 & 1103.3271 & 406.5436 & 1509.8706 & \\
\hline & $7 / 23 / 200821: 00$ & 32 & 205 & 2100 & 6.373 & 6.373 & & 0.2079 & 1.72E-03 & 629.0824 & 231.7984 & 860.8808 & \\
\hline & $7 / 23 / 200822: 00$ & 33 & 205 & 2200 & 7.067 & 7.067 & & 0.0893 & $6.08 \mathrm{E}-04$ & 222.2465 & 81.8913 & 304.1378 & \\
\hline & $7 / 23 / 2008$ 23:00 & 34 & 205 & 2300 & 7.302 & 7.302 & & 0.0542 & 3.29E-04 & 120.3021 & 44.3278 & 164.6298 & \\
\hline & $7 / 24 / 20080: 00$ & 35 & 206 & 0 & 7.359 & 7.359 & 8.11 & 0.0461 & 2.69E-04 & 98.4077 & 36.2603 & 134.6680 & \\
\hline & 7/24/2008 1:00 & 36 & 206 & 100 & 7.466 & 7.466 & & 0.0311 & 1.66E-04 & 60.6110 & 22.3334 & 82.9444 & \\
\hline & 7/24/2008 2:00 & 37 & 206 & 200 & 7.542 & 7.542 & & 0.0207 & 1.00E-04 & 36.7246 & 13.5320 & 50.2566 & \\
\hline & 7/24/2008 3:00 & 38 & 206 & 300 & 7.675 & 7.675 & & 0.0030 & $9.32 \mathrm{E}-06$ & 3.4054 & 1.2548 & 4.6601 & \\
\hline & 7/24/2008 4:00 & 39 & 206 & 400 & 7.736 & 7.736 & & -0.0049 & $0.00 \mathrm{E}+00$ & 0.0000 & 0.0000 & 0.0000 & \\
\hline & $7 / 24 / 20085: 00$ & 40 & 206 & 500 & 7.798 & 7.798 & & -0.0128 & $0.00 \mathrm{E}+00$ & 0.0000 & 0.0000 & 0.0000 & \\
\hline
\end{tabular}




\begin{tabular}{|c|c|c|c|c|c|c|c|c|c|c|c|c|c|}
\hline \multirow[t]{42}{*}{ Tree ID } & TS & RN & JD & HR & $\begin{array}{c}\text { Raw } \\
\text { TC_dTA }\end{array}$ & $\begin{array}{c}\text { Fixed } \\
\text { TC_dT } \Delta\end{array}$ & dTM & $\mathbf{K}$ & $\begin{array}{l}\text { Sap } \\
\text { Velocity } \\
\text { (cm/s) }\end{array}$ & $\begin{array}{l}\text { Probed } \\
\text { Drywood F } \\
\text { (g/hr) }\end{array}$ & $\begin{array}{c}\text { Extrapolated } \\
\text { Wetwood F } \\
(\mathrm{g} / \mathrm{hr})\end{array}$ & $\begin{array}{c}\text { Extrapolated } \\
\text { Total Wood F } \\
(\mathrm{g} / \mathrm{hr})\end{array}$ & $\begin{array}{c}\text { Relative } \\
\text { Humidity, } \\
@ 10 \mathrm{~m}(\%)\end{array}$ \\
\hline & 7/24/2008 6:00 & 41 & 206 & 600 & 7.815 & 7.815 & & -0.0150 & $0.00 \mathrm{E}+00$ & 0.0000 & 0.0000 & 0.0000 & \\
\hline & 7/24/2008 7:00 & 42 & 206 & 700 & 7.694 & 7.694 & & 0.0005 & $1.08 \mathrm{E}-06$ & 0.3942 & 0.1452 & 0.5394 & \\
\hline & 7/24/2008 8:00 & 43 & 206 & 800 & 7.278 & 7.278 & & 0.0577 & $3.55 \mathrm{E}-04$ & 129.8644 & 47.8512 & 177.7156 & \\
\hline & $7 / 24 / 20089: 00$ & 44 & 206 & 900 & 7.074 & 7.074 & & 0.0882 & $5.99 \mathrm{E}-04$ & 218.9484 & 80.6760 & 299.6244 & \\
\hline & $7 / 24 / 2008$ 10:00 & 45 & 206 & 1000 & 7.115 & 7.115 & & 0.0819 & 5.47E-04 & 199.9484 & 73.6751 & 273.6234 & \\
\hline & 7/24/2008 11:00 & 46 & 206 & 1100 & 5.745 & 5.745 & & 0.3399 & $3.15 E-03$ & 1152.3266 & 424.5984 & 1576.9251 & \\
\hline & $7 / 24 / 2008$ 12:00 & 47 & 206 & 1200 & 4.84 & 4.84 & & 0.5905 & $6.22 \mathrm{E}-03$ & 2273.9197 & 837.8725 & 3111.7922 & \\
\hline & 7/24/2008 13:00 & 48 & 206 & 1300 & 5.039 & 5.039 & & 0.5277 & $5.42 \mathrm{E}-03$ & 1979.9286 & 729.5454 & 2709.4740 & \\
\hline & $7 / 24 / 2008$ 14:00 & 49 & 206 & 1400 & 4.93 & 4.93 & & 0.5615 & $5.85 \mathrm{E}-03$ & 2137.0715 & 787.4480 & 2924.5195 & \\
\hline & 7/24/2008 15:00 & 50 & 206 & 1500 & 4.679 & 4.679 & & 0.6452 & 6.94E-03 & 2536.0643 & 934.4650 & 3470.5293 & \\
\hline & $7 / 24 / 2008$ 16:00 & 51 & 206 & 1600 & 4.601 & 4.601 & & 0.6731 & $7.31 E-03$ & 2671.6815 & 984.4360 & 3656.1175 & \\
\hline & $7 / 24 / 2008$ 17:00 & 52 & 206 & 1700 & 4.723 & 4.723 & & 0.6299 & $6.74 \mathrm{E}-03$ & 2462.1093 & 907.2148 & 3369.3241 & \\
\hline & 7/24/2008 18:00 & 53 & 206 & 1800 & 4.688 & 4.688 & & 0.6421 & 6.90E-03 & 2520.7907 & 928.8372 & 3449.6278 & \\
\hline & $7 / 24 / 2008$ 19:00 & 54 & 206 & 1900 & 4.998 & 4.998 & & 0.5402 & 5.58E-03 & 2037.9699 & 750.9319 & 2788.9019 & \\
\hline & $7 / 24 / 200820: 00$ & 55 & 206 & 2000 & 5.571 & 5.571 & & 0.3818 & 3.64E-03 & 1329.3680 & 489.8330 & 1819.2010 & \\
\hline & $7 / 24 / 200821: 00$ & 56 & 206 & 2100 & 6.669 & 6.669 & & 0.1543 & 1.19E-03 & 435.7845 & 160.5738 & 596.3582 & \\
\hline & $7 / 24 / 200822: 00$ & 57 & 206 & 2200 & 7.738 & 7.738 & & -0.0052 & $0.00 \mathrm{E}+00$ & 0.0000 & 0.0000 & 0.0000 & \\
\hline & $7 / 24 / 200823: 00$ & 58 & 206 & 2300 & 8.11 & 8.11 & & -0.0508 & $0.00 \mathrm{E}+00$ & 0.0000 & 0.0000 & 0.0000 & \\
\hline & $7 / 25 / 20080: 00$ & 59 & 207 & 0 & 8.22 & 8.22 & 8.22 & -0.0635 & $0.00 \mathrm{E}+00$ & 0.0000 & 0.0000 & 0.0000 & \\
\hline & 7/25/2008 1:00 & 60 & 207 & 100 & 8.22 & 8.22 & & -0.0635 & $0.00 \mathrm{E}+00$ & 0.0000 & 0.0000 & 0.0000 & \\
\hline & 7/25/2008 2:00 & 61 & 207 & 200 & 8.14 & 8.14 & & -0.0543 & $0.00 E+00$ & 0.0000 & 0.0000 & 0.0000 & \\
\hline & 7/25/2008 3:00 & 62 & 207 & 300 & 8.05 & 8.05 & & -0.0437 & $0.00 \mathrm{E}+00$ & 0.0000 & 0.0000 & 0.0000 & \\
\hline & 7/25/2008 4:00 & 63 & 207 & 400 & 7.964 & 7.964 & & -0.0334 & $0.00 \mathrm{E}+00$ & 0.0000 & 0.0000 & 0.0000 & \\
\hline & 7/25/2008 5:00 & 64 & 207 & 500 & 7.884 & 7.884 & & -0.0236 & $0.00 \mathrm{E}+00$ & 0.0000 & 0.0000 & 0.0000 & \\
\hline & 7/25/2008 6:00 & 65 & 207 & 600 & 7.839 & 7.839 & & -0.0180 & $0.00 \mathrm{E}+00$ & 0.0000 & 0.0000 & 0.0000 & \\
\hline & 7/25/2008 7:00 & 66 & 207 & 700 & 7.776 & 7.776 & & -0.0100 & $0.00 \mathrm{E}+00$ & 0.0000 & 0.0000 & 0.0000 & \\
\hline & 7/25/2008 8:00 & 67 & 207 & 800 & 7.679 & 7.679 & & 0.0025 & $7.36 \mathrm{E}-06$ & 2.6899 & 0.9912 & 3.6811 & \\
\hline & $7 / 25 / 20089: 00$ & 68 & 207 & 900 & 7.6 & 7.6 & & 0.0129 & $5.62 E-05$ & 20.5270 & 7.5636 & 28.0906 & \\
\hline & $7 / 25 / 2008$ 10:00 & 69 & 207 & 1000 & 7.268 & 7.268 & & 0.0592 & 3.66E-04 & 133.9076 & 49.3410 & 183.2485 & \\
\hline & 7/25/2008 11:00 & 70 & 207 & 1100 & 6.39 & 6.39 & & 0.2047 & 1.69E-03 & 617.1344 & 227.3959 & 844.5302 & \\
\hline & $7 / 25 / 2008$ 12:00 & 71 & 207 & 1200 & 5.56 & 5.56 & & 0.3845 & 3.67E-03 & 1341.0951 & 494.1541 & 1835.2492 & \\
\hline & $7 / 25 / 2008$ 13:00 & 72 & 207 & 1300 & 4.916 & 4.916 & & 0.5659 & 5.90E-03 & 2157.9261 & 795.1323 & 2953.0584 & \\
\hline & $7 / 25 / 2008$ 14:00 & 73 & 207 & 1400 & 4.603 & 4.603 & & 0.6724 & 7.30E-03 & 2668.1300 & 983.1274 & 3651.2574 & \\
\hline & $7 / 25 / 2008$ 15:00 & 74 & 207 & 1500 & 4.567 & 4.567 & & 0.6856 & $7.48 \mathrm{E}-03$ & 2732.6704 & 1006.9086 & 3739.5789 & \\
\hline & $7 / 25 / 200816: 00$ & 75 & 207 & 1600 & 4.64 & 4.64 & & 0.6591 & 7.12E-03 & 2603.1373 & 959.1795 & 3562.3167 & \\
\hline & $7 / 25 / 2008$ 17:00 & 76 & 207 & 1700 & 4.483 & 4.483 & & 0.7172 & 7.90E-03 & 2888.4565 & 1064.3112 & 3952.7677 & \\
\hline & $7 / 25 / 2008$ 18:00 & 77 & 207 & 1800 & 4.535 & 4.535 & & 0.6975 & 7.64E-03 & 2791.1464 & 1028.4553 & 3819.6018 & \\
\hline & 7/25/2008 19:00 & 78 & 207 & 1900 & 4.65 & 4.65 & & 0.6555 & 7.08E-03 & 2585.8004 & 952.7913 & 3538.5917 & \\
\hline & $7 / 25 / 200820: 00$ & 79 & 207 & 2000 & 5.21 & 5.21 & & 0.4775 & 4.79E-03 & 1750.9419 & 645.1706 & 2396.1125 & \\
\hline & $7 / 25 / 2008$ 21:00 & 80 & 207 & 2100 & 6.408 & 6.408 & & 0.2013 & 1.65E-03 & 604.5994 & 222.7771 & 827.3765 & \\
\hline & $7 / 25 / 200822: 00$ & 81 & 207 & 2200 & 7.577 & 7.577 & & 0.0160 & 7.31E-05 & 26.7088 & 9.8414 & 36.5502 & \\
\hline
\end{tabular}




\begin{tabular}{|c|c|c|c|c|c|c|c|c|c|c|c|c|c|}
\hline Tree ID & TS & RN & JD & HR & $\begin{array}{c}\text { Raw } \\
\text { TC_dTA }\end{array}$ & $\begin{array}{c}\text { Fixed } \\
\text { TC_dT } \Delta\end{array}$ & dTM & K & $\begin{array}{l}\text { Sap } \\
\text { Velocity } \\
(\mathrm{cm} / \mathrm{s})\end{array}$ & $\begin{array}{c}\text { Probed } \\
\text { Drywood F } \\
(\mathrm{g} / \mathrm{hr})\end{array}$ & $\begin{array}{c}\text { Extrapolated } \\
\text { Wetwood F } \\
(\mathrm{g} / \mathrm{hr})\end{array}$ & $\begin{array}{c}\text { Extrapolated } \\
\text { Total Wood F } \\
(\mathrm{g} / \mathrm{hr})\end{array}$ & $\begin{array}{l}\text { Relative } \\
\text { Humidity, } \\
@ 10 \mathrm{~m}(\%)\end{array}$ \\
\hline & 7/25/2008 23:00 & 82 & 207 & 2300 & \begin{tabular}{|l|}
7.888 \\
\end{tabular} & 7.888 & & -0.0241 & $0.00 \mathrm{E}+00$ & 0.0000 & 0.0000 & 0.0000 & \\
\hline & 7/26/2008 0:00 & 83 & 208 & 0 & 7.858 & 7.858 & 7.86 & -0.0204 & $0.00 \mathrm{E}+00$ & 0.0000 & 0.0000 & 0.0000 & \\
\hline & 7/26/2008 1:00 & 84 & 208 & 100 & 7.72 & 7.72 & & -0.0028 & $0.00 \mathrm{E}+00$ & 0.0000 & 0.0000 & 0.0000 & \\
\hline & $7 / 26 / 20082: 00$ & 85 & 208 & 200 & 7.559 & 7.559 & & 0.0184 & 8.69E-05 & 31.7738 & 11.7077 & 43.4815 & \\
\hline & 7/26/2008 3:00 & 86 & 208 & 300 & 7.42 & 7.42 & & 0.0375 & 2.09E-04 & 76.3061 & 28.1165 & 104.4226 & \\
\hline & 7/26/2008 4:00 & 87 & 208 & 400 & 7.302 & 7.302 & & 0.0542 & $3.29 E-04$ & 120.3021 & 44.3278 & 164.6298 & \\
\hline & 7/26/2008 5:00 & 88 & 208 & 500 & 7.2 & 7.2 & & 0.0692 & 4.44E-04 & 162.3006 & 59.8030 & 222.1036 & \\
\hline & 7/26/2008 6:00 & 89 & 208 & 600 & 7.104 & 7.104 & & 0.0836 & 5.61E-04 & 204.9926 & 75.5337 & 280.5263 & \\
\hline & 7/26/2008 7:00 & 90 & 208 & 700 & 7.044 & 7.044 & & 0.0928 & $6.38 \mathrm{E}-04$ & 233.1942 & 85.9252 & 319.1194 & \\
\hline & 7/26/2008 8:00 & 91 & 208 & 800 & 6.971 & 6.971 & & 0.1043 & 7.36E-04 & 269.0666 & 99.1431 & 368.2097 & \\
\hline & 7/26/2008 9:00 & 92 & 208 & 900 & 5.714 & 5.714 & & 0.3472 & $3.24 \mathrm{E}-03$ & 1182.7352 & 435.8031 & 1618.5383 & \\
\hline & $7 / 26 / 2008$ 10:00 & 93 & 208 & 1000 & 4.586 & 4.586 & & 0.6786 & $7.38 \mathrm{E}-03$ & 2698.4451 & 994.2976 & 3692.7427 & \\
\hline & $7 / 26 / 2008$ 11:00 & 94 & 208 & 1100 & 4.155 & 4.155 & & 0.8527 & $9.78 \mathrm{E}-03$ & 3574.5561 & 1317.1187 & 4891.6748 & \\
\hline & $7 / 26 / 2008$ 12:00 & 95 & 208 & 1200 & 4.443 & 4.443 & & 0.7326 & 8.11E-03 & 2965.2949 & 1092.6239 & 4057.9188 & \\
\hline & $7 / 26 / 2008$ 13:00 & 96 & 208 & 1300 & 4.102 & 4.102 & & 0.8766 & 1.01E-02 & 3698.4823 & 1362.7819 & 5061.2641 & \\
\hline & $7 / 26 / 200814: 00$ & 97 & 208 & 1400 & 4.126 & 4.126 & & 0.8657 & 9.96E-03 & 3641.8721 & 1341.9227 & 4983.7947 & \\
\hline & $7 / 26 / 2008$ 15:00 & 98 & 208 & 1500 & 4.195 & 4.195 & & 0.8350 & 9.53E-03 & 3483.6132 & 1283.6089 & 4767.2221 & \\
\hline & $7 / 26 / 200816: 00$ & 99 & 208 & 1600 & 4.362 & 4.362 & & 0.7648 & 8.55E-03 & 3126.4060 & 1151.9886 & 4278.3946 & \\
\hline & $7 / 26 / 200817: 00$ & 100 & 208 & 1700 & 4.269 & 4.269 & & 0.8032 & 9.09E-03 & 3320.9844 & 1223.6850 & 4544.6695 & \\
\hline & $7 / 26 / 2008$ 18:00 & 101 & 208 & 1800 & 4.423 & 4.423 & & 0.7404 & 8.22E-03 & 3004.3791 & 1107.0253 & 4111.4043 & \\
\hline & $7 / 26 / 2008$ 19:00 & 102 & 208 & 1900 & 4.574 & 4.574 & & 0.6830 & 7.44E-03 & 2720.0185 & 1002.2468 & 3722.2653 & \\
\hline & $7 / 26 / 200820: 00$ & 103 & 208 & 2000 & 4.986 & 4.986 & & 0.5439 & 5.62E-03 & 2055.1982 & 757.2801 & 2812.4783 & \\
\hline & $7 / 26 / 200821: 00$ & 104 & 208 & 2100 & 6.122 & 6.122 & & 0.2574 & 2.24E-03 & 818.3389 & 301.5338 & 1119.8727 & \\
\hline & $7 / 26 / 200822: 00$ & 105 & 208 & 2200 & 7.206 & 7.206 & & 0.0683 & 4.37E-04 & 159.7330 & 58.8569 & 218.5899 & \\
\hline & $7 / 26 / 2008$ 23:00 & 106 & 208 & 2300 & 7.611 & 7.611 & & 0.0114 & 4.84E-05 & 17.6970 & 6.5208 & 24.2179 & \\
\hline & $7 / 27 / 20080: 00$ & 107 & 209 & 0 & 7.65 & 7.65 & 7.65 & 0.0063 & 2.31E-05 & 8.4572 & 3.1162 & 11.5735 & \\
\hline & 7/27/2008 1:00 & 108 & 209 & 100 & 7.616 & 7.616 & & 0.0108 & 4.50E-05 & 16.4402 & 6.0577 & 22.4979 & \\
\hline & 7/27/2008 2:00 & 109 & 209 & 200 & 7.514 & 7.514 & & 0.0245 & 1.24E-04 & 45.2065 & 16.6572 & 61.8637 & \\
\hline & 7/27/2008 3:00 & 110 & 209 & 300 & 7.465 & 7.465 & & 0.0312 & 1.67E-04 & 60.9428 & 22.4556 & 83.3985 & \\
\hline & 7/27/2008 4:00 & 111 & 209 & 400 & 7.433 & 7.433 & & 0.0357 & 1.96E-04 & 71.7827 & 26.4498 & 98.2325 & \\
\hline & 7/27/20085:00 & 112 & 209 & 500 & 7.388 & 7.388 & & 0.0420 & 2.40E-04 & 87.7237 & 32.3236 & 120.0473 & \\
\hline & 7/27/2008 6:00 & 113 & 209 & 600 & 7.327 & 7.327 & & 0.0506 & 3.02E-04 & 110.5560 & 40.7366 & 151.2926 & \\
\hline & 7/27/2008 7:00 & 114 & 209 & 700 & 7.248 & 7.248 & & 0.0621 & 3.89E-04 & 142.0964 & 52.3584 & 194.4548 & \\
\hline & 7/27/2008 8:00 & 115 & 209 & 800 & 6.911 & 6.911 & & 0.1139 & 8.20E-04 & 299.8315 & 110.4791 & 410.3105 & \\
\hline & $7 / 27 / 20089: 00$ & 116 & 209 & 900 & 5.399 & 5.399 & & 0.4258 & $4.16 \mathrm{E}-03$ & 1520.4913 & 560.2563 & 2080.7476 & \\
\hline & $7 / 27 / 2008$ 10:00 & 117 & 209 & 1000 & 4.12 & 4.12 & & 0.8684 & 1.00E-02 & 3655.9475 & 1347.1090 & 5003.0565 & \\
\hline & $7 / 27 / 2008$ 11:00 & 118 & 209 & 1100 & 3.974 & 3.974 & & 0.9371 & 1.10E-02 & 4014.8615 & 1479.3583 & 5494.2198 & \\
\hline & $7 / 27 / 2008$ 12:00 & 119 & 209 & 1200 & 3.949 & 3.949 & & 0.9494 & 1.12E-02 & 4079.6359 & 1503.2258 & 5582.8617 & \\
\hline & $7 / 27 / 200813: 00$ & 120 & 209 & 1300 & 4.06 & 4.06 & & 0.8961 & $1.04 \mathrm{E}-02$ & 3799.5625 & 1400.0270 & 5199.5895 & \\
\hline & $7 / 27 / 2008$ 14:00 & 121 & 209 & 1400 & 4.03 & 4.03 & & 0.9102 & 1.06E-02 & 3873.3715 & 1427.2234 & 5300.5949 & \\
\hline & 7/27/2008 15:00 & 122 & 209 & 1500 & 4.055 & 4.055 & & 0.8984 & 1.04E-02 & 3811.7697 & 1404.5250 & 5216.2947 & \\
\hline
\end{tabular}




\begin{tabular}{|c|c|c|c|c|c|c|c|c|c|c|c|c|c|}
\hline Tree ID & TS & RN & JD & HR & $\begin{array}{c}\text { Raw } \\
\text { TC_dTA }\end{array}$ & $\begin{array}{c}\text { Fixed } \\
\text { TC_dT } \Delta\end{array}$ & dTM & $\mathbf{K}$ & $\begin{array}{l}\text { Sap } \\
\text { Velocity } \\
(\mathrm{cm} / \mathrm{s})\end{array}$ & $\begin{array}{c}\text { Probed } \\
\text { Drywood F } \\
(\mathrm{g} / \mathrm{hr})\end{array}$ & $\begin{array}{c}\text { Extrapolated } \\
\text { Wetwood F } \\
(\mathrm{g} / \mathrm{hr})\end{array}$ & $\begin{array}{c}\text { Extrapolated } \\
\text { Total Wood F } \\
(\mathrm{g} / \mathrm{hr})\end{array}$ & $\begin{array}{l}\text { Relative } \\
\text { Humidity, } \\
@ 10 \mathrm{~m}(\%)\end{array}$ \\
\hline & 7/27/2008 16:00 & 123 & 209 & 1600 & 4.121 & 4.121 & & 0.8680 & 1.00E-02 & 3653.5980 & 1346.2433 & 4999.8413 & \\
\hline & 7/27/2008 17:00 & 124 & 209 & 1700 & 4.122 & 4.122 & & 0.8675 & 9.99E-03 & 3651.2500 & 1345.3781 & 4996.6281 & \\
\hline & $7 / 27 / 2008$ 18:00 & 125 & 209 & 1800 & 4.182 & 4.182 & & 0.8407 & $9.61 \mathrm{E}-03$ & 3512.9307 & 1294.4116 & 4807.3422 & \\
\hline & $7 / 27 / 2008$ 19:00 & 126 & 209 & 1900 & 4.328 & 4.328 & & 0.7787 & 8.75E-03 & 3196.3179 & 1177.7491 & 4374.0669 & \\
\hline & $7 / 27 / 200820: 00$ & 127 & 209 & 2000 & 4.744 & 4.744 & & 0.6227 & 6.64E-03 & 2427.4393 & 894.4399 & 3321.8792 & \\
\hline & $7 / 27 / 2008$ 21:00 & 128 & 209 & 2100 & 5.807 & 5.807 & & 0.3256 & 2.99E-03 & 1092.9234 & 402.7101 & 1495.6336 & \\
\hline & $7 / 27 / 2008$ 22:00 & 129 & 209 & 2200 & 6.783 & 6.783 & & 0.1349 & $1.01 E-03$ & 369.3484 & 136.0940 & 505.4425 & \\
\hline & $7 / 27 / 200823: 00$ & 130 & 209 & 2300 & 7.111 & 7.111 & & 0.0825 & $5.52 \mathrm{E}-04$ & 201.7781 & 74.3493 & 276.1274 & \\
\hline & $7 / 28 / 20080: 00$ & 131 & 210 & 0 & 7.26 & 7.26 & 7.59 & 0.0603 & 3.75E-04 & 137.1667 & 50.5419 & 187.7086 & \\
\hline & 7/28/2008 1:00 & 132 & 210 & 100 & 7.437 & 7.437 & & 0.0351 & 1.93E-04 & 70.4046 & 25.9420 & 96.3466 & \\
\hline & $7 / 28 / 20082: 00$ & 133 & 210 & 200 & 7.468 & 7.468 & & 0.0308 & 1.64E-04 & 59.9487 & 22.0893 & 82.0380 & \\
\hline & 7/28/2008 3:00 & 134 & 210 & 300 & 7.587 & 7.587 & & 0.0146 & $6.56 \mathrm{E}-05$ & 23.9791 & 8.8356 & 32.8147 & \\
\hline & 7/28/2008 4:00 & 135 & 210 & 400 & 7.544 & 7.544 & & 0.0204 & 9.89E-05 & 36.1341 & 13.3144 & 49.4485 & \\
\hline & 7/28/2008 5:00 & 136 & 210 & 500 & 7.568 & 7.568 & & 0.0172 & 7.99E-05 & 29.2177 & 10.7659 & 39.9835 & \\
\hline & 7/28/2008 6:00 & 137 & 210 & 600 & 7.496 & 7.496 & & 0.0269 & 1.39E-04 & 50.8604 & 18.7405 & 69.6009 & \\
\hline & 7/28/2008 7:00 & 138 & 210 & 700 & 7.154 & 7.154 & & 0.0760 & 4.99E-04 & 182.3810 & 67.2020 & 249.5831 & \\
\hline & 7/28/2008 8:00 & 139 & 210 & 800 & 5.808 & 5.808 & & 0.3254 & 2.99E-03 & 1091.9805 & 402.3627 & 1494.3432 & \\
\hline & $7 / 28 / 20089: 00$ & 140 & 210 & 900 & 4.842 & 4.842 & & 0.5898 & $6.21 \mathrm{E}-03$ & 2270.8058 & 836.7251 & 3107.5310 & \\
\hline & $7 / 28 / 2008$ 10:00 & 141 & 210 & 1000 & 4.399 & 4.399 & & 0.7499 & 8.35E-03 & 3051.8773 & 1124.5270 & 4176.4042 & \\
\hline & $7 / 28 / 2008$ 11:00 & 142 & 210 & 1100 & 4.284 & 4.284 & & 0.7969 & $9.00 \mathrm{E}-03$ & 3288.8788 & 1211.8550 & 4500.7338 & \\
\hline & $7 / 28 / 200812: 00$ & 143 & 210 & 1200 & 4.238 & 4.238 & & 0.8164 & $9.27 \mathrm{E}-03$ & 3388.2442 & 1248.4683 & 4636.7125 & \\
\hline & 7/28/2008 13:00 & 144 & 210 & 1300 & 4.248 & 4.248 & & 0.8121 & 9.21E-03 & 3366.4126 & 1240.4240 & 4606.8365 & \\
\hline & $7 / 28 / 2008$ 14:00 & 145 & 210 & 1400 & 4.213 & 4.213 & & 0.8272 & 9.42E-03 & 3443.3939 & 1268.7893 & 4712.1832 & \\
\hline & $7 / 28 / 2008$ 15:00 & 146 & 210 & 1500 & 4.136 & 4.136 & & 0.8612 & $9.90 \mathrm{E}-03$ & 3618.5264 & 1333.3205 & 4951.8468 & \\
\hline & $7 / 28 / 2008$ 16:00 & 147 & 210 & 1600 & 4.182 & 4.182 & & 0.8407 & $9.61 \mathrm{E}-03$ & 3512.9307 & 1294.4116 & 4807.3422 & \\
\hline & 7/28/2008 17:00 & 148 & 210 & 1700 & 4.175 & 4.175 & & 0.8438 & $9.66 \mathrm{E}-03$ & 3528.8118 & 1300.2633 & 4829.0751 & \\
\hline & $7 / 28 / 2008$ 18:00 & 149 & 210 & 1800 & 4.258 & 4.258 & & 0.8079 & $9.15 E-03$ & 3344.7098 & 1232.4271 & 4577.1369 & \\
\hline & $7 / 28 / 2008$ 19:00 & 150 & 210 & 1900 & 4.443 & 4.443 & & 0.7326 & $8.11 \mathrm{E}-03$ & 2965.2949 & 1092.6239 & 4057.9188 & \\
\hline & $7 / 28 / 200820: 00$ & 151 & 210 & 2000 & 4.821 & 4.821 & & 0.5968 & 6.30E-03 & 2303.6703 & 848.8347 & 3152.5050 & \\
\hline & $7 / 28 / 200821: 00$ & 152 & 210 & 2100 & 5.924 & 5.924 & & 0.2995 & $2.70 \mathrm{E}-03$ & 985.7799 & 363.2309 & 1349.0107 & \\
\hline & $7 / 28 / 2008$ 22:00 & 153 & 210 & 2200 & 6.597 & 6.597 & & 0.1669 & 1.31E-03 & 479.9896 & 176.8620 & 656.8516 & \\
\hline & $7 / 28 / 200823: 00$ & 154 & 210 & 2300 & 6.94 & 6.94 & & 0.1092 & 7.79E-04 & 284.8174 & 104.9468 & 389.7642 & \\
\hline & $7 / 29 / 20080: 00$ & 155 & 211 & 0 & 7.173 & 7.173 & 7.48 & 0.0732 & $4.76 \mathrm{E}-04$ & 174.0025 & 64.1148 & 238.1173 & \\
\hline & 7/29/2008 1:00 & 156 & 211 & 100 & 7.313 & 7.313 & & 0.0526 & 3.17E-04 & 115.9866 & 42.7376 & 158.7242 & \\
\hline & 7/29/2008 2:00 & 157 & 211 & 200 & 7.402 & 7.402 & & 0.0400 & 2.26E-04 & 82.6796 & 30.4650 & 113.1446 & \\
\hline & 7/29/2008 3:00 & 158 & 211 & 300 & 7.413 & 7.413 & & 0.0384 & 2.16E-04 & 78.7696 & 29.0243 & 107.7939 & \\
\hline & 7/29/2008 4:00 & 159 & 211 & 400 & 7.48 & 7.48 & & 0.0291 & 1.53E-04 & 56.0111 & 20.6384 & 76.6496 & \\
\hline & 7/29/2008 5:00 & 160 & 211 & 500 & 7.409 & 7.409 & & 0.0390 & 2.19E-04 & 80.1860 & 29.5462 & 109.7321 & \\
\hline & $7 / 29 / 20086: 00$ & 161 & 211 & 600 & 7.251 & 7.251 & & 0.0616 & $3.85 \mathrm{E}-04$ & 140.8594 & 51.9026 & 192.7620 & \\
\hline & $7 / 29 / 20087: 00$ & 162 & 211 & 700 & 6.869 & 6.869 & & 0.1207 & $8.81 \mathrm{E}-04$ & 322.0564 & 118.6683 & 440.7246 & \\
\hline & 7/29/2008 8:00 & 163 & 211 & 800 & 6.021 & 6.021 & & 0.2785 & 2.47E-03 & 901.6444 & 332.2294 & 1233.8738 & \\
\hline
\end{tabular}




\begin{tabular}{|c|c|c|c|c|c|c|c|c|c|c|c|c|c|}
\hline Tree ID & TS & RN & JD & HR & $\begin{array}{c}\text { Raw } \\
\text { TC_dTA }\end{array}$ & $\begin{array}{c}\text { Fixed } \\
\text { TC_dT } \Delta\end{array}$ & dTM & $\mathbf{K}$ & $\begin{array}{l}\text { Sap } \\
\text { Velocity } \\
(\mathrm{cm} / \mathrm{s})\end{array}$ & $\begin{array}{c}\text { Probed } \\
\text { Drywood F } \\
(\mathrm{g} / \mathrm{hr})\end{array}$ & $\begin{array}{c}\text { Extrapolated } \\
\text { Wetwood F } \\
(\mathrm{g} / \mathrm{hr})\end{array}$ & $\begin{array}{c}\text { Extrapolated } \\
\text { Total Wood F } \\
(\mathrm{g} / \mathrm{hr})\end{array}$ & $\begin{array}{l}\text { Relative } \\
\text { Humidity, } \\
@ 10 \mathrm{~m}(\%)\end{array}$ \\
\hline & 7/29/2008 9:00 & 164 & 211 & 900 & \begin{tabular}{|l|}
4.807 \\
\end{tabular} & 4.807 & & 0.6014 & 6.36E-03 & 2325.7890 & 856.9848 & 3182.7739 & \\
\hline & $7 / 29 / 2008$ 10:00 & 165 & 211 & 1000 & 3.816 & 3.816 & & 1.0173 & $1.22 \mathrm{E}-02$ & 4441.9598 & 1636.7314 & 6078.6913 & \\
\hline & $7 / 29 / 2008$ 11:00 & 166 & 211 & 1100 & 4.018 & 4.018 & & 0.9159 & $1.07 E-02$ & 3903.2791 & 1438.2434 & 5341.5225 & \\
\hline & $7 / 29 / 2008$ 12:00 & 167 & 211 & 1200 & 3.656 & 3.656 & & 1.1056 & $1.35 \mathrm{E}-02$ & 4921.1500 & 1813.2989 & 6734.4490 & \\
\hline & 7/29/2008 13:00 & 168 & 211 & 1300 & 3.703 & 3.703 & & 1.0789 & 1.31E-02 & 4775.1246 & 1759.4929 & 6534.6174 & \\
\hline & $7 / 29 / 2008$ 14:00 & 169 & 211 & 1400 & 3.623 & 3.623 & & 1.1248 & 1.38E-02 & 5026.4473 & 1852.0979 & 6878.5452 & \\
\hline & $7 / 29 / 2008$ 15:00 & 170 & 211 & 1500 & 3.811 & 3.811 & & 1.0199 & $1.22 \mathrm{E}-02$ & 4456.1902 & 1641.9749 & 6098.1651 & \\
\hline & $7 / 29 / 2008$ 16:00 & 171 & 211 & 1600 & 3.911 & 3.911 & & 0.9683 & 1.14E-02 & 4180.0587 & 1540.2286 & 5720.2873 & \\
\hline & 7/29/2008 17:00 & 172 & 211 & 1700 & 4.012 & 4.012 & & 0.9187 & 1.07E-02 & 3918.3162 & 1443.7842 & 5362.1004 & \\
\hline & 7/29/2008 18:00 & 173 & 211 & 1800 & 3.882 & 3.882 & & 0.9830 & 1.17E-02 & 4258.3338 & 1569.0707 & 5827.4045 & \\
\hline & $7 / 29 / 2008$ 19:00 & 174 & 211 & 1900 & 3.884 & 3.884 & & 0.9820 & 1.16E-02 & 4252.8892 & 1567.0645 & 5819.9537 & \\
\hline & $7 / 29 / 200820: 00$ & 175 & 211 & 2000 & 4.416 & 4.416 & & 0.7432 & $8.26 \mathrm{E}-03$ & 3018.1650 & 1112.1050 & 4130.2699 & \\
\hline & 7/29/2008 21:00 & 176 & 211 & 2100 & 5.506 & 5.506 & & 0.3981 & 3.83E-03 & 1399.6276 & 515.7216 & 1915.3492 & \\
\hline & $7 / 29 / 2008$ 22:00 & 177 & 211 & 2200 & 6.466 & 6.466 & & 0.1905 & 1.55E-03 & 565.0103 & 208.1897 & 773.2000 & \\
\hline & 7/29/2008 23:00 & 178 & 211 & 2300 & 6.811 & 6.811 & & 0.1302 & $9.68 E-04$ & 353.6866 & 130.3231 & 484.0096 & \\
\hline & $7 / 30 / 20080: 00$ & 179 & 212 & 0 & 6.884 & 6.884 & 6.88 & 0.1182 & 8.59E-04 & 314.0535 & 115.7195 & 429.7730 & \\
\hline & 7/30/2008 1:00 & 180 & 212 & 100 & 6.859 & 6.859 & & 0.1223 & 8.96E-04 & 327.4320 & 120.6490 & 448.0810 & \\
\hline & 7/30/2008 2:00 & 181 & 212 & 200 & 6.819 & 6.819 & & 0.1289 & $9.56 \mathrm{E}-04$ & 349.2588 & 128.6916 & 477.9503 & \\
\hline & 7/30/2008 3:00 & 182 & 212 & 300 & 6.777 & 6.777 & & 0.1359 & $1.02 \mathrm{E}-03$ & 372.7380 & 137.3430 & 510.0809 & \\
\hline & 7/30/2008 4:00 & 183 & 212 & 400 & 6.748 & 6.748 & & 0.1408 & 1.07E-03 & 389.2875 & 143.4410 & 532.7284 & \\
\hline & 7/30/2008 5:00 & 184 & 212 & 500 & 6.706 & 6.706 & & 0.1479 & 1.13E-03 & 413.7485 & 152.4541 & 566.2026 & \\
\hline & 7/30/2008 6:00 & 185 & 212 & 600 & 6.664 & 6.664 & & 0.1552 & 1.20E-03 & 438.7975 & 161.6840 & 600.4815 & \\
\hline & 7/30/2008 7:00 & 186 & 212 & 700 & 6.632 & 6.632 & & 0.1607 & 1.25E-03 & 458.2810 & 168.8631 & 627.1441 & \\
\hline & $7 / 30 / 20088: 00$ & 187 & 212 & 800 & 6.607 & 6.607 & & 0.1651 & $1.30 \mathrm{E}-03$ & 473.7444 & 174.5609 & 648.3053 & \\
\hline & 7/30/2008 9:00 & 188 & 212 & 900 & 5.53 & 5.53 & & 0.3920 & $3.76 \mathrm{E}-03$ & 1373.4141 & 506.0627 & 1879.4768 & \\
\hline & $7 / 30 / 2008$ 10:00 & 189 & 212 & 1000 & 3.839 & 3.839 & & 1.0052 & 1.20E-02 & 4377.0864 & 1612.8275 & 5989.9139 & \\
\hline & 7/30/2008 11:00 & 190 & 212 & 1100 & 3.055 & 3.055 & & 1.5198 & 1.99E-02 & 7280.9415 & 2682.8126 & 9963.7541 & \\
\hline & $7 / 30 / 2008$ 12:00 & 191 & 212 & 1200 & 3.005 & 3.005 & & 1.5617 & 2.06E-02 & 7528.9818 & 2774.2082 & 10303.1900 & \\
\hline & 7/30/2008 13:00 & 192 & 212 & 1300 & 2.98 & 2.98 & & 1.5832 & 2.09E-02 & 7656.7235 & 2821.2772 & 10478.0007 & \\
\hline & $7 / 30 / 2008$ 14:00 & 193 & 212 & 1400 & 2.988 & 2.988 & & 1.5763 & 2.08E-02 & 7615.5696 & 2806.1132 & 10421.6828 & \\
\hline & 7/30/2008 15:00 & 194 & 212 & 1500 & 3.136 & 3.136 & & 1.4547 & 1.89E-02 & 6899.0357 & 2542.0916 & 9441.1273 & \\
\hline & $7 / 30 / 2008$ 16:00 & 195 & 212 & 1600 & 3.109 & 3.109 & & 1.4760 & 1.92E-02 & 7023.7005 & 2588.0269 & 9611.7274 & \\
\hline & $7 / 30 / 2008$ 17:00 & 196 & 212 & 1700 & 3.135 & 3.135 & & 1.4555 & 1.89E-02 & 6903.6072 & 2543.7761 & 9447.3833 & \\
\hline & $7 / 30 / 2008$ 18:00 & 197 & 212 & 1800 & 3.255 & 3.255 & & 1.3650 & $1.75 \mathrm{E}-02$ & 6378.9094 & 2350.4404 & 8729.3498 & \\
\hline & 7/30/2008 19:00 & 198 & 212 & 1900 & 3.346 & 3.346 & & 1.3007 & 1.64E-02 & 6010.9312 & 2214.8512 & 8225.7824 & \\
\hline & $7 / 30 / 200820: 00$ & 199 & 212 & 2000 & 3.804 & 3.804 & & 1.0237 & 1.22E-02 & 4476.1900 & 1649.3443 & 6125.5342 & \\
\hline & 7/30/2008 21:00 & 200 & 212 & 2100 & 5.001 & 5.001 & & 0.5393 & $5.56 \mathrm{E}-03$ & 2033.6800 & 749.3512 & 2783.0312 & \\
\hline & $7 / 30 / 2008$ 22:00 & 201 & 212 & 2200 & 5.931 & 5.931 & & 0.2979 & 2.68E-03 & 979.5687 & 360.9422 & 1340.5109 & \\
\hline & 7/30/2008 23:00 & 202 & 212 & 2300 & 6.328 & 6.328 & & 0.2165 & $1.81 \mathrm{E}-03$ & 661.2279 & 243.6430 & 904.8709 & \\
\hline & $7 / 31 / 20080: 00$ & 203 & 213 & 0 & 6.534 & 6.534 & 6.66 & 0.1781 & 1.42E-03 & 520.1274 & 191.6517 & 711.7791 & \\
\hline & 7/31/2008 1:00 & 204 & 213 & 100 & 6.628 & 6.628 & & 0.1614 & $1.26 \mathrm{E}-03$ & 460.7409 & 169.7694 & 630.5103 & \\
\hline
\end{tabular}




\begin{tabular}{|c|c|c|c|c|c|c|c|c|c|c|c|c|c|}
\hline Tree ID & TS & RN & JD & HR & $\begin{array}{c}\text { Raw } \\
\text { TC_dTA }\end{array}$ & $\begin{array}{c}\text { Fixed } \\
\text { TC_dT } \Delta\end{array}$ & dTM & $\mathbf{K}$ & $\begin{array}{l}\text { Sap } \\
\text { Velocity } \\
(\mathrm{cm} / \mathrm{s})\end{array}$ & $\begin{array}{c}\text { Probed } \\
\text { Drywood F } \\
(\mathrm{g} / \mathrm{hr})\end{array}$ & $\begin{array}{c}\text { Extrapolated } \\
\text { Wetwood F } \\
(\mathrm{g} / \mathrm{hr})\end{array}$ & $\begin{array}{c}\text { Extrapolated } \\
\text { Total Wood F } \\
(\mathrm{g} / \mathrm{hr})\end{array}$ & $\begin{array}{l}\text { Relative } \\
\text { Humidity, } \\
@ 10 \mathrm{~m}(\%)\end{array}$ \\
\hline & 7/31/2008 2:00 & 205 & 213 & 200 & 6.636 & 6.636 & & 0.1600 & 1.25E-03 & 455.8266 & 167.9587 & 623.7853 & \\
\hline & 7/31/2008 3:00 & 206 & 213 & 300 & 6.632 & 6.632 & & 0.1607 & $1.25 \mathrm{E}-03$ & 458.2810 & 168.8631 & 627.1441 & \\
\hline & 7/31/2008 4:00 & 207 & 213 & 400 & 6.657 & 6.657 & & 0.1564 & $1.21 \mathrm{E}-03$ & 443.0300 & 163.2435 & 606.2735 & \\
\hline & 7/31/2008 5:00 & 208 & 213 & 500 & 6.642 & 6.642 & & 0.1590 & $1.24 \mathrm{E}-03$ & 452.1552 & 166.6059 & 618.7611 & \\
\hline & 7/31/2008 6:00 & 209 & 213 & 600 & 6.63 & 6.63 & & 0.1611 & 1.26E-03 & 459.5103 & 169.3160 & 628.8263 & \\
\hline & 7/31/2008 7:00 & 210 & 213 & 700 & 6.616 & 6.616 & & 0.1635 & $1.28 \mathrm{E}-03$ & 468.1531 & 172.5006 & 640.6537 & \\
\hline & $7 / 31 / 2008$ 8:00 & 211 & 213 & 800 & 6.434 & 6.434 & & 0.1965 & 1.61E-03 & 586.7021 & 216.1824 & 802.8845 & \\
\hline & $7 / 31 / 20089: 00$ & 212 & 213 & 900 & 5.123 & 5.123 & & 0.5026 & 5.10E-03 & 1864.8738 & 687.1511 & 2552.0250 & \\
\hline & $7 / 31 / 2008$ 10:00 & 213 & 213 & 1000 & 3.399 & 3.399 & & 1.2648 & 1.59E-02 & 5807.4998 & 2139.8928 & 7947.3926 & \\
\hline & 7/31/2008 11:00 & 214 & 213 & 1100 & 3.05 & 3.05 & & 1.5239 & $2.00 \mathrm{E}-02$ & 7305.3101 & 2691.7917 & 9997.1018 & \\
\hline & $7 / 31 / 2008$ 12:00 & 215 & 213 & 1200 & 2.846 & 2.846 & & 1.7048 & 2.29E-02 & 8387.1132 & 3090.4043 & 11477.5175 & \\
\hline & 7/31/2008 13:00 & 216 & 213 & 1300 & 2.935 & 2.935 & & 1.6228 & $2.16 \mathrm{E}-02$ & 7893.1905 & 2908.4084 & 10801.5988 & \\
\hline & 7/31/2008 14:00 & 217 & 213 & 1400 & 2.866 & 2.866 & & 1.6860 & 2.26E-02 & 8272.9505 & 3048.3388 & 11321.2892 & \\
\hline & 7/31/2008 15:00 & 218 & 213 & 1500 & 2.955 & 2.955 & & 1.6051 & 2.13E-02 & 7787.0380 & 2869.2943 & 10656.3324 & \\
\hline & $7 / 31 / 2008$ 16:00 & 219 & 213 & 1600 & 3.082 & 3.082 & & 1.4977 & 1.96E-02 & 7150.9772 & 2634.9246 & 9785.9018 & \\
\hline & 7/31/2008 17:00 & 220 & 213 & 1700 & 3.113 & 3.113 & & 1.4729 & 1.92E-02 & 7005.0686 & 2581.1616 & 9586.2302 & \\
\hline & $7 / 31 / 2008$ 18:00 & 221 & 213 & 1800 & 3.187 & 3.187 & & 1.4154 & 1.83E-02 & 6670.4279 & 2457.8564 & 9128.2843 & \\
\hline & $7 / 31 / 2008$ 19:00 & 222 & 213 & 1900 & 3.436 & 3.436 & & 1.2404 & $1.55 \mathrm{E}-02$ & 5669.9585 & 2089.2128 & 7759.1713 & \\
\hline & $7 / 31 / 200820: 00$ & 223 & 213 & 2000 & 4.022 & 4.022 & & 0.9140 & 1.07E-02 & 3893.2853 & 1434.5610 & 5327.8463 & \\
\hline & $7 / 31 / 200821: 00$ & 224 & 213 & 2100 & 4.707 & 4.707 & & 0.6354 & $6.81 E-03$ & 2488.7946 & 917.0476 & 3405.8422 & \\
\hline & $7 / 31 / 200822: 00$ & 225 & 213 & 2200 & 5.521 & 5.521 & & 0.3943 & $3.78 \mathrm{E}-03$ & 1383.2066 & 509.6709 & 1892.8775 & \\
\hline & 7/31/2008 23:00 & 226 & 213 & 2300 & 5.62 & 5.62 & & 0.3698 & 3.50E-03 & 1277.9193 & 470.8756 & 1748.7949 & \\
\hline & $8 / 1 / 20080: 00$ & 227 & 214 & 0 & 5.818 & 5.818 & 6.49 & 0.3231 & 2.96E-03 & 1082.5776 & 398.8980 & 1481.4756 & \\
\hline & 8/1/2008 1:00 & 228 & 214 & 100 & 6.208 & 6.208 & & 0.2400 & $2.05 E-03$ & 750.7164 & 276.6169 & 1027.3333 & \\
\hline & 8/1/2008 2:00 & 229 & 214 & 200 & 6.435 & 6.435 & & 0.1963 & 1.60E-03 & 586.0186 & 215.9306 & 801.9492 & \\
\hline & $8 / 1 / 2008$ 3:00 & 230 & 214 & 300 & 6.419 & 6.419 & & 0.1993 & 1.63E-03 & 596.9974 & 219.9760 & 816.9734 & \\
\hline & 8/1/2008 4:00 & 231 & 214 & 400 & 6.471 & 6.471 & & 0.1896 & $1.54 \mathrm{E}-03$ & 561.6542 & 206.9530 & 768.6073 & \\
\hline & $8 / 1 / 20085: 00$ & 232 & 214 & 500 & 6.493 & 6.493 & & 0.1856 & $1.50 \mathrm{E}-03$ & 546.9932 & 201.5509 & 748.5441 & \\
\hline & 8/1/2008 6:00 & 233 & 214 & 600 & 6.474 & 6.474 & & 0.1891 & $1.53 \mathrm{E}-03$ & 559.6448 & 206.2127 & 765.8575 & \\
\hline & 8/1/2008 7:00 & 234 & 214 & 700 & 6.463 & 6.463 & & 0.1911 & $1.55 \mathrm{E}-03$ & 567.0283 & 208.9332 & 775.9616 & \\
\hline & 8/1/2008 8:00 & 235 & 214 & 800 & 6.207 & 6.207 & & 0.2402 & 2.06E-03 & 751.4857 & 276.9004 & 1028.3860 & \\
\hline & 8/1/2008 9:00 & 236 & 214 & 900 & 4.953 & 4.953 & & 0.5542 & $5.75 E-03$ & 2103.1482 & 774.9482 & 2878.0965 & \\
\hline & 8/1/2008 10:00 & 237 & 214 & 1000 & 3.298 & 3.298 & & 1.3341 & 1.70E-02 & 6201.9871 & 2285.2497 & 8487.2368 & \\
\hline & 8/1/2008 11:00 & 238 & 214 & 1100 & 3.099 & 3.099 & & 1.4840 & 1.93E-02 & 7070.5315 & 2605.2827 & 9675.8142 & \\
\hline & 8/1/2008 12:00 & 239 & 214 & 1200 & 3.018 & 3.018 & & 1.5507 & 2.04E-02 & 7463.5497 & 2750.0984 & 10213.6481 & \\
\hline & 8/1/2008 13:00 & 240 & 214 & 1300 & 3.007 & 3.007 & & 1.5600 & 2.06E-02 & 7518.8715 & 2770.4828 & 10289.3543 & \\
\hline & 8/1/2008 14:00 & 241 & 214 & 1400 & 3.011 & 3.011 & & 1.5566 & $2.05 E-02$ & 7498.6988 & 2763.0498 & 10261.7487 & \\
\hline & 8/1/2008 15:00 & 242 & 214 & 1500 & 3.07 & 3.07 & & 1.5075 & 1.97E-02 & 7208.4027 & 2656.0842 & 9864.4869 & \\
\hline & $8 / 1 / 200816: 00$ & 243 & 214 & 1600 & 3.164 & 3.164 & & 1.4330 & $1.85 \mathrm{E}-02$ & 6772.4344 & 2495.4428 & 9267.8772 & \\
\hline & 8/1/2008 17:00 & 244 & 214 & 1700 & 3.224 & 3.224 & & 1.3877 & 1.78E-02 & 6509.9796 & 2398.7359 & 8908.7155 & \\
\hline & 8/1/2008 18:00 & 245 & 214 & 1800 & 3.285 & 3.285 & & 1.3434 & $1.71 \mathrm{E}-02$ & 6254.8887 & 2304.7424 & 8559.6310 & \\
\hline
\end{tabular}




\begin{tabular}{|c|c|c|c|c|c|c|c|c|c|c|c|c|c|}
\hline Tree ID & TS & RN & JD & HR & $\begin{array}{c}\text { Raw } \\
\text { TC_dTA }\end{array}$ & $\begin{array}{c}\text { Fixed } \\
\text { TC_dT } \Delta\end{array}$ & dTM & $\mathbf{K}$ & $\begin{array}{c}\text { Sap } \\
\text { Velocity } \\
(\mathrm{cm} / \mathrm{s})\end{array}$ & $\begin{array}{c}\text { Probed } \\
\text { Drywood F } \\
(\mathrm{g} / \mathrm{hr})\end{array}$ & $\begin{array}{c}\text { Extrapolated } \\
\text { Wetwood F } \\
(\mathrm{g} / \mathrm{hr})\end{array}$ & $\begin{array}{c}\text { Extrapolated } \\
\text { Total Wood F } \\
(\mathrm{g} / \mathrm{hr})\end{array}$ & $\begin{array}{c}\text { Relative } \\
\text { Humidity, } \\
@ 10 \mathrm{~m}(\%)\end{array}$ \\
\hline & 8/1/2008 19:00 & 246 & 214 & 1900 & 3.45 & 3.45 & & 1.2313 & 1.54E-02 & 5618.8442 & 2070.3787 & 7689.2229 & \\
\hline & 8/1/2008 20:00 & 247 & 214 & 2000 & 4.049 & 4.049 & & 0.9012 & 1.05E-02 & 3826.4679 & 1409.9408 & 5236.4087 & \\
\hline & 8/1/2008 21:00 & 248 & 214 & 2100 & 5.296 & 5.296 & & 0.4535 & $4.50 \mathrm{E}-03$ & 1643.2838 & 605.5017 & 2248.7855 & \\
\hline & $8 / 1 / 2008$ 22:00 & 249 & 214 & 2200 & 6.042 & 6.042 & & 0.2741 & $2.42 \mathrm{E}-03$ & 883.9689 & 325.7165 & 1209.6854 & \\
\hline & 8/1/2008 23:00 & 250 & 214 & 2300 & 6.26 & 6.26 & & 0.2297 & 1.95E-03 & 711.2550 & 262.0765 & 973.3316 & \\
\hline & 8/2/2008 0:00 & 251 & 215 & 0 & 6.305 & 6.305 & 6.51 & 0.2209 & $1.85 \mathrm{E}-03$ & 677.9515 & 249.8052 & 927.7567 & \\
\hline & 8/2/2008 1:00 & 252 & 215 & 100 & 6.409 & 6.409 & & 0.2011 & 1.65E-03 & 603.9065 & 222.5218 & 826.4282 & \\
\hline & $8 / 2 / 20082: 00$ & 253 & 215 & 200 & 6.499 & 6.499 & & 0.1845 & 1.49E-03 & 543.0246 & 200.0886 & 743.1132 & \\
\hline & 8/2/2008 3:00 & 254 & 215 & 300 & 6.514 & 6.514 & & 0.1818 & 1.46E-03 & 533.1587 & 196.4533 & 729.6120 & \\
\hline & $8 / 2 / 20084: 00$ & 255 & 215 & 400 & 6.413 & 6.413 & & 0.2004 & 1.64E-03 & 601.1385 & 221.5018 & 822.6403 & \\
\hline & 8/2/2008 5:00 & 256 & 215 & 500 & 6.362 & 6.362 & & 0.2100 & 1.74E-03 & 636.8705 & 234.6680 & 871.5385 & \\
\hline & $8 / 2 / 20086: 00$ & 257 & 215 & 600 & 6.359 & 6.359 & & 0.2106 & $1.75 E-03$ & 639.0023 & 235.4535 & 874.4558 & \\
\hline & 8/2/2008 7:00 & 258 & 215 & 700 & 6.147 & 6.147 & & 0.2523 & $2.18 \mathrm{E}-03$ & 798.3731 & 294.1770 & 1092.5500 & \\
\hline & $8 / 2 / 20088: 00$ & 259 & 215 & 800 & 5.118 & 5.118 & & 0.5041 & $5.12 \mathrm{E}-03$ & 1871.5808 & 689.6224 & 2561.2032 & \\
\hline & 8/2/2008 9:00 & 260 & 215 & 900 & 3.693 & 3.693 & & 1.0845 & 1.31E-02 & 4805.8138 & 1770.8009 & 6576.6147 & \\
\hline & $8 / 2 / 2008$ 10:00 & 261 & 215 & 1000 & 3.277 & 3.277 & & 1.3491 & 1.72E-02 & 6287.6943 & 2316.8303 & 8604.5246 & \\
\hline & $8 / 2 / 2008$ 11:00 & 262 & 215 & 1100 & 3.153 & 3.153 & & 1.4415 & $1.87 E-02$ & 6821.8501 & 2513.6510 & 9335.5011 & \\
\hline & $8 / 2 / 200812: 00$ & 263 & 215 & 1200 & 3.034 & 3.034 & & 1.5372 & $2.02 \mathrm{E}-02$ & 7383.9331 & 2720.7620 & 10104.6951 & \\
\hline & $8 / 2 / 2008$ 13:00 & 264 & 215 & 1300 & 3.052 & 3.052 & & 1.5223 & 2.00E-02 & 7295.5512 & 2688.1959 & 9983.7471 & \\
\hline & $8 / 2 / 2008$ 14:00 & 265 & 215 & 1400 & 3.079 & 3.079 & & 1.5002 & $1.96 \mathrm{E}-02$ & 7165.2835 & 2640.1961 & 9805.4796 & \\
\hline & $8 / 2 / 2008$ 15:00 & 266 & 215 & 1500 & 3.077 & 3.077 & & 1.5018 & $1.96 \mathrm{E}-02$ & 7174.8396 & 2643.7172 & 9818.5568 & \\
\hline & $8 / 2 / 2008$ 16:00 & 267 & 215 & 1600 & 3.106 & 3.106 & & 1.4784 & 1.93E-02 & 7037.7120 & 2593.1897 & 9630.9017 & \\
\hline & $8 / 2 / 2008$ 17:00 & 268 & 215 & 1700 & 3.124 & 3.124 & & 1.4641 & $1.90 \mathrm{E}-02$ & 6954.1246 & 2562.3902 & 9516.5148 & \\
\hline & $8 / 2 / 200818: 00$ & 269 & 215 & 1800 & 3.228 & 3.228 & & 1.3848 & $1.78 \mathrm{E}-02$ & 6492.8976 & 2392.4417 & 8885.3393 & \\
\hline & $8 / 2 / 2008$ 19:00 & 270 & 215 & 1900 & 3.389 & 3.389 & & 1.2715 & $1.60 \mathrm{E}-02$ & 5845.2963 & 2153.8196 & 7999.1159 & \\
\hline & $8 / 2 / 200820: 00$ & 271 & 215 & 2000 & 3.891 & 3.891 & & 0.9784 & 1.16E-02 & 4233.8875 & 1560.0629 & 5793.9504 & \\
\hline & 8/2/2008 21:00 & 272 & 215 & 2100 & 4.712 & 4.712 & & 0.6337 & 6.79E-03 & 2480.4302 & 913.9655 & 3394.3957 & \\
\hline & $8 / 2 / 200822: 00$ & 273 & 215 & 2200 & 5.68 & 5.68 & & 0.3553 & 3.33E-03 & 1216.6406 & 448.2963 & 1664.9369 & \\
\hline & $8 / 2 / 2008$ 23:00 & 274 & 215 & 2300 & 6.1 & 6.1 & & 0.2620 & $2.29 \mathrm{E}-03$ & 836.1210 & 308.0860 & 1144.2070 & \\
\hline & $8 / 3 / 20080: 00$ & 275 & 216 & 0 & 6.034 & 6.034 & 6.33 & 0.2758 & $2.44 \mathrm{E}-03$ & 890.6801 & 328.1894 & 1218.8696 & \\
\hline & 8/3/2008 1:00 & 276 & 216 & 100 & 5.845 & 5.845 & & 0.3170 & 2.89E-03 & 1057.4262 & 389.6304 & 1447.0566 & \\
\hline & $8 / 3 / 20082: 00$ & 277 & 216 & 200 & 5.962 & 5.962 & & 0.2912 & 2.61E-03 & 952.3254 & 350.9039 & 1303.2292 & \\
\hline & 8/3/2008 3:00 & 278 & 216 & 300 & 6.132 & 6.132 & & 0.2554 & $2.22 \mathrm{E}-03$ & 810.3219 & 298.5798 & 1108.9017 & \\
\hline & 8/3/2008 4:00 & 279 & 216 & 400 & 6.259 & 6.259 & & 0.2299 & $1.95 \mathrm{E}-03$ & 712.0040 & 262.3525 & 974.3565 & \\
\hline & $8 / 3 / 20085: 00$ & 280 & 216 & 500 & 6.33 & 6.33 & & 0.2161 & 1.81E-03 & 659.7831 & 243.1107 & 902.8937 & \\
\hline & $8 / 3 / 20086: 00$ & 281 & 216 & 600 & 6.25 & 6.25 & & 0.2317 & 1.97E-03 & 718.7618 & 264.8426 & 983.6043 & \\
\hline & 8/3/2008 7:00 & 282 & 216 & 700 & 6.033 & 6.033 & & 0.2760 & $2.44 \mathrm{E}-03$ & 891.5210 & 328.4992 & 1220.0202 & \\
\hline & $8 / 3 / 20088: 00$ & 283 & 216 & 800 & 5.208 & 5.208 & & 0.4781 & 4.80E-03 & 1753.5033 & 646.1144 & 2399.6177 & \\
\hline & 8/3/2008 9:00 & 284 & 216 & 900 & 3.599 & 3.599 & & 1.1389 & $1.40 \mathrm{E}-02$ & 5104.5073 & 1880.8607 & 6985.3680 & \\
\hline & $8 / 3 / 200810: 00$ & 285 & 216 & 1000 & 3.024 & 3.024 & & 1.5456 & $2.03 E-02$ & 7433.5760 & 2739.0540 & 10172.6299 & \\
\hline & $8 / 3 / 200811: 00$ & 286 & 216 & 1100 & 2.955 & 2.955 & & 1.6051 & $2.13 \mathrm{E}-02$ & 7787.0380 & 2869.2943 & 10656.3324 & \\
\hline
\end{tabular}




\begin{tabular}{|c|c|c|c|c|c|c|c|c|c|c|c|c|c|}
\hline Tree ID & TS & RN & JD & HR & $\begin{array}{c}\text { Raw } \\
\text { TC_dTA }\end{array}$ & $\begin{array}{c}\text { Fixed } \\
\text { TC_dT } \Delta\end{array}$ & dTM & $\mathbf{K}$ & $\begin{array}{l}\text { Sap } \\
\text { Velocity } \\
(\mathrm{cm} / \mathrm{s})\end{array}$ & $\begin{array}{c}\text { Probed } \\
\text { Drywood F } \\
(\mathrm{g} / \mathrm{hr})\end{array}$ & $\begin{array}{c}\text { Extrapolated } \\
\text { Wetwood F } \\
(\mathrm{g} / \mathrm{hr})\end{array}$ & $\begin{array}{c}\text { Extrapolated } \\
\text { Total Wood F } \\
(\mathrm{g} / \mathrm{hr})\end{array}$ & $\begin{array}{l}\text { Relative } \\
\text { Humidity, } \\
@ 10 \mathrm{~m}(\%)\end{array}$ \\
\hline & 8/3/2008 12:00 & 287 & 216 & 1200 & 2.97 & 2.97 & & 1.5919 & 2.11E-02 & 7708.5366 & 2840.3688 & 10548.9054 & \\
\hline & 8/3/2008 13:00 & 288 & 216 & 1300 & 2.973 & 2.973 & & 1.5893 & $2.10 \mathrm{E}-02$ & 7692.9492 & 2834.6253 & 10527.5745 & \\
\hline & $8 / 3 / 2008$ 14:00 & 289 & 216 & 1400 & 2.985 & 2.985 & & 1.5789 & $2.09 \mathrm{E}-02$ & 7630.9716 & 2811.7884 & 10442.7600 & \\
\hline & 8/3/2008 15:00 & 290 & 216 & 1500 & 3.016 & 3.016 & & 1.5524 & 2.04E-02 & 7473.5725 & 2753.7915 & 10227.3640 & \\
\hline & 8/3/2008 16:00 & 291 & 216 & 1600 & 3.067 & 3.067 & & 1.5099 & 1.98E-02 & 7222.8428 & 2661.4050 & 9884.2478 & \\
\hline & 8/3/2008 17:00 & 292 & 216 & 1700 & 3.12 & 3.12 & & 1.4673 & 1.91E-02 & 6972.6000 & 2569.1979 & 9541.7979 & \\
\hline & 8/3/2008 18:00 & 293 & 216 & 1800 & 3.225 & 3.225 & & 1.3870 & 1.78E-02 & 6505.7044 & 2397.1606 & 8902.8649 & \\
\hline & 8/3/2008 19:00 & 294 & 216 & 1900 & 3.402 & 3.402 & & 1.2628 & 1.59E-02 & 5796.2132 & 2135.7340 & 7931.9471 & \\
\hline & 8/3/2008 20:00 & 295 & 216 & 2000 & 3.718 & 3.718 & & 1.0705 & 1.29E-02 & 4729.4689 & 1742.6701 & 6472.1390 & \\
\hline & 8/3/2008 21:00 & 296 & 216 & 2100 & 4.408 & 4.408 & & 0.7464 & 8.30E-03 & 3033.9884 & 1117.9354 & 4151.9239 & \\
\hline & 8/3/2008 22:00 & 297 & 216 & 2200 & 5.029 & 5.029 & & 0.5307 & 5.46E-03 & 1993.9688 & 734.7188 & 2728.6877 & \\
\hline & 8/3/2008 23:00 & 298 & 216 & 2300 & 5.228 & 5.228 & & 0.4725 & $4.73 \mathrm{E}-03$ & 1728.0091 & 636.7205 & 2364.7295 & \\
\hline & 8/4/2008 0:00 & 299 & 217 & 0 & 5.278 & 5.278 & 5.89 & 0.4585 & $4.56 \mathrm{E}-03$ & 1665.4211 & 613.6587 & 2279.0798 & \\
\hline & 8/4/2008 1:00 & 300 & 217 & 100 & 5.293 & 5.293 & & 0.4544 & 4.51E-03 & 1646.9590 & 606.8559 & 2253.8150 & \\
\hline & $8 / 4 / 2008$ 2:00 & 301 & 217 & 200 & 5.071 & 5.071 & & 0.5180 & 5.30E-03 & 1935.4960 & 713.1733 & 2648.6693 & \\
\hline & 8/4/2008 3:00 & 302 & 217 & 300 & 4.895 & 4.895 & & 0.5726 & 5.99E-03 & 2189.5034 & 806.7676 & 2996.2710 & \\
\hline & 8/4/2008 4:00 & 303 & 217 & 400 & 4.705 & 4.705 & & 0.6361 & 6.82E-03 & 2492.1469 & 918.2828 & 3410.4296 & \\
\hline & $8 / 4 / 20085: 00$ & 304 & 217 & 500 & 4.754 & 4.754 & & 0.6193 & $6.60 \mathrm{E}-03$ & 2411.0696 & 888.4082 & 3299.4778 & \\
\hline & 8/4/2008 6:00 & 305 & 217 & 600 & 5.051 & 5.051 & & 0.5241 & 5.37E-03 & 1963.1782 & 723.3734 & 2686.5516 & \\
\hline & 8/4/2008 7:00 & 306 & 217 & 700 & 5.05 & 5.05 & & 0.5244 & $5.38 E-03$ & 1964.5700 & 723.8862 & 2688.4562 & \\
\hline & 8/4/2008 8:00 & 307 & 217 & 800 & 4.83 & 4.83 & & 0.5938 & $6.26 \mathrm{E}-03$ & 2289.5397 & 843.6280 & 3133.1677 & \\
\hline & 8/4/2008 9:00 & 308 & 217 & 900 & 4.506 & 4.506 & & 0.7084 & 7.78E-03 & 2845.0613 & 1048.3213 & 3893.3826 & \\
\hline & 8/4/2008 10:00 & 309 & 217 & 1000 & 3.739 & 3.739 & & 1.0588 & 1.28E-02 & 4666.3030 & 1719.3953 & 6385.6984 & \\
\hline & 8/4/2008 11:00 & 310 & 217 & 1100 & 3.199 & 3.199 & & 1.4064 & 1.81E-02 & 6617.9034 & 2438.5026 & 9056.4060 & \\
\hline & 8/4/2008 12:00 & 311 & 217 & 1200 & 3.066 & 3.066 & & 1.5108 & 1.98E-02 & 7227.6636 & 2663.1813 & 9890.8450 & \\
\hline & 8/4/2008 13:00 & 312 & 217 & 1300 & 3.063 & 3.063 & & 1.5132 & 1.98E-02 & 7242.1487 & 2668.5186 & 9910.6674 & \\
\hline & 8/4/2008 14:00 & 313 & 217 & 1400 & 3.313 & 3.313 & & 1.3236 & 1.68E-02 & 6141.5664 & 2262.9865 & 8404.5529 & \\
\hline & 8/4/2008 15:00 & 314 & 217 & 1500 & 3.541 & 3.541 & & 1.1740 & 1.45E-02 & 5298.4807 & 1952.3342 & 7250.8149 & \\
\hline & 8/4/2008 16:00 & 315 & 217 & 1600 & 3.144 & 3.144 & & 1.4485 & 1.88E-02 & 6862.5888 & 2528.6620 & 9391.2508 & \\
\hline & 8/4/2008 17:00 & 316 & 217 & 1700 & 3.13 & 3.13 & & 1.4594 & $1.90 \mathrm{E}-02$ & 6926.5171 & 2552.2177 & 9478.7347 & \\
\hline & 8/4/2008 18:00 & 317 & 217 & 1800 & 3.25 & 3.25 & & 1.3686 & 1.75E-02 & 6399.8470 & 2358.1552 & 8758.0022 & \\
\hline & 8/4/2008 19:00 & 318 & 217 & 1900 & 3.472 & 3.472 & & 1.2172 & 1.52E-02 & 5539.5277 & 2041.1529 & 7580.6806 & \\
\hline & 8/4/2008 20:00 & 319 & 217 & 2000 & 3.923 & 3.923 & & 0.9623 & 1.13E-02 & 4148.0865 & 1528.4478 & 5676.5342 & \\
\hline & 8/4/2008 21:00 & 320 & 217 & 2100 & 5.032 & 5.032 & & 0.5298 & $5.44 \mathrm{E}-03$ & 1989.7490 & 733.1639 & 2722.9129 & \\
\hline & 8/4/2008 22:00 & 321 & 217 & 2200 & 5.698 & 5.698 & & 0.3510 & $3.28 \mathrm{E}-03$ & 1198.6179 & 441.6554 & 1640.2733 & \\
\hline & 8/4/2008 23:00 & 322 & 217 & 2300 & 5.893 & 5.893 & & 0.3063 & 2.77E-03 & 1013.5530 & 373.4644 & 1387.0174 & \\
\hline & 8/5/2008 0:00 & 323 & 218 & 0 & 6.068 & 6.068 & 6.32 & 0.2686 & $2.36 \mathrm{E}-03$ & 862.3449 & 317.7487 & 1180.0936 & \\
\hline & 8/5/2008 1:00 & 324 & 218 & 100 & 6.179 & 6.179 & & 0.2458 & $2.12 E-03$ & 773.1869 & 284.8966 & 1058.0835 & \\
\hline & 8/5/2008 2:00 & 325 & 218 & 200 & 6.267 & 6.267 & & 0.2283 & 1.93E-03 & 706.0234 & 260.1488 & 966.1722 & \\
\hline & 8/5/2008 3:00 & 326 & 218 & 300 & 6.211 & 6.211 & & 0.2394 & 2.05E-03 & 748.4109 & 275.7674 & 1024.1783 & \\
\hline & 8/5/2008 4:00 & 327 & 218 & 400 & 6.088 & 6.088 & & 0.2645 & $2.31 \mathrm{E}-03$ & 845.9049 & 311.6911 & 1157.5959 & \\
\hline
\end{tabular}




\begin{tabular}{|c|c|c|c|c|c|c|c|c|c|c|c|c|c|}
\hline Tree ID & TS & RN & JD & HR & $\begin{array}{c}\text { Raw } \\
\text { TC_dTA }\end{array}$ & $\begin{array}{c}\text { Fixed } \\
\text { TC_dT } \Delta\end{array}$ & dTM & $\mathbf{K}$ & $\begin{array}{c}\text { Sap } \\
\text { Velocity } \\
(\mathrm{cm} / \mathrm{s})\end{array}$ & $\begin{array}{c}\text { Probed } \\
\text { Drywood F } \\
\text { (g/hr) }\end{array}$ & $\begin{array}{c}\text { Extrapolated } \\
\text { Wetwood F } \\
(\mathrm{g} / \mathrm{hr})\end{array}$ & $\begin{array}{c}\text { Extrapolated } \\
\text { Total Wood F } \\
(\mathrm{g} / \mathrm{hr})\end{array}$ & $\begin{array}{l}\text { Relative } \\
\text { Humidity, } \\
\text { @10m (\%) }\end{array}$ \\
\hline & 8/5/2008 5:00 & 328 & 218 & 500 & \begin{tabular}{|l|} 
\\
6.076 \\
\end{tabular} & 6.076 & & 0.2670 & $2.34 \mathrm{E}-03$ & 855.7488 & 315.3182 & 1171.0670 & \\
\hline & 8/5/2008 6:00 & 329 & 218 & 600 & 6.15 & 6.15 & & 0.2517 & $2.18 \mathrm{E}-03$ & 795.9943 & 293.3005 & 1089.2947 & \\
\hline & 8/5/2008 7:00 & 330 & 218 & 700 & 6.178 & 6.178 & & 0.2460 & $2.12 \mathrm{E}-03$ & 773.9677 & 285.1843 & 1059.1521 & \\
\hline & 8/5/2008 8:00 & 331 & 218 & 800 & 5.905 & 5.905 & & 0.3036 & $2.74 \mathrm{E}-03$ & 1002.7503 & 369.4840 & 1372.2343 & \\
\hline & 8/5/2008 9:00 & 332 & 218 & 900 & 4.752 & 4.752 & & 0.6199 & 6.61E-03 & 2414.3364 & 889.6119 & 3303.9483 & \\
\hline & 8/5/2008 10:00 & 333 & 218 & 1000 & 3.524 & 3.524 & & 1.1844 & 1.47E-02 & 5356.8074 & 1973.8259 & 7330.6332 & \\
\hline & 8/5/2008 11:00 & 334 & 218 & 1100 & 3.567 & 3.567 & & 1.1581 & $1.43 \mathrm{E}-02$ & 5210.5790 & 1919.9450 & 7130.5239 & \\
\hline & 8/5/2008 12:00 & 335 & 218 & 1200 & 3.291 & 3.291 & & 1.3391 & $1.70 \mathrm{E}-02$ & 6230.4101 & 2295.7228 & 8526.1329 & \\
\hline & 8/5/2008 13:00 & 336 & 218 & 1300 & 2.899 & 2.899 & & 1.6554 & 2.21E-02 & 8088.6526 & 2980.4304 & 11069.0830 & \\
\hline & $8 / 5 / 2008$ 14:00 & 337 & 218 & 1400 & 3.261 & 3.261 & & 1.3606 & 1.74E-02 & 6353.8860 & 2341.2200 & 8695.1060 & \\
\hline & 8/5/2008 15:00 & 338 & 218 & 1500 & 3.552 & 3.552 & & 1.1672 & 1.44E-02 & 5261.1008 & 1938.5608 & 7199.6615 & \\
\hline & 8/5/2008 16:00 & 339 & 218 & 1600 & 3.496 & 3.496 & & 1.2019 & 1.49E-02 & 5454.3768 & 2009.7773 & 7464.1541 & \\
\hline & 8/5/2008 17:00 & 340 & 218 & 1700 & 3.149 & 3.149 & & 1.4446 & 1.87E-02 & 6839.9218 & 2520.3099 & 9360.2317 & \\
\hline & 8/5/2008 18:00 & 341 & 218 & 1800 & 2.99 & 2.99 & & 1.5746 & 2.08E-02 & 7605.3220 & 2802.3373 & 10407.6593 & \\
\hline & 8/5/2008 19:00 & 342 & 218 & 1900 & 3.149 & 3.149 & & 1.4446 & 1.87E-02 & 6839.9218 & 2520.3099 & 9360.2317 & \\
\hline & 8/5/2008 20:00 & 343 & 218 & 2000 & 3.872 & 3.872 & & 0.9881 & 1.17E-02 & 4285.6609 & 1579.1399 & 5864.8008 & \\
\hline & 8/5/2008 21:00 & 344 & 218 & 2100 & 5.356 & 5.356 & & 0.4373 & 4.30E-03 & 1570.9627 & 578.8535 & 2149.8163 & \\
\hline & 8/5/2008 22:00 & 345 & 218 & 2200 & 6.078 & 6.078 & & 0.2665 & 2.34E-03 & 854.1040 & 314.7122 & 1168.8161 & \\
\hline & 8/5/2008 23:00 & 346 & 218 & 2300 & 6.316 & 6.316 & & 0.2188 & 1.83E-03 & 669.9283 & 246.8489 & 916.7771 & \\
\hline & 8/6/2008 0:00 & 347 & 219 & 0 & 6.493 & 6.493 & 6.52 & 0.1856 & 1.50E-03 & 546.9932 & 201.5509 & 748.5441 & \\
\hline & 8/6/2008 1:00 & 348 & 219 & 100 & 6.52 & 6.52 & & 0.1807 & 1.45E-03 & 529.2346 & 195.0074 & 724.2420 & \\
\hline & 8/6/2008 2:00 & 349 & 219 & 200 & 6.52 & 6.52 & & 0.1807 & $1.45 \mathrm{E}-03$ & 529.2346 & 195.0074 & 724.2420 & \\
\hline & 8/6/2008 3:00 & 350 & 219 & 300 & 6.508 & 6.508 & & 0.1829 & $1.47 \mathrm{E}-03$ & 537.0955 & 197.9039 & 734.9994 & \\
\hline & 8/6/2008 4:00 & 351 & 219 & 400 & 6.497 & 6.497 & & 0.1849 & $1.49 \mathrm{E}-03$ & 544.3460 & 200.5755 & 744.9215 & \\
\hline & 8/6/2008 5:00 & 352 & 219 & 500 & 6.462 & 6.462 & & 0.1913 & 1.55E-03 & 567.7017 & 209.1814 & 776.8830 & \\
\hline & 8/6/2008 6:00 & 353 & 219 & 600 & 6.441 & 6.441 & & 0.1952 & 1.59E-03 & 581.9255 & 214.4224 & 796.3479 & \\
\hline & $8 / 6 / 20087: 00$ & 354 & 219 & 700 & 6.433 & 6.433 & & 0.1966 & 1.61E-03 & 587.3859 & 216.4344 & 803.8203 & \\
\hline & 8/6/2008 8:00 & 355 & 219 & 800 & 6.419 & 6.419 & & 0.1993 & 1.63E-03 & 596.9974 & 219.9760 & 816.9734 & \\
\hline & 8/6/2008 9:00 & 356 & 219 & 900 & 6.132 & 6.132 & & 0.2554 & $2.22 \mathrm{E}-03$ & 810.3219 & 298.5798 & 1108.9017 & \\
\hline & 8/6/2008 10:00 & 357 & 219 & 1000 & 4.162 & 4.162 & & 0.8496 & $9.74 \mathrm{E}-03$ & 3558.4830 & 1311.1963 & 4869.6793 & \\
\hline & 8/6/2008 11:00 & 358 & 219 & 1100 & 2.876 & 2.876 & & 1.6766 & $2.25 E-02$ & 8216.5734 & 3027.5655 & 11244.1389 & \\
\hline & 8/6/2008 12:00 & 359 & 219 & 1200 & 3.236 & 3.236 & & 1.3789 & 1.77E-02 & 6458.8854 & 2379.9092 & 8838.7946 & \\
\hline & 8/6/2008 13:00 & 360 & 219 & 1300 & 3.59 & 3.59 & & 1.1443 & $1.40 \mathrm{E}-02$ & 5134.1076 & 1891.7675 & 7025.8751 & \\
\hline & $8 / 6 / 2008$ 14:00 & 361 & 219 & 1400 & 3.25 & 3.25 & & 1.3686 & 1.75E-02 & 6399.8470 & 2358.1552 & 8758.0022 & \\
\hline & 8/6/2008 15:00 & 362 & 219 & 1500 & 2.899 & 2.899 & & 1.6554 & 2.21E-02 & 8088.6526 & 2980.4304 & 11069.0830 & \\
\hline & 8/6/2008 16:00 & 363 & 219 & 1600 & 2.897 & 2.897 & & 1.6572 & $2.22 \mathrm{E}-02$ & 8099.6807 & 2984.4939 & 11084.1746 & \\
\hline & 8/6/2008 17:00 & 364 & 219 & 1700 & 2.833 & 2.833 & & 1.7173 & $2.32 \mathrm{E}-02$ & 8462.3428 & 3118.1242 & 11580.4670 & \\
\hline & 8/6/2008 18:00 & 365 & 219 & 1800 & 3.156 & 3.156 & & 1.4392 & $1.86 \mathrm{E}-02$ & 6808.3322 & 2508.6700 & 9317.0023 & \\
\hline & 8/6/2008 19:00 & 366 & 219 & 1900 & 3.352 & 3.352 & & 1.2965 & $1.64 \mathrm{E}-02$ & 5987.5117 & 2206.2218 & 8193.7335 & \\
\hline & 8/6/2008 20:00 & 367 & 219 & 2000 & 3.753 & 3.753 & & 1.0512 & 1.27E-02 & 4624.6729 & 1704.0558 & 6328.7287 & \\
\hline & 8/6/2008 21:00 & 368 & 219 & 2100 & 4.803 & 4.803 & & 0.6027 & $6.38 \mathrm{E}-03$ & 2332.1397 & 859.3248 & 3191.4645 & \\
\hline
\end{tabular}




\begin{tabular}{|c|c|c|c|c|c|c|c|c|c|c|c|c|c|}
\hline Tree ID & TS & RN & JD & HR & $\begin{array}{c}\text { Raw } \\
\text { TC_dTA }\end{array}$ & $\begin{array}{c}\text { Fixed } \\
\text { TC_dT } \Delta\end{array}$ & dTM & $\mathbf{K}$ & $\begin{array}{l}\text { Sap } \\
\text { Velocity } \\
(\mathrm{cm} / \mathrm{s})\end{array}$ & $\begin{array}{c}\text { Probed } \\
\text { Drywood F } \\
(\mathrm{g} / \mathrm{hr})\end{array}$ & $\begin{array}{c}\text { Extrapolated } \\
\text { Wetwood F } \\
(\mathrm{g} / \mathrm{hr})\end{array}$ & $\begin{array}{c}\text { Extrapolated } \\
\text { Total Wood F } \\
(\mathrm{g} / \mathrm{hr})\end{array}$ & $\begin{array}{l}\text { Relative } \\
\text { Humidity, } \\
@ 10 \mathrm{~m}(\%)\end{array}$ \\
\hline & 8/6/2008 22:00 & 369 & 219 & 2200 & \begin{tabular}{|r|}
5.919 \\
\end{tabular} & 5.919 & & 0.3006 & $2.71 \mathrm{E}-03$ & 990.2299 & 364.8706 & 1355.1005 & \\
\hline & 8/6/2008 23:00 & 370 & 219 & 2300 & 6.252 & 6.252 & & 0.2313 & 1.96E-03 & 717.2573 & 264.2882 & 981.5455 & \\
\hline & $8 / 7 / 20080: 00$ & 371 & 220 & 0 & 6.419 & 6.419 & 6.50 & 0.1993 & $1.63 \mathrm{E}-03$ & 596.9974 & 219.9760 & 816.9734 & \\
\hline & 8/7/2008 1:00 & 372 & 220 & 100 & 6.497 & 6.497 & & 0.1849 & 1.49E-03 & 544.3460 & 200.5755 & 744.9215 & \\
\hline & 8/7/2008 2:00 & 373 & 220 & 200 & 6.496 & 6.496 & & 0.1850 & 1.49E-03 & 545.0073 & 200.8191 & 745.8264 & \\
\hline & 8/7/2008 3:00 & 374 & 220 & 300 & 6.485 & 6.485 & & 0.1870 & $1.51 \mathrm{E}-03$ & 552.3046 & 203.5080 & 755.8125 & \\
\hline & 8/7/2008 4:00 & 375 & 220 & 400 & 6.456 & 6.456 & & 0.1924 & $1.56 \mathrm{E}-03$ & 571.7494 & 210.6728 & 782.4223 & \\
\hline & 8/7/2008 5:00 & 376 & 220 & 500 & 6.437 & 6.437 & & 0.1959 & 1.60E-03 & 584.6528 & 215.4273 & 800.0802 & \\
\hline & 8/7/2008 6:00 & 377 & 220 & 600 & 6.42 & 6.42 & & 0.1991 & 1.63E-03 & 596.3085 & 219.7221 & 816.0307 & \\
\hline & 8/7/2008 7:00 & 378 & 220 & 700 & 6.41 & 6.41 & & 0.2009 & 1.65E-03 & 603.2139 & 222.2666 & 825.4805 & \\
\hline & 8/7/2008 8:00 & 379 & 220 & 800 & 6.406 & 6.406 & & 0.2017 & 1.66E-03 & 605.9863 & 223.2881 & 829.2744 & \\
\hline & 8/7/2008 9:00 & 380 & 220 & 900 & 5.696 & 5.696 & & 0.3515 & 3.29E-03 & 1200.6123 & 442.3903 & 1643.0026 & \\
\hline & 8/7/2008 10:00 & 381 & 220 & 1000 & 3.876 & 3.876 & & 0.9861 & 1.17E-02 & 4274.7092 & 1575.1045 & 5849.8137 & \\
\hline & 8/7/2008 11:00 & 382 & 220 & 1100 & 2.785 & 2.785 & & 1.7641 & $2.39 E-02$ & 8747.3239 & 3223.1314 & 11970.4554 & \\
\hline & 8/7/2008 12:00 & 383 & 220 & 1200 & 2.715 & 2.715 & & 1.8354 & $2.51 E-02$ & 9184.3364 & 3384.1576 & 12568.4940 & \\
\hline & 8/7/2008 13:00 & 384 & 220 & 1300 & 2.733 & 2.733 & & 1.8167 & 2.48E-02 & 9069.4380 & 3341.8210 & 12411.2590 & \\
\hline & $8 / 7 / 2008$ 14:00 & 385 & 220 & 1400 & 2.834 & 2.834 & & 1.7163 & $2.31 E-02$ & 8456.5269 & 3115.9813 & 11572.5081 & \\
\hline & 8/7/2008 15:00 & 386 & 220 & 1500 & 2.92 & 2.92 & & 1.6363 & 2.18E-02 & 7973.9385 & 2938.1616 & 10912.1001 & \\
\hline & 8/7/2008 16:00 & 387 & 220 & 1600 & 2.954 & 2.954 & & 1.6060 & 2.13E-02 & 7792.3052 & 2871.2351 & 10663.5403 & \\
\hline & 8/7/2008 17:00 & 388 & 220 & 1700 & 2.96 & 2.96 & & 1.6007 & $2.12 \mathrm{E}-02$ & 7760.7659 & 2859.6138 & 10620.3797 & \\
\hline & 8/7/2008 18:00 & 389 & 220 & 1800 & 3.064 & 3.064 & & 1.5124 & 1.98E-02 & 7237.3166 & 2666.7382 & 9904.0548 & \\
\hline & 8/7/2008 19:00 & 390 & 220 & 1900 & 3.326 & 3.326 & & 1.3145 & 1.67E-02 & 6089.7316 & 2243.8868 & 8333.6184 & \\
\hline & 8/7/2008 20:00 & 391 & 220 & 2000 & 3.756 & 3.756 & & 1.0495 & 1.26E-02 & 4615.8016 & 1700.7870 & 6316.5886 & \\
\hline & 8/7/2008 21:00 & 392 & 220 & 2100 & 4.818 & 4.818 & & 0.5978 & $6.32 \mathrm{E}-03$ & 2308.3959 & 850.5759 & 3158.9718 & \\
\hline & 8/7/2008 22:00 & 393 & 220 & 2200 & 5.574 & 5.574 & & 0.3811 & 3.63E-03 & 1326.1811 & 488.6587 & 1814.8398 & \\
\hline & 8/7/2008 23:00 & 394 & 220 & 2300 & 6.031 & 6.031 & & 0.2764 & 2.44E-03 & 893.2039 & 329.1194 & 1222.3233 & \\
\hline & 8/8/2008 0:00 & 395 & 221 & 0 & 6.282 & 6.282 & 6.53 & 0.2254 & 1.90E-03 & 694.8763 & 256.0414 & 950.9177 & \\
\hline & 8/8/2008 1:00 & 396 & 221 & 100 & 6.311 & 6.311 & & 0.2198 & $1.84 \mathrm{E}-03$ & 673.5695 & 248.1905 & 921.7601 & \\
\hline & 8/8/2008 2:00 & 397 & 221 & 200 & 6.465 & 6.465 & & 0.1907 & $1.55 \mathrm{E}-03$ & 565.6826 & 208.4374 & 774.1200 & \\
\hline & 8/8/2008 3:00 & 398 & 221 & 300 & 6.528 & 6.528 & & 0.1792 & $1.43 E-03$ & 524.0221 & 193.0867 & 717.1088 & \\
\hline & 8/8/2008 4:00 & 399 & 221 & 400 & 6.52 & 6.52 & & 0.1807 & 1.45E-03 & 529.2346 & 195.0074 & 724.2420 & \\
\hline & 8/8/2008 5:00 & 400 & 221 & 500 & 6.495 & 6.495 & & 0.1852 & 1.49E-03 & 545.6689 & 201.0629 & 746.7318 & \\
\hline & 8/8/2008 6:00 & 401 & 221 & 600 & 6.471 & 6.471 & & 0.1896 & 1.54E-03 & 561.6542 & 206.9530 & 768.6073 & \\
\hline & 8/8/2008 7:00 & 402 & 221 & 700 & 6.462 & 6.462 & & 0.1913 & 1.55E-03 & 567.7017 & 209.1814 & 776.8830 & \\
\hline & 8/8/2008 8:00 & 403 & 221 & 800 & 6.33 & 6.33 & & 0.2161 & 1.81E-03 & 659.7831 & 243.1107 & 902.8937 & \\
\hline & 8/8/2008 9:00 & 404 & 221 & 900 & 4.94 & 4.94 & & 0.5583 & 5.81E-03 & 2122.2709 & 781.9944 & 2904.2653 & \\
\hline & 8/8/2008 10:00 & 405 & 221 & 1000 & 3.225 & 3.225 & & 1.3870 & 1.78E-02 & 6505.7044 & 2397.1606 & 8902.8649 & \\
\hline & 8/8/2008 11:00 & 406 & 221 & 1100 & 2.908 & 2.908 & & 1.6472 & $2.20 \mathrm{E}-02$ & 8039.2489 & 2962.2266 & 11001.4754 & \\
\hline & $8 / 8 / 200812: 00$ & 407 & 221 & 1200 & 2.734 & 2.734 & & 1.8157 & $2.48 \mathrm{E}-02$ & 9063.1071 & 3339.4882 & 12402.5953 & \\
\hline & 8/8/2008 13:00 & 408 & 221 & 1300 & 2.718 & 2.718 & & 1.8322 & $2.51 E-02$ & 9165.0620 & 3377.0556 & 12542.1177 & \\
\hline & 8/8/2008 14:00 & 409 & 221 & 1400 & 2.837 & 2.837 & & 1.7134 & $2.31 \mathrm{E}-02$ & 8439.1083 & 3109.5630 & 11548.6714 & \\
\hline
\end{tabular}




\begin{tabular}{|c|c|c|c|c|c|c|c|c|c|c|c|c|c|}
\hline Tree ID & TS & RN & JD & HR & $\begin{array}{c}\text { Raw } \\
\text { TC_dTA }\end{array}$ & $\begin{array}{c}\text { Fixed } \\
\text { TC_dT } \Delta\end{array}$ & dTM & $\mathbf{K}$ & $\begin{array}{l}\text { Sap } \\
\text { Velocity } \\
(\mathrm{cm} / \mathrm{s})\end{array}$ & $\begin{array}{c}\text { Probed } \\
\text { Drywood F } \\
(\mathrm{g} / \mathrm{hr})\end{array}$ & $\begin{array}{c}\text { Extrapolated } \\
\text { Wetwood F } \\
(\mathrm{g} / \mathrm{hr})\end{array}$ & $\begin{array}{c}\text { Extrapolated } \\
\text { Total Wood F } \\
(\mathrm{g} / \mathrm{hr})\end{array}$ & $\begin{array}{l}\text { Relative } \\
\text { Humidity, } \\
@ 10 \mathrm{~m}(\%)\end{array}$ \\
\hline & 8/8/2008 15:00 & 410 & 221 & 1500 & 2.886 & 2.886 & & 1.6674 & 2.23E-02 & 8160.6588 & 3006.9626 & \begin{tabular}{|l|}
11167.6214 \\
\end{tabular} & \\
\hline & 8/8/2008 16:00 & 411 & 221 & 1600 & 2.916 & 2.916 & & 1.6399 & $2.19 \mathrm{E}-02$ & 7995.6378 & 2946.1572 & 10941.7949 & \\
\hline & 8/8/2008 17:00 & 412 & 221 & 1700 & 3.016 & 3.016 & & 1.5524 & $2.04 \mathrm{E}-02$ & 7473.5725 & 2753.7915 & 10227.3640 & \\
\hline & 8/8/2008 18:00 & 413 & 221 & 1800 & 3.226 & 3.226 & & 1.3862 & 1.78E-02 & 6501.4323 & 2395.5864 & 8897.0187 & \\
\hline & 8/8/2008 19:00 & 414 & 221 & 1900 & 3.286 & 3.286 & & 1.3427 & 1.71E-02 & 6250.8015 & 2303.2364 & 8554.0378 & \\
\hline & 8/8/2008 20:00 & 415 & 221 & 2000 & 3.77 & 3.77 & & 1.0419 & 1.25E-02 & 4574.6308 & 1685.6168 & 6260.2477 & \\
\hline & 8/8/2008 21:00 & 416 & 221 & 2100 & 4.685 & 4.685 & & 0.6431 & $6.91 E-03$ & 2525.8734 & 930.7100 & 3456.5834 & \\
\hline & 8/8/2008 22:00 & 417 & 221 & 2200 & 5.418 & 5.418 & & 0.4208 & 4.10E-03 & 1498.5428 & 552.1689 & 2050.7117 & \\
\hline & 8/8/2008 23:00 & 418 & 221 & 2300 & 5.705 & 5.705 & & 0.3493 & $3.26 \mathrm{E}-03$ & 1191.6534 & 439.0892 & 1630.7426 & \\
\hline & 8/9/2008 0:00 & 419 & 222 & 0 & 5.763 & 5.763 & 6.38 & 0.3358 & 3.11E-03 & 1134.8880 & 418.1728 & 1553.0608 & \\
\hline & 8/9/2008 1:00 & 420 & 222 & 100 & 5.773 & 5.773 & & 0.3334 & 3.08E-03 & 1125.2683 & 414.6282 & 1539.8965 & \\
\hline & 8/9/2008 2:00 & 421 & 222 & 200 & 5.829 & 5.829 & & 0.3206 & $2.93 E-03$ & 1072.2892 & 395.1070 & 1467.3962 & \\
\hline & 8/9/2008 3:00 & 422 & 222 & 300 & 5.854 & 5.854 & & 0.3150 & 2.87E-03 & 1049.1185 & 386.5693 & 1435.6878 & \\
\hline & 8/9/2008 4:00 & 423 & 222 & 400 & 5.85 & 5.85 & & 0.3159 & 2.88E-03 & 1052.8062 & 387.9281 & 1440.7342 & \\
\hline & 8/9/2008 5:00 & 424 & 222 & 500 & 5.75 & 5.75 & & 0.3388 & $3.14 \mathrm{E}-03$ & 1147.4666 & 422.8077 & 1570.2743 & \\
\hline & 8/9/2008 6:00 & 425 & 222 & 600 & 5.731 & 5.731 & & 0.3432 & 3.19E-03 & 1166.0004 & 429.6368 & 1595.6372 & \\
\hline & 8/9/2008 7:00 & 426 & 222 & 700 & 5.455 & 5.455 & & 0.4112 & 3.98E-03 & 1456.4100 & 536.6442 & 1993.0542 & \\
\hline & 8/9/2008 8:00 & 427 & 222 & 800 & 4.909 & 4.909 & & 0.5681 & 5.93E-03 & 2168.4123 & 798.9961 & 2967.4084 & \\
\hline & 8/9/2008 9:00 & 428 & 222 & 900 & 5.237 & 5.237 & & 0.4699 & 4.70E-03 & 1716.6229 & 632.5250 & 2349.1480 & \\
\hline & 8/9/2008 10:00 & 429 & 222 & 1000 & 5.11 & 5.11 & & 0.5065 & $5.15 E-03$ & 1882.3486 & 693.5901 & 2575.9387 & \\
\hline & 8/9/2008 11:00 & 430 & 222 & 1100 & 4.608 & 4.608 & & 0.6706 & $7.28 \mathrm{E}-03$ & 2659.2686 & 979.8622 & 3639.1308 & \\
\hline & 8/9/2008 12:00 & 431 & 222 & 1200 & 3.753 & 3.753 & & 1.0512 & 1.27E-02 & 4624.6729 & 1704.0558 & 6328.7287 & \\
\hline & 8/9/2008 13:00 & 432 & 222 & 1300 & 3.406 & 3.406 & & 1.2601 & 1.58E-02 & 5781.2016 & 2130.2026 & 7911.4042 & \\
\hline & 8/9/2008 14:00 & 433 & 222 & 1400 & 2.935 & 2.935 & & 1.6228 & $2.16 \mathrm{E}-02$ & 7893.1905 & 2908.4084 & 10801.5988 & \\
\hline & 8/9/2008 15:00 & 434 & 222 & 1500 & 2.82 & 2.82 & & 1.7298 & $2.34 \mathrm{E}-02$ & 8538.3934 & 3146.1467 & 11684.5401 & \\
\hline & 8/9/2008 16:00 & 435 & 222 & 1600 & 2.969 & 2.969 & & 1.5928 & 2.11E-02 & 7713.7407 & 2842.2864 & 10556.0271 & \\
\hline & 8/9/2008 17:00 & 436 & 222 & 1700 & 3.111 & 3.111 & & 1.4744 & 1.92E-02 & 7014.3774 & 2584.5916 & 9598.9690 & \\
\hline & 8/9/2008 18:00 & 437 & 222 & 1800 & 3.18 & 3.18 & & 1.4208 & 1.83E-02 & 6701.2865 & 2469.2268 & 9170.5133 & \\
\hline & 8/9/2008 19:00 & 438 & 222 & 1900 & 3.38 & 3.38 & & 1.2775 & 1.61E-02 & 5879.5439 & 2166.4389 & 8045.9827 & \\
\hline & 8/9/2008 20:00 & 439 & 222 & 2000 & 3.914 & 3.914 & & 0.9668 & 1.14E-02 & 4172.0430 & 1537.2750 & 5709.3180 & \\
\hline & 8/9/2008 21:00 & 440 & 222 & 2100 & 5.213 & 5.213 & & 0.4767 & 4.78E-03 & 1747.1048 & 643.7567 & 2390.8615 & \\
\hline & 8/9/2008 22:00 & 441 & 222 & 2200 & 6.126 & 6.126 & & 0.2566 & 2.23E-03 & 815.1272 & 300.3504 & 1115.4775 & \\
\hline & 8/9/2008 23:00 & 442 & 222 & 2300 & 6.375 & 6.375 & & 0.2075 & $1.72 \mathrm{E}-03$ & 627.6712 & 231.2784 & 858.9496 & \\
\hline & 8/10/2008 0:00 & 443 & 223 & 0 & 6.417 & 6.417 & 6.44 & 0.1996 & $1.64 \mathrm{E}-03$ & 598.3763 & 220.4841 & 818.8604 & \\
\hline & 8/10/2008 1:00 & 444 & 223 & 100 & 6.441 & 6.441 & & 0.1952 & 1.59E-03 & 581.9255 & 214.4224 & 796.3479 & \\
\hline & 8/10/2008 2:00 & 445 & 223 & 200 & 6.429 & 6.429 & & 0.1974 & 1.61E-03 & 590.1248 & 217.4436 & 807.5684 & \\
\hline & 8/10/2008 3:00 & 446 & 223 & 300 & 6.415 & 6.415 & & 0.2000 & 1.64E-03 & 599.7567 & 220.9927 & 820.7494 & \\
\hline & 8/10/2008 4:00 & 447 & 223 & 400 & 6.407 & 6.407 & & 0.2015 & 1.66E-03 & 605.2927 & 223.0325 & 828.3252 & \\
\hline & $8 / 10 / 20085: 00$ & 448 & 223 & 500 & 6.377 & 6.377 & & 0.2072 & 1.71E-03 & 626.2615 & 230.7589 & 857.0204 & \\
\hline & 8/10/2008 6:00 & 449 & 223 & 600 & 6.357 & 6.357 & & 0.2109 & 1.75E-03 & 640.4254 & 235.9779 & 876.4032 & \\
\hline & 8/10/2008 7:00 & 450 & 223 & 700 & 6.354 & 6.354 & & 0.2115 & $1.76 \mathrm{E}-03$ & 642.5628 & 236.7655 & 879.3282 & \\
\hline
\end{tabular}




\begin{tabular}{|c|c|c|c|c|c|c|c|c|c|c|c|c|c|}
\hline Tree ID & TS & RN & JD & HR & $\begin{array}{c}\text { Raw } \\
\text { TC_dTA }\end{array}$ & $\begin{array}{c}\text { Fixed } \\
\text { TC_dT } \Delta\end{array}$ & dTM & $\mathbf{K}$ & $\begin{array}{l}\text { Sap } \\
\text { Velocity } \\
(\mathrm{cm} / \mathrm{s})\end{array}$ & $\begin{array}{c}\text { Probed } \\
\text { Drywood F } \\
(\mathrm{g} / \mathrm{hr})\end{array}$ & $\begin{array}{c}\text { Extrapolated } \\
\text { Wetwood F } \\
(\mathrm{g} / \mathrm{hr})\end{array}$ & $\begin{array}{c}\text { Extrapolated } \\
\text { Total Wood F } \\
(\mathrm{g} / \mathrm{hr})\end{array}$ & $\begin{array}{l}\text { Relative } \\
\text { Humidity, } \\
@ 10 \mathrm{~m}(\%)\end{array}$ \\
\hline & 8/10/2008 8:00 & 451 & 223 & 800 & $\begin{array}{r}6.342 \\
\end{array}$ & 6.342 & & 0.2138 & $1.78 \mathrm{E}-03$ & 651.1460 & 239.9281 & \begin{tabular}{|r|}
891.0741 \\
\end{tabular} & \\
\hline & 8/10/2008 9:00 & 452 & 223 & 900 & 5.519 & 5.519 & & 0.3948 & $3.79 \mathrm{E}-03$ & 1385.3888 & 510.4750 & 1895.8638 & \\
\hline & $8 / 10 / 2008$ 10:00 & 453 & 223 & 1000 & 3.924 & 3.924 & & 0.9618 & 1.13E-02 & 4145.4330 & 1527.4700 & 5672.9031 & \\
\hline & 8/10/2008 11:00 & 454 & 223 & 1100 & 2.776 & 2.776 & & 1.7731 & 2.41E-02 & 8802.0560 & 3243.2986 & 12045.3546 & \\
\hline & $8 / 10 / 200812: 00$ & 455 & 223 & 1200 & 2.74 & 2.74 & & 1.8095 & 2.47E-02 & 9025.2357 & 3325.5337 & 12350.7694 & \\
\hline & 8/10/2008 13:00 & 456 & 223 & 1300 & 2.726 & 2.726 & & 1.8239 & 2.49E-02 & 9113.9082 & 3358.2069 & 12472.1151 & \\
\hline & $8 / 10 / 2008$ 14:00 & 457 & 223 & 1400 & 2.787 & 2.787 & & 1.7621 & $2.39 \mathrm{E}-02$ & 8735.2179 & 3218.6707 & 11953.8886 & \\
\hline & $8 / 10 / 2008$ 15:00 & 458 & 223 & 1500 & 2.976 & 2.976 & & 1.5867 & $2.10 \mathrm{E}-02$ & 7677.3991 & 2828.8956 & 10506.2947 & \\
\hline & 8/10/2008 16:00 & 459 & 223 & 1600 & 2.935 & 2.935 & & 1.6228 & $2.16 \mathrm{E}-02$ & 7893.1905 & 2908.4084 & 10801.5988 & \\
\hline & 8/10/2008 17:00 & 460 & 223 & 1700 & 3.098 & 3.098 & & 1.4848 & 1.94E-02 & 7075.2344 & 2607.0156 & 9682.2501 & \\
\hline & 8/10/2008 18:00 & 461 & 223 & 1800 & 5.08 & 5.08 & & 0.5154 & $5.26 \mathrm{E}-03$ & 1923.1341 & 708.6183 & 2631.7524 & \\
\hline & $8 / 10 / 2008$ 19:00 & 462 & 223 & 1900 & 5.927 & 5.927 & & 0.2988 & $2.69 \mathrm{E}-03$ & 983.1152 & 362.2490 & 1345.3642 & \\
\hline & 8/10/2008 20:00 & 463 & 223 & 2000 & 5.805 & 5.805 & & 0.3261 & $3.00 \mathrm{E}-03$ & 1094.8107 & 403.4055 & 1498.2162 & \\
\hline & 8/10/2008 21:00 & 464 & 223 & 2100 & 5.692 & 5.692 & & 0.3524 & 3.30E-03 & 1204.6072 & 443.8623 & 1648.4695 & \\
\hline & $8 / 10 / 2008$ 22:00 & 465 & 223 & 2200 & 5.852 & 5.852 & & 0.3154 & 2.88E-03 & 1050.9614 & 387.2483 & 1438.2098 & \\
\hline & 8/10/2008 23:00 & 466 & 223 & 2300 & 5.756 & 5.756 & & 0.3374 & $3.12 \mathrm{E}-03$ & 1141.6508 & 420.6647 & 1562.3155 & \\
\hline & $8 / 11 / 20080: 00$ & 467 & 224 & 0 & 5.71 & 5.71 & 6.23 & 0.3482 & $3.25 E-03$ & 1186.6938 & 437.2617 & 1623.9556 & \\
\hline & 8/11/2008 1:00 & 468 & 224 & 100 & 5.872 & 5.872 & & 0.3110 & 2.83E-03 & 1032.6163 & 380.4887 & 1413.1051 & \\
\hline & 8/11/2008 2:00 & 469 & 224 & 200 & 6.09 & 6.09 & & 0.2640 & 2.31E-03 & 844.2701 & 311.0887 & 1155.3588 & \\
\hline & 8/11/2008 3:00 & 470 & 224 & 300 & 6.165 & 6.165 & & 0.2487 & $2.15 E-03$ & 784.1550 & 288.9380 & 1073.0931 & \\
\hline & 8/11/2008 4:00 & 471 & 224 & 400 & 6.202 & 6.202 & & 0.2412 & 2.07E-03 & 755.3380 & 278.3198 & 1033.6579 & \\
\hline & 8/11/2008 5:00 & 472 & 224 & 500 & 6.168 & 6.168 & & 0.2481 & 2.14E-03 & 781.7981 & 288.0696 & 1069.8677 & \\
\hline & 8/11/2008 6:00 & 473 & 224 & 600 & 6.197 & 6.197 & & 0.2422 & 2.08E-03 & 759.2003 & 279.7430 & 1038.9433 & \\
\hline & 8/11/2008 7:00 & 474 & 224 & 700 & 6.226 & 6.226 & & 0.2364 & $2.02 E-03$ & 736.9368 & 271.5395 & 1008.4763 & \\
\hline & 8/11/2008 8:00 & 475 & 224 & 800 & 6.141 & 6.141 & & 0.2535 & 2.20E-03 & 803.1416 & 295.9340 & 1099.0756 & \\
\hline & 8/11/2008 9:00 & 476 & 224 & 900 & 5.331 & 5.331 & & 0.4440 & 4.38E-03 & 1600.8245 & 589.8567 & 2190.6813 & \\
\hline & $8 / 11 / 2008$ 10:00 & 477 & 224 & 1000 & 3.873 & 3.873 & & 0.9876 & 1.17E-02 & 4282.9203 & 1578.1301 & 5861.0504 & \\
\hline & 8/11/2008 11:00 & 478 & 224 & 1100 & 3.047 & 3.047 & & 1.5264 & 2.00E-02 & 7319.9770 & 2697.1961 & 10017.1731 & \\
\hline & $8 / 11 / 2008$ 12:00 & 479 & 224 & 1200 & 2.995 & 2.995 & & 1.5703 & 2.07E-02 & 7579.7742 & 2792.9237 & 10372.6979 & \\
\hline & 8/11/2008 13:00 & 480 & 224 & 1300 & 3.207 & 3.207 & & 1.4004 & 1.80E-02 & 6583.1484 & 2425.6964 & 9008.8448 & \\
\hline & 8/11/2008 14:00 & 481 & 224 & 1400 & 3.031 & 3.031 & & 1.5398 & 2.02E-02 & 7398.7850 & 2726.2345 & 10125.0196 & \\
\hline & 8/11/2008 15:00 & 482 & 224 & 1500 & 3.007 & 3.007 & & 1.5600 & 2.06E-02 & 7518.8715 & 2770.4828 & 10289.3543 & \\
\hline & $8 / 11 / 2008$ 16:00 & 483 & 224 & 1600 & 3.218 & 3.218 & & 1.3922 & 1.79E-02 & 6535.6981 & 2408.2124 & 8943.9105 & \\
\hline & $8 / 11 / 2008$ 17:00 & 484 & 224 & 1700 & 3.295 & 3.295 & & 1.3363 & $1.70 \mathrm{E}-02$ & 6214.1506 & 2289.7316 & 8503.8822 & \\
\hline & 8/11/2008 18:00 & 485 & 224 & 1800 & 3.515 & 3.515 & & 1.1900 & 1.47E-02 & 5387.9635 & 1985.3060 & 7373.2695 & \\
\hline & $8 / 11 / 2008$ 19:00 & 486 & 224 & 1900 & 3.611 & 3.611 & & 1.1318 & 1.39E-02 & 5065.3194 & 1866.4211 & 6931.7405 & \\
\hline & 8/11/2008 20:00 & 487 & 224 & 2000 & 4.187 & 4.187 & & 0.8385 & $9.58 \mathrm{E}-03$ & 3501.6277 & 1290.2467 & 4791.8745 & \\
\hline & 8/11/2008 21:00 & 488 & 224 & 2100 & 4.911 & 4.911 & & 0.5675 & 5.92E-03 & 2165.4122 & 797.8907 & 2963.3029 & \\
\hline & $8 / 11 / 200822: 00$ & 489 & 224 & 2200 & 5.558 & 5.558 & & 0.3850 & $3.68 \mathrm{E}-03$ & 1343.2344 & 494.9423 & 1838.1767 & \\
\hline & 8/11/2008 23:00 & 490 & 224 & 2300 & 5.826 & 5.826 & & 0.3213 & 2.94E-03 & 1075.0894 & 396.1388 & 1471.2283 & \\
\hline & 8/12/2008 0:00 & 491 & 225 & 0 & 5.976 & 5.976 & 6.32 & 0.2882 & 2.57E-03 & 940.1617 & 346.4219 & 1286.5836 & \\
\hline
\end{tabular}




\begin{tabular}{|c|c|c|c|c|c|c|c|c|c|c|c|c|c|}
\hline Tree ID & TS & RN & JD & HR & $\begin{array}{c}\text { Raw } \\
\text { TC_dTA }\end{array}$ & $\begin{array}{c}\text { Fixed } \\
\text { TC_dT } \Delta\end{array}$ & dTM & $\mathbf{K}$ & $\begin{array}{l}\text { Sap } \\
\text { Velocity } \\
(\mathrm{cm} / \mathrm{s})\end{array}$ & $\begin{array}{c}\text { Probed } \\
\text { Drywood F } \\
(\mathrm{g} / \mathrm{hr})\end{array}$ & $\begin{array}{c}\text { Extrapolated } \\
\text { Wetwood F } \\
(\mathrm{g} / \mathrm{hr})\end{array}$ & $\begin{array}{c}\text { Extrapolated } \\
\text { Total Wood F } \\
(\mathrm{g} / \mathrm{hr})\end{array}$ & $\begin{array}{l}\text { Relative } \\
\text { Humidity, } \\
@ 10 \mathrm{~m}(\%)\end{array}$ \\
\hline & 8/12/2008 1:00 & 492 & 225 & 100 & 6.02 & 6.02 & & 0.2787 & $2.47 \mathrm{E}-03$ & 902.4908 & 332.5413 & 1235.0321 & \\
\hline & 8/12/2008 2:00 & 493 & 225 & 200 & 6.101 & 6.101 & & 0.2618 & $2.29 \mathrm{E}-03$ & 835.3084 & 307.7866 & 1143.0950 & \\
\hline & 8/12/2008 3:00 & 494 & 225 & 300 & 6.22 & 6.22 & & 0.2376 & 2.03E-03 & 741.5158 & 273.2268 & 1014.7426 & \\
\hline & 8/12/2008 4:00 & 495 & 225 & 400 & 6.262 & 6.262 & & 0.2293 & $1.94 \mathrm{E}-03$ & 709.7583 & 261.5251 & 971.2834 & \\
\hline & 8/12/2008 5:00 & 496 & 225 & 500 & 6.291 & 6.291 & & 0.2237 & 1.88E-03 & 688.2295 & 253.5923 & 941.8218 & \\
\hline & 8/12/2008 6:00 & 497 & 225 & 600 & 6.317 & 6.317 & & 0.2186 & 1.83E-03 & 669.2012 & 246.5809 & 915.7821 & \\
\hline & 8/12/2008 7:00 & 498 & 225 & 700 & 6.26 & 6.26 & & 0.2297 & 1.95E-03 & 711.2550 & 262.0765 & 973.3316 & \\
\hline & 8/12/2008 8:00 & 499 & 225 & 800 & 6.22 & 6.22 & & 0.2376 & 2.03E-03 & 741.5158 & 273.2268 & 1014.7426 & \\
\hline & 8/12/2008 9:00 & 500 & 225 & 900 & 5.519 & 5.519 & & 0.3948 & 3.79E-03 & 1385.3888 & 510.4750 & 1895.8638 & \\
\hline & $8 / 12 / 2008$ 10:00 & 501 & 225 & 1000 & 4.346 & 4.346 & & 0.7713 & 8.64E-03 & 3159.1333 & 1164.0476 & 4323.1810 & \\
\hline & $8 / 12 / 2008$ 11:00 & 502 & 225 & 1100 & 3.041 & 3.041 & & 1.5314 & 2.01E-02 & 7349.4143 & 2708.0429 & 10057.4572 & \\
\hline & $8 / 12 / 2008$ 12:00 & 503 & 225 & 1200 & 2.82 & 2.82 & & 1.7298 & 2.34E-02 & 8538.3934 & 3146.1467 & 11684.5401 & \\
\hline & $8 / 12 / 2008$ 13:00 & 504 & 225 & 1300 & 2.75 & 2.75 & & 1.7993 & $2.45 E-02$ & 8962.5495 & 3302.4357 & 12264.9852 & \\
\hline & $8 / 12 / 2008$ 14:00 & 505 & 225 & 1400 & 2.783 & 2.783 & & 1.7661 & $2.40 \mathrm{E}-02$ & 8759.4505 & 3227.5997 & 11987.0502 & \\
\hline & $8 / 12 / 2008$ 15:00 & 506 & 225 & 1500 & 2.85 & 2.85 & & 1.7011 & $2.29 E-02$ & 8364.1289 & 3081.9353 & 11446.0642 & \\
\hline & $8 / 12 / 2008$ 16:00 & 507 & 225 & 1600 & 2.915 & 2.915 & & 1.6408 & 2.19E-02 & 8001.0736 & 2948.1601 & 10949.2338 & \\
\hline & $8 / 12 / 2008$ 17:00 & 508 & 225 & 1700 & 3.002 & 3.002 & & 1.5643 & $2.06 \mathrm{E}-02$ & 7544.1773 & 2779.8073 & 10323.9845 & \\
\hline & $8 / 12 / 2008$ 18:00 & 509 & 225 & 1800 & 3.086 & 3.086 & & 1.4945 & 1.95E-02 & 7131.9536 & 2627.9150 & 9759.8686 & \\
\hline & $8 / 12 / 2008$ 19:00 & 510 & 225 & 1900 & 3.326 & 3.326 & & 1.3145 & 1.67E-02 & 6089.7316 & 2243.8868 & 8333.6184 & \\
\hline & $8 / 12 / 200820: 00$ & 511 & 225 & 2000 & 3.879 & 3.879 & & 0.9845 & 1.17E-02 & 4266.5137 & 1572.0847 & 5838.5984 & \\
\hline & $8 / 12 / 2008$ 21:00 & 512 & 225 & 2100 & 5.272 & 5.272 & & 0.4602 & $4.58 \mathrm{E}-03$ & 1672.8462 & 616.3946 & 2289.2408 & \\
\hline & $8 / 12 / 2008$ 22:00 & 513 & 225 & 2200 & 6.082 & 6.082 & & 0.2657 & 2.33E-03 & 850.8193 & 313.5019 & 1164.3212 & \\
\hline & $8 / 12 / 2008$ 23:00 & 514 & 225 & 2300 & 6.305 & 6.305 & & 0.2209 & 1.85E-03 & 677.9515 & 249.8052 & 927.7567 & \\
\hline & 8/13/2008 0:00 & 515 & 226 & 0 & 6.394 & 6.394 & 6.44 & 0.2039 & $1.68 E-03$ & 614.3386 & 226.3657 & 840.7042 & \\
\hline & 8/13/2008 1:00 & 516 & 226 & 100 & 6.351 & 6.351 & & 0.2121 & $1.76 \mathrm{E}-03$ & 644.7035 & 237.5543 & 882.2578 & \\
\hline & 8/13/2008 2:00 & 517 & 226 & 200 & 6.305 & 6.305 & & 0.2209 & 1.85E-03 & 677.9515 & 249.8052 & 927.7567 & \\
\hline & 8/13/2008 3:00 & 518 & 226 & 300 & 6.377 & 6.377 & & 0.2072 & 1.71E-03 & 626.2615 & 230.7589 & 857.0204 & \\
\hline & 8/13/2008 4:00 & 519 & 226 & 400 & 6.363 & 6.363 & & 0.2098 & $1.74 \mathrm{E}-03$ & 636.1606 & 234.4065 & 870.5671 & \\
\hline & $8 / 13 / 20085: 00$ & 520 & 226 & 500 & 6.364 & 6.364 & & 0.2096 & 1.74E-03 & 635.4511 & 234.1450 & 869.5962 & \\
\hline & 8/13/2008 6:00 & 521 & 226 & 600 & 6.442 & 6.442 & & 0.1950 & 1.59E-03 & 581.2446 & 214.1715 & 795.4161 & \\
\hline & $8 / 13 / 2008$ 7:00 & 522 & 226 & 700 & 6.436 & 6.436 & & 0.1961 & 1.60E-03 & 585.3355 & 215.6789 & 801.0145 & \\
\hline & 8/13/2008 8:00 & 523 & 226 & 800 & 6.182 & 6.182 & & 0.2452 & $2.11 E-03$ & 770.8468 & 284.0344 & 1054.8812 & \\
\hline & 8/13/2008 9:00 & 524 & 226 & 900 & 4.946 & 4.946 & & 0.5564 & 5.78E-03 & 2113.4285 & 778.7362 & 2892.1648 & \\
\hline & 8/13/2008 10:00 & 525 & 226 & 1000 & 3.44 & 3.44 & & 1.2378 & 1.55E-02 & 5655.3031 & 2083.8127 & 7739.1158 & \\
\hline & 8/13/2008 11:00 & 526 & 226 & 1100 & 3.13 & 3.13 & & 1.4594 & 1.90E-02 & 6926.5171 & 2552.2177 & 9478.7347 & \\
\hline & $8 / 13 / 2008$ 12:00 & 527 & 226 & 1200 & 2.775 & 2.775 & & 1.7741 & $2.41 E-02$ & 8808.1632 & 3245.5489 & 12053.7121 & \\
\hline
\end{tabular}




\begin{tabular}{|c|c|c|c|c|c|c|c|c|c|c|c|c|c|}
\hline Tree ID & TS & RN & JD & HR & $\begin{array}{c}\text { Raw } \\
\text { TC_dTA }\end{array}$ & $\begin{array}{c}\text { Fixed } \\
\text { TC_dT } \Delta\end{array}$ & dTM & $\mathbf{K}$ & $\begin{array}{l}\text { Sap } \\
\text { Velocity } \\
(\mathrm{cm} / \mathrm{s})\end{array}$ & $\begin{array}{l}\text { Probed } \\
\text { Drywood F } \\
\text { (g/hr) }\end{array}$ & $\begin{array}{c}\text { Extrapolated } \\
\text { Wetwood F } \\
(\mathrm{g} / \mathrm{hr})\end{array}$ & $\begin{array}{c}\text { Extrapolated } \\
\text { Total Wood F } \\
\text { (g/hr) }\end{array}$ & $\begin{array}{l}\text { Relative } \\
\text { Humidity, } \\
@ 10 m(\%)\end{array}$ \\
\hline & 7/22/2008 13:00 & 0 & 204 & 1300 & 4.924 & 4.924 & 9.40 & 0.9090 & 1.06E-02 & 1810.4596 & 667.1011 & 2477.5607 & \\
\hline \multirow[t]{2}{*}{ W606EC1 } & $7 / 22 / 2008$ 14:00 & 1 & 204 & 1400 & 5.478 & 5.478 & & 0.7160 & $7.89 \mathrm{E}-03$ & 1349.4313 & 497.2257 & 1846.6571 & \\
\hline & $7 / 22 / 2008$ 15:00 & 2 & 204 & 1500 & 5.425 & 5.425 & & 0.7327 & $8.11 \mathrm{E}-03$ & 1388.4319 & 511.5963 & 1900.0282 & \\
\hline \multirow[t]{2}{*}{ Probe \# } & 7/22/2008 16:00 & 3 & 204 & 1600 & 5.368 & 5.368 & & 0.7511 & $8.37 \mathrm{E}-03$ & 1431.4732 & 527.4558 & 1958.9290 & \\
\hline & 7/22/2008 17:00 & 4 & 204 & 1700 & 5.422 & 5.422 & & 0.7337 & 8.13E-03 & 1390.6686 & 512.4204 & 1903.0890 & \\
\hline \multirow[t]{4}{*}{14} & $7 / 22 / 2008$ 18:00 & 5 & 204 & 1800 & 5.47 & 5.47 & & 0.7185 & $7.92 \mathrm{E}-03$ & 1355.2565 & 499.3721 & 1854.6286 & \\
\hline & $7 / 22 / 2008$ 19:00 & 6 & 204 & 1900 & 5.594 & 5.594 & & 0.6804 & 7.41E-03 & 1267.3524 & 466.9821 & 1734.3344 & \\
\hline & 7/22/2008 20:00 & 7 & 204 & 2000 & 5.959 & 5.959 & & 0.5774 & $6.05 E-03$ & 1035.6358 & 381.6013 & 1417.2371 & \\
\hline & 7/22/2008 21:00 & 8 & 204 & 2100 & 7.395 & 7.395 & & 0.2711 & $2.39 \mathrm{E}-03$ & 408.3444 & 150.4629 & \begin{tabular}{|l|}
558.8072 \\
\end{tabular} & \\
\hline \multirow[t]{2}{*}{$\mathrm{DBH}(\mathrm{cm})$} & $7 / 22 / 2008$ 22:00 & 9 & 204 & 2200 & 8.91 & 8.91 & & 0.0550 & 3.35E-04 & 57.2952 & 21.1116 & 78.4069 & \\
\hline & 7/22/2008 23:00 & 10 & 204 & 2300 & 9.4 & 9.4 & & 0.0000 & $0.00 \mathrm{E}+00$ & 0.0000 & 0.0000 & 0.0000 & \\
\hline \multirow[t]{2}{*}{9.7} & 7/23/2008 0:00 & 11 & 205 & 0 & 9.77 & 9.77 & 9.99 & -0.0379 & $0.00 \mathrm{E}+00$ & 0.0000 & 0.0000 & 0.0000 & \\
\hline & 7/23/2008 1:00 & 12 & 205 & 100 & 9.92 & 9.92 & & -0.0524 & $0.00 \mathrm{E}+00$ & 0.0000 & 0.0000 & 0.0000 & \\
\hline Day Count & $7 / 23 / 20082: 00$ & 13 & 205 & 200 & 9.95 & 9.95 & & -0.0553 & $0.00 \mathrm{E}+00$ & 0.0000 & 0.0000 & 0.0000 & \\
\hline \multirow[t]{27}{*}{35} & 7/23/2008 3:00 & 14 & 205 & 300 & 9.99 & 9.99 & & -0.0591 & $0.00 \mathrm{E}+00$ & 0.0000 & 0.0000 & 0.0000 & \\
\hline & 7/23/2008 4:00 & 15 & 205 & 400 & 9.95 & 9.95 & & -0.0553 & $0.00 \mathrm{E}+00$ & 0.0000 & 0.0000 & 0.0000 & \\
\hline & 7/23/2008 5:00 & 16 & 205 & 500 & 9.81 & 9.81 & & -0.0418 & $0.00 E+00$ & 0.0000 & 0.0000 & 0.0000 & \\
\hline & 7/23/2008 6:00 & 17 & 205 & 600 & 9.59 & 9.59 & & -0.0198 & $0.00 \mathrm{E}+00$ & 0.0000 & 0.0000 & 0.0000 & \\
\hline & 7/23/2008 7:00 & 18 & 205 & 700 & 9.07 & 9.07 & & 0.0364 & 2.01E-04 & 34.4558 & 12.6960 & 47.1518 & \\
\hline & 7/23/2008 8:00 & 19 & 205 & 800 & 7.84 & 7.84 & & 0.1990 & 1.63E-03 & 279.0101 & 102.8070 & 381.8172 & \\
\hline & 7/23/2008 9:00 & 20 & 205 & 900 & 6.369 & 6.369 & & 0.4759 & $4.77 \mathrm{E}-03$ & 816.2200 & 300.7531 & 1116.9731 & \\
\hline & 7/23/2008 10:00 & 21 & 205 & 1000 & 5.673 & 5.673 & & 0.6570 & 7.09E-03 & 1213.9102 & 447.2902 & 1661.2003 & \\
\hline & $7 / 23 / 2008$ 11:00 & 22 & 205 & 1100 & 5.61 & 5.61 & & 0.6756 & 7.34E-03 & 1256.3720 & 462.9361 & 1719.3081 & \\
\hline & $7 / 23 / 2008$ 12:00 & 23 & 205 & 1200 & 5.497 & 5.497 & & 0.7100 & 7.81E-03 & 1335.6833 & 492.1600 & 1827.8433 & \\
\hline & $7 / 23 / 2008$ 13:00 & 24 & 205 & 1300 & 5.461 & 5.461 & & 0.7213 & 7.96E-03 & 1361.8358 & 501.7964 & 1863.6323 & \\
\hline & $7 / 23 / 2008$ 14:00 & 25 & 205 & 1400 & 5.454 & 5.454 & & 0.7235 & 7.99E-03 & 1366.9723 & 503.6890 & 1870.6613 & \\
\hline & $7 / 23 / 2008$ 15:00 & 26 & 205 & 1500 & 5.428 & 5.428 & & 0.7318 & 8.10E-03 & 1386.1984 & 510.7733 & 1896.9717 & \\
\hline & 7/23/2008 16:00 & 27 & 205 & 1600 & 5.552 & 5.552 & & 0.6931 & $7.58 \mathrm{E}-03$ & 1296.5634 & 477.7454 & 1774.3088 & \\
\hline & 7/23/2008 17:00 & 28 & 205 & 1700 & 5.906 & 5.906 & & 0.5916 & $6.24 \mathrm{E}-03$ & 1066.9767 & 393.1495 & 1460.1262 & \\
\hline & $7 / 23 / 2008$ 18:00 & 29 & 205 & 1800 & 6.531 & 6.531 & & 0.4393 & 4.32E-03 & 739.6274 & 272.5309 & 1012.1583 & \\
\hline & $7 / 23 / 2008$ 19:00 & 30 & 205 & 1900 & 6.48 & 6.48 & & 0.4506 & 4.46E-03 & 763.1750 & 281.2075 & 1044.3825 & \\
\hline & $7 / 23 / 200820: 00$ & 31 & 205 & 2000 & 6.917 & 6.917 & & 0.3590 & 3.37E-03 & 576.8518 & 212.5529 & 789.4047 & \\
\hline & $7 / 23 / 200821: 00$ & 32 & 205 & 2100 & 7.898 & 7.898 & & 0.1902 & $1.54 \mathrm{E}-03$ & 263.8905 & 97.2359 & 361.1264 & \\
\hline & $7 / 23 / 200822: 00$ & 33 & 205 & 2200 & 8.59 & 8.59 & & 0.0943 & $6.50 \mathrm{E}-04$ & 111.2721 & 41.0005 & 152.2725 & \\
\hline & $7 / 23 / 2008$ 23:00 & 34 & 205 & 2300 & 8.74 & 8.74 & & 0.0755 & 4.95E-04 & 84.6535 & 31.1923 & 115.8458 & \\
\hline & $7 / 24 / 20080: 00$ & 35 & 206 & 0 & 8.72 & 8.72 & 9.92 & 0.0780 & 5.15E-04 & 88.0703 & 32.4513 & 120.5216 & \\
\hline & 7/24/2008 1:00 & 36 & 206 & 100 & 8.84 & 8.84 & & 0.0633 & $3.99 E-04$ & 68.1904 & 25.1262 & 93.3166 & \\
\hline & 7/24/2008 2:00 & 37 & 206 & 200 & 8.95 & 8.95 & & 0.0503 & $3.00 \mathrm{E}-04$ & 51.3094 & 18.9060 & 70.2154 & \\
\hline & 7/24/2008 3:00 & 38 & 206 & 300 & 9.13 & 9.13 & & 0.0296 & $1.56 \mathrm{E}-04$ & 26.6966 & 9.8369 & 36.5335 & \\
\hline & 7/24/2008 4:00 & 39 & 206 & 400 & 9.27 & 9.27 & & 0.0140 & 6.23E-05 & 10.6555 & 3.9263 & 14.5818 & \\
\hline & 7/24/2008 5:00 & 40 & 206 & 500 & 9.4 & 9.4 & & 0.0000 & $0.00 \mathrm{E}+00$ & 0.0000 & 0.0000 & 0.0000 & \\
\hline
\end{tabular}




\begin{tabular}{|c|c|c|c|c|c|c|c|c|c|c|c|c|c|}
\hline \multirow[t]{42}{*}{ Tree ID } & TS & RN & JD & HR & $\begin{array}{c}\text { Raw } \\
\text { TC_dTA }\end{array}$ & $\begin{array}{c}\text { Fixed } \\
\text { TC_dT } \Delta\end{array}$ & dTM & $\mathbf{K}$ & $\begin{array}{l}\text { Sap } \\
\text { Velocity } \\
\text { (cm/s) }\end{array}$ & $\begin{array}{c}\text { Probed } \\
\text { Drywood F } \\
\text { (g/hr) }\end{array}$ & $\begin{array}{c}\text { Extrapolated } \\
\text { Wetwood F } \\
(\mathrm{g} / \mathrm{hr})\end{array}$ & $\begin{array}{c}\text { Extrapolated } \\
\text { Total Wood F } \\
(\mathrm{g} / \mathrm{hr})\end{array}$ & $\begin{array}{c}\text { Relative } \\
\text { Humidity, } \\
@ 10 \mathrm{~m}(\%)\end{array}$ \\
\hline & 7/24/2008 6:00 & 41 & 206 & 600 & 9.48 & 9.48 & & -0.0084 & $0.00 \mathrm{E}+00$ & 0.0000 & 0.0000 & 0.0000 & \\
\hline & 7/24/2008 7:00 & 42 & 206 & 700 & 9.43 & 9.43 & & -0.0032 & $0.00 \mathrm{E}+00$ & 0.0000 & 0.0000 & 0.0000 & \\
\hline & 7/24/2008 8:00 & 43 & 206 & 800 & 9.06 & 9.06 & & 0.0375 & $2.09 \mathrm{E}-04$ & 35.7942 & 13.1891 & 48.9833 & \\
\hline & $7 / 24 / 20089: 00$ & 44 & 206 & 900 & 8.37 & 8.37 & & 0.1231 & 9.03E-04 & 154.4238 & 56.9006 & 211.3244 & \\
\hline & $7 / 24 / 2008$ 10:00 & 45 & 206 & 1000 & 8.44 & 8.44 & & 0.1137 & 8.19E-04 & 140.1635 & 51.6461 & 191.8097 & \\
\hline & 7/24/2008 11:00 & 46 & 206 & 1100 & 6.271 & 6.271 & & 0.4990 & $5.06 \mathrm{E}-03$ & 865.1857 & 318.7955 & 1183.9811 & \\
\hline & $7 / 24 / 2008$ 12:00 & 47 & 206 & 1200 & 5.61 & 5.61 & & 0.6756 & 7.34E-03 & 1256.3720 & 462.9361 & 1719.3081 & \\
\hline & 7/24/2008 13:00 & 48 & 206 & 1300 & 5.924 & 5.924 & & 0.5868 & $6.17 \mathrm{E}-03$ & 1056.2500 & 389.1970 & 1445.4471 & \\
\hline & $7 / 24 / 2008$ 14:00 & 49 & 206 & 1400 & 5.788 & 5.788 & & 0.6240 & $6.66 \mathrm{E}-03$ & 1139.4664 & 419.8598 & 1559.3262 & \\
\hline & 7/24/2008 15:00 & 50 & 206 & 1500 & 5.492 & 5.492 & & 0.7116 & 7.83E-03 & 1339.2894 & 493.4887 & 1832.7782 & \\
\hline & $7 / 24 / 2008$ 16:00 & 51 & 206 & 1600 & 5.48 & 5.48 & & 0.7153 & $7.88 \mathrm{E}-03$ & 1347.9784 & 496.6904 & 1844.6688 & \\
\hline & $7 / 24 / 2008$ 17:00 & 52 & 206 & 1700 & 5.651 & 5.651 & & 0.6634 & $7.18 \mathrm{E}-03$ & 1228.5995 & 452.7027 & 1681.3022 & \\
\hline & 7/24/2008 18:00 & 53 & 206 & 1800 & 5.604 & 5.604 & & 0.6774 & 7.37E-03 & 1260.4802 & 464.4499 & 1724.9301 & \\
\hline & $7 / 24 / 2008$ 19:00 & 54 & 206 & 1900 & 5.807 & 5.807 & & 0.6187 & $6.59 \mathrm{E}-03$ & 1127.5344 & 415.4632 & 1542.9977 & \\
\hline & $7 / 24 / 200820: 00$ & 55 & 206 & 2000 & 6.772 & 6.772 & & 0.3881 & 3.71E-03 & 634.9403 & 233.9568 & 868.8972 & \\
\hline & $7 / 24 / 200821: 00$ & 56 & 206 & 2100 & 8.33 & 8.33 & & 0.1285 & $9.51 \mathrm{E}-04$ & 162.7961 & 59.9856 & 222.7817 & \\
\hline & $7 / 24 / 200822: 00$ & 57 & 206 & 2200 & 9.53 & 9.53 & & -0.0136 & $0.00 \mathrm{E}+00$ & 0.0000 & 0.0000 & 0.0000 & \\
\hline & $7 / 24 / 200823: 00$ & 58 & 206 & 2300 & 9.92 & 9.92 & & -0.0524 & $0.00 \mathrm{E}+00$ & 0.0000 & 0.0000 & 0.0000 & \\
\hline & $7 / 25 / 20080: 00$ & 59 & 207 & 0 & 10.08 & 10.08 & 10.14 & -0.0675 & $0.00 \mathrm{E}+00$ & 0.0000 & 0.0000 & 0.0000 & \\
\hline & 7/25/2008 1:00 & 60 & 207 & 100 & 10.13 & 10.13 & & -0.0721 & $0.00 \mathrm{E}+00$ & 0.0000 & 0.0000 & 0.0000 & \\
\hline & 7/25/2008 2:00 & 61 & 207 & 200 & 10.13 & 10.13 & & -0.0721 & $0.00 E+00$ & 0.0000 & 0.0000 & 0.0000 & \\
\hline & 7/25/2008 3:00 & 62 & 207 & 300 & 10.13 & 10.13 & & -0.0721 & $0.00 \mathrm{E}+00$ & 0.0000 & 0.0000 & 0.0000 & \\
\hline & 7/25/2008 4:00 & 63 & 207 & 400 & 10.14 & 10.14 & & -0.0730 & $0.00 \mathrm{E}+00$ & 0.0000 & 0.0000 & 0.0000 & \\
\hline & 7/25/2008 5:00 & 64 & 207 & 500 & 10.14 & 10.14 & & -0.0730 & $0.00 \mathrm{E}+00$ & 0.0000 & 0.0000 & 0.0000 & \\
\hline & 7/25/2008 6:00 & 65 & 207 & 600 & 10.13 & 10.13 & & -0.0721 & $0.00 \mathrm{E}+00$ & 0.0000 & 0.0000 & 0.0000 & \\
\hline & 7/25/2008 7:00 & 66 & 207 & 700 & 10.09 & 10.09 & & -0.0684 & $0.00 \mathrm{E}+00$ & 0.0000 & 0.0000 & 0.0000 & \\
\hline & 7/25/2008 8:00 & 67 & 207 & 800 & 10.05 & 10.05 & & -0.0647 & $0.00 \mathrm{E}+00$ & 0.0000 & 0.0000 & 0.0000 & \\
\hline & $7 / 25 / 20089: 00$ & 68 & 207 & 900 & 9.97 & 9.97 & & -0.0572 & $0.00 \mathrm{E}+00$ & 0.0000 & 0.0000 & 0.0000 & \\
\hline & $7 / 25 / 2008$ 10:00 & 69 & 207 & 1000 & 9.82 & 9.82 & & -0.0428 & $0.00 \mathrm{E}+00$ & 0.0000 & 0.0000 & 0.0000 & \\
\hline & $7 / 25 / 2008$ 11:00 & 70 & 207 & 1100 & 8.67 & 8.67 & & 0.0842 & $5.66 \mathrm{E}-04$ & 96.7911 & 35.6647 & 132.4558 & \\
\hline & $7 / 25 / 2008$ 12:00 & 71 & 207 & 1200 & 6.62 & 6.62 & & 0.4199 & 4.09E-03 & 699.7286 & 257.8294 & 957.5580 & \\
\hline & $7 / 25 / 2008$ 13:00 & 72 & 207 & 1300 & 5.992 & 5.992 & & 0.5688 & 5.94E-03 & 1016.4892 & 374.5463 & 1391.0355 & \\
\hline & $7 / 25 / 2008$ 14:00 & 73 & 207 & 1400 & 5.637 & 5.637 & & 0.6676 & $7.24 \mathrm{E}-03$ & 1238.0243 & 456.1755 & 1694.1998 & \\
\hline & $7 / 25 / 2008$ 15:00 & 74 & 207 & 1500 & 5.576 & 5.576 & & 0.6858 & $7.48 \mathrm{E}-03$ & 1279.8022 & 471.5694 & 1751.3717 & \\
\hline & $7 / 25 / 200816: 00$ & 75 & 207 & 1600 & 5.646 & 5.646 & & 0.6649 & 7.20E-03 & 1231.9585 & 453.9405 & 1685.8990 & \\
\hline & $7 / 25 / 2008$ 17:00 & 76 & 207 & 1700 & 5.536 & 5.536 & & 0.6980 & 7.64E-03 & 1307.8411 & 481.9010 & 1789.7421 & \\
\hline & $7 / 25 / 2008$ 18:00 & 77 & 207 & 1800 & 5.555 & 5.555 & & 0.6922 & 7.57E-03 & 1294.4581 & 476.9697 & 1771.4278 & \\
\hline & $7 / 25 / 2008$ 19:00 & 78 & 207 & 1900 & 5.582 & 5.582 & & 0.6840 & $7.46 \mathrm{E}-03$ & 1275.6408 & 470.0361 & 1745.6769 & \\
\hline & $7 / 25 / 200820: 00$ & 79 & 207 & 2000 & 6.369 & 6.369 & & 0.4759 & 4.77E-03 & 816.2200 & 300.7531 & 1116.9731 & \\
\hline & $7 / 25 / 2008$ 21:00 & 80 & 207 & 2100 & 7.975 & 7.975 & & 0.1787 & 1.43E-03 & 244.4006 & 90.0544 & 334.4550 & \\
\hline & 7/25/2008 22:00 & 81 & 207 & 2200 & 9.47 & 9.47 & & -0.0074 & $0.00 \mathrm{E}+00$ & 0.0000 & 0.0000 & 0.0000 & \\
\hline
\end{tabular}




\begin{tabular}{|c|c|c|c|c|c|c|c|c|c|c|c|c|c|}
\hline Tree ID & TS & RN & JD & HR & $\begin{array}{c}\text { Raw } \\
\text { TC_dTA }\end{array}$ & $\begin{array}{c}\text { Fixed } \\
\text { TC_dT } \Delta\end{array}$ & dTM & K & $\begin{array}{l}\text { Sap } \\
\text { Velocity } \\
(\mathrm{cm} / \mathrm{s})\end{array}$ & $\begin{array}{c}\text { Probed } \\
\text { Drywood F } \\
(\mathrm{g} / \mathrm{hr})\end{array}$ & $\begin{array}{c}\text { Extrapolated } \\
\text { Wetwood F } \\
(\mathrm{g} / \mathrm{hr})\end{array}$ & $\begin{array}{c}\text { Extrapolated } \\
\text { Total Wood F } \\
(\mathrm{g} / \mathrm{hr})\end{array}$ & $\begin{array}{l}\text { Relative } \\
\text { Humidity, } \\
@ 10 \mathrm{~m}(\%)\end{array}$ \\
\hline & 7/25/2008 23:00 & 82 & 207 & 2300 & $\begin{array}{r}9.86 \\
\end{array}$ & 9.86 & & -0.0467 & $0.00 \mathrm{E}+00$ & 0.0000 & 0.0000 & 0.0000 & \\
\hline & $7 / 26 / 20080: 00$ & 83 & 208 & 0 & 9.97 & 9.97 & 9.98 & -0.0572 & $0.00 \mathrm{E}+00$ & 0.0000 & 0.0000 & 0.0000 & \\
\hline & 7/26/2008 1:00 & 84 & 208 & 100 & 9.98 & 9.98 & & -0.0581 & $0.00 E+00$ & 0.0000 & 0.0000 & 0.0000 & \\
\hline & $7 / 26 / 20082: 00$ & 85 & 208 & 200 & 9.95 & 9.95 & & -0.0553 & $0.00 \mathrm{E}+00$ & 0.0000 & 0.0000 & 0.0000 & \\
\hline & 7/26/2008 3:00 & 86 & 208 & 300 & 9.9 & 9.9 & & -0.0505 & $0.00 \mathrm{E}+00$ & 0.0000 & 0.0000 & 0.0000 & \\
\hline & 7/26/2008 4:00 & 87 & 208 & 400 & 9.84 & 9.84 & & -0.0447 & $0.00 \mathrm{E}+00$ & 0.0000 & 0.0000 & 0.0000 & \\
\hline & 7/26/2008 5:00 & 88 & 208 & 500 & 9.78 & 9.78 & & -0.0389 & $0.00 \mathrm{E}+00$ & 0.0000 & 0.0000 & 0.0000 & \\
\hline & 7/26/2008 6:00 & 89 & 208 & 600 & 9.71 & 9.71 & & -0.0319 & $0.00 \mathrm{E}+00$ & 0.0000 & 0.0000 & 0.0000 & \\
\hline & 7/26/2008 7:00 & 90 & 208 & 700 & 9.66 & 9.66 & & -0.0269 & $0.00 \mathrm{E}+00$ & 0.0000 & 0.0000 & 0.0000 & \\
\hline & 7/26/2008 8:00 & 91 & 208 & 800 & 9.57 & 9.57 & & -0.0178 & $0.00 E+00$ & 0.0000 & 0.0000 & 0.0000 & \\
\hline & 7/26/2008 9:00 & 92 & 208 & 900 & 8.03 & 8.03 & & 0.1706 & 1.35E-03 & 230.8792 & 85.0722 & 315.9514 & \\
\hline & $7 / 26 / 2008$ 10:00 & 93 & 208 & 1000 & 5.752 & 5.752 & & 0.6342 & $6.79 E-03$ & 1162.3559 & 428.2939 & 1590.6498 & \\
\hline & $7 / 26 / 2008$ 11:00 & 94 & 208 & 1100 & 5.759 & 5.759 & & 0.6322 & $6.77 \mathrm{E}-03$ & 1157.8760 & 426.6432 & 1584.5193 & \\
\hline & $7 / 26 / 2008$ 12:00 & 95 & 208 & 1200 & 6.006 & 6.006 & & 0.5651 & 5.89E-03 & 1008.4501 & 371.5842 & 1380.0342 & \\
\hline & $7 / 26 / 2008$ 13:00 & 96 & 208 & 1300 & 5.5 & 5.5 & & 0.7091 & 7.79E-03 & 1333.5237 & 491.3642 & 1824.8879 & \\
\hline & $7 / 26 / 200814: 00$ & 97 & 208 & 1400 & 5.455 & 5.455 & & 0.7232 & 7.99E-03 & 1366.2375 & 503.4183 & 1869.6558 & \\
\hline & $7 / 26 / 2008$ 15:00 & 98 & 208 & 1500 & 5.418 & 5.418 & & 0.7350 & 8.15E-03 & 1393.6557 & 513.5211 & 1907.1768 & \\
\hline & $7 / 26 / 200816: 00$ & 99 & 208 & 1600 & 5.542 & 5.542 & & 0.6961 & 7.62E-03 & 1303.6022 & 480.3390 & 1783.9412 & \\
\hline & $7 / 26 / 200817: 00$ & 100 & 208 & 1700 & 5.43 & 5.43 & & 0.7311 & 8.09E-03 & 1384.7112 & 510.2253 & 1894.9365 & \\
\hline & $7 / 26 / 2008$ 18:00 & 101 & 208 & 1800 & 5.567 & 5.567 & & 0.6885 & 7.52E-03 & 1286.0659 & 473.8774 & 1759.9434 & \\
\hline & $7 / 26 / 2008$ 19:00 & 102 & 208 & 1900 & 5.605 & 5.605 & & 0.6771 & $7.36 \mathrm{E}-03$ & 1259.7947 & 464.1973 & 1723.9920 & \\
\hline & $7 / 26 / 200820: 00$ & 103 & 208 & 2000 & 6.168 & 6.168 & & 0.5240 & 5.37E-03 & 918.9210 & 338.5954 & 1257.5164 & \\
\hline & $7 / 26 / 200821: 00$ & 104 & 208 & 2100 & 7.865 & 7.865 & & 0.1952 & 1.59E-03 & 272.4463 & 100.3885 & 372.8348 & \\
\hline & $7 / 26 / 200822: 00$ & 105 & 208 & 2200 & 9.27 & 9.27 & & 0.0140 & $6.23 \mathrm{E}-05$ & 10.6555 & 3.9263 & 14.5818 & \\
\hline & $7 / 26 / 2008$ 23:00 & 106 & 208 & 2300 & 9.63 & 9.63 & & -0.0239 & $0.00 \mathrm{E}+00$ & 0.0000 & 0.0000 & 0.0000 & \\
\hline & $7 / 27 / 20080: 00$ & 107 & 209 & 0 & 9.73 & 9.73 & 9.77 & -0.0339 & $0.00 \mathrm{E}+00$ & 0.0000 & 0.0000 & 0.0000 & \\
\hline & 7/27/2008 1:00 & 108 & 209 & 100 & 9.77 & 9.77 & & -0.0379 & $0.00 \mathrm{E}+00$ & 0.0000 & 0.0000 & 0.0000 & \\
\hline & 7/27/2008 2:00 & 109 & 209 & 200 & 9.73 & 9.73 & & -0.0339 & $0.00 \mathrm{E}+00$ & 0.0000 & 0.0000 & 0.0000 & \\
\hline & 7/27/2008 3:00 & 110 & 209 & 300 & 9.73 & 9.73 & & -0.0339 & $0.00 \mathrm{E}+00$ & 0.0000 & 0.0000 & 0.0000 & \\
\hline & 7/27/2008 4:00 & 111 & 209 & 400 & 9.73 & 9.73 & & -0.0339 & $0.00 \mathrm{E}+00$ & 0.0000 & 0.0000 & 0.0000 & \\
\hline & 7/27/20085:00 & 112 & 209 & 500 & 9.72 & 9.72 & & -0.0329 & $0.00 \mathrm{E}+00$ & 0.0000 & 0.0000 & 0.0000 & \\
\hline & 7/27/2008 6:00 & 113 & 209 & 600 & 9.68 & 9.68 & & -0.0289 & $0.00 \mathrm{E}+00$ & 0.0000 & 0.0000 & 0.0000 & \\
\hline & 7/27/2008 7:00 & 114 & 209 & 700 & 9.64 & 9.64 & & -0.0249 & $0.00 \mathrm{E}+00$ & 0.0000 & 0.0000 & 0.0000 & \\
\hline & 7/27/2008 8:00 & 115 & 209 & 800 & 9.38 & 9.38 & & 0.0021 & $6.13 \mathrm{E}-06$ & 1.0485 & 0.3863 & 1.4349 & \\
\hline & $7 / 27 / 20089: 00$ & 116 & 209 & 900 & 6.859 & 6.859 & & 0.3705 & 3.50E-03 & 599.6673 & 220.9597 & 820.6270 & \\
\hline & $7 / 27 / 2008$ 10:00 & 117 & 209 & 1000 & 5.269 & 5.269 & & 0.7840 & 8.82E-03 & 1509.0485 & 556.0400 & 2065.0885 & \\
\hline & $7 / 27 / 2008$ 11:00 & 118 & 209 & 1100 & 5.19 & 5.19 & & 0.8112 & $9.20 \mathrm{E}-03$ & 1573.6455 & 579.8421 & 2153.4876 & \\
\hline & $7 / 27 / 2008$ 12:00 & 119 & 209 & 1200 & 5.128 & 5.128 & & 0.8331 & $9.50 \mathrm{E}-03$ & 1626.1018 & 599.1706 & 2225.2724 & \\
\hline & $7 / 27 / 200813: 00$ & 120 & 209 & 1300 & 5.228 & 5.228 & & 0.7980 & $9.01 \mathrm{E}-03$ & 1542.2664 & 568.2798 & 2110.5462 & \\
\hline & $7 / 27 / 2008$ 14:00 & 121 & 209 & 1400 & 5.145 & 5.145 & & 0.8270 & $9.42 \mathrm{E}-03$ & 1611.5606 & 593.8126 & 2205.3732 & \\
\hline & 7/27/2008 15:00 & 122 & 209 & 1500 & 5.155 & 5.155 & & 0.8235 & 9.37E-03 & 1603.0631 & 590.6816 & 2193.7447 & \\
\hline
\end{tabular}




\begin{tabular}{|c|c|c|c|c|c|c|c|c|c|c|c|c|c|}
\hline Tree ID & TS & RN & JD & HR & $\begin{array}{c}\text { Raw } \\
\text { TC_dTA }\end{array}$ & $\begin{array}{c}\text { Fixed } \\
\text { TC_dT } \Delta\end{array}$ & dTM & $\mathbf{K}$ & $\begin{array}{l}\text { Sap } \\
\text { Velocity } \\
(\mathrm{cm} / \mathrm{s})\end{array}$ & $\begin{array}{c}\text { Probed } \\
\text { Drywood F } \\
(\mathrm{g} / \mathrm{hr})\end{array}$ & $\begin{array}{c}\text { Extrapolated } \\
\text { Wetwood F } \\
(\mathrm{g} / \mathrm{hr})\end{array}$ & $\begin{array}{c}\text { Extrapolated } \\
\text { Total Wood F } \\
(\mathrm{g} / \mathrm{hr})\end{array}$ & $\begin{array}{l}\text { Relative } \\
\text { Humidity, } \\
@ 10 \mathrm{~m}(\%)\end{array}$ \\
\hline & 7/27/2008 16:00 & 123 & 209 & 1600 & 5.239 & 5.239 & & 0.7942 & 8.96E-03 & 1533.2899 & 564.9722 & 2098.2621 & \\
\hline & 7/27/2008 17:00 & 124 & 209 & 1700 & 5.299 & 5.299 & & 0.7739 & $8.68 \mathrm{E}-03$ & 1485.1532 & 547.2352 & 2032.3885 & \\
\hline & $7 / 27 / 2008$ 18:00 & 125 & 209 & 1800 & 5.332 & 5.332 & & 0.7629 & $8.53 \mathrm{E}-03$ & 1459.2606 & 537.6946 & 1996.9551 & \\
\hline & $7 / 27 / 2008$ 19:00 & 126 & 209 & 1900 & 5.409 & 5.409 & & 0.7378 & 8.18E-03 & 1400.3973 & 516.0052 & 1916.4025 & \\
\hline & $7 / 27 / 200820: 00$ & 127 & 209 & 2000 & 5.879 & 5.879 & & 0.5989 & 6.33E-03 & 1083.2283 & 399.1377 & 1482.3660 & \\
\hline & $7 / 27 / 2008$ 21:00 & 128 & 209 & 2100 & 7.157 & 7.157 & & 0.3134 & $2.85 E-03$ & 488.0717 & 179.8401 & 667.9117 & \\
\hline & $7 / 27 / 2008$ 22:00 & 129 & 209 & 2200 & 8.38 & 8.38 & & 0.1217 & $8.90 \mathrm{E}-04$ & 152.3562 & 56.1388 & 208.4950 & \\
\hline & $7 / 27 / 200823: 00$ & 130 & 209 & 2300 & 8.7 & 8.7 & & 0.0805 & $5.35 \mathrm{E}-04$ & 91.5280 & 33.7254 & 125.2534 & \\
\hline & $7 / 28 / 20080: 00$ & 131 & 210 & 0 & 8.82 & 8.82 & 9.22 & 0.0658 & 4.17E-04 & 71.3994 & 26.3086 & 97.7080 & \\
\hline & 7/28/2008 1:00 & 132 & 210 & 100 & 9.03 & 9.03 & & 0.0410 & 2.33E-04 & 39.8833 & 14.6958 & 54.5792 & \\
\hline & $7 / 28 / 20082: 00$ & 133 & 210 & 200 & 9.1 & 9.1 & & 0.0330 & 1.78E-04 & 30.5171 & 11.2446 & 41.7617 & \\
\hline & 7/28/2008 3:00 & 134 & 210 & 300 & 9.22 & 9.22 & & 0.0195 & $9.36 \mathrm{E}-05$ & 16.0119 & 5.8999 & 21.9118 & \\
\hline & 7/28/2008 4:00 & 135 & 210 & 400 & 9.12 & 9.12 & & 0.0307 & 1.63E-04 & 27.9566 & 10.3012 & 38.2578 & \\
\hline & 7/28/2008 5:00 & 136 & 210 & 500 & 9.18 & 9.18 & & 0.0240 & 1.20E-04 & 20.6087 & 7.5937 & 28.2023 & \\
\hline & 7/28/2008 6:00 & 137 & 210 & 600 & 9.17 & 9.17 & & 0.0251 & 1.27E-04 & 21.7970 & 8.0316 & 29.8286 & \\
\hline & 7/28/2008 7:00 & 138 & 210 & 700 & 9.01 & 9.01 & & 0.0433 & $2.49 \mathrm{E}-04$ & 42.6698 & 15.7226 & \begin{tabular}{|l|}
58.3924 \\
\end{tabular} & \\
\hline & 7/28/2008 8:00 & 139 & 210 & 800 & 7.088 & 7.088 & & 0.3262 & 3.00E-03 & 512.6973 & 188.9139 & 701.6111 & \\
\hline & $7 / 28 / 20089: 00$ & 140 & 210 & 900 & 5.411 & 5.411 & & 0.7372 & 8.18E-03 & 1398.8967 & 515.4523 & 1914.3490 & \\
\hline & $7 / 28 / 2008$ 10:00 & 141 & 210 & 1000 & 5.081 & 5.081 & & 0.8500 & 9.74E-03 & 1666.9399 & 614.2183 & 2281.1581 & \\
\hline & $7 / 28 / 2008$ 11:00 & 142 & 210 & 1100 & 5.066 & 5.066 & & 0.8555 & $9.82 E-03$ & 1680.1733 & 619.0944 & 2299.2677 & \\
\hline & $7 / 28 / 200812: 00$ & 143 & 210 & 1200 & 5.103 & 5.103 & & 0.8421 & $9.63 \mathrm{E}-03$ & 1647.7068 & 607.1315 & 2254.8383 & \\
\hline & 7/28/2008 13:00 & 144 & 210 & 1300 & 5.103 & 5.103 & & 0.8421 & 9.63E-03 & 1647.7068 & 607.1315 & 2254.8383 & \\
\hline & $7 / 28 / 2008$ 14:00 & 145 & 210 & 1400 & 5.119 & 5.119 & & 0.8363 & 9.55E-03 & 1633.8491 & 602.0253 & 2235.8744 & \\
\hline & $7 / 28 / 2008$ 15:00 & 146 & 210 & 1500 & 5.091 & 5.091 & & 0.8464 & $9.69 \mathrm{E}-03$ & 1658.1718 & 610.9875 & 2269.1593 & \\
\hline & $7 / 28 / 2008$ 16:00 & 147 & 210 & 1600 & 5.153 & 5.153 & & 0.8242 & $9.38 \mathrm{E}-03$ & 1604.7593 & 591.3066 & 2196.0658 & \\
\hline & 7/28/2008 17:00 & 148 & 210 & 1700 & 5.213 & 5.213 & & 0.8032 & 9.09E-03 & 1554.5841 & 572.8185 & 2127.4026 & \\
\hline & $7 / 28 / 2008$ 18:00 & 149 & 210 & 1800 & 5.266 & 5.266 & & 0.7850 & 8.83E-03 & 1511.4570 & 556.9274 & 2068.3844 & \\
\hline & $7 / 28 / 2008$ 19:00 & 150 & 210 & 1900 & 5.409 & 5.409 & & 0.7378 & $8.18 \mathrm{E}-03$ & 1400.3973 & 516.0052 & 1916.4025 & \\
\hline & $7 / 28 / 200820: 00$ & 151 & 210 & 2000 & 5.989 & 5.989 & & 0.5695 & 5.95E-03 & 1018.2183 & 375.1835 & 1393.4018 & \\
\hline & $7 / 28 / 200821: 00$ & 152 & 210 & 2100 & 7.498 & 7.498 & & 0.2537 & $2.20 \mathrm{E}-03$ & 376.2158 & 138.6244 & 514.8402 & \\
\hline & $7 / 28 / 2008$ 22:00 & 153 & 210 & 2200 & 8.28 & 8.28 & & 0.1353 & 1.01E-03 & 173.4917 & 63.9266 & 237.4183 & \\
\hline & $7 / 28 / 200823: 00$ & 154 & 210 & 2300 & 8.45 & 8.45 & & 0.1124 & 8.08E-04 & 138.1668 & 50.9104 & 189.0773 & \\
\hline & $7 / 29 / 20080: 00$ & 155 & 211 & 0 & 8.71 & 8.71 & 9.39 & 0.0792 & $5.25 E-04$ & 89.7940 & 33.0865 & 122.8805 & \\
\hline & 7/29/2008 1:00 & 156 & 211 & 100 & 8.91 & 8.91 & & 0.0550 & $3.35 E-04$ & 57.2952 & 21.1116 & 78.4069 & \\
\hline & 7/29/2008 2:00 & 157 & 211 & 200 & 9.08 & 9.08 & & 0.0352 & 1.94E-04 & 33.1301 & 12.2075 & 45.3376 & \\
\hline & 7/29/2008 3:00 & 158 & 211 & 300 & 9.12 & 9.12 & & 0.0307 & 1.63E-04 & 27.9566 & 10.3012 & 38.2578 & \\
\hline & 7/29/2008 4:00 & 159 & 211 & 400 & 9.2 & 9.2 & & 0.0217 & 1.07E-04 & 18.2781 & 6.7350 & 25.0131 & \\
\hline & 7/29/2008 5:00 & 160 & 211 & 500 & 9.18 & 9.18 & & 0.0240 & 1.20E-04 & 20.6087 & 7.5937 & 28.2023 & \\
\hline & $7 / 29 / 20086: 00$ & 161 & 211 & 600 & 9.11 & 9.11 & & 0.0318 & $1.71 \mathrm{E}-04$ & 29.2302 & 10.7705 & 40.0006 & \\
\hline & 7/29/2008 7:00 & 162 & 211 & 700 & 8.96 & 8.96 & & 0.0491 & 2.91E-04 & 49.8409 & 18.3649 & 68.2058 & \\
\hline & 7/29/2008 8:00 & 163 & 211 & 800 & 8.09 & 8.09 & & 0.1619 & 1.27E-03 & 216.5025 & 79.7748 & 296.2774 & \\
\hline
\end{tabular}




\begin{tabular}{|c|c|c|c|c|c|c|c|c|c|c|c|c|c|}
\hline Tree ID & TS & RN & JD & HR & $\begin{array}{c}\text { Raw } \\
\text { TC_dTA }\end{array}$ & $\begin{array}{c}\text { Fixed } \\
\text { TC_dT } \Delta\end{array}$ & dTM & $\mathbf{K}$ & $\begin{array}{l}\text { Sap } \\
\text { Velocity } \\
(\mathrm{cm} / \mathrm{s})\end{array}$ & $\begin{array}{c}\text { Probed } \\
\text { Drywood F } \\
(\mathrm{g} / \mathrm{hr})\end{array}$ & $\begin{array}{c}\text { Extrapolated } \\
\text { Wetwood F } \\
(\mathrm{g} / \mathrm{hr})\end{array}$ & $\begin{array}{c}\text { Extrapolated } \\
\text { Total Wood F } \\
(\mathrm{g} / \mathrm{hr})\end{array}$ & $\begin{array}{l}\text { Relative } \\
\text { Humidity, } \\
@ 10 \mathrm{~m}(\%)\end{array}$ \\
\hline & 7/29/2008 9:00 & 164 & 211 & 900 & \begin{tabular}{|r|}
5.873 \\
\end{tabular} & 5.873 & & 0.6005 & $6.35 \mathrm{E}-03$ & 1086.8663 & 400.4782 & 1487.3446 & \\
\hline & $7 / 29 / 2008$ 10:00 & 165 & 211 & 1000 & 5.079 & 5.079 & & 0.8508 & $9.75 \mathrm{E}-03$ & 1668.6987 & 614.8663 & 2283.5650 & \\
\hline & $7 / 29 / 2008$ 11:00 & 166 & 211 & 1100 & 5.383 & 5.383 & & 0.7462 & 8.30E-03 & 1420.0342 & 523.2408 & 1943.2750 & \\
\hline & $7 / 29 / 2008$ 12:00 & 167 & 211 & 1200 & 4.924 & 4.924 & & 0.9090 & 1.06E-02 & 1810.4596 & 667.1011 & 2477.5607 & \\
\hline & 7/29/2008 13:00 & 168 & 211 & 1300 & 4.96 & 4.96 & & 0.8952 & 1.04E-02 & 1776.5489 & 654.6060 & 2431.1548 & \\
\hline & $7 / 29 / 2008$ 14:00 & 169 & 211 & 1400 & 4.854 & 4.854 & & 0.9365 & 1.10E-02 & 1878.1907 & 692.0580 & 2570.2486 & \\
\hline & $7 / 29 / 2008$ 15:00 & 170 & 211 & 1500 & 5.063 & 5.063 & & 0.8566 & 9.84E-03 & 1682.8317 & 620.0740 & 2302.9057 & \\
\hline & $7 / 29 / 2008$ 16:00 & 171 & 211 & 1600 & 5.215 & 5.215 & & 0.8025 & 9.08E-03 & 1552.9366 & 572.2114 & 2125.1480 & \\
\hline & 7/29/2008 17:00 & 172 & 211 & 1700 & 5.454 & 5.454 & & 0.7235 & 7.99E-03 & 1366.9723 & 503.6890 & 1870.6613 & \\
\hline & 7/29/2008 18:00 & 173 & 211 & 1800 & 5.488 & 5.488 & & 0.7128 & 7.84E-03 & 1342.1804 & 494.5540 & 1836.7343 & \\
\hline & $7 / 29 / 2008$ 19:00 & 174 & 211 & 1900 & 5.464 & 5.464 & & 0.7204 & 7.95E-03 & 1359.6397 & 500.9872 & 1860.6268 & \\
\hline & $7 / 29 / 200820: 00$ & 175 & 211 & 2000 & 6.455 & 6.455 & & 0.4562 & $4.53 E-03$ & 774.9049 & 285.5296 & 1060.4345 & \\
\hline & 7/29/2008 21:00 & 176 & 211 & 2100 & 8.23 & 8.23 & & 0.1422 & 1.08E-03 & 184.4447 & 67.9625 & 252.4072 & \\
\hline & $7 / 29 / 2008$ 22:00 & 177 & 211 & 2200 & 9.09 & 9.09 & & 0.0341 & 1.86E-04 & 31.8171 & 11.7237 & 43.5408 & \\
\hline & $7 / 29 / 200823: 00$ & 178 & 211 & 2300 & 9.39 & 9.39 & & 0.0011 & $2.61 \mathrm{E}-06$ & 0.4461 & 0.1644 & 0.6105 & \\
\hline & $7 / 30 / 20080: 00$ & 179 & 212 & 0 & 9.44 & 9.44 & 9.45 & -0.0042 & $0.00 \mathrm{E}+00$ & 0.0000 & 0.0000 & 0.0000 & \\
\hline & 7/30/2008 1:00 & 180 & 212 & 100 & 9.45 & 9.45 & & -0.0053 & $0.00 \mathrm{E}+00$ & 0.0000 & 0.0000 & 0.0000 & \\
\hline & 7/30/2008 2:00 & 181 & 212 & 200 & 9.42 & 9.42 & & -0.0021 & $0.00 \mathrm{E}+00$ & 0.0000 & 0.0000 & 0.0000 & \\
\hline & 7/30/2008 3:00 & 182 & 212 & 300 & 9.37 & 9.37 & & 0.0032 & 1.01E-05 & 1.7295 & 0.6373 & 2.3667 & \\
\hline & 7/30/2008 4:00 & 183 & 212 & 400 & 9.27 & 9.27 & & 0.0140 & $6.23 E-05$ & 10.6555 & 3.9263 & 14.5818 & \\
\hline & 7/30/2008 5:00 & 184 & 212 & 500 & 9.15 & 9.15 & & 0.0273 & $1.42 \mathrm{E}-04$ & 24.2182 & 8.9237 & 33.1419 & \\
\hline & 7/30/2008 6:00 & 185 & 212 & 600 & 9.05 & 9.05 & & 0.0387 & 2.17E-04 & 37.1450 & 13.6868 & 50.8318 & \\
\hline & 7/30/2008 7:00 & 186 & 212 & 700 & 8.96 & 8.96 & & 0.0491 & 2.91E-04 & 49.8409 & 18.3649 & 68.2058 & \\
\hline & $7 / 30 / 20088: 00$ & 187 & 212 & 800 & 8.86 & 8.86 & & 0.0609 & 3.80E-04 & 65.0238 & 23.9594 & 88.9832 & \\
\hline & 7/30/2008 9:00 & 188 & 212 & 900 & 7.357 & 7.357 & & 0.2777 & $2.46 \mathrm{E}-03$ & 420.5507 & 154.9606 & 575.5113 & \\
\hline & $7 / 30 / 2008$ 10:00 & 189 & 212 & 1000 & 4.822 & 4.822 & & 0.9494 & 1.12E-02 & 1909.9670 & 703.7666 & 2613.7337 & \\
\hline & 7/30/2008 11:00 & 190 & 212 & 1100 & 4.684 & 4.684 & & 1.0068 & 1.20E-02 & 2053.1781 & 756.5357 & 2809.7138 & \\
\hline & $7 / 30 / 2008$ 12:00 & 191 & 212 & 1200 & 4.725 & 4.725 & & 0.9894 & 1.17E-02 & 2009.5518 & 740.4607 & 2750.0125 & \\
\hline & 7/30/2008 13:00 & 192 & 212 & 1300 & 4.791 & 4.791 & & 0.9620 & 1.13E-02 & 1941.2520 & 715.2942 & 2656.5462 & \\
\hline & $7 / 30 / 2008$ 14:00 & 193 & 212 & 1400 & 4.801 & 4.801 & & 0.9579 & 1.13E-02 & 1931.1055 & 711.5555 & 2642.6610 & \\
\hline & 7/30/2008 15:00 & 194 & 212 & 1500 & 4.962 & 4.962 & & 0.8944 & 1.04E-02 & 1774.6829 & 653.9184 & 2428.6013 & \\
\hline & $7 / 30 / 2008$ 16:00 & 195 & 212 & 1600 & 4.922 & 4.922 & & 0.9098 & 1.06E-02 & 1812.3616 & 667.8019 & 2480.1636 & \\
\hline & $7 / 30 / 2008$ 17:00 & 196 & 212 & 1700 & 5.014 & 5.014 & & 0.8748 & 1.01E-02 & 1726.8167 & 636.2811 & 2363.0978 & \\
\hline & $7 / 30 / 2008$ 18:00 & 197 & 212 & 1800 & 5.17 & 5.17 & & 0.8182 & $9.30 \mathrm{E}-03$ & 1590.3943 & 586.0135 & 2176.4077 & \\
\hline & 7/30/2008 19:00 & 198 & 212 & 1900 & 5.186 & 5.186 & & 0.8126 & 9.22E-03 & 1576.9823 & 581.0716 & 2158.0538 & \\
\hline & $7 / 30 / 200820: 00$ & 199 & 212 & 2000 & 5.84 & 5.84 & & 0.6096 & 6.47E-03 & 1107.0504 & 407.9155 & 1514.9659 & \\
\hline & 7/30/2008 21:00 & 200 & 212 & 2100 & 7.52 & 7.52 & & 0.2500 & $2.16 \mathrm{E}-03$ & 369.5309 & 136.1613 & 505.6922 & \\
\hline & $7 / 30 / 2008$ 22:00 & 201 & 212 & 2200 & 8.7 & 8.7 & & 0.0805 & 5.35E-04 & 91.5280 & 33.7254 & 125.2534 & \\
\hline & 7/30/2008 23:00 & 202 & 212 & 2300 & 9.14 & 9.14 & & 0.0284 & 1.49E-04 & 25.4504 & 9.3777 & 34.8281 & \\
\hline & $7 / 31 / 20080: 00$ & 203 & 213 & 0 & 9.37 & 9.37 & 9.53 & 0.0032 & 1.01E-05 & 1.7295 & 0.6373 & 2.3667 & \\
\hline & 7/31/2008 1:00 & 204 & 213 & 100 & 9.49 & 9.49 & & -0.0095 & $0.00 \mathrm{E}+00$ & 0.0000 & 0.0000 & 0.0000 & \\
\hline
\end{tabular}




\begin{tabular}{|c|c|c|c|c|c|c|c|c|c|c|c|c|c|}
\hline Tree ID & TS & RN & JD & HR & $\begin{array}{c}\text { Raw } \\
\text { TC_dTA }\end{array}$ & $\begin{array}{c}\text { Fixed } \\
\text { TC_dT } \Delta\end{array}$ & dTM & K & $\begin{array}{l}\text { Sap } \\
\text { Velocity } \\
(\mathrm{cm} / \mathrm{s})\end{array}$ & $\begin{array}{c}\text { Probed } \\
\text { Drywood F } \\
(\mathrm{g} / \mathrm{hr})\end{array}$ & $\begin{array}{c}\text { Extrapolated } \\
\text { Wetwood F } \\
(\mathrm{g} / \mathrm{hr})\end{array}$ & $\begin{array}{c}\text { Extrapolated } \\
\text { Total Wood F } \\
(\mathrm{g} / \mathrm{hr})\end{array}$ & $\begin{array}{l}\text { Relative } \\
\text { Humidity, } \\
@ 10 \mathrm{~m}(\%)\end{array}$ \\
\hline & 7/31/2008 2:00 & 205 & 213 & 200 & $\begin{array}{r}9.52 \\
\end{array}$ & 9.52 & & -0.0126 & $0.00 \mathrm{E}+00$ & 0.0000 & 0.0000 & 0.0000 & \\
\hline & 7/31/2008 3:00 & 206 & 213 & 300 & 9.53 & 9.53 & & -0.0136 & $0.00 \mathrm{E}+00$ & 0.0000 & 0.0000 & 0.0000 & \\
\hline & 7/31/2008 4:00 & 207 & 213 & 400 & 9.52 & 9.52 & & -0.0126 & $0.00 E+00$ & 0.0000 & 0.0000 & 0.0000 & \\
\hline & 7/31/2008 5:00 & 208 & 213 & 500 & 9.47 & 9.47 & & -0.0074 & $0.00 \mathrm{E}+00$ & 0.0000 & 0.0000 & 0.0000 & \\
\hline & 7/31/2008 6:00 & 209 & 213 & 600 & 9.42 & 9.42 & & -0.0021 & $0.00 \mathrm{E}+00$ & 0.0000 & 0.0000 & 0.0000 & \\
\hline & 7/31/2008 7:00 & 210 & 213 & 700 & 9.37 & 9.37 & & 0.0032 & $1.01 \mathrm{E}-05$ & 1.7295 & 0.6373 & 2.3667 & \\
\hline & $7 / 31 / 2008$ 8:00 & 211 & 213 & 800 & 9.23 & 9.23 & & 0.0184 & 8.71E-05 & 14.9041 & 5.4917 & 20.3958 & \\
\hline & $7 / 31 / 20089: 00$ & 212 & 213 & 900 & 7.287 & 7.287 & & 0.2900 & 2.59E-03 & 443.5478 & 163.4343 & 606.9821 & \\
\hline & $7 / 31 / 2008$ 10:00 & 213 & 213 & 1000 & 5.134 & 5.134 & & 0.8309 & 9.47E-03 & 1620.9558 & 597.2745 & 2218.2303 & \\
\hline & 7/31/2008 11:00 & 214 & 213 & 1100 & 5.122 & 5.122 & & 0.8352 & 9.53E-03 & 1631.2629 & 601.0724 & 2232.3352 & \\
\hline & $7 / 31 / 2008$ 12:00 & 215 & 213 & 1200 & 4.957 & 4.957 & & 0.8963 & 1.04E-02 & 1779.3514 & 655.6386 & 2434.9900 & \\
\hline & 7/31/2008 13:00 & 216 & 213 & 1300 & 5.137 & 5.137 & & 0.8299 & $9.46 \mathrm{E}-03$ & 1618.3884 & 596.3285 & 2214.7169 & \\
\hline & 7/31/2008 14:00 & 217 & 213 & 1400 & 4.929 & 4.929 & & 0.9071 & 1.06E-02 & 1805.7129 & 665.3521 & 2471.0650 & \\
\hline & 7/31/2008 15:00 & 218 & 213 & 1500 & 4.941 & 4.941 & & 0.9024 & 1.05E-02 & 1794.3696 & 661.1724 & 2455.5420 & \\
\hline & $7 / 31 / 2008$ 16:00 & 219 & 213 & 1600 & 4.998 & 4.998 & & 0.8808 & 1.02E-02 & 1741.4126 & 641.6593 & 2383.0719 & \\
\hline & 7/31/2008 17:00 & 220 & 213 & 1700 & 5.042 & 5.042 & & 0.8643 & 9.95E-03 & 1701.5517 & 626.9717 & 2328.5234 & \\
\hline & $7 / 31 / 2008$ 18:00 & 221 & 213 & 1800 & 5.08 & 5.08 & & 0.8504 & 9.75E-03 & 1667.8190 & 614.5422 & 2282.3613 & \\
\hline & $7 / 31 / 2008$ 19:00 & 222 & 213 & 1900 & 5.317 & 5.317 & & 0.7679 & 8.60E-03 & 1470.9795 & 542.0127 & 2012.9922 & \\
\hline & $7 / 31 / 200820: 00$ & 223 & 213 & 2000 & 6.199 & 6.199 & & 0.5164 & 5.27E-03 & 902.4962 & 332.5433 & 1235.0395 & \\
\hline & $7 / 31 / 200821: 00$ & 224 & 213 & 2100 & 7.2 & 7.2 & & 0.3056 & $2.76 \mathrm{E}-03$ & 473.0779 & 174.3153 & 647.3932 & \\
\hline & $7 / 31 / 200822: 00$ & 225 & 213 & 2200 & 8.06 & 8.06 & & 0.1663 & $1.31 \mathrm{E}-03$ & 223.6424 & 82.4057 & 306.0481 & \\
\hline & 7/31/2008 23:00 & 226 & 213 & 2300 & 7.978 & 7.978 & & 0.1782 & 1.42E-03 & 243.6545 & 89.7795 & 333.4341 & \\
\hline & $8 / 1 / 20080: 00$ & 227 & 214 & 0 & 8.32 & 8.32 & 9.56 & 0.1298 & 9.64E-04 & 164.9147 & 60.7662 & 225.6810 & \\
\hline & 8/1/2008 1:00 & 228 & 214 & 100 & 8.96 & 8.96 & & 0.0491 & 2.91E-04 & 49.8409 & 18.3649 & 68.2058 & \\
\hline & 8/1/2008 2:00 & 229 & 214 & 200 & 9.3 & 9.3 & & 0.0108 & 4.49E-05 & 7.6839 & 2.8313 & 10.5153 & \\
\hline & $8 / 1 / 2008$ 3:00 & 230 & 214 & 300 & 9.37 & 9.37 & & 0.0032 & 1.01E-05 & 1.7295 & 0.6373 & 2.3667 & \\
\hline & 8/1/2008 4:00 & 231 & 214 & 400 & 9.49 & 9.49 & & -0.0095 & $0.00 \mathrm{E}+00$ & 0.0000 & 0.0000 & 0.0000 & \\
\hline & 8/1/2008 5:00 & 232 & 214 & 500 & 9.56 & 9.56 & & -0.0167 & $0.00 \mathrm{E}+00$ & 0.0000 & 0.0000 & 0.0000 & \\
\hline & 8/1/2008 6:00 & 233 & 214 & 600 & 9.56 & 9.56 & & -0.0167 & $0.00 \mathrm{E}+00$ & 0.0000 & 0.0000 & 0.0000 & \\
\hline & 8/1/2008 7:00 & 234 & 214 & 700 & 9.55 & 9.55 & & -0.0157 & $0.00 E+00$ & 0.0000 & 0.0000 & 0.0000 & \\
\hline & 8/1/2008 8:00 & 235 & 214 & 800 & 9.38 & 9.38 & & 0.0021 & $6.13 E-06$ & 1.0485 & 0.3863 & 1.4349 & \\
\hline & 8/1/2008 9:00 & 236 & 214 & 900 & 7.565 & 7.565 & & 0.2426 & 2.08E-03 & 356.0483 & 131.1933 & 487.2416 & \\
\hline & 8/1/2008 10:00 & 237 & 214 & 1000 & 5.328 & 5.328 & & 0.7643 & 8.55E-03 & 1462.3774 & 538.8430 & 2001.2205 & \\
\hline & 8/1/2008 11:00 & 238 & 214 & 1100 & 5.26 & 5.26 & & 0.7871 & 8.86E-03 & 1516.2843 & 558.7061 & 2074.9904 & \\
\hline & 8/1/2008 12:00 & 239 & 214 & 1200 & 5.206 & 5.206 & & 0.8056 & $9.12 \mathrm{E}-03$ & 1560.3630 & 574.9478 & 2135.3108 & \\
\hline & 8/1/2008 13:00 & 240 & 214 & 1300 & 5.125 & 5.125 & & 0.8341 & 9.52E-03 & 1628.6804 & 600.1208 & 2228.8012 & \\
\hline & 8/1/2008 14:00 & 241 & 214 & 1400 & 5.08 & 5.08 & & 0.8504 & $9.75 \mathrm{E}-03$ & 1667.8190 & 614.5422 & 2282.3613 & \\
\hline & 8/1/2008 15:00 & 242 & 214 & 1500 & 5.039 & 5.039 & & 0.8654 & 9.96E-03 & 1704.2419 & 627.9630 & 2332.2049 & \\
\hline & $8 / 1 / 200816: 00$ & 243 & 214 & 1600 & 5.116 & 5.116 & & 0.8374 & $9.56 \mathrm{E}-03$ & 1636.4392 & 602.9797 & 2239.4188 & \\
\hline & 8/1/2008 17:00 & 244 & 214 & 1700 & 5.201 & 5.201 & & 0.8073 & 9.14E-03 & 1564.5027 & 576.4732 & 2140.9759 & \\
\hline & 8/1/2008 18:00 & 245 & 214 & 1800 & 5.208 & 5.208 & & 0.8049 & $9.11 \mathrm{E}-03$ & 1558.7099 & 574.3387 & 2133.0486 & \\
\hline
\end{tabular}




\begin{tabular}{|c|c|c|c|c|c|c|c|c|c|c|c|c|c|}
\hline Tree ID & TS & RN & JD & HR & $\begin{array}{c}\text { Raw } \\
\text { TC_dTA }\end{array}$ & $\begin{array}{c}\text { Fixed } \\
\text { TC_dT } \Delta\end{array}$ & dTM & $\mathbf{K}$ & $\begin{array}{l}\text { Sap } \\
\text { Velocity } \\
(\mathrm{cm} / \mathrm{s})\end{array}$ & $\begin{array}{c}\text { Probed } \\
\text { Drywood F } \\
(\mathrm{g} / \mathrm{hr})\end{array}$ & $\begin{array}{c}\text { Extrapolated } \\
\text { Wetwood F } \\
(\mathrm{g} / \mathrm{hr})\end{array}$ & $\begin{array}{c}\text { Extrapolated } \\
\text { Total Wood F } \\
(\mathrm{g} / \mathrm{hr})\end{array}$ & $\begin{array}{l}\text { Relative } \\
\text { Humidity, } \\
@ 10 \mathrm{~m}(\%)\end{array}$ \\
\hline & 8/1/2008 19:00 & 246 & 214 & 1900 & \begin{tabular}{|r|}
5.318 \\
\end{tabular} & 5.318 & & 0.7676 & $8.59 \mathrm{E}-03$ & 1470.1956 & 541.7238 & 2011.9195 & \\
\hline & 8/1/2008 20:00 & 247 & 214 & 2000 & 6.195 & 6.195 & & 0.5174 & $5.29 \mathrm{E}-03$ & 904.6032 & 333.3197 & 1237.9229 & \\
\hline & 8/1/2008 21:00 & 248 & 214 & 2100 & 8.07 & 8.07 & & 0.1648 & $1.29 \mathrm{E}-03$ & 221.2518 & 81.5248 & 302.7765 & \\
\hline & 8/1/2008 22:00 & 249 & 214 & 2200 & 9.08 & 9.08 & & 0.0352 & $1.94 \mathrm{E}-04$ & 33.1301 & 12.2075 & 45.3376 & \\
\hline & 8/1/2008 23:00 & 250 & 214 & 2300 & 9.32 & 9.32 & & 0.0086 & $3.40 \mathrm{E}-05$ & 5.8229 & 2.1456 & 7.9685 & \\
\hline & 8/2/2008 0:00 & 251 & 215 & 0 & 9.42 & 9.42 & 9.68 & -0.0021 & $0.00 \mathrm{E}+00$ & 0.0000 & 0.0000 & 0.0000 & \\
\hline & 8/2/2008 1:00 & 252 & 215 & 100 & 9.53 & 9.53 & & -0.0136 & $0.00 \mathrm{E}+00$ & 0.0000 & 0.0000 & 0.0000 & \\
\hline & 8/2/2008 2:00 & 253 & 215 & 200 & 9.63 & 9.63 & & -0.0239 & $0.00 \mathrm{E}+00$ & 0.0000 & 0.0000 & 0.0000 & \\
\hline & 8/2/2008 3:00 & 254 & 215 & 300 & 9.68 & 9.68 & & -0.0289 & $0.00 \mathrm{E}+00$ & 0.0000 & 0.0000 & 0.0000 & \\
\hline & 8/2/2008 4:00 & 255 & 215 & 400 & 9.59 & 9.59 & & -0.0198 & $0.00 E+00$ & 0.0000 & 0.0000 & 0.0000 & \\
\hline & 8/2/2008 5:00 & 256 & 215 & 500 & 9.54 & 9.54 & & -0.0147 & $0.00 \mathrm{E}+00$ & 0.0000 & 0.0000 & 0.0000 & \\
\hline & 8/2/2008 6:00 & 257 & 215 & 600 & 9.52 & 9.52 & & -0.0126 & $0.00 E+00$ & 0.0000 & 0.0000 & 0.0000 & \\
\hline & 8/2/2008 7:00 & 258 & 215 & 700 & 9.33 & 9.33 & & 0.0075 & 2.88E-05 & 4.9338 & 1.8179 & 6.7517 & \\
\hline & 8/2/2008 8:00 & 259 & 215 & 800 & 7.75 & 7.75 & & 0.2129 & 1.77E-03 & 303.2348 & 111.7331 & 414.9679 & \\
\hline & 8/2/2008 9:00 & 260 & 215 & 900 & 5.704 & 5.704 & & 0.6480 & 6.98E-03 & 1193.4596 & 439.7547 & 1633.2144 & \\
\hline & 8/2/2008 10:00 & 261 & 215 & 1000 & 5.568 & 5.568 & & 0.6882 & $7.51 \mathrm{E}-03$ & 1285.3687 & 473.6205 & 1758.9892 & \\
\hline & 8/2/2008 11:00 & 262 & 215 & 1100 & 5.437 & 5.437 & & 0.7289 & 8.06E-03 & 1379.5167 & 508.3113 & 1887.8280 & \\
\hline & $8 / 2 / 200812: 00$ & 263 & 215 & 1200 & 5.309 & 5.309 & & 0.7706 & 8.63E-03 & 1477.2639 & 544.3283 & 2021.5922 & \\
\hline & 8/2/2008 13:00 & 264 & 215 & 1300 & 5.272 & 5.272 & & 0.7830 & 8.81E-03 & 1506.6435 & 555.1538 & 2061.7973 & \\
\hline & 8/2/2008 14:00 & 265 & 215 & 1400 & 5.253 & 5.253 & & 0.7895 & 8.90E-03 & 1521.9338 & 560.7878 & 2082.7216 & \\
\hline & $8 / 2 / 2008$ 15:00 & 266 & 215 & 1500 & 5.236 & 5.236 & & 0.7953 & 8.98E-03 & 1535.7333 & 565.8725 & 2101.6059 & \\
\hline & 8/2/2008 16:00 & 267 & 215 & 1600 & 5.254 & 5.254 & & 0.7891 & 8.89E-03 & 1521.1255 & 560.4900 & 2081.6155 & \\
\hline & 8/2/2008 17:00 & 268 & 215 & 1700 & 5.268 & 5.268 & & 0.7844 & 8.82E-03 & 1509.8509 & 556.3356 & 2066.1866 & \\
\hline & $8 / 2 / 200818: 00$ & 269 & 215 & 1800 & 5.358 & 5.358 & & 0.7544 & $8.41 \mathrm{E}-03$ & 1439.1445 & 530.2824 & 1969.4268 & \\
\hline & 8/2/2008 19:00 & 270 & 215 & 1900 & 5.449 & 5.449 & & 0.7251 & 8.01E-03 & 1370.6515 & 505.0447 & 1875.6962 & \\
\hline & 8/2/2008 20:00 & 271 & 215 & 2000 & 6.151 & 6.151 & & 0.5282 & 5.42E-03 & 928.0222 & 341.9489 & 1269.9711 & \\
\hline & 8/2/2008 21:00 & 272 & 215 & 2100 & 7.52 & 7.52 & & 0.2500 & 2.16E-03 & 369.5309 & 136.1613 & 505.6922 & \\
\hline & 8/2/2008 22:00 & 273 & 215 & 2200 & 8.7 & 8.7 & & 0.0805 & $5.35 \mathrm{E}-04$ & 91.5280 & 33.7254 & 125.2534 & \\
\hline & 8/2/2008 23:00 & 274 & 215 & 2300 & 9.18 & 9.18 & & 0.0240 & 1.20E-04 & 20.6087 & 7.5937 & 28.2023 & \\
\hline & 8/3/2008 0:00 & 275 & 216 & 0 & 9.11 & 9.11 & 9.65 & 0.0318 & 1.71E-04 & 29.2302 & 10.7705 & 40.0006 & \\
\hline & 8/3/2008 1:00 & 276 & 216 & 100 & 8.82 & 8.82 & & 0.0658 & 4.17E-04 & 71.3994 & 26.3086 & 97.7080 & \\
\hline & 8/3/2008 2:00 & 277 & 216 & 200 & 9.01 & 9.01 & & 0.0433 & $2.49 \mathrm{E}-04$ & 42.6698 & 15.7226 & 58.3924 & \\
\hline & 8/3/2008 3:00 & 278 & 216 & 300 & 9.34 & 9.34 & & 0.0064 & $2.38 \mathrm{E}-05$ & 4.0756 & 1.5017 & 5.5774 & \\
\hline & 8/3/2008 4:00 & 279 & 216 & 400 & 9.56 & 9.56 & & -0.0167 & $0.00 \mathrm{E}+00$ & 0.0000 & 0.0000 & 0.0000 & \\
\hline & 8/3/2008 5:00 & 280 & 216 & 500 & 9.65 & 9.65 & & -0.0259 & $0.00 \mathrm{E}+00$ & 0.0000 & 0.0000 & 0.0000 & \\
\hline & 8/3/2008 6:00 & 281 & 216 & 600 & 9.54 & 9.54 & & -0.0147 & $0.00 \mathrm{E}+00$ & 0.0000 & 0.0000 & 0.0000 & \\
\hline & 8/3/2008 7:00 & 282 & 216 & 700 & 9.32 & 9.32 & & 0.0086 & 3.40E-05 & 5.8229 & 2.1456 & 7.9685 & \\
\hline & 8/3/2008 8:00 & 283 & 216 & 800 & 7.909 & 7.909 & & 0.1885 & 1.53E-03 & 261.0658 & 96.1951 & 357.2609 & \\
\hline & 8/3/2008 9:00 & 284 & 216 & 900 & 5.735 & 5.735 & & 0.6391 & $6.86 \mathrm{E}-03$ & 1173.2947 & 432.3245 & 1605.6192 & \\
\hline & 8/3/2008 10:00 & 285 & 216 & 1000 & 5.402 & 5.402 & & 0.7401 & $8.22 \mathrm{E}-03$ & 1405.6605 & 517.9445 & 1923.6051 & \\
\hline & 8/3/2008 11:00 & 286 & 216 & 1100 & 5.273 & 5.273 & & 0.7827 & 8.80E-03 & 1505.8426 & 554.8587 & 2060.7013 & \\
\hline
\end{tabular}




\begin{tabular}{|c|c|c|c|c|c|c|c|c|c|c|c|c|c|}
\hline Tree ID & TS & RN & JD & HR & $\begin{array}{c}\text { Raw } \\
\text { TC_dTA }\end{array}$ & $\begin{array}{c}\text { Fixed } \\
\text { TC_dT } \Delta\end{array}$ & dTM & $\mathbf{K}$ & $\begin{array}{l}\text { Sap } \\
\text { Velocity } \\
(\mathrm{cm} / \mathrm{s})\end{array}$ & $\begin{array}{c}\text { Probed } \\
\text { Drywood F } \\
(\mathrm{g} / \mathrm{hr})\end{array}$ & $\begin{array}{c}\text { Extrapolated } \\
\text { Wetwood F } \\
(\mathrm{g} / \mathrm{hr})\end{array}$ & $\begin{array}{c}\text { Extrapolated } \\
\text { Total Wood F } \\
(\mathrm{g} / \mathrm{hr})\end{array}$ & $\begin{array}{l}\text { Relative } \\
\text { Humidity, } \\
@ 10 \mathrm{~m}(\%)\end{array}$ \\
\hline & 8/3/2008 12:00 & 287 & 216 & 1200 & \begin{tabular}{|r|}
5.229 \\
\end{tabular} & 5.229 & & 0.7977 & 9.01E-03 & 1541.4484 & 567.9784 & 2109.4268 & \\
\hline & 8/3/2008 13:00 & 288 & 216 & 1300 & 5.199 & 5.199 & & 0.8080 & $9.15 \mathrm{E}-03$ & 1566.1614 & 577.0844 & 2143.2458 & \\
\hline & $8 / 3 / 2008$ 14:00 & 289 & 216 & 1400 & 5.192 & 5.192 & & 0.8105 & $9.19 \mathrm{E}-03$ & 1571.9796 & 579.2282 & 2151.2078 & \\
\hline & 8/3/2008 15:00 & 290 & 216 & 1500 & 5.28 & 5.28 & & 0.7803 & 8.77E-03 & 1500.2471 & 552.7969 & 2053.0439 & \\
\hline & 8/3/2008 16:00 & 291 & 216 & 1600 & 5.283 & 5.283 & & 0.7793 & 8.75E-03 & 1497.8547 & 551.9154 & 2049.7701 & \\
\hline & 8/3/2008 17:00 & 292 & 216 & 1700 & 5.286 & 5.286 & & 0.7783 & 8.74E-03 & 1495.4658 & 551.0351 & 2046.5009 & \\
\hline & 8/3/2008 18:00 & 293 & 216 & 1800 & 5.327 & 5.327 & & 0.7646 & $8.55 E-03$ & 1463.1576 & 539.1305 & 2002.2881 & \\
\hline & 8/3/2008 19:00 & 294 & 216 & 1900 & 5.407 & 5.407 & & 0.7385 & 8.19E-03 & 1401.8993 & 516.5586 & 1918.4580 & \\
\hline & 8/3/2008 20:00 & 295 & 216 & 2000 & 5.766 & 5.766 & & 0.6302 & $6.74 \mathrm{E}-03$ & 1153.4103 & 424.9977 & 1578.4081 & \\
\hline & 8/3/2008 21:00 & 296 & 216 & 2100 & 6.659 & 6.659 & & 0.4116 & 3.99E-03 & 682.7099 & 251.5585 & 934.2684 & \\
\hline & 8/3/2008 22:00 & 297 & 216 & 2200 & 7.392 & 7.392 & & 0.2716 & 2.39E-03 & 409.3010 & 150.8154 & 560.1164 & \\
\hline & 8/3/2008 23:00 & 298 & 216 & 2300 & 7.532 & 7.532 & & 0.2480 & 2.14E-03 & 365.9106 & 134.8273 & 500.7379 & \\
\hline & 8/4/2008 0:00 & 299 & 217 & 0 & 7.569 & 7.569 & 8.97 & 0.2419 & 2.07E-03 & 354.8621 & 130.7562 & 485.6183 & \\
\hline & 8/4/2008 1:00 & 300 & 217 & 100 & 7.627 & 7.627 & & 0.2325 & 1.97E-03 & 337.8856 & 124.5009 & 462.3865 & \\
\hline & 8/4/2008 2:00 & 301 & 217 & 200 & 7.239 & 7.239 & & 0.2985 & 2.69E-03 & 459.7083 & 169.3890 & 629.0973 & \\
\hline & 8/4/2008 3:00 & 302 & 217 & 300 & 6.986 & 6.986 & & 0.3455 & 3.22E-03 & 550.4158 & 202.8120 & 753.2278 & \\
\hline & 8/4/2008 4:00 & 303 & 217 & 400 & 6.748 & 6.748 & & 0.3930 & 3.77E-03 & 644.8982 & 237.6260 & 882.5242 & \\
\hline & $8 / 4 / 20085: 00$ & 304 & 217 & 500 & 6.95 & 6.95 & & 0.3525 & $3.30 \mathrm{E}-03$ & 564.1139 & 207.8594 & 771.9733 & \\
\hline & 8/4/2008 6:00 & 305 & 217 & 600 & 7.468 & 7.468 & & 0.2587 & $2.25 \mathrm{E}-03$ & 385.4313 & 142.0201 & 527.4513 & \\
\hline & 8/4/2008 7:00 & 306 & 217 & 700 & 7.555 & 7.555 & & 0.2442 & $2.10 \mathrm{E}-03$ & 359.0224 & 132.2892 & 491.3116 & \\
\hline & 8/4/2008 8:00 & 307 & 217 & 800 & 7.359 & 7.359 & & 0.2773 & $2.45 \mathrm{E}-03$ & 419.9035 & 154.7221 & 574.6255 & \\
\hline & 8/4/2008 9:00 & 308 & 217 & 900 & 6.909 & 6.909 & & 0.3605 & 3.39E-03 & 579.9661 & 213.7004 & 793.6666 & \\
\hline & 8/4/2008 10:00 & 309 & 217 & 1000 & 6.077 & 6.077 & & 0.5468 & 5.66E-03 & 968.4319 & 356.8386 & 1325.2705 & \\
\hline & 8/4/2008 11:00 & 310 & 217 & 1100 & 5.628 & 5.628 & & 0.6702 & 7.27E-03 & 1244.1150 & 458.4198 & 1702.5348 & \\
\hline & 8/4/2008 12:00 & 311 & 217 & 1200 & 5.426 & 5.426 & & 0.7324 & 8.11E-03 & 1387.6871 & 511.3218 & 1899.0089 & \\
\hline & 8/4/2008 13:00 & 312 & 217 & 1300 & 5.367 & 5.367 & & 0.7514 & 8.37E-03 & 1432.2387 & 527.7378 & 1959.9766 & \\
\hline & 8/4/2008 14:00 & 313 & 217 & 1400 & 5.639 & 5.639 & & 0.6670 & 7.23E-03 & 1236.6742 & 455.6780 & 1692.3522 & \\
\hline & 8/4/2008 15:00 & 314 & 217 & 1500 & 6.042 & 6.042 & & 0.5558 & $5.77 \mathrm{E}-03$ & 988.0036 & 364.0503 & 1352.0539 & \\
\hline & 8/4/2008 16:00 & 315 & 217 & 1600 & 5.5 & 5.5 & & 0.7091 & 7.79E-03 & 1333.5237 & 491.3642 & 1824.8879 & \\
\hline & 8/4/2008 17:00 & 316 & 217 & 1700 & 5.468 & 5.468 & & 0.7191 & 7.93E-03 & 1356.7162 & 499.9100 & 1856.6262 & \\
\hline & 8/4/2008 18:00 & 317 & 217 & 1800 & 5.529 & 5.529 & & 0.7001 & 7.67E-03 & 1312.8014 & 483.7287 & 1796.5301 & \\
\hline & 8/4/2008 19:00 & 318 & 217 & 1900 & 5.685 & 5.685 & & 0.6535 & 7.05E-03 & 1205.9596 & 444.3606 & 1650.3202 & \\
\hline & 8/4/2008 20:00 & 319 & 217 & 2000 & 6.354 & 6.354 & & 0.4794 & 4.81E-03 & 823.5824 & 303.4659 & 1127.0483 & \\
\hline & 8/4/2008 21:00 & 320 & 217 & 2100 & 7.997 & 7.997 & & 0.1754 & $1.40 \mathrm{E}-03$ & 238.9524 & 88.0469 & 326.9993 & \\
\hline & 8/4/2008 22:00 & 321 & 217 & 2200 & 8.75 & 8.75 & & 0.0743 & 4.85E-04 & 82.9604 & 30.5685 & 113.5289 & \\
\hline & 8/4/2008 23:00 & 322 & 217 & 2300 & 8.97 & 8.97 & & 0.0479 & 2.83E-04 & 48.3837 & 17.8280 & 66.2116 & \\
\hline & 8/5/2008 0:00 & 323 & 218 & 0 & 9.27 & 9.27 & 9.76 & 0.0140 & $6.23 \mathrm{E}-05$ & 10.6555 & 3.9263 & 14.5818 & \\
\hline & 8/5/2008 1:00 & 324 & 218 & 100 & 9.46 & 9.46 & & -0.0063 & $0.00 \mathrm{E}+00$ & 0.0000 & 0.0000 & 0.0000 & \\
\hline & 8/5/2008 2:00 & 325 & 218 & 200 & 9.61 & 9.61 & & -0.0219 & $0.00 \mathrm{E}+00$ & 0.0000 & 0.0000 & 0.0000 & \\
\hline & 8/5/2008 3:00 & 326 & 218 & 300 & 9.58 & 9.58 & & -0.0188 & $0.00 \mathrm{E}+00$ & 0.0000 & 0.0000 & 0.0000 & \\
\hline & 8/5/2008 4:00 & 327 & 218 & 400 & 9.41 & 9.41 & & -0.0011 & $0.00 \mathrm{E}+00$ & 0.0000 & 0.0000 & 0.0000 & \\
\hline
\end{tabular}




\begin{tabular}{|c|c|c|c|c|c|c|c|c|c|c|c|c|c|}
\hline Tree ID & TS & RN & JD & HR & $\begin{array}{c}\text { Raw } \\
\text { TC_dTA }\end{array}$ & $\begin{array}{c}\text { Fixed } \\
\text { TC_dT } \Delta\end{array}$ & dTM & $\mathbf{K}$ & $\begin{array}{l}\text { Sap } \\
\text { Velocity } \\
(\mathrm{cm} / \mathrm{s})\end{array}$ & $\begin{array}{c}\text { Probed } \\
\text { Drywood F } \\
(\mathrm{g} / \mathrm{hr})\end{array}$ & $\begin{array}{c}\text { Extrapolated } \\
\text { Wetwood F } \\
(\mathrm{g} / \mathrm{hr})\end{array}$ & $\begin{array}{c}\text { Extrapolated } \\
\text { Total Wood F } \\
(\mathrm{g} / \mathrm{hr})\end{array}$ & $\begin{array}{l}\text { Relative } \\
\text { Humidity, } \\
@ 10 \mathrm{~m}(\%)\end{array}$ \\
\hline & 8/5/2008 5:00 & 328 & 218 & 500 & $\begin{array}{r}9.38 \\
\end{array}$ & 9.38 & & 0.0021 & $6.13 \mathrm{E}-06$ & 1.0485 & 0.3863 & $\begin{array}{r}1.4349 \\
\end{array}$ & \\
\hline & 8/5/2008 6:00 & 329 & 218 & 600 & 9.49 & 9.49 & & -0.0095 & $0.00 \mathrm{E}+00$ & 0.0000 & 0.0000 & 0.0000 & \\
\hline & 8/5/2008 7:00 & 330 & 218 & 700 & 9.59 & 9.59 & & -0.0198 & $0.00 E+00$ & 0.0000 & 0.0000 & 0.0000 & \\
\hline & 8/5/2008 8:00 & 331 & 218 & 800 & 9.44 & 9.44 & & -0.0042 & $0.00 \mathrm{E}+00$ & 0.0000 & 0.0000 & 0.0000 & \\
\hline & 8/5/2008 9:00 & 332 & 218 & 900 & 7.691 & 7.691 & & 0.2222 & 1.87E-03 & 319.6298 & 117.7742 & 437.4040 & \\
\hline & 8/5/2008 10:00 & 333 & 218 & 1000 & 6.035 & 6.035 & & 0.5576 & $5.80 \mathrm{E}-03$ & 991.9541 & 365.5059 & 1357.4599 & \\
\hline & 8/5/2008 11:00 & 334 & 218 & 1100 & 6.225 & 6.225 & & 0.5100 & $5.20 \mathrm{E}-03$ & 888.8892 & 327.5295 & 1216.4188 & \\
\hline & $8 / 5 / 200812: 00$ & 335 & 218 & 1200 & 5.937 & 5.937 & & 0.5833 & $6.13 \mathrm{E}-03$ & 1048.5560 & 386.3620 & 1434.9181 & \\
\hline & 8/5/2008 13:00 & 336 & 218 & 1300 & 5.564 & 5.564 & & 0.6894 & 7.53E-03 & 1288.1596 & 474.6489 & 1762.8085 & \\
\hline & 8/5/2008 14:00 & 337 & 218 & 1400 & 6.019 & 6.019 & & 0.5617 & 5.85E-03 & 1001.0293 & 368.8498 & 1369.8792 & \\
\hline & 8/5/2008 15:00 & 338 & 218 & 1500 & 6.441 & 6.441 & & 0.4594 & 4.57E-03 & 781.5281 & 287.9701 & 1069.4982 & \\
\hline & 8/5/2008 16:00 & 339 & 218 & 1600 & 6.339 & 6.339 & & 0.4829 & $4.86 \mathrm{E}-03$ & 830.9921 & 306.1961 & 1137.1882 & \\
\hline & 8/5/2008 17:00 & 340 & 218 & 1700 & 5.855 & 5.855 & & 0.6055 & $6.42 \mathrm{E}-03$ & 1097.8390 & 404.5213 & 1502.3603 & \\
\hline & 8/5/2008 18:00 & 341 & 218 & 1800 & 5.626 & 5.626 & & 0.6708 & $7.28 \mathrm{E}-03$ & 1245.4719 & 458.9197 & 1704.3916 & \\
\hline & 8/5/2008 19:00 & 342 & 218 & 1900 & 5.739 & 5.739 & & 0.6379 & 6.84E-03 & 1170.7133 & 431.3734 & 1602.0867 & \\
\hline & 8/5/2008 20:00 & 343 & 218 & 2000 & 6.665 & 6.665 & & 0.4104 & 3.98E-03 & 680.1163 & 250.6028 & 930.7191 & \\
\hline & 8/5/2008 21:00 & 344 & 218 & 2100 & 8.62 & 8.62 & & 0.0905 & $6.18 \mathrm{E}-04$ & 105.7659 & 38.9716 & 144.7375 & \\
\hline & $8 / 5 / 200822: 00$ & 345 & 218 & 2200 & 9.49 & 9.49 & & -0.0095 & $0.00 \mathrm{E}+00$ & 0.0000 & 0.0000 & 0.0000 & \\
\hline & 8/5/2008 23:00 & 346 & 218 & 2300 & 9.76 & 9.76 & & -0.0369 & $0.00 \mathrm{E}+00$ & 0.0000 & 0.0000 & 0.0000 & \\
\hline & 8/6/2008 0:00 & 347 & 219 & 0 & 9.91 & 9.91 & 9.98 & -0.0515 & $0.00 E+00$ & 0.0000 & 0.0000 & 0.0000 & \\
\hline & 8/6/2008 1:00 & 348 & 219 & 100 & 9.94 & 9.94 & & -0.0543 & $0.00 \mathrm{E}+00$ & 0.0000 & 0.0000 & 0.0000 & \\
\hline & $8 / 6 / 2008$ 2:00 & 349 & 219 & 200 & 9.97 & 9.97 & & -0.0572 & $0.00 E+00$ & 0.0000 & 0.0000 & 0.0000 & \\
\hline & 8/6/2008 3:00 & 350 & 219 & 300 & 9.98 & 9.98 & & -0.0581 & $0.00 E+00$ & 0.0000 & 0.0000 & 0.0000 & \\
\hline & 8/6/2008 4:00 & 351 & 219 & 400 & 9.93 & 9.93 & & -0.0534 & $0.00 \mathrm{E}+00$ & 0.0000 & 0.0000 & 0.0000 & \\
\hline & 8/6/2008 5:00 & 352 & 219 & 500 & 9.92 & 9.92 & & -0.0524 & $0.00 \mathrm{E}+00$ & 0.0000 & 0.0000 & 0.0000 & \\
\hline & 8/6/2008 6:00 & 353 & 219 & 600 & 9.88 & 9.88 & & -0.0486 & $0.00 E+00$ & 0.0000 & 0.0000 & 0.0000 & \\
\hline & 8/6/2008 7:00 & 354 & 219 & 700 & 9.86 & 9.86 & & -0.0467 & $0.00 \mathrm{E}+00$ & 0.0000 & 0.0000 & 0.0000 & \\
\hline & 8/6/2008 8:00 & 355 & 219 & 800 & 9.82 & 9.82 & & -0.0428 & $0.00 \mathrm{E}+00$ & 0.0000 & 0.0000 & 0.0000 & \\
\hline & 8/6/2008 9:00 & 356 & 219 & 900 & 9.4 & 9.4 & & 0.0000 & $0.00 \mathrm{E}+00$ & 0.0000 & 0.0000 & 0.0000 & \\
\hline & 8/6/2008 10:00 & 357 & 219 & 1000 & 6.492 & 6.492 & & 0.4479 & 4.43E-03 & 757.5886 & 279.1491 & 1036.7377 & \\
\hline & 8/6/2008 11:00 & 358 & 219 & 1100 & 5.545 & 5.545 & & 0.6952 & 7.61E-03 & 1301.4871 & 479.5597 & 1781.0468 & \\
\hline & 8/6/2008 12:00 & 359 & 219 & 1200 & 6.006 & 6.006 & & 0.5651 & 5.89E-03 & 1008.4501 & 371.5842 & 1380.0342 & \\
\hline & 8/6/2008 13:00 & 360 & 219 & 1300 & 6.448 & 6.448 & & 0.4578 & $4.55 \mathrm{E}-03$ & 778.2116 & 286.7481 & 1064.9596 & \\
\hline & $8 / 6 / 2008$ 14:00 & 361 & 219 & 1400 & 5.953 & 5.953 & & 0.5790 & 6.07E-03 & 1039.1471 & 382.8951 & 1422.0422 & \\
\hline & 8/6/2008 15:00 & 362 & 219 & 1500 & 5.638 & 5.638 & & 0.6673 & 7.23E-03 & 1237.3491 & 455.9267 & 1693.2758 & \\
\hline & 8/6/2008 16:00 & 363 & 219 & 1600 & 5.696 & 5.696 & & 0.6503 & 7.01E-03 & 1198.7096 & 441.6892 & 1640.3988 & \\
\hline & 8/6/2008 17:00 & 364 & 219 & 1700 & 5.62 & 5.62 & & 0.6726 & 7.30E-03 & 1249.5501 & 460.4224 & 1709.9725 & \\
\hline & 8/6/2008 18:00 & 365 & 219 & 1800 & 6.012 & 6.012 & & 0.5635 & 5.87E-03 & 1005.0199 & 370.3202 & 1375.3401 & \\
\hline & 8/6/2008 19:00 & 366 & 219 & 1900 & 6.054 & 6.054 & & 0.5527 & $5.74 \mathrm{E}-03$ & 981.2595 & 361.5652 & 1342.8248 & \\
\hline & $8 / 6 / 200820: 00$ & 367 & 219 & 2000 & 6.419 & 6.419 & & 0.4644 & 4.63E-03 & 792.0159 & 291.8346 & 1083.8505 & \\
\hline & 8/6/2008 21:00 & 368 & 219 & 2100 & 8.06 & 8.06 & & 0.1663 & 1.31E-03 & 223.6424 & 82.4057 & 306.0481 & \\
\hline
\end{tabular}




\begin{tabular}{|c|c|c|c|c|c|c|c|c|c|c|c|c|c|}
\hline Tree ID & TS & RN & JD & HR & $\begin{array}{c}\text { Raw } \\
\text { TC_dTA }\end{array}$ & $\begin{array}{c}\text { Fixed } \\
\text { TC_dT } \Delta\end{array}$ & dTM & $\mathbf{K}$ & $\begin{array}{l}\text { Sap } \\
\text { Velocity } \\
(\mathrm{cm} / \mathrm{s})\end{array}$ & $\begin{array}{c}\text { Probed } \\
\text { Drywood F } \\
(\mathrm{g} / \mathrm{hr})\end{array}$ & $\begin{array}{c}\text { Extrapolated } \\
\text { Wetwood F } \\
(\mathrm{g} / \mathrm{hr})\end{array}$ & $\begin{array}{c}\text { Extrapolated } \\
\text { Total Wood F } \\
(\mathrm{g} / \mathrm{hr})\end{array}$ & $\begin{array}{l}\text { Relative } \\
\text { Humidity, } \\
@ 10 \mathrm{~m}(\%)\end{array}$ \\
\hline & 8/6/2008 22:00 & 369 & 219 & 2200 & $\begin{array}{r}9.36 \\
\end{array}$ & 9.36 & & 0.0043 & 1.44E-05 & 2.4676 & 0.9093 & $\begin{array}{r}3.3769 \\
\end{array}$ & \\
\hline & 8/6/2008 23:00 & 370 & 219 & 2300 & 9.7 & 9.7 & & -0.0309 & $0.00 \mathrm{E}+00$ & 0.0000 & 0.0000 & 0.0000 & \\
\hline & $8 / 7 / 20080: 00$ & 371 & 220 & 0 & 9.87 & 9.87 & 9.97 & -0.0476 & $0.00 E+00$ & 0.0000 & 0.0000 & 0.0000 & \\
\hline & 8/7/2008 1:00 & 372 & 220 & 100 & 9.97 & 9.97 & & -0.0572 & $0.00 \mathrm{E}+00$ & 0.0000 & 0.0000 & 0.0000 & \\
\hline & 8/7/2008 2:00 & 373 & 220 & 200 & 9.97 & 9.97 & & -0.0572 & $0.00 \mathrm{E}+00$ & 0.0000 & 0.0000 & 0.0000 & \\
\hline & 8/7/2008 3:00 & 374 & 220 & 300 & 9.92 & 9.92 & & -0.0524 & $0.00 \mathrm{E}+00$ & 0.0000 & 0.0000 & 0.0000 & \\
\hline & 8/7/2008 4:00 & 375 & 220 & 400 & 9.9 & 9.9 & & -0.0505 & $0.00 \mathrm{E}+00$ & 0.0000 & 0.0000 & 0.0000 & \\
\hline & 8/7/2008 5:00 & 376 & 220 & 500 & 9.87 & 9.87 & & -0.0476 & $0.00 \mathrm{E}+00$ & 0.0000 & 0.0000 & 0.0000 & \\
\hline & 8/7/2008 6:00 & 377 & 220 & 600 & 9.83 & 9.83 & & -0.0437 & $0.00 \mathrm{E}+00$ & 0.0000 & 0.0000 & 0.0000 & \\
\hline & 8/7/2008 7:00 & 378 & 220 & 700 & 9.81 & 9.81 & & -0.0418 & $0.00 E+00$ & 0.0000 & 0.0000 & 0.0000 & \\
\hline & 8/7/2008 8:00 & 379 & 220 & 800 & 9.77 & 9.77 & & -0.0379 & $0.00 \mathrm{E}+00$ & 0.0000 & 0.0000 & 0.0000 & \\
\hline & 8/7/2008 9:00 & 380 & 220 & 900 & 8.75 & 8.75 & & 0.0743 & 4.85E-04 & 82.9604 & 30.5685 & 113.5289 & \\
\hline & 8/7/2008 10:00 & 381 & 220 & 1000 & 6.042 & 6.042 & & 0.5558 & 5.77E-03 & 988.0036 & 364.0503 & 1352.0539 & \\
\hline & 8/7/2008 11:00 & 382 & 220 & 1100 & 5.506 & 5.506 & & 0.7072 & 7.77E-03 & 1329.2134 & 489.7760 & 1818.9894 & \\
\hline & 8/7/2008 12:00 & 383 & 220 & 1200 & 5.477 & 5.477 & & 0.7163 & 7.89E-03 & 1350.1583 & 497.4936 & 1847.6519 & \\
\hline & 8/7/2008 13:00 & 384 & 220 & 1300 & \begin{tabular}{|c|}
5.467 \\
\end{tabular} & 5.467 & & 0.7194 & 7.93E-03 & 1357.4465 & 500.1791 & 1857.6256 & \\
\hline & $8 / 7 / 2008$ 14:00 & 385 & 220 & 1400 & 5.456 & 5.456 & & 0.7229 & 7.98E-03 & 1365.5030 & 503.1477 & 1868.6507 & \\
\hline & 8/7/2008 15:00 & 386 & 220 & 1500 & 5.517 & 5.517 & & 0.7038 & 7.72E-03 & 1321.3423 & 486.8758 & 1808.2181 & \\
\hline & 8/7/2008 16:00 & 387 & 220 & 1600 & 5.488 & 5.488 & & 0.7128 & 7.84E-03 & 1342.1804 & 494.5540 & 1836.7343 & \\
\hline & 8/7/2008 17:00 & 388 & 220 & 1700 & 5.507 & 5.507 & & 0.7069 & 7.76E-03 & 1328.4962 & 489.5117 & 1818.0079 & \\
\hline & 8/7/2008 18:00 & 389 & 220 & 1800 & 5.533 & 5.533 & & 0.6989 & $7.66 \mathrm{E}-03$ & 1309.9650 & 482.6835 & 1792.6485 & \\
\hline & 8/7/2008 19:00 & 390 & 220 & 1900 & 5.739 & 5.739 & & 0.6379 & 6.84E-03 & 1170.7133 & 431.3734 & 1602.0867 & \\
\hline & 8/7/2008 20:00 & 391 & 220 & 2000 & 6.282 & 6.282 & & 0.4963 & 5.02E-03 & 859.5865 & 316.7323 & 1176.3189 & \\
\hline & 8/7/2008 21:00 & 392 & 220 & 2100 & 7.893 & 7.893 & & 0.1909 & 1.55E-03 & 265.1790 & 97.7106 & 362.8896 & \\
\hline & 8/7/2008 22:00 & 393 & 220 & 2200 & 8.89 & 8.89 & & 0.0574 & 3.53E-04 & 60.3542 & 22.2387 & 82.5929 & \\
\hline & 8/7/2008 23:00 & 394 & 220 & 2300 & 9.38 & 9.38 & & 0.0021 & 6.13E-06 & 1.0485 & 0.3863 & 1.4349 & \\
\hline & 8/8/2008 0:00 & 395 & 221 & 0 & 9.66 & 9.66 & 10.03 & -0.0269 & $0.00 \mathrm{E}+00$ & 0.0000 & 0.0000 & 0.0000 & \\
\hline & 8/8/2008 1:00 & 396 & 221 & 100 & 9.69 & 9.69 & & -0.0299 & $0.00 \mathrm{E}+00$ & 0.0000 & 0.0000 & 0.0000 & \\
\hline & 8/8/2008 2:00 & 397 & 221 & 200 & 9.91 & 9.91 & & -0.0515 & $0.00 \mathrm{E}+00$ & 0.0000 & 0.0000 & 0.0000 & \\
\hline & 8/8/2008 3:00 & 398 & 221 & 300 & 10.03 & 10.03 & & -0.0628 & $0.00 \mathrm{E}+00$ & 0.0000 & 0.0000 & 0.0000 & \\
\hline & 8/8/2008 4:00 & 399 & 221 & 400 & 10.01 & 10.01 & & -0.0609 & $0.00 \mathrm{E}+00$ & 0.0000 & 0.0000 & 0.0000 & \\
\hline & 8/8/2008 5:00 & 400 & 221 & 500 & 9.96 & 9.96 & & -0.0562 & $0.00 \mathrm{E}+00$ & 0.0000 & 0.0000 & 0.0000 & \\
\hline & 8/8/2008 6:00 & 401 & 221 & 600 & 9.93 & 9.93 & & -0.0534 & $0.00 \mathrm{E}+00$ & 0.0000 & 0.0000 & 0.0000 & \\
\hline & $8 / 8 / 20087: 00$ & 402 & 221 & 700 & 9.9 & 9.9 & & -0.0505 & $0.00 \mathrm{E}+00$ & 0.0000 & 0.0000 & 0.0000 & \\
\hline & 8/8/2008 8:00 & 403 & 221 & 800 & 9.72 & 9.72 & & -0.0329 & $0.00 \mathrm{E}+00$ & 0.0000 & 0.0000 & 0.0000 & \\
\hline & 8/8/2008 9:00 & 404 & 221 & 900 & 7.446 & 7.446 & & 0.2624 & 2.29E-03 & 392.2632 & 144.5374 & 536.8006 & \\
\hline & 8/8/2008 10:00 & 405 & 221 & 1000 & 5.709 & 5.709 & & 0.6465 & 6.96E-03 & 1190.1880 & 438.5493 & 1628.7373 & \\
\hline & 8/8/2008 11:00 & 406 & 221 & 1100 & 5.675 & 5.675 & & 0.6564 & 7.09E-03 & 1212.5820 & 446.8008 & 1659.3828 & \\
\hline & $8 / 8 / 200812: 00$ & 407 & 221 & 1200 & 5.534 & 5.534 & & 0.6986 & 7.65E-03 & 1309.2567 & 482.4226 & 1791.6793 & \\
\hline & 8/8/2008 13:00 & 408 & 221 & 1300 & 5.473 & 5.473 & & 0.7175 & 7.91E-03 & 1353.0695 & 498.5663 & 1851.6358 & \\
\hline & 8/8/2008 14:00 & 409 & 221 & 1400 & 5.425 & 5.425 & & 0.7327 & 8.11E-03 & 1388.4319 & 511.5963 & 1900.0282 & \\
\hline
\end{tabular}




\begin{tabular}{|c|c|c|c|c|c|c|c|c|c|c|c|c|c|}
\hline Tree ID & TS & RN & JD & HR & $\begin{array}{c}\text { Raw } \\
\text { TC_dTA }\end{array}$ & $\begin{array}{c}\text { Fixed } \\
\text { TC_dT } \Delta\end{array}$ & dTM & $\mathbf{K}$ & $\begin{array}{l}\text { Sap } \\
\text { Velocity } \\
(\mathrm{cm} / \mathrm{s})\end{array}$ & $\begin{array}{c}\text { Probed } \\
\text { Drywood F } \\
(\mathrm{g} / \mathrm{hr})\end{array}$ & $\begin{array}{c}\text { Extrapolated } \\
\text { Wetwood F } \\
(\mathrm{g} / \mathrm{hr})\end{array}$ & $\begin{array}{c}\text { Extrapolated } \\
\text { Total Wood F } \\
(\mathrm{g} / \mathrm{hr})\end{array}$ & $\begin{array}{l}\text { Relative } \\
\text { Humidity, } \\
@ 10 \mathrm{~m}(\%)\end{array}$ \\
\hline & 8/8/2008 15:00 & 410 & 221 & 1500 & 5.46 & 5.46 & & 0.7216 & 7.96E-03 & 1362.5686 & 502.0664 & 1864.6350 & \\
\hline & 8/8/2008 16:00 & 411 & 221 & 1600 & 5.419 & 5.419 & & 0.7346 & $8.14 \mathrm{E}-03$ & 1392.9084 & 513.2458 & 1906.1542 & \\
\hline & 8/8/2008 17:00 & 412 & 221 & 1700 & 5.523 & 5.523 & & 0.7020 & 7.70E-03 & 1317.0659 & 485.3000 & 1802.3660 & \\
\hline & 8/8/2008 18:00 & 413 & 221 & 1800 & 5.692 & 5.692 & & 0.6514 & 7.02E-03 & 1201.3418 & 442.6591 & 1644.0009 & \\
\hline & 8/8/2008 19:00 & 414 & 221 & 1900 & 5.658 & 5.658 & & 0.6614 & 7.15E-03 & 1223.9096 & 450.9747 & 1674.8843 & \\
\hline & 8/8/2008 20:00 & 415 & 221 & 2000 & 6.389 & 6.389 & & 0.4713 & 4.71E-03 & 806.4765 & 297.1629 & 1103.6394 & \\
\hline & 8/8/2008 21:00 & 416 & 221 & 2100 & 7.793 & 7.793 & & 0.2062 & 1.70E-03 & 291.5438 & 107.4253 & 398.9691 & \\
\hline & 8/8/2008 22:00 & 417 & 221 & 2200 & 8.59 & 8.59 & & 0.0943 & $6.50 \mathrm{E}-04$ & 111.2721 & 41.0005 & 152.2725 & \\
\hline & 8/8/2008 23:00 & 418 & 221 & 2300 & 8.84 & 8.84 & & 0.0633 & 3.99E-04 & 68.1904 & 25.1262 & 93.3166 & \\
\hline & 8/9/2008 0:00 & 419 & 222 & 0 & 8.95 & 8.95 & 9.82 & 0.0503 & 3.00E-04 & 51.3094 & 18.9060 & 70.2154 & \\
\hline & 8/9/2008 1:00 & 420 & 222 & 100 & 8.95 & 8.95 & & 0.0503 & 3.00E-04 & 51.3094 & 18.9060 & 70.2154 & \\
\hline & 8/9/2008 2:00 & 421 & 222 & 200 & 9.01 & 9.01 & & 0.0433 & $2.49 E-04$ & 42.6698 & 15.7226 & 58.3924 & \\
\hline & 8/9/2008 3:00 & 422 & 222 & 300 & 9.03 & 9.03 & & 0.0410 & 2.33E-04 & 39.8833 & 14.6958 & 54.5792 & \\
\hline & 8/9/2008 4:00 & 423 & 222 & 400 & 9.02 & 9.02 & & 0.0421 & 2.41E-04 & 41.2706 & 15.2070 & 56.4776 & \\
\hline & $8 / 9 / 20085: 00$ & 424 & 222 & 500 & 8.86 & 8.86 & & 0.0609 & 3.80E-04 & 65.0238 & 23.9594 & 88.9832 & \\
\hline & 8/9/2008 6:00 & 425 & 222 & 600 & 8.84 & 8.84 & & 0.0633 & 3.99E-04 & 68.1904 & 25.1262 & \begin{tabular}{|l|}
93.3166 \\
\end{tabular} & \\
\hline & 8/9/2008 7:00 & 426 & 222 & 700 & 8.43 & 8.43 & & 0.1151 & 8.31E-04 & 142.1703 & 52.3856 & 194.5559 & \\
\hline & 8/9/2008 8:00 & 427 & 222 & 800 & 7.57 & 7.57 & & 0.2417 & 2.07E-03 & 354.5659 & 130.6471 & 485.2130 & \\
\hline & 8/9/2008 9:00 & 428 & 222 & 900 & 8.19 & 8.19 & & 0.1477 & 1.13E-03 & 193.3940 & 71.2600 & 264.6540 & \\
\hline & 8/9/2008 10:00 & 429 & 222 & 1000 & 7.997 & 7.997 & & 0.1754 & 1.40E-03 & 238.9524 & 88.0469 & 326.9993 & \\
\hline & 8/9/2008 11:00 & 430 & 222 & 1100 & 7.112 & 7.112 & & 0.3217 & $2.95 \mathrm{E}-03$ & 504.0518 & 185.7283 & 689.7801 & \\
\hline & 8/9/2008 12:00 & 431 & 222 & 1200 & 6.242 & 6.242 & & 0.5059 & 5.14E-03 & 880.0745 & 324.2816 & 1204.3561 & \\
\hline & 8/9/2008 13:00 & 432 & 222 & 1300 & 6.085 & 6.085 & & 0.5448 & 5.63E-03 & 964.0002 & 355.2057 & 1319.2059 & \\
\hline & 8/9/2008 14:00 & 433 & 222 & 1400 & 5.7 & 5.7 & & 0.6491 & $6.99 \mathrm{E}-03$ & 1196.0822 & 440.7211 & 1636.8033 & \\
\hline & 8/9/2008 15:00 & 434 & 222 & 1500 & 5.619 & 5.619 & & 0.6729 & 7.31E-03 & 1250.2309 & 460.6733 & 1710.9041 & \\
\hline & 8/9/2008 16:00 & 435 & 222 & 1600 & 5.642 & 5.642 & & 0.6661 & $7.22 E-03$ & 1234.6513 & 454.9327 & 1689.5840 & \\
\hline & 8/9/2008 17:00 & 436 & 222 & 1700 & 5.729 & 5.729 & & 0.6408 & 6.88E-03 & 1177.1755 & 433.7545 & 1610.9300 & \\
\hline & 8/9/2008 18:00 & 437 & 222 & 1800 & 5.75 & 5.75 & & 0.6348 & $6.80 \mathrm{E}-03$ & 1163.6385 & 428.7665 & 1592.4050 & \\
\hline & 8/9/2008 19:00 & 438 & 222 & 1900 & 5.886 & 5.886 & & 0.5970 & 6.31E-03 & 1078.9961 & 397.5783 & 1476.5745 & \\
\hline & 8/9/2008 20:00 & 439 & 222 & 2000 & 6.569 & 6.569 & & 0.4310 & $4.22 \mathrm{E}-03$ & 722.4088 & 266.1864 & 988.5952 & \\
\hline & 8/9/2008 21:00 & 440 & 222 & 2100 & 8.51 & 8.51 & & 0.1046 & 7.39E-04 & 126.3987 & 46.5742 & 172.9728 & \\
\hline & 8/9/2008 22:00 & 441 & 222 & 2200 & 9.59 & 9.59 & & -0.0198 & $0.00 \mathrm{E}+00$ & 0.0000 & 0.0000 & 0.0000 & \\
\hline & 8/9/2008 23:00 & 442 & 222 & 2300 & 9.82 & 9.82 & & -0.0428 & $0.00 \mathrm{E}+00$ & 0.0000 & 0.0000 & 0.0000 & \\
\hline & 8/10/2008 0:00 & 443 & 223 & 0 & 9.91 & 9.91 & 9.97 & -0.0515 & $0.00 \mathrm{E}+00$ & 0.0000 & 0.0000 & 0.0000 & \\
\hline & 8/10/2008 1:00 & 444 & 223 & 100 & 9.97 & 9.97 & & -0.0572 & $0.00 \mathrm{E}+00$ & 0.0000 & 0.0000 & 0.0000 & \\
\hline & 8/10/2008 2:00 & 445 & 223 & 200 & 9.97 & 9.97 & & -0.0572 & $0.00 \mathrm{E}+00$ & 0.0000 & 0.0000 & 0.0000 & \\
\hline & 8/10/2008 3:00 & 446 & 223 & 300 & 9.96 & 9.96 & & -0.0562 & $0.00 \mathrm{E}+00$ & 0.0000 & 0.0000 & 0.0000 & \\
\hline & 8/10/2008 4:00 & 447 & 223 & 400 & 9.93 & 9.93 & & -0.0534 & $0.00 \mathrm{E}+00$ & 0.0000 & 0.0000 & 0.0000 & \\
\hline & $8 / 10 / 20085: 00$ & 448 & 223 & 500 & 9.91 & 9.91 & & -0.0515 & $0.00 \mathrm{E}+00$ & 0.0000 & 0.0000 & 0.0000 & \\
\hline & 8/10/2008 6:00 & 449 & 223 & 600 & 9.88 & 9.88 & & -0.0486 & $0.00 \mathrm{E}+00$ & 0.0000 & 0.0000 & 0.0000 & \\
\hline & 8/10/2008 7:00 & 450 & 223 & 700 & 9.86 & 9.86 & & -0.0467 & $0.00 \mathrm{E}+00$ & 0.0000 & 0.0000 & 0.0000 & \\
\hline
\end{tabular}




\begin{tabular}{|c|c|c|c|c|c|c|c|c|c|c|c|c|c|}
\hline Tree ID & TS & RN & JD & HR & $\begin{array}{c}\text { Raw } \\
\text { TC_dTA }\end{array}$ & $\begin{array}{c}\text { Fixed } \\
\text { TC_dT } \Delta\end{array}$ & dTM & K & $\begin{array}{l}\text { Sap } \\
\text { Velocity } \\
(\mathrm{cm} / \mathrm{s})\end{array}$ & $\begin{array}{c}\text { Probed } \\
\text { Drywood F } \\
(\mathrm{g} / \mathrm{hr})\end{array}$ & $\begin{array}{c}\text { Extrapolated } \\
\text { Wetwood F } \\
(\mathrm{g} / \mathrm{hr})\end{array}$ & $\begin{array}{c}\text { Extrapolated } \\
\text { Total Wood F } \\
(\mathrm{g} / \mathrm{hr})\end{array}$ & $\begin{array}{l}\text { Relative } \\
\text { Humidity, } \\
@ 10 \mathrm{~m}(\%)\end{array}$ \\
\hline & 8/10/2008 8:00 & 451 & 223 & 800 & $\begin{array}{r}9.82 \\
\end{array}$ & 9.82 & & -0.0428 & $0.00 \mathrm{E}+00$ & 0.0000 & 0.0000 & 0.0000 & \\
\hline & 8/10/2008 9:00 & 452 & 223 & 900 & 8.64 & 8.64 & & 0.0880 & 5.97E-04 & 102.1456 & 37.6377 & 139.7833 & \\
\hline & $8 / 10 / 2008$ 10:00 & 453 & 223 & 1000 & 6.034 & 6.034 & & 0.5578 & $5.80 \mathrm{E}-03$ & 992.5194 & 365.7142 & 1358.2336 & \\
\hline & 8/10/2008 11:00 & 454 & 223 & 1100 & 5.564 & 5.564 & & 0.6894 & 7.53E-03 & 1288.1596 & 474.6489 & 1762.8085 & \\
\hline & $8 / 10 / 200812: 00$ & 455 & 223 & 1200 & 5.588 & 5.588 & & 0.6822 & 7.43E-03 & 1271.4909 & 468.5070 & 1739.9979 & \\
\hline & 8/10/2008 13:00 & 456 & 223 & 1300 & 5.576 & 5.576 & & 0.6858 & 7.48E-03 & 1279.8022 & 471.5694 & 1751.3717 & \\
\hline & $8 / 10 / 2008$ 14:00 & 457 & 223 & 1400 & 5.542 & 5.542 & & 0.6961 & $7.62 E-03$ & 1303.6022 & 480.3390 & 1783.9412 & \\
\hline & $8 / 10 / 2008$ 15:00 & 458 & 223 & 1500 & 5.699 & 5.699 & & 0.6494 & 6.99E-03 & 1196.7386 & 440.9630 & 1637.7016 & \\
\hline & 8/10/2008 16:00 & 459 & 223 & 1600 & 5.587 & 5.587 & & 0.6825 & 7.44E-03 & 1272.1817 & 468.7615 & 1740.9433 & \\
\hline & 8/10/2008 17:00 & 460 & 223 & 1700 & 5.739 & 5.739 & & 0.6379 & 6.84E-03 & 1170.7133 & 431.3734 & 1602.0867 & \\
\hline & 8/10/2008 18:00 & 461 & 223 & 1800 & 8.42 & 8.42 & & 0.1164 & 8.43E-04 & 144.1872 & 53.1287 & 197.3160 & \\
\hline & $8 / 10 / 2008$ 19:00 & 462 & 223 & 1900 & 9.38 & 9.38 & & 0.0021 & $6.13 E-06$ & 1.0485 & 0.3863 & 1.4349 & \\
\hline & 8/10/2008 20:00 & 463 & 223 & 2000 & 9.12 & 9.12 & & 0.0307 & 1.63E-04 & 27.9566 & 10.3012 & 38.2578 & \\
\hline & 8/10/2008 21:00 & 464 & 223 & 2100 & 9.04 & 9.04 & & 0.0398 & 2.25E-04 & 38.5081 & 14.1891 & 52.6972 & \\
\hline & $8 / 10 / 2008$ 22:00 & 465 & 223 & 2200 & 9.23 & 9.23 & & 0.0184 & 8.71E-05 & 14.9041 & 5.4917 & 20.3958 & \\
\hline & $8 / 10 / 2008$ 23:00 & 466 & 223 & 2300 & 9.17 & 9.17 & & 0.0251 & 1.27E-04 & 21.7970 & 8.0316 & 29.8286 & \\
\hline & $8 / 11 / 20080: 00$ & 467 & 224 & 0 & 9.13 & 9.13 & 10.32 & 0.0296 & 1.56E-04 & 26.6966 & 9.8369 & 36.5335 & \\
\hline & 8/11/2008 1:00 & 468 & 224 & 100 & 9.32 & 9.32 & & 0.0086 & $3.40 \mathrm{E}-05$ & 5.8229 & 2.1456 & 7.9685 & \\
\hline & 8/11/2008 2:00 & 469 & 224 & 200 & 9.6 & 9.6 & & -0.0208 & $0.00 \mathrm{E}+00$ & 0.0000 & 0.0000 & 0.0000 & \\
\hline & 8/11/2008 3:00 & 470 & 224 & 300 & 9.74 & 9.74 & & -0.0349 & $0.00 E+00$ & 0.0000 & 0.0000 & 0.0000 & \\
\hline & 8/11/2008 4:00 & 471 & 224 & 400 & 9.92 & 9.92 & & -0.0524 & $0.00 \mathrm{E}+00$ & 0.0000 & 0.0000 & 0.0000 & \\
\hline & 8/11/2008 5:00 & 472 & 224 & 500 & 9.86 & 9.86 & & -0.0467 & $0.00 E+00$ & 0.0000 & 0.0000 & 0.0000 & \\
\hline & 8/11/2008 6:00 & 473 & 224 & 600 & 9.97 & 9.97 & & -0.0572 & $0.00 \mathrm{E}+00$ & 0.0000 & 0.0000 & 0.0000 & \\
\hline & 8/11/2008 7:00 & 474 & 224 & 700 & 10.12 & 10.12 & & -0.0711 & $0.00 \mathrm{E}+00$ & 0.0000 & 0.0000 & 0.0000 & \\
\hline & 8/11/2008 8:00 & 475 & 224 & 800 & 10.23 & 10.23 & & -0.0811 & $0.00 \mathrm{E}+00$ & 0.0000 & 0.0000 & 0.0000 & \\
\hline & 8/11/2008 9:00 & 476 & 224 & 900 & 9.43 & 9.43 & & -0.0032 & $0.00 \mathrm{E}+00$ & 0.0000 & 0.0000 & 0.0000 & \\
\hline & 8/11/2008 10:00 & 477 & 224 & 1000 & 7.052 & 7.052 & & 0.3330 & 3.07E-03 & 525.8278 & 193.7521 & 719.5799 & \\
\hline & 8/11/2008 11:00 & 478 & 224 & 1100 & 6.627 & 6.627 & & 0.4184 & 4.07E-03 & 696.6534 & 256.6963 & 953.3497 & \\
\hline & $8 / 11 / 2008$ 12:00 & 479 & 224 & 1200 & 7.299 & 7.299 & & 0.2878 & 2.57E-03 & 439.5577 & 161.9641 & 601.5218 & \\
\hline & $8 / 11 / 2008$ 13:00 & 480 & 224 & 1300 & 7.608 & 7.608 & & 0.2355 & 2.01E-03 & 343.4012 & 126.5332 & 469.9344 & \\
\hline & 8/11/2008 14:00 & 481 & 224 & 1400 & 6.974 & 6.974 & & 0.3479 & 3.24E-03 & 554.9591 & 204.4861 & 759.4452 & \\
\hline & 8/11/2008 15:00 & 482 & 224 & 1500 & 7.113 & 7.113 & & 0.3215 & 2.94E-03 & 503.6935 & 185.5962 & 689.2897 & \\
\hline & 8/11/2008 16:00 & 483 & 224 & 1600 & 7.232 & 7.232 & & 0.2998 & $2.70 \mathrm{E}-03$ & 462.0921 & 170.2673 & 632.3594 & \\
\hline & 8/11/2008 17:00 & 484 & 224 & 1700 & 7.717 & 7.717 & & 0.2181 & 1.83E-03 & 312.3540 & 115.0933 & 427.4473 & \\
\hline & 8/11/2008 18:00 & 485 & 224 & 1800 & 8.03 & 8.03 & & 0.1706 & 1.35E-03 & 230.8792 & 85.0722 & 315.9514 & \\
\hline & $8 / 11 / 2008$ 19:00 & 486 & 224 & 1900 & 8.25 & 8.25 & & 0.1394 & 1.05E-03 & 180.0325 & 66.3367 & 246.3692 & \\
\hline & 8/11/2008 20:00 & 487 & 224 & 2000 & 8.78 & 8.78 & & 0.0706 & $4.56 \mathrm{E}-04$ & 77.9434 & 28.7198 & 106.6632 & \\
\hline & 8/11/2008 21:00 & 488 & 224 & 2100 & 9.34 & 9.34 & & 0.0064 & 2.38E-05 & 4.0756 & 1.5017 & 5.5774 & \\
\hline & $8 / 11 / 200822: 00$ & 489 & 224 & 2200 & 10.01 & 10.01 & & -0.0609 & $0.00 \mathrm{E}+00$ & 0.0000 & 0.0000 & 0.0000 & \\
\hline & 8/11/2008 23:00 & 490 & 224 & 2300 & 10.32 & 10.32 & & -0.0891 & $0.00 \mathrm{E}+00$ & 0.0000 & 0.0000 & 0.0000 & \\
\hline & 8/12/2008 0:00 & 491 & 225 & 0 & 10.6 & 10.6 & 11.34 & -0.1132 & $0.00 \mathrm{E}+00$ & 0.0000 & 0.0000 & 0.0000 & \\
\hline
\end{tabular}




\begin{tabular}{|c|c|c|c|c|c|c|c|c|c|c|c|c|c|}
\hline Tree ID & TS & RN & JD & HR & $\begin{array}{c}\text { Raw } \\
\text { TC_dTA }\end{array}$ & $\begin{array}{c}\text { Fixed } \\
\text { TC_dT } \Delta\end{array}$ & dTM & K & $\begin{array}{l}\text { Sap } \\
\text { Velocity } \\
(\mathrm{cm} / \mathrm{s})\end{array}$ & $\begin{array}{c}\text { Probed } \\
\text { Drywood F } \\
(\mathrm{g} / \mathrm{hr})\end{array}$ & $\begin{array}{c}\text { Extrapolated } \\
\text { Wetwood F } \\
(\mathrm{g} / \mathrm{hr})\end{array}$ & $\begin{array}{c}\text { Extrapolated } \\
\text { Total Wood F } \\
(\mathrm{g} / \mathrm{hr})\end{array}$ & $\begin{array}{l}\text { Relative } \\
\text { Humidity, } \\
@ 10 \mathrm{~m}(\%)\end{array}$ \\
\hline & 8/12/2008 1:00 & 492 & 225 & 100 & 10.63 & 10.63 & & -0.1157 & $0.00 \mathrm{E}+00$ & 0.0000 & 0.0000 & 0.0000 & \\
\hline & 8/12/2008 2:00 & 493 & 225 & 200 & 10.4 & 10.4 & & -0.0962 & $0.00 \mathrm{E}+00$ & 0.0000 & 0.0000 & 0.0000 & \\
\hline & 8/12/2008 3:00 & 494 & 225 & 300 & 10.77 & 10.77 & & -0.1272 & $0.00 E+00$ & 0.0000 & 0.0000 & 0.0000 & \\
\hline & 8/12/2008 4:00 & 495 & 225 & 400 & 11.09 & 11.09 & & -0.1524 & $0.00 \mathrm{E}+00$ & 0.0000 & 0.0000 & 0.0000 & \\
\hline & 8/12/2008 5:00 & 496 & 225 & 500 & 11.21 & 11.21 & & -0.1615 & $0.00 \mathrm{E}+00$ & 0.0000 & 0.0000 & 0.0000 & \\
\hline & 8/12/2008 6:00 & 497 & 225 & 600 & 11.21 & 11.21 & & -0.1615 & $0.00 \mathrm{E}+00$ & 0.0000 & 0.0000 & 0.0000 & \\
\hline & 8/12/2008 7:00 & 498 & 225 & 700 & 11.17 & 11.17 & & -0.1585 & $0.00 \mathrm{E}+00$ & 0.0000 & 0.0000 & 0.0000 & \\
\hline & $8 / 12 / 20088: 00$ & 499 & 225 & 800 & 11.34 & 11.34 & & -0.1711 & $0.00 \mathrm{E}+00$ & 0.0000 & 0.0000 & 0.0000 & \\
\hline & 8/12/2008 9:00 & 500 & 225 & 900 & 10.64 & 10.64 & & -0.1165 & $0.00 \mathrm{E}+00$ & 0.0000 & 0.0000 & 0.0000 & \\
\hline & $8 / 12 / 2008$ 10:00 & 501 & 225 & 1000 & 8.5 & 8.5 & & 0.1059 & 7.50E-04 & 128.3348 & 47.2876 & 175.6224 & \\
\hline & $8 / 12 / 2008$ 11:00 & 502 & 225 & 1100 & 7.91 & 7.91 & & 0.1884 & $1.52 E-03$ & 260.8097 & 96.1007 & 356.9104 & \\
\hline & $8 / 12 / 2008$ 12:00 & 503 & 225 & 1200 & 8.14 & 8.14 & & 0.1548 & 1.20E-03 & 204.8161 & 75.4687 & 280.2849 & \\
\hline & $8 / 12 / 2008$ 13:00 & 504 & 225 & 1300 & 7.669 & 7.669 & & 0.2257 & 1.90E-03 & 325.8494 & 120.0659 & 445.9154 & \\
\hline & $8 / 12 / 2008$ 14:00 & 505 & 225 & 1400 & 7.077 & 7.077 & & 0.3282 & $3.02 \mathrm{E}-03$ & 516.6886 & 190.3845 & 707.0731 & \\
\hline & $8 / 12 / 2008$ 15:00 & 506 & 225 & 1500 & 6.987 & 6.987 & & 0.3454 & $3.21 E-03$ & 550.0382 & 202.6729 & 752.7110 & \\
\hline & $8 / 12 / 2008$ 16:00 & 507 & 225 & 1600 & 6.895 & 6.895 & & 0.3633 & $3.42 E-03$ & 585.4412 & 215.7179 & 801.1591 & \\
\hline & $8 / 12 / 2008$ 17:00 & 508 & 225 & 1700 & 6.958 & 6.958 & & 0.3510 & $3.28 \mathrm{E}-03$ & 561.0522 & 206.7312 & 767.7834 & \\
\hline & $8 / 12 / 2008$ 18:00 & 509 & 225 & 1800 & 6.82 & 6.82 & & 0.3783 & $3.60 \mathrm{E}-03$ & 615.3213 & 226.7278 & 842.0491 & \\
\hline & $8 / 12 / 2008$ 19:00 & 510 & 225 & 1900 & 6.975 & 6.975 & & 0.3477 & $3.24 \mathrm{E}-03$ & 554.5796 & 204.3463 & 758.9259 & \\
\hline & $8 / 12 / 200820: 00$ & 511 & 225 & 2000 & 7.519 & 7.519 & & 0.2502 & $2.16 \mathrm{E}-03$ & 369.8335 & 136.2727 & 506.1062 & \\
\hline & $8 / 12 / 2008$ 21:00 & 512 & 225 & 2100 & 9.38 & 9.38 & & 0.0021 & $6.13 \mathrm{E}-06$ & 1.0485 & 0.3863 & 1.4349 & \\
\hline & $8 / 12 / 2008$ 22:00 & 513 & 225 & 2200 & 10.35 & 10.35 & & -0.0918 & $0.00 E+00$ & 0.0000 & 0.0000 & 0.0000 & \\
\hline & $8 / 12 / 2008$ 23:00 & 514 & 225 & 2300 & 10.66 & 10.66 & & -0.1182 & $0.00 \mathrm{E}+00$ & 0.0000 & 0.0000 & 0.0000 & \\
\hline & 8/13/2008 0:00 & 515 & 226 & 0 & 10.74 & 10.74 & 11.04 & -0.1248 & $0.00 E+00$ & 0.0000 & 0.0000 & 0.0000 & \\
\hline & 8/13/2008 1:00 & 516 & 226 & 100 & 10.68 & 10.68 & & -0.1199 & $0.00 \mathrm{E}+00$ & 0.0000 & 0.0000 & 0.0000 & \\
\hline & 8/13/2008 2:00 & 517 & 226 & 200 & 10.79 & 10.79 & & -0.1288 & $0.00 \mathrm{E}+00$ & 0.0000 & 0.0000 & 0.0000 & \\
\hline & 8/13/2008 3:00 & 518 & 226 & 300 & 11.04 & 11.04 & & -0.1486 & $0.00 \mathrm{E}+00$ & 0.0000 & 0.0000 & 0.0000 & \\
\hline & 8/13/2008 4:00 & 519 & 226 & 400 & 10.97 & 10.97 & & -0.1431 & $0.00 \mathrm{E}+00$ & 0.0000 & 0.0000 & 0.0000 & \\
\hline & $8 / 13 / 20085: 00$ & 520 & 226 & 500 & 10.87 & 10.87 & & -0.1352 & $0.00 \mathrm{E}+00$ & 0.0000 & 0.0000 & 0.0000 & \\
\hline & 8/13/2008 6:00 & 521 & 226 & 600 & 10.82 & 10.82 & & -0.1312 & $0.00 \mathrm{E}+00$ & 0.0000 & 0.0000 & 0.0000 & \\
\hline & $8 / 13 / 2008$ 7:00 & 522 & 226 & 700 & 10.71 & 10.71 & & -0.1223 & $0.00 \mathrm{E}+00$ & 0.0000 & 0.0000 & 0.0000 & \\
\hline & 8/13/2008 8:00 & 523 & 226 & 800 & 10.6 & 10.6 & & -0.1132 & $0.00 \mathrm{E}+00$ & 0.0000 & 0.0000 & 0.0000 & \\
\hline & 8/13/2008 9:00 & 524 & 226 & 900 & 8.8 & 8.8 & & 0.0682 & 4.36E-04 & 74.6505 & 27.5065 & 102.1570 & \\
\hline & $8 / 13 / 2008$ 10:00 & 525 & 226 & 1000 & 7.328 & 7.328 & & 0.2828 & $2.51 \mathrm{E}-03$ & 429.9969 & 158.4412 & 588.4381 & \\
\hline & 8/13/2008 11:00 & 526 & 226 & 1100 & 7.555 & 7.555 & & 0.2442 & $2.10 \mathrm{E}-03$ & 359.0224 & 132.2892 & 491.3116 & \\
\hline & $8 / 13 / 2008$ 12:00 & 527 & 226 & 1200 & 7.299 & 7.299 & & 0.2878 & 2.57E-03 & 439.5577 & 161.9641 & 601.5218 & \\
\hline
\end{tabular}




\begin{tabular}{|c|c|c|c|c|c|c|c|c|c|c|c|c|c|}
\hline Tree ID & TS & RN & JD & HR & $\begin{array}{c}\text { Raw } \\
\text { TC_dTA }\end{array}$ & $\begin{array}{c}\text { Fixed } \\
\text { TC_dT } \Delta\end{array}$ & dTM & $\mathbf{K}$ & $\begin{array}{l}\text { Sap } \\
\text { Velocity } \\
(\mathrm{cm} / \mathrm{s})\end{array}$ & $\begin{array}{l}\text { Probed } \\
\text { Drywood F } \\
\text { (g/hr) }\end{array}$ & $\begin{array}{c}\text { Extrapolated } \\
\text { Wetwood F } \\
(\mathrm{g} / \mathrm{hr})\end{array}$ & $\begin{array}{c}\text { Extrapolated } \\
\text { Total Wood F } \\
\text { (g/hr) }\end{array}$ & $\begin{array}{l}\text { Relative } \\
\text { Humidity, } \\
@ 10 m(\%)\end{array}$ \\
\hline & 7/22/2008 13:00 & 0 & 204 & 1300 & 4.864 & 4.864 & 9.09 & 0.8688 & 1.00E-02 & 1601.3983 & 590.0681 & 2191.4664 & \\
\hline \multirow[t]{2}{*}{ W706EC2 } & $7 / 22 / 2008$ 14:00 & 1 & 204 & 1400 & 5.326 & 5.326 & & 0.7067 & 7.76E-03 & 1241.9213 & 457.6114 & 1699.5327 & \\
\hline & $7 / 22 / 2008$ 15:00 & 2 & 204 & 1500 & 5.289 & 5.289 & & 0.7187 & $7.92 \mathrm{E}-03$ & 1267.7997 & 467.1469 & 1734.9466 & \\
\hline \multirow[t]{2}{*}{ Probe \# } & 7/22/2008 16:00 & 3 & 204 & 1600 & 5.22 & 5.22 & & 0.7414 & $8.23 \mathrm{E}-03$ & 1317.3132 & 485.3911 & 1802.7043 & \\
\hline & 7/22/2008 17:00 & 4 & 204 & 1700 & 5.253 & 5.253 & & 0.7304 & 8.08E-03 & 1293.4261 & 476.5894 & 1770.0156 & \\
\hline \multirow[t]{4}{*}{15} & $7 / 22 / 2008$ 18:00 & 5 & 204 & 1800 & 5.434 & 5.434 & & 0.6728 & $7.31 \mathrm{E}-03$ & 1168.9544 & 430.7253 & 1599.6797 & \\
\hline & $7 / 22 / 2008$ 19:00 & 6 & 204 & 1900 & 5.435 & 5.435 & & 0.6725 & 7.30E-03 & 1168.2962 & 430.4827 & 1598.7789 & \\
\hline & 7/22/2008 20:00 & 7 & 204 & 2000 & 5.769 & 5.769 & & 0.5757 & $6.03 E-03$ & 964.7981 & 355.4997 & 1320.2978 & \\
\hline & 7/22/2008 21:00 & 8 & 204 & 2100 & 7.244 & 7.244 & & 0.2548 & $2.21 \mathrm{E}-03$ & 353.8075 & 130.3676 & 484.1751 & \\
\hline \multirow[t]{2}{*}{$\mathrm{DBH}(\mathrm{cm})$} & $7 / 22 / 2008$ 22:00 & 9 & 204 & 2200 & 8.67 & 8.67 & & 0.0484 & 2.86E-04 & 45.8340 & 16.8885 & 62.7225 & \\
\hline & 7/22/2008 23:00 & 10 & 204 & 2300 & 9.09 & 9.09 & & 0.0000 & $0.00 \mathrm{E}+00$ & 0.0000 & 0.0000 & 0.0000 & \\
\hline \multirow[t]{2}{*}{9.4} & 7/23/2008 0:00 & 11 & 205 & 0 & 9.41 & 9.41 & 9.60 & -0.0340 & $0.00 \mathrm{E}+00$ & 0.0000 & 0.0000 & 0.0000 & \\
\hline & 7/23/2008 1:00 & 12 & 205 & 100 & 9.54 & 9.54 & & -0.0472 & $0.00 \mathrm{E}+00$ & 0.0000 & 0.0000 & 0.0000 & \\
\hline Day Count & $7 / 23 / 20082: 00$ & 13 & 205 & 200 & 9.57 & 9.57 & & -0.0502 & $0.00 \mathrm{E}+00$ & 0.0000 & 0.0000 & 0.0000 & \\
\hline \multirow[t]{27}{*}{35} & 7/23/2008 3:00 & 14 & 205 & 300 & 9.6 & 9.6 & & -0.0531 & $0.00 \mathrm{E}+00$ & 0.0000 & 0.0000 & 0.0000 & \\
\hline & 7/23/2008 4:00 & 15 & 205 & 400 & 9.57 & 9.57 & & -0.0502 & $0.00 \mathrm{E}+00$ & 0.0000 & 0.0000 & 0.0000 & \\
\hline & 7/23/2008 5:00 & 16 & 205 & 500 & 9.45 & 9.45 & & -0.0381 & $0.00 E+00$ & 0.0000 & 0.0000 & 0.0000 & \\
\hline & 7/23/2008 6:00 & 17 & 205 & 600 & 9.26 & 9.26 & & -0.0184 & $0.00 \mathrm{E}+00$ & 0.0000 & 0.0000 & 0.0000 & \\
\hline & 7/23/2008 7:00 & 18 & 205 & 700 & 8.81 & 8.81 & & 0.0318 & 1.71E-04 & 27.2807 & 10.0521 & 37.3328 & \\
\hline & 7/23/2008 8:00 & 19 & 205 & 800 & 7.72 & 7.72 & & 0.1775 & 1.42E-03 & 226.6289 & 83.5061 & 310.1350 & \\
\hline & 7/23/2008 9:00 & 20 & 205 & 900 & 6.388 & 6.388 & & 0.4230 & 4.13E-03 & 660.1911 & 243.2610 & 903.4521 & \\
\hline & 7/23/2008 10:00 & 21 & 205 & 1000 & 5.567 & 5.567 & & 0.6328 & $6.78 \mathrm{E}-03$ & 1084.0741 & 399.4494 & 1483.5235 & \\
\hline & $7 / 23 / 2008$ 11:00 & 22 & 205 & 1100 & 5.496 & 5.496 & & 0.6539 & 7.05E-03 & 1128.7254 & 415.9021 & 1544.6276 & \\
\hline & $7 / 23 / 2008$ 12:00 & 23 & 205 & 1200 & 5.402 & 5.402 & & 0.6827 & 7.44E-03 & 1190.1841 & 438.5478 & 1628.7319 & \\
\hline & $7 / 23 / 2008$ 13:00 & 24 & 205 & 1300 & 5.351 & 5.351 & & 0.6987 & 7.65E-03 & 1224.6945 & 451.2639 & 1675.9584 & \\
\hline & $7 / 23 / 200814: 00$ & 25 & 205 & 1400 & 5.339 & 5.339 & & 0.7026 & $7.71 \mathrm{E}-03$ & 1232.9376 & 454.3012 & 1687.2388 & \\
\hline & 7/23/2008 15:00 & 26 & 205 & 1500 & 5.329 & 5.329 & & 0.7058 & 7.75E-03 & 1239.8432 & 456.8457 & 1696.6889 & \\
\hline & 7/23/2008 16:00 & 27 & 205 & 1600 & 5.45 & 5.45 & & 0.6679 & 7.24E-03 & 1158.4597 & 426.8583 & 1585.3180 & \\
\hline & 7/23/2008 17:00 & 28 & 205 & 1700 & 5.825 & 5.825 & & 0.5605 & $5.84 \mathrm{E}-03$ & 933.6415 & 344.0194 & 1277.6609 & \\
\hline & $7 / 23 / 2008$ 18:00 & 29 & 205 & 1800 & 6.479 & 6.479 & & 0.4030 & 3.89E-03 & 622.0025 & 229.1896 & 851.1922 & \\
\hline & $7 / 23 / 2008$ 19:00 & 30 & 205 & 1900 & 6.4 & 6.4 & & 0.4203 & 4.09E-03 & 655.0685 & 241.3735 & 896.4420 & \\
\hline & $7 / 23 / 200820: 00$ & 31 & 205 & 2000 & 6.818 & 6.818 & & 0.3332 & $3.08 E-03$ & 492.2391 & 181.3756 & 673.6147 & \\
\hline & $7 / 23 / 200821: 00$ & 32 & 205 & 2100 & 7.745 & 7.745 & & 0.1737 & $1.38 \mathrm{E}-03$ & 220.6688 & 81.3100 & 301.9788 & \\
\hline & $7 / 23 / 200822: 00$ & 33 & 205 & 2200 & 8.38 & 8.38 & & 0.0847 & 5.70E-04 & 91.2126 & 33.6092 & 124.8218 & \\
\hline & $7 / 23 / 2008$ 23:00 & 34 & 205 & 2300 & 8.47 & 8.47 & & 0.0732 & $4.76 \mathrm{E}-04$ & 76.1867 & 28.0725 & 104.2592 & \\
\hline & $7 / 24 / 20080: 00$ & 35 & 206 & 0 & 8.44 & 8.44 & 9.52 & 0.0770 & 5.07E-04 & 81.1032 & 29.8841 & 110.9873 & \\
\hline & 7/24/2008 1:00 & 36 & 206 & 100 & 8.55 & 8.55 & & 0.0632 & 3.97E-04 & 63.5325 & 23.4098 & 86.9423 & \\
\hline & 7/24/2008 2:00 & 37 & 206 & 200 & 8.67 & 8.67 & & 0.0484 & $2.86 \mathrm{E}-04$ & 45.8340 & 16.8885 & 62.7225 & \\
\hline & 7/24/2008 3:00 & 38 & 206 & 300 & 8.83 & 8.83 & & 0.0294 & $1.55 \mathrm{E}-04$ & 24.8327 & 9.1501 & 33.9828 & \\
\hline & 7/24/2008 4:00 & 39 & 206 & 400 & 8.97 & 8.97 & & 0.0134 & 5.88E-05 & 9.4027 & 3.4646 & 12.8674 & \\
\hline & 7/24/2008 5:00 & 40 & 206 & 500 & 9.1 & 9.1 & & -0.0011 & $0.00 \mathrm{E}+00$ & 0.0000 & 0.0000 & 0.0000 & \\
\hline
\end{tabular}




\begin{tabular}{|c|c|c|c|c|c|c|c|c|c|c|c|c|c|}
\hline \multirow[t]{42}{*}{ Tree ID } & TS & RN & JD & HR & $\begin{array}{c}\text { Raw } \\
\text { TC_dTA }\end{array}$ & $\begin{array}{c}\text { Fixed } \\
\text { TC_dT } \Delta\end{array}$ & dTM & $\mathbf{K}$ & $\begin{array}{l}\text { Sap } \\
\text { Velocity } \\
\text { (cm/s) }\end{array}$ & $\begin{array}{c}\text { Probed } \\
\text { Drywood F } \\
\text { (g/hr) }\end{array}$ & $\begin{array}{c}\text { Extrapolated } \\
\text { Wetwood F } \\
(\mathrm{g} / \mathrm{hr})\end{array}$ & $\begin{array}{c}\text { Extrapolated } \\
\text { Total Wood F } \\
(\mathrm{g} / \mathrm{hr})\end{array}$ & $\begin{array}{c}\text { Relative } \\
\text { Humidity, } \\
@ 10 \mathrm{~m}(\%)\end{array}$ \\
\hline & 7/24/2008 6:00 & 41 & 206 & 600 & 9.17 & 9.17 & & -0.0087 & $0.00 \mathrm{E}+00$ & 0.0000 & 0.0000 & 0.0000 & \\
\hline & 7/24/2008 7:00 & 42 & 206 & 700 & 9.14 & 9.14 & & -0.0055 & $0.00 \mathrm{E}+00$ & 0.0000 & 0.0000 & 0.0000 & \\
\hline & 7/24/2008 8:00 & 43 & 206 & 800 & 8.78 & 8.78 & & 0.0353 & $1.94 \mathrm{E}-04$ & 31.0523 & 11.4419 & 42.4942 & \\
\hline & $7 / 24 / 20089: 00$ & 44 & 206 & 900 & 8.18 & 8.18 & & 0.1112 & 7.97E-04 & 127.5410 & 46.9951 & 174.5361 & \\
\hline & $7 / 24 / 2008$ 10:00 & 45 & 206 & 1000 & 8.26 & 8.26 & & 0.1005 & 7.03E-04 & 112.5258 & 41.4624 & 153.9882 & \\
\hline & 7/24/2008 11:00 & 46 & 206 & 1100 & 6.171 & 6.171 & & 0.4730 & 4.74E-03 & 757.6083 & 279.1564 & 1036.7647 & \\
\hline & $7 / 24 / 2008$ 12:00 & 47 & 206 & 1200 & 5.438 & 5.438 & & 0.6716 & 7.29E-03 & 1166.3233 & 429.7558 & 1596.0791 & \\
\hline & 7/24/2008 13:00 & 48 & 206 & 1300 & 5.765 & 5.765 & & 0.5768 & $6.04 \mathrm{E}-03$ & 967.0542 & 356.3310 & 1323.3851 & \\
\hline & $7 / 24 / 2008$ 14:00 & 49 & 206 & 1400 & 5.645 & 5.645 & & 0.6103 & $6.48 \mathrm{E}-03$ & 1036.6946 & 381.9915 & 1418.6861 & \\
\hline & 7/24/2008 15:00 & 50 & 206 & 1500 & 5.354 & 5.354 & & 0.6978 & 7.64E-03 & 1222.6412 & 450.5073 & 1673.1484 & \\
\hline & $7 / 24 / 2008$ 16:00 & 51 & 206 & 1600 & 5.343 & 5.343 & & 0.7013 & $7.69 \mathrm{E}-03$ & 1230.1846 & 453.2868 & 1683.4715 & \\
\hline & $7 / 24 / 2008$ 17:00 & 52 & 206 & 1700 & 5.482 & 5.482 & & 0.6582 & $7.11 \mathrm{E}-03$ & 1137.7069 & 419.2115 & 1556.9184 & \\
\hline & 7/24/2008 18:00 & 53 & 206 & 1800 & 5.491 & 5.491 & & 0.6554 & 7.07E-03 & 1131.9263 & 417.0815 & 1549.0078 & \\
\hline & $7 / 24 / 2008$ 19:00 & 54 & 206 & 1900 & 5.738 & 5.738 & & 0.5842 & $6.14 \mathrm{E}-03$ & 982.3907 & 361.9821 & 1344.3727 & \\
\hline & $7 / 24 / 200820: 00$ & 55 & 206 & 2000 & 6.633 & 6.633 & & 0.3704 & $3.50 \mathrm{E}-03$ & 560.7031 & 206.6026 & 767.3057 & \\
\hline & $7 / 24 / 200821: 00$ & 56 & 206 & 2100 & 8.1 & 8.1 & & 0.1222 & 8.95E-04 & 143.2027 & 52.7660 & 195.9686 & \\
\hline & $7 / 24 / 200822: 00$ & 57 & 206 & 2200 & 9.2 & 9.2 & & -0.0120 & $0.00 \mathrm{E}+00$ & 0.0000 & 0.0000 & 0.0000 & \\
\hline & $7 / 24 / 200823: 00$ & 58 & 206 & 2300 & 9.52 & 9.52 & & -0.0452 & $0.00 \mathrm{E}+00$ & 0.0000 & 0.0000 & 0.0000 & \\
\hline & $7 / 25 / 20080: 00$ & 59 & 207 & 0 & 9.63 & 9.63 & 9.70 & -0.0561 & $0.00 \mathrm{E}+00$ & 0.0000 & 0.0000 & 0.0000 & \\
\hline & 7/25/2008 1:00 & 60 & 207 & 100 & 9.66 & 9.66 & & -0.0590 & $0.00 \mathrm{E}+00$ & 0.0000 & 0.0000 & 0.0000 & \\
\hline & 7/25/2008 2:00 & 61 & 207 & 200 & 9.66 & 9.66 & & -0.0590 & $0.00 E+00$ & 0.0000 & 0.0000 & 0.0000 & \\
\hline & 7/25/2008 3:00 & 62 & 207 & 300 & 9.67 & 9.67 & & -0.0600 & $0.00 \mathrm{E}+00$ & 0.0000 & 0.0000 & 0.0000 & \\
\hline & 7/25/2008 4:00 & 63 & 207 & 400 & 9.7 & 9.7 & & -0.0629 & $0.00 \mathrm{E}+00$ & 0.0000 & 0.0000 & 0.0000 & \\
\hline & 7/25/2008 5:00 & 64 & 207 & 500 & 9.7 & 9.7 & & -0.0629 & $0.00 \mathrm{E}+00$ & 0.0000 & 0.0000 & 0.0000 & \\
\hline & 7/25/2008 6:00 & 65 & 207 & 600 & 9.69 & 9.69 & & -0.0619 & $0.00 \mathrm{E}+00$ & 0.0000 & 0.0000 & 0.0000 & \\
\hline & 7/25/2008 7:00 & 66 & 207 & 700 & 9.67 & 9.67 & & -0.0600 & $0.00 \mathrm{E}+00$ & 0.0000 & 0.0000 & 0.0000 & \\
\hline & 7/25/2008 8:00 & 67 & 207 & 800 & 9.64 & 9.64 & & -0.0571 & $0.00 \mathrm{E}+00$ & 0.0000 & 0.0000 & 0.0000 & \\
\hline & $7 / 25 / 20089: 00$ & 68 & 207 & 900 & 9.57 & 9.57 & & -0.0502 & $0.00 \mathrm{E}+00$ & 0.0000 & 0.0000 & 0.0000 & \\
\hline & $7 / 25 / 2008$ 10:00 & 69 & 207 & 1000 & 9.43 & 9.43 & & -0.0361 & $0.00 \mathrm{E}+00$ & 0.0000 & 0.0000 & 0.0000 & \\
\hline & $7 / 25 / 2008$ 11:00 & 70 & 207 & 1100 & 8.23 & 8.23 & & 0.1045 & $7.38 \mathrm{E}-04$ & 118.0809 & 43.5093 & 161.5902 & \\
\hline & $7 / 25 / 2008$ 12:00 & 71 & 207 & 1200 & 6.45 & 6.45 & & 0.4093 & 3.96E-03 & 634.0093 & 233.6138 & 867.6231 & \\
\hline & $7 / 25 / 2008$ 13:00 & 72 & 207 & 1300 & 5.896 & 5.896 & & 0.5417 & 5.60E-03 & 895.2601 & 329.8770 & 1225.1372 & \\
\hline & $7 / 25 / 2008$ 14:00 & 73 & 207 & 1400 & 5.475 & 5.475 & & 0.6603 & 7.14E-03 & 1142.2198 & 420.8744 & 1563.0942 & \\
\hline & $7 / 25 / 2008$ 15:00 & 74 & 207 & 1500 & 5.418 & 5.418 & & 0.6777 & 7.37E-03 & 1179.5290 & 434.6217 & 1614.1507 & \\
\hline & $7 / 25 / 200816: 00$ & 75 & 207 & 1600 & 5.496 & 5.496 & & 0.6539 & 7.05E-03 & 1128.7254 & 415.9021 & 1544.6276 & \\
\hline & $7 / 25 / 2008$ 17:00 & 76 & 207 & 1700 & 5.332 & 5.332 & & 0.7048 & 7.74E-03 & 1237.7680 & 456.0811 & 1693.8491 & \\
\hline & $7 / 25 / 2008$ 18:00 & 77 & 207 & 1800 & 5.401 & 5.401 & & 0.6830 & 7.44E-03 & 1190.8528 & 438.7942 & 1629.6470 & \\
\hline & 7/25/2008 19:00 & 78 & 207 & 1900 & 5.411 & 5.411 & & 0.6799 & 7.40E-03 & 1184.1806 & 436.3357 & 1620.5163 & \\
\hline & $7 / 25 / 200820: 00$ & 79 & 207 & 2000 & 6.161 & 6.161 & & 0.4754 & $4.76 \mathrm{E}-03$ & 762.3250 & 280.8943 & 1043.2193 & \\
\hline & $7 / 25 / 2008$ 21:00 & 80 & 207 & 2100 & 7.719 & 7.719 & & 0.1776 & 1.42E-03 & 226.8688 & 83.5945 & 310.4632 & \\
\hline & $7 / 25 / 200822: 00$ & 81 & 207 & 2200 & 9.11 & 9.11 & & -0.0022 & $0.00 \mathrm{E}+00$ & 0.0000 & 0.0000 & 0.0000 & \\
\hline
\end{tabular}




\begin{tabular}{|c|c|c|c|c|c|c|c|c|c|c|c|c|c|}
\hline Tree ID & TS & RN & JD & HR & $\begin{array}{c}\text { Raw } \\
\text { TC_dTA }\end{array}$ & $\begin{array}{c}\text { Fixed } \\
\text { TC_dT } \Delta\end{array}$ & dTM & K & $\begin{array}{l}\text { Sap } \\
\text { Velocity } \\
(\mathrm{cm} / \mathrm{s})\end{array}$ & $\begin{array}{c}\text { Probed } \\
\text { Drywood F } \\
(\mathrm{g} / \mathrm{hr})\end{array}$ & $\begin{array}{c}\text { Extrapolated } \\
\text { Wetwood F } \\
(\mathrm{g} / \mathrm{hr})\end{array}$ & $\begin{array}{c}\text { Extrapolated } \\
\text { Total Wood F } \\
(\mathrm{g} / \mathrm{hr})\end{array}$ & $\begin{array}{l}\text { Relative } \\
\text { Humidity, } \\
@ 10 \mathrm{~m}(\%)\end{array}$ \\
\hline & 7/25/2008 23:00 & 82 & 207 & 2300 & $\begin{array}{r}9.41 \\
\end{array}$ & 9.41 & & -0.0340 & $0.00 \mathrm{E}+00$ & 0.0000 & 0.0000 & 0.0000 & \\
\hline & 7/26/2008 0:00 & 83 & 208 & 0 & 9.49 & 9.49 & 9.49 & -0.0421 & $0.00 \mathrm{E}+00$ & 0.0000 & 0.0000 & 0.0000 & \\
\hline & 7/26/2008 1:00 & 84 & 208 & 100 & 9.49 & 9.49 & & -0.0421 & $0.00 \mathrm{E}+00$ & 0.0000 & 0.0000 & 0.0000 & \\
\hline & $7 / 26 / 20082: 00$ & 85 & 208 & 200 & 9.46 & 9.46 & & -0.0391 & $0.00 \mathrm{E}+00$ & 0.0000 & 0.0000 & 0.0000 & \\
\hline & 7/26/2008 3:00 & 86 & 208 & 300 & 9.4 & 9.4 & & -0.0330 & $0.00 \mathrm{E}+00$ & 0.0000 & 0.0000 & 0.0000 & \\
\hline & 7/26/2008 4:00 & 87 & 208 & 400 & 9.34 & 9.34 & & -0.0268 & $0.00 \mathrm{E}+00$ & 0.0000 & 0.0000 & 0.0000 & \\
\hline & 7/26/2008 5:00 & 88 & 208 & 500 & 9.25 & 9.25 & & -0.0173 & $0.00 \mathrm{E}+00$ & 0.0000 & 0.0000 & 0.0000 & \\
\hline & 7/26/2008 6:00 & 89 & 208 & 600 & 9.17 & 9.17 & & -0.0087 & $0.00 \mathrm{E}+00$ & 0.0000 & 0.0000 & 0.0000 & \\
\hline & 7/26/2008 7:00 & 90 & 208 & 700 & 9.08 & 9.08 & & 0.0011 & $2.72 \mathrm{E}-06$ & 0.4348 & 0.1602 & 0.5950 & \\
\hline & 7/26/2008 8:00 & 91 & 208 & 800 & 8.96 & 8.96 & & 0.0145 & 6.49E-05 & 10.3906 & 3.8286 & 14.2193 & \\
\hline & 7/26/2008 9:00 & 92 & 208 & 900 & 7.292 & 7.292 & & 0.2466 & $2.12 \mathrm{E}-03$ & 339.7435 & 125.1855 & 464.9289 & \\
\hline & $7 / 26 / 2008$ 10:00 & 93 & 208 & 1000 & 5.441 & 5.441 & & 0.6706 & $7.28 \mathrm{E}-03$ & 1164.3532 & 429.0299 & 1593.3831 & \\
\hline & $7 / 26 / 2008$ 11:00 & 94 & 208 & 1100 & 5.58 & 5.58 & & 0.6290 & $6.73 \mathrm{E}-03$ & 1076.0578 & 396.4956 & 1472.5534 & \\
\hline & $7 / 26 / 2008$ 12:00 & 95 & 208 & 1200 & 5.891 & 5.891 & & 0.5430 & 5.61E-03 & 897.9229 & 330.8582 & 1228.7811 & \\
\hline & $7 / 26 / 2008$ 13:00 & 96 & 208 & 1300 & 5.3 & 5.3 & & 0.7151 & 7.88E-03 & 1260.0579 & 464.2942 & 1724.3521 & \\
\hline & $7 / 26 / 2008$ 14:00 & 97 & 208 & 1400 & 5.26 & 5.26 & & 0.7281 & $8.05 \mathrm{E}-03$ & 1288.4082 & 474.7405 & 1763.1486 & \\
\hline & $7 / 26 / 2008$ 15:00 & 98 & 208 & 1500 & 5.207 & 5.207 & & 0.7457 & 8.29E-03 & 1326.8291 & 488.8975 & 1815.7265 & \\
\hline & $7 / 26 / 200816: 00$ & 99 & 208 & 1600 & 5.254 & 5.254 & & 0.7301 & 8.08E-03 & 1292.7082 & 476.3249 & 1769.0331 & \\
\hline & $7 / 26 / 2008$ 17:00 & 100 & 208 & 1700 & 5.13 & 5.13 & & 0.7719 & 8.65E-03 & 1384.4508 & 510.1294 & 1894.5802 & \\
\hline & $7 / 26 / 2008$ 18:00 & 101 & 208 & 1800 & 5.357 & 5.357 & & 0.6968 & 7.63E-03 & 1220.5907 & 449.7518 & 1670.3425 & \\
\hline & $7 / 26 / 2008$ 19:00 & 102 & 208 & 1900 & 5.391 & 5.391 & & 0.6861 & 7.48E-03 & 1197.5567 & 441.2644 & 1638.8211 & \\
\hline & $7 / 26 / 200820: 00$ & 103 & 208 & 2000 & 5.897 & 5.897 & & 0.5415 & 5.59E-03 & 894.7283 & 329.6810 & 1224.4094 & \\
\hline & $7 / 26 / 200821: 00$ & 104 & 208 & 2100 & 7.539 & 7.539 & & 0.2057 & 1.70E-03 & 271.8561 & 100.1710 & 372.0271 & \\
\hline & $7 / 26 / 200822: 00$ & 105 & 208 & 2200 & 8.79 & 8.79 & & 0.0341 & 1.86E-04 & 29.7821 & 10.9738 & 40.7559 & \\
\hline & $7 / 26 / 2008$ 23:00 & 106 & 208 & 2300 & 9.05 & 9.05 & & 0.0044 & 1.50E-05 & 2.4053 & 0.8863 & 3.2916 & \\
\hline & $7 / 27 / 20080: 00$ & 107 & 209 & 0 & 9.11 & 9.11 & 9.11 & -0.0022 & $0.00 \mathrm{E}+00$ & 0.0000 & 0.0000 & 0.0000 & \\
\hline & 7/27/2008 1:00 & 108 & 209 & 100 & 9.11 & 9.11 & & -0.0022 & $0.00 \mathrm{E}+00$ & 0.0000 & 0.0000 & 0.0000 & \\
\hline & 7/27/2008 2:00 & 109 & 209 & 200 & 9.07 & 9.07 & & 0.0022 & 6.39E-06 & 1.0219 & 0.3766 & 1.3985 & \\
\hline & 7/27/2008 3:00 & 110 & 209 & 300 & 9.05 & 9.05 & & 0.0044 & 1.50E-05 & 2.4053 & 0.8863 & 3.2916 & \\
\hline & 7/27/2008 4:00 & 111 & 209 & 400 & 9.05 & 9.05 & & 0.0044 & 1.50E-05 & 2.4053 & 0.8863 & 3.2916 & \\
\hline & 7/27/20085:00 & 112 & 209 & 500 & 9 & 9 & & 0.0100 & 4.11E-05 & 6.5716 & 2.4214 & 8.9930 & \\
\hline & 7/27/2008 6:00 & 113 & 209 & 600 & 8.94 & 8.94 & & 0.0168 & 7.77E-05 & 12.4263 & 4.5787 & 17.0050 & \\
\hline & 7/27/2008 7:00 & 114 & 209 & 700 & 8.88 & 8.88 & & 0.0236 & 1.18E-04 & 18.9594 & 6.9860 & 25.9454 & \\
\hline & 7/27/2008 8:00 & 115 & 209 & 800 & 8.61 & 8.61 & & 0.0557 & $3.41 \mathrm{E}-04$ & 54.4864 & 20.0766 & 74.5631 & \\
\hline & $7 / 27 / 20089: 00$ & 116 & 209 & 900 & 6.179 & 6.179 & & 0.4711 & 4.71E-03 & 753.8499 & 277.7715 & 1031.6214 & \\
\hline & $7 / 27 / 2008$ 10:00 & 117 & 209 & 1000 & 4.877 & 4.877 & & 0.8639 & 9.94E-03 & 1590.1031 & 585.9062 & 2176.0092 & \\
\hline & $7 / 27 / 2008$ 11:00 & 118 & 209 & 1100 & 4.807 & 4.807 & & 0.8910 & 1.03E-02 & 1651.8251 & 608.6489 & 2260.4740 & \\
\hline & $7 / 27 / 2008$ 12:00 & 119 & 209 & 1200 & 4.774 & 4.774 & & 0.9041 & 1.05E-02 & 1681.7066 & 619.6594 & 2301.3659 & \\
\hline & $7 / 27 / 200813: 00$ & 120 & 209 & 1300 & 4.906 & 4.906 & & 0.8528 & $9.78 \mathrm{E}-03$ & 1565.1753 & 576.7210 & 2141.8963 & \\
\hline & $7 / 27 / 200814: 00$ & 121 & 209 & 1400 & 4.806 & 4.806 & & 0.8914 & 1.03E-02 & 1652.7231 & 608.9798 & 2261.7029 & \\
\hline & 7/27/2008 15:00 & 122 & 209 & 1500 & 4.832 & 4.832 & & 0.8812 & 1.02E-02 & 1629.5254 & 600.4322 & 2229.9576 & \\
\hline
\end{tabular}




\begin{tabular}{|c|c|c|c|c|c|c|c|c|c|c|c|c|c|}
\hline Tree ID & TS & RN & JD & HR & $\begin{array}{c}\text { Raw } \\
\text { TC_dTA }\end{array}$ & $\begin{array}{c}\text { Fixed } \\
\text { TC_dT } \Delta\end{array}$ & dTM & $\mathbf{K}$ & $\begin{array}{l}\text { Sap } \\
\text { Velocity } \\
(\mathrm{cm} / \mathrm{s})\end{array}$ & $\begin{array}{c}\text { Probed } \\
\text { Drywood F } \\
(\mathrm{g} / \mathrm{hr})\end{array}$ & $\begin{array}{c}\text { Extrapolated } \\
\text { Wetwood F } \\
(\mathrm{g} / \mathrm{hr})\end{array}$ & $\begin{array}{c}\text { Extrapolated } \\
\text { Total Wood F } \\
(\mathrm{g} / \mathrm{hr})\end{array}$ & $\begin{array}{l}\text { Relative } \\
\text { Humidity, } \\
@ 10 \mathrm{~m}(\%)\end{array}$ \\
\hline & 7/27/2008 16:00 & 123 & 209 & 1600 & \begin{tabular}{|r|}
4.894 \\
\end{tabular} & 4.894 & & 0.8574 & $9.85 \mathrm{E}-03$ & 1575.4454 & 580.5053 & 2155.9507 & \\
\hline & $7 / 27 / 2008$ 17:00 & 124 & 209 & 1700 & 4.917 & 4.917 & & 0.8487 & $9.72 \mathrm{E}-03$ & 1555.8160 & 573.2724 & 2129.0884 & \\
\hline & $7 / 27 / 2008$ 18:00 & 125 & 209 & 1800 & 5.067 & 5.067 & & 0.7940 & $8.96 \mathrm{E}-03$ & 1433.2500 & 528.1104 & 1961.3605 & \\
\hline & $7 / 27 / 2008$ 19:00 & 126 & 209 & 1900 & 5.145 & 5.145 & & 0.7668 & 8.58E-03 & 1373.0542 & 505.9301 & 1878.9843 & \\
\hline & $7 / 27 / 200820: 00$ & 127 & 209 & 2000 & 5.523 & 5.523 & & 0.6458 & 6.95E-03 & 1111.5702 & 409.5809 & 1521.1510 & \\
\hline & $7 / 27 / 2008$ 21:00 & 128 & 209 & 2100 & 6.743 & 6.743 & & 0.3481 & $3.25 E-03$ & 519.3410 & 191.3619 & 710.7029 & \\
\hline & $7 / 27 / 2008$ 22:00 & 129 & 209 & 2200 & 7.941 & 7.941 & & 0.1447 & $1.10 \mathrm{E}-03$ & 176.2694 & 64.9501 & 241.2194 & \\
\hline & $7 / 27 / 200823: 00$ & 130 & 209 & 2300 & 8.12 & 8.12 & & 0.1195 & $8.70 \mathrm{E}-04$ & 139.2265 & 51.3009 & 190.5273 & \\
\hline & $7 / 28 / 20080: 00$ & 131 & 210 & 0 & 8.19 & 8.19 & 8.74 & 0.1099 & 7.85E-04 & 125.6288 & 46.2905 & 171.9193 & \\
\hline & 7/28/2008 1:00 & 132 & 210 & 100 & 8.4 & 8.4 & & 0.0821 & 5.49E-04 & 87.8021 & 32.3525 & 120.1545 & \\
\hline & $7 / 28 / 20082: 00$ & 133 & 210 & 200 & 8.46 & 8.46 & & 0.0745 & $4.86 \mathrm{E}-04$ & 77.8152 & 28.6726 & 106.4878 & \\
\hline & 7/28/2008 3:00 & 134 & 210 & 300 & 8.54 & 8.54 & & 0.0644 & 4.07E-04 & 65.0775 & 23.9792 & 89.0567 & \\
\hline & 7/28/2008 4:00 & 135 & 210 & 400 & 8.46 & 8.46 & & 0.0745 & 4.86E-04 & 77.8152 & 28.6726 & 106.4878 & \\
\hline & 7/28/2008 5:00 & 136 & 210 & 500 & 8.66 & 8.66 & & 0.0497 & 2.95E-04 & 47.2481 & 17.4095 & 64.6576 & \\
\hline & 7/28/2008 6:00 & 137 & 210 & 600 & 8.74 & 8.74 & & 0.0400 & 2.27E-04 & 36.2590 & 13.3604 & 49.6194 & \\
\hline & 7/28/2008 7:00 & 138 & 210 & 700 & 8.59 & 8.59 & & 0.0582 & $3.59 \mathrm{E}-04$ & 57.4587 & 21.1718 & 78.6305 & \\
\hline & 7/28/2008 8:00 & 139 & 210 & 800 & 6.534 & 6.534 & & 0.3912 & 3.75E-03 & 599.6407 & 220.9500 & 820.5907 & \\
\hline & $7 / 28 / 20089: 00$ & 140 & 210 & 900 & 5.278 & 5.278 & & 0.7222 & 7.97E-03 & 1275.5827 & 470.0147 & 1745.5974 & \\
\hline & $7 / 28 / 2008$ 10:00 & 141 & 210 & 1000 & 4.899 & 4.899 & & 0.8555 & $9.82 \mathrm{E}-03$ & 1571.1585 & 578.9257 & 2150.0842 & \\
\hline & $7 / 28 / 2008$ 11:00 & 142 & 210 & 1100 & 4.891 & 4.891 & & 0.8585 & 9.86E-03 & 1578.0228 & 581.4550 & 2159.4778 & \\
\hline & $7 / 28 / 200812: 00$ & 143 & 210 & 1200 & 4.954 & 4.954 & & 0.8349 & $9.53 \mathrm{E}-03$ & 1524.7162 & 561.8131 & 2086.5293 & \\
\hline & 7/28/2008 13:00 & 144 & 210 & 1300 & 4.907 & 4.907 & & 0.8525 & 9.78E-03 & 1564.3222 & 576.4067 & 2140.7289 & \\
\hline & $7 / 28 / 2008$ 14:00 & 145 & 210 & 1400 & 4.891 & 4.891 & & 0.8585 & 9.86E-03 & 1578.0228 & 581.4550 & 2159.4778 & \\
\hline & $7 / 28 / 2008$ 15:00 & 146 & 210 & 1500 & 4.853 & 4.853 & & 0.8731 & 1.01E-02 & 1611.0148 & 593.6115 & 2204.6263 & \\
\hline & $7 / 28 / 2008$ 16:00 & 147 & 210 & 1600 & 4.889 & 4.889 & & 0.8593 & 9.87E-03 & 1579.7433 & 582.0889 & 2161.8322 & \\
\hline & 7/28/2008 17:00 & 148 & 210 & 1700 & 4.871 & 4.871 & & 0.8661 & 9.97E-03 & 1595.3069 & 587.8236 & 2183.1305 & \\
\hline & $7 / 28 / 2008$ 18:00 & 149 & 210 & 1800 & 4.985 & 4.985 & & 0.8235 & 9.37E-03 & 1499.1045 & 552.3759 & 2051.4804 & \\
\hline & $7 / 28 / 2008$ 19:00 & 150 & 210 & 1900 & 5.123 & 5.123 & & 0.7744 & 8.69E-03 & 1389.7981 & 512.0997 & 1901.8978 & \\
\hline & $7 / 28 / 200820: 00$ & 151 & 210 & 2000 & 5.684 & 5.684 & & 0.5992 & 6.34E-03 & 1013.6388 & 373.4961 & 1387.1349 & \\
\hline & $7 / 28 / 200821: 00$ & 152 & 210 & 2100 & 7.159 & 7.159 & & 0.2697 & $2.37 \mathrm{E}-03$ & 379.4407 & 139.8127 & 519.2535 & \\
\hline & $7 / 28 / 2008$ 22:00 & 153 & 210 & 2200 & 7.76 & 7.76 & & 0.1714 & 1.36E-03 & 217.1255 & 80.0044 & 297.1298 & \\
\hline & $7 / 28 / 200823: 00$ & 154 & 210 & 2300 & 7.943 & 7.943 & & 0.1444 & 1.10E-03 & 175.8372 & 64.7908 & 240.6281 & \\
\hline & $7 / 29 / 20080: 00$ & 155 & 211 & 0 & 8.24 & 8.24 & 8.89 & 0.1032 & 7.26E-04 & 116.2191 & 42.8233 & 159.0424 & \\
\hline & 7/29/2008 1:00 & 156 & 211 & 100 & 8.47 & 8.47 & & 0.0732 & $4.76 \mathrm{E}-04$ & 76.1867 & 28.0725 & 104.2592 & \\
\hline & $7 / 29 / 20082: 00$ & 157 & 211 & 200 & 8.66 & 8.66 & & 0.0497 & 2.95E-04 & 47.2481 & 17.4095 & 64.6576 & \\
\hline & 7/29/2008 3:00 & 158 & 211 & 300 & 8.75 & 8.75 & & 0.0389 & 2.18E-04 & 34.9388 & 12.8739 & 47.8127 & \\
\hline & 7/29/2008 4:00 & 159 & 211 & 400 & 8.89 & 8.89 & & 0.0225 & 1.11E-04 & 17.8295 & 6.5696 & 24.3991 & \\
\hline & 7/29/2008 5:00 & 160 & 211 & 500 & 8.87 & 8.87 & & 0.0248 & 1.26E-04 & 20.1047 & 7.4080 & 27.5127 & \\
\hline & $7 / 29 / 20086: 00$ & 161 & 211 & 600 & 8.81 & 8.81 & & 0.0318 & 1.71E-04 & 27.2807 & 10.0521 & 37.3328 & \\
\hline & 7/29/2008 7:00 & 162 & 211 & 700 & 8.6 & 8.6 & & 0.0570 & 3.50E-04 & 55.9671 & 20.6222 & 76.5894 & \\
\hline & 7/29/2008 8:00 & 163 & 211 & 800 & 7.558 & 7.558 & & 0.2027 & 1.67E-03 & 266.9340 & 98.3573 & 365.2913 & \\
\hline
\end{tabular}




\begin{tabular}{|c|c|c|c|c|c|c|c|c|c|c|c|c|c|}
\hline Tree ID & TS & RN & JD & HR & $\begin{array}{c}\text { Raw } \\
\text { TC_dTA }\end{array}$ & $\begin{array}{c}\text { Fixed } \\
\text { TC_dT } \Delta\end{array}$ & dTM & $\mathbf{K}$ & $\begin{array}{l}\text { Sap } \\
\text { Velocity } \\
(\mathrm{cm} / \mathrm{s})\end{array}$ & $\begin{array}{c}\text { Probed } \\
\text { Drywood F } \\
(\mathrm{g} / \mathrm{hr})\end{array}$ & $\begin{array}{c}\text { Extrapolated } \\
\text { Wetwood F } \\
(\mathrm{g} / \mathrm{hr})\end{array}$ & $\begin{array}{c}\text { Extrapolated } \\
\text { Total Wood F } \\
(\mathrm{g} / \mathrm{hr})\end{array}$ & $\begin{array}{l}\text { Relative } \\
\text { Humidity, } \\
@ 10 \mathrm{~m}(\%)\end{array}$ \\
\hline & 7/29/2008 9:00 & 164 & 211 & 900 & \begin{tabular}{|r|}
5.649 \\
\end{tabular} & 5.649 & & 0.6091 & $6.46 \mathrm{E}-03$ & 1034.3108 & 381.1131 & \begin{tabular}{|l|}
1415.4239 \\
\end{tabular} & \\
\hline & $7 / 29 / 2008$ 10:00 & 165 & 211 & 1000 & 4.986 & 4.986 & & 0.8231 & $9.36 \mathrm{E}-03$ & 1498.2850 & 552.0739 & 2050.3589 & \\
\hline & $7 / 29 / 2008$ 11:00 & 166 & 211 & 1100 & 5.249 & 5.249 & & 0.7318 & 8.10E-03 & 1296.3012 & 477.6488 & 1773.9500 & \\
\hline & $7 / 29 / 2008$ 12:00 & 167 & 211 & 1200 & 4.792 & 4.792 & & 0.8969 & 1.04E-02 & 1665.3441 & 613.6303 & 2278.9744 & \\
\hline & 7/29/2008 13:00 & 168 & 211 & 1300 & 4.874 & 4.874 & & 0.8650 & 9.95E-03 & 1592.7030 & 586.8642 & 2179.5671 & \\
\hline & $7 / 29 / 2008$ 14:00 & 169 & 211 & 1400 & 4.728 & 4.728 & & 0.9226 & 1.08E-02 & 1724.2265 & 635.3267 & 2359.5532 & \\
\hline & $7 / 29 / 2008$ 15:00 & 170 & 211 & 1500 & 4.899 & 4.899 & & 0.8555 & $9.82 E-03$ & 1571.1585 & 578.9257 & 2150.0842 & \\
\hline & $7 / 29 / 2008$ 16:00 & 171 & 211 & 1600 & 5.037 & 5.037 & & 0.8046 & 9.11E-03 & 1457.0302 & 536.8727 & 1993.9029 & \\
\hline & 7/29/2008 17:00 & 172 & 211 & 1700 & 5.257 & 5.257 & & 0.7291 & 8.07E-03 & 1290.5566 & 475.5321 & 1766.0888 & \\
\hline & 7/29/2008 18:00 & 173 & 211 & 1800 & 5.26 & 5.26 & & 0.7281 & 8.05E-03 & 1288.4082 & 474.7405 & 1763.1486 & \\
\hline & $7 / 29 / 2008$ 19:00 & 174 & 211 & 1900 & 5.321 & 5.321 & & 0.7083 & 7.78E-03 & 1245.3915 & 458.8901 & 1704.2816 & \\
\hline & $7 / 29 / 200820: 00$ & 175 & 211 & 2000 & 6.256 & 6.256 & & 0.4530 & 4.49E-03 & 718.3435 & 264.6884 & 983.0319 & \\
\hline & 7/29/2008 21:00 & 176 & 211 & 2100 & 7.789 & 7.789 & & 0.1670 & $1.31 \mathrm{E}-03$ & 210.3442 & 77.5057 & 287.8499 & \\
\hline & $7 / 29 / 2008$ 22:00 & 177 & 211 & 2200 & 8.59 & 8.59 & & 0.0582 & 3.59E-04 & 57.4587 & 21.1718 & 78.6305 & \\
\hline & $7 / 29 / 2008$ 23:00 & 178 & 211 & 2300 & 8.85 & 8.85 & & 0.0271 & 1.40E-04 & 22.4400 & 8.2685 & 30.7084 & \\
\hline & $7 / 30 / 20080: 00$ & 179 & 212 & 0 & 8.92 & 8.92 & 8.95 & 0.0191 & 9.09E-05 & 14.5363 & 5.3562 & 19.8924 & \\
\hline & 7/30/2008 1:00 & 180 & 212 & 100 & 8.93 & 8.93 & & 0.0179 & 8.42E-05 & 13.4723 & 4.9642 & 18.4365 & \\
\hline & 7/30/2008 2:00 & 181 & 212 & 200 & 8.93 & 8.93 & & 0.0179 & 8.42E-05 & 13.4723 & 4.9642 & 18.4365 & \\
\hline & 7/30/2008 3:00 & 182 & 212 & 300 & 8.95 & 8.95 & & 0.0156 & 7.12E-05 & 11.3988 & 4.2001 & 15.5989 & \\
\hline & 7/30/2008 4:00 & 183 & 212 & 400 & 8.94 & 8.94 & & 0.0168 & 7.77E-05 & 12.4263 & 4.5787 & 17.0050 & \\
\hline & 7/30/2008 5:00 & 184 & 212 & 500 & 8.92 & 8.92 & & 0.0191 & $9.09 \mathrm{E}-05$ & 14.5363 & 5.3562 & 19.8924 & \\
\hline & 7/30/2008 6:00 & 185 & 212 & 600 & 8.91 & 8.91 & & 0.0202 & 9.76E-05 & 15.6175 & 5.7546 & 21.3720 & \\
\hline & 7/30/2008 7:00 & 186 & 212 & 700 & 8.91 & 8.91 & & 0.0202 & $9.76 \mathrm{E}-05$ & 15.6175 & 5.7546 & 21.3720 & \\
\hline & $7 / 30 / 20088: 00$ & 187 & 212 & 800 & 8.89 & 8.89 & & 0.0225 & 1.11E-04 & 17.8295 & 6.5696 & 24.3991 & \\
\hline & 7/30/2008 9:00 & 188 & 212 & 900 & 7.516 & 7.516 & & 0.2094 & 1.74E-03 & 277.8704 & 102.3871 & 380.2575 & \\
\hline & $7 / 30 / 2008$ 10:00 & 189 & 212 & 1000 & 5.23 & 5.23 & & 0.7380 & 8.19E-03 & 1310.0342 & 482.7090 & 1792.7432 & \\
\hline & $7 / 30 / 2008$ 11:00 & 190 & 212 & 1100 & 4.937 & 4.937 & & 0.8412 & 9.62E-03 & 1538.9328 & 567.0514 & 2105.9842 & \\
\hline & $7 / 30 / 2008$ 12:00 & 191 & 212 & 1200 & 4.942 & 4.942 & & 0.8393 & $9.59 \mathrm{E}-03$ & 1534.7387 & 565.5060 & 2100.2448 & \\
\hline & 7/30/2008 13:00 & 192 & 212 & 1300 & 4.971 & 4.971 & & 0.8286 & 9.44E-03 & 1510.6215 & 556.6195 & 2067.2410 & \\
\hline & $7 / 30 / 2008$ 14:00 & 193 & 212 & 1400 & 4.926 & 4.926 & & 0.8453 & $9.68 \mathrm{E}-03$ & 1548.1973 & 570.4651 & 2118.6625 & \\
\hline & 7/30/2008 15:00 & 194 & 212 & 1500 & 5.048 & 5.048 & & 0.8007 & $9.05 \mathrm{E}-03$ & 1448.2694 & 533.6447 & 1981.9141 & \\
\hline & $7 / 30 / 2008$ 16:00 & 195 & 212 & 1600 & 4.924 & 4.924 & & 0.8461 & $9.69 \mathrm{E}-03$ & 1549.8873 & 571.0879 & 2120.9752 & \\
\hline & $7 / 30 / 2008$ 17:00 & 196 & 212 & 1700 & 4.923 & 4.923 & & 0.8464 & 9.69E-03 & 1550.7330 & 571.3995 & 2122.1325 & \\
\hline & $7 / 30 / 2008$ 18:00 & 197 & 212 & 1800 & 5.113 & 5.113 & & 0.7778 & 8.73E-03 & 1397.4692 & 514.9263 & 1912.3955 & \\
\hline & 7/30/2008 19:00 & 198 & 212 & 1900 & 5.166 & 5.166 & & 0.7596 & 8.48E-03 & 1357.2397 & 500.1029 & 1857.3426 & \\
\hline & $7 / 30 / 200820: 00$ & 199 & 212 & 2000 & 5.72 & 5.72 & & 0.5892 & $6.20 \mathrm{E}-03$ & 992.7208 & 365.7884 & 1358.5092 & \\
\hline & 7/30/2008 21:00 & 200 & 212 & 2100 & 7.235 & 7.235 & & 0.2564 & 2.23E-03 & 356.4772 & 131.3514 & 487.8285 & \\
\hline & $7 / 30 / 2008$ 22:00 & 201 & 212 & 2200 & 8.3 & 8.3 & & 0.0952 & 6.58E-04 & 105.2599 & 38.7852 & 144.0451 & \\
\hline & 7/30/2008 23:00 & 202 & 212 & 2300 & 8.7 & 8.7 & & 0.0448 & $2.60 \mathrm{E}-04$ & 41.6602 & 15.3506 & 57.0108 & \\
\hline & $7 / 31 / 20080: 00$ & 203 & 213 & 0 & 8.97 & 8.97 & 9.31 & 0.0134 & 5.88E-05 & 9.4027 & 3.4646 & 12.8674 & \\
\hline & 7/31/2008 1:00 & 204 & 213 & 100 & 9.12 & 9.12 & & -0.0033 & $0.00 \mathrm{E}+00$ & 0.0000 & 0.0000 & 0.0000 & \\
\hline
\end{tabular}




\begin{tabular}{|c|c|c|c|c|c|c|c|c|c|c|c|c|c|}
\hline Tree ID & TS & RN & JD & HR & $\begin{array}{c}\text { Raw } \\
\text { TC_dTA }\end{array}$ & $\begin{array}{c}\text { Fixed } \\
\text { TC_dT } \Delta\end{array}$ & dTM & K & $\begin{array}{l}\text { Sap } \\
\text { Velocity } \\
(\mathrm{cm} / \mathrm{s})\end{array}$ & $\begin{array}{c}\text { Probed } \\
\text { Drywood F } \\
(\mathrm{g} / \mathrm{hr})\end{array}$ & $\begin{array}{c}\text { Extrapolated } \\
\text { Wetwood F } \\
(\mathrm{g} / \mathrm{hr})\end{array}$ & $\begin{array}{c}\text { Extrapolated } \\
\text { Total Wood F } \\
(\mathrm{g} / \mathrm{hr})\end{array}$ & $\begin{array}{l}\text { Relative } \\
\text { Humidity, } \\
@ 10 \mathrm{~m}(\%)\end{array}$ \\
\hline & 7/31/2008 2:00 & 205 & 213 & 200 & \begin{tabular}{|r|}
9.18 \\
\end{tabular} & 9.18 & & -0.0098 & $0.00 \mathrm{E}+00$ & 0.0000 & 0.0000 & 0.0000 & \\
\hline & 7/31/2008 3:00 & 206 & 213 & 300 & 9.22 & 9.22 & & -0.0141 & $0.00 \mathrm{E}+00$ & 0.0000 & 0.0000 & 0.0000 & \\
\hline & 7/31/2008 4:00 & 207 & 213 & 400 & 9.28 & 9.28 & & -0.0205 & $0.00 E+00$ & 0.0000 & 0.0000 & 0.0000 & \\
\hline & 7/31/2008 5:00 & 208 & 213 & 500 & 9.3 & 9.3 & & -0.0226 & $0.00 \mathrm{E}+00$ & 0.0000 & 0.0000 & 0.0000 & \\
\hline & 7/31/2008 6:00 & 209 & 213 & 600 & 9.31 & 9.31 & & -0.0236 & $0.00 \mathrm{E}+00$ & 0.0000 & 0.0000 & 0.0000 & \\
\hline & 7/31/2008 7:00 & 210 & 213 & 700 & 9.3 & 9.3 & & -0.0226 & $0.00 \mathrm{E}+00$ & 0.0000 & 0.0000 & 0.0000 & \\
\hline & $7 / 31 / 2008$ 8:00 & 211 & 213 & 800 & 9.19 & 9.19 & & -0.0109 & $0.00 \mathrm{E}+00$ & 0.0000 & 0.0000 & 0.0000 & \\
\hline & $7 / 31 / 20089: 00$ & 212 & 213 & 900 & 7.354 & 7.354 & & 0.2361 & 2.01E-03 & 322.0063 & 118.6498 & 440.6561 & \\
\hline & $7 / 31 / 2008$ 10:00 & 213 & 213 & 1000 & 5.395 & 5.395 & & 0.6849 & 7.47E-03 & 1194.8713 & 440.2749 & 1635.1462 & \\
\hline & 7/31/2008 11:00 & 214 & 213 & 1100 & 5.234 & 5.234 & & 0.7367 & 8.17E-03 & 1307.1325 & 481.6398 & 1788.7723 & \\
\hline & $7 / 31 / 2008$ 12:00 & 215 & 213 & 1200 & 5.07 & 5.07 & & 0.7929 & 8.94E-03 & 1430.8915 & 527.2414 & 1958.1329 & \\
\hline & 7/31/2008 13:00 & 216 & 213 & 1300 & 5.254 & 5.254 & & 0.7301 & $8.08 \mathrm{E}-03$ & 1292.7082 & 476.3249 & 1769.0331 & \\
\hline & 7/31/2008 14:00 & 217 & 213 & 1400 & 5.013 & 5.013 & & 0.8133 & $9.23 \mathrm{E}-03$ & 1476.3127 & 543.9778 & 2020.2905 & \\
\hline & 7/31/2008 15:00 & 218 & 213 & 1500 & 5.016 & 5.016 & & 0.8122 & $9.21 \mathrm{E}-03$ & 1473.8897 & 543.0850 & 2016.9747 & \\
\hline & $7 / 31 / 2008$ 16:00 & 219 & 213 & 1600 & 5.071 & 5.071 & & 0.7925 & 8.94E-03 & 1430.1061 & 526.9520 & 1957.0581 & \\
\hline & 7/31/2008 17:00 & 220 & 213 & 1700 & 5.061 & 5.061 & & 0.7961 & 8.99E-03 & 1437.9776 & 529.8524 & 1967.8301 & \\
\hline & $7 / 31 / 2008$ 18:00 & 221 & 213 & 1800 & 5.191 & 5.191 & & 0.7511 & 8.37E-03 & 1338.6241 & 493.2436 & 1831.8677 & \\
\hline & $7 / 31 / 2008$ 19:00 & 222 & 213 & 1900 & 5.389 & 5.389 & & 0.6868 & 7.49E-03 & 1198.9013 & 441.7599 & 1640.6612 & \\
\hline & $7 / 31 / 200820: 00$ & 223 & 213 & 2000 & 6.099 & 6.099 & & 0.4904 & 4.95E-03 & 792.0377 & 291.8426 & 1083.8803 & \\
\hline & $7 / 31 / 200821: 00$ & 224 & 213 & 2100 & 7.04 & 7.04 & & 0.2912 & $2.61 E-03$ & 416.9426 & 153.6311 & 570.5737 & \\
\hline & $7 / 31 / 200822: 00$ & 225 & 213 & 2200 & 7.823 & 7.823 & & 0.1620 & $1.27 \mathrm{E}-03$ & 202.5092 & 74.6187 & 277.1278 & \\
\hline & 7/31/2008 23:00 & 226 & 213 & 2300 & 7.81 & 7.81 & & 0.1639 & 1.28E-03 & 205.4903 & 75.7171 & 281.2074 & \\
\hline & $8 / 1 / 20080: 00$ & 227 & 214 & 0 & 8.18 & 8.18 & 9.30 & 0.1112 & 7.97E-04 & 127.5410 & 46.9951 & 174.5361 & \\
\hline & 8/1/2008 1:00 & 228 & 214 & 100 & 8.77 & 8.77 & & 0.0365 & 2.02E-04 & 32.3353 & 11.9146 & 44.2499 & \\
\hline & 8/1/2008 2:00 & 229 & 214 & 200 & 9.07 & 9.07 & & 0.0022 & 6.39E-06 & 1.0219 & 0.3766 & 1.3985 & \\
\hline & $8 / 1 / 2008$ 3:00 & 230 & 214 & 300 & 9.12 & 9.12 & & -0.0033 & $0.00 E+00$ & 0.0000 & 0.0000 & 0.0000 & \\
\hline & 8/1/2008 4:00 & 231 & 214 & 400 & 9.23 & 9.23 & & -0.0152 & $0.00 \mathrm{E}+00$ & 0.0000 & 0.0000 & 0.0000 & \\
\hline & 8/1/2008 5:00 & 232 & 214 & 500 & 9.3 & 9.3 & & -0.0226 & $0.00 \mathrm{E}+00$ & 0.0000 & 0.0000 & 0.0000 & \\
\hline & 8/1/2008 6:00 & 233 & 214 & 600 & 9.29 & 9.29 & & -0.0215 & $0.00 \mathrm{E}+00$ & 0.0000 & 0.0000 & 0.0000 & \\
\hline & 8/1/2008 7:00 & 234 & 214 & 700 & 9.29 & 9.29 & & -0.0215 & $0.00 \mathrm{E}+00$ & 0.0000 & 0.0000 & 0.0000 & \\
\hline & 8/1/2008 8:00 & 235 & 214 & 800 & 9.16 & 9.16 & & -0.0076 & $0.00 \mathrm{E}+00$ & 0.0000 & 0.0000 & 0.0000 & \\
\hline & 8/1/2008 9:00 & 236 & 214 & 900 & 7.461 & 7.461 & & 0.2183 & 1.83E-03 & 292.5034 & 107.7789 & 400.2823 & \\
\hline & 8/1/2008 10:00 & 237 & 214 & 1000 & 5.448 & 5.448 & & 0.6685 & 7.25E-03 & 1159.7672 & 427.3401 & 1587.1073 & \\
\hline & 8/1/2008 11:00 & 238 & 214 & 1100 & 5.325 & 5.325 & & 0.7070 & 7.77E-03 & 1242.6147 & 457.8669 & 1700.4816 & \\
\hline & 8/1/2008 12:00 & 239 & 214 & 1200 & 5.279 & 5.279 & & 0.7219 & 7.97E-03 & 1274.8735 & 469.7533 & 1744.6268 & \\
\hline & 8/1/2008 13:00 & 240 & 214 & 1300 & 5.17 & 5.17 & & 0.7582 & 8.46E-03 & 1354.2459 & 498.9997 & 1853.2456 & \\
\hline & 8/1/2008 14:00 & 241 & 214 & 1400 & 5.113 & 5.113 & & 0.7778 & 8.73E-03 & 1397.4692 & 514.9263 & 1912.3955 & \\
\hline & 8/1/2008 15:00 & 242 & 214 & 1500 & 5.071 & 5.071 & & 0.7925 & 8.94E-03 & 1430.1061 & 526.9520 & 1957.0581 & \\
\hline & $8 / 1 / 200816: 00$ & 243 & 214 & 1600 & 5.11 & 5.11 & & 0.7789 & $8.75 \mathrm{E}-03$ & 1399.7780 & 515.7770 & 1915.5550 & \\
\hline & 8/1/2008 17:00 & 244 & 214 & 1700 & 5.152 & 5.152 & & 0.7644 & $8.55 \mathrm{E}-03$ & 1367.7646 & 503.9810 & 1871.7456 & \\
\hline & 8/1/2008 18:00 & 245 & 214 & 1800 & 5.291 & 5.291 & & 0.7180 & 7.91E-03 & 1266.3890 & 466.6271 & 1733.0161 & \\
\hline
\end{tabular}




\begin{tabular}{|c|c|c|c|c|c|c|c|c|c|c|c|c|c|}
\hline Tree ID & TS & RN & JD & HR & $\begin{array}{c}\text { Raw } \\
\text { TC_dTA }\end{array}$ & $\begin{array}{c}\text { Fixed } \\
\text { TC_dT } \Delta\end{array}$ & dTM & $\mathbf{K}$ & $\begin{array}{l}\text { Sap } \\
\text { Velocity } \\
(\mathrm{cm} / \mathrm{s})\end{array}$ & $\begin{array}{c}\text { Probed } \\
\text { Drywood F } \\
(\mathrm{g} / \mathrm{hr})\end{array}$ & $\begin{array}{c}\text { Extrapolated } \\
\text { Wetwood F } \\
(\mathrm{g} / \mathrm{hr})\end{array}$ & $\begin{array}{c}\text { Extrapolated } \\
\text { Total Wood F } \\
(\mathrm{g} / \mathrm{hr})\end{array}$ & $\begin{array}{l}\text { Relative } \\
\text { Humidity, } \\
@ 10 \mathrm{~m}(\%)\end{array}$ \\
\hline & 8/1/2008 19:00 & 246 & 214 & 1900 & \begin{tabular}{|r|}
5.352 \\
\end{tabular} & 5.352 & & 0.6984 & 7.65E-03 & 1224.0097 & 451.0116 & 1675.0213 & \\
\hline & 8/1/2008 20:00 & 247 & 214 & 2000 & 6.09 & 6.09 & & 0.4926 & 4.98E-03 & 796.4190 & 293.4570 & 1089.8759 & \\
\hline & 8/1/2008 21:00 & 248 & 214 & 2100 & 7.794 & 7.794 & & 0.1663 & $1.31 \mathrm{E}-03$ & 209.1842 & 77.0782 & 286.2625 & \\
\hline & 8/1/2008 22:00 & 249 & 214 & 2200 & 8.72 & 8.72 & & 0.0424 & 2.43E-04 & 38.9359 & 14.3467 & 53.2826 & \\
\hline & 8/1/2008 23:00 & 250 & 214 & 2300 & 8.96 & 8.96 & & 0.0145 & $6.49 \mathrm{E}-05$ & 10.3906 & 3.8286 & 14.2193 & \\
\hline & 8/2/2008 0:00 & 251 & 215 & 0 & 9.06 & 9.06 & 9.34 & 0.0033 & 1.05E-05 & 1.6857 & 0.6211 & 2.3068 & \\
\hline & 8/2/2008 1:00 & 252 & 215 & 100 & 9.19 & 9.19 & & -0.0109 & $0.00 \mathrm{E}+00$ & 0.0000 & 0.0000 & 0.0000 & \\
\hline & 8/2/2008 2:00 & 253 & 215 & 200 & 9.29 & 9.29 & & -0.0215 & $0.00 \mathrm{E}+00$ & 0.0000 & 0.0000 & 0.0000 & \\
\hline & 8/2/2008 3:00 & 254 & 215 & 300 & 9.34 & 9.34 & & -0.0268 & $0.00 \mathrm{E}+00$ & 0.0000 & 0.0000 & 0.0000 & \\
\hline & 8/2/2008 4:00 & 255 & 215 & 400 & 9.26 & 9.26 & & -0.0184 & $0.00 E+00$ & 0.0000 & 0.0000 & 0.0000 & \\
\hline & 8/2/2008 5:00 & 256 & 215 & 500 & 9.22 & 9.22 & & -0.0141 & $0.00 \mathrm{E}+00$ & 0.0000 & 0.0000 & 0.0000 & \\
\hline & 8/2/2008 6:00 & 257 & 215 & 600 & 9.21 & 9.21 & & -0.0130 & $0.00 E+00$ & 0.0000 & 0.0000 & 0.0000 & \\
\hline & 8/2/2008 7:00 & 258 & 215 & 700 & 9.05 & 9.05 & & 0.0044 & 1.50E-05 & 2.4053 & 0.8863 & 3.2916 & \\
\hline & 8/2/2008 8:00 & 259 & 215 & 800 & 7.493 & 7.493 & & 0.2131 & 1.77E-03 & 283.9464 & 104.6259 & 388.5723 & \\
\hline & 8/2/2008 9:00 & 260 & 215 & 900 & 5.769 & 5.769 & & 0.5757 & 6.03E-03 & 964.7981 & 355.4997 & 1320.2978 & \\
\hline & 8/2/2008 10:00 & 261 & 215 & 1000 & 5.558 & 5.558 & & 0.6355 & 6.81E-03 & 1089.6524 & 401.5048 & 1491.1572 & \\
\hline & 8/2/2008 11:00 & 262 & 215 & 1100 & 5.415 & 5.415 & & 0.6787 & 7.38E-03 & 1181.5206 & 435.3556 & 1616.8762 & \\
\hline & $8 / 2 / 200812: 00$ & 263 & 215 & 1200 & 5.322 & 5.322 & & 0.7080 & 7.78E-03 & 1244.6968 & 458.6341 & 1703.3309 & \\
\hline & 8/2/2008 13:00 & 264 & 215 & 1300 & 5.283 & 5.283 & & 0.7206 & 7.95E-03 & 1272.0398 & 468.7092 & 1740.7491 & \\
\hline & 8/2/2008 14:00 & 265 & 215 & 1400 & 5.272 & 5.272 & & 0.7242 & 8.00E-03 & 1279.8455 & 471.5854 & 1751.4308 & \\
\hline & $8 / 2 / 2008$ 15:00 & 266 & 215 & 1500 & 5.256 & 5.256 & & 0.7295 & 8.07E-03 & 1291.2735 & 475.7963 & 1767.0697 & \\
\hline & 8/2/2008 16:00 & 267 & 215 & 1600 & 5.285 & 5.285 & & 0.7200 & 7.94E-03 & 1270.6251 & 468.1880 & 1738.8130 & \\
\hline & 8/2/2008 17:00 & 268 & 215 & 1700 & 5.29 & 5.29 & & 0.7183 & 7.92E-03 & 1267.0942 & 466.8869 & 1733.9811 & \\
\hline & $8 / 2 / 200818: 00$ & 269 & 215 & 1800 & 5.435 & 5.435 & & 0.6725 & 7.30E-03 & 1168.2962 & 430.4827 & 1598.7789 & \\
\hline & 8/2/2008 19:00 & 270 & 215 & 1900 & 5.52 & 5.52 & & 0.6467 & $6.96 \mathrm{E}-03$ & 1113.4656 & 410.2793 & 1523.7449 & \\
\hline & 8/2/2008 20:00 & 271 & 215 & 2000 & 6.101 & 6.101 & & 0.4899 & 4.94E-03 & 791.0665 & 291.4847 & 1082.5512 & \\
\hline & 8/2/2008 21:00 & 272 & 215 & 2100 & 7.233 & 7.233 & & 0.2567 & $2.23 E-03$ & 357.0719 & 131.5705 & 488.6424 & \\
\hline & 8/2/2008 22:00 & 273 & 215 & 2200 & 8.3 & 8.3 & & 0.0952 & $6.58 \mathrm{E}-04$ & 105.2599 & 38.7852 & 144.0451 & \\
\hline & 8/2/2008 23:00 & 274 & 215 & 2300 & 8.69 & 8.69 & & 0.0460 & 2.69E-04 & 43.0399 & 15.8590 & 58.8989 & \\
\hline & 8/3/2008 0:00 & 275 & 216 & 0 & 8.58 & 8.58 & 9.16 & 0.0594 & 3.69E-04 & 58.9611 & 21.7254 & 80.6865 & \\
\hline & 8/3/2008 1:00 & 276 & 216 & 100 & 8.36 & 8.36 & & 0.0873 & 5.92E-04 & 94.6638 & 34.8808 & 129.5446 & \\
\hline & 8/3/2008 2:00 & 277 & 216 & 200 & 8.67 & 8.67 & & 0.0484 & 2.86E-04 & 45.8340 & 16.8885 & 62.7225 & \\
\hline & 8/3/2008 3:00 & 278 & 216 & 300 & 8.94 & 8.94 & & 0.0168 & 7.77E-05 & 12.4263 & 4.5787 & 17.0050 & \\
\hline & 8/3/2008 4:00 & 279 & 216 & 400 & 9.1 & 9.1 & & -0.0011 & $0.00 \mathrm{E}+00$ & 0.0000 & 0.0000 & 0.0000 & \\
\hline & 8/3/2008 5:00 & 280 & 216 & 500 & 9.16 & 9.16 & & -0.0076 & $0.00 \mathrm{E}+00$ & 0.0000 & 0.0000 & 0.0000 & \\
\hline & 8/3/2008 6:00 & 281 & 216 & 600 & 8.93 & 8.93 & & 0.0179 & 8.42E-05 & 13.4723 & 4.9642 & 18.4365 & \\
\hline & 8/3/2008 7:00 & 282 & 216 & 700 & 8.61 & 8.61 & & 0.0557 & $3.41 \mathrm{E}-04$ & 54.4864 & 20.0766 & 74.5631 & \\
\hline & 8/3/2008 8:00 & 283 & 216 & 800 & 7.351 & 7.351 & & 0.2366 & 2.02E-03 & 322.8535 & 118.9620 & 441.8156 & \\
\hline & 8/3/2008 9:00 & 284 & 216 & 900 & 5.627 & 5.627 & & 0.6154 & $6.55 \mathrm{E}-03$ & 1047.4767 & 385.9643 & 1433.4410 & \\
\hline & $8 / 3 / 2008$ 10:00 & 285 & 216 & 1000 & 5.34 & 5.34 & & 0.7022 & 7.70E-03 & 1232.2489 & 454.0474 & 1686.2963 & \\
\hline & 8/3/2008 11:00 & 286 & 216 & 1100 & 5.248 & 5.248 & & 0.7321 & 8.11E-03 & 1297.0208 & 477.9140 & 1774.9348 & \\
\hline
\end{tabular}




\begin{tabular}{|c|c|c|c|c|c|c|c|c|c|c|c|c|c|}
\hline Tree ID & TS & RN & JD & HR & $\begin{array}{c}\text { Raw } \\
\text { TC_dTA }\end{array}$ & $\begin{array}{c}\text { Fixed } \\
\text { TC_dT } \Delta\end{array}$ & dTM & $\mathbf{K}$ & $\begin{array}{l}\text { Sap } \\
\text { Velocity } \\
(\mathrm{cm} / \mathrm{s})\end{array}$ & $\begin{array}{c}\text { Probed } \\
\text { Drywood F } \\
(\mathrm{g} / \mathrm{hr})\end{array}$ & $\begin{array}{c}\text { Extrapolated } \\
\text { Wetwood F } \\
(\mathrm{g} / \mathrm{hr})\end{array}$ & $\begin{array}{c}\text { Extrapolated } \\
\text { Total Wood F } \\
(\mathrm{g} / \mathrm{hr})\end{array}$ & $\begin{array}{l}\text { Relative } \\
\text { Humidity, } \\
@ 10 \mathrm{~m}(\%)\end{array}$ \\
\hline & 8/3/2008 12:00 & 287 & 216 & 1200 & \begin{tabular}{|r|}
5.227 \\
\end{tabular} & 5.227 & & 0.7390 & $8.20 \mathrm{E}-03$ & 1312.2141 & 483.5123 & \begin{tabular}{|r|}
1795.7264 \\
\end{tabular} & \\
\hline & 8/3/2008 13:00 & 288 & 216 & 1300 & 5.216 & 5.216 & & 0.7427 & $8.25 \mathrm{E}-03$ & 1320.2347 & 486.4676 & 1806.7024 & \\
\hline & $8 / 3 / 2008$ 14:00 & 289 & 216 & 1400 & 5.224 & 5.224 & & 0.7400 & $8.21 \mathrm{E}-03$ & 1314.3973 & 484.3167 & 1798.7140 & \\
\hline & 8/3/2008 15:00 & 290 & 216 & 1500 & 5.3 & 5.3 & & 0.7151 & 7.88E-03 & 1260.0579 & 464.2942 & 1724.3521 & \\
\hline & 8/3/2008 16:00 & 291 & 216 & 1600 & 5.323 & 5.323 & & 0.7077 & 7.77E-03 & 1244.0024 & 458.3783 & 1702.3807 & \\
\hline & 8/3/2008 17:00 & 292 & 216 & 1700 & 5.315 & 5.315 & & 0.7103 & 7.81E-03 & 1249.5668 & 460.4286 & 1709.9954 & \\
\hline & 8/3/2008 18:00 & 293 & 216 & 1800 & 5.437 & 5.437 & & 0.6719 & $7.29 \mathrm{E}-03$ & 1166.9806 & 429.9980 & 1596.9786 & \\
\hline & $8 / 3 / 2008$ 19:00 & 294 & 216 & 1900 & 5.451 & 5.451 & & 0.6676 & $7.24 \mathrm{E}-03$ & 1157.8064 & 426.6176 & 1584.4240 & \\
\hline & 8/3/2008 20:00 & 295 & 216 & 2000 & 5.712 & 5.712 & & 0.5914 & $6.23 E-03$ & 997.3394 & 367.4902 & 1364.8296 & \\
\hline & 8/3/2008 21:00 & 296 & 216 & 2100 & 6.515 & 6.515 & & 0.3952 & 3.80E-03 & 607.3057 & 223.7743 & 831.0800 & \\
\hline & 8/3/2008 22:00 & 297 & 216 & 2200 & 7.145 & 7.145 & & 0.2722 & $2.40 \mathrm{E}-03$ & 383.7536 & 141.4019 & 525.1556 & \\
\hline & 8/3/2008 23:00 & 298 & 216 & 2300 & 7.299 & 7.299 & & 0.2454 & 2.11E-03 & 337.7168 & 124.4387 & 462.1555 & \\
\hline & 8/4/2008 0:00 & 299 & 217 & 0 & 7.379 & 7.379 & 8.56 & 0.2319 & 1.97E-03 & 314.9888 & 116.0641 & 431.0528 & \\
\hline & 8/4/2008 1:00 & 300 & 217 & 100 & 7.456 & 7.456 & & 0.2192 & 1.84E-03 & 293.8514 & 108.2756 & 402.1270 & \\
\hline & $8 / 4 / 2008$ 2:00 & 301 & 217 & 200 & 7.107 & 7.107 & & 0.2790 & 2.47E-03 & 395.5921 & 145.7641 & 541.3562 & \\
\hline & 8/4/2008 3:00 & 302 & 217 & 300 & 6.912 & 6.912 & & 0.3151 & $2.87 \mathrm{E}-03$ & 459.4796 & 169.3047 & 628.7844 & \\
\hline & 8/4/2008 4:00 & 303 & 217 & 400 & 6.71 & 6.71 & & 0.3547 & 3.32E-03 & 531.5450 & 195.8587 & 727.4037 & \\
\hline & $8 / 4 / 20085: 00$ & 304 & 217 & 500 & 6.928 & 6.928 & & 0.3121 & 2.84E-03 & 454.0339 & 167.2981 & 621.3320 & \\
\hline & 8/4/2008 6:00 & 305 & 217 & 600 & 7.42 & 7.42 & & 0.2251 & 1.90E-03 & 303.6449 & 111.8842 & 415.5291 & \\
\hline & 8/4/2008 7:00 & 306 & 217 & 700 & 7.43 & 7.43 & & 0.2234 & $1.88 E-03$ & 300.9089 & 110.8761 & 411.7850 & \\
\hline & 8/4/2008 8:00 & 307 & 217 & 800 & 7.242 & 7.242 & & 0.2552 & $2.21 \mathrm{E}-03$ & 354.3998 & 130.5859 & 484.9857 & \\
\hline & 8/4/2008 9:00 & 308 & 217 & 900 & 6.958 & 6.958 & & 0.3064 & 2.77E-03 & 443.9233 & 163.5727 & 607.4960 & \\
\hline & 8/4/2008 10:00 & 309 & 217 & 1000 & 6.076 & 6.076 & & 0.4961 & 5.02E-03 & 803.2692 & 295.9810 & 1099.2502 & \\
\hline & 8/4/2008 11:00 & 310 & 217 & 1100 & 5.553 & 5.553 & & 0.6370 & $6.83 \mathrm{E}-03$ & 1092.7616 & 402.6505 & 1495.4121 & \\
\hline & 8/4/2008 12:00 & 311 & 217 & 1200 & 5.358 & 5.358 & & 0.6965 & $7.62 \mathrm{E}-03$ & 1219.9079 & 449.5002 & 1669.4081 & \\
\hline & 8/4/2008 13:00 & 312 & 217 & 1300 & 5.311 & 5.311 & & 0.7115 & 7.83E-03 & 1252.3570 & 461.4567 & 1713.8137 & \\
\hline & 8/4/2008 14:00 & 313 & 217 & 1400 & 5.573 & 5.573 & & 0.6311 & $6.75 E-03$ & 1080.3682 & 398.0839 & 1478.4521 & \\
\hline & 8/4/2008 15:00 & 314 & 217 & 1500 & 5.91 & 5.91 & & 0.5381 & $5.55 \mathrm{E}-03$ & 887.8361 & 327.1415 & 1214.9776 & \\
\hline & 8/4/2008 16:00 & 315 & 217 & 1600 & 5.412 & 5.412 & & 0.6796 & 7.40E-03 & 1183.5152 & 436.0905 & 1619.6057 & \\
\hline & 8/4/2008 17:00 & 316 & 217 & 1700 & 5.348 & 5.348 & & 0.6997 & 7.67E-03 & 1226.7509 & 452.0216 & 1678.7724 & \\
\hline & 8/4/2008 18:00 & 317 & 217 & 1800 & 5.505 & 5.505 & & 0.6512 & 7.02E-03 & 1122.9828 & 413.7861 & 1536.7690 & \\
\hline & 8/4/2008 19:00 & 318 & 217 & 1900 & 5.642 & 5.642 & & 0.6111 & 6.49E-03 & 1038.4854 & 382.6513 & 1421.1367 & \\
\hline & 8/4/2008 20:00 & 319 & 217 & 2000 & 6.219 & 6.219 & & 0.4616 & $4.60 \mathrm{E}-03$ & 735.2552 & 270.9199 & 1006.1750 & \\
\hline & 8/4/2008 21:00 & 320 & 217 & 2100 & 7.76 & 7.76 & & 0.1714 & $1.36 \mathrm{E}-03$ & 217.1255 & 80.0044 & 297.1298 & \\
\hline & 8/4/2008 22:00 & 321 & 217 & 2200 & 8.38 & 8.38 & & 0.0847 & 5.70E-04 & 91.2126 & 33.6092 & 124.8218 & \\
\hline & 8/4/2008 23:00 & 322 & 217 & 2300 & 8.56 & 8.56 & & 0.0619 & 3.87E-04 & 61.9980 & 22.8444 & 84.8424 & \\
\hline & 8/5/2008 0:00 & 323 & 218 & 0 & 8.84 & 8.84 & 9.28 & 0.0283 & 1.48E-04 & 23.6293 & 8.7067 & 32.3360 & \\
\hline & 8/5/2008 1:00 & 324 & 218 & 100 & 9.02 & 9.02 & & 0.0078 & 3.01E-05 & 4.8098 & 1.7723 & 6.5820 & \\
\hline & 8/5/2008 2:00 & 325 & 218 & 200 & 9.16 & 9.16 & & -0.0076 & $0.00 \mathrm{E}+00$ & 0.0000 & 0.0000 & 0.0000 & \\
\hline & 8/5/2008 3:00 & 326 & 218 & 300 & 9.14 & 9.14 & & -0.0055 & $0.00 \mathrm{E}+00$ & 0.0000 & 0.0000 & 0.0000 & \\
\hline & 8/5/2008 4:00 & 327 & 218 & 400 & 8.97 & 8.97 & & 0.0134 & 5.88E-05 & 9.4027 & 3.4646 & 12.8674 & \\
\hline
\end{tabular}




\begin{tabular}{|c|c|c|c|c|c|c|c|c|c|c|c|c|c|}
\hline Tree ID & TS & RN & JD & HR & $\begin{array}{c}\text { Raw } \\
\text { TC_dTA }\end{array}$ & $\begin{array}{c}\text { Fixed } \\
\text { TC_dT } \Delta\end{array}$ & dTM & $\mathbf{K}$ & $\begin{array}{l}\text { Sap } \\
\text { Velocity } \\
(\mathrm{cm} / \mathrm{s})\end{array}$ & $\begin{array}{c}\text { Probed } \\
\text { Drywood F } \\
(\mathrm{g} / \mathrm{hr})\end{array}$ & $\begin{array}{c}\text { Extrapolated } \\
\text { Wetwood F } \\
(\mathrm{g} / \mathrm{hr})\end{array}$ & $\begin{array}{c}\text { Extrapolated } \\
\text { Total Wood F } \\
(\mathrm{g} / \mathrm{hr})\end{array}$ & $\begin{array}{l}\text { Relative } \\
\text { Humidity, } \\
@ 10 \mathrm{~m}(\%)\end{array}$ \\
\hline & 8/5/2008 5:00 & 328 & 218 & 500 & 8.96 & 8.96 & & 0.0145 & $6.49 \mathrm{E}-05$ & 10.3906 & 3.8286 & $\begin{array}{r}14.2193 \\
\end{array}$ & \\
\hline & 8/5/2008 6:00 & 329 & 218 & 600 & 9.08 & 9.08 & & 0.0011 & $2.72 \mathrm{E}-06$ & 0.4348 & 0.1602 & 0.5950 & \\
\hline & 8/5/2008 7:00 & 330 & 218 & 700 & 9.17 & 9.17 & & -0.0087 & $0.00 E+00$ & 0.0000 & 0.0000 & 0.0000 & \\
\hline & 8/5/2008 8:00 & 331 & 218 & 800 & 9.01 & 9.01 & & 0.0089 & 3.55E-05 & 5.6768 & 2.0917 & 7.7686 & \\
\hline & 8/5/2008 9:00 & 332 & 218 & 900 & 7.383 & 7.383 & & 0.2312 & 1.96E-03 & 313.8731 & 115.6530 & 429.5260 & \\
\hline & 8/5/2008 10:00 & 333 & 218 & 1000 & 6.009 & 6.009 & & 0.5127 & 5.23E-03 & 836.6488 & 308.2805 & 1144.9293 & \\
\hline & 8/5/2008 11:00 & 334 & 218 & 1100 & 6.17 & 6.17 & & 0.4733 & $4.74 \mathrm{E}-03$ & 758.0790 & 279.3298 & 1037.4088 & \\
\hline & 8/5/2008 12:00 & 335 & 218 & 1200 & 5.857 & 5.857 & & 0.5520 & 5.73E-03 & 916.1902 & 337.5891 & 1253.7793 & \\
\hline & 8/5/2008 13:00 & 336 & 218 & 1300 & 5.5 & 5.5 & & 0.6527 & 7.04E-03 & 1126.1702 & 414.9606 & 1541.1307 & \\
\hline & 8/5/2008 14:00 & 337 & 218 & 1400 & 5.934 & 5.934 & & 0.5319 & 5.47E-03 & 875.2176 & 322.4919 & 1197.7095 & \\
\hline & 8/5/2008 15:00 & 338 & 218 & 1500 & 6.358 & 6.358 & & 0.4297 & $4.21 E-03$ & 673.1152 & 248.0231 & 921.1383 & \\
\hline & 8/5/2008 16:00 & 339 & 218 & 1600 & 6.213 & 6.213 & & 0.4631 & $4.61 E-03$ & 738.0236 & 271.9400 & 1009.9635 & \\
\hline & 8/5/2008 17:00 & 340 & 218 & 1700 & 5.764 & 5.764 & & 0.5770 & $6.05 E-03$ & 967.6188 & 356.5390 & 1324.1579 & \\
\hline & 8/5/2008 18:00 & 341 & 218 & 1800 & 5.546 & 5.546 & & 0.6390 & 6.86E-03 & 1097.1267 & 404.2589 & 1501.3856 & \\
\hline & 8/5/2008 19:00 & 342 & 218 & 1900 & 5.69 & 5.69 & & 0.5975 & 6.31E-03 & 1010.1284 & 372.2026 & 1382.3310 & \\
\hline & 8/5/2008 20:00 & 343 & 218 & 2000 & 6.485 & 6.485 & & 0.4017 & $3.87 \mathrm{E}-03$ & 619.5372 & 228.2812 & 847.8184 & \\
\hline & 8/5/2008 21:00 & 344 & 218 & 2100 & 8.25 & 8.25 & & 0.1018 & 7.15E-04 & 114.3674 & 42.1410 & 156.5084 & \\
\hline & $8 / 5 / 200822: 00$ & 345 & 218 & 2200 & 9.06 & 9.06 & & 0.0033 & 1.05E-05 & 1.6857 & 0.6211 & 2.3068 & \\
\hline & 8/5/2008 23:00 & 346 & 218 & 2300 & 9.28 & 9.28 & & -0.0205 & $0.00 \mathrm{E}+00$ & 0.0000 & 0.0000 & 0.0000 & \\
\hline & 8/6/2008 0:00 & 347 & 219 & 0 & 9.44 & 9.44 & 9.52 & -0.0371 & $0.00 E+00$ & 0.0000 & 0.0000 & 0.0000 & \\
\hline & 8/6/2008 1:00 & 348 & 219 & 100 & 9.47 & 9.47 & & -0.0401 & $0.00 \mathrm{E}+00$ & 0.0000 & 0.0000 & 0.0000 & \\
\hline & $8 / 6 / 2008$ 2:00 & 349 & 219 & 200 & 9.51 & 9.51 & & -0.0442 & $0.00 E+00$ & 0.0000 & 0.0000 & 0.0000 & \\
\hline & 8/6/2008 3:00 & 350 & 219 & 300 & 9.52 & 9.52 & & -0.0452 & $0.00 E+00$ & 0.0000 & 0.0000 & 0.0000 & \\
\hline & 8/6/2008 4:00 & 351 & 219 & 400 & 9.49 & 9.49 & & -0.0421 & $0.00 \mathrm{E}+00$ & 0.0000 & 0.0000 & 0.0000 & \\
\hline & 8/6/2008 5:00 & 352 & 219 & 500 & 9.48 & 9.48 & & -0.0411 & $0.00 \mathrm{E}+00$ & 0.0000 & 0.0000 & 0.0000 & \\
\hline & 8/6/2008 6:00 & 353 & 219 & 600 & 9.46 & 9.46 & & -0.0391 & $0.00 E+00$ & 0.0000 & 0.0000 & 0.0000 & \\
\hline & 8/6/2008 7:00 & 354 & 219 & 700 & 9.44 & 9.44 & & -0.0371 & $0.00 \mathrm{E}+00$ & 0.0000 & 0.0000 & 0.0000 & \\
\hline & 8/6/2008 8:00 & 355 & 219 & 800 & 9.4 & 9.4 & & -0.0330 & $0.00 \mathrm{E}+00$ & 0.0000 & 0.0000 & 0.0000 & \\
\hline & 8/6/2008 9:00 & 356 & 219 & 900 & 8.88 & 8.88 & & 0.0236 & 1.18E-04 & 18.9594 & 6.9860 & 25.9454 & \\
\hline & 8/6/2008 10:00 & 357 & 219 & 1000 & 6.302 & 6.302 & & 0.4424 & $4.36 \mathrm{E}-03$ & 697.6968 & 257.0807 & 954.7775 & \\
\hline & 8/6/2008 11:00 & 358 & 219 & 1100 & 5.5 & 5.5 & & 0.6527 & 7.04E-03 & 1126.1702 & 414.9606 & 1541.1307 & \\
\hline & 8/6/2008 12:00 & 359 & 219 & 1200 & 5.987 & 5.987 & & 0.5183 & 5.30E-03 & 847.8285 & 312.3998 & 1160.2283 & \\
\hline & 8/6/2008 13:00 & 360 & 219 & 1300 & 6.344 & 6.344 & & 0.4328 & $4.25 \mathrm{E}-03$ & 679.2044 & 250.2668 & 929.4713 & \\
\hline & $8 / 6 / 2008$ 14:00 & 361 & 219 & 1400 & 5.882 & 5.882 & & 0.5454 & $5.64 \mathrm{E}-03$ & 902.7311 & 332.6299 & 1235.3610 & \\
\hline & 8/6/2008 15:00 & 362 & 219 & 1500 & 5.62 & 5.62 & & 0.6174 & 6.57E-03 & 1051.6940 & 387.5183 & 1439.2123 & \\
\hline & 8/6/2008 16:00 & 363 & 219 & 1600 & 5.7 & 5.7 & & 0.5947 & $6.28 \mathrm{E}-03$ & 1004.2992 & 370.0547 & 1374.3539 & \\
\hline & 8/6/2008 17:00 & 364 & 219 & 1700 & 5.61 & 5.61 & & 0.6203 & $6.61 \mathrm{E}-03$ & 1057.7426 & 389.7470 & 1447.4896 & \\
\hline & 8/6/2008 18:00 & 365 & 219 & 1800 & 5.996 & 5.996 & & 0.5160 & 5.27E-03 & 843.2417 & 310.7097 & 1153.9514 & \\
\hline & 8/6/2008 19:00 & 366 & 219 & 1900 & 6.042 & 6.042 & & 0.5045 & $5.13 \mathrm{E}-03$ & 820.0837 & 302.1767 & 1122.2604 & \\
\hline & $8 / 6 / 200820: 00$ & 367 & 219 & 2000 & 6.402 & 6.402 & & 0.4199 & 4.09E-03 & 654.2174 & 241.0598 & 895.2772 & \\
\hline & 8/6/2008 21:00 & 368 & 219 & 2100 & 7.675 & 7.675 & & 0.1844 & 1.48E-03 & 237.5302 & 87.5229 & 325.0531 & \\
\hline
\end{tabular}




\begin{tabular}{|c|c|c|c|c|c|c|c|c|c|c|c|c|c|}
\hline Tree ID & TS & RN & JD & HR & $\begin{array}{c}\text { Raw } \\
\text { TC_dTA }\end{array}$ & $\begin{array}{c}\text { Fixed } \\
\text { TC_dT } \Delta\end{array}$ & dTM & $\mathrm{K}$ & $\begin{array}{l}\text { Sap } \\
\text { Velocity } \\
(\mathrm{cm} / \mathrm{s})\end{array}$ & $\begin{array}{c}\text { Probed } \\
\text { Drywood F } \\
(\mathrm{g} / \mathrm{hr})\end{array}$ & $\begin{array}{c}\text { Extrapolated } \\
\text { Wetwood F } \\
(\mathrm{g} / \mathrm{hr})\end{array}$ & $\begin{array}{c}\text { Extrapolated } \\
\text { Total Wood F } \\
(\mathrm{g} / \mathrm{hr})\end{array}$ & $\begin{array}{l}\text { Relative } \\
\text { Humidity, } \\
@ 10 \mathrm{~m}(\%)\end{array}$ \\
\hline & 8/6/2008 22:00 & 369 & 219 & 2200 & 8.85 & 8.85 & & 0.0271 & 1.40E-04 & 22.4400 & 8.2685 & 30.7084 & \\
\hline & 8/6/2008 23:00 & 370 & 219 & 2300 & 9.15 & 9.15 & & -0.0066 & $0.00 \mathrm{E}+00$ & 0.0000 & 0.0000 & 0.0000 & \\
\hline & $8 / 7 / 20080: 00$ & 371 & 220 & 0 & 9.34 & 9.34 & 9.49 & -0.0268 & $0.00 E+00$ & 0.0000 & 0.0000 & 0.0000 & \\
\hline & 8/7/2008 1:00 & 372 & 220 & 100 & 9.49 & 9.49 & & -0.0421 & $0.00 \mathrm{E}+00$ & 0.0000 & 0.0000 & 0.0000 & \\
\hline & 8/7/2008 2:00 & 373 & 220 & 200 & 9.49 & 9.49 & & -0.0421 & $0.00 \mathrm{E}+00$ & 0.0000 & 0.0000 & 0.0000 & \\
\hline & 8/7/2008 3:00 & 374 & 220 & 300 & 9.46 & 9.46 & & -0.0391 & $0.00 \mathrm{E}+00$ & 0.0000 & 0.0000 & 0.0000 & \\
\hline & 8/7/2008 4:00 & 375 & 220 & 400 & 9.44 & 9.44 & & -0.0371 & $0.00 \mathrm{E}+00$ & 0.0000 & 0.0000 & 0.0000 & \\
\hline & 8/7/2008 5:00 & 376 & 220 & 500 & 9.41 & 9.41 & & -0.0340 & $0.00 \mathrm{E}+00$ & 0.0000 & 0.0000 & 0.0000 & \\
\hline & 8/7/2008 6:00 & 377 & 220 & 600 & 9.38 & 9.38 & & -0.0309 & $0.00 \mathrm{E}+00$ & 0.0000 & 0.0000 & 0.0000 & \\
\hline & 8/7/2008 7:00 & 378 & 220 & 700 & 9.35 & 9.35 & & -0.0278 & $0.00 E+00$ & 0.0000 & 0.0000 & 0.0000 & \\
\hline & 8/7/2008 8:00 & 379 & 220 & 800 & 9.32 & 9.32 & & -0.0247 & $0.00 \mathrm{E}+00$ & 0.0000 & 0.0000 & 0.0000 & \\
\hline & 8/7/2008 9:00 & 380 & 220 & 900 & 8.36 & 8.36 & & 0.0873 & 5.92E-04 & 94.6638 & 34.8808 & 129.5446 & \\
\hline & 8/7/2008 10:00 & 381 & 220 & 1000 & 5.984 & 5.984 & & 0.5191 & $5.31 E-03$ & 849.3615 & 312.9647 & 1162.3262 & \\
\hline & 8/7/2008 11:00 & 382 & 220 & 1100 & 5.448 & 5.448 & & 0.6685 & 7.25E-03 & 1159.7672 & 427.3401 & 1587.1073 & \\
\hline & 8/7/2008 12:00 & 383 & 220 & 1200 & 5.461 & 5.461 & & 0.6645 & 7.20E-03 & 1151.2905 & 424.2167 & 1575.5072 & \\
\hline & 8/7/2008 13:00 & 384 & 220 & 1300 & 5.464 & 5.464 & & 0.6636 & $7.18 \mathrm{E}-03$ & 1149.3418 & 423.4986 & 1572.8404 & \\
\hline & $8 / 7 / 2008$ 14:00 & 385 & 220 & 1400 & 5.479 & 5.479 & & 0.6591 & 7.12E-03 & 1139.6392 & 419.9235 & 1559.5626 & \\
\hline & 8/7/2008 15:00 & 386 & 220 & 1500 & 5.512 & 5.512 & & 0.6491 & 6.99E-03 & 1118.5331 & 412.1465 & 1530.6796 & \\
\hline & 8/7/2008 16:00 & 387 & 220 & 1600 & 5.454 & 5.454 & & 0.6667 & 7.22E-03 & 1155.8484 & 425.8961 & 1581.7446 & \\
\hline & 8/7/2008 17:00 & 388 & 220 & 1700 & 5.422 & 5.422 & & 0.6765 & 7.36E-03 & 1176.8778 & 433.6448 & 1610.5226 & \\
\hline & 8/7/2008 18:00 & 389 & 220 & 1800 & 5.614 & 5.614 & & 0.6192 & $6.60 \mathrm{E}-03$ & 1055.3198 & 388.8543 & 1444.1741 & \\
\hline & 8/7/2008 19:00 & 390 & 220 & 1900 & 5.741 & 5.741 & & 0.5833 & 6.13E-03 & 980.6773 & 361.3507 & 1342.0280 & \\
\hline & 8/7/2008 20:00 & 391 & 220 & 2000 & 6.258 & 6.258 & & 0.4525 & 4.48E-03 & 717.4371 & 264.3545 & 981.7916 & \\
\hline & 8/7/2008 21:00 & 392 & 220 & 2100 & 7.539 & 7.539 & & 0.2057 & 1.70E-03 & 271.8561 & 100.1710 & 372.0271 & \\
\hline & 8/7/2008 22:00 & 393 & 220 & 2200 & 8.31 & 8.31 & & 0.0939 & 6.47E-04 & 103.4687 & 38.1252 & 141.5938 & \\
\hline & 8/7/2008 23:00 & 394 & 220 & 2300 & 8.77 & 8.77 & & 0.0365 & 2.02E-04 & 32.3353 & 11.9146 & 44.2499 & \\
\hline & 8/8/2008 0:00 & 395 & 221 & 0 & 9.07 & 9.07 & 9.47 & 0.0022 & 6.39E-06 & 1.0219 & 0.3766 & 1.3985 & \\
\hline & 8/8/2008 1:00 & 396 & 221 & 100 & 9.1 & 9.1 & & -0.0011 & $0.00 \mathrm{E}+00$ & 0.0000 & 0.0000 & 0.0000 & \\
\hline & 8/8/2008 2:00 & 397 & 221 & 200 & 9.32 & 9.32 & & -0.0247 & $0.00 \mathrm{E}+00$ & 0.0000 & 0.0000 & 0.0000 & \\
\hline & 8/8/2008 3:00 & 398 & 221 & 300 & 9.47 & 9.47 & & -0.0401 & $0.00 \mathrm{E}+00$ & 0.0000 & 0.0000 & 0.0000 & \\
\hline & 8/8/2008 4:00 & 399 & 221 & 400 & 9.46 & 9.46 & & -0.0391 & $0.00 \mathrm{E}+00$ & 0.0000 & 0.0000 & 0.0000 & \\
\hline & 8/8/2008 5:00 & 400 & 221 & 500 & 9.43 & 9.43 & & -0.0361 & $0.00 \mathrm{E}+00$ & 0.0000 & 0.0000 & 0.0000 & \\
\hline & 8/8/2008 6:00 & 401 & 221 & 600 & 9.41 & 9.41 & & -0.0340 & $0.00 \mathrm{E}+00$ & 0.0000 & 0.0000 & 0.0000 & \\
\hline & $8 / 8 / 20087: 00$ & 402 & 221 & 700 & 9.38 & 9.38 & & -0.0309 & $0.00 \mathrm{E}+00$ & 0.0000 & 0.0000 & 0.0000 & \\
\hline & 8/8/2008 8:00 & 403 & 221 & 800 & 9.21 & 9.21 & & -0.0130 & $0.00 \mathrm{E}+00$ & 0.0000 & 0.0000 & 0.0000 & \\
\hline & 8/8/20089:00 & 404 & 221 & 900 & 7.131 & 7.131 & & 0.2747 & 2.43E-03 & 388.0927 & 143.0007 & 531.0934 & \\
\hline & 8/8/2008 10:00 & 405 & 221 & 1000 & 5.726 & 5.726 & & 0.5875 & $6.18 \mathrm{E}-03$ & 989.2680 & 364.5161 & 1353.7841 & \\
\hline & 8/8/2008 11:00 & 406 & 221 & 1100 & 5.592 & 5.592 & & 0.6255 & 6.68E-03 & 1068.7010 & 393.7849 & 1462.4859 & \\
\hline & $8 / 8 / 200812: 00$ & 407 & 221 & 1200 & 5.515 & 5.515 & & 0.6482 & $6.98 \mathrm{E}-03$ & 1116.6306 & 411.4455 & 1528.0761 & \\
\hline & 8/8/2008 13:00 & 408 & 221 & 1300 & 5.509 & 5.509 & & 0.6500 & 7.00E-03 & 1120.4383 & 412.8486 & 1533.2869 & \\
\hline & 8/8/2008 14:00 & 409 & 221 & 1400 & 5.473 & 5.473 & & 0.6609 & 7.15E-03 & 1143.5120 & 421.3505 & 1564.8625 & \\
\hline
\end{tabular}




\begin{tabular}{|c|c|c|c|c|c|c|c|c|c|c|c|c|c|}
\hline Tree ID & TS & RN & JD & HR & $\begin{array}{c}\text { Raw } \\
\text { TC_dTA }\end{array}$ & $\begin{array}{c}\text { Fixed } \\
\text { TC_dT } \Delta\end{array}$ & dTM & $\mathbf{K}$ & $\begin{array}{l}\text { Sap } \\
\text { Velocity } \\
(\mathrm{cm} / \mathrm{s})\end{array}$ & $\begin{array}{c}\text { Probed } \\
\text { Drywood F } \\
(\mathrm{g} / \mathrm{hr})\end{array}$ & $\begin{array}{c}\text { Extrapolated } \\
\text { Wetwood F } \\
(\mathrm{g} / \mathrm{hr})\end{array}$ & $\begin{array}{c}\text { Extrapolated } \\
\text { Total Wood F } \\
(\mathrm{g} / \mathrm{hr})\end{array}$ & $\begin{array}{l}\text { Relative } \\
\text { Humidity, } \\
@ 10 \mathrm{~m}(\%)\end{array}$ \\
\hline & 8/8/2008 15:00 & 410 & 221 & 1500 & 5.47 & 5.47 & & 0.6618 & 7.16E-03 & 1145.4525 & 422.0655 & 1567.5180 & \\
\hline & 8/8/2008 16:00 & 411 & 221 & 1600 & 5.441 & 5.441 & & 0.6706 & $7.28 \mathrm{E}-03$ & 1164.3532 & 429.0299 & 1593.3831 & \\
\hline & 8/8/2008 17:00 & 412 & 221 & 1700 & 5.517 & 5.517 & & 0.6476 & $6.97 E-03$ & 1115.3637 & 410.9787 & 1526.3424 & \\
\hline & 8/8/2008 18:00 & 413 & 221 & 1800 & 5.778 & 5.778 & & 0.5732 & $6.00 \mathrm{E}-03$ & 959.7371 & 353.6349 & 1313.3719 & \\
\hline & 8/8/2008 19:00 & 414 & 221 & 1900 & 5.719 & 5.719 & & 0.5894 & $6.21 E-03$ & 993.2972 & 366.0008 & 1359.2980 & \\
\hline & 8/8/2008 20:00 & 415 & 221 & 2000 & 6.304 & 6.304 & & 0.4419 & $4.36 \mathrm{E}-03$ & 696.8085 & 256.7534 & 953.5619 & \\
\hline & 8/8/2008 21:00 & 416 & 221 & 2100 & 7.424 & 7.424 & & 0.2244 & 1.89E-03 & 302.5491 & 111.4804 & 414.0295 & \\
\hline & 8/8/2008 22:00 & 417 & 221 & 2200 & 8.13 & 8.13 & & 0.1181 & 8.58E-04 & 137.2536 & 50.5739 & 187.8275 & \\
\hline & 8/8/2008 23:00 & 418 & 221 & 2300 & 8.29 & 8.29 & & 0.0965 & 6.69E-04 & 107.0613 & 39.4489 & 146.5102 & \\
\hline & 8/9/2008 0:00 & 419 & 222 & 0 & 8.31 & 8.31 & 9.33 & 0.0939 & 6.47E-04 & 103.4687 & 38.1252 & 141.5938 & \\
\hline & 8/9/2008 1:00 & 420 & 222 & 100 & 8.31 & 8.31 & & 0.0939 & 6.47E-04 & 103.4687 & 38.1252 & 141.5938 & \\
\hline & 8/9/2008 2:00 & 421 & 222 & 200 & 8.38 & 8.38 & & 0.0847 & $5.70 \mathrm{E}-04$ & 91.2126 & 33.6092 & 124.8218 & \\
\hline & 8/9/2008 3:00 & 422 & 222 & 300 & 8.42 & 8.42 & & 0.0796 & $5.28 \mathrm{E}-04$ & 84.4322 & 31.1108 & 115.5430 & \\
\hline & 8/9/2008 4:00 & 423 & 222 & 400 & 8.44 & 8.44 & & 0.0770 & 5.07E-04 & 81.1032 & 29.8841 & 110.9873 & \\
\hline & $8 / 9 / 20085: 00$ & 424 & 222 & 500 & 8.31 & 8.31 & & 0.0939 & 6.47E-04 & 103.4687 & 38.1252 & 141.5938 & \\
\hline & 8/9/2008 6:00 & 425 & 222 & 600 & 8.33 & 8.33 & & 0.0912 & $6.24 \mathrm{E}-04$ & 99.9164 & 36.8163 & 136.7326 & \\
\hline & 8/9/2008 7:00 & 426 & 222 & 700 & 7.898 & 7.898 & & 0.1509 & 1.16E-03 & 185.6614 & 68.4108 & 254.0722 & \\
\hline & 8/9/2008 8:00 & 427 & 222 & 800 & 7.179 & 7.179 & & 0.2662 & 2.33E-03 & 373.3244 & 137.5591 & 510.8835 & \\
\hline & 8/9/2008 9:00 & 428 & 222 & 900 & 8.03 & 8.03 & & 0.1320 & 9.84E-04 & 157.4403 & 58.0121 & 215.4524 & \\
\hline & 8/9/2008 10:00 & 429 & 222 & 1000 & 7.659 & 7.659 & & 0.1868 & $1.51 E-03$ & 241.4603 & 88.9710 & 330.4314 & \\
\hline & 8/9/2008 11:00 & 430 & 222 & 1100 & 6.965 & 6.965 & & 0.3051 & $2.76 \mathrm{E}-03$ & 441.5828 & 162.7103 & 604.2931 & \\
\hline & 8/9/2008 12:00 & 431 & 222 & 1200 & 6.258 & 6.258 & & 0.4525 & 4.48E-03 & 717.4371 & 264.3545 & 981.7916 & \\
\hline & 8/9/2008 13:00 & 432 & 222 & 1300 & 6.07 & 6.07 & & 0.4975 & 5.04E-03 & 806.2180 & 297.0676 & 1103.2856 & \\
\hline & 8/9/2008 14:00 & 433 & 222 & 1400 & 5.696 & 5.696 & & 0.5959 & $6.29 \mathrm{E}-03$ & 1006.6276 & 370.9127 & 1377.5403 & \\
\hline & 8/9/2008 15:00 & 434 & 222 & 1500 & 5.621 & 5.621 & & 0.6171 & $6.57 \mathrm{E}-03$ & 1051.0907 & 387.2960 & 1438.3867 & \\
\hline & 8/9/2008 16:00 & 435 & 222 & 1600 & 5.636 & 5.636 & & 0.6128 & 6.51E-03 & 1042.0744 & 383.9738 & 1426.0482 & \\
\hline & 8/9/2008 17:00 & 436 & 222 & 1700 & 5.699 & 5.699 & & 0.5950 & $6.28 \mathrm{E}-03$ & 1004.8809 & 370.2690 & 1375.1499 & \\
\hline & 8/9/2008 18:00 & 437 & 222 & 1800 & 5.816 & 5.816 & & 0.5629 & $5.87 \mathrm{E}-03$ & 938.5954 & 345.8448 & 1284.4402 & \\
\hline & 8/9/2008 19:00 & 438 & 222 & 1900 & 5.944 & 5.944 & & 0.5293 & 5.44E-03 & 869.9999 & 320.5694 & 1190.5693 & \\
\hline & 8/9/2008 20:00 & 439 & 222 & 2000 & 6.531 & 6.531 & & 0.3918 & $3.76 \mathrm{E}-03$ & 600.8468 & 221.3944 & 822.2412 & \\
\hline & 8/9/2008 21:00 & 440 & 222 & 2100 & 8.13 & 8.13 & & 0.1181 & 8.58E-04 & 137.2536 & 50.5739 & 187.8275 & \\
\hline & 8/9/2008 22:00 & 441 & 222 & 2200 & 9.11 & 9.11 & & -0.0022 & $0.00 \mathrm{E}+00$ & 0.0000 & 0.0000 & 0.0000 & \\
\hline & 8/9/2008 23:00 & 442 & 222 & 2300 & 9.33 & 9.33 & & -0.0257 & $0.00 \mathrm{E}+00$ & 0.0000 & 0.0000 & 0.0000 & \\
\hline & 8/10/2008 0:00 & 443 & 223 & 0 & 9.39 & 9.39 & 9.46 & -0.0319 & $0.00 \mathrm{E}+00$ & 0.0000 & 0.0000 & 0.0000 & \\
\hline & 8/10/2008 1:00 & 444 & 223 & 100 & 9.45 & 9.45 & & -0.0381 & $0.00 \mathrm{E}+00$ & 0.0000 & 0.0000 & 0.0000 & \\
\hline & 8/10/2008 2:00 & 445 & 223 & 200 & 9.46 & 9.46 & & -0.0391 & $0.00 \mathrm{E}+00$ & 0.0000 & 0.0000 & 0.0000 & \\
\hline & 8/10/2008 3:00 & 446 & 223 & 300 & 9.46 & 9.46 & & -0.0391 & $0.00 \mathrm{E}+00$ & 0.0000 & 0.0000 & 0.0000 & \\
\hline & 8/10/2008 4:00 & 447 & 223 & 400 & 9.42 & 9.42 & & -0.0350 & $0.00 \mathrm{E}+00$ & 0.0000 & 0.0000 & 0.0000 & \\
\hline & $8 / 10 / 20085: 00$ & 448 & 223 & 500 & 9.4 & 9.4 & & -0.0330 & $0.00 \mathrm{E}+00$ & 0.0000 & 0.0000 & 0.0000 & \\
\hline & 8/10/2008 6:00 & 449 & 223 & 600 & 9.36 & 9.36 & & -0.0288 & $0.00 \mathrm{E}+00$ & 0.0000 & 0.0000 & 0.0000 & \\
\hline & 8/10/2008 7:00 & 450 & 223 & 700 & 9.34 & 9.34 & & -0.0268 & $0.00 \mathrm{E}+00$ & 0.0000 & 0.0000 & 0.0000 & \\
\hline
\end{tabular}




\begin{tabular}{|c|c|c|c|c|c|c|c|c|c|c|c|c|c|}
\hline Tree ID & TS & RN & JD & HR & $\begin{array}{c}\text { Raw } \\
\text { TC_dTA }\end{array}$ & $\begin{array}{c}\text { Fixed } \\
\text { TC_dT } \Delta\end{array}$ & dTM & K & $\begin{array}{l}\text { Sap } \\
\text { Velocity } \\
(\mathrm{cm} / \mathrm{s})\end{array}$ & $\begin{array}{c}\text { Probed } \\
\text { Drywood F } \\
(\mathrm{g} / \mathrm{hr})\end{array}$ & $\begin{array}{c}\text { Extrapolated } \\
\text { Wetwood F } \\
(\mathrm{g} / \mathrm{hr})\end{array}$ & $\begin{array}{c}\text { Extrapolated } \\
\text { Total Wood F } \\
(\mathrm{g} / \mathrm{hr})\end{array}$ & $\begin{array}{l}\text { Relative } \\
\text { Humidity, } \\
@ 10 \mathrm{~m}(\%)\end{array}$ \\
\hline & 8/10/2008 8:00 & 451 & 223 & 800 & \begin{tabular}{|r|}
9.29 \\
\end{tabular} & 9.29 & & -0.0215 & $0.00 \mathrm{E}+00$ & 0.0000 & 0.0000 & 0.0000 & \\
\hline & 8/10/2008 9:00 & 452 & 223 & 900 & 8.2 & 8.2 & & 0.1085 & 7.73E-04 & 123.7267 & 45.5897 & 169.3164 & \\
\hline & $8 / 10 / 2008$ 10:00 & 453 & 223 & 1000 & 5.99 & 5.99 & & 0.5175 & $5.29 \mathrm{E}-03$ & 846.2975 & 311.8357 & 1158.1332 & \\
\hline & 8/10/2008 11:00 & 454 & 223 & 1100 & 5.491 & 5.491 & & 0.6554 & 7.07E-03 & 1131.9263 & 417.0815 & 1549.0078 & \\
\hline & $8 / 10 / 200812: 00$ & 455 & 223 & 1200 & 5.541 & 5.541 & & 0.6405 & 6.88E-03 & 1100.2533 & 405.4110 & 1505.6643 & \\
\hline & 8/10/2008 13:00 & 456 & 223 & 1300 & 5.57 & 5.57 & & 0.6320 & 6.76E-03 & 1082.2199 & 398.7662 & 1480.9860 & \\
\hline & $8 / 10 / 2008$ 14:00 & 457 & 223 & 1400 & 5.555 & 5.555 & & 0.6364 & $6.82 E-03$ & 1091.5170 & 402.1919 & 1493.7089 & \\
\hline & $8 / 10 / 2008$ 15:00 & 458 & 223 & 1500 & 5.692 & 5.692 & & 0.5970 & 6.31E-03 & 1008.9604 & 371.7722 & 1380.7326 & \\
\hline & 8/10/2008 16:00 & 459 & 223 & 1600 & 5.558 & 5.558 & & 0.6355 & $6.81 \mathrm{E}-03$ & 1089.6524 & 401.5048 & 1491.1572 & \\
\hline & 8/10/2008 17:00 & 460 & 223 & 1700 & 5.731 & 5.731 & & 0.5861 & 6.16E-03 & 986.3978 & 363.4586 & 1349.8564 & \\
\hline & 8/10/2008 18:00 & 461 & 223 & 1800 & 8.19 & 8.19 & & 0.1099 & 7.85E-04 & 125.6288 & 46.2905 & 171.9193 & \\
\hline & $8 / 10 / 2008$ 19:00 & 462 & 223 & 1900 & 8.85 & 8.85 & & 0.0271 & 1.40E-04 & 22.4400 & 8.2685 & 30.7084 & \\
\hline & 8/10/2008 20:00 & 463 & 223 & 2000 & 8.49 & 8.49 & & 0.0707 & 4.56E-04 & 72.9605 & 26.8838 & 99.8444 & \\
\hline & 8/10/2008 21:00 & 464 & 223 & 2100 & 8.36 & 8.36 & & 0.0873 & 5.92E-04 & 94.6638 & 34.8808 & 129.5446 & \\
\hline & $8 / 10 / 2008$ 22:00 & 465 & 223 & 2200 & 8.61 & 8.61 & & 0.0557 & $3.41 E-04$ & 54.4864 & 20.0766 & 74.5631 & \\
\hline & $8 / 10 / 2008$ 23:00 & 466 & 223 & 2300 & 8.55 & 8.55 & & 0.0632 & 3.97E-04 & 63.5325 & 23.4098 & 86.9423 & \\
\hline & $8 / 11 / 20080: 00$ & 467 & 224 & 0 & 8.53 & 8.53 & 9.08 & 0.0657 & 4.16E-04 & 66.6332 & 24.5524 & 91.1855 & \\
\hline & 8/11/2008 1:00 & 468 & 224 & 100 & 8.72 & 8.72 & & 0.0424 & $2.43 E-04$ & 38.9359 & 14.3467 & 53.2826 & \\
\hline & 8/11/2008 2:00 & 469 & 224 & 200 & 8.94 & 8.94 & & 0.0168 & 7.77E-05 & 12.4263 & 4.5787 & 17.0050 & \\
\hline & 8/11/2008 3:00 & 470 & 224 & 300 & 9.04 & 9.04 & & 0.0055 & 1.98E-05 & 3.1700 & 1.1680 & 4.3380 & \\
\hline & 8/11/2008 4:00 & 471 & 224 & 400 & 9.07 & 9.07 & & 0.0022 & $6.39 \mathrm{E}-06$ & 1.0219 & 0.3766 & 1.3985 & \\
\hline & 8/11/2008 5:00 & 472 & 224 & 500 & 9.02 & 9.02 & & 0.0078 & 3.01E-05 & 4.8098 & 1.7723 & 6.5820 & \\
\hline & 8/11/2008 6:00 & 473 & 224 & 600 & 9.05 & 9.05 & & 0.0044 & 1.50E-05 & 2.4053 & 0.8863 & 3.2916 & \\
\hline & 8/11/2008 7:00 & 474 & 224 & 700 & 9.08 & 9.08 & & 0.0011 & $2.72 E-06$ & 0.4348 & 0.1602 & 0.5950 & \\
\hline & 8/11/2008 8:00 & 475 & 224 & 800 & 9.05 & 9.05 & & 0.0044 & $1.50 \mathrm{E}-05$ & 2.4053 & 0.8863 & 3.2916 & \\
\hline & 8/11/2008 9:00 & 476 & 224 & 900 & 7.961 & 7.961 & & 0.1418 & 1.07E-03 & 171.9667 & 63.3647 & 235.3313 & \\
\hline & $8 / 11 / 2008$ 10:00 & 477 & 224 & 1000 & 6.096 & 6.096 & & 0.4911 & 4.96E-03 & 793.4962 & 292.3800 & 1085.8762 & \\
\hline & 8/11/2008 11:00 & 478 & 224 & 1100 & 5.686 & 5.686 & & 0.5987 & $6.33 \mathrm{E}-03$ & 1012.4676 & 373.0645 & 1385.5321 & \\
\hline & $8 / 11 / 2008$ 12:00 & 479 & 224 & 1200 & 5.714 & 5.714 & & 0.5908 & $6.23 \mathrm{E}-03$ & 996.1832 & 367.0642 & 1363.2473 & \\
\hline & $8 / 11 / 2008$ 13:00 & 480 & 224 & 1300 & 5.904 & 5.904 & & 0.5396 & 5.57E-03 & 891.0121 & 328.3117 & 1219.3239 & \\
\hline & 8/11/2008 14:00 & 481 & 224 & 1400 & 5.669 & 5.669 & & 0.6035 & 6.39E-03 & 1022.4574 & 376.7454 & 1399.2028 & \\
\hline & 8/11/2008 15:00 & 482 & 224 & 1500 & 5.611 & 5.611 & & 0.6200 & $6.61 \mathrm{E}-03$ & 1057.1365 & 389.5237 & 1446.6602 & \\
\hline & $8 / 11 / 2008$ 16:00 & 483 & 224 & 1600 & 5.772 & 5.772 & & 0.5748 & 6.02E-03 & 963.1088 & 354.8772 & 1317.9860 & \\
\hline & 8/11/2008 17:00 & 484 & 224 & 1700 & 5.852 & 5.852 & & 0.5533 & 5.74E-03 & 918.9003 & 338.5877 & 1257.4881 & \\
\hline & 8/11/2008 18:00 & 485 & 224 & 1800 & 6.099 & 6.099 & & 0.4904 & 4.95E-03 & 792.0377 & 291.8426 & 1083.8803 & \\
\hline & $8 / 11 / 2008$ 19:00 & 486 & 224 & 1900 & 6.216 & 6.216 & & 0.4624 & 4.60E-03 & 736.6385 & 271.4296 & 1008.0680 & \\
\hline & 8/11/2008 20:00 & 487 & 224 & 2000 & 6.865 & 6.865 & & 0.3241 & 2.97E-03 & 475.6943 & 175.2794 & 650.9737 & \\
\hline & 8/11/2008 21:00 & 488 & 224 & 2100 & 7.753 & 7.753 & & 0.1724 & 1.37E-03 & 218.7760 & 80.6125 & 299.3885 & \\
\hline & $8 / 11 / 200822: 00$ & 489 & 224 & 2200 & 8.32 & 8.32 & & 0.0925 & $6.36 \mathrm{E}-04$ & 101.6875 & 37.4688 & 139.1563 & \\
\hline & 8/11/2008 23:00 & 490 & 224 & 2300 & 8.59 & 8.59 & & 0.0582 & 3.59E-04 & 57.4587 & 21.1718 & 78.6305 & \\
\hline & 8/12/2008 0:00 & 491 & 225 & 0 & 8.74 & 8.74 & 8.97 & 0.0400 & 2.27E-04 & 36.2590 & 13.3604 & 49.6194 & \\
\hline
\end{tabular}




\begin{tabular}{|c|c|c|c|c|c|c|c|c|c|c|c|c|c|}
\hline Tree ID & TS & RN & JD & HR & $\begin{array}{c}\text { Raw } \\
\text { TC_dTA }\end{array}$ & $\begin{array}{c}\text { Fixed } \\
\text { TC_dT } \Delta\end{array}$ & dTM & $\mathbf{K}$ & $\begin{array}{l}\text { Sap } \\
\text { Velocity } \\
(\mathrm{cm} / \mathrm{s})\end{array}$ & $\begin{array}{c}\text { Probed } \\
\text { Drywood F } \\
(\mathrm{g} / \mathrm{hr})\end{array}$ & $\begin{array}{c}\text { Extrapolated } \\
\text { Wetwood F } \\
(\mathrm{g} / \mathrm{hr})\end{array}$ & $\begin{array}{c}\text { Extrapolated } \\
\text { Total Wood F } \\
(\mathrm{g} / \mathrm{hr})\end{array}$ & $\begin{array}{l}\text { Relative } \\
\text { Humidity, } \\
@ 10 \mathrm{~m}(\%)\end{array}$ \\
\hline & 8/12/2008 1:00 & 492 & 225 & 100 & 8.75 & 8.75 & & 0.0389 & $2.18 \mathrm{E}-04$ & 34.9388 & 12.8739 & $\begin{array}{r}47.8127 \\
\end{array}$ & \\
\hline & 8/12/2008 2:00 & 493 & 225 & 200 & 8.4 & 8.4 & & 0.0821 & $5.49 \mathrm{E}-04$ & 87.8021 & 32.3525 & 120.1545 & \\
\hline & 8/12/2008 3:00 & 494 & 225 & 300 & 8.59 & 8.59 & & 0.0582 & $3.59 E-04$ & 57.4587 & 21.1718 & 78.6305 & \\
\hline & 8/12/2008 4:00 & 495 & 225 & 400 & 8.83 & 8.83 & & 0.0294 & $1.55 \mathrm{E}-04$ & 24.8327 & 9.1501 & 33.9828 & \\
\hline & 8/12/2008 5:00 & 496 & 225 & 500 & 8.89 & 8.89 & & 0.0225 & 1.11E-04 & 17.8295 & 6.5696 & 24.3991 & \\
\hline & 8/12/2008 6:00 & 497 & 225 & 600 & 8.97 & 8.97 & & 0.0134 & 5.88E-05 & 9.4027 & 3.4646 & 12.8674 & \\
\hline & 8/12/2008 7:00 & 498 & 225 & 700 & 8.97 & 8.97 & & 0.0134 & $5.88 E-05$ & 9.4027 & 3.4646 & 12.8674 & \\
\hline & 8/12/2008 8:00 & 499 & 225 & 800 & 8.94 & 8.94 & & 0.0168 & 7.77E-05 & 12.4263 & 4.5787 & 17.0050 & \\
\hline & 8/12/2008 9:00 & 500 & 225 & 900 & 8.05 & 8.05 & & 0.1292 & $9.58 \mathrm{E}-04$ & 153.3213 & 56.4944 & 209.8157 & \\
\hline & $8 / 12 / 2008$ 10:00 & 501 & 225 & 1000 & 6.165 & 6.165 & & 0.4745 & 4.75E-03 & 760.4358 & 280.1982 & 1040.6340 & \\
\hline & $8 / 12 / 2008$ 11:00 & 502 & 225 & 1100 & 5.561 & 5.561 & & 0.6346 & $6.80 \mathrm{E}-03$ & 1087.7904 & 400.8187 & 1488.6091 & \\
\hline & $8 / 12 / 2008$ 12:00 & 503 & 225 & 1200 & 5.486 & 5.486 & & 0.6569 & 7.09E-03 & 1135.1347 & 418.2637 & 1553.3984 & \\
\hline & $8 / 12 / 2008$ 13:00 & 504 & 225 & 1300 & 5.48 & 5.48 & & 0.6588 & $7.12 \mathrm{E}-03$ & 1138.9948 & 419.6860 & 1558.6808 & \\
\hline & $8 / 12 / 2008$ 14:00 & 505 & 225 & 1400 & 5.487 & 5.487 & & 0.6566 & 7.09E-03 & 1134.4924 & 418.0271 & 1552.5195 & \\
\hline & $8 / 12 / 2008$ 15:00 & 506 & 225 & 1500 & 5.488 & 5.488 & & 0.6563 & 7.09E-03 & 1133.8504 & 417.7905 & 1551.6409 & \\
\hline & $8 / 12 / 2008$ 16:00 & 507 & 225 & 1600 & 5.503 & 5.503 & & 0.6518 & 7.03E-03 & 1124.2569 & 414.2556 & 1538.5124 & \\
\hline & $8 / 12 / 2008$ 17:00 & 508 & 225 & 1700 & 5.527 & 5.527 & & 0.6447 & 6.93E-03 & 1109.0471 & 408.6512 & 1517.6983 & \\
\hline & $8 / 12 / 2008$ 18:00 & 509 & 225 & 1800 & 5.69 & 5.69 & & 0.5975 & $6.31 \mathrm{E}-03$ & 1010.1284 & 372.2026 & 1382.3310 & \\
\hline & $8 / 12 / 2008$ 19:00 & 510 & 225 & 1900 & 5.842 & 5.842 & & 0.5560 & $5.78 E-03$ & 924.3390 & 340.5917 & 1264.9307 & \\
\hline & $8 / 12 / 200820: 00$ & 511 & 225 & 2000 & 6.411 & 6.411 & & 0.4179 & 4.06E-03 & 650.3962 & 239.6519 & 890.0481 & \\
\hline & $8 / 12 / 2008$ 21:00 & 512 & 225 & 2100 & 7.935 & 7.935 & & 0.1456 & 1.11E-03 & 177.5683 & 65.4287 & 242.9970 & \\
\hline & $8 / 12 / 2008$ 22:00 & 513 & 225 & 2200 & 8.64 & 8.64 & & 0.0521 & 3.13E-04 & 50.1101 & 18.4641 & 68.5742 & \\
\hline & $8 / 12 / 2008$ 23:00 & 514 & 225 & 2300 & 8.84 & 8.84 & & 0.0283 & 1.48E-04 & 23.6293 & 8.7067 & 32.3360 & \\
\hline & 8/13/2008 0:00 & 515 & 226 & 0 & 9 & 9 & 9.12 & 0.0100 & 4.11E-05 & 6.5716 & 2.4214 & 8.9930 & \\
\hline & 8/13/2008 1:00 & 516 & 226 & 100 & 8.99 & 8.99 & & 0.0111 & 4.68E-05 & 7.4919 & 2.7605 & 10.2524 & \\
\hline & 8/13/2008 2:00 & 517 & 226 & 200 & 8.99 & 8.99 & & 0.0111 & 4.68E-05 & 7.4919 & 2.7605 & 10.2524 & \\
\hline & 8/13/2008 3:00 & 518 & 226 & 300 & 9.07 & 9.07 & & 0.0022 & 6.39E-06 & 1.0219 & 0.3766 & 1.3985 & \\
\hline & 8/13/2008 4:00 & 519 & 226 & 400 & 9.06 & 9.06 & & 0.0033 & 1.05E-05 & 1.6857 & 0.6211 & 2.3068 & \\
\hline & 8/13/2008 5:00 & 520 & 226 & 500 & 9.03 & 9.03 & & 0.0066 & $2.48 \mathrm{E}-05$ & 3.9730 & 1.4639 & 5.4370 & \\
\hline & 8/13/2008 6:00 & 521 & 226 & 600 & 9.12 & 9.12 & & -0.0033 & $0.00 \mathrm{E}+00$ & 0.0000 & 0.0000 & 0.0000 & \\
\hline & $8 / 13 / 2008$ 7:00 & 522 & 226 & 700 & 9.12 & 9.12 & & -0.0033 & $0.00 \mathrm{E}+00$ & 0.0000 & 0.0000 & 0.0000 & \\
\hline & 8/13/2008 8:00 & 523 & 226 & 800 & 8.78 & 8.78 & & 0.0353 & 1.94E-04 & 31.0523 & 11.4419 & 42.4942 & \\
\hline & 8/13/2008 9:00 & 524 & 226 & 900 & 6.87 & 6.87 & & 0.3231 & $2.96 \mathrm{E}-03$ & 473.9538 & 174.6380 & 648.5918 & \\
\hline & $8 / 13 / 2008$ 10:00 & 525 & 226 & 1000 & 5.782 & 5.782 & & 0.5721 & $5.98 \mathrm{E}-03$ & 957.4944 & 352.8085 & 1310.3029 & \\
\hline & 8/13/2008 11:00 & 526 & 226 & 1100 & 5.704 & 5.704 & & 0.5936 & $6.26 \mathrm{E}-03$ & 1001.9750 & 369.1983 & 1371.1733 & \\
\hline & $8 / 13 / 2008$ 12:00 & 527 & 226 & 1200 & 5.449 & 5.449 & & 0.6682 & 7.24E-03 & 1159.1133 & 427.0991 & 1586.2124 & \\
\hline
\end{tabular}




\begin{tabular}{|c|c|c|c|c|c|c|c|c|c|c|c|c|c|}
\hline Tree ID & TS & RN & JD & HR & $\begin{array}{c}\text { Raw } \\
\text { TC_dTA }\end{array}$ & $\begin{array}{c}\text { Fixed } \\
\text { TC_dT } \Delta\end{array}$ & dTM & $\mathbf{K}$ & $\begin{array}{c}\text { Sap } \\
\text { Velocity } \\
(\mathrm{cm} / \mathrm{s})\end{array}$ & $\begin{array}{c}\text { Probed } \\
\text { Drywood F } \\
(\mathrm{g} / \mathrm{hr})\end{array}$ & $\begin{array}{c}\text { Extrapolated } \\
\text { Wetwood F } \\
(\mathrm{g} / \mathrm{hr})\end{array}$ & $\begin{array}{c}\text { Extrapolated } \\
\text { Total Wood F } \\
(\mathrm{g} / \mathrm{hr})\end{array}$ & $\begin{array}{c}\text { Relative } \\
\text { Humidity, } \\
@ 10 \mathrm{~m}(\%)\end{array}$ \\
\hline & $7 / 22 / 2008$ 13:00 & 0 & 204 & 1300 & 5.004 & 5.004 & 9.50 & 0.8985 & 1.04E-02 & 2421.5040 & 892.2530 & 3313.7570 & \\
\hline \multirow[t]{2}{*}{ W707EC2 } & $7 / 22 / 200814: 00$ & 1 & 204 & 1400 & 5.544 & 5.544 & & 0.7136 & $7.85 \mathrm{E}-03$ & 1823.4410 & 671.8843 & 2495.3253 & \\
\hline & $7 / 22 / 2008$ 15:00 & 2 & 204 & 1500 & 5.496 & 5.496 & & 0.7285 & $8.06 \mathrm{E}-03$ & 1870.6317 & 689.2727 & 2559.9045 & \\
\hline \multirow[t]{2}{*}{ Probe \# } & $7 / 22 / 2008$ 16:00 & 3 & 204 & 1600 & 5.457 & 5.457 & & 0.7409 & $8.23 E-03$ & 1909.7548 & 703.6884 & 2613.4432 & \\
\hline & 7/22/2008 17:00 & 4 & 204 & 1700 & 5.494 & 5.494 & & 0.7292 & 8.07E-03 & 1872.6208 & 690.0057 & 2562.6265 & \\
\hline \multirow[t]{4}{*}{16} & $7 / 22 / 2008$ 18:00 & 5 & 204 & 1800 & 5.601 & 5.601 & & 0.6961 & $7.62 \mathrm{E}-03$ & 1768.7405 & 651.7288 & 2420.4693 & \\
\hline & 7/22/2008 19:00 & 6 & 204 & 1900 & 5.795 & 5.795 & & 0.6393 & 6.86E-03 & 1592.8509 & 586.9187 & 2179.7695 & \\
\hline & $7 / 22 / 200820: 00$ & 7 & 204 & 2000 & 6.253 & 6.253 & & 0.5193 & $5.31 \mathrm{E}-03$ & 1233.0068 & 454.3267 & 1687.3334 & \\
\hline & 7/22/2008 21:00 & 8 & 204 & 2100 & 7.849 & 7.849 & & 0.2103 & 1.75E-03 & 405.3646 & 149.3649 & 554.7295 & \\
\hline \multirow[t]{2}{*}{$\mathrm{DBH}(\mathrm{cm})$} & $7 / 22 / 2008$ 22:00 & 9 & 204 & 2200 & 9.13 & 9.13 & & 0.0405 & $2.30 \mathrm{E}-04$ & 53.3866 & 19.6714 & 73.0580 & \\
\hline & $7 / 22 / 2008$ 23:00 & 10 & 204 & 2300 & 9.5 & 9.5 & & 0.0000 & $0.00 E+00$ & 0.0000 & 0.0000 & 0.0000 & \\
\hline \multirow[t]{2}{*}{11.2} & $7 / 23 / 20080: 00$ & 11 & 205 & 0 & 9.73 & 9.73 & 9.87 & -0.0236 & $0.00 \mathrm{E}+00$ & 0.0000 & 0.0000 & 0.0000 & \\
\hline & 7/23/2008 1:00 & 12 & 205 & 100 & 9.8 & 9.8 & & -0.0306 & $0.00 \mathrm{E}+00$ & 0.0000 & 0.0000 & 0.0000 & \\
\hline Day Count & 7/23/2008 2:00 & 13 & 205 & 200 & 9.83 & 9.83 & & -0.0336 & $0.00 \mathrm{E}+00$ & 0.0000 & 0.0000 & 0.0000 & \\
\hline \multirow{27}{*}{35} & 7/23/2008 3:00 & 14 & 205 & 300 & 9.87 & 9.87 & & -0.0375 & $0.00 \mathrm{E}+00$ & 0.0000 & 0.0000 & 0.0000 & \\
\hline & 7/23/2008 4:00 & 15 & 205 & 400 & 9.85 & 9.85 & & -0.0355 & $0.00 \mathrm{E}+00$ & 0.0000 & 0.0000 & 0.0000 & \\
\hline & 7/23/2008 5:00 & 16 & 205 & 500 & 9.77 & 9.77 & & -0.0276 & $0.00 \mathrm{E}+00$ & 0.0000 & 0.0000 & 0.0000 & \\
\hline & 7/23/2008 6:00 & 17 & 205 & 600 & 9.61 & 9.61 & & -0.0114 & $0.00 \mathrm{E}+00$ & 0.0000 & 0.0000 & 0.0000 & \\
\hline & 7/23/2008 7:00 & 18 & 205 & 700 & 9.22 & 9.22 & & 0.0304 & 1.61E-04 & 37.4269 & 13.7907 & 51.2176 & \\
\hline & 7/23/2008 8:00 & 19 & 205 & 800 & 8.24 & 8.24 & & 0.1529 & 1.18E-03 & 273.7571 & 100.8714 & 374.6285 & \\
\hline & $7 / 23 / 20089: 00$ & 20 & 205 & 900 & 6.851 & 6.851 & & 0.3867 & 3.69E-03 & 857.6624 & 316.0233 & 1173.6857 & \\
\hline & 7/23/2008 10:00 & 21 & 205 & 1000 & 5.846 & 5.846 & & 0.6250 & 6.67E-03 & 1549.1037 & 570.7991 & 2119.9028 & \\
\hline & $7 / 23 / 2008$ 11:00 & 22 & 205 & 1100 & 5.752 & 5.752 & & 0.6516 & 7.02E-03 & 1630.5190 & 600.7983 & 2231.3172 & \\
\hline & 7/23/2008 12:00 & 23 & 205 & 1200 & 5.642 & 5.642 & & 0.6838 & 7.45E-03 & 1730.2680 & 637.5528 & 2367.8209 & \\
\hline & 7/23/2008 13:00 & 24 & 205 & 1300 & 5.579 & 5.579 & & 0.7028 & 7.71E-03 & 1789.6835 & 659.4457 & 2449.1293 & \\
\hline & $7 / 23 / 2008$ 14:00 & 25 & 205 & 1400 & 5.577 & 5.577 & & 0.7034 & $7.72 \mathrm{E}-03$ & 1791.5980 & 660.1511 & 2451.7491 & \\
\hline & $7 / 23 / 2008$ 15:00 & 26 & 205 & 1500 & 5.561 & 5.561 & & 0.7083 & 7.78E-03 & 1806.9767 & 665.8177 & 2472.7944 & \\
\hline & $7 / 23 / 2008$ 16:00 & 27 & 205 & 1600 & 5.74 & 5.74 & & 0.6551 & 7.07E-03 & 1641.1615 & 604.7197 & 2245.8812 & \\
\hline & 7/23/2008 17:00 & 28 & 205 & 1700 & 6.217 & 6.217 & & 0.5281 & 5.42E-03 & 1258.7718 & 463.8204 & 1722.5922 & \\
\hline & $7 / 23 / 2008$ 18:00 & 29 & 205 & 1800 & 6.964 & 6.964 & & 0.3642 & 3.43E-03 & 796.6438 & 293.5398 & 1090.1836 & \\
\hline & $7 / 23 / 2008$ 19:00 & 30 & 205 & 1900 & 6.918 & 6.918 & & 0.3732 & 3.54E-03 & 821.1409 & 302.5662 & 1123.7071 & \\
\hline & $7 / 23 / 200820: 00$ & 31 & 205 & 2000 & 7.345 & 7.345 & & 0.2934 & 2.63E-03 & 610.5951 & 224.9863 & 835.5814 & \\
\hline & $7 / 23 / 200821: 00$ & 32 & 205 & 2100 & 8.28 & 8.28 & & 0.1473 & 1.13E-03 & 261.5346 & 96.3678 & 357.9025 & \\
\hline & $7 / 23 / 200822: 00$ & 33 & 205 & 2200 & 8.87 & 8.87 & & 0.0710 & 4.59E-04 & 106.5148 & 39.2476 & 145.7623 & \\
\hline & $7 / 23 / 2008$ 23:00 & 34 & 205 & 2300 & 8.93 & 8.93 & & 0.0638 & 4.02E-04 & 93.3898 & 34.4114 & 127.8012 & \\
\hline & $7 / 24 / 20080: 00$ & 35 & 206 & 0 & 8.9 & 8.9 & 9.78 & 0.0674 & 4.30E-04 & 99.8897 & 36.8064 & 136.6961 & \\
\hline & 7/24/2008 1:00 & 36 & 206 & 100 & 8.98 & 8.98 & & 0.0579 & 3.57E-04 & 82.8386 & 30.5236 & 113.3621 & \\
\hline & 7/24/2008 2:00 & 37 & 206 & 200 & 9.09 & 9.09 & & 0.0451 & $2.62 E-04$ & 60.9060 & 22.4421 & 83.3481 & \\
\hline & 7/24/2008 3:00 & 38 & 206 & 300 & 9.23 & 9.23 & & 0.0293 & $1.54 \mathrm{E}-04$ & 35.7405 & 13.1693 & 48.9099 & \\
\hline & 7/24/2008 4:00 & 39 & 206 & 400 & 9.33 & 9.33 & & 0.0182 & 8.60E-05 & 19.9560 & 7.3532 & 27.3092 & \\
\hline & $7 / 24 / 20085: 00$ & 40 & 206 & 500 & 9.47 & 9.47 & & 0.0032 & 9.98E-06 & 2.3161 & 0.8534 & 3.1696 & \\
\hline
\end{tabular}




\begin{tabular}{|c|c|c|c|c|c|c|c|c|c|c|c|c|c|}
\hline Tree ID & TS & RN & JD & HR & $\begin{array}{c}\text { Raw } \\
\text { TC_dTA }\end{array}$ & $\begin{array}{c}\text { Fixed } \\
\text { TC_dT } \Delta\end{array}$ & dTM & K & $\begin{array}{l}\text { Sap } \\
\text { Velocity } \\
(\mathrm{cm} / \mathrm{s})\end{array}$ & $\begin{array}{c}\text { Probed } \\
\text { Drywood F } \\
(\mathrm{g} / \mathrm{hr})\end{array}$ & $\begin{array}{c}\text { Extrapolated } \\
\text { Wetwood F } \\
(\mathrm{g} / \mathrm{hr})\end{array}$ & $\begin{array}{c}\text { Extrapolated } \\
\text { Total Wood F } \\
(\mathrm{g} / \mathrm{hr})\end{array}$ & $\begin{array}{l}\text { Relative } \\
\text { Humidity, } \\
@ 10 \mathrm{~m}(\%)\end{array}$ \\
\hline & 7/24/2008 6:00 & 41 & 206 & 600 & 9.52 & 9.52 & & -0.0021 & $0.00 \mathrm{E}+00$ & 0.0000 & 0.0000 & 0.0000 & \\
\hline & 7/24/2008 7:00 & 42 & 206 & 700 & 9.5 & 9.5 & & 0.0000 & $0.00 \mathrm{E}+00$ & 0.0000 & 0.0000 & 0.0000 & \\
\hline & 7/24/2008 8:00 & 43 & 206 & 800 & 9.2 & 9.2 & & 0.0326 & $1.76 \mathrm{E}-04$ & 40.8535 & 15.0533 & 55.9068 & \\
\hline & 7/24/2008 9:00 & 44 & 206 & 900 & 8.73 & 8.73 & & 0.0882 & $5.99 \mathrm{E}-04$ & 139.0584 & 51.2389 & 190.2973 & \\
\hline & $7 / 24 / 2008$ 10:00 & 45 & 206 & 1000 & 8.77 & 8.77 & & 0.0832 & 5.58E-04 & 129.4892 & 47.7130 & 177.2022 & \\
\hline & 7/24/2008 11:00 & 46 & 206 & 1100 & 6.726 & 6.726 & & 0.4124 & 4.00E-03 & 928.5621 & 342.1478 & 1270.7099 & \\
\hline & 7/24/2008 12:00 & 47 & 206 & 1200 & 5.827 & 5.827 & & 0.6303 & $6.74 \mathrm{E}-03$ & 1565.2855 & 576.7616 & 2142.0471 & \\
\hline & $7 / 24 / 2008$ 13:00 & 48 & 206 & 1300 & 6.159 & 6.159 & & 0.5425 & $5.60 \mathrm{E}-03$ & 1301.1294 & 479.4279 & 1780.5573 & \\
\hline & $7 / 24 / 2008$ 14:00 & 49 & 206 & 1400 & 6.041 & 6.041 & & 0.5726 & 5.99E-03 & 1390.6530 & 512.4147 & 1903.0677 & \\
\hline & 7/24/2008 15:00 & 50 & 206 & 1500 & 5.696 & 5.696 & & 0.6678 & $7.24 \mathrm{E}-03$ & 1680.6800 & 619.2811 & 2299.9611 & \\
\hline & 7/24/2008 16:00 & 51 & 206 & 1600 & 5.669 & 5.669 & & 0.6758 & 7.35E-03 & 1705.3221 & 628.3610 & 2333.6831 & \\
\hline & $7 / 24 / 2008$ 17:00 & 52 & 206 & 1700 & 5.884 & 5.884 & & 0.6145 & $6.54 \mathrm{E}-03$ & 1517.1473 & 559.0241 & 2076.1714 & \\
\hline & $7 / 24 / 2008$ 18:00 & 53 & 206 & 1800 & 5.81 & 5.81 & & 0.6351 & 6.81E-03 & 1579.8805 & 582.1395 & 2162.0200 & \\
\hline & $7 / 24 / 2008$ 19:00 & 54 & 206 & 1900 & 6.172 & 6.172 & & 0.5392 & $5.56 \mathrm{E}-03$ & 1291.5433 & 475.8957 & 1767.4390 & \\
\hline & $7 / 24 / 200820: 00$ & 55 & 206 & 2000 & 7.168 & 7.168 & & 0.3253 & 2.99E-03 & 693.4171 & 255.5038 & 948.9208 & \\
\hline & $7 / 24 / 2008$ 21:00 & 56 & 206 & 2100 & 8.64 & 8.64 & & 0.0995 & 6.95E-04 & 161.3745 & 59.4617 & 220.8362 & \\
\hline & $7 / 24 / 2008$ 22:00 & 57 & 206 & 2200 & 9.56 & 9.56 & & -0.0063 & $0.00 E+00$ & 0.0000 & 0.0000 & 0.0000 & \\
\hline & $7 / 24 / 2008$ 23:00 & 58 & 206 & 2300 & 9.78 & 9.78 & & -0.0286 & $0.00 \mathrm{E}+00$ & 0.0000 & 0.0000 & 0.0000 & \\
\hline & 7/25/2008 0:00 & 59 & 207 & 0 & 9.83 & 9.83 & 9.83 & -0.0336 & $0.00 \mathrm{E}+00$ & 0.0000 & 0.0000 & 0.0000 & \\
\hline & 7/25/2008 1:00 & 60 & 207 & 100 & 9.79 & 9.79 & & -0.0296 & $0.00 \mathrm{E}+00$ & 0.0000 & 0.0000 & 0.0000 & \\
\hline & 7/25/2008 2:00 & 61 & 207 & 200 & 9.72 & 9.72 & & -0.0226 & $0.00 E+00$ & 0.0000 & 0.0000 & 0.0000 & \\
\hline & 7/25/2008 3:00 & 62 & 207 & 300 & 9.7 & 9.7 & & -0.0206 & $0.00 \mathrm{E}+00$ & 0.0000 & 0.0000 & 0.0000 & \\
\hline & 7/25/2008 4:00 & 63 & 207 & 400 & 9.72 & 9.72 & & -0.0226 & $0.00 E+00$ & 0.0000 & 0.0000 & 0.0000 & \\
\hline & 7/25/2008 5:00 & 64 & 207 & 500 & 9.72 & 9.72 & & -0.0226 & $0.00 \mathrm{E}+00$ & 0.0000 & 0.0000 & 0.0000 & \\
\hline & 7/25/2008 6:00 & 65 & 207 & 600 & 9.69 & 9.69 & & -0.0196 & $0.00 \mathrm{E}+00$ & 0.0000 & 0.0000 & 0.0000 & \\
\hline & 7/25/2008 7:00 & 66 & 207 & 700 & 9.65 & 9.65 & & -0.0155 & $0.00 \mathrm{E}+00$ & 0.0000 & 0.0000 & 0.0000 & \\
\hline & 7/25/2008 8:00 & 67 & 207 & 800 & 9.6 & 9.6 & & -0.0104 & $0.00 \mathrm{E}+00$ & 0.0000 & 0.0000 & 0.0000 & \\
\hline & 7/25/2008 9:00 & 68 & 207 & 900 & 9.48 & 9.48 & & 0.0021 & $6.05 \mathrm{E}-06$ & 1.4042 & 0.5174 & 1.9216 & \\
\hline & $7 / 25 / 2008$ 10:00 & 69 & 207 & 1000 & 9.38 & 9.38 & & 0.0128 & 5.56E-05 & 12.9124 & 4.7578 & 17.6702 & \\
\hline & 7/25/2008 11:00 & 70 & 207 & 1100 & 8.36 & 8.36 & & 0.1364 & $1.02 \mathrm{E}-03$ & 237.7549 & 87.6057 & 325.3606 & \\
\hline & $7 / 25 / 2008$ 12:00 & 71 & 207 & 1200 & 6.72 & 6.72 & & 0.4137 & $4.01 E-03$ & 932.0585 & 343.4361 & 1275.4947 & \\
\hline & $7 / 25 / 2008$ 13:00 & 72 & 207 & 1300 & 6.074 & 6.074 & & 0.5640 & 5.88E-03 & 1365.1532 & 503.0188 & 1868.1720 & \\
\hline & $7 / 25 / 200814: 00$ & 73 & 207 & 1400 & 5.638 & 5.638 & & 0.6850 & 7.47E-03 & 1733.9899 & 638.9242 & 2372.9141 & \\
\hline & $7 / 25 / 200815: 00$ & 74 & 207 & 1500 & 5.63 & 5.63 & & 0.6874 & $7.50 \mathrm{E}-03$ & 1741.4539 & 641.6745 & 2383.1284 & \\
\hline & $7 / 25 / 2008$ 16:00 & 75 & 207 & 1600 & 5.777 & 5.777 & & 0.6445 & 6.93E-03 & 1608.5306 & 592.6962 & 2201.2268 & \\
\hline & $7 / 25 / 2008$ 17:00 & 76 & 207 & 1700 & 5.59 & 5.59 & & 0.6995 & 7.66E-03 & 1779.1856 & 655.5775 & 2434.7632 & \\
\hline & $7 / 25 / 2008$ 18:00 & 77 & 207 & 1800 & 5.678 & 5.678 & & 0.6731 & 7.31E-03 & 1697.0745 & 625.3220 & 2322.3966 & \\
\hline & $7 / 25 / 2008$ 19:00 & 78 & 207 & 1900 & 5.799 & 5.799 & & 0.6382 & $6.85 E-03$ & 1589.3836 & 585.6411 & 2175.0247 & \\
\hline & $7 / 25 / 200820: 00$ & 79 & 207 & 2000 & 6.661 & 6.661 & & 0.4262 & 4.17E-03 & 966.9080 & 356.2772 & 1323.1852 & \\
\hline & $7 / 25 / 2008$ 21:00 & 80 & 207 & 2100 & 8.29 & 8.29 & & 0.1460 & 1.11E-03 & 258.5138 & 95.2547 & 353.7686 & \\
\hline & $7 / 25 / 200822: 00$ & 81 & 207 & 2200 & 9.46 & 9.46 & & 0.0042 & $1.42 \mathrm{E}-05$ & 3.3047 & 1.2177 & 4.5224 & \\
\hline
\end{tabular}




\begin{tabular}{|c|c|c|c|c|c|c|c|c|c|c|c|c|c|}
\hline Tree ID & TS & RN & JD & HR & $\begin{array}{c}\text { Raw } \\
\text { TC_dTA }\end{array}$ & $\begin{array}{c}\text { Fixed } \\
\text { TC_dT } \Delta\end{array}$ & dTM & K & $\begin{array}{l}\text { Sap } \\
\text { Velocity } \\
(\mathrm{cm} / \mathrm{s})\end{array}$ & $\begin{array}{c}\text { Probed } \\
\text { Drywood F } \\
(\mathrm{g} / \mathrm{hr})\end{array}$ & $\begin{array}{c}\text { Extrapolated } \\
\text { Wetwood F } \\
(\mathrm{g} / \mathrm{hr})\end{array}$ & $\begin{array}{c}\text { Extrapolated } \\
\text { Total Wood F } \\
(\mathrm{g} / \mathrm{hr})\end{array}$ & $\begin{array}{l}\text { Relative } \\
\text { Humidity, } \\
@ 10 \mathrm{~m}(\%)\end{array}$ \\
\hline & 7/25/2008 23:00 & 82 & 207 & 2300 & 9.66 & 9.66 & & -0.0166 & $0.00 \mathrm{E}+00$ & 0.0000 & 0.0000 & 0.0000 & \\
\hline & $7 / 26 / 20080: 00$ & 83 & 208 & 0 & 9.66 & 9.66 & 9.66 & -0.0166 & $0.00 \mathrm{E}+00$ & 0.0000 & 0.0000 & 0.0000 & \\
\hline & 7/26/2008 1:00 & 84 & 208 & 100 & 9.63 & 9.63 & & -0.0135 & $0.00 E+00$ & 0.0000 & 0.0000 & 0.0000 & \\
\hline & $7 / 26 / 20082: 00$ & 85 & 208 & 200 & 9.58 & 9.58 & & -0.0084 & $0.00 \mathrm{E}+00$ & 0.0000 & 0.0000 & 0.0000 & \\
\hline & 7/26/2008 3:00 & 86 & 208 & 300 & 9.52 & 9.52 & & -0.0021 & $0.00 \mathrm{E}+00$ & 0.0000 & 0.0000 & 0.0000 & \\
\hline & 7/26/2008 4:00 & 87 & 208 & 400 & 9.46 & 9.46 & & 0.0042 & 1.42E-05 & 3.3047 & 1.2177 & 4.5224 & \\
\hline & 7/26/2008 5:00 & 88 & 208 & 500 & 9.41 & 9.41 & & 0.0096 & $3.89 E-05$ & 9.0261 & 3.3258 & 12.3519 & \\
\hline & 7/26/2008 6:00 & 89 & 208 & 600 & 9.36 & 9.36 & & 0.0150 & 6.74E-05 & 15.6516 & 5.7672 & 21.4187 & \\
\hline & 7/26/2008 7:00 & 90 & 208 & 700 & 9.3 & 9.3 & & 0.0215 & 1.05E-04 & 24.4727 & 9.0175 & 33.4901 & \\
\hline & 7/26/2008 8:00 & 91 & 208 & 800 & 9.2 & 9.2 & & 0.0326 & 1.76E-04 & 40.8535 & 15.0533 & 55.9068 & \\
\hline & 7/26/2008 9:00 & 92 & 208 & 900 & 7.613 & 7.613 & & 0.2479 & 2.14E-03 & 496.1301 & 182.8093 & 678.9395 & \\
\hline & $7 / 26 / 2008$ 10:00 & 93 & 208 & 1000 & 6.079 & 6.079 & & 0.5628 & 5.86E-03 & 1361.3214 & 501.6069 & 1862.9283 & \\
\hline & 7/26/2008 11:00 & 94 & 208 & 1100 & 6.106 & 6.106 & & 0.5558 & $5.78 \mathrm{E}-03$ & 1340.7730 & 494.0354 & 1834.8084 & \\
\hline & $7 / 26 / 2008$ 12:00 & 95 & 208 & 1200 & 6.364 & 6.364 & & 0.4928 & 4.98E-03 & 1156.0129 & 425.9567 & 1581.9697 & \\
\hline & $7 / 26 / 2008$ 13:00 & 96 & 208 & 1300 & 5.759 & 5.759 & & 0.6496 & 7.00E-03 & 1624.3373 & 598.5205 & 2222.8578 & \\
\hline & $7 / 26 / 200814: 00$ & 97 & 208 & 1400 & 5.671 & 5.671 & & 0.6752 & 7.34E-03 & 1703.4864 & 627.6846 & 2331.1710 & \\
\hline & $7 / 26 / 2008$ 15:00 & 98 & 208 & 1500 & 5.638 & 5.638 & & 0.6850 & 7.47E-03 & 1733.9899 & 638.9242 & 2372.9141 & \\
\hline & $7 / 26 / 200816: 00$ & 99 & 208 & 1600 & 5.729 & 5.729 & & 0.6582 & 7.11E-03 & 1650.9677 & 608.3330 & 2259.3007 & \\
\hline & $7 / 26 / 200817: 00$ & 100 & 208 & 1700 & 5.56 & 5.56 & & 0.7086 & 7.79E-03 & 1807.9416 & 666.1733 & 2474.1148 & \\
\hline & $7 / 26 / 2008$ 18:00 & 101 & 208 & 1800 & 5.745 & 5.745 & & 0.6536 & 7.05E-03 & 1636.7201 & 603.0832 & 2239.8033 & \\
\hline & $7 / 26 / 2008$ 19:00 & 102 & 208 & 1900 & 5.891 & 5.891 & & 0.6126 & $6.51 \mathrm{E}-03$ & 1511.3191 & 556.8766 & 2068.1957 & \\
\hline & $7 / 26 / 200820: 00$ & 103 & 208 & 2000 & 6.503 & 6.503 & & 0.4609 & 4.59E-03 & 1064.5694 & 392.2625 & 1456.8318 & \\
\hline & $7 / 26 / 200821: 00$ & 104 & 208 & 2100 & 8.13 & 8.13 & & 0.1685 & 1.33E-03 & 308.5298 & 113.6842 & 422.2140 & \\
\hline & $7 / 26 / 200822: 00$ & 105 & 208 & 2200 & 9.17 & 9.17 & & 0.0360 & 1.99E-04 & 46.1243 & 16.9954 & 63.1197 & \\
\hline & $7 / 26 / 2008$ 23:00 & 106 & 208 & 2300 & 9.33 & 9.33 & & 0.0182 & 8.60E-05 & 19.9560 & 7.3532 & 27.3092 & \\
\hline & $7 / 27 / 20080: 00$ & 107 & 209 & 0 & 9.32 & 9.32 & 9.32 & 0.0193 & $9.23 E-05$ & 21.4390 & 7.8997 & 29.3387 & \\
\hline & 7/27/2008 1:00 & 108 & 209 & 100 & 9.3 & 9.3 & & 0.0215 & 1.05E-04 & 24.4727 & 9.0175 & 33.4901 & \\
\hline & 7/27/2008 2:00 & 109 & 209 & 200 & 9.26 & 9.26 & & 0.0259 & 1.33E-04 & 30.7934 & 11.3465 & 42.1399 & \\
\hline & 7/27/2008 3:00 & 110 & 209 & 300 & 9.25 & 9.25 & & 0.0270 & 1.40E-04 & 32.4235 & 11.9471 & 44.3705 & \\
\hline & 7/27/2008 4:00 & 111 & 209 & 400 & 9.23 & 9.23 & & 0.0293 & 1.54E-04 & 35.7405 & 13.1693 & 48.9099 & \\
\hline & 7/27/20085:00 & 112 & 209 & 500 & 9.18 & 9.18 & & 0.0349 & 1.91E-04 & 44.3502 & 16.3418 & 60.6920 & \\
\hline & 7/27/2008 6:00 & 113 & 209 & 600 & 9.09 & 9.09 & & 0.0451 & $2.62 \mathrm{E}-04$ & 60.9060 & 22.4421 & 83.3481 & \\
\hline & 7/27/2008 7:00 & 114 & 209 & 700 & 8.99 & 8.99 & & 0.0567 & 3.48E-04 & 80.7712 & 29.7618 & 110.5330 & \\
\hline & 7/27/2008 8:00 & 115 & 209 & 800 & 8.64 & 8.64 & & 0.0995 & $6.95 E-04$ & 161.3745 & 59.4617 & 220.8362 & \\
\hline & $7 / 27 / 20089: 00$ & 116 & 209 & 900 & 6.323 & 6.323 & & 0.5025 & 5.10E-03 & 1184.0291 & 436.2799 & 1620.3089 & \\
\hline & $7 / 27 / 2008$ 10:00 & 117 & 209 & 1000 & 5.255 & 5.255 & & 0.8078 & 9.15E-03 & 2124.2618 & 782.7279 & 2906.9897 & \\
\hline & $7 / 27 / 2008$ 11:00 & 118 & 209 & 1100 & 5.233 & 5.233 & & 0.8154 & $9.26 \mathrm{E}-03$ & 2148.8912 & 791.8032 & 2940.6944 & \\
\hline & $7 / 27 / 2008$ 12:00 & 119 & 209 & 1200 & 5.228 & 5.228 & & 0.8171 & $9.28 \mathrm{E}-03$ & 2154.5252 & 793.8791 & 2948.4043 & \\
\hline & $7 / 27 / 200813: 00$ & 120 & 209 & 1300 & 5.35 & 5.35 & & 0.7757 & $8.70 \mathrm{E}-03$ & 2020.8278 & 744.6156 & 2765.4434 & \\
\hline & $7 / 27 / 200814: 00$ & 121 & 209 & 1400 & 5.232 & 5.232 & & 0.8157 & 9.26E-03 & 2150.0169 & 792.2180 & 2942.2349 & \\
\hline & 7/27/2008 15:00 & 122 & 209 & 1500 & 5.259 & 5.259 & & 0.8064 & 9.13E-03 & 2119.8115 & 781.0882 & 2900.8997 & \\
\hline
\end{tabular}




\begin{tabular}{|c|c|c|c|c|c|c|c|c|c|c|c|c|c|}
\hline Tree ID & TS & RN & JD & HR & $\begin{array}{c}\text { Raw } \\
\text { TC_dTA }\end{array}$ & $\begin{array}{c}\text { Fixed } \\
\text { TC_dT } \Delta\end{array}$ & dTM & $\mathbf{K}$ & $\begin{array}{l}\text { Sap } \\
\text { Velocity } \\
(\mathrm{cm} / \mathrm{s})\end{array}$ & $\begin{array}{c}\text { Probed } \\
\text { Drywood F } \\
(\mathrm{g} / \mathrm{hr})\end{array}$ & $\begin{array}{c}\text { Extrapolated } \\
\text { Wetwood F } \\
(\mathrm{g} / \mathrm{hr})\end{array}$ & $\begin{array}{c}\text { Extrapolated } \\
\text { Total Wood F } \\
(\mathrm{g} / \mathrm{hr})\end{array}$ & $\begin{array}{l}\text { Relative } \\
\text { Humidity, } \\
@ 10 \mathrm{~m}(\%)\end{array}$ \\
\hline & 7/27/2008 16:00 & 123 & 209 & 1600 & 5.347 & 5.347 & & 0.7767 & $8.72 \mathrm{E}-03$ & 2024.0233 & 745.7930 & 2769.8164 & \\
\hline & $7 / 27 / 2008$ 17:00 & 124 & 209 & 1700 & 5.381 & 5.381 & & 0.7655 & $8.56 \mathrm{E}-03$ & 1988.0713 & 732.5458 & 2720.6171 & \\
\hline & $7 / 27 / 2008$ 18:00 & 125 & 209 & 1800 & 5.485 & 5.485 & & 0.7320 & $8.11 \mathrm{E}-03$ & 1881.5947 & 693.3123 & 2574.9070 & \\
\hline & $7 / 27 / 2008$ 19:00 & 126 & 209 & 1900 & 5.647 & 5.647 & & 0.6823 & 7.43E-03 & 1725.6253 & 635.8421 & 2361.4674 & \\
\hline & $7 / 27 / 200820: 00$ & 127 & 209 & 2000 & 6.171 & 6.171 & & 0.5395 & 5.57E-03 & 1292.2788 & 476.1667 & 1768.4455 & \\
\hline & $7 / 27 / 2008$ 21:00 & 128 & 209 & 2100 & 7.477 & 7.477 & & 0.2706 & 2.38E-03 & 552.6347 & 203.6296 & 756.2643 & \\
\hline & $7 / 27 / 2008$ 22:00 & 129 & 209 & 2200 & 8.64 & 8.64 & & 0.0995 & $6.95 E-04$ & 161.3745 & 59.4617 & 220.8362 & \\
\hline & $7 / 27 / 200823: 00$ & 130 & 209 & 2300 & 8.83 & 8.83 & & 0.0759 & $4.98 \mathrm{E}-04$ & 115.5410 & 42.5735 & 158.1144 & \\
\hline & $7 / 28 / 20080: 00$ & 131 & 210 & 0 & 8.86 & 8.86 & 9.21 & 0.0722 & $4.68 \mathrm{E}-04$ & 108.7507 & 40.0714 & 148.8222 & \\
\hline & 7/28/2008 1:00 & 132 & 210 & 100 & 8.99 & 8.99 & & 0.0567 & 3.48E-04 & 80.7712 & 29.7618 & 110.5330 & \\
\hline & $7 / 28 / 20082: 00$ & 133 & 210 & 200 & 9.03 & 9.03 & & 0.0520 & 3.13E-04 & 72.6468 & 26.7682 & 99.4150 & \\
\hline & 7/28/2008 3:00 & 134 & 210 & 300 & 9.13 & 9.13 & & 0.0405 & $2.30 \mathrm{E}-04$ & 53.3866 & 19.6714 & 73.0580 & \\
\hline & 7/28/2008 4:00 & 135 & 210 & 400 & 9.07 & 9.07 & & 0.0474 & 2.79E-04 & 64.7591 & 23.8618 & 88.6209 & \\
\hline & 7/28/2008 5:00 & 136 & 210 & 500 & 9.17 & 9.17 & & 0.0360 & 1.99E-04 & 46.1243 & 16.9954 & 63.1197 & \\
\hline & 7/28/2008 6:00 & 137 & 210 & 600 & 9.21 & 9.21 & & 0.0315 & 1.69E-04 & 39.1313 & 14.4187 & 53.5500 & \\
\hline & 7/28/2008 7:00 & 138 & 210 & 700 & 9.09 & 9.09 & & 0.0451 & $2.62 \mathrm{E}-04$ & 60.9060 & 22.4421 & 83.3481 & \\
\hline & 7/28/2008 8:00 & 139 & 210 & 800 & 7.337 & 7.337 & & 0.2948 & 2.65E-03 & 614.2100 & 226.3183 & 840.5283 & \\
\hline & $7 / 28 / 20089: 00$ & 140 & 210 & 900 & 5.933 & 5.933 & & 0.6012 & $6.36 \mathrm{E}-03$ & 1476.7263 & 544.1302 & 2020.8565 & \\
\hline & $7 / 28 / 2008$ 10:00 & 141 & 210 & 1000 & 5.584 & 5.584 & & 0.7013 & 7.69E-03 & 1784.9052 & 657.6850 & 2442.5902 & \\
\hline & $7 / 28 / 2008$ 11:00 & 142 & 210 & 1100 & 5.578 & 5.578 & & 0.7031 & 7.71E-03 & 1790.6405 & 659.7983 & 2450.4389 & \\
\hline & $7 / 28 / 200812: 00$ & 143 & 210 & 1200 & 5.631 & 5.631 & & 0.6871 & 7.50E-03 & 1740.5194 & 641.3302 & 2381.8496 & \\
\hline & 7/28/2008 13:00 & 144 & 210 & 1300 & 5.545 & 5.545 & & 0.7133 & 7.85E-03 & 1822.4689 & 671.5262 & 2493.9951 & \\
\hline & $7 / 28 / 2008$ 14:00 & 145 & 210 & 1400 & 5.511 & 5.511 & & 0.7238 & 7.99E-03 & 1855.7719 & 683.7973 & 2539.5693 & \\
\hline & $7 / 28 / 2008$ 15:00 & 146 & 210 & 1500 & 5.427 & 5.427 & & 0.7505 & 8.36E-03 & 1940.3368 & 714.9570 & 2655.2937 & \\
\hline & $7 / 28 / 2008$ 16:00 & 147 & 210 & 1600 & 5.494 & 5.494 & & 0.7292 & 8.07E-03 & 1872.6208 & 690.0057 & 2562.6265 & \\
\hline & 7/28/2008 17:00 & 148 & 210 & 1700 & 5.478 & 5.478 & & 0.7342 & 8.14E-03 & 1888.6004 & 695.8937 & 2584.4941 & \\
\hline & $7 / 28 / 2008$ 18:00 & 149 & 210 & 1800 & 5.605 & 5.605 & & 0.6949 & 7.60E-03 & 1764.9553 & 650.3341 & 2415.2894 & \\
\hline & $7 / 28 / 2008$ 19:00 & 150 & 210 & 1900 & 5.839 & 5.839 & & 0.6270 & $6.70 \mathrm{E}-03$ & 1555.0495 & 572.9900 & 2128.0395 & \\
\hline & $7 / 28 / 200820: 00$ & 151 & 210 & 2000 & 6.514 & 6.514 & & 0.4584 & 4.56E-03 & 1057.5589 & 389.6793 & 1447.2383 & \\
\hline & $7 / 28 / 200821: 00$ & 152 & 210 & 2100 & 7.982 & 7.982 & & 0.1902 & 1.54E-03 & 358.0648 & 131.9363 & 490.0011 & \\
\hline & $7 / 28 / 2008$ 22:00 & 153 & 210 & 2200 & 8.6 & 8.6 & & 0.1047 & 7.39E-04 & 171.6408 & 63.2446 & 234.8854 & \\
\hline & $7 / 28 / 200823: 00$ & 154 & 210 & 2300 & 8.8 & 8.8 & & 0.0795 & 5.27E-04 & 122.4540 & 45.1207 & 167.5748 & \\
\hline & $7 / 29 / 20080: 00$ & 155 & 211 & 0 & 9.04 & 9.04 & 9.52 & 0.0509 & 3.04E-04 & 70.6524 & 26.0333 & 96.6858 & \\
\hline & 7/29/2008 1:00 & 156 & 211 & 100 & 9.23 & 9.23 & & 0.0293 & $1.54 \mathrm{E}-04$ & 35.7405 & 13.1693 & 48.9099 & \\
\hline & 7/29/2008 2:00 & 157 & 211 & 200 & 9.39 & 9.39 & & 0.0117 & 4.99E-05 & 11.5856 & 4.2690 & 15.8546 & \\
\hline & 7/29/2008 3:00 & 158 & 211 & 300 & 9.44 & 9.44 & & 0.0064 & $2.35 \mathrm{E}-05$ & 5.4579 & 2.0111 & 7.4690 & \\
\hline & 7/29/2008 4:00 & 159 & 211 & 400 & 9.52 & 9.52 & & -0.0021 & $0.00 \mathrm{E}+00$ & 0.0000 & 0.0000 & 0.0000 & \\
\hline & 7/29/2008 5:00 & 160 & 211 & 500 & 9.5 & 9.5 & & 0.0000 & $0.00 \mathrm{E}+00$ & 0.0000 & 0.0000 & 0.0000 & \\
\hline & $7 / 29 / 20086: 00$ & 161 & 211 & 600 & 9.46 & 9.46 & & 0.0042 & 1.42E-05 & 3.3047 & 1.2177 & 4.5224 & \\
\hline & 7/29/2008 7:00 & 162 & 211 & 700 & 9.29 & 9.29 & & 0.0226 & 1.12E-04 & 26.0220 & 9.5883 & 35.6103 & \\
\hline & 7/29/2008 8:00 & 163 & 211 & 800 & 8.48 & 8.48 & & 0.1203 & 8.78E-04 & 203.7263 & 75.0672 & 278.7935 & \\
\hline
\end{tabular}




\begin{tabular}{|c|c|c|c|c|c|c|c|c|c|c|c|c|c|}
\hline Tree ID & TS & RN & JD & HR & $\begin{array}{c}\text { Raw } \\
\text { TC_dTA }\end{array}$ & $\begin{array}{c}\text { Fixed } \\
\text { TC_dT } \Delta\end{array}$ & dTM & $\mathbf{K}$ & $\begin{array}{l}\text { Sap } \\
\text { Velocity } \\
(\mathrm{cm} / \mathrm{s})\end{array}$ & $\begin{array}{c}\text { Probed } \\
\text { Drywood F } \\
(\mathrm{g} / \mathrm{hr})\end{array}$ & $\begin{array}{c}\text { Extrapolated } \\
\text { Wetwood F } \\
(\mathrm{g} / \mathrm{hr})\end{array}$ & $\begin{array}{c}\text { Extrapolated } \\
\text { Total Wood F } \\
(\mathrm{g} / \mathrm{hr})\end{array}$ & $\begin{array}{l}\text { Relative } \\
\text { Humidity, } \\
@ 10 \mathrm{~m}(\%)\end{array}$ \\
\hline & 7/29/2008 9:00 & 164 & 211 & 900 & \begin{tabular}{|r|}
6.733 \\
\end{tabular} & 6.733 & & 0.4110 & $3.98 \mathrm{E}-03$ & 924.4940 & 340.6488 & 1265.1428 & \\
\hline & 7/29/2008 10:00 & 165 & 211 & 1000 & 5.909 & 5.909 & & 0.6077 & $6.45 \mathrm{E}-03$ & 1496.4150 & 551.3849 & 2047.7999 & \\
\hline & $7 / 29 / 2008$ 11:00 & 166 & 211 & 1100 & 6.201 & 6.201 & & 0.5320 & $5.47 E-03$ & 1270.3513 & 468.0871 & 1738.4384 & \\
\hline & $7 / 29 / 2008$ 12:00 & 167 & 211 & 1200 & 5.692 & 5.692 & & 0.6690 & 7.26E-03 & 1684.3117 & 620.6193 & 2304.9310 & \\
\hline & 7/29/2008 13:00 & 168 & 211 & 1300 & 5.704 & 5.704 & & 0.6655 & 7.21E-03 & 1673.4363 & 616.6120 & 2290.0483 & \\
\hline & $7 / 29 / 2008$ 14:00 & 169 & 211 & 1400 & 5.547 & 5.547 & & 0.7126 & 7.84E-03 & 1820.5261 & 670.8103 & 2491.3364 & \\
\hline & $7 / 29 / 2008$ 15:00 & 170 & 211 & 1500 & 5.718 & 5.718 & & 0.6614 & 7.15E-03 & 1660.8227 & 611.9643 & 2272.7869 & \\
\hline & $7 / 29 / 2008$ 16:00 & 171 & 211 & 1600 & 5.91 & 5.91 & & 0.6074 & 6.44E-03 & 1495.5905 & 551.0811 & 2046.6715 & \\
\hline & 7/29/2008 17:00 & 172 & 211 & 1700 & 6.139 & 6.139 & & 0.5475 & 5.67E-03 & 1315.9827 & 484.9009 & 1800.8835 & \\
\hline & 7/29/2008 18:00 & 173 & 211 & 1800 & 6.138 & 6.138 & & 0.5477 & 5.67E-03 & 1316.7287 & 485.1758 & 1801.9045 & \\
\hline & $7 / 29 / 2008$ 19:00 & 174 & 211 & 1900 & 6.163 & 6.163 & & 0.5415 & 5.59E-03 & 1298.1741 & 478.3390 & 1776.5131 & \\
\hline & $7 / 29 / 200820: 00$ & 175 & 211 & 2000 & 7.105 & 7.105 & & 0.3371 & $3.12 \mathrm{E}-03$ & 724.3781 & 266.9120 & 991.2901 & \\
\hline & 7/29/2008 21:00 & 176 & 211 & 2100 & 8.62 & 8.62 & & 0.1021 & 7.17E-04 & 166.4809 & 61.3433 & 227.8242 & \\
\hline & $7 / 29 / 2008$ 22:00 & 177 & 211 & 2200 & 9.27 & 9.27 & & 0.0248 & 1.26E-04 & 29.1828 & 10.7530 & 39.9358 & \\
\hline & 7/29/2008 23:00 & 178 & 211 & 2300 & 9.45 & 9.45 & & 0.0053 & $1.88 \mathrm{E}-05$ & 4.3550 & 1.6047 & 5.9597 & \\
\hline & $7 / 30 / 20080: 00$ & 179 & 212 & 0 & 9.45 & 9.45 & 9.45 & 0.0053 & 1.88E-05 & 4.3550 & 1.6047 & 5.9597 & \\
\hline & 7/30/2008 1:00 & 180 & 212 & 100 & 9.41 & 9.41 & & 0.0096 & 3.89E-05 & 9.0261 & 3.3258 & 12.3519 & \\
\hline & 7/30/2008 2:00 & 181 & 212 & 200 & 9.35 & 9.35 & & 0.0160 & 7.35E-05 & 17.0614 & 6.2866 & 23.3480 & \\
\hline & 7/30/2008 3:00 & 182 & 212 & 300 & 9.28 & 9.28 & & 0.0237 & 1.19E-04 & 27.5922 & 10.1669 & 37.7591 & \\
\hline & 7/30/2008 4:00 & 183 & 212 & 400 & 9.2 & 9.2 & & 0.0326 & 1.76E-04 & 40.8535 & 15.0533 & 55.9068 & \\
\hline & 7/30/2008 5:00 & 184 & 212 & 500 & 9.1 & 9.1 & & 0.0440 & $2.54 \mathrm{E}-04$ & 59.0026 & 21.7407 & 80.7434 & \\
\hline & 7/30/2008 6:00 & 185 & 212 & 600 & 8.99 & 8.99 & & 0.0567 & 3.48E-04 & 80.7712 & 29.7618 & 110.5330 & \\
\hline & 7/30/2008 7:00 & 186 & 212 & 700 & 8.89 & 8.89 & & 0.0686 & 4.40E-04 & 102.0842 & 37.6150 & 139.6992 & \\
\hline & $7 / 30 / 20088: 00$ & 187 & 212 & 800 & 8.78 & 8.78 & & 0.0820 & $5.48 \mathrm{E}-04$ & 127.1306 & 46.8439 & 173.9745 & \\
\hline & 7/30/2008 9:00 & 188 & 212 & 900 & 7.139 & 7.139 & & 0.3307 & $3.05 E-03$ & 707.5696 & 260.7186 & 968.2882 & \\
\hline & $7 / 30 / 2008$ 10:00 & 189 & 212 & 1000 & 5.283 & 5.283 & & 0.7982 & 9.02E-03 & 2093.2881 & 771.3151 & 2864.6032 & \\
\hline & 7/30/2008 11:00 & 190 & 212 & 1100 & 5.192 & 5.192 & & 0.8297 & 9.46E-03 & 2195.4927 & 808.9744 & 3004.4671 & \\
\hline & $7 / 30 / 2008$ 12:00 & 191 & 212 & 1200 & 5.288 & 5.288 & & 0.7965 & 8.99E-03 & 2087.8006 & 769.2931 & 2857.0937 & \\
\hline & 7/30/2008 13:00 & 192 & 212 & 1300 & 5.35 & 5.35 & & 0.7757 & 8.70E-03 & 2020.8278 & 744.6156 & 2765.4434 & \\
\hline & $7 / 30 / 2008$ 14:00 & 193 & 212 & 1400 & 5.375 & 5.375 & & 0.7674 & 8.59E-03 & 1994.3740 & 734.8681 & 2729.2421 & \\
\hline & 7/30/2008 15:00 & 194 & 212 & 1500 & 5.578 & 5.578 & & 0.7031 & 7.71E-03 & 1790.6405 & 659.7983 & 2450.4389 & \\
\hline & $7 / 30 / 2008$ 16:00 & 195 & 212 & 1600 & 5.499 & 5.499 & & 0.7276 & 8.05E-03 & 1867.6515 & 688.1746 & 2555.8261 & \\
\hline & $7 / 30 / 2008$ 17:00 & 196 & 212 & 1700 & 5.518 & 5.518 & & 0.7216 & 7.96E-03 & 1848.8726 & 681.2551 & 2530.1278 & \\
\hline & $7 / 30 / 2008$ 18:00 & 197 & 212 & 1800 & 5.669 & 5.669 & & 0.6758 & 7.35E-03 & 1705.3221 & 628.3610 & 2333.6831 & \\
\hline & 7/30/2008 19:00 & 198 & 212 & 1900 & 5.783 & 5.783 & & 0.6427 & 6.91E-03 & 1603.2900 & 590.7652 & 2194.0551 & \\
\hline & $7 / 30 / 200820: 00$ & 199 & 212 & 2000 & 6.421 & 6.421 & & 0.4795 & 4.82E-03 & 1117.8642 & 411.9001 & 1529.7643 & \\
\hline & 7/30/2008 21:00 & 200 & 212 & 2100 & 7.93 & 7.93 & & 0.1980 & 1.62E-03 & 376.2381 & 138.6327 & 514.8708 & \\
\hline & $7 / 30 / 2008$ 22:00 & 201 & 212 & 2200 & 8.83 & 8.83 & & 0.0759 & 4.98E-04 & 115.5410 & 42.5735 & 158.1144 & \\
\hline & 7/30/2008 23:00 & 202 & 212 & 2300 & 9.12 & 9.12 & & 0.0417 & $2.38 \mathrm{E}-04$ & 55.2428 & 20.3553 & 75.5981 & \\
\hline & $7 / 31 / 20080: 00$ & 203 & 213 & 0 & 9.27 & 9.27 & 9.32 & 0.0248 & 1.26E-04 & 29.1828 & 10.7530 & 39.9358 & \\
\hline & 7/31/2008 1:00 & 204 & 213 & 100 & 9.32 & 9.32 & & 0.0193 & 9.23E-05 & 21.4390 & 7.8997 & 29.3387 & \\
\hline
\end{tabular}




\begin{tabular}{|c|c|c|c|c|c|c|c|c|c|c|c|c|c|}
\hline Tree ID & TS & RN & JD & HR & $\begin{array}{c}\text { Raw } \\
\text { TC_dTA }\end{array}$ & $\begin{array}{c}\text { Fixed } \\
\text { TC_dT } \Delta\end{array}$ & dTM & $\mathbf{K}$ & $\begin{array}{l}\text { Sap } \\
\text { Velocity } \\
(\mathrm{cm} / \mathrm{s})\end{array}$ & $\begin{array}{c}\text { Probed } \\
\text { Drywood F } \\
(\mathrm{g} / \mathrm{hr})\end{array}$ & $\begin{array}{c}\text { Extrapolated } \\
\text { Wetwood F } \\
(\mathrm{g} / \mathrm{hr})\end{array}$ & $\begin{array}{c}\text { Extrapolated } \\
\text { Total Wood F } \\
(\mathrm{g} / \mathrm{hr})\end{array}$ & $\begin{array}{l}\text { Relative } \\
\text { Humidity, } \\
@ 10 \mathrm{~m}(\%)\end{array}$ \\
\hline & 7/31/2008 2:00 & 205 & 213 & 200 & \begin{tabular}{|r|}
9.29 \\
\end{tabular} & 9.29 & & 0.0226 & 1.12E-04 & 26.0220 & 9.5883 & 35.6103 & \\
\hline & 7/31/2008 3:00 & 206 & 213 & 300 & 9.25 & 9.25 & & 0.0270 & $1.40 \mathrm{E}-04$ & 32.4235 & 11.9471 & 44.3705 & \\
\hline & 7/31/2008 4:00 & 207 & 213 & 400 & 9.21 & 9.21 & & 0.0315 & $1.69 \mathrm{E}-04$ & 39.1313 & 14.4187 & 53.5500 & \\
\hline & 7/31/2008 5:00 & 208 & 213 & 500 & 9.14 & 9.14 & & 0.0394 & $2.22 \mathrm{E}-04$ & 51.5465 & 18.9934 & 70.5399 & \\
\hline & 7/31/2008 6:00 & 209 & 213 & 600 & 9.08 & 9.08 & & 0.0463 & 2.71E-04 & 62.8249 & 23.1491 & 85.9740 & \\
\hline & 7/31/2008 7:00 & 210 & 213 & 700 & 9 & 9 & & 0.0556 & 3.39E-04 & 78.7182 & 29.0053 & 107.7235 & \\
\hline & $7 / 31 / 2008$ 8:00 & 211 & 213 & 800 & 8.79 & 8.79 & & 0.0808 & $5.38 E-04$ & 124.7856 & 45.9798 & 170.7654 & \\
\hline & $7 / 31 / 20089: 00$ & 212 & 213 & 900 & 6.857 & 6.857 & & 0.3854 & 3.68E-03 & 854.3505 & 314.8030 & 1169.1535 & \\
\hline & $7 / 31 / 2008$ 10:00 & 213 & 213 & 1000 & 5.281 & 5.281 & & 0.7989 & 9.03E-03 & 2095.4868 & 772.1252 & 2867.6121 & \\
\hline & 7/31/2008 11:00 & 214 & 213 & 1100 & 5.302 & 5.302 & & 0.7918 & 8.93E-03 & 2072.5048 & 763.6570 & 2836.1619 & \\
\hline & $7 / 31 / 2008$ 12:00 & 215 & 213 & 1200 & 5.191 & 5.191 & & 0.8301 & $9.46 \mathrm{E}-03$ & 2196.6409 & 809.3975 & 3006.0384 & \\
\hline & 7/31/2008 13:00 & 216 & 213 & 1300 & 5.457 & 5.457 & & 0.7409 & $8.23 E-03$ & 1909.7548 & 703.6884 & 2613.4432 & \\
\hline & 7/31/2008 14:00 & 217 & 213 & 1400 & 5.235 & 5.235 & & 0.8147 & $9.25 E-03$ & 2146.6414 & 790.9742 & 2937.6156 & \\
\hline & 7/31/2008 15:00 & 218 & 213 & 1500 & 5.297 & 5.297 & & 0.7935 & 8.95E-03 & 2077.9559 & 765.6656 & 2843.6215 & \\
\hline & $7 / 31 / 2008$ 16:00 & 219 & 213 & 1600 & 5.41 & 5.41 & & 0.7560 & 8.43E-03 & 1957.8579 & 721.4130 & 2679.2709 & \\
\hline & 7/31/2008 17:00 & 220 & 213 & 1700 & 5.428 & 5.428 & & 0.7502 & 8.35E-03 & 1939.3104 & 714.5788 & 2653.8893 & \\
\hline & $7 / 31 / 2008$ 18:00 & 221 & 213 & 1800 & 5.552 & 5.552 & & 0.7111 & 7.82E-03 & 1815.6770 & 669.0235 & 2484.7006 & \\
\hline & $7 / 31 / 2008$ 19:00 & 222 & 213 & 1900 & 5.832 & 5.832 & & 0.6289 & $6.72 \mathrm{E}-03$ & 1561.0138 & 575.1877 & 2136.2015 & \\
\hline & $7 / 31 / 200820: 00$ & 223 & 213 & 2000 & 6.702 & 6.702 & & 0.4175 & 4.06E-03 & 942.6001 & 347.3204 & 1289.9205 & \\
\hline & $7 / 31 / 200821: 00$ & 224 & 213 & 2100 & 7.697 & 7.697 & & 0.2342 & 1.99E-03 & 462.7907 & 170.5247 & 633.3154 & \\
\hline & $7 / 31 / 200822: 00$ & 225 & 213 & 2200 & 8.41 & 8.41 & & 0.1296 & $9.62 \mathrm{E}-04$ & 223.3386 & 82.2937 & 305.6323 & \\
\hline & 7/31/2008 23:00 & 226 & 213 & 2300 & 8.38 & 8.38 & & 0.1337 & 9.99E-04 & 231.9475 & 85.4658 & 317.4133 & \\
\hline & $8 / 1 / 20080: 00$ & 227 & 214 & 0 & 8.68 & 8.68 & 9.32 & 0.0945 & 6.52E-04 & 151.3222 & 55.7578 & 207.0800 & \\
\hline & 8/1/2008 1:00 & 228 & 214 & 100 & 9.09 & 9.09 & & 0.0451 & $2.62 E-04$ & 60.9060 & 22.4421 & 83.3481 & \\
\hline & 8/1/2008 2:00 & 229 & 214 & 200 & 9.27 & 9.27 & & 0.0248 & 1.26E-04 & 29.1828 & 10.7530 & 39.9358 & \\
\hline & $8 / 1 / 2008$ 3:00 & 230 & 214 & 300 & 9.27 & 9.27 & & 0.0248 & 1.26E-04 & 29.1828 & 10.7530 & 39.9358 & \\
\hline & 8/1/2008 4:00 & 231 & 214 & 400 & 9.32 & 9.32 & & 0.0193 & $9.23 E-05$ & 21.4390 & 7.8997 & 29.3387 & \\
\hline & 8/1/2008 5:00 & 232 & 214 & 500 & 9.31 & 9.31 & & 0.0204 & $9.88 \mathrm{E}-05$ & 22.9448 & 8.4545 & 31.3993 & \\
\hline & 8/1/2008 6:00 & 233 & 214 & 600 & 9.23 & 9.23 & & 0.0293 & 1.54E-04 & 35.7405 & 13.1693 & 48.9099 & \\
\hline & 8/1/2008 7:00 & 234 & 214 & 700 & 9.14 & 9.14 & & 0.0394 & $2.22 \mathrm{E}-04$ & 51.5465 & 18.9934 & 70.5399 & \\
\hline & 8/1/2008 8:00 & 235 & 214 & 800 & 8.96 & 8.96 & & 0.0603 & $3.75 E-04$ & 87.0164 & 32.0630 & 119.0794 & \\
\hline & 8/1/2008 9:00 & 236 & 214 & 900 & 7.547 & 7.547 & & 0.2588 & $2.25 E-03$ & 523.1544 & 192.7670 & 715.9213 & \\
\hline & 8/1/2008 10:00 & 237 & 214 & 1000 & 5.613 & 5.613 & & 0.6925 & 7.57E-03 & 1757.4056 & 647.5523 & 2404.9579 & \\
\hline & 8/1/2008 11:00 & 238 & 214 & 1100 & 5.563 & 5.563 & & 0.7077 & 7.78E-03 & 1805.0481 & 665.1071 & 2470.1552 & \\
\hline & 8/1/2008 12:00 & 239 & 214 & 1200 & 5.583 & 5.583 & & 0.7016 & 7.69E-03 & 1785.8600 & 658.0368 & 2443.8968 & \\
\hline & 8/1/2008 13:00 & 240 & 214 & 1300 & 5.5 & 5.5 & & 0.7273 & 8.04E-03 & 1866.6590 & 687.8089 & 2554.4679 & \\
\hline & 8/1/2008 14:00 & 241 & 214 & 1400 & 5.461 & 5.461 & & 0.7396 & $8.21 \mathrm{E}-03$ & 1905.7094 & 702.1978 & 2607.9072 & \\
\hline & 8/1/2008 15:00 & 242 & 214 & 1500 & 5.452 & 5.452 & & 0.7425 & 8.25E-03 & 1914.8221 & 705.5556 & 2620.3776 & \\
\hline & $8 / 1 / 200816: 00$ & 243 & 214 & 1600 & 5.564 & 5.564 & & 0.7074 & 7.77E-03 & 1804.0845 & 664.7520 & 2468.8366 & \\
\hline & 8/1/2008 17:00 & 244 & 214 & 1700 & 5.62 & 5.62 & & 0.6904 & 7.54E-03 & 1750.8223 & 645.1265 & 2395.9487 & \\
\hline & 8/1/2008 18:00 & 245 & 214 & 1800 & 5.7 & 5.7 & & 0.6667 & 7.22E-03 & 1677.0549 & 617.9454 & 2295.0002 & \\
\hline
\end{tabular}




\begin{tabular}{|c|c|c|c|c|c|c|c|c|c|c|c|c|c|}
\hline Tree ID & TS & RN & JD & HR & $\begin{array}{c}\text { Raw } \\
\text { TC_dTA }\end{array}$ & $\begin{array}{c}\text { Fixed } \\
\text { TC_dT } \Delta\end{array}$ & dTM & $\mathbf{K}$ & $\begin{array}{l}\text { Sap } \\
\text { Velocity } \\
(\mathrm{cm} / \mathrm{s})\end{array}$ & $\begin{array}{c}\text { Probed } \\
\text { Drywood F } \\
(\mathrm{g} / \mathrm{hr})\end{array}$ & $\begin{array}{c}\text { Extrapolated } \\
\text { Wetwood F } \\
(\mathrm{g} / \mathrm{hr})\end{array}$ & $\begin{array}{c}\text { Extrapolated } \\
\text { Total Wood F } \\
(\mathrm{g} / \mathrm{hr})\end{array}$ & $\begin{array}{l}\text { Relative } \\
\text { Humidity, } \\
@ 10 \mathrm{~m}(\%)\end{array}$ \\
\hline & 8/1/2008 19:00 & 246 & 214 & 1900 & 5.855 & 5.855 & & 0.6225 & 6.64E-03 & 1541.4863 & 567.9923 & 2109.4786 & \\
\hline & 8/1/2008 20:00 & 247 & 214 & 2000 & 6.697 & 6.697 & & 0.4185 & 4.07E-03 & 945.5424 & 348.4045 & 1293.9469 & \\
\hline & 8/1/2008 21:00 & 248 & 214 & 2100 & 8.41 & 8.41 & & 0.1296 & $9.62 E-04$ & 223.3386 & 82.2937 & 305.6323 & \\
\hline & 8/1/2008 22:00 & 249 & 214 & 2200 & 9.13 & 9.13 & & 0.0405 & $2.30 \mathrm{E}-04$ & 53.3866 & 19.6714 & 73.0580 & \\
\hline & 8/1/2008 23:00 & 250 & 214 & 2300 & 9.28 & 9.28 & & 0.0237 & 1.19E-04 & 27.5922 & 10.1669 & 37.7591 & \\
\hline & 8/2/2008 0:00 & 251 & 215 & 0 & 9.29 & 9.29 & 9.47 & 0.0226 & 1.12E-04 & 26.0220 & 9.5883 & 35.6103 & \\
\hline & 8/2/2008 1:00 & 252 & 215 & 100 & 9.38 & 9.38 & & 0.0128 & $5.56 \mathrm{E}-05$ & 12.9124 & 4.7578 & 17.6702 & \\
\hline & 8/2/2008 2:00 & 253 & 215 & 200 & 9.45 & 9.45 & & 0.0053 & 1.88E-05 & 4.3550 & 1.6047 & 5.9597 & \\
\hline & 8/2/2008 3:00 & 254 & 215 & 300 & 9.47 & 9.47 & & 0.0032 & $9.98 \mathrm{E}-06$ & 2.3161 & 0.8534 & 3.1696 & \\
\hline & 8/2/2008 4:00 & 255 & 215 & 400 & 9.37 & 9.37 & & 0.0139 & 6.15E-05 & 14.2682 & 5.2574 & 19.5256 & \\
\hline & 8/2/2008 5:00 & 256 & 215 & 500 & 9.33 & 9.33 & & 0.0182 & 8.60E-05 & 19.9560 & 7.3532 & 27.3092 & \\
\hline & 8/2/2008 6:00 & 257 & 215 & 600 & 9.31 & 9.31 & & 0.0204 & $9.88 \mathrm{E}-05$ & 22.9448 & 8.4545 & 31.3993 & \\
\hline & 8/2/2008 7:00 & 258 & 215 & 700 & 9.15 & 9.15 & & 0.0383 & $2.14 \mathrm{E}-04$ & 49.7226 & 18.3213 & 68.0439 & \\
\hline & 8/2/2008 8:00 & 259 & 215 & 800 & 7.721 & 7.721 & & 0.2304 & 1.95E-03 & 453.4778 & 167.0932 & 620.5711 & \\
\hline & 8/2/2008 9:00 & 260 & 215 & 900 & 5.956 & 5.956 & & 0.5950 & $6.28 E-03$ & 1458.0525 & 537.2494 & 1995.3019 & \\
\hline & 8/2/2008 10:00 & 261 & 215 & 1000 & 5.836 & 5.836 & & 0.6278 & 6.71E-03 & 1557.6034 & 573.9310 & 2131.5344 & \\
\hline & 8/2/2008 11:00 & 262 & 215 & 1100 & 5.692 & 5.692 & & 0.6690 & 7.26E-03 & 1684.3117 & 620.6193 & 2304.9310 & \\
\hline & $8 / 2 / 200812: 00$ & 263 & 215 & 1200 & 5.593 & 5.593 & & 0.6986 & 7.65E-03 & 1776.3318 & 654.5260 & 2430.8577 & \\
\hline & 8/2/2008 13:00 & 264 & 215 & 1300 & 5.55 & 5.55 & & 0.7117 & 7.83E-03 & 1817.6153 & 669.7377 & 2487.3531 & \\
\hline & 8/2/2008 14:00 & 265 & 215 & 1400 & 5.543 & 5.543 & & 0.7139 & 7.86E-03 & 1824.4135 & 672.2427 & 2496.6562 & \\
\hline & $8 / 2 / 2008$ 15:00 & 266 & 215 & 1500 & 5.541 & 5.541 & & 0.7145 & 7.87E-03 & 1826.3599 & 672.9599 & 2499.3197 & \\
\hline & 8/2/2008 16:00 & 267 & 215 & 1600 & 5.583 & 5.583 & & 0.7016 & 7.69E-03 & 1785.8600 & 658.0368 & 2443.8968 & \\
\hline & 8/2/2008 17:00 & 268 & 215 & 1700 & 5.616 & 5.616 & & 0.6916 & 7.56E-03 & 1754.5816 & 646.5117 & 2401.0933 & \\
\hline & $8 / 2 / 200818: 00$ & 269 & 215 & 1800 & 5.745 & 5.745 & & 0.6536 & 7.05E-03 & 1636.7201 & 603.0832 & 2239.8033 & \\
\hline & 8/2/2008 19:00 & 270 & 215 & 1900 & 5.915 & 5.915 & & 0.6061 & $6.42 \mathrm{E}-03$ & 1491.4733 & 549.5640 & 2041.0373 & \\
\hline & 8/2/2008 20:00 & 271 & 215 & 2000 & 6.614 & 6.614 & & 0.4363 & $4.29 \mathrm{E}-03$ & 995.2885 & 366.7345 & 1362.0230 & \\
\hline & 8/2/2008 21:00 & 272 & 215 & 2100 & 7.831 & 7.831 & & 0.2131 & 1.77E-03 & 411.9745 & 151.8005 & 563.7750 & \\
\hline & 8/2/2008 22:00 & 273 & 215 & 2200 & 8.79 & 8.79 & & 0.0808 & $5.38 \mathrm{E}-04$ & 124.7856 & 45.9798 & 170.7654 & \\
\hline & 8/2/2008 23:00 & 274 & 215 & 2300 & 9.09 & 9.09 & & 0.0451 & $2.62 E-04$ & 60.9060 & 22.4421 & 83.3481 & \\
\hline & 8/3/2008 0:00 & 275 & 216 & 0 & 8.97 & 8.97 & 9.35 & 0.0591 & $3.66 \mathrm{E}-04$ & 84.9203 & 31.2906 & 116.2110 & \\
\hline & 8/3/2008 1:00 & 276 & 216 & 100 & 8.74 & 8.74 & & 0.0870 & 5.89E-04 & 136.6459 & 50.3500 & 186.9959 & \\
\hline & 8/3/2008 2:00 & 277 & 216 & 200 & 8.96 & 8.96 & & 0.0603 & 3.75E-04 & 87.0164 & 32.0630 & 119.0794 & \\
\hline & 8/3/2008 3:00 & 278 & 216 & 300 & 9.18 & 9.18 & & 0.0349 & 1.91E-04 & 44.3502 & 16.3418 & 60.6920 & \\
\hline & 8/3/2008 4:00 & 279 & 216 & 400 & 9.28 & 9.28 & & 0.0237 & $1.19 \mathrm{E}-04$ & 27.5922 & 10.1669 & 37.7591 & \\
\hline & 8/3/2008 5:00 & 280 & 216 & 500 & 9.35 & 9.35 & & 0.0160 & 7.35E-05 & 17.0614 & 6.2866 & 23.3480 & \\
\hline & 8/3/2008 6:00 & 281 & 216 & 600 & 9.2 & 9.2 & & 0.0326 & 1.76E-04 & 40.8535 & 15.0533 & 55.9068 & \\
\hline & 8/3/2008 7:00 & 282 & 216 & 700 & 8.95 & 8.95 & & 0.0615 & 3.84E-04 & 89.1267 & 32.8406 & 121.9673 & \\
\hline & 8/3/2008 8:00 & 283 & 216 & 800 & 7.7 & 7.7 & & 0.2338 & 1.99E-03 & 461.6215 & 170.0939 & 631.7154 & \\
\hline & 8/3/2008 9:00 & 284 & 216 & 900 & 5.893 & 5.893 & & 0.6121 & $6.50 \mathrm{E}-03$ & 1509.6572 & 556.2643 & 2065.9215 & \\
\hline & 8/3/2008 10:00 & 285 & 216 & 1000 & 5.585 & 5.585 & & 0.7010 & 7.68E-03 & 1783.9509 & 657.3334 & 2441.2842 & \\
\hline & 8/3/2008 11:00 & 286 & 216 & 1100 & 5.543 & 5.543 & & 0.7139 & 7.86E-03 & 1824.4135 & 672.2427 & 2496.6562 & \\
\hline
\end{tabular}




\begin{tabular}{|c|c|c|c|c|c|c|c|c|c|c|c|c|c|}
\hline Tree ID & TS & RN & JD & HR & $\begin{array}{c}\text { Raw } \\
\text { TC_dTA }\end{array}$ & $\begin{array}{c}\text { Fixed } \\
\text { TC_dT } \Delta\end{array}$ & dTM & $\mathbf{K}$ & $\begin{array}{l}\text { Sap } \\
\text { Velocity } \\
(\mathrm{cm} / \mathrm{s})\end{array}$ & $\begin{array}{c}\text { Probed } \\
\text { Drywood F } \\
(\mathrm{g} / \mathrm{hr})\end{array}$ & $\begin{array}{c}\text { Extrapolated } \\
\text { Wetwood F } \\
(\mathrm{g} / \mathrm{hr})\end{array}$ & $\begin{array}{c}\text { Extrapolated } \\
\text { Total Wood F } \\
(\mathrm{g} / \mathrm{hr})\end{array}$ & $\begin{array}{l}\text { Relative } \\
\text { Humidity, } \\
@ 10 \mathrm{~m}(\%)\end{array}$ \\
\hline & 8/3/2008 12:00 & 287 & 216 & 1200 & 5.53 & 5.53 & & 0.7179 & 7.91E-03 & 1837.0970 & 676.9162 & 2514.0132 & \\
\hline & 8/3/2008 13:00 & 288 & 216 & 1300 & 5.5 & 5.5 & & 0.7273 & 8.04E-03 & 1866.6590 & 687.8089 & 2554.4679 & \\
\hline & $8 / 3 / 2008$ 14:00 & 289 & 216 & 1400 & 5.526 & 5.526 & & 0.7191 & 7.93E-03 & 1841.0150 & 678.3598 & 2519.3748 & \\
\hline & 8/3/2008 15:00 & 290 & 216 & 1500 & 5.598 & 5.598 & & 0.6970 & 7.63E-03 & 1771.5840 & 652.7766 & 2424.3605 & \\
\hline & 8/3/2008 16:00 & 291 & 216 & 1600 & 5.65 & 5.65 & & 0.6814 & 7.42E-03 & 1722.8447 & 634.8176 & 2357.6623 & \\
\hline & 8/3/2008 17:00 & 292 & 216 & 1700 & 5.647 & 5.647 & & 0.6823 & 7.43E-03 & 1725.6253 & 635.8421 & 2361.4674 & \\
\hline & 8/3/2008 18:00 & 293 & 216 & 1800 & 5.768 & 5.768 & & 0.6470 & $6.96 \mathrm{E}-03$ & 1616.4180 & 595.6025 & 2212.0205 & \\
\hline & 8/3/2008 19:00 & 294 & 216 & 1900 & 5.858 & 5.858 & & 0.6217 & 6.63E-03 & 1538.9539 & 567.0592 & 2106.0131 & \\
\hline & 8/3/2008 20:00 & 295 & 216 & 2000 & 6.23 & 6.23 & & 0.5249 & 5.38E-03 & 1249.4219 & 460.3752 & 1709.7971 & \\
\hline & 8/3/2008 21:00 & 296 & 216 & 2100 & 7.158 & 7.158 & & 0.3272 & 3.01E-03 & 698.2782 & 257.2950 & 955.5732 & \\
\hline & 8/3/2008 22:00 & 297 & 216 & 2200 & 7.759 & 7.759 & & 0.2244 & 1.89E-03 & 438.9227 & 161.7301 & 600.6528 & \\
\hline & 8/3/2008 23:00 & 298 & 216 & 2300 & 7.875 & 7.875 & & 0.2063 & 1.71E-03 & 395.9056 & 145.8796 & 541.7852 & \\
\hline & 8/4/2008 0:00 & 299 & 217 & 0 & 7.916 & 7.916 & 9.06 & 0.2001 & 1.64E-03 & 381.2006 & 140.4612 & 521.6618 & \\
\hline & 8/4/2008 1:00 & 300 & 217 & 100 & 7.954 & 7.954 & & 0.1944 & 1.58E-03 & 367.7999 & 135.5234 & 503.3234 & \\
\hline & $8 / 4 / 2008$ 2:00 & 301 & 217 & 200 & 7.578 & 7.578 & & 0.2536 & $2.20 \mathrm{E}-03$ & 510.3690 & 188.0560 & 698.4250 & \\
\hline & 8/4/2008 3:00 & 302 & 217 & 300 & 7.348 & 7.348 & & 0.2929 & $2.62 \mathrm{E}-03$ & 609.2425 & 224.4879 & 833.7305 & \\
\hline & 8/4/2008 4:00 & 303 & 217 & 400 & 7.101 & 7.101 & & 0.3378 & 3.13E-03 & 726.3711 & 267.6464 & 994.0174 & \\
\hline & $8 / 4 / 20085: 00$ & 304 & 217 & 500 & 7.311 & 7.311 & & 0.2994 & $2.70 \mathrm{E}-03$ & 626.0409 & 230.6777 & 856.7186 & \\
\hline & 8/4/2008 6:00 & 305 & 217 & 600 & 7.775 & 7.775 & & 0.2219 & 1.86E-03 & 432.8633 & 159.4974 & 592.3607 & \\
\hline & 8/4/2008 7:00 & 306 & 217 & 700 & 7.89 & 7.89 & & 0.2041 & $1.68 E-03$ & 390.4959 & 143.8862 & 534.3821 & \\
\hline & 8/4/2008 8:00 & 307 & 217 & 800 & 7.685 & 7.685 & & 0.2362 & $2.01 \mathrm{E}-03$ & 467.4823 & 172.2535 & 639.7357 & \\
\hline & 8/4/2008 9:00 & 308 & 217 & 900 & 7.304 & 7.304 & & 0.3007 & 2.71E-03 & 629.2478 & 231.8593 & 861.1071 & \\
\hline & 8/4/2008 10:00 & 309 & 217 & 1000 & 6.39 & 6.39 & & 0.4867 & 4.90E-03 & 1138.4975 & 419.5028 & 1558.0004 & \\
\hline & 8/4/2008 11:00 & 310 & 217 & 1100 & 5.827 & 5.827 & & 0.6303 & $6.74 \mathrm{E}-03$ & 1565.2855 & 576.7616 & 2142.0471 & \\
\hline & 8/4/2008 12:00 & 311 & 217 & 1200 & 5.673 & 5.673 & & 0.6746 & 7.33E-03 & 1701.6524 & 627.0088 & 2328.6612 & \\
\hline & 8/4/2008 13:00 & 312 & 217 & 1300 & 5.655 & 5.655 & & 0.6799 & 7.40E-03 & 1718.2188 & 633.1131 & 2351.3319 & \\
\hline & 8/4/2008 14:00 & 313 & 217 & 1400 & 6.012 & 6.012 & & 0.5802 & 6.09E-03 & 1413.3669 & 520.7841 & 1934.1509 & \\
\hline & 8/4/2008 15:00 & 314 & 217 & 1500 & 6.373 & 6.373 & & 0.4907 & $4.95 \mathrm{E}-03$ & 1149.9281 & 423.7146 & 1573.6427 & \\
\hline & 8/4/2008 16:00 & 315 & 217 & 1600 & 5.789 & 5.789 & & 0.6410 & $6.88 \mathrm{E}-03$ & 1598.0634 & 588.8393 & 2186.9027 & \\
\hline & 8/4/2008 17:00 & 316 & 217 & 1700 & 5.76 & 5.76 & & 0.6493 & $6.99 \mathrm{E}-03$ & 1623.4558 & 598.1957 & 2221.6515 & \\
\hline & 8/4/2008 18:00 & 317 & 217 & 1800 & 5.893 & 5.893 & & 0.6121 & $6.50 \mathrm{E}-03$ & 1509.6572 & 556.2643 & 2065.9215 & \\
\hline & 8/4/2008 19:00 & 318 & 217 & 1900 & 6.113 & 6.113 & & 0.5541 & $5.75 E-03$ & 1335.4848 & 492.0869 & 1827.5717 & \\
\hline & 8/4/2008 20:00 & 319 & 217 & 2000 & 6.769 & 6.769 & & 0.4035 & 3.89E-03 & 903.7576 & 333.0081 & 1236.7656 & \\
\hline & 8/4/2008 21:00 & 320 & 217 & 2100 & 8.33 & 8.33 & & 0.1405 & $1.06 \mathrm{E}-03$ & 246.5690 & 90.8534 & 337.4224 & \\
\hline & 8/4/2008 22:00 & 321 & 217 & 2200 & 8.92 & 8.92 & & 0.0650 & 4.12E-04 & 95.5424 & 35.2046 & 130.7470 & \\
\hline & 8/4/2008 23:00 & 322 & 217 & 2300 & 9.06 & 9.06 & & 0.0486 & 2.87E-04 & 66.7085 & 24.5801 & 91.2886 & \\
\hline & 8/5/2008 0:00 & 323 & 218 & 0 & 9.22 & 9.22 & 9.51 & 0.0304 & 1.61E-04 & 37.4269 & 13.7907 & 51.2176 & \\
\hline & 8/5/2008 1:00 & 324 & 218 & 100 & 9.3 & 9.3 & & 0.0215 & 1.05E-04 & 24.4727 & 9.0175 & 33.4901 & \\
\hline & 8/5/2008 2:00 & 325 & 218 & 200 & 9.36 & 9.36 & & 0.0150 & $6.74 \mathrm{E}-05$ & 15.6516 & 5.7672 & 21.4187 & \\
\hline & 8/5/2008 3:00 & 326 & 218 & 300 & 9.32 & 9.32 & & 0.0193 & 9.23E-05 & 21.4390 & 7.8997 & 29.3387 & \\
\hline & 8/5/2008 4:00 & 327 & 218 & 400 & 9.2 & 9.2 & & 0.0326 & $1.76 \mathrm{E}-04$ & 40.8535 & 15.0533 & 55.9068 & \\
\hline
\end{tabular}




\begin{tabular}{|c|c|c|c|c|c|c|c|c|c|c|c|c|c|}
\hline Tree ID & TS & RN & JD & HR & $\begin{array}{c}\text { Raw } \\
\text { TC_dTA }\end{array}$ & $\begin{array}{c}\text { Fixed } \\
\text { TC_dT } \Delta\end{array}$ & dTM & $\mathbf{K}$ & $\begin{array}{l}\text { Sap } \\
\text { Velocity } \\
(\mathrm{cm} / \mathrm{s})\end{array}$ & $\begin{array}{c}\text { Probed } \\
\text { Drywood F } \\
(\mathrm{g} / \mathrm{hr})\end{array}$ & $\begin{array}{c}\text { Extrapolated } \\
\text { Wetwood F } \\
(\mathrm{g} / \mathrm{hr})\end{array}$ & $\begin{array}{c}\text { Extrapolated } \\
\text { Total Wood F } \\
(\mathrm{g} / \mathrm{hr})\end{array}$ & $\begin{array}{l}\text { Relative } \\
\text { Humidity, } \\
@ 10 \mathrm{~m}(\%)\end{array}$ \\
\hline & 8/5/2008 5:00 & 328 & 218 & 500 & $\begin{array}{r}9.22 \\
\end{array}$ & 9.22 & & 0.0304 & 1.61E-04 & 37.4269 & 13.7907 & 51.2176 & \\
\hline & 8/5/2008 6:00 & 329 & 218 & 600 & 9.29 & 9.29 & & 0.0226 & $1.12 \mathrm{E}-04$ & 26.0220 & 9.5883 & 35.6103 & \\
\hline & 8/5/2008 7:00 & 330 & 218 & 700 & 9.32 & 9.32 & & 0.0193 & $9.23 \mathrm{E}-05$ & 21.4390 & 7.8997 & 29.3387 & \\
\hline & 8/5/2008 8:00 & 331 & 218 & 800 & 9.18 & 9.18 & & 0.0349 & $1.91 \mathrm{E}-04$ & 44.3502 & 16.3418 & 60.6920 & \\
\hline & 8/5/2008 9:00 & 332 & 218 & 900 & 7.718 & 7.718 & & 0.2309 & 1.96E-03 & 454.6368 & 167.5203 & 622.1571 & \\
\hline & 8/5/2008 10:00 & 333 & 218 & 1000 & 6.282 & 6.282 & & 0.5123 & $5.22 \mathrm{E}-03$ & 1212.5383 & 446.7847 & 1659.3230 & \\
\hline & 8/5/2008 11:00 & 334 & 218 & 1100 & 6.505 & 6.505 & & 0.4604 & $4.58 \mathrm{E}-03$ & 1063.2923 & 391.7919 & 1455.0843 & \\
\hline & 8/5/2008 12:00 & 335 & 218 & 1200 & 6.175 & 6.175 & & 0.5385 & 5.55E-03 & 1289.3387 & 475.0834 & 1764.4221 & \\
\hline & 8/5/2008 13:00 & 336 & 218 & 1300 & 5.784 & 5.784 & & 0.6425 & $6.90 \mathrm{E}-03$ & 1602.4179 & 590.4438 & 2192.8617 & \\
\hline & 8/5/2008 14:00 & 337 & 218 & 1400 & 6.306 & 6.306 & & 0.5065 & 5.15E-03 & 1195.7896 & 440.6133 & 1636.4028 & \\
\hline & 8/5/2008 15:00 & 338 & 218 & 1500 & 6.766 & 6.766 & & 0.4041 & 3.90E-03 & 905.4738 & 333.6404 & 1239.1143 & \\
\hline & 8/5/2008 16:00 & 339 & 218 & 1600 & 6.633 & 6.633 & & 0.4322 & $4.24 \mathrm{E}-03$ & 983.7485 & 362.4824 & 1346.2309 & \\
\hline & 8/5/2008 17:00 & 340 & 218 & 1700 & 6.102 & 6.102 & & 0.5569 & $5.79 \mathrm{E}-03$ & 1343.8021 & 495.1515 & 1838.9536 & \\
\hline & 8/5/2008 18:00 & 341 & 218 & 1800 & 5.905 & 5.905 & & 0.6088 & 6.46E-03 & 1499.7168 & 552.6015 & 2052.3183 & \\
\hline & 8/5/2008 19:00 & 342 & 218 & 1900 & 6.085 & 6.085 & & 0.5612 & 5.84E-03 & 1356.7343 & 499.9166 & 1856.6509 & \\
\hline & 8/5/2008 20:00 & 343 & 218 & 2000 & 6.984 & 6.984 & & 0.3603 & 3.39E-03 & 786.1367 & 289.6683 & 1075.8050 & \\
\hline & 8/5/2008 21:00 & 344 & 218 & 2100 & 8.69 & 8.69 & & 0.0932 & 6.41E-04 & 148.8426 & 54.8441 & 203.6868 & \\
\hline & $8 / 5 / 200822: 00$ & 345 & 218 & 2200 & 9.35 & 9.35 & & 0.0160 & 7.35E-05 & 17.0614 & 6.2866 & 23.3480 & \\
\hline & 8/5/2008 23:00 & 346 & 218 & 2300 & 9.51 & 9.51 & & -0.0011 & $0.00 \mathrm{E}+00$ & 0.0000 & 0.0000 & 0.0000 & \\
\hline & 8/6/2008 0:00 & 347 & 219 & 0 & 9.58 & 9.58 & 9.58 & -0.0084 & $0.00 E+00$ & 0.0000 & 0.0000 & 0.0000 & \\
\hline & 8/6/2008 1:00 & 348 & 219 & 100 & 9.56 & 9.56 & & -0.0063 & $0.00 \mathrm{E}+00$ & 0.0000 & 0.0000 & 0.0000 & \\
\hline & $8 / 6 / 2008$ 2:00 & 349 & 219 & 200 & 9.58 & 9.58 & & -0.0084 & $0.00 E+00$ & 0.0000 & 0.0000 & 0.0000 & \\
\hline & 8/6/2008 3:00 & 350 & 219 & 300 & 9.55 & 9.55 & & -0.0052 & $0.00 E+00$ & 0.0000 & 0.0000 & 0.0000 & \\
\hline & 8/6/2008 4:00 & 351 & 219 & 400 & 9.51 & 9.51 & & -0.0011 & $0.00 \mathrm{E}+00$ & 0.0000 & 0.0000 & 0.0000 & \\
\hline & 8/6/2008 5:00 & 352 & 219 & 500 & 9.46 & 9.46 & & 0.0042 & 1.42E-05 & 3.3047 & 1.2177 & 4.5224 & \\
\hline & 8/6/2008 6:00 & 353 & 219 & 600 & 9.41 & 9.41 & & 0.0096 & 3.89E-05 & 9.0261 & 3.3258 & 12.3519 & \\
\hline & 8/6/2008 7:00 & 354 & 219 & 700 & 9.37 & 9.37 & & 0.0139 & 6.15E-05 & 14.2682 & 5.2574 & 19.5256 & \\
\hline & 8/6/2008 8:00 & 355 & 219 & 800 & 9.32 & 9.32 & & 0.0193 & 9.23E-05 & 21.4390 & 7.8997 & 29.3387 & \\
\hline & 8/6/2008 9:00 & 356 & 219 & 900 & 8.85 & 8.85 & & 0.0734 & 4.78E-04 & 111.0005 & 40.9004 & 151.9009 & \\
\hline & 8/6/2008 10:00 & 357 & 219 & 1000 & 6.351 & 6.351 & & 0.4958 & $5.02 E-03$ & 1164.8433 & 429.2105 & 1594.0538 & \\
\hline & 8/6/2008 11:00 & 358 & 219 & 1100 & 5.569 & 5.569 & & 0.7059 & 7.75E-03 & 1799.2732 & 662.9792 & 2462.2524 & \\
\hline & 8/6/2008 12:00 & 359 & 219 & 1200 & 6.172 & 6.172 & & 0.5392 & $5.56 \mathrm{E}-03$ & 1291.5433 & 475.8957 & 1767.4390 & \\
\hline & 8/6/2008 13:00 & 360 & 219 & 1300 & 6.585 & 6.585 & & 0.4427 & $4.36 \mathrm{E}-03$ & 1013.0795 & 373.2900 & 1386.3694 & \\
\hline & $8 / 6 / 2008$ 14:00 & 361 & 219 & 1400 & 6.092 & 6.092 & & 0.5594 & $5.82 \mathrm{E}-03$ & 1351.3977 & 497.9503 & 1849.3480 & \\
\hline & 8/6/2008 15:00 & 362 & 219 & 1500 & 5.749 & 5.749 & & 0.6525 & 7.03E-03 & 1633.1742 & 601.7766 & 2234.9509 & \\
\hline & 8/6/2008 16:00 & 363 & 219 & 1600 & 5.836 & 5.836 & & 0.6278 & 6.71E-03 & 1557.6034 & 573.9310 & 2131.5344 & \\
\hline & 8/6/2008 17:00 & 364 & 219 & 1700 & 5.753 & 5.753 & & 0.6513 & 7.02E-03 & 1629.6347 & 600.4724 & 2230.1071 & \\
\hline & 8/6/2008 18:00 & 365 & 219 & 1800 & 6.22 & 6.22 & & 0.5273 & 5.41E-03 & 1256.6095 & 463.0236 & 1719.6332 & \\
\hline & 8/6/2008 19:00 & 366 & 219 & 1900 & 6.272 & 6.272 & & 0.5147 & $5.25 \mathrm{E}-03$ & 1219.5678 & 449.3748 & 1668.9426 & \\
\hline & $8 / 6 / 200820: 00$ & 367 & 219 & 2000 & 6.713 & 6.713 & & 0.4152 & 4.03E-03 & 936.1487 & 344.9432 & 1281.0919 & \\
\hline & 8/6/2008 21:00 & 368 & 219 & 2100 & 8.16 & 8.16 & & 0.1642 & 1.29E-03 & 298.8760 & 110.1270 & 409.0030 & \\
\hline
\end{tabular}




\begin{tabular}{|c|c|c|c|c|c|c|c|c|c|c|c|c|c|}
\hline Tree ID & TS & RN & JD & HR & $\begin{array}{c}\text { Raw } \\
\text { TC_dTA }\end{array}$ & $\begin{array}{c}\text { Fixed } \\
\text { TC_dT } \Delta\end{array}$ & dTM & $\mathbf{K}$ & $\begin{array}{l}\text { Sap } \\
\text { Velocity } \\
(\mathrm{cm} / \mathrm{s})\end{array}$ & $\begin{array}{c}\text { Probed } \\
\text { Drywood F } \\
(\mathrm{g} / \mathrm{hr})\end{array}$ & $\begin{array}{c}\text { Extrapolated } \\
\text { Wetwood F } \\
(\mathrm{g} / \mathrm{hr})\end{array}$ & $\begin{array}{c}\text { Extrapolated } \\
\text { Total Wood F } \\
(\mathrm{g} / \mathrm{hr})\end{array}$ & $\begin{array}{l}\text { Relative } \\
\text { Humidity, } \\
@ 10 \mathrm{~m}(\%)\end{array}$ \\
\hline & 8/6/2008 22:00 & 369 & 219 & 2200 & $\begin{array}{r}9.15 \\
\end{array}$ & 9.15 & & 0.0383 & $2.14 \mathrm{E}-04$ & 49.7226 & 18.3213 & $\begin{array}{r}68.0439 \\
\end{array}$ & \\
\hline & 8/6/2008 23:00 & 370 & 219 & 2300 & 9.33 & 9.33 & & 0.0182 & $8.60 \mathrm{E}-05$ & 19.9560 & 7.3532 & 27.3092 & \\
\hline & $8 / 7 / 20080: 00$ & 371 & 220 & 0 & 9.42 & 9.42 & 9.44 & 0.0085 & $3.36 \mathrm{E}-05$ & 7.7976 & 2.8732 & 10.6708 & \\
\hline & 8/7/2008 1:00 & 372 & 220 & 100 & 9.44 & 9.44 & & 0.0064 & 2.35E-05 & 5.4579 & 2.0111 & 7.4690 & \\
\hline & 8/7/2008 2:00 & 373 & 220 & 200 & 9.38 & 9.38 & & 0.0128 & 5.56E-05 & 12.9124 & 4.7578 & 17.6702 & \\
\hline & 8/7/2008 3:00 & 374 & 220 & 300 & 9.33 & 9.33 & & 0.0182 & 8.60E-05 & 19.9560 & 7.3532 & 27.3092 & \\
\hline & 8/7/2008 4:00 & 375 & 220 & 400 & 9.27 & 9.27 & & 0.0248 & 1.26E-04 & 29.1828 & 10.7530 & 39.9358 & \\
\hline & 8/7/2008 5:00 & 376 & 220 & 500 & 9.22 & 9.22 & & 0.0304 & 1.61E-04 & 37.4269 & 13.7907 & 51.2176 & \\
\hline & 8/7/2008 6:00 & 377 & 220 & 600 & 9.17 & 9.17 & & 0.0360 & 1.99E-04 & 46.1243 & 16.9954 & 63.1197 & \\
\hline & 8/7/2008 7:00 & 378 & 220 & 700 & 9.13 & 9.13 & & 0.0405 & 2.30E-04 & 53.3866 & 19.6714 & 73.0580 & \\
\hline & 8/7/2008 8:00 & 379 & 220 & 800 & 9.1 & 9.1 & & 0.0440 & 2.54E-04 & 59.0026 & 21.7407 & 80.7434 & \\
\hline & 8/7/2008 9:00 & 380 & 220 & 900 & 7.901 & 7.901 & & 0.2024 & 1.67E-03 & 386.5507 & 142.4326 & 528.9832 & \\
\hline & 8/7/2008 10:00 & 381 & 220 & 1000 & 5.78 & 5.78 & & 0.6436 & $6.92 \mathrm{E}-03$ & 1605.9085 & 591.7300 & 2197.6385 & \\
\hline & 8/7/2008 11:00 & 382 & 220 & 1100 & 5.318 & 5.318 & & 0.7864 & 8.85E-03 & 2055.1482 & 757.2616 & 2812.4099 & \\
\hline & 8/7/2008 12:00 & 383 & 220 & 1200 & 5.411 & 5.411 & & 0.7557 & 8.43E-03 & 1956.8234 & 721.0318 & 2677.8552 & \\
\hline & 8/7/2008 13:00 & 384 & 220 & 1300 & 5.432 & 5.432 & & 0.7489 & 8.34E-03 & 1935.2100 & 713.0679 & 2648.2779 & \\
\hline & $8 / 7 / 2008$ 14:00 & 385 & 220 & 1400 & 5.488 & 5.488 & & 0.7310 & 8.09E-03 & 1878.5993 & 692.2085 & 2570.8078 & \\
\hline & 8/7/2008 15:00 & 386 & 220 & 1500 & 5.562 & 5.562 & & 0.7080 & 7.78E-03 & 1806.0122 & 665.4623 & 2471.4745 & \\
\hline & 8/7/2008 16:00 & 387 & 220 & 1600 & 5.579 & 5.579 & & 0.7028 & 7.71E-03 & 1789.6835 & 659.4457 & 2449.1293 & \\
\hline & 8/7/2008 17:00 & 388 & 220 & 1700 & 5.555 & 5.555 & & 0.7102 & 7.81E-03 & 1812.7729 & 667.9535 & 2480.7263 & \\
\hline & 8/7/2008 18:00 & 389 & 220 & 1800 & 5.687 & 5.687 & & 0.6705 & 7.27E-03 & 1688.8606 & 622.2954 & 2311.1560 & \\
\hline & 8/7/2008 19:00 & 390 & 220 & 1900 & 5.94 & 5.94 & & 0.5993 & 6.34E-03 & 1471.0229 & 542.0287 & 2013.0516 & \\
\hline & 8/7/2008 20:00 & 391 & 220 & 2000 & 6.578 & 6.578 & & 0.4442 & 4.38E-03 & 1017.4062 & 374.8843 & 1392.2905 & \\
\hline & 8/7/2008 21:00 & 392 & 220 & 2100 & 8.07 & 8.07 & & 0.1772 & 1.41E-03 & 328.2260 & 120.9416 & 449.1676 & \\
\hline & 8/7/2008 22:00 & 393 & 220 & 2200 & 8.72 & 8.72 & & 0.0894 & 6.09E-04 & 141.4843 & 52.1328 & 193.6171 & \\
\hline & 8/7/2008 23:00 & 394 & 220 & 2300 & 9.03 & 9.03 & & 0.0520 & 3.13E-04 & 72.6468 & 26.7682 & 99.4150 & \\
\hline & 8/8/2008 0:00 & 395 & 221 & 0 & 9.14 & 9.14 & 9.24 & 0.0394 & $2.22 \mathrm{E}-04$ & 51.5465 & 18.9934 & 70.5399 & \\
\hline & 8/8/2008 1:00 & 396 & 221 & 100 & 9.1 & 9.1 & & 0.0440 & $2.54 \mathrm{E}-04$ & 59.0026 & 21.7407 & 80.7434 & \\
\hline & 8/8/2008 2:00 & 397 & 221 & 200 & 9.23 & 9.23 & & 0.0293 & 1.54E-04 & 35.7405 & 13.1693 & 48.9099 & \\
\hline & 8/8/2008 3:00 & 398 & 221 & 300 & 9.24 & 9.24 & & 0.0281 & 1.47E-04 & 34.0726 & 12.5548 & 46.6274 & \\
\hline & 8/8/2008 4:00 & 399 & 221 & 400 & 9.14 & 9.14 & & 0.0394 & 2.22E-04 & 51.5465 & 18.9934 & 70.5399 & \\
\hline & 8/8/2008 5:00 & 400 & 221 & 500 & 9.02 & 9.02 & & 0.0532 & 3.22E-04 & 74.6559 & 27.5085 & 102.1644 & \\
\hline & 8/8/2008 6:00 & 401 & 221 & 600 & 8.93 & 8.93 & & 0.0638 & 4.02E-04 & 93.3898 & 34.4114 & 127.8012 & \\
\hline & $8 / 8 / 20087: 00$ & 402 & 221 & 700 & 8.85 & 8.85 & & 0.0734 & $4.78 \mathrm{E}-04$ & 111.0005 & 40.9004 & 151.9009 & \\
\hline & 8/8/2008 8:00 & 403 & 221 & 800 & 8.59 & 8.59 & & 0.1059 & 7.51E-04 & 174.2409 & 64.2026 & 238.4435 & \\
\hline & 8/8/2008 9:00 & 404 & 221 & 900 & 6.464 & 6.464 & & 0.4697 & 4.69E-03 & 1089.6874 & 401.5177 & 1491.2051 & \\
\hline & 8/8/2008 10:00 & 405 & 221 & 1000 & 5.223 & 5.223 & & 0.8189 & $9.31 \mathrm{E}-03$ & 2160.1727 & 795.9601 & 2956.1328 & \\
\hline & 8/8/2008 11:00 & 406 & 221 & 1100 & 5.255 & 5.255 & & 0.8078 & $9.15 E-03$ & 2124.2618 & 782.7279 & 2906.9897 & \\
\hline & $8 / 8 / 200812: 00$ & 407 & 221 & 1200 & 5.235 & 5.235 & & 0.8147 & $9.25 \mathrm{E}-03$ & 2146.6414 & 790.9742 & 2937.6156 & \\
\hline & 8/8/2008 13:00 & 408 & 221 & 1300 & 5.267 & 5.267 & & 0.8037 & 9.09E-03 & 2110.9366 & 777.8180 & 2888.7546 & \\
\hline & 8/8/2008 14:00 & 409 & 221 & 1400 & 5.299 & 5.299 & & 0.7928 & 8.94E-03 & 2075.7739 & 764.8616 & 2840.6355 & \\
\hline
\end{tabular}




\begin{tabular}{|c|c|c|c|c|c|c|c|c|c|c|c|c|c|}
\hline Tree ID & TS & RN & JD & HR & $\begin{array}{c}\text { Raw } \\
\text { TC_dTA }\end{array}$ & $\begin{array}{c}\text { Fixed } \\
\text { TC_dT } \Delta\end{array}$ & dTM & $\mathbf{K}$ & $\begin{array}{l}\text { Sap } \\
\text { Velocity } \\
(\mathrm{cm} / \mathrm{s})\end{array}$ & $\begin{array}{c}\text { Probed } \\
\text { Drywood F } \\
(\mathrm{g} / \mathrm{hr})\end{array}$ & $\begin{array}{c}\text { Extrapolated } \\
\text { Wetwood F } \\
(\mathrm{g} / \mathrm{hr})\end{array}$ & $\begin{array}{c}\text { Extrapolated } \\
\text { Total Wood F } \\
(\mathrm{g} / \mathrm{hr})\end{array}$ & $\begin{array}{l}\text { Relative } \\
\text { Humidity, } \\
@ 10 \mathrm{~m}(\%)\end{array}$ \\
\hline & 8/8/2008 15:00 & 410 & 221 & 1500 & 5.354 & 5.354 & & 0.7744 & $8.69 \mathrm{E}-03$ & 2016.5742 & 743.0482 & 2759.6225 & \\
\hline & 8/8/2008 16:00 & 411 & 221 & 1600 & 5.401 & 5.401 & & 0.7589 & $8.47 \mathrm{E}-03$ & 1967.1905 & 724.8518 & 2692.0423 & \\
\hline & 8/8/2008 17:00 & 412 & 221 & 1700 & 5.556 & 5.556 & & 0.7099 & $7.80 \mathrm{E}-03$ & 1811.8057 & 667.5971 & 2479.4028 & \\
\hline & 8/8/2008 18:00 & 413 & 221 & 1800 & 5.813 & 5.813 & & 0.6343 & $6.79 \mathrm{E}-03$ & 1577.2969 & 581.1875 & 2158.4844 & \\
\hline & 8/8/2008 19:00 & 414 & 221 & 1900 & 5.808 & 5.808 & & 0.6357 & 6.81E-03 & 1581.6049 & 582.7749 & 2164.3798 & \\
\hline & 8/8/2008 20:00 & 415 & 221 & 2000 & 6.56 & 6.56 & & 0.4482 & 4.43E-03 & 1028.5906 & 379.0054 & 1407.5960 & \\
\hline & 8/8/2008 21:00 & 416 & 221 & 2100 & 7.849 & 7.849 & & 0.2103 & $1.75 E-03$ & 405.3646 & 149.3649 & 554.7295 & \\
\hline & 8/8/2008 22:00 & 417 & 221 & 2200 & 8.44 & 8.44 & & 0.1256 & 9.25E-04 & 214.8521 & 79.1667 & 294.0188 & \\
\hline & 8/8/2008 23:00 & 418 & 221 & 2300 & 8.57 & 8.57 & & 0.1085 & 7.73E-04 & 179.4811 & 66.1335 & 245.6146 & \\
\hline & 8/9/2008 0:00 & 419 & 222 & 0 & 8.55 & 8.55 & 9.13 & 0.1111 & 7.96E-04 & 184.7749 & 68.0841 & 252.8591 & \\
\hline & 8/9/2008 1:00 & 420 & 222 & 100 & 8.52 & 8.52 & & 0.1150 & 8.31E-04 & 192.8163 & 71.0471 & 263.8634 & \\
\hline & 8/9/2008 2:00 & 421 & 222 & 200 & 8.54 & 8.54 & & 0.1124 & 8.07E-04 & 187.4420 & 69.0669 & 256.5088 & \\
\hline & 8/9/2008 3:00 & 422 & 222 & 300 & 8.52 & 8.52 & & 0.1150 & 8.31E-04 & 192.8163 & 71.0471 & 263.8634 & \\
\hline & 8/9/2008 4:00 & 423 & 222 & 400 & 8.5 & 8.5 & & 0.1176 & 8.54E-04 & 198.2444 & 73.0472 & 271.2916 & \\
\hline & $8 / 9 / 20085: 00$ & 424 & 222 & 500 & 8.36 & 8.36 & & 0.1364 & 1.02E-03 & 237.7549 & 87.6057 & 325.3606 & \\
\hline & 8/9/2008 6:00 & 425 & 222 & 600 & 8.35 & 8.35 & & 0.1377 & 1.04E-03 & 240.6792 & 88.6832 & 329.3624 & \\
\hline & 8/9/2008 7:00 & 426 & 222 & 700 & 7.968 & 7.968 & & 0.1923 & 1.56E-03 & 362.9177 & 133.7245 & 496.6422 & \\
\hline & 8/9/2008 8:00 & 427 & 222 & 800 & 7.264 & 7.264 & & 0.3078 & $2.79 \mathrm{E}-03$ & 647.7508 & 238.6771 & 886.4279 & \\
\hline & 8/9/2008 9:00 & 428 & 222 & 900 & 7.863 & 7.863 & & 0.2082 & 1.72E-03 & 400.2583 & 147.4834 & 547.7417 & \\
\hline & 8/9/2008 10:00 & 429 & 222 & 1000 & 7.448 & 7.448 & & 0.2755 & $2.43 E-03$ & 565.0997 & 208.2226 & 773.3224 & \\
\hline & 8/9/2008 11:00 & 430 & 222 & 1100 & 6.82 & 6.82 & & 0.3930 & $3.77 \mathrm{E}-03$ & 874.9052 & 322.3768 & 1197.2820 & \\
\hline & 8/9/2008 12:00 & 431 & 222 & 1200 & 6.069 & 6.069 & & 0.5653 & 5.90E-03 & 1368.9933 & 504.4337 & 1873.4270 & \\
\hline & 8/9/2008 13:00 & 432 & 222 & 1300 & 5.873 & 5.873 & & 0.6176 & 6.57E-03 & 1526.3425 & 562.4123 & 2088.7548 & \\
\hline & 8/9/2008 14:00 & 433 & 222 & 1400 & 5.464 & 5.464 & & 0.7387 & $8.20 \mathrm{E}-03$ & 1902.6803 & 701.0817 & 2603.7620 & \\
\hline & 8/9/2008 15:00 & 434 & 222 & 1500 & 5.42 & 5.42 & & 0.7528 & 8.39E-03 & 1947.5345 & 717.6091 & 2665.1436 & \\
\hline & 8/9/2008 16:00 & 435 & 222 & 1600 & 5.521 & 5.521 & & 0.7207 & 7.95E-03 & 1845.9226 & 680.1681 & 2526.0907 & \\
\hline & 8/9/2008 17:00 & 436 & 222 & 1700 & 5.703 & 5.703 & & 0.6658 & 7.21E-03 & 1674.3403 & 616.9451 & 2291.2855 & \\
\hline & 8/9/2008 18:00 & 437 & 222 & 1800 & 5.757 & 5.757 & & 0.6502 & 7.00E-03 & 1626.1015 & 599.1706 & 2225.2721 & \\
\hline & 8/9/2008 19:00 & 438 & 222 & 1900 & 5.993 & 5.993 & & 0.5852 & $6.15 E-03$ & 1428.4052 & 526.3253 & 1954.7305 & \\
\hline & 8/9/2008 20:00 & 439 & 222 & 2000 & 6.657 & 6.657 & & 0.4271 & $4.18 E-03$ & 969.3018 & 357.1592 & 1326.4610 & \\
\hline & 8/9/2008 21:00 & 440 & 222 & 2100 & 8.38 & 8.38 & & 0.1337 & 9.99E-04 & 231.9475 & 85.4658 & 317.4133 & \\
\hline & 8/9/2008 22:00 & 441 & 222 & 2200 & 9.07 & 9.07 & & 0.0474 & 2.79E-04 & 64.7591 & 23.8618 & 88.6209 & \\
\hline & 8/9/2008 23:00 & 442 & 222 & 2300 & 9.13 & 9.13 & & 0.0405 & 2.30E-04 & 53.3866 & 19.6714 & 73.0580 & \\
\hline & 8/10/2008 0:00 & 443 & 223 & 0 & 9.1 & 9.1 & 9.10 & 0.0440 & $2.54 \mathrm{E}-04$ & 59.0026 & 21.7407 & 80.7434 & \\
\hline & 8/10/2008 1:00 & 444 & 223 & 100 & 9.07 & 9.07 & & 0.0474 & 2.79E-04 & 64.7591 & 23.8618 & 88.6209 & \\
\hline & 8/10/2008 2:00 & 445 & 223 & 200 & 9.01 & 9.01 & & 0.0544 & 3.30E-04 & 76.6798 & 28.2542 & 104.9340 & \\
\hline & 8/10/2008 3:00 & 446 & 223 & 300 & 9.02 & 9.02 & & 0.0532 & $3.22 \mathrm{E}-04$ & 74.6559 & 27.5085 & 102.1644 & \\
\hline & 8/10/2008 4:00 & 447 & 223 & 400 & 9.01 & 9.01 & & 0.0544 & 3.30E-04 & 76.6798 & 28.2542 & 104.9340 & \\
\hline & $8 / 10 / 20085: 00$ & 448 & 223 & 500 & 8.96 & 8.96 & & 0.0603 & 3.75E-04 & 87.0164 & 32.0630 & 119.0794 & \\
\hline & 8/10/2008 6:00 & 449 & 223 & 600 & 8.93 & 8.93 & & 0.0638 & 4.02E-04 & 93.3898 & 34.4114 & 127.8012 & \\
\hline & 8/10/2008 7:00 & 450 & 223 & 700 & 8.91 & 8.91 & & 0.0662 & $4.21 \mathrm{E}-04$ & 97.7091 & 36.0029 & 133.7120 & \\
\hline
\end{tabular}




\begin{tabular}{|c|c|c|c|c|c|c|c|c|c|c|c|c|c|}
\hline Tree ID & TS & RN & JD & HR & $\begin{array}{c}\text { Raw } \\
\text { TC_dTA }\end{array}$ & $\begin{array}{c}\text { Fixed } \\
\text { TC_dT } \Delta\end{array}$ & dTM & $\mathbf{K}$ & $\begin{array}{l}\text { Sap } \\
\text { Velocity } \\
(\mathrm{cm} / \mathrm{s})\end{array}$ & $\begin{array}{c}\text { Probed } \\
\text { Drywood F } \\
(\mathrm{g} / \mathrm{hr})\end{array}$ & $\begin{array}{c}\text { Extrapolated } \\
\text { Wetwood F } \\
(\mathrm{g} / \mathrm{hr})\end{array}$ & $\begin{array}{c}\text { Extrapolated } \\
\text { Total Wood F } \\
(\mathrm{g} / \mathrm{hr})\end{array}$ & $\begin{array}{l}\text { Relative } \\
\text { Humidity, } \\
@ 10 \mathrm{~m}(\%)\end{array}$ \\
\hline & 8/10/2008 8:00 & 451 & 223 & 800 & \begin{tabular}{|r|}
8.88 \\
\end{tabular} & 8.88 & & 0.0698 & 4.49E-04 & 104.2926 & 38.4287 & 142.7213 & \\
\hline & 8/10/2008 9:00 & 452 & 223 & 900 & 7.671 & 7.671 & & 0.2384 & $2.04 \mathrm{E}-03$ & 472.9856 & 174.2813 & 647.2668 & \\
\hline & $8 / 10 / 2008$ 10:00 & 453 & 223 & 1000 & 5.684 & 5.684 & & 0.6714 & 7.29E-03 & 1691.5948 & 623.3029 & 2314.8978 & \\
\hline & 8/10/2008 11:00 & 454 & 223 & 1100 & 5.215 & 5.215 & & 0.8217 & $9.34 \mathrm{E}-03$ & 2169.2371 & 799.3000 & 2968.5371 & \\
\hline & $8 / 10 / 200812: 00$ & 455 & 223 & 1200 & 5.354 & 5.354 & & 0.7744 & 8.69E-03 & 2016.5742 & 743.0482 & 2759.6225 & \\
\hline & 8/10/2008 13:00 & 456 & 223 & 1300 & 5.379 & 5.379 & & 0.7661 & 8.57E-03 & 1990.1703 & 733.3192 & 2723.4894 & \\
\hline & $8 / 10 / 2008$ 14:00 & 457 & 223 & 1400 & 5.39 & 5.39 & & 0.7625 & $8.52 E-03$ & 1978.6507 & 729.0745 & 2707.7252 & \\
\hline & $8 / 10 / 2008$ 15:00 & 458 & 223 & 1500 & 5.63 & 5.63 & & 0.6874 & 7.50E-03 & 1741.4539 & 641.6745 & 2383.1284 & \\
\hline & 8/10/2008 16:00 & 459 & 223 & 1600 & 5.508 & 5.508 & & 0.7248 & 8.01E-03 & 1858.7357 & 684.8894 & 2543.6250 & \\
\hline & 8/10/2008 17:00 & 460 & 223 & 1700 & 5.77 & 5.77 & & 0.6464 & 6.96E-03 & 1614.6625 & 594.9556 & 2209.6181 & \\
\hline & 8/10/2008 18:00 & 461 & 223 & 1800 & 8.38 & 8.38 & & 0.1337 & 9.99E-04 & 231.9475 & 85.4658 & 317.4133 & \\
\hline & $8 / 10 / 2008$ 19:00 & 462 & 223 & 1900 & 8.94 & 8.94 & & 0.0626 & 3.93E-04 & 91.2512 & 33.6234 & 124.8746 & \\
\hline & 8/10/2008 20:00 & 463 & 223 & 2000 & 8.74 & 8.74 & & 0.0870 & 5.89E-04 & 136.6459 & 50.3500 & 186.9959 & \\
\hline & 8/10/2008 21:00 & 464 & 223 & 2100 & 8.78 & 8.78 & & 0.0820 & 5.48E-04 & 127.1306 & 46.8439 & 173.9745 & \\
\hline & $8 / 10 / 2008$ 22:00 & 465 & 223 & 2200 & 8.88 & 8.88 & & 0.0698 & 4.49E-04 & 104.2926 & 38.4287 & 142.7213 & \\
\hline & $8 / 10 / 2008$ 23:00 & 466 & 223 & 2300 & 8.77 & 8.77 & & 0.0832 & $5.58 \mathrm{E}-04$ & 129.4892 & 47.7130 & 177.2022 & \\
\hline & $8 / 11 / 20080: 00$ & 467 & 224 & 0 & 8.74 & 8.74 & 9.21 & 0.0870 & 5.89E-04 & 136.6459 & 50.3500 & 186.9959 & \\
\hline & 8/11/2008 1:00 & 468 & 224 & 100 & 8.93 & 8.93 & & 0.0638 & 4.02E-04 & 93.3898 & 34.4114 & 127.8012 & \\
\hline & 8/11/2008 2:00 & 469 & 224 & 200 & 9.12 & 9.12 & & 0.0417 & 2.38E-04 & 55.2428 & 20.3553 & 75.5981 & \\
\hline & 8/11/2008 3:00 & 470 & 224 & 300 & 9.18 & 9.18 & & 0.0349 & 1.91E-04 & 44.3502 & 16.3418 & 60.6920 & \\
\hline & 8/11/2008 4:00 & 471 & 224 & 400 & 9.2 & 9.2 & & 0.0326 & $1.76 \mathrm{E}-04$ & 40.8535 & 15.0533 & 55.9068 & \\
\hline & 8/11/2008 5:00 & 472 & 224 & 500 & 9.17 & 9.17 & & 0.0360 & 1.99E-04 & 46.1243 & 16.9954 & 63.1197 & \\
\hline & 8/11/2008 6:00 & 473 & 224 & 600 & 9.19 & 9.19 & & 0.0337 & 1.83E-04 & 42.5932 & 15.6943 & 58.2876 & \\
\hline & 8/11/2008 7:00 & 474 & 224 & 700 & 9.21 & 9.21 & & 0.0315 & 1.69E-04 & 39.1313 & 14.4187 & 53.5500 & \\
\hline & 8/11/2008 8:00 & 475 & 224 & 800 & 9.18 & 9.18 & & 0.0349 & $1.91 \mathrm{E}-04$ & 44.3502 & 16.3418 & 60.6920 & \\
\hline & 8/11/2008 9:00 & 476 & 224 & 900 & 8.28 & 8.28 & & 0.1473 & 1.13E-03 & 261.5346 & 96.3678 & 357.9025 & \\
\hline & $8 / 11 / 2008$ 10:00 & 477 & 224 & 1000 & 6.301 & 6.301 & & 0.5077 & 5.17E-03 & 1199.2648 & 441.8938 & 1641.1585 & \\
\hline & 8/11/2008 11:00 & 478 & 224 & 1100 & 5.688 & 5.688 & & 0.6702 & $7.27 \mathrm{E}-03$ & 1687.9500 & 621.9599 & 2309.9099 & \\
\hline & $8 / 11 / 2008$ 12:00 & 479 & 224 & 1200 & 5.726 & 5.726 & & 0.6591 & 7.12E-03 & 1653.6506 & 609.3216 & 2262.9721 & \\
\hline & 8/11/2008 13:00 & 480 & 224 & 1300 & 5.952 & 5.952 & & 0.5961 & $6.29 \mathrm{E}-03$ & 1461.2865 & 538.4411 & 1999.7276 & \\
\hline & 8/11/2008 14:00 & 481 & 224 & 1400 & 5.69 & 5.69 & & 0.6696 & 7.26E-03 & 1686.1300 & 621.2893 & 2307.4193 & \\
\hline & 8/11/2008 15:00 & 482 & 224 & 1500 & 5.631 & 5.631 & & 0.6871 & 7.50E-03 & 1740.5194 & 641.3302 & 2381.8496 & \\
\hline & $8 / 11 / 2008$ 16:00 & 483 & 224 & 1600 & 5.877 & 5.877 & & 0.6165 & 6.56E-03 & 1522.9936 & 561.1783 & 2084.1719 & \\
\hline & $8 / 11 / 2008$ 17:00 & 484 & 224 & 1700 & 5.983 & 5.983 & & 0.5878 & $6.19 \mathrm{E}-03$ & 1436.3706 & 529.2603 & 1965.6309 & \\
\hline & 8/11/2008 18:00 & 485 & 224 & 1800 & 6.317 & 6.317 & & 0.5039 & 5.12E-03 & 1188.1701 & 437.8057 & 1625.9758 & \\
\hline & $8 / 11 / 2008$ 19:00 & 486 & 224 & 1900 & 6.472 & 6.472 & & 0.4679 & 4.67E-03 & 1084.5013 & 399.6068 & 1484.1081 & \\
\hline & 8/11/2008 20:00 & 487 & 224 & 2000 & 7.188 & 7.188 & & 0.3216 & $2.95 \mathrm{E}-03$ & 683.7543 & 251.9434 & 935.6977 & \\
\hline & 8/11/2008 21:00 & 488 & 224 & 2100 & 8.18 & 8.18 & & 0.1614 & 1.26E-03 & 292.5114 & 107.7818 & 400.2932 & \\
\hline & $8 / 11 / 200822: 00$ & 489 & 224 & 2200 & 8.72 & 8.72 & & 0.0894 & $6.09 \mathrm{E}-04$ & 141.4843 & 52.1328 & 193.6171 & \\
\hline & 8/11/2008 23:00 & 490 & 224 & 2300 & 8.95 & 8.95 & & 0.0615 & 3.84E-04 & 89.1267 & 32.8406 & 121.9673 & \\
\hline & 8/12/2008 0:00 & 491 & 225 & 0 & 9.08 & 9.08 & 9.29 & 0.0463 & $2.71 \mathrm{E}-04$ & 62.8249 & 23.1491 & 85.9740 & \\
\hline
\end{tabular}




\begin{tabular}{|c|c|c|c|c|c|c|c|c|c|c|c|c|c|}
\hline Tree ID & TS & RN & JD & HR & $\begin{array}{c}\text { Raw } \\
\text { TC_dTA }\end{array}$ & $\begin{array}{c}\text { Fixed } \\
\text { TC_dT } \Delta\end{array}$ & dTM & $\mathbf{K}$ & $\begin{array}{l}\text { Sap } \\
\text { Velocity } \\
(\mathrm{cm} / \mathrm{s})\end{array}$ & $\begin{array}{c}\text { Probed } \\
\text { Drywood F } \\
(\mathrm{g} / \mathrm{hr})\end{array}$ & $\begin{array}{c}\text { Extrapolated } \\
\text { Wetwood F } \\
(\mathrm{g} / \mathrm{hr})\end{array}$ & $\begin{array}{c}\text { Extrapolated } \\
\text { Total Wood F } \\
(\mathrm{g} / \mathrm{hr})\end{array}$ & $\begin{array}{l}\text { Relative } \\
\text { Humidity, } \\
@ 10 \mathrm{~m}(\%)\end{array}$ \\
\hline & 8/12/2008 1:00 & 492 & 225 & 100 & 9.01 & 9.01 & & 0.0544 & 3.30E-04 & 76.6798 & 28.2542 & 104.9340 & \\
\hline & 8/12/2008 2:00 & 493 & 225 & 200 & 8.28 & 8.28 & & 0.1473 & 1.13E-03 & 261.5346 & 96.3678 & 357.9025 & \\
\hline & 8/12/2008 3:00 & 494 & 225 & 300 & 8.4 & 8.4 & & 0.1310 & $9.74 \mathrm{E}-04$ & 226.1946 & 83.3460 & 309.5406 & \\
\hline & 8/12/2008 4:00 & 495 & 225 & 400 & 8.69 & 8.69 & & 0.0932 & $6.41 \mathrm{E}-04$ & 148.8426 & 54.8441 & 203.6868 & \\
\hline & 8/12/2008 5:00 & 496 & 225 & 500 & 8.92 & 8.92 & & 0.0650 & 4.12E-04 & 95.5424 & 35.2046 & 130.7470 & \\
\hline & 8/12/2008 6:00 & 497 & 225 & 600 & 9.09 & 9.09 & & 0.0451 & $2.62 \mathrm{E}-04$ & 60.9060 & 22.4421 & 83.3481 & \\
\hline & 8/12/2008 7:00 & 498 & 225 & 700 & 9.12 & 9.12 & & 0.0417 & $2.38 \mathrm{E}-04$ & 55.2428 & 20.3553 & 75.5981 & \\
\hline & 8/12/2008 8:00 & 499 & 225 & 800 & 9.07 & 9.07 & & 0.0474 & $2.79 E-04$ & 64.7591 & 23.8618 & 88.6209 & \\
\hline & 8/12/2008 9:00 & 500 & 225 & 900 & 8.2 & 8.2 & & 0.1585 & 1.23E-03 & 286.2036 & 105.4576 & 391.6611 & \\
\hline & $8 / 12 / 2008$ 10:00 & 501 & 225 & 1000 & 6.393 & 6.393 & & 0.4860 & 4.90E-03 & 1136.4889 & 418.7627 & 1555.2516 & \\
\hline & $8 / 12 / 2008$ 11:00 & 502 & 225 & 1100 & 5.736 & 5.736 & & 0.6562 & 7.08E-03 & 1644.7217 & 606.0316 & 2250.7533 & \\
\hline & $8 / 12 / 2008$ 12:00 & 503 & 225 & 1200 & 5.671 & 5.671 & & 0.6752 & 7.34E-03 & 1703.4864 & 627.6846 & 2331.1710 & \\
\hline & 8/12/2008 13:00 & 504 & 225 & 1300 & 5.609 & 5.609 & & 0.6937 & 7.59E-03 & 1761.1770 & 648.9419 & 2410.1189 & \\
\hline & $8 / 12 / 2008$ 14:00 & 505 & 225 & 1400 & 5.611 & 5.611 & & 0.6931 & 7.58E-03 & 1759.2905 & 648.2468 & 2407.5372 & \\
\hline & $8 / 12 / 2008$ 15:00 & 506 & 225 & 1500 & 5.631 & 5.631 & & 0.6871 & 7.50E-03 & 1740.5194 & 641.3302 & 2381.8496 & \\
\hline & $8 / 12 / 2008$ 16:00 & 507 & 225 & 1600 & 5.669 & 5.669 & & 0.6758 & 7.35E-03 & 1705.3221 & 628.3610 & 2333.6831 & \\
\hline & $8 / 12 / 2008$ 17:00 & 508 & 225 & 1700 & 5.725 & 5.725 & & 0.6594 & 7.13E-03 & 1654.5457 & 609.6514 & 2264.1970 & \\
\hline & $8 / 12 / 2008$ 18:00 & 509 & 225 & 1800 & 5.828 & 5.828 & & 0.6301 & $6.74 \mathrm{E}-03$ & 1564.4304 & 576.4466 & 2140.8770 & \\
\hline & $8 / 12 / 2008$ 19:00 & 510 & 225 & 1900 & 6.084 & 6.084 & & 0.5615 & 5.85E-03 & 1357.4980 & 500.1980 & 1857.6960 & \\
\hline & $8 / 12 / 200820: 00$ & 511 & 225 & 2000 & 6.756 & 6.756 & & 0.4062 & 3.93E-03 & 911.2100 & 335.7541 & 1246.9641 & \\
\hline & $8 / 12 / 2008$ 21:00 & 512 & 225 & 2100 & 8.47 & 8.47 & & 0.1216 & 8.89E-04 & 206.4875 & 76.0846 & 282.5721 & \\
\hline & $8 / 12 / 2008$ 22:00 & 513 & 225 & 2200 & 9.15 & 9.15 & & 0.0383 & 2.14E-04 & 49.7226 & 18.3213 & 68.0439 & \\
\hline & $8 / 12 / 2008$ 23:00 & 514 & 225 & 2300 & 9.29 & 9.29 & & 0.0226 & 1.12E-04 & 26.0220 & 9.5883 & 35.6103 & \\
\hline & 8/13/2008 0:00 & 515 & 226 & 0 & 9.37 & 9.37 & 9.42 & 0.0139 & $6.15 E-05$ & 14.2682 & 5.2574 & 19.5256 & \\
\hline & 8/13/2008 1:00 & 516 & 226 & 100 & 9.36 & 9.36 & & 0.0150 & $6.74 \mathrm{E}-05$ & 15.6516 & 5.7672 & 21.4187 & \\
\hline & 8/13/2008 2:00 & 517 & 226 & 200 & 9.37 & 9.37 & & 0.0139 & $6.15 E-05$ & 14.2682 & 5.2574 & 19.5256 & \\
\hline & 8/13/2008 3:00 & 518 & 226 & 300 & 9.42 & 9.42 & & 0.0085 & 3.36E-05 & 7.7976 & 2.8732 & 10.6708 & \\
\hline & 8/13/2008 4:00 & 519 & 226 & 400 & 9.4 & 9.4 & & 0.0106 & 4.43E-05 & 10.2895 & 3.7914 & 14.0809 & \\
\hline & 8/13/2008 5:00 & 520 & 226 & 500 & 9.4 & 9.4 & & 0.0106 & 4.43E-05 & 10.2895 & 3.7914 & 14.0809 & \\
\hline & 8/13/2008 6:00 & 521 & 226 & 600 & 9.42 & 9.42 & & 0.0085 & 3.36E-05 & 7.7976 & 2.8732 & 10.6708 & \\
\hline & $8 / 13 / 2008$ 7:00 & 522 & 226 & 700 & 9.4 & 9.4 & & 0.0106 & 4.43E-05 & 10.2895 & 3.7914 & 14.0809 & \\
\hline & 8/13/2008 8:00 & 523 & 226 & 800 & 9.09 & 9.09 & & 0.0451 & $2.62 \mathrm{E}-04$ & 60.9060 & 22.4421 & 83.3481 & \\
\hline & 8/13/2008 9:00 & 524 & 226 & 900 & 7.211 & 7.211 & & 0.3174 & $2.90 \mathrm{E}-03$ & 672.7398 & 247.8848 & 920.6246 & \\
\hline & $8 / 13 / 2008$ 10:00 & 525 & 226 & 1000 & 6.057 & 6.057 & & 0.5684 & $5.94 \mathrm{E}-03$ & 1378.2437 & 507.8422 & 1886.0859 & \\
\hline & 8/13/2008 11:00 & 526 & 226 & 1100 & 5.959 & 5.959 & & 0.5942 & 6.27E-03 & 1455.6306 & 536.3571 & 1991.9877 & \\
\hline & $8 / 13 / 2008$ 12:00 & 527 & 226 & 1200 & 5.64 & 5.64 & & 0.6844 & 7.46E-03 & 1732.1281 & 638.2382 & 2370.3663 & \\
\hline
\end{tabular}




\begin{tabular}{|c|c|c|c|c|c|c|c|c|c|c|c|c|c|}
\hline Tree ID & TS & RN & JD & HR & $\begin{array}{c}\text { Raw } \\
\text { TC_dTA }\end{array}$ & $\begin{array}{c}\text { Fixed } \\
\text { TC_dT } \Delta\end{array}$ & dTM & $\mathbf{K}$ & $\begin{array}{l}\text { Sap } \\
\text { Velocity } \\
(\mathrm{cm} / \mathrm{s})\end{array}$ & $\begin{array}{l}\text { Probed } \\
\text { Drywood F } \\
\text { (g/hr) }\end{array}$ & $\begin{array}{c}\text { Extrapolated } \\
\text { Wetwood F } \\
(\mathrm{g} / \mathrm{hr})\end{array}$ & $\begin{array}{c}\text { Extrapolated } \\
\text { Total Wood F } \\
\text { (g/hr) }\end{array}$ & $\begin{array}{l}\text { Relative } \\
\text { Humidity, } \\
@ 10 \mathrm{~m}(\%)\end{array}$ \\
\hline & 7/22/2008 13:00 & 0 & 204 & 1300 & 5.115 & 5.115 & 9.25 & 0.8084 & 9.16E-03 & 2007.5701 & 739.7305 & 2747.3005 & \\
\hline \multirow[t]{2}{*}{ W810EC1 } & $7 / 22 / 2008$ 14:00 & 1 & 204 & 1400 & 5.669 & 5.669 & & 0.6317 & $6.76 \mathrm{E}-03$ & 1481.8055 & 546.0017 & 2027.8072 & \\
\hline & $7 / 22 / 2008$ 15:00 & 2 & 204 & 1500 & 5.667 & 5.667 & & 0.6323 & $6.77 \mathrm{E}-03$ & 1483.4686 & 546.6145 & 2030.0831 & \\
\hline \multirow[t]{2}{*}{ Probe \# } & 7/22/2008 16:00 & 3 & 204 & 1600 & 5.635 & 5.635 & & 0.6415 & $6.89 \mathrm{E}-03$ & 1510.2861 & 556.4960 & 2066.7821 & \\
\hline & 7/22/2008 17:00 & 4 & 204 & 1700 & 5.612 & 5.612 & & 0.6483 & $6.98 \mathrm{E}-03$ & 1529.8064 & 563.6886 & 2093.4950 & \\
\hline \multirow[t]{4}{*}{17} & $7 / 22 / 2008$ 18:00 & 5 & 204 & 1800 & 5.707 & 5.707 & & 0.6208 & $6.62 \mathrm{E}-03$ & 1450.4946 & 534.4646 & 1984.9592 & \\
\hline & $7 / 22 / 2008$ 19:00 & 6 & 204 & 1900 & 5.83 & 5.83 & & 0.5866 & $6.17 \mathrm{E}-03$ & 1352.7778 & 498.4588 & 1851.2366 & \\
\hline & 7/22/2008 20:00 & 7 & 204 & 2000 & 6.074 & 6.074 & & 0.5229 & $5.36 \mathrm{E}-03$ & 1174.1829 & 432.6518 & 1606.8348 & \\
\hline & 7/22/2008 21:00 & 8 & 204 & 2100 & 7.364 & 7.364 & & 0.2561 & $2.22 \mathrm{E}-03$ & 487.7004 & 179.7032 & \begin{tabular}{|l|}
667.4036 \\
\end{tabular} & \\
\hline \multirow[t]{2}{*}{$\mathrm{DBH}(\mathrm{cm})$} & $7 / 22 / 2008$ 22:00 & 9 & 204 & 2200 & 8.81 & 8.81 & & 0.0499 & 2.97E-04 & 65.1933 & 24.0218 & 89.2152 & \\
\hline & 7/22/2008 23:00 & 10 & 204 & 2300 & 9.25 & 9.25 & & 0.0000 & $0.00 \mathrm{E}+00$ & 0.0000 & 0.0000 & 0.0000 & \\
\hline \multirow[t]{2}{*}{10.9} & 7/23/2008 0:00 & 11 & 205 & 0 & 9.56 & 9.56 & 9.79 & -0.0324 & $0.00 \mathrm{E}+00$ & 0.0000 & 0.0000 & 0.0000 & \\
\hline & 7/23/2008 1:00 & 12 & 205 & 100 & 9.7 & 9.7 & & -0.0464 & $0.00 \mathrm{E}+00$ & 0.0000 & 0.0000 & 0.0000 & \\
\hline Day Count & $7 / 23 / 20082: 00$ & 13 & 205 & 200 & 9.74 & 9.74 & & -0.0503 & $0.00 \mathrm{E}+00$ & 0.0000 & 0.0000 & 0.0000 & \\
\hline \multirow[t]{27}{*}{35} & 7/23/2008 3:00 & 14 & 205 & 300 & 9.79 & 9.79 & & -0.0552 & $0.00 \mathrm{E}+00$ & 0.0000 & 0.0000 & 0.0000 & \\
\hline & 7/23/2008 4:00 & 15 & 205 & 400 & 9.79 & 9.79 & & -0.0552 & $0.00 \mathrm{E}+00$ & 0.0000 & 0.0000 & 0.0000 & \\
\hline & 7/23/2008 5:00 & 16 & 205 & 500 & 9.74 & 9.74 & & -0.0503 & $0.00 E+00$ & 0.0000 & 0.0000 & 0.0000 & \\
\hline & 7/23/2008 6:00 & 17 & 205 & 600 & 9.6 & 9.6 & & -0.0365 & $0.00 \mathrm{E}+00$ & 0.0000 & 0.0000 & 0.0000 & \\
\hline & 7/23/2008 7:00 & 18 & 205 & 700 & 9.22 & 9.22 & & 0.0033 & 1.03E-05 & 2.2601 & 0.8328 & 3.0929 & \\
\hline & 7/23/2008 8:00 & 19 & 205 & 800 & 8.33 & 8.33 & & 0.1104 & 7.90E-04 & 173.1758 & 63.8102 & 236.9860 & \\
\hline & 7/23/2008 9:00 & 20 & 205 & 900 & 6.849 & 6.849 & & 0.3506 & $3.27 \mathrm{E}-03$ & 717.7679 & 264.4763 & 982.2443 & \\
\hline & 7/23/2008 10:00 & 21 & 205 & 1000 & 5.91 & 5.91 & & 0.5651 & 5.89E-03 & 1292.0700 & 476.0898 & 1768.1598 & \\
\hline & $7 / 23 / 2008$ 11:00 & 22 & 205 & 1100 & 5.78 & 5.78 & & 0.6003 & 6.35E-03 & 1391.8446 & 512.8538 & 1904.6984 & \\
\hline & $7 / 23 / 2008$ 12:00 & 23 & 205 & 1200 & 5.698 & 5.698 & & 0.6234 & $6.65 E-03$ & 1457.8613 & 537.1790 & 1995.0403 & \\
\hline & $7 / 23 / 2008$ 13:00 & 24 & 205 & 1300 & 5.647 & 5.647 & & 0.6380 & 6.84E-03 & 1500.1834 & 552.7734 & 2052.9568 & \\
\hline & $7 / 23 / 200814: 00$ & 25 & 205 & 1400 & 5.642 & 5.642 & & 0.6395 & $6.86 \mathrm{E}-03$ & 1504.3861 & 554.3220 & 2058.7081 & \\
\hline & 7/23/2008 15:00 & 26 & 205 & 1500 & 5.639 & 5.639 & & 0.6404 & $6.87 \mathrm{E}-03$ & 1506.9123 & 555.2528 & 2062.1652 & \\
\hline & 7/23/2008 16:00 & 27 & 205 & 1600 & 5.748 & 5.748 & & 0.6093 & $6.47 \mathrm{E}-03$ & 1417.3150 & 522.2388 & 1939.5538 & \\
\hline & 7/23/2008 17:00 & 28 & 205 & 1700 & 6.173 & 6.173 & & 0.4985 & $5.05 E-03$ & 1107.0376 & 407.9108 & 1514.9483 & \\
\hline & $7 / 23 / 2008$ 18:00 & 29 & 205 & 1800 & 6.827 & 6.827 & & 0.3549 & $3.32 \mathrm{E}-03$ & 728.7530 & 268.5240 & 997.2771 & \\
\hline & $7 / 23 / 2008$ 19:00 & 30 & 205 & 1900 & 6.745 & 6.745 & & 0.3714 & $3.52 \mathrm{E}-03$ & 770.6086 & 283.9466 & 1054.5552 & \\
\hline & $7 / 23 / 200820: 00$ & 31 & 205 & 2000 & 7.076 & 7.076 & & 0.3072 & $2.78 \mathrm{E}-03$ & 610.1773 & 224.8324 & 835.0096 & \\
\hline & $7 / 23 / 200821: 00$ & 32 & 205 & 2100 & 7.906 & 7.906 & & 0.1700 & 1.34E-03 & 294.4781 & 108.5065 & 402.9846 & \\
\hline & $7 / 23 / 200822: 00$ & 33 & 205 & 2200 & 8.53 & 8.53 & & 0.0844 & 5.67E-04 & 124.3817 & 45.8310 & 170.2127 & \\
\hline & $7 / 23 / 2008$ 23:00 & 34 & 205 & 2300 & 8.57 & 8.57 & & 0.0793 & 5.26E-04 & 115.2650 & 42.4718 & 157.7367 & \\
\hline & $7 / 24 / 20080: 00$ & 35 & 206 & 0 & 8.54 & 8.54 & 9.67 & 0.0831 & 5.57E-04 & 122.0823 & 44.9837 & 167.0660 & \\
\hline & 7/24/2008 1:00 & 36 & 206 & 100 & 8.63 & 8.63 & & 0.0718 & 4.65E-04 & 101.9960 & 37.5825 & 139.5785 & \\
\hline & 7/24/2008 2:00 & 37 & 206 & 200 & 8.76 & 8.76 & & 0.0559 & $3.42 \mathrm{E}-04$ & 74.9527 & 27.6179 & 102.5705 & \\
\hline & 7/24/2008 3:00 & 38 & 206 & 300 & 8.91 & 8.91 & & 0.0382 & $2.14 \mathrm{E}-04$ & 46.8090 & 17.2477 & 64.0567 & \\
\hline & 7/24/2008 4:00 & 39 & 206 & 400 & 9.07 & 9.07 & & 0.0198 & 9.55E-05 & 20.9317 & 7.7127 & 28.6444 & \\
\hline & 7/24/2008 5:00 & 40 & 206 & 500 & 9.21 & 9.21 & & 0.0043 & 1.47E-05 & 3.2249 & 1.1883 & 4.4131 & \\
\hline
\end{tabular}




\begin{tabular}{|c|c|c|c|c|c|c|c|c|c|c|c|c|c|}
\hline Tree ID & TS & RN & JD & HR & $\begin{array}{c}\text { Raw } \\
\text { TC_dTA }\end{array}$ & $\begin{array}{c}\text { Fixed } \\
\text { TC_dT } \Delta\end{array}$ & dTM & $\mathbf{K}$ & $\begin{array}{l}\text { Sap } \\
\text { Velocity } \\
(\mathrm{cm} / \mathrm{s})\end{array}$ & $\begin{array}{l}\text { Probed } \\
\text { Drywood F } \\
\text { (g/hr) }\end{array}$ & $\begin{array}{c}\text { Extrapolated } \\
\text { Wetwood F } \\
(\mathrm{g} / \mathrm{hr})\end{array}$ & $\begin{array}{c}\text { Extrapolated } \\
\text { Total Wood F } \\
(\mathrm{g} / \mathrm{hr})\end{array}$ & $\begin{array}{l}\text { Relative } \\
\text { Humidity, } \\
@ 10 \mathrm{~m}(\%)\end{array}$ \\
\hline & 7/24/2008 6:00 & 41 & 206 & 600 & $\begin{array}{l}9.29 \\
\end{array}$ & 9.29 & & -0.0043 & $0.00 E+00$ & 0.0000 & 0.0000 & 0.0000 & \\
\hline & 7/24/2008 7:00 & 42 & 206 & 700 & 9.28 & 9.28 & & -0.0032 & $0.00 \mathrm{E}+00$ & 0.0000 & 0.0000 & 0.0000 & \\
\hline & 7/24/2008 8:00 & 43 & 206 & 800 & 8.94 & 8.94 & & 0.0347 & $1.90 \mathrm{E}-04$ & 41.6052 & 15.3303 & 56.9356 & \\
\hline & 7/24/2008 9:00 & 44 & 206 & 900 & 8.39 & 8.39 & & 0.1025 & 7.21E-04 & 157.9774 & 58.2100 & 216.1874 & \\
\hline & $7 / 24 / 2008$ 10:00 & 45 & 206 & 1000 & 8.44 & 8.44 & & 0.0960 & $6.65 E-04$ & 145.6786 & 53.6783 & 199.3569 & \\
\hline & 7/24/2008 11:00 & 46 & 206 & 1100 & 6.631 & 6.631 & & 0.3950 & $3.79 E-03$ & 831.2647 & 306.2966 & 1137.5613 & \\
\hline & 7/24/2008 12:00 & 47 & 206 & 1200 & 5.817 & 5.817 & & 0.5902 & $6.22 \mathrm{E}-03$ & 1362.8506 & 502.1703 & 1865.0209 & \\
\hline & 7/24/2008 13:00 & 48 & 206 & 1300 & 6.039 & 6.039 & & 0.5317 & $5.47 \mathrm{E}-03$ & 1198.6285 & 441.6593 & 1640.2878 & \\
\hline & 7/24/2008 14:00 & 49 & 206 & 1400 & 5.984 & 5.984 & & 0.5458 & $5.65 \mathrm{E}-03$ & 1237.8146 & 456.0983 & 1693.9129 & \\
\hline & 7/24/2008 15:00 & 50 & 206 & 1500 & 5.701 & 5.701 & & 0.6225 & 6.64E-03 & 1455.4024 & 536.2729 & 1991.6753 & \\
\hline & 7/24/2008 16:00 & 51 & 206 & 1600 & 5.686 & 5.686 & & 0.6268 & $6.70 \mathrm{E}-03$ & 1467.7308 & 540.8156 & 2008.5463 & \\
\hline & 7/24/2008 17:00 & 52 & 206 & 1700 & 5.81 & 5.81 & & 0.5921 & $6.24 \mathrm{E}-03$ & 1368.2988 & 504.1778 & 1872.4767 & \\
\hline & $7 / 24 / 2008$ 18:00 & 53 & 206 & 1800 & 5.793 & 5.793 & & 0.5968 & $6.30 \mathrm{E}-03$ & 1381.6022 & 509.0798 & 1890.6820 & \\
\hline & 7/24/2008 19:00 & 54 & 206 & 1900 & 6.078 & 6.078 & & 0.5219 & $5.34 \mathrm{E}-03$ & 1171.4131 & 431.6312 & 1603.0443 & \\
\hline & $7 / 24 / 200820: 00$ & 55 & 206 & 2000 & 6.869 & 6.869 & & 0.3466 & $3.23 \mathrm{E}-03$ & 707.8695 & 260.8291 & 968.6986 & \\
\hline & $7 / 24 / 200821: 00$ & 56 & 206 & 2100 & 8.22 & 8.22 & & 0.1253 & 9.23E-04 & 202.2897 & 74.5378 & 276.8275 & \\
\hline & $7 / 24 / 2008$ 22:00 & 57 & 206 & 2200 & 9.35 & 9.35 & & -0.0107 & $0.00 E+00$ & 0.0000 & 0.0000 & 0.0000 & \\
\hline & $7 / 24 / 200823: 00$ & 58 & 206 & 2300 & 9.67 & 9.67 & & -0.0434 & $0.00 \mathrm{E}+00$ & 0.0000 & 0.0000 & 0.0000 & \\
\hline & 7/25/2008 0:00 & 59 & 207 & 0 & 9.76 & 9.76 & 9.76 & -0.0523 & $0.00 \mathrm{E}+00$ & 0.0000 & 0.0000 & 0.0000 & \\
\hline & 7/25/2008 1:00 & 60 & 207 & 100 & 9.76 & 9.76 & & -0.0523 & $0.00 \mathrm{E}+00$ & 0.0000 & 0.0000 & 0.0000 & \\
\hline & 7/25/2008 2:00 & 61 & 207 & 200 & 9.73 & 9.73 & & -0.0493 & $0.00 \mathrm{E}+00$ & 0.0000 & 0.0000 & 0.0000 & \\
\hline & 7/25/2008 3:00 & 62 & 207 & 300 & 9.69 & 9.69 & & -0.0454 & $0.00 \mathrm{E}+00$ & 0.0000 & 0.0000 & 0.0000 & \\
\hline & 7/25/2008 4:00 & 63 & 207 & 400 & 9.68 & 9.68 & & -0.0444 & $0.00 E+00$ & 0.0000 & 0.0000 & 0.0000 & \\
\hline & 7/25/2008 5:00 & 64 & 207 & 500 & 9.69 & 9.69 & & -0.0454 & $0.00 \mathrm{E}+00$ & 0.0000 & 0.0000 & 0.0000 & \\
\hline & 7/25/2008 6:00 & 65 & 207 & 600 & 9.73 & 9.73 & & -0.0493 & $0.00 \mathrm{E}+00$ & 0.0000 & 0.0000 & 0.0000 & \\
\hline & 7/25/2008 7:00 & 66 & 207 & 700 & 9.71 & 9.71 & & -0.0474 & $0.00 \mathrm{E}+00$ & 0.0000 & 0.0000 & 0.0000 & \\
\hline & 7/25/2008 8:00 & 67 & 207 & 800 & 9.7 & 9.7 & & -0.0464 & $0.00 \mathrm{E}+00$ & 0.0000 & 0.0000 & 0.0000 & \\
\hline & 7/25/2008 9:00 & 68 & 207 & 900 & 9.65 & 9.65 & & -0.0415 & $0.00 \mathrm{E}+00$ & 0.0000 & 0.0000 & 0.0000 & \\
\hline & 7/25/2008 10:00 & 69 & 207 & 1000 & 9.53 & 9.53 & & -0.0294 & $0.00 \mathrm{E}+00$ & 0.0000 & 0.0000 & 0.0000 & \\
\hline & 7/25/2008 11:00 & 70 & 207 & 1100 & 8.72 & 8.72 & & 0.0608 & 3.79E-04 & 83.0207 & 30.5907 & 113.6113 & \\
\hline & $7 / 25 / 2008$ 12:00 & 71 & 207 & 1200 & 7.332 & 7.332 & & 0.2616 & $2.28 \mathrm{E}-03$ & 500.5830 & 184.4501 & 685.0331 & \\
\hline & $7 / 25 / 2008$ 13:00 & 72 & 207 & 1300 & 6.262 & 6.262 & & 0.4772 & 4.79E-03 & 1049.1028 & 386.5635 & 1435.6664 & \\
\hline & $7 / 25 / 200814: 00$ & 73 & 207 & 1400 & 5.825 & 5.825 & & 0.5880 & $6.19 E-03$ & 1356.6450 & 499.8837 & 1856.5287 & \\
\hline & $7 / 25 / 200815: 00$ & 74 & 207 & 1500 & 5.763 & 5.763 & & 0.6051 & $6.41 E-03$ & 1405.3297 & 517.8226 & 1923.1523 & \\
\hline & 7/25/2008 16:00 & 75 & 207 & 1600 & 5.834 & 5.834 & & 0.5855 & 6.16E-03 & 1349.6904 & 497.3212 & 1847.0116 & \\
\hline & $7 / 25 / 2008$ 17:00 & 76 & 207 & 1700 & 5.642 & 5.642 & & 0.6395 & 6.86E-03 & 1504.3861 & 554.3220 & 2058.7081 & \\
\hline & 7/25/2008 18:00 & 77 & 207 & 1800 & 5.719 & 5.719 & & 0.6174 & 6.57E-03 & 1440.7193 & 530.8627 & 1971.5820 & \\
\hline & 7/25/2008 19:00 & 78 & 207 & 1900 & 5.779 & 5.779 & & 0.6006 & $6.35 \mathrm{E}-03$ & 1392.6350 & 513.1450 & 1905.7800 & \\
\hline & $7 / 25 / 200820: 00$ & 79 & 207 & 2000 & 6.418 & 6.418 & & 0.4413 & 4.35E-03 & 952.7876 & 351.0742 & 1303.8617 & \\
\hline & $7 / 25 / 2008$ 21:00 & 80 & 207 & 2100 & 7.943 & 7.943 & & 0.1645 & 1.29E-03 & 282.8998 & 104.2402 & 387.1400 & \\
\hline & $7 / 25 / 200822: 00$ & 81 & 207 & 2200 & 9.32 & 9.32 & & -0.0075 & $0.00 \mathrm{E}+00$ & 0.0000 & 0.0000 & 0.0000 & \\
\hline
\end{tabular}




\begin{tabular}{|c|c|c|c|c|c|c|c|c|c|c|c|c|c|}
\hline Tree ID & TS & RN & JD & HR & $\begin{array}{c}\text { Raw } \\
\text { TC_dTA }\end{array}$ & $\begin{array}{c}\text { Fixed } \\
\text { TC_dT } \Delta\end{array}$ & dTM & K & $\begin{array}{l}\text { Sap } \\
\text { Velocity } \\
(\mathrm{cm} / \mathrm{s})\end{array}$ & $\begin{array}{c}\text { Probed } \\
\text { Drywood F } \\
(\mathrm{g} / \mathrm{hr})\end{array}$ & $\begin{array}{c}\text { Extrapolated } \\
\text { Wetwood F } \\
(\mathrm{g} / \mathrm{hr})\end{array}$ & $\begin{array}{c}\text { Extrapolated } \\
\text { Total Wood F } \\
(\mathrm{g} / \mathrm{hr})\end{array}$ & $\begin{array}{l}\text { Relative } \\
\text { Humidity, } \\
@ 10 \mathrm{~m}(\%)\end{array}$ \\
\hline & 7/25/2008 23:00 & 82 & 207 & 2300 & $\begin{array}{r}9.69 \\
\end{array}$ & 9.69 & & -0.0454 & $0.00 \mathrm{E}+00$ & 0.0000 & 0.0000 & 0.0000 & \\
\hline & 7/26/2008 0:00 & 83 & 208 & 0 & 9.82 & 9.82 & 9.90 & -0.0580 & $0.00 \mathrm{E}+00$ & 0.0000 & 0.0000 & 0.0000 & \\
\hline & 7/26/2008 1:00 & 84 & 208 & 100 & 9.87 & 9.87 & & -0.0628 & $0.00 E+00$ & 0.0000 & 0.0000 & 0.0000 & \\
\hline & $7 / 26 / 20082: 00$ & 85 & 208 & 200 & 9.9 & 9.9 & & -0.0657 & $0.00 \mathrm{E}+00$ & 0.0000 & 0.0000 & 0.0000 & \\
\hline & 7/26/2008 3:00 & 86 & 208 & 300 & 9.9 & 9.9 & & -0.0657 & $0.00 \mathrm{E}+00$ & 0.0000 & 0.0000 & 0.0000 & \\
\hline & 7/26/2008 4:00 & 87 & 208 & 400 & 9.9 & 9.9 & & -0.0657 & $0.00 \mathrm{E}+00$ & 0.0000 & 0.0000 & 0.0000 & \\
\hline & 7/26/2008 5:00 & 88 & 208 & 500 & 9.88 & 9.88 & & -0.0638 & $0.00 \mathrm{E}+00$ & 0.0000 & 0.0000 & 0.0000 & \\
\hline & 7/26/2008 6:00 & 89 & 208 & 600 & 9.84 & 9.84 & & -0.0600 & $0.00 \mathrm{E}+00$ & 0.0000 & 0.0000 & 0.0000 & \\
\hline & 7/26/2008 7:00 & 90 & 208 & 700 & 9.82 & 9.82 & & -0.0580 & $0.00 \mathrm{E}+00$ & 0.0000 & 0.0000 & 0.0000 & \\
\hline & 7/26/2008 8:00 & 91 & 208 & 800 & 9.76 & 9.76 & & -0.0523 & $0.00 E+00$ & 0.0000 & 0.0000 & 0.0000 & \\
\hline & 7/26/2008 9:00 & 92 & 208 & 900 & 8.37 & 8.37 & & 0.1051 & 7.44E-04 & 162.9902 & 60.0571 & 223.0473 & \\
\hline & $7 / 26 / 2008$ 10:00 & 93 & 208 & 1000 & 6.417 & 6.417 & & 0.4415 & 4.35E-03 & 953.3846 & 351.2942 & 1304.6787 & \\
\hline & 7/26/2008 11:00 & 94 & 208 & 1100 & 6.289 & 6.289 & & 0.4708 & $4.71 \mathrm{E}-03$ & 1031.9652 & 380.2488 & 1412.2141 & \\
\hline & $7 / 26 / 2008$ 12:00 & 95 & 208 & 1200 & 6.473 & 6.473 & & 0.4290 & $4.20 \mathrm{E}-03$ & 920.3420 & 339.1189 & 1259.4609 & \\
\hline & $7 / 26 / 2008$ 13:00 & 96 & 208 & 1300 & 5.92 & 5.92 & & 0.5625 & $5.86 \mathrm{E}-03$ & 1284.6333 & 473.3495 & 1757.9828 & \\
\hline & $7 / 26 / 200814: 00$ & 97 & 208 & 1400 & 5.798 & 5.798 & & 0.5954 & $6.29 \mathrm{E}-03$ & 1377.6789 & 507.6341 & 1885.3130 & \\
\hline & $7 / 26 / 2008$ 15:00 & 98 & 208 & 1500 & 5.707 & 5.707 & & 0.6208 & 6.62E-03 & 1450.4946 & 534.4646 & 1984.9592 & \\
\hline & $7 / 26 / 200816: 00$ & 99 & 208 & 1600 & 5.748 & 5.748 & & 0.6093 & 6.47E-03 & 1417.3150 & 522.2388 & 1939.5538 & \\
\hline & $7 / 26 / 200817: 00$ & 100 & 208 & 1700 & 5.633 & 5.633 & & 0.6421 & 6.90E-03 & 1511.9754 & 557.1184 & 2069.0938 & \\
\hline & $7 / 26 / 2008$ 18:00 & 101 & 208 & 1800 & 5.722 & 5.722 & & 0.6166 & $6.56 \mathrm{E}-03$ & 1438.2838 & 529.9653 & 1968.2491 & \\
\hline & $7 / 26 / 2008$ 19:00 & 102 & 208 & 1900 & 5.824 & 5.824 & & 0.5883 & $6.19 \mathrm{E}-03$ & 1357.4195 & 500.1691 & 1857.5886 & \\
\hline & $7 / 26 / 200820: 00$ & 103 & 208 & 2000 & 6.326 & 6.326 & & 0.4622 & 4.60E-03 & 1008.8032 & 371.7143 & 1380.5175 & \\
\hline & $7 / 26 / 200821: 00$ & 104 & 208 & 2100 & 7.902 & 7.902 & & 0.1706 & 1.35E-03 & 295.7415 & 108.9720 & 404.7136 & \\
\hline & $7 / 26 / 200822: 00$ & 105 & 208 & 2200 & 9.27 & 9.27 & & -0.0022 & $0.00 \mathrm{E}+00$ & 0.0000 & 0.0000 & 0.0000 & \\
\hline & $7 / 26 / 2008$ 23:00 & 106 & 208 & 2300 & 9.61 & 9.61 & & -0.0375 & $0.00 \mathrm{E}+00$ & 0.0000 & 0.0000 & 0.0000 & \\
\hline & $7 / 27 / 20080: 00$ & 107 & 209 & 0 & 9.73 & 9.73 & 9.87 & -0.0493 & $0.00 \mathrm{E}+00$ & 0.0000 & 0.0000 & 0.0000 & \\
\hline & 7/27/2008 1:00 & 108 & 209 & 100 & 9.76 & 9.76 & & -0.0523 & $0.00 \mathrm{E}+00$ & 0.0000 & 0.0000 & 0.0000 & \\
\hline & 7/27/2008 2:00 & 109 & 209 & 200 & 9.72 & 9.72 & & -0.0484 & $0.00 \mathrm{E}+00$ & 0.0000 & 0.0000 & 0.0000 & \\
\hline & 7/27/2008 3:00 & 110 & 209 & 300 & 9.76 & 9.76 & & -0.0523 & $0.00 \mathrm{E}+00$ & 0.0000 & 0.0000 & 0.0000 & \\
\hline & 7/27/2008 4:00 & 111 & 209 & 400 & 9.82 & 9.82 & & -0.0580 & $0.00 E+00$ & 0.0000 & 0.0000 & 0.0000 & \\
\hline & 7/27/20085:00 & 112 & 209 & 500 & 9.84 & 9.84 & & -0.0600 & $0.00 \mathrm{E}+00$ & 0.0000 & 0.0000 & 0.0000 & \\
\hline & 7/27/2008 6:00 & 113 & 209 & 600 & 9.87 & 9.87 & & -0.0628 & $0.00 \mathrm{E}+00$ & 0.0000 & 0.0000 & 0.0000 & \\
\hline & 7/27/2008 7:00 & 114 & 209 & 700 & 9.87 & 9.87 & & -0.0628 & $0.00 \mathrm{E}+00$ & 0.0000 & 0.0000 & 0.0000 & \\
\hline & 7/27/2008 8:00 & 115 & 209 & 800 & 9.71 & 9.71 & & -0.0474 & $0.00 \mathrm{E}+00$ & 0.0000 & 0.0000 & 0.0000 & \\
\hline & $7 / 27 / 20089: 00$ & 116 & 209 & 900 & 7.749 & 7.749 & & 0.1937 & 1.58E-03 & 345.8132 & 127.4220 & 473.2352 & \\
\hline & $7 / 27 / 2008$ 10:00 & 117 & 209 & 1000 & 5.885 & 5.885 & & 0.5718 & 5.98E-03 & 1310.8079 & 482.9941 & 1793.8020 & \\
\hline & $7 / 27 / 2008$ 11:00 & 118 & 209 & 1100 & 5.651 & 5.651 & & 0.6369 & 6.83E-03 & 1496.8281 & 551.5371 & 2048.3652 & \\
\hline & $7 / 27 / 2008$ 12:00 & 119 & 209 & 1200 & 5.521 & 5.521 & & 0.6754 & 7.34E-03 & 1609.1062 & 592.9083 & 2202.0145 & \\
\hline & $7 / 27 / 200813: 00$ & 120 & 209 & 1300 & 5.585 & 5.585 & & 0.6562 & 7.08E-03 & 1552.9872 & 572.2301 & 2125.2172 & \\
\hline & $7 / 27 / 200814: 00$ & 121 & 209 & 1400 & 5.428 & 5.428 & & 0.7041 & 7.73E-03 & 1693.7001 & 624.0786 & 2317.7788 & \\
\hline & 7/27/2008 15:00 & 122 & 209 & 1500 & 5.4 & 5.4 & & 0.7130 & $7.85 \mathrm{E}-03$ & 1719.9023 & 633.7334 & 2353.6356 & \\
\hline
\end{tabular}




\begin{tabular}{|c|c|c|c|c|c|c|c|c|c|c|c|c|c|}
\hline Tree ID & TS & RN & JD & HR & $\begin{array}{c}\text { Raw } \\
\text { TC_dTA }\end{array}$ & $\begin{array}{c}\text { Fixed } \\
\text { TC_dT } \Delta\end{array}$ & dTM & $\mathbf{K}$ & $\begin{array}{l}\text { Sap } \\
\text { Velocity } \\
(\mathrm{cm} / \mathrm{s})\end{array}$ & $\begin{array}{c}\text { Probed } \\
\text { Drywood F } \\
(\mathrm{g} / \mathrm{hr})\end{array}$ & $\begin{array}{c}\text { Extrapolated } \\
\text { Wetwood F } \\
(\mathrm{g} / \mathrm{hr})\end{array}$ & $\begin{array}{c}\text { Extrapolated } \\
\text { Total Wood F } \\
(\mathrm{g} / \mathrm{hr})\end{array}$ & $\begin{array}{l}\text { Relative } \\
\text { Humidity, } \\
@ 10 \mathrm{~m}(\%)\end{array}$ \\
\hline & 7/27/2008 16:00 & 123 & 209 & 1600 & \begin{tabular}{|l|}
5.448 \\
\end{tabular} & 5.448 & & 0.6979 & 7.64E-03 & 1675.1950 & 617.2601 & 2292.4551 & \\
\hline & $7 / 27 / 2008$ 17:00 & 124 & 209 & 1700 & 5.434 & 5.434 & & 0.7022 & 7.70E-03 & 1688.1303 & 622.0263 & 2310.1566 & \\
\hline & $7 / 27 / 2008$ 18:00 & 125 & 209 & 1800 & 5.507 & 5.507 & & 0.6797 & $7.40 \mathrm{E}-03$ & 1621.6065 & 597.5143 & 2219.1208 & \\
\hline & $7 / 27 / 2008$ 19:00 & 126 & 209 & 1900 & 5.627 & 5.627 & & 0.6439 & $6.92 \mathrm{E}-03$ & 1517.0524 & 558.9891 & 2076.0415 & \\
\hline & $7 / 27 / 200820: 00$ & 127 & 209 & 2000 & 5.912 & 5.912 & & 0.5646 & 5.89E-03 & 1290.5800 & 475.5407 & 1766.1207 & \\
\hline & $7 / 27 / 2008$ 21:00 & 128 & 209 & 2100 & 7.001 & 7.001 & & 0.3212 & 2.94E-03 & 644.5926 & 237.5134 & 882.1060 & \\
\hline & $7 / 27 / 2008$ 22:00 & 129 & 209 & 2200 & 8.44 & 8.44 & & 0.0960 & $6.65 E-04$ & 145.6786 & 53.6783 & 199.3569 & \\
\hline & $7 / 27 / 200823: 00$ & 130 & 209 & 2300 & 8.69 & 8.69 & & 0.0644 & 4.07E-04 & 89.2205 & 32.8751 & 122.0956 & \\
\hline & $7 / 28 / 20080: 00$ & 131 & 210 & 0 & 8.78 & 8.78 & 9.54 & 0.0535 & 3.24E-04 & 71.0050 & 26.1633 & 97.1683 & \\
\hline & 7/28/2008 1:00 & 132 & 210 & 100 & 8.97 & 8.97 & & 0.0312 & 1.67E-04 & 36.5546 & 13.4693 & 50.0239 & \\
\hline & $7 / 28 / 20082: 00$ & 133 & 210 & 200 & 9.08 & 9.08 & & 0.0187 & 8.89E-05 & 19.4831 & 7.1789 & 26.6620 & \\
\hline & 7/28/2008 3:00 & 134 & 210 & 300 & 9.18 & 9.18 & & 0.0076 & 2.94E-05 & 6.4481 & 2.3760 & 8.8241 & \\
\hline & 7/28/2008 4:00 & 135 & 210 & 400 & 9.15 & 9.15 & & 0.0109 & 4.58E-05 & 10.0431 & 3.7006 & 13.7437 & \\
\hline & 7/28/2008 5:00 & 136 & 210 & 500 & 9.44 & 9.44 & & -0.0201 & $0.00 \mathrm{E}+00$ & 0.0000 & 0.0000 & 0.0000 & \\
\hline & 7/28/2008 6:00 & 137 & 210 & 600 & 9.54 & 9.54 & & -0.0304 & $0.00 \mathrm{E}+00$ & 0.0000 & 0.0000 & 0.0000 & \\
\hline & 7/28/2008 7:00 & 138 & 210 & 700 & 9.43 & 9.43 & & -0.0191 & $0.00 \mathrm{E}+00$ & 0.0000 & 0.0000 & 0.0000 & \\
\hline & 7/28/2008 8:00 & 139 & 210 & 800 & 7.542 & 7.542 & & 0.2265 & 1.91E-03 & 419.1650 & 154.4500 & 573.6149 & \\
\hline & $7 / 28 / 20089: 00$ & 140 & 210 & 900 & 6.216 & 6.216 & & 0.4881 & 4.92E-03 & 1078.7665 & 397.4937 & 1476.2602 & \\
\hline & $7 / 28 / 2008$ 10:00 & 141 & 210 & 1000 & 5.78 & 5.78 & & 0.6003 & $6.35 \mathrm{E}-03$ & 1391.8446 & 512.8538 & 1904.6984 & \\
\hline & $7 / 28 / 2008$ 11:00 & 142 & 210 & 1100 & 5.696 & 5.696 & & 0.6239 & 6.66E-03 & 1459.5025 & 537.7837 & 1997.2862 & \\
\hline & $7 / 28 / 200812: 00$ & 143 & 210 & 1200 & 5.745 & 5.745 & & 0.6101 & $6.48 \mathrm{E}-03$ & 1419.7218 & 523.1257 & 1942.8475 & \\
\hline & 7/28/2008 13:00 & 144 & 210 & 1300 & 5.59 & 5.59 & & 0.6547 & 7.07E-03 & 1548.6726 & 570.6403 & 2119.3129 & \\
\hline & $7 / 28 / 2008$ 14:00 & 145 & 210 & 1400 & 5.527 & 5.527 & & 0.6736 & 7.32E-03 & 1603.7739 & 590.9435 & 2194.7173 & \\
\hline & $7 / 28 / 2008$ 15:00 & 146 & 210 & 1500 & 5.436 & 5.436 & & 0.7016 & 7.69E-03 & 1686.2772 & 621.3435 & 2307.6207 & \\
\hline & $7 / 28 / 2008$ 16:00 & 147 & 210 & 1600 & 5.473 & 5.473 & & 0.6901 & 7.54E-03 & 1652.3070 & 608.8265 & 2261.1335 & \\
\hline & 7/28/2008 17:00 & 148 & 210 & 1700 & 5.438 & 5.438 & & 0.7010 & 7.68E-03 & 1684.4258 & 620.6613 & 2305.0871 & \\
\hline & $7 / 28 / 2008$ 18:00 & 149 & 210 & 1800 & 5.515 & 5.515 & & 0.6772 & 7.37E-03 & 1614.4535 & 594.8786 & 2209.3321 & \\
\hline & $7 / 28 / 2008$ 19:00 & 150 & 210 & 1900 & 5.659 & 5.659 & & 0.6346 & $6.80 \mathrm{E}-03$ & 1490.1361 & 549.0713 & 2039.2074 & \\
\hline & $7 / 28 / 200820: 00$ & 151 & 210 & 2000 & 6.16 & 6.16 & & 0.5016 & 5.09E-03 & 1115.6895 & 411.0988 & 1526.7883 & \\
\hline & $7 / 28 / 200821: 00$ & 152 & 210 & 2100 & 7.471 & 7.471 & & 0.2381 & $2.03 E-03$ & 445.8776 & 164.2928 & 610.1704 & \\
\hline & $7 / 28 / 2008$ 22:00 & 153 & 210 & 2200 & 8.14 & 8.14 & & 0.1364 & 1.02E-03 & 224.4876 & 82.7171 & 307.2046 & \\
\hline & $7 / 28 / 200823: 00$ & 154 & 210 & 2300 & 8.43 & 8.43 & & 0.0973 & $6.76 \mathrm{E}-04$ & 148.1117 & 54.5748 & 202.6865 & \\
\hline & $7 / 29 / 20080: 00$ & 155 & 211 & 0 & 8.75 & 8.75 & 9.82 & 0.0571 & 3.51E-04 & 76.9482 & 28.3531 & 105.3014 & \\
\hline & 7/29/2008 1:00 & 156 & 211 & 100 & 9.04 & 9.04 & & 0.0232 & $1.16 \mathrm{E}-04$ & 25.4090 & 9.3625 & 34.7714 & \\
\hline & 7/29/2008 2:00 & 157 & 211 & 200 & 9.3 & 9.3 & & -0.0054 & $0.00 \mathrm{E}+00$ & 0.0000 & 0.0000 & 0.0000 & \\
\hline & 7/29/2008 3:00 & 158 & 211 & 300 & 9.49 & 9.49 & & -0.0253 & $0.00 \mathrm{E}+00$ & 0.0000 & 0.0000 & 0.0000 & \\
\hline & 7/29/2008 4:00 & 159 & 211 & 400 & 9.74 & 9.74 & & -0.0503 & $0.00 \mathrm{E}+00$ & 0.0000 & 0.0000 & 0.0000 & \\
\hline & 7/29/2008 5:00 & 160 & 211 & 500 & 9.82 & 9.82 & & -0.0580 & $0.00 \mathrm{E}+00$ & 0.0000 & 0.0000 & 0.0000 & \\
\hline & $7 / 29 / 20086: 00$ & 161 & 211 & 600 & 9.8 & 9.8 & & -0.0561 & $0.00 \mathrm{E}+00$ & 0.0000 & 0.0000 & 0.0000 & \\
\hline & 7/29/2008 7:00 & 162 & 211 & 700 & 9.59 & 9.59 & & -0.0355 & $0.00 \mathrm{E}+00$ & 0.0000 & 0.0000 & 0.0000 & \\
\hline & 7/29/2008 8:00 & 163 & 211 & 800 & 8.69 & 8.69 & & 0.0644 & 4.07E-04 & 89.2205 & 32.8751 & 122.0956 & \\
\hline
\end{tabular}




\begin{tabular}{|c|c|c|c|c|c|c|c|c|c|c|c|c|c|}
\hline Tree ID & TS & RN & JD & HR & $\begin{array}{c}\text { Raw } \\
\text { TC_dTA }\end{array}$ & $\begin{array}{c}\text { Fixed } \\
\text { TC_dT } \Delta\end{array}$ & dTM & $\mathbf{K}$ & $\begin{array}{l}\text { Sap } \\
\text { Velocity } \\
(\mathrm{cm} / \mathrm{s})\end{array}$ & $\begin{array}{c}\text { Probed } \\
\text { Drywood F } \\
(\mathrm{g} / \mathrm{hr})\end{array}$ & $\begin{array}{c}\text { Extrapolated } \\
\text { Wetwood F } \\
(\mathrm{g} / \mathrm{hr})\end{array}$ & $\begin{array}{c}\text { Extrapolated } \\
\text { Total Wood F } \\
(\mathrm{g} / \mathrm{hr})\end{array}$ & $\begin{array}{l}\text { Relative } \\
\text { Humidity, } \\
@ 10 \mathrm{~m}(\%)\end{array}$ \\
\hline & 7/29/2008 9:00 & 164 & 211 & 900 & 6.83 & 6.83 & & 0.3543 & 3.32E-03 & 727.2490 & 267.9699 & $\begin{array}{r}995.2189 \\
\end{array}$ & \\
\hline & 7/29/2008 10:00 & 165 & 211 & 1000 & 6.017 & 6.017 & & 0.5373 & $5.54 \mathrm{E}-03$ & 1214.1886 & 447.3928 & 1661.5814 & \\
\hline & $7 / 29 / 2008$ 11:00 & 166 & 211 & 1100 & 6.186 & 6.186 & & 0.4953 & $5.01 E-03$ & 1098.4346 & 404.7408 & 1503.1754 & \\
\hline & $7 / 29 / 2008$ 12:00 & 167 & 211 & 1200 & 5.741 & 5.741 & & 0.6112 & 6.49E-03 & 1422.9361 & 524.3101 & 1947.2461 & \\
\hline & 7/29/2008 13:00 & 168 & 211 & 1300 & 5.706 & 5.706 & & 0.6211 & 6.62E-03 & 1451.3117 & 534.7656 & 1986.0773 & \\
\hline & $7 / 29 / 2008$ 14:00 & 169 & 211 & 1400 & 5.525 & 5.525 & & 0.6742 & 7.32E-03 & 1605.5496 & 591.5978 & 2197.1474 & \\
\hline & $7 / 29 / 2008$ 15:00 & 170 & 211 & 1500 & 5.61 & 5.61 & & 0.6488 & $6.99 \mathrm{E}-03$ & 1531.5136 & 564.3177 & 2095.8313 & \\
\hline & $7 / 29 / 2008$ 16:00 & 171 & 211 & 1600 & 5.815 & 5.815 & & 0.5907 & 6.22E-03 & 1364.4055 & 502.7433 & 1867.1487 & \\
\hline & 7/29/2008 17:00 & 172 & 211 & 1700 & 6.01 & 6.01 & & 0.5391 & $5.56 \mathrm{E}-03$ & 1219.1714 & 449.2288 & 1668.4002 & \\
\hline & 7/29/2008 18:00 & 173 & 211 & 1800 & 6.054 & 6.054 & & 0.5279 & 5.42E-03 & 1188.1056 & 437.7820 & 1625.8876 & \\
\hline & $7 / 29 / 2008$ 19:00 & 174 & 211 & 1900 & 6.011 & 6.011 & & 0.5388 & $5.56 \mathrm{E}-03$ & 1218.4586 & 448.9661 & 1667.4248 & \\
\hline & $7 / 29 / 200820: 00$ & 175 & 211 & 2000 & 6.804 & 6.804 & & 0.3595 & $3.38 \mathrm{E}-03$ & 740.3471 & 272.7961 & 1013.1432 & \\
\hline & 7/29/2008 21:00 & 176 & 211 & 2100 & 8.31 & 8.31 & & 0.1131 & 8.14E-04 & 178.3487 & 65.7162 & 244.0649 & \\
\hline & $7 / 29 / 2008$ 22:00 & 177 & 211 & 2200 & 9.23 & 9.23 & & 0.0022 & $6.25 E-06$ & 1.3702 & 0.5049 & 1.8751 & \\
\hline & $7 / 29 / 200823: 00$ & 178 & 211 & 2300 & 9.61 & 9.61 & & -0.0375 & $0.00 \mathrm{E}+00$ & 0.0000 & 0.0000 & 0.0000 & \\
\hline & $7 / 30 / 20080: 00$ & 179 & 212 & 0 & 9.77 & 9.77 & 9.85 & -0.0532 & $0.00 \mathrm{E}+00$ & 0.0000 & 0.0000 & 0.0000 & \\
\hline & 7/30/2008 1:00 & 180 & 212 & 100 & 9.82 & 9.82 & & -0.0580 & $0.00 \mathrm{E}+00$ & 0.0000 & 0.0000 & 0.0000 & \\
\hline & 7/30/2008 2:00 & 181 & 212 & 200 & 9.84 & 9.84 & & -0.0600 & $0.00 \mathrm{E}+00$ & 0.0000 & 0.0000 & 0.0000 & \\
\hline & 7/30/2008 3:00 & 182 & 212 & 300 & 9.84 & 9.84 & & -0.0600 & $0.00 \mathrm{E}+00$ & 0.0000 & 0.0000 & 0.0000 & \\
\hline & 7/30/2008 4:00 & 183 & 212 & 400 & 9.85 & 9.85 & & -0.0609 & $0.00 E+00$ & 0.0000 & 0.0000 & 0.0000 & \\
\hline & 7/30/2008 5:00 & 184 & 212 & 500 & 9.84 & 9.84 & & -0.0600 & $0.00 \mathrm{E}+00$ & 0.0000 & 0.0000 & 0.0000 & \\
\hline & 7/30/2008 6:00 & 185 & 212 & 600 & 9.81 & 9.81 & & -0.0571 & $0.00 E+00$ & 0.0000 & 0.0000 & 0.0000 & \\
\hline & 7/30/2008 7:00 & 186 & 212 & 700 & 9.79 & 9.79 & & -0.0552 & $0.00 \mathrm{E}+00$ & 0.0000 & 0.0000 & 0.0000 & \\
\hline & $7 / 30 / 20088: 00$ & 187 & 212 & 800 & 9.75 & 9.75 & & -0.0513 & $0.00 \mathrm{E}+00$ & 0.0000 & 0.0000 & 0.0000 & \\
\hline & 7/30/2008 9:00 & 188 & 212 & 900 & 8.48 & 8.48 & & 0.0908 & $6.21 \mathrm{E}-04$ & 136.0796 & 50.1413 & 186.2210 & \\
\hline & $7 / 30 / 2008$ 10:00 & 189 & 212 & 1000 & 6.11 & 6.11 & & 0.5139 & 5.24E-03 & 1149.4286 & 423.5306 & 1572.9592 & \\
\hline & 7/30/2008 11:00 & 190 & 212 & 1100 & 5.709 & 5.709 & & 0.6202 & 6.61E-03 & 1448.8617 & 533.8629 & 1982.7246 & \\
\hline & $7 / 30 / 2008$ 12:00 & 191 & 212 & 1200 & 5.672 & 5.672 & & 0.6308 & $6.75 \mathrm{E}-03$ & 1479.3138 & 545.0836 & 2024.3973 & \\
\hline & 7/30/2008 13:00 & 192 & 212 & 1300 & 5.64 & 5.64 & & 0.6401 & 6.87E-03 & 1506.0699 & 554.9424 & 2061.0123 & \\
\hline & $7 / 30 / 2008$ 14:00 & 193 & 212 & 1400 & 5.574 & 5.574 & & 0.6595 & 7.13E-03 & 1562.5145 & 575.7406 & 2138.2550 & \\
\hline & 7/30/2008 15:00 & 194 & 212 & 1500 & 5.721 & 5.721 & & 0.6169 & 6.57E-03 & 1439.0953 & 530.2643 & 1969.3596 & \\
\hline & $7 / 30 / 2008$ 16:00 & 195 & 212 & 1600 & 5.617 & 5.617 & & 0.6468 & $6.96 \mathrm{E}-03$ & 1525.5452 & 562.1185 & 2087.6638 & \\
\hline & $7 / 30 / 2008$ 17:00 & 196 & 212 & 1700 & 5.534 & 5.534 & & 0.6715 & 7.29E-03 & 1597.5716 & 588.6581 & 2186.2297 & \\
\hline & $7 / 30 / 2008$ 18:00 & 197 & 212 & 1800 & 5.627 & 5.627 & & 0.6439 & $6.92 \mathrm{E}-03$ & 1517.0524 & 558.9891 & 2076.0415 & \\
\hline & 7/30/2008 19:00 & 198 & 212 & 1900 & 5.723 & 5.723 & & 0.6163 & $6.56 \mathrm{E}-03$ & 1437.4728 & 529.6664 & 1967.1392 & \\
\hline & $7 / 30 / 200820: 00$ & 199 & 212 & 2000 & 6.175 & 6.175 & & 0.4980 & 5.04E-03 & 1105.7109 & 407.4219 & 1513.1328 & \\
\hline & 7/30/2008 21:00 & 200 & 212 & 2100 & 7.513 & 7.513 & & 0.2312 & $1.96 \mathrm{E}-03$ & 429.9774 & 158.4340 & 588.4114 & \\
\hline & $7 / 30 / 2008$ 22:00 & 201 & 212 & 2200 & 8.66 & 8.66 & & 0.0681 & 4.36E-04 & 95.5460 & 35.2059 & 130.7519 & \\
\hline & 7/30/2008 23:00 & 202 & 212 & 2300 & 9.14 & 9.14 & & 0.0120 & $5.16 \mathrm{E}-05$ & 11.3086 & 4.1669 & 15.4755 & \\
\hline & $7 / 31 / 20080: 00$ & 203 & 213 & 0 & 9.46 & 9.46 & 9.83 & -0.0222 & $0.00 \mathrm{E}+00$ & 0.0000 & 0.0000 & 0.0000 & \\
\hline & 7/31/2008 1:00 & 204 & 213 & 100 & 9.62 & 9.62 & & -0.0385 & $0.00 \mathrm{E}+00$ & 0.0000 & 0.0000 & 0.0000 & \\
\hline
\end{tabular}




\begin{tabular}{|c|c|c|c|c|c|c|c|c|c|c|c|c|c|}
\hline Tree ID & TS & RN & JD & HR & $\begin{array}{c}\text { Raw } \\
\text { TC_dTA }\end{array}$ & $\begin{array}{c}\text { Fixed } \\
\text { TC_dT } \Delta\end{array}$ & dTM & K & $\begin{array}{l}\text { Sap } \\
\text { Velocity } \\
(\mathrm{cm} / \mathrm{s})\end{array}$ & $\begin{array}{c}\text { Probed } \\
\text { Drywood F } \\
(\mathrm{g} / \mathrm{hr})\end{array}$ & $\begin{array}{c}\text { Extrapolated } \\
\text { Wetwood F } \\
(\mathrm{g} / \mathrm{hr})\end{array}$ & $\begin{array}{c}\text { Extrapolated } \\
\text { Total Wood F } \\
(\mathrm{g} / \mathrm{hr})\end{array}$ & $\begin{array}{l}\text { Relative } \\
\text { Humidity, } \\
@ 10 \mathrm{~m}(\%)\end{array}$ \\
\hline & 7/31/2008 2:00 & 205 & 213 & 200 & $\begin{array}{r}9.67 \\
\end{array}$ & 9.67 & & -0.0434 & $0.00 \mathrm{E}+00$ & 0.0000 & 0.0000 & 0.0000 & \\
\hline & 7/31/2008 3:00 & 206 & 213 & 300 & 9.72 & 9.72 & & -0.0484 & $0.00 \mathrm{E}+00$ & 0.0000 & 0.0000 & 0.0000 & \\
\hline & 7/31/2008 4:00 & 207 & 213 & 400 & 9.8 & 9.8 & & -0.0561 & $0.00 E+00$ & 0.0000 & 0.0000 & 0.0000 & \\
\hline & 7/31/2008 5:00 & 208 & 213 & 500 & 9.81 & 9.81 & & -0.0571 & $0.00 \mathrm{E}+00$ & 0.0000 & 0.0000 & 0.0000 & \\
\hline & 7/31/2008 6:00 & 209 & 213 & 600 & 9.82 & 9.82 & & -0.0580 & $0.00 \mathrm{E}+00$ & 0.0000 & 0.0000 & 0.0000 & \\
\hline & 7/31/2008 7:00 & 210 & 213 & 700 & 9.83 & 9.83 & & -0.0590 & $0.00 \mathrm{E}+00$ & 0.0000 & 0.0000 & 0.0000 & \\
\hline & $7 / 31 / 2008$ 8:00 & 211 & 213 & 800 & 9.7 & 9.7 & & -0.0464 & $0.00 \mathrm{E}+00$ & 0.0000 & 0.0000 & 0.0000 & \\
\hline & $7 / 31 / 20089: 00$ & 212 & 213 & 900 & 8.15 & 8.15 & & 0.1350 & 1.01E-03 & 221.6653 & 81.6771 & 303.3424 & \\
\hline & $7 / 31 / 2008$ 10:00 & 213 & 213 & 1000 & 5.927 & 5.927 & & 0.5607 & 5.84E-03 & 1279.4473 & 471.4387 & 1750.8859 & \\
\hline & 7/31/2008 11:00 & 214 & 213 & 1100 & 5.724 & 5.724 & & 0.6160 & $6.55 E-03$ & 1436.6620 & 529.3677 & 1966.0297 & \\
\hline & $7 / 31 / 2008$ 12:00 & 215 & 213 & 1200 & 5.515 & 5.515 & & 0.6772 & 7.37E-03 & 1614.4535 & 594.8786 & 2209.3321 & \\
\hline & $7 / 31 / 2008$ 13:00 & 216 & 213 & 1300 & 5.657 & 5.657 & & 0.6351 & 6.81E-03 & 1491.8068 & 549.6869 & 2041.4937 & \\
\hline & 7/31/2008 14:00 & 217 & 213 & 1400 & 5.366 & 5.366 & & 0.7238 & 7.99E-03 & 1752.1895 & 645.6303 & 2397.8198 & \\
\hline & 7/31/2008 15:00 & 218 & 213 & 1500 & 5.363 & 5.363 & & 0.7248 & 8.01E-03 & 1755.0635 & 646.6892 & 2401.7527 & \\
\hline & $7 / 31 / 2008$ 16:00 & 219 & 213 & 1600 & 5.426 & 5.426 & & 0.7048 & 7.74E-03 & 1695.5602 & 624.7640 & 2320.3243 & \\
\hline & 7/31/2008 17:00 & 220 & 213 & 1700 & 5.408 & 5.408 & & 0.7104 & 7.81E-03 & 1712.3805 & 630.9618 & 2343.3424 & \\
\hline & $7 / 31 / 2008$ 18:00 & 221 & 213 & 1800 & 5.503 & 5.503 & & 0.6809 & 7.41E-03 & 1625.1931 & 598.8358 & 2224.0289 & \\
\hline & $7 / 31 / 2008$ 19:00 & 222 & 213 & 1900 & 5.743 & 5.743 & & 0.6107 & 6.48E-03 & 1421.3282 & 523.7176 & 1945.0458 & \\
\hline & $7 / 31 / 200820: 00$ & 223 & 213 & 2000 & 6.406 & 6.406 & & 0.4440 & 4.38E-03 & 959.9689 & 353.7203 & 1313.6891 & \\
\hline & $7 / 31 / 200821: 00$ & 224 & 213 & 2100 & 7.306 & 7.306 & & 0.2661 & 2.33E-03 & 511.1799 & 188.3547 & 699.5346 & \\
\hline & $7 / 31 / 200822: 00$ & 225 & 213 & 2200 & 8.13 & 8.13 & & 0.1378 & $1.04 \mathrm{E}-03$ & 227.3235 & 83.7620 & 311.0855 & \\
\hline & 7/31/2008 23:00 & 226 & 213 & 2300 & 8.24 & 8.24 & & 0.1226 & 8.98E-04 & 196.8754 & 72.5428 & 269.4182 & \\
\hline & $8 / 1 / 20080: 00$ & 227 & 214 & 0 & 8.61 & 8.61 & 9.75 & 0.0743 & 4.85E-04 & 106.3646 & 39.1922 & 145.5568 & \\
\hline & 8/1/2008 1:00 & 228 & 214 & 100 & 9.14 & 9.14 & & 0.0120 & 5.16E-05 & 11.3086 & 4.1669 & 15.4755 & \\
\hline & 8/1/2008 2:00 & 229 & 214 & 200 & 9.44 & 9.44 & & -0.0201 & $0.00 \mathrm{E}+00$ & 0.0000 & 0.0000 & 0.0000 & \\
\hline & $8 / 1 / 2008$ 3:00 & 230 & 214 & 300 & 9.51 & 9.51 & & -0.0273 & $0.00 E+00$ & 0.0000 & 0.0000 & 0.0000 & \\
\hline & 8/1/2008 4:00 & 231 & 214 & 400 & 9.65 & 9.65 & & -0.0415 & $0.00 \mathrm{E}+00$ & 0.0000 & 0.0000 & 0.0000 & \\
\hline & 8/1/2008 5:00 & 232 & 214 & 500 & 9.74 & 9.74 & & -0.0503 & $0.00 \mathrm{E}+00$ & 0.0000 & 0.0000 & 0.0000 & \\
\hline & 8/1/2008 6:00 & 233 & 214 & 600 & 9.75 & 9.75 & & -0.0513 & $0.00 \mathrm{E}+00$ & 0.0000 & 0.0000 & 0.0000 & \\
\hline & 8/1/2008 7:00 & 234 & 214 & 700 & 9.72 & 9.72 & & -0.0484 & $0.00 \mathrm{E}+00$ & 0.0000 & 0.0000 & 0.0000 & \\
\hline & 8/1/2008 8:00 & 235 & 214 & 800 & 9.54 & 9.54 & & -0.0304 & $0.00 \mathrm{E}+00$ & 0.0000 & 0.0000 & 0.0000 & \\
\hline & 8/1/2008 9:00 & 236 & 214 & 900 & 8.05 & 8.05 & & 0.1491 & 1.14E-03 & 250.5046 & 92.3036 & 342.8082 & \\
\hline & 8/1/2008 10:00 & 237 & 214 & 1000 & 5.884 & 5.884 & & 0.5721 & 5.98E-03 & 1311.5618 & 483.2719 & 1794.8337 & \\
\hline & 8/1/2008 11:00 & 238 & 214 & 1100 & 5.703 & 5.703 & & 0.6220 & $6.63 \mathrm{E}-03$ & 1453.7650 & 535.6696 & 1989.4346 & \\
\hline & 8/1/2008 12:00 & 239 & 214 & 1200 & 5.649 & 5.649 & & 0.6375 & 6.84E-03 & 1498.5050 & 552.1550 & 2050.6599 & \\
\hline & 8/1/2008 13:00 & 240 & 214 & 1300 & 5.54 & 5.54 & & 0.6697 & 7.26E-03 & 1592.2714 & 586.7051 & 2178.9765 & \\
\hline & 8/1/2008 14:00 & 241 & 214 & 1400 & 5.476 & 5.476 & & 0.6892 & 7.53E-03 & 1649.5784 & 607.8211 & 2257.3995 & \\
\hline & 8/1/2008 15:00 & 242 & 214 & 1500 & 5.432 & 5.432 & & 0.7029 & 7.71E-03 & 1689.9851 & 622.7098 & 2312.6949 & \\
\hline & $8 / 1 / 200816: 00$ & 243 & 214 & 1600 & 5.509 & 5.509 & & 0.6791 & 7.39E-03 & 1619.8158 & 596.8544 & 2216.6702 & \\
\hline & 8/1/2008 17:00 & 244 & 214 & 1700 & 5.547 & 5.547 & & 0.6676 & 7.24E-03 & 1586.1065 & 584.4336 & 2170.5401 & \\
\hline & 8/1/2008 18:00 & 245 & 214 & 1800 & 5.595 & 5.595 & & 0.6533 & 7.05E-03 & 1544.3680 & 569.0541 & 2113.4221 & \\
\hline
\end{tabular}




\begin{tabular}{|c|c|c|c|c|c|c|c|c|c|c|c|c|c|}
\hline Tree ID & TS & RN & JD & HR & $\begin{array}{c}\text { Raw } \\
\text { TC_dTA }\end{array}$ & $\begin{array}{c}\text { Fixed } \\
\text { TC_dT } \Delta\end{array}$ & dTM & $\mathbf{K}$ & $\begin{array}{l}\text { Sap } \\
\text { Velocity } \\
(\mathrm{cm} / \mathrm{s})\end{array}$ & $\begin{array}{c}\text { Probed } \\
\text { Drywood F } \\
(\mathrm{g} / \mathrm{hr})\end{array}$ & $\begin{array}{c}\text { Extrapolated } \\
\text { Wetwood F } \\
(\mathrm{g} / \mathrm{hr})\end{array}$ & $\begin{array}{c}\text { Extrapolated } \\
\text { Total Wood F } \\
(\mathrm{g} / \mathrm{hr})\end{array}$ & $\begin{array}{l}\text { Relative } \\
\text { Humidity, } \\
@ 10 \mathrm{~m}(\%)\end{array}$ \\
\hline & 8/1/2008 19:00 & 246 & 214 & 1900 & \begin{tabular}{|r|}
5.754 \\
\end{tabular} & 5.754 & & 0.6076 & $6.44 \mathrm{E}-03$ & 1412.5111 & 520.4688 & 1932.9798 & \\
\hline & 8/1/2008 20:00 & 247 & 214 & 2000 & 6.411 & 6.411 & & 0.4428 & 4.37E-03 & 956.9721 & 352.6161 & 1309.5882 & \\
\hline & 8/1/2008 21:00 & 248 & 214 & 2100 & 8.14 & 8.14 & & 0.1364 & $1.02 \mathrm{E}-03$ & 224.4876 & 82.7171 & 307.2046 & \\
\hline & 8/1/2008 22:00 & 249 & 214 & 2200 & 9.14 & 9.14 & & 0.0120 & $5.16 \mathrm{E}-05$ & 11.3086 & 4.1669 & 15.4755 & \\
\hline & 8/1/2008 23:00 & 250 & 214 & 2300 & 9.38 & 9.38 & & -0.0139 & $0.00 \mathrm{E}+00$ & 0.0000 & 0.0000 & 0.0000 & \\
\hline & 8/2/2008 0:00 & 251 & 215 & 0 & 9.45 & 9.45 & 9.73 & -0.0212 & $0.00 \mathrm{E}+00$ & 0.0000 & 0.0000 & 0.0000 & \\
\hline & 8/2/2008 1:00 & 252 & 215 & 100 & 9.54 & 9.54 & & -0.0304 & $0.00 \mathrm{E}+00$ & 0.0000 & 0.0000 & 0.0000 & \\
\hline & 8/2/2008 2:00 & 253 & 215 & 200 & 9.66 & 9.66 & & -0.0424 & $0.00 \mathrm{E}+00$ & 0.0000 & 0.0000 & 0.0000 & \\
\hline & 8/2/2008 3:00 & 254 & 215 & 300 & 9.73 & 9.73 & & -0.0493 & $0.00 \mathrm{E}+00$ & 0.0000 & 0.0000 & 0.0000 & \\
\hline & 8/2/2008 4:00 & 255 & 215 & 400 & 9.64 & 9.64 & & -0.0405 & $0.00 E+00$ & 0.0000 & 0.0000 & 0.0000 & \\
\hline & 8/2/2008 5:00 & 256 & 215 & 500 & 9.6 & 9.6 & & -0.0365 & $0.00 \mathrm{E}+00$ & 0.0000 & 0.0000 & 0.0000 & \\
\hline & 8/2/2008 6:00 & 257 & 215 & 600 & 9.6 & 9.6 & & -0.0365 & $0.00 E+00$ & 0.0000 & 0.0000 & 0.0000 & \\
\hline & 8/2/2008 7:00 & 258 & 215 & 700 & 9.41 & 9.41 & & -0.0170 & $0.00 \mathrm{E}+00$ & 0.0000 & 0.0000 & 0.0000 & \\
\hline & 8/2/2008 8:00 & 259 & 215 & 800 & 8 & 8 & & 0.1563 & $1.21 \mathrm{E}-03$ & 265.4427 & 97.8078 & 363.2505 & \\
\hline & 8/2/2008 9:00 & 260 & 215 & 900 & 6.161 & 6.161 & & 0.5014 & 5.09E-03 & 1115.0223 & 410.8529 & 1525.8751 & \\
\hline & 8/2/2008 10:00 & 261 & 215 & 1000 & 5.963 & 5.963 & & 0.5512 & 5.72E-03 & 1253.0303 & 461.7048 & 1714.7351 & \\
\hline & 8/2/2008 11:00 & 262 & 215 & 1100 & 5.759 & 5.759 & & 0.6062 & 6.43E-03 & 1408.5178 & 518.9974 & 1927.5152 & \\
\hline & $8 / 2 / 200812: 00$ & 263 & 215 & 1200 & 5.615 & 5.615 & & 0.6474 & 6.97E-03 & 1527.2485 & 562.7461 & 2089.9946 & \\
\hline & 8/2/2008 13:00 & 264 & 215 & 1300 & 5.53 & 5.53 & & 0.6727 & 7.30E-03 & 1601.1133 & 589.9631 & 2191.0764 & \\
\hline & 8/2/2008 14:00 & 265 & 215 & 1400 & 5.46 & 5.46 & & 0.6941 & 7.59E-03 & 1664.1752 & 613.1996 & 2277.3748 & \\
\hline & $8 / 2 / 2008$ 15:00 & 266 & 215 & 1500 & 5.431 & 5.431 & & 0.7032 & $7.71 \mathrm{E}-03$ & 1690.9132 & 623.0518 & 2313.9650 & \\
\hline & 8/2/2008 16:00 & 267 & 215 & 1600 & 5.464 & 5.464 & & 0.6929 & 7.58E-03 & 1660.5158 & 611.8512 & 2272.3669 & \\
\hline & 8/2/2008 17:00 & 268 & 215 & 1700 & 5.453 & 5.453 & & 0.6963 & $7.62 E-03$ & 1670.5959 & 615.5654 & 2286.1613 & \\
\hline & $8 / 2 / 200818: 00$ & 269 & 215 & 1800 & 5.56 & 5.56 & & 0.6637 & $7.18 E-03$ & 1574.7105 & 580.2345 & 2154.9450 & \\
\hline & 8/2/2008 19:00 & 270 & 215 & 1900 & 5.712 & 5.712 & & 0.6194 & $6.60 \mathrm{E}-03$ & 1446.4151 & 532.9614 & 1979.3765 & \\
\hline & 8/2/2008 20:00 & 271 & 215 & 2000 & 6.263 & 6.263 & & 0.4769 & 4.78E-03 & 1048.4645 & 386.3283 & 1434.7928 & \\
\hline & 8/2/2008 21:00 & 272 & 215 & 2100 & 7.38 & 7.38 & & 0.2534 & $2.20 \mathrm{E}-03$ & 481.3245 & 177.3539 & 658.6784 & \\
\hline & 8/2/2008 22:00 & 273 & 215 & 2200 & 8.5 & 8.5 & & 0.0882 & $5.99 \mathrm{E}-04$ & 131.3603 & 48.4024 & 179.7627 & \\
\hline & 8/2/2008 23:00 & 274 & 215 & 2300 & 8.93 & 8.93 & & 0.0358 & 1.98E-04 & 43.3231 & 15.9633 & 59.2864 & \\
\hline & 8/3/2008 0:00 & 275 & 216 & 0 & 8.81 & 8.81 & 9.38 & 0.0499 & 2.97E-04 & 65.1933 & 24.0218 & 89.2152 & \\
\hline & 8/3/2008 1:00 & 276 & 216 & 100 & 8.53 & 8.53 & & 0.0844 & 5.67E-04 & 124.3817 & 45.8310 & 170.2127 & \\
\hline & 8/3/2008 2:00 & 277 & 216 & 200 & 8.79 & 8.79 & & 0.0523 & $3.15 E-04$ & 69.0531 & 25.4440 & 94.4971 & \\
\hline & 8/3/2008 3:00 & 278 & 216 & 300 & 9.12 & 9.12 & & 0.0143 & $6.35 \mathrm{E}-05$ & 13.9280 & 5.1321 & 19.0601 & \\
\hline & 8/3/2008 4:00 & 279 & 216 & 400 & 9.3 & 9.3 & & -0.0054 & $0.00 \mathrm{E}+00$ & 0.0000 & 0.0000 & 0.0000 & \\
\hline & 8/3/2008 5:00 & 280 & 216 & 500 & 9.38 & 9.38 & & -0.0139 & $0.00 \mathrm{E}+00$ & 0.0000 & 0.0000 & 0.0000 & \\
\hline & 8/3/2008 6:00 & 281 & 216 & 600 & 9.06 & 9.06 & & 0.0210 & 1.02E-04 & 22.4026 & 8.2547 & 30.6574 & \\
\hline & 8/3/2008 7:00 & 282 & 216 & 700 & 8.57 & 8.57 & & 0.0793 & $5.26 \mathrm{E}-04$ & 115.2650 & 42.4718 & 157.7367 & \\
\hline & 8/3/2008 8:00 & 283 & 216 & 800 & 7.477 & 7.477 & & 0.2371 & 2.02E-03 & 443.5886 & 163.4493 & 607.0379 & \\
\hline & 8/3/2008 9:00 & 284 & 216 & 900 & 5.977 & 5.977 & & 0.5476 & $5.67 \mathrm{E}-03$ & 1242.8708 & 457.9613 & 1700.8321 & \\
\hline & 8/3/2008 10:00 & 285 & 216 & 1000 & 5.615 & 5.615 & & 0.6474 & $6.97 \mathrm{E}-03$ & 1527.2485 & 562.7461 & 2089.9946 & \\
\hline & 8/3/2008 11:00 & 286 & 216 & 1100 & 5.439 & 5.439 & & 0.7007 & 7.68E-03 & 1683.5008 & 620.3205 & 2303.8212 & \\
\hline
\end{tabular}




\begin{tabular}{|c|c|c|c|c|c|c|c|c|c|c|c|c|c|}
\hline Tree ID & TS & RN & JD & HR & $\begin{array}{c}\text { Raw } \\
\text { TC_dTA }\end{array}$ & $\begin{array}{c}\text { Fixed } \\
\text { TC_dT } \Delta\end{array}$ & dTM & $\mathbf{K}$ & $\begin{array}{l}\text { Sap } \\
\text { Velocity } \\
(\mathrm{cm} / \mathrm{s})\end{array}$ & $\begin{array}{c}\text { Probed } \\
\text { Drywood F } \\
(\mathrm{g} / \mathrm{hr})\end{array}$ & $\begin{array}{c}\text { Extrapolated } \\
\text { Wetwood F } \\
(\mathrm{g} / \mathrm{hr})\end{array}$ & $\begin{array}{c}\text { Extrapolated } \\
\text { Total Wood F } \\
(\mathrm{g} / \mathrm{hr})\end{array}$ & $\begin{array}{l}\text { Relative } \\
\text { Humidity, } \\
@ 10 \mathrm{~m}(\%)\end{array}$ \\
\hline & 8/3/2008 12:00 & 287 & 216 & 1200 & $\begin{array}{r}5.395 \\
\end{array}$ & 5.395 & & 0.7146 & 7.87E-03 & 1724.6178 & 635.4709 & 2360.0887 & \\
\hline & 8/3/2008 13:00 & 288 & 216 & 1300 & 5.316 & 5.316 & & 0.7400 & $8.21 \mathrm{E}-03$ & 1800.6293 & 663.4789 & 2464.1083 & \\
\hline & $8 / 3 / 2008$ 14:00 & 289 & 216 & 1400 & 5.353 & 5.353 & & 0.7280 & 8.05E-03 & 1764.6731 & 650.2301 & 2414.9031 & \\
\hline & 8/3/2008 15:00 & 290 & 216 & 1500 & 5.425 & 5.425 & & 0.7051 & 7.74E-03 & 1696.4910 & 625.1070 & 2321.5980 & \\
\hline & 8/3/2008 16:00 & 291 & 216 & 1600 & 5.485 & 5.485 & & 0.6864 & 7.49E-03 & 1641.4157 & 604.8134 & 2246.2291 & \\
\hline & 8/3/2008 17:00 & 292 & 216 & 1700 & 5.494 & 5.494 & & 0.6837 & $7.45 \mathrm{E}-03$ & 1633.2873 & 601.8183 & 2235.1056 & \\
\hline & 8/3/2008 18:00 & 293 & 216 & 1800 & 5.556 & 5.556 & & 0.6649 & $7.20 \mathrm{E}-03$ & 1578.2096 & 581.5238 & 2159.7334 & \\
\hline & 8/3/2008 19:00 & 294 & 216 & 1900 & 5.669 & 5.669 & & 0.6317 & 6.76E-03 & 1481.8055 & 546.0017 & 2027.8072 & \\
\hline & 8/3/2008 20:00 & 295 & 216 & 2000 & 5.905 & 5.905 & & 0.5665 & 5.91E-03 & 1295.8008 & 477.4645 & 1773.2653 & \\
\hline & 8/3/2008 21:00 & 296 & 216 & 2100 & 6.717 & 6.717 & & 0.3771 & 3.58E-03 & 785.2364 & 289.3365 & 1074.5728 & \\
\hline & 8/3/2008 22:00 & 297 & 216 & 2200 & 7.359 & 7.359 & & 0.2570 & 2.23E-03 & 489.7018 & 180.4407 & 670.1424 & \\
\hline & 8/3/2008 23:00 & 298 & 216 & 2300 & 7.487 & 7.487 & & 0.2355 & $2.01 E-03$ & 439.7866 & 162.0484 & 601.8350 & \\
\hline & 8/4/2008 0:00 & 299 & 217 & 0 & 7.533 & 7.533 & 8.93 & 0.2279 & 1.93E-03 & 422.5061 & 155.6811 & 578.1872 & \\
\hline & 8/4/2008 1:00 & 300 & 217 & 100 & 7.596 & 7.596 & & 0.2177 & 1.82E-03 & 399.3884 & 147.1629 & 546.5513 & \\
\hline & $8 / 4 / 2008$ 2:00 & 301 & 217 & 200 & 7.261 & 7.261 & & 0.2739 & $2.42 E-03$ & 529.7990 & 195.2153 & 725.0143 & \\
\hline & 8/4/2008 3:00 & 302 & 217 & 300 & 7.064 & 7.064 & & 0.3095 & 2.81E-03 & 615.6109 & 226.8345 & 842.4453 & \\
\hline & 8/4/2008 4:00 & 303 & 217 & 400 & 6.853 & 6.853 & & 0.3498 & 3.27E-03 & 715.7816 & 263.7444 & 979.5260 & \\
\hline & $8 / 4 / 20085: 00$ & 304 & 217 & 500 & 7.02 & 7.02 & & 0.3177 & $2.90 \mathrm{E}-03$ & 635.7709 & 234.2629 & 870.0337 & \\
\hline & 8/4/2008 6:00 & 305 & 217 & 600 & 7.436 & 7.436 & & 0.2439 & 2.10E-03 & 459.3482 & 169.2563 & 628.6045 & \\
\hline & 8/4/2008 7:00 & 306 & 217 & 700 & 7.453 & 7.453 & & 0.2411 & 2.07E-03 & 452.7802 & 166.8362 & 619.6163 & \\
\hline & 8/4/2008 8:00 & 307 & 217 & 800 & 7.315 & 7.315 & & 0.2645 & $2.32 \mathrm{E}-03$ & 507.4985 & 186.9983 & 694.4968 & \\
\hline & 8/4/2008 9:00 & 308 & 217 & 900 & 7.101 & 7.101 & & 0.3026 & 2.73E-03 & 598.9451 & 220.6937 & 819.6388 & \\
\hline & 8/4/2008 10:00 & 309 & 217 & 1000 & 6.305 & 6.305 & & 0.4671 & $4.66 \mathrm{E}-03$ & 1021.9037 & 376.5414 & 1398.4452 & \\
\hline & 8/4/2008 11:00 & 310 & 217 & 1100 & 5.732 & 5.732 & & 0.6137 & $6.52 \mathrm{E}-03$ & 1430.1895 & 526.9827 & 1957.1723 & \\
\hline & 8/4/2008 12:00 & 311 & 217 & 1200 & 5.477 & 5.477 & & 0.6889 & 7.52E-03 & 1648.6698 & 607.4863 & 2256.1561 & \\
\hline & 8/4/2008 13:00 & 312 & 217 & 1300 & 5.411 & 5.411 & & 0.7095 & 7.80E-03 & 1709.5672 & 629.9252 & 2339.4924 & \\
\hline & 8/4/2008 14:00 & 313 & 217 & 1400 & 5.762 & 5.762 & & 0.6053 & $6.41 E-03$ & 1406.1262 & 518.1161 & 1924.2423 & \\
\hline & 8/4/2008 15:00 & 314 & 217 & 1500 & 6.242 & 6.242 & & 0.4819 & 4.84E-03 & 1061.9273 & 391.2890 & 1453.2163 & \\
\hline & 8/4/2008 16:00 & 315 & 217 & 1600 & 5.708 & 5.708 & & 0.6205 & 6.61E-03 & 1449.6780 & 534.1637 & 1983.8417 & \\
\hline & 8/4/2008 17:00 & 316 & 217 & 1700 & 5.604 & 5.604 & & 0.6506 & 7.01E-03 & 1536.6447 & 566.2083 & 2102.8530 & \\
\hline & 8/4/2008 18:00 & 317 & 217 & 1800 & 5.682 & 5.682 & & 0.6279 & 6.71E-03 & 1471.0326 & 542.0322 & 2013.0648 & \\
\hline & 8/4/2008 19:00 & 318 & 217 & 1900 & 5.899 & 5.899 & & 0.5681 & 5.93E-03 & 1300.2889 & 479.1182 & 1779.4071 & \\
\hline & 8/4/2008 20:00 & 319 & 217 & 2000 & 6.403 & 6.403 & & 0.4446 & 4.39E-03 & 961.7700 & 354.3839 & 1316.1539 & \\
\hline & 8/4/2008 21:00 & 320 & 217 & 2100 & 7.952 & 7.952 & & 0.1632 & $1.28 \mathrm{E}-03$ & 280.1129 & 103.2133 & 383.3262 & \\
\hline & 8/4/2008 22:00 & 321 & 217 & 2200 & 8.74 & 8.74 & & 0.0584 & 3.60E-04 & 78.9581 & 29.0937 & 108.0518 & \\
\hline & 8/4/2008 23:00 & 322 & 217 & 2300 & 8.93 & 8.93 & & 0.0358 & 1.98E-04 & 43.3231 & 15.9633 & 59.2864 & \\
\hline & 8/5/2008 0:00 & 323 & 218 & 0 & 9.14 & 9.14 & 9.59 & 0.0120 & 5.16E-05 & 11.3086 & 4.1669 & 15.4755 & \\
\hline & 8/5/2008 1:00 & 324 & 218 & 100 & 9.27 & 9.27 & & -0.0022 & $0.00 \mathrm{E}+00$ & 0.0000 & 0.0000 & 0.0000 & \\
\hline & 8/5/2008 2:00 & 325 & 218 & 200 & 9.39 & 9.39 & & -0.0149 & $0.00 \mathrm{E}+00$ & 0.0000 & 0.0000 & 0.0000 & \\
\hline & 8/5/2008 3:00 & 326 & 218 & 300 & 9.35 & 9.35 & & -0.0107 & $0.00 \mathrm{E}+00$ & 0.0000 & 0.0000 & 0.0000 & \\
\hline & 8/5/2008 4:00 & 327 & 218 & 400 & 9.18 & 9.18 & & 0.0076 & 2.94E-05 & 6.4481 & 2.3760 & 8.8241 & \\
\hline
\end{tabular}




\begin{tabular}{|c|c|c|c|c|c|c|c|c|c|c|c|c|c|}
\hline Tree ID & TS & RN & JD & HR & $\begin{array}{c}\text { Raw } \\
\text { TC_dTA }\end{array}$ & $\begin{array}{c}\text { Fixed } \\
\text { TC_dT } \Delta\end{array}$ & dTM & $\mathbf{K}$ & $\begin{array}{l}\text { Sap } \\
\text { Velocity } \\
(\mathrm{cm} / \mathrm{s})\end{array}$ & $\begin{array}{c}\text { Probed } \\
\text { Drywood F } \\
(\mathrm{g} / \mathrm{hr})\end{array}$ & $\begin{array}{c}\text { Extrapolated } \\
\text { Wetwood F } \\
(\mathrm{g} / \mathrm{hr})\end{array}$ & $\begin{array}{c}\text { Extrapolated } \\
\text { Total Wood F } \\
(\mathrm{g} / \mathrm{hr})\end{array}$ & $\begin{array}{l}\text { Relative } \\
\text { Humidity, } \\
@ 10 \mathrm{~m}(\%)\end{array}$ \\
\hline & 8/5/2008 5:00 & 328 & 218 & 500 & $\begin{array}{r}9.15 \\
\end{array}$ & 9.15 & & 0.0109 & 4.58E-05 & 10.0431 & 3.7006 & \begin{tabular}{|r|}
13.7437 \\
\end{tabular} & \\
\hline & 8/5/2008 6:00 & 329 & 218 & 600 & 9.25 & 9.25 & & 0.0000 & $0.00 \mathrm{E}+00$ & 0.0000 & 0.0000 & 0.0000 & \\
\hline & 8/5/2008 7:00 & 330 & 218 & 700 & 9.33 & 9.33 & & -0.0086 & $0.00 E+00$ & 0.0000 & 0.0000 & 0.0000 & \\
\hline & 8/5/2008 8:00 & 331 & 218 & 800 & 9.21 & 9.21 & & 0.0043 & 1.47E-05 & 3.2249 & 1.1883 & 4.4131 & \\
\hline & 8/5/2008 9:00 & 332 & 218 & 900 & 7.797 & 7.797 & & 0.1864 & 1.50E-03 & 329.7346 & 121.4975 & 451.2321 & \\
\hline & 8/5/2008 10:00 & 333 & 218 & 1000 & 6.247 & 6.247 & & 0.4807 & 4.83E-03 & 1058.7107 & 390.1037 & 1448.8145 & \\
\hline & 8/5/2008 11:00 & 334 & 218 & 1100 & 6.407 & 6.407 & & 0.4437 & $4.38 E-03$ & 959.3690 & 353.4992 & 1312.8682 & \\
\hline & $8 / 5 / 200812: 00$ & 335 & 218 & 1200 & 6.095 & 6.095 & & 0.5176 & $5.29 \mathrm{E}-03$ & 1159.6953 & 427.3136 & 1587.0089 & \\
\hline & 8/5/2008 13:00 & 336 & 218 & 1300 & 5.69 & 5.69 & & 0.6257 & $6.68 \mathrm{E}-03$ & 1464.4349 & 539.6012 & 2004.0361 & \\
\hline & 8/5/2008 14:00 & 337 & 218 & 1400 & 6.149 & 6.149 & & 0.5043 & 5.12E-03 & 1123.0489 & 413.8105 & 1536.8594 & \\
\hline & 8/5/2008 15:00 & 338 & 218 & 1500 & 6.57 & 6.57 & & 0.4079 & $3.95 E-03$ & 864.9466 & 318.7074 & 1183.6540 & \\
\hline & 8/5/2008 16:00 & 339 & 218 & 1600 & 6.485 & 6.485 & & 0.4264 & $4.17 \mathrm{E}-03$ & 913.3639 & 336.5477 & 1249.9117 & \\
\hline & 8/5/2008 17:00 & 340 & 218 & 1700 & 6.033 & 6.033 & & 0.5332 & $5.49 \mathrm{E}-03$ & 1202.8572 & 443.2175 & 1646.0746 & \\
\hline & 8/5/2008 18:00 & 341 & 218 & 1800 & 5.842 & 5.842 & & 0.5834 & 6.13E-03 & 1343.5321 & 495.0520 & 1838.5842 & \\
\hline & 8/5/2008 19:00 & 342 & 218 & 1900 & 5.918 & 5.918 & & 0.5630 & 5.87E-03 & 1286.1180 & 473.8966 & 1760.0146 & \\
\hline & 8/5/2008 20:00 & 343 & 218 & 2000 & 6.59 & 6.59 & & 0.4036 & 3.90E-03 & 853.8069 & 314.6027 & 1168.4097 & \\
\hline & 8/5/2008 21:00 & 344 & 218 & 2100 & 8.38 & 8.38 & & 0.1038 & 7.32E-04 & 160.4771 & 59.1311 & 219.6082 & \\
\hline & $8 / 5 / 200822: 00$ & 345 & 218 & 2200 & 9.36 & 9.36 & & -0.0118 & $0.00 \mathrm{E}+00$ & 0.0000 & 0.0000 & 0.0000 & \\
\hline & 8/5/2008 23:00 & 346 & 218 & 2300 & 9.59 & 9.59 & & -0.0355 & $0.00 \mathrm{E}+00$ & 0.0000 & 0.0000 & 0.0000 & \\
\hline & 8/6/2008 0:00 & 347 & 219 & 0 & 9.72 & 9.72 & 9.73 & -0.0484 & $0.00 E+00$ & 0.0000 & 0.0000 & 0.0000 & \\
\hline & 8/6/2008 1:00 & 348 & 219 & 100 & 9.72 & 9.72 & & -0.0484 & $0.00 \mathrm{E}+00$ & 0.0000 & 0.0000 & 0.0000 & \\
\hline & $8 / 6 / 2008$ 2:00 & 349 & 219 & 200 & 9.73 & 9.73 & & -0.0493 & $0.00 E+00$ & 0.0000 & 0.0000 & 0.0000 & \\
\hline & 8/6/2008 3:00 & 350 & 219 & 300 & 9.73 & 9.73 & & -0.0493 & $0.00 E+00$ & 0.0000 & 0.0000 & 0.0000 & \\
\hline & 8/6/2008 4:00 & 351 & 219 & 400 & 9.71 & 9.71 & & -0.0474 & $0.00 \mathrm{E}+00$ & 0.0000 & 0.0000 & 0.0000 & \\
\hline & 8/6/2008 5:00 & 352 & 219 & 500 & 9.68 & 9.68 & & -0.0444 & $0.00 \mathrm{E}+00$ & 0.0000 & 0.0000 & 0.0000 & \\
\hline & 8/6/2008 6:00 & 353 & 219 & 600 & 9.64 & 9.64 & & -0.0405 & $0.00 E+00$ & 0.0000 & 0.0000 & 0.0000 & \\
\hline & 8/6/2008 7:00 & 354 & 219 & 700 & 9.61 & 9.61 & & -0.0375 & $0.00 \mathrm{E}+00$ & 0.0000 & 0.0000 & 0.0000 & \\
\hline & 8/6/2008 8:00 & 355 & 219 & 800 & 9.54 & 9.54 & & -0.0304 & $0.00 \mathrm{E}+00$ & 0.0000 & 0.0000 & 0.0000 & \\
\hline & 8/6/2008 9:00 & 356 & 219 & 900 & 9.03 & 9.03 & & 0.0244 & 1.23E-04 & 26.9432 & 9.9278 & 36.8710 & \\
\hline & 8/6/2008 10:00 & 357 & 219 & 1000 & 6.471 & 6.471 & & 0.4295 & $4.20 \mathrm{E}-03$ & 921.5085 & 339.5488 & 1261.0572 & \\
\hline & 8/6/2008 11:00 & 358 & 219 & 1100 & 5.549 & 5.549 & & 0.6670 & 7.23E-03 & 1584.3488 & 583.7859 & 2168.1347 & \\
\hline & 8/6/2008 12:00 & 359 & 219 & 1200 & 5.99 & 5.99 & & 0.5442 & 5.63E-03 & 1233.4933 & 454.5060 & 1687.9992 & \\
\hline & 8/6/2008 13:00 & 360 & 219 & 1300 & 6.308 & 6.308 & & 0.4664 & $4.65 \mathrm{E}-03$ & 1020.0249 & 375.8492 & 1395.8741 & \\
\hline & $8 / 6 / 2008$ 14:00 & 361 & 219 & 1400 & 5.94 & 5.94 & & 0.5572 & $5.79 \mathrm{E}-03$ & 1269.8589 & 467.9056 & 1737.7646 & \\
\hline & 8/6/2008 15:00 & 362 & 219 & 1500 & 5.692 & 5.692 & & 0.6251 & 6.67E-03 & 1462.7893 & 538.9948 & 2001.7841 & \\
\hline & 8/6/2008 16:00 & 363 & 219 & 1600 & 5.745 & 5.745 & & 0.6101 & 6.48E-03 & 1419.7218 & 523.1257 & 1942.8475 & \\
\hline & 8/6/2008 17:00 & 364 & 219 & 1700 & 5.688 & 5.688 & & 0.6262 & $6.69 \mathrm{E}-03$ & 1466.0821 & 540.2081 & 2006.2902 & \\
\hline & 8/6/2008 18:00 & 365 & 219 & 1800 & 6.097 & 6.097 & & 0.5171 & 5.28E-03 & 1158.3225 & 426.8078 & 1585.1303 & \\
\hline & 8/6/2008 19:00 & 366 & 219 & 1900 & 6.192 & 6.192 & & 0.4939 & 4.99E-03 & 1094.4804 & 403.2838 & 1497.7642 & \\
\hline & $8 / 6 / 200820: 00$ & 367 & 219 & 2000 & 6.429 & 6.429 & & 0.4388 & 4.32E-03 & 946.2371 & 348.6605 & 1294.8976 & \\
\hline & 8/6/2008 21:00 & 368 & 219 & 2100 & 7.579 & 7.579 & & 0.2205 & 1.85E-03 & 405.5646 & 149.4386 & 555.0033 & \\
\hline
\end{tabular}




\begin{tabular}{|c|c|c|c|c|c|c|c|c|c|c|c|c|c|}
\hline Tree ID & TS & RN & JD & HR & $\begin{array}{c}\text { Raw } \\
\text { TC_dTA }\end{array}$ & $\begin{array}{c}\text { Fixed } \\
\text { TC_dT } \Delta\end{array}$ & dTM & $\mathbf{K}$ & $\begin{array}{l}\text { Sap } \\
\text { Velocity } \\
(\mathrm{cm} / \mathrm{s})\end{array}$ & $\begin{array}{c}\text { Probed } \\
\text { Drywood F } \\
(\mathrm{g} / \mathrm{hr})\end{array}$ & $\begin{array}{c}\text { Extrapolated } \\
\text { Wetwood F } \\
(\mathrm{g} / \mathrm{hr})\end{array}$ & $\begin{array}{c}\text { Extrapolated } \\
\text { Total Wood F } \\
(\mathrm{g} / \mathrm{hr})\end{array}$ & $\begin{array}{l}\text { Relative } \\
\text { Humidity, } \\
@ 10 \mathrm{~m}(\%)\end{array}$ \\
\hline & 8/6/2008 22:00 & 369 & 219 & 2200 & 8.9 & 8.9 & & 0.0393 & 2.22E-04 & 48.5766 & 17.8990 & 66.4756 & \\
\hline & 8/6/2008 23:00 & 370 & 219 & 2300 & 9.24 & 9.24 & & 0.0011 & $2.66 \mathrm{E}-06$ & 0.5830 & 0.2148 & 0.7978 & \\
\hline & $8 / 7 / 20080: 00$ & 371 & 220 & 0 & 9.48 & 9.48 & 9.67 & -0.0243 & $0.00 E+00$ & 0.0000 & 0.0000 & 0.0000 & \\
\hline & 8/7/2008 1:00 & 372 & 220 & 100 & 9.66 & 9.66 & & -0.0424 & $0.00 \mathrm{E}+00$ & 0.0000 & 0.0000 & 0.0000 & \\
\hline & 8/7/2008 2:00 & 373 & 220 & 200 & 9.67 & 9.67 & & -0.0434 & $0.00 \mathrm{E}+00$ & 0.0000 & 0.0000 & 0.0000 & \\
\hline & 8/7/2008 3:00 & 374 & 220 & 300 & 9.64 & 9.64 & & -0.0405 & $0.00 \mathrm{E}+00$ & 0.0000 & 0.0000 & 0.0000 & \\
\hline & 8/7/2008 4:00 & 375 & 220 & 400 & 9.59 & 9.59 & & -0.0355 & $0.00 \mathrm{E}+00$ & 0.0000 & 0.0000 & 0.0000 & \\
\hline & 8/7/2008 5:00 & 376 & 220 & 500 & 9.53 & 9.53 & & -0.0294 & $0.00 \mathrm{E}+00$ & 0.0000 & 0.0000 & 0.0000 & \\
\hline & 8/7/2008 6:00 & 377 & 220 & 600 & 9.46 & 9.46 & & -0.0222 & $0.00 \mathrm{E}+00$ & 0.0000 & 0.0000 & 0.0000 & \\
\hline & 8/7/2008 7:00 & 378 & 220 & 700 & 9.4 & 9.4 & & -0.0160 & $0.00 E+00$ & 0.0000 & 0.0000 & 0.0000 & \\
\hline & 8/7/2008 8:00 & 379 & 220 & 800 & 9.34 & 9.34 & & -0.0096 & $0.00 \mathrm{E}+00$ & 0.0000 & 0.0000 & 0.0000 & \\
\hline & 8/7/2008 9:00 & 380 & 220 & 900 & 8.32 & 8.32 & & 0.1118 & $8.02 \mathrm{E}-04$ & 175.7555 & 64.7607 & 240.5163 & \\
\hline & 8/7/2008 10:00 & 381 & 220 & 1000 & 5.946 & 5.946 & & 0.5557 & 5.77E-03 & 1265.4522 & 466.2819 & 1731.7342 & \\
\hline & 8/7/2008 11:00 & 382 & 220 & 1100 & 5.293 & 5.293 & & 0.7476 & 8.32E-03 & 1823.3033 & 671.8336 & 2495.1368 & \\
\hline & 8/7/2008 12:00 & 383 & 220 & 1200 & 5.295 & 5.295 & & 0.7469 & 8.31E-03 & 1821.3217 & 671.1034 & 2492.4251 & \\
\hline & 8/7/2008 13:00 & 384 & 220 & 1300 & 5.312 & 5.312 & & 0.7413 & $8.23 \mathrm{E}-03$ & 1804.5547 & 664.9253 & 2469.4800 & \\
\hline & $8 / 7 / 2008$ 14:00 & 385 & 220 & 1400 & 5.361 & 5.361 & & 0.7254 & 8.02E-03 & 1756.9818 & 647.3961 & 2404.3778 & \\
\hline & 8/7/2008 15:00 & 386 & 220 & 1500 & 5.444 & 5.444 & & 0.6991 & 7.66E-03 & 1678.8821 & 618.6186 & 2297.5008 & \\
\hline & 8/7/2008 16:00 & 387 & 220 & 1600 & 5.478 & 5.478 & & 0.6886 & 7.52E-03 & 1647.7615 & 607.1516 & 2254.9132 & \\
\hline & 8/7/2008 17:00 & 388 & 220 & 1700 & 5.438 & 5.438 & & 0.7010 & 7.68E-03 & 1684.4258 & 620.6613 & 2305.0871 & \\
\hline & 8/7/2008 18:00 & 389 & 220 & 1800 & 5.558 & 5.558 & & 0.6643 & 7.19E-03 & 1576.4593 & 580.8788 & 2157.3381 & \\
\hline & 8/7/2008 19:00 & 390 & 220 & 1900 & 5.778 & 5.778 & & 0.6009 & 6.36E-03 & 1393.4257 & 513.4364 & 1906.8620 & \\
\hline & 8/7/2008 20:00 & 391 & 220 & 2000 & 6.209 & 6.209 & & 0.4898 & 4.94E-03 & 1083.3328 & 399.1762 & 1482.5090 & \\
\hline & 8/7/2008 21:00 & 392 & 220 & 2100 & 7.495 & 7.495 & & 0.2342 & 1.99E-03 & 436.7567 & 160.9320 & 597.6887 & \\
\hline & 8/7/2008 22:00 & 393 & 220 & 2200 & 8.4 & 8.4 & & 0.1012 & 7.09E-04 & 155.4910 & 57.2939 & 212.7848 & \\
\hline & 8/7/2008 23:00 & 394 & 220 & 2300 & 8.92 & 8.92 & & 0.0370 & $2.06 \mathrm{E}-04$ & 45.0578 & 16.6025 & 61.6603 & \\
\hline & 8/8/2008 0:00 & 395 & 221 & 0 & 9.21 & 9.21 & 9.57 & 0.0043 & 1.47E-05 & 3.2249 & 1.1883 & 4.4131 & \\
\hline & 8/8/2008 1:00 & 396 & 221 & 100 & 9.17 & 9.17 & & 0.0087 & 3.47E-05 & 7.6104 & 2.8042 & 10.4146 & \\
\hline & 8/8/2008 2:00 & 397 & 221 & 200 & 9.41 & 9.41 & & -0.0170 & $0.00 \mathrm{E}+00$ & 0.0000 & 0.0000 & 0.0000 & \\
\hline & 8/8/2008 3:00 & 398 & 221 & 300 & 9.57 & 9.57 & & -0.0334 & $0.00 \mathrm{E}+00$ & 0.0000 & 0.0000 & 0.0000 & \\
\hline & 8/8/2008 4:00 & 399 & 221 & 400 & 9.57 & 9.57 & & -0.0334 & $0.00 \mathrm{E}+00$ & 0.0000 & 0.0000 & 0.0000 & \\
\hline & 8/8/2008 5:00 & 400 & 221 & 500 & 9.53 & 9.53 & & -0.0294 & $0.00 \mathrm{E}+00$ & 0.0000 & 0.0000 & 0.0000 & \\
\hline & 8/8/2008 6:00 & 401 & 221 & 600 & 9.47 & 9.47 & & -0.0232 & $0.00 \mathrm{E}+00$ & 0.0000 & 0.0000 & 0.0000 & \\
\hline & $8 / 8 / 20087: 00$ & 402 & 221 & 700 & 9.43 & 9.43 & & -0.0191 & $0.00 \mathrm{E}+00$ & 0.0000 & 0.0000 & 0.0000 & \\
\hline & 8/8/2008 8:00 & 403 & 221 & 800 & 9.2 & 9.2 & & 0.0054 & 1.94E-05 & 4.2500 & 1.5660 & 5.8160 & \\
\hline & 8/8/20089:00 & 404 & 221 & 900 & 7.246 & 7.246 & & 0.2766 & $2.45 E-03$ & 536.0847 & 197.5314 & 733.6161 & \\
\hline & 8/8/2008 10:00 & 405 & 221 & 1000 & 5.523 & 5.523 & & 0.6748 & 7.33E-03 & 1607.3271 & 592.2527 & 2199.5798 & \\
\hline & 8/8/2008 11:00 & 406 & 221 & 1100 & 5.395 & 5.395 & & 0.7146 & 7.87E-03 & 1724.6178 & 635.4709 & 2360.0887 & \\
\hline & $8 / 8 / 200812: 00$ & 407 & 221 & 1200 & 5.288 & 5.288 & & 0.7492 & $8.34 \mathrm{E}-03$ & 1828.2655 & 673.6620 & 2501.9276 & \\
\hline & 8/8/2008 13:00 & 408 & 221 & 1300 & 5.282 & 5.282 & & 0.7512 & 8.37E-03 & 1834.2360 & 675.8620 & 2510.0980 & \\
\hline & 8/8/2008 14:00 & 409 & 221 & 1400 & 5.256 & 5.256 & & 0.7599 & 8.49E-03 & 1860.3082 & 685.4688 & 2545.7771 & \\
\hline
\end{tabular}




\begin{tabular}{|c|c|c|c|c|c|c|c|c|c|c|c|c|c|}
\hline Tree ID & TS & RN & JD & HR & $\begin{array}{c}\text { Raw } \\
\text { TC_dTA }\end{array}$ & $\begin{array}{c}\text { Fixed } \\
\text { TC_dT } \Delta\end{array}$ & dTM & $\mathbf{K}$ & $\begin{array}{l}\text { Sap } \\
\text { Velocity } \\
(\mathrm{cm} / \mathrm{s})\end{array}$ & $\begin{array}{c}\text { Probed } \\
\text { Drywood F } \\
(\mathrm{g} / \mathrm{hr})\end{array}$ & $\begin{array}{c}\text { Extrapolated } \\
\text { Wetwood F } \\
(\mathrm{g} / \mathrm{hr})\end{array}$ & $\begin{array}{c}\text { Extrapolated } \\
\text { Total Wood F } \\
(\mathrm{g} / \mathrm{hr})\end{array}$ & $\begin{array}{l}\text { Relative } \\
\text { Humidity, } \\
@ 10 \mathrm{~m}(\%)\end{array}$ \\
\hline & 8/8/2008 15:00 & 410 & 221 & 1500 & 5.287 & 5.287 & & 0.7496 & 8.35E-03 & 1829.2594 & 674.0283 & \begin{tabular}{|r|}
2503.2877 \\
\end{tabular} & \\
\hline & 8/8/2008 16:00 & 411 & 221 & 1600 & 5.314 & 5.314 & & 0.7407 & $8.22 \mathrm{E}-03$ & 1802.5911 & 664.2018 & 2466.7928 & \\
\hline & 8/8/2008 17:00 & 412 & 221 & 1700 & 5.404 & 5.404 & & 0.7117 & $7.83 E-03$ & 1716.1379 & 632.3463 & 2348.4841 & \\
\hline & 8/8/2008 18:00 & 413 & 221 & 1800 & 5.668 & 5.668 & & 0.6320 & $6.76 \mathrm{E}-03$ & 1482.6368 & 546.3080 & 2028.9449 & \\
\hline & 8/8/2008 19:00 & 414 & 221 & 1900 & 5.69 & 5.69 & & 0.6257 & 6.68E-03 & 1464.4349 & 539.6012 & 2004.0361 & \\
\hline & 8/8/2008 20:00 & 415 & 221 & 2000 & 6.243 & 6.243 & & 0.4817 & 4.84E-03 & 1061.2834 & 391.0517 & 1452.3351 & \\
\hline & 8/8/2008 21:00 & 416 & 221 & 2100 & 7.392 & 7.392 & & 0.2514 & 2.17E-03 & 476.5710 & 175.6024 & 652.1734 & \\
\hline & 8/8/2008 22:00 & 417 & 221 & 2200 & 8.19 & 8.19 & & 0.1294 & $9.60 \mathrm{E}-04$ & 210.5122 & 77.5676 & 288.0798 & \\
\hline & 8/8/2008 23:00 & 418 & 221 & 2300 & 8.34 & 8.34 & & 0.1091 & 7.78E-04 & 170.6094 & 62.8645 & 233.4739 & \\
\hline & 8/9/2008 0:00 & 419 & 222 & 0 & 8.27 & 8.27 & 9.27 & 0.1185 & 8.62E-04 & 188.8549 & 69.5875 & 258.4424 & \\
\hline & 8/9/2008 1:00 & 420 & 222 & 100 & 8.22 & 8.22 & & 0.1253 & $9.23 \mathrm{E}-04$ & 202.2897 & 74.5378 & 276.8275 & \\
\hline & 8/9/2008 2:00 & 421 & 222 & 200 & 8.25 & 8.25 & & 0.1212 & 8.86E-04 & 194.1885 & 71.5527 & 265.7413 & \\
\hline & 8/9/2008 3:00 & 422 & 222 & 300 & 8.27 & 8.27 & & 0.1185 & 8.62E-04 & 188.8549 & 69.5875 & 258.4424 & \\
\hline & 8/9/2008 4:00 & 423 & 222 & 400 & 8.24 & 8.24 & & 0.1226 & 8.98E-04 & 196.8754 & 72.5428 & 269.4182 & \\
\hline & $8 / 9 / 20085: 00$ & 424 & 222 & 500 & 8.1 & 8.1 & & 0.1420 & 1.08E-03 & 235.9132 & 86.9271 & 322.8403 & \\
\hline & 8/9/2008 6:00 & 425 & 222 & 600 & 8.12 & 8.12 & & 0.1392 & 1.05E-03 & 230.1730 & 84.8120 & 314.9850 & \\
\hline & 8/9/2008 7:00 & 426 & 222 & 700 & 7.714 & 7.714 & & 0.1991 & 1.63E-03 & 357.7542 & 131.8219 & 489.5760 & \\
\hline & 8/9/2008 8:00 & 427 & 222 & 800 & 7.155 & 7.155 & & 0.2928 & 2.62E-03 & 575.0842 & 211.9016 & 786.9857 & \\
\hline & 8/9/2008 9:00 & 428 & 222 & 900 & 7.743 & 7.743 & & 0.1946 & 1.59E-03 & 347.8471 & 128.1714 & 476.0186 & \\
\hline & 8/9/2008 10:00 & 429 & 222 & 1000 & 7.271 & 7.271 & & 0.2722 & $2.40 \mathrm{E}-03$ & 525.6307 & 193.6794 & 719.3101 & \\
\hline & 8/9/2008 11:00 & 430 & 222 & 1100 & 6.739 & 6.739 & & 0.3726 & 3.53E-03 & 773.7286 & 285.0962 & 1058.8248 & \\
\hline & 8/9/2008 12:00 & 431 & 222 & 1200 & 6.089 & 6.089 & & 0.5191 & 5.31E-03 & 1163.8210 & 428.8338 & 1592.6548 & \\
\hline & 8/9/2008 13:00 & 432 & 222 & 1300 & 5.875 & 5.875 & & 0.5745 & 6.01E-03 & 1318.3620 & 485.7776 & 1804.1395 & \\
\hline & 8/9/2008 14:00 & 433 & 222 & 1400 & 5.458 & 5.458 & & 0.6948 & 7.60E-03 & 1666.0076 & 613.8747 & 2279.8823 & \\
\hline & 8/9/2008 15:00 & 434 & 222 & 1500 & 5.339 & 5.339 & & 0.7325 & 8.11E-03 & 1778.2035 & 655.2157 & 2433.4192 & \\
\hline & 8/9/2008 16:00 & 435 & 222 & 1600 & 5.413 & 5.413 & & 0.7088 & 7.79E-03 & 1707.6939 & 629.2349 & 2336.9288 & \\
\hline & 8/9/2008 17:00 & 436 & 222 & 1700 & 5.565 & 5.565 & & 0.6622 & 7.16E-03 & 1570.3457 & 578.6262 & 2148.9718 & \\
\hline & 8/9/2008 18:00 & 437 & 222 & 1800 & 5.65 & 5.65 & & 0.6372 & $6.83 \mathrm{E}-03$ & 1497.6663 & 551.8460 & 2049.5123 & \\
\hline & 8/9/2008 19:00 & 438 & 222 & 1900 & 5.841 & 5.841 & & 0.5836 & 6.13E-03 & 1344.3007 & 495.3352 & 1839.6359 & \\
\hline & 8/9/2008 20:00 & 439 & 222 & 2000 & 6.296 & 6.296 & & 0.4692 & 4.69E-03 & 1027.5548 & 378.6237 & 1406.1784 & \\
\hline & 8/9/2008 21:00 & 440 & 222 & 2100 & 7.919 & 7.919 & & 0.1681 & 1.32E-03 & 290.3878 & 106.9993 & 397.3871 & \\
\hline & 8/9/2008 22:00 & 441 & 222 & 2200 & 9.06 & 9.06 & & 0.0210 & 1.02E-04 & 22.4026 & 8.2547 & 30.6574 & \\
\hline & 8/9/2008 23:00 & 442 & 222 & 2300 & 9.27 & 9.27 & & -0.0022 & $0.00 \mathrm{E}+00$ & 0.0000 & 0.0000 & 0.0000 & \\
\hline & 8/10/2008 0:00 & 443 & 223 & 0 & 9.33 & 9.33 & 9.36 & -0.0086 & $0.00 \mathrm{E}+00$ & 0.0000 & 0.0000 & 0.0000 & \\
\hline & 8/10/2008 1:00 & 444 & 223 & 100 & 9.36 & 9.36 & & -0.0118 & $0.00 \mathrm{E}+00$ & 0.0000 & 0.0000 & 0.0000 & \\
\hline & 8/10/2008 2:00 & 445 & 223 & 200 & 9.34 & 9.34 & & -0.0096 & $0.00 \mathrm{E}+00$ & 0.0000 & 0.0000 & 0.0000 & \\
\hline & 8/10/2008 3:00 & 446 & 223 & 300 & 9.31 & 9.31 & & -0.0064 & $0.00 \mathrm{E}+00$ & 0.0000 & 0.0000 & 0.0000 & \\
\hline & 8/10/2008 4:00 & 447 & 223 & 400 & 9.27 & 9.27 & & -0.0022 & $0.00 \mathrm{E}+00$ & 0.0000 & 0.0000 & 0.0000 & \\
\hline & $8 / 10 / 20085: 00$ & 448 & 223 & 500 & 9.19 & 9.19 & & 0.0065 & $2.43 \mathrm{E}-05$ & 5.3265 & 1.9627 & 7.2891 & \\
\hline & 8/10/2008 6:00 & 449 & 223 & 600 & 9.11 & 9.11 & & 0.0154 & 6.97E-05 & 15.2790 & 5.6299 & 20.9089 & \\
\hline & 8/10/2008 7:00 & 450 & 223 & 700 & 9.04 & 9.04 & & 0.0232 & 1.16E-04 & 25.4090 & 9.3625 & 34.7714 & \\
\hline
\end{tabular}




\begin{tabular}{|c|c|c|c|c|c|c|c|c|c|c|c|c|c|}
\hline Tree ID & TS & RN & JD & HR & $\begin{array}{c}\text { Raw } \\
\text { TC_dTA }\end{array}$ & $\begin{array}{c}\text { Fixed } \\
\text { TC_dT } \Delta\end{array}$ & dTM & $\mathbf{K}$ & $\begin{array}{l}\text { Sap } \\
\text { Velocity } \\
(\mathrm{cm} / \mathrm{s})\end{array}$ & $\begin{array}{c}\text { Probed } \\
\text { Drywood F } \\
(\mathrm{g} / \mathrm{hr})\end{array}$ & $\begin{array}{c}\text { Extrapolated } \\
\text { Wetwood F } \\
(\mathrm{g} / \mathrm{hr})\end{array}$ & $\begin{array}{c}\text { Extrapolated } \\
\text { Total Wood F } \\
(\mathrm{g} / \mathrm{hr})\end{array}$ & $\begin{array}{l}\text { Relative } \\
\text { Humidity, } \\
@ 10 \mathrm{~m}(\%)\end{array}$ \\
\hline & 8/10/2008 8:00 & 451 & 223 & 800 & $\begin{array}{r}8.92 \\
\end{array}$ & 8.92 & & 0.0370 & 2.06E-04 & 45.0578 & 16.6025 & 61.6603 & \\
\hline & 8/10/2008 9:00 & 452 & 223 & 900 & 7.769 & 7.769 & & 0.1906 & 1.55E-03 & 339.0722 & 124.9381 & 464.0103 & \\
\hline & $8 / 10 / 2008$ 10:00 & 453 & 223 & 1000 & 5.515 & 5.515 & & 0.6772 & 7.37E-03 & 1614.4535 & 594.8786 & 2209.3321 & \\
\hline & 8/10/2008 11:00 & 454 & 223 & 1100 & 4.96 & 4.96 & & 0.8649 & 9.95E-03 & 2181.7014 & 803.8928 & 2985.5942 & \\
\hline & $8 / 10 / 200812: 00$ & 455 & 223 & 1200 & 4.896 & 4.896 & & 0.8893 & 1.03E-02 & 2257.6427 & 831.8749 & 3089.5176 & \\
\hline & 8/10/2008 13:00 & 456 & 223 & 1300 & 4.881 & 4.881 & & 0.8951 & 1.04E-02 & 2275.8010 & 838.5657 & 3114.3667 & \\
\hline & $8 / 10 / 2008$ 14:00 & 457 & 223 & 1400 & 4.856 & 4.856 & & 0.9049 & $1.05 E-02$ & 2306.3754 & 849.8315 & 3156.2069 & \\
\hline & $8 / 10 / 2008$ 15:00 & 458 & 223 & 1500 & 5.077 & 5.077 & & 0.8219 & 9.35E-03 & 2049.0279 & 755.0065 & 2804.0344 & \\
\hline & 8/10/2008 16:00 & 459 & 223 & 1600 & 5.029 & 5.029 & & 0.8393 & 9.59E-03 & 2102.5229 & 774.7178 & 2877.2407 & \\
\hline & 8/10/2008 17:00 & 460 & 223 & 1700 & 5.23 & 5.23 & & 0.7686 & 8.61E-03 & 1886.7094 & 695.1969 & 2581.9063 & \\
\hline & 8/10/2008 18:00 & 461 & 223 & 1800 & 7.708 & 7.708 & & 0.2001 & 1.64E-03 & 359.8197 & 132.5830 & 492.4027 & \\
\hline & $8 / 10 / 2008$ 19:00 & 462 & 223 & 1900 & 8.55 & 8.55 & & 0.0819 & $5.47 \mathrm{E}-04$ & 119.7964 & 44.1414 & 163.9378 & \\
\hline & 8/10/2008 20:00 & 463 & 223 & 2000 & 8.26 & 8.26 & & 0.1199 & 8.74E-04 & 191.5150 & 70.5676 & 262.0827 & \\
\hline & 8/10/2008 21:00 & 464 & 223 & 2100 & 8.2 & 8.2 & & 0.1280 & 9.48E-04 & 207.7579 & 76.5527 & 284.3105 & \\
\hline & $8 / 10 / 2008$ 22:00 & 465 & 223 & 2200 & 8.37 & 8.37 & & 0.1051 & 7.44E-04 & 162.9902 & 60.0571 & 223.0473 & \\
\hline & 8/10/2008 23:00 & 466 & 223 & 2300 & 8.28 & 8.28 & & 0.1171 & 8.50E-04 & 186.2083 & 68.6123 & 254.8206 & \\
\hline & $8 / 11 / 20080: 00$ & 467 & 224 & 0 & 8.25 & 8.25 & 8.53 & 0.1212 & 8.86E-04 & 194.1885 & 71.5527 & 265.7413 & \\
\hline & 8/11/2008 1:00 & 468 & 224 & 100 & 8.34 & 8.34 & & 0.1091 & 7.78E-04 & 170.6094 & 62.8645 & 233.4739 & \\
\hline & 8/11/2008 2:00 & 469 & 224 & 200 & 8.48 & 8.48 & & 0.0908 & $6.21 E-04$ & 136.0796 & 50.1413 & 186.2210 & \\
\hline & 8/11/2008 3:00 & 470 & 224 & 300 & 8.53 & 8.53 & & 0.0844 & 5.67E-04 & 124.3817 & 45.8310 & 170.2127 & \\
\hline & 8/11/2008 4:00 & 471 & 224 & 400 & 8.48 & 8.48 & & 0.0908 & $6.21 \mathrm{E}-04$ & 136.0796 & 50.1413 & 186.2210 & \\
\hline & 8/11/2008 5:00 & 472 & 224 & 500 & 8.39 & 8.39 & & 0.1025 & 7.21E-04 & 157.9774 & 58.2100 & 216.1874 & \\
\hline & 8/11/2008 6:00 & 473 & 224 & 600 & 8.38 & 8.38 & & 0.1038 & 7.32E-04 & 160.4771 & 59.1311 & 219.6082 & \\
\hline & 8/11/2008 7:00 & 474 & 224 & 700 & 8.34 & 8.34 & & 0.1091 & 7.78E-04 & 170.6094 & 62.8645 & 233.4739 & \\
\hline & 8/11/2008 8:00 & 475 & 224 & 800 & 8.24 & 8.24 & & 0.1226 & 8.98E-04 & 196.8754 & 72.5428 & 269.4182 & \\
\hline & 8/11/2008 9:00 & 476 & 224 & 900 & 7.276 & 7.276 & & 0.2713 & 2.39E-03 & 523.5531 & 192.9139 & 716.4671 & \\
\hline & 8/11/2008 10:00 & 477 & 224 & 1000 & 5.312 & 5.312 & & 0.7413 & 8.23E-03 & 1804.5547 & 664.9253 & 2469.4800 & \\
\hline & 8/11/2008 11:00 & 478 & 224 & 1100 & 4.812 & 4.812 & & 0.9223 & $1.08 \mathrm{E}-02$ & 2361.1472 & 870.0132 & 3231.1604 & \\
\hline & $8 / 11 / 2008$ 12:00 & 479 & 224 & 1200 & 4.709 & 4.709 & & 0.9643 & 1.14E-02 & 2494.3455 & 919.0929 & 3413.4384 & \\
\hline & 8/11/2008 13:00 & 480 & 224 & 1300 & 4.863 & 4.863 & & 0.9021 & $1.05 E-02$ & 2297.7752 & 846.6625 & 3144.4377 & \\
\hline & 8/11/2008 14:00 & 481 & 224 & 1400 & 4.643 & 4.643 & & 0.9922 & 1.18E-02 & 2583.5506 & 951.9624 & 3535.5130 & \\
\hline & 8/11/2008 15:00 & 482 & 224 & 1500 & 4.573 & 4.573 & & 1.0227 & 1.22E-02 & 2681.6401 & 988.1054 & 3669.7455 & \\
\hline & $8 / 11 / 2008$ 16:00 & 483 & 224 & 1600 & 4.836 & 4.836 & & 0.9127 & 1.06E-02 & 2331.1182 & 858.9485 & 3190.0667 & \\
\hline & 8/11/2008 17:00 & 484 & 224 & 1700 & 4.988 & 4.988 & & 0.8545 & 9.81E-03 & 2149.2406 & 791.9319 & 2941.1725 & \\
\hline & 8/11/2008 18:00 & 485 & 224 & 1800 & 5.279 & 5.279 & & 0.7522 & 8.38E-03 & 1837.2278 & 676.9644 & 2514.1921 & \\
\hline & $8 / 11 / 2008$ 19:00 & 486 & 224 & 1900 & 5.418 & 5.418 & & 0.7073 & 7.77E-03 & 1703.0183 & 627.5121 & 2330.5304 & \\
\hline & 8/11/2008 20:00 & 487 & 224 & 2000 & 6.064 & 6.064 & & 0.5254 & 5.39E-03 & 1181.1289 & 435.2112 & 1616.3402 & \\
\hline & 8/11/2008 21:00 & 488 & 224 & 2100 & 6.933 & 6.933 & & 0.3342 & 3.09E-03 & 676.7500 & 249.3625 & 926.1125 & \\
\hline & $8 / 11 / 200822: 00$ & 489 & 224 & 2200 & 7.534 & 7.534 & & 0.2278 & 1.93E-03 & 422.1342 & 155.5440 & 577.6783 & \\
\hline & 8/11/2008 23:00 & 490 & 224 & 2300 & 7.813 & 7.813 & & 0.1839 & 1.48E-03 & 324.4508 & 119.5506 & 444.0014 & \\
\hline & 8/12/2008 0:00 & 491 & 225 & 0 & 7.983 & 7.983 & 8.44 & 0.1587 & 1.23E-03 & 270.6013 & 99.7086 & 370.3099 & \\
\hline
\end{tabular}




\begin{tabular}{|c|c|c|c|c|c|c|c|c|c|c|c|c|c|}
\hline Tree ID & TS & RN & JD & HR & $\begin{array}{c}\text { Raw } \\
\text { TC_dTA }\end{array}$ & $\begin{array}{c}\text { Fixed } \\
\text { TC_dT } \Delta\end{array}$ & dTM & $\mathbf{K}$ & $\begin{array}{l}\text { Sap } \\
\text { Velocity } \\
(\mathrm{cm} / \mathrm{s})\end{array}$ & $\begin{array}{c}\text { Probed } \\
\text { Drywood F } \\
(\mathrm{g} / \mathrm{hr})\end{array}$ & $\begin{array}{c}\text { Extrapolated } \\
\text { Wetwood F } \\
(\mathrm{g} / \mathrm{hr})\end{array}$ & $\begin{array}{c}\text { Extrapolated } \\
\text { Total Wood F } \\
(\mathrm{g} / \mathrm{hr})\end{array}$ & $\begin{array}{l}\text { Relative } \\
\text { Humidity, } \\
@ 10 \mathrm{~m}(\%)\end{array}$ \\
\hline & 8/12/2008 1:00 & 492 & 225 & 100 & 8.05 & 8.05 & & 0.1491 & $1.14 \mathrm{E}-03$ & 250.5046 & 92.3036 & 342.8082 & \\
\hline & 8/12/2008 2:00 & 493 & 225 & 200 & 8.18 & 8.18 & & 0.1308 & 9.73E-04 & 213.2802 & 78.5875 & 291.8676 & \\
\hline & 8/12/2008 3:00 & 494 & 225 & 300 & 8.43 & 8.43 & & 0.0973 & $6.76 \mathrm{E}-04$ & 148.1117 & 54.5748 & 202.6865 & \\
\hline & 8/12/2008 4:00 & 495 & 225 & 400 & 8.44 & 8.44 & & 0.0960 & 6.65E-04 & 145.6786 & 53.6783 & 199.3569 & \\
\hline & 8/12/2008 5:00 & 496 & 225 & 500 & 8.38 & 8.38 & & 0.1038 & 7.32E-04 & 160.4771 & 59.1311 & 219.6082 & \\
\hline & 8/12/2008 6:00 & 497 & 225 & 600 & 8.31 & 8.31 & & 0.1131 & 8.14E-04 & 178.3487 & 65.7162 & 244.0649 & \\
\hline & 8/12/2008 7:00 & 498 & 225 & 700 & 8.31 & 8.31 & & 0.1131 & $8.14 \mathrm{E}-04$ & 178.3487 & 65.7162 & 244.0649 & \\
\hline & 8/12/2008 8:00 & 499 & 225 & 800 & 8.25 & 8.25 & & 0.1212 & 8.86E-04 & 194.1885 & 71.5527 & 265.7413 & \\
\hline & 8/12/2008 9:00 & 500 & 225 & 900 & 7.43 & 7.43 & & 0.2450 & 2.11E-03 & 461.6778 & 170.1147 & 631.7925 & \\
\hline & $8 / 12 / 2008$ 10:00 & 501 & 225 & 1000 & 5.48 & 5.48 & & 0.6880 & 7.51E-03 & 1645.9463 & 606.4828 & 2252.4291 & \\
\hline & $8 / 12 / 2008$ 11:00 & 502 & 225 & 1100 & 4.663 & 4.663 & & 0.9837 & 1.17E-02 & 2556.1897 & 941.8807 & 3498.0704 & \\
\hline & $8 / 12 / 2008$ 12:00 & 503 & 225 & 1200 & 4.559 & 4.559 & & 1.0290 & 1.23E-02 & 2701.7030 & 995.4981 & 3697.2011 & \\
\hline & $8 / 12 / 2008$ 13:00 & 504 & 225 & 1300 & 4.455 & 4.455 & & 1.0763 & 1.30E-02 & 2855.6014 & 1052.2051 & 3907.8064 & \\
\hline & $8 / 12 / 2008$ 14:00 & 505 & 225 & 1400 & 4.408 & 4.408 & & 1.0985 & 1.34E-02 & 2928.0767 & 1078.9101 & 4006.9868 & \\
\hline & $8 / 12 / 2008$ 15:00 & 506 & 225 & 1500 & 4.419 & 4.419 & & 1.0932 & 1.33E-02 & 2910.9455 & 1072.5977 & 3983.5433 & \\
\hline & $8 / 12 / 2008$ 16:00 & 507 & 225 & 1600 & 4.464 & 4.464 & & 1.0721 & 1.30E-02 & 2841.9357 & 1047.1697 & 3889.1053 & \\
\hline & $8 / 12 / 2008$ 17:00 & 508 & 225 & 1700 & 4.511 & 4.511 & & 1.0505 & 1.26E-02 & 2771.6528 & 1021.2725 & 3792.9252 & \\
\hline & $8 / 12 / 2008$ 18:00 & 509 & 225 & 1800 & 4.607 & 4.607 & & 1.0078 & $1.20 \mathrm{E}-02$ & 2633.5386 & 970.3815 & 3603.9201 & \\
\hline & $8 / 12 / 2008$ 19:00 & 510 & 225 & 1900 & 4.829 & 4.829 & & 0.9155 & 1.07E-02 & 2339.8384 & 862.1616 & 3202.0000 & \\
\hline & $8 / 12 / 200820: 00$ & 511 & 225 & 2000 & 5.294 & 5.294 & & 0.7473 & 8.31E-03 & 1822.3122 & 671.4684 & 2493.7807 & \\
\hline & $8 / 12 / 2008$ 21:00 & 512 & 225 & 2100 & 6.822 & 6.822 & & 0.3559 & 3.34E-03 & 731.2639 & 269.4492 & 1000.7131 & \\
\hline & $8 / 12 / 2008$ 22:00 & 513 & 225 & 2200 & 7.711 & 7.711 & & 0.1996 & 1.64E-03 & 358.7863 & 132.2022 & 490.9884 & \\
\hline & $8 / 12 / 2008$ 23:00 & 514 & 225 & 2300 & 7.862 & 7.862 & & 0.1765 & 1.41E-03 & 308.5024 & 113.6741 & 422.1765 & \\
\hline & 8/13/2008 0:00 & 515 & 226 & 0 & 8.03 & 8.03 & 8.27 & 0.1519 & 1.17E-03 & 256.4380 & 94.4899 & 350.9279 & \\
\hline & 8/13/2008 1:00 & 516 & 226 & 100 & 8.04 & 8.04 & & 0.1505 & $1.16 \mathrm{E}-03$ & 253.4644 & 93.3942 & 346.8585 & \\
\hline & 8/13/2008 2:00 & 517 & 226 & 200 & 8.06 & 8.06 & & 0.1476 & 1.13E-03 & 247.5587 & 91.2181 & 338.7768 & \\
\hline & 8/13/2008 3:00 & 518 & 226 & 300 & 8.17 & 8.17 & & 0.1322 & 9.86E-04 & 216.0616 & 79.6123 & 295.6740 & \\
\hline & 8/13/2008 4:00 & 519 & 226 & 400 & 8.18 & 8.18 & & 0.1308 & $9.73 E-04$ & 213.2802 & 78.5875 & 291.8676 & \\
\hline & 8/13/2008 5:00 & 520 & 226 & 500 & 8.13 & 8.13 & & 0.1378 & $1.04 \mathrm{E}-03$ & 227.3235 & 83.7620 & 311.0855 & \\
\hline & 8/13/2008 6:00 & 521 & 226 & 600 & 8.26 & 8.26 & & 0.1199 & 8.74E-04 & 191.5150 & 70.5676 & 262.0827 & \\
\hline & $8 / 13 / 2008$ 7:00 & 522 & 226 & 700 & 8.27 & 8.27 & & 0.1185 & $8.62 E-04$ & 188.8549 & 69.5875 & 258.4424 & \\
\hline & 8/13/2008 8:00 & 523 & 226 & 800 & 7.96 & 7.96 & & 0.1621 & 1.27E-03 & 277.6453 & 102.3041 & 379.9494 & \\
\hline & 8/13/2008 9:00 & 524 & 226 & 900 & 6.087 & 6.087 & & 0.5196 & $5.32 \mathrm{E}-03$ & 1165.1986 & 429.3414 & 1594.5401 & \\
\hline & $8 / 13 / 2008$ 10:00 & 525 & 226 & 1000 & 4.744 & 4.744 & & 0.9498 & 1.12E-02 & 2448.2805 & 902.1193 & 3350.3999 & \\
\hline & 8/13/2008 11:00 & 526 & 226 & 1100 & 4.666 & 4.666 & & 0.9824 & 1.16E-02 & 2552.1105 & 940.3776 & 3492.4881 & \\
\hline & $8 / 13 / 2008$ 12:00 & 527 & 226 & 1200 & 4.266 & 4.266 & & 1.1683 & 1.44E-02 & 3158.9389 & 1163.9760 & 4322.9149 & \\
\hline
\end{tabular}




\begin{tabular}{|c|c|c|c|c|c|c|c|c|c|c|c|c|c|}
\hline Tree ID & TS & RN & JD & HR & $\begin{array}{c}\text { Raw } \\
\text { TC_dTA }\end{array}$ & $\begin{array}{c}\text { Fixed } \\
\text { TC_dT } \Delta\end{array}$ & dTM & $\mathbf{K}$ & $\begin{array}{c}\text { Sap } \\
\text { Velocity } \\
(\mathrm{cm} / \mathrm{s})\end{array}$ & $\begin{array}{c}\text { Probed } \\
\text { Drywood F } \\
(\mathrm{g} / \mathrm{hr})\end{array}$ & $\begin{array}{c}\text { Extrapolated } \\
\text { Wetwood F } \\
(\mathrm{g} / \mathrm{hr})\end{array}$ & $\begin{array}{c}\text { Extrapolated } \\
\text { Total Wood F } \\
(\mathrm{g} / \mathrm{hr})\end{array}$ & $\begin{array}{c}\text { Relative } \\
\text { Humidity, } \\
@ 10 \mathrm{~m}(\%)\end{array}$ \\
\hline & $7 / 22 / 2008$ 13:00 & 0 & 204 & 1300 & 4.974 & 4.974 & 9.14 & 0.8376 & 9.57E-03 & 2435.4512 & 897.3921 & 3332.8433 & \\
\hline \multirow[t]{2}{*}{ W809EC1 } & $7 / 22 / 200814: 00$ & 1 & 204 & 1400 & 5.478 & 5.478 & & 0.6685 & $7.25 \mathrm{E}-03$ & 1845.1998 & 679.9018 & 2525.1016 & \\
\hline & $7 / 22 / 2008$ 15:00 & 2 & 204 & 1500 & 5.401 & 5.401 & & 0.6923 & 7.57E-03 & 1926.3540 & 709.8048 & 2636.1588 & \\
\hline \multirow[t]{2}{*}{ Probe \# } & $7 / 22 / 2008$ 16:00 & 3 & 204 & 1600 & 5.299 & 5.299 & & 0.7249 & 8.01E-03 & 2038.5345 & 751.1400 & 2789.6745 & \\
\hline & 7/22/2008 17:00 & 4 & 204 & 1700 & 5.327 & 5.327 & & 0.7158 & $7.88 \mathrm{E}-03$ & 2007.1927 & 739.5914 & 2746.7842 & \\
\hline \multirow[t]{4}{*}{18} & $7 / 22 / 2008$ 18:00 & 5 & 204 & 1800 & 5.458 & 5.458 & & 0.6746 & $7.33 \mathrm{E}-03$ & 1865.9960 & 687.5646 & 2553.5606 & \\
\hline & 7/22/2008 19:00 & 6 & 204 & 1900 & 5.717 & 5.717 & & 0.5987 & 6.33E-03 & 1611.1304 & 593.6541 & 2204.7845 & \\
\hline & $7 / 22 / 200820: 00$ & 7 & 204 & 2000 & 6.085 & 6.085 & & 0.5021 & $5.10 \mathrm{E}-03$ & 1297.1012 & 477.9436 & 1775.0448 & \\
\hline & 7/22/2008 21:00 & 8 & 204 & 2100 & 7.496 & 7.496 & & 0.2193 & 1.84E-03 & 467.9611 & 172.4299 & 640.3910 & \\
\hline \multirow[t]{2}{*}{$\mathrm{DBH}(\mathrm{cm})$} & $7 / 22 / 2008$ 22:00 & 9 & 204 & 2200 & 8.69 & 8.69 & & 0.0518 & $3.11 \mathrm{E}-04$ & 79.1628 & 29.1692 & 108.3320 & \\
\hline & $7 / 22 / 2008$ 23:00 & 10 & 204 & 2300 & 9.14 & 9.14 & & 0.0000 & $0.00 E+00$ & 0.0000 & 0.0000 & 0.0000 & \\
\hline \multirow[t]{2}{*}{11.7} & $7 / 23 / 20080: 00$ & 11 & 205 & 0 & 9.45 & 9.45 & 9.64 & -0.0328 & $0.00 \mathrm{E}+00$ & 0.0000 & 0.0000 & 0.0000 & \\
\hline & 7/23/2008 1:00 & 12 & 205 & 100 & 9.57 & 9.57 & & -0.0449 & $0.00 \mathrm{E}+00$ & 0.0000 & 0.0000 & 0.0000 & \\
\hline Day Count & 7/23/2008 2:00 & 13 & 205 & 200 & 9.62 & 9.62 & & -0.0499 & $0.00 \mathrm{E}+00$ & 0.0000 & 0.0000 & 0.0000 & \\
\hline \multirow{27}{*}{35} & 7/23/2008 3:00 & 14 & 205 & 300 & 9.64 & 9.64 & & -0.0519 & $0.00 \mathrm{E}+00$ & 0.0000 & 0.0000 & 0.0000 & \\
\hline & 7/23/2008 4:00 & 15 & 205 & 400 & 9.61 & 9.61 & & -0.0489 & $0.00 \mathrm{E}+00$ & 0.0000 & 0.0000 & 0.0000 & \\
\hline & 7/23/2008 5:00 & 16 & 205 & 500 & 9.51 & 9.51 & & -0.0389 & $0.00 \mathrm{E}+00$ & 0.0000 & 0.0000 & 0.0000 & \\
\hline & 7/23/2008 6:00 & 17 & 205 & 600 & 9.34 & 9.34 & & -0.0214 & $0.00 \mathrm{E}+00$ & 0.0000 & 0.0000 & 0.0000 & \\
\hline & 7/23/2008 7:00 & 18 & 205 & 700 & 9 & 9 & & 0.0156 & 7.08E-05 & 18.0120 & 6.6369 & 24.6488 & \\
\hline & 7/23/2008 8:00 & 19 & 205 & 800 & 8.23 & 8.23 & & 0.1106 & 7.91E-04 & 201.4061 & 74.2122 & 275.6183 & \\
\hline & $7 / 23 / 20089: 00$ & 20 & 205 & 900 & 6.802 & 6.802 & & 0.3437 & $3.20 \mathrm{E}-03$ & 813.6202 & 299.7951 & 1113.4153 & \\
\hline & $7 / 23 / 2008$ 10:00 & 21 & 205 & 1000 & 5.817 & 5.817 & & 0.5713 & 5.97E-03 & 1520.5795 & 560.2888 & 2080.8683 & \\
\hline & $7 / 23 / 2008$ 11:00 & 22 & 205 & 1100 & 5.718 & 5.718 & & 0.5985 & $6.33 \mathrm{E}-03$ & 1610.2043 & 593.3129 & 2203.5172 & \\
\hline & 7/23/2008 12:00 & 23 & 205 & 1200 & 5.586 & 5.586 & & 0.6362 & 6.82E-03 & 1736.2080 & 639.7415 & 2375.9495 & \\
\hline & 7/23/2008 13:00 & 24 & 205 & 1300 & 5.482 & 5.482 & & 0.6673 & 7.23E-03 & 1841.0640 & 678.3779 & 2519.4419 & \\
\hline & $7 / 23 / 2008$ 14:00 & 25 & 205 & 1400 & 5.456 & 5.456 & & 0.6752 & 7.34E-03 & 1868.0864 & 688.3348 & 2556.4212 & \\
\hline & $7 / 23 / 2008$ 15:00 & 26 & 205 & 1500 & 5.433 & 5.433 & & 0.6823 & 7.43E-03 & 1892.2685 & 697.2453 & 2589.5138 & \\
\hline & $7 / 23 / 2008$ 16:00 & 27 & 205 & 1600 & 5.575 & 5.575 & & 0.6395 & $6.86 \mathrm{E}-03$ & 1747.0595 & 643.7400 & 2390.7995 & \\
\hline & 7/23/2008 17:00 & 28 & 205 & 1700 & 6.065 & 6.065 & & 0.5070 & 5.16E-03 & 1312.8722 & 483.7548 & 1796.6270 & \\
\hline & $7 / 23 / 2008$ 18:00 & 29 & 205 & 1800 & 6.822 & 6.822 & & 0.3398 & $3.15 E-03$ & 802.1566 & 295.5711 & 1097.7277 & \\
\hline & $7 / 23 / 2008$ 19:00 & 30 & 205 & 1900 & 6.722 & 6.722 & & 0.3597 & 3.38E-03 & 860.4663 & 317.0565 & 1177.5228 & \\
\hline & $7 / 23 / 200820: 00$ & 31 & 205 & 2000 & 7.134 & 7.134 & & 0.2812 & 2.50E-03 & 635.4277 & 234.1364 & 869.5641 & \\
\hline & $7 / 23 / 200821: 00$ & 32 & 205 & 2100 & 7.908 & 7.908 & & 0.1558 & $1.21 \mathrm{E}-03$ & 307.1649 & 113.1812 & 420.3461 & \\
\hline & $7 / 23 / 200822: 00$ & 33 & 205 & 2200 & 8.44 & 8.44 & & 0.0829 & $5.55 \mathrm{E}-04$ & 141.3637 & 52.0884 & 193.4521 & \\
\hline & $7 / 23 / 2008$ 23:00 & 34 & 205 & 2300 & 8.48 & 8.48 & & 0.0778 & $5.14 \mathrm{E}-04$ & 130.7233 & 48.1677 & 178.8910 & \\
\hline & $7 / 24 / 20080: 00$ & 35 & 206 & 0 & 8.45 & 8.45 & 9.51 & 0.0817 & 5.45E-04 & 138.6796 & 51.0993 & 189.7789 & \\
\hline & 7/24/2008 1:00 & 36 & 206 & 100 & 8.52 & 8.52 & & 0.0728 & 4.73E-04 & 120.3408 & 44.3420 & 164.6828 & \\
\hline & 7/24/2008 2:00 & 37 & 206 & 200 & 8.63 & 8.63 & & 0.0591 & $3.66 \mathrm{E}-04$ & 93.1407 & 34.3196 & 127.4603 & \\
\hline & 7/24/2008 3:00 & 38 & 206 & 300 & 8.79 & 8.79 & & 0.0398 & $2.25 \mathrm{E}-04$ & 57.2858 & 21.1081 & 78.3940 & \\
\hline & 7/24/2008 4:00 & 39 & 206 & 400 & 8.96 & 8.96 & & 0.0201 & 9.69E-05 & 24.6774 & 9.0929 & 33.7703 & \\
\hline & $7 / 24 / 20085: 00$ & 40 & 206 & 500 & 9.1 & 9.1 & & 0.0044 & 1.49E-05 & 3.8011 & 1.4006 & 5.2017 & \\
\hline
\end{tabular}




\begin{tabular}{|c|c|c|c|c|c|c|c|c|c|c|c|c|c|}
\hline \multirow[t]{42}{*}{ Tree ID } & TS & RN & JD & HR & $\begin{array}{c}\text { Raw } \\
\text { TC_dTA }\end{array}$ & $\begin{array}{c}\text { Fixed } \\
\text { TC_dT } \Delta\end{array}$ & dTM & $\mathbf{K}$ & $\begin{array}{l}\text { Sap } \\
\text { Velocity } \\
\text { (cm/s) }\end{array}$ & $\begin{array}{c}\text { Probed } \\
\text { Drywood F } \\
\text { (g/hr) }\end{array}$ & $\begin{array}{c}\text { Extrapolated } \\
\text { Wetwood F } \\
(\mathrm{g} / \mathrm{hr})\end{array}$ & $\begin{array}{c}\text { Extrapolated } \\
\text { Total Wood F } \\
(\mathrm{g} / \mathrm{hr})\end{array}$ & $\begin{array}{c}\text { Relative } \\
\text { Humidity, } \\
@ 10 \mathrm{~m}(\%)\end{array}$ \\
\hline & 7/24/2008 6:00 & 41 & 206 & 600 & $\begin{array}{r}9.2 \\
\end{array}$ & 9.2 & & -0.0065 & $0.00 \mathrm{E}+00$ & 0.0000 & 0.0000 & 0.0000 & \\
\hline & 7/24/2008 7:00 & 42 & 206 & 700 & 9.19 & 9.19 & & -0.0054 & $0.00 \mathrm{E}+00$ & 0.0000 & 0.0000 & 0.0000 & \\
\hline & 7/24/2008 8:00 & 43 & 206 & 800 & 8.91 & 8.91 & & 0.0258 & $1.32 \mathrm{E}-04$ & 33.5999 & 12.3806 & 45.9804 & \\
\hline & $7 / 24 / 20089: 00$ & 44 & 206 & 900 & 8.42 & 8.42 & & 0.0855 & 5.77E-04 & 146.7800 & 54.0841 & 200.8641 & \\
\hline & $7 / 24 / 2008$ 10:00 & 45 & 206 & 1000 & 8.46 & 8.46 & & 0.0804 & 5.34E-04 & 136.0114 & 50.1162 & 186.1276 & \\
\hline & $7 / 24 / 2008$ 11:00 & 46 & 206 & 1100 & 6.584 & 6.584 & & 0.3882 & $3.71 E-03$ & 945.1403 & 348.2564 & 1293.3967 & \\
\hline & $7 / 24 / 2008$ 12:00 & 47 & 206 & 1200 & 5.709 & 5.709 & & 0.6010 & 6.36E-03 & 1618.5545 & 596.3897 & 2214.9442 & \\
\hline & 7/24/2008 13:00 & 48 & 206 & 1300 & 6.017 & 6.017 & & 0.5190 & 5.31E-03 & 1351.2981 & 497.9136 & 1849.2116 & \\
\hline & $7 / 24 / 2008$ 14:00 & 49 & 206 & 1400 & 5.913 & 5.913 & & 0.5457 & $5.65 \mathrm{E}-03$ & 1437.4272 & 529.6496 & 1967.0769 & \\
\hline & 7/24/2008 15:00 & 50 & 206 & 1500 & 5.576 & 5.576 & & 0.6392 & $6.86 \mathrm{E}-03$ & 1746.0707 & 643.3757 & 2389.4463 & \\
\hline & $7 / 24 / 2008$ 16:00 & 51 & 206 & 1600 & 5.522 & 5.522 & & 0.6552 & 7.07E-03 & 1800.1304 & 663.2951 & 2463.4255 & \\
\hline & $7 / 24 / 2008$ 17:00 & 52 & 206 & 1700 & 5.708 & 5.708 & & 0.6013 & $6.36 \mathrm{E}-03$ & 1619.4844 & 596.7324 & 2216.2168 & \\
\hline & 7/24/2008 18:00 & 53 & 206 & 1800 & 5.637 & 5.637 & & 0.6214 & 6.63E-03 & 1686.6133 & 621.4674 & 2308.0806 & \\
\hline & $7 / 24 / 2008$ 19:00 & 54 & 206 & 1900 & 6.044 & 6.044 & & 0.5122 & 5.22E-03 & 1329.5829 & 489.9121 & 1819.4950 & \\
\hline & $7 / 24 / 200820: 00$ & 55 & 206 & 2000 & 6.994 & 6.994 & & 0.3068 & $2.78 \mathrm{E}-03$ & 707.5038 & 260.6943 & 968.1981 & \\
\hline & $7 / 24 / 200821: 00$ & 56 & 206 & 2100 & 8.26 & 8.26 & & 0.1065 & $7.56 \mathrm{E}-04$ & 192.4002 & 70.8938 & 263.2940 & \\
\hline & $7 / 24 / 200822: 00$ & 57 & 206 & 2200 & 9.19 & 9.19 & & -0.0054 & $0.00 \mathrm{E}+00$ & 0.0000 & 0.0000 & 0.0000 & \\
\hline & $7 / 24 / 200823: 00$ & 58 & 206 & 2300 & 9.51 & 9.51 & & -0.0389 & $0.00 \mathrm{E}+00$ & 0.0000 & 0.0000 & 0.0000 & \\
\hline & $7 / 25 / 20080: 00$ & 59 & 207 & 0 & 9.65 & 9.65 & 9.72 & -0.0528 & $0.00 \mathrm{E}+00$ & 0.0000 & 0.0000 & 0.0000 & \\
\hline & 7/25/2008 1:00 & 60 & 207 & 100 & 9.7 & 9.7 & & -0.0577 & $0.00 \mathrm{E}+00$ & 0.0000 & 0.0000 & 0.0000 & \\
\hline & 7/25/2008 2:00 & 61 & 207 & 200 & 9.71 & 9.71 & & -0.0587 & $0.00 E+00$ & 0.0000 & 0.0000 & 0.0000 & \\
\hline & 7/25/2008 3:00 & 62 & 207 & 300 & 9.71 & 9.71 & & -0.0587 & $0.00 \mathrm{E}+00$ & 0.0000 & 0.0000 & 0.0000 & \\
\hline & 7/25/2008 4:00 & 63 & 207 & 400 & 9.72 & 9.72 & & -0.0597 & $0.00 \mathrm{E}+00$ & 0.0000 & 0.0000 & 0.0000 & \\
\hline & 7/25/2008 5:00 & 64 & 207 & 500 & 9.71 & 9.71 & & -0.0587 & $0.00 \mathrm{E}+00$ & 0.0000 & 0.0000 & 0.0000 & \\
\hline & 7/25/2008 6:00 & 65 & 207 & 600 & 9.7 & 9.7 & & -0.0577 & $0.00 \mathrm{E}+00$ & 0.0000 & 0.0000 & 0.0000 & \\
\hline & 7/25/2008 7:00 & 66 & 207 & 700 & 9.65 & 9.65 & & -0.0528 & $0.00 \mathrm{E}+00$ & 0.0000 & 0.0000 & 0.0000 & \\
\hline & 7/25/2008 8:00 & 67 & 207 & 800 & 9.6 & 9.6 & & -0.0479 & $0.00 \mathrm{E}+00$ & 0.0000 & 0.0000 & 0.0000 & \\
\hline & $7 / 25 / 20089: 00$ & 68 & 207 & 900 & 9.55 & 9.55 & & -0.0429 & $0.00 \mathrm{E}+00$ & 0.0000 & 0.0000 & 0.0000 & \\
\hline & $7 / 25 / 2008$ 10:00 & 69 & 207 & 1000 & 9.37 & 9.37 & & -0.0245 & $0.00 \mathrm{E}+00$ & 0.0000 & 0.0000 & 0.0000 & \\
\hline & $7 / 25 / 2008$ 11:00 & 70 & 207 & 1100 & 8.22 & 8.22 & & 0.1119 & 8.03E-04 & 204.4398 & 75.3300 & 279.7698 & \\
\hline & $7 / 25 / 2008$ 12:00 & 71 & 207 & 1200 & 7.024 & 7.024 & & 0.3013 & 2.72E-03 & 691.6941 & 254.8689 & 946.5631 & \\
\hline & $7 / 25 / 2008$ 13:00 & 72 & 207 & 1300 & 6.202 & 6.202 & & 0.4737 & 4.74E-03 & 1207.5775 & 444.9567 & 1652.5342 & \\
\hline & $7 / 25 / 2008$ 14:00 & 73 & 207 & 1400 & 5.788 & 5.788 & & 0.5791 & 6.07E-03 & 1546.4165 & 569.8090 & 2116.2254 & \\
\hline & $7 / 25 / 2008$ 15:00 & 74 & 207 & 1500 & 5.7 & 5.7 & & 0.6035 & $6.39 \mathrm{E}-03$ & 1626.9392 & 599.4792 & 2226.4185 & \\
\hline & $7 / 25 / 200816: 00$ & 75 & 207 & 1600 & 5.785 & 5.785 & & 0.5799 & 6.09E-03 & 1549.1087 & 570.8010 & 2119.9097 & \\
\hline & $7 / 25 / 2008$ 17:00 & 76 & 207 & 1700 & 5.583 & 5.583 & & 0.6371 & $6.83 E-03$ & 1739.1620 & 640.8300 & 2379.9920 & \\
\hline & 7/25/2008 18:00 & 77 & 207 & 1800 & 5.663 & 5.663 & & 0.6140 & $6.53 \mathrm{E}-03$ & 1661.7761 & 612.3156 & 2274.0916 & \\
\hline & 7/25/2008 19:00 & 78 & 207 & 1900 & 5.814 & 5.814 & & 0.5721 & 5.98E-03 & 1523.2366 & 561.2679 & 2084.5044 & \\
\hline & $7 / 25 / 200820: 00$ & 79 & 207 & 2000 & 6.537 & 6.537 & & 0.3982 & 3.83E-03 & 975.1415 & 359.3109 & 1334.4524 & \\
\hline & $7 / 25 / 2008$ 21:00 & 80 & 207 & 2100 & 7.91 & 7.91 & & 0.1555 & 1.20E-03 & 306.4558 & 112.9199 & 419.3757 & \\
\hline & $7 / 25 / 200822: 00$ & 81 & 207 & 2200 & 9.07 & 9.07 & & 0.0077 & 2.99E-05 & 7.6007 & 2.8006 & 10.4013 & \\
\hline
\end{tabular}




\begin{tabular}{|c|c|c|c|c|c|c|c|c|c|c|c|c|c|}
\hline Tree ID & TS & RN & JD & HR & $\begin{array}{c}\text { Raw } \\
\text { TC_dTA }\end{array}$ & $\begin{array}{c}\text { Fixed } \\
\text { TC_dT } \Delta\end{array}$ & dTM & K & $\begin{array}{l}\text { Sap } \\
\text { Velocity } \\
(\mathrm{cm} / \mathrm{s})\end{array}$ & $\begin{array}{c}\text { Probed } \\
\text { Drywood F } \\
(\mathrm{g} / \mathrm{hr})\end{array}$ & $\begin{array}{c}\text { Extrapolated } \\
\text { Wetwood F } \\
(\mathrm{g} / \mathrm{hr})\end{array}$ & $\begin{array}{c}\text { Extrapolated } \\
\text { Total Wood F } \\
(\mathrm{g} / \mathrm{hr})\end{array}$ & $\begin{array}{l}\text { Relative } \\
\text { Humidity, } \\
@ 10 \mathrm{~m}(\%)\end{array}$ \\
\hline & 7/25/2008 23:00 & 82 & 207 & 2300 & \begin{tabular}{|r|}
9.43 \\
\end{tabular} & 9.43 & & -0.0308 & $0.00 \mathrm{E}+00$ & 0.0000 & 0.0000 & 0.0000 & \\
\hline & $7 / 26 / 20080: 00$ & 83 & 208 & 0 & 9.54 & 9.54 & 9.56 & -0.0419 & $0.00 \mathrm{E}+00$ & 0.0000 & 0.0000 & 0.0000 & \\
\hline & 7/26/2008 1:00 & 84 & 208 & 100 & 9.56 & 9.56 & & -0.0439 & $0.00 \mathrm{E}+00$ & 0.0000 & 0.0000 & 0.0000 & \\
\hline & $7 / 26 / 20082: 00$ & 85 & 208 & 200 & 9.56 & 9.56 & & -0.0439 & $0.00 \mathrm{E}+00$ & 0.0000 & 0.0000 & 0.0000 & \\
\hline & 7/26/2008 3:00 & 86 & 208 & 300 & 9.53 & 9.53 & & -0.0409 & $0.00 \mathrm{E}+00$ & 0.0000 & 0.0000 & 0.0000 & \\
\hline & 7/26/2008 4:00 & 87 & 208 & 400 & 9.5 & 9.5 & & -0.0379 & $0.00 \mathrm{E}+00$ & 0.0000 & 0.0000 & 0.0000 & \\
\hline & 7/26/2008 5:00 & 88 & 208 & 500 & 9.44 & 9.44 & & -0.0318 & $0.00 \mathrm{E}+00$ & 0.0000 & 0.0000 & 0.0000 & \\
\hline & 7/26/2008 6:00 & 89 & 208 & 600 & 9.37 & 9.37 & & -0.0245 & $0.00 \mathrm{E}+00$ & 0.0000 & 0.0000 & 0.0000 & \\
\hline & 7/26/2008 7:00 & 90 & 208 & 700 & 9.32 & 9.32 & & -0.0193 & $0.00 \mathrm{E}+00$ & 0.0000 & 0.0000 & 0.0000 & \\
\hline & 7/26/2008 8:00 & 91 & 208 & 800 & 9.21 & 9.21 & & -0.0076 & $0.00 E+00$ & 0.0000 & 0.0000 & 0.0000 & \\
\hline & 7/26/2008 9:00 & 92 & 208 & 900 & 7.671 & 7.671 & & 0.1915 & 1.56E-03 & 396.0052 & 145.9163 & 541.9214 & \\
\hline & $7 / 26 / 2008$ 10:00 & 93 & 208 & 1000 & 6.054 & 6.054 & & 0.5097 & 5.19E-03 & 1321.6060 & 486.9729 & 1808.5789 & \\
\hline & $7 / 26 / 2008$ 11:00 & 94 & 208 & 1100 & 5.966 & 5.966 & & 0.5320 & 5.47E-03 & 1393.0345 & 513.2922 & 1906.3268 & \\
\hline & $7 / 26 / 2008$ 12:00 & 95 & 208 & 1200 & 6.22 & 6.22 & & 0.4695 & 4.69E-03 & 1194.2085 & 440.0307 & 1634.2392 & \\
\hline & $7 / 26 / 2008$ 13:00 & 96 & 208 & 1300 & 5.688 & 5.688 & & 0.6069 & 6.44E-03 & 1638.1729 & 603.6185 & 2241.7913 & \\
\hline & $7 / 26 / 200814: 00$ & 97 & 208 & 1400 & 5.62 & 5.62 & & 0.6263 & 6.69E-03 & 1703.0149 & 627.5109 & 2330.5258 & \\
\hline & $7 / 26 / 2008$ 15:00 & 98 & 208 & 1500 & 5.566 & 5.566 & & 0.6421 & 6.90E-03 & 1755.9794 & 647.0267 & 2403.0061 & \\
\hline & $7 / 26 / 200816: 00$ & 99 & 208 & 1600 & 5.6 & 5.6 & & 0.6321 & 6.77E-03 & 1722.4769 & 634.6820 & 2357.1589 & \\
\hline & $7 / 26 / 2008$ 17:00 & 100 & 208 & 1700 & 5.492 & 5.492 & & 0.6642 & 7.19E-03 & 1830.7585 & 674.5806 & 2505.3391 & \\
\hline & $7 / 26 / 2008$ 18:00 & 101 & 208 & 1800 & 5.697 & 5.697 & & 0.6044 & $6.40 \mathrm{E}-03$ & 1629.7419 & 600.5119 & 2230.2538 & \\
\hline & $7 / 26 / 2008$ 19:00 & 102 & 208 & 1900 & 5.854 & 5.854 & & 0.5613 & $5.85 \mathrm{E}-03$ & 1488.1041 & 548.3226 & 2036.4266 & \\
\hline & $7 / 26 / 200820: 00$ & 103 & 208 & 2000 & 6.385 & 6.385 & & 0.4315 & 4.23E-03 & 1076.4317 & 396.6334 & 1473.0650 & \\
\hline & $7 / 26 / 200821: 00$ & 104 & 208 & 2100 & 7.869 & 7.869 & & 0.1615 & 1.26E-03 & 321.1264 & 118.3256 & 439.4521 & \\
\hline & $7 / 26 / 200822: 00$ & 105 & 208 & 2200 & 8.98 & 8.98 & & 0.0178 & 8.36E-05 & 21.2882 & 7.8441 & 29.1322 & \\
\hline & $7 / 26 / 2008$ 23:00 & 106 & 208 & 2300 & 9.31 & 9.31 & & -0.0183 & $0.00 \mathrm{E}+00$ & 0.0000 & 0.0000 & 0.0000 & \\
\hline & $7 / 27 / 20080: 00$ & 107 & 209 & 0 & 9.38 & 9.38 & 9.38 & -0.0256 & $0.00 \mathrm{E}+00$ & 0.0000 & 0.0000 & 0.0000 & \\
\hline & 7/27/2008 1:00 & 108 & 209 & 100 & 9.35 & 9.35 & & -0.0225 & $0.00 \mathrm{E}+00$ & 0.0000 & 0.0000 & 0.0000 & \\
\hline & 7/27/2008 2:00 & 109 & 209 & 200 & 9.24 & 9.24 & & -0.0108 & $0.00 \mathrm{E}+00$ & 0.0000 & 0.0000 & 0.0000 & \\
\hline & 7/27/2008 3:00 & 110 & 209 & 300 & 9.22 & 9.22 & & -0.0087 & $0.00 \mathrm{E}+00$ & 0.0000 & 0.0000 & 0.0000 & \\
\hline & 7/27/2008 4:00 & 111 & 209 & 400 & 9.23 & 9.23 & & -0.0098 & $0.00 \mathrm{E}+00$ & 0.0000 & 0.0000 & 0.0000 & \\
\hline & 7/27/20085:00 & 112 & 209 & 500 & 9.2 & 9.2 & & -0.0065 & $0.00 \mathrm{E}+00$ & 0.0000 & 0.0000 & 0.0000 & \\
\hline & 7/27/2008 6:00 & 113 & 209 & 600 & 9.15 & 9.15 & & -0.0011 & $0.00 \mathrm{E}+00$ & 0.0000 & 0.0000 & 0.0000 & \\
\hline & 7/27/2008 7:00 & 114 & 209 & 700 & 9.07 & 9.07 & & 0.0077 & 2.99E-05 & 7.6007 & 2.8006 & 10.4013 & \\
\hline & 7/27/2008 8:00 & 115 & 209 & 800 & 8.83 & 8.83 & & 0.0351 & 1.93E-04 & 49.0612 & 18.0776 & 67.1389 & \\
\hline & $7 / 27 / 20089: 00$ & 116 & 209 & 900 & 6.759 & 6.759 & & 0.3523 & $3.29 \mathrm{E}-03$ & 838.6010 & 308.9998 & 1147.6008 & \\
\hline & $7 / 27 / 2008$ 10:00 & 117 & 209 & 1000 & 5.193 & 5.193 & & 0.7601 & 8.49E-03 & 2161.0993 & 796.3015 & 2957.4007 & \\
\hline & $7 / 27 / 2008$ 11:00 & 118 & 209 & 1100 & 5.14 & 5.14 & & 0.7782 & 8.74E-03 & 2224.7955 & 819.7716 & 3044.5671 & \\
\hline & $7 / 27 / 2008$ 12:00 & 119 & 209 & 1200 & 5.071 & 5.071 & & 0.8024 & 9.08E-03 & 2310.2499 & 851.2591 & 3161.5089 & \\
\hline & $7 / 27 / 200813: 00$ & 120 & 209 & 1300 & 5.172 & 5.172 & & 0.7672 & $8.59 \mathrm{E}-03$ & 2186.1398 & 805.5282 & 2991.6679 & \\
\hline & $7 / 27 / 200814: 00$ & 121 & 209 & 1400 & 5.11 & 5.11 & & 0.7886 & 8.88E-03 & 2261.5918 & 833.3300 & 3094.9218 & \\
\hline & 7/27/2008 15:00 & 122 & 209 & 1500 & 5.155 & 5.155 & & 0.7730 & 8.67E-03 & 2206.6000 & 813.0672 & 3019.6672 & \\
\hline
\end{tabular}




\begin{tabular}{|c|c|c|c|c|c|c|c|c|c|c|c|c|c|}
\hline Tree ID & TS & RN & JD & HR & $\begin{array}{c}\text { Raw } \\
\text { TC_dTA }\end{array}$ & $\begin{array}{c}\text { Fixed } \\
\text { TC_dT } \Delta\end{array}$ & dTM & $\mathbf{K}$ & $\begin{array}{l}\text { Sap } \\
\text { Velocity } \\
(\mathrm{cm} / \mathrm{s})\end{array}$ & $\begin{array}{c}\text { Probed } \\
\text { Drywood F } \\
(\mathrm{g} / \mathrm{hr})\end{array}$ & $\begin{array}{c}\text { Extrapolated } \\
\text { Wetwood F } \\
(\mathrm{g} / \mathrm{hr})\end{array}$ & $\begin{array}{c}\text { Extrapolated } \\
\text { Total Wood F } \\
(\mathrm{g} / \mathrm{hr})\end{array}$ & $\begin{array}{l}\text { Relative } \\
\text { Humidity, } \\
@ 10 \mathrm{~m}(\%)\end{array}$ \\
\hline & 7/27/2008 16:00 & 123 & 209 & 1600 & \begin{tabular}{|r|}
5.214 \\
\end{tabular} & 5.214 & & 0.7530 & 8.39E-03 & 2136.3142 & 787.1689 & 2923.4831 & \\
\hline & $7 / 27 / 2008$ 17:00 & 124 & 209 & 1700 & 5.303 & 5.303 & & 0.7236 & 7.99E-03 & 2034.0313 & 749.4806 & 2783.5119 & \\
\hline & $7 / 27 / 2008$ 18:00 & 125 & 209 & 1800 & 5.421 & 5.421 & & 0.6860 & $7.48 \mathrm{E}-03$ & 1904.9901 & 701.9328 & 2606.9228 & \\
\hline & $7 / 27 / 2008$ 19:00 & 126 & 209 & 1900 & 5.602 & 5.602 & & 0.6316 & $6.76 \mathrm{E}-03$ & 1720.5225 & 633.9619 & 2354.4844 & \\
\hline & $7 / 27 / 200820: 00$ & 127 & 209 & 2000 & 5.964 & 5.964 & & 0.5325 & 5.48E-03 & 1394.6907 & 513.9025 & 1908.5931 & \\
\hline & $7 / 27 / 2008$ 21:00 & 128 & 209 & 2100 & 7.018 & 7.018 & & 0.3024 & 2.73E-03 & 694.8399 & 256.0281 & 950.8680 & \\
\hline & $7 / 27 / 2008$ 22:00 & 129 & 209 & 2200 & 8.14 & 8.14 & & 0.1229 & $9.01 E-04$ & 229.2827 & 84.4839 & 313.7666 & \\
\hline & $7 / 27 / 200823: 00$ & 130 & 209 & 2300 & 8.34 & 8.34 & & 0.0959 & 6.64E-04 & 169.0823 & 62.3019 & 231.3842 & \\
\hline & $7 / 28 / 20080: 00$ & 131 & 210 & 0 & 8.43 & 8.43 & 8.88 & 0.0842 & 5.66E-04 & 144.0639 & 53.0833 & 197.1472 & \\
\hline & 7/28/2008 1:00 & 132 & 210 & 100 & 8.56 & 8.56 & & 0.0678 & 4.33E-04 & 110.2185 & 40.6123 & 150.8308 & \\
\hline & $7 / 28 / 20082: 00$ & 133 & 210 & 200 & 8.59 & 8.59 & & 0.0640 & 4.04E-04 & 102.7994 & 37.8786 & 140.6780 & \\
\hline & 7/28/2008 3:00 & 134 & 210 & 300 & 8.68 & 8.68 & & 0.0530 & $3.20 \mathrm{E}-04$ & 81.4492 & 30.0116 & 111.4609 & \\
\hline & 7/28/2008 4:00 & 135 & 210 & 400 & 8.65 & 8.65 & & 0.0566 & 3.47E-04 & 88.4126 & 32.5775 & 120.9901 & \\
\hline & 7/28/2008 5:00 & 136 & 210 & 500 & 8.88 & 8.88 & & 0.0293 & 1.54E-04 & 39.2361 & 14.4573 & 53.6934 & \\
\hline & 7/28/2008 6:00 & 137 & 210 & 600 & 8.87 & 8.87 & & 0.0304 & $1.62 E-04$ & 41.1590 & 15.1659 & 56.3249 & \\
\hline & 7/28/2008 7:00 & 138 & 210 & 700 & 8.7 & 8.7 & & 0.0506 & 3.02E-04 & 76.8939 & 28.3331 & 105.2270 & \\
\hline & 7/28/2008 8:00 & 139 & 210 & 800 & 7.087 & 7.087 & & 0.2897 & 2.59E-03 & 659.1456 & 242.8758 & 902.0214 & \\
\hline & $7 / 28 / 20089: 00$ & 140 & 210 & 900 & 5.747 & 5.747 & & 0.5904 & $6.22 \mathrm{E}-03$ & 1583.5306 & 583.4844 & 2167.0150 & \\
\hline & $7 / 28 / 2008$ 10:00 & 141 & 210 & 1000 & 5.376 & 5.376 & & 0.7001 & 7.67E-03 & 1953.3460 & 719.7505 & 2673.0964 & \\
\hline & $7 / 28 / 2008$ 11:00 & 142 & 210 & 1100 & 5.387 & 5.387 & & 0.6967 & 7.63E-03 & 1941.4300 & 715.3598 & 2656.7897 & \\
\hline & $7 / 28 / 200812: 00$ & 143 & 210 & 1200 & 5.417 & 5.417 & & 0.6873 & 7.50E-03 & 1909.2467 & 703.5012 & 2612.7479 & \\
\hline & 7/28/2008 13:00 & 144 & 210 & 1300 & 5.319 & 5.319 & & 0.7184 & 7.92E-03 & 2016.1046 & 742.8752 & 2758.9798 & \\
\hline & $7 / 28 / 2008$ 14:00 & 145 & 210 & 1400 & 5.3 & 5.3 & & 0.7245 & 8.00E-03 & 2037.4079 & 750.7248 & 2788.1327 & \\
\hline & $7 / 28 / 2008$ 15:00 & 146 & 210 & 1500 & 5.24 & 5.24 & & 0.7443 & 8.27E-03 & 2105.9767 & 775.9904 & 2881.9671 & \\
\hline & $7 / 28 / 2008$ 16:00 & 147 & 210 & 1600 & 5.263 & 5.263 & & 0.7367 & 8.17E-03 & 2079.4568 & 766.2186 & 2845.6754 & \\
\hline & 7/28/2008 17:00 & 148 & 210 & 1700 & 5.303 & 5.303 & & 0.7236 & 7.99E-03 & 2034.0313 & 749.4806 & 2783.5119 & \\
\hline & $7 / 28 / 2008$ 18:00 & 149 & 210 & 1800 & 5.395 & 5.395 & & 0.6942 & 7.59E-03 & 1932.8029 & 712.1810 & 2644.9838 & \\
\hline & $7 / 28 / 2008$ 19:00 & 150 & 210 & 1900 & 5.621 & 5.621 & & 0.6260 & $6.69 \mathrm{E}-03$ & 1702.0466 & 627.1541 & 2329.2006 & \\
\hline & $7 / 28 / 200820: 00$ & 151 & 210 & 2000 & 6.172 & 6.172 & & 0.4809 & 4.83E-03 & 1230.0948 & 453.2537 & 1683.3486 & \\
\hline & $7 / 28 / 200821: 00$ & 152 & 210 & 2100 & 7.436 & 7.436 & & 0.2292 & 1.94E-03 & 493.9354 & 182.0006 & 675.9360 & \\
\hline & $7 / 28 / 2008$ 22:00 & 153 & 210 & 2200 & 7.92 & 7.92 & & 0.1540 & 1.19E-03 & 302.9201 & 111.6171 & 414.5372 & \\
\hline & $7 / 28 / 200823: 00$ & 154 & 210 & 2300 & 8.14 & 8.14 & & 0.1229 & $9.01 \mathrm{E}-04$ & 229.2827 & 84.4839 & 313.7666 & \\
\hline & $7 / 29 / 20080: 00$ & 155 & 211 & 0 & 8.39 & 8.39 & 9.16 & 0.0894 & 6.09E-04 & 155.0241 & 57.1218 & 212.1459 & \\
\hline & 7/29/2008 1:00 & 156 & 211 & 100 & 8.59 & 8.59 & & 0.0640 & 4.04E-04 & 102.7994 & 37.8786 & 140.6780 & \\
\hline & 7/29/2008 2:00 & 157 & 211 & 200 & 8.8 & 8.8 & & 0.0386 & 2.17E-04 & 55.2004 & 20.3397 & 75.5401 & \\
\hline & 7/29/2008 3:00 & 158 & 211 & 300 & 8.93 & 8.93 & & 0.0235 & 1.18E-04 & 29.9574 & 11.0384 & 40.9958 & \\
\hline & 7/29/2008 4:00 & 159 & 211 & 400 & 9.11 & 9.11 & & 0.0033 & 1.05E-05 & 2.6639 & 0.9816 & 3.6455 & \\
\hline & 7/29/2008 5:00 & 160 & 211 & 500 & 9.16 & 9.16 & & -0.0022 & $0.00 \mathrm{E}+00$ & 0.0000 & 0.0000 & 0.0000 & \\
\hline & $7 / 29 / 20086: 00$ & 161 & 211 & 600 & 9.09 & 9.09 & & 0.0055 & 1.97E-05 & 5.0094 & 1.8458 & 6.8553 & \\
\hline & 7/29/2008 7:00 & 162 & 211 & 700 & 8.87 & 8.87 & & 0.0304 & 1.62E-04 & 41.1590 & 15.1659 & 56.3249 & \\
\hline & 7/29/2008 8:00 & 163 & 211 & 800 & 8.1 & 8.1 & & 0.1284 & 9.51E-04 & 242.0878 & 89.2022 & 331.2900 & \\
\hline
\end{tabular}




\begin{tabular}{|c|c|c|c|c|c|c|c|c|c|c|c|c|c|}
\hline Tree ID & TS & RN & JD & HR & $\begin{array}{c}\text { Raw } \\
\text { TC_dTA }\end{array}$ & $\begin{array}{c}\text { Fixed } \\
\text { TC_dT } \Delta\end{array}$ & dTM & $\mathbf{K}$ & $\begin{array}{l}\text { Sap } \\
\text { Velocity } \\
(\mathrm{cm} / \mathrm{s})\end{array}$ & $\begin{array}{c}\text { Probed } \\
\text { Drywood F } \\
(\mathrm{g} / \mathrm{hr})\end{array}$ & $\begin{array}{c}\text { Extrapolated } \\
\text { Wetwood F } \\
(\mathrm{g} / \mathrm{hr})\end{array}$ & $\begin{array}{c}\text { Extrapolated } \\
\text { Total Wood F } \\
(\mathrm{g} / \mathrm{hr})\end{array}$ & $\begin{array}{l}\text { Relative } \\
\text { Humidity, } \\
@ 10 \mathrm{~m}(\%)\end{array}$ \\
\hline & 7/29/2008 9:00 & 164 & 211 & 900 & 6.305 & 6.305 & & 0.4496 & 4.45E-03 & 1132.4794 & 417.2853 & 1549.7647 & \\
\hline & $7 / 29 / 2008$ 10:00 & 165 & 211 & 1000 & 5.544 & 5.544 & & 0.6486 & $6.98 \mathrm{E}-03$ & 1777.9416 & 655.1191 & 2433.0607 & \\
\hline & $7 / 29 / 2008$ 11:00 & 166 & 211 & 1100 & 5.846 & 5.846 & & 0.5635 & $5.87 E-03$ & 1495.0799 & 550.8929 & 2045.9728 & \\
\hline & $7 / 29 / 2008$ 12:00 & 167 & 211 & 1200 & 5.385 & 5.385 & & 0.6973 & 7.63E-03 & 1943.5919 & 716.1564 & 2659.7483 & \\
\hline & 7/29/2008 13:00 & 168 & 211 & 1300 & 5.409 & 5.409 & & 0.6898 & 7.53E-03 & 1917.7841 & 706.6470 & 2624.4311 & \\
\hline & $7 / 29 / 2008$ 14:00 & 169 & 211 & 1400 & 5.264 & 5.264 & & 0.7363 & $8.16 \mathrm{E}-03$ & 2078.3104 & 765.7962 & 2844.1066 & \\
\hline & $7 / 29 / 2008$ 15:00 & 170 & 211 & 1500 & 5.428 & 5.428 & & 0.6839 & $7.45 E-03$ & 1897.5604 & 699.1952 & 2596.7555 & \\
\hline & $7 / 29 / 2008$ 16:00 & 171 & 211 & 1600 & 5.577 & 5.577 & & 0.6389 & 6.86E-03 & 1745.0823 & 643.0115 & 2388.0938 & \\
\hline & 7/29/2008 17:00 & 172 & 211 & 1700 & 5.764 & 5.764 & & 0.5857 & $6.16 \mathrm{E}-03$ & 1568.0577 & 577.7831 & 2145.8408 & \\
\hline & 7/29/2008 18:00 & 173 & 211 & 1800 & 5.778 & 5.778 & & 0.5819 & 6.11E-03 & 1555.4049 & 573.1209 & 2128.5259 & \\
\hline & $7 / 29 / 2008$ 19:00 & 174 & 211 & 1900 & 5.874 & 5.874 & & 0.5560 & $5.78 \mathrm{E}-03$ & 1470.7744 & 541.9371 & 2012.7115 & \\
\hline & $7 / 29 / 200820: 00$ & 175 & 211 & 2000 & 6.733 & 6.733 & & 0.3575 & $3.35 \mathrm{E}-03$ & 853.9297 & 314.6479 & 1168.5776 & \\
\hline & 7/29/2008 21:00 & 176 & 211 & 2100 & 8.05 & 8.05 & & 0.1354 & $1.02 \mathrm{E}-03$ & 258.4563 & 95.2335 & 353.6898 & \\
\hline & $7 / 29 / 2008$ 22:00 & 177 & 211 & 2200 & 8.8 & 8.8 & & 0.0386 & 2.17E-04 & 55.2004 & 20.3397 & 75.5401 & \\
\hline & $7 / 29 / 200823: 00$ & 178 & 211 & 2300 & 9.12 & 9.12 & & 0.0022 & $6.34 \mathrm{E}-06$ & 1.6150 & 0.5951 & 2.2100 & \\
\hline & $7 / 30 / 20080: 00$ & 179 & 212 & 0 & 9.25 & 9.25 & 9.27 & -0.0119 & $0.00 \mathrm{E}+00$ & 0.0000 & 0.0000 & 0.0000 & \\
\hline & 7/30/2008 1:00 & 180 & 212 & 100 & 9.27 & 9.27 & & -0.0140 & $0.00 \mathrm{E}+00$ & 0.0000 & 0.0000 & 0.0000 & \\
\hline & 7/30/2008 2:00 & 181 & 212 & 200 & 9.26 & 9.26 & & -0.0130 & $0.00 \mathrm{E}+00$ & 0.0000 & 0.0000 & 0.0000 & \\
\hline & 7/30/2008 3:00 & 182 & 212 & 300 & 9.24 & 9.24 & & -0.0108 & $0.00 \mathrm{E}+00$ & 0.0000 & 0.0000 & 0.0000 & \\
\hline & 7/30/2008 4:00 & 183 & 212 & 400 & 9.19 & 9.19 & & -0.0054 & $0.00 E+00$ & 0.0000 & 0.0000 & 0.0000 & \\
\hline & 7/30/2008 5:00 & 184 & 212 & 500 & 9.12 & 9.12 & & 0.0022 & $6.34 \mathrm{E}-06$ & 1.6150 & 0.5951 & 2.2100 & \\
\hline & 7/30/2008 6:00 & 185 & 212 & 600 & 9.04 & 9.04 & & 0.0111 & 4.65E-05 & 11.8388 & 4.3622 & 16.2010 & \\
\hline & 7/30/2008 7:00 & 186 & 212 & 700 & 8.98 & 8.98 & & 0.0178 & 8.36E-05 & 21.2882 & 7.8441 & 29.1322 & \\
\hline & $7 / 30 / 20088: 00$ & 187 & 212 & 800 & 8.94 & 8.94 & & 0.0224 & 1.11E-04 & 28.1722 & 10.3806 & 38.5529 & \\
\hline & 7/30/2008 9:00 & 188 & 212 & 900 & 7.653 & 7.653 & & 0.1943 & $1.58 \mathrm{E}-03$ & 403.1511 & 148.5493 & 551.7004 & \\
\hline & $7 / 30 / 2008$ 10:00 & 189 & 212 & 1000 & 5.548 & 5.548 & & 0.6474 & 6.97E-03 & 1773.9317 & 653.6416 & 2427.5733 & \\
\hline & 7/30/2008 11:00 & 190 & 212 & 1100 & 5.268 & 5.268 & & 0.7350 & 8.15E-03 & 2073.7305 & 764.1087 & 2837.8392 & \\
\hline & $7 / 30 / 2008$ 12:00 & 191 & 212 & 1200 & 5.26 & 5.26 & & 0.7376 & $8.18 \mathrm{E}-03$ & 2082.8992 & 767.4870 & 2850.3862 & \\
\hline & 7/30/2008 13:00 & 192 & 212 & 1300 & 5.25 & 5.25 & & 0.7410 & 8.23E-03 & 2094.4100 & 771.7284 & 2866.1384 & \\
\hline & $7 / 30 / 2008$ 14:00 & 193 & 212 & 1400 & 5.218 & 5.218 & & 0.7516 & 8.37E-03 & 2131.6219 & 785.4399 & 2917.0618 & \\
\hline & 7/30/2008 15:00 & 194 & 212 & 1500 & 5.367 & 5.367 & & 0.7030 & 7.71E-03 & 1963.1420 & 723.3600 & 2686.5020 & \\
\hline & $7 / 30 / 2008$ 16:00 & 195 & 212 & 1600 & 5.223 & 5.223 & & 0.7500 & 8.35E-03 & 2125.7693 & 783.2834 & 2909.0527 & \\
\hline & $7 / 30 / 2008$ 17:00 & 196 & 212 & 1700 & 5.253 & 5.253 & & 0.7400 & 8.21E-03 & 2090.9509 & 770.4539 & 2861.4047 & \\
\hline & $7 / 30 / 2008$ 18:00 & 197 & 212 & 1800 & 5.395 & 5.395 & & 0.6942 & $7.59 \mathrm{E}-03$ & 1932.8029 & 712.1810 & 2644.9838 & \\
\hline & 7/30/2008 19:00 & 198 & 212 & 1900 & 5.566 & 5.566 & & 0.6421 & 6.90E-03 & 1755.9794 & 647.0267 & 2403.0061 & \\
\hline & $7 / 30 / 200820: 00$ & 199 & 212 & 2000 & 6.063 & 6.063 & & 0.5075 & 5.16E-03 & 1314.4570 & 484.3387 & 1798.7957 & \\
\hline & 7/30/2008 21:00 & 200 & 212 & 2100 & 7.354 & 7.354 & & 0.2429 & 2.08E-03 & 530.5488 & 195.4916 & 726.0404 & \\
\hline & $7 / 30 / 2008$ 22:00 & 201 & 212 & 2200 & 8.28 & 8.28 & & 0.1039 & 7.33E-04 & 186.4756 & 68.7108 & 255.1864 & \\
\hline & 7/30/2008 23:00 & 202 & 212 & 2300 & 8.72 & 8.72 & & 0.0482 & $2.84 \mathrm{E}-04$ & 72.4092 & 26.6807 & 99.0899 & \\
\hline & $7 / 31 / 20080: 00$ & 203 & 213 & 0 & 9.01 & 9.01 & 9.31 & 0.0144 & $6.45 \mathrm{E}-05$ & 16.4190 & 6.0499 & 22.4690 & \\
\hline & 7/31/2008 1:00 & 204 & 213 & 100 & 9.14 & 9.14 & & 0.0000 & $0.00 \mathrm{E}+00$ & 0.0000 & 0.0000 & 0.0000 & \\
\hline
\end{tabular}




\begin{tabular}{|c|c|c|c|c|c|c|c|c|c|c|c|c|c|}
\hline Tree ID & TS & RN & JD & HR & $\begin{array}{c}\text { Raw } \\
\text { TC_dTA }\end{array}$ & $\begin{array}{c}\text { Fixed } \\
\text { TC_dT } \Delta\end{array}$ & dTM & K & $\begin{array}{l}\text { Sap } \\
\text { Velocity } \\
(\mathrm{cm} / \mathrm{s})\end{array}$ & $\begin{array}{c}\text { Probed } \\
\text { Drywood F } \\
(\mathrm{g} / \mathrm{hr})\end{array}$ & $\begin{array}{c}\text { Extrapolated } \\
\text { Wetwood F } \\
(\mathrm{g} / \mathrm{hr})\end{array}$ & $\begin{array}{c}\text { Extrapolated } \\
\text { Total Wood F } \\
(\mathrm{g} / \mathrm{hr})\end{array}$ & $\begin{array}{l}\text { Relative } \\
\text { Humidity, } \\
@ 10 \mathrm{~m}(\%)\end{array}$ \\
\hline & 7/31/2008 2:00 & 205 & 213 & 200 & \begin{tabular}{|r|}
9.18 \\
\end{tabular} & 9.18 & & -0.0044 & $0.00 \mathrm{E}+00$ & 0.0000 & 0.0000 & 0.0000 & \\
\hline & 7/31/2008 3:00 & 206 & 213 & 300 & 9.22 & 9.22 & & -0.0087 & $0.00 \mathrm{E}+00$ & 0.0000 & 0.0000 & 0.0000 & \\
\hline & 7/31/2008 4:00 & 207 & 213 & 400 & 9.28 & 9.28 & & -0.0151 & $0.00 E+00$ & 0.0000 & 0.0000 & 0.0000 & \\
\hline & 7/31/2008 5:00 & 208 & 213 & 500 & 9.29 & 9.29 & & -0.0161 & $0.00 \mathrm{E}+00$ & 0.0000 & 0.0000 & 0.0000 & \\
\hline & 7/31/2008 6:00 & 209 & 213 & 600 & 9.31 & 9.31 & & -0.0183 & $0.00 \mathrm{E}+00$ & 0.0000 & 0.0000 & 0.0000 & \\
\hline & 7/31/2008 7:00 & 210 & 213 & 700 & 9.31 & 9.31 & & -0.0183 & $0.00 \mathrm{E}+00$ & 0.0000 & 0.0000 & 0.0000 & \\
\hline & $7 / 31 / 2008$ 8:00 & 211 & 213 & 800 & 9.18 & 9.18 & & -0.0044 & $0.00 \mathrm{E}+00$ & 0.0000 & 0.0000 & 0.0000 & \\
\hline & $7 / 31 / 20089: 00$ & 212 & 213 & 900 & 7.625 & 7.625 & & 0.1987 & 1.63E-03 & 414.3817 & 152.6874 & 567.0691 & \\
\hline & $7 / 31 / 2008$ 10:00 & 213 & 213 & 1000 & 5.603 & 5.603 & & 0.6313 & $6.75 \mathrm{E}-03$ & 1719.5461 & 633.6021 & 2353.1482 & \\
\hline & 7/31/2008 11:00 & 214 & 213 & 1100 & 5.513 & 5.513 & & 0.6579 & 7.11E-03 & 1809.2737 & 666.6641 & 2475.9378 & \\
\hline & $7 / 31 / 2008$ 12:00 & 215 & 213 & 1200 & 5.296 & 5.296 & & 0.7258 & 8.02E-03 & 2041.9176 & 752.3865 & 2794.3042 & \\
\hline & 7/31/2008 13:00 & 216 & 213 & 1300 & 5.465 & 5.465 & & 0.6725 & $7.30 \mathrm{E}-03$ & 1858.6950 & 684.8744 & 2543.5694 & \\
\hline & 7/31/2008 14:00 & 217 & 213 & 1400 & 5.199 & 5.199 & & 0.7580 & 8.46E-03 & 2153.9919 & 793.6826 & 2947.6745 & \\
\hline & 7/31/2008 15:00 & 218 & 213 & 1500 & 5.207 & 5.207 & & 0.7553 & 8.42E-03 & 2144.5477 & 790.2027 & 2934.7504 & \\
\hline & $7 / 31 / 2008$ 16:00 & 219 & 213 & 1600 & 5.233 & 5.233 & & 0.7466 & 8.30E-03 & 2114.1068 & 778.9861 & 2893.0929 & \\
\hline & 7/31/2008 17:00 & 220 & 213 & 1700 & 5.272 & 5.272 & & 0.7337 & 8.13E-03 & 2069.1595 & 762.4244 & 2831.5838 & \\
\hline & $7 / 31 / 2008$ 18:00 & 221 & 213 & 1800 & 5.372 & 5.372 & & 0.7014 & 7.69E-03 & 1957.6945 & 721.3528 & 2679.0474 & \\
\hline & $7 / 31 / 2008$ 19:00 & 222 & 213 & 1900 & 5.628 & 5.628 & & 0.6240 & $6.66 \mathrm{E}-03$ & 1695.2805 & 624.6610 & 2319.9414 & \\
\hline & $7 / 31 / 200820: 00$ & 223 & 213 & 2000 & 6.286 & 6.286 & & 0.4540 & 4.50E-03 & 1146.0797 & 422.2966 & 1568.3763 & \\
\hline & $7 / 31 / 200821: 00$ & 224 & 213 & 2100 & 7.152 & 7.152 & & 0.2780 & $2.46 \mathrm{E}-03$ & 626.4698 & 230.8357 & 857.3055 & \\
\hline & $7 / 31 / 200822: 00$ & 225 & 213 & 2200 & 7.877 & 7.877 & & 0.1603 & $1.25 \mathrm{E}-03$ & 318.2418 & 117.2627 & 435.5045 & \\
\hline & 7/31/2008 23:00 & 226 & 213 & 2300 & 7.926 & 7.926 & & 0.1532 & 1.18E-03 & 300.8067 & 110.8384 & 411.6451 & \\
\hline & $8 / 1 / 20080: 00$ & 227 & 214 & 0 & 8.3 & 8.3 & 9.50 & 0.1012 & 7.09E-04 & 180.6145 & 66.5511 & 247.1656 & \\
\hline & 8/1/2008 1:00 & 228 & 214 & 100 & 8.85 & 8.85 & & 0.0328 & 1.77E-04 & 45.0687 & 16.6065 & 61.6751 & \\
\hline & 8/1/2008 2:00 & 229 & 214 & 200 & 9.14 & 9.14 & & 0.0000 & $0.00 \mathrm{E}+00$ & 0.0000 & 0.0000 & 0.0000 & \\
\hline & $8 / 1 / 2008$ 3:00 & 230 & 214 & 300 & 9.21 & 9.21 & & -0.0076 & $0.00 E+00$ & 0.0000 & 0.0000 & 0.0000 & \\
\hline & 8/1/2008 4:00 & 231 & 214 & 400 & 9.36 & 9.36 & & -0.0235 & $0.00 \mathrm{E}+00$ & 0.0000 & 0.0000 & 0.0000 & \\
\hline & 8/1/2008 5:00 & 232 & 214 & 500 & 9.45 & 9.45 & & -0.0328 & $0.00 \mathrm{E}+00$ & 0.0000 & 0.0000 & 0.0000 & \\
\hline & 8/1/2008 6:00 & 233 & 214 & 600 & 9.49 & 9.49 & & -0.0369 & $0.00 \mathrm{E}+00$ & 0.0000 & 0.0000 & 0.0000 & \\
\hline & 8/1/2008 7:00 & 234 & 214 & 700 & 9.5 & 9.5 & & -0.0379 & $0.00 \mathrm{E}+00$ & 0.0000 & 0.0000 & 0.0000 & \\
\hline & 8/1/2008 8:00 & 235 & 214 & 800 & 9.35 & 9.35 & & -0.0225 & $0.00 \mathrm{E}+00$ & 0.0000 & 0.0000 & 0.0000 & \\
\hline & 8/1/2008 9:00 & 236 & 214 & 900 & 7.776 & 7.776 & & 0.1754 & 1.40E-03 & 355.4556 & 130.9749 & 486.4306 & \\
\hline & 8/1/2008 10:00 & 237 & 214 & 1000 & 5.74 & 5.74 & & 0.5923 & $6.25 E-03$ & 1589.9367 & 585.8449 & 2175.7816 & \\
\hline & 8/1/2008 11:00 & 238 & 214 & 1100 & 5.609 & 5.609 & & 0.6295 & $6.73 \mathrm{E}-03$ & 1713.6967 & 631.4468 & 2345.1435 & \\
\hline & 8/1/2008 12:00 & 239 & 214 & 1200 & 5.544 & 5.544 & & 0.6486 & 6.98E-03 & 1777.9416 & 655.1191 & 2433.0607 & \\
\hline & 8/1/2008 13:00 & 240 & 214 & 1300 & 5.404 & 5.404 & & 0.6913 & 7.55E-03 & 1923.1365 & 708.6192 & 2631.7557 & \\
\hline & 8/1/2008 14:00 & 241 & 214 & 1400 & 5.353 & 5.353 & & 0.7075 & 7.77E-03 & 1978.4640 & 729.0058 & 2707.4698 & \\
\hline & 8/1/2008 15:00 & 242 & 214 & 1500 & 5.302 & 5.302 & & 0.7239 & 7.99E-03 & 2035.1563 & 749.8952 & 2785.0514 & \\
\hline & $8 / 1 / 200816: 00$ & 243 & 214 & 1600 & 5.34 & 5.34 & & 0.7116 & 7.83E-03 & 1992.7837 & 734.2821 & 2727.0658 & \\
\hline & 8/1/2008 17:00 & 244 & 214 & 1700 & 5.386 & 5.386 & & 0.6970 & 7.63E-03 & 1942.5107 & 715.7580 & 2658.2687 & \\
\hline & 8/1/2008 18:00 & 245 & 214 & 1800 & 5.472 & 5.472 & & 0.6703 & 7.27E-03 & 1851.4181 & 682.1931 & 2533.6112 & \\
\hline
\end{tabular}




\begin{tabular}{|c|c|c|c|c|c|c|c|c|c|c|c|c|c|}
\hline Tree ID & TS & RN & JD & HR & $\begin{array}{c}\text { Raw } \\
\text { TC_dTA }\end{array}$ & $\begin{array}{c}\text { Fixed } \\
\text { TC_dT } \Delta\end{array}$ & dTM & $\mathbf{K}$ & $\begin{array}{l}\text { Sap } \\
\text { Velocity } \\
(\mathrm{cm} / \mathrm{s})\end{array}$ & $\begin{array}{c}\text { Probed } \\
\text { Drywood F } \\
(\mathrm{g} / \mathrm{hr})\end{array}$ & $\begin{array}{c}\text { Extrapolated } \\
\text { Wetwood F } \\
(\mathrm{g} / \mathrm{hr})\end{array}$ & $\begin{array}{c}\text { Extrapolated } \\
\text { Total Wood F } \\
(\mathrm{g} / \mathrm{hr})\end{array}$ & $\begin{array}{l}\text { Relative } \\
\text { Humidity, } \\
@ 10 \mathrm{~m}(\%)\end{array}$ \\
\hline & 8/1/2008 19:00 & 246 & 214 & 1900 & 5.697 & 5.697 & & 0.6044 & $6.40 \mathrm{E}-03$ & 1629.7419 & 600.5119 & 2230.2538 & \\
\hline & 8/1/2008 20:00 & 247 & 214 & 2000 & 6.388 & 6.388 & & 0.4308 & 4.22E-03 & 1074.3675 & 395.8728 & 1470.2403 & \\
\hline & 8/1/2008 21:00 & 248 & 214 & 2100 & 7.964 & 7.964 & & 0.1477 & $1.13 \mathrm{E}-03$ & 287.5601 & 105.9574 & 393.5176 & \\
\hline & 8/1/2008 22:00 & 249 & 214 & 2200 & 8.78 & 8.78 & & 0.0410 & 2.33E-04 & 59.3904 & 21.8836 & 81.2741 & \\
\hline & 8/1/2008 23:00 & 250 & 214 & 2300 & 9.09 & 9.09 & & 0.0055 & 1.97E-05 & 5.0094 & 1.8458 & 6.8553 & \\
\hline & 8/2/2008 0:00 & 251 & 215 & 0 & 9.22 & 9.22 & 9.46 & -0.0087 & $0.00 \mathrm{E}+00$ & 0.0000 & 0.0000 & 0.0000 & \\
\hline & 8/2/2008 1:00 & 252 & 215 & 100 & 9.32 & 9.32 & & -0.0193 & $0.00 \mathrm{E}+00$ & 0.0000 & 0.0000 & 0.0000 & \\
\hline & 8/2/2008 2:00 & 253 & 215 & 200 & 9.4 & 9.4 & & -0.0277 & $0.00 \mathrm{E}+00$ & 0.0000 & 0.0000 & 0.0000 & \\
\hline & 8/2/2008 3:00 & 254 & 215 & 300 & 9.46 & 9.46 & & -0.0338 & $0.00 \mathrm{E}+00$ & 0.0000 & 0.0000 & 0.0000 & \\
\hline & 8/2/2008 4:00 & 255 & 215 & 400 & 9.37 & 9.37 & & -0.0245 & $0.00 E+00$ & 0.0000 & 0.0000 & 0.0000 & \\
\hline & 8/2/2008 5:00 & 256 & 215 & 500 & 9.33 & 9.33 & & -0.0204 & $0.00 \mathrm{E}+00$ & 0.0000 & 0.0000 & 0.0000 & \\
\hline & 8/2/2008 6:00 & 257 & 215 & 600 & 9.31 & 9.31 & & -0.0183 & $0.00 E+00$ & 0.0000 & 0.0000 & 0.0000 & \\
\hline & 8/2/2008 7:00 & 258 & 215 & 700 & 9.12 & 9.12 & & 0.0022 & 6.34E-06 & 1.6150 & 0.5951 & 2.2100 & \\
\hline & 8/2/2008 8:00 & 259 & 215 & 800 & 7.756 & 7.756 & & 0.1784 & 1.43E-03 & 363.0314 & 133.7664 & 496.7978 & \\
\hline & 8/2/2008 9:00 & 260 & 215 & 900 & 5.986 & 5.986 & & 0.5269 & 5.41E-03 & 1376.5541 & 507.2197 & 1883.7738 & \\
\hline & 8/2/2008 10:00 & 261 & 215 & 1000 & 5.813 & 5.813 & & 0.5723 & 5.99E-03 & 1524.1231 & 561.5945 & 2085.7176 & \\
\hline & 8/2/2008 11:00 & 262 & 215 & 1100 & 5.634 & 5.634 & & 0.6223 & 6.64E-03 & 1689.4983 & 622.5304 & 2312.0288 & \\
\hline & $8 / 2 / 200812: 00$ & 263 & 215 & 1200 & 5.521 & 5.521 & & 0.6555 & 7.08E-03 & 1801.1444 & 663.6687 & 2464.8131 & \\
\hline & 8/2/2008 13:00 & 264 & 215 & 1300 & 5.429 & 5.429 & & 0.6836 & 7.45E-03 & 1896.5010 & 698.8048 & 2595.3058 & \\
\hline & 8/2/2008 14:00 & 265 & 215 & 1400 & 5.413 & 5.413 & & 0.6885 & 7.52E-03 & 1913.5113 & 705.0726 & 2618.5840 & \\
\hline & $8 / 2 / 2008$ 15:00 & 266 & 215 & 1500 & 5.38 & 5.38 & & 0.6989 & 7.66E-03 & 1949.0056 & 718.1512 & 2667.1569 & \\
\hline & 8/2/2008 16:00 & 267 & 215 & 1600 & 5.368 & 5.368 & & 0.7027 & 7.71E-03 & 1962.0514 & 722.9582 & 2685.0096 & \\
\hline & 8/2/2008 17:00 & 268 & 215 & 1700 & 5.41 & 5.41 & & 0.6895 & 7.53E-03 & 1916.7152 & 706.2531 & 2622.9683 & \\
\hline & $8 / 2 / 200818: 00$ & 269 & 215 & 1800 & 5.493 & 5.493 & & 0.6639 & 7.19E-03 & 1829.7306 & 674.2019 & 2503.9325 & \\
\hline & 8/2/2008 19:00 & 270 & 215 & 1900 & 5.666 & 5.666 & & 0.6131 & $6.52 \mathrm{E}-03$ & 1658.9293 & 611.2666 & 2270.1959 & \\
\hline & 8/2/2008 20:00 & 271 & 215 & 2000 & 6.192 & 6.192 & & 0.4761 & 4.77E-03 & 1215.0503 & 447.7103 & 1662.7606 & \\
\hline & 8/2/2008 21:00 & 272 & 215 & 2100 & 7.261 & 7.261 & & 0.2588 & $2.25 E-03$ & 573.6765 & 211.3829 & 785.0595 & \\
\hline & 8/2/2008 22:00 & 273 & 215 & 2200 & 8.21 & 8.21 & & 0.1133 & $8.15 \mathrm{E}-04$ & 207.4894 & 76.4537 & 283.9431 & \\
\hline & 8/2/2008 23:00 & 274 & 215 & 2300 & 8.61 & 8.61 & & 0.0616 & 3.85E-04 & 97.9365 & 36.0867 & 134.0232 & \\
\hline & 8/3/2008 0:00 & 275 & 216 & 0 & 8.54 & 8.54 & 9.20 & 0.0703 & 4.53E-04 & 115.2469 & 42.4651 & 157.7120 & \\
\hline & 8/3/2008 1:00 & 276 & 216 & 100 & 8.36 & 8.36 & & 0.0933 & 6.42E-04 & 163.4114 & 60.2123 & 223.6237 & \\
\hline & 8/3/2008 2:00 & 277 & 216 & 200 & 8.63 & 8.63 & & 0.0591 & $3.66 \mathrm{E}-04$ & 93.1407 & 34.3196 & 127.4603 & \\
\hline & 8/3/2008 3:00 & 278 & 216 & 300 & 8.92 & 8.92 & & 0.0247 & 1.25E-04 & 31.7668 & 11.7051 & 43.4719 & \\
\hline & 8/3/2008 4:00 & 279 & 216 & 400 & 9.09 & 9.09 & & 0.0055 & 1.97E-05 & 5.0094 & 1.8458 & 6.8553 & \\
\hline & 8/3/2008 5:00 & 280 & 216 & 500 & 9.2 & 9.2 & & -0.0065 & $0.00 \mathrm{E}+00$ & 0.0000 & 0.0000 & 0.0000 & \\
\hline & 8/3/2008 6:00 & 281 & 216 & 600 & 8.99 & 8.99 & & 0.0167 & 7.71E-05 & 19.6354 & 7.2351 & 26.8705 & \\
\hline & 8/3/2008 7:00 & 282 & 216 & 700 & 8.66 & 8.66 & & 0.0554 & 3.38E-04 & 86.0743 & 31.7158 & 117.7901 & \\
\hline & 8/3/2008 8:00 & 283 & 216 & 800 & 7.61 & 7.61 & & 0.2011 & 1.65E-03 & 420.4559 & 154.9256 & 575.3816 & \\
\hline & 8/3/2008 9:00 & 284 & 216 & 900 & 5.945 & 5.945 & & 0.5374 & $5.54 \mathrm{E}-03$ & 1410.4982 & 519.7271 & 1930.2253 & \\
\hline & $8 / 3 / 2008$ 10:00 & 285 & 216 & 1000 & 5.591 & 5.591 & & 0.6348 & 6.80E-03 & 1731.2937 & 637.9308 & 2369.2245 & \\
\hline & 8/3/2008 11:00 & 286 & 216 & 1100 & 5.505 & 5.505 & & 0.6603 & 7.14E-03 & 1817.4334 & 669.6707 & 2487.1041 & \\
\hline
\end{tabular}




\begin{tabular}{|c|c|c|c|c|c|c|c|c|c|c|c|c|c|}
\hline Tree ID & TS & RN & JD & HR & $\begin{array}{c}\text { Raw } \\
\text { TC_dTA }\end{array}$ & $\begin{array}{c}\text { Fixed } \\
\text { TC_dT } \Delta\end{array}$ & dTM & $\mathbf{K}$ & $\begin{array}{l}\text { Sap } \\
\text { Velocity } \\
(\mathrm{cm} / \mathrm{s})\end{array}$ & $\begin{array}{c}\text { Probed } \\
\text { Drywood F } \\
(\mathrm{g} / \mathrm{hr})\end{array}$ & $\begin{array}{c}\text { Extrapolated } \\
\text { Wetwood F } \\
(\mathrm{g} / \mathrm{hr})\end{array}$ & $\begin{array}{c}\text { Extrapolated } \\
\text { Total Wood F } \\
(\mathrm{g} / \mathrm{hr})\end{array}$ & $\begin{array}{l}\text { Relative } \\
\text { Humidity, } \\
@ 10 \mathrm{~m}(\%)\end{array}$ \\
\hline & 8/3/2008 12:00 & 287 & 216 & 1200 & \begin{tabular}{|r|}
5.427 \\
\end{tabular} & 5.427 & & 0.6842 & 7.46E-03 & 1898.6203 & 699.5857 & 2598.2060 & \\
\hline & 8/3/2008 13:00 & 288 & 216 & 1300 & 5.377 & 5.377 & & 0.6998 & 7.67E-03 & 1952.2601 & 719.3504 & 2671.6105 & \\
\hline & $8 / 3 / 2008$ 14:00 & 289 & 216 & 1400 & 5.393 & 5.393 & & 0.6948 & $7.60 \mathrm{E}-03$ & 1934.9566 & 712.9745 & 2647.9311 & \\
\hline & 8/3/2008 15:00 & 290 & 216 & 1500 & 5.428 & 5.428 & & 0.6839 & 7.45E-03 & 1897.5604 & 699.1952 & 2596.7555 & \\
\hline & 8/3/2008 16:00 & 291 & 216 & 1600 & 5.437 & 5.437 & & 0.6811 & 7.42E-03 & 1888.0440 & 695.6887 & 2583.7327 & \\
\hline & 8/3/2008 17:00 & 292 & 216 & 1700 & 5.469 & 5.469 & & 0.6712 & 7.29E-03 & 1854.5338 & 683.3411 & 2537.8750 & \\
\hline & 8/3/2008 18:00 & 293 & 216 & 1800 & 5.52 & 5.52 & & 0.6558 & 7.08E-03 & 1802.1589 & 664.0425 & 2466.2014 & \\
\hline & 8/3/2008 19:00 & 294 & 216 & 1900 & 5.651 & 5.651 & & 0.6174 & 6.57E-03 & 1673.2024 & 616.5258 & 2289.7282 & \\
\hline & 8/3/2008 20:00 & 295 & 216 & 2000 & 5.922 & 5.922 & & 0.5434 & 5.62E-03 & 1429.8143 & 526.8445 & 1956.6589 & \\
\hline & 8/3/2008 21:00 & 296 & 216 & 2100 & 6.728 & 6.728 & & 0.3585 & 3.37E-03 & 856.8971 & 315.7413 & 1172.6384 & \\
\hline & 8/3/2008 22:00 & 297 & 216 & 2200 & 7.261 & 7.261 & & 0.2588 & $2.25 E-03$ & 573.6765 & 211.3829 & 785.0595 & \\
\hline & 8/3/2008 23:00 & 298 & 216 & 2300 & 7.41 & 7.41 & & 0.2335 & 1.99E-03 & 505.4037 & 186.2264 & 691.6300 & \\
\hline & 8/4/2008 0:00 & 299 & 217 & 0 & 7.485 & 7.485 & 8.65 & 0.2211 & 1.86E-03 & 472.6722 & 174.1658 & 646.8380 & \\
\hline & 8/4/2008 1:00 & 300 & 217 & 100 & 7.569 & 7.569 & & 0.2076 & 1.72E-03 & 437.2666 & 161.1199 & 598.3865 & \\
\hline & $8 / 4 / 2008$ 2:00 & 301 & 217 & 200 & 7.278 & 7.278 & & 0.2558 & $2.22 \mathrm{E}-03$ & 565.6633 & 208.4303 & 774.0935 & \\
\hline & 8/4/2008 3:00 & 302 & 217 & 300 & 7.099 & 7.099 & & 0.2875 & $2.57 \mathrm{E}-03$ & 653.0446 & 240.6277 & 893.6723 & \\
\hline & 8/4/2008 4:00 & 303 & 217 & 400 & 6.901 & 6.901 & & 0.3244 & $2.98 \mathrm{E}-03$ & 757.8194 & 279.2341 & 1037.0536 & \\
\hline & $8 / 4 / 20085: 00$ & 304 & 217 & 500 & 7.073 & 7.073 & & 0.2922 & $2.62 \mathrm{E}-03$ & 666.3031 & 245.5131 & 911.8162 & \\
\hline & 8/4/2008 6:00 & 305 & 217 & 600 & 7.492 & 7.492 & & 0.2200 & 1.84E-03 & 469.6716 & 173.0602 & 642.7318 & \\
\hline & 8/4/2008 7:00 & 306 & 217 & 700 & 7.597 & 7.597 & & 0.2031 & $1.67 E-03$ & 425.7531 & 156.8775 & 582.6306 & \\
\hline & 8/4/2008 8:00 & 307 & 217 & 800 & 7.534 & 7.534 & & 0.2132 & $1.78 \mathrm{E}-03$ & 451.8602 & 166.4972 & 618.3574 & \\
\hline & 8/4/2008 9:00 & 308 & 217 & 900 & 7.187 & 7.187 & & 0.2717 & 2.39E-03 & 609.2481 & 224.4900 & 833.7380 & \\
\hline & 8/4/2008 10:00 & 309 & 217 & 1000 & 6.344 & 6.344 & & 0.4407 & 4.34E-03 & 1104.9129 & 407.1279 & 1512.0407 & \\
\hline & 8/4/2008 11:00 & 310 & 217 & 1100 & 5.782 & 5.782 & & 0.5808 & $6.10 \mathrm{E}-03$ & 1551.8046 & 571.7943 & 2123.5990 & \\
\hline & 8/4/2008 12:00 & 311 & 217 & 1200 & 5.53 & 5.53 & & 0.6528 & 7.04E-03 & 1792.0353 & 660.3123 & 2452.3476 & \\
\hline & 8/4/2008 13:00 & 312 & 217 & 1300 & 5.487 & 5.487 & & 0.6658 & 7.21E-03 & 1835.9052 & 676.4770 & 2512.3822 & \\
\hline & 8/4/2008 14:00 & 313 & 217 & 1400 & 5.805 & 5.805 & & 0.5745 & 6.02E-03 & 1531.2295 & 564.2130 & 2095.4425 & \\
\hline & 8/4/2008 15:00 & 314 & 217 & 1500 & 6.153 & 6.153 & & 0.4855 & 4.89E-03 & 1244.5100 & 458.5653 & 1703.0754 & \\
\hline & 8/4/2008 16:00 & 315 & 217 & 1600 & 5.589 & 5.589 & & 0.6354 & 6.81E-03 & 1733.2581 & 638.6546 & 2371.9126 & \\
\hline & 8/4/2008 17:00 & 316 & 217 & 1700 & 5.538 & 5.538 & & 0.6504 & 7.01E-03 & 1783.9705 & 657.3406 & 2441.3111 & \\
\hline & 8/4/2008 18:00 & 317 & 217 & 1800 & 5.636 & 5.636 & & 0.6217 & 6.63E-03 & 1687.5745 & 621.8215 & 2309.3961 & \\
\hline & 8/4/2008 19:00 & 318 & 217 & 1900 & 5.903 & 5.903 & & 0.5484 & 5.68E-03 & 1445.9221 & 532.7797 & 1978.7019 & \\
\hline & 8/4/2008 20:00 & 319 & 217 & 2000 & 6.469 & 6.469 & & 0.4129 & 4.01E-03 & 1019.6353 & 375.7056 & 1395.3409 & \\
\hline & 8/4/2008 21:00 & 320 & 217 & 2100 & 7.835 & 7.835 & & 0.1666 & 1.31E-03 & 333.5066 & 122.8873 & 456.3939 & \\
\hline & 8/4/2008 22:00 & 321 & 217 & 2200 & 8.41 & 8.41 & & 0.0868 & 5.87E-04 & 149.5121 & 55.0908 & 204.6029 & \\
\hline & 8/4/2008 23:00 & 322 & 217 & 2300 & 8.65 & 8.65 & & 0.0566 & 3.47E-04 & 88.4126 & 32.5775 & 120.9901 & \\
\hline & 8/5/2008 0:00 & 323 & 218 & 0 & 8.94 & 8.94 & 9.44 & 0.0224 & 1.11E-04 & 28.1722 & 10.3806 & 38.5529 & \\
\hline & 8/5/2008 1:00 & 324 & 218 & 100 & 9.12 & 9.12 & & 0.0022 & 6.34E-06 & 1.6150 & 0.5951 & 2.2100 & \\
\hline & 8/5/2008 2:00 & 325 & 218 & 200 & 9.27 & 9.27 & & -0.0140 & $0.00 \mathrm{E}+00$ & 0.0000 & 0.0000 & 0.0000 & \\
\hline & 8/5/2008 3:00 & 326 & 218 & 300 & 9.21 & 9.21 & & -0.0076 & $0.00 \mathrm{E}+00$ & 0.0000 & 0.0000 & 0.0000 & \\
\hline & 8/5/2008 4:00 & 327 & 218 & 400 & 9.03 & 9.03 & & 0.0122 & 5.24E-05 & 13.3307 & 4.9120 & 18.2427 & \\
\hline
\end{tabular}




\begin{tabular}{|c|c|c|c|c|c|c|c|c|c|c|c|c|c|}
\hline Tree ID & TS & RN & JD & HR & $\begin{array}{c}\text { Raw } \\
\text { TC_dTA }\end{array}$ & $\begin{array}{c}\text { Fixed } \\
\text { TC_dT } \Delta\end{array}$ & dTM & $\mathbf{K}$ & $\begin{array}{l}\text { Sap } \\
\text { Velocity } \\
(\mathrm{cm} / \mathrm{s})\end{array}$ & $\begin{array}{c}\text { Probed } \\
\text { Drywood F } \\
(\mathrm{g} / \mathrm{hr})\end{array}$ & $\begin{array}{c}\text { Extrapolated } \\
\text { Wetwood F } \\
(\mathrm{g} / \mathrm{hr})\end{array}$ & $\begin{array}{c}\text { Extrapolated } \\
\text { Total Wood F } \\
(\mathrm{g} / \mathrm{hr})\end{array}$ & $\begin{array}{l}\text { Relative } \\
\text { Humidity, } \\
@ 10 \mathrm{~m}(\%)\end{array}$ \\
\hline & 8/5/2008 5:00 & 328 & 218 & 500 & $\begin{array}{r}9.02 \\
\end{array}$ & 9.02 & & 0.0133 & $5.84 \mathrm{E}-05$ & 14.8581 & 5.4748 & 20.3328 & \\
\hline & 8/5/2008 6:00 & 329 & 218 & 600 & 9.16 & 9.16 & & -0.0022 & $0.00 \mathrm{E}+00$ & 0.0000 & 0.0000 & 0.0000 & \\
\hline & 8/5/2008 7:00 & 330 & 218 & 700 & 9.27 & 9.27 & & -0.0140 & $0.00 E+00$ & 0.0000 & 0.0000 & 0.0000 & \\
\hline & 8/5/2008 8:00 & 331 & 218 & 800 & 9.16 & 9.16 & & -0.0022 & $0.00 \mathrm{E}+00$ & 0.0000 & 0.0000 & 0.0000 & \\
\hline & 8/5/2008 9:00 & 332 & 218 & 900 & 7.741 & 7.741 & & 0.1807 & 1.45E-03 & 368.7587 & 135.8767 & 504.6354 & \\
\hline & 8/5/2008 10:00 & 333 & 218 & 1000 & 6.27 & 6.27 & & 0.4577 & $4.55 E-03$ & 1157.6202 & 426.5490 & 1584.1692 & \\
\hline & 8/5/2008 11:00 & 334 & 218 & 1100 & 6.457 & 6.457 & & 0.4155 & 4.04E-03 & 1027.6234 & 378.6490 & 1406.2724 & \\
\hline & 8/5/2008 12:00 & 335 & 218 & 1200 & 6.139 & 6.139 & & 0.4888 & 4.93E-03 & 1255.2091 & 462.5076 & 1717.7168 & \\
\hline & 8/5/2008 13:00 & 336 & 218 & 1300 & 5.687 & 5.687 & & 0.6072 & 6.44E-03 & 1639.1118 & 603.9645 & 2243.0762 & \\
\hline & 8/5/2008 14:00 & 337 & 218 & 1400 & 6.178 & 6.178 & & 0.4794 & 4.81E-03 & 1225.5676 & 451.5856 & 1677.1532 & \\
\hline & 8/5/2008 15:00 & 338 & 218 & 1500 & 6.582 & 6.582 & & 0.3886 & $3.72 E-03$ & 946.4046 & 348.7223 & 1295.1269 & \\
\hline & 8/5/2008 16:00 & 339 & 218 & 1600 & 6.418 & 6.418 & & 0.4241 & 4.14E-03 & 1053.8725 & 388.3210 & 1442.1935 & \\
\hline & 8/5/2008 17:00 & 340 & 218 & 1700 & 5.97 & 5.97 & & 0.5310 & $5.46 \mathrm{E}-03$ & 1389.7267 & 512.0734 & 1901.8000 & \\
\hline & 8/5/2008 18:00 & 341 & 218 & 1800 & 5.751 & 5.751 & & 0.5893 & $6.21 E-03$ & 1579.8791 & 582.1389 & 2162.0181 & \\
\hline & 8/5/2008 19:00 & 342 & 218 & 1900 & 5.941 & 5.941 & & 0.5385 & 5.55E-03 & 1413.8432 & 520.9596 & 1934.8029 & \\
\hline & 8/5/2008 20:00 & 343 & 218 & 2000 & 6.73 & 6.73 & & 0.3581 & 3.36E-03 & 855.7093 & 315.3037 & 1171.0131 & \\
\hline & 8/5/2008 21:00 & 344 & 218 & 2100 & 8.4 & 8.4 & & 0.0881 & 5.98E-04 & 152.2602 & 56.1034 & 208.3635 & \\
\hline & $8 / 5 / 200822: 00$ & 345 & 218 & 2200 & 9.17 & 9.17 & & -0.0033 & $0.00 \mathrm{E}+00$ & 0.0000 & 0.0000 & 0.0000 & \\
\hline & 8/5/2008 23:00 & 346 & 218 & 2300 & 9.44 & 9.44 & & -0.0318 & $0.00 \mathrm{E}+00$ & 0.0000 & 0.0000 & 0.0000 & \\
\hline & 8/6/2008 0:00 & 347 & 219 & 0 & 9.62 & 9.62 & 9.74 & -0.0499 & $0.00 E+00$ & 0.0000 & 0.0000 & 0.0000 & \\
\hline & 8/6/2008 1:00 & 348 & 219 & 100 & 9.67 & 9.67 & & -0.0548 & $0.00 \mathrm{E}+00$ & 0.0000 & 0.0000 & 0.0000 & \\
\hline & $8 / 6 / 2008$ 2:00 & 349 & 219 & 200 & 9.72 & 9.72 & & -0.0597 & $0.00 E+00$ & 0.0000 & 0.0000 & 0.0000 & \\
\hline & 8/6/2008 3:00 & 350 & 219 & 300 & 9.74 & 9.74 & & -0.0616 & $0.00 E+00$ & 0.0000 & 0.0000 & 0.0000 & \\
\hline & 8/6/2008 4:00 & 351 & 219 & 400 & 9.72 & 9.72 & & -0.0597 & $0.00 \mathrm{E}+00$ & 0.0000 & 0.0000 & 0.0000 & \\
\hline & 8/6/2008 5:00 & 352 & 219 & 500 & 9.71 & 9.71 & & -0.0587 & $0.00 \mathrm{E}+00$ & 0.0000 & 0.0000 & 0.0000 & \\
\hline & 8/6/2008 6:00 & 353 & 219 & 600 & 9.69 & 9.69 & & -0.0568 & $0.00 E+00$ & 0.0000 & 0.0000 & 0.0000 & \\
\hline & 8/6/2008 7:00 & 354 & 219 & 700 & 9.68 & 9.68 & & -0.0558 & $0.00 \mathrm{E}+00$ & 0.0000 & 0.0000 & 0.0000 & \\
\hline & 8/6/2008 8:00 & 355 & 219 & 800 & 9.64 & 9.64 & & -0.0519 & $0.00 \mathrm{E}+00$ & 0.0000 & 0.0000 & 0.0000 & \\
\hline & 8/6/2008 9:00 & 356 & 219 & 900 & 9.11 & 9.11 & & 0.0033 & 1.05E-05 & 2.6639 & 0.9816 & 3.6455 & \\
\hline & 8/6/2008 10:00 & 357 & 219 & 1000 & 6.552 & 6.552 & & 0.3950 & $3.79 \mathrm{E}-03$ & 965.5007 & 355.7586 & 1321.2593 & \\
\hline & 8/6/2008 11:00 & 358 & 219 & 1100 & 5.681 & 5.681 & & 0.6089 & 6.46E-03 & 1644.7544 & 606.0436 & 2250.7980 & \\
\hline & 8/6/2008 12:00 & 359 & 219 & 1200 & 6.179 & 6.179 & & 0.4792 & 4.81E-03 & 1224.8142 & 451.3080 & 1676.1222 & \\
\hline & 8/6/2008 13:00 & 360 & 219 & 1300 & 6.466 & 6.466 & & 0.4135 & 4.01E-03 & 1021.6285 & 376.4400 & 1398.0685 & \\
\hline & $8 / 6 / 2008$ 14:00 & 361 & 219 & 1400 & 6.06 & 6.06 & & 0.5083 & 5.17E-03 & 1316.8369 & 485.2156 & 1802.0525 & \\
\hline & 8/6/2008 15:00 & 362 & 219 & 1500 & 5.73 & 5.73 & & 0.5951 & $6.28 \mathrm{E}-03$ & 1599.1240 & 589.2301 & 2188.3541 & \\
\hline & 8/6/2008 16:00 & 363 & 219 & 1600 & 5.788 & 5.788 & & 0.5791 & 6.07E-03 & 1546.4165 & 569.8090 & 2116.2254 & \\
\hline & 8/6/2008 17:00 & 364 & 219 & 1700 & 5.7 & 5.7 & & 0.6035 & $6.39 \mathrm{E}-03$ & 1626.9392 & 599.4792 & 2226.4185 & \\
\hline & 8/6/2008 18:00 & 365 & 219 & 1800 & 6.155 & 6.155 & & 0.4850 & 4.88E-03 & 1242.9870 & 458.0041 & 1700.9911 & \\
\hline & 8/6/2008 19:00 & 366 & 219 & 1900 & 6.2 & 6.2 & & 0.4742 & $4.75 \mathrm{E}-03$ & 1209.0694 & 445.5065 & 1654.5759 & \\
\hline & $8 / 6 / 200820: 00$ & 367 & 219 & 2000 & 6.589 & 6.589 & & 0.3872 & 3.70E-03 & 941.9842 & 347.0934 & 1289.0776 & \\
\hline & 8/6/2008 21:00 & 368 & 219 & 2100 & 7.761 & 7.761 & & 0.1777 & 1.42E-03 & 361.1310 & 133.0661 & 494.1972 & \\
\hline
\end{tabular}




\begin{tabular}{|c|c|c|c|c|c|c|c|c|c|c|c|c|c|}
\hline Tree ID & TS & RN & JD & HR & $\begin{array}{c}\text { Raw } \\
\text { TC_dTA }\end{array}$ & $\begin{array}{c}\text { Fixed } \\
\text { TC_dT } \Delta\end{array}$ & dTM & $\mathbf{K}$ & $\begin{array}{l}\text { Sap } \\
\text { Velocity } \\
(\mathrm{cm} / \mathrm{s})\end{array}$ & $\begin{array}{c}\text { Probed } \\
\text { Drywood F } \\
(\mathrm{g} / \mathrm{hr})\end{array}$ & $\begin{array}{c}\text { Extrapolated } \\
\text { Wetwood F } \\
(\mathrm{g} / \mathrm{hr})\end{array}$ & $\begin{array}{c}\text { Extrapolated } \\
\text { Total Wood F } \\
(\mathrm{g} / \mathrm{hr})\end{array}$ & $\begin{array}{l}\text { Relative } \\
\text { Humidity, } \\
@ 10 \mathrm{~m}(\%)\end{array}$ \\
\hline & 8/6/2008 22:00 & 369 & 219 & 2200 & 8.87 & 8.87 & & 0.0304 & 1.62E-04 & 41.1590 & 15.1659 & $\begin{array}{r}56.3249 \\
\end{array}$ & \\
\hline & 8/6/2008 23:00 & 370 & 219 & 2300 & 9.24 & 9.24 & & -0.0108 & $0.00 \mathrm{E}+00$ & 0.0000 & 0.0000 & 0.0000 & \\
\hline & $8 / 7 / 20080: 00$ & 371 & 220 & 0 & 9.53 & 9.53 & 9.73 & -0.0409 & $0.00 E+00$ & 0.0000 & 0.0000 & 0.0000 & \\
\hline & 8/7/2008 1:00 & 372 & 220 & 100 & 9.7 & 9.7 & & -0.0577 & $0.00 \mathrm{E}+00$ & 0.0000 & 0.0000 & 0.0000 & \\
\hline & 8/7/2008 2:00 & 373 & 220 & 200 & 9.73 & 9.73 & & -0.0606 & $0.00 \mathrm{E}+00$ & 0.0000 & 0.0000 & 0.0000 & \\
\hline & 8/7/2008 3:00 & 374 & 220 & 300 & 9.72 & 9.72 & & -0.0597 & $0.00 \mathrm{E}+00$ & 0.0000 & 0.0000 & 0.0000 & \\
\hline & 8/7/2008 4:00 & 375 & 220 & 400 & 9.7 & 9.7 & & -0.0577 & $0.00 \mathrm{E}+00$ & 0.0000 & 0.0000 & 0.0000 & \\
\hline & 8/7/2008 5:00 & 376 & 220 & 500 & 9.68 & 9.68 & & -0.0558 & $0.00 \mathrm{E}+00$ & 0.0000 & 0.0000 & 0.0000 & \\
\hline & 8/7/2008 6:00 & 377 & 220 & 600 & 9.65 & 9.65 & & -0.0528 & $0.00 \mathrm{E}+00$ & 0.0000 & 0.0000 & 0.0000 & \\
\hline & 8/7/2008 7:00 & 378 & 220 & 700 & 9.63 & 9.63 & & -0.0509 & $0.00 E+00$ & 0.0000 & 0.0000 & 0.0000 & \\
\hline & 8/7/2008 8:00 & 379 & 220 & 800 & 9.6 & 9.6 & & -0.0479 & $0.00 \mathrm{E}+00$ & 0.0000 & 0.0000 & 0.0000 & \\
\hline & 8/7/2008 9:00 & 380 & 220 & 900 & 8.62 & 8.62 & & 0.0603 & 3.75E-04 & 95.5302 & 35.2001 & 130.7302 & \\
\hline & 8/7/2008 10:00 & 381 & 220 & 1000 & 6.267 & 6.267 & & 0.4584 & $4.56 \mathrm{E}-03$ & 1159.7931 & 427.3496 & 1587.1427 & \\
\hline & 8/7/2008 11:00 & 382 & 220 & 1100 & 5.644 & 5.644 & & 0.6194 & 6.60E-03 & 1679.8970 & 618.9926 & 2298.8896 & \\
\hline & 8/7/2008 12:00 & 383 & 220 & 1200 & 5.618 & 5.618 & & 0.6269 & 6.70E-03 & 1704.9530 & 628.2250 & 2333.1780 & \\
\hline & 8/7/2008 13:00 & 384 & 220 & 1300 & 5.547 & 5.547 & & 0.6477 & $6.97 \mathrm{E}-03$ & 1774.9334 & 654.0107 & 2428.9442 & \\
\hline & $8 / 7 / 2008$ 14:00 & 385 & 220 & 1400 & 5.554 & 5.554 & & 0.6457 & 6.94E-03 & 1767.9308 & 651.4305 & 2419.3613 & \\
\hline & 8/7/2008 15:00 & 386 & 220 & 1500 & 5.552 & 5.552 & & 0.6463 & $6.95 \mathrm{E}-03$ & 1769.9292 & 652.1668 & 2422.0961 & \\
\hline & 8/7/2008 16:00 & 387 & 220 & 1600 & 5.485 & 5.485 & & 0.6664 & 7.22E-03 & 1837.9673 & 677.2368 & 2515.2041 & \\
\hline & 8/7/2008 17:00 & 388 & 220 & 1700 & 5.474 & 5.474 & & 0.6697 & 7.26E-03 & 1849.3434 & 681.4286 & 2530.7720 & \\
\hline & 8/7/2008 18:00 & 389 & 220 & 1800 & 5.599 & 5.599 & & 0.6324 & $6.77 \mathrm{E}-03$ & 1723.4547 & 635.0423 & 2358.4970 & \\
\hline & 8/7/2008 19:00 & 390 & 220 & 1900 & 5.905 & 5.905 & & 0.5478 & 5.67E-03 & 1444.2201 & 532.1526 & 1976.3727 & \\
\hline & 8/7/2008 20:00 & 391 & 220 & 2000 & 6.438 & 6.438 & & 0.4197 & 4.09E-03 & 1040.3562 & 383.3406 & 1423.6968 & \\
\hline & 8/7/2008 21:00 & 392 & 220 & 2100 & 7.672 & 7.672 & & 0.1913 & 1.55E-03 & 395.6099 & 145.7706 & 541.3805 & \\
\hline & 8/7/2008 22:00 & 393 & 220 & 2200 & 8.45 & 8.45 & & 0.0817 & $5.45 \mathrm{E}-04$ & 138.6796 & 51.0993 & 189.7789 & \\
\hline & 8/7/2008 23:00 & 394 & 220 & 2300 & 8.98 & 8.98 & & 0.0178 & 8.36E-05 & 21.2882 & 7.8441 & 29.1322 & \\
\hline & 8/8/2008 0:00 & 395 & 221 & 0 & 9.28 & 9.28 & 9.70 & -0.0151 & $0.00 \mathrm{E}+00$ & 0.0000 & 0.0000 & 0.0000 & \\
\hline & 8/8/2008 1:00 & 396 & 221 & 100 & 9.29 & 9.29 & & -0.0161 & $0.00 \mathrm{E}+00$ & 0.0000 & 0.0000 & 0.0000 & \\
\hline & 8/8/2008 2:00 & 397 & 221 & 200 & 9.52 & 9.52 & & -0.0399 & $0.00 \mathrm{E}+00$ & 0.0000 & 0.0000 & 0.0000 & \\
\hline & 8/8/2008 3:00 & 398 & 221 & 300 & 9.68 & 9.68 & & -0.0558 & $0.00 \mathrm{E}+00$ & 0.0000 & 0.0000 & 0.0000 & \\
\hline & 8/8/2008 4:00 & 399 & 221 & 400 & 9.7 & 9.7 & & -0.0577 & $0.00 \mathrm{E}+00$ & 0.0000 & 0.0000 & 0.0000 & \\
\hline & 8/8/2008 5:00 & 400 & 221 & 500 & 9.68 & 9.68 & & -0.0558 & $0.00 \mathrm{E}+00$ & 0.0000 & 0.0000 & 0.0000 & \\
\hline & 8/8/2008 6:00 & 401 & 221 & 600 & 9.66 & 9.66 & & -0.0538 & $0.00 \mathrm{E}+00$ & 0.0000 & 0.0000 & 0.0000 & \\
\hline & $8 / 8 / 20087: 00$ & 402 & 221 & 700 & 9.65 & 9.65 & & -0.0528 & $0.00 \mathrm{E}+00$ & 0.0000 & 0.0000 & 0.0000 & \\
\hline & 8/8/2008 8:00 & 403 & 221 & 800 & 9.49 & 9.49 & & -0.0369 & $0.00 \mathrm{E}+00$ & 0.0000 & 0.0000 & 0.0000 & \\
\hline & 8/8/2008 9:00 & 404 & 221 & 900 & 7.463 & 7.463 & & 0.2247 & 1.89E-03 & 482.1627 & 177.6628 & 659.8254 & \\
\hline & 8/8/2008 10:00 & 405 & 221 & 1000 & 5.811 & 5.811 & & 0.5729 & 5.99E-03 & 1525.8973 & 562.2482 & 2088.1455 & \\
\hline & 8/8/2008 11:00 & 406 & 221 & 1100 & 5.741 & 5.741 & & 0.5921 & $6.24 \mathrm{E}-03$ & 1589.0203 & 585.5072 & 2174.5276 & \\
\hline & $8 / 8 / 200812: 00$ & 407 & 221 & 1200 & 5.598 & 5.598 & & 0.6327 & $6.77 \mathrm{E}-03$ & 1724.4330 & 635.4028 & 2359.8358 & \\
\hline & 8/8/2008 13:00 & 408 & 221 & 1300 & 5.513 & 5.513 & & 0.6579 & 7.11E-03 & 1809.2737 & 666.6641 & 2475.9378 & \\
\hline & 8/8/2008 14:00 & 409 & 221 & 1400 & 5.47 & 5.47 & & 0.6709 & 7.28E-03 & 1853.4948 & 682.9583 & 2536.4530 & \\
\hline
\end{tabular}




\begin{tabular}{|c|c|c|c|c|c|c|c|c|c|c|c|c|c|}
\hline Tree ID & TS & RN & JD & HR & $\begin{array}{c}\text { Raw } \\
\text { TC_dTA }\end{array}$ & $\begin{array}{c}\text { Fixed } \\
\text { TC_dT } \Delta\end{array}$ & dTM & $\mathbf{K}$ & $\begin{array}{l}\text { Sap } \\
\text { Velocity } \\
(\mathrm{cm} / \mathrm{s})\end{array}$ & $\begin{array}{c}\text { Probed } \\
\text { Drywood F } \\
(\mathrm{g} / \mathrm{hr})\end{array}$ & $\begin{array}{c}\text { Extrapolated } \\
\text { Wetwood F } \\
(\mathrm{g} / \mathrm{hr})\end{array}$ & $\begin{array}{c}\text { Extrapolated } \\
\text { Total Wood F } \\
(\mathrm{g} / \mathrm{hr})\end{array}$ & $\begin{array}{l}\text { Relative } \\
\text { Humidity, } \\
@ 10 \mathrm{~m}(\%)\end{array}$ \\
\hline & 8/8/2008 15:00 & 410 & 221 & 1500 & 5.436 & 5.436 & & 0.6814 & $7.42 \mathrm{E}-03$ & 1889.0994 & 696.0775 & 2585.1769 & \\
\hline & 8/8/2008 16:00 & 411 & 221 & 1600 & 5.401 & 5.401 & & 0.6923 & 7.57E-03 & 1926.3540 & 709.8048 & 2636.1588 & \\
\hline & 8/8/2008 17:00 & 412 & 221 & 1700 & 5.521 & 5.521 & & 0.6555 & 7.08E-03 & 1801.1444 & 663.6687 & 2464.8131 & \\
\hline & 8/8/2008 18:00 & 413 & 221 & 1800 & 5.722 & 5.722 & & 0.5973 & 6.31E-03 & 1606.5041 & 591.9495 & 2198.4536 & \\
\hline & 8/8/2008 19:00 & 414 & 221 & 1900 & 5.763 & 5.763 & & 0.5860 & 6.16E-03 & 1568.9645 & 578.1172 & 2147.0818 & \\
\hline & 8/8/2008 20:00 & 415 & 221 & 2000 & 6.343 & 6.343 & & 0.4410 & 4.34E-03 & 1105.6139 & 407.3862 & 1513.0000 & \\
\hline & 8/8/2008 21:00 & 416 & 221 & 2100 & 7.481 & 7.481 & & 0.2218 & 1.86E-03 & 474.3910 & 174.7991 & 649.1901 & \\
\hline & 8/8/2008 22:00 & 417 & 221 & 2200 & 8.19 & 8.19 & & 0.1160 & 8.39E-04 & 213.6363 & 78.7187 & 292.3550 & \\
\hline & 8/8/2008 23:00 & 418 & 221 & 2300 & 8.38 & 8.38 & & 0.0907 & $6.20 \mathrm{E}-04$ & 157.8040 & 58.1461 & 215.9501 & \\
\hline & 8/9/2008 0:00 & 419 & 222 & 0 & 8.35 & 8.35 & 9.47 & 0.0946 & 6.53E-04 & 166.2389 & 61.2542 & 227.4931 & \\
\hline & 8/9/2008 1:00 & 420 & 222 & 100 & 8.33 & 8.33 & & 0.0972 & $6.75 E-04$ & 171.9416 & 63.3554 & 235.2970 & \\
\hline & 8/9/2008 2:00 & 421 & 222 & 200 & 8.39 & 8.39 & & 0.0894 & $6.09 E-04$ & 155.0241 & 57.1218 & 212.1459 & \\
\hline & 8/9/2008 3:00 & 422 & 222 & 300 & 8.45 & 8.45 & & 0.0817 & $5.45 E-04$ & 138.6796 & 51.0993 & 189.7789 & \\
\hline & 8/9/2008 4:00 & 423 & 222 & 400 & 8.46 & 8.46 & & 0.0804 & 5.34E-04 & 136.0114 & 50.1162 & 186.1276 & \\
\hline & $8 / 9 / 20085: 00$ & 424 & 222 & 500 & 8.36 & 8.36 & & 0.0933 & 6.42E-04 & 163.4114 & 60.2123 & 223.6237 & \\
\hline & 8/9/2008 6:00 & 425 & 222 & 600 & 8.42 & 8.42 & & 0.0855 & 5.77E-04 & 146.7800 & 54.0841 & 200.8641 & \\
\hline & 8/9/2008 7:00 & 426 & 222 & 700 & 8.1 & 8.1 & & 0.1284 & 9.51E-04 & 242.0878 & 89.2022 & 331.2900 & \\
\hline & 8/9/2008 8:00 & 427 & 222 & 800 & 7.485 & 7.485 & & 0.2211 & $1.86 \mathrm{E}-03$ & 472.6722 & 174.1658 & 646.8380 & \\
\hline & 8/9/2008 9:00 & 428 & 222 & 900 & 8.14 & 8.14 & & 0.1229 & 9.01E-04 & 229.2827 & 84.4839 & 313.7666 & \\
\hline & 8/9/2008 10:00 & 429 & 222 & 1000 & 7.731 & 7.731 & & 0.1823 & $1.46 \mathrm{E}-03$ & 372.5985 & 137.2916 & 509.8901 & \\
\hline & 8/9/2008 11:00 & 430 & 222 & 1100 & 7.13 & 7.13 & & 0.2819 & $2.50 \mathrm{E}-03$ & 637.4278 & 234.8734 & 872.3011 & \\
\hline & 8/9/2008 12:00 & 431 & 222 & 1200 & 6.43 & 6.43 & & 0.4215 & 4.11E-03 & 1045.7487 & 385.3276 & 1431.0763 & \\
\hline & 8/9/2008 13:00 & 432 & 222 & 1300 & 6.219 & 6.219 & & 0.4697 & 4.69E-03 & 1194.9485 & 440.3033 & 1635.2518 & \\
\hline & 8/9/2008 14:00 & 433 & 222 & 1400 & 5.75 & 5.75 & & 0.5896 & $6.21 \mathrm{E}-03$ & 1580.7914 & 582.4751 & 2163.2664 & \\
\hline & 8/9/2008 15:00 & 434 & 222 & 1500 & 5.61 & 5.61 & & 0.6292 & $6.73 \mathrm{E}-03$ & 1712.7234 & 631.0881 & 2343.8115 & \\
\hline & 8/9/2008 16:00 & 435 & 222 & 1600 & 5.58 & 5.58 & & 0.6380 & 6.84E-03 & 1742.1201 & 641.9200 & 2384.0401 & \\
\hline & 8/9/2008 17:00 & 436 & 222 & 1700 & 5.718 & 5.718 & & 0.5985 & 6.33E-03 & 1610.2043 & 593.3129 & 2203.5172 & \\
\hline & 8/9/2008 18:00 & 437 & 222 & 1800 & 5.75 & 5.75 & & 0.5896 & $6.21 \mathrm{E}-03$ & 1580.7914 & 582.4751 & 2163.2664 & \\
\hline & 8/9/2008 19:00 & 438 & 222 & 1900 & 6.01 & 6.01 & & 0.5208 & 5.33E-03 & 1356.9706 & 500.0037 & 1856.9744 & \\
\hline & 8/9/2008 20:00 & 439 & 222 & 2000 & 6.59 & 6.59 & & 0.3869 & $3.70 \mathrm{E}-03$ & 941.3537 & 346.8611 & 1288.2149 & \\
\hline & 8/9/2008 21:00 & 440 & 222 & 2100 & 8.19 & 8.19 & & 0.1160 & 8.39E-04 & 213.6363 & 78.7187 & 292.3550 & \\
\hline & 8/9/2008 22:00 & 441 & 222 & 2200 & 9.19 & 9.19 & & -0.0054 & $0.00 \mathrm{E}+00$ & 0.0000 & 0.0000 & 0.0000 & \\
\hline & 8/9/2008 23:00 & 442 & 222 & 2300 & 9.47 & 9.47 & & -0.0348 & $0.00 \mathrm{E}+00$ & 0.0000 & 0.0000 & 0.0000 & \\
\hline & 8/10/2008 0:00 & 443 & 223 & 0 & 9.6 & 9.6 & 9.74 & -0.0479 & $0.00 \mathrm{E}+00$ & 0.0000 & 0.0000 & 0.0000 & \\
\hline & 8/10/2008 1:00 & 444 & 223 & 100 & 9.69 & 9.69 & & -0.0568 & $0.00 \mathrm{E}+00$ & 0.0000 & 0.0000 & 0.0000 & \\
\hline & 8/10/2008 2:00 & 445 & 223 & 200 & 9.72 & 9.72 & & -0.0597 & $0.00 \mathrm{E}+00$ & 0.0000 & 0.0000 & 0.0000 & \\
\hline & 8/10/2008 3:00 & 446 & 223 & 300 & 9.74 & 9.74 & & -0.0616 & $0.00 \mathrm{E}+00$ & 0.0000 & 0.0000 & 0.0000 & \\
\hline & 8/10/2008 4:00 & 447 & 223 & 400 & 9.73 & 9.73 & & -0.0606 & $0.00 \mathrm{E}+00$ & 0.0000 & 0.0000 & 0.0000 & \\
\hline & 8/10/2008 5:00 & 448 & 223 & 500 & 9.7 & 9.7 & & -0.0577 & $0.00 \mathrm{E}+00$ & 0.0000 & 0.0000 & 0.0000 & \\
\hline & 8/10/2008 6:00 & 449 & 223 & 600 & 9.68 & 9.68 & & -0.0558 & $0.00 \mathrm{E}+00$ & 0.0000 & 0.0000 & 0.0000 & \\
\hline & 8/10/2008 7:00 & 450 & 223 & 700 & 9.67 & 9.67 & & -0.0548 & $0.00 \mathrm{E}+00$ & 0.0000 & 0.0000 & 0.0000 & \\
\hline
\end{tabular}




\begin{tabular}{|c|c|c|c|c|c|c|c|c|c|c|c|c|c|}
\hline Tree ID & TS & RN & JD & HR & $\begin{array}{c}\text { Raw } \\
\text { TC_dTA }\end{array}$ & $\begin{array}{c}\text { Fixed } \\
\text { TC_dT } \Delta\end{array}$ & dTM & K & $\begin{array}{l}\text { Sap } \\
\text { Velocity } \\
(\mathrm{cm} / \mathrm{s})\end{array}$ & $\begin{array}{c}\text { Probed } \\
\text { Drywood F } \\
(\mathrm{g} / \mathrm{hr})\end{array}$ & $\begin{array}{c}\text { Extrapolated } \\
\text { Wetwood F } \\
(\mathrm{g} / \mathrm{hr})\end{array}$ & $\begin{array}{c}\text { Extrapolated } \\
\text { Total Wood F } \\
(\mathrm{g} / \mathrm{hr})\end{array}$ & $\begin{array}{l}\text { Relative } \\
\text { Humidity, } \\
@ 10 \mathrm{~m}(\%)\end{array}$ \\
\hline & 8/10/2008 8:00 & 451 & 223 & 800 & $\begin{array}{r}9.64 \\
\end{array}$ & 9.64 & & -0.0519 & $0.00 \mathrm{E}+00$ & 0.0000 & 0.0000 & 0.0000 & \\
\hline & 8/10/2008 9:00 & 452 & 223 & 900 & 8.52 & 8.52 & & 0.0728 & $4.73 \mathrm{E}-04$ & 120.3408 & 44.3420 & 164.6828 & \\
\hline & $8 / 10 / 2008$ 10:00 & 453 & 223 & 1000 & 6.344 & 6.344 & & 0.4407 & 4.34E-03 & 1104.9129 & 407.1279 & 1512.0407 & \\
\hline & 8/10/2008 11:00 & 454 & 223 & 1100 & 5.706 & 5.706 & & 0.6018 & 6.37E-03 & 1621.3456 & 597.4181 & 2218.7637 & \\
\hline & $8 / 10 / 200812: 00$ & 455 & 223 & 1200 & 5.701 & 5.701 & & 0.6032 & 6.39E-03 & 1626.0059 & 599.1353 & 2225.1412 & \\
\hline & 8/10/2008 13:00 & 456 & 223 & 1300 & 5.632 & 5.632 & & 0.6229 & 6.64E-03 & 1691.4239 & 623.2399 & 2314.6639 & \\
\hline & $8 / 10 / 2008$ 14:00 & 457 & 223 & 1400 & 5.597 & 5.597 & & 0.6330 & $6.78 E-03$ & 1725.4117 & 635.7634 & 2361.1752 & \\
\hline & $8 / 10 / 2008$ 15:00 & 458 & 223 & 1500 & 5.728 & 5.728 & & 0.5957 & 6.29E-03 & 1600.9665 & 589.9090 & 2190.8755 & \\
\hline & 8/10/2008 16:00 & 459 & 223 & 1600 & 5.536 & 5.536 & & 0.6510 & 7.02E-03 & 1785.9839 & 658.0825 & 2444.0664 & \\
\hline & 8/10/2008 17:00 & 460 & 223 & 1700 & 5.768 & 5.768 & & 0.5846 & $6.15 E-03$ & 1564.4344 & 576.4480 & 2140.8824 & \\
\hline & 8/10/2008 18:00 & 461 & 223 & 1800 & 8.23 & 8.23 & & 0.1106 & 7.91E-04 & 201.4061 & 74.2122 & 275.6183 & \\
\hline & $8 / 10 / 2008$ 19:00 & 462 & 223 & 1900 & 8.89 & 8.89 & & 0.0281 & 1.47E-04 & 37.3350 & 13.7568 & 51.0918 & \\
\hline & 8/10/2008 20:00 & 463 & 223 & 2000 & 8.62 & 8.62 & & 0.0603 & $3.75 E-04$ & 95.5302 & 35.2001 & 130.7302 & \\
\hline & 8/10/2008 21:00 & 464 & 223 & 2100 & 8.7 & 8.7 & & 0.0506 & $3.02 E-04$ & 76.8939 & 28.3331 & 105.2270 & \\
\hline & $8 / 10 / 2008$ 22:00 & 465 & 223 & 2200 & 8.94 & 8.94 & & 0.0224 & 1.11E-04 & 28.1722 & 10.3806 & 38.5529 & \\
\hline & $8 / 10 / 2008$ 23:00 & 466 & 223 & 2300 & 8.93 & 8.93 & & 0.0235 & 1.18E-04 & 29.9574 & 11.0384 & 40.9958 & \\
\hline & $8 / 11 / 20080: 00$ & 467 & 224 & 0 & 8.93 & 8.93 & 9.37 & 0.0235 & 1.18E-04 & 29.9574 & 11.0384 & 40.9958 & \\
\hline & 8/11/2008 1:00 & 468 & 224 & 100 & 9.04 & 9.04 & & 0.0111 & $4.65 \mathrm{E}-05$ & 11.8388 & 4.3622 & 16.2010 & \\
\hline & 8/11/2008 2:00 & 469 & 224 & 200 & 9.2 & 9.2 & & -0.0065 & $0.00 \mathrm{E}+00$ & 0.0000 & 0.0000 & 0.0000 & \\
\hline & 8/11/2008 3:00 & 470 & 224 & 300 & 9.33 & 9.33 & & -0.0204 & $0.00 E+00$ & 0.0000 & 0.0000 & 0.0000 & \\
\hline & 8/11/2008 4:00 & 471 & 224 & 400 & 9.35 & 9.35 & & -0.0225 & $0.00 \mathrm{E}+00$ & 0.0000 & 0.0000 & 0.0000 & \\
\hline & 8/11/2008 5:00 & 472 & 224 & 500 & 9.33 & 9.33 & & -0.0204 & $0.00 E+00$ & 0.0000 & 0.0000 & 0.0000 & \\
\hline & 8/11/2008 6:00 & 473 & 224 & 600 & 9.37 & 9.37 & & -0.0245 & $0.00 \mathrm{E}+00$ & 0.0000 & 0.0000 & 0.0000 & \\
\hline & 8/11/2008 7:00 & 474 & 224 & 700 & 9.37 & 9.37 & & -0.0245 & $0.00 \mathrm{E}+00$ & 0.0000 & 0.0000 & 0.0000 & \\
\hline & 8/11/2008 8:00 & 475 & 224 & 800 & 9.32 & 9.32 & & -0.0193 & $0.00 \mathrm{E}+00$ & 0.0000 & 0.0000 & 0.0000 & \\
\hline & 8/11/2008 9:00 & 476 & 224 & 900 & 8.34 & 8.34 & & 0.0959 & 6.64E-04 & 169.0823 & 62.3019 & 231.3842 & \\
\hline & 8/11/2008 10:00 & 477 & 224 & 1000 & 6.384 & 6.384 & & 0.4317 & 4.23E-03 & 1077.1203 & 396.8871 & 1474.0075 & \\
\hline & 8/11/2008 11:00 & 478 & 224 & 1100 & 5.9 & 5.9 & & 0.5492 & $5.69 \mathrm{E}-03$ & 1448.4781 & 533.7215 & 1982.1996 & \\
\hline & $8 / 11 / 2008$ 12:00 & 479 & 224 & 1200 & 5.843 & 5.843 & & 0.5643 & 5.88E-03 & 1497.7023 & 551.8592 & 2049.5615 & \\
\hline & 8/11/2008 13:00 & 480 & 224 & 1300 & 6.014 & 6.014 & & 0.5198 & $5.32 E-03$ & 1353.7270 & 498.8086 & 1852.5356 & \\
\hline & 8/11/2008 14:00 & 481 & 224 & 1400 & 5.74 & 5.74 & & 0.5923 & $6.25 \mathrm{E}-03$ & 1589.9367 & 585.8449 & 2175.7816 & \\
\hline & 8/11/2008 15:00 & 482 & 224 & 1500 & 5.659 & 5.659 & & 0.6151 & $6.54 \mathrm{E}-03$ & 1665.5778 & 613.7164 & 2279.2942 & \\
\hline & $8 / 11 / 2008$ 16:00 & 483 & 224 & 1600 & 5.854 & 5.854 & & 0.5613 & 5.85E-03 & 1488.1041 & 548.3226 & 2036.4266 & \\
\hline & $8 / 11 / 2008$ 17:00 & 484 & 224 & 1700 & 5.919 & 5.919 & & 0.5442 & $5.63 \mathrm{E}-03$ & 1432.3486 & 527.7783 & 1960.1268 & \\
\hline & 8/11/2008 18:00 & 485 & 224 & 1800 & 6.197 & 6.197 & & 0.4749 & 4.76E-03 & 1211.3098 & 446.3320 & 1657.6418 & \\
\hline & $8 / 11 / 2008$ 19:00 & 486 & 224 & 1900 & 6.335 & 6.335 & & 0.4428 & 4.37E-03 & 1111.2329 & 409.4566 & 1520.6896 & \\
\hline & 8/11/2008 20:00 & 487 & 224 & 2000 & 6.978 & 6.978 & & 0.3098 & 2.81E-03 & 716.0187 & 263.8318 & 979.8505 & \\
\hline & 8/11/2008 21:00 & 488 & 224 & 2100 & 7.845 & 7.845 & & 0.1651 & 1.30E-03 & 329.8451 & 121.5382 & 451.3833 & \\
\hline & $8 / 11 / 200822: 00$ & 489 & 224 & 2200 & 8.43 & 8.43 & & 0.0842 & $5.66 \mathrm{E}-04$ & 144.0639 & 53.0833 & 197.1472 & \\
\hline & 8/11/2008 23:00 & 490 & 224 & 2300 & 8.73 & 8.73 & & 0.0470 & 2.76E-04 & 70.1937 & 25.8643 & 96.0580 & \\
\hline & 8/12/2008 0:00 & 491 & 225 & 0 & 8.91 & 8.91 & 9.56 & 0.0258 & 1.32E-04 & 33.5999 & 12.3806 & 45.9804 & \\
\hline
\end{tabular}




\begin{tabular}{|c|c|c|c|c|c|c|c|c|c|c|c|c|c|}
\hline Tree ID & TS & RN & JD & HR & $\begin{array}{c}\text { Raw } \\
\text { TC_dTA }\end{array}$ & $\begin{array}{c}\text { Fixed } \\
\text { TC_dT } \Delta\end{array}$ & dTM & $\mathbf{K}$ & $\begin{array}{l}\text { Sap } \\
\text { Velocity } \\
(\mathrm{cm} / \mathrm{s})\end{array}$ & $\begin{array}{c}\text { Probed } \\
\text { Drywood F } \\
(\mathrm{g} / \mathrm{hr})\end{array}$ & $\begin{array}{c}\text { Extrapolated } \\
\text { Wetwood F } \\
(\mathrm{g} / \mathrm{hr})\end{array}$ & $\begin{array}{c}\text { Extrapolated } \\
\text { Total Wood F } \\
(\mathrm{g} / \mathrm{hr})\end{array}$ & $\begin{array}{l}\text { Relative } \\
\text { Humidity, } \\
@ 10 \mathrm{~m}(\%)\end{array}$ \\
\hline & 8/12/2008 1:00 & 492 & 225 & 100 & 9 & 9 & & 0.0156 & $7.08 \mathrm{E}-05$ & 18.0120 & 6.6369 & $\begin{array}{r}24.6488 \\
\end{array}$ & \\
\hline & 8/12/2008 2:00 & 493 & 225 & 200 & 9.24 & 9.24 & & -0.0108 & $0.00 \mathrm{E}+00$ & 0.0000 & 0.0000 & 0.0000 & \\
\hline & 8/12/2008 3:00 & 494 & 225 & 300 & 9.23 & 9.23 & & -0.0098 & $0.00 E+00$ & 0.0000 & 0.0000 & 0.0000 & \\
\hline & 8/12/2008 4:00 & 495 & 225 & 400 & 9.23 & 9.23 & & -0.0098 & $0.00 \mathrm{E}+00$ & 0.0000 & 0.0000 & 0.0000 & \\
\hline & 8/12/2008 5:00 & 496 & 225 & 500 & 9.31 & 9.31 & & -0.0183 & $0.00 \mathrm{E}+00$ & 0.0000 & 0.0000 & 0.0000 & \\
\hline & 8/12/2008 6:00 & 497 & 225 & 600 & 9.4 & 9.4 & & -0.0277 & $0.00 \mathrm{E}+00$ & 0.0000 & 0.0000 & 0.0000 & \\
\hline & 8/12/2008 7:00 & 498 & 225 & 700 & 9.56 & 9.56 & & -0.0439 & $0.00 \mathrm{E}+00$ & 0.0000 & 0.0000 & 0.0000 & \\
\hline & 8/12/2008 8:00 & 499 & 225 & 800 & 9.55 & 9.55 & & -0.0429 & $0.00 \mathrm{E}+00$ & 0.0000 & 0.0000 & 0.0000 & \\
\hline & 8/12/2008 9:00 & 500 & 225 & 900 & 8.75 & 8.75 & & 0.0446 & $2.59 \mathrm{E}-04$ & 65.8171 & 24.2516 & 90.0687 & \\
\hline & $8 / 12 / 2008$ 10:00 & 501 & 225 & 1000 & 6.774 & 6.774 & & 0.3493 & 3.26E-03 & 829.8346 & 305.7696 & 1135.6043 & \\
\hline & $8 / 12 / 2008$ 11:00 & 502 & 225 & 1100 & 5.919 & 5.919 & & 0.5442 & $5.63 E-03$ & 1432.3486 & 527.7783 & 1960.1268 & \\
\hline & $8 / 12 / 2008$ 12:00 & 503 & 225 & 1200 & 5.83 & 5.83 & & 0.5678 & 5.93E-03 & 1509.1073 & 556.0616 & 2065.1689 & \\
\hline & $8 / 12 / 2008$ 13:00 & 504 & 225 & 1300 & 5.719 & 5.719 & & 0.5982 & $6.32 \mathrm{E}-03$ & 1609.2786 & 592.9718 & 2202.2504 & \\
\hline & $8 / 12 / 2008$ 14:00 & 505 & 225 & 1400 & 5.695 & 5.695 & & 0.6049 & 6.41E-03 & 1631.6124 & 601.2011 & 2232.8135 & \\
\hline & $8 / 12 / 2008$ 15:00 & 506 & 225 & 1500 & 5.624 & 5.624 & & 0.6252 & 6.67E-03 & 1699.1441 & 626.0846 & 2325.2287 & \\
\hline & $8 / 12 / 2008$ 16:00 & 507 & 225 & 1600 & 5.586 & 5.586 & & 0.6362 & 6.82E-03 & 1736.2080 & 639.7415 & 2375.9495 & \\
\hline & $8 / 12 / 2008$ 17:00 & 508 & 225 & 1700 & 5.648 & 5.648 & & 0.6183 & $6.58 \mathrm{E}-03$ & 1676.0689 & 617.5821 & 2293.6509 & \\
\hline & $8 / 12 / 2008$ 18:00 & 509 & 225 & 1800 & 5.733 & 5.733 & & 0.5943 & $6.27 \mathrm{E}-03$ & 1596.3634 & 588.2129 & 2184.5763 & \\
\hline & $8 / 12 / 2008$ 19:00 & 510 & 225 & 1900 & 6.017 & 6.017 & & 0.5190 & $5.31 E-03$ & 1351.2981 & 497.9136 & 1849.2116 & \\
\hline & $8 / 12 / 200820: 00$ & 511 & 225 & 2000 & 6.588 & 6.588 & & 0.3874 & 3.70E-03 & 942.6148 & 347.3258 & 1289.9407 & \\
\hline & $8 / 12 / 2008$ 21:00 & 512 & 225 & 2100 & 8.04 & 8.04 & & 0.1368 & 1.03E-03 & 261.7785 & 96.4577 & 358.2362 & \\
\hline & $8 / 12 / 2008$ 22:00 & 513 & 225 & 2200 & 8.8 & 8.8 & & 0.0386 & 2.17E-04 & 55.2004 & 20.3397 & 75.5401 & \\
\hline & $8 / 12 / 2008$ 23:00 & 514 & 225 & 2300 & 8.99 & 8.99 & & 0.0167 & 7.71E-05 & 19.6354 & 7.2351 & 26.8705 & \\
\hline & 8/13/2008 0:00 & 515 & 226 & 0 & 9.21 & 9.21 & 9.58 & -0.0076 & $0.00 E+00$ & 0.0000 & 0.0000 & 0.0000 & \\
\hline & 8/13/2008 1:00 & 516 & 226 & 100 & 9.24 & 9.24 & & -0.0108 & $0.00 \mathrm{E}+00$ & 0.0000 & 0.0000 & 0.0000 & \\
\hline & 8/13/2008 2:00 & 517 & 226 & 200 & 9.25 & 9.25 & & -0.0119 & $0.00 \mathrm{E}+00$ & 0.0000 & 0.0000 & 0.0000 & \\
\hline & 8/13/2008 3:00 & 518 & 226 & 300 & 9.41 & 9.41 & & -0.0287 & $0.00 \mathrm{E}+00$ & 0.0000 & 0.0000 & 0.0000 & \\
\hline & 8/13/2008 4:00 & 519 & 226 & 400 & 9.41 & 9.41 & & -0.0287 & $0.00 E+00$ & 0.0000 & 0.0000 & 0.0000 & \\
\hline & $8 / 13 / 20085: 00$ & 520 & 226 & 500 & 9.39 & 9.39 & & -0.0266 & $0.00 \mathrm{E}+00$ & 0.0000 & 0.0000 & 0.0000 & \\
\hline & 8/13/2008 6:00 & 521 & 226 & 600 & 9.56 & 9.56 & & -0.0439 & $0.00 \mathrm{E}+00$ & 0.0000 & 0.0000 & 0.0000 & \\
\hline & $8 / 13 / 2008$ 7:00 & 522 & 226 & 700 & 9.58 & 9.58 & & -0.0459 & $0.00 \mathrm{E}+00$ & 0.0000 & 0.0000 & 0.0000 & \\
\hline & 8/13/2008 8:00 & 523 & 226 & 800 & 9.31 & 9.31 & & -0.0183 & $0.00 \mathrm{E}+00$ & 0.0000 & 0.0000 & 0.0000 & \\
\hline & 8/13/2008 9:00 & 524 & 226 & 900 & 7.57 & 7.57 & & 0.2074 & $1.72 \mathrm{E}-03$ & 436.8530 & 160.9675 & 597.8204 & \\
\hline & $8 / 13 / 2008$ 10:00 & 525 & 226 & 1000 & 6.144 & 6.144 & & 0.4876 & 4.92E-03 & 1251.3805 & 461.0969 & 1712.4773 & \\
\hline & 8/13/2008 11:00 & 526 & 226 & 1100 & 6.108 & 6.108 & & 0.4964 & 5.02E-03 & 1279.1362 & 471.3240 & 1750.4602 & \\
\hline & $8 / 13 / 2008$ 12:00 & 527 & 226 & 1200 & 5.71 & 5.71 & & 0.6007 & 6.35E-03 & 1617.6250 & 596.0472 & 2213.6722 & \\
\hline
\end{tabular}




\begin{tabular}{|c|c|c|c|c|c|c|c|c|c|c|c|c|c|}
\hline Tree ID & TS & RN & JD & HR & $\begin{array}{c}\text { Raw } \\
\text { TC_dTA }\end{array}$ & $\begin{array}{c}\text { Fixed } \\
\text { TC_dT } \Delta\end{array}$ & dTM & $\mathbf{K}$ & $\begin{array}{c}\text { Sap } \\
\text { Velocity } \\
(\mathrm{cm} / \mathrm{s})\end{array}$ & $\begin{array}{c}\text { Probed } \\
\text { Drywood F } \\
(\mathrm{g} / \mathrm{hr})\end{array}$ & $\begin{array}{c}\text { Extrapolated } \\
\text { Wetwood F } \\
(\mathrm{g} / \mathrm{hr})\end{array}$ & $\begin{array}{c}\text { Extrapolated } \\
\text { Total Wood F } \\
(\mathrm{g} / \mathrm{hr})\end{array}$ & $\begin{array}{c}\text { Relative } \\
\text { Humidity, } \\
@ 10 \mathrm{~m}(\%)\end{array}$ \\
\hline & $7 / 22 / 2008$ 13:00 & 0 & 204 & 1300 & 5.127 & 5.127 & 9.52 & 0.8568 & 9.84E-03 & 912.5376 & 336.2433 & 1248.7809 & \\
\hline \multirow[t]{2}{*}{ W708EC1 } & $7 / 22 / 200814: 00$ & 1 & 204 & 1400 & 5.639 & 5.639 & & 0.6882 & $7.51 \mathrm{E}-03$ & 696.8083 & 256.7534 & 953.5617 & \\
\hline & $7 / 22 / 2008$ 15:00 & 2 & 204 & 1500 & 5.588 & 5.588 & & 0.7037 & $7.72 \mathrm{E}-03$ & 716.0611 & 263.8474 & 979.9086 & \\
\hline \multirow[t]{2}{*}{ Probe \# } & $7 / 22 / 2008$ 16:00 & 3 & 204 & 1600 & 5.542 & 5.542 & & 0.7178 & 7.91E-03 & 733.8163 & 270.3897 & 1004.2060 & \\
\hline & 7/22/2008 17:00 & 4 & 204 & 1700 & 5.589 & 5.589 & & 0.7033 & $7.72 \mathrm{E}-03$ & 715.6793 & 263.7068 & 979.3860 & \\
\hline \multirow[t]{4}{*}{19} & $7 / 22 / 2008$ 18:00 & 5 & 204 & 1800 & 5.636 & 5.636 & & 0.6891 & $7.52 \mathrm{E}-03$ & 697.9285 & 257.1661 & 955.0946 & \\
\hline & 7/22/2008 19:00 & 6 & 204 & 1900 & 5.846 & 5.846 & & 0.6285 & $6.72 \mathrm{E}-03$ & 623.0696 & 229.5828 & 852.6525 & \\
\hline & $7 / 22 / 200820: 00$ & 7 & 204 & 2000 & 6.274 & 6.274 & & 0.5174 & $5.29 \mathrm{E}-03$ & 490.3952 & 180.6962 & 671.0914 & \\
\hline & 7/22/2008 21:00 & 8 & 204 & 2100 & 7.882 & 7.882 & & 0.2078 & $1.72 \mathrm{E}-03$ & 159.5565 & 58.7919 & 218.3483 & \\
\hline \multirow[t]{2}{*}{$\mathrm{DBH}(\mathrm{cm})$} & $7 / 22 / 2008$ 22:00 & 9 & 204 & 2200 & 9.19 & 9.19 & & 0.0359 & $1.98 \mathrm{E}-04$ & 18.3782 & 6.7718 & 25.1500 & \\
\hline & $7 / 22 / 2008$ 23:00 & 10 & 204 & 2300 & 9.52 & 9.52 & & 0.0000 & $0.00 E+00$ & 0.0000 & 0.0000 & 0.0000 & \\
\hline \multirow[t]{2}{*}{7.1} & $7 / 23 / 20080: 00$ & 11 & 205 & 0 & 9.76 & 9.76 & 9.91 & -0.0246 & $0.00 \mathrm{E}+00$ & 0.0000 & 0.0000 & 0.0000 & \\
\hline & 7/23/2008 1:00 & 12 & 205 & 100 & 9.84 & 9.84 & & -0.0325 & $0.00 \mathrm{E}+00$ & 0.0000 & 0.0000 & 0.0000 & \\
\hline Day Count & 7/23/2008 2:00 & 13 & 205 & 200 & 9.87 & 9.87 & & -0.0355 & $0.00 \mathrm{E}+00$ & 0.0000 & 0.0000 & 0.0000 & \\
\hline \multirow{27}{*}{35} & 7/23/2008 3:00 & 14 & 205 & 300 & 9.91 & 9.91 & & -0.0394 & $0.00 \mathrm{E}+00$ & 0.0000 & 0.0000 & 0.0000 & \\
\hline & 7/23/2008 4:00 & 15 & 205 & 400 & 9.91 & 9.91 & & -0.0394 & $0.00 \mathrm{E}+00$ & 0.0000 & 0.0000 & 0.0000 & \\
\hline & 7/23/2008 5:00 & 16 & 205 & 500 & 9.89 & 9.89 & & -0.0374 & $0.00 \mathrm{E}+00$ & 0.0000 & 0.0000 & 0.0000 & \\
\hline & 7/23/2008 6:00 & 17 & 205 & 600 & 9.79 & 9.79 & & -0.0276 & $0.00 \mathrm{E}+00$ & 0.0000 & 0.0000 & 0.0000 & \\
\hline & 7/23/2008 7:00 & 18 & 205 & 700 & 9.51 & 9.51 & & 0.0011 & $2.57 \mathrm{E}-06$ & 0.2381 & 0.0877 & 0.3258 & \\
\hline & 7/23/2008 8:00 & 19 & 205 & 800 & 8.68 & 8.68 & & 0.0968 & 6.71E-04 & 62.2764 & 22.9470 & 85.2234 & \\
\hline & $7 / 23 / 20089: 00$ & 20 & 205 & 900 & 7.232 & 7.232 & & 0.3164 & 2.89E-03 & 267.6682 & 98.6278 & 366.2960 & \\
\hline & $7 / 23 / 2008$ 10:00 & 21 & 205 & 1000 & 6.159 & 6.159 & & 0.5457 & $5.65 E-03$ & 523.6597 & 192.9532 & 716.6129 & \\
\hline & 7/23/2008 11:00 & 22 & 205 & 1100 & 6.051 & 6.051 & & 0.5733 & $6.00 \mathrm{E}-03$ & 556.4367 & 205.0305 & 761.4672 & \\
\hline & 7/23/2008 12:00 & 23 & 205 & 1200 & 5.882 & 5.882 & & 0.6185 & 6.59E-03 & 610.9282 & 225.1091 & 836.0372 & \\
\hline & 7/23/2008 13:00 & 24 & 205 & 1300 & 5.753 & 5.753 & & 0.6548 & 7.07E-03 & 655.3512 & 241.4776 & 896.8289 & \\
\hline & $7 / 23 / 2008$ 14:00 & 25 & 205 & 1400 & 5.749 & 5.749 & & 0.6559 & $7.08 \mathrm{E}-03$ & 656.7701 & 242.0004 & 898.7705 & \\
\hline & $7 / 23 / 2008$ 15:00 & 26 & 205 & 1500 & 5.737 & 5.737 & & 0.6594 & 7.13E-03 & 661.0419 & 243.5745 & 904.6164 & \\
\hline & $7 / 23 / 2008$ 16:00 & 27 & 205 & 1600 & 5.887 & 5.887 & & 0.6171 & 6.57E-03 & 609.2572 & 224.4933 & 833.7505 & \\
\hline & 7/23/2008 17:00 & 28 & 205 & 1700 & 6.422 & 6.422 & & 0.4824 & 4.85E-03 & 449.9172 & 165.7812 & 615.6984 & \\
\hline & $7 / 23 / 2008$ 18:00 & 29 & 205 & 1800 & 7.228 & 7.228 & & 0.3171 & $2.89 \mathrm{E}-03$ & 268.4271 & 98.9075 & 367.3346 & \\
\hline & $7 / 23 / 2008$ 19:00 & 30 & 205 & 1900 & 7.16 & 7.16 & & 0.3296 & 3.04E-03 & 281.5206 & 103.7321 & 385.2527 & \\
\hline & $7 / 23 / 200820: 00$ & 31 & 205 & 2000 & 7.614 & 7.614 & & 0.2503 & 2.16E-03 & 200.6408 & 73.9302 & 274.5711 & \\
\hline & 7/23/2008 21:00 & 32 & 205 & 2100 & 8.49 & 8.49 & & 0.1213 & 8.87E-04 & 82.2566 & 30.3091 & 112.5657 & \\
\hline & $7 / 23 / 200822: 00$ & 33 & 205 & 2200 & 9.01 & 9.01 & & 0.0566 & 3.47E-04 & 32.1815 & 11.8579 & 44.0394 & \\
\hline & $7 / 23 / 2008$ 23:00 & 34 & 205 & 2300 & 9.06 & 9.06 & & 0.0508 & 3.04E-04 & 28.1503 & 10.3726 & 38.5229 & \\
\hline & $7 / 24 / 20080: 00$ & 35 & 206 & 0 & 9.06 & 9.06 & 9.72 & 0.0508 & 3.04E-04 & 28.1503 & 10.3726 & 38.5229 & \\
\hline & 7/24/2008 1:00 & 36 & 206 & 100 & 9.13 & 9.13 & & 0.0427 & $2.45 \mathrm{E}-04$ & 22.7570 & 8.3853 & 31.1422 & \\
\hline & 7/24/2008 2:00 & 37 & 206 & 200 & 9.24 & 9.24 & & 0.0303 & 1.61E-04 & 14.9129 & 5.4950 & 20.4079 & \\
\hline & 7/24/2008 3:00 & 38 & 206 & 300 & 9.36 & 9.36 & & 0.0171 & $7.95 \mathrm{E}-05$ & 7.3703 & 2.7157 & 10.0860 & \\
\hline & 7/24/2008 4:00 & 39 & 206 & 400 & 9.46 & 9.46 & & 0.0063 & $2.34 \mathrm{E}-05$ & 2.1749 & 0.8014 & 2.9763 & \\
\hline & $7 / 24 / 20085: 00$ & 40 & 206 & 500 & 9.58 & 9.58 & & -0.0063 & $0.00 \mathrm{E}+00$ & 0.0000 & 0.0000 & 0.0000 & \\
\hline
\end{tabular}




\begin{tabular}{|c|c|c|c|c|c|c|c|c|c|c|c|c|c|}
\hline \multirow[t]{42}{*}{ Tree ID } & TS & RN & JD & HR & $\begin{array}{c}\text { Raw } \\
\text { TC_dTA }\end{array}$ & $\begin{array}{c}\text { Fixed } \\
\text { TC_dT } \Delta\end{array}$ & dTM & $\mathbf{K}$ & $\begin{array}{l}\text { Sap } \\
\text { Velocity } \\
\text { (cm/s) }\end{array}$ & $\begin{array}{l}\text { Probed } \\
\text { Drywood F } \\
\text { (g/hr) }\end{array}$ & $\begin{array}{c}\text { Extrapolated } \\
\text { Wetwood F } \\
(\mathrm{g} / \mathrm{hr})\end{array}$ & $\begin{array}{c}\text { Extrapolated } \\
\text { Total Wood F } \\
(\mathrm{g} / \mathrm{hr})\end{array}$ & $\begin{array}{c}\text { Relative } \\
\text { Humidity, } \\
@ 10 \mathrm{~m}(\%)\end{array}$ \\
\hline & 7/24/2008 6:00 & 41 & 206 & 600 & 9.63 & 9.63 & & -0.0114 & $0.00 \mathrm{E}+00$ & 0.0000 & 0.0000 & 0.0000 & \\
\hline & 7/24/2008 7:00 & 42 & 206 & 700 & 9.64 & 9.64 & & -0.0124 & $0.00 \mathrm{E}+00$ & 0.0000 & 0.0000 & 0.0000 & \\
\hline & 7/24/2008 8:00 & 43 & 206 & 800 & 9.41 & 9.41 & & 0.0117 & $4.98 \mathrm{E}-05$ & 4.6166 & 1.7011 & 6.3176 & \\
\hline & $7 / 24 / 20089: 00$ & 44 & 206 & 900 & 8.92 & 8.92 & & 0.0673 & 4.29E-04 & 39.7978 & 14.6643 & 54.4621 & \\
\hline & $7 / 24 / 2008$ 10:00 & 45 & 206 & 1000 & 8.99 & 8.99 & & 0.0590 & 3.65E-04 & 33.8344 & 12.4670 & 46.3014 & \\
\hline & 7/24/2008 11:00 & 46 & 206 & 1100 & 7.087 & 7.087 & & 0.3433 & $3.19 E-03$ & 295.9887 & 109.0631 & 405.0519 & \\
\hline & $7 / 24 / 2008$ 12:00 & 47 & 206 & 1200 & 6.097 & 6.097 & & 0.5614 & 5.85E-03 & 542.2884 & 199.8173 & 742.1058 & \\
\hline & 7/24/2008 13:00 & 48 & 206 & 1300 & 6.452 & 6.452 & & 0.4755 & 4.77E-03 & 442.0167 & 162.8701 & 604.8868 & \\
\hline & $7 / 24 / 2008$ 14:00 & 49 & 206 & 1400 & 6.342 & 6.342 & & 0.5011 & $5.08 \mathrm{E}-03$ & 471.4813 & 173.7270 & 645.2082 & \\
\hline & 7/24/2008 15:00 & 50 & 206 & 1500 & 5.955 & 5.955 & & 0.5987 & $6.33 \mathrm{E}-03$ & 586.8935 & 216.2530 & 803.1465 & \\
\hline & $7 / 24 / 2008$ 16:00 & 51 & 206 & 1600 & 5.882 & 5.882 & & 0.6185 & $6.59 \mathrm{E}-03$ & 610.9282 & 225.1091 & 836.0372 & \\
\hline & $7 / 24 / 2008$ 17:00 & 52 & 206 & 1700 & 6.085 & 6.085 & & 0.5645 & $5.89 \mathrm{E}-03$ & 545.9521 & 201.1673 & 747.1194 & \\
\hline & 7/24/2008 18:00 & 53 & 206 & 1800 & 6.046 & 6.046 & & 0.5746 & 6.02E-03 & 557.9917 & 205.6035 & 763.5952 & \\
\hline & $7 / 24 / 2008$ 19:00 & 54 & 206 & 1900 & 6.39 & 6.39 & & 0.4898 & 4.94E-03 & 458.4553 & 168.9273 & 627.3826 & \\
\hline & $7 / 24 / 200820: 00$ & 55 & 206 & 2000 & 7.376 & 7.376 & & 0.2907 & $2.60 \mathrm{E}-03$ & 241.1591 & 88.8600 & 330.0191 & \\
\hline & $7 / 24 / 200821: 00$ & 56 & 206 & 2100 & 8.69 & 8.69 & & 0.0955 & $6.61 \mathrm{E}-04$ & 61.2781 & 22.5792 & 83.8573 & \\
\hline & $7 / 24 / 200822: 00$ & 57 & 206 & 2200 & 9.55 & 9.55 & & -0.0031 & $0.00 \mathrm{E}+00$ & 0.0000 & 0.0000 & 0.0000 & \\
\hline & $7 / 24 / 200823: 00$ & 58 & 206 & 2300 & 9.72 & 9.72 & & -0.0206 & $0.00 \mathrm{E}+00$ & 0.0000 & 0.0000 & 0.0000 & \\
\hline & $7 / 25 / 20080: 00$ & 59 & 207 & 0 & 9.74 & 9.74 & 9.78 & -0.0226 & $0.00 \mathrm{E}+00$ & 0.0000 & 0.0000 & 0.0000 & \\
\hline & 7/25/2008 1:00 & 60 & 207 & 100 & 9.68 & 9.68 & & -0.0165 & $0.00 \mathrm{E}+00$ & 0.0000 & 0.0000 & 0.0000 & \\
\hline & 7/25/2008 2:00 & 61 & 207 & 200 & 9.64 & 9.64 & & -0.0124 & $0.00 E+00$ & 0.0000 & 0.0000 & 0.0000 & \\
\hline & 7/25/2008 3:00 & 62 & 207 & 300 & 9.61 & 9.61 & & -0.0094 & $0.00 \mathrm{E}+00$ & 0.0000 & 0.0000 & 0.0000 & \\
\hline & 7/25/2008 4:00 & 63 & 207 & 400 & 9.6 & 9.6 & & -0.0083 & $0.00 \mathrm{E}+00$ & 0.0000 & 0.0000 & 0.0000 & \\
\hline & 7/25/2008 5:00 & 64 & 207 & 500 & 9.62 & 9.62 & & -0.0104 & $0.00 \mathrm{E}+00$ & 0.0000 & 0.0000 & 0.0000 & \\
\hline & 7/25/2008 6:00 & 65 & 207 & 600 & 9.67 & 9.67 & & -0.0155 & $0.00 \mathrm{E}+00$ & 0.0000 & 0.0000 & 0.0000 & \\
\hline & 7/25/2008 7:00 & 66 & 207 & 700 & 9.63 & 9.63 & & -0.0114 & $0.00 \mathrm{E}+00$ & 0.0000 & 0.0000 & 0.0000 & \\
\hline & 7/25/2008 8:00 & 67 & 207 & 800 & 9.6 & 9.6 & & -0.0083 & $0.00 \mathrm{E}+00$ & 0.0000 & 0.0000 & 0.0000 & \\
\hline & $7 / 25 / 20089: 00$ & 68 & 207 & 900 & 9.53 & 9.53 & & -0.0010 & $0.00 \mathrm{E}+00$ & 0.0000 & 0.0000 & 0.0000 & \\
\hline & $7 / 25 / 2008$ 10:00 & 69 & 207 & 1000 & 9.46 & 9.46 & & 0.0063 & 2.34E-05 & 2.1749 & 0.8014 & 2.9763 & \\
\hline & 7/25/2008 11:00 & 70 & 207 & 1100 & 8.71 & 8.71 & & 0.0930 & 6.39E-04 & 59.2975 & 21.8494 & 81.1468 & \\
\hline & $7 / 25 / 2008$ 12:00 & 71 & 207 & 1200 & 7.406 & 7.406 & & 0.2854 & $2.54 \mathrm{E}-03$ & 235.8306 & 86.8966 & 322.7273 & \\
\hline & $7 / 25 / 2008$ 13:00 & 72 & 207 & 1300 & 6.734 & 6.734 & & 0.4137 & 4.02E-03 & 372.4097 & 137.2220 & 509.6318 & \\
\hline & $7 / 25 / 2008$ 14:00 & 73 & 207 & 1400 & 6.201 & 6.201 & & 0.5352 & 5.51E-03 & 511.3203 & 188.4065 & 699.7268 & \\
\hline & $7 / 25 / 2008$ 15:00 & 74 & 207 & 1500 & 6.155 & 6.155 & & 0.5467 & $5.66 \mathrm{E}-03$ & 524.8466 & 193.3905 & 718.2371 & \\
\hline & $7 / 25 / 200816: 00$ & 75 & 207 & 1600 & 6.254 & 6.254 & & 0.5222 & 5.35E-03 & 496.0633 & 182.7847 & 678.8480 & \\
\hline & $7 / 25 / 2008$ 17:00 & 76 & 207 & 1700 & 6.031 & 6.031 & & 0.5785 & 6.07E-03 & 562.6769 & 207.3299 & 770.0068 & \\
\hline & $7 / 25 / 2008$ 18:00 & 77 & 207 & 1800 & 6.026 & 6.026 & & 0.5798 & $6.08 \mathrm{E}-03$ & 564.2455 & 207.9079 & 772.1534 & \\
\hline & 7/25/2008 19:00 & 78 & 207 & 1900 & 6.114 & 6.114 & & 0.5571 & 5.79E-03 & 537.1308 & 197.9169 & 735.0477 & \\
\hline & $7 / 25 / 200820: 00$ & 79 & 207 & 2000 & 6.912 & 6.912 & & 0.3773 & $3.58 \mathrm{E}-03$ & 332.4880 & 122.5120 & 455.0000 & \\
\hline & $7 / 25 / 2008$ 21:00 & 80 & 207 & 2100 & 8.42 & 8.42 & & 0.1306 & $9.71 \mathrm{E}-04$ & 90.1050 & 33.2010 & 123.3060 & \\
\hline & $7 / 25 / 200822: 00$ & 81 & 207 & 2200 & 9.54 & 9.54 & & -0.0021 & $0.00 \mathrm{E}+00$ & 0.0000 & 0.0000 & 0.0000 & \\
\hline
\end{tabular}




\begin{tabular}{|c|c|c|c|c|c|c|c|c|c|c|c|c|c|}
\hline Tree ID & TS & RN & JD & HR & $\begin{array}{c}\text { Raw } \\
\text { TC_dTA }\end{array}$ & $\begin{array}{c}\text { Fixed } \\
\text { TC_dT } \Delta\end{array}$ & dTM & K & $\begin{array}{l}\text { Sap } \\
\text { Velocity } \\
(\mathrm{cm} / \mathrm{s})\end{array}$ & $\begin{array}{c}\text { Probed } \\
\text { Drywood F } \\
(\mathrm{g} / \mathrm{hr})\end{array}$ & $\begin{array}{c}\text { Extrapolated } \\
\text { Wetwood F } \\
(\mathrm{g} / \mathrm{hr})\end{array}$ & $\begin{array}{c}\text { Extrapolated } \\
\text { Total Wood F } \\
(\mathrm{g} / \mathrm{hr})\end{array}$ & $\begin{array}{l}\text { Relative } \\
\text { Humidity, } \\
@ 10 \mathrm{~m}(\%)\end{array}$ \\
\hline & 7/25/2008 23:00 & 82 & 207 & 2300 & \begin{tabular}{|r|}
9.78 \\
\end{tabular} & 9.78 & & -0.0266 & $0.00 \mathrm{E}+00$ & 0.0000 & 0.0000 & 0.0000 & \\
\hline & $7 / 26 / 20080: 00$ & 83 & 208 & 0 & 9.84 & 9.84 & 9.84 & -0.0325 & $0.00 \mathrm{E}+00$ & 0.0000 & 0.0000 & 0.0000 & \\
\hline & 7/26/2008 1:00 & 84 & 208 & 100 & 9.84 & 9.84 & & -0.0325 & $0.00 \mathrm{E}+00$ & 0.0000 & 0.0000 & 0.0000 & \\
\hline & $7 / 26 / 20082: 00$ & 85 & 208 & 200 & 9.82 & 9.82 & & -0.0305 & $0.00 \mathrm{E}+00$ & 0.0000 & 0.0000 & 0.0000 & \\
\hline & 7/26/2008 3:00 & 86 & 208 & 300 & 9.8 & 9.8 & & -0.0286 & $0.00 \mathrm{E}+00$ & 0.0000 & 0.0000 & 0.0000 & \\
\hline & 7/26/2008 4:00 & 87 & 208 & 400 & 9.76 & 9.76 & & -0.0246 & $0.00 \mathrm{E}+00$ & 0.0000 & 0.0000 & 0.0000 & \\
\hline & 7/26/2008 5:00 & 88 & 208 & 500 & 9.72 & 9.72 & & -0.0206 & $0.00 \mathrm{E}+00$ & 0.0000 & 0.0000 & 0.0000 & \\
\hline & 7/26/2008 6:00 & 89 & 208 & 600 & 9.65 & 9.65 & & -0.0135 & $0.00 \mathrm{E}+00$ & 0.0000 & 0.0000 & 0.0000 & \\
\hline & 7/26/2008 7:00 & 90 & 208 & 700 & 9.61 & 9.61 & & -0.0094 & $0.00 \mathrm{E}+00$ & 0.0000 & 0.0000 & 0.0000 & \\
\hline & 7/26/2008 8:00 & 91 & 208 & 800 & 9.54 & 9.54 & & -0.0021 & $0.00 E+00$ & 0.0000 & 0.0000 & 0.0000 & \\
\hline & 7/26/2008 9:00 & 92 & 208 & 900 & 8.31 & 8.31 & & 0.1456 & 1.11E-03 & 102.9754 & 37.9434 & 140.9188 & \\
\hline & $7 / 26 / 2008$ 10:00 & 93 & 208 & 1000 & 6.674 & 6.674 & & 0.4264 & 4.17E-03 & 386.5425 & 142.4295 & 528.9720 & \\
\hline & $7 / 26 / 2008$ 11:00 & 94 & 208 & 1100 & 6.728 & 6.728 & & 0.4150 & 4.03E-03 & 373.8072 & 137.7370 & 511.5442 & \\
\hline & $7 / 26 / 2008$ 12:00 & 95 & 208 & 1200 & 6.996 & 6.996 & & 0.3608 & 3.39E-03 & 314.6410 & 115.9360 & 430.5770 & \\
\hline & $7 / 26 / 2008$ 13:00 & 96 & 208 & 1300 & 6.377 & 6.377 & & 0.4929 & 4.98E-03 & 461.9571 & 170.2176 & 632.1746 & \\
\hline & $7 / 26 / 200814: 00$ & 97 & 208 & 1400 & 6.243 & 6.243 & & 0.5249 & 5.38E-03 & 499.2014 & 183.9410 & 683.1424 & \\
\hline & $7 / 26 / 2008$ 15:00 & 98 & 208 & 1500 & 6.126 & 6.126 & & 0.5540 & 5.75E-03 & 533.5128 & 196.5838 & 730.0966 & \\
\hline & $7 / 26 / 200816: 00$ & 99 & 208 & 1600 & 6.197 & 6.197 & & 0.5362 & 5.53E-03 & 512.4859 & 188.8360 & 701.3219 & \\
\hline & $7 / 26 / 200817: 00$ & 100 & 208 & 1700 & 6.034 & 6.034 & & 0.5777 & 6.06E-03 & 561.7374 & 206.9837 & 768.7211 & \\
\hline & $7 / 26 / 2008$ 18:00 & 101 & 208 & 1800 & 6.098 & 6.098 & & 0.5612 & 5.84E-03 & 541.9840 & 199.7051 & 741.6891 & \\
\hline & $7 / 26 / 2008$ 19:00 & 102 & 208 & 1900 & 6.239 & 6.239 & & 0.5259 & $5.39 \mathrm{E}-03$ & 500.3462 & 184.3628 & 684.7091 & \\
\hline & $7 / 26 / 200820: 00$ & 103 & 208 & 2000 & 6.785 & 6.785 & & 0.4031 & 3.89E-03 & 360.6701 & 132.8963 & 493.5664 & \\
\hline & $7 / 26 / 200821: 00$ & 104 & 208 & 2100 & 8.32 & 8.32 & & 0.1442 & 1.10E-03 & 101.7780 & 37.5022 & 139.2802 & \\
\hline & $7 / 26 / 200822: 00$ & 105 & 208 & 2200 & 9.43 & 9.43 & & 0.0095 & 3.88E-05 & 3.5967 & 1.3253 & 4.9220 & \\
\hline & $7 / 26 / 2008$ 23:00 & 106 & 208 & 2300 & 9.66 & 9.66 & & -0.0145 & $0.00 \mathrm{E}+00$ & 0.0000 & 0.0000 & 0.0000 & \\
\hline & $7 / 27 / 20080: 00$ & 107 & 209 & 0 & 9.73 & 9.73 & 9.82 & -0.0216 & $0.00 \mathrm{E}+00$ & 0.0000 & 0.0000 & 0.0000 & \\
\hline & 7/27/2008 1:00 & 108 & 209 & 100 & 9.77 & 9.77 & & -0.0256 & $0.00 \mathrm{E}+00$ & 0.0000 & 0.0000 & 0.0000 & \\
\hline & 7/27/2008 2:00 & 109 & 209 & 200 & 9.77 & 9.77 & & -0.0256 & $0.00 \mathrm{E}+00$ & 0.0000 & 0.0000 & 0.0000 & \\
\hline & 7/27/2008 3:00 & 110 & 209 & 300 & 9.79 & 9.79 & & -0.0276 & $0.00 \mathrm{E}+00$ & 0.0000 & 0.0000 & 0.0000 & \\
\hline & 7/27/2008 4:00 & 111 & 209 & 400 & 9.81 & 9.81 & & -0.0296 & $0.00 \mathrm{E}+00$ & 0.0000 & 0.0000 & 0.0000 & \\
\hline & 7/27/20085:00 & 112 & 209 & 500 & 9.82 & 9.82 & & -0.0305 & $0.00 \mathrm{E}+00$ & 0.0000 & 0.0000 & 0.0000 & \\
\hline & 7/27/2008 6:00 & 113 & 209 & 600 & 9.78 & 9.78 & & -0.0266 & $0.00 \mathrm{E}+00$ & 0.0000 & 0.0000 & 0.0000 & \\
\hline & 7/27/2008 7:00 & 114 & 209 & 700 & 9.75 & 9.75 & & -0.0236 & $0.00 \mathrm{E}+00$ & 0.0000 & 0.0000 & 0.0000 & \\
\hline & 7/27/2008 8:00 & 115 & 209 & 800 & 9.63 & 9.63 & & -0.0114 & $0.00 \mathrm{E}+00$ & 0.0000 & 0.0000 & 0.0000 & \\
\hline & $7 / 27 / 20089: 00$ & 116 & 209 & 900 & 7.761 & 7.761 & & 0.2266 & 1.91E-03 & 177.5363 & 65.4169 & 242.9531 & \\
\hline & $7 / 27 / 2008$ 10:00 & 117 & 209 & 1000 & 6.241 & 6.241 & & 0.5254 & 5.39E-03 & 499.7736 & 184.1518 & 683.9254 & \\
\hline & $7 / 27 / 2008$ 11:00 & 118 & 209 & 1100 & 6.141 & 6.141 & & 0.5502 & $5.70 \mathrm{E}-03$ & 529.0168 & 194.9271 & 723.9439 & \\
\hline & $7 / 27 / 2008$ 12:00 & 119 & 209 & 1200 & 5.944 & 5.944 & & 0.6016 & 6.37E-03 & 590.4659 & 217.5693 & 808.0352 & \\
\hline & $7 / 27 / 200813: 00$ & 120 & 209 & 1300 & 5.98 & 5.98 & & 0.5920 & $6.24 \mathrm{E}-03$ & 578.8384 & 213.2849 & 792.1233 & \\
\hline & $7 / 27 / 200814: 00$ & 121 & 209 & 1400 & 5.778 & 5.778 & & 0.6476 & 6.97E-03 & 646.5410 & 238.2313 & 884.7724 & \\
\hline & 7/27/2008 15:00 & 122 & 209 & 1500 & 5.721 & 5.721 & & 0.6640 & 7.19E-03 & 666.7736 & 245.6865 & 912.4601 & \\
\hline
\end{tabular}




\begin{tabular}{|c|c|c|c|c|c|c|c|c|c|c|c|c|c|}
\hline Tree ID & TS & RN & JD & HR & $\begin{array}{c}\text { Raw } \\
\text { TC_dTA }\end{array}$ & $\begin{array}{c}\text { Fixed } \\
\text { TC_dT } \Delta\end{array}$ & dTM & $\mathbf{K}$ & $\begin{array}{l}\text { Sap } \\
\text { Velocity } \\
(\mathrm{cm} / \mathrm{s})\end{array}$ & $\begin{array}{c}\text { Probed } \\
\text { Drywood F } \\
(\mathrm{g} / \mathrm{hr})\end{array}$ & $\begin{array}{c}\text { Extrapolated } \\
\text { Wetwood F } \\
(\mathrm{g} / \mathrm{hr})\end{array}$ & $\begin{array}{c}\text { Extrapolated } \\
\text { Total Wood F } \\
(\mathrm{g} / \mathrm{hr})\end{array}$ & $\begin{array}{l}\text { Relative } \\
\text { Humidity, } \\
@ 10 \mathrm{~m}(\%)\end{array}$ \\
\hline & 7/27/2008 16:00 & 123 & 209 & 1600 & \begin{tabular}{|r|}
5.741 \\
\end{tabular} & 5.741 & & 0.6582 & 7.11E-03 & 659.6154 & 243.0489 & $\begin{array}{r}902.6642 \\
\end{array}$ & \\
\hline & $7 / 27 / 2008$ 17:00 & 124 & 209 & 1700 & 5.768 & 5.768 & & 0.6505 & 7.01E-03 & 650.0533 & 239.5255 & 889.5788 & \\
\hline & $7 / 27 / 2008$ 18:00 & 125 & 209 & 1800 & 5.819 & 5.819 & & 0.6360 & $6.82 \mathrm{E}-03$ & 632.3040 & 232.9854 & 865.2895 & \\
\hline & $7 / 27 / 2008$ 19:00 & 126 & 209 & 1900 & 5.98 & 5.98 & & 0.5920 & $6.24 \mathrm{E}-03$ & 578.8384 & 213.2849 & 792.1233 & \\
\hline & $7 / 27 / 200820: 00$ & 127 & 209 & 2000 & 6.414 & 6.414 & & 0.4843 & 4.87E-03 & 452.0409 & 166.5638 & 618.6047 & \\
\hline & $7 / 27 / 2008$ 21:00 & 128 & 209 & 2100 & 7.629 & 7.629 & & 0.2479 & $2.14 \mathrm{E}-03$ & 198.2180 & 73.0375 & 271.2555 & \\
\hline & $7 / 27 / 2008$ 22:00 & 129 & 209 & 2200 & 8.89 & 8.89 & & 0.0709 & $4.58 \mathrm{E}-04$ & 42.4369 & 15.6367 & 58.0736 & \\
\hline & $7 / 27 / 200823: 00$ & 130 & 209 & 2300 & 9.09 & 9.09 & & 0.0473 & 2.78E-04 & 25.8025 & 9.5074 & 35.3099 & \\
\hline & $7 / 28 / 20080: 00$ & 131 & 210 & 0 & 9.15 & 9.15 & 9.77 & 0.0404 & 2.29E-04 & 21.2716 & 7.8380 & 29.1096 & \\
\hline & 7/28/2008 1:00 & 132 & 210 & 100 & 9.31 & 9.31 & & 0.0226 & 1.12E-04 & 10.3688 & 3.8206 & 14.1894 & \\
\hline & $7 / 28 / 20082: 00$ & 133 & 210 & 200 & 9.4 & 9.4 & & 0.0128 & $5.55 E-05$ & 5.1452 & 1.8959 & 7.0411 & \\
\hline & 7/28/2008 3:00 & 134 & 210 & 300 & 9.55 & 9.55 & & -0.0031 & $0.00 \mathrm{E}+00$ & 0.0000 & 0.0000 & 0.0000 & \\
\hline & 7/28/2008 4:00 & 135 & 210 & 400 & 9.58 & 9.58 & & -0.0063 & $0.00 \mathrm{E}+00$ & 0.0000 & 0.0000 & 0.0000 & \\
\hline & 7/28/2008 5:00 & 136 & 210 & 500 & 9.72 & 9.72 & & -0.0206 & $0.00 \mathrm{E}+00$ & 0.0000 & 0.0000 & 0.0000 & \\
\hline & 7/28/2008 6:00 & 137 & 210 & 600 & 9.77 & 9.77 & & -0.0256 & $0.00 \mathrm{E}+00$ & 0.0000 & 0.0000 & 0.0000 & \\
\hline & 7/28/2008 7:00 & 138 & 210 & 700 & 9.73 & 9.73 & & -0.0216 & $0.00 \mathrm{E}+00$ & 0.0000 & 0.0000 & 0.0000 & \\
\hline & 7/28/2008 8:00 & 139 & 210 & 800 & 8.17 & 8.17 & & 0.1652 & $1.30 \mathrm{E}-03$ & 120.3231 & 44.3355 & 164.6586 & \\
\hline & $7 / 28 / 20089: 00$ & 140 & 210 & 900 & 6.762 & 6.762 & & 0.4079 & $3.95 E-03$ & 365.9338 & 134.8358 & 500.7696 & \\
\hline & $7 / 28 / 2008$ 10:00 & 141 & 210 & 1000 & 6.278 & 6.278 & & 0.5164 & $5.28 \mathrm{E}-03$ & 489.2674 & 180.2806 & 669.5480 & \\
\hline & $7 / 28 / 2008$ 11:00 & 142 & 210 & 1100 & 6.197 & 6.197 & & 0.5362 & 5.53E-03 & 512.4859 & 188.8360 & 701.3219 & \\
\hline & $7 / 28 / 200812: 00$ & 143 & 210 & 1200 & 6.152 & 6.152 & & 0.5475 & $5.67 \mathrm{E}-03$ & 525.7381 & 193.7190 & 719.4571 & \\
\hline & 7/28/2008 13:00 & 144 & 210 & 1300 & 6.01 & 6.01 & & 0.5840 & 6.14E-03 & 569.2881 & 209.7659 & 779.0540 & \\
\hline & $7 / 28 / 2008$ 14:00 & 145 & 210 & 1400 & 5.935 & 5.935 & & 0.6040 & $6.40 \mathrm{E}-03$ & 593.4016 & 218.6510 & 812.0527 & \\
\hline & $7 / 28 / 2008$ 15:00 & 146 & 210 & 1500 & 5.784 & 5.784 & & 0.6459 & $6.95 \mathrm{E}-03$ & 644.4412 & 237.4576 & 881.8989 & \\
\hline & $7 / 28 / 2008$ 16:00 & 147 & 210 & 1600 & 5.801 & 5.801 & & 0.6411 & $6.88 \mathrm{E}-03$ & 638.5223 & 235.2767 & 873.7990 & \\
\hline & 7/28/2008 17:00 & 148 & 210 & 1700 & 5.825 & 5.825 & & 0.6343 & $6.80 \mathrm{E}-03$ & 630.2423 & 232.2258 & 862.4681 & \\
\hline & $7 / 28 / 2008$ 18:00 & 149 & 210 & 1800 & 5.849 & 5.849 & & 0.6276 & 6.71E-03 & 622.0504 & 229.2073 & 851.2577 & \\
\hline & $7 / 28 / 2008$ 19:00 & 150 & 210 & 1900 & 6.047 & 6.047 & & 0.5743 & $6.01 \mathrm{E}-03$ & 557.6804 & 205.4888 & 763.1692 & \\
\hline & $7 / 28 / 200820: 00$ & 151 & 210 & 2000 & 6.704 & 6.704 & & 0.4200 & 4.09E-03 & 379.4321 & 139.8096 & 519.2417 & \\
\hline & $7 / 28 / 200821: 00$ & 152 & 210 & 2100 & 8.13 & 8.13 & & 0.1710 & 1.35E-03 & 125.4825 & 46.2366 & 171.7191 & \\
\hline & $7 / 28 / 2008$ 22:00 & 153 & 210 & 2200 & 8.73 & 8.73 & & 0.0905 & 6.18E-04 & 57.3382 & 21.1274 & 78.4656 & \\
\hline & $7 / 28 / 200823: 00$ & 154 & 210 & 2300 & 8.94 & 8.94 & & 0.0649 & $4.10 \mathrm{E}-04$ & 38.0660 & 14.0262 & 52.0922 & \\
\hline & $7 / 29 / 20080: 00$ & 155 & 211 & 0 & 9.2 & 9.2 & 9.89 & 0.0348 & 1.91E-04 & 17.6714 & 6.5114 & 24.1828 & \\
\hline & 7/29/2008 1:00 & 156 & 211 & 100 & 9.42 & 9.42 & & 0.0106 & 4.42E-05 & 4.1001 & 1.5108 & 5.6109 & \\
\hline & 7/29/2008 2:00 & 157 & 211 & 200 & 9.61 & 9.61 & & -0.0094 & $0.00 \mathrm{E}+00$ & 0.0000 & 0.0000 & 0.0000 & \\
\hline & 7/29/2008 3:00 & 158 & 211 & 300 & 9.73 & 9.73 & & -0.0216 & $0.00 \mathrm{E}+00$ & 0.0000 & 0.0000 & 0.0000 & \\
\hline & 7/29/2008 4:00 & 159 & 211 & 400 & 9.85 & 9.85 & & -0.0335 & $0.00 \mathrm{E}+00$ & 0.0000 & 0.0000 & 0.0000 & \\
\hline & 7/29/2008 5:00 & 160 & 211 & 500 & 9.89 & 9.89 & & -0.0374 & $0.00 \mathrm{E}+00$ & 0.0000 & 0.0000 & 0.0000 & \\
\hline & 7/29/2008 6:00 & 161 & 211 & 600 & 9.88 & 9.88 & & -0.0364 & $0.00 \mathrm{E}+00$ & 0.0000 & 0.0000 & 0.0000 & \\
\hline & 7/29/2008 7:00 & 162 & 211 & 700 & 9.77 & 9.77 & & -0.0256 & $0.00 \mathrm{E}+00$ & 0.0000 & 0.0000 & 0.0000 & \\
\hline & 7/29/2008 8:00 & 163 & 211 & 800 & 9.14 & 9.14 & & 0.0416 & 2.37E-04 & 22.0111 & 8.1105 & 30.1216 & \\
\hline
\end{tabular}




\begin{tabular}{|c|c|c|c|c|c|c|c|c|c|c|c|c|c|}
\hline Tree ID & TS & RN & JD & HR & $\begin{array}{c}\text { Raw } \\
\text { TC_dTA }\end{array}$ & $\begin{array}{c}\text { Fixed } \\
\text { TC_dT } \Delta\end{array}$ & dTM & $\mathbf{K}$ & $\begin{array}{l}\text { Sap } \\
\text { Velocity } \\
(\mathrm{cm} / \mathrm{s})\end{array}$ & $\begin{array}{c}\text { Probed } \\
\text { Drywood F } \\
(\mathrm{g} / \mathrm{hr})\end{array}$ & $\begin{array}{c}\text { Extrapolated } \\
\text { Wetwood F } \\
(\mathrm{g} / \mathrm{hr})\end{array}$ & $\begin{array}{c}\text { Extrapolated } \\
\text { Total Wood F } \\
(\mathrm{g} / \mathrm{hr})\end{array}$ & $\begin{array}{l}\text { Relative } \\
\text { Humidity, } \\
@ 10 \mathrm{~m}(\%)\end{array}$ \\
\hline & 7/29/2008 9:00 & 164 & 211 & 900 & \begin{tabular}{|r|}
7.493 \\
\end{tabular} & 7.493 & & 0.2705 & $2.38 \mathrm{E}-03$ & 220.7442 & 81.3377 & $\begin{array}{r}302.0819 \\
\end{array}$ & \\
\hline & $7 / 29 / 2008$ 10:00 & 165 & 211 & 1000 & 6.627 & 6.627 & & 0.4365 & 4.29E-03 & 397.8618 & 146.6004 & 544.4621 & \\
\hline & $7 / 29 / 2008$ 11:00 & 166 & 211 & 1100 & 6.89 & 6.89 & & 0.3817 & $3.64 \mathrm{E}-03$ & 337.2649 & 124.2722 & 461.5371 & \\
\hline & $7 / 29 / 2008$ 12:00 & 167 & 211 & 1200 & 6.312 & 6.312 & & 0.5082 & $5.17 \mathrm{E}-03$ & 479.7582 & 176.7768 & 656.5350 & \\
\hline & 7/29/2008 13:00 & 168 & 211 & 1300 & 6.253 & 6.253 & & 0.5225 & 5.35E-03 & 496.3479 & 182.8896 & 679.2375 & \\
\hline & $7 / 29 / 2008$ 14:00 & 169 & 211 & 1400 & 6.014 & 6.014 & & 0.5830 & $6.12 \mathrm{E}-03$ & 568.0241 & 209.3002 & 777.3243 & \\
\hline & $7 / 29 / 2008$ 15:00 & 170 & 211 & 1500 & 6.133 & 6.133 & & 0.5523 & $5.73 E-03$ & 531.4110 & 195.8093 & 727.2204 & \\
\hline & $7 / 29 / 2008$ 16:00 & 171 & 211 & 1600 & 6.331 & 6.331 & & 0.5037 & 5.12E-03 & 474.5039 & 174.8407 & 649.3446 & \\
\hline & 7/29/2008 17:00 & 172 & 211 & 1700 & 6.569 & 6.569 & & 0.4492 & 4.44E-03 & 412.1393 & 151.8612 & 564.0005 & \\
\hline & 7/29/2008 18:00 & 173 & 211 & 1800 & 6.614 & 6.614 & & 0.4394 & 4.32E-03 & 401.0319 & 147.7685 & 548.8004 & \\
\hline & $7 / 29 / 2008$ 19:00 & 174 & 211 & 1900 & 6.572 & 6.572 & & 0.4486 & 4.44E-03 & 411.3923 & 151.5859 & 562.9782 & \\
\hline & $7 / 29 / 200820: 00$ & 175 & 211 & 2000 & 7.412 & 7.412 & & 0.2844 & $2.53 E-03$ & 234.7728 & 86.5069 & 321.2796 & \\
\hline & 7/29/2008 21:00 & 176 & 211 & 2100 & 8.85 & 8.85 & & 0.0757 & 4.96E-04 & 46.0325 & 16.9616 & 62.9941 & \\
\hline & $7 / 29 / 2008$ 22:00 & 177 & 211 & 2200 & 9.52 & 9.52 & & 0.0000 & $0.00 \mathrm{E}+00$ & 0.0000 & 0.0000 & 0.0000 & \\
\hline & $7 / 29 / 2008$ 23:00 & 178 & 211 & 2300 & 9.75 & 9.75 & & -0.0236 & $0.00 \mathrm{E}+00$ & 0.0000 & 0.0000 & 0.0000 & \\
\hline & $7 / 30 / 20080: 00$ & 179 & 212 & 0 & 9.84 & 9.84 & 9.87 & -0.0325 & $0.00 \mathrm{E}+00$ & 0.0000 & 0.0000 & 0.0000 & \\
\hline & 7/30/2008 1:00 & 180 & 212 & 100 & 9.87 & 9.87 & & -0.0355 & $0.00 \mathrm{E}+00$ & 0.0000 & 0.0000 & 0.0000 & \\
\hline & 7/30/2008 2:00 & 181 & 212 & 200 & 9.87 & 9.87 & & -0.0355 & $0.00 \mathrm{E}+00$ & 0.0000 & 0.0000 & 0.0000 & \\
\hline & 7/30/2008 3:00 & 182 & 212 & 300 & 9.87 & 9.87 & & -0.0355 & $0.00 \mathrm{E}+00$ & 0.0000 & 0.0000 & 0.0000 & \\
\hline & 7/30/2008 4:00 & 183 & 212 & 400 & 9.86 & 9.86 & & -0.0345 & $0.00 E+00$ & 0.0000 & 0.0000 & 0.0000 & \\
\hline & 7/30/2008 5:00 & 184 & 212 & 500 & 9.83 & 9.83 & & -0.0315 & $0.00 \mathrm{E}+00$ & 0.0000 & 0.0000 & 0.0000 & \\
\hline & 7/30/2008 6:00 & 185 & 212 & 600 & 9.79 & 9.79 & & -0.0276 & $0.00 E+00$ & 0.0000 & 0.0000 & 0.0000 & \\
\hline & 7/30/2008 7:00 & 186 & 212 & 700 & 9.77 & 9.77 & & -0.0256 & $0.00 \mathrm{E}+00$ & 0.0000 & 0.0000 & 0.0000 & \\
\hline & $7 / 30 / 20088: 00$ & 187 & 212 & 800 & 9.74 & 9.74 & & -0.0226 & $0.00 \mathrm{E}+00$ & 0.0000 & 0.0000 & 0.0000 & \\
\hline & 7/30/2008 9:00 & 188 & 212 & 900 & 8.56 & 8.56 & & 0.1121 & 8.05E-04 & 74.6714 & 27.5142 & 102.1856 & \\
\hline & $7 / 30 / 2008$ 10:00 & 189 & 212 & 1000 & 6.552 & 6.552 & & 0.4530 & 4.49E-03 & 416.3900 & 153.4275 & 569.8175 & \\
\hline & 7/30/2008 11:00 & 190 & 212 & 1100 & 6.314 & 6.314 & & 0.5078 & 5.17E-03 & 479.2031 & 176.5722 & 655.7754 & \\
\hline & $7 / 30 / 2008$ 12:00 & 191 & 212 & 1200 & 6.215 & 6.215 & & 0.5318 & $5.47 \mathrm{E}-03$ & 507.2564 & 186.9090 & 694.1654 & \\
\hline & 7/30/2008 13:00 & 192 & 212 & 1300 & 6.152 & 6.152 & & 0.5475 & 5.67E-03 & 525.7381 & 193.7190 & 719.4571 & \\
\hline & $7 / 30 / 2008$ 14:00 & 193 & 212 & 1400 & 6.106 & 6.106 & & 0.5591 & $5.82 E-03$ & 539.5532 & 198.8095 & 738.3626 & \\
\hline & 7/30/2008 15:00 & 194 & 212 & 1500 & 6.237 & 6.237 & & 0.5264 & $5.40 \mathrm{E}-03$ & 500.9193 & 184.5740 & 685.4934 & \\
\hline & $7 / 30 / 2008$ 16:00 & 195 & 212 & 1600 & 6.093 & 6.093 & & 0.5624 & $5.86 \mathrm{E}-03$ & 543.5075 & 200.2665 & 743.7741 & \\
\hline & $7 / 30 / 2008$ 17:00 & 196 & 212 & 1700 & 6.09 & 6.09 & & 0.5632 & 5.87E-03 & 544.4232 & 200.6039 & 745.0272 & \\
\hline & 7/30/2008 18:00 & 197 & 212 & 1800 & 6.146 & 6.146 & & 0.5490 & 5.69E-03 & 527.5245 & 194.3773 & 721.9018 & \\
\hline & $7 / 30 / 2008$ 19:00 & 198 & 212 & 1900 & 6.23 & 6.23 & & 0.5281 & 5.42E-03 & 502.9292 & 185.3146 & 688.2438 & \\
\hline & $7 / 30 / 200820: 00$ & 199 & 212 & 2000 & 6.788 & 6.788 & & 0.4025 & 3.88E-03 & 359.9872 & 132.6447 & 492.6319 & \\
\hline & 7/30/2008 21:00 & 200 & 212 & 2100 & 8.19 & 8.19 & & 0.1624 & 1.27E-03 & 117.7775 & 43.3975 & 161.1751 & \\
\hline & $7 / 30 / 2008$ 22:00 & 201 & 212 & 2200 & 9.15 & 9.15 & & 0.0404 & $2.29 \mathrm{E}-04$ & 21.2716 & 7.8380 & 29.1096 & \\
\hline & 7/30/2008 23:00 & 202 & 212 & 2300 & 9.46 & 9.46 & & 0.0063 & $2.34 \mathrm{E}-05$ & 2.1749 & 0.8014 & 2.9763 & \\
\hline & $7 / 31 / 20080: 00$ & 203 & 213 & 0 & 9.67 & 9.67 & 9.89 & -0.0155 & $0.00 \mathrm{E}+00$ & 0.0000 & 0.0000 & 0.0000 & \\
\hline & 7/31/2008 1:00 & 204 & 213 & 100 & 9.78 & 9.78 & & -0.0266 & $0.00 \mathrm{E}+00$ & 0.0000 & 0.0000 & 0.0000 & \\
\hline
\end{tabular}




\begin{tabular}{|c|c|c|c|c|c|c|c|c|c|c|c|c|c|}
\hline Tree ID & TS & RN & JD & HR & $\begin{array}{c}\text { Raw } \\
\text { TC_dTA }\end{array}$ & $\begin{array}{c}\text { Fixed } \\
\text { TC_dT } \Delta\end{array}$ & dTM & K & $\begin{array}{l}\text { Sap } \\
\text { Velocity } \\
(\mathrm{cm} / \mathrm{s})\end{array}$ & $\begin{array}{c}\text { Probed } \\
\text { Drywood F } \\
(\mathrm{g} / \mathrm{hr})\end{array}$ & $\begin{array}{c}\text { Extrapolated } \\
\text { Wetwood F } \\
(\mathrm{g} / \mathrm{hr})\end{array}$ & $\begin{array}{c}\text { Extrapolated } \\
\text { Total Wood F } \\
(\mathrm{g} / \mathrm{hr})\end{array}$ & $\begin{array}{l}\text { Relative } \\
\text { Humidity, } \\
@ 10 \mathrm{~m}(\%)\end{array}$ \\
\hline & 7/31/2008 2:00 & 205 & 213 & 200 & $\begin{array}{r}9.81 \\
\end{array}$ & 9.81 & & -0.0296 & $0.00 \mathrm{E}+00$ & 0.0000 & 0.0000 & 0.0000 & \\
\hline & 7/31/2008 3:00 & 206 & 213 & 300 & 9.84 & 9.84 & & -0.0325 & $0.00 \mathrm{E}+00$ & 0.0000 & 0.0000 & 0.0000 & \\
\hline & 7/31/2008 4:00 & 207 & 213 & 400 & 9.88 & 9.88 & & -0.0364 & $0.00 E+00$ & 0.0000 & 0.0000 & 0.0000 & \\
\hline & 7/31/2008 5:00 & 208 & 213 & 500 & 9.89 & 9.89 & & -0.0374 & $0.00 \mathrm{E}+00$ & 0.0000 & 0.0000 & 0.0000 & \\
\hline & 7/31/2008 6:00 & 209 & 213 & 600 & 9.88 & 9.88 & & -0.0364 & $0.00 \mathrm{E}+00$ & 0.0000 & 0.0000 & 0.0000 & \\
\hline & 7/31/2008 7:00 & 210 & 213 & 700 & 9.87 & 9.87 & & -0.0355 & $0.00 \mathrm{E}+00$ & 0.0000 & 0.0000 & 0.0000 & \\
\hline & $7 / 31 / 2008$ 8:00 & 211 & 213 & 800 & 9.78 & 9.78 & & -0.0266 & $0.00 \mathrm{E}+00$ & 0.0000 & 0.0000 & 0.0000 & \\
\hline & $7 / 31 / 20089: 00$ & 212 & 213 & 900 & 8.32 & 8.32 & & 0.1442 & 1.10E-03 & 101.7780 & 37.5022 & 139.2802 & \\
\hline & $7 / 31 / 2008$ 10:00 & 213 & 213 & 1000 & 6.463 & 6.463 & & 0.4730 & 4.73E-03 & 439.1448 & 161.8119 & 600.9567 & \\
\hline & 7/31/2008 11:00 & 214 & 213 & 1100 & 6.353 & 6.353 & & 0.4985 & 5.05E-03 & 468.4727 & 172.6184 & 641.0911 & \\
\hline & $7 / 31 / 2008$ 12:00 & 215 & 213 & 1200 & 6.063 & 6.063 & & 0.5702 & $5.96 \mathrm{E}-03$ & 552.7185 & 203.6605 & 756.3790 & \\
\hline & 7/31/2008 13:00 & 216 & 213 & 1300 & 6.256 & 6.256 & & 0.5217 & $5.34 \mathrm{E}-03$ & 495.4943 & 182.5751 & 678.0693 & \\
\hline & 7/31/2008 14:00 & 217 & 213 & 1400 & 5.905 & 5.905 & & 0.6122 & $6.50 \mathrm{E}-03$ & 603.2719 & 222.2879 & 825.5599 & \\
\hline & 7/31/2008 15:00 & 218 & 213 & 1500 & 5.875 & 5.875 & & 0.6204 & 6.61E-03 & 613.2739 & 225.9734 & 839.2472 & \\
\hline & $7 / 31 / 2008$ 16:00 & 219 & 213 & 1600 & 5.914 & 5.914 & & 0.6097 & 6.47E-03 & 600.2971 & 221.1918 & 821.4889 & \\
\hline & 7/31/2008 17:00 & 220 & 213 & 1700 & 5.956 & 5.956 & & 0.5984 & 6.32E-03 & 586.5696 & 216.1336 & 802.7033 & \\
\hline & $7 / 31 / 2008$ 18:00 & 221 & 213 & 1800 & 5.999 & 5.999 & & 0.5869 & 6.18E-03 & 572.7753 & 211.0508 & 783.8261 & \\
\hline & $7 / 31 / 2008$ 19:00 & 222 & 213 & 1900 & 6.283 & 6.283 & & 0.5152 & $5.26 \mathrm{E}-03$ & 487.8603 & 179.7622 & 667.6225 & \\
\hline & $7 / 31 / 200820: 00$ & 223 & 213 & 2000 & 7.076 & 7.076 & & 0.3454 & $3.22 \mathrm{E}-03$ & 298.2066 & 109.8804 & 408.0870 & \\
\hline & $7 / 31 / 200821: 00$ & 224 & 213 & 2100 & 7.974 & 7.974 & & 0.1939 & $1.58 \mathrm{E}-03$ & 146.4896 & 53.9771 & 200.4668 & \\
\hline & $7 / 31 / 200822: 00$ & 225 & 213 & 2200 & 8.72 & 8.72 & & 0.0917 & $6.29 \mathrm{E}-04$ & 58.3152 & 21.4874 & 79.8026 & \\
\hline & 7/31/2008 23:00 & 226 & 213 & 2300 & 8.78 & 8.78 & & 0.0843 & 5.66E-04 & 52.5335 & 19.3570 & 71.8905 & \\
\hline & $8 / 1 / 20080: 00$ & 227 & 214 & 0 & 9.08 & 9.08 & 9.83 & 0.0485 & 2.87E-04 & 26.5791 & 9.7936 & 36.3727 & \\
\hline & 8/1/2008 1:00 & 228 & 214 & 100 & 9.49 & 9.49 & & 0.0032 & 9.95E-06 & 0.9229 & 0.3401 & 1.2630 & \\
\hline & 8/1/2008 2:00 & 229 & 214 & 200 & 9.66 & 9.66 & & -0.0145 & $0.00 \mathrm{E}+00$ & 0.0000 & 0.0000 & 0.0000 & \\
\hline & $8 / 1 / 2008$ 3:00 & 230 & 214 & 300 & 9.71 & 9.71 & & -0.0196 & $0.00 E+00$ & 0.0000 & 0.0000 & 0.0000 & \\
\hline & 8/1/2008 4:00 & 231 & 214 & 400 & 9.79 & 9.79 & & -0.0276 & $0.00 \mathrm{E}+00$ & 0.0000 & 0.0000 & 0.0000 & \\
\hline & 8/1/2008 5:00 & 232 & 214 & 500 & 9.83 & 9.83 & & -0.0315 & $0.00 \mathrm{E}+00$ & 0.0000 & 0.0000 & 0.0000 & \\
\hline & 8/1/2008 6:00 & 233 & 214 & 600 & 9.78 & 9.78 & & -0.0266 & $0.00 \mathrm{E}+00$ & 0.0000 & 0.0000 & 0.0000 & \\
\hline & 8/1/2008 7:00 & 234 & 214 & 700 & 9.72 & 9.72 & & -0.0206 & $0.00 \mathrm{E}+00$ & 0.0000 & 0.0000 & 0.0000 & \\
\hline & 8/1/2008 8:00 & 235 & 214 & 800 & 9.65 & 9.65 & & -0.0135 & $0.00 \mathrm{E}+00$ & 0.0000 & 0.0000 & 0.0000 & \\
\hline & 8/1/2008 9:00 & 236 & 214 & 900 & 8.41 & 8.41 & & 0.1320 & 9.84E-04 & 91.2478 & 33.6221 & 124.8699 & \\
\hline & 8/1/2008 10:00 & 237 & 214 & 1000 & 6.494 & 6.494 & & 0.4660 & $4.65 \mathrm{E}-03$ & 431.1223 & 158.8559 & 589.9782 & \\
\hline & 8/1/2008 11:00 & 238 & 214 & 1100 & 6.356 & 6.356 & & 0.4978 & $5.04 \mathrm{E}-03$ & 467.6546 & 172.3170 & 639.9716 & \\
\hline & 8/1/2008 12:00 & 239 & 214 & 1200 & 6.262 & 6.262 & & 0.5203 & 5.32E-03 & 493.7902 & 181.9472 & 675.7374 & \\
\hline & 8/1/2008 13:00 & 240 & 214 & 1300 & 6.069 & 6.069 & & 0.5686 & 5.94E-03 & 550.8667 & 202.9782 & 753.8449 & \\
\hline & 8/1/2008 14:00 & 241 & 214 & 1400 & 5.991 & 5.991 & & 0.5891 & $6.20 \mathrm{E}-03$ & 575.3220 & 211.9892 & 787.3113 & \\
\hline & 8/1/2008 15:00 & 242 & 214 & 1500 & 5.924 & 5.924 & & 0.6070 & 6.44E-03 & 597.0056 & 219.9790 & 816.9846 & \\
\hline & $8 / 1 / 200816: 00$ & 243 & 214 & 1600 & 6.002 & 6.002 & & 0.5861 & $6.17 \mathrm{E}-03$ & 571.8226 & 210.6998 & 782.5224 & \\
\hline & 8/1/2008 17:00 & 244 & 214 & 1700 & 6.095 & 6.095 & & 0.5619 & 5.85E-03 & 542.8977 & 200.0418 & 742.9396 & \\
\hline & 8/1/2008 18:00 & 245 & 214 & 1800 & 6.091 & 6.091 & & 0.5630 & 5.87E-03 & 544.1179 & 200.4914 & 744.6093 & \\
\hline
\end{tabular}




\begin{tabular}{|c|c|c|c|c|c|c|c|c|c|c|c|c|c|}
\hline Tree ID & TS & RN & JD & HR & $\begin{array}{c}\text { Raw } \\
\text { TC_dTA }\end{array}$ & $\begin{array}{c}\text { Fixed } \\
\text { TC_dT } \Delta\end{array}$ & dTM & $\mathbf{K}$ & $\begin{array}{l}\text { Sap } \\
\text { Velocity } \\
(\mathrm{cm} / \mathrm{s})\end{array}$ & $\begin{array}{c}\text { Probed } \\
\text { Drywood F } \\
(\mathrm{g} / \mathrm{hr})\end{array}$ & $\begin{array}{c}\text { Extrapolated } \\
\text { Wetwood F } \\
(\mathrm{g} / \mathrm{hr})\end{array}$ & $\begin{array}{c}\text { Extrapolated } \\
\text { Total Wood F } \\
(\mathrm{g} / \mathrm{hr})\end{array}$ & $\begin{array}{l}\text { Relative } \\
\text { Humidity, } \\
@ 10 \mathrm{~m}(\%)\end{array}$ \\
\hline & 8/1/2008 19:00 & 246 & 214 & 1900 & \begin{tabular}{|r|}
6.278 \\
\end{tabular} & 6.278 & & 0.5164 & $5.28 \mathrm{E}-03$ & 489.2674 & 180.2806 & 669.5480 & \\
\hline & 8/1/2008 20:00 & 247 & 214 & 2000 & 7.023 & 7.023 & & 0.3555 & 3.33E-03 & 309.0340 & 113.8699 & 422.9040 & \\
\hline & 8/1/2008 21:00 & 248 & 214 & 2100 & 8.61 & 8.61 & & 0.1057 & $7.48 \mathrm{E}-04$ & 69.4136 & 25.5769 & 94.9905 & \\
\hline & 8/1/2008 22:00 & 249 & 214 & 2200 & 9.42 & 9.42 & & 0.0106 & $4.42 \mathrm{E}-05$ & 4.1001 & 1.5108 & 5.6109 & \\
\hline & 8/1/2008 23:00 & 250 & 214 & 2300 & 9.58 & 9.58 & & -0.0063 & $0.00 \mathrm{E}+00$ & 0.0000 & 0.0000 & 0.0000 & \\
\hline & 8/2/2008 0:00 & 251 & 215 & 0 & 9.65 & 9.65 & 9.88 & -0.0135 & $0.00 \mathrm{E}+00$ & 0.0000 & 0.0000 & 0.0000 & \\
\hline & 8/2/2008 1:00 & 252 & 215 & 100 & 9.76 & 9.76 & & -0.0246 & $0.00 \mathrm{E}+00$ & 0.0000 & 0.0000 & 0.0000 & \\
\hline & 8/2/2008 2:00 & 253 & 215 & 200 & 9.86 & 9.86 & & -0.0345 & $0.00 \mathrm{E}+00$ & 0.0000 & 0.0000 & 0.0000 & \\
\hline & 8/2/2008 3:00 & 254 & 215 & 300 & 9.88 & 9.88 & & -0.0364 & $0.00 \mathrm{E}+00$ & 0.0000 & 0.0000 & 0.0000 & \\
\hline & 8/2/2008 4:00 & 255 & 215 & 400 & 9.84 & 9.84 & & -0.0325 & $0.00 E+00$ & 0.0000 & 0.0000 & 0.0000 & \\
\hline & 8/2/2008 5:00 & 256 & 215 & 500 & 9.82 & 9.82 & & -0.0305 & $0.00 \mathrm{E}+00$ & 0.0000 & 0.0000 & 0.0000 & \\
\hline & 8/2/2008 6:00 & 257 & 215 & 600 & 9.84 & 9.84 & & -0.0325 & $0.00 E+00$ & 0.0000 & 0.0000 & 0.0000 & \\
\hline & 8/2/2008 7:00 & 258 & 215 & 700 & 9.77 & 9.77 & & -0.0256 & $0.00 \mathrm{E}+00$ & 0.0000 & 0.0000 & 0.0000 & \\
\hline & 8/2/2008 8:00 & 259 & 215 & 800 & 8.63 & 8.63 & & 0.1031 & $7.26 \mathrm{E}-04$ & 67.3478 & 24.8157 & 92.1635 & \\
\hline & 8/2/2008 9:00 & 260 & 215 & 900 & 6.8 & 6.8 & & 0.4000 & 3.85E-03 & 357.2641 & 131.6413 & 488.9054 & \\
\hline & 8/2/2008 10:00 & 261 & 215 & 1000 & 6.551 & 6.551 & & 0.4532 & 4.49E-03 & 416.6410 & 153.5199 & 570.1609 & \\
\hline & 8/2/2008 11:00 & 262 & 215 & 1100 & 6.396 & 6.396 & & 0.4884 & 4.93E-03 & 456.8456 & 168.3342 & 625.1798 & \\
\hline & $8 / 2 / 200812: 00$ & 263 & 215 & 1200 & 6.199 & 6.199 & & 0.5357 & 5.52E-03 & 511.9028 & 188.6211 & 700.5240 & \\
\hline & 8/2/2008 13:00 & 264 & 215 & 1300 & 6.091 & 6.091 & & 0.5630 & 5.87E-03 & 544.1179 & 200.4914 & 744.6093 & \\
\hline & 8/2/2008 14:00 & 265 & 215 & 1400 & 6.065 & 6.065 & & 0.5697 & 5.95E-03 & 552.1007 & 203.4329 & 755.5336 & \\
\hline & $8 / 2 / 2008$ 15:00 & 266 & 215 & 1500 & 6.017 & 6.017 & & 0.5822 & $6.11 \mathrm{E}-03$ & 567.0776 & 208.9514 & 776.0290 & \\
\hline & 8/2/2008 16:00 & 267 & 215 & 1600 & 6.014 & 6.014 & & 0.5830 & $6.12 \mathrm{E}-03$ & 568.0241 & 209.3002 & 777.3243 & \\
\hline & 8/2/2008 17:00 & 268 & 215 & 1700 & 6.044 & 6.044 & & 0.5751 & 6.02E-03 & 558.6146 & 205.8330 & 764.4476 & \\
\hline & $8 / 2 / 200818: 00$ & 269 & 215 & 1800 & 6.14 & 6.14 & & 0.5505 & $5.71 \mathrm{E}-03$ & 529.3156 & 195.0372 & 724.3528 & \\
\hline & 8/2/2008 19:00 & 270 & 215 & 1900 & 6.307 & 6.307 & & 0.5094 & $5.19 \mathrm{E}-03$ & 481.1480 & 177.2889 & 658.4369 & \\
\hline & 8/2/2008 20:00 & 271 & 215 & 2000 & 6.918 & 6.918 & & 0.3761 & 3.57E-03 & 331.1927 & 122.0347 & 453.2274 & \\
\hline & 8/2/2008 21:00 & 272 & 215 & 2100 & 8.03 & 8.03 & & 0.1856 & $1.50 \mathrm{E}-03$ & 138.7845 & 51.1380 & 189.9225 & \\
\hline & 8/2/2008 22:00 & 273 & 215 & 2200 & 8.97 & 8.97 & & 0.0613 & $3.83 \mathrm{E}-04$ & 35.5101 & 13.0844 & 48.5946 & \\
\hline & 8/2/2008 23:00 & 274 & 215 & 2300 & 9.28 & 9.28 & & 0.0259 & 1.32E-04 & 12.2699 & 4.5211 & 16.7910 & \\
\hline & 8/3/2008 0:00 & 275 & 216 & 0 & 9.24 & 9.24 & 9.77 & 0.0303 & 1.61E-04 & 14.9129 & 5.4950 & 20.4079 & \\
\hline & 8/3/2008 1:00 & 276 & 216 & 100 & 9.08 & 9.08 & & 0.0485 & 2.87E-04 & 26.5791 & 9.7936 & 36.3727 & \\
\hline & 8/3/2008 2:00 & 277 & 216 & 200 & 9.28 & 9.28 & & 0.0259 & 1.32E-04 & 12.2699 & 4.5211 & 16.7910 & \\
\hline & 8/3/2008 3:00 & 278 & 216 & 300 & 9.53 & 9.53 & & -0.0010 & $0.00 \mathrm{E}+00$ & 0.0000 & 0.0000 & 0.0000 & \\
\hline & 8/3/2008 4:00 & 279 & 216 & 400 & 9.67 & 9.67 & & -0.0155 & $0.00 \mathrm{E}+00$ & 0.0000 & 0.0000 & 0.0000 & \\
\hline & 8/3/2008 5:00 & 280 & 216 & 500 & 9.77 & 9.77 & & -0.0256 & $0.00 \mathrm{E}+00$ & 0.0000 & 0.0000 & 0.0000 & \\
\hline & 8/3/2008 6:00 & 281 & 216 & 600 & 9.67 & 9.67 & & -0.0155 & $0.00 \mathrm{E}+00$ & 0.0000 & 0.0000 & 0.0000 & \\
\hline & 8/3/2008 7:00 & 282 & 216 & 700 & 9.47 & 9.47 & & 0.0053 & 1.87E-05 & 1.7354 & 0.6394 & 2.3748 & \\
\hline & 8/3/2008 8:00 & 283 & 216 & 800 & 8.44 & 8.44 & & 0.1280 & 9.47E-04 & 87.8356 & 32.3648 & 120.2004 & \\
\hline & 8/3/2008 9:00 & 284 & 216 & 900 & 6.63 & 6.63 & & 0.4359 & $4.28 \mathrm{E}-03$ & 397.1326 & 146.3317 & 543.4643 & \\
\hline & 8/3/2008 10:00 & 285 & 216 & 1000 & 6.205 & 6.205 & & 0.5342 & $5.50 \mathrm{E}-03$ & 510.1567 & 187.9777 & 698.1344 & \\
\hline & 8/3/2008 11:00 & 286 & 216 & 1100 & 6.092 & 6.092 & & 0.5627 & 5.86E-03 & 543.8126 & 200.3790 & 744.1916 & \\
\hline
\end{tabular}




\begin{tabular}{|c|c|c|c|c|c|c|c|c|c|c|c|c|c|}
\hline Tree ID & TS & RN & JD & HR & $\begin{array}{c}\text { Raw } \\
\text { TC_dTA }\end{array}$ & $\begin{array}{c}\text { Fixed } \\
\text { TC_dT } \Delta\end{array}$ & dTM & $\mathbf{K}$ & $\begin{array}{l}\text { Sap } \\
\text { Velocity } \\
(\mathrm{cm} / \mathrm{s})\end{array}$ & $\begin{array}{c}\text { Probed } \\
\text { Drywood F } \\
(\mathrm{g} / \mathrm{hr})\end{array}$ & $\begin{array}{c}\text { Extrapolated } \\
\text { Wetwood F } \\
(\mathrm{g} / \mathrm{hr})\end{array}$ & $\begin{array}{c}\text { Extrapolated } \\
\text { Total Wood F } \\
(\mathrm{g} / \mathrm{hr})\end{array}$ & $\begin{array}{l}\text { Relative } \\
\text { Humidity, } \\
@ 10 \mathrm{~m}(\%)\end{array}$ \\
\hline & 8/3/2008 12:00 & 287 & 216 & 1200 & 5.967 & 5.967 & & 0.5954 & $6.29 \mathrm{E}-03$ & 583.0160 & 214.8243 & \begin{tabular}{|l|}
797.8403 \\
\end{tabular} & \\
\hline & 8/3/2008 13:00 & 288 & 216 & 1300 & 5.908 & 5.908 & & 0.6114 & $6.49 \mathrm{E}-03$ & 602.2790 & 221.9221 & 824.2011 & \\
\hline & $8 / 3 / 2008$ 14:00 & 289 & 216 & 1400 & 5.941 & 5.941 & & 0.6024 & $6.38 E-03$ & 591.4432 & 217.9294 & 809.3726 & \\
\hline & 8/3/2008 15:00 & 290 & 216 & 1500 & 5.988 & 5.988 & & 0.5898 & $6.21 E-03$ & 576.2794 & 212.3420 & 788.6214 & \\
\hline & 8/3/2008 16:00 & 291 & 216 & 1600 & 6.009 & 6.009 & & 0.5843 & 6.14E-03 & 569.6044 & 209.8825 & 779.4868 & \\
\hline & 8/3/2008 17:00 & 292 & 216 & 1700 & 6.038 & 6.038 & & 0.5767 & 6.04E-03 & 560.4867 & 206.5228 & 767.0095 & \\
\hline & 8/3/2008 18:00 & 293 & 216 & 1800 & 6.085 & 6.085 & & 0.5645 & $5.89 E-03$ & 545.9521 & 201.1673 & 747.1194 & \\
\hline & 8/3/2008 19:00 & 294 & 216 & 1900 & 6.192 & 6.192 & & 0.5375 & 5.54E-03 & 513.9457 & 189.3739 & 703.3196 & \\
\hline & 8/3/2008 20:00 & 295 & 216 & 2000 & 6.488 & 6.488 & & 0.4673 & $4.66 \mathrm{E}-03$ & 432.6669 & 159.4250 & 592.0919 & \\
\hline & 8/3/2008 21:00 & 296 & 216 & 2100 & 7.373 & 7.373 & & 0.2912 & 2.61E-03 & 241.6956 & 89.0577 & 330.7533 & \\
\hline & 8/3/2008 22:00 & 297 & 216 & 2200 & 8.03 & 8.03 & & 0.1856 & 1.50E-03 & 138.7845 & 51.1380 & 189.9225 & \\
\hline & 8/3/2008 23:00 & 298 & 216 & 2300 & 8.18 & 8.18 & & 0.1638 & 1.28E-03 & 119.0475 & 43.8655 & 162.9130 & \\
\hline & 8/4/2008 0:00 & 299 & 217 & 0 & 8.28 & 8.28 & 9.27 & 0.1498 & 1.15E-03 & 106.6007 & 39.2792 & 145.8800 & \\
\hline & 8/4/2008 1:00 & 300 & 217 & 100 & 8.39 & 8.39 & & 0.1347 & 1.01E-03 & 93.5496 & 34.4703 & 128.0199 & \\
\hline & $8 / 4 / 2008$ 2:00 & 301 & 217 & 200 & 8.13 & 8.13 & & 0.1710 & 1.35E-03 & 125.4825 & 46.2366 & 171.7191 & \\
\hline & 8/4/2008 3:00 & 302 & 217 & 300 & 7.938 & 7.938 & & 0.1993 & $1.63 \mathrm{E}-03$ & 151.5418 & 55.8387 & 207.3804 & \\
\hline & 8/4/2008 4:00 & 303 & 217 & 400 & 7.748 & 7.748 & & 0.2287 & 1.94E-03 & 179.5229 & 66.1489 & 245.6718 & \\
\hline & $8 / 4 / 20085: 00$ & 304 & 217 & 500 & 7.933 & 7.933 & & 0.2001 & 1.64E-03 & 152.2496 & 56.0995 & 208.3491 & \\
\hline & 8/4/2008 6:00 & 305 & 217 & 600 & 8.39 & 8.39 & & 0.1347 & 1.01E-03 & 93.5496 & 34.4703 & 128.0199 & \\
\hline & 8/4/2008 7:00 & 306 & 217 & 700 & 8.48 & 8.48 & & 0.1226 & 8.99E-04 & 83.3616 & 30.7163 & 114.0779 & \\
\hline & 8/4/2008 8:00 & 307 & 217 & 800 & 8.29 & 8.29 & & 0.1484 & $1.14 \mathrm{E}-03$ & 105.3868 & 38.8319 & 144.2187 & \\
\hline & 8/4/2008 9:00 & 308 & 217 & 900 & 7.901 & 7.901 & & 0.2049 & 1.69E-03 & 156.8158 & 57.7820 & 214.5978 & \\
\hline & 8/4/2008 10:00 & 309 & 217 & 1000 & 6.971 & 6.971 & & 0.3657 & $3.45 E-03$ & 319.8884 & 117.8695 & 437.7579 & \\
\hline & 8/4/2008 11:00 & 310 & 217 & 1100 & 6.427 & 6.427 & & 0.4813 & 4.84E-03 & 448.5935 & 165.2935 & 613.8870 & \\
\hline & 8/4/2008 12:00 & 311 & 217 & 1200 & 6.13 & 6.13 & & 0.5530 & $5.74 \mathrm{E}-03$ & 532.3110 & 196.1410 & 728.4520 & \\
\hline & 8/4/2008 13:00 & 312 & 217 & 1300 & 6.075 & 6.075 & & 0.5671 & 5.92E-03 & 549.0197 & 202.2976 & 751.3173 & \\
\hline & 8/4/2008 14:00 & 313 & 217 & 1400 & 6.441 & 6.441 & & 0.4780 & 4.80E-03 & 444.9019 & 163.9333 & 608.8352 & \\
\hline & 8/4/2008 15:00 & 314 & 217 & 1500 & 6.83 & 6.83 & & 0.3939 & $3.78 \mathrm{E}-03$ & 350.5150 & 129.1545 & 479.6695 & \\
\hline & 8/4/2008 16:00 & 315 & 217 & 1600 & 6.28 & 6.28 & & 0.5159 & 5.27E-03 & 488.7042 & 180.0731 & 668.7773 & \\
\hline & 8/4/2008 17:00 & 316 & 217 & 1700 & 6.213 & 6.213 & & 0.5323 & $5.48 E-03$ & 507.8355 & 187.1224 & 694.9579 & \\
\hline & 8/4/2008 18:00 & 317 & 217 & 1800 & 6.264 & 6.264 & & 0.5198 & 5.32E-03 & 493.2232 & 181.7382 & 674.9614 & \\
\hline & 8/4/2008 19:00 & 318 & 217 & 1900 & 6.502 & 6.502 & & 0.4642 & 4.63E-03 & 429.0689 & 158.0993 & 587.1682 & \\
\hline & 8/4/2008 20:00 & 319 & 217 & 2000 & 7.08 & 7.08 & & 0.3446 & $3.21 E-03$ & 297.3989 & 109.5828 & 406.9817 & \\
\hline & 8/4/2008 21:00 & 320 & 217 & 2100 & 8.55 & 8.55 & & 0.1135 & 8.17E-04 & 75.7389 & 27.9076 & 103.6465 & \\
\hline & 8/4/2008 22:00 & 321 & 217 & 2200 & 9.14 & 9.14 & & 0.0416 & 2.37E-04 & 22.0111 & 8.1105 & 30.1216 & \\
\hline & 8/4/2008 23:00 & 322 & 217 & 2300 & 9.27 & 9.27 & & 0.0270 & 1.39E-04 & 12.9194 & 4.7604 & 17.6798 & \\
\hline & 8/5/2008 0:00 & 323 & 218 & 0 & 9.43 & 9.43 & 9.76 & 0.0095 & 3.88E-05 & 3.5967 & 1.3253 & 4.9220 & \\
\hline & 8/5/2008 1:00 & 324 & 218 & 100 & 9.53 & 9.53 & & -0.0010 & $0.00 \mathrm{E}+00$ & 0.0000 & 0.0000 & 0.0000 & \\
\hline & 8/5/2008 2:00 & 325 & 218 & 200 & 9.61 & 9.61 & & -0.0094 & $0.00 \mathrm{E}+00$ & 0.0000 & 0.0000 & 0.0000 & \\
\hline & 8/5/2008 3:00 & 326 & 218 & 300 & 9.59 & 9.59 & & -0.0073 & $0.00 \mathrm{E}+00$ & 0.0000 & 0.0000 & 0.0000 & \\
\hline & 8/5/2008 4:00 & 327 & 218 & 400 & 9.53 & 9.53 & & -0.0010 & $0.00 \mathrm{E}+00$ & 0.0000 & 0.0000 & 0.0000 & \\
\hline
\end{tabular}




\begin{tabular}{|c|c|c|c|c|c|c|c|c|c|c|c|c|c|}
\hline Tree ID & TS & RN & JD & HR & $\begin{array}{c}\text { Raw } \\
\text { TC_dTA }\end{array}$ & $\begin{array}{c}\text { Fixed } \\
\text { TC_dT } \Delta\end{array}$ & dTM & K & $\begin{array}{l}\text { Sap } \\
\text { Velocity } \\
(\mathrm{cm} / \mathrm{s})\end{array}$ & $\begin{array}{c}\text { Probed } \\
\text { Drywood F } \\
(\mathrm{g} / \mathrm{hr})\end{array}$ & $\begin{array}{c}\text { Extrapolated } \\
\text { Wetwood F } \\
(\mathrm{g} / \mathrm{hr})\end{array}$ & $\begin{array}{c}\text { Extrapolated } \\
\text { Total Wood F } \\
(\mathrm{g} / \mathrm{hr})\end{array}$ & $\begin{array}{l}\text { Relative } \\
\text { Humidity, } \\
@ 10 \mathrm{~m}(\%)\end{array}$ \\
\hline & 8/5/2008 5:00 & 328 & 218 & 500 & 9.56 & 9.56 & & -0.0042 & $0.00 \mathrm{E}+00$ & 0.0000 & 0.0000 & 0.0000 & \\
\hline & 8/5/2008 6:00 & 329 & 218 & 600 & 9.62 & 9.62 & & -0.0104 & $0.00 \mathrm{E}+00$ & 0.0000 & 0.0000 & 0.0000 & \\
\hline & 8/5/2008 7:00 & 330 & 218 & 700 & 9.65 & 9.65 & & -0.0135 & $0.00 E+00$ & 0.0000 & 0.0000 & 0.0000 & \\
\hline & 8/5/2008 8:00 & 331 & 218 & 800 & 9.58 & 9.58 & & -0.0063 & $0.00 \mathrm{E}+00$ & 0.0000 & 0.0000 & 0.0000 & \\
\hline & 8/5/2008 9:00 & 332 & 218 & 900 & 8.4 & 8.4 & & 0.1333 & 9.96E-04 & 92.3960 & 34.0452 & 126.4412 & \\
\hline & 8/5/2008 10:00 & 333 & 218 & 1000 & 6.86 & 6.86 & & 0.3878 & $3.71 E-03$ & 343.8490 & 126.6982 & 470.5473 & \\
\hline & 8/5/2008 11:00 & 334 & 218 & 1100 & 7.115 & 7.115 & & 0.3380 & 3.13E-03 & 290.3881 & 106.9995 & 397.3876 & \\
\hline & 8/5/2008 12:00 & 335 & 218 & 1200 & 6.797 & 6.797 & & 0.4006 & 3.86E-03 & 357.9436 & 131.8917 & 489.8353 & \\
\hline & 8/5/2008 13:00 & 336 & 218 & 1300 & 6.355 & 6.355 & & 0.4980 & 5.05E-03 & 467.9272 & 172.4174 & 640.3446 & \\
\hline & 8/5/2008 14:00 & 337 & 218 & 1400 & 6.873 & 6.873 & & 0.3851 & 3.68E-03 & 340.9859 & 125.6433 & 466.6292 & \\
\hline & 8/5/2008 15:00 & 338 & 218 & 1500 & 7.254 & 7.254 & & 0.3124 & 2.84E-03 & 263.5163 & 97.0980 & 360.6143 & \\
\hline & 8/5/2008 16:00 & 339 & 218 & 1600 & 7.151 & 7.151 & & 0.3313 & $3.05 E-03$ & 283.2811 & 104.3807 & 387.6618 & \\
\hline & 8/5/2008 17:00 & 340 & 218 & 1700 & 6.658 & 6.658 & & 0.4299 & $4.21 E-03$ & 390.3710 & 143.8402 & 534.2113 & \\
\hline & 8/5/2008 18:00 & 341 & 218 & 1800 & 6.442 & 6.442 & & 0.4778 & 4.79E-03 & 444.6391 & 163.8364 & 608.4755 & \\
\hline & 8/5/2008 19:00 & 342 & 218 & 1900 & 6.562 & 6.562 & & 0.4508 & 4.46E-03 & 413.8859 & 152.5048 & 566.3907 & \\
\hline & 8/5/2008 20:00 & 343 & 218 & 2000 & 7.313 & 7.313 & & 0.3018 & 2.72E-03 & 252.5646 & 93.0626 & 345.6272 & \\
\hline & 8/5/2008 21:00 & 344 & 218 & 2100 & 8.92 & 8.92 & & 0.0673 & 4.29E-04 & 39.7978 & 14.6643 & 54.4621 & \\
\hline & $8 / 5 / 200822: 00$ & 345 & 218 & 2200 & 9.61 & 9.61 & & -0.0094 & $0.00 \mathrm{E}+00$ & 0.0000 & 0.0000 & 0.0000 & \\
\hline & 8/5/2008 23:00 & 346 & 218 & 2300 & 9.76 & 9.76 & & -0.0246 & $0.00 \mathrm{E}+00$ & 0.0000 & 0.0000 & 0.0000 & \\
\hline & 8/6/2008 0:00 & 347 & 219 & 0 & 9.85 & 9.85 & 9.85 & -0.0335 & $0.00 E+00$ & 0.0000 & 0.0000 & 0.0000 & \\
\hline & 8/6/2008 1:00 & 348 & 219 & 100 & 9.85 & 9.85 & & -0.0335 & $0.00 \mathrm{E}+00$ & 0.0000 & 0.0000 & 0.0000 & \\
\hline & $8 / 6 / 2008$ 2:00 & 349 & 219 & 200 & 9.84 & 9.84 & & -0.0325 & $0.00 E+00$ & 0.0000 & 0.0000 & 0.0000 & \\
\hline & 8/6/2008 3:00 & 350 & 219 & 300 & 9.81 & 9.81 & & -0.0296 & $0.00 E+00$ & 0.0000 & 0.0000 & 0.0000 & \\
\hline & 8/6/2008 4:00 & 351 & 219 & 400 & 9.77 & 9.77 & & -0.0256 & $0.00 \mathrm{E}+00$ & 0.0000 & 0.0000 & 0.0000 & \\
\hline & 8/6/2008 5:00 & 352 & 219 & 500 & 9.75 & 9.75 & & -0.0236 & $0.00 \mathrm{E}+00$ & 0.0000 & 0.0000 & 0.0000 & \\
\hline & 8/6/2008 6:00 & 353 & 219 & 600 & 9.7 & 9.7 & & -0.0186 & $0.00 E+00$ & 0.0000 & 0.0000 & 0.0000 & \\
\hline & 8/6/2008 7:00 & 354 & 219 & 700 & 9.64 & 9.64 & & -0.0124 & $0.00 \mathrm{E}+00$ & 0.0000 & 0.0000 & 0.0000 & \\
\hline & 8/6/2008 8:00 & 355 & 219 & 800 & 9.59 & 9.59 & & -0.0073 & $0.00 \mathrm{E}+00$ & 0.0000 & 0.0000 & 0.0000 & \\
\hline & 8/6/2008 9:00 & 356 & 219 & 900 & 9.28 & 9.28 & & 0.0259 & 1.32E-04 & 12.2699 & 4.5211 & 16.7910 & \\
\hline & 8/6/2008 10:00 & 357 & 219 & 1000 & 6.993 & 6.993 & & 0.3614 & $3.40 \mathrm{E}-03$ & 315.2679 & 116.1669 & 431.4348 & \\
\hline & 8/6/2008 11:00 & 358 & 219 & 1100 & 6.157 & 6.157 & & 0.5462 & 5.65E-03 & 524.2529 & 193.1718 & 717.4246 & \\
\hline & 8/6/2008 12:00 & 359 & 219 & 1200 & 6.746 & 6.746 & & 0.4112 & 3.99E-03 & 369.6251 & 136.1960 & 505.8211 & \\
\hline & 8/6/2008 13:00 & 360 & 219 & 1300 & 7.155 & 7.155 & & 0.3305 & 3.05E-03 & 282.4979 & 104.0921 & 386.5900 & \\
\hline & $8 / 6 / 2008$ 14:00 & 361 & 219 & 1400 & 6.726 & 6.726 & & 0.4154 & 4.04E-03 & 374.2739 & 137.9089 & 512.1828 & \\
\hline & 8/6/2008 15:00 & 362 & 219 & 1500 & 6.38 & 6.38 & & 0.4922 & 4.97E-03 & 461.1473 & 169.9192 & 631.0664 & \\
\hline & 8/6/2008 16:00 & 363 & 219 & 1600 & 6.442 & 6.442 & & 0.4778 & 4.79E-03 & 444.6391 & 163.8364 & 608.4755 & \\
\hline & 8/6/2008 17:00 & 364 & 219 & 1700 & 6.379 & 6.379 & & 0.4924 & 4.97E-03 & 461.4171 & 170.0186 & 631.4357 & \\
\hline & 8/6/2008 18:00 & 365 & 219 & 1800 & 6.773 & 6.773 & & 0.4056 & 3.92E-03 & 363.4101 & 133.9059 & 497.3160 & \\
\hline & 8/6/2008 19:00 & 366 & 219 & 1900 & 6.832 & 6.832 & & 0.3934 & 3.77E-03 & 350.0681 & 128.9898 & 479.0578 & \\
\hline & $8 / 6 / 200820: 00$ & 367 & 219 & 2000 & 7.119 & 7.119 & & 0.3373 & 3.12E-03 & 289.5933 & 106.7066 & 396.2999 & \\
\hline & 8/6/2008 21:00 & 368 & 219 & 2100 & 8.4 & 8.4 & & 0.1333 & $9.96 \mathrm{E}-04$ & 92.3960 & 34.0452 & 126.4412 & \\
\hline
\end{tabular}




\begin{tabular}{|c|c|c|c|c|c|c|c|c|c|c|c|c|c|}
\hline Tree ID & TS & RN & JD & HR & $\begin{array}{c}\text { Raw } \\
\text { TC_dTA }\end{array}$ & $\begin{array}{c}\text { Fixed } \\
\text { TC_dT } \Delta\end{array}$ & dTM & $\mathbf{K}$ & $\begin{array}{l}\text { Sap } \\
\text { Velocity } \\
(\mathrm{cm} / \mathrm{s})\end{array}$ & $\begin{array}{c}\text { Probed } \\
\text { Drywood F } \\
(\mathrm{g} / \mathrm{hr})\end{array}$ & $\begin{array}{c}\text { Extrapolated } \\
\text { Wetwood F } \\
(\mathrm{g} / \mathrm{hr})\end{array}$ & $\begin{array}{c}\text { Extrapolated } \\
\text { Total Wood F } \\
(\mathrm{g} / \mathrm{hr})\end{array}$ & $\begin{array}{l}\text { Relative } \\
\text { Humidity, } \\
@ 10 \mathrm{~m}(\%)\end{array}$ \\
\hline & 8/6/2008 22:00 & 369 & 219 & 2200 & $\begin{array}{r}9.48 \\
\end{array}$ & 9.48 & & 0.0042 & 1.42E-05 & 1.3169 & 0.4852 & $\begin{array}{r}1.8021 \\
\end{array}$ & \\
\hline & 8/6/2008 23:00 & 370 & 219 & 2300 & 9.7 & 9.7 & & -0.0186 & $0.00 \mathrm{E}+00$ & 0.0000 & 0.0000 & 0.0000 & \\
\hline & $8 / 7 / 20080: 00$ & 371 & 220 & 0 & 9.79 & 9.79 & 9.79 & -0.0276 & $0.00 E+00$ & 0.0000 & 0.0000 & 0.0000 & \\
\hline & 8/7/2008 1:00 & 372 & 220 & 100 & 9.79 & 9.79 & & -0.0276 & $0.00 \mathrm{E}+00$ & 0.0000 & 0.0000 & 0.0000 & \\
\hline & 8/7/2008 2:00 & 373 & 220 & 200 & 9.74 & 9.74 & & -0.0226 & $0.00 \mathrm{E}+00$ & 0.0000 & 0.0000 & 0.0000 & \\
\hline & 8/7/2008 3:00 & 374 & 220 & 300 & 9.67 & 9.67 & & -0.0155 & $0.00 \mathrm{E}+00$ & 0.0000 & 0.0000 & 0.0000 & \\
\hline & 8/7/2008 4:00 & 375 & 220 & 400 & 9.6 & 9.6 & & -0.0083 & $0.00 \mathrm{E}+00$ & 0.0000 & 0.0000 & 0.0000 & \\
\hline & 8/7/2008 5:00 & 376 & 220 & 500 & 9.53 & 9.53 & & -0.0010 & $0.00 \mathrm{E}+00$ & 0.0000 & 0.0000 & 0.0000 & \\
\hline & 8/7/2008 6:00 & 377 & 220 & 600 & 9.47 & 9.47 & & 0.0053 & 1.87E-05 & 1.7354 & 0.6394 & 2.3748 & \\
\hline & 8/7/2008 7:00 & 378 & 220 & 700 & 9.41 & 9.41 & & 0.0117 & 4.98E-05 & 4.6166 & 1.7011 & 6.3176 & \\
\hline & 8/7/2008 8:00 & 379 & 220 & 800 & 9.32 & 9.32 & & 0.0215 & 1.05E-04 & 9.7515 & 3.5931 & 13.3446 & \\
\hline & 8/7/2008 9:00 & 380 & 220 & 900 & 8.56 & 8.56 & & 0.1121 & 8.05E-04 & 74.6714 & 27.5142 & 102.1856 & \\
\hline & 8/7/2008 10:00 & 381 & 220 & 1000 & 6.335 & 6.335 & & 0.5028 & $5.10 \mathrm{E}-03$ & 473.4031 & 174.4351 & 647.8382 & \\
\hline & 8/7/2008 11:00 & 382 & 220 & 1100 & 5.831 & 5.831 & & 0.6327 & 6.77E-03 & 628.1862 & 231.4681 & 859.6543 & \\
\hline & 8/7/2008 12:00 & 383 & 220 & 1200 & 5.913 & 5.913 & & 0.6100 & 6.48E-03 & 600.6271 & 221.3134 & 821.9404 & \\
\hline & 8/7/2008 13:00 & 384 & 220 & 1300 & 5.946 & 5.946 & & 0.6011 & $6.36 \mathrm{E}-03$ & 589.8151 & 217.3295 & 807.1446 & \\
\hline & $8 / 7 / 2008$ 14:00 & 385 & 220 & 1400 & 6.039 & 6.039 & & 0.5764 & 6.04E-03 & 560.1743 & 206.4077 & 766.5821 & \\
\hline & 8/7/2008 15:00 & 386 & 220 & 1500 & 6.107 & 6.107 & & 0.5589 & 5.81E-03 & 539.2499 & 198.6977 & 737.9476 & \\
\hline & 8/7/2008 16:00 & 387 & 220 & 1600 & 6.109 & 6.109 & & 0.5584 & 5.81E-03 & 538.6438 & 198.4744 & 737.1182 & \\
\hline & 8/7/2008 17:00 & 388 & 220 & 1700 & 6.087 & 6.087 & & 0.5640 & 5.88E-03 & 545.3402 & 200.9418 & 746.2819 & \\
\hline & 8/7/2008 18:00 & 389 & 220 & 1800 & 6.148 & 6.148 & & 0.5485 & $5.68 \mathrm{E}-03$ & 526.9285 & 194.1576 & 721.0862 & \\
\hline & 8/7/2008 19:00 & 390 & 220 & 1900 & 6.406 & 6.406 & & 0.4861 & 4.90E-03 & 454.1718 & 167.3489 & 621.5208 & \\
\hline & 8/7/2008 20:00 & 391 & 220 & 2000 & 6.887 & 6.887 & & 0.3823 & 3.64E-03 & 337.9197 & 124.5134 & 462.4331 & \\
\hline & 8/7/2008 21:00 & 392 & 220 & 2100 & 8.25 & 8.25 & & 0.1539 & 1.19E-03 & 110.2760 & 40.6335 & 150.9095 & \\
\hline & 8/7/2008 22:00 & 393 & 220 & 2200 & 9.09 & 9.09 & & 0.0473 & $2.78 \mathrm{E}-04$ & 25.8025 & 9.5074 & 35.3099 & \\
\hline & 8/7/2008 23:00 & 394 & 220 & 2300 & 9.45 & 9.45 & & 0.0074 & 2.84E-05 & 2.6328 & 0.9701 & 3.6029 & \\
\hline & 8/8/2008 0:00 & 395 & 221 & 0 & 9.65 & 9.65 & 9.77 & -0.0135 & $0.00 \mathrm{E}+00$ & 0.0000 & 0.0000 & 0.0000 & \\
\hline & 8/8/2008 1:00 & 396 & 221 & 100 & 9.65 & 9.65 & & -0.0135 & $0.00 \mathrm{E}+00$ & 0.0000 & 0.0000 & 0.0000 & \\
\hline & 8/8/2008 2:00 & 397 & 221 & 200 & 9.75 & 9.75 & & -0.0236 & $0.00 \mathrm{E}+00$ & 0.0000 & 0.0000 & 0.0000 & \\
\hline & 8/8/2008 3:00 & 398 & 221 & 300 & 9.77 & 9.77 & & -0.0256 & $0.00 \mathrm{E}+00$ & 0.0000 & 0.0000 & 0.0000 & \\
\hline & 8/8/2008 4:00 & 399 & 221 & 400 & 9.7 & 9.7 & & -0.0186 & $0.00 \mathrm{E}+00$ & 0.0000 & 0.0000 & 0.0000 & \\
\hline & 8/8/2008 5:00 & 400 & 221 & 500 & 9.62 & 9.62 & & -0.0104 & $0.00 \mathrm{E}+00$ & 0.0000 & 0.0000 & 0.0000 & \\
\hline & 8/8/2008 6:00 & 401 & 221 & 600 & 9.54 & 9.54 & & -0.0021 & $0.00 \mathrm{E}+00$ & 0.0000 & 0.0000 & 0.0000 & \\
\hline & $8 / 8 / 20087: 00$ & 402 & 221 & 700 & 9.46 & 9.46 & & 0.0063 & 2.34E-05 & 2.1749 & 0.8014 & 2.9763 & \\
\hline & 8/8/2008 8:00 & 403 & 221 & 800 & 9.33 & 9.33 & & 0.0204 & 9.86E-05 & 9.1427 & 3.3688 & 12.5115 & \\
\hline & 8/8/20089:00 & 404 & 221 & 900 & 7.547 & 7.547 & & 0.2614 & $2.28 \mathrm{E}-03$ & 211.6483 & 77.9862 & 289.6344 & \\
\hline & 8/8/2008 10:00 & 405 & 221 & 1000 & 6.036 & 6.036 & & 0.5772 & $6.05 E-03$ & 561.1118 & 206.7532 & 767.8649 & \\
\hline & 8/8/2008 11:00 & 406 & 221 & 1100 & 6.073 & 6.073 & & 0.5676 & 5.93E-03 & 549.6349 & 202.5243 & 752.1591 & \\
\hline & $8 / 8 / 200812: 00$ & 407 & 221 & 1200 & 5.987 & 5.987 & & 0.5901 & $6.22 \mathrm{E}-03$ & 576.5988 & 212.4597 & 789.0584 & \\
\hline & 8/8/2008 13:00 & 408 & 221 & 1300 & 5.989 & 5.989 & & 0.5896 & $6.21 \mathrm{E}-03$ & 575.9601 & 212.2244 & 788.1845 & \\
\hline & 8/8/2008 14:00 & 409 & 221 & 1400 & 6.01 & 6.01 & & 0.5840 & 6.14E-03 & 569.2881 & 209.7659 & 779.0540 & \\
\hline
\end{tabular}




\begin{tabular}{|c|c|c|c|c|c|c|c|c|c|c|c|c|c|}
\hline Tree ID & TS & RN & JD & HR & $\begin{array}{c}\text { Raw } \\
\text { TC_dTA }\end{array}$ & $\begin{array}{c}\text { Fixed } \\
\text { TC_dT } \Delta\end{array}$ & dTM & $\mathbf{K}$ & $\begin{array}{l}\text { Sap } \\
\text { Velocity } \\
(\mathrm{cm} / \mathrm{s})\end{array}$ & $\begin{array}{c}\text { Probed } \\
\text { Drywood F } \\
(\mathrm{g} / \mathrm{hr})\end{array}$ & $\begin{array}{c}\text { Extrapolated } \\
\text { Wetwood F } \\
(\mathrm{g} / \mathrm{hr})\end{array}$ & $\begin{array}{c}\text { Extrapolated } \\
\text { Total Wood F } \\
(\mathrm{g} / \mathrm{hr})\end{array}$ & $\begin{array}{l}\text { Relative } \\
\text { Humidity, } \\
@ 10 \mathrm{~m}(\%)\end{array}$ \\
\hline & 8/8/2008 15:00 & 410 & 221 & 1500 & 5.976 & 5.976 & & 0.5930 & $6.25 \mathrm{E}-03$ & 580.1213 & 213.7576 & \begin{tabular}{|r|}
793.8789 \\
\end{tabular} & \\
\hline & 8/8/2008 16:00 & 411 & 221 & 1600 & 5.952 & 5.952 & & 0.5995 & $6.34 \mathrm{E}-03$ & 587.8661 & 216.6114 & 804.4775 & \\
\hline & 8/8/2008 17:00 & 412 & 221 & 1700 & 6.089 & 6.089 & & 0.5635 & $5.87 E-03$ & 544.7288 & 200.7165 & 745.4453 & \\
\hline & 8/8/2008 18:00 & 413 & 221 & 1800 & 6.309 & 6.309 & & 0.5090 & $5.18 \mathrm{E}-03$ & 480.5917 & 177.0839 & 657.6756 & \\
\hline & 8/8/2008 19:00 & 414 & 221 & 1900 & 6.305 & 6.305 & & 0.5099 & 5.19E-03 & 481.7047 & 177.4940 & 659.1988 & \\
\hline & 8/8/2008 20:00 & 415 & 221 & 2000 & 6.928 & 6.928 & & 0.3741 & $3.55 E-03$ & 329.0409 & 121.2419 & 450.2828 & \\
\hline & 8/8/2008 21:00 & 416 & 221 & 2100 & 8.13 & 8.13 & & 0.1710 & 1.35E-03 & 125.4825 & 46.2366 & 171.7191 & \\
\hline & 8/8/2008 22:00 & 417 & 221 & 2200 & 8.84 & 8.84 & & 0.0769 & 5.06E-04 & 46.9450 & 17.2978 & 64.2428 & \\
\hline & 8/8/2008 23:00 & 418 & 221 & 2300 & 9.02 & 9.02 & & 0.0554 & 3.38E-04 & 31.3636 & 11.5566 & 42.9202 & \\
\hline & 8/9/2008 0:00 & 419 & 222 & 0 & 9.02 & 9.02 & 9.62 & 0.0554 & 3.38E-04 & 31.3636 & 11.5566 & 42.9202 & \\
\hline & 8/9/2008 1:00 & 420 & 222 & 100 & 8.99 & 8.99 & & 0.0590 & 3.65E-04 & 33.8344 & 12.4670 & 46.3014 & \\
\hline & 8/9/2008 2:00 & 421 & 222 & 200 & 9.02 & 9.02 & & 0.0554 & $3.38 \mathrm{E}-04$ & 31.3636 & 11.5566 & 42.9202 & \\
\hline & 8/9/2008 3:00 & 422 & 222 & 300 & 9.04 & 9.04 & & 0.0531 & $3.21 E-04$ & 29.7453 & 10.9603 & 40.7055 & \\
\hline & 8/9/2008 4:00 & 423 & 222 & 400 & 9.03 & 9.03 & & 0.0543 & 3.29E-04 & 30.5515 & 11.2573 & 41.8089 & \\
\hline & $8 / 9 / 20085: 00$ & 424 & 222 & 500 & 8.94 & 8.94 & & 0.0649 & 4.10E-04 & 38.0660 & 14.0262 & 52.0922 & \\
\hline & 8/9/2008 6:00 & 425 & 222 & 600 & 8.96 & 8.96 & & 0.0625 & $3.92 \mathrm{E}-04$ & 36.3565 & 13.3963 & 49.7528 & \\
\hline & 8/9/2008 7:00 & 426 & 222 & 700 & 8.66 & 8.66 & & 0.0993 & 6.93E-04 & 64.2890 & 23.6886 & 87.9776 & \\
\hline & 8/9/2008 8:00 & 427 & 222 & 800 & 7.979 & 7.979 & & 0.1931 & 1.57E-03 & 145.7941 & 53.7208 & 199.5149 & \\
\hline & 8/9/2008 9:00 & 428 & 222 & 900 & 8.56 & 8.56 & & 0.1121 & 8.05E-04 & 74.6714 & 27.5142 & 102.1856 & \\
\hline & 8/9/2008 10:00 & 429 & 222 & 1000 & 8.3 & 8.3 & & 0.1470 & 1.12E-03 & 104.1783 & 38.3866 & 142.5650 & \\
\hline & 8/9/2008 11:00 & 430 & 222 & 1100 & 7.606 & 7.606 & & 0.2516 & $2.18 \mathrm{E}-03$ & 201.9392 & 74.4086 & 276.3478 & \\
\hline & 8/9/2008 12:00 & 431 & 222 & 1200 & 6.912 & 6.912 & & 0.3773 & 3.58E-03 & 332.4880 & 122.5120 & 455.0000 & \\
\hline & 8/9/2008 13:00 & 432 & 222 & 1300 & 6.713 & 6.713 & & 0.4181 & 4.07E-03 & 377.3162 & 139.0299 & 516.3462 & \\
\hline & 8/9/2008 14:00 & 433 & 222 & 1400 & 6.292 & 6.292 & & 0.5130 & $5.23 \mathrm{E}-03$ & 485.3352 & 178.8317 & 664.1669 & \\
\hline & 8/9/2008 15:00 & 434 & 222 & 1500 & 6.182 & 6.182 & & 0.5400 & 5.57E-03 & 516.8748 & 190.4532 & 707.3280 & \\
\hline & 8/9/2008 16:00 & 435 & 222 & 1600 & 6.191 & 6.191 & & 0.5377 & 5.54E-03 & 514.2381 & 189.4816 & 703.7197 & \\
\hline & 8/9/2008 17:00 & 436 & 222 & 1700 & 6.373 & 6.373 & & 0.4938 & 4.99E-03 & 463.0384 & 170.6160 & 633.6544 & \\
\hline & 8/9/2008 18:00 & 437 & 222 & 1800 & 6.399 & 6.399 & & 0.4877 & $4.92 \mathrm{E}-03$ & 456.0423 & 168.0382 & 624.0804 & \\
\hline & 8/9/2008 19:00 & 438 & 222 & 1900 & 6.581 & 6.581 & & 0.4466 & 4.41E-03 & 409.1569 & 150.7623 & 559.9192 & \\
\hline & 8/9/2008 20:00 & 439 & 222 & 2000 & 7.087 & 7.087 & & 0.3433 & $3.19 \mathrm{E}-03$ & 295.9887 & 109.0631 & 405.0519 & \\
\hline & 8/9/2008 21:00 & 440 & 222 & 2100 & 8.66 & 8.66 & & 0.0993 & $6.93 \mathrm{E}-04$ & 64.2890 & 23.6886 & 87.9776 & \\
\hline & 8/9/2008 22:00 & 441 & 222 & 2200 & 9.54 & 9.54 & & -0.0021 & $0.00 \mathrm{E}+00$ & 0.0000 & 0.0000 & 0.0000 & \\
\hline & 8/9/2008 23:00 & 442 & 222 & 2300 & 9.62 & 9.62 & & -0.0104 & $0.00 \mathrm{E}+00$ & 0.0000 & 0.0000 & 0.0000 & \\
\hline & 8/10/2008 0:00 & 443 & 223 & 0 & 9.6 & 9.6 & 9.60 & -0.0083 & $0.00 \mathrm{E}+00$ & 0.0000 & 0.0000 & 0.0000 & \\
\hline & 8/10/2008 1:00 & 444 & 223 & 100 & 9.58 & 9.58 & & -0.0063 & $0.00 \mathrm{E}+00$ & 0.0000 & 0.0000 & 0.0000 & \\
\hline & 8/10/2008 2:00 & 445 & 223 & 200 & 9.52 & 9.52 & & 0.0000 & $0.00 \mathrm{E}+00$ & 0.0000 & 0.0000 & 0.0000 & \\
\hline & 8/10/2008 3:00 & 446 & 223 & 300 & 9.47 & 9.47 & & 0.0053 & 1.87E-05 & 1.7354 & 0.6394 & 2.3748 & \\
\hline & 8/10/2008 4:00 & 447 & 223 & 400 & 9.38 & 9.38 & & 0.0149 & 6.72E-05 & 6.2367 & 2.2980 & 8.5348 & \\
\hline & $8 / 10 / 20085: 00$ & 448 & 223 & 500 & 9.28 & 9.28 & & 0.0259 & 1.32E-04 & 12.2699 & 4.5211 & 16.7910 & \\
\hline & 8/10/2008 6:00 & 449 & 223 & 600 & 9.2 & 9.2 & & 0.0348 & 1.91E-04 & 17.6714 & 6.5114 & 24.1828 & \\
\hline & 8/10/2008 7:00 & 450 & 223 & 700 & 9.12 & 9.12 & & 0.0439 & 2.53E-04 & 23.5091 & 8.6624 & 32.1715 & \\
\hline
\end{tabular}




\begin{tabular}{|c|c|c|c|c|c|c|c|c|c|c|c|c|c|}
\hline Tree ID & TS & RN & JD & HR & $\begin{array}{c}\text { Raw } \\
\text { TC_dTA }\end{array}$ & $\begin{array}{c}\text { Fixed } \\
\text { TC_dT } \Delta\end{array}$ & dTM & $\mathbf{K}$ & $\begin{array}{l}\text { Sap } \\
\text { Velocity } \\
(\mathrm{cm} / \mathrm{s})\end{array}$ & $\begin{array}{c}\text { Probed } \\
\text { Drywood F } \\
(\mathrm{g} / \mathrm{hr})\end{array}$ & $\begin{array}{c}\text { Extrapolated } \\
\text { Wetwood F } \\
(\mathrm{g} / \mathrm{hr})\end{array}$ & $\begin{array}{c}\text { Extrapolated } \\
\text { Total Wood F } \\
(\mathrm{g} / \mathrm{hr})\end{array}$ & $\begin{array}{l}\text { Relative } \\
\text { Humidity, } \\
@ 10 \mathrm{~m}(\%)\end{array}$ \\
\hline & 8/10/2008 8:00 & 451 & 223 & 800 & \begin{tabular}{|r|}
9.03 \\
\end{tabular} & 9.03 & & 0.0543 & 3.29E-04 & 30.5515 & 11.2573 & $\begin{array}{r}41.8089 \\
\end{array}$ & \\
\hline & 8/10/2008 9:00 & 452 & 223 & 900 & 8.16 & 8.16 & & 0.1667 & $1.31 \mathrm{E}-03$ & 121.6044 & 44.8076 & 166.4120 & \\
\hline & $8 / 10 / 2008$ 10:00 & 453 & 223 & 1000 & 6.031 & 6.031 & & 0.5785 & $6.07 E-03$ & 562.6769 & 207.3299 & 770.0068 & \\
\hline & 8/10/2008 11:00 & 454 & 223 & 1100 & 5.64 & 5.64 & & 0.6879 & 7.51E-03 & 696.4353 & 256.6159 & 953.0512 & \\
\hline & $8 / 10 / 200812: 00$ & 455 & 223 & 1200 & 5.753 & 5.753 & & 0.6548 & 7.07E-03 & 655.3512 & 241.4776 & 896.8289 & \\
\hline & 8/10/2008 13:00 & 456 & 223 & 1300 & 5.858 & 5.858 & & 0.6251 & 6.67E-03 & 619.0009 & 228.0836 & 847.0845 & \\
\hline & $8 / 10 / 2008$ 14:00 & 457 & 223 & 1400 & 5.931 & 5.931 & & 0.6051 & $6.41 E-03$ & 594.7101 & 219.1332 & 813.8433 & \\
\hline & $8 / 10 / 2008$ 15:00 & 458 & 223 & 1500 & 6.164 & 6.164 & & 0.5445 & 5.63E-03 & 522.1790 & 192.4076 & 714.5866 & \\
\hline & 8/10/2008 16:00 & 459 & 223 & 1600 & 6.035 & 6.035 & & 0.5775 & $6.05 E-03$ & 561.4245 & 206.8684 & 768.2930 & \\
\hline & 8/10/2008 17:00 & 460 & 223 & 1700 & 6.243 & 6.243 & & 0.5249 & 5.38E-03 & 499.2014 & 183.9410 & 683.1424 & \\
\hline & 8/10/2008 18:00 & 461 & 223 & 1800 & 8.6 & 8.6 & & 0.1070 & 7.60E-04 & 70.4545 & 25.9604 & 96.4149 & \\
\hline & $8 / 10 / 2008$ 19:00 & 462 & 223 & 1900 & 9.24 & 9.24 & & 0.0303 & 1.61E-04 & 14.9129 & 5.4950 & 20.4079 & \\
\hline & 8/10/2008 20:00 & 463 & 223 & 2000 & 9.01 & 9.01 & & 0.0566 & 3.47E-04 & 32.1815 & 11.8579 & 44.0394 & \\
\hline & 8/10/2008 21:00 & 464 & 223 & 2100 & 8.92 & 8.92 & & 0.0673 & $4.29 \mathrm{E}-04$ & 39.7978 & 14.6643 & 54.4621 & \\
\hline & $8 / 10 / 2008$ 22:00 & 465 & 223 & 2200 & 9.03 & 9.03 & & 0.0543 & $3.29 E-04$ & 30.5515 & 11.2573 & 41.8089 & \\
\hline & $8 / 10 / 2008$ 23:00 & 466 & 223 & 2300 & 8.94 & 8.94 & & 0.0649 & $4.10 \mathrm{E}-04$ & 38.0660 & 14.0262 & 52.0922 & \\
\hline & $8 / 11 / 20080: 00$ & 467 & 224 & 0 & 8.94 & 8.94 & 9.27 & 0.0649 & 4.10E-04 & 38.0660 & 14.0262 & 52.0922 & \\
\hline & 8/11/2008 1:00 & 468 & 224 & 100 & 9.14 & 9.14 & & 0.0416 & 2.37E-04 & 22.0111 & 8.1105 & 30.1216 & \\
\hline & 8/11/2008 2:00 & 469 & 224 & 200 & 9.27 & 9.27 & & 0.0270 & 1.39E-04 & 12.9194 & 4.7604 & 17.6798 & \\
\hline & 8/11/2008 3:00 & 470 & 224 & 300 & 9.26 & 9.26 & & 0.0281 & 1.46E-04 & 13.5765 & 5.0025 & 18.5790 & \\
\hline & 8/11/2008 4:00 & 471 & 224 & 400 & 9.24 & 9.24 & & 0.0303 & 1.61E-04 & 14.9129 & 5.4950 & 20.4079 & \\
\hline & 8/11/2008 5:00 & 472 & 224 & 500 & 9.19 & 9.19 & & 0.0359 & 1.98E-04 & 18.3782 & 6.7718 & 25.1500 & \\
\hline & 8/11/2008 6:00 & 473 & 224 & 600 & 9.2 & 9.2 & & 0.0348 & 1.91E-04 & 17.6714 & 6.5114 & 24.1828 & \\
\hline & 8/11/2008 7:00 & 474 & 224 & 700 & 9.19 & 9.19 & & 0.0359 & 1.98E-04 & 18.3782 & 6.7718 & 25.1500 & \\
\hline & 8/11/2008 8:00 & 475 & 224 & 800 & 9.13 & 9.13 & & 0.0427 & $2.45 E-04$ & 22.7570 & 8.3853 & 31.1422 & \\
\hline & 8/11/2008 9:00 & 476 & 224 & 900 & 8.24 & 8.24 & & 0.1553 & 1.20E-03 & 111.5122 & 41.0890 & 152.6012 & \\
\hline & $8 / 11 / 2008$ 10:00 & 477 & 224 & 1000 & 6.419 & 6.419 & & 0.4831 & 4.86E-03 & 450.7127 & 166.0744 & 616.7871 & \\
\hline & 8/11/2008 11:00 & 478 & 224 & 1100 & 5.978 & 5.978 & & 0.5925 & $6.25 \mathrm{E}-03$ & 579.4796 & 213.5212 & 793.0007 & \\
\hline & $8 / 11 / 2008$ 12:00 & 479 & 224 & 1200 & 6.055 & 6.055 & & 0.5723 & 5.99E-03 & 555.1951 & 204.5731 & 759.7682 & \\
\hline & 8/11/2008 13:00 & 480 & 224 & 1300 & 6.344 & 6.344 & & 0.5006 & $5.08 \mathrm{E}-03$ & 470.9332 & 173.5250 & 644.4582 & \\
\hline & 8/11/2008 14:00 & 481 & 224 & 1400 & 6.109 & 6.109 & & 0.5584 & 5.81E-03 & 538.6438 & 198.4744 & 737.1182 & \\
\hline & 8/11/2008 15:00 & 482 & 224 & 1500 & 6.037 & 6.037 & & 0.5769 & $6.05 E-03$ & 560.7992 & 206.6380 & 767.4371 & \\
\hline & $8 / 11 / 2008$ 16:00 & 483 & 224 & 1600 & 6.261 & 6.261 & & 0.5205 & 5.33E-03 & 494.0739 & 182.0517 & 676.1256 & \\
\hline & $8 / 11 / 2008$ 17:00 & 484 & 224 & 1700 & 6.367 & 6.367 & & 0.4952 & $5.01 \mathrm{E}-03$ & 464.6639 & 171.2150 & 635.8788 & \\
\hline & 8/11/2008 18:00 & 485 & 224 & 1800 & 6.63 & 6.63 & & 0.4359 & $4.28 \mathrm{E}-03$ & 397.1326 & 146.3317 & 543.4643 & \\
\hline & $8 / 11 / 2008$ 19:00 & 486 & 224 & 1900 & 6.791 & 6.791 & & 0.4019 & 3.87E-03 & 359.3051 & 132.3934 & 491.6985 & \\
\hline & 8/11/2008 20:00 & 487 & 224 & 2000 & 7.434 & 7.434 & & 0.2806 & 2.49E-03 & 230.9162 & 85.0858 & 316.0020 & \\
\hline & 8/11/2008 21:00 & 488 & 224 & 2100 & 8.32 & 8.32 & & 0.1442 & 1.10E-03 & 101.7780 & 37.5022 & 139.2802 & \\
\hline & $8 / 11 / 200822: 00$ & 489 & 224 & 2200 & 8.78 & 8.78 & & 0.0843 & $5.66 \mathrm{E}-04$ & 52.5335 & 19.3570 & 71.8905 & \\
\hline & 8/11/2008 23:00 & 490 & 224 & 2300 & 8.97 & 8.97 & & 0.0613 & 3.83E-04 & 35.5101 & 13.0844 & 48.5946 & \\
\hline & 8/12/2008 0:00 & 491 & 225 & 0 & 9.06 & 9.06 & 9.24 & 0.0508 & 3.04E-04 & 28.1503 & 10.3726 & 38.5229 & \\
\hline
\end{tabular}




\begin{tabular}{|c|c|c|c|c|c|c|c|c|c|c|c|c|c|}
\hline Tree ID & TS & RN & JD & HR & $\begin{array}{c}\text { Raw } \\
\text { TC_dTA }\end{array}$ & $\begin{array}{c}\text { Fixed } \\
\text { TC_dT } \Delta\end{array}$ & dTM & $\mathbf{K}$ & $\begin{array}{l}\text { Sap } \\
\text { Velocity } \\
(\mathrm{cm} / \mathrm{s})\end{array}$ & $\begin{array}{c}\text { Probed } \\
\text { Drywood F } \\
(\mathrm{g} / \mathrm{hr})\end{array}$ & $\begin{array}{c}\text { Extrapolated } \\
\text { Wetwood F } \\
(\mathrm{g} / \mathrm{hr})\end{array}$ & $\begin{array}{c}\text { Extrapolated } \\
\text { Total Wood F } \\
(\mathrm{g} / \mathrm{hr})\end{array}$ & $\begin{array}{l}\text { Relative } \\
\text { Humidity, } \\
@ 10 \mathrm{~m}(\%)\end{array}$ \\
\hline & 8/12/2008 1:00 & 492 & 225 & 100 & 9.08 & 9.08 & & 0.0485 & 2.87E-04 & 26.5791 & 9.7936 & $\begin{array}{r}36.3727 \\
\end{array}$ & \\
\hline & 8/12/2008 2:00 & 493 & 225 & 200 & 8.86 & 8.86 & & 0.0745 & 4.87E-04 & 45.1254 & 16.6274 & 61.7528 & \\
\hline & 8/12/2008 3:00 & 494 & 225 & 300 & 8.73 & 8.73 & & 0.0905 & $6.18 E-04$ & 57.3382 & 21.1274 & 78.4656 & \\
\hline & 8/12/2008 4:00 & 495 & 225 & 400 & 8.71 & 8.71 & & 0.0930 & 6.39E-04 & 59.2975 & 21.8494 & 81.1468 & \\
\hline & 8/12/2008 5:00 & 496 & 225 & 500 & 8.79 & 8.79 & & 0.0830 & 5.56E-04 & 51.5886 & 19.0089 & 70.5975 & \\
\hline & 8/12/2008 6:00 & 497 & 225 & 600 & 8.86 & 8.86 & & 0.0745 & 4.87E-04 & 45.1254 & 16.6274 & 61.7528 & \\
\hline & 8/12/2008 7:00 & 498 & 225 & 700 & 8.83 & 8.83 & & 0.0781 & $5.16 \mathrm{E}-04$ & 47.8629 & 17.6361 & 65.4990 & \\
\hline & 8/12/2008 8:00 & 499 & 225 & 800 & 8.72 & 8.72 & & 0.0917 & $6.29 E-04$ & 58.3152 & 21.4874 & 79.8026 & \\
\hline & 8/12/2008 9:00 & 500 & 225 & 900 & 7.934 & 7.934 & & 0.1999 & 1.64E-03 & 152.1079 & 56.0473 & 208.1552 & \\
\hline & $8 / 12 / 2008$ 10:00 & 501 & 225 & 1000 & 6.198 & 6.198 & & 0.5360 & 5.52E-03 & 512.1943 & 188.7285 & 700.9228 & \\
\hline & $8 / 12 / 2008$ 11:00 & 502 & 225 & 1100 & 5.65 & 5.65 & & 0.6850 & 7.47E-03 & 692.7141 & 255.2448 & 947.9589 & \\
\hline & $8 / 12 / 2008$ 12:00 & 503 & 225 & 1200 & 5.679 & 5.679 & & 0.6764 & 7.35E-03 & 682.0179 & 251.3035 & 933.3214 & \\
\hline & 8/12/2008 13:00 & 504 & 225 & 1300 & 5.692 & 5.692 & & 0.6725 & 7.30E-03 & 677.2685 & 249.5535 & 926.8220 & \\
\hline & $8 / 12 / 2008$ 14:00 & 505 & 225 & 1400 & 5.784 & 5.784 & & 0.6459 & 6.95E-03 & 644.4412 & 237.4576 & 881.8989 & \\
\hline & $8 / 12 / 2008$ 15:00 & 506 & 225 & 1500 & 5.844 & 5.844 & & 0.6290 & 6.73E-03 & 623.7499 & 229.8335 & 853.5833 & \\
\hline & $8 / 12 / 2008$ 16:00 & 507 & 225 & 1600 & 5.915 & 5.915 & & 0.6095 & 6.47E-03 & 599.9673 & 221.0703 & 821.0376 & \\
\hline & $8 / 12 / 2008$ 17:00 & 508 & 225 & 1700 & 6.033 & 6.033 & & 0.5780 & 6.06E-03 & 562.0505 & 207.0991 & 769.1495 & \\
\hline & $8 / 12 / 2008$ 18:00 & 509 & 225 & 1800 & 6.081 & 6.081 & & 0.5655 & 5.90E-03 & 547.1775 & 201.6188 & 748.7964 & \\
\hline & $8 / 12 / 2008$ 19:00 & 510 & 225 & 1900 & 6.335 & 6.335 & & 0.5028 & $5.10 \mathrm{E}-03$ & 473.4031 & 174.4351 & 647.8382 & \\
\hline & $8 / 12 / 200820: 00$ & 511 & 225 & 2000 & 6.854 & 6.854 & & 0.3890 & $3.72 E-03$ & 345.1756 & 127.1871 & 472.3627 & \\
\hline & $8 / 12 / 2008$ 21:00 & 512 & 225 & 2100 & 8.4 & 8.4 & & 0.1333 & 9.96E-04 & 92.3960 & 34.0452 & 126.4412 & \\
\hline & $8 / 12 / 2008$ 22:00 & 513 & 225 & 2200 & 9.17 & 9.17 & & 0.0382 & 2.14E-04 & 19.8118 & 7.3001 & 27.1119 & \\
\hline & $8 / 12 / 2008$ 23:00 & 514 & 225 & 2300 & 9.24 & 9.24 & & 0.0303 & 1.61E-04 & 14.9129 & 5.4950 & 20.4079 & \\
\hline & 8/13/2008 0:00 & 515 & 226 & 0 & 9.25 & 9.25 & 9.25 & 0.0292 & $1.54 \mathrm{E}-04$ & 14.2410 & 5.2474 & 19.4884 & \\
\hline & 8/13/2008 1:00 & 516 & 226 & 100 & 9.18 & 9.18 & & 0.0370 & 2.06E-04 & 19.0917 & 7.0347 & 26.1264 & \\
\hline & 8/13/2008 2:00 & 517 & 226 & 200 & 9.14 & 9.14 & & 0.0416 & 2.37E-04 & 22.0111 & 8.1105 & 30.1216 & \\
\hline & 8/13/2008 3:00 & 518 & 226 & 300 & 9.17 & 9.17 & & 0.0382 & 2.14E-04 & 19.8118 & 7.3001 & 27.1119 & \\
\hline & 8/13/2008 4:00 & 519 & 226 & 400 & 9.1 & 9.1 & & 0.0462 & $2.70 \mathrm{E}-04$ & 25.0319 & 9.2235 & 34.2554 & \\
\hline & 8/13/2008 5:00 & 520 & 226 & 500 & 9.04 & 9.04 & & 0.0531 & $3.21 \mathrm{E}-04$ & 29.7453 & 10.9603 & 40.7055 & \\
\hline & 8/13/2008 6:00 & 521 & 226 & 600 & 9.03 & 9.03 & & 0.0543 & $3.29 E-04$ & 30.5515 & 11.2573 & 41.8089 & \\
\hline & $8 / 13 / 2008$ 7:00 & 522 & 226 & 700 & 8.91 & 8.91 & & 0.0685 & 4.39E-04 & 40.6720 & 14.9864 & 55.6584 & \\
\hline & 8/13/2008 8:00 & 523 & 226 & 800 & 8.65 & 8.65 & & 0.1006 & 7.04E-04 & 65.3033 & 24.0623 & 89.3656 & \\
\hline & 8/13/2008 9:00 & 524 & 226 & 900 & 7.043 & 7.043 & & 0.3517 & 3.29E-03 & 304.9205 & 112.3542 & 417.2748 & \\
\hline & $8 / 13 / 2008$ 10:00 & 525 & 226 & 1000 & 5.724 & 5.724 & & 0.6632 & $7.18 \mathrm{E}-03$ & 665.6958 & 245.2893 & 910.9851 & \\
\hline & 8/13/2008 11:00 & 526 & 226 & 1100 & 5.797 & 5.797 & & 0.6422 & $6.90 \mathrm{E}-03$ & 639.9110 & 235.7884 & 875.6993 & \\
\hline & $8 / 13 / 2008$ 12:00 & 527 & 226 & 1200 & 5.554 & 5.554 & & 0.7141 & 7.86E-03 & 729.1483 & 268.6697 & 997.8179 & \\
\hline
\end{tabular}




\begin{tabular}{|c|c|c|c|c|c|c|c|c|c|c|c|c|c|}
\hline Tree ID & TS & RN & JD & HR & $\begin{array}{c}\text { Raw } \\
\text { TC_dTA }\end{array}$ & $\begin{array}{c}\text { Fixed } \\
\text { TC_dT } \Delta\end{array}$ & dTM & $\mathbf{K}$ & $\begin{array}{c}\text { Sap } \\
\text { Velocity } \\
(\mathrm{cm} / \mathrm{s})\end{array}$ & $\begin{array}{c}\text { Probed } \\
\text { Drywood F } \\
(\mathrm{g} / \mathrm{hr})\end{array}$ & $\begin{array}{c}\text { Extrapolated } \\
\text { Wetwood F } \\
(\mathrm{g} / \mathrm{hr})\end{array}$ & $\begin{array}{c}\text { Extrapolated } \\
\text { Total Wood F } \\
(\mathrm{g} / \mathrm{hr})\end{array}$ & $\begin{array}{c}\text { Relative } \\
\text { Humidity, } \\
@ 10 \mathrm{~m}(\%)\end{array}$ \\
\hline & $7 / 22 / 2008$ 13:00 & 0 & 204 & 1300 & 16.55 & 16.55 & 25.76 & 0.5565 & $5.78 \mathrm{E}-03$ & 55.3654 & 31.1430 & 86.5084 & \\
\hline \multirow[t]{2}{*}{ W707GA } & $7 / 22 / 200814: 00$ & 1 & 204 & 1400 & 18.11 & 18.11 & & 0.4224 & $4.12 \mathrm{E}-03$ & 39.4334 & 22.1813 & 61.6147 & \\
\hline & $7 / 22 / 2008$ 15:00 & 2 & 204 & 1500 & 17.86 & 17.86 & & 0.4423 & $4.36 \mathrm{E}-03$ & 41.7338 & 23.4753 & 65.2091 & \\
\hline \multirow[t]{2}{*}{ Probe \# } & $7 / 22 / 2008$ 16:00 & 3 & 204 & 1600 & 17.58 & 17.58 & & 0.4653 & 4.64E-03 & 44.4177 & 24.9850 & 69.4027 & \\
\hline & 7/22/2008 17:00 & 4 & 204 & 1700 & 17.62 & 17.62 & & 0.4620 & 4.60E-03 & 44.0271 & 24.7653 & 68.7924 & \\
\hline \multirow[t]{4}{*}{20} & $7 / 22 / 2008$ 18:00 & 5 & 204 & 1800 & 18.75 & 18.75 & & 0.3739 & $3.54 \mathrm{E}-03$ & 33.9304 & 19.0859 & 53.0163 & \\
\hline & 7/22/2008 19:00 & 6 & 204 & 1900 & 19.03 & 19.03 & & 0.3537 & 3.31E-03 & 31.6864 & 17.8236 & 49.5099 & \\
\hline & $7 / 22 / 200820: 00$ & 7 & 204 & 2000 & 20.14 & 20.14 & & 0.2790 & $2.47 \mathrm{E}-03$ & 23.6703 & 13.3145 & 36.9848 & \\
\hline & 7/22/2008 21:00 & 8 & 204 & 2100 & 23.18 & 23.18 & & 0.1113 & 7.98E-04 & 7.6353 & 4.2948 & 11.9301 & \\
\hline \multirow[t]{2}{*}{$\mathrm{DBH}(\mathrm{cm})$} & $7 / 22 / 2008$ 22:00 & 9 & 204 & 2200 & 25.11 & 25.11 & & 0.0259 & $1.32 \mathrm{E}-04$ & 1.2678 & 0.7132 & 1.9810 & \\
\hline & $7 / 22 / 2008$ 23:00 & 10 & 204 & 2300 & 25.76 & 25.76 & & 0.0000 & $0.00 E+00$ & 0.0000 & 0.0000 & 0.0000 & \\
\hline \multirow[t]{2}{*}{2.5} & $7 / 23 / 20080: 00$ & 11 & 205 & 0 & 26.21 & 26.21 & 26.30 & -0.0172 & $0.00 \mathrm{E}+00$ & 0.0000 & 0.0000 & 0.0000 & \\
\hline & 7/23/2008 1:00 & 12 & 205 & 100 & 26.3 & 26.3 & & -0.0205 & $0.00 \mathrm{E}+00$ & 0.0000 & 0.0000 & 0.0000 & \\
\hline Day Count & 7/23/2008 2:00 & 13 & 205 & 200 & 26.11 & 26.11 & & -0.0134 & $0.00 \mathrm{E}+00$ & 0.0000 & 0.0000 & 0.0000 & \\
\hline \multirow{27}{*}{35} & 7/23/2008 3:00 & 14 & 205 & 300 & 26.19 & 26.19 & & -0.0164 & $0.00 \mathrm{E}+00$ & 0.0000 & 0.0000 & 0.0000 & \\
\hline & 7/23/2008 4:00 & 15 & 205 & 400 & 26.05 & 26.05 & & -0.0111 & $0.00 \mathrm{E}+00$ & 0.0000 & 0.0000 & 0.0000 & \\
\hline & 7/23/2008 5:00 & 16 & 205 & 500 & 25.8 & 25.8 & & -0.0016 & $0.00 \mathrm{E}+00$ & 0.0000 & 0.0000 & 0.0000 & \\
\hline & 7/23/2008 6:00 & 17 & 205 & 600 & 25.53 & 25.53 & & 0.0090 & $3.61 \mathrm{E}-05$ & 0.3458 & 0.1945 & 0.5403 & \\
\hline & 7/23/2008 7:00 & 18 & 205 & 700 & 25.07 & 25.07 & & 0.0275 & 1.43E-04 & 1.3672 & 0.7691 & 2.1363 & \\
\hline & 7/23/2008 8:00 & 19 & 205 & 800 & 24.16 & 24.16 & & 0.0662 & $4.21 E-04$ & 4.0295 & 2.2666 & 6.2961 & \\
\hline & $7 / 23 / 20089: 00$ & 20 & 205 & 900 & 22.12 & 22.12 & & 0.1646 & 1.29E-03 & 12.3555 & 6.9500 & 19.3055 & \\
\hline & $7 / 23 / 2008$ 10:00 & 21 & 205 & 1000 & 20.05 & 20.05 & & 0.2848 & $2.54 \mathrm{E}-03$ & 24.2712 & 13.6525 & 37.9237 & \\
\hline & $7 / 23 / 2008$ 11:00 & 22 & 205 & 1100 & 19.57 & 19.57 & & 0.3163 & $2.89 \mathrm{E}-03$ & 27.6184 & 15.5353 & 43.1537 & \\
\hline & 7/23/2008 12:00 & 23 & 205 & 1200 & 19.04 & 19.04 & & 0.3529 & 3.30E-03 & 31.6080 & 17.7795 & 49.3874 & \\
\hline & 7/23/2008 13:00 & 24 & 205 & 1300 & 18.54 & 18.54 & & 0.3894 & $3.73 \mathrm{E}-03$ & 35.6772 & 20.0684 & 55.7457 & \\
\hline & $7 / 23 / 2008$ 14:00 & 25 & 205 & 1400 & 18.37 & 18.37 & & 0.4023 & $3.88 \mathrm{E}-03$ & 37.1328 & 20.8872 & 58.0200 & \\
\hline & $7 / 23 / 2008$ 15:00 & 26 & 205 & 1500 & 18.3 & 18.3 & & 0.4077 & 3.94E-03 & 37.7432 & 21.2306 & 58.9738 & \\
\hline & $7 / 23 / 2008$ 16:00 & 27 & 205 & 1600 & 18.8 & 18.8 & & 0.3702 & $3.50 \mathrm{E}-03$ & 33.5227 & 18.8565 & 52.3792 & \\
\hline & 7/23/2008 17:00 & 28 & 205 & 1700 & 19.79 & 19.79 & & 0.3017 & 2.72E-03 & 26.0540 & 14.6554 & 40.7094 & \\
\hline & $7 / 23 / 2008$ 18:00 & 29 & 205 & 1800 & 21.22 & 21.22 & & 0.2139 & $1.78 \mathrm{E}-03$ & 17.0682 & 9.6009 & 26.6690 & \\
\hline & $7 / 23 / 2008$ 19:00 & 30 & 205 & 1900 & 21.27 & 21.27 & & 0.2111 & 1.75E-03 & 16.7884 & 9.4435 & 26.2318 & \\
\hline & $7 / 23 / 200820: 00$ & 31 & 205 & 2000 & 21.83 & 21.83 & & 0.1800 & 1.44E-03 & 13.8006 & 7.7628 & 21.5634 & \\
\hline & 7/23/2008 21:00 & 32 & 205 & 2100 & 23.07 & 23.07 & & 0.1166 & 8.45E-04 & 8.0852 & 4.5479 & 12.6331 & \\
\hline & $7 / 23 / 200822: 00$ & 33 & 205 & 2200 & 24.22 & 24.22 & & 0.0636 & $4.00 \mathrm{E}-04$ & 3.8326 & 2.1558 & 5.9885 & \\
\hline & $7 / 23 / 2008$ 23:00 & 34 & 205 & 2300 & 24.14 & 24.14 & & 0.0671 & $4.28 \mathrm{E}-04$ & 4.0958 & 2.3039 & 6.3997 & \\
\hline & $7 / 24 / 20080: 00$ & 35 & 206 & 0 & 24.02 & 24.02 & 26.24 & 0.0724 & 4.70E-04 & 4.4999 & 2.5312 & 7.0311 & \\
\hline & 7/24/2008 1:00 & 36 & 206 & 100 & 24.25 & 24.25 & & 0.0623 & $3.90 \mathrm{E}-04$ & 3.7352 & 2.1011 & 5.8363 & \\
\hline & 7/24/2008 2:00 & 37 & 206 & 200 & 24.54 & 24.54 & & 0.0497 & $2.96 \mathrm{E}-04$ & 2.8311 & 1.5925 & 4.4235 & \\
\hline & 7/24/2008 3:00 & 38 & 206 & 300 & 24.84 & 24.84 & & 0.0370 & $2.06 \mathrm{E}-04$ & 1.9705 & 1.1084 & 3.0788 & \\
\hline & 7/24/2008 4:00 & 39 & 206 & 400 & 25.21 & 25.21 & & 0.0218 & 1.07E-04 & 1.0271 & 0.5778 & 1.6049 & \\
\hline & $7 / 24 / 20085: 00$ & 40 & 206 & 500 & 25.45 & 25.45 & & 0.0122 & 5.24E-05 & 0.5012 & 0.2819 & 0.7832 & \\
\hline
\end{tabular}




\begin{tabular}{|c|c|c|c|c|c|c|c|c|c|c|c|c|c|}
\hline Tree ID & TS & RN & JD & HR & $\begin{array}{c}\text { Raw } \\
\text { TC_dTA }\end{array}$ & $\begin{array}{c}\text { Fixed } \\
\text { TC_dT } \Delta\end{array}$ & dTM & $\mathbf{K}$ & $\begin{array}{l}\text { Sap } \\
\text { Velocity } \\
(\mathrm{cm} / \mathrm{s})\end{array}$ & $\begin{array}{c}\text { Probed } \\
\text { Drywood F } \\
(\mathrm{g} / \mathrm{hr})\end{array}$ & $\begin{array}{c}\text { Extrapolated } \\
\text { Wetwood F } \\
(\mathrm{g} / \mathrm{hr})\end{array}$ & $\begin{array}{c}\text { Extrapolated } \\
\text { Total Wood F } \\
(\mathrm{g} / \mathrm{hr})\end{array}$ & $\begin{array}{l}\text { Relative } \\
\text { Humidity, } \\
@ 10 \mathrm{~m}(\%)\end{array}$ \\
\hline & 7/24/2008 6:00 & 41 & 206 & 600 & 25.41 & 25.41 & & 0.0138 & 6.09E-05 & 0.5831 & 0.3280 & 0.9111 & \\
\hline & 7/24/2008 7:00 & 42 & 206 & 700 & 25.42 & 25.42 & & 0.0134 & $5.88 \mathrm{E}-05$ & 0.5624 & 0.3164 & 0.8788 & \\
\hline & 7/24/2008 8:00 & 43 & 206 & 800 & 24.71 & 24.71 & & 0.0425 & $2.44 \mathrm{E}-04$ & 2.3336 & 1.3127 & 3.6463 & \\
\hline & 7/24/2008 9:00 & 44 & 206 & 900 & 24.09 & 24.09 & & 0.0693 & 4.45E-04 & 4.2628 & 2.3978 & 6.6607 & \\
\hline & $7 / 24 / 2008$ 10:00 & 45 & 206 & 1000 & 24.08 & 24.08 & & 0.0698 & 4.49E-04 & 4.2965 & 2.4168 & 6.7132 & \\
\hline & 7/24/2008 11:00 & 46 & 206 & 1100 & 20.97 & 20.97 & & 0.2284 & $1.93 E-03$ & 18.5004 & 10.4065 & 28.9068 & \\
\hline & 7/24/2008 12:00 & 47 & 206 & 1200 & 19.11 & 19.11 & & 0.3480 & $3.24 \mathrm{E}-03$ & 31.0625 & 17.4727 & 48.5352 & \\
\hline & 7/24/2008 13:00 & 48 & 206 & 1300 & $\mid 19.44$ & 19.44 & & 0.3251 & $2.98 \mathrm{E}-03$ & 28.5675 & 16.0692 & 44.6368 & \\
\hline & 7/24/2008 14:00 & 49 & 206 & 1400 & $\mid 19.04$ & 19.04 & & 0.3529 & $3.30 \mathrm{E}-03$ & 31.6080 & 17.7795 & 49.3874 & \\
\hline & $7 / 24 / 2008$ 15:00 & 50 & 206 & 1500 & 18.21 & 18.21 & & 0.4146 & 4.03E-03 & 38.5377 & 21.6775 & 60.2152 & \\
\hline & 7/24/2008 16:00 & 51 & 206 & 1600 & 18.11 & 18.11 & & 0.4224 & $4.12 \mathrm{E}-03$ & 39.4334 & 22.1813 & 61.6147 & \\
\hline & 7/24/2008 17:00 & 52 & 206 & 1700 & 18.51 & 18.51 & & 0.3917 & 3.75E-03 & 35.9314 & 20.2114 & 56.1428 & \\
\hline & $7 / 24 / 2008$ 18:00 & 53 & 206 & 1800 & 18.89 & 18.89 & & 0.3637 & $3.43 E-03$ & 32.7965 & 18.4480 & 51.2445 & \\
\hline & 7/24/2008 19:00 & 54 & 206 & 1900 & 19.83 & 19.83 & & 0.2990 & $2.69 \mathrm{E}-03$ & 25.7751 & 14.4985 & 40.2737 & \\
\hline & $7 / 24 / 200820: 00$ & 55 & 206 & 2000 & 21.66 & 21.66 & & 0.1893 & $1.53 \mathrm{E}-03$ & 14.6797 & 8.2573 & 22.9370 & \\
\hline & $7 / 24 / 200821: 00$ & 56 & 206 & 2100 & 24.3 & 24.3 & & 0.0601 & 3.73E-04 & 3.5745 & 2.0106 & 5.5851 & \\
\hline & $7 / 24 / 200822: 00$ & 57 & 206 & 2200 & 25.9 & 25.9 & & -0.0054 & $0.00 E+00$ & 0.0000 & 0.0000 & 0.0000 & \\
\hline & $7 / 24 / 200823: 00$ & 58 & 206 & 2300 & 26.24 & 26.24 & & -0.0183 & $0.00 \mathrm{E}+00$ & 0.0000 & 0.0000 & 0.0000 & \\
\hline & 7/25/2008 0:00 & 59 & 207 & 0 & 26.28 & 26.28 & 26.28 & -0.0198 & $0.00 \mathrm{E}+00$ & 0.0000 & 0.0000 & 0.0000 & \\
\hline & 7/25/2008 1:00 & 60 & 207 & 100 & 26.17 & 26.17 & & -0.0157 & $0.00 \mathrm{E}+00$ & 0.0000 & 0.0000 & 0.0000 & \\
\hline & 7/25/2008 2:00 & 61 & 207 & 200 & 26.04 & 26.04 & & -0.0108 & $0.00 \mathrm{E}+00$ & 0.0000 & 0.0000 & 0.0000 & \\
\hline & 7/25/2008 3:00 & 62 & 207 & 300 & 26.04 & 26.04 & & -0.0108 & $0.00 \mathrm{E}+00$ & 0.0000 & 0.0000 & 0.0000 & \\
\hline & 7/25/2008 4:00 & 63 & 207 & 400 & 26.15 & 26.15 & & -0.0149 & $0.00 E+00$ & 0.0000 & 0.0000 & 0.0000 & \\
\hline & 7/25/2008 5:00 & 64 & 207 & 500 & 26.24 & 26.24 & & -0.0183 & $0.00 \mathrm{E}+00$ & 0.0000 & 0.0000 & 0.0000 & \\
\hline & 7/25/2008 6:00 & 65 & 207 & 600 & 26.27 & 26.27 & & -0.0194 & $0.00 \mathrm{E}+00$ & 0.0000 & 0.0000 & 0.0000 & \\
\hline & 7/25/2008 7:00 & 66 & 207 & 700 & 26.22 & 26.22 & & -0.0175 & $0.00 \mathrm{E}+00$ & 0.0000 & 0.0000 & 0.0000 & \\
\hline & 7/25/2008 8:00 & 67 & 207 & 800 & 26.08 & 26.08 & & -0.0123 & $0.00 \mathrm{E}+00$ & 0.0000 & 0.0000 & 0.0000 & \\
\hline & 7/25/2008 9:00 & 68 & 207 & 900 & 25.71 & 25.71 & & 0.0019 & 5.47E-06 & 0.0524 & 0.0295 & 0.0818 & \\
\hline & 7/25/2008 10:00 & 69 & 207 & 1000 & 25.29 & 25.29 & & 0.0186 & 8.81E-05 & 0.8431 & 0.4743 & 1.3174 & \\
\hline & 7/25/2008 11:00 & 70 & 207 & 1100 & 23.29 & 23.29 & & 0.1061 & 7.52E-04 & 7.1945 & 4.0469 & 11.2414 & \\
\hline & $7 / 25 / 2008$ 12:00 & 71 & 207 & 1200 & 21.43 & 21.43 & & 0.2021 & 1.66E-03 & 15.9076 & 8.9480 & 24.8555 & \\
\hline & $7 / 25 / 2008$ 13:00 & 72 & 207 & 1300 & 20.05 & 20.05 & & 0.2848 & 2.54E-03 & 24.2712 & 13.6525 & 37.9237 & \\
\hline & $7 / 25 / 200814: 00$ & 73 & 207 & 1400 & 18.72 & 18.72 & & 0.3761 & $3.57 \mathrm{E}-03$ & 34.1766 & 19.2243 & 53.4009 & \\
\hline & $7 / 25 / 200815: 00$ & 74 & 207 & 1500 & 18.28 & 18.28 & & 0.4092 & $3.96 \mathrm{E}-03$ & 37.9189 & 21.3294 & 59.2482 & \\
\hline & 7/25/2008 16:00 & 75 & 207 & 1600 & 18.54 & 18.54 & & 0.3894 & 3.73E-03 & 35.6772 & 20.0684 & 55.7457 & \\
\hline & $7 / 25 / 2008$ 17:00 & 76 & 207 & 1700 & 17.78 & 17.78 & & 0.4488 & 4.44E-03 & 42.4888 & 23.9000 & 66.3888 & \\
\hline & $7 / 25 / 2008$ 18:00 & 77 & 207 & 1800 & 18.65 & 18.65 & & 0.3812 & 3.63E-03 & 34.7553 & 19.5498 & 54.3051 & \\
\hline & 7/25/2008 19:00 & 78 & 207 & 1900 & 18.78 & 18.78 & & 0.3717 & $3.52 \mathrm{E}-03$ & 33.6854 & 18.9480 & 52.6334 & \\
\hline & $7 / 25 / 200820: 00$ & 79 & 207 & 2000 & 20.68 & 20.68 & & 0.2456 & $2.11 \mathrm{E}-03$ & 20.2325 & 11.3808 & 31.6134 & \\
\hline & $7 / 25 / 2008$ 21:00 & 80 & 207 & 2100 & 23.99 & 23.99 & & 0.0738 & 4.81E-04 & 4.6027 & 2.5890 & 7.1917 & \\
\hline & $7 / 25 / 200822: 00$ & 81 & 207 & 2200 & 25.97 & 25.97 & & -0.0081 & $0.00 \mathrm{E}+00$ & 0.0000 & 0.0000 & 0.0000 & \\
\hline
\end{tabular}




\begin{tabular}{|c|c|c|c|c|c|c|c|c|c|c|c|c|c|}
\hline Tree ID & TS & RN & JD & HR & $\begin{array}{c}\text { Raw } \\
\text { TC_dTA }\end{array}$ & $\begin{array}{c}\text { Fixed } \\
\text { TC_dT } \Delta\end{array}$ & dTM & K & $\begin{array}{l}\text { Sap } \\
\text { Velocity } \\
(\mathrm{cm} / \mathrm{s})\end{array}$ & $\begin{array}{c}\text { Probed } \\
\text { Drywood F } \\
(\mathrm{g} / \mathrm{hr})\end{array}$ & $\begin{array}{c}\text { Extrapolated } \\
\text { Wetwood F } \\
(\mathrm{g} / \mathrm{hr})\end{array}$ & $\begin{array}{c}\text { Extrapolated } \\
\text { Total Wood F } \\
(\mathrm{g} / \mathrm{hr})\end{array}$ & $\begin{array}{l}\text { Relative } \\
\text { Humidity, } \\
@ 10 \mathrm{~m}(\%)\end{array}$ \\
\hline & 7/25/2008 23:00 & 82 & 207 & 2300 & \begin{tabular}{|r|}
26.19 \\
\end{tabular} & 26.19 & & -0.0164 & $0.00 \mathrm{E}+00$ & 0.0000 & 0.0000 & 0.0000 & \\
\hline & $7 / 26 / 20080: 00$ & 83 & 208 & 0 & 26.19 & 26.19 & 26.19 & -0.0164 & $0.00 \mathrm{E}+00$ & 0.0000 & 0.0000 & 0.0000 & \\
\hline & 7/26/2008 1:00 & 84 & 208 & 100 & 26.15 & 26.15 & & -0.0149 & $0.00 E+00$ & 0.0000 & 0.0000 & 0.0000 & \\
\hline & $7 / 26 / 20082: 00$ & 85 & 208 & 200 & 26.11 & 26.11 & & -0.0134 & $0.00 \mathrm{E}+00$ & 0.0000 & 0.0000 & 0.0000 & \\
\hline & 7/26/2008 3:00 & 86 & 208 & 300 & 26.09 & 26.09 & & -0.0126 & $0.00 \mathrm{E}+00$ & 0.0000 & 0.0000 & 0.0000 & \\
\hline & 7/26/2008 4:00 & 87 & 208 & 400 & 26.09 & 26.09 & & -0.0126 & $0.00 \mathrm{E}+00$ & 0.0000 & 0.0000 & 0.0000 & \\
\hline & 7/26/2008 5:00 & 88 & 208 & 500 & 26.07 & 26.07 & & -0.0119 & $0.00 \mathrm{E}+00$ & 0.0000 & 0.0000 & 0.0000 & \\
\hline & 7/26/2008 6:00 & 89 & 208 & 600 & 26 & 26 & & -0.0092 & $0.00 \mathrm{E}+00$ & 0.0000 & 0.0000 & 0.0000 & \\
\hline & 7/26/2008 7:00 & 90 & 208 & 700 & 25.96 & 25.96 & & -0.0077 & $0.00 \mathrm{E}+00$ & 0.0000 & 0.0000 & 0.0000 & \\
\hline & 7/26/2008 8:00 & 91 & 208 & 800 & 25.86 & 25.86 & & -0.0039 & $0.00 E+00$ & 0.0000 & 0.0000 & 0.0000 & \\
\hline & 7/26/2008 9:00 & 92 & 208 & 900 & 25.25 & 25.25 & & 0.0202 & 9.76E-05 & 0.9341 & 0.5255 & 1.4596 & \\
\hline & $7 / 26 / 2008$ 10:00 & 93 & 208 & 1000 & 21.34 & 21.34 & & 0.2071 & 1.71E-03 & 16.4003 & 9.2252 & 25.6255 & \\
\hline & $7 / 26 / 2008$ 11:00 & 94 & 208 & 1100 & 20.52 & 20.52 & & 0.2554 & $2.22 \mathrm{E}-03$ & 21.2218 & 11.9372 & 33.1590 & \\
\hline & $7 / 26 / 2008$ 12:00 & 95 & 208 & 1200 & 20.62 & 20.62 & & 0.2493 & 2.15E-03 & 20.6007 & 11.5879 & 32.1885 & \\
\hline & $7 / 26 / 2008$ 13:00 & 96 & 208 & 1300 & 19.09 & 19.09 & & 0.3494 & $3.26 \mathrm{E}-03$ & 31.2178 & 17.5600 & 48.7778 & \\
\hline & $7 / 26 / 200814: 00$ & 97 & 208 & 1400 & 18.88 & 18.88 & & 0.3644 & 3.43E-03 & 32.8767 & 18.4931 & 51.3698 & \\
\hline & $7 / 26 / 2008$ 15:00 & 98 & 208 & 1500 & 18.43 & 18.43 & & 0.3977 & 3.82E-03 & 36.6148 & 20.5958 & 57.2106 & \\
\hline & $7 / 26 / 200816: 00$ & 99 & 208 & 1600 & 18.55 & 18.55 & & 0.3887 & $3.72 \mathrm{E}-03$ & 35.5928 & 20.0209 & 55.6137 & \\
\hline & $7 / 26 / 200817: 00$ & 100 & 208 & 1700 & 17.62 & 17.62 & & 0.4620 & 4.60E-03 & 44.0271 & 24.7653 & 68.7924 & \\
\hline & $7 / 26 / 2008$ 18:00 & 101 & 208 & 1800 & 18.97 & 18.97 & & 0.3579 & 3.36E-03 & 32.1592 & 18.0896 & 50.2488 & \\
\hline & $7 / 26 / 2008$ 19:00 & 102 & 208 & 1900 & 19.25 & 19.25 & & 0.3382 & $3.13 E-03$ & 29.9888 & 16.8687 & 46.8575 & \\
\hline & $7 / 26 / 200820: 00$ & 103 & 208 & 2000 & 20.91 & 20.91 & & 0.2319 & 1.97E-03 & 18.8524 & 10.6045 & 29.4569 & \\
\hline & $7 / 26 / 200821: 00$ & 104 & 208 & 2100 & 24.01 & 24.01 & & 0.0729 & 4.74E-04 & 4.5341 & 2.5504 & 7.0845 & \\
\hline & $7 / 26 / 200822: 00$ & 105 & 208 & 2200 & 25.76 & 25.76 & & 0.0000 & $0.00 E+00$ & 0.0000 & 0.0000 & 0.0000 & \\
\hline & $7 / 26 / 2008$ 23:00 & 106 & 208 & 2300 & 26.1 & 26.1 & & -0.0130 & $0.00 \mathrm{E}+00$ & 0.0000 & 0.0000 & 0.0000 & \\
\hline & $7 / 27 / 20080: 00$ & 107 & 209 & 0 & 26.14 & 26.14 & 26.14 & -0.0145 & $0.00 \mathrm{E}+00$ & 0.0000 & 0.0000 & 0.0000 & \\
\hline & 7/27/2008 1:00 & 108 & 209 & 100 & 26.09 & 26.09 & & -0.0126 & $0.00 \mathrm{E}+00$ & 0.0000 & 0.0000 & 0.0000 & \\
\hline & 7/27/2008 2:00 & 109 & 209 & 200 & 25.94 & 25.94 & & -0.0069 & $0.00 \mathrm{E}+00$ & 0.0000 & 0.0000 & 0.0000 & \\
\hline & 7/27/2008 3:00 & 110 & 209 & 300 & 25.82 & 25.82 & & -0.0023 & $0.00 \mathrm{E}+00$ & 0.0000 & 0.0000 & 0.0000 & \\
\hline & 7/27/2008 4:00 & 111 & 209 & 400 & 25.96 & 25.96 & & -0.0077 & $0.00 \mathrm{E}+00$ & 0.0000 & 0.0000 & 0.0000 & \\
\hline & 7/27/20085:00 & 112 & 209 & 500 & 26.12 & 26.12 & & -0.0138 & $0.00 \mathrm{E}+00$ & 0.0000 & 0.0000 & 0.0000 & \\
\hline & 7/27/2008 6:00 & 113 & 209 & 600 & 26.13 & 26.13 & & -0.0142 & $0.00 \mathrm{E}+00$ & 0.0000 & 0.0000 & 0.0000 & \\
\hline & 7/27/2008 7:00 & 114 & 209 & 700 & 26.1 & 26.1 & & -0.0130 & $0.00 \mathrm{E}+00$ & 0.0000 & 0.0000 & 0.0000 & \\
\hline & 7/27/2008 8:00 & 115 & 209 & 800 & 26.01 & 26.01 & & -0.0096 & $0.00 \mathrm{E}+00$ & 0.0000 & 0.0000 & 0.0000 & \\
\hline & $7 / 27 / 20089: 00$ & 116 & 209 & 900 & 23.87 & 23.87 & & 0.0792 & 5.24E-04 & 5.0207 & 2.8241 & 7.8448 & \\
\hline & $7 / 27 / 2008$ 10:00 & 117 & 209 & 1000 & 20.03 & 20.03 & & 0.2861 & 2.55E-03 & 24.4059 & 13.7283 & 38.1342 & \\
\hline & $7 / 27 / 2008$ 11:00 & 118 & 209 & 1100 & 19.36 & 19.36 & & 0.3306 & 3.05E-03 & 29.1610 & 16.4030 & 45.5640 & \\
\hline & $7 / 27 / 2008$ 12:00 & 119 & 209 & 1200 & 18.29 & 18.29 & & 0.4084 & 3.95E-03 & 37.8310 & 21.2799 & 59.1109 & \\
\hline & $7 / 27 / 200813: 00$ & 120 & 209 & 1300 & 18.04 & 18.04 & & 0.4279 & $4.19 \mathrm{E}-03$ & 40.0687 & 22.5386 & 62.6073 & \\
\hline & $7 / 27 / 2008$ 14:00 & 121 & 209 & 1400 & 17.57 & 17.57 & & 0.4661 & $4.65 \mathrm{E}-03$ & 44.5157 & 25.0401 & 69.5558 & \\
\hline & 7/27/2008 15:00 & 122 & 209 & 1500 & 17.21 & 17.21 & & 0.4968 & 5.03E-03 & 48.1481 & 27.0833 & 75.2314 & \\
\hline
\end{tabular}




\begin{tabular}{|c|c|c|c|c|c|c|c|c|c|c|c|c|c|}
\hline Tree ID & TS & RN & JD & HR & $\begin{array}{c}\text { Raw } \\
\text { TC_dTA }\end{array}$ & $\begin{array}{c}\text { Fixed } \\
\text { TC_dT } \Delta\end{array}$ & dTM & $\mathbf{K}$ & $\begin{array}{l}\text { Sap } \\
\text { Velocity } \\
(\mathrm{cm} / \mathrm{s})\end{array}$ & $\begin{array}{c}\text { Probed } \\
\text { Drywood F } \\
(\mathrm{g} / \mathrm{hr})\end{array}$ & $\begin{array}{c}\text { Extrapolated } \\
\text { Wetwood F } \\
(\mathrm{g} / \mathrm{hr})\end{array}$ & $\begin{array}{c}\text { Extrapolated } \\
\text { Total Wood F } \\
(\mathrm{g} / \mathrm{hr})\end{array}$ & $\begin{array}{l}\text { Relative } \\
\text { Humidity, } \\
@ 10 \mathrm{~m}(\%)\end{array}$ \\
\hline & 7/27/2008 16:00 & 123 & 209 & 1600 & \begin{tabular}{|r|}
17.42 \\
\end{tabular} & 17.42 & & 0.4788 & 4.81E-03 & 46.0045 & 25.8775 & 71.8820 & \\
\hline & $7 / 27 / 2008$ 17:00 & 124 & 209 & 1700 & 17.23 & 17.23 & & 0.4951 & $5.01 \mathrm{E}-03$ & 47.9409 & 26.9667 & 74.9076 & \\
\hline & $7 / 27 / 2008$ 18:00 & 125 & 209 & 1800 & 17.58 & 17.58 & & 0.4653 & $4.64 \mathrm{E}-03$ & 44.4177 & 24.9850 & 69.4027 & \\
\hline & $7 / 27 / 2008$ 19:00 & 126 & 209 & 1900 & 17.89 & 17.89 & & 0.4399 & 4.33E-03 & 41.4531 & 23.3173 & 64.7704 & \\
\hline & $7 / 27 / 200820: 00$ & 127 & 209 & 2000 & 19.19 & 19.19 & & 0.3424 & 3.18E-03 & 30.4462 & 17.1260 & 47.5721 & \\
\hline & $7 / 27 / 2008$ 21:00 & 128 & 209 & 2100 & 22.29 & 22.29 & & 0.1557 & $1.21 \mathrm{E}-03$ & 11.5398 & 6.4911 & 18.0309 & \\
\hline & $7 / 27 / 2008$ 22:00 & 129 & 209 & 2200 & 24.64 & 24.64 & & 0.0455 & $2.65 E-04$ & 2.5354 & 1.4262 & 3.9616 & \\
\hline & $7 / 27 / 200823: 00$ & 130 & 209 & 2300 & 24.86 & 24.86 & & 0.0362 & 2.00E-04 & 1.9160 & 1.0777 & 2.9937 & \\
\hline & $7 / 28 / 20080: 00$ & 131 & 210 & 0 & 24.65 & 24.65 & 26.03 & 0.0450 & 2.62E-04 & 2.5063 & 1.4098 & 3.9162 & \\
\hline & 7/28/2008 1:00 & 132 & 210 & 100 & 25.19 & 25.19 & & 0.0226 & 1.12E-04 & 1.0743 & 0.6043 & 1.6787 & \\
\hline & $7 / 28 / 20082: 00$ & 133 & 210 & 200 & 25.21 & 25.21 & & 0.0218 & 1.07E-04 & 1.0271 & 0.5778 & 1.6049 & \\
\hline & 7/28/2008 3:00 & 134 & 210 & 300 & 25.84 & 25.84 & & -0.0031 & $0.00 \mathrm{E}+00$ & 0.0000 & 0.0000 & 0.0000 & \\
\hline & 7/28/2008 4:00 & 135 & 210 & 400 & 25.94 & 25.94 & & -0.0069 & $0.00 \mathrm{E}+00$ & 0.0000 & 0.0000 & 0.0000 & \\
\hline & 7/28/2008 5:00 & 136 & 210 & 500 & 26.03 & 26.03 & & -0.0104 & $0.00 \mathrm{E}+00$ & 0.0000 & 0.0000 & 0.0000 & \\
\hline & 7/28/2008 6:00 & 137 & 210 & 600 & 25.93 & 25.93 & & -0.0066 & $0.00 \mathrm{E}+00$ & 0.0000 & 0.0000 & 0.0000 & \\
\hline & 7/28/2008 7:00 & 138 & 210 & 700 & 25.41 & 25.41 & & 0.0138 & $6.09 \mathrm{E}-05$ & 0.5831 & 0.3280 & 0.9111 & \\
\hline & 7/28/2008 8:00 & 139 & 210 & 800 & 22.1 & 22.1 & & 0.1656 & 1.30E-03 & 12.4530 & 7.0048 & 19.4578 & \\
\hline & $7 / 28 / 20089: 00$ & 140 & 210 & 900 & 19.95 & 19.95 & & 0.2912 & 2.61E-03 & 24.9486 & 14.0336 & 38.9822 & \\
\hline & $7 / 28 / 2008$ 10:00 & 141 & 210 & 1000 & 18.41 & 18.41 & & 0.3992 & 3.84E-03 & 36.7869 & 20.6926 & 57.4796 & \\
\hline & $7 / 28 / 2008$ 11:00 & 142 & 210 & 1100 & 18.04 & 18.04 & & 0.4279 & 4.19E-03 & 40.0687 & 22.5386 & 62.6073 & \\
\hline & $7 / 28 / 200812: 00$ & 143 & 210 & 1200 & 17.73 & 17.73 & & 0.4529 & 4.49E-03 & 42.9655 & 24.1681 & 67.1335 & \\
\hline & 7/28/2008 13:00 & 144 & 210 & 1300 & 17.66 & 17.66 & & 0.4587 & 4.56E-03 & 43.6390 & 24.5469 & 68.1859 & \\
\hline & $7 / 28 / 2008$ 14:00 & 145 & 210 & 1400 & 17.41 & 17.41 & & 0.4796 & 4.82E-03 & 46.1050 & 25.9340 & 72.0390 & \\
\hline & $7 / 28 / 2008$ 15:00 & 146 & 210 & 1500 & 17.11 & 17.11 & & 0.5056 & $5.14 \mathrm{E}-03$ & 49.1939 & 27.6716 & 76.8654 & \\
\hline & $7 / 28 / 2008$ 16:00 & 147 & 210 & 1600 & 17.29 & 17.29 & & 0.4899 & 4.94E-03 & 47.3232 & 26.6193 & 73.9425 & \\
\hline & 7/28/2008 17:00 & 148 & 210 & 1700 & 17.13 & 17.13 & & 0.5038 & 5.12E-03 & 48.9834 & 27.5532 & 76.5366 & \\
\hline & $7 / 28 / 2008$ 18:00 & 149 & 210 & 1800 & 17.54 & 17.54 & & 0.4686 & 4.68E-03 & 44.8107 & 25.2060 & 70.0167 & \\
\hline & $7 / 28 / 2008$ 19:00 & 150 & 210 & 1900 & 18.11 & 18.11 & & 0.4224 & $4.12 \mathrm{E}-03$ & 39.4334 & 22.1813 & 61.6147 & \\
\hline & $7 / 28 / 200820: 00$ & 151 & 210 & 2000 & 20.75 & 20.75 & & 0.2414 & 2.07E-03 & 19.8073 & 11.1416 & 30.9490 & \\
\hline & $7 / 28 / 200821: 00$ & 152 & 210 & 2100 & 23.9 & 23.9 & & 0.0778 & $5.13 E-04$ & 4.9152 & 2.7648 & 7.6799 & \\
\hline & $7 / 28 / 2008$ 22:00 & 153 & 210 & 2200 & 23.97 & 23.97 & & 0.0747 & 4.88E-04 & 4.6716 & 2.6278 & 7.2994 & \\
\hline & $7 / 28 / 200823: 00$ & 154 & 210 & 2300 & 24.01 & 24.01 & & 0.0729 & 4.74E-04 & 4.5341 & 2.5504 & 7.0845 & \\
\hline & $7 / 29 / 20080: 00$ & 155 & 211 & 0 & 24.59 & 24.59 & 26.05 & 0.0476 & 2.80E-04 & 2.6822 & 1.5087 & 4.1909 & \\
\hline & 7/29/2008 1:00 & 156 & 211 & 100 & 25.05 & 25.05 & & 0.0283 & $1.48 \mathrm{E}-04$ & 1.4176 & 0.7974 & 2.2149 & \\
\hline & 7/29/2008 2:00 & 157 & 211 & 200 & 25.5 & 25.5 & & 0.0102 & $4.21 E-05$ & 0.4027 & 0.2265 & 0.6292 & \\
\hline & 7/29/2008 3:00 & 158 & 211 & 300 & 25.61 & 25.61 & & 0.0059 & 2.13E-05 & 0.2035 & 0.1145 & 0.3180 & \\
\hline & 7/29/2008 4:00 & 159 & 211 & 400 & 25.99 & 25.99 & & -0.0088 & $0.00 \mathrm{E}+00$ & 0.0000 & 0.0000 & 0.0000 & \\
\hline & 7/29/2008 5:00 & 160 & 211 & 500 & 26.05 & 26.05 & & -0.0111 & $0.00 \mathrm{E}+00$ & 0.0000 & 0.0000 & 0.0000 & \\
\hline & $7 / 29 / 20086: 00$ & 161 & 211 & 600 & 25.94 & 25.94 & & -0.0069 & $0.00 \mathrm{E}+00$ & 0.0000 & 0.0000 & 0.0000 & \\
\hline & 7/29/2008 7:00 & 162 & 211 & 700 & 25.15 & 25.15 & & 0.0243 & $1.22 \mathrm{E}-04$ & 1.1702 & 0.6582 & 1.8284 & \\
\hline & 7/29/2008 8:00 & 163 & 211 & 800 & 23.39 & 23.39 & & 0.1013 & 7.11E-04 & 6.8016 & 3.8259 & 10.6276 & \\
\hline
\end{tabular}




\begin{tabular}{|c|c|c|c|c|c|c|c|c|c|c|c|c|c|}
\hline Tree ID & TS & RN & JD & HR & $\begin{array}{c}\text { Raw } \\
\text { TC_dTA }\end{array}$ & $\begin{array}{c}\text { Fixed } \\
\text { TC_dT } \Delta\end{array}$ & dTM & $\mathbf{K}$ & $\begin{array}{l}\text { Sap } \\
\text { Velocity } \\
(\mathrm{cm} / \mathrm{s})\end{array}$ & $\begin{array}{c}\text { Probed } \\
\text { Drywood F } \\
(\mathrm{g} / \mathrm{hr})\end{array}$ & $\begin{array}{c}\text { Extrapolated } \\
\text { Wetwood F } \\
(\mathrm{g} / \mathrm{hr})\end{array}$ & $\begin{array}{c}\text { Extrapolated } \\
\text { Total Wood F } \\
(\mathrm{g} / \mathrm{hr})\end{array}$ & $\begin{array}{l}\text { Relative } \\
\text { Humidity, } \\
@ 10 \mathrm{~m}(\%)\end{array}$ \\
\hline & 7/29/2008 9:00 & 164 & 211 & 900 & 21.47 & 21.47 & & 0.1998 & 1.64E-03 & 15.6908 & 8.8261 & $\begin{array}{r}24.5169 \\
\end{array}$ & \\
\hline & $7 / 29 / 2008$ 10:00 & 165 & 211 & 1000 & 19.65 & 19.65 & & 0.3109 & 2.83E-03 & 27.0435 & 15.2120 & 42.2554 & \\
\hline & 7/29/2008 11:00 & 166 & 211 & 1100 & 19.83 & 19.83 & & 0.2990 & 2.69E-03 & 25.7751 & 14.4985 & 40.2737 & \\
\hline & $7 / 29 / 2008$ 12:00 & 167 & 211 & 1200 & 18.48 & 18.48 & & 0.3939 & $3.78 \mathrm{E}-03$ & 36.1867 & 20.3550 & 56.5417 & \\
\hline & 7/29/2008 13:00 & 168 & 211 & 1300 & 18.34 & 18.34 & & 0.4046 & 3.91E-03 & 37.3936 & 21.0339 & 58.4275 & \\
\hline & $7 / 29 / 2008$ 14:00 & 169 & 211 & 1400 & 17.82 & 17.82 & & 0.4456 & 4.40E-03 & 42.1101 & 23.6870 & 65.7971 & \\
\hline & $7 / 29 / 2008$ 15:00 & 170 & 211 & 1500 & 18.15 & 18.15 & & 0.4193 & $4.08 E-03$ & 39.0735 & 21.9788 & 61.0524 & \\
\hline & $7 / 29 / 2008$ 16:00 & 171 & 211 & 1600 & 18.39 & 18.39 & & 0.4008 & 3.86E-03 & 36.9596 & 20.7898 & 57.7494 & \\
\hline & 7/29/2008 17:00 & 172 & 211 & 1700 & 18.9 & 18.9 & & 0.3630 & $3.42 \mathrm{E}-03$ & 32.7164 & 18.4030 & 51.1194 & \\
\hline & 7/29/2008 18:00 & 173 & 211 & 1800 & 19.1 & 19.1 & & 0.3487 & $3.25 E-03$ & 31.1401 & 17.5163 & 48.6564 & \\
\hline & $7 / 29 / 2008$ 19:00 & 174 & 211 & 1900 & 19.02 & 19.02 & & 0.3544 & $3.32 E-03$ & 31.7649 & 17.8677 & 49.6326 & \\
\hline & $7 / 29 / 200820: 00$ & 175 & 211 & 2000 & 20.82 & 20.82 & & 0.2373 & 2.03E-03 & 19.3867 & 10.9050 & 30.2917 & \\
\hline & 7/29/2008 21:00 & 176 & 211 & 2100 & 23.66 & 23.66 & & 0.0888 & 6.04E-04 & 5.7785 & 3.2504 & 9.0289 & \\
\hline & $7 / 29 / 2008$ 22:00 & 177 & 211 & 2200 & 25.46 & 25.46 & & 0.0118 & 5.03E-05 & 0.4812 & 0.2707 & 0.7518 & \\
\hline & 7/29/2008 23:00 & 178 & 211 & 2300 & 25.97 & 25.97 & & -0.0081 & $0.00 \mathrm{E}+00$ & 0.0000 & 0.0000 & 0.0000 & \\
\hline & $7 / 30 / 20080: 00$ & 179 & 212 & 0 & 26.07 & 26.07 & 26.07 & -0.0119 & $0.00 \mathrm{E}+00$ & 0.0000 & 0.0000 & 0.0000 & \\
\hline & 7/30/2008 1:00 & 180 & 212 & 100 & 26.05 & 26.05 & & -0.0111 & $0.00 \mathrm{E}+00$ & 0.0000 & 0.0000 & 0.0000 & \\
\hline & 7/30/2008 2:00 & 181 & 212 & 200 & 26.06 & 26.06 & & -0.0115 & $0.00 \mathrm{E}+00$ & 0.0000 & 0.0000 & 0.0000 & \\
\hline & 7/30/2008 3:00 & 182 & 212 & 300 & 26.05 & 26.05 & & -0.0111 & $0.00 \mathrm{E}+00$ & 0.0000 & 0.0000 & 0.0000 & \\
\hline & 7/30/2008 4:00 & 183 & 212 & 400 & 26.02 & 26.02 & & -0.0100 & $0.00 E+00$ & 0.0000 & 0.0000 & 0.0000 & \\
\hline & 7/30/2008 5:00 & 184 & 212 & 500 & 25.96 & 25.96 & & -0.0077 & $0.00 \mathrm{E}+00$ & 0.0000 & 0.0000 & 0.0000 & \\
\hline & 7/30/2008 6:00 & 185 & 212 & 600 & 25.95 & 25.95 & & -0.0073 & $0.00 E+00$ & 0.0000 & 0.0000 & 0.0000 & \\
\hline & 7/30/2008 7:00 & 186 & 212 & 700 & 25.99 & 25.99 & & -0.0088 & $0.00 \mathrm{E}+00$ & 0.0000 & 0.0000 & 0.0000 & \\
\hline & $7 / 30 / 20088: 00$ & 187 & 212 & 800 & 25.93 & 25.93 & & -0.0066 & $0.00 \mathrm{E}+00$ & 0.0000 & 0.0000 & 0.0000 & \\
\hline & 7/30/2008 9:00 & 188 & 212 & 900 & 24.53 & 24.53 & & 0.0501 & 2.99E-04 & 2.8611 & 1.6094 & 4.4704 & \\
\hline & $7 / 30 / 2008$ 10:00 & 189 & 212 & 1000 & 20.32 & 20.32 & & 0.2677 & 2.35E-03 & 22.4928 & 12.6522 & 35.1450 & \\
\hline & 7/30/2008 11:00 & 190 & 212 & 1100 & 19.2 & 19.2 & & 0.3417 & $3.17 \mathrm{E}-03$ & 30.3697 & 17.0829 & 47.4526 & \\
\hline & $7 / 30 / 2008$ 12:00 & 191 & 212 & 1200 & 18.72 & 18.72 & & 0.3761 & $3.57 \mathrm{E}-03$ & 34.1766 & 19.2243 & 53.4009 & \\
\hline & 7/30/2008 13:00 & 192 & 212 & 1300 & 18.22 & 18.22 & & 0.4138 & 4.02E-03 & 38.4489 & 21.6275 & 60.0764 & \\
\hline & $7 / 30 / 2008$ 14:00 & 193 & 212 & 1400 & 17.77 & 17.77 & & 0.4496 & 4.45E-03 & 42.5838 & 23.9534 & 66.5373 & \\
\hline & 7/30/2008 15:00 & 194 & 212 & 1500 & 17.9 & 17.9 & & 0.4391 & 4.32E-03 & 41.3598 & 23.2649 & 64.6246 & \\
\hline & $7 / 30 / 2008$ 16:00 & 195 & 212 & 1600 & 17.74 & 17.74 & & 0.4521 & 4.48E-03 & 42.8698 & 24.1143 & 66.9841 & \\
\hline & 7/30/2008 17:00 & 196 & 212 & 1700 & 17.52 & 17.52 & & 0.4703 & $4.70 \mathrm{E}-03$ & 45.0081 & 25.3171 & 70.3252 & \\
\hline & 7/30/2008 18:00 & 197 & 212 & 1800 & 18.26 & 18.26 & & 0.4107 & 3.98E-03 & 38.0950 & 21.4284 & 59.5234 & \\
\hline & $7 / 30 / 2008$ 19:00 & 198 & 212 & 1900 & 18.25 & 18.25 & & 0.4115 & 3.99E-03 & 38.1833 & 21.4781 & 59.6614 & \\
\hline & $7 / 30 / 200820: 00$ & 199 & 212 & 2000 & 19.8 & 19.8 & & 0.3010 & 2.71E-03 & 25.9841 & 14.6161 & 40.6002 & \\
\hline & 7/30/2008 21:00 & 200 & 212 & 2100 & 23.02 & 23.02 & & 0.1190 & 8.66E-04 & 8.2927 & 4.6646 & 12.9573 & \\
\hline & $7 / 30 / 2008$ 22:00 & 201 & 212 & 2200 & 24.84 & 24.84 & & 0.0370 & 2.06E-04 & 1.9705 & 1.1084 & 3.0788 & \\
\hline & 7/30/2008 23:00 & 202 & 212 & 2300 & 25.38 & 25.38 & & 0.0150 & $6.75 \mathrm{E}-05$ & 0.6462 & 0.3635 & 1.0097 & \\
\hline & $7 / 31 / 20080: 00$ & 203 & 213 & 0 & 25.63 & 25.63 & 25.84 & 0.0051 & 1.78E-05 & 0.1705 & 0.0959 & 0.2664 & \\
\hline & 7/31/2008 1:00 & 204 & 213 & 100 & 25.79 & 25.79 & & -0.0012 & $0.00 \mathrm{E}+00$ & 0.0000 & 0.0000 & 0.0000 & \\
\hline
\end{tabular}




\begin{tabular}{|c|c|c|c|c|c|c|c|c|c|c|c|c|c|}
\hline Tree ID & TS & RN & JD & HR & $\begin{array}{c}\text { Raw } \\
\text { TC_dTA }\end{array}$ & $\begin{array}{c}\text { Fixed } \\
\text { TC_dT } \Delta\end{array}$ & dTM & $\mathrm{K}$ & $\begin{array}{l}\text { Sap } \\
\text { Velocity } \\
(\mathrm{cm} / \mathrm{s})\end{array}$ & $\begin{array}{c}\text { Probed } \\
\text { Drywood F } \\
(\mathrm{g} / \mathrm{hr})\end{array}$ & $\begin{array}{c}\text { Extrapolated } \\
\text { Wetwood F } \\
(\mathrm{g} / \mathrm{hr})\end{array}$ & $\begin{array}{c}\text { Extrapolated } \\
\text { Total Wood F } \\
(\mathrm{g} / \mathrm{hr})\end{array}$ & $\begin{array}{l}\text { Relative } \\
\text { Humidity, } \\
@ 10 \mathrm{~m}(\%)\end{array}$ \\
\hline & 7/31/2008 2:00 & 205 & 213 & 200 & 25.75 & 25.75 & & 0.0004 & 7.53E-07 & 0.0072 & 0.0041 & 0.0113 & \\
\hline & 7/31/2008 3:00 & 206 & 213 & 300 & 25.67 & 25.67 & & 0.0035 & 1.13E-05 & 0.1082 & 0.0609 & 0.1691 & \\
\hline & 7/31/2008 4:00 & 207 & 213 & 400 & 25.84 & 25.84 & & -0.0031 & $0.00 E+00$ & 0.0000 & 0.0000 & 0.0000 & \\
\hline & 7/31/2008 5:00 & 208 & 213 & 500 & 25.78 & 25.78 & & -0.0008 & $0.00 \mathrm{E}+00$ & 0.0000 & 0.0000 & 0.0000 & \\
\hline & 7/31/2008 6:00 & 209 & 213 & 600 & 25.73 & 25.73 & & 0.0012 & 2.91E-06 & 0.0279 & 0.0157 & 0.0436 & \\
\hline & 7/31/2008 7:00 & 210 & 213 & 700 & 25.79 & 25.79 & & -0.0012 & $0.00 \mathrm{E}+00$ & 0.0000 & 0.0000 & 0.0000 & \\
\hline & $7 / 31 / 2008$ 8:00 & 211 & 213 & 800 & 25.61 & 25.61 & & 0.0059 & 2.13E-05 & 0.2035 & 0.1145 & 0.3180 & \\
\hline & $7 / 31 / 20089: 00$ & 212 & 213 & 900 & 23.17 & 23.17 & & 0.1118 & 8.02E-04 & 7.6758 & 4.3176 & 11.9934 & \\
\hline & $7 / 31 / 2008$ 10:00 & 213 & 213 & 1000 & 19.6 & 19.6 & & 0.3143 & $2.86 \mathrm{E}-03$ & 27.4020 & 15.4136 & 42.8156 & \\
\hline & 7/31/2008 11:00 & 214 & 213 & 1100 & 18.73 & 18.73 & & 0.3753 & 3.56E-03 & 34.0944 & 19.1781 & 53.2725 & \\
\hline & $7 / 31 / 2008$ 12:00 & 215 & 213 & 1200 & 17.9 & 17.9 & & 0.4391 & 4.32E-03 & 41.3598 & 23.2649 & 64.6246 & \\
\hline & 7/31/2008 13:00 & 216 & 213 & 1300 & 17.87 & 17.87 & & 0.4415 & $4.35 \mathrm{E}-03$ & 41.6401 & 23.4225 & 65.0626 & \\
\hline & 7/31/2008 14:00 & 217 & 213 & 1400 & 17.25 & 17.25 & & 0.4933 & 4.99E-03 & 47.7343 & 26.8506 & 74.5849 & \\
\hline & 7/31/2008 15:00 & 218 & 213 & 1500 & 16.93 & 16.93 & & 0.5216 & 5.34E-03 & 51.1182 & 28.7540 & 79.8723 & \\
\hline & $7 / 31 / 200816: 00$ & 219 & 213 & 1600 & 17.23 & 17.23 & & 0.4951 & 5.01E-03 & 47.9409 & 26.9667 & 74.9076 & \\
\hline & 7/31/2008 17:00 & 220 & 213 & 1700 & 17.05 & 17.05 & & 0.5109 & 5.21E-03 & 49.8293 & 28.0290 & 77.8583 & \\
\hline & $7 / 31 / 2008$ 18:00 & 221 & 213 & 1800 & 17.4 & 17.4 & & 0.4805 & 4.83E-03 & 46.2056 & 25.9907 & 72.1963 & \\
\hline & $7 / 31 / 2008$ 19:00 & 222 & 213 & 1900 & 17.98 & 17.98 & & 0.4327 & 4.24E-03 & 40.6186 & 22.8480 & 63.4665 & \\
\hline & $7 / 31 / 200820: 00$ & 223 & 213 & 2000 & 20.24 & 20.24 & & 0.2727 & 2.40E-03 & 23.0121 & 12.9443 & 35.9565 & \\
\hline & $7 / 31 / 200821: 00$ & 224 & 213 & 2100 & 22.03 & 22.03 & & 0.1693 & 1.34E-03 & 12.7967 & 7.1981 & 19.9948 & \\
\hline & $7 / 31 / 200822: 00$ & 225 & 213 & 2200 & 23.74 & 23.74 & & 0.0851 & $5.73 \mathrm{E}-04$ & 5.4859 & 3.0858 & 8.5717 & \\
\hline & 7/31/2008 23:00 & 226 & 213 & 2300 & 23.61 & 23.61 & & 0.0911 & $6.23 E-04$ & 5.9638 & 3.3547 & 9.3185 & \\
\hline & $8 / 1 / 20080: 00$ & 227 & 214 & 0 & 24.44 & 24.44 & 25.98 & 0.0540 & $3.28 E-04$ & 3.1351 & 1.7635 & 4.8986 & \\
\hline & 8/1/2008 1:00 & 228 & 214 & 100 & 25.52 & 25.52 & & 0.0094 & 3.81E-05 & 0.3645 & 0.2051 & 0.5696 & \\
\hline & 8/1/2008 2:00 & 229 & 214 & 200 & 25.79 & 25.79 & & -0.0012 & $0.00 \mathrm{E}+00$ & 0.0000 & 0.0000 & 0.0000 & \\
\hline & $8 / 1 / 2008$ 3:00 & 230 & 214 & 300 & 25.74 & 25.74 & & 0.0008 & 1.77E-06 & 0.0169 & 0.0095 & 0.0265 & \\
\hline & 8/1/2008 4:00 & 231 & 214 & 400 & 25.92 & 25.92 & & -0.0062 & $0.00 \mathrm{E}+00$ & 0.0000 & 0.0000 & 0.0000 & \\
\hline & 8/1/2008 5:00 & 232 & 214 & 500 & 25.98 & 25.98 & & -0.0085 & $0.00 \mathrm{E}+00$ & 0.0000 & 0.0000 & 0.0000 & \\
\hline & 8/1/2008 6:00 & 233 & 214 & 600 & 25.82 & 25.82 & & -0.0023 & $0.00 \mathrm{E}+00$ & 0.0000 & 0.0000 & 0.0000 & \\
\hline & 8/1/2008 7:00 & 234 & 214 & 700 & 25.8 & 25.8 & & -0.0016 & $0.00 \mathrm{E}+00$ & 0.0000 & 0.0000 & 0.0000 & \\
\hline & 8/1/2008 8:00 & 235 & 214 & 800 & 25.71 & 25.71 & & 0.0019 & 5.47E-06 & 0.0524 & 0.0295 & 0.0818 & \\
\hline & 8/1/2008 9:00 & 236 & 214 & 900 & 22.55 & 22.55 & & 0.1424 & 1.08E-03 & 10.3362 & 5.8141 & 16.1503 & \\
\hline & 8/1/2008 10:00 & 237 & 214 & 1000 & 19.31 & 19.31 & & 0.3340 & 3.09E-03 & 29.5355 & 16.6137 & 46.1493 & \\
\hline & 8/1/2008 11:00 & 238 & 214 & 1100 & 18.78 & 18.78 & & 0.3717 & $3.52 \mathrm{E}-03$ & 33.6854 & 18.9480 & 52.6334 & \\
\hline & 8/1/2008 12:00 & 239 & 214 & 1200 & 18.52 & 18.52 & & 0.3909 & 3.74E-03 & 35.8465 & 20.1637 & 56.0102 & \\
\hline & 8/1/2008 13:00 & 240 & 214 & 1300 & 17.91 & 17.91 & & 0.4383 & 4.31E-03 & 41.2666 & 23.2125 & 64.4791 & \\
\hline & 8/1/2008 14:00 & 241 & 214 & 1400 & 17.62 & 17.62 & & 0.4620 & $4.60 \mathrm{E}-03$ & 44.0271 & 24.7653 & 68.7924 & \\
\hline & 8/1/2008 15:00 & 242 & 214 & 1500 & 17.11 & 17.11 & & 0.5056 & 5.14E-03 & 49.1939 & 27.6716 & 76.8654 & \\
\hline & $8 / 1 / 200816: 00$ & 243 & 214 & 1600 & 17.47 & 17.47 & & 0.4745 & $4.75 \mathrm{E}-03$ & 45.5044 & 25.5962 & 71.1006 & \\
\hline & 8/1/2008 17:00 & 244 & 214 & 1700 & 17.38 & 17.38 & & 0.4822 & 4.85E-03 & 46.4074 & 26.1042 & 72.5115 & \\
\hline & 8/1/2008 18:00 & 245 & 214 & 1800 & 18.16 & 18.16 & & 0.4185 & 4.07E-03 & 38.9839 & 21.9284 & 60.9123 & \\
\hline
\end{tabular}




\begin{tabular}{|c|c|c|c|c|c|c|c|c|c|c|c|c|c|}
\hline Tree ID & TS & RN & JD & HR & $\begin{array}{c}\text { Raw } \\
\text { TC_dTA }\end{array}$ & $\begin{array}{c}\text { Fixed } \\
\text { TC_dT } \Delta\end{array}$ & dTM & $\mathbf{K}$ & $\begin{array}{l}\text { Sap } \\
\text { Velocity } \\
(\mathrm{cm} / \mathrm{s})\end{array}$ & $\begin{array}{c}\text { Probed } \\
\text { Drywood F } \\
(\mathrm{g} / \mathrm{hr})\end{array}$ & $\begin{array}{c}\text { Extrapolated } \\
\text { Wetwood F } \\
(\mathrm{g} / \mathrm{hr})\end{array}$ & $\begin{array}{c}\text { Extrapolated } \\
\text { Total Wood F } \\
(\mathrm{g} / \mathrm{hr})\end{array}$ & $\begin{array}{l}\text { Relative } \\
\text { Humidity, } \\
@ 10 \mathrm{~m}(\%)\end{array}$ \\
\hline & 8/1/2008 19:00 & 246 & 214 & 1900 & 18.62 & 18.62 & & 0.3835 & $3.66 \mathrm{E}-03$ & 35.0052 & 19.6904 & 54.6956 & \\
\hline & 8/1/2008 20:00 & 247 & 214 & 2000 & 21.34 & 21.34 & & 0.2071 & 1.71E-03 & 16.4003 & 9.2252 & 25.6255 & \\
\hline & 8/1/2008 21:00 & 248 & 214 & 2100 & 24.86 & 24.86 & & 0.0362 & $2.00 \mathrm{E}-04$ & 1.9160 & 1.0777 & 2.9937 & \\
\hline & 8/1/2008 22:00 & 249 & 214 & 2200 & 25.77 & 25.77 & & -0.0004 & $0.00 \mathrm{E}+00$ & 0.0000 & 0.0000 & 0.0000 & \\
\hline & 8/1/2008 23:00 & 250 & 214 & 2300 & 25.94 & 25.94 & & -0.0069 & $0.00 \mathrm{E}+00$ & 0.0000 & 0.0000 & 0.0000 & \\
\hline & 8/2/2008 0:00 & 251 & 215 & 0 & 25.89 & 25.89 & 26.06 & -0.0050 & $0.00 \mathrm{E}+00$ & 0.0000 & 0.0000 & 0.0000 & \\
\hline & 8/2/2008 1:00 & 252 & 215 & 100 & 26.01 & 26.01 & & -0.0096 & $0.00 \mathrm{E}+00$ & 0.0000 & 0.0000 & 0.0000 & \\
\hline & 8/2/2008 2:00 & 253 & 215 & 200 & 26.06 & 26.06 & & -0.0115 & $0.00 \mathrm{E}+00$ & 0.0000 & 0.0000 & 0.0000 & \\
\hline & 8/2/2008 3:00 & 254 & 215 & 300 & 26.06 & 26.06 & & -0.0115 & $0.00 \mathrm{E}+00$ & 0.0000 & 0.0000 & 0.0000 & \\
\hline & 8/2/2008 4:00 & 255 & 215 & 400 & 25.84 & 25.84 & & -0.0031 & $0.00 E+00$ & 0.0000 & 0.0000 & 0.0000 & \\
\hline & 8/2/2008 5:00 & 256 & 215 & 500 & 25.74 & 25.74 & & 0.0008 & 1.77E-06 & 0.0169 & 0.0095 & 0.0265 & \\
\hline & 8/2/2008 6:00 & 257 & 215 & 600 & 25.77 & 25.77 & & -0.0004 & $0.00 \mathrm{E}+00$ & 0.0000 & 0.0000 & 0.0000 & \\
\hline & 8/2/2008 7:00 & 258 & 215 & 700 & 25.37 & 25.37 & & 0.0154 & 6.97E-05 & 0.6675 & 0.3755 & 1.0430 & \\
\hline & 8/2/2008 8:00 & 259 & 215 & 800 & 23.42 & 23.42 & & 0.0999 & 6.98E-04 & 6.6853 & 3.7605 & 10.4457 & \\
\hline & 8/2/2008 9:00 & 260 & 215 & 900 & 20.8 & 20.8 & & 0.2385 & $2.04 \mathrm{E}-03$ & 19.5064 & 10.9723 & 30.4787 & \\
\hline & 8/2/2008 10:00 & 261 & 215 & 1000 & 19.44 & 19.44 & & 0.3251 & 2.98E-03 & 28.5675 & 16.0692 & 44.6368 & \\
\hline & 8/2/2008 11:00 & 262 & 215 & 1100 & 18.76 & 18.76 & & 0.3731 & 3.54E-03 & 33.8486 & 19.0399 & 52.8885 & \\
\hline & $8 / 2 / 200812: 00$ & 263 & 215 & 1200 & 18.46 & 18.46 & & 0.3954 & 3.80E-03 & 36.3575 & 20.4511 & 56.8086 & \\
\hline & 8/2/2008 13:00 & 264 & 215 & 1300 & 18.01 & 18.01 & & 0.4303 & 4.21E-03 & 40.3430 & 22.6929 & 63.0359 & \\
\hline & 8/2/2008 14:00 & 265 & 215 & 1400 & 17.59 & 17.59 & & 0.4645 & $4.63 E-03$ & 44.3198 & 24.9299 & 69.2497 & \\
\hline & $8 / 2 / 2008$ 15:00 & 266 & 215 & 1500 & 17.23 & 17.23 & & 0.4951 & $5.01 \mathrm{E}-03$ & 47.9409 & 26.9667 & 74.9076 & \\
\hline & 8/2/2008 16:00 & 267 & 215 & 1600 & 17.35 & 17.35 & & 0.4847 & 4.88E-03 & 46.7112 & 26.2751 & 72.9863 & \\
\hline & 8/2/2008 17:00 & 268 & 215 & 1700 & 17.16 & 17.16 & & 0.5012 & 5.08E-03 & 48.6689 & 27.3763 & 76.0452 & \\
\hline & $8 / 2 / 200818: 00$ & 269 & 215 & 1800 & 17.46 & 17.46 & & 0.4754 & $4.76 \mathrm{E}-03$ & 45.6041 & 25.6523 & 71.2564 & \\
\hline & 8/2/2008 19:00 & 270 & 215 & 1900 & 17.97 & 17.97 & & 0.4335 & $4.25 E-03$ & 40.7107 & 22.8998 & 63.6105 & \\
\hline & 8/2/2008 20:00 & 271 & 215 & 2000 & 19.86 & 19.86 & & 0.2971 & 2.67E-03 & 25.5671 & 14.3815 & 39.9486 & \\
\hline & 8/2/2008 21:00 & 272 & 215 & 2100 & 22.37 & 22.37 & & 0.1515 & 1.17E-03 & 11.1638 & 6.2796 & 17.4434 & \\
\hline & 8/2/2008 22:00 & 273 & 215 & 2200 & 24.3 & 24.3 & & 0.0601 & 3.73E-04 & 3.5745 & 2.0106 & 5.5851 & \\
\hline & 8/2/2008 23:00 & 274 & 215 & 2300 & 24.96 & 24.96 & & 0.0321 & $1.72 \mathrm{E}-04$ & 1.6492 & 0.9277 & 2.5769 & \\
\hline & 8/3/2008 0:00 & 275 & 216 & 0 & 24.46 & 24.46 & 25.46 & 0.0531 & $3.21 E-04$ & 3.0736 & 1.7289 & 4.8025 & \\
\hline & 8/3/2008 1:00 & 276 & 216 & 100 & 23.92 & 23.92 & & 0.0769 & 5.06E-04 & 4.8452 & 2.7254 & 7.5706 & \\
\hline & 8/3/2008 2:00 & 277 & 216 & 200 & 24.74 & 24.74 & & 0.0412 & $2.35 \mathrm{E}-04$ & 2.2485 & 1.2648 & 3.5132 & \\
\hline & 8/3/2008 3:00 & 278 & 216 & 300 & 25.22 & 25.22 & & 0.0214 & 1.05E-04 & 1.0037 & 0.5646 & 1.5683 & \\
\hline & 8/3/2008 4:00 & 279 & 216 & 400 & 25.46 & 25.46 & & 0.0118 & $5.03 E-05$ & 0.4812 & 0.2707 & 0.7518 & \\
\hline & 8/3/2008 5:00 & 280 & 216 & 500 & 25.46 & 25.46 & & 0.0118 & 5.03E-05 & 0.4812 & 0.2707 & 0.7518 & \\
\hline & 8/3/2008 6:00 & 281 & 216 & 600 & 25.12 & 25.12 & & 0.0255 & 1.30E-04 & 1.2432 & 0.6993 & 1.9426 & \\
\hline & 8/3/2008 7:00 & 282 & 216 & 700 & 24.62 & 24.62 & & 0.0463 & $2.71 E-04$ & 2.5939 & 1.4591 & 4.0529 & \\
\hline & 8/3/2008 8:00 & 283 & 216 & 800 & 23.33 & 23.33 & & 0.1042 & 7.35E-04 & 7.0364 & 3.9580 & 10.9944 & \\
\hline & 8/3/2008 9:00 & 284 & 216 & 900 & 19.67 & 19.67 & & 0.3096 & $2.81 \mathrm{E}-03$ & 26.9008 & 15.1317 & 42.0325 & \\
\hline & 8/3/2008 10:00 & 285 & 216 & 1000 & 17.98 & 17.98 & & 0.4327 & $4.24 \mathrm{E}-03$ & 40.6186 & 22.8480 & 63.4665 & \\
\hline & 8/3/2008 11:00 & 286 & 216 & 1100 & 17.59 & 17.59 & & 0.4645 & 4.63E-03 & 44.3198 & 24.9299 & 69.2497 & \\
\hline
\end{tabular}




\begin{tabular}{|c|c|c|c|c|c|c|c|c|c|c|c|c|c|}
\hline Tree ID & TS & RN & JD & HR & $\begin{array}{c}\text { Raw } \\
\text { TC_dTA }\end{array}$ & $\begin{array}{c}\text { Fixed } \\
\text { TC_dT } \Delta\end{array}$ & dTM & $\mathbf{K}$ & $\begin{array}{l}\text { Sap } \\
\text { Velocity } \\
(\mathrm{cm} / \mathrm{s})\end{array}$ & $\begin{array}{c}\text { Probed } \\
\text { Drywood F } \\
(\mathrm{g} / \mathrm{hr})\end{array}$ & $\begin{array}{c}\text { Extrapolated } \\
\text { Wetwood F } \\
(\mathrm{g} / \mathrm{hr})\end{array}$ & $\begin{array}{c}\text { Extrapolated } \\
\text { Total Wood F } \\
(\mathrm{g} / \mathrm{hr})\end{array}$ & $\begin{array}{l}\text { Relative } \\
\text { Humidity, } \\
@ 10 \mathrm{~m}(\%)\end{array}$ \\
\hline & 8/3/2008 12:00 & 287 & 216 & 1200 & \begin{tabular}{|l|}
17.37 \\
\end{tabular} & 17.37 & & 0.4830 & 4.86E-03 & 46.5085 & 26.1610 & 72.6695 & \\
\hline & 8/3/2008 13:00 & 288 & 216 & 1300 & 17.14 & 17.14 & & 0.5029 & $5.11 \mathrm{E}-03$ & 48.8784 & 27.4941 & 76.3725 & \\
\hline & $8 / 3 / 2008$ 14:00 & 289 & 216 & 1400 & 16.86 & 16.86 & & 0.5279 & $5.42 E-03$ & 51.8815 & 29.1833 & 81.0648 & \\
\hline & 8/3/2008 15:00 & 290 & 216 & 1500 & 16.78 & 16.78 & & 0.5352 & 5.51E-03 & 52.7642 & 29.6799 & 82.4440 & \\
\hline & 8/3/2008 16:00 & 291 & 216 & 1600 & 16.95 & 16.95 & & 0.5198 & 5.32E-03 & 50.9017 & 28.6322 & 79.5339 & \\
\hline & 8/3/2008 17:00 & 292 & 216 & 1700 & 16.8 & 16.8 & & 0.5333 & 5.49E-03 & 52.5425 & 29.5551 & 82.0976 & \\
\hline & 8/3/2008 18:00 & 293 & 216 & 1800 & 17.09 & 17.09 & & 0.5073 & $5.16 \mathrm{E}-03$ & 49.4050 & 27.7903 & 77.1953 & \\
\hline & 8/3/2008 19:00 & 294 & 216 & 1900 & 17.59 & 17.59 & & 0.4645 & 4.63E-03 & 44.3198 & 24.9299 & 69.2497 & \\
\hline & 8/3/2008 20:00 & 295 & 216 & 2000 & 18.7 & 18.7 & & 0.3775 & 3.59E-03 & 34.3413 & 19.3170 & 53.6583 & \\
\hline & 8/3/2008 21:00 & 296 & 216 & 2100 & 20.42 & 20.42 & & 0.2615 & $2.28 \mathrm{E}-03$ & 21.8524 & 12.2920 & 34.1444 & \\
\hline & 8/3/2008 22:00 & 297 & 216 & 2200 & 21.58 & 21.58 & & 0.1937 & 1.58E-03 & 15.1017 & 8.4947 & 23.5964 & \\
\hline & 8/3/2008 23:00 & 298 & 216 & 2300 & 21.85 & 21.85 & & 0.1789 & 1.43E-03 & 13.6987 & 7.7055 & 21.4042 & \\
\hline & 8/4/2008 0:00 & 299 & 217 & 0 & 21.95 & 21.95 & 24.61 & 0.1736 & 1.38E-03 & 13.1943 & 7.4218 & 20.6162 & \\
\hline & 8/4/2008 1:00 & 300 & 217 & 100 & 22.06 & 22.06 & & 0.1677 & 1.32E-03 & 12.6489 & 7.1150 & 19.7639 & \\
\hline & 8/4/2008 2:00 & 301 & 217 & 200 & 21.22 & 21.22 & & 0.2139 & $1.78 \mathrm{E}-03$ & 17.0682 & 9.6009 & 26.6690 & \\
\hline & 8/4/2008 3:00 & 302 & 217 & 300 & 20.87 & 20.87 & & 0.2343 & $1.99 \mathrm{E}-03$ & 19.0890 & 10.7375 & 29.8265 & \\
\hline & 8/4/2008 4:00 & 303 & 217 & 400 & 20.45 & 20.45 & & 0.2597 & 2.26E-03 & 21.6622 & 12.1850 & 33.8472 & \\
\hline & $8 / 4 / 20085: 00$ & 304 & 217 & 500 & 21.24 & 21.24 & & 0.2128 & 1.77E-03 & 16.9560 & 9.5377 & 26.4937 & \\
\hline & 8/4/2008 6:00 & 305 & 217 & 600 & 22.11 & 22.11 & & 0.1651 & 1.30E-03 & 12.4042 & 6.9774 & 19.3816 & \\
\hline & 8/4/2008 7:00 & 306 & 217 & 700 & 22.19 & 22.19 & & 0.1609 & 1.26E-03 & 12.0168 & 6.7595 & 18.7763 & \\
\hline & 8/4/2008 8:00 & 307 & 217 & 800 & 22.65 & 22.65 & & 0.1373 & 1.03E-03 & 9.8872 & 5.5616 & 15.4488 & \\
\hline & 8/4/2008 9:00 & 308 & 217 & 900 & 22.06 & 22.06 & & 0.1677 & $1.32 \mathrm{E}-03$ & 12.6489 & 7.1150 & 19.7639 & \\
\hline & 8/4/2008 10:00 & 309 & 217 & 1000 & 20.33 & 20.33 & & 0.2671 & 2.34E-03 & 22.4283 & 12.6159 & 35.0442 & \\
\hline & 8/4/2008 11:00 & 310 & 217 & 1100 & 19.16 & 19.16 & & 0.3445 & $3.20 \mathrm{E}-03$ & 30.6764 & 17.2555 & 47.9319 & \\
\hline & 8/4/2008 12:00 & 311 & 217 & 1200 & 17.99 & 17.99 & & 0.4319 & 4.23E-03 & 40.5266 & 22.7962 & 63.3228 & \\
\hline & 8/4/2008 13:00 & 312 & 217 & 1300 & 17.68 & 17.68 & & 0.4570 & 4.54E-03 & 43.4458 & 24.4383 & 67.8840 & \\
\hline & 8/4/2008 14:00 & 313 & 217 & 1400 & 18.31 & 18.31 & & 0.4069 & 3.93E-03 & 37.6556 & 21.1813 & 58.8369 & \\
\hline & 8/4/2008 15:00 & 314 & 217 & 1500 & 19.21 & 19.21 & & 0.3410 & $3.16 \mathrm{E}-03$ & 30.2932 & 17.0400 & 47.3332 & \\
\hline & 8/4/2008 16:00 & 315 & 217 & 1600 & 17.83 & 17.83 & & 0.4448 & 4.39E-03 & 42.0158 & 23.6339 & 65.6497 & \\
\hline & 8/4/2008 17:00 & 316 & 217 & 1700 & 17.51 & 17.51 & & 0.4712 & $4.71 E-03$ & 45.1071 & 25.3727 & 70.4798 & \\
\hline & 8/4/2008 18:00 & 317 & 217 & 1800 & 18.21 & 18.21 & & 0.4146 & 4.03E-03 & 38.5377 & 21.6775 & 60.2152 & \\
\hline & 8/4/2008 19:00 & 318 & 217 & 1900 & 18.68 & 18.68 & & 0.3790 & $3.60 \mathrm{E}-03$ & 34.5065 & 19.4099 & 53.9164 & \\
\hline & 8/4/2008 20:00 & 319 & 217 & 2000 & 20.3 & 20.3 & & 0.2690 & $2.36 \mathrm{E}-03$ & 22.6220 & 12.7249 & 35.3469 & \\
\hline & 8/4/2008 21:00 & 320 & 217 & 2100 & 23.16 & 23.16 & & 0.1123 & 8.06E-04 & 7.7164 & 4.3405 & 12.0569 & \\
\hline & 8/4/2008 22:00 & 321 & 217 & 2200 & 24.19 & 24.19 & & 0.0649 & 4.11E-04 & 3.9307 & 2.2110 & 6.1417 & \\
\hline & 8/4/2008 23:00 & 322 & 217 & 2300 & 24.61 & 24.61 & & 0.0467 & 2.74E-04 & 2.6232 & 1.4756 & 4.0988 & \\
\hline & 8/5/2008 0:00 & 323 & 218 & 0 & 25.21 & 25.21 & 26.04 & 0.0218 & 1.07E-04 & 1.0271 & 0.5778 & 1.6049 & \\
\hline & 8/5/2008 1:00 & 324 & 218 & 100 & 25.32 & 25.32 & & 0.0174 & 8.11E-05 & 0.7762 & 0.4366 & 1.2129 & \\
\hline & 8/5/2008 2:00 & 325 & 218 & 200 & 25.4 & 25.4 & & 0.0142 & $6.31 \mathrm{E}-05$ & 0.6040 & 0.3397 & 0.9437 & \\
\hline & 8/5/2008 3:00 & 326 & 218 & 300 & 25.15 & 25.15 & & 0.0243 & 1.22E-04 & 1.1702 & 0.6582 & 1.8284 & \\
\hline & 8/5/2008 4:00 & 327 & 218 & 400 & 24.56 & 24.56 & & 0.0489 & 2.89E-04 & 2.7713 & 1.5588 & 4.3301 & \\
\hline
\end{tabular}




\begin{tabular}{|c|c|c|c|c|c|c|c|c|c|c|c|c|c|}
\hline Tree ID & TS & RN & JD & HR & $\begin{array}{c}\text { Raw } \\
\text { TC_dTA }\end{array}$ & $\begin{array}{c}\text { Fixed } \\
\text { TC_dT } \Delta\end{array}$ & dTM & $\mathbf{K}$ & $\begin{array}{l}\text { Sap } \\
\text { Velocity } \\
(\mathrm{cm} / \mathrm{s})\end{array}$ & $\begin{array}{c}\text { Probed } \\
\text { Drywood F } \\
(\mathrm{g} / \mathrm{hr})\end{array}$ & $\begin{array}{c}\text { Extrapolated } \\
\text { Wetwood F } \\
(\mathrm{g} / \mathrm{hr})\end{array}$ & $\begin{array}{c}\text { Extrapolated } \\
\text { Total Wood F } \\
(\mathrm{g} / \mathrm{hr})\end{array}$ & $\begin{array}{l}\text { Relative } \\
\text { Humidity, } \\
@ 10 \mathrm{~m}(\%)\end{array}$ \\
\hline & 8/5/2008 5:00 & 328 & 218 & 500 & 24.74 & 24.74 & & 0.0412 & 2.35E-04 & 2.2485 & 1.2648 & $\begin{array}{r}3.5132 \\
\end{array}$ & \\
\hline & 8/5/2008 6:00 & 329 & 218 & 600 & 25.06 & 25.06 & & 0.0279 & $1.45 \mathrm{E}-04$ & 1.3923 & 0.7832 & 2.1755 & \\
\hline & 8/5/2008 7:00 & 330 & 218 & 700 & 25.14 & 25.14 & & 0.0247 & $1.25 E-04$ & 1.1944 & 0.6719 & 1.8663 & \\
\hline & 8/5/2008 8:00 & 331 & 218 & 800 & 24.75 & 24.75 & & 0.0408 & $2.32 \mathrm{E}-04$ & 2.2203 & 1.2489 & 3.4692 & \\
\hline & 8/5/2008 9:00 & 332 & 218 & 900 & 22.53 & 22.53 & & 0.1434 & 1.09E-03 & 10.4269 & 5.8651 & 16.2920 & \\
\hline & 8/5/2008 10:00 & 333 & 218 & 1000 & 19.97 & 19.97 & & 0.2899 & $2.59 \mathrm{E}-03$ & 24.8123 & 13.9569 & 38.7692 & \\
\hline & 8/5/2008 11:00 & 334 & 218 & 1100 & 20.04 & 20.04 & & 0.2854 & 2.54E-03 & 24.3385 & 13.6904 & 38.0289 & \\
\hline & 8/5/2008 12:00 & 335 & 218 & 1200 & 19.24 & 19.24 & & 0.3389 & 3.14E-03 & 30.0647 & 16.9114 & 46.9761 & \\
\hline & 8/5/2008 13:00 & 336 & 218 & 1300 & 18.06 & 18.06 & & 0.4264 & 4.17E-03 & 39.8865 & 22.4361 & 62.3226 & \\
\hline & 8/5/2008 14:00 & 337 & 218 & 1400 & 19.01 & 19.01 & & 0.3551 & 3.33E-03 & 31.8435 & 17.9120 & 49.7555 & \\
\hline & 8/5/2008 15:00 & 338 & 218 & 1500 & 20.19 & 20.19 & & 0.2759 & 2.44E-03 & 23.3400 & 13.1287 & 36.4687 & \\
\hline & 8/5/2008 16:00 & 339 & 218 & 1600 & 19.6 & 19.6 & & 0.3143 & 2.86E-03 & 27.4020 & 15.4136 & 42.8156 & \\
\hline & 8/5/2008 17:00 & 340 & 218 & 1700 & 18.84 & 18.84 & & 0.3673 & 3.47E-03 & 33.1987 & 18.6743 & 51.8730 & \\
\hline & 8/5/2008 18:00 & 341 & 218 & 1800 & 18.38 & 18.38 & & 0.4015 & 3.87E-03 & 37.0461 & 20.8385 & 57.8846 & \\
\hline & 8/5/2008 19:00 & 342 & 218 & 1900 & 18.79 & 18.79 & & 0.3709 & 3.51E-03 & 33.6040 & 18.9022 & 52.5062 & \\
\hline & 8/5/2008 20:00 & 343 & 218 & 2000 & 21.65 & 21.65 & & 0.1898 & 1.54E-03 & 14.7321 & 8.2868 & 23.0190 & \\
\hline & 8/5/2008 21:00 & 344 & 218 & 2100 & 25.03 & 25.03 & & 0.0292 & 1.53E-04 & 1.4683 & 0.8259 & 2.2942 & \\
\hline & $8 / 5 / 200822: 00$ & 345 & 218 & 2200 & 25.87 & 25.87 & & -0.0043 & $0.00 \mathrm{E}+00$ & 0.0000 & 0.0000 & 0.0000 & \\
\hline & 8/5/2008 23:00 & 346 & 218 & 2300 & 26.04 & 26.04 & & -0.0108 & $0.00 \mathrm{E}+00$ & 0.0000 & 0.0000 & 0.0000 & \\
\hline & 8/6/2008 0:00 & 347 & 219 & 0 & 26.05 & 26.05 & 26.05 & -0.0111 & $0.00 E+00$ & 0.0000 & 0.0000 & 0.0000 & \\
\hline & 8/6/2008 1:00 & 348 & 219 & 100 & 25.98 & 25.98 & & -0.0085 & $0.00 \mathrm{E}+00$ & 0.0000 & 0.0000 & 0.0000 & \\
\hline & $8 / 6 / 2008$ 2:00 & 349 & 219 & 200 & 25.93 & 25.93 & & -0.0066 & $0.00 E+00$ & 0.0000 & 0.0000 & 0.0000 & \\
\hline & 8/6/2008 3:00 & 350 & 219 & 300 & 25.91 & 25.91 & & -0.0058 & $0.00 E+00$ & 0.0000 & 0.0000 & 0.0000 & \\
\hline & 8/6/2008 4:00 & 351 & 219 & 400 & 25.82 & 25.82 & & -0.0023 & $0.00 \mathrm{E}+00$ & 0.0000 & 0.0000 & 0.0000 & \\
\hline & 8/6/2008 5:00 & 352 & 219 & 500 & 25.86 & 25.86 & & -0.0039 & $0.00 \mathrm{E}+00$ & 0.0000 & 0.0000 & 0.0000 & \\
\hline & 8/6/2008 6:00 & 353 & 219 & 600 & 25.81 & 25.81 & & -0.0019 & $0.00 E+00$ & 0.0000 & 0.0000 & 0.0000 & \\
\hline & 8/6/2008 7:00 & 354 & 219 & 700 & 25.82 & 25.82 & & -0.0023 & $0.00 \mathrm{E}+00$ & 0.0000 & 0.0000 & 0.0000 & \\
\hline & 8/6/2008 8:00 & 355 & 219 & 800 & 25.73 & 25.73 & & 0.0012 & $2.91 \mathrm{E}-06$ & 0.0279 & 0.0157 & 0.0436 & \\
\hline & 8/6/2008 9:00 & 356 & 219 & 900 & 25.63 & 25.63 & & 0.0051 & 1.78E-05 & 0.1705 & 0.0959 & 0.2664 & \\
\hline & 8/6/2008 10:00 & 357 & 219 & 1000 & 22.09 & 22.09 & & 0.1661 & 1.31E-03 & 12.5018 & 7.0323 & 19.5341 & \\
\hline & 8/6/2008 11:00 & 358 & 219 & 1100 & 19.08 & 19.08 & & 0.3501 & 3.27E-03 & 31.2956 & 17.6038 & 48.8993 & \\
\hline & 8/6/2008 12:00 & 359 & 219 & 1200 & 19.55 & 19.55 & & 0.3176 & 2.90E-03 & 27.7632 & 15.6168 & 43.3800 & \\
\hline & 8/6/2008 13:00 & 360 & 219 & 1300 & 20.32 & 20.32 & & 0.2677 & $2.35 \mathrm{E}-03$ & 22.4928 & 12.6522 & 35.1450 & \\
\hline & $8 / 6 / 2008$ 14:00 & 361 & 219 & 1400 & 18.85 & 18.85 & & 0.3666 & $3.46 \mathrm{E}-03$ & 33.1180 & 18.6289 & 51.7469 & \\
\hline & 8/6/2008 15:00 & 362 & 219 & 1500 & 18.11 & 18.11 & & 0.4224 & $4.12 E-03$ & 39.4334 & 22.1813 & 61.6147 & \\
\hline & 8/6/2008 16:00 & 363 & 219 & 1600 & 18.61 & 18.61 & & 0.3842 & 3.67E-03 & 35.0888 & 19.7374 & 54.8262 & \\
\hline & 8/6/2008 17:00 & 364 & 219 & 1700 & 18.25 & 18.25 & & 0.4115 & 3.99E-03 & 38.1833 & 21.4781 & 59.6614 & \\
\hline & 8/6/2008 18:00 & 365 & 219 & 1800 & 19.78 & 19.78 & & 0.3023 & 2.73E-03 & 26.1240 & 14.6947 & 40.8187 & \\
\hline & 8/6/2008 19:00 & 366 & 219 & 1900 & 20.1 & 20.1 & & 0.2816 & $2.50 \mathrm{E}-03$ & 23.9363 & 13.4642 & 37.4005 & \\
\hline & $8 / 6 / 200820: 00$ & 367 & 219 & 2000 & 21.52 & 21.52 & & 0.1970 & $1.61 \mathrm{E}-03$ & 15.4217 & 8.6747 & 24.0965 & \\
\hline & 8/6/2008 21:00 & 368 & 219 & 2100 & 24.07 & 24.07 & & 0.0702 & $4.52 \mathrm{E}-04$ & 4.3302 & 2.4357 & 6.7659 & \\
\hline
\end{tabular}




\begin{tabular}{|c|c|c|c|c|c|c|c|c|c|c|c|c|c|}
\hline Tree ID & TS & RN & JD & HR & $\begin{array}{c}\text { Raw } \\
\text { TC_dTA }\end{array}$ & $\begin{array}{c}\text { Fixed } \\
\text { TC_dT } \Delta\end{array}$ & dTM & K & $\begin{array}{l}\text { Sap } \\
\text { Velocity } \\
(\mathrm{cm} / \mathrm{s})\end{array}$ & $\begin{array}{c}\text { Probed } \\
\text { Drywood F } \\
(\mathrm{g} / \mathrm{hr})\end{array}$ & $\begin{array}{c}\text { Extrapolated } \\
\text { Wetwood F } \\
(\mathrm{g} / \mathrm{hr})\end{array}$ & $\begin{array}{c}\text { Extrapolated } \\
\text { Total Wood F } \\
(\mathrm{g} / \mathrm{hr})\end{array}$ & $\begin{array}{l}\text { Relative } \\
\text { Humidity, } \\
@ 10 \mathrm{~m}(\%)\end{array}$ \\
\hline & 8/6/2008 22:00 & 369 & 219 & 2200 & 25.77 . & 25.77 & & -0.0004 & $0.00 \mathrm{E}+00$ & 0.0000 & 0.0000 & 0.0000 & \\
\hline & 8/6/2008 23:00 & 370 & 219 & 2300 & 25.99 & 25.99 & & -0.0088 & $0.00 \mathrm{E}+00$ & 0.0000 & 0.0000 & 0.0000 & \\
\hline & $8 / 7 / 20080: 00$ & 371 & 220 & 0 & 26 & 26 & 26.00 & -0.0092 & $0.00 E+00$ & 0.0000 & 0.0000 & 0.0000 & \\
\hline & 8/7/2008 1:00 & 372 & 220 & 100 & 25.96 & 25.96 & & -0.0077 & $0.00 \mathrm{E}+00$ & 0.0000 & 0.0000 & 0.0000 & \\
\hline & 8/7/2008 2:00 & 373 & 220 & 200 & 25.93 & 25.93 & & -0.0066 & $0.00 \mathrm{E}+00$ & 0.0000 & 0.0000 & 0.0000 & \\
\hline & 8/7/2008 3:00 & 374 & 220 & 300 & 25.8 & 25.8 & & -0.0016 & $0.00 \mathrm{E}+00$ & 0.0000 & 0.0000 & 0.0000 & \\
\hline & 8/7/2008 4:00 & 375 & 220 & 400 & 25.89 & 25.89 & & -0.0050 & $0.00 \mathrm{E}+00$ & 0.0000 & 0.0000 & 0.0000 & \\
\hline & 8/7/2008 5:00 & 376 & 220 & 500 & 25.88 & 25.88 & & -0.0046 & $0.00 \mathrm{E}+00$ & 0.0000 & 0.0000 & 0.0000 & \\
\hline & 8/7/2008 6:00 & 377 & 220 & 600 & 25.86 & 25.86 & & -0.0039 & $0.00 \mathrm{E}+00$ & 0.0000 & 0.0000 & 0.0000 & \\
\hline & 8/7/2008 7:00 & 378 & 220 & 700 & 25.84 & 25.84 & & -0.0031 & $0.00 E+00$ & 0.0000 & 0.0000 & 0.0000 & \\
\hline & 8/7/2008 8:00 & 379 & 220 & 800 & 25.67 & 25.67 & & 0.0035 & 1.13E-05 & 0.1082 & 0.0609 & 0.1691 & \\
\hline & 8/7/2008 9:00 & 380 & 220 & 900 & 25.05 & 25.05 & & 0.0283 & 1.48E-04 & 1.4176 & 0.7974 & 2.2149 & \\
\hline & 8/7/2008 10:00 & 381 & 220 & 1000 & 20.67 & 20.67 & & 0.2463 & $2.12 \mathrm{E}-03$ & 20.2937 & 11.4152 & 31.7088 & \\
\hline & 8/7/2008 11:00 & 382 & 220 & 1100 & 19.17 & 19.17 & & 0.3438 & $3.20 \mathrm{E}-03$ & 30.5996 & 17.2122 & 47.8118 & \\
\hline & 8/7/2008 12:00 & 383 & 220 & 1200 & 18.63 & 18.63 & & 0.3827 & $3.65 E-03$ & 34.9218 & 19.6435 & 54.5653 & \\
\hline & 8/7/2008 13:00 & 384 & 220 & 1300 & $\mid 18.07$ & $\mid 18.07$ & & 0.4256 & $4.16 \mathrm{E}-03$ & 39.7956 & 22.3850 & 62.1806 & \\
\hline & $8 / 7 / 2008$ 14:00 & 385 & 220 & 1400 & 17.66 & 17.66 & & 0.4587 & 4.56E-03 & 43.6390 & 24.5469 & 68.1859 & \\
\hline & 8/7/2008 15:00 & 386 & 220 & 1500 & 17.41 & 17.41 & & 0.4796 & $4.82 E-03$ & 46.1050 & 25.9340 & 72.0390 & \\
\hline & 8/7/2008 16:00 & 387 & 220 & 1600 & 17.61 & 17.61 & & 0.4628 & 4.61E-03 & 44.1245 & 24.8201 & 68.9446 & \\
\hline & 8/7/2008 17:00 & 388 & 220 & 1700 & 17.21 & 17.21 & & 0.4968 & 5.03E-03 & 48.1481 & 27.0833 & 75.2314 & \\
\hline & 8/7/2008 18:00 & 389 & 220 & 1800 & 18.12 & 18.12 & & 0.4216 & $4.11 \mathrm{E}-03$ & 39.3432 & 22.1306 & 61.4738 & \\
\hline & 8/7/2008 19:00 & 390 & 220 & 1900 & 19.09 & 19.09 & & 0.3494 & 3.26E-03 & 31.2178 & 17.5600 & 48.7778 & \\
\hline & 8/7/2008 20:00 & 391 & 220 & 2000 & 21.27 & 21.27 & & 0.2111 & 1.75E-03 & 16.7884 & 9.4435 & 26.2318 & \\
\hline & 8/7/2008 21:00 & 392 & 220 & 2100 & 24.33 & 24.33 & & 0.0588 & 3.63E-04 & 3.4790 & 1.9569 & 5.4359 & \\
\hline & 8/7/2008 22:00 & 393 & 220 & 2200 & 25.49 & 25.49 & & 0.0106 & 4.41E-05 & 0.4220 & 0.2374 & 0.6594 & \\
\hline & 8/7/2008 23:00 & 394 & 220 & 2300 & 25.9 & 25.9 & & -0.0054 & $0.00 E+00$ & 0.0000 & 0.0000 & 0.0000 & \\
\hline & 8/8/2008 0:00 & 395 & 221 & 0 & 26.02 & 26.02 & 26.02 & -0.0100 & $0.00 \mathrm{E}+00$ & 0.0000 & 0.0000 & 0.0000 & \\
\hline & 8/8/2008 1:00 & 396 & 221 & 100 & 26 & 26 & & -0.0092 & $0.00 \mathrm{E}+00$ & 0.0000 & 0.0000 & 0.0000 & \\
\hline & 8/8/2008 2:00 & 397 & 221 & 200 & 25.89 & 25.89 & & -0.0050 & $0.00 \mathrm{E}+00$ & 0.0000 & 0.0000 & 0.0000 & \\
\hline & 8/8/2008 3:00 & 398 & 221 & 300 & 25.93 & 25.93 & & -0.0066 & $0.00 \mathrm{E}+00$ & 0.0000 & 0.0000 & 0.0000 & \\
\hline & 8/8/2008 4:00 & 399 & 221 & 400 & 25.9 & 25.9 & & -0.0054 & $0.00 \mathrm{E}+00$ & 0.0000 & 0.0000 & 0.0000 & \\
\hline & 8/8/2008 5:00 & 400 & 221 & 500 & 25.87 & 25.87 & & -0.0043 & $0.00 \mathrm{E}+00$ & 0.0000 & 0.0000 & 0.0000 & \\
\hline & 8/8/2008 6:00 & 401 & 221 & 600 & 25.79 & 25.79 & & -0.0012 & $0.00 \mathrm{E}+00$ & 0.0000 & 0.0000 & 0.0000 & \\
\hline & 8/8/2008 7:00 & 402 & 221 & 700 & 25.86 & 25.86 & & -0.0039 & $0.00 \mathrm{E}+00$ & 0.0000 & 0.0000 & 0.0000 & \\
\hline & 8/8/2008 8:00 & 403 & 221 & 800 & 25.77 & 25.77 & & -0.0004 & $0.00 \mathrm{E}+00$ & 0.0000 & 0.0000 & 0.0000 & \\
\hline & 8/8/20089:00 & 404 & 221 & 900 & 23.54 & 23.54 & & 0.0943 & $6.50 \mathrm{E}-04$ & 6.2265 & 3.5024 & 9.7289 & \\
\hline & 8/8/2008 10:00 & 405 & 221 & 1000 & 19.42 & 19.42 & & 0.3265 & $3.00 \mathrm{E}-03$ & 28.7152 & 16.1523 & 44.8675 & \\
\hline & 8/8/2008 11:00 & 406 & 221 & 1100 & 18.86 & 18.86 & & 0.3659 & $3.45 E-03$ & 33.0374 & 18.5836 & 51.6210 & \\
\hline & $8 / 8 / 200812: 00$ & 407 & 221 & 1200 & 18.25 & 18.25 & & 0.4115 & 3.99E-03 & 38.1833 & 21.4781 & 59.6614 & \\
\hline & 8/8/2008 13:00 & 408 & 221 & 1300 & 17.82 & 17.82 & & 0.4456 & 4.40E-03 & 42.1101 & 23.6870 & 65.7971 & \\
\hline & 8/8/2008 14:00 & 409 & 221 & 1400 & 17.36 & 17.36 & & 0.4839 & 4.87E-03 & 46.6098 & 26.2180 & 72.8278 & \\
\hline
\end{tabular}




\begin{tabular}{|c|c|c|c|c|c|c|c|c|c|c|c|c|c|}
\hline Tree ID & TS & RN & JD & HR & $\begin{array}{c}\text { Raw } \\
\text { TC_dTA }\end{array}$ & $\begin{array}{c}\text { Fixed } \\
\text { TC_dT } \Delta\end{array}$ & dTM & $\mathbf{K}$ & $\begin{array}{l}\text { Sap } \\
\text { Velocity } \\
(\mathrm{cm} / \mathrm{s})\end{array}$ & $\begin{array}{c}\text { Probed } \\
\text { Drywood F } \\
(\mathrm{g} / \mathrm{hr})\end{array}$ & $\begin{array}{c}\text { Extrapolated } \\
\text { Wetwood F } \\
(\mathrm{g} / \mathrm{hr})\end{array}$ & $\begin{array}{c}\text { Extrapolated } \\
\text { Total Wood F } \\
(\mathrm{g} / \mathrm{hr})\end{array}$ & $\begin{array}{l}\text { Relative } \\
\text { Humidity, } \\
@ 10 \mathrm{~m}(\%)\end{array}$ \\
\hline & 8/8/2008 15:00 & 410 & 221 & 1500 & \begin{tabular}{|r|}
17.15 \\
\end{tabular} & 17.15 & & 0.5020 & $5.10 \mathrm{E}-03$ & 48.7736 & 27.4351 & $\begin{array}{r}76.2087 \\
\end{array}$ & \\
\hline & 8/8/2008 16:00 & 411 & 221 & 1600 & 17.34 & 17.34 & & 0.4856 & 4.89E-03 & 46.8128 & 26.3322 & 73.1450 & \\
\hline & 8/8/2008 17:00 & 412 & 221 & 1700 & 17.23 & 17.23 & & 0.4951 & $5.01 E-03$ & 47.9409 & 26.9667 & 74.9076 & \\
\hline & 8/8/2008 18:00 & 413 & 221 & 1800 & 17.99 & 17.99 & & 0.4319 & 4.23E-03 & 40.5266 & 22.7962 & 63.3228 & \\
\hline & 8/8/2008 19:00 & 414 & 221 & 1900 & 18.48 & 18.48 & & 0.3939 & 3.78E-03 & 36.1867 & 20.3550 & 56.5417 & \\
\hline & 8/8/2008 20:00 & 415 & 221 & 2000 & 20.32 & 20.32 & & 0.2677 & $2.35 \mathrm{E}-03$ & 22.4928 & 12.6522 & 35.1450 & \\
\hline & 8/8/2008 21:00 & 416 & 221 & 2100 & 22.65 & 22.65 & & 0.1373 & 1.03E-03 & 9.8872 & 5.5616 & 15.4488 & \\
\hline & 8/8/2008 22:00 & 417 & 221 & 2200 & 24.16 & 24.16 & & 0.0662 & $4.21 E-04$ & 4.0295 & 2.2666 & 6.2961 & \\
\hline & 8/8/2008 23:00 & 418 & 221 & 2300 & 24.11 & 24.11 & & 0.0684 & 4.38E-04 & 4.1958 & 2.3601 & 6.5559 & \\
\hline & 8/9/2008 0:00 & 419 & 222 & 0 & 23.64 & 23.64 & 25.97 & 0.0897 & 6.11E-04 & 5.8524 & 3.2920 & 9.1444 & \\
\hline & 8/9/2008 1:00 & 420 & 222 & 100 & 23.33 & 23.33 & & 0.1042 & 7.35E-04 & 7.0364 & 3.9580 & 10.9944 & \\
\hline & 8/9/2008 2:00 & 421 & 222 & 200 & 23.56 & 23.56 & & 0.0934 & $6.43 E-04$ & 6.1511 & 3.4600 & 9.6110 & \\
\hline & 8/9/2008 3:00 & 422 & 222 & 300 & 23.53 & 23.53 & & 0.0948 & $6.54 \mathrm{E}-04$ & 6.2643 & 3.5237 & 9.7879 & \\
\hline & 8/9/2008 4:00 & 423 & 222 & 400 & 23.76 & 23.76 & & 0.0842 & 5.66E-04 & 5.4135 & 3.0451 & 8.4586 & \\
\hline & $8 / 9 / 20085: 00$ & 424 & 222 & 500 & 23.34 & 23.34 & & 0.1037 & 7.31E-04 & 6.9971 & 3.9359 & 10.9330 & \\
\hline & 8/9/2008 6:00 & 425 & 222 & 600 & 23.41 & 23.41 & & 0.1004 & 7.02E-04 & 6.7240 & 3.7822 & 10.5062 & \\
\hline & 8/9/2008 7:00 & 426 & 222 & 700 & 22.66 & 22.66 & & 0.1368 & 1.03E-03 & 9.8427 & 5.5365 & 15.3793 & \\
\hline & 8/9/2008 8:00 & 427 & 222 & 800 & 22.05 & 22.05 & & 0.1683 & 1.33E-03 & 12.6981 & 7.1427 & 19.8408 & \\
\hline & 8/9/2008 9:00 & 428 & 222 & 900 & 23.67 & 23.67 & & 0.0883 & 6.00E-04 & 5.7417 & 3.2297 & 8.9713 & \\
\hline & 8/9/2008 10:00 & 429 & 222 & 1000 & 22.59 & 22.59 & & 0.1403 & 1.06E-03 & 10.1557 & 5.7126 & 15.8682 & \\
\hline & 8/9/2008 11:00 & 430 & 222 & 1100 & 21.33 & 21.33 & & 0.2077 & $1.72 \mathrm{E}-03$ & 16.4555 & 9.2562 & 25.7117 & \\
\hline & 8/9/2008 12:00 & 431 & 222 & 1200 & 19.82 & 19.82 & & 0.2997 & 2.70E-03 & 25.8447 & 14.5376 & 40.3823 & \\
\hline & 8/9/2008 13:00 & 432 & 222 & 1300 & 19.37 & 19.37 & & 0.3299 & 3.04E-03 & 29.0864 & 16.3611 & 45.4475 & \\
\hline & 8/9/2008 14:00 & 433 & 222 & 1400 & 18.19 & 18.19 & & 0.4162 & 4.04E-03 & 38.7158 & 21.7776 & 60.4934 & \\
\hline & 8/9/2008 15:00 & 434 & 222 & 1500 & 17.53 & 17.53 & & 0.4695 & 4.69E-03 & 44.9093 & 25.2615 & 70.1708 & \\
\hline & 8/9/2008 16:00 & 435 & 222 & 1600 & 17.71 & 17.71 & & 0.4545 & 4.51E-03 & 43.1571 & 24.2759 & 67.4330 & \\
\hline & 8/9/2008 17:00 & 436 & 222 & 1700 & 17.71 & 17.71 & & 0.4545 & 4.51E-03 & 43.1571 & 24.2759 & 67.4330 & \\
\hline & 8/9/2008 18:00 & 437 & 222 & 1800 & 18.56 & 18.56 & & 0.3879 & $3.71 \mathrm{E}-03$ & 35.5085 & 19.9735 & 55.4820 & \\
\hline & 8/9/2008 19:00 & 438 & 222 & 1900 & 19.19 & 19.19 & & 0.3424 & $3.18 \mathrm{E}-03$ & 30.4462 & 17.1260 & 47.5721 & \\
\hline & 8/9/2008 20:00 & 439 & 222 & 2000 & 20.92 & 20.92 & & 0.2314 & $1.96 \mathrm{E}-03$ & 18.7935 & 10.5714 & 29.3649 & \\
\hline & 8/9/2008 21:00 & 440 & 222 & 2100 & 24.41 & 24.41 & & 0.0553 & 3.37E-04 & 3.2279 & 1.8157 & 5.0436 & \\
\hline & 8/9/2008 22:00 & 441 & 222 & 2200 & 25.83 & 25.83 & & -0.0027 & $0.00 \mathrm{E}+00$ & 0.0000 & 0.0000 & 0.0000 & \\
\hline & 8/9/2008 23:00 & 442 & 222 & 2300 & 25.97 & 25.97 & & -0.0081 & $0.00 \mathrm{E}+00$ & 0.0000 & 0.0000 & 0.0000 & \\
\hline & 8/10/2008 0:00 & 443 & 223 & 0 & 26 & 26 & 26.00 & -0.0092 & $0.00 \mathrm{E}+00$ & 0.0000 & 0.0000 & 0.0000 & \\
\hline & 8/10/2008 1:00 & 444 & 223 & 100 & 25.99 & 25.99 & & -0.0088 & $0.00 \mathrm{E}+00$ & 0.0000 & 0.0000 & 0.0000 & \\
\hline & 8/10/2008 2:00 & 445 & 223 & 200 & 25.94 & 25.94 & & -0.0069 & $0.00 \mathrm{E}+00$ & 0.0000 & 0.0000 & 0.0000 & \\
\hline & 8/10/2008 3:00 & 446 & 223 & 300 & 25.93 & 25.93 & & -0.0066 & $0.00 \mathrm{E}+00$ & 0.0000 & 0.0000 & 0.0000 & \\
\hline & 8/10/2008 4:00 & 447 & 223 & 400 & 25.82 & 25.82 & & -0.0023 & $0.00 \mathrm{E}+00$ & 0.0000 & 0.0000 & 0.0000 & \\
\hline & $8 / 10 / 20085: 00$ & 448 & 223 & 500 & 25.86 & 25.86 & & -0.0039 & $0.00 \mathrm{E}+00$ & 0.0000 & 0.0000 & 0.0000 & \\
\hline & 8/10/2008 6:00 & 449 & 223 & 600 & 25.82 & 25.82 & & -0.0023 & $0.00 \mathrm{E}+00$ & 0.0000 & 0.0000 & 0.0000 & \\
\hline & 8/10/2008 7:00 & 450 & 223 & 700 & 25.86 & 25.86 & & -0.0039 & $0.00 \mathrm{E}+00$ & 0.0000 & 0.0000 & 0.0000 & \\
\hline
\end{tabular}




\begin{tabular}{|c|c|c|c|c|c|c|c|c|c|c|c|c|c|}
\hline Tree ID & TS & RN & JD & HR & $\begin{array}{c}\text { Raw } \\
\text { TC_dTA }\end{array}$ & $\begin{array}{c}\text { Fixed } \\
\text { TC_dT } \Delta\end{array}$ & dTM & K & $\begin{array}{l}\text { Sap } \\
\text { Velocity } \\
(\mathrm{cm} / \mathrm{s})\end{array}$ & $\begin{array}{c}\text { Probed } \\
\text { Drywood F } \\
(\mathrm{g} / \mathrm{hr})\end{array}$ & $\begin{array}{c}\text { Extrapolated } \\
\text { Wetwood F } \\
(\mathrm{g} / \mathrm{hr})\end{array}$ & $\begin{array}{c}\text { Extrapolated } \\
\text { Total Wood F } \\
(\mathrm{g} / \mathrm{hr})\end{array}$ & $\begin{array}{l}\text { Relative } \\
\text { Humidity, } \\
@ 10 \mathrm{~m}(\%)\end{array}$ \\
\hline & 8/10/2008 8:00 & 451 & 223 & 800 & 25.77 . & 25.77 & & -0.0004 & $0.00 \mathrm{E}+00$ & 0.0000 & 0.0000 & 0.0000 & \\
\hline & 8/10/2008 9:00 & 452 & 223 & 900 & 25.29 & 25.29 & & 0.0186 & 8.81E-05 & 0.8431 & 0.4743 & 1.3174 & \\
\hline & $8 / 10 / 2008$ 10:00 & 453 & 223 & 1000 & 20.99 & 20.99 & & 0.2273 & $1.92 \mathrm{E}-03$ & 18.3837 & 10.3409 & 28.7246 & \\
\hline & 8/10/2008 11:00 & 454 & 223 & 1100 & 19.25 & 19.25 & & 0.3382 & 3.13E-03 & 29.9888 & 16.8687 & 46.8575 & \\
\hline & $8 / 10 / 200812: 00$ & 455 & 223 & 1200 & 18.73 & 18.73 & & 0.3753 & 3.56E-03 & 34.0944 & 19.1781 & 53.2725 & \\
\hline & 8/10/2008 13:00 & 456 & 223 & 1300 & 18.24 & 18.24 & & 0.4123 & $4.00 \mathrm{E}-03$ & 38.2717 & 21.5278 & 59.7995 & \\
\hline & $8 / 10 / 2008$ 14:00 & 457 & 223 & 1400 & 17.84 & 17.84 & & 0.4439 & $4.38 E-03$ & 41.9217 & 23.5809 & 65.5026 & \\
\hline & $8 / 10 / 2008$ 15:00 & 458 & 223 & 1500 & 18.04 & 18.04 & & 0.4279 & 4.19E-03 & 40.0687 & 22.5386 & 62.6073 & \\
\hline & 8/10/2008 16:00 & 459 & 223 & 1600 & 17.93 & 17.93 & & 0.4367 & $4.29 \mathrm{E}-03$ & 41.0808 & 23.1079 & 64.1887 & \\
\hline & 8/10/2008 17:00 & 460 & 223 & 1700 & 18.33 & 18.33 & & 0.4053 & 3.92E-03 & 37.4808 & 21.0830 & 58.5638 & \\
\hline & 8/10/2008 18:00 & 461 & 223 & 1800 & 23.99 & 23.99 & & 0.0738 & 4.81E-04 & 4.6027 & 2.5890 & 7.1917 & \\
\hline & $8 / 10 / 2008$ 19:00 & 462 & 223 & 1900 & 25.45 & 25.45 & & 0.0122 & $5.24 \mathrm{E}-05$ & 0.5012 & 0.2819 & 0.7832 & \\
\hline & 8/10/2008 20:00 & 463 & 223 & 2000 & 25.52 & 25.52 & & 0.0094 & 3.81E-05 & 0.3645 & 0.2051 & 0.5696 & \\
\hline & 8/10/2008 21:00 & 464 & 223 & 2100 & 25.56 & 25.56 & & 0.0078 & 3.04E-05 & 0.2907 & 0.1635 & 0.4542 & \\
\hline & 8/10/2008 22:00 & 465 & 223 & 2200 & 25.62 & 25.62 & & 0.0055 & 1.95E-05 & 0.1869 & 0.1051 & 0.2920 & \\
\hline & $8 / 10 / 2008$ 23:00 & 466 & 223 & 2300 & 25.09 & 25.09 & & 0.0267 & 1.38E-04 & 1.3173 & 0.7410 & 2.0583 & \\
\hline & $8 / 11 / 20080: 00$ & 467 & 224 & 0 & 24.56 & 24.56 & 25.47 & 0.0489 & 2.89E-04 & 2.7713 & 1.5588 & 4.3301 & \\
\hline & 8/11/2008 1:00 & 468 & 224 & 100 & 24.93 & 24.93 & & 0.0333 & $1.81 E-04$ & 1.7282 & 0.9721 & 2.7003 & \\
\hline & 8/11/2008 2:00 & 469 & 224 & 200 & 25.39 & 25.39 & & 0.0146 & 6.53E-05 & 0.6250 & 0.3516 & 0.9766 & \\
\hline & 8/11/2008 3:00 & 470 & 224 & 300 & 25.47 & 25.47 & & 0.0114 & $4.82 \mathrm{E}-05$ & 0.4613 & 0.2595 & 0.7208 & \\
\hline & 8/11/2008 4:00 & 471 & 224 & 400 & 25.42 & 25.42 & & 0.0134 & $5.88 \mathrm{E}-05$ & 0.5624 & 0.3164 & 0.8788 & \\
\hline & 8/11/2008 5:00 & 472 & 224 & 500 & 25.25 & 25.25 & & 0.0202 & 9.76E-05 & 0.9341 & 0.5255 & 1.4596 & \\
\hline & 8/11/2008 6:00 & 473 & 224 & 600 & 25.36 & 25.36 & & 0.0158 & 7.20E-05 & 0.6890 & 0.3875 & 1.0765 & \\
\hline & 8/11/2008 7:00 & 474 & 224 & 700 & 25.32 & 25.32 & & 0.0174 & $8.11 \mathrm{E}-05$ & 0.7762 & 0.4366 & 1.2129 & \\
\hline & 8/11/2008 8:00 & 475 & 224 & 800 & 25.15 & 25.15 & & 0.0243 & 1.22E-04 & 1.1702 & 0.6582 & 1.8284 & \\
\hline & 8/11/2008 9:00 & 476 & 224 & 900 & 23.04 & 23.04 & & 0.1181 & 8.58E-04 & 8.2094 & 4.6178 & 12.8272 & \\
\hline & 8/11/2008 10:00 & 477 & 224 & 1000 & 20.27 & 20.27 & & 0.2708 & $2.38 \mathrm{E}-03$ & 22.8166 & 12.8344 & 35.6510 & \\
\hline & 8/11/2008 11:00 & 478 & 224 & 1100 & 19.33 & 19.33 & & 0.3326 & $3.07 \mathrm{E}-03$ & 29.3854 & 16.5293 & 45.9147 & \\
\hline & $8 / 11 / 2008$ 12:00 & 479 & 224 & 1200 & 18.82 & 18.82 & & 0.3688 & 3.49E-03 & 33.3604 & 18.7652 & 52.1257 & \\
\hline & $8 / 11 / 2008$ 13:00 & 480 & 224 & 1300 & 19.03 & 19.03 & & 0.3537 & 3.31E-03 & 31.6864 & 17.8236 & 49.5099 & \\
\hline & 8/11/2008 14:00 & 481 & 224 & 1400 & 18.32 & 18.32 & & 0.4061 & $3.92 \mathrm{E}-03$ & 37.5682 & 21.1321 & 58.7003 & \\
\hline & 8/11/2008 15:00 & 482 & 224 & 1500 & 17.96 & 17.96 & & 0.4343 & 4.26E-03 & 40.8030 & 22.9517 & 63.7547 & \\
\hline & $8 / 11 / 2008$ 16:00 & 483 & 224 & 1600 & 18.44 & 18.44 & & 0.3970 & 3.82E-03 & 36.5289 & 20.5475 & 57.0764 & \\
\hline & 8/11/2008 17:00 & 484 & 224 & 1700 & 18.86 & 18.86 & & 0.3659 & 3.45E-03 & 33.0374 & 18.5836 & 51.6210 & \\
\hline & 8/11/2008 18:00 & 485 & 224 & 1800 & 19.52 & 19.52 & & 0.3197 & 2.92E-03 & 27.9812 & 15.7394 & 43.7206 & \\
\hline & $8 / 11 / 2008$ 19:00 & 486 & 224 & 1900 & 19.53 & 19.53 & & 0.3190 & 2.92E-03 & 27.9084 & 15.6985 & 43.6069 & \\
\hline & 8/11/2008 20:00 & 487 & 224 & 2000 & 21.17 & 21.17 & & 0.2168 & $1.81 \mathrm{E}-03$ & 17.3502 & 9.7595 & 27.1097 & \\
\hline & 8/11/2008 21:00 & 488 & 224 & 2100 & 22.49 & 22.49 & & 0.1454 & 1.11E-03 & 10.6092 & 5.9677 & 16.5769 & \\
\hline & $8 / 11 / 200822: 00$ & 489 & 224 & 2200 & 23.74 & 23.74 & & 0.0851 & 5.73E-04 & 5.4859 & 3.0858 & 8.5717 & \\
\hline & 8/11/2008 23:00 & 490 & 224 & 2300 & 24.02 & 24.02 & & 0.0724 & 4.70E-04 & 4.4999 & 2.5312 & 7.0311 & \\
\hline & 8/12/2008 0:00 & 491 & 225 & 0 & 24.23 & 24.23 & 25.83 & 0.0631 & 3.97E-04 & 3.8001 & 2.1375 & 5.9376 & \\
\hline
\end{tabular}




\begin{tabular}{|c|c|c|c|c|c|c|c|c|c|c|c|c|c|}
\hline Tree ID & TS & RN & JD & HR & $\begin{array}{c}\text { Raw } \\
\text { TC_dTA }\end{array}$ & $\begin{array}{c}\text { Fixed } \\
\text { TC_dT } \Delta\end{array}$ & dTM & $\mathrm{K}$ & $\begin{array}{l}\text { Sap } \\
\text { Velocity } \\
(\mathrm{cm} / \mathrm{s})\end{array}$ & $\begin{array}{c}\text { Probed } \\
\text { Drywood F } \\
(\mathrm{g} / \mathrm{hr})\end{array}$ & $\begin{array}{c}\text { Extrapolated } \\
\text { Wetwood F } \\
(\mathrm{g} / \mathrm{hr})\end{array}$ & $\begin{array}{c}\text { Extrapolated } \\
\text { Total Wood F } \\
(\mathrm{g} / \mathrm{hr})\end{array}$ & $\begin{array}{l}\text { Relative } \\
\text { Humidity, } \\
@ 10 \mathrm{~m}(\%)\end{array}$ \\
\hline & 8/12/2008 1:00 & 492 & 225 & 100 & 24.26 & 24.26 & & 0.0618 & 3.87E-04 & 3.7029 & 2.0829 & $\begin{array}{r}5.7858 \\
\end{array}$ & \\
\hline & 8/12/2008 2:00 & 493 & 225 & 200 & 25.01 & 25.01 & & 0.0300 & $1.59 \mathrm{E}-04$ & 1.5195 & 0.8547 & 2.3742 & \\
\hline & 8/12/2008 3:00 & 494 & 225 & 300 & 25 & 25 & & 0.0304 & $1.61 E-04$ & 1.5452 & 0.8692 & 2.4144 & \\
\hline & 8/12/2008 4:00 & 495 & 225 & 400 & 25.08 & 25.08 & & 0.0271 & $1.40 \mathrm{E}-04$ & 1.3422 & 0.7550 & 2.0972 & \\
\hline & 8/12/2008 5:00 & 496 & 225 & 500 & 25.12 & 25.12 & & 0.0255 & 1.30E-04 & 1.2432 & 0.6993 & 1.9426 & \\
\hline & 8/12/2008 6:00 & 497 & 225 & 600 & 25.2 & 25.2 & & 0.0222 & 1.10E-04 & 1.0507 & 0.5910 & 1.6417 & \\
\hline & 8/12/2008 7:00 & 498 & 225 & 700 & 25.36 & 25.36 & & 0.0158 & 7.20E-05 & 0.6890 & 0.3875 & 1.0765 & \\
\hline & 8/12/2008 8:00 & 499 & 225 & 800 & 25.44 & 25.44 & & 0.0126 & 5.45E-05 & 0.5215 & 0.2933 & 0.8148 & \\
\hline & 8/12/2008 9:00 & 500 & 225 & 900 & 24.46 & 24.46 & & 0.0531 & $3.21 \mathrm{E}-04$ & 3.0736 & 1.7289 & 4.8025 & \\
\hline & $8 / 12 / 2008$ 10:00 & 501 & 225 & 1000 & 21.48 & 21.48 & & 0.1993 & 1.63E-03 & 15.6368 & 8.7957 & 24.4325 & \\
\hline & $8 / 12 / 2008$ 11:00 & 502 & 225 & 1100 & 20 & 20 & & 0.2880 & 2.57E-03 & 24.6086 & 13.8423 & 38.4510 & \\
\hline & $8 / 12 / 2008$ 12:00 & 503 & 225 & 1200 & 19.02 & 19.02 & & 0.3544 & $3.32 \mathrm{E}-03$ & 31.7649 & 17.8677 & 49.6326 & \\
\hline & 8/12/2008 13:00 & 504 & 225 & 1300 & 18.36 & 18.36 & & 0.4031 & 3.89E-03 & 37.2196 & 20.9360 & 58.1557 & \\
\hline & $8 / 12 / 2008$ 14:00 & 505 & 225 & 1400 & 17.92 & 17.92 & & 0.4375 & 4.30E-03 & 41.1736 & 23.1602 & 64.3338 & \\
\hline & $8 / 12 / 2008$ 15:00 & 506 & 225 & 1500 & 17.46 & 17.46 & & 0.4754 & $4.76 \mathrm{E}-03$ & 45.6041 & 25.6523 & 71.2564 & \\
\hline & $8 / 12 / 2008$ 16:00 & 507 & 225 & 1600 & 17.99 & 17.99 & & 0.4319 & 4.23E-03 & 40.5266 & 22.7962 & 63.3228 & \\
\hline & $8 / 12 / 2008$ 17:00 & 508 & 225 & 1700 & 17.73 & 17.73 & & 0.4529 & 4.49E-03 & 42.9655 & 24.1681 & 67.1335 & \\
\hline & $8 / 12 / 2008$ 18:00 & 509 & 225 & 1800 & 18.4 & 18.4 & & 0.4000 & $3.85 E-03$ & 36.8732 & 20.7412 & 57.6144 & \\
\hline & $8 / 12 / 2008$ 19:00 & 510 & 225 & 1900 & 19.29 & 19.29 & & 0.3354 & $3.10 \mathrm{E}-03$ & 29.6862 & 16.6985 & 46.3846 & \\
\hline & $8 / 12 / 200820: 00$ & 511 & 225 & 2000 & 21.73 & 21.73 & & 0.1855 & 1.50E-03 & 14.3148 & 8.0521 & 22.3669 & \\
\hline & $8 / 12 / 2008$ 21:00 & 512 & 225 & 2100 & 24.7 & 24.7 & & 0.0429 & $2.47 \mathrm{E}-04$ & 2.3622 & 1.3287 & 3.6909 & \\
\hline & $8 / 12 / 2008$ 22:00 & 513 & 225 & 2200 & 25.74 & 25.74 & & 0.0008 & 1.77E-06 & 0.0169 & 0.0095 & 0.0265 & \\
\hline & $8 / 12 / 2008$ 23:00 & 514 & 225 & 2300 & 25.83 & 25.83 & & -0.0027 & $0.00 \mathrm{E}+00$ & 0.0000 & 0.0000 & 0.0000 & \\
\hline & 8/13/2008 0:00 & 515 & 226 & 0 & 25.81 & 25.81 & 25.88 & -0.0019 & $0.00 E+00$ & 0.0000 & 0.0000 & 0.0000 & \\
\hline & 8/13/2008 1:00 & 516 & 226 & 100 & 25.81 & 25.81 & & -0.0019 & $0.00 \mathrm{E}+00$ & 0.0000 & 0.0000 & 0.0000 & \\
\hline & 8/13/2008 2:00 & 517 & 226 & 200 & 25.53 & 25.53 & & 0.0090 & 3.61E-05 & 0.3458 & 0.1945 & 0.5403 & \\
\hline & 8/13/2008 3:00 & 518 & 226 & 300 & 25.8 & 25.8 & & -0.0016 & $0.00 \mathrm{E}+00$ & 0.0000 & 0.0000 & 0.0000 & \\
\hline & 8/13/2008 4:00 & 519 & 226 & 400 & 25.63 & 25.63 & & 0.0051 & $1.78 \mathrm{E}-05$ & 0.1705 & 0.0959 & 0.2664 & \\
\hline & $8 / 13 / 20085: 00$ & 520 & 226 & 500 & 25.44 & 25.44 & & 0.0126 & 5.45E-05 & 0.5215 & 0.2933 & 0.8148 & \\
\hline & 8/13/2008 6:00 & 521 & 226 & 600 & 25.83 & 25.83 & & -0.0027 & $0.00 \mathrm{E}+00$ & 0.0000 & 0.0000 & 0.0000 & \\
\hline & $8 / 13 / 2008$ 7:00 & 522 & 226 & 700 & 25.88 & 25.88 & & -0.0046 & $0.00 \mathrm{E}+00$ & 0.0000 & 0.0000 & 0.0000 & \\
\hline & 8/13/2008 8:00 & 523 & 226 & 800 & 25.84 & 25.84 & & -0.0031 & $0.00 E+00$ & 0.0000 & 0.0000 & 0.0000 & \\
\hline & 8/13/2008 9:00 & 524 & 226 & 900 & 23.22 & 23.22 & & 0.1094 & 7.81E-04 & 7.4739 & 4.2041 & 11.6780 & \\
\hline & $8 / 13 / 2008$ 10:00 & 525 & 226 & 1000 & 20.17 & 20.17 & & 0.2771 & $2.45 E-03$ & 23.4718 & 13.2029 & 36.6747 & \\
\hline & 8/13/2008 11:00 & 526 & 226 & 1100 & 19.83 & 19.83 & & 0.2990 & 2.69E-03 & 25.7751 & 14.4985 & 40.2737 & \\
\hline & $8 / 13 / 2008$ 12:00 & 527 & 226 & 1200 & 18.84 & 18.84 & & 0.3673 & 3.47E-03 & 33.1987 & 18.6743 & 51.8730 & \\
\hline
\end{tabular}




\begin{tabular}{|c|c|c|c|c|c|c|c|c|c|c|c|c|c|}
\hline Tree ID & TS & RN & JD & HR & $\begin{array}{c}\text { Raw } \\
\text { TC_dTA }\end{array}$ & $\begin{array}{l}\text { Fixed } \\
\text { TC_dT } \Delta\end{array}$ & dTM & $\mathbf{K}$ & $\begin{array}{c}\text { Sap } \\
\text { Velocity } \\
(\mathrm{cm} / \mathrm{s})\end{array}$ & $\begin{array}{c}\text { Probed Drywood } \\
F(g / h r)\end{array}$ & $\begin{array}{c}\text { Extrapolated } \\
\text { Wetwood F } \\
(\mathrm{g} / \mathrm{hr})\end{array}$ & $\begin{array}{c}\text { Extrapolated } \\
\text { Total Wood F } \\
(\mathrm{g} / \mathrm{hr})\end{array}$ & $\begin{array}{c}\text { Relative } \\
\text { Humidity, } \\
@ 10 \mathrm{~m}(\%)\end{array}$ \\
\hline & $7 / 22 / 2008$ 13:00 & 0 & 204 & 1300 & 0.068 & 0.068 & 4.91 & 71.1471 & $2.27 E+00$ & 526405.9871 & 193965.1124 & 720371.0996 & \\
\hline \multirow[t]{2}{*}{ W708EC2 } & $7 / 22 / 2008$ 14:00 & 1 & 204 & 1400 & 4.827 & 4.827 & & 0.0164 & $7.53 \mathrm{E}-05$ & 17.4859 & 6.4430 & 23.9289 & \\
\hline & $7 / 22 / 2008$ 15:00 & 2 & 204 & 1500 & 4.906 & 4.906 & & 0.0000 & $0.00 \mathrm{E}+00$ & 0.0000 & 0.0000 & 0.0000 & \\
\hline \multirow[t]{2}{*}{ Probe \# } & $7 / 22 / 2008$ 16:00 & 3 & 204 & 1600 & 4.669 & 4.669 & & 0.0508 & $3.03 E-04$ & 70.4395 & 25.9549 & 96.3944 & \\
\hline & $7 / 22 / 2008$ 17:00 & 4 & 204 & 1700 & 2.617 & 2.617 & & 0.8747 & $1.01 E-02$ & 2342.7352 & 863.2290 & 3205.9642 & \\
\hline \multirow[t]{4}{*}{21} & $7 / 22 / 2008$ 18:00 & 5 & 204 & 1800 & 2.804 & 2.804 & & 0.7496 & 8.35E-03 & 1937.5894 & 713.9447 & 2651.5341 & \\
\hline & $7 / 22 / 2008$ 19:00 & 6 & 204 & 1900 & 2.86 & 2.86 & & 0.7154 & 7.88E-03 & 1829.1691 & 673.9950 & 2503.1641 & \\
\hline & $7 / 22 / 200820: 00$ & 7 & 204 & 2000 & 2.937 & 2.937 & & 0.6704 & 7.27E-03 & 1688.6604 & 622.2217 & 2310.8821 & \\
\hline & 7/22/2008 21:00 & 8 & 204 & 2100 & 3.66 & 3.66 & & 0.3404 & 3.16E-03 & 733.2517 & 270.1817 & 1003.4334 & \\
\hline \multirow[t]{2}{*}{$\mathrm{DBH}(\mathrm{cm})$} & $7 / 22 / 200822: 00$ & 9 & 204 & 2200 & 4.28 & 4.28 & & 0.1463 & 1.12E-03 & 259.1739 & 95.4980 & 354.6719 & \\
\hline & 7/22/2008 23:00 & 10 & 204 & 2300 & 4.489 & 4.489 & & 0.0929 & 6.38E-04 & 148.2200 & 54.6147 & 202.8348 & \\
\hline \multirow[t]{2}{*}{11.2} & $7 / 23 / 20080: 00$ & 11 & 205 & 0 & 4.658 & 4.658 & 4.77 & 0.0532 & $3.22 \mathrm{E}-04$ & 74.7020 & 27.5255 & 102.2275 & \\
\hline & 7/23/2008 1:00 & 12 & 205 & 100 & 4.721 & 4.721 & & 0.0392 & $2.21 E-04$ & 51.2234 & 18.8743 & 70.0977 & \\
\hline Day Count & 7/23/2008 2:00 & 13 & 205 & 200 & 4.771 & 4.771 & & 0.0283 & $1.48 \mathrm{E}-04$ & 34.3074 & 12.6413 & 46.9487 & \\
\hline \multirow[t]{27}{*}{35} & 7/23/2008 3:00 & 14 & 205 & 300 & 4.772 & 4.772 & & 0.0281 & 1.46E-04 & 33.9861 & 12.5229 & 46.5090 & \\
\hline & 7/23/2008 4:00 & 15 & 205 & 400 & 4.76 & 4.76 & & 0.0307 & $1.63 \mathrm{E}-04$ & 37.8878 & 13.9606 & 51.8484 & \\
\hline & 7/23/2008 5:00 & 16 & 205 & 500 & 4.719 & 4.719 & & 0.0396 & 2.24E-04 & 51.9330 & 19.1358 & 71.0688 & \\
\hline & 7/23/2008 6:00 & 17 & 205 & 600 & 4.648 & 4.648 & & 0.0555 & 3.39E-04 & 78.6348 & 28.9746 & 107.6094 & \\
\hline & 7/23/2008 7:00 & 18 & 205 & 700 & 4.474 & 4.474 & & 0.0966 & 6.70E-04 & 155.4495 & 57.2786 & 212.7281 & \\
\hline & 7/23/2008 8:00 & 19 & 205 & 800 & 4.017 & 4.017 & & 0.2213 & 1.86E-03 & 431.5295 & 159.0059 & 590.5355 & \\
\hline & $7 / 23 / 20089: 00$ & 20 & 205 & 900 & 3.202 & 3.202 & & 0.5322 & $5.47 \mathrm{E}-03$ & 1270.8112 & 468.2565 & 1739.0677 & \\
\hline & $7 / 23 / 2008$ 10:00 & 21 & 205 & 1000 & 2.641 & 2.641 & & 0.8576 & $9.85 E-03$ & 2286.6920 & 842.5787 & 3129.2707 & \\
\hline & 7/23/2008 11:00 & 22 & 205 & 1100 & 2.706 & 2.706 & & 0.8130 & $9.22 \mathrm{E}-03$ & 2141.1270 & 788.9423 & 2930.0692 & \\
\hline & 7/23/2008 12:00 & 23 & 205 & 1200 & 2.729 & 2.729 & & 0.7977 & 9.01E-03 & 2091.6980 & 770.7292 & 2862.4272 & \\
\hline & $7 / 23 / 2008$ 13:00 & 24 & 205 & 1300 & 2.718 & 2.718 & & 0.8050 & $9.11 \mathrm{E}-03$ & 2115.2066 & 779.3914 & 2894.5979 & \\
\hline & $7 / 23 / 2008$ 14:00 & 25 & 205 & 1400 & 2.715 & 2.715 & & 0.8070 & $9.14 \mathrm{E}-03$ & 2121.6596 & 781.7691 & 2903.4288 & \\
\hline & 7/23/2008 15:00 & 26 & 205 & 1500 & 2.683 & 2.683 & & 0.8286 & 9.44E-03 & 2191.6240 & 807.5489 & 2999.1729 & \\
\hline & $7 / 23 / 2008$ 16:00 & 27 & 205 & 1600 & 2.756 & 2.756 & & 0.7801 & 8.77E-03 & 2034.9964 & 749.8363 & 2784.8327 & \\
\hline & $7 / 23 / 2008$ 17:00 & 28 & 205 & 1700 & 2.937 & 2.937 & & 0.6704 & 7.27E-03 & 1688.6604 & 622.2217 & 2310.8821 & \\
\hline & $7 / 23 / 2008$ 18:00 & 29 & 205 & 1800 & 3.271 & 3.271 & & 0.4998 & 5.07E-03 & 1176.4791 & 433.4979 & 1609.9770 & \\
\hline & $7 / 23 / 2008$ 19:00 & 30 & 205 & 1900 & 3.215 & 3.215 & & 0.5260 & 5.40E-03 & 1252.6237 & 461.5550 & 1714.1786 & \\
\hline & $7 / 23 / 200820: 00$ & 31 & 205 & 2000 & 3.387 & 3.387 & & 0.4485 & $4.43 E-03$ & 1029.4630 & 379.3268 & 1408.7898 & \\
\hline & $7 / 23 / 2008$ 21:00 & 32 & 205 & 2100 & 3.819 & 3.819 & & 0.2846 & 2.53E-03 & 588.2124 & 216.7389 & 804.9513 & \\
\hline & $7 / 23 / 200822: 00$ & 33 & 205 & 2200 & 4.114 & 4.114 & & 0.1925 & 1.57E-03 & 363.4854 & 133.9337 & 497.4191 & \\
\hline & $7 / 23 / 200823: 00$ & 34 & 205 & 2300 & 4.185 & 4.185 & & 0.1723 & 1.37E-03 & 317.0493 & 116.8233 & 433.8726 & \\
\hline & $7 / 24 / 20080: 00$ & 35 & 206 & 0 & 4.203 & 4.203 & 4.58 & 0.1673 & 1.32E-03 & 305.7145 & 112.6468 & 418.3613 & \\
\hline & 7/24/2008 1:00 & 36 & 206 & 100 & 4.23 & 4.23 & & 0.1598 & $1.25 \mathrm{E}-03$ & 289.0380 & 106.5020 & 395.5401 & \\
\hline & 7/24/2008 2:00 & 37 & 206 & 200 & 4.271 & 4.271 & & 0.1487 & 1.14E-03 & 264.4528 & 97.4431 & 361.8959 & \\
\hline & 7/24/2008 3:00 & 38 & 206 & 300 & 4.336 & 4.336 & & 0.1315 & 9.79E-04 & 227.2692 & 83.7420 & 311.0113 & \\
\hline & 7/24/2008 4:00 & 39 & 206 & 400 & 4.392 & 4.392 & & 0.1170 & 8.48E-04 & 196.9672 & 72.5766 & 269.5438 & \\
\hline & 7/24/2008 5:00 & 40 & 206 & 500 & 4.457 & 4.457 & & 0.1007 & 7.05E-04 & 163.7794 & 60.3479 & 224.1273 & \\
\hline
\end{tabular}




\begin{tabular}{|c|c|c|c|c|c|c|c|c|c|c|c|c|c|}
\hline Tree ID & TS & RN & JD & HR & $\begin{array}{c}\text { Raw } \\
\text { TC_dTA }\end{array}$ & $\begin{array}{c}\text { Fixed } \\
\text { TC_dT } \Delta\end{array}$ & dTM & $\mathbf{K}$ & $\begin{array}{l}\text { Sap } \\
\text { Velocity } \\
\text { (cm/s) }\end{array}$ & $\begin{array}{c}\text { Probed Drywood } \\
\mathrm{F}(\mathrm{g} / \mathrm{hr})\end{array}$ & $\begin{array}{c}\text { Extrapolated } \\
\text { Wetwood F } \\
(\mathrm{g} / \mathrm{hr})\end{array}$ & $\begin{array}{c}\text { Extrapolated } \\
\text { Total Wood F } \\
(\mathrm{g} / \mathrm{hr})\end{array}$ & $\begin{array}{l}\text { Relative } \\
\text { Humidity, } \\
@ 10 \mathrm{~m} \text { (\%) }\end{array}$ \\
\hline & 7/24/2008 6:00 & 41 & 206 & 600 & 4.515 & 4.515 & & 0.0866 & 5.86E-04 & 135.9570 & 50.0961 & 186.0531 & \\
\hline & 7/24/2008 7:00 & 42 & 206 & 700 & 4.517 & 4.517 & & 0.0861 & $5.82 \mathrm{E}-04$ & 135.0278 & 49.7538 & 184.7815 & \\
\hline & 7/24/2008 8:00 & 43 & 206 & 800 & 4.439 & 4.439 & & 0.1052 & 7.44E-04 & 172.7574 & 63.6560 & 236.4134 & \\
\hline & 7/24/2008 9:00 & 44 & 206 & 900 & 4.218 & 4.218 & & 0.1631 & $1.28 \mathrm{E}-03$ & 296.4018 & 109.2153 & 405.6171 & \\
\hline & 7/24/2008 10:00 & 45 & 206 & 1000 & 4.183 & 4.183 & & 0.1728 & 1.37E-03 & 318.3195 & 117.2914 & 435.6109 & \\
\hline & 7/24/2008 11:00 & 46 & 206 & 1100 & 3.155 & 3.155 & & 0.5550 & $5.76 \mathrm{E}-03$ & 1338.2358 & 493.1005 & 1831.3363 & \\
\hline & 7/24/2008 12:00 & 47 & 206 & 1200 & 2.681 & 2.681 & & 0.8299 & $9.46 \mathrm{E}-03$ & 2196.0665 & 809.1859 & 3005.2523 & \\
\hline & 7/24/2008 13:00 & 48 & 206 & 1300 & 2.957 & 2.957 & & 0.6591 & $7.12 \mathrm{E}-03$ & 1653.6973 & 609.3388 & 2263.0360 & \\
\hline & 7/24/2008 14:00 & 49 & 206 & 1400 & 2.892 & 2.892 & & 0.6964 & $7.62 \mathrm{E}-03$ & 1769.6106 & 652.0494 & 2421.6601 & \\
\hline & 7/24/2008 15:00 & 50 & 206 & 1500 & 2.748 & 2.748 & & 0.7853 & $8.84 \mathrm{E}-03$ & 2051.6503 & 755.9728 & 2807.6231 & \\
\hline & 7/24/2008 16:00 & 51 & 206 & 1600 & 2.773 & 2.773 & & 0.7692 & $8.62 \mathrm{E}-03$ & 2000.0095 & 736.9446 & 2736.9542 & \\
\hline & 7/24/2008 17:00 & 52 & 206 & 1700 & 2.847 & 2.847 & & 0.7232 & 7.99E-03 & 1853.8543 & 683.0908 & 2536.9451 & \\
\hline & 7/24/2008 18:00 & 53 & 206 & 1800 & 2.866 & 2.866 & & 0.7118 & 7.83E-03 & 1817.8723 & 669.8324 & 2487.7047 & \\
\hline & 7/24/2008 19:00 & 54 & 206 & 1900 & 3.129 & 3.129 & & 0.5679 & 5.93E-03 & 1376.6914 & 507.2702 & 1883.9616 & \\
\hline & $7 / 24 / 200820: 00$ & 55 & 206 & 2000 & 3.44 & 3.44 & & 0.4262 & $4.16 \mathrm{E}-03$ & 966.7698 & 356.2262 & 1322.9961 & \\
\hline & 7/24/2008 21:00 & 56 & 206 & 2100 & 4.078 & 4.078 & & 0.2030 & $1.67 \mathrm{E}-03$ & 388.1061 & 143.0057 & 531.1117 & \\
\hline & 7/24/2008 22:00 & 57 & 206 & 2200 & 4.501 & 4.501 & & 0.0900 & $6.14 \mathrm{E}-04$ & 142.5179 & 52.5136 & 195.0315 & \\
\hline & $7 / 24 / 200823: 00$ & 58 & 206 & 2300 & 4.584 & 4.584 & & 0.0702 & 4.53E-04 & 105.0737 & 38.7166 & 143.7903 & \\
\hline & 7/25/2008 0:00 & 59 & 207 & 0 & 4.585 & 4.585 & 4.59 & 0.0700 & $4.51 \mathrm{E}-04$ & 104.6441 & 38.5583 & 143.2023 & \\
\hline & 7/25/2008 1:00 & 60 & 207 & 100 & 4.534 & 4.534 & & 0.0820 & 5.48E-04 & 127.2112 & 46.8736 & 174.0847 & \\
\hline & 7/25/2008 2:00 & 61 & 207 & 200 & 4.477 & 4.477. & & 0.0958 & $6.63 \mathrm{E}-04$ & 153.9946 & 56.7425 & 210.7370 & \\
\hline & 7/25/2008 3:00 & 62 & 207 & 300 & 4.445 & 4.445 & & 0.1037 & 7.31E-04 & 169.7467 & 62.5467 & 232.2933 & \\
\hline & 7/25/2008 4:00 & 63 & 207 & 400 & 4.449 & 4.449 & & 0.1027 & $7.23 \mathrm{E}-04$ & 167.7496 & 61.8108 & 229.5604 & \\
\hline & $7 / 25 / 20085: 00$ & 64 & 207 & 500 & 4.471 & 4.471 & & 0.0973 & $6.76 \mathrm{E}-04$ & 156.9090 & 57.8163 & 214.7253 & \\
\hline & 7/25/2008 6:00 & 65 & 207 & 600 & 4.5 & 4.5 & & 0.0902 & $6.16 \mathrm{E}-04$ & 142.9903 & 52.6877 & 195.6780 & \\
\hline & 7/25/2008 7:00 & 66 & 207 & 700 & 4.499 & 4.499 & & 0.0905 & $6.18 \mathrm{E}-04$ & 143.4632 & 52.8620 & 196.3252 & \\
\hline & 7/25/2008 8:00 & 67. & 207 & 800 & 4.488 & 4.488 & & 0.0931 & $6.41 \mathrm{E}-04$ & 148.6985 & 54.7910 & 203.4895 & \\
\hline & 7/25/2008 9:00 & 68 & 207 & 900 & 4.406 & 4.406 & & 0.1135 & 8.17E-04 & 189.6396 & 69.8766 & 259.5161 & \\
\hline & $7 / 25 / 2008$ 10:00 & 69 & 207 & 1000 & 4.389 & 4.389 & & 0.1178 & 8.55E-04 & 198.5503 & 73.1599 & 271.7102 & \\
\hline & 7/25/2008 11:00 & 70 & 207 & 1100 & 3.97 & 3.97 & & 0.2358 & $2.01 \mathrm{E}-03$ & 466.4929 & 171.8889 & 638.3818 & \\
\hline & $7 / 25 / 2008$ 12:00 & 71 & 207 & 1200 & 3.182 & 3.182 & & 0.5418 & $5.60 \mathrm{E}-03$ & 1299.1792 & 478.7093 & 1777.8885 & \\
\hline & 7/25/2008 13:00 & 72 & 207 & 1300 & 2.887 & 2.887 & & 0.6993 & $7.66 \mathrm{E}-03$ & 1778.8054 & 655.4374 & 2434.2428 & \\
\hline & 7/25/2008 14:00 & 73 & 207 & 1400 & 2.777 & 2.777 & & 0.7667 & $8.58 \mathrm{E}-03$ & 1991.8560 & 733.9403 & 2725.7963 & \\
\hline & 7/25/2008 15:00 & 74 & 207 & 1500 & 2.824 & 2.824 & & 0.7373 & $8.18 \mathrm{E}-03$ & 1898.2393 & 699.4453 & 2597.6845 & \\
\hline & 7/25/2008 16:00 & 75 & 207 & 1600 & 2.879 & 2.879 & & 0.7041 & 7.73E-03 & 1793.6020 & 660.8896 & 2454.4916 & \\
\hline & 7/25/2008 17:00 & 76 & 207 & 1700 & 2.794 & 2.794 & & 0.7559 & $8.43 \mathrm{E}-03$ & 1957.5331 & 721.2933 & 2678.8265 & \\
\hline & 7/25/2008 18:00 & 77. & 207 & 1800 & 2.856 & 2.856 & & 0.7178 & 7.91E-03 & 1836.7340 & 676.7824 & 2513.5164 & \\
\hline & 7/25/2008 19:00 & 78 & 207 & 1900 & 2.954 & 2.954 & & 0.6608 & 7.15E-03 & 1658.9028 & 611.2569 & 2270.1597 & \\
\hline & $7 / 25 / 200820: 00$ & 79 & 207 & 2000 & 3.199 & 3.199 & & 0.5336 & $5.49 \mathrm{E}-03$ & 1275.0363 & 469.8133 & 1744.8496 & \\
\hline & 7/25/2008 21:00 & 80 & 207 & 2100 & 3.91 & 3.91 & & 0.2547 & 2.21E-03 & 513.1015 & 189.0628 & 702.1643 & \\
\hline & 7/25/2008 22:00 & 81 & 207 & 2200 & 4.444 & 4.444 & & 0.1040 & 7.33E-04 & 170.2472 & 62.7311 & 232.9783 & \\
\hline
\end{tabular}




\begin{tabular}{|c|c|c|c|c|c|c|c|c|c|c|c|c|c|}
\hline Tree ID & TS & RN & JD & HR & $\begin{array}{c}\text { Raw } \\
\text { TC_dTA }\end{array}$ & $\begin{array}{c}\text { Fixed } \\
\text { TC_dT } \Delta\end{array}$ & dTM & $\mathbf{K}$ & $\begin{array}{l}\text { Sap } \\
\text { Velocity } \\
\text { (cm/s) }\end{array}$ & $\begin{array}{c}\text { Probed Drywood } \\
F(\mathrm{~g} / \mathrm{hr})\end{array}$ & $\begin{array}{c}\text { Extrapolated } \\
\text { Wetwood F } \\
\text { (g/hr) }\end{array}$ & $\begin{array}{c}\text { Extrapolated } \\
\text { Total Wood F } \\
(\mathrm{g} / \mathrm{hr})\end{array}$ & $\begin{array}{l}\text { Relative } \\
\text { Humidity, } \\
\text { @10m (\%) }\end{array}$ \\
\hline & $7 / 25 / 200823: 00$ & 82 & 207 & 2300 & \begin{tabular}{|r|}
4.543 \\
\end{tabular} & 4.543 & & 0.0799 & 5.30E-04 & 123.1322 & 45.3706 & 168.5028 & \\
\hline & $7 / 26 / 20080: 00$ & 83 & 208 & 0 & 4.56 & 4.56 & 4.56 & 0.0759 & 4.98E-04 & 115.5401 & 42.5731 & 158.1132 & \\
\hline & 7/26/2008 1:00 & 84 & 208 & 100 & 4.559 & 4.559 & & 0.0761 & 5.00E-04 & 115.9826 & 42.7362 & 158.7187 & \\
\hline & $7 / 26 / 20082: 00$ & 85 & 208 & 200 & 4.547 & 4.547 & & 0.0790 & $5.23 E-04$ & 121.3326 & 44.7075 & 166.0401 & \\
\hline & 7/26/2008 3:00 & 86 & 208 & 300 & 4.537 & 4.537 & & 0.0813 & $5.42 \mathrm{E}-04$ & 125.8470 & 46.3709 & 172.2179 & \\
\hline & 7/26/2008 4:00 & 87 & 208 & 400 & 4.517 & 4.517 & & 0.0861 & 5.82E-04 & 135.0278 & 49.7538 & 184.7815 & \\
\hline & 7/26/2008 5:00 & 88 & 208 & 500 & 4.493 & 4.493 & & 0.0919 & $6.30 \mathrm{E}-04$ & 146.3113 & 53.9114 & 200.2227 & \\
\hline & 7/26/2008 6:00 & 89 & 208 & 600 & 4.468 & 4.468 & & 0.0980 & $6.82 \mathrm{E}-04$ & 158.3729 & 58.3558 & 216.7287 & \\
\hline & 7/26/2008 7:00 & 90 & 208 & 700 & 4.449 & 4.449 & & 0.1027 & $7.23 \mathrm{E}-04$ & 167.7496 & 61.8108 & 229.5604 & \\
\hline & 7/26/2008 8:00 & 91 & 208 & 800 & 4.44 & 4.44 & & 0.1050 & 7.42E-04 & 172.2544 & 63.4707 & 235.7250 & \\
\hline & $7 / 26 / 20089: 00$ & 92 & 208 & 900 & 3.831 & 3.831 & & 0.2806 & 2.49E-03 & 577.9925 & 212.9732 & 790.9657 & \\
\hline & $7 / 26 / 2008$ 10:00 & 93 & 208 & 1000 & 2.628 & 2.628 & & 0.8668 & 9.98E-03 & 2316.8900 & 853.7058 & 3170.5958 & \\
\hline & 7/26/2008 11:00 & 94 & 208 & 1100 & 2.772 & 2.772 & & 0.7698 & $8.62 \mathrm{E}-03$ & 2002.0526 & 737.6974 & 2739.7500 & \\
\hline & $7 / 26 / 2008$ 12:00 & 95 & 208 & 1200 & 3.049 & 3.049 & & 0.6091 & 6.46E-03 & 1500.4630 & 552.8764 & 2053.3394 & \\
\hline & 7/26/2008 13:00 & 96 & 208 & 1300 & 2.823 & 2.823 & & 0.7379 & $8.19 \mathrm{E}-03$ & 1900.1899 & 700.1641 & 2600.3540 & \\
\hline & 7/26/2008 14:00 & 97 & 208 & 1400 & 2.844 & 2.844 & & 0.7250 & $8.01 \mathrm{E}-03$ & 1859.5918 & 685.2049 & 2544.7967 & \\
\hline & 7/26/2008 15:00 & 98 & 208 & 1500 & 2.826 & 2.826 & & 0.7360 & 8.16E-03 & 1894.3432 & 698.0097 & 2592.3528 & \\
\hline & $7 / 26 / 2008$ 16:00 & 99 & 208 & 1600 & 2.847 & 2.847 & & 0.7232 & 7.99E-03 & 1853.8543 & 683.0908 & 2536.9451 & \\
\hline & 7/26/2008 17:00 & 100 & 208 & 1700 & 2.787 & 2.787 & & 0.7603 & 8.49E-03 & 1971.6018 & 726.4772 & 2698.0791 & \\
\hline & $7 / 26 / 2008$ 18:00 & 101 & 208 & 1800 & 2.925 & 2.925 & & 0.6773 & 7.37E-03 & 1709.9344 & 630.0605 & 2339.9949 & \\
\hline & 7/26/2008 19:00 & 102 & 208 & 1900 & 3.015 & 3.015 & & 0.6272 & $6.70 \mathrm{E}-03$ & 1555.6797 & 573.2222 & 2128.9019 & \\
\hline & 7/26/2008 20:00 & 103 & 208 & 2000 & 3.167 & 3.167 & & 0.5491 & $5.69 \mathrm{E}-03$ & 1320.7683 & 486.6642 & 1807.4325 & \\
\hline & 7/26/2008 21:00 & 104 & 208 & 2100 & 3.876 & 3.876 & & 0.2657 & $2.33 \mathrm{E}-03$ & 540.5275 & 199.1685 & 739.6960 & \\
\hline & $7 / 26 / 200822: 00$ & 105 & 208 & 2200 & 4.359 & 4.359 & & 0.1255 & $9.25 \mathrm{E}-04$ & 214.6312 & 79.0853 & 293.7164 & \\
\hline & 7/26/2008 23:00 & 106 & 208 & 2300 & 4.471 & 4.471 & & 0.0973 & $6.76 \mathrm{E}-04$ & 156.9090 & 57.8163 & 214.7253 & \\
\hline & $7 / 27 / 20080: 00$ & 107 & 209 & 0 & 4.49 & 4.49 & 4.49 & 0.0927 & 6.36E-04 & 147.7421 & 54.4386 & 202.1807 & \\
\hline & 7/27/2008 1:00 & 108 & 209 & 100 & 4.493 & 4.493 & & 0.0919 & $6.30 \mathrm{E}-04$ & 146.3113 & 53.9114 & 200.2227 & \\
\hline & 7/27/2008 2:00 & 109 & 209 & 200 & 4.488 & 4.488 & & 0.0931 & 6.41E-04 & 148.6985 & 54.7910 & 203.4895 & \\
\hline & 7/27/2008 3:00 & 110 & 209 & 300 & 4.459 & 4.459 & & 0.1002 & 7.01E-04 & 162.7919 & 59.9840 & 222.7760 & \\
\hline & 7/27/2008 4:00 & 111 & 209 & 400 & 4.44 & 4.44 & & 0.1050 & 7.42E-04 & 172.2544 & 63.4707 & 235.7250 & \\
\hline & 7/27/2008 5:00 & 112 & 209 & 500 & 4.41 & 4.41 & & 0.1125 & 8.08E-04 & 187.5641 & 69.1118 & 256.6759 & \\
\hline & 7/27/2008 6:00 & 113 & 209 & 600 & 4.365 & 4.365 & & 0.1239 & $9.11 \mathrm{E}-04$ & 211.3785 & 77.8868 & 289.2653 & \\
\hline & 7/27/2008 7:00 & 114 & 209 & 700 & 4.327 & 4.327 & & 0.1338 & 1.00E-03 & 232.2880 & 85.5913 & 317.8794 & \\
\hline & 7/27/2008 8:00 & 115 & 209 & 800 & 4.281 & 4.281 & & 0.1460 & 1.11E-03 & 258.5900 & 95.2828 & 353.8728 & \\
\hline & $7 / 27 / 20089: 00$ & 116 & 209 & 900 & 3.372 & 3.372 & & 0.4549 & 4.51E-03 & 1047.7002 & 386.0467 & 1433.7469 & \\
\hline & $7 / 27 / 2008$ 10:00 & 117 & 209 & 1000 & 2.486 & 2.486 & & 0.9735 & 1.15E-02 & 2672.5783 & 984.7665 & 3657.3448 & \\
\hline & 7/27/2008 11:00 & 118 & 209 & 1100 & 2.601 & 2.601 & & 0.8862 & 1.03E-02 & 2380.8156 & 877.2605 & 3258.0760 & \\
\hline & 7/27/2008 12:00 & 119 & 209 & 1200 & 2.666 & 2.666 & & 0.8402 & $9.60 \mathrm{E}-03$ & 2229.6520 & 821.5611 & 3051.2131 & \\
\hline & 7/27/2008 13:00 & 120 & 209 & 1300 & 2.723 & 2.723 & & 0.8017 & $9.07 \mathrm{E}-03$ & 2104.4912 & 775.4431 & 2879.9343 & \\
\hline & 7/27/2008 14:00 & 121 & 209 & 1400 & 2.713 & 2.713 & & 0.8083 & $9.16 \mathrm{E}-03$ & 2125.9717 & 783.3580 & 2909.3297 & \\
\hline & 7/27/2008 15:00 & 122 & 209 & 1500 & 2.701 & 2.701 & & 0.8164 & $9.27 \mathrm{E}-03$ & 2152.0127 & 792.9533 & 2944.9660 & \\
\hline
\end{tabular}




\begin{tabular}{|c|c|c|c|c|c|c|c|c|c|c|c|c|c|}
\hline Tree ID & TS & RN & JD & HR & $\begin{array}{c}\text { Raw } \\
\text { TC_dTA }\end{array}$ & $\begin{array}{c}\text { Fixed } \\
\text { TC_dT } \Delta\end{array}$ & dTM & $\mathbf{K}$ & $\begin{array}{l}\text { Sap } \\
\text { Velocity } \\
(\mathrm{cm} / \mathrm{s})\end{array}$ & $\begin{array}{c}\text { Probed Drywood } \\
\text { F (g/hr) }\end{array}$ & $\begin{array}{c}\text { Extrapolated } \\
\text { Wetwood F } \\
(\mathrm{g} / \mathrm{hr})\end{array}$ & $\begin{array}{c}\text { Extrapolated } \\
\text { Total Wood F } \\
(\mathrm{g} / \mathrm{hr})\end{array}$ & $\begin{array}{l}\text { Relative } \\
\text { Humidity, } \\
@ 10 \mathrm{~m}(\%)\end{array}$ \\
\hline & 7/27/2008 16:00 & 123 & 209 & 1600 & \begin{tabular}{|r|}
2.729 \\
\end{tabular} & 2.729 & & 0.7977 & $9.01 \mathrm{E}-03$ & 2091.6980 & 770.7292 & 2862.4272 & \\
\hline & 7/27/2008 17:00 & 124 & 209 & 1700 & 2.743 & 2.743 & & 0.7886 & $8.88 \mathrm{E}-03$ & 2062.1214 & 759.8310 & 2821.9524 & \\
\hline & $7 / 27 / 2008$ 18:00 & 125 & 209 & 1800 & 2.842 & 2.842 & & 0.7262 & $8.03 \mathrm{E}-03$ & 1863.4254 & 686.6174 & 2550.0428 & \\
\hline & $7 / 27 / 2008$ 19:00 & 126 & 209 & 1900 & 2.975 & 2.975 & & 0.6491 & 6.99E-03 & 1622.7482 & 597.9349 & 2220.6831 & \\
\hline & $7 / 27 / 200820: 00$ & 127 & 209 & 2000 & 3.063 & 3.063 & & 0.6017 & 6.37E-03 & 1478.1903 & 544.6696 & 2022.8599 & \\
\hline & $7 / 27 / 2008$ 21:00 & 128 & 209 & 2100 & 3.585 & 3.585 & & 0.3685 & 3.48E-03 & 808.2967 & 297.8336 & 1106.1303 & \\
\hline & $7 / 27 / 2008$ 22:00 & 129 & 209 & 2200 & 4.056 & 4.056 & & 0.2096 & $1.74 \mathrm{E}-03$ & 403.5169 & 148.6841 & 552.2010 & \\
\hline & $7 / 27 / 200823: 00$ & 130 & 209 & 2300 & 4.166 & 4.166 & & 0.1776 & 1.42E-03 & 329.2043 & 121.3021 & 450.5063 & \\
\hline & $7 / 28 / 20080: 00$ & 131 & 210 & 0 & 4.206 & 4.206 & 4.38 & 0.1664 & 1.31E-03 & 303.8423 & 111.9569 & 415.7992 & \\
\hline & 7/28/2008 1:00 & 132 & 210 & 100 & 4.285 & 4.285 & & 0.1449 & 1.10E-03 & 256.2595 & 94.4241 & 350.6835 & \\
\hline & 7/28/2008 2:00 & 133 & 210 & 200 & 4.3 & 4.3 & & 0.1409 & 1.07E-03 & 247.5938 & 91.2310 & 338.8248 & \\
\hline & 7/28/2008 3:00 & 134 & 210 & 300 & 4.33 & 4.33 & & 0.1330 & 9.93E-04 & 230.6105 & 84.9732 & 315.5837 & \\
\hline & 7/28/2008 4:00 & 135 & 210 & 400 & 4.351 & 4.351 & & 0.1276 & 9.43E-04 & 218.9965 & 80.6938 & 299.6902 & \\
\hline & 7/28/2008 5:00 & 136 & 210 & 500 & 4.358 & 4.358 & & 0.1257 & 9.27E-04 & 215.1750 & 79.2857 & 294.4607 & \\
\hline & 7/28/2008 6:00 & 137 & 210 & 600 & 4.376 & 4.376 & & 0.1211 & $8.85 \mathrm{E}-04$ & 205.4627 & 75.7070 & 281.1697 & \\
\hline & 7/28/2008 7:00 & 138 & 210 & 700 & 4.331 & 4.331 & & 0.1328 & 9.91E-04 & 230.0524 & 84.7675 & 314.8199 & \\
\hline & 7/28/2008 8:00 & 139 & 210 & 800 & 3.638 & 3.638 & & 0.3485 & $3.25 E-03$ & 754.8027 & 278.1226 & 1032.9253 & \\
\hline & $7 / 28 / 20089: 00$ & 140 & 210 & 900 & 2.82 & 2.82 & & 0.7397 & $8.21 E-03$ & 1906.0525 & 702.3243 & 2608.3768 & \\
\hline & $7 / 28 / 2008$ 10:00 & 141 & 210 & 1000 & 2.569 & 2.569 & & 0.9097 & 1.06E-02 & 2458.7528 & 905.9781 & 3364.7309 & \\
\hline & $7 / 28 / 2008$ 11:00 & 142 & 210 & 1100 & 2.674 & 2.674 & & 0.8347 & 9.53E-03 & 2211.6809 & 814.9393 & 3026.6203 & \\
\hline & $7 / 28 / 200812: 00$ & 143 & 210 & 1200 & 2.684 & 2.684 & & 0.8279 & $9.43 \mathrm{E}-03$ & 2189.4058 & 806.7316 & 2996.1375 & \\
\hline & 7/28/2008 13:00 & 144 & 210 & 1300 & 2.714 & 2.714 & & 0.8077 & 9.15E-03 & 2123.8147 & 782.5632 & 2906.3778 & \\
\hline & $7 / 28 / 2008$ 14:00 & 145 & 210 & 1400 & 2.735 & 2.735 & & 0.7938 & 8.96E-03 & 2078.9755 & 766.0413 & 2845.0168 & \\
\hline & $7 / 28 / 2008$ 15:00 & 146 & 210 & 1500 & 2.713 & 2.713 & & 0.8083 & $9.16 \mathrm{E}-03$ & 2125.9717 & 783.3580 & 2909.3297 & \\
\hline & $7 / 28 / 2008$ 16:00 & 147 & 210 & 1600 & 2.739 & 2.739 & & 0.7912 & 8.92E-03 & 2070.5329 & 762.9304 & 2833.4634 & \\
\hline & $7 / 28 / 2008$ 17:00 & 148 & 210 & 1700 & 2.753 & 2.753 & & 0.7821 & 8.79E-03 & 2041.2273 & 752.1322 & 2793.3595 & \\
\hline & $7 / 28 / 2008$ 18:00 & 149 & 210 & 1800 & 2.854 & 2.854 & & 0.7190 & 7.93E-03 & 1840.5266 & 678.1799 & 2518.7065 & \\
\hline & $7 / 28 / 2008$ 19:00 & 150 & 210 & 1900 & 2.988 & 2.988 & & 0.6419 & 6.90E-03 & 1600.6955 & 589.8092 & 2190.5047 & \\
\hline & $7 / 28 / 200820: 00$ & 151 & 210 & 2000 & 3.163 & 3.163 & & 0.5511 & 5.71E-03 & 1326.5713 & 488.8025 & 1815.3738 & \\
\hline & $7 / 28 / 2008$ 21:00 & 152 & 210 & 2100 & 3.782 & 3.782 & & 0.2972 & 2.67E-03 & 620.3457 & 228.5791 & 848.9248 & \\
\hline & $7 / 28 / 2008$ 22:00 & 153 & 210 & 2200 & 4.006 & 4.006 & & 0.2247 & 1.89E-03 & 439.5932 & 161.9772 & 601.5704 & \\
\hline & $7 / 28 / 200823: 00$ & 154 & 210 & 2300 & 4.102 & 4.102 & & 0.1960 & 1.60E-03 & 371.6107 & 136.9276 & 508.5382 & \\
\hline & $7 / 29 / 20080: 00$ & 155 & 211 & 0 & 4.209 & 4.209 & 4.43 & 0.1656 & $1.30 \mathrm{E}-03$ & 301.9749 & 111.2689 & 413.2438 & \\
\hline & 7/29/2008 1:00 & 156 & 211 & 100 & 4.289 & 4.289 & & 0.1439 & $1.09 \mathrm{E}-03$ & 253.9372 & 93.5684 & 347.5056 & \\
\hline & 7/29/2008 2:00 & 157 & 211 & 200 & 4.367 & 4.367 & & 0.1234 & 9.06E-04 & 210.2984 & 77.4888 & 287.7871 & \\
\hline & 7/29/2008 3:00 & 158 & 211 & 300 & 4.4 & 4.4 & & 0.1150 & 8.30E-04 & 192.7679 & 71.0293 & 263.7972 & \\
\hline & 7/29/2008 4:00 & 159 & 211 & 400 & 4.427 & 4.427 & & 0.1082 & 7.70E-04 & 178.8331 & 65.8947 & 244.7278 & \\
\hline & 7/29/2008 5:00 & 160 & 211 & 500 & 4.399 & 4.399 & & 0.1153 & 8.33E-04 & 193.2910 & 71.2221 & 264.5131 & \\
\hline & $7 / 29 / 20086: 00$ & 161 & 211 & 600 & 4.352 & 4.352 & & 0.1273 & $9.41 \mathrm{E}-04$ & 218.4490 & 80.4920 & 298.9411 & \\
\hline & 7/29/2008 7:00 & 162 & 211 & 700 & 4.262 & 4.262 & & 0.1511 & 1.16E-03 & 269.7739 & 99.4037 & 369.1777 & \\
\hline & 7/29/2008 8:00 & 163 & 211 & 800 & 3.933 & 3.933 & & 0.2474 & 2.13E-03 & 494.9682 & 182.3812 & 677.3495 & \\
\hline
\end{tabular}




\begin{tabular}{|c|c|c|c|c|c|c|c|c|c|c|c|c|c|}
\hline Tree ID & TS & RN & JD & HR & $\begin{array}{c}\text { Raw } \\
\text { TC_dTA }\end{array}$ & $\begin{array}{c}\text { Fixed } \\
\text { TC_dT } \Delta\end{array}$ & dTM & $\mathbf{K}$ & $\begin{array}{l}\text { Sap } \\
\text { Velocity } \\
\text { (cm/s) }\end{array}$ & $\begin{array}{c}\text { Probed Drywood } \\
F(\mathrm{~g} / \mathrm{hr})\end{array}$ & $\begin{array}{c}\text { Extrapolated } \\
\text { Wetwood F } \\
\text { (g/hr) }\end{array}$ & $\begin{array}{c}\text { Extrapolated } \\
\text { Total Wood F } \\
(\mathrm{g} / \mathrm{hr})\end{array}$ & $\begin{array}{l}\text { Relative } \\
\text { Humidity, } \\
\text { @10m (\%) }\end{array}$ \\
\hline & $7 / 29 / 20089: 00$ & 164 & 211 & 900 & \begin{tabular}{|l|}
3.064 \\
\end{tabular} & 3.064 & & 0.6012 & 6.36E-03 & 1476.6096 & 544.0872 & 2020.6967 & \\
\hline & $7 / 29 / 2008$ 10:00 & 165 & 211 & 1000 & 2.658 & 2.658 & & 0.8457 & 9.68E-03 & 2247.7587 & 828.2329 & 3075.9916 & \\
\hline & 7/29/2008 11:00 & 166 & 211 & 1100 & 2.879 & 2.879 & & 0.7041 & 7.73E-03 & 1793.6020 & 660.8896 & 2454.4916 & \\
\hline & $7 / 29 / 200812: 00$ & 167 & 211 & 1200 & 2.669 & 2.669 & & 0.8381 & $9.58 \mathrm{E}-03$ & 2222.8970 & 819.0721 & 3041.9692 & \\
\hline & 7/29/2008 13:00 & 168 & 211 & 1300 & 2.714 & 2.714 & & 0.8077 & $9.15 \mathrm{E}-03$ & 2123.8147 & 782.5632 & 2906.3778 & \\
\hline & 7/29/2008 14:00 & 169 & 211 & 1400 & 2.672 & 2.672 & & 0.8361 & 9.55E-03 & 2216.1611 & 816.5901 & 3032.7512 & \\
\hline & 7/29/2008 15:00 & 170 & 211 & 1500 & 2.797 & 2.797 & & 0.7540 & $8.41 \mathrm{E}-03$ & 1951.5310 & 719.0817 & 2670.6127 & \\
\hline & 7/29/2008 16:00 & 171 & 211 & 1600 & 2.811 & 2.811 & & 0.7453 & $8.29 \mathrm{E}-03$ & 1923.7359 & 708.8401 & 2632.5760 & \\
\hline & 7/29/2008 17:00 & 172 & 211 & 1700 & 2.896 & 2.896 & & 0.6941 & $7.59 \mathrm{E}-03$ & 1762.2842 & 649.3498 & 2411.6340 & \\
\hline & $7 / 29 / 2008$ 18:00 & 173 & 211 & 1800 & 2.831 & 2.831 & & 0.7330 & 8.12E-03 & 1884.6335 & 694.4320 & 2579.0655 & \\
\hline & $7 / 29 / 2008$ 19:00 & 174 & 211 & 1900 & 3.016 & 3.016 & & 0.6267 & 6.69E-03 & 1554.0326 & 572.6153 & 2126.6478 & \\
\hline & 7/29/2008 20:00 & 175 & 211 & 2000 & 3.312 & 3.312 & & 0.4813 & $4.84 \mathrm{E}-03$ & 1122.9167 & 413.7617 & 1536.6784 & \\
\hline & 7/29/2008 21:00 & 176 & 211 & 2100 & 3.992 & 3.992 & & 0.2290 & 1.94E-03 & 449.9610 & 165.7974 & 615.7583 & \\
\hline & $7 / 29 / 200822: 00$ & 177 & 211 & 2200 & 4.312 & 4.312 & & 0.1378 & 1.04E-03 & 240.7449 & 88.7074 & 329.4524 & \\
\hline & 7/29/2008 23:00 & 178 & 211 & 2300 & 4.391 & 4.391 & & 0.1173 & $8.51 \mathrm{E}-04$ & 197.4944 & 72.7709 & 270.2652 & \\
\hline & $7 / 30 / 20080: 00$ & 179 & 212 & 0 & 4.368 & 4.368 & 4.37 & 0.1232 & 9.04E-04 & 209.7590 & 77.2900 & 287.0491 & \\
\hline & 7/30/2008 1:00 & 180 & 212 & 100 & 4.32 & 4.32 & & 0.1356 & $1.02 \mathrm{E}-03$ & 236.2202 & 87.0402 & 323.2604 & \\
\hline & 7/30/2008 2:00 & 181 & 212 & 200 & 4.264 & 4.264 & & 0.1506 & 1.16E-03 & 268.5878 & 98.9667 & 367.5545 & \\
\hline & 7/30/2008 3:00 & 182 & 212 & 300 & 4.225 & 4.225 & & 0.1612 & 1.26E-03 & 292.0970 & 107.6291 & 399.7261 & \\
\hline & 7/30/2008 4:00 & 183 & 212 & 400 & 4.173 & 4.173 & & 0.1757 & 1.40E-03 & 324.7033 & 119.6436 & 444.3468 & \\
\hline & 7/30/2008 5:00 & 184 & 212 & 500 & 4.122 & 4.122 & & 0.1902 & 1.54E-03 & 358.1135 & 131.9543 & 490.0679 & \\
\hline & 7/30/2008 6:00 & 185 & 212 & 600 & 4.071 & 4.071 & & 0.2051 & 1.69E-03 & 392.9792 & 144.8013 & 537.7805 & \\
\hline & 7/30/2008 7:00 & 186 & 212 & 700 & 4.023 & 4.023 & & 0.2195 & $1.84 \mathrm{E}-03$ & 427.1615 & 157.3965 & 584.5580 & \\
\hline & 7/30/2008 8:00 & 187 & 212 & 800 & 3.994 & 3.994 & & 0.2283 & $1.93 \mathrm{E}-03$ & 448.4726 & 165.2490 & 613.7216 & \\
\hline & 7/30/2008 9:00 & 188 & 212 & 900 & 3.514 & 3.514 & & 0.3961 & 3.81E-03 & 883.5956 & 325.5790 & 1209.1746 & \\
\hline & $7 / 30 / 2008$ 10:00 & 189 & 212 & 1000 & 2.286 & 2.286 & & 1.1461 & 1.41E-02 & 3267.5457 & 1203.9944 & 4471.5401 & \\
\hline & 7/30/2008 11:00 & 190 & 212 & 1100 & 2.353 & 2.353 & & 1.0850 & $1.32 \mathrm{E}-02$ & 3054.4187 & 1125.4634 & 4179.8821 & \\
\hline & $7 / 30 / 2008$ 12:00 & 191 & 212 & 1200 & 2.467 & 2.467 & & 0.9887 & 1.17E-02 & 2724.0377 & 1003.7277 & 3727.7654 & \\
\hline & $7 / 30 / 2008$ 13:00 & 192 & 212 & 1300 & 2.488 & 2.488 & & 0.9719 & 1.15E-02 & 2667.2179 & 982.7913 & 3650.0092 & \\
\hline & $7 / 30 / 2008$ 14:00 & 193 & 212 & 1400 & 2.526 & 2.526 & & 0.9422 & 1.11E-02 & 2567.3578 & 945.9958 & 3513.3536 & \\
\hline & $7 / 30 / 2008$ 15:00 & 194 & 212 & 1500 & 2.586 & 2.586 & & 0.8971 & 1.04E-02 & 2417.0499 & 890.6117 & 3307.6616 & \\
\hline & $7 / 30 / 2008$ 16:00 & 195 & 212 & 1600 & 2.55 & 2.55 & & 0.9239 & $1.08 \mathrm{E}-02$ & 2506.1810 & 923.4539 & 3429.6349 & \\
\hline & $7 / 30 / 2008$ 17:00 & 196 & 212 & 1700 & 2.562 & 2.562 & & 0.9149 & 1.07E-02 & 2476.1248 & 912.3791 & 3388.5039 & \\
\hline & $7 / 30 / 2008$ 18:00 & 197 & 212 & 1800 & 2.714 & 2.714 & & 0.8077 & $9.15 E-03$ & 2123.8147 & 782.5632 & 2906.3778 & \\
\hline & 7/30/2008 19:00 & 198 & 212 & 1900 & 2.861 & 2.861 & & 0.7148 & 7.87E-03 & 1827.2821 & 673.2997 & 2500.5818 & \\
\hline & $7 / 30 / 200820: 00$ & 199 & 212 & 2000 & 2.976 & 2.976 & & 0.6485 & $6.98 \mathrm{E}-03$ & 1621.0430 & 597.3066 & 2218.3496 & \\
\hline & 7/30/2008 21:00 & 200 & 212 & 2100 & 3.602 & 3.602 & & 0.3620 & 3.41E-03 & 790.8917 & 291.4203 & 1082.3120 & \\
\hline & 7/30/2008 22:00 & 201 & 212 & 2200 & 4.026 & 4.026 & & 0.2186 & 1.83E-03 & 424.9856 & 156.5947 & 581.5802 & \\
\hline & 7/30/2008 23:00 & 202 & 212 & 2300 & 4.18 & 4.18 & & 0.1737 & $1.38 \mathrm{E}-03$ & 320.2289 & 117.9949 & 438.2239 & \\
\hline & 7/31/2008 0:00 & 203 & 213 & 0 & 4.263 & 4.263 & 4.28 & 0.1508 & 1.16E-03 & 269.1806 & 99.1851 & 368.3657 & \\
\hline & 7/31/2008 1:00 & 204 & 213 & 100 & 4.284 & 4.284 & & 0.1452 & 1.11E-03 & 256.8413 & 94.6385 & 351.4798 & \\
\hline
\end{tabular}




\begin{tabular}{|c|c|c|c|c|c|c|c|c|c|c|c|c|c|}
\hline Tree ID & TS & RN & JD & HR & $\begin{array}{c}\text { Raw } \\
\text { TC_dTA }\end{array}$ & $\begin{array}{c}\text { Fixed } \\
\text { TC_dT } \Delta\end{array}$ & dTM & $\mathbf{K}$ & $\begin{array}{l}\text { Sap } \\
\text { Velocity } \\
(\mathrm{cm} / \mathrm{s})\end{array}$ & $\begin{array}{c}\text { Probed Drywood } \\
\text { F (g/hr) }\end{array}$ & $\begin{array}{c}\text { Extrapolated } \\
\text { Wetwood F } \\
(\mathrm{g} / \mathrm{hr})\end{array}$ & $\begin{array}{c}\text { Extrapolated } \\
\text { Total Wood F } \\
(\mathrm{g} / \mathrm{hr})\end{array}$ & $\begin{array}{l}\text { Relative } \\
\text { Humidity, } \\
@ 10 \mathrm{~m}(\%)\end{array}$ \\
\hline & 7/31/2008 2:00 & 205 & 213 & 200 & $\begin{array}{r}4.255 \\
\end{array}$ & 4.255 & & 0.1530 & 1.18E-03 & 273.9419 & 100.9395 & \begin{tabular}{|l|}
374.8814 \\
\end{tabular} & \\
\hline & 7/31/2008 3:00 & 206 & 213 & 300 & 4.212 & 4.212 & & 0.1648 & $1.29 \mathrm{E}-03$ & 300.1124 & 110.5826 & 410.6950 & \\
\hline & 7/31/2008 4:00 & 207 & 213 & 400 & 4.171 & 4.171 & & 0.1762 & $1.40 \mathrm{E}-03$ & 325.9865 & 120.1164 & 446.1030 & \\
\hline & 7/31/2008 5:00 & 208 & 213 & 500 & 4.117 & 4.117 & & 0.1916 & $1.56 \mathrm{E}-03$ & 361.4668 & 133.1899 & 494.6566 & \\
\hline & 7/31/2008 6:00 & 209 & 213 & 600 & 4.073 & 4.073 & & 0.2045 & 1.69E-03 & 391.5840 & 144.2872 & 535.8712 & \\
\hline & 7/31/2008 7:00 & 210 & 213 & 700 & 4.023 & 4.023 & & 0.2195 & 1.84E-03 & 427.1615 & 157.3965 & 584.5580 & \\
\hline & $7 / 31 / 2008$ 8:00 & 211 & 213 & 800 & 3.965 & 3.965 & & 0.2373 & 2.03E-03 & 470.2914 & 173.2886 & 643.5800 & \\
\hline & $7 / 31 / 20089: 00$ & 212 & 213 & 900 & 3.282 & 3.282 & & 0.4948 & 5.01E-03 & 1161.9312 & 428.1375 & 1590.0687 & \\
\hline & $7 / 31 / 2008$ 10:00 & 213 & 213 & 1000 & 2.255 & 2.255 & & 1.1756 & 1.45E-02 & 3371.3946 & 1242.2597 & 4613.6543 & \\
\hline & 7/31/2008 11:00 & 214 & 213 & 1100 & 2.379 & 2.379 & & 1.0622 & 1.28E-02 & 2975.6449 & 1096.4376 & 4072.0825 & \\
\hline & $7 / 31 / 2008$ 12:00 & 215 & 213 & 1200 & 2.359 & 2.359 & & 1.0797 & 1.31E-02 & 3036.0516 & 1118.6957 & 4154.7473 & \\
\hline & 7/31/2008 13:00 & 216 & 213 & 1300 & 2.495 & 2.495 & & 0.9663 & $1.14 \mathrm{E}-02$ & 2648.5400 & 975.9090 & 3624.4490 & \\
\hline & $7 / 31 / 2008$ 14:00 & 217 & 213 & 1400 & 2.433 & 2.433 & & 1.0164 & $1.21 \mathrm{E}-02$ & 2818.6008 & 1038.5714 & 3857.1722 & \\
\hline & $7 / 31 / 2008$ 15:00 & 218 & 213 & 1500 & 2.469 & 2.469 & & 0.9870 & 1.17E-02 & 2718.5749 & 1001.7148 & 3720.2898 & \\
\hline & 7/31/2008 16:00 & 219 & 213 & 1600 & 2.53 & 2.53 & & 0.9391 & 1.10E-02 & 2557.0617 & 942.2020 & 3499.2637 & \\
\hline & 7/31/2008 17:00 & 220 & 213 & 1700 & 2.571 & 2.571 & & 0.9082 & 1.06E-02 & 2453.8110 & 904.1571 & 3357.9682 & \\
\hline & $7 / 31 / 2008$ 18:00 & 221 & 213 & 1800 & 2.684 & 2.684 & & 0.8279 & 9.43E-03 & 2189.4058 & 806.7316 & 2996.1375 & \\
\hline & $7 / 31 / 2008$ 19:00 & 222 & 213 & 1900 & 2.86 & 2.86 & & 0.7154 & $7.88 \mathrm{E}-03$ & 1829.1691 & 673.9950 & 2503.1641 & \\
\hline & $7 / 31 / 200820: 00$ & 223 & 213 & 2000 & 3.085 & 3.085 & & 0.5903 & $6.22 \mathrm{E}-03$ & 1443.7236 & 531.9696 & 1975.6932 & \\
\hline & $7 / 31 / 200821: 00$ & 224 & 213 & 2100 & 3.411 & 3.411 & & 0.4383 & $4.31 E-03$ & 1000.7406 & 368.7435 & 1369.4841 & \\
\hline & $7 / 31 / 200822: 00$ & 225 & 213 & 2200 & 3.73 & 3.73 & & 0.3153 & 2.87E-03 & 667.1346 & 245.8195 & 912.9540 & \\
\hline & 7/31/2008 23:00 & 226 & 213 & 2300 & 3.735 & 3.735 & & 0.3135 & 2.85E-03 & 662.5512 & 244.1306 & 906.6818 & \\
\hline & $8 / 1 / 20080: 00$ & 227 & 214 & 0 & 3.877 & 3.877 & 4.17 & 0.2654 & 2.32E-03 & 539.7102 & 198.8673 & 738.5775 & \\
\hline & 8/1/2008 1:00 & 228 & 214 & 100 & 4.079 & 4.079 & & 0.2027 & 1.67E-03 & 387.4122 & 142.7500 & 530.1622 & \\
\hline & 8/1/2008 2:00 & 229 & 214 & 200 & 4.15 & 4.15 & & 0.1822 & $1.46 \mathrm{E}-03$ & 339.5930 & 125.1300 & 464.7230 & \\
\hline & 8/1/2008 3:00 & 230 & 214 & 300 & 4.157 & 4.157 & & 0.1802 & 1.44E-03 & 335.0306 & 123.4489 & 458.4796 & \\
\hline & 8/1/2008 4:00 & 231 & 214 & 400 & 4.169 & 4.169 & & 0.1768 & 1.41E-03 & 327.2720 & 120.5901 & 447.8621 & \\
\hline & 8/1/2008 5:00 & 232 & 214 & 500 & 4.144 & 4.144 & & 0.1839 & 1.48E-03 & 343.5251 & 126.5789 & 470.1040 & \\
\hline & 8/1/2008 6:00 & 233 & 214 & 600 & 4.056 & 4.056 & & 0.2096 & 1.74E-03 & 403.5169 & 148.6841 & 552.2010 & \\
\hline & 8/1/2008 7:00 & 234 & 214 & 700 & 3.962 & 3.962 & & 0.2383 & 2.04E-03 & 472.5779 & 174.1311 & 646.7090 & \\
\hline & 8/1/2008 8:00 & 235 & 214 & 800 & 3.912 & 3.912 & & 0.2541 & 2.20E-03 & 511.5113 & 188.4769 & 699.9882 & \\
\hline & 8/1/2008 9:00 & 236 & 214 & 900 & 3.325 & 3.325 & & 0.4755 & 4.77E-03 & 1106.3059 & 407.6412 & 1513.9470 & \\
\hline & 8/1/2008 10:00 & 237 & 214 & 1000 & 2.293 & 2.293 & & 1.1396 & $1.40 \mathrm{E}-02$ & 3244.5677 & 1195.5277 & 4440.0953 & \\
\hline & 8/1/2008 11:00 & 238 & 214 & 1100 & 2.366 & 2.366 & & 1.0735 & $1.30 \mathrm{E}-02$ & 3014.7671 & 1110.8530 & 4125.6201 & \\
\hline & 8/1/2008 12:00 & 239 & 214 & 1200 & 2.44 & 2.44 & & 1.0107 & 1.21E-02 & 2798.8667 & 1031.3000 & 3830.1666 & \\
\hline & 8/1/2008 13:00 & 240 & 214 & 1300 & 2.439 & 2.439 & & 1.0115 & 1.21E-02 & 2801.6773 & 1032.3356 & 3834.0129 & \\
\hline & 8/1/2008 14:00 & 241 & 214 & 1400 & 2.451 & 2.451 & & 1.0016 & 1.19E-02 & 2768.1358 & 1019.9766 & 3788.1123 & \\
\hline & 8/1/2008 15:00 & 242 & 214 & 1500 & 2.459 & 2.459 & & 0.9951 & 1.18E-02 & 2745.9984 & 1011.8196 & 3757.8180 & \\
\hline & $8 / 1 / 200816: 00$ & 243 & 214 & 1600 & 2.492 & 2.492 & & 0.9687 & $1.14 \mathrm{E}-02$ & 2656.5289 & 978.8527 & 3635.3817 & \\
\hline & 8/1/2008 17:00 & 244 & 214 & 1700 & 2.51 & 2.51 & & 0.9546 & 1.12E-02 & 2608.9486 & 961.3208 & 3570.2694 & \\
\hline & 8/1/2008 18:00 & 245 & 214 & 1800 & 2.704 & 2.704 & & 0.8143 & $9.24 \mathrm{E}-03$ & 2145.4752 & 790.5445 & 2936.0196 & \\
\hline
\end{tabular}




\begin{tabular}{|c|c|c|c|c|c|c|c|c|c|c|c|c|c|}
\hline Tree ID & TS & RN & JD & HR & $\begin{array}{c}\text { Raw } \\
\text { TC_dTA }\end{array}$ & $\begin{array}{c}\text { Fixed } \\
\text { TC_dT } \Delta\end{array}$ & dTM & $\mathbf{K}$ & $\begin{array}{l}\text { Sap } \\
\text { Velocity } \\
(\mathrm{cm} / \mathrm{s})\end{array}$ & $\begin{array}{c}\text { Probed Drywood } \\
\text { F (g/hr) }\end{array}$ & $\begin{array}{c}\text { Extrapolated } \\
\text { Wetwood F } \\
(\mathrm{g} / \mathrm{hr})\end{array}$ & $\begin{array}{c}\text { Extrapolated } \\
\text { Total Wood F } \\
(\mathrm{g} / \mathrm{hr})\end{array}$ & $\begin{array}{l}\text { Relative } \\
\text { Humidity, } \\
@ 10 \mathrm{~m}(\%)\end{array}$ \\
\hline & 8/1/2008 19:00 & 246 & 214 & 1900 & \begin{tabular}{|r|}
2.793 \\
\end{tabular} & 2.793 & & 0.7565 & $8.44 \mathrm{E}-03$ & 1959.5375 & 722.0319 & 2681.5694 & \\
\hline & 8/1/2008 20:00 & 247 & 214 & 2000 & 3.018 & 3.018 & & 0.6256 & $6.68 \mathrm{E}-03$ & 1550.7425 & 571.4030 & 2122.1454 & \\
\hline & 8/1/2008 21:00 & 248 & 214 & 2100 & 3.719 & 3.719 & & 0.3192 & $2.92 \mathrm{E}-03$ & 677.2825 & 249.5586 & 926.8411 & \\
\hline & 8/1/2008 22:00 & 249 & 214 & 2200 & 4.041 & 4.041 & & 0.2141 & $1.78 \mathrm{E}-03$ & 414.1853 & 152.6151 & 566.8004 & \\
\hline & 8/1/2008 23:00 & 250 & 214 & 2300 & 4.115 & 4.115 & & 0.1922 & 1.56E-03 & 362.8120 & 133.6855 & 496.4975 & \\
\hline & 8/2/2008 0:00 & 251 & 215 & 0 & 4.144 & 4.144 & 4.24 & 0.1839 & 1.48E-03 & 343.5251 & 126.5789 & 470.1040 & \\
\hline & 8/2/2008 1:00 & 252 & 215 & 100 & 4.201 & 4.201 & & 0.1678 & 1.32E-03 & 306.9653 & 113.1077 & 420.0730 & \\
\hline & 8/2/2008 2:00 & 253 & 215 & 200 & 4.237 & 4.237 & & 0.1579 & 1.23E-03 & 284.7778 & 104.9323 & 389.7101 & \\
\hline & 8/2/2008 3:00 & 254 & 215 & 300 & 4.225 & 4.225 & & 0.1612 & 1.26E-03 & 292.0970 & 107.6291 & 399.7261 & \\
\hline & 8/2/2008 4:00 & 255 & 215 & 400 & 4.167 & 4.167 & & 0.1773 & 1.42E-03 & 328.5596 & 121.0645 & 449.6242 & \\
\hline & 8/2/2008 5:00 & 256 & 215 & 500 & 4.113 & 4.113 & & 0.1928 & 1.57E-03 & 364.1594 & 134.1820 & 498.3414 & \\
\hline & 8/2/2008 6:00 & 257 & 215 & 600 & 4.067 & 4.067 & & 0.2063 & 1.70E-03 & 395.7766 & 145.8320 & 541.6086 & \\
\hline & 8/2/2008 7:00 & 258 & 215 & 700 & 3.966 & 3.966 & & 0.2370 & 2.02E-03 & 469.5305 & 173.0082 & 642.5387 & \\
\hline & 8/2/2008 8:00 & 259 & 215 & 800 & 3.414 & 3.414 & & 0.4370 & 4.30E-03 & 997.1894 & 367.4349 & 1364.6243 & \\
\hline & 8/2/2008 9:00 & 260 & 215 & 900 & 2.515 & 2.515 & & 0.9507 & 1.12E-02 & 2595.8812 & 956.5058 & 3552.3870 & \\
\hline & 8/2/2008 10:00 & 261 & 215 & 1000 & 2.349 & 2.349 & & 1.0885 & 1.32E-02 & 3066.7272 & 1129.9987 & 4196.7259 & \\
\hline & 8/2/2008 11:00 & 262 & 215 & 1100 & 2.389 & 2.389 & & 1.0536 & 1.27E-02 & 2945.9052 & 1085.4794 & 4031.3846 & \\
\hline & $8 / 2 / 200812: 00$ & 263 & 215 & 1200 & 2.41 & 2.41 & & 1.0357 & 1.24E-02 & 2884.4347 & 1062.8293 & 3947.2640 & \\
\hline & 8/2/2008 13:00 & 264 & 215 & 1300 & 2.454 & 2.454 & & 0.9992 & 1.19E-02 & 2759.8134 & 1016.9100 & 3776.7235 & \\
\hline & 8/2/2008 14:00 & 265 & 215 & 1400 & 2.519 & 2.519 & & 0.9476 & 1.11E-02 & 2585.4734 & 952.6708 & 3538.1442 & \\
\hline & $8 / 2 / 2008$ 15:00 & 266 & 215 & 1500 & 2.529 & 2.529 & & 0.9399 & $1.10 \mathrm{E}-02$ & 2559.6320 & 943.1490 & 3502.7810 & \\
\hline & 8/2/2008 16:00 & 267 & 215 & 1600 & 2.568 & 2.568 & & 0.9104 & 1.06E-02 & 2461.2273 & 906.8898 & 3368.1172 & \\
\hline & 8/2/2008 17:00 & 268 & 215 & 1700 & 2.59 & 2.59 & & 0.8942 & 1.04E-02 & 2407.3363 & 887.0326 & 3294.3689 & \\
\hline & $8 / 2 / 200818: 00$ & 269 & 215 & 1800 & 2.672 & 2.672 & & 0.8361 & $9.55 \mathrm{E}-03$ & 2216.1611 & 816.5901 & 3032.7512 & \\
\hline & 8/2/2008 19:00 & 270 & 215 & 1900 & 2.842 & 2.842 & & 0.7262 & 8.03E-03 & 1863.4254 & 686.6174 & 2550.0428 & \\
\hline & 8/2/2008 20:00 & 271 & 215 & 2000 & 2.961 & 2.961 & & 0.6569 & 7.09E-03 & 1646.7777 & 606.7891 & 2253.5668 & \\
\hline & 8/2/2008 21:00 & 272 & 215 & 2100 & 3.374 & 3.374 & & 0.4541 & 4.50E-03 & 1045.2557 & 385.1460 & 1430.4017 & \\
\hline & 8/2/2008 22:00 & 273 & 215 & 2200 & 3.782 & 3.782 & & 0.2972 & 2.67E-03 & 620.3457 & 228.5791 & 848.9248 & \\
\hline & 8/2/2008 23:00 & 274 & 215 & 2300 & 3.945 & 3.945 & & 0.2436 & 2.09E-03 & 485.6398 & 178.9440 & 664.5837 & \\
\hline & 8/3/2008 0:00 & 275 & 216 & 0 & 3.907 & 3.907 & 4.15 & 0.2557 & $2.22 \mathrm{E}-03$ & 515.4915 & 189.9434 & 705.4349 & \\
\hline & 8/3/2008 1:00 & 276 & 216 & 100 & 3.861 & 3.861 & & 0.2707 & 2.38E-03 & 552.8665 & 203.7150 & 756.5816 & \\
\hline & 8/3/2008 2:00 & 277 & 216 & 200 & 3.958 & 3.958 & & 0.2395 & 2.05E-03 & 475.6352 & 175.2576 & 650.8928 & \\
\hline & 8/3/2008 3:00 & 278 & 216 & 300 & 4.081 & 4.081 & & 0.2022 & $1.66 \mathrm{E}-03$ & 386.0262 & 142.2393 & 528.2654 & \\
\hline & 8/3/2008 4:00 & 279 & 216 & 400 & 4.129 & 4.129 & & 0.1882 & 1.52E-03 & 353.4426 & 130.2332 & 483.6757 & \\
\hline & 8/3/2008 5:00 & 280 & 216 & 500 & 4.151 & 4.151 & & 0.1819 & 1.46E-03 & 338.9396 & 124.8893 & 463.8288 & \\
\hline & 8/3/2008 6:00 & 281 & 216 & 600 & 4.092 & 4.092 & & 0.1989 & 1.63E-03 & 378.4439 & 139.4454 & 517.8893 & \\
\hline & 8/3/2008 7:00 & 282 & 216 & 700 & 3.989 & 3.989 & & 0.2299 & 1.95E-03 & 452.1980 & 166.6217 & 618.8197 & \\
\hline & 8/3/2008 8:00 & 283 & 216 & 800 & 3.551 & 3.551 & & 0.3816 & 3.63E-03 & 843.8227 & 310.9238 & 1154.7465 & \\
\hline & 8/3/2008 9:00 & 284 & 216 & 900 & 2.549 & 2.549 & & 0.9247 & 1.08E-02 & 2508.7015 & 924.3827 & 3433.0842 & \\
\hline & $8 / 3 / 2008$ 10:00 & 285 & 216 & 1000 & 2.367 & 2.367 & & 1.0727 & 1.30E-02 & 3011.7391 & 1109.7372 & 4121.4763 & \\
\hline & 8/3/2008 11:00 & 286 & 216 & 1100 & 2.471 & 2.471 & & 0.9854 & 1.17E-02 & 2713.1230 & 999.7060 & 3712.8290 & \\
\hline
\end{tabular}




\begin{tabular}{|c|c|c|c|c|c|c|c|c|c|c|c|c|c|}
\hline Tree ID & TS & RN & JD & HR & $\begin{array}{c}\text { Raw } \\
\text { TC_dTA }\end{array}$ & $\begin{array}{c}\text { Fixed } \\
\text { TC_dT } \Delta\end{array}$ & dTM & $\mathbf{K}$ & $\begin{array}{l}\text { Sap } \\
\text { Velocity } \\
(\mathrm{cm} / \mathrm{s})\end{array}$ & $\begin{array}{c}\text { Probed Drywood } \\
\text { F (g/hr) }\end{array}$ & $\begin{array}{c}\text { Extrapolated } \\
\text { Wetwood F } \\
(\mathrm{g} / \mathrm{hr})\end{array}$ & $\begin{array}{c}\text { Extrapolated } \\
\text { Total Wood F } \\
(\mathrm{g} / \mathrm{hr})\end{array}$ & $\begin{array}{l}\text { Relative } \\
\text { Humidity, } \\
@ 10 \mathrm{~m}(\%)\end{array}$ \\
\hline & 8/3/2008 12:00 & 287 & 216 & 1200 & 2.477 & 2.477 & & 0.9806 & 1.16E-02 & 2696.8325 & 993.7034 & 3690.5359 & \\
\hline & 8/3/2008 13:00 & 288 & 216 & 1300 & 2.54 & 2.54 & & 0.9315 & 1.09E-02 & 2531.4972 & 932.7822 & 3464.2794 & \\
\hline & $8 / 3 / 2008$ 14:00 & 289 & 216 & 1400 & 2.58 & 2.58 & & 0.9016 & $1.05 \mathrm{E}-02$ & 2431.6906 & 896.0064 & 3327.6970 & \\
\hline & 8/3/2008 15:00 & 290 & 216 & 1500 & 2.585 & 2.585 & & 0.8979 & 1.04E-02 & 2419.4841 & 891.5087 & 3310.9928 & \\
\hline & 8/3/2008 16:00 & 291 & 216 & 1600 & 2.661 & 2.661 & & 0.8437 & 9.65E-03 & 2240.9527 & 825.7251 & 3066.6778 & \\
\hline & 8/3/2008 17:00 & 292 & 216 & 1700 & 2.671 & 2.671 & & 0.8368 & $9.56 \mathrm{E}-03$ & 2218.4043 & 817.4167 & 3035.8210 & \\
\hline & 8/3/2008 18:00 & 293 & 216 & 1800 & 2.742 & 2.742 & & 0.7892 & 8.89E-03 & 2064.2213 & 760.6048 & 2824.8262 & \\
\hline & 8/3/2008 19:00 & 294 & 216 & 1900 & 2.867 & 2.867 & & 0.7112 & 7.82E-03 & 1815.9953 & 669.1408 & 2485.1362 & \\
\hline & 8/3/2008 20:00 & 295 & 216 & 2000 & 2.895 & 2.895 & & 0.6946 & 7.60E-03 & 1764.1133 & 650.0238 & 2414.1372 & \\
\hline & 8/3/2008 21:00 & 296 & 216 & 2100 & 3.193 & 3.193 & & 0.5365 & 5.53E-03 & 1283.5183 & 472.9387 & 1756.4570 & \\
\hline & 8/3/2008 22:00 & 297 & 216 & 2200 & 3.413 & 3.413 & & 0.4374 & $4.30 \mathrm{E}-03$ & 998.3722 & 367.8708 & 1366.2429 & \\
\hline & 8/3/2008 23:00 & 298 & 216 & 2300 & 3.45 & 3.45 & & 0.4220 & $4.11 \mathrm{E}-03$ & 955.2388 & 351.9774 & 1307.2162 & \\
\hline & 8/4/2008 0:00 & 299 & 217 & 0 & 3.441 & 3.441 & 3.89 & 0.4257 & $4.16 \mathrm{E}-03$ & 965.6125 & 355.7998 & 1321.4123 & \\
\hline & 8/4/2008 1:00 & 300 & 217 & 100 & 3.492 & 3.492 & & 0.4049 & 3.91E-03 & 907.8089 & 334.5009 & 1242.3098 & \\
\hline & $8 / 4 / 2008$ 2:00 & 301 & 217 & 200 & 3.388 & 3.388 & & 0.4481 & 4.43E-03 & 1028.2550 & 378.8817 & 1407.1367 & \\
\hline & 8/4/2008 3:00 & 302 & 217 & 300 & 3.266 & 3.266 & & 0.5021 & 5.10E-03 & 1183.1355 & 435.9506 & 1619.0861 & \\
\hline & 8/4/2008 4:00 & 303 & 217 & 400 & 3.161 & 3.161 & & 0.5520 & 5.73E-03 & 1329.4801 & 489.8743 & 1819.3544 & \\
\hline & $8 / 4 / 20085: 00$ & 304 & 217 & 500 & 3.245 & 3.245 & & 0.5119 & $5.22 \mathrm{E}-03$ & 1211.3938 & 446.3629 & 1657.7567 & \\
\hline & 8/4/2008 6:00 & 305 & 217 & 600 & 3.513 & 3.513 & & 0.3965 & 3.81E-03 & 884.6870 & 325.9811 & 1210.6681 & \\
\hline & 8/4/2008 7:00 & 306 & 217 & 700 & 3.599 & 3.599 & & 0.3632 & $3.42 E-03$ & 793.9461 & 292.5458 & 1086.4918 & \\
\hline & 8/4/2008 8:00 & 307 & 217 & 800 & 3.523 & 3.523 & & 0.3926 & $3.76 \mathrm{E}-03$ & 873.8125 & 321.9742 & 1195.7867 & \\
\hline & 8/4/2008 9:00 & 308 & 217 & 900 & 3.295 & 3.295 & & 0.4889 & 4.93E-03 & 1144.9070 & 421.8645 & 1566.7715 & \\
\hline & 8/4/2008 10:00 & 309 & 217 & 1000 & 2.734 & 2.734 & & 0.7944 & 8.96E-03 & 2081.0911 & 766.8208 & 2847.9119 & \\
\hline & 8/4/2008 11:00 & 310 & 217 & 1100 & 2.591 & 2.591 & & 0.8935 & 1.04E-02 & 2404.9137 & 886.1399 & 3291.0537 & \\
\hline & 8/4/2008 12:00 & 311 & 217 & 1200 & 2.631 & 2.631 & & 0.8647 & $9.95 \mathrm{E}-03$ & 2309.8882 & 851.1258 & 3161.0140 & \\
\hline & 8/4/2008 13:00 & 312 & 217 & 1300 & 2.649 & 2.649 & & 0.8520 & 9.77E-03 & 2268.2926 & 835.7991 & 3104.0916 & \\
\hline & 8/4/2008 14:00 & 313 & 217 & 1400 & 2.755 & 2.755 & & 0.7808 & 8.77E-03 & 2037.0715 & 750.6009 & 2787.6723 & \\
\hline & 8/4/2008 15:00 & 314 & 217 & 1500 & 2.834 & 2.834 & & 0.7311 & 8.09E-03 & 1878.8287 & 692.2931 & 2571.1218 & \\
\hline & 8/4/2008 16:00 & 315 & 217 & 1600 & 2.655 & 2.655 & & 0.8478 & 9.71E-03 & 2254.5840 & 830.7478 & 3085.3318 & \\
\hline & 8/4/2008 17:00 & 316 & 217 & 1700 & 2.657 & 2.657 & & 0.8464 & 9.69E-03 & 2250.0317 & 829.0704 & 3079.1021 & \\
\hline & 8/4/2008 18:00 & 317 & 217 & 1800 & 2.757 & 2.757 & & 0.7795 & 8.76E-03 & 2032.9232 & 749.0724 & 2781.9956 & \\
\hline & 8/4/2008 19:00 & 318 & 217 & 1900 & 2.886 & 2.886 & & 0.6999 & 7.67E-03 & 1780.6492 & 656.1168 & 2436.7660 & \\
\hline & 8/4/2008 20:00 & 319 & 217 & 2000 & 3.026 & 3.026 & & 0.6213 & 6.62E-03 & 1537.6386 & 566.5746 & 2104.2132 & \\
\hline & 8/4/2008 21:00 & 320 & 217 & 2100 & 3.604 & 3.604 & & 0.3613 & $3.40 \mathrm{E}-03$ & 788.8595 & 290.6715 & 1079.5310 & \\
\hline & 8/4/2008 22:00 & 321 & 217 & 2200 & 3.84 & 3.84 & & 0.2776 & 2.46E-03 & 570.3914 & 210.1725 & 780.5639 & \\
\hline & 8/4/2008 23:00 & 322 & 217 & 2300 & 3.893 & 3.893 & & 0.2602 & 2.27E-03 & 526.7211 & 194.0812 & 720.8024 & \\
\hline & 8/5/2008 0:00 & 323 & 218 & 0 & 3.987 & 3.987 & 4.05 & 0.2305 & 1.95E-03 & 453.6924 & 167.1723 & 620.8647 & \\
\hline & 8/5/2008 1:00 & 324 & 218 & 100 & 4.034 & 4.034 & & 0.2162 & $1.81 \mathrm{E}-03$ & 419.2089 & 154.4662 & 573.6751 & \\
\hline & 8/5/2008 2:00 & 325 & 218 & 200 & 4.049 & 4.049 & & 0.2117 & 1.76E-03 & 408.4791 & 150.5125 & 558.9917 & \\
\hline & 8/5/2008 3:00 & 326 & 218 & 300 & 4.012 & 4.012 & & 0.2228 & 1.87E-03 & 435.1859 & 160.3532 & 595.5391 & \\
\hline & 8/5/2008 4:00 & 327 & 218 & 400 & 3.942 & 3.942 & & 0.2445 & $2.10 \mathrm{E}-03$ & 487.9634 & 179.8002 & 667.7636 & \\
\hline
\end{tabular}




\begin{tabular}{|c|c|c|c|c|c|c|c|c|c|c|c|c|c|}
\hline Tree ID & TS & RN & JD & HR & $\begin{array}{c}\text { Raw } \\
\text { TC_dTA }\end{array}$ & $\begin{array}{c}\text { Fixed } \\
\text { TC_dT } \Delta\end{array}$ & dTM & $\mathbf{K}$ & $\begin{array}{l}\text { Sap } \\
\text { Velocity } \\
(\mathrm{cm} / \mathrm{s})\end{array}$ & $\begin{array}{c}\text { Probed Drywood } \\
\text { F (g/hr) }\end{array}$ & $\begin{array}{c}\text { Extrapolated } \\
\text { Wetwood F } \\
(\mathrm{g} / \mathrm{hr})\end{array}$ & $\begin{array}{c}\text { Extrapolated } \\
\text { Total Wood F } \\
(\mathrm{g} / \mathrm{hr})\end{array}$ & $\begin{array}{l}\text { Relative } \\
\text { Humidity, } \\
@ 10 \mathrm{~m}(\%)\end{array}$ \\
\hline & 8/5/2008 5:00 & 328 & 218 & 500 & 3.923 & 3.923 & & 0.2506 & 2.17E-03 & 502.8111 & 185.2711 & $\begin{array}{r}688.0822 \\
\end{array}$ & \\
\hline & 8/5/2008 6:00 & 329 & 218 & 600 & 3.914 & 3.914 & & 0.2534 & $2.20 \mathrm{E}-03$ & 509.9238 & 187.8919 & 697.8157 & \\
\hline & 8/5/2008 7:00 & 330 & 218 & 700 & 3.896 & 3.896 & & 0.2592 & $2.26 \mathrm{E}-03$ & 524.3042 & 193.1906 & 717.4948 & \\
\hline & 8/5/2008 8:00 & 331 & 218 & 800 & 3.836 & 3.836 & & 0.2789 & 2.47E-03 & 573.7629 & 211.4148 & 785.1777 & \\
\hline & 8/5/2008 9:00 & 332 & 218 & 900 & 3.271 & 3.271 & & 0.4998 & 5.07E-03 & 1176.4791 & 433.4979 & 1609.9770 & \\
\hline & 8/5/2008 10:00 & 333 & 218 & 1000 & 2.438 & 2.438 & & 1.0123 & 1.21E-02 & 2804.4908 & 1033.3723 & 3837.8631 & \\
\hline & 8/5/2008 11:00 & 334 & 218 & 1100 & 2.606 & 2.606 & & 0.8826 & 1.02E-02 & 2368.8528 & 872.8525 & 3241.7054 & \\
\hline & 8/5/2008 12:00 & 335 & 218 & 1200 & 2.512 & 2.512 & & 0.9530 & 1.12E-02 & 2603.7139 & 959.3919 & 3563.1059 & \\
\hline & 8/5/2008 13:00 & 336 & 218 & 1300 & 2.366 & 2.366 & & 1.0735 & 1.30E-02 & 3014.7671 & 1110.8530 & 4125.6201 & \\
\hline & 8/5/2008 14:00 & 337 & 218 & 1400 & 2.61 & 2.61 & & 0.8797 & 1.02E-02 & 2359.3237 & 869.3414 & 3228.6651 & \\
\hline & 8/5/2008 15:00 & 338 & 218 & 1500 & 2.743 & 2.743 & & 0.7886 & 8.88E-03 & 2062.1214 & 759.8310 & 2821.9524 & \\
\hline & 8/5/2008 16:00 & 339 & 218 & 1600 & 2.677 & 2.677 & & 0.8326 & $9.50 \mathrm{E}-03$ & 2204.9765 & 812.4689 & 3017.4454 & \\
\hline & 8/5/2008 17:00 & 340 & 218 & 1700 & 2.492 & 2.492 & & 0.9687 & 1.14E-02 & 2656.5289 & 978.8527 & 3635.3817 & \\
\hline & 8/5/2008 18:00 & 341 & 218 & 1800 & 2.479 & 2.479 & & 0.9790 & 1.16E-02 & 2691.4239 & 991.7105 & 3683.1344 & \\
\hline & 8/5/2008 19:00 & 342 & 218 & 1900 & 2.626 & 2.626 & & 0.8682 & $1.00 \mathrm{E}-02$ & 2321.5691 & 855.4299 & 3176.9989 & \\
\hline & 8/5/2008 20:00 & 343 & 218 & 2000 & 2.844 & 2.844 & & 0.7250 & 8.01E-03 & 1859.5918 & 685.2049 & 2544.7967 & \\
\hline & 8/5/2008 21:00 & 344 & 218 & 2100 & 3.63 & 3.63 & & 0.3515 & 3.29E-03 & 762.7334 & 281.0448 & 1043.7781 & \\
\hline & $8 / 5 / 200822: 00$ & 345 & 218 & 2200 & 3.889 & 3.889 & & 0.2615 & $2.28 \mathrm{E}-03$ & 529.9528 & 195.2720 & 725.2248 & \\
\hline & 8/5/2008 23:00 & 346 & 218 & 2300 & 3.879 & 3.879 & & 0.2648 & 2.32E-03 & 538.0774 & 198.2657 & 736.3431 & \\
\hline & 8/6/2008 0:00 & 347 & 219 & 0 & 3.835 & 3.835 & 3.84 & 0.2793 & $2.48 E-03$ & 574.6075 & 211.7259 & 786.3334 & \\
\hline & 8/6/2008 1:00 & 348 & 219 & 100 & 3.801 & 3.801 & & 0.2907 & $2.60 \mathrm{E}-03$ & 603.7266 & 222.4555 & 826.1821 & \\
\hline & $8 / 6 / 2008$ 2:00 & 349 & 219 & 200 & 3.786 & 3.786 & & 0.2958 & 2.66E-03 & 616.8260 & 227.2822 & 844.1082 & \\
\hline & 8/6/2008 3:00 & 350 & 219 & 300 & 3.766 & 3.766 & & 0.3027 & 2.73E-03 & 634.5368 & 233.8081 & 868.3449 & \\
\hline & 8/6/2008 4:00 & 351 & 219 & 400 & 3.773 & 3.773 & & 0.3003 & 2.71E-03 & 628.3060 & 231.5123 & 859.8182 & \\
\hline & 8/6/2008 5:00 & 352 & 219 & 500 & 3.719 & 3.719 & & 0.3192 & 2.92E-03 & 677.2825 & 249.5586 & 926.8411 & \\
\hline & 8/6/2008 6:00 & 353 & 219 & 600 & 3.697 & 3.697 & & 0.3270 & 3.01E-03 & 697.8462 & 257.1358 & 954.9820 & \\
\hline & 8/6/2008 7:00 & 354 & 219 & 700 & 3.665 & 3.665 & & 0.3386 & $3.14 \mathrm{E}-03$ & 728.4062 & 268.3962 & 996.8024 & \\
\hline & 8/6/2008 8:00 & 355 & 219 & 800 & 3.655 & 3.655 & & 0.3423 & 3.18E-03 & 738.1166 & 271.9743 & 1010.0909 & \\
\hline & 8/6/2008 9:00 & 356 & 219 & 900 & 3.665 & 3.665 & & 0.3386 & 3.14E-03 & 728.4062 & 268.3962 & 996.8024 & \\
\hline & 8/6/2008 10:00 & 357 & 219 & 1000 & 2.528 & 2.528 & & 0.9407 & $1.10 \mathrm{E}-02$ & 2562.2047 & 944.0970 & 3506.3018 & \\
\hline & 8/6/2008 11:00 & 358 & 219 & 1100 & 2.006 & 2.006 & & 1.4457 & 1.87E-02 & 4348.6873 & 1602.3633 & 5951.0505 & \\
\hline & 8/6/2008 12:00 & 359 & 219 & 1200 & 2.347 & 2.347 & & 1.0903 & 1.32E-02 & 3072.9007 & 1132.2735 & 4205.1741 & \\
\hline & 8/6/2008 13:00 & 360 & 219 & 1300 & 2.535 & 2.535 & & 0.9353 & 1.10E-02 & 2544.2482 & 937.4806 & 3481.7288 & \\
\hline & $8 / 6 / 2008$ 14:00 & 361 & 219 & 1400 & 2.325 & 2.325 & & 1.1101 & 1.35E-02 & 3141.6658 & 1157.6114 & 4299.2772 & \\
\hline & 8/6/2008 15:00 & 362 & 219 & 1500 & 2.206 & 2.206 & & 1.2239 & 1.53E-02 & 3542.7950 & 1305.4157 & 4848.2107 & \\
\hline & 8/6/2008 16:00 & 363 & 219 & 1600 & 2.264 & 2.264 & & 1.1670 & 1.44E-02 & 3340.8889 & 1231.0192 & 4571.9081 & \\
\hline & 8/6/2008 17:00 & 364 & 219 & 1700 & 2.208 & 2.208 & & 1.2219 & $1.52 \mathrm{E}-02$ & 3535.6185 & 1302.7713 & 4838.3898 & \\
\hline & 8/6/2008 18:00 & 365 & 219 & 1800 & 2.455 & 2.455 & & 0.9984 & 1.19E-02 & 2757.0449 & 1015.8899 & 3772.9348 & \\
\hline & 8/6/2008 19:00 & 366 & 219 & 1900 & 2.366 & 2.366 & & 1.0735 & 1.30E-02 & 3014.7671 & 1110.8530 & 4125.6201 & \\
\hline & $8 / 6 / 200820: 00$ & 367 & 219 & 2000 & 2.521 & 2.521 & & 0.9461 & 1.11E-02 & 2580.2848 & 950.7590 & 3531.0438 & \\
\hline & 8/6/2008 21:00 & 368 & 219 & 2100 & 3.174 & 3.174 & & 0.5457 & 5.65E-03 & 1310.6597 & 482.9395 & 1793.5992 & \\
\hline
\end{tabular}




\begin{tabular}{|c|c|c|c|c|c|c|c|c|c|c|c|c|c|}
\hline Tree ID & TS & RN & JD & HR & $\begin{array}{c}\text { Raw } \\
\text { TC_dTA }\end{array}$ & $\begin{array}{c}\text { Fixed } \\
\text { TC_dT } \Delta\end{array}$ & dTM & $\mathbf{K}$ & $\begin{array}{l}\text { Sap } \\
\text { Velocity } \\
(\mathrm{cm} / \mathrm{s})\end{array}$ & $\begin{array}{c}\text { Probed Drywood } \\
\text { F (g/hr) }\end{array}$ & $\begin{array}{c}\text { Extrapolated } \\
\text { Wetwood F } \\
(\mathrm{g} / \mathrm{hr})\end{array}$ & $\begin{array}{c}\text { Extrapolated } \\
\text { Total Wood F } \\
(\mathrm{g} / \mathrm{hr})\end{array}$ & $\begin{array}{l}\text { Relative } \\
\text { Humidity, } \\
@ 10 \mathrm{~m}(\%)\end{array}$ \\
\hline & 8/6/2008 22:00 & 369 & 219 & 2200 & 3.612 & 3.612 & & 0.3583 & 3.36E-03 & 780.7630 & 287.6882 & 1068.4512 & \\
\hline & 8/6/2008 23:00 & 370 & 219 & 2300 & 3.7 & 3.7 & & 0.3259 & $2.99 \mathrm{E}-03$ & 695.0208 & 256.0947 & 951.1156 & \\
\hline & $8 / 7 / 20080: 00$ & 371 & 220 & 0 & 3.73 & 3.73 & 3.73 & 0.3153 & $2.87 \mathrm{E}-03$ & 667.1346 & 245.8195 & 912.9540 & \\
\hline & 8/7/2008 1:00 & 372 & 220 & 100 & 3.716 & 3.716 & & 0.3202 & 2.93E-03 & 680.0655 & 250.5841 & 930.6496 & \\
\hline & 8/7/2008 2:00 & 373 & 220 & 200 & 3.693 & 3.693 & & 0.3285 & 3.02E-03 & 701.6238 & 258.5277 & 960.1516 & \\
\hline & 8/7/2008 3:00 & 374 & 220 & 300 & 3.7 & 3.7 & & 0.3259 & 2.99E-03 & 695.0208 & 256.0947 & 951.1156 & \\
\hline & 8/7/2008 4:00 & 375 & 220 & 400 & 3.646 & 3.646 & & 0.3456 & $3.22 E-03$ & 746.9223 & 275.2189 & 1022.1412 & \\
\hline & 8/7/2008 5:00 & 376 & 220 & 500 & 3.63 & 3.63 & & 0.3515 & 3.29E-03 & 762.7334 & 281.0448 & 1043.7781 & \\
\hline & 8/7/2008 6:00 & 377 & 220 & 600 & 3.609 & 3.609 & & 0.3594 & 3.38E-03 & 783.7932 & 288.8047 & 1072.5979 & \\
\hline & 8/7/2008 7:00 & 378 & 220 & 700 & 3.601 & 3.601 & & 0.3624 & 3.41E-03 & 791.9090 & 291.7952 & 1083.7042 & \\
\hline & 8/7/2008 8:00 & 379 & 220 & 800 & 3.605 & 3.605 & & 0.3609 & $3.39 E-03$ & 787.8446 & 290.2976 & 1078.1422 & \\
\hline & 8/7/2008 9:00 & 380 & 220 & 900 & 3.405 & 3.405 & & 0.4408 & 4.34E-03 & 1007.8690 & 371.3701 & 1379.2390 & \\
\hline & 8/7/2008 10:00 & 381 & 220 & 1000 & 2.157 & 2.157 & & 1.2745 & 1.60E-02 & 3723.6615 & 1372.0596 & 5095.7211 & \\
\hline & 8/7/2008 11:00 & 382 & 220 & 1100 & 2.004 & 2.004 & & 1.4481 & 1.88E-02 & 4357.7271 & 1605.6942 & 5963.4213 & \\
\hline & 8/7/2008 12:00 & 383 & 220 & 1200 & 2.12 & 2.12 & & 1.3142 & 1.67E-02 & 3866.9443 & 1424.8552 & 5291.7995 & \\
\hline & 8/7/2008 13:00 & 384 & 220 & 1300 & 2.131 & 2.131 & & 1.3022 & 1.65E-02 & 3823.7204 & 1408.9284 & 5232.6488 & \\
\hline & $8 / 7 / 2008$ 14:00 & 385 & 220 & 1400 & 2.16 & 2.16 & & 1.2713 & 1.60E-02 & 3712.3029 & 1367.8743 & 5080.1772 & \\
\hline & 8/7/2008 15:00 & 386 & 220 & 1500 & 2.183 & 2.183 & & 1.2474 & $1.56 \mathrm{E}-02$ & 3626.4702 & 1336.2475 & 4962.7177 & \\
\hline & 8/7/2008 16:00 & 387 & 220 & 1600 & 2.193 & 2.193 & & 1.2371 & 1.55E-02 & 3589.8289 & 1322.7463 & 4912.5752 & \\
\hline & 8/7/2008 17:00 & 388 & 220 & 1700 & 2.114 & 2.114 & & 1.3207 & 1.68E-02 & 3890.7494 & 1433.6266 & 5324.3760 & \\
\hline & 8/7/2008 18:00 & 389 & 220 & 1800 & 2.254 & 2.254 & & 1.1766 & $1.45 \mathrm{E}-02$ & 3374.8024 & 1243.5154 & 4618.3178 & \\
\hline & 8/7/2008 19:00 & 390 & 220 & 1900 & 2.423 & 2.423 & & 1.0248 & 1.23E-02 & 2847.0356 & 1049.0488 & 3896.0845 & \\
\hline & 8/7/2008 20:00 & 391 & 220 & 2000 & 2.491 & 2.491 & & 0.9695 & 1.15E-02 & 2659.1972 & 979.8359 & 3639.0331 & \\
\hline & 8/7/2008 21:00 & 392 & 220 & 2100 & 3.11 & 3.11 & & 0.5775 & 6.05E-03 & 1405.3311 & 517.8232 & 1923.1543 & \\
\hline & 8/7/2008 22:00 & 393 & 220 & 2200 & 3.438 & 3.438 & & 0.4270 & 4.17E-03 & 969.0872 & 357.0801 & 1326.1673 & \\
\hline & 8/7/2008 23:00 & 394 & 220 & 2300 & 3.573 & 3.573 & & 0.3731 & 3.54E-03 & 820.7254 & 302.4132 & 1123.1386 & \\
\hline & 8/8/2008 0:00 & 395 & 221 & 0 & 3.657 & 3.657 & 3.76 & 0.3415 & 3.17E-03 & 736.1684 & 271.2564 & 1007.4247 & \\
\hline & 8/8/2008 1:00 & 396 & 221 & 100 & 3.682 & 3.682 & & 0.3324 & 3.07E-03 & 712.0744 & 262.3785 & 974.4529 & \\
\hline & 8/8/2008 2:00 & 397 & 221 & 200 & 3.764 & 3.764 & & 0.3034 & 2.74E-03 & 636.3234 & 234.4665 & 870.7899 & \\
\hline & 8/8/2008 3:00 & 398 & 221 & 300 & 3.726 & 3.726 & & 0.3167 & 2.89E-03 & 670.8145 & 247.1754 & 917.9898 & \\
\hline & 8/8/2008 4:00 & 399 & 221 & 400 & 3.694 & 3.694 & & 0.3281 & 3.02E-03 & 700.6783 & 258.1793 & 958.8576 & \\
\hline & 8/8/2008 5:00 & 400 & 221 & 500 & 3.656 & 3.656 & & 0.3419 & 3.18E-03 & 737.1421 & 271.6152 & 1008.7573 & \\
\hline & 8/8/2008 6:00 & 401 & 221 & 600 & 3.632 & 3.632 & & 0.3508 & $3.28 \mathrm{E}-03$ & 760.7460 & 280.3125 & 1041.0584 & \\
\hline & 8/8/2008 7:00 & 402 & 221 & 700 & 3.599 & 3.599 & & 0.3632 & $3.42 \mathrm{E}-03$ & 793.9461 & 292.5458 & 1086.4918 & \\
\hline & 8/8/2008 8:00 & 403 & 221 & 800 & 3.599 & 3.599 & & 0.3632 & 3.42E-03 & 793.9461 & 292.5458 & 1086.4918 & \\
\hline & 8/8/2008 9:00 & 404 & 221 & 900 & 2.876 & 2.876 & & 0.7058 & 7.75E-03 & 1799.1780 & 662.9441 & 2462.1221 & \\
\hline & 8/8/2008 10:00 & 405 & 221 & 1000 & 1.963 & 1.963 & & 1.4992 & $1.96 \mathrm{E}-02$ & 4547.9066 & 1675.7697 & 6223.6763 & \\
\hline & 8/8/2008 11:00 & 406 & 221 & 1100 & 2.02 & 2.02 & & 1.4287 & 1.85E-02 & 4286.0068 & 1579.2674 & 5865.2742 & \\
\hline & $8 / 8 / 200812: 00$ & 407 & 221 & 1200 & 2.094 & 2.094 & & 1.3429 & $1.71 \mathrm{E}-02$ & 3971.2861 & 1463.3020 & 5434.5882 & \\
\hline & 8/8/2008 13:00 & 408 & 221 & 1300 & 2.119 & 2.119 & & 1.3152 & 1.67E-02 & 3870.9006 & 1426.3129 & 5297.2135 & \\
\hline & 8/8/2008 14:00 & 409 & 221 & 1400 & 2.116 & 2.116 & & 1.3185 & 1.67E-02 & 3882.7963 & 1430.6961 & 5313.4924 & \\
\hline
\end{tabular}




\begin{tabular}{|c|c|c|c|c|c|c|c|c|c|c|c|c|c|}
\hline Tree ID & TS & RN & JD & HR & $\begin{array}{c}\text { Raw } \\
\text { TC_dTA }\end{array}$ & $\begin{array}{c}\text { Fixed } \\
\text { TC_dT } \Delta\end{array}$ & dTM & $\mathbf{K}$ & $\begin{array}{l}\text { Sap } \\
\text { Velocity } \\
(\mathrm{cm} / \mathrm{s})\end{array}$ & $\begin{array}{c}\text { Probed Drywood } \\
F(\mathrm{~g} / \mathrm{hr})\end{array}$ & $\begin{array}{c}\text { Extrapolated } \\
\text { Wetwood F } \\
\text { (g/hr) }\end{array}$ & $\begin{array}{c}\text { Extrapolated } \\
\text { Total Wood F } \\
(\mathrm{g} / \mathrm{hr})\end{array}$ & $\begin{array}{l}\text { Relative } \\
\text { Humidity, } \\
\text { @10m (\%) }\end{array}$ \\
\hline & 8/8/2008 15:00 & 410 & 221 & 1500 & \begin{tabular}{|l|}
2.099 \\
\end{tabular} & 2.099 & & 1.3373 & 1.70E-02 & 3950.9789 & 1455.8194 & 5406.7983 & \\
\hline & 8/8/2008 16:00 & 411 & 221 & 1600 & 2.133 & 2.133 & & 1.3000 & 1.64E-02 & 3815.9191 & 1406.0539 & 5221.9730 & \\
\hline & 8/8/2008 17:00 & 412 & 221 & 1700 & 2.161 & 2.161 & & 1.2702 & 1.60E-02 & 3708.5251 & 1366.4824 & 5075.0075 & \\
\hline & 8/8/2008 18:00 & 413 & 221 & 1800 & 2.278 & 2.278 & & 1.1536 & 1.42E-02 & 3294.0168 & 1213.7482 & 4507.7650 & \\
\hline & 8/8/2008 19:00 & 414 & 221 & 1900 & 2.347 & 2.347 & & 1.0903 & 1.32E-02 & 3072.9007 & 1132.2735 & 4205.1741 & \\
\hline & 8/8/2008 20:00 & 415 & 221 & 2000 & 2.497 & 2.497 & & 0.9648 & 1.14E-02 & 2643.2272 & 973.9514 & 3617.1786 & \\
\hline & 8/8/2008 21:00 & 416 & 221 & 2100 & 3.016 & 3.016 & & 0.6267 & $6.69 \mathrm{E}-03$ & 1554.0326 & 572.6153 & 2126.6478 & \\
\hline & 8/8/2008 22:00 & 417 & 221 & 2200 & 3.275 & 3.275 & & 0.4980 & $5.04 \mathrm{E}-03$ & 1171.1737 & 431.5430 & 1602.7168 & \\
\hline & 8/8/2008 23:00 & 418 & 221 & 2300 & 3.411 & 3.411 & & 0.4383 & $4.31 \mathrm{E}-03$ & 1000.7406 & 368.7435 & 1369.4841 & \\
\hline & $8 / 9 / 20080: 00$ & 419 & 222 & 0 & 3.397 & 3.397 & 3.60 & 0.4442 & 4.38E-03 & 1017.4274 & 374.8921 & 1392.3195 & \\
\hline & 8/9/2008 1:00 & 420 & 222 & 100 & 3.376 & 3.376 & & 0.4532 & 4.49E-03 & 1042.8152 & 384.2467 & 1427.0619 & \\
\hline & 8/9/2008 2:00 & 421 & 222 & 200 & 3.356 & 3.356 & & 0.4619 & 4.60E-03 & 1067.3998 & 393.3054 & 1460.7052 & \\
\hline & 8/9/2008 3:00 & 422 & 222 & 300 & 3.326 & 3.326 & & 0.4750 & 4.76E-03 & 1105.0354 & 407.1730 & 1512.2085 & \\
\hline & 8/9/2008 4:00 & 423 & 222 & 400 & 3.304 & 3.304 & & 0.4849 & 4.88E-03 & 1133.2269 & 417.5608 & 1550.7877 & \\
\hline & 8/9/2008 5:00 & 424 & 222 & 500 & 3.237 & 3.237 & & 0.5156 & $5.27 \mathrm{E}-03$ & 1222.2884 & 450.3773 & 1672.6657 & \\
\hline & 8/9/2008 6:00 & 425 & 222 & 600 & 3.238 & 3.238 & & 0.5151 & 5.26E-03 & 1220.9226 & 449.8741 & 1670.7967 & \\
\hline & $8 / 9 / 20087: 00$ & 426 & 222 & 700 & 3.09 & 3.09 & & 0.5877 & $6.19 \mathrm{E}-03$ & 1435.9798 & 529.1163 & 1965.0961 & \\
\hline & 8/9/2008 8:00 & 427 & 222 & 800 & 2.829 & 2.829 & & 0.7342 & 8.13E-03 & 1888.5121 & 695.8611 & 2584.3733 & \\
\hline & 8/9/2008 9:00 & 428 & 222 & 900 & 3.033 & 3.033 & & 0.6175 & $6.57 \mathrm{E}-03$ & 1526.2465 & 562.3769 & 2088.6234 & \\
\hline & 8/9/2008 10:00 & 429 & 222 & 1000 & 2.935 & 2.935 & & 0.6716 & 7.29E-03 & 1692.1906 & 623.5224 & 2315.7130 & \\
\hline & 8/9/2008 11:00 & 430 & 222 & 1100 & 2.623 & 2.623 & & 0.8704 & $1.00 \mathrm{E}-02$ & 2328.6043 & 858.0221 & 3186.6264 & \\
\hline & 8/9/2008 12:00 & 431 & 222 & 1200 & 2.256 & 2.256 & & 1.1746 & $1.45 \mathrm{E}-02$ & 3367.9905 & 1241.0054 & 4608.9959 & \\
\hline & 8/9/2008 13:00 & 432 & 222 & 1300 & 2.165 & 2.165 & & 1.2661 & $1.59 \mathrm{E}-02$ & 3693.4563 & 1360.9299 & 5054.3862 & \\
\hline & 8/9/2008 14:00 & 433 & 222 & 1400 & 2.031 & 2.031 & & 1.4156 & 1.83E-02 & 4237.4822 & 1561.3875 & 5798.8697 & \\
\hline & 8/9/2008 15:00 & 434 & 222 & 1500 & 2.09 & 2.09 & & 1.3474 & 1.72E-02 & 3987.6160 & 1469.3191 & 5456.9351 & \\
\hline & 8/9/2008 16:00 & 435 & 222 & 1600 & 2.147 & 2.147 & & 1.2850 & 1.62E-02 & 3761.8001 & 1386.1126 & 5147.9127 & \\
\hline & 8/9/2008 17:00 & 436 & 222 & 1700 & 2.176 & 2.176 & & 1.2546 & 1.57E-02 & 3652.3613 & 1345.7877 & 4998.1490 & \\
\hline & 8/9/2008 18:00 & 437 & 222 & 1800 & 2.22 & 2.22 & & 1.2099 & 1.50E-02 & 3492.8875 & 1287.0262 & 4779.9137 & \\
\hline & 8/9/2008 19:00 & 438 & 222 & 1900 & 2.405 & 2.405 & & 1.0399 & $1.25 \mathrm{E}-02$ & 2898.9512 & 1068.1782 & 3967.1294 & \\
\hline & 8/9/2008 20:00 & 439 & 222 & 2000 & 2.489 & 2.489 & & 0.9711 & 1.15E-02 & 2664.5417 & 981.8052 & 3646.3469 & \\
\hline & 8/9/2008 21:00 & 440 & 222 & 2100 & 3.205 & 3.205 & & 0.5307 & $5.46 \mathrm{E}-03$ & 1266.5966 & 466.7036 & 1733.3001 & \\
\hline & 8/9/2008 22:00 & 441 & 222 & 2200 & 3.555 & 3.555 & & 0.3800 & $3.62 \mathrm{E}-03$ & 839.5930 & 309.3653 & 1148.9583 & \\
\hline & 8/9/2008 23:00 & 442 & 222 & 2300 & 3.601 & 3.601 & & 0.3624 & $3.41 E-03$ & 791.9090 & 291.7952 & 1083.7042 & \\
\hline & 8/10/2008 0:00 & 443 & 223 & 0 & 3.614 & 3.614 & 3.62 & 0.3575 & 3.35E-03 & 778.7469 & 286.9453 & 1065.6923 & \\
\hline & 8/10/2008 1:00 & 444 & 223 & 100 & 3.621 & 3.621 & & 0.3549 & 3.32E-03 & 771.7159 & 284.3546 & 1056.0705 & \\
\hline & 8/10/2008 2:00 & 445 & 223 & 200 & 3.607 & 3.607 & & 0.3601 & 3.38E-03 & 785.8173 & 289.5505 & 1075.3678 & \\
\hline & 8/10/2008 3:00 & 446 & 223 & 300 & 3.593 & 3.593 & & 0.3654 & 3.45E-03 & 800.0768 & 294.8047 & 1094.8815 & \\
\hline & 8/10/2008 4:00 & 447 & 223 & 400 & 3.615 & 3.615 & & 0.3571 & $3.35 \mathrm{E}-03$ & 777.7401 & 286.5743 & 1064.3145 & \\
\hline & 8/10/2008 5:00 & 448 & 223 & 500 & 3.564 & 3.564 & & 0.3765 & $3.58 \mathrm{E}-03$ & 830.1253 & 305.8767 & 1136.0021 & \\
\hline & 8/10/2008 6:00 & 449 & 223 & 600 & 3.551 & 3.551 & & 0.3816 & 3.63E-03 & 843.8227 & 310.9238 & 1154.7465 & \\
\hline & $8 / 10 / 20087: 00$ & 450 & 223 & 700 & 3.538 & 3.538 & & 0.3867 & 3.69E-03 & 857.6631 & 316.0236 & 1173.6867 & \\
\hline
\end{tabular}




\begin{tabular}{|c|c|c|c|c|c|c|c|c|c|c|c|c|c|}
\hline Tree ID & TS & RN & JD & HR & $\begin{array}{c}\text { Raw } \\
\text { TC_dTA }\end{array}$ & $\begin{array}{c}\text { Fixed } \\
\text { TC_dT } \Delta\end{array}$ & dTM & $\mathbf{K}$ & $\begin{array}{c}\text { Sap } \\
\text { Velocity } \\
(\mathrm{cm} / \mathrm{s})\end{array}$ & $\begin{array}{c}\text { Probed Drywood } \\
\text { F (g/hr) }\end{array}$ & $\begin{array}{c}\text { Extrapolated } \\
\text { Wetwood F } \\
(\mathbf{g} / \mathrm{hr})\end{array}$ & $\begin{array}{c}\text { Extrapolated } \\
\text { Total Wood F } \\
\text { (g/hr) }\end{array}$ & $\begin{array}{l}\text { Relative } \\
\text { Humidity, } \\
@ 10 \mathrm{~m}(\%)\end{array}$ \\
\hline & 8/10/2008 8:00 & 451 & 223 & 800 & 3.543 & 3.543 & & 0.3847 & 3.67E-03 & 852.3228 & 314.0559 & 1166.3787 & \\
\hline & 8/10/2008 9:00 & 452 & 223 & 900 & 3.366 & 3.366 & & 0.4575 & 4.54E-03 & 1055.0575 & 388.7577 & 1443.8152 & \\
\hline & 8/10/2008 10:00 & 453 & 223 & 1000 & 2.108 & 2.108 & & 1.3273 & 1.69E-02 & 3914.7175 & 1442.4582 & 5357.1757 & \\
\hline & 8/10/2008 11:00 & 454 & 223 & 1100 & 1.97 & 1.97 & & 1.4904 & 1.94E-02 & 4514.7675 & 1663.5589 & 6178.3264 & \\
\hline & $8 / 10 / 200812: 00$ & 455 & 223 & 1200 & 2.088 & 2.088 & & 1.3496 & $1.72 \mathrm{E}-02$ & 3995.8091 & 1472.3380 & 5468.1471 & \\
\hline & $8 / 10 / 2008$ 13:00 & 456 & 223 & 1300 & 2.105 & 2.105 & & 1.3306 & $1.69 \mathrm{E}-02$ & 3926.7632 & 1446.8967 & 5373.6599 & \\
\hline & $8 / 10 / 2008$ 14:00 & 457 & 223 & 1400 & 2.126 & 2.126 & & 1.3076 & $1.66 \mathrm{E}-02$ & 3843.3009 & 1416.1432 & 5259.4441 & \\
\hline & $8 / 10 / 2008$ 15:00 & 458 & 223 & 1500 & 2.179 & 2.179 & & 1.2515 & 1.57E-02 & 3641.2405 & 1341.6900 & 4982.9305 & \\
\hline & 8/10/2008 16:00 & 459 & 223 & 1600 & 2.14 & 2.14 & & 1.2925 & 1.63E-02 & 3788.7529 & 1396.0439 & 5184.7969 & \\
\hline & 8/10/2008 17:00 & 460 & 223 & 1700 & 2.168 & 2.168 & & 1.2629 & 1.59E-02 & 3682.1986 & 1356.7818 & 5038.9804 & \\
\hline & 8/10/2008 18:00 & 461 & 223 & 1800 & 3.093 & 3.093 & & 0.5862 & $6.17 \mathrm{E}-03$ & 1431.3493 & 527.4101 & 1958.7594 & \\
\hline & $8 / 10 / 2008$ 19:00 & 462 & 223 & 1900 & 3.406 & 3.406 & & 0.4404 & 4.34E-03 & 1006.6785 & 370.9314 & 1377.6099 & \\
\hline & 8/10/2008 20:00 & 463 & 223 & 2000 & 3.365 & 3.365 & & 0.4579 & 4.55E-03 & 1056.2873 & 389.2108 & 1445.4980 & \\
\hline & $8 / 10 / 200821: 00$ & 464 & 223 & 2100 & 3.342 & 3.342 & & 0.4680 & 4.67E-03 & 1084.8486 & 399.7348 & 1484.5833 & \\
\hline & $8 / 10 / 200822: 00$ & 465 & 223 & 2200 & 3.39 & 3.39 & & 0.4472 & 4.42E-03 & 1025.8421 & 377.9926 & 1403.8347 & \\
\hline & $8 / 10 / 2008$ 23:00 & 466 & 223 & 2300 & 3.34 & 3.34 & & 0.4689 & $4.68 \mathrm{E}-03$ & 1087.3575 & 400.6592 & 1488.0168 & \\
\hline & $8 / 11 / 20080: 00$ & 467 & 224 & 0 & 3.371 & 3.371 & 3.62 & 0.4554 & 4.52E-03 & 1048.9240 & 386.4976 & 1435.4216 & \\
\hline & 8/11/2008 1:00 & 468 & 224 & 100 & 3.51 & 3.51 & & 0.3977 & $3.82 E-03$ & 887.9663 & 327.1895 & 1215.1558 & \\
\hline & 8/11/2008 2:00 & 469 & 224 & 200 & 3.595 & 3.595 & & 0.3647 & $3.44 \mathrm{E}-03$ & 798.0299 & 294.0505 & 1092.0805 & \\
\hline & 8/11/2008 3:00 & 470 & 224 & 300 & 3.618 & 3.618 & & 0.3560 & $3.34 \mathrm{E}-03$ & 774.7244 & 285.4631 & 1060.1876 & \\
\hline & 8/11/2008 4:00 & 471 & 224 & 400 & 3.621 & 3.621 & & 0.3549 & $3.32 \mathrm{E}-03$ & 771.7159 & 284.3546 & 1056.0705 & \\
\hline & 8/11/2008 5:00 & 472 & 224 & 500 & 3.583 & 3.583 & & 0.3692 & $3.49 E-03$ & 810.3599 & 298.5938 & 1108.9537 & \\
\hline & 8/11/2008 6:00 & 473 & 224 & 600 & 3.591 & 3.591 & & 0.3662 & $3.46 \mathrm{E}-03$ & 802.1268 & 295.5601 & 1097.6870 & \\
\hline & 8/11/2008 7:00 & 474 & 224 & 700 & 3.588 & 3.588 & & 0.3673 & 3.47E-03 & 805.2081 & 296.6955 & 1101.9036 & \\
\hline & 8/11/2008 8:00 & 475 & 224 & 800 & 3.575 & 3.575 & & 0.3723 & 3.53E-03 & 818.6457 & 301.6468 & 1120.2925 & \\
\hline & $8 / 11 / 20089: 00$ & 476 & 224 & 900 & 3.225 & 3.225 & & 0.5212 & $5.34 \mathrm{E}-03$ & 1238.7663 & 456.4489 & 1695.2152 & \\
\hline & $8 / 11 / 2008$ 10:00 & 477 & 224 & 1000 & 2.249 & 2.249 & & 1.1814 & 1.46E-02 & 3391.8965 & 1249.8140 & 4641.7106 & \\
\hline & 8/11/2008 11:00 & 478 & 224 & 1100 & 1.988 & 1.988 & & 1.4678 & 1.91E-02 & 4430.8295 & 1632.6303 & 6063.4598 & \\
\hline & $8 / 11 / 200812: 00$ & 479 & 224 & 1200 & 2.081 & 2.081 & & 1.3575 & $1.73 \mathrm{E}-02$ & 4024.6340 & 1482.9592 & 5507.5932 & \\
\hline & $8 / 11 / 2008$ 13:00 & 480 & 224 & 1300 & 2.245 & 2.245 & & 1.1853 & $1.47 \mathrm{E}-02$ & 3405.6384 & 1254.8775 & 4660.5159 & \\
\hline & $8 / 11 / 2008$ 14:00 & 481 & 224 & 1400 & 2.127 & 2.127 & & 1.3065 & 1.65E-02 & 3839.3759 & 1414.6970 & 5254.0729 & \\
\hline & $8 / 11 / 2008$ 15:00 & 482 & 224 & 1500 & 2.128 & 2.128 & & 1.3055 & 1.65E-02 & 3835.4554 & 1413.2524 & 5248.7078 & \\
\hline & 8/11/2008 16:00 & 483 & 224 & 1600 & 2.201 & 2.201 & & 1.2290 & $1.53 \mathrm{E}-02$ & 3560.8055 & 1312.0520 & 4872.8575 & \\
\hline & $8 / 11 / 2008$ 17:00 & 484 & 224 & 1700 & 2.202 & 2.202 & & 1.2280 & $1.53 E-02$ & 3557.1955 & 1310.7218 & 4867.9173 & \\
\hline & 8/11/2008 18:00 & 485 & 224 & 1800 & 2.358 & 2.358 & & 1.0806 & 1.31E-02 & 3039.1049 & 1119.8207 & 4158.9255 & \\
\hline & 8/11/2008 19:00 & 486 & 224 & 1900 & 2.354 & 2.354 & & 1.0841 & $1.31 \mathrm{E}-02$ & 3051.3496 & 1124.3325 & 4175.6821 & \\
\hline & $8 / 11 / 200820: 00$ & 487 & 224 & 2000 & 2.619 & 2.619 & & 0.8732 & $1.01 \mathrm{E}-02$ & 2338.0159 & 861.4900 & 3199.5060 & \\
\hline & $8 / 11 / 2008$ 21:00 & 488 & 224 & 2100 & 2.996 & 2.996 & & 0.6375 & $6.84 \mathrm{E}-03$ & 1587.2478 & 584.8541 & 2172.1018 & \\
\hline & $8 / 11 / 2008$ 22:00 & 489 & 224 & 2200 & 3.255 & 3.255 & & 0.5072 & $5.16 \mathrm{E}-03$ & 1197.8763 & 441.3822 & 1639.2585 & \\
\hline & $8 / 11 / 2008$ 23:00 & 490 & 224 & 2300 & 3.385 & 3.385 & & 0.4493 & 4.44E-03 & 1031.8818 & 380.2181 & 1412.0999 & \\
\hline & 8/12/2008 0:00 & 491 & 225 & 0 & 3.46 & 3.46 & 3.47 & 0.4179 & 4.07E-03 & 943.8003 & 347.7626 & 1291.5629 & \\
\hline
\end{tabular}




\begin{tabular}{|c|c|c|c|c|c|c|c|c|c|c|c|c|c|}
\hline Tree ID & TS & RN & JD & HR & $\begin{array}{c}\text { Raw } \\
\text { TC_dTA }\end{array}$ & $\begin{array}{c}\text { Fixed } \\
\text { TC_dT } \Delta\end{array}$ & dTM & $\mathbf{K}$ & $\begin{array}{c}\text { Sap } \\
\text { Velocity } \\
(\mathrm{cm} / \mathrm{s})\end{array}$ & $\begin{array}{c}\text { Probed Drywood } \\
F(g / h r)\end{array}$ & $\begin{array}{c}\text { Extrapolated } \\
\text { Wetwood F } \\
(\mathrm{g} / \mathrm{hr})\end{array}$ & $\begin{array}{c}\text { Extrapolated } \\
\text { Total Wood F } \\
\text { (g/hr) }\end{array}$ & $\begin{array}{c}\text { Relative } \\
\text { Humidity, } \\
@ 10 \mathrm{~m}(\%)\end{array}$ \\
\hline & 8/12/2008 1:00 & 492 & 225 & 100 & \begin{tabular}{|r|}
3.467 \\
\end{tabular} & 3.467 & & 0.4151 & 4.03E-03 & $\begin{array}{r}935.8479 \\
\end{array}$ & 344.8324 & 1280.6803 & \\
\hline & 8/12/2008 2:00 & 493 & 225 & 200 & 3.241 & 3.241 & & 0.5137 & $5.24 \mathrm{E}-03$ & 1216.8321 & 448.3668 & 1665.1989 & \\
\hline & 8/12/2008 3:00 & 494 & 225 & 300 & 3.24 & 3.24 & & 0.5142 & 5.25E-03 & 1218.1945 & 448.8688 & 1667.0633 & \\
\hline & 8/12/2008 4:00 & 495 & 225 & 400 & 3.328 & 3.328 & & 0.4742 & 4.75E-03 & 1102.4976 & 406.2379 & 1508.7356 & \\
\hline & 8/12/2008 5:00 & 496 & 225 & 500 & 3.373 & 3.373 & & 0.4545 & $4.51 \mathrm{E}-03$ & 1046.4775 & 385.5962 & 1432.0736 & \\
\hline & $8 / 12 / 20086: 00$ & 497 & 225 & 600 & 3.419 & 3.419 & & 0.4349 & 4.27E-03 & 991.2898 & 365.2611 & 1356.5509 & \\
\hline & 8/12/2008 7:00 & 498 & 225 & 700 & 3.432 & 3.432 & & 0.4295 & 4.20E-03 & 976.0618 & 359.6500 & 1335.7118 & \\
\hline & 8/12/2008 8:00 & 499 & 225 & 800 & 3.431 & 3.431 & & 0.4299 & 4.21E-03 & 977.2275 & 360.0796 & 1337.3071 & \\
\hline & 8/12/2008 9:00 & 500 & 225 & 900 & 3.213 & 3.213 & & 0.5269 & 5.41E-03 & 1255.4090 & 462.5813 & 1717.9902 & \\
\hline & $8 / 12 / 2008$ 10:00 & 501 & 225 & 1000 & 2.288 & 2.288 & & 1.1442 & 1.40E-02 & 3260.9631 & 1201.5689 & 4462.5320 & \\
\hline & 8/12/2008 11:00 & 502 & 225 & 1100 & 1.971 & 1.971 & & 1.4891 & 1.94E-02 & 4510.0562 & 1661.8230 & 6171.8792 & \\
\hline & $8 / 12 / 200812: 00$ & 503 & 225 & 1200 & 2 & 2 & & 1.4530 & 1.88E-02 & 4375.8717 & 1612.3799 & 5988.2517 & \\
\hline & 8/12/2008 13:00 & 504 & 225 & 1300 & 1.98 & 1.98 & & 1.4778 & 1.92E-02 & 4467.9104 & 1646.2935 & 6114.2039 & \\
\hline & $8 / 12 / 2008$ 14:00 & 505 & 225 & 1400 & 2.01 & 2.01 & & 1.4408 & 1.87E-02 & 4330.6720 & 1595.7252 & 5926.3971 & \\
\hline & $8 / 12 / 2008$ 15:00 & 506 & 225 & 1500 & 2.024 & 2.024 & & 1.4239 & 1.84E-02 & 4268.2885 & 1572.7387 & 5841.0271 & \\
\hline & $8 / 12 / 2008$ 16:00 & 507 & 225 & 1600 & 2.043 & 2.043 & & 1.4014 & 1.80E-02 & 4185.2589 & 1542.1447 & 5727.4037 & \\
\hline & $8 / 12 / 2008$ 17:00 & 508 & 225 & 1700 & 2.007 & 2.007 & & 1.4444 & 1.87E-02 & 4344.1754 & 1600.7008 & 5944.8762 & \\
\hline & $8 / 12 / 2008$ 18:00 & 509 & 225 & 1800 & 2.114 & 2.114 & & 1.3207 & 1.68E-02 & 3890.7494 & 1433.6266 & 5324.3760 & \\
\hline & $8 / 12 / 2008$ 19:00 & 510 & 225 & 1900 & 2.274 & 2.274 & & 1.1574 & 1.42E-02 & 3307.3372 & 1218.6564 & 4525.9937 & \\
\hline & $8 / 12 / 200820: 00$ & 511 & 225 & 2000 & 2.353 & 2.353 & & 1.0850 & 1.32E-02 & 3054.4187 & 1125.4634 & 4179.8821 & \\
\hline & 8/12/2008 21:00 & 512 & 225 & 2100 & 3.066 & 3.066 & & 0.6001 & $6.35 \mathrm{E}-03$ & 1473.4521 & 542.9237 & 2016.3759 & \\
\hline & $8 / 12 / 200822: 00$ & 513 & 225 & 2200 & 3.406 & 3.406 & & 0.4404 & $4.34 \mathrm{E}-03$ & 1006.6785 & 370.9314 & 1377.6099 & \\
\hline & $8 / 12 / 200823: 00$ & 514 & 225 & 2300 & 3.465 & 3.465 & & 0.4159 & 4.04E-03 & 938.1154 & 345.6679 & 1283.7834 & \\
\hline & $8 / 13 / 20080: 00$ & 515 & 226 & 0 & 3.486 & 3.486 & 3.54 & 0.4073 & 3.94E-03 & 914.4870 & 336.9616 & 1251.4486 & \\
\hline & 8/13/2008 1:00 & 516 & 226 & 100 & 3.496 & 3.496 & & 0.4033 & 3.89E-03 & 903.3747 & 332.8670 & 1236.2417 & \\
\hline & $8 / 13 / 20082: 00$ & 517 & 226 & 200 & 3.511 & 3.511 & & 0.3973 & 3.82E-03 & 886.8723 & 326.7864 & 1213.6587 & \\
\hline & 8/13/2008 3:00 & 518 & 226 & 300 & 3.541 & 3.541 & & 0.3855 & 3.68E-03 & 854.4564 & 314.8420 & 1169.2984 & \\
\hline & 8/13/2008 4:00 & 519 & 226 & 400 & 3.534 & 3.534 & & 0.3882 & 3.71E-03 & 861.9507 & 317.6034 & 1179.5541 & \\
\hline & $8 / 13 / 2008$ 5:00 & 520 & 226 & 500 & 3.526 & 3.526 & & 0.3914 & 3.75E-03 & 870.5671 & 320.7784 & 1191.3454 & \\
\hline & $8 / 13 / 20086: 00$ & 521 & 226 & 600 & 3.527 & 3.527 & & 0.3910 & $3.75 E-03$ & 869.4870 & 320.3804 & 1189.8674 & \\
\hline & $8 / 13 / 20087: 00$ & 522 & 226 & 700 & 3.505 & 3.505 & & 0.3997 & 3.85E-03 & 893.4495 & 329.2098 & 1222.6593 & \\
\hline & 8/13/2008 8:00 & 523 & 226 & 800 & 3.462 & 3.462 & & 0.4171 & 4.06E-03 & 941.5236 & 346.9237 & 1288.4473 & \\
\hline & 8/13/2008 9:00 & 524 & 226 & 900 & 2.807 & 2.807 & & 0.7478 & 8.32E-03 & 1931.6414 & 711.7530 & 2643.3945 & \\
\hline & 8/13/2008 10:00 & 525 & 226 & 1000 & 1.955 & 1.955 & & 1.5095 & $1.98 \mathrm{E}-02$ & 4586.1267 & 1689.8527 & 6275.9794 & \\
\hline & 8/13/2008 11:00 & 526 & 226 & 1100 & 2.02 & 2.02 & & 1.4287 & 1.85E-02 & 4286.0068 & 1579.2674 & 5865.2742 & \\
\hline & 8/13/2008 12:00 & 527 & 226 & 1200 & 1.95 & 1.95 & & 1.5159 & 1.99E-02 & 4610.2042 & 1698.7245 & 6308.9287 & \\
\hline
\end{tabular}


tree stand water use - summary

\begin{tabular}{|c|c|c|c|c|c|c|c|c|c|c|c|}
\hline tree spp & count & $\begin{array}{l}\text { mean } \\
\text { diameter } \\
\text { cm }\end{array}$ & $\begin{array}{l}\text { mean sap } \\
\text { flux } \\
\mathrm{mL} / \mathrm{cm} 2 / \mathrm{hr}\end{array}$ & $\begin{array}{l}\text { sap flux } \\
\text { stdev }\end{array}$ & $\begin{array}{l}\text { sapwooa } \\
\text { diameter } \\
\text { (minus } \\
\text { bark) } \mathrm{cm}\end{array}$ & $\begin{array}{l}\text { tree basal } \\
\text { area cm2 }\end{array}$ & $\begin{array}{l}\text { stand basal } \\
\text { area } \mathrm{cm} 2\end{array}$ & $\begin{array}{l}\text { sap flow } \\
\text { L/day }\end{array}$ & $\begin{array}{l}\text { sap flow } \\
\text { gals/ day }\end{array}$ & $\begin{array}{l}\text { yearly sap } \\
\text { flow m3 }\end{array}$ & $\begin{array}{l}\text { yearly sap } \\
\text { flow gal }\end{array}$ \\
\hline EC & 735 & 9 & 16.4 & 6.5 & $5 \quad 8$ & 55 & 40,748 & 8,019 & 2,110 & 1,443 & 379,854 \\
\hline WN & 924 & 15 & 24.4 & 4.3 & 15 & 167 & 154,751 & 45,311 & 11,924 & 8,156 & $2,146,320$ \\
\hline BW & 35 & 7 & 19.5 & 6.5 & 7 & 36 & 1,272 & 298 & 78 & 54 & 14,109 \\
\hline $\mathrm{HP}$ & 10 & 13 & 16.4 & 4.3 & 13 & 123 & 1,228 & 242 & 64 & 43 & 11,444 \\
\hline GA & 149 & 2 & 6.5 & 3.0 & 2 & 3 & 423 & 33 & 9 & 6 & 1,569 \\
\hline NC & 56 & 3 & 6.5 & 3.0 & 2 & 4 & 233 & 18 & 5 & 3 & 860 \\
\hline total & 1,909 & & & & & 389 & 198,654 & 53,921 & 14,190 & 9,706 & $2,554,156$ \\
\hline
\end{tabular}

italics: estimates

\begin{tabular}{|c|c|c|c|c|c|c|c|c|c|c|}
\hline \multirow{2}{*}{ 5th \%ile } & \multirow[b]{2}{*}{ count } & \multirow[b]{2}{*}{$\begin{array}{l}\text { mean } \\
\text { diameter } \\
\text { cm }\end{array}$} & \multirow[b]{2}{*}{$\begin{array}{l}\text { 5th \%ile sap } \\
\text { flux } \\
\mathrm{mL} / \mathrm{cm} 2 / \mathrm{hr}\end{array}$} & \multirow[b]{2}{*}{$\begin{array}{l}\text { sapwooa } \\
\text { diameter } \\
\text { (minus } \\
\text { bark) }\end{array}$} & \multirow[b]{2}{*}{$\begin{array}{l}\text { tree basal } \\
\text { area } \mathrm{cm} 2\end{array}$} & \multirow[b]{2}{*}{$\begin{array}{l}\text { stand basal } \\
\text { area } \mathrm{cm} 2\end{array}$} & \multirow[b]{2}{*}{$\begin{array}{l}\text { sap flow } \\
\text { L/day }\end{array}$} & \multirow[b]{2}{*}{$\begin{array}{l}\text { sap flow } \\
\text { gals/ day }\end{array}$} & \multirow[b]{2}{*}{$\begin{array}{l}\text { yearly sap } \\
\text { flow m3 }\end{array}$} & \multirow[b]{2}{*}{$\begin{array}{l}\text { yearly sap } \\
\text { flow gal }\end{array}$} \\
\hline & & & & & & & & & & \\
\hline EC & 735 & 9 & 3.4 & 8 & 55 & 40,748 & 1,663 & 438 & 299 & 78,750 \\
\hline WN & 924 & 15 & 15.8 & 15 & 167 & 154,751 & 29,341 & 7,721 & 5,281 & $1,389,830$ \\
\hline BW & 35 & 7 & 6.5 & 7 & 36 & 1,272 & 99 & 26 & 18 & 4,713 \\
\hline HP & 10 & 13 & 7.8 & 13 & 123 & 1,228 & 115 & 30 & 21 & 5,443 \\
\hline GA & 149 & 2 & 0.5 & 2 & 3 & 423 & 3 & 1 & 0 & 127 \\
\hline NC & 56 & 3 & 0.5 & 2 & 4 & 233 & 1 & 0 & 0 & 66 \\
\hline total & 1,909 & & & & & 198,654 & 31,222 & 8,216 & 5,620 & $1,478,929$ \\
\hline
\end{tabular}

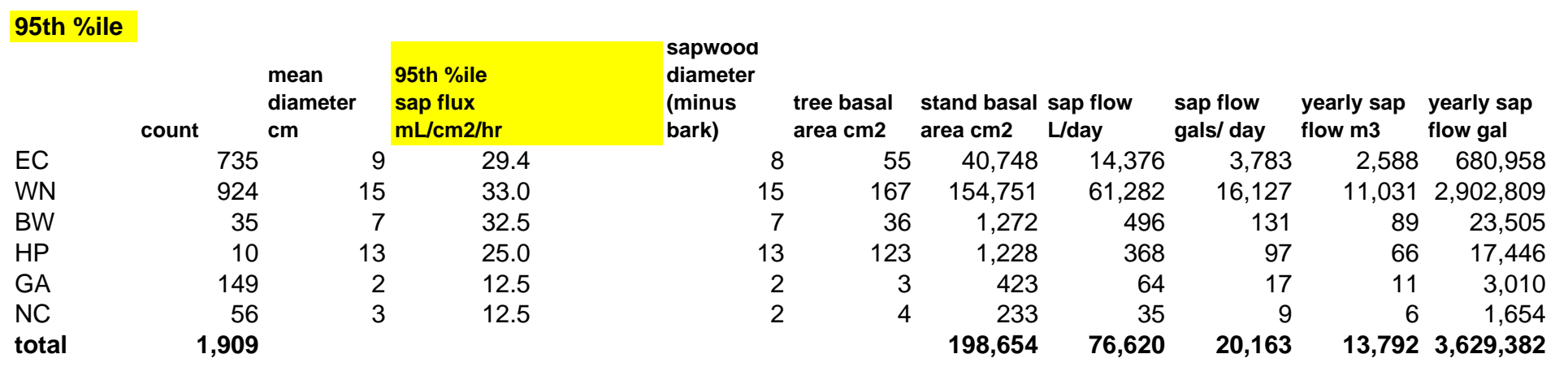

\begin{tabular}{|c|c|c|c|c|}
\hline tree spp & count & $\begin{array}{l}\text { Stand-k } \\
\text { mean } \\
\text { sap flow } \\
\text { gals/ day }\end{array}$ & $\begin{array}{l}\text { sis daily s } \\
5 \text { th \%ile } \\
\text { sap flow } \\
\text { gals/ day }\end{array}$ & $\begin{array}{l}\text { ap flow } \\
\text { 95th \%ile } \\
\text { sap flow } \\
\text { gals/ day }\end{array}$ \\
\hline EC & 735 & 2,110 & 438 & 3,783 \\
\hline WN & 924 & 11,924 & 7,721 & 16,127 \\
\hline BW & 35 & 78 & 26 & 131 \\
\hline HP & 10 & 64 & 30 & 9 \\
\hline GA & 149 & 9 & 1 & 17 \\
\hline NC & 56 & 5 & 0 & \\
\hline total & 1,909 & 14,190 & 8,216 & 20,163 \\
\hline
\end{tabular}

\begin{tabular}{|c|c|c|c|c|}
\hline \multirow[b]{2}{*}{ tree spp } & \multirow[b]{2}{*}{ count } & \multicolumn{3}{|c|}{ Stand-basis yearly sapflow } \\
\hline & & $\begin{array}{l}\text { mean } \\
\text { sap flow } \\
\text { gal/yr }\end{array}$ & $\begin{array}{l}\text { 5th \%ile } \\
\text { sap flow } \\
\text { gal/yr }\end{array}$ & $\begin{array}{l}\text { 95th \%ile } \\
\text { sap flow } \\
\text { gal/yr }\end{array}$ \\
\hline EC & 735 & 379,854 & 78,750 & 680,958 \\
\hline WN & 924 & $2,146,320$ & $1,389,830$ & $2,902,809$ \\
\hline BW & 35 & 14,109 & 4,713 & $3 \quad 23,505$ \\
\hline HP & 10 & 11,444 & 5,443 & 17,446 \\
\hline GA & 149 & 1,569 & 127 & 3,010 \\
\hline NC & 56 & 860 & 66 & $\quad 1,654$ \\
\hline total & 1,909 & $2,554,156$ & $1,478,929$ & $3,629,382$ \\
\hline
\end{tabular}

tree area $=4.5$ acres

Liters/yr $\quad \begin{array}{ll}1.8 & 1 \text { acre=Ha }\end{array}$

liters/Ha \#\#\#\#\#\#

$\mathrm{mm} 549$

\section{sap flow gals/ day}

$\square \mathrm{EC} \backsim \mathrm{WN} \backsim \mathrm{BW}$ O\%P $\square \mathrm{GA} \backsim \mathrm{NC}$

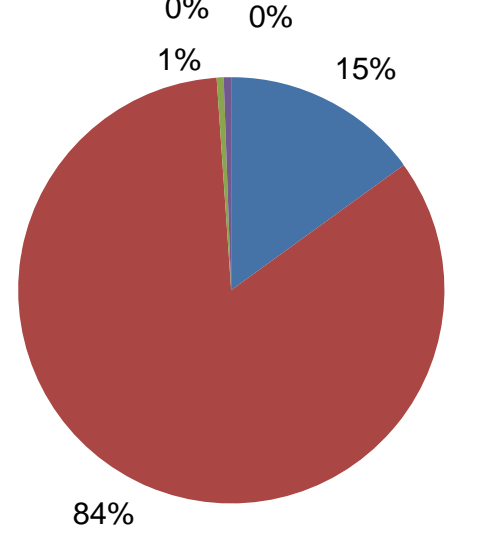


Argonne

Environmental Science Division

Argonne National Laboratory

9700 South Cass Avenue, Bldg. 203

Argonne, IL 60439-4843

www.anl.gov 